\title{
IUPAC-NIST Solubility Data Series. 99. Solubility of Benzoic Acid and Substituted Benzoic Acids in Both Neat Organic Solvents and Organic Solvent Mixtures
}

\author{
William E. Acree, Jr. ${ }^{\text {a) }}$ \\ Department of Chemistry, University of North Texas, Denton, Texas 76203, USA
}

(Received 10 June 2013; accepted 17 June 2013; published online 30 August 2013)

\begin{abstract}
Solubility data are compiled and reviewed for benzoic acid and 63 substituted benzoic acids dissolved in neat organic solvents and well-defined binary and ternary organic solvent mixtures. The compiled solubility data were retrieved from the published chemical and pharmaceutical literature covering the period from 1900 to the beginning of 2013. ( 2013 AIP Publishing LLC. [http://dx.doi.org/10.1063/1.4816161]
\end{abstract}

Key words: alcohols; alkanes; alkanenitriles; alkoxyalcohols; alkyl ethanoates; benzoic acid; chloroalkanes; dialkyl ethers; solubility; solvents; substituted benzoic acids.

\section{CONTENTS}

1. Preface $\ldots \ldots \ldots \ldots \ldots \ldots \ldots+10$

1.1. Scope of this volume ................ 10

1.2. Concentration units for nonelectrolyte solutions ...

1.3. Procedures used in critical evaluation of published solubility data ............ 11

2. Solubility of Benzoic Acid in Organic Solvents. 16

2.1. Critical evaluation of experimental solubility data

10

(10)

10

11

2.2. Benzoic acid solubility data in saturated hydrocarbons (including cycloalkanes) ....

2.3. Benzoic acid solubility data in aromatic hydrocarbons.......................

2.4. Benzoic acid solubility data in esters......

2.5. Benzoic acid solubility data in ethers .....

2.6. Benzoic acid solubility data in haloalkanes, haloalkenes, and haloaromatic hydrocarbons

2.7. Benzoic acid solubility data in alcohols ...

2.8. Benzoic acid solubility data in alkoxyalcohols

2.9. Benzoic acid solubility data in ketones....

2.10. Benzoic acid solubility data in miscellaneous organic solvents ..........

2.11. Benzoic acid solubility data in binary organic solvent mixtures................

3. Solubility of 2-Acetoxybenzoic Acid in Organic

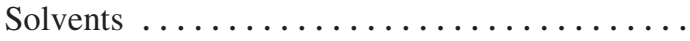

3.1. Critical evaluation of experimental solubility data ......................

3.2. 2-Acetoxybenzoic acid solubility data in aromatic hydrocarbons.................

3.3. 2-Acetoxybenzoic acid solubility data in esters

26

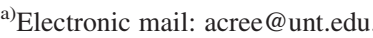

(C) 2013 AIP Publishing LLC.

3.4. 2-Acetoxybenzoic acid solubility data in ethers. ...

3.5. 2-Acetoxybenzoic acid solubility data in haloalkanes, haloalkenes, and haloaromatic hydrocarbons......................

3.6. 2-Acetoxybenzoic acid solubility data in alcohols .........................

3.7. 2-Acetoxybenzoic acid solubility data in ketones ...........................

3.8. 2-Acetoxybenzoic acid solubility data in miscellaneous organic solvents...........

4. Solubility of 4-Acetoxybenzoic Acid in Organic Solvents .......................

4.1. Critical evaluation of experimental solubility data......................

4.2. 4-Acetoxybenzoic acid solubility data in alcohols .........................

5. Solubility of 4-Acetylbenzoic Acid in Organic

Solvents .......................

5.1. Critical evaluation of experimental solubility data .......................

5.2. 4-Acetylbenzoic acid solubility data in aromatic hydrocarbons.................

5.3. 4-Acetylbenzoic acid solubility data in miscellaneous organic solvents...........

6. Solubility of 2-Aminobenzoic Acid in Organic

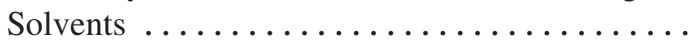

6.1. Critical evaluation of experimental solubility data .......................

6.2. 2-Aminobenzoic acid solubility data in aromatic hydrocarbons................ 107

6.3. 2-Aminobenzoic acid solubility data in esters

6.4. 2-Aminobenzoic acid solubility data in haloalkanes, haloalkenes, and haloaromatic hydrocarbons.......................

6.5. 2-Aminobenzoic acid solubility data in alcohols .........................

7. Solubility of 3-Aminobenzoic Acid in Organic

Solvents ..................... 110 
7.1. Critical evaluation of experimental solubility data

7.2. 3-Aminobenzoic acid solubility data in saturated hydrocarbons (including cycloalkanes $). \ldots \ldots \ldots \ldots \ldots \ldots \ldots \ldots$

7.3. 3-Aminobenzoic acid solubility data in aromatic hydrocarbons.

7.4. 3-Aminobenzoic acid solubility data in esters

7.5. 3-Aminobenzoic acid solubility data in haloalkanes, haloalkenes, and haloaromatic

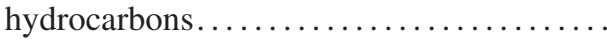

7.6. 3-Aminobenzoic acid solubility data in alcohols

7.7. 3-Aminobenzoic acid solubility data in miscellaneous organic solvents.

112

8. Solubility of 4-Aminobenzoic Acid in Organic

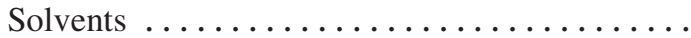

8.1. Critical evaluation of experimental

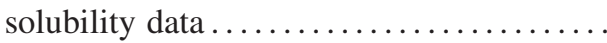

8.2. 4-Aminobenzoic acid solubility data in saturated hydrocarbons (including cycloalkanes $). \ldots \ldots \ldots \ldots \ldots \ldots \ldots \ldots \ldots$

8.3. 4-Aminobenzoic acid solubility data in aromatic hydrocarbons...............

8.4. 4-Aminobenzoic acid solubility data in esters

8.5. 4-Aminobenzoic acid solubility data in ethers

8.6. 4-Aminobenzoic acid solubility data in haloalkanes, haloalkenes, and haloaromatic

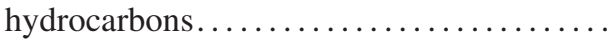

8.7. 4-Aminobenzoic acid solubility data

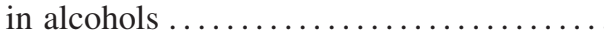

8.8. 4-Aminobenzoic acid solubility data in alkoxyalcohols ....................

8.9. 4-Aminobenzoic acid solubility data in

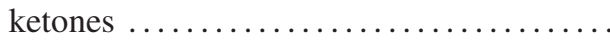

8.10. 4-Aminobenzoic acid solubility data in miscellaneous organic solvents .........

9. Solubility of 4-Amino-2-hydroxybenzoic Acid in Organic Solvents ................

9.1. Critical evaluation of experimental

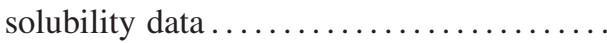

9.2. 4-Amino-2-hydroxybenzoic acid solubility

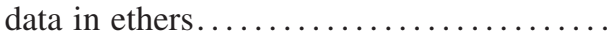

10. Solubility of 5-Amino-2-hydroxybenzoic Acid in Organic Solvents ...............

10.1. Critical evaluation of experimental solubility data....................

10.2. 5-Amino-2-hydroxybenzoic acid solubility data in ethers .....................

10.3. 5-Amino-2-hydroxybenzoic acid solubility data in haloalkanes, haloalkenes, and haloaromatic hydrocarbons .............

10.4. 5-Amino-2-hydroxybenzoic acid solubility data in alcohols

11. Solubility of 1,2-Benzenedicarboxylic Acid in Organic Solvents ...............

11.1. Critical evaluation of experimental solubility data....................
11.2. 1,2-Benzenedicarboxylic acid solubility data in aromatic hydrocarbons ..........

11.3. 1,2-Benzenedicarboxylic acid solubility

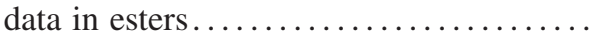

11.4. 1,2-Benzenedicarboxylic acid solubility data in ethers

11.5. 1,2-Benzenedicarboxylic acid solubility data in haloalkanes, haloalkenes, and haloaromatic hydrocarbons ............

11.6. 1,2-Benzenedicarboxylic acid solubility data in alcohols ...................

11.7. 1,2-Benzenedicarboxylic acid solubility data in ketones...................

11.8. 1,2-Benzenedicarboxylic acid solubility data in miscellaneous organic solvents ...

11.9. 1,2-Benzenedicarboxylic acid solubility data in binary organic solvent mixtures ..

12. Solubility of 1,3 -Benzenedicarboxylic Acid in Organic Solvents .................

12.1. Critical evaluation of experimental solubility data.

12.2. 1,3-Benzenedicarboxylic acid solubility data in esters . . . . . . . . . . . . . . . . . . .

12.3. 1,3-Benzenedicarboxylic acid solubilities

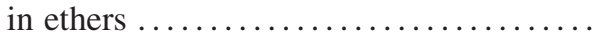

12.4. 1,3-Benzenedicarboxylic acid solubility data in alcohols ....................

12.5. 1,3-Benzenedicarboxylic acid solubility data in ketones....................

12.6. 1,3-Benzenedicarboxylic acid solubility data in miscellaneous organic solvents ...

13. Solubility of 1,4-Benzenedicarboxylic Acid in Organic Solvents ...............

13.1. Critical evaluation of experimental solubility data.

13.2. 1,4-Benzenedicarboxylic acid solubility data in aromatic hydrocarbons ..........

13.3. 1,4-Benzenedicarboxylic acid solubility data in esters .....................

13.4. 1,4-Benzenedicarboxylic acid solubility

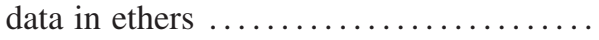

13.5. 1,4-Benzenedicarboxylic acid solubility data in alcohols ....................

13.6. 1,4-Benzenedicarboxylic acid solubility data in ketones.....................

13.7. 1,4-Benzenedicarboxylic acid solubility data in miscellaneous organic solvents ...

14. Solubility of 1,3,5-Benzenetricarboxylic Acid in Organic Solvents ................

14.1. Critical evaluation of experimental solubility data.

14.2. 1,3,5-Benzenetricarboxylic acid solubility data in miscellaneous organic solvents ...

15. Solubility of 2-Bromobenzoic Acid in Organic

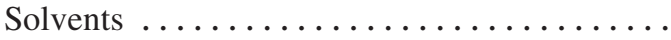

15.1. Critical evaluation of experimental solubility data.................... 
15.2. 2-Bromobenzoic acid solubility data in alcohols

16. Solubility of 3-Bromobenzoic Acid in Organic

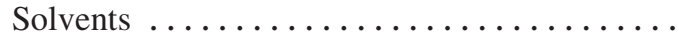

16.1. Critical evaluation of experimental solubility data

16.2. 3-Bromobenzoic acid solubility data in saturated hydrocarbons (including cycloalkanes) ......................

16.3. 3-Bromobenzoic acid solubility data in aromatic hydrocarbons..............

16.4. 3-Bromobenzoic acid solubility data in ethers..........................

16.5. 3-Bromobenzoic acid solubility data in alcohols ........................

16.6. 3-Bromobenzoic acid solubility data in miscellaneous organic solvents........

17. Solubility of 4-Bromobenzoic Acid in Organic Solvents ......................

17.1. Critical evaluation of experimental solubility data......................

17.2. 4-Bromobenzoic acid solubility data in saturated hydrocarbons (including cycloalkanes) .....................

17.3. 4-Bromobenzoic acid solubility data in aromatic hydrocarbons..............

17.4. 4-Bromobenzoic acid solubility data in alcohols ........................

17.5. 4-Bromobenzoic acid solubility data in miscellaneous organic solvents .........

18. Solubility of 2-Chlorobenzoic Acid in Organic

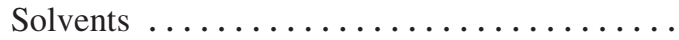

18.1. Critical evaluation of experimental solubility data......................

18.2. 2-Chlorobenzoic acid solubility data in saturated hydrocarbons (including cycloalkanes) .......................

18.3. 2-Chlorobenzoic acid solubility data in aromatic hydrocarbons...............

18.4. 2-Chlorobenzoic acid solubility data in alcohols ........................

18.5. 2-Chlorobenzoic acid solubility data in miscellaneous organic solvents........

19. Solubility of 3-Chlorobenzoic Acid in Organic Solvents .......................

19.1. Critical evaluation of experimental solubility data.

19.2. 3-Chlorobenzoic acid solubility data in saturated hydrocarbons (including cycloalkanes) .....................

19.3. 3-Chlorobenzoic acid solubility data in aromatic hydrocarbons...............

19.4. 3-Chlorobenzoic acid solubility data in esters ...........................

19.5. 3-Chlorobenzoic acid solubility data in ethers ............................

19.6. 3-Chlorobenzoic acid solubility data in alcohols .........................
20. Solubility of 4-Chlorobenzoic Acid in Organic Solvents ......................

20.1. Critical evaluation of experimental solubility data....

20.2. 4-Chlorobenzoic acid solubility data in saturated hydrocarbons (including cycloalkanes) ......................

20.3. 4-Chlorobenzoic acid solubility data in aromatic hydrocarbons..............

20.4. 4-Chlorobenzoic acid solubility data in esters ..........................

20.5. 4-Chlorobenzoic acid solubility data in ethers...........................

20.6. 4-Chlorobenzoic acid solubility in haloalkanes, haloalkenes, haloaromatic hydrocarbons ....................

20.7. 4-Chlorobenzoic acid solubility data in alcohols ........................

20.8. 4-Chlorobenzoic acid solubility data in alkoxyalcohols .....................

20.9. 4-Chlorobenzoic acid solubility data in miscellaneous organic solvents........

21. Solubility of 2-Chloro-5-nitrobenzoic Acid in Organic Solvents ...................

21.1. Critical evaluation of experimental solubility data.....................

21.2. 2-Chloro-5-nitrobenzoic acid solubility data

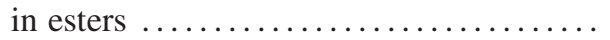

21.3. 2-Chloro-5-nitrobenzoic acid solubility data in ethers .......................

21.4. 2-Chloro-5-nitrobenzoic acid solubility data in alcohols....

22. Solubility of 4-Chloro-3,5-dinitrobenzoic Acid

in Organic Solvents .................

22.1. Critical evaluation of experimental solubility data.....................

22.2. 4-Chloro-3,5-dinitrobenzoic acid solubility data in alcohols ....................

22.3. 4-Chloro-3,5-dinitrobenzoic acid solubility data in miscellaneous organic solvents ...

23. Solubility of 4-Chloro-3-nitrobenzoic Acid in Organic Solvents ...................

23.1. Critical evaluation of experimental solubility data.....................

23.2. 4-Chloro-3-nitrobenzoic acid solubility data in saturated hydrocarbons (including cycloalkanes) .....................

23.3. 4-Chloro-3-nitrobenzoic acid solubility data in aromatic hydrocarbons...............

23.4. 4-Chloro-3-nitrobenzoic acid solubility data in esters .........................

23.5. 4-Chloro-3-nitrobenzoic acid solubility data in ethers $\ldots \ldots \ldots \ldots \ldots \ldots \ldots \ldots \ldots$.

23.6. 4-Chloro-3-nitrobenzoic acid solubility data in alcohols ........................

23.7. 4-Chloro-3-nitrobenzoic acid solubility data in miscellaneous organic solvents........ 
24. Solubility of 4-Cyanobenzoic Acid in Organic Solvents ................

24.1. Critical evaluation of experimental solubility data

24.2. 4-Cyanobenzoic acid solubility data in alcohols ......................

25. Solubility of 3,5-Diaminobenzoic Acid in Organic Solvents ...............

25.1. Critical evaluation of experimental solubility data.

25.2. 3,5-Diaminobenzoic acid solubility data

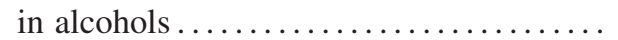

26. Solubility of 2,4-Dichlorobenzoic Acid in Organic Solvents ................

26.1. Critical evaluation of experimental solubility data.

26.2. 2,4-Dichlorobenzoic acid solubility data in alcohols .

26.3. 2,4-Dichlorobenzoic acid solubility data in miscellaneous organic solvents

27. Solubility of 3,4-Dichlorobenzoic Acid in Organic Solvents .................

27.1. Critical evaluation of experimental solubility data.

27.2. 3,4-Dichlorobenzoic acid solubility data in saturated hydrocarbons (including

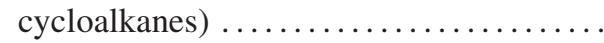

27.3. 3,4-Dichlorobenzoic acid solubility data in aromatic hydrocarbons............

27.4. 3,4-Dichlorobenzoic acid solubility data in esters $\ldots \ldots \ldots \ldots \ldots \ldots \ldots \ldots \ldots . . . \ldots \ldots$

27.5. 3,4-Dichlorobenzoic acid solubility data in ethers

27.6. 3,4-Dichlorobenzoic acid solubility data in alcohols

27.7. 3,4-Dichlorobenzoic acid solubility data in alkoxyalcohols

27.8. 3,4-Dichlorobenzoic acid solubility data in miscellaneous organic solvents .........

28. Solubility of 3,5-Dichlorobenzoic Acid in Organic Solvents ...............

28.1. Critical evaluation of experimental

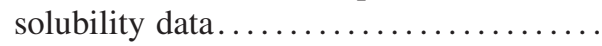

28.2. 3,5-Dichlorobenzoic acid solubility data

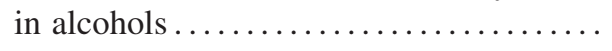

28.3. 3,5-Dichlorobenzoic acid solubility data in miscellaneous organic solvents ..........

29. Solubility of 2,4-Dihydroxybenzoic Acid in Organic Solvents ................

29.1. Critical evaluation of experimental solubility data

29.2. 2,4-Dihydroxybenzoic acid solubility data in saturated hydrocarbons (including

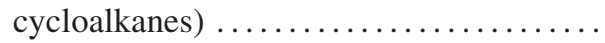

29.3. 2,4-Dihydroxybenzoic acid solubility data in aromatic hydrocarbons.
29.4. 2,4-Dihydroxybenzoic acid solubility data in haloalkanes, haloalkenes, and

haloaromatic hydrocarbons

30. Solubility of 2,5-Dihydroxybenzoic Acid in

Organic Solvents ................

30.1. Critical evaluation of experimental solubility data......................

30.2. 2,5-Dihydroxybenzoic acid solubility data in aromatic hydrocarbons............

30.3. 2,5-Dihydroxybenzoic acid solubility data in haloalkanes, haloalkenes, and haloaromatic hydrocarbons ............

30.4. 2,5-Dihydroxybenzoic acid solubility data in alcohols ........................

31. Solubility of 2,6-Dihydroxybenzoic Acid in Organic Solvents ................

31.1. Critical evaluation of experimental solubility data.

31.2. 2,6-Dihydroxybenzoic acid solubility data in saturated hydrocarbons (including

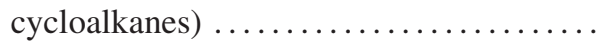

31.3. 2,6-Dihydroxybenzoic acid solubility data in aromatic hydrocarbons.............

31.4. 2,6-Dihydroxybenzoic acid solubility data in haloalkanes, haloalkenes, and haloaromatic hydrocarbons ............

32. Solubility of 3,4-Dihydroxybenzoic Acid in Organic Solvents ..............

32.1. Critical evaluation of experimental

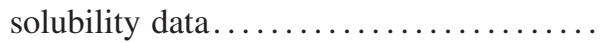

32.2. 3,4-Dihydroxybenzoic acid solubility data in haloalkanes, haloalkenes, and haloaromatic hydrocarbons

33. Solubility of 2,6-Dimethoxybenzoic Acid in Organic Solvents ...............

33.1. Critical evaluation of experimental solubility data......................

33.2. 2,6-Dimethoxybenzoic acid solubility data in saturated hydrocarbons (including

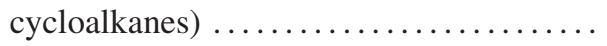

33.3. 2,6-Dimethoxybenzoic acid solubility data in aromatic hydrocarbons.............

33.4. 2,6-Dimethoxybenzoic acid solubility data in haloalkanes, haloalkenes, and haloaromatic hydrocarbons ............

34. Solubility of 3,4-Dimethoxybenzoic Acid in Organic Solvents .................

34.1. Critical evaluation of experimental solubility data....................

34.2. 3,4-Dimethoxybenzoic acid solubility data in esters

34.3. 3,4-Dimethoxybenzoic acid solubility data in ethers .........................

34.4. 3,4-Dimethoxybenzoic acid solubility data in alcohols ........................
34.5. 3,4-Dimethoxybenzoic acid solubility in alkoxyalcohols ..................... 
34.6. 3,4-Dimethoxybenzoic acid solubility data in alkanones ......................

34.7. 3,4-Dimethoxybenzoic acid solubility data in miscellaneous organic solvents........

34.8. 3,4-Dimethoxybenzoic acid solubility data in binary organic solvent mixtures .......

35. Solubility of 3,5-Dimethoxybenzoic Acid in Organic Solvents ..................

35.1. Critical evaluation of experimental solubility data.....................

35.2. 3,5-Dimethoxybenzoic acid solubility data in alcohols ........................

36. Solubility of 3-(Dimethylamino)benzoic Acid in Organic Solvents ..................

36.1. Critical evaluation of experimental solubility data......................

36.2. 3-(Dimethylamino)benzoic acid solubility data in saturated hydrocarbons (including cycloalkanes)

281

282

284

284

284

285

285

285

36.3. 3-(Dimethylamino)benzoic acid solubility data in aromatic hydrocarbons

37. Solubility of 4-(Dimethylamino)benzoic Acid in Organic Solvents ..................

37.1. Critical evaluation of experimental solubility data.....................

37.2. 4-(Dimethylamino)benzoic acid solubility data in saturated hydrocarbons (including cycloalkanes) .....................

37.3. 4-(Dimethylamino)benzoic acid solubility data in aromatic hydrocarbons..........

37.4. 4-(Dimethylamino)benzoic acid solubility data in alcohols .....................

37.5. 4-(Dimethylamino)benzoic acid solubility data in miscellaneous organic solvents ...

38. Solubility of 3,4-Dimethylbenzoic Acid in Organic Solvents ..................

38.1. Critical evaluation of experimental solubility data.....................

38.2. 3,4-Dimethylbenzoic acid solubility data in alcohols .......................

38.3. 3,4-Dimethylbenzoic acid solubility data in miscellaneous organic solvents ..........

39. Solubility of 2,4-Dinitrobenzoic Acid in Organic

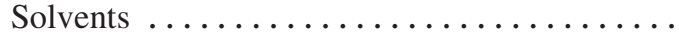

39.1. Critical evaluation of experimental solubility data.

39.2. 2,4-Dinitrobenzoic acid solubility data in alcohols .......................

39.3. 2,4-Dinitrobenzoic acid solubility data in miscellaneous organic solvents........

40. Solubility of 3,5-Dinitrobenzoic Acid in Organic

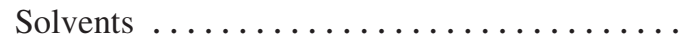

40.1. Critical evaluation of experimental solubility data.....................

40.2. 3,5-Dinitrobenzoic acid solubility data in saturated hydrocarbons (including cycloalkanes)
40.3. 3,5-Dinitrobenzoic acid solubility data in aromatic hydrocarbons ................

40.4. 3,5-Dinitrobenzoic acid solubility data in esters ..........................

40.5. 3,5-Dinitrobenzoic acid solubility data in ethers ............................

40.6. 3,5-Dinitrobenzoic acid solubility data in alcohols .........................

40.7. 3,5-Dinitrobenzoic acid solubility data in miscellaneous organic solvents .........

41. Solubility of 3,5-Dinitro-2-methylbenzoic Acid in Organic Solvents ................

41.1. Critical evaluation of experimental solubility data.....................

41.2. 3,5-Dinitro-2-methylbenzoic acid solubility data in esters.....................

41.3. 3,5-Dinitro-2-methylbenzoic acid solubility data in ethers

41.4. 3,5-Dinitro-2-methylbenzoic acid solubility data in alcohols

42. Solubility of 2-Fluorobenzoic Acid in Organic Solvents .......................

42.1. Critical evaluation of experimental solubility data.

42.2. 2-Fluorobenzoic acid solubility data in alcohols .......................

43. Solubility of 3-Fluorobenzoic Acid in Organic

Solvents .........................

43.1. Critical evaluation of experimental solubility data.....................

43.2. 3-Fluorobenzoic acid solubility data in alcohols ........................

44. Solubility of 4-Fluorobenzoic Acid in Organic

Solvents ......................

44.1. Critical evaluation of experimental solubility data.

44.2. 4-Fluorobenzoic acid solubility data in ethers ..........................

44.3. 4-Fluorobenzoic acid solubility data in alcohols ........................

45. Solubility of 4-Formylbenzoic Acid in Organic

Solvents .......................

45.1. Critical evaluation of experimental solubility data.....................

45.2. 4-Formylbenzoic acid solubility data in miscellaneous organic solvents ..........

46. Solubility of 2-Hydroxybenzoic Acid in Organic

Solvents ......................

46.1. Critical evaluation of experimental solubility data......................

46.2. 2-Hydroxybenzoic acid solubility data in saturated hydrocarbons (including cycloalkanes) .....................

46.3. 2-Hydroxybenzoic acid solubility data in aromatic hydrocarbons ................

46.4. 2-Hydroxybenzoic acid solubility data in

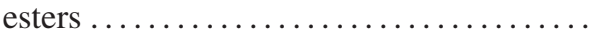


46.5. 2-Hydroxybenzoic acid solubility data in

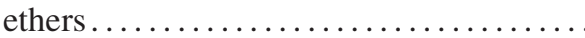

46.6. 2-Hydroxybenzoic acid solubility data in haloalkanes, haloalkenes, and haloaromatic hydrocarbons

46.7. 2-Hydroxybenzoic acid solubility data in alcohols ........................

46.8. 2-Hydroxybenzoic acid solubility data in alkoxyalcohols .................

46.9. 2-Hydroxybenzoic acid solubility data

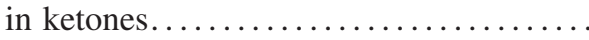

46.10. 2-Hydroxybenzoic acid solubility data in miscellaneous organic solvents.......

46.11. 2-Hydroxybenzoic acid solubility data in binary organic solvent mixtures......

47. Solubility of 3-Hydroxybenzoic Acid in Organic Solvents .......................

47.1. Critical evaluation of experimental solubility data.

47.2. 3-Hydroxybenzoic acid solubility data in saturated hydrocarbons (including cycloalkanes)

47.3. 3-Hydroxybenzoic acid solubility data in aromatic hydrocarbons

47.4. 3-Hydroxybenzoic acid solubility data in esters ...........................

47.5. 3-Hydroxybenzoic acid solubility data in

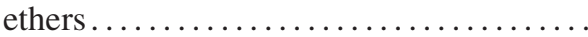

47.6. 3-Hydroxybenzoic acid solubility data in haloalkanes, haloalkenes, and haloaromatic hydrocarbons

47.7. 3-Hydroxybenzoic acid solubility data in alcohols

47.8. 3-Hydroxybenzoic acid solubility data in ketones

47.9. 3-Hydroxybenzoic acid solubility data in miscellaneous organic solvents ..........

48. Solubility of 4-Hydroxybenzoic Acid in Organic

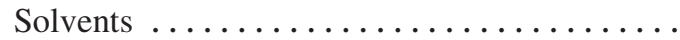

48.1. Critical evaluation of experimental solubility data....................

48.2. 4-Hydroxybenzoic acid solubility data in saturated hydrocarbons (including cycloalkanes) .....................

48.3. 4-Hydroxybenzoic acid solubility data in aromatic hydrocarbons ..............

48.4. 4-Hydroxybenzoic acid solubility data in

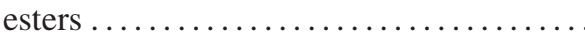

48.5. 4-Hydroxybenzoic acid solubility data in ethers .............................

48.6. 4-Hydroxybenzoic acid solubility data in haloalkanes, haloalkenes, and haloaromatic hydrocarbons ....................

48.7. 4-Hydroxybenzoic acid solubility data

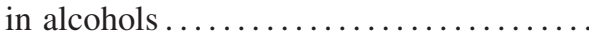

48.8. 4-Hydroxybenzoic acid solubility data in

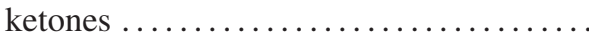

48.9. 4-Hydroxybenzoic acid solubility data in miscellaneous organic solvents .........

49. Solubility of 2-Hydroxy-3-methylbenzoic Acid in Organic Solvents ..............

49.1. Critical evaluation of experimental solubility data.

49.2. 2-Hydroxy-3-methylbenzoic acid solubility data in saturated hydrocarbons (including cycloalkanes)

49.3. 2-Hydroxy-3-methylbenzoic acid solubility data in aromatic hydrocarbons ..........

50. Solubility of 2-Hydroxy-4-methylbenzoic Acid in Organic Solvents ..............

50.1. Critical evaluation of experimental

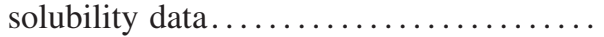

50.2. 2-Hydroxy-4-methylbenzoic acid solubility data in saturated hydrocarbons (including cycloalkanes)

50.3. 2-Hydroxy-4-methylbenzoic acid solubility data in aromatic hydrocarbons

51. Solubility of 2-Hydroxy-5-methylbenzoic Acid in Organic Solvents .............

51.1. Critical evaluation of experimental solubility data.

51.2. 2-Hydroxy-5-methylbenzoic acid solubility data in saturated hydrocarbons (including

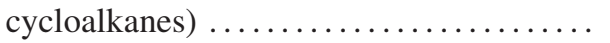

51.3. 2-Hydroxy-5-methylbenzoic acid solubility data in aromatic hydrocarbons .

52. Solubility of 3-Hydroxy-4-methylbenzoic Acid in Organic Solvents ...............

52.1. Critical evaluation of experimental solubility data.

52.2. 3-Hydroxy-4-methylbenzoic acid solubility data in aromatic hydrocarbons

53. Solubility of 4-Hydroxy-3-methoxybenzoic Acid in Organic Solvents ................

53.1. Critical evaluation of experimental solubility data...................

53.2. 4-Hydroxy-3- methoxybenzoic acid solubility data in miscellaneous organic

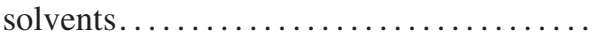

54. Solubility of 4-Hydroxy-3-methylbenzoic Acid in Organic Solvents ..............

54.1. Critical evaluation of experimental solubility data.

54.2. 4-Hydroxy-3-methylbenzoic acid solubility data in aromatic hydrocarbons

55. Solubility of 4-Iodobenzoic Acid in Organic Solvents .................

55.1. Critical evaluation of experimental solubility data.....................

55.2. 4-Iodobenzoic acid solubility data in alcohols ......................

55.3. 4-Iodobenzoic acid solubility data in miscellaneous organic solvents .........

56. Solubility of 2-Methoxybenzoic Acid in Organic Solvents ................ 
56.1. Critical evaluation of experimental solubility data.

56.2. 2-Methoxybenzoic acid solubility data in saturated hydrocarbons (including cycloalkanes)

56.3. 2-Methoxybenzoic acid solubility data in aromatic hydrocarbons

56.4. 2-Methoxybenzoic acid solubility data in esters ............................

56.5. 2-Methoxybenzoic acid solubility data in ethers...........................

56.6. 2-Methoxybenzoic acid solubility data in haloalkanes, haloalkenes, and haloaromatic hydrocarbons

56.7. 2-Methoxybenzoic acid solubility data in alcohols ........................

56.8. 2-Methoxybenzoic acid solubility data in alkoxyalcohols

56.9. 2-Methoxybenzoic acid solubility data in miscellaneous organic solvents ..........

57. Solubility of 3-Methoxybenzoic Acid in Organic Solvents .......................

57.1. Critical evaluation of experimental solubility data......................

57.2. 3-Methoxybenzoic acid solubility data in saturated hydrocarbons (including cycloalkanes) .....................

57.3. 3-Methoxybenzoic acid solubility data in aromatic hydrocarbons ................

57.4. 3-Methoxybenzoic acid solubility data in ethers...........................

57.5. 3-Methoxybenzoic acid solubility data in haloalkanes, haloalkenes, and haloaromatic hydrocarbons

57.6. 3-Methoxybenzoic acid solubility data in alcohols ..........................

58. Solubility of 4-Methoxybenzoic Acid in Organic Solvents ......................

58.1. Critical evaluation of experimental solubility data......................

58.2. 4-Methoxybenzoic acid solubility data in saturated hydrocarbons (including cycloalkanes) .....................

58.3. 4-Methoxybenzoic acid solubility data in aromatic hydrocarbons ...............

58.4. 4-Methoxybenzoic acid solubility data in esters ..........................

58.5. 4-Methoxybenzoic acid solubility data in ethers............................

58.6. 4-Methoxybenzoic acid solubility data in haloalkanes, haloalkenes, and haloaromatic hydrocarbons .....................

58.7. 4-Methoxybenzoic acid solubility data in alcohols ........................

58.8. 4-Methoxybenzoic acid solubility data in alkoxyalcohols ......................

59. Solubility of 2-Methylbenzoic Acid in Organic Solvents ......................
59.1. Critical evaluation of experimental solubility data......................

59.2. 2-Methylbenzoic acid solubility data in saturated hydrocarbons (including cycloalkanes) ......................

59.3. 2-Methylbenzoic acid solubility data in aromatic hydrocarbons .................

59.4. 2-Methylbenzoic acid solubility data in esters ...........................

59.5. 2-Methylbenzoic acid solubility data in ethers..........................

59.6. 2-Methylbenzoic acid solubility data in haloalkanes, haloalkenes, and haloaromatic hydrocarbons ....................

59.7. 2-Methylbenzoic acid solubility data in alcohols .........................

59.8. 2-Methylbenzoic acid solubility data in miscellaneous organic solvents .........

59.9. 2-Methylbenzoic acid solubility data in binary organic solvent mixtures..........

60. Solubility of 3-Methylbenzoic Acid in Organic Solvents .......................

60.1. Critical evaluation of experimental solubility data.....................

60.2. 3-Methylbenzoic acid solubility data in saturated hydrocarbons (including cycloalkanes) .....................

60.3. 3-Methylbenzoic acid solubility data in aromatic hydrocarbons ................

60.4. 3-Methylbenzoic acid solubility data in esters ...........................

60.5. 3-Methylbenzoic acid solubility data in ethers ...........................

60.6. 3-Methylbenzoic acid solubility data in alcohols ..........................

60.7. 3-Methylbenzoic acid solubility data in alkoxyalcohols .....................

60.8. 3-Methylbenzoic acid solubility data in miscellaneous organic solvents ..........

60.9. 3-Methylbenzoic acid solubility data in binary organic solvent mixtures..........

61. Solubility of 4-Methylbenzoic Acid in Organic

Solvents ........................

61.1. Critical evaluation of experimental solubility data.....................

61.2. 4-Methylbenzoic acid solubility data in saturated hydrocarbons (including cycloalkanes)

61.3. 4-Methylbenzoic acid solubility data in aromatic hydrocarbons

61.4. 4-Methylbenzoic acid solubility data in esters ............................

61.5. 4-Methylbenzoic acid solubility data in ethers..........................

61.6. 4-Methylbenzoic acid solubility data in alcohols ........................

61.7. 4-Methylbenzoic acid solubility data in miscellaneous organic solvents .......... 
62. Solubility of 2-Nitrobenzoic Acid in Organic

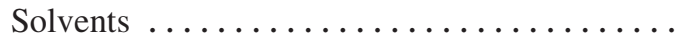

62.1. Critical evaluation of experimental solubility data

62.2. 2-Nitrobenzoic acid solubility data in aromatic hydrocarbons

62.3. 2-Nitrobenzoic acid solubility data in ethers

62.4. 2-Nitrobenzoic acid solubility data in haloalkanes, haloalkenes, and haloaromatic hydrocarbons ..................

62.5. 2-Nitrobenzoic acid solubility data in alcohols .......................

62.6. 2-Nitrobenzoic acid solubility data in ketones

62.7. 2-Nitrobenzoic acid solubility data in miscellaneous organic solvents .........

63. Solubility of 3-Nitrobenzoic Acid in Organic

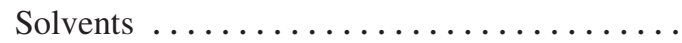

63.1. Critical evaluation of experimental solubility data...................

63.2. 3-Nitrobenzoic acid solubility data in saturated hydrocarbons (including cycloalkanes $)$.....................

63.3. 3-Nitrobenzoic acid solubility data in aromatic hydrocarbons ..............

63.4. 3-Nitrobenzoic acid solubility data in esters

63.5. 3-Nitrobenzoic acid solubility data in ethers

63.6. 3-Nitrobenzoic acid solubility data in haloalkanes, haloalkenes, and haloaromatic hydrocarbons

63.7. 3-Nitrobenzoic acid solubility data

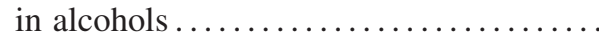

63.8. 3-Nitrobenzoic acid solubility data in ketones ........................

63.9. 3-Nitrobenzoic acid solubility data in miscellaneous organic solvents ..........

64. Solubility of 4-Nitrobenzoic Acid in Organic

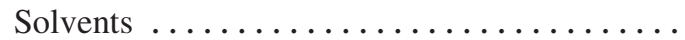

64.1. Critical evaluation of experimental solubility data....................

64.2. 4-Nitrobenzoic acid solubility data in saturated hydrocarbons (including

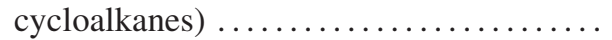

64.3. 4-Nitrobenzoic acid solubility data in aromatic hydrocarbons ..............

64.4. 4-Nitrobenzoic acid solubility data in esters

64.5. 4-Nitrobenzoic acid solubility data in ethers

64.6. 4-Nitrobenzoic acid solubility data in haloalkanes, haloalkenes, and haloaromatic hydrocarbons

64.7. 4-Nitrobenzoic acid solubility data in alcohols ........................

64.8. 4-Nitrobenzoic acid solubility data in alkoxyalcohols ...................

64.9. 4-Nitrobenzoic acid solubility data in ketones .......................

64.10. 4-Nitrobenzoic acid solubility data in miscellaneous organic solvents ........
65. Solubility of 3-Nitro-1,2-benzenedicarboxylic Acid in Organic Solvents..............

65.1. Critical evaluation of experimental solubility data....................

65.2. 3-Nitro-1,2-benzenedicarboxylic acid solubility data in esters ..............

65.3. 3-Nitro-1,2-benzenedicarboxylic acid solubility data in ethers...............

65.4. 3-Nitro-1,2-benzenedicarboxylic acid solubility data in ketones .............

\section{List of Tables}

1. Abraham model equation coefficients describing solute transfer to an organic solvent from water [Eq. (10) $\ldots \ldots \ldots \ldots \ldots \ldots \ldots \ldots \ldots \ldots \ldots \ldots$

2. Abraham model equation coefficients describing solute transfer to an organic solvent from gas phase $[$ Eq. (11) $\ldots \ldots \ldots \ldots \ldots \ldots \ldots \ldots \ldots \ldots$

3. Comparison between observed and predicted molar solubilities of benzoic acid based on the Abraham model, Eq. (20) .................

4. Recommended mole fraction solubilities for benzoic acid in select solvents..............

5. Parameters of Eq. (22) for describing the solubility of benzoic acid in various organic solvents ....

6. Parameters of the Van't Hoff-type equation for describing the solubility of benzoic acid in various

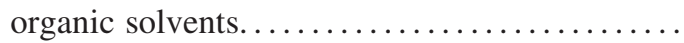

7. Parameters of the Buchowski $\lambda$ h equation for describing the solubility of benzoic acid in various

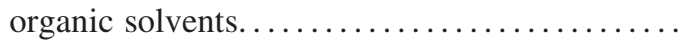

8. Comparison between observed and calculated molar solubilities of 2-acetoxybenzoic acid based on the Abraham model, Eqs. (20) and (21)....

9. Parameters of the Modified Apelblat equation for describing the solubility of 2-acetoxybenzoic acid in various organic solvents ...

10. Parameters of the Modified Apelblat equation for describing the solubility of 4-acetoxybenzoic acid in ethanol...........................

11. Parameters of the Modified Apelblat equation for describing the solubility of 4-acetylbenzoic acid in various organic solvents...............

12. Parameters of the Buchowski $\lambda \mathrm{h}$ equation for describing the solubility of 4-acetylbenzoic acid in various organic solvents...............

13. Parameters of Eq. (22) describing the solubility of 3-aminobenzoic acid in ethyl ethanoate, ethanenitrile, and methanol

14. Comparison between observed and calculated molar solubilities of 4-aminobenzoic acid based on the Abraham model, Eq. (20)............ 
15. Parameters of the Modified Apelblat equation for describing the solubility of 5-amino-2-hydroxybenzoic acid in various organic solvents .......

16. Parameters of the Modified Apelblat equation for describing the solubility of 1,2-benzenedicarboxylic acid in various organic solvents .......

17. Parameters of the Modified Apelblat equation for describing the solubility of 1,3-benzenedicarboxylic acid in various organic solvents .......

18. Parameters of the Buchowski $\lambda \mathrm{h}$ equation for describing the solubility of 1,3-benzenedicarboxylic acid in several organic solvents........

19. Parameters of the Modified Apelblat equation for describing the solubility of 1,4-benzenedicarboxylic acid in various organic solvents .......

20. Parameters of the Buchowski $\lambda \mathrm{h}$ equation for describing the solubility of 1,4-benzenedicarboxylic acid in organic solvents .............

21. Comparison between observed and calculated molar solubilities of 3-chlorobenzoic acid based on the Abraham model, Eqs. (20) and (21).....

22. Comparison between observed and calculated molar solubilities of 4-chlorobenzoic acid based on the Abraham model, Eqs. (20) and (21).....

23. Comparison between observed and calculated molar solubilities of 2-chloro-5-nitrobenzoic acid based on the Abraham model, Eqs. (20) and (21)

24. Comparison between observed and calculated molar solubilities of 4-chloro-3-nitrobenzoic acid based on the Abraham model, Eqs. (20) and (21)

25. Parameters of the Modified Apelblat equation for describing the solubility of 4-cyanobenzoic acid in ethanol ................................

26. Parameters of the Modified Apelblat equation for describing the solubility of 3,5-diaminobenzoic acid in ethanol..........................

27. Parameters of the Modified Apelblat equation for describing the solubility of 2,4-dichlorobenzoic acid in ethanol.........................

28. Comparison between observed and predicted molar solubilities of 3,4-dichlorobenzoic acid based on the Abraham model, Eqs. (20) and (21)

29. Comparison between observed and calculated molar solubilities of 3,4-dimethoxybenzoic acid based on the Abraham model, Eqs. (20) and (21)

30. Parameters of the Modified Apelblat equation for describing the solubility of 3,4-dimethoxybenzoic acid in various organic solvents .............

31. Parameters of the Modified Apelblat equation for describing the solubility of 3,5-dimethoxybenzoic acid in ethanol ..........................

32. Comparison between observed and calculated molar solubilities of 3,5-dinitrobenzoic acid based on the Abraham model, Eqs. (20) and (21).....
33. Comparison between observed and calculated molar solubilities of 3,5-dinitro-2-methylbenzoic acid based on the Abraham model, Eqs. (20)

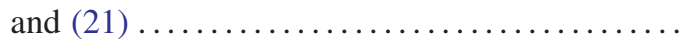

34. Comparison between observed and predicted 142 molar solubilities of 2-hydroxybenzoic acid based on the Abraham model, Eq. (20)..............

35. Recommended mole fraction solubilites for 2hydroxybenzoic acid in selected solvents ......

36. Parameters of Eq. (49) for describing the solubility of 2-hydroxybenzoic acid in various organic solvents ..............................

37. Parameters of the Modified Apelblat-type equation for describing the solubility of 2-hydroxybenzoic acid in various organic solvents..........

38. Comparison between observed and predicted molar solubilities of 4-hydroxybenzoic acid based on the Abraham model, Eqs. (20) and (21).....

39. Parameters of Eq. (53) for describing the solubility of 4-hydroxybenzoic acid in various organic solvents ..............................

40. Comparison between observed and calculated molar solubilities of 2-methoxybenzoic acid based on the Abraham model, Eqs. (20) and (21)....

41. Comparison between observed and calculated molar solubilities of 4-methoxybenzoic acid based on the Abraham model, Eqs. (20) and (21).....

42. Comparison between observed and calculated molar solubilities of 2-methylbenzoic acid based on the Abraham model, Eqs. (20) and (21).....

43. Parameters of the Buchowski $\lambda \mathrm{h}$ model for describing the solubility of 2-methylbenzoic acid in various organic solvents .................

44. Comparison between observed and calculated molar solubilities of 3-methylbenzoic acid based on the Abraham model, Eqs. (20) and (21)....

45. Parameters of the Modified Apelblat equation for describing the solubility of 2-nitrobenzoic acid in various organic solvents...................

46. Comparison between observed and calculated molar solubilities of 3-nitrobenzoic acid based on the Abraham model, Eqs. (20) and (21)........

47. Parameters of the Modified Apelblat equation for describing the solubility of 3-nitrobenzoic acid in various organic solvents...................

48. Comparison between observed and calculated molar solubilities of 4-nitrobenzoic acid based on the Abraham model, Eqs. (20) and (21)........

49. Parameters of the Modified Apelblat equation for describing the solubility of 4-nitrobenzoic acid in various organic solvents..................

50. Parameters of the Modified Apelblat equation for describing the solubility of 3-nitro-1,2-benzenedicarboxylic acid in various organic solvents... 


\section{Preface}

\subsection{Scope of this volume}

This volume reviews experimentally determined solubility data for benzoic acid and 63 substituted benzoic acids dissolved in neat organic solvents and well-defined binary and ternary organic solvent mixtures retrieved from the published chemical and pharmaceutical literature covering the period from 1900 to the end of 2012. Solubility data are compiled and critically reviewed for benzoic acid, 2-acetoxybenzoic acid, 4-acetoxybenzoic acid, 4-acetylbenzoic acid, 2-aminobenzoic acid, 3-aminobenzoic acid, 4-aminobenzoic acid, 4-amino-2-hydroxybenzoic acid, 5-amino-2hydroxybenzoic acid, 1,2-benzenedicarboxylic acid, 1,3benzenedicarboxylic acid, 1,4-benzenedicarboxylic acid, 1,3,5-benzenetricarboxylic acid, 2-bromobenzic acid, 3-bromobenzoic acid, 4-bromobenzoic acid, 2-chlorobenzoic acid, 3-chlorobenzoic acid, 4-chlorobenzoic acid, 2chloro-5-nitrobenzoic acid, 4-chloro-3,5-dinitrobenzoic acid, 4-chloro-3-nitrobenzoic acid, 4-cyanobenzoic acid, 3,5-diaminobenzoic acid, 2,4-dichlorobenzoic acid, 3,5dichlorobenzoic acid, 2,4-dihydroxybenzoic acid, 2,5-dihydroxybenzoic acid, 2,6-dihydroxybenzoic acid, 3,4-dihydroxybenzoic acid, 2,6-dimethoxybenzoic acid, 3,4dimethoxybenzoic acid, 3,5-dimethoxybenzoic acid, 3(dimethylamino)benzoic acid, 4-(dimethylamino)benzoic acid, 3,4-dimethylbenzoic acid, 2,4-dinitrobenzoic acid, 3,5-dinitrobenzoic acid, 3,5-dinitro-2-methylbenzoic acid, 2-fluorobenzoic acid, 3-fluorobenzoic acid, 4-fluorobenzoic acid, 4-formylbenzoic acid, 2-hydroxybenzoic acid, 3hydroxybenzoic acid, 4-hydroxybenzoic acid, 2-hydroxy3-methylbenzoic acid, 2-hydroxy-4-methylbenzoic acid, 2hydroxy-5-methylbenzoic acid, 3-hydroxy-4-methylbenzoic acid, 4-hydroxy-3-methoxybenzoic acid, 4-hydroxy-3methylbenzoic acid, 4-iodobenzoic acid, 2-methoxybenzoic acid, 3-methoxybenzoic acid, 4-methoxybenzoic acid, 2methylbenzoic acid, 3-methylbenzoic acid, 4-methylbenzoic acid, 2-nitrobenzoic acid, 3-nitrobenzoic acid, 4-nitrobenzoic acid, and 3-nitro-1,2-benzenedicarboxylic acid. Aqueous systems and inorganic systems (namely supercritical carbon dioxide) are not included in this volume. Readers wishing solubility data for aqueous and inorganic systems are referred to Vol. 90 in the IUPAC-NIST Solubility Data Series, ${ }^{1,2}$ which dealt with the solubility of hydroxybenzoic acid derivatives in binary, ternary and multicomponent systems. Here one will find solubility data for 2-hydroxybenzoic acid, 3-hydroxybenzoic acid and 4hydroxybenzoic acid, as well as solubility data for several 4hydroxybenzoate alkyl esters (parabens) and hydroxybenzoic acid salts. As an informational note, Vol. 90 contains solubility data for the three hydroxybenzoic acids dissolved in organic solvents. The solubility data reported in the current volume for organic solvents are slightly more extensive and include references that were either published after or overlooked in the preparation of the earlier volume.

Several substituted benzoic acids and benzoic acid derivatives exhibit therapeutic properties. For example, 2-hydroxy- benzoic acid (commonly known as salicylic acid) is a nonsteroidal anti-inflammatory drug (NSAID) used to alleviate aches and pains, and is a key ingredient in many "overthe-counter" and prescription skin-care products for the treatment of acne, psoriasis, corns, warts, calluses, and seborrhoeic dermatitis. Salicylic acid is also an ingredient in shampoos added to control dandruff and dry scalp. Its methyl ester, methyl salicylate, can be applied topically as a liniment to relieve joint and muscle pains. 2-Acetoxybenzoic acid (aspirin) is widely used and prescribed in the treatment of a number of medical conditions, including fever, muscle aches and pains, rheumatoid arthritis, and pericarditis. 2-Acetoxybenzoic acid has also been used long-term, at low doses, to help prevent heart attacks, strokes, and blood clot formation in individuals at high risk of developing blood clots. 4-Aminobenzoic acid is one of the components of the folic acid molecule, and is an effective chemical sunscreen (absorbs ultraviolet and visible sun light) when applied topically. 4Aminosalicylic acid is an antibiotic, prescribed alone or in combination with other drugs, in the treatment of tuberculosis. 5-Amino-2-hydroxybenzoic acid is a drug used for treating ulcerative colitis, which is a chronic inflammation of the large intestine. 4-Hydroxy-3-methoxybenzoic acid has been used as a flavoring agent for many years, and is known for its anthelmintic properties.

\subsection{Concentration units for nonelectrolyte solutions}

Composition of a liquid nonelectrolyte solution can be expressed in a variety of ways, as (1) the ratio of the number of moles of one component to the number of moles of a second component, $n_{1} / n_{2}$, etc., (2) molar concentration

$$
c_{i}=[i]=\frac{n_{i}}{V} \quad \text { SI base units : } \mathrm{mol} \mathrm{dm}^{-3},
$$

(3) mole fraction

$$
x_{i}=\frac{n_{i}}{n_{1}+n_{2}+\ldots .+n_{i}+\ldots},
$$

or (4) volume fraction

$$
\phi_{i}=\frac{n_{i} V_{i}}{n_{1} V_{1}+n_{2} V_{2}+\ldots .+n_{i} V_{i}+\ldots .} .
$$

Strictly speaking, the true volume of a real solution is not equal to the sum of the volumes of the individual components but is the mole-fraction sum of partial volumes, which for a ternary solution is $V=x_{1} V_{1}+x_{2} V_{2}+x_{3} V_{3}$. For purposes of this study, volume fractions are defined in terms of the molar volumes of the pure unmixed components, $V_{\mathrm{m}, i}$ (molar mass of component $i$ divided by density of component $i$ )

$$
\varphi_{i}=\frac{n_{i} V_{\mathrm{m}, i}}{n_{1} V_{\mathrm{m}, 1}+n_{2} V_{\mathrm{m}, 2}+\ldots . .+n_{i} V_{\mathrm{m}, i}+\ldots .},
$$

as this quantity serves as an input parameter in expressions for estimating solubilities in mixed solvents since it requires no $a$ priori knowledge concerning volumetric behavior. Solute solubilities can be found in the chemical literature in terms 
of any of the aforementioned concentration variables, or as molality, $m_{i}$, which is the number of moles of solute $i$ divided by the mass of the solvent

$$
m_{i}=\frac{n_{i}}{n_{\text {solvent }} M_{\text {solvent }}} \quad \text { SI base units : } \mathrm{mol} \mathrm{kg}^{-1},
$$

where $M_{\text {solvent }}$ is the molar mass of the solvent.

\subsection{Procedures used in critical evaluation of published solubility data}

Procedures used in the critical evaluation of published solubility data for crystalline nonelectrolytes dissolved in organic monosolvents and organic solvent mixtures depend to a large extent on the quantity and type of data to be evaluated. In those instances where independent experimental measurements exist, one can compute the mean value and standard deviation for each set of replicate values (or set of values) differing from the rest. This type of analysis will be limited primarily to the neat mono-solvents as published data for binary and ternary solvent mixtures are relatively scarce compared to solubility data for solutes dissolved in single solvent systems. Given the scarcity of binary solvent and ternary solvent solubility data, researchers have tended to perform measurements on new mixtures as opposed to repeating measurements on already studied mixtures, even if measured at different temperatures.

Published solubility data may be found for a given solutesolvent system measured at several different temperatures. The temperature variation can be critically evaluated using standard thermodynamic relationships based on the ideal mole fraction solubility of a solid solute, $x_{1}{ }^{\text {ideal soly }}$ in a liquid solvent ${ }^{3}$

$$
\begin{aligned}
-\ln x_{1}{ }^{\text {ideal soly }}= & \frac{\Delta H_{1}^{\text {fus }}}{R T}\left[1-\frac{T}{T_{\mathrm{mp}}}\right]+\frac{\Delta C_{p, 1}}{R}\left(\frac{T_{\mathrm{mp}}-T}{T}\right) \\
& +\frac{\Delta C_{p, 1}}{R} \ln \left(\frac{T_{\mathrm{mp}}}{T}\right),
\end{aligned}
$$

where $\Delta H_{1}$ fus is the standard molar enthalpy of fusion of the solute at its normal melting point temperature, $T_{\mathrm{mp}}, \Delta \mathrm{C}_{p, 1}$ is the difference in the molar heat capacities of the liquid and crystalline forms of the solute (i.e., $\Delta C_{p, 1}=C_{p \text {,liquid }}-C_{p \text {,solid }}$ ) and $R$ is the universal gas constant. Through suitable algebraic manipulations, Eq. (6) can be rearranged to give

$$
\begin{aligned}
\ln x_{1}{ }^{\text {ideal soly }}= & {\left[\frac{\Delta H_{1}^{\text {fus }}}{R T_{\mathrm{mp}}}+\frac{\Delta C_{p, 1}}{R}\left(1+\ln T_{\mathrm{mp}}\right)\right] } \\
& -\left(\frac{\Delta H_{1}^{\text {fus }}}{R}+\frac{\Delta C_{p, 1} T_{\mathrm{mp}}}{R}\right) \frac{1}{T}+\frac{\Delta C_{p, 1}}{R} \ln T,
\end{aligned}
$$

which has the generalized mathematical form of

$$
\ln x_{1}=A+\frac{B}{T}+C \ln T .
$$

Though derived for an ideal solution, Eq. (8) has been used successfully to describe solute solubility in many nonideal solutions. The equation is commonly referred to as the Modified Apelblat equation in the published literature.
The $\lambda \mathrm{h}$ model, developed by Buchowski et al., ${ }^{4,5}$ is

$$
\ln \left[1+\frac{\lambda\left(1-x_{1}\right)}{x_{1}}\right]=\lambda h\left(\frac{1}{T}-\frac{1}{T_{\mathrm{mp}}}\right),
$$

a second popular mathematical representation for describing how the mole-fraction solubility varies with solution temperature. In Eq. (9), $T$ and $T_{\mathrm{mp}}$ refer to the solution temperature and melting-point temperature of the solute, respectively. The two model parameters, $\lambda$ and $h$, are determined by least-squares analyses using the measured mole-fraction solubilities. Experimental solubility data are considered to be internally consistent if the measured $x_{i}$ values can be accurately described by Eqs. (8) and/or (9).

Solution models have been used with success to rationalize the solubility behavior of a given solute molecule in a series of organic solvents. Of the models developed in recent years, the general solvation parameter developed by Abraham and coworkers $^{6-14}$ is probably the most widely used approach in correlating the solubilities of substituted benzoic acids. The model is based on two linear free energy relationships describing solute transfer between two immiscible phases. The first expression quantifies solute transfer between two condensed phases

$$
\begin{aligned}
\log _{10}(S R \text { or } P)= & c_{\mathrm{p}}+e_{\mathrm{p}} \cdot E+s_{\mathrm{p}} \cdot S+a_{\mathrm{p}} \cdot A+b_{\mathrm{p}} \cdot B \\
& +v_{\mathrm{p}} \cdot V,
\end{aligned}
$$

and the second expression involves solute transfer from the gas phase

$$
\begin{aligned}
\log _{10}(G S R \text { or } K)= & c_{\mathrm{k}}+e_{\mathrm{k}} \cdot E+s_{\mathrm{k}} \cdot S+a_{\mathrm{k}} \cdot A \\
& +b_{\mathrm{k}} \cdot B+l_{\mathrm{k}} \cdot L,
\end{aligned}
$$

where $P$ is the water-to-organic solvent partition coefficient or nonpolar organic solvent-to-polar organic solvent partition coefficient, and $K$ is the gas-to-organic solvent partition coefficient. For solubility predictions, the Abraham model uses the solubility ratio which is given by the ratio of the molar solubilities of the solute in the organic solvent, $c_{1, \mathrm{~S}}^{\mathrm{sat}}$, and in water, $c_{1, \mathrm{~W}}^{\text {sat }}$ (i.e., $\left.S R=c_{1, \mathrm{~S}}^{\text {sat }} / c_{1, \mathrm{~W}}^{\mathrm{sat}}\right)$. The gas phase solubility ratio is similarly calculated as the molar solubility in the organic solvent divided by the solute gas phase concentration (i.e., $G S R=c_{1, \mathrm{~S}}^{\mathrm{sat}} / c_{1, \mathrm{G}}$ ), the latter value calculable from the solute vapor pressure above the solid at the solution temperature.

The dependent variables in Eqs. (10) and (11) are solute descriptors as follows: $E$ is the solute excess molar refraction (in units of $\mathrm{cm}^{3} \mathrm{~mol}^{-1} / 10$ ), $S$ refers to the solute dipolarity/ polarizability, $A$ and $B$ represent the overall solute hydrogen bond acidity and basicity, $V$ denotes the solute's McGowan characteristic molecular volume (in units of $\mathrm{cm}^{3} \mathrm{~mol}^{-1} / 100$ ), and $L$ is the logarithm of the gas-to-hexadecane partition coefficient measured at $298 \mathrm{~K}$. The lower-case regression coefficients and constants $\left(c_{\mathrm{p}}, e_{\mathrm{p}}, s_{\mathrm{p}}, a_{\mathrm{p}}, b_{\mathrm{p}}, v_{\mathrm{p}}, c_{\mathrm{k}}, e_{\mathrm{k}}, s_{\mathrm{k}}, a_{\mathrm{k}}\right.$, $b_{\mathrm{k}}$, and $l_{\mathrm{k}}$ ) in Eqs. (10) and (11) are obtained by multiple linear regression analysis of experimental partition coefficient data and solubility ratios for a specific biphasic system. To date Abraham model correlations have been developed for predicting the solubility of crystalline nonelectrolytes in more than 70 different organic solvents, ${ }^{15-22}$ for predicting the 
water-to-organic solvent and gas-to-organic solvent partition coefficient for more than 70 different biphasic systems, ${ }^{15-24}$ and for predicting the partition coefficients of organic vapors and gaseous solutes into aqueous micellar solvent media, ${ }^{25,26}$ into humic acid, ${ }^{27}$ and into various body tissues and fluids. ${ }^{28-34}$ Each of the aforementioned predictions requires a priori knowledge of the compound's solute descriptors as input parameters.

Equation (10) correlates experimental partition coefficients and/or solubility ratios, and for select organic solvents both "dry" and "wet" equation coefficients have been reported. For solvents that are partially miscible with water, such as 1pentanol and butyl ethanoate, solubility ratios calculated as the molar solute solubility in the organic solvent divided by the solute's aqueous molar solubility are not the same as those obtained from direct partition between water (saturated with the organic solvent) and organic solvent (saturated with water). Care must be taken not to confuse the two sets of transfer process. There should be no confusion in the case of solvents that are fully miscible with water, such as ethanol. Only one set of equation coefficients has been published, and the dependent variable is the logarithm of the solubility ratio. And for solvents that are "almost" completely immiscible with water, such as alkylbenzenes (benzene, toluene, etc.) and chloroalkanes (1,2-dichloroethane, chloroform), there should be no confusion because the solubility ratio [see Eq. (3)] will be nearly identical to the practical partition coefficient.

Applicability of the Abraham solvation parameter model is fairly straightforward. One starts with the set of equations that have been obtained for the ratio of the molar solubilities of the solute in the organic solvent and in water (i.e., $S R=$ $c_{1, \mathrm{~S}}^{\mathrm{sat}} / c_{1, \mathrm{~W}}^{\mathrm{sat}}$ ). Table 1 lists the coefficients in Eq. (10) for transfer processes that shall be considered in the present volume. It is noted that coefficients are periodically revised when additional experimental data become available. Thus, if $c_{1, \mathrm{~W}}^{\mathrm{sat}}$ is known, predicted $\log _{10} S R$ values based upon Eq. (10) will lead to predicted molar solubilities in organic solvents through $S R=$ $c_{1, \mathrm{~S}}^{\mathrm{sat}} / c_{1, \mathrm{~W}}^{\mathrm{sat}}$.

Solubilities in organic solvents can also be predicted and correlated with Eq. (11). Listed in Table 2 are the equation coefficients that have been previously determined for the gas phase solubility ratio, $G S R=c_{1, \mathrm{~S}}^{\mathrm{sat}} / c_{1, \mathrm{G}}$. Predicted $\log _{10} G S R$ values can also be converted to saturation molar solubilities, provided that the saturated vapor pressure above the crystalline solute at $298.15 \mathrm{~K}, \mathrm{VP}^{\circ}$, is known. $\mathrm{VP}^{\circ}$ is transformed into the solute's gas phase molar concentration, $c_{1, \mathrm{G}}$, which is then used to calculate the respective gas-to-water and gas-to-solvent partition coefficients, $G S R_{\mathrm{W}}$ and $G S R_{\mathrm{S}}$ :

$$
\begin{gathered}
G S R_{\mathrm{W}}=c_{1, \mathrm{~W}}^{\mathrm{sat}} / c_{1, \mathrm{G}}^{\mathrm{sat}} \quad \text { or } \\
\log _{10} G S R_{\mathrm{W}}=\log _{10} c_{1, \mathrm{~W}}^{\mathrm{sat}}-\log _{10} c_{1, \mathrm{G}}, \\
G S R_{\mathrm{S}}=K_{\mathrm{S}}=c_{1, \mathrm{~S}}^{\mathrm{sat}} / c_{1, \mathrm{G}} \quad \text { or } \\
\log _{10} G S R_{\mathrm{S}}=\log _{10} c_{1, \mathrm{~S}}^{\mathrm{sat}}-\log _{10} c_{1, \mathrm{G}} .
\end{gathered}
$$

An estimated value of $c_{1, \mathrm{G}}$ can be assumed in the preliminary calculations if an experimental vapor pressure cannot be located in the published literature for the solute at $298.15 \mathrm{~K}$. The value can be adjusted if necessary in order to reduce the $\log _{10} G S R$ deviations, and to make the $\log _{10} S R$ and $\log _{10} G S R$ computations internally consistent as discussed in several previous publications.

Three specific conditions must be met in order to use the Abraham solvation parameter model to predict saturation solubilities. First, the same solid phase must be in equilibrium with the saturated solutions in the organic solvent and in water (i.e., there should be no solvate or hydrate formation). Second, the secondary medium activity coefficient of the solid in the saturated solutions must be unity (or near unity). This condition generally restricts the method to those solutes that are sparingly soluble in water and nonaqueous solvents. Finally, for solutes that are ionized in aqueous solution, $c_{\mathrm{A} \text {,water }}$ refers to the solubility of the neutral monomeric form. In the case of substituted benzoic acids, this will limit the model to solvents such as alcohols, short alkyl chain ethers, alkyl alkanoates and propylene carbonate. Carboxylic acids are known to dimerize in alkane and nonpolar aromatic solvents, and the solute descriptors that have been calculated for the benzoic acid derivatives pertain to the monomeric form. The second restriction involving the secondary medium activity coefficient may not be as important as initially believed. The Abraham solvation parameter model has shown remarkable success in correlating the solubility of several very soluble crystalline solutes. For example, Eqs. (10) and (11) described the molar solubility of benzil in 24 organic solvents to within overall standard deviations of 0.124 and $0.109 \log _{10}$ units, respectively. ${ }^{35}$ Standard deviations for acetylsalicylic acid dissolved in 13 alcohols, 4 ethers, and ethyl ethanoate were 0.123 and $0.138 \log _{10}$ units. $^{12}$ Benzil $^{35}$ and acetylsalicylic acid ${ }^{12}$ exhibited solubilities exceeding 1 molar in several of the organic solvents studied. In the case of acetylsalicylic acid, it could be argued that the model's success relates back to when the equation coefficients were originally calculated for the dry solvents. The databases used in the regression analyses contained very few carboxylic acid solutes (benzoic acid, 2-hydroxybenzoic acid, and 4-hydroxybenzoic acid). Most of the experimental data for carboxylic acids and other very acidic solutes was in the form of saturation solubilities, which were also in the 1-3 molar range. Such arguments do not explain why Eqs. (10) and (11) described the measured benzil solubility data. The benzil solubilities were measured after most of the equation coefficients were first determined.

Numerical values of solute descriptors exist for more than 5000 different organic and organometallic compounds, and if not readily available are easily calculable from measured partition coefficient and solubility data. ${ }^{7,15,36,37}$ The McGowan volume solute descriptor, $V$, is calculated from the molecular formula and the number of chemical bonds in the solute as follows: ${ }^{38}$

$$
V=\sum_{i}^{\text {atoms }} n_{i} A V_{i}-6.56 n_{\text {bonds }}
$$

where $n_{i}$ and $A V_{i}$ denote the number of atoms and atomic volume of element $i$ in the solute molecule, respectively, and 
TABLE 1. Abraham model equation coefficients describing solute transfer to an organic solvent from water [Eq. (10)]

\begin{tabular}{|c|c|c|c|c|c|c|}
\hline Organic solvent & $c_{\mathrm{p}}$ & $e_{\mathrm{p}}$ & $s_{\mathrm{p}}$ & $a_{\mathrm{p}}$ & $b_{\mathrm{p}}$ & $v_{\mathrm{p}}$ \\
\hline Dichloromethane & 0.319 & 0.102 & -0.187 & -3.058 & -4.090 & 4.324 \\
\hline Trichloromethane & 0.191 & 0.105 & -0.403 & -3.112 & -3.514 & 4.395 \\
\hline Tetrachloromethane & 0.199 & 0.523 & -1.159 & -3.560 & -4.594 & 4.618 \\
\hline 1,2-Dichloroethane & 0.183 & 0.294 & -0.134 & -2.801 & -4.291 & 4.180 \\
\hline 1-Chlorobutane & 0.222 & 0.273 & -0.569 & -2.918 & -4.883 & 4.456 \\
\hline Hexane & 0.333 & 0.560 & -1.710 & -3.578 & -4.939 & 4.463 \\
\hline Heptane & 0.297 & 0.634 & -1.755 & -3.571 & -4.946 & 4.488 \\
\hline Octane & 0.241 & 0.690 & -1.769 & -3.545 & -5.011 & 4.511 \\
\hline Decane & 0.172 & 0.726 & -1.750 & -3.446 & -4.496 & 4.489 \\
\hline Undecane & 0.058 & 0.603 & -1.661 & -3.421 & -5.120 & 4.619 \\
\hline Dodecane & 0.114 & 0.668 & -1.644 & -3.545 & -5.006 & 4.459 \\
\hline Hexadecane & 0.087 & 0.667 & -1.617 & -3.587 & -4.869 & 4.433 \\
\hline Cyclohexane & 0.159 & 0.784 & -1.678 & -3.740 & -4.929 & 4.577 \\
\hline Methylcyclohexane & 0.246 & 0.782 & -1.982 & -3.517 & -4.293 & 4.528 \\
\hline 2,2,4-Trimethylpentane & 0.318 & 0.555 & -1.737 & -3.677 & -4.864 & 4.417 \\
\hline Benzene & 0.142 & 0.464 & -0.588 & -3.099 & -4.625 & 4.491 \\
\hline Toluene & 0.125 & 0.431 & -0.644 & -3.002 & -4.748 & 4.524 \\
\hline Ethylbenzene & 0.093 & 0.467 & -0.723 & -3.001 & -4.844 & 5.514 \\
\hline 1,2-Dimethylbenzene & 0.083 & 0.518 & -0.813 & -2.884 & -4.821 & 4.559 \\
\hline 1,3-Dimethylbenzene & 0.122 & 0.377 & -0.603 & -2.981 & -4.961 & 4.535 \\
\hline 1,4-Dimethylbenzene & 0.166 & 0.477 & -0.812 & -2.939 & -4.874 & 4.532 \\
\hline Fluorobenzene & 0.139 & 0.152 & -0.374 & -3.030 & -4.601 & 4.540 \\
\hline Chlorobenzene & 0.065 & 0.381 & -0.521 & -3.183 & -4.700 & 4.614 \\
\hline Bromobenzene & -0.017 & 0.436 & -0.424 & -3.174 & -4.558 & 4.445 \\
\hline Iodobenzene & -0.192 & 0.298 & -0.308 & -3.213 & -4.653 & 4.588 \\
\hline Nitrobenzene & -0.152 & 0.525 & 0.081 & -2.332 & -4.494 & 4.187 \\
\hline Benzonitrile & 0.097 & 0.285 & 0.059 & -1.605 & -4.562 & 4.028 \\
\hline Olive oil & -0.035 & 0.574 & -0.798 & -1.422 & -4.984 & 4.210 \\
\hline Carbon disulfide & 0.047 & 0.686 & -0.943 & -3.603 & -5.818 & 4.921 \\
\hline Isopropyl myristate & -0.605 & 0.930 & -1.153 & -1.682 & -4.093 & 4.249 \\
\hline Triolein & 0.385 & 0.983 & -2.083 & -2.007 & -3.452 & 4.072 \\
\hline Methanol & 0.276 & 0.334 & -0.714 & 0.243 & -3.320 & 3.549 \\
\hline Ethanol & 0.222 & 0.471 & -1.035 & 0.326 & -3.596 & 3.857 \\
\hline Propan-1-ol & 0.139 & 0.405 & -1.029 & 0.247 & -3.767 & 3.986 \\
\hline Butan-1-ol & 0.165 & 0.401 & -1.011 & 0.056 & -3.958 & 4.044 \\
\hline Pentan-1-ol & 0.150 & 0.536 & -1.229 & 0.141 & -3.864 & 4.077 \\
\hline Hexan-1-ol & 0.115 & 0.492 & -1.164 & 0.054 & -3.978 & 4.131 \\
\hline Heptan-1-ol & 0.035 & 0.398 & -1.063 & 0.002 & -4.342 & 4.317 \\
\hline Octan-1-ol & -0.034 & 0.489 & -1.044 & -0.024 & -4.235 & 4.218 \\
\hline Decan-1-ol & -0.058 & 0.616 & -1.319 & 0.026 & -4.153 & 4.279 \\
\hline Propan-2-ol & 0.102 & 0.315 & -1.020 & 0.532 & -3.865 & 4.023 \\
\hline 2-Methylpropan-1-ol & 0.161 & 0.310 & -1.069 & 0.183 & -3.774 & 4.040 \\
\hline 2-Butanol & 0.194 & 0.383 & -0.956 & 0.134 & -3.606 & 3.829 \\
\hline 2-Methylpropan-2-ol & 0.197 & 0.136 & -0.916 & 0.318 & -4.031 & 4.112 \\
\hline 3-Methylbutan-1-ol & 0.123 & 0.370 & -1.243 & 0.074 & -3.781 & 4.208 \\
\hline 2-Pentanol & 0.115 & 0.455 & -1.331 & 0.206 & -3.745 & 4.201 \\
\hline Ethylene glycol & -0.270 & 0.578 & -0.511 & 0.715 & -2.619 & 2.729 \\
\hline 2,2,2-Trifluoroethanol & 0.395 & -0.094 & -0.594 & -1.280 & -1.274 & 3.088 \\
\hline 1,1'-Oxybisethane & 0.330 & 0.401 & -0.814 & -0.457 & -4.959 & 4.320 \\
\hline Tetrahydrofuran & 0.207 & 0.372 & -0.392 & -0.236 & -4.934 & 4.447 \\
\hline Dioxane & 0.098 & 0.350 & -0.083 & -0.556 & -4.826 & 4.172 \\
\hline $1,1^{\prime}-$ Oxybisbutane & 0.203 & 0.369 & -0.954 & -1.488 & -5.426 & 4.508 \\
\hline 2-Methoxy-2-methylpropane & 0.376 & 0.264 & -0.788 & -1.078 & -5.030 & 4.410 \\
\hline Methyl ethanoate & 0.351 & 0.223 & -0.150 & -1.035 & -4.527 & 3.972 \\
\hline Ethyl ethanoate & 0.328 & 0.369 & -0.446 & -0.700 & -4.904 & 4.150 \\
\hline Propyl ethanoate & 0.288 & 0.363 & -0.474 & -0.784 & -4.939 & 4.216 \\
\hline Butyl ethanoate & 0.248 & 0.356 & -0.501 & -0.867 & -4.973 & 4.281 \\
\hline Propanone & 0.313 & 0.312 & -0.121 & -0.608 & -4.753 & 3.942 \\
\hline Butanone & 0.246 & 0.256 & -0.080 & -0.767 & -4.855 & 4.148 \\
\hline Cyclohexanone & 0.038 & 0.225 & 0.058 & -0.976 & -4.842 & 4.315 \\
\hline Propylene carbonate & 0.004 & 0.168 & 0.504 & -1.283 & -4.407 & 3.421 \\
\hline Dimethylformamide & -0.305 & -0.058 & 0.343 & 0.358 & -4.865 & 4.486 \\
\hline Dimethylacetamide & -0.271 & 0.084 & 0.209 & 0.915 & -5.003 & 4.557 \\
\hline Diethylacetamide & 0.213 & 0.034 & 0.089 & 1.342 & -5.084 & 4.088 \\
\hline Dibutylformamide & 0.332 & 0.302 & -0.436 & 0.358 & -4.902 & 3.952 \\
\hline$N$-Methylpyrolidinone & 0.147 & 0.532 & 0.225 & 0.840 & -4.794 & 3.674 \\
\hline
\end{tabular}


TABLE 1. Abraham model equation coefficients describing solute transfer to an organic solvent from water [Eq. (10)] - Continued

\begin{tabular}{|c|c|c|c|c|c|c|}
\hline Organic solvent & $c_{\mathrm{p}}$ & $e_{\mathrm{p}}$ & $s_{\mathrm{p}}$ & $a_{\mathrm{p}}$ & $b_{\mathrm{p}}$ & $v_{\mathrm{p}}$ \\
\hline$N$-Methyl-2-piperidone & 0.056 & 0.332 & 0.257 & 1.556 & -5.035 & 3.983 \\
\hline$N$-Formylmorpholine & -0.032 & 0.696 & -0.062 & 0.014 & -4.092 & 3.405 \\
\hline$N$-Methylformamide & 0.114 & 0.407 & -0.287 & 0.542 & -4.085 & 3.471 \\
\hline$N$-Ethylformamide & 0.220 & 0.034 & -0.166 & 0.935 & -4.589 & 3.730 \\
\hline$N$-Methylacetamide & 0.090 & 0.205 & -0.172 & 1.305 & -4.589 & 3.833 \\
\hline$N$-Ethylacetamide & 0.284 & 0.128 & -0.442 & 1.180 & -4.728 & 3.856 \\
\hline Formamide & -0.171 & 0.070 & 0.308 & 0.589 & -3.152 & 2.432 \\
\hline Acetonitrile & 0.413 & 0.077 & 0.326 & -1.566 & -4.391 & 3.364 \\
\hline Nitromethane & 0.023 & -0.091 & 0.793 & -1.463 & -4.364 & 3.460 \\
\hline Dimethylsulfoxide & -0.194 & 0.327 & 0.791 & 1.260 & -4.540 & 3.361 \\
\hline Sulfolane (303 K) & 0.000 & 0.147 & 0.601 & -0.318 & -4.541 & 3.290 \\
\hline Tributylphosphate & 0.327 & 0.570 & -0.837 & -1.069 & -4.333 & 3.919 \\
\hline Gas-water & -0.994 & 0.577 & 2.549 & 3.813 & 4.841 & -0.869 \\
\hline
\end{tabular}

$n_{\text {bonds }}$ is the number of chemical bonds. The bond contribution is $6.56 \mathrm{~cm}^{3} \mathrm{~mol}^{-1}$ for each bond, no matter whether single, double, or triple, to be subtracted. In other words, double and triple bonds count as one bond. Numerical values of $A V_{i}$ for elements present in substituted benzoic acids are: $A V_{\mathrm{C}}=$ $16.35 \mathrm{~cm}^{3} \mathrm{~mol}^{-1} ; A V_{\mathrm{H}}=8.71 \mathrm{~cm}^{3} \mathrm{~mol}^{-1} ; A V_{\mathrm{N}}=14.39 \mathrm{~cm}^{3}$ $\mathrm{mol}^{-1} ; A V_{\mathrm{O}}=12.43 \mathrm{~cm}^{3} \mathrm{~mol}^{-1} ; A V_{\mathrm{F}}=10.48 \mathrm{~cm}^{3} \mathrm{~mol}^{-1}$;
$A V_{\mathrm{Cl}}=20.95 \mathrm{~cm}^{3} \mathrm{~mol}^{-1} ; A V_{\mathrm{Br}}=26.21 \mathrm{~cm}^{3} \mathrm{~mol}^{-1} ; A V_{\mathrm{I}}=$ $34.53 \mathrm{~cm}^{3} \mathrm{~mol}^{-1} ; A V_{\mathrm{S}}=22.91 \mathrm{~cm}^{3} \mathrm{~mol}^{-1}$; and $A V_{\mathrm{P}}=24.87$ $\mathrm{cm}^{3} \mathrm{~mol}^{-1}$.

The numerical value of the excess molar refraction solute descriptor, $E$, is also fairly easy to calculate. It is defined as the molar refraction of the solute using McGowan's volume, $M R_{X}$, minus the molar refraction of an alkane

TABLE 2. Abraham model equation coefficients describing solute transfer to an organic solvent from gas phase [Eq. (11)]

\begin{tabular}{|c|c|c|c|c|c|c|}
\hline Organic solvent & $c_{\mathrm{k}}$ & $e_{\mathrm{k}}$ & $s_{\mathrm{k}}$ & $a_{\mathrm{k}}$ & $b_{\mathrm{k}}$ & $l_{\mathrm{k}}$ \\
\hline Oleyl alcohol & -0.268 & -0.392 & 0.800 & 3.117 & 0.978 & 0.918 \\
\hline Dichloromethane & 0.192 & -0.572 & 1.492 & 0.460 & 0.847 & 0.965 \\
\hline Trichloromethane & 0.157 & -0.560 & 1.259 & 0.374 & 1.333 & 0.976 \\
\hline Tetrachloromethane & 0.217 & -0.435 & 0.554 & 0.000 & 0.000 & 1.069 \\
\hline 1,2-Dichloroethane & 0.017 & -0.337 & 1.600 & 0.774 & 0.637 & 0.921 \\
\hline 1-Chlorobutane & 0.130 & -0.581 & 1.114 & 0.724 & 0.000 & 1.016 \\
\hline Hexane & 0.320 & 0.000 & 0.000 & 0.000 & 0.000 & 0.945 \\
\hline Heptane & 0.284 & 0.000 & 0.000 & 0.000 & 0.000 & 0.950 \\
\hline Octane & 0.219 & 0.000 & 0.000 & 0.000 & 0.000 & 0.960 \\
\hline Decane & 0.159 & 0.000 & 0.000 & 0.000 & 0.000 & 0.972 \\
\hline Undecane & 0.113 & 0.000 & 0.000 & 0.000 & 0.000 & 0.971 \\
\hline Dodecane & 0.053 & 0.000 & 0.000 & 0.000 & 0.000 & 0.986 \\
\hline Hexadecane & 0.000 & 0.000 & 0.000 & 0.000 & 0.000 & 1.000 \\
\hline Cyclohexane & 0.163 & -0.110 & 0.000 & 0.000 & 0.000 & 1.013 \\
\hline Methylcyclohexane & 0.318 & -0.215 & 0.000 & 0.000 & 0.000 & 1.012 \\
\hline 2,2,4-Trimethylpentane & 0.264 & -0.230 & 0.000 & 0.000 & 0.000 & 0.975 \\
\hline Benzene & 0.107 & -0.313 & 1.053 & 0.457 & 0.169 & 1.020 \\
\hline Toluene & 0.085 & -0.400 & 1.060 & 0.501 & 0.154 & 1.011 \\
\hline Ethylbenzene & 0.059 & -0.295 & 0.924 & 0.537 & 0.098 & 1.010 \\
\hline 1,2-Dimethylbenzene & 0.064 & -0.296 & 0.934 & 0.647 & 0.000 & 1.010 \\
\hline 1,3-Dimethylbenzene & 0.071 & -0.423 & 1.068 & 0.552 & 0.000 & 1.014 \\
\hline 1,4-Dimethylbenzene & 0.113 & -0.302 & 0.826 & 0.651 & 0.000 & 1.011 \\
\hline Fluorobenzene & 0.181 & -0.621 & 1.432 & 0.647 & 0.000 & 0.986 \\
\hline Chlorobenzene & 0.064 & -0.399 & 1.151 & 0.313 & 0.171 & 1.032 \\
\hline Bromobenzene & -0.064 & -0.326 & 1.261 & 0.323 & 0.292 & 1.002 \\
\hline Iodobenzene & -0.171 & -0.192 & 1.197 & 0.245 & 0.245 & 1.002 \\
\hline Nitrobenzene & -0.295 & 0.121 & 1.682 & 1.247 & 0.370 & 0.915 \\
\hline Benzonitrile & -0.075 & -0.341 & 1.798 & 2.030 & 0.291 & 0.880 \\
\hline Olive oil & -0.159 & -0.277 & 0.904 & 1.695 & -0.090 & 0.876 \\
\hline Carbon disulfide & 0.101 & 0.251 & 0.177 & 0.027 & 0.095 & 1.068 \\
\hline Triolein & 0.147 & 0.254 & -0.246 & 1.520 & 1.473 & 0.918 \\
\hline Methanol & -0.039 & -0.338 & 1.317 & 3.826 & 1.396 & 0.773 \\
\hline Ethanol & 0.017 & -0.232 & 0.867 & 3.894 & 1.192 & 0.846 \\
\hline Propan-1-ol & -0.042 & -0.246 & 0.749 & 3.888 & 1.076 & 0.874 \\
\hline Butan-1-ol & -0.004 & -0.285 & 0.768 & 3.705 & 0.879 & 0.890 \\
\hline Pentan-1-ol & -0.002 & -0.161 & 0.535 & 3.778 & 0.960 & 0.900 \\
\hline
\end{tabular}


TABLE 2. Abraham model equation coefficients describing solute transfer to an organic solvent from gas phase [Eq. (11)] - Continued

\begin{tabular}{|c|c|c|c|c|c|c|}
\hline Organic solvent & $c_{\mathrm{k}}$ & $e_{\mathrm{k}}$ & $s_{\mathrm{k}}$ & $a_{\mathrm{k}}$ & $b_{\mathrm{k}}$ & $l_{\mathrm{k}}$ \\
\hline Hexan-1-ol & -0.014 & -0.205 & 0.583 & 3.621 & 0.891 & 0.913 \\
\hline Heptan-1-ol & -0.056 & -0.216 & 0.554 & 3.596 & 0.803 & 0.933 \\
\hline Octan-1-ol & -0.147 & -0.214 & 0.561 & 3.507 & 0.749 & 0.943 \\
\hline Decan-1-ol & -0.139 & -0.090 & 0.356 & 3.547 & 0.727 & 0.958 \\
\hline Propan-2-ol & -0.062 & -0.327 & 0.707 & 4.024 & 1.072 & 0.886 \\
\hline 2-Methylpropan-1-ol & 0.012 & -0.407 & 0.670 & 3.645 & 1.283 & 0.895 \\
\hline Butan-2-ol & -0.017 & -0.376 & 0.852 & 3.740 & 1.161 & 0.867 \\
\hline 2-Methylpropan-2-ol & 0.071 & -0.538 & 0.818 & 3.951 & 0.823 & 0.905 \\
\hline 3-Methylbutan-1-ol & -0.014 & -0.341 & 0.525 & 3.666 & 1.096 & 0.925 \\
\hline 2-Pentanol & -0.031 & -0.325 & 0.496 & 3.792 & 1.024 & 0.934 \\
\hline Ethylene glycol & -0.887 & 0.132 & 1.657 & 4.457 & 2.325 & 0.565 \\
\hline 2,2,2-Trifluoroethanol & -0.092 & -0.547 & 1.339 & 2.213 & 3.807 & 0.645 \\
\hline 1,1'-Oxybisethane & 0.288 & -0.347 & 0.775 & 2.985 & 0.000 & 0.973 \\
\hline Tetrahydrofuran & 0.189 & -0.347 & 1.238 & 3.289 & 0.000 & 0.982 \\
\hline Dioxane & -0.034 & -0.354 & 1.674 & 3.021 & 0.000 & 0.919 \\
\hline 1,1'-Oxybisbutane & 0.165 & -0.421 & 0.760 & 2.102 & -0.664 & 1.002 \\
\hline 2-Methoxy-2-methylpropane & 0.278 & -0.489 & 0.801 & 2.495 & 0.000 & 0.993 \\
\hline Methyl ethanoate & 0.129 & -0.447 & 1.675 & 2.625 & 0.213 & 0.874 \\
\hline Ethyl ethanoate & 0.182 & -0.352 & 1.316 & 2.891 & 0.000 & 0.916 \\
\hline Propyl ethanoate & 0.165 & -0.383 & 1.264 & 2.757 & 0.000 & 0.954 \\
\hline Butyl ethanoate & 0.147 & -0.414 & 1.212 & 2.623 & 0.000 & 0.954 \\
\hline Propanone & 0.127 & -0.387 & 1.733 & 3.060 & 0.000 & 0.866 \\
\hline Butanone & 0.112 & -0.474 & 1.671 & 2.878 & 0.000 & 0.916 \\
\hline Cyclohexanone & -0.086 & -0.441 & 1.725 & 2.786 & 0.000 & 0.957 \\
\hline Propylene carbonate & -0.356 & -0.413 & 2.587 & 2.207 & 0.455 & 0.719 \\
\hline Dimethylformamide & -0.391 & -0.869 & 2.107 & 3.774 & 0.000 & 1.011 \\
\hline Dimethylacetamide & -0.308 & -0.736 & 1.802 & 4.361 & 0.000 & 1.028 \\
\hline Diethylacetamide & -0.075 & -0.434 & 1.911 & 4.801 & 0.000 & 0.899 \\
\hline Dibutylformamide & -0.002 & -0.239 & 1.402 & 4.029 & 0.000 & 0.900 \\
\hline$N$-Methylpyrrolidinone & -0.128 & -0.029 & 2.217 & 4.429 & 0.000 & 0.777 \\
\hline$N$-Methyl-2-piperidone & -0.264 & -0.171 & 2.086 & 5.056 & 0.000 & 0.883 \\
\hline$N$-Formylmorpholine & -0.437 & 0.024 & 2.631 & 4.318 & 0.000 & 0.712 \\
\hline$N$-Methylformamide & -0.249 & -0.142 & 1.661 & 4.147 & 0.817 & 0.739 \\
\hline$N$-Ethylformamide & -0.220 & -0.302 & 1.743 & 4.498 & 0.480 & 0.824 \\
\hline$N$-Methylacetamide & -0.197 & -0.175 & 1.608 & 4.867 & 0.375 & 0.837 \\
\hline$N$-Ethylacetamide & -0.018 & -0.157 & 1.352 & 4.588 & 0.357 & 0.824 \\
\hline Formamide & -0.800 & 0.310 & 2.292 & 4.130 & 1.933 & 0.442 \\
\hline Acetonitrile & -0.007 & -0.595 & 2.461 & 2.085 & 0.418 & 0.738 \\
\hline Nitromethane & -0.340 & -0.297 & 2.689 & 2.193 & 0.514 & 0.728 \\
\hline Dimethylsulfoxide & -0.556 & -0.223 & 2.903 & 5.036 & 0.000 & 0.719 \\
\hline Sulfolane (303 K) & -0.414 & 0.084 & 2.396 & 3.144 & 0.420 & 0.684 \\
\hline Tributylphosphate & 0.097 & -0.098 & 1.103 & 2.411 & 0.588 & 0.844 \\
\hline Gas-water & -1.271 & 0.822 & 2.743 & 3.904 & 4.814 & -0.213 \\
\hline
\end{tabular}

having the same McGowan volume. The molar refraction is given by $^{7}$

$$
M R_{X}=10\left[\frac{\left(\eta^{2}-1\right)}{\left(\eta^{2}+2\right)}\right] V
$$

where $\eta$ is the refractive index of the solute as a pure liquid at $293 \mathrm{~K}$, and $V$ is in units of $\left(\mathrm{cm}^{3} \mathrm{~mol}^{-1}\right) / 100$. For compounds that are solid at $293 \mathrm{~K}$, a refractive index for the liquid at $293 \mathrm{~K}$ can be calculated by commercial software; ${ }^{39}$ or alternatively $E$ can be computed by summing fragment groups in the molecule ${ }^{40}$ or by using the PharmaAlgorithm commercial software. ${ }^{41}$ The molar refraction is one of the few properties that is very nearly the same for a given molecule in both the gaseous and liquid state, even for associated liquid molecules such as water. The numerical value of molar refraction of the alkane molecule needed in the computation of $E$ is given by ${ }^{7}$

$$
\left(M R_{X}\right)_{\text {alkane }}=2.83195 \mathrm{~V}-0.52553,
$$

where $V$ is the characteristic McGowan volume described above. The remaining four solute descriptors, $S, A, B$, and $L$, are calculated by solving a series of simultaneous $\log _{10} P$ and $\log _{10} K$ equations for which both experimental partition coefficient data and solvent equation coefficients $\left(c_{\mathrm{p}}, e_{\mathrm{p}}, s_{\mathrm{p}}\right.$, $a_{\mathrm{p}}, b_{\mathrm{p}}, v_{\mathrm{p}}, c_{\mathrm{k}}, e_{\mathrm{k}}, s_{\mathrm{k}}, a_{\mathrm{k}}, b_{\mathrm{k}}$, and $\left.l_{\mathrm{k}}\right)$ are known. The computation method is illustrated in several published papers and will not be repeated here.

The Abraham general solvation parameter model has been used successfully to correlate the solubility behavior of several substituted benzoic acids (namely, 2-acetoxybenzoic acid, 4aminobenzoic acid, 3-chlorobenzoic acid, 4-chlorobenzoic acid, 2-chloro-5-nitrobenzoic acid, 4-chloro-3-nitrobenzoic 
acid, 3,4-dichlorobenzoic acid, 3,5-dinitrobenzoic acid, 3,5dinitro-2-methylbenzoic acid, 2-methoxybenzoic acid, 4methoxybenzoic acid, 2-methylbenzoic acid, 3-methylbenzoic acid, 3-nitrobenzoic acid, 4-nitrobenzoic acid) dissolved in a series of alcohols, dialkyl ethers, and alkyl alkanoates. Equations (10) and (11) described the experimental solubility data to within a standard deviation of $\pm 0.15 \log _{10}$ units. Past experience in using various solution models has been that the better solution will generally give predicted values that fall with $\pm 40 \%$ or so (about $\pm 0.15 \log _{10}$ units) of the observed solute solubilities. The Abraham model will be used to assess the experimental solubility data for a few select substituted benzoic acids, and to identify possible values that need to be remeasured. More detailed information concerning the model will be given later in this work where actual experimental solubility is being evaluated.

The solvent composition dependence upon solubility is generally evaluated using semi-theoretical solution models. During the past 50 years, more than 100 solution models have developed for describing variation of solubility with solvent composition based on different assumptions regarding how molecules interact in solution. Predictive expressions derived from several of the proposed solution models have served as mathematical representations for isothermal solubility data in binary and ternary solvent mixtures, and for identifying experimental data points in need of redetermination. The Combined Nearly Ideal Binary Solvent (NIBS)/Redlich-Kister equation ${ }^{42,43}$

$$
\begin{aligned}
\ln x_{1}^{\mathrm{sat}}= & x_{2}{ }^{(\mathrm{s})} \ln \left(x_{1}^{\mathrm{sat}}\right)_{2}+x_{3}{ }^{(\mathrm{s})} \ln \left(x_{1}^{\mathrm{sat}}\right)_{3} \\
& +x_{2}{ }^{(\mathrm{s})} x_{3}{ }^{(\mathrm{s})} \sum_{j=0}^{r} S_{23, j}\left(x_{2}{ }^{(\mathrm{s})}-x_{3}{ }^{(\mathrm{s})}\right)^{j}
\end{aligned}
$$

is likely the most popular of the proposed mathematical representations. In the above equation, $x_{i}{ }^{(\mathrm{s})}$ refers to the initial mole fraction solvent composition of component $i$ calculated as if the solute were not present, and $\left(x_{\mathrm{A}}^{\text {sat }}\right)_{i}$ denotes the measured solute solubility in pure solvent $i$. The summation in the last term on the right-hand side of Eq. (17) includes as many curve-fit $S_{23, j}$ parameters as are needed to accurately describe the observed solubility data. Generally no more than three parameters will be needed in a given mathematical representation. The various $S_{23, j}$ parameters are determined by regression analysis.

The popularity of the Combined NIBS/Redlich-Kister model results from the fact that the computed $S_{I J, i}$ parameters can be used to predict solute solubility in ternary solvent systems:

$$
\begin{aligned}
\ln x_{1}^{\mathrm{sat}}= & x_{2}{ }^{(\mathrm{s})} \ln \left(x_{1}^{\mathrm{sat}}\right)_{2}+x_{3}{ }^{(\mathrm{s})} \ln \left(x_{1}^{\mathrm{sat}}\right)_{3}+x_{4}{ }^{(\mathrm{s})} \ln \left(x_{1}^{\mathrm{sat}}\right)_{4} \\
& +x_{2}{ }^{(\mathrm{s})} x_{3}{ }^{(\mathrm{s})} \sum_{j=0}^{r} S_{23, j}\left(x_{2}{ }^{(\mathrm{s})}-x_{3}{ }^{(\mathrm{s})}\right)^{j} \\
& +x_{2}{ }^{(\mathrm{s})} x_{4}{ }^{(\mathrm{s})} \sum_{k=0}^{s} S_{24, k}\left(x_{2}{ }^{(\mathrm{s})}-x_{4}{ }^{(\mathrm{s})}\right)^{k} \\
& +x_{3}{ }^{(\mathrm{s})} x_{4}^{(\mathrm{s})} \sum_{l=0}^{t} S_{34, l}\left(x_{3}{ }^{(\mathrm{s})}-x_{4}{ }^{(\mathrm{s})}\right)^{l}
\end{aligned}
$$

and in higher-order multicomponent solvent systems:

$$
\ln x_{1}^{\mathrm{sat}}=\sum_{I}^{\text {Solvents Solvents }} \sum_{J>I}\left[x_{I}^{(\mathrm{s})} x_{J}{ }^{(\mathrm{s})} \sum_{k=0}^{n} S_{I J, k}\left(x_{I}{ }^{(\mathrm{s})}-x_{J}{ }^{(\mathrm{s})}\right)^{k}\right] .
$$

Equation (18) is referred to as the Combined Nearly Ideal Ternary Solvent (NITS)/Redlich-Kister model. To date Eq. (18) has been shown to provide very accurate predictions for the solubility of anthracene and/or pyrene in 114 different ternary solvent mixtures including several alcohol + hydrocarbon + hydrocarbon, alcohol + alcohol + hydrocarbon, alkoxyalcohol + alcohol + hydrocarbon, alkoxyalcohol + alcohol + alcohol, and alkyl ether + alcohol + hydrocarbon solvent systems. ${ }^{44-46}$

\section{Solubility of Benzoic Acid in Organic Solvents}

\subsection{Critical evaluation of experimental solubility data}

There have been numerous studies ${ }^{47-91}$ involving the solubility of benzoic acid in organic solvents, particularly at $298 \mathrm{~K}$. Most notably, Beerbower et al. ${ }^{47}$ measured the solubility in 57 different organic solvents, including six saturated hydrocarbons (pentane, hexane, heptane, nonane, decane, and cyclohexane), two aromatic hydrocarbons (benzene and methylbenzene), one dialkyl ether (1,1'-oxybisethane) and one cyclic ether (1,4-dioxane), two alkyl alkanoates (ethyl ethanoate and butyl ethanoate), three chloroalkanes (trichloromethane, tetrachloromethane, and 1,2-dichloroethane) and one chlorinated aromatic hydrocarbon (chlorobenzene), 12 alcohols (methanol, ethanol, 1-propanol, 2-propanol, 1-butanol, 2-methyl-1-propanol, 1-pentanol, 1-hexanol, 1-octanol, 1,2-ethanediol, 1,2,3-propanetriol, and benzenemethanol), one alkanone (propanone) and one aromatic ketone (acetophenone), and nine miscellaneous organic solvents (nitrobenzene, ethanoic acid, propanoic acid, dimethyl sulfoxide, pyridine, formamide, $N$-methylformamide, $N, N$-dimethylformamide, and $N, N$-dimethylacetamide). The experimental data were used to test the limitations and applications of the Expanded Solubility Parameter Approach. Restaino and Martin ${ }^{75}$ published solubility data for benzoic acid in 1-propanol, 1-butanol, 1-pentanol, 1-hexanol, and 1-octanol. Acree and Bertrand, ${ }^{49}$ Perlovich and Bauer-Brandl, ${ }^{53}$ Gomaa, ${ }^{64}$ Ongley, ${ }^{56}$ Thuaire, ${ }^{72}$ Yurquina et al. ${ }^{78}$ and Chantooni and Kolthoff ${ }^{76}$ have also performed benzoic acid solubility measurements at $298 \mathrm{~K}$.

The Abraham solvation parameter model can provide an indication of the quality of experimental solubility data for benzoic acid dissolved in a series of organic solvents of varying polarity and hydrogen bonding character. As discussed above, the evaluation will be restricted to those solvents where dimerization is not likely to occur and to solvents where benzoic acid does not form a solid solvate. Expressions based on the Abraham model have been shown to provide reasonably accurate mathematical correlations for the solubility behavior of numerous crystalline nonelectrolyte solutes, with deviations between observed and calculated values on the order of 
$0.15 \log _{10}$ units or less. Several of the published studies have involved substituted benzoic acids. Results of these studies will be discussed later in the volume as the solubility data of the respective solutes are presented.

The Abraham model is based on two linear free energy relationships that describe solute transfer to organic solvents from water and from the gas phase. Expressed in terms of molar solubility, the linear free energy relationships take the following mathematical forms

$$
\begin{aligned}
\log _{10}\left(c_{1, \mathrm{~S}}^{\mathrm{sat}} / c_{1, \mathrm{~W}}^{\mathrm{sat}}\right)= & c_{\mathrm{p}}+e_{\mathrm{p}} \cdot E+s_{\mathrm{p}} \cdot S+a_{\mathrm{p}} \cdot A \\
& +b_{\mathrm{p}} \cdot B+v_{\mathrm{p}} \cdot V, \\
\log _{10}\left(c_{1, \mathrm{~S}}^{\mathrm{sat}} / c_{1, \mathrm{G}}\right)= & c_{\mathrm{k}}+e_{\mathrm{k}} \cdot E+s_{\mathrm{k}} \cdot S+a_{\mathrm{k}} \cdot A \\
& +b_{\mathrm{k}} \cdot B+l_{\mathrm{k}} \cdot L,
\end{aligned}
$$

where $c_{1, \mathrm{~S}}^{\mathrm{sat}}$ and $c_{1, \mathrm{~W}}^{\mathrm{sat}}$ are the molar solubilities of the solute in the organic solvent and in water, respectively, and $c_{1, \mathrm{G}}$ is the molar concentration of the solute in the gas phase. The molar concentrations are expressed in units of mol dm ${ }^{-3}$. For notational simplicity the "sat" superscript will be dropped in subsequent discussions, and the quantities simply denoted as $c_{1}$ and $c_{1, \mathrm{w}}$. The Abraham model solvent equation coefficients that are given in Tables 1 and 2 pertain to $298 \mathrm{~K}$ unless otherwise noted. For a given solute-solvent system, Eqs. (20) and (21) give calculated $c_{1}$ values that differ from one another by only a few hundredths of a $\log _{10}$ unit.

Numerical values of the solute descriptors for benzoic acid are known $(E=0.730, S=0.900, A=0.590, B=0.400$, and $V$ $=0.9317$ ), so that combination of these descriptors with the coefficients listed in Table 1 permit the prediction of $\log _{10}$ $\left(c_{1, \mathrm{~S}}^{\mathrm{sat}} / c_{1, \mathrm{~W}}^{\mathrm{sat}}\right)$. The molar solubility of molecular benzoic acid in water, $\log _{10} c_{1, \mathrm{~W}}^{\mathrm{sat}}=-1.55$, is available to convert the predicted $\left(c_{1, \mathrm{~S}}^{\mathrm{sat}} / c_{1, \mathrm{~W}}^{\mathrm{sat}}\right)$ solubility ratios to $c_{1, \mathrm{~S}}^{\mathrm{sat}}$ values. For carboxylic acid solutes, $c_{1, \mathrm{~W}}^{\mathrm{sat}}$ corresponds to the aqueous solubility of the molecular, nonionized form of the solute.

The predicted molar solubilities of benzoic acid in methanol, ethanol, 1-propanol, 1-butanol, 1-pentanol, 1-hexanol, 1-heptanol, 1-octanol, 1-decanol, 2-propanol, 2-butanol, 2-pentanol, 2methyl-1-propanol, 2-methyl-2-propanol, 3-methyl-1-butanol, 1,2-ethanediol, 1,1'-oxybisethane, 2-methoxy-2-methylpropane, tetrahydrofuran, 1,4-dioxane, methyl ethanoate, ethyl ethanoate, propyl ethanoate, butyl ethanoate, and propanone based on Eq. (20) are listed in the second column of Table 3. Alkane and aromatic hydrocarbon solvents are excluded from consideration because of dimerization concerns. The numerical values represent outright solubility predictions in that none of the experimental data was used in the determination of the

\begin{tabular}{|c|c|c|c|c|c|}
\hline Solvent & $\log _{10} c_{1}{ }^{\text {calc }}$; Eq. (20) & $\log _{10} c_{1} \exp$ & $\log _{10} c_{1} \exp$ & $\log _{10} c_{1} \exp$ & $\log _{10} c_{1} \exp$ \\
\hline Methanol & 0.449 & $0.504^{\mathrm{a}}$ & & $0.228^{\mathrm{b}}$ & \\
\hline Ethanol & 0.432 & $0.427^{\mathrm{a}}$ & & $0.292^{\mathrm{b}}$ & $0.441^{\mathrm{c}}$ \\
\hline 1-Propanol & 0.311 & $0.348^{\mathrm{a}}$ & $0.366^{\mathrm{d}}$ & & \\
\hline 2-Propanol & 0.322 & $0.372^{\mathrm{a}}$ & & & \\
\hline 1-Butanol & 0.215 & $0.329^{\mathrm{a}}$ & $0.332^{\mathrm{d}}$ & & \\
\hline 2-Butanol & 0.257 & & & & \\
\hline 2-Methyl-1-propanol & 0.178 & $0.209^{\mathrm{a}}$ & & & \\
\hline 2-Methyl-2-propanol & 0.323 & $0.398^{\mathrm{e}}$ & & & \\
\hline 1-Pentanol & 0.221 & $0.232^{\mathrm{a}}$ & $0.322^{\mathrm{d}}$ & & \\
\hline 2-Pentanol & 0.237 & & & & \\
\hline 3-Methyl-1-butanol & 0.166 & & & & \\
\hline 1-Hexanol & 0.166 & $0.196^{\mathrm{a}}$ & $0.220^{\mathrm{d}}$ & & \\
\hline 1-Heptanol & 0.105 & $0.160^{f}$ & & & \\
\hline 1-Octanol & 0.055 & $0.129^{\mathrm{a}}$ & $-0.070^{\mathrm{d}}$ & & \\
\hline 1-Decanol & -0.005 & & & & \\
\hline 1,2-Ethanediol & 0.068 & $0.167^{\mathrm{a}}$ & $0.176^{\mathrm{h}}$ & & \\
\hline $1,1^{\prime}$-Oxybisethane & 0.047 & $0.243^{\mathrm{a}}$ & & & \\
\hline 2-Methoxy-2- & -0.001 & & & & \\
\hline methylpropane & & & & & \\
\hline Tetrahydrofuran & 0.625 & & & & \\
\hline 1,4-Dioxane & 0.359 & $0.496^{\mathrm{a}}$ & & $0.371^{\mathrm{b}}$ & \\
\hline Methyl ethanoate & 0.108 & & & & \\
\hline Ethyl ethanoate & 0.138 & $0.220^{\mathrm{a}}$ & & & \\
\hline Propyl ethanoate & 0.067 & & & & \\
\hline Butyl ethanoate & -0.005 & $0.124^{\mathrm{a}}$ & & & \\
\hline Propanone & 0.295 & $0.368^{\mathrm{a}}$ & $0.382^{\mathrm{g}}$ & & \\
\hline
\end{tabular}
molecular solute descriptors. For comparison purposes, the measured mole fraction solubilities of benzoic acid, $x_{1}$, given

TABLE 3. Comparison between observed and predicted molar solubilities of benzoic acid based on the Abraham model, Eq. (20)

${ }^{\mathrm{a}}$ Experimental value is taken from Beerbower et al. ${ }^{4}$

${ }^{\mathrm{b}}$ Experimental value is taken from Gomaa. ${ }^{64}$

${ }^{\mathrm{c}}$ Experimental value is taken from Thuaire. ${ }^{72}$

${ }^{\mathrm{d}}$ Experimental value is taken from Restaino and Martin. ${ }^{75}$

${ }^{\mathrm{e}}$ Experimental value is from Chantooni and Kolthoff. ${ }^{76}$

${ }^{\mathrm{f}}$ Experimental value is from Perlovich and Bauer-Brandl. ${ }^{53}$

${ }^{\mathrm{g}}$ Experimental value is from Long et al. ${ }^{55}$

${ }^{\mathrm{h}}$ Experimental value is from Yurquina et al. ${ }^{78}$ 
TABLE 4. Recommended mole fraction solubilities for benzoic acid in select solvents

\begin{tabular}{|c|c|c|c|}
\hline Solvent & Recommended value & Individual values & Outliers \\
\hline Cyclohexane & 0.0107 & $0.0102,,^{47} 0.01095,{ }^{55} 0.0100,^{56} 0.0115$ (Ref. 49) & \\
\hline Benzene & 0.0731 & $0.073,{ }^{59} 0.0728,,^{58} 0.0734$ (Ref. 47 ) & \\
\hline Methylbenzene & 0.0728 & $0.0734,^{47} 0.071,{ }^{48} 0.0741$ (Ref. 58 ) & \\
\hline Tetrachloromethane & 0.0495 & $0.0494,{ }^{47} 0.0500,{ }^{67} 0.0492$ (Ref. 49 ) & \\
\hline Methanol & 0.163 & $0.1632,{ }^{47} 0.1569,{ }^{51} 0.1693$ (Ref. 68) & 0.0771 (Ref. 64) \\
\hline Ethanol & 0.183 & $0.1789,^{47} 0.1808,^{71} 0.1855,^{72} 0.1882$ (Ref. 50 ) & 0.1264 (Ref. 64) \\
\hline 1-Propanol & 0.180 & $0.1791,,^{47} 0.1743,,^{51} 0.187,,^{75} 0.1810$ (Ref. 50 ) & \\
\hline Propanone & 0.196 & $0.1857,,^{47} 0.1925,,^{55} 0.209$ (Ref. 79) & \\
\hline Ethanoic acid & 0.156 & $0.1675,,^{47} 0.148,{ }^{55} 0.151$ (Ref. 80 ) & 0.1097 (Ref. 79) \\
\hline$N, N$-Dimethylformamide & 0.478 & $0.4909,,^{47} 0.4724,{ }^{80} 0.470$ (Ref. 82) & 0.2228 (Ref. 64) \\
\hline
\end{tabular}

in Secs. 2.2-2.10 were converted into molar solubilities by dividing $x_{1}$ by the ideal molar volume of the saturated solution (i.e., $\left.c_{1}{ }^{\text {sat }}=x_{1} /\left[x_{1} V_{1}+\left(1-x_{1}\right) V_{\text {solvent }}\right]\right)$. The molar volume of the hypothetical subcooled liquid benzoic acid is taken to be $V_{\text {solute }}$ $=104.38 \mathrm{~cm}^{3} \mathrm{~mol}^{-1}$. Any errors resulting from the estimation of benzoic acid's hypothetical subcooled liquid molar volume, $V_{\text {solute, }}$, or the ideal molar volume approximation will have negligible effect of the calculated $c_{1}$ values because benzoic acid is not overly soluble in many of the solvents considered. From a mathematical standpoint, the $x_{1}{ }^{\exp } V_{\text {solute }}$ term contributes very little to the molar volumes of the saturated solutions.

Examination of the numerical entries in Table 3 reveals that expressions based on the Abraham model provide a very reasonable estimate of the solubility behavior of benzoic acid in 1,1'-oxybisethane, 1,4-dioxane, propanone and in 12 alcohol and two alkyl ethanoate solvents. Standard deviations between predicted and observed values were on the order of $0.12 \log _{10}$ units or less for most solvents. Differences in chemical purities and experimental methodologies can account for differences of several percent between values determined by different research groups.

Solution models, such as the Abraham solvation parameter model, prove useful in screening datasets for obvious outliers, particularly in cases where there are only one or two experimental data points for a given solute-solvent system. Readers are reminded that such models are only able to identify those outliers that fall outside of the model's expected predictive applicability. There is a sufficient number of data points for several of the solvents in Secs. 2.2-2.11 to compute a recommended value. The criterion that is used here to give a recommended value is that there must be at least three independent solubility measurements that differ from each other by no more than approximately 15 relative percent. Moreover, there must not be too many data points that fall outside of this range. The recommended mole fraction solubilities were calculated as an arithmetic average and are given in Table 4 for the solvents that had three or more close solubility measurements. Included in the table are the individual mole fraction solubilities that went into the calculation, and any suspected outlier values. There are a couple of instances were two independent experimental values are close and a third value differs by more than 30 relative percent. For example, in the case of ethyl ethanoate there are two close values $x_{1}=0.1649$ (Ref. 47) and $x_{1}=0.1637$ (Ref. 31) which differ significantly from a third value of $x_{1}=0.229,{ }^{57}$ in the case of trichloromethane there are two close values of $x_{1}=0.1283($ Ref. 47$)$ and $x_{1}=0.132$ (Ref. 48) which differ significantly from a third value of $x_{1}=0.273,{ }^{57}$ and in the case of 1-octanol there are two close values of $x_{1}=0.1987$ (Ref. 47) and $x_{1}=0.185$ (Ref. 77) which differ significantly from a third value of $x_{1}=0.129 .^{75}$ The latter value in each of the three listings is believed to be an outlier.

There have been several experimental studies examining the solubility of benzoic acid in different organic solvents as a function of temperature. Thati et al. ${ }^{48}$ determined benzoic acid solubilities in pentane, heptane, cyclohexane, methylbenzene, ethanol, and trichloromethane, and in binary heptane + ethanol and methylbenzene + ethanol solvent mixtures, in the approximate temperature range from 278 to $323 \mathrm{~K}$. The authors described the variation of $\ln x_{1}$ with temperature in terms of

$$
\ln x_{1}=A+\frac{B}{T}+C T
$$

a relatively simple nonlinear equation. The calculated regression coefficients $(A, B$, and $C$ ) are listed in Table 5 , along with the actual temperature range for each of the six monosolvents. The authors noted that the squared correlation coefficient, $R^{2}$, exceeded 0.9996. The graphical comparison provided in the paper indicated very good agreement between the experimental $x_{1}$ data and back-calculated values based on Eq. (22).

Zhao et al. ${ }^{60}$ measured the solubility of benzoic acid in isobutyl ethanoate at 18 temperatures between 300 and $343 \mathrm{~K}$. The experimental data were correlated with the Wilson and UNIQUAC models. Interaction coefficients calculated from the experimental solid-liquid equilibrium data provided a reasonably accurate mathematical description of the measured values. The mean absolute relative deviation between calculated and observed values was $0.85 \%$ (Wilson model) and $1.06 \%$ (UNIQUAC model).

TABLE 5. Parameters of Eq. (22) for describing the solubility of benzoic acid in various organic solvents ${ }^{\mathrm{a}}$

\begin{tabular}{lcrrr}
\hline \hline Solvent & $T / \mathrm{K}$ & \multicolumn{1}{c}{$A$} & \multicolumn{1}{c}{$B$} & \multicolumn{1}{c}{$C$} \\
\hline Pentane & $278-303$ & -20.9245 & 444.93 & 0.04932 \\
Heptane & $278-323$ & -8.4537 & -3803.41 & -0.00032 \\
Cyclohexane & $283-323$ & -7.9394 & -1683.88 & 0.03071 \\
Methylbenzene & $278-323$ & -0.4993 & -1927.02 & 0.01466 \\
Trichloromethane & $278-323$ & -0.2008 & -1492.75 & 0.01066 \\
Ethanol & $278-323$ & -6.1372 & -102.31 & 0.01599
\end{tabular}

${ }^{\mathrm{a}}$ Values of the coefficients were taken from Thati et al. ${ }^{48}$ 
TABle 6. Parameters of the Van't Hoff-type equation for describing the solubility of benzoic acid in various organic solvents

\begin{tabular}{lcrrrc}
\hline \hline Solvent & \multicolumn{1}{c}{$T / \mathrm{K}$} & \multicolumn{1}{c}{$A$} & \multicolumn{1}{c}{$B$} & \multicolumn{1}{c}{$C$} & MRD (\%) \\
\hline Cyclohexane $^{\mathrm{a}}$ & $283-343$ & -8.5248 & 8766.1 & 2259000 & 2.75 \\
2-Propanol $^{\mathrm{a}}$ & $278-343$ & 3.9085 & -11624.0 & -9250 & 0.54 \\
Propanone $^{\mathrm{a}}$ & $278-323$ & 6.0996 & -2925.6 & 184060 & 0.63 \\
Ethanoic acid $^{\mathrm{a}}$ & $293-346$ & 4.8518 & -1721.8 & -88300 & 0.62 \\
\hline
\end{tabular}

${ }^{\mathrm{a}}$ Values of the coefficients and mean relative deviation were taken from Long et $a l^{55}$

Long et al..$^{55}$ published experimental solubility data for benzoic acid in cyclohexane, 2-propanol, propanone, and ethanoic acid over the approximate temperature range between 277 and $346 \mathrm{~K}$ at $5 \mathrm{~K}$ intervals using a gravimetric method. Experimental measurements showed that the benzoic acid solubility was greatest in propanone, followed by 2-propanol, ethanoic acid, and cyclohexane in descending order. The authors correlated the temperature dependence with a Van't Hoff-type relation:

$$
\ln x_{1}=A+\frac{B}{T}+\frac{C}{T^{2}},
$$

where $A, B$, and $C$ represent the empirical curve-fit parameters determined by regression analysis of $\ln x_{1}$ data in accordance with Eq. (23). The calculated equation coefficients and the mean relative deviation (MRD) defined by Eq. (24) below

$$
\operatorname{MRD}(\%)=\frac{100}{N} \sum\left|\frac{\left(x_{1}^{\text {exp }}-x_{1}^{\text {calc }}\right)}{x_{1}^{\exp }}\right|
$$

are tabulated in Table 6. In Eq. (24), $N$ denotes the number of experimental solubility measurements in an individual solutesolvent dataset. Examination of the numerical values in the last column of Table 6 indicates that Eq. (23) does provide a reasonably accurate mathematical description of how the solubility varies with temperature. The small mean relative deviations suggest that the experimental values in each solvent dataset are internally consistent.

$\mathrm{Ma}$ and $\mathrm{Xia}^{80}$ determined the solubility of benzoic acid in both ethanoic acid and $\mathrm{N}, \mathrm{N}$-dimethylformamide. The internal consistency of the two datasets was assessed by curve-fitting the measured mole fraction solubility data to the Buchowski $\lambda \mathrm{h}$ model [Eq. (9)]. The values of the equation coefficients $(\lambda$ and $h$ ) are given in Table 7 , along with the mean relative

TABLE 7. Parameters of the Buchowski $\lambda$ h equation for describing the solubility of benzoic acid in various organic solvents

\begin{tabular}{lcrlc}
\hline \hline Solvent & $T / \mathrm{K}$ & $\lambda$ & \multicolumn{1}{c}{$h$} & MRD (\%) \\
\hline Ethanoic acid $^{\mathrm{a}}$ & $291-334$ & 0.7767 & 2614.61 & 0.67 \\
Ethanoic acid $^{\mathrm{b}}$ & $291-356$ & 0.8061 & 2594.9 & 0.58 \\
$N, N$-Dimethylformamide $^{\mathrm{a}}$ & $299-343$ & -0.6448 & 2379.67 & 0.13 \\
Benzene $^{\mathrm{b}}$ & $291-356$ & 1.5203 & 2438.3 & 0.36 \\
Ethyl ethanoate $^{\mathrm{b}}$ & $291-356$ & 0.6058 & 2656.9 & 0.31 \\
Ethanol $^{\mathrm{b}}$ & $291-356$ & 0.3918 & 3212.2 & 0.28 \\
\hline
\end{tabular}

${ }^{\mathrm{a}}$ Values of the coefficients and mean relative deviation were taken from $\mathrm{Ma}$ and Xia. ${ }^{80}$

${ }^{\mathrm{b}}$ Values of the coefficients and mean relative deviation were taken from Cheng et al. ${ }^{90}$ deviation. Also included in Table 7 are the equation coefficients reported by Cheng et al. ${ }^{90}$ for the solubility of benzoic acid in ethanol, benzene, ethanoic acid, and ethyl ethanoate. The authors performed solubility measurements over the temperature range of $291-356 \mathrm{~K}$ and reported their results only in the form of curve-fit equation coefficients. The actual mole fraction solubility data was not given in the published paper. The small mean relative deviations suggest that the experimental values in each solvent dataset are internally consistent. Readers are reminded that Eq. (9) can only check data points for internal consistency with respect to temperature. The fact that one can accurately describe the experimental data does not mean that the data are accurate. In the case of ethanoic acid, there are three sets of independent solubility data in the published literature. A point-by-point comparison will not be performed here; however, one does note that the experimental solubilities reported by Long et al. ${ }^{55}$ and Ma and $\mathrm{Xia}^{80}$ are in reasonably good agreement with each other at $298 \mathrm{~K}\left[x_{1}=0.1481\left(\right.\right.$ Ref. 55) versus $x_{1}=0.1448$ (Ref. 80) $]$ and $318 \mathrm{~K}\left[x_{1}=0.2367\right.$ (Ref. 55) and $x_{1}=0.2384$ (Ref. 80)], and both sets of values differ from those reported by Wang et al. ${ }^{79}$ which are $x_{1}=0.1097$ at $298 \mathrm{~K}$ and $x_{1}=0.1576$ at $318 \mathrm{~K}$.

Li et al. ${ }^{84}$ determined the solubility of benzoic acid in $N$ methyl-2-pyrrolidone from 296 to $371 \mathrm{~K}$ using a synthetic method with laser monitoring to determine when the last amount of solid solute dissolved. The authors employed a polynomial expression in temperature,

$$
\begin{aligned}
x_{1}= & -6.0581+0.06480 T-2.17813 \times 10^{-4} T \\
& +2.49784 \times 10^{-7} T^{2},
\end{aligned}
$$

to represent the measured mole fraction solubility data. The root-mean-square deviation between the observed $x_{1}$ and calculated values from Eq. (25) was on the order of 0.0012 mole fraction.

The experimental solubility data for benzoic acid in organic

\begin{tabular}{|c|c|}
\hline $\begin{array}{l}\text { Components: } \\
\text { (1) Benzoic acid; } \mathrm{C}_{7} \mathrm{H}_{6} \mathrm{O}_{2} \text {; } \\
\text { [65-85-0] } \\
\text { (2) Pentane; } \mathrm{C}_{5} \mathrm{H}_{12} ;[109-66-0]\end{array}$ & $\begin{array}{l}\text { Original Measurements: } \\
{ }^{47} \text { A. Beerbower, P. L. Wu, and A. } \\
\text { Martin, J. Pharm. Sci. 73, } 179 \text { (1984) }\end{array}$ \\
\hline $\begin{array}{l}\text { Variables: } \\
T / \mathrm{K}=298.15\end{array}$ & $\begin{array}{l}\text { Prepared by: } \\
\text { W. E. Acree, Jr. }\end{array}$ \\
\hline
\end{tabular}
solvents are in Secs. 2.2-2.11.

\subsection{Benzoic acid solubility data in saturated hydrocarbons (including cycloalkanes)}

Experimental Values

\begin{tabular}{lc}
\hline \hline$x_{2}{ }^{\mathrm{a}}$ & $x_{1}{ }^{\mathrm{b}}$ \\
\hline 0.9941 & 0.0059 \\
\hline${ }^{\mathrm{a}} x_{2}:$ mole fraction of component 2 in the saturated solution. & \\
${ }^{\mathrm{b}} x_{1}:$ mole fraction solubility of the solute. &
\end{tabular}




\section{Auxiliary Information}

\section{Method/Apparatus/Procedure:}

Constant-temperature bath and ultraviolet/visible spectrophotometer.

Excess solute and solvent were placed in screw-capped vials. The vials were sealed and submerged in a constant-temperature water bath and shaken at 100 cycles/min for $24 \mathrm{~h}$. After the $24 \mathrm{~h}$ equilibration period, the vial was removed, wiped dry, and the contents analyzed. The solutions were transferred to a syringe and filtered through a $0.1 \mu \mathrm{m}$ pore size filter. The solutions were diluted and the absorbances recorded at the maximum absorption wavelength of benzoic acid. The solubility was determined at least six times.

\section{Source and Purity of Chemicals:}

(1) Purity not given, Chemical source not specified, no information provided concerning purification.

(2) Spectrophotometric or ACS Reagent grade, Chemical source not specified, redistilled before use.

\section{Estimated Error:}

Temperature: $\pm 0.2 \mathrm{~K}$.

$x_{1}: \pm 3 \%$ (relative error).

\begin{tabular}{ll}
\hline \hline Components: & Original Measurements: \\
$\begin{array}{l}\text { (1) Benzoic acid; } \mathrm{C}_{7} \mathrm{H}_{6} \mathrm{O}_{2} ; \\
\text { [65-85-0] }\end{array}$ & $\begin{array}{l}{ }^{2} \text { J. Thati, F. L. Nordström, and A. C. } \\
\text { (2) Pentane; } \mathrm{C}_{5} \mathrm{H}_{12} ;[109-66-0]\end{array}$ \\
\hline Variables: & 5124 (2010). \\
Temperature & Prepared by: \\
\hline
\end{tabular}

Experimental Values

\begin{tabular}{lcc}
\hline \hline$T / \mathrm{K}$ & $x_{2}{ }^{\mathrm{a}}$ & $x_{1}{ }^{\mathrm{b}}$ \\
\hline 278.15 & 0.996 & 0.004 \\
283.15 & 0.995 & 0.005 \\
293.15 & 0.991 & 0.009 \\
303.15 & 0.989 & 0.011
\end{tabular}

${ }^{\mathrm{a}} x_{2}$ : mole fraction of component 2 in the saturated solution.

${ }^{b} x_{1}$ : mole fraction solubility of the solute.

\section{Auxiliary Information}

\section{Method/Apparatus/Procedure:}

Constant-temperature bath, magnetic stirrer, analytical balance, and ventilated laboratory hood.

Solubilities were determined by a gravimetric method. Excess solute and solvent were placed in sealed bottles and allowed to equilibrate in a constanttemperature bath for $2 \mathrm{~h}$ with stirring. The stirring was discontinued and the solid was allowed to settle to the bottom of the container. After 30 min, an aliquot of the clear solution was removed by syringe, filtered through a $0.2 \mu \mathrm{m}$ PTFE filter, and transferred to a preweighed glass vial. The glass vial was then weighed, and the solvent was allowed to evaporate in a ventilated laboratory hood at ambient room temperature. Once the solvent had evaporated, the vial with solid residue was weighed until constant weight was obtained. The solubility was calculated from the mass of the solid residue and mass of sample analyzed.

\section{Source and Purity of Chemicals:}

(1) $99.7 \%$, Merck Chemical Company, Germany, was used as received.

(2) $95+\%$, VWR Scientific, USA, used as received.

\section{Estimated Error:}

Temperature: $\pm 0.01 \mathrm{~K}$.

$x_{1}: \pm 1 \%$ (relative error).

\begin{tabular}{|c|c|}
\hline $\begin{array}{l}\text { Components: } \\
\text { (1) Benzoic acid; } \mathrm{C}_{7} \mathrm{H}_{6} \mathrm{O}_{2} \text {; } \\
\text { [65-85-0] } \\
\text { (2) Hexane; } \mathrm{C}_{6} \mathrm{H}_{14} ;[110-54-3]\end{array}$ & $\begin{array}{l}\text { Original Measurements: } \\
{ }^{47} \text { A. Beerbower, P. L. Wu, and A. } \\
\text { Martin, J. Pharm. Sci. 73, } 179 \text { (1984). }\end{array}$ \\
\hline $\begin{array}{l}\text { Variables: } \\
T / \mathrm{K}=298.15\end{array}$ & $\begin{array}{l}\text { Prepared by: } \\
\text { W. E. Acree, Jr. }\end{array}$ \\
\hline
\end{tabular}

Experimental Values

\begin{tabular}{lc}
\hline \hline$x_{2}{ }^{\mathrm{a}}$ & $x_{1}{ }^{\mathrm{b}}$ \\
\hline 0.9905 & 0.0095
\end{tabular}

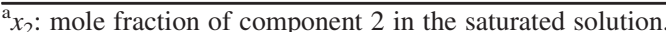

${ }^{\mathrm{b}} x_{1}$ : mole fraction solubility of the solute.

\section{Auxiliary Information}

\section{Method/Apparatus/Procedure:}

Constant-temperature bath and ultraviolet/visible spectrophotometer.

Excess solute and solvent were placed in screw-capped vials. The vials were sealed and submerged in a constant-temperature water bath and shaken at 100 cycles/min for $24 \mathrm{~h}$. After the $24 \mathrm{~h}$ equilibration period, the vial was removed, wiped dry, and the contents analyzed. The solutions were transferred to a syringe and filtered through a $0.1 \mu \mathrm{m}$ pore size filter. The solutions were diluted and the absorbances recorded at the maximum absorption wavelength of benzoic acid. The solubility was determined at least six times.

Source and Purity of Chemicals:

(1) Purity not given, Chemical source not specified, no information provided concerning purification.

(2) Spectrophotometric or ACS Reagent grade, Chemical source not specified, redistilled before use.

\section{Estimated Error:}

Temperature: $\pm 0.2 \mathrm{~K}$.

$x_{1}: \pm 3 \%$ (relative error)

\begin{tabular}{ll}
\hline \hline Components: & Original Measurements: \\
$\begin{array}{l}\text { (1) Benzoic acid; } \mathrm{C}_{7} \mathrm{H}_{6} \mathrm{O}_{2} ; \\
{[65-85-0]}\end{array}$ & ${ }^{49}$ W. E. Acree, Jr. and G. L. Bertrand, J. \\
(2) Hexane; $\mathrm{C}_{6} \mathrm{H}_{14} ;[110-54-3]$ & Pharm. Sci. 70, 1033 (1981). \\
\hline Variables: & Prepared by: \\
Temperature & W. E. Acree, Jr. \\
\hline
\end{tabular}

Experimental Values

\begin{tabular}{lcc}
\hline \hline$T / \mathrm{K}$ & $x_{2}{ }^{\mathrm{a}}$ & $x_{1}{ }^{\mathrm{b}}$ \\
\hline 298.15 & 0.9900 & 0.0100 \\
303.15 & 0.9874 & 0.0126 \\
\hline${ }^{\mathrm{a}} x_{2}:$ mole fraction of component 2 in the saturated solution. & \\
${ }^{\mathrm{b}} x_{1}:$ mole fraction solubility of the solute.
\end{tabular}




\section{Auxiliary Information}

\section{Method/Apparatus/Procedure:}

Constant-temperature water bath and an analytical balance.

Excess solute and solvent and were placed in brown glass containers and allowed to equilibrate in a constant-temperature water bath for several days. Attainment of equilibrium was verified by repetitive measurements after several additional days. Solubility was determined by transferring a weighed aliquot through a coarse filter into a flask containing blank nonaqueous titration solvent. The solutions were titrated with freshly standardized sodium methoxide to the thymol blue endpoint.

Source and Purity of Chemicals:

(1) $99 \%$, Chemical source not given, was dried at $333 \mathrm{~K}$ for several hours before use.

(2) $99 \%$, Chemical source not given, stored over molecular sieves and distilled before use.

Estimated Error:

Temperature: $\pm 0.01 \mathrm{~K}$.

$x_{1}: \pm 1 \%$ (relative error).

\begin{tabular}{|c|c|}
\hline $\begin{array}{l}\text { Components: } \\
\text { (1) Benzoic acid; } \mathrm{C}_{7} \mathrm{H}_{6} \mathrm{O}_{2} \text {; } \\
\text { [65-85-0] } \\
\text { (2) Hexane; } \mathrm{C}_{6} \mathrm{H}_{14} ;[110-54-3]\end{array}$ & $\begin{array}{l}\text { Original Measurements: } \\
{ }^{50} \text { P. G. Desai and A. M. Patel, J. Indian } \\
\text { Chem. Soc. 12, } 131 \text { (1935). }\end{array}$ \\
\hline $\begin{array}{l}\text { Variables: } \\
T / \mathrm{K}=301.2\end{array}$ & $\begin{array}{l}\text { Prepared by: } \\
\text { W. E. Acree, Jr. }\end{array}$ \\
\hline
\end{tabular}

\section{Experimental Values}

\begin{tabular}{lc}
\hline \hline$x_{2}{ }^{\mathrm{a}}$ & $x_{1}{ }^{\mathrm{b}}$ \\
\hline 0.9860 & 0.01399 \\
\hline
\end{tabular}

${ }^{a} x_{2}$ : mole fraction of component 2 in the saturated solution.

${ }^{b} x_{1}$ : mole fraction solubility of the solute.

\section{Auxiliary Information}

\section{Method/Apparatus/Procedure:}

Excess solute and solvent were placed in a glass flask which was kept revolving on a wheel in an air thermostat for two days. After two days of equilibration, the solution was filtered. To minimize absorption of the solute onto the filter paper, a $100 \mathrm{ml}$ portion of fresh saturated solution was first filtered through the paper before filtering the sample to be analyzed. An aliquot of the filtrate was titrated with barium hydroxide using phenolphthalein as the endpoint indictor. The barium hydroxide titrant was previously standardized against a solution of succinic acid.

\section{Source and Purity of Chemicals:}

(1) Purity not given, Chemical source not specified, no information provided concerning purification.

(2) Purity not given, Chemical source not specified, no information provided concerning purification.

Estimated Error:

Temperature: No information given.

$x_{1}: \pm 3 \%$ (relative error, estimated by compiler).

\begin{tabular}{|c|c|}
\hline $\begin{array}{l}\text { Components: } \\
\text { (1) Benzoic acid; } \mathrm{C}_{7} \mathrm{H}_{6} \mathrm{O}_{2} \text {; } \\
{[65-85-0]} \\
\text { (2) Hexane; } \mathrm{C}_{6} \mathrm{H}_{14} ;[110-54-3]\end{array}$ & $\begin{array}{l}\text { Original Measurements: } \\
\text { 51 H.-M. Lin and R. A. Nash, J. Pharm. } \\
\text { Sci. 82, } 1018 \text { (1993). }\end{array}$ \\
\hline $\begin{array}{l}\text { Variables: } \\
T / \mathrm{K}=298.15\end{array}$ & $\begin{array}{l}\text { Prepared by: } \\
\text { W. E. Acree, Jr. }\end{array}$ \\
\hline
\end{tabular}

Experimental Values

\begin{tabular}{lc}
\hline \hline$x_{2}{ }^{\mathrm{a}}$ & $x_{1}{ }^{\mathrm{b}}$ \\
\hline 0.9870 & 0.0130 \\
\hline$x_{2}$.
\end{tabular}

${ }^{a} x_{2}$ : mole fraction of component 2 in the saturated solution.

${ }^{\mathrm{b}} x_{1}$ : mole fraction solubility of the solute.

\section{Auxiliary Information}

\section{Method/Apparatus/Procedure:}

Constant-temperature bath and high-performance liquid chromatograph. Excess solute and solvent were placed in screw-capped vials. The vials were sealed with several turns of electrical tape, warmed to about $323 \mathrm{~K}$, and then shaken in a constant-temperature bath for at least $24 \mathrm{~h}$ at $298 \mathrm{~K}$. After equilibrium was reached, the sample was filtered through a 0.45 or $1.0 \mu \mathrm{m}$ filter, and an aliquot was diluted appropriately for chromatographic analysis. Concentrations were determined by a high-performance liquid chromatographic method.

\section{Source and Purity of Chemicals:}

(1) Purity not given, Amend Drug and Chemical Company, no information provided concerning purification.

(2) HPLC grade, Aldrich Chemical Company, Milwaukee, WI, USA, no purification details were provided.

\section{Estimated Error:}

Temperature: $\pm 0.2 \mathrm{~K}$ (estimated by compiler). $x_{1}: \pm 3 \%$ (relative error, estimated by compiler).

\begin{tabular}{|c|c|}
\hline $\begin{array}{l}\text { Components: } \\
\text { (1) Benzoic acid; } \mathrm{C}_{7} \mathrm{H}_{6} \mathrm{O}_{2} \text {; } \\
\text { [65-85-0] } \\
\text { (2) Hexane; } \mathrm{C}_{6} \mathrm{H}_{14} ;[110-54-3]\end{array}$ & $\begin{array}{l}\text { Original Measurements: } \\
\text { 52 A. Ksiazczak, Fluid Phase Equilib. } \\
\text { 28, } 57 \text { (1986). }\end{array}$ \\
\hline $\begin{array}{l}\text { Variables: } \\
\text { Temperature }\end{array}$ & $\begin{array}{l}\text { Prepared by: } \\
\text { W. E. Acree, Jr. }\end{array}$ \\
\hline
\end{tabular}

Experimental Values

\begin{tabular}{lcc}
\hline \hline$T / \mathrm{K}$ & $x_{2}{ }^{\mathrm{a}}$ & $x_{1}{ }^{\mathrm{b}}$ \\
\hline 312.55 & 0.9812 & 0.0188 \\
316.30 & 0.9773 & 0.0227 \\
319.57 & 0.9737 & 0.0263 \\
319.85 & 0.9734 & 0.0264 \\
321.48 & 0.9711 & 0.0289 \\
321.52 & 0.9709 & 0.0291 \\
322.85 & 0.9698 & 0.0302 \\
323.48 & 0.9685 & 0.0315 \\
325.50 & 0.9660 & 0.0340 \\
325.80 & 0.9652 & 0.0348 \\
327.49 & 0.9630 & 0.0370 \\
327.50 & 0.9630 & 0.0370 \\
329.47 & 0.9595 & 0.0405
\end{tabular}




\begin{tabular}{lcc}
\hline \hline$T / \mathrm{K}$ & $x_{2}{ }^{\mathrm{a}}$ & $x_{1}{ }^{\mathrm{b}}$ \\
\hline 331.45 & 0.9564 & 0.0436 \\
333.48 & 0.9530 & 0.0470 \\
333.50 & 0.9525 & 0.0475 \\
335.48 & 0.9476 & 0.0524 \\
336.10 & 0.9454 & 0.0546 \\
336.15 & 0.9445 & 0.0555 \\
338.47 & 0.9395 & 0.0605 \\
339.50 & 0.9363 & 0.0637 \\
341.55 & 0.9299 & 0.0701 \\
341.65 & 0.9291 & 0.0709 \\
343.52 & 0.9230 & 0.0770 \\
344.50 & 0.9198 & 0.0802 \\
344.60 & 0.9186 & 0.0814 \\
345.48 & 0.9152 & 0.0848 \\
347.40 & 0.9065 & 0.0935 \\
347.46 & 0.9067 & 0.0933 \\
349.30 & 0.899 & 0.101 \\
350.30 & 0.893 & 0.107 \\
351.41 & 0.888 & 0.112 \\
353.50 & 0.876 & 0.124 \\
356.45 & 0.855 & 0.145 \\
359.40 & 0.832 & 0.168 \\
362.60 & 0.798 & 0.202 \\
\hline a & m m &
\end{tabular}

${ }^{\mathrm{a}} x_{2}$ : mole fraction of component 2 in the saturated solution.

${ }^{\mathrm{b}} x_{1}$ : mole fraction solubility of the solute.

\section{Auxiliary Information}

\section{Method/Apparatus/Procedure:}

Constant-temperature bath and ultraviolet/visible spectrophotometer. Excess solute and solvent were placed in screw-capped vials. The vials were sealed and submerged in a constant-temperature water bath and shaken at 100 cycles/min for $24 \mathrm{~h}$. After the $24 \mathrm{~h}$ equilibration period, the vial was removed, wiped dry and the contents analyzed. The solutions were transferred to a syringe and filtered through a $0.1 \mu \mathrm{m}$ pore size filter. The solutions were diluted and the absorbances recorded at the maximum absorption wavelength of benzoic acid. The solubility was determined at least six times.

\section{Source and Purity of Chemicals:}

(1) Purity not given, Chemical source not specified, no information provided concerning purification.

(2) Spectrophotometric or ACS Reagent grade, Chemical source not specified, redistilled before use.

\section{Estimated Error:}

Temperature: $\pm 0.2 \mathrm{~K}$.

$x_{1}: \pm 3 \%$ (relative error).

\begin{tabular}{|c|c|}
\hline $\begin{array}{l}\text { Components: } \\
\text { (1) Benzoic acid; } \mathrm{C}_{7} \mathrm{H}_{6} \mathrm{O}_{2} \text {; } \\
\text { [65-85-0] } \\
\text { (2) Heptane; } \mathrm{C}_{7} \mathrm{H}_{16} ;[142-82-5]\end{array}$ & $\begin{array}{l}\text { Original Measurements: } \\
{ }^{48} \text { J. Thati, F. L. Nordström, and A. C. } \\
\text { Rasmuson, J. Chem. Eng. Data 55, } \\
5124 \text { (2010). }\end{array}$ \\
\hline $\begin{array}{l}\text { Variables: } \\
\text { Temperature }\end{array}$ & $\begin{array}{l}\text { Prepared by: } \\
\text { W. E. Acree, Jr. }\end{array}$ \\
\hline
\end{tabular}

\section{Auxiliary Information}

\section{Method/Apparatus/Procedure:}

Solubilities were determined using a dynamic method. Known amounts of solute and solvent were placed in a container which was then sealed. The temperature of the solution was slowly increased until the last amount of solid dissolved. The temperature at which the last crystals disappeared was taken as the temperature of the solution-crystal equilibrium.

\section{Source and Purity of Chemicals:}

(1) Purity not given, Chemical source not given, no purification details were provided.

(2) Purity not given, POCh Gliwice, Poland, was dried over phosphorous pentoxide and distilled before use.

Estimated Error:

Temperature: $\pm 0.1 \mathrm{~K}$ (estimated by compiler). $x_{1}: \pm 3 \%$ (relative error, estimated by compiler).

\begin{tabular}{|c|c|}
\hline $\begin{array}{l}\text { Components: } \\
\text { (1) Benzoic acid; } \mathrm{C}_{7} \mathrm{H}_{6} \mathrm{O}_{2} \text {; } \\
\text { [65-85-0] } \\
\text { (2) Heptane; } \mathrm{C}_{7} \mathrm{H}_{16} ;[142-82-5]\end{array}$ & $\begin{array}{l}\text { Original Measurements: } \\
{ }^{47} \text { A. Beerbower, P. L. Wu, and A. } \\
\text { Martin, J. Pharm. Sci. 73, } 179 \text { (1984) }\end{array}$ \\
\hline $\begin{array}{l}\text { Variables: } \\
T / \mathrm{K}=298.15\end{array}$ & $\begin{array}{l}\text { Prepared by: } \\
\text { W. E. Acree, Jr. }\end{array}$ \\
\hline
\end{tabular}

\section{Experimental Values}

\begin{tabular}{lc}
\hline \hline$x_{2}^{\mathrm{a}}$ & $x_{1}{ }^{\mathrm{b}}$ \\
\hline 0.9883 & 0.0117 \\
\hline${ }^{\mathrm{a}} x_{2}:$ mole fraction of component 2 in the saturated solution. & \\
${ }^{\mathrm{b}} x_{1}:$ mole fraction solubility of the solute. &
\end{tabular}

Experimental Values

\begin{tabular}{lcc}
\hline \hline$T / \mathrm{K}$ & $x_{2}{ }^{\mathrm{a}}$ & $x_{1}{ }^{\mathrm{b}}$ \\
\hline 278.15 & 0.995 & 0.005 \\
283.15 & 0.994 & 0.006 \\
293.15 & 0.990 & 0.010 \\
303.15 & 0.985 & 0.015 \\
313.15 & 0.978 & 0.022 \\
323.15 & 0.967 & 0.033
\end{tabular}

${ }^{\mathrm{a}} x_{2}$ : mole fraction of component 2 in the saturated solution.

${ }^{\mathrm{b}} x_{1}$ : mole fraction solubility of the solute.

\section{Auxiliary Information}

\section{Method/Apparatus/Procedure:}

Constant-temperature bath, magnetic stirrer, analytical balance, and ventilated laboratory hood.

Solubilities were determined by a gravimetric method. Excess solute and solvent were placed in sealed bottles and allowed to equilibrate in a constanttemperature bath for $2 \mathrm{~h}$ with stirring. The stirring was discontinued and the solid was allowed to settle to the bottom of the container. After $30 \mathrm{~min}$, an aliquot of the clear solution was removed by syringe, filtered through a $0.2 \mu \mathrm{m}$ PTFE filter, and transferred to a preweighed glass vial. The glass vial was then weighed, and the solvent was allowed to evaporate in a ventilated laboratory hood at ambient room temperature. Once the solvent had evaporated, the vial with solid residue was weighed until constant weight was obtained. The solubility was calculated from the mass of the solid residue and mass of sample analyzed.

Source and Purity of Chemicals:

(1) $99.7 \%$, Merck Chemical Company, Germany, was used as received.

(2) $99 \%$, VWR Scientific, USA, used as received.

Estimated Error:

Temperature: $\pm 0.01 \mathrm{~K}$.

$x_{1}: \pm 1 \%$ (relative error) 


\section{Components:}

(1) Benzoic acid; $\mathrm{C}_{7} \mathrm{H}_{6} \mathrm{O}_{2}$;

[65-85-0]

(2) Heptane; $\mathrm{C}_{7} \mathrm{H}_{16} ;[142-82-5]$

\section{Variables:}

Temperature
Original Measurements:

${ }^{49}$ W. E. Acree, Jr. and G. L. Bertrand, J.
Pharm. Sci. 70, 1033 (1981).

\section{Prepared by:}

W. E. Acree, Jr.

Experimental Values

\begin{tabular}{lcc}
\hline \hline$T / \mathrm{K}$ & $x_{2}{ }^{\mathrm{a}}$ & $x_{1}{ }^{\mathrm{b}}$ \\
\hline 298.15 & 0.9886 & 0.0114 \\
303.15 & 0.9853 & 0.0147
\end{tabular}

${ }^{\mathrm{a}} x_{2}$ : mole fraction of component 2 in the saturated solution.

${ }^{\mathrm{b}} \mathrm{x}_{1}$ : mole fraction solubility of the solute.

\section{Auxiliary Information}

\section{Method/Apparatus/Procedure:}

Constant-temperature water bath and an analytical balance.

Excess solute and solvent and were placed in brown glass containers and allowed to equilibrate in a constant-temperature water bath for several days. Attainment of equilibrium was verified by repetitive measurements after several additional days. Solubility was determined by transferring a weighed aliquot through a coarse filter into a flask containing blank nonaqueous titration solvent. The solutions were titrated with freshly standardized sodium methoxide to the thymol blue endpoint.

\section{Source and Purity of Chemicals:}

(1) $99 \%$, Chemical source not given, was dried at $333 \mathrm{~K}$ for several hours before use.

(2) $99+\%$, Chemical source not given, stored over molecular sieves and distilled before use.

Estimated Error:

Temperature: $\pm 0.01 \mathrm{~K}$.

$x_{1}: \pm 1 \%$ (relative error).

\begin{tabular}{ll}
\hline \hline Components: & Original Measurements: \\
(1) Benzoic acid; $\mathrm{C}_{7} \mathrm{H}_{6} \mathrm{O}_{2} ;$ & ${ }^{53}$ G. L. Perlovich and A. Bauer-Brandl, \\
65-85-0] & Pharm. Res. 20, 471 (2003). \\
(2) Octane; $\mathrm{C}_{8} \mathrm{H}_{18} ;[111-65-9]$ & \\
\hline Variables: & Prepared by: \\
$T / \mathrm{K}=298.15$ & W. E. Acree, Jr. \\
\hline
\end{tabular}

Experimental Values

\begin{tabular}{lc}
\hline \hline$x_{2}{ }^{\mathrm{a}}$ & $x_{1}{ }^{\mathrm{b}}$ \\
\hline 0.9871 & 0.0129
\end{tabular}

${ }^{\mathrm{a}} x_{2}$ : mole fraction of component 2 in the saturated solution.

${ }^{b} x_{1}$ : mole fraction solubility of the solute.

\section{Auxiliary Information}

Method/Apparatus/Procedure:

Thermostated constant-temperature bath, centrifuge, and analytical balance. Very little experimental details were given in the paper. The solubility of the solute was by a weighing method.

Source and Purity of Chemicals:

(1) Analytical Reagent grade, Norsk Medisinaldepot, Oslo, Norway, no purification details were provided.

(2) Analytical Reagent grade, Sigma Chemical Company, USA, no purification details were provided.

\section{Estimated Error:}

Temperature: $\pm 0.01 \mathrm{~K}$.

$x_{1}: \pm 3.0 \%$ (relative error).

\begin{tabular}{|c|c|}
\hline $\begin{array}{l}\text { Components: } \\
\text { (1) Benzoic acid; } \mathrm{C}_{7} \mathrm{H}_{6} \mathrm{O}_{2} \text {; } \\
\text { [65-85-0] } \\
\text { (2) Nonane; } \mathrm{C}_{9} \mathrm{H}_{20} ;[111-84-2]\end{array}$ & $\begin{array}{l}\text { Original Measurements: } \\
{ }^{47} \text { A. Beerbower, P. L. Wu, and A. } \\
\text { Martin, J. Pharm. Sci. 73, } 179 \text { (1984). }\end{array}$ \\
\hline $\begin{array}{l}\text { Variables: } \\
T / \mathrm{K}=298.15\end{array}$ & $\begin{array}{l}\text { Prepared by: } \\
\text { W. E. Acree, Jr. }\end{array}$ \\
\hline
\end{tabular}

\section{Experimental Values}

\begin{tabular}{lc}
\hline \hline$x_{2}{ }^{\mathrm{a}}$ & $x_{1}{ }^{\mathrm{b}}$ \\
\hline 0.9859 & 0.0141 \\
\hline${ }^{\mathrm{a}} x_{2}:$ mole fraction of component 2 in the saturated solution. & \\
${ }^{\mathrm{b}} x_{1}:$ mole fraction solubility of the solute. &
\end{tabular}

\section{Auxiliary Information}

Method/Apparatus/Procedure:

Constant-temperature bath and ultraviolet/visible spectrophotometer.

Excess solute and solvent were placed in screw-capped vials. The vials were sealed and submerged in a constant-temperature water bath and shaken at 100 cycles/min for $24 \mathrm{~h}$. After the $24 \mathrm{~h}$ equilibration period, the vial was removed, wiped dry, and the contents analyzed. The solutions were transferred to a syringe and filtered through a $0.1 \mu \mathrm{m}$ pore size filter. The solutions were diluted and the absorbances recorded at the maximum absorption wavelength of benzoic acid. The solubility was determined at least six times.

\section{Source and Purity of Chemicals:}

(1) Purity not given, Chemical source not specified, no information provided concerning purification.

(2) Spectrophotometric or ACS Reagent grade, Chemical source not specified, redistilled before use.

\section{Estimated Error:}

Temperature: $\pm 0.2 \mathrm{~K}$.

$x_{1}: \pm 3 \%$ (relative error).

\begin{tabular}{|c|c|}
\hline $\begin{array}{l}\text { Components: } \\
\text { (1) Benzoic acid; } \mathrm{C}_{7} \mathrm{H}_{6} \mathrm{O}_{2} \text {; } \\
\text { [65-85-0] } \\
\text { (2) Decane; } \mathrm{C}_{10} \mathrm{H}_{22} ;[124-18-5]\end{array}$ & $\begin{array}{l}\text { Original Measurements: } \\
{ }^{47} \text { A. Beerbower, P. L. Wu, and A. } \\
\text { Martin, J. Pharm. Sci. 73, } 179 \text { (1984) }\end{array}$ \\
\hline $\begin{array}{l}\text { Variables: } \\
T / \mathrm{K}=298.15\end{array}$ & $\begin{array}{l}\text { Prepared by: } \\
\text { W. E. Acree, Jr. }\end{array}$ \\
\hline
\end{tabular}




\section{Experimental Values}

\begin{tabular}{lc}
\hline \hline$x_{2}{ }^{\mathrm{a}}$ & $x_{1}{ }^{\mathrm{b}}$ \\
\hline 0.9898 & 0.0102
\end{tabular}

${ }^{\mathrm{a}} x_{2}$ : mole fraction of component 2 in the saturated solution.

${ }^{\mathrm{b}} \mathrm{x}_{1}$ : mole fraction solubility of the solute.

\section{Auxiliary Information}

\section{Method/Apparatus/Procedure:}

Constant-temperature bath and ultraviolet/visible spectrophotometer.

Excess solute and solvent were placed in screw-capped vials. The vials were sealed and submerged in a constant-temperature water bath and shaken at 100 cycles/min for $24 \mathrm{~h}$. After the $24 \mathrm{~h}$ equilibration period, the vial was removed, wiped dry, and the contents analyzed. The solutions were transferred to a syringe and filtered through a $0.1 \mu \mathrm{m}$ pore size filter. The solutions were diluted and the absorbances recorded at the maximum absorption wavelength of benzoic acid. The solubility was determined at least six times.

\section{Source and Purity of Chemicals:}

(1) Purity not given, Chemical source not specified, no information provided concerning purification.

(2) Spectrophotometric or ACS Reagent grade, Chemical source not specified, redistilled before use.

Estimated Error:

Temperature: $\pm 0.2 \mathrm{~K}$

$x_{1}: \pm 3 \%$ (relative error)

\begin{tabular}{ll}
\hline \hline Components: & Original Measurements: \\
$\begin{array}{l}\text { (1) Benzoic acid; } \mathrm{C}_{7} \mathrm{H}_{6} \mathrm{O}_{2} ; \\
{[65-85-0]}\end{array}$ & $\begin{array}{l}{ }^{47} \text { A. Beerbower, P. L. Wu, and A. } \\
\text { (2) Cyclohexane; } \mathrm{C}_{6} \mathrm{H}_{12} ;\end{array}$ \\
{$[110-82-7]$} & \\
\hline Variables: & Prepared by: \\
$T / \mathrm{K}=298.15$ & W. E. Acree, Jr. \\
\hline
\end{tabular}

\section{Experimental Values}

\begin{tabular}{lc}
\hline \hline$x_{2}{ }^{\mathrm{a}}$ & $x_{1}{ }^{\mathrm{b}}$ \\
\hline 0.9898 & 0.0102 \\
\hline
\end{tabular}

${ }^{a} x_{2}$ : mole fraction of component 2 in the saturated solution.

${ }^{\mathrm{b}} \mathrm{x}_{1}$ : mole fraction solubility of the solute.

\section{Auxiliary Information}

\section{Method/Apparatus/Procedure:}

Constant-temperature bath and ultraviolet/visible spectrophotometer.

Excess solute and solvent were placed in screw-capped vials. The vials were sealed and submerged in a constant-temperature water bath and shaken at 100 cycles/min for $24 \mathrm{~h}$. After the $24 \mathrm{~h}$ equilibration period, the vial was removed, wiped dry, and the contents analyzed. The solutions were transferred to a syringe and filtered through a $0.1 \mu \mathrm{m}$ pore size filter. The solutions were diluted and the absorbances recorded at the maximum absorption wavelength of benzoic acid. The solubility was determined at least six times.

Source and Purity of Chemicals:

(1) Purity not given, Chemical source not specified, no information provided concerning purification.

(2) Spectrophotometric or ACS Reagent grade, Chemical source not specified, redistilled before use.
Estimated Error:

Temperature: $\pm 0.2 \mathrm{~K}$.

$x_{1}: \pm 3 \%$ (relative error)

\begin{tabular}{|c|c|}
\hline $\begin{array}{l}\text { Components: } \\
\text { (1) Benzoic acid; } \mathrm{C}_{7} \mathrm{H}_{6} \mathrm{O}_{2} \text {; } \\
\text { [65-85-0] } \\
\text { (2) Cyclohexane; } \mathrm{C}_{6} \mathrm{H}_{12} \text {; } \\
\text { [110-82-7] }\end{array}$ & $\begin{array}{l}\text { Original Measurements: } \\
{ }^{54} \text { C. K. Hancock, J. N. Pawloski, and J. } \\
\text { P. Idoux, J. Org. Chem. 31, } 3801 \\
\text { (1966). }\end{array}$ \\
\hline $\begin{array}{l}\text { Variables: } \\
T / \mathrm{K}=303.15\end{array}$ & $\begin{array}{l}\text { Prepared by: } \\
\text { W. E. Acree, Jr. }\end{array}$ \\
\hline
\end{tabular}

\section{Experimental Values}

\begin{tabular}{lc}
\hline \hline$x_{2}{ }^{\mathrm{a}}$ & $x_{1}{ }^{\mathrm{b}}$ \\
\hline 0.9857 & 0.0143
\end{tabular}

${ }^{a} x_{2}$ : mole fraction of component 2 in the saturated solution.

${ }^{\mathrm{b}} x_{1}$ : mole fraction solubility of the solute.

\section{Auxiliary Information}

\section{Method/Apparatus/Procedure:}

Constant-temperature bath, Soxhlet thimble, conical flask, and analytical balance.

Excess solute and solvent were placed in a conical flask and allowed to equilibrate for several days at constant temperature. Attainment of equilibrium was verified by several repetitive measurements and by approaching equilibrium from supersaturation. Aliquots of saturated solutions were transferred using a Soxhlet thimble equipped with a one-hole cork stopper and an inverted U-shaped delivery tube to a second ground-glass stoppered flask suspended in the $303 \mathrm{~K}$ water bath. Successive portions of the contents were evaporated at room temperature in a tared aluminum foil weighing dish under a bell jar through which a slow stream of dry air was passed. The second flask plus unused saturated solution and the aluminum foil dish plus residue were weighed. The saturation solubility of the solute was calculated from the recorded mass data and molar masses of the solute and solvent.

\section{Source and Purity of Chemicals:}

(1) Purity not given, Chemical source not specified, was recrystallized several times from aqueous-ethanol mixture, and then dried under vacuum over phosphorous pentoxide.

(2) Reagent grade, Chemical source not specified, refluxed over phosphorous pentoxide for $24 \mathrm{~h}$, and then distilled through a $3 \times 80 \mathrm{~cm}$ column filled with $0.32-\mathrm{cm}$ glass helices.

\section{Estimated Error:}

Temperature: $\pm 0.02 \mathrm{~K}$

$x_{1}: \pm 1.5 \%$ (relative error)

\begin{tabular}{ll}
\hline \hline Components: & Original Measurements: \\
$\begin{array}{l}\text { (1) Benzoic acid; } \mathrm{C}_{7} \mathrm{H}_{6} \mathrm{O}_{2} ; \\
{[65-85-0]}\end{array}$ & $\begin{array}{l}{ }^{55} \text { B. Long, J. Li, R. Zhang, and L. Wan, } \\
\text { (2) Cyclohexane; } \mathrm{C}_{6} \mathrm{H}_{12} ;\end{array}$ \\
{$[110-82-7]$} & \\
\hline Variables: & Prepared by: \\
Temperature & W. E. Acree, Jr. \\
\hline
\end{tabular}


Experimental Values

\begin{tabular}{lcl}
\hline \hline$T / \mathrm{K}$ & $x_{2}{ }^{\mathrm{a}}$ & \multicolumn{1}{c}{$x_{1}{ }^{\mathrm{b}}$} \\
\hline 283.15 & 0.9968 & 0.003179 \\
287.95 & 0.9953 & 0.004724 \\
293.16 & 0.9926 & 0.007413 \\
298.00 & 0.9890 & 0.01095 \\
303.30 & 0.9847 & 0.01528 \\
308.02 & 0.9795 & 0.02052 \\
313.18 & 0.9698 & 0.03023 \\
317.75 & 0.9630 & 0.03701 \\
322.91 & 0.9532 & 0.04678 \\
328.18 & 0.9434 & 0.05656 \\
333.11 & 0.9275 & 0.07254 \\
337.98 & 0.9066 & 0.09341 \\
342.80 & 0.8777 & 0.1223 \\
\hline
\end{tabular}

${ }^{\mathrm{a}} x_{2}$ : mole fraction of component 2 in the saturated solution.

${ }^{\mathrm{b}} x_{1}$ : mole fraction solubility of the solute.

\section{Auxiliary Information}

\section{Method/Apparatus/Procedure:}

Double layer jacketed glass equilibrium cell, circulating water bath, analytical balance, and drying oven.

Excess solute and solvent and were placed in an equilibrium vessel, which was connected to a circulating water bath. The solution was allowed to equilibrate at constant temperature with stirring for $3 \mathrm{~h}$. The stirring was discontinued and the solution was allowed to stand for $1 \mathrm{~h}$ to allow the undissolved solid to settle to the bottom portion of the equilibrium vessel. An aliquot of the clear solution was transferred to a preweighed vial by a warm syringe. The vial was tightly closed and reweighed to determine the mass of the sample transferred. The vial was then put into a drying oven with the cap half-closed to permit complete evaporation of the solvent. The vial was covered with a piece of stainless steel filter cloth to prevent dust contamination. After the solvent had evaporated, the vial was removed from the drying oven and placed in a desiccator with silica gel for another $2 \mathrm{~h}$ to reach ambient room temperature. The vial with solid residue was weighed until constant weight was obtained. The solubility was calculated from the mass of the solid residue and mass of sample analyzed.

\section{Source and Purity of Chemicals:}

(1) $99.5 \%$, Analytical grade, Shantou Xilong Chemical Company, China, was used as received.

(2) $99.5 \%$, Analytical grade, Beijing Chemical Reagent Company, China, used as received.

\section{Estimated Error:}

Temperature: $\pm 0.05 \mathrm{~K}$.

$x_{1}: \pm 1 \%$ (relative error).

\begin{tabular}{ll}
\hline \hline Components: & Original Measurements: \\
$\begin{array}{l}\text { (1) Benzoic acid; } \mathrm{C}_{7} \mathrm{H}_{6} \mathrm{O}_{2} ; \\
\text { [65-85-0] }\end{array}$ & $\begin{array}{l}\text { 28. Ksiazczak, Fluid Phase Equilib. } \\
\text { (2) Cyclohexane; } \mathrm{C}_{6} \mathrm{H}_{12} ;\end{array}$ \\
{$[110-82-7]$} & \\
\hline Variables: & Prepared by: \\
Temperature & W. E. Acree, Jr. \\
\hline
\end{tabular}

Experimental Values

\begin{tabular}{lcc}
\hline \hline$T / \mathrm{K}$ & $x_{2}{ }^{\mathrm{a}}$ & $x_{1}{ }^{\mathrm{b}}$ \\
\hline 309.65 & 0.9802 & 0.0198 \\
315.45 & 0.9745 & 0.0255 \\
318.20 & 0.9700 & 0.0300 \\
320.95 & 0.9654 & 0.0346 \\
325.35 & 0.9564 & 0.0436 \\
329.00 & 0.9494 & 0.0506 \\
332.60 & 0.9366 & 0.0634 \\
334.45 & 0.9302 & 0.0698 \\
338.80 & 0.9118 & 0.0882 \\
339.00 & 0.9110 & 0.0890 \\
341.35 & 0.9005 & 0.0995 \\
342.95 & 0.890 & 0.110 \\
343.55 & 0.888 & 0.112 \\
345.55 & 0.873 & 0.127 \\
346.55 & 0.867 & 0.133 \\
347.30 & 0.863 & 0.137 \\
349.35 & 0.846 & 0.154 \\
349.50 & 0.841 & 0.159 \\
351.15 & 0.830 & 0.170 \\
351.55 & 0.823 & 0.177 \\
354.50 & 0.786 & 0.214 \\
360.65 & 0.695 & 0.305 \\
366.35 & 0.574 & 0.426 \\
369.40 & 0.514 & 0.486 \\
372.35 & 0.448 & 0.552 \\
375.50 & 0.391 & 0.609 \\
379.40 & 0.305 & 0.695 \\
383.80 & 0.235 & 0.765 \\
389.85 & 0.115 & 0.885 \\
\hline & 2.50 &
\end{tabular}

${ }^{a} x_{2}$ : mole fraction of component 2 in the saturated solution.

${ }^{\mathrm{b}} x_{1}$ : mole fraction solubility of the solute.

\section{Auxiliary Information}

\section{Method/Apparatus/Procedure:}

Solubilities were determined using a dynamic method. Known amounts of solute and solvent were placed in a container which was then sealed. The temperature of the solution was slowly increased until the last amount of solid dissolved. The temperature at which the last crystals disappeared was taken as the temperature of the solution-crystal equilibrium.

Source and Purity of Chemicals:

(1) Purity not given, Chemical source not given, no purification details were provided.

(2) Purity not given, POCh Gliwice, Poland, was dried over phosphorous pentoxide and distilled before use.

\section{Estimated Error:}

Temperature: $\pm 0.1 \mathrm{~K}$ (estimated by compiler).

$x_{1}: \pm 3 \%$ (relative error, estimated by compiler).

\begin{tabular}{ll}
\hline \hline Components: & Original Measurements: \\
$\begin{array}{l}\text { (1) Benzoic acid; } \mathrm{C}_{7} \mathrm{H}_{6} \mathrm{O}_{2} ; \\
{[65-85-0]}\end{array}$ & $\begin{array}{l}{ }^{49} \text { W. E. Acree, Jr. and G. L. Bertrand, J. } \\
\text { (2) Cyclohexane; } \mathrm{C}_{6} \mathrm{H}_{12} ;\end{array}$ \\
{$[110-82-7]$} & \\
\hline Variables: & \\
Temperature & Prepared by: \\
\hline
\end{tabular}


Experimental Values

\begin{tabular}{lcc}
\hline \hline$T / \mathrm{K}$ & $x_{2}{ }^{\mathrm{a}}$ & $x_{1}{ }^{\mathrm{b}}$ \\
\hline 298.15 & 0.9885 & 0.0115 \\
303.15 & 0.9854 & 0.0146
\end{tabular}

${ }^{\mathrm{a}} x_{2}$ : mole fraction of component 2 in the saturated solution.

${ }^{\mathrm{b}} x_{1}$ : mole fraction solubility of the solute.

\section{Auxiliary Information}

\section{Method/Apparatus/Procedure:}

Constant-temperature water bath and an analytical balance.

Excess solute and solvent and were placed in brown glass containers and allowed to equilibrate in a constant-temperature water bath for several days. Attainment of equilibrium was verified by repetitive measurements after several additional days. Solubility was determined by transferring a weighed aliquot through a coarse filter into a flask containing blank nonaqueous titration solvent. The solutions were titrated with freshly standardized sodium methoxide to the thymol blue endpoint.

\section{Source and Purity of Chemicals:}

(1) $99 \%$, Chemical source not given, was dried at $333 \mathrm{~K}$ for several hours before use.

(2) $99+\%$, Chemical source not given, stored over molecular sieves and distilled before use.

\section{Estimated Error:}

Temperature: $\pm 0.01 \mathrm{~K}$.

$x_{1}: \pm 1 \%$ (relative error).

\begin{tabular}{ll}
\hline \hline Components: & Original Measurements: \\
$\begin{array}{l}\text { (1) Benzoic acid; } \mathrm{C}_{7} \mathrm{H}_{6} \mathrm{O}_{2} ; \\
{[65-85-0]}\end{array}$ & $\begin{array}{l}{ }^{48} \text { J. Thati, F. L. Nordström, and A. C. } \\
\text { (2) Cyclohexane; } \mathrm{C}_{6} \mathrm{H}_{12} ;\end{array}$ \\
$\begin{array}{ll}\text { Rasmuson, J. Chem. Eng. Data 55, } \\
\text { Var-82-7] }\end{array}$ & 5124 (2010). \\
\hline Variables: & Prepared by: \\
Temperature & W. E. Acree, Jr. \\
\hline
\end{tabular}

Experimental Values

\begin{tabular}{lcc}
\hline \hline$T / \mathrm{K}$ & $x_{2}{ }^{\mathrm{a}}$ & $x_{1}{ }^{\mathrm{b}}$ \\
\hline 283.15 & 0.994 & 0.006 \\
293.15 & 0.991 & 0.009 \\
303.15 & 0.985 & 0.015 \\
313.15 & 0.975 & 0.025 \\
323.15 & 0.961 & 0.039 \\
\hline
\end{tabular}

${ }^{\mathrm{a}} x_{2}$ : mole fraction of component 2 in the saturated solution.

${ }^{b} x_{1}$ : mole fraction solubility of the solute.

\section{Auxiliary Information}

\section{Method/Apparatus/Procedure:}

Constant-temperature bath, magnetic stirrer, analytical balance, and ventilated laboratory hood.

Solubilities were determined by a gravimetric method. Excess solute and solvent were placed in sealed bottles and allowed to equilibrate in a constanttemperature bath for $2 \mathrm{~h}$ with stirring. The stirring was discontinued and the solid was allowed to settle to the bottom of the container. After $30 \mathrm{~min}$, an aliquot of the clear solution was removed by syringe, filtered through a $0.2 \mu \mathrm{m}$ PTFE filter, and transferred to a preweighed glass vial. The glass vial was then weighed, and the solvent was allowed to evaporate in a ventilated laboratory hood at ambient room temperature. Once the solvent had evaporated, the vial with solid residue was weighed until constant weight was obtained. The solubility was calculated from the mass of the solid residue and mass of sample analyzed.

Source and Purity of Chemicals:

(1) $99.7 \%$, Merck Chemical Company, Germany, was used as received.

(2) $99.5 \%$, VWR Scientific, USA, used as received.

Estimated Error:

Temperature: $\pm 0.01 \mathrm{~K}$.

$x_{1}: \pm 1 \%$ (relative error)

\begin{tabular}{ll}
\hline \hline Components: & Original Measurements: \\
$\begin{array}{l}\text { (1) Benzoic acid; } \mathrm{C}_{7} \mathrm{H}_{6} \mathrm{O}_{2} ; \\
{[65-85-0]}\end{array}$ & 3634. \\
$\begin{array}{l}\text { (2) Cyclohexane; } \mathrm{C}_{6} \mathrm{H}_{12} ; \\
{[110-82-7]}\end{array}$ & \\
\hline Variables: & Prepared by: \\
$T / \mathrm{K}=298.15$ & W. E. Acree, Jr. \\
\hline
\end{tabular}

\section{Experimental Values}

The measured solubility was reported to be $-\log _{10} c_{1}=1.038$, which corresponds to a solubility of $c_{1}=0.0916 \mathrm{~mol} \mathrm{dm}^{-3}$.

\section{Auxiliary Information}

\section{Method/Apparatus/Procedure:}

Very little experimental details were provided. Excess solute and solvent were placed in tubes, which were rotated for at least $8 \mathrm{~h}$ at $298 \mathrm{~K}$. The solutions were then removed, concentrated if necessary, and titrated with standard alkali using a mixed (bromothymol blue + neutral red) indicator.

Source and Purity of Chemicals:

(1) Purity not given, Chemical source not given, solute was either "AnalaR" grade or recrystallized prior to use.

(2) Purity not given, Chemical source not given, no purification details were provided.

\section{Estimated Error:}

Temperature: No information was given. $c_{1}: \pm 4 \%$ (relative error, estimated by compiler).

\subsection{Benzoic acid solubility data in aromatic hydrocarbons}

\begin{tabular}{ll}
\hline \hline Components: & Original Measurements: \\
$\begin{array}{l}\text { (1) Benzoic acid; } \mathrm{C}_{7} \mathrm{H}_{6} \mathrm{O}_{2} ; \\
{[65-85-0]}\end{array}$ & $\begin{array}{l}{ }^{47} \text { A. Beerbower, P. L. Wu, and A. } \\
\text { (2) Benzene; } \mathrm{C}_{6} \mathrm{H}_{6} ;[71-43-2]\end{array}$ \\
\hline Variables: & Prepared by: \\
$T / \mathrm{K}=298.15$ & W. E. Acree, Jr. \\
\hline
\end{tabular}

Experimental Values

\begin{tabular}{lc}
\hline \hline$x_{2}{ }^{\mathrm{a}}$ & $x_{1}{ }^{\mathrm{b}}$ \\
\hline 0.9311 & 0.0689 \\
\hline${ }^{\mathrm{a}} x_{2}:$ mole fraction of component 2 in the saturated solution. & \\
${ }^{\mathrm{b}} x_{1}:$ mole fraction solubility of the solute. &
\end{tabular}




\section{Auxiliary Information}

\section{Method/Apparatus/Procedure:}

Constant-temperature bath and ultraviolet/visible spectrophotometer.

Excess solute and solvent were placed in screw-capped vials. The vials were sealed and submerged in a constant-temperature water bath and shaken at 100 cycles/min for $24 \mathrm{~h}$. After the $24 \mathrm{~h}$ equilibration period, the vial was removed, wiped dry, and the contents analyzed. The solutions were transferred to a syringe and filtered through a $0.1 \mu \mathrm{m}$ pore size filter. The solutions were diluted and the absorbances recorded at the maximum absorption wavelength of benzoic acid. The solubility was determined at least six times.

\section{Source and Purity of Chemicals:}

(1) Purity not given, Chemical source not specified, no information provided concerning purification.

(2) Spectrophotometric or ACS Reagent grade, Chemical source not specified, redistilled before use.

\section{Estimated Error:}

Temperature: $\pm 0.2 \mathrm{~K}$

$x_{1}: \pm 3 \%$ (relative error).

\begin{tabular}{ll}
\hline \hline Components: & Original Measurements: \\
$\begin{array}{l}\text { (1) Benzoic acid; } \mathrm{C}_{7} \mathrm{H}_{6} \mathrm{O}_{2} ; \\
{[65-85-0]}\end{array}$ & ${ }^{54} \mathrm{C}$. K. Hancock, J. N. Pawloski, and J. \\
(2) Benzene; $\mathrm{C}_{6} \mathrm{H}_{6} ;[71-43-2]$ & $(1966)$. \\
\hline Variables: & Prepared by: \\
$T / \mathrm{K}=303.15$ & W. E. Acree, Jr. \\
\hline
\end{tabular}

Experimental Values

\begin{tabular}{lc}
\hline \hline$x_{2}{ }^{\mathrm{a}}$ & $x_{1}{ }^{\mathrm{b}}$ \\
\hline 0.9168 & 0.0832
\end{tabular}

${ }^{\mathrm{a}} x_{2}$ : mole fraction of component 2 in the saturated solution.

${ }^{\mathrm{b}} x_{1}$ : mole fraction solubility of the solute.

\section{Auxiliary Information}

\section{Method/Apparatus/Procedure:}

Constant-temperature bath, Soxhlet thimble, conical flask, and analytical balance. Excess solute and solvent were placed in a conical flask and allowed to equilibrate for several days at constant temperature. Attainment of equilibrium was verified by several repetitive measurements and by approaching equilibrium from supersaturation. Aliquots of saturated solutions were transferred using a Soxhlet thimble equipped with a one-hole cork stopper and an inverted U-shaped delivery tube to a second ground-glass stoppered flask suspended in the $303 \mathrm{~K}$ water bath. Successive portions of the contents were evaporated at room temperature in a tared aluminum foil weighing dish under a bell jar through which a slow stream of dry air was passed. The second flask plus unused saturated solution and the aluminum foil dish plus residue were weighed. The saturation solubility of the solute was calculated from the recorded mass data and molar masses of the solute and solvent.

\section{Source and Purity of Chemicals:}

(1) Purity not given, Chemical source not specified, was recrystallized severa times from aqueous-ethanol mixture, and then dried under vacuum over phosphorous pentoxide.

(2) Reagent grade, Chemical source not specified, refluxed over phosphorous pentoxide for $24 \mathrm{~h}$, and then distilled through a $3 \times 80 \mathrm{~cm}$ column filled with 0.32 -cm glass helices.

\section{Estimated Error:}

Temperature: $\pm 0.02 \mathrm{~K}$.

$x_{1}: \pm 1.5 \%$ (relative error)

\begin{tabular}{ll}
\hline \hline Components: & Original Measurements: \\
(1) Benzoic acid; $\mathrm{C}_{7} \mathrm{H}_{6} \mathrm{O}_{2} ;$ & ${ }^{56} \mathrm{P}$. A. Ongley, J. Chem. Soc. 1954, \\
{$[65-85-0]$} & 3634. \\
(2) Benzene; $\mathrm{C}_{6} \mathrm{H}_{6} ;[71-43-2]$ & \\
\hline Variables: & Prepared by: \\
$T / \mathrm{K}=298.15$ & W. E. Acree, Jr. \\
\hline
\end{tabular}

\section{Experimental Values}

The measured solubility was reported to be $-\log _{10} c_{1}=0.118$, which corresponds to a solubility of $c_{1}=0.762 \mathrm{~mol} \mathrm{dm}^{-3}$.

\section{Auxiliary Information}

\section{Method/Apparatus/Procedure:}

Very little experimental details were provided. Excess solute and solvent were placed in tubes, which were rotated for at least $8 \mathrm{~h}$ at $298 \mathrm{~K}$. The solutions were then removed, concentrated if necessary, and titrated with standard alkali using a mixed (bromothymol blue + neutral red) indicator.

\section{Source and Purity of Chemicals:}

(1) Purity not given, Chemical source not given, solute was either "AnalaR" grade or recrystallized prior to use.

(2) Purity not given, Chemical source not given, no purification details were provided.

\section{Estimated Error:}

Temperature: No information was given $c_{1}: \pm 4 \%$ (relative error, estimated by compiler).

\begin{tabular}{|c|c|}
\hline $\begin{array}{l}\text { Components: } \\
\text { (1) Benzoic acid; } \mathrm{C}_{7} \mathrm{H}_{6} \mathrm{O}_{2} \text {; } \\
\text { [65-85-0] } \\
\text { (2) Benzene; } \mathrm{C}_{6} \mathrm{H}_{6} ;[71-43-2]\end{array}$ & $\begin{array}{l}\text { Original Measurements: } \\
{ }^{57} \text { J. W. Marden and M. V. Dover, J. } \\
\text { Am. Chem. Soc. 38, } 1235 \text { (1916). }\end{array}$ \\
\hline $\begin{array}{l}\text { Variables: } \\
T / \mathrm{K}=298.15\end{array}$ & $\begin{array}{l}\text { Prepared by: } \\
\text { W. E. Acree, Jr. }\end{array}$ \\
\hline
\end{tabular}

\section{Experimental Values}

\begin{tabular}{lc}
\hline \hline$x_{2}{ }^{\mathrm{a}}$ & $x_{1}{ }^{\mathrm{b}}$ \\
\hline 0.931 & 0.069 \\
\hline
\end{tabular}

${ }^{a} x_{2}$ : mole fraction of component 2 in the saturated solution.

${ }^{\mathrm{b}} x_{1}$ : mole fraction solubility of the solute. Experimental solubility data were given as grams of solute per $100 \mathrm{~g}$ of solvent. Mole fraction solubility was calculated by the compiler. 


\section{Auxiliary Information}

\section{Method/Apparatus/Procedure:}

Constant-temperature thermostat, analytical balance and a steam bath Excess solute and solvent were placed in a bottle with ground glass stopper which was protected by a coating of beeswax and rosin, over which was tied a piece of rubber sheeting. The sealed mixture was shaken in a constanttemperature thermostat for 8-20 h. An aliquot of the saturated solution was withdrawn through a glass wool filter into a weighing pipet from which the solutions were weighed directly into small glass evaporating dishes. The solvent was removed on a steam bath, and the sample further dried in a sulfuric acid desiccator. The solubility was calculated from the mass of the solid residue and the mass of the saturated solution removed for analysis.

\section{Source and Purity of Chemicals:}

(1) Purity not given, Chemical source not given, no purification details were provided.

(2) Purity not given, Chemical source not given, no purification details were provided.

\section{Estimated Error:}

Temperature: $\pm 0.1 \mathrm{~K}$.

$x_{1}: \pm 10.0 \%$ (relative error, estimated by compiler).

\begin{tabular}{|c|c|}
\hline $\begin{array}{l}\text { Components: } \\
\text { (1) Benzoic acid; } \mathrm{C}_{7} \mathrm{H}_{6} \mathrm{O}_{2} \text {; } \\
\text { [65-85-0] } \\
\text { (2) Benzene; } \mathrm{C}_{6} \mathrm{H}_{6} ;[71-43-2]\end{array}$ & $\begin{array}{l}\text { Original Measurements: } \\
\text { }{ }^{50} \text { P. G. Desai and A. M. Patel, J. Indian } \\
\text { Chem. Soc. 12, } 131 \text { (1935). }\end{array}$ \\
\hline $\begin{array}{l}\text { Variables: } \\
T / \mathrm{K}=301.2\end{array}$ & $\begin{array}{l}\text { Prepared by: } \\
\text { W. E. Acree, Jr. }\end{array}$ \\
\hline
\end{tabular}

Experimental Values

\begin{tabular}{lc}
\hline \hline$x_{2}^{\mathrm{a}}$ & $x_{1}{ }^{\mathrm{b}}$ \\
\hline 0.9181 & 0.08191 \\
\hline${ }^{\mathrm{a}} x_{2}:$ mole fraction of component 2 in the saturated solution. & \\
${ }^{\mathrm{b}} x_{1}:$ mole fraction solubility of the solute. &
\end{tabular}

\section{Auxiliary Information}

\section{Method/Apparatus/Procedure:}

Excess solute and solvent were placed in a glass flask which was kept revolving on a wheel in an air thermostat for two days. After two days of equilibration, the solution was filtered. To minimize absorption of the solute onto the filter paper, a $100 \mathrm{ml}$ portion of fresh saturated solution was first filtered through the paper before filtering the sample to be analyzed. An aliquot of the filtrate was titrated with barium hydroxide using phenolphthalein as the endpoint indictor. The barium hydroxide titrant was previously standardized against a solution of succinic acid.

\section{Source and Purity of Chemicals:}

(1) Purity not given, Chemical source not specified, no information provided concerning purification.

(2) Purity not given, Chemical source not specified, no information provided concerning purification.

\section{Estimated Error:}

Temperature: No information given.

$x_{1}: \pm 3 \%$ (relative error, estimated by compiler).

\begin{tabular}{|c|c|}
\hline $\begin{array}{l}\text { Components: } \\
\text { (1) Benzoic acid; } \mathrm{C}_{7} \mathrm{H}_{6} \mathrm{O}_{2} \text {; } \\
\text { [65-85-0] } \\
\text { (2) Benzene; } \mathrm{C}_{6} \mathrm{H}_{6} ;[71-43-2]\end{array}$ & $\begin{array}{l}\text { Original Measurements: } \\
{ }^{58} \text { J. Chipman, J. Am. Chem. Soc. 46, } \\
2445 \text { (1924). }\end{array}$ \\
\hline $\begin{array}{l}\text { Variables: } \\
\text { Temperature }\end{array}$ & $\begin{array}{l}\text { Prepared by: } \\
\text { W. E. Acree, Jr. }\end{array}$ \\
\hline
\end{tabular}

Experimental Values (Interpolated Values)

\begin{tabular}{lcc}
\hline \hline$T / \mathrm{K}$ & $x_{2}{ }^{\mathrm{a}}$ & $x_{1}{ }^{\mathrm{b}}$ \\
\hline 277.5 & 0.967 & 0.0332 \\
283.2 & 0.966 & 0.0339 \\
293.2 & 0.941 & 0.0594 \\
298.2 & 0.927 & 0.0728 \\
303.2 & 0.912 & 0.0879 \\
313.2 & 0.874 & 0.1261 \\
323.2 & 0.821 & 0.1794 \\
333.2 & 0.751 & 0.249 \\
343.2 & 0.663 & 0.337 \\
353.2 & 0.556 & 0.444 \\
363.2 & 0.432 & 0.568 \\
373.2 & 0.302 & 0.698 \\
383.2 & 0.165 & 0.835
\end{tabular}

${ }^{\mathrm{a}} x_{2}$ : mole fraction of component 2 in the saturated solution.

${ }^{b} x_{1}$ : mole fraction solubility of the solute. The solid-liquid equilibrium temperatures and mole fraction compositions represent interpolated values obtained from the actual experimental data. The actual experimental data were not given in the paper.

\section{Auxiliary Information}

\section{Method/Apparatus/Procedure:}

Water bath, glycerol bath, and stirrer.

Solubilities were determined in a $2.5 \times 15 \mathrm{~cm}$ air-jacketed test-tubes using short-stemmed thermometers. The tube, together with the thermometer, cork stopper, and stirrer were weighed, benzoic acid was then added, and the entire assembly reweighed again. The total weight of the mixture was determined again after the solid-liquid equilibrium temperature had been recorded in order to avoid errors due to solvent evaporation. The mixture of benzoic acid and solvent was heated in a water bath or glycerol bath until entirely homogeneous. The mixture cooled with rapid stirring to form a suspension of minute crystals, and then warmed slowly (about $0.5 \mathrm{~K} / \mathrm{min}$ ) with continuous stirring until all of the crystals dissolved. The temperature at which the solution became clear was recorded as the solid-liquid equilibrium temperature.

The experimental solubilities at the three lower temperatures were determined by titration. Glass-stoppered bottles containing excess solute and solvent were allowed to equilibrate at constant temperature in a thermostat. An aliquot of the clear saturated solution was removed and transferred into a glass-stoppered Erlenmeyer flask and weighed. A measured amount of slight excess sodium hydroxide solution was added, and the excess sodium hydroxide was determined by back titration with a standardized acetic acid solution with phenolphthalein being used to detect the endpoint. The solvent was evaporated in a current of carbon dioxide-free air.

\section{Source and Purity of Chemicals:}

(1) Purity not given, Chemical source not given, was recrystallized successively from $50 \%$ aqueous-acetic acid solution, benzene, three times from $30 \%$ aqueous-alcohol, and twice from pure benzene. The melting point of the purified sample was $394.9 \mathrm{~K}$

(2) Purity not given, Chemical source not given, was shaken repeatedly with sulfuric acid, and then successively with water, dilute aqueous sodium hydroxide solution, water, and saturated calcium chloride solution. The sample was then fractionally distilled over solid calcium chloride, then frozen out and finally distilled from metallic sodium. 
Estimated Error:

Temperature: Unable to determine. $x_{1}$ : Unable to determine.

\begin{tabular}{|c|c|}
\hline $\begin{array}{l}\text { Components: } \\
\text { (1) Benzoic acid; } \mathrm{C}_{7} \mathrm{H}_{6} \mathrm{O}_{2} \text {; } \\
{[65-85-0]} \\
\text { (2) Benzene; } \mathrm{C}_{6} \mathrm{H}_{6} ;[71-43-2]\end{array}$ & $\begin{array}{l}\text { Original Measurements: } \\
{ }^{59} \text { H. Buchowski, J. Solution Chem. 20, } \\
139 \text { (1991). }\end{array}$ \\
\hline $\begin{array}{l}\text { Variables: } \\
\text { Temperature }\end{array}$ & $\begin{array}{l}\text { Prepared by: } \\
\text { W. E. Acree, Jr. }\end{array}$ \\
\hline
\end{tabular}

Experimental Values (Interpolated Values)

\begin{tabular}{lcc}
\hline \hline$T / \mathrm{K}$ & $x_{2}{ }^{\mathrm{a}}$ & $x_{1}{ }^{\mathrm{b}}$ \\
\hline 298.15 & 0.927 & 0.073 \\
300.15 & 0.921 & 0.079 \\
302.15 & 0.915 & 0.085 \\
313.15 & 0.874 & 0.126 \\
318.15 & 0.850 & 0.150 \\
323.15 & 0.821 & 0.179 \\
328.15 & 0.790 & 0.210 \\
333.15 & 0.751 & 0.249 \\
\hline
\end{tabular}

${ }^{\mathrm{a}} x_{2}$ : mole fraction of component 2 in the saturated solution.

${ }^{\mathrm{b}} x_{1}$ : mole fraction solubility of the solute.

\section{Auxiliary Information}

\section{Method/Apparatus/Procedure:}

Very little experimental details were provided in the paper. In the author's earlier studies, solubilities were determined using a dynamic solubility method where known amounts of solute and solvent were placed in containers which were then sealed. The temperature was then slowly increased until all of the solid solute dissolved. The compiler believes that the benzoic acid solubilities in the present study may have been determined by a similar method. The tabulated numerical values might be interpolated values obtained from a smooth mole fraction versus temperature curve.

Source and Purity of Chemicals:

(1) $99.5 \%$, Argon, Lodz, Poland, purified by at least two vacuum sublimations. (2) $99.5 \%$, POCh, Gliwice, Poland, was dried over phosphorous pentoxide and distilled before use.

\section{Estimated Error:}

Temperature: $\pm 0.1 \mathrm{~K}$ (estimated by compiler).

$x_{1}: \pm 3 \%$ (relative error, estimated by compiler).

\begin{tabular}{|c|c|}
\hline $\begin{array}{l}\text { Components: } \\
\text { (1) Benzoic acid; } \mathrm{C}_{7} \mathrm{H}_{6} \mathrm{O}_{2} \text {; } \\
\text { [65-85-0] } \\
\text { (2) Methylbenzene; } \mathrm{C}_{7} \mathrm{H}_{8} \text {; } \\
\text { [108-88-3] }\end{array}$ & $\begin{array}{l}\text { Original Measurements: } \\
\text { 47 A. Beerbower, P. L. Wu, and A. } \\
\text { Martin, J. Pharm. Sci. 73, } 179 \text { (1984). }\end{array}$ \\
\hline $\begin{array}{l}\text { Variables: } \\
T / \mathrm{K}=298.15\end{array}$ & $\begin{array}{l}\text { Prepared by: } \\
\text { W. E. Acree, Jr. }\end{array}$ \\
\hline
\end{tabular}

Experimental Values

\begin{tabular}{lc}
\hline \hline$x_{2}{ }^{\mathrm{a}}$ & $x_{1}{ }^{\mathrm{b}}$ \\
\hline 0.9266 & 0.0734 \\
\hline${ }^{\mathrm{a}} x_{2}:$ mole fraction of component 2 in the saturated solution. & \\
${ }^{\mathrm{b}} x_{1}:$ mole fraction solubility of the solute. &
\end{tabular}

\section{Auxiliary Information}

\section{Method/Apparatus/Procedure: \\ Constant-temperature bath and ultraviolet/visible spectrophotometer.} Excess solute and solvent were placed in screw-capped vials. The vials were sealed and submerged in a constant-temperature water bath and shaken at 100 cycles/min for $24 \mathrm{~h}$. After the $24 \mathrm{~h}$ equilibration period, the vial was removed, wiped dry, and the contents analyzed. The solutions were transferred to a syringe and filtered through a $0.1 \mu \mathrm{m}$ pore size filter. The solutions were diluted and the absorbances recorded at the maximum absorption wavelength of benzoic acid. The solubility was determined at least six times.

Source and Purity of Chemicals:

(1) Purity not given, Chemical source not specified, no information provided concerning purification.

(2) Spectrophotometric or ACS Reagent grade, Chemical source not specified, redistilled before use.

Estimated Error:

Temperature: $\pm 0.2 \mathrm{~K}$.

$x_{1}: \pm 3 \%$ (relative error).

\begin{tabular}{|c|c|}
\hline $\begin{array}{l}\text { Components: } \\
\text { (1) Benzoic acid; } \mathrm{C}_{7} \mathrm{H}_{6} \mathrm{O}_{2} \text {; } \\
\text { [65-85-0] } \\
\text { (2) Methylbenzene; } \mathrm{C}_{7} \mathrm{H}_{8} \text {; } \\
\text { [108-88-3] }\end{array}$ & $\begin{array}{l}\text { Original Measurements: } \\
{ }^{50} \text { P. G. Desai and A. M. Patel, J. Indian } \\
\text { Chem. Soc. 12, } 131 \text { (1935). }\end{array}$ \\
\hline $\begin{array}{l}\text { Variables: } \\
T / \mathrm{K}=301.2\end{array}$ & $\begin{array}{l}\text { Prepared by: } \\
\text { W. E. Acree, Jr. }\end{array}$ \\
\hline
\end{tabular}

\section{Experimental Values}

\begin{tabular}{lc}
\hline \hline$x_{2}{ }^{\mathrm{a}}$ & $x_{1}{ }^{\mathrm{b}}$ \\
\hline 0.9145 & 0.08553 \\
\hline${ }^{\mathrm{a}} x_{2}:$ mole fraction of component 2 in the saturated solution. & \\
${ }^{\mathrm{b}} x_{1}$ : mole fraction solubility of the solute. &
\end{tabular}

\section{Auxiliary Information}

\section{Method/Apparatus/Procedure:}

Excess solute and solvent were placed in a glass flask which was kept revolving on a wheel in an air thermostat for two days. After two days of equilibration, the solution was filtered. To minimize absorption of the solute onto the filter paper, a $100 \mathrm{ml}$ portion of fresh saturated solution was first filtered through the paper before filtering the sample to be analyzed. An aliquot of the filtrate was titrated with barium hydroxide using phenolphthalein as the endpoint indictor. The barium hydroxide titrant was previously standardized against a solution of succinic acid.

\section{Source and Purity of Chemicals:}

(1) Purity not given, Chemical source not specified, no information provided concerning purification.

(2) Purity not given, Chemical source not specified, no information provided concerning purification. 
Estimated Error:

Temperature: No information given. $x_{1}: \pm 3 \%$ (relative error, estimated by compiler).

\begin{tabular}{|c|c|}
\hline $\begin{array}{l}\text { Components: } \\
\text { (1) Benzoic acid; } \mathrm{C}_{7} \mathrm{H}_{6} \mathrm{O}_{2} \text {; } \\
\text { [65-85-0] } \\
\text { (2) Methylbenzene; } \mathrm{C}_{7} \mathrm{H}_{8} \text {; } \\
\text { [108-88-3] }\end{array}$ & $\begin{array}{l}\text { Original Measurements: } \\
{ }^{48} \text { J. Thati, F. L. Nordström, and A. C. } \\
\text { Rasmuson, J. Chem. Eng. Data 55, } \\
5124 \text { (2010). }\end{array}$ \\
\hline $\begin{array}{l}\text { Variables: } \\
\text { Temperature }\end{array}$ & $\begin{array}{l}\text { Prepared by: } \\
\text { W. E. Acree, Jr. }\end{array}$ \\
\hline
\end{tabular}

Experimental Values

\begin{tabular}{lcc}
\hline \hline$T / \mathrm{K}$ & $x_{2}{ }^{\mathrm{a}}$ & $x_{1}{ }^{\mathrm{b}}$ \\
\hline 278.15 & 0.965 & 0.035 \\
283.15 & 0.957 & 0.043 \\
293.15 & 0.938 & 0.062 \\
303.15 & 0.911 & 0.089 \\
313.15 & 0.872 & 0.128 \\
323.15 & 0.822 & 0.178 \\
\hline
\end{tabular}

${ }^{\mathrm{a}} x_{2}$ : mole fraction of component 2 in the saturated solution.

${ }^{\mathrm{b}} x_{1}$ : mole fraction solubility of the solute.

\section{Auxiliary Information}

\section{Method/Apparatus/Procedure:}

Constant-temperature bath, magnetic stirrer, analytical balance, and ventilated laboratory hood.

Solubilities were determined by a gravimetric method. Excess solute and solvent were placed in sealed bottles and allowed to equilibrate in a constanttemperature bath for $2 \mathrm{~h}$ with stirring. The stirring was discontinued and the solid was allowed to settle to the bottom of the container. After $30 \mathrm{~min}$, an aliquot of the clear solution was removed by syringe, filtered through a $0.2 \mu \mathrm{m}$ PTFE filter, and transferred to a preweighed glass vial. The glass vial was then weighed, and the solvent was allowed to evaporate in a ventilated laboratory hood at ambient room temperature. Once the solvent had evaporated, the vial with solid residue was weighed until constant weight was obtained. The solubility was calculated from the mass of the solid residue and mass of sample analyzed.

\section{Source and Purity of Chemicals:}

(1) $99.7 \%$, Merck Chemical Company, Germany, was used as received.

(2) $99+\%$, Merck Chemical Company, used as received.

Estimated Error:

Temperature: $\pm 0.01 \mathrm{~K}$.

$x_{1}: \pm 1 \%$ (relative error).

\begin{tabular}{|c|c|}
\hline $\begin{array}{l}\text { Components: } \\
\text { (1) Benzoic acid; } \mathrm{C}_{7} \mathrm{H}_{6} \mathrm{O}_{2} \text {; } \\
\text { [65-85-0] } \\
\text { (2) Methylbenzene; } \mathrm{C}_{7} \mathrm{H}_{8} \text {; } \\
\text { [108-88-3] }\end{array}$ & $\begin{array}{l}\text { Original Measurements: } \\
\text { 58 J. Chipman, J. Am. Chem. Soc. 46, } \\
2445 \text { (1924). }\end{array}$ \\
\hline $\begin{array}{l}\text { Variables: } \\
\text { Temnerature }\end{array}$ & Prepared by: \\
\hline
\end{tabular}

Experimental Values (Interpolated Values)

\begin{tabular}{lcc}
\hline \hline$T / \mathrm{K}$ & $x_{2}{ }^{\mathrm{a}}$ & $x_{1}{ }^{\mathrm{b}}$ \\
\hline 273.2 & 0.964 & 0.0339 \\
283.2 & 0.958 & 0.0423 \\
293.2 & 0.938 & 0.0618 \\
298.2 & 0.926 & 0.0741 \\
303.2 & 0.911 & 0.0891 \\
313.2 & 0.873 & 0.1270 \\
323.2 & 0.821 & 0.1790 \\
333.2 & 0.753 & 0.247 \\
343.2 & 0.667 & 0.333 \\
353.2 & 0.561 & 0.439 \\
363.2 & 0.439 & 0.561 \\
373.2 & 0.304 & 0.696 \\
383.2 & 0.166 & 0.834 \\
\hline
\end{tabular}

${ }^{\mathrm{a}} x_{2}$ : mole fraction of component 2 in the saturated solution.

${ }^{b} x_{1}$ : mole fraction solubility of the solute. The solid-liquid equilibrium temperatures and mole fraction compositions represent interpolated values obtained from the actual experimental data. The actual experimental data were not given in the paper.

\section{Auxiliary Information}

\section{Method/Apparatus/Procedure:}

Water bath, glycerol bath, and stirrer.

Solubilities were determined in a $2.5 \times 15 \mathrm{~cm}$ air-jacketed test-tubes using short-stemmed thermometers. The tube, together with the thermometer, cork stopper, and stirrer were weighed, benzoic acid was then added, and the entire assembly reweighed again. The total weight of the mixture was determined again after the solid-liquid equilibrium temperature had been recorded in order to avoid errors due to solvent evaporation. The mixture of benzoic acid and solvent was heated in a water bath or glycerol bath until entirely homogeneous. The mixture cooled with rapid stirring to form a suspension of minute crystals, and then warmed slowly (about $0.5 \mathrm{~K} / \mathrm{min}$ ) with continuous stirring until all of the crystals dissolved. The temperature at which the solution became clear was recorded as the solid-liquid equilibrium temperature.

The experimental solubilities at the three lower temperatures were determined by titration. Glass-stoppered bottles containing excess solute and solvent were allowed to equilibrate at constant temperature in a thermostat. An aliquot of the clear saturated solution was removed and transferred into a glass-stoppered Erlenmeyer flask and weighed. A measured amount of slight excess sodium hydroxide solution was added, and the excess sodium hydroxide was determined by back titration with a standardized acetic acid solution with phenolphthalein being used to detect the endpoint. The solvent was evaporated in a current of carbon dioxide-free air.

\section{Source and Purity of Chemicals:}

(1) Purity not given, Chemical source not given, was recrystallized successively from $50 \%$ aqueous-acetic acid solution, benzene, three times from $30 \%$ aqueous-alcohol, and twice from pure benzene. The melting point of the purified sample was $394.9 \mathrm{~K}$.

(2) Purity not given, Chemical source not given, was shaken repeatedly with sulfuric acid, and then successively with water, dilute aqueous sodium hydroxide solution, water, and saturated calcium chloride solution. The sample was then fractionally distilled over solid calcium chloride, and then distilled from metallic sodium.

\section{Estimated Error:}

Temperature: Unable to determine.

$x_{1}$ : Unable to determine. 


\section{Components:}

(1) Benzoic acid; $\mathrm{C}_{7} \mathrm{H}_{6} \mathrm{O}_{2}$; [65-85-0]

(2) 1,3-Dimethylbenzene; $\mathrm{C}_{8} \mathrm{H}_{10}$; [108-38-3]

\begin{tabular}{ll}
\hline Variables: & Prepared by: \\
$T / \mathrm{K}=301.2$ & W. E. Acree, Jr. \\
\hline
\end{tabular}

\section{Experimental Values}

\begin{tabular}{lc}
\hline \hline$x_{2}{ }^{\mathrm{a}}$ & $x_{1}{ }^{\mathrm{b}}$ \\
\hline 0.9111 & 0.0889 \\
\hline${ }^{\mathrm{a}} x_{2}:$ mole fraction of component 2 in the saturated solution. & \\
${ }^{\mathrm{b}} x_{1}:$ mole fraction solubility of the solute. &
\end{tabular}

\section{Auxiliary Information}

\section{Method/Apparatus/Procedure:}

Excess solute and solvent were placed in a glass flask which was kept revolving on a wheel in an air thermostat for two days. After two days of equilibration, the solution was filtered. To minimize absorption of the solute onto the filter paper, a $100 \mathrm{ml}$ portion of fresh saturated solution was first filtered through the paper before filtering the sample to be analyzed. An aliquot of the filtrate was titrated with barium hydroxide using phenolphthalein as the endpoint indictor. The barium hydroxide titrant was previously standardized against a solution of succinic acid.

\section{Source and Purity of Chemicals:}

(1) Purity not given, Chemical source not specified, no information provided concerning purification.

(2) Purity not given, Chemical source not specified, no information provided concerning purification.

\section{Estimated Error:}

Temperature: No information given.

$x_{1}: \pm 3 \%$ (relative error, estimated by compiler).

\subsection{Benzoic acid solubility data in esters}

\begin{tabular}{|c|c|}
\hline $\begin{array}{l}\text { Components: } \\
\text { (1) Benzoic acid; } \mathrm{C}_{7} \mathrm{H}_{6} \mathrm{O}_{2} \text {; } \\
\text { [65-85-0] } \\
\text { (2) Ethyl ethanoate; } \mathrm{C}_{4} \mathrm{H}_{8} \mathrm{O}_{2} \text {; } \\
\text { [141-78-6] }\end{array}$ & $\begin{array}{l}\text { Original Measurements: } \\
{ }^{47} \text { A. Beerbower, P. L. Wu, and A. } \\
\text { Martin, J. Pharm. Sci. 73, } 179 \text { (1984) }\end{array}$ \\
\hline $\begin{array}{l}\text { Variables: } \\
T / \mathrm{K}=298.15\end{array}$ & $\begin{array}{l}\text { Prepared by: } \\
\text { W. E. Acree, Jr. }\end{array}$ \\
\hline
\end{tabular}

Experimental Values

\begin{tabular}{lc}
\hline \hline$x_{2}^{\mathrm{a}}$ & $x_{1}{ }^{\mathrm{b}}$ \\
\hline 0.8351 & 0.1649 \\
\hline${ }^{\mathrm{a}} x_{2}:$ mole fraction of component 2 in the saturated solution. & \\
${ }^{\mathrm{b}} x_{1}:$ mole fraction solubility of the solute. &
\end{tabular}

\section{Auxiliary Information}

Method/Apparatus/Procedure:

Constant-temperature bath and ultraviolet/visible spectrophotometer.

Excess solute and solvent were placed in screw-capped vials. The vials were sealed and submerged in a constant-temperature water bath and shaken at 100 cycles/min for $24 \mathrm{~h}$. After the $24 \mathrm{~h}$ equilibration period, the vial was removed, wiped dry, and the contents analyzed. The solutions were transferred to a syringe and filtered through a $0.1 \mu \mathrm{m}$ pore size filter. The solutions were diluted and the absorbances recorded at the maximum absorption wavelength of benzoic acid. The solubility was determined at least six times.

Source and Purity of Chemicals:

(1) Purity not given, Chemical source not specified, no information provided concerning purification.

(2) Spectrophotometric or ACS Reagent grade, Chemical source not specified, redistilled before use.

Estimated Error:

Temperature: $\pm 0.2 \mathrm{~K}$.

$x_{1}: \pm 3 \%$ (relative error).

\begin{tabular}{|c|c|}
\hline $\begin{array}{l}\text { Components: } \\
\text { (1) Benzoic acid; } \mathrm{C}_{7} \mathrm{H}_{6} \mathrm{O}_{2} \text {; } \\
\text { [65-85-0] } \\
\text { (2) Ethyl ethanoate; } \mathrm{C}_{4} \mathrm{H}_{8} \mathrm{O}_{2} \text {; } \\
\text { [141-78-6] }\end{array}$ & $\begin{array}{l}\text { Original Measurements: } \\
\text { }{ }^{51} \text { H.-M. Lin and R. A. Nash, J. Pharm. } \\
\text { Sci. 82, } 1018 \text { (1993). }\end{array}$ \\
\hline $\begin{array}{l}\text { Variables: } \\
T / \mathrm{K}=298.15\end{array}$ & $\begin{array}{l}\text { Prepared by: } \\
\text { W. E. Acree, Jr. }\end{array}$ \\
\hline
\end{tabular}

Experimental Values

\begin{tabular}{lc}
\hline \hline$x_{2}{ }^{\mathrm{a}}$ & $x_{1}{ }^{\mathrm{b}}$ \\
\hline 0.8363 & 0.1637 \\
\hline${ }^{\mathrm{a}} x_{2}:$ mole fraction of component 2 in the saturated solution. & \\
${ }^{\mathrm{b}} x_{1}:$ mole fraction solubility of the solute. &
\end{tabular}

Auxiliary Information

\section{Method/Apparatus/Procedure:}

Constant-temperature bath and high-performance liquid chromatograph. Excess solute and solvent were placed in screw-capped vials. The vials were sealed with several turns of electrical tape, warmed to about $323 \mathrm{~K}$, and then shaken in a constant-temperature bath for at least $24 \mathrm{~h}$ at $298 \mathrm{~K}$. After equilibrium was reached, the sample was filtered through a 0.45 or $1.0 \mu \mathrm{m}$ filter, and an aliquot was diluted appropriately for chromatographic analysis. Concentrations were determined by a high-performance liquid chromatographic method.

Source and Purity of Chemicals:

(1) Purity not given, Amend Drug and Chemical Company, no information provided concerning purification.

(2) HPLC grade, Aldrich Chemical Company, Milwaukee, WI, USA, no purification details were provided.

\section{Estimated Error:}

Temperature: $\pm 0.2 \mathrm{~K}$ (estimated by compiler). $x_{1}: \pm 3 \%$ (relative error, estimated by compiler). 


\section{Components:}

(1) Benzoic acid; $\mathrm{C}_{7} \mathrm{H}_{6} \mathrm{O}_{2}$;

[65-85-0]

(2) Ethyl ethanoate; $\mathrm{C}_{4} \mathrm{H}_{8} \mathrm{O}_{2}$;

[141-78-6]

\begin{tabular}{ll}
\hline Variables: & Prepared by: \\
$T / \mathrm{K}=298.15$ & W. E. Acree, Jr. \\
\hline
\end{tabular}

\section{Experimental Values}

\begin{tabular}{lc}
\hline \hline$x_{2}{ }^{\mathrm{a}}$ & $x_{1}{ }^{\mathrm{b}}$ \\
\hline 0.771 & 0.229 \\
\hline
\end{tabular}

${ }^{\mathrm{a}} x_{2}$ : mole fraction of component 2 in the saturated solution.

${ }^{b} x_{1}$ : mole fraction solubility of the solute. Experimental solubility data were given as grams of solute per $100 \mathrm{~g}$ of solvent. Mole fraction solubility was calculated by the compiler.

\section{Auxiliary Information}

\section{Method/Apparatus/Procedure:}

Constant-temperature thermostat, analytical balance, and a steam bath Excess solute and solvent were placed in a bottle with ground glass stopper which was protected by a coating of beeswax and rosin, over which was tied a piece of rubber sheeting. The sealed mixture was shaken in a constanttemperature thermostat for 8-20 h. An aliquot of the saturated solution was withdrawn through a glass wool filter into a weighing pipet from which the solutions were weighed directly into small glass evaporating dishes. The solvent was removed on a steam bath, and the sample further dried in a sulfuric acid desiccator. The solubility was calculated from the mass of the solid residue and the mass of the saturated solution removed for analysis.

\section{Source and Purity of Chemicals:}

(1) Purity not given, Chemical source not given, no purification details were provided.

(2) Purity not given, Chemical source not given, no purification details were provided.

\section{Estimated Error:}

Temperature: $\pm 0.1 \mathrm{~K}$.

$x_{1}: \pm 10.0 \%$ (relative error, estimated by compiler).

\begin{tabular}{|c|c|}
\hline $\begin{array}{l}\text { Components: } \\
\text { (1) Benzoic acid; } \mathrm{C}_{7} \mathrm{H}_{6} \mathrm{O}_{2} \text {; } \\
\text { [65-85-0] } \\
\text { (2) Ethyl ethanoate; } \mathrm{C}_{4} \mathrm{H}_{8} \mathrm{O}_{2} \text {; } \\
\text { [141-78-6] }\end{array}$ & $\begin{array}{l}\text { Original Measurements: } \\
\text { 89 S. J. Lloyd, J. Phys. Chem. 22, } 300 \\
\text { (1918). }\end{array}$ \\
\hline $\begin{array}{l}\text { Variables: } \\
\text { Temperature }\end{array}$ & $\begin{array}{l}\text { Prepared by: } \\
\text { W. E. Acree, } \mathrm{J}_{1}\end{array}$ \\
\hline
\end{tabular}

Experimental Values

\begin{tabular}{ll}
\hline \hline$T / \mathrm{K}$ & $c_{1}{ }^{\mathrm{a}}$ \\
\hline 266.7 & 0.0655 \\
294.7 & 0.309 \\
348.2 & 0.784 \\
\hline${ }^{{ }^{a} c_{1}: \text { molar solubility of the solute expressed in units of } \mathrm{mol} \mathrm{dm}^{-3} .}$ &
\end{tabular}

\section{Auxiliary Information}

Method/Apparatus/Procedure:

Experimental details were not provided in the paper. Solubility measurements were performed as part of a study of the electrolytic decomposition of benzoic acid in ethyl ethanoate.

Source and Purity of Chemicals:

(1) Purity not given, Chemical source not given, purification details were not provided.

(2) Purity not given, Chemical source not given, purification details were not provided.

Estimated Error:

Temperature: Insufficient information to estimate.

$c_{1}$ : Insufficient information to estimate.

\begin{tabular}{|c|c|}
\hline $\begin{array}{l}\text { Components: } \\
\text { (1) Benzoic acid; } \mathrm{C}_{7} \mathrm{H}_{6} \mathrm{O}_{2} \text {; } \\
\text { [65-85-0] } \\
\text { (2) Butyl ethanoate; } \mathrm{C}_{6} \mathrm{H}_{12} \mathrm{O}_{2} \text {; } \\
\text { [123-86-4] }\end{array}$ & $\begin{array}{l}\text { Original Measurements: } \\
{ }^{47} \text { A. Beerbower, P. L. Wu, and A. } \\
\text { Martin, J. Pharm. Sci. 73, } 179 \text { (1984). }\end{array}$ \\
\hline $\begin{array}{l}\text { Variables: } \\
T / \mathrm{K}=298.15\end{array}$ & $\begin{array}{l}\text { Prepared by: } \\
\text { W. E. Acree, Jr. }\end{array}$ \\
\hline
\end{tabular}

Experimental Values

\begin{tabular}{lc}
\hline \hline$x_{2}^{\mathrm{a}}$ & $x_{1}{ }^{\mathrm{b}}$ \\
\hline 0.8301 & 0.1699 \\
\hline${ }^{\mathrm{a}} x_{2}:$ mole fraction of component 2 in the saturated solution. & \\
${ }^{\mathrm{b}} x_{1}:$ mole fraction solubility of the solute. &
\end{tabular}

\section{Auxiliary Information}

\section{Method/Apparatus/Procedure:}

Constant-temperature bath and ultraviolet/visible spectrophotometer.

Excess solute and solvent were placed in screw-capped vials. The vials were sealed and submerged in a constant-temperature water bath and shaken at 100 cycles/min for $24 \mathrm{~h}$. After the $24 \mathrm{~h}$ equilibration period, the vial was removed, wiped dry and the contents analyzed. The solutions were transferred to a syringe and filtered through a $0.1 \mu \mathrm{m}$ pore size filter. The solutions were diluted and the absorbances recorded at the maximum absorption wavelength of benzoic acid. The solubility was determined at least six times.

\section{Source and Purity of Chemicals:}

(1) Purity not given, Chemical source not specified, no information provided concerning purification.

(2) Spectrophotometric or ACS Reagent grade, Chemical source not specified, redistilled before use.

\section{Estimated Error:}

Temperature: $\pm 0.2 \mathrm{~K}$.

$x_{1}: \pm 3 \%$ (relative error).

\begin{tabular}{ll}
\hline \hline Components: & $\begin{array}{l}\text { Original Measurements: } \\
\text { (1) Benzoic acid; } \mathrm{C}_{7} \mathrm{H}_{6} \mathrm{O}_{2} ;\end{array}$ \\
$\begin{array}{l}{ }^{87} \text { M. Dias, J. Hadgraft, and M. E. Lane, } \\
\text { [65-85-0] }\end{array}$ & Int. J. Pharm. 336, 108 (2007). \\
$\begin{array}{l}\text { (2) Butyl ethanoate; } \mathrm{C}_{6} \mathrm{H}_{12} \mathrm{O}_{2} ; \\
{[123-86-4]}\end{array}$ & Prepared by: \\
\hline Variables: & W. E. Acree, Jr. \\
$T / \mathrm{K}=305.2$ &
\end{tabular}




\section{Experimental Values}

The measured solubility was reported to be $197.80 \mathrm{mg} / \mathrm{ml}$, which corresponds to a molar solubility of $c_{1}=1.620 \mathrm{~mol} \mathrm{dm}^{-3}$.

\section{Auxiliary Information}

\section{Method/Apparatus/Procedure:}

Constant-temperature bath, magnetic stirrer, centrifuge, and an ultraviolet/ visible spectrophotometer.

Excess solute and solvent were equilibrated in a constant-temperature bath with stirring for $48 \mathrm{~h}$. The saturated solution was then centrifuged for $10 \mathrm{~min}$, and an aliquot of the supernatant solution was removed and diluted quantitatively for spectrophotometric analysis. The concentration of the dissolved solute was determined from the measured absorbance.

\section{Source and Purity of Chemicals:}

(1) Purity not given, Fisons Scientific Equipment, UK, no purification details were provided.

(2) Purity not given, Aldrich Chemical Company, UK, no purification details were provided.

Estimated Error:

Temperature: Insufficient experimental details to estimate. $c_{1}: \pm 0.27$

\begin{tabular}{ll}
\hline \hline Components: & Original Measurements: \\
(1) Benzoic acid; $\mathrm{C}_{7} \mathrm{H}_{6} \mathrm{O}_{2} ;$ & ${ }^{60}$ S. Zhao, X. Chen, Q. Dai, and L. \\
[65-85-0] & Wang, J. Chem. Eng. Data 56, 2399 \\
(2) Isobutyl ethanoate; & $(2011)$. \\
$\mathrm{C}_{6} \mathrm{H}_{12} \mathrm{O}_{2} ;[110-19-0]$ & \\
\hline Variables: & Prepared by: \\
Temperature & W. E. Acree, Jr. \\
\hline
\end{tabular}

\section{Experimental Values}

\begin{tabular}{lcc}
\hline \hline$T / \mathrm{K}$ & $x_{2}{ }^{\mathrm{a}}$ & $x_{1}{ }^{\mathrm{b}}$ \\
\hline 299.73 & 0.860 & 0.140 \\
302.46 & 0.851 & 0.149 \\
307.34 & 0.822 & 0.178 \\
313.61 & 0.792 & 0.208 \\
322.93 & 0.746 & 0.254 \\
328.98 & 0.707 & 0.293 \\
332.24 & 0.689 & 0.311 \\
334.34 & 0.675 & 0.325 \\
335.58 & 0.663 & 0.337 \\
336.03 & 0.662 & 0.338 \\
337.33 & 0.651 & 0.349 \\
337.67 & 0.649 & 0.351 \\
338.76 & 0.642 & 0.358 \\
339.37 & 0.638 & 0.362 \\
339.86 & 0.631 & 0.369 \\
341.48 & 0.621 & 0.379 \\
342.69 & 0.610 & 0.390 \\
343.67 & 0.601 & 0.399 \\
\hline
\end{tabular}

${ }^{a} x_{2}$ : mole fraction of component 2 in the saturated solution.

${ }^{\mathrm{b}} x_{1}$ : mole fraction solubility of the solute.

\section{Auxiliary Information}

Method/Apparatus/Procedure:

Equilibrium jacketed glass vessel, thermoelectric circulating water temperature controller, electromagnetic stirrer, analytical balance, laser monitoring system.

Experimental solubilities were determined by a dynamic method. Preweighed amounts of solute and solvent and were placed in an equilibrium vessel, which was connected to a circulating water bath. The solution was stirred and the temperature gradually increased at a rate $1-2 \mathrm{~K} / \mathrm{h}(0.5-1.0 \mathrm{~K} / \mathrm{h}$ or slower near saturation temperature) until all of the solid solute dissolved. The temperature at which all of the solute dissolved was determined using laser monitoring.

Source and Purity of Chemicals:

(1) Analytical Reagent grade, Sinopharm Chemical Reagent Co. Ltd., China, was used as received.

(2) Analytical Reagent grade, Sinopharm Chemical Reagent Co. Ltd., China, was used as received.

Estimated Error:

Temperature: $\pm 0.05 \mathrm{~K}$.

$x_{1}: \pm 0.001$

\begin{tabular}{ll}
\hline \hline Components: & Original Measurements: \\
(1) Benzoic acid; $\mathrm{C}_{7} \mathrm{H}_{6} \mathrm{O}_{2} ;$ & ${ }^{61} \mathrm{E}$. R. Cooper, J. Controlled Release \\
[65-85-0] & $\mathbf{1}, 153$ (1984). \\
$(2)$ 1-Methylethyl tetradecanoate; & \\
$\mathrm{C}_{17} \mathrm{H}_{34} \mathrm{O}_{2} ;[110-27-0]$ & \\
\hline Variables: & Prepared by: \\
$T / \mathrm{K}=295$ & W. E. Acree, Jr. \\
\hline
\end{tabular}

Experimental Values

\begin{tabular}{lc}
\hline \hline$x_{2}{ }^{\mathrm{a}}$ & $x_{1}{ }^{\mathrm{b}}$ \\
\hline 0.896 & 0.104 \\
${ }^{\mathrm{a}}{ }_{x_{2}}:$ mole fraction of component 2 in the saturated solution. \\
${ }^{\mathrm{b}} x_{1}$ : mole fraction solubility of the solute. Solubility data reported in terms of \\
mass percent. Mole fraction solubility calculated by the compiler. \\
Auxiliary Information
\end{tabular}

\section{Method/Apparatus/Procedure:}

Very little experimental details provided. Solubility determinations were made by stirring the solvent with excess solute for two days, centrifuging the sample, and analyzing the supernatant.

\section{Source and Purity of Chemicals:}

(1) Reagent grade, Matheson, Coleman, and Bell, Cincinnati, OH, USA, no purification details provided.

(2) Reagent grade, Wickhen Corporation, Huguenot, NJ, USA, no purification details provided.

Estimated Error:

Temperature: No information given.

$x_{1}$ : No information given.

\begin{tabular}{ll}
\hline \hline Components: & Original Measurements: \\
$\begin{array}{l}\text { (1) Benzoic acid; } \mathrm{C}_{7} \mathrm{H}_{6} \mathrm{O}_{2} ; \\
{[65-85-0]}\end{array}$ & $\begin{array}{l}{ }^{87} \text { M. Dias, J. Hadgraft, and M. E. Lane, } \\
\text { Int. J. Pharm. 336, } 108 \text { (2007). }\end{array}$ \\
$\mathrm{C}_{17} \mathrm{H}_{34} \mathrm{O}_{2} ;[110-27-0]$ & \\
\hline Variables: & Prepared by: \\
$T / \mathrm{K}=305.2$ & W. E. Acree, Jr. \\
\hline
\end{tabular}




\section{Experimental Values}

The measured solubility was reported to be $41.30 \mathrm{mg} / \mathrm{ml}$, which corresponds to a molar solubility of $c_{1}=0.338 \mathrm{~mol} \mathrm{dm}^{-3}$.

\section{Auxiliary Information}

\section{Method/Apparatus/Procedure:}

Constant-temperature bath, magnetic stirrer, centrifuge, and an ultraviolet/ visible spectrophotometer.

Excess solute and solvent were equilibrated in a constant-temperature bath with stirring for $48 \mathrm{~h}$. The saturated solution was then centrifuged for $10 \mathrm{~min}$, and an aliquot of the supernatant solution was removed and diluted quantitatively for spectrophotometric analysis. The concentration of the dissolved solute was determined from the measured absorbance.

\section{Source and Purity of Chemicals:}

(1) Purity not given, Fisons Scientific Equipment, UK, no purification details were provided.

(2) Purity not given, Croda Universal Ltd., no purification details were provided.

Estimated Error:

Temperature: Insufficient experimental details to estimate. $c_{1}: \pm 0.0066$.

\begin{tabular}{ll}
\hline \hline Components: & Original Measurements: \\
$\begin{array}{l}\text { (1) Benzoic acid; } \mathrm{C}_{7} \mathrm{H}_{6} \mathrm{O}_{2} ; \\
{[65-85-0]}\end{array}$ & ${ }^{51} \mathrm{H} .-\mathrm{M}$. Lin and R. A. Nash, J. Pharm. \\
$\begin{array}{ll}\text { Sci. 82, } 1018 \text { (1993). } \\
\mathrm{C}_{9} \mathrm{H}_{14} \mathrm{O}_{6} ;[102-76-1]\end{array}$ & \\
\hline Variables: & Prepared by: \\
$T / \mathrm{K}=298.15$ & W. E. Acree, Jr. \\
\hline
\end{tabular}

Experimental Values

\begin{tabular}{lc}
\hline \hline$x_{2}{ }^{\mathrm{a}}$ & $x_{1}{ }^{\mathrm{b}}$ \\
\hline 0.8588 & 0.1412
\end{tabular}

${ }^{a_{1}} x_{2}$ mole fraction of component 2 in the saturated solution.

${ }^{\mathrm{b}} x_{1}$ : mole fraction solubility of the solute.

\section{Auxiliary Information}

\section{Method/Apparatus/Procedure:}

Constant-temperature bath and high-performance liquid chromatograph. Excess solute and solvent were placed in screw-capped vials. The vials were sealed with several turns of electrical tape, warmed to about $323 \mathrm{~K}$, and then shaken in a constant-temperature bath for at least $24 \mathrm{~h}$ at $298 \mathrm{~K}$. After equilibrium was reached, the sample was filtered through a 0.45 or $1.0 \mu \mathrm{m}$ filter, and an aliquot was diluted appropriately for chromatographic analysis. Concentrations were determined by a high-performance liquid chromatographic method.

\section{Source and Purity of Chemicals:}

(1) Purity not given, Amend Drug and Chemical Company, no information provided concerning purification.

(2) Purity not given, Unichema International, no purification details were provided.

Estimated Error:

Temperature: $\pm 0.2 \mathrm{~K}$ (estimated by compiler). $x_{1}: \pm 3 \%$ (relative error, estimated by compiler).

\subsection{Benzoic acid solubility data in ethers}

\begin{tabular}{ll}
\hline \hline Components: & Original Measurements: \\
$\begin{array}{l}\text { (1) Benzoic acid; } \mathrm{C}_{7} \mathrm{H}_{6} \mathrm{O}_{2} ; \\
{[65-85-0]}\end{array}$ & ${ }^{47}$ A. Beerbower, P. L. Wu, and A. \\
(2) $1,1^{\prime}$-Oxybisethane; & \\
$\mathrm{C}_{4} \mathrm{H}_{10} \mathrm{O} ;[60-29-7]$ & \\
\hline Variables: & \\
$T / \mathrm{K}=298.15$ & Prepared byarm. Sci. 73, 179 (1984). \\
\hline
\end{tabular}

Experimental Values

\begin{tabular}{lc}
\hline \hline$x_{2}{ }^{\mathrm{a}}$ & $x_{1}{ }^{\mathrm{b}}$ \\
\hline 0.8163 & 0.1837 \\
\hline${ }^{\mathrm{a}} x_{2}:$ mole fraction of component 2 in the saturated solution. & \\
${ }^{\mathrm{b}} x_{1}:$ mole fraction solubility of the solute. &
\end{tabular}

Auxiliary Information

Method/Apparatus/Procedure:

Constant-temperature bath and ultraviolet/visible spectrophotometer.

Excess solute and solvent were placed in screw-capped vials. The vials were sealed and submerged in a constant-temperature water bath and shaken at 100 cycles/min for $24 \mathrm{~h}$. After the $24 \mathrm{~h}$ equilibration period, the vial was removed, wiped dry, and the contents analyzed. The solutions were transferred to a syringe and filtered through a $0.1 \mu \mathrm{m}$ pore size filter. The solutions were diluted and the absorbances recorded at the maximum absorption wavelength of benzoic acid. The solubility was determined at least six times.

\section{Source and Purity of Chemicals:}

(1) Purity not given, Chemical source not specified, no information provided concerning purification.

(2) Spectrophotometric or ACS Reagent grade, Chemical source not specified, redistilled before use.

\section{Estimated Error:}

Temperature: $\pm 0.2 \mathrm{~K}$.

$x_{1}: \pm 3 \%$ (relative error)

\begin{tabular}{ll}
\hline \hline Components: & Original Measurements: \\
$\begin{array}{l}\text { (1) Benzoic acid; } \mathrm{C}_{7} \mathrm{H}_{6} \mathrm{O}_{2} ; \\
{[65-85-0]}\end{array}$ & $\begin{array}{l}\text { 57. W. Marden and M. V. Dover, J. } \\
(2) 1,1^{\prime}-\text { Oxybisethane; } \mathrm{C}_{4} \mathrm{H}_{10} \mathrm{O} ;\end{array}$ \\
{$[60-29-7]$} & \\
\hline Variables: & Prepared by: Soc. 38, 1235 (1916). \\
$T / \mathrm{K}=298.15$ & W. E. Acree, Jr. \\
\hline
\end{tabular}

Experimental Values

\begin{tabular}{lc}
\hline \hline$x_{2}{ }^{\mathrm{a}}$ & $x_{1}{ }^{\mathrm{b}}$ \\
\hline 0.917 & 0.083 \\
${ }^{\mathrm{a}}{ }_{x_{2}: \text { mole fraction of component } 2 \text { in the saturated solution. }}$ \\
${ }^{\mathrm{b}}{ }_{x_{1}}:$ mole fraction solubility of the solute. Experimental solubility data were \\
given as grams of solute per $100 \mathrm{~g}$ of solvent. Mole fraction solubility was \\
calculated by the compiler.
\end{tabular}




\section{Auxiliary Information}

\section{Method/Apparatus/Procedure:}

Constant-temperature thermostat, analytical balance, and a steam bath. Excess solute and solvent were placed in a bottle with ground glass stopper which was protected by a coating of beeswax and rosin, over which was tied a piece of rubber sheeting. The sealed mixture was shaken in a constanttemperature thermostat for 8-20 h. An aliquot of the saturated solution was withdrawn through a glass wool filter into a weighing pipet from which the solutions were weighed directly into small glass evaporating dishes. The solvent was removed on a steam bath, and the sample further dried in a sulfuric acid desiccator. The solubility was calculated from the mass of the solid residue and the mass of the saturated solution removed for analysis.

\section{Source and Purity of Chemicals:}

(1) Purity not given, Chemical source not given, no purification details were provided.

(2) Purity not given, Chemical source not given, no purification details were provided.

\section{Estimated Error:}

Temperature: $\pm 0.1 \mathrm{~K}$.

$x_{1}: \pm 10.0 \%$ (relative error, estimated by compiler).

\begin{tabular}{ll}
\hline \hline Components: & Original Measurements: \\
$\begin{array}{l}\text { (1) Benzoic acid; } \mathrm{C}_{7} \mathrm{H}_{6} \mathrm{O}_{2} ; \\
\text { [65-85-0] }\end{array}$ & ${ }^{62} \mathrm{~S}$. H. Ghosh and D. K. Hazra, J. \\
$\begin{array}{l}\text { Chem. Soc., Perkin Trans. 2 1989, } \\
\text { (1,2-Dimethoxyethane; }\end{array}$ & 1021. \\
$\mathrm{C}_{4} \mathrm{H}_{10} \mathrm{O}_{2} ;[110-71-4]$ & \\
\hline Variables: & Prepared by: \\
$T / \mathrm{K}=298.15$ & W. E. Acree, Jr. \\
\hline
\end{tabular}

\section{Experimental Values}

The measured solubility was reported to be $c_{1}=3.2025$ mol dm ${ }^{-3}$.

\section{Auxiliary Information}

\section{Method/Apparatus/Procedure:}

Mechanical shaker and a constant-temperature thermostat.

Excess solute and solvent were placed in a Campbell solubility apparatus and allowed to equilibrate at $298 \mathrm{~K}$ for $24 \mathrm{~h}$. An aliquot of the saturated solution was then removed and filtered. The concentration of the dissolved solute was determined by titration with standard caustic soda using phenolphthalein as indicator. Solubility was also determined spectrophotometrically from absorbance measurements made at an analysis wavelength of $272 \mathrm{~nm}$.

\section{Source and Purity of Chemicals:}

(1) G.R.E, Merck Chemical Company, was recrystallized from alcohol and then dried.

(2) Purum, Fluka, was shaken with ferrous sulfate and then distilled. The distillate was further purified by refluxing for $12 \mathrm{~h}$ and distilling over metallic sodium.

Estimated Error:

Temperature: $\pm 0.01 \mathrm{~K}$.

$c_{1}: \pm .5 \%$ (relative error, estimated by compiler).

\begin{tabular}{ll}
\hline \hline Components: & $\begin{array}{l}\text { Original Measurements: } \\
\text { (1) Benzoic acid; } \mathrm{C}_{7} \mathrm{H}_{6} \mathrm{O}_{2} ;\end{array}$ \\
$\begin{array}{l}{ }^{63} \mathrm{C} . \text { K. Hancock, J. N. Pawloski, and J. } \\
\text { [65-85-0] }\end{array}$ & P. Idoux, J. Org. Chem. 32, 1931 \\
$\begin{array}{l}\text { (2) Tetrahydrofuran; } \mathrm{C}_{4} \mathrm{H}_{8} \mathrm{O} ; \\
{[109-99-9]}\end{array}$ & $(1967)$. \\
\hline Variables: & Prepared by: \\
$T / \mathrm{K}=303.15$ & W. E. Acree, Jr. \\
\hline
\end{tabular}

\section{Experimental Values}

\begin{tabular}{lc}
\hline \hline$x_{2}{ }^{\mathrm{a}}$ & $x_{1}{ }^{\mathrm{b}}$ \\
\hline 0.620 & 0.380 \\
\hline
\end{tabular}

${ }^{\mathrm{a}} x_{2}$ : mole fraction of component 2 in the saturated solution.

${ }^{\mathrm{b}} x_{1}$ : mole fraction solubility of the solute.

\section{Auxiliary Information}

\section{Method/Apparatus/Procedure:}

Constant-temperature bath, Soxhlet thimble, conical flask, and analytical balance.

Excess solute and solvent were placed in a conical flask and allowed to equilibrate for several days at constant temperature. Attainment of equilibrium was verified by several repetitive measurements and by approaching equilibrium from supersaturation. Aliquots of saturated solutions were transferred using a Soxhlet thimble equipped with a one-hole cork stopper and an inverted U-shaped delivery tube to a second ground-glass stoppered flask suspended in the $303 \mathrm{~K}$ water bath. Successive portions of the contents were evaporated at room temperature in a tared aluminum foil weighing dish under a bell jar through which a slow stream of dry air was passed. The second flask plus unused saturated solution and the aluminum foil dish plus residue were weighed. The saturation solubility of the solute was calculated from the recorded mass data and molar masses of the solute and solvent.

\section{Source and Purity of Chemicals:}

(1) Purity not given, Chemical source not specified, was recrystallized several times from aqueous-ethanol mixture, and then dried under vacuum over phosphorous pentoxide.

(2) Practical grade, Chemical source not specified, stored over sodium hydroxide pellets for $24 \mathrm{~h}$, and then passed through $2 \times 70-\mathrm{cm}$ chromatographic adsorption columns containing activated alumina. After this treatment, the purified solvent was stored over copper in a nitrogen atmosphere.

\section{Estimated Error:}

Temperature: $\pm 0.02 \mathrm{~K}$.

$x_{1}: \pm 1.0 \%$ (relative error).

\begin{tabular}{|c|c|}
\hline $\begin{array}{l}\text { Components: } \\
\text { (1) Benzoic acid; } \mathrm{C}_{7} \mathrm{H}_{6} \mathrm{O}_{2} \text {; } \\
\text { [65-85-0] } \\
\text { (2) Tetrahydrofuran; } \mathrm{C}_{4} \mathrm{H}_{8} \mathrm{O} \text {; } \\
\text { [109-99-9] }\end{array}$ & $\begin{array}{l}\text { Original Measurements: } \\
\text { 51 H.-M. Lin and R. A. Nash, J. Pharm. } \\
\text { Sci. 82, } 1018 \text { (1993). }\end{array}$ \\
\hline $\begin{array}{l}\text { Variables: } \\
T / \mathrm{K}=298.15\end{array}$ & $\begin{array}{l}\text { Prepared by: } \\
\text { W. E. Acree, Jr. }\end{array}$ \\
\hline
\end{tabular}

Experimental Values

\begin{tabular}{lc}
\hline \hline$x_{2}{ }^{\mathrm{a}}$ & $x_{1}{ }^{\mathrm{b}}$ \\
\hline 0.6652 & 0.3348 \\
\hline${ }^{\mathrm{a}} x_{2}:$ mole fraction of component 2 in the saturated solution. & \\
${ }^{\mathrm{b}} x_{1}:$ mole fraction solubility of the solute. &
\end{tabular}




\section{Auxiliary Information}

\section{Method/Apparatus/Procedure:}

Constant-temperature bath and high-performance liquid chromatograph. Excess solute and solvent were placed in screw-capped vials. The vials were sealed with several turns of electrical tape, warmed to about $323 \mathrm{~K}$, and then shaken in a constant-temperature bath for at least $24 \mathrm{~h}$ at $298 \mathrm{~K}$. After equilibrium was reached, the sample was filtered through a 0.45 or $1.0 \mu \mathrm{m}$ filter, and an aliquot was diluted appropriately for chromatographic analysis Concentrations were determined by a high-performance liquid

chromatographic method.

\section{Source and Purity of Chemicals:}

(1) Purity not given, Amend Drug and Chemical Company, no information provided concerning purification.

(2) Reagent grade, Aldrich Chemical Company, Milwaukee, WI, USA, no purification details were provided.

\section{Estimated Error:}

Temperature: $\pm 0.2 \mathrm{~K}$ (estimated by compiler). $x_{1}: \pm 3 \%$ (relative error, estimated by compiler).

\begin{tabular}{|c|c|}
\hline $\begin{array}{l}\text { Components: } \\
\text { (1) Benzoic acid; } \mathrm{C}_{7} \mathrm{H}_{6} \mathrm{O}_{2} \text {; } \\
\text { [65-85-0] } \\
\text { (2) 1,4-Dioxane; } \mathrm{C}_{4} \mathrm{H}_{8} \mathrm{O}_{2} \text {; } \\
\text { [123-91-1] }\end{array}$ & $\begin{array}{l}\text { Original Measurements: } \\
{ }^{47} \text { A. Beerbower, P. L. Wu, and A. } \\
\text { Martin, J. Pharm. Sci. 73, } 179 \text { (1984). }\end{array}$ \\
\hline $\begin{array}{l}\text { Variables: } \\
T / \mathrm{K}=298.15\end{array}$ & $\begin{array}{l}\text { Prepared by: } \\
\text { W. E. Acree, Jr. }\end{array}$ \\
\hline
\end{tabular}

Experimental Values

\begin{tabular}{lc}
\hline \hline$x_{2}{ }^{\mathrm{a}}$ & $x_{1}{ }^{\mathrm{b}}$ \\
\hline 0.7147 & 0.2853
\end{tabular}

${ }^{\mathrm{a}} x_{2}$ : mole fraction of component 2 in the saturated solution.

${ }^{b} x_{1}$ : mole fraction solubility of the solute.

\section{Auxiliary Information}

\section{Method/Apparatus/Procedure:}

Constant-temperature bath and ultraviolet/visible spectrophotometer.

Excess solute and solvent were placed in screw-capped vials. The vials were sealed and submerged in a constant-temperature water bath and shaken at 100 cycles/min for $24 \mathrm{~h}$. After the $24 \mathrm{~h}$ equilibration period, the vial was removed, wiped dry, and the contents analyzed. The solutions were transferred to a syringe and filtered through a $0.1 \mu \mathrm{m}$ pore size filter. The solutions were diluted and the absorbances recorded at the maximum absorption wavelength of benzoic acid. The solubility was determined at least six times.

\section{Source and Purity of Chemicals:}

(1) Purity not given, Chemical source not specified, no information provided concerning purification.

(2) Spectrophotometric or ACS Reagent grade, Chemical source not specified, redistilled before use.

\section{Estimated Error:}

Temperature: $\pm 0.2 \mathrm{~K}$.

$x_{1}: \pm 3 \%$ (relative error).

\begin{tabular}{ll}
\hline \hline Components: & $\begin{array}{l}\text { Original Measurements: } \\
\text { (1) Benzoic acid; } \mathrm{C}_{7} \mathrm{H}_{6} \mathrm{O}_{2} ;\end{array}$ \\
$\begin{array}{l}64 \mathrm{E} \text {. A. Gomaa, Phys. Chem. Liq. 50, } \\
\text { [65-85-0] }\end{array}$ & $279(2012)$. \\
$\begin{array}{l}\text { (2) 1,4-Dioxane; } \mathrm{C}_{4} \mathrm{H}_{8} \mathrm{O}_{2} ; \\
{[123-91-1]}\end{array}$ & \\
\hline Variables: & Prepared by: \\
$T / \mathrm{K}=298.15$ & W. E. Acree, Jr. \\
\hline
\end{tabular}

\section{Experimental Values}

\begin{tabular}{lc}
\hline \hline$x_{2}{ }^{\mathrm{a}}$ & $x_{1}{ }^{\mathrm{b}}$ \\
\hline 0.7893 & 0.2107 \\
${ }^{\mathrm{a}} x_{2}:$ mole fraction of component 2 in the saturated solution. \\
${ }^{\mathrm{b}} x_{1}:$ mole fraction solubility of the solute, computed by the compiler. The \\
original solubility data were given in units of moles per kilogram of solvent.
\end{tabular}

\section{Auxiliary Information}

\section{Method/Apparatus/Procedure:}

Constant-temperature shaker bath and analytical balance.

Excess solute and solvent were placed in closed test tubes and allowed to equilibrate in a constant-temperature shaker bath for several days. The solubility was determined gravimetrically by transferring $1 \mathrm{ml}$ of the saturated solution to an aluminum disk. The solvent was removed by heating with an infrared lamp. The solubility was calculated from the mass of the solid residue and amount of saturated solution analyzed.

\section{Source and Purity of Chemicals:}

(1) Purity not given, Merck Chemicals, Germany, no information provided concerning purification.

(2) Purity not given, Merck Chemicals, Germany, no information provided concerning purification.

\section{Estimated Error:}

Temperature: $\pm 0.1 \mathrm{~K}$ (estimated by compiler). $x_{1}: \pm 3 \%$ (relative error, estimated by compiler).

\begin{tabular}{ll}
\hline \hline Components: & Original Measurements: \\
$\begin{array}{l}\text { (1) Benzoic acid; } \mathrm{C}_{7} \mathrm{H}_{6} \mathrm{O}_{2} ; \\
{[65-85-0]}\end{array}$ & ${ }^{63}$ C. K. Hancock, J. N. Pawloski, and J. \\
$\begin{array}{ll}\text { P. Idoux, J. Org. Chem. 32, 1931 } \\
{[123-91-1]}\end{array}$ & $(1967)$. \\
\hline Variables: & $\mathrm{C}_{4} \mathrm{H}_{8} \mathrm{O}_{2} ;$ \\
$T / \mathrm{K}=303.15$ & Prepared by: \\
\hline
\end{tabular}

\section{Experimental Values}

\begin{tabular}{lc}
\hline \hline$x_{2}^{\mathrm{a}}$ & $x_{1}{ }^{\mathrm{b}}$ \\
\hline 0.701 & 0.299 \\
\hline${ }^{\mathrm{a}} x_{2}:$ mole fraction of component 2 in the saturated solution. & \\
${ }^{\mathrm{b}} x_{1}:$ mole fraction solubility of the solute. &
\end{tabular}




\section{Auxiliary Information}

\section{Method/Apparatus/Procedure:}

Constant-temperature bath, Soxhlet thimble, conical flask, and analytical balance.

Excess solute and solvent were placed in a conical flask and allowed to equilibrate for several days at constant temperature. Attainment of equilibrium was verified by several repetitive measurements and by approaching equilibrium from supersaturation. Weighed aliquots of saturated solutions were removed and titrated with a standardized sodium hydroxide solution (carbonate free) using a pH meter. The endpoint of the titration was determined by computing the second derivative in the $\mathrm{pH}$ versus volume of sodium hydroxide added.

\section{Source and Purity of Chemicals:}

(1) Purity not given, Chemical source not specified, was recrystallized several times from aqueous-ethanol mixture, and then dried under vacuum over phosphorous pentoxide.

(2) Practical grade, Chemical source not specified, stored over sodium hydroxide pellets for $24 \mathrm{~h}$, and then passed through $2 \times 70-\mathrm{cm}$ chromatographic adsorption columns containing activated alumina. After this treatment, the purified solvent was stored over copper in a nitrogen atmosphere.

\section{Estimated Error:}

Temperature: $\pm 0.02 \mathrm{~K}$.

$x_{1}: \pm 1.0 \%$ (relative error).

\begin{tabular}{|c|c|}
\hline $\begin{array}{l}\text { Components: } \\
\text { (1) Benzoic acid; } \mathrm{C}_{7} \mathrm{H}_{6} \mathrm{O}_{2} \text {; } \\
\text { [65-85-0] } \\
\text { (2) Methyl nonafluorobutyl ether; } \\
\mathrm{C}_{5} \mathrm{H}_{3} \mathrm{~F}_{9} \mathrm{O} ;[163702-07-6]\end{array}$ & $\begin{array}{l}\text { Original Measurements: } \\
{ }^{65} \text { A. F. Lagalante, A. Abdulagatov, and } \\
\text { T. J. Bruno, J. Chem. Eng. Data 47, } 47 \\
(2002) \text {. }\end{array}$ \\
\hline $\begin{array}{l}\text { Variables: } \\
\text { Temperature }\end{array}$ & $\begin{array}{l}\text { Prepared by: } \\
\text { W. E. Acree, Jr. }\end{array}$ \\
\hline
\end{tabular}

Experimental Values (Interpolated Values)

\begin{tabular}{lcc}
\hline \hline$T / \mathrm{K}$ & $x_{2}{ }^{\mathrm{a}}$ & $x_{1}{ }^{\mathrm{b}}$ \\
\hline 259.7 & 0.9999 & 0.000138 \\
268.2 & 0.9998 & 0.000172 \\
283.2 & 0.9997 & 0.000272 \\
298.2 & 0.9994 & 0.000560 \\
313.3 & 0.9989 & 0.00110 \\
328.2 & 0.9976 & 0.00235
\end{tabular}

${ }^{\mathrm{a}} x_{2}$ : mole fraction of component 2 in the saturated solution.

${ }^{\mathrm{b}} x_{1}$ : mole fraction solubility of the solute.

\section{Auxiliary Information}

Method/Apparatus/Procedure:

$\mathrm{UV} /$ visible spectrophotometer.

Solubilities were determined by supersaturating the solvent with naphthalene and measuring the amount of dissolved solute using a spectroscopic method. Aliquots of the saturated solution were pipetted into a volumetric flask, and diluted with acetonitrile. If necessary, the sample was further diluted to bring the measured absorbance to within the linear range of the absorbance versus calibration curve obtained from absorbance measurements on solutions of known naphthalene concentration. Absorbance measurements recorded at $272 \mathrm{~nm}$.
Source and Purity of Chemicals:

(1) Purity and Chemical source were not given, was used as received.

(2) $99 \%$, Name of commercial supplier was not given, used as received.

Estimated Error:

Temperature: $\pm 0.1 \mathrm{~K}$.

$x_{1}: 1.0 \%$ (relative error).

\begin{tabular}{|c|c|}
\hline $\begin{array}{l}\text { Components: } \\
\text { (1) Benzoic acid; } \mathrm{C}_{7} \mathrm{H}_{6} \mathrm{O}_{2} \text {; } \\
\text { [65-85-0] } \\
\text { (2) Ethyl nonafluorobutyl ether; } \\
\mathrm{C}_{6} \mathrm{H}_{5} \mathrm{~F}_{9} \mathrm{O} ;[163702-05-4]\end{array}$ & $\begin{array}{l}\text { Original Measurements: } \\
{ }^{65} \text { A. F. Lagalante, A. Abdulagatov, and } \\
\text { T. J. Bruno, J. Chem. Eng. Data 47, } 47 \\
\text { (2002). }\end{array}$ \\
\hline $\begin{array}{l}\text { Variables: } \\
\text { Temperature }\end{array}$ & $\begin{array}{l}\text { Prepared by: } \\
\text { W. E. Acree, Jr. }\end{array}$ \\
\hline
\end{tabular}

Experimental Values (Interpolated)

\begin{tabular}{lcc}
\hline \hline$T / \mathrm{K}$ & $x_{2}{ }^{\mathrm{a}}$ & $x_{1}{ }^{\mathrm{b}}$ \\
\hline 259.7 & 0.9997 & 0.000282 \\
268.2 & 0.9997 & 0.000297 \\
283.2 & 0.9995 & 0.000459 \\
298.2 & 0.9991 & 0.000857 \\
313.3 & 0.9987 & 0.00133 \\
328.2 & 0.9976 & 0.00242 \\
343.2 & 0.9954 & 0.00463
\end{tabular}

${ }^{\mathrm{a}} x_{2}$ : mole fraction of component 2 in the saturated solution.

${ }^{\mathrm{b}} x_{1}$ : mole fraction solubility of the solute.

\section{Auxiliary Information}

\section{Method/Apparatus/Procedure:}

$\mathrm{UV} /$ visible spectrophotometer.

Solubilities were determined by supersaturating the solvent with naphthalene and measuring the amount of dissolved solute using a spectroscopic method. Aliquots of the saturated solution were pipetted into a volumetric flask, and diluted with acetonitrile. If necessary, the sample was further diluted to bring the measured absorbance to within the linear range of the absorbance versus calibration curve obtained from absorbance measurements on solutions of known naphthalene concentration. Absorbance measurements recorded at $272 \mathrm{~nm}$.

Source and Purity of Chemicals:

(1) Purity and Chemical source were not given, used as received.

(2) $99 \%$, Name of commercial supplier was not given, used as received.

Estimated Error:

Temperature: $\pm 0.1 \mathrm{~K}$.

$x_{1}: 1.0 \%$ (relative error).

\subsection{Benzoic acid solubility data in haloalkanes, haloalkenes, and haloaromatic hydrocarbons}

\begin{tabular}{ll}
\hline \hline Components: & Original Measurements: \\
$\begin{array}{l}\text { (1) Benzoic acid; } \mathrm{C}_{7} \mathrm{H}_{6} \mathrm{O}_{2} ; \\
\text { [65-85-0] }\end{array}$ & ${ }^{47} \mathrm{~A}$. Beerbower, P. L. Wu, and A. \\
$\begin{array}{l}\text { (2) Trichloromethane; } \mathrm{CHCl}_{3} ; \\
\text { [67-66-3] }\end{array}$ & \\
\hline Variables: & \\
$T / \mathrm{K}=298.15$ & Prepared by: \\
\hline
\end{tabular}




\section{Experimental Values}

\begin{tabular}{lc}
\hline \hline$x_{2}{ }^{\mathrm{a}}$ & $x_{1}{ }^{\mathrm{b}}$ \\
\hline 0.8717 & 0.1283 \\
\hline$x_{2}:$
\end{tabular}

${ }^{\mathrm{a}} x_{2}$ : mole fraction of component 2 in the saturated solution.

${ }^{\mathrm{b}} x_{1}$ : mole fraction solubility of the solute.

\section{Auxiliary Information}

\section{Method/Apparatus/Procedure:}

Constant-temperature bath and ultraviolet/visible spectrophotometer.

Excess solute and solvent were placed in screw-capped vials. The vials were sealed and submerged in a constant-temperature water bath and shaken at 100 cycles/min for $24 \mathrm{~h}$. After the $24 \mathrm{~h}$ equilibration period, the vial was removed, wiped dry, and the contents analyzed. The solutions were transferred to a syringe and filtered through a $0.1 \mu \mathrm{m}$ pore size filter. The solutions were diluted and the absorbances recorded at the maximum absorption wavelength of benzoic acid. The solubility was determined at least six times.

Source and Purity of Chemicals:

(1) Purity not given, Chemical source not specified, no information provided concerning purification.

(2) Spectrophotometric or ACS Reagent grade, Chemical source not specified, redistilled before use.

Estimated Error:

Temperature: $\pm 0.2 \mathrm{~K}$.

$x_{1}: \pm 3 \%$ (relative error).

\begin{tabular}{|c|c|}
\hline $\begin{array}{l}\text { Components: } \\
\text { (1) Benzoic acid; } \mathrm{C}_{7} \mathrm{H}_{6} \mathrm{O}_{2} \text {; } \\
\text { [65-85-0] } \\
\text { (2) Trichloromethane; } \mathrm{CHCl}_{3} \text {; } \\
\text { [67-66-3] }\end{array}$ & $\begin{array}{l}\text { Original Measurements: } \\
{ }^{48} \text { J. Thati, F. L. Nordström, and A. C. } \\
\text { Rasmuson, J. Chem. Eng. Data 55, } \\
5124 \text { (2010). }\end{array}$ \\
\hline $\begin{array}{l}\text { Variables: } \\
\text { Temperature }\end{array}$ & $\begin{array}{l}\text { Prepared by: } \\
\text { W. E. Acree, Jr. }\end{array}$ \\
\hline
\end{tabular}

Experimental Values

\begin{tabular}{lcc}
\hline \hline$T / \mathrm{K}$ & $x_{2}{ }^{\mathrm{a}}$ & $x_{1}{ }^{\mathrm{b}}$ \\
\hline 278.15 & 0.926 & 0.074 \\
283.15 & 0.914 & 0.086 \\
293.15 & 0.886 & 0.114 \\
303.15 & 0.849 & 0.151 \\
313.15 & 0.804 & 0.196 \\
323.15 & 0.747 & 0.253 \\
\hline
\end{tabular}

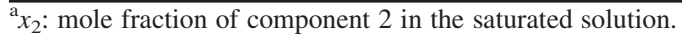

${ }^{\mathrm{b}} x_{1}$ : mole fraction solubility of the solute.

\section{Auxiliary Information}

\section{Method/Apparatus/Procedure:}

Constant-temperature bath, magnetic stirrer, analytical balance, and ventilated laboratory hood.

Solubilities were determined by a gravimetric method. Excess solute and solvent were placed in sealed bottles and allowed to equilibrate in a constanttemperature bath for $2 \mathrm{~h}$ with stirring. The stirring was discontinued and the solid was allowed to settle to the bottom of the container. After $30 \mathrm{~min}$, an aliquot of the clear solution was removed by syringe, filtered through a $0.2 \mu \mathrm{m}$ PTFE filter, and transferred to a preweighed glass vial. The glass vial was then weighed, and the solvent was allowed to evaporate in a ventilated laboratory hood at ambient room temperature. Once the solvent had evaporated, the vial with solid residue was weighed until constant weight was obtained. The solubility was calculated from the mass of the solid residue and mass of sample analyzed.

\section{Source and Purity of Chemicals:}

(1) $99.7 \%$, Merck Chemical Company, Germany, was used as received.

(2) $99+\%$, Merck Chemical Company, used as received.

Estimated Error:

Temperature: $\pm 0.01 \mathrm{~K}$.

$x_{1}: \pm 1 \%$ (relative error)

\begin{tabular}{|c|c|}
\hline $\begin{array}{l}\text { Components: } \\
\text { (1) Benzoic acid; } \mathrm{C}_{7} \mathrm{H}_{6} \mathrm{O}_{2} \text {; } \\
\text { [65-85-0] } \\
\text { (2) Trichloromethane; } \mathrm{CHCl}_{3} \text {; } \\
\text { [67-66-3] }\end{array}$ & $\begin{array}{l}\text { Original Measurements: } \\
\text { 56 P. A. Ongley, J. Chem. Soc. 1954, } \\
3634 \text {. }\end{array}$ \\
\hline $\begin{array}{l}\text { Variables: } \\
T / \mathrm{K}=298.15\end{array}$ & $\begin{array}{l}\text { Prepared by: } \\
\text { W. E. Acree, Jr. }\end{array}$ \\
\hline
\end{tabular}

\section{Experimental Values}

The measured solubility was reported to be $-\log _{10} c_{1}=-0.188$, which corresponds to a solubility of $c_{1}=1.542 \mathrm{~mol} \mathrm{dm}^{-3}$.

\section{Auxiliary Information}

\section{Method/Apparatus/Procedure:}

Very little experimental details were provided. Excess solute and solvent were placed in tubes, which were rotated for at least $8 \mathrm{~h}$ at $298 \mathrm{~K}$. The solutions were then removed, concentrated if necessary, and titrated with standard alkali using a mixed (bromothymol blue + neutral red) indicator.

Source and Purity of Chemicals:

(1) Purity not given, Chemical source not given, solute was either "AnalaR" grade or recrystallized prior to use.

(2) Purity not given, Chemical source not given, no purification details were provided.

Estimated Error:

Temperature: No information was given.

$c_{1}: \pm 4 \%$ (relative error, estimated by compiler).

\begin{tabular}{ll}
\hline \hline Components: & Original Measurements: \\
$\begin{array}{l}\text { (1) Benzoic acid; } \mathrm{C}_{7} \mathrm{H}_{6} \mathrm{O}_{2} ; \\
{[65-85-0]}\end{array}$ & ${ }^{50} \mathrm{P}$. G. Desai and A. M. Patel, J. Indian \\
$\begin{array}{l}\text { Chem. Soc. 12, 131 (1935). } \\
{[67-66-3]}\end{array}$ & \\
\hline Variables: & Prepared by: \\
$T / \mathrm{K}=301.2$ & W. E. Acree, Jr. \\
\hline
\end{tabular}

\section{Experimental Values}

\begin{tabular}{lc}
\hline \hline$x_{2}{ }^{\mathrm{a}}$ & $x_{1}{ }^{\mathrm{b}}$ \\
\hline 0.8505 & 0.1495 \\
\hline${ }^{\mathrm{a}} x_{2}:$ mole fraction of component 2 in the saturated solution. & \\
${ }^{\mathrm{b}} x_{1}:$ mole fraction solubility of the solute. &
\end{tabular}


Auxiliary Information

\section{Method/Apparatus/Procedure:}

Excess solute and solvent were placed in a glass flask which was kept revolving on a wheel in an air thermostat for two days. After two days of equilibration, the solution was filtered. To minimize absorption of the solute onto the filter paper, a $100 \mathrm{ml}$ portion of fresh saturated solution was first filtered through the paper before filtering the sample to be analyzed. An aliquot of the filtrate was titrated with barium hydroxide using phenolphthalein as the endpoint indictor. The barium hydroxide titrant was previously standardized against a solution of succinic acid.

\section{Source and Purity of Chemicals:}

(1) Purity not given, Chemical source not specified, no information provided concerning purification.

(2) Purity not given, Chemical source not specified, no information provided concerning purification.

\section{Estimated Error:}

Temperature: No information given.

$x_{1}: \pm 3 \%$ (relative error, estimated by compiler).

\begin{tabular}{|c|c|}
\hline $\begin{array}{l}\text { Components: } \\
\text { (1) Benzoic acid; } \mathrm{C}_{7} \mathrm{H}_{6} \mathrm{O}_{2} \text {; } \\
\text { [65-85-0] } \\
\text { (2) Trichloromethane; } \mathrm{CHCl}_{3} \text {; } \\
\text { [67-66-3] }\end{array}$ & $\begin{array}{l}\text { Original Measurements: } \\
\text { 66 W. Herz and W. Rathmann, Z. } \\
\text { Elektrochem. 19, } 887 \text { (1913). }\end{array}$ \\
\hline $\begin{array}{l}\text { Variables: } \\
T / \mathrm{K}=298.15\end{array}$ & $\begin{array}{l}\text { Prepared by: } \\
\text { W. E. Acree, Jr. }\end{array}$ \\
\hline
\end{tabular}

\section{Experimental Values}

The measured molar solubility was $c_{1}=1.477 \mathrm{~mol} \mathrm{dm}^{-3}$.

\section{Auxiliary Information}

\section{Method/Apparatus/Procedure:}

Very little experimental details were provided. Excess solute and solvent were shaken in a thermostat at $298 \mathrm{~K}$. After equilibrium was reached and the solid phase settled to the bottom of the container, an aliquot of the saturated clear solution was removed by pipet. The concentration of the solute in the saturated solution was determined by titration.

\section{Source and Purity of Chemicals:}

(1) Purity not given, Chemical source not given, no purification details were provided.

(2) Purity not given, Chemical source not given, no purification details were provided.

\section{Estimated Error:}

Temperature: No information was given. $c_{1}: \pm 4 \%$ (relative error, estimated by compiler).

\begin{tabular}{|c|c|}
\hline $\begin{array}{l}\text { Components: } \\
\text { (1) Benzoic acid; } \mathrm{C}_{7} \mathrm{H}_{6} \mathrm{O}_{2} \text {; } \\
\text { [65-85-0] } \\
\text { (2) Trichloromethane; } \mathrm{CHCl}_{3} \text {; } \\
\text { [67-66-3] }\end{array}$ & $\begin{array}{l}\text { Original Measurements: } \\
{ }^{57} \text { J. W. Marden and M. V. Dover, J. } \\
\text { Am. Chem. Soc. 38, } 1235 \text { (1916). }\end{array}$ \\
\hline $\begin{array}{l}\text { Variables: } \\
T / \mathrm{K}=298.15\end{array}$ & $\begin{array}{l}\text { Prepared by: } \\
\text { W. E. Acree, Jr. }\end{array}$ \\
\hline
\end{tabular}

Experimental Values

\begin{tabular}{lc}
\hline \hline$x_{2}{ }^{\mathrm{a}}$ & $x_{1}{ }^{\mathrm{b}}$ \\
\hline 0.727 & 0.273
\end{tabular}

${ }^{\mathrm{a}} x_{2}$ : mole fraction of component 2 in the saturated solution.

${ }^{b} x_{1}$ : mole fraction solubility of the solute. Experimental solubility data were given as grams of solute per $100 \mathrm{~g}$ of solvent. Mole fraction solubility was calculated by the compiler.

\section{Auxiliary Information}

\section{Method/Apparatus/Procedure:}

Constant-temperature thermostat, analytical balance, and a steam bath. Excess solute and solvent were placed in a bottle with ground glass stopper which was protected by a coating of beeswax and rosin, over which was tied a piece of rubber sheeting. The sealed mixture was shaken in a constanttemperature thermostat for 8-20 h. An aliquot of the saturated solution was withdrawn through a glass wool filter into a weighing pipet from which the solutions were weighed directly into small glass evaporating dishes. The solvent was removed on a steam bath, and the sample further dried in a sulfuric acid desiccator. The solubility was calculated from the mass of the solid residue and the mass of the saturated solution removed for analysis.

\section{Source and Purity of Chemicals:}

(1) Purity not given, Chemical source not given, no purification details were provided.

(2) Purity not given, Chemical source not given, no purification details were provided.

Estimated Error:

Temperature: $\pm 0.1 \mathrm{~K}$.

$x_{1}: \pm 10.0 \%$ (relative error, estimated by compiler).

\begin{tabular}{ll}
\hline \hline Components: & Original Measurements: \\
$\begin{array}{l}\text { (1) Benzoic acid; } \mathrm{C}_{7} \mathrm{H}_{6} \mathrm{O}_{2} ; \\
\text { [65-85-0] }\end{array}$ & $\begin{array}{l}{ }^{47} \mathrm{~A} . \text { Beerbower, P. L. Wu, and A. } \\
\text { (2) Tetrachloromethane; } \mathrm{CCl}_{4} ;\end{array}$ \\
[56-23-5] & \\
\hline Vartin, J. Pharm. Sci. 73, 179 (1984). \\
$T / \mathrm{K}=298.15$ & Prepared by: \\
\hline
\end{tabular}

Experimental Values

\begin{tabular}{lc}
\hline \hline$x_{2}{ }^{\mathrm{a}}$ & $x_{1}{ }^{\mathrm{b}}$ \\
\hline 0.9506 & 0.0494 \\
\hline${ }^{\mathrm{a}} x_{2}:$ mole fraction of component 2 in the saturated solution. & \\
${ }^{\mathrm{b}} x_{1}:$ mole fraction solubility of the solute. &
\end{tabular}

\section{Auxiliary Information}

\section{Method/Apparatus/Procedure:}

Constant-temperature bath and ultraviolet/visible spectrophotometer.

Excess solute and solvent were placed in screw-capped vials. The vials were sealed and submerged in a constant-temperature water bath and shaken at 100 cycles/min for $24 \mathrm{~h}$. After the $24 \mathrm{~h}$ equilibration period, the vial was removed, wiped dry, and the contents analyzed. The solutions were transferred to a syringe and filtered through a $0.1 \mu \mathrm{m}$ pore size filter. The solutions were diluted and the absorbances recorded at the maximum absorption wavelength of benzoic acid. The solubility was determined at least six times. 
Source and Purity of Chemicals:

(1) Purity not given, Chemical source not specified, no information provided concerning purification.

(2) Spectrophotometric or ACS Reagent grade, Chemical source not specified, redistilled before use.

Estimated Error:

Temperature: $\pm 0.2 \mathrm{~K}$.

$x_{1}: \pm 3 \%$ (relative error).

\begin{tabular}{|c|c|}
\hline $\begin{array}{l}\text { Components: } \\
\text { (1) Benzoic acid; } \mathrm{C}_{7} \mathrm{H}_{6} \mathrm{O}_{2} \text {; } \\
\text { [65-85-0] } \\
\text { (2) Tetrachloromethane; } \mathrm{CCl}_{4} \text {; } \\
\text { [56-23-5] }\end{array}$ & $\begin{array}{l}\text { Original Measurements: } \\
\text { }{ }^{67} \text { M. Davies and D. M. L. Griffiths, J. } \\
\text { Chem. Soc. 1955, } 132 \text {. }\end{array}$ \\
\hline $\begin{array}{l}\text { Variables: } \\
T / \mathrm{K}=298.15\end{array}$ & $\begin{array}{l}\text { Prepared by: } \\
\text { W. E. Acree, Jr. }\end{array}$ \\
\hline
\end{tabular}

Experimental Values

\begin{tabular}{lc}
\hline \hline$x_{2}^{\mathrm{a}}$ & $x_{1}{ }^{\mathrm{b}}$ \\
\hline 0.9500 & 0.0500
\end{tabular}

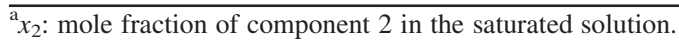

${ }^{\mathrm{b}} x_{1}$ : mole fraction solubility of the solute.

\section{Auxiliary Information}

\section{Method/Apparatus/Procedure:}

Experimental solubility was determined by percolating the solvent at an adjustable rate through a column of solute in one limb of an U-shaped tube, a plug of cotton wool serving as support and filter. The saturated solution collects in the parallel arm and the whole is assembly is immersed in a thermostat bath except for the stoppered ends. Samples of the saturated solutions were removed and titrated with standardized carbonate-free alkali using phenolphthalein as the indicator.

\section{Source and Purity of Chemicals:}

(1) Purity not given, Chemical source not specified, no information provided concerning purification.

(2) Purity not given, Chemical source not specified, no information provided concerning purification.

\section{Estimated Error:}

Temperature: $\pm 0.02 \mathrm{~K}$.

$x_{1}: \pm 3 \%$ (relative error, estimated by compiler).

\begin{tabular}{|c|c|}
\hline $\begin{array}{l}\text { Components: } \\
\text { (1) Benzoic acid; } \mathrm{C}_{7} \mathrm{H}_{6} \mathrm{O}_{2} \text {; } \\
\text { [65-85-0] } \\
\text { (2) Tetrachloromethane; } \mathrm{CCl}_{4} \text {; } \\
\text { [56-23-5] }\end{array}$ & $\begin{array}{l}\text { Original Measurements: } \\
\text { W9 W. E. Acree, Jr. and G. L. Bertrand, J. } \\
\text { Pharm. Sci. 70, } 1033 \text { (1981). }\end{array}$ \\
\hline $\begin{array}{l}\text { Variables: } \\
\text { Temperature }\end{array}$ & $\begin{array}{l}\text { Prepared by: } \\
\text { W. E. Acree, Jr. }\end{array}$ \\
\hline
\end{tabular}

Experimental Values

\begin{tabular}{lcc}
\hline \hline$T / \mathrm{K}$ & $x_{2}{ }^{\mathrm{a}}$ & $x_{1}{ }^{\mathrm{b}}$ \\
\hline 298.15 & 0.9508 & 0.0492 \\
303.15 & 0.9402 & 0.0598
\end{tabular}

${ }^{\mathrm{a}} x_{2}$ : mole fraction of component 2 in the saturated solution.

${ }^{\mathrm{b}} x_{1}$ : mole fraction solubility of the solute.

\section{Auxiliary Information}

\section{Method/Apparatus/Procedure:}

Constant-temperature water bath and an analytical balance.

Excess solute and solvent and were placed in brown glass containers and allowed to equilibrate in a constant-temperature water bath for several days. Attainment of equilibrium was verified by repetitive measurements after several additional days. Solubility was determined by transferring a weighed aliquot through a coarse filter into a flask containing blank nonaqueous titration solvent. The solutions were titrated with freshly standardized sodium methoxide to the thymol blue endpoint.

Source and Purity of Chemicals:

(1) $99 \%$, Chemical Source not given, was dried at $333 \mathrm{~K}$ for several h before use.

(2) $99+\%$, Chemical source not given, refluxed for $11 \mathrm{~h}$ over an aqueous solution $10 \%$ in potassium permanganate and $10 \%$ in sodium hydroxide. The tetrachloromethane was distilled off and dried with calcium hydroxide, stored in contact with mercury under an argon atmosphere, and then distilled shortly before use.

\section{Estimated Error:}

Temperature: $\pm 0.01 \mathrm{~K}$.

$x_{1}: \pm 1 \%$ (relative error)

\begin{tabular}{|c|c|}
\hline $\begin{array}{l}\text { Components: } \\
\text { (1) Benzoic acid; } \mathrm{C}_{7} \mathrm{H}_{6} \mathrm{O}_{2} \text {; } \\
\text { [65-85-0] } \\
\text { (2) Tetrachloromethane; } \mathrm{CCl}_{4} \text {; } \\
\text { [56-23-5] }\end{array}$ & $\begin{array}{l}\text { Original Measurements: } \\
{ }^{56} \text { P. A. Ongley, J. Chem. Soc. 1954, } \\
3634 \text {. }\end{array}$ \\
\hline $\begin{array}{l}\text { Variables: } \\
T / \mathrm{K}=298.15\end{array}$ & $\begin{array}{l}\text { Prepared by: } \\
\text { W. E. Acree, Jr. }\end{array}$ \\
\hline
\end{tabular}

\section{Experimental Values}

The measured solubility was reported to be $-\log _{10} c_{1}=0.291$, which corresponds to a solubility of $c_{1}=0.512 \mathrm{~mol} \mathrm{dm}^{-3}$.

\section{Auxiliary Information}

\section{Method/Apparatus/Procedure:}

Very little experimental details were provided. Excess solute and solvent were placed in tubes, which were rotated for at least $8 \mathrm{~h}$ at $298 \mathrm{~K}$. The solutions were then removed, concentrated if necessary, and titrated with standard alkali using a mixed (bromothymol blue + neutral red) indicator.

\section{Source and Purity of Chemicals:}

(1) Purity not given, Chemical source not given, solute was either "AnalaR" grade or recrystallized prior to use.

(2) Purity not given, Chemical source not given, no purification details were provided.

\section{Estimated Error:}

Temperature: No information was given.

$c_{1}: \pm 4 \%$ (relative error, estimated by compiler). 


\section{Components:}

(1) Benzoic acid; $\mathrm{C}_{7} \mathrm{H}_{6} \mathrm{O}_{2}$;

[65-85-0]

(2) Tetrachloromethane; $\mathrm{CCl}_{4}$;

[56-23-5]

\begin{tabular}{ll}
\hline Variables: & Prepared by: \\
$T / \mathrm{K}=301.2$ & W. E. Acree, Jr.
\end{tabular}

Experimental Values

\begin{tabular}{lc}
\hline \hline$x_{2}{ }^{\mathrm{a}}$ & $x_{1}{ }^{\mathrm{b}}$ \\
\hline 0.9407 & 0.0593
\end{tabular}

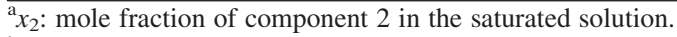

${ }^{\mathrm{b}} x_{1}$ : mole fraction solubility of the solute.

\section{Auxiliary Information}

\section{Method/Apparatus/Procedure:}

Excess solute and solvent were placed in a glass flask which was kept revolving on a wheel in an air thermostat for two days. After two days of equilibration, the solution was filtered. To minimize absorption of the solute onto the filter paper, a $100 \mathrm{ml}$ portion of fresh saturated solution was first filtered through the paper before filtering the sample to be analyzed. An aliquot of the filtrate was titrated with barium hydroxide using phenolphthalein as the endpoint indictor. The barium hydroxide titrant was previously standardized against a solution of succinic acid.

Source and Purity of Chemicals:

(1) Purity not given, Chemical source not specified, no information provided concerning purification.

(2) Purity not given, Chemical source not specified, no information provided concerning purification.

\section{Estimated Error:}

Temperature: No information given.

$x_{1}: \pm 3 \%$ (relative error, estimated by compiler).

\begin{tabular}{|c|c|}
\hline $\begin{array}{l}\text { Components: } \\
\text { (1) Benzoic acid; } \mathrm{C}_{7} \mathrm{H}_{6} \mathrm{O}_{2} \text {; } \\
\text { [65-85-0] } \\
\text { (2) Tetrachloromethane; } \mathrm{CCl}_{4} \text {; } \\
\text { [56-23-5] }\end{array}$ & $\begin{array}{l}\text { Original Measurements: } \\
{ }^{66} \mathrm{~W} . \text { Herz and W. Rathmann, Z. } \\
\text { Elektrochem. 19, } 887 \text { (1913). }\end{array}$ \\
\hline $\begin{array}{l}\text { Variables: } \\
T / \mathrm{K}=298.15\end{array}$ & $\begin{array}{l}\text { Prepared by: } \\
\text { W. E. Acree, Jr. }\end{array}$ \\
\hline
\end{tabular}

\section{Experimental Values}

The measured molar solubility was $c_{1}=0.549 \mathrm{~mol} \mathrm{dm}^{-3}$.

\section{Auxiliary Information}

\section{Method/Apparatus/Procedure:}

Very little experimental details were provided. Excess solute and solvent were shaken in a thermostat at $298 \mathrm{~K}$. After equilibrium was reached and the solid phase settled to the bottom of the container, an aliquot of the saturated clear solution was removed by pipet. The concentration of the solute in the saturated solution was determined by titration.
Source and Purity of Chemicals:

(1) Purity not given, Chemical source not given, no purification details were provided.

(2) Purity not given, Chemical source not given, no purification details were provided.

\section{Estimated Error:}

Temperature: No information was given. $c_{1}: \pm 4 \%$ (relative error, estimated by compiler).

\begin{tabular}{|c|c|}
\hline $\begin{array}{l}\text { Components: } \\
\text { (1) Benzoic acid; } \mathrm{C}_{7} \mathrm{H}_{6} \mathrm{O}_{2} \text {; } \\
\text { [65-85-0] } \\
\text { (2) 1,2-Dichloroethane; } \mathrm{C}_{2} \mathrm{H}_{4} \mathrm{Cl}_{2} \text {; } \\
\text { [107-06-2] }\end{array}$ & $\begin{array}{l}\text { Original Measurements: } \\
{ }^{47} \text { A. Beerbower, P. L. Wu, and A. } \\
\text { Martin, J. Pharm. Sci. 73, } 179 \text { (1984). }\end{array}$ \\
\hline $\begin{array}{l}\text { Variables: } \\
T / \mathrm{K}=298.15\end{array}$ & $\begin{array}{l}\text { Prepared by: } \\
\text { W. E. Acree, Jr. }\end{array}$ \\
\hline
\end{tabular}

\section{Experimental Values}

\begin{tabular}{lc}
\hline \hline$x_{2}{ }^{\mathrm{a}}$ & $x_{1}^{\mathrm{b}}$ \\
\hline 0.9305 & 0.0695 \\
\hline${ }^{\mathrm{a}} x_{2}:$ mole fraction of component 2 in the saturated solution. & \\
${ }^{\mathrm{b}} x_{1}:$ mole fraction solubility of the solute. &
\end{tabular}

\section{Auxiliary Information}

\section{Method/Apparatus/Procedure:}

Constant-temperature bath and ultraviolet/visible spectrophotometer.

Excess solute and solvent were placed in screw-capped vials. The vials were sealed and submerged in a constant-temperature water bath and shaken at 100 cycles/min for $24 \mathrm{~h}$. After the $24 \mathrm{~h}$ equilibration period, the vial was removed, wiped dry, and the contents analyzed. The solutions were transferred to a syringe and filtered through a $0.1 \mu \mathrm{m}$ pore size filter. The solutions were diluted and the absorbances recorded at the maximum absorption wavelength of benzoic acid. The solubility was determined at least six times.

\section{Source and Purity of Chemicals:}

(1) Purity not given, Chemical source not specified, no information provided concerning purification.

(2) Spectrophotometric or ACS Reagent grade, Chemical source not specified, redistilled before use.

\section{Estimated Error:}

Temperature: $\pm 0.2 \mathrm{~K}$.

$x_{1}: \pm 3 \%$ (relative error).

\begin{tabular}{|c|c|}
\hline $\begin{array}{l}\text { Components: } \\
\text { (1) Benzoic acid; } \mathrm{C}_{7} \mathrm{H}_{6} \mathrm{O}_{2} \text {; } \\
\text { [65-85-0] } \\
\text { (2) } 1,1,2,2 \text {-Tetrachloroethane; } \\
\mathrm{C}_{2} \mathrm{H}_{2} \mathrm{Cl}_{4} ;[79-34-5]\end{array}$ & $\begin{array}{l}\text { Original Measurements: } \\
\text { 66 W. Herz and W. Rathmann, Z. } \\
\text { Elektrochem. 19, } 887 \text { (1913). }\end{array}$ \\
\hline $\begin{array}{l}\text { Variables: } \\
T / \mathrm{K}=298.15\end{array}$ & $\begin{array}{l}\text { Prepared by: } \\
\text { W. E. Acree, Jr. }\end{array}$ \\
\hline
\end{tabular}

\section{Experimental Values}

The measured molar solubility was $c_{1}=1.243 \mathrm{~mol} \mathrm{dm}^{-3}$. 


\section{Auxiliary Information}

\section{Method/Apparatus/Procedure:}

Very little experimental details were provided. Excess solute and solvent were shaken in a thermostat at $298 \mathrm{~K}$. After equilibrium was reached and the solid phase settled to the bottom of the container, an aliquot of the saturated clear solution was removed by pipet. The concentration of the solute in the saturated solution was determined by titration.

\section{Source and Purity of Chemicals:}

(1) Purity not given, Chemical source not given, no purification details were provided.

(2) Purity not given, Chemical source not given, no purification details were provided.

\section{Estimated Error:}

Temperature: No information was given. $c_{1}: \pm 4 \%$ (relative error, estimated by compiler).

\begin{tabular}{ll}
\hline \hline Components: & Original Measurements: \\
(1) Benzoic acid; $\mathrm{C}_{7} \mathrm{H}_{6} \mathrm{O}_{2} ;$ & ${ }^{66} \mathrm{~W}$. Herz and W. Rathmann, Z. \\
{$[65-85-0]$} & Elektrochem. 19, 887 (1913). \\
$(2) 1,1,1,2,2-P e n t a c h l o r o e t h a n e ;$ & \\
$\mathrm{C}_{2} \mathrm{HCl}_{5} ;[76-01-7]$ & \\
\hline Variables: & Prepared by: \\
$T / \mathrm{K}=298.15$ & W. E. Acree, Jr. \\
\hline
\end{tabular}

\section{Experimental Values}

The measured molar solubility was $c_{1}=0.893 \mathrm{~mol} \mathrm{dm}^{-3}$.

\section{Auxiliary Information}

\section{Method/Apparatus/Procedure:}

Very little experimental details were provided. Excess solute and solvent were shaken in a thermostat at $298 \mathrm{~K}$. After equilibrium was reached and the solid phase settled to the bottom of the container, an aliquot of the saturated clear solution was removed by pipet. The concentration of the solute in the saturated solution was determined by titration.

\section{Source and Purity of Chemicals:}

(1) Purity not given, Chemical source not given, no purification details were provided.

(2) Purity not given, Chemical source not given, no purification details were provided.

\section{Estimated Error:}

Temperature: No information was given. $c_{1}: \pm 4 \%$ (relative error, estimated by compiler).

\begin{tabular}{ll}
\hline \hline Components: & Original Measurements: \\
$\begin{array}{l}\text { (1) Benzoic acid; } \mathrm{C}_{7} \mathrm{H}_{6} \mathrm{O}_{2} ; \\
\text { [65-85-0] }\end{array}$ & ${ }^{86} \mathrm{D}$. H. Wester and A. Bruins, Pharm. \\
$\begin{array}{l}\text { (2) Dichloroethene; } \mathrm{C}_{2} \mathrm{H}_{2} \mathrm{Cl}_{2} ; \\
\text { isomer was not given }\end{array}$ & \\
\hline Variables: & Prepared by: \\
$T / \mathrm{K}=288.15$ & W. E. Acree, Jr. \\
\hline
\end{tabular}

\section{Experimental Values}

\begin{tabular}{lc}
\hline \hline$x_{2}{ }^{\mathrm{a}}$ & $x_{1}{ }^{\mathrm{b}}$ \\
\hline 0.9218 & 0.0782 \\
\hline${ }^{\mathrm{a}} x_{2}:$ mole fraction of component 2 in the saturated solution. & \\
${ }^{\mathrm{b}} x_{1}:$ mole fraction solubility of the solute. &
\end{tabular}

\section{Auxiliary Information}

\section{Method/Apparatus/Procedure:}

Excess solute and solvent were continuously shaken in a water bath at $303 \mathrm{~K}$ for $1 \mathrm{~h}$. The solution was then transferred to a cellar which was maintained at a constant temperature of $288 \mathrm{~K}$. The solution was allowed to equilibrate in the cellar for at least two days with repeated shaking. The concentration of the dissolved solute was determined; however, the analytical method was not described.

\section{Source and Purity of Chemicals:}

(1) Purity not given, Chemical source not specified, no information provided concerning purification.

(2) Purity not given, Chemical source not specified, no information provided concerning purification.

\section{Estimated Error:}

Temperature: $\pm 0.2 \mathrm{~K}$.

$x_{1}$ : Not enough information in paper to estimate an uncertainty.

\begin{tabular}{ll}
\hline \hline Components: & Original Measurements: \\
$\begin{array}{l}\text { (1) Benzoic acid; } \mathrm{C}_{7} \mathrm{H}_{6} \mathrm{O}_{2} ; \\
{[65-85-0]}\end{array}$ & $\begin{array}{l}{ }^{66} \mathrm{~W} . \text { Herz and W. Rathmann, Z. } \\
\text { Elektrochem. 19, } 887(1913) .\end{array}$ \\
[79-06-1] & \\
\hline Variables: & Prepared by: \\
$T / \mathrm{K}=298.15$ & W. E. Acree, Jr. \\
\hline
\end{tabular}

\section{Experimental Values}

The measured molar solubility was $c_{1}=1.116 \mathrm{~mol} \mathrm{dm}^{-3}$.

\section{Auxiliary Information}

\section{Method/Apparatus/Procedure:}

Very little experimental details were provided. Excess solute and solvent were shaken in a thermostat at $298 \mathrm{~K}$. After equilibrium was reached and the solid phase settled to the bottom of the container, an aliquot of the saturated clear solution was removed by pipet. The concentration of the solute in the saturated solution was determined by titration.

\section{Source and Purity of Chemicals:}

(1) Purity not given, Chemical source not given, no purification details were provided.

(2) Purity not given, Chemical source not given, no purification details were provided.

\section{Estimated Error:}

Temperature: No information was given $c_{1}: \pm 4 \%$ (relative error, estimated by compiler). 


\section{Components:}

(1) Benzoic acid; $\mathrm{C}_{7} \mathrm{H}_{6} \mathrm{O}_{2}$;

[65-85-0]

(2) Trichloroethene; $\mathrm{C}_{2} \mathrm{HCl}_{3}$;

[79-06-1]

\begin{tabular}{ll}
\hline Variables: & Prepared by: \\
$T / \mathrm{K}=288.15$ & W. E. Acree, Jr.
\end{tabular}

\section{Experimental Values}

\begin{tabular}{lc}
\hline \hline$x_{2}{ }^{\mathrm{a}}$ & $x_{1}{ }^{\mathrm{b}}$ \\
\hline 0.9307 & 0.0693
\end{tabular}

${ }^{a_{X_{2}}}$ : mole fraction of component 2 in the saturated solution.

${ }^{\mathrm{b}} x_{1}$ : mole fraction solubility of the solute.

\section{Auxiliary Information}

\begin{abstract}
Method/Apparatus/Procedure:
Excess solute and solvent were continuously shaken in a water bath at $303 \mathrm{~K}$ for $1 \mathrm{~h}$. The solution was then transferred to a cellar which was maintained at a constant temperature of $288 \mathrm{~K}$. The solution was allowed to equilibrate in the cellar for at least two days with repeated shaking. The concentration of the dissolved solute was determined; however, the analytical method was not described.
\end{abstract}

\section{Source and Purity of Chemicals:}

(1) Purity not given, Chemical source not specified, no information provided concerning purification.

(2) Purity not given, Chemical source not specified, no information provided concerning purification.

\section{Estimated Error:}

Temperature: $\pm 0.2 \mathrm{~K}$

$x_{1}$ : Not enough information in paper to estimate an uncertainty.

\begin{tabular}{|c|c|}
\hline $\begin{array}{l}\text { Components: } \\
\text { (1) Benzoic acid; } \mathrm{C}_{7} \mathrm{H}_{6} \mathrm{O}_{2} \text {; } \\
\text { [65-85-0] } \\
\text { (2) Tetrachloroethene; } \mathrm{C}_{2} \mathrm{Cl}_{4} \text {; } \\
\text { [127-18-4] }\end{array}$ & $\begin{array}{l}\text { Original Measurements: } \\
\text { 66 W. Herz and W. Rathmann, Z. } \\
\text { Elektrochem. 19, } 887 \text { (1913). }\end{array}$ \\
\hline $\begin{array}{l}\text { Variables: } \\
T / \mathrm{K}=298.15\end{array}$ & $\begin{array}{l}\text { Prepared by: } \\
\text { W. E. Acree, Jr. }\end{array}$ \\
\hline
\end{tabular}

\section{Experimental Values}

The measured molar solubility was $c_{1}=0.660 \mathrm{~mol} \mathrm{dm}^{-3}$.

\section{Auxiliary Information}

\section{Method/Apparatus/Procedure:}

Very little experimental details were provided. Excess solute and solvent were shaken in a thermostat at $298 \mathrm{~K}$. After equilibrium was reached and the solid phase settled to the bottom of the container, an aliquot of the saturated clear solution was removed by pipet. The concentration of the solute in the saturated solution was determined by titration.

Source and Purity of Chemicals:

(1) Purity not given, Chemical source not given, no purification details were provided.

(2) Purity not given, Chemical source not given, no purification details were provided.
Estimated Error:

Temperature: No information was given

$c_{1}: \pm 4 \%$ (relative error, estimated by compiler).

\begin{tabular}{ll}
\hline \hline Components: & Original Measurements: \\
(1) Benzoic acid; $\mathrm{C}_{7} \mathrm{H}_{6} \mathrm{O}_{2} ;$ & ${ }^{47}$ A. Beerbower, P. L. Wu, and A. \\
{$[65-85-0]$} & Martin, J. Pharm. Sci. 73, 179 (1984). \\
$\begin{array}{l}\text { (2) Chlorobenzene; } \mathrm{C}_{6} \mathrm{H}_{5} \mathrm{Cl} ; \\
{[108-90-7]}\end{array}$ & \\
\hline Variables: & Prepared by: \\
$T / \mathrm{K}=298.15$ & W. E. Acree, Jr. \\
\hline
\end{tabular}

Experimental Values

\begin{tabular}{lc}
\hline \hline$x_{2}{ }^{\mathrm{a}}$ & $x_{1}{ }^{\mathrm{b}}$ \\
\hline 0.9138 & 0.0862
\end{tabular}

${ }^{\mathrm{a}} x_{2}$ : mole fraction of component 2 in the saturated solution.

${ }^{\mathrm{b}} x_{1}$ : mole fraction solubility of the solute.

\section{Auxiliary Information}

\section{Method/Apparatus/Procedure:}

Constant-temperature bath and ultraviolet/visible spectrophotometer.

Excess solute and solvent were placed in screw-capped vials. The vials were sealed and submerged in a constant-temperature water bath and shaken at 100 cycles/min for $24 \mathrm{~h}$. After the $24 \mathrm{~h}$ equilibration period, the vial was removed, wiped dry, and the contents analyzed. The solutions were transferred to a syringe and filtered through a $0.1 \mu \mathrm{m}$ pore size filter. The solutions were diluted and the absorbances recorded at the maximum absorption wavelength of benzoic acid. The solubility was determined at least six times.

\section{Source and Purity of Chemicals:}

(1) Purity not given, Chemical source not specified, no information provided concerning purification.

(2) Spectrophotometric or ACS Reagent grade, Chemical source not specified, redistilled before use.

\section{Estimated Error:}

Temperature: $\pm 0.2 \mathrm{~K}$.

$x_{1}: \pm 3 \%$ (relative error).

\begin{tabular}{ll}
\hline \hline Components: & Original Measurements: \\
$\begin{array}{l}\text { (1) Benzoic acid; } \mathrm{C}_{7} \mathrm{H}_{6} \mathrm{O}_{2} ; \\
{[65-85-0]}\end{array}$ & ${ }^{50}$ P. G. Desai and A. M. Patel, J. Indian \\
$\begin{array}{l}\text { Chem. Soc. 12, 131 (1935). } \\
{[108-90-7]}\end{array}$ & \\
\hline Variables: & Prepared by: \\
$T / \mathrm{K}=301.2$ & W. E. Acree, Jr. \\
\hline
\end{tabular}

\section{Experimental Values}

\begin{tabular}{lc}
\hline \hline$x_{2}{ }^{\mathrm{a}}$ & $x_{1}{ }^{\mathrm{b}}$ \\
\hline 0.8953 & 0.1047 \\
\hline${ }^{\mathrm{a}} x_{2}:$ mole fraction of component 2 in the saturated solution. & \\
${ }^{\mathrm{b}} x_{1}:$ mole fraction solubility of the solute. &
\end{tabular}




\section{Auxiliary Information}

\section{Method/Apparatus/Procedure:}

Excess solute and solvent were placed in a glass flask which was kept revolving on a wheel in an air thermostat for two days. After two days of equilibration, the solution was filtered. To minimize absorption of the solute onto the filter paper, a $100 \mathrm{ml}$ portion of fresh saturated solution was first filtered through the paper before filtering the sample to be analyzed. An aliquot of the filtrate was titrated with barium hydroxide using phenolphthalein as the endpoint indictor. The barium hydroxide titrant was previously standardized against a solution of succinic acid.

\section{Source and Purity of Chemicals:}

(1) Purity not given, Chemical source not specified, no information provided concerning purification.

(2) Purity not given, Chemical source not specified, no information provided concerning purification.

\section{Estimated Error:}

Temperature: No information given.

$x_{1}: \pm 3 \%$ (relative error, estimated by compiler).

\subsection{Benzoic acid solubility data in alcohols}

\begin{tabular}{|c|c|}
\hline $\begin{array}{l}\text { Components: } \\
\text { (1) Benzoic acid; } \mathrm{C}_{7} \mathrm{H}_{6} \mathrm{O}_{2} \text {; } \\
\text { [65-85-0] } \\
\text { (2) Methanol; } \mathrm{CH}_{4} \mathrm{O} ;[67-56-1]\end{array}$ & $\begin{array}{l}\text { Original Measurements: } \\
{ }^{47} \text { A. Beerbower, P. L. Wu, and A. } \\
\text { Martin, J. Pharm. Sci. 73, } 179 \text { (1984). }\end{array}$ \\
\hline $\begin{array}{l}\text { Variables: } \\
T / \mathrm{K}=298.15\end{array}$ & $\begin{array}{l}\text { Prepared by: } \\
\text { W. E. Acree, Jr. }\end{array}$ \\
\hline
\end{tabular}

\section{Experimental Values}

\begin{tabular}{lc}
\hline \hline$x_{2}{ }^{\mathrm{a}}$ & $x_{1}{ }^{\mathrm{b}}$ \\
\hline 0.8368 & 0.1632 \\
\hline${ }^{\mathrm{a}} x_{2}:$ mole fraction of component 2 in the saturated solution. & \\
${ }^{\mathrm{b}} x_{1}:$ mole fraction solubility of the solute. &
\end{tabular}

\section{Auxiliary Information}

\section{Method/Apparatus/Procedure:}

Constant-temperature bath and ultraviolet/visible spectrophotometer.

Excess solute and solvent were placed in screw-capped vials. The vials were sealed and submerged in a constant-temperature water bath and shaken at 100 cycles/min for $24 \mathrm{~h}$. After the $24 \mathrm{~h}$ equilibration period, the vial was removed, wiped dry, and the contents analyzed. The solutions were transferred to a syringe and filtered through a $0.1 \mu \mathrm{m}$ pore size filter. The solutions were diluted and the absorbances recorded at the maximum absorption wavelength of benzoic acid. The solubility was determined at least six times.

\section{Source and Purity of Chemicals:}

(1) Purity not given, Chemical source not specified, no information provided concerning purification.

(2) Spectrophotometric or ACS Reagent grade, Chemical source not specified, redistilled before use.

\section{Estimated Error:}

Temperature: $\pm 0.2 \mathrm{~K}$.

$x_{1}: \pm 3 \%$ (relative error).

\begin{tabular}{|c|c|}
\hline $\begin{array}{l}\text { Components: } \\
\text { (1) Benzoic acid; } \mathrm{C}_{7} \mathrm{H}_{6} \mathrm{O}_{2} \text {; } \\
\text { [65-85-0] } \\
\text { (2) Methanol; } \mathrm{CH}_{4} \mathrm{O} ;[67-56-1]\end{array}$ & $\begin{array}{l}\text { Original Measurements: } \\
{ }^{51} \text { H.-M. Lin and R. A. Nash, J. Pharm } \\
\text { Sci. 82, } 1018 \text { (1993). }\end{array}$ \\
\hline $\begin{array}{l}\text { Variables: } \\
T / \mathrm{K}=298.15\end{array}$ & $\begin{array}{l}\text { Prepared by: } \\
\text { W. E. Acree, Jr. }\end{array}$ \\
\hline
\end{tabular}

Experimental Values

\begin{tabular}{lc}
\hline \hline$x_{2}{ }^{\mathrm{a}}$ & $x_{1}{ }^{\mathrm{b}}$ \\
\hline 0.8431 & 0.1569 \\
\hline${ }_{{ }^{\mathrm{a}} x_{2}: \text { mole fraction of component } 2 \text { in the saturated solution. }}$ & \\
${ }^{\mathrm{b}} x_{1}:$ mole fraction solubility of the solute. &
\end{tabular}

Auxiliary Information

\section{Method/Apparatus/Procedure:}

Constant-temperature bath and high-performance liquid chromatograph. Excess solute and solvent were placed in screw-capped vials. The vials were sealed with several turns of electrical tape, warmed to about $323 \mathrm{~K}$, and then shaken in a constant-temperature bath for at least $24 \mathrm{~h}$ at $298 \mathrm{~K}$. After equilibrium was reached, the sample was filtered through a 0.45 or $1.0 \mu \mathrm{m}$ filter, and an aliquot was diluted appropriately for chromatographic analysis. Concentrations were determined by a high-performance liquid chromatographic method.

\section{Source and Purity of Chemicals:}

(1) Purity not given, Amend Drug and Chemical Company, no information provided concerning purification.

(2) HPLC grade, Aldrich Chemical Company, Milwaukee, WI, USA, no purification details were provided.

\section{Estimated Error:}

Temperature: $\pm 0.2 \mathrm{~K}$ (estimated by compiler). $x_{1}: \pm 3 \%$ (relative error, estimated by compiler).

\begin{tabular}{ll}
\hline \hline Components: & Original Measurements: \\
$\begin{array}{l}\text { (1) Benzoic acid; } \mathrm{C}_{7} \mathrm{H}_{6} \mathrm{O}_{2} ; \\
\text { [65-85-0] }\end{array}$ & ${ }^{68} \mathrm{~K}$. K. Kundu, A. L. De, and M. N. \\
Das, J. Chem. Soc. Dalton Trans. 1972, \\
\hline Variables: & 386. \\
$T / \mathrm{K}=298.15$ & Prepared by: \\
\hline
\end{tabular}

Experimental Values

\begin{tabular}{lc}
\hline \hline$x_{2}{ }^{\mathrm{a}}$ & $x_{1}{ }^{\mathrm{b}}$ \\
\hline 0.8307 & 0.1693 \\
\hline${ }^{\mathrm{a}} x_{2}:$ mole fraction of component 2 in the saturated solution. & \\
${ }^{\mathrm{b}} x_{1}:$ mole fraction solubility of the solute. &
\end{tabular}

\section{Auxiliary Information}

\section{Method/Apparatus/Procedure:}

Very little experimental details are given in paper. Authors state that a saturated solution can be obtained by mild shaking for approximately $24 \mathrm{~h}$. Concentrations determined by titration with standard aqueous alkali. 
Source and Purity of Chemicals:

(1) G.R. grade, Merck Chemical Company, was used as received.

(2) Purity not given, Chemical source not given, no purification details were provided.

\section{Estimated Error:}

Temperature: $\pm 0.2 \mathrm{~K}$ (estimated by compiler). $x_{1}: \pm 3 \%$ (relative error, estimated by compiler).

\begin{tabular}{|c|c|}
\hline $\begin{array}{l}\text { Components: } \\
\text { (1) Benzoic acid; } \mathrm{C}_{7} \mathrm{H}_{6} \mathrm{O}_{2} \text {; } \\
\text { [65-85-0] } \\
\text { (2) Methanol; } \mathrm{CH}_{4} \mathrm{O} ;[67-56-1]\end{array}$ & $\begin{array}{l}\text { Original Measurements: } \\
{ }^{64} \text { E. A. Gomaa, Phys. Chem. Liq. 50, } \\
279 \text { (2012). }\end{array}$ \\
\hline $\begin{array}{l}\text { Variables: } \\
T / \mathrm{K}=298.15\end{array}$ & $\begin{array}{l}\text { Prepared by: } \\
\text { W. E. Acree, Jr. }\end{array}$ \\
\hline
\end{tabular}

\section{Experimental Values}

\begin{tabular}{lc}
\hline \hline$x_{2}{ }^{\mathrm{a}}$ & $x_{1}{ }^{\mathrm{b}}$ \\
\hline 0.9229 & 0.0771
\end{tabular}

${ }^{\mathrm{a}} x_{2}$ : mole fraction of component 2 in the saturated solution.

${ }^{\mathrm{b}} x_{1}$ : mole fraction solubility of the solute, computed by the compiler. The

original solubility data were given in units of moles per kilogram of solvent.

\section{Auxiliary Information}

\section{Method/Apparatus/Procedure:}

Constant-temperature shaker bath and analytical balance.

Excess solute and solvent were placed in closed test tubes and allowed to equilibrate in a constant-temperature shaker bath for several days. The solubility was determined gravimetrically by transferring $1 \mathrm{ml}$ of the saturated solution to an aluminum disk. The solvent was removed by heating with an infrared lamp. The solubility was calculated from the mass of the solid residue and amount of saturated solution analyzed.

\section{Source and Purity of Chemicals:}

(1) Purity not given, Merck Chemicals, Germany, no information provided concerning purification.

(2) Purity not given, Merck Chemicals, Germany, no information provided concerning purification.

\section{Estimated Error:}

Temperature: $\pm 0.1 \mathrm{~K}$ (estimated by compiler). $x_{1}: \pm 3 \%$ (relative error, estimated by compiler).

\begin{tabular}{ll}
\hline \hline Components: & Original Measurements: \\
$\begin{array}{l}\text { (1) Benzoic acid; } \mathrm{C}_{7} \mathrm{H}_{6} \mathrm{O}_{2} ; \\
\text { 65-85-0] }\end{array}$ & $\begin{array}{l}{ }^{9} \mathrm{C} . \mathrm{M} . \text { McLoughlin, W. A. M. } \\
\text { McMinn, and T. R. A. Magee, Powder }\end{array}$ \\
(2) Methanol; $\mathrm{CH}_{4} \mathrm{O} ;[67-56-1]$ & Technol. 134, 40 (2003). \\
\hline Variables: & Prepared by: \\
$T / \mathrm{K}=293$ and 333 & W. E. Acree, Jr. \\
\hline
\end{tabular}

\section{Experimental Values}

The measured solubility was reported to be 650 and $1200 \mathrm{~g} / \mathrm{l}$ at 293 and $333 \mathrm{~K}$, respectively. The authors did not specify whether the values were per liter of solvent or per liter of saturated solution.

\section{Auxiliary Information}

\section{Method/Apparatus/Procedure:}

Very little experimental details were provided. Solubility was determined by adding $0.50 \pm 0.1 \mathrm{~g}$ amounts of the powdered solute to $100 \pm 1 \mathrm{ml}$ of solvent until a saturated solution was obtained. The mass of the powder was recorded.

Source and Purity of Chemicals:

(1) Purity not given, Sigma-Aldrich, Poole, England, UK, no purification details were provided.

(2) Purity not given, Sigma-Aldrich, no purification details were provided.

\section{Estimated Error:}

Temperature: Insufficient experimental details to estimate.

Solubility: $\pm 5 \mathrm{~g} / \mathrm{l}$ (estimated by compiler assuming solute added in increments of $0.50 \mathrm{~g}$ ).

\begin{tabular}{|c|c|}
\hline $\begin{array}{l}\text { Components: } \\
\text { (1) Benzoic acid; } \mathrm{C}_{7} \mathrm{H}_{6} \mathrm{O}_{2} \text {; } \\
\text { [65-85-0] } \\
\text { (2) Methanol; } \mathrm{CH}_{4} \mathrm{O} ;[67-56-1]\end{array}$ & $\begin{array}{l}\text { Original Measurements: } \\
\text { 50 P. G. Desai and A. M. Patel, J. Indian } \\
\text { Chem. Soc. 12, } 131 \text { (1935). }\end{array}$ \\
\hline $\begin{array}{l}\text { Variables: } \\
T / \mathrm{K}=301.2\end{array}$ & $\begin{array}{l}\text { Prepared by: } \\
\text { W. E. Acree, Jr. }\end{array}$ \\
\hline
\end{tabular}

\section{Experimental Values}

\begin{tabular}{lc}
\hline \hline$x_{2}{ }^{\mathrm{a}}$ & $x_{1}{ }^{\mathrm{b}}$ \\
\hline 0.8311 & 0.1689 \\
\hline${ }^{\mathrm{a}} x_{2}:$ mole fraction of component 2 in the saturated solution. & \\
${ }^{\mathrm{b}} x_{1}:$ mole fraction solubility of the solute. &
\end{tabular}

\section{Auxiliary Information}

\section{Method/Apparatus/Procedure:}

Excess solute and solvent were placed in a glass flask which was kept revolving on a wheel in an air thermostat for two days. After two days of equilibration, the solution was filtered. To minimize absorption of the solute onto the filter paper, a $100 \mathrm{ml}$ portion of fresh saturated solution was first filtered through the paper before filtering the sample to be analyzed. An aliquot of the filtrate was titrated with barium hydroxide using phenolphthalein as the endpoint indictor. The barium hydroxide titrant was previously standardized against a solution of succinic acid.

\section{Source and Purity of Chemicals:}

(1) Purity not given, Chemical source not specified, no information provided concerning purification.

(2) Purity not given, Chemical source not specified, no information provided concerning purification.

\section{Estimated Error:}

Temperature: No information given.

$x_{1}: \pm 3 \%$ (relative error, estimated by compiler).

\begin{tabular}{|c|c|}
\hline $\begin{array}{l}\text { Components: } \\
\text { (1) Benzoic acid; } \mathrm{C}_{7} \mathrm{H}_{6} \mathrm{O}_{2} \text {; } \\
\text { [65-85-0] } \\
\text { (2) Methanol; } \mathrm{CH}_{4} \mathrm{O} ;[67-56-1]\end{array}$ & $\begin{array}{l}\text { Original Measurements: } \\
\text { 70 J. M. Jessy, J. Indian Chem. Soc. 75, } \\
352 \text { (1998). }\end{array}$ \\
\hline $\begin{array}{l}\text { Variables: } \\
T / \mathrm{K}=308.15\end{array}$ & $\begin{array}{l}\text { Prepared by: } \\
\text { W. E. Acree, Jr. }\end{array}$ \\
\hline
\end{tabular}




\section{Experimental Values}

The measured molar solubility was $c_{1}=0.2223 \mathrm{~mol} \mathrm{dm}^{-3}$.

\section{Auxiliary Information}

\section{Method/Apparatus/Procedure:}

Very little experimental details were provided. A convenient amount of benzoic acid in the form of fine powder was placed in a conical flask. Alcohol was added such that the volume was approximately half of that required to make the saturated solution. The flask was tightly stoppered and thermostated at $308 \mathrm{~K}$ for $15 \mathrm{~min}$. Then, keeping the flask still partially immersed in the temperature bath, more alcohol was added dropwise with vigorous shaking by rotary motion until the last speck of solid dissolved. Repeated trials showed that the endpoint was quite reproducible. The experiment was performed a minimum of three times.

\section{Source and Purity of Chemicals:}

(1) Purity not given, Chemical source not given, purification method was referenced to a published method [A. I. Vogel, A Text Book of Practical Organic Chemistry Including Qualitative Organic Analysis (Longmans, London, 1968)].

(2) Purity not given, Chemical source not given, purification method was referenced to a published method [A. I. Vogel, A Text Book of Practical Organic Chemistry Including Qualitative Organic Analysis (Longmans, London, 1968)].

\section{Estimated Error:}

Temperature: \pm 0.2 (estimated by compiler). $c_{1}: \pm 4 \%$ (relative error, estimated by compiler).

\begin{tabular}{|c|c|}
\hline $\begin{array}{l}\text { Components: } \\
\text { (1) Benzoic acid; } \mathrm{C}_{7} \mathrm{H}_{6} \mathrm{O}_{2} \text {; } \\
\text { [65-85-0] } \\
\text { (2) Ethanol; } \mathrm{C}_{2} \mathrm{H}_{6} \mathrm{O} ;[64-17-5]\end{array}$ & $\begin{array}{l}\text { Original Measurements: } \\
{ }^{64} \text { E. A. Gomaa, Phys. Chem. Liq. 50, } \\
279 \text { (2012). }\end{array}$ \\
\hline $\begin{array}{l}\text { Variables: } \\
T / \mathrm{K}=298.15\end{array}$ & $\begin{array}{l}\text { Prepared by: } \\
\text { W. E. Acree, Jr. }\end{array}$ \\
\hline
\end{tabular}

Experimental Values

\begin{tabular}{lc}
\hline \hline$x_{2}{ }^{\mathrm{a}}$ & $x_{1}{ }^{\mathrm{b}}$ \\
\hline 0.8736 & 0.1264 \\
${ }^{\mathrm{a}} x_{2}:$ mole fraction of component 2 in the saturated solution. \\
${ }^{\mathrm{b}} x_{1}:$ mole fraction solubility of the solute, computed by the compiler. The \\
original solubility data were given in units of moles per kilogram of solvent.
\end{tabular}

\section{Auxiliary Information}

\section{Method/Apparatus/Procedure:}

Constant-temperature shaker bath and analytical balance.

Excess solute and solvent were placed in closed test tubes and allowed to equilibrate in a constant-temperature shaker bath for several days. The solubility was determined gravimetrically by transferring $1 \mathrm{ml}$ of the saturated solution to an aluminum disk. The solvent was removed by heating with an infrared lamp. The solubility was calculated from the mass of the solid residue and amount of saturated solution analyzed.

\section{Source and Purity of Chemicals:}

(1) Purity not given, Merck Chemicals, Germany, no information provided concerning purification.

(2) Purity not given, Merck Chemicals, Germany, no information provided concerning purification.
Estimated Error:

Temperature: $\pm 0.1 \mathrm{~K}$ (estimated by compiler).

$x_{1}: \pm 3 \%$ (relative error, estimated by compiler).

\begin{tabular}{ll}
\hline \hline Components: & Original Measurements: \\
$\begin{array}{l}\text { (1) Benzoic acid; } \mathrm{C}_{7} \mathrm{H}_{6} \mathrm{O}_{2} ; \\
{[65-85-0]}\end{array}$ & $\begin{array}{l}{ }^{47} \text { A. Beerbower, P. L. Wu, and A. } \\
\text { (2) Ethanol; } \mathrm{C}_{2} \mathrm{H}_{6} \mathrm{O} ;[64-17-5]\end{array}$ \\
\hline Variables: & Prepared by: \\
$T / \mathrm{K}=298.15$ & W. E. Acree, Jr. \\
\hline
\end{tabular}

Experimental Values

\begin{tabular}{lc}
\hline \hline$x_{2}{ }^{\mathrm{a}}$ & $x_{1}{ }^{\mathrm{b}}$ \\
\hline 0.8211 & 0.1789
\end{tabular}

${ }^{\mathrm{a}} x_{2}$ : mole fraction of component 2 in the saturated solution.

${ }^{\mathrm{b}} x_{1}$ : mole fraction solubility of the solute.

\section{Auxiliary Information}

\section{Method/Apparatus/Procedure:}

Constant-temperature bath and ultraviolet/visible spectrophotometer.

Excess solute and solvent were placed in screw-capped vials. The vials were sealed and submerged in a constant-temperature water bath and shaken at 100 cycles/min for $24 \mathrm{~h}$. After the $24 \mathrm{~h}$ equilibration period, the vial was removed, wiped dry, and the contents analyzed. The solutions were transferred to a syringe and filtered through a $0.1 \mu \mathrm{m}$ pore size filter. The solutions were diluted and the absorbances recorded at the maximum absorption wavelength of benzoic acid. The solubility was determined at least six times.

\section{Source and Purity of Chemicals:}

(1) Purity not given, Chemical source not specified, no information provided concerning purification.

(2) Spectrophotometric or ACS Reagent grade, Chemical source not specified, redistilled before use.

\section{Estimated Error:}

Temperature: $\pm 0.2 \mathrm{~K}$.

$x_{1}: \pm 3 \%$ (relative error).

\begin{tabular}{|c|c|}
\hline $\begin{array}{l}\text { Components: } \\
\text { (1) Benzoic acid; } \mathrm{C}_{7} \mathrm{H}_{6} \mathrm{O}_{2} \text {; } \\
\text { [65-85-0] } \\
\text { (2) Ethanol; } \mathrm{C}_{2} \mathrm{H}_{6} \mathrm{O} ;[64-17-5]\end{array}$ & $\begin{array}{l}\text { Original Measurements: } \\
{ }^{71} \text { A. Seidell, Trans. Am. Electrochem. } \\
\text { Soc. 13, } 319 \text { (1908). }\end{array}$ \\
\hline $\begin{array}{l}\text { Variables: } \\
T / \mathrm{K}=298.15\end{array}$ & $\begin{array}{l}\text { Prepared by: } \\
\text { W. E. Acree, Jr. }\end{array}$ \\
\hline
\end{tabular}

Experimental Values

\begin{tabular}{lc}
\hline \hline$x_{2}{ }^{\mathrm{a}}$ & $x_{1}{ }^{\mathrm{b}}$ \\
\hline 0.8192 & 0.1808 \\
\hline${ }^{\mathrm{a}} x_{2}:$ mole fraction of component 2 in the saturated solution. & \\
${ }^{\mathrm{b}} x_{1}:$ mole fraction solubility of the solute. &
\end{tabular}




\section{Auxiliary Information}

\section{Method/Apparatus/Procedure:}

Excess solute and solvent were placed in a test tube and sealed with a rubber stopper. The test tube was attached to a rotating frame immersed in a constanttemperature water bath and revolved upon the axis parallel to the diameter of the test tube at the rate of approximately six revolutions per minute. The duration of the rotation was between four and seven days. At the end of the rotation period the test tube was placed in an upright position in the bath with the stopper and a very small part of the test tube protruding from the bath, until the undissolved solid settled to the bottom of the test tube. A portion of the clear supernatant solution was withdrawn into a pipette having a cotton plug attached. The cotton plug served as a coarse filter. The concentration of the solution was determined by titration with standard alkali solution. The author determined the solubility of benzoic acid in several aqueous-alcohol solvent mixtures having ethanol concentrations between $0.00 \%$ and $99.8 \%$ by mass The value for $100 \%$ ethanol was obtained from the curve of solubility versus alcohol concentration.

\section{Source and Purity of Chemicals:}

(1) Purity not given, Chemical source not specified, no information provided concerning purification.

(2) Purity not given, Chemical source not specified, no information provided concerning purification.

\section{Estimated Error:}

Temperature: $\pm 0.2 \mathrm{~K}$ (estimated by compiler). $x_{1}: \pm 3 \%$ (relative error, estimated by compiler).

\begin{tabular}{ll}
\hline \hline Components: & Original Measurements: \\
$\begin{array}{l}\text { (1) Benzoic acid; } \mathrm{C}_{7} \mathrm{H}_{6} \mathrm{O}_{2} ; \\
\text { [65-85-0] }\end{array}$ & $\begin{array}{l}{ }^{48} \text { J. Thati, F. L. Nordström, and A. C. } \\
\text { Rasmuson, J. Chem. Eng. Data 55, }\end{array}$ \\
\hline 2) Ethanol; $\mathrm{C}_{2} \mathrm{H}_{6} \mathrm{O} ;[64-17-5]$ & $5124(2010)$. \\
\hline Variables: & Prepared by: \\
Temperature & W. E. Acree, Jr. \\
\hline
\end{tabular}

Experimental Values

\begin{tabular}{lcc}
\hline \hline$T / \mathrm{K}$ & $x_{2}{ }^{\mathrm{a}}$ & $x_{1}{ }^{\mathrm{b}}$ \\
\hline 278.15 & 0.872 & 0.128 \\
283.15 & 0.861 & 0.139 \\
293.15 & 0.634 & 0.166 \\
303.15 & 0.804 & 0.196 \\
313.15 & 0.766 & 0.234 \\
323.15 & 0.724 & 0.276 \\
\hline
\end{tabular}

${ }^{\mathrm{a}} x_{2}$ : mole fraction of component 2 in the saturated solution.

${ }^{\mathrm{b}} \mathrm{x}_{1}$ : mole fraction solubility of the solute.

\section{Auxiliary Information}

\section{Method/Apparatus/Procedure:}

Constant-temperature bath, magnetic stirrer, analytical balance, and ventilated laboratory hood.

Solubilities were determined by a gravimetric method. Excess solute and solvent were placed in sealed bottles and allowed to equilibrate in a constanttemperature bath for $2 \mathrm{~h}$ with stirring. The stirring was discontinued and the solid was allowed to settle to the bottom of the container. After $30 \mathrm{~min}$, an aliquot of the clear solution was removed by syringe, filtered through a $0.2 \mu \mathrm{m}$ PTFE filter, and transferred to a preweighed glass vial. The glass vial was then weighed, and the solvent was allowed to evaporate in a ventilated laboratory hood at ambient room temperature. Once the solvent had evaporated, the vial with solid residue was weighed until constant weight was obtained. The solubility was calculated from the mass of the solid residue and mass of sample analyzed.
Source and Purity of Chemicals:

(1) $99.7 \%$, Merck Chemical Company, Germany, used as received.

(2) $99+\%$, Solveco Chemicals, used as received.

\section{Estimated Error:}

Temperature: $\pm 0.01 \mathrm{~K}$.

$x_{1}: \pm 1 \%$ (relative error).

\begin{tabular}{|c|c|}
\hline $\begin{array}{l}\text { Components: } \\
\text { (1) Benzoic acid; } \mathrm{C}_{7} \mathrm{H}_{6} \mathrm{O}_{2} \text {; } \\
\text { [65-85-0] } \\
\text { (2) Ethanol; } \mathrm{C}_{2} \mathrm{H}_{6} \mathrm{O} ;[64-17-5]\end{array}$ & $\begin{array}{l}\text { Original Measurements: } \\
{ }^{72} \text { R. Thuaire, Bull. Soc. Chim. Fr. } 3815 \\
\text { (1971). }\end{array}$ \\
\hline $\begin{array}{l}\text { Variables: } \\
T / \mathrm{K}=298.15\end{array}$ & $\begin{array}{l}\text { Prepared by: } \\
\text { W. E. Acree, Jr. }\end{array}$ \\
\hline
\end{tabular}

\section{Experimental Values}

The measured solubility was reported to be $4.945 \mathrm{~mol} / \mathrm{kg}$ of solvent, which corresponds to a mole fraction solubility of $x_{1}=$ 0.1855 .

\section{Auxiliary Information}

\section{Method/Apparatus/Procedure:}

Very little experimental details were provided. Solubility was determined by a gravimetric method. The saturated solution was evaporated to dryness and the remaining solid residue was weighed. The solubility was calculated from the mass of the solid residue and mass of saturated solution analyzed.

\section{Source and Purity of Chemicals:}

(1) Purity not given, Chemical source not given, no purification details were provided.

(2) Purity not given, Chemical source not given, no purification details were provided.

\section{Estimated Error:}

Temperature: $\pm 0.05 \mathrm{~K}$

$x_{1}: \pm 4 \%$ (relative error, estimated by compiler).

\begin{tabular}{|c|c|}
\hline $\begin{array}{l}\text { Components: } \\
\text { (1) Benzoic acid; } \mathrm{C}_{7} \mathrm{H}_{6} \mathrm{O}_{2} \text {; } \\
\text { [65-85-0] } \\
\text { (2) Ethanol; } \mathrm{C}_{2} \mathrm{H}_{6} \mathrm{O} ;[64-17-5]\end{array}$ & $\begin{array}{l}\text { Original Measurements: } \\
{ }^{69} \text { C. M. McLoughlin, W. A. M. } \\
\text { McMinn, and T. R. A. Magee, Powder } \\
\text { Technol. 134, } 40 \text { (2003). }\end{array}$ \\
\hline $\begin{array}{l}\text { Variables: } \\
T / \mathrm{K}=293 \text { and } 333\end{array}$ & $\begin{array}{l}\text { Prepared by: } \\
\text { W. E. Acree, Jr. }\end{array}$ \\
\hline
\end{tabular}

\section{Experimental Values}

The measured solubility was reported to be 750 and $1600 \mathrm{~g} / \mathrm{l}$ at 293 and $333 \mathrm{~K}$, respectively. The authors did not specify whether the values were per liter of solvent or per liter of saturated solution. 


\section{Auxiliary Information}

\section{Method/Apparatus/Procedure:}

Very little experimental details were provided. Solubility was determined by adding $0.50 \pm 0.1 \mathrm{~g}$ amounts of the powdered solute to $100 \pm 1 \mathrm{ml}$ of solvent until a saturated solution was obtained. The mass of the powder was recorded.

\section{Source and Purity of Chemicals:}

(1) Purity not given, Sigma-Aldrich, Poole, England, UK, no purification details were provided.

(2) Purity not given, Sigma-Aldrich, no purification details were provided.

\section{Estimated Error:}

Temperature: Insufficient experimental details to estimate.

Solubility: $\pm 5 \mathrm{~g} / \mathrm{l}$ (estimated by compiler assuming solute added in increments of $0.50 \mathrm{~g}$ ).

\begin{tabular}{|c|c|}
\hline $\begin{array}{l}\text { Components: } \\
\text { (1) Benzoic acid; } \mathrm{C}_{7} \mathrm{H}_{6} \mathrm{O}_{2} \text {; } \\
\text { [65-85-0] } \\
\text { (2) Ethanol; } \mathrm{C}_{2} \mathrm{H}_{6} \mathrm{O} ;[64-17-5]\end{array}$ & $\begin{array}{l}\text { Original Measurements: } \\
{ }^{73} \text { M. Dias, S. L. Raghavan, and J. } \\
\text { Hadgraft, Int. J. Pharm. 216, } 51 \text { (2001) }\end{array}$ \\
\hline $\begin{array}{l}\text { Variables: } \\
T / \mathrm{K}=305.2\end{array}$ & $\begin{array}{l}\text { Prepared by: } \\
\text { W. E. Acree, Jr. }\end{array}$ \\
\hline
\end{tabular}

\section{Experimental Values}

The measured solubility was reported to be $340.1 \mathrm{mg} / \mathrm{ml}$, which corresponds to a molar solubility of $c_{1}=2.785$ mol dm ${ }^{-3}$.

\section{Auxiliary Information}

\section{Method/Apparatus/Procedure:}

Constant-temperature bath, magnetic stirrer, centrifuge, and an ultraviolet/ visible spectrophotometer.

Excess solute and solvent were equilibrated in a constant-temperature bath with stirring for $48 \mathrm{~h}$. The saturated solution was then centrifuged for $10 \mathrm{~min}$ and an aliquot of the supernatant solution was removed and diluted quantitatively for spectrophotometric analysis. The concentration of the dissolved solute was determined from the measured absorbance.

\section{Source and Purity of Chemicals:}

(1) Purity not given, Fisons Scientific Equipment, UK, no purification details were provided.

(2) Purity not given, Fisher Scientific, UK, no purification details were provided.

\section{Estimated Error:}

Temperature: Insufficient experimental details to estimate. $c_{1}: \pm 0.13$

\begin{tabular}{|c|c|}
\hline $\begin{array}{l}\text { Components: } \\
\text { (1) Benzoic acid; } \mathrm{C}_{7} \mathrm{H}_{6} \mathrm{O}_{2} \text {; } \\
\text { [65-85-0] } \\
\text { (2) Ethanol; } \mathrm{C}_{2} \mathrm{H}_{6} \mathrm{O} ;[64-17-5]\end{array}$ & $\begin{array}{l}\text { Original Measurements: } \\
{ }^{50} \text { P. G. Desai and A. M. Patel, J. Indian } \\
\text { Chem. Soc. 12, } 131 \text { (1935). }\end{array}$ \\
\hline Variables: & Prepared by: \\
\hline$T / \mathrm{K}=301.2$ & W. E. Acree, Jr. \\
\hline
\end{tabular}

Experimental Values

\begin{tabular}{lc}
\hline \hline$x_{2}{ }^{\mathrm{a}}$ & $x_{1}{ }^{\mathrm{b}}$ \\
\hline 0.8118 & 0.1882 \\
\hline${ }^{\mathrm{a}} x_{2}:$ mole fraction of component 2 in the saturated solution. & \\
${ }^{\mathrm{b}}{ }_{x_{1}}:$ mole fraction solubility of the solute. &
\end{tabular}

\section{Auxiliary Information}

\section{Method/Apparatus/Procedure:}

Excess solute and solvent were placed in a glass flask which was kept revolving on a wheel in an air thermostat for two days. After two days of equilibration, the solution was filtered. To minimize absorption of the solute onto the filter paper, a $100 \mathrm{ml}$ portion of fresh saturated solution was first filtered through the paper before filtering the sample to be analyzed. An aliquot of the filtrate was titrated with barium hydroxide using phenolphthalein as the endpoint indictor. The barium hydroxide titrant was previously standardized against a solution of succinic acid.

\section{Source and Purity of Chemicals:}

(1) Purity not given, Chemical source not specified, no information provided concerning purification.

(2) Purity not given, Chemical source not specified, no information provided concerning purification.

\section{Estimated Error:}

Temperature: No information given.

$x_{1}: \pm 3 \%$ (relative error, estimated by compiler).

\begin{tabular}{|c|c|}
\hline $\begin{array}{l}\text { Components: } \\
\text { (1) Benzoic acid; } \mathrm{C}_{7} \mathrm{H}_{6} \mathrm{O}_{2} \text {; } \\
\text { [65-85-0] } \\
\text { (2) Ethanol; } \mathrm{C}_{2} \mathrm{H}_{6} \mathrm{O} ;[64-17-5]\end{array}$ & $\begin{array}{l}\text { Original Measurements: } \\
{ }^{74} \text { A. Pal and S. C. Lahiri, Indian J. } \\
\text { Chem. 28A, } 276 \text { (1989). }\end{array}$ \\
\hline $\begin{array}{l}\text { Variables: } \\
\text { Temperature }\end{array}$ & $\begin{array}{l}\text { Prepared by: } \\
\text { W. E. Acree, Jr. }\end{array}$ \\
\hline
\end{tabular}

Experimental Values

\begin{tabular}{lc}
\hline \hline$T / \mathrm{K}$ & $c_{1}{ }^{\mathrm{a}}$ \\
\hline 288 & 2.2439 \\
293 & 2.5042 \\
298 & 2.7481 \\
\hline${ }^{\mathrm{a}} c_{1}$ : mole fraction solubility of the solute.
\end{tabular}

Auxiliary Information

\section{Method/Apparatus/Procedure:}

A clear solution of benzoic acid, saturated at a slightly higher temperature (about $1 \mathrm{~K}$ higher than that of the experimental temperature) was placed in a Campbell solubility apparatus, which was then put in the experimental thermostat. The solution was allowed to equilibrate at constant temperature for approximately $4 \mathrm{~h}$. An aliquot of the solution was removed and filtered. The concentration of the solute in the saturated solution was determined by titration with standardized sodium hydroxide solution.

Source and Purity of Chemicals:

(1) G.R. grade, Merck Chemical Company, Germany, was used as received. (2) Purity not given, Chemical source not given, was purified by the standard procedure. The authors did not specify what was the standard procedure nor did was any literature method referenced. 
Estimated Error:

Temperature: $\pm 0.01 \mathrm{~K}$.

$c_{1}: \pm 1 \%$ (relative error).

\begin{tabular}{ll}
\hline \hline Components: & Original Measurements: \\
$\begin{array}{l}\text { (1) Benzoic acid; } \mathrm{C}_{7} \mathrm{H}_{6} \mathrm{O}_{2} ; \\
\text { [65-85-0] }\end{array}$ & $\begin{array}{l}{ }^{48} \text { J. Thati, F. L. Nordström, and A. C. } \\
\text { (2) Ethanol; } \mathrm{C}_{2} \mathrm{H}_{6} \mathrm{O} ;[64-17-5]\end{array}$ \\
\hline $\begin{array}{l}\text { Rasmuson, J. Chem. Eng. Data 55, } \\
\text { Temiables: }\end{array}$ & 5124 (2010). \\
\hline & Prepared by: \\
\hline
\end{tabular}

Experimental Values

\begin{tabular}{lcc}
\hline \hline$T / \mathrm{K}$ & $x_{2}{ }^{\mathrm{a}}$ & $x_{1}{ }^{\mathrm{b}}$ \\
\hline 278.15 & 0.872 & 0.128 \\
283.15 & 0.861 & 0.139 \\
293.15 & 0.834 & 0.166 \\
303.15 & 0.804 & 0.196 \\
313.15 & 0.766 & 0.234 \\
323.15 & 0.724 & 0.276
\end{tabular}

${ }^{\mathrm{a}} x_{2}$ : mole fraction of component 2 in the saturated solution.

${ }^{\mathrm{b}} x_{1}$ : mole fraction solubility of the solute.

\section{Auxiliary Information}

\section{Method/Apparatus/Procedure:}

Constant-temperature bath, magnetic stirrer, analytical balance, and ventilated laboratory hood.

Solubilities were determined by a gravimetric method. Excess solute and solvent were placed in sealed bottles and allowed to equilibrate in a constanttemperature bath for $2 \mathrm{~h}$ with stirring. The stirring was discontinued and the solid was allowed to settle to the bottom of the container. After $30 \mathrm{~min}$, an aliquot of the clear solution was removed by syringe, filtered through a $0.2 \mu \mathrm{m}$ PTFE filter, and transferred to a preweighed glass vial. The glass vial was then weighed, and the solvent was allowed to evaporate in a ventilated laboratory hood at ambient room temperature. Once the solvent had evaporated, the vial with solid residue was weighed until constant weight was obtained. The solubility was calculated from the mass of the solid residue and mass of sample analyzed.

Source and Purity of Chemicals:

(1) $99.7 \%$, Merck Chemical Company, Germany, was used as received.

(2) $99+\%$, Solveco Chemicals, used as received.

Estimated Error:

Temperature: $\pm 0.01 \mathrm{~K}$.

$x_{1}: \pm 1 \%$ (relative error)

\begin{tabular}{|c|c|}
\hline $\begin{array}{l}\text { Components: } \\
\text { (1) Benzoic acid; } \mathrm{C}_{7} \mathrm{H}_{6} \mathrm{O}_{2} \text {; } \\
\text { [65-85-0] } \\
\text { (2) Ethanol; } \mathrm{C}_{2} \mathrm{H}_{6} \mathrm{O} ;[64-17-5]\end{array}$ & $\begin{array}{l}\text { Original Measurements: } \\
{ }^{70} \text { J. M. Jessy, J. Indian Chem. Soc. 75, } \\
352 \text { (1998). }\end{array}$ \\
\hline $\begin{array}{l}\text { Variables: } \\
T / \mathrm{K}=308.15\end{array}$ & $\begin{array}{l}\text { Prepared by: } \\
\text { W. E. Acree, Jr. }\end{array}$ \\
\hline
\end{tabular}

\author{
Experimental Values \\ The measured molar solubility was $c_{1}=0.2566 \mathrm{~mol} \mathrm{dm}^{-3}$.

\section{Auxiliary Information}

\section{Method/Apparatus/Procedure:}

Very little experimental details were provided. A convenient amount of benzoic acid in the form of fine powder was placed in a conical flask. Alcohol was added such that the volume was approximately half of that required to make the saturated solution. The flask was tightly stoppered and thermostated at $308 \mathrm{~K}$ for $15 \mathrm{~min}$. Then, keeping the flask still partially immersed in the temperature bath, more alcohol was added dropwise with vigorous shaking by rotary motion until the last speck of solid dissolved. Repeated trials showed that the endpoint was quite reproducible. The experiment was performed a minimum of three times.

Source and Purity of Chemicals:

(1) Purity not given, Chemical source not given, purification method was referenced to a published method [A. I. Vogel, A Text Book of Practical Organic Chemistry Including Qualitative Organic Analysis (Longmans, London, 1968)].

(2) Purity not given, Chemical source not given, purification method was referenced to a published method [A. I. Vogel, A Text Book of Practical Organic Chemistry Including Qualitative Organic Analysis (Longmans, London, 1968)].

Estimated Error:

Temperature: \pm 0.2 (estimated by compiler).

$x_{1}: \pm 4 \%$ (relative error, estimated by compiler).

\begin{tabular}{|c|c|}
\hline $\begin{array}{l}\text { Components: } \\
\text { (1) Benzoic acid; } \mathrm{C}_{7} \mathrm{H}_{6} \mathrm{O}_{2} \text {; } \\
\text { [65-85-0] } \\
\text { (2) 1-Propanol; } \mathrm{C}_{3} \mathrm{H}_{8} \mathrm{O} ;[71-23-8]\end{array}$ & $\begin{array}{l}\text { Original Measurements: } \\
{ }^{47} \text { A. Beerbower, P. L. Wu, and A. } \\
\text { Martin, J. Pharm. Sci. 73, } 179 \text { (1984). }\end{array}$ \\
\hline $\begin{array}{l}\text { Variables: } \\
T / \mathrm{K}=298.15\end{array}$ & $\begin{array}{l}\text { Prepared by: } \\
\text { W. E. Acree, Jr. }\end{array}$ \\
\hline
\end{tabular}

Experimental Values

\begin{tabular}{lc}
\hline \hline$x_{2}{ }^{\mathrm{a}}$ & $\mathrm{x}_{1}{ }^{\mathrm{b}}$ \\
\hline 0.8209 & 0.1791 \\
\hline${ }^{\mathrm{a}} x_{2}:$ mole fraction of component 2 in the saturated solution. & \\
${ }^{\mathrm{b}} x_{1}:$ mole fraction solubility of the solute. &
\end{tabular}

Auxiliary Information

\section{Method/Apparatus/Procedure:}

Constant-temperature bath and ultraviolet/visible spectrophotometer.

Excess solute and solvent were placed in screw-capped vials. The vials were sealed and submerged in a constant-temperature water bath and shaken at 100 cycles/min for $24 \mathrm{~h}$. After the $24 \mathrm{~h}$ equilibration period, the vial was removed, wiped dry, and the contents analyzed. The solutions were transferred to a syringe and filtered through a $0.1 \mu \mathrm{m}$ pore size filter. The solutions were diluted and the absorbances recorded at the maximum absorption wavelength of benzoic acid. The solubility was determined at least six times.

\section{Source and Purity of Chemicals:}

(1) Purity not given, Chemical source not specified, no information provided concerning purification.

(2) Spectrophotometric or ACS Reagent grade, Chemical source not specified, redistilled before use. 
Estimated Error:

Temperature: $\pm 0.2 \mathrm{~K}$.

$x_{1}: \pm 3 \%$ (relative error).

\begin{tabular}{|c|c|}
\hline $\begin{array}{l}\text { Components: } \\
\text { (1) Benzoic acid; } \mathrm{C}_{7} \mathrm{H}_{6} \mathrm{O}_{2} \text {; } \\
\text { [65-85-0] } \\
\text { (2) 1-Propanol; } \mathrm{C}_{3} \mathrm{H}_{8} \mathrm{O} ;[71-23-8]\end{array}$ & $\begin{array}{l}\text { Original Measurements: } \\
{ }^{51} \text { H.-M. Lin and R. A. Nash, J. Pharm. } \\
\text { Sci. 82, } 1018 \text { (1993). }\end{array}$ \\
\hline $\begin{array}{l}\text { Variables: } \\
T / \mathrm{K}=298.15\end{array}$ & $\begin{array}{l}\text { Prepared by: } \\
\text { W. E. Acree, Jr. }\end{array}$ \\
\hline
\end{tabular}

Experimental Values

\begin{tabular}{lc}
\hline \hline$x_{2}^{\mathrm{a}}$ & $x_{1}{ }^{\mathrm{b}}$ \\
\hline 0.8257 & 0.1743
\end{tabular}

${ }^{\mathrm{a}} x_{2}$ : mole fraction of component 2 in the saturated solution.

${ }^{b_{1}}$ : mole fraction solubility of the solute.

\section{Auxiliary Information}

\section{Method/Apparatus/Procedure:}

Constant-temperature bath and high-performance liquid chromatograph.

Excess solute and solvent were placed in screw-capped vials. The vials were sealed with several turns of electrical tape, warmed to about $323 \mathrm{~K}$, and then shaken in a constant-temperature bath for at least $24 \mathrm{~h}$ at $298 \mathrm{~K}$. After equilibrium was reached, the sample was filtered through a 0.45 or $1.0 \mu \mathrm{m}$ filter, and an aliquot was diluted appropriately for chromatographic analysis. Concentrations were determined by a high-performance liquid chromatographic method.

Source and Purity of Chemicals:

(1) Purity not given, Amend Drug and Chemical Company, no information provided concerning purification.

(2) HPLC grade, Aldrich Chemical Company, Milwaukee, WI, USA, no purification details were provided.

\section{Estimated Error:}

Temperature: $\pm 0.2 \mathrm{~K}$ (estimated by compiler). $x_{1}: \pm 3 \%$ (relative error, estimated by compiler).

\begin{tabular}{|c|c|}
\hline $\begin{array}{l}\text { Components: } \\
\text { (1) Benzoic acid; } \mathrm{C}_{7} \mathrm{H}_{6} \mathrm{O}_{2} \text {; } \\
\text { [65-85-0] } \\
\text { (2) 1-Propanol; } \mathrm{C}_{3} \mathrm{H}_{8} \mathrm{O} ;[71-23-8]\end{array}$ & $\begin{array}{l}\text { Original Measurements: } \\
\text { }{ }^{75} \text { F. A. Restaino and A. N. Martin, J. } \\
\text { Pharm. Sci. 53, } 636 \text { (1964). }\end{array}$ \\
\hline $\begin{array}{l}\text { Variables: } \\
T / \mathrm{K}=298.15\end{array}$ & $\begin{array}{l}\text { Prepared by: } \\
\text { W. E. Acree, Jr. }\end{array}$ \\
\hline
\end{tabular}

Experimental Values

\begin{tabular}{lc}
\hline \hline$x_{2}{ }^{\mathrm{a}}$ & $x_{1}{ }^{\mathrm{b}}$ \\
\hline 0.813 & 0.187
\end{tabular}

${ }^{\mathrm{a}} x_{2}$ : mole fraction of component 2 in the saturated solution.

${ }^{\mathrm{b}} x_{1}$ : mole fraction solubility of the solute.

\section{Auxiliary Information}

\section{Method/Apparatus/Procedure:}

Constant-temperature bath, shaker, analytical balance, constant-temperature bath, and ultraviolet/visible spectrophotometer.

Excess solute and solvent were placed in screw-capped vials and allowed to equilibrate in a constant-temperature bath with shaking for $48 \mathrm{~h}$. An aliquot of the equilibrated sample was removed and filtered. Concentration of the dissolved solute was determined gravimetrically and/or spectrophotometrically. The authors did not specify which of the two methods was used for the different solutes studied.

Source and Purity of Chemicals:

(1) Certified Reagent grade, Fisher Scientific, USA, was recrsytallized from trichloromethane.

(2) Certified Reagent grade, Fisher Scientific, was used as received.

Estimated Error:

Temperature: $\pm 0.05 \mathrm{~K}$

$x_{1}: \pm 3 \%$ (relative error, estimated by compiler)

\begin{tabular}{|c|c|}
\hline $\begin{array}{l}\text { Components: } \\
\text { (1) Benzoic acid; } \mathrm{C}_{7} \mathrm{H}_{6} \mathrm{O}_{2} \text {; } \\
\text { [65-85-0] } \\
\text { (2) 1-Propanol; } \mathrm{C}_{3} \mathrm{H}_{8} \mathrm{O} ;[71-23-8]\end{array}$ & $\begin{array}{l}\text { Original Measurements: } \\
{ }^{50} \text { P. G. Desai and A. M. Patel, J. Indian } \\
\text { Chem. Soc. 12, } 131 \text { (1935). }\end{array}$ \\
\hline $\begin{array}{l}\text { Variables: } \\
T / \mathrm{K}=301.2\end{array}$ & $\begin{array}{l}\text { Prepared by: } \\
\text { W. E. Acree, Jr. }\end{array}$ \\
\hline
\end{tabular}

\section{Experimental Values}

\begin{tabular}{lc}
\hline \hline$x_{2}{ }^{\mathrm{a}}$ & $x_{1}{ }^{\mathrm{b}}$ \\
\hline 0.8190 & 0.1810 \\
\hline${ }^{\mathrm{a}} x_{2}:$ mole fraction of component 2 in the saturated solution. & \\
${ }^{\mathrm{b}} x_{1}:$ mole fraction solubility of the solute. &
\end{tabular}

\section{Auxiliary Information}

\section{Method/Apparatus/Procedure:}

Excess solute and solvent were placed in a glass flask which was kept revolving on a wheel in an air thermostat for two days. After two days of equilibration, the solution was filtered. To minimize absorption of the solute onto the filter paper, a $100 \mathrm{ml}$ portion of fresh saturated solution was first filtered through the paper before filtering the sample to be analyzed. An aliquot of the filtrate was titrated with barium hydroxide using phenolphthalein as the endpoint indictor. The barium hydroxide titrant was previously standardized against a solution of succinic acid.

\section{Source and Purity of Chemicals:}

(1) Purity not given, Chemical source not specified, no information provided concerning purification.

(2) Purity not given, Chemical source not specified, no information provided concerning purification.

\section{Estimated Error:}

Temperature: No information given.

$x_{1}: \pm 3 \%$ (relative error, estimated by compiler) 


\section{Components:}

(1) Benzoic acid; $\mathrm{C}_{7} \mathrm{H}_{6} \mathrm{O}_{2}$; [65-85-0]

(2) 1-Propanol; $\mathrm{C}_{3} \mathrm{H}_{8} \mathrm{O}$;

[71-23-8]

\begin{tabular}{ll}
\hline Variables: & Prepared by: \\
$T / \mathrm{K}=308.15$ & W. E. Acree, Jr. \\
\hline
\end{tabular}

\section{Experimental Values}

The measured molar solubility was $c_{1}=0.2838 \mathrm{~mol} \mathrm{dm}^{-3}$.

\section{Auxiliary Information}

\section{Method/Apparatus/Procedure:}

Very little experimental details were provided. A convenient amount of benzoic acid in the form of fine powder was placed in a conical flask. Alcohol was added such that the volume was approximately half of that required to make the saturated solution. The flask was tightly stoppered and thermostated at $308 \mathrm{~K}$ for $15 \mathrm{~min}$. Then, keeping the flask still partially immersed in the temperature bath, more alcohol was added dropwise with vigorous shaking by rotary motion until the last speck of solid dissolved. Repeated trials showed that the endpoint was quite reproducible. The experiment was performed a minimum of three times.

\section{Source and Purity of Chemicals:}

(1) Purity not given, Chemical source not given, purification method was referenced to a published method [A. I. Vogel, A Text Book of Practical Organic Chemistry Including Qualitative Organic Analysis (Longmans, London, 1968)].

(2) Purity not given, Chemical Source not given, purification method was referenced to a published method [A. I. Vogel, A Text Book of Practical Organic Chemistry Including Qualitative Organic Analysis (Longmans, London, 1968)].

\section{Estimated Error:}

Temperature: \pm 0.2 (estimated by compiler).

$c_{1}: \pm 4 \%$ (relative error, estimated by compiler).

\begin{tabular}{|c|c|}
\hline $\begin{array}{l}\text { Components: } \\
\text { (1) Benzoic acid; } \mathrm{C}_{7} \mathrm{H}_{6} \mathrm{O}_{2} \text {; } \\
\text { [65-85-0] } \\
\text { (2) 2-Propanol; } \mathrm{C}_{3} \mathrm{H}_{8} \mathrm{O} \text {; } \\
\text { [67-63-0] }\end{array}$ & $\begin{array}{l}\text { Original Measurements: } \\
{ }^{47} \text { A. Beerbower, P. L. Wu, and A. } \\
\text { Martin, J. Pharm. Sci. 73, } 179 \text { (1984). }\end{array}$ \\
\hline $\begin{array}{l}\text { Variables: } \\
\mathrm{T} / \mathrm{K}=298.15\end{array}$ & $\begin{array}{l}\text { Prepared by: } \\
\text { W. E. Acree, Jr. }\end{array}$ \\
\hline
\end{tabular}

Experimental Values

\begin{tabular}{lc}
\hline \hline$x_{2}{ }^{\mathrm{a}}$ & $x_{1}{ }^{\mathrm{b}}$ \\
\hline 0.8063 & 0.1937 \\
\hline${ }^{\mathrm{a}} x_{2}:$ mole fraction of component 2 in the saturated solution. \\
${ }^{\mathrm{b}} x_{1}:$ mole fraction solubility of the solute.
\end{tabular}

\section{Auxiliary Information}

Method/Apparatus/Procedure:

Constant-temperature bath and ultraviolet/visible spectrophotometer.

Excess solute and solvent were placed in screw-capped vials. The vials were sealed and submerged in a constant-temperature water bath and shaken at 100 cycles/min for $24 \mathrm{~h}$. After the $24 \mathrm{~h}$ equilibration period, the vial was removed, wiped dry, and the contents analyzed. The solutions were transferred to a syringe and filtered through a $0.1 \mu \mathrm{m}$ pore size filter. The solutions were diluted and the absorbances recorded at the maximum absorption wavelength of benzoic acid. The solubility was determined at least six times.

Source and Purity of Chemicals:

(1) Purity not given, Chemical source not specified, no information provided concerning purification.

(2) Spectrophotometric or ACS Reagent grade, Chemical source not specified, redistilled before use.

Estimated Error:

Temperature: $\pm 0.2 \mathrm{~K}$.

$x_{1}: \pm 3 \%$ (relative error).

\begin{tabular}{ll}
\hline \hline Components: & Original Measurements: \\
$\begin{array}{l}\text { (1) Benzoic acid; } \mathrm{C}_{7} \mathrm{H}_{6} \mathrm{O}_{2} ; \\
\text { [65-85-0] }\end{array}$ & ${ }^{55} \mathrm{~B}$. Long, J. Li, R. Zhang, and L. Wan, \\
$\begin{array}{l}\text { (2) 2-Propanol; } \mathrm{C}_{3} \mathrm{H}_{8} \mathrm{O} ; \\
\text { [67-63-0] }\end{array}$ & \\
\hline Variables: & Prepared by: \\
Temperature & W. E. Acree, Jr. \\
\hline
\end{tabular}

Experimental Values

\begin{tabular}{lcc}
\hline \hline$T / \mathrm{K}$ & $x_{2}{ }^{\mathrm{a}}$ & $x_{1}{ }^{\mathrm{b}}$ \\
\hline 278.06 & 0.8714 & 0.1286 \\
282.96 & 0.8571 & 0.1429 \\
288.15 & 0.8405 & 0.1595 \\
293.11 & 0.8262 & 0.1738 \\
297.79 & 0.8078 & 0.1922 \\
303.45 & 0.7854 & 0.2146 \\
308.09 & 0.7679 & 0.2321 \\
312.80 & 0.7475 & 0.2525 \\
317.85 & 0.7220 & 0.2780 \\
322.78 & 0.7041 & 0.2959 \\
328.20 & 0.6749 & 0.3251 \\
333.20 & 0.6535 & 0.3465 \\
338.15 & 0.6265 & 0.3735 \\
343.10 & 0.5893 & 0.4107 \\
\hline
\end{tabular}

${ }^{a} x_{2}$ : mole fraction of component 2 in the saturated solution.

${ }^{b} x_{1}$ : mole fraction solubility of the solute. 


\section{Auxiliary Information}

\section{Method/Apparatus/Procedure:}

Double layer jacketed glass equilibrium cell, circulating water bath, analytical balance, and drying oven.

Excess solute and solvent and were placed in an equilibrium vessel, which was connected to a circulating water bath. The solution was allowed to equilibrate at constant temperature with stirring for $3 \mathrm{~h}$. The stirring was discontinued and the solution was allowed to stand for $1 \mathrm{~h}$ to allow the undissolved solid to settle to the bottom portion of the equilibrium vessel. An aliquot of the clear solution was transferred to a preweighed vial by a warm syringe. The vial was tightly closed and reweighed to determine the mass of the sample transferred. The vial was then put into a drying oven with the cap half-closed to permit complete evaporation of the solvent. The vial was covered with a piece of stainless steel filter cloth to prevent dust contamination. After the solvent had evaporated, the vial was removed from the drying oven and placed in a desiccator with silica gel for another $2 \mathrm{~h}$ to reach ambient room temperature. The vial with solid residue was weighed until constant weight was obtained. The solubility was calculated from the mass of the solid residue and mass of sample analyzed.

\section{Source and Purity of Chemicals:}

(1) $99.5 \%$, Analytical grade, Shantou Xilong Chemical Company, China, used as received.

(2) $99.5 \%$, Analytical grade, Beijing Chemical Reagent Company, China, used as received.

\section{Estimated Error:}

Temperature: $\pm 0.05 \mathrm{~K}$.

$x_{1}: \pm 1 \%$ (relative error).

\begin{tabular}{|c|c|}
\hline $\begin{array}{l}\text { Components: } \\
\text { (1) Benzoic acid; } \mathrm{C}_{7} \mathrm{H}_{6} \mathrm{O}_{2} \text {; } \\
\text { [65-85-0] } \\
\text { (2) 2-Propanol; } \mathrm{C}_{3} \mathrm{H}_{8} \mathrm{O} \text {; } \\
\text { [67-63-0] }\end{array}$ & $\begin{array}{l}\text { Original Measurements: } \\
{ }^{70} \text { J. M. Jessy, J. Indian Chem. Soc. 75, } \\
352 \text { (1998). }\end{array}$ \\
\hline $\begin{array}{l}\text { Variables: } \\
T / \mathrm{K}=308.15\end{array}$ & $\begin{array}{l}\text { Prepared by: } \\
\text { W. E. Acree, Jr. }\end{array}$ \\
\hline
\end{tabular}

Experimental Values
The measured molar solubility was $c_{1}=0.2873 \mathrm{~mol} \mathrm{dm}^{-3}$.

\section{Auxiliary Information}

\begin{abstract}
Method/Apparatus/Procedure:
Very little experimental details were provided. A convenient amount of benzoic acid in the form of fine powder was placed in a conical flask. Alcohol was added such that the volume was approximately half of that required to make the saturated solution. The flask was tightly stoppered and thermostated at $308 \mathrm{~K}$ for $15 \mathrm{~min}$. Then, keeping the flask still partially immersed in the temperature bath, more alcohol was added dropwise with vigorous shaking by rotary motion until the last speck of solid dissolved. Repeated trials showed that the endpoint was quite reproducible. The experiment was performed a minimum of three times.
\end{abstract}

\section{Source and Purity of Chemicals:}

(1) Purity not given, Chemical Source not given, purification method was referenced to a published method [A. I. Vogel, A Text Book of Practical Organic Chemistry Including Qualitative Organic Analysis (Longmans, London, 1968)].

(2) Purity not given, Chemical Source not given, purification method was referenced to a published method [A. I. Vogel, A Text Book of Practical Organic Chemistry Including Qualitative Organic Analysis (Longmans, London, 1968)].

\section{Estimated Error:}

Temperature: \pm 0.2 (estimated by compiler). $c_{1}: \pm 4 \%$ (relative error, estimated by compiler).

\begin{tabular}{|c|c|}
\hline $\begin{array}{l}\text { Components: } \\
\text { (1) Benzoic acid; } \mathrm{C}_{7} \mathrm{H}_{6} \mathrm{O}_{2} \text {; } \\
\text { [65-85-0] } \\
\text { (2) 1-Butanol; } \mathrm{C}_{4} \mathrm{H}_{10} \mathrm{O} \text {; } \\
\text { [71-36-3] }\end{array}$ & $\begin{array}{l}\text { Original Measurements: } \\
{ }^{47} \text { A. Beerbower, P. L. Wu, and A. } \\
\text { Martin, J. Pharm. Sci. 73, } 179 \text { (1984) }\end{array}$ \\
\hline $\begin{array}{l}\text { Variables: } \\
T / \mathrm{K}=298.15\end{array}$ & $\begin{array}{l}\text { Prepared by: } \\
\text { W. E. Acree, Jr. }\end{array}$ \\
\hline
\end{tabular}

Experimental Values

\begin{tabular}{lc}
\hline \hline$x_{2}{ }^{\mathrm{a}}$ & $x_{1}{ }^{\mathrm{b}}$ \\
\hline 0.7984 & 0.2016 \\
\hline${ }_{{ }^{\mathrm{a}} x_{2}: \text { mole fraction of component } 2 \text { in the saturated solution. }}$ & \\
${ }^{\mathrm{b}}{ }_{x_{1}}:$ mole fraction solubility of the solute. &
\end{tabular}

\section{Auxiliary Information}

\section{Method/Apparatus/Procedure:}

Constant-temperature bath and ultraviolet/visible spectrophotometer. Excess solute and solvent were placed in screw-capped vials. The vials were sealed and submerged in a constant-temperature water bath and shaken at 100 cycles/min for $24 \mathrm{~h}$. After the $24 \mathrm{~h}$ equilibration period, the vial was removed, wiped dry, and the contents analyzed. The solutions were transferred to a syringe and filtered through a $0.1 \mu \mathrm{m}$ pore size filter. The solutions were diluted and the absorbances recorded at the maximum absorption wavelength of benzoic acid. The solubility was determined at least six times.

\section{Source and Purity of Chemicals:}

(1) Purity not given, Chemical source not specified, no information provided concerning purification.

(2) Spectrophotometric or ACS Reagent grade, Chemical source not specified, redistilled before use.

\section{Estimated Error:}

Temperature: $\pm 0.2 \mathrm{~K}$.

$x_{1}: \pm 3 \%$ (relative error).

\begin{tabular}{|c|c|}
\hline $\begin{array}{l}\text { Components: } \\
\text { (1) Benzoic acid; } \mathrm{C}_{7} \mathrm{H}_{6} \mathrm{O}_{2} \text {; } \\
\text { [65-85-0] } \\
\text { (2) } 1-\mathrm{Butanol} ; \mathrm{C}_{4} \mathrm{H}_{10} \mathrm{O} \text {; } \\
\text { [71-36-3] }\end{array}$ & $\begin{array}{l}\text { Original Measurements: } \\
{ }^{75} \text { F. A. Restaino and A. N. Martin, J. } \\
\text { Pharm. Sci. 53, } 636 \text { (1964). }\end{array}$ \\
\hline $\begin{array}{l}\text { Variables: } \\
T / \mathrm{K}=298.15\end{array}$ & $\begin{array}{l}\text { Prepared by: } \\
\text { W. E. Acree, Jr. }\end{array}$ \\
\hline
\end{tabular}

\section{Experimental Values}

\begin{tabular}{lc}
\hline \hline$x_{2}{ }^{\mathrm{a}}$ & $x_{1}{ }^{\mathrm{b}}$ \\
\hline 0.797 & 0.203 \\
\hline${ }^{a} x_{2}:$ mole fraction of component 2 in the saturated solution. & \\
${ }^{\mathrm{b}} x_{1}:$ mole fraction solubility of the solute. &
\end{tabular}




\section{Auxiliary Information}

\section{Method/Apparatus/Procedure:}

Constant-temperature bath, shaker, analytical balance, constant-temperature bath, and ultraviolet/visible spectrophotometer.

Excess solute and solvent were placed in screw-capped vials and allowed to equilibrate in a constant-temperature bath with shaking for $48 \mathrm{~h}$. An aliquot of the equilibrated sample was removed and filtered. Concentration of the dissolved solute was determined gravimetrically and/or spectrophotometrically. The authors did not specify which of the two methods was used for the different solutes studied.

\section{Source and Purity of Chemicals:}

(1) Certified Reagent grade, Fisher Scientific, USA, was recrsytallized from trichloromethane.

(2) Certified Reagent grade, Fisher Scientific, was used as received.

Estimated Error:

Temperature: $\pm 0.05 \mathrm{~K}$.

$x_{1}: \pm 3 \%$ (relative error, estimated by compiler).

\begin{tabular}{ll}
\hline \hline Components: & Original Measurements: \\
$\begin{array}{l}\text { (1) Benzoic acid; } \mathrm{C}_{7} \mathrm{H}_{6} \mathrm{O}_{2} ; \\
\text { [65-85-0] }\end{array}$ & $\begin{array}{l}73 \text { M. Dias, S. L. Raghavan, and J. } \\
\text { (2) 1-Butanol; } \mathrm{C}_{4} \mathrm{H}_{10} \mathrm{O} ;\end{array}$ \\
{$[71-36-3]$} & \\
\hline Variables: & Prepared by: \\
$T / \mathrm{K}=305.2$ & W. E. Acree, Jr. Jharm. 216, 51 (2001). \\
\hline
\end{tabular}

\section{Experimental Values}

The measured solubility was reported to be $239.7 \mathrm{mg} / \mathrm{ml}$, which corresponds to a molar solubility of $c_{1}=1.963$ mol dm ${ }^{-3}$.

\section{Auxiliary Information}

\section{Method/Apparatus/Procedure:}

Constant-temperature bath, magnetic stirrer, centrifuge, and an ultraviolet/ visible spectrophotometer.

Excess solute and solvent were equilibrated in a constant-temperature bath with stirring for $48 \mathrm{~h}$. The saturated solution was then centrifuged for $10 \mathrm{~min}$, and an aliquot of the supernatant solution was removed and diluted quantitatively for spectrophotometric analysis. The concentration of the dissolved solute was determined from the measured absorbance.

\section{Source and Purity of Chemicals:}

(1) Purity not given, Fisons Scientific Equipment, UK, no purification details were provided.

(2) Purity not given, Fisher Scientific, UK, no purification details were provided.

\section{Estimated Error:}

Temperature: Insufficient experimental details to estimate. $c_{1}: \pm 0.097$.

\begin{tabular}{ll}
\hline \hline Components: & $\begin{array}{l}\text { Original Measurements: } \\
\text { (1) Benzoic acid; } \mathrm{C}_{7} \mathrm{H}_{6} \mathrm{O}_{2} ;\end{array}$ \\
$\begin{array}{ll}{ }^{50} \mathrm{P} . \mathrm{G} \text {. Desai and A. M. Patel, J. Indian } \\
\text { C65-85-0] }\end{array}$ & $\begin{array}{l}\text { Chem. Soc. 12, 131 (1935). } \\
\text { (2) 1-Butanol; } \mathrm{C}_{4} \mathrm{H}_{10} \mathrm{O} ;[71-36-3]\end{array}$ \\
\hline Variables: & Prepared by: \\
$T / \mathrm{K}=301.2$ & W. E. Acree, Jr. \\
\hline
\end{tabular}

Experimental Values

\begin{tabular}{lc}
\hline \hline$x_{2}{ }^{\mathrm{a}}$ & $x_{1}{ }^{\mathrm{b}}$ \\
\hline 0.8032 & 0.1968 \\
\hline${ }^{\mathrm{a}} x_{2}:$ mole fraction of component 2 in the saturated solution. & \\
${ }^{\mathrm{b}} x_{1}:$ mole fraction solubility of the solute. &
\end{tabular}

\section{Auxiliary Information}

\begin{abstract}
Method/Apparatus/Procedure:
Excess solute and solvent were placed in a glass flask which was kept revolving on a wheel in an air thermostat for two days. After two days of equilibration, the solution was filtered. To minimize absorption of the solute onto the filter paper, a $100 \mathrm{ml}$ portion of fresh saturated solution was first filtered through the paper before filtering the sample to be analyzed. An aliquot of the filtrate was titrated with barium hydroxide using phenolphthalein as the endpoint indictor. The barium hydroxide titrant was previously standardized against a solution of succinic acid.
\end{abstract}

Source and Purity of Chemicals:

(1) Purity not given, Chemical source not specified, no information provided concerning purification.

(2) Purity not given, Chemical source not specified, no information provided concerning purification.

\section{Estimated Error:}

Temperature: No information given.

$x_{1}: \pm 3 \%$ (relative error, estimated by compiler).

\begin{tabular}{ll}
\hline \hline Components: & Original Measurements: \\
$\begin{array}{l}\text { (1) Benzoic acid; } \mathrm{C}_{7} \mathrm{H}_{6} \mathrm{O}_{2} ; \\
\text { [65-85-0] }\end{array}$ & ${ }^{70}$ J. M. Jessy, J. Indian Chem. Soc. 75, \\
$\begin{array}{l}\text { (2) 1-Butanol; } \mathrm{C}_{4} \mathrm{H}_{10} \mathrm{O} ; \\
{[71-36-3]}\end{array}$ & \\
\hline Variables: & Prepared by: \\
$T / \mathrm{K}=308.15$ & W. E. Acree, Jr. \\
\hline
\end{tabular}

\section{Experimental Values}

The measured molar solubility was $c_{1}=0.2529 \mathrm{~mol} \mathrm{dm}^{-3}$.

\section{Auxiliary Information}

\section{Method/Apparatus/Procedure:}

Very little experimental details were provided. A convenient amount of benzoic acid in the form of fine powder was placed in a conical flask. Alcohol was added such that the volume was approximately half of that required to make the saturated solution. The flask was tightly stoppered and thermostated at $308 \mathrm{~K}$ for $15 \mathrm{~min}$. Then, keeping the flask still partially immersed in the temperature bath, more alcohol was added dropwise with vigorous shaking by rotary motion until the last speck of solid dissolved. Repeated trials showed that the endpoint was quite reproducible. The experiment was performed a minimum of three times.

\section{Source and Purity of Chemicals:}

(1) Purity not given, Chemical source not given, purification method was referenced to a published method [A. I. Vogel, A Text Book of Practical Organic Chemistry Including Qualitative Organic Analysis (Longmans, London, 1968)].

(2) Purity not given, Chemical source not given, purification method was referenced to a published method [A. I. Vogel, A Text Book of Practical Organic Chemistry Including Qualitative Organic Analysis (Longmans, London, 1968)]. 
Estimated Error:

Temperature: \pm 0.2 (estimated by compiler). $c_{1}: \pm 4 \%$ (relative error, estimated by compiler).

\begin{tabular}{|c|c|}
\hline $\begin{array}{l}\text { Components: } \\
\text { (1) Benzoic acid; } \mathrm{C}_{7} \mathrm{H}_{6} \mathrm{O}_{2} \text {; } \\
{[65-85-0]} \\
\text { (2) } 1 \text {-Butanol; } \mathrm{C}_{4} \mathrm{H}_{10} \mathrm{O} \text {; } \\
{[71-36-3]}\end{array}$ & $\begin{array}{l}\text { Original Measurements: } \\
{ }^{91} \text { J. Bradil, J. Malek, and V. Bazant, } \\
\text { Chem. Prumysl 20, } 117 \text { (1970). }\end{array}$ \\
\hline $\begin{array}{l}\text { Variables: } \\
\text { Temperature }\end{array}$ & $\begin{array}{l}\text { Prepared by: } \\
\text { W. E. Acree, Jr. }\end{array}$ \\
\hline
\end{tabular}

Experimental Values

\begin{tabular}{lcc}
\hline \hline$T / \mathrm{K}$ & $x_{2}{ }^{\mathrm{a}}$ & $x_{1}{ }^{\mathrm{b}}$ \\
\hline 273.2 & 0.9355 & 0.0645 \\
290.2 & 0.8915 & 0.1085 \\
298.2 & 0.8596 & 0.1404 \\
323.6 & 0.7545 & 0.2455 \\
333.9 & 0.6824 & 0.3176 \\
348.4 & 0.6147 & 0.3853 \\
363.2 & 0.3878 & 0.6122
\end{tabular}

${ }^{a} x_{2}$ : mole fraction of component 2 in the saturated solution.

${ }^{b} x_{1}$ : mole fraction solubility of the solute. Solubility data were reported in terms of grams of dissolved solute per $100 \mathrm{~g}$ of solution. Mole fraction solubilities calculated by the compiler.

\section{Auxiliary Information}

\section{Method/Apparatus/Procedure:}

Excess solute and solvent and were placed in a flask and equilibrated at constant temperature with stirring. After $90 \mathrm{~min}$ the stirring was discontinued, and the solution was allowed to stand for $30 \mathrm{~min}$ to allow the undissolved solid to settle to the bottom of the flask. An aliquot of the saturated solution was removed by pipette fitted with a filtering device. The mass of the aliquot was determined by weighing. The concentration of the dissolved solute was determined by titration using sodium hydroxide, with phenolphthalein being the endpoint indicator.

Source and Purity of Chemicals:

(1) Purity not given, Argon, Lodz, Poland, no purification details were provided.

(2) Purity not given, Spolana, Neratovice, Czechoslovakia, was distilled before use.

\section{Estimated Error:}

Temperature: $\pm 0.1 \mathrm{~K}$.

$x_{1}: \pm 1 \%$ (relative error).

\begin{tabular}{|c|c|}
\hline $\begin{array}{l}\text { Components: } \\
\text { (1) Benzoic acid; } \mathrm{C}_{7} \mathrm{H}_{6} \mathrm{O}_{2} \text {; } \\
\text { [65-85-0] } \\
\text { (2) 2-Methyl-1-propanol; } \\
\mathrm{C}_{4} \mathrm{H}_{10} \mathrm{O} \text {; [78-83-1] }\end{array}$ & $\begin{array}{l}\text { Original Measurements: } \\
{ }^{47} \text { A. Beerbower, P. L. Wu, and A. } \\
\text { Martin, J. Pharm. Sci. 73, } 179 \text { (1984). }\end{array}$ \\
\hline $\begin{array}{l}\text { Variables: } \\
T / \mathrm{K}=298.15\end{array}$ & $\begin{array}{l}\text { Prepared by: } \\
\text { W. E. Acree, Jr. }\end{array}$ \\
\hline
\end{tabular}

Experimental Values

\begin{tabular}{lc}
\hline \hline$x_{2}{ }^{\mathrm{a}}$ & $x_{1}{ }^{\mathrm{b}}$ \\
\hline 0.8476 & 0.1524 \\
\hline${ }^{\mathrm{a}} x_{2}:$ mole fraction of component 2 in the saturated solution. \\
${ }^{\mathrm{b}} x_{1}$ : mole fraction solubility of the solute. \\
\\
\multicolumn{2}{c}{ Auxiliary Information } \\
\hline
\end{tabular}

\section{Method/Apparatus/Procedure:}

Constant-temperature bath and ultraviolet/visible spectrophotometer. Excess solute and solvent were placed in screw-capped vials. The vials were sealed and submerged in a constant-temperature water bath and shaken at 100 cycles/min for $24 \mathrm{~h}$. After the $24 \mathrm{~h}$ equilibration period, the vial was removed, wiped dry, and the contents analyzed. The solutions were transferred to a syringe and filtered through a $0.1 \mu \mathrm{m}$ pore size filter. The solutions were diluted and the absorbances recorded at the maximum absorption wavelength of benzoic acid. The solubility was determined at least six times.

\section{Source and Purity of Chemicals:}

(1) Purity not given, Chemical source not specified, no information provided concerning purification.

(2) Spectrophotometric or ACS Reagent grade, Chemical source not specified, redistilled before use.

Estimated Error:

Temperature: $\pm 0.2 \mathrm{~K}$.

$x_{1}: \pm 3 \%$ (relative error).

\begin{tabular}{|c|c|}
\hline $\begin{array}{l}\text { Components: } \\
\text { (1) Benzoic acid; } \mathrm{C}_{7} \mathrm{H}_{6} \mathrm{O}_{2} \text {; } \\
\text { [65-85-0] } \\
\text { (2) 2-Methyl-2-propanol; } \\
\mathrm{C}_{4} \mathrm{H}_{10} \mathrm{O} \text {; [75-65-0] }\end{array}$ & $\begin{array}{l}\text { Original Measurements: } \\
{ }^{76} \text { M. K. Chantooni and I. M. Kolthoff, } \\
\text { Anal. Chem. 51, } 133 \text { (1979). }\end{array}$ \\
\hline $\begin{array}{l}\text { Variables: } \\
T / \mathrm{K}=298.15\end{array}$ & $\begin{array}{l}\text { Prepared by: } \\
\text { W. E. Acree, Jr. }\end{array}$ \\
\hline
\end{tabular}

\section{Experimental Values}

The measured solubility was reported to be $2.50 \mathrm{~mol} \mathrm{dm}^{-3}$. The authors did not give the temperature at which the solubility was measured. Based on the experimental description given in an earlier paper [I. M. Kolthoff, J. J. Lingane, and W. Larson, J. Am. Chem. Soc. 60, 2512 (1938)], the compiler believes the temperature to be $298.15 \mathrm{~K}$.

\section{Auxiliary Information}

\section{Method/Apparatus/Procedure:}

Very little experimental details were provided. Solubility was determined by titrating a filtered aliquot of the saturated solution alkalimetrically in an aqueous-ethanol mixture using phenolphthalein as the acid-base indicator.

\section{Source and Purity of Chemicals:}

(1) High Purity, National Bureau of Standards, USA, was dried in vacuo at 383 $\mathrm{K}$ before use.

(2) White Label, Eastman Kodak Chemical Company, Rochester, USA, shaken with calcium hydride and distilled before use.

\section{Estimated Error:}

Temperature: $\pm 0.1 \mathrm{~K}$ (estimated by compiler). $c_{1}: \pm 2 \%$ (relative error, estimated by compiler). 


\section{Components:}

(1) Benzoic acid; $\mathrm{C}_{7} \mathrm{H}_{6} \mathrm{O}_{2}$; [65-85-0]

(2) 2-Methyl-2-propanol;

$\mathrm{C}_{4} \mathrm{H}_{10} \mathrm{O}$; [75-65-0]

\begin{tabular}{ll}
\hline Variables: & Prepared by: \\
$T / \mathrm{K}=308.15$ & W. E. Acree, Jr. \\
\hline
\end{tabular}

\section{Experimental Values}

The measured molar solubility was $c_{1}=0.3092 \mathrm{~mol} \mathrm{dm}^{-3}$.

\section{Auxiliary Information}

\section{Method/Apparatus/Procedure:}

Very little experimental details were provided. A convenient amount of benzoic acid in the form of fine powder was placed in a conical flask. Alcohol was added such that the volume was approximately half of that required to make the saturated solution. The flask was tightly stoppered and thermostated at $308 \mathrm{~K}$ for $15 \mathrm{~min}$. Then, keeping the flask still partially immersed in the temperature bath, more alcohol was added dropwise with vigorous shaking by rotary motion until the last speck of solid dissolved. Repeated trials showed that the endpoint was quite reproducible. The experiment was performed a minimum of three times.

\section{Source and Purity of Chemicals:}

(1) Purity not given, Chemical source not given, purification method was referenced to a published method [A. I. Vogel, A Text Book of Practical Organic Chemistry Including Qualitative Organic Analysis (Longmans, London, 1968)].

(2) Purity not given, Chemical source not given, purification method was referenced to a published method [A. I. Vogel, A Text Book of Practical Organic Chemistry Including Qualitative Organic Analysis (Longmans, London, 1968)].

\section{Estimated Error:}

Temperature: \pm 0.2 (estimated by compiler).

$c_{1}: \pm 4 \%$ (relative error, estimated by compiler).

\begin{tabular}{ll}
\hline \hline Components: & Original Measurements: \\
(1) Benzoic acid; $\mathrm{C}_{7} \mathrm{H}_{6} \mathrm{O}_{2} ;$ & ${ }^{47}$ A. Beerbower, P. L. Wu, and A. \\
$\begin{array}{l}\text { 65-85-0] } \\
\text { (2) 1-Pentanol; } \mathrm{C}_{5} \mathrm{H}_{12} \mathrm{O} ;\end{array}$ & Martin, J. Pharm. Sci. 73, 179 (1984). \\
{$[71-41-0]$} & \\
\hline Variables: & Prepared by: \\
$T / \mathrm{K}=298.15$ & W. E. Acree, Jr. \\
\hline
\end{tabular}

Experimental Values

\begin{tabular}{lc}
\hline \hline$x_{2}{ }^{\mathrm{a}}$ & $x_{1}{ }^{\mathrm{b}}$ \\
\hline 0.8161 & 0.1839 \\
\hline${ }^{\mathrm{a}} x_{2}:$ mole fraction of component 2 in the saturated solution. & \\
${ }^{\mathrm{b}} x_{1}:$ mole fraction solubility of the solute. &
\end{tabular}

\section{Auxiliary Information}

Method/Apparatus/Procedure:

Constant-temperature bath and ultraviolet/visible spectrophotometer. Excess solute and solvent were placed in screw-capped vials. The vials were sealed and submerged in a constant-temperature water bath and shaken at 100 cycles/min for $24 \mathrm{~h}$. After the $24 \mathrm{~h}$ equilibration period, the vial was removed, wiped dry, and the contents analyzed. The solutions were transferred to a syringe and filtered through a $0.1 \mu \mathrm{m}$ pore size filter. The solutions were diluted and the absorbances recorded at the maximum absorption wavelength of benzoic acid. The solubility was determined at least six times.

Source and Purity of Chemicals:

(1) Purity not given, Chemical source not specified, no information provided concerning purification.

(2) Spectrophotometric or ACS Reagent grade, Chemical source not specified, redistilled before use.

Estimated Error:

Temperature: $\pm 0.2 \mathrm{~K}$.

$x_{1}: \pm 3 \%$ (relative error).

\begin{tabular}{|c|c|}
\hline $\begin{array}{l}\text { Components: } \\
\text { (1) Benzoic acid; } \mathrm{C}_{7} \mathrm{H}_{6} \mathrm{O}_{2} \text {; } \\
\text { [65-85-0] } \\
\text { (2) 1-Pentanol; } \mathrm{C}_{5} \mathrm{H}_{12} \mathrm{O} \text {; } \\
\text { [71-41-0] }\end{array}$ & $\begin{array}{l}\text { Original Measurements: } \\
{ }^{75} \text { F. A. Restaino and A. N. Martin, J. } \\
\text { Pharm. Sci. 53, } 636 \text { (1964). }\end{array}$ \\
\hline $\begin{array}{l}\text { Variables: } \\
T / \mathrm{K}=298.15\end{array}$ & $\begin{array}{l}\text { Prepared by: } \\
\text { W. E. Acree, Jr. }\end{array}$ \\
\hline
\end{tabular}

\section{Experimental Values}

\begin{tabular}{lc}
\hline \hline$x_{2}{ }^{\mathrm{a}}$ & $x_{1}{ }^{\mathrm{b}}$ \\
\hline 0.774 & 0.226 \\
\hline${ }^{\mathrm{a}} x_{2}:$ mole fraction of component 2 in the saturated solution. & \\
${ }^{\mathrm{b}} x_{1}:$ mole fraction solubility of the solute. &
\end{tabular}

${ }^{b} x_{1}$ : mole fraction solubility of the solute.

\section{Auxiliary Information}

\section{Method/Apparatus/Procedure:}

Constant-temperature bath, shaker, analytical balance, constant-temperature bath, and ultraviolet/visible spectrophotometer.

Excess solute and solvent were placed in screw-capped vials and allowed to equilibrate in a constant-temperature bath with shaking for $48 \mathrm{~h}$. An aliquot of the equilibrated sample was removed and filtered. Concentration of the dissolved solute was determined gravimetrically and/or spectrophotometrically. The authors did not specify which of the two methods was used for the different solutes studied.

\section{Source and Purity of Chemicals:}

(1) Certified Reagent grade, Fisher Scientific, USA, was recrsytallized from trichloromethane.

(2) Certified Reagent grade, Fisher Scientific, was used as received.

Estimated Error:

Temperature: $\pm 0.05 \mathrm{~K}$

$x_{1}: \pm 3 \%$ (relative error, estimated by compiler). 


\section{Components:}

(1) Benzoic acid; $\mathrm{C}_{7} \mathrm{H}_{6} \mathrm{O}_{2}$;

[65-85-0]

(2) 1-Pentanol; $\mathrm{C}_{5} \mathrm{H}_{12} \mathrm{O}$

[71-41-0]

\begin{tabular}{ll}
\hline Variables: & Prepared by: \\
$T / \mathrm{K}=308.15$ & W. E. Acree, Jr. \\
\hline
\end{tabular}

\section{Experimental Values}

The measured molar solubility was $c_{1}=0.2919 \mathrm{~mol} \mathrm{dm}^{-3}$.

\section{Auxiliary Information}

\section{Method/Apparatus/Procedure:}

Very little experimental details were provided. A convenient amount of benzoic acid in the form of fine powder was placed in a conical flask. Alcohol was added such that the volume was approximately half of that required to make the saturated solution. The flask was tightly stoppered and thermostated at $308 \mathrm{~K}$ for $15 \mathrm{~min}$. Then, keeping the flask still partially immersed in the temperature bath, more alcohol was added dropwise with vigorous shaking by rotary motion until the last speck of solid dissolved. Repeated trials showed that the endpoint was quite reproducible. The experiment was performed a minimum of three times.

\section{Source and Purity of Chemicals:}

(1) Purity not given, Chemical source not given, purification method was referenced to a published method [A. I. Vogel, A Text Book of Practical Organic Chemistry Including Qualitative Organic Analysis (Longmans, London, 1968)].

(2) Purity not given, Chemical source not given, purification method was referenced to a published method [A. I. Vogel, A Text Book of Practical Organic Chemistry Including Qualitative Organic Analysis (Longmans, London, 1968)].

\section{Estimated Error:}

Temperature: \pm 0.2 (estimated by compiler).

$c_{1}: \pm 4 \%$ (relative error, estimated by compiler).

\begin{tabular}{ll}
\hline \hline Components: & Original Measurements: \\
(1) Benzoic acid; $\mathrm{C}_{7} \mathrm{H}_{6} \mathrm{O}_{2} ;$ & ${ }^{47}$ A. Beerbower, P. L. Wu, and A. \\
{$[65-85-0]$} & Martin, J. Pharm. Sci. 73, 179 (1984). \\
$\begin{array}{l}\text { (2) 1-Hexanol; } \mathrm{C}_{6} \mathrm{H}_{14} \mathrm{O} ; \\
{[111-27-3]}\end{array}$ \\
\hline $\begin{array}{l}\text { Variables: } \\
T / \mathrm{K}=298.15\end{array}$ & Prepared by: \\
\hline
\end{tabular}

Experimental Values

\begin{tabular}{lc}
\hline \hline$x_{2}{ }^{\mathrm{a}}$ & $x_{1}{ }^{\mathrm{b}}$ \\
\hline 0.8095 & 0.1905
\end{tabular}

${ }^{\mathrm{a}} x_{2}$ : mole fraction of component 2 in the saturated solution.

${ }^{\mathrm{b}} x_{1}$ : mole fraction solubility of the solute.

\section{Auxiliary Information}

\section{Method/Apparatus/Procedure:}

Constant-temperature bath and ultraviolet/visible spectrophotometer Excess solute and solvent were placed in screw-capped vials. The vials were sealed and submerged in a constant-temperature water bath and shaken at 100 cycles/min for $24 \mathrm{~h}$. After the $24 \mathrm{~h}$ equilibration period, the vial was removed, wiped dry, and the contents analyzed. The solutions were transferred to a syringe and filtered through a $0.1 \mu \mathrm{m}$ pore size filter. The solutions were diluted and the absorbances recorded at the maximum absorption wavelength of benzoic acid. The solubility was determined at least six times.

\section{Source and Purity of Chemicals:}

(1) Purity not given, Chemical source not specified, no information provided concerning purification.

(2) Spectrophotometric or ACS Reagent grade, Chemical source not specified, redistilled before use.

\section{Estimated Error:}

Temperature: $\pm 0.2 \mathrm{~K}$.

$x_{1}: \pm 3 \%$ (relative error)

\begin{tabular}{|c|c|}
\hline $\begin{array}{l}\text { Components: } \\
\text { (1) Benzoic acid; } \mathrm{C}_{7} \mathrm{H}_{6} \mathrm{O}_{2} \text {; } \\
\text { [65-85-0] } \\
\text { (2) } 1-\mathrm{Hexanol} ; \mathrm{C}_{6} \mathrm{H}_{14} \mathrm{O} \text {; } \\
\text { [111-27-3] }\end{array}$ & $\begin{array}{l}\text { Original Measurements: } \\
{ }^{75} \text { F. A. Restaino and A. N. Martin, J. } \\
\text { Pharm. Sci. 53, } 636 \text { (1964). }\end{array}$ \\
\hline $\begin{array}{l}\text { Variables: } \\
T / \mathrm{K}=298.15\end{array}$ & $\begin{array}{l}\text { Prepared by: } \\
\text { W. E. Acree, Jr. }\end{array}$ \\
\hline
\end{tabular}

Experimental Values

\begin{tabular}{lc}
\hline \hline$x_{2}{ }^{\mathrm{a}}$ & $x_{1}{ }^{\mathrm{b}}$ \\
\hline 0.799 & 0.201
\end{tabular}

${ }^{a} x_{2}$ : mole fraction of component 2 in the saturated solution.

${ }^{\mathrm{b}} x_{1}$ : mole fraction solubility of the solute.

\section{Auxiliary Information}

\section{Method/Apparatus/Procedure:}

Constant-temperature bath, shaker, analytical balance, constant-temperature bath, and ultraviolet/visible spectrophotometer.

Excess solute and solvent were placed in screw-capped vials and allowed to equilibrate in a constant-temperature bath with shaking for $48 \mathrm{~h}$. An aliquot of the equilibrated sample was removed and filtered. Concentration of the dissolved solute was determined gravimetrically and/or spectrophotometrically. The authors did not specify which of the two methods was used for the different solutes studied.

\section{Source and Purity of Chemicals:}

(1) Certified Reagent grade, Fisher Scientific, USA, was recrsytallized from trichloromethane.

(2) Purity not given, Eastman Organic Chemicals, USA, was redistilled before use.

\section{Estimated Error:}

Temperature: $\pm 0.05 \mathrm{~K}$

$x_{1}: \pm 3 \%$ (relative error, estimated by compiler)

\begin{tabular}{ll}
\hline \hline Components: & $\begin{array}{l}\text { Original Measurements: } \\
\text { (1) Benzoic acid; } \mathrm{C}_{7} \mathrm{H}_{6} \mathrm{O}_{2} ;\end{array}$ \\
$\begin{array}{l}70 \text { J. M. Jessy, J. Indian Chem. Soc. 75, } \\
\text { (2) 1-Hexanol; } \mathrm{C}_{6} \mathrm{H}_{14} \mathrm{O} ;\end{array}$ & 352 (1998). \\
{$[111-27-3]$} & \\
\hline Variables: & Prepared by: \\
$T / \mathrm{K}=308.15$ & W. E. Acree, Jr. \\
\hline
\end{tabular}




\section{Experimental Values}

The measured molar solubility was $c_{1}=0.2301 \mathrm{~mol} \mathrm{dm}^{-3}$.

\section{Auxiliary Information}

\section{Method/Apparatus/Procedure:}

Very little experimental details were provided. A convenient amount of benzoic acid in the form of fine powder was placed in a conical flask. Alcohol was added such that the volume was approximately half of that required to make the saturated solution. The flask was tightly stoppered and thermostated at $308 \mathrm{~K}$ for $15 \mathrm{~min}$. Then, keeping the flask still partially immersed in the temperature bath, more alcohol was added dropwise with vigorous shaking by rotary motion until the last speck of solid dissolved. Repeated trials showed that the endpoint was quite reproducible. The experiment was performed a minimum of three times.

\section{Source and Purity of Chemicals:}

(1) Purity not given, Chemical source not given, purification method was referenced to a published method [A. I. Vogel, A Text Book of Practical Organic Chemistry Including Qualitative Organic Analysis (Longmans, London, 1968)].

(2) Purity not given, Chemical source not given, purification method was referenced to a published method [A. I. Vogel, A Text Book of Practical Organic Chemistry Including Qualitative Organic Analysis (Longmans, London, 1968)].

\section{Estimated Error:}

Temperature: \pm 0.2 (estimated by compiler). $c_{1}: \pm 4 \%$ (relative error, estimated by compiler).

\begin{tabular}{|c|c|}
\hline $\begin{array}{l}\text { Components: } \\
\text { (1) Benzoic acid; } \mathrm{C}_{7} \mathrm{H}_{6} \mathrm{O}_{2} \text {; } \\
\text { [65-85-0] } \\
\text { (2) 3-Methyl-3-pentanol; } \\
\mathrm{C}_{6} \mathrm{H}_{14} \mathrm{O} \text {; [77-74-3] }\end{array}$ & $\begin{array}{l}\text { Original Measurements: } \\
\text { 51 H.-M. Lin and R. A. Nash, J. Pharm. } \\
\text { Sci. 82, } 1018 \text { (1993). }\end{array}$ \\
\hline $\begin{array}{l}\text { Variables: } \\
T / \mathrm{K}=298.15\end{array}$ & $\begin{array}{l}\text { Prepared by: } \\
\text { W. E. Acree, Jr. }\end{array}$ \\
\hline
\end{tabular}

Experimental Values

\begin{tabular}{lc}
\hline \hline$x_{2}{ }^{\mathrm{a}}$ & $x_{1}{ }^{\mathrm{b}}$ \\
\hline 0.7284 & 0.2716 \\
\hline$x_{2}$.
\end{tabular}

${ }^{\mathrm{a}} x_{2}$ : mole fraction of component 2 in the saturated solution.

${ }^{\mathrm{b}} x_{1}$ : mole fraction solubility of the solute.

\section{Auxiliary Information}

\footnotetext{
Method/Apparatus/Procedure:

Constant-temperature bath and high-performance liquid chromatograph. Excess solute and solvent were placed in screw-capped vials. The vials were sealed with several turns of electrical tape, warmed to about $323 \mathrm{~K}$, and then shaken in a constant-temperature bath for at least $24 \mathrm{~h}$ at $298 \mathrm{~K}$. After equilibrium was reached, the sample was filtered through a 0.45 or $1.0 \mu \mathrm{m}$ filter, and an aliquot was diluted appropriately for chromatographic analysis. Concentrations were determined by a high-performance liquid chromatographic method.
}

Source and Purity of Chemicals:

(1) Purity not given, Amend Drug and Chemical Company, no information provided concerning purification.

(2) Reagent grade, Aldrich Chemical Company, Milwaukee, WI, USA, no purification details were provided.
Estimated Error:

Temperature: $\pm 0.2 \mathrm{~K}$ (estimated by compiler). $x_{1}: \pm 3 \%$ (relative error, estimated by compiler).

\begin{tabular}{ll}
\hline \hline Components: & $\begin{array}{l}\text { Original Measurements: } \\
\text { (1) Benzoic acid; } \mathrm{C}_{7} \mathrm{H}_{6} \mathrm{O}_{2} ;\end{array}$ \\
$\begin{array}{l}{ }^{53} \mathrm{G} . \text { L. Perlovich and A. Bauer-Brandl, } \\
\text { [65-85-0] }\end{array}$ & Pharm. Res. 20, 471 (2003). \\
(2) 1-Heptanol; $\mathrm{C}_{7} \mathrm{H}_{16} \mathrm{O} ;$ & \\
{$[111-70-6]$} & \\
\hline Variables: & Prepared by: \\
$T / \mathrm{K}=298.15$ & W. E. Acree, Jr. \\
\hline
\end{tabular}

\section{Experimental Values}

\begin{tabular}{lc}
\hline \hline$x_{2}{ }^{\mathrm{a}}$ & $x_{1}{ }^{\mathrm{b}}$ \\
\hline 0.8054 & 0.1946 \\
\hline${ }^{\mathrm{a}} x_{2}:$ mole fraction of component 2 in the saturated solution. \\
${ }^{\mathrm{b}} x_{1}:$ mole fraction solubility of the solute.
\end{tabular}

\section{Auxiliary Information}

\section{Method/Apparatus/Procedure:}

Thermostated constant-temperature bath, centrifuge, and analytical balance. Very little experimental details were given in the paper. The solubility of the solute was by a weighing method.

\section{Source and Purity of Chemicals:}

(1) Analytical Reagent grade, Norsk Medisinaldepot, Oslo, Norway, no purification details were provided.

(2) Analytical Reagent grade, Sigma Chemical Company, USA, no purification details were provided.

\section{Estimated Error:}

Temperature: $\pm 0.01 \mathrm{~K}$.

$x_{1}: \pm 3.0 \%$ (relative error)

\begin{tabular}{ll}
\hline \hline Components: & Original Measurements: \\
$\begin{array}{l}\text { (1) Benzoic acid; } \mathrm{C}_{7} \mathrm{H}_{6} \mathrm{O}_{2} ; \\
\text { [65-85-0] }\end{array}$ & ${ }^{70}$ J. M. Jessy, J. Indian Chem. Soc. 75, \\
$\begin{array}{l}\text { (2) 1-Heptanol; } \mathrm{C}_{7} \mathrm{H}_{16} \mathrm{O} ; \\
{[111-70-6]}\end{array}$ & \\
\hline Variables: & Prepared by: \\
$T / \mathrm{K}=308.15$ & W. E. Acree, Jr. \\
\hline
\end{tabular}

\section{Experimental Values}

The measured molar solubility was $c_{1}=0.2542 \mathrm{~mol} \mathrm{dm}^{-3}$. 


\section{Auxiliary Information}

\section{Method/Apparatus/Procedure:}

Very little experimental details were provided. A convenient amount of benzoic acid in the form of fine powder was placed in a conical flask. Alcohol was added such that the volume was approximately half of that required to make the saturated solution. The flask was tightly stoppered and thermostated at $308 \mathrm{~K}$ for $15 \mathrm{~min}$. Then, keeping the flask still partially immersed in the temperature bath, more alcohol was added dropwise with vigorous shaking by rotary motion until the last speck of solid dissolved. Repeated trials showed that the endpoint was quite reproducible. The experiment was performed a minimum of three times.

Source and Purity of Chemicals:

(1) Purity not given, Chemical source not given, purification method was referenced to a published method [A. I. Vogel, A Text Book of Practical Organic Chemistry Including Qualitative Organic Analysis (Longmans, London, 1968)]. referenced to a published method [A. I. Vogel, A Text Book of Practical Organic Chemistry Including Qualitative Organic Analysis (Longmans, London, 1968)].

Estimated Error:

Temperature: \pm 0.2 (estimated by compiler). $c_{1}: \pm 4 \%$ (relative error, estimated by compiler).

\begin{tabular}{ll}
\hline \hline Components: & Original Measurements: \\
(1) Benzoic acid; $\mathrm{C}_{7} \mathrm{H}_{6} \mathrm{O}_{2} ;$ & ${ }^{47}$ A. Beerbower, P. L. Wu, and A. \\
[65-85-0] & Martin, J. Pharm. Sci. 73, 179 (1984). \\
$\begin{array}{l}\text { (2) 1-Octanol; } \mathrm{C}_{8} \mathrm{H}_{18} \mathrm{O} ; \\
{[111-87-5]}\end{array}$ & \\
\hline Variables: & Prepared by: \\
$T / \mathrm{K}=298.15$ & W. E. Acree, Jr. \\
\hline
\end{tabular}

\section{Experimental Values}

\begin{tabular}{lc}
\hline \hline$x_{2}{ }^{\mathrm{a}}$ & $x_{1}{ }^{\mathrm{b}}$ \\
\hline 0.8013 & 0.1987 \\
\hline${ }_{{ }^{\mathrm{a}} x_{2}: \text { mole fraction of component } 2 \text { in the saturated solution. }}$ & \\
${ }^{\mathrm{b}} x_{1}:$ mole fraction solubility of the solute. &
\end{tabular}

\section{Auxiliary Information}

\section{Method/Apparatus/Procedure:}

Constant-temperature bath and ultraviolet/visible spectrophotometer. Excess solute and solvent were placed in screw-capped vials. The vials were sealed and submerged in a constant-temperature water bath and shaken at 100 cycles/min for $24 \mathrm{~h}$. After the $24 \mathrm{~h}$ equilibration period, the vial was removed, wiped dry, and the contents analyzed. The solutions were transferred to a syringe and filtered through a $0.1 \mu \mathrm{m}$ pore size filter. The solutions were diluted and the absorbances recorded at the maximum absorption wavelength of benzoic acid. The solubility was determined at least six times.

\section{Source and Purity of Chemicals:}

(1) Purity not given, Chemical source not specified, no information provided concerning purification.

(2) Spectrophotometric or ACS Reagent grade, Chemical source not specified, redistilled before use.

\section{Estimated Error:}

Temperature: $\pm 0.2 \mathrm{~K}$
(2) Purity not given, Chemical source not given, purification method was

$x_{1}: \pm 3 \%$ (relative error).

\begin{tabular}{ll}
\hline \hline Components: & Original Measurements: \\
(1) Benzoic acid; $\mathrm{C}_{7} \mathrm{H}_{6} \mathrm{O}_{2} ;$ & ${ }^{75} \mathrm{~F}$. A. Restaino and A. N. Martin, J. \\
{$[65-85-0]$} & Pharm. Sci. 53, 636 (1964). \\
(2) 1-Octanol; $\mathrm{C}_{8} \mathrm{H}_{18} \mathrm{O} ;[111-87-5]$ & \\
\hline Variables: & Prepared by: \\
$T / \mathrm{K}=298.15$ & W. E. Acree, Jr. \\
\hline
\end{tabular}

\section{Experimental Values}

\begin{tabular}{lc}
\hline \hline$x_{2}{ }^{\mathrm{a}}$ & $x_{1}{ }^{\mathrm{b}}$ \\
\hline 0.871 & 0.129
\end{tabular}

${ }^{\mathrm{a}} x_{2}$ : mole fraction of component 2 in the saturated solution.

${ }^{\mathrm{b}} x_{1}$ : mole fraction solubility of the solute.

\section{Auxiliary Information}

\section{Method/Apparatus/Procedure:}

Constant-temperature bath, shaker, analytical balance, constant-temperature bath, and ultraviolet/visible spectrophotometer.

Excess solute and solvent were placed in screw-capped vials and allowed to equilibrate in a constant-temperature bath with shaking for $48 \mathrm{~h}$. An aliquot of the equilibrated sample was removed and filtered. Concentration of the dissolved solute was determined gravimetrically and/or spectrophotometrically. The authors did not specify which of the two methods was used for the different solutes studied.

\section{Source and Purity of Chemicals:}

(1) Certified Reagent grade, Fisher Scientific, USA, was recrsytallized from trichloromethane.

(2) Purity not given, Eastman Organic Chemicals, USA, was redistilled before use.

\section{Estimated Error:}

Temperature: $\pm 0.05 \mathrm{~K}$.

$x_{1}: \pm 3 \%$ (relative error, estimated by compiler).

\begin{tabular}{|c|c|}
\hline Components: & Original Measurements: \\
\hline $\begin{array}{l}\text { (1) Benzoic acid; } \mathrm{C}_{7} \mathrm{H}_{6} \mathrm{O}_{2} \text {; } \\
\text { [65-85-0] }\end{array}$ & $\begin{array}{l}{ }^{77} \text { J. Qingzhu, M. Peisheng, Y. Shouzhi, } \\
\text { W. Qiang, W. Chang, and L. Guiju, J. }\end{array}$ \\
\hline (2) 1-Octanol; $\mathrm{C}_{8} \mathrm{H}_{18} \mathrm{O}$; [111-87-5] & Chem. Eng. Data 53, 1278 (2008). \\
\hline Variables: & Prepared by: \\
\hline Temperature & W. E. Acree, Jr. \\
\hline
\end{tabular}

Experimental Values

\begin{tabular}{lcc}
\hline \hline$T / \mathrm{K}$ & $x_{2}{ }^{\mathrm{a}}$ & $x_{1}{ }^{\mathrm{b}}$ \\
\hline 295.30 & 0.8335 & 0.1665 \\
297.65 & 0.8199 & 0.1801 \\
300.15 & 0.8078 & 0.1922 \\
301.85 & 0.7967 & 0.2033 \\
304.25 & 0.7886 & 0.2114 \\
305.25 & 0.7806 & 0.2194 \\
307.75 & 0.7726 & 0.2274 \\
309.35 & 0.7650 & 0.2350 \\
312.30 & 0.7501 & 0.2499 \\
313.95 & 0.7345 & 0.2655 \\
317.15 & 0.7207 & 0.2793 \\
320.85 & 0.7103 & 0.2897 \\
322.15 & 0.7000 & 0.3000 \\
\hline${ }^{\mathrm{a}} X_{2}:$ mole fraction of component 2 in the saturated solution. \\
${ }^{\mathrm{b}}{ }_{x_{1}: \text { mole fraction solubility of the solute. }}$
\end{tabular}


Auxiliary Information

\section{Method/Apparatus/Procedure:}

Circulating water bath, analytical balance, laser monitoring system. Preweighed amounts of solute and solvent and were placed in an equilibrium vessel, which was connected to a circulating water bath. The solution was stirred and the temperature gradually increased at a rate $0.5 \mathrm{~K} / 20 \mathrm{~min}(0.2 \mathrm{~K} / 20$ min or slower near saturation temperature) until all of the solid solute dissolved. The temperature at which all of the solute dissolved was determined using laser monitoring.

Source and Purity of Chemicals:

(1) $<99 \%$, Chemical source not specified, used as received.

(2) $<99 \%$, Chemical source not specified, used as received.

\section{Estimated Error:}

Temperature: $\pm 0.05 \mathrm{~K}$

$x_{1}: \pm 0.0005$.

\begin{tabular}{|c|c|}
\hline $\begin{array}{l}\text { Components: } \\
\text { (1) Benzoic acid; } \mathrm{C}_{7} \mathrm{H}_{6} \mathrm{O}_{2} \text {; } \\
\text { [65-85-0] } \\
\text { (2) } 1-\text { Octanol; } \mathrm{C}_{8} \mathrm{H}_{18} \mathrm{O} ; \\
\text { [111-87-5] }\end{array}$ & $\begin{array}{l}\text { Original Measurements: } \\
{ }^{73} \text { M. Dias, S. L. Raghavan, and J. } \\
\text { Hadgraft, Int. J. Pharm. 216, } 51 \text { (2001). }\end{array}$ \\
\hline $\begin{array}{l}\text { Variables: } \\
T / \mathrm{K}=305.2\end{array}$ & $\begin{array}{l}\text { Prepared by: } \\
\text { W. E. Acree, Jr. }\end{array}$ \\
\hline
\end{tabular}

\section{Experimental Values}

The measured solubility was reported to be $152.6 \mathrm{mg} / \mathrm{ml}$, which corresponds to a molar solubility of $c_{1}=1.250$ mol $\mathrm{dm}^{-3}$.

\section{Auxiliary Information}

\section{Method/Apparatus/Procedure:}

Constant-temperature bath, magnetic stirrer, centrifuge, and an ultraviolet/ visible spectrophotometer.

Excess solute and solvent were equilibrated in a constant-temperature bath with stirring for $48 \mathrm{~h}$. The saturated solution was then centrifuged for $10 \mathrm{~min}$ and an aliquot of the supernatant solution was removed and diluted quantitatively for spectrophotometric analysis. The concentration of the dissolved solute was determined from the measured absorbance.

\section{Source and Purity of Chemicals:}

(1) Purity not given, Fisons Scientific Equipment, UK, no purification details were provided.

(2) Purity not given, Fisher Scientific, UK, no purification details were provided.

\section{Estimated Error:}

Temperature: Insufficient experimental details to estimate. $c_{1}: \pm 0.078$.

\section{Original Measurements:}

${ }^{70}$ J. M. Jessy, J. Indian Chem. Soc. 75, 352 (1998).

[65-85-0]

(2) 1-Octanol; $\mathrm{C}_{8} \mathrm{H}_{18} \mathrm{O}$

[111-87-5]

\begin{tabular}{ll}
\hline Variables: & Prepared by: \\
$T / \mathrm{K}=308.15$ & W. E. Acree, Jr. \\
\hline
\end{tabular}

\section{Experimental Values}

The measured molar solubility was $c_{1}=0.2674 \mathrm{~mol} \mathrm{dm}^{-3}$.

\section{Auxiliary Information}

\section{Method/Apparatus/Procedure:}

Very little experimental details were provided. A convenient amount of benzoic acid in the form of fine powder was placed in a conical flask. Alcohol was added such that the volume was approximately half of that required to make the saturated solution. The flask was tightly stoppered and thermostated at $308 \mathrm{~K}$ for $15 \mathrm{~min}$. Then, keeping the flask still partially immersed in the temperature bath, more alcohol was added dropwise with vigorous shaking by rotary motion until the last speck of solid dissolved. Repeated trials showed that the endpoint was quite reproducible. The experiment was performed a minimum of three times.

Source and Purity of Chemicals:

(1) Purity not given, Chemical source not given, purification method was referenced to a published method [A. I. Vogel, A Text Book of Practical Organic Chemistry Including Qualitative Organic Analysis (Longmans, London, 1968)].

(2) Purity not given, Chemical source not given, purification method was referenced to a published method [A. I. Vogel, A Text Book of Practical Organic Chemistry Including Qualitative Organic Analysis (Longmans, London, 1968)].

\section{Estimated Error:}

Temperature: \pm 0.2 (estimated by compiler). $c_{1}: \pm 4 \%$ (relative error, estimated by compiler).

\begin{tabular}{|c|c|}
\hline $\begin{array}{l}\text { Components: } \\
\text { (1) Benzoic acid; } \mathrm{C}_{7} \mathrm{H}_{6} \mathrm{O}_{2} \text {; } \\
\text { [65-85-0] } \\
\text { (2) 1-Nonanol; } \mathrm{C}_{9} \mathrm{H}_{20} \mathrm{O} \text {; } \\
\text { [143-08-8] }\end{array}$ & $\begin{array}{l}\text { Original Measurements: } \\
\text { 70 J. M. Jessy, J. Indian Chem. Soc. 75, } \\
352 \text { (1998). }\end{array}$ \\
\hline $\begin{array}{l}\text { Variables: } \\
T / \mathrm{K}=308.15\end{array}$ & $\begin{array}{l}\text { Prepared by: } \\
\text { W. E. Acree, Jr. }\end{array}$ \\
\hline
\end{tabular}

Experimental Values

The measured molar solubility was $c_{1}=0.2439 \mathrm{~mol} \mathrm{dm}^{-3}$.

\section{Auxiliary Information}

\section{Method/Apparatus/Procedure:}

Very little experimental details were provided. A convenient amount of benzoic acid in the form of fine powder was placed in a conical flask. Alcohol was added such that the volume was approximately half of that required to make the saturated solution. The flask was tightly stoppered and thermostated at $308 \mathrm{~K}$ for $15 \mathrm{~min}$. Then, keeping the flask still partially immersed in the temperature bath, more alcohol was added dropwise with vigorous shaking by rotary motion until the last speck of solid dissolved. Repeated trials showed that the endpoint was quite reproducible. The experiment was performed a minimum of three times.

\section{Source and Purity of Chemicals:}

(1) Purity not given, Chemical source not given, purification method was referenced to a published method [A. I. Vogel, A Text Book of Practical Organic Chemistry Including Qualitative Organic Analysis (Longmans, London, 1968)].

(2) Purity not given, Chemical source not given, purification method was referenced to a published method [A. I. Vogel, A Text Book of Practical Organic Chemistry Including Qualitative Organic Analysis (Longmans, London, 1968)]. 


\section{Estimated Error:}

Temperature: \pm 0.2 (estimated by compiler). $c_{1}: \pm 4 \%$ (relative error, estimated by compiler).

\begin{tabular}{|c|c|}
\hline $\begin{array}{l}\text { Components: } \\
\text { (1) Benzoic acid; } \mathrm{C}_{7} \mathrm{H}_{6} \mathrm{O}_{2} \text {; } \\
\text { [65-85-0] } \\
\text { (2) } 1-\text { Decanol; } \mathrm{C}_{10} \mathrm{H}_{22} \mathrm{O} \text {; } \\
\text { [112-30-1] }\end{array}$ & $\begin{array}{l}\text { Original Measurements: } \\
{ }^{73} \text { M. Dias, S. L. Raghavan, and J. } \\
\text { Hadgraft, Int. J. Pharm. 216, } 51 \text { (2001). }\end{array}$ \\
\hline $\begin{array}{l}\text { Variables: } \\
T / \mathrm{K}=305.2\end{array}$ & $\begin{array}{l}\text { Prepared by: } \\
\text { W. E. Acree, Jr. }\end{array}$ \\
\hline
\end{tabular}

\section{Experimental Values}

The measured solubility was reported to be $123.8 \mathrm{mg} / \mathrm{ml}$, which corresponds to a molar solubility of $c_{1}=1.014 \mathrm{~mol} \mathrm{dm}^{-3}$.

\section{Auxiliary Information}

\begin{abstract}
Method/Apparatus/Procedure:
Constant-temperature bath, magnetic stirrer, centrifuge, and an ultraviolet/ visible spectrophotometer.

Excess solute and solvent were equilibrated in a constant-temperature bath with stirring for $48 \mathrm{~h}$. The saturated solution was then centrifuged for $10 \mathrm{~min}$, and an aliquot of the supernatant solution was removed and diluted quantitatively for spectrophotometric analysis. The concentration of the dissolved solute was determined from the measured absorbance.
\end{abstract}

\section{Source and Purity of Chemicals:}

(1) Purity not given, Fisons Scientific Equipment, UK, no purification details were provided.

(2) Purity not given, Fisher Scientific, UK, no purification details were provided.

\section{Estimated Error:}

Temperature: Insufficient experimental details to estimate. $c_{1}: \pm 0.18$.

\begin{tabular}{|c|c|}
\hline $\begin{array}{l}\text { Components: } \\
\text { (1) Benzoic acid; } \mathrm{C}_{7} \mathrm{H}_{6} \mathrm{O}_{2} \text {; } \\
\text { [65-85-0] } \\
\text { (2) 1-Decanol; } \mathrm{C}_{10} \mathrm{H}_{22} \mathrm{O} \text {; } \\
\text { [112-30-1] }\end{array}$ & $\begin{array}{l}\text { Original Measurements: } \\
{ }^{70} \text { J. M. Jessy, J. Indian Chem. Soc. 75, } \\
352 \text { (1998). }\end{array}$ \\
\hline $\begin{array}{l}\text { Variables: } \\
T / \mathrm{K}=308.15\end{array}$ & $\begin{array}{l}\text { Prepared by: } \\
\text { W. E. Acree, Jr. }\end{array}$ \\
\hline
\end{tabular}

\section{Experimental Values \\ The measured molar solubility was $c_{1}=0.2420 \mathrm{~mol} \mathrm{dm}^{-3}$.}

\section{Auxiliary Information}

\footnotetext{
Method/Apparatus/Procedure:

Very little experimental details were provided. A convenient amount of benzoic acid in the form of fine powder was placed in a conical flask. Alcohol was added such that the volume was approximately half of that required to make the saturated solution. The flask was tightly stoppered and thermostated at $308 \mathrm{~K}$ for $15 \mathrm{~min}$. Then, keeping the flask still partially immersed in the temperature bath, more alcohol was added dropwise with vigorous shaking by rotary motion until the last speck of solid dissolved. Repeated trials showed that the endpoint was quite reproducible. The experiment was performed a minimum of three times.
}

Source and Purity of Chemicals:

(1) Purity not given, Chemical source not given, purification method was referenced to a published method [A. I. Vogel, A Text Book of Practical Organic Chemistry Including Qualitative Organic Analysis (Longmans, London, 1968)].

(2) Purity not given, Chemical source not given, purification method was referenced to a published method [A. I. Vogel, A Text Book of Practical Organic Chemistry Including Qualitative Organic Analysis (Longmans, London, 1968)].

Estimated Error:

Temperature: \pm 0.2 (estimated by compiler). $c_{1}: \pm 4 \%$ (relative error, estimated by compiler).

\begin{tabular}{|c|c|}
\hline $\begin{array}{l}\text { Components: } \\
\text { (1) Benzoic acid; } \mathrm{C}_{7} \mathrm{H}_{6} \mathrm{O}_{2} \text {; } \\
\text { [65-85-0] } \\
\text { (2) Benzenemethanol; } \mathrm{C}_{7} \mathrm{H}_{8} \mathrm{O} \text {; } \\
\text { [100-51-6] }\end{array}$ & $\begin{array}{l}\text { Original Measurements: } \\
\text { }{ }^{47} \text { A. Beerbower, P. L. Wu, and A. } \\
\text { Martin, J. Pharm. Sci. 73, } 179 \text { (1984). }\end{array}$ \\
\hline $\begin{array}{l}\text { Variables: } \\
T / \mathrm{K}=298.15\end{array}$ & $\begin{array}{l}\text { Prepared by: } \\
\text { W. E. Acree, Jr. }\end{array}$ \\
\hline
\end{tabular}

Experimental Values

\begin{tabular}{lc}
\hline \hline$x_{2}{ }^{\mathrm{a}}$ & $x_{1}{ }^{\mathrm{b}}$ \\
\hline 0.8559 & 0.1441 \\
\hline${ }^{\mathrm{a}} x_{2}:$ mole fraction of component 2 in the saturated solution. & \\
${ }^{\mathrm{b}} x_{1}:$ mole fraction solubility of the solute. &
\end{tabular}

\section{Auxiliary Information}

\section{Method/Apparatus/Procedure:}

Constant-temperature bath and ultraviolet/visible spectrophotometer. Excess solute and solvent were placed in screw-capped vials. The vials were sealed and submerged in a constant-temperature water bath and shaken at 100 cycles/min for $24 \mathrm{~h}$. After the $24 \mathrm{~h}$ equilibration period, the vial was removed, wiped dry, and the contents analyzed. The solutions were transferred to a syringe and filtered through a $0.1 \mu \mathrm{m}$ pore size filter. The solutions were diluted and the absorbances recorded at the maximum absorption wavelength of benzoic acid. The solubility was determined at least six times.

\section{Source and Purity of Chemicals:}

(1) Purity not given, Chemical source not specified, no information provided concerning purification.

(2) Spectrophotometric or ACS Reagent grade, Chemical source not specified, redistilled before use.

\section{Estimated Error:}

Temperature: $\pm 0.2 \mathrm{~K}$.

$x_{1}: \pm 3 \%$ (relative error)

\begin{tabular}{|c|c|}
\hline $\begin{array}{l}\text { Components: } \\
\text { (1) Benzoic acid; } \mathrm{C}_{7} \mathrm{H}_{6} \mathrm{O}_{2} \text {; } \\
\text { [65-85-0] } \\
\text { (2) } 1,2 \text {-Ethanediol; } \mathrm{C}_{2} \mathrm{H}_{6} \mathrm{O}_{2} \text {; } \\
\text { [107-21-1] }\end{array}$ & $\begin{array}{l}\text { Original Measurements: } \\
{ }^{47} \text { A. Beerbower, P. L. Wu, and A. } \\
\text { Martin, J. Pharm. Sci. 73, } 179 \text { (1984). }\end{array}$ \\
\hline $\begin{array}{l}\text { Variables: } \\
T / \mathrm{K}=298.15\end{array}$ & $\begin{array}{l}\text { Prepared by: } \\
\text { W. E. Acree, Jr. }\end{array}$ \\
\hline
\end{tabular}


Experimental Values

\begin{tabular}{lc}
\hline \hline$x_{2}{ }^{\mathrm{a}}$ & $x_{1}{ }^{\mathrm{b}}$ \\
\hline 0.9116 & 0.0884 \\
\hline${ }^{\mathrm{a}} x_{2}:$ mole fraction of component 2 in the saturated solution. \\
${ }^{\mathrm{b}}{ }_{x_{1}}$ : mole fraction solubility of the solute.
\end{tabular}

\section{Auxiliary Information}

\section{Method/Apparatus/Procedure:}

Constant-temperature bath and ultraviolet/visible spectrophotometer.

Excess solute and solvent were placed in screw-capped vials. The vials were sealed and submerged in a constant-temperature water bath and shaken at 100 cycles/min for $24 \mathrm{~h}$. After the $24 \mathrm{~h}$ equilibration period, the vial was removed, wiped dry, and the contents analyzed. The solutions were transferred to a syringe and filtered through a $0.1 \mu \mathrm{m}$ pore size filter. The solutions were diluted and the absorbances recorded at the maximum absorption wavelength of benzoic acid. The solubility was determined at least six times.

Source and Purity of Chemicals:

(1) Purity not given, Chemical source not specified, no information provided concerning purification.

(2) Spectrophotometric or ACS Reagent grade, Chemical source not specified, redistilled before use.

Estimated Error:

Temperature: $\pm 0.2 \mathrm{~K}$.

$x_{1}: \pm 3 \%$ (relative error).

\begin{tabular}{|c|c|}
\hline $\begin{array}{l}\text { Components: } \\
\text { (1) Benzoic acid; } \mathrm{C}_{7} \mathrm{H}_{6} \mathrm{O}_{2} \text {; } \\
\text { [65-85-0] } \\
\text { (2) } 1,2-\text { Ethanediol; } \mathrm{C}_{2} \mathrm{H}_{6} \mathrm{O}_{2} \text {; } \\
\text { [107-21-1] }\end{array}$ & $\begin{array}{l}\text { Original Measurements: } \\
{ }^{78} \text { A. Yurquina, M. E. Manzur, P. Brito, } \\
\text { R. Manzo, and M. A. A. Molina, J. Mol. } \\
\text { Liq. 108, } 119 \text { (2003). }\end{array}$ \\
\hline $\begin{array}{l}\text { Variables: } \\
T / \mathrm{K}=298.15\end{array}$ & $\begin{array}{l}\text { Prepared by: } \\
\text { W. E. Acree, Jr. }\end{array}$ \\
\hline
\end{tabular}

\section{Experimental Values}

The measured solubility was reported to be $1.501 \mathrm{~mol} \mathrm{dm}^{-3}$.

\section{Auxiliary Information}

\section{Method/Apparatus/Procedure:}

Constant-temperature bath and an ultraviolet/visible spectrophotometer. Very little experimental details were provided. Authors state that after equilibrium is obtained, the solution was filtered through Millipore filters having a porous size of $1 \mu \mathrm{m}$. Concentrations of the saturated solution were determined from spectroscopic measurements at an analysis wavelength of $227 \mathrm{~nm}$.

\section{Source and Purity of Chemicals:}

(1) Purity not given, Chemical source not given, no purification details were provided.

(2) Purity not given, Merck Chemical Company, no purification details were provided.

\section{Estimated Error:}

Temperature: $\pm 0.2 \mathrm{~K}$.

$c_{1}: \pm 2 \%$ (relative error, estimated by compiler).

\begin{tabular}{|c|c|}
\hline $\begin{array}{l}\text { Components: } \\
\text { (1) Benzoic acid; } \mathrm{C}_{7} \mathrm{H}_{6} \mathrm{O}_{2} \text {; } \\
\text { [65-85-0] } \\
\text { (2) 1,2-Propanediol; } \mathrm{C}_{3} \mathrm{H}_{8} \mathrm{O}_{2} \text {; } \\
\text { [57-55-6] }\end{array}$ & $\begin{array}{l}\text { Original Measurements: } \\
{ }^{73} \text { M. Dias, S. L. Raghavan, and J. } \\
\text { Hadgraft, Int. J. Pharm. 216, } 51 \text { (2001). }\end{array}$ \\
\hline $\begin{array}{l}\text { Variables: } \\
T / \mathrm{K}=305.2\end{array}$ & $\begin{array}{l}\text { Prepared by: } \\
\text { W. E. Acree, Jr. }\end{array}$ \\
\hline
\end{tabular}

\section{Experimental Values}

The measured solubility was reported to be $240.8 \mathrm{mg} / \mathrm{ml}$, which corresponds to a molar solubility of $c_{1}=1.972 \mathrm{~mol} \mathrm{dm}^{-3}$.

\section{Auxiliary Information}

\section{Method/Apparatus/Procedure:}

Constant-temperature bath, magnetic stirrer, centrifuge, and an ultraviolet/ visible spectrophotometer.

Excess solute and solvent were equilibrated in a constant-temperature bath with stirring for $48 \mathrm{~h}$. The saturated solution was then centrifuged for $10 \mathrm{~min}$, and an aliquot of the supernatant solution was removed and diluted quantitatively for spectrophotometric analysis. The concentration of the dissolved solute was determined from the measured absorbance.

\section{Source and Purity of Chemicals:}

(1) Purity not given, Fisons Scientific Equipment, UK, no purification details were provided.

(2) Purity not given, Fisher Scientific, UK, no purification details were provided.

\section{Estimated Error:}

Temperature: Insufficient experimental details to estimate. $c_{1}: \pm 0.077$.

\begin{tabular}{|c|c|}
\hline $\begin{array}{l}\text { Components: } \\
\text { (1) Benzoic acid; } \mathrm{C}_{7} \mathrm{H}_{6} \mathrm{O}_{2} \text {; } \\
\text { [65-85-0] } \\
\text { (2) 1,2-Propanediol; } \mathrm{C}_{3} \mathrm{H}_{8} \mathrm{O}_{2} \text {; } \\
\text { [57-55-6] }\end{array}$ & $\begin{array}{l}\text { Original Measurements: } \\
{ }^{88} \text { B. J. Aungst, J. A. Blake, and A. } \\
\text { Hussain, Pharm. Res. 7, } 712 \text { (1990). }\end{array}$ \\
\hline $\begin{array}{l}\text { Variables: } \\
T / \mathrm{K}=\text { Not given }\end{array}$ & $\begin{array}{l}\text { Prepared by: } \\
\text { W. E. Acree, Jr. }\end{array}$ \\
\hline
\end{tabular}

\section{Experimental Values}

The measured solubility was reported to be $250 \mathrm{mg} / \mathrm{ml}$, which corresponds to a molar solubility of $c_{1}=2.047 \mathrm{~mol} \mathrm{dm}^{-3}$.

\section{Auxiliary Information}

\section{Method/Apparatus/Procedure:}

The solubility measurements were performed as part of a study involving drug solubility, partitioning and skin permeation. There were very little experimental details given in regards to the solubility measurement. The authors simply state "Drug solubilities in the vehicles were determined after filtration and dilution or extraction." For the skin permeation measurements the reservoir was maintained at $310 \mathrm{~K}$. The solubility measurement may have been performed at $310 \mathrm{~K}$ as well.

\section{Source and Purity of Chemicals:}

(1) Purity not given, Fisher Scientific, USA, no purification details were provided.

(2) U.S.P. grade, Fisher Scientific, USA, no purification details were provided. 
Estimated Error:

Temperature: Insufficient experimental details to estimate. $c_{1}$ : Insufficient experimental details to estimate.

\begin{tabular}{|c|c|}
\hline $\begin{array}{l}\text { Components: } \\
\text { (1) Benzoic acid; } \mathrm{C}_{7} \mathrm{H}_{6} \mathrm{O}_{2} \text {; } \\
\text { [65-85-0] } \\
\text { (2) 1,2-Propanediol; } \mathrm{C}_{3} \mathrm{H}_{8} \mathrm{O}_{2} \text {; } \\
\text { [57-55-6] }\end{array}$ & $\begin{array}{l}\text { Original Measurements: } \\
{ }^{61} \text { E. R. Cooper, J. Controlled Release } \\
\mathbf{1}, 153 \text { (1984). }\end{array}$ \\
\hline $\begin{array}{l}\text { Variables: } \\
T / \mathrm{K}=295\end{array}$ & Prepared by: \\
\hline
\end{tabular}

Experimental Values

\begin{tabular}{lc}
\hline \hline$x_{2}{ }^{\mathrm{a}}$ & $x_{1}{ }^{\mathrm{b}}$ \\
\hline 0.8652 & 0.1348
\end{tabular}

${ }^{\mathrm{a}} x_{2}$ : mole fraction of component 2 in the saturated solution.

${ }^{b} x_{1}$ : mole fraction solubility of the solute. Solubility data reported in terms of mass percent. Mole fraction solubility calculated by the compiler.

\section{Auxiliary Information}

\section{Method/Apparatus/Procedure:}

Very little experimental details provided. Solubility determinations were made by stirring the solvent with excess solute for two days, centrifuging the sample, and analyzing the supernatant.

Source and Purity of Chemicals:

(1) Reagent grade, Matheson, Coleman, and Bell, Cincinnati, OH, USA, no purification details provided.

(2) Reagent grade, J.T. Baker Chemical Company, Phillipsburg, NJ, USA, no purification details provided.

\section{Estimated Error:}

Temperature: No information given.

$x_{1}$ : No information given.

\begin{tabular}{|c|c|}
\hline $\begin{array}{l}\text { Components: } \\
\text { (1) Benzoic acid; } \mathrm{C}_{7} \mathrm{H}_{6} \mathrm{O}_{2} \text {; } \\
\text { [65-85-0] } \\
\text { (2) 1,2-Propanediol; } \mathrm{C}_{3} \mathrm{H}_{8} \mathrm{O}_{2} \text {; } \\
\text { [57-55-6] }\end{array}$ & $\begin{array}{l}\text { Original Measurements: } \\
{ }^{51} \text { H.-M. Lin and R. A. Nash, J. Pharm. } \\
\text { Sci. 82, } 1018 \text { (1993). }\end{array}$ \\
\hline $\begin{array}{l}\text { Variables: } \\
T / \mathrm{K}=298.15\end{array}$ & $\begin{array}{l}\text { Prepared by: } \\
\text { W. E. Acree, Jr. }\end{array}$ \\
\hline
\end{tabular}

\section{Experimental Values}

\begin{tabular}{lc}
\hline \hline$x_{2}{ }^{\mathrm{a}}$ & $x_{1}{ }^{\mathrm{b}}$ \\
\hline 0.8464 & 0.1536 \\
\hline${ }^{\mathrm{a}} x_{2}:$ mole fraction of component 2 in the saturated solution. & \\
${ }^{\mathrm{b}} x_{1}:$ mole fraction solubility of the solute. &
\end{tabular}

\section{Auxiliary Information}

Method/Apparatus/Procedure:

Constant-temperature bath and high-performance liquid chromatograph. Excess solute and solvent were placed in screw-capped vials. The vials were sealed with several turns of electrical tape, warmed to about $323 \mathrm{~K}$, and then shaken in a constant-temperature bath for at least $24 \mathrm{~h}$ at $298 \mathrm{~K}$. After equilibrium was reached, the sample was filtered through a 0.45 or $1.0 \mu \mathrm{m}$ filter, and an aliquot was diluted appropriately for chromatographic analysis. Concentrations were determined by a high-performance liquid

chromatographic method.

Source and Purity of Chemicals:

(1) Purity not given, Amend Drug and Chemical Company, no information provided concerning purification.

(2) Reagent grade, Aldrich Chemical Company, Milwaukee, WI, USA, no purification details were provided.

\section{Estimated Error:}

Temperature: $\pm 0.2 \mathrm{~K}$ (estimated by compiler). $x_{1}: \pm 3 \%$ (relative error, estimated by compiler).

\begin{tabular}{|c|c|}
\hline $\begin{array}{l}\text { Components: } \\
\text { (1) Benzoic acid; } \mathrm{C}_{7} \mathrm{H}_{6} \mathrm{O}_{2} \text {; } \\
\text { [65-85-0] } \\
\text { (2) 1,2-Propanediol; } \mathrm{C}_{3} \mathrm{H}_{8} \mathrm{O}_{2} \text {; } \\
\text { [57-55-6] }\end{array}$ & $\begin{array}{l}\text { Original Measurements: } \\
{ }^{68} \text { K. K. Kundu, A. L. De, and M. N. } \\
\text { Das, J. Chem. Soc. Dalton Trans. } 1972 \\
386 .\end{array}$ \\
\hline $\begin{array}{l}\text { Variables: } \\
T / \mathrm{K}=298.15\end{array}$ & $\begin{array}{l}\text { Prepared by: } \\
\text { W. E. Acree, Jr. }\end{array}$ \\
\hline
\end{tabular}

\section{Experimental Values}

\begin{tabular}{lc}
\hline \hline$x_{2}{ }^{\mathrm{a}}$ & $x_{1}{ }^{\mathrm{b}}$ \\
\hline 0.8300 & 0.1700 \\
\hline${ }^{\mathrm{a}} x_{2}:$ mole fraction of component 2 in the saturated solution. & \\
${ }^{\mathrm{b}} x_{1}:$ mole fraction solubility of the solute. &
\end{tabular}

\section{Auxiliary Information}

\section{Method/Apparatus/Procedure:}

Very little experimental details are given in paper. Authors state that a saturated solution can be obtained by mild shaking for approximately $24 \mathrm{~h}$. Concentrations determined by titration with standard aqueous alkali.

Source and Purity of Chemicals:

(1) G.R. grade, Merck Chemical Company, was used as received.

(2) Purity not given, Chemical source not given, no purification details were provided.

Estimated Error:

Temperature: $\pm 0.2 \mathrm{~K}$ (estimated by compiler). $x_{1}: \pm 3 \%$ (relative error, estimated by compiler).

\begin{tabular}{|c|c|}
\hline $\begin{array}{l}\text { Components: } \\
\text { (1) Benzoic acid; } \mathrm{C}_{7} \mathrm{H}_{6} \mathrm{O}_{2} \text {; } \\
\text { [65-85-0] } \\
\text { (2) 1,3-Propanediol; } \mathrm{C}_{3} \mathrm{H}_{8} \mathrm{O}_{2} \text {; } \\
\text { [504-63-2] }\end{array}$ & $\begin{array}{l}\text { Original Measurements: } \\
{ }^{51} \text { H.-M. Lin and R. A. Nash, J. Pharm } \\
\text { Sci. 82, } 1018 \text { (1993). }\end{array}$ \\
\hline $\begin{array}{l}\text { Variables: } \\
T / \mathrm{K}=298.15\end{array}$ & $\begin{array}{l}\text { Prepared by: } \\
\text { W. E. Acree, Jr. }\end{array}$ \\
\hline
\end{tabular}


Experimental Values

\begin{tabular}{lc}
\hline \hline$x_{2}{ }^{\mathrm{a}}$ & $x_{1}{ }^{\mathrm{b}}$ \\
\hline 0.8826 & 0.1174 \\
\hline${ }^{\mathrm{a}} x_{2}:$ mole fraction of component 2 in the saturated solution. & \\
${ }^{\mathrm{b}}{ }_{x_{1}}:$ mole fraction solubility of the solute. &
\end{tabular}

Auxiliary Information

\footnotetext{
Method/Apparatus/Procedure:

Constant-temperature bath and high-performance liquid chromatograph. Excess solute and solvent were placed in screw-capped vials. The vials were sealed with several turns of electrical tape, warmed to about $323 \mathrm{~K}$, and then shaken in a constant-temperature bath for at least $24 \mathrm{~h}$ at $298 \mathrm{~K}$. After equilibrium was reached, the sample was filtered through a 0.45 or $1.0 \mu \mathrm{m}$ filter, and an aliquot was diluted appropriately for chromatographic analysis. Concentrations were determined by a high-performance liquid chromatographic method.
}

Source and Purity of Chemicals:

(1) Purity not given, Amend Drug and Chemical Company, no information provided concerning purification.

(2) Reagent grade, Aldrich Chemical Company, Milwaukee, WI, USA, no purification details were provided.

Estimated Error:

Temperature: $\pm 0.2 \mathrm{~K}$ (estimated by compiler).

$x_{1}: \pm 3 \%$ (relative error, estimated by compiler).

\begin{tabular}{ll}
\hline \hline Components: & Original Measurements: \\
(1) Benzoic acid; $\mathrm{C}_{7} \mathrm{H}_{6} \mathrm{O}_{2} ;$ & ${ }^{47}$ A. Beerbower, P. L. Wu, and A. \\
[65-85-0] & Martin, J. Pharm. Sci. 73, 179 (1984). \\
$(2) 1,2,3-$ Propanetriol (Glycerol); & \\
$\mathrm{C}_{3} \mathrm{H}_{8} \mathrm{O}_{3} ;[56-81-5]$ & \\
\hline Variables: & Prepared by: \\
$T / \mathrm{K}=298.15$ & W. E. Acree, Jr. \\
\hline
\end{tabular}

\section{Experimental Values}

\begin{tabular}{lc}
\hline \hline$x_{2}{ }^{\mathrm{a}}$ & $x_{1}{ }^{\mathrm{b}}$ \\
\hline 0.9836 & 0.0164 \\
\hline${ }^{\mathrm{a}} x_{2}:$ mole fraction of component 2 in the saturated solution. & \\
${ }^{\mathrm{b}} x_{1}:$ mole fraction solubility of the solute. &
\end{tabular}

\section{Auxiliary Information}

\section{Method/Apparatus/Procedure:}

Constant-temperature bath and ultraviolet/visible spectrophotometer.

Excess solute and solvent were placed in screw-capped vials. The vials were sealed and submerged in a constant-temperature water bath and shaken at 100 cycles/min for $24 \mathrm{~h}$. After the $24 \mathrm{~h}$ equilibration period, the vial was removed, wiped dry, and the contents analyzed. The solutions were transferred to a syringe and filtered through a $0.1 \mu \mathrm{m}$ pore size filter. The solutions were diluted and the absorbances recorded at the maximum absorption wavelength of benzoic acid. The solubility was determined at least six times.
}

Source and Purity of Chemicals:

(1) Purity not given, Chemical source not specified, no information provided concerning purification.

(2) Spectrophotometric or ACS Reagent grade, Chemical source not specified, redistilled before use.

\section{Estimated Error:}

Temperature: $\pm 0.2 \mathrm{~K}$.

$x_{1}: \pm 3 \%$ (relative error).

\begin{tabular}{|c|c|}
\hline $\begin{array}{l}\text { Components: } \\
\text { (1) Benzoic acid; } \mathrm{C}_{7} \mathrm{H}_{6} \mathrm{O}_{2} \text {; } \\
\text { [65-85-0] } \\
\text { (2) } 1,2,3-\text { Propanetriol (Glycerol); } \\
\left.\mathrm{C}_{3} \mathrm{H}_{8} \mathrm{O}_{3} ; \text {; } 56-81-5\right]\end{array}$ & $\begin{array}{l}\text { Original Measurements: } \\
{ }^{87} \text { M. Dias, J. Hadgraft, and M. E. Lane } \\
\text { Int. J. Pharm. 336, } 108 \text { (2007). }\end{array}$ \\
\hline $\begin{array}{l}\text { Variables: } \\
T / \mathrm{K}=305.2\end{array}$ & $\begin{array}{l}\text { Prepared by: } \\
\text { W. E. Acree, Jr. }\end{array}$ \\
\hline
\end{tabular}

\section{Experimental Values}

The measured solubility was reported to be $22.60 \mathrm{mg} / \mathrm{ml}$, which corresponds to a molar solubility of $c_{1}=0.185 \mathrm{~mol} \mathrm{dm}^{-3}$.

\section{Auxiliary Information}

\section{Method/Apparatus/Procedure:}

Constant-temperature bath, magnetic stirrer, centrifuge, and an ultraviolet/ visible spectrophotometer.

Excess solute and solvent were equilibrated in a constant-temperature bath with stirring for $48 \mathrm{~h}$. The saturated solution was then centrifuged for $10 \mathrm{~min}$, and an aliquot of the supernatant solution was removed and diluted quantitatively for spectrophotometric analysis. The concentration of the dissolved solute was determined from the measured absorbance.

Source and Purity of Chemicals:

(1) Purity not given, Fisons Scientific Equipment, UK, no purification details were provided.

(2) Purity not given, ICN Biochemicals, no purification details were provided.

\section{Estimated Error:}

Temperature: Insufficient experimental details to estimate.

$c_{1}: \pm 0.0041$.

\subsection{Benzoic acid solubility data in alkoxyalcohols}

\section{Components:}

(1) Benzoic acid; $\mathrm{C}_{7} \mathrm{H}_{6} \mathrm{O}_{2}$; [65-85-0]

(2) 2-Methoxyethanol; $\mathrm{C}_{3} \mathrm{H}_{8} \mathrm{O}_{2}$; [109-86-4]

\begin{tabular}{ll}
\hline Variables: & Prepared by: \\
$T / \mathrm{K}=298.15$ & W. E. Acree, Jr. \\
\hline
\end{tabular}

Experimental Values

The measured solubility was reported to be $c_{1}=$ $3.0535 \mathrm{~mol} \mathrm{dm}^{-3}$.

\section{Original Measurements:}

${ }^{62}$ S. H. Ghosh and D. K. Hazra, J.

Chem. Soc., Perkin Trans. 21989 1021.

W. E. Acree, Jr. 


\section{Auxiliary Information}

\section{Method/Apparatus/Procedure:}

Mechanical shaker and a constant-temperature thermostat.

Excess solute and solvent were placed in a Campbell solubility apparatus and allowed to equilibrate at $298 \mathrm{~K}$ for $24 \mathrm{~h}$. An aliquot of the saturated solution was then removed and filtered. The concentration of the dissolved solute was determined by titration with standard caustic soda using phenolphthalein as indicator. Solubility was also determined spectrophotometrically from absorbance measurements made at an analysis wavelength of $272 \mathrm{~nm}$.

\section{Source and Purity of Chemicals:}

(1) G.R.E, Merck Chemical Company, was recrystallized from alcohol and then dried.

(2) Purity not given, Merck Chemical Company, was distilled twice before use.

\section{Estimated Error:}

Temperature: $\pm 0.01 \mathrm{~K}$.

$c_{1}: \pm 5 \%$ (relative error, estimated by compiler).

\begin{tabular}{|c|c|}
\hline $\begin{array}{l}\text { Components: } \\
\text { (1) Benzoic acid; } \mathrm{C}_{7} \mathrm{H}_{6} \mathrm{O}_{2} \text {; } \\
\text { [65-85-0] } \\
\text { (2) 2-Ethoxyethanol; } \mathrm{C}_{4} \mathrm{H}_{10} \mathrm{O}_{2} \text {; } \\
\text { [110-80-5] }\end{array}$ & $\begin{array}{l}\text { Original Measurements: } \\
{ }^{51} \text { H.-M. Lin and R. A. Nash, J. Pharm. } \\
\text { Sci. 82, } 1018 \text { (1993). }\end{array}$ \\
\hline $\begin{array}{l}\text { Variables: } \\
T / \mathrm{K}=298.15\end{array}$ & $\begin{array}{l}\text { Prepared by: } \\
\text { W. E. Acree, Jr. }\end{array}$ \\
\hline
\end{tabular}

Experimental Values

\begin{tabular}{lc}
\hline \hline$x_{2}{ }^{\mathrm{a}}$ & $x_{1}{ }^{\mathrm{b}}$ \\
\hline 0.7164 & 0.2836 \\
\hline${ }^{\mathrm{a}} x_{2}:$ mole fraction of component 2 in the saturated solution. & \\
${ }^{\mathrm{b}} x_{1}:$ mole fraction solubility of the solute. &
\end{tabular}

\section{Auxiliary Information}

\section{Method/Apparatus/Procedure:}

Constant-temperature bath and high-performance liquid chromatograph. Excess solute and solvent were placed in screw-capped vials. The vials were sealed with several turns of electrical tape, warmed to about $323 \mathrm{~K}$, and then shaken in a constant-temperature bath for at least $24 \mathrm{~h}$ at $298 \mathrm{~K}$. After equilibrium was reached, the sample was filtered through a 0.45 or $1.0 \mu \mathrm{m}$ filter, and an aliquot was diluted appropriately for chromatographic analysis. Concentrations were determined by a high-performance liquid chromatographic method.

\section{Source and Purity of Chemicals:}

(1) Purity not given, Amend Drug and Chemical Company, no information provided concerning purification.

(2) HPLC grade, Aldrich Chemical Company, Milwaukee, WI, USA, no purification details were provided.

\section{Estimated Error:}

Temperature: $\pm 0.2 \mathrm{~K}$ (estimated by compiler). $x_{1}: \pm 3 \%$ (relative error, estimated by compiler).

\subsection{Benzoic acid solubility data in ketones}

\begin{tabular}{ll}
\hline \hline Components: & Original Measurements: \\
$\begin{array}{l}\text { (1) Benzoic acid; } \mathrm{C}_{7} \mathrm{H}_{6} \mathrm{O}_{2} ; \\
\text { [65-85-0] }\end{array}$ & ${ }^{47}$ A. Beerbower, P. L. Wu, and A. \\
$\begin{array}{l}\text { (2) Propanone; } \mathrm{C}_{3} \mathrm{H}_{6} \mathrm{O} ; \\
{[67-64-1]}\end{array}$ & \\
\hline Variables: & Prepared byarm. Sci. 73, 179 (1984). \\
$T / \mathrm{K}=298.15$ & W. E. Acree, Jr. \\
\hline
\end{tabular}

Experimental Values

\begin{tabular}{lc}
\hline \hline$x_{2}{ }^{\mathrm{a}}$ & $x_{1}{ }^{\mathrm{b}}$ \\
\hline 0.8143 & 0.1857 \\
\hline${ }^{\mathrm{a}} x_{2}:$ mole fraction of component 2 in the saturated solution. & \\
${ }^{\mathrm{b}} x_{1}:$ mole fraction solubility of the solute. &
\end{tabular}

\section{Auxiliary Information}

\section{Method/Apparatus/Procedure:}

Constant-temperature bath and ultraviolet/visible spectrophotometer.

Excess solute and solvent were placed in screw-capped vials. The vials were sealed and submerged in a constant-temperature water bath and shaken at 100 cycles/min for $24 \mathrm{~h}$. After the $24 \mathrm{~h}$ equilibration period, the vial was removed, wiped dry, and the contents analyzed. The solutions were transferred to a syringe and filtered through a $0.1 \mu \mathrm{m}$ pore size filter. The solutions were diluted and the absorbances recorded at the maximum absorption wavelength of benzoic acid. The solubility was determined at least six times.

Source and Purity of Chemicals:

(1) Purity not given, Chemical source not specified, no information provided concerning purification.

(2) Spectrophotometric or ACS Reagent grade, Chemical source not specified, redistilled before use.

\section{Estimated Error:}

Temperature: $\pm 0.2 \mathrm{~K}$.

$x_{1}: \pm 3 \%$ (relative error).

\begin{tabular}{ll}
\hline \hline Components: & Original Measurements: \\
$\begin{array}{l}\text { (1) Benzoic acid; } \mathrm{C}_{7} \mathrm{H}_{6} \mathrm{O}_{2} ; \\
\text { [65-85-0] }\end{array}$ & $\begin{array}{l}\text { McMinn, and T. R. A. Magee, Powder } \\
\text { (2) Propanone; } \mathrm{C}_{3} \mathrm{H}_{6} \mathrm{O} ;\end{array}$ \\
[67-64-1] & Technol. 134, 40 (2003). \\
\hline Variables: & Prepared by: \\
$T / \mathrm{K}=293$ & W. E. Acree, Jr. \\
\hline
\end{tabular}

\section{Experimental Values}

The measured solubility was reported to be $550 \mathrm{~g} / \mathrm{l}$. The authors did not specify whether the values were per liter of solvent or per liter of saturated solution. 


\section{Auxiliary Information}

\section{Method/Apparatus/Procedure:}

Very little experimental details were provided. Solubility was determined by adding $(0.50 \pm 0.1) \mathrm{g}$ amounts of the powdered solute to $(100 \pm 1) \mathrm{ml}$ of solvent until a saturated solution was obtained. The mass of the powder was recorded.

Source and Purity of Chemicals:

(1) Purity not given, Sigma-Aldrich, Poole, England, UK, no purification details were provided.

(2) Purity not given, Sigma-Aldrich, no purification details were provided.

Estimated Error:

Temperature: Insufficient experimental details to estimate.

Solubility: $\pm 5 \mathrm{~g} / \mathrm{l}$ (estimated by compiler assuming solute added in increments of $0.50 \mathrm{~g}$ ).

\begin{tabular}{|c|c|}
\hline $\begin{array}{l}\text { Components: } \\
\text { (1) Benzoic acid; } \mathrm{C}_{7} \mathrm{H}_{6} \mathrm{O}_{2} \text {; } \\
\text { [65-85-0] } \\
\text { (2) Propanone; } \mathrm{C}_{3} \mathrm{H}_{6} \mathrm{O} \text {; } \\
\text { [67-64-1] }\end{array}$ & $\begin{array}{l}\text { Original Measurements: } \\
{ }^{55} \text { B. Long, J. Li, R. Zhang, and L. Wan, } \\
\text { Fluid Phase Equilib. 297, } 113 \text { (2010). }\end{array}$ \\
\hline $\begin{array}{l}\text { Variables: } \\
\text { Temperature }\end{array}$ & $\begin{array}{l}\text { Prepared by: } \\
\text { W. E. Acree, Jr. }\end{array}$ \\
\hline
\end{tabular}

Experimental Values

\begin{tabular}{lcc}
\hline \hline$T / \mathrm{K}$ & $x_{2}{ }^{\mathrm{a}}$ & $x_{1}{ }^{\mathrm{b}}$ \\
\hline 277.59 & 0.8733 & 0.1267 \\
282.21 & 0.8570 & 0.1430 \\
287.03 & 0.8424 & 0.1576 \\
287.19 & 0.8649 & 0.1571 \\
293.20 & 0.8247 & 0.1753 \\
298.15 & 0.8075 & 0.1925 \\
302.95 & 0.7889 & 0.2111 \\
307.75 & 0.7688 & 0.2312 \\
313.05 & 0.7469 & 0.2531 \\
317.63 & 0.7235 & 0.2765 \\
322.72 & 0.6967 & 0.3033
\end{tabular}

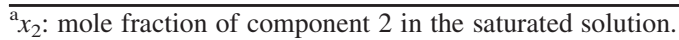

${ }^{b} x_{1}$ : mole fraction solubility of the solute.

\section{Auxiliary Information}

\section{Method/Apparatus/Procedure:}

Double layer jacketed glass equilibrium cell, circulating water bath, analytical balance, and drying oven.

Excess solute and solvent and were placed in an equilibrium vessel, which was connected to a circulating water bath. The solution was allowed to equilibrate at constant temperature with stirring for $3 \mathrm{~h}$. The stirring was discontinued and the solution was allowed to stand for $1 \mathrm{~h}$ to allow the undissolved solid to settle to the bottom portion of the equilibrium vessel. An aliquot of the clear solution was transferred to a preweighed vial by a warm syringe. The vial was tightly closed and reweighed to determine the mass of the sample transferred. The vial was then put into a drying oven with the cap half-closed to permit complete evaporation of the solvent. The vial was covered with a piece of stainless steel filter cloth to prevent dust contamination. After the solvent had evaporated, the vial was removed from the drying oven and placed in a desiccator with silica gel for another $2 \mathrm{~h}$ to reach ambient room temperature. The vial with solid residue was weighed until constant weight was obtained. The solubility was calculated from the mass of the solid residue and mass of sample analyzed.
Source and Purity of Chemicals:

(1) $99.5 \%$, Analytical grade, Shantou Xilong Chemical Company, China, used as received.

(2) $99.5 \%$, Analytical grade, Beijing Chemical Reagent Company, China, used as received.

\section{Estimated Error:}

Temperature: $\pm 0.05 \mathrm{~K}$.

$x_{1}: \pm 1 \%$ (relative error)

\begin{tabular}{ll}
\hline \hline Components: & $\begin{array}{l}\text { Original Measurements: } \\
\text { (1) Benzoic acid; } \mathrm{C}_{7} \mathrm{H}_{6} \mathrm{O}_{2} ;\end{array}$ \\
$\begin{array}{l}\text { 57 J. W. Marden and M. V. Dover, J. } \\
\text { (2) Pr-0] }\end{array}$ & Am. Chem. Soc. 38, 1235 (1916). \\
[67-64-1] & \\
\hline Variables: $\mathrm{C}_{3} \mathrm{H}_{6} \mathrm{O} ;$ & Prepared by: \\
$T / \mathrm{K}=298.15$ & W. E. Acree, Jr. \\
\hline
\end{tabular}

\section{Experimental Values}

\begin{tabular}{lc}
\hline \hline$x_{2}{ }^{\mathrm{a}}$ & $x_{1}{ }^{\mathrm{b}}$ \\
\hline 0.791 & 0.209 \\
\hline
\end{tabular}

${ }^{a} x_{2}$ : mole fraction of component 2 in the saturated solution.

${ }^{\mathrm{b}} \mathrm{x}_{1}$ : mole fraction solubility of the solute. Experimental solubility data were given as grams of solute per $100 \mathrm{~g}$ of solvent. Mole fraction solubility was calculated by the compiler.

\section{Auxiliary Information}

\section{Method/Apparatus/Procedure:}

Constant-temperature thermostat, analytical balance, and a steam bath. Excess solute and solvent were placed in a bottle with ground glass stopper which was protected by a coating of beeswax and rosin, over which was tied a piece of rubber sheeting. The sealed mixture was shaken in a constanttemperature thermostat for 8-20 h. An aliquot of the saturated solution was withdrawn through a glass wool filter into a weighing pipet from which the solutions were weighed directly into small glass evaporating dishes. The solvent was removed on a steam bath, and the sample further dried in a sulfuric acid desiccator. The solubility was calculated from the mass of the solid residue and the mass of the saturated solution removed for analysis.

\section{Source and Purity of Chemicals:}

(1) Purity not given, Chemical source not given, no purification details were provided.

(2) Purity not given, Chemical source not given, no purification details were provided.

\section{Estimated Error:}

Temperature: $\pm 0.1 \mathrm{~K}$.

$x_{1}: \pm 10.0 \%$ (relative error, estimated by compiler).

\begin{tabular}{|c|c|}
\hline $\begin{array}{l}\text { Components: } \\
\text { (1) Benzoic acid; } \mathrm{C}_{7} \mathrm{H}_{6} \mathrm{O}_{2} \text {; } \\
\text { [65-85-0] } \\
\text { (2) Propanone; } \mathrm{C}_{3} \mathrm{H}_{6} \mathrm{O} \text {; } \\
\text { [67-64-1] }\end{array}$ & $\begin{array}{l}\text { Original Measurements: } \\
\text { }{ }^{50} \text { P. G. Desai and A. M. Patel, J. Indian } \\
\text { Chem. Soc. 12, } 131 \text { (1935). }\end{array}$ \\
\hline $\begin{array}{l}\text { Variables: } \\
T / \mathrm{K}=301.2\end{array}$ & $\begin{array}{l}\text { Prepared by: } \\
\text { W. E. Acree, Jr. }\end{array}$ \\
\hline
\end{tabular}


Experimental Values

\begin{tabular}{lc}
\hline \hline$x_{2}^{\mathrm{a}}$ & $x_{1}^{\mathrm{b}}$ \\
\hline 0.7859 & 0.2141
\end{tabular}

${ }^{\mathrm{a}} x_{2}$ : mole fraction of component 2 in the saturated solution.

${ }^{\mathrm{b}} x_{1}$ : mole fraction solubility of the solute.

\section{Auxiliary Information}

\section{Method/Apparatus/Procedure:}

Excess solute and solvent were placed in a glass flask which was kept revolving on a wheel in an air thermostat for two days. After two days of equilibration, the solution was filtered. To minimize absorption of the solute onto the filter paper, a $100 \mathrm{ml}$ portion of fresh saturated solution was first filtered through the paper before filtering the sample to be analyzed. An aliquot of the filtrate was titrated with barium hydroxide using phenolphthalein as the endpoint indictor. The barium hydroxide titrant was previously standardized against a solution of succinic acid.

Source and Purity of Chemicals:

(1) Purity not given, Chemical source not specified, no information provided concerning purification.

(2) Purity not given, Chemical source not specified, no information provided concerning purification.

Estimated Error:

Temperature: No information given.

$x_{1}: \pm 3 \%$ (relative error, estimated by compiler).

\begin{tabular}{ll}
\hline \hline Components: & Original Measurements: \\
$\begin{array}{l}\text { (1) Benzoic acid; } \mathrm{C}_{7} \mathrm{H}_{6} \mathrm{O}_{2} ; \\
\text { [65-85-0] }\end{array}$ & $\begin{array}{l}{ }^{47} \text { A. Beerbower, P. L. Wu, and A. } \\
\text { (2) Acetophenone; } \mathrm{C}_{8} \mathrm{H}_{8} \mathrm{O} ;\end{array}$ \\
[98-86-2] & \\
\hline Variables: & Prepared by: \\
$T / \mathrm{K}=298.15$ & W. E. Acree, Jr. \\
\hline
\end{tabular}

Experimental Values

\begin{tabular}{lc}
\hline \hline$x_{2}^{\mathrm{a}}$ & $x_{1}{ }^{\mathrm{b}}$ \\
\hline 0.8122 & 0.1878 \\
\hline${ }^{\mathrm{a}} x_{2}:$ mole fraction of component 2 in the saturated solution. & \\
${ }^{\mathrm{b}} x_{1}:$ mole fraction solubility of the solute. &
\end{tabular}

\section{Auxiliary Information}

\section{Method/Apparatus/Procedure:}

Constant-temperature bath and ultraviolet/visible spectrophotometer.

Excess solute and solvent were placed in screw-capped vials. The vials were sealed and submerged in a constant-temperature water bath and shaken at 100 cycles/min for $24 \mathrm{~h}$. After the $24 \mathrm{~h}$ equilibration period, the vial was removed, wiped dry, and the contents analyzed. The solutions were transferred to a syringe and filtered through a $0.1 \mu \mathrm{m}$ pore size filter. The solutions were diluted and the absorbances recorded at the maximum absorption wavelength of benzoic acid. The solubility was determined at least six times.

Source and Purity of Chemicals:

(1) Purity not given, Chemical source not specified, no information provided concerning purification.

(2) Spectrophotometric or ACS Reagent grade, Chemical source not specified, redistilled before use

Estimated Error:

Temperature: $\pm 0.2 \mathrm{~K}$.

$x_{1}: \pm 3 \%$ (relative error)

\subsection{Benzoic acid solubility data in miscellaneous organic solvents}

\begin{tabular}{ll}
\hline \hline Components: & $\begin{array}{l}\text { Original Measurements: } \\
\text { (1) Benzoic acid; } \mathrm{C}_{7} \mathrm{H}_{6} \mathrm{O}_{2} ;\end{array}$ \\
$\begin{array}{l}{ }^{47} \text { A. Beerbower, P. L. Wu, and A. } \\
\text { [65-85-0] }\end{array}$ & Martin, J. Pharm. Sci. 73, 179 (1984). \\
(2) Nitrobenzene; $\mathrm{C}_{6} \mathrm{H}_{5} \mathrm{NO}_{2} ;$ & \\
\hline $98-95-3]$ & Prepared by: \\
\hline Variables: & W. E. Acree, Jr. \\
\hline$/ \mathrm{K}=298.15$ &
\end{tabular}

Experimental Values

\begin{tabular}{lc}
\hline \hline$x_{2}{ }^{\mathrm{a}}$ & $x_{1}{ }^{\mathrm{b}}$ \\
\hline 0.9103 & 0.0897 \\
\hline${ }^{\mathrm{a}} x_{2}:$ mole fraction of component 2 in the saturated solution. & \\
${ }^{\mathrm{b}} x_{1}:$ mole fraction solubility of the solute. &
\end{tabular}

Auxiliary Information

\section{Method/Apparatus/Procedure:}

Constant-temperature bath and ultraviolet/visible spectrophotometer.

Excess solute and solvent were placed in screw-capped vials. The vials were sealed and submerged in a constant-temperature water bath and shaken at 100 cycles/min for $24 \mathrm{~h}$. After the $24 \mathrm{~h}$ equilibration period, the vial was removed, wiped dry, and the contents analyzed. The solutions were transferred to a syringe and filtered through a $0.1 \mu \mathrm{m}$ pore size filter. The solutions were diluted and the absorbances recorded at the maximum absorption wavelength of benzoic acid. The solubility was determined at least six times.

Source and Purity of Chemicals:

(1) Purity not given, Chemical source not specified, no information provided concerning purification.

(2) Spectrophotometric or ACS Reagent grade, Chemical source not specified, redistilled before use.

\section{Estimated Error:}

Temperature: $\pm 0.2 \mathrm{~K}$.

$x_{1}: \pm 3 \%$ (relative error).

\section{Components:}

(1) Benzoic acid; $\mathrm{C}_{7} \mathrm{H}_{6} \mathrm{O}_{2}$; [65-85-0]

(2) Nitrobenzene; $\mathrm{C}_{6} \mathrm{H}_{5} \mathrm{NO}_{2}$; [98-95-3]

\begin{tabular}{ll}
\hline Variables: & Prepared by: \\
$T / \mathrm{K}=301.2$ & W. E. Acree, Jr. \\
\hline
\end{tabular}

\section{Experimental Values}

\begin{tabular}{lc}
\hline \hline$x_{2}{ }^{\mathrm{a}}$ & $x_{1}{ }^{\mathrm{b}}$ \\
\hline 0.8919 & 0.1081 \\
\hline${ }^{\mathrm{a}} x_{2}:$ mole fraction of component 2 in the saturated solution. & \\
${ }^{\mathrm{b}} x_{1}:$ mole fraction solubility of the solute. &
\end{tabular}




\section{Auxiliary Information}

\section{Method/Apparatus/Procedure:}

Excess solute and solvent were placed in a glass flask which was kept revolving on a wheel in an air thermostat for two days. After two days of equilibration, the solution was filtered. To minimize absorption of the solute onto the filter paper, a $100 \mathrm{ml}$ portion of fresh saturated solution was first filtered through the paper before filtering the sample to be analyzed. An aliquot of the filtrate was titrated with barium hydroxide using phenolphthalein as the endpoint indictor. The barium hydroxide titrant was previously standardized against a solution of succinic acid.

\section{Source and Purity of Chemicals:}

(1) Purity not given, Chemical source not specified, no information provided concerning purification.

(2) Purity not given, Chemical source not specified, no information provided concerning purification.

\section{Estimated Error:}

Temperature: No information given.

$x_{1}: \pm 3 \%$ (relative error, estimated by compiler).

\begin{tabular}{|c|c|}
\hline $\begin{array}{l}\text { Components: } \\
\text { (1) Benzoic acid; } \mathrm{C}_{7} \mathrm{H}_{6} \mathrm{O}_{2} \text {; } \\
\text { [65-85-0] } \\
\text { (2) Pyridine; } \mathrm{C}_{5} \mathrm{H}_{5} \mathrm{~N} \text {; } \\
\text { [110-86-1] }\end{array}$ & $\begin{array}{l}\text { Original Measurements: } \\
{ }^{47} \text { A. Beerbower, P. L. Wu, and A. } \\
\text { Martin, J. Pharm. Sci. 73, } 179 \text { (1984) }\end{array}$ \\
\hline $\begin{array}{l}\text { Variables: } \\
T / \mathrm{K}=298.15\end{array}$ & $\begin{array}{l}\text { Prepared by: } \\
\text { W. E. Acree, Jr. }\end{array}$ \\
\hline
\end{tabular}

Experimental Values

\begin{tabular}{lc}
\hline \hline$x_{2}{ }^{\mathrm{a}}$ & $x_{1}{ }^{\mathrm{b}}$ \\
\hline 0.4652 & 0.5348
\end{tabular}

${ }^{\mathrm{a}} x_{2}$ : mole fraction of component 2 in the saturated solution.

${ }^{\mathrm{b}} x_{1}$ : mole fraction solubility of the solute.

\section{Auxiliary Information}

\section{Method/Apparatus/Procedure:}

Constant-temperature bath and ultraviolet/visible spectrophotometer.

Excess solute and solvent were placed in screw-capped vials. The vials were sealed and submerged in a constant-temperature water bath and shaken at 100 cycles/min for $24 \mathrm{~h}$. After the $24 \mathrm{~h}$ equilibration period, the vial was removed, wiped dry, and the contents analyzed. The solutions were transferred to a syringe and filtered through a $0.1 \mu \mathrm{m}$ pore size filter. The solutions were diluted and the absorbances recorded at the maximum absorption wavelength of benzoic acid. The solubility was determined at least six times.

Source and Purity of Chemicals:

(1) Purity not given, Chemical source not specified, no information provided concerning purification.

(2) Spectrophotometric or ACS Reagent grade, Chemical source not specified, redistilled before use.

Estimated Error:

Temperature: $\pm 0.2 \mathrm{~K}$.

$x_{1}: \pm 3 \%$ (relative error).

\begin{tabular}{ll}
\hline \hline Components: & Original Measurements: \\
$\begin{array}{l}\text { (1) Benzoic acid; } \mathrm{C}_{7} \mathrm{H}_{6} \mathrm{O}_{2} ; \\
\text { [65-85-0] }\end{array}$ & ${ }^{47}$ A. Beerbower, P. L. Wu, and A. \\
$\begin{array}{l}\text { (2) Ethanoic acid; } \mathrm{C}_{2} \mathrm{H}_{4} \mathrm{O}_{2} ; \\
\text { [64-19-7] }\end{array}$ & \\
\hline Variables: & Prepared by: \\
$T / \mathrm{K}=298.15$ & W. E. Acree, Jr. \\
\hline
\end{tabular}

Experimental Values

\begin{tabular}{lc}
\hline \hline$x_{2}{ }^{\mathrm{a}}$ & $x_{1}{ }^{\mathrm{b}}$ \\
\hline 0.8325 & 0.1675 \\
\hline
\end{tabular}

${ }^{a} x_{2}$ : mole fraction of component 2 in the saturated solution.

${ }^{\mathrm{b}}{ }_{x_{1}}$ : mole fraction solubility of the solute.

\section{Auxiliary Information}

Method/Apparatus/Procedure:

Constant-temperature bath and ultraviolet/visible spectrophotometer.

Excess solute and solvent were placed in screw-capped vials. The vials were sealed and submerged in a constant-temperature water bath and shaken at 100 cycles/min for $24 \mathrm{~h}$. After the $24 \mathrm{~h}$ equilibration period, the vial was removed, wiped dry, and the contents analyzed. The solutions were transferred to a syringe and filtered through a $0.1 \mu \mathrm{m}$ pore size filter. The solutions were diluted and the absorbances recorded at the maximum absorption wavelength of benzoic acid. The solubility was determined at least six times.

\section{Source and Purity of Chemicals:}

(1) Purity not given, Chemical source not specified, no information provided concerning purification.

(2) Spectrophotometric or ACS Reagent grade, Chemical source not specified, redistilled before use.

Estimated Error:

Temperature: $\pm 0.2 \mathrm{~K}$.

$x_{1}: \pm 3 \%$ (relative error).

\begin{tabular}{|c|c|}
\hline $\begin{array}{l}\text { Components: } \\
\text { (1) Benzoic acid; } \mathrm{C}_{7} \mathrm{H}_{6} \mathrm{O}_{2} \text {; } \\
\text { [65-85-0] } \\
\text { (2) Ethanoic acid; } \mathrm{C}_{2} \mathrm{H}_{4} \mathrm{O}_{2} \text {; } \\
\text { [64-19-7] }\end{array}$ & $\begin{array}{l}\text { Original Measurements: } \\
\text { 55 B. Long, J. Li, R. Zhang, and L. Wan, } \\
\text { Fluid Phase Equilib. 297, } 113 \text { (2010). }\end{array}$ \\
\hline Variables: & Prepared by: \\
\hline Temperature & W. E. Acree, Jr. \\
\hline
\end{tabular}

Experimental Values

\begin{tabular}{|c|c|c|}
\hline$T / \mathrm{K}$ & $x_{2}{ }^{\mathrm{a}}$ & $x_{1}{ }^{\mathrm{b}}$ \\
\hline 292.95 & 0.8715 & 0.1285 \\
\hline 298.45 & 0.8519 & 0.1481 \\
\hline 303.05 & 0.8345 & 0.1655 \\
\hline 307.75 & 0.8345 & 0.1877 \\
\hline 312.15 & 0.7893 & 0.2107 \\
\hline 318.35 & 0.7633 & 0.2367 \\
\hline 323.15 & 0.7338 & 0.2662 \\
\hline 327.95 & 0.7033 & 0.2967 \\
\hline 332.75 & 0.6719 & 0.3281 \\
\hline 337.15 & 0.6464 & 0.3536 \\
\hline 342.75 & 0.5992 & 0.4008 \\
\hline 346.65 & 0.5750 & 0.4250 \\
\hline
\end{tabular}




\section{Auxiliary Information}

\section{Method/Apparatus/Procedure:}

Double layer jacketed glass equilibrium cell, circulating water bath, analytical balance, and drying oven.

Excess solute and solvent and were placed in an equilibrium vessel, which was connected to a circulating water bath. The solution was allowed to equilibrate at constant temperature with stirring for $3 \mathrm{~h}$. The stirring was discontinued and the solution was allowed to stand for $1 \mathrm{~h}$ to allow the undissolved solid to settle to the bottom portion of the equilibrium vessel. An aliquot of the clear solution was transferred to a preweighed vial by a warm syringe. The vial was tightly closed and reweighed to determine the mass of the sample transferred. The vial was then put into a drying oven with the cap half-closed to permit complete evaporation of the solvent. The vial was covered with a piece of stainless steel filter cloth to prevent dust contamination. After the solvent had evaporated the vial was removed from the drying oven and placed in a desiccator with silica gel for another $2 \mathrm{~h}$ to reach ambient room temperature. The vial with solid residue was weighed until constant weight was obtained. The solubility was calculated from the mass of the solid residue and mass of sample analyzed.

\section{Source and Purity of Chemicals:}

(1) $99.5 \%$, Analytical grade, Shantou Xilong Chemical Company, China, was used as received.

(2) $99.5 \%$, Analytical grade, Beijing Chemical Reagent Company, China, used as received.

Estimated Error:

Temperature: $\pm 0.05 \mathrm{~K}$.

$x_{1}: \pm 1 \%$ (relative error)

\begin{tabular}{|c|c|}
\hline $\begin{array}{l}\text { Components: } \\
\text { (1) Benzoic acid; } \mathrm{C}_{7} \mathrm{H}_{6} \mathrm{O}_{2} \text {; } \\
\text { [65-85-0] } \\
\text { (2) Ethanoic acid; } \mathrm{C}_{2} \mathrm{H}_{4} \mathrm{O}_{2} \text {; } \\
\text { [64-19-7] }\end{array}$ & $\begin{array}{l}\text { Original Measurements: } \\
{ }^{79} \text { Q. Wang, L. Hou, Y. Cheng, and X. } \\
\text { Li, J. Chem. Eng. Data 52, } 936 \text { (2007). }\end{array}$ \\
\hline $\begin{array}{l}\text { Variables: } \\
\text { Temperature }\end{array}$ & $\begin{array}{l}\text { Prepared by: } \\
\text { W. E. Acree, Jr. }\end{array}$ \\
\hline
\end{tabular}

Experimental Values

\begin{tabular}{lcc}
\hline \hline$T / \mathrm{K}$ & $x_{2}{ }^{\mathrm{a}}$ & $x_{1}{ }^{\mathrm{b}, \mathrm{c}}$ \\
\hline 298.3 & 0.8903 & 0.1097 \\
308.4 & 0.8689 & 0.1311 \\
318.4 & 0.8424 & 0.1576 \\
328.2 & 0.8199 & 0.1801 \\
338.0 & 0.7928 & 0.2072 \\
348.3 & 0.7753 & 0.2247 \\
358.6 & 0.7446 & 0.2554
\end{tabular}

${ }^{a} x_{2}$ : mole fraction of component 2 in the saturated solution.

${ }^{\mathrm{b}} x_{1}$ : mole fraction solubility of the solute.

${ }^{\mathrm{c}}$ Solubility data were given in units of grams per $100 \mathrm{~g}$ of solvent. Mole fraction solubilities were calculated by compiler.

\section{Auxiliary Information}

\section{Method/Apparatus/Procedure:}

Constant-temperature bath and analytical balance.

Solubilities were determined by a static method. Excess solute and solvent were placed in sealed bottles and allowed to equilibrate in a constanttemperature bath for at least $24 \mathrm{~h}$. An aliquot of the clear solution was removed by syringe and transferred to a preweighed glass vial. The glass vial was then weighed, and the solvent was evaporated in in vacuo at $323 \mathrm{~K}$ for more than $3 \mathrm{~h}$ Once the solvent had evaporated, the vial with solid residue was weighed. The solubility was calculated from the mass of the solid residue and mass of sample analyzed.

Source and Purity of Chemicals:

(1) $99 \%$, Shanghai Fine Chemical Reagent Company, China, no purification details provided.

(2) Analytical Reagent grade, Hangzhou Chemical Reagent Company, China, no purification details provided.

Estimated Error:

Temperature: $\pm 0.1 \mathrm{~K}$.

$x_{1}: \pm 3 \%$ (relative error).

\begin{tabular}{|c|c|}
\hline $\begin{array}{l}\text { Components: } \\
\text { (1) Benzoic acid; } \mathrm{C}_{7} \mathrm{H}_{6} \mathrm{O}_{2} \text {; } \\
\text { [65-85-0] } \\
\text { (2) Ethanoic acid; } \mathrm{C}_{2} \mathrm{H}_{4} \mathrm{O}_{2} \text {; } \\
\text { [64-19-7] }\end{array}$ & $\begin{array}{l}\text { Original Measurements: } \\
{ }^{80} \text { P. Ma and Q. Xia, Chin. J. Chem. } \\
\text { Eng. 9, } 39 \text { (2001). }\end{array}$ \\
\hline $\begin{array}{l}\text { Variables: } \\
\text { Temperature }\end{array}$ & $\begin{array}{l}\text { Prepared by: } \\
\text { W. E. Acree, Jr. }\end{array}$ \\
\hline
\end{tabular}

Experimental Values

\begin{tabular}{lcc}
\hline \hline$T / \mathrm{K}$ & $x_{2}{ }^{\mathrm{a}}$ & $x_{1}{ }^{\mathrm{b}}$ \\
\hline 291.4 & 0.8709 & 0.1291 \\
296.1 & 0.8559 & 0.1441 \\
299.0 & 0.8456 & 0.1544 \\
302.1 & 0.8336 & 0.1664 \\
305.3 & 0.8252 & 0.1748 \\
306.3 & 0.8191 & 0.1809 \\
308.1 & 0.8130 & 0.1870 \\
310.5 & 0.8005 & 0.1995 \\
313.5 & 0.7874 & 0.2126 \\
316.2 & 0.7753 & 0.2247 \\
318.2 & 0.7616 & 0.2384 \\
320.7 & 0.7494 & 0.2506 \\
322.8 & 0.7359 & 0.2641 \\
325.5 & 0.7226 & 0.2774 \\
328.2 & 0.7061 & 0.2939 \\
330.3 & 0.6923 & 0.3077 \\
332.1 & 0.6806 & 0.3194 \\
333.9 & 0.6684 & 0.3316 \\
\hline
\end{tabular}

${ }^{\mathrm{a}} x_{2}$ : mole fraction of component 2 in the saturated solution.

${ }^{\mathrm{b}} x_{1}$ : mole fraction solubility of the solute.

\section{Auxiliary Information}

\section{Method/Apparatus/Procedure:}

Titanium solid-liquid equilibrium cell, analytical balance, magnetic stirring system, temperature controlling system, and a laser monitoring system.

Solubilities were determined using a dynamic method. Weighed amounts of solute and solvent were sealed in a titanium solid-liquid equilibrium cell, and the temperature slowly increased until the solid phase completely disappeared. Near the solid-liquid equilibrium temperature, the rate of temperature increase was $0.1 \mathrm{~K} / 10 \mathrm{~min}$. The disappearance of the solid solute was detected by a laser monitoring system.

Source and Purity of Chemicals:

(1) Purity not given, Chemical source not given, no purification details were provided.

(2) Purity not given, Chemical source not given, no purification details were provided. 
Estimated Error:

Temperature: $\pm 0.1 \mathrm{~K}$ (estimated by compiler).

$x_{1}: \pm 3 \%$ (relative error, estimated by compiler).

\begin{tabular}{ll}
\hline \hline Components: & Original Measurements: \\
$\begin{array}{l}\text { (1) Benzoic acid; } \mathrm{C}_{7} \mathrm{H}_{6} \mathrm{O}_{2} ; \\
\text { [65-85-0] }\end{array}$ & ${ }^{47}$ A. Beerbower, P. L. Wu, and A. \\
$\begin{array}{l}\text { (2) Propanoic acid; } \mathrm{C}_{3} \mathrm{H}_{6} \mathrm{O}_{2} ; \\
\text { [79-09-4] }\end{array}$ & \\
\hline Variables: & Prepared by: \\
$T / \mathrm{K}=298.15$ & W. E. Acree, Jr. \\
\hline
\end{tabular}

Experimental Values

\begin{tabular}{lc}
\hline \hline$x_{2}{ }^{\mathrm{a}}$ & $x_{1}{ }^{\mathrm{b}}$ \\
\hline 0.8113 & 0.1887 \\
\hline
\end{tabular}

${ }^{a} x_{2}$ : mole fraction of component 2 in the saturated solution.

${ }^{\mathrm{b}} x_{1}$ : mole fraction solubility of the solute.

\section{Auxiliary Information}

\section{Method/Apparatus/Procedure:}

Constant-temperature bath and ultraviolet/visible spectrophotometer.

Excess solute and solvent were placed in screw-capped vials. The vials were sealed and submerged in a constant-temperature water bath and shaken at 100 cycles/min for $24 \mathrm{~h}$. After the $24 \mathrm{~h}$ equilibration period, the vial was removed, wiped dry, and the contents analyzed. The solutions were transferred to a syringe and filtered through a $0.1 \mu \mathrm{m}$ pore size filter. The solutions were diluted and the absorbances recorded at the maximum absorption wavelength of benzoic acid. The solubility was determined at least six times.

\section{Source and Purity of Chemicals:}

(1) Purity not given, Chemical source not specified, no information provided concerning purification.

(2) Spectrophotometric or ACS Reagent grade, Chemical source not specified, redistilled before use.

\section{Estimated Error:}

Temperature: $\pm 0.2 \mathrm{~K}$

$x_{1}: \pm 3 \%$ (relative error).

\begin{tabular}{ll}
\hline \hline Components: & Original Measurements: \\
(1) Benzoic acid; $\mathrm{C}_{7} \mathrm{H}_{6} \mathrm{O}_{2} ;$ & ${ }^{47}$ A. Beerbower, P. L. Wu, and A. \\
[65-85-0] & Martin, J. Pharm. Sci. 73, 179 (1984). \\
$\begin{array}{l}\text { (2) Dimethyl sulfoxide; } \\
\mathrm{C}_{2} \mathrm{H}_{6} \mathrm{OS} ;[67-68-5]\end{array}$ & \\
\hline Variables: & Prepared by: \\
$T / \mathrm{K}=298.15$ & W. E. Acree, Jr. \\
\hline
\end{tabular}

\section{Experimental Values}

\begin{tabular}{lc}
\hline \hline$x_{2}^{\mathrm{a}}$ & $x_{1}^{\mathrm{b}}$ \\
\hline 0.4898 & 0.5102 \\
\hline
\end{tabular}

${ }^{\mathrm{a}} x_{2}$ : mole fraction of component 2 in the saturated solution.

${ }^{b} x_{1}$ : mole fraction solubility of the solute.
Auxiliary Information

Method/Apparatus/Procedure:

Constant-temperature bath and ultraviolet/visible spectrophotometer.

Excess solute and solvent were placed in screw-capped vials. The vials were sealed and submerged in a constant-temperature water bath and shaken at 100 cycles/min for $24 \mathrm{~h}$. After the $24 \mathrm{~h}$ equilibration period, the vial was removed, wiped dry, and the contents analyzed. The solutions were transferred to a syringe and filtered through a $0.1 \mu \mathrm{m}$ pore size filter. The solutions were diluted and the absorbances recorded at the maximum absorption wavelength of benzoic acid. The solubility was determined at least six times.

Source and Purity of Chemicals:

(1) Purity not given, Chemical source not specified, no information provided concerning purification.

(2) Spectrophotometric or ACS Reagent grade, Chemical source not specified, redistilled before use

\section{Estimated Error:}

Temperature: $\pm 0.2 \mathrm{~K}$.

$x_{1}: \pm 3 \%$ (relative error).

\begin{tabular}{ll}
\hline \hline Components: & Original Measurements: \\
$\begin{array}{l}\text { (1) Benzoic acid; } \mathrm{C}_{7} \mathrm{H}_{6} \mathrm{O}_{2} ; \\
\text { [65-85-0] }\end{array}$ & D. G. G. Zakharov, M. I. Voronova, \\
$\begin{array}{l}\text { (2) Dimethyl sulfoxide; } \\
\mathrm{C}_{2} \mathrm{H}_{6} \mathrm{OS} ; \text { [67-68-5] }\end{array}$ & J. Phys. Chem. 85, 408 (2011). \\
\hline Variables: & \\
$T / \mathrm{K}=298$ & Prepared by: \\
\hline
\end{tabular}

\section{Experimental Values}

The authors reported the solubility data graphically. The compiler estimated a solubility of 14 molal $(14 \mathrm{~mol} / \mathrm{kg}$ of solvent) from the graph, which would correspond to a mole fraction solubility of $x_{1}=0.522$.

\section{Auxiliary Information}

Method/Apparatus/Procedure:

Very little experimental details were provided. The authors stated that the solubility was determined at $298 \mathrm{~K}$ gravimetrically, and referenced a paper by Shalmashi and Eliassi [J. Chem. Eng. Data 53, 199 (2008)].

Source and Purity of Chemicals:

(1) $99 \%$, Sigma Aldrich Chemical Company, no purification details were provided.

(2) $99.5 \%$, Lab-Scan, used as received.

Estimated Error:

Temperature: $\pm 0.2 \mathrm{~K}$ (estimated by compiler). $x_{1}: \pm 5$ to $6 \%$ (relative error).

\begin{tabular}{|c|c|}
\hline $\begin{array}{l}\text { Components: } \\
\text { (1) Benzoic acid; } \mathrm{C}_{7} \mathrm{H}_{6} \mathrm{O}_{2} \text {; } \\
\text { [65-85-0] } \\
\text { (2) Dimethyl sulfoxide; } \\
\mathrm{C}_{2} \mathrm{H}_{6} \mathrm{OS} \text {; [67-68-5] }\end{array}$ & $\begin{array}{l}\text { Original Measurements: } \\
{ }^{64} \text { E. A. Gomaa, Phys. Chem. Liq. 50, } \\
279 \text { (2012). }\end{array}$ \\
\hline $\begin{array}{l}\text { Variables: } \\
T / \mathrm{K}=298.15\end{array}$ & $\begin{array}{l}\text { Prepared by: } \\
\text { W. E. Acree, Jr. }\end{array}$ \\
\hline
\end{tabular}


Experimental Values

\begin{tabular}{lc}
\hline \hline$x_{2}^{\mathrm{a}}$ & $x_{1}{ }^{\mathrm{b}}$ \\
\hline 0.7165 & 0.2835
\end{tabular}

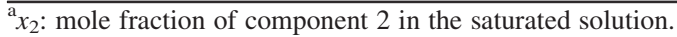

${ }^{\mathrm{b}} x_{1}$ : mole fraction solubility of the solute, computed by the compiler. The original solubility data were given in units of moles per kilogram of solvent.

\section{Auxiliary Information}

\section{Method/Apparatus/Procedure:}

Constant-temperature shaker bath and analytical balance.

Excess solute and solvent were placed in closed test tubes and allowed to equilibrate in a constant-temperature shaker bath for several days. The solubility was determined gravimetrically by transferring $1 \mathrm{ml}$ of the saturated solution to an aluminum disk. The solvent was removed by heating with an infrared lamp. The solubility was calculated from the mass of the solid residue and amount of saturated solution analyzed.

Source and Purity of Chemicals:

(1) Purity not given, Merck Chemicals, Germany, no information provided concerning purification.

(2) Purity not given, Merck Chemicals, Germany, no information provided concerning purification.

\section{Estimated Error:}

Temperature: $\pm 0.1 \mathrm{~K}$ (estimated by compiler). $x_{1}: \pm 3 \%$ (relative error, estimated by compiler).

\begin{tabular}{|c|c|}
\hline $\begin{array}{l}\text { Components: } \\
\text { (1) Benzoic acid; } \mathrm{C}_{7} \mathrm{H}_{6} \mathrm{O}_{2} \text {; } \\
\text { [65-85-0] } \\
\text { (2) Formamide; } \mathrm{CH}_{3} \mathrm{NO} ;[75-12-7]\end{array}$ & $\begin{array}{l}\text { Original Measurements: } \\
\text { 47 A. Beerbower, P. L. Wu, and A. } \\
\text { Martin, J. Pharm. Sci. 73, } 179 \text { (1984) }\end{array}$ \\
\hline $\begin{array}{l}\text { Variables: } \\
T / \mathrm{K}=298.15\end{array}$ & $\begin{array}{l}\text { Prepared by: } \\
\text { W. E. Acree, Jr. }\end{array}$ \\
\hline
\end{tabular}

Experimental Values

\begin{tabular}{lc}
\hline \hline$x_{2}{ }^{\mathrm{a}}$ & $x_{1}{ }^{\mathrm{b}}$ \\
\hline 0.8475 & 0.1525
\end{tabular}

${ }^{a} x_{2}$ : mole fraction of component 2 in the saturated solution.

${ }^{\mathrm{b}} x_{1}$ : mole fraction solubility of the solute.

\section{Auxiliary Information}

\section{Method/Apparatus/Procedure:}

Constant-temperature bath and ultraviolet/visible spectrophotometer.

Excess solute and solvent were placed in screw-capped vials. The vials were sealed and submerged in a constant-temperature water bath and shaken at 100 cycles/min for $24 \mathrm{~h}$. After the $24 \mathrm{~h}$ equilibration period, the vial was removed, wiped dry, and the contents analyzed. The solutions were transferred to a syringe and filtered through a $0.1 \mu \mathrm{m}$ pore size filter. The solutions were diluted and the absorbances recorded at the maximum absorption wavelength of benzoic acid. The solubility was determined at least six times.

\section{Source and Purity of Chemicals:}

(1) Purity not given, Chemical source not specified, no information provided concerning purification.

(2) Spectrophotometric or ACS Reagent grade, Chemical source not specified, redistilled before use.
Estimated Error:

Temperature: $\pm 0.2 \mathrm{~K}$.

$x_{1}: \pm 3 \%$ (relative error).

\begin{tabular}{|c|c|}
\hline $\begin{array}{l}\text { Components: } \\
\text { (1) Benzoic acid; } \mathrm{C}_{7} \mathrm{H}_{6} \mathrm{O}_{2} \text {; } \\
\text { [65-85-0] } \\
\text { (2) } \mathrm{N} \text {-Methylformamide; } \\
\mathrm{C}_{2} \mathrm{H}_{5} \mathrm{NO} \text {; [123-39-7] }\end{array}$ & $\begin{array}{l}\text { Original Measurements: } \\
{ }^{47} \text { A. Beerbower, P. L. Wu, and A. } \\
\text { Martin, J. Pharm. Sci. 73, } 179 \text { (1984). }\end{array}$ \\
\hline $\begin{array}{l}\text { Variables: } \\
T / \mathrm{K}=298.15\end{array}$ & $\begin{array}{l}\text { Prepared by: } \\
\text { W. E. Acree, Jr. }\end{array}$ \\
\hline
\end{tabular}

Experimental Values

\begin{tabular}{lc}
\hline \hline$x_{2}{ }^{\mathrm{a}}$ & $x_{1}{ }^{\mathrm{b}}$ \\
\hline 0.6572 & 0.3428 \\
\hline
\end{tabular}

${ }^{\mathrm{a}} x_{2}$ : mole fraction of component 2 in the saturated solution.

${ }^{\mathrm{b}} x_{1}$ : mole fraction solubility of the solute.

\section{Auxiliary Information}

\section{Method/Apparatus/Procedure:}

Constant-temperature bath and ultraviolet/visible spectrophotometer.

Excess solute and solvent were placed in screw-capped vials. The vials were sealed and submerged in a constant-temperature water bath and shaken at 100 cycles/min for $24 \mathrm{~h}$. After the $24 \mathrm{~h}$ equilibration period, the vial was removed, wiped dry, and the contents analyzed. The solutions were transferred to a syringe and filtered through a $0.1 \mu \mathrm{m}$ pore size filter. The solutions were diluted and the absorbances recorded at the maximum absorption wavelength of benzoic acid. The solubility was determined at least six times.

Source and Purity of Chemicals:

(1) Purity not given, Chemical source not specified, no information provided concerning purification.

(2) Spectrophotometric or ACS Reagent grade, Chemical source not specified, redistilled before use.

\section{Estimated Error:}

Temperature: $\pm 0.2 \mathrm{~K}$.

$x_{1}: \pm 3 \%$ (relative error)

\begin{tabular}{|c|c|}
\hline $\begin{array}{l}\text { Components: } \\
\text { (1) Benzoic acid; } \mathrm{C}_{7} \mathrm{H}_{6} \mathrm{O}_{2} \text {; } \\
\text { [65-85-0] } \\
\text { (2) } \mathrm{N}, \mathrm{N} \text {-Dimethylformamide; } \\
\mathrm{C}_{3} \mathrm{H}_{7} \mathrm{NO} \text {; [64-19-7] }\end{array}$ & $\begin{array}{l}\text { Original Measurements: } \\
{ }^{47} \text { A. Beerbower, P. L. Wu, and A. } \\
\text { Martin, J. Pharm. Sci. 73, } 179 \text { (1984). }\end{array}$ \\
\hline $\begin{array}{l}\text { Variables: } \\
T / \mathrm{K}=298.15\end{array}$ & $\begin{array}{l}\text { Prepared by: } \\
\text { W. E. Acree, Jr. }\end{array}$ \\
\hline
\end{tabular}

\section{Experimental Values}

\begin{tabular}{lc}
\hline \hline$x_{2}{ }^{\mathrm{a}}$ & $x_{1}{ }^{\mathrm{b}}$ \\
\hline 0.5091 & 0.4909 \\
\hline${ }^{\mathrm{a}} x_{2}:$ mole fraction of component 2 in the saturated solution. & \\
${ }^{\mathrm{b}} x_{1}:$ mole fraction solubility of the solute. &
\end{tabular}




\section{Auxiliary Information}

\section{Method/Apparatus/Procedure:}

Constant-temperature bath and ultraviolet/visible spectrophotometer.

Excess solute and solvent were placed in screw-capped vials. The vials were sealed and submerged in a constant-temperature water bath and shaken at 100 cycles/min for $24 \mathrm{~h}$. After the $24 \mathrm{~h}$ equilibration period, the vial was removed, wiped dry, and the contents analyzed. The solutions were transferred to a syringe and filtered through a $0.1 \mu \mathrm{m}$ pore size filter. The solutions were diluted and the absorbances recorded at the maximum absorption wavelength of benzoic acid. The solubility was determined at least six times.

Source and Purity of Chemicals:

(1) Purity not given, Chemical source not specified, no information provided concerning purification.

(2) Spectrophotometric or ACS Reagent grade, Chemical source not specified, redistilled before use.

Estimated Error:

Temperature: $\pm 0.2 \mathrm{~K}$.

$x_{1}: \pm 3 \%$ (relative error).

\begin{tabular}{|c|c|}
\hline $\begin{array}{l}\text { Components: } \\
\text { (1) Benzoic acid; } \mathrm{C}_{7} \mathrm{H}_{6} \mathrm{O}_{2} \text {; } \\
\text { [65-85-0] } \\
\text { (2) N,N-Dimethylformamide; } \\
\mathrm{C}_{3} \mathrm{H}_{7} \mathrm{NO} \text {; [64-19-7] }\end{array}$ & $\begin{array}{l}\text { Original Measurements: } \\
{ }^{64} \text { E. A. Gomaa, Phys. Chem. Liq. 50, } \\
279 \text { (2012). }\end{array}$ \\
\hline $\begin{array}{l}\text { Variables: } \\
T / \mathrm{K}=298.15\end{array}$ & $\begin{array}{l}\text { Prepared by: } \\
\text { W. E. Acree, Jr. }\end{array}$ \\
\hline
\end{tabular}

Experimental Values

\begin{tabular}{lc}
\hline \hline$x_{2}{ }^{\mathrm{a}}$ & $x_{1}{ }^{\mathrm{b}}$ \\
\hline 0.7772 & 0.2228
\end{tabular}

${ }^{\mathrm{a}} x_{2}$ : mole fraction of component 2 in the saturated solution.

${ }^{b} x_{1}$ : mole fraction solubility of the solute, computed by the compiler. The

original solubility data were given in units of moles per kilogram of solvent.

\section{Auxiliary Information}

\section{Method/Apparatus/Procedure:}

Constant-temperature shaker bath and analytical balance.

Excess solute and solvent were placed in closed test tubes and allowed to equilibrate in a constant-temperature shaker bath for several days. The solubility was determined gravimetrically by transferring $1 \mathrm{ml}$ of the saturated solution to an aluminum disk. The solvent was removed by heating with an infrared lamp. The solubility was calculated from the mass of the solid residue and amount of saturated solution analyzed.

\section{Source and Purity of Chemicals:}

(1) Purity not given, Merck Chemicals, Germany, no information provided concerning purification.

(2) Purity not given, Merck Chemicals, Germany, no information provided concerning purification.

\section{Estimated Error:}

Temperature: $\pm 0.1 \mathrm{~K}$ (estimated by compiler).

$x_{1}: \pm 3 \%$ (relative error, estimated by compiler).

\begin{tabular}{|c|c|}
\hline $\begin{array}{l}\text { Components: } \\
\text { (1) Benzoic acid; } \mathrm{C}_{7} \mathrm{H}_{6} \mathrm{O}_{2} \text {; } \\
\text { [65-85-0] } \\
\text { (2) N,N-Dimethylformamide; } \\
\mathrm{C}_{3} \mathrm{H}_{7} \mathrm{NO} \text {; [64-19-7] }\end{array}$ & $\begin{array}{l}\text { Original Measurements: } \\
{ }^{80} \text { P. Ma and Q. Xia, Chin. J. Chem. } \\
\text { Eng. 9, } 39 \text { (2001). }\end{array}$ \\
\hline $\begin{array}{l}\text { Variables: } \\
\text { Temperature }\end{array}$ & $\begin{array}{l}\text { Prepared by: } \\
\text { W. E. Acree, Jr. }\end{array}$ \\
\hline
\end{tabular}

Experimental Values

\begin{tabular}{lcc}
\hline \hline$T / \mathrm{K}$ & $x_{2}{ }^{\mathrm{a}}$ & $x_{1}{ }^{\mathrm{b}}$ \\
\hline 298.8 & 0.5276 & 0.4724 \\
300.7 & 0.5227 & 0.4773 \\
301.1 & 0.5224 & 0.4776 \\
302.7 & 0.5190 & 0.4810 \\
304.6 & 0.5161 & 0.4839 \\
306.3 & 0.5119 & 0.4881 \\
308.2 & 0.5088 & 0.4912 \\
310.1 & 0.5043 & 0.4957 \\
312.2 & 0.4996 & 0.5004 \\
316.5 & 0.4896 & 0.5104 \\
319.4 & 0.4824 & 0.5176 \\
321.8 & 0.4762 & 0.5238 \\
323.4 & 0.4719 & 0.5281 \\
325.7 & 0.4662 & 0.5338 \\
329.1 & 0.4572 & 0.5428 \\
331.6 & 0.4480 & 0.5520 \\
334.3 & 0.4416 & 0.5584 \\
336.4 & 0.4358 & 0.5642 \\
340.1 & 0.4226 & 0.5774 \\
343.1 & 0.4098 & 0.5902 \\
\hline
\end{tabular}

${ }^{\mathrm{a}} x_{2}$ : mole fraction of component 2 in the saturated solution.

${ }^{\mathrm{b}} x_{1}$ : mole fraction solubility of the solute.

\section{Auxiliary Information}

\section{Method/Apparatus/Procedure:}

Titanium solid-liquid equilibrium cell, analytical balance, magnetic stirring system, temperature controlling system, and a laser monitoring system.

Solubilities were determined using a dynamic method. Weighed amounts of solute and solvent were sealed in a titanium solid-liquid equilibrium cell, and the temperature slowly increased until the solid phase completely disappeared. Near the solid-liquid equilibrium temperature, the rate of temperature increase was $0.1 \mathrm{~K}$ per $10 \mathrm{~min}$. The disappearance of the solid solute was detected by a laser monitoring system.

\section{Source and Purity of Chemicals:}

(1) Purity not given, Chemical source not given, no purification details were provided.

(2) Purity not given, Chemical source not given, no purification details were provided.

\section{Estimated Error:}

Temperature: $\pm 0.1 \mathrm{~K}$ (estimated by compiler).

$x_{1}: \pm 3 \%$ (relative error, estimated by compiler).

\begin{tabular}{|c|c|}
\hline $\begin{array}{l}\text { Components: } \\
\text { (1) Benzoic acid; } \mathrm{C}_{7} \mathrm{H}_{6} \mathrm{O}_{2} \text {; } \\
\text { [65-85-0] } \\
\text { (2) } \mathrm{N}, \mathrm{N} \text {-Dimethylformamide; } \\
\mathrm{C}_{3} \mathrm{H}_{7} \mathrm{NO} \text {; [64-19-7] }\end{array}$ & $\begin{array}{l}\text { Original Measurements: } \\
\text { 82 L. Dian-Qing, L. Jiang-Chu, L. Da- } \\
\text { Zhuang, and W. Fu-An, Fluid Phase } \\
\text { Equilib. 200, } 69 \text { (2002). }\end{array}$ \\
\hline $\begin{array}{l}\text { Variables: } \\
\text { Temperature }\end{array}$ & $\begin{array}{l}\text { Prepared by: } \\
\text { W. E. Acree, Jr. }\end{array}$ \\
\hline
\end{tabular}


Experimental Values

\begin{tabular}{lcc}
\hline \hline$T / \mathrm{K}$ & $x_{2}{ }^{\mathrm{a}}$ & $x_{1}{ }^{\mathrm{b}}$ \\
\hline 294.75 & 0.5357 & 0.4643 \\
298.75 & 0.5273 & 0.4727 \\
301.05 & 0.5224 & 0.4776 \\
302.15 & 0.5186 & 0.4814 \\
306.35 & 0.5119 & 0.4881 \\
310.05 & 0.5043 & 0.4957 \\
312.15 & 0.4996 & 0.5004 \\
316.55 & 0.4896 & 0.5104 \\
319.35 & 0.4824 & 0.5176 \\
323.35 & 0.4719 & 0.5281 \\
325.65 & 0.4662 & 0.5338 \\
329.05 & 0.4572 & 0.5428 \\
331.85 & 0.4480 & 0.5520 \\
336.45 & 0.4336 & 0.5664 \\
340.05 & 0.4226 & 0.5774 \\
343.35 & 0.4098 & 0.5902 \\
346.05 & 0.3985 & 0.6015 \\
348.75 & 0.3865 & 0.6135 \\
351.25 & 0.3757 & 0.6243 \\
355.55 & 0.3528 & 0.6472 \\
358.05 & 0.3395 & 0.6605 \\
362.85 & 0.3129 & 0.6871 \\
366.85 & 0.2874 & 0.7126 \\
369.65 & 0.2665 & 0.7335 \\
\hline & &
\end{tabular}

${ }^{\mathrm{a}} x_{2}$ : mole fraction of component 2 in the saturated solution.

${ }^{\mathrm{b}} x_{1}$ : mole fraction solubility of the solute.

\section{Auxiliary Information}

Method/Apparatus/Procedure:

Jacketed solid-liquid equilibrium cell, analytical balance, magnetic stirring system, temperature controlling system, and a laser monitoring system. Solubilities were determined using a synthetic method. Weighed amounts of solute and solvent were sealed in a jacketed equilibrium vessel, and the temperature slowly increased until the solid phase completely disappeared. The disappearance of the solid solute was detected by a laser monitoring system. Measurements were repeated two or three times to check the reproducibility.

Source and Purity of Chemicals:

(1) Analytical Reagent, Shanghai Chemical Reagent Company, China, used as received.

(2) Analytical Reagent, Shanghai Chemical Reagent Company, used as received.

Estimated Error:

Temperature: $\pm 0.05 \mathrm{~K}$ (estimated by compiler).

$x_{1}: \pm 0.0005$ or less.

\begin{tabular}{|c|c|}
\hline $\begin{array}{l}\text { Components: } \\
\text { (1) Benzoic acid; } \mathrm{C}_{7} \mathrm{H}_{6} \mathrm{O}_{2} \text {; } \\
\text { [65-85-0] } \\
\text { (2) N,N-Dimethylformamide; } \\
\mathrm{C}_{3} \mathrm{H}_{7} \mathrm{NO} ;[64-19-7]\end{array}$ & $\begin{array}{l}\text { Original Measurements: } \\
{ }^{83} \text { M. K. Chantooni and I. M. Kolthoff, } \\
\text { J. Phys. Chem. 77, } 527 \text { (1973). }\end{array}$ \\
\hline $\begin{array}{l}\text { Variables: } \\
T / \mathrm{K}=298.15\end{array}$ & $\begin{array}{l}\text { Prepared by: } \\
\text { W. E. Acree, Jr. }\end{array}$ \\
\hline
\end{tabular}

\section{Experimental Values}

The measured solubility was reported to be $5.35 \mathrm{~mol} \mathrm{dm}^{-3}$. The authors did not give the temperature at which the solubility was measured. Based on the experimental description given in an earlier paper [I. M. Kolthoff, J. J. Lingane, and W. Larson, J. Am. Chem. Soc. 60, 2512 (1938)], the compiler believes the temperature to be $298.15 \mathrm{~K}$.

\section{Auxiliary Information}

\section{Method/Apparatus/Procedure:}

Very little experimental details were provided. Solubility was determined by titrating a filtered aliquot of the saturated solution alkalimetrically in an aqueous-ethanol mixture using phenolphthalein as the acid-base indicator.

Source and Purity of Chemicals:

(1) High purity, National Bureau of Standards, USA, no purification details given.

(2) Purity not given, Chemical source not given, was shaken first with phosphorous pentoxide and then with potassium hydroxide pellets. Solvent was distilled shortly before use.

Estimated Error:

Temperature: $\pm 0.1 \mathrm{~K}$ (estimated by compiler). $c_{1}: \pm 2 \%$ (relative error, estimated by compiler).

\begin{tabular}{|c|c|}
\hline $\begin{array}{l}\text { Components: } \\
\text { (1) Benzoic acid; } \mathrm{C}_{7} \mathrm{H}_{6} \mathrm{O}_{2} \text {; } \\
\text { [65-85-0] } \\
\text { (2) } N, N \text {-Dimethylacetamide; } \\
\mathrm{C}_{4} \mathrm{H}_{9} \mathrm{NO} ;[127-19-5]\end{array}$ & $\begin{array}{l}\text { Original Measurements: } \\
{ }^{47} \text { A. Beerbower, P. L. Wu, and A. } \\
\text { Martin, J. Pharm. Sci. 73, } 179 \text { (1984) }\end{array}$ \\
\hline $\begin{array}{l}\text { Variables: } \\
T / \mathrm{K}=298.15\end{array}$ & $\begin{array}{l}\text { Prepared by: } \\
\text { W. E. Acree, Jr. }\end{array}$ \\
\hline
\end{tabular}

\section{Experimental Values}

\begin{tabular}{lc}
\hline \hline$x_{2}{ }^{\mathrm{a}}$ & $x_{1}{ }^{\mathrm{b}}$ \\
\hline 0.4755 & 0.5245
\end{tabular}

${ }^{\mathrm{a}} x_{2}$ : mole fraction of component 2 in the saturated solution.

${ }^{b} x_{1}$ : mole fraction solubility of the solute.

\section{Auxiliary Information}

\section{Method/Apparatus/Procedure:}

Constant-temperature bath and ultraviolet/visible spectrophotometer. Excess solute and solvent were placed in screw-capped vials. The vials were sealed and submerged in a constant-temperature water bath and shaken at 100 cycles/min for $24 \mathrm{~h}$. After the $24 \mathrm{~h}$ equilibration period, the vial was removed, wiped dry, and the contents analyzed. The solutions were transferred to a syringe and filtered through a $0.1 \mu \mathrm{m}$ pore size filter. The solutions were diluted and the absorbances recorded at the maximum absorption wavelength of benzoic acid. The solubility was determined at least six times.

Source and Purity of Chemicals:

(1) Purity not given, Chemical source not specified, no information provided concerning purification.

(2) Spectrophotometric or ACS Reagent grade, Chemical source not specified, redistilled before use. 
Estimated Error:

Temperature: $\pm 0.2 \mathrm{~K}$.

$x_{1}: \pm 3 \%$ (relative error).

\begin{tabular}{|c|c|}
\hline $\begin{array}{l}\text { Components: } \\
\text { (1) Benzoic acid; } \mathrm{C}_{7} \mathrm{H}_{6} \mathrm{O}_{2} \text {; } \\
\text { [65-85-0] } \\
\text { (2) Ethanenitrile; } \mathrm{C}_{2} \mathrm{H}_{3} \mathrm{~N} \text {; } \\
\text { [75-05-8] }\end{array}$ & $\begin{array}{l}\text { Original Measurements: } \\
{ }^{53} \text { G. L. Perlovich and A. Bauer-Brandl, } \\
\text { Pharm. Res. 20, } 471 \text { (2003). }\end{array}$ \\
\hline $\begin{array}{l}\text { Variables: } \\
T / \mathrm{K}=298.15\end{array}$ & $\begin{array}{l}\text { Prepared by: } \\
\text { W. E. Acree, Jr. }\end{array}$ \\
\hline
\end{tabular}

Experimental Values

\begin{tabular}{lc}
\hline \hline$x_{2}{ }^{\mathrm{a}}$ & $x_{1}{ }^{\mathrm{b}}$ \\
\hline 0.9461 & 0.0539
\end{tabular}

${ }^{a} x_{2}$ : mole fraction of component 2 in the saturated solution.

${ }^{\mathrm{b}} x_{1}$ : mole fraction solubility of the solute.

\section{Auxiliary Information}

\section{Method/Apparatus/Procedure:}

Thermostated constant-temperature bath, centrifuge, and analytical balance. Very little experimental details were given in the paper. The solubility of the solute was by a weighing method.

Source and Purity of Chemicals:

(1) Analytical Reagent grade, Norsk Medisinaldepot, Oslo, Norway, no purification details were provided.

(2) HPLC grade, Merck Chemicals, Germany, no purification details were provided.

\section{Estimated Error:}

Temperature: $\pm 0.01 \mathrm{~K}$.

$x_{1}: \pm 3.0 \%$ (relative error).

\begin{tabular}{ll}
\hline \hline Components: & Original Measurements: \\
$\begin{array}{l}\text { (1) Benzoic acid; } \mathrm{C}_{7} \mathrm{H}_{6} \mathrm{O}_{2} ; \\
\text { [65-85-0] }\end{array}$ & 279 (2012). A. Gomaa, Phys. Chem. Liq. 50, \\
$\begin{array}{l}\text { (2) Ethanenitrile; } \mathrm{C}_{2} \mathrm{H}_{3} \mathrm{~N} ; \\
{[75-05-8]}\end{array}$ & \\
\hline $\begin{array}{l}\text { Variables: } \\
T / \mathrm{K}=298.15\end{array}$ & Prepared by: \\
\hline
\end{tabular}

Experimental Values

\begin{tabular}{lc}
\hline \hline$x_{2}{ }^{\mathrm{a}}$ & $x_{1}{ }^{\mathrm{b}}$ \\
\hline 0.9577 & 0.0423 \\
\hline${ }^{\mathrm{a}} x_{2}:$ mole fraction of component 2 in the saturated solution. \\
${ }^{\mathrm{b}} x_{1}:$ mole fraction solubility of the solute, computed by the compiler. The \\
original solubility data were given in units of moles per kilogram of solvent.
\end{tabular}

\section{Auxiliary Information}

Method/Apparatus/Procedure:

Constant-temperature shaker bath and analytical balance.

Excess solute and solvent were placed in closed test tubes and allowed to equilibrate in a constant-temperature shaker bath for several days. The solubility was determined gravimetrically by transferring $1 \mathrm{ml}$ of the saturated solution to an aluminum disk. The solvent was removed by heating with an infrared lamp. The solubility was calculated from the mass of the solid residue and amount of saturated solution analyzed.

Source and Purity of Chemicals:

(1) Purity not given, Merck Chemicals, Germany, no information provided concerning purification.

(2) Purity not given, Merck Chemicals, Germany, no information provided concerning purification.

Estimated Error:

Temperature: $\pm 0.1 \mathrm{~K}$ (estimated by compiler).

$x_{1}: \pm 3 \%$ (relative error, estimated by compiler).

\begin{tabular}{ll}
\hline \hline Components: & Original Measurements: \\
(1) Benzoic acid; $\mathrm{C}_{7} \mathrm{H}_{6} \mathrm{O}_{2} ;$ & ${ }^{83}$ M. K. Chantooni and I. M. Kolthoff, \\
$\begin{array}{l}\text { [65-85-0] } \\
\text { (2) Ethanenitrile; } \mathrm{C}_{2} \mathrm{H}_{3} \mathrm{~N} ;\end{array}$ & J. Phys. Chem. 77, 527 (1973). \\
[75-05-8] & \\
\hline Variables: & Prepared by: \\
$T / \mathrm{K}=298.15 \mathrm{~K}$ & W. E. Acree, Jr. \\
\hline
\end{tabular}

Experimental Values

The measured solubility was reported to be $0.85 \mathrm{~mol} \mathrm{dm}^{-3}$. The authors did not give the temperature at which the solubility was measured. Based on the experimental description given in an earlier paper [I. M. Kolthoff, J. J. Lingane, and W. Larson, J. Am. Chem. Soc. 60, 2512 (1938)], the compiler believes the temperature to be $298.15 \mathrm{~K}$.

\section{Auxiliary Information}

Method/Apparatus/Procedure:

Very little experimental details were provided. Solubility was determined by titrating a filtered aliquot of the saturated solution alkalimetrically in an aqueous-ethanol mixture using phenolphthalein as the acid-base indicator.

Source and Purity of Chemicals:

(1) High Purity, National Bureau of Standards, USA, was dried at $383 \mathrm{~K}$ before use.

(2) Purity not given, Chemical source not given, was purified by shaking with saturated potassium hydroxide, followed by activated alumina, and then anhydrous calcium chloride to remove water. Ethanenitrile was further dried over anhydrous magnesium sulfate and then phosphorous pentoxide. The sample was distilled shortly before use.

\section{Estimated Error:}

Temperature: $\pm 0.1 \mathrm{~K}$ (estimated by compiler).

$c_{1}: \pm 2 \%$ (relative error, estimated by compiler). 


\section{Components:}

(1) Benzoic acid; $\mathrm{C}_{7} \mathrm{H}_{6} \mathrm{O}_{2}$; [65-85-0]

(2) Ethanenitrile; $\mathrm{C}_{2} \mathrm{H}_{3} \mathrm{~N}$; [75-05-8]

\begin{tabular}{ll}
\hline Variables: & Prepared by: \\
$T / \mathrm{K}=298$ & W. E. Acree, Jr. \\
\hline
\end{tabular}

\section{Experimental Values}

The authors reported the solubility data graphically. The compiler estimated a solubility of $1 \mathrm{molal}$ ( $1 \mathrm{~mol} / \mathrm{kg}$ of solvent) from the graph, which would correspond to a mole fraction solubility of $x_{1}=0.0394$.

\section{Auxiliary Information}

Method/Apparatus/Procedure:

Very little experimental details were provided. The authors stated that the solubility was determined at $298 \mathrm{~K}$ gravimetrically, and referenced a paper by Shalmashi and Eliassi [J. Chem. Eng. Data 53, 199 (2008)].

Source and Purity of Chemicals:

(1) $99 \%$, Sigma Aldrich Chemical Company, no purification details were provided

(2) $99.9 \%$, Lab-Scan, used as received.

Estimated Error:

Temperature: $\pm 0.2 \mathrm{~K}$ (estimated by compiler). $x_{1}: \pm 5$ to $6 \%$ (relative error).

\begin{tabular}{ll}
\hline \hline Components: & $\begin{array}{l}\text { Original Measurements: } \\
\text { (1) Benzoic acid; } \mathrm{C}_{7} \mathrm{H}_{6} \mathrm{O}_{2} ;\end{array}$ \\
$\begin{array}{ll}{ }^{64} \mathrm{E} \text {. A. Gomaa, Phys. Chem. Liq. 50, } \\
\text { (2) Hexamethylphosphortriamide; }\end{array}$ & 279 (2012). \\
$\mathrm{C}_{6} \mathrm{H}_{18} \mathrm{~N}_{3} \mathrm{OP} ;[680-31-9]$ & \\
\hline Variables: & Prepared by: \\
$T / \mathrm{K}=298.15$ & W. E. Acree, Jr. \\
\hline
\end{tabular}

\section{Experimental Values}

\begin{tabular}{lc}
\hline$\frac{x_{2}{ }^{\mathrm{a}}}{0.9857}$ & $x_{1}{ }^{\mathrm{b}}$ \\
\hline${ }^{\mathrm{a}} x_{2}:$ mole fraction of component 2 in the saturated solution. \\
${ }^{\mathrm{b}} x_{1}:$ mole fraction solubility of the solute, computed by the compiler. The \\
original solubility data were given in units of moles per kilogram of solvent.
\end{tabular}

\section{Auxiliary Information}

\begin{abstract}
Method/Apparatus/Procedure:
Constant-temperature shaker bath and analytical balance.

Excess solute and solvent were placed in closed test tubes and allowed to equilibrate in a constant-temperature shaker bath for several days. The solubility was determined gravimetrically by transferring $1 \mathrm{ml}$ of the saturated solution to an aluminum disk. The solvent was removed by heating with an infrared lamp. The solubility was calculated from the mass of the solid residue and amount of saturated solution analyzed.
\end{abstract}

Source and Purity of Chemicals:

(1) Purity not given, Merck Chemicals, Germany, no information provided concerning purification.
(2) Purity not given, Merck Chemicals, Germany, no information provided concerning purification.

\section{Estimated Error:}

Temperature: $\pm 0.1 \mathrm{~K}$ (estimated by compiler). $x_{1}: \pm 3 \%$ (relative error, estimated by compiler).

\begin{tabular}{|c|c|}
\hline $\begin{array}{l}\text { Components: } \\
\text { (1) Benzoic acid; } \mathrm{C}_{7} \mathrm{H}_{6} \mathrm{O}_{2} \text {; } \\
\text { [65-85-0] } \\
\text { (2) N-Methyl-2-pyrrolidone; } \\
\mathrm{C}_{5} \mathrm{H}_{9} \mathrm{NO} \text {; [872-50-4] }\end{array}$ & $\begin{array}{l}\text { Original Measurements: } \\
{ }^{84} \text { D.-Q. Li, D.-Z. Liu, and F.-A. Wang, } \\
\text { J. Chem. Eng. Data 46, } 172 \text { (2001). }\end{array}$ \\
\hline $\begin{array}{l}\text { Variables: } \\
\text { Temperature }\end{array}$ & $\begin{array}{l}\text { Prepared by: } \\
\text { W. E. Acree, Jr. }\end{array}$ \\
\hline
\end{tabular}

\begin{tabular}{lcc}
\hline \hline$T / \mathrm{K}$ & $x_{2}{ }^{\mathrm{a}}$ & $x_{1}{ }^{\mathrm{b}}$ \\
\hline 296.35 & 0.4838 & 0.5162 \\
301.15 & 0.4765 & 0.5235 \\
305.05 & 0.4698 & 0.5302 \\
307.95 & 0.4647 & 0.5353 \\
311.05 & 0.4591 & 0.5409 \\
314.25 & 0.4530 & 0.5470 \\
318.35 & 0.4465 & 0.5535 \\
321.95 & 0.4393 & 0.5607 \\
327.35 & 0.4256 & 0.5744 \\
333.55 & 0.4087 & 0.5913 \\
338.85 & 0.3918 & 0.6082 \\
343.25 & 0.3772 & 0.6228 \\
347.75 & 0.3605 & 0.6395 \\
351.15 & 0.3465 & 0.6535 \\
355.05 & 0.3309 & 0.6691 \\
359.85 & 0.3067 & 0.6933 \\
364.55 & 0.2819 & 0.7181 \\
369.05 & 0.2553 & 0.7447 \\
371.35 & 0.2408 & 0.7592 \\
\hline
\end{tabular}

${ }^{\mathrm{a}} x_{2}$ : mole fraction of component 2 in the saturated solution.

${ }^{\mathrm{b}} x_{1}$ : mole fraction solubility of the solute.

\section{Auxiliary Information}

\section{Method/Apparatus/Procedure:}

Jacketed solid-liquid equilibrium cell, analytical balance, magnetic stirring system, temperature controlling system, and a laser monitoring system. Solubilities were determined using a synthetic method. Weighed amounts of solute and solvent were sealed in a jacketed equilibrium vessel, and the temperature slowly increased until the solid phase completely disappeared. The disappearance of the solid solute was detected by a laser monitoring system. Measurements were repeated two or three times to check the reproducibility.

\section{Source and Purity of Chemicals:}

(1) $99.5 \%$, Analytical Reagent, Shanghai Chemical Reagent Company, China, used as received.

(2) $99.0 \%$, Analytical Reagent, Shanghai Chemical Reagent Company, used as received.

\section{Estimated Error:}

Temperature: $\pm 0.05 \mathrm{~K}$ (estimated by compiler).

$x_{1}: \pm 0.0005$ or less. 


\begin{tabular}{|c|c|}
\hline $\begin{array}{l}\text { Components: } \\
\text { (1) Benzoic acid; } \mathrm{C}_{7} \mathrm{H}_{6} \mathrm{O}_{2} \text {; } \\
\text { [65-85-0] } \\
\text { (2) Ethyl 2-hydroxypropanoate; } \\
\mathrm{C}_{5} \mathrm{H}_{10} \mathrm{O}_{3} \text {; [97-64-3] }\end{array}$ & $\begin{array}{l}\text { Original Measurements: } \\
{ }^{87} \text { M. Dias, J. Hadgraft, and M. E. Lane, } \\
\text { Int. J. Pharm. 336, } 108 \text { (2007). }\end{array}$ \\
\hline $\begin{array}{l}\text { Variables: } \\
T / \mathrm{K}=305.2\end{array}$ & $\begin{array}{l}\text { Prepared by: } \\
\text { W. E. Acree, Jr. }\end{array}$ \\
\hline
\end{tabular}

\section{Experimental Values}

The measured solubility was reported to be $164.70 \mathrm{mg} / \mathrm{ml}$, which corresponds to a molar solubility of $c_{1}=1.349$ mol dm ${ }^{-3}$.

\section{Auxiliary Information}

\section{Method/Apparatus/Procedure:}

Constant-temperature bath, magnetic stirrer, centrifuge, and an ultraviolet/ visible spectrophotometer.

Excess solute and solvent were equilibrated in a constant-temperature bath with stirring for $48 \mathrm{~h}$. The saturated solution was then centrifuged for $10 \mathrm{~min}$, and an aliquot of the supernatant solution was removed and diluted quantitatively for spectrophotometric analysis. The concentration of the dissolved solute was determined from the measured absorbance.

\section{Source and Purity of Chemicals:}

(1) Purity not given, Fisons Scientific Equipment, UK, no purification details were provided.

(2) Purity not given, Purac Biochem, Gorinchem, Netherlands, no purification details were provided.

\section{Estimated Error:}

Temperature: Insufficient experimental details to estimate. $c_{1}: \pm 0.20$.

\begin{tabular}{ll}
\hline \hline Components: & Original Measurements: \\
(1) Benzoic acid; $\mathrm{C}_{7} \mathrm{H}_{6} \mathrm{O}_{2} ;$ & ${ }^{87}$ M. Dias, J. Hadgraft, and M. E. Lane, \\
65-85-0] & Int. J. Pharm. 336, 108 (2007). \\
$\begin{array}{l}\text { (2) 1-Methylethyl 2- } \\
\text { hydroxypropanoate; } \mathrm{C}_{6} \mathrm{H}_{12} \mathrm{O}_{3} ;\end{array}$ & \\
{$[617-64-3]$} & \\
\hline Variables: & Prepared by: \\
$T / \mathrm{K}=305.2$ & W. E. Acree, Jr. \\
\hline
\end{tabular}

\section{Experimental Values}

The measured solubility was reported to be $239.70 \mathrm{mg} / \mathrm{ml}$, which corresponds to a molar solubility of $c_{1}=1.963$ mol dm ${ }^{-3}$.

\section{Auxiliary Information}

\section{Method/Apparatus/Procedure:}

Constant-temperature bath, magnetic stirrer, centrifuge, and an ultraviolet/ visible spectrophotometer.

Excess solute and solvent were equilibrated in a constant-temperature bath with stirring for $48 \mathrm{~h}$. The saturated solution was then centrifuged for $10 \mathrm{~min}$, and an aliquot of the supernatant solution was removed and diluted quantitatively for spectrophotometric analysis. The concentration of the dissolved solute was determined from the measured absorbance.

Source and Purity of Chemicals:

(1) Purity not given, Fisons Scientific Equipment, UK, no purification details were provided.

(2) Purity not given, Purac Biochem, Gorinchem, Netherlands, no purification details were provided.

\section{Estimated Error:}

Temperature: Insufficient experimental details to estimate. $c_{1}: \pm 0.095$.

\section{Components:}

(1) Benzoic acid; $\mathrm{C}_{7} \mathrm{H}_{6} \mathrm{O}_{2}$;

[65-85-0]

(2) Butyl 2-hydroxypropanoate;

$\mathrm{C}_{7} \mathrm{H}_{14} \mathrm{O}_{3} ;[138-22-7]$

\begin{tabular}{ll}
\hline Variables: & Prepared by: \\
$T / \mathrm{K}=305.2$ & W. E. Acree, Jr.
\end{tabular}

\section{Experimental Values}

The measured solubility was reported to be $135.80 \mathrm{mg} / \mathrm{ml}$, which corresponds to a molar solubility of $c_{1}=1.112$ mol $\mathrm{dm}^{-3}$.

\section{Auxiliary Information}

\section{Method/Apparatus/Procedure:}

Constant-temperature bath, magnetic stirrer, centrifuge, and an ultraviolet/ visible spectrophotometer.

Excess solute and solvent were equilibrated in a constant-temperature bath with stirring for $48 \mathrm{~h}$. The saturated solution was then centrifuged for $10 \mathrm{~min}$, and an aliquot of the supernatant solution was removed and diluted quantitatively for spectrophotometric analysis. The concentration of the dissolved solute was determined from the measured absorbance.

Source and Purity of Chemicals:

(1) Purity not given, Fisons Scientific Equipment, UK, no purification details were provided.

(2) Purity not given, Purac Biochem, Gorinchem, Netherlands, no purification details were provided.

\section{Estimated Error:}

Temperature: Insufficient experimental details to estimate. $c_{1}: \pm 0.038$.

\section{Components:}

(1) Benzoic acid; $\mathrm{C}_{7} \mathrm{H}_{6} \mathrm{O}_{2}$; [65-85-0]

(2) Butyl 2-hydroxypropanoate; $\mathrm{C}_{7} \mathrm{H}_{14} \mathrm{O}_{3} ;[128-22-7]$

\begin{tabular}{ll}
\hline Variables: & Prepared by: \\
$T / \mathrm{K}=298.15$ & W. E. Acree, Jr.
\end{tabular}

\section{Original Measurements:}

${ }^{51}$ H.-M. Lin and R. A. Nash, J. Pharm. Sci. 82, 1018 (1993).
Experimental Values

\begin{tabular}{lc}
\hline \hline$x_{2}{ }^{\mathrm{a}}$ & $x_{1}{ }^{\mathrm{b}}$ \\
\hline 0.7987 & 0.2013 \\
\hline${ }^{\mathrm{a}} x_{2}:$ mole fraction of component 2 in the saturated solution. & \\
${ }^{\mathrm{b}} x_{1}:$ mole fraction solubility of the solute. &
\end{tabular}




\section{Auxiliary Information}

\section{Method/Apparatus/Procedure:}

Constant-temperature bath and high-performance liquid chromatograph. Excess solute and solvent were placed in screw-capped vials. The vials were sealed with several turns of electrical tape, warmed to about $323 \mathrm{~K}$, and then shaken in a constant-temperature bath for at least $24 \mathrm{~h}$ at $298 \mathrm{~K}$. After equilibrium was reached, the sample was filtered through a 0.45 or $1.0 \mu \mathrm{m}$ filter, and an aliquot was diluted appropriately for chromatographic analysis Concentrations were determined by a high-performance liquid chromatographic method.

\section{Source and Purity of Chemicals:}

(1) Purity not given, Amend Drug and Chemical Company, no information provided concerning purification.

(2) Purity not given, Purac Inc., no purification details were provided.

\section{Estimated Error:}

Temperature: $\pm 0.2 \mathrm{~K}$ (estimated by compiler). $x_{1}: \pm 3 \%$ (relative error, estimated by compiler).

\begin{tabular}{|c|c|}
\hline $\begin{array}{l}\text { Components: } \\
\text { (1) Benzoic acid; } \mathrm{C}_{7} \mathrm{H}_{6} \mathrm{O}_{2} \text {; } \\
\text { [65-85-0] } \\
\text { (2) } \gamma \text {-Butyrolactone; } \mathrm{C}_{4} \mathrm{H}_{6} \mathrm{O}_{2} \text {; } \\
\text { [96-48-0] }\end{array}$ & $\begin{array}{l}\text { Original Measurements: } \\
{ }^{51} \text { H.-M. Lin and R. A. Nash, J. Pharm. } \\
\text { Sci. 82, } 1018 \text { (1993). }\end{array}$ \\
\hline $\begin{array}{l}\text { Variables: } \\
T / \mathrm{K}=298.15\end{array}$ & $\begin{array}{l}\text { Prepared by: } \\
\text { W. E. Acree, Jr. }\end{array}$ \\
\hline
\end{tabular}

Experimental Values

\begin{tabular}{lc}
\hline \hline$x_{2}{ }^{\mathrm{a}}$ & $x_{1}{ }^{\mathrm{b}}$ \\
\hline 0.8434 & 0.1566
\end{tabular}

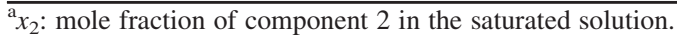

${ }^{b} x_{1}$ : mole fraction solubility of the solute.

\section{Auxiliary Information}

\footnotetext{
Method/Apparatus/Procedure:

Constant-temperature bath and high-performance liquid chromatograph. Excess solute and solvent were placed in screw-capped vials. The vials were sealed with several turns of electrical tape, warmed to about $323 \mathrm{~K}$, and then shaken in a constant-temperature bath for at least $24 \mathrm{~h}$ at $298 \mathrm{~K}$. After equilibrium was reached, the sample was filtered through a 0.45 or $1.0 \mu \mathrm{m}$ filter, and an aliquot was diluted appropriately for chromatographic analysis. Concentrations were determined by a high-performance liquid chromatographic method.
}

\section{Source and Purity of Chemicals:}

(1) Purity not given, Amend Drug and Chemical Company, no information provided concerning purification.

(2) Reagent grade, Aldrich Chemical Company, Milwaukee, WI, USA, no purification details were provided.

\section{Estimated Error:}

Temperature: $\pm 0.2 \mathrm{~K}$ (estimated by compiler). $x_{1}: \pm 3 \%$ (relative error, estimated by compiler).

\begin{tabular}{ll}
\hline \hline Components: & Original Measurements: \\
(1) Benzoic acid; $\mathrm{C}_{7} \mathrm{H}_{6} \mathrm{O}_{2} ;$ & ${ }^{51} \mathrm{H} .-\mathrm{M}$. Lin and R. A. Nash, J. Pharm. \\
[65-85-0] & Sci. 82, 1018 (1993). \\
(2) Dimethyl isosorbide; & \\
$\mathrm{C}_{8} \mathrm{H}_{14} \mathrm{O}_{4} ;[5306-85-4]$ & \\
\hline Variables: & Prepared by: \\
$T / \mathrm{K}=298.15$ & W. E. Acree, Jr. \\
\hline
\end{tabular}

\section{Experimental Values}

\begin{tabular}{lc}
\hline \hline$x_{2}{ }^{\mathrm{a}}$ & $x_{1}{ }^{\mathrm{b}}$ \\
\hline 0.6810 & 0.3190 \\
\hline${ }^{\mathrm{a}} x_{2}:$ mole fraction of component 2 in the saturated solution. & \\
${ }^{\mathrm{b}} x_{1}:$ mole fraction solubility of the solute. &
\end{tabular}

\section{Auxiliary Information}

\section{Method/Apparatus/Procedure:}

Constant-temperature bath and high-performance liquid chromatograph. Excess solute and solvent were placed in screw-capped vials. The vials were sealed with several turns of electrical tape, warmed to about $323 \mathrm{~K}$, and then shaken in a constant-temperature bath for at least $24 \mathrm{~h}$ at $298 \mathrm{~K}$. After equilibrium was reached, the sample was filtered through a 0.45 or $1.0 \mu \mathrm{m}$ filter, and an aliquot was diluted appropriately for chromatographic analysis. Concentrations were determined by a high-performance liquid chromatographic method.

\section{Source and Purity of Chemicals:}

(1) Purity not given, Amend Drug and Chemical Company, no information provided concerning purification.

(2) Reagent grade, Aldrich Chemical Company, Milwaukee, WI, USA, no purification details were provided.

\section{Estimated Error:}

Temperature: $\pm 0.2 \mathrm{~K}$ (estimated by compiler). $x_{1}: \pm 3 \%$ (relative error, estimated by compiler).

\begin{tabular}{|c|c|}
\hline $\begin{array}{l}\text { Components: } \\
\text { (1) Benzoic acid; } \mathrm{C}_{7} \mathrm{H}_{6} \mathrm{O}_{2} \text {; } \\
{[65-85-0]} \\
\text { (2) } 9(\mathrm{Z}) \text {-Octadecenoic acid } \\
\text { (Oleic acid); } \mathrm{C}_{18} \mathrm{H}_{34} \mathrm{O}_{2} ;[112-80-1]\end{array}$ & $\begin{array}{l}\text { Original Measurements: } \\
{ }^{87} \text { M. Dias, J. Hadgraft, and M. E. Lane, } \\
\text { Int. J. Pharm. 336, } 108 \text { (2007). }\end{array}$ \\
\hline $\begin{array}{l}\text { Variables: } \\
T / \mathrm{K}=305.2\end{array}$ & $\begin{array}{l}\text { Prepared by: } \\
\text { W. E. Acree, Jr. }\end{array}$ \\
\hline
\end{tabular}

\section{Experimental Values}

The measured solubility was reported to be $245.50 \mathrm{mg} / \mathrm{ml}$, which corresponds to a molar solubility of $c_{1}=2.010$ mol dm ${ }^{-3}$. 


\section{Auxiliary Information}

\section{Method/Apparatus/Procedure:}

Constant-temperature bath, magnetic stirrer, centrifuge, and an ultraviolet/ visible spectrophotometer.

Excess solute and solvent were equilibrated in a constant-temperature bath with stirring for $48 \mathrm{~h}$. The saturated solution was then centrifuged for $10 \mathrm{~min}$, and an aliquot of the supernatant solution was removed and diluted quantitatively for spectrophotometric analysis. The concentration of the dissolved solute was determined from the measured absorbance.

\section{Source and Purity of Chemicals:}

(1) Purity not given, Fisons Scientific Equipment, UK, no purification details were provided.

(2) Purity not given, Chemical source not given, no purification details were provided.

\section{Estimated Error:}

Temperature: Insufficient experimental details to estimate. $c_{1}: \pm 0.16$.

\begin{tabular}{ll}
\hline \hline Components: & Original Measurements: \\
(1) Benzoic acid; $\mathrm{C}_{7} \mathrm{H}_{6} \mathrm{O}_{2} ;$ & ${ }^{85} \mathrm{~K}$. Mislow, J. Phys. Colloid Chem. \\
$\begin{array}{l}\text { 65-85-0] } \\
\text { (2) 2-Furancarboxylic acid; }\end{array}$ & $\mathbf{5 2}, 729(1948)$. \\
$\mathrm{C}_{5} \mathrm{H}_{4} \mathrm{O}_{3} ;[88-14-2]$ & \\
\hline Variables: & Prepared by: \\
Temperature & W. E. Acree, Jr. \\
\hline
\end{tabular}

\begin{tabular}{lcc}
\hline & & \\
& & \\
& & \\
\hline \hline$t /{ }^{\circ} \mathrm{C}$ (thawing) & $t /{ }^{\circ} \mathrm{C}($ melting) & $w_{1}{ }^{\mathrm{a}}$ \\
\hline 132 & 133 & 0.000 \\
85.6 & 127.2 & 0.104 \\
85.1 & 124.0 & 0.217 \\
85.0 & 121.5 & 0.289 \\
85.3 & 114.5 & 0.420 \\
85.5 & 100.2 & 0.486 \\
85.0 & 95.2 & 0.570 \\
85.0 & 96.0 & 0.669 \\
85.1 & 105.5 & 0.775 \\
85.1 & 110.8 & 0.865 \\
121 & 122 & 1.000 \\
\hline
\end{tabular}

${ }^{\mathrm{a}} w_{1}$ : mass fraction solubility of the solute.

The author reports that the binary system forms a simple eutectic at $w_{1}=0.600$ and $t=85^{\circ} \mathrm{C}$.

\section{Auxiliary Information}

\section{Method/Apparatus/Procedure:}

Experimental method involved observing the thawing-melting behavior of binary mixtures of known composition. Thoroughly mixed samples were placed in capillaries, and sealed at one end. The capillaries were placed next to the junction of a thermocouple in a hot stage, which was mounted on a polarizing microscope. Heating rates were kept at $(2 \pm 1)^{\circ} \mathrm{C} / \mathrm{min}$. The thawing temperature was observed by reflected light, while the melting points were observed by transmitted light. Accuracy of the method, as judged by the reproducibility of the measured data, was estimated to be $\pm 2{ }^{\circ} \mathrm{C}$ for thawing and melting temperatures.
Source and Purity of Chemicals:

(1) Purity not given, Chemical source not given, recrystallized from aqueous solution.

(2) Purity not given, Chemical source not given, recrystallized from aqueous solution.

\section{Estimated Error:}

Temperature: $2^{\circ} \mathrm{C}$

$w_{1}: \pm 0.002$ (estimated by compiler).

\begin{tabular}{|c|c|}
\hline $\begin{array}{l}\text { Components: } \\
\text { (1) Benzoic acid; } \mathrm{C}_{7} \mathrm{H}_{6} \mathrm{O}_{2} \text {; } \\
\text { [65-85-0] } \\
\text { (2) 2-Pyridinecarboxylic acid; } \\
\mathrm{C}_{6} \mathrm{H}_{5} \mathrm{NO}_{2} ; \text { [98-98-6] }\end{array}$ & $\begin{array}{l}\text { Original Measurements: } \\
{ }^{85} \mathrm{~K} \text {. Mislow, J. Phys. Colloid Chem. } \\
\mathbf{5 2}, 729 \text { (1948). }\end{array}$ \\
\hline $\begin{array}{l}\text { Variables: } \\
\text { Temperature }\end{array}$ & $\begin{array}{l}\text { Prepared by: } \\
\text { W. E. Acree, Jr. }\end{array}$ \\
\hline
\end{tabular}

\begin{tabular}{lcc}
\hline \hline$t /{ }^{\circ} \mathrm{C}$ (thawing) & $t /{ }^{\circ} \mathrm{C}$ (melting) & $w_{1}{ }^{\mathrm{a}}$ \\
\hline 136 & 137 & 0.000 \\
88.4 & 134.2 & 0.116 \\
87.6 & 131.0 & 0.186 \\
86.4 & 123.8 & 0.303 \\
87.0 & 116.0 & 0.383 \\
87.2 & 107.4 & 0.465 \\
87.4 & 102.4 & 0.588 \\
87.0 & 107.4 & 0.672 \\
87.6 & 111.4 & 0.755 \\
87.6 & 116.2 & 0.866 \\
121 & 122 & 1.000 \\
\hline
\end{tabular}

${ }^{\mathrm{a}} w_{1}$ : mass fraction solubility of the solute.

The author reports that the binary system forms a simple eutectic at $w_{1}=0.530$ and $t=115^{\circ} \mathrm{C}$.

\section{Auxiliary Information}

\section{Method/Apparatus/Procedure:}

Experimental method involved observing the thawing-melting behavior of binary mixtures of known composition. Thoroughly mixed samples were placed in capillaries, and sealed at one end. The capillaries were placed next to the junction of a thermocouple in a hot stage, which was mounted on a polarizing microscope. Heating rates were kept at $(2 \pm 1)^{\circ} \mathrm{C} / \mathrm{min}$. The thawing temperature was observed by reflected light, while the melting points were observed by transmitted light. Accuracy of the method, as judged by the reproducibility of the measured data, was estimated to be $\pm 2{ }^{\circ} \mathrm{C}$ for thawing and melting temperatures.

\section{Source and Purity of Chemicals:}

(1) Purity not given, Chemical source not given, recrystallized from aqueous solution.

(2) Purity not given, Chemical source not given, recrystallized from aqueous solution.

\section{Estimated Error:}

Temperature: $2{ }^{\circ} \mathrm{C}$

$w_{1}: \pm 0.002$ (estimated by compiler). 


\begin{tabular}{|c|c|}
\hline $\begin{array}{l}\text { Components: } \\
\text { (1) Benzoic acid; } \mathrm{C}_{7} \mathrm{H}_{6} \mathrm{O}_{2} \text {; } \\
\text { [65-85-0] } \\
\text { (2) 3-Pyridinecarboxylic acid; } \\
\mathrm{C}_{6} \mathrm{H}_{5} \mathrm{NO}_{2} ;[59-67-6]\end{array}$ & $\begin{array}{l}\text { Original Measurements: } \\
\text { 85 K. Mislow, J. Phys. Colloid Chem. } \\
\text { 52, } 729 \text { (1948). }\end{array}$ \\
\hline $\begin{array}{l}\text { Variables: } \\
\text { Temperature }\end{array}$ & $\begin{array}{l}\text { Prepared by: } \\
\text { W. E. Acree, Jr. }\end{array}$ \\
\hline
\end{tabular}

Experimental Values

\begin{tabular}{lcc}
\hline \hline$t /{ }^{\circ} \mathrm{C}$ (thawing) & $t /{ }^{\circ} \mathrm{C}$ (melting) & $w_{1}{ }^{\mathrm{a}}$ \\
\hline 233 & 233 & 0.000 \\
115.0 & 227.6 & 0.090 \\
115.4 & 217.6 & 0.200 \\
115.4 & 211.2 & 0.286 \\
114.2 & 195.8 & 0.454 \\
115.0 & 184.0 & 0.505 \\
114.6 & 169.8 & 0.666 \\
114.0 & 142.2 & 0.708 \\
113.6 & 117.8 & 0.890 \\
114.0 & 120.4 & 0.968 \\
121 & 122 & 1.000 \\
\hline
\end{tabular}

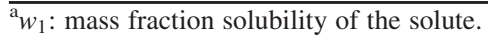

The author reports that the binary system forms a simple eutectic at $w_{1}=0.900$ and $t=115^{\circ} \mathrm{C}$.

\section{Auxiliary Information}

\section{Method/Apparatus/Procedure:}

Experimental method involved observing the thawing-melting behavior of binary mixtures of known composition. Thoroughly mixed samples were placed in capillaries, and sealed at one end. The capillaries were placed next to the junction of a thermocouple in a hot stage, which was mounted on a polarizing microscope. Heating rates were kept at $(2 \pm 1)^{\circ} \mathrm{C} / \mathrm{min}$. The thawing temperature was observed by reflected light, while the melting points were observed by transmitted light. Accuracy of the method, as judged by the reproducibility of the measured data, was estimated to be $\pm 2{ }^{\circ} \mathrm{C}$ for thawing and melting temperatures.

\section{Source and Purity of Chemicals:}

(1) Purity not given, Chemical source not given, recrystallized from aqueous solution.

(2) Purity not given, Chemical source not given, recrystallized from aqueous solution.

\section{Estimated Error:}

Temperature: $2^{\circ} \mathrm{C}$.

$w_{1}: \pm 0.002$ (estimated by compiler).
Experimental Values

\begin{tabular}{llc}
\hline \hline$t /{ }^{\circ} \mathrm{C}$ (thawing) & \multicolumn{1}{c}{${ }^{\circ} \mathrm{C}$ (melting) } & $w_{1}{ }^{\mathrm{a}}$ \\
\hline 312 & 314 (decomposed) & 0.000 \\
11.6 & 304 (decomposed) & 0.081 \\
115.4 & 282.8 & 0.368 \\
115.0 & 265.4 & 0.539 \\
115.0 & 264.2 & 0.618 \\
115.0 & 255.4 & 0.684 \\
114.8 & 225.2 & 0.818 \\
115.4 & 199.4 & 0.894 \\
115.4 & 158.8 & 0.961 \\
121 & 122 & 1.000 \\
\hline
\end{tabular}

${ }^{\mathrm{a}} w_{1}$ : mass fraction solubility of the solute.

The author reports that the binary system forms a simple eutectic at $w_{1}=0.990$ and $t=115^{\circ} \mathrm{C}$.

\section{Auxiliary Information}

\section{Method/Apparatus/Procedure:}

Experimental method involved observing the thawing-melting behavior of binary mixtures of known composition. Thoroughly mixed samples were placed in capillaries, and sealed at one end. The capillaries were placed next to the junction of a thermocouple in a hot stage, which was mounted on a polarizing microscope. Heating rates were kept at $(2 \pm 1)^{\circ} \mathrm{C} / \mathrm{min}$. The thawing temperature was observed by reflected light, while the melting points were observed by transmitted light. Accuracy of the method, as judged by the reproducibility of the measured data, was estimated to be $\pm 2{ }^{\circ} \mathrm{C}$ for thawing and melting temperatures.

\section{Source and Purity of Chemicals:}

(1) Purity not given, Chemical source not given, recrystallized from aqueous solution.

(2) Purity not given, Chemical source not given, recrystallized from aqueous solution.

\section{Estimated Error:}

Temperature: $2{ }^{\circ} \mathrm{C}$.

$w_{1}: \pm 0.002$ (estimated by compiler).

\begin{tabular}{|c|c|}
\hline $\begin{array}{l}\text { Components: } \\
\text { (1) Benzoic acid; } \mathrm{C}_{7} \mathrm{H}_{6} \mathrm{O}_{2} \text {; } \\
\text { [65-85-0] } \\
\text { (2) 2-Thiophenecarboxylic acid; } \\
\mathrm{C}_{5} \mathrm{H}_{4} \mathrm{O}_{2} \mathrm{~S} ;[527-72-0]\end{array}$ & $\begin{array}{l}\text { Original Measurements: } \\
{ }^{85} \text { K. Mislow, J. Phys. Colloid Chem } \\
\text { 52, } 729 \text { (1948). }\end{array}$ \\
\hline $\begin{array}{l}\text { Variables: } \\
\text { Temperature }\end{array}$ & $\begin{array}{l}\text { Prepared by: } \\
\text { W. E. Acree, Jr. }\end{array}$ \\
\hline
\end{tabular}

\begin{tabular}{ll}
\hline \hline Components: & Original Measurements: \\
(1) Benzoic acid; $\mathrm{C}_{7} \mathrm{H}_{6} \mathrm{O}_{2} ;$ & ${ }^{85} \mathrm{~K}$. Mislow, J. Phys. Colloid Chem. \\
65-85-0] & $\mathbf{5 2}, 729(1948)$. \\
(2) 4-Pyridinecarboxylic acid; & \\
$\mathrm{C}_{6} \mathrm{H}_{5} \mathrm{NO}_{2} ;[55-22-1]$ & \\
\hline Variables: & Prepared by: \\
Temperature & W. E. Acree, Jr. \\
\hline
\end{tabular}


Experimental Values

\begin{tabular}{lcc}
\hline \hline$t /{ }^{\circ} \mathrm{C}$ (thawing) & $t /{ }^{\circ} \mathrm{C}($ melting) & $w_{1}{ }^{\mathrm{a}}$ \\
\hline 127 & 128 & 0.000 \\
94.6 & 123.8 & 0.084 \\
94.8 & 120.4 & 0.171 \\
94.8 & 118.6 & 0.207 \\
95.4 & 115.2 & 0.308 \\
94.8 & 112.0 & 0.383 \\
95.8 & 110.2 & 0.415 \\
95.2 & 109.2 & 0.445 \\
95.4 & 104.8 & 0.555 \\
94.4 & 109.2 & 0.710 \\
94.8 & 111.6 & 0.767 \\
95.6 & 116.2 & 0.835 \\
95.6 & 117.0 & 0.860 \\
97.8 & 120.6 & 0.903 \\
121 & 122 & 1.000 \\
\hline
\end{tabular}

${ }^{\mathrm{a}} w_{1}$ : mass fraction solubility of the solute.

The author reports that the binary system forms a simple eutectic at $w_{1}=0.630$ and $t=95^{\circ} \mathrm{C}$, with an indication of solid solution formation at $w_{1}>0.90$.

\section{Auxiliary Information}

\section{Method/Apparatus/Procedure:}

Experimental method involved observing the thawing-melting behavior of binary mixtures of known composition. Thoroughly mixed samples were placed in capillaries, and sealed at one end. The capillaries were placed next to the junction of a thermocouple in a hot stage, which was mounted on a polarizing microscope. Heating rates were kept at $(2 \pm 1)^{\circ} \mathrm{C} / \mathrm{min}$. The thawing temperature was observed by reflected light, while the melting points were observed by transmitted light. Accuracy of the method, as judged by the reproducibility of the measured data, was estimated to be $\pm 2{ }^{\circ} \mathrm{C}$ for thawing and melting temperatures.

\section{Source and Purity of Chemicals:}

(1) Purity not given, Chemical source not given, recrystallized from aqueous solution.

(2) Purity not given, prepared by the authors, synthesized by iodinating thiophene, and then converting the resulting 2-iodothiophene into the desired acid by the Grignard synthesis. The sample was purified by successive recrystallizations from aqueous solution and from ligroin-benzene mixture.

Estimated Error:

Temperature: $2^{\circ} \mathrm{C}$.

$w_{1}: \pm 0.002$ (estimated by compiler).

\subsection{Benzoic acid solubility data in binary organic solvent mixtures}

\begin{tabular}{l}
\hline \hline Components: \\
(1) Benzoic acid; $\mathrm{C}_{7} \mathrm{H}_{6} \mathrm{O}_{2}$; \\
[65-85-0] \\
(2) Cyclohexane; $\mathrm{C}_{6} \mathrm{H}_{12}$; \\
[110-82-7] \\
(3) Hexane; $\mathrm{C}_{6} \mathrm{H}_{14} ;$; $\left.110-54-3\right]$
\end{tabular}

\begin{tabular}{ll}
\hline Variables: & Prepared by: \\
Temperature; Solvent Composition & W. E. Acree, Jr.
\end{tabular}

\author{
Original Measurements: \\ ${ }^{49}$ W. E. Acree, Jr. and G. L. Bertrand, J. \\ Pharm. Sci. 70, 1033 (1981).
}

W. E. Acree, Jr.
Experimental Values

\begin{tabular}{lcc}
\hline \hline$T / \mathrm{K}$ & $x_{2}{ }^{(\mathrm{s}) \mathrm{a}}$ & $x_{1}{ }^{\mathrm{b}}$ \\
\hline 298.15 & 0.0000 & 0.0100 \\
298.15 & 0.2573 & 0.0108 \\
298.15 & 0.4699 & 0.0113 \\
298.15 & 0.5342 & 0.0115 \\
298.15 & 0.6566 & 0.0118 \\
298.15 & 0.8044 & 0.0117 \\
298.15 & 1.0000 & 0.0115 \\
303.15 & 0.0000 & 0.0126 \\
303.15 & 0.2341 & 0.0136 \\
303.15 & 0.2845 & 0.0138 \\
303.15 & 0.5059 & 0.0146 \\
303.15 & 0.6670 & 0.0150 \\
303.15 & 0.7821 & 0.0151 \\
303.15 & 1.0000 & 0.0146 \\
\hline${ }^{\mathrm{a}} x_{2}{ }^{(\mathrm{s})}:$ initial mole fraction of component 2 in the binary solvent mixture. \\
${ }^{\mathrm{b}} x_{1}:$ mole fraction solubility of the solute.
\end{tabular}

\section{Auxiliary Information}

\section{Method/Apparatus/Procedure:}

Constant-temperature water bath and an analytical balance.

Excess solute and solvent and were placed in brown glass containers and allowed to equilibrate in a constant-temperature water bath for several days. Attainment of equilibrium was verified by repetitive measurements after several additional days. Solubility was determined by transferring a weighed aliquot through a coarse filter into a flask containing blank nonaqueous titration solvent. The solutions were titrated with freshly standardized sodium methoxide to the thymol blue endpoint.

Source and Purity of Chemicals:

(1) $99 \%$, Chemical source not given, was dried at $333 \mathrm{~K}$ for several hours before use.

(2) $99+\%$, Chemical source not given, was dried over molecular sieves and distilled before use.

(3) $99 \%$, Chemical source not given, was dried over molecular sieves and distilled before use.

\section{Estimated Error:}

Temperature: $\pm 0.01 \mathrm{~K}$.

$x_{2}{ }^{(\mathrm{s})}: \pm 0.0001$.

$x_{1}: \pm 1 \%$ (relative error).

\begin{tabular}{|c|c|}
\hline $\begin{array}{l}\text { Components: } \\
\text { (1) Benzoic acid; } \mathrm{C}_{7} \mathrm{H}_{6} \mathrm{O}_{2} \text {; } \\
\text { [65-85-0] } \\
\text { (2) Cyclohexane; } \mathrm{C}_{6} \mathrm{H}_{12} \text {; } \\
\text { [110-82-7] } \\
\text { (3) Heptane; } \mathrm{C}_{7} \mathrm{H}_{16} ;[142-82-5]\end{array}$ & $\begin{array}{l}\text { Original Measurements: } \\
{ }^{49} \text { W. E. Acree, Jr. and G. L. Bertrand, J. } \\
\text { Pharm. Sci. 70, } 1033 \text { (1981). }\end{array}$ \\
\hline $\begin{array}{l}\text { Variables: } \\
T / \mathrm{K}=298.15 \text {; Solvent Composition }\end{array}$ & $\begin{array}{l}\text { Prepared by: } \\
\text { W. E. Acree, Jr. }\end{array}$ \\
\hline
\end{tabular}


Experimental Values

\begin{tabular}{lcc}
\hline \hline$x_{2}{ }^{(\mathrm{s}) \mathrm{a}}$ & $x_{2}{ }^{\mathrm{b}}$ & $x_{1}{ }^{\mathrm{c}}$ \\
\hline 0.0000 & 0.0000 & 0.0114 \\
0.2781 & 0.2748 & 0.0119 \\
0.4602 & 0.4546 & 0.0121 \\
0.5713 & 0.5644 & 0.0121 \\
0.6825 & 0.4742 & 0.0122 \\
0.8142 & 0.4045 & 0.0119 \\
1.0000 & 0.9885 & 0.0115 \\
\hline
\end{tabular}

${ }^{\mathrm{a}} x_{2}{ }^{(\mathrm{s})}$ : initial mole fraction of component 2 in the binary solvent mixture.

${ }^{\mathrm{b}} x_{2}$ : mole fraction of component 2 in the saturated solution.

${ }^{c} x_{1}$ : mole fraction solubility of the solute.

\section{Auxiliary Information}

\section{Method/Apparatus/Procedure:}

Constant-temperature water bath and an analytical balance.

Excess solute and solvent and were placed in brown glass containers and allowed to equilibrate in a constant-temperature water bath for several days. Attainment of equilibrium was verified by repetitive measurements after several additional days. Solubility was determined by transferring a weighed aliquot through a coarse filter into a flask containing blank nonaqueous titration solvent. The solutions were titrated with freshly standardized sodium methoxide to the thymol blue endpoint.

\section{Source and Purity of Chemicals:}

(1) $99 \%$, Chemical source not given, was dried at $333 \mathrm{~K}$ for several hours before use.

(2) $99+\%$, Chemical source not given, was dried over molecular sieves and distilled before use.

(3) $99+\%$, Chemical source not given, was dried over molecular sieves and distilled before use.

\section{Estimated Error:}

Temperature: $\pm 0.01 \mathrm{~K}$.

$x_{2}{ }^{(\mathrm{s})}: \pm 0.0001$.

$x_{1}: \pm 1 \%$ (relative error).

\begin{tabular}{|c|c|}
\hline $\begin{array}{l}\text { Components: } \\
\text { (1) Benzoic acid; } \mathrm{C}_{7} \mathrm{H}_{6} \mathrm{O}_{2} \text {; } \\
\text { [65-85-0] } \\
\text { (2) Hexane; } \mathrm{C}_{6} \mathrm{H}_{14} ;[110-54-3] \\
\text { (3) Tetrachloromethane; } \mathrm{CCl}_{4} \text {; } \\
\text { [56-23-5] }\end{array}$ & $\begin{array}{l}\text { Original Measurements: } \\
\text { }{ }^{49} \text { W. E. Acree, Jr. and G. L. Bertrand, J. } \\
\text { Pharm. Sci. 70, } 1033 \text { (1981). }\end{array}$ \\
\hline $\begin{array}{l}\text { Variables: } \\
\text { Temperature; Solvent Composition }\end{array}$ & $\begin{array}{l}\text { Prepared by: } \\
\text { W. E. Acree, Jr. }\end{array}$ \\
\hline
\end{tabular}

Experimental Values

\begin{tabular}{lcc}
\hline \hline$T / \mathrm{K}$ & $x_{2}{ }^{(\mathrm{s}) \mathrm{a}}$ & $x_{1}{ }^{\mathrm{b}}$ \\
\hline 298.15 & 0.0000 & 0.0492 \\
298.15 & 0.1550 & 0.0395 \\
298.15 & 0.3419 & 0.0297 \\
298.15 & 0.4408 & 0.0251 \\
298.15 & 0.5951 & 0.0195 \\
298.15 & 0.8028 & 0.0137 \\
298.15 & 1.0000 & 0.0100 \\
303.15 & 0.0000 & 0.0598 \\
303.15 & 0.2255 & 0.0483
\end{tabular}

\begin{tabular}{lcc}
\hline \hline$T / \mathrm{K}$ & $x_{2}{ }^{(\mathrm{s}) \mathrm{a}}$ & $x_{1}{ }^{\mathrm{b}}$ \\
\hline 303.15 & 0.3078 & 0.0391 \\
303.15 & 0.4556 & 0.0304 \\
303.15 & 0.6438 & 0.0226 \\
303.15 & 0.7564 & 0.0185 \\
303.15 & 1.0000 & 0.0126
\end{tabular}

${ }^{a} x_{2}{ }^{(\mathrm{s})}$ : initial mole fraction of component 2 in the binary solvent mixture.

${ }^{b} x_{1}$ : mole fraction solubility of the solute.

\section{Auxiliary Information}

\section{Method/Apparatus/Procedure:}

Constant-temperature water bath and an analytical balance.

Excess solute and solvent and were placed in brown glass containers and allowed to equilibrate in a constant-temperature water bath for several days. Attainment of equilibrium was verified by repetitive measurements after several additional days. Solubility was determined by transferring a weighed aliquot through a coarse filter into a flask containing blank nonaqueous titration solvent. The solutions were titrated with freshly standardized sodium methoxide to the thymol blue endpoint.

\section{Source and Purity of Chemicals:}

(1) $99 \%$, Chemical source not given, was dried at $333 \mathrm{~K}$ for several hours before use.

(2) $99 \%$, Chemical source not given, was dried over molecular sieves and distilled before use.

(3) $99+\%$, Chemical source not given, refluxed for $11 \mathrm{~h}$ over an aqueous solution $10 \%$ in potassium permanganate and $10 \%$ in sodium hydroxide. The tetrachloromethane was distilled off and dried with calcium hydroxide, stored in contact with mercury under an argon atmosphere, and then distilled shortly before use.

Estimated Error:

Temperature: $\pm 0.01 \mathrm{~K}$.

$x_{2}{ }^{(\mathrm{s})}: \pm 0.0001$.

$x_{1}: \pm 1 \%$ (relative error)

\begin{tabular}{|c|c|}
\hline $\begin{array}{l}\text { Components: } \\
\text { (1) Benzoic acid; } \mathrm{C}_{7} \mathrm{H}_{6} \mathrm{O}_{2} \text {; } \\
\text { [65-85-0] } \\
\text { (2) Heptane; } \mathrm{C}_{7} \mathrm{H}_{16} ;[142-82-5] \\
\text { (3) Tetrachloromethane; } \mathrm{CCl}_{4} \text {; } \\
\text { [56-23-5] }\end{array}$ & $\begin{array}{l}\text { Original Measurements: } \\
{ }^{49} \text { W. E. Acree, Jr. and G. L. Bertrand, J. } \\
\text { Pharm. Sci. 70, } 1033 \text { (1981). }\end{array}$ \\
\hline $\begin{array}{l}\text { Variables: } \\
\text { Temperature; Solvent Composition }\end{array}$ & $\begin{array}{l}\text { Prepared by: } \\
\text { W. E. Acree, Jr. }\end{array}$ \\
\hline
\end{tabular}

\section{Components:}

[65-85-0] 
Experimental Values

\begin{tabular}{lcc}
\hline \hline$T / \mathrm{K}$ & $x_{2}{ }^{(\mathrm{s}) \mathrm{a}}$ & $x_{1}{ }^{\mathrm{b}}$ \\
\hline 298.15 & 0.0000 & 0.0492 \\
298.15 & 0.1784 & 0.0375 \\
298.15 & 0.2303 & 0.0347 \\
298.15 & 0.3348 & 0.0295 \\
298.15 & 0.3930 & 0.0270 \\
298.15 & 0.3932 & 0.0269 \\
298.15 & 0.4708 & 0.0240 \\
298.15 & 0.5433 & 0.0214 \\
298.15 & 0.7013 & 0.0170 \\
298.15 & 1.0000 & 0.0114 \\
303.15 & 0.0000 & 0.0598 \\
303.15 & 0.2421 & 0.0423 \\
303.15 & 0.4269 & 0.0322 \\
303.15 & 0.6237 & 0.0241 \\
303.15 & 0.7346 & 0.0206 \\
303.15 & 1.0000 & 0.0147 \\
${ }^{\mathrm{a}}{ }_{x_{2}}{ }^{(\mathrm{s})}:$ initial mole fraction of component 2 in the binary solvent mixture. \\
${ }^{\mathrm{b}} x_{1}:$ mole fraction solubility of the solute.
\end{tabular}

\section{Auxiliary Information}

\section{Method/Apparatus/Procedure:}

Constant-temperature water bath and an analytical balance.

Excess solute and solvent and were placed in brown glass containers and allowed to equilibrate in a constant-temperature water bath for several days. Attainment of equilibrium was verified by repetitive measurements after several additional days. Solubility was determined by transferring a weighed aliquot through a coarse filter into a flask containing blank nonaqueous titration solvent. The solutions were titrated with freshly standardized sodium methoxide to the thymol blue endpoint.

\section{Source and Purity of Chemicals:}

(1) $99 \%$, Chemical source not given, was dried at $333 \mathrm{~K}$ for several hours before use.

(2) $99+\%$, Chemical source not given, was dried over molecular sieves and distilled before use.

(3) $99+\%$, Chemical source not given, refluxed for $11 \mathrm{~h}$ over an aqueous solution $10 \%$ in potassium permanganate and $10 \%$ in sodium hydroxide. The tetrachloromethane was distilled off and dried with calcium hydroxide, stored in contact with mercury under an argon atmosphere, and then distilled shortly before use.

\section{Estimated Error:}

Temperature: $\pm 0.01 \mathrm{~K}$.

$x_{2}{ }^{(\mathrm{s})}: \pm 0.0001$.

$x_{1}: \pm 1 \%$ (relative error).

\begin{tabular}{ll}
\hline \hline Components: & Original Measurements: \\
(1) Benzoic acid; $\mathrm{C}_{7} \mathrm{H}_{6} \mathrm{O}_{2} ;$ & ${ }^{49}$ W. E. Acree, Jr. and G. L. Bertrand, J. \\
[65-85-0] & Pharm. Sci. 70, 1033 (1981). \\
(2) Cyclohexane; $\mathrm{C}_{6} \mathrm{H}_{12} ;$ & \\
{$[110-82-7]$} & \\
(3) Tetrachloromethane; $\mathrm{CCl}_{4} ;$ & \\
[56-23-5] & \\
\hline
\end{tabular}

Variables:

Prepared by:

$T / \mathrm{K}=298.15$; Solvent Composition W. E. Acree, Jr.
Experimental Values

\begin{tabular}{lcc}
\hline \hline$x_{2}{ }^{(\mathrm{s}) \mathrm{a}}$ & $x_{2}{ }^{\mathrm{b}}$ & $x_{1}{ }^{\mathrm{c}}$ \\
\hline 0.0000 & 0.0000 & 0.0492 \\
0.1847 & 0.1775 & 0.0389 \\
0.3681 & 0.3568 & 0.0307 \\
0.5207 & 0.5079 & 0.0246 \\
0.6226 & 0.6094 & 0.0212 \\
0.8084 & 0.7958 & 0.0156 \\
1.0000 & 0.9885 & 0.0115 \\
\hline
\end{tabular}

${ }^{\mathrm{a}} x_{2}{ }^{(\mathrm{s})}$ : initial mole fraction of component 2 in the binary solvent mixture.

${ }^{\mathrm{b}} x_{2}$ : mole fraction of component 2 in the saturated solution.

${ }^{c} x_{1}$ : mole fraction solubility of the solute.

\section{Auxiliary Information}

\section{Method/Apparatus/Procedure:}

Constant-temperature water bath and an analytical balance.

Excess solute and solvent and were placed in brown glass containers and allowed to equilibrate in a constant-temperature water bath for several days. Attainment of equilibrium was verified by repetitive measurements after several additional days. Solubility was determined by transferring a weighed aliquot through a coarse filter into a flask containing blank nonaqueous titration solvent. The solutions were titrated with freshly standardized sodium methoxide to the thymol blue endpoint.

\section{Source and Purity of Chemicals:}

(1) $99 \%$, Chemical source not given, was dried at $333 \mathrm{~K}$ for several hours before use.

(2) $99+\%$, Chemical source not given, was dried over molecular sieves and distilled before use.

(3) $99+\%$, Chemical source not given, refluxed for $11 \mathrm{~h}$ over an aqueous solution $10 \%$ in potassium permanganate and $10 \%$ in sodium hydroxide. The tetrachloromethane was distilled off and dried with calcium hydroxide, stored in contact with mercury under an argon atmosphere, and then distilled shortly before use.

\section{Estimated Error:}

Temperature: $\pm 0.01 \mathrm{~K}$.

$x_{2}{ }^{(\mathrm{s})}: \pm 0.0001$.

$x_{1}: \pm 1 \%$ (relative error).

\begin{tabular}{|c|c|}
\hline $\begin{array}{l}\text { Components: } \\
\text { (1) Benzoic acid; } \mathrm{C}_{7} \mathrm{H}_{6} \mathrm{O}_{2} \text {; } \\
\text { [65-85-0] } \\
\text { (2) Heptane; } \mathrm{C}_{7} \mathrm{H}_{16} ;[142-82-5] \\
\text { (3) Ethanol; } \mathrm{C}_{2} \mathrm{H}_{6} \mathrm{O} ;[64-17-5]\end{array}$ & $\begin{array}{l}\text { Original Measurements: } \\
{ }^{48} \text { J. Thati, F. L. Nordström, and A. C. } \\
\text { Rasmuson, J. Chem. Eng. Data 55, } \\
5124 \text { (2010). }\end{array}$ \\
\hline $\begin{array}{l}\text { Variables: } \\
\text { Temperature; Solvent Composition }\end{array}$ & $\begin{array}{l}\text { Prepared by: } \\
\text { W. E. Acree, Jr. }\end{array}$ \\
\hline
\end{tabular}


Experimental Values

\begin{tabular}{|c|c|c|}
\hline$\underline{T / \mathrm{K}}$ & $m_{2}{ }^{(\mathrm{s}) \mathrm{a}}$ & $x_{1}{ }^{\mathrm{b}}$ \\
\hline 278.15 & 0.00 & 0.128 \\
\hline 278.15 & 0.10 & 0.121 \\
\hline 278.15 & 0.20 & 0.112 \\
\hline 278.15 & 0.30 & 0.102 \\
\hline 278.15 & 0.40 & 0.092 \\
\hline 278.15 & 0.50 & 0.079 \\
\hline 278.15 & 1.00 & 0.005 \\
\hline 283.15 & 0.00 & 0.139 \\
\hline 283.15 & 0.10 & 0.132 \\
\hline 283.15 & 0.20 & 0.123 \\
\hline 283.15 & 0.30 & 0.111 \\
\hline 283.15 & 0.40 & 0.101 \\
\hline 283.15 & 0.50 & 0.087 \\
\hline 283.15 & 1.00 & 0.006 \\
\hline 293.15 & 0.00 & 0.166 \\
\hline 293.15 & 0.10 & 0.158 \\
\hline 293.15 & 0.20 & 0.147 \\
\hline 293.15 & 0.30 & 0.135 \\
\hline 293.15 & 0.40 & 0.124 \\
\hline 293.15 & 0.50 & 0.107 \\
\hline 293.15 & 1.00 & 0.010 \\
\hline 303.15 & 0.00 & 0.196 \\
\hline 303.15 & 0.10 & 0.188 \\
\hline 303.15 & 0.20 & 0.178 \\
\hline 303.15 & 0.30 & 0.164 \\
\hline 303.15 & 0.40 & 0.145 \\
\hline 303.15 & 0.50 & 0.132 \\
\hline 303.15 & 1.00 & 0.015 \\
\hline 313.15 & 0.00 & 0.234 \\
\hline 313.15 & 0.10 & 0.227 \\
\hline 313.15 & 0.20 & 0.216 \\
\hline 313.15 & 0.30 & 0.203 \\
\hline 313.15 & 0.40 & 0.187 \\
\hline 313.15 & 0.50 & 0.165 \\
\hline 313.15 & 1.00 & 0.022 \\
\hline 323.15 & 0.00 & 0.276 \\
\hline 323.15 & 0.10 & 0.271 \\
\hline 323.15 & 0.20 & 0.261 \\
\hline 323.15 & 0.30 & 0.246 \\
\hline 323.15 & 0.40 & 0.224 \\
\hline 323.15 & 0.50 & 0.203 \\
\hline 323.15 & 1.00 & 0.033 \\
\hline
\end{tabular}

\section{Auxiliary Information}

\section{Method/Apparatus/Procedure:}

Constant-temperature bath, magnetic stirrer, analytical balance, and ventilated laboratory hood.

Solubilities were determined by a gravimetric method. Binary solvent mixtures were prepared by mass and allowed to equilibrate in sealed bottles in a constant-temperature bath for $2 \mathrm{~h}$ with stirring. The stirring was discontinued and the solid was allowed to settle to the bottom of the container. After $30 \mathrm{~min}$, an aliquot of the clear solution was removed by syringe, filtered through a $0.2 \mu \mathrm{m}$ PTFE filter, and transferred to a preweighed glass vial. The glass vial was then weighed, and the solvent was allowed to evaporate in a ventilated laboratory hood at ambient room temperature. Once the solvent had evaporated, the vial with solid residue was weighed until constant weight was obtained. The solubility was calculated from the mass of the solid residue and mass of sample analyzed.

Source and Purity of Chemicals:

(1) $99.7 \%$, Merck Chemical Company, Germany, was used as received.
(2) $99 \%$, VWR Scientific, USA, used as received

(3) $99+\%$, Solveco Chemicals, used as received.

\section{Estimated Error:}

Temperature: $\pm 0.01 \mathrm{~K}$

$m_{2}{ }^{(\mathrm{s})}: \pm 0.01$

$x_{1}: \pm 1 \%$ (relative error).

\begin{tabular}{|c|c|}
\hline Components: & \multirow{3}{*}{$\begin{array}{l}\text { Original Measurements: } \\
{ }^{48} \text { J. Thati, F. L. Nordström, and A. C. } \\
\text { Rasmuson, J. Chem. Eng. Data 55, } \\
5124 \text { (2010). }\end{array}$} \\
\hline $\begin{array}{l}\text { (1) Benzoic acid; } \mathrm{C}_{7} \mathrm{H}_{6} \mathrm{O}_{2} \text {; } \\
\text { [65-85-0] }\end{array}$ & \\
\hline $\begin{array}{l}\text { (2) Methylbenzene; } \mathrm{C}_{7} \mathrm{H}_{8} \text {; } \\
\text { [108-88-3] } \\
\text { (3) Ethanol; } \mathrm{C}_{2} \mathrm{H}_{6} \mathrm{O} ;[64-17-5]\end{array}$ & \\
\hline Variables: & Prepared by: \\
\hline Temperature; Solvent Composition & W. E. Acree, Jr. \\
\hline
\end{tabular}

Experimental Values

\begin{tabular}{|c|c|c|}
\hline$T / \mathrm{K}$ & $m_{2}{ }^{(\mathrm{s}) \mathrm{a}}$ & $x_{1}{ }^{b}$ \\
\hline 278.15 & 0.00 & 0.128 \\
\hline 278.15 & 0.10 & 0.127 \\
\hline 278.15 & 0.20 & 0.125 \\
\hline 278.15 & 0.30 & 0.123 \\
\hline 278.15 & 0.40 & 0.119 \\
\hline 278.15 & 0.50 & 0.112 \\
\hline 278.15 & 1.00 & 0.035 \\
\hline 283.15 & 0.00 & 0.139 \\
\hline 283.15 & 0.10 & 0.138 \\
\hline 283.15 & 0.20 & 0.137 \\
\hline 283.15 & 0.30 & 0.133 \\
\hline 283.15 & 0.40 & 0.129 \\
\hline 283.15 & 0.50 & 0.122 \\
\hline 283.15 & 1.00 & 0.043 \\
\hline 293.15 & 0.00 & 0.166 \\
\hline 293.15 & 0.10 & 0.163 \\
\hline 293.15 & 0.20 & 0.161 \\
\hline 293.15 & 0.30 & 0.157 \\
\hline 293.15 & 0.40 & 0.152 \\
\hline 293.15 & 0.50 & 0.145 \\
\hline 293.15 & 1.00 & 0.062 \\
\hline 303.15 & 0.00 & 0.196 \\
\hline 303.15 & 0.10 & 0.194 \\
\hline 303.15 & 0.20 & 0.191 \\
\hline 303.15 & 0.30 & 0.186 \\
\hline 303.15 & 0.40 & 0.181 \\
\hline 303.15 & 0.50 & 0.172 \\
\hline 303.15 & 1.00 & 0.089 \\
\hline 313.15 & 0.00 & 0.234 \\
\hline 313.15 & 0.10 & 0.231 \\
\hline 313.15 & 0.20 & 0.227 \\
\hline 313.15 & 0.30 & 0.222 \\
\hline 313.15 & 0.40 & 0.214 \\
\hline 313.15 & 0.50 & 0.203 \\
\hline 313.15 & 1.00 & 0.128 \\
\hline 323.15 & 0.00 & 0.276 \\
\hline 323.15 & 0.10 & 0.273 \\
\hline 323.15 & 0.20 & 0.266 \\
\hline 323.15 & 0.30 & 0.262 \\
\hline 323.15 & 0.40 & 0.254 \\
\hline 323.15 & 0.50 & 0.234 \\
\hline 323.15 & 1.00 & 0.178 \\
\hline
\end{tabular}

${ }^{\mathrm{a}} m_{2}{ }^{(\mathrm{s})}$ : initial mass fraction of component 2 in the binary solvent mixture.

${ }^{b} x_{1}$ : mole fraction solubility of the solute. 


\section{Auxiliary Information}

\section{Method/Apparatus/Procedure:}

Constant-temperature bath, magnetic stirrer, analytical balance, and ventilated laboratory hood.

Solubilities were determined by a gravimetric method. Binary solvent mixtures were prepared by mass and allowed to equilibrate in sealed bottles in a constant-temperature bath for $2 \mathrm{~h}$ with stirring. The stirring was discontinued and the solid was allowed to settle to the bottom of the container. After $30 \mathrm{~min}$, an aliquot of the clear solution was removed by syringe, filtered through a $0.2 \mu \mathrm{m}$ PTFE filter, and transferred to a preweighed glass vial. The glass vial was then weighed, and the solvent was allowed to evaporate in a ventilated laboratory hood at ambient room temperature. Once the solvent had evaporated, the vial with solid residue was weighed until constant weight was obtained. The solubility was calculated from the mass of the solid residue and mass of sample analyzed.

\section{Source and Purity of Chemicals:}

(1) $99.7 \%$, Merck Chemical Company, Germany, was used as received.

(2) $99+\%$, Merck Chemical Company, used as received.

(3) $99+\%$, Solveco Chemicals, used as received.

\section{Estimated Error:}

Temperature: $\pm 0.01 \mathrm{~K}$.

$m_{2}{ }^{(\mathrm{s})}: \pm 0.01$.

$x_{1}: \pm 1 \%$ (relative error). steam bath, and the sample further dried in a sulfuric acid desiccator. The solubility was calculated from the mass of the solid residue and the mass of the saturated solution removed for analysis.

\section{Source and Purity of Chemicals:}

(1) Purity not given, Chemical source not given, purification details were not provided.

(2) Purity not given, Chemical source not given, purification details were not provided.

(3) Purity not given, Chemical source not given, purification details were not provided.

\section{Estimated Error:}

Temperature: $\pm 0.1 \mathrm{~K}$.

$w_{2}^{(\mathrm{s})}: \pm 0.01$.

$m_{1}: \pm 10 \%$ (relative error).

\begin{tabular}{ll}
\hline \hline Components: & Original Measurements: \\
(1) Benzoic acid; $\mathrm{C}_{7} \mathrm{H}_{6} \mathrm{O}_{2} ;$ & ${ }^{57}$ J. W. Marden and M. V. Dover, J. \\
[65-85-0] & Am. Chem. Soc. 38, 1235 (1917). \\
(2) Benzene; $\mathrm{C}_{6} \mathrm{H}_{6} ;[71-43-2]$ & \\
(3) Propanone; $\mathrm{C}_{3} \mathrm{H}_{6} \mathrm{O} ;[67-64-1]$ & \\
\hline
\end{tabular}

Variables:

Prepared by:

$T / \mathrm{K}=298.15$; Solvent Composition W. E. Acree, Jr.

Experimental Values

\begin{tabular}{lcc}
\hline \hline$w_{2}^{(\mathrm{s}) \mathrm{a}}$ & $s_{1}{ }^{\mathrm{b}}$ & $m_{1}{ }^{\mathrm{c}}$ \\
\hline 0.00 & 55.6 & 4.553 \\
0.10 & 51.3 & 4.201 \\
0.20 & 49.2 & 4.029 \\
0.40 & 42.2 & 3.456 \\
0.60 & 33.5 & 2.743 \\
0.80 & 24.1 & 1.973 \\
0.90 & 18.3 & 1.499 \\
1.00 & 11.6 & 0.950 \\
\hline
\end{tabular}

${ }^{\mathrm{a}} w_{2}^{(\mathrm{s})}$ : initial mass fraction of component 2 in the binary solvent mixture.

$\mathrm{b}_{s_{1}}$ : solubility of the solute given as grams of solute per $100 \mathrm{~g}$ of solvent.

${ }^{c} m_{1}$ : solubility of the solute given as moles of solute per kilogram of solvent.

\section{Auxiliary Information}

\section{Method/Apparatus/Procedure:}

Constant-temperature thermostat, analytical balance, and a steam bath. Binary solvent mixtures were prepared by mass. Excess solute and solvent were placed in a bottle with ground glass stopper which was protected by a coating of beeswax and rosin, over which was tied a piece of rubber sheeting. The sealed mixture was shaken in a constant-temperature thermostat for 8-20 h. An aliquot of the saturated solution was withdrawn through a glass wool filter into a weighing pipet from which the solutions were weighed directly into small glass evaporating dishes. The solvent was removed on a steam bath, and the sample further dried in a sulfuric acid desiccator. The solubility was calculated from the mass of the solid residue and the mass of the saturated solution removed for analysis.

Source and Purity of Chemicals:

(1) Purity not given, Chemical source not given, purification details were not provided.

(2) Purity not given, Chemical source not given, purification details were not provided.

(3) Purity not given, Chemical source not given, purification details were not provided. 
Estimated Error:

Temperature: $\pm 0.1 \mathrm{~K}$

$w_{2}^{(\mathrm{s})}: \pm 0.01$.

$m_{1}: \pm 10 \%$ (relative error)
Original Measurements:

${ }^{57}$ J. W. Marden and M. V. Dover, J.

Am. Chem. Soc. 38, 1235 (1917).

(2) Benzene; $\mathrm{C}_{6} \mathrm{H}_{6}$; [71-43-2]

(3) Ethyl ethanoate; $\mathrm{C}_{4} \mathrm{H}_{8} \mathrm{O}_{2}$;

[141-78-6]

Variables:

Prepared by:

$T / \mathrm{K}=298.15$; Solvent Composition W. E. Acree, Jr.

Experimental Values

\begin{tabular}{lcc}
\hline \hline$w_{2}^{(\mathrm{s}) \mathrm{a}}$ & $s_{1}{ }^{\mathrm{b}}$ & $m_{1}{ }^{\mathrm{c}}$ \\
\hline 0.00 & 41.2 & 3.374 \\
0.10 & 28.1 & 2.301 \\
0.20 & 29.0 & 2.375 \\
0.40 & 23.9 & 1.957 \\
0.60 & 20.4 & 1.670 \\
0.80 & 16.5 & 1.351 \\
0.90 & 14.0 & 1.146 \\
1.00 & 11.6 & 0.950 \\
\hline
\end{tabular}

${ }^{\mathrm{a}} w_{2}^{(\mathrm{s})}$ : initial mass fraction of component 2 in the binary solvent mixture.

${ }^{\mathrm{b}} s_{1}$ : solubility of the solute given as grams of solute per $100 \mathrm{~g}$ of solvent.

${ }^{\mathrm{c}} m_{1}$ : solubility of the solute given as moles of solute per kilogram of solvent.

\section{Auxiliary Information}

\section{Method/Apparatus/Procedure:}

Constant-temperature thermostat, analytical balance, and a steam bath. Binary solvent mixtures were prepared by mass. Excess solute and solvent were placed in a bottle with ground glass stopper which was protected by a coating of beeswax and rosin, over which was tied a piece of rubber sheeting. The sealed mixture was shaken in a constant-temperature thermostat for 8-20 h. An aliquot of the saturated solution was withdrawn through a glass wool filter into a weighing pipet from which the solutions were weighed directly into small glass evaporating dishes. The solvent was removed on a steam bath, and the sample further dried in a sulfuric acid desiccator. The solubility was calculated from the mass of the solid residue and the mass of the saturated solution removed for analysis.

Source and Purity of Chemicals:

(1) Purity not given, Chemical source not given, purification details were not provided.

(2) Purity not given, Chemical source not given, purification details were not provided.

(3) Purity not given, Chemical source not given, purification details were not provided.

\section{Estimated Error:}

Temperature: $\pm 0.1 \mathrm{~K}$.

$w_{2}^{(\mathrm{s})}: \pm 0.01$.

$m_{1}: \pm 10 \%$ (relative error).

\section{Solubility of 2-Acetoxybenzoic Acid in Organic Solvents}

\subsection{Critical evaluation of experimental solubility data}

There have been several published studies ${ }^{12,53,69,92-94}$ investigating the solubility behavior of 2-acetoxybenzoic acid in organic solvents of varying polarity and hydrogen-bonding capability. Charlton et al. ${ }^{12}$ measured the solubility of 2acetoxybenzoic acid in 19 alcohols (1-propanol, 2-propanol, 1-butanol, 2-butanol, 2-methyl-1-propanol, 2-methyl-2-propanol, 1-pentanol, 2-pentanol, 2-methyl-1-butanol, 3-methyl1-butanol, 2-methyl-2-butanol, 1-hexanol, 2-methyl-1-pentanol, 4-methyl-2-pentanol, 1-heptanol, 1-octanol, 2-ethyl-1hexanol, 1-decanol, and 3,7-dimethyl-1-octanol), in four dia$1 \mathrm{kyl}$ ethers $\left(1,1^{\prime}\right.$-oxybisethane, $2,2^{\prime}$-oxybispropane, 1,1'-oxybisbutane, and 2-methoxy-2-methylpropane (also called methyl tert-butyl ether) and two cyclic ethers (tetrahydrofuran and 1,4-dioxane), in five alkyl alkanoates (methyl ethanoate, ethyl ethanoate, butyl ethanoate, pentyl ethanoate and methyl butanoate), and in propylene carbonate at $298 \mathrm{~K}$. Results of the experimental measurements were used to update the existing Abraham solute descriptors that the authors had for 2-acetoxybenzoic acid. The authors were able to assemble a total of $44 \log _{10}\left(S R\right.$ or $P$ ) and $\log _{10}(G S R$ or $K$ ) equations for which experimental partition coefficient data, solubility ratios, Abraham model equation coefficients and aqueous molar solubility were available. The logarithm of the aqueous molar solubility of 2-acetoxybenzoic acid is $\log _{10} c_{1, \mathrm{~W}}=-1.68 .{ }^{95}$ The McGowan volume of 2-acetoxybenzoic acid, $V=1.2879$, was calculated from the number of chemical bonds in the molecule and the individual atomic group volumes, $A V_{i}$, given in Sec. 1.3. The excess molar refraction solute descriptor was estimated as $E=0.780$. This left four solute descriptors ( $S, A$, $B$, and $L$ ) still to be determined. The 44 equations were then solved using the Microsoft "SOLVER" program to yield numerical values of the remaining four solute descriptors, $S=1.690$, $A=0.710, B=0.670$, and $L=6.2789$, that best described the $\log _{10}(S R$ or $P)$ and $\log _{10}(G S R$ or $K)$ values. The computation treated $\log _{10} c_{1, \mathrm{G}}$ as a floating parameter to be determined as part of the regression analyses. The data analyses returned a value of $\log _{10} c_{1, \mathrm{G}}=-10.18$ for the gas-phase solute concentration that made the $\log _{10}\left(S R\right.$ or $P$ ) and $\log _{10}(G S R$ or $K$ ) predictions internally consistent. The calculated molecular solute descriptors reproduced the 44 combined $\log _{10}$ ( $S R$ or $P)$ and $\log _{10}(G S R$ or $K)$ values to within an average standard deviation of $0.129 \log _{10}$ units. The authors excluded 2-methoxy-2-methylpropane from the standard deviation computations because of concerns that trace water in the solvent might adversely affect the measured solubility. Diaz and Drogos ${ }^{96}$ reported that 2-methoxy-2-methylpropane has limited stability in water acidified with either mineral acids or organic acids. The authors noted that the reaction proceeds even in very dilute solutions with weak acids, such as acetylsalicylic acid. The reaction produces methanol, which would enhance the saturation solubility. Acetylsalicylic acid is more soluble in methanol than in 2-methoxy-2-methylpropane. Such chemical 
TABLE 8. Comparison between observed and calculated molar solubilities of 2 acetoxybenzoic acid based on the Abraham model, Eqs. (20) and (21)

\begin{tabular}{|c|c|c|c|}
\hline Solvent & $\log _{10} c_{1}{ }^{\exp }$ & $\begin{array}{c}\log _{10} c_{1}{ }^{\text {calc }} \\
\text { Eq. } \\
\text { (20) }\end{array}$ & $\begin{array}{c}\log _{10} c_{1}{ }^{\text {calc }} ; \\
\text { Eq. }(21)\end{array}$ \\
\hline Methanol & $0.176^{\mathrm{a}}$ & 0.169 & -0.248 \\
\hline Ethanol & $0.114^{\mathrm{a}}$ & -0.050 & -0.004 \\
\hline 1-Propanol & -0.197 & -0.179 & -0.179 \\
\hline 2-Propanol & -0.186 & -0.167 & -0.153 \\
\hline 1-Butanol & -0.324 & -0.314 & -0.301 \\
\hline 2-Butanol & -0.256 & -0.195 & -0.237 \\
\hline 2-Methyl-1-propanol & -0.471 & -0.366 & -0.361 \\
\hline 2-Methyl-2-propanol & -0.154 & -0.146 & -0.147 \\
\hline 1-Pentanol & -0.443 & -0.426 & -0.427 \\
\hline 2-Pentanol & -0.404 & -0.411 & -0.384 \\
\hline 3-Methyl-1-butanol & -0.462 & -0.436 & -0.399 \\
\hline 1-Hexanol & -0.505 & -0.455 & -0.468 \\
\hline 1-Heptanol & -0.565 & -0.478 & -0.519 \\
\hline 1-Octanol & -0.665 & -0.519 & -0.633 \\
\hline 1-Decanol & -0.718 & -0.739 & -0.767 \\
\hline 1,1'-Oxybisethane & -0.478 & -0.572 & -0.503 \\
\hline Tetrahydrofuran & 0.312 & 0.435 & 0.363 \\
\hline 1,4-Dioxane & 0.134 & 0.316 & 0.307 \\
\hline Methyl ethanoate & -0.192 & -0.061 & -0.061 \\
\hline Ethyl ethanoate & -0.351 & -0.255 & -0.245 \\
\hline Butyl ethanoate & -0.597 & -0.435 & -0.456 \\
\hline Propanone & $0.017^{\mathrm{a}}$ & 0.133 & 0.184 \\
\hline
\end{tabular}

${ }^{\mathrm{a}}$ Experimental solubility data taken from Perlovich and Bauer-Brandl. ${ }^{53}$ All other experimental data were taken from Charlton et al. ${ }^{12}$

reaction effects are expected to be fairly small if water is needed for the reaction to proceed.

After the 2-acetoxybenzoic acid solubility study was published, Abraham model correlations have been developed for 3methyl-1-butanol, 2-pentanol, methyl ethanoate, and butyl ethanoate, and equation coefficients for a few solvents were updated based on additional experimental data. The new correlations (listed in Tables 1 and 2) will be used in illustrating the ability of the Abraham model to correlate the experimental 2acetoxybenzoic acid solubility data. Table 8 compares the experimental $\log _{10} c_{1}$ values to calculated values based on Eqs. (20) and (21) of the Abraham model. For comparison purposes, the measured mole fraction solubilities of 2-acetoxybenzoic acid, $x_{1}$, determined by Charlton et al. ${ }^{12}$ were converted into molar solubilities by dividing $x_{1}$ by the ideal molar volume of the saturated solution (i.e., $c_{1}^{\text {sat }}=x_{1} /\left[x_{1} V_{1}+\left(1-x_{1}\right) V_{\text {solvent }}\right]$ ). The molar volume of the hypothetical subcooled liquid 2-acetoxybenzoic acid is $V_{\text {solute }}=126.89 \mathrm{~cm}^{3} \mathrm{~mol}^{-1}$. Examination of the numerical entries in Table 8 reveals that the Abraham model provides a reasonably accurate mathematical description of the observed solubility data, suggesting that there are no obvious outliers in the dataset.

Independent 2-acetoxybenzoic acid solubility measurements do exist for several solvents. Previously Perlovich and Bauer-Brandl $^{53}$ had measured the solubility of 2-acetoxybenzoic acid in eight 1-alkanols (methanol through 1-octanol), and in benzene, methylbenzene, ethyl ethanoate, 1,4-dioxane, trichloromethane, propanone, and ethanenitrile. Except for 1,4-dioxane, the two sets of solubility data are in reasonably good agreement for the most part, $x_{1}=0.04976$ (Ref. 91)
TABle 9. Parameters of the Modified Apelblat equation for describing the solubility of 2-acetoxybenzoic acid in various organic solvents ${ }^{\mathrm{a}}$

\begin{tabular}{lrrrrr}
\hline \hline Solvent & $T / \mathrm{K}$ & $A$ & $B$ & \multicolumn{1}{c}{$C$} & APLD (\%) \\
\hline Ethanol & $276-336$ & 63.952 & -5759.6 & -8.323 & 0.679 \\
2-Propanol & $282-330$ & 588.32 & -30787.7 & -85.663 & 3.372 \\
1,2- & $296-334$ & 477.25 & -27072.7 & -68.500 & 0.674 \\
$\begin{array}{l}\text { Propanediol } \\
\text { Propanone }\end{array}$ & $282-326$ & 588.32 & 257.07 & 8.125 & 0.550 \\
\hline
\end{tabular}

${ }^{\mathrm{a}}$ Values of the coefficients and average percent logarithmic deviation were taken from Maia and Giulietti. ${ }^{92}$

versus $x_{1}=0.0418$ (Ref. 53) for 1-propanol, $x_{1}=0.04616$ (Ref. 12) versus $x_{1}=0.0453$ (Ref. 53) for 1-butanol, $x_{1}=$ 0.03966 (Ref. 91) versus $x_{1}=0.0395$ (Ref. 53) for 1-pentanol, $x_{1}=0.03973$ (Ref. 12) versus $x_{1}=0.0393$ (Ref. 53) for 1hexanol, $x_{1}=0.03892$ (Ref. 12) versus $x_{1}=0.0386$ (Ref. 53) for 1-heptanol, $x_{1}=0.03581$ (Ref. 12) versus $x_{1}=0.0341$ (Ref. 53) for 1-octanol, $x_{1}=0.04583$ (Ref. 12) versus $x_{1}=$ 0.0448 (Ref. 53) for ethyl ethanoate, and $x_{1}=0.1263$ (Ref. 12) versus $x_{1}=0.0516$ (Ref. 53) for 1,4-dioxane. Differences in chemical purities and experimental methodologies can lead to differences of a few percent between values determined by two different research groups.

There has been one experimental study examining the solubility of 2-acetoxybenzoic acid as a function of temperature. Maina and Giulietti ${ }^{92}$ measured the solubility of 2acetoxybenzoic acid in ethanol, 2-propanol, 1,2-propanediol, and propanone at several temperatures in the range of about 280-330 K. The internal consistency of the datasets was assessed by curve-fitting the measured mole fraction solubility data to Eq. (8). The values of the equation coefficients $(A, B$, and $C$ ) are given in Table 9, along with the average percent logarithmic deviation (APLD) calculated according to

$$
\mathrm{APLD}=\frac{100}{N} \sum_{i=1}^{N}\left|\ln \left(\frac{x_{i}^{\mathrm{exp}}}{x_{i}^{\mathrm{calc}}}\right)\right|
$$

where $N$ is the number of experimental solubility measurements in an individual solute-solvent dataset. Examination of the numerical entries in the last column of Table 9 reveals that the largest APLD between the back-calculated values based on Eq. (8) and experimental data is $3.5 \%$. Results of the mathematical representation analyses indicate that the experimental data for all four 2-acetoxybenzoic acid - organic solvent systems are internally consistent.

The experimental solubility data for 2-acetoxybenzoic acid in organic solvents are in Secs. 3.2-3.8.

\subsection{2-Acetoxybenzoic acid solubility data in aromatic hydrocarbons}

\begin{tabular}{|c|c|}
\hline $\begin{array}{l}\text { Components: } \\
\text { (1) 2-Acetoxybenzoic acid; } \\
\mathrm{C}_{9} \mathrm{H}_{8} \mathrm{O}_{4} ;[50-78-2] \\
\text { (2) Benzene; } \mathrm{C}_{6} \mathrm{H}_{6} ;[71-43-2]\end{array}$ & $\begin{array}{l}\text { Original Measurements: } \\
{ }^{53} \text { G. L. Perlovich and A. Bauer-Brandl, } \\
\text { Pharm. Res. 20, } 471 \text { (2003). }\end{array}$ \\
\hline $\begin{array}{l}\text { Variables: } \\
T / \mathrm{K}=298.15\end{array}$ & $\begin{array}{l}\text { Prepared by: } \\
\text { W. E. Acree, Jr. }\end{array}$ \\
\hline
\end{tabular}


Experimental Values

\begin{tabular}{lc}
\hline \hline$x_{2}{ }^{\mathrm{a}}$ & $x_{1}{ }^{\mathrm{b}}$ \\
\hline 0.9990 & 0.00101
\end{tabular}

${ }^{a} x_{2}$ : mole fraction of component 2 in the saturated solution.

${ }^{\mathrm{b}} x_{1}$ : mole fraction solubility of the solute.

\section{Auxiliary Information}

\section{Method/Apparatus/Procedure:}

Thermostated constant-temperature bath, centrifuge, and an ultraviolet/visible spectrophotometer.

Excess solute and solvent were placed in a glass ampoule and allowed to equilibrate in a thermostated temperature bath with mixing (at a speed of $25 \mathrm{rpm}$ ) for a minimum of four days. After suitable equilibration the saturated solution was centrifuged, the supernatant liquid collected, quantitatively diluted, and the absorbance recorded using an ultraviolet/visible spectrophotometer. The solubility of the solute was calculated from the measured absorbance.

Source and Purity of Chemicals:

(1) Analytical Reagent grade, Norsk Medisinaldepot, Oslo, Norway, no purification details were provided.

(2) Analytical Reagent grade, Merck Chemicals, Germany, no purification details were provided.

Estimated Error:

Temperature: $\pm 0.01 \mathrm{~K}$.

$x_{1}: \pm 2.5 \%$ (relative error).

\section{Components:}

(1) 2-Acetoxybenzoic acid;

$\mathrm{C}_{9} \mathrm{H}_{8} \mathrm{O}_{4}$; [50-78-2]

(2) Methylbenzene; $\mathrm{C}_{7} \mathrm{H}_{8}$;

[108-88-3]

\begin{tabular}{ll}
\hline Variables: & Prepared by: \\
$T / \mathrm{K}=298.15$ & W. E. Acree, Jr. \\
\hline
\end{tabular}

Experimental Values

\begin{tabular}{lc}
\hline \hline$x_{2}{ }^{\mathrm{a}}$ & $x_{1}{ }^{\mathrm{b}}$ \\
\hline 0.9987 & 0.00129 \\
\hline
\end{tabular}

${ }^{\mathrm{a}} x_{2}$ : mole fraction of component 2 in the saturated solution.

${ }^{b} x_{1}$ : mole fraction solubility of the solute.

\section{Auxiliary Information}

\section{Method/Apparatus/Procedure:}

Thermostated constant-temperature bath, centrifuge, and an ultraviolet/visible spectrophotometer.

Excess solute and solvent were placed in a glass ampoule and allowed to equilibrate in a thermostated temperature bath with mixing (at a speed of $25 \mathrm{rpm}$ ) for a minimum of four days. After suitable equilibration the saturated solution was centrifuged, the supernatant liquid collected, quantitatively diluted, and the absorbance recorded using an ultraviolet/visible spectrophotometer. The solubility of the solute was calculated from the measured absorbance.
Source and Purity of Chemicals:

(1) Analytical Reagent grade, Norsk Medisinaldepot, Oslo, Norway, no purification details were provided.

(2) Analytical Reagent grade, Merck Chemicals, Germany, no purification details were provided.

\section{Estimated Error:}

Temperature: $\pm 0.01 \mathrm{~K}$.

$x_{1}: \pm 2.5 \%$ (relative error).

\subsection{2-Acetoxybenzoic acid solubility data in esters}

\begin{tabular}{|c|c|}
\hline $\begin{array}{l}\text { Components: } \\
\text { (1) 2-Acetoxybenzoic acid; } \\
\mathrm{C}_{9} \mathrm{H}_{8} \mathrm{O}_{4} ;[50-78-2] \\
\text { (2) Methyl ethanoate; } \mathrm{C}_{3} \mathrm{H}_{6} \mathrm{O}_{2} \text {; } \\
\text { [79-20-9] }\end{array}$ & $\begin{array}{l}\text { Original Measurements: } \\
{ }^{12} \text { A. K. Charlton, C. R. Daniels, W. E. } \\
\text { Acree, Jr., and M. H. Abraham, J. } \\
\text { Solution Chem. 32, } 1087 \text { (2003). }\end{array}$ \\
\hline $\begin{array}{l}\text { Variables: } \\
T / \mathrm{K}=298.15\end{array}$ & $\begin{array}{l}\text { Prepared by: } \\
\text { W. E. Acree, Jr. }\end{array}$ \\
\hline
\end{tabular}

Experimental Values

\begin{tabular}{lc}
\hline \hline$x_{2}{ }^{\mathrm{a}}$ & $x_{1}{ }^{\mathrm{b}}$ \\
\hline 0.9471 & 0.05287 \\
\hline${ }^{\mathrm{a}} x_{2}:$ mole fraction of component 2 in the saturated solution. & \\
${ }^{\mathrm{b}} x_{1}:$ mole fraction solubility of the solute. &
\end{tabular}

\section{Auxiliary Information}

\section{Method/Apparatus/Procedure:}

Constant-temperature bath, calorimetric thermometer, and an ultraviolet/ visible spectrophotometer.

Excess solute and solvent were placed in amber glass bottles and allowed to equilibrate for several days at constant temperature. Attainment of equilibrium was verified by several repetitive measurements and by approaching equilibrium from supersaturation. Aliquots of saturated solutions were transferred through a coarse filter into tared volumetric flasks, weighed, and diluted with methanol. Concentrations were determined by spectrophotometric measurements at $272 \mathrm{~nm}$.

Source and Purity of Chemicals:

(1) $99 \%$, Aldrich Chemical Company, Milwaukee, WI, USA, was used as received.

(2) $99.5 \%$, anhydrous, Aldrich Chemical Company, stored over molecular sieves and distilled shortly before use.

Estimated Error:

Temperature: $\pm 0.1 \mathrm{~K}$.

$x_{1}: \pm 1.5 \%$ (relative error).

\begin{tabular}{ll}
\hline \hline Components: & Original Measurements: \\
(1) 2-Acetoxybenzoic acid; & ${ }^{12}$ A. K. Charlton, C. R. Daniels, W. E. \\
$\mathrm{C}_{9} \mathrm{H}_{8} \mathrm{O}_{4} ;$ [50-78-2] & Acree, Jr., and M. H. Abraham, J. \\
$\begin{array}{l}\text { (2) Ethyl ethanoate; } \mathrm{C}_{4} \mathrm{H}_{8} \mathrm{O}_{2} ; \\
{[141-78-6]}\end{array}$ & Solution Chem. 32, 1087 (2003). \\
\hline Variables: & Prepared by: \\
$T / \mathrm{K}=298.15$ & W. E. Acree, Jr. \\
\hline
\end{tabular}


Experimental Values

\begin{tabular}{lc}
\hline \hline$x_{2}{ }^{\mathrm{a}}$ & $x_{1}{ }^{\mathrm{b}}$ \\
\hline 0.9542 & 0.04583 \\
\hline${ }^{\mathrm{a}} x_{2}:$ mole fraction of component 2 in the saturated solution. \\
${ }^{\mathrm{b}} x_{1}:$ mole fraction solubility of the solute.
\end{tabular}

\section{Auxiliary Information}

\section{Method/Apparatus/Procedure:}

Constant-temperature bath, calorimetric thermometer, and an ultraviolet/ visible spectrophotometer.

Excess solute and solvent were placed in amber glass bottles and allowed to equilibrate for several days at constant temperature. Attainment of equilibrium was verified by several repetitive measurements and by approaching equilibrium from supersaturation. Aliquots of saturated solutions were transferred through a coarse filter into tared volumetric flasks, weighed, and diluted with methanol. Concentrations were determined by

spectrophotometric measurements at $272 \mathrm{~nm}$.

Source and Purity of Chemicals:

(1) $99 \%$, Aldrich Chemical Company, Milwaukee, WI, USA, was used as received.

(2) $99.9 \%$, HPLC grade, Aldrich Chemical Company, stored over molecular sieves and distilled shortly before use.

\section{Estimated Error:}

Temperature: $\pm 0.1 \mathrm{~K}$

$x_{1}: \pm 1.5 \%$ (relative error).

\begin{tabular}{ll}
\hline \hline Components: & Original Measurements: \\
(1) 2-Acetoxybenzoic acid; & ${ }^{53} \mathrm{G}$. L. Perlovich and A. Bauer-Brandl, \\
$\mathrm{C}_{9} \mathrm{H}_{8} \mathrm{O}_{4} ;[50-78-2]$ & Pharm. Res. 20, 471 (2003). \\
$\begin{array}{l}\text { (2) Ethyl ethanoate; } \mathrm{C}_{4} \mathrm{H}_{8} \mathrm{O}_{2} ; \\
{[141-78-6]}\end{array}$ & \\
\hline Variables: & Prepared by: \\
$T / \mathrm{K}=298.15$ & W. E. Acree, Jr. \\
\hline
\end{tabular}

\section{Experimental Values}

\begin{tabular}{lc}
\hline \hline$x_{2}{ }^{\mathrm{a}}$ & $x_{1}{ }^{\mathrm{b}}$ \\
\hline 0.9552 & 0.0448
\end{tabular}

${ }^{\mathrm{a}} x_{2}$ : mole fraction of component 2 in the saturated solution.

${ }^{\mathrm{b}} x_{1}$ : mole fraction solubility of the solute.

\section{Auxiliary Information}

\section{Method/Apparatus/Procedure:}

Thermostated constant-temperature bath, centrifuge, and an ultraviolet/visible spectrophotometer.

Excess solute and solvent were placed in a glass ampoule and allowed to equilibrate in a thermostated temperature bath with mixing (at a speed of $25 \mathrm{rpm}$ ) for a minimum of four days. After suitable equilibration the saturated solution was centrifuged, the supernatant liquid collected, quantitatively diluted, and the absorbance recorded using an ultraviolet/visible spectrophotometer. The solubility of the solute was calculated from the measured absorbance.
Source and Purity of Chemicals:

(1) Analytical Reagent grade, Norsk Medisinaldepot, Oslo, Norway, no purification details were provided.

(2) Analytical Reagent grade, Merck Chemicals, Germany, no purification details were provided.

\section{Estimated Error:}

Temperature: $\pm 0.01 \mathrm{~K}$.

$x_{1}: \pm 2.5 \%$ (relative error).

\begin{tabular}{|c|c|}
\hline Components: & Original Measurements: \\
\hline $\begin{array}{l}\text { (1) 2-Acetoxybenzoic acid; } \\
\mathrm{C}_{9} \mathrm{H}_{8} \mathrm{O}_{4} ;[50-78-2]\end{array}$ & $\begin{array}{l}{ }^{12} \text { A. K. Charlton, C. R. Daniels, W. E } \\
\text { Acree, Jr., and M. H. Abraham, J. }\end{array}$ \\
\hline $\begin{array}{l}\text { (2) Butyl ethanoate; } \mathrm{C}_{6} \mathrm{H}_{12} \mathrm{O}_{2} \text {; } \\
\text { [123-86-4] }\end{array}$ & Solution Chem. 32, 1087 (2003). \\
\hline Variables: & Prepared by: \\
\hline$T / \mathrm{K}=298.15$ & W. E. Acree, Jr. \\
\hline
\end{tabular}

Experimental Values

\begin{tabular}{lc}
\hline \hline$x_{2}{ }^{\mathrm{a}}$ & $x_{1}{ }^{\mathrm{b}}$ \\
\hline 0.9665 & 0.03345 \\
\hline${ }^{\mathrm{a}} x_{2}:$ mole fraction of component 2 in the saturated solution. & \\
${ }^{\mathrm{b}} x_{1}:$ mole fraction solubility of the solute. &
\end{tabular}

\section{Auxiliary Information}

\section{Method/Apparatus/Procedure:}

Constant-temperature bath, calorimetric thermometer, and an ultraviolet/ visible spectrophotometer.

Excess solute and solvent were placed in amber glass bottles and allowed to equilibrate for several days at constant temperature. Attainment of equilibrium was verified by several repetitive measurements and by approaching equilibrium from supersaturation. Aliquots of saturated solutions were transferred through a coarse filter into tared volumetric flasks, weighed, and diluted with methanol. Concentrations were determined by spectrophotometric measurements at $272 \mathrm{~nm}$.

Source and Purity of Chemicals:

(1) $99 \%$, Aldrich Chemical Company, Milwaukee, WI, USA, was used as received.

(2) $99.8 \%$, HPLC grade, Aldrich Chemical Company, stored over molecular sieves and distilled shortly before use.

\section{Estimated Error:}

Temperature: $\pm 0.1 \mathrm{~K}$.

$x_{1}: \pm 1.5 \%$ (relative error).

\begin{tabular}{ll}
\hline \hline Components: & Original Measurements: \\
(1) 2-Acetoxybenzoic acid; & ${ }^{12}$ A. K. Charlton, C. R. Daniels, W. E. \\
$\mathrm{C}_{9} \mathrm{H}_{8} \mathrm{O}_{4} ;$ [50-78-2] & Acree, Jr., and M. H. Abraham, J. \\
$\begin{array}{l}\text { (2) Pentyl ethanoate; } \mathrm{C}_{7} \mathrm{H}_{14} \mathrm{O}_{2} ; \\
{[628-63-7]}\end{array}$ & Solution Chem. 32, 1087 (2003). \\
\hline Variables: & Prepared by: \\
$T / \mathrm{K}=298.15$ & W. E. Acree, Jr. \\
\hline
\end{tabular}


Experimental Values

\begin{tabular}{lc}
\hline \hline$x_{2}{ }^{\mathrm{a}}$ & $x_{1}{ }^{\mathrm{b}}$ \\
\hline 0.9732 & 0.02677
\end{tabular}

${ }^{\mathrm{a}} x_{2}$ : mole fraction of component 2 in the saturated solution.

${ }^{\mathrm{b}} x_{1}$ : mole fraction solubility of the solute.

\section{Auxiliary Information}

\section{Method/Apparatus/Procedure:}

Constant-temperature bath, calorimetric thermometer, and an ultraviolet/ visible spectrophotometer.

Excess solute and solvent were placed in amber glass bottles and allowed to equilibrate for several days at constant temperature. Attainment of equilibrium was verified by several repetitive measurements and by approaching equilibrium from supersaturation. Aliquots of saturated solutions were transferred through a coarse filter into tared volumetric flasks, weighed, and diluted with methanol. Concentrations were determined by

spectrophotometric measurements at $272 \mathrm{~nm}$.

\section{Source and Purity of Chemicals:}

(1) $99 \%$, Aldrich Chemical Company, Milwaukee, WI, USA, was used as received.

(2) $99 \%$, Aldrich Chemical Company, stored over molecular sieves and distilled shortly before use.

\section{Estimated Error:}

Temperature: $\pm 0.1 \mathrm{~K}$

$x_{1}: \pm 1.5 \%$ (relative error).

\section{Components:}

(1) 2-Acetoxybenzoic acid; $\mathrm{C}_{9} \mathrm{H}_{8} \mathrm{O}_{4} ;[50-78-2]$

(2) Methyl butanoate; $\mathrm{C}_{5} \mathrm{H}_{10} \mathrm{O}_{2}$; [623-42-7]

\begin{tabular}{ll}
\hline Variables: & Prepared by: \\
$T / \mathrm{K}=298.15$ & W. E. Acree, Jr. \\
\hline
\end{tabular}

\section{Experimental Values}

\begin{tabular}{lc}
\hline \hline$x_{2}^{\mathrm{a}}$ & $x_{1}{ }^{\mathrm{b}}$ \\
\hline 0.9703 & 0.02970 \\
\hline$x_{2}: \operatorname{mole}$ fraction of compont
\end{tabular}

${ }^{\mathrm{a}} x_{2}$ : mole fraction of component 2 in the saturated solution.

${ }^{b} x_{1}$ : mole fraction solubility of the solute.

\section{Auxiliary Information}

\section{Method/Apparatus/Procedure:}

Constant-temperature bath, calorimetric thermometer, and an ultraviolet/ visible spectrophotometer.

Excess solute and solvent were placed in amber glass bottles and allowed to equilibrate for several days at constant temperature. Attainment of equilibrium was verified by several repetitive measurements and by approaching equilibrium from supersaturation. Aliquots of saturated solutions were transferred through a coarse filter into tared volumetric flasks, weighed, and diluted with methanol. Concentrations were determined by spectrophotometric measurements at $272 \mathrm{~nm}$.
Source and Purity of Chemicals:

(1) $99 \%$, Aldrich Chemical Company, Milwaukee, WI, USA, was used as received.

(2) $99 \%$, Aldrich Chemical Company, stored over molecular sieves and distilled shortly before use.

\section{Estimated Error:}

Temperature: $\pm 0.1 \mathrm{~K}$.

$x_{1}: \pm 1.5 \%$ (relative error)

\subsection{2-Acetoxybenzoic acid solubility data in ethers}

\begin{tabular}{ll}
\hline \hline Components: & Original Measurements: \\
(1) 2-Acetoxybenzoic acid; & ${ }^{12}$ A. K. Charlton, C. R. Daniels, W. E. \\
$\mathrm{C}_{9} \mathrm{H}_{8} \mathrm{O}_{4} ;[50-78-2]$ & Acree, Jr., and M. H. Abraham, J. \\
$\begin{array}{l}\text { (2) } 1,1^{\prime}-\text {-Oxybisethane; } \mathrm{C}_{4} \mathrm{H}_{10} \mathrm{O} ; \\
{[60-29-7]}\end{array}$ & Solution Chem. 32, 1087 (2003). \\
\hline Variables: & Prepared by: \\
$T / \mathrm{K}=298.15$ & W. E. Acree, Jr. \\
\hline
\end{tabular}

\section{Experimental Values}

\begin{tabular}{lc}
\hline \hline$x_{2}{ }^{\mathrm{a}}$ & $x_{1}{ }^{\mathrm{b}}$ \\
\hline 0.9647 & 0.03529 \\
\hline${ }^{\mathrm{a}} x_{2}:$ mole fraction of component 2 in the saturated solution. & \\
${ }^{\mathrm{b}} x_{1}:$ mole fraction solubility of the solute. &
\end{tabular}

\section{Auxiliary Information}

\section{Method/Apparatus/Procedure:}

Constant-temperature bath, calorimetric thermometer, and an ultraviolet/ visible spectrophotometer.

Excess solute and solvent were placed in amber glass bottles and allowed to equilibrate for several days at constant temperature. Attainment of equilibrium was verified by several repetitive measurements and by approaching equilibrium from supersaturation. Aliquots of saturated solutions were transferred through a coarse filter into tared volumetric flasks, weighed, and diluted with methanol. Concentrations were determined by spectrophotometric measurements at $272 \mathrm{~nm}$.

\section{Source and Purity of Chemicals:}

(1) $99 \%$, Aldrich Chemical Company, Milwaukee, WI, USA, was used as received.

(2) 99+\%, anhydrous, Aldrich Chemical Company, stored over molecular sieves and distilled shortly before use.

Estimated Error:

Temperature: $\pm 0.1 \mathrm{~K}$.

$x_{1}: \pm 1.5 \%$ (relative error)

Components: Original Measurements:

(1) 2-Acetoxybenzoic acid; $\mathrm{C}_{9} \mathrm{H}_{8} \mathrm{O}_{4} ;{ }^{12}$ A. K. Charlton, C. R. Daniels, W. E. [50-78-2]

(2) 2,2'-Oxybispropane; $\mathrm{C}_{6} \mathrm{H}_{14} \mathrm{O}$; Acree, Jr., and M. H. Abraham, J. [108-20-3]

Variables:

$T / \mathrm{K}=298.15$

Prepared by:

W. E. Acree, Jr. 
Experimental Values

\begin{tabular}{lc}
\hline \hline$x_{2}{ }^{\mathrm{a}}$ & $x_{1}{ }^{\mathrm{b}}$ \\
\hline 0.9878 & 0.01224 \\
${ }^{\mathrm{a}} x_{2}:$ mole fraction of component 2 in the saturated solution. & \\
${ }^{\mathrm{b}} x_{1}:$ mole fraction solubility of the solute. &
\end{tabular}

\section{Auxiliary Information}

\section{Method/Apparatus/Procedure:}

Constant-temperature bath, calorimetric thermometer, and an ultraviolet/ visible spectrophotometer.

Excess solute and solvent were placed in amber glass bottles and allowed to equilibrate for several days at constant temperature. Attainment of equilibrium was verified by several repetitive measurements and by approaching equilibrium from supersaturation. Aliquots of saturated solutions were transferred through a coarse filter into tared volumetric flasks, weighed, and diluted with methanol. Concentrations were determined by

spectrophotometric measurements at $272 \mathrm{~nm}$.

Source and Purity of Chemicals:

(1) $99 \%$, Aldrich Chemical Company, Milwaukee, WI, USA, was used as received.

(2) $99 \%$, anhydrous, Aldrich Chemical Company, stored over molecular sieves and distilled shortly before use.

\section{Estimated Error:}

Temperature: $\pm 0.1 \mathrm{~K}$

$x_{1}: \pm 1.5 \%$ (relative error).

\begin{tabular}{|c|c|}
\hline $\begin{array}{l}\text { Components: } \\
\text { (1) 2-Acetoxybenzoic acid; } \\
\mathrm{C}_{9} \mathrm{H}_{8} \mathrm{O}_{4} ;[50-78-2] \\
\text { (2) } 1,1^{\prime} \text {-Oxybisbutane; } \mathrm{C}_{8} \mathrm{H}_{18} \mathrm{O} \text {; } \\
\text { [142-96-1] }\end{array}$ & $\begin{array}{l}\text { Original Measurements: } \\
{ }^{12} \text { A. K. Charlton, C. R. Daniels, W. E. } \\
\text { Acree, Jr., and M. H. Abraham, J. } \\
\text { Solution Chem. 32, } 1087 \text { (2003). }\end{array}$ \\
\hline $\begin{array}{l}\text { Variables: } \\
T / \mathrm{K}=298.15\end{array}$ & $\begin{array}{l}\text { Prepared by: } \\
\text { W. E. Acree, Jr. }\end{array}$ \\
\hline
\end{tabular}

Experimental Values

\begin{tabular}{lc}
\hline \hline$x_{2}{ }^{\mathrm{a}}$ & $x_{1}{ }^{\mathrm{b}}$ \\
\hline 0.9929 & 0.007095 \\
\hline$x_{2}: \operatorname{molefraction}$ of compong 2 inthe sacted
\end{tabular}

${ }^{\mathrm{a}} x_{2}$ : mole fraction of component 2 in the saturated solution.

${ }^{\mathrm{b}} x_{1}$ : mole fraction solubility of the solute.

\section{Auxiliary Information}

\footnotetext{
Method/Apparatus/Procedure:

Constant-temperature bath, calorimetric thermometer, and an ultraviolet/ visible spectrophotometer.

Excess solute and solvent were placed in amber glass bottles and allowed to equilibrate for several days at constant temperature. Attainment of equilibrium was verified by several repetitive measurements and by approaching equilibrium from supersaturation. Aliquots of saturated solutions were transferred through a coarse filter into tared volumetric flasks, weighed, and diluted with methanol. Concentrations were determined by spectrophotometric measurements at $272 \mathrm{~nm}$.
}

Source and Purity of Chemicals:

(1) $99 \%$, Aldrich Chemical Company, Milwaukee, WI, USA, was used as received.

(2) $99.3 \%$, anhydrous, Aldrich Chemical Company, stored over molecular sieves and distilled shortly before use.

\section{Estimated Error:}

Temperature: $\pm 0.1 \mathrm{~K}$.

$x_{1}: \pm 1.5 \%$ (relative error).

\begin{tabular}{|c|c|}
\hline $\begin{array}{l}\text { Components: } \\
\text { (1) 2-Acetoxybenzoic acid; } \\
\mathrm{C}_{9} \mathrm{H}_{8} \mathrm{O}_{4} ;[50-78-2] \\
\text { (2) 2-Methoxy-2-methylpropane; } \\
\mathrm{C}_{5} \mathrm{H}_{12} \mathrm{O} \text {; [1634-04-4] }\end{array}$ & $\begin{array}{l}\text { Original Measurements: } \\
{ }^{12} \text { A. K. Charlton, C. R. Daniels, W. E } \\
\text { Acree, Jr., and M. H. Abraham, J. } \\
\text { Solution Chem. 32, } 1087 \text { (2003). }\end{array}$ \\
\hline $\begin{array}{l}\text { Variables: } \\
T / \mathrm{K}=298.15\end{array}$ & $\begin{array}{l}\text { Prepared by: } \\
\text { W. E. Acree, Jr. }\end{array}$ \\
\hline
\end{tabular}

\section{Experimental Values}

\begin{tabular}{lc}
\hline \hline$x_{2}{ }^{\mathrm{a}}$ & $x_{1}{ }^{\mathrm{b}}$ \\
\hline 0.9599 & 0.04013 \\
\hline${ }^{\mathrm{a}} x_{2}:$ mole fraction of component 2 in the saturated solution. & \\
${ }^{\mathrm{b}}{ }_{x_{1}}$ : mole fraction solubility of the solute. &
\end{tabular}

\section{Auxiliary Information}

\section{Method/Apparatus/Procedure:}

Constant-temperature bath, calorimetric thermometer, and an ultraviolet/ visible spectrophotometer.

Excess solute and solvent were placed in amber glass bottles and allowed to equilibrate for several days at constant temperature. Attainment of equilibrium was verified by several repetitive measurements and by approaching equilibrium from supersaturation. Aliquots of saturated solutions were transferred through a coarse filter into tared volumetric flasks, weighed, and diluted with methanol. Concentrations were determined by spectrophotometric measurements at $272 \mathrm{~nm}$.

\section{Source and Purity of Chemicals:}

(1) $99 \%$, Aldrich Chemical Company, Milwaukee, WI, USA, was used as received.

(2) $99+\%$, Arco Chemical Company, USA, stored over molecular sieves and distilled shortly before use.

\section{Estimated Error:}

Temperature: $\pm 0.1 \mathrm{~K}$.

$x_{1}: \pm 1.5 \%$ (relative error)

\begin{tabular}{ll}
\hline \hline Components: & Original Measurements: \\
(1) 2-Acetoxybenzoic acid; & ${ }^{12}$ A. K. Charlton, C. R. Daniels, W. E. \\
$\mathrm{C}_{9} \mathrm{H}_{8} \mathrm{O}_{4} ;$ [50-78-2] & Acree, Jr., and M. H. Abraham, J. \\
$\begin{array}{l}\text { (2) Tetrahydrofuran; } \mathrm{C}_{4} \mathrm{H}_{8} \mathrm{O} ; \\
\text { [109-99-9] }\end{array}$ & Solution Chem. 32, 1087 (2003). \\
\hline Variables: & Prepared by: \\
$T / \mathrm{K}=298.15$ & W. E. Acree, Jr. \\
\hline
\end{tabular}


Experimental Values

\begin{tabular}{lc}
\hline \hline$x_{2}{ }^{\mathrm{a}}$ & $x_{1}{ }^{\mathrm{b}}$ \\
\hline 0.8096 & 0.1904 \\
\hline${ }^{\mathrm{a}} x_{2}:$ mole fraction of component 2 in the saturated solution. \\
${ }^{\mathrm{b}}{ }_{x_{1}: \text { mole fraction solubility of the solute. }}$
\end{tabular}

\section{Auxiliary Information}

\section{Method/Apparatus/Procedure:}

Constant-temperature bath, calorimetric thermometer, and an ultraviolet/ visible spectrophotometer.

Excess solute and solvent were placed in amber glass bottles and allowed to equilibrate for several days at constant temperature. Attainment of equilibrium was verified by several repetitive measurements and by approaching equilibrium from supersaturation. Aliquots of saturated solutions were transferred through a coarse filter into tared volumetric flasks, weighed, and diluted with methanol. Concentrations were determined by spectrophotometric measurements at $272 \mathrm{~nm}$.

\section{Source and Purity of Chemicals:}

(1) $99 \%$, Aldrich Chemical Company, Milwaukee, WI, USA, was used as received.

(2) $99.9 \%$, anhydrous, Aldrich Chemical Company, stored over molecular sieves and distilled shortly before use.

\section{Estimated Error:}

Temperature: $\pm 0.1 \mathrm{~K}$

$x_{1}: \pm 2.0 \%$ (relative error)

\begin{tabular}{|c|c|}
\hline $\begin{array}{l}\text { Components: } \\
\text { (1) 2-Acetoxybenzoic acid; } \\
\mathrm{C}_{9} \mathrm{H}_{8} \mathrm{O}_{4} ;[50-78-2] \\
\text { (2) } 1,4-\text {-Dioxane; } \mathrm{C}_{4} \mathrm{H}_{8} \mathrm{O}_{2} \text {; } \\
\text { [123-91-1] }\end{array}$ & $\begin{array}{l}\text { Original Measurements: } \\
{ }^{12} \text { A. K. Charlton, C. R. Daniels, W. E. } \\
\text { Acree, Jr., and M. H. Abraham, J. } \\
\text { Solution Chem. 32, } 1087 \text { (2003). }\end{array}$ \\
\hline $\begin{array}{l}\text { Variables: } \\
T / \mathrm{K}=298.15\end{array}$ & $\begin{array}{l}\text { Prepared by: } \\
\text { W. E. Acree, Jr. }\end{array}$ \\
\hline
\end{tabular}

Experimental Values

\begin{tabular}{lc}
\hline \hline$x_{2}{ }^{\mathrm{a}}$ & $x_{1}{ }^{\mathrm{b}}$ \\
\hline 0.8737 & 0.1263 \\
\hline${ }^{\mathrm{a}} x_{2}:$ mole fraction of component 2 in the saturated solution. &
\end{tabular}

${ }^{\mathrm{b}} x_{1}$ : mole fraction solubility of the solute.

\section{Auxiliary Information}

\footnotetext{
Method/Apparatus/Procedure:

Constant-temperature bath, calorimetric thermometer, and an ultraviolet/ visible spectrophotometer.

Excess solute and solvent were placed in amber glass bottles and allowed to equilibrate for several days at constant temperature. Attainment of equilibrium was verified by several repetitive measurements and by approaching equilibrium from supersaturation. Aliquots of saturated solutions were transferred through a coarse filter into tared volumetric flasks, weighed, and diluted with methanol. Concentrations were determined by spectrophotometric measurements at $272 \mathrm{~nm}$.
}

Source and Purity of Chemicals:

(1) $99 \%$, Aldrich Chemical Company, Milwaukee, WI, USA, was used as received.

(2) $99.8 \%$, anhydrous, Aldrich Chemical Company, stored over molecular sieves and distilled shortly before use.

\section{Estimated Error:}

Temperature: $\pm 0.1 \mathrm{~K}$.

$x_{1}: \pm 2.0 \%$ (relative error).

\begin{tabular}{|c|c|}
\hline $\begin{array}{l}\text { Components: } \\
\text { (1) 2-Acetoxybenzoic acid; } \\
\mathrm{C}_{9} \mathrm{H}_{8} \mathrm{O}_{4} ;[50-78-2] \\
\text { (2) } 1,4-\text {-Dioxane; } \mathrm{C}_{4} \mathrm{H}_{8} \mathrm{O}_{2} \text {; } \\
\text { [123-91-1] }\end{array}$ & $\begin{array}{l}\text { Original Measurements: } \\
{ }^{53} \text { G. L. Perlovich and A. Bauer-Brandl, } \\
\text { Pharm. Res. 20, } 471 \text { (2003). }\end{array}$ \\
\hline $\begin{array}{l}\text { Variables: } \\
T / \mathrm{K}=298.15\end{array}$ & $\begin{array}{l}\text { Prepared by: } \\
\text { W. E. Acree, Jr. }\end{array}$ \\
\hline
\end{tabular}

\section{Experimental Values}

\begin{tabular}{lc}
\hline \hline$x_{2}{ }^{\mathrm{a}}$ & $x_{1}{ }^{\mathrm{b}}$ \\
\hline 0.9484 & 0.0516 \\
\hline${ }^{\mathrm{a}} x_{2}:$ mole fraction of component 2 in the saturated solution. & \\
${ }^{\mathrm{b}} x_{1}:$ mole fraction solubility of the solute. &
\end{tabular}

${ }^{b} x_{1}$ : mole fraction solubility of the solute.

\section{Auxiliary Information}

\section{Method/Apparatus/Procedure:}

Thermostated constant-temperature bath, centrifuge, and an ultraviolet/visible spectrophotometer.

Excess solute and solvent were placed in a glass ampoule and allowed to equilibrate in a thermostated temperature bath with mixing (at a speed of 25 rpm) for a minimum of four days. After suitable equilibration the saturated solution was centrifuged, the supernatant liquid collected, quantitatively diluted, and the absorbance recorded using an ultraviolet/visible spectrophotometer. The solubility of the solute was calculated from the measured absorbance.

\section{Source and Purity of Chemicals:}

(1) Analytical Reagent grade, Norsk Medisinaldepot, Oslo, Norway, no purification details were provided.

(2) Analytical Reagent grade, Sigma Chemical Company, USA, no purification details were provided.

\section{Estimated Error:}

Temperature: $\pm 0.01 \mathrm{~K}$.

$x_{1}: \pm 2.5 \%$ (relative error). 


\subsection{2-Acetoxybenzoic acid solubility data in haloalkanes, haloalkenes, and haloaromatic hydrocarbons}

\begin{tabular}{ll}
\hline \hline Components: & Original Measurements: \\
(1) 2-Acetoxybenzoic acid; & ${ }^{53}$ G. L. Perlovich and A. Bauer-Brandl, \\
$\mathrm{C}_{9} \mathrm{H}_{8} \mathrm{O}_{4} ;[50-78-2]$ & Pharm. Res. 20, 471 (2003). \\
$\begin{array}{l}\text { (2) Trichloromethane; } \mathrm{CHCl}_{3} ; \\
\text { 67-66-3] }\end{array}$ & \\
\hline Variables: & Prepared by: \\
$T / \mathrm{K}=298.15$ & W. E. Acree, Jr. \\
\hline
\end{tabular}

Experimental Values

\begin{tabular}{lc}
\hline \hline$x_{2}{ }^{\mathrm{a}}$ & $x_{1}{ }^{\mathrm{b}}$ \\
\hline 0.794 & 0.206 \\
\hline
\end{tabular}

${ }^{\mathrm{a}} x_{2}$ : mole fraction of component 2 in the saturated solution.

${ }^{\mathrm{b}} x_{1}$ : mole fraction solubility of the solute.

\section{Auxiliary Information}

\section{Method/Apparatus/Procedure:}

Thermostated constant-temperature bath, centrifuge, and an ultraviolet/visible spectrophotometer.

Excess solute and solvent were placed in a glass ampoule and allowed to equilibrate in a thermostated temperature bath with mixing (at a speed of 25 $\mathrm{rpm}$ ) for a minimum of four days. After suitable equilibration the saturated solution was centrifuged, the supernatant liquid collected, quantitatively diluted, and the absorbance recorded using an ultraviolet/visible spectrophotometer. The solubility of the solute was calculated from the measured absorbance.

\section{Source and Purity of Chemicals:}

(1) Analytical Reagent grade, Norsk Medisinaldepot, Oslo, Norway, no purification details were provided.

(2) Analytical Reagent grade, Merck Chemicals, Germany, no purification details were provided.

Estimated Error:

Temperature: $\pm 0.01 \mathrm{~K}$.

$x_{1}: \pm 2.5 \%$ (relative error).

\subsection{2-Acetoxybenzoic acid solubility data in alcohols}

\begin{tabular}{|c|c|}
\hline $\begin{array}{l}\text { Components: } \\
\text { (1) 2-Acetoxybenzoic acid; } \\
\mathrm{C}_{9} \mathrm{H}_{8} \mathrm{O}_{4} ;[50-78-2] \\
\text { (2) Methanol; } \mathrm{CH}_{4} \mathrm{O} \text {; } \\
\text { [67-56-1] }\end{array}$ & $\begin{array}{l}\text { Original Measurements: } \\
{ }^{53} \text { G. L. Perlovich and A. Bauer-Brandl, } \\
\text { Pharm. Res. 20, } 471 \text { (2003). }\end{array}$ \\
\hline $\begin{array}{l}\text { Variables: } \\
T / \mathrm{K}=298.15\end{array}$ & $\begin{array}{l}\text { Prepared by: } \\
\text { W. E. Acree, Jr. }\end{array}$ \\
\hline
\end{tabular}

Experimental Values

\begin{tabular}{lc}
\hline \hline$x_{2}{ }^{\mathrm{a}}$ & $x_{1}{ }^{\mathrm{b}}$ \\
\hline 0.9281 & 0.0719 \\
\hline${ }^{\mathrm{a}} x_{2}:$ mole fraction of component 2 in the saturated solution. & \\
${ }^{\mathrm{b}}{ }_{x_{1}}:$ mole fraction solubility of the solute. &
\end{tabular}

\section{Auxiliary Information}

\section{Method/Apparatus/Procedure:}

Thermostated constant-temperature bath, centrifuge, and an ultraviolet/visible spectrophotometer.

Excess solute and solvent were placed in a glass ampoule and allowed to equilibrate in a thermostated temperature bath with mixing (at a speed of 25 $\mathrm{rpm}$ ) for a minimum of four days. After suitable equilibration the saturated solution was centrifuged, the supernatant liquid collected, quantitatively diluted, and the absorbance recorded using an ultraviolet/visible spectrophotometer. The solubility of the solute was calculated from the measured absorbance.

\section{Source and Purity of Chemicals:}

(1) Analytical Reagent grade, Norsk Medisinaldepot, Oslo, Norway, no purification details were provided.

(2) HPLC grade, Merck Chemicals, Germany, no purification details were provided.

Estimated Error:

Temperature: $\pm 0.01 \mathrm{~K}$.

$x_{1}: \pm 2.5 \%$ (relative error).

\begin{tabular}{ll}
\hline \hline Components: & Original Measurements: \\
$\begin{array}{l}\text { (1) 2-Acetoxybenzoic acid; } \\
\mathrm{C}_{9} \mathrm{H}_{8} \mathrm{O}_{4} ;[50-78-2]\end{array}$ & ${ }^{69} \mathrm{C}$. M. McLoughlin, W. A. M. \\
(2) Methanol; $\mathrm{CH}_{4} \mathrm{O} ;[67-56-1]$ & Techinn, and T. R. A. Magee, Powder \\
\hline Variables: & Prepared by: \\
$T / \mathrm{K}=293$ and 333 & W. E. Acree, Jr. \\
\hline
\end{tabular}

\section{Experimental Values}

The measured solubility was reported to be 140 and $580 \mathrm{~g} / \mathrm{l}$ at 293 and $333 \mathrm{~K}$, respectively. The authors did not specify whether the values were per liter of solvent or per liter of saturated solution.

\section{Auxiliary Information}

\section{Method/Apparatus/Procedure:}

Very little experimental details were provided. Solubility was determined by adding $(0.50 \pm 0.1) \mathrm{g}$ amounts of the powdered solute to $(100 \pm 1) \mathrm{ml}$ of solvent until a saturated solution was obtained. The mass of the powder was recorded.

Source and Purity of Chemicals:

(1) Purity not given, Sigma-Aldrich, Poole, England, UK, no purification details were provided.

(2) Purity not given, Sigma-Aldrich, no purification details were provided.

Estimated Error:

Temperature: Insufficient experimental details to estimate.

Solubility: $\pm 5 \mathrm{~g} / \mathrm{l}$ (estimated by compiler assuming solute added in increments of $0.50 \mathrm{~g}$ ). 


\begin{tabular}{|c|c|}
\hline $\begin{array}{l}\text { Components: } \\
\text { (1) 2-Acetoxybenzoic acid; } \\
\mathrm{C}_{9} \mathrm{H}_{8} \mathrm{O}_{4} ;[50-78-2] \\
\text { (2) Ethanol; } \mathrm{C}_{2} \mathrm{H}_{6} \mathrm{O} ;[64-17-5]\end{array}$ & $\begin{array}{l}\text { Original Measurements: } \\
\text { }{ }^{53} \text { G. L. Perlovich and A. Bauer-Brandl, } \\
\text { Pharm. Res. 20, } 471 \text { (2003). }\end{array}$ \\
\hline $\begin{array}{l}\text { Variables: } \\
T / \mathrm{K}=298.15\end{array}$ & $\begin{array}{l}\text { Prepared by: } \\
\text { W. E. Acree, Jr. }\end{array}$ \\
\hline
\end{tabular}

Experimental Values

\begin{tabular}{lc}
\hline \hline$x_{2}^{\mathrm{a}}$ & $x_{1}{ }^{\mathrm{b}}$ \\
\hline 0.9145 & 0.0855
\end{tabular}

${ }^{\mathrm{a}} x_{2}$ : mole fraction of component 2 in the saturated solution.

${ }^{\mathrm{b}} x_{1}$ : mole fraction solubility of the solute.

\section{Auxiliary Information}

Method/Apparatus/Procedure:
Thermostated constant-temperature bath, centrifuge, and an ultraviolet/visible
spectrophotometer.
Excess solute and solvent were placed in a glass ampoule and allowed to
equilibrate in a thermostated temperature bath with mixing (at a speed of
$25 \mathrm{rpm}$ ) for a minimum of four days. After suitable equilibration the saturated
solution was centrifuged, the supernatant liquid collected, quantitatively
diluted, and the absorbance recorded using an ultraviolet/visible
spectrophotometer. The solubility of the solute was calculated from the
measured absorbance.
Source and Purity of Chemicals:
(1) Analytical Reagent grade, Norsk Medisinaldepot, Oslo, Norway, no
purification details were provided.
(2) $99.6 \%$, Chemical source not given, no purification details were provided.
Estimated Error:
Temperature: $\pm 0.01 \mathrm{~K}$.
$x_{1}: \pm 2.5 \%$ (relative error).

\begin{tabular}{ll}
\hline \hline Components: & Original Measurements: \\
(1) 2-Acetoxybenzoic acid; & ${ }^{69} \mathrm{C}$. M. McLoughlin, W. A. M. \\
$\mathrm{C}_{9} \mathrm{H}_{8} \mathrm{O}_{4} ;[50-78-2]$ & McMinn, and T. R. A. Magee, Powder \\
$(2)$ Ethanol; $\mathrm{C}_{2} \mathrm{H}_{6} \mathrm{O} ;[64-17-5]$ & Technol. 134, 40 (2003). \\
\hline Variables: & Prepared by: \\
$T / \mathrm{K}=293$ and 333 & W. E. Acree, Jr. \\
\hline
\end{tabular}

\section{Experimental Values}

The measured solubility was reported to be 240 and $720 \mathrm{~g} / \mathrm{l}$ at 293 and $333 \mathrm{~K}$, respectively. The authors did not specify whether the values were per liter of solvent or per liter of saturated solution.

\section{Auxiliary Information}

\section{Method/Apparatus/Procedure:}

Very little experimental details were provided. Solubility was determined by adding $(0.50 \pm 0.1) \mathrm{g}$ amounts of the powdered solute to $(100 \pm 1) \mathrm{ml}$ of solvent until a saturated solution was obtained. The mass of the powder was recorded.
Source and Purity of Chemicals:

(1) Purity not given, Sigma-Aldrich, Poole, England, UK, no purification details were provided.

(2) Purity not given, Sigma-Aldrich, no purification details were provided.

\section{Estimated Error:}

Temperature: Insufficient experimental details to estimate.

Solubility: $\pm 5 \mathrm{~g} / \mathrm{l}$ (estimated by compiler assuming solute added in increments of $0.50 \mathrm{~g}$ ).

\begin{tabular}{|c|c|}
\hline $\begin{array}{l}\text { Components: } \\
\text { (1) 2-Acetoxybenzoic acid; } \\
\mathrm{C}_{9} \mathrm{H}_{8} \mathrm{O}_{4} ;[50-78-2] \\
\text { (2) Ethanol; } \mathrm{C}_{2} \mathrm{H}_{6} \mathrm{O} ;[64-17-5]\end{array}$ & $\begin{array}{l}\text { Original Measurements: } \\
{ }^{92} \text { G. D. Maia and M. Giulietti, J. Chem } \\
\text { Eng. Data 53, } 256 \text { (2008). }\end{array}$ \\
\hline $\begin{array}{l}\text { Variables: } \\
\text { Temperature }\end{array}$ & $\begin{array}{l}\text { Prepared by: } \\
\text { W. E. Acree, Jr. }\end{array}$ \\
\hline
\end{tabular}

Experimental Values

\begin{tabular}{|c|c|c|}
\hline$T / \mathrm{K}$ & $x_{2}{ }^{a}$ & $x_{1}{ }^{\mathrm{b}}$ \\
\hline 276.2 & 0.975 & 0.025 \\
\hline 291.9 & 0.951 & 0.049 \\
\hline 302.4 & 0.929 & 0.071 \\
\hline 310.0 & 0.907 & 0.093 \\
\hline 316.5 & 0.887 & 0.113 \\
\hline 321.5 & 0.867 & 0.133 \\
\hline 325.9 & 0.848 & 0.152 \\
\hline 330.4 & 0.830 & 0.170 \\
\hline 333.4 & 0.813 & 0.187 \\
\hline 336.6 & 0.796 & 0.204 \\
\hline
\end{tabular}

\section{Auxiliary Information}

\section{Method/Apparatus/Procedure:}

Circulating thermostated water bath, analytical balance, and drying oven. Known amounts of solute and solvent were placed in the equilibrium vessel which was connected to the thermostated water bath. The vessel was agitated at $750 \mathrm{rpm}$ as temperature was increased and decreased. By regulating the bath temperature, the authors were able to determine the temperature at which complete dissolution occurred (heating mode) and the temperature at which the first crystals formed (cooling mode).

\section{Source and Purity of Chemicals:}

(1) $99.5 \%$, Anidrol Quimica, Diadema, Brazil, no purification details were given.

(2) $99.5 \%$, Synthon Chemicals, no purification details were given.

\section{Estimated Error:}

Temperature: $\pm 0.2 \mathrm{~K}$ (estimated by compiler). $x_{1}: \pm 3 \%$ (relative error, estimated by compiler).

\begin{tabular}{ll}
\hline \hline Components: & Original Measurements: \\
$\begin{array}{l}\text { (1) 2-Acetoxybenzoic acid; } \\
\mathrm{C}_{9} \mathrm{H}_{8} \mathrm{O}_{4} ;[50-78-2]\end{array}$ & ${ }^{93}$ C. Lindenberg, M. Krättli, J. Cornel, \\
(2) Ethanol; $\mathrm{C}_{2} \mathrm{H}_{6} \mathrm{O} ;[64-17-5]$ & Mrowth Des. 9, 1124 (2009). \\
\hline Variables: & Prepared by: \\
Temperature & W. E. Acree, Jr. \\
\hline
\end{tabular}


Experimental Values

\begin{tabular}{lcc}
\hline \hline$T / \mathrm{K}$ & $x_{2}{ }^{\mathrm{a}}$ & $x_{1}{ }^{\mathrm{b}}$ \\
\hline 298 & 0.9427 & 0.0573 \\
308 & 0.9118 & 0.0882 \\
323 & 0.8578 & 0.1422
\end{tabular}

${ }^{\mathrm{a}} x_{2}$ : mole fraction of component 2 in the saturated solution.

${ }^{\mathrm{b}} \mathrm{x}_{1}$ : mole fraction solubility of the solute determined by in situ ATR-FTIR spectroscopic method. Experimental data are reported as grams of solute per kilogram of solvent. Mole fraction solubilities calculated by the compiler.

\begin{tabular}{lcc}
\hline \hline$T / \mathrm{K}$ & $x_{2}{ }^{\mathrm{a}}$ & $x_{1}{ }^{\mathrm{b}}$ \\
\hline 298 & 0.9425 & 0.0575 \\
308 & 0.9124 & 0.0876 \\
323 & 0.8549 & 0.1451
\end{tabular}

${ }^{a} x_{2}$ : mole fraction of component 2 in the saturated solution.

${ }^{\mathrm{b}} x_{1}$ : mole fraction solubility of the solute determined by a gravimetric method of analysis. Experimental data are reported as grams of solute per kilogram of solvent. Mole fraction solubilities calculated by the compiler.

\section{Auxiliary Information}

\section{Method/Apparatus/Procedure:}

Jacketed glass equilibrium cell equipped with a four-blade glass impeller and including blades, temperature controlling system, and an attenuated total reflectance-Fourier transform infrared (ATR-FTIR) probe.

Very little experimental details are given. Excess solute and solvent were placed in the equilibrium cell. The concentration of the dissolved solute was determined by ATR-FTIR spectroscopic methods. Solubilities were also determined by a gravimetric method.

Source and Purity of Chemicals:

(1) $99+\%$, Sigma-Aldrich, Switzerland, no purification details were given.

(2) $99.9 \%$, Merck KGaA, Germany, no purification details were given.

Estimated Error:

Temperature: $\pm 0.2 \mathrm{~K}$ (estimated by compiler).

$x_{1}: \pm 3 \%$ (relative error, estimated by compiler).

\begin{tabular}{|c|c|}
\hline $\begin{array}{l}\text { Components: } \\
\text { (1) 2-Acetoxybenzoic acid; } \\
\mathrm{C}_{9} \mathrm{H}_{8} \mathrm{O}_{4} ;[50-78-2] \\
\text { (2) 1-Propanol; } \mathrm{C}_{3} \mathrm{H}_{8} \mathrm{O} \text {; } \\
\text { [71-23-8] }\end{array}$ & $\begin{array}{l}\text { Original Measurements: } \\
{ }^{12} \text { A. K. Charlton, C. R. Daniels, W. E. } \\
\text { Acree, Jr., and M. H. Abraham, J. } \\
\text { Solution Chem. 32, } 1087 \text { (2003). }\end{array}$ \\
\hline $\begin{array}{l}\text { Variables: } \\
T / \mathrm{K}=298.15\end{array}$ & $\begin{array}{l}\text { Prepared by: } \\
\text { W. E. Acree, Jr. }\end{array}$ \\
\hline
\end{tabular}

Experimental Values

\begin{tabular}{lc}
\hline \hline$x_{2}{ }^{\mathrm{a}}$ & $x_{1}^{\mathrm{b}}$ \\
\hline 0.9502 & 0.04976 \\
\hline${ }^{\mathrm{a}} x_{2}:$ mole fraction of component 2 in the saturated solution. \\
${ }^{\mathrm{b}} x_{1}:$ mole fraction solubility of the solute.
\end{tabular}

\section{Auxiliary Information}

\section{Method/Apparatus/Procedure:}

Constant-temperature bath, calorimetric thermometer, and an ultraviolet/ visible spectrophotometer.

Excess solute and solvent were placed in amber glass bottles and allowed to equilibrate for several days at constant temperature. Attainment of equilibrium was verified by several repetitive measurements and by approaching equilibrium from supersaturation. Aliquots of saturated solutions were transferred through a coarse filter into tared volumetric flasks, weighed, and diluted with methanol. Concentrations were determined by spectrophotometric measurements at $272 \mathrm{~nm}$.

\section{Source and Purity of Chemicals:}

(1) $99 \%$, Aldrich Chemical Company, Milwaukee, WI, USA, was used as received.

(2) $99+\%$, anhydrous, Aldrich Chemical Company, stored over molecular sieves and distilled shortly before use.

Estimated Error:

Temperature: $\pm 0.1 \mathrm{~K}$.

$x_{1}: \pm 1.5 \%$ (relative error)

\begin{tabular}{|c|c|}
\hline $\begin{array}{l}\text { Components: } \\
\text { (1) 2-Acetoxybenzoic acid; } \\
\mathrm{C}_{9} \mathrm{H}_{8} \mathrm{O}_{4} ;[50-78-2] \\
\text { (2) 1-Propanol; } \mathrm{C}_{3} \mathrm{H}_{8} \mathrm{O} \text {; } \\
\text { [71-23-8] }\end{array}$ & $\begin{array}{l}\text { Original Measurements: } \\
{ }^{53} \text { G. L. Perlovich and A. Bauer-Brandl, } \\
\text { Pharm. Res. 20, } 471 \text { (2003). }\end{array}$ \\
\hline $\begin{array}{l}\text { Variables: } \\
T / \mathrm{K}=298.15\end{array}$ & $\begin{array}{l}\text { Prepared by: } \\
\text { W. E. Acree, Jr. }\end{array}$ \\
\hline
\end{tabular}

Experimental Values

\begin{tabular}{lc}
\hline \hline$x_{2}{ }^{\mathrm{a}}$ & $x_{1}{ }^{\mathrm{b}}$ \\
\hline 0.9582 & 0.0418 \\
\hline$x_{2}{ }^{2} \mathrm{~mole}$ fraction of component
\end{tabular}

${ }^{a_{x_{2}}}$ : mole fraction of component 2 in the saturated solution.

${ }^{\mathrm{b}} x_{1}$ : mole fraction solubility of the solute.

\section{Auxiliary Information}

\section{Method/Apparatus/Procedure:}

Thermostated constant-temperature bath, centrifuge, and an ultraviolet/visible spectrophotometer.

Excess solute and solvent were placed in a glass ampoule and allowed to equilibrate in a thermostated temperature bath with mixing (at a speed of $25 \mathrm{rpm}$ ) for a minimum of four days. After suitable equilibration the saturated solution was centrifuged, the supernatant liquid collected, quantitatively diluted, and the absorbance recorded using an ultraviolet/visible spectrophotometer. The solubility of the solute was calculated from the measured absorbance.

\section{Source and Purity of Chemicals:}

(1) Analytical Reagent grade, Norsk Medisinaldepot, Oslo, Norway, no purification details were provided.

(2) HPLC grade, Aldrich Chemical Company, Germany, no purification details were provided.

\section{Estimated Error:}

Temperature: $\pm 0.01 \mathrm{~K}$.

$x_{1}: \pm 2.5 \%$ (relative error). 


\begin{tabular}{ll}
\hline \hline Components: & Original Measurements: \\
(1) 2-Acetoxybenzoic acid; & ${ }^{12}$ A. K. Charlton, C. R. Daniels, W. E. \\
$\mathrm{C}_{9} \mathrm{H}_{8} \mathrm{O}_{4} ;[50-78-2]$ & Acree, Jr., and M. H. Abraham, J. \\
$\begin{array}{l}\text { (2) 2-Propanol; } \mathrm{C}_{3} \mathrm{H}_{8} \mathrm{O} ; \\
{[67-63-0]}\end{array}$ & Solution Chem. 32, 1087 (2003). \\
\hline Variables: & \\
$T / \mathrm{K}=298.15$ & Prepared by: \\
\hline
\end{tabular}

Experimental Values

\begin{tabular}{lc}
\hline \hline$x_{2}{ }^{\mathrm{a}}$ & $x_{1}{ }^{\mathrm{b}}$ \\
\hline 0.9477 & 0.05232
\end{tabular}

${ }^{\mathrm{a}} x_{2}$ : mole fraction of component 2 in the saturated solution.

${ }^{\mathrm{b}} x_{1}$ : mole fraction solubility of the solute.

\section{Auxiliary Information}

\section{Method/Apparatus/Procedure:}

Constant-temperature bath, calorimetric thermometer, and an ultraviolet/ visible spectrophotometer.

Excess solute and solvent were placed in amber glass bottles and allowed to equilibrate for several days at constant temperature. Attainment of equilibrium was verified by several repetitive measurements and by approaching equilibrium from supersaturation. Aliquots of saturated solutions were transferred through a coarse filter into tared volumetric flasks, weighed, and diluted with methanol. Concentrations were determined by spectrophotometric measurements at $272 \mathrm{~nm}$.

\section{Source and Purity of Chemicals:}

(1) $99 \%$, Aldrich Chemical Company, Milwaukee, WI, USA, was used as received.

(2) 99+\%, anhydrous, Aldrich Chemical Company, stored over molecular sieves and distilled shortly before use.

\section{Estimated Error:}

Temperature: $\pm 0.1 \mathrm{~K}$.

$x_{1}: \pm 1.5 \%$ (relative error).

\begin{tabular}{|c|c|}
\hline $\begin{array}{l}\text { Components: } \\
\text { (1) 2-Acetoxybenzoic acid; } \\
\mathrm{C}_{9} \mathrm{H}_{8} \mathrm{O}_{4} ;[50-78-2] \\
\text { (2) 2-Propanol; } \mathrm{C}_{3} \mathrm{H}_{8} \mathrm{O} ;[67-63-0]\end{array}$ & $\begin{array}{l}\text { Original Measurements: } \\
{ }^{92} \text { G. D. Maia and M. Giulietti, J. Chem. } \\
\text { Eng. Data 53, } 256 \text { (2008). }\end{array}$ \\
\hline $\begin{array}{l}\text { Variables: } \\
\text { Temperature }\end{array}$ & $\begin{array}{l}\text { Prepared by: } \\
\text { W. E. Acree, Jr. }\end{array}$ \\
\hline
\end{tabular}

Experimental Values

\begin{tabular}{lcc}
\hline \hline$T / \mathrm{K}$ & $x_{2}{ }^{\mathrm{a}}$ & $x_{1}{ }^{\mathrm{b}}$ \\
\hline 281.6 & 0.987 & 0.013 \\
291.8 & 0.968 & 0.032 \\
304.1 & 0.937 & 0.063 \\
312.9 & 0.909 & 0.091 \\
316.1 & 0.898 & 0.102 \\
320.4 & 0.882 & 0.118 \\
322.7 & 0.872 & 0.128
\end{tabular}

\begin{tabular}{lcc}
\hline \hline$T / \mathrm{K}$ & $x_{2}{ }^{\mathrm{a}}$ & $x_{1}{ }^{\mathrm{b}}$ \\
\hline 325.8 & 0.857 & 0.143 \\
327.7 & 0.847 & 0.153 \\
330.2 & 0.833 & 0.167 \\
\hline${ }^{\mathrm{a}} x_{2}:$ mole fraction of component 2 in the saturated solution. \\
${ }^{\mathrm{b}}{ }_{x_{1}: \text { mole fraction solubility of the solute. }}$
\end{tabular}

\section{Auxiliary Information}

\section{Method/Apparatus/Procedure:}

Circulating thermostated water bath, analytical balance, and drying oven. Known amounts of solute and solvent were placed in the equilibrium vessel which was connected to the thermostated water bath. The vessel was agitated at $750 \mathrm{rpm}$ as temperature was increased and decreased. By regulating the bath temperature, the authors were able to determine the temperature at which complete dissolution occurred (heating mode) and the temperature at which the first crystals formed (cooling mode).

Source and Purity of Chemicals:

(1) $99.5 \%$, Anidrol Quimica, Diadema, Brazil, no purification details were given.

(2) $99.9 \%$, Synthon Chemicals, no purification details were given.

Estimated Error:

Temperature: $\pm 0.2 \mathrm{~K}$ (estimated by compiler). $x_{1}: \pm 3 \%$ (relative error, estimated by compiler).

\begin{tabular}{ll}
\hline \hline Components: & Original Measurements: \\
$\begin{array}{l}\text { (1) 2-Acetoxybenzoic acid; } \\
\mathrm{C}_{9} \mathrm{H}_{8} \mathrm{O}_{4} ;[\text { [50-78-2] }\end{array}$ & ${ }^{12}$ A. K. Charlton, C. R. Daniels, W. E. \\
$(2)$ 1-Butanol; $\mathrm{C}_{4} \mathrm{H}_{10} \mathrm{O} ;$ [71-36-3] & Acree, Jr., and M. H. Abraham, J. \\
\hline Variables: & Preparion Chem. 32, 1087 (2003). \\
$T / \mathrm{K}=298.15$ & W. E. Acree, Jr. \\
\hline
\end{tabular}

\section{Experimental Values}

\begin{tabular}{lc}
\hline \hline$x_{2}{ }^{\mathrm{a}}$ & $x_{1}{ }^{\mathrm{b}}$ \\
\hline 0.9538 & 0.04616 \\
\hline
\end{tabular}

${ }^{\mathrm{a}} x_{2}$ : mole fraction of component 2 in the saturated solution.

${ }^{\mathrm{b}} x_{1}$ : mole fraction solubility of the solute.

\section{Auxiliary Information}

\section{Method/Apparatus/Procedure:}

Constant-temperature bath, calorimetric thermometer, and an ultraviolet/ visible spectrophotometer.

Excess solute and solvent were placed in amber glass bottles and allowed to equilibrate for several days at constant temperature. Attainment of equilibrium was verified by several repetitive measurements and by approaching equilibrium from supersaturation. Aliquots of saturated solutions were transferred through a coarse filter into tared volumetric flasks, weighed, and diluted with methanol. Concentrations were determined by spectrophotometric measurements at $272 \mathrm{~nm}$.

\section{Source and Purity of Chemicals:}

(1) $99 \%$, Aldrich Chemical Company, Milwaukee, WI, USA, was used as received.

(2) $99.8+\%$, HPLC grade, Aldrich Chemical Company, stored over molecular sieves and distilled shortly before use. 
Estimated Error:

Temperature: $\pm 0.1 \mathrm{~K}$.

$x_{1}: \pm 1.5 \%$ (relative error).

\begin{tabular}{ll}
\hline \hline Components: & Original Measurements: \\
(1) 2-Acetoxybenzoic acid; & ${ }^{53}$ G. L. Perlovich and A. Bauer-Brandl, \\
$\mathrm{C}_{9} \mathrm{H}_{8} \mathrm{O}_{4} ;[50-78-2]$ & Pharm. Res. 20, 471 (2003). \\
(2) $1-\mathrm{Butanol} ; \mathrm{C}_{4} \mathrm{H}_{10} \mathrm{O} ;$ & \\
{$[71-36-3]$} & \\
\hline Variables: & Prepared by: \\
$T / \mathrm{K}=298.15$ & W. E. Acree, Jr. \\
\hline
\end{tabular}

Experimental Values

\begin{tabular}{lc}
\hline \hline$x_{2}{ }^{\mathrm{a}}$ & $x_{1}{ }^{\mathrm{b}}$ \\
\hline 0.9547 & 0.0453 \\
\hline
\end{tabular}

${ }^{a} x_{2}$ : mole fraction of component 2 in the saturated solution.

${ }^{\mathrm{b}} x_{1}$ : mole fraction solubility of the solute.

\section{Auxiliary Information}

\section{Method/Apparatus/Procedure:}

Thermostated constant-temperature bath, centrifuge, and an ultraviolet/visible spectrophotometer.

Excess solute and solvent were placed in a glass ampoule and allowed to equilibrate in a thermostated temperature bath with mixing (at a speed of 25 $\mathrm{rpm}$ ) for a minimum of four days. After suitable equilibration the saturated solution was centrifuged, the supernatant liquid collected, quantitatively diluted, and the absorbance recorded using an ultraviolet/visible spectrophotometer. The solubility of the solute was calculated from the measured absorbance.

Source and Purity of Chemicals:

(1) Analytical Reagent grade, Norsk Medisinaldepot, Oslo, Norway, no purification details were provided.

(2) Analytical Reagent grade, Merck Chemicals, Germany, no purification details were provided.

Estimated Error:

Temperature: $\pm 0.01 \mathrm{~K}$.

$x_{1}: \pm 2.5 \%$ (relative error).

\begin{tabular}{ll}
\hline \hline Components: & Original Measurements: \\
$(1)$ 2-Acetoxybenzoic acid; & ${ }^{12}$ A. K. Charlton, C. R. Daniels, W. E. \\
$\mathrm{C}_{9} \mathrm{H}_{8} \mathrm{O}_{4} ;[50-78-2]$ & Acree, Jr., and M. H. Abraham, J. \\
$(2)$ 2-Butanol; $\mathrm{C}_{4} \mathrm{H}_{10} \mathrm{O} ;[78-92-2]$ & Solution Chem. 32, 1087 (2003). \\
\hline Variables: & Prepared by: \\
$T / \mathrm{K}=298.15$ & W. E. Acree, Jr. \\
\hline
\end{tabular}

\section{Experimental Values}

\begin{tabular}{lc}
\hline \hline$x_{2}^{\mathrm{a}}$ & $x_{1}^{\mathrm{b}}$ \\
\hline 0.9464 & 0.05360 \\
\hline${ }^{\mathrm{a}} x_{2}:$ mole fraction of component 2 in the saturated solution. & \\
${ }^{\mathrm{b}}{ }_{x_{1}: \text { mole fraction solubility of the solute. }}$ &
\end{tabular}

\section{Auxiliary Information}

Method/Apparatus/Procedure:

Constant-temperature bath, calorimetric thermometer, and an ultraviolet/ visible spectrophotometer.

Excess solute and solvent were placed in amber glass bottles and allowed to equilibrate for several days at constant temperature. Attainment of equilibrium was verified by several repetitive measurements and by approaching equilibrium from supersaturation. Aliquots of saturated solutions were transferred through a coarse filter into tared volumetric flasks, weighed, and diluted with methanol. Concentrations were determined by spectrophotometric measurements at $272 \mathrm{~nm}$.

Source and Purity of Chemicals:

(1) $99 \%$, Aldrich Chemical Company, Milwaukee, WI, USA, was used as received.

(2) $99+\%$, Aldrich Chemical Company, stored over molecular sieves and distilled shortly before use.

\section{Estimated Error:}

Temperature: $\pm 0.1 \mathrm{~K}$.

$x_{1}: \pm 1.5 \%$ (relative error).

\begin{tabular}{ll}
\hline \hline Components: & Original Measurements: \\
(1) 2-Acetoxybenzoic acid; & ${ }^{12}$ A. K. Charlton, C. R. Daniels, W. E. \\
$\mathrm{C}_{9} \mathrm{H}_{8} \mathrm{O}_{4} ;$ [50-78-2] & Acree, Jr., and M. H. Abraham, J. \\
$(2)$ 2-Methyl-1-propanol; & Solution Chem. 32, 1087 (2003). \\
$\mathrm{C}_{4} \mathrm{H}_{10} \mathrm{O} ;[78-83-1]$ & \\
\hline Variables: & Prepared by: \\
$T / \mathrm{K}=298.15$ & W. E. Acree, Jr. \\
\hline
\end{tabular}

\section{Experimental Values}

\begin{tabular}{lc}
\hline \hline$x_{2}{ }^{\mathrm{a}}$ & $x_{1}{ }^{\mathrm{b}}$ \\
\hline 0.9681 & 0.03186 \\
\hline${ }^{\mathrm{a}} x_{2}:$ mole fraction of component 2 in the saturated solution. & \\
${ }^{\mathrm{b}} x_{1}:$ mole fraction solubility of the solute. &
\end{tabular}

\section{Auxiliary Information}

Method/Apparatus/Procedure:

Constant-temperature bath, calorimetric thermometer, and an ultraviolet/ visible spectrophotometer.

Excess solute and solvent were placed in amber glass bottles and allowed to equilibrate for several days at constant temperature. Attainment of equilibrium was verified by several repetitive measurements and by approaching equilibrium from supersaturation. Aliquots of saturated solutions were transferred through a coarse filter into tared volumetric flasks, weighed, and diluted with methanol. Concentrations were determined by spectrophotometric measurements at $272 \mathrm{~nm}$.

\section{Source and Purity of Chemicals:}

(1) $99 \%$, Aldrich Chemical Company, Milwaukee, WI, USA, was used as received.

(2) 99+\%, anhydrous, Aldrich Chemical Company, stored over molecular sieves and distilled shortly before use.

\section{Estimated Error:}

Temperature: $\pm 0.1 \mathrm{~K}$.

$x_{1}: \pm 1.5 \%$ (relative error) 


\begin{tabular}{ll}
\hline \hline Components: & Original Measurements: \\
(1) 2-Acetoxybenzoic acid; & ${ }^{12}$ A. K. Charlton, C. R. Daniels, W. E. \\
$\mathrm{C}_{9} \mathrm{H}_{8} \mathrm{O}_{4} ;[50-78-2]$ & Acree, Jr., and M. H. Abraham, J. \\
$(2)$ 2-Methyl-2-propanol; & Solution Chem. 32, 1087 (2003). \\
$\mathrm{C}_{4} \mathrm{H}_{10} \mathrm{O} ;$ [78-83-1] & \\
\hline Variables: & Prepared by: \\
$T / \mathrm{K}=298.15$ & W. E. Acree, Jr. \\
\hline
\end{tabular}

Experimental Values

\begin{tabular}{lc}
\hline \hline$x_{2}{ }^{\mathrm{a}}$ & $x_{1}{ }^{\mathrm{b}}$ \\
\hline 0.9316 & 0.06844
\end{tabular}

${ }^{\mathrm{a}} x_{2}$ : mole fraction of component 2 in the saturated solution.

${ }^{\mathrm{b}} x_{1}$ : mole fraction solubility of the solute.

\section{Auxiliary Information}

\section{Method/Apparatus/Procedure:}

Constant-temperature bath, calorimetric thermometer, and an ultraviolet/ visible spectrophotometer.

Excess solute and solvent were placed in amber glass bottles and allowed to equilibrate for several days at constant temperature. Attainment of equilibrium was verified by several repetitive measurements and by approaching equilibrium from supersaturation. Aliquots of saturated solutions were transferred through a coarse filter into tared volumetric flasks, weighed, and diluted with methanol. Concentrations were determined by spectrophotometric measurements at $272 \mathrm{~nm}$.

\section{Source and Purity of Chemicals:}

(1) $99 \%$, Aldrich Chemical Company, Milwaukee, WI, USA, was used as received.

(2) $99+\%$, Arco Chemical Company, USA, stored over molecular sieves and distilled shortly before use.

\section{Estimated Error:}

Temperature: $\pm 0.1 \mathrm{~K}$.

$x_{1}: \pm 2.0 \%$ (relative error).

\begin{tabular}{|c|c|}
\hline $\begin{array}{l}\text { Components: } \\
\text { (1) 2-Acetoxybenzoic acid; } \\
\mathrm{C}_{9} \mathrm{H}_{8} \mathrm{O}_{4} ;[50-78-2] \\
\text { (2) 1-Pentanol; } \mathrm{C}_{5} \mathrm{H}_{12} \mathrm{O} \text {; } \\
\text { [71-41-0] }\end{array}$ & $\begin{array}{l}\text { Original Measurements: } \\
{ }^{12} \text { A. K. Charlton, C. R. Daniels, W. E. } \\
\text { Acree, Jr., and M. H. Abraham, J. } \\
\text { Solution Chem. 32, } 1087 \text { (2003). }\end{array}$ \\
\hline $\begin{array}{l}\text { Variables: } \\
T / \mathrm{K}=298.15\end{array}$ & $\begin{array}{l}\text { Prepared by: } \\
\text { W. E. Acree, Jr. }\end{array}$ \\
\hline
\end{tabular}

Experimental Values

\begin{tabular}{lc}
\hline \hline$x_{2}^{\mathrm{a}}$ & $x_{1}{ }^{\mathrm{b}}$ \\
\hline 0.9603 & 0.03966 \\
\hline${ }^{\mathrm{a}} x_{2}:$ mole fraction of component 2 in the saturated solution. \\
${ }^{\mathrm{b}} x_{1}:$ mole fraction solubility of the solute.
\end{tabular}

\section{Auxiliary Information}

\section{Method/Apparatus/Procedure:}

Constant-temperature bath, calorimetric thermometer, and an ultraviolet/ visible spectrophotometer.

Excess solute and solvent were placed in amber glass bottles and allowed to equilibrate for several days at constant temperature. Attainment of equilibrium was verified by several repetitive measurements and by approaching equilibrium from supersaturation. Aliquots of saturated solutions were transferred through a coarse filter into tared volumetric flasks, weighed, and diluted with methanol. Concentrations were determined by spectrophotometric measurements at $272 \mathrm{~nm}$.

\section{Source and Purity of Chemicals:}

(1) $99 \%$, Aldrich Chemical Company, Milwaukee, WI, USA, was used as received.

(2) $99+\%$, Aldrich Chemical Company, stored over molecular sieves and distilled shortly before use.

Estimated Error:

Temperature: $\pm 0.1 \mathrm{~K}$.

$x_{1}: \pm 1.5 \%$ (relative error)

\begin{tabular}{|c|c|}
\hline $\begin{array}{l}\text { Components: } \\
\text { (1) 2-Acetoxybenzoic acid; } \\
\mathrm{C}_{9} \mathrm{H}_{8} \mathrm{O}_{4} ;[50-78-2] \\
\text { (2) } 1-P e n t a n o l ; \mathrm{C}_{5} \mathrm{H}_{12} \mathrm{O} \text {; } \\
\text { [71-41-0] }\end{array}$ & $\begin{array}{l}\text { Original Measurements: } \\
{ }^{53} \text { G. L. Perlovich and A. Bauer-Brandl } \\
\text { Pharm. Res. 20, } 471 \text { (2003). }\end{array}$ \\
\hline $\begin{array}{l}\text { Variables: } \\
T / \mathrm{K}=298.15\end{array}$ & $\begin{array}{l}\text { Prepared by: } \\
\text { W. E. Acree, Jr. }\end{array}$ \\
\hline
\end{tabular}

\section{Experimental Values}

\begin{tabular}{lc}
\hline \hline$x_{2}{ }^{\mathrm{a}}$ & $x_{1}{ }^{\mathrm{b}}$ \\
\hline 0.9605 & 0.0395 \\
\hline${ }^{\mathrm{a}} x_{2}:$ mole fraction of component 2 in the saturated solution. & \\
${ }^{\mathrm{b}} x_{1}:$ mole fraction solubility of the solute. &
\end{tabular}

${ }^{\mathrm{b}} x_{1}$ : mole fraction solubility of the solute.

\section{Auxiliary Information}

\section{Method/Apparatus/Procedure:}

Thermostated constant-temperature bath, centrifuge, and an ultraviolet/visible spectrophotometer.

Excess solute and solvent were placed in a glass ampoule and allowed to equilibrate in a thermostated temperature bath with mixing (at a speed of 25 rpm) for a minimum of four days. After suitable equilibration the saturated solution was centrifuged, the supernatant liquid collected, quantitatively diluted, and the absorbance recorded using an ultraviolet/visible spectrophotometer. The solubility of the solute was calculated from the measured absorbance.

\section{Source and Purity of Chemicals:}

(1) Analytical Reagent grade, Norsk Medisinaldepot, Oslo, Norway, no purification details were provided.

(2) Analytical Reagent grade, Aldrich Chemical Company, Germany, no purification details were provided.

\section{Estimated Error:}

Temperature: $\pm 0.01 \mathrm{~K}$.

$x_{1}: \pm 2.5 \%$ (relative error) 


\begin{tabular}{ll}
\hline \hline Components: & Original Measurements: \\
(1) 2-Acetoxybenzoic acid; & ${ }^{12}$ A. K. Charlton, C. R. Daniels, W. E. \\
$\mathrm{C}_{9} \mathrm{H}_{8} \mathrm{O}_{4} ;[50-78-2]$ & Acree, Jr., and M. H. Abraham, J. \\
$\begin{array}{l}\text { (2) 2-Pentanol; } \mathrm{C}_{5} \mathrm{H}_{12} \mathrm{O} ; \\
{[6032-29-7]}\end{array}$ & Solution Chem. 32, 1087 (2003). \\
\hline Variables: & \\
$T / \mathrm{K}=298.15$ & Prepared by: \\
\hline
\end{tabular}

Experimental Values

\begin{tabular}{lc}
\hline \hline$x_{2}{ }^{\mathrm{a}}$ & $x_{1}{ }^{\mathrm{b}}$ \\
\hline 0.9565 & 0.04345
\end{tabular}

${ }^{\mathrm{a}} x_{2}$ : mole fraction of component 2 in the saturated solution.

${ }^{\mathrm{b}} x_{1}$ : mole fraction solubility of the solute.

\section{Auxiliary Information}

\section{Method/Apparatus/Procedure:}

Constant-temperature bath, calorimetric thermometer, and an ultraviolet/ visible spectrophotometer.

Excess solute and solvent were placed in amber glass bottles and allowed to equilibrate for several days at constant temperature. Attainment of equilibrium was verified by several repetitive measurements and by approaching equilibrium from supersaturation. Aliquots of saturated solutions were transferred through a coarse filter into tared volumetric flasks, weighed, and diluted with methanol. Concentrations were determined by spectrophotometric measurements at $272 \mathrm{~nm}$.

Source and Purity of Chemicals:

(1) $99 \%$, Aldrich Chemical Company, Milwaukee, WI, USA, was used as received.

(2) $99+\%$, Acros Organics, USA, stored over molecular sieves and distilled shortly before use.

Estimated Error:

Temperature: $\pm 0.1 \mathrm{~K}$.

$x_{1}: \pm 1.5 \%$ (relative error).

\begin{tabular}{ll}
\hline \hline Components: & Original Measurements: \\
(1) 2-Acetoxybenzoic acid; & ${ }^{12}$ A. K. Charlton, C. R. Daniels, W. E. \\
$\mathrm{C}_{9} \mathrm{H}_{8} \mathrm{O}_{4} ;$ [ [50-78-2] & Acree, Jr., and M. H. Abraham, J. \\
(2) 2-Methyl-1-butanol; & Solution Chem. 32, 1087 (2003). \\
$\mathrm{C}_{5} \mathrm{H}_{12} \mathrm{O} ;[137-32-6]$ & \\
\hline Variables: & Prepared by: \\
$T / \mathrm{K}=298.15$ & W. E. Acree, Jr. \\
\hline
\end{tabular}

Experimental Values

\begin{tabular}{lc}
\hline \hline$x_{2}^{\mathrm{a}}$ & $x_{1}^{\mathrm{b}}$ \\
\hline 0.9656 & 0.03444 \\
\hline${ }^{\mathrm{a}} x_{2}:$ mole fraction of component 2 in the saturated solution. \\
${ }^{\mathrm{b}}{ }_{x_{1}: \text { mole fraction solubility of the solute. }}$
\end{tabular}

\section{Auxiliary Information}

Method/Apparatus/Procedure:

Constant-temperature bath, calorimetric thermometer, and an ultraviolet/ visible spectrophotometer.

Excess solute and solvent were placed in amber glass bottles and allowed to equilibrate for several days at constant temperature. Attainment of equilibrium was verified by several repetitive measurements and by approaching equilibrium from supersaturation. Aliquots of saturated solutions were transferred through a coarse filter into tared volumetric flasks, weighed, and diluted with methanol. Concentrations were determined by spectrophotometric measurements at $272 \mathrm{~nm}$.

\section{Source and Purity of Chemicals:}

(1) $99 \%$, Aldrich Chemical Company, Milwaukee, WI, USA, was used as received.

(2) $99+\%$, Aldrich Chemical Company, stored over molecular sieves and distilled shortly before use.

Estimated Error:

Temperature: $\pm 0.1 \mathrm{~K}$.

$x_{1}: \pm 1.5 \%$ (relative error).

\begin{tabular}{ll}
\hline \hline Components: & Original Measurements: \\
$\begin{array}{l}\text { (1) 2-Acetoxybenzoic acid; } \\
\mathrm{C}_{9} \mathrm{H}_{8} \mathrm{O}_{4} ;[50-78-2]\end{array}$ & ${ }^{12}$ A. K. Charlton, C. R. Daniels, W. E. \\
(2) 3-Methyl-1-butanol; & Acree, Jr., and M. H. Abraham, J. \\
$\mathrm{C}_{5} \mathrm{H}_{12} \mathrm{O} ;[123-51-3]$ & Solution Chem. 32, 1087 (2003). \\
\hline Variables: & \\
$T / \mathrm{K}=298.15$ & Prepared by: \\
\hline
\end{tabular}

Experimental Values

\begin{tabular}{lc}
\hline \hline$x_{2}{ }^{\mathrm{a}}$ & $x_{1}{ }^{\mathrm{b}}$ \\
\hline 0.9619 & 0.03812 \\
\hline${ }_{{ }^{\mathrm{a}} x_{2}: \text { mole fraction of component } 2 \text { in the saturated solution. }}$ & \\
${ }^{\mathrm{b}} x_{1}:$ mole fraction solubility of the solute. &
\end{tabular}

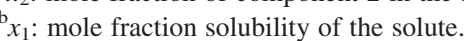

\section{Method/Apparatus/Procedure:}

Constant-temperature bath, calorimetric thermometer, and an ultraviolet/ visible spectrophotometer.

Excess solute and solvent were placed in amber glass bottles and allowed to equilibrate for several days at constant temperature. Attainment of equilibrium was verified by several repetitive measurements and by approaching equilibrium from supersaturation. Aliquots of saturated solutions were transferred through a coarse filter into tared volumetric flasks, weighed, and diluted with methanol. Concentrations were determined by spectrophotometric measurements at $272 \mathrm{~nm}$.

Source and Purity of Chemicals:

(1) $99 \%$, Aldrich Chemical Company, Milwaukee, WI, USA, was used as received.

(2) $99+\%$, anhydrous, Aldrich Chemical Company, stored over molecular sieves and distilled shortly before use.

Estimated Error:

Temperature: $\pm 0.1 \mathrm{~K}$.

$x_{1}: \pm 1.5 \%$ (relative error). 


\begin{tabular}{ll}
\hline \hline Components: & Original Measurements: \\
$(1)$ 2-Acetoxybenzoic acid; & ${ }^{12}$ A. K. Charlton, C. R. Daniels, W. E. \\
$\mathrm{C}_{9} \mathrm{H}_{8} \mathrm{O}_{4} ;$ [50-78-2] & Acree, Jr., and M. H. Abraham, J. \\
$(2)$ 2-Methyl-2-butanol; & Solution Chem. 32, 1087 (2003). \\
$\mathrm{C}_{5} \mathrm{H}_{12} \mathrm{O} ;[75-85-4]$ & \\
\hline Variables: & Prepared by: \\
$T / \mathrm{K}=298.15$ & W. E. Acree, Jr. \\
\hline
\end{tabular}

Experimental Values

\begin{tabular}{lc}
\hline \hline$x_{2}{ }^{\mathrm{a}}$ & $x_{1}{ }^{\mathrm{b}}$ \\
\hline 0.9182 & 0.08176
\end{tabular}

${ }^{\mathrm{a}} x_{2}$ : mole fraction of component 2 in the saturated solution.

${ }^{\mathrm{b}} x_{1}$ : mole fraction solubility of the solute.

\section{Auxiliary Information}

\section{Method/Apparatus/Procedure:}

Constant-temperature bath, calorimetric thermometer, and an ultraviolet/ visible spectrophotometer.

Excess solute and solvent were placed in amber glass bottles and allowed to equilibrate for several days at constant temperature. Attainment of equilibrium was verified by several repetitive measurements and by approaching equilibrium from supersaturation. Aliquots of saturated solutions were transferred through a coarse filter into tared volumetric flasks, weighed, and diluted with methanol. Concentrations were determined by spectrophotometric measurements at $272 \mathrm{~nm}$.

\section{Source and Purity of Chemicals:}

(1) $99 \%$, Aldrich Chemical Company, Milwaukee, WI, USA, was used as received.

(2) $99+\%$, Acros Organics, USA, stored over molecular sieves and distilled shortly before use.

Estimated Error:

Temperature: $\pm 0.1 \mathrm{~K}$.

$x_{1}: \pm 2.0 \%$ (relative error).

\begin{tabular}{ll}
\hline \hline Components: & Original Measurements: \\
(1) 2-Acetoxybenzoic acid; & ${ }^{12}$ A. K. Charlton, C. R. Daniels, W. E. \\
$\mathrm{C}_{9} \mathrm{H}_{8} \mathrm{O}_{4} ;[50-78-2]$ & Acree, Jr., and M. H. Abraham, J. \\
$(2) 1-\mathrm{Hexanol} ; \mathrm{C}_{6} \mathrm{H}_{14} \mathrm{O} ;$ & Solution Chem. 32, 1087 (2003). \\
{$[111-27-3]$} & \\
\hline Variables: & Prepared by: \\
$T / \mathrm{K}=298.15$ & W. E. Acree, Jr. \\
\hline
\end{tabular}

Experimental Values

\begin{tabular}{lc}
\hline \hline$x_{2}^{\mathrm{a}}$ & $x_{1}{ }^{\mathrm{b}}$ \\
\hline 0.9603 & 0.03973 \\
\hline${ }^{\mathrm{a}} x_{2}:$ mole fraction of component 2 in the saturated solution. \\
${ }^{\mathrm{b}} x_{1}:$ mole fraction solubility of the solute.
\end{tabular}

\section{Auxiliary Information}

\section{Method/Apparatus/Procedure:}

Constant-temperature bath, calorimetric thermometer, and an ultraviolet/ visible spectrophotometer.

Excess solute and solvent were placed in amber glass bottles and allowed to equilibrate for several days at constant temperature. Attainment of equilibrium was verified by several repetitive measurements and by approaching equilibrium from supersaturation. Aliquots of saturated solutions were transferred through a coarse filter into tared volumetric flasks, weighed, and diluted with methanol. Concentrations were determined by spectrophotometric measurements at $272 \mathrm{~nm}$.

\section{Source and Purity of Chemicals:}

(1) $99 \%$, Aldrich Chemical Company, Milwaukee, WI, USA, was used as received.

(2) $99+\%$, Alfa Aesar, USA, stored over molecular sieves and distilled shortly before use.

Estimated Error:

Temperature: $\pm 0.1 \mathrm{~K}$.

$x_{1}: \pm 1.5 \%$ (relative error).

\begin{tabular}{ll}
\hline \hline Components: & Original Measurements: \\
$\begin{array}{l}\text { (1) 2-Acetoxybenzoic acid; } \\
\mathrm{C}_{9} \mathrm{H}_{8} \mathrm{O}_{4} ;[50-78-2]\end{array}$ & ${ }^{53} \mathrm{G}$. L. Perlovich and A. Bauer-Brandl, \\
$\begin{array}{l}\text { (2) } 1 \text { - } \mathrm{Hexanol} ; \mathrm{C}_{6} \mathrm{H}_{14} \mathrm{O} ; \\
{[111-27-3]}\end{array}$ & \\
\hline Variables: & \\
$T / \mathrm{K}=298.15$ & Prepared by: \\
\hline
\end{tabular}

\section{Experimental Values}

\begin{tabular}{lc}
\hline \hline$x_{2}{ }^{\mathrm{a}}$ & $x_{1}{ }^{\mathrm{b}}$ \\
\hline 0.9607 & 0.0393
\end{tabular}

${ }^{a} x_{2}$ : mole fraction of component 2 in the saturated solution.

${ }^{\mathrm{b}} x_{1}$ : mole fraction solubility of the solute.

\section{Auxiliary Information}

\section{Method/Apparatus/Procedure:}

Thermostated constant-temperature bath, centrifuge, and an ultraviolet/visible spectrophotometer.

Excess solute and solvent were placed in a glass ampoule and allowed to equilibrate in a thermostated temperature bath with mixing (at a speed of 25 rpm) for a minimum of four days. After suitable equilibration the saturated solution was centrifuged, the supernatant liquid collected, quantitatively diluted, and the absorbance recorded using an ultraviolet/visible spectrophotometer. The solubility of the solute was calculated from the measured absorbance.

\section{Source and Purity of Chemicals:}

(1) Analytical Reagent grade, Norsk Medisinaldepot, Oslo, Norway, no purification details were provided.

(2) Analytical Reagent grade, Merck Chemicals, Germany, no purification details were provided.

\section{Estimated Error:}

Temperature: $\pm 0.01 \mathrm{~K}$.

$x_{1}: \pm 2.5 \%$ (relative error) 


\begin{tabular}{ll}
\hline \hline Components: & Original Measurements: \\
$(1)$ 2-Acetoxybenzoic acid; & ${ }^{12}$ A. K. Charlton, C. R. Daniels, W. E. \\
$\mathrm{C}_{9} \mathrm{H}_{8} \mathrm{O}_{4} ;[50-78-2]$ & Acree, Jr., and M. H. Abraham, J. \\
(2) 2-Methyl-1-pentanol; & Solution Chem. 32, 1087 (2003). \\
$\mathrm{C}_{6} \mathrm{H}_{14} \mathrm{O} ;[105-30-6]$ & \\
\hline Variables: & Prepared by: \\
$T / \mathrm{K}=298.15$ & W. E. Acree, Jr. \\
\hline
\end{tabular}

Experimental Values

\begin{tabular}{lc}
\hline \hline$x_{2}{ }^{\mathrm{a}}$ & $x_{1}{ }^{\mathrm{b}}$ \\
\hline 0.9679 & 0.03205
\end{tabular}

${ }^{\mathrm{a}} x_{2}$ : mole fraction of component 2 in the saturated solution.

${ }^{\mathrm{b}} x_{1}$ : mole fraction solubility of the solute.

\section{Auxiliary Information}

\section{Method/Apparatus/Procedure:}

Constant-temperature bath, calorimetric thermometer, and an ultraviolet/ visible spectrophotometer.

Excess solute and solvent were placed in amber glass bottles and allowed to equilibrate for several days at constant temperature. Attainment of equilibrium was verified by several repetitive measurements and by approaching equilibrium from supersaturation. Aliquots of saturated solutions were transferred through a coarse filter into tared volumetric flasks, weighed, and diluted with methanol. Concentrations were determined by spectrophotometric measurements at $272 \mathrm{~nm}$.

\section{Source and Purity of Chemicals:}

(1) $99 \%$, Aldrich Chemical Company, Milwaukee, WI, USA, was used as received.

(2) $99 \%$, Aldrich Chemical Company, stored over molecular sieves and distilled shortly before use.

\section{Estimated Error:}

Temperature: $\pm 0.1 \mathrm{~K}$.

$x_{1}: \pm 1.5 \%$ (relative error).

\begin{tabular}{ll}
\hline \hline Components: & Original Measurements: \\
(1) 2-Acetoxybenzoic acid; & ${ }^{12}$ A. K. Charlton, C. R. Daniels, W. E. \\
$\mathrm{C}_{9} \mathrm{H}_{8} \mathrm{O}_{4} ;$; [50-78-2] & Acree, Jr., and M. H. Abraham, J. \\
(2) 4-Methyl-2-pentanol; & Solution Chem. 32, 1087 (2003). \\
$\mathrm{C}_{6} \mathrm{H}_{14} \mathrm{O} ;[108-11-2]$ & \\
\hline Variables: & Prepared by: \\
$T / \mathrm{K}=298.15$ & W. E. Acree, Jr. \\
\hline
\end{tabular}

Experimental Values

\begin{tabular}{lc}
\hline \hline$x_{2}^{\mathrm{a}}$ & $x_{1}{ }^{\mathrm{b}}$ \\
\hline 0.9607 & 0.03931 \\
\hline${ }^{\mathrm{a}} x_{2}:$ mole fraction of component 2 in the saturated solution. \\
${ }^{\mathrm{b}} x_{1}:$ mole fraction solubility of the solute.
\end{tabular}

\section{Auxiliary Information}

\section{Method/Apparatus/Procedure:}

Constant-temperature bath, calorimetric thermometer, and an ultraviolet/ visible spectrophotometer.

Excess solute and solvent were placed in amber glass bottles and allowed to equilibrate for several days at constant temperature. Attainment of equilibrium was verified by several repetitive measurements and by approaching equilibrium from supersaturation. Aliquots of saturated solutions were transferred through a coarse filter into tared volumetric flasks, weighed, and diluted with methanol. Concentrations were determined by spectrophotometric measurements at $272 \mathrm{~nm}$.

\section{Source and Purity of Chemicals:}

(1) $99 \%$, Aldrich Chemical Company, Milwaukee, WI, USA, was used as received.

(2) $99+\%$, Acros Organics, USA, stored over molecular sieves and distilled shortly before use.

Estimated Error:

Temperature: $\pm 0.1 \mathrm{~K}$.

$x_{1}: \pm 1.5 \%$ (relative error).

\begin{tabular}{|c|c|}
\hline $\begin{array}{l}\text { Components: } \\
\text { (1) 2-Acetoxybenzoic acid; } \\
\mathrm{C}_{9} \mathrm{H}_{8} \mathrm{O}_{4} ;[50-78-2] \\
\text { (2) } 1 \text {-Heptanol; } \mathrm{C}_{7} \mathrm{H}_{16} \mathrm{O} \text {; } \\
\text { [111-70-6] }\end{array}$ & $\begin{array}{l}\text { Original Measurements: } \\
{ }^{12} \text { A. K. Charlton, C. R. Daniels, W. E } \\
\text { Acree, Jr., and M. H. Abraham, J. } \\
\text { Solution Chem. 32, } 1087 \text { (2003). }\end{array}$ \\
\hline $\begin{array}{l}\text { Variables: } \\
T / \mathrm{K}=298.15\end{array}$ & $\begin{array}{l}\text { Prepared by: } \\
\text { W. E. Acree, Jr. }\end{array}$ \\
\hline
\end{tabular}

Experimental Values

\begin{tabular}{lc}
\hline \hline$x_{2}{ }^{\mathrm{a}}$ & $x_{1}{ }^{\mathrm{b}}$ \\
\hline 0.9611 & 0.03892 \\
\hline${ }^{\mathrm{a}} x_{2}:$ mole fraction of component 2 in the saturated solution. & \\
${ }^{\mathrm{b}} x_{1}:$ mole fraction solubility of the solute. &
\end{tabular}

${ }^{\mathrm{b}} x_{1}$ : mole fraction solubility of the solute.

\section{Auxiliary Information}

\section{Method/Apparatus/Procedure:}

Constant-temperature bath, calorimetric thermometer, and an ultraviolet/ visible spectrophotometer.

Excess solute and solvent were placed in amber glass bottles and allowed to equilibrate for several days at constant temperature. Attainment of equilibrium was verified by several repetitive measurements and by approaching equilibrium from supersaturation. Aliquots of saturated solutions were transferred through a coarse filter into tared volumetric flasks, weighed, and diluted with methanol. Concentrations were determined by spectrophotometric measurements at $272 \mathrm{~nm}$.

Source and Purity of Chemicals:

(1) $99 \%$, Aldrich Chemical Company, Milwaukee, WI, USA, was used as received.

(2) $99+\%$, Alfa Aesar, USA, stored over molecular sieves and distilled shortly before use.

\section{Estimated Error:}

Temperature: $\pm 0.1 \mathrm{~K}$.

$x_{1}: \pm 1.5 \%$ (relative error). 


\begin{tabular}{ll}
\hline \hline Components: & Original Measurements: \\
(1) 2-Acetoxybenzoic acid; & ${ }^{53}$ G. L. Perlovich and A. Bauer-Brandl, \\
$\mathrm{C}_{9} \mathrm{H}_{8} \mathrm{O}_{4} ;[50-78-2]$ & Pharm. Res. 20, 471 (2003). \\
$\begin{array}{l}\text { (2) } 1-\mathrm{Heptanol} ; \mathrm{C}_{7} \mathrm{H}_{16} \mathrm{O} ; \\
{[111-37-3]}\end{array}$ & \\
\hline Variables: & Prepared by: \\
$T / \mathrm{K}=298.15$ & W. E. Acree, Jr. \\
\hline
\end{tabular}

\section{Experimental Values}

\begin{tabular}{lc}
\hline \hline$x_{2}{ }^{\mathrm{a}}$ & $x_{1}{ }^{\mathrm{b}}$ \\
\hline 0.9614 & 0.0386 \\
\hline${ }^{\mathrm{a}} x_{2}:$ mole fraction of component 2 in the saturated solution. & \\
${ }^{\mathrm{b}} x_{1}:$ mole fraction solubility of the solute. &
\end{tabular}

\section{Auxiliary Information}

\section{Method/Apparatus/Procedure:}

Thermostated constant-temperature bath, centrifuge, and an ultraviolet/visible spectrophotometer.

Excess solute and solvent were placed in a glass ampoule and allowed to equilibrate in a thermostated temperature bath with mixing (at a speed of $25 \mathrm{rpm}$ ) for a minimum of four days. After suitable equilibration the saturated solution was centrifuged, the supernatant liquid collected, quantitatively diluted, and the absorbance recorded using an ultraviolet/visible spectrophotometer. The solubility of the solute was calculated from the measured absorbance.

\section{Source and Purity of Chemicals:}

(1) Analytical Reagent grade, Norsk Medisinaldepot, Oslo, Norway, no purification details were provided.

(2) Analytical Reagent grade, Sigma Chemical Company, USA, no

purification details were provided.

Estimated Error:

Temperature: $\pm 0.01 \mathrm{~K}$.

$x_{1}: \pm 2.5 \%$ (relative error).

\begin{tabular}{|c|c|}
\hline $\begin{array}{l}\text { Components: } \\
\text { (1) 2-Acetoxybenzoic acid; } \\
\mathrm{C}_{9} \mathrm{H}_{8} \mathrm{O}_{4} ;[50-78-2] \\
\text { (2) } 1-\text { Octanol; } \mathrm{C}_{8} \mathrm{H}_{18} \mathrm{O} \text {; } \\
\text { [111-87-5] }\end{array}$ & $\begin{array}{l}\text { Original Measurements: } \\
{ }^{12} \text { A. K. Charlton, C. R. Daniels, W. E. } \\
\text { Acree, Jr., and M. H. Abraham, J. } \\
\text { Solution Chem. 32, } 1087 \text { (2003). }\end{array}$ \\
\hline $\begin{array}{l}\text { Variables: } \\
T / \mathrm{K}=298.15\end{array}$ & $\begin{array}{l}\text { Prepared by: } \\
\text { W. E. Acree, Jr. }\end{array}$ \\
\hline
\end{tabular}

Experimental Values

\begin{tabular}{lc}
\hline \hline$x_{2}^{\mathrm{a}}$ & $x_{1}{ }^{\mathrm{b}}$ \\
\hline 0.9642 & 0.03581
\end{tabular}

${ }^{a} x_{2}$ : mole fraction of component 2 in the saturated solution.

${ }^{\mathrm{b}} x_{1}$ : mole fraction solubility of the solute.

\section{Auxiliary Information}

\section{Method/Apparatus/Procedure:}

Constant-temperature bath, calorimetric thermometer, and an ultraviolet/ visible spectrophotometer.

Excess solute and solvent were placed in amber glass bottles and allowed to equilibrate for several days at constant temperature. Attainment of equilibrium was verified by several repetitive measurements and by approaching equilibrium from supersaturation. Aliquots of saturated solutions were transferred through a coarse filter into tared volumetric flasks, weighed, and diluted with methanol. Concentrations were determined by spectrophotometric measurements at $272 \mathrm{~nm}$.

\section{Source and Purity of Chemicals:}

(1) $99 \%$, Aldrich Chemical Company, Milwaukee, WI, USA, was used as received.

(2) $99+\%$, anhydrous, Aldrich Chemical Company, stored over molecular sieves and distilled shortly before use.

Estimated Error:

Temperature: $\pm 0.1 \mathrm{~K}$.

$x_{1}: \pm 1.5 \%$ (relative error)

\begin{tabular}{|c|c|}
\hline $\begin{array}{l}\text { Components: } \\
\text { (1) 2-Acetoxybenzoic acid; } \\
\mathrm{C}_{9} \mathrm{H}_{8} \mathrm{O}_{4} ;[50-78-2] \\
\text { (2) } 1-\text { Octanol; } \mathrm{C}_{8} \mathrm{H}_{18} \mathrm{O} \text {; } \\
\text { [111-87-5] }\end{array}$ & $\begin{array}{l}\text { Original Measurements: } \\
{ }^{53} \text { G. L. Perlovich and A. Bauer-Brandl } \\
\text { Pharm. Res. 20, } 471 \text { (2003). }\end{array}$ \\
\hline $\begin{array}{l}\text { Variables: } \\
T / \mathrm{K}=298.15\end{array}$ & $\begin{array}{l}\text { Prepared by: } \\
\text { W. E. Acree, Jr. }\end{array}$ \\
\hline
\end{tabular}

Experimental Values

\begin{tabular}{lc}
\hline \hline$x_{2}{ }^{\mathrm{a}}$ & $x_{1}{ }^{\mathrm{b}}$ \\
\hline 0.9659 & 0.0341 \\
\hline${ }^{\mathrm{a}} x_{2}:$ mole fraction of component 2 in the saturated solution. & \\
${ }^{\mathrm{b}} x_{1}:$ mole fraction solubility of the solute. &
\end{tabular}

${ }^{b} x_{1}$ : mole fraction solubility of the solute.

\section{Auxiliary Information}

\section{Method/Apparatus/Procedure:}

Thermostated constant-temperature bath, centrifuge, and an ultraviolet/visible spectrophotometer.

Excess solute and solvent were placed in a glass ampoule and allowed to equilibrate in a thermostated temperature bath with mixing (at a speed of $25 \mathrm{rpm}$ ) for a minimum of four days. After suitable equilibration the saturated solution was centrifuged, the supernatant liquid collected, quantitatively diluted, and the absorbance recorded using an ultraviolet/visible spectrophotometer. The solubility of the solute was calculated from the measured absorbance.

\section{Source and Purity of Chemicals:}

(1) Analytical Reagent grade, Norsk Medisinaldepot, Oslo, Norway, no purification details were provided.

(2) Analytical Reagent grade, Sigma Chemical Company, USA, no purification details were provided.

\section{Estimated Error:}

Temperature: $\pm 0.01 \mathrm{~K}$.

$x_{1}: \pm 2.5 \%$ (relative error) 


\section{Components:}

(1) 2-Acetoxybenzoic acid;

$\mathrm{C}_{9} \mathrm{H}_{8} \mathrm{O}_{4} ;[50-78-2]$

(2) $1-$ Octanol; $\mathrm{C}_{8} \mathrm{H}_{18} \mathrm{O}$;

[111-87-5]

\begin{tabular}{ll}
\hline Variables: & Prepared by: \\
$T / \mathrm{K}=310$ & W. E. Acree, Jr. \\
\hline
\end{tabular}

\section{Experimental Values}

The measured solubility was reported to be $42.6 \mathrm{~g} / \mathrm{l}$ of saturated solution, which corresponds to a solubility of $c_{1}=0.24 \mathrm{~mol} \mathrm{dm}^{-3}$.

\section{Auxiliary Information}

\section{Method/Apparatus/Procedure:}

Very little experimental details were provided. Saturated solution was prepared at $310 \mathrm{~K}$, and the concentration of the dissolved solute determined spectrophotometrically by reference to an absorbance curve obtained from absorbance measurements of standard solutions of known concentration.

\section{Source and Purity of Chemicals:}

(1) $98 \%$ to $100 \%$, Eli Lilly Drug Company, USA, used as received. The authors studied the solubility of several drugs, and their only statement regarding purity was that all drugs were used as supplied at a purity of $90 \%-$ $100 \%$.

(2) Purity not given, Chemical source not given, no purification details were provided.

Estimated Error:

Temperature: No information was given. $c_{1}: \pm 5 \%$ (relative error, estimated by compiler).

\begin{tabular}{ll}
\hline \hline Components: & Original Measurements: \\
(1) 2-Acetoxybenzoic acid; & ${ }^{12}$ A. K. Charlton, C. R. Daniels, W. E. \\
$\mathrm{C}_{9} \mathrm{H}_{8} \mathrm{O}_{4}$; [50-78-2] & Acree, Jr., and M. H. Abraham, J. \\
$\begin{array}{l}\text { (2) 2-Ethyl-1-hexanol; } \mathrm{C}_{8} \mathrm{H}_{18} \mathrm{O} ; \\
{[104-76-7]}\end{array}$ & Solution Chem. 32, 1087 (2003). \\
\hline Variables: & Prepared by: \\
$T / \mathrm{K}=298.15$ & W. E. Acree, Jr. \\
\hline
\end{tabular}

\section{Experimental Values}

\begin{tabular}{lc}
\hline \hline$x_{2}{ }^{\mathrm{a}}$ & $x_{1}{ }^{\mathrm{b}}$ \\
\hline 0.9697 & 0.03033
\end{tabular}

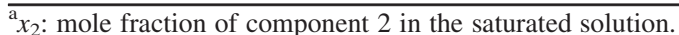

${ }^{b} x_{1}$ : mole fraction solubility of the solute.

\section{Auxiliary Information}

\section{Method/Apparatus/Procedure:}

Constant-temperature bath, calorimetric thermometer, and an ultraviolet/ visible spectrophotometer.

Excess solute and solvent were placed in amber glass bottles and allowed to equilibrate for several days at constant temperature. Attainment of equilibrium was verified by several repetitive measurements and by approaching equilibrium from supersaturation. Aliquots of saturated solutions were transferred through a coarse filter into tared volumetric flasks, weighed, and diluted with methanol. Concentrations were determined by

spectrophotometric measurements at $272 \mathrm{~nm}$.
Source and Purity of Chemicals:

(1) $99 \%$, Aldrich Chemical Company, Milwaukee, WI, USA, was used as received.

(2) $99+\%$, anhydrous, Aldrich Chemical Company, stored over molecular sieves and distilled shortly before use.

\section{Estimated Error:}

Temperature: $\pm 0.1 \mathrm{~K}$.

$x_{1}: \pm 1.5 \%$ (relative error)

\begin{tabular}{ll}
\hline \hline Components: & Original Measurements: \\
(1) 2-Acetoxybenzoic acid; & ${ }^{12}$ A. K. Charlton, C. R. Daniels, W. E. \\
$\mathrm{C}_{9} \mathrm{H}_{8} \mathrm{O}_{4} ;[50-78-2]$ & Acree, Jr., and M. H. Abraham, J. \\
$\begin{array}{l}\text { (2) 1-Decanol; } \mathrm{C}_{10} \mathrm{H}_{22} \mathrm{O} ; \\
{[112-30-1]}\end{array}$ & Solution Chem. 32, 1087 (2003). \\
\hline Variables: & \\
$T / \mathrm{K}=298.15$ & Prepared by: \\
\hline
\end{tabular}

Experimental Values

\begin{tabular}{lc}
\hline \hline$x_{2}{ }^{\mathrm{a}}$ & $x_{1}{ }^{\mathrm{b}}$ \\
\hline 0.9635 & 0.03652
\end{tabular}

${ }^{\mathrm{a}} x_{2}$ : mole fraction of component 2 in the saturated solution.

${ }^{\mathrm{b}} x_{1}$ : mole fraction solubility of the solute.

\section{Auxiliary Information}

\section{Method/Apparatus/Procedure:}

Constant-temperature bath, calorimetric thermometer, and an ultraviolet/ visible spectrophotometer.

Excess solute and solvent were placed in amber glass bottles and allowed to equilibrate for several days at constant temperature. Attainment of equilibrium was verified by several repetitive measurements and by approaching equilibrium from supersaturation. Aliquots of saturated solutions were transferred through a coarse filter into tared volumetric flasks, weighed, and diluted with methanol. Concentrations were determined by

spectrophotometric measurements at $272 \mathrm{~nm}$.

\section{Source and Purity of Chemicals:}

(1) $99 \%$, Aldrich Chemical Company, Milwaukee, WI, USA, was used as received.

(2) $99+\%$, Alfa Aesar, USA, stored over molecular sieves and distilled shortly before use.

Estimated Error:

Temperature: $\pm 0.1 \mathrm{~K}$.

$x_{1}: \pm 1.5 \%$ (relative error)

\begin{tabular}{ll}
\hline \hline Components: & Original Measurements: \\
$\begin{array}{l}\text { (1) 2-Acetoxybenzoic acid; } \\
\mathrm{C}_{9} \mathrm{H}_{8} \mathrm{O}_{4} ;[50-78-2]\end{array}$ & ${ }^{12}$ A. K. Charlton, C. R. Daniels, W. E. \\
$(2)$ Acree, Jr., and M. H. Abraham, J. \\
$\mathrm{C}_{10} \mathrm{H}_{22} \mathrm{O}$; [106-21-8] & Solution Chem. 32, 1087 (2003). \\
\hline Variables: & \\
$T / \mathrm{K}=298.15$ & Prepared by: \\
\hline
\end{tabular}


Experimental Values

\begin{tabular}{lc}
\hline \hline$x_{2}{ }^{\mathrm{a}}$ & $x_{1}{ }^{\mathrm{b}}$ \\
\hline 0.9696 & 0.03039 \\
${ }^{\mathrm{a}} x_{2}:$ mole fraction of component 2 in the saturated solution. & \\
${ }^{\mathrm{b}} x_{1}:$ mole fraction solubility of the solute. &
\end{tabular}

\section{Auxiliary Information}

\section{Method/Apparatus/Procedure:}

Constant-temperature bath, calorimetric thermometer, and an ultraviolet/ visible spectrophotometer.

Excess solute and solvent were placed in amber glass bottles and allowed to equilibrate for several days at constant temperature. Attainment of equilibrium was verified by several repetitive measurements and by approaching equilibrium from supersaturation. Aliquots of saturated solutions were transferred through a coarse filter into tared volumetric flasks, weighed, and diluted with methanol. Concentrations were determined by spectrophotometric measurements at $272 \mathrm{~nm}$.

Source and Purity of Chemicals:

(1) $99 \%$, Aldrich Chemical Company, Milwaukee, WI, USA, was used as received.

(2) $99 \%$, Aldrich Chemical Company, stored over molecular sieves and distilled shortly before use.

\section{Estimated Error:}

Temperature: $\pm 0.1 \mathrm{~K}$

$x_{1}: \pm 1.5 \%$ (relative error).

\section{Components:}

(1) 2-Acetoxybenzoic acid; $\mathrm{C}_{9} \mathrm{H}_{8} \mathrm{O}_{4} ;[50-78-2]$

(2) 1,2-Propanediol; $\mathrm{C}_{3} \mathrm{H}_{8} \mathrm{O}_{2}$; [57-55-6]

\begin{tabular}{ll}
\hline Variables: & Prepared by: \\
Temperature & W. E. Acree, Jr. \\
\hline
\end{tabular}

Experimental Values

\begin{tabular}{lcc}
\hline \hline$T / \mathrm{K}$ & $x_{2}{ }^{\mathrm{a}}$ & $x_{1}{ }^{\mathrm{b}}$ \\
\hline 295.7 & 0.983 & 0.017 \\
301.1 & 0.975 & 0.025 \\
305.1 & 0.967 & 0.033 \\
308.2 & 0.959 & 0.041 \\
313.6 & 0.944 & 0.056 \\
318.3 & 0.929 & 0.071 \\
323.7 & 0.908 & 0.092 \\
327.9 & 0.888 & 0.112 \\
330.6 & 0.874 & 0.126 \\
333.9 & 0.855 & 0.145
\end{tabular}

${ }^{\mathrm{a}} x_{2}$ : mole fraction of component 2 in the saturated solution.

${ }^{\mathrm{b}} x_{1}$ : mole fraction solubility of the solute.

\section{Auxiliary Information}

\section{Method/Apparatus/Procedure:}

Circulating thermostated water bath, analytical balance, and drying oven. Known amounts of solute and solvent were placed in the equilibrium vessel which was connected to the thermostated water bath. The vessel was agitated at $750 \mathrm{rpm}$ as temperature was increased and decreased. By regulating the bath temperature, the authors were able to determine the temperature at which complete dissolution occurred (heating mode) and the temperature at which the first crystals formed (cooling mode).

Source and Purity of Chemicals:

(1) $99.5 \%$, Anidrol Quimica, Diadema, Brazil, no purification details were given.

(2) $99.9 \%$, Synthon Chemicals, no purification details were given.

\section{Estimated Error:}

Temperature: $\pm 0.2 \mathrm{~K}$ (estimated by compiler).

$x_{1}: \pm 3 \%$ (relative error, estimated by compiler).

\subsection{2-Acetoxybenzoic acid solubility data in ketones}

\begin{tabular}{|c|c|}
\hline $\begin{array}{l}\text { Components: } \\
\text { (1) 2-Acetoxybenzoic acid; } \\
\mathrm{C}_{9} \mathrm{H}_{8} \mathrm{O}_{4} ;[50-78-2] \\
\text { (2) Propanone; } \mathrm{C}_{3} \mathrm{H}_{6} \mathrm{O} \text {; } \\
\text { [67-64-1] }\end{array}$ & $\begin{array}{l}\text { Original Measurements: } \\
{ }^{53} \text { G. L. Perlovich and A. Bauer-Brandl, } \\
\text { Pharm. Res. 20, } 471 \text { (2003). }\end{array}$ \\
\hline $\begin{array}{l}\text { Variables: } \\
T / \mathrm{K}=298.15\end{array}$ & $\begin{array}{l}\text { Prepared by: } \\
\text { W. E. Acree, Jr. }\end{array}$ \\
\hline
\end{tabular}

Experimental Values

\begin{tabular}{lc}
\hline \hline$x_{2}{ }^{\mathrm{a}}$ & $x_{1}{ }^{\mathrm{b}}$ \\
\hline 0.9172 & 0.0828
\end{tabular}

${ }^{a} x_{2}$ : mole fraction of component 2 in the saturated solution.

${ }^{\mathrm{b}} x_{1}$ : mole fraction solubility of the solute.

\section{Auxiliary Information}

Method/Apparatus/Procedure:

Thermostated constant-temperature bath, centrifuge, and an ultraviolet/visible spectrophotometer.

Excess solute and solvent were placed in a glass ampoule and allowed to equilibrate in a thermostated temperature bath with mixing (at a speed of $25 \mathrm{rpm}$ ) for a minimum of four days. After suitable equilibration the saturated solution was centrifuged, the supernatant liquid collected, quantitatively diluted, and the absorbance recorded using an ultraviolet/visible spectrophotometer. The solubility of the solute was calculated from the measured absorbance.

Source and Purity of Chemicals:

(1) Analytical Reagent grade, Norsk Medisinaldepot, Oslo, Norway, no purification details were provided.

(2) Analytical Reagent grade, SDS, Peypin, France, no purification details were provided.

Estimated Error:

Temperature: $\pm 0.01 \mathrm{~K}$.

$x_{1}: \pm 2.5 \%$ (relative error) 


\begin{tabular}{|c|c|}
\hline $\begin{array}{l}\text { Components: } \\
\text { (1) 2-Acetoxybenzoic acid; } \\
\mathrm{C}_{9} \mathrm{H}_{8} \mathrm{O}_{4} ;[50-78-2] \\
\text { (2) Propanone; } \mathrm{C}_{3} \mathrm{H}_{6} \mathrm{O} \text {; } \\
\text { [67-64-1] }\end{array}$ & $\begin{array}{l}\text { Original Measurements: } \\
{ }^{69} \text { C. M. McLoughlin, W. A. M. } \\
\text { McMinn, and T. R. A. Magee, Powder } \\
\text { Technol. 134, } 40 \text { (2003). }\end{array}$ \\
\hline $\begin{array}{l}\text { Variables: } \\
T / \mathrm{K}=293\end{array}$ & $\begin{array}{l}\text { Prepared by: } \\
\text { W. E. Acree, Jr. }\end{array}$ \\
\hline
\end{tabular}

\section{Experimental Values}

The measured solubility was reported to be $250 \mathrm{~g} / \mathrm{l}$. The authors did not specify whether the value was per liter of solvent or per liter of saturated solution.

\section{Auxiliary Information}

\section{Method/Apparatus/Procedure:}

Very little experimental details were provided. Solubility was determined by adding $(0.50 \pm 0.1) \mathrm{g}$ amounts of the powdered solute to $(100 \pm 1) \mathrm{ml}$ of solvent until a saturated solution was obtained. The mass of the powder was recorded

Source and Purity of Chemicals:

(1) Purity not given, Sigma-Aldrich, Poole, England, UK, no purification details were provided.

(2) Purity not given, Sigma-Aldrich, no purification details were provided.

\section{Estimated Error:}

Temperature: Insufficient experimental details to estimate.

Solubility: $\pm 5 \mathrm{~g} / \mathrm{l}$ (estimated by compiler assuming solute added in increments of $0.50 \mathrm{~g}$ ).

\section{Auxiliary Information}

Method/Apparatus/Procedure:

Circulating thermostated water bath, analytical balance, and drying oven. Known amounts of solute and solvent were placed in the equilibrium vessel which was connected to the thermostated water bath. The vessel was agitated at $750 \mathrm{rpm}$ as temperature was increased and decreased. By regulating the bath temperature, the authors were able to determine the temperature at which complete dissolution occurred (heating mode) and the temperature at which the first crystals formed (cooling mode).

Source and Purity of Chemicals:

(1) $99.5 \%$, Anidrol Quimica, Diadema, Brazil, no purification details were given.

(2) $99.9 \%$, Synthon Chemicals, no purification details were given.

\section{Estimated Error:}

Temperature: $\pm 0.2 \mathrm{~K}$ (estimated by compiler). $x_{1}: \pm 3 \%$ (relative error, estimated by compiler).

\subsection{2-Acetoxybenzoic acid solubility data in miscellaneous organic solvents}

\begin{tabular}{ll}
\hline \hline Components: & Original Measurements: \\
(1) 2-Acetoxybenzoic acid; & ${ }^{12}$ A. K. Charlton, C. R. Daniels, W. E. \\
$\mathrm{C}_{9} \mathrm{H}_{8} \mathrm{O}_{4} ;[50-78-2]$ & Acree, Jr., and M. H. Abraham, J. \\
$\begin{array}{l}\text { (2) Propylene carbonate; } \\
\mathrm{C}_{4} \mathrm{H}_{6} \mathrm{O}_{3} ;[108-32-7]\end{array}$ & Solution Chem. 32, 1087 (2003). \\
\hline Variables: & \\
$T / \mathrm{K}=298.15$ & Prepared by: \\
\hline
\end{tabular}

\begin{tabular}{ll}
\hline \hline Components: & Original Measurements: \\
$\begin{array}{l}\text { (1) 2-Acetoxybenzoic acid; } \\
\mathrm{C}_{9} \mathrm{H}_{8} \mathrm{O}_{4} ;[50-78-2]\end{array}$ & ${ }^{91}$ G. D. Maia and M. Giulietti, J. Chem. \\
$\begin{array}{l}\text { (2) Propanone; } \mathrm{C}_{3} \mathrm{H}_{6} \mathrm{O} ; \\
{[67-64-1]}\end{array}$ & \\
\hline Variables: & Prepared by: \\
Temperature & W. E. Acree, Jr. \\
\hline
\end{tabular}

Experimental Values

\begin{tabular}{lcc}
\hline \hline$T / \mathrm{K}$ & $x_{2}{ }^{\mathrm{a}}$ & $x_{1}{ }^{\mathrm{b}}$ \\
\hline 281.9 & 0.939 & 0.061 \\
290.6 & 0.925 & 0.075 \\
297.9 & 0.912 & 0.088 \\
304.4 & 0.899 & 0.101 \\
310.6 & 0.886 & 0.114 \\
315.3 & 0.873 & 0.127 \\
319.8 & 0.861 & 0.139 \\
323.3 & 0.849 & 0.151 \\
326.3 & 0.838 & 0.162 \\
\hline
\end{tabular}

${ }^{\mathrm{a}} x_{2}$ : mole fraction of component 2 in the saturated solution.

${ }^{\mathrm{b}} x_{1}$ : mole fraction solubility of the solute.

\section{Experimental Values}

\begin{tabular}{lc}
\hline \hline$x_{2}{ }^{\mathrm{a}}$ & $x_{1}{ }^{\mathrm{b}}$ \\
\hline 0.9687 & 0.03133
\end{tabular}

${ }^{\mathrm{a}} x_{2}$ : mole fraction of component 2 in the saturated solution.

${ }^{\mathrm{b}} x_{1}$ : mole fraction solubility of the solute.

\section{Auxiliary Information}

\section{Method/Apparatus/Procedure:}

Constant-temperature bath, calorimetric thermometer, and an ultraviolet/ visible spectrophotometer.

Excess solute and solvent were placed in amber glass bottles and allowed to equilibrate for several days at constant temperature. Attainment of equilibrium was verified by several repetitive measurements and by approaching equilibrium from supersaturation. Aliquots of saturated solutions were diluted with methanol. Concentrations were determined by spectrophotometric measurements at $272 \mathrm{~nm}$.

\section{Source and Purity of Chemicals:}

(1) $99 \%$, Aldrich Chemical Company, Milwaukee, WI, USA, was used as received.

(2) 99.7\%, anhydrous, Aldrich Chemical Company, stored over molecular sieves and distilled shortly before use.

\section{Estimated Error:}

Temperature: $\pm 0.1 \mathrm{~K}$.

$x_{1}: \pm 1.5 \%$ (relative error) transferred through a coarse filter into tared volumetric flasks, weighed, and 


\begin{tabular}{|c|c|}
\hline $\begin{array}{l}\text { Components: } \\
\text { (1) 2-Acetoxybenzoic acid; } \\
\mathrm{C}_{9} \mathrm{H}_{8} \mathrm{O}_{4} ;[50-78-2] \\
\text { (2) Ethanenitrile; } \mathrm{C}_{2} \mathrm{H}_{3} \mathrm{~N} \text {; } \\
\text { [75-05-8] }\end{array}$ & $\begin{array}{l}\text { Original Measurements: } \\
\text { 53 G. L. Perlovich and A. Bauer-Brandl, } \\
\text { Pharm. Res. 20, } 471 \text { (2003). }\end{array}$ \\
\hline $\begin{array}{l}\text { Variables: } \\
T / \mathrm{K}=298.15\end{array}$ & $\begin{array}{l}\text { Prepared by: } \\
\text { W. E. Acree, Jr. }\end{array}$ \\
\hline
\end{tabular}

Experimental Values

\begin{tabular}{lc}
\hline \hline$x_{2}{ }^{\mathrm{a}}$ & $x_{1}{ }^{\mathrm{b}}$ \\
\hline 0.9815 & 0.0185 \\
\hline${ }^{a} x_{2}:$ mole fraction of component 2 in the saturated solution. & \\
${ }^{\mathrm{b}} x_{1}:$ mole fraction solubility of the solute. &
\end{tabular}

\section{Auxiliary Information}

\section{Method/Apparatus/Procedure:}

Thermostated constant-temperature bath, centrifuge, and an ultraviolet/visible spectrophotometer.

Excess solute and solvent were placed in a glass ampoule and allowed to equilibrate in a thermostated temperature bath with mixing (at a speed of $25 \mathrm{rpm}$ ) for a minimum of four days. After suitable equilibration the saturated solution was centrifuged, the supernatant liquid collected, quantitatively diluted, and the absorbance recorded using an ultraviolet/visible spectrophotometer. The solubility of the solute was calculated from the measured absorbance.

Source and Purity of Chemicals:

(1) Analytical Reagent grade, Norsk Medisinaldepot, Oslo, Norway, no purification details were provided.

(2) HPLC grade, Merck Chemicals, Germany, no purification details were provided.

Estimated Error:

Temperature: $\pm 0.01 \mathrm{~K}$.

$x_{1}: \pm 2.5 \%$ (relative error).

\section{Solubility of 4-Acetoxybenzoic Acid in Organic Solvents}

\subsection{Critical evaluation of experimental solubility data}

There is only a single published study regarding the solubility behavior of 4-acetoxybenzoic acid in organic solvents. Wang and Zhang ${ }^{97}$ measured the mole fraction solubilities in ethanol as a function of temperature using a static and dynamic method. The internal consistency of the two datasets was assessed by curve-fitting the measured mole fraction solubility data to Eq. (8). The values of the equation coefficients $(A, B$, and $C$ ) are given in Table 10, along with the MRD calculated according to Eq. (24). Examination of the numerical entries in the last column of Table 10 reveals that the static experimental method yielded the more consistent experimental values. Readers are reminded that Eq. (8) can only check data points for internal consistency with respect to temperature. The fact that one can accurately describe the experimental data does not
TABLE 10. Parameters of the Modified Apelblat equation for describing the solubility of 4-acetoxybenzoic acid in ethanol ${ }^{\mathrm{a}}$

\begin{tabular}{lrrrc}
\hline \hline Solvent & \multicolumn{1}{c}{$A$} & \multicolumn{1}{c}{$B$} & \multicolumn{1}{c}{$C$} & MRD (\%) \\
\hline Ethanol (dynamic method) & 24.84 & -4110 & -1.781 & 5.599 \\
Ethanol (static method) & -26.18 & -1737 & 5.776 & 0.674 \\
\hline
\end{tabular}

${ }^{\mathrm{a}}$ Values of the coefficients and the mean relative deviations were taken from Wang and Zhang. ${ }^{97}$

mean that the data are accurate. In the case of the 4-acetoxybenzoic acid solubility data, there is a noticeable difference between the two experimental methods. The relative difference can be fairly large at times. For example the static method gave a mole fraction solubility of $x_{1}=0.002489$ for 4acetoxybenzoic acid dissolved in ethanol at $298.15 \mathrm{~K}$, versus a value of $x_{1}=0.002193$ determined with the dynamic method. The calculated relative difference between the two reported values is more than $10 \%$.

The experimental solubility data for 4-acetoxybenzoic acid in ethanol are in Sec. 4.2.

\subsection{4-Acetoxybenzoic acid solubility data in alcohols}

\begin{tabular}{|c|c|}
\hline $\begin{array}{l}\text { Components: } \\
\text { (1) 4-Acetoxybenzoic acid; } \\
\mathrm{C}_{9} \mathrm{H}_{8} \mathrm{O}_{4} ;[2345-34-8] \\
\text { (2) Ethanol; } \mathrm{C}_{2} \mathrm{H}_{6} \mathrm{O} ; \\
\text { [64-17-5] }\end{array}$ & $\begin{array}{l}\text { Original Measurements: } \\
{ }^{97} \mathrm{H} \text {. Wang and W. Zhang, J. Chem. } \\
\text { Eng. Data 54, } 1942 \text { (2009). }\end{array}$ \\
\hline $\begin{array}{l}\text { Variables: } \\
\text { Temperature }\end{array}$ & $\begin{array}{l}\text { Prepared by: } \\
\text { W. E. Acree, Jr. }\end{array}$ \\
\hline
\end{tabular}

Experimental Values

\begin{tabular}{lcc}
\hline \hline$T / \mathrm{K}$ & $x_{2}{ }^{\mathrm{a}}$ & $x_{1}{ }^{\mathrm{b}, \mathrm{c}}$ \\
\hline 298.2 & 0.9751 & 0.02489 \\
303.2 & 0.9698 & 0.03018 \\
308.2 & 0.9642 & 0.03584 \\
313.2 & 0.9569 & 0.04315 \\
318.2 & 0.9478 & 0.05217 \\
\hline
\end{tabular}

${ }^{\mathrm{a}} x_{2}$ : mole fraction of component 2 in the saturated solution.

${ }^{\mathrm{b}} x_{1}$ : mole fraction solubility of the solute.

${ }^{\mathrm{c}}$ Solubility determined by the static method.

Experimental Values

\begin{tabular}{lcc}
\hline \hline$T / \mathrm{K}$ & $x_{2}{ }^{\mathrm{a}}$ & $x_{1}{ }^{\mathrm{b}, \mathrm{c}}$ \\
\hline 298.2 & 0.9781 & 0.02193 \\
303.2 & 0.9685 & 0.03149 \\
308.2 & 0.9612 & 0.03876 \\
313.2 & 0.9549 & 0.04514 \\
318.2 & 0.9488 & 0.05117 \\
${ }^{\mathrm{a}}{ }^{2}:$ mole fraction of component 2 in the saturated solution. \\
${ }^{\mathrm{b}}{ }_{1}:$ mole fraction solubility of the solute. \\
${ }^{\mathrm{c}}$ Solubility determined by the dynamic method.
\end{tabular}




\section{Auxiliary Information}

\section{Method/Apparatus/Procedure:}

Thermostated water-jacketed glass vessel, thermometer, analytical balance, laser monitoring system.

Solubilities were determined using both a static and dynamic method. In the static method, excess solute and solvent were placed in a water-jacketed vessel allowed to equilibrate with stirring for three days at constant temperature. Samples of the saturated liquid phase were decanted into Petri dishes of known mass and weighed. The samples were then evaporated to dryness and the solubility determined from the mass of the solid residue and the mass of the sample analyzed. In the dynamic solid method, an excess of carboxylic acid was added to a known mass of ethanol The resulting suspension was stirred in a water-jacketed vessel for $1 \mathrm{~h}$ at constant temperature. A known mass of ethanol to the vessel through a buret until the solute completely dissolved. The dissolution was monitored with a laser monitoring system. When the last portion of solid solute disappeared, the penetrated light intensity reached its maximum value. The mass of the added solvent was recorded. The solubility of the carboxylic acid was calculated from the known masses of solute and ethanol.

\section{Source and Purity of Chemicals:}

(1) $98 \%$, Sigma-Aldrich Chemical Company, Milwaukee, WI, USA, no purification details provided.

(2) $99.55 \%$, Tianjin Kewei Chemical Reagent, China, no information given regarding any further purification.

\section{Estimated Error:}

Temperature: $\pm 0.1 \mathrm{~K}$

$x_{1}$ : Authors report that the uncertainty in the solubility values is estimated to be $\pm 0.5 \%$. The differences between the experimental values based on the static and dynamic solubility methods are considerably larger, and more on the order of $\pm 5 \%$ to $10 \%$ (relative error).

\section{Solubility of 4-Acetylbenzoic Acid in Organic Solvents}

\subsection{Critical evaluation of experimental solubility data}

Only a single published paper was found in the chemical and engineering literature regarding the solubility of 4-acetylbenzoic acid in organic solvents. Sunsandee et al. ${ }^{98}$ measured the solubility of 4-acetylbenzoic acid in 1-methyl-4-(1-methylethyl)benzene (also called $p$-cymene), tert-butylbenzene, and ethanoic acid from 303 to $473 \mathrm{~K}$. The authors described the observed solubility data in terms of both the Modified Apelblat model [Eq. (8)] and Buchowski $\lambda$ h-model [Eq. (9)]. The calculated curve-fit equation coefficients and squared correlation coefficients are given in Tables 11 and 12 for the Modified Apelblat and Buchowski $\lambda \mathrm{h}$-models, respectively. The experimental solubility data are internally consistent as evidenced by the near unity values of the squared correlation coefficients.

The experimental solubility data for 4-acetylbenzoic acid in organic solvents are given in Secs. 5.2 and 5.3.

TABLE 11. Parameters of the Modified Apelblat equation for describing the solubility of 4-acetylbenzoic acid in various organic solvents

\begin{tabular}{lccccc}
\hline \hline Solvent & $T / \mathrm{K}$ & $A$ & $B$ & $C$ & $R^{2}$ \\
\hline p-Cymene $^{\mathrm{a}}$ & $303-453$ & -125.42 & 3239.20 & 18.99 & 0.9990 \\
tert-Butylbenzene $^{\mathrm{a}}$ & $303-453$ & -116.93 & 2858.70 & 17.73 & 0.9990 \\
Ethanoic acid $^{\mathrm{a}}$ & $303-453$ & -114.80 & 3288.30 & 17.12 & 0.9980 \\
\hline
\end{tabular}

${ }^{\mathrm{a}}$ Values of the coefficients and squared correlation coefficients were taken from Sunsandee et $_{\text {al. }}{ }^{98}$
TABLE 12. Parameters of the Buchowski $\lambda$ h equation for describing the solubility of 4-acetylbenzoic acid in various organic solvents

\begin{tabular}{lcccc}
\hline \hline Solvent & $T / \mathrm{K}$ & $\lambda$ & $h$ & $R^{2}$ \\
\hline p-Cymene $^{\mathrm{a}}$ & $303-453$ & 0.045739 & 47820.72 & 0.9980 \\
tert-Butylbenzene $^{\mathrm{a}}$ & $303-453$ & 0.042173 & 42168.65 & 0.9990 \\
Ethanoic acid $^{\mathrm{a}}$ & $303-453$ & 0.036581 & 70114.79 & 0.9990 \\
\hline
\end{tabular}

${ }^{a}$ Values of the coefficients and squared correlation coefficients were taken from Sunsandee et al. ${ }^{98}$

\subsection{4-Acetylbenzoic acid solubility data in aromatic hydrocarbons}

\begin{tabular}{ll}
\hline \hline Components: & Original Measurements: \\
(1) 4-Acetylbenzoic acid; & ${ }^{98}$ N. Sunsandee, M. Hronec, M. \\
$\mathrm{C}_{9} \mathrm{H}_{7} \mathrm{O}_{3} ;[586-89-0]$ & Stolcova, N. Leepipatpiboon, and U. \\
$\begin{array}{l}\text { (2) 1-Methyl-4-(1-methylethyl) } \\
\text { benzene; } \mathrm{C}_{10} \mathrm{H}_{14} ; \text { [99-87-6] }\end{array}$ & Pancharoen, J. Mol. Liq. 180, 252 \\
\hline Variables: & (2013). \\
Temperature & Prepared by: \\
\hline
\end{tabular}

Experimental Values

\begin{tabular}{lcc}
\hline \hline$T / \mathrm{K}$ & $x_{2}{ }^{\mathrm{a}}$ & $x_{1}{ }^{\mathrm{b}}$ \\
\hline 303.15 & 0.9980 & 0.00199 \\
313.15 & 0.9974 & 0.00260 \\
323.15 & 0.9965 & 0.00346 \\
333.15 & 0.9955 & 0.00451 \\
343.15 & 0.9940 & 0.00602 \\
353.15 & 0.9920 & 0.00802 \\
363.15 & 0.9895 & 0.01050 \\
373.15 & 0.9860 & 0.01398 \\
383.15 & 0.9817 & 0.01828 \\
393.15 & 0.9758 & 0.02417 \\
403.15 & 0.9687 & 0.03134 \\
413.15 & 0.9589 & 0.04114 \\
423.15 & 0.9459 & 0.05413 \\
433.15 & 0.9295 & 0.07046 \\
443.15 & 0.9080 & 0.09202 \\
453.15 & 0.8794 & 0.12063 \\
\hline
\end{tabular}

${ }^{a} x_{2}$ : mole fraction of component 2 in the saturated solution.

${ }^{\mathrm{b}} x_{1}$ : mole fraction of the solute.

Auxiliary Information

Method/Apparatus/Procedure:

Stainless steel closed equilibrium vessel equipped with magnetic stirrer, analytical balance, high-performance liquid chromatographic system, thermostated silicone oil bath, and digital density meter.

Excess solute and solvent were allowed to equilibrate with stirring in a thermostated silicone oil bath for $90 \mathrm{~min}$. The stirring was suspended and the suspended particles were allowed to settle to the bottom of the vessel for 120 $\min$. An aliquot of the saturated solution was withdrawn, transferred to a tared volumetric flask, and then weighed to determine the amount of sample withdrawn. The concentration of the dissolved solute was determined by highperformance liquid chromatographic analysis. The density of the saturated was also measured to allow the measured solubilities in units of molarity to be converted to mole fraction solubilities. 
Source and Purity of Chemicals:

(1) $98 \%$, Sigma-Aldrich Chemical Company, was used as received.

(2) $99.9 \%$ (by HPLC analysis), Sigma-Aldrich Chemical Company, was used as received.

Estimated Error:

Temperature: $\pm 0.1 \mathrm{~K}$

$x_{1}: \pm 2 \%$ (relative error, estimated by compiler).

\begin{tabular}{ll}
\hline \hline Components: & Original Measurements: \\
(1) 4-Acetylbenzoic acid; & ${ }^{98}$ N. Sunsandee, M. Hronec, M. \\
$\mathrm{C}_{9} \mathrm{H}_{7} \mathrm{O}_{3} ;$ [586-89-0] & Stolcova, N. Leepipatpiboon, and U. \\
(2) tert-Butylbenzene; $\mathrm{C}_{10} \mathrm{H}_{14} ;$ & Pancharoen, J. Mol. Liq. 180, 252 \\
[98-06-6] & $(2013)$. \\
\hline Variables: & Prepared by: \\
Temperature & W. E. Acree, Jr. \\
\hline
\end{tabular}

Experimental Values

\begin{tabular}{lcc}
\hline \hline$T / \mathrm{K}$ & $x_{2}{ }^{\mathrm{a}}$ & $x_{1}{ }^{\mathrm{b}}$ \\
\hline 303.15 & 0.9979 & 0.00205 \\
313.15 & 0.9973 & 0.00271 \\
323.15 & 0.9964 & 0.00357 \\
333.15 & 0.9953 & 0.00473 \\
343.15 & 0.9938 & 0.00618 \\
353.15 & 0.9918 & 0.00817 \\
363.15 & 0.9894 & 0.01063 \\
373.15 & 0.9862 & 0.01384 \\
383.15 & 0.9818 & 0.01822 \\
393.15 & 0.9761 & 0.02387 \\
403.15 & 0.9690 & 0.03098 \\
413.15 & 0.9600 & 0.03999 \\
423.15 & 0.9480 & 0.05198 \\
433.15 & 0.9321 & 0.06785 \\
443.15 & 0.9123 & 0.08767 \\
453.15 & 0.8860 & 0.11403 \\
\hline
\end{tabular}

${ }^{\mathrm{a}} x_{2}$ : mole fraction of component 2 in the saturated solution.

${ }^{\mathrm{b}} \mathrm{x}_{1}$ : mole fraction of the solute.

\section{Auxiliary Information}

\section{Method/Apparatus/Procedure:}

Stainless steel closed equilibrium vessel equipped with magnetic stirrer, analytical balance, high-performance liquid chromatographic system, thermostated silicone oil bath, and digital density meter.

Excess solute and solvent were allowed to equilibrate with stirring in a thermostated silicone oil bath for $90 \mathrm{~min}$. The stirring was suspended and the suspended particles were allowed to settle to the bottom of the vessel for 120 min. An aliquot of the saturated solution was withdrawn, transferred to a tared volumetric flask, and then weighed to determine the amount of sample withdrawn. The concentration of the dissolved solute was determined by highperformance liquid chromatographic analysis. The density of the saturated was also measured to allow the measured solubilities in units of molarity to be converted to mole fraction solubilities.

Source and Purity of Chemicals:

(1) $98 \%$, Sigma-Aldrich Chemical Company, was used as received.

(2) $99.9 \%$ (by HPLC analysis), Sigma-Aldrich Chemical Company, was used as received.

\section{Estimated Error:}

Temperature: $\pm 0.1 \mathrm{~K}$.

$x_{1}: \pm 2 \%$ (relative error, estimated by compiler).

\subsection{4-Acetylbenzoic acid solubility data in miscellaneous organic solvents}

\begin{tabular}{ll}
\hline \hline Components: & $\begin{array}{l}\text { Original Measurements: } \\
\text { (1) 4-Acetylbenzoic acid; }\end{array}$ \\
${ }^{98}$ N. Sunsandee, M. Hronec, M. \\
$\mathrm{C}_{9} \mathrm{H}_{7} \mathrm{O}_{3} ;$ [586-89-0] & Stolcova, N. Leepipatpiboon, and U. \\
(2) Ethanoic acid; $\mathrm{C}_{2} \mathrm{H}_{4} \mathrm{O}_{2} ;$ & Pancharoen, J. Mol. Liq. 180, 252 \\
[64-19-7] & $(2013)$. \\
\hline Variables: & Prepared by: \\
Temperature & W. E. Acree, Jr. \\
\hline
\end{tabular}

Experimental Values

\begin{tabular}{lcc}
\hline \hline$T / \mathrm{K}$ & $x_{2}{ }^{\mathrm{a}}$ & $x_{1}{ }^{\mathrm{b}}$ \\
\hline 303.15 & 0.9978 & 0.00218 \\
313.15 & 0.9973 & 0.00269 \\
323.15 & 0.9967 & 0.00332 \\
333.15 & 0.9958 & 0.00416 \\
343.15 & 0.9948 & 0.00515 \\
353.15 & 0.9936 & 0.00644 \\
363.15 & 0.9919 & 0.00807 \\
373.15 & 0.9899 & 0.01005 \\
383.15 & 0.9875 & 0.01253 \\
393.15 & 0.9843 & 0.01567 \\
403.15 & 0.9805 & 0.01953 \\
413.15 & 0.9755 & 0.02453 \\
423.15 & 0.9695 & 0.03047 \\
433.15 & 0.9610 & 0.03896 \\
443.15 & 0.9527 & 0.04731 \\
453.15 & 0.9417 & 0.05833 \\
\hline
\end{tabular}

${ }^{\mathrm{a}} x_{2}$ : mole fraction of component 2 in the saturated solution.

${ }^{\mathrm{b}} x_{1}$ : mole fraction of the solute.

\section{Auxiliary Information}

\section{Method/Apparatus/Procedure:}

Stainless steel closed equilibrium vessel equipped with magnetic stirrer, analytical balance, high-performance liquid chromatographic system, thermostated silicone oil bath, and digital density meter.

Excess solute and solvent were allowed to equilibrate with stirring in a thermostated silicone oil bath for $90 \mathrm{~min}$. The stirring was suspended and the suspended particles were allowed to settle to the bottom of the vessel for $120 \mathrm{~min}$. An aliquot of the saturated solution was withdrawn, transferred to a tared volumetric flask, and then weighed to determine the amount of sample withdrawn. The concentration of the dissolved solute was determined by highperformance liquid chromatographic analysis. The density of the saturated was also measured to allow the measured solubilities in units of molarity to be converted to mole fraction solubilities.

Source and Purity of Chemicals:

(1) $98 \%$, Sigma-Aldrich Chemical Company, was used as received.

(2) $99.8 \%$ (by HPLC analysis), Sigma-Aldrich Chemical Company, was used as received.

Estimated Error:

Temperature: $\pm 0.1 \mathrm{~K}$.

$x_{1}: \pm 2 \%$ (relative error, estimated by compiler). 


\section{Solubility of 2-Aminobenzoic Acid in Organic Solvents}

\subsection{Critical evaluation of experimental solubility data}

Only two published papers were found in the chemical and engineering literature regarding the solubility of 2-aminobenzoic acid in organic solvents. Jia et al. ${ }^{99}$ determined the solubility of 2-aminobenzoic acid in 1-octanol in the temperature range of 293-323 K. The calculated curve-fit parameters from the Buchowski $\lambda$ h-model [Eq. (9)] of $\lambda=0.4895$ and $h=$ 4520.42 described the observed solubility data to within a mean relative deviation of $0.9 \%$.

Lazzell and Johnston ${ }^{100}$ measured the solubility of 2-aminobenzoic acid in benzene, ethyl ethanoate, trichloromethane, methanol, ethanol, and 1-butanol as a function of temperature. Except for 1-butanol, the measurements went from $298 \mathrm{~K}$ to well above the normal boiling temperature of the solvent. The internal consistency of the benzene, trichloromethane, and ethanol datasets was assessed by curve-fitting the measured mole fraction solubility data to the Modified Apelblat model to yield the following representations:

$$
\begin{aligned}
\ln x_{1}(\text { in benzene })=-92.104+ & \frac{113.71}{T}+15.248 \ln T, \\
\ln x_{1}(\text { in trichloromethane })= & -80.747+\frac{113.94}{T} \\
& +13.370 \ln T \\
\ln x_{1}(\text { in ethanol })=-48.945+ & \frac{114.60}{T}+8.074 \ln T .
\end{aligned}
$$

There were too few data points in the ethyl ethanoate, methanol, and 1-butanol datasets to perform a meaningful regression analysis. The average absolute deviations between the observed experimental data and back-calculated values based on Eqs. (27)-(29) (of 5.36\%, 3.61\%, and $0.62 \%$, respectively) are quite small given the range of mole fraction solubilities covered by each of the three datasets. In the case of benzene, the observed solubilities ranged from $x_{1}=0.0081$ to $x_{1}=$ 0.8064 . It is more difficult to curve-fit experimental solubility data covering large mole fraction ranges.

The experimental solubility data for 2 -aminobenzoic acid in

\begin{tabular}{|c|c|}
\hline $\begin{array}{l}\text { Components: } \\
\text { (1) 2-Aminobenzoic acid; } \\
\mathrm{C}_{7} \mathrm{H}_{7} \mathrm{NO}_{2} ;[118-92-3] \\
\text { (2) Benzene; } \mathrm{C}_{6} \mathrm{H}_{6} ;[71-43-2]\end{array}$ & $\begin{array}{l}\text { Original Measurements: } \\
{ }^{100} \text { C. L. Lazzell and J. Johnston, J. } \\
\text { Phys. Chem. 32, } 1331 \text { (1928). }\end{array}$ \\
\hline $\begin{array}{l}\text { Variables: } \\
\text { Temperature }\end{array}$ & $\begin{array}{l}\text { Prepared by: } \\
\text { W. E. Acree, Jr. }\end{array}$ \\
\hline
\end{tabular}
organic solvents are given in Secs. 6.2-6.5.

\subsection{2-Aminobenzoic acid solubility data in aromatic hydrocarbons}

Experimental Values

\begin{tabular}{lcc}
\hline \hline$T / \mathrm{K}$ & $x_{2}{ }^{\mathrm{a}}$ & $x_{1}{ }^{\mathrm{b}}$ \\
\hline 298.2 & 0.9919 & 0.0081 \\
348.9 & 0.9129 & 0.0871 \\
362.7 & 0.8687 & 0.1313 \\
366.5 & 0.8364 & 0.1636 \\
374.8 & 0.7778 & 0.2222 \\
381.5 & 0.7074 & 0.2926 \\
383.5 & 0.6504 & 0.3496 \\
392.8 & 0.4912 & 0.5088 \\
392.5 & 0.4897 & 0.5103 \\
398.3 & 0.3826 & 0.6174 \\
399.5 & 0.3531 & 0.6469 \\
403.1 & 0.2874 & 0.7126 \\
408.4 & 0.1936 & 0.8064
\end{tabular}

${ }^{\mathrm{a}} x_{2}$ : mole fraction of component 2 in the saturated solution.

${ }^{b} x_{1}$ : mole fraction solubility of the solute. The solubility is given as molal percentage, which, based on the description of 100 times the mole fraction, is taken to be mole percentage.

Auxiliary Information

Method/Apparatus/Procedure:

Preweighed quantities of solute and solvent were placed in bulbs, which were then sealed and heated in a suitable bath. The temperature at which the solid completely dissolved was recorded, and was taken to be the solid-liquid equilibrium temperature. In a few instances near room temperature, the solubility was determined by agitating the solvent in a thermostat with an excess of solute. After equilibrium had been established, an aliquot of the saturated solution was pipetted, weighed, and the amount of dissolved solute determined with a solution of sodium hydroxide standardized against the pure acid.

Source and Purity of Chemicals:

(1) Certified Pure, Chemical source not given, was recrystallized several times from hot water, then from chloroform to give a sample having a melting point of $419.3 \mathrm{~K}$.

(2) Thiophene-free, Chemical source not given, was dried over sodium and distilled before use.

Estimated Error:

Temperature: Not given in paper.

$x_{1}: \pm 5 \%$ (relative error, estimated by compiler).

\subsection{2-Aminobenzoic acid solubility data in esters}

\section{Components:}

(1) 2-Aminobenzoic acid; $\mathrm{C}_{7} \mathrm{H}_{7} \mathrm{NO}_{2}$; [118-92-3]

(2) Ethyl ethanoate; $\mathrm{C}_{4} \mathrm{H}_{8} \mathrm{O}_{2}$; [141-78-6]

Variables: $\begin{array}{ll}\text { Temperature } & \text { W. E. Acree, Jr. }\end{array}$
Original Measurements:

${ }^{100}$ C. L. Lazzell and J. Johnston, J.

Phys. Chem. 32, 1331 (1928). 
Experimental Values

\begin{tabular}{lcc}
\hline \hline$T / \mathrm{K}$ & $x_{2}{ }^{\mathrm{a}}$ & $x_{1}{ }^{\mathrm{b}}$ \\
\hline 298.2 & 0.8529 & 0.1471 \\
350.0 & 0.7027 & 0.2973 \\
381.2 & 0.4718 & 0.5282 \\
394.0 & 0.3497 & 0.6503
\end{tabular}

${ }^{a} x_{2}$ : mole fraction of component 2 in the saturated solution.

${ }^{b} x_{1}$ : mole fraction solubility of the solute. The solubility is given as molal percentage, which, based on the description of 100 times the mole fraction, is taken to be mole percentage.

Auxiliary Information

\section{Method/Apparatus/Procedure:}

Preweighed quantities of solute and solvent were placed in bulbs, which were then sealed and heated in a suitable bath. The temperature at which the solid completely dissolved was recorded, and was taken to be the solid-liquid equilibrium temperature. In a few instances near room temperature, the solubility was determined by agitating the solvent in a thermostat with an excess of solute. After equilibrium had been established, an aliquot of the saturated solution was pipetted, weighed, and the amount of dissolved solute determined with a solution of sodium hydroxide standardized against the pure acid.

\section{Source and Purity of Chemicals:}

(1) Certified Pure, Chemical source not given, was recrystallized several times from hot water, then from chloroform to give a sample having a melting point of $419.3 \mathrm{~K}$.

(2) USP grade, Chemical source not given, washed several times with aqueous sodium carbonate, and then with water until the aqueous layer was neutral. The sample was further purified by drying over calcium chloride and then distilling two times before use.

\section{Estimated Error:}

Temperature: Not given in paper.

$x_{1}: \pm 5 \%$ (relative error, estimated by compiler).

\subsection{2-Aminobenzoic acid solubility data in haloalkanes, haloalkenes, and haloaromatic hydrocarbons}

\begin{tabular}{|c|c|}
\hline $\begin{array}{l}\text { Components: } \\
\text { (1) 2-Aminobenzoic acid; } \\
\mathrm{C}_{7} \mathrm{H}_{7} \mathrm{NO}_{2} ;[118-92-3] \\
\text { (2) Trichloromethane; } \mathrm{CHCl}_{3} \text {; } \\
\text { [67-66-3] }\end{array}$ & $\begin{array}{l}\text { Original Measurements: } \\
{ }^{100} \text { C. L. Lazzell and J. Johnston, J. } \\
\text { Phys. Chem. 32, } 1331 \text { (1928). }\end{array}$ \\
\hline $\begin{array}{l}\text { Variables: } \\
\text { Temperature }\end{array}$ & $\begin{array}{l}\text { Prepared by: } \\
\text { W. E. Acree, Jr. }\end{array}$ \\
\hline
\end{tabular}

Experimental Values

\begin{tabular}{lcc}
\hline \hline$T / \mathrm{K}$ & $x_{2}{ }^{\mathrm{a}}$ & $x_{1}{ }^{\mathrm{b}}$ \\
\hline 298.2 & 0.9843 & 0.0157 \\
349.7 & 0.8876 & 0.1124 \\
362.1 & 0.8117 & 0.1883 \\
373.6 & 0.7093 & 0.2907 \\
384.0 & 0.5686 & 0.4314 \\
\hline
\end{tabular}

${ }^{\mathrm{a}} x_{2}$ : mole fraction of component 2 in the saturated solution.

${ }^{b} x_{1}$ : mole fraction solubility of the solute. The solubility is given as molal percentage, which, based on the description of 100 times the mole fraction, is taken to be mole percentage.

\section{Auxiliary Information}

\section{Method/Apparatus/Procedure:}

Preweighed quantities of solute and solvent were placed in bulbs, which were then sealed and heated in a suitable bath. The temperature at which the solid completely dissolved was recorded, and was taken to be the solid-liquid equilibrium temperature. In a few instances near room temperature, the solubility was determined by agitating the solvent in a thermostat with an excess of solute. After equilibrium had been established, an aliquot of the saturated solution was pipetted, weighed, and the amount of dissolved solute determined with a solution of sodium hydroxide standardized against the pure acid.

Source and Purity of Chemicals:

(1) Certified Pure, Chemical source not given, was recrystallized several times from hot water, then from chloroform to give a sample having a melting point of $419.3 \mathrm{~K}$.

(2) Certified Pure, Chemical source not given, was dried over calcium chloride and distilled before use.

Estimated Error:

Temperature: Not given in paper.

$x_{1}: \pm 5 \%$ (relative error, estimated by compiler).

\subsection{2-Aminobenzoic acid solubility data in alcohols}

\begin{tabular}{|c|c|}
\hline $\begin{array}{l}\text { Components: } \\
\text { (1) 2-Aminobenzoic acid; } \\
\mathrm{C}_{7} \mathrm{H}_{7} \mathrm{NO}_{2} ;[118-92-3] \\
\text { (2) Methanol; } \mathrm{CH}_{4} \mathrm{O} ;[67-56-1]\end{array}$ & $\begin{array}{l}\text { Original Measurements: } \\
{ }^{100} \text { C. L. Lazzell and J. Johnston, J. } \\
\text { Phys. Chem. 32, } 1331 \text { (1928). }\end{array}$ \\
\hline $\begin{array}{l}\text { Variables: } \\
\text { Temperature }\end{array}$ & $\begin{array}{l}\text { Prepared by: } \\
\text { W. E. Acree, Jr. }\end{array}$ \\
\hline
\end{tabular}

Experimental Values

\begin{tabular}{|c|c|c|}
\hline$T / \mathrm{K}$ & $x_{2}{ }^{\mathrm{a}}$ & $x_{1}{ }^{\mathrm{b}}$ \\
\hline 298.2 & 0.9238 & 0.0762 \\
\hline 323.9 & 0.8630 & 0.1370 \\
\hline 350.3 & 0.7408 & 0.2592 \\
\hline
\end{tabular}




\section{Auxiliary Information}

\section{Method/Apparatus/Procedure:}

Preweighed quantities of solute and solvent were placed in bulbs, which were then sealed and heated in a suitable bath. The temperature at which the solid completely dissolved was recorded, and was taken to be the solid-liquid equilibrium temperature. In a few instances near room temperature, the solubility was determined by agitating the solvent in a thermostat with an excess of solute. After equilibrium had been established, an aliquot of the saturated solution was pipetted, weighed, and the amount of dissolved solute determined with a solution of sodium hydroxide standardized against the pure acid.

\section{Source and Purity of Chemicals:}

(1) Certified Pure, Chemical source not given, was recrystallized several times from hot water, then from chloroform to give a sample having a melting point of $419.3 \mathrm{~K}$.

(2) Purity not given, Chemical source not given, no purification details were provided.

\section{Estimated Error:}

Temperature: Not given in paper.

$x_{1}: \pm 5 \%$ (relative error, estimated by compiler).

\begin{tabular}{|c|c|}
\hline $\begin{array}{l}\text { Components: } \\
\text { (1) 2-Aminobenzoic acid; } \\
\mathrm{C}_{7} \mathrm{H}_{7} \mathrm{NO}_{2} ;[118-92-3] \\
\text { (2) Ethanol; } \mathrm{C}_{2} \mathrm{H}_{6} \mathrm{O} ;[64-17-5]\end{array}$ & $\begin{array}{l}\text { Original Measurements: } \\
{ }^{100} \text { C. L. Lazzell and J. Johnston, J. } \\
\text { Phys. Chem. 32, } 1331 \text { (1928). }\end{array}$ \\
\hline $\begin{array}{l}\text { Variables: } \\
\text { Temperature }\end{array}$ & $\begin{array}{l}\text { Prepared by: } \\
\text { W. E. Acree, Jr. }\end{array}$ \\
\hline
\end{tabular}

Experimental Values

\begin{tabular}{lcc}
\hline \hline$T / \mathrm{K}$ & $x_{2}{ }^{\mathrm{a}}$ & $x_{1}{ }^{\mathrm{b}}$ \\
\hline 298.2 & 0.9225 & 0.0775 \\
341.1 & 0.7781 & 0.2219 \\
350.5 & 0.7306 & 0.2694 \\
359.7 & 0.6697 & 0.3303 \\
367.1 & 0.6181 & 0.3819 \\
398.7 & 0.2608 & 0.7392
\end{tabular}

${ }^{a_{X_{2}}}$ : mole fraction of component 2 in the saturated solution.

${ }^{b} x_{1}$ : mole fraction solubility of the solute. The solubility is given as molal percentage, which, based on the description of 100 times the mole fraction, is taken to be mole percentage.

\section{Auxiliary Information}

\section{Method/Apparatus/Procedure:}

Preweighed quantities of solute and solvent were placed in bulbs, which were then sealed and heated in a suitable bath. The temperature at which the solid completely dissolved was recorded, and was taken to be the solid-liquid equilibrium temperature. In a few instances near room temperature, the solubility was determined by agitating the solvent in a thermostat with an excess of solute. After equilibrium had been established, an aliquot of the saturated solution was pipetted, weighed, and the amount of dissolved solute determined with a solution of sodium hydroxide standardized against the pure acid.

Source and Purity of Chemicals:

(1) Certified Pure, Chemical source not given, was recrystallized several times from hot water, then from chloroform to give a sample having a melting point of $419.3 \mathrm{~K}$.

(2) $95 \%$, Chemical source not given, was refluxed with lime and distilled. The sample was further purified by drying over sodium and distilling twice before use.
Estimated Error:

Temperature: Not given in paper.

$x_{1}: \pm 5 \%$ (relative error, estimated by compiler).

\begin{tabular}{|c|c|}
\hline $\begin{array}{l}\text { Components: } \\
\text { (1) 2-Aminobenzoic acid; } \\
\mathrm{C}_{7} \mathrm{H}_{7} \mathrm{NO}_{2} ;[118-92-3] \\
\text { (2) 1-Butanol; } \mathrm{C}_{4} \mathrm{H}_{10} \mathrm{O} \text {; } \\
\text { [71-36-3] }\end{array}$ & $\begin{array}{l}\text { Original Measurements: } \\
{ }^{100} \text { C. L. Lazzell and J. Johnston, J. } \\
\text { Phys. Chem. 32, } 1331 \text { (1928). }\end{array}$ \\
\hline $\begin{array}{l}\text { Variables: } \\
\text { Temperature }\end{array}$ & $\begin{array}{l}\text { Prepared by: } \\
\text { W. E. Acree, Jr. }\end{array}$ \\
\hline
\end{tabular}

Experimental Values

\begin{tabular}{lcc}
\hline \hline$T / \mathrm{K}$ & $x_{2}{ }^{\mathrm{a}}$ & $x_{1}{ }^{\mathrm{b}}$ \\
\hline 353.3 & 0.7320 & 0.2680 \\
377.8 & 0.5514 & 0.4486 \\
\hline
\end{tabular}

${ }^{a} x_{2}$ : mole fraction of component 2 in the saturated solution.

${ }^{b} x_{1}$ : mole fraction solubility of the solute. The solubility is given as molal percentage, which, based on the description of 100 times the mole fraction, is taken to be mole percentage.

\section{Auxiliary Information}

\section{Method/Apparatus/Procedure:}

Preweighed quantities of solute and solvent were placed in bulbs, which were then sealed and heated in a suitable bath. The temperature at which the solid completely dissolved was recorded, and was taken to be the solid-liquid equilibrium temperature.

\section{Source and Purity of Chemicals:}

(1) Certified Pure, Chemical source not given, was recrystallized several times from hot water, then from chloroform to give a sample having a melting point of $419.3 \mathrm{~K}$.

(2) Purity not given, Chemical source not given, no purification details were provided.

\section{Estimated Error:}

Temperature: Not given in paper.

$x_{1}: \pm 5 \%$ (relative error, estimated by compiler).

\begin{tabular}{|c|c|}
\hline $\begin{array}{l}\text { Components: } \\
\text { (1) 2-Aminobenzoic acid; } \\
\mathrm{C}_{7} \mathrm{H}_{7} \mathrm{NO}_{2} ;[118-92-3] \\
\text { (2) } 1-\text { Octanol; } \mathrm{C}_{8} \mathrm{H}_{18} \mathrm{O} \text {; } \\
\text { [111-87-5] }\end{array}$ & $\begin{array}{l}\text { Original Measurements: } \\
{ }^{99} \text { Q. Jia, P. Ma, S. Ma, and C. Wang, } \\
\text { Chin. J. Chem. Eng. 15, } 710 \text { (2007). }\end{array}$ \\
\hline $\begin{array}{l}\text { Variables: } \\
\text { Temperature }\end{array}$ & $\begin{array}{l}\text { Prepared by: } \\
\text { W. E. Acree, Jr. }\end{array}$ \\
\hline
\end{tabular}


Experimental Values

\begin{tabular}{lcc}
\hline \hline$T / \mathrm{K}$ & $x_{2}{ }^{\mathrm{a}}$ & $x_{1}{ }^{\mathrm{b}}$ \\
\hline 295.30 & 0.9444 & 0.05555 \\
298.45 & 0.9384 & 0.06159 \\
299.95 & 0.9352 & 0.06478 \\
301.80 & 0.9318 & 0.06817 \\
303.15 & 0.9288 & 0.07116 \\
305.05 & 0.9214 & 0.07512 \\
307.05 & 0.9214 & 0.07856 \\
308.55 & 0.9185 & 0.08146 \\
309.65 & 0.9161 & 0.08392 \\
311.35 & 0.9124 & 0.08761 \\
313.35 & 0.9081 & 0.09187 \\
315.95 & 0.9026 & 0.09735 \\
317.75 & 0.8987 & 0.1013 \\
320.15 & 0.8924 & 0.1076 \\
322.25 & 0.8891 & 0.1109 \\
\hline
\end{tabular}

${ }^{\mathrm{a}} x_{2}$ : mole fraction of component 2 in the saturated solution.

${ }^{\mathrm{b}} x_{1}$ : mole fraction solubility of the solute.

Auxiliary Information

\begin{abstract}
Method/Apparatus/Procedure:
Constant-temperature circulating bath, stirrer, analytical balance, and laser monitoring system.

Solubilities were determined by a dynamic method. Preweighed amounts of solute and solvent were placed in a stoppered equilibrium vessel, which was connected to a circulating constant-temperature water bath. The solution was stirred and the temperature slowly increased until all of the solid dissolved. Near the dissolution temperature, the temperature was increased at a rate of $0.2 \mathrm{~K}$ per $20 \mathrm{~min}$. Complete dissolution was determined using a laser monitoring system.
\end{abstract}

Source and Purity of Chemicals:

(1) $99+\%$, Chemical source not specified, no purification details were provided.

(2) $99+\%$, Chemical source not specified, no purification details were provided.

Estimated Error:

Temperature: $\pm 0.05 \mathrm{~K}$.

$x_{1}: \pm 1 \%$ (relative error).

\section{Solubility of 3-Aminobenzoic Acid in Organic Solvents}

\subsection{Critical evaluation of experimental solubility data}

The have been only three published studies involving the solubility of 3-aminobenzoic acid in organic solvents. Hancock et al. ${ }^{54}$ measured the solubility of 3 -aminobenzoic acid in cyclohexane and benzene at $303 \mathrm{~K}$ based on a gravimetric method. Svärd et al. ${ }^{101}$ determined the solubility of two polymorphic forms of 3-aminobenzoic acid in ethyl ethanoate, methanol, and ethanenitrile using a gravimetric method. Differential scanning calorimetry showed both polymorphs melting without any prior solid-solid transformations, with measured enthalpies of fusion and melting temperatures of
$\Delta H_{1}$ fus $=35.51 \mathrm{~kJ} \mathrm{~mol}^{-1}$ and $T_{\mathrm{mp}}=445.2 \mathrm{~K}$, and $\Delta H_{1}{ }^{\text {fus }}=$ $26.74 \mathrm{~kJ} \mathrm{~mol}^{-1}$ and $T_{\mathrm{mp}}=451.2 \mathrm{~K}$ for the forms I and II, respectively. Form I was characterized by dendritic growth, forming small cascades of threads, possibly due to twinning. Form II, on the other hand, developed into needle-like crystals that can be grown to several millimeters in length. Form II was the more soluble of the two polymorphs as one might expect from its lower enthalpy of fusion. The authors fit the solubility data for each solvent and each polymorph to Eq. (22). The calculated equation coefficients $(A, B$, and $C)$ are given in Table 13, along with the $\chi^{2}$ "goodness-of-fit" calculated by the Origin 6.1 statistical software. Graphs of the mole fraction solubility versus temperature given in the paper fell on smooth curves, and did not indicate any obvious outlier data points.

Lazzell and Johnston ${ }^{100}$ measured the solubility of 3-aminobenzoic acid in benzene, ethyl ethanoate, trichloromethane, methanol, ethanol, and 1-butanol as a function of temperature. Except for 1-butanol, the measurements went from $298 \mathrm{~K}$ to well above the normal boiling temperature of the solvent. The internal consistency of the benzene and ethanol datasets was assessed by curve-fitting the measured mole fraction solubility data to the Modified Apelblat model to yield the following representations:

$$
\begin{aligned}
\ln x_{1}(\text { in benzene })= & -255.914+\frac{110.95}{T} \\
& +41.973 \ln T,
\end{aligned}
$$

$$
\ln x_{1}(\text { in ethanol })=-65.997+\frac{114.72}{T}+10.772 \ln T .
$$

The experimental value at $298 \mathrm{~K}$ had to be removed from the benzene dataset in order to obtain a reasonable correlation equation. There were too few data points in the ethyl ethanoate, trichloromethane, methanol, and 1-butanol datasets to perform a meaningful regression analysis. The average absolute deviations between the observed experimental data and back-calculated values based on Eqs. (30) and (31) of $18.36 \%$ and $7.88 \%$ are larger than desired. The large deviation may be due in part to the fact that the solution temperature is above the solvent's normal boiling point temperature, to uncertainties in estimating the amount of solvent that remained in the liquid phase, and the large range covered by the experimental values, $x_{1}=0.0423$ to $x_{1}=0.6704$ for benzene and $x_{1}=0.0152$ to $x_{1}=$ 0.7806 for ethanol. It is more difficult to curve-fit experimental solubility data covering large mole fraction ranges.

The experimental solubility data for 3-aminobenzoic acid in organic solvents are given in Secs. 7.2-7.6.

TABLE 13. Parameters of Eq. (22) describing the solubility of 3-aminobenzoic acid in ethyl ethanoate, ethanenitrile, and methanol ${ }^{\mathrm{a}}$

\begin{tabular}{lrrrc}
\hline \hline Solvent & \multicolumn{1}{c}{$A$} & \multicolumn{1}{c}{$B$} & \multicolumn{1}{c}{$C$} & $\chi^{2}$ \\
\hline Ethyl ethanoate (Form I) & -31.57 & 2471.76 & 0.06134 & 0.00048 \\
Ethyl ethanoate (Form II) & -8.55 & -242.26 & 0.01841 & 0.00014 \\
Methanol (Form I) & -11.93 & -279.14 & 0.02891 & 0.00031 \\
Ethanenitrile (Form I) & -36.07 & 2604.92 & 0.07192 & 0.00187 \\
Ethanenitrile (Form II) & 16.47 & -4464.59 & -0.02117 & 0.00009
\end{tabular}

${ }^{\mathrm{a}}$ Values of the coefficients and goodness-of-fit were taken from Svärd et al. ${ }^{101}$ 


\subsection{3-Aminobenzoic acid solubility data in saturated hydrocarbons (including cycloalkanes)}

\begin{tabular}{|c|c|}
\hline $\begin{array}{l}\text { Components: } \\
\text { (1) 3-Aminobenzoic acid; } \\
\mathrm{C}_{7} \mathrm{H}_{7} \mathrm{NO}_{2} ; \text { [99-05-8] } \\
\text { (2) Cyclohexane; } \mathrm{C}_{6} \mathrm{H}_{12} \text {; } \\
{[110-82-7]}\end{array}$ & $\begin{array}{l}\text { Original Measurements: } \\
{ }^{54} \text { C. K. Hancock, J. N. Pawloski, and J. } \\
\text { P. Idoux, J. Org. Chem. 31, } 3801 \\
\text { (1966). }\end{array}$ \\
\hline $\begin{array}{l}\text { Variables: } \\
T / \mathrm{K}=303.15\end{array}$ & $\begin{array}{l}\text { Prepared by: } \\
\text { W. E. Acree, Jr. }\end{array}$ \\
\hline
\end{tabular}

Experimental Values

\begin{tabular}{lc}
\hline \hline$x_{2}{ }^{\mathrm{a}}$ & $x_{1}{ }^{\mathrm{b}}$ \\
\hline 0.9999 & 0.0000275
\end{tabular}

${ }^{a_{2}}$ : mole fraction of component 2 in the saturated solution.

${ }^{\mathrm{b}} x_{1}$ : mole fraction solubility of the solute.

\section{Auxiliary Information}

\section{Method/Apparatus/Procedure:}

Constant-temperature bath, Soxhlet thimble, conical flask, and analytical balance.

Excess solute and solvent were placed in a conical flask and allowed to equilibrate for several days at constant temperature. Attainment of equilibrium was verified by several repetitive measurements and by approaching equilibrium from supersaturation. Aliquots of saturated solutions were transferred using a Soxhlet thimble equipped with a one-hole cork stopper and an inverted U-shaped delivery tube to a second ground-glass stoppered flask suspended in the $303 \mathrm{~K}$ water bath. Successive portions of the contents were evaporated at room temperature in a tared aluminum foil weighing dish under a bell jar through which a slow stream of dry air was passed. The second flask plus unused saturated solution and the aluminum foil dish plus residue were weighed. The saturation solubility of the solute was calculated from the recorded mass data and molar masses of the solute and solvent.

\section{Source and Purity of Chemicals:}

(1) Purity not given, Chemical source not specified, was recrystallized several times from aqueous-ethanol mixture, and then dried under vacuum over phosphorous pentoxide.

(2) Reagent grade, Chemical source not specified, refluxed over phosphorous pentoxide for $24 \mathrm{~h}$, and then distilled through a $3 \times 80 \mathrm{~cm}$ column filled with $0.32-\mathrm{cm}$ glass helices.

\section{Estimated Error:}

Temperature: $\pm 0.02 \mathrm{~K}$.

$x_{1}: \pm 10 \%$ (relative error, by compiler).

\subsection{3-Aminobenzoic acid solubility data in aromatic hydrocarbons}

\begin{tabular}{|c|c|}
\hline $\begin{array}{l}\text { Components: } \\
\text { (1) 3-Aminobenzoic acid; } \\
\mathrm{C}_{7} \mathrm{H}_{7} \mathrm{NO}_{2} ;[99-05-8] \\
\text { (2) Benzene; } \mathrm{C}_{6} \mathrm{H}_{6} ;[71-43-2]\end{array}$ & $\begin{array}{l}\text { Original Measurements: } \\
{ }^{54} \text { C. K. Hancock, J. N. Pawloski, and J. } \\
\text { P. Idoux, J. Org. Chem. 31, } 3801 \\
\text { (1966). }\end{array}$ \\
\hline $\begin{array}{l}\text { Variables: } \\
T / \mathrm{K}=303.15\end{array}$ & $\begin{array}{l}\text { Prepared by: } \\
\text { W. E. Acree, Jr. }\end{array}$ \\
\hline
\end{tabular}

Experimental Values

\begin{tabular}{lc}
\hline \hline$x_{2}{ }^{\mathrm{a}}$ & $x_{1}{ }^{\mathrm{b}}$ \\
\hline 0.9999 & 0.0000771 \\
\hline${ }^{\mathrm{a}} x_{2}:$ mole fraction of component 2 in the saturated solution. \\
${ }^{\mathrm{b}}{ }_{x_{1}: \text { mole fraction solubility of the solute. }}$
\end{tabular}

\section{Auxiliary Information}

Method/Apparatus/Procedure:

Constant-temperature bath, Soxhlet thimble, conical flask, and analytical balance.

Excess solute and solvent were placed in a conical flask and allowed to equilibrate for several days at constant temperature. Attainment of equilibrium was verified by several repetitive measurements and by approaching equilibrium from supersaturation. Aliquots of saturated solutions were transferred using a Soxhlet thimble equipped with a one-hole cork stopper and an inverted U-shaped delivery tube to a second ground-glass stoppered flask suspended in the $303 \mathrm{~K}$ water bath. Successive portions of the contents were evaporated at room temperature in a tared aluminum foil weighing dish under a bell jar through which a slow stream of dry air was passed. The second flask plus unused saturated solution and the aluminum foil dish plus residue were weighed. The saturation solubility of the solute was calculated from the recorded mass data and molar masses of the solute and solvent.

\section{Source and Purity of Chemicals:}

(1) Purity not given, Chemical source not specified, was recrystallized several times from aqueous-ethanol mixture, and then dried under vacuum over phosphorous pentoxide.

(2) Reagent grade, Chemical source not specified, refluxed over phosphorous pentoxide for $24 \mathrm{~h}$, and then distilled through a $3 \times 80 \mathrm{~cm}$ column filled with 0.32 -cm glass helices.

\section{Estimated Error:}

Temperature: $\pm 0.02 \mathrm{~K}$.

$x_{1}: \pm 10 \%$ (relative error, by compiler).

\begin{tabular}{|c|c|}
\hline $\begin{array}{l}\text { Components: } \\
\text { (1) 3-Aminobenzoic acid; } \\
\mathrm{C}_{7} \mathrm{H}_{7} \mathrm{NO}_{2} ;[99-05-8] \\
\text { (2) Benzene; } \mathrm{C}_{6} \mathrm{H}_{6} ;[71-43-2]\end{array}$ & $\begin{array}{l}\text { Original Measurements: } \\
{ }^{100} \text { C. L. Lazzell and J. Johnston, J } \\
\text { Phys. Chem. 32, } 1331 \text { (1928). }\end{array}$ \\
\hline $\begin{array}{l}\text { Variables: } \\
\text { Temperature }\end{array}$ & $\begin{array}{l}\text { Prepared by: } \\
\text { W. E. Acree, Jr. }\end{array}$ \\
\hline
\end{tabular}

Experimental Values

\begin{tabular}{lcc}
\hline \hline$T / \mathrm{K}$ & $x_{2}{ }^{\mathrm{a}}$ & $x_{1}{ }^{\mathrm{b}}$ \\
\hline 298.2 & 0.9992 & 0.0008 \\
407.6 & 0.9577 & 0.0423 \\
411.7 & 0.9447 & 0.0553 \\
416.9 & 0.9246 & 0.0754 \\
423.6 & 0.8740 & 0.1260 \\
426.7 & 0.8046 & 0.1954 \\
429.9 & 0.6508 & 0.3492 \\
434.9 & 0.3296 & 0.6704 \\
\hline
\end{tabular}

${ }^{a} x_{2}$ : mole fraction of component 2 in the saturated solution.

${ }^{\mathrm{b}} x_{1}$ : mole fraction solubility of the solute. The solubility is given as molal percentage, which, based on the description of 100 times the mole fraction, is taken to be mole percentage. 


\section{Auxiliary Information}

\section{Method/Apparatus/Procedure:}

Preweighed quantities of solute and solvent were placed in bulbs, which were then sealed and heated in a suitable bath. The temperature at which the solid completely dissolved was recorded, and was taken to be the solid-liquid equilibrium temperature. In a few instances near room temperature, the solubility was determined by agitating the solvent in a thermostat with an excess of solute. After equilibrium had been established, an aliquot of the saturated solution was pipetted, weighed, and the amount of dissolved solute determined with a solution of sodium hydroxide standardized against the pure acid.

Source and Purity of Chemicals:

(1) Certified Pure, was recrystallized several times from hot water, then from $95 \%$ ethanol, and finally from boiling water. The purified sample had a melting point temperature of $451.1 \mathrm{~K}$.

(2) Thiophene-free, Chemical Source not given, was dried over sodium and distilled before use.

\section{Estimated Error:}

Temperature: Not given in paper.

$x_{1}: \pm 5 \%$ (relative error, estimated by compiler).

\subsection{3-Aminobenzoic acid solubility data in esters}

\begin{tabular}{ll}
\hline \hline Components: & Original Measurements: \\
$\begin{array}{l}\text { (1) 3-Aminobenzoic acid; } \\
\left.\mathrm{C}_{7} \mathrm{H}_{7} \mathrm{NO}_{2} ; \text {; } 99-05-8\right]\end{array}$ & ${ }^{101}$ M. Svärd, F. L. Nordström, and A. \\
$\begin{array}{l}\text { (2) Ethyl ethanoate; } \mathrm{C}_{4} \mathrm{H}_{8} \mathrm{O}_{2} ; \\
{[141-78-6]}\end{array}$ & $195(2010)$. \\
\hline Variables: & Prepared by: \\
Temperature & W. E. Acree, Jr. \\
\hline
\end{tabular}

Experimental Values

\begin{tabular}{lcc}
\hline \hline$T / \mathrm{K}$ & $x_{2}{ }^{\mathrm{a}}$ & $x_{1}{ }^{\mathrm{b}}$ \\
\hline 283.2 & 0.9957 & 0.00429 \\
288.2 & 0.9951 & 0.00490 \\
293.3 & 0.9944 & 0.00561 \\
298.2 & 0.9933 & 0.00670 \\
303.2 & 0.9917 & 0.00830 \\
308.2 & 0.9903 & 0.00972 \\
313.2 & 0.9883 & 0.01170 \\
318.2 & 0.9864 & 0.01362 \\
323.2 & 0.9835 & 0.01654
\end{tabular}

${ }^{\mathrm{a}} x_{2}$ : mole fraction of component 2 in the saturated solution.

${ }^{b} x_{1}$ : mole fraction solubility of the solute. The authors noted that the solid existed in different polymorphic forms. The solubility data are for polymorph form I. Solubilities were reported as grams of solute per kilogram of solvent. Mole fraction values were calculated by the compiler.

Experimental Values

\begin{tabular}{lcc}
\hline \hline$T / \mathrm{K}$ & $x_{2}{ }^{\mathrm{a}}$ & $x_{1}{ }^{\mathrm{b}}$ \\
\hline 273.2 & 0.9877 & 0.01227 \\
278.2 & 0.9866 & 0.01338 \\
283.2 & 0.9848 & 0.01522
\end{tabular}

\begin{tabular}{lcc}
\hline \hline$T / \mathrm{K}$ & $x_{2}{ }^{\mathrm{a}}$ & $x_{1}{ }^{\mathrm{b}}$ \\
\hline 288.2 & 0.9832 & 0.01675 \\
293.3 & 0.9813 & 0.01867 \\
298.2 & 0.9789 & 0.02106 \\
303.2 & 0.9771 & 0.02293
\end{tabular}

${ }^{a} x_{2}$ : mole fraction of component 2 in the saturated solution.

${ }^{b} x_{1}$ : mole fraction solubility of the solute. The authors noted that the solid existed in different polymorphic forms. The solubility data are for polymorph form II. Solubilities were reported as grams of solute per kilogram of solvent. Mole fraction values were calculated by the compiler.

\section{Auxiliary Information}

\section{Method/Apparatus/Procedure:}

Constant-temperature bath, magnetic stirrer, analytical balance, and ventilated laboratory hood.

Solubilities were determined by a gravimetric method. Excess solute and solvent were placed in sealed bottles and allowed to equilibrate in a constanttemperature bath for at least $24 \mathrm{~h}$ with stirring. The stirring was discontinued and the solid was allowed to settle to the bottom of the container. An aliquot of the clear solution was removed by syringe, filtered through a $0.2 \mu \mathrm{m}$ PTFE filter, and transferred to a preweighed glass vial. The glass vial was then weighed, and the solvent was allowed to evaporate in a ventilated laboratory hood at ambient room temperature. Once the solvent had evaporated, the vial with solid residue was weighed until constant weight was obtained. The solubility was calculated from the mass of the solid residue and mass of sample analyzed.

\section{Source and Purity of Chemicals:}

(1) $99.8 \%$, Sigma-Aldrich Chemical Company, USA, was used as received. (2) $99.8 \%$, HiperSolv, VWR Scientific, USA, no purification details were provided.

\section{Estimated Error:}

Temperature: $\pm 0.01 \mathrm{~K}$.

$x_{1}: \pm 2 \%$ (relative error)

\begin{tabular}{|c|c|}
\hline $\begin{array}{l}\text { Components: } \\
\text { (1) 3-Aminobenzoic acid; } \\
\mathrm{C}_{7} \mathrm{H}_{7} \mathrm{NO}_{2} \text {; [99-05-8] } \\
\text { (2) Ethyl ethanoate; } \mathrm{C}_{4} \mathrm{H}_{8} \mathrm{O}_{2} \text {; } \\
\text { [141-78-6] }\end{array}$ & $\begin{array}{l}\text { Original Measurements: } \\
{ }^{100} \text { C. L. Lazzell and J. Johnston, J. } \\
\text { Phys. Chem. 32, } 1331 \text { (1928). }\end{array}$ \\
\hline $\begin{array}{l}\text { Variables: } \\
\text { Temperature }\end{array}$ & $\begin{array}{l}\text { Prepared by: } \\
\text { W. E. Acree, Jr. }\end{array}$ \\
\hline
\end{tabular}

Experimental Values

\begin{tabular}{lcc}
\hline \hline$T / \mathrm{K}$ & $x_{2}{ }^{\mathrm{a}}$ & $x_{1}{ }^{\mathrm{b}}$ \\
\hline 298.2 & 0.9870 & 0.0130 \\
383.7 & 0.8824 & 0.1176 \\
418.3 & 0.5281 & 0.4719
\end{tabular}

${ }^{\mathrm{a}} x_{2}$ : mole fraction of component 2 in the saturated solution.

${ }^{b} x_{1}$ : mole fraction solubility of the solute. The solubility is given as molal percentage, which, based on the description of 100 times the mole fraction, is taken to be mole percentage. 


\section{Auxiliary Information}

\section{Method/Apparatus/Procedure:}

Preweighed quantities of solute and solvent were placed in bulbs, which were then sealed and heated in a suitable bath. The temperature at which the solid completely dissolved was recorded, and was taken to be the solid-liquid equilibrium temperature. In a few instances near room temperature, the solubility was determined by agitating the solvent in a thermostat with an excess of solute. After equilibrium had been established, an aliquot of the saturated solution was pipetted, weighed, and the amount of dissolved solute determined with a solution of sodium hydroxide standardized against the pure acid.

Source and Purity of Chemicals:

(1) Certified Pure, was recrystallized several times from hot water, then from $95 \%$ ethanol, and finally from boiling water. The purified sample had a melting point temperature of $451.1 \mathrm{~K}$.

(2) USP grade, Chemical source not given, washed several times with aqueous sodium carbonate, and then with water until the aqueous layer was neutral. The sample was further purified by drying over calcium chloride and then distilling two times before use.

\section{Estimated Error:}

Temperature: Not given in paper.

$x_{1}: \pm 5 \%$ (relative error, estimated by compiler).

\subsection{3-Aminobenzoic acid solubility data in haloalkanes, haloalkenes, and haloaromatic hydrocarbons}

\section{Components: \\ (1) 3-Aminobenzoic acid; \\ $\mathrm{C}_{7} \mathrm{H}_{7} \mathrm{NO}_{2}$; [99-05-8] \\ (2) Trichloromethane; $\mathrm{CHCl}_{3}$; \\ [67-66-3]}

\section{Variables:}

Temperature

\section{Original Measurements:}

${ }^{100} \mathrm{C}$. L. Lazzell and J. Johnston, J.

Phys. Chem. 32, 1331 (1928).

W. E. Acree, Jr.

Experimental Values

\begin{tabular}{lcc}
\hline \hline$T / \mathrm{K}$ & $x_{2}{ }^{\mathrm{a}}$ & $x_{1}{ }^{\mathrm{b}}$ \\
\hline 298.2 & 0.9995 & 0.0005 \\
398.0 & 0.9635 & 0.0365 \\
406.1 & 0.9437 & 0.0563 \\
425.9 & 0.6787 & 0.3213 \\
\hline
\end{tabular}

${ }^{\mathrm{a}} x_{2}$ : mole fraction of component 2 in the saturated solution.

${ }^{b} x_{1}$ : mole fraction solubility of the solute. The solubility is given as molal percentage, which, based on the description of 100 times the mole fraction, is taken to be mole percentage.

\section{Auxiliary Information}

\section{Method/Apparatus/Procedure:}

Preweighed quantities of solute and solvent were placed in bulbs, which were then sealed and heated in a suitable bath. The temperature at which the solid completely dissolved was recorded, and was taken to be the solid-liquid equilibrium temperature. In a few instances near room temperature, the solubility was determined by agitating the solvent in a thermostat with an excess of solute. After equilibrium had been established, an aliquot of the saturated solution was pipetted, weighed, and the amount of dissolved solute determined with a solution of sodium hydroxide standardized against the pure acid.
Source and Purity of Chemicals:

(1) Certified Pure, was recrystallized several times from hot water, then from $95 \%$ ethanol, and finally from boiling water. The purified sample had a melting point temperature of $451.1 \mathrm{~K}$.

(2) Certified Pure, Chemical source not given, was dried over calcium chloride and distilled before use.

\section{Estimated Error:}

Temperature: Not given in paper.

$x_{1}: \pm 5 \%$ (relative error, estimated by compiler).

\subsection{3-Aminobenzoic acid solubility data in alcohols}

\begin{tabular}{ll}
\hline \hline Components: & Original Measurements: \\
$\begin{array}{l}\text { (1) 3-Aminobenzoic acid; } \\
\mathrm{C}_{7} \mathrm{H}_{7} \mathrm{NO}_{2} ;[\text { [99-05-8] }\end{array}$ & ${ }^{101} \mathrm{M}$. Svärd, F. L. Nordström, and A. \\
(2) Methanol; $\mathrm{CH}_{4} \mathrm{O} ;[67-56-1]$ & $195(2010)$. \\
\hline Variables: & Prepared by: \\
Temperature & W. E. Acree, Jr. \\
\hline
\end{tabular}

Experimental Values

\begin{tabular}{lcc}
\hline \hline$T / \mathrm{K}$ & $x_{2}{ }^{\mathrm{a}}$ & $x_{1}{ }^{\mathrm{b}}$ \\
\hline 283.2 & 0.9911 & 0.00893 \\
288.2 & 0.9897 & 0.01025 \\
293.3 & 0.9881 & 0.01194 \\
298.2 & 0.9860 & 0.01399 \\
303.2 & 0.9831 & 0.01691 \\
308.2 & 0.9798 & 0.02016 \\
313.2 & 0.9771 & 0.02294 \\
318.2 & 0.9731 & 0.02686 \\
323.2 & 0.9686 & 0.03135 \\
\hline
\end{tabular}

${ }^{a} x_{2}$ : mole fraction of component 2 in the saturated solution.

${ }^{b} x_{1}$ : mole fraction solubility of the solute. The authors noted that the solid existed in different polymorphic forms. The solubility data are for polymorph form I. Solubilities were reported as grams of solute per kilogram of solvent. Mole fraction values were calculated by the compiler.

\section{Auxiliary Information}

\section{Method/Apparatus/Procedure:}

Constant-temperature bath, magnetic stirrer, analytical balance, and ventilated laboratory hood.

Solubilities were determined by a gravimetric method. Excess solute and solvent were placed in sealed bottles and allowed to equilibrate in a constanttemperature bath for at least $24 \mathrm{~h}$ with stirring. The stirring was discontinued and the solid was allowed to settle to the bottom of the container. An aliquot of the clear solution was removed by syringe, filtered through a $0.2 \mu \mathrm{m}$ PTFE filter, and transferred to a preweighed glass vial. The glass vial was then weighed, and the solvent was allowed to evaporate in a ventilated laboratory hood at ambient room temperature. Once the solvent had evaporated, the vial with solid residue was weighed until constant weight was obtained. The solubility was calculated from the mass of the solid residue and mass of sample analyzed.

\section{Source and Purity of Chemicals:}

(1) $99.7 \%$, Sigma-Aldrich Chemical Company, USA, used as received.

(2) $99.9+\%$, VWR Scientific, USA, used as received.

\section{Estimated Error:}

Temperature: $\pm 0.01 \mathrm{~K}$.

$x_{1}: \pm 2 \%$ (relative error) 


\begin{tabular}{|c|c|}
\hline $\begin{array}{l}\text { Components: } \\
\text { (1) 3-Aminobenzoic acid; } \\
\mathrm{C}_{7} \mathrm{H}_{7} \mathrm{NO}_{2} ;[99-05-8] \\
\text { (2) Methanol; } \mathrm{CH}_{4} \mathrm{O} ;[67-56-1]\end{array}$ & $\begin{array}{l}\text { Original Measurements: } \\
{ }^{100} \text { C. L. Lazzell and J. Johnston, J. } \\
\text { Phys. Chem. 32, } 1331 \text { (1928). }\end{array}$ \\
\hline Variables: & Prepared by: \\
\hline Temperature & W. E. Acree, Jr. \\
\hline
\end{tabular}

Experimental Values

\begin{tabular}{lcc}
\hline \hline$T / \mathrm{K}$ & $x_{2}{ }^{\mathrm{a}}$ & $x_{1}{ }^{\mathrm{b}}$ \\
\hline 298.2 & 0.9830 & 0.0170 \\
359.4 & 0.9027 & 0.0973 \\
382.8 & 0.7993 & 0.2007
\end{tabular}

${ }^{a} x_{2}$ : mole fraction of component 2 in the saturated solution.

${ }^{\mathrm{b}} x_{1}$ : mole fraction solubility of the solute. The solubility is given as molal percentage, which, based on the description of 100 times the mole fraction, is taken to be mole percentage.

\section{Auxiliary Information}

\section{Method/Apparatus/Procedure:}

Preweighed quantities of solute and solvent were placed in bulbs, which were then sealed and heated in a suitable bath. The temperature at which the solid completely dissolved was recorded, and was taken to be the solid-liquid equilibrium temperature. In a few instances near room temperature, the solubility was determined by agitating the solvent in a thermostat with an excess of solute. After equilibrium had been established, an aliquot of the saturated solution was pipetted, weighed, and the amount of dissolved solute determined with a solution of sodium hydroxide standardized against the pure acid.

\section{Source and Purity of Chemicals:}

(1) Certified Pure, was recrystallized several times from hot water, then from $95 \%$ ethanol, and finally from boiling water. The purified sample had a melting point temperature of $451.1 \mathrm{~K}$.

(2) Purity not given, Chemical source not given, no purification details were provided.

\section{Estimated Error:}

Temperature: Not given in paper.

$x_{1}: \pm 5 \%$ (relative error, estimated by compiler).

\begin{tabular}{|c|c|}
\hline $\begin{array}{l}\text { Components: } \\
\text { (1) 3-Aminobenzoic acid; } \\
\mathrm{C}_{7} \mathrm{H}_{7} \mathrm{NO}_{2} ;[99-05-8] \\
\text { (2) Ethanol; } \mathrm{C}_{2} \mathrm{H}_{6} \mathrm{O} ;[64-17-5]\end{array}$ & $\begin{array}{l}\text { Original Measurements: } \\
{ }^{100} \text { C. L. Lazzell and J. Johnston, J. } \\
\text { Phys. Chem. 32, } 1331 \text { (1928). }\end{array}$ \\
\hline $\begin{array}{l}\text { Variables: } \\
\text { Temperature }\end{array}$ & $\begin{array}{l}\text { Prepared by: } \\
\text { W. E. Acree, Jr. }\end{array}$ \\
\hline
\end{tabular}

Experimental Values

\begin{tabular}{lcc}
\hline \hline$T / \mathrm{K}$ & $x_{2}{ }^{\mathrm{a}}$ & $x_{1}{ }^{\mathrm{b}}$ \\
\hline 298.2 & 0.9848 & 0.0152 \\
371.8 & 0.8811 & 0.1189 \\
388.7 & 0.7589 & 0.2411 \\
413.7 & 0.5208 & 0.4792 \\
436.8 & 0.2194 & 0.7806 \\
\hline
\end{tabular}

${ }^{\mathrm{a}} x_{2}$ : mole fraction of component 2 in the saturated solution.

${ }^{b} x_{1}$ : mole fraction solubility of the solute. The solubility is given as molal percentage, which, based on the description of 100 times the mole fraction, is taken to be mole percentage.

\section{Auxiliary Information}

\section{Method/Apparatus/Procedure:}

Preweighed quantities of solute and solvent were placed in bulbs, which were then sealed and heated in a suitable bath. The temperature at which the solid completely dissolved was recorded, and was taken to be the solid-liquid equilibrium temperature. In a few instances near room temperature, the solubility was determined by agitating the solvent in a thermostat with an excess of solute. After equilibrium had been established, an aliquot of the saturated solution was pipetted, weighed, and the amount of dissolved solute determined with a solution of sodium hydroxide standardized against the pure acid.

\section{Source and Purity of Chemicals:}

(1) Certified Pure, was recrystallized several times from hot water, then from $95 \%$ ethanol, and finally from boiling water. The purified sample had a melting point temperature of $451.1 \mathrm{~K}$.

(2) $95 \%$, Chemical source not given, was refluxed with lime and distilled. The sample was further purified by drying over sodium and distilling twice before use.

\section{Estimated Error:}

Temperature: Not given in paper.

$x_{1}: \pm 5 \%$ (relative error, estimated by compiler).

\begin{tabular}{|c|c|}
\hline $\begin{array}{l}\text { Components: } \\
\text { (1) 3-Aminobenzoic acid; } \\
\mathrm{C}_{7} \mathrm{H}_{7} \mathrm{NO}_{2} \text {; [99-05-8] } \\
\text { (2) 1-Butanol; } \mathrm{C}_{4} \mathrm{H}_{10} \mathrm{O} ;[71-36-3]\end{array}$ & $\begin{array}{l}\text { Original Measurements: } \\
{ }^{100} \text { C. L. Lazzell and J. Johnston, J. } \\
\text { Phys. Chem. 32, } 1331 \text { (1928). }\end{array}$ \\
\hline $\begin{array}{l}\text { Variables: } \\
\text { Temperature }\end{array}$ & $\begin{array}{l}\text { Prepared by: } \\
\text { W. E. Acree, Jr. }\end{array}$ \\
\hline
\end{tabular}

Experimental Values

\begin{tabular}{lcc}
\hline \hline$T / \mathrm{K}$ & $x_{2}{ }^{\mathrm{a}}$ & $x_{1}{ }^{\mathrm{b}}$ \\
\hline 400.4 & 0.7928 & 0.2072 \\
411.9 & 0.7065 & 0.2935 \\
\hline${ }^{\mathrm{a}} X_{2}:$ mole fraction of component 2 in the saturated solution. \\
${ }^{\mathrm{b}}{ }_{X_{1}}:$ mole fraction solubility of the solute. The solubility is given as molal \\
percentage, which, based on the description of 100 times the mole fraction, is \\
taken to be mole percentage.
\end{tabular}




\section{Auxiliary Information}

\section{Method/Apparatus/Procedure:}

Preweighed quantities of solute and solvent were placed in bulbs, which were then sealed and heated in a suitable bath. The temperature at which the solid completely dissolved was recorded, and was taken to be the solid-liquid equilibrium temperature.

Source and Purity of Chemicals:

(1) Certified Pure, was recrystallized several times from hot water, then from $95 \%$ ethanol, and finally from boiling water. The purified sample had a melting point temperature of $451.1 \mathrm{~K}$.

(2) Purity not given, Chemical source not given, no purification details were provided.

\section{Estimated Error:}

Temperature: Not given in paper.

$x_{1}: \pm 5 \%$ (relative error, estimated by compiler).

\subsection{3-Aminobenzoic acid solubility data in miscellaneous organic solvents}

\begin{tabular}{|c|c|}
\hline $\begin{array}{l}\text { Components: } \\
\text { (1) 3-Aminobenzoic acid; } \\
\mathrm{C}_{7} \mathrm{H}_{7} \mathrm{NO}_{2} \text {; [99-05-8] } \\
\text { (2) Ethanenitrile; } \mathrm{C}_{2} \mathrm{H}_{3} \mathrm{~N} \text {; } \\
\text { [75-05-8] }\end{array}$ & $\begin{array}{l}\text { Original Measurements: } \\
{ }^{101} \text { M. Svärd, F. L. Nordström, and A. } \\
\text { C. Rasmuson, Cryst. Growth Des. 10, } \\
195 \text { (2010). }\end{array}$ \\
\hline $\begin{array}{l}\text { Variables: } \\
\text { Temperature }\end{array}$ & $\begin{array}{l}\text { Prepared by: } \\
\text { W. E. Acree, Jr. }\end{array}$ \\
\hline
\end{tabular}

Experimental Values

\begin{tabular}{lcc}
\hline \hline$T / \mathrm{K}$ & $x_{2}{ }^{\mathrm{a}}$ & $x_{1}{ }^{\mathrm{b}}$ \\
\hline 283.2 & 0.9984 & 0.00157 \\
288.2 & 0.9982 & 0.00177 \\
293.3 & 0.9979 & 0.00214 \\
298.2 & 0.9972 & 0.00282 \\
303.2 & 0.9966 & 0.00337 \\
308.2 & 0.9955 & 0.00447 \\
313.2 & 0.9944 & 0.00561 \\
318.2 & 0.9933 & 0.00673 \\
323.2 & 0.9917 & 0.00830 \\
\hline
\end{tabular}

${ }^{\mathrm{a}} x_{2}$ : mole fraction of component 2 in the saturated solution.

${ }^{b} x_{1}$ : mole fraction solubility of the solute. The authors noted that the solid existed in different polymorphic forms. The solubility data are for polymorph form I. Solubilities were reported as grams of solute per kilogram of solvent. Mole fraction values were calculated by the compiler.

Experimental Values

\begin{tabular}{lcc}
\hline \hline$T / \mathrm{K}$ & $x_{2}{ }^{\mathrm{a}}$ & $x_{1}{ }^{\mathrm{b}}$ \\
\hline 283.2 & 0.9958 & 0.00423 \\
288.2 & 0.9950 & 0.00501 \\
293.3 & 0.9940 & 0.00603 \\
298.2 & 0.9930 & 0.00695 \\
303.2 & 0.9919 & 0.00813
\end{tabular}

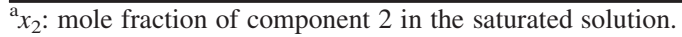

${ }^{b} x_{1}$ : mole fraction solubility of the solute. The authors noted that the solid existed in different polymorphic forms. The solubility data are for polymorph form II. Solubilities were reported as grams of solute per kilogram of solvent. Mole fraction values were calculated by the compiler.
Auxiliary Information

\section{Method/Apparatus/Procedure:}

Constant-temperature bath, magnetic stirrer, analytical balance, and ventilated laboratory hood.

Solubilities were determined by a gravimetric method. Excess solute and solvent were placed in sealed bottles and allowed to equilibrate in a constanttemperature bath for at least $24 \mathrm{~h}$ with stirring. The stirring was discontinued and the solid was allowed to settle to the bottom of the container. An aliquot of the clear solution was removed by syringe, filtered through a $0.2 \mu \mathrm{m}$ PTFE filter, and transferred to a preweighed glass vial. The glass vial was then weighed, and the solvent was allowed to evaporate in a ventilated laboratory hood at ambient room temperature. Once the solvent had evaporated, the vial with solid residue was weighed until constant weight was obtained. The solubility was calculated from the mass of the solid residue and mass of sample analyzed.

Source and Purity of Chemicals:

(1) $99.8 \%$, Sigma-Aldrich Chemical Company, USA, was used as received. (2) $99.8 \%$, LiChrosolv, VWR Scientific, USA, no purification details were provided.

Estimated Error:

Temperature: $\pm 0.01 \mathrm{~K}$.

$x_{1}: \pm 2 \%$ (relative error)

\section{Solubility of 4-Aminobenzoic Acid in Organic Solvents}

\subsection{Critical evaluation of experimental solubility data}

There have been several published studies ${ }^{51,102-105}$ investigating the solubility behavior of 4-aminobenzoic acid in organic solvents of varying polarity and hydrogen-bonding capability. Daniels et al. ${ }^{103}$ measured the solubility of 4 aminobenzoic acid in 13 alcohols (ethanol, 1-propanol, 2propanol, 1-butanol, 2-butanol, 2-methyl-1-propanol, 1-pentanol, 2-pentanol, 3-methyl-1-butanol, 1-hexanol, 1-heptanol, 1-octanol, and 1-decanol), and in one cyclic ether (1,4-dioxane) at $298 \mathrm{~K}$. Results of the experimental measurements were used to calculate the Abraham solute descriptors of 4-aminobenzoic acid. The authors were able to assemble a total of 26 $\log _{10}(S R$ or $P$ ) equations for which experimental partition coefficient data, solubility ratios, Abraham model equation coefficients and aqueous molar solubility were available. The logarithm of the aqueous molar solubility of 4-aminobenzoic acid is $\log _{10} c_{1, \mathrm{w}}=-1.37 .{ }^{106}$ The McGowan volume of 4aminobenzoic acid, $V=1.0315$, was calculated from the number of chemical bonds in the molecule and the individual atomic group volumes, $A V_{i}$, given in Sec. 1.3. The excess molar refraction solute descriptor was estimated as $E=1.075$. This left three solute descriptors $(S, A$, and $B$ ) still to be determined. The 26 equations were then solved using the Microsoft "SOLVER" program to yield numerical values of the remaining four solute descriptors, $S=1.650, A=0.940$, and $B$ $=0.600$, that best described the $\log _{10}(S R$ or $P)$ values. The calculated molecular solute descriptors reproduced the $\log _{10}$ ( $S R$ or $P$ ) values to within an average standard deviation of $0.120 \log _{10}$ units. 
TABLE 14. Comparison between observed and calculated molar solubilities of 4-aminobenzoic acid based on the Abraham model, Eq. (20)

\begin{tabular}{lcc}
\hline \hline Solvent & $\log _{10} c_{1}{ }^{\text {exp }}$ & $\log _{10} c_{1}{ }^{\text {calc}} ;$ Eq. (20) \\
\hline Ethanol & -0.082 & -0.222 \\
1-Propanol & -0.360 & -0.410 \\
2-Propanol & -0.384 & -0.387 \\
1-Butanol & -0.470 & -0.593 \\
2-Butanol & -0.520 & -0.519 \\
2-Methyl-1-propanol & -0.724 & -0.675 \\
1-Pentanol & -0.616 & -0.652 \\
2-Pentanol & -0.673 & -0.682 \\
3-Methyl-1-butanol & -0.742 & -0.780 \\
1-Hexanol & -0.670 & -0.722 \\
1-Heptanol & -0.792 & -0.811 \\
1-Octanol & -0.877 & -0.814 \\
1-Decanol & -1.040 & -0.996 \\
1,1'-Oxybisethane & $-0.888^{\mathrm{a}}$ & -1.027 \\
1,4-Dioxane & -0.096 & -0.122 \\
Propanone & $-0.158^{\mathrm{a}}$ & -0.278 \\
\hline
\end{tabular}

${ }^{\mathrm{a}}$ Experimental data from a study by Barra et al. ${ }^{102}$ All other experimental values are from Daniels et al. ${ }^{103}$

After the 4-aminobenzoic acid solubility study was published, Abraham model correlations have been developed for 3-methyl-1-butanol and 2-pentanol, and equation coefficients for a few solvents were updated based on additional experimental data. The new correlations (listed in Table 1) will be used in illustrating the ability of the Abraham model to correlate the experimental 4-aminobenzoic acid solubility data. Table 14 compares the experimental $\log _{10} c_{1}$ values to calculated values based on Eq. (20) of the Abraham model.

For comparison purposes, the measured mole fraction solubilities of 4-aminobenzoic acid, $x_{1}$, determined by Daniels et al. ${ }^{103}$ were converted into molar solubilities by dividing $x_{1}$ by the ideal molar volume of the saturated solution (i.e., $c_{1}{ }^{\text {sat }}=$ $\left.x_{1} /\left[x_{1} V_{1}+\left(1-x_{1}\right) V_{\text {solvent }}\right]\right)$. The molar volume of the hypothetical subcooled liquid 4-aminobenzoic acid is $V_{\text {solute }}=106.49$ $\mathrm{cm}^{3} \mathrm{~mol}^{-1}$. Examination of the numerical entries in Table 14 reveals that the Abraham model provides a reasonably accurate mathematical description of the observed solubility data, suggesting that there are no obvious outliers in the dataset. Included in Table 14 are experimental solubility measurements for 4-aminobenzoic acid dissolved in 1,1'-oxybisethane and propanone from the solubility study of Barra et al. ${ }^{102}$

Barra et al. ${ }^{102}$ published solubility data for 4 -aminobenzoic acid in two saturated hydrocarbons (heptane and cyclohexane), in one aromatic hydrocarbon (benzene), in one alkyl alkanoate (ethyl ethanoate), in one alkyl ether (1,1'-oxybisethane) and one cyclic ether (1,4-dioxane), in two chloroalkanes (trichloromethane and 1,2-dichloroethane) and one chloroaromatic hydrocarbon (chlorobenzene), in seven alcohols [methanol, ethanol, 1-pentanol, 1-octanol, 1,2-ethanediol, 1,2-propanediol, and 1,2,3-propanetriol (also referred to as glycerol)], in one alkanone (propanone) and one aromatic ketone (acetophenone), and in four miscellaneous organic solvents (ethanoic acid, propanoic acid, formamide, and $N$, $\mathrm{N}$-dimethylformamide) at $298 \mathrm{~K}$. Lin and $\mathrm{Nash}^{51}$ determined 4-aminobenzoic acid solubilities at $298 \mathrm{~K}$ in 16 different organic solvents, including hexane, ethyl ethanoate, tetrahy- drofuran, methanol, 1-propanol, 3-methyl-3-pentanol, 1,2propanediol, 1,3-propanediol, butyl 2-hydroxypropanoate (also called butyl lactate), and 2-ethoxyethanol. Abraham model equation coefficients are available for several of the solvents studied by Barra et al. ${ }^{102}$ and by Lin and Nash. ${ }^{51}$ Several solvents were not included in the Table 13 comparison because of the likelihood of dimerization in the nonpolar alkane and aromatic hydrocarbon solvents, or concerns regarding the formation of possible solid solvates. The Abraham model requires that the solute exist in the same crystalline form in both water and the organic solvent. The measurements of Barra et al. ${ }^{102}$ do provide independent experimental values for the solubility of 4-aminobenzoic acid in ethanol, 1-pentanol, 1-octanol, and 1,4-dioxane. The mole fraction solubilities reported by Barra et al. ${ }^{102}$ are about $10 \%$ less than the published values of Daniels et al. ${ }^{103}$ namely, $x_{1}=0.0465$ versus $x_{1}=0.0506$ for ethanol, $x_{1}=0.0234$ versus $x_{1}=0.0263$ for 1-pentanol, $x_{1}=0.0181$ versus $x_{1}=0.0209$ for 1-octanol, and $x_{1}=0.0632$ versus $x_{1}=0.0700$ for 1,4-dioxane.

There are three or more experimental data points in a few solvents listed in Secs. 8.2-8.10 to compute recommended values. The recommended mole fraction solubilities of 4aminobenzoic acid at $298 \mathrm{~K}$ are as follows: $x_{1}=0.0566$ for ethyl ethanoate [arithmetic average of $x_{1}=0.0576,{ }^{102}$ $0.0532,{ }^{51} 0.0574,{ }^{104}$ and 0.0589 (Ref. 104)]; $x_{1}=0.0570$ for methanol [arithmetic average of $x_{1}=0.0539,{ }^{102} 0.0576,{ }^{51}$ and 0.0594 (Ref. 100)]; and $x_{1}=0.192$ for 1-octanol [arithmetic average of $x_{1}=0.0187,{ }^{99} 0.02088,{ }^{103}$ and 0.01806 (Ref. 102)].

Jia et al..$^{99}$ determined the solubility of 4-aminobenzoic acid in 1-octanol in the temperature range of 293-323 K. The calculated curve-fit parameters from the Buchowski $\lambda \mathrm{h}$-model [Eq. (9)] of $\lambda=0.2257$ and $h=9393.6$ described the observed solubility data to within a mean relative deviation of $0.9 \%$.

Lazzell and Johnston ${ }^{100}$ measured the solubility of 4-aminobenzoic acid in benzene, ethyl ethanoate, trichloromethane, methanol, ethanol, and 1-butanol as a function of temperature. Except for ethyl ethanoate and 1-butanol, the measurements went from $298 \mathrm{~K}$ to well above the normal boiling temperature of the solvent. The internal consistency of the benzene, ethyl ethanoate, and ethanol datasets was assessed by curve-fitting the measured mole fraction solubility data to the Modified Apelblat model to yield the following representations:

$$
\begin{aligned}
\ln x_{1}(\text { in benzene })= & -185.559+\frac{112.06}{T} \\
+ & 30.304 \ln T, \\
\ln x_{1}(\text { in ethyl ethanoate })= & -54.622+\frac{114.72}{T} \\
& +8.884 \ln T, \\
\ln x_{1}(\text { in ethanol })=-45.766+ & \frac{114.89}{T}+7.429 \ln T .
\end{aligned}
$$

The experimental value at $298 \mathrm{~K}$ had to be removed from the benzene dataset in order to obtain a reasonable correlation equation. There were too few data points in the trichloromethane, methanol, and 1-butanol datasets to perform a 
meaningful regression analysis. The average absolute deviations between the observed experimental data and back-calculated values based on Eqs. (32)-(34) of 15.00\%, 3.07\%, and $4.29 \%$ are larger than desired. The large deviation may be due in part to the fact that the solution temperature is above the solvent's normal boiling point temperature, to uncertainties in estimating the amount of solvent that remained in the liquid phase, and the large range covered by the experimental values, $x_{1}=0.0211$ to $x_{1}=0.5342$ for benzene, $x_{1}=0.1219$ to $x_{1}=$ 0.4840 for ethyl ethanoate, and $x_{1}=0.0497$ to $x_{1}=0.6552$ for ethanol. It is more difficult to curve-fit experimental solubility data covering large mole fraction ranges.

The experimental solubility data for 4-aminobenzoic acid in the different organic solvents are in Secs. 8.2-8.10.

\subsection{4-Aminobenzoic acid solubility data in saturated hydrocarbons (including cycloalkanes)}

\begin{tabular}{|c|c|}
\hline $\begin{array}{l}\text { Components: } \\
\text { (1) 4-Aminobenzoic acid; } \\
\mathrm{C}_{7} \mathrm{H}_{7} \mathrm{NO}_{2} ;[150-13-0] \\
\text { (2) Hexane; } \mathrm{C}_{6} \mathrm{H}_{14} ;[110-54-3]\end{array}$ & $\begin{array}{l}\text { Original Measurements: } \\
\text { 51 H.-M. Lin and R. A. Nash, J. Pharm. } \\
\text { Sci. 82, } 1018 \text { (1993). }\end{array}$ \\
\hline $\begin{array}{l}\text { Variables: } \\
T / \mathrm{K}=298.15\end{array}$ & $\begin{array}{l}\text { Prepared by: } \\
\text { W. E. Acree, Jr. }\end{array}$ \\
\hline
\end{tabular}

Experimental Values

\begin{tabular}{lc}
\hline \hline$x_{2}{ }^{\mathrm{a}}$ & $x_{1}{ }^{\mathrm{b}}$ \\
\hline 0.9999 & 0.000001 \\
\hline${ }^{\mathrm{a}} x_{2}:$ mole fraction of component 2 in the saturated solution. \\
${ }^{\mathrm{b}}{ }_{x_{1}: \text { mole fraction solubility of the solute. }}$
\end{tabular}

\section{Auxiliary Information}

\section{Method/Apparatus/Procedure:}

Constant-temperature bath, and high-performance liquid chromatograph.

Excess solute and solvent were placed in screw-capped vials. The vials were sealed with several turns of electrical tape, warmed to about $323 \mathrm{~K}$, and then shaken in a constant-temperature bath for at least $24 \mathrm{~h}$ at $298 \mathrm{~K}$. After equilibrium was reached, the sample was filtered through a 0.45 or $1.0 \mu \mathrm{m}$ filter, and an aliquot was diluted appropriately for chromatographic analysis. Concentrations were determined by a high-performance liquid chromatographic method.

Source and Purity of Chemicals:

(1) Purity not given, Eastman Kodak Chemical Company, Rochester, NY, USA, no information provided concerning purification.

(2) HPLC grade, Aldrich Chemical Company, Milwaukee, WI, USA, no purification details were provided.

\section{Estimated Error:}

Temperature: $\pm 0.2 \mathrm{~K}$ (estimated by compiler).

$x_{1}: \pm 3 \%$ (relative error, estimated by compiler).

\begin{tabular}{ll}
\hline \hline Components: & $\begin{array}{l}\text { Original Measurements: } \\
(1) \text { 4-Aminobenzoic acid; }\end{array}$ \\
${ }_{\mathrm{C}_{7} \mathrm{H}_{7} \mathrm{NO}_{2} ;[150-13-0]}$ J. Barra, M.-A. Peña, and P. \\
(2) Heptane; $\mathrm{C}_{7} \mathrm{H}_{16} ;[142-82-5]$ & $(2000)$. \\
\hline Variables: & Prepared by: \\
$T / \mathrm{K}=298.15$ & W. E. Acree, Jr. \\
\hline
\end{tabular}

Experimental Values

\begin{tabular}{lc}
\hline \hline$x_{2}{ }^{\mathrm{a}}$ & $x_{1}{ }^{\mathrm{b}}$ \\
\hline 0.9999 & 0.0000061 \\
\hline${ }^{\mathrm{a}} x_{2}:$ mole fraction of component 2 in the saturated solution. \\
${ }^{\mathrm{b}} x_{1}:$ mole fraction solubility of the solute.
\end{tabular}

\section{Auxiliary Information}

\section{Method/Apparatus/Procedure:}

Constant-temperature bath, analytical balance, and an ultraviolet/visible spectrophotometer.

Excess solute and solvent were placed in a flask and allowed to equilibrate for several days at constant temperature. The solid phase was removed by filtration, and the clear solution was diluted quantitatively with $96 \%$ ethanol. The concentration of the diluted solution was determined by spectrophotometric measurement at the wavelength of maximum absorption. In instances where the solvent interfered with the spectrophotometric determination, the samples were evaporated to dryness and the residue diluted with $96 \%$ ethanol.

\section{Source and Purity of Chemicals:}

(1) Purity not given, Sigma Chemical Company, USA, no purification details were provided.

(2) Spectrophotometric or Analytical grade, Chemical source not specified, no purification details were provided.

Estimated Error:

Temperature: $\pm 0.2 \mathrm{~K}$.

$x_{1}: \pm 10.0 \%$ (relative error, estimated by compiler).

\begin{tabular}{|c|c|}
\hline $\begin{array}{l}\text { Components: } \\
\text { (1) 4-Aminobenzoic acid; } \\
\mathrm{C}_{7} \mathrm{H}_{7} \mathrm{NO}_{2} ;[150-13-0] \\
\text { (2) Cyclohexane; } \mathrm{C}_{6} \mathrm{H}_{12} \text {; } \\
\text { [110-82-7] }\end{array}$ & $\begin{array}{l}\text { Original Measurements: } \\
{ }^{54} \text { C. K. Hancock, J. N. Pawloski, and J. } \\
\text { P. Idoux, J. Org. Chem. 31, } 3801 \\
\text { (1966). }\end{array}$ \\
\hline $\begin{array}{l}\text { Variables: } \\
T / \mathrm{K}=303.15\end{array}$ & $\begin{array}{l}\text { Prepared by: } \\
\text { W. E. Acree, Jr. }\end{array}$ \\
\hline
\end{tabular}

Experimental Values

\begin{tabular}{lc}
\hline \hline$x_{2}{ }^{\mathrm{a}}$ & $x_{1}{ }^{\mathrm{b}}$ \\
\hline 0.9999 & 0.0000116 \\
\hline${ }^{\mathrm{a}} x_{2}:$ mole fraction of component 2 in the saturated solution. \\
${ }^{\mathrm{b}} x_{1}:$ mole fraction solubility of the solute.
\end{tabular}




\section{Auxiliary Information}

\section{Method/Apparatus/Procedure:}

Constant-temperature bath, Soxhlet thimble, conical flask, and analytical balance.

Excess solute and solvent were placed in a conical flask and allowed to equilibrate for several days at constant temperature. Attainment of equilibrium was verified by several repetitive measurements and by approaching equilibrium from supersaturation. Aliquots of saturated solutions were transferred using a Soxhlet thimble equipped with a one-hole cork stopper and an inverted U-shaped delivery tube to a second ground-glass stoppered flask suspended in the $303 \mathrm{~K}$ water bath. Successive portions of the contents were evaporated at room temperature in a tared aluminum foil weighing dish under a bell jar through which a slow stream of dry air was passed. The second flask plus unused saturated solution and the aluminum foil dish plus residue were weighed. The saturation solubility of the solute was calculated from the recorded mass data and molar masses of the solute and solvent.

Source and Purity of Chemicals:

(1) Purity not given, Chemical source not specified, was recrystallized several times from aqueous-ethanol mixture, and then dried under vacuum over phosphorous pentoxide.

(2) Reagent grade, Chemical source not specified, refluxed over phosphorous pentoxide for $24 \mathrm{~h}$, and then distilled through a $3 \times 80 \mathrm{~cm}$ column filled with 0.32 -cm glass helices.

\section{Estimated Error:}

Temperature: $\pm 0.02 \mathrm{~K}$.

$x_{1}: \pm 10 \%$ (relative error, by compiler).

\begin{tabular}{ll}
\hline \hline Components: & Original Measurements: \\
(1) 4-Aminobenzoic acid; & ${ }^{102}$ J. Barra, M.-A. Peña, and P. \\
$\mathrm{C}_{7} \mathrm{H}_{7} \mathrm{NO}_{2} ;[150-13-0]$ & Bustamante, Eur. J. Pharm. Sci. 10, 153 \\
$\begin{array}{l}\text { (2) Cyclohexane; } \mathrm{C}_{6} \mathrm{H}_{12} ; \\
{[110-82-7]}\end{array}$ & $(2000)$. \\
\hline Variables: & Prepared by: \\
$T / \mathrm{K}=298.15$ & W. E. Acree, Jr. \\
\hline
\end{tabular}

Experimental Values

\begin{tabular}{lc}
\hline \hline$x_{2}{ }^{\mathrm{a}}$ & $x_{1}{ }^{\mathrm{b}}$ \\
\hline 0.9999 & 0.00000087 \\
\hline
\end{tabular}

${ }^{a} x_{2}$ : mole fraction of component 2 in the saturated solution.

${ }^{\mathrm{b}} x_{1}$ : mole fraction solubility of the solute.

\section{Auxiliary Information}

\section{Method/Apparatus/Procedure:}

Constant-temperature bath, analytical balance, and an ultraviolet/visible spectrophotometer.

Excess solute and solvent were placed in a flask and allowed to equilibrate for several days at constant temperature. The solid phase was removed by filtration, and the clear solution was diluted quantitatively with $96 \%$ ethanol. The concentration of the diluted solution was determined by spectrophotometric measurement at the wavelength of maximum absorption. In instances where the solvent interfered with the spectrophotometric determination, the samples were evaporated to dryness and the residue diluted with $96 \%$ ethanol.

\section{Source and Purity of Chemicals:}

(1) Purity not given, Sigma Chemical Company, USA, no purification details were provided.

(2) Spectrophotometric or Analytical grade, Chemical source not specified, no purification details were provided.
Estimated Error:

Temperature: $\pm 0.2 \mathrm{~K}$.

$x_{1}: \pm 10.0 \%$ (relative error, estimated by compiler).

\subsection{4-Aminobenzoic acid solubility data in aromatic hydrocarbons}

\begin{tabular}{ll}
\hline \hline Components: & Original Measurements: \\
$\begin{array}{l}\text { (1) 4-Aminobenzoic acid; } \\
\mathrm{C}_{7} \mathrm{H}_{7} \mathrm{NO}_{2} ;[150-13-0]\end{array}$ & ${ }^{54} \mathrm{C}$. K. Hancock, J. N. Pawloski, and J. \\
(2) Benzene; $\mathrm{C}_{6} \mathrm{H}_{6} ;[71-43-2]$ & P. Idoux, J. Org. Chem. 31, 3801 \\
\hline Variables: & $(1966)$. \\
$T / \mathrm{K}=303.15$ & Prepared by: \\
\hline
\end{tabular}

Experimental Values

\begin{tabular}{lc}
\hline \hline$x_{2}{ }^{\mathrm{a}}$ & $x_{1}{ }^{\mathrm{b}}$ \\
\hline 0.9996 & 0.000427 \\
\hline${ }^{\mathrm{a}} x_{2}:$ mole fraction of component 2 in the saturated solution. & \\
${ }^{\mathrm{b}} x_{1}:$ mole fraction solubility of the solute. &
\end{tabular}

\section{Auxiliary Information}

\section{Method/Apparatus/Procedure:}

Constant-temperature bath, Soxhlet thimble, conical flask, and analytical balance.

Excess solute and solvent were placed in a conical flask and allowed to equilibrate for several days at constant temperature. Attainment of equilibrium was verified by several repetitive measurements and by approaching equilibrium from supersaturation. Aliquots of saturated solutions were transferred using a Soxhlet thimble equipped with a one-hole cork stopper and an inverted $\mathrm{U}$-shaped delivery tube to a second ground-glass stoppered flask suspended in the $303 \mathrm{~K}$ water bath. Successive portions of the contents were evaporated at room temperature in a tared aluminum foil weighing dish under a bell jar through which a slow stream of dry air was passed. The second flask plus unused saturated solution and the aluminum foil dish plus residue were weighed. The saturation solubility of the solute was calculated from the recorded mass data and molar masses of the solute and solvent.

\section{Source and Purity of Chemicals:}

(1) Purity not given, Chemical source not specified, was recrystallized several times from aqueous-ethanol mixture, and then dried under vacuum over phosphorous pentoxide.

(2) Reagent grade, Chemical source not specified, refluxed over phosphorous pentoxide for $24 \mathrm{~h}$, and then distilled through a $3 \times 80 \mathrm{~cm}$ column filled with $0.32-\mathrm{cm}$ glass helices.

\section{Estimated Error:}

Temperature: $\pm 0.02 \mathrm{~K}$.

$x_{1}: \pm 5 \%$ (relative error, by compiler).

\begin{tabular}{ll}
\hline \hline Components: & Original Measurements: \\
$\begin{array}{l}\text { (1) 4-Aminobenzoic acid; } \\
\mathrm{C}_{7} \mathrm{H}_{7} \mathrm{NO}_{2} ;[150-13-0]\end{array}$ & $\begin{array}{l}{ }^{\text {J. }} \text { Barra, M.-A. Peña, and P. } \\
\text { (2) Benzene; } \mathrm{C}_{6} \mathrm{H}_{6} ;[71-43-2]\end{array}$ \\
\hline Variables: & $(2000)$. \\
$T / \mathrm{K}=298.15$ & Prepared by: \\
\hline
\end{tabular}


Experimental Values

\begin{tabular}{lc}
\hline \hline$x_{2}{ }^{\mathrm{a}}$ & $x_{1}{ }^{\mathrm{b}}$ \\
\hline 0.9998 & 0.000210 \\
${ }^{\mathrm{a}} x_{2}:$ mole fraction of component 2 in the saturated solution. \\
${ }^{\mathrm{b}} x_{1}:$ mole fraction solubility of the solute.
\end{tabular}

\section{Auxiliary Information}

\section{Method/Apparatus/Procedure:}

Constant-temperature bath, analytical balance, and an ultraviolet/visible spectrophotometer.

Excess solute and solvent were placed in a flask and allowed to equilibrate for several days at constant temperature. The solid phase was removed by filtration, and the clear solution was diluted quantitatively with $96 \%$ ethanol. The concentration of the diluted solution was determined by spectrophotometric measurement at the wavelength of maximum absorption. In instances where the solvent interfered with the spectrophotometric determination, the samples were evaporated to dryness and the residue diluted with $96 \%$ ethanol.

\section{Source and Purity of Chemicals:}

(1) Purity not given, Sigma Chemical Company, USA, no purification details were provided.

(2) Spectrophotometric or Analytical grade, Chemical source not specified, no purification details were provided.

\section{Estimated Error:}

Temperature: $\pm 0.2 \mathrm{~K}$.

$x_{1}: \pm 3.0 \%$ (relative error, estimated by compiler).

\begin{tabular}{|c|c|}
\hline $\begin{array}{l}\text { Components: } \\
\text { (1) 4-Aminobenzoic acid; } \\
\mathrm{C}_{7} \mathrm{H}_{7} \mathrm{NO}_{2} ;[150-13-0] \\
\text { (2) Benzene; } \mathrm{C}_{6} \mathrm{H}_{6} ;[71-43-2]\end{array}$ & $\begin{array}{l}\text { Original Measurements: } \\
\text { 100 C. L. Lazzell and J. Johnston, J. } \\
\text { Phys. Chem. 32, } 1331 \text { (1928). }\end{array}$ \\
\hline Variables: & Prepared by: \\
\hline Temperature & W. E. Acree, Jr. \\
\hline
\end{tabular}

Experimental Values

\begin{tabular}{lcc}
\hline \hline$T / \mathrm{K}$ & $x_{2}{ }^{\mathrm{a}}$ & $x_{1}{ }^{\mathrm{b}}$ \\
\hline 298.2 & 0.9996 & 0.0004 \\
394.9 & 0.9789 & 0.0211 \\
413.1 & 0.9502 & 0.0498 \\
428.0 & 0.8586 & 0.1414 \\
428.4 & 0.8434 & 0.1566 \\
429.7 & 0.8176 & 0.1824 \\
433.0 & 0.7150 & 0.2850 \\
433.4 & 0.6763 & 0.3237 \\
438.2 & 0.4658 & 0.5342
\end{tabular}

${ }^{\mathrm{a}} x_{2}$ : mole fraction of component 2 in the saturated solution.

${ }^{b} x_{1}$ : mole fraction solubility of the solute. The solubility is given as molal percentage, which, based on the description of 100 times the mole fraction, is taken to be mole percentage.

\section{Auxiliary Information}

Method/Apparatus/Procedure:

Preweighed quantities of solute and solvent were placed in bulbs, which were then sealed and heated in a suitable bath. The temperature at which the solid completely dissolved was recorded, and was taken to be the solid-liquid equilibrium temperature. In a few instances near room temperature, the solubility was determined by agitating the solvent in a thermostat with an excess of solute. After equilibrium had been established, an aliquot of the saturated solution was pipetted, weighed, and the amount of dissolved solute determined with a solution of sodium hydroxide standardized against the pure acid.

Source and Purity of Chemicals:

(1) Certified Pure, Chemical source not given, was recrystallized several times from hot aqueous solution, then from $95 \%$ ethanol, and finally from hot water. The purified solute had a melting point temperature of $460.2 \mathrm{~K}$.

(2) Thiophene-free, Chemical source not given, was dried over sodium and distilled before use.

\section{Estimated Error:}

Temperature: Not given in paper.

$x_{1}: \pm 5 \%$ (relative error, estimated by compiler).

\subsection{4-Aminobenzoic acid solubility data in esters}

\begin{tabular}{ll}
\hline \hline Components: & $\begin{array}{l}\text { Original Measurements: } \\
\text { (1) 4-Aminobenzoic acid; }\end{array}$ \\
$\mathrm{C}_{7} \mathrm{H}_{7} \mathrm{NO}_{2} ;$ [150-13-0] & $\begin{array}{l}\text { J. Barra, M.-A. Peña, and P. } \\
\text { (2) Ethyl ethanoate; } \mathrm{C}_{4} \mathrm{H}_{8} \mathrm{O}_{2} ;\end{array}$ \\
[141-78-6] & $(2000)$. \\
\hline Variables: & Prepared byr. J. Pharm. Sci. 10, 153 \\
$T / \mathrm{K}=298.15$ & W. E. Acree, Jr. \\
\hline
\end{tabular}

\section{Experimental Values}

\begin{tabular}{lc}
\hline \hline$x_{2}^{\mathrm{a}}$ & $x_{1}{ }^{\mathrm{b}}$ \\
\hline 0.9424 & 0.0576 \\
\hline$x_{2}: \operatorname{lngle}$ fracton of conponent 2 in
\end{tabular}

${ }^{a} x_{2}$ : mole fraction of component 2 in the saturated solution.

${ }^{\mathrm{b}} x_{1}$ : mole fraction solubility of the solute.

\section{Auxiliary Information}

\section{Method/Apparatus/Procedure:}

Constant-temperature bath, analytical balance, and an ultraviolet/visible spectrophotometer.

Excess solute and solvent were placed in a flask and allowed to equilibrate for several days at constant temperature. The solid phase was removed by filtration, and the clear solution was diluted quantitatively with $96 \%$ ethanol. The concentration of the diluted solution was determined by spectrophotometric measurement at the wavelength of maximum absorption. In instances where the solvent interfered with the spectrophotometric determination, the samples were evaporated to dryness and the residue diluted with $96 \%$ ethanol.

Source and Purity of Chemicals:

(1) Purity not given, Sigma Chemical Company, USA, no purification details were provided.

(2) Spectrophotometric or Analytical grade, Chemical source not specified, no purification details were provided. 
Estimated Error:

Temperature: $\pm 0.2 \mathrm{~K}$.

$x_{1}: \pm 3.0 \%$ (relative error, estimated by compiler).

\begin{tabular}{|c|c|}
\hline $\begin{array}{l}\text { Components: } \\
\text { (1) 4-Aminobenzoic acid; } \\
\mathrm{C}_{7} \mathrm{H}_{7} \mathrm{NO}_{2} ;[150-13-0] \\
\text { (2) Ethyl ethanoate; } \mathrm{C}_{4} \mathrm{H}_{8} \mathrm{O}_{2} \text {; } \\
\text { [141-78-6] }\end{array}$ & $\begin{array}{l}\text { Original Measurements: } \\
\text { 51 H.-M. Lin and R. A. Nash, J. Pharm. } \\
\text { Sci. 82, } 1018 \text { (1993). }\end{array}$ \\
\hline $\begin{array}{l}\text { Variables: } \\
T / \mathrm{K}=298.15\end{array}$ & $\begin{array}{l}\text { Prepared by: } \\
\text { W. E. Acree, Jr. }\end{array}$ \\
\hline
\end{tabular}

Experimental Values

\begin{tabular}{lc}
\hline \hline$x_{2}{ }^{\mathrm{a}}$ & $x_{1}{ }^{\mathrm{b}}$ \\
\hline 0.9468 & 0.0532 \\
\hline$x_{2}$.
\end{tabular}

${ }^{a} x_{2}$ : mole fraction of component 2 in the saturated solution.

${ }^{\mathrm{b}} x_{1}$ : mole fraction solubility of the solute.

\section{Auxiliary Information}

\section{Method/Apparatus/Procedure:}

Constant-temperature bath, and high-performance liquid chromatograph.

Excess solute and solvent were placed in screw-capped vials. The vials were sealed with several turns of electrical tape, warmed to about $323 \mathrm{~K}$, and then shaken in a constant-temperature bath for at least $24 \mathrm{~h}$ at $298 \mathrm{~K}$. After equilibrium was reached, the sample was filtered through a 0.45 or $1.0 \mu \mathrm{m}$ filter, and an aliquot was diluted appropriately for chromatographic analysis. Concentrations were determined by a high-performance liquid chromatographic method.

\section{Source and Purity of Chemicals:}

(1) Purity not given, Eastman Kodak Chemical Company, Rochester, NY, USA, no information provided concerning purification.

(2) HPLC grade, Aldrich Chemical Company, Milwaukee, WI, USA, no purification details were provided.

\section{Estimated Error:}

Temperature: $\pm 0.2 \mathrm{~K}$ (estimated by compiler).

$x_{1}: \pm 3 \%$ (relative error, estimated by compiler).

\begin{tabular}{|c|c|}
\hline $\begin{array}{l}\text { Components: } \\
\text { (1) 4-Aminobenzoic acid; } \\
\mathrm{C}_{7} \mathrm{H}_{7} \mathrm{NO}_{2} ;[150-13-0] \\
\text { (2) Ethyl ethanoate; } \mathrm{C}_{4} \mathrm{H}_{8} \mathrm{O}_{2} \text {; } \\
\text { [141-78-6] }\end{array}$ & $\begin{array}{l}\text { Original Measurements: } \\
{ }^{104} \text { S. Gracin and A. C. Rasmuson, } \\
\text { Cryst. Growth Des. 4, } 1013 \text { (2004). }\end{array}$ \\
\hline $\begin{array}{l}\text { Variables: } \\
\text { Temperature }\end{array}$ & $\begin{array}{l}\text { Prepared by: } \\
\text { W. E. Acree, Jr. }\end{array}$ \\
\hline
\end{tabular}

Experimental Values

\begin{tabular}{lcc}
\hline \hline$T / \mathrm{K}$ & $x_{2}{ }^{\mathrm{a}}$ & $x_{1}{ }^{\mathrm{b}}$ \\
\hline 268.2 & 0.9554 & 0.0446 \\
273.2 & 0.9554 & 0.0446 \\
278.2 & 0.9541 & 0.0459 \\
283.2 & 0.9522 & 0.0478 \\
288.2 & 0.9491 & 0.0509 \\
293.2 & 0.9459 & 0.0541 \\
297.2 & 0.9431 & 0.0569 \\
301.2 & 0.9411 & 0.0589 \\
305.2 & 0.9383 & 0.0617 \\
309.2 & 0.9360 & 0.0640 \\
315.2 & 0.9282 & 0.0718 \\
323.2 & 0.9235 & 0.0765 \\
\hline
\end{tabular}

${ }^{a} x_{2}$ : mole fraction of component 2 in the saturated solution.

${ }^{b} x_{1}$ : mole fraction solubility of the solute. The authors noted that the solid existed in different polymorphic forms. The solubility data are for polymorph $\alpha$ (needles). Solubilities were reported as grams of solute per kilogram of solvent. Mole fraction values were calculated by the compiler.

Experimental Values

\begin{tabular}{lcc}
\hline \hline$T / \mathrm{K}$ & $x_{2}{ }^{\mathrm{a}}$ & $x_{1}{ }^{\mathrm{b}}$ \\
\hline 262.2 & 0.9608 & 0.0392 \\
268.2 & 0.9608 & 0.0392 \\
273.2 & 0.9608 & 0.0392 \\
278.2 & 0.9581 & 0.0419 \\
283.2 & 0.9551 & 0.0449 \\
288.2 & 0.9508 & 0.0492 \\
293.2 & 0.9470 & 0.0530 \\
297.2 & 0.9428 & 0.0572
\end{tabular}

${ }^{\mathrm{a}} x_{2}$ : mole fraction of component 2 in the saturated solution.

${ }_{x_{1}}$ : mole fraction solubility of the solute. The authors noted that the solid existed in different polymorphic forms. The solubility data are for polymorph form $\beta$ (prisms). Solubilities were reported as grams of solute per kilogram of solvent. Mole fraction values were calculated by the compiler.

\section{Auxiliary Information}

\section{Method/Apparatus/Procedure:}

Constant-temperature bath, magnetic stirrer, analytical balance, oven, and a heating and refrigeration circulator.

Solubilities were determined by a gravimetric method. Excess solute and solvent were placed in sealed bottles and allowed to equilibrate in a constanttemperature bath for at least $24 \mathrm{~h}$ with stirring. The stirring was discontinued and the solid was allowed to settle to the bottom of the container. An aliquot of the clear solution was removed by syringe, filtered through a $0.2 \mu \mathrm{m}$ PTFE filter, and transferred to a preweighed glass vial. The glass vial was then weighed, and the solvent was allowed to evaporate in an oven. Once the solvent had evaporated, the vial with solid residue was weighed until constant weight was obtained. The solubility was calculated from the mass of the solid residue and mass of sample analyzed.

\section{Source and Purity of Chemicals:}

(1) $99.8 \%$, Sigma-Aldrich Chemical Company, USA, was used as received. (2) $99.8 \%$, HiperSolv, VWR Scientific, USA, no purification details were provided.

\section{Estimated Error:}

Temperature: $\pm 0.01 \mathrm{~K}$.

$x_{1}: \pm 2 \%$ (relative error). 


\section{Components:}

(1) 4-Aminobenzoic acid;

$\mathrm{C}_{7} \mathrm{H}_{7} \mathrm{NO}_{2} ;[150-13-0]$

(2) Ethyl ethanoate; $\mathrm{C}_{4} \mathrm{H}_{8} \mathrm{O}_{2}$;

[141-78-6]

\begin{tabular}{ll}
\hline Variables: & Prepared by: \\
Temperature & W. E. Acree, Jr. \\
\hline
\end{tabular}

Experimental Values

\begin{tabular}{lcc}
\hline \hline$T / \mathrm{K}$ & $x_{2}{ }^{\mathrm{a}}$ & $x_{1}{ }^{\mathrm{b}}$ \\
\hline 355.3 & 0.8781 & 0.1219 \\
385.3 & 0.7693 & 0.2307 \\
404.4 & 0.6525 & 0.3475 \\
409.8 & 0.5805 & 0.4195 \\
417.2 & 0.5160 & 0.4840
\end{tabular}

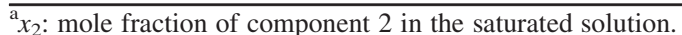

${ }^{b} x_{1}$ : mole fraction solubility of the solute. The solubility is given as molal percentage, which, based on the description of 100 times the mole fraction, is taken to be mole percentage.

\section{Auxiliary Information}

\section{Method/Apparatus/Procedure:}

Preweighed quantities of solute and solvent were placed in bulbs, which were then sealed and heated in a suitable bath. The temperature at which the solid completely dissolved was recorded, and was taken to be the solid-liquid equilibrium temperature. In a few instances near room temperature, the solubility was determined by agitating the solvent in a thermostat with an excess of solute. After equilibrium had been established, an aliquot of the saturated solution was pipetted, weighed, and the amount of dissolved solute determined with a solution of sodium hydroxide standardized against the pure acid.

\section{Source and Purity of Chemicals:}

(1) Certified Pure, Chemical source not given, was recrystallized several times from hot aqueous solution, then from $95 \%$ ethanol, and finally from hot water. The purified solute had a melting point temperature of $460.2 \mathrm{~K}$.

(2) USP grade, Chemical source not given, washed several times with aqueous sodium carbonate, and then with water until the aqueous layer was neutral. The sample was further purified by drying over calcium chloride and then distilling two times before use.

\section{Estimated Error:}

Temperature: Not given in paper.

$x_{1}: \pm 5 \%$ (relative error, estimated by compiler).

\begin{tabular}{ll}
\hline \hline Components: & Original Measurements: \\
(1) $4-\mathrm{Aminobenzoic} \mathrm{acid;}_{\mathrm{C}_{7} \mathrm{H}_{2} ;[150-13-0]}$ & ${ }^{51} \mathrm{H} .-\mathrm{M}$. Lin and R. A. Nash, J. Pharm. \\
$(2) 1,2,3-$ Triacetoxypropane; & Sci. 82, 1018 (1993). \\
$\mathrm{C}_{9} \mathrm{H}_{14} \mathrm{O}_{6} ;[102-76-1]$ & \\
\hline Variables: & \\
$T / \mathrm{K}=298.15$ & Prepared by: \\
\hline
\end{tabular}

Experimental Values

\begin{tabular}{lc}
\hline \hline$x_{2}{ }^{\mathrm{a}}$ & $x_{1}{ }^{\mathrm{b}}$ \\
\hline 0.9224 & 0.0776 \\
\hline$x_{2}$.
\end{tabular}

${ }^{\mathrm{a}} x_{2}$ : mole fraction of component 2 in the saturated solution.

${ }^{\mathrm{b}} x_{1}$ : mole fraction solubility of the solute.

\section{Auxiliary Information}

\section{Method/Apparatus/Procedure:}

Constant-temperature bath, and high-performance liquid chromatograph. Excess solute and solvent were placed in screw-capped vials. The vials were sealed with several turns of electrical tape, warmed to about $323 \mathrm{~K}$, and then shaken in a constant-temperature bath for at least $24 \mathrm{~h}$ at $298 \mathrm{~K}$. After equilibrium was reached, the sample was filtered through a 0.45 or $1.0 \mu \mathrm{m}$ filter, and an aliquot was diluted appropriately for chromatographic analysis. Concentrations were determined by a high-performance liquid chromatographic method.

\section{Source and Purity of Chemicals:}

(1) Purity not given, Eastman Kodak Chemical Company, Rochester, NY, USA, no information provided concerning purification.

(2) Purity not given, Unichema International, no purification details were provided.

\section{Estimated Error:}

Temperature: $\pm 0.2 \mathrm{~K}$ (estimated by compiler). $x_{1}: \pm 3 \%$ (relative error, estimated by compiler).

\subsection{4-Aminobenzoic acid solubility data in ethers}

\begin{tabular}{|c|c|}
\hline $\begin{array}{l}\text { Components: } \\
\text { (1) 4-Aminobenzoic acid; } \\
\mathrm{C}_{7} \mathrm{H}_{7} \mathrm{NO}_{2} ;[150-13-0] \\
\text { (2) } 1,1^{\prime} \text {-Oxybisethane; } \\
\mathrm{C}_{4} \mathrm{H}_{10} \mathrm{O} \text {; [60-29-7] }\end{array}$ & $\begin{array}{l}\text { Original Measurements: } \\
{ }^{102} \text { J. Barra, M.-A. Peña, and P. } \\
\text { Bustamante, Eur. J. Pharm. Sci. 10, } 153 \\
(2000) .\end{array}$ \\
\hline $\begin{array}{l}\text { Variables: } \\
T / \mathrm{K}=298.15\end{array}$ & $\begin{array}{l}\text { Prepared by: } \\
\text { W. E. Acree, Jr. }\end{array}$ \\
\hline
\end{tabular}

Experimental Values

\begin{tabular}{lc}
\hline \hline$x_{2}{ }^{\mathrm{a}}$ & $x_{1}^{\mathrm{b}}$ \\
\hline 0.9864 & 0.0136 \\
\hline${ }^{\mathrm{a}} x_{2}:$ mole fraction of component 2 in the saturated solution. & \\
${ }^{\mathrm{b}} x_{1}:$ mole fraction solubility of the solute. &
\end{tabular}

\section{Auxiliary Information}

\section{Method/Apparatus/Procedure:}

Constant-temperature bath, analytical balance, and an ultraviolet/visible spectrophotometer.

Excess solute and solvent were placed in a flask and allowed to equilibrate for several days at constant temperature. The solid phase was removed by filtration, and the clear solution was diluted quantitatively with $96 \%$ ethanol. The concentration of the diluted solution was determined by spectrophotometric measurement at the wavelength of maximum absorption. In instances where the solvent interfered with the spectrophotometric determination, the samples were evaporated to dryness and the residue diluted with $96 \%$ ethanol.

Source and Purity of Chemicals:

(1) Purity not given, Sigma Chemical Company, USA, no purification details were provided.

(2) Spectrophotometric or Analytical grade, Chemical source not specified, no purification details were provided.

\section{Estimated Error:}

Temperature: $\pm 0.2 \mathrm{~K}$.

$x_{1}: \pm 3.0 \%$ (relative error, estimated by compiler). 


\begin{tabular}{|c|c|}
\hline $\begin{array}{l}\text { Components: } \\
\text { (1) 4-Aminobenzoic acid; } \\
\mathrm{C}_{7} \mathrm{H}_{7} \mathrm{NO}_{2} \text {; [150-13-0] } \\
\text { (2) } 1,1^{\prime} \text {-Oxybisethane; } \\
\mathrm{C}_{4} \mathrm{H}_{10} \mathrm{O} \text {; }[60-29-7]\end{array}$ & $\begin{array}{l}\text { Original Measurements: } \\
{ }^{105} \text { P. K. Takayama, N. Nambu, and T. } \\
\text { Nagai, Chem. Pharm. Bull. 25, } 879 \\
\text { (1977). }\end{array}$ \\
\hline $\begin{array}{l}\text { Variables: } \\
T / \mathrm{K}=283\end{array}$ & $\begin{array}{l}\text { Prepared by: } \\
\text { W. E. Acree, Jr. }\end{array}$ \\
\hline
\end{tabular}

\section{Experimental Values}

The solubility was given graphically in the paper as a plot of molar concentration of the solute versus the concentration of ligand added. From the graph the compiler estimated a molar solubility of $c_{1}=0.102 \mathrm{~mol} \mathrm{dm}^{-3}$.

\section{Auxiliary Information}

\section{Method/Apparatus/Procedure:}

Very little experimental details were provided. Excess solute and solvent were sealed in vials and incubated for $48 \mathrm{~h}$ at $283 \mathrm{~K}$. A $1 \mathrm{ml}$ sample of the supernatant was withdrawn and the solvent evaporated at $323 \mathrm{~K}$. The residue was dissolved in water or ethanol, and the concentration of the solute determined from spectroscopic absorbance measurements.

\section{Source and Purity of Chemicals:}

(1) Purity not given, Chemical source not given, was recrystallized from water before use.

(2) Purity not given, Chemical source not given, no purification details were provided.

\section{Estimated Error:}

Temperature: No information was given. $c_{1}: \pm 5 \%$ (relative error, estimated by compiler).

\begin{tabular}{|c|c|}
\hline $\begin{array}{l}\text { Components: } \\
\text { (1) 4-Aminobenzoic acid; } \\
\mathrm{C}_{7} \mathrm{H}_{7} \mathrm{NO}_{2} ; \text { [150-13-0] } \\
\text { (2) Tetrahydrofuran; } \mathrm{C}_{4} \mathrm{H}_{8} \mathrm{O} \text {; } \\
\text { [109-99-9] }\end{array}$ & $\begin{array}{l}\text { Original Measurements: } \\
6{ }^{6} \text { C. K. Hancock, J. N. Pawloski, and J. } \\
\text { P. Idoux, J. Org. Chem. 32, } 1931 \\
\text { (1967). }\end{array}$ \\
\hline $\begin{array}{l}\text { Variables: } \\
T / \mathrm{K}=303.15\end{array}$ & $\begin{array}{l}\text { Prepared by: } \\
\text { W. E. Acree, Jr. }\end{array}$ \\
\hline
\end{tabular}

Experimental Values

\begin{tabular}{lc}
\hline \hline$x_{2}^{\mathrm{a}}$ & $x_{1}{ }^{\mathrm{b}}$ \\
\hline 0.767 & 0.233 \\
\hline${ }^{\mathrm{a}} x_{2}:$ mole fraction of component 2 in the saturated solution. & \\
${ }^{\mathrm{b}} x_{1}:$ mole fraction solubility of the solute. &
\end{tabular}

\section{Auxiliary Information}

\section{Method/Apparatus/Procedure:}

Constant-temperature bath, Soxhlet thimble, conical flask, and analytical balance.

Excess solute and solvent were placed in a conical flask and allowed to equilibrate for several days at constant temperature. Attainment of equilibrium was verified by several repetitive measurements and by approaching equilibrium from supersaturation. Aliquots of saturated solutions were transferred using a Soxhlet thimble equipped with a one-hole cork stopper and an inverted U-shaped delivery tube to a second ground-glass stoppered flask suspended in the $303 \mathrm{~K}$ water bath. Successive portions of the contents were evaporated at room temperature in a tared aluminum foil weighing dish under a bell jar through which a slow stream of dry air was passed. The second flask plus unused saturated solution and the aluminum foil dish plus residue were weighed. The saturation solubility of the solute was calculated from the recorded mass data and molar masses of the solute and solvent.

\section{Source and Purity of Chemicals:}

(1) Purity not given, Chemical source not specified, was recrystallized several times from aqueous-ethanol mixture, and then dried under vacuum over phosphorous pentoxide.

(2) Practical grade, Chemical source not specified, stored over sodium hydroxide pellets for $24 \mathrm{~h}$, and then passed through $2 \times 70-\mathrm{cm}$ chromatographic adsorption columns containing activated alumina. After this treatment, the purified solvent was stored over copper in a nitrogen atmosphere.

\section{Estimated Error:}

Temperature: $\pm 0.02 \mathrm{~K}$

$x_{1}: \pm 1.0 \%$ (relative error)

\begin{tabular}{ll}
\hline \hline Components: & Original Measurements: \\
$\begin{array}{l}\text { (1) 4-Aminobenzoic acid; } \\
\mathrm{C}_{7} \mathrm{H}_{7} \mathrm{NO}_{2} ;[\text { [150-13-0] }\end{array}$ & Sci. 82, Lin and R. A. Nash, J. Pharm. \\
$\begin{array}{l}\text { (2) Tetrahydrofuran; } \mathrm{C}_{4} \mathrm{H}_{8} \mathrm{O} ; \\
{[109-99-9]}\end{array}$ & \\
\hline Variables: & Prepared by: \\
$T / \mathrm{K}=298.15$ & W. E. Acree, Jr. \\
\hline
\end{tabular}

\section{Experimental Values}

\begin{tabular}{lc}
\hline \hline$x_{2}{ }^{\mathrm{a}}$ & $x_{1}{ }^{\mathrm{b}}$ \\
\hline 0.7830 & 0.2170 \\
\hline${ }_{x_{2}: \text { mole fraction of component } 2 \text { in the saturated solution. }}$ & \\
${ }^{\mathrm{b}} x_{1}:$ mole fraction solubility of the solute. &
\end{tabular}

${ }^{b} x_{1}$ : mole fraction solubility of the solute.

\section{Auxiliary Information}

\section{Method/Apparatus/Procedure:}

Constant-temperature bath, and high-performance liquid chromatograph. Excess solute and solvent were placed in screw-capped vials. The vials were sealed with several turns of electrical tape, warmed to about $323 \mathrm{~K}$, and then shaken in a constant-temperature bath for at least $24 \mathrm{~h}$ at $298 \mathrm{~K}$. After equilibrium was reached, the sample was filtered through a 0.45 or $1.0 \mu \mathrm{m}$ filter, and an aliquot was diluted appropriately for chromatographic analysis. Concentrations were determined by a high-performance liquid chromatographic method.

\section{Source and Purity of Chemicals:}

(1) Purity not given, Eastman Kodak Chemical Company, Rochester, NY, USA, no information provided concerning purification.

(2) Reagent grade, Aldrich Chemical Company, Milwaukee, WI, USA, no purification details were provided.

\section{Estimated Error:}

Temperature: $\pm 0.2 \mathrm{~K}$ (estimated by compiler). $x_{1}: \pm 3 \%$ (relative error, estimated by compiler). 


\section{Components:}

(1) 4-Aminobenzoic acid;

$\mathrm{C}_{7} \mathrm{H}_{7} \mathrm{NO}_{2} ;[150-13-0]$

(2) 1,4-Dioxane; $\mathrm{C}_{4} \mathrm{H}_{8} \mathrm{O}_{2}$; [123-91-1]

\begin{tabular}{ll}
\hline Variables: & Prepared by: \\
$T / \mathrm{K}=298.15$ & W. E. Acree, Jr.
\end{tabular}

\section{Experimental Values}

\begin{tabular}{lc}
\hline \hline$x_{2}{ }^{\mathrm{a}}$ & $x_{1}{ }^{\mathrm{b}}$ \\
\hline 0.9300 & 0.06998
\end{tabular}

${ }^{\mathrm{a}} x_{2}$ : mole fraction of component 2 in the saturated solution.

${ }^{\mathrm{b}} x_{1}$ : mole fraction solubility of the solute.

\section{Auxiliary Information}

\section{Method/Apparatus/Procedure:}

Constant-temperature bath, calorimetric thermometer, and an ultraviolet/ visible spectrophotometer.

Excess solute and solvent were placed in amber glass bottles and allowed to equilibrate for several days at constant temperature. Attainment of equilibrium was verified by several repetitive measurements and by approaching equilibrium from supersaturation. Aliquots of saturated solutions were transferred through a coarse filter into tared volumetric flasks, weighed, and diluted with methanol. Concentrations were determined by spectrophotometric measurements at $289 \mathrm{~nm}$.

\section{Source and Purity of Chemicals:}

(1) $99 \%$, Aldrich Chemical Company, Milwaukee, WI, USA, was used as received.

(2) $99.8 \%$, anhydrous, Aldrich Chemical Company, stored over molecular sieves and distilled shortly before use.

Estimated Error:

Temperature: $\pm 0.1 \mathrm{~K}$.

$x_{1}: \pm 1.5 \%$ (relative error).

\begin{tabular}{|c|c|}
\hline $\begin{array}{l}\text { Components: } \\
\text { (1) 4-Aminobenzoic acid; } \\
\mathrm{C}_{7} \mathrm{H}_{7} \mathrm{NO}_{2} \text {; [150-13-0] } \\
\text { (2) 1,4-Dioxane; } \mathrm{C}_{4} \mathrm{H}_{8} \mathrm{O}_{2} \text {; } \\
\text { [123-91-1] }\end{array}$ & $\begin{array}{l}\text { Original Measurements: } \\
{ }^{102} \text { J. Barra, M.-A. Peña, and P. } \\
\text { Bustamante, Eur. J. Pharm. Sci. 10, } 153 \\
(2000) \text {. }\end{array}$ \\
\hline $\begin{array}{l}\text { Variables: } \\
T / \mathrm{K}=298.15\end{array}$ & $\begin{array}{l}\text { Prepared by: } \\
\text { W. E. Acree, Jr. }\end{array}$ \\
\hline
\end{tabular}

Experimental Values

\begin{tabular}{lc}
\hline \hline$x_{2}{ }^{\mathrm{a}}$ & $x_{1}{ }^{\mathrm{b}}$ \\
\hline 0.9368 & 0.0632 \\
${ }^{\mathrm{a}} x_{2}:$ mole fraction of component 2 in the saturated solution. \\
${ }^{\mathrm{b}} x_{1}:$ mole fraction solubility of the solute.
\end{tabular}

\section{Auxiliary Information}

\section{Method/Apparatus/Procedure:}

Constant-temperature bath, analytical balance, and an ultraviolet/visible spectrophotometer.

Excess solute and solvent were placed in a flask and allowed to equilibrate for several days at constant temperature. The solid phase was removed by filtration, and the clear solution was diluted quantitatively with $96 \%$ ethanol. The concentration of the diluted solution was determined by spectrophotometric measurement at the wavelength of maximum absorption. In instances where the solvent interfered with the spectrophotometric determination, the samples were evaporated to dryness and the residue diluted with $96 \%$ ethanol.

Source and Purity of Chemicals:

(1) Purity not given, Sigma Chemical Company, USA, no purification details were provided.

(2) Spectrophotometric or Analytical grade, Chemical source not specified, no purification details were provided.

\section{Estimated Error:}

Temperature: $\pm 0.2 \mathrm{~K}$.

$x_{1}: \pm 3.0 \%$ (relative error, estimated by compiler).

\section{Components:}

(1) 4-Aminobenzoic acid;

$\mathrm{C}_{7} \mathrm{H}_{7} \mathrm{NO}_{2} ;[150-13-0]$

(2) 1,4-Dioxane; $\mathrm{C}_{4} \mathrm{H}_{8} \mathrm{O}_{2}$; [123-91-1]

\begin{tabular}{ll}
\hline Variables: & Prepared by: \\
$T / \mathrm{K}=303.15$ & W. E. Acree, Jr.
\end{tabular}

\section{Experimental Values}

\begin{tabular}{lc}
\hline \hline$x_{2}{ }^{\mathrm{a}}$ & $x_{1}{ }^{\mathrm{b}}$ \\
\hline 0.922 & 0.078 \\
\hline${ }^{\mathrm{a}} x_{2}:$ mole fraction of component 2 in the saturated solution. & \\
${ }^{\mathrm{b}} x_{1}:$ mole fraction solubility of the solute. &
\end{tabular}

\section{Auxiliary Information}

\section{Method/Apparatus/Procedure:}

Constant-temperature bath, Soxhlet thimble, conical flask, and analytical balance.

Excess solute and solvent were placed in a conical flask and allowed to equilibrate for several days at constant temperature. Attainment of equilibrium was verified by several repetitive measurements and by approaching equilibrium from supersaturation. Weighed aliquots of saturated solutions were removed and titrated with a standardized sodium hydroxide solution (carbonate free) using a $\mathrm{pH}$ meter. The endpoint of the titration was determined by computing the second derivative in the $\mathrm{pH}$ versus volume of sodium hydroxide added.

\section{Source and Purity of Chemicals:}

(1) Purity not given, Chemical source not specified, was recrystallized several times from aqueous-ethanol mixture, and then dried under vacuum over phosphorous pentoxide.

(2) Practical grade, Chemical source not specified, stored over sodium hydroxide pellets for $24 \mathrm{~h}$, and then passed through $2 \times 70-\mathrm{cm}$ chromatographic adsorption columns containing activated alumina. After this treatment, the purified solvent was stored over copper in a nitrogen atmosphere. 
Estimated Error:

Temperature: $\pm 0.02 \mathrm{~K}$

$x_{1}: \pm 1.0 \%$ (relative error).

\begin{tabular}{|c|c|}
\hline $\begin{array}{l}\text { Components: } \\
\text { (1) 4-Aminobenzoic acid; } \\
\mathrm{C}_{7} \mathrm{H}_{7} \mathrm{NO}_{2} ;[150-13-0] \\
\text { (2) } 1,2-\text { Dimethoxyethane; } \\
\mathrm{C}_{4} \mathrm{H}_{10} \mathrm{O}_{2} ;[110-71-4]\end{array}$ & $\begin{array}{l}\text { Original Measurements: } \\
\text { 51 H.-M. Lin and R. A. Nash, J. Pharm. } \\
\text { Sci. 82, } 1018 \text { (1993). }\end{array}$ \\
\hline $\begin{array}{l}\text { Variables: } \\
T / \mathrm{K}=298.15\end{array}$ & $\begin{array}{l}\text { Prepared by: } \\
\text { W. E. Acree, Jr. }\end{array}$ \\
\hline
\end{tabular}

Experimental Values

\begin{tabular}{lc}
\hline \hline$x_{2}{ }^{\mathrm{a}}$ & $x_{1}{ }^{\mathrm{b}}$ \\
\hline 0.7872 & 0.2128 \\
\hline
\end{tabular}

${ }^{\mathrm{a}} x_{2}$ : mole fraction of component 2 in the saturated solution.

${ }^{\mathrm{b}} x_{1}$ : mole fraction solubility of the solute.

\section{Auxiliary Information}

\section{Method/Apparatus/Procedure:}

Constant-temperature bath, and high-performance liquid chromatograph. Excess solute and solvent were placed in screw-capped vials. The vials were sealed with several turns of electrical tape, warmed to about $323 \mathrm{~K}$, and then shaken in a constant-temperature bath for at least $24 \mathrm{~h}$ at $298 \mathrm{~K}$. After equilibrium was reached, the sample was filtered through a 0.45 or $1.0 \mu \mathrm{m}$ filter, and an aliquot was diluted appropriately for chromatographic analysis. Concentrations were determined by a high-performance liquid chromatographic method.

Source and Purity of Chemicals:

(1) Purity not given, Eastman Kodak Chemical Company, Rochester, NY,

USA, no information provided concerning purification.

(2) Purity not given, Hoechst Chemikalien, no purification details were provided.

\section{Estimated Error:}

Temperature: $\pm 0.2 \mathrm{~K}$ (estimated by compiler). $x_{1}: \pm 3 \%$ (relative error, estimated by compiler).

\begin{tabular}{|c|c|}
\hline $\begin{array}{l}\text { Components: } \\
\text { (1) 4-Aminobenzoic acid; } \\
\mathrm{C}_{7} \mathrm{H}_{7} \mathrm{NO}_{2} ;[150-13-0] \\
\text { (2) } 2,5,8,11 \text {-Tetraoxadodecane; } \\
\mathrm{C}_{8} \mathrm{H}_{18} \mathrm{O}_{4} ;[112-49-2]\end{array}$ & $\begin{array}{l}\text { Original Measurements: } \\
\text { 51 H.-M. Lin and R. A. Nash, J. Pharm. } \\
\text { Sci. 82, } 1018 \text { (1993). }\end{array}$ \\
\hline $\begin{array}{l}\text { Variables: } \\
T / \mathrm{K}=298.15\end{array}$ & $\begin{array}{l}\text { Prepared by: } \\
\text { W. E. Acree, Jr. }\end{array}$ \\
\hline
\end{tabular}

Experimental Values

\begin{tabular}{lc}
\hline \hline$x_{2}^{\mathrm{a}}$ & $x_{1}{ }^{\mathrm{b}}$ \\
\hline 0.6435 & 0.3565 \\
\hline${ }^{\mathrm{a}} x_{2}:$ mole fraction of component 2 in the saturated solution. & \\
${ }^{\mathrm{b}}{ }_{x_{1}: \text { mole fraction solubility of the solute. }}$ &
\end{tabular}

Auxiliary Information

Method/Apparatus/Procedure:

Constant-temperature bath, and high-performance liquid chromatograph. Excess solute and solvent were placed in screw-capped vials. The vials were sealed with several turns of electrical tape, warmed to about $323 \mathrm{~K}$, and then shaken in a constant-temperature bath for at least $24 \mathrm{~h}$ at $298 \mathrm{~K}$. After equilibrium was reached, the sample was filtered through a 0.45 or $1.0 \mu \mathrm{m}$ filter, and an aliquot was diluted appropriately for chromatographic analysis. Concentrations were determined by a high-performance liquid chromatographic method.

\section{Source and Purity of Chemicals:}

(1) Purity not given, Eastman Kodak Chemical Company, Rochester, NY, USA, no information provided concerning purification.

(2) Purity not given, Chemical source not given, no purification details were provided.

\section{Estimated Error:}

Temperature: $\pm 0.2 \mathrm{~K}$ (estimated by compiler). $x_{1}: \pm 3 \%$ (relative error, estimated by compiler).

\subsection{4-Aminobenzoic acid solubility data in haloalkanes, haloalkenes, and haloaromatic hydrocarbons}

\begin{tabular}{ll}
\hline \hline Components: & Original Measurements: \\
(1) 4-Aminobenzoic acid; & $\begin{array}{l}{ }^{102} \text { J. Barra, M.-A. Peña, and P. } \\
\mathrm{C}_{7} \mathrm{H}_{7} \mathrm{NO}_{2} ;[150-13-0]\end{array}$ \\
$\begin{array}{l}\text { (2) Trichloromethane; } \mathrm{CHCl}_{3} ; \\
\text { Bustamante, Eur. J. Pharm. Sci. 10, 153 } \\
\text { (2000). }\end{array}$ \\
\hline Variables: & Prepared by: \\
$T / \mathrm{K}=298.15$ & W. E. Acree, Jr. \\
\hline
\end{tabular}

\section{Experimental Values}

\begin{tabular}{lc}
\hline \hline$x_{2}{ }^{\mathrm{a}}$ & $x_{1}{ }^{\mathrm{b}}$ \\
\hline 0.9984 & 0.001558 \\
\hline${ }^{\mathrm{a}} x_{2}:$ mole fraction of component 2 in the saturated solution. \\
${ }^{\mathrm{b}}{ }_{x_{1}: \text { mole fraction solubility of the solute. }}$
\end{tabular}

\section{Auxiliary Information}

\section{Method/Apparatus/Procedure:}

Constant-temperature bath, analytical balance, and an ultraviolet/visible spectrophotometer.

Excess solute and solvent were placed in a flask and allowed to equilibrate for several days at constant temperature. The solid phase was removed by filtration, and the clear solution was diluted quantitatively with $96 \%$ ethanol. The concentration of the diluted solution was determined by spectrophotometric measurement at the wavelength of maximum absorption. In instances where the solvent interfered with the spectrophotometric determination, the samples were evaporated to dryness and the residue diluted with $96 \%$ ethanol.

Source and Purity of Chemicals:

(1) Purity not given, Sigma Chemical Company, USA, no purification details were provided.

(2) Spectrophotometric or Analytical grade, Chemical source not specified, no purification details were provided. 
Estimated Error:

Temperature: $\pm 0.2 \mathrm{~K}$

$x_{1}: \pm 3.0 \%$ (relative error, estimated by compiler).

\begin{tabular}{|c|c|}
\hline $\begin{array}{l}\text { Components: } \\
\text { (1) 4-Aminobenzoic acid; } \\
\mathrm{C}_{7} \mathrm{H}_{7} \mathrm{NO}_{2} ;[150-13-0] \\
\text { (2) Trichloromethane; } \mathrm{CHCl}_{3} \text {; } \\
\text { [67-66-3] }\end{array}$ & $\begin{array}{l}\text { Original Measurements: } \\
{ }^{100} \text { C. L. Lazzell and J. Johnston, J. } \\
\text { Phys. Chem. 32, } 1331 \text { (1928). }\end{array}$ \\
\hline $\begin{array}{l}\text { Variables: } \\
\text { Temperature }\end{array}$ & $\begin{array}{l}\text { Prepared by: } \\
\text { W. E. Acree, Jr. }\end{array}$ \\
\hline
\end{tabular}

Experimental Values

\begin{tabular}{lcc}
\hline \hline$T / \mathrm{K}$ & $x_{2}{ }^{\mathrm{a}}$ & $x_{1}{ }^{\mathrm{b}}$ \\
\hline 298.2 & 0.9987 & 0.0013 \\
409.9 & 0.9475 & 0.0525 \\
429.6 & 0.7010 & 0.2990 \\
\hline
\end{tabular}

${ }^{a} x_{2}$ : mole fraction of component 2 in the saturated solution.

${ }^{b} x_{1}$ : mole fraction solubility of the solute. The solubility is given as molal percentage, which, based on the description of 100 times the mole fraction, is taken to be mole percentage.

\section{Auxiliary Information}

\section{Method/Apparatus/Procedure:}

Preweighed quantities of solute and solvent were placed in bulbs, which were then sealed and heated in a suitable bath. The temperature at which the solid completely dissolved was recorded, and was taken to be the solid-liquid equilibrium temperature. In a few instances near room temperature, the solubility was determined by agitating the solvent in a thermostat with an excess of solute. After equilibrium had been established, an aliquot of the saturated solution was pipetted, weighed, and the amount of dissolved solute determined with a solution of sodium hydroxide standardized against the pure acid.

\section{Source and Purity of Chemicals:}

(1) Certified Pure, Chemical source not given, was recrystallized several times from hot aqueous solution, then from $95 \%$ ethanol, and finally from hot water. The purified solute had a melting point temperature of $460.2 \mathrm{~K}$.

(2) Certified Pure, Chemical source not given, was dried over calcium chloride and distilled before use.

\section{Estimated Error:}

Temperature: Not given in paper.

$x_{1}: \pm 5 \%$ (relative error, estimated by compiler).

\begin{tabular}{|c|c|}
\hline $\begin{array}{l}\text { Components: } \\
\text { (1) 4-Aminobenzoic acid; } \\
\mathrm{C}_{7} \mathrm{H}_{7} \mathrm{NO}_{2} ;[150-13-0] \\
\text { (2) 1,2-Dichloroethane; } \\
\mathrm{C}_{2} \mathrm{H}_{4} \mathrm{Cl}_{2} ;[107-06-2]\end{array}$ & $\begin{array}{l}\text { Original Measurements: } \\
{ }^{102} \text { J. Barra, M.-A. Peña, and P. } \\
\text { Bustamante, Eur. J. Pharm. Sci. 10, } 153 \\
(2000) \text {. }\end{array}$ \\
\hline $\begin{array}{l}\text { Variables: } \\
T / \mathrm{K}=298.15\end{array}$ & $\begin{array}{l}\text { Prepared by: } \\
\text { W. E. Acree, Jr. }\end{array}$ \\
\hline
\end{tabular}

Experimental Values

\begin{tabular}{lc}
\hline \hline$x_{2}^{\mathrm{a}}$ & $x_{1}{ }^{\mathrm{b}}$ \\
\hline 0.9971 & 0.00292 \\
\hline${ }^{\mathrm{a}} x_{2}:$ mole fraction of component 2 in the saturated solution. & \\
${ }^{\mathrm{b}} x_{1}:$ mole fraction solubility of the solute. &
\end{tabular}

\section{Auxiliary Information}

\section{Method/Apparatus/Procedure:}

Constant-temperature bath, analytical balance, and an ultraviolet/visible spectrophotometer.

Excess solute and solvent were placed in a flask and allowed to equilibrate for several days at constant temperature. The solid phase was removed by filtration, and the clear solution was diluted quantitatively with $96 \%$ ethanol. The concentration of the diluted solution was determined by spectrophotometric measurement at the wavelength of maximum absorption. In instances where the solvent interfered with the spectrophotometric determination, the samples were evaporated to dryness and the residue diluted with $96 \%$ ethanol.

\section{Source and Purity of Chemicals:}

(1) Purity not given, Sigma Chemical Company, USA, no purification details were provided.

(2) Spectrophotometric or Analytical grade, Chemical source not specified, no purification details were provided.

\section{Estimated Error:}

Temperature: $\pm 0.2 \mathrm{~K}$.

$x_{1}: \pm 3.0 \%$ (relative error, estimated by compiler).

\begin{tabular}{ll}
\hline \hline Components: & $\begin{array}{l}\text { Original Measurements: } \\
(1) \text { 4-Aminobenzoic acid; }\end{array}$ \\
$\mathrm{C}_{7} \mathrm{H}_{7} \mathrm{NO}_{2} ;$ [150-13-0] Barra, M.-A. Peña, and P. \\
$\begin{array}{l}\text { (2) Chlorobenzene; } \mathrm{C}_{6} \mathrm{H}_{5} \mathrm{Cl} ; \\
\text { Bustamante, Eur. J. Pharm. Sci. 10, 153 } \\
\text { (108-90-7] }\end{array}$ \\
\hline Variables: & Prepared by: \\
$T / \mathrm{K}=298.15$ & W. E. Acree, Jr. \\
\hline
\end{tabular}

Experimental Values

\begin{tabular}{lc}
\hline \hline$x_{2}{ }^{\mathrm{a}}$ & $x_{1}{ }^{\mathrm{b}}$ \\
\hline 0.9995 & 0.000485 \\
\hline${ }^{\mathrm{a}} x_{2}:$ mole fraction of component 2 in the saturated solution. & \\
${ }^{\mathrm{b}} x_{1}:$ mole fraction solubility of the solute. &
\end{tabular}

\section{Auxiliary Information}

\section{Method/Apparatus/Procedure:}

Constant-temperature bath, analytical balance, and an ultraviolet/visible spectrophotometer.

Excess solute and solvent were placed in a flask and allowed to equilibrate for several days at constant temperature. The solid phase was removed by filtration, and the clear solution was diluted quantitatively with $96 \%$ ethanol. The concentration of the diluted solution was determined by spectrophotometric measurement at the wavelength of maximum absorption. In instances where the solvent interfered with the spectrophotometric determination, the samples were evaporated to dryness and the residue diluted with $96 \%$ ethanol. 
Source and Purity of Chemicals:

(1) Purity not given, Sigma Chemical Company, USA, no purification details were provided.

(2) Spectrophotometric or Analytical grade, Chemical source not specified, no purification details were provided.

\section{Estimated Error:}

Temperature: $\pm 0.2 \mathrm{~K}$.

$x_{1}: \pm 3.0 \%$ (relative error, estimated by compiler).

\subsection{4-Aminobenzoic acid solubility data in alcohols}

\begin{tabular}{ll}
\hline \hline Components: & Original Measurements: \\
(1) 4-Aminobenzoic acid; & ${ }^{102}$ J. Barra, M.-A. Peña, and P. \\
$\mathrm{C}_{7} \mathrm{H}_{7} \mathrm{NO}_{2} ;[150-13-0]$ & Bustamante, Eur. J. Pharm. Sci. 10, 153 \\
(2) Methanol; $\mathrm{CH}_{4} \mathrm{O} ;[67-56-1]$ & $(2000)$. \\
\hline Variables: & Prepared by: \\
$T / \mathrm{K}=298.15$ & W. E. Acree, Jr. \\
\hline
\end{tabular}

Experimental Values

\begin{tabular}{lc}
\hline \hline$x_{2}{ }^{\mathrm{a}}$ & $x_{1}{ }^{\mathrm{b}}$ \\
\hline 0.9461 & 0.0539 \\
\hline${ }^{\mathrm{a}} x_{2}:$ mole fraction of component 2 in the saturated solution. & \\
${ }^{\mathrm{b}} x_{1}:$ mole fraction solubility of the solute. &
\end{tabular}

\section{Auxiliary Information}

\section{Method/Apparatus/Procedure:}

Constant-temperature bath, analytical balance, and an ultraviolet/visible spectrophotometer.

Excess solute and solvent were placed in a flask and allowed to equilibrate for several days at constant temperature. The solid phase was removed by filtration, and the clear solution was diluted quantitatively with $96 \%$ ethanol. The concentration of the diluted solution was determined by spectrophotometric measurement at the wavelength of maximum absorption. In instances where the solvent interfered with the spectrophotometric determination, the samples were evaporated to dryness and the residue diluted with $96 \%$ ethanol.

Source and Purity of Chemicals:

(1) Purity not given, Sigma Chemical Company, USA, no purification details were provided.

(2) Spectrophotometric or Analytical grade, Chemical source not specified, no purification details were provided.

Estimated Error:

Temperature: $\pm 0.2 \mathrm{~K}$.

$x_{1}: \pm 3.0 \%$ (relative error, estimated by compiler).

\begin{tabular}{|c|c|}
\hline $\begin{array}{l}\text { Components: } \\
\text { (1) 4-Aminobenzoic acid; } \\
\mathrm{C}_{7} \mathrm{H}_{7} \mathrm{NO}_{2} ;[150-13-0] \\
\text { (2) Methanol; } \mathrm{CH}_{4} \mathrm{O} ;[67-56-1]\end{array}$ & $\begin{array}{l}\text { Original Measurements: } \\
\text { 51 H.-M. Lin and R. A. Nash, J. Pharm. } \\
\text { Sci. 82, } 1018 \text { (1993). }\end{array}$ \\
\hline $\begin{array}{l}\text { Variables: } \\
T / \mathrm{K}=298.15\end{array}$ & $\begin{array}{l}\text { Prepared by: } \\
\text { W. E. Acree, Jr. }\end{array}$ \\
\hline
\end{tabular}

Experimental Values

\begin{tabular}{lc}
\hline \hline$x_{2}{ }^{\mathrm{a}}$ & $x_{1}{ }^{\mathrm{b}}$ \\
\hline 0.9424 & 0.0576 \\
\hline${ }^{\mathrm{a}} x_{2}:$ mole fraction of component 2 in the saturated solution. & \\
${ }^{\mathrm{b}}{ }_{x_{1}}:$ mole fraction solubility of the solute. &
\end{tabular}

\section{Auxiliary Information}

\section{Method/Apparatus/Procedure:}

Constant-temperature bath, and high-performance liquid chromatograph. Excess solute and solvent were placed in screw-capped vials. The vials were sealed with several turns of electrical tape, warmed to about $323 \mathrm{~K}$, and then shaken in a constant-temperature bath for at least $24 \mathrm{~h}$ at $298 \mathrm{~K}$. After equilibrium was reached, the sample was filtered through a 0.45 or $1.0 \mu \mathrm{m}$ filter, and an aliquot was diluted appropriately for chromatographic analysis. Concentrations were determined by a high-performance liquid chromatographic method.

Source and Purity of Chemicals:

(1) Purity not given, Eastman Kodak Chemical Company, Rochester, NY, USA, no information provided concerning purification.

(2) HPLC grade, Aldrich Chemical Company, Milwaukee, WI, USA, no purification details were provided.

\section{Estimated Error:}

Temperature: $\pm 0.2 \mathrm{~K}$ (estimated by compiler). $x_{1}: \pm 3 \%$ (relative error, estimated by compiler).

\begin{tabular}{|c|c|}
\hline $\begin{array}{l}\text { Components: } \\
\text { (1) 4-Aminobenzoic acid; } \\
\mathrm{C}_{7} \mathrm{H}_{7} \mathrm{NO}_{2} ;[150-13-0] \\
\text { (2) Methanol; } \mathrm{CH}_{4} \mathrm{O} ;[67-56-1]\end{array}$ & $\begin{array}{l}\text { Original Measurements: } \\
{ }^{100} \text { C. L. Lazzell and J. Johnston, J. } \\
\text { Phys. Chem. 32, } 1331 \text { (1928). }\end{array}$ \\
\hline $\begin{array}{l}\text { Variables: } \\
\text { Temperature }\end{array}$ & $\begin{array}{l}\text { Prepared by: } \\
\text { W. E. Acree, Jr. }\end{array}$ \\
\hline
\end{tabular}

Experimental Values

\begin{tabular}{lcc}
\hline \hline$T / \mathrm{K}$ & $x_{2}{ }^{\mathrm{a}}$ & $x_{1}{ }^{\mathrm{b}}$ \\
\hline 298.2 & 0.9406 & 0.0594 \\
359.7 & 0.8225 & 0.1775 \\
381.5 & 0.7313 & 0.2687
\end{tabular}

${ }^{a} x_{2}$ : mole fraction of component 2 in the saturated solution.

${ }^{b} x_{1}$ : mole fraction solubility of the solute. The solubility is given as molal percentage, which, based on the description of 100 times the mole fraction, is taken to be mole percentage.

\section{Auxiliary Information}

\section{Method/Apparatus/Procedure:}

Preweighed quantities of solute and solvent were placed in bulbs, which were then sealed and heated in a suitable bath. The temperature at which the solid completely dissolved was recorded, and was taken to be the solid-liquid equilibrium temperature. In a few instances near room temperature, the solubility was determined by agitating the solvent in a thermostat with an excess of solute. After equilibrium had been established, an aliquot of the saturated solution was pipetted, weighed, and the amount of dissolved solute determined with a solution of sodium hydroxide standardized against the pure acid. 
Source and Purity of Chemicals:

(1) Certified Pure, Chemical source not given, was recrystallized several times from hot aqueous solution, then from $95 \%$ ethanol, and finally from hot water. The purified solute had a melting point temperature of $460.2 \mathrm{~K}$.

(2) Purity not given, Chemical source not given, purification details were not provided.

\section{Estimated Error:}

Temperature: Not given in paper.

$x_{1}: \pm 5 \%$ (relative error, estimated by compiler).

\begin{tabular}{ll}
\hline \hline Components: & Original Measurements: \\
$\begin{array}{l}\text { (1) } 4-\mathrm{Aminobenzoic} \mathrm{acid;} \\
\mathrm{C}_{7} \mathrm{H}_{7} \mathrm{NO}_{2} ;[150-13-0]\end{array}$ & C. R. Daniels, A. K. Charlton, R. M. \\
(2) Ethanol; $\mathrm{C}_{2} \mathrm{H}_{6} \mathrm{O} ;[64-17-5]$ & $\begin{array}{l}\text { and M. J. Moreno, W. E. Acree, Jr., } \\
\end{array}$ \\
\hline Variables: 633 (2004). \\
$T / \mathrm{K}=298.15$ & Prepared by: \\
\hline
\end{tabular}

Experimental Values

\begin{tabular}{lc}
\hline \hline$x_{2}^{\mathrm{a}}$ & $x_{1}{ }^{\mathrm{b}}$ \\
\hline 0.9494 & 0.05062
\end{tabular}

${ }^{\mathrm{a}} x_{2}$ : mole fraction of component 2 in the saturated solution.

${ }^{\mathrm{b}} x_{1}$ : mole fraction solubility of the solute.

\section{Auxiliary Information}

\section{Method/Apparatus/Procedure:}

Constant-temperature bath, calorimetric thermometer, and an ultraviolet/ visible spectrophotometer.

Excess solute and solvent were placed in amber glass bottles and allowed to equilibrate for several days at constant temperature. Attainment of equilibrium was verified by several repetitive measurements and by approaching equilibrium from supersaturation. Aliquots of saturated solutions were transferred through a coarse filter into tared volumetric flasks, weighed, and diluted with methanol. Concentrations were determined by

spectrophotometric measurements at $289 \mathrm{~nm}$.

Source and Purity of Chemicals:

(1) $99 \%$, Aldrich Chemical Company, Milwaukee, WI, USA, was used as received.

(2) $99 \%$, Aaper Alcohol and Chemical Company, USA, stored over molecular sieves and distilled shortly before use.

\section{Estimated Error:}

Temperature: $\pm 0.1 \mathrm{~K}$.

$x_{1}: \pm 1.5 \%$ (relative error).

\begin{tabular}{|c|c|}
\hline $\begin{array}{l}\text { Components: } \\
\text { (1) 4-Aminobenzoic acid; } \\
\mathrm{C}_{7} \mathrm{H}_{7} \mathrm{NO}_{2} ;[150-13-0] \\
\text { (2) Ethanol; } \mathrm{C}_{2} \mathrm{H}_{6} \mathrm{O} ;[64-17-5]\end{array}$ & $\begin{array}{l}\text { Original Measurements: } \\
{ }^{102} \text { J. Barra, M.-A. Peña, and P. } \\
\text { Bustamante, Eur. J. Pharm. Sci. 10, } 153 \\
\text { (2000). }\end{array}$ \\
\hline $\begin{array}{l}\text { Variables: } \\
T / \mathrm{K}=298.15\end{array}$ & $\begin{array}{l}\text { Prepared by: } \\
\text { W. E. Acree, Jr. }\end{array}$ \\
\hline
\end{tabular}

Experimental Values

\begin{tabular}{lc}
\hline \hline$x_{2}{ }^{\mathrm{a}}$ & $x_{1}{ }^{\mathrm{b}}$ \\
\hline 0.9535 & 0.0465 \\
\hline${ }^{\mathrm{a}} x_{2}:$ mole fraction of component 2 in the saturated solution. & \\
${ }^{\mathrm{b}} x_{1}:$ mole fraction solubility of the solute. &
\end{tabular}

\section{Auxiliary Information}

\section{Method/Apparatus/Procedure:}

Constant-temperature bath, analytical balance, and an ultraviolet/visible spectrophotometer.

Excess solute and solvent were placed in a flask and allowed to equilibrate for several days at constant temperature. The solid phase was removed by filtration, and the clear solution was diluted quantitatively with $96 \%$ ethanol. The concentration of the diluted solution was determined by spectrophotometric measurement at the wavelength of maximum absorption. In instances where the solvent interfered with the spectrophotometric determination, the samples were evaporated to dryness and the residue diluted with $96 \%$ ethanol.

\section{Source and Purity of Chemicals:}

(1) Purity not given, Sigma Chemical Company, USA, no purification details were provided.

(2) Spectrophotometric or Analytical grade, Chemical source not specified, no purification details were provided.

\section{Estimated Error:}

Temperature: $\pm 0.2 \mathrm{~K}$.

$x_{1}: \pm 3.0 \%$ (relative error, estimated by compiler).

\begin{tabular}{|c|c|}
\hline $\begin{array}{l}\text { Components: } \\
\text { (1) 4-Aminobenzoic acid; } \\
\mathrm{C}_{7} \mathrm{H}_{7} \mathrm{NO}_{2} ;[150-13-0] \\
\text { (2) Ethanol; } \mathrm{C}_{2} \mathrm{H}_{6} \mathrm{O} ;[64-17-5]\end{array}$ & $\begin{array}{l}\text { Original Measurements: } \\
{ }^{100} \text { C. L. Lazzell and J. Johnston, J. } \\
\text { Phys. Chem. 32, } 1331 \text { (1928). }\end{array}$ \\
\hline $\begin{array}{l}\text { Variables: } \\
\text { Temperature }\end{array}$ & $\begin{array}{l}\text { Prepared by: } \\
\text { W. E. Acree, Jr. }\end{array}$ \\
\hline
\end{tabular}

Experimental Values

\begin{tabular}{lcc}
\hline \hline$T / \mathrm{K}$ & $x_{2}{ }^{\mathrm{a}}$ & $x_{1}{ }^{\mathrm{b}}$ \\
\hline 298.2 & 0.9503 & 0.0497 \\
349.0 & 0.8644 & 0.1356 \\
354.5 & 0.8471 & 0.1529 \\
361.9 & 0.7996 & 0.2004 \\
372.2 & 0.7796 & 0.2204 \\
388.2 & 0.7000 & 0.3000 \\
413.3 & 0.5199 & 0.4801 \\
428.4 & 0.3448 & 0.6552
\end{tabular}

${ }^{\mathrm{a}} x_{2}$ : mole fraction of component 2 in the saturated solution.

${ }^{b} x_{1}$ : mole fraction solubility of the solute. The solubility is given as molal percentage, which, based on the description of 100 times the mole fraction, is taken to be mole percentage. 


\section{Auxiliary Information}

\section{Method/Apparatus/Procedure:}

Preweighed quantities of solute and solvent were placed in bulbs, which were then sealed and heated in a suitable bath. The temperature at which the solid completely dissolved was recorded, and was taken to be the solid-liquid equilibrium temperature. In a few instances near room temperature, the solubility was determined by agitating the solvent in a thermostat with an excess of solute. After equilibrium had been established, an aliquot of the saturated solution was pipetted, weighed, and the amount of dissolved solute determined with a solution of sodium hydroxide standardized against the pure acid.

Source and Purity of Chemicals:

(1) Certified Pure, Chemical source not given, was recrystallized several times from hot aqueous solution, then from $95 \%$ ethanol, and finally from hot water. The purified solute had a melting point temperature of $460.2 \mathrm{~K}$.

(2) $95 \%$, Chemical source not given, was refluxed with lime and distilled. The sample was further purified by drying over sodium and distilling twice before use.

\section{Estimated Error:}

Temperature: Not given in paper.

$x_{1}: \pm 5 \%$ (relative error, estimated by compiler).

\begin{tabular}{ll}
\hline \hline Components: & Original Measurements: \\
(1) 4-Aminobenzoic acid; & ${ }^{103}$ C. R. Daniels, A. K. Charlton, R. M. \\
$\mathrm{C}_{7} \mathrm{H}_{7} \mathrm{NO}_{2} ;[150-13-0]$ & Wold, R. J. Moreno, W. E. Acree, Jr., \\
$\begin{array}{l}\text { (2) 1-Propanol; } \mathrm{C}_{3} \mathrm{H}_{8} \mathrm{O} ; \\
\text { [71-23-8] }\end{array}$ & $\begin{array}{l}\text { and M. H. Abraham, Phys. Chem. Liq. } \\
\mathbf{4 2}, 633 \text { (2004). }\end{array}$ \\
\hline Variables: & Prepared by: \\
$T / \mathrm{K}=298.15$ & W. E. Acree, Jr. \\
\hline
\end{tabular}

Experimental Values

\begin{tabular}{lc}
\hline \hline$x_{2}{ }^{\mathrm{a}}$ & $x_{1}^{\mathrm{b}}$ \\
\hline 0.9668 & 0.03316 \\
\hline${ }^{\mathrm{a}} x_{2}:$ mole fraction of component 2 in the saturated solution. & \\
${ }^{\mathrm{b}} x_{1}:$ mole fraction solubility of the solute. &
\end{tabular}

\section{Auxiliary Information}

\section{Method/Apparatus/Procedure:}

Constant-temperature bath, calorimetric thermometer, and an ultraviolet/ visible spectrophotometer.

Excess solute and solvent were placed in amber glass bottles and allowed to equilibrate for several days at constant temperature. Attainment of equilibrium was verified by several repetitive measurements and by approaching equilibrium from supersaturation. Aliquots of saturated solutions were transferred through a coarse filter into tared volumetric flasks, weighed, and diluted with methanol. Concentrations were determined by spectrophotometric measurements at $289 \mathrm{~nm}$.

Source and Purity of Chemicals:

(1) $99 \%$, Aldrich Chemical Company, Milwaukee, WI, USA, was used as received.

(2) $99+\%$, anhydrous, Aldrich Chemical Company, stored over molecular sieves and distilled shortly before use.

Estimated Error:

Temperature: $\pm 0.1 \mathrm{~K}$.

$x_{1}: \pm 1.5 \%$ (relative error).

\begin{tabular}{|c|c|}
\hline $\begin{array}{l}\text { Components: } \\
\text { (1) 4-Aminobenzoic acid; } \\
\mathrm{C}_{7} \mathrm{H}_{7} \mathrm{NO}_{2} ;[150-13-0] \\
\text { (2) 1-Propanol; } \mathrm{C}_{3} \mathrm{H}_{8} \mathrm{O} ;[71-23-8]\end{array}$ & $\begin{array}{l}\text { Original Measurements: } \\
{ }^{51} \text { H.-M. Lin and R. A. Nash, J. Pharm } \\
\text { Sci. 82, } 1018 \text { (1993). }\end{array}$ \\
\hline $\begin{array}{l}\text { Variables: } \\
T / \mathrm{K}=298.15\end{array}$ & $\begin{array}{l}\text { Prepared by: } \\
\text { W. E. Acree, Jr. }\end{array}$ \\
\hline
\end{tabular}

Experimental Values

\begin{tabular}{lc}
\hline \hline$x_{2}{ }^{\mathrm{a}}$ & $x_{1}{ }^{\mathrm{b}}$ \\
\hline 0.9671 & 0.0329
\end{tabular}

${ }^{a} x_{2}$ : mole fraction of component 2 in the saturated solution.

${ }^{\mathrm{b}} x_{1}$ : mole fraction solubility of the solute.

\section{Auxiliary Information}

\section{Method/Apparatus/Procedure:}

Constant-temperature bath, and high-performance liquid chromatograph Excess solute and solvent were placed in screw-capped vials. The vials were sealed with several turns of electrical tape, warmed to about $323 \mathrm{~K}$, and then shaken in a constant-temperature bath for at least $24 \mathrm{~h}$ at $298 \mathrm{~K}$. After equilibrium was reached, the sample was filtered through a 0.45 or $1.0 \mu \mathrm{m}$ filter, and an aliquot was diluted appropriately for chromatographic analysis. Concentrations were determined by a high-performance liquid chromatographic method.

\section{Source and Purity of Chemicals:}

(1) Purity not given, Eastman Kodak Chemical Company, Rochester, NY, USA, no information provided concerning purification.

(2) HPLC grade, Aldrich Chemical Company, Milwaukee, WI, USA, no purification details were provided.

Estimated Error:

Temperature: $\pm 0.2 \mathrm{~K}$ (estimated by compiler). $x_{1}: \pm 3 \%$ (relative error, estimated by compiler).

\begin{tabular}{ll}
\hline \hline Components: & Original Measurements: \\
(1) 4-Aminobenzoic acid; & ${ }^{103}$ C. R. Daniels, A. K. Charlton, R. M. \\
$\mathrm{C}_{7} \mathrm{H}_{7} \mathrm{NO}_{2} ;[150-13-0]$ & Wold, R. J. Moreno, W. E. Acree, Jr., \\
$\begin{array}{l}\text { (2) 2-Propanol; } \mathrm{C}_{3} \mathrm{H}_{8} \mathrm{O} ; \\
\text { [67-63-0] }\end{array}$ & $\begin{array}{l}\text { and M. H. Abraham, Phys. Chem. Liq. } \\
\mathbf{4 2} 633(2004) .\end{array}$ \\
\hline Variables: & Prepared by: \\
$T / \mathrm{K}=298.15$ & W. E. Acree, Jr. \\
\hline
\end{tabular}

\section{Experimental Values}

\begin{tabular}{lc}
\hline \hline$x_{2}{ }^{\mathrm{a}}$ & $x_{1}{ }^{\mathrm{b}}$ \\
\hline 0.9678 & 0.03218 \\
\hline${ }^{\mathrm{a}} x_{2}:$ mole fraction of component 2 in the saturated solution. & \\
${ }^{\mathrm{b}} x_{1}:$ mole fraction solubility of the solute. &
\end{tabular}




\section{Auxiliary Information}

\section{Method/Apparatus/Procedure:}

Constant-temperature bath, calorimetric thermometer, and an ultraviolet/ visible spectrophotometer.

Excess solute and solvent were placed in amber glass bottles and allowed to equilibrate for several days at constant temperature. Attainment of equilibrium was verified by several repetitive measurements and by approaching equilibrium from supersaturation. Aliquots of saturated solutions were transferred through a coarse filter into tared volumetric flasks, weighed, and diluted with methanol. Concentrations were determined by

spectrophotometric measurements at $289 \mathrm{~nm}$.

Source and Purity of Chemicals:

(1) $99 \%$, Aldrich Chemical Company, Milwaukee, WI, USA, was used as received.

(2) 99+\%, anhydrous, Aldrich Chemical Company, stored over molecular sieves and distilled shortly before use.

Estimated Error:

Temperature: $\pm 0.1 \mathrm{~K}$.

$x_{1}: \pm 1.5 \%$ (relative error).

\begin{tabular}{ll}
\hline \hline Components: & Original Measurements: \\
(1) 4-Aminobenzoic acid; & ${ }^{103}$ C. R. Daniels, A. K. Charlton, R. M. \\
$\mathrm{C}_{7} \mathrm{H}_{7} \mathrm{NO}_{2} ;[$ [150-13-0] & Wold, R. J. Moreno, W. E. Acree, Jr., \\
(2) 1-Butanol; $\mathrm{C}_{4} \mathrm{H}_{10} \mathrm{O} ;[71-36-3]$ & $\begin{array}{l}\text { and M. H. Abraham, Phys. Chem. Liq. } \\
\mathbf{4 2 , 6 3 3} \text { (2004). }\end{array}$ \\
\hline Variables: & Prepared by: \\
$T / \mathrm{K}=298.15$ & W. E. Acree, Jr. \\
\hline
\end{tabular}

Experimental Values

\begin{tabular}{lc}
\hline \hline$x_{2}^{\mathrm{a}}$ & $x_{1}{ }^{\mathrm{b}}$ \\
\hline 0.9686 & 0.03139 \\
\hline${ }^{\mathrm{a}} x_{2}:$ mole fraction of component 2 in the saturated solution. \\
${ }^{\mathrm{b}} x_{1}:$ mole fraction solubility of the solute.
\end{tabular}

\footnotetext{
${ }^{\mathrm{b}} x_{1}$ : mole fraction solubility of the solute.
}

\section{Auxiliary Information}

\footnotetext{
Method/Apparatus/Procedure:

Constant-temperature bath, calorimetric thermometer, and an ultraviolet/ visible spectrophotometer.

Excess solute and solvent were placed in amber glass bottles and allowed to equilibrate for several days at constant temperature. Attainment of equilibrium was verified by several repetitive measurements and by approaching equilibrium from supersaturation. Aliquots of saturated solutions were transferred through a coarse filter into tared volumetric flasks, weighed, and diluted with methanol. Concentrations were determined by spectrophotometric measurements at $289 \mathrm{~nm}$.
}

Source and Purity of Chemicals:

(1) $99 \%$, Aldrich Chemical Company, Milwaukee, WI, USA, was used as received.

(2) $99.8+\%$, HPLC grade, Aldrich Chemical Company, stored over molecular sieves and distilled shortly before use.

\section{Estimated Error:}

Temperature: $\pm 0.1 \mathrm{~K}$.

$x_{1}: \pm 1.5 \%$ (relative error).

\begin{tabular}{|c|c|}
\hline $\begin{array}{l}\text { Components: } \\
\text { (1) 4-Aminobenzoic acid; } \\
\mathrm{C}_{7} \mathrm{H}_{7} \mathrm{NO}_{2} ;[150-13-0] \\
\text { (2) 1-Butanol; } \mathrm{C}_{4} \mathrm{H}_{10} \mathrm{O} \text {; } \\
\text { [71-36-3] }\end{array}$ & $\begin{array}{l}\text { Original Measurements: } \\
{ }^{100} \text { C. L. Lazzell and J. Johnston, J. } \\
\text { Phys. Chem. 32, } 1331 \text { (1928). }\end{array}$ \\
\hline $\begin{array}{l}\text { Variables: } \\
\text { Temperature }\end{array}$ & $\begin{array}{l}\text { Prepared by: } \\
\text { W. E. Acree, Jr. }\end{array}$ \\
\hline
\end{tabular}

Experimental Values

\begin{tabular}{lcc}
\hline \hline$T / \mathrm{K}$ & \multicolumn{1}{c}{$x_{2}{ }^{\mathrm{a}}$} & $x_{1}{ }^{\mathrm{b}}$ \\
\hline 407.7 & 0.6664 & 0.3336 \\
426.4 & 0.4490 & 0.5510 \\
\hline${ }^{\mathrm{a}} x_{2}:$ mole fraction of component 2 in the saturated solution. \\
${ }^{\mathrm{b}} x_{1}:$ mole fraction solubility of the solute. The solubility is given as molal \\
percentage, which, based on the description of 100 times the mole fraction, is \\
taken to be mole percentage.
\end{tabular}

\section{Auxiliary Information}

\begin{abstract}
Method/Apparatus/Procedure:
Preweighed quantities of solute and solvent were placed in bulbs, which were then sealed and heated in a suitable bath. The temperature at which the solid completely dissolved was recorded, and was taken to be the solid-liquid equilibrium temperature. In a few instances near room temperature, the solubility was determined by agitating the solvent in a thermostat with an excess of solute. After equilibrium had been established, an aliquot of the saturated solution was pipetted, weighed, and the amount of dissolved solute determined with a solution of sodium hydroxide standardized against the pure acid.
\end{abstract}

Source and Purity of Chemicals:

(1) Certified Pure, Chemical source not given, was recrystallized several times from hot aqueous solution, then from $95 \%$ ethanol, and finally from hot water. The purified solute had a melting point temperature of $460.2 \mathrm{~K}$.

(2) Purity not given, Chemical source not given, no purification details were provided.

Estimated Error:

Temperature: Not given in paper.

$x_{1}: \pm 5 \%$ (relative error, estimated by compiler).

\begin{tabular}{ll}
\hline \hline Components: & Original Measurements: \\
$(1) 4-A_{10}$ inobenzoic acid; & ${ }^{103}$ C. R. Daniels, A. K. Charlton, R. M. \\
$\mathrm{C}_{7} \mathrm{H}_{7} \mathrm{NO}_{2} ;[150-13-0]$ & Wold, R. J. Moreno, W. E. Acree, Jr., \\
(2) 2-Butanol; $\mathrm{C}_{4} \mathrm{H}_{10} \mathrm{O} ;[78-92-2]$ & $\begin{array}{l}\text { and M. H. Abraham, Phys. Chem. Liq. } \\
\mathbf{4 2}, 633 \text { (2004). }\end{array}$ \\
\hline Variables: & Prepared by: \\
$T / \mathrm{K}=298.15$ & W. E. Acree, Jr. \\
\hline
\end{tabular}

Experimental Values

\begin{tabular}{lc}
\hline \hline$x_{2}{ }^{\mathrm{a}}$ & $x_{1}{ }^{\mathrm{b}}$ \\
\hline 0.9719 & 0.02808 \\
\hline${ }^{\mathrm{a}} x_{2}:$ mole fraction of component 2 in the saturated solution. \\
${ }^{\mathrm{b}} x_{1}:$ mole fraction solubility of the solute.
\end{tabular}




\section{Auxiliary Information}

\section{Method/Apparatus/Procedure:}

Constant-temperature bath, calorimetric thermometer, and an ultraviolet/ visible spectrophotometer.

Excess solute and solvent were placed in amber glass bottles and allowed to equilibrate for several days at constant temperature. Attainment of equilibrium was verified by several repetitive measurements and by approaching equilibrium from supersaturation. Aliquots of saturated solutions were transferred through a coarse filter into tared volumetric flasks, weighed, and diluted with methanol. Concentrations were determined by spectrophotometric measurements at $289 \mathrm{~nm}$.

Source and Purity of Chemicals:

(1) $99 \%$, Aldrich Chemical Company, Milwaukee, WI, USA, was used as received.

(2) $99+\%$, anhydrous, Aldrich Chemical Company, stored over molecular sieves and distilled shortly before use.

Estimated Error:

Temperature: $\pm 0.1 \mathrm{~K}$.

$x_{1}: \pm 1.5 \%$ (relative error)

\begin{tabular}{ll}
\hline \hline Components: & Original Measurements: \\
(1) 4-Aminobenzoic acid; & ${ }^{103}$ C. R. Daniels, A. K. Charlton, R. M. \\
$\mathrm{C}_{7} \mathrm{H}_{7} \mathrm{NO}_{2}$; [150-13-0] & Wold, R. J. Moreno, W. E. Acree, Jr., \\
$\begin{array}{l}\text { (2) 2-Methyl-1-propanol; } \\
\mathrm{C}_{4} \mathrm{H}_{10} \mathrm{O} ;[\text { [78-83-1] }\end{array}$ & and M. H. Abraham, Phys. Chem. Liq. \\
\hline Variables: & $\mathbf{4 2 , 6 3 3 ~ ( 2 0 0 4 ) .}$ \\
$T / \mathrm{K}=298.15$ & Prepared by: \\
\hline
\end{tabular}

Experimental Values

\begin{tabular}{lc}
\hline \hline$x_{2}{ }^{\mathrm{a}}$ & $x_{1}{ }^{\mathrm{b}}$ \\
\hline 0.9825 & 0.01751
\end{tabular}

${ }^{\mathrm{a}} x_{2}$ : mole fraction of component 2 in the saturated solution.

${ }^{\mathrm{b}} x_{1}$ : mole fraction solubility of the solute.

\section{Auxiliary Information}

\section{Method/Apparatus/Procedure:}

Constant-temperature bath, calorimetric thermometer, and an ultraviolet/ visible spectrophotometer.

Excess solute and solvent were placed in amber glass bottles and allowed to equilibrate for several days at constant temperature. Attainment of equilibrium was verified by several repetitive measurements and by approaching equilibrium from supersaturation. Aliquots of saturated solutions were transferred through a coarse filter into tared volumetric flasks, weighed, and diluted with methanol. Concentrations were determined by spectrophotometric measurements at $289 \mathrm{~nm}$.

\section{Source and Purity of Chemicals:}

(1) $99 \%$, Aldrich Chemical Company, Milwaukee, WI, USA, was used as received.

(2) 99+\%, anhydrous, Aldrich Chemical Company, stored over molecular sieves and distilled shortly before use.

\section{Estimated Error:}

Temperature: $\pm 0.1 \mathrm{~K}$.

$x_{1}: \pm 1.5 \%$ (relative error).

\begin{tabular}{|c|c|}
\hline $\begin{array}{l}\text { Components: } \\
\text { (1) 4-Aminobenzoic acid; } \\
\mathrm{C}_{7} \mathrm{H}_{7} \mathrm{NO}_{2} ;[150-13-0] \\
\text { (2) 1-Pentanol; } \mathrm{C}_{5} \mathrm{H}_{12} \mathrm{O} ;[71-41-0]\end{array}$ & $\begin{array}{l}\text { Original Measurements: } \\
{ }^{103} \text { C. R. Daniels, A. K. Charlton, R. M. } \\
\text { Wold, R. J. Moreno, W. E. Acree, Jr., } \\
\text { and M. H. Abraham, Phys. Chem. Liq. } \\
\text { 42, } 633 \text { (2004). }\end{array}$ \\
\hline
\end{tabular}

\begin{tabular}{ll}
\hline Variables: & Prepared by: \\
$T / \mathrm{K}=298.15$ & W. E. Acree, Jr.
\end{tabular}

\section{Experimental Values}

\begin{tabular}{lc}
\hline \hline$x_{2}{ }^{\mathrm{a}}$ & $x_{1}{ }^{\mathrm{b}}$ \\
\hline 0.9737 & 0.02630 \\
\hline
\end{tabular}

${ }^{\mathrm{a}} x_{2}$ : mole fraction of component 2 in the saturated solution.

${ }^{\mathrm{b}} x_{1}$ : mole fraction solubility of the solute.

\section{Auxiliary Information}

\section{Method/Apparatus/Procedure:}

Constant-temperature bath, calorimetric thermometer, and an ultraviolet/ visible spectrophotometer.

Excess solute and solvent were placed in amber glass bottles and allowed to equilibrate for several days at constant temperature. Attainment of equilibrium was verified by several repetitive measurements and by approaching equilibrium from supersaturation. Aliquots of saturated solutions were transferred through a coarse filter into tared volumetric flasks, weighed, and diluted with methanol. Concentrations were determined by spectrophotometric measurements at $289 \mathrm{~nm}$.

\section{Source and Purity of Chemicals:}

(1) $99 \%$, Aldrich Chemical Company, Milwaukee, WI, USA, was used as received.

(2) $99+\%$, Aldrich Chemical Company, stored over molecular sieves and distilled shortly before use.

\section{Estimated Error:}

Temperature: $\pm 0.1 \mathrm{~K}$.

$x_{1}: \pm 1.5 \%$ (relative error).

\begin{tabular}{ll}
\hline \hline Components: & Original Measurements: \\
$(1)$ 4-Aminobenzoic acid; & $\begin{array}{l}{ }^{102} \text { J. Barra, M.-A. Peña, and P. } \\
\mathrm{C}_{7} \mathrm{H}_{7} \mathrm{NO}_{2} ;[150-13-0]\end{array}$ \\
$\begin{array}{l}\text { Bustamante, Eur. J. Pharm. Sci. 10, 153 } \\
\text { (2) 1-Pentanol; } \mathrm{C}_{5} \mathrm{H}_{12} \mathrm{O} ;[71-41-0]\end{array}$ & $(2000)$. \\
\hline Variables: & Prepared by: \\
$T / \mathrm{K}=298.15$ & W. E. Acree, Jr. \\
\hline
\end{tabular}

\section{Experimental Values}

\begin{tabular}{|c|c|}
\hline$x_{2}{ }^{\mathrm{a}}$ & $x_{1}{ }^{\mathrm{b}}$ \\
\hline 0.9766 & 0.02338 \\
\hline
\end{tabular}




\section{Auxiliary Information}

\section{Method/Apparatus/Procedure:}

Constant-temperature bath, analytical balance, and an ultraviolet/visible spectrophotometer.

Excess solute and solvent were placed in a flask and allowed to equilibrate for several days at constant temperature. The solid phase was removed by filtration, and the clear solution was diluted quantitatively with $96 \%$ ethanol. The concentration of the diluted solution was determined by spectrophotometric measurement at the wavelength of maximum absorption. In instances where the solvent interfered with the spectrophotometric determination, the samples were evaporated to dryness and the residue diluted with $96 \%$ ethanol.

Source and Purity of Chemicals:

(1) Purity not given, Sigma Chemical Company, USA, no purification details were provided.

(2) Spectrophotometric or Analytical grade, Chemical source not specified, no purification details were provided.

\section{Estimated Error:}

Temperature: $\pm 0.2 \mathrm{~K}$.

$x_{1}: \pm 3.0 \%$ (relative error, estimated by compiler).

\begin{tabular}{ll}
\hline \hline Components: & Original Measurements: \\
(1) 4-Aminobenzoic acid; & ${ }^{103}$ C. R. Daniels, A. K. Charlton, R. M. \\
$\mathrm{C}_{7} \mathrm{H}_{7} \mathrm{NO}_{2} ;[150-13-0]$ & Wold, R. J. Moreno, W. E. Acree, Jr., \\
$\begin{array}{l}\text { (2) 2-Pentanol; } \mathrm{C}_{5} \mathrm{H}_{12} \mathrm{O} ; \\
\text { [6032-29-7] }\end{array}$ & \begin{tabular}{l}
$\mathbf{4 2}, 633$ (2004). \\
\hline Variables:
\end{tabular} \\
$T / \mathrm{K}=298.15$ & Prepared by: \\
\hline
\end{tabular}

Experimental Values

\begin{tabular}{lc}
\hline \hline$x_{2}{ }^{\mathrm{a}}$ & $x_{1}{ }^{\mathrm{b}}$ \\
\hline 0.9767 & 0.02325 \\
\hline
\end{tabular}

${ }^{\mathrm{a}} x_{2}$ : mole fraction of component 2 in the saturated solution.

${ }^{\mathrm{b}} x_{1}$ : mole fraction solubility of the solute.

\section{Auxiliary Information}

\section{Method/Apparatus/Procedure:}

Constant-temperature bath, calorimetric thermometer, and an ultraviolet/ visible spectrophotometer.

Excess solute and solvent were placed in amber glass bottles and allowed to equilibrate for several days at constant temperature. Attainment of equilibrium was verified by several repetitive measurements and by approaching equilibrium from supersaturation. Aliquots of saturated solutions were transferred through a coarse filter into tared volumetric flasks, weighed, and diluted with methanol. Concentrations were determined by

spectrophotometric measurements at $289 \mathrm{~nm}$.

Source and Purity of Chemicals:

(1) $99 \%$, Aldrich Chemical Company, Milwaukee, WI, USA, was used as received.

(2) $99+\%$, Acros Organics, USA, stored over molecular sieves and distilled shortly before use.

\section{Estimated Error:}

Temperature: $\pm 0.1 \mathrm{~K}$

$x_{1}: \pm 1.5 \%$ (relative error).

\begin{tabular}{ll}
\hline \hline Components: & Original Measurements: \\
(1) 4-Aminobenzoic acid; & ${ }^{103}$ C. R. Daniels, A. K. Charlton, R. M. \\
$\mathrm{C}_{7} \mathrm{H}_{7} \mathrm{NO}_{2} ;[150-13-0]$ & Wold, R. J. Moreno, W. E. Acree, Jr., \\
(2) 3-Methyl-1-butanol; & and M. H. Abraham, Phys. Chem. Liq. \\
$\mathrm{C}_{5} \mathrm{H}_{12} \mathrm{O} ;[123-51-3]$ & $\mathbf{4 2}, 633$ (2004).
\end{tabular}

\begin{tabular}{ll}
\hline Variables: & Prepared by: \\
$T / \mathrm{K}=298.15$ & W. E. Acree, Jr. \\
\hline
\end{tabular}

Experimental Values

\begin{tabular}{lc}
\hline \hline$x_{2}{ }^{\mathrm{a}}$ & $x_{1}{ }^{\mathrm{b}}$ \\
\hline 0.9801 & 0.01989
\end{tabular}

${ }^{\mathrm{a}} x_{2}$ : mole fraction of component 2 in the saturated solution.

${ }^{b} x_{1}$ : mole fraction solubility of the solute.

\section{Auxiliary Information}

\section{Method/Apparatus/Procedure:}

Constant-temperature bath, calorimetric thermometer, and an ultraviolet/ visible spectrophotometer.

Excess solute and solvent were placed in amber glass bottles and allowed to equilibrate for several days at constant temperature. Attainment of equilibrium was verified by several repetitive measurements and by approaching equilibrium from supersaturation. Aliquots of saturated solutions were transferred through a coarse filter into tared volumetric flasks, weighed, and diluted with methanol. Concentrations were determined by spectrophotometric measurements at $289 \mathrm{~nm}$.

\section{Source and Purity of Chemicals:}

(1) $99 \%$, Aldrich Chemical Company, Milwaukee, WI, USA, was used as received.

(2) $99 \%$, anhydrous, Aldrich Chemical Company, stored over molecular sieves and distilled shortly before use.

\section{Estimated Error:}

Temperature: $\pm 0.1 \mathrm{~K}$.

$x_{1}: \pm 1.5 \%$ (relative error).

\begin{tabular}{|c|c|}
\hline $\begin{array}{l}\text { Components: } \\
\text { (1) 4-Aminobenzoic acid; } \\
\mathrm{C}_{7} \mathrm{H}_{7} \mathrm{NO}_{2} ;[150-13-0] \\
\text { (2) } 1-\mathrm{Hexanol} ; \mathrm{C}_{6} \mathrm{H}_{14} \mathrm{O} ; \\
\text { [111-27-3] }\end{array}$ & $\begin{array}{l}\text { Original Measurements: } \\
{ }^{103} \text { C. R. Daniels, A. K. Charlton, R. M. } \\
\text { Wold, R. J. Moreno, W. E. Acree, Jr., } \\
\text { and M. H. Abraham, Phys. Chem. Liq. } \\
\text { 42, } 633 \text { (2004). }\end{array}$ \\
\hline $\begin{array}{l}\text { Variables: } \\
T / \mathrm{K}=298.15\end{array}$ & $\begin{array}{l}\text { Prepared by: } \\
\text { W. E. Acree, Jr. }\end{array}$ \\
\hline
\end{tabular}

Experimental Values

\begin{tabular}{lc}
\hline \hline$x_{2}{ }^{\mathrm{a}}$ & $x_{1}{ }^{\mathrm{b}}$ \\
\hline 0.9734 & 0.02664 \\
\hline${ }^{\mathrm{a}} x_{2}:$ mole fraction of component 2 in the saturated solution. & \\
${ }^{\mathrm{b}} x_{1}:$ mole fraction solubility of the solute. &
\end{tabular}




\section{Auxiliary Information}

\section{Method/Apparatus/Procedure:}

Constant-temperature bath, calorimetric thermometer, and an ultraviolet/ visible spectrophotometer.

Excess solute and solvent were placed in amber glass bottles and allowed to equilibrate for several days at constant temperature. Attainment of equilibrium was verified by several repetitive measurements and by approaching equilibrium from supersaturation. Aliquots of saturated solutions were transferred through a coarse filter into tared volumetric flasks, weighed, and diluted with methanol. Concentrations were determined by spectrophotometric measurements at $289 \mathrm{~nm}$.

Source and Purity of Chemicals:

(1) $99 \%$, Aldrich Chemical Company, Milwaukee, WI, USA, was used as received.

(2) $99+\%$, Alfa Aesar, USA, stored over molecular sieves and distilled shortly before use.

Estimated Error:

Temperature: $\pm 0.1 \mathrm{~K}$.

$x_{1}: \pm 1.5 \%$ (relative error).

\begin{tabular}{|c|c|}
\hline $\begin{array}{l}\text { Components: } \\
\text { (1) 4-Aminobenzoic acid; } \\
\mathrm{C}_{7} \mathrm{H}_{7} \mathrm{NO}_{2} \text {; [150-13-0] } \\
\text { (2) 3-Methyl-3-pentanol; } \\
\mathrm{C}_{6} \mathrm{H}_{14} \mathrm{O} \text {; [77-74-3] }\end{array}$ & $\begin{array}{l}\text { Original Measurements: } \\
\text { 51 H.-M. Lin and R. A. Nash, J. Pharm. } \\
\text { Sci. 82, } 1018 \text { (1993). }\end{array}$ \\
\hline $\begin{array}{l}\text { Variables: } \\
T / \mathrm{K}=298.15\end{array}$ & $\begin{array}{l}\text { Prepared by: } \\
\text { W. E. Acree, Jr. }\end{array}$ \\
\hline
\end{tabular}

Experimental Values

\begin{tabular}{lc}
\hline \hline$x_{2}{ }^{\mathrm{a}}$ & $x_{1}{ }^{\mathrm{b}}$ \\
\hline 0.9639 & 0.0361 \\
\hline$x_{2}$ m
\end{tabular}

${ }^{\mathrm{a}} x_{2}$ : mole fraction of component 2 in the saturated solution.

${ }^{\mathrm{b}} x_{1}$ : mole fraction solubility of the solute.

\section{Auxiliary Information}

\begin{abstract}
Method/Apparatus/Procedure:
Constant-temperature bath, and high-performance liquid chromatograph. Excess solute and solvent were placed in screw-capped vials. The vials were sealed with several turns of electrical tape, warmed to about $323 \mathrm{~K}$, and then shaken in a constant-temperature bath for at least $24 \mathrm{~h}$ at $298 \mathrm{~K}$. After equilibrium was reached, the sample was filtered through a 0.45 or $1.0 \mu \mathrm{m}$ filter, and an aliquot was diluted appropriately for chromatographic analysis. Concentrations were determined by a high-performance liquid

chromatographic method.
\end{abstract}

Source and Purity of Chemicals:

(1) Purity not given, Eastman Kodak Chemical Company, Rochester, NY, USA, no information provided concerning purification.

(2) Reagent grade, Aldrich Chemical Company, Milwaukee, WI, USA, no purification details were provided.

\section{Estimated Error:}

Temperature: $\pm 0.2 \mathrm{~K}$ (estimated by compiler).

$x_{1}: \pm 3 \%$ (relative error, estimated by compiler).

\begin{tabular}{|c|c|}
\hline $\begin{array}{l}\text { Components: } \\
\text { (1) 4-Aminobenzoic acid; } \\
\mathrm{C}_{7} \mathrm{H}_{7} \mathrm{NO}_{2} ;[150-13-0] \\
\text { (2) 1-Heptanol; } \mathrm{C}_{7} \mathrm{H}_{16} \mathrm{O} \text {; } \\
\text { [111-70-6] }\end{array}$ & $\begin{array}{l}\text { Original Measurements: } \\
{ }^{103} \text { C. R. Daniels, A. K. Charlton, R. M. } \\
\text { Wold, R. J. Moreno, W. E. Acree, Jr., } \\
\text { and M. H. Abraham, Phys. Chem. Liq. } \\
\text { 42, } 633 \text { (2004). }\end{array}$ \\
\hline $\begin{array}{l}\text { Variables: } \\
T / \mathrm{K}=298.15\end{array}$ & $\begin{array}{l}\text { Prepared by: } \\
\text { W. E. Acree, Jr. }\end{array}$ \\
\hline
\end{tabular}

Experimental Values

\begin{tabular}{lc}
\hline \hline$x_{2}{ }^{\mathrm{a}}$ & $x_{1}{ }^{\mathrm{b}}$ \\
\hline 0.9772 & 0.02277 \\
\hline${ }^{\mathrm{a}} x_{2}:$ mole fraction of component 2 in the saturated solution. & \\
${ }^{\mathrm{b}} x_{1}:$ mole fraction solubility of the solute. &
\end{tabular}

\section{Auxiliary Information}

\section{Method/Apparatus/Procedure:}

Constant-temperature bath, calorimetric thermometer, and an ultraviolet visible spectrophotometer.

Excess solute and solvent were placed in amber glass bottles and allowed to equilibrate for several days at constant temperature. Attainment of equilibrium was verified by several repetitive measurements and by approaching equilibrium from supersaturation. Aliquots of saturated solutions were transferred through a coarse filter into tared volumetric flasks, weighed, and diluted with methanol. Concentrations were determined by spectrophotometric measurements at $289 \mathrm{~nm}$.

\section{Source and Purity of Chemicals:}

(1) $99 \%$, Aldrich Chemical Company, Milwaukee, WI, USA, was used as received.

(2) $99+\%$, Alfa Aesar, USA, stored over molecular sieves and distilled shortly before use.

\section{Estimated Error:}

Temperature: $\pm 0.1 \mathrm{~K}$.

$x_{1}: \pm 1.5 \%$ (relative error).

\begin{tabular}{|c|c|}
\hline $\begin{array}{l}\text { Components: } \\
\text { (1) 4-Aminobenzoic acid; } \\
\mathrm{C}_{7} \mathrm{H}_{7} \mathrm{NO}_{2} ;[150-13-0] \\
\text { (2) 1-Octanol; } \mathrm{C}_{8} \mathrm{H}_{18} \mathrm{O} \text {; } \\
\text { [111-87-5] }\end{array}$ & $\begin{array}{l}\text { Original Measurements: } \\
{ }^{103} \text { C. R. Daniels, A. K. Charlton, R. M. } \\
\text { Wold, R. J. Moreno, W. E. Acree, Jr., } \\
\text { and M. H. Abraham, Phys. Chem. Liq. } \\
\text { 42, } 633 \text { (2004). }\end{array}$ \\
\hline $\begin{array}{l}\text { Variables: } \\
T / \mathrm{K}=298.15\end{array}$ & $\begin{array}{l}\text { Prepared by: } \\
\text { W. E. Acree, Jr. }\end{array}$ \\
\hline
\end{tabular}

\section{Experimental Values}

\begin{tabular}{lc}
\hline \hline$x_{2}{ }^{\mathrm{a}}$ & $x_{1}{ }^{\mathrm{b}}$ \\
\hline 0.9791 & 0.02088 \\
\hline${ }^{\mathrm{a}} x_{2}:$ mole fraction of component 2 in the saturated solution. & \\
${ }^{\mathrm{b}} x_{1}:$ mole fraction solubility of the solute. &
\end{tabular}




\section{Auxiliary Information}

\section{Method/Apparatus/Procedure:}

Constant-temperature bath, calorimetric thermometer, and an ultraviolet/ visible spectrophotometer.

Excess solute and solvent were placed in amber glass bottles and allowed to equilibrate for several days at constant temperature. Attainment of equilibrium was verified by several repetitive measurements and by approaching equilibrium from supersaturation. Aliquots of saturated solutions were transferred through a coarse filter into tared volumetric flasks, weighed, and diluted with methanol. Concentrations were determined by

spectrophotometric measurements at $289 \mathrm{~nm}$.

Source and Purity of Chemicals:

(1) $99 \%$, Aldrich Chemical Company, Milwaukee, WI, USA, was used as received.

(2) 99+\%, anhydrous, Aldrich Chemical Company, stored over molecular sieves and distilled shortly before use.

Estimated Error:

Temperature: $\pm 0.1 \mathrm{~K}$.

$x_{1}: \pm 1.5 \%$ (relative error).

\begin{tabular}{|c|c|}
\hline $\begin{array}{l}\text { Components: } \\
\text { (1) 4-Aminobenzoic acid; } \\
\mathrm{C}_{7} \mathrm{H}_{7} \mathrm{NO}_{2} ;[150-13-0] \\
\text { (2) 1-Octanol; } \mathrm{C}_{8} \mathrm{H}_{18} \mathrm{O} ; \\
\text { [111-87-5] }\end{array}$ & $\begin{array}{l}\text { Original Measurements: } \\
{ }^{102} \text { J. Barra, M.-A. Peña, and P. } \\
\text { Bustamante, Eur. J. Pharm. Sci. 10, } 153 \\
(2000) .\end{array}$ \\
\hline $\begin{array}{l}\text { Variables: } \\
T / \mathrm{K}=298.15\end{array}$ & $\begin{array}{l}\text { Prepared by: } \\
\text { W. E. Acree, Jr. }\end{array}$ \\
\hline
\end{tabular}

Experimental Values

\begin{tabular}{lc}
\hline \hline$x_{2}^{\mathrm{a}}$ & $x_{1}{ }^{\mathrm{b}}$ \\
\hline 0.9819 & 0.01806
\end{tabular}

${ }^{a} x_{2}$ : mole fraction of component 2 in the saturated solution.

${ }^{\mathrm{b}} x_{1}$ : mole fraction solubility of the solute.

\section{Auxiliary Information}

\section{Method/Apparatus/Procedure:}

Constant-temperature bath, analytical balance, and an ultraviolet/visible spectrophotometer.

Excess solute and solvent were placed in a flask and allowed to equilibrate for several days at constant temperature. The solid phase was removed by filtration, and the clear solution was diluted quantitatively with $96 \%$ ethanol. The concentration of the diluted solution was determined by

spectrophotometric measurement at the wavelength of maximum absorption In instances where the solvent interfered with the spectrophotometric determination, the samples were evaporated to dryness and the residue diluted with $96 \%$ ethanol.

\section{Source and Purity of Chemicals:}

(1) Purity not given, Sigma Chemical Company, USA, no purification details were provided.

(2) Spectrophotometric or Analytical grade, Chemical source not specified, no purification details were provided.

\section{Estimated Error:}

Temperature: $\pm 0.2 \mathrm{~K}$.

$x_{1}: \pm 3.0 \%$ (relative error, estimated by compiler).

\begin{tabular}{|c|c|}
\hline $\begin{array}{l}\text { Components: } \\
\text { (1) 4-Aminobenzoic acid; } \\
\mathrm{C}_{7} \mathrm{H}_{7} \mathrm{NO}_{2} ;[150-13-0] \\
\text { (2) } 1-\text { Octanol; } \mathrm{C}_{8} \mathrm{H}_{18} \mathrm{O} \text {; } \\
\text { [111-87-5] }\end{array}$ & $\begin{array}{l}\text { Original Measurements: } \\
\text { 99 Q. Jia, P. Ma, S. Ma, and C. Wang, } \\
\text { Chin. J. Chem. Eng. 15, } 710 \text { (2007). }\end{array}$ \\
\hline $\begin{array}{l}\text { Variables: } \\
\text { Temperature }\end{array}$ & $\begin{array}{l}\text { Prepared by: } \\
\text { W. E. Acree, Jr. }\end{array}$ \\
\hline
\end{tabular}

Experimental Values

\begin{tabular}{lcc}
\hline \hline$T / \mathrm{K}$ & $x_{2}{ }^{\mathrm{a}}$ & $x_{1}{ }^{\mathrm{b}}$ \\
\hline 297.95 & 0.9815 & 0.01849 \\
300.85 & 0.9798 & 0.02076 \\
303.55 & 0.9781 & 0.02186 \\
303.45 & 0.9774 & 0.02257 \\
305.80 & 0.9769 & 0.02307 \\
307.65 & 0.9751 & 0.02493 \\
308.85 & 0.9745 & 0.02550 \\
309.90 & 0.9739 & 0.02605 \\
310.85 & 0.9734 & 0.02659 \\
311.75 & 0.9729 & 0.02710 \\
313.35 & 0.9719 & 0.02814 \\
315.15 & 0.9707 & 0.02926 \\
318.35 & 0.9683 & 0.03165 \\
320.05 & 0.9672 & 0.03276 \\
\hline
\end{tabular}

${ }^{a} x_{2}$ : mole fraction of component 2 in the saturated solution.

${ }^{\mathrm{b}} x_{1}$ : mole fraction solubility of the solute.

\section{Auxiliary Information}

\section{Method/Apparatus/Procedure:}

Constant-temperature circulating bath, stirrer, analytical balance, and laser monitoring system.

Solubilities were determined by a dynamic method. Preweighed amounts of solute and solvent were placed in a stoppered equilibrium vessel, which was connected to a circulating constant-temperature water bath. The solution was stirred and the temperature slowly increased until all of the solid dissolved. Near the dissolution temperature, the temperature was increased at a rate of $0.2 \mathrm{~K} / 20 \mathrm{~min}$. Complete dissolution was determined using a laser monitoring system.

\section{Source and Purity of Chemicals:}

(1) $99+\%$, Chemical source not specified, no purification details were provided.

(2) $99+\%$, Chemical source not specified, no purification details were provided.

Estimated Error:

Temperature: $\pm 0.05 \mathrm{~K}$.

$x_{1}: \pm 1 \%$ (relative error).

\begin{tabular}{|c|c|}
\hline $\begin{array}{l}\text { Components: } \\
\text { (1) 4-Aminobenzoic acid; } \\
\mathrm{C}_{7} \mathrm{H}_{7} \mathrm{NO}_{2} ;[150-13-0] \\
\text { (2) } 1-\text { Decanol; } \mathrm{C}_{10} \mathrm{H}_{22} \mathrm{O} \text {; } \\
\text { [112-30-1] }\end{array}$ & $\begin{array}{l}\text { Original Measurements: } \\
{ }^{103} \text { C. R. Daniels, A. K. Charlton, R. M. } \\
\text { Wold, R. J. Moreno, W. E. Acree, Jr., } \\
\text { and M. H. Abraham, Phys. Chem. Liq. } \\
\text { 42, } 633 \text { (2004). }\end{array}$ \\
\hline $\begin{array}{l}\text { Variables: } \\
T / \mathrm{K}=298.15\end{array}$ & $\begin{array}{l}\text { Prepared by: } \\
\text { W. E. Acree, Jr. }\end{array}$ \\
\hline
\end{tabular}


Experimental Values

\begin{tabular}{lc}
\hline \hline$x_{2}{ }^{\mathrm{a}}$ & $x_{1}{ }^{\mathrm{b}}$ \\
\hline 0.9826 & 0.01736 \\
\hline
\end{tabular}

${ }^{\mathrm{a}} x_{2}$ : mole fraction of component 2 in the saturated solution.

${ }^{\mathrm{b}}{ }_{x_{1}}$ : mole fraction solubility of the solute.

\section{Auxiliary Information}

\section{Method/Apparatus/Procedure:}

Constant-temperature bath, calorimetric thermometer, and an ultraviolet/ visible spectrophotometer.

Excess solute and solvent were placed in amber glass bottles and allowed to equilibrate for several days at constant temperature. Attainment of equilibrium was verified by several repetitive measurements and by approaching equilibrium from supersaturation. Aliquots of saturated solutions were transferred through a coarse filter into tared volumetric flasks, weighed, and diluted with methanol. Concentrations were determined by

spectrophotometric measurements at $289 \mathrm{~nm}$.

Source and Purity of Chemicals:

(1) $99 \%$, Aldrich Chemical Company, Milwaukee, WI, USA, was used as received.

(2) $99+\%$, Alfa Aesar, USA, stored over molecular sieves and distilled shortly before use.

Estimated Error:

Temperature: $\pm 0.1 \mathrm{~K}$.

$x_{1}: \pm 1.5 \%$ (relative error).

\begin{tabular}{ll}
\hline \hline Components: & Original Measurements: \\
$\begin{array}{l}\text { (1) 4-Aminobenzoic acid; } \\
\mathrm{C}_{7} \mathrm{H}_{7} \mathrm{NO}_{2} ;[150-13-0]\end{array}$ & $\begin{array}{l}102 \text { J. Barra, M.-A. Peña, and P. } \\
\text { (2) } 1,2-\text { Ethanediol; } \mathrm{C}_{2} \mathrm{H}_{6} \mathrm{O}_{2} ;\end{array}$ \\
{$[107-21-1]$} & $(2000)$. \\
\hline Variables: & \\
$T / \mathrm{K}=298.15$ & Prepared by: \\
\hline
\end{tabular}

Experimental Values

\begin{tabular}{lc}
\hline \hline$x_{2}^{\mathrm{a}}$ & $x_{1}{ }^{\mathrm{b}}$ \\
\hline 0.9307 & 0.06932 \\
\hline${ }^{\mathrm{a}} x_{2}:$ mole fraction of component 2 in the saturated solution. & \\
${ }^{\mathrm{b}} x_{1}:$ mole fraction solubility of the solute. &
\end{tabular}

\section{Auxiliary Information}

\footnotetext{
Method/Apparatus/Procedure:

Constant-temperature bath, analytical balance, and an ultraviolet/visible spectrophotometer.

Excess solute and solvent were placed in a flask and allowed to equilibrate for several days at constant temperature. The solid phase was removed by filtration, and the clear solution was diluted quantitatively with $96 \%$ ethanol. The concentration of the diluted solution was determined by spectrophotometric measurement at the wavelength of maximum absorption. In instances where the solvent interfered with the spectrophotometric determination, the samples were evaporated to dryness and the residue diluted with $96 \%$ ethanol.
}

Source and Purity of Chemicals:

(1) Purity not given, Sigma Chemical Company, USA, no purification details were provided.

(2) Spectrophotometric or Analytical grade, Chemical source not specified, no purification details were provided.

\section{Estimated Error:}

Temperature: $\pm 0.2 \mathrm{~K}$.

$x_{1}: \pm 3.0 \%$ (relative error, estimated by compiler).

\begin{tabular}{ll}
\hline \hline Components: & Original Measurements: \\
(1) 4-Aminobenzoic acid; & 102J. Barra, M.-A. Peña, and P. \\
$\mathrm{C}_{7} \mathrm{H}_{7} \mathrm{NO}_{2} ;[150-13-0]$ & Bustamante, Eur. J. Pharm. Sci. 10, 153 \\
(2) $1,2-$ Propanediol; $\mathrm{C}_{3} \mathrm{H}_{8} \mathrm{O}_{2} ;$ & $(2000)$. \\
{$[57-55-6]$} & \\
\hline Variables: & Prepared by: \\
$T / \mathrm{K}=298.15$ & W. E. Acree, Jr. \\
\hline
\end{tabular}

Experimental Values

\begin{tabular}{lc}
\hline \hline$x_{2}{ }^{\mathrm{a}}$ & $x_{1}{ }^{\mathrm{b}}$ \\
\hline 0.9173 & 0.08266 \\
\hline${ }^{a} x_{2}:$ mole fraction of component 2 in the saturated solution. & \\
${ }^{\mathrm{b}} x_{1}:$ mole fraction solubility of the solute. &
\end{tabular}

\section{Auxiliary Information}

\section{Method/Apparatus/Procedure:}

Constant-temperature bath, analytical balance, and an ultraviolet/visible spectrophotometer.

Excess solute and solvent were placed in a flask and allowed to equilibrate for several days at constant temperature. The solid phase was removed by filtration, and the clear solution was diluted quantitatively with $96 \%$ ethanol. The concentration of the diluted solution was determined by spectrophotometric measurement at the wavelength of maximum absorption. In instances where the solvent interfered with the spectrophotometric determination, the samples were evaporated to dryness and the residue diluted with $96 \%$ ethanol.

Source and Purity of Chemicals:

(1) Purity not given, Sigma Chemical Company, USA, no purification details were provided.

(2) Spectrophotometric or Analytical grade, Chemical source not specified, no purification details were provided.

\section{Estimated Error:}

Temperature: $\pm 0.2 \mathrm{~K}$.

$x_{1}: \pm 3.0 \%$ (relative error, estimated by compiler).

\begin{tabular}{|c|c|}
\hline $\begin{array}{l}\text { Components: } \\
\text { (1) 4-Aminobenzoic acid; } \\
\mathrm{C}_{7} \mathrm{H}_{7} \mathrm{NO}_{2} ;[150-13-0] \\
\text { (2) 1,2-Propanediol; } \mathrm{C}_{3} \mathrm{H}_{8} \mathrm{O}_{2} \text {; } \\
\text { [57-55-6] }\end{array}$ & $\begin{array}{l}\text { Original Measurements: } \\
{ }^{51} \text { H.-M. Lin and R. A. Nash, J. Pharm. } \\
\text { Sci. 82, } 1018 \text { (1993). }\end{array}$ \\
\hline $\begin{array}{l}\text { Variables: } \\
T / \mathrm{K}=298.15\end{array}$ & $\begin{array}{l}\text { Prepared by: } \\
\text { W. E. Acree, Jr. }\end{array}$ \\
\hline
\end{tabular}


Experimental Values

\begin{tabular}{lc}
\hline \hline$x_{2}{ }^{\mathrm{a}}$ & $x_{1}{ }^{\mathrm{b}}$ \\
\hline 0.9207 & 0.0793 \\
\hline${ }^{\mathrm{a}} x_{2}:$ mole fraction of component 2 in the saturated solution. \\
${ }^{\mathrm{b}} x_{1}:$ mole fraction solubility of the solute.
\end{tabular}

\section{Auxiliary Information}

\section{Method/Apparatus/Procedure:}

Constant-temperature bath, and high-performance liquid chromatograph. Excess solute and solvent were placed in screw-capped vials. The vials were sealed with several turns of electrical tape, warmed to about $323 \mathrm{~K}$, and then shaken in a constant-temperature bath for at least $24 \mathrm{~h}$ at $298 \mathrm{~K}$. After equilibrium was reached, the sample was filtered through a 0.45 or $1.0 \mu \mathrm{m}$ filter, and an aliquot was diluted appropriately for chromatographic analysis. Concentrations were determined by a high-performance liquid chromatographic method.

\section{Source and Purity of Chemicals:}

(1) Purity not given, Eastman Kodak Chemical Company, Rochester, NY, USA, no information provided concerning purification.

(2) Reagent grade, Aldrich Chemical Company, Milwaukee, WI, USA, no purification details were provided.

\section{Estimated Error:}

Temperature: $\pm 0.2 \mathrm{~K}$ (estimated by compiler). $x_{1}: \pm 3 \%$ (relative error, estimated by compiler).

\begin{tabular}{|c|c|}
\hline $\begin{array}{l}\text { Components: } \\
\text { (1) 4-Aminobenzoic acid; } \\
\mathrm{C}_{7} \mathrm{H}_{7} \mathrm{NO}_{2} ;[150-13-0] \\
\text { (2) 1,3-Propanediol; } \mathrm{C}_{3} \mathrm{H}_{8} \mathrm{O}_{2} \text {; } \\
\text { [504-63-2] }\end{array}$ & $\begin{array}{l}\text { Original Measurements: } \\
\text { 51 H.-M. Lin and R. A. Nash, J. Pharm. } \\
\text { Sci. 82, } 1018 \text { (1993). }\end{array}$ \\
\hline $\begin{array}{l}\text { Variables: } \\
T / \mathrm{K}=298.15\end{array}$ & $\begin{array}{l}\text { Prepared by: } \\
\text { W. E. Acree, Jr. }\end{array}$ \\
\hline
\end{tabular}

Experimental Values

\begin{tabular}{lc}
\hline \hline$x_{2}{ }^{\mathrm{a}}$ & $x_{1}{ }^{\mathrm{b}}$ \\
\hline 0.9331 & 0.0669 \\
\hline${ }^{\mathrm{a}} x_{2}:$ mole fraction of component 2 in the saturated solution. & \\
${ }^{\mathrm{b}} x_{1}:$ mole fraction solubility of the solute. &
\end{tabular}

\section{Auxiliary Information}

\section{Method/Apparatus/Procedure:}

Constant-temperature bath, and high-performance liquid chromatograph. Excess solute and solvent were placed in screw-capped vials. The vials were sealed with several turns of electrical tape, warmed to about $323 \mathrm{~K}$, and then shaken in a constant-temperature bath for at least $24 \mathrm{~h}$ at $298 \mathrm{~K}$. After equilibrium was reached, the sample was filtered through a 0.45 or $1.0 \mu \mathrm{m}$ filter, and an aliquot was diluted appropriately for chromatographic analysis. Concentrations were determined by a high-performance liquid chromatographic method.

\section{Source and Purity of Chemicals:}

(1) Purity not given, Eastman Kodak Chemical Company, Rochester, NY, USA, no information provided concerning purification.

(2) Reagent grade, Aldrich Chemical Company, Milwaukee, WI, USA, no purification details were provided.
Estimated Error:

Temperature: $\pm 0.2 \mathrm{~K}$ (estimated by compiler).

$x_{1}: \pm 3 \%$ (relative error, estimated by compiler).

\begin{tabular}{|c|c|}
\hline $\begin{array}{l}\text { Components: } \\
\text { (1) 4-Aminobenzoic acid; } \\
\mathrm{C}_{7} \mathrm{H}_{7} \mathrm{NO}_{2} ;[150-13-0] \\
\text { (2) } 1,2,3 \text {-Propanetriol (Glycerol); } \\
\mathrm{C}_{3} \mathrm{H}_{8} \mathrm{O}_{3} ;[60-29-7]\end{array}$ & $\begin{array}{l}\text { Original Measurements: } \\
{ }^{102} \text { J. Barra, M.-A. Peña, and P. } \\
\text { Bustamante, Eur. J. Pharm. Sci. 10, } 153 \\
\text { (2000). }\end{array}$ \\
\hline $\begin{array}{l}\text { Variables: } \\
T / \mathrm{K}=298.15\end{array}$ & $\begin{array}{l}\text { Prepared by: } \\
\text { W. E. Acree, Jr. }\end{array}$ \\
\hline
\end{tabular}

Experimental Values

\begin{tabular}{lc}
\hline \hline$x_{2}{ }^{\mathrm{a}}$ & $x_{1}{ }^{\mathrm{b}}$ \\
\hline 0.9282 & 0.07179
\end{tabular}

${ }^{\mathrm{a}} x_{2}$ : mole fraction of component 2 in the saturated solution.

${ }^{b} x_{1}$ : mole fraction solubility of the solute.

Auxiliary Information

Method/Apparatus/Procedure:

Constant-temperature bath, analytical balance, and an ultraviolet/visible spectrophotometer.

Excess solute and solvent were placed in a flask and allowed to equilibrate for several days at constant temperature. The solid phase was removed by filtration, and the clear solution was diluted quantitatively with $96 \%$ ethanol. The concentration of the diluted solution was determined by spectrophotometric measurement at the wavelength of maximum absorption. In instances where the solvent interfered with the spectrophotometric determination, the samples were evaporated to dryness and the residue diluted with $96 \%$ ethanol.

Source and Purity of Chemicals:

(1) Purity not given, Sigma Chemical Company, USA, no purification details were provided.

(2) Spectrophotometric or Analytical grade, Chemical source not specified, no purification details were provided.

\section{Estimated Error:}

Temperature: $\pm 0.2 \mathrm{~K}$.

$x_{1}: \pm 3.0 \%$ (relative error, estimated by compiler).

\subsection{4-Aminobenzoic acid solubility data in alkoxyalcohols}

\begin{tabular}{ll}
\hline \hline Components: & Original Measurements: \\
(1) 4-Aminobenzoic acid; & ${ }^{51} \mathrm{H} .-\mathrm{M}$. Lin and R. A. Nash, J. Pharm. \\
$\mathrm{C}_{7} \mathrm{H}_{7} \mathrm{NO}_{2} ;[150-13-0]$ & Sci. 82, 1018 (1993). \\
$(2)$ 2-Ethoxyethanol; & \\
$\mathrm{C}_{4} \mathrm{H}_{10} \mathrm{O}_{2} ;[110-85-5]$ & \\
\hline Variables: & Prepared by: \\
$T / \mathrm{K}=298.15$ & W. E. Acree, Jr. \\
\hline
\end{tabular}


Experimental Values

\begin{tabular}{lc}
\hline \hline$x_{2}{ }^{\mathrm{a}}$ & $x_{1}{ }^{\mathrm{b}}$ \\
\hline 0.8169 & 0.1831 \\
${ }^{\mathrm{a}} x_{2}:$ mole fraction of component 2 in the saturated solution. \\
${ }^{\mathrm{b}} x_{1}:$ mole fraction solubility of the solute.
\end{tabular}

\section{Auxiliary Information}

\section{Method/Apparatus/Procedure:}

Constant-temperature bath, and high-performance liquid chromatograph. Excess solute and solvent were placed in screw-capped vials. The vials were sealed with several turns of electrical tape, warmed to about $323 \mathrm{~K}$, and then shaken in a constant-temperature bath for at least $24 \mathrm{~h}$ at $298 \mathrm{~K}$. After equilibrium was reached, the sample was filtered through a 0.45 or $1.0 \mu \mathrm{m}$ filter, and an aliquot was diluted appropriately for chromatographic analysis. Concentrations were determined by a high-performance liquid chromatographic method.

Source and Purity of Chemicals:

(1) Purity not given, Eastman Kodak Chemical Company, Rochester, NY, USA, no information provided concerning purification.

(2) HPLC grade, Aldrich Chemical Company, Milwaukee, WI, USA, no purification details were provided.

\section{Estimated Error:}

Temperature: $\pm 0.2 \mathrm{~K}$ (estimated by compiler). $x_{1}: \pm 3 \%$ (relative error, estimated by compiler).

\subsection{4-Aminobenzoic acid solubility data in ketones}

\begin{tabular}{|c|c|}
\hline $\begin{array}{l}\text { Components: } \\
\text { (1) 4-Aminobenzoic acid; } \\
\mathrm{C}_{7} \mathrm{H}_{7} \mathrm{NO}_{2} ;[150-13-0] \\
\text { (2) Propanone; } \mathrm{C}_{3} \mathrm{H}_{6} \mathrm{O} ; \\
\text { [67-64-1] }\end{array}$ & $\begin{array}{l}\text { Original Measurements: } \\
{ }^{102} \text { J. Barra, M.-A. Peña, and P. } \\
\text { Bustamante, Eur. J. Pharm. Sci. 10, } 153 \\
(2000) \text {. }\end{array}$ \\
\hline $\begin{array}{l}\text { Variables: } \\
T / \mathrm{K}=298.15\end{array}$ & $\begin{array}{l}\text { Prepared by: } \\
\text { W. E. Acree, Jr. }\end{array}$ \\
\hline
\end{tabular}

Experimental Values

\begin{tabular}{lc}
\hline \hline$x_{2}^{\mathrm{a}}$ & $x_{1}{ }^{\mathrm{b}}$ \\
\hline 0.9474 & 0.05265 \\
\hline${ }^{\mathrm{a}} x_{2}:$ mole fraction of component 2 in the saturated solution. & \\
${ }^{\mathrm{b}} x_{1}:$ mole fraction solubility of the solute. &
\end{tabular}

\section{Auxiliary Information}

\footnotetext{
Method/Apparatus/Procedure:

Constant-temperature bath, analytical balance, and an ultraviolet/visible spectrophotometer.

Excess solute and solvent were placed in a flask and allowed to equilibrate for several days at constant temperature. The solid phase was removed by filtration, and the clear solution was diluted quantitatively with $96 \%$ ethanol. The concentration of the diluted solution was determined by spectrophotometric measurement at the wavelength of maximum absorption. In instances where the solvent interfered with the spectrophotometric determination, the samples were evaporated to dryness and the residue diluted with $96 \%$ ethanol.
}

Source and Purity of Chemicals:

(1) Purity not given, Sigma Chemical Company, USA, no purification details were provided.

(2) Spectrophotometric or Analytical grade, Chemical source not specified, no purification details were provided.

Estimated Error:

Temperature: $\pm 0.2 \mathrm{~K}$.

$x_{1}: \pm 3.0 \%$ (relative error, estimated by compiler).

\begin{tabular}{ll}
\hline \hline Components: & Original Measurements: \\
(1) 4-Aminobenzoic acid; & ${ }^{102}$ J. Barra, M.-A. Peña, and P. \\
$\mathrm{C}_{7} \mathrm{H}_{7} \mathrm{NO}_{2} ;[150-13-0]$ & Bustamante, Eur. J. Pharm. Sci. 10, 153 \\
$\begin{array}{l}\text { (2) Acetophenone; } \mathrm{C}_{8} \mathrm{H}_{8} \mathrm{O} ; \\
\text { [98-86-2] }\end{array}$ & $(2000)$. \\
\hline Variables: & Prepared by: \\
$T / \mathrm{K}=298.15$ & W. E. Acree, Jr. \\
\hline
\end{tabular}

Experimental Values

\begin{tabular}{|c|c|}
\hline$x_{2}{ }^{\mathrm{a}}$ & $x_{1}{ }^{\mathrm{b}}$ \\
\hline 0.9957 & 0.004335 \\
\hline
\end{tabular}

\section{Auxiliary Information}

\section{Method/Apparatus/Procedure:}

Constant-temperature bath, analytical balance, and an ultraviolet/visible spectrophotometer.

Excess solute and solvent were placed in a flask and allowed to equilibrate for several days at constant temperature. The solid phase was removed by filtration, and the clear solution was diluted quantitatively with $96 \%$ ethanol. The concentration of the diluted solution was determined by spectrophotometric measurement at the wavelength of maximum absorption. In instances where the solvent interfered with the spectrophotometric determination, the samples were evaporated to dryness and the residue diluted with $96 \%$ ethanol.

Source and Purity of Chemicals:

(1) Purity not given, Sigma Chemical Company, USA, no purification details were provided.

(2) Spectrophotometric or Analytical grade, Chemical source not specified, no purification details were provided.

\section{Estimated Error:}

Temperature: $\pm 0.2 \mathrm{~K}$.

$x_{1}: \pm 3.0 \%$ (relative error, estimated by compiler).

\subsection{4-Aminobenzoic acid solubility data in miscellaneous organic solvents}

\begin{tabular}{|c|c|}
\hline $\begin{array}{l}\text { Components: } \\
\text { (1) 4-Aminobenzoic acid; } \\
\mathrm{C}_{7} \mathrm{H}_{7} \mathrm{NO}_{2} ;[150-13-0] \\
\text { (2) Ethanoic acid; } \mathrm{C}_{2} \mathrm{H}_{4} \mathrm{O}_{2} \text {; } \\
\text { [64-19-7] }\end{array}$ & $\begin{array}{l}\text { Original Measurements: } \\
{ }^{102} \text { J. Barra, M.-A. Peña, and P. } \\
\text { Bustamante, Eur. J. Pharm. Sci. 10, } 153 \\
(2000) \text {. }\end{array}$ \\
\hline $\begin{array}{l}\text { Variables: } \\
T / \mathrm{K}=298.15\end{array}$ & $\begin{array}{l}\text { Prepared by: } \\
\text { W. E. Acree, Jr. }\end{array}$ \\
\hline
\end{tabular}


Experimental Values

\begin{tabular}{lc}
\hline \hline$x_{2}^{\mathrm{a}}$ & $x_{1}{ }^{\mathrm{b}}$ \\
\hline 0.9229 & 0.0771
\end{tabular}

${ }^{\mathrm{a}} x_{2}$ : mole fraction of component 2 in the saturated solution.

${ }^{\mathrm{b}} x_{1}$ : mole fraction solubility of the solute.

\section{Auxiliary Information}

\section{Method/Apparatus/Procedure:}

Constant-temperature bath, analytical balance, and an ultraviolet/visible spectrophotometer.

Excess solute and solvent were placed in a flask and allowed to equilibrate for several days at constant temperature. The solid phase was removed by filtration, and the clear solution was diluted quantitatively with $96 \%$ ethanol. The concentration of the diluted solution was determined by spectrophotometric measurement at the wavelength of maximum absorption. In instances where the solvent interfered with the spectrophotometric determination, the samples were evaporated to dryness and the residue diluted with $96 \%$ ethanol.

Source and Purity of Chemicals:

(1) Purity not given, Sigma Chemical Company, USA, no purification details were provided.

(2) Spectrophotometric or Analytical grade, Chemical source not specified, no purification details were provided.

\section{Estimated Error:}

Temperature: $\pm 0.2 \mathrm{~K}$.

$x_{1}: \pm 3.0 \%$ (relative error, estimated by compiler).
Source and Purity of Chemicals:

(1) Purity not given, Sigma Chemical Company, USA, no purification details were provided.

(2) Spectrophotometric or Analytical grade, Chemical source not specified, no purification details were provided.

\section{Estimated Error:}

Temperature: $\pm 0.2 \mathrm{~K}$.

$x_{1}: \pm 3.0 \%$ (relative error, estimated by compiler).

\begin{tabular}{ll}
\hline \hline $\begin{array}{l}\text { Components: } \\
\text { (1) 4-Aminobenzoic acid; }\end{array}$ & $\begin{array}{l}\text { Original Measurements: } \\
{ }^{102} \text { J. Barra, M.-A. Peña, and P. }\end{array}$ \\
$\mathrm{C}_{7} \mathrm{H}_{7} \mathrm{NO}_{2} ;[150-13-0]$ & $\begin{array}{l}\text { Bustamante, Eur. J. Pharm. Sci. 10, 153 } \\
\text { (2) Formamide; } \mathrm{CH}_{3} \mathrm{NO} ;\end{array}$ \\
[75-12-7] & \\
\hline Variables: & Prepared by: \\
$T / \mathrm{K}=298.15$ & W. E. Acree, Jr. \\
\hline
\end{tabular}

Experimental Values

\begin{tabular}{lc}
\hline \hline$x_{2}{ }^{\mathrm{a}}$ & $x_{1}{ }^{\mathrm{b}}$ \\
\hline 0.9878 & 0.01223 \\
\hline${ }^{a} x_{2}:$ mole fraction of component 2 in the saturated solution. & \\
${ }^{\mathrm{b}} x_{1}:$ mole fraction solubility of the solute. &
\end{tabular}

\section{Auxiliary Information}

\begin{tabular}{|c|c|}
\hline $\begin{array}{l}\text { Components: } \\
\text { (1) 4-Aminobenzoic acid; } \\
\mathrm{C}_{7} \mathrm{H}_{7} \mathrm{NO}_{2} ;[150-13-0] \\
\text { (2) Propanoic acid; } \mathrm{C}_{3} \mathrm{H}_{6} \mathrm{O}_{2} \text {; } \\
\text { [79-09-4] }\end{array}$ & $\begin{array}{l}\text { Original Measurements: } \\
{ }^{102} \text { J. Barra, M.-A. Peña, and P. } \\
\text { Bustamante, Eur. J. Pharm. Sci. 10, } 153 \\
(2000) \text {. }\end{array}$ \\
\hline $\begin{array}{l}\text { Variables: } \\
T / \mathrm{K}=298.15\end{array}$ & $\begin{array}{l}\text { Prepared by: } \\
\text { W. E. Acree, Jr. }\end{array}$ \\
\hline
\end{tabular}

\section{Experimental Values}

\begin{tabular}{lc}
\hline \hline$x_{2}^{\mathrm{a}}$ & $x_{1}{ }^{\mathrm{b}}$ \\
\hline 0.9510 & 0.0490 \\
\hline${ }^{\mathrm{a}} x_{2}:$ mole fraction of component 2 in the saturated solution. \\
${ }^{\mathrm{b}} x_{1}:$ mole fraction solubility of the solute.
\end{tabular}

\section{Auxiliary Information}

\footnotetext{
Method/Apparatus/Procedure:

Constant-temperature bath, analytical balance, and an ultraviolet/visible spectrophotometer.

Excess solute and solvent were placed in a flask and allowed to equilibrate for several days at constant temperature. The solid phase was removed by filtration, and the clear solution was diluted quantitatively with $96 \%$ ethanol. The concentration of the diluted solution was determined by spectrophotometric measurement at the wavelength of maximum absorption. In instances where the solvent interfered with the spectrophotometric determination, the samples were evaporated to dryness and the residue diluted with $96 \%$ ethanol.
}

\begin{abstract}
Method/Apparatus/Procedure:
Constant-temperature bath, analytical balance, and an ultraviolet/visible spectrophotometer.

Excess solute and solvent were placed in a flask and allowed to equilibrate for several days at constant temperature. The solid phase was removed by filtration, and the clear solution was diluted quantitatively with $96 \%$ ethanol. The concentration of the diluted solution was determined by spectrophotometric measurement at the wavelength of maximum absorption. In instances where the solvent interfered with the spectrophotometric determination, the samples were evaporated to dryness and the residue diluted with $96 \%$ ethanol.
\end{abstract}

Source and Purity of Chemicals:

(1) Purity not given, Sigma Chemical Company, USA, no purification details were provided.

(2) Spectrophotometric or Analytical grade, Chemical source not specified, no purification details were provided.

\section{Estimated Error:}

Temperature: $\pm 0.2 \mathrm{~K}$.

\begin{tabular}{|c|c|}
\hline $\begin{array}{l}\text { Components: } \\
\text { (1) 4-Aminobenzoic acid; } \\
\mathrm{C}_{7} \mathrm{H}_{7} \mathrm{NO}_{2} ;[150-13-0] \\
\text { (2) } \mathrm{N}, \mathrm{N} \text {-Dimethylformamide; } \\
\mathrm{C}_{3} \mathrm{H}_{7} \mathrm{NO} ;[68-12-2]\end{array}$ & $\begin{array}{l}\text { Original Measurements: } \\
{ }^{102} \text { J. Barra, M.-A. Peña, and P. } \\
\text { Bustamante, Eur. J. Pharm. Sci. 10, } 153 \\
(2000) \text {. }\end{array}$ \\
\hline $\begin{array}{l}\text { Variables: } \\
T / \mathrm{K}=298.15\end{array}$ & $\begin{array}{l}\text { Prepared by: } \\
\text { W. E. Acree, Jr. }\end{array}$ \\
\hline
\end{tabular}

$x_{1}: \pm 3.0 \%$ (relative error, estimated by compiler). 
Experimental Values

\begin{tabular}{lc}
\hline \hline$x_{2}{ }^{\mathrm{a}}$ & $x_{1}{ }^{\mathrm{b}}$ \\
\hline 0.9991 & 0.000904 \\
${ }^{\mathrm{a}} x_{2}:$ mole fraction of component 2 in the saturated solution. \\
${ }^{\mathrm{b}}{ }_{x_{1}}$ : mole fraction solubility of the solute.
\end{tabular}

\section{Auxiliary Information}

\section{Method/Apparatus/Procedure:}

Constant-temperature bath, analytical balance, and an ultraviolet/visible spectrophotometer.

Excess solute and solvent were placed in a flask and allowed to equilibrate for several days at constant temperature. The solid phase was removed by filtration, and the clear solution was diluted quantitatively with $96 \%$ ethanol. The concentration of the diluted solution was determined by spectrophotometric measurement at the wavelength of maximum absorption In instances where the solvent interfered with the spectrophotometric determination, the samples were evaporated to dryness and the residue diluted with $96 \%$ ethanol.

Source and Purity of Chemicals:

(1) Purity not given, Sigma Chemical Company, USA, no purification details were provided.

(2) Spectrophotometric or Analytical grade, Chemical source not specified, no purification details were provided.

Estimated Error:

Temperature: $\pm 0.2 \mathrm{~K}$.

$x_{1}: \pm 3.0 \%$ (relative error, estimated by compiler).

\begin{tabular}{|c|c|}
\hline $\begin{array}{l}\text { Components: } \\
\text { (1) 4-Aminobenzoic acid; } \\
\mathrm{C}_{7} \mathrm{H}_{7} \mathrm{NO}_{2} ;[150-13-0] \\
\text { (2) Butyl 2-hydroxypropanoate; } \\
\mathrm{C}_{7} \mathrm{H}_{14} \mathrm{O}_{3} ;[128-22-7]\end{array}$ & $\begin{array}{l}\text { Original Measurements: } \\
\text { 51 H.-M. Lin and R. A. Nash, J. Pharm. } \\
\text { Sci. 82, } 1018 \text { (1993). }\end{array}$ \\
\hline $\begin{array}{l}\text { Variables: } \\
T / \mathrm{K}=298.15\end{array}$ & $\begin{array}{l}\text { Prepared by: } \\
\text { W. E. Acree, Jr. }\end{array}$ \\
\hline
\end{tabular}

Experimental Values

\begin{tabular}{lc}
\hline \hline$x_{2}{ }^{\mathrm{a}}$ & $x_{1}{ }^{\mathrm{b}}$ \\
\hline 0.9015 & 0.0985 \\
\hline${ }^{\mathrm{a}} x_{2}:$ mole fraction of component 2 in the saturated solution. & \\
${ }^{\mathrm{b}} x_{1}:$ mole fraction solubility of the solute. &
\end{tabular}

\section{Auxiliary Information}

\section{Method/Apparatus/Procedure:}

Constant-temperature bath, and high-performance liquid chromatograph. Excess solute and solvent were placed in screw-capped vials. The vials were sealed with several turns of electrical tape, warmed to about $323 \mathrm{~K}$, and then shaken in a constant-temperature bath for at least $24 \mathrm{~h}$ at $298 \mathrm{~K}$. After equilibrium was reached, the sample was filtered through a 0.45 or $1.0 \mu \mathrm{m}$ filter, and an aliquot was diluted appropriately for chromatographic analysis. Concentrations were determined by a high-performance liquid chromatographic method.

\section{Source and Purity of Chemicals:}

(1) Purity not given, Eastman Kodak Chemical Company, Rochester, NY,

USA, no information provided concerning purification.

(2) Purity not given, Purac Inc., no purification details were provided.
Estimated Error:

Temperature: $\pm 0.2 \mathrm{~K}$ (estimated by compiler).

$x_{1}: \pm 3 \%$ (relative error, estimated by compiler).

\begin{tabular}{|c|c|}
\hline $\begin{array}{l}\text { Components: } \\
\text { (1) 4-Aminobenzoic acid; } \\
\mathrm{C}_{7} \mathrm{H}_{7} \mathrm{NO}_{2} ;[150-13-0] \\
\text { (2) } \gamma \text {-Butyrolactone; } \mathrm{C}_{4} \mathrm{H}_{6} \mathrm{O}_{2} \text {; } \\
\text { [96-48-0] }\end{array}$ & $\begin{array}{l}\text { Original Measurements: } \\
{ }^{51} \text { H.-M. Lin and R. A. Nash, J. Pharm } \\
\text { Sci. 82, } 1018 \text { (1993). }\end{array}$ \\
\hline $\begin{array}{l}\text { Variables: } \\
T / \mathrm{K}=298.15\end{array}$ & $\begin{array}{l}\text { Prepared by: } \\
\text { W. E. Acree, Jr. }\end{array}$ \\
\hline
\end{tabular}

Experimental Values

\begin{tabular}{lc}
\hline \hline$x_{2}^{\mathrm{a}}$ & $x_{1}^{\mathrm{b}}$ \\
\hline 0.8335 & 0.1665 \\
\hline${ }^{\mathrm{a}}{ }_{2}:$ mole fraction of component 2 in the saturated solution. & \\
${ }^{\mathrm{b}} x_{1}:$ mole fraction solubility of the solute. &
\end{tabular}

Auxiliary Information

Method/Apparatus/Procedure:

Constant-temperature bath, and high-performance liquid chromatograph. Excess solute and solvent were placed in screw-capped vials. The vials were sealed with several turns of electrical tape, warmed to about $323 \mathrm{~K}$, and then shaken in a constant-temperature bath for at least $24 \mathrm{~h}$ at $298 \mathrm{~K}$. After equilibrium was reached, the sample was filtered through a 0.45 or $1.0 \mu \mathrm{m}$ filter, and an aliquot was diluted appropriately for chromatographic analysis. Concentrations were determined by a high-performance liquid chromatographic method.

Source and Purity of Chemicals:

(1) Purity not given, Eastman Kodak Chemical Company, Rochester, NY, USA, no information provided concerning purification.

(2) Reagent grade, Aldrich Chemical Company, Milwaukee, WI, USA, no purification details were provided.

\section{Estimated Error:}

Temperature: $\pm 0.2 \mathrm{~K}$ (estimated by compiler).

$x_{1}: \pm 3 \%$ (relative error, estimated by compiler).

\section{Solubility of 4-Amino-2-hydroxybenzoic Acid in Organic Solvents}

\subsection{Critical evaluation of experimental solubility data}

There has been only a single publication that has reported solubility data for 4-amino-2-hydroxybenzoic acid. Takayama et al. ${ }^{105}$ measured the solubility of 4-amino-2-hydroxybenzoic acid in 1,1'-oxybisethane as part of an experimental study aimed at studying the interactions between $N$-methyl-2-pyrrolidone and 4-aminobenzoic acids. Solubilities were measured as a function of $N$-methyl-2-pyrrolidone with 1,1'oxybisethane serving as the solvent in the complexation study. 4-Amino-2-hydroxybenzoic acid and $\mathrm{N}$-methyl-2-pyrrolidone 
were found to form a slightly soluble 1:1 association complex having a calculated stability constant of $K=24.44 \mathrm{dm}^{3} \mathrm{~mol}^{-1}$. It is not possible to perform a critical evaluation of the experimental data as measurements were made at only a single temperature, and there is no independent experimental 4amino-2-hydroxybenzoic acid solubility data in $1,1^{\prime}$ oxybisethane.

The experimental solubility data for 4-amino-2-hydroxybenzoic acid in 1,1'-oxybisethane is given in Sec. 9.2.

\subsection{4-Amino-2-hydroxybenzoic acid solubility data in ethers}

\begin{tabular}{ll}
\hline \hline Components: & Original Measurements: \\
(1) 4-Amino-2-hydroxybenzoic & ${ }^{105} \mathrm{P} . \mathrm{K}$. Takayama, N. Nambu, and \\
acid; $\mathrm{C}_{7} \mathrm{H}_{7} \mathrm{NO}_{3} ;$ [65-49-6] & T. Nagai, Chem. Pharm. Bull. 25, 879 \\
(2) 1,1'-Oxybisethane; $\mathrm{C}_{4} \mathrm{H}_{10} \mathrm{O} ;$ & $(1977)$. \\
[60-29-7] &
\end{tabular}

Variables:

$T / \mathrm{K}=283$

Prepared by:

W. E. Acree, Jr.

The solubility was given graphically as a plot of molar concentration of the solute versus the concentration of ligand added. From the graph, the compiler estimated a molar solubility of $c_{1}=0.076 \mathrm{~mol} \mathrm{dm}^{-3}$.

\section{Auxiliary Information}

\section{Method/Apparatus/Procedure:}

Very little experimental details were provided. Excess solute and solvent were sealed in vials and incubated for $48 \mathrm{~h}$ at $283 \mathrm{~K}$. A $1 \mathrm{ml}$ sample of the supernatant was withdrawn and the solvent evaporated at $323 \mathrm{~K}$. The residue was dissolved in water or ethanol, and the concentration of the solute determined from spectroscopic absorbance measurements.

Source and Purity of Chemicals:

(1) Purity not given, Chemical source not given, was recrystallized from water before use.

(2) Purity not given, Chemical source not given, no purification details were provided.

\section{Estimated Error:}

Temperature: No information was given.

$c_{1}: \pm 5 \%$ (relative error, estimated by compiler).

\section{Solubility of 5-Amino-2-hydroxybenzoic Acid in Organic Solvents}

\subsection{Critical evaluation of experimental solubility data}

There has been only a single publication that has reported solubility data for 5-amino-2-hydroxybenzoic acid. Patel et al. ${ }^{107}$ measured the solubility of 5-amino-2-hydroxybenzoic acid in tetrahydrofuran, tetrachloromethane, methanol, and ethanol at 11 temperatures between 293 and $313 \mathrm{~K}$. The internal consistency of the dataset was assessed by curvefitting the measured mole fraction solubility data to Eq. (8). The values of the equation coefficients $(A, B$, and $C$ ) are given in Table 15, along with the root-mean-square deviation (RMSD) defined by

$$
\operatorname{RMSD}=\left[\sum_{i=1}^{N} \frac{\left(x_{1}{ }^{\text {exp }}-x_{1}^{\text {calc }}\right)^{2}}{N-1}\right]^{1 / 2},
$$

and the MRD given by Eq. (24). In Eq. (35), $N$ is the number of experimental solubility measurements in an individual solutesolvent data set. Examination of the entries in the last column of Table 15 reveals that the largest average relative deviation between the back-calculated values based on Eq. (8) and experimental data is less than $4 \%$. Results of the mathematical representation analyses indicate that the experimental data for all four 5-amino-2-hydroxybenzoic acid - organic solvent systems are internally consistent.

The experimental solubility data for 5-amino-2-hydroxybenzoic acid in the different organic solvents are in Secs. 10.210.4 .

\subsection{5-Amino-2-hydroxybenzoic acid solubility data in ethers}

\begin{tabular}{|c|c|}
\hline $\begin{array}{l}\text { Components: } \\
\text { (1) 5-Amino-2-hydroxybenzoic } \\
\left.\text { acid; } \mathrm{C}_{7} \mathrm{H}_{7} \mathrm{NO}_{3} ; \text { [ } 89-57-6\right] \\
\text { (2) Tetrahydrofuran; } \mathrm{C}_{4} \mathrm{H}_{8} \mathrm{O} \text {; } \\
\text { [109-99-9] }\end{array}$ & $\begin{array}{l}\text { Original Measurements: } \\
{ }^{107} \text { A. Patel, A. Vaghasiya, R. Gajera, } \\
\text { and S. Baluja, J. Chem. Eng. Data 55, } \\
1453 \text { (2010). }\end{array}$ \\
\hline $\begin{array}{l}\text { Variables: } \\
\text { Temperature }\end{array}$ & $\begin{array}{l}\text { Prepared by: } \\
\text { W. E. Acree, Jr. }\end{array}$ \\
\hline
\end{tabular}

TABLE 15. Parameters of the Modified Apelblat equation for describing the solubility of 5-amino-2-hydroxybenzoic acid in various organic solvents

\begin{tabular}{lcccc}
\hline \hline Solvent & $A$ & $B$ & $C$ & $10^{6}$ RMSD \\
\hline Tetrahydrofuran $^{\mathrm{a}}$ & -0.9140 & 0.0024 & -0.1237 & 17.9244 \\
Tetrachloromethane $^{\mathrm{a}}$ & -9.3301 & 0.0118 & 0 & 0.2812 \\
Methanol $^{\mathrm{a}}$ & -18.053 & 0.0399 & 0 & 0.1036 \\
Ethanol $^{\mathrm{a}}$ & -15.354 & 0.0313 & 0 & -3.86 \\
\hline
\end{tabular}

${ }^{\mathrm{a}}$ Values of the coefficients, root-mean-square deviation, and mean relative deviation were taken from Patel et al. ${ }^{107}$ 
Experimental Values

\begin{tabular}{lcc}
\hline \hline$T / \mathrm{K}$ & $x_{2}{ }^{\mathrm{a}}$ & $x_{1}{ }^{\mathrm{b}}$ \\
\hline 293.15 & 0.1845 & 0.8155 \\
295.15 & 0.1803 & 0.8197 \\
297.15 & 0.1764 & 0.8236 \\
299.15 & 0.1724 & 0.8276 \\
301.15 & 0.1683 & 0.8317 \\
303.15 & 0.1643 & 0.8357 \\
305.15 & 0.1603 & 0.8397 \\
307.15 & 0.1562 & 0.8438 \\
309.15 & 0.1521 & 0.8479 \\
311.15 & 0.1480 & 0.8520 \\
313.15 & 0.1440 & 0.8560
\end{tabular}

${ }^{a_{2}}{ }_{2}$ : mole fraction of component 2 in the saturated solution.

${ }^{\mathrm{b}} x_{1}$ : mole fraction solubility of the solute.

\section{Auxiliary Information}

\section{Method/Apparatus/Procedure:}

Constant-temperature water bath, stirrer, and analytical balance.

Solubilities were determined by a gravimetric method. Excess solute and solvent were placed in an equilibrium cell and allowed to equilibrate in a constant-temperature water bath with continuous stirring for at least $3 \mathrm{~h}$. The stirring was then stopped and the solution was allowed to equilibrate an additional $2 \mathrm{~h}$ to allow suspended solid to settle to the bottom of the container. An aliquot of the saturated solution was removed, filtered, and transferred to a tared vial. The vial was tightly closed and quickly weighed to determine the amount of sample transferred. The vial was then opened and the solvent allowed to evaporate at ambient room temperature. After the solvent had evaporated, the vial was dried and reweighed. The solubility was calculated from the mass of the solid residue and mass of sample transferred.

Source and Purity of Chemicals:

(1) $99.5 \%$, Hiran Orgochem Ltd., Ankleshwar, India, was recrystallized before use.

(2) Analytical grade, Chemical source not specified, no purification details were provided.

Estimated Error:

Temperature: $\pm 0.01 \mathrm{~K}$.

$x_{1}: \pm<1.0 \%$ (relative error)

\subsection{5-Amino-2-hydroxybenzoic acid solubility data in haloalkanes, haloalkenes, and haloaromatic hydrocarbons}

\begin{tabular}{|c|c|}
\hline $\begin{array}{l}\text { Components: } \\
\text { (1) 5-Amino-2-hydroxybenzoic } \\
\text { acid; } \mathrm{C}_{7} \mathrm{H}_{7} \mathrm{NO}_{3} ; \text { [89-57-6] } \\
\text { (2) Tetrachloromethane; } \\
\mathrm{CCl}_{4} ;[56-23-5]\end{array}$ & $\begin{array}{l}\text { Original Measurements: } \\
{ }^{107} \text { A. Patel, A. Vaghasiya, R. Gajera, } \\
\text { and S. Baluja, J. Chem. Eng. Data 55, } \\
1453 \text { (2010). }\end{array}$ \\
\hline $\begin{array}{l}\text { Variables: } \\
\text { Temperature }\end{array}$ & $\begin{array}{l}\text { Prepared by: } \\
\text { W. E. Acree, Jr. }\end{array}$ \\
\hline
\end{tabular}

Experimental Values

\begin{tabular}{lcc}
\hline \hline$T / \mathrm{K}$ & $x_{2}{ }^{\mathrm{a}}$ & $x_{1}{ }^{\mathrm{b}}$ \\
\hline 293.15 & 0.9971 & 0.002902 \\
295.15 & 0.9970 & 0.003027 \\
297.15 & 0.9969 & 0.003098 \\
299.15 & 0.9969 & 0.003116 \\
301.15 & 0.9968 & 0.003159 \\
303.15 & 0.9968 & 0.003187 \\
305.15 & 0.9967 & 0.003313 \\
307.15 & 0.9966 & 0.003446 \\
309.15 & 0.9964 & 0.003619 \\
311.15 & 0.9963 & 0.003705 \\
313.15 & 0.9962 & 0.003827
\end{tabular}

${ }^{a} x_{2}$ : mole fraction of component 2 in the saturated solution.

${ }^{b} x_{1}$ : mole fraction solubility of the solute.
Method/Apparatus/Procedure:

Constant-temperature water bath, stirrer, and analytical balance.

Solubilities were determined by a gravimetric method. Excess solute and solvent were placed in an equilibrium cell and allowed to equilibrate in a constant-temperature water bath with continuous stirring for at least $3 \mathrm{~h}$. The stirring was then stopped and the solution was allowed to equilibrate an additional $2 \mathrm{~h}$ to allow suspended solid to settle to the bottom of the container. An aliquot of the saturated solution was removed, filtered, and transferred to a tared vial. The vial was tightly closed and quickly weighed to determine the amount of sample transferred. The vial was then opened and the solvent allowed to evaporate at ambient room temperature. After the solvent had evaporated, the vial was dried and reweighed. The solubility was calculated from the mass of the solid residue and mass of sample transferred.

Source and Purity of Chemicals:

(1) $99.5 \%$, Hiran Orgochem Ltd., Ankleshwar, India, was recrystallized before use.

(2) Analytical grade, Chemical source not specified, no purification details were provided.

Estimated Error:

Temperature: $\pm 0.01 \mathrm{~K}$

$x_{1}: \pm<3.0 \%$ (relative error).

\subsection{5-Amino-2-hydroxybenzoic acid solubility data in alcohols}

\begin{tabular}{ll}
\hline \hline Components: & Original Measurements: \\
$\begin{array}{l}\text { (1) 5-Amino-2-hydroxybenzoic } \\
\text { acid; } \mathrm{C}_{7} \mathrm{H}_{7} \mathrm{NO}_{3} ;[89-57-6]\end{array}$ & $\begin{array}{l}{ }^{107} \text { A. Patel, A. Vaghasiya, R. Gajera, } \\
\text { and S. Baluja, J. Chem. Eng. Data 55, }\end{array}$ \\
(2) Methanol; $\mathrm{CH}_{4} \mathrm{O} ;[67-56-1]$ & $1453(2010)$. \\
\hline Variables: & Prepared by: \\
Temperature & W. E. Acree, Jr. \\
\hline
\end{tabular}


Experimental Values

\begin{tabular}{lcc}
\hline \hline$T / \mathrm{K}$ & $x_{2}{ }^{\mathrm{a}}$ & $x_{1}{ }^{\mathrm{b}}$ \\
\hline 293.15 & 0.9986 & 0.001408 \\
295.15 & 0.9984 & 0.001551 \\
297.15 & 0.9983 & 0.001705 \\
299.15 & 0.9981 & 0.001876 \\
301.15 & 0.9980 & 0.002018 \\
303.15 & 0.9978 & 0.002154 \\
305.15 & 0.9976 & 0.002401 \\
307.15 & 0.9974 & 0.002642 \\
309.15 & 0.9922 & 0.002837 \\
311.15 & 0.9970 & 0.003021 \\
313.15 & 0.9968 & 0.003162 \\
\hline${ }^{\mathrm{a}} x_{2}:$ mole fraction of component 2 in the saturated solution. & \\
${ }^{\mathrm{b}}{ }_{x_{1}: \text { mole fraction solubility of the solute. }}$
\end{tabular}

\section{Auxiliary Information}

\section{Method/Apparatus/Procedure:}

Constant-temperature water bath, stirrer, and analytical balance.

Solubilities were determined by a gravimetric method. Excess solute and solvent were placed in an equilibrium cell and allowed to equilibrate in a constant-temperature water bath with continuous stirring for at least $3 \mathrm{~h}$. The stirring was then stopped and the solution was allowed to equilibrate an additional $2 \mathrm{~h}$ to allow suspended solid to settle to the bottom of the container. An aliquot of the saturated solution was removed, filtered, and transferred to a tared vial. The vial was tightly closed and quickly weighed to determine the amount of sample transferred. The vial was then opened and the solvent allowed to evaporate at ambient room temperature. After the solvent had evaporated, the vial was dried and reweighed. The solubility was calculated from the mass of the solid residue and mass of sample transferred.

Source and Purity of Chemicals:

(1) $99.5 \%$, Hiran Orgochem Ltd., Ankleshwar, India, was recrystallized before use.

(2) Analytical grade, Chemical source not specified, no purification details were provided

Estimated Error:

Temperature: $\pm 0.01 \mathrm{~K}$.

$x_{1}: \pm<3.0 \%$ (relative error)

\begin{tabular}{ll}
\hline \hline Components: & Original Measurements: \\
$\begin{array}{l}\text { (1) 5-Amino-2-hydroxybenzoic } \\
\text { acid; } \mathrm{C}_{7} \mathrm{H}_{7} \mathrm{NO}_{3} ;[89-57-6]\end{array}$ & ${ }^{107}$ A. Patel, A. Vaghasiya, R. Gajera, \\
(2) Ethanol; $\mathrm{C}_{2} \mathrm{H}_{6} \mathrm{O} ;[64-17-5]$ & $1453(2010)$. \\
\hline $\begin{array}{l}\text { Variables: } \\
\text { Temperature }\end{array}$ & $\begin{array}{l}\text { Prepared by: } \\
\text { W. E. Acree, Jr. }\end{array}$ \\
\hline
\end{tabular}

Experimental Values

\begin{tabular}{lcc}
\hline \hline$T / \mathrm{K}$ & $x_{2}{ }^{\mathrm{a}}$ & $x_{1}{ }^{\mathrm{b}}$ \\
\hline 293.15 & 0.9982 & 0.001765 \\
295.15 & 0.9981 & 0.001931 \\
297.15 & 0.9979 & 0.002117 \\
299.15 & 0.9977 & 0.002254 \\
301.15 & 0.9977 & 0.002334 \\
303.15 & 0.9975 & 0.002491 \\
305.15 & 0.9973 & 0.002738
\end{tabular}

\begin{tabular}{lcc}
\hline \hline$T / \mathrm{K}$ & $x_{2}{ }^{\mathrm{a}}$ & $x_{1}{ }^{\mathrm{b}}$ \\
\hline 307.15 & 0.9971 & 0.002946 \\
309.15 & 0.9969 & 0.003087 \\
311.15 & 0.9968 & 0.003227 \\
313.15 & 0.9965 & 0.003454 \\
\hline
\end{tabular}

${ }^{\mathrm{a}} x_{2}$ : mole fraction of component 2 in the saturated solution.

${ }^{\mathrm{b}} x_{1}$ : mole fraction solubility of the solute.

Auxiliary Information

\begin{abstract}
Method/Apparatus/Procedure:
Constant-temperature water bath, stirrer, and analytical balance.

Solubilities were determined by a gravimetric method. Excess solute and solvent were placed in an equilibrium cell and allowed to equilibrate in a constant-temperature water bath with continuous stirring for at least $3 \mathrm{~h}$. The stirring was then stopped and the solution was allowed to equilibrate an additional $2 \mathrm{~h}$ to allow suspended solid to settle to the bottom of the container. An aliquot of the saturated solution was removed, filtered, and transferred to a tared vial. The vial was tightly closed and quickly weighed to determine the amount of sample transferred. The vial was then opened and the solvent allowed to evaporate at ambient room temperature. After the solvent had evaporated, the vial was dried and reweighed. The solubility was calculated from the mass of the solid residue and mass of sample transferred.
\end{abstract}

Source and Purity of Chemicals:

(1) $99.5 \%$, Hiran Orgochem Ltd., Ankleshwar, India, was recrystallized before use.

(2) Analytical grade, Chemical source not specified, no purification details were provided.

Estimated Error:

Temperature: $\pm 0.01 \mathrm{~K}$.

$x_{1}: \pm<4.0 \%$ (relative error).

\section{Solubility of 1,2-Benzenedicarboxylic Acid in Organic Solvents}

\subsection{Critical evaluation of experimental solubility data}

Several research groups ${ }^{50,60,76,79,108-111}$ have investigated the solubility behavior of 1,2-benzenedicarboxylic acid as a function of temperature. Ren et al. ${ }^{108}$ employed a dynamic method with laser monitoring to study the solubility of 1,2benzenedicarboxylic acid in butyl ethanoate and methanol. Che et al. ${ }^{109}$ determined the solubility of 1,2-benzenedicarboxylic acid in tetrahydrofuran, 1,2-diethoxyethane, cyclohexanone, and acetophenone at several temperatures.

The internal consistency of the six datasets of measured 1,2benzenedicarboxylic acid solubilities was assessed by curvefitting the measured mole fraction solubility data to Eq. (8). The values of the equation coefficients $(A, B$, and $C)$ are given in Table 16, along with the MRD calculated according to Eq. (24). Examination of the numerical entries in the last column of Table 16 reveals that the largest mean relative deviation between the back-calculated values based on Eq. (8) and experimental data is only $0.8 \%$, which is less than the experimental uncertainties in the measured values. Results of the mathematical representation analyses indicate that the 
TABLE 16. Parameters of the Modified Apelblat equation for describing the solubility of 1,2-benzenedicarboxylic acid in various organic solvents

\begin{tabular}{lrrcc}
\hline \hline Solvent & \multicolumn{1}{c}{$A$} & \multicolumn{1}{c}{$B$} & \multicolumn{1}{c}{$C$} & MRD (\%) \\
\hline Butyl ethanoate $^{\mathrm{a}}$ & -146.09 & 4646.2 & 21.836 & 0.82 \\
Methanol $^{\mathrm{a}}$ & 15.381 & -2208.7 & -1.9198 & 0.03 \\
1,2-Diethoxyethane $^{\mathrm{b}}$ & -59.994 & 1482.7 & 8.9357 & 0.44 \\
Tetrahydrofuran $^{\mathrm{b}}$ & -78.560 & 2796.4 & 11.682 & 0.31 \\
Cyclohexanone $^{\mathrm{b}}$ & -78.708 & 1933.1 & 11.984 & 0.70 \\
Acetophenone $^{\mathrm{b}}$ & -78.865 & 1321.7 & 12.131 & 0.29
\end{tabular}

${ }^{\mathrm{a}}$ Values of the coefficients and the mean relative deviations were taken from Ren et al. ${ }^{108}$

${ }^{\mathrm{b}}$ Values of the coefficients and the mean relative deviations were taken from Che et al. ${ }^{109}$

experimental data for all six 1,2-benzenedicarboxylic acid organic solvent systems are internally consistent.

Zhao et al. ${ }^{60}$ measured the solubility of 1,2-benzenedicarboxylic acid in isobutyl ethanoate at 12 temperatures between 301 and $349 \mathrm{~K}$. The experimental data were correlated with the UNIQUAC model. Interaction coefficients calculated from the experimental solid-liquid equilibrium data provided a reasonably accurate mathematical description of the measured values. The mean absolute relative deviation between calculated and observed values was less than $6.4 \%$.

The experimental solubility data for 1,2-benzenedicarboxylic acid in the different organic solvents are in Secs. 11.211.9 .

\subsection{1,2-Benzenedicarboxylic acid solubility data in aromatic hydrocarbons}

\begin{tabular}{|c|c|}
\hline $\begin{array}{l}\text { Components: } \\
\text { (1) } 1,2 \text {-Benzenedicarboxylic acid; } \\
\mathrm{C}_{8} \mathrm{H}_{6} \mathrm{O}_{4} ;[88-99-3] \\
\text { (2) Benzene; } \mathrm{C}_{6} \mathrm{H}_{6} ;[71-43-2]\end{array}$ & $\begin{array}{l}\text { Original Measurements: } \\
{ }^{50} \text { P. G. Desai and A. M. Patel, J. Indian } \\
\text { Chem. Soc. 12, } 131 \text { (1935). }\end{array}$ \\
\hline $\begin{array}{l}\text { Variables: } \\
T / \mathrm{K}=301.2\end{array}$ & $\begin{array}{l}\text { Prepared by: } \\
\text { W. E. Acree, Jr. }\end{array}$ \\
\hline
\end{tabular}

Experimental Values

\begin{tabular}{lc}
\hline \hline$x_{2}{ }^{\mathrm{a}}$ & $x_{1}{ }^{\mathrm{b}}$ \\
\hline 0.9999 & 0.0000447 \\
${ }^{\mathrm{a}} x_{2}:$ mole fraction of component 2 in the saturated solution. \\
${ }^{\mathrm{b}}{ }_{x_{1}}:$ mole fraction solubility of the solute.
\end{tabular}

\section{Auxiliary Information}

Method/Apparatus/Procedure:

Excess solute and solvent were placed in a glass flask which was kept revolving on a wheel in an air thermostat for two days. After two days of equilibration, the solution was filtered. To minimize absorption of the solute onto the filter paper, a $100 \mathrm{ml}$ portion of fresh saturated solution was first filtered through the paper before filtering the sample to be analyzed. An aliquot of the filtrate was titrated with barium hydroxide using phenolphthalein as the endpoint indictor. The barium hydroxide titrant was previously standardized against a solution of succinic acid.
Source and Purity of Chemicals:

(1) Purity not given, Chemical source not specified, no information provided concerning purification.

(2) Purity not given, Chemical source not specified, no information provided concerning purification.

Estimated Error:

Temperature: No information given.

$x_{1}: \pm 3 \%$ (relative error, estimated by compiler).

\begin{tabular}{|c|c|}
\hline $\begin{array}{l}\text { Components: } \\
\text { (1) } 1,2-\text {-Benzenedicarboxylic acid; } \\
\left.\mathrm{C}_{8} \mathrm{H}_{6} \mathrm{O}_{4} ; \text {; } 88-99-3\right] \\
\text { (2) Methylbenzene; } \mathrm{C}_{7} \mathrm{H}_{8} \text {; } \\
\text { [108-88-8] }\end{array}$ & $\begin{array}{l}\text { Original Measurements: } \\
{ }^{50} \text { P. G. Desai and A. M. Patel, J. Indian } \\
\text { Chem. Soc. 12, } 131 \text { (1935). }\end{array}$ \\
\hline $\begin{array}{l}\text { Variables: } \\
T / \mathrm{K}=301.2\end{array}$ & $\begin{array}{l}\text { Prepared by: } \\
\text { W. E. Acree, Jr. }\end{array}$ \\
\hline
\end{tabular}

\section{Experimental Values}

\begin{tabular}{lc}
\hline \hline$x_{2}{ }^{\mathrm{a}}$ & $x_{1}{ }^{\mathrm{b}}$ \\
\hline 0.9999 & 0.0000465 \\
\hline${ }^{\mathrm{a}} x_{2}:$ mole fraction of component 2 in the saturated solution. \\
${ }^{\mathrm{b}} x_{1}:$ mole fraction solubility of the solute.
\end{tabular}

\section{Auxiliary Information}

\section{Method/Apparatus/Procedure:}

Excess solute and solvent were placed in a glass flask which was kept revolving on a wheel in an air thermostat for two days. After two days of equilibration, the solution was filtered. To minimize absorption of the solute onto the filter paper, a $100 \mathrm{ml}$ portion of fresh saturated solution was first filtered through the paper before filtering the sample to be analyzed. An aliquot of the filtrate was titrated with barium hydroxide using phenolphthalein as the endpoint indictor. The barium hydroxide titrant was previously standardized against a solution of succinic acid.

\section{Source and Purity of Chemicals:}

(1) Purity not given, Chemical source not specified, no information provided concerning purification.

(2) Purity not given, Chemical source not specified, no information provided concerning purification.

\section{Estimated Error:}

Temperature: No information given.

$x_{1}: \pm 3 \%$ (relative error, estimated by compiler).

\begin{tabular}{|c|c|}
\hline $\begin{array}{l}\text { Components: } \\
\text { (1) } 1,2 \text {-Benzenedicarboxylic acid; } \\
\mathrm{C}_{8} \mathrm{H}_{6} \mathrm{O}_{4} \text {; [88-99-3] } \\
\text { (2) 1,3-Dimethylbenzene; } \mathrm{C}_{8} \mathrm{H}_{10} \text {; } \\
\text { [108-38-3] }\end{array}$ & $\begin{array}{l}\text { Original Measurements: } \\
\text { }{ }^{50} \text { P. G. Desai and A. M. Patel, J. Indian } \\
\text { Chem. Soc. 12, } 131 \text { (1935). }\end{array}$ \\
\hline $\begin{array}{l}\text { Variables: } \\
T / \mathrm{K}=301.2\end{array}$ & $\begin{array}{l}\text { Prepared by: } \\
\text { W. E. Acree, Jr. }\end{array}$ \\
\hline
\end{tabular}


Experimental Values

\begin{tabular}{lc}
\hline \hline$x_{2}{ }^{\mathrm{a}}$ & $x_{1}{ }^{\mathrm{b}}$ \\
\hline 0.9999 & 0.0000465 \\
${ }^{\mathrm{a}} x_{2}:$ mole fraction of component 2 in the saturated solution. \\
${ }^{\mathrm{b}} x_{1}:$ mole fraction solubility of the solute.
\end{tabular}

\section{Auxiliary Information}

Method/Apparatus/Procedure:

Excess solute and solvent were placed in a glass flask which was kept revolving on a wheel in an air thermostat for two days. After two days of equilibration, the solution was filtered. To minimize absorption of the solute onto the filter paper, a $100 \mathrm{ml}$ portion of fresh saturated solution was first filtered through the paper before filtering the sample to be analyzed. An aliquot of the filtrate was titrated with barium hydroxide using phenolphthalein as the endpoint indictor. The barium hydroxide titrant was previously standardized against a solution of succinic acid.

Source and Purity of Chemicals:

(1) Purity not given, Chemical source not specified, no information provided concerning purification.

(2) Purity not given, Chemical source not specified, no information provided concerning purification.

Estimated Error:

Temperature: No information given.

$x_{1}: \pm 3 \%$ (relative error, estimated by compiler).

\subsection{1,2-Benzenedicarboxylic acid solubility data in esters}

\begin{tabular}{|c|c|}
\hline $\begin{array}{l}\text { Components: } \\
\text { (1) 1,2-Benzenedicarboxylic acid; } \\
\left.\mathrm{C}_{8} \mathrm{H}_{6} \mathrm{O}_{4} ; \text {; } 88-99-3\right] \\
\text { (2) Butyl ethanoate; } \mathrm{C}_{6} \mathrm{H}_{12} \mathrm{O}_{2} \text {; } \\
\text { [123-86-4] }\end{array}$ & $\begin{array}{l}\text { Original Measurements: } \\
{ }^{108} \text { B.-Z. Ren, C.-H. Hou, H.-G. Chong, } \\
\text { W.-R. Li, and H.-J. Song, J. Chem. } \\
\text { Eng. Data 51, } 2022 \text { (2006). }\end{array}$ \\
\hline $\begin{array}{l}\text { Variables: } \\
\text { Temperature; Solvent Composition }\end{array}$ & $\begin{array}{l}\text { Prepared by: } \\
\text { W. E. Acree, Jr. }\end{array}$ \\
\hline
\end{tabular}

Experimental Values

\begin{tabular}{lcc}
\hline \hline$T / \mathrm{K}$ & $x_{2}{ }^{\mathrm{a}}$ & $x_{1}{ }^{\mathrm{b}}$ \\
\hline 304.89 & 0.9972 & 0.002753 \\
310.12 & 0.9970 & 0.002958 \\
315.87 & 0.9967 & 0.003294 \\
320.35 & 0.9964 & 0.003640 \\
325.33 & 0.9959 & 0.004080 \\
330.25 & 0.9954 & 0.004587 \\
334.75 & 0.9949 & 0.005060 \\
338.99 & 0.9944 & 0.005583 \\
341.55 & 0.9939 & 0.006117 \\
345.83 & 0.9933 & 0.006724 \\
352.01 & 0.9922 & 0.007751 \\
356.07 & 0.9915 & 0.008515 \\
359.75 & 0.9906 & 0.009362
\end{tabular}

${ }^{a} x_{2}$ : mole fraction of component 2 in the saturated solution.

${ }^{\mathrm{b}} x_{1}$ : mole fraction solubility of the solute.
Auxiliary Information

\section{Method/Apparatus/Procedure:}

Equilibrium jacketed glass vessel, thermoelectric circulating water temperature controller, electromagnetic stirrer, analytical balance, laser monitoring system.

Experimental solubilities were determined by a dynamic method. Preweighed amounts of solute and solvent and were placed in an equilibrium vessel, which was connected to a circulating water bath. The solution was stirred and the temperature gradually increased until all of the solid solute dissolved. The temperature at which all of the solute dissolved was determined using laser monitoring.

Source and Purity of Chemicals:

(1) $99.8 \%$, CP grade, Shanghai Chemical Reagent Research Institute, China, no purification details were provided.

(2) $99.0 \%$, Analytical Reagent grade, Tianjing Huadong Chemical Reagent Factory, distilled prior to use.

\section{Estimated Error:}

Temperature: $\pm 0.05 \mathrm{~K}$ (estimated by compiler). $x_{1}: \pm 0.001$.

\begin{tabular}{|c|c|}
\hline $\begin{array}{l}\text { Components: } \\
\text { (1) } 1,2-\text {-Benzenedicarboxylic acid; } \\
\left.\mathrm{C}_{8} \mathrm{H}_{6} \mathrm{O}_{4} ; \text {; } 88-99-3\right] \\
\text { (2) Isobutyl ethanoate; } \mathrm{C}_{6} \mathrm{H}_{12} \mathrm{O}_{2} \text {; } \\
\text { [110-19-0] }\end{array}$ & $\begin{array}{l}\text { Original Measurements: } \\
{ }^{60} \text { S. Zhao, X. Chen, Q. Dai, and L. } \\
\text { Wang, J. Chem. Eng. Data 56, } 2399 \\
(2011) \text {. }\end{array}$ \\
\hline $\begin{array}{l}\text { Variables: } \\
\text { Temperature }\end{array}$ & Prepared by: \\
\hline
\end{tabular}

Experimental Values

\begin{tabular}{lcc}
\hline \hline$T / \mathrm{K}$ & $x_{2}{ }^{\mathrm{a}}$ & $x_{1}{ }^{\mathrm{b}}$ \\
\hline 300.68 & 0.9989 & 0.0011 \\
302.37 & 0.9988 & 0.0012 \\
304.42 & 0.9988 & 0.0012 \\
309.58 & 0.9984 & 0.0016 \\
312.72 & 0.9978 & 0.0022 \\
317.36 & 0.9974 & 0.0026 \\
326.85 & 0.9968 & 0.0032 \\
331.86 & 0.9963 & 0.0037 \\
335.27 & 0.9950 & 0.0050 \\
340.04 & 0.9938 & 0.0062 \\
342.69 & 0.9923 & 0.0077 \\
348.70 & 0.9908 & 0.0092 \\
\hline
\end{tabular}

${ }^{a} x_{2}$ : mole fraction of component 2 in the saturated solution.

${ }^{\mathrm{b}} x_{1}$ : mole fraction solubility of the solute.

\section{Auxiliary Information}

\section{Method/Apparatus/Procedure:}

Equilibrium jacketed glass vessel, thermoelectric circulating water temperature controller, electromagnetic stirrer, analytical balance, laser monitoring system.

Experimental solubilities were determined by a dynamic method. Preweighed amounts of solute and solvent and were placed in an equilibrium vessel, which was connected to a circulating water bath. The solution was stirred and the temperature gradually increased at a rate $1-2 \mathrm{~K} / \mathrm{h}(0.5-1.0 \mathrm{~K} / \mathrm{h}$ or slower near saturation temperature) until all of the solid solute dissolved. The temperature at which all of the solute dissolved was determined using laser monitoring. 
Source and Purity of Chemicals:

(1) Analytical Reagent grade, Sinopharm Chemical Reagent Co. Ltd., China, was used as received.

(2) Analytical Reagent grade, Sinopharm Chemical Reagent Co. Ltd., China, was used as received.

Estimated Error:

Temperature: $\pm 0.05 \mathrm{~K}$.

$x_{1}: \pm 0.0002$.

\subsection{1,2-Benzenedicarboxylic acid solubility data in ethers}

\begin{tabular}{|c|c|}
\hline $\begin{array}{l}\text { Components: } \\
\text { (1) } 1,2-\text { Benzenedicarboxylic acid; } \\
\mathrm{C}_{8} \mathrm{H}_{6} \mathrm{O}_{4} \text {; [88-99-3] } \\
\text { (2) Tetrahydrofuran; } \mathrm{C}_{4} \mathrm{H}_{8} \mathrm{O} \text {; } \\
\text { [109-99-9] }\end{array}$ & $\begin{array}{l}\text { Original Measurements: } \\
{ }^{109} \text { Y.-K. Che, Y.-X. Qu, and S. Wang, } \\
\text { J. Chem. Eng. Data 54, } 3130 \text { (2009). }\end{array}$ \\
\hline $\begin{array}{l}\text { Variables: } \\
\text { Temperature }\end{array}$ & $\begin{array}{l}\text { Prepared by: } \\
\text { W. E. Acree, Jr. }\end{array}$ \\
\hline
\end{tabular}

Experimental Values

\begin{tabular}{lcc}
\hline \hline$T / \mathrm{K}$ & $x_{2}{ }^{\mathrm{a}}$ & $x_{1}{ }^{\mathrm{b}}$ \\
\hline 293.25 & 0.9303 & 0.06973 \\
303.15 & 0.9246 & 0.07543 \\
313.45 & 0.9174 & 0.08263 \\
323.15 & 0.9101 & 0.08991 \\
329.05 & 0.9051 & 0.09493
\end{tabular}

${ }^{\mathrm{a}} x_{2}$ : mole fraction of component 2 in the saturated solution.

${ }^{b} x_{1}$ : mole fraction solubility of the solute.

\section{Auxiliary Information}

\section{Method/Apparatus/Procedure:}

Equilibrium jacketed glass vessel, thermostated circulating water bath, electromagnetic stirrer, analytical balance, laser monitoring system. Experimental solubilities were determined by a synthetic method. Preweighed amounts of solute and solvent and were placed in an equilibrium vessel, which was connected to a circulating constant-temperature water bath. The solution was stirred and small amounts of solute were incrementally added until no further solid dissolved. The dissolution of the solid was determined using laser monitoring. The total amount of solute dissolved was recorded. Experimental measurement was repeated three times.

\section{Source and Purity of Chemicals:}

(1) $99.8 \%$, Sinopharm Chemical Reagent Company, Ltd., China, no purification details were provided.

(2) $99 \%$, Kermel Chemical Reagents Development Centre, China, no purification details were provided.

\section{Estimated Error:}

Temperature: $\pm 0.05 \mathrm{~K}$

$x_{1}: \pm 0.5 \%$ (relative error).

\begin{tabular}{|c|c|}
\hline $\begin{array}{l}\text { Components: } \\
\text { (1) 1,2-Benzenedicarboxylic acid; } \\
\mathrm{C}_{8} \mathrm{H}_{6} \mathrm{O}_{4} \text {; [88-99-3] } \\
\text { (2) 1,2-Diethoxyethane; } \mathrm{C}_{6} \mathrm{H}_{14} \mathrm{O}_{2} \text {; } \\
\text { [629-14-1] }\end{array}$ & $\begin{array}{l}\text { Original Measurements: } \\
{ }^{109} \text { Y.-K. Che, Y.-X. Qu, and S. Wang } \\
\text { J. Chem. Eng. Data 54, } 3130 \text { (2009). }\end{array}$ \\
\hline $\begin{array}{l}\text { Variables: } \\
\text { Temperature }\end{array}$ & $\begin{array}{l}\text { Prepared by: } \\
\text { W. E. Acree, Jr. }\end{array}$ \\
\hline
\end{tabular}

Experimental Values

\begin{tabular}{lcc}
\hline \hline$T / \mathrm{K}$ & $x_{2}{ }^{\mathrm{a}}$ & $x_{1}{ }^{\mathrm{b}}$ \\
\hline 293.15 & 0.9846 & 0.01544 \\
303.25 & 0.9823 & 0.01767 \\
313.20 & 0.9800 & 0.02003 \\
323.05 & 0.9772 & 0.02283 \\
333.15 & 0.9739 & 0.02613 \\
343.05 & 0.9698 & 0.03017 \\
\hline
\end{tabular}

${ }^{a} x_{2}$ : mole fraction of component 2 in the saturated solution.

$\mathrm{b}_{x_{1}}$ : mole fraction solubility of the solute.

\section{Auxiliary Information}

\section{Method/Apparatus/Procedure:}

Equilibrium jacketed glass vessel, thermostated circulating water bath, electromagnetic stirrer, analytical balance, laser monitoring system. Experimental solubilities were determined by a synthetic method. Preweighed amounts of solute and solvent and were placed in an equilibrium vessel, which was connected to a circulating constant-temperature water bath. The solution was stirred and small amounts of solute were incrementally added until no further solid dissolved. The dissolution of the solid was determined using laser monitoring. The total amount of solute dissolved was recorded. Experimental measurement was repeated three times.

Source and Purity of Chemicals:

(1) $99.8 \%$, Sinopharm Chemical Reagent Company, Ltd., China, no purification details were provided.

(2) $99+\%$, Tianjin Guangfu Fine Chemical Research Institute, China, no purification details were provided.

Estimated Error:

Temperature: $\pm 0.05 \mathrm{~K}$

$x_{1}: \pm 0.5 \%$ (relative error).

\subsection{1,2-Benzenedicarboxylic acid solubility data in haloalkanes, haloalkenes, and haloaromatic hydrocarbons}

\begin{tabular}{|c|c|}
\hline $\begin{array}{l}\text { Components: } \\
\text { (1) } 1,2-\text {-Benzenedicarboxylic acid; } \\
\left.\mathrm{C}_{8} \mathrm{H}_{6} \mathrm{O}_{4} ; \text {; } 88-99-3\right] \\
\text { (2) Trichloromethane; } \mathrm{CHCl}_{3} \text {; } \\
\text { [67-66-3] }\end{array}$ & $\begin{array}{l}\text { Original Measurements: } \\
{ }^{50} \mathrm{P} \text {. G. Desai and A. M. Patel, J. Indian } \\
\text { Chem. Soc. 12, } 131 \text { (1935). }\end{array}$ \\
\hline $\begin{array}{l}\text { Variables: } \\
T / \mathrm{K}=301.2\end{array}$ & $\begin{array}{l}\text { Prepared by: } \\
\text { W. E. Acree, Jr. }\end{array}$ \\
\hline
\end{tabular}


Experimental Values

\begin{tabular}{lc}
\hline \hline$x_{2}{ }^{\mathrm{a}}$ & $x_{1}{ }^{\mathrm{b}}$ \\
\hline 0.9998 & 0.000184 \\
\hline${ }^{\mathrm{a}} x_{2}:$ mole fraction of component 2 in the saturated solution. & \\
${ }^{\mathrm{b}} x_{1}:$ mole fraction solubility of the solute. &
\end{tabular}

\section{Auxiliary Information}

\section{Method/Apparatus/Procedure:}

Excess solute and solvent were placed in a glass flask which was kept revolving on a wheel in an air thermostat for two days. After two days of equilibration, the solution was filtered. To minimize absorption of the solute onto the filter paper, a $100 \mathrm{ml}$ portion of fresh saturated solution was first filtered through the paper before filtering the sample to be analyzed. An aliquot of the filtrate was titrated with barium hydroxide using phenolphthalein as the endpoint indictor. The barium hydroxide titrant was previously standardized against a solution of succinic acid.

Source and Purity of Chemicals:

(1) Purity not given, Chemical source not specified, no information provided concerning purification.

(2) Purity not given, Chemical source not specified, no information provided concerning purification.

Estimated Error:

Temperature: No information given.

$x_{1}: \pm 3 \%$ (relative error, estimated by compiler).

\begin{tabular}{|c|c|}
\hline $\begin{array}{l}\text { Components: } \\
\text { (1) } 1,2-\text {-Benzenedicarboxylic acid; } \\
\left.\mathrm{C}_{8} \mathrm{H}_{6} \mathrm{O}_{4} ; \text {; } 88-99-3\right] \\
\text { (2) Tetrachloromethane; } \mathrm{CCl}_{4} \text {; } \\
\text { [56-23-5] }\end{array}$ & $\begin{array}{l}\text { Original Measurements: } \\
\text { 50 P. G. Desai and A. M. Patel, J. Indian } \\
\text { Chem. Soc. 12, } 131 \text { (1935). }\end{array}$ \\
\hline $\begin{array}{l}\text { Variables: } \\
T / \mathrm{K}=301.2\end{array}$ & $\begin{array}{l}\text { Prepared by: } \\
\text { W. E. Acree, Jr. }\end{array}$ \\
\hline
\end{tabular}

Experimental Values

\begin{tabular}{lc}
\hline \hline$x_{2}{ }^{\mathrm{a}}$ & $x_{1}{ }^{\mathrm{b}}$ \\
\hline 0.9999 & 0.0000244 \\
\hline
\end{tabular}

${ }^{\mathrm{a}} x_{2}$ : mole fraction of component 2 in the saturated solution.

${ }^{\mathrm{b}} x_{1}$ : mole fraction solubility of the solute.

\section{Auxiliary Information}

\section{Method/Apparatus/Procedure:}

Excess solute and solvent were placed in a glass flask which was kept revolving on a wheel in an air thermostat for two days. After two days of equilibration, the solution was filtered. To minimize absorption of the solute onto the filter paper, a $100 \mathrm{ml}$ portion of fresh saturated solution was first filtered through the paper before filtering the sample to be analyzed. An aliquot of the filtrate was titrated with barium hydroxide using phenolphthalein as the endpoint indictor. The barium hydroxide titrant was previously standardized against a solution of succinic acid.
Source and Purity of Chemicals:

(1) Purity not given, Chemical source not specified, no information provided concerning purification.

(2) Purity not given, Chemical source not specified, no information provided concerning purification.

\section{Estimated Error:}

Temperature: No information given.

$x_{1}: \pm 3 \%$ (relative error, estimated by compiler).

\begin{tabular}{|c|c|}
\hline $\begin{array}{l}\text { Components: } \\
\text { (1) 1,2-Benzenedicarboxylic acid; } \\
\left.\mathrm{C}_{8} \mathrm{H}_{6} \mathrm{O}_{4} ; \text {; } 88-99-3\right] \\
\text { (2) Chlorobenzene; } \mathrm{C}_{6} \mathrm{H}_{5} \mathrm{Cl} \text {; } \\
{[108-90-7]}\end{array}$ & $\begin{array}{l}\text { Original Measurements: } \\
\text { 50 P. G. Desai and A. M. Patel, J. Indian } \\
\text { Chem. Soc. 12, } 131 \text { (1935). }\end{array}$ \\
\hline $\begin{array}{l}\text { Variables: } \\
T / \mathrm{K}=301.2\end{array}$ & $\begin{array}{l}\text { Prepared by: } \\
\text { W. E. Acree, Jr. }\end{array}$ \\
\hline
\end{tabular}

Experimental Values

\begin{tabular}{lc}
\hline \hline$x_{2}{ }^{\mathrm{a}}$ & $x_{1}{ }^{\mathrm{b}}$ \\
\hline 0.9999 & 0.0000577 \\
\hline${ }^{\mathrm{a}} x_{2}:$ mole fraction of component 2 in the saturated solution. \\
${ }^{\mathrm{b}}{ }_{x_{1}}$ : mole fraction solubility of the solute.
\end{tabular}

Auxiliary Information

\section{Method/Apparatus/Procedure:}

Excess solute and solvent were placed in a glass flask which was kept revolving on a wheel in an air thermostat for two days. After two days of equilibration, the solution was filtered. To minimize absorption of the solute onto the filter paper, a $100 \mathrm{ml}$ portion of fresh saturated solution was first filtered through the paper before filtering the sample to be analyzed. An aliquot of the filtrate was titrated with barium hydroxide using phenolphthalein as the endpoint indictor. The barium hydroxide titrant was previously standardized against a solution of succinic acid.

Source and Purity of Chemicals:

(1) Purity not given, Chemical source not specified, no information provided concerning purification.

(2) Purity not given, Chemical source not specified, no information provided concerning purification.

\section{Estimated Error:}

Temperature: No information given.

$x_{1}: \pm 3 \%$ (relative error, estimated by compiler).

\subsection{1,2-Benzenedicarboxylic acid solubility data in alcohols}

\begin{tabular}{|c|c|}
\hline $\begin{array}{l}\text { Components: } \\
\text { (1) } 1,2 \text {-Benzenedicarboxylic acid; } \\
\mathrm{C}_{8} \mathrm{H}_{6} \mathrm{O}_{4} \text {; [88-99-3] } \\
\text { (2) Methanol; } \mathrm{CH}_{4} \mathrm{O} ;[67-56-1]\end{array}$ & $\begin{array}{l}\text { Original Measurements: } \\
{ }^{108} \text { B.-Z. Ren, C.-H. Hou, H.-G. Chong, } \\
\text { W.-R. Li, and H.-J. Song, J. Chem. } \\
\text { Eng. Data 51, } 2022 \text { (2006). }\end{array}$ \\
\hline $\begin{array}{l}\text { Variables: } \\
\text { Temperature; Solvent Composition }\end{array}$ & $\begin{array}{l}\text { Prepared by: } \\
\text { W. E. Acree, Jr. }\end{array}$ \\
\hline
\end{tabular}


Experimental Values

\begin{tabular}{lcc}
\hline \hline$T / \mathrm{K}$ & $x_{2}{ }^{\mathrm{a}}$ & $x_{1}{ }^{\mathrm{b}}$ \\
\hline 302.67 & 0.9441 & 0.05591 \\
305.75 & 0.9410 & 0.05902 \\
308.63 & 0.9379 & 0.06209 \\
311.55 & 0.9349 & 0.06513 \\
314.35 & 0.9318 & 0.06817 \\
317.03 & 0.9288 & 0.07121 \\
319.71 & 0.9257 & 0.07427 \\
322.29 & 0.9227 & 0.07730 \\
324.85 & 0.9197 & 0.08034 \\
327.35 & 0.9166 & 0.08342 \\
329.83 & 0.9135 & 0.08650 \\
332.25 & 0.9105 & 0.08953 \\
\hline
\end{tabular}

${ }^{a} x_{2}$ : mole fraction of component 2 in the saturated solution.

${ }^{\mathrm{b}} x_{1}$ : mole fraction solubility of the solute.

\section{Auxiliary Information}

\section{Method/Apparatus/Procedure:}

Equilibrium jacketed glass vessel, thermoelectric circulating water temperature controller, electromagnetic stirrer, analytical balance, laser monitoring system.

Experimental solubilities were determined by a dynamic method. Preweighed amounts of solute and solvent and were placed in an equilibrium vessel, which was connected to a circulating water bath. The solution was stirred and the temperature gradually increased until all of the solid solute dissolved. The temperature at which all of the solute dissolved was determined using laser monitoring.

\section{Source and Purity of Chemicals:}

(1) $99.8 \%$, CP grade, Shanghai Chemical Reagent Research Institute, China, no purification details were provided.

(2) $99.5 \%$, Analytical Reagent grade, Tianjing Huadong Chemical Reagent Factory, China, no purification details were provided.

\section{Estimated Error:}

Temperature: $\pm 0.05 \mathrm{~K}$ (estimated by compiler).

$x_{1}: \pm 0.001$.

\begin{tabular}{|c|c|}
\hline $\begin{array}{l}\text { Components: } \\
\text { (1) } 1,2 \text {-Benzenedicarboxylic acid; } \\
\left.\mathrm{C}_{8} \mathrm{H}_{6} \mathrm{O}_{4} ; \text {; } 88-99-3\right] \\
\text { (2) Methanol; } \mathrm{CH}_{4} \mathrm{O} ;[67-56-1]\end{array}$ & $\begin{array}{l}\text { Original Measurements: } \\
{ }^{110} \text { M. K. Chantooni, Jr. and I. M. } \\
\text { Kolthoff, J. Phys. Chem. 79, } 1176 \\
\text { (1975). }\end{array}$ \\
\hline $\begin{array}{l}\text { Variables: } \\
T / \mathrm{K}=298.15\end{array}$ & $\begin{array}{l}\text { Prepared by: } \\
\text { W. E. Acree, Jr. }\end{array}$ \\
\hline
\end{tabular}

\section{Experimental Values}

The measured solubility was reported to be $1.15 \mathrm{~mol} \mathrm{dm}^{-3}$. The authors did not give the temperature at which the solubility was measured. Based on the experimental description given in an earlier paper [I. M. Kolthoff, J. J. Lingane, and W. Larson, J. Am. Chem. Soc. 60, 2512 (1938)], the compiler believes the temperature to be $298.15 \mathrm{~K}$.

\section{Auxiliary Information}

\section{Method/Apparatus/Procedure:}

Very little experimental details were provided. Solubility was determined by titrating a filtered aliquot of the saturated solution alkalimetrically in an aqueous-ethanol mixture using phenolphthalein as the acid-base indicator.

\section{Source and Purity of Chemicals:}

(1) Purity not given, Matheson Coleman and Bell, USA, was recrystallized from acetonitrile.

(2) Purity not given, Chemical source not given, was dehydrated and then distilled over sulfanilic acid to remove alkaline impurities.

Estimated Error:

Temperature: $\pm 0.1 \mathrm{~K}$ (estimated by compiler).

$c_{1}: \pm 2 \%$ (relative error, estimated by compiler).

\begin{tabular}{|c|c|}
\hline $\begin{array}{l}\text { Components: } \\
\text { (1) } 1 \text {,2-Benzenedicarboxylic acid; } \\
\mathrm{C}_{8} \mathrm{H}_{6} \mathrm{O}_{4} ;[88-99-3] \\
\text { (2) Methanol; } \mathrm{CH}_{4} \mathrm{O} ;[67-56-1]\end{array}$ & $\begin{array}{l}\text { Original Measurements: } \\
\text { }{ }^{50} \text { P. G. Desai and A. M. Patel, J. Indian } \\
\text { Chem. Soc. 12, } 131 \text { (1935). }\end{array}$ \\
\hline $\begin{array}{l}\text { Variables: } \\
T / \mathrm{K}=301.2\end{array}$ & $\begin{array}{l}\text { Prepared by: } \\
\text { W. E. Acree, Jr. }\end{array}$ \\
\hline
\end{tabular}

Experimental Values

\begin{tabular}{lc}
\hline \hline$x_{2}{ }^{\mathrm{a}}$ & $x_{1}{ }^{\mathrm{b}}$ \\
\hline 0.9487 & 0.05125 \\
\hline${ }^{\mathrm{a}} x_{2}:$ mole fraction of component 2 in the saturated solution. & \\
${ }^{\mathrm{b}} x_{1}:$ mole fraction solubility of the solute. &
\end{tabular}

\section{Auxiliary Information}

\section{Method/Apparatus/Procedure:}

Excess solute and solvent were placed in a glass flask which was kept revolving on a wheel in an air thermostat for two days. After two days of equilibration, the solution was filtered. To minimize absorption of the solute onto the filter paper, a $100 \mathrm{ml}$ portion of fresh saturated solution was first filtered through the paper before filtering the sample to be analyzed. An aliquot of the filtrate was titrated with barium hydroxide using phenolphthalein as the endpoint indictor. The barium hydroxide titrant was previously standardized against a solution of succinic acid.

\section{Source and Purity of Chemicals:}

(1) Purity not given, Chemical source not specified, no information provided concerning purification.

(2) Purity not given, Chemical source not specified, no information provided concerning purification.

\section{Estimated Error:}

Temperature: No information given.

$x_{1}: \pm 3 \%$ (relative error, estimated by compiler).

\begin{tabular}{|c|c|}
\hline $\begin{array}{l}\text { Components: } \\
\text { (1) } 1,2 \text {-Benzenedicarboxylic acid; } \\
\mathrm{C}_{8} \mathrm{H}_{6} \mathrm{O}_{4} ;[88-99-3] \\
\text { (2) Ethanol; } \mathrm{C}_{2} \mathrm{H}_{6} \mathrm{O} ;[64-17-5]\end{array}$ & $\begin{array}{l}\text { Original Measurements: } \\
{ }^{50} \text { P. G. Desai and A. M. Patel, J. Indian } \\
\text { Chem. Soc. 12, } 131 \text { (1935). }\end{array}$ \\
\hline $\begin{array}{l}\text { Variables: } \\
T / \mathrm{K}=301.2\end{array}$ & $\begin{array}{l}\text { Prepared by: } \\
\text { W. E. Acree, Jr. }\end{array}$ \\
\hline
\end{tabular}


Experimental Values

\begin{tabular}{lc}
\hline \hline$x_{2}{ }^{\mathrm{a}}$ & $x_{1}{ }^{\mathrm{b}}$ \\
\hline 0.9573 & 0.04265 \\
\hline${ }^{\mathrm{a}} x_{2}:$ mole fraction of component 2 in the saturated solution. & \\
${ }^{\mathrm{b}}{ }_{1}:$ mole fraction solubility of the solute. &
\end{tabular}

\section{Auxiliary Information}

\section{Method/Apparatus/Procedure:}

Excess solute and solvent were placed in a glass flask which was kept revolving on a wheel in an air thermostat for two days. After two days of equilibration, the solution was filtered. To minimize absorption of the solute onto the filter paper, a $100 \mathrm{ml}$ portion of fresh saturated solution was first filtered through the paper before filtering the sample to be analyzed. An aliquot of the filtrate was titrated with barium hydroxide using phenolphthalein as the endpoint indictor. The barium hydroxide titrant was previously standardized against a solution of succinic acid.

Source and Purity of Chemicals:

(1) Purity not given, Chemical source not specified, no information provided concerning purification.

(2) Purity not given, Chemical source not specified, no information provided concerning purification.

Estimated Error:

Temperature: No information given. $x_{1}: \pm 3 \%$ (relative error, estimated by compiler).

\begin{tabular}{|c|c|}
\hline $\begin{array}{l}\text { Components: } \\
\text { (1) } 1,2 \text {-Benzenedicarboxylic acid; } \\
\mathrm{C}_{8} \mathrm{H}_{6} \mathrm{O}_{4} ;[88-99-3] \\
\text { (2) 1-Propanol; } \mathrm{C}_{3} \mathrm{H}_{8} \mathrm{O} ;[71-23-8]\end{array}$ & $\begin{array}{l}\text { Original Measurements: } \\
\text { 50 P. G. Desai and A. M. Patel, J. Indian } \\
\text { Chem. Soc. 12, } 131 \text { (1935). }\end{array}$ \\
\hline $\begin{array}{l}\text { Variables: } \\
T / \mathrm{K}=301.2\end{array}$ & $\begin{array}{l}\text { Prepared by: } \\
\text { W. E. Acree, Jr. }\end{array}$ \\
\hline
\end{tabular}

\section{Experimental Values}

\begin{tabular}{lc}
\hline \hline$x_{2}{ }^{\mathrm{a}}$ & $x_{1}{ }^{\mathrm{b}}$ \\
\hline 0.9727 & 0.02732 \\
\hline
\end{tabular}

${ }^{\mathrm{a}} x_{2}$ : mole fraction of component 2 in the saturated solution.

${ }^{\mathrm{b}} x_{1}$ : mole fraction solubility of the solute.

\section{Auxiliary Information}

\section{Method/Apparatus/Procedure:}

Excess solute and solvent were placed in a glass flask which was kept revolving on a wheel in an air thermostat for two days. After two days of equilibration, the solution was filtered. To minimize absorption of the solute onto the filter paper, a $100 \mathrm{ml}$ portion of fresh saturated solution was first filtered through the paper before filtering the sample to be analyzed. An aliquot of the filtrate was titrated with barium hydroxide using phenolphthalein as the endpoint indictor. The barium hydroxide titrant was previously standardized against a solution of succinic acid.

\section{Source and Purity of Chemicals:}

(1) Purity not given, Chemical source not specified, no information provided concerning purification.

(2) Purity not given, Chemical source not specified, no information provided concerning purification.
Estimated Error:

Temperature: No information given.

$x_{1}: \pm 3 \%$ (relative error, estimated by compiler).

\begin{tabular}{|c|c|}
\hline $\begin{array}{l}\text { Components: } \\
\text { (1) 1,2-Benzenedicarboxylic acid; } \\
\mathrm{C}_{8} \mathrm{H}_{6} \mathrm{O}_{4} ;[88-99-3] \\
\text { (2) } 1 \text {-Butanol; } \mathrm{C}_{4} \mathrm{H}_{10} \mathrm{O} ;[71-36-3]\end{array}$ & $\begin{array}{l}\text { Original Measurements: } \\
{ }^{50} \text { P. G. Desai and A. M. Patel, J. Indian } \\
\text { Chem. Soc. 12, } 131 \text { (1935). }\end{array}$ \\
\hline $\begin{array}{l}\text { Variables: } \\
T / \mathrm{K}=301.2\end{array}$ & $\begin{array}{l}\text { Prepared by: } \\
\text { W. E. Acree, Jr. }\end{array}$ \\
\hline
\end{tabular}

Experimental Values

\begin{tabular}{lc}
\hline \hline$x_{2}{ }^{\mathrm{a}}$ & $x_{1}{ }^{\mathrm{b}}$ \\
\hline 0.9777 & 0.02231
\end{tabular}

${ }^{\mathrm{a}} x_{2}$ : mole fraction of component 2 in the saturated solution.

${ }^{\mathrm{b}} x_{1}$ : mole fraction solubility of the solute.

\section{Auxiliary Information}

\section{Method/Apparatus/Procedure:}

Excess solute and solvent were placed in a glass flask which was kept revolving on a wheel in an air thermostat for two days. After two days of equilibration, the solution was filtered. To minimize absorption of the solute onto the filter paper, a $100 \mathrm{ml}$ portion of fresh saturated solution was first filtered through the paper before filtering the sample to be analyzed. An aliquot of the filtrate was titrated with barium hydroxide using phenolphthalein as the endpoint indictor. The barium hydroxide titrant was previously standardized against a solution of succinic acid.

\section{Source and Purity of Chemicals:}

(1) Purity not given, Chemical source not specified, no information provided concerning purification

(2) Purity not given, Chemical source not specified, no information provided concerning purification.

\section{Estimated Error:}

Temperature: No information given.

$x_{1}: \pm 3 \%$ (relative error, estimated by compiler).

\begin{tabular}{|c|c|}
\hline $\begin{array}{l}\text { Components: } \\
\text { (1) 1,2-Benzenedicarboxylic acid; } \\
\mathrm{C}_{8} \mathrm{H}_{6} \mathrm{O}_{4} \text {; [88-99-3] } \\
\text { (2) 2-Methyl-2-propanol; } \mathrm{C}_{4} \mathrm{H}_{10} \mathrm{O} \text {; } \\
\text { [75-65-0] }\end{array}$ & $\begin{array}{l}\text { Original Measurements: } \\
{ }^{76} \text { M. K. Chantooni and I. M. Kolthoff, } \\
\text { Anal. Chem. 51, } 133 \text { (1979). }\end{array}$ \\
\hline $\begin{array}{l}\text { Variables: } \\
T / \mathrm{K}=298.15\end{array}$ & $\begin{array}{l}\text { Prepared by: } \\
\text { W. E. Acree, Jr. }\end{array}$ \\
\hline
\end{tabular}

\section{Experimental Values}

The measured solubility was reported to be $0.354 \mathrm{~mol} \mathrm{dm}^{-3}$. The authors did not give the temperature at which the solubility was measured. Based on the experimental description given in an earlier paper [I. M. Kolthoff, J. J. Lingane, and W. Larson, J. Am. Chem. Soc. 60, 2512 (1938)], the compiler believes the temperature to be $298.15 \mathrm{~K}$. 


\section{Auxiliary Information}

\section{Method/Apparatus/Procedure:}

Very little experimental details were provided. Solubility was determined by titrating a filtered aliquot of the saturated solution alkalimetrically in an aqueous-ethanol mixture using phenolphthalein as the acid-base indicator.

\section{Source and Purity of Chemicals:}

(1) Purity not given, Matheson Coleman and Bell, USA, was recrystallized from acetonitrile.

(2) White Label, Eastman Kodak Chemical Company, Rochester, NY, USA shaken with calcium hydride and distilled before use.

Estimated Error:

Temperature: $\pm 0.1 \mathrm{~K}$ (estimated by compiler).

$c_{1}: \pm 2 \%$ (relative error, estimated by compiler).

\begin{tabular}{|c|c|}
\hline $\begin{array}{l}\text { Components: } \\
\text { (1) } 1,2 \text {-Benzenedicarboxylic acid; } \\
\mathrm{C}_{8} \mathrm{H}_{6} \mathrm{O}_{4} \text {; }[88-99-3] \\
\text { (2) } 1,2 \text {-Ethanediol; } \mathrm{C}_{2} \mathrm{H}_{6} \mathrm{O}_{2} \text {; } \\
\text { [107-21-1] }\end{array}$ & $\begin{array}{l}\text { Original Measurements: } \\
{ }^{111} \text { B. K. Dolui, S. K. Bhattacharya, and } \\
\text { K. K. Kundu, J. Solution Chem. 37, } 987 \\
(2008) \text {. }\end{array}$ \\
\hline $\begin{array}{l}\text { Variables: } \\
T / \mathrm{K}=298.15\end{array}$ & $\begin{array}{l}\text { Prepared by: } \\
\text { W. E. Acree, Jr. }\end{array}$ \\
\hline
\end{tabular}

Experimental Values

\begin{tabular}{lc}
\hline \hline$x_{2}{ }^{\mathrm{a}}$ & $x_{1}{ }^{\mathrm{b}}$ \\
\hline 0.9533 & 0.04669 \\
\hline${ }^{\mathrm{a}} x_{2}:$ mole fraction of component 2 in the saturated solution. \\
${ }^{\mathrm{b}} x_{1}:$ mole fraction solubility of the solute. Solubility was reported in the paper \\
as 0.789 mol/kg of solvent. Mole fraction value calculated by the compiler.
\end{tabular}

\section{Auxiliary Information}

\section{Method/Apparatus/Procedure:}

Mechanical shaker and a thermostated temperature control system.

Excess solute and solvent were placed in a well-stoppered bottle and shaken in a mechanical shaker at low speed for $12 \mathrm{~h}$ at ambient room temperature. The bottle was then thermostated at $298 \mathrm{~K}$ for several days for the solution to reach equilibrium. Aliquots of the solution were withdrawn every two days by a specially constructed pipette fitted with G3-Gooch disk, and then weighed. The concentration in the saturated solution was determined by titrating with standardized potassium hydroxide solution using phenolphthalein as the endpoint indicator. The equilibration and analysis continued until successive readings agreed to within $\pm 1 \%$.

Source and Purity of Chemicals:

(1) G.R. grade, Merck Chemical Company, Germany, was used as received. (2) L.R. grade, BDH Chemicals, was purified by methods detailed in an earlier publication [B. K. Dolui, S. K. Bhattacharya, and S. S. Kundu, Indian J. Chem. Sect. A 45A, 2607 (2006)].

\section{Estimated Error:}

Temperature: $\pm 0.01 \mathrm{~K}$.

$x_{1}: \pm 1 \%$ (relative error).

\subsection{1,2-Benzenedicarboxylic acid solubility data in ketones}

\begin{tabular}{|c|c|}
\hline $\begin{array}{l}\text { Components: } \\
\text { (1) } 1,2 \text {-Benzenedicarboxylic acid; } \\
\mathrm{C}_{8} \mathrm{H}_{6} \mathrm{O}_{4} ;[88-99-3] \\
\text { (2) Propanone; } \mathrm{C}_{3} \mathrm{H}_{6} \mathrm{O} ;[67-64-1]\end{array}$ & $\begin{array}{l}\text { Original Measurements: } \\
\text { 50 P. G. Desai and A. M. Patel, J. Indian } \\
\text { Chem. Soc. 12, } 131 \text { (1935). }\end{array}$ \\
\hline $\begin{array}{l}\text { Variables: } \\
T / \mathrm{K}=301.2\end{array}$ & $\begin{array}{l}\text { Prepared by: } \\
\text { W. E. Acree, Jr. }\end{array}$ \\
\hline
\end{tabular}

\section{Experimental Values}

\begin{tabular}{lc}
\hline \hline$x_{2}{ }^{\mathrm{a}}$ & $x_{1}{ }^{\mathrm{b}}$ \\
\hline 0.9710 & 0.02896
\end{tabular}

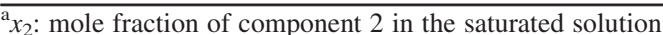

${ }^{\mathrm{b}} x_{1}$ : mole fraction solubility of the solute.

\section{Auxiliary Information}

\section{Method/Apparatus/Procedure:}

Excess solute and solvent were placed in a glass flask which was kept revolving on a wheel in an air thermostat for two days. After two days of equilibration, the solution was filtered. To minimize absorption of the solute onto the filter paper, a $100 \mathrm{ml}$ portion of fresh saturated solution was first filtered through the paper before filtering the sample to be analyzed. An aliquot of the filtrate was titrated with barium hydroxide using phenolphthalein as the endpoint indictor. The barium hydroxide titrant was previously standardized against a solution of succinic acid.

\section{Source and Purity of Chemicals:}

(1) Purity not given, Chemical source not specified, no information provided concerning purification.

(2) Purity not given, Chemical source not specified, no information provided concerning purification.

Estimated Error:

Temperature: No information given.

$x_{1}: \pm 3 \%$ (relative error, estimated by compiler).

\begin{tabular}{|c|c|}
\hline $\begin{array}{l}\text { Components: } \\
\text { (1) 1,2-Benzenedicarboxylic acid; } \\
\mathrm{C}_{8} \mathrm{H}_{6} \mathrm{O}_{4} \text {; [88-99-3] } \\
\text { (2) Cyclohexanone; } \mathrm{C}_{6} \mathrm{H}_{10} \mathrm{O} \text {; } \\
\text { [108-94-1] }\end{array}$ & $\begin{array}{l}\text { Original Measurements: } \\
{ }^{109} \text { Y.-K. Che, Y.-X. Qu, and S. Wang } \\
\text { J. Chem. Eng. Data 54, } 3130 \text { (2009). }\end{array}$ \\
\hline Variables: & Prepared by: \\
\hline Temperature & \\
\hline
\end{tabular}

Experimental Values

\begin{tabular}{lcc}
\hline \hline$T / \mathrm{K}$ & $x_{2}{ }^{\mathrm{a}}$ & $x_{1}{ }^{\mathrm{b}}$ \\
\hline 293.15 & 0.9824 & 0.01763 \\
303.15 & 0.9788 & 0.02117 \\
313.15 & 0.9743 & 0.02568 \\
323.15 & 0.9690 & 0.03096 \\
333.15 & 0.9630 & 0.03697 \\
343.40 & 0.9541 & 0.04591 \\
${ }^{\mathrm{a}}{ }^{2}{ }_{2}:$ mole fraction of component 2 in the saturated solution. \\
${ }^{\mathrm{b}}{ }_{x_{1}: \text { mole fraction solubility of the solute. }}$
\end{tabular}


Auxiliary Information

\section{Method/Apparatus/Procedure:}

Equilibrium jacketed glass vessel, thermostated circulating water bath, electromagnetic stirrer, analytical balance, laser monitoring system.

Experimental solubilities were determined by a synthetic method. Preweighed amounts of solute and solvent and were placed in an equilibrium vessel, which was connected to a circulating constant-temperature water bath. The solution was stirred and small amounts of solute were incrementally added until no further solid dissolved. The dissolution of the solid was determined using laser monitoring. The total amount of solute dissolved was recorded. Experimental measurement was repeated three times.

Source and Purity of Chemicals:

(1) $99.8 \%$, Sinopharm Chemical Reagent Company, Ltd., China, no purification details were provided.

(2) 99+\%, Tianjin Guangfu Fine Chemical Research Institute, China, no purification details were provided.

Estimated Error:

Temperature: $\pm 0.05 \mathrm{~K}$.

$x_{1}: \pm 0.5 \%$ (relative error).

\begin{tabular}{|c|c|}
\hline $\begin{array}{l}\text { Components: } \\
\text { (1) } 1,2 \text {-Benzenedicarboxylic acid; } \\
\left.\mathrm{C}_{8} \mathrm{H}_{6} \mathrm{O}_{4} ; \text {; } 88-99-3\right] \\
\text { (2) Acetophenone; } \mathrm{C}_{8} \mathrm{H}_{8} \mathrm{O} \text {; } \\
\text { [98-86-2] }\end{array}$ & $\begin{array}{l}\text { Original Measurements: } \\
{ }^{109} \text { Y.-K. Che, Y.-X. Qu, and S. Wang, } \\
\text { J. Chem. Eng. Data 54, } 3130 \text { (2009). }\end{array}$ \\
\hline $\begin{array}{l}\text { Variables: } \\
\text { Temperature }\end{array}$ & $\begin{array}{l}\text { Prepared by: } \\
\text { W. E. Acree, Jr. }\end{array}$ \\
\hline
\end{tabular}

Experimental Values

\begin{tabular}{lcc}
\hline \hline$T / \mathrm{K}$ & $x_{2}{ }^{\mathrm{a}}$ & $x_{1}{ }^{\mathrm{b}}$ \\
\hline 303.35 & 0.9944 & 0.005601 \\
313.35 & 0.9928 & 0.007236 \\
323.35 & 0.9907 & 0.009333 \\
332.95 & 0.9881 & 0.01186 \\
343.55 & 0.9848 & 0.01525
\end{tabular}

${ }^{a} x_{2}$ : mole fraction of component 2 in the saturated solution.

${ }^{\mathrm{b}} x_{1}$ : mole fraction solubility of the solute.

\section{Auxiliary Information}

\section{Method/Apparatus/Procedure:}

Equilibrium jacketed glass vessel, thermostated circulating water bath, electromagnetic stirrer, analytical balance, laser monitoring system. Experimental solubilities were determined by a synthetic method. Preweighed amounts of solute and solvent and were placed in an equilibrium vessel, which was connected to a circulating constant-temperature water bath. The solution was stirred and small amounts of solute were incrementally added until no further solid dissolved. The dissolution of the solid was determined using laser monitoring. The total amount of solute dissolved was recorded. Experimental measurement was repeated three times.

Source and Purity of Chemicals:

(1) $99.8 \%$, Sinopharm Chemical Reagent Company, Ltd., China, no purification details were provided.

(2) $99+\%$, Tianjin Guangfu Fine Chemical Research Institute, China, no purification details were provided.
Estimated Error:

Temperature: $\pm 0.05 \mathrm{~K}$.

$x_{1}: \pm 0.5 \%$ (relative error).

\subsection{1,2-Benzenedicarboxylic acid solubility data in miscellaneous organic solvents}

\begin{tabular}{|c|c|}
\hline $\begin{array}{l}\text { Components: } \\
\text { (1) 1,2-Benzenedicarboxylic acid; } \\
\left.\mathrm{C}_{8} \mathrm{H}_{6} \mathrm{O}_{4} ; \text {; } 88-99-3\right] \\
\text { (2) Ethanoic acid; } \mathrm{C}_{2} \mathrm{H}_{4} \mathrm{O}_{2} \text {; } \\
\text { [64-19-7] }\end{array}$ & $\begin{array}{l}\text { Original Measurements: } \\
{ }^{79} \text { Q. Wang, L. Hou, Y. Cheng, and X. } \\
\text { Li, J. Chem. Eng. Data 52, } 936 \text { (2007). }\end{array}$ \\
\hline $\begin{array}{l}\text { Variables: } \\
\text { Temperature }\end{array}$ & $\begin{array}{l}\text { Prepared by: } \\
\text { W. E. Acree, Jr. }\end{array}$ \\
\hline
\end{tabular}

Experimental Values

\begin{tabular}{lcc}
\hline \hline$T / \mathrm{K}$ & $x_{2}{ }^{\mathrm{a}}$ & $x_{1}{ }^{\mathrm{b}, \mathrm{c}}$ \\
\hline 298.3 & 0.9894 & 0.0106 \\
308.4 & 0.9850 & 0.0150 \\
318.3 & 0.9778 & 0.0222 \\
328.2 & 0.9692 & 0.0308 \\
338.0 & 0.9583 & 0.0417 \\
348.3 & 0.9443 & 0.0557 \\
358.6 & 0.9278 & 0.0722 \\
367.9 & 0.9095 & 0.0905 \\
\hline
\end{tabular}

${ }^{\mathrm{a}} x_{2}$ : mole fraction of component 2 in the saturated solution.

${ }^{b} x_{1}$ : mole fraction solubility of the solute.

${ }^{\mathrm{c}}$ Solubility data were given in units of grams per $100 \mathrm{~g}$ of solvent. Mole fraction solubilities were calculated by compiler.

\section{Auxiliary Information}

\section{Method/Apparatus/Procedure:}

Constant-temperature bath and analytical balance.

Solubilities were determined by a static method. Excess solute and solvent were placed in sealed bottles and allowed to equilibrate in a constanttemperature bath for at least $24 \mathrm{~h}$. An aliquot of the clear solution was removed by syringe and transferred to a preweighed glass vial. The glass vial was then weighed, and the solvent was evaporated in in vacuo at $323 \mathrm{~K}$ for more than $3 \mathrm{~h}$. Once the solvent had evaporated, the vial with solid residue was weighed. The solubility was calculated from the mass of the solid residue and mass of sample analyzed.

Source and Purity of Chemicals:

(1) $98.5 \%$, Shanghai Fine Chemical Reagent Company, China, no purification details provided.

(2) Analytical Reagent grade, Hangzhou Chemical Reagent Company, China, no purification details provided.

\section{Estimated Error:}

Temperature: $\pm 0.1 \mathrm{~K}$.

$x_{1}: \pm 3 \%$ (relative error) 


\begin{tabular}{|c|c|}
\hline $\begin{array}{l}\text { Components: } \\
\text { (1) 1,2-Benzenedicarboxylic acid; } \\
\mathrm{C}_{8} \mathrm{H}_{6} \mathrm{O}_{4} \text {; [88-99-3] } \\
\text { (2) Ethanenitrile; } \mathrm{C}_{2} \mathrm{H}_{3} \mathrm{~N} ;[75-05-8]\end{array}$ & $\begin{array}{l}\text { Original Measurements: } \\
{ }^{110} \text { M. K. Chantooni, Jr. and I. M. } \\
\text { Kolthoff, J. Phys. Chem. 79, } 1176 \\
\text { (1975). }\end{array}$ \\
\hline $\begin{array}{l}\text { Variables: } \\
T / \mathrm{K}=298.15\end{array}$ & $\begin{array}{l}\text { Prepared by: } \\
\text { W. E. Acree, Jr. }\end{array}$ \\
\hline
\end{tabular}

\section{Experimental Values}

The measured solubility was reported to be $0.0245 \mathrm{~mol} \mathrm{dm}^{-3}$. The authors did not give the temperature at which the solubility was measured. Based on the experimental description given in an earlier paper [I. M. Kolthoff, J. J. Lingane, and W. Larson, J. Am. Chem. Soc. 60, 2512 (1938)], the compiler believes the temperature to be $298.15 \mathrm{~K}$.

\section{Auxiliary Information}

\section{Method/Apparatus/Procedure:}

Very little experimental details were provided. Solubility was determined by titrating a filtered aliquot of the saturated solution alkalimetrically in an aqueous-ethanol mixture using phenolphthalein as the acid-base indicator.

\section{Source and Purity of Chemicals:}

(1) Purity not given, Matheson Coleman and Bell, USA, was recrystallized from acetonitrile.

(2) Purity not given, Chemical source not given, was purified by shaking with saturated potassium hydroxide, followed by activated alumina, and then anhydrous calcium chloride to remove water. Ethanenitrile was further dried over anhydrous magnesium sulfate and then phosphorous pentoxide. The sample was distilled shortly before use.

Estimated Error:

Temperature: $\pm 0.1 \mathrm{~K}$ (estimated by compiler). $c_{1}: \pm 2 \%$ (relative error, estimated by compiler).

\begin{tabular}{|c|c|}
\hline $\begin{array}{l}\text { Components: } \\
\text { (1) 1,2-Benzenedicarboxylic acid; } \\
\left.\mathrm{C}_{8} \mathrm{H}_{6} \mathrm{O}_{4} ; \text {; } 88-99-3\right] \\
\text { (2) Dimethyl sulfoxide; } \mathrm{C}_{2} \mathrm{H}_{6} \mathrm{OS} \text {; } \\
\text { [67-68-5] }\end{array}$ & $\begin{array}{l}\text { Original Measurements: } \\
{ }^{110} \text { M. K. Chantooni, Jr. and I. M. } \\
\text { Kolthoff, J. Phys. Chem. 79, } 1176 \\
\text { (1975). }\end{array}$ \\
\hline $\begin{array}{l}\text { Variables: } \\
T / \mathrm{K}=298.15\end{array}$ & $\begin{array}{l}\text { Prepared by: } \\
\text { W. E. Acree, Jr. }\end{array}$ \\
\hline
\end{tabular}

\section{Experimental Values}

The measured solubility was reported to be $3.77 \mathrm{~mol} \mathrm{dm}^{-3}$. The authors did not give the temperature at which the solubility was measured. Based on the experimental description given in an earlier paper [I. M. Kolthoff, J. J. Lingane, and W. Larson, J. Am. Chem. Soc. 60, 2512 (1938)], the compiler believes the temperature to be $298.15 \mathrm{~K}$.

\section{Auxiliary Information}

\section{Method/Apparatus/Procedure:}

Very little experimental details were provided. Solubility was determined by titrating a filtered aliquot of the saturated solution alkalimetrically in an aqueous-ethanol mixture using phenolphthalein as the acid-base indicator.
Source and Purity of Chemicals:

(1) Purity not given, Matheson Coleman and Bell, USA, was recrystallized from acetonitrile.

(2) Purity not given, Chemical Source not given, was shaken with activated alumina and then distilled before use.

\section{Estimated Error:}

Temperature: $\pm 0.1 \mathrm{~K}$ (estimated by compiler). $c_{1}: \pm 2 \%$ (relative error, estimated by compiler).

\begin{tabular}{|c|c|}
\hline $\begin{array}{l}\text { Components: } \\
\text { (1) } 1,2-\text {-Benzenedicarboxylic acid; } \\
\left.\mathrm{C}_{8} \mathrm{H}_{6} \mathrm{O}_{4} ; \text {; } 88-99-3\right] \\
\text { (2) Nitrobenzene; } \mathrm{C}_{6} \mathrm{H}_{5} \mathrm{NO}_{2} \text {; } \\
\text { [98-95-3] }\end{array}$ & $\begin{array}{l}\text { Original Measurements: } \\
\text { }{ }^{50} \text { P. G. Desai and A. M. Patel, J. Indian } \\
\text { Chem. Soc. 12, } 131 \text { (1935). }\end{array}$ \\
\hline $\begin{array}{l}\text { Variables: } \\
T / \mathrm{K}=301.2\end{array}$ & $\begin{array}{l}\text { Prepared by: } \\
\text { W. E. Acree, Jr. }\end{array}$ \\
\hline
\end{tabular}

Experimental Values

\begin{tabular}{lc}
\hline \hline$x_{2}{ }^{\mathrm{a}}$ & $x_{1}{ }^{\mathrm{b}}$ \\
\hline 0.9999 & 0.0000898 \\
\hline${ }^{\mathrm{a}} x_{2}:$ mole fraction of component 2 in the saturated solution. \\
${ }^{\mathrm{b}}{ }_{x_{1}}$ : mole fraction solubility of the solute.
\end{tabular}

Auxiliary Information

\begin{abstract}
Method/Apparatus/Procedure:
Excess solute and solvent were placed in a glass flask which was kept revolving on a wheel in an air thermostat for two days. After two days of equilibration, the solution was filtered. To minimize absorption of the solute onto the filter paper, a $100 \mathrm{ml}$ portion of fresh saturated solution was first filtered through the paper before filtering the sample to be analyzed. An aliquot of the filtrate was titrated with barium hydroxide using phenolphthalein as the endpoint indictor. The barium hydroxide titrant was previously standardized against a solution of succinic acid.
\end{abstract}

Source and Purity of Chemicals:

(1) Purity not given, Chemical source not specified, no information provided concerning purification.

(2) Purity not given, Chemical source not specified, no information provided concerning purification.

Estimated Error:

Temperature: No information given.

$x_{1}: \pm 3 \%$ (relative error, estimated by compiler).

\subsection{1,2-Benzenedicarboxylic acid solubility data in binary organic solvent mixtures}

\section{Components:}

(1) 1,2-Benzenedicarboxylic acid;

$\mathrm{C}_{8} \mathrm{H}_{6} \mathrm{O}_{4}$; [88-99-3]

(2) Methanol; $\mathrm{CH}_{4} \mathrm{O} ;[67-56-1]$

(3) Butyl ethanoate; $\mathrm{C}_{6} \mathrm{H}_{12} \mathrm{O}_{2}$;

[123-86-4]

Variables:

Temperature; Solvent Composition
Original Measurements:

${ }^{108}$ B.-Z. Ren, C.-H. Hou, H.-G. Chong, W.-R. Li, and H.-J. Song, J. Chem. Eng. Data 51, 2022 (2006). 
Experimental Values

\begin{tabular}{|c|c|c|}
\hline$T / \mathrm{K}$ & $m_{2}^{(\mathrm{s}) \mathrm{a}}$ & $x_{1}{ }^{\mathrm{b}}$ \\
\hline 304.89 & 0.0000 & 0.002753 \\
\hline 310.12 & 0.0000 & 0.002958 \\
\hline 315.87 & 0.0000 & 0.003294 \\
\hline 320.35 & 0.0000 & 0.003640 \\
\hline 325.33 & 0.0000 & 0.004080 \\
\hline 330.25 & 0.0000 & 0.004587 \\
\hline 334.75 & 0.0000 & 0.005060 \\
\hline 338.99 & 0.0000 & 0.005583 \\
\hline 341.55 & 0.0000 & 0.006117 \\
\hline 345.83 & 0.0000 & 0.006724 \\
\hline 352.01 & 0.0000 & 0.007751 \\
\hline 356.07 & 0.0000 & 0.008515 \\
\hline 359.75 & 0.0000 & 0.009362 \\
\hline 303.10 & 0.1997 & 0.01863 \\
\hline 305.82 & 0.1997 & 0.01953 \\
\hline 309.65 & 0.1997 & 0.02082 \\
\hline 313.56 & 0.1997 & 0.02182 \\
\hline 316.52 & 0.1997 & 0.02276 \\
\hline 320.44 & 0.1997 & 0.02377 \\
\hline 324.07 & 0.1997 & 0.02478 \\
\hline 327.66 & 0.1997 & 0.02586 \\
\hline 332.67 & 0.1997 & 0.02704 \\
\hline 334.30 & 0.1997 & 0.02792 \\
\hline 304.57 & 0.3992 & 0.03555 \\
\hline 307.20 & 0.3992 & 0.03711 \\
\hline 310.62 & 0.3992 & 0.03879 \\
\hline 314.04 & 0.3992 & 0.04053 \\
\hline 317.66 & 0.3992 & 0.04230 \\
\hline 320.77 & 0.3992 & 0.04409 \\
\hline 324.25 & 0.3992 & 0.04592 \\
\hline 327.82 & 0.3992 & 0.04783 \\
\hline 330.86 & 0.3992 & 0.04981 \\
\hline 334.26 & 0.3992 & 0.05187 \\
\hline 300.46 & 0.6001 & 0.04255 \\
\hline 303.37 & 0.6001 & 0.04471 \\
\hline 306.50 & 0.6001 & 0.04687 \\
\hline 309.74 & 0.6001 & 0.04906 \\
\hline 312.88 & 0.6001 & 0.05125 \\
\hline 315.77 & 0.6001 & 0.05344 \\
\hline 318.86 & 0.6001 & 0.05573 \\
\hline 321.70 & 0.6001 & 0.05792 \\
\hline 324.82 & 0.6001 & 0.06020 \\
\hline 327.74 & 0.6001 & 0.06248 \\
\hline 330.77 & 0.6001 & 0.06480 \\
\hline 333.94 & 0.6001 & 0.06717 \\
\hline 303.62 & 0.8000 & 0.05167 \\
\hline 306.24 & 0.8000 & 0.05412 \\
\hline 309.46 & 0.8000 & 0.05662 \\
\hline 312.13 & 0.8000 & 0.05910 \\
\hline 315.22 & 0.8000 & 0.06168 \\
\hline 317.91 & 0.8000 & 0.06429 \\
\hline 320.96 & 0.8000 & 0.06696 \\
\hline 324.22 & 0.8000 & 0.06975 \\
\hline 326.24 & 0.8000 & 0.07268 \\
\hline 328.99 & 0.8000 & 0.07576 \\
\hline 332.06 & 0.8000 & 0.07901 \\
\hline 335.20 & 0.8000 & 0.08247 \\
\hline 302.67 & 1.0000 & 0.05591 \\
\hline 305.75 & 1.0000 & 0.05902 \\
\hline 308.63 & 1.0000 & 0.06209 \\
\hline
\end{tabular}

\begin{tabular}{|c|c|c|}
\hline$T / \mathrm{K}$ & $m_{2}{ }^{(\mathrm{s}) \mathrm{a}}$ & $x_{1}{ }^{\mathrm{b}}$ \\
\hline 311.55 & 1.0000 & 0.06513 \\
\hline 314.35 & 1.0000 & 0.06817 \\
\hline 317.03 & 1.0000 & 0.07121 \\
\hline 319.71 & 1.0000 & 0.07427 \\
\hline 322.29 & 1.0000 & 0.07730 \\
\hline 324.85 & 1.0000 & 0.08034 \\
\hline 327.35 & 1.0000 & 0.08342 \\
\hline 329.83 & 1.0000 & 0.08650 \\
\hline 332.25 & 1.0000 & 0.08953 \\
\hline
\end{tabular}

Auxiliary Information

Method/Apparatus/Procedure:

Equilibrium jacketed glass vessel, thermoelectric circulating water temperature controller, electromagnetic stirrer, analytical balance, laser monitoring system.

Experimental solubilities were determined by a dynamic method. Preweighed amounts of solute and solvent and were placed in an equilibrium vessel, which was connected to a circulating water bath. The solution was stirred and the temperature gradually increased until all of the solid solute dissolved. The temperature at which all of the solute dissolved was determined using laser monitoring.

Source and Purity of Chemicals:

(1) $99.8 \%$, CP grade, Shanghai Chemical Reagent Research Institute, China, no purification details were provided.

(2) $99.5 \%$, Analytical Reagent grade, Tianjing Huadong Chemical Reagent Factory, China, no purification details were provided.

(3) $99.0 \%$, Analytical Reagent grade, Tianjing Huadong Chemical Reagent Factory, was distilled prior to use.

Estimated Error:

Temperature: $\pm 0.05 \mathrm{~K}$ (estimated by compiler).

$m_{2}{ }^{(\mathrm{s})}: 0.0001$

$x_{1}: \pm 0.001$

\section{Solubility of 1,3-Benzenedicarboxylic Acid in Organic Solvents}

\subsection{Critical evaluation of experimental solubility data}

Several research groups ${ }^{60,63,82,84,91,109,112-117}$ have investigated the solubility behavior of 1,3-benzenedicarboxylic acid as a function of temperature. Che et al. ${ }^{109}$ determined the solubility of 1,3-benzenedicarboxylic acid in tetrahydrofuran, 1,2-diethoxyethane, cyclohexanone, and acetophenone at several temperatures. The internal consistency of the four sets of measured 1,3-benzenedicarboxylic acid solubilities were assessed by curve-fitting the measured mole fraction solubility data to Eq. (8). The values of the equation coefficients $(A, B$, and $C$ ) are given in Table 17, along with the MRD calculated according to Eq. (24). Examination of the entries in the last column of Table 17 reveals that the largest mean relative deviation between the back-calculated values based on Eq. (8) and experimental data is only $1.2 \%$,which is less than the experimental uncertainties in the measured values. Results of 
TABLE 17. Parameters of the Modified Apelblat equation for describing the solubility of 1,3-benzenedicarboxylic acid in various organic solvents

\begin{tabular}{lcccc}
\hline \hline Solvent & $A$ & $B$ & $C$ & MRD (\%) \\
\hline 1,2-Diethoxyethane $^{\mathrm{a}}$ & -139.95 & 5175.9 & 20.483 & 0.17 \\
Tetrahydrofuran $^{\mathrm{a}}$ & -79.312 & 2713.1 & 11.583 & 0.20 \\
Cyclohexanone $^{\mathrm{a}}$ & -58.711 & 679.31 & 8.8686 & 1.18 \\
Acetophenone $^{\mathrm{a}}$ & -272.87 & 10667 & 40.328 & 0.59
\end{tabular}

${ }^{\mathrm{a}}$ Values of the coefficients and the mean relative deviations were taken from Che et al. ${ }^{109}$

the mathematical representation analyses indicate that the experimental data for all four 1,3-benzenedicarboxylic acid - organic solvent systems are internally consistent.

Long et al. ${ }^{112}$ used a gravimetric method to examine the solubility behavior of 1,3-benzenedicarboxylic acid in seven monobasic alcohols (methanol, 1-propanol, 2-propanol, 1butanol, 2-butanol, 1-pentanol, and 1-hexanol) in the temperature range of about $278-352 \mathrm{~K}$. Long and Yang ${ }^{116}$ had previously measured the solubility of 1,3-benzenedicarboxylic acid in ethanol, propanone, and ethyl ethanoate. For the alcohol solvents, solubilities were found to decrease with increasing number of carbon atoms, except for 1-pentanol where the solubility was comparable to that observed in 1butanol and higher than the measured solubility in 2-butanol. Methanol exhibited the highest ability to dissolve the dicarboxylic acid solute, whereas 1-hexanol showed the lowest solubility. The internal consistency of the ten datasets of measured 1,3-benzenedicarboxylic acid solubilities mentioned above, along with the measured values of Feng et al. ${ }^{117}$ for 1,3-benzenedicarboxylic acid dissolved in ethanoic acid, were assessed by curve-fitting the measured mole fraction solubility data to Eq. (9). The values of the equation coefficients $(\lambda$ and $h)$ are given in Table 18, along with the MRD.

TABLE 18. Parameters of the Buchowski $\lambda \mathrm{h}$ equation for describing the solubility of 1,3-benzenedicarboxylic acid in several organic solvents

\begin{tabular}{lcllc}
\hline \hline Solvent & $T / \mathrm{K}$ & \multicolumn{1}{c}{$\lambda$} & $10^{-4} h$ & MRD (\%) \\
\hline Methanol $^{\mathrm{a}}$ & $278-337$ & 0.1016 & 1.865 & 2.62 \\
Ethanol $^{\mathrm{b}}$ & $278-350$ & 0.1696 & 1.223 & 1.57 \\
1-Propanol $^{\mathrm{a}}$ & $278-358$ & 0.3630 & 0.7471 & 4.05 \\
2-Propanol $^{\mathrm{a}}$ & $278-353$ & 0.4151 & 0.6431 & 2.01 \\
1-Butanol $^{\mathrm{a}}$ & $278-358$ & 0.2366 & 1.049 & 4.33 \\
2-Butanol $^{\mathrm{a}}$ & $283-352$ & 0.2356 & 1.124 & 3.80 \\
1-Pentanol $^{\mathrm{a}}$ & $303-348$ & 0.2362 & 1.000 & 3.03 \\
1-Hexanol $^{\mathrm{a}}$ & $278-353$ & 0.3793 & 0.7523 & 3.52 \\
Propanone $^{\mathrm{b}}$ & $278-328$ & 1.2853 & 0.2840 & 2.62 \\
Ethyl ethanoate $^{\mathrm{b}}$ & $278-338$ & 0.0658 & 4.1360 & 9.87 \\
Ethanoic acid $^{\mathrm{c}}$ & $313-363$ & 0.0434 & 6.7978 & 3.72 \\
\hline
\end{tabular}

${ }^{\mathrm{a}}$ Values of the coefficients and mean relative deviations were taken from Long et al. ${ }^{112}$

${ }^{b}$ Values of the coefficients and mean relative deviations were taken from Long and Yang. ${ }^{116}$

${ }^{\mathrm{c}}$ Values of the coefficients and mean relative deviation were taken from Feng et al. ${ }^{113}$
Zhao et al. ${ }^{60}$ measured the solubility of 1,3-benzenedicarboxylic acid in isobutyl ethanoate at ten temperatures between 308 and $353 \mathrm{~K}$. The experimental data were correlated with both the UNIQUAC and Wilson models. Interaction coefficients calculated from the experimental solid-liquid equilibrium data provided a reasonably accurate mathematical description of the measured values. The mean absolute relative deviation between calculated and observed values was $11.56 \%$ (Wilson model) and $10.90 \%$ (UNIQUAC model).

Li et al. ${ }^{84}$ determined the solubility of 1,3-benzenedicarboxylic acid in $N$-methyl-2-pyrrolidone from 296 to $346 \mathrm{~K}$ using a synthetic method with laser monitoring to determine when the last amount of solid solute dissolved. The authors employed a polynomial expression in temperature

$$
\begin{aligned}
x_{1}= & -6.9703+0.06877 T-2.29731 \times 10^{-4} T \\
& +2.63346 \times 10^{-7} T^{2}
\end{aligned}
$$

to represent the measured mole fraction solubility data. The root-mean-square deviation between the observed $x_{1}$ data and calculated values from Eq. (36) was on the order of 0.0014 mole fraction.

Feng et al. ${ }^{113}$ studied the water-to-propyl ethanoate partitioning behavior of 1,3-benzenedicarboxylic acid as well as the solute's solubility in propyl ethanoate from 303 to $363 \mathrm{~K}$. The authors described the logarithm of the observed mole fraction solubilities with a simple linear relationship

$$
\log _{10} x_{1}=-5.2411+0.00705 T \text {. }
$$

The mean absolute deviation between the observed mole fraction solubilities and back-calculated values based on Eq. (37) was less than $2.6 \%$.

The experimental solubility data for 1,3-benzenedicarboxylic acid in the different organic solvents are in Secs. 12.212.6.

\begin{tabular}{|c|c|}
\hline $\begin{array}{l}\text { Components: } \\
\text { (1) } 1,3 \text {-Benzenedicarboxylic acid; } \\
\mathrm{C}_{8} \mathrm{H}_{6} \mathrm{O}_{4} ;[121-91-5] \\
\text { (2) Ethyl ethanoate; } \mathrm{C}_{4} \mathrm{H}_{8} \mathrm{O}_{2} \text {; } \\
\text { [141-78-6] }\end{array}$ & $\begin{array}{l}\text { Original Measurements: } \\
{ }^{116} \text { B. Long and Z. Yang, Fluid Phase } \\
\text { Equilib. 266, } 38 \text { (2008). }\end{array}$ \\
\hline $\begin{array}{l}\text { Variables: } \\
\text { Temperature }\end{array}$ & Prepared by: \\
\hline
\end{tabular}

\subsection{1,3-Benzenedicarboxylic acid solubility data in esters}


Experimental Values

\begin{tabular}{lc}
\hline \hline$T / \mathrm{K}$ & $100 s_{1}{ }^{\mathrm{a}}$ \\
\hline 278.05 & 0.2754 \\
282.95 & 0.3939 \\
287.85 & 0.5311 \\
293.05 & 0.6039 \\
303.05 & 0.8864 \\
307.75 & 1.0034 \\
312.85 & 1.0819 \\
323.65 & 1.3660 \\
333.15 & 1.6203 \\
338.15 & 1.7177 \\
\hline
\end{tabular}

${ }^{\mathrm{a}} s_{1}$ : solubility of the solute in units of moles per kilogram. The authors description of how the solubilities are reported is contradictory. In the manuscript text, the authors state molarity, and moles per kilogram of solution. Compiler has assumed that the units are moles per kilogram, which would be consistent with the column heading of the table in the published paper.

\section{Auxiliary Information}

\section{Method/Apparatus/Procedure:}

Jacketed equilibrium cell, constant-temperature circulating water bath, analytical balance, oven, and magnetic stirrer.

Excess solute and solvent were placed in a jacketed equilibrium cell and allowed to equilibrate with stirring at constant temperature for at least $5 \mathrm{~h}$. After sufficient equilibration, the stirrer was stopped and the solution was kept still for at least $1 \mathrm{~h}$ to allow suspended solid to settle to the bottom of the cell. An aliquot of the clear upper saturated solution was removed with a warm pipet, and transferred to a weighed vial. The vial was weighed, uncovered, and placed in an oven. The solvent was allowed to evaporate in the oven at $323 \mathrm{~K}$. The vial was covered with a piece of filter cloth to prevent dust contamination. After the solvent had evaporated, the vial was reweighed. The solubility was calculated from the mass of the solid residue and mass of saturated solution analyzed.

Source and Purity of Chemicals:

(1) $99.8 \%$, Beijing Yanshan Petrochemical Company, China, no purification details provided.

(2) $99.5 \%$, Beijing Chemical Reagent Company, China, used as received.

Estimated Error:

Temperature: $\pm 0.1 \mathrm{~K}$.

$s_{1}: \pm 2 \%$ (relative error)

\begin{tabular}{|c|c|}
\hline $\begin{array}{l}\text { Components: } \\
\text { (1) } 1,3-\text { Benzenedicarboxylic acid; } \\
\mathrm{C}_{8} \mathrm{H}_{6} \mathrm{O}_{4} ;[121-91-5] \\
\text { (2) Propyl ethanoate; } \mathrm{C}_{5} \mathrm{H}_{10} \mathrm{O}_{2} \text {; } \\
\text { [109-60-4] }\end{array}$ & $\begin{array}{l}\text { Original Measurements: } \\
{ }^{113} \text { L. Feng, L. Wang, G. Peng, X. } \\
\text { Guo, and X. Li, J. Chem. Eng. Data } \\
\text { 55, } 500 \text { (2010). }\end{array}$ \\
\hline $\begin{array}{l}\text { Variables: } \\
\text { Temperature }\end{array}$ & $\begin{array}{l}\text { Prepared by: } \\
\text { W. E. Acree, Jr. }\end{array}$ \\
\hline
\end{tabular}

Experimental Values

\begin{tabular}{lcc}
\hline \hline$T / \mathrm{K}$ & $x_{2}{ }^{\mathrm{a}}$ & $x_{1}{ }^{\mathrm{b}}$ \\
\hline 303.2 & 0.9992 & 0.000791 \\
313.2 & 0.9991 & 0.000934 \\
323.2 & 0.9989 & 0.001082 \\
333.2 & 0.9987 & 0.001321 \\
343.2 & 0.9986 & 0.001379
\end{tabular}

\begin{tabular}{lcc}
\hline \hline$T / \mathrm{K}$ & $x_{2}{ }^{\mathrm{a}}$ & $x_{1}{ }^{\mathrm{b}}$ \\
\hline 353.2 & 0.9982 & 0.001797 \\
363.2 & 0.9979 & 0.002145
\end{tabular}

${ }^{a} x_{2}$ : mole fraction of component 2 in the saturated solution.

${ }^{b} x_{1}$ : mole fraction solubility of the solute.

\section{Auxiliary Information}

\section{Method/Apparatus/Procedure:}

Thermoelectric temperature controlling system and a high-performance liquid chromatograph.

Experimental solubilities were determined by a static method. A sealed bottle containing excess solute and solvent was allowed to equilibrate at constant temperature for at least $24 \mathrm{~h}$. A $3 \mathrm{ml}$ aliquot of the saturated solution was removed with a preheated syringe, and injected into a test tube which contained $3 \mathrm{ml}$ of dimethyl sulfoxide. The concentration of the solute was determined by high-performance liquid chromatographic method.

Source and Purity of Chemicals:

(1) $98 \%$, Tokyo Kasei Kogyo Company, Ltd., Japan, used as received.

(2) 99\%, Hangzhou Chemical Reagent Company, China, used as received.

\section{Estimated Error:}

Temperature: $\pm 0.1 \mathrm{~K}$.

$x_{1}: \pm 3 \%$ (relative error).

\begin{tabular}{ll}
\hline \hline Components: & Original Measurements: \\
$(1) 1,3-$ Benzenedicarboxylic acid; & ${ }^{60}$ S. Zhao, X. Chen, Q. Dai, and L. \\
$\mathrm{C}_{8} \mathrm{H}_{6} \mathrm{O}_{4} ;[121-91-5]$ & Wang, J. Chem. Eng. Data 56, 2399 \\
$\begin{array}{l}\text { (2) Isobutyl ethanoate; } \mathrm{C}_{6} \mathrm{H}_{12} \mathrm{O}_{2} ; \\
\text { [110-19-0] }\end{array}$ & $(2011)$. \\
\hline Variables: & Prepared by: \\
Temperature & W. E. Acree, Jr. \\
\hline
\end{tabular}

Experimental Values

\begin{tabular}{lcc}
\hline \hline$T / \mathrm{K}$ & $x_{2}{ }^{\mathrm{a}}$ & $x_{1}{ }^{\mathrm{b}}$ \\
\hline 308.15 & 0.9996 & 0.000352 \\
313.15 & 0.9996 & 0.000388 \\
318.15 & 0.9996 & 0.000438 \\
323.15 & 0.9995 & 0.000507 \\
328.15 & 0.9993 & 0.000691 \\
333.15 & 0.9991 & 0.000917 \\
338.15 & 0.9987 & 0.001300 \\
343.15 & 0.9983 & 0.001663 \\
348.15 & 0.9980 & 0.002009 \\
353.15 & 0.9978 & 0.002209
\end{tabular}

${ }^{\mathrm{a}} x_{2}$ : mole fraction of component 2 in the saturated solution.

${ }^{\mathrm{b}} x_{1}$ : mole fraction solubility of the solute. 


\section{Auxiliary Information}

\section{Method/Apparatus/Procedure:}

Thermostated temperature controller, electromagnetic stirrer, analytical balance, an ultraviolet-visible spectrophotometer.

Experimental solubilities were determined by a static method. A sealed flask containing excess solute and solvent was allowed to equilibrate at constant temperature with continuous stirring for $2 \mathrm{~h}$. The stirring was stopped and the excess solid was permitted to settle to the bottom of the flask. Aliquots of the saturated solution were removed, diluted quantitatively with isobutyl ethanoate, and the absorbance recorded at $280 \mathrm{~nm}$. The solubility was calculated based on the Beer-Lambert law using measured absorbances for standard solutions of known concentration.

\section{Source and Purity of Chemicals:}

(1) Analytical Reagent grade, Sinopharm Chemical Reagent Co. Ltd., China, was used as received.

(2) Analytical Reagent grade, Sinopharm Chemical Reagent Co. Ltd., China, was used as received.

\section{Estimated Error:}

Temperature: $\pm 0.05 \mathrm{~K}$. $x_{1}: \pm 0.00005$.

\subsection{1,3-Benzenedicarboxylic acid solubilities in ethers}

\begin{tabular}{|c|c|}
\hline $\begin{array}{l}\text { Components: } \\
\text { (1) 1,3-Benzenedicarboxylic acid; } \\
\left.\mathrm{C}_{8} \mathrm{H}_{6} \mathrm{O}_{4} ; \text {; } 121-91-5\right] \\
\text { (2) Tetrahydrofuran; } \mathrm{C}_{4} \mathrm{H}_{8} \mathrm{O} \text {; } \\
\text { [109-99-9] }\end{array}$ & $\begin{array}{l}\text { Original Measurements: } \\
6{ }^{6} \text { C. K. Hancock, J. N. Pawloski, and J. } \\
\text { P. Idoux, J. Org. Chem. 32, } 1931 \\
\text { (1967). }\end{array}$ \\
\hline $\begin{array}{l}\text { Variables: } \\
T / \mathrm{K}=303.15\end{array}$ & $\begin{array}{l}\text { Prepared by: } \\
\text { W. E. Acree, Jr. }\end{array}$ \\
\hline
\end{tabular}

Experimental Values

\begin{tabular}{lc}
\hline \hline$x_{2}{ }^{\mathrm{a}}$ & $x_{1}{ }^{\mathrm{b}}$ \\
\hline 0.984 & 0.016 \\
\hline${ }^{\mathrm{a}} x_{2}:$ mole fraction of component 2 in the saturated solution. \\
${ }^{\mathrm{b}}{ }_{x_{1}: \text { mole fraction solubility of the solute. }}$
\end{tabular}

\section{Auxiliary Information}

\footnotetext{
Method/Apparatus/Procedure:

Constant-temperature bath, Soxhlet thimble, conical flask, and analytical balance.

Excess solute and solvent were placed in a conical flask and allowed to equilibrate for several days at constant temperature. Attainment of equilibrium was verified by several repetitive measurements and by approaching equilibrium from supersaturation. Aliquots of saturated solutions were transferred using a Soxhlet thimble equipped with a one-hole cork stopper and an inverted U-shaped delivery tube to a second ground-glass stoppered flask suspended in the $303 \mathrm{~K}$ water bath. Successive portions of the contents were evaporated at room temperature in a tared aluminum foil weighing dish under a bell jar through which a slow stream of dry air was passed. The second flask plus unused saturated solution and the aluminum foil dish plus residue were weighed. The saturation solubility of the solute was calculated from the recorded mass data and molar masses of the solute and solvent.
}

Source and Purity of Chemicals:

(1) Purity not given, Chemical source not specified, was recrystallized several times from aqueous-ethanol mixture, and then dried under vacuum over phosphorous pentoxide.

(2) Practical grade, Chemical source not specified, stored over sodium hydroxide pellets for $24 \mathrm{~h}$, and then passed through $2 \times 70-\mathrm{cm}$ chromatographic adsorption columns containing activated alumina. After this treatment, the purified solvent was stored over copper in a nitrogen atmosphere.

Estimated Error:

Temperature: $\pm 0.02 \mathrm{~K}$

$x_{1}: \pm 1.7 \%$ (relative error)

\begin{tabular}{|c|c|}
\hline $\begin{array}{l}\text { Components: } \\
\text { (1) 1,3-Benzenedicarboxylic acid; } \\
\mathrm{C}_{8} \mathrm{H}_{6} \mathrm{O}_{4} ;[121-91-5] \\
\text { (2) Tetrahydrofuran; } \mathrm{C}_{4} \mathrm{H}_{8} \mathrm{O} \text {; } \\
\text { [109-99-9] }\end{array}$ & $\begin{array}{l}\text { Original Measurements: } \\
{ }^{109} \text { Y.-K. Che, Y.-X. Qu, and S. Wang, } \\
\text { J. Chem. Eng. Data 54, } 3130 \text { (2009). }\end{array}$ \\
\hline $\begin{array}{l}\text { Variables: } \\
\text { Temperature }\end{array}$ & $\begin{array}{l}\text { Prepared by: } \\
\text { W. E. Acree, Jr. }\end{array}$ \\
\hline
\end{tabular}

Experimental Values

\begin{tabular}{lcc}
\hline \hline$T / \mathrm{K}$ & $x_{2}{ }^{\mathrm{a}}$ & $x_{1}{ }^{\mathrm{b}}$ \\
\hline 293.35 & 0.9859 & 0.01414 \\
303.15 & 0.9846 & 0.01541 \\
313.45 & 0.9831 & 0.01693 \\
322.85 & 0.9815 & 0.01849 \\
328.75 & 0.9804 & 0.01956 \\
\hline
\end{tabular}

${ }^{a} x_{2}$ : mole fraction of component 2 in the saturated solution.

${ }^{\mathrm{b}} x_{1}$ : mole fraction solubility of the solute.

\section{Auxiliary Information}

\section{Method/Apparatus/Procedure:}

Equilibrium jacketed glass vessel, thermostated circulating water bath, electromagnetic stirrer, analytical balance, laser monitoring system. Experimental solubilities were determined by a synthetic method. Preweighed amounts of solute and solvent and were placed in an equilibrium vessel, which was connected to a circulating constant-temperature water bath. The solution was stirred and small amounts of solute were incrementally added until no further solid dissolved. The dissolution of the solid was determined using laser monitoring. The total amount of solute dissolved was recorded. Experimental measurement was repeated three times.

Source and Purity of Chemicals:

(1) $99 \%$, Sinopharm Chemical Reagent Company, Ltd., China, no purification details were provided.

(2) $99 \%$, Kermel Chemical Reagents Development Centre, China, no purification details were provided.

\section{Estimated Error:}

Temperature: $\pm 0.05 \mathrm{~K}$.

$x_{1}: \pm 0.5 \%$ (relative error) 


\begin{tabular}{|c|c|}
\hline $\begin{array}{l}\text { Components: } \\
\text { (1) 1,3-Benzenedicarboxylic acid; } \\
\mathrm{C}_{8} \mathrm{H}_{6} \mathrm{O}_{4} ;[121-91-5] \\
\text { (2) 1,4-Dioxane; } \mathrm{C}_{4} \mathrm{H}_{8} \mathrm{O}_{2} \\
\text { [123-91-1] }\end{array}$ & $\begin{array}{l}\text { Original Measurements: } \\
{ }^{63} \text { C. K. Hancock, J. N. Pawloski, and } \\
\text { J. P. Idoux, J. Org. Chem. 32, } 1931 \\
\text { (1967). }\end{array}$ \\
\hline $\begin{array}{l}\text { Variables: } \\
T / \mathrm{K}=303.15\end{array}$ & $\begin{array}{l}\text { Prepared by: } \\
\text { W. E. Acree, Jr. }\end{array}$ \\
\hline
\end{tabular}

Experimental Values

\begin{tabular}{lc}
\hline \hline$x_{2}{ }^{\mathrm{a}}$ & $x_{1}{ }^{\mathrm{b}}$ \\
\hline 0.994 & 0.006 \\
\hline$x_{2}:$ mole fraction of component 2 in the sater
\end{tabular}

${ }^{\mathrm{a}} x_{2}$ : mole fraction of component 2 in the saturated solution.

${ }^{\mathrm{b}} x_{1}$ : mole fraction solubility of the solute.

\section{Auxiliary Information}

Method/Apparatus/Procedure:

Constant-temperature bath, Soxhlet thimble, conical flask, and analytical balance.

Excess solute and solvent were placed in a conical flask and allowed to equilibrate for several days at constant temperature. Attainment of equilibrium was verified by several repetitive measurements and by approaching equilibrium from supersaturation. Weighed aliquots of saturated solutions were removed and titrated with a standardized sodium hydroxide solution (carbonate free) using a $\mathrm{pH}$ meter. The endpoint of the titration was determined by computing the second derivative in the $\mathrm{pH}$ versus volume of sodium hydroxide added.

\section{Source and Purity of Chemicals:}

(1) Purity not given, Chemical source not specified, was recrystallized several times from aqueous-ethanol mixture, and then dried under vacuum over phosphorous pentoxide.

(2) Practical grade, Chemical source not specified, stored over sodium hydroxide pellets for $24 \mathrm{~h}$, and then passed through $2 \times 70-\mathrm{cm}$ chromatographic adsorption columns containing activated alumina. After this treatment, the purified solvent was stored over copper in a nitrogen atmosphere.

Estimated Error:

Temperature: $\pm 0.02 \mathrm{~K}$.

$x_{1}: \pm 3.6 \%$ (relative error).

\section{Components:}

(1) 1,3-Benzenedicarboxylic acid; $\mathrm{C}_{8} \mathrm{H}_{6} \mathrm{O}_{4} ;$; 121-91-5]

(2) 1,2-Diethoxyethane; $\mathrm{C}_{6} \mathrm{H}_{14} \mathrm{O}_{2}$;

[629-14-1]

Variables:

Temperature

\section{Original Measurements:}

${ }^{109}$ Y.-K. Che, Y.-X. Qu, and S. Wang,

J. Chem. Eng. Data 54, 3130 (2009).

Prepared by:

W. E. Acree, Jr.
Experimental Values

\begin{tabular}{lcc}
\hline \hline$T / \mathrm{K}$ & $x_{2}{ }^{\mathrm{a}}$ & $x_{1}{ }^{\mathrm{b}}$ \\
\hline 293.30 & 0.9973 & 0.002651 \\
303.15 & 0.9971 & 0.002929 \\
313.15 & 0.9967 & 0.003297 \\
322.75 & 0.9962 & 0.003759
\end{tabular}

\begin{tabular}{lcc}
\hline \hline$T / \mathrm{K}$ & $x_{2}{ }^{\mathrm{a}}$ & $x_{1}{ }^{\mathrm{b}}$ \\
\hline 332.65 & 0.9957 & 0.004331 \\
342.45 & 0.9950 & 0.005017
\end{tabular}

${ }^{a} x_{2}$ : mole fraction of component 2 in the saturated solution.

${ }^{b} x_{1}$ : mole fraction solubility of the solute.

\section{Auxiliary Information}

Method/Apparatus/Procedure:

Equilibrium jacketed glass vessel, thermostated circulating water bath, electromagnetic stirrer, analytical balance, laser monitoring system.

Experimental solubilities were determined by a synthetic method. Preweighed amounts of solute and solvent and were placed in an equilibrium vessel, which was connected to a circulating constant-temperature water bath. The solution was stirred and small amounts of solute were incrementally added until no further solid dissolved. The dissolution of the solid was determined using laser monitoring. The total amount of solute dissolved was recorded. Experimental measurement was repeated three times.

Source and Purity of Chemicals:

(1) $99 \%$, Sinopharm Chemical Reagent Company, Ltd., China, no purification details were provided.

(2) $99+\%$, Tianjin Guangfu Fine Chemical Research Institute, China, no purification details were provided.

Estimated Error:

Temperature: $\pm 0.05 \mathrm{~K}$.

$x_{1}: \pm 0.5 \%$ (relative error)

\subsection{1,3-Benzenedicarboxylic acid solubility data in alcohols}

\begin{tabular}{|c|c|}
\hline $\begin{array}{l}\text { Components: } \\
\text { (1) } 1,3 \text {-Benzenedicarboxylic acid; } \\
\mathrm{C}_{8} \mathrm{H}_{6} \mathrm{O}_{4} ;[121-91-5] \\
\text { (2) Methanol; } \mathrm{CH}_{4} \mathrm{O} ;[67-56-1]\end{array}$ & $\begin{array}{l}\text { Original Measurements: } \\
{ }^{114} \text { G. N. Friedlin and V. N. Davydov, } \\
\text { Zh. Prikl. Khim. 35, } 2530 \text { (1962). }\end{array}$ \\
\hline $\begin{array}{l}\text { Variables: } \\
\text { Temnerature }\end{array}$ & Prepared by: \\
\hline Temperature & W. E. Acree, Jr. \\
\hline
\end{tabular}

\section{Experimental Values}

The authors report a solubility of $1.753 \mathrm{~g}$ of solute per $100 \mathrm{ml}$ of solvent at $293 \mathrm{~K}$, and a solubility of $3.990 \mathrm{~g}$ of solute per $100 \mathrm{ml}$ of solvent at $338 \mathrm{~K}$.

\section{Auxiliary Information}

\section{Method/Apparatus/Procedure:}

Experimental details were not provided.

Source and Purity of Chemicals:

(1) Purity not given, Chemical source not given, no purification details were provided.

(2) Purity not given, Chemical source not given, no purification details were provided.

Estimated Error:

Temperature: No information given.

Solubility: $\pm 1 \%$ (relative error) 


\begin{tabular}{|c|c|}
\hline $\begin{array}{l}\text { Components: } \\
\text { (1) } 1,3 \text {-Benzenedicarboxylic acid; } \\
\mathrm{C}_{8} \mathrm{H}_{6} \mathrm{O}_{4} ;[121-91-5] \\
\text { (2) Methanol; } \mathrm{CH}_{4} \mathrm{O} ;[67-56-1]\end{array}$ & $\begin{array}{l}\text { Original Measurements: } \\
{ }^{115} \text { G. N. Freidlin and V. N. Davydov, } \\
\text { Dopov. Akad. Nauk Ukr. RSR } 622 \\
\text { (1962). }\end{array}$ \\
\hline Variables: & Prepared by: \\
\hline Temperature & W. E. Acree, Jr. \\
\hline
\end{tabular}

Experimental Values

\begin{tabular}{lcc}
\hline \hline$T / \mathrm{K}$ & $x_{2}{ }^{\mathrm{a}}$ & $x_{1}{ }^{\mathrm{b}}$ \\
\hline 293 & 0.9957 & 0.00426 \\
313 & 0.9937 & 0.00633 \\
323 & 0.9924 & 0.00758 \\
330 & 0.9917 & 0.00834 \\
337 & 0.9895 & 0.01051 \\
\hline
\end{tabular}

${ }^{\mathrm{a}} x_{2}$ : mole fraction of component 2 in the saturated solution.

${ }^{b} x_{1}$ : mole fraction solubility of the solute. Solubility data were expressed as grams of solute per $100 \mathrm{~g}$ of solvent. Mole fraction values calculated by compiler.

\section{Auxiliary Information}

\section{Method/Apparatus/Procedure:}

Experimental details were not provided.

Source and Purity of Chemicals:

(1) Purity not given, Chemical source not given, no purification details were provided.

(2) Purity not given, Chemical source not given, no purification details were provided.

Estimated Error:

Temperature: No information given.

$x_{1}: \pm 2 \%$ (relative error).

\begin{tabular}{|c|c|}
\hline $\begin{array}{l}\text { Components: } \\
\text { (1) } 1,3 \text {-Benzenedicarboxylic acid; } \\
\mathrm{C}_{8} \mathrm{H}_{6} \mathrm{O}_{4} ;[121-91-5] \\
\text { (2) Methanol; } \mathrm{CH}_{4} \mathrm{O} ;[67-56-1]\end{array}$ & $\begin{array}{l}\text { Original Measurements: } \\
{ }^{112} \text { B. Long, Y. Wang, R. Zhang, and J. } \\
\text { Xu, J. Chem. Eng. Data 54, } 1764 \\
(2009) .\end{array}$ \\
\hline $\begin{array}{l}\text { Variables: } \\
\text { Temperature }\end{array}$ & $\begin{array}{l}\text { Prepared by: } \\
\text { W. E. Acree, Jr. }\end{array}$ \\
\hline
\end{tabular}

Experimental Values

\begin{tabular}{lcc}
\hline \hline$T / \mathrm{K}$ & $x_{2}{ }^{\mathrm{a}}$ & $x_{1}{ }^{\mathrm{b}}$ \\
\hline 278.15 & 0.9975 & 0.002530 \\
283.25 & 0.9972 & 0.002755 \\
288.05 & 0.9969 & 0.003142 \\
293.25 & 0.9965 & 0.003455 \\
298.75 & 0.9962 & 0.003787 \\
302.65 & 0.9959 & 0.004110 \\
307.95 & 0.9954 & 0.004555 \\
313.45 & 0.9949 & 0.005118 \\
318.45 & 0.9942 & 0.005784 \\
323.25 & 0.9934 & 0.006589 \\
328.25 & 0.9929 & 0.007144
\end{tabular}

\begin{tabular}{lcc}
\hline \hline$T / \mathrm{K}$ & $x_{2}{ }^{\mathrm{a}}$ & $x_{1}{ }^{\mathrm{b}}$ \\
\hline 333.15 & 0.9921 & 0.007913 \\
337.25 & 0.9912 & 0.008823
\end{tabular}

${ }^{a} x_{2}$ : mole fraction of component 2 in the saturated solution.

${ }^{\mathrm{b}} x_{1}$ : mole fraction solubility of the solute.

\section{Auxiliary Information}

\section{Method/Apparatus/Procedure:}

Jacketed equilibrium cell, constant-temperature circulating water bath, analytical balance, oven, and magnetic stirrer.

Excess solute and solvent were placed in a jacketed equilibrium cell and allowed to equilibrate with stirring at constant temperature for $2 \mathrm{~h}$. After sufficient equilibration, the stirrer was stopped and the solution was kept still for at least $1 \mathrm{~h}$ to allow suspended solid to settle to the bottom of the cell. An aliquot of the clear upper saturated solution was removed with a warm pipet and transferred to a weighed vial. The vial was weighed, uncovered, and placed in an oven. The solvent was allowed to evaporate in the oven at $323 \mathrm{~K}$. The vial was covered with a piece of filter cloth to prevent dust contamination. After the solvent had evaporated, the vial was reweighed. The solubility was calculated from the mass of the solid residue and mass of saturated solution analyzed.

\section{Source and Purity of Chemicals:}

(1) $99.8 \%$, Beijing Yanshan Petrochemical Company, China, no purification details provided.

(2) $99+\%$, Beijing Chemical Reagent Company, China, used as received.

\section{Estimated Error:}

Temperature: $\pm 0.1 \mathrm{~K}$.

$x_{1}: \pm 2 \%$ (relative error)

\begin{tabular}{|c|c|}
\hline $\begin{array}{l}\text { Components: } \\
\text { (1) } 1,3 \text {-Benzenedicarboxylic acid; } \\
\mathrm{C}_{8} \mathrm{H}_{6} \mathrm{O}_{4} ;[121-91-5] \\
\text { (2) Ethanol; } \mathrm{C}_{2} \mathrm{H}_{6} \mathrm{O} ;[64-17-5]\end{array}$ & $\begin{array}{l}\text { Original Measurements: } \\
{ }^{116} \text { B. Long and Z. Yang, Fluid Phase } \\
\text { Equilib. 266, } 38 \text { (2008). }\end{array}$ \\
\hline $\begin{array}{l}\text { Variables: } \\
\text { Temperature }\end{array}$ & $\begin{array}{l}\text { Prepared by: } \\
\text { W. E. Acree, Jr. }\end{array}$ \\
\hline
\end{tabular}

Experimental Values

\begin{tabular}{lr}
\hline \hline$T / \mathrm{K}$ & $100 s_{1}{ }^{\mathrm{a}}$ \\
\hline 278.54 & 6.2361 \\
283.33 & 6.9042 \\
288.33 & 7.8402 \\
293.05 & 8.9950 \\
297.45 & 9.8858 \\
302.66 & 10.6423 \\
308.55 & 12.7611 \\
313.15 & 13.9168 \\
318.45 & 15.7286 \\
323.45 & 16.8814 \\
328.15 & 18.4253 \\
333.05 & 20.9806 \\
338.15 & 22.9459 \\
343.65 & 25.9315 \\
349.35 & 29.3565 \\
\hline${ }^{2} s_{1}:$ solubility of the solute in units of moles per kilogram. The authors' \\
description of how the solubilities are reported is contradictory. In the \\
manuscript text, the authors state molarity, and moles per kilogram of solution. \\
Compiler has assumed that the units are moles per kilogram, which would be \\
consistent with the column heading of the table in the published paper.
\end{tabular}




\section{Auxiliary Information}

\section{Method/Apparatus/Procedure:}

Jacketed equilibrium cell, constant temperature circulating water bath, analytical balance, oven, and magnetic stirrer.

Excess solute and solvent were placed in a jacketed equilibrium cell and allowed to equilibrate with stirring at constant temperature for at least $5 \mathrm{~h}$. After sufficient equilibration, the stirrer was stopped and the solution was kep still for at least $1 \mathrm{~h}$ to allow suspended solid to settle to the bottom of the cell. An aliquot of the clear upper saturated solution was removed with a warm pipet and transferred to a weighed vial. The vial was weighed, uncovered, and placed in an oven. The solvent was allowed to evaporate in the oven at $323 \mathrm{~K}$. The vial was covered with a piece of filter cloth to prevent dust contamination. After the solvent had evaporated, the vial was reweighed. The solubility was calculated from the mass of the solid residue and mass of saturated solution analyzed.

\section{Source and Purity of Chemicals:}

(1) $99.8 \%$, Beijing Yanshan Petrochemical Company, China, no purification details provided.

(2) $99.5 \%$, Beijing Chemical Reagent Company, China, used as received.

\section{Estimated Error:}

Temperature: $\pm 0.1 \mathrm{~K}$.

$s_{1}: \pm 2 \%$ (relative error)

\section{Auxiliary Information}

\section{Method/Apparatus/Procedure:}

Jacketed equilibrium cell, constant temperature circulating water bath, analytical balance, oven, and magnetic stirrer.

Excess solute and solvent were placed in a jacketed equilibrium cell and allowed to equilibrate with stirring at constant temperature for $2 \mathrm{~h}$. After sufficient equilibration, the stirrer was stopped and the solution was kept still for at least $1 \mathrm{~h}$ to allow suspended solid to settle to the bottom of the cell. An aliquot of the clear upper saturated solution was removed with a warm pipet and transferred to a weighed vial. The vial was weighed, uncovered, and placed in an oven. The solvent was allowed to evaporate in the oven at $323 \mathrm{~K}$. The vial was covered with a piece of filter cloth to prevent dust contamination. After the solvent had evaporated, the vial was reweighed. The solubility was calculated from the mass of the solid residue and mass of saturated solution analyzed.

\section{Source and Purity of Chemicals:}

(1) $99.8 \%$, Beijing Yanshan Petrochemical Company, China, no purification details provided.

(2) $99+\%$, Beijing Chemical Reagent Company, China, used as received.

\section{Estimated Error:}

Temperature: $\pm 0.1 \mathrm{~K}$.

$x_{1}: \pm 2 \%$ (relative error).

\begin{tabular}{|c|c|}
\hline $\begin{array}{l}\text { Components: } \\
\text { (1) 1,3-Benzenedicarboxylic acid; } \\
\mathrm{C}_{8} \mathrm{H}_{6} \mathrm{O}_{4} ;[121-91-5] \\
\text { (2) 1-Propanol; } \mathrm{C}_{3} \mathrm{H}_{8} \mathrm{O} ;[71-23-8]\end{array}$ & $\begin{array}{l}\text { Original Measurements: } \\
115 \text { G. N. Freidlin and V. N. Davydov, } \\
\text { Dopov. Akad. Nauk Ukr. RSR } 622 \\
\text { (1962). }\end{array}$ \\
\hline $\begin{array}{l}\text { Variables: } \\
\text { Temperature }\end{array}$ & $\begin{array}{l}\text { Prepared by: } \\
\text { W. E. Acree, Jr. }\end{array}$ \\
\hline
\end{tabular}

Experimental Values

Experimental Values

\begin{tabular}{lcc}
\hline \hline$T / \mathrm{K}$ & $x_{2}{ }^{\mathrm{a}}$ & $x_{1}{ }^{\mathrm{b}}$ \\
\hline 278.15 & 0.9983 & 0.001685 \\
280.84 & 0.9981 & 0.001901 \\
283.34 & 0.9978 & 0.002198 \\
288.17 & 0.9975 & 0.002493 \\
293.15 & 0.9973 & 0.002677 \\
298.35 & 0.9968 & 0.003205 \\
304.05 & 0.9962 & 0.003769 \\
308.25 & 0.9958 & 0.004248 \\
313.15 & 0.9953 & 0.004731 \\
317.55 & 0.9947 & 0.005281 \\
320.17 & 0.9944 & 0.005636 \\
323.50 & 0.9933 & 0.006461 \\
328.20 & 0.9929 & 0.007128 \\
333.15 & 0.9914 & 0.008576 \\
338.15 & 0.9903 & 0.009711 \\
342.65 & 0.9895 & 0.01047 \\
349.05 & 0.9878 & 0.01215 \\
349.37 & 0.9875 & 0.01245 \\
353.55 & 0.9852 & 0.01480 \\
358.75 & 0.9826 & 0.01744 \\
\hline
\end{tabular}

${ }^{\mathrm{a}} x_{2}$ : mole fraction of component 2 in the saturated solution.

${ }^{\mathrm{b}} x_{1}$ : mole fraction solubility of the solute.

Original Measurements:

(1) 1,3-Benzenedicarboxylic acid;

$\mathrm{C}_{8} \mathrm{H}_{6} \mathrm{O}_{4} ;[121-91-5]$

Zhang, and (2009)

\begin{tabular}{ll}
\hline Variables: & Prepared by: \\
Temperature & W. E. Acree, Jr. \\
\hline
\end{tabular}

\begin{tabular}{lcc}
\hline \hline$T / \mathrm{K}$ & $x_{2}{ }^{\mathrm{a}}$ & $x_{1}{ }^{\mathrm{b}}$ \\
\hline 293 & 0.9941 & 0.00590 \\
323 & 0.9901 & 0.00994 \\
343 & 0.9877 & 0.01234 \\
370 & 0.9751 & 0.02492
\end{tabular}

${ }^{a} x_{2}$ : mole fraction of component 2 in the saturated solution.

${ }^{b} x_{1}$ : mole fraction solubility of the solute. Solubility data were expressed as grams of solute per $100 \mathrm{~g}$ of solvent. Mole fraction values calculated by compiler.

\section{Auxiliary Information}

Method/Apparatus/Procedure:

Experimental details were not provided.

Source and Purity of Chemicals:

(1) Purity not given, Chemical source not given, no purification details were provided.

(2) Purity not given, Chemical source not given, no purification details were provided.

\section{Estimated Error:}

Temperature: No information given.

$x_{1}: \pm 10 \%$ for $293 \mathrm{~K}$ value, less than $2 \%$ at the other temperatures (relative error). 


\begin{tabular}{|c|c|}
\hline $\begin{array}{l}\text { Components: } \\
\text { (1) 1,3-Benzenedicarboxylic acid; } \\
\mathrm{C}_{8} \mathrm{H}_{6} \mathrm{O}_{4} ;[121-91-5] \\
\text { (2) 2-Propanol; } \mathrm{C}_{3} \mathrm{H}_{8} \mathrm{O} ;[67-63-0]\end{array}$ & $\begin{array}{l}\text { Original Measurements: } \\
{ }^{112} \text { B. Long, Y. Wang, R. Zhang, and J. } \\
\text { Xu, J. Chem. Eng. Data 54, } 1764 \\
(2009) .\end{array}$ \\
\hline Variables: & Prepared by: \\
\hline Temperature & W. E. Acree, Jr. \\
\hline
\end{tabular}

Experimental Values

\begin{tabular}{lcc}
\hline \hline$T / \mathrm{K}$ & $x_{2}{ }^{\mathrm{a}}$ & $x_{1}{ }^{\mathrm{b}}$ \\
\hline 278.55 & 0.9978 & 0.002185 \\
279.87 & 0.9977 & 0.002260 \\
283.25 & 0.9974 & 0.002550 \\
288.15 & 0.9991 & 0.002910 \\
293.15 & 0.9967 & 0.003253 \\
298.15 & 0.9960 & 0.003961 \\
303.05 & 0.9954 & 0.004594 \\
308.25 & 0.9947 & 0.005346 \\
313.15 & 0.9939 & 0.006074 \\
318.25 & 0.9932 & 0.006754 \\
319.56 & 0.9930 & 0.007035 \\
323.95 & 0.9919 & 0.008105 \\
328.15 & 0.9909 & 0.009110 \\
333.15 & 0.9898 & 0.01020 \\
338.35 & 0.9889 & 0.01105 \\
343.25 & 0.9866 & 0.01343 \\
348.15 & 0.9852 & 0.01477 \\
349.85 & 0.9849 & 0.01513 \\
353.15 & 0.9828 & 0.01715 \\
\hline
\end{tabular}

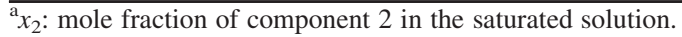

${ }^{\mathrm{b}} x_{1}$ : mole fraction solubility of the solute.

\section{Auxiliary Information}

\begin{abstract}
Method/Apparatus/Procedure:
Jacketed equilibrium cell, constant temperature circulating water bath, analytical balance, oven, and magnetic stirrer.

Excess solute and solvent were placed in a jacketed equilibrium cell and allowed to equilibrate with stirring at constant temperature for $2 \mathrm{~h}$. After sufficient equilibration, the stirrer was stopped and the solution was kept still for at least $1 \mathrm{~h}$ to allow suspended solid to settle to the bottom of the cell. An aliquot of the clear upper saturated solution was removed with a warm pipet and transferred to a weighed vial. The vial was weighed, uncovered, and placed in an oven. The solvent was allowed to evaporate in the oven at $323 \mathrm{~K}$. The vial was covered with a piece of filter cloth to prevent dust contamination. After the solvent had evaporated, the vial was reweighed. The solubility was calculated from the mass of the solid residue and mass of saturated solution analyzed.
\end{abstract}

\section{Source and Purity of Chemicals:}

(1) $99.8 \%$, Beijing Yanshan Petrochemical Company, China, no purification details provided.

(2) $99+\%$, Beijing Chemical Reagent Company, China, used as received.

\section{Estimated Error:}

Temperature: $\pm 0.1 \mathrm{~K}$.

$x_{1}: \pm 2 \%$ (relative error).

\begin{tabular}{|c|c|}
\hline $\begin{array}{l}\text { Components: } \\
\text { (1) } 1,3 \text {-Benzenedicarboxylic acid; } \\
\mathrm{C}_{8} \mathrm{H}_{6} \mathrm{O}_{4} ;[121-91-5] \\
\text { (2) } 1 \text {-Butanol; } \mathrm{C}_{4} \mathrm{H}_{10} \mathrm{O} ;[71-36-3]\end{array}$ & $\begin{array}{l}\text { Original Measurements: } \\
{ }^{112} \text { B. Long, Y. Wang, R. Zhang, and J. } \\
\text { Xu, J. Chem. Eng. Data 54, } 1764 \\
(2009) \text {. }\end{array}$ \\
\hline $\begin{array}{l}\text { Variables: } \\
\text { Temperature }\end{array}$ & $\begin{array}{l}\text { Prepared by: } \\
\text { W. E. Acree, Jr. }\end{array}$ \\
\hline
\end{tabular}

Experimental Values

\begin{tabular}{lcc}
\hline \hline$T / \mathrm{K}$ & $x_{2}{ }^{\mathrm{a}}$ & $x_{1}{ }^{\mathrm{b}}$ \\
\hline 278.06 & 0.9981 & 0.001872 \\
283.11 & 0.9978 & 0.002157 \\
288.27 & 0.9977 & 0.002339 \\
293.30 & 0.9974 & 0.002649 \\
298.06 & 0.9969 & 0.003149 \\
303.66 & 0.9963 & 0.003664 \\
308.36 & 0.9961 & 0.003860 \\
312.66 & 0.9953 & 0.004736 \\
317.94 & 0.9948 & 0.005200 \\
322.25 & 0.9943 & 0.005661 \\
328.54 & 0.9939 & 0.006333 \\
334.57 & 0.9923 & 0.007655 \\
337.96 & 0.9918 & 0.008166 \\
338.64 & 0.9911 & 0.008924 \\
343.20 & 0.9905 & 0.009537 \\
347.67 & 0.9889 & 0.01106 \\
353.44 & 0.9870 & 0.01304 \\
358.67 & 0.9856 & 0.01437 \\
\hline
\end{tabular}

${ }^{\mathrm{a}} x_{2}$ : mole fraction of component 2 in the saturated solution.

${ }^{\mathrm{b}} x_{1}$ : mole fraction solubility of the solute.

\section{Auxiliary Information}

\section{Method/Apparatus/Procedure:}

Jacketed equilibrium cell, constant temperature circulating water bath, analytical balance, oven, and magnetic stirrer.

Excess solute and solvent were placed in a jacketed equilibrium cell and allowed to equilibrate with stirring at constant temperature for at least $5 \mathrm{~h}$. After sufficient equilibration, the stirrer was stopped and the solution was kept still for at least $1 \mathrm{~h}$ to allow suspended solid to settle to the bottom of the cell. An aliquot of the clear upper saturated solution was removed with a warm pipet and transferred to a weighed vial. The vial was weighed, uncovered, and placed in an oven. The solvent was allowed to evaporate in the oven at $323 \mathrm{~K}$. The vial was covered with a piece of filter cloth to prevent dust contamination. After the solvent had evaporated, the vial was reweighed. The solubility was calculated from the mass of the solid residue and mass of saturated solution analyzed.

Source and Purity of Chemicals:

(1) $99.8 \%$, Beijing Yanshan Petrochemical Company, China, no purification details provided.

(2) $99+\%$, Beijing Chemical Reagent Company, China, used as received.

Estimated Error:

Temperature: $\pm 0.1 \mathrm{~K}$.

$x_{1}: \pm 2 \%$ (relative error).

\begin{tabular}{|c|c|}
\hline $\begin{array}{l}\text { Components: } \\
\text { (1) } 1,3 \text {-Benzenedicarboxylic acid; } \\
\mathrm{C}_{8} \mathrm{H}_{6} \mathrm{O}_{4} ;[121-91-5] \\
\text { (2) } 1 \text {-Butanol; } \mathrm{C}_{4} \mathrm{H}_{10} \mathrm{O} ;[71-36-3]\end{array}$ & $\begin{array}{l}\text { Original Measurements: } \\
{ }^{91} \text { J. Bradil, J. Malek, and V. Bazant, } \\
\text { Chem. Prumysl 20, } 117 \text { (1970). }\end{array}$ \\
\hline $\begin{array}{l}\text { Variables: } \\
\text { Temperature }\end{array}$ & $\begin{array}{l}\text { Prepared by: } \\
\text { W. E. Acree, Jr. }\end{array}$ \\
\hline
\end{tabular}


Experimental Values

\begin{tabular}{lcc}
\hline \hline$T / \mathrm{K}$ & $x_{2}{ }^{\mathrm{a}}$ & $x_{1}{ }^{\mathrm{b}}$ \\
\hline 273.2 & 0.9977 & 0.00225 \\
298.2 & 0.9942 & 0.00575 \\
323.2 & 0.9921 & 0.00788 \\
343.6 & 0.9877 & 0.01228 \\
358.2 & 0.9823 & 0.01774 \\
362.7 & 0.9785 & 0.02151 \\
\hline
\end{tabular}

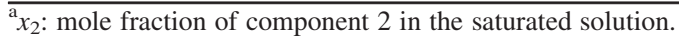

${ }^{b} x_{1}$ : mole fraction solubility of the solute. Solubility data were reported in terms of grams of dissolved solute per $100 \mathrm{~g}$ of solution. Mole fraction solubilities calculated by the compiler.

\section{Auxiliary Information}

\section{Method/Apparatus/Procedure:}

Excess solute and solvent and were placed in a flask and equilibrated at constant temperature with stirring. After $90 \mathrm{~min}$ the stirring was discontinued, and the solution was allowed to stand for $30 \mathrm{~min}$ to allow the undissolved solid to settle to the bottom of the flask. An aliquot of the saturated solution was removed by pipette fitted with a filtering device. The mass of the aliquot was determined by weighing. The concentration of the dissolved solute was determined by titration using sodium hydroxide, with phenolphthalein being the endpoint indicator.

Source and Purity of Chemicals:

(1) Purity not given, Amoco Chemical Corporation, Chicago, IL, USA, recrystallized three times from a mixture of methylbenzene and tetrachloromethane.

(2) Purity not given, Spolana, Neratovice, Czechoslovakia, was distilled before use.

Estimated Error:

Temperature: $\pm 0.1 \mathrm{~K}$.

$x_{1}: \pm 1 \%$ (relative error).

\begin{tabular}{|c|c|}
\hline $\begin{array}{l}\text { Components: } \\
\text { (1) 1,3-Benzenedicarboxylic acid; } \\
\mathrm{C}_{8} \mathrm{H}_{6} \mathrm{O}_{4} \text {; [121-91-5] } \\
\text { (2) 2-Butanol; } \mathrm{C}_{4} \mathrm{H}_{10} \mathrm{O} \text {; [78-92-2] }\end{array}$ & $\begin{array}{l}\text { Original Measurements: } \\
{ }^{112} \text { B. Long, Y. Wang, R. Zhang, and J. } \\
\text { Xu, J. Chem. Eng. Data 54, } 1764 \\
(2009) \text {. }\end{array}$ \\
\hline $\begin{array}{l}\text { Variables: } \\
\text { Temperature }\end{array}$ & $\begin{array}{l}\text { Prepared by: } \\
\text { W. E. Acree, Jr. }\end{array}$ \\
\hline
\end{tabular}

Experimental Values

\begin{tabular}{lcc}
\hline \hline$T / \mathrm{K}$ & $x_{2}{ }^{\mathrm{a}}$ & $x_{1}{ }^{\mathrm{b}}$ \\
\hline 283.05 & 0.9985 & 0.001455 \\
288.05 & 0.9983 & 0.001721 \\
293.15 & 0.9979 & 0.002085 \\
298.25 & 0.9977 & 0.002349 \\
303.45 & 0.9974 & 0.002625 \\
308.25 & 0.9969 & 0.003113 \\
313.25 & 0.9965 & 0.003457 \\
318.15 & 0.9961 & 0.003910 \\
322.95 & 0.9959 & 0.004134 \\
327.95 & 0.9950 & 0.005035 \\
332.65 & 0.9944 & 0.005634 \\
337.55 & 0.9932 & 0.006785 \\
342.25 & 0.9925 & 0.007479
\end{tabular}

\begin{tabular}{lcc}
\hline \hline$T / \mathrm{K}$ & $x_{2}{ }^{\mathrm{a}}$ & \multicolumn{1}{c}{$x_{1}{ }^{\mathrm{b}}$} \\
\hline 347.35 & 0.9913 & 0.008695 \\
350.65 & 0.9904 & 0.009585 \\
352.65 & 0.9898 & 0.01023 \\
\hline
\end{tabular}

${ }^{a} x_{2}$ : mole fraction of component 2 in the saturated solution.

${ }^{\mathrm{b}} x_{1}$ : mole fraction solubility of the solute.

\section{Auxiliary Information}

\section{Method/Apparatus/Procedure:}

Jacketed equilibrium cell, constant temperature circulating water bath, analytical balance, oven, and magnetic stirrer.

Excess solute and solvent were placed in a jacketed equilibrium cell and allowed to equilibrate with stirring at constant temperature for at least $5 \mathrm{~h}$. After sufficient equilibration, the stirrer was stopped and the solution was kept still for at least $1 \mathrm{~h}$ to allow suspended solid to settle to the bottom of the cell. An aliquot of the clear upper saturated solution was removed with a warm pipet and transferred to a weighed vial. The vial was weighed, uncovered, and placed in an oven. The solvent was allowed to evaporate in the oven at $323 \mathrm{~K}$. The vial was covered with a piece of filter cloth to prevent dust contamination. After the solvent had evaporated, the vial was reweighed. The solubility was calculated from the mass of the solid residue and mass of saturated solution analyzed.

Source and Purity of Chemicals:

(1) $99.8 \%$, Beijing Yanshan Petrochemical Company, China, no purification details provided.

(2) 99+\%, Beijing Chemical Reagent Company, China, used as received.

Estimated Error:

Temperature: $\pm 0.1 \mathrm{~K}$.

$x_{1}: \pm 2 \%$ (relative error).

\begin{tabular}{ll}
\hline \hline Components: & Original Measurements: \\
$(1)$ 1,3-Benzenedicarboxylic acid; & ${ }^{112}$ B. Long, Y. Wang, R. Zhang, and J. \\
$\mathrm{C}_{8} \mathrm{H}_{6} \mathrm{O}_{4} ;[121-91-5]$ & Xu, J. Chem. Eng. Data 54, 1764 \\
$(2)$ 1-Pentanol; $\mathrm{C}_{5} \mathrm{H}_{12} \mathrm{O} ;$ [71-41-0] & $(2009)$. \\
\hline Variables: & Prepared by: \\
Temperature & W. E. Acree, Jr. \\
\hline
\end{tabular}

Experimental Values

\begin{tabular}{|c|c|c|}
\hline$T / \mathrm{K}$ & $x_{2}{ }^{\mathrm{a}}$ & $x_{1}{ }^{\mathrm{b}}$ \\
\hline 303.05 & 0.9959 & 0.004081 \\
\hline 308.05 & 0.9947 & 0.005323 \\
\hline 313.15 & 0.9941 & 0.005935 \\
\hline 318.15 & 0.9935 & 0.006513 \\
\hline 323.05 & 0.9926 & 0.007402 \\
\hline 328.15 & 0.9918 & 0.008232 \\
\hline 334.95 & 0.9906 & 0.009400 \\
\hline 340.35 & 0.9897 & 0.01028 \\
\hline 344.25 & 0.9883 & 0.01173 \\
\hline 348.85 & 0.9877 & 0.01232 \\
\hline
\end{tabular}




\section{Auxiliary Information}

\section{Method/Apparatus/Procedure:}

Jacketed equilibrium cell, constant temperature circulating water bath, analytical balance, oven, and magnetic stirrer.

Excess solute and solvent were placed in a jacketed equilibrium cell and allowed to equilibrate with stirring at constant temperature for at least $5 \mathrm{~h}$. After sufficient equilibration, the stirrer was stopped and the solution was kept still for at least $1 \mathrm{~h}$ to allow suspended solid to settle to the bottom of the cell. An aliquot of the clear upper saturated solution was removed with a warm pipet and transferred to a weighed vial. The vial was weighed, uncovered, and placed in an oven. The solvent was allowed to evaporate in the oven at $323 \mathrm{~K}$. The vial was covered with a piece of filter cloth to prevent dust contamination. After the solvent had evaporated, the vial was reweighed. The solubility was calculated from the mass of the solid residue and mass of saturated solution analyzed.

\section{Source and Purity of Chemicals:}

(1) $99.8 \%$, Beijing Yanshan Petrochemical Company, China, no purification details provided.

(2) 99+\%, Beijing Chemical Reagent Company, China, used as received.

\section{Estimated Error:}

Temperature: $\pm 0.1 \mathrm{~K}$.

$x_{1}: \pm 2 \%$ (relative error). solvent had evaporated, the vial was reweighed. The solubility was calculated from the mass of the solid residue and mass of saturated solution analyzed.

Source and Purity of Chemicals:

(1) $99.8 \%$, Beijing Yanshan Petrochemical Company, China, no purification details provided.

(2) $99+\%$, Beijing Chemical Reagent Company, China, used as received.

Estimated Error:

Temperature: $\pm 0.1 \mathrm{~K}$.

$x_{1}: \pm 2 \%$ (relative error)

\subsection{1,3-Benzenedicarboxylic acid solubility data in ketones}

\begin{tabular}{|c|c|}
\hline $\begin{array}{l}\text { Components: } \\
\text { (1) 1,3-Benzenedicarboxylic acid; } \\
\mathrm{C}_{8} \mathrm{H}_{6} \mathrm{O}_{4} ;[121-91-5] \\
\text { (2) Propanone; } \mathrm{C}_{3} \mathrm{H}_{6} \mathrm{O} ;[67-64-1]\end{array}$ & $\begin{array}{l}\text { Original Measurements: } \\
{ }^{116} \text { B. Long and Z. Yang, Fluid Phase } \\
\text { Equilib. 266, } 38 \text { (2008). }\end{array}$ \\
\hline $\begin{array}{l}\text { Variables: } \\
\text { Temperature }\end{array}$ & $\begin{array}{l}\text { Prepared by: } \\
\text { W. E. Acree, Jr. }\end{array}$ \\
\hline
\end{tabular}

\begin{tabular}{|c|c|}
\hline $\begin{array}{l}\text { Components: } \\
\text { (1) } 1,3 \text {-Benzenedicarboxylic acid; } \\
\mathrm{C}_{8} \mathrm{H}_{6} \mathrm{O}_{4} ;[121-91-5] \\
\text { (2) } 1-\mathrm{Hexanol} ; \mathrm{C}_{6} \mathrm{H}_{14} \mathrm{O} ;[111-27-3]\end{array}$ & $\begin{array}{l}\text { Original Measurements: } \\
{ }^{112} \text { B. Long, Y. Wang, R. Zhang, and } \\
\text { J. Xu, J. Chem. Eng. Data 54, } 1764 \\
(2009) \text {. }\end{array}$ \\
\hline $\begin{array}{l}\text { Variables: } \\
\text { Temperature }\end{array}$ & $\begin{array}{l}\text { Prepared by: } \\
\text { W. E. Acree, Jr. }\end{array}$ \\
\hline
\end{tabular}

Experimental Values

\begin{tabular}{lcc}
\hline \hline$T / \mathrm{K}$ & $x_{2}{ }^{\mathrm{a}}$ & $x_{1}{ }^{\mathrm{b}}$ \\
\hline 298.48 & 0.9976 & 0.002435 \\
302.97 & 0.9968 & 0.003204 \\
308.15 & 0.9964 & 0.003617 \\
312.70 & 0.9958 & 0.004235 \\
317.67 & 0.9952 & 0.004766 \\
320.84 & 0.9946 & 0.005439 \\
326.96 & 0.9941 & 0.005930 \\
332.56 & 0.9926 & 0.007372 \\
337.65 & 0.9914 & 0.008570 \\
343.76 & 0.9906 & 0.009458 \\
349.25 & 0.9896 & 0.01042 \\
353.94 & 0.9884 & 0.01159
\end{tabular}

${ }^{\mathrm{a}} x_{2}$ : mole fraction of component 2 in the saturated solution.

${ }^{\mathrm{b}} \mathrm{x}_{1}$ : mole fraction solubility of the solute.

\section{Auxiliary Information}

\section{Method/Apparatus/Procedure:}

Jacketed equilibrium cell, constant temperature circulating water bath, analytical balance, oven, and magnetic stirrer.

Excess solute and solvent were placed in a jacketed equilibrium cell and allowed to equilibrate with stirring at constant temperature for at least $5 \mathrm{~h}$. After sufficient equilibration, the stirrer was stopped and the solution was kept still for at least $1 \mathrm{~h}$ to allow suspended solid to settle to the bottom of the cell. An aliquot of the clear upper saturated solution was removed with a warm pipet and transferred to a weighed vial. The vial was weighed, uncovered, and placed in an oven. The solvent was allowed to evaporate in the oven at $323 \mathrm{~K}$. The vial was covered with a piece of filter cloth to prevent dust contamination. After the
Experimental Values

\begin{tabular}{lc}
\hline \hline$T / \mathrm{K}$ & $100 s_{1}{ }^{\mathrm{a}}$ \\
\hline 278.25 & 1.7634 \\
283.05 & 1.9788 \\
288.05 & 2.4777 \\
292.95 & 2.7495 \\
297.65 & 3.5875 \\
303.05 & 4.2633 \\
308.05 & 5.3245 \\
313.05 & 6.6758 \\
318.05 & 8.0520 \\
323.05 & 9.9103 \\
327.85 & 12.3663 \\
\hline${ }^{a} s_{1}:$ solubility of the solute in units of moles per kilogram. The authors' \\
description of how the solubilities are reported is contradictory. In the \\
manuscript text, the authors state molarity, and moles per kilogram of solution. \\
Compiler has assumed that the units are moles per kilogram, which would be \\
consistent with the column heading of the table in the published paper.
\end{tabular}

\section{Auxiliary Information}

\section{Method/Apparatus/Procedure:}

Jacketed equilibrium cell, constant temperature circulating water bath, analytical balance, oven, and magnetic stirrer.

Excess solute and solvent were placed in a jacketed equilibrium cell and allowed to equilibrate with stirring at constant temperature for at least $5 \mathrm{~h}$. After sufficient equilibration, the stirrer was stopped and the solution was kept still for at least $1 \mathrm{~h}$ to allow suspended solid to settle to the bottom of the cell. An aliquot of the clear upper saturated solution was removed with a warm pipet and transferred to a weighed vial. The vial was weighed, uncovered, and placed in an oven. The solvent was allowed to evaporate in the oven at $323 \mathrm{~K}$. The vial was covered with a piece of filter cloth to prevent dust contamination. After the solvent had evaporated, the vial was reweighed. The solubility was calculated from the mass of the solid residue and mass of saturated solution analyzed.

\section{Source and Purity of Chemicals:}

(1) $99.8 \%$, Beijing Yanshan Petrochemical Company, China, no purification details provided.

(2) $99.5 \%$, Beijing Chemical Reagent Company, China, used as received. 
Estimated Error:

Temperature: $\pm 0.1 \mathrm{~K}$.

$s_{1}: \pm 2 \%$ (relative error).

\begin{tabular}{|c|c|}
\hline $\begin{array}{l}\text { Components: } \\
\text { (1) } 1,3 \text {-Benzenedicarboxylic acid; } \\
\mathrm{C}_{8} \mathrm{H}_{6} \mathrm{O}_{4} ;[121-91-5] \\
\text { (2) Cyclohexanone; } \mathrm{C}_{6} \mathrm{H}_{10} \mathrm{O} \text {; } \\
\text { [108-94-1] }\end{array}$ & $\begin{array}{l}\text { Original Measurements: } \\
{ }^{109} \text { Y.-K. Che, Y.-X. Qu, and S. Wang, } \\
\text { J. Chem. Eng. Data 54, } 3130 \text { (2009). }\end{array}$ \\
\hline $\begin{array}{l}\text { Variables: } \\
\text { Temperature }\end{array}$ & $\begin{array}{l}\text { Prepared by: } \\
\text { W. E. Acree, Jr. }\end{array}$ \\
\hline
\end{tabular}

Experimental Values

\begin{tabular}{lcc}
\hline \hline$T / \mathrm{K}$ & $x_{2}{ }^{\mathrm{a}}$ & $x_{1}{ }^{\mathrm{b}}$ \\
\hline 293.35 & 0.9976 & 0.002392 \\
303.15 & 0.9970 & 0.003017 \\
313.15 & 0.9962 & 0.003847 \\
322.75 & 0.9953 & 0.004693 \\
332.75 & 0.9943 & 0.005710 \\
342.85 & 0.9930 & 0.006969
\end{tabular}

${ }^{\mathrm{a}} x_{2}$ : mole fraction of component 2 in the saturated solution.

${ }^{\mathrm{b}} x_{1}$ : mole fraction solubility of the solute.

\section{Auxiliary Information}

\section{Method/Apparatus/Procedure:}

Equilibrium jacketed glass vessel, thermostated circulating water bath, electromagnetic stirrer, analytical balance, laser monitoring system.

Experimental solubilities were determined by a synthetic method. Preweighed amounts of solute and solvent and were placed in an equilibrium vessel, which was connected to a circulating constant-temperature water bath. The solution was stirred and small amounts of solute were incrementally added until no further solid dissolved. The dissolution of the solid was determined using laser monitoring. The total amount of solute dissolved was recorded. Experimental measurement was repeated three times.

Source and Purity of Chemicals:

(1) $99 \%$, Sinopharm Chemical Reagent Company, Ltd., China, no purification details were provided.

(2) 99+\%, Tianjin Guangfu Fine Chemical Research Institute, China, no purification details were provided.

Estimated Error:

Temperature: $\pm 0.05 \mathrm{~K}$.

$x_{1}: \pm 0.5 \%$ (relative error).

\begin{tabular}{|c|c|}
\hline $\begin{array}{l}\text { Components: } \\
\text { (1) } 1,3 \text {-Benzenedicarboxylic acid; } \\
\mathrm{C}_{8} \mathrm{H}_{6} \mathrm{O}_{4} ;[121-91-5] \\
\text { (2) Acetophenone; } \mathrm{C}_{8} \mathrm{H}_{8} \mathrm{O} \text {; } \\
\text { [98-86-2] }\end{array}$ & $\begin{array}{l}\text { Original Measurements: } \\
{ }^{109} \text { Y.-K. Che, Y.-X. Qu, and S. Wang, } \\
\text { J. Chem. Eng. Data 54, } 3130 \text { (2009). }\end{array}$ \\
\hline $\begin{array}{l}\text { Variables: } \\
\text { Temperature }\end{array}$ & $\begin{array}{l}\text { Prepared by: } \\
\text { W. E. Acree, Jr. }\end{array}$ \\
\hline
\end{tabular}

Experimental Values

\begin{tabular}{lcc}
\hline \hline$T / \mathrm{K}$ & $x_{2}{ }^{\mathrm{a}}$ & \multicolumn{1}{c}{$x_{1}{ }^{\mathrm{b}}$} \\
\hline 303.15 & 0.9993 & 0.0007249 \\
313.15 & 0.9991 & 0.0008585 \\
323.05 & 0.9989 & 0.001054 \\
332.95 & 0.9986 & 0.001350 \\
342.95 & 0.9982 & 0.001748 \\
\hline
\end{tabular}

${ }^{\mathrm{a}} x_{2}$ : mole fraction of component 2 in the saturated solution.

${ }^{\mathrm{b}} x_{1}$ : mole fraction solubility of the solute.

\section{Auxiliary Information}

\section{Method/Apparatus/Procedure:}

Equilibrium jacketed glass vessel, thermostated circulating water bath, electromagnetic stirrer, analytical balance, laser monitoring system. Experimental solubilities were determined by a synthetic method. Preweighed amounts of solute and solvent and were placed in an equilibrium vessel, which was connected to a circulating constant-temperature water bath. The solution was stirred and small amounts of solute were incrementally added until no further solid dissolved. The dissolution of the solid was determined using laser monitoring. The total amount of solute dissolved was recorded. Experimental measurement was repeated three times.

\section{Source and Purity of Chemicals:}

(1) $99 \%$, Sinopharm Chemical Reagent Company, Ltd., China, no purification details were provided.

(2) $99+\%$, Tianjin Guangfu Fine Chemical Research Institute, China, no purification details were provided.

Estimated Error:

Temperature: $\pm 0.05 \mathrm{~K}$.

$x_{1}: \pm 0.5 \%$ (relative error).

\subsection{1,3-Benzenedicarboxylic acid solubility data in miscellaneous organic solvents}

\begin{tabular}{|c|c|}
\hline Components: & Original Measurements: \\
\hline $\begin{array}{l}\text { (1) 1,3-Benzenedicarboxylic acid; } \\
\mathrm{C}_{8} \mathrm{H}_{6} \mathrm{O}_{4} ;[121-91-5]\end{array}$ & $\begin{array}{l}{ }^{82} \text { L. Dian-Qing, L. Jiang-Chu, Liu Da- } \\
\text { Zhuang, and W. Fu-An, Fluid Phase }\end{array}$ \\
\hline $\begin{array}{l}\text { (2) } N, N \text {-Dimethylformamide; } \\
\mathrm{C}_{3} \mathrm{H}_{7} \mathrm{NO} ;[64-19-7]\end{array}$ & Equilib. 200, 69 (2002). \\
\hline Variables: & \\
\hline Temperature & W. E. Acree, Jr. \\
\hline
\end{tabular}

\begin{tabular}{lcc} 
& & \\
& & \\
& & \\
& & \\
\hline \hline$T / \mathrm{K}$ & $x_{2}{ }^{\mathrm{a}}$ & $x_{1}{ }^{\mathrm{b}}$ \\
\hline \hline 295.85 & 0.8482 & 0.1518 \\
299.65 & 0.8477 & 0.1523 \\
303.55 & 0.8471 & 0.1529 \\
309.65 & 0.8461 & 0.1539 \\
313.35 & 0.8454 & 0.1546 \\
317.25 & 0.8446 & 0.1554 \\
322.55 & 0.8434 & 0.1566 \\
329.15 & 0.8419 & 0.1581 \\
332.55 & 0.8410 & 0.1590 \\
337.15 & 0.8397 & 0.1603
\end{tabular}




\begin{tabular}{lcc}
\hline \hline$T / \mathrm{K}$ & $x_{2}{ }^{\mathrm{a}}$ & $x_{1}{ }^{\mathrm{b}}$ \\
\hline 341.35 & 0.8383 & 0.1617 \\
345.75 & 0.8368 & 0.1632 \\
349.75 & 0.8354 & 0.1646 \\
351.95 & 0.8346 & 0.1654 \\
354.55 & 0.8336 & 0.1664 \\
357.95 & 0.8322 & 0.1678 \\
362.75 & 0.8301 & 0.1699 \\
365.05 & 0.8291 & 0.1709 \\
370.25 & 0.8266 & 0.1734 \\
\hline
\end{tabular}

${ }^{a} x_{2}$ : mole fraction of component 2 in the saturated solution.

${ }^{\mathrm{b}} x_{1}$ : mole fraction solubility of the solute.

\section{Auxiliary Information}

\section{Method/Apparatus/Procedure:}

Titanium solid-liquid equilibrium cell, analytical balance, magnetic stirring system, temperature controlling system, and a laser monitoring system. Solubilities were determined using a synthetic method. Weighed amounts of solute and solvent were sealed in a jacketed equilibrium vessel, and the temperature slowly increased until the solid phase completely disappeared. The disappearance of the solid solute was detected by a laser monitoring system. Measurements were repeated two or three times to check the reproducibility.

\section{Source and Purity of Chemicals:}

(1) Analytical Reagent, Shanghai Chemical Reagent Company, used as received.

(2) Analytical Reagent, Shanghai Chemical Reagent Company, used as received.

\section{Estimated Error:}

Temperature: $\pm 0.05 \mathrm{~K}$ (estimated by compiler).

$x_{1}: \pm 0.0005$ or less.

\begin{tabular}{|c|c|}
\hline $\begin{array}{l}\text { Components: } \\
\text { (1) } 1,3 \text {-Benzenedicarboxylic acid; } \\
\mathrm{C}_{8} \mathrm{H}_{6} \mathrm{O}_{4} ;[121-91-5] \\
\text { (2) } \mathrm{N} \text {-Methyl-2-pyrrolidone; } \\
\mathrm{C}_{5} \mathrm{H}_{9} \mathrm{NO} ;[872-50-4]\end{array}$ & $\begin{array}{l}\text { Original Measurements: } \\
{ }^{84} \text { D.-Q. Li, D.-Z. Liu, and F.-A. Wang, } \\
\text { J. Chem. Eng. Data 46, } 172 \text { (2001). }\end{array}$ \\
\hline $\begin{array}{l}\text { Variables: } \\
\text { Temperature }\end{array}$ & $\begin{array}{l}\text { Prepared by: } \\
\text { W. E. Acree, Jr. }\end{array}$ \\
\hline
\end{tabular}

Experimental Values

\begin{tabular}{lcc}
\hline \hline$T / \mathrm{K}$ & $x_{2}{ }^{\mathrm{a}}$ & $x_{1}{ }^{\mathrm{b}}$ \\
\hline 296.35 & 0.9107 & 0.0893 \\
298.85 & 0.9057 & 0.0943 \\
303.55 & 0.8963 & 0.1037 \\
307.05 & 0.8885 & 0.1115 \\
310.25 & 0.8817 & 0.1183 \\
313.65 & 0.8732 & 0.1268 \\
316.95 & 0.8654 & 0.1346 \\
320.45 & 0.8563 & 0.1437 \\
323.55 & 0.8482 & 0.1518 \\
326.55 & 0.8392 & 0.1608 \\
331.25 & 0.8244 & 0.1756 \\
336.25 & 0.8075 & 0.1925 \\
340.75 & 0.7905 & 0.2095 \\
343.55 & 0.7787 & 0.2213
\end{tabular}

\begin{tabular}{lcc}
\hline \hline$T / \mathrm{K}$ & $x_{2}{ }^{\mathrm{a}}$ & $x_{1}{ }^{\mathrm{b}}$ \\
\hline 345.25 & 0.7722 & 0.2278 \\
346.55 & 0.7660 & 0.2340 \\
\hline${ }^{\mathrm{a}}{ }_{X_{2}: \text { mole fraction of component } 2 \text { in the saturated solution. }}$ \\
${ }^{{ } x_{1}: \text { mole fraction solubility of the solute. }}$ \\
& \\
& \\
& Auxiliary Information & \\
\hline
\end{tabular}

\section{Method/Apparatus/Procedure:}

Jacketed solid-liquid equilibrium cell, analytical balance, magnetic stirring system, temperature controlling system, and a laser monitoring system. Solubilities were determined using a synthetic method. Weighed amounts of solute and solvent were sealed in a jacketed equilibrium vessel, and the temperature slowly increased until the solid phase completely disappeared. The disappearance of the solid solute was detected by a laser monitoring system. Measurements were repeated two or three times to check the reproducibility.

\section{Source and Purity of Chemicals:}

(1) $99.5 \%$, Analytical Reagent, Shanghai Chemical Reagent Company, China, used as received.

(2) $99.0 \%$, Analytical Reagent, Shanghai Chemical Reagent Company, used as received.

\section{Estimated Error:}

Temperature: $\pm 0.05 \mathrm{~K}$ (estimated by compiler). $x_{1}: \pm 0.0005$ or less.

\begin{tabular}{|c|c|}
\hline $\begin{array}{l}\text { Components: } \\
\text { (1) } 1,3 \text {-Benzenedicarboxylic acid; } \\
\mathrm{C}_{8} \mathrm{H}_{6} \mathrm{O}_{4} ;[121-91-5] \\
\text { (2) Ethanoic acid; } \mathrm{C}_{2} \mathrm{H}_{4} \mathrm{O}_{2} ; \text { [64-19-7] }\end{array}$ & $\begin{array}{l}\text { Original Measurements: } \\
{ }^{117} \text { L. Feng, Q. Wang, and X. Li, J. } \\
\text { Chem. Eng. Data 53, } 2501 \text { (2008). }\end{array}$ \\
\hline $\begin{array}{l}\text { Variables: } \\
\text { Temperature }\end{array}$ & $\begin{array}{l}\text { Prepared by: } \\
\text { W. E. Acree, Jr. }\end{array}$ \\
\hline
\end{tabular}

Experimental Values

\begin{tabular}{lcc}
\hline \hline$T / \mathrm{K}$ & $x_{2}{ }^{\mathrm{a}}$ & $x_{1}{ }^{\mathrm{b}}$ \\
\hline 313.2 & 0.9988 & 0.001181 \\
323.2 & 0.9986 & 0.001436 \\
333.2 & 0.9982 & 0.001849 \\
343.2 & 0.9974 & 0.002550 \\
353.2 & 0.9969 & 0.003145 \\
363.2 & 0.9958 & 0.004225 \\
\hline
\end{tabular}

${ }^{a} x_{2}$ : mole fraction of component 2 in the saturated solution.

${ }^{\mathrm{b}} x_{1}$ : mole fraction solubility of the solute. Solubility data were reported as grams of solute per kilogram of solvent. Mole fraction solubilities were calculated by the compiler.

\section{Auxiliary Information}

\section{Method/Apparatus/Procedure:}

Jacketed equilibrium glass bottle, analytical balance, thermoelectric temperature controlling system, and high-performance liquid chromatographic system.

Solubilities were determined using a static method. Excess solute and solvent were sealed in a jacketed equilibrium bottle and allowed to equilibrate for at least $24 \mathrm{~h}$ at constant temperature. After equilibrium was obtained, an aliquot of the clear saturated solution was removed by syringe and deposited into a clear test tube that contained about $3 \mathrm{ml}$ of dimethyl sulfoxide. The concentration of the solute was determined by high-performance liquid chromatographic analysis. 
Source and Purity of Chemicals:

(1) $99 \%$, Tokyo Kasei Kogyo Company, Ltd., Japan, was used as received. (2) Purity not given, Hanzhou Chemical Reagent Company, China, was used as received.

Estimated Error:

Temperature: $\pm 0.1 \mathrm{~K}$ (estimated by compiler).

$x_{1}: \pm 3 \%$ (relative error, estimated by compiler).

\section{Solubility of 1,4-Benzenedicarboxylic Acid in Organic Solvents}

\subsection{Critical evaluation of experimental solubility data}

Several research groups ${ }^{60,63,80,82,84,91,109,114,115,118-124}$ have investigated the solubility behavior of 1,4-benzenedicarboxylic acid as a function of temperature. Che et al. ${ }^{109}$ determined the solubility of 1,4-benzenedicarboxylic acid in tetrahydrofuran, 1,2-diethoxyethane, cyclohexanone, and acetophenone at several temperatures. The internal consistency of the four datasets of measured 1,4-benzenedicarboxylic acid solubilities were assessed by curve-fitting the measured mole fraction solubility data to Eq. (8). The values of the equation coefficients $(A, B$, and $C$ ) are given in Table 19, along with the MRD calculated according to Eq. (24). Examination of the numerical entries in the last column of Table 19 reveals that the largest mean relative deviation between the back-calculated values based on Eq. (8) and experimental data is $2.63 \%$. A significant part of the deviation results from the experimental solubility measurement at $303 \mathrm{~K}$, which is about $10 \%$ larger than the calculated value. Results of the mathematical representation analyses indicate that the experimental data for all four 1,4-benzenedicarboxylic acid - organic solvent systems are internally consistent.

Zhao et al. ${ }^{60}$ measured the solubility of 1,4-benzenedicarboxylic acid in isobutyl ethanoate at ten temperatures between $305 \mathrm{~K}$ and $348 \mathrm{~K}$. The experimental data were correlated with the UNIQUAC model. Interaction coefficients calculated from the experimental solid-liquid equilibrium data provided a reasonably accurate mathematical description of the measured values. The mean absolute relative deviation between calculated and observed values was less than $6.5 \%$.

$\mathrm{Ma}$ and $\mathrm{Xia}^{80}$ and $\mathrm{Ma}$ and Chen ${ }^{121}$ both determined the solubility of 1,4-benzenedicarboxylic acid in $N, N$-dimethylformamide, $N, N$-dimethylacetamide, and $N$-methyl-2-pyrrolidone. Ma and $\mathrm{Xia}^{80}$ also performed solubility measurements in ethanoic acid, while $\mathrm{Ma}$ and $\mathrm{Chen}^{121}$ also studied the

TABLE 19. Parameters of the Modified Apelblat equation for describing the solubility of 1,4-benzenedicarboxylic acid in various organic solvents

\begin{tabular}{|c|c|c|c|c|}
\hline Solvent & $A$ & $B$ & $C$ & MRD (\%) \\
\hline 1,2-Diethoxyethane ${ }^{a}$ & -61.352 & 1131.2 & 8.6320 & 0.46 \\
\hline Tetrahydrofuran $^{\mathrm{a}}$ & -397.52 & 16627 & -58.757 & 0.80 \\
\hline Cyclohexanone $^{\mathrm{a}}$ & -256.51 & 9662.9 & 37.813 & 2.50 \\
\hline Acetophenone $^{\mathrm{a}}$ & -90.392 & -1694.1 & 14.774 & 2.63 \\
\hline
\end{tabular}

${ }^{\mathrm{a}}$ Values of the coefficients and the mean relative deviations were taken from Che et al. ${ }^{109}$
TABLE 20. Parameters of the Buchowski $\lambda$ h equation for describing the solubility of 1,4-benzenedicarboxylic acid in organic solvents

\begin{tabular}{|c|c|c|c|c|}
\hline Solvent & $T / \mathrm{K}$ & $\lambda$ & $h$ & $\operatorname{MRD}(\%)$ \\
\hline Ethanoic acid $^{\mathrm{a}}$ & $306-353$ & 0.1448 & 30386.1 & 8.90 \\
\hline$N, N$-Dimethylformamide ${ }^{\mathrm{a}}$ & $293-429$ & 0.0046 & 16554.7 & 0.47 \\
\hline$N, N$-Dimethylformamide ${ }^{\mathrm{b}}$ & $296-370$ & 0.0336 & 11862.9 & 0.18 \\
\hline$N, N$-Dimethylformamide ${ }^{\mathrm{c}}$ & $298-363$ & 0.1760 & 5379.87 & 0.51 \\
\hline$N, N$-Dimethylacetamide ${ }^{\mathrm{a}}$ & $293-365$ & 38.47 & 122.49 & 1.15 \\
\hline$N, N$-Dimethylacetamide $\mathrm{c}^{\mathrm{c}}$ & $293-364$ & 20.06 & 203.78 & 1.60 \\
\hline$N$-Methyl-2-pyrrolidone ${ }^{\mathrm{a}}$ & $298-363$ & 14.13 & 212.27 & 1.50 \\
\hline$N$-Methyl-2-pyrrolidone ${ }^{\mathrm{c}}$ & $296-363$ & 12.94 & 232.83 & 1.35 \\
\hline Dimethyl sulfoxide ${ }^{c}$ & $304-362$ & 0.3166 & 1926.48 & 1.35 \\
\hline
\end{tabular}

${ }^{\mathrm{a}}$ Values of the coefficients and mean relative deviations were taken from $\mathrm{Ma}$ and Xia. ${ }^{80}$

${ }^{b}$ Values of the coefficients and mean relative deviation were taken from Dian-Qing et al. ${ }^{82}$

${ }^{\mathrm{c}}$ Values of the coefficients and mean relative deviations were taken from $\mathrm{Ma}$ and Chen. ${ }^{121}$

solubility of 1,4-benzenedicarboxylic acid in dimethyl sulfoxide. Dian-Qing et al. ${ }^{82}$ conducted solubility measurements in $N, N$-dimethylformamide. The internal consistency of the nine datasets was assessed by curve-fitting the measured mole fraction solubility data to the Buchowski $\lambda \mathrm{h}$ model [Eq. (9)]. The values of the equation coefficients $(\lambda$ and $h)$ are given in Table 20, along with the mean relative deviation. The large deviation between observed and back-calculated values noted in the 1,4-benzenedicarboxylic acid-ethanoic acid system is likely due to the extremely small mole fraction solubilities.

Li et al. ${ }^{84}$ also determined the solubility of 1,4-benzenedicarboxylic acid in $\mathrm{N}$-methyl-2-pyrrolidone using a synthetic method with laser monitoring to determine when the last amount of solid solute dissolved. The authors employed a polynomial expression in temperature

$$
\begin{aligned}
x_{1}= & 0.8315+0.00602 T+0.09145 \times 10^{-4} T^{2} \\
& +0.06873 \times 10^{-7} T^{3}
\end{aligned}
$$

to represent the measured mole fraction solubility data from 297 to $332 \mathrm{~K}$. The root-mean-square deviation between the observed $x_{1}$ data and calculated values from Eq. (38) was on the order of 0.0007 mole fraction. While there is no theoretical basis for this type of polynomial representation in temperature, expressions like Eq. (38) do provide a convenient means for researchers to describe the observed solubility behavior at different temperatures. Wang et al. ${ }^{122}$ correlated the molar solubility of 1,4-benzenedicarboxylic acid in dimethyl sulfoxide (from 302 to $373 \mathrm{~K}$ ) and in $N, N$-dimethylformamide (from 302 to $373 \mathrm{~K}$ ) using

$$
\begin{aligned}
c_{1}(\text { in DMSO })= & -59.10+0.5414 T-1.648 \times 10^{-3} T^{2} \\
& +1.714 \times 10^{-6} T^{3} \\
c_{1}(\text { in DMF })= & -0.2047+0.002860 T-0.826 \times 10^{-5} T^{2} \\
& +1.740 \times 10^{-8} T^{3},
\end{aligned}
$$

and similar third-degree polynomial equations. 
The experimental solubility data for 1,4-benzenedicarboxylic acid in the different organic solvents are in Secs. 13.213.7 .

\subsection{1,4-Benzenedicarboxylic acid solubility data in aromatic hydrocarbons}

\section{Components:}

(1) 1,4-Benzenedicarboxylic acid $\mathrm{C}_{8} \mathrm{H}_{6} \mathrm{O}_{4}$; [100-21-0]

(2) Methylbenzene; $\mathrm{C}_{7} \mathrm{H}_{8} ;[108-88-8]$

\begin{tabular}{ll}
\hline Variables: & Prepared by: \\
Temperature & W. E. Acree, Jr. \\
\hline
\end{tabular}

Experimental Values

\begin{tabular}{lcc}
\hline \hline$T / \mathrm{K}$ & $x_{2}{ }^{\mathrm{a}}$ & $x_{1}{ }^{\mathrm{b}}$ \\
\hline 485.2 & 0.9998 & 0.000230 \\
494.2 & 0.9997 & 0.000319 \\
500.2 & 0.9996 & 0.000431 \\
\hline
\end{tabular}

${ }^{\mathrm{a}} x_{2}$ : mole fraction of component 2 in the saturated solution.

${ }^{\mathrm{b}} x_{1}$ : mole fraction solubility of the solute.

\section{Auxiliary Information}

\section{Method/Apparatus/Procedure:}

Solubilities were determined by placing known amounts of solute and solvent into a glass tube, which was then sealed and submerged in a constanttemperature bath. The temperature of the bath was increased at a rate of $1 \mathrm{~K} /$ $10 \mathrm{~min}$, and the tube was shaken until all of the solute dissolved. The temperature at which all of the solute dissolved was taken to be the solid-liquid equilibrium temperature.

Source and Purity of Chemicals:

(1) $99.8 \%$, Chemical Source not specified, no purification details were provided.

(2) Purity not given, Chemical source not given, no purification details were provided.

Estimated Error:

Temperature: $\pm 0.05 \mathrm{~K}$.

$x_{1}: \pm 5 \%$ (relative error)

\subsection{1,4-Benzenedicarboxylic acid solubility data in esters}

\begin{tabular}{|c|c|}
\hline $\begin{array}{l}\text { Components: } \\
\text { (1) 1,4-Benzenedicarboxylic acid; } \\
\mathrm{C}_{8} \mathrm{H}_{6} \mathrm{O}_{4} ;[100-21-0] \\
\text { (2) Isobutyl ethanoate; } \mathrm{C}_{6} \mathrm{H}_{12} \mathrm{O}_{2} \text {; } \\
\text { [110-19-0] }\end{array}$ & $\begin{array}{l}\text { Original Measurements: } \\
{ }^{60} \text { S. Zhao, X. Chen, Q. Dai, and L. } \\
\text { Wang, J. Chem. Eng. Data 56, } 2399 \\
(2011) \text {. }\end{array}$ \\
\hline $\begin{array}{l}\text { Variables: } \\
\text { Temperatur }\end{array}$ & Preparec \\
\hline
\end{tabular}

Experimental Values

\begin{tabular}{lcc}
\hline \hline$T / \mathrm{K}$ & $x_{2}{ }^{\mathrm{a}}$ & $x_{1}{ }^{\mathrm{b}}$ \\
\hline 305.15 & 0.9999 & 0.0000238 \\
308.15 & 0.9999 & 0.0000257 \\
313.15 & 0.9999 & 0.0000322 \\
318.15 & 0.9999 & 0.0000341 \\
323.15 & 0.9999 & 0.0000386 \\
328.15 & 0.9999 & 0.0000578 \\
333.15 & 0.9999 & 0.0000707 \\
338.15 & 0.9999 & 0.0000825 \\
343.15 & 0.9999 & 0.0000891 \\
348.15 & 0.9999 & 0.0001043
\end{tabular}

${ }^{a} x_{2}$ : mole fraction of component 2 in the saturated solution.

${ }^{\mathrm{b}} x_{1}$ : mole fraction solubility of the solute.

\section{Auxiliary Information}

\section{Method/Apparatus/Procedure:}

Thermostated temperature controller, electromagnetic stirrer, analytical balance, an ultraviolet/visible spectrophotometer.

Experimental solubilities were determined by a static method. A sealed flask containing excess solute and solvent was allowed to equilibrate at constant temperature with continuous stirring for $2 \mathrm{~h}$. The stirring was stopped and the excess solid was permitted to settle to the bottom of the flask. Aliquots of the saturated solution were removed, diluted quantitatively with isobutyl ethanoate, and the absorbance recorded at $252 \mathrm{~nm}$. The solubility was calculated based on the Beer-Lambert law using measured absorbances for standard solutions of known concentration.

Source and Purity of Chemicals:

(1) Analytical Reagent grade, Sinopharm Chemical Reagent Co. Ltd., China, was used as received.

(2) Analytical Reagent grade, Sinopharm Chemical Reagent Co. Ltd., China, was used as received.

\section{Estimated Error:}

Temperature: $\pm 0.05 \mathrm{~K}$

$x_{1}: \pm 0.000002$

\subsection{1,4-Benzenedicarboxylic acid solubility data in ethers}

\begin{tabular}{|c|c|}
\hline $\begin{array}{l}\text { Components: } \\
\text { (1) 1,4-Benzenedicarboxylic acid; } \\
\left.\mathrm{C}_{8} \mathrm{H}_{6} \mathrm{O}_{4} ; \text {; } 100-21-0\right] \\
\text { (2) Tetrahydrofuran; } \mathrm{C}_{4} \mathrm{H}_{8} \mathrm{O} \text {; } \\
\text { [109-99-9] }\end{array}$ & $\begin{array}{l}\text { Original Measurements: } \\
{ }^{63} \text { C. K. Hancock, J. N. Pawloski, and J. } \\
\text { P. Idoux, J. Org. Chem. 32, } 1931 \\
\text { (1967). }\end{array}$ \\
\hline $\begin{array}{l}\text { Variables: } \\
T / \mathrm{K}=303.15\end{array}$ & $\begin{array}{l}\text { Prepared by: } \\
\text { W. E. Acree, Jr. }\end{array}$ \\
\hline
\end{tabular}

\section{Experimental Values}

\begin{tabular}{lc}
\hline \hline$x_{2}{ }^{\mathrm{a}}$ & $x_{1}{ }^{\mathrm{b}}$ \\
\hline 0.9988 & 0.0012 \\
\hline${ }^{\mathrm{a}} x_{2}:$ mole fraction of component 2 in the saturated solution. & \\
${ }^{\mathrm{b}} x_{1}:$ mole fraction solubility of the solute. &
\end{tabular}




\section{Auxiliary Information}

\section{Method/Apparatus/Procedure:}

Constant-temperature bath, Soxhlet thimble, conical flask, and analytical balance.

Excess solute and solvent were placed in a conical flask and allowed to equilibrate for several days at constant temperature. Attainment of equilibrium was verified by several repetitive measurements and by approaching equilibrium from supersaturation. Aliquots of saturated solutions were transferred using a Soxhlet thimble equipped with a one-hole cork stopper and an inverted U-shaped delivery tube to a second ground-glass stoppered flask suspended in the $303 \mathrm{~K}$ water bath. Successive portions of the contents were evaporated at room temperature in a tared aluminum foil weighing dish under a bell jar through which a slow stream of dry air was passed. The second flask plus unused saturated solution and the aluminum foil dish plus residue were weighed. The saturation solubility of the solute was calculated from the recorded mass data and molar masses of the solute and solvent.

\section{Source and Purity of Chemicals:}

(1) Purity not given, Chemical source not specified, was recrystallized several times from aqueous-ethanol mixture, and then dried under vacuum over phosphorous pentoxide.

(2) Practical grade, Chemical source not specified, stored over sodium hydroxide pellets for $24 \mathrm{~h}$, and then passed through $2 \times 70-\mathrm{cm}$ chromatographic adsorption columns containing activated alumina. After this treatment, the purified solvent was stored over copper in a nitrogen atmosphere.

\section{Estimated Error:}

Temperature: $\pm 0.02 \mathrm{~K}$.

$x_{1}: \pm 7.8 \%$ (relative error).

\begin{tabular}{|c|c|}
\hline $\begin{array}{l}\text { Components: } \\
\text { (1) 1,4-Benzenedicarboxylic acid; } \\
\mathrm{C}_{8} \mathrm{H}_{6} \mathrm{O}_{4} ;[100-21-0] \\
\text { (2) Tetrahydrofuran; } \mathrm{C}_{4} \mathrm{H}_{8} \mathrm{O} \text {; } \\
\text { [109-99-9] }\end{array}$ & $\begin{array}{l}\text { Original Measurements: } \\
{ }^{109} \text { Y.-K. Che, Y.-X. Qu, and S. Wang, } \\
\text { J. Chem. Eng. Data 54, } 3130 \text { (2009). }\end{array}$ \\
\hline $\begin{array}{l}\text { Variables: } \\
\text { Temperature }\end{array}$ & $\begin{array}{l}\text { Prepared by: } \\
\text { W. E. Acree Jr }\end{array}$ \\
\hline
\end{tabular}

Experimental Values

\begin{tabular}{lcc}
\hline \hline$T / \mathrm{K}$ & $x_{2}{ }^{\mathrm{a}}$ & $x_{1}{ }^{\mathrm{b}}$ \\
\hline 293.15 & 0.9991 & 0.0008816 \\
303.15 & 0.9990 & 0.0009952 \\
313.45 & 0.9989 & 0.001143 \\
323.35 & 0.9986 & 0.001398 \\
329.35 & 0.9984 & 0.001640 \\
\hline
\end{tabular}

${ }^{\mathrm{a}} x_{2}$ : mole fraction of component 2 in the saturated solution.

${ }^{b} x_{1}$ : mole fraction solubility of the solute.

\section{Auxiliary Information}

\section{Method/Apparatus/Procedure:}

Equilibrium jacketed glass vessel, thermostated circulating water bath, electromagnetic stirrer, analytical balance, laser monitoring system.

Experimental solubilities were determined by a synthetic method. Preweighed amounts of solute and solvent and were placed in an equilibrium vessel, which was connected to a circulating constant-temperature water bath. The solution was stirred and small amounts of solute were incrementally added until no further solid dissolved. The dissolution of the solid was determined using laser monitoring. The total amount of solute dissolved was recorded. Experimental measurement was repeated three times.
Source and Purity of Chemicals:

(1) $99 \%$, Sinopharm Chemical Reagent Company, Ltd., China, no purification details were provided.

(2) $99 \%$, Kermel Chemical Reagents Development Centre, China, no purification details were provided.

\section{Estimated Error:}

Temperature: $\pm 0.05 \mathrm{~K}$

$x_{1}: \pm 0.5 \%$ (relative error).

\begin{tabular}{|c|c|}
\hline $\begin{array}{l}\text { Components: } \\
\text { (1) 1,4-Benzenedicarboxylic acid; } \\
\mathrm{C}_{8} \mathrm{H}_{6} \mathrm{O}_{4} ;[100-21-0] \\
\text { (2) 1,4-Dioxane; } \mathrm{C}_{4} \mathrm{H}_{8} \mathrm{O}_{2} \text {; } \\
\text { [123-91-1] }\end{array}$ & $\begin{array}{l}\text { Original Measurements: } \\
{ }^{63} \text { C. K. Hancock, J. N. Pawloski, } \\
\text { and J. P. Idoux, J. Org. Chem. 32, } \\
1931 \text { (1967). }\end{array}$ \\
\hline $\begin{array}{l}\text { Variables: } \\
T / \mathrm{K}=303.15\end{array}$ & $\begin{array}{l}\text { Prepared by: } \\
\text { W. E. Acree, Jr. }\end{array}$ \\
\hline
\end{tabular}

Experimental Values

\begin{tabular}{lc}
\hline \hline$x_{2}{ }^{\mathrm{a}}$ & $x_{1}{ }^{\mathrm{b}}$ \\
\hline 0.9996 & 0.00042 \\
\hline${ }^{\mathrm{a}} x_{2}:$ mole fraction of component 2 in the saturated solution. \\
${ }^{\mathrm{b}} x_{1}:$ mole fraction solubility of the solute.
\end{tabular}

\section{Auxiliary Information}

\section{Method/Apparatus/Procedure:}

Constant-temperature bath, Soxhlet thimble, conical flask, and analytical balance.

Excess solute and solvent were placed in a conical flask and allowed to equilibrate for several days at constant temperature. Attainment of equilibrium was verified by several repetitive measurements and by approaching equilibrium from supersaturation. Aliquots of saturated solutions were transferred using a Soxhlet thimble equipped with a one-hole cork stopper and an inverted U-shaped delivery tube to a second ground-glass stoppered flask suspended in the $303 \mathrm{~K}$ water bath. Successive portions of the contents were evaporated at room temperature in a tared aluminum foil weighing dish under a bell jar through which a slow stream of dry air was passed. The second flask plus unused saturated solution and the aluminum foil dish plus residue were weighed. The saturation solubility of the solute was calculated from the recorded mass data and molar masses of the solute and solvent.

\section{Source and Purity of Chemicals:}

(1) Purity not given, Chemical source not specified, was recrystallized several times from aqueous-ethanol mixture, and then dried under vacuum over phosphorous pentoxide.

(2) Practical grade, Chemical source not specified, stored over sodium hydroxide pellets for $24 \mathrm{~h}$, and then passed through $2 \times 70-\mathrm{cm}$ chromatographic adsorption columns containing activated alumina. After this treatment, the purified solvent was stored over copper in a nitrogen atmosphere.

\section{Estimated Error:}

Temperature: $\pm 0.02 \mathrm{~K}$

$x_{1}: \pm 4.5 \%$ (relative error).

\section{Components:}

(1) 1,4-Benzenedicarboxylic acid;

$\mathrm{C}_{8} \mathrm{H}_{6} \mathrm{O}_{4}$; [100-21-0]

(2) 1,4-Dioxane; $\mathrm{C}_{4} \mathrm{H}_{8} \mathrm{O}_{2}$; [123-91-1]

Variables:

Temperature

Prepared by:

W. E. Acree, Jr. 
Experimental Values

\begin{tabular}{lcc}
\hline \hline$T / \mathrm{K}$ & $x_{2}{ }^{\mathrm{a}}$ & \multicolumn{1}{c}{$x_{1}{ }^{\mathrm{b}}$} \\
\hline 397.2 & 0.9966 & 0.00337 \\
433.7 & 0.9933 & 0.00672 \\
471.7 & 0.9869 & 0.0131 \\
\hline
\end{tabular}

${ }^{\mathrm{a}} x_{2}$ : mole fraction of component 2 in the saturated solution.

${ }^{\mathrm{b}} x_{1}$ : mole fraction solubility of the solute.

\section{Auxiliary Information}

\section{Method/Apparatus/Procedure:}

Solubilities were determined by placing known amounts of solute and solvent into a glass tube, which was then sealed and submerged in a constanttemperature bath. The temperature of the bath was increased at a rate of $1 \mathrm{~K} /$ $10 \mathrm{~min}$, and the tube was shaken until all of the solute dissolved. The temperature at which all of the solute dissolved was taken to be the solid-liquid equilibrium temperature.

\section{Source and Purity of Chemicals:}

(1) $99.8 \%$, Chemical source not specified, no purification details were provided.

(2) Purity not given, Chemical source not given, no purification details were provided.

\section{Estimated Error:}

Temperature: $\pm 0.05 \mathrm{~K}$.

$x_{1}: \pm 5 \%$ (relative error).

\begin{tabular}{|c|c|}
\hline $\begin{array}{l}\text { Components: } \\
\text { (1) } 1,4-\text {-Benzenedicarboxylic acid; } \\
\mathrm{C}_{8} \mathrm{H}_{6} \mathrm{O}_{4} \text {; [100-21-0] } \\
\text { (2) 1,2-Diethoxyethane; } \mathrm{C}_{6} \mathrm{H}_{14} \mathrm{O}_{2} \text {; } \\
\text { [629-14-1] }\end{array}$ & $\begin{array}{l}\text { Original Measurements: } \\
{ }^{109} \text { Y.-K. Che, Y.-X. Qu, and S. Wang, } \\
\text { J. Chem. Eng. Data 54, } 3130 \text { (2009). }\end{array}$ \\
\hline $\begin{array}{l}\text { Variables: } \\
\text { Temperature }\end{array}$ & $\begin{array}{l}\text { Prepared by: } \\
\text { W. E. Acree, Jr. }\end{array}$ \\
\hline
\end{tabular}

Experimental Values

\begin{tabular}{lcc}
\hline \hline$T / \mathrm{K}$ & $x_{2}{ }^{\mathrm{a}}$ & $x_{1}{ }^{\mathrm{b}}$ \\
\hline 293.15 & 0.9998 & 0.0002137 \\
303.30 & 0.9997 & 0.0002513 \\
313.25 & 0.9997 & 0.0002936 \\
323.35 & 0.9997 & 0.0003439 \\
333.65 & 0.9996 & 0.0004034 \\
343.55 & 0.9995 & 0.0004770
\end{tabular}

${ }^{\mathrm{a}} x_{2}$ : mole fraction of component 2 in the saturated solution.

${ }^{\mathrm{b}} x_{1}$ : mole fraction solubility of the solute.

\section{Auxiliary Information}

\section{Method/Apparatus/Procedure:}

Equilibrium jacketed glass vessel, thermostated circulating water bath, electromagnetic stirrer, analytical balance, laser monitoring system.

Experimental solubilities were determined by a synthetic method. Preweighed amounts of solute and solvent and were placed in an equilibrium vessel, which was connected to a circulating constant-temperature water bath. The solution was stirred and small amounts of solute were incrementally added until no further solid dissolved. The dissolution of the solid was determined using laser monitoring. The total amount of solute dissolved was recorded. Experimental measurement was repeated three times.
Source and Purity of Chemicals:

(1) $99 \%$, Sinopharm Chemical Reagent Company, Ltd., China, no purification details were provided.

(2) $99+\%$, Tianjin Guangfu Fine Chemical Research Institute, China, no purification details were provided.

Estimated Error:

Temperature: $\pm 0.05 \mathrm{~K}$

$x_{1}: \pm 0.5 \%$ (relative error)

\begin{tabular}{|c|c|}
\hline $\begin{array}{l}\text { Components: } \\
\text { (1) } 1,4-\text { Benzenedicarboxylic acid; } \\
\mathrm{C}_{8} \mathrm{H}_{6} \mathrm{O}_{4} ;[100-21-0] \\
\text { (2) Methoxybenzene; } \mathrm{C}_{7} \mathrm{H}_{8} \mathrm{O} \text {; } \\
{[100-66-3]}\end{array}$ & $\begin{array}{l}\text { Original Measurements: } \\
{ }^{118} \text { J. J. Harper and P. Janik, J. Chem } \\
\text { Eng. Data 15, } 439 \text { (1970). }\end{array}$ \\
\hline $\begin{array}{l}\text { Variables: } \\
\text { Temperature }\end{array}$ & $\begin{array}{l}\text { Prepared by: } \\
\text { W. E. Acree, Jr. }\end{array}$ \\
\hline
\end{tabular}

Experimental Values

\begin{tabular}{lcc}
\hline \hline$T / \mathrm{K}$ & $x_{2}{ }^{\mathrm{a}}$ & $x_{1}{ }^{\mathrm{b}}$ \\
\hline 488.2 & 0.9905 & 0.00947 \\
513.2 & 0.9775 & 0.0225 \\
518.7 & 0.9732 & 0.0268
\end{tabular}

${ }^{\mathrm{a}} x_{2}$ : mole fraction of component 2 in the saturated solution.

${ }^{b} x_{1}$ : mole fraction solubility of the solute.

\section{Auxiliary Information}

\section{Method/Apparatus/Procedure:}

Solubilities were determined by placing known amounts of solute and solvent into a glass tube, which was then sealed and submerged in a constanttemperature bath. The temperature of the bath was increased at a rate of $1 \mathrm{~K} / 10$ min, and the tube was shaken until all of the solute dissolved. The temperature at which all of the solute dissolved was taken to be the solid-liquid equilibrium temperature.

Source and Purity of Chemicals:

(1) $99.8 \%$, Chemical source not specified, no purification details were provided.

(2) Purity not given, Chemical source not given, no purification details were provided.

\section{Estimated Error:}

Temperature: $\pm 0.05 \mathrm{~K}$.

$x_{1}: \pm 5 \%$ (relative error).

\subsection{1,4-Benzenedicarboxylic acid solubility data in alcohols}

\begin{tabular}{|c|c|}
\hline $\begin{array}{l}\text { Components: } \\
\text { (1) } 1,4-\text {-Benzenedicarboxylic acid; } \\
\mathrm{C}_{8} \mathrm{H}_{6} \mathrm{O}_{4} ;[100-21-0] \\
\text { (2) Methanol; } \mathrm{CH}_{4} \mathrm{O} ;[67-56-1]\end{array}$ & $\begin{array}{l}\text { Original Measurements: } \\
\text { '114G. N. Friedlin and V. N. Davydov, } \\
\text { Zh. Prikl. Khim. 35, } 2530 \text { (1962). }\end{array}$ \\
\hline $\begin{array}{l}\text { Variables: } \\
\text { Temperature }\end{array}$ & $\begin{array}{l}\text { Prepared by: } \\
\text { W. E. Acree, Jr. }\end{array}$ \\
\hline
\end{tabular}




\section{Experimental Values}

Authors report a solubility of $0.141 \mathrm{~g}$ of solute per $100 \mathrm{ml}$ of solvent at $293 \mathrm{~K}$, and a solubility of $0.260 \mathrm{~g}$ of solute per $100 \mathrm{ml}$ of solvent at $338 \mathrm{~K}$.

\section{Auxiliary Information}

Method/Apparatus/Procedure:

Experimental details were not provided.

Source and Purity of Chemicals:

(1) Purity not given, Chemical source not given, no purification details were provided.

(2) Purity not given, Chemical source not given, no purification details were provided.

Estimated Error:

Temperature: No information given.

Solubility: $\pm 1 \%$ (relative error).

\begin{tabular}{ll}
\hline \hline Components: & Original Measurements: \\
$(1) 1$ 1,4-Benzenedicarboxylic acid; & ${ }^{115}$ G. N. Freidlin and V. N. Davydov, \\
$\mathrm{C}_{8} \mathrm{H}_{6} \mathrm{O}_{4} ;[100-21-0]$ & Dopov. Akad. Nauk Ukr. RSR 622 \\
(2) Methanol; $\mathrm{CH}_{4} \mathrm{O} ;[67-56-1]$ & $(1962)$. \\
\hline Variables: & Prepared by: \\
Temperature & W. E. Acree, Jr. \\
\hline
\end{tabular}

Experimental Values

\begin{tabular}{lcc}
\hline \hline$T / \mathrm{K}$ & $x_{2}{ }^{\mathrm{a}}$ & $x_{1}{ }^{\mathrm{b}}$ \\
\hline 293 & 0.9997 & 0.000343 \\
313 & 0.9996 & 0.000438 \\
323 & 0.9995 & 0.000480 \\
330 & 0.9995 & 0.000532 \\
337 & 0.9994 & 0.000634 \\
${ }^{\mathrm{a}} x_{2}:$ mole fraction of component 2 in the saturated solution. \\
${ }^{\mathrm{b}} x_{1}:$ mole fraction solubility of the solute. Solubility data were expressed as \\
grams of solute per 100 g of solvent. Mole fraction values calculated by \\
compiler.
\end{tabular}

\section{Auxiliary Information}

Method/Apparatus/Procedure:

Experimental details were not provided.

Source and Purity of Chemicals:

(1) Purity not given, Chemical source not given, no purification details were provided.

(2) Purity not given, Chemical source not given, no purification details were provided.

Estimated Error:

Temperature: No information given.

$x_{1}: \pm 2 \%$ (relative error).

\begin{tabular}{ll}
\hline \hline Components: & Original Measurements: \\
(1) 1,4-Benzenedicarboxylic acid; & ${ }^{115}$ G. N. Freidlin and V. N. Davydov, \\
$\mathrm{C}_{8} \mathrm{H}_{6} \mathrm{O}_{4} ;[100-21-0]$ & $\begin{array}{l}\text { Dopov. Akad. Nauk Ukr. RSR 622 } \\
\text { (2) 1-Propanol; } \mathrm{C}_{3} \mathrm{H}_{8} \mathrm{O} ;[71-23-8]\end{array}$ \\
\hline Variables: & Prepared by: \\
Temperature & W. E. Acree, Jr. \\
\hline
\end{tabular}

Experimental Values

\begin{tabular}{lcc}
\hline \hline$T / \mathrm{K}$ & $x_{2}{ }^{\mathrm{a}}$ & $x_{1}{ }^{\mathrm{b}}$ \\
\hline 293 & 0.9996 & 0.000401 \\
323 & 0.9992 & 0.000813 \\
343 & 0.9984 & 0.001571 \\
370 & 0.9983 & 0.001734 \\
\hline
\end{tabular}

${ }^{\mathrm{a}} x_{2}$ : mole fraction of component 2 in the saturated solution.

${ }^{b} x_{1}$ : mole fraction solubility of the solute. Solubility data were expressed as grams of solute per $100 \mathrm{~g}$ of solvent. Mole fraction values calculated by compiler.

\section{Auxiliary Information}

\section{Method/Apparatus/Procedure:}

Experimental details were not provided.

Source and Purity of Chemicals:

(1) Purity not given, Chemical source not given, no purification details were provided.

(2) Purity not given, Chemical source not given, no purification details were provided.

\section{Estimated Error:}

Temperature: No information given.

$x_{1}: \pm 2 \%$ for $293 \mathrm{~K}$ value, less than $2 \%$ at the other temperatures (relative error).

\begin{tabular}{|c|c|}
\hline $\begin{array}{l}\text { Components: } \\
\text { (1) 1,4-Benzenedicarboxylic acid; } \\
\mathrm{C}_{8} \mathrm{H}_{6} \mathrm{O}_{4} ;[100-21-0] \\
\text { (2) 2-Propanol; } \mathrm{C}_{3} \mathrm{H}_{8} \mathrm{O} ;[67-63-0]\end{array}$ & $\begin{array}{l}\text { Original Measurements: } \\
{ }^{91} \text { J. Bradil, J. Malek, and V. Bazant, } \\
\text { Chem. Prumysl 20, } 117 \text { (1970). }\end{array}$ \\
\hline Variables: & Prepared by: \\
\hline Temperature & W. E. Acree, Jr. \\
\hline
\end{tabular}

Experimental Values

\begin{tabular}{lcc}
\hline \hline$T / \mathrm{K}$ & $x_{2}{ }^{\mathrm{a}}$ & $x_{1}{ }^{\mathrm{b}}$ \\
\hline 273.2 & 0.9998 & 0.000172 \\
298.2 & 0.9996 & 0.000398 \\
323.2 & 0.9994 & 0.000561 \\
343.2 & 0.9992 & 0.000844 \\
\hline
\end{tabular}

${ }^{\mathrm{a}} x_{2}$ : mole fraction of component 2 in the saturated solution.

${ }^{\mathrm{b}} x_{1}$ : mole fraction solubility of the solute. Solubility data were reported in terms of grams of dissolved solute per $100 \mathrm{~g}$ of solution. Mole fraction solubilities calculated by the compiler. 


\section{Auxiliary Information}

\section{Method/Apparatus/Procedure:}

Excess solute and solvent and were placed in a flask and equilibrated at constant temperature with stirring. After 90 min the stirring was discontinued, and the solution was allowed to stand for $30 \mathrm{~min}$ to allow the undissolved solid to settle to the bottom of the flask. An aliquot of the saturated solution was removed by pipette fitted with a filtering device. The mass of the aliquot was determined by weighing. The concentration of the dissolved solute was determined by titration using sodium hydroxide, with phenolphthalein being the endpoint indicator.

Source and Purity of Chemicals:

(1) Purity not given, Synthesized in authors' laboratory, prepared from dimethyl-1,4-benzenedicarboxylate.

(2) Purity not given, Lachema, Brono, Czechoslovakia, was distilled before use.

Estimated Error:

Temperature: $\pm 0.1 \mathrm{~K}$.

$x_{1}: \pm 1 \%$ (relative error).

\subsection{1,4-Benzenedicarboxylic acid solubility data in ketones}

\begin{tabular}{|c|c|}
\hline $\begin{array}{l}\text { Components: } \\
\text { (1) } 1,4-\text { Benzenedicarboxylic acid; } \\
\mathrm{C}_{8} \mathrm{H}_{6} \mathrm{O}_{4} ;[100-21-0] \\
\text { (2) 3-Pentanone; } \mathrm{C}_{5} \mathrm{H}_{10} \mathrm{O} \text {; } \\
\text { [99-22-0] }\end{array}$ & $\begin{array}{l}\text { Original Measurements: } \\
{ }^{118} \text { J. J. Harper and P. Janik, J. Chem. } \\
\text { Eng. Data 15, } 439 \text { (1970). }\end{array}$ \\
\hline $\begin{array}{l}\text { Variables: } \\
\text { Temperature }\end{array}$ & $\begin{array}{l}\text { Prepared by: } \\
\text { W. E. Acree, Jr. }\end{array}$ \\
\hline
\end{tabular}

Experimental Values

\begin{tabular}{lcc}
\hline \hline$T / \mathrm{K}$ & $x_{2}{ }^{\mathrm{a}}$ & $x_{1}{ }^{\mathrm{b}}$ \\
\hline 417.2 & 0.9908 & 0.00919 \\
448.2 & 0.9822 & 0.0178 \\
458.7 & 0.9788 & 0.0212
\end{tabular}

${ }^{\mathrm{a}} x_{2}$ : mole fraction of component 2 in the saturated solution.

${ }^{\mathrm{b}} x_{1}$ : mole fraction solubility of the solute.

\section{Auxiliary Information}

\section{Method/Apparatus/Procedure:}

Solubilities were determined by placing known amounts of solute and solvent into a glass tube, which was then sealed and submerged in a constant-

temperature bath. The temperature of the bath was increased at a rate of $1 \mathrm{~K} /$ $10 \mathrm{~min}$, and the tube was shaken until all of the solute dissolved. The temperature at which all of the solute dissolved was taken to be the solid-liquid equilibrium temperature.

Source and Purity of Chemicals:

(1) $99.8 \%$, Chemical source not specified, no purification details were provided.

(2) Purity not given, Chemical source not given, no purification details were provided.

\section{Estimated Error:}

Temperature: $\pm 0.05 \mathrm{~K}$.

$x_{1}: \pm 5 \%$ (relative error).

\begin{tabular}{|c|c|}
\hline $\begin{array}{l}\text { Components: } \\
\text { (1) 1,4-Benzenedicarboxylic acid; } \\
\mathrm{C}_{8} \mathrm{H}_{6} \mathrm{O}_{4} ;[100-21-0] \\
\text { (2) Cyclohexanone; } \mathrm{C}_{6} \mathrm{H}_{10} \mathrm{O} \text {; } \\
{[108-94-1]}\end{array}$ & $\begin{array}{l}\text { Original Measurements: } \\
{ }^{109} \text { Y.-K. Che, Y.-X. Qu, and S. Wang, } \\
\text { J. Chem. Eng. Data 54, } 3130 \text { (2009). }\end{array}$ \\
\hline $\begin{array}{l}\text { Variables: } \\
\text { Temperature }\end{array}$ & $\begin{array}{l}\text { Prepared by: } \\
\text { W. E. Acree, Jr. }\end{array}$ \\
\hline
\end{tabular}

Experimental Values

\begin{tabular}{lcc}
\hline \hline$T / \mathrm{K}$ & $x_{2}{ }^{\mathrm{a}}$ & $x_{1}{ }^{\mathrm{b}}$ \\
\hline 293.15 & 0.9998 & 0.0001671 \\
303.15 & 0.9998 & 0.0001834 \\
313.15 & 0.9998 & 0.0002311 \\
323.30 & 0.9997 & 0.0003019 \\
333.40 & 0.9996 & 0.0003855 \\
343.85 & 0.9995 & 0.0005302 \\
\hline${ }^{\mathrm{a}} x_{2}:$ mole fraction of component 2 in the saturated solution. \\
${ }^{\mathrm{b}}{ }_{X_{1}: \text { mole fraction solubility of the solute. }}$
\end{tabular}

Auxiliary Information

Method/Apparatus/Procedure:

Equilibrium jacketed glass vessel, thermostated circulating water bath, electromagnetic stirrer, analytical balance, laser monitoring system. Experimental solubilities were determined by a synthetic method. Preweighed amounts of solute and solvent and were placed in an equilibrium vessel, which was connected to a circulating constant-temperature water bath. The solution was stirred and small amounts of solute were incrementally added until no further solid dissolved. The dissolution of the solid was determined using laser monitoring. The total amount of solute dissolved was recorded. Experimental measurement was repeated three times.

Source and Purity of Chemicals:

(1) $99 \%$, Sinopharm Chemical Reagent Company, Ltd., China, no purification details were provided.

(2) 99+\%, Tianjin Guangfu Fine Chemical Research Institute, China, no purification details were provided.

\section{Estimated Error:}

Temperature: $\pm 0.05 \mathrm{~K}$.

$x_{1}: \pm 0.5 \%$ (relative error).

\begin{tabular}{|c|c|}
\hline $\begin{array}{l}\text { Components: } \\
\text { (1) 1,4-Benzenedicarboxylic acid; } \\
\mathrm{C}_{8} \mathrm{H}_{6} \mathrm{O}_{4} ;[100-21-0] \\
\text { (2) Acetophenone; } \mathrm{C}_{8} \mathrm{H}_{8} \mathrm{O} \text {; } \\
\text { [98-86-2] }\end{array}$ & $\begin{array}{l}\text { Original Measurements: } \\
{ }^{109} \text { Y.-K. Che, Y.-X. Qu, and S. Wang, } \\
\text { J. Chem. Eng. Data 54, } 3130 \text { (2009). }\end{array}$ \\
\hline $\begin{array}{l}\text { Variables: } \\
\text { Temperature }\end{array}$ & $\begin{array}{l}\text { Prepared by: } \\
\text { W. E. Acree, Jr. }\end{array}$ \\
\hline
\end{tabular}


Experimental Values

\begin{tabular}{lcc}
\hline \hline$T / \mathrm{K}$ & $x_{2}{ }^{\mathrm{a}}$ & $x_{1}{ }^{\mathrm{b}}$ \\
\hline 303.15 & 0.9999 & 0.0000107 \\
313.15 & 0.9999 & 0.0000187 \\
323.15 & 0.9999 & 0.0000347 \\
332.85 & 0.9999 & 0.0000617 \\
343.30 & 0.9999 & 0.0001158 \\
\hline
\end{tabular}

${ }^{\mathrm{a}} x_{2}$ : mole fraction of component 2 in the saturated solution.

${ }^{\mathrm{b}} x_{1}$ : mole fraction solubility of the solute.

\section{Auxiliary Information}

\begin{abstract}
Method/Apparatus/Procedure:
Equilibrium jacketed glass vessel, thermostated circulating water bath, electromagnetic stirrer, analytical balance, laser monitoring system. Experimental solubilities were determined by a synthetic method. Preweighed amounts of solute and solvent and were placed in an equilibrium vessel, which was connected to a circulating constant-temperature water bath. The solution was stirred and small amounts of solute were incrementally added until no further solid dissolved. The dissolution of the solid was determined using laser monitoring. The total amount of solute dissolved was recorded. Experimental measurement was repeated three times.
\end{abstract}

Source and Purity of Chemicals:

(1) $99 \%$, Sinopharm Chemical Reagent Company, Ltd., China, no purification details were provided.

(2) 99+\%, Tianjin Guangfu Fine Chemical Research Institute, China, no purification details were provided.

Estimated Error:

Temperature: $\pm 0.05 \mathrm{~K}$.

$x_{1}: \pm 0.5 \%$ (relative error).

\subsection{1,4-Benzenedicarboxylic acid solubility data in miscellaneous organic solvents}

\begin{tabular}{|c|c|}
\hline $\begin{array}{l}\text { Components: } \\
\text { (1) } 1,4-\text { Benzenedicarboxylic acid; } \\
\mathrm{C}_{8} \mathrm{H}_{6} \mathrm{O}_{4} ;[100-21-0] \\
\text { (2) Ethanoic acid; } \mathrm{C}_{2} \mathrm{H}_{4} \mathrm{O}_{2} \text {; } \\
\text { [64-19-7] }\end{array}$ & $\begin{array}{l}\text { Original Measurements: } \\
{ }^{80} \text { P. Ma and Q. Xia, Chin. J. Chem. } \\
\text { Eng. 9, } 39 \text { (2001). }\end{array}$ \\
\hline $\begin{array}{l}\text { Variables: } \\
\text { Temperature }\end{array}$ & $\begin{array}{l}\text { Prepared by: } \\
\text { W. E. Acree, Jr. }\end{array}$ \\
\hline
\end{tabular}

Experimental Values

\begin{tabular}{lcc}
\hline \hline$T / \mathrm{K}$ & $x_{2}{ }^{\mathrm{a}}$ & \multicolumn{1}{c}{$x_{1}{ }^{\mathrm{b}}$} \\
\hline 305.6 & 0.9999 & 0.00003904 \\
310.3 & 0.9999 & 0.00004497 \\
315.2 & 0.9999 & 0.00005495 \\
319.4 & 0.9999 & 0.00006402 \\
325.7 & 0.9999 & 0.00008026 \\
329.3 & 0.9999 & 0.00008998 \\
334.4 & 0.9999 & 0.0001053 \\
339.3 & 0.9999 & 0.0001244 \\
343.3 & 0.9999 & 0.0001433 \\
348.3 & 0.9998 & 0.0001682
\end{tabular}

\begin{tabular}{lcc}
\hline \hline$T / \mathrm{K}$ & $x_{2}{ }^{\mathrm{a}}$ & $x_{1}{ }^{\mathrm{b}}$ \\
\hline 355.0 & 0.9998 & 0.0002071 \\
357.3 & 0.9998 & 0.0002262 \\
363.1 & 0.9997 & 0.0002700 \\
\hline${ }^{\mathrm{a}} x_{2}:$ mole fraction of component 2 in the saturated solution. \\
${ }^{\mathrm{b}}{ }_{x_{1}: \text { mole fraction solubility of the solute. }}$
\end{tabular}

Auxiliary Information

Method/Apparatus/Procedure:

Titanium solid-liquid equilibrium cell, analytical balance, magnetic stirring system, temperature controlling system, and a laser monitoring system. Solubilities were determined using a dynamic method. Weighed amounts of solute and solvent were sealed in a titanium solid-liquid equilibrium cell, and the temperature slowly increased until the solid phase completely disappeared. Near the solid-liquid equilibrium temperature, the rate of temperature increase was $0.1 \mathrm{~K} / 10 \mathrm{~min}$. The disappearance of the solid solute was detected by a laser monitoring system.

Source and Purity of Chemicals:

(1) Purity not given, Chemical source not given, no purification details were provided.

(2) Purity not given, Chemical source not given, no purification details were provided.

Estimated Error:

Temperature: $\pm 0.1 \mathrm{~K}$ (estimated by compiler).

$x_{1}: \pm 10 \%$ (relative error, estimated by compiler)

\begin{tabular}{|c|c|}
\hline $\begin{array}{l}\text { Components: } \\
\text { (1) 1,4-Benzenedicarboxylic acid; } \\
\mathrm{C}_{8} \mathrm{H}_{6} \mathrm{O}_{4} ;[100-21-0] \\
\text { (2) Ethanoic acid; } \mathrm{C}_{2} \mathrm{H}_{4} \mathrm{O}_{2} \text {; } \\
\text { [64-19-7] }\end{array}$ & $\begin{array}{l}\text { Original Measurements: } \\
{ }^{119} \text { M.-M. Chen, P.-S. Ma, L. Wang, } \\
\text { and F. Chen, Acta Phys. Chim. Sin. } 20 \\
445 \text { (2004). }\end{array}$ \\
\hline $\begin{array}{l}\text { Variables: } \\
\text { Temperature }\end{array}$ & $\begin{array}{l}\text { Prepared by: } \\
\text { W. E. Acree, Jr. }\end{array}$ \\
\hline
\end{tabular}

Experimental Values

\begin{tabular}{lcl}
\hline \hline$T / \mathrm{K}$ & $x_{2}{ }^{\mathrm{a}}$ & \multicolumn{1}{c}{$x_{1}{ }^{\mathrm{b}}$} \\
\hline 311.57 & 0.9999 & 0.00000470 \\
315.15 & 0.9999 & 0.00000670 \\
319.79 & 0.9999 & 0.0000110 \\
327.95 & 0.9999 & 0.0000229 \\
333.95 & 0.9999 & 0.0000402 \\
336.55 & 0.9999 & 0.0000512 \\
337.95 & 0.9999 & 0.0000582 \\
342.25 & 0.9999 & 0.0000864 \\
350.15 & 0.9999 & 0.0001370 \\
367.75 & 0.9997 & 0.0002569 \\
383.15 & 0.9996 & 0.0004287 \\
395.55 & 0.9994 & 0.0006344 \\
405.35 & 0.9992 & 0.0008559 \\
421.95 & 0.9986 & 0.001396 \\
435.75 & 0.9979 & 0.002073 \\
444.85 & 0.9973 & 0.002673 \\
\hline${ }^{\mathrm{a}}{ }_{X_{2}: \text { mole fraction of component } 2 \text { in the saturated solution. }}$ \\
${ }^{{ }_{x_{1}}: \text { mole fraction solubility of the solute. }}$
\end{tabular}




\section{Auxiliary Information}

\section{Method/Apparatus/Procedure:}

Titanium solid-liquid equilibrium cell with quartz windows enabling laser monitoring of the inside cell contents, analytical balance, magnetic stirring system, temperature controlling system, and a laser monitoring system. Solubilities were determined using a dynamic method. Weighed amounts of solute and solvent were sealed in a titanium solid-liquid equilibrium cell, and the temperature slowly increased until the solid phase completely disappeared. The disappearance of the solid solute was detected by a laser monitoring system.

\section{Source and Purity of Chemicals:}

(1) $99.95 \%$, Chemical source given only as the factory, no purification details were provided.

(2) $99.9 \%$, Chemical source not given, authors state that ethanoic acid was purified to $99.9 \%$, but no purification details were provided.

\section{Estimated Error:}

Temperature: $\pm 0.05 \mathrm{~K}$.

$x_{1}: \pm 5 \%$ (relative error, estimated by compiler).
Source and Purity of Chemicals:

(1) $99.5 \%$, Shanghai Chemical Reagent Company, China, no purification details were provided.

(2) $99 \%$, Hangzhou Chemical Reagent Company, China, no purification details were provided.

Estimated Error:

Temperature: $\pm 0.1 \mathrm{~K}$ (estimated by compiler). $x_{1}: \pm 3 \%$ (relative error, estimated by compiler).

\begin{tabular}{|c|c|}
\hline $\begin{array}{l}\text { Components: } \\
\text { (1) 1,4-Benzenedicarboxylic acid; } \\
\mathrm{C}_{8} \mathrm{H}_{6} \mathrm{O}_{4} ;[100-21-0] \\
\text { (2) } N, N \text {-Dimethylformamide; } \\
\mathrm{C}_{3} \mathrm{H}_{7} \mathrm{NO} ;[68-12-2]\end{array}$ & $\begin{array}{l}\text { Original Measurements: } \\
{ }^{80} \text { P. Ma and Q. Xia, Chin. J. Chem. } \\
\text { Eng. 9, } 39 \text { (2001). }\end{array}$ \\
\hline $\begin{array}{l}\text { Variables: } \\
\text { Temperature }\end{array}$ & $\begin{array}{l}\text { Prepared by: } \\
\text { W. E. Acree, Jr. }\end{array}$ \\
\hline
\end{tabular}

Experimental Values

\begin{tabular}{|c|c|}
\hline $\begin{array}{l}\text { Components: } \\
\text { (1) 1,4-Benzenedicarboxylic acid; } \\
\mathrm{C}_{8} \mathrm{H}_{6} \mathrm{O}_{4} ;[100-21-0] \\
\text { (2) Ethanoic acid; } \mathrm{C}_{2} \mathrm{H}_{4} \mathrm{O}_{2} \text {; } \\
\text { [64-19-7] }\end{array}$ & $\begin{array}{l}\text { Original Measurements: } \\
{ }^{120} \text { Q. Wang, H. Xu, and X. Li, J. Chem. } \\
\text { Eng. Data 50, } 258 \text { (2005). }\end{array}$ \\
\hline $\begin{array}{l}\text { Variables: } \\
\text { Temperature }\end{array}$ & $\begin{array}{l}\text { Prepared by: } \\
\text { W. E. Acree, Jr. }\end{array}$ \\
\hline
\end{tabular}

Experimental Values

\begin{tabular}{lcc}
\hline \hline$T / \mathrm{K}$ & $x_{2}{ }^{\mathrm{a}}$ & $x_{1}{ }^{\mathrm{b}}$ \\
\hline 433.2 & 0.9978 & 0.00220 \\
443.2 & 0.9970 & 0.00296 \\
453.2 & 0.9963 & 0.00367 \\
463.2 & 0.9954 & 0.00457 \\
473.2 & 0.9943 & 0.00571 \\
483.2 & 0.9929 & 0.00711 \\
493.2 & 0.9911 & 0.00888 \\
503.2 & 0.9889 & 0.01105 \\
513.2 & 0.9862 & 0.01376
\end{tabular}

${ }^{a} x_{2}$ : mole fraction of component 2 in the saturated solution.

${ }^{b} x_{1}$ : mole fraction solubility of the solute. Solubility data are given as grams of dissolved solute per $100 \mathrm{~g}$ of solvent. Mole fraction solubilities calculated by the compiler.

\section{Auxiliary Information}

\section{Method/Apparatus/Procedure:}

Titanium solid-liquid equilibrium cell, analytical balance, and a highperformance liquid chromatographic system.

Solubilities were determined using a steady-state method. Excess solute and solvent were sealed in a titanium solid-liquid equilibrium cell and the temperature slowly increased (rate of less than $1 \mathrm{~K} / \mathrm{min}$ ) until the desired temperature was reached. Stirring was continuous during the period of increasing temperature, but was stopped when the experiment temperature was reached in order to allow the suspended particles to settle to the bottom of the container. An aliquot of the saturated solution was removed, and quickly cooled to room temperature to prevent evaporation of the solvent. The concentration of the solute in the sample was determined by a high performance liquid chromatographic method. A more detailed description of experimental apparatus and experimental methodology is given in the paper.
${ }^{a} x_{2}$ : mole fraction of component 2 in the saturated solution.

${ }^{\mathrm{b}} x_{1}$ : mole fraction solubility of the solute.

\section{Auxiliary Information}

\section{Method/Apparatus/Procedure:}

Titanium solid-liquid equilibrium cell, analytical balance, magnetic stirring system, temperature controlling system, and a laser monitoring system.

Solubilities were determined using a dynamic method. Weighed amounts of solute and solvent were sealed in a titanium solid-liquid equilibrium cell, and the temperature slowly increased until the solid phase completely disappeared. Near the solid-liquid equilibrium temperature, the rate of temperature increase was $0.1 \mathrm{~K} / 10 \mathrm{~min}$. The disappearance of the solid solute was detected by a laser monitoring system.

\section{Source and Purity of Chemicals:}

(1) Purity not given, Chemical source not given, no purification details were provided.

(2) Purity not given, Chemical source not given, no purification details were provided.

\begin{tabular}{lcc}
\hline \hline$T / \mathrm{K}$ & $x_{2}{ }^{\mathrm{a}}$ & $x_{1}{ }^{\mathrm{b}}$ \\
\hline 293.0 & 0.9737 & 0.02633 \\
298.1 & 0.9731 & 0.02685 \\
304.2 & 0.9720 & 0.02803 \\
308.1 & 0.9714 & 0.02861 \\
319.0 & 0.9693 & 0.03066 \\
329.8 & 0.9671 & 0.03293 \\
344.2 & 0.9660 & 0.03403 \\
355.3 & 0.9629 & 0.03711 \\
359.2 & 0.9618 & 0.03818 \\
363.7 & 0.9612 & 0.03884 \\
368.8 & 0.9601 & 0.03994 \\
376.0 & 0.9584 & 0.04156 \\
382.1 & 0.9570 & 0.04297 \\
387.0 & 0.9559 & 0.04414 \\
393.4 & 0.9543 & 0.04571 \\
398.5 & 0.9530 & 0.04703 \\
406.6 & 0.9508 & 0.04917 \\
413.8 & 0.9488 & 0.05115 \\
417.9 & 0.9477 & 0.05232 \\
420.4 & 0.9470 & 0.05304 \\
423.4 & 0.9461 & 0.05392 \\
427.4 & 0.9449 & 0.05512 \\
429.3 & 0.9443 & 0.05571 \\
\hline
\end{tabular}


Estimated Error:

Temperature: $\pm 0.1 \mathrm{~K}$ (estimated by compiler). $x_{1}: \pm 3 \%$ (relative error, estimated by compiler).

\begin{tabular}{|c|c|}
\hline $\begin{array}{l}\text { Components: } \\
\text { (1) } 1,4-\text { Benzenedicarboxylic acid; } \\
\mathrm{C}_{8} \mathrm{H}_{6} \mathrm{O}_{4} \text {; [100-21-0] } \\
\text { (2) } N, N \text {-Dimethylformamide; } \\
\mathrm{C}_{3} \mathrm{H}_{7} \mathrm{NO} ;[68-12-2]\end{array}$ & $\begin{array}{l}\text { Original Measurements: } \\
{ }^{121} \text { P. Ma and M. Chen, Chin. J. Chem } \\
\text { Eng. 11, } 334 \text { (2003). }\end{array}$ \\
\hline Variables: & Prepared by: \\
\hline Temperature & W. E. Acree, Jr. \\
\hline
\end{tabular}

Experimental Values

\begin{tabular}{lcc}
\hline \hline$T / \mathrm{K}$ & $x_{2}{ }^{\mathrm{a}}$ & $x_{1}{ }^{\mathrm{b}}$ \\
\hline 298.10 & 0.9737 & 0.02629 \\
304.15 & 0.9716 & 0.02840 \\
308.10 & 0.9708 & 0.02919 \\
312.25 & 0.9700 & 0.03001 \\
316.75 & 0.9679 & 0.03210 \\
319.04 & 0.9675 & 0.03247 \\
321.64 & 0.9665 & 0.03352 \\
329.78 & 0.9643 & 0.03569 \\
330.35 & 0.9643 & 0.03565 \\
340.95 & 0.9613 & 0.03872 \\
344.15 & 0.9600 & 0.04000 \\
351.15 & 0.9580 & 0.04198 \\
354.55 & 0.9570 & 0.04301 \\
355.25 & 0.9567 & 0.04333 \\
359.15 & 0.9555 & 0.04450 \\
360.23 & 0.9550 & 0.04497 \\
363.71 & 0.9536 & 0.04637 \\
\hline
\end{tabular}

${ }^{a} x_{2}$ : mole fraction of component 2 in the saturated solution.

${ }^{\mathrm{b}} x_{1}$ : mole fraction solubility of the solute.

\section{Auxiliary Information}

\section{Method/Apparatus/Procedure:}

Titanium solid-liquid equilibrium cell, analytical balance, magnetic stirring system, temperature controlling system, and a laser monitoring system.

Solubilities were determined using a dynamic method. Weighed amounts of solute and solvent were sealed in a solubility cell, and the temperature slowly increased until the solid phase completely disappeared. The rate of temperature increase was $0.1 \mathrm{~K} / \mathrm{min}$. The disappearance of the solid solute was observed visually.

\section{Source and Purity of Chemicals:}

(1) $99.8 \%$, Chemical source not given, used as received.

(2) $99.5 \%$, Chemical source not given, used as received.

\section{Estimated Error:}

Temperature: $\pm 0.1 \mathrm{~K}$ (estimated by compiler).

$x_{1}: \pm 3 \%$ (relative error, estimated by compiler).

\begin{tabular}{|c|c|}
\hline $\begin{array}{l}\text { Components: } \\
\text { (1) 1,4-Benzenedicarboxylic acid; } \\
\mathrm{C}_{8} \mathrm{H}_{6} \mathrm{O}_{4} ;[100-21-0] \\
\text { (2) } \mathrm{N}, \mathrm{N} \text {-Dimethylformamide; } \\
\mathrm{C}_{3} \mathrm{H}_{7} \mathrm{NO} ;[64-19-7]\end{array}$ & $\begin{array}{l}\text { Original Measurements: } \\
{ }^{82} \text { L. Dian-Qing, L. Jiang-Chu, L. } \\
\text { Da-Zhuang, and W. Fu-An, Fluid } \\
\text { Phase Equilib. 200, } 69 \text { (2002). }\end{array}$ \\
\hline $\begin{array}{l}\text { Variables: } \\
\text { Temperature }\end{array}$ & $\begin{array}{l}\text { Prepared by: } \\
\text { W. E. Acree, Jr. }\end{array}$ \\
\hline
\end{tabular}

Experimental Values

\begin{tabular}{lcc}
\hline \hline$T / \mathrm{K}$ & $x_{2}{ }^{\mathrm{a}}$ & $x_{1}{ }^{\mathrm{b}}$ \\
\hline 296.05 & 0.9722 & 0.0278 \\
298.85 & 0.9717 & 0.0283 \\
301.25 & 0.9711 & 0.0289 \\
304.65 & 0.9704 & 0.0296 \\
307.35 & 0.9697 & 0.0303 \\
311.85 & 0.9684 & 0.0316 \\
314.45 & 0.9680 & 0.0320 \\
318.05 & 0.9671 & 0.0329 \\
320.25 & 0.9667 & 0.0333 \\
325.75 & 0.9652 & 0.0348 \\
328.25 & 0.9644 & 0.0356 \\
331.55 & 0.9635 & 0.0365 \\
336.95 & 0.9621 & 0.0379 \\
340.25 & 0.9611 & 0.0389 \\
343.15 & 0.9603 & 0.0397 \\
345.55 & 0.9596 & 0.0404 \\
351.65 & 0.9579 & 0.0421 \\
353.75 & 0.9571 & 0.0429 \\
356.65 & 0.9562 & 0.0438 \\
359.95 & 0.9553 & 0.0447 \\
365.65 & 0.9534 & 0.0466 \\
370.45 & 0.9517 & 0.0483 \\
\hline${ }^{a} x_{2}:$ mole fraction of component 2 in the saturated solution. & \\
${ }^{\mathrm{b}} x_{1}:$ mole fraction solubility of the solute. &
\end{tabular}

\section{Auxiliary Information}

\section{Method/Apparatus/Procedure:}

Jacketed solid-liquid equilibrium cell, analytical balance, magnetic stirring system, temperature controlling system, and a laser monitoring system. Solubilities were determined using a synthetic method. Weighed amounts of solute and solvent were sealed in a jacketed equilibrium vessel, and the temperature slowly increased until the solid phase completely disappeared. The disappearance of the solid solute was detected by a laser monitoring system. Measurements were repeated two or three times to check the reproducibility.

Source and Purity of Chemicals:

(1) Analytical Reagent, Shanghai Chemical Reagent Company, used as received.

(2) Analytical Reagent, Shanghai Chemical Reagent Company, used as received.

\section{Estimated Error:}

Temperature: $\pm 0.05 \mathrm{~K}$ (estimated by compiler). $x_{1}: \pm 0.0005$ or less. 


\begin{tabular}{|c|c|}
\hline $\begin{array}{l}\text { Components: } \\
\text { (1) } 1,4 \text {-Benzenedicarboxylic acid; } \\
\mathrm{C}_{8} \mathrm{H}_{6} \mathrm{O}_{4} \text {; [100-21-0] } \\
\text { (2) N,N-Dimethylformamide; } \\
\mathrm{C}_{3} \mathrm{H}_{7} \mathrm{NO} ;[64-19-7]\end{array}$ & $\begin{array}{l}\text { Original Measurements: } \\
{ }^{122} \text { Q. Wang, H. Xu, and X. Li, J. Chem. } \\
\text { Eng. Data 50, } 719 \text { (2005). }\end{array}$ \\
\hline $\begin{array}{l}\text { Variables: } \\
\text { Temperature }\end{array}$ & $\begin{array}{l}\text { Prepared by: } \\
\text { W. E. Acree, Jr. }\end{array}$ \\
\hline
\end{tabular}

Experimental Values

\begin{tabular}{|c|c|}
\hline$T / \mathrm{K}$ & $c_{1}{ }^{\mathrm{a}}$ \\
\hline 301.9 & 0.381 \\
\hline 307.5 & 0.392 \\
\hline 315.0 & 0.418 \\
\hline 323.0 & 0.440 \\
\hline 328.6 & 0.452 \\
\hline 334.7 & 0.474 \\
\hline 340.4 & 0.490 \\
\hline 344.2 & 0.520 \\
\hline 351.5 & 0.539 \\
\hline 357.9 & 0.552 \\
\hline 363.6 & 0.575 \\
\hline 369.4 & 0.611 \\
\hline 373.5 & 0.621 \\
\hline
\end{tabular}

${ }^{\mathrm{a}} c_{1}$ : molar solubility of the solute expressed in units of $\mathrm{mol} \mathrm{dm}^{-3}$.

\section{Auxiliary Information}

\section{Method/Apparatus/Procedure:}

Solubilities were measured by the static analytical method. Excess solute and solvent were placed in a jacketed glass bottle. The solution was allowed to equilibrate at constant temperature with stirring for several hours. Attainment of equilibrium was verified by repetitive measurements until the results were reproducible to within $\pm 0.5 \%$. Concentration of the dissolved solute was determined by titration with a standardized sodium hydroxide using phenolphthalein as the endpoint indicator.

Source and Purity of Chemicals:

(1) $99.5 \%$, Shanghai Chemical Reagent Company, China, no purification details were provided.

(2) $99+\%$, Hanzhou Chemical Reagent Company, China, no purification details were provided.

\section{Estimated Error:}

Temperature: $\pm 0.1 \mathrm{~K}$.

$c_{1}: \pm 1 \%$ (relative error, estimated by compiler).

\begin{tabular}{|c|c|}
\hline $\begin{array}{l}\text { Components: } \\
\text { (1) } 1,4-\text {-Benzenedicarboxylic acid; } \\
\mathrm{C}_{8} \mathrm{H}_{6} \mathrm{O}_{4} \text {; [100-21-0] } \\
\text { (2) } N, N \text {-Dimethylacetamide; } \\
\mathrm{C}_{4} \mathrm{H}_{9} \mathrm{NO} ;[127-19-5]\end{array}$ & $\begin{array}{l}\text { Original Measurements: } \\
{ }^{80} \text { P. Ma and Q. Xia, Chin. J. Chem. } \\
\text { Eng. 9, } 39 \text { (2001). }\end{array}$ \\
\hline $\begin{array}{l}\text { Variables: } \\
\text { Temperature }\end{array}$ & $\begin{array}{l}\text { Prepared by: } \\
\text { W. E. Acree, Jr. }\end{array}$ \\
\hline
\end{tabular}

Experimental Values

\begin{tabular}{lcc}
\hline \hline$T / \mathrm{K}$ & $x_{2}{ }^{\mathrm{a}}$ & $x_{1}{ }^{\mathrm{b}}$ \\
\hline 293.2 & 0.9951 & 0.004914 \\
296.3 & 0.9942 & 0.005761 \\
298.5 & 0.9937 & 0.006311 \\
303.0 & 0.9921 & 0.007914 \\
307.8 & 0.9904 & 0.009613 \\
311.5 & 0.9884 & 0.01161 \\
320.0 & 0.9834 & 0.01663 \\
326.2 & 0.9779 & 0.02206 \\
333.7 & 0.9710 & 0.02899 \\
340.3 & 0.9638 & 0.03622 \\
349.9 & 0.9501 & 0.04989 \\
356.3 & 0.9362 & 0.06381 \\
362.4 & 0.9239 & 0.07614 \\
365.3 & 0.9116 & 0.08838 \\
\hline${ }^{\mathrm{a}}{ }_{x_{2}: \text { mole fraction of component } 2 \text { in the saturated solution. }}$ \\
${ }^{\mathrm{b}}{ }_{x_{1}}:$ mole fraction solubility of the solute.
\end{tabular}

\section{Auxiliary Information}

\section{Method/Apparatus/Procedure:}

Titanium solid-liquid equilibrium cell, analytical balance, magnetic stirring system, temperature controlling system, and a laser monitoring system.

Solubilities were determined using a dynamic method. Weighed amounts of solute and solvent were sealed in a titanium solid-liquid equilibrium cell, and the temperature slowly increased until the solid phase completely disappeared. Near the solid-liquid equilibrium temperature, the rate of temperature increase was $0.1 \mathrm{~K} / 10 \mathrm{~min}$. The disappearance of the solid solute was detected by a laser monitoring system.

Source and Purity of Chemicals:

(1) Purity not given, Chemical source not given, no purification details were provided.

(2) Purity not given, Chemical source not given, no purification details were provided.

Estimated Error:

Temperature: $\pm 0.1 \mathrm{~K}$ (estimated by compiler).

$x_{1}: \pm 3 \%$ (relative error, estimated by compiler).

\begin{tabular}{|c|c|}
\hline $\begin{array}{l}\text { Components: } \\
\text { (1) 1,4-Benzenedicarboxylic acid; } \\
\mathrm{C}_{8} \mathrm{H}_{6} \mathrm{O}_{4} ;[100-21-0] \\
\text { (2) } N, N \text {-Dimethylacetamide; } \\
\mathrm{C}_{4} \mathrm{H}_{9} \mathrm{NO} ;[127-19-5]\end{array}$ & $\begin{array}{l}\text { Original Measurements: } \\
{ }^{121} \text { P. Ma and M. Chen, Chin. J. Chem. } \\
\text { Eng. 11, } 334 \text { (2003). }\end{array}$ \\
\hline $\begin{array}{l}\text { Variables: } \\
\text { Temperature }\end{array}$ & $\begin{array}{l}\text { Prepared by: } \\
\text { W. E. Acree, Jr. }\end{array}$ \\
\hline
\end{tabular}


Experimental Values

\begin{tabular}{lcc}
\hline \hline$T / \mathrm{K}$ & $x_{2}{ }^{\mathrm{a}}$ & $x_{1}{ }^{\mathrm{b}}$ \\
\hline 293.19 & 0.9951 & 0.004910 \\
296.30 & 0.9940 & 0.006000 \\
299.07 & 0.9933 & 0.006700 \\
301.76 & 0.9925 & 0.007450 \\
305.23 & 0.9914 & 0.008590 \\
310.61 & 0.9894 & 0.01060 \\
317.42 & 0.9858 & 0.01420 \\
326.22 & 0.9804 & 0.01960 \\
334.29 & 0.9730 & 0.02700 \\
341.70 & 0.9642 & 0.03580 \\
348.57 & 0.9577 & 0.04230 \\
357.98 & 0.9412 & 0.05880 \\
361.75 & 0.9333 & 0.06670 \\
364.44 & 0.9290 & 0.07100 \\
\hline
\end{tabular}

${ }^{\mathrm{a}} x_{2}$ : mole fraction of component 2 in the saturated solution.

${ }^{\mathrm{b}} x_{1}$ : mole fraction solubility of the solute.

\section{Auxiliary Information}

\section{Method/Apparatus/Procedure:}

Titanium solid-liquid equilibrium cell, analytical balance, magnetic stirring system, temperature controlling system, and a laser monitoring system.

Solubilities were determined using a dynamic method. Weighed amounts of solute and solvent were sealed in a solubility cell, and the temperature slowly increased until the solid phase completely disappeared. The rate of temperature increase was $0.1 \mathrm{~K} / \mathrm{min}$. The disappearance of the solid solute was observed visually.

\section{Source and Purity of Chemicals:}

(1) $99.8 \%$, Chemical source not given, used as received.

(2) $99.5 \%$, Chemical source not given, used as received.

\section{Estimated Error:}

Temperature: $\pm 0.1 \mathrm{~K}$ (estimated by compiler).

$x_{1}: \pm 3 \%$ (relative error, estimated by compiler).

\begin{tabular}{|c|c|}
\hline $\begin{array}{l}\text { Components: } \\
\text { (1) } 1,4 \text {-Benzenedicarboxylic acid; } \\
\mathrm{C}_{8} \mathrm{H}_{6} \mathrm{O}_{4} ;[100-21-0] \\
\text { (2) } N, N \text {-Dimethylacetamide; } \\
\mathrm{C}_{4} \mathrm{H}_{9} \mathrm{NO} ;[127-19-5]\end{array}$ & $\begin{array}{l}\text { Original Measurements: } \\
{ }^{123} \text { X. Guo, Y.-W. Cheng, L.-J. Wang, } \\
\text { and X. Li, J. Chem. Eng. Data 53, } 1421 \\
(2008) \text {. }\end{array}$ \\
\hline Variables: & Prepared by: \\
\hline Temperature & W. E. Acree, Jr. \\
\hline
\end{tabular}

Experimental Values

\begin{tabular}{lcc}
\hline \hline$T / \mathrm{K}$ & $x_{2}{ }^{\mathrm{a}}$ & $x_{1}{ }^{\mathrm{b}}$ \\
\hline 303.2 & 0.9840 & 0.01597 \\
313.2 & 0.9789 & 0.02109 \\
323.2 & 0.9731 & 0.02690 \\
333.2 & 0.9669 & 0.03311 \\
343.2 & 0.9615 & 0.03850 \\
353.2 & 0.9548 & 0.04516 \\
363.2 & 0.9476 & 0.05237 \\
\hline
\end{tabular}

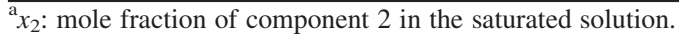

${ }^{\mathrm{b}} x_{1}$ : mole fraction solubility of the solute.

\section{Auxiliary Information}

\section{Method/Apparatus/Procedure:}

Analytical balance, magnetic stirring system, thermoelectric temperature controlling system, and a high-performance liquid chromatograph.

Excess solute and solvent were placed in a sealed glass bottle and allowed to equilibrate at constant temperature with stirring for $48 \mathrm{~h}$. Attainment of equilibrium was verified by repetitive measurements after an additional $48 \mathrm{~h}$ equilibration period and by approaching equilibrium from supersaturation by pre-equilibrating the solutions at a higher temperature. An aliquot of the clear saturated solution was transferred to a volumetric flask and diluted quantitatively with the solvent being studied. The concentration of the diluted solution was determined by high-performance liquid chromatographic analysis.

\section{Source and Purity of Chemicals:}

(1) $99.5 \%$, Shanghai Chemical Reagent Company, China, no purification details were provided.

(2) $99.5 \%$, Analytical Reagent grade, Tianjin Chemical Reagent Company, China, no purification details were provided.

\section{Estimated Error:}

Temperature: $\pm 0.1 \mathrm{~K}$.

$x_{1}: \pm 3 \%$ (relative error, estimated by compiler).

\begin{tabular}{|c|c|}
\hline $\begin{array}{l}\text { Components: } \\
\text { (1) } 1,4-\text {-Benzenedicarboxylic acid; } \\
\mathrm{C}_{8} \mathrm{H}_{6} \mathrm{O}_{4} \text {; [100-21-0] } \\
\text { (2) } \mathrm{N} \text {-Methyl-2-pyrrolidone; } \\
\mathrm{C}_{5} \mathrm{H}_{9} \mathrm{NO} ;[872-50-4]\end{array}$ & $\begin{array}{l}\text { Original Measurements: } \\
{ }^{80} \text { P. Ma and Q. Xia, Chin. J. Chem. } \\
\text { Eng. 9, } 39 \text { (2001). }\end{array}$ \\
\hline $\begin{array}{l}\text { Variables: } \\
\text { Temperature }\end{array}$ & $\begin{array}{l}\text { Prepared by: } \\
\text { W. E. Acree, Jr. }\end{array}$ \\
\hline
\end{tabular}

Experimental Values

\begin{tabular}{lcc}
\hline \hline$T / \mathrm{K}$ & $x_{2}{ }^{\mathrm{a}}$ & $x_{1}{ }^{\mathrm{b}}$ \\
\hline 298.1 & 0.9673 & 0.03266 \\
310.4 & 0.9529 & 0.04705 \\
314.2 & 0.9465 & 0.05347 \\
318.7 & 0.9387 & 0.06130 \\
324.9 & 0.9284 & 0.07157 \\
331.1 & 0.9149 & 0.08510 \\
337.2 & 0.9018 & 0.09824 \\
343.3 & 0.8904 & 0.1096 \\
349.7 & 0.8744 & 0.1256 \\
355.7 & 0.8522 & 0.1478 \\
363.7 & 0.8176 & 0.1824 \\
\hline
\end{tabular}

${ }^{a} x_{2}$ : mole fraction of component 2 in the saturated solution.

${ }^{\mathrm{b}} x_{1}$ : mole fraction solubility of the solute.

\section{Auxiliary Information}

\section{Method/Apparatus/Procedure:}

Titanium solid-liquid equilibrium cell, analytical balance, magnetic stirring system, temperature controlling system, and a laser monitoring system.

Solubilities were determined using a dynamic method. Weighed amounts of solute and solvent were sealed in a titanium solid-liquid equilibrium cell, and the temperature slowly increased until the solid phase completely disappeared. Near the solid-liquid equilibrium temperature, the rate of temperature increase was $0.1 \mathrm{~K} / 10 \mathrm{~min}$. The disappearance of the solid solute was detected by a laser monitoring system. 
Source and Purity of Chemicals:

(1) Purity not given, Chemical source not given, no purification details were provided.

(2) Purity not given, Chemical source not given, no purification details were provided.

\section{Estimated Error:}

Temperature: $\pm 0.1 \mathrm{~K}$ (estimated by compiler).

$x_{1}: \pm 3 \%$ (relative error, estimated by compiler).

\begin{tabular}{|c|c|}
\hline $\begin{array}{l}\text { Components: } \\
\text { (1) 1,4-Benzenedicarboxylic acid; } \\
\mathrm{C}_{8} \mathrm{H}_{6} \mathrm{O}_{4} ;[100-21-0] \\
\text { (2) } \mathrm{N} \text {-Methyl-2-pyrrolidone; } \\
\mathrm{C}_{5} \mathrm{H}_{9} \mathrm{NO} ;[872-50-4]\end{array}$ & $\begin{array}{l}\text { Original Measurements: } \\
{ }^{121} \text { P. Ma and M. Chen, Chin. J. Chem. } \\
\text { Eng. 11, } 334 \text { (2003). }\end{array}$ \\
\hline $\begin{array}{l}\text { Variables: } \\
\text { Temperature }\end{array}$ & $\begin{array}{l}\text { Prepared by: } \\
\text { W. E. Acree, Jr. }\end{array}$ \\
\hline
\end{tabular}

Experimental Values

\begin{tabular}{lcc}
\hline \hline$T / \mathrm{K}$ & $x_{2}{ }^{\mathrm{a}}$ & $x_{1}{ }^{\mathrm{b}}$ \\
\hline 295.60 & 0.9669 & 0.03307 \\
302.86 & 0.9602 & 0.03977 \\
310.79 & 0.9513 & 0.04865 \\
317.54 & 0.9423 & 0.05774 \\
325.18 & 0.9299 & 0.07011 \\
332.82 & 0.9149 & 0.08513 \\
339.35 & 0.8995 & 0.1005 \\
346.72 & 0.8788 & 0.1212 \\
351.49 & 0.8632 & 0.1368 \\
357.76 & 0.8396 & 0.1604 \\
363.28 & 0.8155 & 0.1845 \\
\hline
\end{tabular}

${ }^{\mathrm{a}} x_{2}$ : mole fraction of component 2 in the saturated solution.

${ }^{\mathrm{b}} x_{1}$ : mole fraction solubility of the solute.

\section{Auxiliary Information}

\section{Method/Apparatus/Procedure:}

Titanium solid-liquid equilibrium cell, analytical balance, magnetic stirring system, temperature controlling system, and a laser monitoring system. Solubilities were determined using a dynamic method. Weighed amounts of solute and solvent were sealed in a solubility cell, and the temperature slowly increased until the solid phase completely disappeared. The rate of temperature increase was $0.1 \mathrm{~K} / \mathrm{min}$. The disappearance of the solid solute was observed visually.

\section{Source and Purity of Chemicals:}

(1) $99.8 \%$, Chemical source not given, used as received.

(2) $99.5 \%$, Chemical source not given, used as received.

\section{Estimated Error:}

Temperature: $\pm 0.1 \mathrm{~K}$ (estimated by compiler).

$x_{1}: \pm 3 \%$ (relative error, estimated by compiler).

\begin{tabular}{|c|c|}
\hline $\begin{array}{l}\text { Components: } \\
\text { (1) } 1,4-\text {-Benzenedicarboxylic acid; } \\
\mathrm{C}_{8} \mathrm{H}_{6} \mathrm{O}_{4} ;[100-21-0] \\
\text { (2) } \mathrm{N} \text {-Methyl-2-pyrrolidone; } \\
\mathrm{C}_{5} \mathrm{H}_{9} \mathrm{NO} ;[872-50-4]\end{array}$ & $\begin{array}{l}\text { Original Measurements: } \\
\text { 84 D.-Q. Li, D.-Z. Liu, and F.-A. Wang, } \\
\text { J. Chem. Eng. Data 46, } 172 \text { (2001). }\end{array}$ \\
\hline Variables: & Prepared by: \\
\hline Temperature & W. E. Acree, Jr. \\
\hline
\end{tabular}

Experimental Values

\begin{tabular}{lcc}
\hline \hline$T / \mathrm{K}$ & $x_{2}{ }^{\mathrm{a}}$ & $x_{1}{ }^{\mathrm{b}}$ \\
\hline 296.35 & 0.9699 & 0.0301 \\
299.55 & 0.9659 & 0.0341 \\
303.75 & 0.9599 & 0.0401 \\
307.05 & 0.9549 & 0.0451 \\
309.75 & 0.9511 & 0.0489 \\
312.75 & 0.9461 & 0.0539 \\
316.25 & 0.9395 & 0.0605 \\
319.65 & 0.9332 & 0.0668 \\
324.05 & 0.9243 & 0.0757 \\
328.45 & 0.9151 & 0.0849 \\
331.55 & 0.9078 & 0.0922 \\
332.65 & 0.9055 & 0.0945 \\
\hline
\end{tabular}

${ }^{\mathrm{a}} x_{2}$ : mole fraction of component 2 in the saturated solution.

${ }^{\mathrm{b}} x_{1}$ : mole fraction solubility of the solute.

\section{Auxiliary Information}

\section{Method/Apparatus/Procedure:}

Jacketed solid-liquid equilibrium cell, analytical balance, magnetic stirring system, temperature controlling system, and a laser monitoring system. Solubilities were determined using a synthetic method. Weighed amounts of solute and solvent were sealed in a jacketed equilibrium vessel, and the temperature slowly increased until the solid phase completely disappeared. The disappearance of the solid solute was detected by a laser monitoring system. Measurements were repeated two or three times to check the reproducibility.

\section{Source and Purity of Chemicals:}

(1) $99.5 \%$, Analytical Reagent, Shanghai Chemical Reagent Company, China, used as received.

(2) $99.0 \%$, Analytical Reagent, Shanghai Chemical Reagent Company, used as received.

\section{Estimated Error:}

Temperature: $\pm 0.05 \mathrm{~K}$ (estimated by compiler). $x_{1}: \pm 0.0005$ or less.

\begin{tabular}{|c|c|}
\hline $\begin{array}{l}\text { Components: } \\
\text { (1) 1,4-Benzenedicarboxylic acid; } \\
\mathrm{C}_{8} \mathrm{H}_{6} \mathrm{O}_{4} ;[100-21-0] \\
\text { (2) } N \text {-Methyl-2-pyrrolidone; } \\
\mathrm{C}_{5} \mathrm{H}_{9} \mathrm{NO} ;[872-50-4]\end{array}$ & $\begin{array}{l}\text { Original Measurements: } \\
{ }^{123} \text { X. Guo, Y.-W. Cheng, L.-J. Wang, } \\
\text { and X. Li, J. Chem. Eng. Data 53, } 1421 \\
(2008) \text {. }\end{array}$ \\
\hline $\begin{array}{l}\text { Variables: } \\
\text { Temperature }\end{array}$ & $\begin{array}{l}\text { Prepared by: } \\
\text { W. E. Acree, Jr. }\end{array}$ \\
\hline
\end{tabular}

\section{Experimental Values}

\begin{tabular}{|c|c|c|}
\hline$T / \mathrm{K}$ & $x_{2}{ }^{\mathrm{a}}$ & $x_{1}{ }^{b}$ \\
\hline 303.2 & 0.9867 & 0.01334 \\
\hline 313.2 & 0.9845 & 0.01552 \\
\hline 323.2 & 0.9811 & 0.01885 \\
\hline 333.2 & 0.9784 & 0.02165 \\
\hline 343.2 & 0.9744 & 0.02559 \\
\hline 353.2 & 0.9713 & 0.02869 \\
\hline 363.2 & 0.9672 & 0.03276 \\
\hline
\end{tabular}




\section{Auxiliary Information}

\section{Method/Apparatus/Procedure:}

Analytical balance, magnetic stirring system, thermoelectric temperature controlling system, and a high-performance liquid chromatograph.

Excess solute and solvent were placed in a sealed glass bottle and allowed to equilibrate at constant temperature with stirring for $48 \mathrm{~h}$. Attainment of equilibrium was verified by repetitive measurements after an additional $48 \mathrm{~h}$ equilibration period and by approaching equilibrium from supersaturation by pre-equilibrating the solutions at a higher temperature. An aliquot of the clear saturated solution was transferred to a volumetric flask and diluted quantitatively with the solvent being studied. The concentration of the diluted solution was determined by high-performance liquid chromatographic analysis.

Source and Purity of Chemicals:

(1) $99.5 \%$, Shanghai Chemical Reagent Company, China, no purification details were provided.

(2) $99.5 \%$, Analytical Reagent grade, Tianjin Chemical Reagent Company, China, no purification details were provided.

Estimated Error:

Temperature: $\pm 0.1 \mathrm{~K}$.

$x_{1}: \pm 3 \%$ (relative error, estimated by compiler).

\begin{tabular}{|c|c|}
\hline $\begin{array}{l}\text { Components: } \\
\text { (1) } 1,4-\text {-Benzenedicarboxylic acid; } \\
\mathrm{C}_{8} \mathrm{H}_{6} \mathrm{O}_{4} \text {; [100-21-0] } \\
\text { (2) } \mathrm{N} \text {-Methyl-2-pyrrolidone; } \\
\mathrm{C}_{5} \mathrm{H}_{9} \mathrm{NO} ;[872-50-4]\end{array}$ & $\begin{array}{l}\text { Original Measurements: } \\
{ }^{124} \mathrm{Q} \text {. Wang, H. Xu, and X. Li, J. Chem. } \\
\text { Eng. Data 50, } 243 \text { (2005). }\end{array}$ \\
\hline $\begin{array}{l}\text { Variables: } \\
\text { Temperature }\end{array}$ & $\begin{array}{l}\text { Prepared by: } \\
\text { W. E. Acree, Jr. }\end{array}$ \\
\hline
\end{tabular}

Experimental Values

\begin{tabular}{lcc}
\hline \hline$T / \mathrm{K}$ & $x_{2}{ }^{\mathrm{a}}$ & $x_{1}{ }^{\mathrm{b}}$ \\
\hline 343.2 & 0.9389 & 0.0611 \\
348.2 & 0.9311 & 0.0689 \\
353.2 & 0.9265 & 0.0735 \\
358.2 & 0.9179 & 0.0821 \\
363.2 & 0.9134 & 0.0866 \\
368.2 & 0.9074 & 0.0926 \\
373.2 & 0.9040 & 0.0960 \\
378.2 & 0.8958 & 0.1042 \\
383.2 & 0.8868 & 0.1132 \\
388.2 & 0.8789 & 0.1211 \\
393.2 & 0.8657 & 0.1343 \\
398.2 & 0.8604 & 0.1396 \\
403.2 & 0.8473 & 0.1527 \\
408.2 & 0.8326 & 0.1674 \\
413.2 & 0.8260 & 0.1740 \\
418.2 & 0.8093 & 0.1907 \\
423.2 & 0.7970 & 0.2030 \\
428.2 & 0.7817 & 0.2183 \\
433.2 & 0.7723 & 0.2277 \\
438.2 & 0.7509 & 0.2491 \\
443.2 & 0.7319 & 0.2681 \\
448.2 & 0.7175 & 0.2825 \\
453.2 & 0.6963 & 0.3037 \\
458.2 & 0.6699 & 0.3301 \\
463.2 & 0.6451 & 0.3549 \\
468.2 & 0.6180 & 0.3820 \\
\hline & &
\end{tabular}

${ }^{\mathrm{a}} x_{2}$ : mole fraction of component 2 in the saturated solution.

${ }^{\mathrm{b}} x_{1}$ : mole fraction solubility of the solute. The numerical values were calculated by the compiler. The solubility data were given in the paper as grams of solute per $100 \mathrm{~g}$ of solvent.

\section{Auxiliary Information}

\section{Method/Apparatus/Procedure:}

Titanium solid-liquid equilibrium cell, analytical balance, and temperature controlling system.

Solubilities were determined using a steady-state method. Excess solute and solvent were placed in a titanium solid-liquid equilibrium cell and allowed to equilibrate at constant temperature. After equilibrium was reached, an aliquot of the saturated solution was removed and filtered through a porous stainless steel filter with an internal aperture size of $1 \mu \mathrm{m}$. The mass of the transferred solution was determined by weighing. The solvent was removed by drying in vacuo at $323.2 \mathrm{~K}$. The solubility was calculated from the mass of the solid residue and the mass of the saturated solution analyzed.

\section{Source and Purity of Chemicals:}

(1) $99.5 \%$, Shanghai Chemical Reagent Company, China, no purification details were provided.

(2) Purity not given, Chemical source not given, no purification details were provided.

Estimated Error:

Temperature: $\pm 0.5 \mathrm{~K}$.

$x_{1}: \pm 5 \%$ (relative error, estimated by compiler).

\begin{tabular}{|c|c|}
\hline $\begin{array}{l}\text { Components: } \\
\text { (1) } 1,4-\text { Benzenedicarboxylic acid; } \\
\mathrm{C}_{8} \mathrm{H}_{6} \mathrm{O}_{4} ;[100-21-0] \\
\text { (2) Dimethyl sulfoxide; } \\
\mathrm{C}_{2} \mathrm{H}_{6} \mathrm{OS} ;[67-68-5]\end{array}$ & $\begin{array}{l}\text { Original Measurements: } \\
{ }^{122} \mathrm{Q} \text {. Wang, H. Xu, and X. Li, J. Chem } \\
\text { Eng. Data 50, } 719 \text { (2005). }\end{array}$ \\
\hline $\begin{array}{l}\text { Variables: } \\
\text { Temperature }\end{array}$ & $\begin{array}{l}\text { Prepared by: } \\
\text { W. E. Acree, Jr. }\end{array}$ \\
\hline
\end{tabular}

Experimental Values

\begin{tabular}{lc}
\hline \hline$T / \mathrm{K}$ & $c_{1}{ }^{\mathrm{a}}$ \\
\hline 301.9 & 1.249 \\
307.5 & 1.330 \\
315.0 & 1.491 \\
323.0 & 1.569 \\
328.6 & 1.650 \\
334.7 & 1.704 \\
340.4 & 1.997 \\
344.2 & 1.876 \\
351.5 & 1.989 \\
357.9 & 2.102 \\
363.6 & 2.244 \\
369.4 & 2.378 \\
373.5 & 2.491 \\
\hline${ }^{\mathrm{a}}{ }^{1}$ : molar solubility of the solute expressed in units of $\mathrm{mol} \mathrm{dm}^{-3}$. &
\end{tabular}

Auxiliary Information

\section{Method/Apparatus/Procedure:}

Solubilities were measured by the static analytical method. Excess solute and solvent were placed in a jacketed glass bottle. The solution was allowed to equilibrate at constant temperature with stirring for several hours. Attainment of equilibrium was verified by repetitive measurements until the results were reproducible to within $\pm 0.5 \%$. Concentration of the dissolved solute was determined by titration with a standardized sodium hydroxide using phenolphthalein as the endpoint indicator. 
Source and Purity of Chemicals:

(1) $99.5 \%$, Shanghai Chemical Reagent Company, China, no purification details were provided.

(2) $99+\%$, Hanzhou Chemical Reagent Company, China, no purification details were provided.

Estimated Error:

Temperature: $\pm 0.1 \mathrm{~K}$.

$c_{1}: \pm 1 \%$ (relative error, estimated by compiler).

\begin{tabular}{|c|c|}
\hline $\begin{array}{l}\text { Components: } \\
\text { (1) } 1,4-\text { Benzenedicarboxylic acid; } \\
\mathrm{C}_{8} \mathrm{H}_{6} \mathrm{O}_{4} ;[100-21-0] \\
\text { (2) Dimethyl sulfoxide; } \mathrm{C}_{2} \mathrm{H}_{6} \mathrm{OS} \text {; } \\
\text { [67-68-5] }\end{array}$ & $\begin{array}{l}\text { Original Measurements: } \\
{ }^{121} \text { P. Ma and M. Chen, Chin. J. Chem. } \\
\text { Eng. 11, } 334 \text { (2003). }\end{array}$ \\
\hline $\begin{array}{l}\text { Variables: } \\
\text { Temperature }\end{array}$ & $\begin{array}{l}\text { Prepared by: } \\
\text { W. E. Acree, Jr. }\end{array}$ \\
\hline
\end{tabular}

Experimental Values

\begin{tabular}{lcc}
\hline \hline$T / \mathrm{K}$ & $x_{2}{ }^{\mathrm{a}}$ & $x_{1}^{\mathrm{b}}$ \\
\hline 303.95 & 0.9154 & 0.08459 \\
311.55 & 0.9069 & 0.09309 \\
317.78 & 0.9032 & 0.09679 \\
325.41 & 0.8980 & 0.1020 \\
331.55 & 0.8958 & 0.1042 \\
335.52 & 0.8919 & 0.1081 \\
341.30 & 0.8884 & 0.1116 \\
346.15 & 0.8863 & 0.1137 \\
348.64 & 0.8856 & 0.1144 \\
354.02 & 0.8845 & 0.1155 \\
357.45 & 0.8834 & 0.1166 \\
362.49 & 0.8822 & 0.1178 \\
\hline
\end{tabular}

${ }^{\mathrm{a}} x_{2}$ : mole fraction of component 2 in the saturated solution.

${ }^{\mathrm{b}} x_{1}$ : mole fraction solubility of the solute.

\section{Auxiliary Information}

\section{Method/Apparatus/Procedure:}

Titanium solid-liquid equilibrium cell, analytical balance, magnetic stirring system, temperature controlling system, and a laser monitoring system.

Solubilities were determined using a dynamic method. Weighed amounts of solute and solvent were sealed in a solubility cell, and the temperature slowly increased until the solid phase completely disappeared. The rate of temperature increase was $0.1 \mathrm{~K} / \mathrm{min}$. The disappearance of the solid solute was observed visually.

Source and Purity of Chemicals:

(1) $99.8 \%$, Chemical source not given, used as received.

(2) $99.5 \%$, Chemical source not given, used as received.

\section{Estimated Error:}

Temperature: $\pm 0.1 \mathrm{~K}$ (estimated by compiler).

$x_{1}: \pm 3 \%$ (relative error, estimated by compiler).

\begin{tabular}{|c|c|}
\hline $\begin{array}{l}\text { Components: } \\
\text { (1) } 1,4-\text { Benzenedicarboxylic acid; } \\
\mathrm{C}_{8} \mathrm{H}_{6} \mathrm{O}_{4} ;[100-21-0] \\
\text { (2) Dimethyl sulfoxide; } \mathrm{C}_{2} \mathrm{H}_{6} \mathrm{OS} \text {; } \\
\text { [67-68-5] }\end{array}$ & $\begin{array}{l}\text { Original Measurements: } \\
{ }^{91} \text { J. Bradil, J. Malek, and V. Bazant, } \\
\text { Chem. Prumys1 20, } 117 \text { (1970). }\end{array}$ \\
\hline $\begin{array}{l}\text { Variables: } \\
\text { Temperature }\end{array}$ & $\begin{array}{l}\text { Prepared by: } \\
\text { W. E. Acree, Jr. }\end{array}$ \\
\hline
\end{tabular}

Experimental Values

\begin{tabular}{lcc}
\hline \hline$T / \mathrm{K}$ & $x_{2}{ }^{\mathrm{a}}$ & $x_{1}{ }^{\mathrm{b}}$ \\
\hline 313.2 & 0.6607 & 0.3393 \\
333.2 & 0.5574 & 0.4426 \\
343.2 & 0.4340 & 0.5660 \\
\hline
\end{tabular}

${ }^{a} x_{2}$ : mole fraction of component 2 in the saturated solution.

${ }^{b} x_{1}$ : mole fraction solubility of the solute. Solubility data were reported in terms of grams of dissolved solute per $100 \mathrm{~g}$ of solution. Mole fraction solubilities calculated by the compiler.

Auxiliary Information

\begin{abstract}
Method/Apparatus/Procedure:
Excess solute and solvent and were placed in a flask and equilibrated at constant temperature with stirring. After $90 \mathrm{~min}$ the stirring was discontinued, and the solution was allowed to stand for $30 \mathrm{~min}$ to allow the undissolved solid to settle to the bottom of the flask. An aliquot of the saturated solution was removed by a pipette fitted with a filtering device. The mass of the aliquot was determined by weighing. The concentration of the dissolved solute was determined by titration using sodium hydroxide, with phenolphthalein being the endpoint indicator.
\end{abstract}

Source and Purity of Chemicals:

(1) Purity not given, Synthesized in authors' laboratory, prepared from dimethyl-1,4-benzenedicarboxylate.

(2) Purity not given, Sojuz Chemical Export, Moscow, Soviet Union, mixed with activated carbon and distilled before use.

Estimated Error:

Temperature: $\pm 0.1 \mathrm{~K}$.

$x_{1}: \pm 1 \%$ (relative error).

\section{Solubility of 1,3,5-Benzenetricarboxylic Acid in Organic Solvents}

\subsection{Critical evaluation of experimental solubility data}

There is only a single published study regarding the solubility behavior of 1,3,5-benzenetricarboxylic acid in organic solvents. Feng et al. ${ }^{117}$ determined the solubility of 1,3,5benzenetricarboxylic acid in ethanoic acid in the temperature range of 313-363 K. The calculated curve-fit parameters from the Buchowski $\lambda$ h-model [Eq. (9)] of $\lambda=0.0170$ and $h=$ 131653.7 described the observed solubility data to within a mean relative deviation of $2.9 \%$.

The experimental solubility data for 1,3,5-benzenetricarboxylic acid in ethanoic acid are given in Sec. 14.2. 


\subsection{1,3,5-Benzenetricarboxylic acid solubility data in miscellaneous organic solvents}

\begin{tabular}{|c|c|}
\hline $\begin{array}{l}\text { Components: } \\
\text { (1) } 1,3,5 \text {-Benzenetricarboxylic acid; } \\
\mathrm{C}_{9} \mathrm{H}_{6} \mathrm{O}_{6} ;[554-95-0] \\
\text { (2) Ethanoic acid; } \mathrm{C}_{2} \mathrm{H}_{4} \mathrm{O}_{2} \text {; } \\
\text { [64-19-7] }\end{array}$ & $\begin{array}{l}\text { Original Measurements: } \\
{ }^{117} \text { L. Feng, Q. Wang, and X. Li, J. } \\
\text { Chem. Eng. Data 53, } 2501 \text { (2008). }\end{array}$ \\
\hline $\begin{array}{l}\text { Variables: } \\
\text { Temperature }\end{array}$ & $\begin{array}{l}\text { Prepared by: } \\
\text { W. E. Acree, Jr. }\end{array}$ \\
\hline
\end{tabular}

Experimental Values

\begin{tabular}{lcc}
\hline \hline$T / \mathrm{K}$ & $x_{2}{ }^{\mathrm{a}}$ & $x_{1}{ }^{\mathrm{b}}$ \\
\hline 313.2 & 0.9988 & 0.001200 \\
323.2 & 0.9987 & 0.001340 \\
333.2 & 0.9983 & 0.001691 \\
343.2 & 0.9980 & 0.002000 \\
353.2 & 0.9975 & 0.002489 \\
363.2 & 0.9968 & 0.003173 \\
\hline
\end{tabular}

${ }^{a} x_{2}$ : mole fraction of component 2 in the saturated solution.

${ }^{b} x_{1}$ : mole fraction solubility of the solute. Solubility data were reported as grams of dissolved solute per kilogram of solvent. Mole fraction solubilities were calculated by the compiler.

\section{Auxiliary Information}

\begin{abstract}
Method/Apparatus/Procedure:
Jacketed equilibrium glass bottle, analytical balance, thermoelectric temperature controlling system, and high-performance liquid chromatographic system.

Solubilities were determined using a static method. Excess solute and solvent were sealed in a jacketed equilibrium bottle and allowed to equilibrate for at least $24 \mathrm{~h}$ at constant temperature. After equilibrium was obtained, an aliquot of the clear saturated solution was removed by syringe and deposited into a clear test tube that contained about $3 \mathrm{ml}$ of dimethyl sulfoxide. The concentration of the solute was determined by high-performance liquid chromatographic analysis.
\end{abstract}

Source and Purity of Chemicals:

(1) $98 \%$, Alfa Aesar, used as received.

(2) Purity not given, Hanzhou Chemical Reagent Company, China, used as received.

\section{Estimated Error:}

Temperature: $\pm 0.1 \mathrm{~K}$ (estimated by compiler).

$x_{1}: \pm 3 \%$ (relative error, estimated by compiler).

\section{Solubility of 2-Bromobenzoic Acid in Organic Solvents}

\subsection{Critical evaluation of experimental solubility data}

There have been only two publications ${ }^{72,125}$ reporting the solubility of 2-bromobenzoic acid in organic solvents. Thuaire $^{72}$ determined the solubility of 2-bromobenzoic acid in ethanol at $298 \mathrm{~K}$ as part of study involving solubilities of benzoic acids in binary aqueous-ethanol solvent mixtures. Pinsuwan et al. ${ }^{125}$ measured the solubility of 2-bromobenzoic acid in 1-octanol to examine correlations between experimental octanol/water solubility ratios and measured water-to-1octanol practical partition coefficients. It is not possible to perform a critical evaluation of the experimental data as measurements were made at only a single temperature, and there are no independent experimental 2-bromobenzoic acid solubility data in either ethanol or 1-octanol.

The experimental solubility data for 2-bromobenzoic acid in organic solvents are given in Sec. 15.2.

\subsection{2-Bromobenzoic acid solubility data in alcohols}

\begin{tabular}{|c|c|}
\hline $\begin{array}{l}\text { Components: } \\
\text { (1) 2-Bromobenzoic acid; } \\
\mathrm{C}_{7} \mathrm{H}_{5} \mathrm{BrO}_{2} ;[88-65-3] \\
\text { (2) Ethanol; } \mathrm{C}_{2} \mathrm{H}_{6} \mathrm{O} ;[64-17-5]\end{array}$ & $\begin{array}{l}\text { Original Measurements: } \\
{ }^{72} \text { R. Thuaire, Bull. Soc. Chim. Fr. } 3815 \\
\text { (1971). }\end{array}$ \\
\hline $\begin{array}{l}\text { Variables: } \\
T / \mathrm{K}=298.15\end{array}$ & $\begin{array}{l}\text { Prepared by: } \\
\text { W. E. Acree, Jr. }\end{array}$ \\
\hline
\end{tabular}

Experimental Values

The measured solubility was reported to be $3.250 \mathrm{~mol} / \mathrm{kg}$ of solvent, which corresponds to a mole fraction solubility of $x_{1}=0.1302$

\section{Auxiliary Information}

\section{Method/Apparatus/Procedure:}

Very little experimental details were provided. Solubility was determined by a gravimetric method. The saturated solution was evaporated to dryness and the remaining solid residue was weighed. The solubility was calculated from the mass of the solid residue and mass of saturated solution analyzed.

\section{Source and Purity of Chemicals:}

(1) Purity not given, Chemical source not given, no purification details were provided.

(2) Purity not given, Chemical source not given, no purification details were provided.

Estimated Error:

Temperature: $\pm 0.05 \mathrm{~K}$.

$x_{1}: \pm 4 \%$ (relative error, estimated by compiler).

\begin{tabular}{|c|c|}
\hline $\begin{array}{l}\text { Components: } \\
\text { (1) 2-Bromobenzoic acid; } \\
\mathrm{C}_{7} \mathrm{H}_{5} \mathrm{BrO}_{2} ;[88-65-3] \\
\text { (2) } 1 \text {-Octanol; } \mathrm{C}_{8} \mathrm{H}_{18} \mathrm{O} ;[111-87-5]\end{array}$ & $\begin{array}{l}\text { Original Measurements: } \\
{ }^{125} \text { S. Pinsuwan, A. Li, and S. H. } \\
\text { Yalkowsky, J. Chem. Eng. Data 40, } \\
623 \text { (1995). }\end{array}$ \\
\hline $\begin{array}{l}\text { Variables: } \\
T / \mathrm{K}=298.15\end{array}$ & $\begin{array}{l}\text { Prepared by: } \\
\text { W. E. Acree, Jr. }\end{array}$ \\
\hline
\end{tabular}

\section{Experimental Values}

The measured solubility was reported to be $\log _{10} c_{1}=$ -0.12 , which corresponds to a molar solubility of $c_{1}=0.759$. 
Auxiliary Information

Method/Apparatus/Procedure:

Constant-temperature bath, analytical balance, mechanical stirrer, and ultraviolet/visible spectrophotometer.

Excess solute and solvent were equilibrated at $(298 \pm 2) \mathrm{K}$ in a sealed vial for 48-72 h. The solution was mixed using an end-over-end mechanical stirrer. After equilibrium was obtained, the saturated sample was vacuum filtered through a $5.0 \mu \mathrm{m}$ fritted glass filter. An aliquot of the filtrate was diluted quantitatively with 2-propanol, and the absorbance of the diluted sample was recorded. The solubility was calculated from the measured absorbance.

Source and Purity of Chemicals:

(1) Purity not given, Aldrich Chemical Company, Milwaukee, WI, USA, used as received.

(2) Purity not given, Chemical source not given, used as received.

Estimated Error:

Temperature: $\pm 2 \mathrm{~K}$.

$c_{1}: \pm 10 \%$ (relative error, estimated by compiler given the large uncertainty in temperature).

\section{Solubility of 3-Bromobenzoic Acid in Organic Solvents}

\subsection{Critical evaluation of experimental solubility data}

There have been several publications ${ }^{54,63,72,76,83,125}$ reporting the solubility of 3-bromobenzoic acid in organic solvents. Chantooni and Kolthoff ${ }^{83}$ determined the solubility of 3bromobenzoic acid in methanol and ethanenitrile at $298 \mathrm{~K}$. In a followup study ${ }^{76}$ the authors extended their measurements to include 2-propanol, 2-methyl-2-propanol, and 1hexanol. Hancock et $a l .{ }^{54,63}$ measured the solubility of 3bromobenzoic acid in cyclohexane, benzene, tetrahydrofuran, and 1,4-dioxane at $303 \mathrm{~K}$ based on a gravimetric method. Thuaire ${ }^{72}$ determined the solubility of 3-bromobenzoic acid in ethanol at $298 \mathrm{~K}$ as part of study involving solubilities of benzoic acids in binary aqueous-ethanol solvent mixtures. Pinsuwan et al. ${ }^{125}$ measured the solubility of 3-bromobenzoic acid in 1-octanol to examine correlations between experimental octanol/water solubility ratios and measured water-to-1octanol practical partition coefficients. It is not possible to perform a critical evaluation of the experimental data as each of the research groups performed measurements at only a single temperature, and there are no independent experimental 3-bromobenzoic acid solubility data in any of the 11 aforementioned organic solvents.

The experimental solubility data for 3-bromobenzoic acid in organic solvents are given in Secs. 16.2-16.6.
16.2. 3-Bromobenzoic acid solubility data in saturated hydrocarbons (including cycloalkanes)

\begin{tabular}{|c|c|}
\hline $\begin{array}{l}\text { Components: } \\
\text { (1) 3-Bromobenzoic acid; } \\
\mathrm{C}_{7} \mathrm{H}_{5} \mathrm{BrO}_{2} ;[585-76-2] \\
\text { (2) Cyclohexane; } \mathrm{C}_{6} \mathrm{H}_{12} \text {; } \\
\text { [110-82-7] }\end{array}$ & $\begin{array}{l}\text { Original Measurements: } \\
{ }^{54} \text { C. K. Hancock, J. N. Pawloski, and J. } \\
\text { P. Idoux, J. Org. Chem. 31, } 3801 \\
\text { (1966). }\end{array}$ \\
\hline $\begin{array}{l}\text { Variables: } \\
T / \mathrm{K}=303.15\end{array}$ & $\begin{array}{l}\text { Prepared by: } \\
\text { W. E. Acree, Jr. }\end{array}$ \\
\hline
\end{tabular}

Experimental Values

\begin{tabular}{lc}
\hline \hline$x_{2}{ }^{\mathrm{a}}$ & $x_{1}{ }^{\mathrm{b}}$ \\
\hline 0.9984 & 0.00160 \\
\hline${ }^{\mathrm{a}} x_{2}:$ mole fraction of component 2 in the saturated solution. & \\
${ }^{\mathrm{b}} x_{1}:$ mole fraction solubility of the solute. &
\end{tabular}

Auxiliary Information

Method/Apparatus/Procedure:

Constant-temperature bath, Soxhlet thimble, conical flask, and analytical balance.

Excess solute and solvent were placed in a conical flask and allowed to equilibrate for several days at constant temperature. Attainment of equilibrium was verified by several repetitive measurements and by approaching equilibrium from supersaturation. Aliquots of saturated solutions were transferred using a Soxhlet thimble equipped with a one-hole cork stopper and an inverted U-shaped delivery tube to a second ground-glass stoppered flask suspended in the $303 \mathrm{~K}$ water bath. Successive portions of the contents were evaporated at room temperature in a tared aluminum foil weighing dish under a bell jar through which a slow stream of dry air was passed. The second flask plus unused saturated solution and the aluminum foil dish plus residue were weighed. The saturation solubility of the solute was calculated from the recorded mass data and molar masses of the solute and solvent.

Source and Purity of Chemicals:

(1) Purity not given, Chemical source not specified, was recrystallized several times from aqueous-ethanol mixture, and then dried under vacuum over phosphorous pentoxide.

(2) Reagent grade, Chemical source not specified, refluxed over phosphorous pentoxide for $24 \mathrm{~h}$, and then distilled through a $3 \times 80 \mathrm{~cm}$ column filled with $0.32-\mathrm{cm}$ glass helices.

Estimated Error:

Temperature: $\pm 0.02 \mathrm{~K}$.

$x_{1}: \pm 3 \%$ (relative error).

\subsection{3-Bromobenzoic acid solubility data in aromatic hydrocarbons}

\begin{tabular}{ll}
\hline \hline Components: & Original Measurements: \\
(1) 3-Bromobenzoic acid; & ${ }^{54} \mathrm{C}$. K. Hancock, J. N. Pawloski, and J. \\
$\mathrm{C}_{7} \mathrm{H}_{5} \mathrm{BrO}_{2} ;[585-76-2]$ & P. Idoux, J. Org. Chem. 31, 3801 \\
$(2)$ Benzene; $\mathrm{C}_{6} \mathrm{H}_{6} ;[71-43-2]$ & $(1966)$. \\
\hline Variables: & Prepared by: \\
$T / \mathrm{K}=303.15$ & W. E. Acree, Jr. \\
\hline
\end{tabular}


Experimental Values

\begin{tabular}{lc}
\hline \hline$x_{2}{ }^{\mathrm{a}}$ & $x_{1}{ }^{\mathrm{b}}$ \\
\hline 0.9898 & 0.0102 \\
\hline${ }^{\mathrm{a}} x_{2}:$ mole fraction of component 2 in the saturated solution. \\
${ }^{\mathrm{b}}{ }_{x_{1}: \text { mole fraction solubility of the solute. }}$
\end{tabular}

\section{Auxiliary Information}

\section{Method/Apparatus/Procedure:}

Constant-temperature bath, Soxhlet thimble, conical flask, and analytical balance.

Excess solute and solvent were placed in a conical flask and allowed to equilibrate for several days at constant temperature. Attainment of equilibrium was verified by several repetitive measurements and by approaching equilibrium from supersaturation. Aliquots of saturated solutions were transferred using a Soxhlet thimble equipped with a one-hole cork stopper and an inverted U-shaped delivery tube to a second ground-glass stoppered flask suspended in the $303 \mathrm{~K}$ water bath. Successive portions of the contents were evaporated at room temperature in a tared aluminum foil weighing dish under a bell jar through which a slow stream of dry air was passed. The second flask plus unused saturated solution and the aluminum foil dish plus residue were weighed. The saturation solubility of the solute was calculated from the recorded mass data and molar masses of the solute and solvent.

\section{Source and Purity of Chemicals:}

(1) Purity not given, Chemical source not specified, was recrystallized several times from aqueous-ethanol mixture, and then dried under vacuum over phosphorous pentoxide.

(2) Reagent grade, Chemical source not specified, refluxed over phosphorous pentoxide for $24 \mathrm{~h}$, and then distilled through a $3 \times 80 \mathrm{~cm}$ column filled with 0.32 -cm glass helices.

Estimated Error:

Temperature: $\pm 0.02 \mathrm{~K}$.

$x_{1}: \pm 3 \%$ (relative error).

\subsection{3-Bromobenzoic acid solubility data in ethers}

\begin{tabular}{|c|c|}
\hline $\begin{array}{l}\text { Components: } \\
\text { (1) 3-Bromobenzoic acid; } \\
\mathrm{C}_{7} \mathrm{H}_{5} \mathrm{BrO}_{2} ; \text { [585-76-2] } \\
\text { (2) Tetrahydrofuran; } \mathrm{C}_{4} \mathrm{H}_{8} \mathrm{O} \text {; } \\
\text { [109-99-9] }\end{array}$ & $\begin{array}{l}\text { Original Measurements: } \\
{ }^{63} \text { C. K. Hancock, J. N. Pawloski, and J. } \\
\text { P. Idoux, J. Org. Chem. 32, } 1931 \\
\text { (1967). }\end{array}$ \\
\hline $\begin{array}{l}\text { Variables: } \\
T / \mathrm{K}=303.15\end{array}$ & $\begin{array}{l}\text { Prepared by: } \\
\text { W. E. Acree, Jr. }\end{array}$ \\
\hline
\end{tabular}

\section{Experimental Values}

\begin{tabular}{lc}
\hline \hline$x_{2}{ }^{\mathrm{a}}$ & $x_{1}{ }^{\mathrm{b}}$ \\
\hline 0.751 & 0.249 \\
\hline${ }^{a} x_{2}:$ mole fraction of component 2 in the saturated solution. & \\
${ }^{\mathrm{b}} x_{1}:$ mole fraction solubility of the solute. &
\end{tabular}

\section{Auxiliary Information}

Method/Apparatus/Procedure:

Constant-temperature bath, Soxhlet thimble, conical flask, and analytical balance.

Excess solute and solvent were placed in a conical flask and allowed to equilibrate for several days at constant temperature. Attainment of equilibrium was verified by several repetitive measurements and by approaching equilibrium from supersaturation. Aliquots of saturated solutions were transferred using a Soxhlet thimble equipped with a one-hole cork stopper and an inverted U-shaped delivery tube to a second ground-glass stoppered flask suspended in the $303 \mathrm{~K}$ water bath. Successive portions of the contents were evaporated at room temperature in a tared aluminum foil weighing dish under a bell jar through which a slow stream of dry air was passed. The second flask plus unused saturated solution and the aluminum foil dish plus residue were weighed. The saturation solubility of the solute was calculated from the recorded mass data and molar masses of the solute and solvent.

\section{Source and Purity of Chemicals:}

(1) Purity not given, Chemical source not specified, was recrystallized several times from aqueous-ethanol mixture, and then dried under vacuum over phosphorous pentoxide.

(2) Practical grade, Chemical source not specified, stored over sodium hydroxide pellets for $24 \mathrm{~h}$, and then passed through $2 \times 70-\mathrm{cm}$ chromatographic adsorption columns containing activated alumina. After this treatment, the purified solvent was stored over copper in a nitrogen atmosphere.

\section{Estimated Error:}

Temperature: $\pm 0.02 \mathrm{~K}$.

$x_{1}: \pm 1.0 \%$ (relative error)

\begin{tabular}{|c|c|}
\hline $\begin{array}{l}\text { Components: } \\
\text { (1) 3-Bromobenzoic acid; } \\
\mathrm{C}_{7} \mathrm{H}_{5} \mathrm{BrO}_{2} \text {; [585-76-2] } \\
\text { (2) 1,4-Dioxane; } \mathrm{C}_{4} \mathrm{H}_{8} \mathrm{O}_{2} \text {; } \\
\text { [123-91-1] }\end{array}$ & $\begin{array}{l}\text { Original Measurements: } \\
{ }^{63} \text { C. K. Hancock, J. N. Pawloski, and J } \\
\text { P. Idoux, J. Org. Chem. 32, } 1931 \\
\text { (1967). }\end{array}$ \\
\hline $\begin{array}{l}\text { Variables: } \\
T / \mathrm{K}=303.15\end{array}$ & $\begin{array}{l}\text { Prepared by: } \\
\text { W. E. Acree, Jr. }\end{array}$ \\
\hline
\end{tabular}

\section{Experimental Values}

\begin{tabular}{lc}
\hline \hline$x_{2}{ }^{\mathrm{a}}$ & $x_{1}{ }^{\mathrm{b}}$ \\
\hline 0.817 & 0.183 \\
\hline${ }^{\mathrm{a}} x_{2}:$ mole fraction of component 2 in the saturated solution. & \\
${ }^{\mathrm{b}} x_{1}:$ mole fraction solubility of the solute. &
\end{tabular}

\section{Auxiliary Information}

\section{Method/Apparatus/Procedure:}

Constant-temperature bath, Soxhlet thimble, conical flask, and analytical balance.

Excess solute and solvent were placed in a conical flask and allowed to equilibrate for several days at constant temperature. Attainment of equilibrium was verified by several repetitive measurements and by approaching equilibrium from supersaturation. Weighed aliquots of saturated solutions were removed and titrated with a standardized sodium hydroxide solution (carbonate free) using a pH meter. The endpoint of the titration was determined by computing the second derivative in the $\mathrm{pH}$ versus volume of sodium hydroxide added. 


\section{Source and Purity of Chemicals:}

(1) Purity not given, Chemical source not specified, was recrystallized several times from aqueous-ethanol mixture, and then dried under vacuum over phosphorous pentoxide.

(2) Practical grade, Chemical source not specified, stored over sodium hydroxide pellets for $24 \mathrm{~h}$, and then passed through $2 \times 70-\mathrm{cm}$ chromatographic adsorption columns containing activated alumina. After this treatment, the purified solvent was stored over copper in a nitrogen atmosphere.

Estimated Error:

Temperature: $\pm 0.02 \mathrm{~K}$.

$x_{1}: \pm 1.0 \%$ (relative error).

\subsection{3-Bromobenzoic acid solubility data in alcohols}

\begin{tabular}{|c|c|}
\hline $\begin{array}{l}\text { Components: } \\
\text { (1) 3-Bromobenzoic acid; } \\
\mathrm{C}_{7} \mathrm{H}_{5} \mathrm{BrO}_{2} ;[585-76-2] \\
\text { (2) Methanol; } \mathrm{CH}_{4} \mathrm{O} ;[67-56-1]\end{array}$ & $\begin{array}{l}\text { Original Measurements: } \\
{ }^{83} \text { M. K. Chantooni and I. M. Kolthoff, } \\
\text { J. Phys. Chem. 77, } 527 \text { (1973). }\end{array}$ \\
\hline $\begin{array}{l}\text { Variables: } \\
T / \mathrm{K}=298.15\end{array}$ & $\begin{array}{l}\text { Prepared by: } \\
\text { W. E. Acree, Jr. }\end{array}$ \\
\hline
\end{tabular}

\section{Experimental Values}

The measured solubility was reported to be $1.51 \mathrm{~mol} \mathrm{dm}^{-3}$. The authors did not give the temperature at which the solubility was measured. Based on the experimental description given in an earlier paper [I. M. Kolthoff, J. J. Lingane, and W. Larson, J. Am. Chem. Soc. 60, 2512 (1938)], the compiler believes the temperature to be $298.15 \mathrm{~K}$.

\section{Auxiliary Information}

\section{Method/Apparatus/Procedure:}

Very little experimental details were provided. Solubility was determined by titrating a filtered aliquot of the saturated solution alkalimetrically in an aqueous-ethanol mixture using phenolphthalein as the acid-base indicator.

\section{Source and Purity of Chemicals:}

(1) White Label, Eastman Kodak Chemical Company, Rochester, NY, USA, was recrystallized from acetone and dried in vacuo at $343 \mathrm{~K}$.

(2) Purity not given, Chemical source not given, was dehydrated and then distilled over sulfanilic acid to remove alkaline impurities.

\section{Estimated Error:}

Temperature: $\pm 0.1 \mathrm{~K}$ (estimated by compiler). $c_{1}: \pm 2 \%$ (relative error, estimated by compiler).

\begin{tabular}{|c|c|}
\hline $\begin{array}{l}\text { Components: } \\
\text { (1) 3-Bromobenzoic acid; } \\
\mathrm{C}_{7} \mathrm{H}_{5} \mathrm{BrO}_{2} ;[585-76-2] \\
\text { (2) Ethanol; } \mathrm{C}_{2} \mathrm{H}_{6} \mathrm{O} ;[64-17-5]\end{array}$ & $\begin{array}{l}\text { Original Measurements: } \\
{ }^{72} \text { R. Thuaire, Bull. Soc. Chim. Fr. } 3815 \\
\text { (1971). }\end{array}$ \\
\hline $\begin{array}{l}\text { Variables: } \\
T / \mathrm{K}=298.15\end{array}$ & $\begin{array}{l}\text { Prepared by: } \\
\text { W. E. Acree, Jr. }\end{array}$ \\
\hline
\end{tabular}

\section{Experimental Values}

The measured solubility was reported to be $1.955 \mathrm{~mol} / \mathrm{kg}$ of solvent, which corresponds to a mole fraction solubility of $x_{1}=0.0826$.

\section{Auxiliary Information}

\begin{abstract}
Method/Apparatus/Procedure:
Very little experimental details were provided. Solubility was determined by a gravimetric method. The saturated solution was evaporated to dryness and the remaining solid residue was weighed. The solubility was calculated from the mass of the solid residue and mass of saturated solution analyzed.
\end{abstract}

\section{Source and Purity of Chemicals:}

(1) Purity not given, Chemical source not given, no purification details were provided.

(2) Purity not given, Chemical source not given, no purification details were provided.

\section{Estimated Error:}

Temperature: $\pm 0.05 \mathrm{~K}$.

$x_{1}: \pm 4 \%$ (relative error, estimated by compiler).

\begin{tabular}{|c|c|}
\hline $\begin{array}{l}\text { Components: } \\
\text { (1) 3-Bromobenzoic acid; } \\
\mathrm{C}_{7} \mathrm{H}_{5} \mathrm{BrO}_{2} \text {; [585-76-2] } \\
\text { (2) 2-Propanol; } \mathrm{C}_{3} \mathrm{H}_{8} \mathrm{O} ;[67-63-0]\end{array}$ & $\begin{array}{l}\text { Original Measurements: } \\
{ }^{76} \text { M. K. Chantooni and I. M. Kolthoff, } \\
\text { Anal. Chem. 51, } 133 \text { (1979). }\end{array}$ \\
\hline $\begin{array}{l}\text { Variables: } \\
T / \mathrm{K}=298.15\end{array}$ & $\begin{array}{l}\text { Prepared by: } \\
\text { W. E. Acree, Jr. }\end{array}$ \\
\hline
\end{tabular}

\section{Experimental Values}

The measured solubility was reported to be $1.20 \mathrm{~mol} \mathrm{dm}^{-3}$. The authors did not give the temperature at which the solubility was measured. Based on the experimental description given in an earlier paper [I. M. Kolthoff, J. J. Lingane, and W. Larson, J. Am. Chem. Soc. 60, 2512 (1938)], the compiler believes the temperature to be $298.15 \mathrm{~K}$.

\section{Auxiliary Information}

Method/Apparatus/Procedure:
Very little experimental details were provided. Solubility was determined by
titrating a filtered aliquot of the saturated solution alkalimetrically in an
aqueous-ethanol mixture using phenolphthalein as the acid-base indicator.
Source and Purity of Chemicals:
(1) White Label, Eastman Kodak Chemical Company, Rochester, NY, USA,
was recrystallized from acetone and dried in vacuo at $343 \mathrm{~K}$.
(2) No Purity given, Fisher Scientific, USA, shaken with calcium hydride and
distilled before use.
Estimated Error:
Temperature: $\pm 0.1 \mathrm{~K}$ (estimated by compiler).
$c_{1}: \pm 2 \%$ (relative error, estimated by compiler).




\begin{tabular}{|c|c|}
\hline $\begin{array}{l}\text { Components: } \\
\text { (1) 3-Bromobenzoic acid; } \\
\mathrm{C}_{7} \mathrm{H}_{5} \mathrm{BrO}_{2} \text {; [585-76-2] } \\
\text { (2) 2-Methyl-2-propanol; } \\
\mathrm{C}_{4} \mathrm{H}_{10} \mathrm{O} ;[75-65-0]\end{array}$ & $\begin{array}{l}\text { Original Measurements: } \\
{ }^{76} \text { M. K. Chantooni and I. M. Kolthoff, } \\
\text { Anal. Chem. 51, } 133 \text { (1979). }\end{array}$ \\
\hline $\begin{array}{l}\text { Variables: } \\
T / \mathrm{K}=298.15\end{array}$ & $\begin{array}{l}\text { Prepared by: } \\
\text { W. E. Acree, Jr. }\end{array}$ \\
\hline
\end{tabular}

Experimental Values

The measured solubility was reported to be $1.35 \mathrm{~mol} \mathrm{dm}^{-3}$. The authors did not give the temperature at which the solubility was measured. Based on the experimental description given in an earlier paper [I. M. Kolthoff, J. J. Lingane, and W. Larson, J. Am. Chem. Soc. 60, 2512 (1938)], the compiler believes the temperature to be $298.15 \mathrm{~K}$.

\section{Auxiliary Information}

\section{Method/Apparatus/Procedure:}

Very little experimental details were provided. Solubility was determined by titrating a filtered aliquot of the saturated solution alkalimetrically in an aqueous-ethanol mixture using phenolphthalein as the acid-base indicator.

Source and Purity of Chemicals:

(1) White Label, Eastman Kodak Chemical Company, Rochester, NY, USA, was recrystallized from acetone and dried in vacuo at $343 \mathrm{~K}$.

(2) White Label, Eastman Kodak Chemical Company, Rochester, NY, USA, shaken with calcium hydride and distilled before use.

\section{Estimated Error:}

Temperature: $\pm 0.1 \mathrm{~K}$ (estimated by compiler).

$c_{1}: \pm 2 \%$ (relative error, estimated by compiler).

\begin{tabular}{|c|c|}
\hline $\begin{array}{l}\text { Components: } \\
\text { (1) 3-Bromobenzoic acid; } \\
\mathrm{C}_{7} \mathrm{H}_{5} \mathrm{BrO}_{2} ;[585-76-2] \\
\text { (2) } 1-\mathrm{Hexanol} ; \mathrm{C}_{6} \mathrm{H}_{14} \mathrm{O} \text {; } \\
\text { [111-27-3] }\end{array}$ & $\begin{array}{l}\text { Original Measurements: } \\
{ }^{76} \text { M. K. Chantooni and I. M. Kolthoff, } \\
\text { Anal. Chem. 51, } 133 \text { (1979). }\end{array}$ \\
\hline $\begin{array}{l}\text { Variables: } \\
T / \mathrm{K}=298.15\end{array}$ & $\begin{array}{l}\text { Prepared by: } \\
\text { W. E. Acree, Jr. }\end{array}$ \\
\hline
\end{tabular}

Experimental Values

The measured solubility was reported to be $0.99 \mathrm{~mol} \mathrm{dm}^{-3}$. The authors did not give the temperature at which the solubility was measured. Based on the experimental description given in an earlier paper [I. M. Kolthoff, J. J. Lingane, and W. Larson, J. Am. Chem. Soc. 60, 2512 (1938)], the compiler believes the temperature to be $298.15 \mathrm{~K}$.

\section{Auxiliary Information}

\footnotetext{
Method/Apparatus/Procedure:

Very little experimental details were provided. Solubility was determined by titrating a filtered aliquot of the saturated solution alkalimetrically in an aqueous-ethanol mixture using phenolphthalein as the acid-base indicator.
}

Source and Purity of Chemicals:

(1) White Label, Eastman Kodak Chemical Company, Rochester, NY, USA, was recrystallized from acetone and dried in vacuo at $343 \mathrm{~K}$.
(2) Yellow Label, Eastman Kodak Chemical Company, Rochester, NY, USA, shaken with calcium hydride and distilled before use.

Estimated Error:

Temperature: $\pm 0.1 \mathrm{~K}$ (estimated by compiler). $c_{1}: \pm 2 \%$ (relative error, estimated by compiler).

\begin{tabular}{|c|c|}
\hline $\begin{array}{l}\text { Components: } \\
\text { (1) 3-Bromobenzoic acid; } \\
\left.\mathrm{C}_{7} \mathrm{H}_{5} \mathrm{BrO}_{2} ; \text {; } 585-76-2\right] \\
\text { (2) } 1-O c t a n o l ; \mathrm{C}_{8} \mathrm{H}_{18} \mathrm{O} \text {; } \\
\text { [111-87-5] }\end{array}$ & $\begin{array}{l}\text { Original Measurements: } \\
{ }^{125} \text { S. Pinsuwan, A. Li, and S. H. } \\
\text { Yalkowsky, J. Chem. Eng. Data 40, } \\
623 \text { (1995). }\end{array}$ \\
\hline $\begin{array}{l}\text { Variables: } \\
T / \mathrm{K}=298.15\end{array}$ & $\begin{array}{l}\text { Prepared by: } \\
\text { W. E. Acree, Jr. }\end{array}$ \\
\hline
\end{tabular}

\section{Experimental Values}

The measured solubility was reported to be $\log _{10} c_{1}=$ -0.07 , which corresponds to a molar solubility of $c_{1}=0.851$.

\section{Auxiliary Information}

\section{Method/Apparatus/Procedure:}

Constant-temperature bath, analytical balance, mechanical stirrer, and ultraviolet/visible spectrophotometer.

Excess solute and solvent were equilibrated at $(298 \pm 2) \mathrm{K}$ in a sealed vial for 48 to $72 \mathrm{~h}$. The solution was mixed using an end-over-end mechanical stirrer. After equilibrium was obtained, the saturated sample was vacuum filtered through a $5.0 \mu \mathrm{m}$ fritted glass filter. An aliquot of the filtrate was diluted quantitatively with 2-propanol, and the absorbance of the diluted sample was recorded. The solubility was calculated from the measured absorbance.

Source and Purity of Chemicals:

(1) Purity not given, Aldrich Chemical Company, Milwaukee, WI, USA, used as received.

(2) Purity not given, Chemical source not given, used as received.

\section{Estimated Error:}

Temperature: $\pm 2 \mathrm{~K}$.

$c_{1}: \pm 10 \%$ (relative error, estimated by compiler given the large uncertainty in temperature).

\subsection{3-Bromobenzoic acid solubility data in miscellaneous organic solvents}

\begin{tabular}{|c|c|}
\hline $\begin{array}{l}\text { Components: } \\
\text { (1) 3-Bromobenzoic acid; } \\
\mathrm{C}_{7} \mathrm{H}_{5} \mathrm{BrO}_{2} ;[585-76-2] \\
\text { (2) Ethanenitrile; } \mathrm{C}_{2} \mathrm{H}_{3} \mathrm{~N} \text {; } \\
\text { [75-05-8] }\end{array}$ & $\begin{array}{l}\text { Original Measurements: } \\
{ }^{83} \text { M. K. Chantooni and I. M. Kolthoff, } \\
\text { J. Phys. Chem. 77, } 527 \text { (1973). }\end{array}$ \\
\hline $\begin{array}{l}\text { Variables: } \\
T / \mathrm{K}=298.15\end{array}$ & $\begin{array}{l}\text { Prepared by: } \\
\text { W. E. Acree, Jr. }\end{array}$ \\
\hline
\end{tabular}

Experimental Values

The measured solubility was reported to be $0.167 \mathrm{~mol} \mathrm{dm}^{-3}$. The authors did not give the temperature at which the solubility was measured. Based on the experimental description given in an earlier paper [I. M. Kolthoff, J. J. Lingane, and W. Larson, 
J. Am. Chem. Soc. 60, 2512 (1938)], the compiler believes the temperature to be $298.15 \mathrm{~K}$.

\section{Auxiliary Information}

\begin{abstract}
Method/Apparatus/Procedure:
Very little experimental details were provided. Solubility was determined by titrating a filtered aliquot of the saturated solution alkalimetrically in an aqueous-ethanol mixture using phenolphthalein as the acid-base indicator.
\end{abstract}

Source and Purity of Chemicals:

(1) White Label, Eastman Kodak Chemical Company, Rochester, NY, USA, was recrystallized from acetone and dried in vacuo at $343 \mathrm{~K}$.

(2) Purity not given, Chemical source not given, was purified by shaking with saturated potassium hydroxide, followed by activated alumina, and then anhydrous calcium chloride to remove water. Ethanenitrile was further dried over anhydrous magnesium sulfate and then phosphorous pentoxide. The sample was distilled shortly before use.

Estimated Error:

Temperature: $\pm 0.1 \mathrm{~K}$ (estimated by compiler).

$c_{1}: \pm 2 \%$ (relative error, estimated by compiler).

\section{Solubility of 4-Bromobenzoic Acid in Organic Solvents}

\subsection{Critical evaluation of experimental solubility data}

There have been several publications ${ }^{54,72,76,83,125}$ reporting the solubility of 4-bromobenzoic acid in organic solvents. Chantooni and Kolthoff ${ }^{83}$ determined the solubility of 4bromobenzoic acid in methanol, ethanenitrile, dimethyl sulfoxide, and $N, N$-dimethylformamide at $298 \mathrm{~K}$. In a followup study, the authors ${ }^{76}$ extended their measurements to include 2propanol, 2-methyl-2-propanol, and 1-hexanol. Hancock et $a l .{ }^{54}$ measured the solubility of 4-bromobenzoic acid in cyclohexane and benzene at $303 \mathrm{~K}$ based on a gravimetric method. Thuaire ${ }^{72}$ determined the solubility of 4-bromobenzoic acid in ethanol at $298 \mathrm{~K}$ as part of a study involving solubilities of benzoic acids in binary aqueous-ethanol solvent mixtures. Pinsuwan et al. ${ }^{125}$ measured the solubility of 4bromobenzoic acid in 1-octanol to examine correlations between experimental octanol/water solubility ratios and measured water-to-1-octanol practical partition coefficients. It is not possible to perform a critical evaluation of the experimental data as each of the research groups performed measurements at only a single temperature, and there are no independent experimental 4-bromobenzoic acid solubility data in any of the 11 organic solvents.

The experimental solubility data for 4-bromobenzoic acid in organic solvents are given in Secs. 17.2-17.5.
17.2. 4-Bromobenzoic acid solubility data in saturated hydrocarbons (including cycloalkanes)

\begin{tabular}{ll}
\hline \hline Components: & Original Measurements: \\
$(1)$ 4-Bromobenzoic acid; & ${ }^{54} \mathrm{C} . \mathrm{K}$. Hancock, J. N. Pawloski, and J. \\
$\mathrm{C}_{7} \mathrm{H}_{5} \mathrm{BrO}_{2} ;[586-76-5]$ & P. Idoux, J. Org. Chem. 31, 3801 \\
$\begin{array}{l}(2) \text { Cyclohexane; } \mathrm{C}_{6} \mathrm{H}_{12} ; \\
{[110-82-7]}\end{array}$ & $(1966)$. \\
\hline Variables: & Prepared by: \\
$T / \mathrm{K}=303.15$ & W. E. Acree, Jr. \\
\hline
\end{tabular}

Experimental Values

\begin{tabular}{lc}
\hline \hline$x_{2}{ }^{\mathrm{a}}$ & $x_{1}{ }^{\mathrm{b}}$ \\
\hline 0.9999 & 0.0000362 \\
\hline${ }^{\mathrm{a}} x_{2}:$ mole fraction of component 2 in the saturated solution. & \\
${ }^{\mathrm{b}} x_{1}:$ mole fraction solubility of the solute. &
\end{tabular}

Auxiliary Information

Method/Apparatus/Procedure:

Constant-temperature bath, Soxhlet thimble, conical flask, and analytical balance.

Excess solute and solvent were placed in a conical flask and allowed to equilibrate for several days at constant temperature. Attainment of equilibrium was verified by several repetitive measurements and by approaching equilibrium from supersaturation. Aliquots of saturated solutions were transferred using a Soxhlet thimble equipped with a one-hole cork stopper and an inverted U-shaped delivery tube to a second ground-glass stoppered flask suspended in the $303 \mathrm{~K}$ water bath. Successive portions of the contents were evaporated at room temperature in a tared aluminum foil weighing dish under a bell jar through which a slow stream of dry air was passed. The second flask plus unused saturated solution and the aluminum foil dish plus residue were weighed. The saturation solubility of the solute was calculated from the recorded mass data and molar masses of the solute and solvent.

Source and Purity of Chemicals:

(1) Purity not given, Chemical source not specified, was recrystallized several times from aqueous-ethanol mixture, and then dried under vacuum over phosphorous pentoxide.

(2) Reagent grade, Chemical source not specified, refluxed over phosphorous pentoxide for $24 \mathrm{~h}$, and then distilled through a $3 \times 80 \mathrm{~cm}$ column filled with 0.32 -cm glass helices.

Estimated Error:

Temperature: $\pm 0.02 \mathrm{~K}$

$x_{1}: \pm 10 \%$ (relative error, by compiler).

\subsection{4-Bromobenzoic acid solubility data in aromatic hydrocarbons}

\begin{tabular}{|c|c|}
\hline $\begin{array}{l}\text { Components: } \\
\text { (1) 4-Bromobenzoic acid; } \\
\mathrm{C}_{7} \mathrm{H}_{5} \mathrm{BrO}_{2} ;[586-76-5] \\
\text { (2) Benzene; } \mathrm{C}_{6} \mathrm{H}_{6} ;[71-43-2]\end{array}$ & $\begin{array}{l}\text { Original Measurements: } \\
{ }^{54} \text { C. K. Hancock, J. N. Pawloski, and J. } \\
\text { P. Idoux, J. Org. Chem. 31, } 3801 \\
\text { (1966). }\end{array}$ \\
\hline $\begin{array}{l}\text { Variables: } \\
T / \mathrm{K}=303.15\end{array}$ & $\begin{array}{l}\text { Prepared by: } \\
\text { W. E. Acree, Jr. }\end{array}$ \\
\hline
\end{tabular}


Experimental Values

\begin{tabular}{lc}
\hline \hline$x_{2}{ }^{\mathrm{a}}$ & $x_{1}{ }^{\mathrm{b}}$ \\
\hline 0.9997 & 0.000272 \\
${ }^{\mathrm{a}} x_{2}:$ mole fraction of component 2 in the saturated solution. & \\
${ }^{\mathrm{b}} x_{1}:$ mole fraction solubility of the solute. &
\end{tabular}

\section{Auxiliary Information}

\section{Method/Apparatus/Procedure:}

Constant-temperature bath, Soxhlet thimble, conical flask, and analytical balance.

Excess solute and solvent were placed in a conical flask and allowed to equilibrate for several days at constant temperature. Attainment of equilibrium was verified by several repetitive measurements and by approaching equilibrium from supersaturation. Aliquots of saturated solutions were transferred using a Soxhlet thimble equipped with a one-hole cork stopper and an inverted U-shaped delivery tube to a second ground-glass stoppered flask suspended in the $303 \mathrm{~K}$ water bath. Successive portions of the contents were evaporated at room temperature in a tared aluminum foil weighing dish under a bell jar through which a slow stream of dry air was passed. The second flask plus unused saturated solution and the aluminum foil dish plus residue were weighed. The saturation solubility of the solute was calculated from the recorded mass data and molar masses of the solute and solvent.

\section{Source and Purity of Chemicals:}

(1) Purity not given, Chemical source not specified, was recrystallized several times from aqueous-ethanol mixture, and then dried under vacuum over phosphorous pentoxide.

(2) Reagent grade, Chemical source not specified, refluxed over phosphorous pentoxide for $24 \mathrm{~h}$, and then distilled through a $3 \times 80 \mathrm{~cm}$ column filled with 0.32 -cm glass helices.

\section{Estimated Error:}

Temperature: $\pm 0.02 \mathrm{~K}$.

$x_{1}: \pm 5 \%$ (relative error, by compiler).

\subsection{4-Bromobenzoic acid solubility data in alcohols}

\begin{tabular}{|c|c|}
\hline $\begin{array}{l}\text { Components: } \\
\text { (1) 4-Bromobenzoic acid; } \\
\mathrm{C}_{7} \mathrm{H}_{5} \mathrm{BrO}_{2} ;[586-76-5] \\
\text { (2) Methanol; } \mathrm{CH}_{4} \mathrm{O} ;[67-56-1]\end{array}$ & $\begin{array}{l}\text { Original Measurements: } \\
{ }^{83} \text { M. K. Chantooni and I. M. Kolthoff, } \\
\text { J. Phys. Chem. 77, } 527 \text { (1973). }\end{array}$ \\
\hline $\begin{array}{l}\text { Variables: } \\
T / \mathrm{K}=298.15\end{array}$ & $\begin{array}{l}\text { Prepared by: } \\
\text { W. E. Acree, Jr. }\end{array}$ \\
\hline
\end{tabular}

\section{Experimental Values}

The measured solubility was reported to be $0.12 \mathrm{~mol} \mathrm{dm}^{-3}$. The authors did not give the temperature at which the solubility was measured. Based on the experimental description given in an earlier paper [I. M. Kolthoff, J. J. Lingane, and W. Larson, J. Am. Chem. Soc. 60, 2512 (1938)], the compiler believes the temperature to be $298.15 \mathrm{~K}$.

\section{Auxiliary Information}

\section{Method/Apparatus/Procedure:}

Very little experimental details were provided. Solubility was determined by titrating a filtered aliquot of the saturated solution alkalimetrically in an aqueous-ethanol mixture using phenolphthalein as the acid-base indicator.

Source and Purity of Chemicals:

(1) Purity not given, Chemical source not given, was recrystallized from either aqueous or aqueous-ethanol solution and dried in vacuo at $333 \mathrm{~K}$.

(2) Purity not given, Chemical source not given, was dehydrated and then distilled over sulfanilic acid to remove alkaline impurities.

Estimated Error:

Temperature: $\pm 0.1 \mathrm{~K}$ (estimated by compiler). $c_{1}: \pm 2 \%$ (relative error, estimated by compiler).

\begin{tabular}{|c|c|}
\hline $\begin{array}{l}\text { Components: } \\
\text { (1) 4-Bromobenzoic acid; } \\
\mathrm{C}_{7} \mathrm{H}_{5} \mathrm{BrO}_{2} ;[586-76-5] \\
\text { (2) Ethanol; } \mathrm{C}_{2} \mathrm{H}_{6} \mathrm{O} ;[64-17-5]\end{array}$ & $\begin{array}{l}\text { Original Measurements: } \\
{ }^{72} \text { R. Thuaire, Bull. Soc. Chim. Fr. } 3815 \\
\text { (1971). }\end{array}$ \\
\hline $\begin{array}{l}\text { Variables: } \\
T / \mathrm{K}=298.15\end{array}$ & $\begin{array}{l}\text { Prepared by: } \\
\text { W. E. Acree, Jr. }\end{array}$ \\
\hline
\end{tabular}

\section{Experimental Values}

The measured solubility was reported to be $0.164 \mathrm{~mol} / \mathrm{kg}$ of solvent, which corresponds to a mole fraction solubility of $x_{1}=0.00750$.

\section{Auxiliary Information}

\section{Method/Apparatus/Procedure:}

Very little experimental details were provided. Solubility was determined by a gravimetric method. The saturated solution was evaporated to dryness and the remaining solid residue was weighed. The solubility was calculated from the mass of the solid residue and mass of saturated solution analyzed.

Source and Purity of Chemicals:

(1) Purity not given, Chemical source not given, no purification details were provided.

(2) Purity not given, Chemical source not given, no purification details were provided.

\section{Estimated Error:}

Temperature: $\pm 0.05 \mathrm{~K}$

$x_{1}: \pm 4 \%$ (relative error, estimated by compiler).

\begin{tabular}{|c|c|}
\hline $\begin{array}{l}\text { Components: } \\
\text { (1) 4-Bromobenzoic acid; } \\
\mathrm{C}_{7} \mathrm{H}_{5} \mathrm{BrO}_{2} ;[586-76-5] \\
\text { (2) 2-Propanol; } \mathrm{C}_{3} \mathrm{H}_{8} \mathrm{O} ;[67-63-0]\end{array}$ & $\begin{array}{l}\text { Original Measurements: } \\
{ }^{76} \text { M. K. Chantooni and I. M. Kolthoff, } \\
\text { Anal. Chem. 51, } 133 \text { (1979). }\end{array}$ \\
\hline $\begin{array}{l}\text { Variables: } \\
T / \mathrm{K}=298.15\end{array}$ & $\begin{array}{l}\text { Prepared by: } \\
\text { W. E. Acree, Jr. }\end{array}$ \\
\hline
\end{tabular}

Experimental Values

The measured solubility was reported to be $0.112 \mathrm{~mol} \mathrm{dm}^{-3}$. The authors did not give the temperature at which the solubility was measured. Based on the experimental description given in an earlier paper [I. M. Kolthoff, J. J. Lingane, and W. Larson, 
J. Am. Chem. Soc. 60, 2512 (1938)], the compiler believes the temperature to be $298.15 \mathrm{~K}$.

\section{Auxiliary Information}

\section{Method/Apparatus/Procedure:}

Very little experimental details were provided. Solubility was determined by titrating a filtered aliquot of the saturated solution alkalimetrically in an aqueous-ethanol mixture using phenolphthalein as the acid-base indicator.

\section{Source and Purity of Chemicals:}

(1) Purity not given, Chemical source not given, was recrystallized from either aqueous or aqueous-ethanol solution and dried in vacuo at $333 \mathrm{~K}$.

(2) No Purity given, Fisher Scientific, USA, shaken with calcium hydride and distilled before use.

\section{Estimated Error:}

Temperature: $\pm 0.1 \mathrm{~K}$ (estimated by compiler).

$c_{1}: \pm 2 \%$ (relative error, estimated by compiler).

\begin{tabular}{|c|c|}
\hline $\begin{array}{l}\text { Components: } \\
\text { (1) 4-Bromobenzoic acid; } \\
\mathrm{C}_{7} \mathrm{H}_{5} \mathrm{BrO}_{2} \text {; [586-76-5] } \\
\text { (2) 2-Methyl-2-propanol; } \\
\mathrm{C}_{4} \mathrm{H}_{10} \mathrm{O} \text {; [75-65-0] }\end{array}$ & $\begin{array}{l}\text { Original Measurements: } \\
{ }^{76} \text { M. K. Chantooni and I. M. Kolthoff, } \\
\text { Anal. Chem. 51, } 133 \text { (1979). }\end{array}$ \\
\hline $\begin{array}{l}\text { Variables: } \\
T / \mathrm{K}=298.15 \mathrm{~K}\end{array}$ & $\begin{array}{l}\text { Prepared by: } \\
\text { W. E. Acree, Jr. }\end{array}$ \\
\hline
\end{tabular}

\section{Experimental Values}

The measured solubility was reported to be $0.125 \mathrm{~mol} \mathrm{dm}^{-3}$. The authors did not give the temperature at which the solubility was measured. Based on the experimental description given in an earlier paper [I. M. Kolthoff, J. J. Lingane, and W. Larson, J. Am. Chem. Soc. 60, 2512 (1938)], the compiler believes the temperature to be $298.15 \mathrm{~K}$.

\section{Auxiliary Information}

Method/Apparatus/Procedure:
Very little experimental details were provided. Solubility was determined by
titrating a filtered aliquot of the saturated solution alkalimetrically in an
aqueous-ethanol mixture using phenolphthalein as the acid-base indicator.
Source and Purity of Chemicals:
(1) Purity not given, Chemical source not given, was recrystallized from either
aqueous or aqueous-ethanol solution and dried in vacuo at $333 \mathrm{~K}$.
(2) White Label, Eastman Kodak Chemical Company, Rochester, NY, USA,
shaken with calcium hydride and distilled before use.
Estimated Error:
Temperature: $\pm 0.1 \mathrm{~K}$ (estimated by compiler).
$c_{1}: \pm 2 \%$ (relative error, estimated by compiler).

\begin{tabular}{|c|c|}
\hline $\begin{array}{l}\text { Components: } \\
\text { (1) 4-Bromobenzoic acid; } \\
\mathrm{C}_{7} \mathrm{H}_{5} \mathrm{BrO}_{2} ;[586-76-5] \\
\text { (2) } 1-\mathrm{Hexanol} ; \mathrm{C}_{6} \mathrm{H}_{14} \mathrm{O} ;[111-27-3]\end{array}$ & $\begin{array}{l}\text { Original Measurements: } \\
{ }^{76} \text { M. K. Chantooni and I. M. Kolthoff, } \\
\text { Anal. Chem. 51, } 133 \text { (1979). }\end{array}$ \\
\hline $\begin{array}{l}\text { Variables: } \\
T / \mathrm{K}=298.15\end{array}$ & $\begin{array}{l}\text { Prepared by: } \\
\text { W. E. Acree, Jr. }\end{array}$ \\
\hline
\end{tabular}

\section{Experimental Values}

The measured solubility was reported to be $0.103 \mathrm{~mol} \mathrm{dm}^{-3}$. The authors did not give the temperature at which the solubility was measured. Based on the experimental description given in an earlier paper [I. M. Kolthoff, J. J. Lingane, and W. Larson, J. Am. Chem. Soc. 60, 2512 (1938)], the compiler believes the temperature to be $298.15 \mathrm{~K}$.

\section{Auxiliary Information}

\section{Method/Apparatus/Procedure:}

Very little experimental details were provided. Solubility was determined by titrating a filtered aliquot of the saturated solution alkalimetrically in an aqueous-ethanol mixture using phenolphthalein as the acid-base indicator.

Source and Purity of Chemicals:

(1) Purity not given, Chemical source not given, was recrystallized from either aqueous or aqueous-ethanol solution and dried in vасио at $333 \mathrm{~K}$.

(2) Yellow Label, Eastman Kodak Chemical Company, Rochester, NY, USA, shaken with calcium hydride and distilled before use.

\section{Estimated Error:}

Temperature: $\pm 0.1 \mathrm{~K}$ (estimated by compiler). $c_{1}: \pm 2 \%$ (relative error, estimated by compiler).

\begin{tabular}{|c|c|}
\hline $\begin{array}{l}\text { Components: } \\
\text { (1) 4-Bromobenzoic acid; } \\
\mathrm{C}_{7} \mathrm{H}_{5} \mathrm{BrO}_{2} ;[586-76-5] \\
\text { (2) } 1-\text { Octanol; } \mathrm{C}_{8} \mathrm{H}_{18} \mathrm{O} ;[111-87-5]\end{array}$ & $\begin{array}{l}\text { Original Measurements: } \\
{ }^{125} \text { S. Pinsuwan, A. Li, and S. H. } \\
\text { Yalkowsky, J. Chem. Eng. Data 40, } \\
623 \text { (1995). }\end{array}$ \\
\hline $\begin{array}{l}\text { Variables: } \\
T / \mathrm{K}=298.15\end{array}$ & $\begin{array}{l}\text { Prepared by: } \\
\text { W. E. Acree, Jr. }\end{array}$ \\
\hline
\end{tabular}

\section{Experimental Values}

The measured solubility was reported to be $\log _{10} c_{1}=$ -1.11 , which corresponds to a molar solubility of $c_{1}=0.0776$.

\section{Auxiliary Information}

\section{Method/Apparatus/Procedure:}

Constant-temperature bath, analytical balance, mechanical stirrer, and ultraviolet/visible spectrophotometer.

Excess solute and solvent were equilibrated at $(298 \pm 2) \mathrm{K}$ in a sealed vial for 48 to $72 \mathrm{~h}$. The solution was mixed using an end-over-end mechanical stirrer. After equilibrium was obtained, the saturated sample was vacuum filtered through a $5.0 \mu \mathrm{m}$ fritted glass filter. An aliquot of the filtrate was diluted quantitatively with 2-propanol, and the absorbance of the diluted sample was recorded. The solubility was calculated from the measured absorbance.

\section{Source and Purity of Chemicals:}

(1) Purity not given, Aldrich Chemical Company, Milwaukee, WI, USA, used as received.

(2) Purity not given, Chemical source not given, used as received.

\section{Estimated Error:}

Temperature: $\pm 2 \mathrm{~K}$.

$c_{1}: \pm 10 \%$ (relative error, estimated by compiler given the large uncertainty in temperature). 


\subsection{4-Bromobenzoic acid solubility data in miscellaneous organic solvents}

\begin{tabular}{ll}
\hline \hline Components: & Original Measurements: \\
$\begin{array}{l}\text { (1) 4-Bromobenzoic acid; } \\
\mathrm{C}_{7} \mathrm{H}_{5} \mathrm{BrO}_{2} ;[586-76-5]\end{array}$ & ${ }^{83} \mathrm{M}$. K. Chantooni and I. M. Kolthoff, \\
$\begin{array}{l}\text { (2) Ethanenitrile; } \mathrm{C}_{2} \mathrm{H}_{3} \mathrm{~N} ; \\
{[75-05-8]}\end{array}$ & J. Phys. Chem. 77, 527 (1973). \\
\hline Variables: & Prepared by: \\
$T / \mathrm{K}=298.15$ & W. E. Acree, Jr. \\
\hline
\end{tabular}

Experimental Values

The measured solubility was reported to be $0.018 \mathrm{~mol} \mathrm{dm}^{-3}$. The authors did not give the temperature at which the solubility was measured. Based on the experimental description given in an earlier paper [I. M. Kolthoff, J. J. Lingane, and W. Larson, J. Am. Chem. Soc. 60, 2512 (1938)], the compiler believes the temperature to be $298.15 \mathrm{~K}$.

\section{Auxiliary Information}

\section{Method/Apparatus/Procedure:}

Very little experimental details were provided. Solubility was determined by titrating a filtered aliquot of the saturated solution alkalimetrically in an aqueous-ethanol mixture using phenolphthalein as the acid-base indicator.

\section{Source and Purity of Chemicals:}

(1) Purity not given, Chemical source not given, was recrystallized from either aqueous or aqueous-ethanol solution and dried in vacuo at $333 \mathrm{~K}$.

(2) Purity not given, Chemical source not given, was purified by shaking with saturated potassium hydroxide, followed by activated alumina, and then anhydrous calcium chloride to remove water. Ethanenitrile was further dried over anhydrous magnesium sulfate and then phosphorous pentoxide. The sample was distilled shortly before use.

Estimated Error:

Temperature: $\pm 0.1 \mathrm{~K}$ (estimated by compiler). $c_{1}: \pm 2 \%$ (relative error, estimated by compiler).

\begin{tabular}{|c|c|}
\hline $\begin{array}{l}\text { Components: } \\
\text { (1) } 4-\text {-Bromobenzoic acid; } \\
\mathrm{C}_{7} \mathrm{H}_{5} \mathrm{BrO}_{2} \text {; [586-76-5] } \\
\text { (2) Dimethyl sulfoxide; } \\
\mathrm{C}_{2} \mathrm{H}_{6} \mathrm{OS} ;[67-68-5]\end{array}$ & $\begin{array}{l}\text { Original Measurements: } \\
{ }^{83} \text { M. K. Chantooni and I. M. Kolthoff, } \\
\text { J. Phys. Chem. 77, } 527 \text { (1973). }\end{array}$ \\
\hline $\begin{array}{l}\text { Variables: } \\
T / \mathrm{K}=298.15\end{array}$ & $\begin{array}{l}\text { Prepared by: } \\
\text { W. E. Acree, Jr. }\end{array}$ \\
\hline
\end{tabular}

Experimental Values

The measured solubility was reported to be $2.45 \mathrm{~mol} \mathrm{dm}^{-3}$. The authors did not give the temperature at which the solubility was measured. Based on the experimental description given in an earlier paper [I. M. Kolthoff, J. J. Lingane, and W. Larson, J. Am. Chem. Soc. 60, 2512 (1938)], the compiler believes the temperature to be $298.15 \mathrm{~K}$.

\section{Auxiliary Information}

Method/Apparatus/Procedure:

Very little experimental details were provided. Solubility was determined by titrating a filtered aliquot of the saturated solution alkalimetrically in an aqueous-ethanol mixture using phenolphthalein as the acid-base indicator.

Source and Purity of Chemicals:

(1) Purity not given, Chemical source not given, was recrystallized from either aqueous or aqueous-ethanol solution and dried in vacuo at $333 \mathrm{~K}$.

(2) Purity not given, Chemical source not given, was shaken with activated alumina and then distilled before use.

Estimated Error:

Temperature: $\pm 0.1 \mathrm{~K}$ (estimated by compiler). $c_{1}: \pm 2 \%$ (relative error, estimated by compiler).

\begin{tabular}{|c|c|}
\hline $\begin{array}{l}\text { Components: } \\
\text { (1) 4-Bromobenzoic acid; } \\
\mathrm{C}_{7} \mathrm{H}_{5} \mathrm{BrO}_{2} \text {; [586-76-5] } \\
\text { (2) } \mathrm{N}, \mathrm{N} \text {-Dimethylformamide; } \\
\mathrm{C}_{3} \mathrm{H}_{7} \mathrm{NO} \text {; [64-19-7] }\end{array}$ & $\begin{array}{l}\text { Original Measurements: } \\
{ }^{83} \text { M. K. Chantooni and I. M. Kolthoff, } \\
\text { J. Phys. Chem. 77, } 527 \text { (1973). }\end{array}$ \\
\hline $\begin{array}{l}\text { Variables: } \\
T / \mathrm{K}=298.15\end{array}$ & $\begin{array}{l}\text { Prepared by: } \\
\text { W. E. Acree, Jr. }\end{array}$ \\
\hline
\end{tabular}

Experimental Values

The measured solubility was reported to be $0.93 \mathrm{~mol} \mathrm{dm}^{-3}$. The authors did not give the temperature at which the solubility was measured. Based on the experimental description given in an earlier paper [I. M. Kolthoff, J. J. Lingane, and W. Larson, J. Am. Chem. Soc. 60, 2512 (1938)], the compiler believes the temperature to be $298.15 \mathrm{~K}$.

\section{Auxiliary Information}

\section{Method/Apparatus/Procedure:}

Very little experimental details were provided. Solubility was determined by titrating a filtered aliquot of the saturated solution alkalimetrically in an aqueous-ethanol mixture using phenolphthalein as the acid-base indicator.

Source and Purity of Chemicals:

(1) Purity not given, Chemical source not given, was recrystallized from either aqueous or aqueous-ethanol solution and dried in vacuo at $333 \mathrm{~K}$.

(2) Purity not given, Chemical source not given, was shaken first with phosphorous pentoxide and then with potassium hydroxide pellets. Solvent was distilled shortly before use.

Estimated Error:

Temperature: $\pm 0.1 \mathrm{~K}$ (estimated by compiler). $c_{1}: \pm 2 \%$ (relative error, estimated by compiler).

\section{Solubility of 2-Chlorobenzoic Acid in Organic Solvents}

\subsection{Critical evaluation of experimental solubility data}

There have been several published studies $54,72,83,99,126,127$ investigating the solubility behavior of 2-chlorobenzoic acid in organic solvents of varying polarity and hydrogen-bonding capability. Chantooni and Kolthoff ${ }^{83}$ determined the solubility 
of 2-chlorobenzoic acid in methanol, ethanenitrile, and $\mathrm{N}, \mathrm{N}$ dimethylformamide at $298 \mathrm{~K}$. Hancock et al. ${ }^{54}$ measured the solubility of 2-chlorobenzoic acid in cyclohexane and benzene at $303 \mathrm{~K}$ based on a gravimetric method. Thuaire ${ }^{72}$ determined the solubility of 2-chlorobenzoic acid in ethanol at $298 \mathrm{~K}$ as part of study involving solubilities of benzoic acids in binary aqueous-ethanol solvent mixtures. Biswas et al. ${ }^{126}$ also measured the solubility of 2-chlorobenzoic acid in ethanol at $298 \mathrm{~K}$. The two independent experimental determinations of the mole fraction solubility of 2-chlorobenzoic acid in ethanol differ significantly from one another, $x_{1}=0.0708$ (Biswas value, Ref. 126) versus $x_{1}=0.1424$ (Thuaire value, Ref. 72). Given only two independent measurements it is normally impossible to know of the two values is best. However, in the present case, both research groups have performed independent experimental measurements for other substituted benzoic acid derivatives in ethanol. For several of the other solutes (e.g., 3-chlorobenzoic acid, 4-chlorobenzoic acid, and 4-nitrobenzoic acid), there does exist at least one additional set of observed values. One finds that the measured values reported by Thuaire $^{72}$ are in better agreement with experimental data reported by other research groups than are the observed values reported by Biswas et al. ${ }^{126}$

Sidgwick and Ewbank ${ }^{127}$ measured the solubility of 2chlorobenzoic acid in heptane and benzene as a function of temperature. The internal consistency of the two datasets was assessed by curve-fitting the measured mole fraction solubility data to the Modified Apelblat model to yield the following two representations:

$$
\begin{aligned}
& \ln x_{1}=-167.18+\frac{112.27}{T}+27.681 \ln T, \\
& \ln x_{1}=-93.217+\frac{113.76}{T}+15.491 \ln T,
\end{aligned}
$$

for solubilities in heptane and benzene, respectively. The average absolute deviations between the observed experimental data and back-calculated values based on Eqs. (41) and (42) of $26.1 \%$ and $8.3 \%$ are larger than desired. The large deviation may be due in part to the fact that the solution temperature is above the solvent's normal boiling point temperature, to uncertainties in estimating the amount of solvent that remained in the liquid phase, and the large range covered by the experimental values, $x_{1}=0.0166$ to $x_{1}=0.834$ for heptane and $x_{1}=0.0100$ to $x_{1}=0.833$ for benzene. It is more difficult to curve-fit experimental solubility data covering large mole fraction ranges.

Jia et al. ${ }^{99}$ determined the solubility of 2-chlorobenzoic acid in 1-octanol in the temperature range of 293-323 K. The calculated curve-fit parameters from the Buchowski $\lambda$ h-model [Eq. (9)] of $\lambda=1.885$ and $h=1567.8$ described the observed solubility data to within a mean relative deviation of $1.7 \%$.

The experimental solubility data for 2-chlorobenzoic acid in organic solvents are in Secs. 18.2-18.5.
18.2. 2-Chlorobenzoic acid solubility data in

\begin{tabular}{|c|c|}
\hline $\begin{array}{l}\text { Components: } \\
\text { (1) 2-Chlorobenzoic acid; } \\
\mathrm{C}_{7} \mathrm{H}_{5} \mathrm{ClO}_{2} ;[118-91-2] \\
\text { (2) Heptane; } \mathrm{C}_{7} \mathrm{H}_{16} ;[142-82-5]\end{array}$ & $\begin{array}{l}\text { Original Measurements: } \\
\text { 127 N. V. Sidgwick and E. K. Ewbank, J. } \\
\text { Chem. Soc. Trans. 119, } 979 \text { (1921). }\end{array}$ \\
\hline $\begin{array}{l}\text { Variables: } \\
\text { Temperature }\end{array}$ & $\begin{array}{l}\text { Prepared by: } \\
\text { W. E. Acree, Jr. }\end{array}$ \\
\hline
\end{tabular}
saturated hydrocarbons (including cycloalkanes)

Experimental Values

\begin{tabular}{lcc}
\hline \hline$T / \mathrm{K}$ & $x_{2}{ }^{\mathrm{a}}$ & $x_{1}{ }^{\mathrm{b}}$ \\
\hline 352.2 & 0.983 & 0.0166 \\
368.0 & 0.970 & 0.0300 \\
382.0 & 0.931 & 0.0693 \\
386.0 & 0.916 & 0.0841 \\
399.2 & 0.728 & 0.272 \\
401.2 & 0.591 & 0.409 \\
403.0 & 0.415 & 0.585 \\
407.9 & 0.166 & 0.834 \\
\hline
\end{tabular}

${ }^{a} x_{2}$ : mole fraction of component 2 in the saturated solution.

${ }^{\mathrm{b}} x_{1}$ : mole fraction solubility of the solute. Solubility data reported in units of mass percent. Mole fraction values calculated by the compiler.

\section{Auxiliary Information}

\begin{abstract}
Method/Apparatus/Procedure:
Preweighed quantities of solute and solvent were placed in bulbs, which were then sealed and heated in a suitable bath. At the higher temperatures, the concentration of solvent in the liquid solution was corrected for the amount of solvent vapor in the bulb. The correction assumed that the vapor pressure of the saturated solution was one half that of the pure solvent at the solution temperature. The temperature at which the solid completely dissolved was recorded, and was taken to be the solid-liquid equilibrium temperature.
\end{abstract}

Source and Purity of Chemicals:

(1) Purity not given, prepared and purified by the authors using a published synthetic procedure [see J. Walker and J. K. Wood, J. Chem. Soc. 117, 40 (1920) for synthetic details]. Melting point of the purified solute was $413.5 \mathrm{~K}$. (2) Purity not given, sample isolated from the resin of Pinus sabiniana, purified by treatment with sulfuric acid and nitric acid, and then distilled before use.

\section{Estimated Error:}

Temperature: Not given in paper.

$x_{1}: \pm 10 \%$ (relative error, estimated by compiler).

\subsection{2-Chlorobenzoic acid solubility data

\begin{tabular}{|c|c|}
\hline $\begin{array}{l}\text { Components: } \\
\text { (1) 2-Chlorobenzoic acid; } \\
\mathrm{C}_{7} \mathrm{H}_{5} \mathrm{ClO}_{2} ;[118-91-2] \\
\text { (2) Benzene; } \mathrm{C}_{6} \mathrm{H}_{6} ;[71-43-2]\end{array}$ & $\begin{array}{l}\text { Original Measurements: } \\
{ }^{127} \text { N. V. Sidgwick and E. K. Ewbank, J. } \\
\text { Chem. Soc. Trans. 119, } 979 \text { (1921). }\end{array}$ \\
\hline $\begin{array}{l}\text { Variables: } \\
\text { Temperature }\end{array}$ & $\begin{array}{l}\text { Prepared by: } \\
\text { W. E. Acree, Jr. }\end{array}$ \\
\hline
\end{tabular} in aromatic hydrocarbons}


Experimental Values

\begin{tabular}{lcc}
\hline \hline$T / \mathrm{K}$ & $x_{2}{ }^{\mathrm{a}}$ & $x_{1}{ }^{\mathrm{b}}$ \\
\hline 299.2 & 0.990 & 0.0100 \\
318.0 & 0.974 & 0.0264 \\
330.9 & 0.948 & 0.0520 \\
355.9 & 0.825 & 0.175 \\
372.8 & 0.666 & 0.334 \\
387.1 & 0.457 & 0.543 \\
402.7 & 0.167 & 0.833 \\
\hline
\end{tabular}

${ }^{\mathrm{a}} x_{2}$ : mole fraction of component 2 in the saturated solution.

${ }^{b} x_{1}$ : mole fraction solubility of the solute. Solubility data reported in units of mass percent. Mole fraction values calculated by the compiler.

\section{Auxiliary Information}

\section{Method/Apparatus/Procedure:}

Preweighed quantities of solute and solvent were placed in bulbs, which were then sealed and heated in a suitable bath. At the higher temperatures, the concentration of solvent in the liquid solution was corrected for the amount of solvent vapor in the bulb. The correction assumed that the vapor pressure of the saturated solution was one half that of the pure solvent at the solution temperature. The temperature at which the solid completely dissolved was recorded, and was taken to be the solid-liquid equilibrium temperature.

\section{Source and Purity of Chemicals:}

(1) Purity not given, prepared and purified by the authors using a published synthetic procedure [see J. Walker and J. K. Wood, J. Chem. Soc. 117, 40 (1920) for synthetic details]. Melting point of the purified solute was $413.5 \mathrm{~K}$. (2) Purity not given, Chemical source not given, thiophene was removed by treatment with sulfuric acid. Sample was further purified by freezing several times.

\section{Estimated Error:}

Temperature: Not given in paper.

$x_{1}: \pm 10 \%$ (relative error, estimated by compiler).

\subsection{2-Chlorobenzoic acid solubility data in alcohols}

\begin{tabular}{|c|c|}
\hline $\begin{array}{l}\text { Components: } \\
\text { (1) 2-Chlorobenzoic acid; } \\
\mathrm{C}_{7} \mathrm{H}_{5} \mathrm{ClO}_{2} ;[118-91-2] \\
\text { (2) Methanol; } \mathrm{CH}_{4} \mathrm{O} ;[67-56-1]\end{array}$ & $\begin{array}{l}\text { Original Measurements: } \\
{ }^{83} \text { M. K. Chantooni and I. M. Kolthoff, } \\
\text { J. Phys. Chem. 77, } 527 \text { (1973). }\end{array}$ \\
\hline $\begin{array}{l}\text { Variables: } \\
T / \mathrm{K}=298.15\end{array}$ & $\begin{array}{l}\text { Prepared by: } \\
\text { W. E. Acree, Jr. }\end{array}$ \\
\hline
\end{tabular}

\section{Experimental Values}

The measured solubility was reported to be $2.53 \mathrm{~mol} \mathrm{dm}^{-3}$. The authors did not give the temperature at which the solubility was measured. Based on the experimental description given in an earlier paper [I. M. Kolthoff, J. J. Lingane, and W. Larson, J. Am. Chem. Soc. 60, 2512 (1938)], the compiler believes the temperature to be $298.15 \mathrm{~K}$.

\section{Auxiliary Information}

\section{Method/Apparatus/Procedure:}

Very little experimental details were provided. Solubility was determined by titrating a filtered aliquot of the saturated solution alkalimetrically in an aqueous-ethanol mixture using phenolphthalein as the acid-base indicator.

Source and Purity of Chemicals:

(1) White Label, Eastman Kodak Chemical Company, Rochester, NY, USA, was recrystallized from either aqueous or aqueous-ethanol solution and dried in vacuo at $333 \mathrm{~K}$.

(2) Purity not given, Chemical source not given, was dehydrated and then distilled over sulfanilic acid to remove alkaline impurities.

Estimated Error:

Temperature: $\pm 0.1 \mathrm{~K}$ (estimated by compiler). $c_{1}: \pm 2 \%$ (relative error, estimated by compiler).

\begin{tabular}{ll}
\hline \hline Components: & Original Measurements: \\
(1) 2-Chlorobenzoic acid; & ${ }^{126} \mathrm{P}$. K. Biswas, S. C. Lahiri, and B. P. \\
$\mathrm{C}_{7} \mathrm{H}_{5} \mathrm{ClO}_{2} ;[118-91-2]$ & Dey, Bull. Chem. Soc. Jpn. 66, 2785 \\
(2) Ethanol; $\mathrm{C}_{2} \mathrm{H}_{6} \mathrm{O} ;[64-17-5]$ & $(1993)$. \\
\hline Variables: & Prepared by: \\
$T / \mathrm{K}=298.15$ & W. E. Acree, Jr. \\
\hline
\end{tabular}

Experimental Values

\begin{tabular}{lcc}
\hline \hline$c_{1}^{\mathrm{a}}$ & $x_{2}{ }^{\mathrm{b}}$ & $x_{1}{ }^{\mathrm{c}}$ \\
\hline 1.1275 & 0.9292 & 0.0708
\end{tabular}

${ }^{\mathrm{a}} c_{1}$ : solubility of the solute expressed in units of $\mathrm{mol} \mathrm{dm}{ }^{-3}$.

${ }^{\mathrm{b}} x_{2}$ : mole fraction of component 2 in the saturated solution.

${ }^{c} x_{1}$ : mole fraction solubility of the solute calculated by the compiler.

Auxiliary Information

Method/Apparatus/Procedure:

Analytical balance and an ultraviolet/visible spectrophotometer.

Solutions containing excess solute and solvent were allowed to equilibrate at constant temperature for at least $24 \mathrm{~h}$. An aliquot of the saturated solution was removed, filtered, and the absorbance recorded. Solubility was calculated from the measured absorbance.

\section{Source and Purity of Chemicals:}

(1) Puris grade, Fluka Chemicals, recrystallized from aqueous ethanol mixture and dried in an air oven at $390 \mathrm{~K}$. The purified compound was stored in vacuum desiccators.

(2) Absolute, B.C.P.W., Calcutta, India, was distilled twice before use.

\section{Estimated Error:}

Temperature: $\pm 0.2 \mathrm{~K}$ (estimated by compiler).

$c_{1}: \pm 1.0 \%$ (relative error, estimated by compiler).

$x_{1}: \pm 2.5 \%$ (relative error, estimated by compiler).

\begin{tabular}{|c|c|}
\hline $\begin{array}{l}\text { Components: } \\
\text { (1) 2-Chlorobenzoic acid; } \\
\mathrm{C}_{7} \mathrm{H}_{5} \mathrm{ClO}_{2} ;[118-91-2] \\
\text { (2) Ethanol; } \mathrm{C}_{2} \mathrm{H}_{6} \mathrm{O} ;[64-17-5]\end{array}$ & $\begin{array}{l}\text { Original Measurements: } \\
{ }^{72} \text { R. Thuaire, Bull. Soc. Chim. Fr. } 3815 \\
\text { (1971). }\end{array}$ \\
\hline $\begin{array}{l}\text { Variables: } \\
T / \mathrm{K}=298.15\end{array}$ & $\begin{array}{l}\text { Prepared by: } \\
\text { W. E. Acree, Jr. }\end{array}$ \\
\hline
\end{tabular}




\section{Experimental Values}

The measured solubility was reported to be $3.607 \mathrm{~mol} / \mathrm{kg}$ of solvent, which corresponds to a mole fraction solubility of $x_{1}=0.1424$.

\section{Auxiliary Information}

\section{Method/Apparatus/Procedure:}

Very little experimental details were provided. Solubility was determined by a gravimetric method. The saturated solution was evaporated to dryness and the remaining solid residue was weighed. The solubility was calculated from the mass of the solid residue and mass of saturated solution analyzed.

\section{Source and Purity of Chemicals:}

(1) Purity not given, Chemical source not given, no purification details were provided.

(2) Purity not given, Chemical source not given, no purification details were provided.

\section{Estimated Error:}

Temperature: $\pm 0.05 \mathrm{~K}$.

$x_{1}: \pm 4 \%$ (relative error, estimated by compiler).

\begin{tabular}{|c|c|}
\hline $\begin{array}{l}\text { Components: } \\
\text { (1) 2-Chlorobenzoic acid; } \\
\mathrm{C}_{7} \mathrm{H}_{5} \mathrm{ClO}_{2} ;[118-91-2] \\
\text { (2) 1-Octanol; } \mathrm{C}_{8} \mathrm{H}_{18} \mathrm{O} ;[111-87-5]\end{array}$ & $\begin{array}{l}\text { Original Measurements: } \\
{ }^{99} \text { Q. Jia, P. Ma, S. Ma, and C. Wang, } \\
\text { Chin. J. Chem. Eng. 15, } 710 \text { (2007). }\end{array}$ \\
\hline $\begin{array}{l}\text { Variables: } \\
\text { Temperature }\end{array}$ & $\begin{array}{l}\text { Prepared by: } \\
\text { W. E Acree Jr }\end{array}$ \\
\hline
\end{tabular}

Experimental Values

\begin{tabular}{lcc}
\hline \hline$T / \mathrm{K}$ & $x_{2}{ }^{\mathrm{a}}$ & $x_{1}{ }^{\mathrm{b}}$ \\
\hline 297.65 & 0.8914 & 0.1086 \\
298.55 & 0.8877 & 0.1123 \\
298.90 & 0.8864 & 0.1136 \\
299.10 & 0.8856 & 0.1144 \\
304.75 & 0.8602 & 0.1398 \\
306.40 & 0.8527 & 0.1473 \\
308.00 & 0.8454 & 0.1546 \\
309.55 & 0.8380 & 0.1620 \\
311.15 & 0.8309 & 0.1691 \\
313.05 & 0.8240 & 0.1760 \\
315.10 & 0.8172 & 0.1828 \\
316.70 & 0.8103 & 0.1897 \\
318.65 & 0.8035 & 0.1965 \\
319.95 & 0.7994 & 0.2006
\end{tabular}

${ }^{a} x_{2}$ : mole fraction of component 2 in the saturated solution.

${ }^{b} x_{1}$ : mole fraction solubility of the solute.

\section{Auxiliary Information}

\footnotetext{
Method/Apparatus/Procedure:

Constant-temperature circulating bath, stirrer, analytical balance, and laser monitoring system.

Solubilities were determined by a dynamic method. Preweighed amounts of solute and solvent were placed in a stoppered equilibrium vessel, which was connected to a circulating constant-temperature water bath. The solution was stirred and the temperature slowly increased until all of the solid dissolved. Near the dissolution temperature, the temperature was increased at a rate of $0.2 \mathrm{~K} / 20 \mathrm{~min}$. Complete dissolution was determined using a laser monitoring system.
}

Source and Purity of Chemicals:

(1) $99+\%$, Chemical source not specified, no purification details were provided.

(2) $99+\%$, Chemical source not specified, no purification details were provided.

\section{Estimated Error:}

Temperature: $\pm 0.05 \mathrm{~K}$.

$x_{1}: \pm 1 \%$ (relative error).

\subsection{2-Chlorobenzoic acid solubility data in miscellaneous organic solvents}

\begin{tabular}{|c|c|}
\hline $\begin{array}{l}\text { Components: } \\
\text { (1) 2-Chlorobenzoic acid; } \\
\mathrm{C}_{7} \mathrm{H}_{5} \mathrm{ClO}_{2} ;[118-91-2] \\
\text { (2) Ethanenitrile; } \mathrm{C}_{2} \mathrm{H}_{3} \mathrm{~N} \text {; } \\
\text { [75-05-8] }\end{array}$ & $\begin{array}{l}\text { Original Measurements: } \\
{ }^{83} \text { M. K. Chantooni and I. M. Kolthoff, } \\
\text { J. Phys. Chem. 77, } 527 \text { (1973). }\end{array}$ \\
\hline $\begin{array}{l}\text { Variables: } \\
T / \mathrm{K}=298.15\end{array}$ & $\begin{array}{l}\text { Prepared by: } \\
\text { W. E. Acree, Jr. }\end{array}$ \\
\hline
\end{tabular}

Experimental Values

The measured solubility was reported to be $0.53 \mathrm{~mol} \mathrm{dm}^{-3}$. The authors did not give the temperature at which the solubility was measured. Based on the experimental description given in an earlier paper [I. M. Kolthoff, J. J. Lingane, and W. Larson, J. Am. Chem. Soc. 60, 2512 (1938)], the compiler believes the temperature to be $298.15 \mathrm{~K}$.

\section{Auxiliary Information}

\section{Method/Apparatus/Procedure:}

Very little experimental details were provided. Solubility was determined by titrating a filtered aliquot of the saturated solution alkalimetrically in an aqueous-ethanol mixture using phenolphthalein as the acid-base indicator.

\section{Source and Purity of Chemicals:}

(1) White Label, Eastman Kodak Chemical Company, Rochester, NY, USA, was recrystallized from either aqueous or aqueous-ethanol solution and dried in vacuo at $333 \mathrm{~K}$.

(2) Purity not given, Chemical source not given, was purified by shaking with saturated potassium hydroxide, followed by activated alumina, and then anhydrous calcium chloride to remove water. Ethanenitrile was further dried over anhydrous magnesium sulfate and then phosphorous pentoxide. The sample was distilled shortly before use.

\section{Estimated Error:}

Temperature: $\pm 0.1 \mathrm{~K}$ (estimated by compiler). $c_{1}: \pm 2 \%$ (relative error, estimated by compiler).

\begin{tabular}{|c|c|}
\hline $\begin{array}{l}\text { Components: } \\
\text { (1) 2-Chlorobenzoic acid; } \\
\mathrm{C}_{7} \mathrm{H}_{5} \mathrm{ClO}_{2} \text {; [118-91-2] } \\
\text { (2) } \mathrm{N}, \mathrm{N} \text {-Dimethylformamide; } \\
\mathrm{C}_{3} \mathrm{H}_{7} \mathrm{NO} ;[64-19-7]\end{array}$ & $\begin{array}{l}\text { Original Measurements: } \\
{ }^{83} \text { M. K. Chantooni and I. M. Kolthoff, } \\
\text { J. Phys. Chem. 77, } 527 \text { (1973). }\end{array}$ \\
\hline $\begin{array}{l}\text { Variables: } \\
T / \mathrm{K}=298.15\end{array}$ & $\begin{array}{l}\text { Prepared by: } \\
\text { W. E. Acree, Jr. }\end{array}$ \\
\hline
\end{tabular}




\section{Experimental Values}

The measured solubility was reported to be $4.74 \mathrm{~mol} \mathrm{dm}^{-3}$. The authors did not give the temperature at which the solubility was measured. Based on the experimental description given in an earlier paper [I. M. Kolthoff, J. J. Lingane, and W. Larson, J. Am. Chem. Soc. 60, 2512 (1938)], the compiler believes the temperature to be $298.15 \mathrm{~K}$.

\section{Auxiliary Information}

\section{Method/Apparatus/Procedure:}

Very little experimental details were provided. Solubility was determined by titrating a filtered aliquot of the saturated solution alkalimetrically in an aqueous-ethanol mixture using phenolphthalein as the acid-base indicator.

\section{Source and Purity of Chemicals:}

(1) White Label, Eastman Kodak Chemical Company, Rochester, NY, USA, was recrystallized from either aqueous or aqueous-ethanol solution and dried in vacuo at $333 \mathrm{~K}$

(2) Purity not given, Chemical source not given, was shaken first with phosphorous pentoxide and then with potassium hydroxide pellets. Solvent was distilled shortly before use.

\section{Estimated Error:}

Temperature: $\pm 0.1 \mathrm{~K}$ (estimated by compiler). $c_{1}: \pm 2 \%$ (relative error, estimated by compiler).

\section{Solubility of 3-Chlorobenzoic Acid in Organic Solvents}

\subsection{Critical evaluation of experimental solubility data}

There have been several published studies $8,18,54,61,72,126,127$ investigating the solubility behavior of 3-chlorobenzoic acid in organic solvents of varying polarity and hydrogen-bonding capability. Hoover et al. ${ }^{8}$ measured the solubility of 3-chlorobenzoic acid in 11 alcohols (1-propanol, 2-propanol, 1-butanol, 2-butanol, 2-methyl-1-propanol, 1-pentanol, 3-methyl-1-butanol, 1-hexanol, 1-heptanol, 1-octanol, and 1-decanol), in three dialkyl ethers (1,1'-oxybisethane, 2,2'-oxybispropane, and 1,1'oxybisbutane) and one cyclic ether (tetrahydrofuran), and in three alkyl alkanoates (ethyl ethanoate, butyl ethanoate, and pentyl ethanoate) at $298 \mathrm{~K}$. Results of the experimental measurements were used to calculate the Abraham solute descriptors of 3chlorobenzoic acid. The authors were able to assemble a total of $37 \log _{10}(S R$ or $P)$ and $\log _{10}(G S R$ or $K$ ) equations for which experimental partition coefficient data, solubility ratios, Abraham Model equation coefficients and aqueous molar solubility were available. The logarithm of the aqueous molar solubility of 3-chlorobenzoic acid is $\log _{10} c_{1, \mathrm{w}}=-2.65 .^{72}$ The McGowan volume of 3-chlorobenzoic acid, $V=1.0541$, was calculated from the number of chemical bonds in the molecule and the individual atomic group volumes, $A V_{i}$, given in Sec. 1.3. The excess molar refraction solute descriptor was estimated as $E=0.840$. This left four solute descriptors $(S, A, B$ and $L$ ) still to be determined. The 37 equations were then solved using the Microsoft "SOLVER" program to yield numerical values of the remaining four solute descriptors, $S=0.950, A=0.630$, $B=0.320$, and $L=5.197$, that best described the $\log _{10}(S R$ or $P)$
TABLE 21. Comparison between observed and calculated molar solubilities of 3-chlorobenzoic acid based on the Abraham model, Eqs. (20) and (21)

\begin{tabular}{lrrr}
\hline \hline Solvent & $\log _{10} c_{1}{ }^{\text {exp }}$ & $\begin{array}{c}\log _{10} c_{1}{ }^{\text {calc }} ; \\
\text { Eq. }(20)\end{array}$ & $\begin{array}{r}\log _{10} c_{1}{ }^{\text {calc}} \text {; } \\
\text { Eq. }(21)\end{array}$ \\
\hline Ethanol & $0.073^{\mathrm{a}}$ & 0.105 & 0.077 \\
1-Propanol & $0.042^{\mathrm{b}}$ & 0.003 & -0.001 \\
2-Propanol & $0.054^{\mathrm{b}}$ & 0.024 & -0.032 \\
1-Butanol & $-0.009^{\mathrm{b}}$ & -0.077 & -0.073 \\
2-Butanol & $-0.018^{\mathrm{b}}$ & -0.044 & -0.071 \\
2-Methyl-1-propanol & $-0.135^{\mathrm{b}}$ & -0.165 & -0.196 \\
1-Pentanol & $-0.035^{\mathrm{b}}$ & -0.067 & -0.064 \\
3-Methyl-1-butanol & $-0.098^{\mathrm{b}}$ & -0.129 & -0.142 \\
1-Hexanol & $-0.069^{\mathrm{b}}$ & -0.112 & -0.121 \\
1-Heptanol & $-0.127^{\mathrm{b}}$ & -0.128 & -0.140 \\
1-Octanol & $-0.146^{\mathrm{b}}$ & -0.189 & -0.244 \\
1-Decanol & $-0.220^{\mathrm{b}}$ & -0.246 & -0.230 \\
1,1'-Oxybisethane & $-0.121^{\mathrm{b}}$ & -0.149 & -0.009 \\
Tetrahydrofuran & $0.400^{\mathrm{b}}$ & 0.476 & 0.463 \\
1,4-Dioxane & $0.215^{\mathrm{c}}$ & 0.160 & 0.152 \\
Ethyl ethanoate & $-0.185^{\mathrm{b}}$ & -0.072 & -0.082 \\
Butyl ethanoate & $-0.298^{\mathrm{b}}$ & -0.204 & -0.239 \\
\hline
\end{tabular}

${ }^{\mathrm{a}}$ Experimental value is from the study of Thuaire. ${ }^{72}$

${ }^{\mathrm{b}}$ Experimental values are from Hoover et al. ${ }^{8}$

${ }^{c}$ Experimental value is from the study of Saifullah et al. ${ }^{18}$

and $\log _{10}(G S R$ or $K)$ values. The computation treated $\log _{10} c_{1, \mathrm{G}}$ as a floating parameter to be determined as part of the regression analyses. The data analyses returned a value of $\log _{10}$ $c_{1, \mathrm{G}}=-7.80$ for the logarithm of the gas-phase solute concentration that made the $\log _{10}\left(S R\right.$ or $P$ ) and $\log _{10}(G S R$ or $K)$ predictions internally consistent. The calculated molecular solute descriptors reproduced the $\log _{10}\left(S R\right.$ or $P$ ) and $\log _{10}(G S R$ or $K$ ) values to within an average standard deviation of 0.081 and $0.088 \log _{10}$ units, respectively.

After the 3-chlorobenzoic acid solubility study was published, Abraham model correlations have been developed for 3-methyl-1-butanol and butyl ethanoate, and equation coefficients for a few solvents were updated based on additional experimental data. The new correlations (listed in Tables 1 and 2) will be used in illustrating the ability of the Abraham model to correlate the experimental 3-chlorobenzoic acid solubility data. Table 21 compares the experimental $\log _{10} c_{1}$ values to calculated values based on Eqs. (20) and (21) of the Abraham model. For comparison purposes, the measured mole fraction solubilities of 3chlorobenzoic acid, $x_{1}$, determined by Hoover et al. ${ }^{8}$ were converted into molar solubilities by dividing $x_{1}$ by the ideal molar volume of the saturated solution (i.e., $c_{1}{ }^{\text {sat }}=x_{1} /\left[x_{1} V_{1}\right.$ $\left.\left.+\left(1-x_{1}\right) V_{\text {solvent }}\right]\right)$. The molar volume of the hypothetical subcooled liquid 3-chlorobenzoic acid is $V_{\text {solute }}=117.08$ $\mathrm{cm}^{3} \mathrm{~mol}^{-1}$. Examination of the numerical entries in Table 21 reveals that the Abraham model provides a reasonably accurate mathematical description of the observed solubility data, suggesting that there are no obvious outliers in the dataset.

Sidgwick and Ewbank ${ }^{127}$ measured the solubility of 3chlorobenzoic acid in heptane and benzene as a function of temperature. The internal consistency of the two datasets was assessed by curve-fitting the measured mole fraction solubility 
data to the Modified Apelblat model to yield the following two representations:

$$
\begin{gathered}
\ln x_{1}=-133.498+\frac{112.89}{T}+22.0061 \ln T, \\
\ln x_{1}=-91.101+\frac{113.72}{T}+15.0631 \ln T,
\end{gathered}
$$

for solubilities in heptane and benzene, respectively. The average absolute deviations between the observed experimental data and back-calculated values based on Eqs. (43) and (44) of $12.8 \%$ and $9.4 \%$ are larger than desired. The large deviations may be due in part to the fact that the solution temperature is above the solvent's normal boiling point temperature, to uncertainties in estimating the amount of solvent that remained in the liquid phase, and the large range covered by the experimental values, $x_{1}=0.0124$ to $x_{1}=0.850$ for heptane and $x_{1}=0.0114$ to $x_{1}=0.821$ for benzene. It is more difficult to curve-fit experimental solubility data covering large mole fraction ranges.

The experimental solubility data for 3-chlorobenzoic acid in organic solvents are in Secs. 19.2-19.6.

\subsection{3-Chlorobenzoic acid solubility data in

\begin{tabular}{|c|c|}
\hline $\begin{array}{l}\text { Components: } \\
\text { (1) 3-Chlorobenzoic acid; } \\
\mathrm{C}_{7} \mathrm{H}_{5} \mathrm{ClO}_{2} ;[535-80-8] \\
\text { (2) Heptane; } \mathrm{C}_{7} \mathrm{H}_{16} ;[142-82-5]\end{array}$ & $\begin{array}{l}\text { Original Measurements: } \\
{ }^{127} \text { N. V. Sidgwick and E. K. Ewbank, J. } \\
\text { Chem. Soc. Trans. 119, } 979 \text { (1921). }\end{array}$ \\
\hline $\begin{array}{l}\text { Variables: } \\
\text { Temperature }\end{array}$ & $\begin{array}{l}\text { Prepared by: } \\
\text { W. E. Acree, Jr. }\end{array}$ \\
\hline
\end{tabular} saturated hydrocarbons (including cycloalkanes)}

Experimental Values

\begin{tabular}{lcc}
\hline \hline$T / \mathrm{K}$ & $x_{2}{ }^{\mathrm{a}}$ & $x_{1}{ }^{\mathrm{b}}$ \\
\hline 345.4 & 0.988 & 0.0124 \\
362.8 & 0.971 & 0.0291 \\
379.0 & 0.934 & 0.0663 \\
401.3 & 0.780 & 0.220 \\
407.4 & 0.609 & 0.391 \\
413.3 & 0.400 & 0.600 \\
420.9 & 0.150 & 0.850 \\
\hline
\end{tabular}

${ }^{\mathrm{a}} x_{2}$ : mole fraction of component 2 in the saturated solution.

${ }^{\mathrm{b}} x_{1}$ : mole fraction solubility of the solute. Solubility data reported in units of mass percent. Mole fraction values calculated by the compiler.

\section{Auxiliary Information}

\footnotetext{
Method/Apparatus/Procedure:

Preweighed quantities of solute and solvent were placed in bulbs, which were then sealed and heated in a suitable bath. At the higher temperatures, the concentration of solvent in the liquid solution was corrected for the amount of solvent vapor in the bulb. The correction assumed that the vapor pressure of the saturated solution was one half that of the pure solvent at the solution temperature. The temperature at which the solid completely dissolved was recorded, and was taken to be the solid-liquid equilibrium temperature.
}

Source and Purity of Chemicals:

(1) Purity not given, prepared and purified by the authors using a published synthetic procedure [see J. Walker and J. K. Wood, J. Chem. Soc. 117, 40 (1920) for synthetic details]. Melting point of the purified solute was $427.7 \mathrm{~K}$. (2) Purity not given, sample isolated from the resin of Pinus sabiniana, purified by treatment with sulfuric acid and nitric acid, and then distilled before use.

\section{Estimated Error:}

Temperature: Not given in paper.

$x_{1}: \pm 10 \%$ (relative error, estimated by compiler).

\begin{tabular}{ll}
\hline \hline Components: & Original Measurements: \\
(1) 3-Chlorobenzoic acid; & ${ }^{54} \mathrm{C} . \mathrm{K}$. Hancock, J. N. Pawloski, and J. \\
$\mathrm{C}_{7} \mathrm{H}_{5} \mathrm{ClO}_{2} ;[535-80-8]$ & P. Idoux, J. Org. Chem. 31, 3801 \\
$\begin{array}{l}(2) \mathrm{Cyclohexane} ; \mathrm{C}_{6} \mathrm{H}_{12} ; \\
{[110-82-7]}\end{array}$ & $(1966)$. \\
\hline Variables: & Prepared by: \\
$T / \mathrm{K}=303.15$ & W. E. Acree, Jr. \\
\hline
\end{tabular}

\section{Experimental Values}

\begin{tabular}{lc}
\hline \hline$x_{2}{ }^{\mathrm{a}}$ & $x_{1}{ }^{\mathrm{b}}$ \\
\hline 0.9985 & 0.00145 \\
\hline${ }^{a} x_{2}:$ mole fraction of component 2 in the saturated solution. & \\
${ }^{\mathrm{b}} x_{1}:$ mole fraction solubility of the solute. &
\end{tabular}

${ }^{b} x_{1}$ : mole fraction solubility of the solute.

\section{Auxiliary Information}

\section{Method/Apparatus/Procedure:}

Constant-temperature bath, Soxhlet thimble, conical flask, and analytical balance.

Excess solute and solvent were placed in a conical flask and allowed to equilibrate for several days at constant temperature. Attainment of equilibrium was verified by several repetitive measurements and by approaching equilibrium from supersaturation. Aliquots of saturated solutions were transferred using a Soxhlet thimble equipped with a one-hole cork stopper and an inverted U-shaped delivery tube to a second ground-glass stoppered flask suspended in the $303 \mathrm{~K}$ water bath. Successive portions of the contents were evaporated at room temperature in a tared aluminum foil weighing dish under a bell jar through which a slow stream of dry air was passed. The second flask plus unused saturated solution and the aluminum foil dish plus residue were weighed. The saturation solubility of the solute was calculated from the recorded mass data and molar masses of the solute and solvent.

\section{Source and Purity of Chemicals:}

(1) Purity not given, Chemical source not specified, was recrystallized several times from aqueous-ethanol mixture, and then dried under vacuum over phosphorous pentoxide.

(2) Reagent grade, Chemical source not specified, refluxed over phosphorous pentoxide for $24 \mathrm{~h}$, and then distilled through a $3 \times 80 \mathrm{~cm}$ column filled with $0.32-\mathrm{cm}$ glass helices.

\section{Estimated Error:}

Temperature: $\pm 0.02 \mathrm{~K}$.

$x_{1}: \pm 3 \%$ (relative error) 


\subsection{3-Chlorobenzoic acid solubility data in aromatic hydrocarbons}

\begin{tabular}{ll}
\hline \hline Components: & Original Measurements: \\
$\begin{array}{l}\text { (1) 3-Chlorobenzoic acid; } \\
\mathrm{C}_{7} \mathrm{H}_{5} \mathrm{ClO}_{2} ;[535-80-8]\end{array}$ & ${ }^{54} \mathrm{C}$. K. Hancock, J. N. Pawloski, and J. \\
(2) Benzene; $\mathrm{C}_{6} \mathrm{H}_{6} ;[71-43-2]$ & P. Idoux, J. Org. Chem. 31, 3801 \\
\hline Variables: & Prepared by: \\
$T / \mathrm{K}=303.15$ & W. E. Acree, Jr. \\
\hline
\end{tabular}

\section{Experimental Values}

\begin{tabular}{lc}
\hline \hline$x_{2}{ }^{\mathrm{a}}$ & $x_{1}{ }^{\mathrm{b}}$ \\
\hline 0.9905 & 0.00948
\end{tabular}

${ }^{\mathrm{a}} x_{2}$ : mole fraction of component 2 in the saturated solution.

${ }^{\mathrm{b}} x_{1}$ : mole fraction solubility of the solute.

\section{Auxiliary Information}

\section{Method/Apparatus/Procedure:}

Constant-temperature bath, Soxhlet thimble, conical flask, and analytical balance.

Excess solute and solvent were placed in a conical flask and allowed to equilibrate for several days at constant temperature. Attainment of equilibrium was verified by several repetitive measurements and by approaching equilibrium from supersaturation. Aliquots of saturated solutions were transferred using a Soxhlet thimble equipped with a one-hole cork stopper and an inverted U-shaped delivery tube to a second ground-glass stoppered flask suspended in the $303 \mathrm{~K}$ water bath. Successive portions of the contents were evaporated at room temperature in a tared aluminum foil weighing dish under a bell jar through which a slow stream of dry air was passed. The second flask plus unused saturated solution and the aluminum foil dish plus residue were weighed. The saturation solubility of the solute was calculated from the recorded mass data and molar masses of the solute and solvent.

\section{Source and Purity of Chemicals:}

(1) Purity not given, Chemical source not specified, was recrystallized several times from aqueous-ethanol mixture, and then dried under vacuum over phosphorous pentoxide.

(2) Reagent grade, Chemical source not specified, refluxed over phosphorous pentoxide for $24 \mathrm{~h}$, and then distilled through a $3 \times 80 \mathrm{~cm}$ column filled with 0.32 -cm glass helices.

Estimated Error:

Temperature: $\pm 0.02 \mathrm{~K}$.

$x_{1}: \pm 3 \%$ (relative error).

\begin{tabular}{|c|c|}
\hline $\begin{array}{l}\text { Components: } \\
\text { (1) 3-Chlorobenzoic acid; } \\
\mathrm{C}_{7} \mathrm{H}_{5} \mathrm{ClO}_{2} ;[535-80-8] \\
\text { (2) Benzene; } \mathrm{C}_{6} \mathrm{H}_{6} ;[71-43-2]\end{array}$ & $\begin{array}{l}\text { Original Measurements: } \\
{ }^{127} \text { N. V. Sidgwick and E. K. Ewbank, J. } \\
\text { Chem. Soc. Trans. 119, } 979 \text { (1921). }\end{array}$ \\
\hline Variables: & Prepared by: \\
\hline Temperature & W. E. Acree, Jr. \\
\hline
\end{tabular}

Experimental Values

\begin{tabular}{lcc}
\hline \hline$T / \mathrm{K}$ & $x_{2}{ }^{\mathrm{a}}$ & $x_{1}{ }^{\mathrm{b}}$ \\
\hline 309.0 & 0.989 & 0.0114 \\
324.4 & 0.975 & 0.0254 \\
338.7 & 0.949 & 0.0507 \\
366.9 & 0.823 & 0.177 \\
381.2 & 0.676 & 0.324 \\
398.5 & 0.448 & 0.552 \\
415.7 & 0.179 & 0.821 \\
\hline
\end{tabular}

${ }^{\mathrm{a}} x_{2}$ : mole fraction of component 2 in the saturated solution.

${ }^{\mathrm{b}} x_{1}$ : mole fraction solubility of the solute. Solubility data reported in units of mass percent. Mole fraction values calculated by the compiler.

\section{Auxiliary Information}

\section{Method/Apparatus/Procedure:}

Preweighed quantities of solute and solvent were placed in bulbs, which were then sealed and heated in a suitable bath. At the higher temperatures, the concentration of solvent in the liquid solution was corrected for the amount of solvent vapor in the bulb. The correction assumed that the vapor pressure of the saturated solution was one half that of the pure solvent at the solution temperature. The temperature at which the solid completely dissolved was recorded, and was taken to be the solid-liquid equilibrium temperature.

Source and Purity of Chemicals:

(1) Purity not given, prepared and purified by the authors using a published synthetic procedure [see J. Walker and J. K. Wood, J. Chem. Soc. 117, 40 (1920) for synthetic details]. Melting point of the purified solute was $427.7 \mathrm{~K}$. (2) Purity not given, Chemical source not given, thiophene was removed by treatment with sulfuric acid. Sample was further purified by freezing several times.

Estimated Error:

Temperature: Not given in paper.

$x_{1}: \pm 10 \%$ (relative error, estimated by compiler).

\subsection{3-Chlorobenzoic acid solubility data in esters}

\begin{tabular}{|c|c|}
\hline $\begin{array}{l}\text { Components: } \\
\text { (1) 3-Chlorobenzoic acid; } \\
\mathrm{C}_{7} \mathrm{H}_{5} \mathrm{ClO}_{2} ; \text { [535-80-8] } \\
\text { (2) Ethyl ethanoate; } \mathrm{C}_{4} \mathrm{H}_{8} \mathrm{O}_{2} \text {; } \\
\text { [141-78-6] }\end{array}$ & $\begin{array}{l}\text { Original Measurements: } \\
{ }^{8} \text { K. R. Hoover, K. Pop, W. E. Acree, Jr., } \\
\text { and M. H. Abraham, S. Afr. J. Chem. } \\
\mathbf{5 8 ,} 25 \text { (2005). }\end{array}$ \\
\hline $\begin{array}{l}\text { Variables: } \\
T / \mathrm{K}=298.15\end{array}$ & $\begin{array}{l}\text { Prepared by: } \\
\text { W. E. Acree, Jr. }\end{array}$ \\
\hline
\end{tabular}

\section{Experimental Values}

\begin{tabular}{lc}
\hline \hline$x_{2}{ }^{\mathrm{a}}$ & $x_{1}{ }^{\mathrm{b}}$ \\
\hline 0.9348 & 0.0652
\end{tabular}

${ }^{\mathrm{a}} x_{2}$ : mole fraction of component 2 in the saturated solution.

${ }^{\mathrm{b}} x_{1}$ : mole fraction solubility of the solute. 


\section{Auxiliary Information}

\section{Method/Apparatus/Procedure:}

Constant-temperature bath, calorimetric thermometer, and an ultraviolet/ visible spectrophotometer.

Excess solute and solvent were placed in amber glass bottles and allowed to equilibrate for several days at constant temperature. Attainment of equilibrium was verified by several repetitive measurements and by approaching equilibrium from supersaturation. Aliquots of saturated solutions were transferred through a coarse filter into tared volumetric flasks, weighed, and diluted with methanol. Concentrations were determined by spectrophotometric measurements at $280 \mathrm{~nm}$.

Source and Purity of Chemicals:

(1) $99 \%$, Aldrich Chemical Company, Milwaukee, WI, USA, was used as received.

(2) $99.9 \%$, HPLC grade, Aldrich Chemical Company, stored over molecular sieves and distilled shortly before use.

Estimated Error:

Temperature: $\pm 0.1 \mathrm{~K}$.

$x_{1}: \pm 1.5 \%$ (relative error)

\begin{tabular}{|c|c|}
\hline $\begin{array}{l}\text { Components: } \\
\text { (1) 3-Chlorobenzoic acid; } \\
\mathrm{C}_{7} \mathrm{H}_{5} \mathrm{ClO}_{2} \text {; [535-80-8] } \\
\text { (2) Butyl ethanoate; } \mathrm{C}_{6} \mathrm{H}_{12} \mathrm{O}_{2} \text {; } \\
\text { [123-86-4] }\end{array}$ & $\begin{array}{l}\text { Original Measurements: } \\
{ }^{8} \text { K. R. Hoover, K. Pop, W. E. Acree, Jr., } \\
\text { and M. H. Abraham, S. Afr. J. Chem. } \\
\text { 58, } 25 \text { (2005). }\end{array}$ \\
\hline $\begin{array}{l}\text { Variables: } \\
T / \mathrm{K}=298.15\end{array}$ & $\begin{array}{l}\text { Prepared by: } \\
\text { W. E. Acree, Jr. }\end{array}$ \\
\hline
\end{tabular}

Experimental Values

\begin{tabular}{lc}
\hline \hline$x_{2}{ }^{\mathrm{a}}$ & $x_{1}{ }^{\mathrm{b}}$ \\
\hline 0.9338 & 0.0662
\end{tabular}

${ }^{a} x_{2}$ : mole fraction of component 2 in the saturated solution.

${ }^{\mathrm{b}} x_{1}$ : mole fraction solubility of the solute.

\section{Auxiliary Information}

\section{Method/Apparatus/Procedure:}

Constant-temperature bath, calorimetric thermometer, and an ultraviolet/ visible spectrophotometer.

Excess solute and solvent were placed in amber glass bottles and allowed to equilibrate for several days at constant temperature. Attainment of equilibrium was verified by several repetitive measurements and by approaching equilibrium from supersaturation. Aliquots of saturated solutions were transferred through a coarse filter into tared volumetric flasks, weighed, and diluted with methanol. Concentrations were determined by spectrophotometric measurements at $280 \mathrm{~nm}$.

Source and Purity of Chemicals:

(1) $99 \%$, Aldrich Chemical Company, Milwaukee, WI, USA, was used as received.

(2) $99.7 \%$, HPLC grade, Aldrich Chemical Company, stored over molecular sieves and distilled shortly before use.

\section{Estimated Error:}

Temperature: $\pm 0.1 \mathrm{~K}$.

$x_{1}: \pm 1.5 \%$ (relative error).

\begin{tabular}{|c|c|}
\hline $\begin{array}{l}\text { Components: } \\
\text { (1) 3-Chlorobenzoic acid; } \\
\mathrm{C}_{7} \mathrm{H}_{5} \mathrm{ClO}_{2} ; \text { [535-80-8] } \\
\text { (2) Pentyl ethanoate; } \mathrm{C}_{7} \mathrm{H}_{14} \mathrm{O}_{2} \text {; } \\
\text { [628-63-7] }\end{array}$ & $\begin{array}{l}\text { Original Measurements: } \\
{ }^{8} \text { K. R. Hoover, K. Pop, W. E. Acree, Jr., } \\
\text { and M. H. Abraham, S. Afr. J. Chem. } \\
\text { 58, } 25 \text { (2005). }\end{array}$ \\
\hline $\begin{array}{l}\text { Variables: } \\
T / \mathrm{K}=298.15\end{array}$ & $\begin{array}{l}\text { Prepared by: } \\
\text { W. E. Acree, Jr. }\end{array}$ \\
\hline
\end{tabular}

Experimental Values

\begin{tabular}{lc}
\hline \hline$x_{2}{ }^{\mathrm{a}}$ & $x_{1}{ }^{\mathrm{b}}$ \\
\hline 0.9368 & 0.0632
\end{tabular}

${ }^{\mathrm{a}} x_{2}$ : mole fraction of component 2 in the saturated solution.

${ }^{\mathrm{b}} x_{1}$ : mole fraction solubility of the solute.

\section{Auxiliary Information}

\section{Method/Apparatus/Procedure:}

Constant-temperature bath, calorimetric thermometer, and an ultraviolet visible spectrophotometer.

Excess solute and solvent were placed in amber glass bottles and allowed to equilibrate for several days at constant temperature. Attainment of equilibrium was verified by several repetitive measurements and by approaching equilibrium from supersaturation. Aliquots of saturated solutions were transferred through a coarse filter into tared volumetric flasks, weighed, and diluted with methanol. Concentrations were determined by spectrophotometric measurements at $280 \mathrm{~nm}$.

\section{Source and Purity of Chemicals:}

(1) $99 \%$, Aldrich Chemical Company, Milwaukee, WI, USA, was used as received.

(2) $99.9 \%$, Aldrich Chemical Company, stored over molecular sieves and distilled shortly before use.

\section{Estimated Error:}

Temperature: $\pm 0.1 \mathrm{~K}$.

$x_{1}: \pm 1.5 \%$ (relative error)

\begin{tabular}{|c|c|}
\hline $\begin{array}{l}\text { Components: } \\
\text { (1) 3-Chlorobenzoic acid; } \\
\mathrm{C}_{7} \mathrm{H}_{5} \mathrm{ClO}_{2} ;[535-80-8] \\
\text { (2) 1-Methylethyl tetradecanoate; } \\
\mathrm{C}_{17} \mathrm{H}_{34} \mathrm{O}_{2} ;[110-27-0]\end{array}$ & $\begin{array}{l}\text { Original Measurements: } \\
{ }^{61} \text { E. R. Cooper, J. Control. Release 1, } \\
153 \text { (1984). }\end{array}$ \\
\hline $\begin{array}{l}\text { Variables: } \\
T / \mathrm{K}=295\end{array}$ & $\begin{array}{l}\text { Prepared by: } \\
\text { W. E. Acree, Jr. }\end{array}$ \\
\hline
\end{tabular}

Experimental Values

\begin{tabular}{lc}
\hline \hline$x_{2}{ }^{\mathrm{a}}$ & $x_{1}{ }^{\mathrm{b}}$ \\
\hline 0.956 & 0.044
\end{tabular}

${ }^{\mathrm{a}} x_{2}$ : mole fraction of component 2 in the saturated solution.

${ }^{b} x_{1}$ : mole fraction solubility of the solute. Solubility data reported in terms of mass percent. Mole fraction solubility calculated by the compiler. 


\section{Auxiliary Information}

\section{Method/Apparatus/Procedure:}

Very little experimental details provided. Solubility determinations were made by stirring the solvent with excess solute for two days, centrifuging the sample, and analyzing the supernatant.

\section{Source and Purity of Chemicals:}

(1) Reagent grade, Aldrich Chemical Company, Metuchen, NJ, USA, no purification details provided.

(2) Reagent grade, Wickhen Corporation, Huguenot, NJ, USA, no purification details provided.

Estimated Error:

Temperature: No information given.

$x_{1}$ : No information given.

\begin{tabular}{ll}
\hline \hline Components: & Original Measurements: \\
$\begin{array}{l}\text { (1) 3-Chlorobenzoic acid; } \\
\mathrm{C}_{7} \mathrm{H}_{5} \mathrm{ClO}_{2} ;[535-80-8]\end{array}$ & W. E. Acree, Jr., unpublished data. \\
$\begin{array}{l}\text { (2) } 1,2,3-T \text {-Tiacetoxypropane } \\
\text { (Triacetin); } \mathrm{C}_{9} \mathrm{H}_{14} \mathrm{O}_{6} ;[102-76-1]\end{array}$ & \\
\hline Variables: & Prepared by: \\
$T / \mathrm{K}=298.15$ & W. E. Acree, Jr. \\
\hline
\end{tabular}

Experimental Values

\begin{tabular}{lc}
\hline \hline$x_{2}{ }^{\mathrm{a}}$ & $x_{1}^{\mathrm{b}}$ \\
\hline 0.9458 & 0.05424 \\
\hline${ }^{\mathrm{a}} x_{2}:$ mole fraction of component 2 in the saturated solution. \\
${ }^{\mathrm{b}} x_{1}:$ mole fraction solubility of the solute.
\end{tabular}

\section{Auxiliary Information}

\section{Method: Apparatus/Procedure:}

Constant-temperature bath, calorimetric thermometer, and an ultraviolet/ visible spectrophotometer

Excess solute and solvent were placed in amber glass bottles and allowed to equilibrate for several days at constant temperature. Attainment of equilibrium was verified by several repetitive measurements and by approaching equilibrium from supersaturation. Aliquots of saturated solutions were transferred through a coarse filter into tared volumetric flasks, weighed, and diluted with 2-propanol. Concentrations were determined by spectrophotometric measurements at $280 \mathrm{~nm}$.

Source and Purity of Chemicals:

(1) $99 \%$, Aldrich Chemical Company, Milwaukee, WI, USA, was used as received.

(2) $99 \%$, Acros Organics, USA, stored over molecular sieves before use.

\section{Estimated Error:}

Temperature: $\pm 0.1 \mathrm{~K}$.

$x_{1}: \pm 1.5 \%$ (relative error)

\subsection{3-Chlorobenzoic acid solubility data in ethers}

\begin{tabular}{|c|c|}
\hline $\begin{array}{l}\text { Components: } \\
\text { (1) 3-Chlorobenzoic acid; } \\
\mathrm{C}_{7} \mathrm{H}_{5} \mathrm{ClO}_{2} ;[535-80-8] \\
\text { (2) } 1,1^{\prime}-\mathrm{O} \text { xybisethane; } \mathrm{C}_{4} \mathrm{H}_{10} \mathrm{O} \text {; } \\
\text { [60-29-7] }\end{array}$ & $\begin{array}{l}\text { Original Measurements: } \\
{ }^{8} \text { K. R. Hoover, K. Pop, W. E. Acree, Jr., } \\
\text { and M. H. Abraham, S. Afr. J. Chem. } \\
\text { 58, } 25 \text { (2005). }\end{array}$ \\
\hline $\begin{array}{l}\text { Variables: } \\
T / \mathrm{K}=298.15\end{array}$ & $\begin{array}{l}\text { Prepared by: } \\
\text { W. E. Acree, Jr. }\end{array}$ \\
\hline
\end{tabular}

\section{Experimental Values}

\begin{tabular}{lc}
\hline \hline$x_{2}{ }^{\mathrm{a}}$ & $x_{1}{ }^{\mathrm{b}}$ \\
\hline 0.9199 & 0.0801 \\
\hline${ }^{\mathrm{a}} x_{2}:$ mole fraction of component 2 in the saturated solution. & \\
${ }^{\mathrm{b}} x_{1}:$ mole fraction solubility of the solute. &
\end{tabular}

\section{Auxiliary Information}

\section{Method/Apparatus/Procedure:}

Constant-temperature bath, calorimetric thermometer, and an ultraviolet/ visible spectrophotometer.

Excess solute and solvent were placed in amber glass bottles and allowed to equilibrate for several days at constant temperature. Attainment of equilibrium was verified by several repetitive measurements and by approaching equilibrium from supersaturation. Aliquots of saturated solutions were transferred through a coarse filter into tared volumetric flasks, weighed, and diluted with methanol. Concentrations were determined by spectrophotometric measurements at $280 \mathrm{~nm}$.

Source and Purity of Chemicals:

(1) $99 \%$, Aldrich Chemical Company, Milwaukee, WI, USA, was used as received.

(2) $99+\%$, anhydrous, Aldrich Chemical Company, stored over molecular sieves and distilled shortly before use.

\section{Estimated Error:}

Temperature: $\pm 0.1 \mathrm{~K}$.

$x_{1}: \pm 1.5 \%$ (relative error).

\begin{tabular}{|c|c|}
\hline $\begin{array}{l}\text { Components: } \\
\text { (1) 3-Chlorobenzoic acid; } \\
\mathrm{C}_{7} \mathrm{H}_{5} \mathrm{ClO}_{2} \text {; [535-80-8] } \\
\text { (2) 2,2'-Oxybispropane; } \mathrm{C}_{6} \mathrm{H}_{14} \mathrm{O} \text {; } \\
\text { [108-20-3] }\end{array}$ & $\begin{array}{l}\text { Original Measurements: } \\
{ }^{8} \text { K. R. Hoover, K. Pop, W. E. Acree, Jr. } \\
\text { and M. H. Abraham, S. Afr. J. Chem. } \\
\text { 58, } 25 \text { (2005). }\end{array}$ \\
\hline $\begin{array}{l}\text { Variables: } \\
T / \mathrm{K}=298.15\end{array}$ & $\begin{array}{l}\text { Prepared by: } \\
\text { W. E. Acree, Jr. }\end{array}$ \\
\hline
\end{tabular}

\section{Experimental Values}

\begin{tabular}{lc}
\hline \hline$x_{2}{ }^{\mathrm{a}}$ & $x_{1}{ }^{\mathrm{b}}$ \\
\hline 0.9449 & 0.0551 \\
\hline${ }^{\mathrm{a}} x_{2}:$ mole fraction of component 2 in the saturated solution. & \\
${ }^{\mathrm{b}} x_{1}:$ mole fraction solubility of the solute. &
\end{tabular}




\section{Auxiliary Information}

\section{Method/Apparatus/Procedure:}

Constant-temperature bath, calorimetric thermometer, and an ultraviolet/ visible spectrophotometer.

Excess solute and solvent were placed in amber glass bottles and allowed to equilibrate for several days at constant temperature. Attainment of equilibrium was verified by several repetitive measurements and by approaching equilibrium from supersaturation. Aliquots of saturated solutions were transferred through a coarse filter into tared volumetric flasks, weighed, and diluted with methanol. Concentrations were determined by spectrophotometric measurements at $280 \mathrm{~nm}$.

Source and Purity of Chemicals:

(1) $99 \%$, Aldrich Chemical Company, Milwaukee, WI, USA, was used as received.

(2) $99 \%$, anhydrous, Aldrich Chemical Company, stored over molecular sieves and distilled shortly before use.

Estimated Error:

Temperature: $\pm 0.1 \mathrm{~K}$.

$x_{1}: \pm 1.5 \%$ (relative error).

\begin{tabular}{|c|c|}
\hline $\begin{array}{l}\text { Components: } \\
\text { (1) 3-Chlorobenzoic acid; } \\
\mathrm{C}_{7} \mathrm{H}_{5} \mathrm{ClO}_{2} ;[535-80-8] \\
\text { (2) } 1,1^{\prime} \text {-Oxybisbutane; } \mathrm{C}_{8} \mathrm{H}_{18} \mathrm{O} \text {; } \\
\text { [142-96-1] }\end{array}$ & $\begin{array}{l}\text { Original Measurements: } \\
{ }^{8} \text { K. R. Hoover, K. Pop, W. E. Acree, Jr., } \\
\text { and M. H. Abraham, S. Afr. J. Chem. } \\
\text { 58, } 25 \text { (2005). }\end{array}$ \\
\hline $\begin{array}{l}\text { Variables: } \\
T / \mathrm{K}=298.15\end{array}$ & $\begin{array}{l}\text { Prepared by: } \\
\text { W. E. Acree, Jr. }\end{array}$ \\
\hline
\end{tabular}

Experimental Values

\begin{tabular}{lc}
\hline \hline$x_{2}^{\mathrm{a}}$ & $x_{1}^{\mathrm{b}}$ \\
\hline 0.9621 & 0.0379
\end{tabular}

${ }^{\mathrm{a}} x_{2}$ : mole fraction of component 2 in the saturated solution.

${ }^{\mathrm{b}} x_{1}$ : mole fraction solubility of the solute.

\section{Auxiliary Information}

\section{Method/Apparatus/Procedure:}

Constant-temperature bath, calorimetric thermometer, and an ultraviolet/ visible spectrophotometer.

Excess solute and solvent were placed in amber glass bottles and allowed to equilibrate for several days at constant temperature. Attainment of equilibrium was verified by several repetitive measurements and by approaching equilibrium from supersaturation. Aliquots of saturated solutions were transferred through a coarse filter into tared volumetric flasks, weighed, and diluted with methanol. Concentrations were determined by

spectrophotometric measurements at $280 \mathrm{~nm}$.

Source and Purity of Chemicals:

(1) $99 \%$, Aldrich Chemical Company, Milwaukee, WI, USA, was used as received.

(2) $99.3 \%$, anhydrous, Aldrich Chemical Company, stored over molecular sieves and distilled shortly before use.

\section{Estimated Error:}

Temperature: $\pm 0.1 \mathrm{~K}$.

$x_{1}: \pm 1.5 \%$ (relative error).

\begin{tabular}{|c|c|}
\hline $\begin{array}{l}\text { Components: } \\
\text { (1) 3-Chlorobenzoic acid; } \\
\mathrm{C}_{7} \mathrm{H}_{5} \mathrm{ClO}_{2} \text {; [535-80-8] } \\
\text { (2) Tetrahydrofuran; } \mathrm{C}_{4} \mathrm{H}_{8} \mathrm{O} \text {; } \\
\text { [109-99-9] }\end{array}$ & $\begin{array}{l}\text { Original Measurements: } \\
{ }^{8} \text { K. R. Hoover, K. Pop, W. E. Acree, Jr., } \\
\text { and M. H. Abraham, S. Afr. J. Chem. } \\
\text { 58, } 25 \text { (2005). }\end{array}$ \\
\hline $\begin{array}{l}\text { Variables: } \\
T / \mathrm{K}=298.15\end{array}$ & $\begin{array}{l}\text { Prepared by: } \\
\text { W. E. Acree, Jr. }\end{array}$ \\
\hline
\end{tabular}

Experimental Values

\begin{tabular}{lc}
\hline \hline$x_{2}{ }^{\mathrm{a}}$ & $x_{1}{ }^{\mathrm{b}}$ \\
\hline 0.7755 & 0.2245
\end{tabular}

${ }^{\mathrm{a}} x_{2}$ : mole fraction of component 2 in the saturated solution.

${ }^{\mathrm{b}} x_{1}$ : mole fraction solubility of the solute.

\section{Auxiliary Information}

\section{Method/Apparatus/Procedure:}

Constant-temperature bath, calorimetric thermometer, and an ultraviolet visible spectrophotometer.

Excess solute and solvent were placed in amber glass bottles and allowed to equilibrate for several days at constant temperature. Attainment of equilibrium was verified by several repetitive measurements and by approaching equilibrium from supersaturation. Aliquots of saturated solutions were transferred through a coarse filter into tared volumetric flasks, weighed, and diluted with methanol. Concentrations were determined by spectrophotometric measurements at $280 \mathrm{~nm}$.

\section{Source and Purity of Chemicals:}

(1) $99 \%$, Aldrich Chemical Company, Milwaukee, WI, USA, was used as received.

(2) $99.9 \%$, anhydrous, Aldrich Chemical Company, stored over molecular sieves and distilled shortly before use.

\section{Estimated Error:}

Temperature: $\pm 0.1 \mathrm{~K}$.

$x_{1}: \pm 1.5 \%$ (relative error)

\begin{tabular}{|c|c|}
\hline $\begin{array}{l}\text { Components: } \\
\text { (1) 3-Chlorobenzoic acid; } \\
\mathrm{C}_{7} \mathrm{H}_{5} \mathrm{ClO}_{2} ;[535-80-8] \\
\text { (2) 1,4-Dioxane; } \mathrm{C}_{4} \mathrm{H}_{8} \mathrm{O}_{2} \text {; } \\
\text { [123-91-1] }\end{array}$ & $\begin{array}{l}\text { Original Measurements: } \\
{ }^{18} \text { M. Saifullah, S. Ye, L. M. Grubbs, N. } \\
\text { E. De La Rosa, W. E. Acree, Jr., and M. } \\
\text { H. Abraham, J. Solution Chem. 40, } \\
2082 \text { (2011). }\end{array}$ \\
\hline $\begin{array}{l}\text { Variables: } \\
T / \mathrm{K}=298.15\end{array}$ & $\begin{array}{l}\text { Prepared by: } \\
\text { W. E. Acree, Jr. }\end{array}$ \\
\hline
\end{tabular}

Experimental Values

\begin{tabular}{lc}
\hline \hline$x_{2}{ }^{\mathrm{a}}$ & $x_{1}{ }^{\mathrm{b}}$ \\
\hline 0.8515 & 0.1485 \\
\hline${ }_{{ }_{2}}:$ mole fraction of component 2 in the saturated solution. & \\
${ }^{\mathrm{b}} x_{1}:$ mole fraction solubility of the solute. &
\end{tabular}




\section{Auxiliary Information}

\section{Method/Apparatus/Procedure:}

Constant-temperature bath, calorimetric thermometer, and an ultraviolet/ visible spectrophotometer.

Excess solute and solvent were placed in amber glass bottles and allowed to equilibrate for several days at constant temperature. Attainment of equilibrium was verified by several repetitive measurements and by approaching equilibrium from supersaturation. Aliquots of saturated solutions were transferred through a coarse filter into tared volumetric flasks, weighed, and diluted with methanol. Concentrations were determined by spectrophotometric measurements at $280 \mathrm{~nm}$.

Source and Purity of Chemicals:

(1) $99 \%$, Aldrich Chemical Company, Milwaukee, WI, USA, was used as received.

(2) $99.8 \%$, anhydrous, Aldrich Chemical Company, stored over molecular sieves and distilled shortly before use.

Estimated Error:

Temperature: $\pm 0.1 \mathrm{~K}$.

$x_{1}: \pm 1.5 \%$ (relative error).

\subsection{3-Chlorobenzoic acid solubility data in alcohols}

\begin{tabular}{ll}
\hline \hline Components: & Original Measurements: \\
(1) 3-Chlorobenzoic acid; & ${ }^{126} \mathrm{P}$. K. Biswas, S. C. Lahiri, and B. P. \\
$\mathrm{C}_{7} \mathrm{H}_{5} \mathrm{ClO}_{2} ;[535-80-8]$ & Dey, Bull. Chem. Soc. Jpn. 66, 2785 \\
(2) Ethanol; $\mathrm{C}_{2} \mathrm{H}_{6} \mathrm{O} ;[64-17-5]$ & $(1993)$. \\
\hline Variables: & Prepared by: \\
$T / \mathrm{K}=298.15$ & W. E. Acree, Jr. \\
\hline
\end{tabular}

Experimental Values

\begin{tabular}{|c|c|c|}
\hline$c_{1}{ }^{\mathrm{a}}$ & $x_{2}{ }^{\mathrm{b}}$ & $x_{1}{ }^{\mathrm{c}}$ \\
\hline 0.6480 & 0.9605 & 0.0395 \\
\hline
\end{tabular}

\section{Auxiliary Information}

\section{Method/Apparatus/Procedure:}

Analytical balance and an ultraviolet/visible spectrophotometer.

Solutions containing excess solute and solvent were allowed to equilibrate at constant temperature for at least $24 \mathrm{~h}$. An aliquot of the saturated solution was removed, filtered, and the absorbance recorded. Solubility was calculated from the measured absorbance.

\section{Source and Purity of Chemicals:}

(1) Puris grade, Fluka Chemicals, recrystallized from aqueous ethanol mixture and dried in an air oven at $390 \mathrm{~K}$. The purified compound was stored in vacuum desiccators.

(2) Absolute, B.C.P.W., Calcutta, India, was distilled twice before use.

\section{Estimated Error:}

Temperature: $\pm 0.2 \mathrm{~K}$ (estimated by compiler). $c_{1}: \pm 1.0 \%$ (relative error, estimated by compiler). $x_{1}: \pm 2.5 \%$ (relative error, estimated by compiler).

\begin{tabular}{ll}
\hline \hline Components: & $\begin{array}{l}\text { Original Measurements: } \\
\text { (1) 3-Chlorobenzoic acid; }\end{array}$ \\
${ }^{72}$ R. Thuaire, Bull. Soc. Chim. Fr. 3815 \\
$\mathrm{C}_{7} \mathrm{H}_{5} \mathrm{ClO}_{2} ;[535-80-8]$ & $(1971)$. \\
(2) Ethanol; $\mathrm{C}_{2} \mathrm{H}_{6} \mathrm{O} ;[64-17-5]$ & \\
\hline Variables: & Prepared by: \\
$T / \mathrm{K}=298.15$ & W. E. Acree, Jr. \\
\hline
\end{tabular}

\section{Experimental Values}

The measured solubility was reported to be $1.750 \mathrm{~mol} / \mathrm{kg}$ of solvent, which corresponds to a mole fraction solubility of $x_{1}=0.0746$.

\section{Auxiliary Information}

\section{Method/Apparatus/Procedure:}

Very little experimental details were provided. Solubility was determined by a gravimetric method. The saturated solution was evaporated to dryness and the remaining solid residue was weighed. The solubility was calculated from the mass of the solid residue and mass of saturated solution analyzed.

Source and Purity of Chemicals:

(1) Purity not given, Chemical source not given, no purification details were provided.

(2) Purity not given, Chemical source not given, no purification details were provided.

\section{Estimated Error:}

Temperature: $\pm 0.05 \mathrm{~K}$

$x_{1}: \pm 4 \%$ (relative error, estimated by compiler).

\begin{tabular}{ll}
\hline \hline Components: & Original Measurements: \\
(1) 3-Chlorobenzoic acid; & ${ }^{8}$ K. R. Hoover, K. Pop, W. E. Acree, Jr., \\
$\mathrm{C}_{7} \mathrm{H}_{5} \mathrm{ClO}_{2} ;[535-80-8]$ & and M. H. Abraham, S. Afr. J. Chem. \\
(2) 1-Propanol; $\mathrm{C}_{3} \mathrm{H}_{8} \mathrm{O} ;[71-23-8]$ & $\mathbf{5 8}, 25$ (2005). \\
\hline Variables: & Prepared by: \\
$T / \mathrm{K}=298.15$ & W. E. Acree, Jr. \\
\hline
\end{tabular}

\section{Experimental Values}

\begin{tabular}{lc}
\hline \hline$x_{2}{ }^{\mathrm{a}}$ & $x_{1}{ }^{\mathrm{b}}$ \\
\hline 0.9133 & 0.0867 \\
\hline${ }^{\mathrm{a}} x_{2}:$ mole fraction of component 2 in the saturated solution. & \\
${ }^{\mathrm{b}} x_{1}:$ mole fraction solubility of the solute. &
\end{tabular}

\section{Auxiliary Information}

\section{Method/Apparatus/Procedure:}

Constant-temperature bath, calorimetric thermometer, and an ultraviolet/ visible spectrophotometer.

Excess solute and solvent were placed in amber glass bottles and allowed to equilibrate for several days at constant temperature. Attainment of equilibrium was verified by several repetitive measurements and by approaching equilibrium from supersaturation. Aliquots of saturated solutions were transferred through a coarse filter into tared volumetric flasks, weighed, and diluted with methanol. Concentrations were determined by spectrophotometric measurements at $280 \mathrm{~nm}$.

Source and Purity of Chemicals:

(1) $99 \%$, Aldrich Chemical Company, Milwaukee, WI, USA, was used as received.

(2) $99+\%$, anhydrous, Aldrich Chemical Company, stored over molecular sieves and distilled shortly before use. 
Estimated Error:

Temperature: $\pm 0.1 \mathrm{~K}$.

$x_{1}: \pm 1.5 \%$ (relative error).

\begin{tabular}{ll}
\hline \hline Components: & Original Measurements: \\
$\begin{array}{l}\text { (1) 3-Chlorobenzoic acid; } \\
\mathrm{C}_{7} \mathrm{H}_{5} \mathrm{ClO}_{2} ;[535-80-8]\end{array}$ & ${ }^{8}$ K. R. Hoover, K. Pop, W. E. Acree, Jr., \\
(2) 2-Propanol; $\mathrm{C}_{3} \mathrm{H}_{8} \mathrm{O} ;[67-63-0]$ & and M. H. Abraham, S. Afr. J. Chem. \\
\hline Variables: & Prepared by: \\
$T / \mathrm{K}=298.15$ & W. E. Acree, Jr. \\
\hline
\end{tabular}

\section{Experimental Values}

\begin{tabular}{lc}
\hline \hline$x_{2}{ }^{\mathrm{a}}$ & $x_{1}{ }^{\mathrm{b}}$ \\
\hline 0.9087 & 0.0913
\end{tabular}

${ }^{\mathrm{a}} x_{2}$ : mole fraction of component 2 in the saturated solution.

${ }^{\mathrm{b}} x_{1}$ : mole fraction solubility of the solute.

\section{Auxiliary Information}

\section{Method/Apparatus/Procedure:}

Constant-temperature bath, calorimetric thermometer, and an ultraviolet/ visible spectrophotometer.

Excess solute and solvent were placed in amber glass bottles and allowed to equilibrate for several days at constant temperature. Attainment of equilibrium was verified by several repetitive measurements and by approaching equilibrium from supersaturation. Aliquots of saturated solutions were transferred through a coarse filter into tared volumetric flasks, weighed, and diluted with methanol. Concentrations were determined by

spectrophotometric measurements at $280 \mathrm{~nm}$.

\section{Source and Purity of Chemicals:}

(1) $99 \%$, Aldrich Chemical Company, Milwaukee, WI, USA, was used as received.

(2) $99+\%$, anhydrous, Aldrich Chemical Company, stored over molecular sieves and distilled shortly before use.

Estimated Error:

Temperature: $\pm 0.1 \mathrm{~K}$

$x_{1}: \pm 1.5 \%$ (relative error).

\begin{tabular}{|c|c|}
\hline Components: & Original Measurements: \\
\hline $\begin{array}{l}\text { (1) 3-Chlorobenzoic acid; } \\
\mathrm{C}_{7} \mathrm{H}_{5} \mathrm{ClO}_{2} ;[535-80-8] \\
\text { (2) 1-Butanol; } \mathrm{C}_{4} \mathrm{H}_{10} \mathrm{O} ;[71-36-3]\end{array}$ & $\begin{array}{l}{ }^{8} \text { K. R. Hoover, K. Pop, W. E. Acree, Jr., } \\
\text { and M. H. Abraham, S. Afr. J. Chem. } \\
\text { 58, } 25 \text { (2005). }\end{array}$ \\
\hline $\begin{array}{l}\text { Variables: } \\
T / \mathrm{K}=298.15\end{array}$ & $\begin{array}{l}\text { Prepared by: } \\
\text { W. E. Acree, Jr. }\end{array}$ \\
\hline
\end{tabular}

Experimental Values

\begin{tabular}{lc}
\hline \hline$x_{2}{ }^{\mathrm{a}}$ & $x_{1}{ }^{\mathrm{b}}$ \\
\hline 0.9075 & 0.0925 \\
\hline${ }^{\mathrm{a}} x_{2}:$ mole fraction of component 2 in the saturated solution. \\
${ }^{\mathrm{b}} x_{1}:$ mole fraction solubility of the solute.
\end{tabular}

\section{Auxiliary Information}

Method/Apparatus/Procedure:

Constant-temperature bath, calorimetric thermometer, and an ultraviolet/ visible spectrophotometer.

Excess solute and solvent were placed in amber glass bottles and allowed to equilibrate for several days at constant temperature. Attainment of equilibrium was verified by several repetitive measurements and by approaching equilibrium from supersaturation. Aliquots of saturated solutions were transferred through a coarse filter into tared volumetric flasks, weighed, and diluted with methanol. Concentrations were determined by spectrophotometric measurements at $280 \mathrm{~nm}$.

Source and Purity of Chemicals:

(1) $99 \%$, Aldrich Chemical Company, Milwaukee, WI, USA, was used as received.

(2) $99.8+\%$, HPLC grade, Aldrich Chemical Company, stored over molecular sieves and distilled shortly before use.

Estimated Error:

Temperature: $\pm 0.1 \mathrm{~K}$.

$x_{1}: \pm 1.5 \%$ (relative error)

\begin{tabular}{ll}
\hline \hline Components: & Original Measurements: \\
$(1)$ 3-Chlorobenzoic acid; & ${ }^{8} \mathrm{~K}$. R. Hoover, K. Pop, W. E. Acree, Jr., \\
$\mathrm{C}_{7} \mathrm{H}_{5} \mathrm{ClO}_{2} ;[535-80-8]$ & and M. H. Abraham, S. Afr. J. Chem. \\
(2) 2-Butanol; $\mathrm{C}_{4} \mathrm{H}_{10} \mathrm{O} ;[78-92-2]$ & $\mathbf{5 8 , 2 5}(2005)$. \\
\hline Variables: & Prepared by: \\
$T / \mathrm{K}=298.15$ & W. E. Acree, Jr. \\
\hline
\end{tabular}

\section{Experimental Values}

\begin{tabular}{lc}
\hline \hline$x_{2}{ }^{\mathrm{a}}$ & $x_{1}{ }^{\mathrm{b}}$ \\
\hline 0.9093 & 0.0907 \\
\hline${ }^{\mathrm{a}} x_{2}:$ mole fraction of component 2 in the saturated solution. & \\
${ }^{\mathrm{b}} x_{1}:$ mole fraction solubility of the solute. &
\end{tabular}

${ }^{\mathrm{a}} x_{2}:$ mole fraction of component 2 in the
${ }^{\mathrm{b}} x_{1}$ : mole fraction solubility of the solute.

\section{Auxiliary Information}

\section{Method/Apparatus/Procedure:}

Constant-temperature bath, calorimetric thermometer, and an ultraviolet/ visible spectrophotometer.

Excess solute and solvent were placed in amber glass bottles and allowed to equilibrate for several days at constant temperature. Attainment of equilibrium was verified by several repetitive measurements and by approaching equilibrium from supersaturation. Aliquots of saturated solutions were transferred through a coarse filter into tared volumetric flasks, weighed, and diluted with methanol. Concentrations were determined by spectrophotometric measurements at $280 \mathrm{~nm}$.

\section{Source and Purity of Chemicals:}

(1) $99 \%$, Aldrich Chemical Company, Milwaukee, WI, USA, was used as received.

(2) $99+\%$, Aldrich Chemical Company, stored over molecular sieves and distilled shortly before use.

\section{Estimated Error:}

Temperature: $\pm 0.1 \mathrm{~K}$.

$x_{1}: \pm 1.5 \%$ (relative error). 


\section{Components:}

(1) 3-Chlorobenzoic acid;

$\mathrm{C}_{7} \mathrm{H}_{5} \mathrm{ClO}_{2} ;$; [535-80-8]

(2) 2-Methyl-1-propanol;

$\mathrm{C}_{4} \mathrm{H}_{10} \mathrm{O}$; [78-83-1]

\begin{tabular}{ll}
\hline Variables: & Prepared by: \\
$T / \mathrm{K}=298.15$ & W. E. Acree, Jr. \\
\hline
\end{tabular}

Experimental Values

\begin{tabular}{lc}
\hline \hline$x_{2}{ }^{\mathrm{a}}$ & $x_{1}{ }^{\mathrm{b}}$ \\
\hline 0.9308 & 0.0692
\end{tabular}

${ }^{\mathrm{a}} x_{2}$ : mole fraction of component 2 in the saturated solution.

${ }^{\mathrm{b}} x_{1}$ : mole fraction solubility of the solute.

\section{Auxiliary Information}

\section{Method/Apparatus/Procedure:}

Constant-temperature bath, calorimetric thermometer, and an ultraviolet/ visible spectrophotometer.

Excess solute and solvent were placed in amber glass bottles and allowed to equilibrate for several days at constant temperature. Attainment of equilibrium was verified by several repetitive measurements and by approaching equilibrium from supersaturation. Aliquots of saturated solutions were transferred through a coarse filter into tared volumetric flasks, weighed, and diluted with methanol. Concentrations were determined by spectrophotometric measurements at $280 \mathrm{~nm}$.

Source and Purity of Chemicals:

(1) $99 \%$, Aldrich Chemical Company, Milwaukee, WI, USA, was used as received.

(2) $99+\%$, anhydrous, Aldrich Chemical Company, stored over molecular sieves and distilled shortly before use.

Estimated Error:

Temperature: $\pm 0.1 \mathrm{~K}$.

$x_{1}: \pm 1.5 \%$ (relative error)

\begin{tabular}{|c|c|}
\hline $\begin{array}{l}\text { Components: } \\
\text { (1) 3-Chlorobenzoic acid; } \\
\mathrm{C}_{7} \mathrm{H}_{5} \mathrm{ClO}_{2} ;[535-80-8] \\
\text { (2) 1-Pentanol; } \mathrm{C}_{5} \mathrm{H}_{12} \mathrm{O} \text {; } \\
\text { [71-41-0] }\end{array}$ & $\begin{array}{l}\text { Original Measurements: } \\
{ }^{8} \text { K. R. Hoover, K. Pop, W. E. Acree, Jr., } \\
\text { and M. H. Abraham, S. Afr. J. Chem. } \\
\text { 58, } 25 \text { (2005). }\end{array}$ \\
\hline $\begin{array}{l}\text { Variables: } \\
T / \mathrm{K}=298.15\end{array}$ & $\begin{array}{l}\text { Prepared by: } \\
\text { W. E. Acree, Jr. }\end{array}$ \\
\hline
\end{tabular}

Experimental Values

\begin{tabular}{lc}
\hline \hline$x_{2}{ }^{\mathrm{a}}$ & $x_{1}{ }^{\mathrm{b}}$ \\
\hline 0.8990 & 0.1010 \\
\hline${ }^{\mathrm{a}}{ }_{x_{2}}:$ mole fraction of component 2 in the saturated solution. \\
${ }^{\mathrm{b}}{ }_{x_{1}}:$ mole fraction solubility of the solute.
\end{tabular}

\section{Auxiliary Information}

Method/Apparatus/Procedure:

Constant-temperature bath, calorimetric thermometer, and an ultraviolet/ visible spectrophotometer.

Excess solute and solvent were placed in amber glass bottles and allowed to equilibrate for several days at constant temperature. Attainment of equilibrium was verified by several repetitive measurements and by approaching equilibrium from supersaturation. Aliquots of saturated solutions were transferred through a coarse filter into tared volumetric flasks, weighed, and diluted with methanol. Concentrations were determined by spectrophotometric measurements at $280 \mathrm{~nm}$.

Source and Purity of Chemicals:

(1) $99 \%$, Aldrich Chemical Company, Milwaukee, WI, USA, was used as received.

(2) $99+\%$, Aldrich Chemical Company, stored over molecular sieves and distilled shortly before use.

Estimated Error:

Temperature: $\pm 0.1 \mathrm{~K}$.

$x_{1}: \pm 1.5 \%$ (relative error).

\begin{tabular}{|c|c|}
\hline $\begin{array}{l}\text { Components: } \\
\text { (1) 3-Chlorobenzoic acid; } \\
\mathrm{C}_{7} \mathrm{H}_{5} \mathrm{ClO}_{2} \text {; [535-80-8] } \\
\text { (2) 3-Methyl-1-butanol; } \mathrm{C}_{5} \mathrm{H}_{10} \mathrm{O} \text {; } \\
\text { [123-51-3] }\end{array}$ & $\begin{array}{l}\text { Original Measurements: } \\
{ }^{8} \text { K. R. Hoover, K. Pop, W. E. Acree, Jr., } \\
\text { and M. H. Abraham, S. Afr. J. Chem. } \\
\text { 58, } 25 \text { (2005). }\end{array}$ \\
\hline $\begin{array}{l}\text { Variables: } \\
T / \mathrm{K}=298.15\end{array}$ & $\begin{array}{l}\text { Prepared by: } \\
\text { W. E. Acree, Jr. }\end{array}$ \\
\hline
\end{tabular}

Experimental Values

\begin{tabular}{lc}
\hline \hline$x_{2}{ }^{\mathrm{a}}$ & $x_{1}{ }^{\mathrm{b}}$ \\
\hline 0.9118 & 0.0882
\end{tabular}

${ }^{\mathrm{a}} x_{2}$ : mole fraction of component 2 in the saturated solution.

${ }^{\mathrm{b}} x_{1}$ : mole fraction solubility of the solute.

\section{Auxiliary Information}

\section{Method/Apparatus/Procedure:}

Constant-temperature bath, calorimetric thermometer, and an ultraviolet/ visible spectrophotometer.

Excess solute and solvent were placed in amber glass bottles and allowed to equilibrate for several days at constant temperature. Attainment of equilibrium was verified by several repetitive measurements and by approaching equilibrium from supersaturation. Aliquots of saturated solutions were transferred through a coarse filter into tared volumetric flasks, weighed, and diluted with methanol. Concentrations were determined by spectrophotometric measurements at $280 \mathrm{~nm}$.

Source and Purity of Chemicals:

(1) $99 \%$, Aldrich Chemical Company, Milwaukee, WI, USA, was used as received.

(2) $99 \%$, anhydrous, Aldrich Chemical Company, stored over molecular sieves and distilled shortly before use.

Estimated Error:

Temperature: $\pm 0.1 \mathrm{~K}$.

$x_{1}: \pm 1.5 \%$ (relative error). 


\section{Components:}

(1) 3-Chlorobenzoic acid;

$\mathrm{C}_{7} \mathrm{H}_{5} \mathrm{ClO}_{2} ;[535-80-8]$

(2) $1-\mathrm{Hexanol} ; \mathrm{C}_{6} \mathrm{H}_{14} \mathrm{O}$;

[111-27-3]

\begin{tabular}{ll}
\hline Variables: & Prepared by: \\
$T / \mathrm{K}=298.15$ & W. E. Acree, Jr. \\
\hline
\end{tabular}

Experimental Values

\begin{tabular}{lc}
\hline \hline$x_{2}{ }^{\mathrm{a}}$ & $x_{1}{ }^{\mathrm{b}}$ \\
\hline 0.8940 & 0.1060
\end{tabular}

${ }^{\mathrm{a}} x_{2}$ : mole fraction of component 2 in the saturated solution.

${ }^{\mathrm{b}} x_{1}$ : mole fraction solubility of the solute.

\section{Auxiliary Information}

\section{Method/Apparatus/Procedure:}

Constant-temperature bath, calorimetric thermometer, and an ultraviolet/ visible spectrophotometer.

Excess solute and solvent were placed in amber glass bottles and allowed to equilibrate for several days at constant temperature. Attainment of equilibrium was verified by several repetitive measurements and by approaching equilibrium from supersaturation. Aliquots of saturated solutions were transferred through a coarse filter into tared volumetric flasks, weighed, and diluted with methanol. Concentrations were determined by spectrophotometric measurements at $280 \mathrm{~nm}$.

Source and Purity of Chemicals:

(1) $99 \%$, Aldrich Chemical Company, Milwaukee, WI, USA, was used as received.

(2) $99+\%$, Alfa Aesar, USA, stored over molecular sieves and distilled shortly before use.

Estimated Error:

Temperature: $\pm 0.1 \mathrm{~K}$.

$x_{1}: \pm 1.5 \%$ (relative error)

\begin{tabular}{|c|c|}
\hline $\begin{array}{l}\text { Components: } \\
\text { (1) 3-Chlorobenzoic acid; } \\
\mathrm{C}_{7} \mathrm{H}_{5} \mathrm{ClO}_{2} ;[535-80-8] \\
\text { (2) 1-Heptanol; } \mathrm{C}_{7} \mathrm{H}_{16} \mathrm{O} \text {; } \\
\text { [111-70-6] }\end{array}$ & $\begin{array}{l}\text { Original Measurements: } \\
{ }^{8} \text { K. R. Hoover, K. Pop, W. E. Acree, Jr., } \\
\text { and M. H. Abraham, S. Afr. J. Chem. } \\
\text { 58, } 25 \text { (2005). }\end{array}$ \\
\hline $\begin{array}{l}\text { Variables: } \\
T / \mathrm{K}=298.15\end{array}$ & $\begin{array}{l}\text { Prepared by: } \\
\text { W. E. Acree, Jr. }\end{array}$ \\
\hline
\end{tabular}

Experimental Values

\begin{tabular}{lc}
\hline \hline$x_{2}{ }^{\mathrm{a}}$ & $x_{1}{ }^{\mathrm{b}}$ \\
\hline 0.8961 & 0.1039 \\
\hline${ }^{\mathrm{a}}{ }_{x_{2}}:$ mole fraction of component 2 in the saturated solution. \\
${ }^{\mathrm{b}}{ }_{x_{1}}:$ mole fraction solubility of the solute.
\end{tabular}

\section{Auxiliary Information}

\section{Method/Apparatus/Procedure:}

Constant-temperature bath, calorimetric thermometer, and an ultraviolet/ visible spectrophotometer.

Excess solute and solvent were placed in amber glass bottles and allowed to equilibrate for several days at constant temperature. Attainment of equilibrium was verified by several repetitive measurements and by approaching equilibrium from supersaturation. Aliquots of saturated solutions were transferred through a coarse filter into tared volumetric flasks, weighed, and diluted with methanol. Concentrations were determined by spectrophotometric measurements at $280 \mathrm{~nm}$.

Source and Purity of Chemicals:

(1) $99 \%$, Aldrich Chemical Company, Milwaukee, WI, USA, was used as received.

(2) $99+\%$, Alfa Aesar, USA, stored over molecular sieves and distilled shortly before use.

Estimated Error:

Temperature: $\pm 0.1 \mathrm{~K}$.

$x_{1}: \pm 1.5 \%$ (relative error).

\begin{tabular}{ll}
\hline \hline Components: & Original Measurements: \\
(1) 3-Chlorobenzoic acid; & ${ }^{8}$ K. R. Hoover, K. Pop, W. E. Acree, Jr., \\
$\mathrm{C}_{7} \mathrm{H}_{5} \mathrm{ClO}_{2} ;[535-80-8]$ & and M. H. Abraham, S. Afr. J. Chem. \\
(2) 1-Octanol; $\mathrm{C}_{8} \mathrm{H}_{18} \mathrm{O} ;$ & $\mathbf{5 8}, 25$ (2005). \\
{$[111-87-5]$} & \\
\hline Variables: & Prepared by: \\
$T / \mathrm{K}=298.15$ & W. E. Acree, Jr. \\
\hline
\end{tabular}

\section{Experimental Values}

\begin{tabular}{lc}
\hline \hline$x_{2}{ }^{\mathrm{a}}$ & $x_{1}{ }^{\mathrm{b}}$ \\
\hline 0.8901 & 0.1099 \\
\hline${ }^{\mathrm{a}} x_{2}:$ mole fraction of component 2 in the saturated solution. & \\
${ }^{\mathrm{b}} x_{1}:$ mole fraction solubility of the solute. &
\end{tabular}

${ }^{\mathrm{b}} x_{1}$ : mole fraction solubility of the solute.

\section{Auxiliary Information}

\section{Method/Apparatus/Procedure:}

Constant-temperature bath, calorimetric thermometer, and an ultraviolet/ visible spectrophotometer.

Excess solute and solvent were placed in amber glass bottles and allowed to equilibrate for several days at constant temperature. Attainment of equilibrium was verified by several repetitive measurements and by approaching equilibrium from supersaturation. Aliquots of saturated solutions were transferred through a coarse filter into tared volumetric flasks, weighed, and diluted with methanol. Concentrations were determined by spectrophotometric measurements at $280 \mathrm{~nm}$.

Source and Purity of Chemicals:

(1) $99 \%$, Aldrich Chemical Company, Milwaukee, WI, USA, was used as received.

(2) $99+\%$, anhydrous, Aldrich Chemical Company, stored over molecular sieves and distilled shortly before use.

Estimated Error:

Temperature: $\pm 0.1 \mathrm{~K}$.

$x_{1}: \pm 1.5 \%$ (relative error). 


\section{Components:}

(1) 3-Chlorobenzoic acid;

$\mathrm{C}_{7} \mathrm{H}_{5} \mathrm{ClO}_{2} ;[535-80-8]$

(2) 1-Decanol; $\mathrm{C}_{10} \mathrm{H}_{22} \mathrm{O}$;

[112-30-1]

\begin{tabular}{ll}
\hline Variables: & Prepared by: \\
$T / \mathrm{K}=298.15$ & W. E. Acree, Jr. \\
\hline
\end{tabular}

Experimental Values

\begin{tabular}{lc}
\hline \hline$x_{2}{ }^{\mathrm{a}}$ & $x_{1}{ }^{\mathrm{b}}$ \\
\hline 0.8896 & 0.1104
\end{tabular}

${ }^{\mathrm{a}} x_{2}$ : mole fraction of component 2 in the saturated solution.

${ }^{\mathrm{b}} x_{1}$ : mole fraction solubility of the solute.

\section{Auxiliary Information}

\section{Method/Apparatus/Procedure:}

Constant-temperature bath, calorimetric thermometer, and an ultraviolet/ visible spectrophotometer.

Excess solute and solvent were placed in amber glass bottles and allowed to equilibrate for several days at constant temperature. Attainment of equilibrium was verified by several repetitive measurements and by approaching equilibrium from supersaturation. Aliquots of saturated solutions were transferred through a coarse filter into tared volumetric flasks, weighed, and diluted with methanol. Concentrations were determined by spectrophotometric measurements at $280 \mathrm{~nm}$.

Source and Purity of Chemicals:

(1) $99 \%$, Aldrich Chemical Company, Milwaukee, WI, USA, was used as received.

(2) $99+\%$, Alfa Aesar, USA, stored over molecular sieves and distilled shortly before use.

Estimated Error:

Temperature: $\pm 0.1 \mathrm{~K}$.

$x_{1}: \pm 1.5 \%$ (relative error).

\begin{tabular}{|c|c|}
\hline $\begin{array}{l}\text { Components: } \\
\text { (1) 3-Chlorobenzoic acid; } \\
\mathrm{C}_{7} \mathrm{H}_{5} \mathrm{ClO}_{2} ;[535-80-8] \\
\text { (2) 1,2-Propanediol; } \mathrm{C}_{3} \mathrm{H}_{8} \mathrm{O}_{2} \text {; } \\
\text { [57-55-6] }\end{array}$ & $\begin{array}{l}\text { Original Measurements: } \\
{ }^{61} \text { E. R. Cooper, J. Control. Release 1, } \\
153 \text { (1984). }\end{array}$ \\
\hline $\begin{array}{l}\text { Variables: } \\
T / \mathrm{K}=295\end{array}$ & $\begin{array}{l}\text { Prepared by: } \\
\text { W. E. Acree, Jr. }\end{array}$ \\
\hline
\end{tabular}

Experimental Values

\begin{tabular}{lc}
\hline \hline$x_{2}{ }^{\mathrm{a}}$ & $x_{1}{ }^{\mathrm{b}}$ \\
\hline 0.949 & 0.051 \\
\hline${ }^{\mathrm{a}} x_{2}:$ mole fraction of component 2 in the saturated solution. \\
${ }^{\mathrm{b}} x_{1}:$ mole fraction solubility of the solute. Solubility data reported in terms of \\
mass percent. Mole fraction solubility calculated by the compiler.
\end{tabular}

\section{Auxiliary Information}

Method/Apparatus/Procedure:

Very little experimental details provided. Solubility determinations were made by stirring the solvent with excess solute for two days, centrifuging the sample, and analyzing the supernatant.

Source and Purity of Chemicals:

(1) Reagent grade, Aldrich Chemical Company, Metuchen, NJ, USA, no purification details provided.

(2) Reagent grade, J.T. Baker Chemical Company, Phillipsburg, NJ, USA, no purification details provided.

Estimated Error:

Temperature: No information given.

$x_{1}$ : No information given.

\section{Solubility of 4-Chlorobenzoic Acid in Organic Solvents}

\subsection{Critical evaluation of experimental solubility data}

There have been several published studies ${ }^{14,54,61,127-130}$ investigating the solubility behavior of 4-chlorobenzoic acid in organic solvents of varying polarity and hydrogen-bonding capability. Daniels et al. ${ }^{14}$ measured the solubility of 4-chlorobenzoic acid in 16 alcohols (methanol, ethanol, 1-propanol, 2propanol, 1-butanol, 2-butanol, 2-methyl-1-propanol, 2-methyl2-propanol, 1-pentanol, 2-pentanol, 2-methyl-1-butanol, 3methyl-1-butanol, 1-hexanol, 1-heptanol, 1-octanol, and 1decanol), in two dialkyl ethers (2,2'-oxybispropane and 1,1'oxybisbutane) and two cyclic ethers (tetrahydrofuran and 1,4dioxane), and in three alkanoates (methyl ethanoate, ethyl ethanoate, and butyl ethanoate), and in propylene carbonate at 298 K. Results of the experimental measurements were used to calculate the Abraham solute descriptors of 4-chlorobenzoic acid. The authors were able to assemble a total of $49 \log _{10}(S R$ or $P$ ) and $\log _{10}(G S R$ or $K)$ equations for which experimental partition coefficient data, solubility ratios, Abraham model equation coefficients and aqueous molar solubility were available. The logarithm of the aqueous molar solubility of 4-chlorobenzoic acid is $\log _{10} c_{1, \mathrm{w}}=-3.56 .{ }^{131-135}$ The McGowan volume of 4-chlorobenzoic acid, $V=1.0541$, was calculated from the number of chemical bonds in the molecule and the individual atomic group volumes, $A V_{i}$, given in Sec. 1.3. The excess molar refraction solute descriptor was estimated as $E=0.840$. This left four solute descriptors $(S, A, B$, and $L$ ) still to be determined. The 49 equations were then solved using the Microsoft "SOLVER" program to yield numerical values of the remaining four solute descriptors, $S=1.020$, $A=0.630, B=0.270$, and $L=4.9474$, that best described the $\log _{10}(S R$ or $P)$ and $\log _{10}(G S R$ or $K)$ values. The computation treated $\log _{10} c_{1, \mathrm{G}}$ as a floating parameter to be determined as part of the regression analyses. The data analyses returned a value of $\log _{10} c_{1, \mathrm{G}}=-8.360$ for the gas-phase solute concentration that made the $\log _{10}\left(S R\right.$ or $P$ ) and $\log _{10}(G S R$ or $K)$ predictions internally consistent. The calculated molecular solute descriptors reproduced the $\log _{10}(S R$ or $P)$ and $\log _{10}(G S R$ or $K)$ values to 
TABLE 22. Comparison between observed and calculated molar solubilities of 4-chlorobenzoic acid based on the Abraham model, Eqs. (20) and (21)

\begin{tabular}{|c|c|c|c|}
\hline Solvent & $\log _{10} c_{1}^{\text {exp,a }}$ & $\begin{array}{c}\log _{10} c_{1}{ }^{\text {calc }} \text {; } \\
\text { Eq. (20) }\end{array}$ & $\begin{array}{c}\log _{10} c_{1}{ }^{\text {calc }} \text {; } \\
\text { Eq. (21) }\end{array}$ \\
\hline Methanol & -0.701 & -0.734 & -0.728 \\
\hline Ethanol & -0.767 & -0.698 & -0.693 \\
\hline 1-Propanol & -0.800 & -0.790 & -0.781 \\
\hline 2-Propanol & -0.780 & -0.768 & -0.752 \\
\hline 1-Butanol & -0.840 & -0.860 & -0.845 \\
\hline 2-Butanol & -0.800 & -0.828 & -0.861 \\
\hline 2-Methyl-1-propanol & -0.990 & -0.976 & -0.990 \\
\hline 2-Methyl-2-propanol & -0.670 & -0.734 & -0.704 \\
\hline 1-Pentanol & -0.860 & -0.870 & -0.860 \\
\hline 2-Pentanol & -0.821 & -0.874 & -0.872 \\
\hline 3-Methyl-1-butanol & -0.919 & -0.940 & -0.939 \\
\hline 1-Hexanol & -0.880 & -0.905 & -0.913 \\
\hline 1-Heptanol & -0.900 & -0.895 & -0.934 \\
\hline 1-Octanol & -0.920 & -0.960 & -1.038 \\
\hline 1-Decanol & -0.988 & -1.040 & -1.041 \\
\hline Tetrahydrofuran & -0.153 & -0.215 & -0.258 \\
\hline 1,4-Dioxane & -0.465 & -0.512 & -0.518 \\
\hline Methyl ethanoate & -0.951 & -0.862 & -0.822 \\
\hline Ethyl ethanoate & -0.950 & -0.768 & -0.780 \\
\hline Butyl ethanoate & -1.155 & -0.900 & -0.952 \\
\hline
\end{tabular}

${ }^{\mathrm{a}}$ All experimental data were taken from Daniels et al. ${ }^{14}$

within an average standard deviation of 0.078 and $0.098 \log _{10}$ units, respectively.

After the 4-chlorobenzoic acid solubility study was published, Abraham model correlations have been developed for 2-pentanol, 3-methyl-1-butanol, methyl ethanoate, and butyl ethanoate, and equation coefficients for a few solvents were updated based on additional experimental data. The new correlations (listed in Tables 1 and 2) will be used in illustrating the ability of the Abraham model to correlate the experimental 4-chlorobenzoic acid solubility data. Table 22 compares the experimental $\log _{10} c_{1}$ values to calculated values based on Eqs. (20) and (21) of the Abraham model. For comparison purposes, the measured mole fraction solubilities of 4-chlorobenzoic acid, $x_{1}$, determined by Daniel et al. ${ }^{14}$ were converted into molar solubilities by dividing $x_{1}$ by the ideal molar volume of the saturated solution (i.e., $c_{1}{ }^{\text {sat }}=x_{1} /\left[x_{1} V_{1}+\right.$ $\left.\left.\left(1-x_{1}\right) V_{\text {solvent }}\right]\right)$. The molar volume of the hypothetical subcooled liquid 4-chlorobenzoic acid is $V_{\text {solute }}=117.08 \mathrm{~cm}^{3}$ $\mathrm{mol}^{-1}$. Examination of the numerical entries in Table 22 reveals that the Abraham model provides a reasonably accurate mathematical description of the observed solubility data, suggesting that there are no obvious outliers in the dataset.

Sidgwick and Ewbank ${ }^{127}$ measured the solubility of 4chlorobenzoic acid in heptane and benzene as a function of temperature. The internal consistency of the two datasets was assessed by curve-fitting the measured mole fraction solubility data to the Modified Apelblat model to yield the following representations:

$$
\begin{gathered}
\ln x_{1}=-130.159+\frac{113.10}{T}+20.8141 \ln T, \\
\ln x_{1}=-89.694+\frac{113.78}{T}+14.3691 \ln T,
\end{gathered}
$$

for solubilities in heptane and benzene, respectively. The average absolute deviations between the observed experimental data and back-calculated values based on Eqs. (45) and (46) of $12.4 \%$ and $6.7 \%$ are larger than desired. The large deviations may be due in part to the fact that the solution temperature is above the solvent's normal boiling point temperature, to uncertainties in estimating the amount of solvent that remained in the liquid phase, and the large range covered by the experimental values, $x_{1}=0.0109$ to $x_{1}=0.680$ for heptane and $x_{1}=0.0100$ to $x_{1}=0.855$ for benzene. It is more difficult to curve-fit experimental solubility data covering large mole fraction ranges.

The experimental solubility data for 4-chlorobenzoic acid in organic solvents are in Secs. 20.2-20.9.

\subsection{4-Chlorobenzoic acid solubility data in}

\begin{tabular}{|c|c|}
\hline $\begin{array}{l}\text { Components: } \\
\text { (1) 4-Chlorobenzoic acid; } \\
\mathrm{C}_{7} \mathrm{H}_{5} \mathrm{ClO}_{2} ;[74-11-3] \\
\text { (2) Heptane; } \mathrm{C}_{7} \mathrm{H}_{16} ;[142-82-5]\end{array}$ & $\begin{array}{l}\text { Original Measurements: } \\
{ }^{127} \text { N. V. Sidgwick and E. K. Ewbank } \\
\text { J. Chem. Soc. Trans. 119, } 979 \text { (1921) }\end{array}$ \\
\hline $\begin{array}{l}\text { Variables: } \\
\text { Temperature }\end{array}$ & $\begin{array}{l}\text { Prepared by: } \\
\text { W. E. Acree, Jr. }\end{array}$ \\
\hline
\end{tabular}
saturated hydrocarbons (including cycloalkanes)

Experimental Values

\begin{tabular}{lcc}
\hline \hline$T / \mathrm{K}$ & $x_{2}{ }^{\mathrm{a}}$ & \multicolumn{1}{c}{$x_{1}{ }^{\mathrm{b}}$} \\
\hline 409.3 & 0.989 & 0.0109 \\
438.5 & 0.968 & 0.0323 \\
454.1 & 0.933 & 0.0670 \\
480.4 & 0.775 & 0.225 \\
491.5 & 0.597 & 0.403 \\
500.8 & 0.320 & 0.680 \\
\hline
\end{tabular}

${ }^{\mathrm{a}} x_{2}$ : mole fraction of component 2 in the saturated solution.

${ }^{b} x_{1}$ : mole fraction solubility of the solute. Solubility data reported in units of mass percent. Mole fraction values calculated by the compiler.

\section{Auxiliary Information}

\section{Method/Apparatus/Procedure:}

Preweighed quantities of solute and solvent were placed in bulbs, which were then sealed and heated in a suitable bath. At the higher temperatures, the concentration of solvent in the liquid solution was corrected for the amount of solvent vapor in the bulb. The correction assumed that the vapor pressure of the saturated solution was one half that of the pure solvent at the solution temperature. The temperature at which the solid completely dissolved was recorded, and was taken to be the solid-liquid equilibrium temperature.

\section{Source and Purity of Chemicals:}

(1) Purity not given, prepared and purified by the authors using a published synthetic procedure [see J. Walker and J. K. Wood, J. Chem. Soc. 117, 40 (1920) for synthetic details]. Melting point of the purified solute was $514.7 \mathrm{~K}$. (2) Purity not given, sample isolated from the resin of Pinus sabiniana, purified by treatment with sulfuric acid and nitric acid, and then distilled before use.

Estimated Error:

Temperature: Not given in paper.

$x_{1}: \pm 10 \%$ (relative error, estimated by compiler). 


\begin{tabular}{ll}
\hline \hline Components: & Original Measurements: \\
$\begin{array}{l}\text { (1) 4-Chlorobenzoic acid; } \\
\mathrm{C}_{7} \mathrm{H}_{5} \mathrm{ClO}_{2} ;[74-11-3]\end{array}$ & $\begin{array}{l}{ }^{4} \mathrm{C} . \mathrm{K} . \text { Hancock, J. N. Pawloski, and } \\
(2) \mathrm{Cyclohexane} ; \mathrm{C}_{6} \mathrm{H}_{12} ;\end{array}$ \\
$\begin{array}{ll}\text { J. P. Idoux, J. Org. Chem. 31, 3801 } \\
\text { (110-82-7] }\end{array}$ & \\
\hline Variables: & Prepared by: \\
$T / \mathrm{K}=303.15$ & W. E. Acree, Jr. \\
\hline
\end{tabular}

Experimental Values

\begin{tabular}{|c|c|}
\hline$x_{2}{ }^{a}$ & $x_{1}{ }^{\mathrm{b}}$ \\
\hline 0.9999 & 0.0000719 \\
\hline
\end{tabular}

${ }^{\mathrm{a}} x_{2}$ : mole fraction of component 2 in the saturated solution.

${ }^{\mathrm{b}} x_{1}$ : mole fraction solubility of the solute.

\section{Auxiliary Information}

\begin{abstract}
Method/Apparatus/Procedure:
Constant-temperature bath, Soxhlet thimble, conical flask, and analytical balance.

Excess solute and solvent were placed in a conical flask and allowed to equilibrate for several days at constant temperature. Attainment of equilibrium was verified by several repetitive measurements and by approaching equilibrium from supersaturation. Aliquots of saturated solutions were transferred using a Soxhlet thimble equipped with a one-hole cork stopper and an inverted U-shaped delivery tube to a second ground-glass stoppered flask suspended in the $303 \mathrm{~K}$ water bath. Successive portions of the contents were evaporated at room temperature in a tared aluminum foil weighing dish under a bell jar through which a slow stream of dry air was passed. The second flask plus unused saturated solution and the aluminum foil dish plus residue were weighed. The saturation solubility of the solute was calculated from the recorded mass data and molar masses of the solute and solvent.
\end{abstract}

Source and Purity of Chemicals:

(1) Purity not given, Chemical source not specified, was recrystallized several times from aqueous-ethanol mixture, and then dried under vacuum over phosphorous pentoxide.

(2) Reagent grade, Chemical source not specified, refluxed over phosphorous pentoxide for $24 \mathrm{~h}$, and then distilled through a $3 \times 80 \mathrm{~cm}$ column filled with 0.32 -cm glass helices.

\section{Estimated Error:}

Temperature: $\pm 0.02 \mathrm{~K}$.

$x_{1}: \pm 10 \%$ (relative error, by compiler).

\begin{tabular}{|c|c|}
\hline $\begin{array}{l}\text { Components: } \\
\text { (1) 4-Chlorobenzoic acid; } \\
\mathrm{C}_{7} \mathrm{H}_{5} \mathrm{ClO}_{2} ;[74-11-3] \\
\text { (2) Cyclohexane; } \mathrm{C}_{6} \mathrm{H}_{12} \text {; } \\
{[110-82-7]}\end{array}$ & $\begin{array}{l}\text { Original Measurements: } \\
{ }^{128} \text { G. S. Krasil'nikova, E. G. Freidlin, } \\
\text { and Y. N. Pirig, Zh. Prikl. Khim. 60, } \\
2519 \text { (1987). }\end{array}$ \\
\hline $\begin{array}{l}\text { Variables: } \\
T / \mathrm{K}=293 \\
\end{array}$ & $\begin{array}{l}\text { Prepared by: } \\
\text { W. E. Acree, Jr. }\end{array}$ \\
\hline
\end{tabular}

\section{Experimental Values}

The measured solubility was reported to be $0.1 \mathrm{~g} / 1$, which corresponds to a molar solubility of $c_{1}=0.00064$.

\section{Auxiliary Information}

Method/Apparatus/Procedure:

No information given.

Source and Purity of Chemicals:

(1) Purity not given, Chemical source not given, no purification details were provided.

(2) Purity not given, Chemical source not given, no purification details were provided.

Estimated Error:

Temperature: No information given.

$c_{1}$ : No information given.

\subsection{4-Chlorobenzoic acid solubility data in aromatic hydrocarbons}

\begin{tabular}{ll}
\hline \hline Components: & Original Measurements: \\
$\begin{array}{l}\text { (1) 4-Chlorobenzoic acid; } \\
\mathrm{C}_{7} \mathrm{H}_{5} \mathrm{ClO}_{2} ;[74-11-3]\end{array}$ & ${ }^{54} \mathrm{C}$. K. Hancock, J. N. Pawloski, and J. \\
(2) Benzene; $\mathrm{C}_{6} \mathrm{H}_{6} ;[71-43-2]$ & $(1966)$. \\
\hline Variables: & Prepared by: \\
$T / \mathrm{K}=303.15$ & W. E. Acree, Jr. \\
\hline
\end{tabular}

\section{Experimental Values}

\begin{tabular}{lc}
\hline \hline$x_{2}{ }^{\mathrm{a}}$ & $x_{1}{ }^{\mathrm{b}}$ \\
\hline 0.9995 & 0.000489 \\
\hline${ }^{\mathrm{a}} x_{2}:$ mole fraction of component 2 in the saturated solution. \\
${ }^{\mathrm{b}} x_{1}:$ mole fraction solubility of the solute.
\end{tabular}

Auxiliary Information

\section{Method/Apparatus/Procedure:}

Constant-temperature bath, Soxhlet thimble, conical flask, and analytical balance.

Excess solute and solvent were placed in a conical flask and allowed to equilibrate for several days at constant temperature. Attainment of equilibrium was verified by several repetitive measurements and by approaching equilibrium from supersaturation. Aliquots of saturated solutions were transferred using a Soxhlet thimble equipped with a one-hole cork stopper and an inverted U-shaped delivery tube to a second ground-glass stoppered flask suspended in the $303 \mathrm{~K}$ water bath. Successive portions of the contents were evaporated at room temperature in a tared aluminum foil weighing dish under a bell jar through which a slow stream of dry air was passed. The second flask plus unused saturated solution and the aluminum foil dish plus residue were weighed. The saturation solubility of the solute was calculated from the recorded mass data and molar masses of the solute and solvent.

\section{Source and Purity of Chemicals:}

(1) Purity not given, Chemical source not specified, was recrystallized several times from aqueous-ethanol mixture, and then dried under vacuum over phosphorous pentoxide.

(2) Reagent grade, Chemical source not specified, refluxed over phosphorous pentoxide for $24 \mathrm{~h}$, and then distilled through a $3 \times 80 \mathrm{~cm}$ column filled with $0.32-\mathrm{cm}$ glass helices.

\section{Estimated Error:}

Temperature: $\pm 0.02 \mathrm{~K}$.

$x_{1}: \pm 3 \%$ (relative error, by compiler). 


\begin{tabular}{|c|c|}
\hline $\begin{array}{l}\text { Components: } \\
\text { (1) 4-Chlorobenzoic acid; } \\
\mathrm{C}_{7} \mathrm{H}_{5} \mathrm{ClO}_{2} ;[74-11-3] \\
\text { (2) Benzene; } \mathrm{C}_{6} \mathrm{H}_{6} ;[71-43-2]\end{array}$ & $\begin{array}{l}\text { Original Measurements: } \\
{ }^{127} \text { N. V. Sidgwick and E. K. Ewbank, J. } \\
\text { Chem. Soc. Trans. 119, } 979 \text { (1921). }\end{array}$ \\
\hline $\begin{array}{l}\text { Variables: } \\
\text { Temperature }\end{array}$ & $\begin{array}{l}\text { Prepared by: } \\
\text { W. E. Acree, Jr. }\end{array}$ \\
\hline
\end{tabular}

Experimental Values

\begin{tabular}{lcc}
\hline \hline$T / \mathrm{K}$ & $x_{2}{ }^{\mathrm{a}}$ & $x_{1}{ }^{\mathrm{b}}$ \\
\hline 366.8 & 0.990 & 0.0100 \\
392.6 & 0.973 & 0.0272 \\
410.6 & 0.946 & 0.0542 \\
445.7 & 0.820 & 0.1796 \\
467.2 & 0.645 & 0.355 \\
485.9 & 0.427 & 0.573 \\
505.7 & 0.145 & 0.855
\end{tabular}

${ }^{\mathrm{a}} x_{2}$ : mole fraction of component 2 in the saturated solution.

${ }^{b} x_{1}$ : mole fraction solubility of the solute. Solubility data reported in units of mass percent. Mole fraction values calculated by the compiler.

\section{Auxiliary Information}

\section{Method/Apparatus/Procedure:}

Preweighed quantities of solute and solvent were placed in bulbs, which were then sealed and heated in a suitable bath. At the higher temperatures, the concentration of solvent in the liquid solution was corrected for the amount of solvent vapor in the bulb. The correction assumed that the vapor pressure of the saturated solution was one half that of the pure solvent at the solution temperature. The temperature at which the solid completely dissolved was recorded, and was taken to be the solid-liquid equilibrium temperature.

\section{Source and Purity of Chemicals:}

(1) Purity not given, prepared and purified by the authors using a published synthetic procedure [see J. Walker and J. K. Wood, J. Chem. Soc. 117, 40 (1920) for synthetic details]. Melting point of the purified solute was $514.7 \mathrm{~K}$. (2) Purity not given, Chemical source not given, thiophene was removed by treatment with sulfuric acid. Sample was further purified by freezing several times.

\section{Estimated Error:}

Temperature: Not given in paper.

$x_{1}: \pm 10 \%$ (relative error, estimated by compiler).

\begin{tabular}{ll}
\hline \hline Components: & Original Measurements: \\
$\begin{array}{l}\text { (1) 4-Chlorobenzoic acid; } \\
\mathrm{C}_{7} \mathrm{H}_{5} \mathrm{ClO}_{2} ;[74-11-3]\end{array}$ & ${ }^{128}$ G. S. Krasil'nikova, E. G. Freidlin, \\
(2) Benzene; $\mathrm{C}_{6} \mathrm{H}_{6} ;[71-43-2]$ & and Y. N. Pirig, Zh. Prikl. Khim. 60, \\
\hline Variables: & $2519(1987)$. \\
$T / \mathrm{K}=293$ & Prepared by: \\
\hline
\end{tabular}

\section{Experimental Values}

The measured solubility was reported to be $0.5 \mathrm{~g} / \mathrm{l}$, which corresponds to a molar solubility of $c_{1}=0.0032$.

\section{Auxiliary Information}

Method/Apparatus/Procedure:

No information given.

Source and Purity of Chemicals:

(1) Purity not given, Chemical source not given, no purification details were provided.

(2) Purity not given, Chemical source not given, no purification details were provided.

Estimated Error:

Temperature: No information given.

$c_{1}$ : No information given.

\subsection{4-Chlorobenzoic acid solubility data in esters}

\begin{tabular}{ll}
\hline \hline Components: & Original Measurements: \\
(1) 4-Chlorobenzoic acid; & ${ }^{14}$ C. R. Daniels, A. K. Charlton, R. M. \\
$\mathrm{C}_{7} \mathrm{H}_{5} \mathrm{ClO}_{2} ;$ [74-11-3] & Wold, W. E. Acree, Jr., and M. H. \\
$\begin{array}{l}\text { (2) Methyl ethanoate; } \mathrm{C}_{3} \mathrm{H}_{6} \mathrm{O}_{2} ; \\
\text { [79-20-9] }\end{array}$ & $\begin{array}{l}\text { Abraham, Can. J. Chem. 81, 1492 } \\
(2003) .\end{array}$ \\
\hline Variables: & Prepared by: \\
$T / \mathrm{K}=298.15$ & W. E. Acree, Jr. \\
\hline
\end{tabular}

Experimental Values

\begin{tabular}{lc}
\hline \hline$x_{2}{ }^{\mathrm{a}}$ & $x_{1}{ }^{\mathrm{b}}$ \\
\hline 0.9910 & 0.008963
\end{tabular}

${ }^{\mathrm{a}} x_{2}$ : mole fraction of component 2 in the saturated solution.

${ }^{\mathrm{b}} x_{1}$ : mole fraction solubility of the solute.

\section{Auxiliary Information}

\section{Method/Apparatus/Procedure:}

Constant-temperature bath, calorimetric thermometer, and an ultraviolet/ visible spectrophotometer.

Excess solute and solvent were placed in amber glass bottles and allowed to equilibrate for several days at constant temperature. Attainment of equilibrium was verified by several repetitive measurements and by approaching equilibrium from supersaturation. Aliquots of saturated solutions were transferred through a coarse filter into tared volumetric flasks, weighed, and diluted with methanol. Concentrations were determined by

spectrophotometric measurements at $272 \mathrm{~nm}$.

\section{Source and Purity of Chemicals:}

(1) $99 \%$, Acros Organics, USA, was used as received.

(2) $99.5 \%$, anhydrous, Aldrich Chemical Company, Milwaukee, WI, USA, stored over molecular sieves and distilled shortly before use.

\section{Estimated Error:}

Temperature: $\pm 0.1 \mathrm{~K}$.

$x_{1}: \pm 1.5 \%$ (relative error) 


\begin{tabular}{ll}
\hline \hline Components: & Original Measurements: \\
$\begin{array}{l}\text { (1) 4-Chlorobenzoic acid; } \\
\mathrm{C}_{7} \mathrm{H}_{5} \mathrm{ClO}_{2} ; \text { [74-11-3] }\end{array}$ & ${ }^{14} \mathrm{C}$. R. Daniels, A. K. Charlton, R. M. \\
(2) Ethyl ethanoate; $\mathrm{C}_{4} \mathrm{H}_{8} \mathrm{O}_{2} ;$ & Wold, W. E. Acree, Jr., and M. H. \\
[141-78-6] & Abraham, Can. J. Chem. 81, 1492 \\
\hline Variables: & $(2003)$. \\
$T / \mathrm{K}=298.15$ & Prepared by: \\
\hline
\end{tabular}

Experimental Values

\begin{tabular}{lc}
\hline \hline$x_{2}{ }^{\mathrm{a}}$ & $x_{1}{ }^{\mathrm{b}}$ \\
\hline 0.9889 & 0.01111
\end{tabular}

${ }^{\mathrm{a}} x_{2}$ : mole fraction of component 2 in the saturated solution.

${ }^{\mathrm{b}} x_{1}$ : mole fraction solubility of the solute.

\section{Auxiliary Information}

\section{Method/Apparatus/Procedure:}

Constant-temperature bath, calorimetric thermometer, and an ultraviolet/ visible spectrophotometer.

Excess solute and solvent were placed in amber glass bottles and allowed to equilibrate for several days at constant temperature. Attainment of equilibrium was verified by several repetitive measurements and by approaching equilibrium from supersaturation. Aliquots of saturated solutions were transferred through a coarse filter into tared volumetric flasks, weighed, and diluted with methanol. Concentrations were determined by spectrophotometric measurements at $272 \mathrm{~nm}$.

Source and Purity of Chemicals:

(1) $99 \%$, Acros Organics, USA, was used as received.

(2) $99.9 \%$, HPLC grade, Aldrich Chemical Company, Milwaukee, WI, USA, stored over molecular sieves and distilled shortly before use.

\section{Estimated Error:}

Temperature: $\pm 0.1 \mathrm{~K}$.

$x_{1}: \pm 1.5 \%$ (relative error).

\begin{tabular}{ll}
\hline \hline Components: & Original Measurements: \\
(1) 4-Chlorobenzoic acid; & ${ }^{14}$ C. R. Daniels, A. K. Charlton, R. M. \\
$\mathrm{C}_{7} \mathrm{H}_{5} \mathrm{ClO}_{2} ;$ [74-11-3] & Wold, W. E. Acree, Jr., and M. H. \\
(2) Butyl ethanoate; $\mathrm{C}_{6} \mathrm{H}_{12} \mathrm{O}_{2} ;$ & Abraham, Can. J. Chem. 81, 1492 \\
[123-86-4] & $(2003)$. \\
\hline Variables: & Prepared by: \\
$T / \mathrm{K}=298.15$ & W. E. Acree, Jr. \\
\hline
\end{tabular}

\section{Experimental Values}

\begin{tabular}{lc}
\hline \hline$x_{2}^{\mathrm{a}}$ & $x_{1}{ }^{\mathrm{b}}$ \\
\hline 0.9907 & 0.00926 \\
\hline${ }^{a} x_{2}:$ mole fraction of component 2 in the saturated solution. & \\
${ }^{\mathrm{b}} x_{1}:$ mole fraction solubility of the solute. &
\end{tabular}

\section{Auxiliary Information}

\section{Method/Apparatus/Procedure:}

Constant-temperature bath, calorimetric thermometer, and an ultraviolet/ visible spectrophotometer.

Excess solute and solvent were placed in amber glass bottles and allowed to equilibrate for several days at constant temperature. Attainment of equilibrium was verified by several repetitive measurements and by approaching equilibrium from supersaturation. Aliquots of saturated solutions were transferred through a coarse filter into tared volumetric flasks, weighed, and diluted with methanol. Concentrations were determined by spectrophotometric measurements at $272 \mathrm{~nm}$.

\section{Source and Purity of Chemicals:}

(1) $99 \%$, Acros Organics, USA, was used as received

(2) $99.7 \%$, HPLC grade, Aldrich Chemical Company, Milwaukee, WI, USA, stored over molecular sieves and distilled shortly before use.

\section{Estimated Error:}

Temperature: $\pm 0.1 \mathrm{~K}$.

$x_{1}: \pm 1.5 \%$ (relative error).

\begin{tabular}{ll}
\hline \hline Components: & Original Measurements: \\
(1) 4-Chlorobenzoic acid; & ${ }^{61}$ E. R. Cooper, J. Control. Release 1, \\
$\mathrm{C}_{7} \mathrm{H}_{5} \mathrm{ClO}_{2} ;[74-11-3]$ & $153(1984)$. \\
$(2) 1-\mathrm{Methylethyl} \mathrm{tetradecanoate;}$ & \\
$\mathrm{C}_{17} \mathrm{H}_{34} \mathrm{O}_{2} ;[110-27-0]$ & \\
\hline Variables: & Prepared by: \\
$T / \mathrm{K}=295$ & W. E. Acree, Jr. \\
\hline
\end{tabular}

\section{Experimental Values}

\begin{tabular}{lc}
\hline \hline$x_{2}{ }^{\mathrm{a}}$ & $x_{1}{ }^{\mathrm{b}}$ \\
\hline 0.9914 & 0.0086 \\
\hline${ }^{\mathrm{a}} x_{2}:$ mole fraction of component 2 in the saturated solution. \\
${ }^{\mathrm{b}} x_{1}:$ mole fraction solubility of the solute. Solubility data reported in terms of \\
mass percent. Mole fraction solubility calculated by the compiler.
\end{tabular}

\section{Auxiliary Information}

\author{
Method/Apparatus/Procedure: \\ Very little experimental details provided. Solubility determinations were made \\ by stirring the solvent with excess solute for two days, centrifuging the sample, \\ and analyzing the supernatant.
}

Source and Purity of Chemicals:

(1) Reagent grade, Aldrich Chemical Company, Metuchen, NJ, USA, no purification details provided.

(2) Reagent grade, Wickhen Corporation, Huguenot, NJ, USA, no purification details provided.

\section{Estimated Error:}

Temperature: No information given. $x_{1}$ : No information given. 


\begin{tabular}{|c|c|}
\hline $\begin{array}{l}\text { Components: } \\
\text { (1) 4-Chlorobenzoic acid; } \\
\mathrm{C}_{7} \mathrm{H}_{5} \mathrm{ClO}_{2} ;[74-11-3] \\
\text { (2) } 1,2,3-\text { Triacetoxypropane } \\
\text { (Triacetin); } \mathrm{C}_{9} \mathrm{H}_{14} \mathrm{O}_{6} ;[102-76-1]\end{array}$ & $\begin{array}{l}\text { Original Measurements: } \\
\text { W. E. Acree, Jr., unpublished data. }\end{array}$ \\
\hline $\begin{array}{l}\text { Variables: } \\
T / \mathrm{K}=298.15\end{array}$ & $\begin{array}{l}\text { Prepared by: } \\
\text { W. E. Acree, Jr. }\end{array}$ \\
\hline
\end{tabular}

Experimental Values

\begin{tabular}{lc}
\hline \hline$x_{2}{ }^{\mathrm{a}}$ & $x_{1}{ }^{\mathrm{b}}$ \\
\hline 0.9894 & 0.01060 \\
\hline
\end{tabular}

${ }^{\mathrm{a}} x_{2}$ : mole fraction of component 2 in the saturated solution.

${ }^{\mathrm{b}} x_{1}$ : mole fraction solubility of the solute.

\section{Auxiliary Information}

\section{Method: Apparatus/Procedure:}

Constant-temperature bath, calorimetric thermometer, and an ultraviolet/ visible spectrophotometer

Excess solute and solvent were placed in amber glass bottles and allowed to equilibrate for several days at constant temperature. Attainment of equilibrium was verified by several repetitive measurements and by approaching equilibrium from supersaturation. Aliquots of saturated solutions were transferred through a coarse filter into tared volumetric flasks, weighed, and diluted with 2-propanol. Concentrations were determined by spectrophotometric measurements at $272 \mathrm{~nm}$.

Source and Purity of Chemicals:

(1) $99 \%$, Acros Organics, USA, was used as received.

(2) $99 \%$, Acros Organics, USA, stored over molecular sieves before use.

Estimated Error:

Temperature: $\pm 0.1 \mathrm{~K}$.

$x_{1}: \pm 1.5 \%$ (relative error).

\subsection{4-Chlorobenzoic acid solubility data in ethers}

\begin{tabular}{|c|c|}
\hline $\begin{array}{l}\text { Components: } \\
\text { (1) 4-Chlorobenzoic acid; } \\
\mathrm{C}_{7} \mathrm{H}_{5} \mathrm{ClO}_{2} ; \text { [74-11-3] } \\
\text { (2) } 1,1^{\prime} \text {-Oxybisethane; } \mathrm{C}_{4} \mathrm{H}_{10} \mathrm{O} \text {; } \\
\text { [60-29-7] }\end{array}$ & $\begin{array}{l}\text { Original Measurements: } \\
{ }^{128} \text { G. S. Krasil'nikova, E. G. Freidlin, } \\
\text { and Y. N. Pirig, Zh. Prikl. Khim. 60, } \\
2519 \text { (1987). }\end{array}$ \\
\hline $\begin{array}{l}\text { Variables: } \\
T / \mathrm{K}=293\end{array}$ & $\begin{array}{l}\text { Prepared by: } \\
\text { W. E. Acree, Jr. }\end{array}$ \\
\hline
\end{tabular}

\section{Experimental Values}

The measured solubility was reported to be $15.2 \mathrm{~g} / \mathrm{l}$, which corresponds to a molar solubility of $c_{1}=0.097$.

\section{Auxiliary Information}

Method/Apparatus/Procedure:

No information given.

Source and Purity of Chemicals:

(1) Purity not given, Chemical source not given, no purification details were provided.

(2) Purity not given, Chemical source not given, no purification details were provided.

Estimated Error:

Temperature: No information given.

$c_{1}$ : No information given.

\begin{tabular}{ll}
\hline \hline Components: & Original Measurements: \\
(1) 4-Chlorobenzoic acid; & ${ }^{14} \mathrm{C}$. R. Daniels, A. K. Charlton, R. M. \\
$\mathrm{C}_{7} \mathrm{H}_{5} \mathrm{ClO}_{2} ;[74-11-3]$ & Wold, W. E. Acree, Jr., and M. H. \\
$(2) 2,2^{\prime}-$ Oxybispropane; & Abraham, Can. J. Chem. 81, 1492 \\
$\mathrm{C}_{6} \mathrm{H}_{14} \mathrm{O} ;[108-20-3]$ & $(2003)$. \\
\hline Variables: & Prepared by: \\
$T / \mathrm{K}=298.15$ & W. E. Acree, Jr. \\
\hline
\end{tabular}

Experimental Values

\begin{tabular}{lc}
\hline \hline$x_{2}{ }^{\mathrm{a}}$ & $x_{1}{ }^{\mathrm{b}}$ \\
\hline 0.9932 & 0.006780 \\
\hline${ }^{\mathrm{a}} x_{2}:$ mole fraction of component 2 in the saturated solution. \\
${ }^{\mathrm{b}} x_{1}:$ mole fraction solubility of the solute.
\end{tabular}

Auxiliary Information

Method/Apparatus/Procedure:

Constant-temperature bath, calorimetric thermometer, and an ultraviolet/ visible spectrophotometer.

Excess solute and solvent were placed in amber glass bottles and allowed to equilibrate for several days at constant temperature. Attainment of equilibrium was verified by several repetitive measurements and by approaching equilibrium from supersaturation. Aliquots of saturated solutions were transferred through a coarse filter into tared volumetric flasks, weighed, and diluted with methanol. Concentrations were determined by spectrophotometric measurements at $272 \mathrm{~nm}$.

Source and Purity of Chemicals:

(1) $99 \%$, Acros Organics, USA, was used as received.

(2) $99 \%$, anhydrous, Aldrich Chemical Company, Milwaukee, WI, USA, stored over molecular sieves and distilled shortly before use.

Estimated Error:

Temperature: $\pm 0.1 \mathrm{~K}$.

$x_{1}: \pm 1.5 \%$ (relative error).

\begin{tabular}{ll}
\hline \hline Components: & Original Measurements: \\
$(1)$ 4-Chlorobenzoic acid; & ${ }^{14} \mathrm{C}$. R. Daniels, A. K. Charlton, R. M. \\
$\mathrm{C}_{7} \mathrm{H}_{5} \mathrm{ClO}_{2} ;[74-11-3]$ & Wold, W. E. Acree, Jr., and M. H. \\
$(2) 1,1^{\prime}-$ Oxybisbutane; & Abraham, Can. J. Chem. 81, 1492 \\
$\mathrm{C}_{8} \mathrm{H}_{18} \mathrm{O} ;[142-96-1]$ & $(2003)$. \\
\hline Variables: & Prepared by: \\
$T / \mathrm{K}=298.15$ & W. E. Acree, Jr. \\
\hline
\end{tabular}


Experimental Values

\begin{tabular}{lc}
\hline \hline$x_{2}{ }^{\mathrm{a}}$ & $x_{1}{ }^{\mathrm{b}}$ \\
\hline 0.9942 & 0.005833 \\
${ }^{\mathrm{a}} x_{2}:$ mole fraction of component 2 in the saturated solution. \\
${ }^{\mathrm{b}} x_{1}:$ mole fraction solubility of the solute.
\end{tabular}

\section{Auxiliary Information}

\section{Method/Apparatus/Procedure:}

Constant-temperature bath, calorimetric thermometer, and an ultraviolet/ visible spectrophotometer.

Excess solute and solvent were placed in amber glass bottles and allowed to equilibrate for several days at constant temperature. Attainment of equilibrium was verified by several repetitive measurements and by approaching equilibrium from supersaturation. Aliquots of saturated solutions were transferred through a coarse filter into tared volumetric flasks, weighed, and diluted with methanol. Concentrations were determined by spectrophotometric measurements at $272 \mathrm{~nm}$.

\section{Source and Purity of Chemicals:}

(1) $99 \%$, Acros Organics, USA, was used as received.

(2) $99.3 \%$, anhydrous, Aldrich Chemical Company, Milwaukee, WI, USA, stored over molecular sieves and distilled shortly before use.

\section{Estimated Error:}

Temperature: $\pm 0.1 \mathrm{~K}$

$x_{1}: \pm 1.5 \%$ (relative error).

\begin{tabular}{ll}
\hline \hline Components: & Original Measurements: \\
$(1)$ 4-Chlorobenzoic acid; & ${ }^{129}$ S. H. Ghosh and D. K. Hazra, J. Ind. \\
$\mathrm{C}_{7} \mathrm{H}_{5} \mathrm{ClO}_{2} ;[74-11-3]$ & Chem. Soc. 65, 620 (1988). \\
$(2) 1,2-$ Dimethoxyethane; & \\
$\mathrm{C}_{4} \mathrm{H}_{10} \mathrm{O}_{2} ;[110-71-4]$ & \\
\hline Variables: & Prepared by: \\
$T / \mathrm{K}=298.15$ & W. E. Acree, Jr. \\
\hline
\end{tabular}

\section{Experimental Values}

The measured solubility was reported to be $c_{1}=$ $1.0369 \mathrm{~mol} \mathrm{dm}^{-3}$.

\section{Auxiliary Information}

\section{Method/Apparatus/Procedure:}

Mechanical shaker and a constant-temperature thermostat.

Excess solute and solvent were placed in a bottle and stoppered. The solution was shaken in a mechanical shaker for $24 \mathrm{~h}$ at ambient room temperature. The solution was then thermostated at $298 \mathrm{~K}$ for $24 \mathrm{~h}$ with occasional shaking. An aliquot of the saturated solution was then removed and filtered. The concentration of the dissolved solute was determined by titration with standard caustic soda using phenolphthalein as indicator.

\section{Source and Purity of Chemicals:}

(1) Puriss, Fluka, distilled from aqueous-ethanol mixture.

(2) Purum, Fluka, was shaken with ferrous sulfate and then distilled. The distillate was further purified by refluxing for $12 \mathrm{~h}$ and distilling over metallic sodium.

\section{Estimated Error:}

Temperature: $\pm 0.01 \mathrm{~K}$.

$c_{1}: \pm 1.5 \%$ (relative error, estimated by compiler).

\begin{tabular}{ll}
\hline \hline Components: & Original Measurements: \\
(1) 4-Chlorobenzoic acid; & ${ }^{14} \mathrm{C} . \mathrm{R}$. Daniels, A. K. Charlton, R. M. \\
$\mathrm{C}_{7} \mathrm{H}_{5} \mathrm{ClO}_{2} ;$ [74-11-3] & Wold, W. E. Acree, Jr., and M. H. \\
$\begin{array}{l}\text { (2) Tetrahydrofuran; } \mathrm{C}_{4} \mathrm{H}_{8} \mathrm{O} ; \\
{[109-99-9]}\end{array}$ & $\begin{array}{l}\text { Abraham, Can. J. Chem. 81, 1492 } \\
\text { (2003). }\end{array}$ \\
\hline Variables: & Prepared by: \\
$T / \mathrm{K}=298.15$ & W. E. Acree, Jr. \\
\hline
\end{tabular}

Experimental Values

\begin{tabular}{lc}
\hline \hline$x_{2}{ }^{\mathrm{a}}$ & $x_{1}{ }^{\mathrm{b}}$ \\
\hline 0.9413 & 0.05874 \\
\hline${ }^{\mathrm{a}} x_{2}:$ mole fraction of component 2 in the saturated solution. & \\
${ }^{\mathrm{b}} x_{1}:$ mole fraction solubility of the solute. &
\end{tabular}

\section{Auxiliary Information}

\section{Method/Apparatus/Procedure:}

Constant-temperature bath, calorimetric thermometer, and an ultraviolet/ visible spectrophotometer.

Excess solute and solvent were placed in amber glass bottles and allowed to equilibrate for several days at constant temperature. Attainment of equilibrium was verified by several repetitive measurements and by approaching equilibrium from supersaturation. Aliquots of saturated solutions were transferred through a coarse filter into tared volumetric flasks, weighed, and diluted with methanol. Concentrations were determined by spectrophotometric measurements at $272 \mathrm{~nm}$.

\section{Source and Purity of Chemicals:}

(1) $99 \%$, Acros Organics, USA, was used as received.

(2) $99.9 \%$, anhydrous, Aldrich Chemical Company, Milwaukee, WI, USA, stored over molecular sieves and distilled shortly before use.

\section{Estimated Error:}

Temperature: $\pm 0.1 \mathrm{~K}$.

$x_{1}: \pm 2.0 \%$ (relative error).

\begin{tabular}{ll}
\hline \hline Components: & Original Measurements: \\
(1) 4-Chlorobenzoic acid; & ${ }^{14}$ C. R. Daniels, A. K. Charlton, R. M. \\
$\mathrm{C}_{7} \mathrm{H}_{5} \mathrm{ClO}_{2} ;$ [74-11-3] & Wold, W. E. Acree, Jr., and M. H. \\
$(2) 1,4-D i o x a n e ; \mathrm{C}_{4} \mathrm{H}_{8} \mathrm{O}_{2} ;$ & Abraham, Can. J. Chem. 81, 1492 \\
{$[123-91-1]$} & $(2003)$. \\
\hline Variables: & Prepared by: \\
$T / \mathrm{K}=298.15$ & W. E. Acree, Jr. \\
\hline
\end{tabular}

\section{Experimental Values}

\begin{tabular}{lc}
\hline \hline$x_{2}{ }^{\mathrm{a}}$ & $x_{1}{ }^{\mathrm{b}}$ \\
\hline 0.9703 & 0.02974 \\
\hline${ }^{\mathrm{a}} x_{2}:$ mole fraction of component 2 in the saturated solution. & \\
${ }^{\mathrm{b}}{ }_{x_{1}: \text { mole fraction solubility of the solute. }}$ &
\end{tabular}




\section{Auxiliary Information}

\section{Method/Apparatus/Procedure:}

Constant-temperature bath, calorimetric thermometer, and an ultraviolet/ visible spectrophotometer.

Excess solute and solvent were placed in amber glass bottles and allowed to equilibrate for several days at constant temperature. Attainment of equilibrium was verified by several repetitive measurements and by approaching equilibrium from supersaturation. Aliquots of saturated solutions were transferred through a coarse filter into tared volumetric flasks, weighed, and diluted with methanol. Concentrations were determined by spectrophotometric measurements at $272 \mathrm{~nm}$.

Source and Purity of Chemicals:

(1) $99 \%$, Acros Organics, USA, was used as received.

(2) $99.8 \%$, anhydrous, Aldrich Chemical Company, Milwaukee, WI, USA, stored over molecular sieves and distilled shortly before use.

Estimated Error:

Temperature: $\pm 0.1 \mathrm{~K}$.

$x_{1}: \pm 2.0 \%$ (relative error)

\begin{tabular}{|c|c|}
\hline $\begin{array}{l}\text { Components: } \\
\text { (1) 4-Chlorobenzoic acid; } \\
\mathrm{C}_{7} \mathrm{H}_{5} \mathrm{ClO}_{2} \text {; [74-11-3] } \\
\text { (2) 1,4-Dioxane; } \mathrm{C}_{4} \mathrm{H}_{8} \mathrm{O}_{2} \text {; } \\
\text { [123-91-1] }\end{array}$ & $\begin{array}{l}\text { Original Measurements: } \\
{ }^{128} \text { G. S. Krasil'nikova, E. G. Freidlin, } \\
\text { and Y. N. Pirig, Zh. Prikl. Khim. 60, } \\
2519 \text { (1987). }\end{array}$ \\
\hline $\begin{array}{l}\text { Variables: } \\
T / \mathrm{K}=293\end{array}$ & $\begin{array}{l}\text { Prepared by: } \\
\text { W. E. Acree, Jr. }\end{array}$ \\
\hline
\end{tabular}

\section{Experimental Values}

The measured solubility was reported to be $53.0 \mathrm{~g} / \mathrm{l}$, which corresponds to a molar solubility of $c_{1}=0.339$.

\section{Auxiliary Information}

Method/Apparatus/Procedure:

No information given.

Source and Purity of Chemicals:

(1) Purity not given, Chemical source not given, no purification details were provided.

(2) Purity not given, Chemical source not given, no purification details were provided.

Estimated Error:

Temperature: No information given.

$c_{1}$ : No information given.

\subsection{4-Chlorobenzoic acid solubility in haloalkanes, haloalkenes, haloaromatic hydrocarbons}

\begin{tabular}{|c|c|}
\hline $\begin{array}{l}\text { Components: } \\
\text { (1) 4-Chlorobenzoic acid; } \\
\mathrm{C}_{7} \mathrm{H}_{5} \mathrm{ClO}_{2} ; \text { [74-11-3] } \\
\text { (2) Trichloromethane; } \mathrm{CHCl}_{3} \text {; } \\
\text { [67-66-3] }\end{array}$ & $\begin{array}{l}\text { Original Measurements: } \\
{ }^{128} \text { G. S. Krasil'nikova, E. G. Freidlin, } \\
\text { and Y. N. Pirig, Zh. Prikl. Khim. 60, } \\
2519 \text { (1987). }\end{array}$ \\
\hline $\begin{array}{l}\text { Variables: } \\
T / \mathrm{K}=293\end{array}$ & $\begin{array}{l}\text { Prepared by: } \\
\text { W. E. Acree, Jr. }\end{array}$ \\
\hline
\end{tabular}

\section{Experimental Values}

The measured solubility was reported to be $2.1 \mathrm{~g} / \mathrm{l}$, which corresponds to a molar solubility of $c_{1}=0.0134$.

\section{Auxiliary Information}

Method/Apparatus/Procedure:

No information given.

Source and Purity of Chemicals:

(1) Purity not given, Chemical source not given, no purification details were provided.

(2) Purity not given, Chemical source not given, no purification details were provided.

\section{Estimated Error:}

Temperature: No information given.

$c_{1}$ : No information given.

\begin{tabular}{ll}
\hline \hline Components: & Original Measurements: \\
$\begin{array}{l}\text { (1) 4-Chlorobenzoic acid; } \\
\mathrm{C}_{7} \mathrm{H}_{5} \mathrm{ClO}_{2} ;[74-11-3]\end{array}$ & ${ }^{128}$ G. S. Krasil'nikova, E. G. Freidlin, \\
(2) Tetrachloromethane; $\mathrm{CCl}_{4} ;$ & 2519 (1987). \\
[56-23-5] & \\
\hline Variables: & Prepared by: \\
$T / \mathrm{K}=293$ & W. E. Acree, Jr. \\
\hline
\end{tabular}

\section{Experimental Values}

The measured solubility was reported to be $0.2 \mathrm{~g} / 1$, which corresponds to a molar solubility of $c_{1}=0.0013$.

\section{Auxiliary Information}

\section{Method/Apparatus/Procedure:}

No information given.

Source and Purity of Chemicals:

(1) Purity not given, Chemical source not given, no purification details were provided.

(2) Purity not given, Chemical source not given, no purification details were provided.

Estimated Error:

Temperature: No information given.

$c_{1}$ : No information given.

\begin{tabular}{ll}
\hline \hline Components: & Original Measurements: \\
(1) 4-Chlorobenzoic acid; & ${ }^{128}$ G. S. Krasil'nikova, E. G. Freidlin, \\
$\mathrm{C}_{7} \mathrm{H}_{5} \mathrm{ClO}_{2} ;[74-11-3]$ & and Y. N. Pirig, Zh. Prikl. Khim. 60, \\
$(2) 1,2$-Dichloroethane; & 2519 (1987). \\
$\mathrm{C}_{2} \mathrm{H}_{4} \mathrm{Cl}_{2} ;[107-06-2]$ & \\
\hline Variables: & Prepared by: \\
$T / \mathrm{K}=293$ & W. E. Acree, Jr. \\
\hline
\end{tabular}

\section{Experimental Values}

The measured solubility was reported to be $1.4 \mathrm{~g} / \mathrm{l}$, which corresponds to a molar solubility of $c_{1}=0.0089$. 


\section{Auxiliary Information}

\section{Method/Apparatus/Procedure:}

No information given.

Source and Purity of Chemicals:

(1) Purity not given, Chemical source not given, no purification details were provided

(2) Purity not given, Chemical source not given, no purification details were provided.

Estimated Error:

Temperature: No information given.

$c_{1}$ : No information given.

\begin{tabular}{ll}
\hline \hline Components: & Original Measurements: \\
$(1)$ 4-Chlorobenzoic acid; & ${ }^{128}$ G. S. Krasil'nikova, E. G. Freidlin, \\
$\mathrm{C}_{7} \mathrm{H}_{5} \mathrm{ClO}_{2} ;[74-11-3]$ & and Y. N. Pirig, Zh. Prikl. Khim. 60, \\
$(2) 1,2-D i c h l o r o e t h a n e ;$ & $2519(1987)$. \\
$\mathrm{C}_{2} \mathrm{H}_{4} \mathrm{Cl}_{2} ;[107-06-2]$ & \\
\hline Variables: & Prepared by: \\
$T / \mathrm{K}=293$ & W. E. Acree, Jr. \\
\hline
\end{tabular}

Experimental Values

The measured solubility was reported to be $1.4 \mathrm{~g} / \mathrm{l}$, which corresponds to a molar solubility of $c_{1}=0.0089$.

\section{Auxiliary Information}

\section{Method/Apparatus/Procedure:}

No information given.

Source and Purity of Chemicals:

(1) Purity not given, Chemical source not given, no purification details were provided.

(2) Purity not given, Chemical source not given, no purification details were provided.

\section{Estimated Error:}

Temperature: No information given.

$c_{1}$ : No information given.

\begin{tabular}{|c|c|}
\hline $\begin{array}{l}\text { Components: } \\
\text { (1) 4-Chlorobenzoic acid; } \\
\mathrm{C}_{7} \mathrm{H}_{5} \mathrm{ClO}_{2} ; \text { [74-11-3] } \\
\text { (2) Chlorobenzene; } \mathrm{C}_{6} \mathrm{H}_{5} \mathrm{Cl} \text {; } \\
\text { [108-90-7] }\end{array}$ & $\begin{array}{l}\text { Original Measurements: } \\
{ }^{128} \text { G. S. Krasil'nikova, E. G. Freidlin, } \\
\text { and Y. N. Pirig, Zh. Prikl. Khim. 60, } \\
2519 \text { (1987). }\end{array}$ \\
\hline $\begin{array}{l}\text { Variables: } \\
T / \mathrm{K}=293\end{array}$ & $\begin{array}{l}\text { Prepared by: } \\
\text { W. E. Acree, Jr. }\end{array}$ \\
\hline
\end{tabular}

\section{Experimental Values}

The measured solubility was reported to be $0.7 \mathrm{~g} / \mathrm{l}$, which corresponds to a molar solubility of $c_{1}=0.0045$.

\section{Auxiliary Information}

Method/Apparatus/Procedure:

No information given.

Source and Purity of Chemicals:

(1) Purity not given, Chemical source not given, no purification details were provided.

(2) Purity not given, Chemical source not given, no purification details were provided.

Estimated Error:

Temperature: No information given.

$c_{1}$ : No information given.

\subsection{4-Chlorobenzoic acid solubility data in alcohols}

\begin{tabular}{ll}
\hline \hline Components: & Original Measurements: \\
(1) 4-Chlorobenzoic acid; & ${ }^{14} \mathrm{C}$. R. Daniels, A. K. Charlton, R. M. \\
$\mathrm{C}_{7} \mathrm{H}_{5} \mathrm{ClO}_{2}$; [74-11-3] & Wold, W. E. Acree, Jr., and M. H. \\
(2) Methanol; $\mathrm{CH}_{4} \mathrm{O} ;$ [67-56-1] & $\begin{array}{l}\text { Abraham, Can. J. Chem. 81, 1492 } \\
(2003) .\end{array}$ \\
\hline Variables: & Prepared by: \\
$T / \mathrm{K}=298.15$ & W. E. Acree, Jr. \\
\hline
\end{tabular}

Experimental Values

\begin{tabular}{lc}
\hline \hline$x_{2}{ }^{\mathrm{a}}$ & $x_{1}{ }^{\mathrm{b}}$ \\
\hline 0.9918 & 0.00823
\end{tabular}

${ }^{\mathrm{a}} x_{2}$ : mole fraction of component 2 in the saturated solution.

${ }^{\mathrm{b}} x_{1}$ : mole fraction solubility of the solute.

\section{Auxiliary Information}

\section{Method/Apparatus/Procedure:}

Constant-temperature bath, calorimetric thermometer, and an ultraviolet/ visible spectrophotometer.

Excess solute and solvent were placed in amber glass bottles and allowed to equilibrate for several days at constant temperature. Attainment of equilibrium was verified by several repetitive measurements and by approaching equilibrium from supersaturation. Aliquots of saturated solutions were transferred through a coarse filter into tared volumetric flasks, weighed, and diluted with methanol. Concentrations were determined by spectrophotometric measurements at $272 \mathrm{~nm}$.

\section{Source and Purity of Chemicals:}

(1) $99 \%$, Acros Organics, USA, was used as received

(2) $99.8 \%$, anhydrous, Aldrich Chemical Company, Milwaukee, WI, USA, stored over molecular sieves and distilled shortly before use.

Estimated Error:

Temperature: $\pm 0.1 \mathrm{~K}$.

$x_{1}: \pm 1.5 \%$ (relative error). 


\begin{tabular}{ll}
\hline \hline Components: & Original Measurements: \\
(1) 4-Chlorobenzoic acid; & ${ }^{14} \mathrm{C}$. R. Daniels, A. K. Charlton, R. M. \\
$\mathrm{C}_{7} \mathrm{H}_{5} \mathrm{ClO}_{2} ;[74-11-3]$ & Wold, W. E. Acree, Jr., and M. H. \\
(2) Ethanol; $\mathrm{C}_{2} \mathrm{H}_{6} \mathrm{O} ;[64-17-5]$ & $\begin{array}{l}\text { Abraham, Can. J. Chem. 81, 1492 } \\
\text { (2003). }\end{array}$ \\
\hline Variables: & Prepared by: \\
$T / \mathrm{K}=298.15$ & W. E. Acree, Jr. \\
\hline
\end{tabular}

Experimental Values

\begin{tabular}{lc}
\hline \hline$x_{2}{ }^{\mathrm{a}}$ & $x_{1}{ }^{\mathrm{b}}$ \\
\hline 0.9899 & 0.01013 \\
\hline
\end{tabular}

${ }^{\mathrm{a}} x_{2}$ : mole fraction of component 2 in the saturated solution.

${ }^{\mathrm{b}} x_{1}$ : mole fraction solubility of the solute.

\section{Auxiliary Information}

\section{Method/Apparatus/Procedure:}

Constant-temperature bath, calorimetric thermometer, and an ultraviolet/ visible spectrophotometer.

Excess solute and solvent were placed in amber glass bottles and allowed to equilibrate for several days at constant temperature. Attainment of equilibrium was verified by several repetitive measurements and by approaching equilibrium from supersaturation. Aliquots of saturated solutions were transferred through a coarse filter into tared volumetric flasks, weighed, and diluted with methanol. Concentrations were determined by spectrophotometric measurements at $272 \mathrm{~nm}$.

Source and Purity of Chemicals:

(1) $99 \%$, Acros Organics, USA, was used as received.

(2) Absolute, Aaper Alcohol and Chemical Company, USA, stored over molecular sieves and distilled shortly before use.

\section{Estimated Error:}

Temperature: $\pm 0.1 \mathrm{~K}$

$x_{1}: \pm 1.5 \%$ (relative error).

\begin{tabular}{ll}
\hline \hline Components: & Original Measurements: \\
$\begin{array}{l}\text { (1) 4-Chlorobenzoic acid; } \\
\mathrm{C}_{7} \mathrm{H}_{5} \mathrm{ClO}_{2} ;[74-11-3]\end{array}$ & $\begin{array}{l}{ }^{126} \mathrm{P} . \mathrm{K} \text {. Biswas, S. C. Lahiri, and B. P. } \\
\text { (2) Ethanol; } \mathrm{C}_{2} \mathrm{H}_{6} \mathrm{O} ;[64-17-5]\end{array}$ \\
\hline Variables: & $(1993)$. \\
$T / \mathrm{K}=298.15$ & Prepared by: \\
\hline
\end{tabular}

Experimental Values

\begin{tabular}{|c|c|c|}
\hline$c_{1}^{\mathrm{a}}$ & $x_{2}{ }^{\mathrm{b}}$ & $x_{1}{ }^{\mathrm{c}}$ \\
\hline 0.2895 & 0.9827 & 0.0173 \\
\hline
\end{tabular}

\section{Auxiliary Information}

Method/Apparatus/Procedure:

Analytical balance and an ultraviolet/visible spectrophotometer.

Solutions containing excess solute and solvent were allowed to equilibrate at constant temperature for at least $24 \mathrm{~h}$. An aliquot of the saturated solution was removed, filtered, and the absorbance recorded. Solubility was calculated from the measured absorbance.

Source and Purity of Chemicals:

(1) Puris grade, Fluka Chemicals, recrystallized from aqueous ethanol mixture and dried in an air oven at $390 \mathrm{~K}$. The purified compound was stored in vacuum desiccators.

(2) Absolute, B.C.P.W., Calcutta, India, was distilled twice before use.

\section{Estimated Error:}

Temperature: $\pm 0.2 \mathrm{~K}$ (estimated by compiler). $c_{1}: \pm 1.0 \%$ (relative error, estimated by compiler). $x_{1}: \pm 2.5 \%$ (relative error, estimated by compiler).

\begin{tabular}{|c|c|}
\hline $\begin{array}{l}\text { Components: } \\
\text { (1) 4-Chlorobenzoic acid; } \\
\mathrm{C}_{7} \mathrm{H}_{5} \mathrm{ClO}_{2} ;[74-11-3] \\
\text { (2) Ethanol; } \mathrm{C}_{2} \mathrm{H}_{6} \mathrm{O} ;[64-17-5]\end{array}$ & $\begin{array}{l}\text { Original Measurements: } \\
{ }^{72} \text { R. Thuaire, Bull. Soc. Chim. Fr. } 3815 \\
\text { (1971). }\end{array}$ \\
\hline $\begin{array}{l}\text { Variables: } \\
T / \mathrm{K}=298.15\end{array}$ & $\begin{array}{l}\text { Prepared by: } \\
\text { W. E. Acree, Jr. }\end{array}$ \\
\hline
\end{tabular}

\section{Experimental Values}

The measured solubility was reported to be $0.270 \mathrm{~mol} / \mathrm{kg}$ of solvent, which corresponds to a mole fraction solubility of $x_{1}=0.01229$.

\section{Auxiliary Information}

\section{Method/Apparatus/Procedure:}

Very little experimental details were provided. Solubility was determined by a gravimetric method. The saturated solution was evaporated to dryness and the remaining solid residue was weighed. The solubility was calculated from the mass of the solid residue and mass of saturated solution analyzed.

Source and Purity of Chemicals:

(1) Purity not given, Chemical source not given, no purification details were provided.

(2) Purity not given, Chemical source not given, no purification details were provided.

\section{Estimated Error:}

Temperature: $\pm 0.05 \mathrm{~K}$

$x_{1}: \pm 4 \%$ (relative error, estimated by compiler).

\begin{tabular}{|c|c|}
\hline $\begin{array}{l}\text { Components: } \\
\text { (1) 4-Chlorobenzoic acid; } \\
\mathrm{C}_{7} \mathrm{H}_{5} \mathrm{ClO}_{2} ;[74-11-3] \\
\text { (2) Ethanol; } \mathrm{C}_{2} \mathrm{H}_{6} \mathrm{O} ;[64-17-5]\end{array}$ & $\begin{array}{l}\text { Original Measurements: } \\
{ }^{128} \text { G. S. Krasil'nikova, E. G. Freidlin, } \\
\text { and Y. N. Pirig, Zh. Prikl. Khim. 60, } \\
2519 \text { (1987). }\end{array}$ \\
\hline $\begin{array}{l}\text { Variables: } \\
T / \mathrm{K}=293\end{array}$ & $\begin{array}{l}\text { Prepared by: } \\
\text { W. E. Acree, Jr. }\end{array}$ \\
\hline
\end{tabular}

\section{Experimental Values}

The measured solubility was reported to be $25.5 \mathrm{~g} / \mathrm{l}$, which corresponds to a molar solubility of $c_{1}=0.163$. 


\section{Auxiliary Information}

\section{Method/Apparatus/Procedure:}

No information given.

Source and Purity of Chemicals:

(1) Purity not given, Chemical source not given, no purification details were provided.

(2) Purity not given, Chemical source not given, no purification details were provided.

Estimated Error:

Temperature: No information given.

$c_{1}$ : No information given.

\begin{tabular}{ll}
\hline \hline Components: & Original Measurements: \\
$\begin{array}{l}\text { (1) 4-Chlorobenzoic acid; } \\
\mathrm{C}_{7} \mathrm{H}_{5} \mathrm{ClO}_{2} ;[74-11-3]\end{array}$ & Wold, W. E. Acree, Jr., and M. H. \\
(2) 1-Propanol; $\mathrm{C}_{3} \mathrm{H}_{8} \mathrm{O} ;[$ [71-23-8] & $\begin{array}{l}\text { Abraham, Can. J. Chem. 81, } 1492 \\
(2003) .\end{array}$ \\
\hline Variables: & Prepared by: \\
$T / \mathrm{K}=298.15$ & W. E. Acree, Jr. \\
\hline
\end{tabular}

Experimental Values

\begin{tabular}{lc}
\hline \hline$x_{2}^{\mathrm{a}}$ & $x_{1}{ }^{\mathrm{b}}$ \\
\hline 0.9881 & 0.01188
\end{tabular}

${ }^{\mathrm{a}} x_{2}$ : mole fraction of component 2 in the saturated solution.

${ }^{\mathrm{b}} x_{1}$ : mole fraction solubility of the solute.

\section{Auxiliary Information}

\section{Method/Apparatus/Procedure:}

Constant-temperature bath, calorimetric thermometer, and an ultraviolet/ visible spectrophotometer.

Excess solute and solvent were placed in amber glass bottles and allowed to equilibrate for several days at constant temperature. Attainment of equilibrium was verified by several repetitive measurements and by approaching equilibrium from supersaturation. Aliquots of saturated solutions were transferred through a coarse filter into tared volumetric flasks, weighed, and diluted with methanol. Concentrations were determined by

spectrophotometric measurements at $272 \mathrm{~nm}$.

\section{Source and Purity of Chemicals:}

(1) $99 \%$, Acros Organics, USA, was used as received.

(2) $99+\%$, anhydrous, Aldrich Chemical Company, Milwaukee, WI, USA, stored over molecular sieves and distilled shortly before use.

\section{Estimated Error:}

Temperature: $\pm 0.1 \mathrm{~K}$.

$x_{1}: \pm 1.5 \%$ (relative error).

\begin{tabular}{ll}
\hline \hline Components: & Original Measurements: \\
$\begin{array}{l}\text { (1) 4-Chlorobenzoic acid; } \\
\mathrm{C}_{7} \mathrm{H}_{5} \mathrm{ClO}_{2} ;[74-11-3]\end{array}$ & ${ }^{128}$ G. S. Krasil'nikova, E. G. Freidlin, \\
$\begin{array}{l}\text { (2) 1-Propanol; } \mathrm{C}_{3} \mathrm{H}_{8} \mathrm{O} ; \\
\text { [71-23-8] }\end{array}$ & 2519 (1987). \\
\hline Variables: & \\
$T / \mathrm{K}=293$ & Prepared by: \\
\hline
\end{tabular}

\section{Experimental Values}

The measured solubility was reported to be $23.8 \mathrm{~g} / \mathrm{l}$, which corresponds to a molar solubility of $c_{1}=0.152$.

\section{Auxiliary Information}

Method/Apparatus/Procedure:

No information given.

Source and Purity of Chemicals:

(1) Purity not given, Chemical source not given, no purification details were provided.

(2) Purity not given, Chemical source not given, no purification details were provided.

\section{Estimated Error:}

Temperature: No information given.

$c_{1}$ : No information given.

\begin{tabular}{ll}
\hline \hline Components: & Original Measurements: \\
(1) 4-Chlorobenzoic acid; & ${ }^{14}$ C. R. Daniels, A. K. Charlton, R. M. \\
$\mathrm{C}_{7} \mathrm{H}_{5} \mathrm{ClO}_{2} ;$ [74-11-3] & Wold, W. E. Acree, Jr., and M. H. \\
$\begin{array}{l}\text { (2) 2-Propanol; } \mathrm{C}_{3} \mathrm{H}_{8} \mathrm{O} ; \\
\text { [71-23-8] }\end{array}$ & $\begin{array}{l}\text { Abraham, Can. J. Chem. 81, 1492 } \\
\text { (2003). }\end{array}$ \\
\hline Variables: & Prepared by: \\
$T / \mathrm{K}=298.15$ & W. E. Acree, Jr. \\
\hline
\end{tabular}

\section{Experimental Values}

\begin{tabular}{lc}
\hline \hline$x_{2}{ }^{\mathrm{a}}$ & $x_{1}{ }^{\mathrm{b}}$ \\
\hline 0.9873 & 0.01274 \\
\hline${ }^{a} x_{2}:$ mole fraction of component 2 in the saturated solution. & \\
${ }^{\mathrm{b}} x_{1}:$ mole fraction solubility of the solute. &
\end{tabular}

\section{Auxiliary Information}

\section{Method/Apparatus/Procedure:}

Constant-temperature bath, calorimetric thermometer, and an ultraviolet/ visible spectrophotometer.

Excess solute and solvent were placed in amber glass bottles and allowed to equilibrate for several days at constant temperature. Attainment of equilibrium was verified by several repetitive measurements and by approaching equilibrium from supersaturation. Aliquots of saturated solutions were transferred through a coarse filter into tared volumetric flasks, weighed, and diluted with methanol. Concentrations were determined by spectrophotometric measurements at $272 \mathrm{~nm}$.

\section{Source and Purity of Chemicals:}

(1) $99 \%$, Acros Organics, USA, was used as received

(2) $99+\%$, anhydrous, Aldrich Chemical Company, Milwaukee, WI, USA, stored over molecular sieves and distilled shortly before use.

\section{Estimated Error:}

Temperature: $\pm 0.1 \mathrm{~K}$.

$x_{1}: \pm 1.5 \%$ (relative error). 


\begin{tabular}{|c|c|}
\hline $\begin{array}{l}\text { Components: } \\
\text { (1) 4-Chlorobenzoic acid; } \\
\mathrm{C}_{7} \mathrm{H}_{5} \mathrm{ClO}_{2} ;[74-11-3] \\
\text { (2) } 1-\mathrm{Butanol} ; \mathrm{C}_{4} \mathrm{H}_{10} \mathrm{O} ;[71-36-3]\end{array}$ & $\begin{array}{l}\text { Original Measurements: } \\
{ }^{14} \text { C. R. Daniels, A. K. Charlton, R. M. } \\
\text { Wold, W. E. Acree, Jr., and M. H. } \\
\text { Abraham, Can. J. Chem. 81, } 1492 \\
\text { (2003). }\end{array}$ \\
\hline $\begin{array}{l}\text { Variables: } \\
T / \mathrm{K}=298.15\end{array}$ & $\begin{array}{l}\text { Prepared by: } \\
\text { W. E. Acree, Jr. }\end{array}$ \\
\hline
\end{tabular}

\section{Experimental Values}

\begin{tabular}{lc}
\hline \hline$x_{2}{ }^{\mathrm{a}}$ & $x_{1}{ }^{\mathrm{b}}$ \\
\hline 0.9865 & 0.01350 \\
\hline
\end{tabular}

${ }^{\mathrm{a}} x_{2}$ : mole fraction of component 2 in the saturated solution.

${ }^{\mathrm{b}} x_{1}$ : mole fraction solubility of the solute.

\section{Auxiliary Information}

\section{Method/Apparatus/Procedure:}

Constant-temperature bath, calorimetric thermometer, and an ultraviolet/ visible spectrophotometer.

Excess solute and solvent were placed in amber glass bottles and allowed to equilibrate for several days at constant temperature. Attainment of equilibrium was verified by several repetitive measurements and by approaching equilibrium from supersaturation. Aliquots of saturated solutions were transferred through a coarse filter into tared volumetric flasks, weighed, and diluted with methanol. Concentrations were determined by spectrophotometric measurements at $272 \mathrm{~nm}$.

\section{Source and Purity of Chemicals:}

(1) $99 \%$, Acros Organics, USA, was used as received.

(2) $99.8 \%$, HPLC grade, Aldrich Chemical Company, Milwaukee, WI, USA, stored over molecular sieves and distilled shortly before use.

\section{Estimated Error:}

Temperature: $\pm 0.1 \mathrm{~K}$

$x_{1}: \pm 1.5 \%$ (relative error).

\begin{tabular}{ll}
\hline \hline Components: & Original Measurements: \\
$\begin{array}{l}\text { (1) 4-Chlorobenzoic acid; } \\
\mathrm{C}_{7} \mathrm{H}_{5} \mathrm{ClO}_{2} ; \text { [74-11-3] }\end{array}$ & Wold, W. Daniels, A. K. Charlton, R. M. \\
$\begin{array}{l}\text { (2) 2-Butanol; } \mathrm{C}_{4} \mathrm{H}_{10} \mathrm{O} ; \\
{[78-92-2]}\end{array}$ & $\begin{array}{l}\text { Abraham, Can. J. Chem. and M. H. } \\
\text { (2003). }\end{array}$ \\
\hline Variables: & Prepared by: \\
$T / \mathrm{K}=298.15$ & W. E. Acree, Jr. \\
\hline
\end{tabular}

Experimental Values

\begin{tabular}{lc}
\hline \hline$x_{2}{ }^{\mathrm{a}}$ & $x_{1}{ }^{\mathrm{b}}$ \\
\hline 0.9852 & 0.01480 \\
\hline${ }^{\mathrm{a}} x_{2}:$ mole fraction of component 2 in the saturated solution. \\
${ }^{\mathrm{b}} x_{1}:$ mole fraction solubility of the solute.
\end{tabular}

\section{Auxiliary Information}

\section{Method/Apparatus/Procedure:}

Constant-temperature bath, calorimetric thermometer, and an ultraviolet/ visible spectrophotometer.

Excess solute and solvent were placed in amber glass bottles and allowed to equilibrate for several days at constant temperature. Attainment of equilibrium was verified by several repetitive measurements and by approaching equilibrium from supersaturation. Aliquots of saturated solutions were transferred through a coarse filter into tared volumetric flasks, weighed, and diluted with methanol. Concentrations were determined by spectrophotometric measurements at $272 \mathrm{~nm}$.

\section{Source and Purity of Chemicals:}

(1) $99 \%$, Acros Organics, USA, was used as received.

(2) $99+\%$, anhydrous, Aldrich Chemical Company, Milwaukee, WI, USA, stored over molecular sieves and distilled shortly before use.

\section{Estimated Error:}

Temperature: $\pm 0.1 \mathrm{~K}$.

$x_{1}: \pm 1.5 \%$ (relative error).

\begin{tabular}{ll}
\hline \hline Components: & Original Measurements: \\
(1) 4-Chlorobenzoic acid; & ${ }^{14} \mathrm{C}$. R. Daniels, A. K. Charlton, R. M. \\
$\mathrm{C}_{7} \mathrm{H}_{5} \mathrm{ClO}_{2}$; [74-11-3] & Wold, W. E. Acree, Jr., and M. H. \\
$\begin{array}{l}\text { (2) 2-Methyl-1-propanol; } \mathrm{C}_{4} \mathrm{H}_{10} \mathrm{O} ; \\
\text { [78-83-1] }\end{array}$ & $\begin{array}{l}\text { Abraham, Can. J. Chem. 81, 1492 } \\
(2003) .\end{array}$ \\
\hline Variables: & Prepared by: \\
$T / \mathrm{K}=298.15$ & W. E. Acree, Jr. \\
\hline
\end{tabular}

\section{Experimental Values}

\begin{tabular}{lc}
\hline \hline$x_{2}{ }^{\mathrm{a}}$ & $x_{1}{ }^{\mathrm{b}}$ \\
\hline 0.9904 & 0.009578 \\
\hline${ }^{\mathrm{a}} x_{2}:$ mole fraction of component 2 in the saturated solution. \\
${ }^{\mathrm{b}} x_{1}:$ mole fraction solubility of the solute.
\end{tabular}

\section{Auxiliary Information}

\section{Method/Apparatus/Procedure:}

Constant-temperature bath, calorimetric thermometer, and an ultraviolet/ visible spectrophotometer.

Excess solute and solvent were placed in amber glass bottles and allowed to equilibrate for several days at constant temperature. Attainment of equilibrium was verified by several repetitive measurements and by approaching equilibrium from supersaturation. Aliquots of saturated solutions were transferred through a coarse filter into tared volumetric flasks, weighed, and diluted with methanol. Concentrations were determined by spectrophotometric measurements at $272 \mathrm{~nm}$.

\section{Source and Purity of Chemicals:}

(1) $99 \%$, Acros Organics, USA, was used as received.

(2) $99+\%$, anhydrous, Aldrich Chemical Company, Milwaukee, WI, USA, stored over molecular sieves and distilled shortly before use.

\section{Estimated Error:}

Temperature: $\pm 0.1 \mathrm{~K}$.

$x_{1}: \pm 1.5 \%$ (relative error). 


\section{Components:}

(1) 4-Chlorobenzoic acid;

$\mathrm{C}_{7} \mathrm{H}_{5} \mathrm{ClO}_{2} ;$ [74-11-3]

(2) 2-Methyl-2-propanol; $\mathrm{C}_{4} \mathrm{H}_{10} \mathrm{O}$; [75-65-0]

Original Measurements:

${ }^{14}$ C. R. Daniels, A. K. Charlton, R. M. Wold, W. E. Acree, Jr., and M. H. Abraham, Can. J. Chem. 81, 1492 (2003).

\begin{tabular}{ll}
\hline Variables: & Prepared by: \\
$T / \mathrm{K}=298.15$ & W. E. Acree, Jr.
\end{tabular}

Experimental Values

\begin{tabular}{lc}
\hline \hline$x_{2}{ }^{\mathrm{a}}$ & $x_{1}{ }^{\mathrm{b}}$ \\
\hline 0.9798 & 0.02021
\end{tabular}

${ }^{\mathrm{a}} x_{2}$ : mole fraction of component 2 in the saturated solution.

${ }^{\mathrm{b}} x_{1}$ : mole fraction solubility of the solute.

\section{Auxiliary Information}

\section{Method/Apparatus/Procedure:}

Constant-temperature bath, calorimetric thermometer, and an ultraviolet/ visible spectrophotometer.

Excess solute and solvent were placed in amber glass bottles and allowed to equilibrate for several days at constant temperature. Attainment of equilibrium was verified by several repetitive measurements and by approaching equilibrium from supersaturation. Aliquots of saturated solutions were transferred through a coarse filter into tared volumetric flasks, weighed, and diluted with methanol. Concentrations were determined by spectrophotometric measurements at $272 \mathrm{~nm}$.

Source and Purity of Chemicals:

(1) $99 \%$, Acros Organics, USA, was used as received.

(2) $99+\%$, Arco Chemical Company, USA, stored over molecular sieves and distilled shortly before use.

Estimated Error:

Temperature: $\pm 0.1 \mathrm{~K}$.

$x_{1}: \pm 1.5 \%$ (relative error)

\begin{tabular}{|c|c|}
\hline $\begin{array}{l}\text { Components: } \\
\text { (1) 4-Chlorobenzoic acid; } \\
\mathrm{C}_{7} \mathrm{H}_{5} \mathrm{ClO}_{2} ;[74-11-3] \\
\text { (2) 2-Methyl-2-propanol; } \\
\mathrm{C}_{4} \mathrm{H}_{10} \mathrm{O} ;[75-65-0]\end{array}$ & $\begin{array}{l}\text { Original Measurements: } \\
{ }^{128} \text { G. S. Krasil'nikova, E. G. Freidlin, } \\
\text { and Y. N. Pirig, Zh. Prikl. Khim. 60, } \\
2519 \text { (1987). }\end{array}$ \\
\hline $\begin{array}{l}\text { Variables: } \\
T / \mathrm{K}=293\end{array}$ & $\begin{array}{l}\text { Prepared by: } \\
\text { W. E. Acree, Jr. }\end{array}$ \\
\hline
\end{tabular}

\section{Experimental Values}

The measured solubility was reported to be $30.7 \mathrm{~g} / \mathrm{l}$, which corresponds to a molar solubility of $c_{1}=0.196$.

\section{Auxiliary Information}

Method/Apparatus/Procedure:

No information given.

Source and Purity of Chemicals:

(1) Purity not given, Chemical source not given, no purification details were provided.

(2) Purity not given, Chemical source not given, no purification details were provided.
Estimated Error:

Temperature: No information given.

$c_{1}$ : No information given.

\begin{tabular}{ll}
\hline \hline Components: & Original Measurements: \\
(1) 4-Chlorobenzoic acid; & ${ }^{14} \mathrm{C}$. R. Daniels, A. K. Charlton, R. M. \\
$\mathrm{C}_{7} \mathrm{H}_{5} \mathrm{ClO}_{2} ;[74-11-3]$ & Wold, W. E. Acree, Jr., and M. H. \\
$\begin{array}{l}\text { (2) 1-Pentanol; } \mathrm{C}_{5} \mathrm{H}_{12} \mathrm{O} ; \\
{[71-41-0]}\end{array}$ & Abraham, Can. J. Chem. 81, 1492 \\
\hline Variables: & $(2003)$. \\
$T / \mathrm{K}=298.15$ & Prepared by: \\
\hline
\end{tabular}

Experimental Values

\begin{tabular}{lc}
\hline \hline$x_{2}{ }^{\mathrm{a}}$ & $x_{1}{ }^{\mathrm{b}}$ \\
\hline 0.9848 & 0.01517
\end{tabular}

${ }^{a} x_{2}$ : mole fraction of component 2 in the saturated solution.

${ }^{\mathrm{b}} x_{1}$ : mole fraction solubility of the solute.

\section{Auxiliary Information}

\section{Method/Apparatus/Procedure:}

Constant-temperature bath, calorimetric thermometer, and an ultraviolet/ visible spectrophotometer.

Excess solute and solvent were placed in amber glass bottles and allowed to equilibrate for several days at constant temperature. Attainment of equilibrium was verified by several repetitive measurements and by approaching equilibrium from supersaturation. Aliquots of saturated solutions were transferred through a coarse filter into tared volumetric flasks, weighed, and diluted with methanol. Concentrations were determined by spectrophotometric measurements at $272 \mathrm{~nm}$.

Source and Purity of Chemicals:

(1) $99 \%$, Acros Organics, USA, was used as received. (2) $99+\%$, Aldrich Chemical Company, Milwaukee, WI, USA, stored over molecular sieves and distilled shortly before use.

\section{Estimated Error:}

Temperature: $\pm 0.1 \mathrm{~K}$.

$x_{1}: \pm 1.5 \%$ (relative error)

\begin{tabular}{ll}
\hline \hline Components: & Original Measurements: \\
$\begin{array}{l}\text { (1) 4-Chlorobenzoic acid; } \\
\mathrm{C}_{7} \mathrm{H}_{5} \mathrm{ClO}_{2} ;[74-11-3]\end{array}$ & $\begin{array}{l}{ }^{14} \mathrm{C} . \text { R. Daniels, A. K. Charlton, R. M. } \\
\text { (2) 2-Pentanol; } \mathrm{C}_{5} \mathrm{H}_{12} \mathrm{O} ;\end{array}$ \\
$\begin{array}{l}\text { Wold, W. E. Acree, Jr., and M. H. } \\
\text { [6032-29-7] }\end{array}$ & $\begin{array}{l}\text { Abraham, Can. J. Chem. 81, 1492 } \\
(2003) .\end{array}$ \\
\hline Variables: & Prepared by: \\
$T / \mathrm{K}=298.15$ & W. E. Acree, Jr. \\
\hline
\end{tabular}

\section{Experimental Values}

\begin{tabular}{lc}
\hline \hline$x_{2}{ }^{\mathrm{a}}$ & $x_{1}{ }^{\mathrm{b}}$ \\
\hline 0.9835 & 0.01655
\end{tabular}

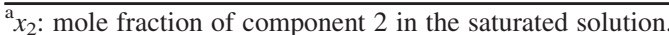

${ }^{\mathrm{b}} x_{1}$ : mole fraction solubility of the solute. 


\section{Auxiliary Information}

\section{Method/Apparatus/Procedure:}

Constant-temperature bath, calorimetric thermometer, and an ultraviolet/ visible spectrophotometer.

Excess solute and solvent were placed in amber glass bottles and allowed to equilibrate for several days at constant temperature. Attainment of equilibrium was verified by several repetitive measurements and by approaching equilibrium from supersaturation. Aliquots of saturated solutions were transferred through a coarse filter into tared volumetric flasks, weighed, and diluted with methanol. Concentrations were determined by spectrophotometric measurements at $272 \mathrm{~nm}$.

\section{Source and Purity of Chemicals:}

(1) $99 \%$, Acros Organics, USA, was used as received.

(2) $99+\%$, Acros Organics, USA, stored over molecular sieves and distilled shortly before use.

\section{Estimated Error:}

Temperature: $\pm 0.1 \mathrm{~K}$

$x_{1}: \pm 1.5 \%$ (relative error).

\begin{tabular}{ll}
\hline \hline Components: & Original Measurements: \\
$(1)$ 4-Chlorobenzoic acid; & ${ }^{14} \mathrm{C} . \mathrm{R}$. Daniels, A. K. Charlton, R. M. \\
$\mathrm{C}_{7} \mathrm{H}_{5} \mathrm{ClO}_{2} ;$ [74-11-3] & Wold, W. E. Acree, Jr., and M. H. \\
$(2)$ 2-Methyl-1-butanol; & Abraham, Can. J. Chem. 81, 1492 \\
$\mathrm{C}_{5} \mathrm{H}_{12} \mathrm{O} ;[137-32-6]$ & $(2003)$. \\
\hline Variables: & Prepared by: \\
$T / \mathrm{K}=298.15$ & W. E. Acree, Jr. \\
\hline
\end{tabular}

Experimental Values

\begin{tabular}{lc}
\hline \hline$x_{2}{ }^{\mathrm{a}}$ & $x_{1}{ }^{\mathrm{b}}$ \\
\hline 0.9887 & 0.01133 \\
\hline
\end{tabular}

${ }^{a} x_{2}$ : mole fraction of component 2 in the saturated solution.

${ }^{\mathrm{b}} x_{1}$ : mole fraction solubility of the solute.

\section{Auxiliary Information}

\section{Method/Apparatus/Procedure:}

Constant-temperature bath, calorimetric thermometer, and an ultraviolet/ visible spectrophotometer.

Excess solute and solvent were placed in amber glass bottles and allowed to equilibrate for several days at constant temperature. Attainment of equilibrium was verified by several repetitive measurements and by approaching equilibrium from supersaturation. Aliquots of saturated solutions were transferred through a coarse filter into tared volumetric flasks, weighed, and diluted with methanol. Concentrations were determined by spectrophotometric measurements at $272 \mathrm{~nm}$.

\section{Source and Purity of Chemicals:}

(1) $99 \%$, Acros Organics, USA, was used as received.

(2) $99 \%$, Aldrich Chemical Company, Milwaukee, WI, USA, stored over molecular sieves and distilled shortly before use.

\section{Estimated Error:}

Temperature: $\pm 0.1 \mathrm{~K}$

$x_{1}: \pm 1.5 \%$ (relative error).

\begin{tabular}{ll}
\hline \hline Components: & Original Measurements: \\
(1) 4-Chlorobenzoic acid; & ${ }^{14} \mathrm{C} . \mathrm{R}$. Daniels, A. K. Charlton, R. M. \\
$\mathrm{C}_{7} \mathrm{H}_{5} \mathrm{ClO}_{2}$; [74-11-3] & Wold, W. E. Acree, Jr., and M. H. \\
$(2)$ 3-Methyl-1-butanol; & Abraham, Can. J. Chem. 81, 1492 \\
$\mathrm{C}_{5} \mathrm{H}_{12} \mathrm{O} ;$ [123-51-3] & $(2003)$. \\
\hline Variables: & Prepared by: \\
$T / \mathrm{K}=298.15$ & W. E. Acree, Jr. \\
\hline
\end{tabular}

Experimental Values

\begin{tabular}{lc}
\hline \hline$x_{2}{ }^{\mathrm{a}}$ & $x_{1}{ }^{\mathrm{b}}$ \\
\hline 0.9868 & 0.01323
\end{tabular}

${ }^{\mathrm{a}} x_{2}$ : mole fraction of component 2 in the saturated solution.

${ }^{b} x_{1}$ : mole fraction solubility of the solute.

\section{Auxiliary Information}

\section{Method/Apparatus/Procedure:}

Constant-temperature bath, calorimetric thermometer, and an ultraviolet/ visible spectrophotometer.

Excess solute and solvent were placed in amber glass bottles and allowed to equilibrate for several days at constant temperature. Attainment of equilibrium was verified by several repetitive measurements and by approaching equilibrium from supersaturation. Aliquots of saturated solutions were transferred through a coarse filter into tared volumetric flasks, weighed, and diluted with methanol. Concentrations were determined by spectrophotometric measurements at $272 \mathrm{~nm}$.

\section{Source and Purity of Chemicals:}

(1) $99 \%$, Acros Organics, USA, was used as received.

(2) $99 \%$, anhydrous, Aldrich Chemical Company, Milwaukee, WI, USA, stored over molecular sieves and distilled shortly before use.

\section{Estimated Error:}

Temperature: $\pm 0.1 \mathrm{~K}$.

$x_{1}: \pm 1.5 \%$ (relative error).

\begin{tabular}{|c|c|}
\hline $\begin{array}{l}\text { Components: } \\
\text { (1) 4-Chlorobenzoic acid; } \\
\mathrm{C}_{7} \mathrm{H}_{5} \mathrm{ClO}_{2} ;[74-11-3] \\
\text { (2) } 1-\mathrm{Hexanol} ; \mathrm{C}_{6} \mathrm{H}_{14} \mathrm{O} \text {; } \\
\text { [111-27-3] }\end{array}$ & $\begin{array}{l}\text { Original Measurements: } \\
{ }^{14} \text { C. R. Daniels, A. K. Charlton, R. M. } \\
\text { Wold, W. E. Acree, Jr., and M. H. } \\
\text { Abraham, Can. J. Chem. 81, } 1492 \\
\text { (2003). }\end{array}$ \\
\hline $\begin{array}{l}\text { Variables: } \\
T / \mathrm{K}=298.15\end{array}$ & $\begin{array}{l}\text { Prepared by: } \\
\text { W. E. Acree, Jr. }\end{array}$ \\
\hline
\end{tabular}

\section{Experimental Values}

\begin{tabular}{lc}
\hline \hline$x_{2}{ }^{\mathrm{a}}$ & $x_{1}{ }^{\mathrm{b}}$ \\
\hline 0.9835 & 0.01646 \\
\hline${ }^{\mathrm{a}} x_{2}:$ mole fraction of component 2 in the saturated solution. & \\
${ }^{\mathrm{b}} x_{1}:$ mole fraction solubility of the solute. &
\end{tabular}




\section{Auxiliary Information}

\section{Method/Apparatus/Procedure:}

Constant-temperature bath, calorimetric thermometer, and an ultraviolet/ visible spectrophotometer.

Excess solute and solvent were placed in amber glass bottles and allowed to was verified by several repetitive measurements and by approaching equilibrium from supersaturation. Aliquots of saturated solutions were diluted with methanol. Concentrations were determined by spectrophotometric measurements at $272 \mathrm{~nm}$.

(1) $99 \%$, Acros Organics, USA, was used as received.

(2) $99+\%$, Alfa Aesar, USA, stored over molecular sieves and distilled shortly before use.

Estimated Error:

Temperature: $\pm 0.1 \mathrm{~K}$.

$x_{1}: \pm 1.5 \%$ (relative error) equilibrate for several days at constant temperature. Attainment of equilibrium transferred through a coarse filter into tared volumetric flasks, weighed, and

Source and Purity of Chemicals:

\begin{tabular}{|c|c|}
\hline $\begin{array}{l}\text { Components: } \\
\text { (1) 4-Chlorobenzoic acid; } \\
\mathrm{C}_{7} \mathrm{H}_{5} \mathrm{ClO}_{2} ;[74-11-3] \\
\text { (2) } 1-\text { Octanol; } \mathrm{C}_{8} \mathrm{H}_{18} \mathrm{O} \text {; } \\
\text { [111-87-5] }\end{array}$ & $\begin{array}{l}\text { Original Measurements: } \\
{ }^{14} \text { C. R. Daniels, A. K. Charlton, R. M. } \\
\text { Wold, W. E. Acree, Jr., and M. H. } \\
\text { Abraham, Can. J. Chem. 81, } 1492 \\
\text { (2003). }\end{array}$ \\
\hline $\begin{array}{l}\text { Variables: } \\
T / \mathrm{K}=298.15\end{array}$ & $\begin{array}{l}\text { Prepared by: } \\
\text { W. E. Acree, Jr. }\end{array}$ \\
\hline
\end{tabular}

Experimental Values

\begin{tabular}{lc}
\hline \hline$x_{2}{ }^{\mathrm{a}}$ & $x_{1}{ }^{\mathrm{b}}$ \\
\hline 0.9811 & 0.01885
\end{tabular}

${ }^{a} x_{2}$ : mole fraction of component 2 in the saturated solution.

${ }^{b} x_{1}$ : mole fraction solubility of the solute.

\section{Auxiliary Information}

\begin{tabular}{|c|c|}
\hline $\begin{array}{l}\text { Components: } \\
\text { (1) 4-Chlorobenzoic acid; } \\
\mathrm{C}_{7} \mathrm{H}_{5} \mathrm{ClO}_{2} ;[74-11-3] \\
\text { (2) } 1-\mathrm{Heptanol} ; \mathrm{C}_{7} \mathrm{H}_{16} \mathrm{O} ; \\
{[111-70-6]}\end{array}$ & $\begin{array}{l}\text { Original Measurements: } \\
{ }^{14} \text { C. R. Daniels, A. K. Charlton, R. M. } \\
\text { Wold, W. E. Acree, Jr., and M. H. } \\
\text { Abraham, Can. J. Chem. 81, } 1492 \\
\text { (2003). }\end{array}$ \\
\hline $\begin{array}{l}\text { Variables: } \\
T / \mathrm{K}=298.15\end{array}$ & $\begin{array}{l}\text { Prepared by: } \\
\text { W. E. Acree, Jr. }\end{array}$ \\
\hline
\end{tabular}

Experimental Values

\begin{tabular}{lc}
\hline \hline$x_{2}{ }^{\mathrm{a}}$ & $x_{1}{ }^{\mathrm{b}}$ \\
\hline 0.9822 & 0.01777 \\
\hline
\end{tabular}

${ }^{\mathrm{a}} x_{2}$ : mole fraction of component 2 in the saturated solution.

${ }^{\mathrm{b}} x_{1}$ : mole fraction solubility of the solute.

\section{Auxiliary Information}

\section{Method/Apparatus/Procedure:}

Constant-temperature bath, calorimetric thermometer, and an ultraviolet/ visible spectrophotometer.

Excess solute and solvent were placed in amber glass bottles and allowed to equilibrate for several days at constant temperature. Attainment of equilibrium was verified by several repetitive measurements and by approaching equilibrium from supersaturation. Aliquots of saturated solutions were transferred through a coarse filter into tared volumetric flasks, weighed, and diluted with methanol. Concentrations were determined by

spectrophotometric measurements at $272 \mathrm{~nm}$.

\section{Source and Purity of Chemicals:}

(1) $99 \%$, Acros Organics, USA, was used as received.

(2) $99+\%$, Alfa Aesar, USA, stored over molecular sieves and distilled shortly before use.

\section{Estimated Error:}

Temperature: $\pm 0.1 \mathrm{~K}$.

$x_{1}: \pm 1.5 \%$ (relative error).

\author{
Method/Apparatus/Procedure: \\ Constant-temperature bath, calorimetric thermometer, and an ultraviolet/ \\ visible spectrophotometer. \\ Excess solute and solvent were placed in amber glass bottles and allowed to \\ equilibrate for several days at constant temperature. Attainment of equilibrium \\ was verified by several repetitive measurements and by approaching \\ equilibrium from supersaturation. Aliquots of saturated solutions were \\ transferred through a coarse filter into tared volumetric flasks, weighed, and \\ diluted with methanol. Concentrations were determined by \\ spectrophotometric measurements at $272 \mathrm{~nm}$.

\section{Source and Purity of Chemicals:} \\ (1) $99 \%$, Acros Organics, USA, was used as received. \\ (2) $99+\%$, anhydrous, Aldrich Chemical Company, Milwaukee, WI, USA, \\ stored over molecular sieves and distilled shortly before use.

\section{Estimated Error:} \\ Temperature: $\pm 0.1 \mathrm{~K}$. \\ $x_{1}: \pm 1.5 \%$ (relative error).
}

\begin{tabular}{ll}
\hline \hline Components: & Original Measurements: \\
(1) 4-Chlorobenzoic acid; & ${ }^{14} \mathrm{C}$. R. Daniels, A. K. Charlton, R. M. \\
$\mathrm{C}_{7} \mathrm{H}_{5} \mathrm{ClO}_{2} ;[74-11-3]$ & Wold, W. E. Acree, Jr., and M. H. \\
$(2)$ 1-Decanol; $\mathrm{C}_{10} \mathrm{H}_{22} \mathrm{O} ;$ & Abraham, Can. J. Chem. 81, 1492 \\
{$[112-30-1]$} & $(2003)$. \\
\hline Variables: & Prepared by: \\
$T / \mathrm{K}=298.15$ & W. E. Acree, Jr. \\
\hline
\end{tabular}

\section{Experimental Values}

\begin{tabular}{lc}
\hline \hline$x_{2}{ }^{\mathrm{a}}$ & $x_{1}^{\mathrm{b}}$ \\
\hline 0.9804 & 0.01956 \\
\hline${ }^{\mathrm{a}} x_{2}:$ mole fraction of component 2 in the saturated solution. & \\
${ }^{\mathrm{b}} x_{1}:$ mole fraction solubility of the solute. &
\end{tabular}




\section{Auxiliary Information}

\section{Method/Apparatus/Procedure:}

Constant-temperature bath, calorimetric thermometer, and an ultraviolet/ visible spectrophotometer.

Excess solute and solvent were placed in amber glass bottles and allowed to equilibrate for several days at constant temperature. Attainment of equilibrium was verified by several repetitive measurements and by approaching equilibrium from supersaturation. Aliquots of saturated solutions were transferred through a coarse filter into tared volumetric flasks, weighed, and diluted with methanol. Concentrations were determined by spectrophotometric measurements at $272 \mathrm{~nm}$.

Source and Purity of Chemicals:

(1) $99 \%$, Acros Organics, USA, was used as received.

(2) 99+\%, anhydrous, Aldrich Chemical Company, Milwaukee, WI, USA, stored over molecular sieves and distilled shortly before use.

Estimated Error:

Temperature: $\pm 0.1 \mathrm{~K}$.

$x_{1}: \pm 1.5 \%$ (relative error)

\begin{tabular}{|c|c|}
\hline $\begin{array}{l}\text { Components: } \\
\text { (1) 4-Chlorobenzoic acid; } \\
\mathrm{C}_{7} \mathrm{H}_{5} \mathrm{ClO}_{2} ;[74-11-3] \\
\text { (2) } 1,2-\text { Propanediol; } \mathrm{C}_{3} \mathrm{H}_{8} \mathrm{O}_{2} \text {; } \\
\text { [57-55-6] }\end{array}$ & $\begin{array}{l}\text { Original Measurements: } \\
{ }^{61} \text { E. R. Cooper, J. Control. Release 1, } \\
153 \text { (1984). }\end{array}$ \\
\hline Variables: & Prepared by: \\
\hline$T / \mathrm{K}=295$ & W. E. Acree, Jr. \\
\hline
\end{tabular}

Experimental Values

\begin{tabular}{lc}
\hline \hline$x_{2}{ }^{\mathrm{a}}$ & $x_{1}{ }^{\mathrm{cb}}$ \\
\hline 0.9902 & 0.0098
\end{tabular}

${ }^{a} x_{2}$ : mole fraction of component 2 in the saturated solution.

${ }^{b} x_{1}$ : mole fraction solubility of the solute. Solubility data reported in terms of mass percent. Mole fraction solubility calculated by the compiler.

\section{Auxiliary Information}

\section{Method/Apparatus/Procedure:}

Very little experimental details provided. Solubility determinations were made by stirring the solvent with excess solute for two days, centrifuging the sample, and analyzing the supernatant.

\section{Source and Purity of Chemicals:}

(1) Reagent grade, Aldrich Chemical Company, Metuchen, NJ, USA, no purification details provided.

(2) Reagent grade, J.T. Baker Chemical Company, Phillipsburg, NJ, USA, no purification details provided.

\section{Estimated Error:}

Temperature: No information given.

$x_{1}$ : No information given.

\subsection{4-Chlorobenzoic acid solubility data in alkoxyalcohols}

\begin{tabular}{|c|c|}
\hline $\begin{array}{l}\text { Components: } \\
\text { (1) 4-Chlorobenzoic acid; } \\
\mathrm{C}_{7} \mathrm{H}_{5} \mathrm{ClO}_{2} ; \text { [74-11-3] } \\
\text { (2) 2-Methoxyethanol; } \mathrm{C}_{3} \mathrm{H}_{8} \mathrm{O}_{2} \text {; } \\
\text { [109-86-4] }\end{array}$ & $\begin{array}{l}\text { Original Measurements: } \\
{ }^{129} \text { S. H. Ghosh and D. K. Hazra, J. } \\
\text { Indian Chem. Soc. 65, } 620 \text { (1988). }\end{array}$ \\
\hline $\begin{array}{l}\text { Variables: } \\
T / \mathrm{K}=298.15\end{array}$ & $\begin{array}{l}\text { Prepared by: } \\
\text { W. E. Acree, Jr. }\end{array}$ \\
\hline
\end{tabular}

Experimental Values

The measured solubility was reported to be $c_{1}=0.4031$ mol dm ${ }^{-3}$.

\section{Auxiliary Information}

\section{Method/Apparatus/Procedure:}

Mechanical shaker and a constant-temperature thermostat.

Excess solute and solvent were placed in a bottle and stoppered. The solution was shaken in a mechanical shaker for $24 \mathrm{~h}$ at ambient room temperature. The solution was then thermostated at $298 \mathrm{~K}$ for $24 \mathrm{~h}$ with occasional shaking. An aliquot of the saturated solution was then removed and filtered. The concentration of the dissolved solute was determined by titration with standard caustic soda using phenolphthalein as indicator.

Source and Purity of Chemicals:

(1) Puriss, Fluka, distilled from aqueous-ethanol mixture.

(2) G. R., Merck Chemical Company, was distilled twice before use.

\section{Estimated Error:}

Temperature: $\pm 0.01 \mathrm{~K}$

$c_{1}: \pm 1.5 \%$ (relative error, estimated by compiler).

\begin{tabular}{|c|c|}
\hline $\begin{array}{l}\text { Components: } \\
\text { (1) 4-Chlorobenzoic acid; } \\
\mathrm{C}_{7} \mathrm{H}_{5} \mathrm{ClO}_{2} ; \text { [74-11-3] } \\
\text { (2) 2-Ethoxyethanol; } \mathrm{C}_{4} \mathrm{H}_{10} \mathrm{O}_{2} \text {; } \\
\text { [110-80-5] }\end{array}$ & $\begin{array}{l}\text { Original Measurements: } \\
{ }^{130} \text { L. M. Grubbs, M. Saifullah, N. E. De } \\
\text { La Rosa, S. Ye, S. S. Achi, W. E. Acree, } \\
\text { Jr., and M. H. Abraham, Fluid Phase } \\
\text { Equilib. 298, } 48 \text { (2010). }\end{array}$ \\
\hline $\begin{array}{l}\text { Variables: } \\
T / \mathrm{K}=298.15\end{array}$ & $\begin{array}{l}\text { Prepared by: } \\
\text { W. E. Acree, Jr. }\end{array}$ \\
\hline
\end{tabular}

Experimental Values

\begin{tabular}{lc}
\hline \hline$x_{2}{ }^{\mathrm{a}}$ & $x_{1}{ }^{\mathrm{b}}$ \\
\hline 0.9663 & 0.0337 \\
\hline${ }^{\mathrm{a}} x_{2}:$ mole fraction of component 2 in the saturated solution. & \\
${ }^{\mathrm{b}} x_{1}:$ mole fraction solubility of the solute. &
\end{tabular}




\section{Auxiliary Information}

\section{Method/Apparatus/Procedure:}

Constant-temperature bath, calorimetric thermometer, and an ultraviolet/ visible spectrophotometer.

Excess solute and solvent were placed in amber glass bottles and allowed to equilibrate for several days at constant temperature. Attainment of equilibrium was verified by several repetitive measurements and by approaching equilibrium from supersaturation. Aliquots of saturated solutions were transferred through a coarse filter into tared volumetric flasks, weighed, and diluted with methanol. Concentrations were determined by spectrophotometric measurements at $272 \mathrm{~nm}$.

Source and Purity of Chemicals:

(1) $99 \%$, Acros Organics, USA, was used as received.

(2) $99 \%$, Aldrich Chemical Company, Milwaukee, WI, USA, stored over molecular sieves and distilled shortly before use.

Estimated Error:

Temperature: $\pm 0.1 \mathrm{~K}$.

$x_{1}: \pm 1.5 \%$ (relative error)

\begin{tabular}{ll}
\hline \hline Components: & Original Measurements: \\
(1) 4-Chlorobenzoic acid; & ${ }^{130}$ L. M. Grubbs, M. Saifullah, N. E. De \\
$\mathrm{C}_{7} \mathrm{H}_{5} \mathrm{ClO}_{2} ;[74-11-3]$ & La Rosa, S. Ye, S. S. Achi, W. E. Acree, \\
(2) 2-Isopropoxyethanol; & Jr., and M. H. Abraham, Fluid Phase \\
$\mathrm{C}_{5} \mathrm{H}_{12} \mathrm{O}_{2} ;[$ [109-59-1] & Equilib. 298, 48 (2010). \\
\hline Variables: & Prepared by: \\
$T / \mathrm{K}=298.15$ & W. E. Acree, Jr. \\
\hline
\end{tabular}

Experimental Values

\begin{tabular}{lc}
\hline \hline$x_{2}{ }^{\mathrm{a}}$ & $x_{1}{ }^{\mathrm{b}}$ \\
\hline 0.9646 & 0.0354
\end{tabular}

${ }^{a} x_{2}$ : mole fraction of component 2 in the saturated solution.

${ }^{b} x_{1}$ : mole fraction solubility of the solute.

\section{Auxiliary Information}

\begin{tabular}{|c|c|}
\hline $\begin{array}{l}\text { Components: } \\
\text { (1) 4-Chlorobenzoic acid; } \\
\mathrm{C}_{7} \mathrm{H}_{5} \mathrm{ClO}_{2} ; \text { [74-11-3] } \\
\text { (2) 2-Propoxyethanol; } \mathrm{C}_{5} \mathrm{H}_{12} \mathrm{O}_{2} \text {; } \\
\text { [2807-30-9] }\end{array}$ & $\begin{array}{l}\text { Original Measurements: } \\
{ }^{130} \text { L. M. Grubbs, M. Saifullah, N. E. De } \\
\text { La Rosa, S. Ye, S. S. Achi, W. E. Acree, } \\
\text { Jr., and M. H. Abraham, Fluid Phase } \\
\text { Equilib. 298, } 48 \text { (2010). }\end{array}$ \\
\hline $\begin{array}{l}\text { Variables: } \\
T / \mathrm{K}=298.15\end{array}$ & $\begin{array}{l}\text { Prepared by: } \\
\text { W. E. Acree, Jr. }\end{array}$ \\
\hline
\end{tabular}

Experimental Values

\begin{tabular}{lc}
\hline \hline$x_{2}{ }^{\mathrm{a}}$ & $x_{1}{ }^{\mathrm{b}}$ \\
\hline 0.9691 & 0.0309 \\
\hline${ }^{\mathrm{a}} x_{2}:$ mole fraction of component 2 in the saturated solution. & \\
${ }^{\mathrm{b}}{ }_{x_{1}}:$ mole fraction solubility of the solute. &
\end{tabular}

\section{Auxiliary Information}

\section{Method/Apparatus/Procedure:}

Constant-temperature bath, calorimetric thermometer, and an ultraviolet/ visible spectrophotometer.

Excess solute and solvent were placed in amber glass bottles and allowed to equilibrate for several days at constant temperature. Attainment of equilibrium was verified by several repetitive measurements and by approaching equilibrium from supersaturation. Aliquots of saturated solutions were transferred through a coarse filter into tared volumetric flasks, weighed, and diluted with methanol. Concentrations were determined by

spectrophotometric measurements at $272 \mathrm{~nm}$.

\section{Source and Purity of Chemicals:}

(1) $99 \%$, Acros Organics, USA, was used as received.

(2) $99+\%$, Aldrich Chemical Company, Milwaukee, WI, USA, stored over molecular sieves and distilled shortly before use.

\section{Estimated Error:}

Temperature: $\pm 0.1 \mathrm{~K}$.

$x_{1}: \pm 1.5 \%$ (relative error).
Constant-temperature bath, calorimetric thermometer, and an ultraviolet/ visible spectrophotometer.

Excess solute and solvent were placed in amber glass bottles and allowed to equilibrate for several days at constant temperature. Attainment of equilibrium was verified by several repetitive measurements and by approaching equilibrium from supersaturation. Aliquots of saturated solutions were transferred through a coarse filter into tared volumetric flasks, weighed, and diluted with methanol. Concentrations were determined by spectrophotometric measurements at $272 \mathrm{~nm}$.

Source and Purity of Chemicals:

(1) $99 \%$, Acros Organics, USA, was used as received.

(2) $99 \%$, Aldrich Chemical Company, Milwaukee, WI, USA, stored over molecular sieves and distilled shortly before use.

\section{Estimated Error:}

Temperature: $\pm 0.1 \mathrm{~K}$.

$x_{1}: \pm 1.5 \%$ (relative error).

\begin{tabular}{ll}
\hline \hline Components: & Original Measurements: \\
$(1)$ 4-Chlorobenzoic acid; & ${ }^{130}$ L. M. Grubbs, M. Saifullah, N. E. De \\
$\mathrm{C}_{7} \mathrm{H}_{5} \mathrm{ClO}_{2} ;$ [74-11-3] & La Rosa, S. Ye, S. S. Achi, W. E. Acree, \\
$\begin{array}{l}\text { (2) 2-Butoxyethanol; } \mathrm{C}_{6} \mathrm{H}_{14} \mathrm{O}_{2} ; \\
\text { [111-76-2] }\end{array}$ & Jr., and M. H. Abraham, Fluid Phase \\
\hline Variables: & Equilib. 298, 48 (2010). \\
$T / \mathrm{K}=298.15$ & Prepared by: \\
\hline
\end{tabular}

\section{Experimental Values}

\begin{tabular}{lc}
\hline \hline$x_{2}{ }^{\mathrm{a}}$ & $x_{1}{ }^{\mathrm{b}}$ \\
\hline 0.9701 & 0.0299 \\
\hline${ }^{\mathrm{a}} x_{2}:$ mole fraction of component 2 in the saturated solution. & \\
${ }^{\mathrm{b}} x_{1}:$ mole fraction solubility of the solute. &
\end{tabular}

\section{Method/Apparatus/Procedure:}




\section{Auxiliary Information}

\section{Method/Apparatus/Procedure:}

Constant-temperature bath, calorimetric thermometer, and an ultraviolet/ visible spectrophotometer.

Excess solute and solvent were placed in amber glass bottles and allowed to equilibrate for several days at constant temperature. Attainment of equilibrium was verified by several repetitive measurements and by approaching equilibrium from supersaturation. Aliquots of saturated solutions were transferred through a coarse filter into tared volumetric flasks, weighed, and diluted with methanol. Concentrations were determined by spectrophotometric measurements at $272 \mathrm{~nm}$.

Source and Purity of Chemicals:

(1) $99 \%$, Acros Organics, USA, was used as received.

(2) $99 \%$, Acros Organics, stored over molecular sieves and distilled shortly before use.

Estimated Error:

Temperature: $\pm 0.1 \mathrm{~K}$.

$x_{1}: \pm 1.5 \%$ (relative error).

\subsection{4-Chlorobenzoic acid solubility data in miscellaneous organic solvents}

\begin{tabular}{ll}
\hline \hline Components: & Original Measurements: \\
$(1)$ 4-Chlorobenzoic acid; & ${ }^{14}$ C. R. Daniels, A. K. Charlton, R. M. \\
$\mathrm{C}_{7} \mathrm{H}_{5} \mathrm{ClO}_{2}$; [74-11-3] & Wold, W. E. Acree, Jr., and M. H. \\
(2) $\mathrm{Propylene} \mathrm{carbonate;}_{\mathrm{C}_{4} \mathrm{H}_{6} \mathrm{O}_{3} ;[108-32-7]}$ & Abraham, Can. J. Chem. 81, 1492 \\
\hline Variables: & $(2003)$. \\
$T / \mathrm{K}=298.15$ & Prepared by: \\
\hline
\end{tabular}

Experimental Values

\begin{tabular}{lc}
\hline \hline$x_{2}{ }^{\mathrm{a}}$ & $x_{1}{ }^{\mathrm{b}}$ \\
\hline 0.9967 & 0.003247 \\
${ }^{\mathrm{a}} x_{2}:$ mole fraction of component 2 in the saturated solution. \\
${ }^{\mathrm{b}} x_{1}:$ mole fraction solubility of the solute.
\end{tabular}

\section{Auxiliary Information}

\footnotetext{
Method/Apparatus/Procedure:

Constant-temperature bath, calorimetric thermometer, and an ultraviolet/ visible spectrophotometer.

Excess solute and solvent were placed in amber glass bottles and allowed to equilibrate for several days at constant temperature. Attainment of equilibrium was verified by several repetitive measurements and by approaching equilibrium from supersaturation. Aliquots of saturated solutions were transferred through a coarse filter into tared volumetric flasks, weighed, and diluted with methanol. Concentrations were determined by spectrophotometric measurements at $272 \mathrm{~nm}$.
}

\section{Source and Purity of Chemicals:}

(1) $99 \%$, Acros Organics, USA, was used as received.

(2) $99+\%$, anhydrous, Aldrich Chemical Company, Milwaukee, WI, USA, stored over molecular sieves and distilled shortly before use.

\section{Estimated Error:}

Temperature: $\pm 0.1 \mathrm{~K}$

$x_{1}: \pm 1.5 \%$ (relative error).

\section{Solubility of 2-Chloro-5-nitrobenzoic Acid in Organic Solvents}

\subsection{Critical evaluation of experimental solubility data}

There has been only one published study investigating the solubility behavior of 2-chloro-5-nitrobenzoic acid in organic solvents of varying polarity and hydrogen-bonding capability. Stovall et al. ${ }^{13}$ measured the solubility of 2-chloro-5-nitrobenzoic acid in 14 alcohols (ethanol, 1-propanol, 2-propanol, 1-butanol, 2-butanol, 2-methyl-1-propanol, 2-methyl-2-propanol, 1-pentanol, 2-pentanol, 3-methyl-1-butanol, 1-hexanol, 1-heptanol, 1-octanol, and 1-decanol), in three dialkyl ethers (1,1'-oxybisethane, 2,2'-oxybispropane, and 1,1'-oxybisbutane) and one cyclic ether (tetrahydrofuran), and in four alkyl alkanoates (methyl ethanoate, ethyl ethanoate, propyl ethanoate, and butyl ethanoate) at $298 \mathrm{~K}$. Results of the experimental measurements were used to calculate the Abraham solute descriptors of 2-chloro-5-nitrobenzoic acid. The authors were able to assemble a total of $35 \log _{10}\left(S R\right.$ or $P$ ) and $\log _{10}$ (GSR or $K$ ) equations for which experimental partition coefficient data, solubility ratios, Abraham Model equation coefficients and aqueous molar solubility were available. The logarithm of the aqueous molar solubility of 2-chloro-5-nitrobenzoic acid, $\log _{10} c_{1, \mathrm{~W}}=-2.588$ (corrected for ionization), was determined by the authors as part of their experimental study. The McGowan volume of 2-chloro-5-nitrobenzoic acid, $V=$ 1.2283, was calculated from the number of chemical bonds in the molecule and the individual atomic group volumes, $A V_{i}$, given in Sec. 1.3. The excess molar refraction solute descriptor was estimated as $E=1.250$. This left four solute descriptors ( $S$, $A, B$, and $L$ ) still to be determined. The 35 equations were then solved using the Microsoft "SOLVER" program to yield numerical values of the remaining four solute descriptors, $S=1.400$, $A=0.670, B=0.460$, and $L=6.513$, that best described the $\log _{10}(S R$ or $P)$ and $\log _{10}(G S R$ or $K$ ) values. The computation treated $\log _{10} c_{1, \mathrm{G}}$ as a floating parameter to be determined as part of the regression analyses. The data analyses returned a value of $\log _{10} c_{1, \mathrm{G}}=-9.538$ for the logarithm of the gas-phase solute concentration that made the $\log _{10}\left(S R\right.$ or $P$ ) and $\log _{10}$ (GSR or $K$ ) predictions internally consistent. The calculated molecular solute descriptors reproduced the $\log _{10}(S R$ or $P$ ) and $\log _{10}(G S R$ or $K)$ values to within an average standard deviation of 0.104 and $0.124 \log _{10}$ units, respectively.

After the 2-chloro-5-nitrobenzoic acid solubility study was published, Abraham model correlations were developed for 2pentanol, 3-methyl-1-butanol, methyl ethanoate, propyl ethanoate, and butyl ethanoate, and equation coefficients for a few solvents were updated based on additional experimental data. The new correlations (listed in Tables 1 and 2) will be used in illustrating the ability of the Abraham model to correlate the experimental 2-chloro-5-nitrobenzoic acid solubility data. Table 23 compares the experimental $\log _{10} c_{1}$ values to calculated values based on Eqs. (20) and (21) of the Abraham model. For comparison purposes, the measured mole fraction solubilities of 2-chloro-5-nitrobenzoic acid, $x_{1}$, determined by Stovall et al., ${ }^{13}$ were converted into molar 
TABLE 23. Comparison between observed and calculated molar solubilities of 2-chloro-5-nitrobenzoic acid based on the Abraham model, Eqs. (20) and (21)

\begin{tabular}{lcrr}
\hline \hline Solvent & & $\log _{10} c_{1}{ }^{\text {calc}} ;$ & $\log _{10} c_{1}{ }^{\text {calc }} ;$ \\
Ethanol & $\log _{10} c_{1}{ }^{\text {exp }}$ & Eq. (20) & Eq. $(21)$ \\
1-Propanol & 0.146 & 0.076 & 0.070 \\
2-Propanol & -0.022 & -0.055 & -0.047 \\
1-Butanol & 0.045 & -0.062 & -0.046 \\
2-Butanol & -0.111 & -0.153 & -0.140 \\
2-Methyl-1-propanol & -0.134 & -0.138 & -0.151 \\
2-Methyl-2-propanol & -0.204 & -0.207 & -0.288 \\
1-Pentanol & 0.070 & -0.099 & -0.050 \\
2-Pentanol & -0.134 & -0.164 & -0.158 \\
3-Methyl-1-butanol & -0.125 & -0.192 & -0.186 \\
1-Hexanol & -0.173 & -0.273 & -0.264 \\
1-Heptanol & -0.198 & -0.207 & -0.210 \\
1-Octanol & -0.269 & -0.237 & -0.233 \\
1-Decanol & -0.324 & -0.256 & -0.331 \\
1,1'-Oxybisethane & -0.411 & -0.360 & -0.341 \\
Tetrahydrofuran & -0.171 & -0.269 & -0.178 \\
Methyl ethanoate & 0.461 & 0.589 & 0.563 \\
Ethyl ethanoate & -0.070 & -0.065 & -0.052 \\
Propyl ethanoate & -0.126 & -0.051 & -0.051 \\
Butyl ethanoate & -0.289 & -0.128 & -0.146 \\
\hline
\end{tabular}

solubilities by dividing $x_{1}$ by the ideal molar volume of the saturated solution (i.e., $c_{1}{ }^{\text {sat }}=x_{1} /\left[x_{1} V_{1}+\left(1-x_{1}\right) V_{\text {solvent }}\right]$ ). The molar volume of the hypothetical subcooled liquid 2-chloro-5nitrobenzoic acid is $V_{\text {solute }}=130.38 \mathrm{~cm}^{3} \mathrm{~mol}^{-1}$. Examination of the numerical entries in Table 23 reveals that the Abraham model provides a reasonably accurate mathematical description of the observed solubility data, suggesting that there are no obvious outliers in the dataset.

The experimental solubility data for 2-chloro-5-nitrobenzoic acid in organic solvents are in Secs. 21.2-21.4.

\subsection{2-Chloro-5-nitrobenzoic acid solubility data in esters}

\begin{tabular}{|c|c|}
\hline $\begin{array}{l}\text { Components: } \\
\text { (1) 2-Chloro-5-nitrobenzoic acid; } \\
\left.\mathrm{C}_{7} \mathrm{H}_{4} \mathrm{ClNO}_{4} ; \text {; } 2516-96-3\right] \\
\text { (2) Methyl ethanoate; } \mathrm{C}_{3} \mathrm{H}_{6} \mathrm{O}_{2} \text {; } \\
\text { [79-20-9] }\end{array}$ & $\begin{array}{l}\text { Original Measurements: } \\
{ }^{13} \text { D. M. Stovall, C. Givens, S. Keown, } \\
\text { K. R. Hoover, R. Barnes, C. Harris, J. } \\
\text { Lozano, M. Nguyen, E. Rodriguez, W. } \\
\text { E. Acree, Jr., and M. H. Abraham, } \\
\text { Phys. Chem. Liq. 43, } 351 \text { (2005). }\end{array}$ \\
\hline $\begin{array}{l}\text { Variables: } \\
T / \mathrm{K}=298.15\end{array}$ & $\begin{array}{l}\text { Prepared by: } \\
\text { W. E. Acree, Jr. }\end{array}$ \\
\hline
\end{tabular}

Experimental Values

\begin{tabular}{lc}
\hline \hline$x_{2}{ }^{\mathrm{a}}$ & $x_{1}{ }^{\mathrm{b}}$ \\
\hline 0.9290 & 0.07096
\end{tabular}

${ }^{\mathrm{a}} x_{2}$ : mole fraction of component 2 in the saturated solution.

${ }^{\mathrm{b}} x_{1}$ : mole fraction solubility of the solute.

\section{Auxiliary Information}

\begin{tabular}{|c|c|}
\hline $\begin{array}{l}\text { Method/Apparatus/Procedure: } \\
\text { Constant-temperature bath, calorir } \\
\text { visible spectrophotometer. } \\
\text { Excess solute and solvent were pl } \\
\text { equilibrate for several days at const } \\
\text { was verified by several repetitive } \\
\text { equilibrium from supersaturation. } \\
\text { transferred through a coarse filter } \\
\text { diluted with methanol. Concentrat } \\
\text { spectrophotometric measurements }\end{array}$ & $\begin{array}{l}\text { tric thermometer, and an ultraviolet/ } \\
\text { t in amber glass bottles and allowed to } \\
\text { temperature. Attainment of equilibrium } \\
\text { iquots of saturated solutions were } \\
\text { o tared volumetric flasks, weighed, and } \\
\text { ts were determined by } \\
280 \mathrm{~nm} \text {. }\end{array}$ \\
\hline $\begin{array}{l}\text { Source and Purity of Chemicals } \\
\text { (1) } 99+\% \text {, Acros Organics, USA, } \\
\text { (2) } 99.5 \% \text {, anhydrous, Aldrich Ch } \\
\text { stored over molecular sieves and }\end{array}$ & $\begin{array}{l}\text { s used as received. } \\
\text { ical Company, Milwaukee, WI, USA, } \\
\text { tilled shortly before use. }\end{array}$ \\
\hline $\begin{array}{l}\text { Estimated Error: } \\
\text { Temperature: } \pm 0.1 \mathrm{~K} . \\
x_{1}: \pm 1.5 \% \text { (relative error). }\end{array}$ & \\
\hline $\begin{array}{l}\text { Components: } \\
\text { (1) 2-Chloro-5-nitrobenzoic acid; } \\
\mathrm{C}_{7} \mathrm{H}_{4} \mathrm{ClNO}_{4} ; \text { [2516-96-3] } \\
\text { (2) Ethyl ethanoate; } \mathrm{C}_{4} \mathrm{H}_{8} \mathrm{O}_{2} \text {; } \\
\text { [141-78-6] }\end{array}$ & $\begin{array}{l}\text { Original Measurements: } \\
{ }^{13} \text { D. M. Stovall, C. Givens, S. Keown, } \\
\text { K. R. Hoover, R. Barnes, C. Harris, J. } \\
\text { Lozano, M. Nguyen, E. Rodriguez, W. } \\
\text { E. Acree, Jr., and M. H. Abraham, } \\
\text { Phys. Chem. Liq. 43, } 351 \text { (2005). }\end{array}$ \\
\hline $\begin{array}{l}\text { Variables: } \\
T / \mathrm{K}=298.15\end{array}$ & $\begin{array}{l}\text { Prepared by: } \\
\text { W. E. Acree, Jr. }\end{array}$ \\
\hline
\end{tabular}

Experimental Values

\begin{tabular}{lc}
\hline \hline$x_{2}{ }^{\mathrm{a}}$ & $x_{1}{ }^{\mathrm{b}}$ \\
\hline 0.9244 & 0.07561 \\
\hline${ }^{\mathrm{a}} x_{2}:$ mole fraction of component 2 in the saturated solution. & \\
${ }^{\mathrm{b}} x_{1}:$ mole fraction solubility of the solute. &
\end{tabular}

\section{Auxiliary Information}

\section{Method/Apparatus/Procedure:}

Constant-temperature bath, calorimetric thermometer, and an ultraviolet/ visible spectrophotometer.

Excess solute and solvent were placed in amber glass bottles and allowed to equilibrate for several days at constant temperature. Attainment of equilibrium was verified by several repetitive measurements and by approaching equilibrium from supersaturation. Aliquots of saturated solutions were transferred through a coarse filter into tared volumetric flasks, weighed, and diluted with methanol. Concentrations were determined by spectrophotometric measurements at $280 \mathrm{~nm}$.

Source and Purity of Chemicals:

(1) $99+\%$, Acros Organics, USA, was used as received.

(2) $99.9 \%$, HPLC grade, Aldrich Chemical Company, Milwaukee, WI, USA, stored over molecular sieves and distilled shortly before use.

\section{Estimated Error:}

Temperature: $\pm 0.1 \mathrm{~K}$.

$x_{1}: \pm 1.5 \%$ (relative error) 


\section{Components:}

(1) 2-Chloro-5-nitrobenzoic acid;

$\mathrm{C}_{7} \mathrm{H}_{4} \mathrm{ClNO}_{4}$; [2516-96-3]

(2) Propyl ethanoate; $\mathrm{C}_{5} \mathrm{H}_{10} \mathrm{O}_{2}$; [109-60-4]

\section{Variables:}

$T / \mathrm{K}=298.15$

Original Measurements:

${ }^{13}$ D. M. Stovall, C. Givens, S. Keown,

K. R. Hoover, R. Barnes, C. Harris, J.

Lozano, M. Nguyen, E. Rodriguez, W.

E. Acree, Jr., and M. H. Abraham,

Phys. Chem. Liq. 43, 351 (2005).

Prepared by:

W. E. Acree, Jr.

Experimental Values

\begin{tabular}{lc}
\hline \hline$x_{2}{ }^{\mathrm{a}}$ & $x_{1}{ }^{\mathrm{b}}$ \\
\hline 0.9401 & 0.05988 \\
\hline${ }^{\mathrm{a}} x_{2}:$ mole fraction of component 2 in the saturated solution. & \\
${ }^{\mathrm{b}} x_{1}:$ mole fraction solubility of the solute. &
\end{tabular}

\section{Auxiliary Information}

\section{Method/Apparatus/Procedure:}

Constant-temperature bath, calorimetric thermometer, and an ultraviolet/ visible spectrophotometer.

Excess solute and solvent were placed in amber glass bottles and allowed to equilibrate for several days at constant temperature. Attainment of equilibrium was verified by several repetitive measurements and by approaching equilibrium from supersaturation. Aliquots of saturated solutions were transferred through a coarse filter into tared volumetric flasks, weighed, and diluted with methanol. Concentrations were determined by

spectrophotometric measurements at $280 \mathrm{~nm}$.

\section{Source and Purity of Chemicals:}

(1) $99+\%$, Acros Organics, USA, was used as received.

(2) $99.5 \%$, Aldrich Chemical Company, Milwaukee, WI, USA, stored over molecular sieves and distilled shortly before use.

\section{Estimated Error:}

Temperature: $\pm 0.1 \mathrm{~K}$

$x_{1}: \pm 1.5 \%$ (relative error).

\begin{tabular}{ll}
\hline \hline Components: & Original Measurements: \\
$(1)$ 2-Chloro-5-nitrobenzoic acid; & ${ }^{13}$ D. M. Stovall, C. Givens, S. Keown, \\
$\mathrm{C}_{7} \mathrm{H}_{4} \mathrm{ClNO}_{4} ;$ [2516-96-3] & K. R. Hoover, R. Barnes, C. Harris, J. \\
$(2)$ Butyl ethanoate; $\mathrm{C}_{6} \mathrm{H}_{12} \mathrm{O}_{2} ;$ & Lozano, M. Nguyen, E. Rodriguez, W. \\
[123-86-4] & E. Acree, Jr., and M. H. Abraham, \\
& Phys. Chem. Liq. 43, 351 (2005). \\
\hline Variables: & Prepared by: \\
$T / \mathrm{K}=298.15$ & W. E. Acree, Jr. \\
\hline
\end{tabular}

\section{Experimental Values}

\begin{tabular}{lc}
\hline \hline$x_{2}{ }^{\mathrm{a}}$ & $x_{1}{ }^{\mathrm{b}}$ \\
\hline 0.9374 & 0.06260 \\
\hline
\end{tabular}

${ }^{\mathrm{a}} x_{2}$ : mole fraction of component 2 in the saturated solution.

${ }^{b} x_{1}$ : mole fraction solubility of the solute.

\section{Auxiliary Information}

\section{Method/Apparatus/Procedure:}

Constant-temperature bath, calorimetric thermometer, and an ultraviolet/ visible spectrophotometer.

Excess solute and solvent were placed in amber glass bottles and allowed to equilibrate for several days at constant temperature. Attainment of equilibrium was verified by several repetitive measurements and by approaching equilibrium from supersaturation. Aliquots of saturated solutions were transferred through a coarse filter into tared volumetric flasks, weighed, and diluted with methanol. Concentrations were determined by spectrophotometric measurements at $280 \mathrm{~nm}$.

\section{Source and Purity of Chemicals:}

(1) $99+\%$, Acros Organics, USA, was used as received.

(2) $99.7 \%$, HPLC grade, Aldrich Chemical Company, Milwaukee, WI, USA, stored over molecular sieves and distilled shortly before use.

\section{Estimated Error:}

Temperature: $\pm 0.1 \mathrm{~K}$.

$x_{1}: \pm 1.5 \%$ (relative error).

\begin{tabular}{|c|c|}
\hline $\begin{array}{l}\text { Components: } \\
\text { (1) 2-Chloro-5-nitrobenzoic acid; } \\
\mathrm{C}_{7} \mathrm{H}_{4} \mathrm{ClNO}_{4} ;[2516-96-3] \\
\text { (2) } 1,2,3-\text { Triacetoxypropane } \\
\text { (Triacetin); } \mathrm{C}_{9} \mathrm{H}_{14} \mathrm{O}_{6} ;[102-76-1]\end{array}$ & $\begin{array}{l}\text { Original Measurements: } \\
\text { W. E. Acree, Jr., unpublished data. }\end{array}$ \\
\hline $\begin{array}{l}\text { Variables: } \\
T / \mathrm{K}=298.15\end{array}$ & $\begin{array}{l}\text { Prepared by: } \\
\text { W. E. Acree, Jr. }\end{array}$ \\
\hline
\end{tabular}

\section{Experimental Values}

\begin{tabular}{lc}
\hline \hline$x_{2}{ }^{\mathrm{a}}$ & $x_{1}{ }^{\mathrm{b}}$ \\
\hline 0.9170 & 0.08295 \\
\hline${ }^{\mathrm{a}} x_{2}:$ mole fraction of component 2 in the saturated solution. & \\
${ }^{\mathrm{b}} x_{1}:$ mole fraction solubility of the solute. &
\end{tabular}

\section{Auxiliary Information}

\section{Method/Apparatus/Procedure:}

Constant-temperature bath, calorimetric thermometer, and an ultraviolet/ visible spectrophotometer

Excess solute and solvent were placed in amber glass bottles and allowed to equilibrate for several days at constant temperature. Attainment of equilibrium was verified by several repetitive measurements and by approaching equilibrium from supersaturation. Aliquots of saturated solutions were transferred through a coarse filter into tared volumetric flasks, weighed, and diluted with 2-propanol. Concentrations were determined by spectrophotometric measurements at $280 \mathrm{~nm}$.

Source and Purity of Chemicals:

(1) $99+\%$, Acros Organics, USA, was used as received.

(2) $99 \%$, Acros Organics, USA, stored over molecular sieves before use.

\section{Estimated Error:}

Temperature: $\pm 0.1 \mathrm{~K}$.

$x_{1}: \pm 1.5 \%$ (relative error) 


\subsection{2-Chloro-5-nitrobenzoic acid solubility data in ethers}

\begin{tabular}{|c|c|}
\hline $\begin{array}{l}\text { Components: } \\
\text { (1) 2-Chloro-5-nitrobenzoic acid; } \\
\mathrm{C}_{7} \mathrm{H}_{4} \mathrm{ClNO}_{4} ; \text { [2516-96-3] } \\
\text { (2) 1,1'-Oxybisethane; } \mathrm{C}_{4} \mathrm{H}_{10} \mathrm{O} \text {; } \\
\text { [60-29-7] }\end{array}$ & $\begin{array}{l}\text { Original Measurements: } \\
{ }^{13} \text { D. M. Stovall, C. Givens, S. Keown, } \\
\text { K. R. Hoover, R. Barnes, C. Harris, J. } \\
\text { Lozano, M. Nguyen, E. Rodriguez, W. } \\
\text { E. Acree, Jr., and M. H. Abraham, } \\
\text { Phys. Chem. Liq. 43, } 351 \text { (2005). }\end{array}$ \\
\hline $\begin{array}{l}\text { Variables: } \\
T / \mathrm{K}=298.15\end{array}$ & $\begin{array}{l}\text { Prepared by: } \\
\text { W. E. Acree, Jr. }\end{array}$ \\
\hline
\end{tabular}

\section{Experimental Values}

\begin{tabular}{lc}
\hline \hline$x_{2}{ }^{\mathrm{a}}$ & $x_{1}{ }^{\mathrm{b}}$ \\
\hline 0.9415 & 0.05852 \\
\hline
\end{tabular}

${ }^{a} x_{2}$ : mole fraction of component 2 in the saturated solution.

${ }^{b} x_{1}$ : mole fraction solubility of the solute.

\section{Auxiliary Information}

\section{Method/Apparatus/Procedure:}

Constant-temperature bath, calorimetric thermometer, and an ultraviolet/ visible spectrophotometer.

Excess solute and solvent were placed in amber glass bottles and allowed to equilibrate for several days at constant temperature. Attainment of equilibrium was verified by several repetitive measurements and by approaching equilibrium from supersaturation. Aliquots of saturated solutions were transferred through a coarse filter into tared volumetric flasks, weighed, and diluted with methanol. Concentrations were determined by spectrophotometric measurements at $280 \mathrm{~nm}$.

\section{Source and Purity of Chemicals:}

(1) $99+\%$, Acros Organics, USA, was used as received.

(2) $99+\%$, anhydrous, Aldrich Chemical Company, Milwaukee, WI, USA, stored over molecular sieves and distilled shortly before use.

Estimated Error:

Temperature: $\pm 0.1 \mathrm{~K}$.

$x_{1}: \pm 1.5 \%$ (relative error).

\begin{tabular}{ll}
\hline \hline Components: & Original Measurements: \\
$\begin{array}{l}\text { (1) 2-Chloro-5-nitrobenzoic acid; } \\
\mathrm{C}_{7} \mathrm{H}_{4} \mathrm{ClNO}_{4} \text {; [2516-96-3] }\end{array}$ & ${ }^{13}$ D. M. Stovall, C. Givens, S. Keown, \\
$\begin{array}{l}\text { (2) } 2,2^{\prime}-\text {-Oxybispropane; } \mathrm{C}_{6} \mathrm{H}_{14} \mathrm{O} ; \\
{[108-20-3]}\end{array}$ & Lozano, M. Nguyen, R. Barnes, C. Harris, J. \\
& E. Acree, Jr., and M. H. Abraham, \\
& Phys. Chem. Liq. 43, 351 (2005). \\
\hline Variables: & Prepared by: \\
$T / \mathrm{K}=298.15$ & W. E. Acree, Jr. \\
\hline
\end{tabular}

\section{Experimental Values}

\begin{tabular}{lc}
\hline \hline$x_{2}{ }^{\mathrm{a}}$ & $x_{1}{ }^{\mathrm{b}}$ \\
\hline 0.9738 & 0.02621 \\
\hline${ }_{{ }_{2} x_{2}: \text { mole fraction of component } 2 \text { in the saturated solution. }}$ & \\
${ }^{\mathrm{b}} x_{1}:$ mole fraction solubility of the solute. &
\end{tabular}

\section{Auxiliary Information}

\section{Method/Apparatus/Procedure:}

Constant-temperature bath, calorimetric thermometer, and an ultraviolet/ visible spectrophotometer.

Excess solute and solvent were placed in amber glass bottles and allowed to equilibrate for several days at constant temperature. Attainment of equilibrium was verified by several repetitive measurements and by approaching equilibrium from supersaturation. Aliquots of saturated solutions were transferred through a coarse filter into tared volumetric flasks, weighed, and diluted with methanol. Concentrations were determined by spectrophotometric measurements at $280 \mathrm{~nm}$.

Source and Purity of Chemicals:

(1) $99+\%$, Acros Organics, USA, was used as received.

(2) $99 \%$, Aldrich Chemical Company, Milwaukee, WI, USA, stored over molecular sieves and distilled shortly before use.

\section{Estimated Error:}

Temperature: $\pm 0.1 \mathrm{~K}$.

$x_{1}: \pm 1.5 \%$ (relative error)

\begin{tabular}{ll}
\hline \hline Components: & Original Measurements: \\
(1) 2-Chloro-5-nitrobenzoic acid; & ${ }^{13}$ D. M. Stovall, C. Givens, S. Keown, \\
$\mathrm{C}_{7} \mathrm{H}_{4} \mathrm{ClNO}_{4}$; [2516-96-3] & K. R. Hoover, R. Barnes, C. Harris, J. \\
$\begin{array}{l}\text { (2) } 1,1^{\prime}-\text {-Oxybisbutane; } \mathrm{C}_{8} \mathrm{H}_{18} \mathrm{O} ; \\
\text { [142-96-1] }\end{array}$ & Lozano, M. Nguyen, E. Rodriguez, W. \\
& E. Acree, Jr., and M. H. Abraham, \\
& Phys. Chem. Liq. 43, 351 (2005). \\
\hline Variables: & Prepared by: \\
$T / \mathrm{K}=298.15$ & W. E. Acree, Jr.
\end{tabular}

\section{Experimental Values}

\begin{tabular}{lc}
\hline \hline$x_{2}{ }^{\mathrm{a}}$ & $x_{1}{ }^{\mathrm{b}}$ \\
\hline 0.9837 & 0.01630 \\
\hline${ }^{\mathrm{a}} x_{2}:$ mole fraction of component 2 in the saturated solution. & \\
${ }^{\mathrm{b}} x_{1}:$ mole fraction solubility of the solute. &
\end{tabular}

\section{Auxiliary Information}

\section{Method/Apparatus/Procedure:}

Constant-temperature bath, calorimetric thermometer, and an ultraviolet/ visible spectrophotometer.

Excess solute and solvent were placed in amber glass bottles and allowed to equilibrate for several days at constant temperature. Attainment of equilibrium was verified by several repetitive measurements and by approaching equilibrium from supersaturation. Aliquots of saturated solutions were transferred through a coarse filter into tared volumetric flasks, weighed, and diluted with methanol. Concentrations were determined by spectrophotometric measurements at $280 \mathrm{~nm}$.

\section{Source and Purity of Chemicals:}

(1) $99+\%$, Acros Organics, USA, was used as received.

(2) $99.3 \%$, anhydrous, Aldrich Chemical Company, Milwaukee, WI, USA, stored over molecular sieves and distilled shortly before use.

\section{Estimated Error:}

Temperature: $\pm 0.1 \mathrm{~K}$.

$x_{1}: \pm 1.5 \%$ (relative error). 


\section{Components:}

(1) 2-Chloro-5-nitrobenzoic acid;

$\mathrm{C}_{7} \mathrm{H}_{4} \mathrm{ClNO}_{4}$; [2516-96-3]

(2) Tetrahydrofuran; $\mathrm{C}_{4} \mathrm{H}_{8} \mathrm{O}$; [109-99-9]

\section{Variables:}

$T / \mathrm{K}=298.15$

Original Measurements:

${ }^{13}$ D. M. Stovall, C. Givens, S. Keown,

K. R. Hoover, R. Barnes, C. Harris, J.

Lozano, M. Nguyen, E. Rodriguez, W.

E. Acree, Jr., and M. H. Abraham,

Phys. Chem. Liq. 43, 351 (2005).

Prepared by:

W. E. Acree, Jr.

Experimental Values

\begin{tabular}{lc}
\hline \hline$x_{2}{ }^{\mathrm{a}}$ & $x_{1}{ }^{\mathrm{b}}$ \\
\hline 0.7256 & 0.2744
\end{tabular}

${ }^{a} x_{2}$ : mole fraction of component 2 in the saturated solution.

${ }^{\mathrm{b}} \mathrm{x}_{1}$ : mole fraction solubility of the solute.

\section{Auxiliary Information}

\section{Method/Apparatus/Procedure:}

Constant-temperature bath, calorimetric thermometer, and an ultraviolet/ visible spectrophotometer.

Excess solute and solvent were placed in amber glass bottles and allowed to equilibrate for several days at constant temperature. Attainment of equilibrium was verified by several repetitive measurements and by approaching equilibrium from supersaturation. Aliquots of saturated solutions were transferred through a coarse filter into tared volumetric flasks, weighed, and diluted with methanol. Concentrations were determined by

spectrophotometric measurements at $280 \mathrm{~nm}$.

Source and Purity of Chemicals:

(1) $99+\%$, Acros Organics, USA, was used as received.

(2) $99.9 \%$, anhydrous, Aldrich Chemical Company, Milwaukee, WI, USA, stored over molecular sieves and distilled shortly before use.

Estimated Error:

Temperature: $\pm 0.1 \mathrm{~K}$.

$x_{1}: \pm 1.5 \%$ (relative error)
Auxiliary Information

\section{Method/Apparatus/Procedure:}

Constant-temperature bath, calorimetric thermometer, and an ultraviolet/ visible spectrophotometer.

Excess solute and solvent were placed in amber glass bottles and allowed to equilibrate for several days at constant temperature. Attainment of equilibrium was verified by several repetitive measurements and by approaching equilibrium from supersaturation. Aliquots of saturated solutions were transferred through a coarse filter into tared volumetric flasks, weighed, and diluted with methanol. Concentrations were determined by spectrophotometric measurements at $280 \mathrm{~nm}$.

\section{Source and Purity of Chemicals:}

(1) $99+\%$, Acros Organics, USA, was used as received.

(2) Absolute, Aaper Alcohol and Chemical Company, USA, stored over molecular sieves and distilled shortly before use.

\section{Estimated Error:}

Temperature: $\pm 0.1 \mathrm{~K}$.

$x_{1}: \pm 1.5 \%$ (relative error)

\begin{tabular}{ll}
\hline \hline Components: & Original Measurements: \\
(1) 2-Chloro-5-nitrobenzoic acid; & ${ }^{13}$ D. M. Stovall, C. Givens, S. Keown \\
$\mathrm{C}_{7} \mathrm{H}_{4} \mathrm{ClNO}_{4} ;$ [2516-96-3] & K. R. Hoover, R. Barnes, C. Harris, J. \\
(2) 1-Propanol; $\mathrm{C}_{3} \mathrm{H}_{8} \mathrm{O}$; [71-23-8] & Lozano, M. Nguyen, E. Rodriguez, W \\
& E. Acree, Jr., and M. H. Abraham, \\
& Phys. Chem. Liq. 43, 351 (2005). \\
\hline Variables: & Prepared by: \\
$T / \mathrm{K}=298.15$ & W. E. Acree, Jr. \\
\hline
\end{tabular}

\section{Experimental Values}

\begin{tabular}{lc}
\hline \hline$x_{2}{ }^{\mathrm{a}}$ & $x_{1}{ }^{\mathrm{b}}$ \\
\hline 0.9245 & 0.07550
\end{tabular}

${ }^{\mathrm{a}} x_{2}$ : mole fraction of component 2 in the saturated solution.

${ }^{\mathrm{b}} x_{1}$ : mole fraction solubility of the solute.

\section{Auxiliary Information}

\section{Method/Apparatus/Procedure:}

Constant-temperature bath, calorimetric thermometer, and an ultraviolet/ visible spectrophotometer.

Excess solute and solvent were placed in amber glass bottles and allowed to equilibrate for several days at constant temperature. Attainment of equilibrium was verified by several repetitive measurements and by approaching equilibrium from supersaturation. Aliquots of saturated solutions were transferred through a coarse filter into tared volumetric flasks, weighed, and diluted with methanol. Concentrations were determined by spectrophotometric measurements at $280 \mathrm{~nm}$.

\section{Source and Purity of Chemicals:}

(1) $99+\%$, Acros Organics, USA, was used as received.

(2) $99+\%$, anhydrous, Aldrich Chemical Company, Milwaukee, WI, USA, stored over molecular sieves and distilled shortly before use.

\section{Estimated Error:}

Temperature: $\pm 0.1 \mathrm{~K}$.

$x_{1}: \pm 1.5 \%$ (relative error) 


\section{Components:}

(1) 2-Chloro-5-nitrobenzoic acid;

$\mathrm{C}_{7} \mathrm{H}_{4} \mathrm{ClNO}_{4}$; [2516-96-3]

(2) 2-Propanol; $\mathrm{C}_{3} \mathrm{H}_{8} \mathrm{O}$; [67-63-0]

Original Measurements:

${ }^{13}$ D. M. Stovall, C. Givens, S. Keown,

K. R. Hoover, R. Barnes, C. Harris, J.

Lozano, M. Nguyen, E. Rodriguez, W.

E. Acree, Jr., and M. H. Abraham,

Phys. Chem. Liq. 43, 351 (2005).

\begin{tabular}{ll}
\hline Variables: & Prepared by: \\
$T / \mathrm{K}=298.15$ & W. E. Acree, Jr.
\end{tabular}

Experimental Values

\begin{tabular}{lc}
\hline \hline$x_{2}{ }^{\mathrm{a}}$ & $x_{1}{ }^{\mathrm{b}}$ \\
\hline 0.9025 & 0.09750
\end{tabular}

${ }^{a} x_{2}$ : mole fraction of component 2 in the saturated solution.

${ }^{\mathrm{b}} x_{1}$ : mole fraction solubility of the solute.

\section{Auxiliary Information}

\section{Method/Apparatus/Procedure:}

Constant-temperature bath, calorimetric thermometer, and an ultraviolet/ visible spectrophotometer.

Excess solute and solvent were placed in amber glass bottles and allowed to equilibrate for several days at constant temperature. Attainment of equilibrium was verified by several repetitive measurements and by approaching equilibrium from supersaturation. Aliquots of saturated solutions were transferred through a coarse filter into tared volumetric flasks, weighed, and diluted with methanol. Concentrations were determined by spectrophotometric measurements at $280 \mathrm{~nm}$.

\section{Source and Purity of Chemicals:}

(1) $99+\%$, Acros Organics, USA, was used as received.

(2) $99+\%$, anhydrous, Aldrich Chemical Company, Milwaukee, WI, USA, stored over molecular sieves and distilled shortly before use.

\section{Estimated Error:}

Temperature: $\pm 0.1 \mathrm{~K}$

$x_{1}: \pm 1.5 \%$ (relative error).

\begin{tabular}{|c|c|}
\hline $\begin{array}{l}\text { Components: } \\
\text { (1) 2-Chloro-5-nitrobenzoic acid; } \\
\mathrm{C}_{7} \mathrm{H}_{4} \mathrm{ClNO}_{4} \text {; [2516-96-3] } \\
\text { (2) } 1 \text {-Butanol; } \mathrm{C}_{4} \mathrm{H}_{10} \mathrm{O} \text {; [71-36-3] }\end{array}$ & $\begin{array}{l}\text { Original Measurements: } \\
{ }^{13} \text { D. M. Stovall, C. Givens, S. Keown, } \\
\text { K. R. Hoover, R. Barnes, C. Harris, J. } \\
\text { Lozano, M. Nguyen, E. Rodriguez, W. } \\
\text { E. Acree, Jr., and M. H. Abraham, } \\
\text { Phys. Chem. Liq. 43, } 351 \text { (2005). }\end{array}$ \\
\hline $\begin{array}{l}\text { Variables: } \\
T / \mathrm{K}=298.15\end{array}$ & $\begin{array}{l}\text { Prepared by: } \\
\text { W. E. Acree, Jr. }\end{array}$ \\
\hline
\end{tabular}

Experimental Values

\begin{tabular}{lc}
\hline \hline$x_{2}{ }^{\mathrm{a}}$ & $x_{1}{ }^{\mathrm{b}}$ \\
\hline 0.9265 & 0.07350 \\
\hline
\end{tabular}

\section{Auxiliary Information}

\section{Method/Apparatus/Procedure:}

Constant-temperature bath, calorimetric thermometer, and an ultraviolet/ visible spectrophotometer.

Excess solute and solvent were placed in amber glass bottles and allowed to equilibrate for several days at constant temperature. Attainment of equilibrium was verified by several repetitive measurements and by approaching equilibrium from supersaturation. Aliquots of saturated solutions were transferred through a coarse filter into tared volumetric flasks, weighed, and diluted with methanol. Concentrations were determined by spectrophotometric measurements at $280 \mathrm{~nm}$.

\section{Source and Purity of Chemicals:}

(1) $99+\%$, Acros Organics, USA, was used as received.

(2) $99.8+\%$, HPLC grade, Aldrich Chemical Company, Milwaukee, WI, USA, stored over molecular sieves and distilled shortly before use.

\section{Estimated Error:}

Temperature: $\pm 0.1 \mathrm{~K}$.

$x_{1}: \pm 1.5 \%$ (relative error)

\begin{tabular}{ll}
\hline \hline Components: & Original Measurements: \\
(1) 2-Chloro-5-nitrobenzoic acid; & ${ }^{13}$ D. M. Stovall, C. Givens, S. Keown, \\
$\mathrm{C}_{7} \mathrm{H}_{4} \mathrm{ClNO}_{4}$; [2516-96-3] & K. R. Hoover, R. Barnes, C. Harris, J. \\
(2) 2-Butanol; $\mathrm{C}_{4} \mathrm{H}_{10} \mathrm{O} ;$ [78-92-2] & Lozano, M. Nguyen, E. Rodriguez, W. \\
& E. Acree, Jr., and M. H. Abraham, \\
& Phys. Chem. Liq. 43, 351 (2005). \\
\hline Variables: & Prepared by: \\
$T / \mathrm{K}=298.15$ & W. E. Acree, Jr. \\
\hline
\end{tabular}

Experimental Values

\begin{tabular}{lc}
\hline \hline$x_{2}{ }^{\mathrm{a}}$ & $x_{1}{ }^{\mathrm{b}}$ \\
\hline 0.9131 & 0.08685 \\
\hline${ }^{\mathrm{a}} x_{2}:$ mole fraction of component 2 in the saturated solution. & \\
${ }^{\mathrm{b}} x_{1}:$ mole fraction solubility of the solute. &
\end{tabular}

${ }^{\mathrm{b}} x_{1}$ : mole fraction solubility of the solute.

\section{Auxiliary Information}

\section{Method/Apparatus/Procedure:}

Constant-temperature bath, calorimetric thermometer, and an ultraviolet/ visible spectrophotometer.

Excess solute and solvent were placed in amber glass bottles and allowed to equilibrate for several days at constant temperature. Attainment of equilibrium was verified by several repetitive measurements and by approaching equilibrium from supersaturation. Aliquots of saturated solutions were transferred through a coarse filter into tared volumetric flasks, weighed, and diluted with methanol. Concentrations were determined by spectrophotometric measurements at $280 \mathrm{~nm}$.

\section{Source and Purity of Chemicals:}

(1) $99+\%$, Acros Organics, USA, was used as received.

(2) $99+\%$, anhydrous, Aldrich Chemical Company, Milwaukee, WI, USA, stored over molecular sieves and distilled shortly before use.

\section{Estimated Error:}

Temperature: $\pm 0.1 \mathrm{~K}$.

$x_{1}: \pm 1.5 \%$ (relative error). 


\section{Components:}

(1) 2-Chloro-5-nitrobenzoic acid;

$\mathrm{C}_{7} \mathrm{H}_{4} \mathrm{ClNO}_{4}$; [2516-96-3]

(2) 2-Methyl-1-propanol; $\mathrm{C}_{4} \mathrm{H}_{10} \mathrm{O} ;$; $\left.78-83-1\right]$

\section{Variables:}

$T / \mathrm{K}=298.15$

Original Measurements:

${ }^{13}$ D. M. Stovall, C. Givens, S. Keown,

K. R. Hoover, R. Barnes, C. Harris, J.

Lozano, M. Nguyen, E. Rodriguez, W.

E. Acree, Jr., and M. H. Abraham,

Phys. Chem. Liq. 43, 351 (2005).

Prepared by:

W. E. Acree, Jr.

Experimental Values

\begin{tabular}{lc}
\hline \hline$x_{2}{ }^{\mathrm{a}}$ & $x_{1}{ }^{\mathrm{b}}$ \\
\hline 0.9405 & 0.05947
\end{tabular}

${ }^{\mathrm{a}} x_{2}$ : mole fraction of component 2 in the saturated solution.

${ }^{\mathrm{b}} x_{1}$ : mole fraction solubility of the solute.

\section{Auxiliary Information}

\section{Method/Apparatus/Procedure:}

Constant-temperature bath, calorimetric thermometer, and an ultraviolet/ visible spectrophotometer.

Excess solute and solvent were placed in amber glass bottles and allowed to equilibrate for several days at constant temperature. Attainment of equilibrium was verified by several repetitive measurements and by approaching equilibrium from supersaturation. Aliquots of saturated solutions were transferred through a coarse filter into tared volumetric flasks, weighed, and diluted with methanol. Concentrations were determined by

spectrophotometric measurements at $280 \mathrm{~nm}$.

\section{Source and Purity of Chemicals:}

(1) $99+\%$, Acros Organics, USA, was used as received.

(2) $99+\%$, anhydrous, Aldrich Chemical Company, Milwaukee, WI, USA, stored over molecular sieves and distilled shortly before use.

Estimated Error:

Temperature: $\pm 0.1 \mathrm{~K}$.

$x_{1}: \pm 1.5 \%$ (relative error).

\begin{tabular}{ll}
\hline \hline Components: & Original Measurements: \\
(1) 2-Chloro-5-nitrobenzoic acid; & ${ }^{13}$ D. M. Stovall, C. Givens, S. Keown, \\
$\mathrm{C}_{7} \mathrm{H}_{4} \mathrm{ClNO}_{4}$; [2516-96-3] & K. R. Hoover, R. Barnes, C. Harris, J. \\
(2) 2-Methyl-2-propanol; $\mathrm{C}_{4} \mathrm{H}_{10} \mathrm{O} ;$ & Lozano, M. Nguyen, E. Rodriguez, W. \\
[75-65-0] & E. Acree, Jr., and M. H. Abraham, \\
& Phys. Chem. Liq. 43, 351 (2005). \\
\hline Variables: & Prepared by: \\
$T / \mathrm{K}=298.15$ & W. E. Acree, Jr. \\
\hline
\end{tabular}

\section{Experimental Values}

\begin{tabular}{lc}
\hline \hline$x_{2}{ }^{\mathrm{a}}$ & $x_{1}{ }^{\mathrm{b}}$ \\
\hline 0.8842 & 0.1158
\end{tabular}

${ }^{\mathrm{a}} x_{2}$ : mole fraction of component 2 in the saturated solution.

${ }^{\mathrm{b}} x_{1}$ : mole fraction solubility of the solute.

\section{Auxiliary Information}

\section{Method/Apparatus/Procedure:}

Constant-temperature bath, calorimetric thermometer, and an ultraviolet/ visible spectrophotometer.

Excess solute and solvent were placed in amber glass bottles and allowed to equilibrate for several days at constant temperature. Attainment of equilibrium was verified by several repetitive measurements and by approaching equilibrium from supersaturation. Aliquots of saturated solutions were transferred through a coarse filter into tared volumetric flasks, weighed, and diluted with methanol. Concentrations were determined by spectrophotometric measurements at $280 \mathrm{~nm}$.

\section{Source and Purity of Chemicals:}

(1) $99+\%$, Acros Organics, USA, was used as received.

(2) $99+\%$, Arco Chemical Company, USA, stored over molecular sieves and distilled shortly before use.

\section{Estimated Error:}

Temperature: $\pm 0.1 \mathrm{~K}$.

$x_{1}: \pm 1.5 \%$ (relative error).

\begin{tabular}{ll}
\hline \hline Components: & Original Measurements: \\
(1) 2-Chloro-5-nitrobenzoic acid; & ${ }^{13}$ D. M. Stovall, C. Givens, S. Keown, \\
$\mathrm{C}_{7} \mathrm{H}_{4} \mathrm{ClNO}_{4}$; [2516-96-3] & K. R. Hoover, R. Barnes, C. Harris, J. \\
(2) 1-Pentanol; $\mathrm{C}_{5} \mathrm{H}_{12} \mathrm{O} ;$ [71-41-0] & Lozano, M. Nguyen, E. Rodriguez, W. \\
& E. Acree, Jr., and M. H. Abraham, \\
& Phys. Chem. Liq. 43, 351 (2005). \\
\hline Variables: & Prepared by: \\
$T / \mathrm{K}=298.15$ & W. E. Acree, Jr. \\
\hline
\end{tabular}

Experimental Values

\begin{tabular}{lc}
\hline \hline$x_{2}{ }^{\mathrm{a}}$ & $x_{1}{ }^{\mathrm{b}}$ \\
\hline 0.9189 & 0.08109 \\
\hline
\end{tabular}

\section{Auxiliary Information}

\section{Method/Apparatus/Procedure:}

Constant-temperature bath, calorimetric thermometer, and an ultraviolet/ visible spectrophotometer.

Excess solute and solvent were placed in amber glass bottles and allowed to equilibrate for several days at constant temperature. Attainment of equilibrium was verified by several repetitive measurements and by approaching equilibrium from supersaturation. Aliquots of saturated solutions were transferred through a coarse filter into tared volumetric flasks, weighed, and diluted with methanol. Concentrations were determined by spectrophotometric measurements at $280 \mathrm{~nm}$.

\section{Source and Purity of Chemicals:}

(1) $99+\%$, Acros Organics, USA, was used as received.

(2) $99+\%$, Aldrich Chemical Company, Milwaukee, WI, USA, stored over molecular sieves and distilled shortly before use.

\section{Estimated Error:}

Temperature: $\pm 0.1 \mathrm{~K}$.

$x_{1}: \pm 1.5 \%$ (relative error). 


\section{Components:}

(1) 2-Chloro-5-nitrobenzoic acid;

$\mathrm{C}_{7} \mathrm{H}_{4} \mathrm{ClNO}_{4}$; [2516-96-3]

(2) 2-Pentanol; $\mathrm{C}_{5} \mathrm{H}_{12} \mathrm{O}$;

[6032-29-7]

\section{Variables:}

$T / \mathrm{K}=298.15$

Original Measurements:

${ }^{13}$ D. M. Stovall, C. Givens, S. Keown,

K. R. Hoover, R. Barnes, C. Harris, J.

Lozano, M. Nguyen, E. Rodriguez, W.

E. Acree, Jr., and M. H. Abraham,

Phys. Chem. Liq. 43, 351 (2005).

Prepared by:

W. E. Acree, Jr.

Experimental Values

\begin{tabular}{lc}
\hline \hline$x_{2}{ }^{\mathrm{a}}$ & $x_{1}{ }^{\mathrm{b}}$ \\
\hline 0.9165 & 0.08349
\end{tabular}

${ }^{\mathrm{a}} x_{2}$ : mole fraction of component 2 in the saturated solution.

${ }^{\mathrm{b}} x_{1}$ : mole fraction solubility of the solute.

\section{Auxiliary Information}

\section{Method/Apparatus/Procedure:}

Constant-temperature bath, calorimetric thermometer, and an ultraviolet/ visible spectrophotometer.

Excess solute and solvent were placed in amber glass bottles and allowed to equilibrate for several days at constant temperature. Attainment of equilibrium was verified by several repetitive measurements and by approaching equilibrium from supersaturation. Aliquots of saturated solutions were transferred through a coarse filter into tared volumetric flasks, weighed, and diluted with methanol. Concentrations were determined by spectrophotometric measurements at $280 \mathrm{~nm}$.

\section{Source and Purity of Chemicals:}

(1) $99+\%$, Acros Organics, USA, was used as received.

(2) $99+\%$, Acros Organics, stored over molecular sieves and distilled shortly before use.

Estimated Error:

Temperature: $\pm 0.1 \mathrm{~K}$.

$x_{1}: \pm 1.5 \%$ (relative error).

\begin{tabular}{ll}
\hline \hline Components: & Original Measurements: \\
$\begin{array}{l}\text { (1) 2-Chloro-5-nitrobenzoic acid; } \\
\mathrm{C}_{7} \mathrm{H}_{4} \mathrm{ClNO}_{4} \text {; [2516-96-3] }\end{array}$ & K. M. R. Stovall, C. Givens, S. Keown, \\
$\begin{array}{l}\text { (2) 3-Methyl-1-butanol; } \mathrm{C}_{5} \mathrm{H}_{12} \mathrm{O} ; \\
\text { [123-51-3] }\end{array}$ & Lozano, M. Nguyen, E. Rodriguez, W. \\
& E. Acree, Jr., and M. H. Abraham, \\
& Phys. Chem. Liq. 43, 351 (2005). \\
\hline Variables: & Prepared by: \\
$T / \mathrm{K}=298.15$ & W. E. Acree, Jr. \\
\hline
\end{tabular}

\section{Experimental Values}

\begin{tabular}{lc}
\hline \hline$x_{2}^{\mathrm{a}}$ & $x_{1}{ }^{\mathrm{b}}$ \\
\hline 0.9252 & 0.07483
\end{tabular}

${ }^{\mathrm{a}} x_{2}$ : mole fraction of component 2 in the saturated solution.

${ }^{\mathrm{b}} x_{1}$ : mole fraction solubility of the solute.

\section{Auxiliary Information}

Method/Apparatus/Procedure:

Constant-temperature bath, calorimetric thermometer, and an ultraviolet/ visible spectrophotometer.

Excess solute and solvent were placed in amber glass bottles and allowed to equilibrate for several days at constant temperature. Attainment of equilibrium was verified by several repetitive measurements and by approaching equilibrium from supersaturation. Aliquots of saturated solutions were transferred through a coarse filter into tared volumetric flasks, weighed, and diluted with methanol. Concentrations were determined by spectrophotometric measurements at $280 \mathrm{~nm}$.

\section{Source and Purity of Chemicals:}

(1) $99+\%$, Acros Organics, USA, was used as received.

(2) $99 \%$, anhydrous, Aldrich Chemical Company, Milwaukee, WI, USA, stored over molecular sieves and distilled shortly before use.

\section{Estimated Error:}

Temperature: $\pm 0.1 \mathrm{~K}$.

$x_{1}: \pm 1.5 \%$ (relative error).

\begin{tabular}{ll}
\hline \hline Components: & Original Measurements: \\
(1) 2-Chloro-5-nitrobenzoic acid; & ${ }^{13}$ D. M. Stovall, C. Givens, S. Keown, \\
$\mathrm{C}_{7} \mathrm{H}_{4} \mathrm{ClNO}_{4} ;$ [2516-96-3] & K. R. Hoover, R. Barnes, C. Harris, J. \\
(2) 1-Hexanol; $\mathrm{C}_{6} \mathrm{H}_{14} \mathrm{O}$; [111-27-3] & Lozano, M. Nguyen, E. Rodriguez, W. \\
& E. Acree, Jr., and M. H. Abraham, \\
& Phys. Chem. Liq. 43, 351 (2005). \\
\hline Variables: & Prepared by: \\
$T / \mathrm{K}=298.15$ & W. E. Acree, Jr. \\
\hline
\end{tabular}

Experimental Values

\begin{tabular}{lc}
\hline \hline$x_{2}{ }^{\mathrm{a}}$ & $x_{1}{ }^{\mathrm{b}}$ \\
\hline 0.9202 & 0.07980 \\
\hline${ }^{\mathrm{a}} x_{2}:$ mole fraction of component 2 in the saturated solution. & \\
${ }^{\mathrm{b}} x_{1}:$ mole fraction solubility of the solute. &
\end{tabular}

${ }^{\mathrm{b}} x_{1}$ : mole fraction solubility of the solute.

\section{Auxiliary Information}

\section{Method/Apparatus/Procedure:}

Constant-temperature bath, calorimetric thermometer, and an ultraviolet/ visible spectrophotometer.

Excess solute and solvent were placed in amber glass bottles and allowed to equilibrate for several days at constant temperature. Attainment of equilibrium was verified by several repetitive measurements and by approaching equilibrium from supersaturation. Aliquots of saturated solutions were transferred through a coarse filter into tared volumetric flasks, weighed, and diluted with methanol. Concentrations were determined by spectrophotometric measurements at $280 \mathrm{~nm}$.

\section{Source and Purity of Chemicals:}

(1) $99+\%$, Acros Organics, USA, was used as received.

(2) $99+\%$, Alfa Aesar, USA, stored over molecular sieves and distilled shortly before use.

\section{Estimated Error:}

Temperature: $\pm 0.1 \mathrm{~K}$.

$x_{1}: \pm 1.5 \%$ (relative error). 


\section{Components:}

(1) 2-Chloro-5-nitrobenzoic acid;

$\mathrm{C}_{7} \mathrm{H}_{4} \mathrm{ClNO}_{4}$; [2516-96-3]

(2) 1-Heptanol; $\mathrm{C}_{7} \mathrm{H}_{16} \mathrm{O}$;

[111-70-6]

\section{Variables:}

$T / \mathrm{K}=298.15$

Original Measurements:

${ }^{13}$ D. M. Stovall, C. Givens, S. Keown,

K. R. Hoover, R. Barnes, C. Harris, J.

Lozano, M. Nguyen, E. Rodriguez, W.

E. Acree, Jr., and M. H. Abraham,

Phys. Chem. Liq. 43, 351 (2005).

Prepared by:

W. E. Acree, Jr.

Experimental Values

\begin{tabular}{lc}
\hline \hline$x_{2}{ }^{\mathrm{a}}$ & $x_{1}{ }^{\mathrm{b}}$ \\
\hline 0.9242 & 0.07584
\end{tabular}

${ }^{\mathrm{a}} x_{2}$ : mole fraction of component 2 in the saturated solution.

${ }^{\mathrm{b}} x_{1}$ : mole fraction solubility of the solute.

\section{Auxiliary Information}

\section{Method/Apparatus/Procedure:}

Constant-temperature bath, calorimetric thermometer, and an ultraviolet/ visible spectrophotometer.

Excess solute and solvent were placed in amber glass bottles and allowed to equilibrate for several days at constant temperature. Attainment of equilibrium was verified by several repetitive measurements and by approaching equilibrium from supersaturation. Aliquots of saturated solutions were transferred through a coarse filter into tared volumetric flasks, weighed, and diluted with methanol. Concentrations were determined by spectrophotometric measurements at $280 \mathrm{~nm}$.

\section{Source and Purity of Chemicals:}

(1) $99+\%$, Acros Organics, USA, was used as received.

(2) $99+\%$, Alfa Aesar, USA, stored over molecular sieves and distilled shortly before use.

Estimated Error:

Temperature: $\pm 0.1 \mathrm{~K}$.

$x_{1}: \pm 1.5 \%$ (relative error).

\begin{tabular}{|c|c|}
\hline $\begin{array}{l}\text { Components: } \\
\text { (1) 2-Chloro-5-nitrobenzoic acid; } \\
\mathrm{C}_{7} \mathrm{H}_{4} \mathrm{ClNO}_{4} ;[2516-96-3] \\
\text { (2) 1-Octanol; } \mathrm{C}_{8} \mathrm{H}_{18} \mathrm{O} ; \\
\text { [111-87-5] }\end{array}$ & $\begin{array}{l}\text { Original Measurements: } \\
{ }^{13} \text { D. M. Stovall, C. Givens, S. Keown, } \\
\text { K. R. Hoover, R. Barnes, C. Harris, J. } \\
\text { Lozano, M. Nguyen, E. Rodriguez, W. } \\
\text { E. Acree, Jr., and M. H. Abraham, } \\
\text { Phys. Chem. Liq. 43, } 351 \text { (2005). }\end{array}$ \\
\hline $\begin{array}{l}\text { Variables: } \\
T / \mathrm{K}=298.15\end{array}$ & $\begin{array}{l}\text { Prepared by: } \\
\text { W. E. Acree, Jr. }\end{array}$ \\
\hline
\end{tabular}

\section{Experimental Values}

\begin{tabular}{lc}
\hline \hline$x_{2}^{\mathrm{a}}$ & $x_{1}^{\mathrm{b}}$ \\
\hline 0.9259 & 0.07409
\end{tabular}

${ }^{\mathrm{a}} x_{2}$ : mole fraction of component 2 in the saturated solution.

${ }^{b} x_{1}$ : mole fraction solubility of the solute.

\section{Auxiliary Information}

\section{Method/Apparatus/Procedure:}

Constant-temperature bath, calorimetric thermometer, and an ultraviolet/ visible spectrophotometer.

Excess solute and solvent were placed in amber glass bottles and allowed to equilibrate for several days at constant temperature. Attainment of equilibrium was verified by several repetitive measurements and by approaching equilibrium from supersaturation. Aliquots of saturated solutions were transferred through a coarse filter into tared volumetric flasks, weighed, and diluted with methanol. Concentrations were determined by spectrophotometric measurements at $280 \mathrm{~nm}$.

\section{Source and Purity of Chemicals:}

(1) $99+\%$, Acros Organics, USA, was used as received.

(2) $99+\%$, anhydrous, Aldrich Chemical Company, Milwaukee, WI, USA, stored over molecular sieves and distilled shortly before use.

\section{Estimated Error:}

Temperature: $\pm 0.1 \mathrm{~K}$.

$x_{1}: \pm 1.5 \%$ (relative error)

\begin{tabular}{ll}
\hline \hline Components: & Original Measurements: \\
(1) 2-Chloro-5-nitrobenzoic acid; & ${ }^{13}$ D. M. Stovall, C. Givens, S. Keown, \\
$\mathrm{C}_{7} \mathrm{H}_{4} \mathrm{ClNO}_{4} ;$ [2516-96-3] & K. R. Hoover, R. Barnes, C. Harris, J. \\
$(2)$ 1-Decanol; $\mathrm{C}_{10} \mathrm{H}_{22} \mathrm{O} ;$ & Lozano, M. Nguyen, E. Rodriguez, W. \\
[112-30-1] & E. Acree, Jr., and M. H. Abraham, \\
& Phys. Chem. Liq. 43, 351 (2005). \\
\hline Variables: & Prepared by: \\
$T / \mathrm{K}=298.15$ & W. E. Acree, Jr. \\
\hline
\end{tabular}

\section{Experimental Values}

\begin{tabular}{lc}
\hline \hline$x_{2}{ }^{\mathrm{a}}$ & $x_{1}{ }^{\mathrm{b}}$ \\
\hline 0.9274 & 0.07261 \\
\hline${ }^{\mathrm{a}} x_{2}:$ mole fraction of component 2 in the saturated solution. & \\
${ }^{\mathrm{b}} x_{1}:$ mole fraction solubility of the solute. &
\end{tabular}

${ }^{\mathrm{b}} x_{1}$ : mole fraction solubility of the solute.

\section{Auxiliary Information}

\section{Method/Apparatus/Procedure:}

Constant-temperature bath, calorimetric thermometer, and an ultraviolet/ visible spectrophotometer.

Excess solute and solvent were placed in amber glass bottles and allowed to equilibrate for several days at constant temperature. Attainment of equilibrium was verified by several repetitive measurements and by approaching equilibrium from supersaturation. Aliquots of saturated solutions were transferred through a coarse filter into tared volumetric flasks, weighed, and diluted with methanol. Concentrations were determined by spectrophotometric measurements at $280 \mathrm{~nm}$.

\section{Source and Purity of Chemicals:}

(1) $99+\%$, Acros Organics, USA, was used as received.

(2) $99+\%$, Alfa Aesar, USA, stored over molecular sieves and distilled shortly before use.

\section{Estimated Error:}

Temperature: $\pm 0.1 \mathrm{~K}$.

$x_{1}: \pm 1.5 \%$ (relative error). 


\section{Solubility of 4-Chloro-3,5- dinitrobenzoic Acid in Organic Solvents}

\subsection{Critical evaluation of experimental solubility data}

There has been only a single publication reporting the solubility of 4-chloro-3,5-dinitrobenzoic acid in organic solvents. Chantooni and Kolthoff ${ }^{83}$ determined the solubility of 4-chloro-3,5-dinitrobenzoic acid in methanol and ethanenitrile at $298 \mathrm{~K}$. It is not possible to perform a critical evaluation of the experimental data as measurements at only a single temperature, and there are no independent experimental solubility data for 4-chloro-3,5-dinitrobenzoic acid in either methanol or ethanenitrile.

The experimental solubility data for 4-chloro-3,5-dinitrobenzoic acid in organic solvents are in Secs. 22.2 and 22.3.

\subsection{4-Chloro-3,5-dinitrobenzoic acid solubility data in alcohols}

\begin{tabular}{|c|c|}
\hline $\begin{array}{l}\text { Components: } \\
\text { (1) 4-Chloro-3,5-dinitrobenzoic } \\
\text { acid; } \mathrm{C}_{7} \mathrm{H}_{3} \mathrm{ClN}_{2} \mathrm{O}_{6} ;[118-97-8] \\
\text { (2) Methanol; } \mathrm{CH}_{4} \mathrm{O} ;[67-56-1]\end{array}$ & $\begin{array}{l}\text { Original Measurements: } \\
{ }^{83} \text { M. K. Chantooni and I. M. Kolthoff, } \\
\text { J. Phys. Chem. 77, } 527 \text { (1973). }\end{array}$ \\
\hline $\begin{array}{l}\text { Variables: } \\
T / \mathrm{K}=298.15\end{array}$ & $\begin{array}{l}\text { Prepared by: } \\
\text { W. E. Acree, Jr. }\end{array}$ \\
\hline
\end{tabular}

Experimental Values

The measured solubility was reported to be $1.55 \mathrm{~mol} \mathrm{dm}^{-3}$. The authors did not give the temperature at which the solubility was measured. Based on the experimental description given in an earlier paper [I. M. Kolthoff, J. J. Lingane, and W. Larson, J. Am. Chem. Soc. 60, 2512 (1938)], the compiler believes the temperature to be $298.15 \mathrm{~K}$.

\section{Auxiliary Information}

\section{Method/Apparatus/Procedure:}

Very little experimental details were provided. Solubility was determined by titrating a filtered aliquot of the saturated solution alkalimetrically in an aqueous-ethanol mixture using phenolphthalein as the acid-base indicator.

\section{Source and Purity of Chemicals:}

(1) Purity not given, Chemical source not given, was recrystallized from either aqueous or aqueous-ethanol solution and dried in vacuo at $333 \mathrm{~K}$.

(2) Purity not given, Chemical source not given, was dehydrated and then distilled over sulfanilic acid to remove alkaline impurities.

\section{Estimated Error:}

Temperature: $\pm 0.1 \mathrm{~K}$ (estimated by compiler). $c_{1}: \pm 2 \%$ (relative error, estimated by compiler).

\subsection{4-Chloro-3,5-dinitrobenzoic acid solubility data in miscellaneous organic solvents}

\begin{tabular}{|c|c|}
\hline $\begin{array}{l}\text { Components: } \\
\text { (1) 4-Chloro-3,5-dinitrobenzoic } \\
\text { acid; } \mathrm{C}_{7} \mathrm{H}_{3} \mathrm{ClN}_{2} \mathrm{O}_{6} ;[118-97-8] \\
\text { (2) Ethanenitrile; } \mathrm{C}_{2} \mathrm{H}_{3} \mathrm{~N} ;[75-05-8]\end{array}$ & $\begin{array}{l}\text { Original Measurements: } \\
{ }^{83} \text { M. K. Chantooni and I. M. Kolthoff, } \\
\text { J. Phys. Chem. 77, } 527 \text { (1973). }\end{array}$ \\
\hline $\begin{array}{l}\text { Variables: } \\
T / \mathrm{K}=298.15\end{array}$ & $\begin{array}{l}\text { Prepared by: } \\
\text { W. E. Acree, Jr. }\end{array}$ \\
\hline
\end{tabular}

Experimental Values

The measured solubility was reported to be $1.22 \mathrm{~mol} \mathrm{dm}^{-3}$. The authors did not give the temperature at which the solubility was measured. Based on the experimental description given in an earlier paper [I. M. Kolthoff, J. J. Lingane, and W. Larson, J. Am. Chem. Soc. 60, 2512 (1938)], the compiler believes the temperature to be $298.15 \mathrm{~K}$.

\section{Auxiliary Information}

\section{Method/Apparatus/Procedure:}

Very little experimental details were provided. Solubility was determined by titrating a filtered aliquot of the saturated solution alkalimetrically in an aqueous-ethanol mixture using phenolphthalein as the acid-base indicator.

Source and Purity of Chemicals:

(1) Purity not given, Aldrich Chemical Company, Milwaukee, WI, USA, was recrystallized from aqueous ethanol solution.

(2) Purity not given, Chemical source not given, was purified by shaking with saturated potassium hydroxide, followed by activated alumina, and then anhydrous calcium chloride to remove water. Ethanenitrile was further dried over anhydrous magnesium sulfate and then phosphorous pentoxide. The sample was distilled shortly before use.

\section{Estimated Error:}

Temperature: $\pm 0.1 \mathrm{~K}$ (estimated by compiler). $c_{1}: \pm 2 \%$ (relative error, estimated by compiler).

\section{Solubility of 4-Chloro-3-nitrobenzoic Acid in Organic Solvents}

\subsection{Critical evaluation of experimental solubility data}

There have been several published studies ${ }^{13,54,63,76,83}$ investigating the solubility behavior of 4-chloro-3-nitrobenzoic acid in organic solvents of varying polarity and hydrogenbonding capability. Stovall et al. ${ }^{13}$ measured the solubility of 4-chloro-3-nitrobenzoic acid in 17 alcohols (ethanol, 1-propanol, 2-propanol, 1-butanol, 2-butanol, 2-methyl-1-propanol, 2-methyl-2-propanol, 1-pentanol, 2-pentanol, 3-methyl-1butanol, 1-hexanol, 2-methyl-1-pentanol, 4-methyl-2-pentanol, 1-heptanol, 1-octanol, 2-ethyl-1-hexanol, and 1-decanol), in three dialkyl ethers (1,1'-oxybisethane, $2,2^{\prime}$-oxybispropane, and $1,1^{\prime}$-oxybisbutane), and two cyclic ethers (tetrahydrofuran and 1,4-dioxane), in six alkyl alkanoates (methyl ethanoate, ethyl ethanoate, propyl ethanoate, butyl ethanoate, pentyl ethanoate, and methyl butanoate), and in propylene carbonate 
at $298 \mathrm{~K}$. Results of the experimental measurements were used to calculate the Abraham solute descriptors of 4-chloro-3nitrobenzoic acid. The authors were able to assemble a total of $37 \log _{10}(S R$ or $P)$ and $\log _{10}(G S R$ or $K)$ equations for which experimental partition coefficient data, solubility ratios, Abraham model equation coefficients and aqueous molar solubility were available. The logarithm of the aqueous molar solubility of 4-chloro-3-nitrobenzoic acid is $\log _{10} c_{1, \mathrm{~W}}=-3.00 .^{136}$ The McGowan volume of 4-chloro-3-nitrobenzoic acid, $V=$ 1.2283 , was calculated from the number of chemical bonds in the molecule and the individual atomic group volumes, $A V_{i}$, given in Sec. 1.3. The excess molar refraction solute descriptor was estimated as $E=1.250$. This left four solute descriptors $(S$, $A, B$, and $L$ ) still to be determined. The 37 equations were then solved using the Microsoft "SOLVER" program to yield numerical values of the remaining four solute descriptors, $S=1.470$, $A=0.700, B=0.440$, and $L=6.685$, that best described the $\log _{10}(S R$ or $P)$ and $\log _{10}(G S R$ or $K)$ values. The computation treated $\log _{10} c_{1, \mathrm{G}}$ as a floating parameter to be determined as part of the regression analyses. The data analyses returned a value of $\log _{10} c_{1, \mathrm{G}}=-10.21$ for the logarithm of the gas-phase solute concentration that made the $\log _{10}(S R$ or $P)$ and $\log _{10}$ (GSR or $K$ ) predictions internally consistent. The calculated molecular solute descriptors reproduced the $\log _{10}(S R$ or $P$ ) and $\log _{10}(G S R$ or $K)$ values to within an average standard deviation of 0.063 and $0.072 \log _{10}$ units, respectively.

After the 4-chloro-3-nitrobenzoic acid solubility study was published, several additional Abraham model correlations have been developed, and equation coefficients for a few solvents were updated based on additional experimental data. The new correlations (listed in Tables 1 and 2) will be used in illustrating the ability of the Abraham model to correlate the experimental 4-chloro-3-nitrobenzoic acid solubility data. Table 24 compares the experimental $\log _{10} c_{1}$ values to calculated values based on Eqs. (20) and (21) of the Abraham model. For comparison purposes, the measured mole fraction solubilities of 4-chloro-3-nitrobenzoic acid, $x_{1}$, determined by Stovall et al. $^{13}$ were converted into molar solubilities by dividing $x_{1}$ by the ideal molar volume of the saturated solution (i.e., $\left.c_{1}{ }^{\text {sat }}=x_{1} /\left[x_{1} V_{1}+\left(1-x_{1}\right) V_{\text {solvent }}\right]\right)$. The molar volume of the hypothetical subcooled liquid 4-chloro-3-nitrobenzoic acid is $V_{\text {solute }}=130.38 \mathrm{~cm}^{3} \mathrm{~mol}^{-1}$. Examination of the numerical entries in Table 24 reveals that the Abraham model provides a reasonably accurate mathematical description of the observed solubility data, suggesting that there are no obvious outliers in the dataset.

Chantooni and Kolthoff ${ }^{76,83}$ determined the solubility of 4chloro-3-nitrobenzoic acid in methanol, 2-propanol, 2-methyl2-propanol, 1-hexanol, and ethanenitrile by titrating an aliquot of the saturated solutions with a standardized base titrant. The molar solubilities reported by the authors for 4-chloro-3nitrobenzoic acid dissolved in 2-propanol, $c_{1}=0.436 \mathrm{~mol}$ $\mathrm{dm}^{-3}$, in 2-methyl-2-propanol, $c_{1}=0.511 \mathrm{~mol} \mathrm{dm}^{-3}$, and in 1hexanol, $c_{1}=0.283 \mathrm{~mol} \mathrm{dm}^{-3}$, are in reasonable agreement with the values of $c_{1}=0.386 \mathrm{~mol} \mathrm{dm}^{-3}$ (for 2propanol), $c_{1}=0.367 \mathrm{~mol} \mathrm{dm}^{-3}$ (for 2-methyl-2-propanol), and $c_{1}=0.265 \mathrm{~mol} \mathrm{dm}^{-3}$ (for 1-hexanol) calculated from the mole fraction solubility data of Stovall et al. ${ }^{13}$ The deviations
TABLE 24. Comparison between observed and calculated molar solubilities of 4-chloro-3-nitrobenzoic acid based on the Abraham model, Eqs. (20) and (21)

\begin{tabular}{lccc}
\hline \hline & & $\log _{10} c_{1}{ }^{\text {calc }} ;$ & $\log _{10} c_{1}{ }^{\text {calc}} ;$ \\
Solvent & $\log _{10} c_{1}{ }^{\text {exp }}$ & Eq. $(20)$ & Eq. $(21)$ \\
\hline Methanol & $-0.152^{\mathrm{a}}$ & -0.288 & -0.276 \\
Ethanol & -0.290 & -0.327 & -0.303 \\
1-Propanol & -0.433 & -0.456 & -0.421 \\
2-Propanol & -0.413 & -0.459 & -0.416 \\
1-Butanol & -0.510 & -0.555 & -0.511 \\
2-Butanol & -0.499 & -0.536 & -0.527 \\
2-Methyl-1-propanol & -0.728 & -0.689 & -0.657 \\
2-Methyl-2-propanol & -0.435 & -0.486 & -0.414 \\
1-Pentanol & -0.561 & -0.580 & -0.543 \\
2-Pentanol & -0.525 & -0.616 & -0.569 \\
3-Methyl-1-butanol & -0.627 & -0.696 & -0.640 \\
1-Hexanol & -0.577 & -0.619 & -0.593 \\
1-Heptanol & -0.609 & -0.637 & -0.614 \\
1-Octanol & -0.642 & -0.657 & -0.711 \\
1-Decanol & -0.733 & -0.780 & -0.731 \\
1,1'-Oxybisethane & -0.632 & -0.657 & -0.533 \\
Tetrahydrofuran & 0.280 & 0.242 & 0.247 \\
1,4-Dioxane & 0.165 & 0.033 & 0.060 \\
Methyl ethanoate & -0.365 & -0.428 & -0.371 \\
Ethyl ethanoate & -0.450 & -0.417 & -0.386 \\
Propyl ethanoate & -0.592 & -0.498 & -0.585 \\
Butyl ethanoate & -0.641 & -0.580 & \\
\hline Experimental & & & \\
\hline
\end{tabular}

${ }^{a}$ Experimental value is from Chantooni and Kolthoff. ${ }^{83}$

between the two sets of experimental measurements are attributed in part to differences in chemical purities and experimental methodologies.

The experimental solubility data for 4-chloro-3-nitrobenzoic acid in organic solvents are in Secs. 23.2-23.7.

\subsection{4-Chloro-3-nitrobenzoic acid solubility data in saturated hydrocarbons (including cycloalkanes)}

\begin{tabular}{|c|c|}
\hline $\begin{array}{l}\text { Components: } \\
\text { (1) 4-Chloro-3-nitrobenzoic acid; } \\
\mathrm{C}_{7} \mathrm{H}_{4} \mathrm{ClNO}_{4} ;[96-99-1] \\
\text { (2) Cyclohexane; } \mathrm{C}_{6} \mathrm{H}_{12} \text {; } \\
{[110-82-7]}\end{array}$ & $\begin{array}{l}\text { Original Measurements: } \\
{ }^{54} \text { C. K. Hancock, J. N. Pawloski, and J. } \\
\text { P. Idoux, J. Org. Chem. 31, } 3801 \\
\text { (1966). }\end{array}$ \\
\hline $\begin{array}{l}\text { Variables: } \\
T / \mathrm{K}=303.15\end{array}$ & $\begin{array}{l}\text { Prepared by: } \\
\text { W. E. Acree, Jr. }\end{array}$ \\
\hline
\end{tabular}

Experimental Values

\begin{tabular}{lc}
\hline \hline$x_{2}{ }^{\mathrm{a}}$ & $x_{1}{ }^{\mathrm{b}}$ \\
\hline 0.9999 & 0.0000218 \\
\hline${ }^{\mathrm{a}} x_{2}:$ mole fraction of component 2 in the saturated solution. \\
${ }^{\mathrm{b}} x_{1}:$ mole fraction solubility of the solute.
\end{tabular}




\section{Auxiliary Information}

\section{Method/Apparatus/Procedure:}

Constant-temperature bath, Soxhlet thimble, conical flask, and analytical balance.

Excess solute and solvent were placed in a conical flask and allowed to equilibrate for several days at constant temperature. Attainment of equilibrium was verified by several repetitive measurements and by approaching equilibrium from supersaturation. Aliquots of saturated solutions were transferred using a Soxhlet thimble equipped with a one-hole cork stopper and an inverted U-shaped delivery tube to a second ground-glass stoppered flask suspended in the $303 \mathrm{~K}$ water bath. Successive portions of the contents were evaporated at room temperature in a tared aluminum foil weighing dish under a bell jar through which a slow stream of dry air was passed. The second flask plus unused saturated solution and the aluminum foil dish plus residue were weighed. The saturation solubility of the solute was calculated from the recorded mass data and molar masses of the solute and solvent.

\section{Source and Purity of Chemicals:}

(1) Purity not given, Chemical source not specified, was recrystallized several times from aqueous-ethanol mixture, and then dried under vacuum over phosphorous pentoxide.

(2) Reagent grade, Chemical source not specified, refluxed over phosphorous pentoxide for $24 \mathrm{~h}$, and then distilled through a $3 \times 80 \mathrm{~cm}$ column filled with $0.32-\mathrm{cm}$ glass helices.

\section{Estimated Error:}

Temperature: $\pm 0.02 \mathrm{~K}$.

$x_{1}: \pm 10 \%$ (relative error, by compiler).

\subsection{4-Chloro-3-nitrobenzoic acid solubility data in aromatic hydrocarbons}

\begin{tabular}{|c|c|}
\hline $\begin{array}{l}\text { Components: } \\
\text { (1) 4-Chloro-3-nitrobenzoic acid; } \\
\mathrm{C}_{7} \mathrm{H}_{4} \mathrm{ClNO}_{4} ;[96-99-1] \\
\text { (2) Benzene; } \mathrm{C}_{6} \mathrm{H}_{6} ;[71-43-2]\end{array}$ & $\begin{array}{l}\text { Original Measurements: } \\
{ }^{54} \text { C. K. Hancock, J. N. Pawloski, and J. } \\
\text { P. Idoux, J. Org. Chem. 31, } 3801 \\
\text { (1966). }\end{array}$ \\
\hline $\begin{array}{l}\text { Variables: } \\
T / \mathrm{K}=303.15\end{array}$ & $\begin{array}{l}\text { Prepared by: } \\
\text { W. E. Acree, Jr. }\end{array}$ \\
\hline
\end{tabular}

\section{Experimental Values}

\begin{tabular}{lc}
\hline \hline$x_{2}{ }^{\mathrm{a}}$ & $x_{1}{ }^{\mathrm{b}}$ \\
\hline 0.9993 & 0.000678 \\
\hline${ }^{\mathrm{a}} x_{2}:$ mole fraction of component 2 in the saturated solution. \\
${ }^{\mathrm{b}} x_{1}:$ mole fraction solubility of the solute.
\end{tabular}

\section{Auxiliary Information}

\section{Method/Apparatus/Procedure:}

Constant-temperature bath, Soxhlet thimble, conical flask, and analytical balance.

Excess solute and solvent were placed in a conical flask and allowed to equilibrate for several days at constant temperature. Attainment of equilibrium was verified by several repetitive measurements and by approaching equilibrium from supersaturation. Aliquots of saturated solutions were transferred using a Soxhlet thimble equipped with a one-hole cork stopper and an inverted U-shaped delivery tube to a second ground-glass stoppered flask suspended in the $303 \mathrm{~K}$ water bath. Successive portions of the contents were evaporated at room temperature in a tared aluminum foil weighing dish under a bell jar through which a slow stream of dry air was passed. The second flask plus unused saturated solution and the aluminum foil dish plus residue were weighed. The saturation solubility of the solute was calculated from the recorded mass data and molar masses of the solute and solvent.

\section{Source and Purity of Chemicals:}

(1) Purity not given, Chemical source not specified, was recrystallized several times from aqueous-ethanol mixture, and then dried under vacuum over phosphorous pentoxide.

(2) Reagent grade, Chemical source not specified, refluxed over phosphorous pentoxide for $24 \mathrm{~h}$, and then distilled through a $3 \times 80 \mathrm{~cm}$ column filled with $0.32-\mathrm{cm}$ glass helices.

\section{Estimated Error:}

Temperature: $\pm 0.02 \mathrm{~K}$

$x_{1}: \pm 3 \%$ (relative error, by compiler).

\subsection{4-Chloro-3-nitrobenzoic acid solubility data in esters}

\begin{tabular}{ll}
\hline \hline Components: & Original Measurements: \\
(1) 4-Chloro-3-nitrobenzoic acid; & ${ }^{13}$ D. M. Stovall, C. Givens, S. Keown, \\
$\mathrm{C}_{7} \mathrm{H}_{4} \mathrm{ClNO}_{4}$; [96-99-1] & K. R. Hoover, R. Barnes, C. Harris, J. \\
$\begin{array}{l}\text { (2) Methyl ethanoate; } \mathrm{C}_{3} \mathrm{H}_{6} \mathrm{O}_{2} ; \\
\text { [79-20-9] }\end{array}$ & Lozano, M. Nguyen, E. Rodriguez, W. \\
& E. Acree, Jr., and M. H. Abraham, \\
& Phys. Chem. Liq. 43, 351 (2005). \\
\hline Variables: & Prepared by: \\
$T / \mathrm{K}=298.15$ & W. E. Acree, Jr. \\
\hline
\end{tabular}

\section{Experimental Values}

\begin{tabular}{lc}
\hline \hline$x_{2}{ }^{\mathrm{a}}$ & $x_{1}{ }^{\mathrm{b}}$ \\
\hline 0.9648 & 0.03520 \\
\hline$x_{2}:$ mole fraction of compont 2 inthe saturated solution
\end{tabular}

${ }^{a} x_{2}$ : mole fraction of component 2 in the saturated solution.

${ }^{\mathrm{b}} x_{1}$ : mole fraction solubility of the solute.

\section{Auxiliary Information}

\section{Method/Apparatus/Procedure:}

Constant-temperature bath, calorimetric thermometer, and an ultraviolet/ visible spectrophotometer.

Excess solute and solvent were placed in amber glass bottles and allowed to equilibrate for several days at constant temperature. Attainment of equilibrium was verified by several repetitive measurements and by approaching equilibrium from supersaturation. Aliquots of saturated solutions were transferred through a coarse filter into tared volumetric flasks, weighed, and diluted with methanol. Concentrations were determined by spectrophotometric measurements at $292 \mathrm{~nm}$.

\section{Source and Purity of Chemicals:}

(1) $99.5 \%$, Acros Organics, USA, was used as received.

(2) $99.5 \%$, anhydrous, Aldrich Chemical Company, Milwaukee, WI, USA, stored over molecular sieves and distilled shortly before use.

\section{Estimated Error:}

Temperature: $\pm 0.1 \mathrm{~K}$.

$x_{1}: \pm 1.5 \%$ (relative error). 


\section{Components:}

(1) 4-Chloro-3-nitrobenzoic acid;

$\mathrm{C}_{7} \mathrm{H}_{4} \mathrm{ClNO}_{4}$; [96-99-1]

(2) Ethyl ethanoate; $\mathrm{C}_{4} \mathrm{H}_{8} \mathrm{O}_{2}$; [141-78-6]

\section{Variables:}

$T / \mathrm{K}=298.15$

Original Measurements:

${ }^{13}$ D. M. Stovall, C. Givens, S. Keown,

K. R. Hoover, R. Barnes, C. Harris, J.

Lozano, M. Nguyen, E. Rodriguez, W.

E. Acree, Jr., and M. H. Abraham,

Phys. Chem. Liq. 43, 351 (2005).

Prepared by:

W. E. Acree, Jr.

Experimental Values

\begin{tabular}{lc}
\hline \hline$x_{2}{ }^{\mathrm{a}}$ & $x_{1}{ }^{\mathrm{b}}$ \\
\hline 0.9647 & 0.03534
\end{tabular}

${ }^{\mathrm{a}} x_{2}$ : mole fraction of component 2 in the saturated solution.

${ }^{\mathrm{b}} x_{1}$ : mole fraction solubility of the solute.

\section{Auxiliary Information}

\section{Method/Apparatus/Procedure:}

Constant-temperature bath, calorimetric thermometer, and an ultraviolet/ visible spectrophotometer.

Excess solute and solvent were placed in amber glass bottles and allowed to equilibrate for several days at constant temperature. Attainment of equilibrium was verified by several repetitive measurements and by approaching equilibrium from supersaturation. Aliquots of saturated solutions were transferred through a coarse filter into tared volumetric flasks, weighed, and diluted with methanol. Concentrations were determined by

spectrophotometric measurements at $292 \mathrm{~nm}$.

Source and Purity of Chemicals:

(1) $99.5 \%$, Acros Organics, USA, was used as received.

(2) $99.9 \%$, HPLC grade, Aldrich Chemical Company, Milwaukee, WI, USA, stored over molecular sieves and distilled shortly before use.

Estimated Error:

Temperature: $\pm 0.1 \mathrm{~K}$.

$x_{1}: \pm 1.5 \%$ (relative error).

\begin{tabular}{ll}
\hline \hline Components: & Original Measurements: \\
(1) 4-Chloro-3-nitrobenzoic acid; & ${ }^{13}$ D. M. Stovall, C. Givens, S. Keown, \\
$\mathrm{C}_{7} \mathrm{H}_{4} \mathrm{ClNO}_{4}$; [96-99-1] & K. R. Hoover, R. Barnes, C. Harris, J. \\
(2) Propyl ethanoate; $\mathrm{C}_{5} \mathrm{H}_{10} \mathrm{O}_{2} ;$ & Lozano, M. Nguyen, E. Rodriguez, W. \\
[109-60-4] & E. Acree, Jr., and M. H. Abraham, \\
& Phys. Chem. Liq. 43, 351 (2005). \\
\hline Variables: & Prepared by: \\
$T / \mathrm{K}=298.15$ & W. E. Acree, Jr. \\
\hline
\end{tabular}

\section{Experimental Values}

\begin{tabular}{lc}
\hline \hline$x_{2}{ }^{\mathrm{a}}$ & $x_{1}{ }^{\mathrm{b}}$ \\
\hline 0.9703 & 0.02966 \\
\hline
\end{tabular}

${ }^{\mathrm{a}} x_{2}$ : mole fraction of component 2 in the saturated solution.

${ }^{\mathrm{b}} x_{1}$ : mole fraction solubility of the solute.

\section{Auxiliary Information}

\section{Method/Apparatus/Procedure:}

Constant-temperature bath, calorimetric thermometer, and an ultraviolet/ visible spectrophotometer.

Excess solute and solvent were placed in amber glass bottles and allowed to equilibrate for several days at constant temperature. Attainment of equilibrium was verified by several repetitive measurements and by approaching equilibrium from supersaturation. Aliquots of saturated solutions were transferred through a coarse filter into tared volumetric flasks, weighed, and diluted with methanol. Concentrations were determined by spectrophotometric measurements at $292 \mathrm{~nm}$.

\section{Source and Purity of Chemicals:}

(1) $99.5 \%$, Acros Organics, USA, was used as received

(2) $99.5 \%$, Aldrich Chemical Company, Milwaukee, WI, USA, stored over molecular sieves and distilled shortly before use.

\section{Estimated Error:}

Temperature: $\pm 0.1 \mathrm{~K}$.

$x_{1}: \pm 1.5 \%$ (relative error)

\begin{tabular}{ll}
\hline \hline Components: & Original Measurements: \\
(1) 4-Chloro-3-nitrobenzoic acid; & ${ }^{13}$ D. M. Stovall, C. Givens, S. Keown, \\
$\mathrm{C}_{7} \mathrm{H}_{4} \mathrm{ClNO}_{4}$; [96-99-1] & K. R. Hoover, R. Barnes, C. Harris, J. \\
$\begin{array}{l}\text { (2) Butyl ethanoate; } \mathrm{C}_{6} \mathrm{H}_{12} \mathrm{O}_{2} ; \\
\text { [123-86-4] }\end{array}$ & Lozano, M. Nguyen, E. Rodriguez, W. \\
& E. Acree, Jr., and M. H. Abraham, \\
& Phys. Chem. Liq. 43, 351 (2005). \\
\hline Variables: & Prepared by: \\
$T / \mathrm{K}=298.15$ & W. E. Acree, Jr. \\
\hline
\end{tabular}

\section{Experimental Values}

\begin{tabular}{lc}
\hline \hline$x_{2}{ }^{\mathrm{a}}$ & $x_{1}{ }^{\mathrm{b}}$ \\
\hline 0.9697 & 0.03029 \\
\hline${ }^{\mathrm{a}} x_{2}:$ mole fraction of component 2 in the saturated solution. & \\
${ }^{\mathrm{b}} x_{1}:$ mole fraction solubility of the solute. &
\end{tabular}

${ }^{\mathrm{b}} x_{1}$ : mole fraction solubility of the solute.

\section{Auxiliary Information}

\section{Method/Apparatus/Procedure:}

Constant-temperature bath, calorimetric thermometer, and an ultraviolet/ visible spectrophotometer.

Excess solute and solvent were placed in amber glass bottles and allowed to equilibrate for several days at constant temperature. Attainment of equilibrium was verified by several repetitive measurements and by approaching equilibrium from supersaturation. Aliquots of saturated solutions were transferred through a coarse filter into tared volumetric flasks, weighed, and diluted with methanol. Concentrations were determined by spectrophotometric measurements at $292 \mathrm{~nm}$.

\section{Source and Purity of Chemicals:}

(1) $99.5 \%$, Acros Organics, USA, was used as received.

(2) $99.7 \%$, HPLC grade, Aldrich Chemical Company, Milwaukee, WI, USA, stored over molecular sieves and distilled shortly before use.

\section{Estimated Error:}

Temperature: $\pm 0.1 \mathrm{~K}$.

$x_{1}: \pm 1.5 \%$ (relative error). 


\section{Components:}

(1) 4-Chloro-3-nitrobenzoic acid;

$\mathrm{C}_{7} \mathrm{H}_{4} \mathrm{ClNO}_{4}$; [96-99-1]

(2) Pentyl ethanoate; $\mathrm{C}_{7} \mathrm{H}_{14} \mathrm{O}_{2}$; [628-63-7]

\section{Variables:}

$T / \mathrm{K}=298.15$

Original Measurements:

${ }^{13}$ D. M. Stovall, C. Givens, S. Keown,

K. R. Hoover, R. Barnes, C. Harris, J.

Lozano, M. Nguyen, E. Rodriguez, W.

E. Acree, Jr., and M. H. Abraham,

Phys. Chem. Liq. 43, 351 (2005).

Prepared by:

W. E. Acree, Jr.

Experimental Values

\begin{tabular}{lc}
\hline \hline$x_{2}{ }^{\mathrm{a}}$ & $x_{1}{ }^{\mathrm{b}}$ \\
\hline 0.9758 & 0.02420
\end{tabular}

${ }^{\mathrm{a}} x_{2}$ : mole fraction of component 2 in the saturated solution.

${ }^{\mathrm{b}} x_{1}$ : mole fraction solubility of the solute.

\section{Auxiliary Information}

\section{Method/Apparatus/Procedure:}

Constant-temperature bath, calorimetric thermometer, and an ultraviolet/ visible spectrophotometer.

Excess solute and solvent were placed in amber glass bottles and allowed to equilibrate for several days at constant temperature. Attainment of equilibrium was verified by several repetitive measurements and by approaching equilibrium from supersaturation. Aliquots of saturated solutions were transferred through a coarse filter into tared volumetric flasks, weighed, and diluted with methanol. Concentrations were determined by

spectrophotometric measurements at $292 \mathrm{~nm}$.

Source and Purity of Chemicals:

(1) $99.5 \%$, Acros Organics, USA, was used as received.

(2) $99 \%$, Aldrich Chemical Company, Milwaukee, WI, USA, stored over molecular sieves and distilled shortly before use.

Estimated Error:

Temperature: $\pm 0.1 \mathrm{~K}$

$x_{1}: \pm 1.5 \%$ (relative error).

\begin{tabular}{ll}
\hline \hline Components: & Original Measurements: \\
(1) 4-Chloro-3-nitrobenzoic acid; & ${ }^{13}$ D. M. Stovall, C. Givens, S. Keown, \\
$\mathrm{C}_{7} \mathrm{H}_{4} \mathrm{ClNO}_{4}$; [96-99-1] & K. R. Hoover, R. Barnes, C. Harris, J. \\
(2) Methyl butanoate; $\mathrm{C}_{5} \mathrm{H}_{10} \mathrm{O}_{2} ;$ & Lozano, M. Nguyen, E. Rodriguez, W. \\
[623-42-7] & E. Acree, Jr., and M. H. Abraham, \\
& Phys. Chem. Liq. 43, 351 (2005). \\
\hline Variables: & Prepared by: \\
$T / \mathrm{K}=298.15$ & W. E. Acree, Jr. \\
\hline
\end{tabular}

Experimental Values

\begin{tabular}{lc}
\hline \hline$x_{2}{ }^{\mathrm{a}}$ & $x_{1}{ }^{\mathrm{b}}$ \\
\hline 0.9759 & 0.02410 \\
\hline
\end{tabular}

${ }^{\mathrm{a}} x_{2}$ : mole fraction of component 2 in the saturated solution.

${ }^{b} x_{1}$ : mole fraction solubility of the solute.

\section{Auxiliary Information}

Method/Apparatus/Procedure:

Constant-temperature bath, calorimetric thermometer, and an ultraviolet/ visible spectrophotometer.

Excess solute and solvent were placed in amber glass bottles and allowed to equilibrate for several days at constant temperature. Attainment of equilibrium was verified by several repetitive measurements and by approaching equilibrium from supersaturation. Aliquots of saturated solutions were transferred through a coarse filter into tared volumetric flasks, weighed, and diluted with methanol. Concentrations were determined by spectrophotometric measurements at $292 \mathrm{~nm}$.

\section{Source and Purity of Chemicals:}

(1) $99.5 \%$, Acros Organics, USA, was used as received.

(2) $99 \%$, Aldrich Chemical Company, Milwaukee, WI, USA, stored over molecular sieves and distilled shortly before use.

\section{Estimated Error:}

Temperature: $\pm 0.1 \mathrm{~K}$.

$x_{1}: \pm 1.5 \%$ (relative error)

\begin{tabular}{|c|c|}
\hline $\begin{array}{l}\text { Components: } \\
\text { (1) 4-Chloro-3-nitrobenzoic acid; } \\
\mathrm{C}_{7} \mathrm{H}_{4} \mathrm{ClNO}_{4} \text {; [96-99-1] } \\
\text { (2) 1,2,3-Triacetoxypropane } \\
\text { (Triacetin); } \mathrm{C}_{9} \mathrm{H}_{14} \mathrm{O}_{6} ;[102-76-1]\end{array}$ & $\begin{array}{l}\text { Original Measurements: } \\
\text { W. E. Acree, Jr., unpublished data. }\end{array}$ \\
\hline $\begin{array}{l}\text { Variables: } \\
T / \mathrm{K}=298.15\end{array}$ & $\begin{array}{l}\text { Prepared by: } \\
\text { W. E. Acree, Jr. }\end{array}$ \\
\hline
\end{tabular}

\section{Experimental Values}

\begin{tabular}{lc}
\hline \hline$x_{2}{ }^{\mathrm{a}}$ & $x_{1}{ }^{\mathrm{b}}$ \\
\hline 0.9637 & 0.03631 \\
\hline${ }^{\mathrm{a}} x_{2}:$ mole fraction of component 2 in the saturated solution. & \\
${ }^{\mathrm{b}} x_{1}:$ mole fraction solubility of the solute. &
\end{tabular}

\section{Auxiliary Information}

\section{Method: Apparatus/Procedure:}

Constant-temperature bath, calorimetric thermometer, and an ultraviolet/ visible spectrophotometer

Excess solute and solvent were placed in amber glass bottles and allowed to equilibrate for several days at constant temperature. Attainment of equilibrium was verified by several repetitive measurements and by approaching equilibrium from supersaturation. Aliquots of saturated solutions were transferred through a coarse filter into tared volumetric flasks, weighed, and diluted with 2-propanol. Concentrations were determined by spectrophotometric measurements at $292 \mathrm{~nm}$.

Source and Purity of Chemicals:

(1) $99.5 \%$, Acros Organics, USA, was used as received.

(2) $99 \%$, Acros Organics, USA, stored over molecular sieves before use.

\section{Estimated Error:}

Temperature: $\pm 0.1 \mathrm{~K}$.

$x_{1}: \pm 1.5 \%$ (relative error). 


\subsection{4-Chloro-3-nitrobenzoic acid solubility data in ethers}

\begin{tabular}{ll}
\hline \hline Components: & Original Measurements: \\
$\begin{array}{l}\text { (1) 4-Chloro-3-nitrobenzoic acid; } \\
\mathrm{C}_{7} \mathrm{H}_{4} \mathrm{ClNO}_{4} \text {; [96-99-1] }\end{array}$ & ${ }^{13}$ D. M. Stovall, C. Givens, S. Keown, \\
(2) $1,1^{\prime}-$ Oxybisethane; $\mathrm{C}_{4} \mathrm{H}_{10} \mathrm{O} ;$ & K. R. Hoover, R. Barnes, C. Harris, J. \\
[60-29-7] & E. Acree, Jr., and M. H. Abraham, \\
& Phys. Chem. Liq. 43, 351 (2005). \\
\hline Variables: & Prepared by: \\
$T / \mathrm{K}=298.15$ & W. E. Acree, Jr. \\
\hline
\end{tabular}

\section{Experimental Values}

\begin{tabular}{lc}
\hline \hline$x_{2}{ }^{\mathrm{a}}$ & $x_{1}{ }^{\mathrm{b}}$ \\
\hline 0.9754 & 0.02459
\end{tabular}

${ }^{\mathrm{a}} x_{2}$ : mole fraction of component 2 in the saturated solution.

${ }^{\mathrm{b}} x_{1}$ : mole fraction solubility of the solute.

\section{Auxiliary Information}

\section{Method/Apparatus/Procedure:}

Constant-temperature bath, calorimetric thermometer, and an ultraviolet/ visible spectrophotometer.

Excess solute and solvent were placed in amber glass bottles and allowed to equilibrate for several days at constant temperature. Attainment of equilibrium was verified by several repetitive measurements and by approaching equilibrium from supersaturation. Aliquots of saturated solutions were transferred through a coarse filter into tared volumetric flasks, weighed, and diluted with methanol. Concentrations were determined by spectrophotometric measurements at $292 \mathrm{~nm}$.

Source and Purity of Chemicals:

(1) $99.5 \%$, Acros Organics, USA, was used as received.

(2) $99+\%$, anhydrous, Aldrich Chemical Company, Milwaukee, WI, USA, stored over molecular sieves and distilled shortly before use.

Estimated Error:

Temperature: $\pm 0.1 \mathrm{~K}$.

$x_{1}: \pm 1.5 \%$ (relative error).

\begin{tabular}{|c|c|}
\hline $\begin{array}{l}\text { Components: } \\
\text { (1) 4-Chloro-3-nitrobenzoic acid; } \\
\mathrm{C}_{7} \mathrm{H}_{4} \mathrm{ClNO}_{4} \text {; [96-99-1] } \\
\text { (2) 2,2'-Oxybispropane; } \mathrm{C}_{6} \mathrm{H}_{14} \mathrm{O} \text {; } \\
\text { [108-20-3] }\end{array}$ & $\begin{array}{l}\text { Original Measurements: } \\
{ }^{13} \text { D. M. Stovall, C. Givens, S. Keown, } \\
\text { K. R. Hoover, R. Barnes, C. Harris, J. } \\
\text { Lozano, M. Nguyen, E. Rodriguez, W. } \\
\text { E. Acree, Jr., and M. H. Abraham, } \\
\text { Phys. Chem. Liq. 43, } 351 \text { (2005). }\end{array}$ \\
\hline $\begin{array}{l}\text { Variables: } \\
T / \mathrm{K}=298.15\end{array}$ & $\begin{array}{l}\text { Prepared by: } \\
\text { W. E. Acree, Jr. }\end{array}$ \\
\hline
\end{tabular}

\section{Experimental Values}

\begin{tabular}{lc}
\hline \hline$x_{2}{ }^{\mathrm{a}}$ & $x_{1}{ }^{\mathrm{b}}$ \\
\hline 0.9871 & 0.01289 \\
\hline${ }^{\mathrm{a}} x_{2}:$ mole fraction of component 2 in the saturated solution. & \\
${ }^{\mathrm{b}} x_{1}:$ mole fraction solubility of the solute. &
\end{tabular}

Auxiliary Information

\section{Method/Apparatus/Procedure:}

Constant-temperature bath, calorimetric thermometer, and an ultraviolet/ visible spectrophotometer.

Excess solute and solvent were placed in amber glass bottles and allowed to equilibrate for several days at constant temperature. Attainment of equilibrium was verified by several repetitive measurements and by approaching equilibrium from supersaturation. Aliquots of saturated solutions were transferred through a coarse filter into tared volumetric flasks, weighed, and diluted with methanol. Concentrations were determined by spectrophotometric measurements at $292 \mathrm{~nm}$.

\section{Source and Purity of Chemicals:}

(1) $99.5 \%$, Acros Organics, USA, was used as received.

(2) 99\%, anhydrous, Aldrich Chemical Company, Milwaukee, WI, USA, stored over molecular sieves and distilled shortly before use.

\section{Estimated Error:}

Temperature: $\pm 0.1 \mathrm{~K}$.

$x_{1}: \pm 1.5 \%$ (relative error).

\begin{tabular}{ll}
\hline \hline Components: & Original Measurements: \\
(1) 4-Chloro-3-nitrobenzoic acid; & ${ }^{13}$ D. M. Stovall, C. Givens, S. Keown, \\
$\mathrm{C}_{7} \mathrm{H}_{4} \mathrm{ClNO}_{4}$; [96-99-1] & K. R. Hoover, R. Barnes, C. Harris, J. \\
$(2) 1,1^{\prime}$-Oxybisbutane; $\mathrm{C}_{8} \mathrm{H}_{18} \mathrm{O}$; & Lozano, M. Nguyen, E. Rodriguez, W. \\
{$[142-96-1]$} & E. Acree, Jr., and M. H. Abraham, \\
& Phys. Chem. Liq. 43, 351 (2005). \\
\hline Variables: & Prepared by: \\
$T / \mathrm{K}=298.15$ & W. E. Acree, Jr. \\
\hline
\end{tabular}

Experimental Values

\begin{tabular}{lc}
\hline \hline$x_{2}{ }^{\mathrm{a}}$ & $x_{1}{ }^{\mathrm{b}}$ \\
\hline 0.9913 & 0.008711 \\
\hline${ }^{\mathrm{a}} x_{2}:$ mole fraction of component 2 in the saturated solution. & \\
${ }^{\mathrm{b}} x_{1}:$ mole fraction solubility of the solute. &
\end{tabular}

${ }^{b} x_{1}$ : mole fraction solubility of the solute.

\section{Auxiliary Information}

\section{Method/Apparatus/Procedure:}

Constant-temperature bath, calorimetric thermometer, and an ultraviolet/ visible spectrophotometer.

Excess solute and solvent were placed in amber glass bottles and allowed to equilibrate for several days at constant temperature. Attainment of equilibrium was verified by several repetitive measurements and by approaching equilibrium from supersaturation. Aliquots of saturated solutions were transferred through a coarse filter into tared volumetric flasks, weighed, and diluted with methanol. Concentrations were determined by spectrophotometric measurements at $292 \mathrm{~nm}$.

Source and Purity of Chemicals:

(1) $99.5 \%$, Acros Organics, USA, was used as received.

(2) $99.3 \%$, anhydrous, Aldrich Chemical Company, Milwaukee, WI, USA stored over molecular sieves and distilled shortly before use.

\section{Estimated Error:}

Temperature: $\pm 0.1 \mathrm{~K}$.

$x_{1}: \pm 1.5 \%$ (relative error) 


\section{Components:}

(1) 4-Chloro-3-nitrobenzoic acid;

$\mathrm{C}_{7} \mathrm{H}_{4} \mathrm{ClNO}_{4}$; [96-99-1]

(2) Tetrahydrofuran; $\mathrm{C}_{4} \mathrm{H}_{8} \mathrm{O}$; [109-99-9]

\section{Variables:}

$T / \mathrm{K}=298.15$

Original Measurements:

${ }^{13}$ D. M. Stovall, C. Givens, S. Keown,

K. R. Hoover, R. Barnes, C. Harris, J.

Lozano, M. Nguyen, E. Rodriguez, W.

E. Acree, Jr., and M. H. Abraham,

Phys. Chem. Liq. 43, 351 (2005).

Prepared by:

W. E. Acree, Jr.

Experimental Values

\begin{tabular}{lc}
\hline \hline$x_{2}{ }^{\mathrm{a}}$ & $x_{1}{ }^{\mathrm{b}}$ \\
\hline 0.8290 & 0.1710
\end{tabular}

${ }^{a} x_{2}$ : mole fraction of component 2 in the saturated solution.

${ }^{b} x_{1}$ : mole fraction solubility of the solute.

\section{Auxiliary Information}

\section{Method/Apparatus/Procedure:}

Constant-temperature bath, calorimetric thermometer, and an ultraviolet/ visible spectrophotometer.

Excess solute and solvent were placed in amber glass bottles and allowed to equilibrate for several days at constant temperature. Attainment of equilibrium was verified by several repetitive measurements and by approaching equilibrium from supersaturation. Aliquots of saturated solutions were transferred through a coarse filter into tared volumetric flasks, weighed, and diluted with methanol. Concentrations were determined by

spectrophotometric measurements at $292 \mathrm{~nm}$.

\section{Source and Purity of Chemicals:}

(1) $99.5 \%$, Acros Organics, USA, was used as received.

(2) $99.9 \%$, anhydrous, Aldrich Chemical Company, Milwaukee, WI, USA, stored over molecular sieves and distilled shortly before use.

Estimated Error:

Temperature: $\pm 0.1 \mathrm{~K}$.

$x_{1}: \pm 1.5 \%$ (relative error).

\begin{tabular}{|c|c|}
\hline $\begin{array}{l}\text { Components: } \\
\text { (1) 4-Chloro-3-nitrobenzoic acid; } \\
\mathrm{C}_{7} \mathrm{H}_{4} \mathrm{ClNO}_{4} \text {; [96-99-1] } \\
\text { (2) Tetrahydrofuran; } \mathrm{C}_{4} \mathrm{H}_{8} \mathrm{O} \text {; } \\
\text { [109-99-9] }\end{array}$ & $\begin{array}{l}\text { Original Measurements: } \\
{ }^{63} \text { C. K. Hancock, J. N. Pawloski, and J. } \\
\text { P. Idoux, J. Org. Chem. 32, } 1931 \\
\text { (1967). }\end{array}$ \\
\hline $\begin{array}{l}\text { Variables: } \\
T / \mathrm{K}=303.15\end{array}$ & $\begin{array}{l}\text { Prepared by: } \\
\text { W. E. Acree, Jr. }\end{array}$ \\
\hline
\end{tabular}

Experimental Values

\begin{tabular}{lc}
\hline \hline$x_{2}{ }^{\mathrm{a}}$ & $x_{1}{ }^{\mathrm{b}}$ \\
\hline 0.802 & 0.198 \\
\hline${ }^{\mathrm{a}} x_{2}:$ mole fraction of component 2 in the saturated solution. \\
${ }^{\mathrm{b}}{ }_{x_{1}: \text { mole fraction solubility of the solute. }}$
\end{tabular}

\section{Auxiliary Information}

Method/Apparatus/Procedure:

Constant-temperature bath, Soxhlet thimble, conical flask, and analytical balance.

Excess solute and solvent were placed in a conical flask and allowed to equilibrate for several days at constant temperature. Attainment of equilibrium was verified by several repetitive measurements and by approaching equilibrium from supersaturation. Aliquots of saturated solutions were transferred using a Soxhlet thimble equipped with a one-hole cork stopper and an inverted U-shaped delivery tube to a second ground-glass stoppered flask suspended in the $303 \mathrm{~K}$ water bath. Successive portions of the contents were evaporated at room temperature in a tared aluminum foil weighing dish under a bell jar through which a slow stream of dry air was passed. The second flask plus unused saturated solution and the aluminum foil dish plus residue were weighed. The saturation solubility of the solute was calculated from the recorded mass data and molar masses of the solute and solvent.

\section{Source and Purity of Chemicals:}

(1) Purity not given, Chemical source not specified, was recrystallized several times from aqueous-ethanol mixture, and then dried under vacuum over phosphorous pentoxide.

(2) Practical grade, Chemical source not specified, stored over sodium hydroxide pellets for $24 \mathrm{~h}$, and then passed through $2 \times 70-\mathrm{cm}$ chromatographic adsorption columns containing activated alumina. After this treatment, the purified solvent was stored over copper in a nitrogen atmosphere.

\section{Estimated Error:}

Temperature: $\pm 0.02 \mathrm{~K}$

$x_{1}: \pm 1.0 \%$ (relative error).

\begin{tabular}{ll}
\hline \hline Components: & Original Measurements: \\
(1) 4-Chloro-3-nitrobenzoic acid; & ${ }^{13}$ D. M. Stovall, C. Givens, S. Keown, \\
$\mathrm{C}_{7} \mathrm{H}_{4} \mathrm{ClNO}_{4} ;$ [96-99-1] & K. R. Hoover, R. Barnes, C. Harris, J. \\
$\begin{array}{l}\text { (2) 1,4-Dioxane; } \mathrm{C}_{4} \mathrm{H}_{8} \mathrm{O}_{2} ; \\
\text { [123-91-1] }\end{array}$ & Lozano, M. Nguyen, E. Rodriguez, W. \\
& E. Acree, Jr., and M. H. Abraham, \\
& Phys. Chem. Liq. 43, 351 (2005). \\
\hline Variables: & Prepared by: \\
$T / \mathrm{K}=298.15$ & W. E. Acree, Jr. \\
\hline
\end{tabular}

\section{Experimental Values}

\begin{tabular}{lc}
\hline \hline$x_{2}{ }^{\mathrm{a}}$ & $x_{1}{ }^{\mathrm{b}}$ \\
\hline 0.8661 & 0.1339 \\
\hline${ }^{\mathrm{a}} x_{2}:$ mole fraction of component 2 in the saturated solution. & \\
${ }^{\mathrm{b}} x_{1}:$ mole fraction solubility of the solute. &
\end{tabular}

\section{Auxiliary Information}

\section{Method/Apparatus/Procedure:}

Constant-temperature bath, calorimetric thermometer, and an ultraviolet/ visible spectrophotometer.

Excess solute and solvent were placed in amber glass bottles and allowed to equilibrate for several days at constant temperature. Attainment of equilibrium was verified by several repetitive measurements and by approaching equilibrium from supersaturation. Aliquots of saturated solutions were transferred through a coarse filter into tared volumetric flasks, weighed, and diluted with methanol. Concentrations were determined by

spectrophotometric measurements at $292 \mathrm{~nm}$. 
Source and Purity of Chemicals:

(1) $99.5 \%$, Acros Organics, USA, was used as received.

(2) $99.8 \%$, anhydrous, Aldrich Chemical Company, Milwaukee, WI, USA, stored over molecular sieves and distilled shortly before use.

Estimated Error:

Temperature: $\pm 0.1 \mathrm{~K}$.

$x_{1}: \pm 1.5 \%$ (relative error).

\begin{tabular}{|c|c|}
\hline $\begin{array}{l}\text { Components: } \\
\text { (1) 4-Chloro-3-nitrobenzoic acid; } \\
\mathrm{C}_{7} \mathrm{H}_{4} \mathrm{ClNO}_{4} ; \text { [96-99-1] } \\
\text { (2) 1,4-Dioxane; } \mathrm{C}_{4} \mathrm{H}_{8} \mathrm{O}_{2} \text {; } \\
\text { [123-91-1] }\end{array}$ & $\begin{array}{l}\text { Original Measurements: } \\
{ }^{63} \text { C. K. Hancock, J. N. Pawloski, and J. } \\
\text { P. Idoux, J. Org. Chem. 32, } 1931 \\
(1967) .\end{array}$ \\
\hline $\begin{array}{l}\text { Variables: } \\
T / \mathrm{K}=303.15\end{array}$ & $\begin{array}{l}\text { Prepared by: } \\
\text { W. E. Acree, Jr. }\end{array}$ \\
\hline
\end{tabular}

Experimental Values

\begin{tabular}{lc}
\hline \hline$x_{2}{ }^{\mathrm{a}}$ & $x_{1}{ }^{\mathrm{b}}$ \\
\hline 0.852 & 0.148
\end{tabular}

${ }^{\mathrm{a}} x_{2}$ : mole fraction of component 2 in the saturated solution.

${ }^{\mathrm{b}} x_{1}$ : mole fraction solubility of the solute.

\section{Auxiliary Information}

\section{Method/Apparatus/Procedure:}

Constant-temperature bath, Soxhlet thimble, conical flask, and analytical balance.

Excess solute and solvent were placed in a conical flask and allowed to equilibrate for several days at constant temperature. Attainment of equilibrium was verified by several repetitive measurements and by approaching equilibrium from supersaturation. Weighed aliquots of saturated solutions were removed and titrated with a standardized sodium hydroxide solution (carbonate free) using a pH meter. The endpoint of the titration was determined by computing the second derivative in the $\mathrm{pH}$ versus volume of sodium hydroxide added.

Source and Purity of Chemicals:

(1) Purity not given, Chemical source not specified, was recrystallized several times from aqueous-ethanol mixture, and then dried under vacuum over phosphorous pentoxide.

(2) Practical grade, Chemical source not specified, stored over sodium hydroxide pellets for $24 \mathrm{~h}$, and then passed through $2 \times 70-\mathrm{cm}$ chromatographic adsorption columns containing activated alumina. After this treatment, the purified solvent was stored over copper in a nitrogen atmosphere.

\section{Estimated Error:}

Temperature: $\pm 0.02 \mathrm{~K}$.

$x_{1}: \pm 1.0 \%$ (relative error).

\subsection{4-Chloro-3-nitrobenzoic acid solubility data in alcohols}

\begin{tabular}{|c|c|}
\hline $\begin{array}{l}\text { Components: } \\
\text { (1) 4-Chloro-3-nitrobenzoic acid; } \\
\mathrm{C}_{7} \mathrm{H}_{4} \mathrm{ClNO}_{4} ;[96-99-1] \\
\text { (2) Methanol; } \mathrm{CH}_{4} \mathrm{O} ;[67-56-1]\end{array}$ & $\begin{array}{l}\text { Original Measurements: } \\
{ }^{83} \text { M. K. Chantooni and I. M. Kolthoff } \\
\text { J. Phys. Chem. 77, } 527 \text { (1973). }\end{array}$ \\
\hline $\begin{array}{l}\text { Variables: } \\
T / \mathrm{K}=298.15\end{array}$ & $\begin{array}{l}\text { Prepared by: } \\
\text { W. E. Acree, Jr. }\end{array}$ \\
\hline
\end{tabular}

\section{Experimental Values}

The measured solubility was reported to be $0.705 \mathrm{~mol} \mathrm{dm}^{-3}$. The authors did not give the temperature at which the solubility was measured. Based on the experimental description given in an earlier paper [I. M. Kolthoff, J. J. Lingane, and W. Larson, J. Am. Chem. Soc. 60, 2512 (1938)], the compiler believes the temperature to be $298.15 \mathrm{~K}$.

\section{Auxiliary Information}

\section{Method/Apparatus/Procedure:}

Very little experimental details were provided. Solubility was determined by titrating a filtered aliquot of the saturated solution alkalimetrically in an aqueous-ethanol mixture using phenolphthalein as the acid-base indicator.

Source and Purity of Chemicals:

(1) Purity not given, Baker Chemical Company, USA, was recrystallized from either aqueous or aqueous-ethanol solution and dried in vacuo at $343 \mathrm{~K}$. (2) Purity not given, Chemical source not given, was dehydrated and then distilled over sulfanilic acid to remove alkaline impurities.

Estimated Error:

Temperature: $\pm 0.1 \mathrm{~K}$ (estimated by compiler). $c_{1}: \pm 2 \%$ (relative error, estimated by compiler).

\begin{tabular}{ll}
\hline \hline Components: & Original Measurements: \\
(1) 4-Chloro-3-nitrobenzoic acid; & ${ }^{13}$ D. M. Stovall, C. Givens, S. Keown, \\
$\mathrm{C}_{7} \mathrm{H}_{4} \mathrm{ClNO}_{4} ;$ [96-99-1] & K. R. Hoover, R. Barnes, C. Harris, J. \\
(2) Ethanol; $\mathrm{C}_{2} \mathrm{H}_{6} \mathrm{O} ;$ [54-17-5] & Lozano, M. Nguyen, E. Rodriguez, W. \\
& E. Acree, Jr., and M. H. Abraham, \\
& Phys. Chem. Liq. 43, 351 (2005). \\
\hline Variables: & Prepared by: \\
$T / \mathrm{K}=298.15$ & W. E. Acree, Jr. \\
\hline
\end{tabular}

Experimental Values

\begin{tabular}{lc}
\hline \hline$x_{2}{ }^{\mathrm{a}}$ & $x_{1}{ }^{\mathrm{b}}$ \\
\hline 0.9665 & 0.03348 \\
\hline${ }^{\mathrm{a}} x_{2}:$ mole fraction of component 2 in the saturated solution. & \\
${ }^{\mathrm{b}} x_{1}:$ mole fraction solubility of the solute. &
\end{tabular}




\section{Auxiliary Information}

\section{Method/Apparatus/Procedure:}

Constant-temperature bath, calorimetric thermometer, and an ultraviolet/ visible spectrophotometer.

Excess solute and solvent were placed in amber glass bottles and allowed to equilibrate for several days at constant temperature. Attainment of equilibrium was verified by several repetitive measurements and by approaching equilibrium from supersaturation. Aliquots of saturated solutions were transferred through a coarse filter into tared volumetric flasks, weighed, and diluted with methanol. Concentrations were determined by

spectrophotometric measurements at $292 \mathrm{~nm}$.

Source and Purity of Chemicals:

(1) $99.5 \%$, Acros Organics, USA, was used as received.

(2) Absolute, Aaper Alcohol and Chemical Company, USA, stored over molecular sieves and distilled shortly before use.

Estimated Error:

Temperature: $\pm 0.1 \mathrm{~K}$.

$x_{1}: \pm 1.5 \%$ (relative error)

\section{Components:}

Original Measurements:

(1) 4-Chloro-3-nitrobenzoic acid;

$\mathrm{C}_{7} \mathrm{H}_{4} \mathrm{ClNO}_{4}$; [96-99-1]

(2) 1-Propanol; $\mathrm{C}_{3} \mathrm{H}_{8} \mathrm{O}$; [71-23-8]

${ }^{2}$ D. M. Stovall, C. Givens, S. Keown,

K. R. Hoover, R. Barnes, C. Harris, J.

Lozano, M. Nguyen, E. Rodriguez, W

E. Acree, Jr., and M. H. Abraham,

Phys. Chem. Liq. 43, 351 (2005).

\begin{tabular}{ll}
\hline Variables: & Prepared by: \\
$T / \mathrm{K}=298.15$ & W. E. Acree, Jr.
\end{tabular}

Experimental Values

\begin{tabular}{lc}
\hline \hline$x_{2}{ }^{\mathrm{a}}$ & $x_{1}{ }^{\mathrm{b}}$ \\
\hline 0.9719 & 0.02812
\end{tabular}

${ }^{a} x_{2}$ : mole fraction of component 2 in the saturated solution.

${ }^{\mathrm{b}} x_{1}$ : mole fraction solubility of the solute.

\section{Auxiliary Information}

Method/Apparatus/Procedure:
Constant-temperature bath, calorimetric thermometer, and an ultraviolet/
visible spectrophotometer.
Excess solute and solvent were placed in amber glass bottles and allowed to
equilibrate for several days at constant temperature. Attainment of equilibrium
was verified by several repetitive measurements and by approaching
equilibrium from supersaturation. Aliquots of saturated solutions were
transferred through a coarse filter into tared volumetric flasks, weighed, and
diluted with methanol. Concentrations were determined by
spectrophotometric measurements at 292 nm.
Source and Purity of Chemicals:
(1) $99.5 \%$, Acros Organics, USA, was used as received.
(2) $99+\%$, anhydrous, Aldrich Chemical Company, Milwaukee, WI, USA,
stored over molecular sieves and distilled shortly before use.
Estimated Error:
Temperature: $\pm 0.1 \mathrm{~K}$.
$x_{1}: \pm 1.5 \%$ (relative error).

\begin{tabular}{ll}
\hline \hline Components: & Original Measurements: \\
(1) 4-Chloro-3-nitrobenzoic acid; & ${ }^{13}$ D. M. Stovall, C. Givens, S. Keown, \\
$\mathrm{C}_{7} \mathrm{H}_{4} \mathrm{ClNO}_{4}$; [96-99-1] & K. R. Hoover, R. Barnes, C. Harris, J. \\
(2) 2-Propanol; $\mathrm{C}_{3} \mathrm{H}_{8} \mathrm{O} ;$ [67-63-0] & Lozano, M. Nguyen, E. Rodriguez, W. \\
& E. Acree, Jr., and M. H. Abraham, \\
& Phys. Chem. Liq. 43, 351 (2005). \\
\hline Variables: & Prepared by: \\
$T / \mathrm{K}=298.15$ & W. E. Acree, Jr. \\
\hline
\end{tabular}

Experimental Values

\begin{tabular}{|c|c|}
\hline$x_{2}{ }^{\mathrm{a}}$ & $x_{1}{ }^{\mathrm{b}}$ \\
\hline 0.9696 & 0.03036 \\
\hline
\end{tabular}

\section{Auxiliary Information}

\section{Method/Apparatus/Procedure:}

Constant-temperature bath, calorimetric thermometer, and an ultraviolet/ visible spectrophotometer.

Excess solute and solvent were placed in amber glass bottles and allowed to equilibrate for several days at constant temperature. Attainment of equilibrium was verified by several repetitive measurements and by approaching equilibrium from supersaturation. Aliquots of saturated solutions were transferred through a coarse filter into tared volumetric flasks, weighed, and diluted with methanol. Concentrations were determined by spectrophotometric measurements at $292 \mathrm{~nm}$.

Source and Purity of Chemicals:

(1) $99.5 \%$, Acros Organics, USA, was used as received.

(2) $99+\%$, anhydrous, Aldrich Chemical Company, Milwaukee, WI, USA, stored over molecular sieves and distilled shortly before use.

\section{Estimated Error:}

Temperature: $\pm 0.1 \mathrm{~K}$.

$x_{1}: \pm 1.5 \%$ (relative error).

\begin{tabular}{|c|c|}
\hline $\begin{array}{l}\text { Components: } \\
\text { (1) 4-Chloro-3-nitrobenzoic acid; } \\
\mathrm{C}_{7} \mathrm{H}_{4} \mathrm{ClNO}_{4} \text {; [96-99-1] } \\
\text { (2) 2-Propanol; } \mathrm{C}_{3} \mathrm{H}_{8} \mathrm{O} ;[67-63-0]\end{array}$ & $\begin{array}{l}\text { Original Measurements: } \\
{ }^{76} \text { M. K. Chantooni and I. M. Kolthoff, } \\
\text { Anal. Chem. 51, } 133 \text { (1979). }\end{array}$ \\
\hline $\begin{array}{l}\text { Variables: } \\
T / \mathrm{K}=298.15\end{array}$ & $\begin{array}{l}\text { Prepared by: } \\
\text { W. E. Acree, Jr. }\end{array}$ \\
\hline
\end{tabular}

\section{Experimental Values}

The measured solubility was reported to be $0.436 \mathrm{~mol} \mathrm{dm}^{-3}$. The authors did not give the temperature at which the solubility was measured. Based on the experimental description given in an earlier paper [I. M. Kolthoff, J. J. Lingane, and W. Larson, J. Am. Chem. Soc. 60, 2512 (1938)], the compiler believes the temperature to be $298.15 \mathrm{~K}$. 


\section{Auxiliary Information}

\section{Method/Apparatus/Procedure:}

Very little experimental details were provided. Solubility was determined by titrating a filtered aliquot of the saturated solution alkalimetrically in an aqueous-ethanol mixture using phenolphthalein as the acid-base indicator.

\section{Source and Purity of Chemicals:}

(1) Purity not given, Baker Chemical Company, USA, was recrystallized from either aqueous or aqueous-ethanol solution and dried in vacuo at $333 \mathrm{~K}$ before use.

(2) Purity not given, Fisher Scientific, USA, shaken with calcium hydride and distilled before use.

\section{Estimated Error:}

Temperature: $\pm 0.1 \mathrm{~K}$ (estimated by compiler). $c_{1}: \pm 2 \%$ (relative error, estimated by compiler).

\begin{tabular}{ll}
\hline \hline Components: & Original Measurements: \\
(1) 4-Chloro-3-nitrobenzoic acid; & ${ }^{13}$ D. M. Stovall, C. Givens, S. Keown, \\
$\mathrm{C}_{7} \mathrm{H}_{4} \mathrm{ClNO}_{4}$; [96-99-1] & K. R. Hoover, R. Barnes, C. Harris, J. \\
(2) 1-Butanol; $\mathrm{C}_{4} \mathrm{H}_{10} \mathrm{O} ;$ [71-36-3] & Lozano, M. Nguyen, E. Rodriguez, W. \\
& E. Acree, Jr., and M. H. Abraham, \\
& Phys. Chem. Liq. 43, 351 (2005). \\
\hline Variables: & Prepared by: \\
$T / \mathrm{K}=298.15$ & W. E. Acree, Jr. \\
\hline
\end{tabular}

\section{Experimental Values}

\begin{tabular}{lc}
\hline \hline$x_{2}^{\mathrm{a}}$ & $x_{1}{ }^{\mathrm{b}}$ \\
\hline 0.9713 & 0.02874 \\
\hline
\end{tabular}

${ }^{\mathrm{a}} x_{2}$ : mole fraction of component 2 in the saturated solution.

${ }^{\mathrm{b}} x_{1}$ : mole fraction solubility of the solute.

\section{Auxiliary Information}

\section{Method/Apparatus/Procedure:}

Constant-temperature bath, calorimetric thermometer, and an ultraviolet/ visible spectrophotometer.

Excess solute and solvent were placed in amber glass bottles and allowed to equilibrate for several days at constant temperature. Attainment of equilibrium was verified by several repetitive measurements and by approaching equilibrium from supersaturation. Aliquots of saturated solutions were transferred through a coarse filter into tared volumetric flasks, weighed, and diluted with methanol. Concentrations were determined by spectrophotometric measurements at $292 \mathrm{~nm}$.

\section{Source and Purity of Chemicals:}

(1) $99.5 \%$, Acros Organics, USA, was used as received.

(2) $99.8 \%$, HPLC grade, Aldrich Chemical Company, Milwaukee, WI, USA, stored over molecular sieves and distilled shortly before use.

\section{Estimated Error:}

Temperature: $\pm 0.1 \mathrm{~K}$.

$x_{1}: \pm 1.5 \%$ (relative error).

\begin{tabular}{ll}
\hline \hline Components: & Original Measurements: \\
$\begin{array}{l}\text { (1) 4-Chloro-3-nitrobenzoic acid; } \\
\mathrm{C}_{7} \mathrm{H}_{4} \mathrm{ClNO}_{4} \text {; [96-99-1] }\end{array}$ & K. M. Stovall, C. Givens, S. Keown, \\
(2) 2-Butanol; $\mathrm{C}_{4} \mathrm{H}_{10} \mathrm{O} ;$ [78-92-2] & Lozano, M. Nguyen, E. Rodriguez, W \\
& E. Acree, Jr., and M. H. Abraham, \\
& Phys. Chem. Liq. 43, 351 (2005). \\
\hline Variables: & Prepared by: \\
$T / \mathrm{K}=298.15$ & W. E. Acree, Jr. \\
\hline
\end{tabular}

Experimental Values

\begin{tabular}{lc}
\hline \hline$x_{2}{ }^{\mathrm{a}}$ & $x_{1}{ }^{\mathrm{b}}$ \\
\hline 0.9704 & 0.02962 \\
\hline${ }^{\mathrm{a}} x_{2}:$ mole fraction of component 2 in the saturated solution. & \\
${ }^{\mathrm{b}} x_{1}:$ mole fraction solubility of the solute. &
\end{tabular}

\section{Auxiliary Information}

\section{Method/Apparatus/Procedure:}

Constant-temperature bath, calorimetric thermometer, and an ultraviolet/ visible spectrophotometer.

Excess solute and solvent were placed in amber glass bottles and allowed to equilibrate for several days at constant temperature. Attainment of equilibrium was verified by several repetitive measurements and by approaching equilibrium from supersaturation. Aliquots of saturated solutions were transferred through a coarse filter into tared volumetric flasks, weighed, and diluted with methanol. Concentrations were determined by spectrophotometric measurements at $292 \mathrm{~nm}$.

\section{Source and Purity of Chemicals:}

(1) $99.5 \%$, Acros Organics, USA, was used as received.

(2) $99+\%$, anhydrous, Aldrich Chemical Company, Milwaukee, WI, USA, stored over molecular sieves and distilled shortly before use.

\section{Estimated Error:}

Temperature: $\pm 0.1 \mathrm{~K}$.

$x_{1}: \pm 1.5 \%$ (relative error).

\begin{tabular}{ll}
\hline \hline Components: & Original Measurements: \\
(1) 4-Chloro-3-nitrobenzoic acid; & ${ }^{13}$ D. M. Stovall, C. Givens, S. Keown, \\
$\mathrm{C}_{7} \mathrm{H}_{4} \mathrm{ClNO}_{4}$; [96-99-1] & K. R. Hoover, R. Barnes, C. Harris, J. \\
(2) 2-Methyl-1-propanol; & Lozano, M. Nguyen, E. Rodriguez, W. \\
$\mathrm{C}_{4} \mathrm{H}_{10} \mathrm{O}$; [78-83-1] & E. Acree, Jr., and M. H. Abraham, \\
& Phys. Chem. Liq. 43, 351 (2005). \\
\hline Variables: & Prepared by: \\
$T / \mathrm{K}=298.15$ & W. E. Acree, Jr. \\
\hline
\end{tabular}

\section{Experimental Values}

\begin{tabular}{lc}
\hline \hline$x_{2}^{\mathrm{a}}$ & $x_{1}^{\mathrm{b}}$ \\
\hline 0.9825 & 0.01747 \\
\hline${ }^{\mathrm{a}} x_{2}:$ mole fraction of component 2 in the saturated solution. & \\
${ }^{\mathrm{b}} x_{1}:$ mole fraction solubility of the solute. &
\end{tabular}




\section{Auxiliary Information}

\section{Method/Apparatus/Procedure:}

Constant-temperature bath, calorimetric thermometer, and an ultraviolet/ visible spectrophotometer.

Excess solute and solvent were placed in amber glass bottles and allowed to equilibrate for several days at constant temperature. Attainment of equilibrium was verified by several repetitive measurements and by approaching equilibrium from supersaturation. Aliquots of saturated solutions were transferred through a coarse filter into tared volumetric flasks, weighed, and diluted with methanol. Concentrations were determined by spectrophotometric measurements at $292 \mathrm{~nm}$.

Source and Purity of Chemicals:

(1) $99.5 \%$, Acros Organics, USA, was used as received.

(2) $99+\%$, anhydrous, Aldrich Chemical Company, Milwaukee, WI, USA, stored over molecular sieves and distilled shortly before use.

Estimated Error:

Temperature: $\pm 0.1 \mathrm{~K}$.

$x_{1}: \pm 1.5 \%$ (relative error)

\begin{tabular}{ll}
\hline \hline Components: & Original Measurements: \\
(1) 4-Chloro-3-nitrobenzoic acid; & ${ }^{13}$ D. M. Stovall, C. Givens, S. Keown, \\
$\mathrm{C}_{7} \mathrm{H}_{4} \mathrm{ClNO}_{4}$; [96-99-1] & K. R. Hoover, R. Barnes, C. Harris, J. \\
(2) 2-Methyl-2-propanol; & Lozano, M. Nguyen, E. Rodriguez, W. \\
$\mathrm{C}_{4} \mathrm{H}_{10} \mathrm{O}$; [75-65-0] & E. Acree, Jr., and M. H. Abraham, \\
& Phys. Chem. Liq. 43, 351 (2005). \\
\hline Variables: & Prepared by: \\
$T / \mathrm{K}=298.15$ & W. E. Acree, Jr. \\
\hline
\end{tabular}

Experimental Values

\begin{tabular}{lc}
\hline \hline$x_{2}{ }^{\mathrm{a}}$ & $x_{1}{ }^{\mathrm{b}}$ \\
\hline 0.9649 & 0.03509 \\
\hline$x_{2}:$ mole fration of componen 2 inthe sats
\end{tabular}

${ }^{\mathrm{a}} x_{2}$ : mole fraction of component 2 in the saturated solution.

${ }^{\mathrm{b}} x_{1}$ : mole fraction solubility of the solute.

\section{Auxiliary Information}

\footnotetext{
Method/Apparatus/Procedure:

Constant-temperature bath, calorimetric thermometer, and an ultraviolet/ visible spectrophotometer.

Excess solute and solvent were placed in amber glass bottles and allowed to equilibrate for several days at constant temperature. Attainment of equilibrium was verified by several repetitive measurements and by approaching equilibrium from supersaturation. Aliquots of saturated solutions were transferred through a coarse filter into tared volumetric flasks, weighed, and diluted with methanol. Concentrations were determined by spectrophotometric measurements at $292 \mathrm{~nm}$.
}

Source and Purity of Chemicals:

(1) $99.5 \%$, Acros Organics, USA, was used as received.

(2) $99+\%$, Arco Chemical Company, USA, stored over molecular sieves and distilled shortly before use.

\section{Estimated Error:}

Temperature: $\pm 0.1 \mathrm{~K}$.

$x_{1}: \pm 1.5 \%$ (relative error).

\begin{tabular}{|c|c|}
\hline $\begin{array}{l}\text { Components: } \\
\text { (1) 4-Chloro-3-nitrobenzoic acid; } \\
\mathrm{C}_{7} \mathrm{H}_{4} \mathrm{ClNO}_{4} \text {; [96-99-1] } \\
\text { (2) 2-Methyl-2-propanol; } \mathrm{C}_{4} \mathrm{H}_{10} \mathrm{O} \text {; } \\
\text { [75-65-0] }\end{array}$ & $\begin{array}{l}\text { Original Measurements: } \\
{ }^{76} \text { M. K. Chantooni and I. M. Kolthoff, } \\
\text { Anal. Chem. 51, } 133 \text { (1979). }\end{array}$ \\
\hline $\begin{array}{l}\text { Variables: } \\
T / \mathrm{K}=298.15\end{array}$ & $\begin{array}{l}\text { Prepared by: } \\
\text { W. E. Acree, Jr. }\end{array}$ \\
\hline
\end{tabular}

\section{Experimental Values}

The measured solubility was reported to be $0.511 \mathrm{~mol} \mathrm{dm}^{-3}$. The authors did not give the temperature at which the solubility was measured. Based on the experimental description given in an earlier paper [I. M. Kolthoff, J. J. Lingane, and W. Larson, J. Am. Chem. Soc. 60, 2512 (1938)], the compiler believes the temperature to be $298.15 \mathrm{~K}$.

\section{Auxiliary Information}

\section{Method/Apparatus/Procedure:}

Very little experimental details were provided. Solubility was determined by titrating a filtered aliquot of the saturated solution alkalimetrically in an aqueous-ethanol mixture using phenolphthalein as the acid-base indicator.

\section{Source and Purity of Chemicals:}

(1) Purity not given, Baker Chemical Company, USA, was recrystallized from either aqueous or aqueous-ethanol solution and dried in vacuo at $333 \mathrm{~K}$ before use.

(2) White Label, Eastman Kodak Chemical Company, Rochester, NY, USA, shaken with calcium hydride and distilled before use.

\section{Estimated Error:}

Temperature: $\pm 0.1 \mathrm{~K}$ (estimated by compiler). $c_{1}: \pm 2 \%$ (relative error, estimated by compiler).

\begin{tabular}{ll}
\hline \hline Components: & Original Measurements: \\
(1) 4-Chloro-3-nitrobenzoic acid; & ${ }^{13}$ D. M. Stovall, C. Givens, S. Keown, \\
$\mathrm{C}_{7} \mathrm{H}_{4} \mathrm{ClNO}_{4}$; [96-99-1] & K. R. Hoover, R. Barnes, C. Harris, J. \\
(2) 1-Pentanol; $\mathrm{C}_{5} \mathrm{H}_{12} \mathrm{O} ;$ [71-41-0] & Lozano, M. Nguyen, E. Rodriguez, W. \\
& E. Acree, Jr., and M. H. Abraham, \\
& Phys. Chem. Liq. 43, 351 (2005). \\
\hline Variables: & Prepared by: \\
$T / \mathrm{K}=298.15$ & W. E. Acree, Jr. \\
\hline
\end{tabular}

\section{Experimental Values}

\begin{tabular}{lc}
\hline \hline$x_{2}{ }^{\mathrm{a}}$ & $x_{1}{ }^{\mathrm{b}}$ \\
\hline 0.9700 & 0.03001 \\
\hline${ }^{\mathrm{a}} x_{2}:$ mole fraction of component 2 in the saturated solution. & \\
${ }^{\mathrm{b}} x_{1}:$ mole fraction solubility of the solute. &
\end{tabular}




\section{Auxiliary Information}

\section{Method/Apparatus/Procedure:}

Constant-temperature bath, calorimetric thermometer, and an ultraviolet/ visible spectrophotometer.

Excess solute and solvent were placed in amber glass bottles and allowed to equilibrate for several days at constant temperature. Attainment of equilibrium was verified by several repetitive measurements and by approaching equilibrium from supersaturation. Aliquots of saturated solutions were transferred through a coarse filter into tared volumetric flasks, weighed, and diluted with methanol. Concentrations were determined by spectrophotometric measurements at $292 \mathrm{~nm}$.

Source and Purity of Chemicals:

(1) $99.5 \%$, Acros Organics, USA, was used as received.

(2) $99+\%$, Aldrich Chemical Company, Milwaukee, WI, USA, stored over molecular sieves and distilled shortly before use.

\section{Estimated Error:}

Temperature: $\pm 0.1 \mathrm{~K}$.

$x_{1}: \pm 1.5 \%$ (relative error)

\begin{tabular}{ll}
\hline \hline Components: & Original Measurements: \\
$\begin{array}{l}\text { (1) 4-Chloro-3-nitrobenzoic acid; } \\
\mathrm{C}_{7} \mathrm{H}_{4} \mathrm{ClNO}_{4} \text {; [96-99-1] }\end{array}$ & ${ }^{13}$ D. M. Stovall, C. Givens, S. Keown, \\
(2) 2-Pentanol; $\mathrm{C}_{5} \mathrm{H}_{12} \mathrm{O} ;$ & K. R. Hoover, R. Barnes, C. Harris, J. \\
6032-29-7] & Lozano, M. Nguyen, E. Rodriguez, W. \\
& E. Acree, Jr., and M. H. Abraham, \\
\hline Variables: & Phys. Chem. Liq. 43, 351 (2005). \\
$T / \mathrm{K}=298.15$ & Prepared by: \\
\hline
\end{tabular}

\section{Experimental Values}

\begin{tabular}{lc}
\hline \hline$x_{2}{ }^{\mathrm{a}}$ & $x_{1}{ }^{\mathrm{b}}$ \\
\hline 0.9671 & 0.03290 \\
\hline$x_{2}$ m
\end{tabular}

${ }^{\mathrm{a}} x_{2}$ : mole fraction of component 2 in the saturated solution.

${ }^{\mathrm{b}}{ }_{x_{1}}$ : mole fraction solubility of the solute.

\section{Auxiliary Information}

Method/Apparatus/Procedure:
Constant-temperature bath, calorimetric thermometer, and an ultraviolet/
visible spectrophotometer.
Excess solute and solvent were placed in amber glass bottles and allowed to
equilibrate for several days at constant temperature. Attainment of equilibrium
was verified by several repetitive measurements and by approaching
equilibrium from supersaturation. Aliquots of saturated solutions were
transferred through a coarse filter into tared volumetric flasks, weighed, and
diluted with methanol. Concentrations were determined by
spectrophotometric measurements at 292 nm.
Source and Purity of Chemicals:
(1) $99.5 \%$, Acros Organics, USA, was used as received.
(2) $99+\%$, Acros Organics, stored over molecular sieves and distilled shortly
before use.
Estimated Error:
Temperature: $\pm 0.1 \mathrm{~K}$.
$x_{1}: \pm 1.5 \%$ (relative error).

\begin{tabular}{|c|c|}
\hline \multirow{6}{*}{$\begin{array}{l}\text { Components: } \\
\text { (1) 4-Chloro-3-nitrobenzoic acid; } \\
\mathrm{C}_{7} \mathrm{H}_{4} \mathrm{ClNO}_{4} \text {; [96-99-1] } \\
\text { (2) 3-Methyl-1-butanol; } \mathrm{C}_{5} \mathrm{H}_{12} \mathrm{O} \text {; } \\
\text { [123-51-3] }\end{array}$} & \multirow{6}{*}{$\begin{array}{l}\text { Original Measurements: } \\
{ }^{13} \text { D. M. Stovall, C. Givens, S. Keown, } \\
\text { K. R. Hoover, R. Barnes, C. Harris, J. } \\
\text { Lozano, M. Nguyen, E. Rodriguez, W. } \\
\text { E. Acree, Jr., and M. H. Abraham, } \\
\text { Phys. Chem. Liq. 43, } 351 \text { (2005). }\end{array}$} \\
\hline & \\
\hline & \\
\hline & \\
\hline & \\
\hline & \\
\hline Variables: & Prepar \\
\hline$T / \mathrm{K}=298.15$ & W. E. Acree, Jr. \\
\hline
\end{tabular}

Experimental Values

\begin{tabular}{lc}
\hline \hline$x_{2}{ }^{\mathrm{a}}$ & $x_{1}{ }^{\mathrm{b}}$ \\
\hline 0.9739 & 0.02606 \\
\hline${ }^{a} x_{2}:$ mole fraction of component 2 in the saturated solution. & \\
${ }^{\mathrm{b}} x_{1}:$ mole fraction solubility of the solute. &
\end{tabular}

\section{Auxiliary Information}

\section{Method/Apparatus/Procedure:}

Constant-temperature bath, calorimetric thermometer, and an ultraviolet/ visible spectrophotometer.

Excess solute and solvent were placed in amber glass bottles and allowed to equilibrate for several days at constant temperature. Attainment of equilibrium was verified by several repetitive measurements and by approaching equilibrium from supersaturation. Aliquots of saturated solutions were transferred through a coarse filter into tared volumetric flasks, weighed, and diluted with methanol. Concentrations were determined by spectrophotometric measurements at $292 \mathrm{~nm}$.

Source and Purity of Chemicals:

(1) $99.5 \%$, Acros Organics, USA, was used as received.

(2) 99\%, anhydrous, Aldrich Chemical Company, Milwaukee, WI, USA, stored over molecular sieves and distilled shortly before use.

\section{Estimated Error:}

Temperature: $\pm 0.1 \mathrm{~K}$.

$x_{1}: \pm 1.5 \%$ (relative error).

\begin{tabular}{|c|c|}
\hline $\begin{array}{l}\text { Components: } \\
\text { (1) 4-Chloro-3-nitrobenzoic acid; } \\
\mathrm{C}_{7} \mathrm{H}_{4} \mathrm{ClNO}_{4} ;[96-99-1] \\
\text { (2) 1-Hexanol; } \mathrm{C}_{6} \mathrm{H}_{14} \mathrm{O} ;[111-27-3]\end{array}$ & $\begin{array}{l}\text { Original Measurements: } \\
{ }^{13} \text { D. M. Stovall, C. Givens, S. Keown, } \\
\text { K. R. Hoover, R. Barnes, C. Harris, J. } \\
\text { Lozano, M. Nguyen, E. Rodriguez, W. } \\
\text { E. Acree, Jr., and M. H. Abraham, } \\
\text { Phys. Chem. Liq. 43, } 351 \text { (2005). }\end{array}$ \\
\hline $\begin{array}{l}\text { Variables: } \\
T / \mathrm{K}=298.15\end{array}$ & $\begin{array}{l}\text { Prepared by: } \\
\text { W. E. Acree, Jr. }\end{array}$ \\
\hline
\end{tabular}

\section{Experimental Values}

\begin{tabular}{lc}
\hline \hline$x_{2}{ }^{\mathrm{a}}$ & $x_{1}{ }^{\mathrm{b}}$ \\
\hline 0.9668 & 0.03320 \\
\hline${ }^{\mathrm{a}} x_{2}:$ mole fraction of component 2 in the saturated solution. & \\
${ }^{\mathrm{b}} x_{1}:$ mole fraction solubility of the solute. &
\end{tabular}




\section{Auxiliary Information}

\section{Method/Apparatus/Procedure:}

Constant-temperature bath, calorimetric thermometer, and an ultraviolet/ visible spectrophotometer.

Excess solute and solvent were placed in amber glass bottles and allowed to equilibrate for several days at constant temperature. Attainment of equilibrium was verified by several repetitive measurements and by approaching equilibrium from supersaturation. Aliquots of saturated solutions were transferred through a coarse filter into tared volumetric flasks, weighed, and diluted with methanol. Concentrations were determined by

spectrophotometric measurements at $292 \mathrm{~nm}$.

Source and Purity of Chemicals:

(1) $99.5 \%$, Acros Organics, USA, was used as received.

(2) $99+\%$, Alfa Aesar, USA, stored over molecular sieves and distilled shortly before use.

Estimated Error:

Temperature: $\pm 0.1 \mathrm{~K}$.

$x_{1}: \pm 1.5 \%$ (relative error)

\section{Components:}

Original Measurements:

(1) 4-Chloro-3-nitrobenzoic acid;

$\mathrm{C}_{7} \mathrm{H}_{4} \mathrm{ClNO}_{4}$; [96-99-1]

(2) 1-Hexanol; $\mathrm{C}_{6} \mathrm{H}_{14} \mathrm{O}$; [111-27-3]

\begin{tabular}{ll}
\hline Variables: & Prepared by: \\
$T / \mathrm{K}=298.15$ & W. E. Acree, Jr. \\
\hline
\end{tabular}

\section{Experimental Values}

The measured solubility was reported to be $0.283 \mathrm{~mol} \mathrm{dm}^{-3}$. The authors did not give the temperature at which the solubility was measured. Based on the experimental description given in an earlier paper [I. M. Kolthoff, J. J. Lingane, and W. Larson, J. Am. Chem. Soc. 60, 2512 (1938)], the compiler believes the temperature to be $298.15 \mathrm{~K}$.

\section{Auxiliary Information}

Method/Apparatus/Procedure:

Very little experimental details were provided. Solubility was determined by titrating a filtered aliquot of the saturated solution alkalimetrically in an aqueous-ethanol mixture using phenolphthalein as the acid-base indicator.

Source and Purity of Chemicals:

(1) Purity not given, Baker Chemical Company, USA, was recrystallized from either aqueous or aqueous-ethanol solution and dried in vacuo at $333 \mathrm{~K}$ before use.

(2) Yellow Label, Eastman Kodak Chemical Company, Rochester, NY, USA, shaken with calcium hydride and distilled before use.

\section{Estimated Error:}

Temperature: $\pm 0.1 \mathrm{~K}$ (estimated by compiler).

$c_{1}: \pm 2 \%$ (relative error, estimated by compiler).

\begin{tabular}{ll}
\hline \hline Components: & Original Measurements: \\
(1) 4-Chloro-3-nitrobenzoic acid; & ${ }^{13}$ D. M. Stovall, C. Givens, S. Keown, \\
$\mathrm{C}_{7} \mathrm{H}_{4} \mathrm{ClNO}_{4}$; [96-99-1] & K. R. Hoover, R. Barnes, C. Harris, J. \\
$\begin{array}{l}\text { (2) 2-Methyl-1-pentanol; } \mathrm{C}_{6} \mathrm{H}_{14} \mathrm{O} ; \\
\text { [105-30-6] }\end{array}$ & Lozano, M. Nguyen, E. Rodriguez, W. \\
& E. Acree, Jr., and M. H. Abraham, \\
& Phys. Chem. Liq. 43, 351 (2005). \\
\hline Variables: & Prepared by: \\
$T / \mathrm{K}=298.15$ & W. E. Acree, Jr. \\
\hline
\end{tabular}

Experimental Values

\begin{tabular}{lc}
\hline \hline$x_{2}{ }^{\mathrm{a}}$ & $x_{1}{ }^{\mathrm{b}}$ \\
\hline 0.9764 & 0.02356 \\
\hline${ }^{\mathrm{a}} x_{2}:$ mole fraction of component 2 in the saturated solution. & \\
${ }^{\mathrm{b}} x_{1}:$ mole fraction solubility of the solute. &
\end{tabular}

\section{Auxiliary Information}

\section{Method/Apparatus/Procedure:}

Constant-temperature bath, calorimetric thermometer, and an ultraviolet/ visible spectrophotometer.

Excess solute and solvent were placed in amber glass bottles and allowed to equilibrate for several days at constant temperature. Attainment of equilibrium was verified by several repetitive measurements and by approaching equilibrium from supersaturation. Aliquots of saturated solutions were transferred through a coarse filter into tared volumetric flasks, weighed, and diluted with methanol. Concentrations were determined by spectrophotometric measurements at $292 \mathrm{~nm}$.

Source and Purity of Chemicals:

(1) $99.5 \%$, Acros Organics, USA, was used as received.

(2) $99 \%$, Aldrich Chemical Company, Milwaukee, WI, USA, stored over molecular sieves and distilled shortly before use.

Estimated Error:

Temperature: $\pm 0.1 \mathrm{~K}$.

$x_{1}: \pm 1.5 \%$ (relative error).

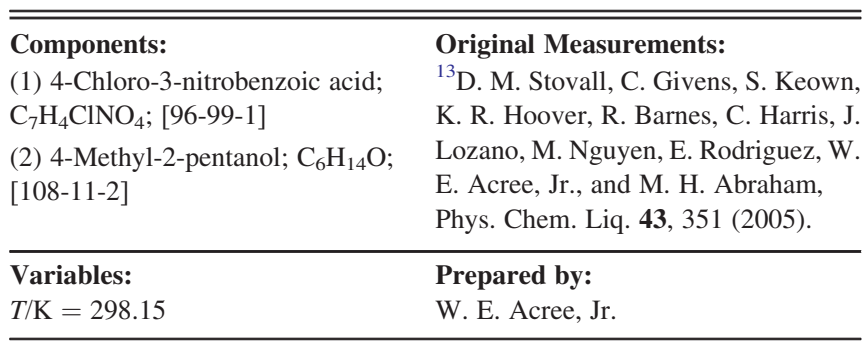

\section{Experimental Values}

\begin{tabular}{lc}
\hline \hline$x_{2}{ }^{\mathrm{a}}$ & $x_{1}{ }^{\mathrm{b}}$ \\
\hline 0.9711 & 0.02890 \\
\hline${ }^{\mathrm{a}} x_{2}:$ mole fraction of component 2 in the saturated solution. & \\
${ }^{\mathrm{b}} x_{1}:$ mole fraction solubility of the solute. &
\end{tabular}




\section{Auxiliary Information}

\section{Method/Apparatus/Procedure:}

Constant-temperature bath, calorimetric thermometer, and an ultraviolet/ visible spectrophotometer.

Excess solute and solvent were placed in amber glass bottles and allowed to equilibrate for several days at constant temperature. Attainment of equilibrium was verified by several repetitive measurements and by approaching equilibrium from supersaturation. Aliquots of saturated solutions were transferred through a coarse filter into tared volumetric flasks, weighed, and diluted with methanol. Concentrations were determined by spectrophotometric measurements at $292 \mathrm{~nm}$.

Source and Purity of Chemicals:

(1) $99.5 \%$, Acros Organics, USA, was used as received.

(2) $99+\%$, Acros Organics, stored over molecular sieves and distilled shortly before use.

Estimated Error:

Temperature: $\pm 0.1 \mathrm{~K}$.

$x_{1}: \pm 1.5 \%$ (relative error).

\section{Components:}

Original Measurements:

(1) 4-Chloro-3-nitrobenzoic acid;

$\mathrm{C}_{7} \mathrm{H}_{4} \mathrm{ClNO}_{4}$; [96-99-1]

(2) 1-Heptanol; $\mathrm{C}_{7} \mathrm{H}_{16} \mathrm{O}$; [111-70-6] Lozano, M. Nguyen, E. Rodriguez, W E. Acree, Jr., and M. H. Abraham, Phys. Chem. Liq. 43, 351 (2005).

\begin{tabular}{ll}
\hline Variables: & Prepared by: \\
$T / \mathrm{K}=298.15$ & W. E. Acree, Jr.
\end{tabular}

Experimental Values

\begin{tabular}{lc}
\hline \hline$x_{2}{ }^{\mathrm{a}}$ & $x_{1}{ }^{\mathrm{b}}$ \\
\hline 0.9652 & 0.03481 \\
\hline$x_{2}:$ mole fration of componen 2 inthe sats
\end{tabular}

${ }^{\mathrm{a}} x_{2}$ : mole fraction of component 2 in the saturated solution.

${ }^{\mathrm{b}} x_{1}$ : mole fraction solubility of the solute.

\section{Auxiliary Information}

Method/Apparatus/Procedure:
Constant-temperature bath, calorimetric thermometer, and an ultraviolet/
visible spectrophotometer.
Excess solute and solvent were placed in amber glass bottles and allowed to
equilibrate for several days at constant temperature. Attainment of equilibrium
was verified by several repetitive measurements and by approaching
equilibrium from supersaturation. Aliquots of saturated solutions were
transferred through a coarse filter into tared volumetric flasks, weighed, and
diluted with methanol. Concentrations were determined by
spectrophotometric measurements at 292 nm.
Source and Purity of Chemicals:
(1) $99.5 \%$, Acros Organics, USA, was used as received.
(2) $99+\%$, Alfa Aesar, USA, stored over molecular sieves and distilled shortly
before use.
Estimated Error:
Temperature: $\pm 0.1 \mathrm{~K}$.
$x_{1}: \pm 1.5 \%$ (relative error).

\begin{tabular}{|c|c|}
\hline $\begin{array}{l}\text { Components: } \\
\text { (1) 4-Chloro-3-nitrobenzoic acid; } \\
\mathrm{C}_{7} \mathrm{H}_{4} \mathrm{ClNO}_{4} \text {; [96-99-1] } \\
\text { (2) 1-Octanol; } \mathrm{C}_{8} \mathrm{H}_{18} \mathrm{O} ;[111-87-5]\end{array}$ & $\begin{array}{l}\text { Original Measurements: } \\
{ }^{13} \text { D. M. Stovall, C. Givens, S. Keown, } \\
\text { K. R. Hoover, R. Barnes, C. Harris, J. } \\
\text { Lozano, M. Nguyen, E. Rodriguez, W. } \\
\text { E. Acree, Jr., and M. H. Abraham, } \\
\text { Phys. Chem. Liq. 43, } 351 \text { (2005). }\end{array}$ \\
\hline $\begin{array}{l}\text { Variables: } \\
T / \mathrm{K}=298.15\end{array}$ & $\begin{array}{l}\text { Prepared by: } \\
\text { W. E. Acree, Jr. }\end{array}$ \\
\hline
\end{tabular}

Experimental Values

\begin{tabular}{lc}
\hline \hline$x_{2}{ }^{\mathrm{a}}$ & $x_{1}{ }^{\mathrm{b}}$ \\
\hline 0.9641 & 0.03589 \\
\hline${ }^{a} x_{2}:$ mole fraction of component 2 in the saturated solution. & \\
${ }^{\mathrm{b}} x_{1}:$ mole fraction solubility of the solute. &
\end{tabular}

\section{Auxiliary Information}

\section{Method/Apparatus/Procedure:}

Constant-temperature bath, calorimetric thermometer, and an ultraviolet/ visible spectrophotometer.

Excess solute and solvent were placed in amber glass bottles and allowed to equilibrate for several days at constant temperature. Attainment of equilibrium was verified by several repetitive measurements and by approaching equilibrium from supersaturation. Aliquots of saturated solutions were transferred through a coarse filter into tared volumetric flasks, weighed, and diluted with methanol. Concentrations were determined by spectrophotometric measurements at $292 \mathrm{~nm}$.

Source and Purity of Chemicals:

(1) $99.5 \%$, Acros Organics, USA, was used as received.

(2) $99+\%$, anhydrous, Aldrich Chemical Company, Milwaukee, WI, USA, stored over molecular sieves and distilled shortly before use.

\section{Estimated Error:}

Temperature: $\pm 0.1 \mathrm{~K}$.

$x_{1}: \pm 1.5 \%$ (relative error).

\begin{tabular}{ll}
\hline \hline Components: & Original Measurements: \\
(1) 4-Chloro-3-nitrobenzoic acid; & ${ }^{13}$ D. M. Stovall, C. Givens, S. Keown, \\
$\mathrm{C}_{7} \mathrm{H}_{4} \mathrm{ClNO}_{4}$; [96-99-1] & K. R. Hoover, R. Barnes, C. Harris, J. \\
$\begin{array}{l}\text { (2) 2-Ethyl-1-hexanol; } \mathrm{C}_{8} \mathrm{H}_{18} \mathrm{O} ; \\
\text { [104-76-7] }\end{array}$ & Lozano, M. Nguyen, E. Rodriguez, W. \\
& E. Acree, Jr., and M. H. Abraham, \\
& Phys. Chem. Liq. 43, 351 (2005). \\
\hline Variables: & Prepared by: \\
$T / \mathrm{K}=298.15$ & W. E. Acree, Jr. \\
\hline
\end{tabular}

\section{Experimental Values}

\begin{tabular}{lc}
\hline \hline$x_{2}{ }^{\mathrm{a}}$ & $x_{1}{ }^{\mathrm{b}}$ \\
\hline 0.9760 & 0.02399 \\
\hline${ }^{\mathrm{a}} x_{2}:$ mole fraction of component 2 in the saturated solution. & \\
${ }^{\mathrm{b}}{ }_{x_{1}: \text { mole fraction solubility of the solute. }}$ &
\end{tabular}




\section{Auxiliary Information}

Method/Apparatus/Procedure:

Constant-temperature bath, calorimetric thermometer, and an ultraviolet/ visible spectrophotometer.

Excess solute and solvent were placed in amber glass bottles and allowed to equilibrate for several days at constant temperature. Attainment of equilibrium was verified by several repetitive measurements and by approaching equilibrium from supersaturation. Aliquots of saturated solutions were transferred through a coarse filter into tared volumetric flasks, weighed, and diluted with methanol. Concentrations were determined by spectrophotometric measurements at $292 \mathrm{~nm}$.

Source and Purity of Chemicals:

(1) $99.5 \%$, Acros Organics, USA, was used as received.

(2) $99 \%$, Aldrich Chemical Company, Milwaukee, WI, USA, stored over molecular sieves and distilled shortly before use.

Estimated Error:

Temperature: $\pm 0.1 \mathrm{~K}$.

$x_{1}: \pm 1.5 \%$ (relative error)

\begin{tabular}{ll}
\hline \hline Components: & Original Measurements: \\
$\begin{array}{l}\text { (1) 4-Chloro-3-nitrobenzoic acid; } \\
\mathrm{C}_{7} \mathrm{H}_{4} \mathrm{ClNO}_{4} \text {; [96-99-1] }\end{array}$ & K. M. Stovall, C. Givens, S. Keown, \\
$(2) 1-$-Decanol; $\mathrm{C}_{10} \mathrm{H}_{22} \mathrm{O} ;$ & Lozano, R. B. Ngurnes, C. Harris, J. \\
{$[112-30-1]$} & E. Acree, Jr., and M. H. Abraham, \\
& Phys. Chem. Liq. 43, 351 (2005). \\
\hline Variables: & Prepared by: \\
$T / \mathrm{K}=298.15$ & W. E. Acree, Jr. \\
\hline
\end{tabular}

Experimental Values

\begin{tabular}{lc}
\hline \hline$x_{2}{ }^{\mathrm{a}}$ & $x_{1}{ }^{\mathrm{b}}$ \\
\hline 0.9649 & 0.03507 \\
\hline$x_{2}$ m
\end{tabular}

${ }^{\mathrm{a}} x_{2}$ : mole fraction of component 2 in the saturated solution.

${ }^{\mathrm{b}} x_{1}$ : mole fraction solubility of the solute.

\section{Auxiliary Information}

Method/Apparatus/Procedure:
Constant-temperature bath, calorimetric thermometer, and an ultraviolet/
visible spectrophotometer.
Excess solute and solvent were placed in amber glass bottles and allowed to
equilibrate for several days at constant temperature. Attainment of equilibrium
was verified by several repetitive measurements and by approaching
equilibrium from supersaturation. Aliquots of saturated solutions were
transferred through a coarse filter into tared volumetric flasks, weighed, and
diluted with methanol. Concentrations were determined by
spectrophotometric measurements at 292 nm.
Source and Purity of Chemicals:
(1) $99.5 \%$, Acros Organics, USA, was used as received.
(2) $99+\%$, Alfa Aesar, USA, stored over molecular sieves and distilled shortly
before use.
Estimated Error:
Temperature: $\pm 0.1 \mathrm{~K}$.
$x_{1}: \pm 1.5 \%$ (relative error).

\subsection{4-Chloro-3-nitrobenzoic acid solubility data in miscellaneous organic solvents}

\begin{tabular}{ll}
\hline \hline Components: & Original Measurements: \\
(1) 4-Chloro-3-nitrobenzoic acid; & ${ }^{13}$ D. M. Stovall, C. Givens, S. Keown, \\
$\mathrm{C}_{7} \mathrm{H}_{4} \mathrm{ClNO}_{4}$; [96-99-1] & K. R. Hoover, R. Barnes, C. Harris, J. \\
$\begin{array}{l}\text { (2) Propylene carbonate; } \mathrm{C}_{4} \mathrm{H}_{6} \mathrm{O}_{3} ; \\
\text { [108-32-7] }\end{array}$ & Lozano, M. Nguyen, E. Rodriguez, W. \\
& $\begin{array}{l}\text { E. Acree, Jr., and M. H. Abraham, } \\
\text { Phys. Chem. Liq. 43, 351 (2005). }\end{array}$ \\
\hline Variables: & Prepared by: \\
$T / \mathrm{K}=298.15$ & W. E. Acree, Jr. \\
\hline
\end{tabular}

\section{Experimental Values}

\begin{tabular}{lc}
\hline \hline$x_{2}{ }^{\mathrm{a}}$ & $x_{1}{ }^{\mathrm{b}}$ \\
\hline 0.9866 & 0.01343 \\
\hline${ }^{\mathrm{a}} x_{2}:$ mole fraction of component 2 in the saturated solution. \\
${ }^{\mathrm{b}}{ }_{x_{1}: \text { mole fraction solubility of the solute. }}$
\end{tabular}

\section{Auxiliary Information}

\section{Method/Apparatus/Procedure:}

Constant-temperature bath, calorimetric thermometer, and an ultraviolet/ visible spectrophotometer.

Excess solute and solvent were placed in amber glass bottles and allowed to equilibrate for several days at constant temperature. Attainment of equilibrium was verified by several repetitive measurements and by approaching equilibrium from supersaturation. Aliquots of saturated solutions were transferred through a coarse filter into tared volumetric flasks, weighed, and diluted with methanol. Concentrations were determined by

spectrophotometric measurements at $292 \mathrm{~nm}$.

\section{Source and Purity of Chemicals:}

(1) $99.5 \%$, Acros Organics, USA, was used as received.

(2) $99.7 \%$, anhydrous, Aldrich Chemical Company, Milwaukee, WI, USA, stored over molecular sieves and distilled shortly before use.

\section{Estimated Error:}

Temperature: $\pm 0.1 \mathrm{~K}$.

$x_{1}: \pm 1.5 \%$ (relative error).

\begin{tabular}{|c|c|}
\hline $\begin{array}{l}\text { Components: } \\
\text { (1) 4-Chloro-3-nitrobenzoic acid; } \\
\mathrm{C}_{7} \mathrm{H}_{4} \mathrm{ClNO}_{4} \text {; [96-99-1] } \\
\text { (2) Ethanenitrile; } \mathrm{C}_{2} \mathrm{H}_{3} \mathrm{~N} ;[75-05-8]\end{array}$ & $\begin{array}{l}\text { Original Measurements: } \\
{ }^{83} \text { M. K. Chantooni and I. M. Kolthoff, } \\
\text { J. Phys. Chem. 77, } 527 \text { (1973). }\end{array}$ \\
\hline $\begin{array}{l}\text { Variables: } \\
T / \mathrm{K}=298.15\end{array}$ & $\begin{array}{l}\text { Prepared by: } \\
\text { W. E. Acree, Jr. }\end{array}$ \\
\hline
\end{tabular}

\section{Experimental Values}

The measured solubility was reported to be $0.133 \mathrm{~mol} \mathrm{dm}^{-3}$. The authors did not give the temperature at which the solubility was measured. Based on the experimental description given in an earlier paper [I. M. Kolthoff, J. J. Lingane, and W. Larson, J. Am. Chem. Soc. 60, 2512 (1938)], the compiler believes the temperature to be $298.15 \mathrm{~K}$. 


\section{Auxiliary Information}

\section{Method/Apparatus/Procedure:}

Very little experimental details were provided. Solubility was determined by titrating a filtered aliquot of the saturated solution alkalimetrically in an aqueous-ethanol mixture using phenolphthalein as the acid-base indicator.

\section{Source and Purity of Chemicals:}

(1) Purity not given, Baker Chemical Company, USA, was recrystallized from either aqueous or aqueous-ethanol solution and dried in vacuo at $333 \mathrm{~K}$ before use.

(2) Purity not given, Chemical source not given, was purified by shaking with saturated potassium hydroxide, followed by activated alumina, and then anhydrous calcium chloride to remove water. Ethanenitrile was further dried over anhydrous magnesium sulfate and then phosphorous pentoxide. The sample was distilled shortly before use.

\section{Estimated Error:}

Temperature: $\pm 0.1 \mathrm{~K}$ (estimated by compiler). $c_{1}: \pm 2 \%$ (relative error, estimated by compiler).

\section{Solubility of 4-Cyanobenzoic Acid in Organic Solvents}

\subsection{Critical evaluation of experimental solubility data}

There is only a single published study regarding the solubility behavior of 4-cyanobenzoic acid in organic solvents. Wang and Zhang ${ }^{97}$ measured the mole fraction solubilities in ethanol as a function of temperature using a static and dynamic method. The internal consistency of the two datasets was assessed by curve-fitting the measured mole fraction solubility data to Eq. (8). The values of the equation coefficients ( $A, B$, and $C$ ) are given in Table 25, along with the MRD calculated according to Eq. (24). Examination of the numerical entries in the last column of Table 25 reveals that the static experimental method yielded slightly more consistent experimental values as evidenced by the smaller MRD. In the case of the 4cyanobenzoic acid solubility data, there is a noticeable difference between the two experimental methods. The relative difference can be fairly large at times. For example, the static method gave a mole fraction solubility of $x_{1}=0.03533$ for 4 cyanobenzoic acid dissolved in ethanol at $313.15 \mathrm{~K}$, versus a value of $x_{1}=0.03879$ determined with the dynamic method. The calculated relative difference between the two reported values is just under $10 \%$.

The experimental solubility data for 4-cyanobenzoic acid in organic solvents are in Sec. 24.2.

TABLE 25. Parameters of the Modified Apelblat equation for describing the solubility of 4-cyanobenzoic acid in ethanol ${ }^{\mathrm{a}}$

\begin{tabular}{lcccc}
\hline \hline Solvent & $A$ & $B$ & $C$ & MRD (\%) \\
\hline Ethanol (dynamic method) & -2.476 & -2523 & 2.062 & 3.794 \\
Ethanol (static method) & -4.654 & -2487 & 2.420 & 2.992
\end{tabular}

${ }^{\mathrm{a}}$ Values of the coefficients and the mean relative deviations were taken from Wang and Zhang. ${ }^{97}$

\subsection{4-Cyanobenzoic acid solubility data in alcohols}

\begin{tabular}{|c|c|}
\hline $\begin{array}{l}\text { Components: } \\
\text { (1) 4-Cyanobenzoic acid; } \\
\mathrm{C}_{8} \mathrm{H}_{5} \mathrm{NO}_{2} ;[619-65-8] \\
\text { (2) Ethanol; } \mathrm{C}_{2} \mathrm{H}_{6} \mathrm{O} ;[64-17-5]\end{array}$ & $\begin{array}{l}\text { Original Measurements: } \\
{ }^{97} \mathrm{H} \text {. Wang and W. Zhang, J. Chem. } \\
\text { Eng. Data 54, } 1942 \text { (2009). }\end{array}$ \\
\hline $\begin{array}{l}\text { Variables: } \\
\text { Temperature }\end{array}$ & $\begin{array}{l}\text { Prepared by: } \\
\text { W. E. Acree, Jr. }\end{array}$ \\
\hline
\end{tabular}

Experimental Values

\begin{tabular}{lcc}
\hline \hline$T / \mathrm{K}$ & $x_{2}{ }^{\mathrm{a}}$ & $x_{1}{ }^{\mathrm{b}, \mathrm{c}}$ \\
\hline 298.2 & 0.9786 & 0.02136 \\
303.2 & 0.9734 & 0.02657 \\
308.2 & 0.9669 & 0.03305 \\
313.2 & 0.9647 & 0.03533 \\
318.2 & 0.9560 & 0.04404 \\
\hline
\end{tabular}

${ }^{a} x_{2}$ : mole fraction of component 2 in the saturated solution.

${ }^{b} x_{1}$ : mole fraction solubility of the solute.

${ }^{\mathrm{c}}$ Solubility determined by the static method.

Experimental Values

\begin{tabular}{lcc}
\hline \hline$T / \mathrm{K}$ & $x_{2}{ }^{\mathrm{a}}$ & $x_{1}{ }^{\mathrm{b}, \mathrm{c}}$ \\
\hline 298.2 & 0.9764 & 0.02363 \\
303.2 & 0.9748 & 0.02518 \\
308.2 & 0.9693 & 0.03075 \\
313.2 & 0.9612 & 0.03879 \\
318.2 & 0.9569 & 0.04311
\end{tabular}

${ }^{a} x_{2}$ : mole fraction of component 2 in the saturated solution.

${ }^{b} x_{1}$ : mole fraction solubility of the solute.

${ }^{\mathrm{c}}$ Solubility determined by the dynamic method.

\section{Auxiliary Information}

\section{Method/Apparatus/Procedure:}

Thermostated water-jacketed glass vessel, thermometer, analytical balance, laser monitoring system.

Solubilities were determined using both a static and dynamic method. In the static method, excess solute and solvent were placed in a water-jacketed vessel allowed to equilibrate with stirring for three days at constant temperature. Samples of the saturated liquid phase were decanted into Petri dishes of known mass and weighed. The samples were then evaporated to dryness and the solubility determined from the mass of the solid residue and the mass of the sample analyzed. In the dynamic method, an excess of carboxylic acid was added to a known mass of ethanol. The resulting suspension was stirred in a water-jacketed vessel for $1 \mathrm{~h}$ at constant temperature. A known mass of ethanol was added to the vessel through a buret until the solute completely dissolved. The dissolution was monitored with a laser monitoring system. When the last portion of solid solute disappeared, the penetrated light intensity reached its maximum value. The mass of the added solvent was recorded. The solubility of the carboxylic acid was calculated from the known masses of solute and ethanol.

Source and Purity of Chemicals:

(1) $98 \%$, Sigma-Aldrich Chemical Company, Milwaukee, WI, USA, no purification details provided.

(2) $99.55 \%$, Tianjin Kewei Chemical Reagent, China, no information given regarding any further purification. 
Estimated Error:

Temperature: $\pm 0.1 \mathrm{~K}$.

$x_{1}$ : Authors report that the uncertainty in the solubility values is estimated to be $\pm 0.5 \%$. The differences between the experimental values based on the static and dynamic solubility methods are considerably larger, and more on the order of $\pm 5 \%$ to $10 \%$ (relative error).

\section{Solubility of 3,5-Diaminobenzoic Acid in Organic Solvents}

\subsection{Critical evaluation of experimental solubility data}

There is only a single published study regarding the solubility behavior of 3,5-diaminobenzoic acid in organic solvents. Wang and Zhang ${ }^{97}$ measured the mole fraction solubilities in ethanol as a function of temperature using a static and dynamic method. The internal consistency of the two datasets was assessed by curve-fitting the measured mole fraction solubility data to Eq. (8). The values of the equation coefficients ( $A, B$, and $C$ ) are given in Table 26, along with the MRD calculated according to Eq. (24). Examination of the numerical entries in the last column of Table 26 reveals that the static experimental method yielded more consistent experimental values as documented by the smaller MRD. In the case of the 3,5-diaminobenzoic acid solubility data, there is a noticeable difference between the two experimental methods. The relative difference can be fairly large at times. For example, the static method gave a mole fraction solubility of $x_{1}=0.003336$ for 3,5-diaminobenzoic acid in ethanol at $298.15 \mathrm{~K}$, versus a value of $x_{1}=0.002112$ determined with the dynamic method. The calculated relative difference between the two reported values is more than $35 \%$.

The experimental solubility data for 3,5-diaminobenzoic acid in organic solvents are in Sec. 25.2.

\subsection{3,5-Diaminobenzoic acid solubility data in alcohols}

\begin{tabular}{|c|c|}
\hline $\begin{array}{l}\text { Components: } \\
\text { (1) 3,5-Diaminobenzoic acid; } \\
\mathrm{C}_{7} \mathrm{H}_{8} \mathrm{~N}_{2} \mathrm{O}_{2} ;[535-87-5] \\
\text { (2) Ethanol; } \mathrm{C}_{2} \mathrm{H}_{6} \mathrm{O} ;[64-17-5]\end{array}$ & $\begin{array}{l}\text { Original Measurements: } \\
\text { 97 H. Wang and W. Zhang, J. Chem. } \\
\text { Eng. Data 54, } 1942 \text { (2009). }\end{array}$ \\
\hline $\begin{array}{l}\text { Variables: } \\
\text { Temperature }\end{array}$ & $\begin{array}{l}\text { Prepared by: } \\
\text { W. E. Acree, Jr. }\end{array}$ \\
\hline
\end{tabular}

TABLE 26. Parameters of the Modified Apelblat equation for describing the solubility of 3,5-diaminobenzoic acid in ethanol ${ }^{\mathrm{a}}$

\begin{tabular}{lcccc}
\hline \hline Solvent & $A$ & $B$ & $C$ & MRD (\%) \\
\hline $\begin{array}{l}\text { Ethanol (dynamic } \\
\text { method) }\end{array}$ & 44.98 & -4433 & -5.516 & 10.43 \\
$\begin{array}{l}\text { Ethanol (static } \\
\text { method) }\end{array}$ & -8.322 & -1580 & 2.188 & 3.305 \\
\hline
\end{tabular}

${ }^{\mathrm{a}}$ Values of the coefficients and the mean relative deviations were taken from Wang and Zhang. ${ }^{97}$
Experimental Values

\begin{tabular}{lcc}
\hline \hline$T / \mathrm{K}$ & $x_{2}{ }^{\mathrm{a}}$ & $x_{1}^{\mathrm{b}, \mathrm{c}}$ \\
\hline 298.2 & 0.9967 & 0.003336 \\
303.2 & 0.9966 & 0.003419 \\
308.2 & 0.9960 & 0.003999 \\
313.2 & 0.9956 & 0.004405 \\
318.2 & 0.9948 & 0.005192
\end{tabular}

${ }^{a} x_{2}$ : mole fraction of component 2 in the saturated solution.

${ }^{\mathrm{b}} x_{1}$ : mole fraction solubility of the solute.

${ }^{\mathrm{c}}$ Solubility determined by the static method.

Experimental Values

\begin{tabular}{lcc}
\hline \hline$T / \mathrm{K}$ & $x_{2}{ }^{\mathrm{a}}$ & $x_{1}{ }^{\mathrm{b}, \mathrm{c}}$ \\
\hline 298.2 & 0.9979 & 0.002112 \\
303.2 & 0.9963 & 0.003737 \\
308.2 & 0.9962 & 0.003776 \\
313.2 & 0.9960 & 0.003994 \\
318.2 & 0.9953 & 0.004716 \\
\hline
\end{tabular}

${ }^{\mathrm{a}} \mathrm{x}_{2}$ : mole fraction of component 2 in the saturated solution.

${ }^{\mathrm{b}} x_{1}$ : mole fraction solubility of the solute.

${ }^{\mathrm{c}}$ Solubility determined by the dynamic method.

\section{Auxiliary Information}

\section{Method/Apparatus/Procedure:}

Thermostated water-jacketed glass vessel, thermometer, analytical balance, laser monitoring system.

Solubilities were determined using both a static and dynamic method. In the static method, excess solute and solvent were placed in a water-jacketed vessel allowed to equilibrate with stirring for three days at constant temperature. Samples of the saturated liquid phase were decanted into Petri dishes of known mass and weighed. The samples were then evaporated to dryness and the solubility determined from the mass of the solid residue and the mass of the sample analyzed. In the dynamic method, an excess of carboxylic acid was added to a known mass of ethanol. The resulting suspension was stirred in a water-jacketed vessel for $1 \mathrm{~h}$ at constant temperature. A known mass of ethanol was added to the vessel through a buret until the solute completely dissolved. The dissolution was monitored with a laser monitoring system. When the last portion of solid solute disappeared, the penetrated light intensity reached its maximum value. The mass of the added solvent was recorded. The solubility of the carboxylic acid was calculated from the known masses of solute and ethanol.

Source and Purity of Chemicals:

(1) $98 \%$, Sigma-Aldrich Chemical Company, Milwaukee, WI, USA, no purification details provided.

(2) $99.55 \%$, Tianjin Kewei Chemical Reagent, China, no information given regarding any further purification.

Estimated Error:

Temperature: $\pm 0.1 \mathrm{~K}$.

$x_{1}$ : Authors report that the uncertainty in the solubility values is estimated to be $\pm 0.5 \%$. The differences between the experimental values based on the static and dynamic solubility methods are considerably larger, and more on the order of $\pm 10 \%$ to $30 \%$ (relative error). 


\section{Solubility of 2,4-Dichlorobenzoic Acid in Organic Solvents}

\subsection{Critical evaluation of experimental solubility data}

There have been very few published solubility studies ${ }^{83,97}$ involving 2,4-dichlorobenzoic acid in organic solvents. Chantooni and Kolthoff ${ }^{83}$ determined the solubility of 2,4-dichlorobenzoic acid in methanol, ethanenitrile and $N, N$ dimethylformamide at $298.15 \mathrm{~K}$. Wang and Zhang ${ }^{97}$ measured the mole fraction solubilities in ethanol as a function of temperature using a static and dynamic method. The internal consistency of the ethanol datasets was assessed by curvefitting the measured mole fraction solubility data to Eq. (8). The values of the equation coefficients $(A, B$, and $C)$ are given in Table 27, along with the MRD calculated according to Eq. (24). Examination of the numerical entries in the last column of Table 27 reveals that the static experimental method yielded more consistent experimental values. In the case of the 2,4-dichlorobenzoic acid solubility data, there is a noticeable difference between the two experimental methods. The relative difference can be fairly large at times. For example, the static method gave a mole fraction solubility of $x_{1}=0.06087$ for 2,4-dichlorobenzoic acid in ethanol at $298.15 \mathrm{~K}$, versus a value of $x_{1}=0.05012$ determined with the dynamic method. The calculated relative difference between the two reported values is more than $15 \%$.

The experimental solubility data for 2,4-dichlorobenzoic acid in organic solvents are in Secs. 26.2 and 26.3.

\subsection{2,4-Dichlorobenzoic acid solubility data in alcohols}

\begin{tabular}{|c|c|}
\hline $\begin{array}{l}\text { Components: } \\
\text { (1) 2,4-Dichlorobenzoic acid; } \\
\mathrm{C}_{7} \mathrm{H}_{4} \mathrm{Cl}_{2} \mathrm{O}_{2} ;[50-84-0] \\
\text { (2) Methanol; } \mathrm{CH}_{4} \mathrm{O} ;[67-56-1]\end{array}$ & $\begin{array}{l}\text { Original Measurements: } \\
{ }^{83} \text { M. K. Chantooni and I. M. Kolthoff, } \\
\text { J. Phys. Chem. 77, } 527 \text { (1973). }\end{array}$ \\
\hline $\begin{array}{l}\text { Variables: } \\
T / \mathrm{K}=298.15\end{array}$ & $\begin{array}{l}\text { Prepared by: } \\
\text { W. E. Acree, Jr. }\end{array}$ \\
\hline
\end{tabular}

\section{Experimental Values}

The measured solubility was reported to be $1.34 \mathrm{~mol} \mathrm{dm}^{-3}$. The authors did not give the temperature at which the solubility was measured. Based on the experimental description given in an earlier paper [I. M. Kolthoff, J. J. Lingane, and W. Larson, J.

TABLE 27. Parameters of the Modified Apelblat equation for describing the solubility of 2,4-dichlorobenzoic acid in ethanol ${ }^{\mathrm{a}}$

\begin{tabular}{lrccc}
\hline \hline Solvent & \multicolumn{1}{c}{$A$} & \multicolumn{1}{c}{$B$} & $C$ & MRD (\%) \\
\hline Ethanol (dynamic method) & -1.902 & -2652 & 2.183 & 5.661 \\
Ethanol (static method) & 3.819 & -2942 & 1.367 & 0.995
\end{tabular}

${ }^{\mathrm{a}}$ Values of the coefficients and the mean relative deviations were taken from Wang and Zhang. ${ }^{97}$
Am. Chem. Soc. 60, 2512 (1938)], the compiler believes the temperature to be $298.15 \mathrm{~K}$.

\section{Auxiliary Information}

\begin{abstract}
Method/Apparatus/Procedure:
Very little experimental details were provided. Solubility was determined by titrating a filtered aliquot of the saturated solution alkalimetrically in an aqueous-ethanol mixture using phenolphthalein as the acid-base indicator.
\end{abstract}

Source and Purity of Chemicals:

(1) Purity not given, Chemical source not given, was recrystallized from either aqueous or aqueous-ethanol solution and dried in vacuo at $333 \mathrm{~K}$.

(2) Purity not given, Chemical source not given, was dehydrated and then distilled over sulfanilic acid to remove alkaline impurities.

\section{Estimated Error:}

Temperature: $\pm 0.1 \mathrm{~K}$ (estimated by compiler). $c_{1}: \pm 2 \%$ (relative error, estimated by compiler).

\begin{tabular}{|c|c|}
\hline $\begin{array}{l}\text { Components: } \\
\text { (1) 2,4-Dichlorobenzoic acid; } \\
\mathrm{C}_{7} \mathrm{H}_{4} \mathrm{Cl}_{2} \mathrm{O}_{2} ;[50-84-0] \\
\text { (2) Ethanol; } \mathrm{C}_{2} \mathrm{H}_{6} \mathrm{O} ;[64-17-5]\end{array}$ & $\begin{array}{l}\text { Original Measurements: } \\
{ }^{97} \mathrm{H} \text {. Wang and W. Zhang, J. Chem. } \\
\text { Eng. Data 54, } 1942 \text { (2009). }\end{array}$ \\
\hline $\begin{array}{l}\text { Variables: } \\
\text { Temperature }\end{array}$ & $\begin{array}{l}\text { Prepared by: } \\
\text { W. E. Acree, Jr. }\end{array}$ \\
\hline
\end{tabular}

Experimental Values

\begin{tabular}{lcc}
\hline \hline$T / \mathrm{K}$ & $x_{2}{ }^{\mathrm{a}}$ & $x_{1}{ }^{\mathrm{b}, \mathrm{c}}$ \\
\hline 298.2 & 0.9419 & 0.05812 \\
303.2 & 0.9307 & 0.06933 \\
308.2 & 0.9183 & 0.08166 \\
313.2 & 0.9047 & 0.09534 \\
318.2 & 0.8821 & 0.1179 \\
\hline
\end{tabular}

${ }^{a} x_{2}$ : mole fraction of component 2 in the saturated solution.

${ }^{\mathrm{b}} x_{1}$ : mole fraction solubility of the solute.

${ }^{\mathrm{c}}$ Solubility determined by the static method.

Experimental Values

\begin{tabular}{lcc}
\hline \hline$T / \mathrm{K}$ & $x_{2}{ }^{\mathrm{a}}$ & $x_{1}^{\mathrm{b}, \mathrm{c}}$ \\
\hline 298.2 & 0.9519 & 0.04814 \\
303.2 & 0.9322 & 0.06785 \\
308.2 & 0.9291 & 0.07093 \\
313.2 & 0.9193 & 0.08072 \\
318.2 & 0.8944 & 0.1056 \\
\hline
\end{tabular}

${ }^{a_{2}}{ }_{2}$ : mole fraction of component 2 in the saturated solution.

${ }^{b} x_{1}$ : mole fraction solubility of the solute.

${ }^{\mathrm{c}}$ Solubility determined by the dynamic method. 


\section{Auxiliary Information}

\section{Method/Apparatus/Procedure:}

Thermostated water-jacketed glass vessel, thermometer, analytical balance, laser monitoring system.

Solubilities were determined using both a static and dynamic method. In the static method, excess solute and solvent were placed in a water-jacketed vessel allowed to equilibrate with stirring for three days at constant temperature. Samples of the saturated liquid phase were decanted into Petri dishes of known mass and weighed. The samples were then evaporated to dryness and the solubility determined from the mass of the solid residue and the mass of the sample analyzed. In the dynamic method, an excess of carboxylic acid was added to a known mass of ethanol. The resulting suspension was stirred in a water-jacketed vessel for $1 \mathrm{~h}$ at constant temperature. A known mass of ethanol was added to the vessel through a buret until the solute completely dissolved. The dissolution was monitored with a laser monitoring system. When the last portion of solid solute disappeared, the penetrated light intensity reached its maximum value. The mass of the added solvent was recorded. The solubility of the carboxylic acid was calculated from the known masses of solute and ethanol.

\section{Source and Purity of Chemicals:}

(1) $98 \%$, Sigma-Aldrich Chemical Company, Milwaukee, WI, USA, no purification details provided.

(2) $99.55 \%$, Tianjin Kewei Chemical Reagent, China, no information given regarding any further purification.

\section{Estimated Error:}

Temperature: $\pm 0.1 \mathrm{~K}$

$x_{1}$ : Authors report that the uncertainty in the solubility values is estimated to be $\pm 0.5 \%$. The differences between the experimental values based on the static and dynamic solubility methods are considerably larger, and more on the order of $\pm 5 \%$ to $15 \%$ (relative error).

\subsection{2,4-Dichlorobenzoic acid solubility data in miscellaneous organic solvents}

\begin{tabular}{|c|c|}
\hline $\begin{array}{l}\text { Components: } \\
\text { (1) } 2,4-\text { Dichlorobenzoic acid; } \\
\mathrm{C}_{7} \mathrm{H}_{4} \mathrm{Cl}_{2} \mathrm{O}_{2} ;[50-84-0] \\
\text { (2) Ethanenitrile; } \mathrm{C}_{2} \mathrm{H}_{3} \mathrm{~N} \text {; } \\
\text { [75-05-8] }\end{array}$ & $\begin{array}{l}\text { Original Measurements: } \\
{ }^{83} \text { M. K. Chantooni and I. M. Kolthoff, } \\
\text { J. Phys. Chem. 77, } 527 \text { (1973). }\end{array}$ \\
\hline $\begin{array}{l}\text { Variables: } \\
T / \mathrm{K}=298.15\end{array}$ & $\begin{array}{l}\text { Prepared by: } \\
\text { W. E. Acree, Jr. }\end{array}$ \\
\hline
\end{tabular}

\section{Experimental Values}

The measured solubility was reported to be $0.168 \mathrm{~mol} \mathrm{dm}^{-3}$. The authors did not give the temperature at which the solubility was measured. Based on the experimental description given in an earlier paper [I. M. Kolthoff, J. J. Lingane, and W. Larson, J. Am. Chem. Soc. 60, 2512 (1938)], the compiler believes the temperature to be $298.15 \mathrm{~K}$.

\section{Auxiliary Information}

\section{Method/Apparatus/Procedure:}

Very little experimental details were provided. Solubility was determined by titrating a filtered aliquot of the saturated solution alkalimetrically in an aqueous-ethanol mixture using phenolphthalein as the acid-base indicator.
Source and Purity of Chemicals:

(1) Purity not given, Chemical source not given, was recrystallized from either aqueous or aqueous-ethanol solution and dried in vacuo at $333 \mathrm{~K}$.

(2) Purity not given, Chemical source not given, was purified by shaking with saturated potassium hydroxide, followed by activated alumina, and then anhydrous calcium chloride to remove water. Ethanenitrile was further dried over anhydrous magnesium sulfate and then phosphorous pentoxide. The sample was distilled shortly before use.

Estimated Error:

Temperature: $\pm 0.1 \mathrm{~K}$ (estimated by compiler). $c_{1}: \pm 2 \%$ (relative error, estimated by compiler).

\begin{tabular}{|c|c|}
\hline $\begin{array}{l}\text { Components: } \\
\text { (1) 2,4-Dichlorobenzoic acid; } \\
\mathrm{C}_{7} \mathrm{H}_{4} \mathrm{Cl}_{2} \mathrm{O}_{2} \text {; [50-84-0] } \\
\text { (2) N,N-Dimethylformamide; } \\
\mathrm{C}_{3} \mathrm{H}_{7} \mathrm{NO} \text {; [64-19-7] }\end{array}$ & $\begin{array}{l}\text { Original Measurements: } \\
{ }^{83} \text { M. K. Chantooni and I. M. Kolthoff } \\
\text { J. Phys. Chem. 77, } 527 \text { (1973). }\end{array}$ \\
\hline $\begin{array}{l}\text { Variables: } \\
T / \mathrm{K}=298.15\end{array}$ & $\begin{array}{l}\text { Prepared by: } \\
\text { W. E. Acree, Jr. }\end{array}$ \\
\hline
\end{tabular}

\section{Experimental Values}

The measured solubility was reported to be $2.93 \mathrm{~mol} \mathrm{dm}^{-3}$. The authors did not give the temperature at which the solubility was measured. Based on the experimental description given in an earlier paper [I. M. Kolthoff, J. J. Lingane, and W. Larson, J. Am. Chem. Soc. 60, 2512 (1938)], the compiler believes the temperature to be $298.15 \mathrm{~K}$.

\section{Auxiliary Information}

Method/Apparatus/Procedure:

Very little experimental details were provided. Solubility was determined by titrating a filtered aliquot of the saturated solution alkalimetrically in an aqueous-ethanol mixture using phenolphthalein as the acid-base indicator.

Source and Purity of Chemicals:

(1) Purity not given, Chemical source not given, was recrystallized from either aqueous or aqueous-ethanol solution and dried in vacuo at $333 \mathrm{~K}$.

(2) Purity not given, Chemical source not given, was shaken first with phosphorous pentoxide and then with potassium hydroxide pellets. Solvent was distilled shortly before use.

Estimated Error:

Temperature: $\pm 0.1 \mathrm{~K}$ (estimated by compiler). $c_{1}: \pm 2 \%$ (relative error, estimated by compiler).

\section{Solubility of 3,4-Dichlorobenzoic Acid in Organic Solvents}

\subsection{Critical evaluation of experimental solubility data}

There have been several studies $54,63,76,83,137$ that have reported the solubility of 3,4-dichlorobenzoic acid in organic solvents. Wilson et al. ${ }^{137}$ determined the solubility of 3,4dichlorobenzoic acid in 16 alcohols (ethanol, 1-propanol, 2propanol, 1-butanol, 2-butanol, 2-methyl-1-propanol, 2methyl-2-propanol, 1-pentanol, 2-pentanol, 2-methyl-1-butanol, 3-methyl-1-butanol, 1-hexanol, 2-methyl-1-pentanol, 
4-methyl-2-pentanol, 1-heptanol and 1-octanol), in six alkyl alkanoates (methyl ethanoate, ethyl ethanoate, propyl ethanoate, butyl ethanoate, pentyl ethanoate and methyl butanoate), in four dialkyl ethers (1,1'-oxybisethane, 2,2'-oxybispropane, 1,1'-oxybisbutane, and 2-methoxy-2-methylpropane) and two cyclic ethers (tetrahydrofuran and 1,4-dioxane), and in five alkoxyalcohols (2-ethoxyethanol, 2-propoxyethanol, 2-isopropoxyethanol, 2-butoxyethanol and 3-methoxy-1-butanol). The solubilities were measured at single temperature of $298 \mathrm{~K}$. The authors determined the solubilities to check the numerical values of the solute descriptors of 3,4-dichlorobenzoic acid that had been calculated previously based on limited water-toorganic solvent partition coefficient data.

An indication of the consistency of the experimental data can be gained by comparing the measured solubility data to predicted values based on the Abraham model Eqs. (20) and (21).

Numerical values of the solute descriptors for 3,4-dichlorobenzoic acid are known $(E=0.950, S=0.920, A=0.670, B$ $=0.260, V=1.1766$ and $L=5.623$ ), so that combination of these descriptors with the coefficients listed in Tables 1 and 2 enables the prediction of $\log _{10}\left(c_{1, \mathrm{~S}} / c_{1, \mathrm{~W}}\right)$ and $\log _{10}\left(c_{1, \mathrm{~S}} / c_{1, \mathrm{G}}\right)$. The molar solubility of molecular 3,4-dichlorobenzoic acid in water, $\log _{10} c_{1, \mathrm{~W}}=-3.98$, is available to convert the predicted $\left(c_{1, \mathrm{~S}} / c_{1, \mathrm{~W}}\right)$ solubility ratios to $c_{1, \mathrm{~S}}$ values. For carboxylic acid solutes, $c_{1, \mathrm{~W}}$ corresponds to the aqueous solubility of the molecular, nonionized form of the solute. The aqueous solubility was determined by adding two drops of hydrochloric acid to suppress ionization. Similarly, the numerical value of $\log _{10} c_{1, \mathrm{G}}$ is known $\left(\log _{10} c_{1, \mathrm{G}}=-8.72\right)$ for converting predicted $\left(c_{1, \mathrm{~S}} / c_{1, \mathrm{G}}\right)$ solubility ratios to $c_{1, \mathrm{~S}}$ values.

The predicted molar solubilities of 3,4-dichlorobenzoic acid in methanol, ethanol, 1-propanol, 1-butanol, 1-pentanol, 1hexanol, 1-heptanol, 1-octanol, 2-propanol, 2-butanol, 2-pentanol, 2-methyl-1-propanol, 2-methyl-2-propanol, 3-methyl1-butanol, 1,1'-oxybisethane, 2-methoxy-2-methylpropane, tetrahydrofuran, 1,4-dioxane, methyl ethanoate, ethyl ethanoate, propyl ethanoate and butyl ethanoate are listed in the third and fourth columns of Table 28. The numerical values represent outright solubility predictions in that none of the experimental data was used in the determination of the molecular solute descriptors. For comparison purposes, the measured mole fraction solubilities of 3,4-dichlorobenzoic acid, $x_{1}$, determined by Wilson et al. ${ }^{137}$ were converted into molar solubilities by dividing $x_{1}$ by the ideal molar volume of the saturated solution (i.e., $\left.c_{1}{ }^{\text {sat }}=x_{1} /\left[x_{1} V_{1}+\left(1-x_{1}\right) V_{\text {solvent }}\right]\right)$. The molar volume of the hypothetical subcooled liquid 3,4dichlorobenzoic acid, $V_{\text {solute }}=129.78 \mathrm{~cm}^{3} \mathrm{~mol}^{-1}$, was estimated as the molar volume of benzoic acid ( $V_{\text {benzoic acid }}=$ $104.38 \mathrm{~cm}^{3} \mathrm{~mol}^{-1}$ ) plus two times the molar volume of chlorobenzene $\left(V_{\text {chlorobenzene }}=102.1 \mathrm{~cm}^{3} \mathrm{~mol}^{-1}\right)$ minus two times the molar volume of benzene $\left(V_{\text {benzene }}=89.40 \mathrm{~cm}^{3}\right.$ $\left.\mathrm{mol}^{-1}\right)$. Any errors resulting from the estimation of 3,4dichlorobenzoic acid's hypothetical subcooled liquid molar volume, $V_{\text {Solute }}$, or the ideal molar volume approximation will have negligible effect of the calculated $c_{1}$ values because 3,4dichlorobenzoic acid is not overly soluble in many of the solvents considered. From a mathematical standpoint, the
TABLE 28. Comparison between observed and predicted molar solubilities of 3,4-dichlorobenzoic acid based on the Abraham model, Eqs. (20) and (21)

\begin{tabular}{|c|c|c|c|}
\hline Solvent & $\log _{10} c_{1} \exp$ & $\begin{array}{c}\log _{10} c_{1}{ }^{\text {calc }} ; \\
\text { Eq. (20) }\end{array}$ & $\begin{array}{c}\log _{10} c_{1}{ }^{\text {calc. }} ; \\
\text { Eq. }(21)\end{array}$ \\
\hline Methanol & -0.569 & -0.568 & -0.596 \\
\hline Ethanol & -0.539 & -0.441 & -0.450 \\
\hline 1-Propanol & -0.615 & -0.527 & -0.507 \\
\hline 2-Propanol & -0.644 & -0.497 & -0.471 \\
\hline 1-Butanol & -0.661 & -0.598 & -0.573 \\
\hline 2-Butanol & -0.691 & -0.573 & -0.588 \\
\hline 2-Methyl-1-propanol & -0.826 & -0.720 & -0.732 \\
\hline 2-Methyl-2-propanol & -0.442 & -0.483 & -0.418 \\
\hline 1-Pentanol & -0.699 & -0.565 & -0.541 \\
\hline 2-Pentanol & -0.647 & -0.550 & -0.545 \\
\hline 3-Methyl-1-butanol & -0.708 & -0.628 & -0.639 \\
\hline 1-Hexanol & -0.722 & -0.606 & -0.601 \\
\hline 1-Heptanol & -0.752 & -0.593 & -0.607 \\
\hline 1-Octanol & -0.814 & -0.664 & -0.707 \\
\hline 1,1'-Oxybisethane & -0.535 & -0.609 & -0.547 \\
\hline $\begin{array}{l}\text { 2-Methoxy-2- } \\
\text { methylpropane }\end{array}$ & -0.523 & -0.632 & -0.805 \\
\hline Tetrahydrofuran & 0.067 & 0.029 & 0.017 \\
\hline 1,4-Dioxane & -0.251 & -0.362 & -0.350 \\
\hline Methyl ethanoate & -0.829 & -0.752 & -0.710 \\
\hline Ethyl ethanoate & -0.781 & -0.573 & -0.574 \\
\hline Propyl ethanoate & -0.818 & -0.632 & -0.652 \\
\hline Butyl ethanoate & -0.809 & -0.692 & -0.730 \\
\hline
\end{tabular}

$x_{1}{ }^{\exp } V_{\text {Solute }}$ term contributes very little to the molar volumes of the saturated solutions. The molar solubility of 3,4-dichlorobenzoic acid, $\log _{10} c_{1}=-0.569$, in methanol in Table 28 was determined by Chantooni and Kolthoff. ${ }^{83}$

Examination of the numerical entries in Table 28 reveals that expressions based on the Abraham model provide a very reasonable estimation of the solubility behavior of 3,4-dichlorobenzoic acid in 1,1'-oxybisethane, 2-methoxy-2-methylpropane, tetrahydrofuran, 1,4-dioxane, and in 15 alcohol and four alkyl ethanoate solvents. Standard deviations between predicted and observed values were on the order of 0.12 and $0.13 \log _{10}$ units for Eqs. (20) and (21), respectively. 1,1'Oxybisbutane was excluded from the comparison because of concerns regarding possible dimerization of 3,4-dichlorobenzoic acid due to the presence of the larger alkyl chains. Solubility predictions were not made for 2-methyl-1-butanol, 2-methyl-1-pentanol, 4-methyl-2-pentanol, 2,2'-oxybispropane, pentyl ethanoate, methyl butanoate, and the five alkoxyalcohols due to lack of Abraham model equation coefficients.

Hancock et al. ${ }^{54,63}$ measured the solubility of 3,4-dichlorobenzoic acid in cyclohexane, benzene, tetrahydrofuran and 1,4-dioxane at $303 \mathrm{~K}$. Chantooni and Kolthoff ${ }^{76,83}$ determined the solubility of 3,4-dichlorobenzoic acid in methanol, 2propanol, 2-methyl-2-propanol, 1-hexanol, ethanenitrile, dimethyl sulfoxide, and $N, N$-dimethylformamide at $298 \mathrm{~K}$. The solubility data of Chantooni and Kolthoff of $\log _{10} c_{1}=$ -0.499 for 2-propanol, $\log _{10} c_{1}=-0.438$ for 2-methyl-2propanol, and $\log _{10} c_{1}=-0.569$ for 1 -hexanol are in reasonably good agreement with the experimental values of Wilson et al. ${ }^{137}$ and predicted values based on Eqs. (20) and (21) which are given in Table 28. The deviations between the two sets of experimental measurements are attributed in part to differences in chemical purities and experimental methodologies. 
The experimental solubility data for 3,4-dichlorobenzoic acid in organic solvents are in Secs. 27.2-27.8.

\subsection{3,4-Dichlorobenzoic acid solubility data in saturated hydrocarbons (including cycloalkanes)}

\begin{tabular}{|c|c|}
\hline $\begin{array}{l}\text { Components: } \\
\text { (1) } 3,4-\text { Dichlorobenzoic acid; } \\
\mathrm{C}_{7} \mathrm{H}_{4} \mathrm{Cl}_{2} \mathrm{O}_{2} \text {; [51-44-5] } \\
\text { (2) Cyclohexane; } \mathrm{C}_{6} \mathrm{H}_{12} \text {; } \\
\text { [110-82-7] }\end{array}$ & $\begin{array}{l}\text { Original Measurements: } \\
{ }^{54} \text { C. K. Hancock, J. N. Pawloski, and J. } \\
\text { P. Idoux, J. Org. Chem. 31, } 3801 \\
\text { (1966). }\end{array}$ \\
\hline $\begin{array}{l}\text { Variables: } \\
T / \mathrm{K}=303.15\end{array}$ & $\begin{array}{l}\text { Prepared by: } \\
\text { W. E. Acree, Jr. }\end{array}$ \\
\hline
\end{tabular}

Experimental Values

\begin{tabular}{lc}
\hline \hline$x_{2}{ }^{\mathrm{a}}$ & $x_{1}{ }^{\mathrm{b}}$ \\
\hline 0.9999 & 0.0000578
\end{tabular}

${ }^{\mathrm{a}} x_{2}$ : mole fraction of component 2 in the saturated solution.

${ }^{\mathrm{b}} x_{1}$ : mole fraction solubility of the solute.

\section{Auxiliary Information}

\section{Method/Apparatus/Procedure:}

Constant-temperature bath, Soxhlet thimble, conical flask, and analytical balance.

Excess solute and solvent were placed in a conical flask and allowed to equilibrate for several days at constant temperature. Attainment of equilibrium was verified by several repetitive measurements and by approaching equilibrium from supersaturation. Aliquots of saturated solutions were transferred using a Soxhlet thimble equipped with a one-hole cork stopper and an inverted $\mathrm{U}$-shaped delivery tube to a second ground-glass stoppered flask suspended in the $303 \mathrm{~K}$ water bath. Successive portions of the contents were evaporated at room temperature in a tared aluminum foil weighing dish under a bell jar through which a slow stream of dry air was passed. The second flask plus unused saturated solution and the aluminum foil dish plus residue were weighed. The saturation solubility of the solute was calculated from the recorded mass data and molar masses of the solute and solvent.

Source and Purity of Chemicals:

(1) Purity not given, Chemical source not specified, was recrystallized several times from aqueous-ethanol mixture, and then dried under vacuum over phosphorous pentoxide.

(2) Reagent grade, Chemical source not specified, refluxed over phosphorous pentoxide for $24 \mathrm{~h}$, and then distilled through a $3 \times 80 \mathrm{~cm}$ column filled with $0.32-\mathrm{cm}$ glass helices.

\section{Estimated Error:}

Temperature: $\pm 0.02 \mathrm{~K}$.

$x_{1}: \pm 10 \%$ (relative error, by compiler)

\subsection{3,4-Dichlorobenzoic acid solubility data in aromatic hydrocarbons}

\begin{tabular}{ll}
\hline \hline Components: & Original Measurements: \\
(1) 3,4-Dichlorobenzoic acid; & ${ }^{54} \mathrm{C}$. K. Hancock, J. N. Pawloski, and J. \\
$\mathrm{C}_{7} \mathrm{H}_{4} \mathrm{Cl}_{2} \mathrm{O}_{2} ;[51-44-5]$ & P. Idoux, J. Org. Chem. 31, 3801 \\
(2) Benzene; $\mathrm{C}_{6} \mathrm{H}_{6} ;[71-43-2]$ & $(1966)$. \\
\hline Variables: & Prepared by: \\
$T / \mathrm{K}=303.15$ & W. E. Acree, Jr. \\
\hline
\end{tabular}

Experimental Values

\begin{tabular}{lc}
\hline \hline$x_{2}{ }^{\mathrm{a}}$ & $x_{1}{ }^{\mathrm{b}}$ \\
\hline 0.9993 & 0.000734 \\
\hline${ }^{\mathrm{a}} x_{2}:$ mole fraction of component 2 in the saturated solution. \\
${ }^{\mathrm{b}} x_{1}:$ mole fraction solubility of the solute.
\end{tabular}

Auxiliary Information

\section{Method/Apparatus/Procedure:}

Constant-temperature bath, Soxhlet thimble, conical flask, and analytical balance.

Excess solute and solvent were placed in a conical flask and allowed to equilibrate for several days at constant temperature. Attainment of equilibrium was verified by several repetitive measurements and by approaching equilibrium from supersaturation. Aliquots of saturated solutions were transferred using a Soxhlet thimble equipped with a one-hole cork stopper and an inverted U-shaped delivery tube to a second ground-glass stoppered flask suspended in the $303 \mathrm{~K}$ water bath. Successive portions of the contents were evaporated at room temperature in a tared aluminum foil weighing dish under a bell jar through which a slow stream of dry air was passed. The second flask plus unused saturated solution and the aluminum foil dish plus residue were weighed. The saturation solubility of the solute was calculated from the recorded mass data and molar masses of the solute and solvent.

Source and Purity of Chemicals:

(1) Purity not given, Chemical source not specified, was recrystallized several times from aqueous-ethanol mixture, and then dried under vacuum over phosphorous pentoxide.

(2) Reagent grade, Chemical source not specified, refluxed over phosphorous pentoxide for $24 \mathrm{~h}$, and then distilled through a $3 \times 80 \mathrm{~cm}$ column filled with $0.32-\mathrm{cm}$ glass helices.

Estimated Error:

Temperature: $\pm 0.02 \mathrm{~K}$.

$x_{1}: \pm 3 \%$ (relative error, by compiler).

\subsection{3,4-Dichlorobenzoic acid solubility data in esters}

\begin{tabular}{|c|c|}
\hline $\begin{array}{l}\text { Components: } \\
\text { (1) 3,4-Dichlorobenzoic acid; } \\
\mathrm{C}_{7} \mathrm{H}_{4} \mathrm{Cl}_{2} \mathrm{O}_{2} \text {; [51-44-5] } \\
\text { (2) Methyl ethanoate; } \mathrm{C}_{3} \mathrm{H}_{6} \mathrm{O}_{2} \text {; } \\
\text { [79-20-9] }\end{array}$ & $\begin{array}{l}\text { Original Measurements: } \\
{ }^{137} \text { A. Wilson, A. Tian, V. Chou, A. N. } \\
\text { Quay, W. E. Acree, Jr., and M. H. } \\
\text { Abraham, Phys. Chem. Liq. 50, } 324 \\
\text { (2012). }\end{array}$ \\
\hline $\begin{array}{l}\text { Variables: } \\
T / \mathrm{K}=298.15\end{array}$ & $\begin{array}{l}\text { Prepared by: } \\
\text { W. E. Acree, Jr. }\end{array}$ \\
\hline
\end{tabular}


Experimental Values

\begin{tabular}{lc}
\hline \hline$x_{2}{ }^{\mathrm{b}}$ & $x_{1}{ }^{\mathrm{c}}$ \\
\hline 0.9881 & 0.01192
\end{tabular}

${ }^{\mathrm{a}} x_{2}$ : mole fraction of component 2 in the saturated solution.

${ }^{\mathrm{b}}{ }_{x_{1}}$ : mole fraction solubility of the solute.

\section{Auxiliary Information}

\section{Method/Apparatus/Procedure:}

Constant-temperature bath, calorimetric thermometer, and an ultraviolet/ visible spectrophotometer.

Excess solute and solvent were placed in amber glass bottles and allowed to equilibrate for several days at constant temperature. Attainment of equilibrium was verified by several repetitive measurements and by approaching equilibrium from supersaturation. Aliquots of saturated solutions were transferred through a coarse filter into tared volumetric flasks, weighed, and diluted with 2-propanol. Concentrations were determined by spectrophotometric measurements at $280 \mathrm{~nm}$.

\section{Source and Purity of Chemicals:}

(1) $99 \%$, Aldrich Chemical Company, Milwaukee, WI, USA, was used as received.

(2) $99.5 \%$, anhydrous, Aldrich Chemical Company, stored over molecular sieves and distilled shortly before use.

\section{Estimated Error:}

Temperature: $\pm 0.1 \mathrm{~K}$.

$x_{1}: \pm 1.5 \%$ (relative error)

\begin{tabular}{ll}
\hline \hline Components: & Original Measurements: \\
(1) 3,4-Dichlorobenzoic acid; & ${ }^{137}$ A. Wilson, A. Tian, V. Chou, A. N. \\
$\mathrm{C}_{7} \mathrm{H}_{4} \mathrm{Cl}_{2} \mathrm{O}_{2} ;$ [51-44-5] & Quay, W. E. Acree, Jr., and M. H. \\
(2) Ethyl ethanoate; $\mathrm{C}_{4} \mathrm{H}_{8} \mathrm{O}_{2} ;$ & Abraham, Phys. Chem. Liq. 50, 324 \\
{$[141-78-6]$} & $(2012)$. \\
\hline Variables: & Prepared by: \\
$T / \mathrm{K}=298.15$ & W. E. Acree, Jr. \\
\hline
\end{tabular}

Experimental Values

\begin{tabular}{lc}
\hline \hline$x_{2}^{\mathrm{a}}$ & $x_{1}{ }^{\mathrm{b}}$ \\
\hline 0.9836 & 0.01641
\end{tabular}

${ }^{\mathrm{a}} x_{2}$ : mole fraction of component 2 in the saturated solution.

${ }^{\mathrm{b}} \mathrm{x}_{1}$ : mole fraction solubility of the solute.

\section{Auxiliary Information}

\section{Method/Apparatus/Procedure:}

Constant-temperature bath, calorimetric thermometer, and an ultraviolet/ visible spectrophotometer.

Excess solute and solvent were placed in amber glass bottles and allowed to equilibrate for several days at constant temperature. Attainment of equilibrium was verified by several repetitive measurements and by approaching equilibrium from supersaturation. Aliquots of saturated solutions were transferred through a coarse filter into tared volumetric flasks, weighed, and diluted with 2-propanol. Concentrations were determined by spectrophotometric measurements at $280 \mathrm{~nm}$.
Source and Purity of Chemicals:

(1) $99 \%$, Aldrich Chemical Company, Milwaukee, WI, USA, was used as received.

(2) $99.8 \%$, anhydrous, Aldrich Chemical Company, stored over molecular sieves and distilled shortly before use.

\section{Estimated Error:}

Temperature: $\pm 0.1 \mathrm{~K}$.

$x_{1}: \pm 1.5 \%$ (relative error)

\begin{tabular}{ll}
\hline \hline Components: & $\begin{array}{l}\text { Original Measurements: } \\
\text { (1) 3,4-Dichlorobenzoic acid; }\end{array}$ \\
${ }^{137}$ A. Wilson, A. Tian, V. Chou, A. N. \\
$\mathrm{C}_{7} \mathrm{H}_{4} \mathrm{Cl}_{2} \mathrm{O}_{2} ;$ [51-44-5] & Quay, W. E. Acree, Jr., and M. H. \\
$\begin{array}{l}\text { (2) Propyl ethanoate; } \mathrm{C}_{5} \mathrm{H}_{10} \mathrm{O}_{2} ; \\
\text { [109-60-4] }\end{array}$ & $\begin{array}{l}\text { Abraham, Phys. Chem. Liq. 50, 324 } \\
\text { (2012). }\end{array}$ \\
\hline Variables: & Prepared by: \\
$T / \mathrm{K}=298.15$ & W. E. Acree, Jr. \\
\hline
\end{tabular}

\section{Experimental Values}

\begin{tabular}{lc}
\hline \hline$x_{2}{ }^{\mathrm{a}}$ & $x_{1}{ }^{\mathrm{b}}$ \\
\hline 0.9824 & 0.01761 \\
\hline${ }^{\mathrm{a}} x_{2}:$ mole fraction of component 2 in the saturated solution. & \\
${ }^{\mathrm{b}}{ }_{x_{1}}:$ mole fraction solubility of the solute. &
\end{tabular}

\section{Auxiliary Information}

\section{Method/Apparatus/Procedure:}

Constant-temperature bath, calorimetric thermometer, and an ultraviolet/ visible spectrophotometer.

Excess solute and solvent were placed in amber glass bottles and allowed to equilibrate for several days at constant temperature. Attainment of equilibrium was verified by several repetitive measurements and by approaching equilibrium from supersaturation. Aliquots of saturated solutions were transferred through a coarse filter into tared volumetric flasks, weighed, and diluted with 2-propanol. Concentrations were determined by spectrophotometric measurements at $280 \mathrm{~nm}$.

\section{Source and Purity of Chemicals:}

(1) $99 \%$, Aldrich Chemical Company, Milwaukee, WI, USA, was used as received.

(2) $99.5 \%$, Aldrich Chemical Company, stored over molecular sieves and distilled shortly before use.

\section{Estimated Error:}

Temperature: $\pm 0.1 \mathrm{~K}$.

$x_{1}: \pm 1.5 \%$ (relative error).

\begin{tabular}{|c|c|}
\hline $\begin{array}{l}\text { Components: } \\
\text { (1) 3,4-Dichlorobenzoic acid; } \\
\left.\mathrm{C}_{7} \mathrm{H}_{4} \mathrm{Cl}_{2} \mathrm{O}_{2} ; \text {; } 51-44-5\right] \\
\text { (2) Butyl ethanoate; } \mathrm{C}_{6} \mathrm{H}_{12} \mathrm{O}_{2} \text {; } \\
\text { [123-86-4] }\end{array}$ & $\begin{array}{l}\text { Original Measurements: } \\
{ }^{137} \text { A. Wilson, A. Tian, V. Chou, A. N. } \\
\text { Quay, W. E. Acree, Jr., and M. H. } \\
\text { Abraham, Phys. Chem. Liq. 50, } 324 \\
\text { (2012). }\end{array}$ \\
\hline $\begin{array}{l}\text { Variables: } \\
T / \mathrm{K}=298.15\end{array}$ & $\begin{array}{l}\text { Prepared by: } \\
\text { W. E. Acree, Jr. }\end{array}$ \\
\hline
\end{tabular}


Experimental Values

\begin{tabular}{lc}
\hline \hline$x_{2}{ }^{\mathrm{a}}$ & $x_{1}{ }^{\mathrm{b}}$ \\
\hline 0.9794 & 0.02056 \\
${ }^{\mathrm{a}} x_{2}:$ mole fraction of component 2 in the saturated solution. & \\
${ }^{\mathrm{b}} x_{1}:$ mole fraction solubility of the solute. &
\end{tabular}

Auxiliary Information

Method/Apparatus/Procedure:

Constant-temperature bath, calorimetric thermometer, and an ultraviolet/ visible spectrophotometer.

Excess solute and solvent were placed in amber glass bottles and allowed to equilibrate for several days at constant temperature. Attainment of equilibrium was verified by several repetitive measurements and by approaching equilibrium from supersaturation. Aliquots of saturated solutions were transferred through a coarse filter into tared volumetric flasks, weighed, and diluted with 2-propanol. Concentrations were determined by

spectrophotometric measurements at $280 \mathrm{~nm}$.

Source and Purity of Chemicals:

(1) $99 \%$, Aldrich Chemical Company, Milwaukee, WI, USA, was used as received.

(2) $99.7 \%$, Aldrich Chemical Company, stored over molecular sieves and distilled shortly before use.

\section{Estimated Error:}

Temperature: $\pm 0.1 \mathrm{~K}$.

$x_{1}: \pm 1.5 \%$ (relative error).

\begin{tabular}{ll}
\hline \hline Components: & Original Measurements: \\
$\begin{array}{l}\text { (1) 3,4-Dichlorobenzoic acid; } \\
\left.\mathrm{C}_{7} \mathrm{H}_{4} \mathrm{Cl}_{2} \mathrm{O}_{2} ; \text {; } 51-44-5\right]\end{array}$ & Qu. Wilson, A. Tian, V. Chou, A. N. \\
$\begin{array}{l}\text { (2) } \mathrm{Pentyl} \text { ethanoate; } \mathrm{C}_{7} \mathrm{H}_{14} \mathrm{O}_{2} ; \\
\text { [628-63-7] }\end{array}$ & $\begin{array}{l}\text { Abraham, Phys. Chem. Lr., and M. H. } \\
\text { (2012). }\end{array}$ \\
\hline Variables: & \\
$T / \mathrm{K}=298.15$ & $\begin{array}{l}\text { Prepared by: } \\
\text { W. E. Acree, Jr. }\end{array}$ \\
\hline
\end{tabular}

Experimental Values

\begin{tabular}{lc}
\hline \hline$x_{2}{ }^{\mathrm{a}}$ & $x_{1}{ }^{\mathrm{b}}$ \\
\hline 0.9784 & 0.02156 \\
\hline
\end{tabular}

${ }^{\mathrm{a}} x_{2}$ : mole fraction of component 2 in the saturated solution.

${ }^{\mathrm{b}} x_{1}$ : mole fraction solubility of the solute.

\section{Auxiliary Information}

\section{Method/Apparatus/Procedure:}

Constant-temperature bath, calorimetric thermometer, and an ultraviolet/ visible spectrophotometer.

Excess solute and solvent were placed in amber glass bottles and allowed to equilibrate for several days at constant temperature. Attainment of equilibrium was verified by several repetitive measurements and by approaching equilibrium from supersaturation. Aliquots of saturated solutions were transferred through a coarse filter into tared volumetric flasks, weighed, and diluted with 2-propanol. Concentrations were determined by spectrophotometric measurements at $280 \mathrm{~nm}$.
Source and Purity of Chemicals:

(1) $99 \%$, Aldrich Chemical Company, Milwaukee, WI, USA, was used as received.

(2) $99 \%$, Aldrich Chemical Company, stored over molecular sieves and distilled shortly before use.

\section{Estimated Error:}

Temperature: $\pm 0.1 \mathrm{~K}$.

$x_{1}: \pm 1.5 \%$ (relative error).

\begin{tabular}{ll}
\hline \hline Components: & Original Measurements: \\
(1) 3,4-Dichlorobenzoic acid; & ${ }^{137}$ A. Wilson, A. Tian, V. Chou, A. N. \\
$\mathrm{C}_{7} \mathrm{H}_{4} \mathrm{Cl}_{2} \mathrm{O}_{2} ;$ [51-44-5] & Quay, W. E. Acree, Jr., and M. H. \\
$\begin{array}{l}\text { (2) Methyl butanoate; } \mathrm{C}_{5} \mathrm{H}_{10} \mathrm{O}_{2} ; \\
\text { [623-42-7] }\end{array}$ & $\begin{array}{l}\text { Abraham, Phys. Chem. Liq. 50, 324 } \\
\text { (2012). }\end{array}$ \\
\hline Variables: & Prepared by: \\
$T / \mathrm{K}=298.15$ & W. E. Acree, Jr. \\
\hline
\end{tabular}

\section{Experimental Values}

\begin{tabular}{lc}
\hline \hline$x_{2}{ }^{\mathrm{a}}$ & $x_{1}{ }^{\mathrm{b}}$ \\
\hline 0.9857 & 0.01430 \\
\hline${ }^{\mathrm{a}} x_{2}:$ mole fraction of component 2 in the saturated solution. & \\
${ }^{\mathrm{b}} x_{1}:$ mole fraction solubility of the solute. &
\end{tabular}

\section{Auxiliary Information}

\begin{abstract}
Method/Apparatus/Procedure:
Constant-temperature bath, calorimetric thermometer, and an ultraviolet/ visible spectrophotometer.

Excess solute and solvent were placed in amber glass bottles and allowed to equilibrate for several days at constant temperature. Attainment of equilibrium was verified by several repetitive measurements and by approaching equilibrium from supersaturation. Aliquots of saturated solutions were transferred through a coarse filter into tared volumetric flasks, weighed, and diluted with 2-propanol. Concentrations were determined by spectrophotometric measurements at $280 \mathrm{~nm}$.
\end{abstract}

Source and Purity of Chemicals:

(1) $99 \%$, Aldrich Chemical Company, Milwaukee, WI, USA, was used as received.

(2) $99 \%$, Aldrich Chemical Company, stored over molecular sieves and distilled shortly before use.

\section{Estimated Error:}

Temperature: $\pm 0.1 \mathrm{~K}$.

$x_{1}: \pm 1.5 \%$ (relative error).

\subsection{3,4-Dichlorobenzoic acid solubility data in ethers}

\begin{tabular}{|c|c|}
\hline $\begin{array}{l}\text { Components: } \\
\text { (1) 3,4-Dichlorobenzoic acid; } \\
\mathrm{C}_{7} \mathrm{H}_{4} \mathrm{Cl}_{2} \mathrm{O}_{2} ; \text { [51-44-5] } \\
\text { (2) } 1,1^{\prime} \text {-Oxybisethane; } \mathrm{C}_{4} \mathrm{H}_{10} \mathrm{O} \text {; } \\
\text { [60-29-7] }\end{array}$ & $\begin{array}{l}\text { Original Measurements: } \\
{ }^{137} \text { A. Wilson, A. Tian, V. Chou, A. N } \\
\text { Quay, W. E. Acree, Jr., and M. H. } \\
\text { Abraham, Phys. Chem. Liq. 50, } 324 \\
\text { (2012). }\end{array}$ \\
\hline $\begin{array}{l}\text { Variables: } \\
T / \mathrm{K}=298.15\end{array}$ & $\begin{array}{l}\text { Prepared by: } \\
\text { W. E. Acree, Jr. }\end{array}$ \\
\hline
\end{tabular}


Experimental Values

\begin{tabular}{lc}
\hline \hline$x_{2}{ }^{\mathrm{a}}$ & $x_{1}{ }^{\mathrm{b}}$ \\
\hline 0.9692 & 0.03077
\end{tabular}

${ }^{\mathrm{a}} x_{2}$ : mole fraction of component 2 in the saturated solution.

${ }^{\mathrm{b}} x_{1}$ : mole fraction solubility of the solute.

\section{Auxiliary Information}

\section{Method/Apparatus/Procedure:}

Constant-temperature bath, calorimetric thermometer, and an ultraviolet/ visible spectrophotometer.

Excess solute and solvent were placed in amber glass bottles and allowed to equilibrate for several days at constant temperature. Attainment of equilibrium was verified by several repetitive measurements and by approaching equilibrium from supersaturation. Aliquots of saturated solutions were transferred through a coarse filter into tared volumetric flasks, weighed, and diluted with 2-propanol. Concentrations were determined by

spectrophotometric measurements at $280 \mathrm{~nm}$.

Source and Purity of Chemicals:

(1) $99 \%$, Aldrich Chemical Company, Milwaukee, WI, USA, was used as received.

(2) $99+\%$, anhydrous, Aldrich Chemical Company, stored over molecular sieves and distilled shortly before use.

Estimated Error:

Temperature: $\pm 0.1 \mathrm{~K}$.

$x_{1}: \pm 1.5 \%$ (relative error).

\begin{tabular}{ll}
\hline \hline Components: & Original Measurements: \\
(1) 3,4-Dichlorobenzoic acid; & ${ }^{137}$ A. Wilson, A. Tian, V. Chou, A. N. \\
$\mathrm{C}_{7} \mathrm{H}_{4} \mathrm{Cl}_{2} \mathrm{O}_{2} ;[51-44-5]$ & Quay, W. E. Acree, Jr., and M. H. \\
(2) $2,2^{\prime}-$ Oxybispropane; & Abraham, Phys. Chem. Liq. 50, 324 \\
$\mathrm{C}_{6} \mathrm{H}_{14} \mathrm{O} ;[108-20-3]$ & $(2012)$. \\
\hline Variables: & Prepared by: \\
$T / \mathrm{K}=298.15$ & W. E. Acree, Jr. \\
\hline
\end{tabular}

Experimental Values

\begin{tabular}{lc}
\hline \hline$x_{2}{ }^{\mathrm{a}}$ & $x_{1}{ }^{\mathrm{b}}$ \\
\hline 0.9874 & 0.01256
\end{tabular}

${ }^{a} x_{2}$ : mole fraction of component 2 in the saturated solution.

${ }^{\mathrm{b}} x_{1}$ : mole fraction solubility of the solute.

\section{Auxiliary Information}

\footnotetext{
Method/Apparatus/Procedure:

Constant-temperature bath, calorimetric thermometer, and an ultraviolet/ visible spectrophotometer.

Excess solute and solvent were placed in amber glass bottles and allowed to equilibrate for several days at constant temperature. Attainment of equilibrium was verified by several repetitive measurements and by approaching equilibrium from supersaturation. Aliquots of saturated solutions were transferred through a coarse filter into tared volumetric flasks, weighed, and diluted with 2-propanol. Concentrations were determined by

spectrophotometric measurements at $280 \mathrm{~nm}$.
}

Source and Purity of Chemicals:

(1) $99 \%$, Aldrich Chemical Company, Milwaukee, WI, USA, was used as received.

(2) $99 \%$, anhydrous, Aldrich Chemical Company, stored over molecular sieves and distilled shortly before use.

\section{Estimated Error:}

Temperature: $\pm 0.1 \mathrm{~K}$.

$x_{1}: \pm 1.5 \%$ (relative error).

\begin{tabular}{ll}
\hline \hline Components: & Original Measurements: \\
(1) $3,4-$-Dichlorobenzoic acid; & ${ }^{137}$ A. Wilson, A. Tian, V. Chou, A. N. \\
$\mathrm{C}_{7} \mathrm{H}_{4} \mathrm{Cl}_{2} \mathrm{O}_{2} ;$ [51-44-5] & Quay, W. E. Acree, Jr., and M. H. \\
$\begin{array}{l}\text { (2) } 1,1^{\prime}-\text {-Oxybisbutane; } \mathrm{C}_{8} \mathrm{H}_{18} \mathrm{O} ; \\
{[142-96-1]}\end{array}$ & Abraham, Phys. Chem. Liq. 50, 324 \\
\hline Variables: & $(2012)$. \\
$T / \mathrm{K}=298.15$ & Prepared by: \\
\hline
\end{tabular}

Experimental Values

\begin{tabular}{lc}
\hline \hline$x_{2}{ }^{\mathrm{a}}$ & $x_{1}{ }^{\mathrm{b}}$ \\
\hline 0.9903 & 0.00969 \\
\hline${ }^{\mathrm{a}} x_{2}:$ mole fraction of component 2 in the saturated solution. & \\
${ }^{\mathrm{b}} x_{1}:$ mole fraction solubility of the solute. &
\end{tabular}

\section{Auxiliary Information}

\section{Method/Apparatus/Procedure:}

Constant-temperature bath, calorimetric thermometer, and an ultraviolet/ visible spectrophotometer.

Excess solute and solvent were placed in amber glass bottles and allowed to equilibrate for several days at constant temperature. Attainment of equilibrium was verified by several repetitive measurements and by approaching equilibrium from supersaturation. Aliquots of saturated solutions were transferred through a coarse filter into tared volumetric flasks, weighed, and diluted with 2-propanol. Concentrations were determined by spectrophotometric measurements at $280 \mathrm{~nm}$.

\section{Source and Purity of Chemicals:}

(1) $99 \%$, Aldrich Chemical Company, Milwaukee, WI, USA, was used as received.

(2) $99+\%$, Acros Organics, USA, stored over molecular sieves and distilled shortly before use.

\section{Estimated Error:}

Temperature: $\pm 0.1 \mathrm{~K}$.

$x_{1}: \pm 1.5 \%$ (relative error).

\begin{tabular}{ll}
\hline \hline Components: & Original Measurements: \\
(1) 3,4-Dichlorobenzoic acid; & ${ }^{137}$ A. Wilson, A. Tian, V. Chou, A. N. \\
$\mathrm{C}_{7} \mathrm{H}_{4} \mathrm{Cl}_{2} \mathrm{O}_{2} ;$ [51-44-5] & Quay, W. E. Acree, Jr., and M. H. \\
(2) 2-Methoxy-2-methylpropane; & Abraham, Phys. Chem. Liq. 50, 324 \\
$\mathrm{C}_{5} \mathrm{H}_{12} \mathrm{O} ;[1634-04-4]$ & $(2012)$. \\
\hline Variables: & Prepared by: \\
$T / \mathrm{K}=298.15$ & W. E. Acree, Jr. \\
\hline
\end{tabular}


Experimental Values

\begin{tabular}{lc}
\hline \hline$x_{2}{ }^{\mathrm{a}}$ & $x_{1}{ }^{\mathrm{b}}$ \\
\hline 0.9642 & 0.03583 \\
\hline${ }^{\mathrm{a}} x_{2}:$ mole fraction of component 2 in the saturated solution. & \\
${ }^{\mathrm{b}} x_{1}:$ mole fraction solubility of the solute. &
\end{tabular}

\section{Auxiliary Information}

\section{Method/Apparatus/Procedure:}

Constant-temperature bath, calorimetric thermometer, and an ultraviolet/ visible spectrophotometer.

Excess solute and solvent were placed in amber glass bottles and allowed to equilibrate for several days at constant temperature. Attainment of equilibrium was verified by several repetitive measurements and by approaching equilibrium from supersaturation. Aliquots of saturated solutions were transferred through a coarse filter into tared volumetric flasks, weighed, and diluted with 2-propanol. Concentrations were determined by

spectrophotometric measurements at $280 \mathrm{~nm}$.

\section{Source and Purity of Chemicals:}

(1) $99 \%$, Aldrich Chemical Company, Milwaukee, WI, USA, was used as received.

(2) $99.9+\%$, Arco Chemical Company, USA, stored over molecular sieves and distilled shortly before use.

\section{Estimated Error:}

Temperature: $\pm 0.1 \mathrm{~K}$

$x_{1}: \pm 1.5 \%$ (relative error).

\begin{tabular}{|c|c|}
\hline $\begin{array}{l}\text { Components: } \\
\text { (1) 3,4-Dichlorobenzoic acid; } \\
\mathrm{C}_{7} \mathrm{H}_{4} \mathrm{Cl}_{2} \mathrm{O}_{2} ; \text { [51-44-5] } \\
\text { (2) Tetrahydrofuran; } \mathrm{C}_{4} \mathrm{H}_{8} \mathrm{O} \text {; } \\
\text { [109-99-9] }\end{array}$ & $\begin{array}{l}\text { Original Measurements: } \\
{ }^{137} \text { A. Wilson, A. Tian, V. Chou, A. N. } \\
\text { Quay, W. E. Acree, Jr., and M. H. } \\
\text { Abraham, Phys. Chem. Liq. 50, } 324 \\
\text { (2012). }\end{array}$ \\
\hline $\begin{array}{l}\text { Variables: } \\
T / \mathrm{K}=298.15\end{array}$ & $\begin{array}{l}\text { Prepared by: } \\
\text { W. E. Acree, Jr. }\end{array}$ \\
\hline
\end{tabular}

\section{Experimental Values}

\begin{tabular}{lc}
\hline \hline$x_{2}^{\mathrm{a}}$ & $x_{1}^{\mathrm{b}}$ \\
\hline 0.8993 & 0.1007 \\
\hline${ }^{\mathrm{a}} x_{2}:$ mole fraction of component 2 in the saturated solution. & \\
${ }^{\mathrm{b}} x_{1}:$ mole fraction solubility of the solute. &
\end{tabular}

\section{Auxiliary Information}

\footnotetext{
Method/Apparatus/Procedure:

Constant-temperature bath, calorimetric thermometer, and an ultraviolet/ visible spectrophotometer.

Excess solute and solvent were placed in amber glass bottles and allowed to equilibrate for several days at constant temperature. Attainment of equilibrium was verified by several repetitive measurements and by approaching equilibrium from supersaturation. Aliquots of saturated solutions were transferred through a coarse filter into tared volumetric flasks, weighed, and diluted with 2-propanol. Concentrations were determined by spectrophotometric measurements at $280 \mathrm{~nm}$.
}

Source and Purity of Chemicals:

(1) $99 \%$, Aldrich Chemical Company, Milwaukee, WI, USA, was used as received.

(2) $99.9 \%$, anhydrous, Aldrich Chemical Company, stored over molecular sieves and distilled shortly before use.

\section{Estimated Error:}

Temperature: $\pm 0.1 \mathrm{~K}$.

$x_{1}: \pm 1.5 \%$ (relative error).

\begin{tabular}{|c|c|}
\hline $\begin{array}{l}\text { Components: } \\
\text { (1) 3,4-Dichlorobenzoic acid; } \\
\mathrm{C}_{7} \mathrm{H}_{4} \mathrm{Cl}_{2} \mathrm{O}_{2} \text {; [51-44-5] } \\
\text { (2) Tetrahydrofuran; } \mathrm{C}_{4} \mathrm{H}_{8} \mathrm{O} \text {; } \\
\text { [109-99-9] }\end{array}$ & $\begin{array}{l}\text { Original Measurements: } \\
{ }^{63} \text { C. K. Hancock, J. N. Pawloski, and J } \\
\text { P. Idoux, J. Org. Chem. 32, } 1931 \\
\text { (1967). }\end{array}$ \\
\hline $\begin{array}{l}\text { Variables: } \\
T / \mathrm{K}=303.15\end{array}$ & $\begin{array}{l}\text { Prepared by: } \\
\text { W. E. Acree, Jr. }\end{array}$ \\
\hline
\end{tabular}

\section{Experimental Values}

\begin{tabular}{lc}
\hline \hline$x_{2}{ }^{\mathrm{a}}$ & $x_{1}{ }^{\mathrm{b}}$ \\
\hline 0.875 & 0.125 \\
\hline${ }^{\mathrm{a}} x_{2}:$ mole fraction of component 2 in the saturated solution. & \\
${ }^{\mathrm{b}} x_{1}:$ mole fraction solubility of the solute. &
\end{tabular}

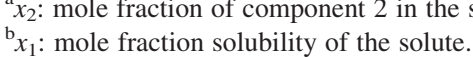

\section{Auxiliary Information}

\section{Method/Apparatus/Procedure:}

Constant-temperature bath, Soxhlet thimble, conical flask, and analytical balance.

Excess solute and solvent were placed in a conical flask and allowed to equilibrate for several days at constant temperature. Attainment of equilibrium was verified by several repetitive measurements and by approaching equilibrium from supersaturation. Aliquots of saturated solutions were transferred using a Soxhlet thimble equipped with a one-hole cork stopper and an inverted $\mathrm{U}$-shaped delivery tube to a second ground-glass stoppered flask suspended in the $303 \mathrm{~K}$ water bath. Successive portions of the contents were evaporated at room temperature in a tared aluminum foil weighing dish under a bell jar through which a slow stream of dry air was passed. The second flask plus unused saturated solution and the aluminum foil dish plus residue were weighed. The saturation solubility of the solute was calculated from the recorded mass data and molar masses of the solute and solvent.

\section{Source and Purity of Chemicals:}

(1) Purity not given, Chemical source not specified, was recrystallized several times from aqueous-ethanol mixture, and then dried under vacuum over phosphorous pentoxide.

(2) Practical grade, Chemical source not specified, stored over sodium hydroxide pellets for $24 \mathrm{~h}$, and then passed through $2 \times 70-\mathrm{cm}$ chromatographic adsorption columns containing activated alumina. After this treatment, the purified solvent was stored over copper in a nitrogen atmosphere.

\section{Estimated Error:}

Temperature: $\pm 0.02 \mathrm{~K}$.

$x_{1}: \pm 1.0 \%$ (relative error) 


\begin{tabular}{|c|c|}
\hline $\begin{array}{l}\text { Components: } \\
\text { (1) 3,4-Dichlorobenzoic acid; } \\
\mathrm{C}_{7} \mathrm{H}_{4} \mathrm{Cl}_{2} \mathrm{O}_{2} ;[51-44-5] \\
\text { (2) 1,4-Dioxane; } \mathrm{C}_{4} \mathrm{H}_{8} \mathrm{O}_{2} \text {; } \\
\text { [123-91-1] }\end{array}$ & $\begin{array}{l}\text { Original Measurements: } \\
{ }^{137} \text { A. Wilson, A. Tian, V. Chou, A. N. } \\
\text { Quay, W. E. Acree, Jr., and M. H. } \\
\text { Abraham, Phys. Chem. Liq. 50, } 324 \\
(2012) .\end{array}$ \\
\hline $\begin{array}{l}\text { Variables: } \\
T / \mathrm{K}=298.15\end{array}$ & $\begin{array}{l}\text { Prepared by: } \\
\text { W. E. Acree, Jr. }\end{array}$ \\
\hline
\end{tabular}

Experimental Values

\begin{tabular}{lc}
\hline \hline$x_{2}^{\mathrm{a}}$ & $x_{1}{ }^{\mathrm{b}}$ \\
\hline 0.9507 & 0.04931 \\
\hline
\end{tabular}

${ }^{\mathrm{a}} x_{2}$ : mole fraction of component 2 in the saturated solution.

${ }^{\mathrm{b}} x_{1}$ : mole fraction solubility of the solute.

\section{Auxiliary Information}

\section{Method/Apparatus/Procedure:}

Constant-temperature bath, calorimetric thermometer, and an ultraviolet/ visible spectrophotometer.

Excess solute and solvent were placed in amber glass bottles and allowed to equilibrate for several days at constant temperature. Attainment of equilibrium was verified by several repetitive measurements and by approaching equilibrium from supersaturation. Aliquots of saturated solutions were transferred through a coarse filter into tared volumetric flasks, weighed, and diluted with 2-propanol. Concentrations were determined by spectrophotometric measurements at $280 \mathrm{~nm}$.

Source and Purity of Chemicals:

(1) $99 \%$, Aldrich Chemical Company, Milwaukee, WI, USA, was used as received.

(2) $99.9 \%$, anhydrous, Aldrich Chemical Company, stored over molecular sieves and distilled shortly before use.

Estimated Error:

Temperature: $\pm 0.1 \mathrm{~K}$.

$x_{1}: \pm 1.5 \%$ (relative error).

\begin{tabular}{|c|c|}
\hline $\begin{array}{l}\text { Components: } \\
\text { (1) 3,4-Dichlorobenzoic acid; } \\
\mathrm{C}_{7} \mathrm{H}_{4} \mathrm{Cl}_{2} \mathrm{O}_{2} ;[51-44-5] \\
\text { (2) 1,4-Dioxane; } \mathrm{C}_{4} \mathrm{H}_{8} \mathrm{O}_{2} \text {; } \\
\text { [123-91-1] }\end{array}$ & $\begin{array}{l}\text { Original Measurements: } \\
{ }^{63} \text { C. K. Hancock, J. N. Pawloski, and J. } \\
\text { P. Idoux, J. Org. Chem. 32, } 1931 \\
\text { (1967). }\end{array}$ \\
\hline $\begin{array}{l}\text { Variables: } \\
T / \mathrm{K}=303.15\end{array}$ & $\begin{array}{l}\text { Prepared by: } \\
\text { W. E. Acree, Jr. }\end{array}$ \\
\hline
\end{tabular}

Experimental Values

\begin{tabular}{lc}
\hline \hline$x_{2}^{\mathrm{a}}$ & $x_{1}{ }^{\mathrm{b}}$ \\
\hline 0.943 & 0.057 \\
\hline${ }^{\mathrm{a}} x_{2}:$ mole fraction of component 2 in the saturated solution. & \\
${ }^{\mathrm{b}} x_{1}:$ mole fraction solubility of the solute. &
\end{tabular}

\section{Auxiliary Information}

\section{Method/Apparatus/Procedure:}

Constant-temperature bath, Soxhlet thimble, conical flask, and analytical balance.

Excess solute and solvent were placed in a conical flask and allowed to equilibrate for several days at constant temperature. Attainment of equilibrium was verified by several repetitive measurements and by approaching equilibrium from supersaturation. Weighed aliquots of saturated solutions were removed and titrated with a standardized sodium hydroxide solution (carbonate free) using a $\mathrm{pH}$ meter. The endpoint of the titration was determined by computing the second derivative in the $\mathrm{pH}$ versus volume of sodium hydroxide added.

\section{Source and Purity of Chemicals:}

(1) Purity not given, Chemical source not specified, was recrystallized several times from aqueous-ethanol mixture, and then dried under vacuum over phosphorous pentoxide.

(2) Practical grade, Chemical source not specified, stored over sodium hydroxide pellets for $24 \mathrm{~h}$, and then passed through $2 \times 70-\mathrm{cm}$ chromatographic adsorption columns containing activated alumina. After this treatment, the purified solvent was stored over copper in a nitrogen atmosphere.

\section{Estimated Error:}

Temperature: $\pm 0.02 \mathrm{~K}$

$x_{1}: \pm 1.0 \%$ (relative error)

\subsection{3,4-Dichlorobenzoic acid solubility data in alcohols}

\begin{tabular}{|c|c|}
\hline $\begin{array}{l}\text { Components: } \\
\text { (1) 3,4-Dichlorobenzoic acid; } \\
\mathrm{C}_{7} \mathrm{H}_{4} \mathrm{Cl}_{2} \mathrm{O}_{2} ;[51-44-5] \\
\text { (2) Methanol; } \mathrm{CH}_{4} \mathrm{O} ;[67-56-1]\end{array}$ & $\begin{array}{l}\text { Original Measurements: } \\
{ }^{83} \text { M. K. Chantooni and I. M. Kolthoff, } \\
\text { J. Phys. Chem. 77, } 527 \text { (1973). }\end{array}$ \\
\hline $\begin{array}{l}\text { Variables: } \\
T / \mathrm{K}=298.15\end{array}$ & $\begin{array}{l}\text { Prepared by: } \\
\text { W. E. Acree, Jr. }\end{array}$ \\
\hline
\end{tabular}

\section{Experimental Values}

The measured solubility was reported to be $0.27 \mathrm{~mol} \mathrm{dm}^{-3}$. The authors did not give the temperature at which the solubility was measured. Based on the experimental description given in an earlier paper [I. M. Kolthoff, J. J. Lingane, and W. Larson, J. Am. Chem. Soc. 60, 2512 (1938)], the compiler believes the temperature to be $298.15 \mathrm{~K}$.

\section{Auxiliary Information}

\section{Method/Apparatus/Procedure:}

Very little experimental details were provided. Solubility was determined by titrating a filtered aliquot of the saturated solution alkalimetrically in an aqueous-ethanol mixture using phenolphthalein as the acid-base indicator.

\section{Source and Purity of Chemicals:}

(1) Purity not given, synthesized by the authors by the oxidation of 3,4dichlorobenzaldehyde with hydrogen peroxide in dilute aqueous sodium hydroxide solution. The sample was further purified by recrystallization from either aqueous or aqueous-ethanol solution and dried in vacuo at $333 \mathrm{~K}$. (2) Purity not given, Chemical source not given, was dehydrated and then distilled over sulfanilic acid to remove alkaline impurities.

Estimated Error:

Temperature: $\pm 0.1 \mathrm{~K}$ (estimated by compiler).

$c_{1}: \pm 2 \%$ (relative error, estimated by compiler). 


\begin{tabular}{ll}
\hline \hline Components: & Original Measurements: \\
$(1)$ 3,4-Dichlorobenzoic acid; & ${ }^{137}$ A. Wilson, A. Tian, V. Chou, A. N. \\
$\mathrm{C}_{7} \mathrm{H}_{4} \mathrm{Cl}_{2} \mathrm{O}_{2} ;[51-44-5]$ & Quay, W. E. Acree, Jr., and M. H. \\
(2) Ethanol; $\mathrm{C}_{2} \mathrm{H}_{6} \mathrm{O} ;[64-17-5]$ & Abraham, Phys. Chem. Liq. 50, 324 \\
& $(2012)$. \\
\hline Variables: & Prepared by: \\
$T / \mathrm{K}=298.15$ & W. E. Acree, Jr. \\
\hline
\end{tabular}

Experimental Values

\begin{tabular}{lc}
\hline \hline$x_{2}{ }^{\mathrm{a}}$ & $x_{1}{ }^{\mathrm{b}}$ \\
\hline 0.9827 & 0.01732 \\
\hline
\end{tabular}

${ }^{a} x_{2}$ : mole fraction of component 2 in the saturated solution.

${ }^{\mathrm{b}} x_{1}$ : mole fraction solubility of the solute.

\section{Auxiliary Information}

\section{Method/Apparatus/Procedure:}

Constant-temperature bath, calorimetric thermometer, and an ultraviolet/ visible spectrophotometer.

Excess solute and solvent were placed in amber glass bottles and allowed to equilibrate for several days at constant temperature. Attainment of equilibrium was verified by several repetitive measurements and by approaching equilibrium from supersaturation. Aliquots of saturated solutions were transferred through a coarse filter into tared volumetric flasks, weighed, and diluted with 2-propanol. Concentrations were determined by spectrophotometric measurements at $280 \mathrm{~nm}$.

Source and Purity of Chemicals:

(1) $99 \%$, Aldrich Chemical Company, Milwaukee, WI, USA, was used as received.

(2) Absolute, Aaper Alcohol Chemical Company, USA, stored over molecular sieves and distilled shortly before use.

\section{Estimated Error:}

Temperature: $\pm 0.1 \mathrm{~K}$.

$x_{1}: \pm 1.5 \%$ (relative error).

\begin{tabular}{ll}
\hline \hline Components: & Original Measurements: \\
$\begin{array}{l}\text { (1) 3,4-Dichlorobenzoic acid; } \\
\mathrm{C}_{7} \mathrm{H}_{4} \mathrm{Cl}_{2} \mathrm{O}_{2} ; \text {; [51-44-5] }\end{array}$ & Quay, Wilson, A. Tian, V. Chou, A. N. \\
(2) 1-Propanol; $\mathrm{C}_{3} \mathrm{H}_{8} \mathrm{O} ;$ [71-23-8] $]$ & $\begin{array}{l}\text { Abraham, and M. H. } \\
\text { (2012). }\end{array}$ \\
\hline Variables: & Prepared by: \\
$T / \mathrm{K}=298.15$ & W. E. Acree, Jr. \\
\hline
\end{tabular}

\section{Experimental Values}

\begin{tabular}{lc}
\hline \hline$x_{2}{ }^{\mathrm{a}}$ & $x_{1}{ }^{\mathrm{b}}$ \\
\hline 0.9815 & 0.01846 \\
\hline${ }^{\mathrm{a}} x_{2}:$ mole fraction of component 2 in the saturated solution. \\
${ }^{\mathrm{b}} x_{1}:$ mole fraction solubility of the solute.
\end{tabular}

\section{Auxiliary Information}

\section{Method/Apparatus/Procedure:}

Constant-temperature bath, calorimetric thermometer, and an ultraviolet/ visible spectrophotometer.

Excess solute and solvent were placed in amber glass bottles and allowed to equilibrate for several days at constant temperature. Attainment of equilibrium was verified by several repetitive measurements and by approaching equilibrium from supersaturation. Aliquots of saturated solutions were transferred through a coarse filter into tared volumetric flasks, weighed, and diluted with 2-propanol. Concentrations were determined by spectrophotometric measurements at $280 \mathrm{~nm}$.

\section{Source and Purity of Chemicals:}

(1) $99 \%$, Aldrich Chemical Company, Milwaukee, WI, USA, was used as received.

(2) $99+\%$, anhydrous, Aldrich Chemical Company, stored over molecular sieves and distilled shortly before use.

Estimated Error:

Temperature: $\pm 0.1 \mathrm{~K}$.

$x_{1}: \pm 1.5 \%$ (relative error)

\begin{tabular}{ll}
\hline \hline Components: & $\begin{array}{l}\text { Original Measurements: } \\
\text { (1) 3,4-Dichlorobenzoic acid; }\end{array}$ \\
${ }^{137}$ A. Wilson, A. Tian, V. Chou, A. N. \\
$\mathrm{C}_{7} \mathrm{H}_{4} \mathrm{Cl}_{2} \mathrm{O}_{2} ;[51-44-5]$ & Quay, W. E. Acree, Jr., and M. H. \\
(2) 2-Propanol; $\mathrm{C}_{3} \mathrm{H}_{8} \mathrm{O} ;$ [67-63-0] & $\begin{array}{l}\text { Abraham, Phys. Chem. Liq. 50, 324 } \\
\text { (2012). }\end{array}$ \\
\hline Variables: & Prepared by: \\
$T / \mathrm{K}=298.15$ & W. E. Acree, Jr. \\
\hline
\end{tabular}

Experimental Values

\begin{tabular}{lc}
\hline \hline$x_{2}{ }^{\mathrm{a}}$ & $x_{1}{ }^{\mathrm{b}}$ \\
\hline 0.9823 & 0.01767 \\
\hline${ }^{\mathrm{a}} x_{2}:$ mole fraction of component 2 in the saturated solution. & \\
${ }^{\mathrm{b}}{ }_{x_{1}: \text { mole fraction solubility of the solute. }}$ &
\end{tabular}

\section{Auxiliary Information}

\section{Method/Apparatus/Procedure:}

Constant-temperature bath, calorimetric thermometer, and an ultraviolet/ visible spectrophotometer.

Excess solute and solvent were placed in amber glass bottles and allowed to equilibrate for several days at constant temperature. Attainment of equilibrium was verified by several repetitive measurements and by approaching equilibrium from supersaturation. Aliquots of saturated solutions were transferred through a coarse filter into tared volumetric flasks, weighed, and diluted with 2-propanol. Concentrations were determined by spectrophotometric measurements at $280 \mathrm{~nm}$.

Source and Purity of Chemicals:

(1) $99 \%$, Aldrich Chemical Company, Milwaukee, WI, USA, was used as received.

(2) 99+\%, anhydrous, Aldrich Chemical Company, stored over molecular sieves and distilled shortly before use.

\section{Estimated Error:}

Temperature: $\pm 0.1 \mathrm{~K}$.

$x_{1}: \pm 1.5 \%$ (relative error). 


\begin{tabular}{|c|c|}
\hline $\begin{array}{l}\text { Components: } \\
\text { (1) 3,4-Dichlorobenzoic acid; } \\
\mathrm{C}_{7} \mathrm{H}_{4} \mathrm{Cl}_{2} \mathrm{O}_{2} ;[51-44-5] \\
\text { (2) 2-Propanol; } \mathrm{C}_{3} \mathrm{H}_{8} \mathrm{O} ;[67-63-0]\end{array}$ & $\begin{array}{l}\text { Original Measurements: } \\
{ }^{76} \text { M. K. Chantooni and I. M. Kolthoff, } \\
\text { Anal. Chem. 51, } 133 \text { (1979). }\end{array}$ \\
\hline $\begin{array}{l}\text { Variables: } \\
T / \mathrm{K}=298.15\end{array}$ & $\begin{array}{l}\text { Prepared by: } \\
\text { W. E. Acree, Jr. }\end{array}$ \\
\hline
\end{tabular}

Experimental Values

The measured solubility was reported to be $0.317 \mathrm{~mol} \mathrm{dm}^{-3}$. The authors did not give the temperature at which the solubility was measured. Based on the experimental description given in an earlier paper [I. M. Kolthoff, J. J. Lingane, and W. Larson, J. Am. Chem. Soc. 60, 2512 (1938)], the compiler believes the temperature to be $298.15 \mathrm{~K}$.

\section{Auxiliary Information}

Method/Apparatus/Procedure:

Very little experimental details were provided. Solubility was determined by titrating a filtered aliquot of the saturated solution alkalimetrically in an aqueous-ethanol mixture using phenolphthalein as the acid-base indicator.

Source and Purity of Chemicals:

(1) Purity not given, synthesized by the authors by the oxidation of 3,4dichlorobenzaldehyde with hydrogen peroxide in dilute aqueous sodium hydroxide solution. The sample was further purified by recrystallization from either aqueous or aqueous-ethanol solution and dried in vасио at $333 \mathrm{~K}$. (2) Purity not given, Fisher Scientific, USA, shaken with calcium hydride and distilled before use.

\section{Estimated Error:}

Temperature: $\pm 0.1 \mathrm{~K}$ (estimated by compiler).

$c_{1}: \pm 2 \%$ (relative error, estimated by compiler).

\begin{tabular}{ll}
\hline \hline Components: & Original Measurements: \\
(1) 3,4-Dichlorobenzoic acid; & ${ }^{137}$ A. Wilson, A. Tian, V. Chou, A. N. \\
$\mathrm{C}_{7} \mathrm{H}_{4} \mathrm{Cl}_{2} \mathrm{O}_{2} ;$; 51-44-5] & Quay, W. E. Acree, Jr., and M. H. \\
(2) 1-Butanol; $\mathrm{C}_{4} \mathrm{H}_{10} \mathrm{O} ;$ [71-36-3] & $\begin{array}{l}\text { Abraham, Phys. Chem. Liq. 50, 324 } \\
\text { (2012). }\end{array}$ \\
\hline Variables: & Prepared by: \\
$T / \mathrm{K}=298.15$ & W. E. Acree, Jr. \\
\hline
\end{tabular}

\section{Experimental Values}

\begin{tabular}{lc}
\hline \hline$x_{2}{ }^{\mathrm{a}}$ & $x_{1}{ }^{\mathrm{b}}$ \\
\hline 0.9797 & 0.02026 \\
\hline
\end{tabular}

${ }^{\mathrm{a}} x_{2}$ : mole fraction of component 2 in the saturated solution.

${ }^{b} x_{1}$ : mole fraction solubility of the solute.

\section{Auxiliary Information}

\section{Method/Apparatus/Procedure:}

Constant-temperature bath, calorimetric thermometer, and an ultraviolet/ visible spectrophotometer.

Excess solute and solvent were placed in amber glass bottles and allowed to equilibrate for several days at constant temperature. Attainment of equilibrium was verified by several repetitive measurements and by approaching equilibrium from supersaturation. Aliquots of saturated solutions were transferred through a coarse filter into tared volumetric flasks, weighed, and diluted with 2-propanol. Concentrations were determined by spectrophotometric measurements at $280 \mathrm{~nm}$.

Source and Purity of Chemicals:

(1) $99 \%$, Aldrich Chemical Company, Milwaukee, WI, USA, was used as received.

(2) $99.8+\%$, HPLC grade, Aldrich Chemical Company, stored over molecular sieves and distilled shortly before use.

Estimated Error:

Temperature: $\pm 0.1 \mathrm{~K}$.

$x_{1}: \pm 1.5 \%$ (relative error)

\begin{tabular}{ll}
\hline \hline Components: & Original Measurements: \\
$\begin{array}{l}\text { (1) 3,4-Dichlorobenzoic acid; } \\
\mathrm{C}_{7} \mathrm{H}_{4} \mathrm{Cl}_{2} \mathrm{O}_{2} ;[51-44-5]\end{array}$ & $\begin{array}{l}{ }^{137} \text { A. Wilson, A. Tian, V. Chou, A. N. } \\
\text { Quay, W. E. Acree, Jr., and M. H. }\end{array}$ \\
(2) 2-Butanol; $\mathrm{C}_{4} \mathrm{H}_{10} \mathrm{O} ;$ [78-92-2] & $\begin{array}{l}\text { Abraham, Phys. Chem. Liq. 50, 324 } \\
\text { (2012). }\end{array}$ \\
\hline Variables: & $\begin{array}{l}\text { Prepared by: } \\
T / \mathrm{K}=298.15\end{array}$ \\
\hline
\end{tabular}

Experimental Values

\begin{tabular}{lc}
\hline \hline$x_{2}{ }^{\mathrm{a}}$ & $x_{1}{ }^{\mathrm{b}}$ \\
\hline 0.9810 & 0.01898
\end{tabular}

${ }^{a} x_{2}$ : mole fraction of component 2 in the saturated solution.

${ }^{\mathrm{b}} x_{1}$ : mole fraction solubility of the solute.

\section{Auxiliary Information}

Method/Apparatus/Procedure:

Constant-temperature bath, calorimetric thermometer, and an ultraviolet/ visible spectrophotometer.

Excess solute and solvent were placed in amber glass bottles and allowed to equilibrate for several days at constant temperature. Attainment of equilibrium was verified by several repetitive measurements and by approaching equilibrium from supersaturation. Aliquots of saturated solutions were transferred through a coarse filter into tared volumetric flasks, weighed, and diluted with 2-propanol. Concentrations were determined by spectrophotometric measurements at $280 \mathrm{~nm}$.

Source and Purity of Chemicals:

(1) $99 \%$, Aldrich Chemical Company, Milwaukee, WI, USA, was used as received.

(2) $99+\%$, anhydrous, Aldrich Chemical Company, stored over molecular sieves and distilled shortly before use.

\section{Estimated Error:}

Temperature: $\pm 0.1 \mathrm{~K}$.

$x_{1}: \pm 1.5 \%$ (relative error) 


\begin{tabular}{|c|c|}
\hline $\begin{array}{l}\text { Components: } \\
\text { (1) 3,4-Dichlorobenzoic acid; } \\
\mathrm{C}_{7} \mathrm{H}_{4} \mathrm{Cl}_{2} \mathrm{O}_{2} \text {; [51-44-5] } \\
\text { (2) 2-Methyl-1-propanol; } \mathrm{C}_{4} \mathrm{H}_{10} \mathrm{O} \text {; } \\
\text { [78-83-1] }\end{array}$ & $\begin{array}{l}\text { Original Measurements: } \\
{ }^{137} \text { A. Wilson, A. Tian, V. Chou, A. N. } \\
\text { Quay, W. E. Acree, Jr., and M. H. } \\
\text { Abraham, Phys. Chem. Liq. 50, } 324 \\
\text { (2012). }\end{array}$ \\
\hline $\begin{array}{l}\text { Variables: } \\
T / \mathrm{K}=298.15\end{array}$ & $\begin{array}{l}\text { Prepared by: } \\
\text { W. E. Acree, Jr. }\end{array}$ \\
\hline
\end{tabular}

Experimental Values

\begin{tabular}{lc}
\hline \hline$x_{2}{ }^{\mathrm{a}}$ & $x_{1}{ }^{\mathrm{b}}$ \\
\hline 0.9861 & 0.01389 \\
\hline${ }^{\mathrm{a}} x_{2}:$ mole fraction of component 2 in the saturated solution. & \\
${ }^{\mathrm{b}} x_{1}:$ mole fraction solubility of the solute. &
\end{tabular}

\section{Auxiliary Information}

Method/Apparatus/Procedure:

Constant-temperature bath, calorimetric thermometer, and an ultraviolet/ visible spectrophotometer.

Excess solute and solvent were placed in amber glass bottles and allowed to equilibrate for several days at constant temperature. Attainment of equilibrium was verified by several repetitive measurements and by approaching equilibrium from supersaturation. Aliquots of saturated solutions were transferred through a coarse filter into tared volumetric flasks, weighed, and diluted with 2-propanol. Concentrations were determined by

spectrophotometric measurements at $280 \mathrm{~nm}$.

Source and Purity of Chemicals:

(1) $99 \%$, Aldrich Chemical Company, Milwaukee, WI, USA, was used as received.

(2) $99+\%$, anhydrous, Aldrich Chemical Company, stored over molecular sieves and distilled shortly before use.

Estimated Error:

Temperature: $\pm 0.1 \mathrm{~K}$.

$x_{1}: \pm 1.5 \%$ (relative error).

\begin{tabular}{|c|c|}
\hline $\begin{array}{l}\text { Components: } \\
\text { (1) 3,4-Dichlorobenzoic acid; } \\
\mathrm{C}_{7} \mathrm{H}_{4} \mathrm{Cl}_{2} \mathrm{O}_{2} \text {; [51-44-5] } \\
\text { (2) 2-Methyl-2-propanol; } \mathrm{C}_{4} \mathrm{H}_{10} \mathrm{O} \text {; } \\
\text { [75-65-0] }\end{array}$ & $\begin{array}{l}\text { Original Measurements: } \\
{ }^{137} \text { A. Wilson, A. Tian, V. Chou, A. N. } \\
\text { Quay, W. E. Acree, Jr., and M. H. } \\
\text { Abraham, Phys. Chem. Liq. 50, } 324 \\
\text { (2012). }\end{array}$ \\
\hline $\begin{array}{l}\text { Variables: } \\
T / \mathrm{K}=298.15\end{array}$ & $\begin{array}{l}\text { Prepared by: } \\
\text { W. E. Acree, Jr. }\end{array}$ \\
\hline
\end{tabular}

Experimental Values

\begin{tabular}{lc}
\hline \hline$x_{2}{ }^{\mathrm{a}}$ & $x_{1}{ }^{\mathrm{b}}$ \\
\hline 0.9655 & 0.03449 \\
\hline${ }^{\mathrm{a}} x_{2}:$ mole fraction of component 2 in the saturated solution. & \\
${ }^{\mathrm{b}} x_{1}:$ mole fraction solubility of the solute. &
\end{tabular}

\section{Auxiliary Information}

Method/Apparatus/Procedure:

Constant-temperature bath, calorimetric thermometer, and an ultraviolet/ visible spectrophotometer.

Excess solute and solvent were placed in amber glass bottles and allowed to equilibrate for several days at constant temperature. Attainment of equilibrium was verified by several repetitive measurements and by approaching equilibrium from supersaturation. Aliquots of saturated solutions were transferred through a coarse filter into tared volumetric flasks, weighed, and diluted with 2-propanol. Concentrations were determined by spectrophotometric measurements at $280 \mathrm{~nm}$.

Source and Purity of Chemicals:

(1) $99 \%$, Aldrich Chemical Company, Milwaukee, WI, USA, was used as received.

(2) $99+\%$, Arco Chemical Company, USA, stored over molecular sieves and distilled shortly before use.

Estimated Error:

Temperature: $\pm 0.1 \mathrm{~K}$.

$x_{1}: \pm 1.5 \%$ (relative error).

\begin{tabular}{|c|c|}
\hline $\begin{array}{l}\text { Components: } \\
\text { (1) 3,4-Dichlorobenzoic acid; } \\
\left.\mathrm{C}_{7} \mathrm{H}_{4} \mathrm{Cl}_{2} \mathrm{O}_{2} ; \text {; } 51-44-5\right] \\
\text { (2) 2-Methyl-2-propanol; } \mathrm{C}_{4} \mathrm{H}_{10} \mathrm{O} \text {; } \\
\text { [75-65-0] }\end{array}$ & $\begin{array}{l}\text { Original Measurements: } \\
{ }^{76} \text { M. K. Chantooni and I. M. Kolthoff, } \\
\text { Anal. Chem. 51, } 133 \text { (1979). }\end{array}$ \\
\hline $\begin{array}{l}\text { Variables: } \\
T / \mathrm{K}=298.15\end{array}$ & $\begin{array}{l}\text { Prepared by: } \\
\text { W. E. Acree, Jr. }\end{array}$ \\
\hline
\end{tabular}

Experimental Values

The measured solubility was reported to be $0.365 \mathrm{~mol} \mathrm{dm}^{-3}$. The authors did not give the temperature at which the solubility was measured. Based on the experimental description given in an earlier paper [I. M. Kolthoff, J. J. Lingane, and W. Larson, J. Am. Chem. Soc. 60, 2512 (1938)], the compiler believes the temperature to be $298.15 \mathrm{~K}$.

\section{Auxiliary Information}

\section{Method/Apparatus/Procedure:}

Very little experimental details were provided. Solubility was determined by titrating a filtered aliquot of the saturated solution alkalimetrically in an aqueous-ethanol mixture using phenolphthalein as the acid-base indicator.

\section{Source and Purity of Chemicals:}

(1) Purity not given, synthesized by the authors by the oxidation of 3,4dichlorobenzaldehyde with hydrogen peroxide in dilute aqueous sodium hydroxide solution. The sample was further purified by recrystallization from either aqueous or aqueous-ethanol solution and dried in vacuo at $333 \mathrm{~K}$.

(2) White Label, Eastman Kodak Chemical Company, Rochester, NY, USA, was shaken with calcium hydride and distilled before use.

\section{Estimated Error:}

Temperature: $\pm 0.1 \mathrm{~K}$ (estimated by compiler).

$c_{1}: \pm 2 \%$ (relative error, estimated by compiler). 
Experimental Values

\begin{tabular}{lc}
\hline \hline$x_{2}{ }^{\mathrm{a}}$ & $x_{1}{ }^{\mathrm{b}}$ \\
\hline 0.9782 & 0.02180 \\
\hline$x_{2}:$
\end{tabular}

${ }^{a} x_{2}$ : mole fraction of component 2 in the saturated solution.

${ }^{\mathrm{b}} x_{1}$ : mole fraction solubility of the solute.

\section{Auxiliary Information}

\section{Method/Apparatus/Procedure:}

Constant-temperature bath, calorimetric thermometer, and an ultraviolet/ visible spectrophotometer.

Excess solute and solvent were placed in amber glass bottles and allowed to equilibrate for several days at constant temperature. Attainment of equilibrium was verified by several repetitive measurements and by approaching equilibrium from supersaturation. Aliquots of saturated solutions were transferred through a coarse filter into tared volumetric flasks, weighed, and diluted with 2-propanol. Concentrations were determined by spectrophotometric measurements at $280 \mathrm{~nm}$.

\section{Source and Purity of Chemicals:}

(1) $99 \%$, Aldrich Chemical Company, Milwaukee, WI, USA, was used as received.

(2) $99+\%$, Aldrich Chemical Company, stored over molecular sieves and distilled shortly before use.

Estimated Error:

Temperature: $\pm 0.1 \mathrm{~K}$.

$x_{1}: \pm 1.5 \%$ (relative error).

\begin{tabular}{ll}
\hline \hline Components: & Original Measurements: \\
$\begin{array}{l}\text { (1) 3,4-Dichlorobenzoic acid; } \\
\mathrm{C}_{7} \mathrm{H}_{4} \mathrm{Cl}_{2} \mathrm{O}_{2} ;[51-44-5]\end{array}$ & ${ }^{137}$ A. Wilson, A. Tian, V. Chou, A. N. \\
(2) 1-Pentanol; $\mathrm{C}_{5} \mathrm{H}_{12} \mathrm{O} ;$ [71-41-0] $]$ & $\begin{array}{l}\text { Abraham, W. E. Acree, Jr., and M. H. } \\
\text { (2012). }\end{array}$ \\
\hline Variables: & Prepared by: \\
$T / \mathrm{K}=298.15$ & W. E. Acree, Jr. \\
\hline
\end{tabular}

\section{Auxiliary Information}

\section{Method/Apparatus/Procedure:}

Constant-temperature bath, calorimetric thermometer, and an ultraviolet/ visible spectrophotometer.

Excess solute and solvent were placed in amber glass bottles and allowed to equilibrate for several days at constant temperature. Attainment of equilibrium was verified by several repetitive measurements and by approaching equilibrium from supersaturation. Aliquots of saturated solutions were transferred through a coarse filter into tared volumetric flasks, weighed, and diluted with 2-propanol. Concentrations were determined by spectrophotometric measurements at $280 \mathrm{~nm}$.

\section{Source and Purity of Chemicals:}

(1) $99 \%$, Aldrich Chemical Company, Milwaukee, WI, USA, was used as received.

(2) $99+\%$, Acros Organics, USA, stored over molecular sieves and distilled shortly before use.

Estimated Error:

Temperature: $\pm 0.1 \mathrm{~K}$.

$x_{1}: \pm 1.5 \%$ (relative error)

\begin{tabular}{|c|c|}
\hline Components: & Original Measurements: \\
\hline (1) 3,4-Dichlorobenzoic acid; & ${ }^{137}$ A. Wilson, A. Tian, V. Chou, A. N \\
\hline $\mathrm{C}_{7} \mathrm{H}_{4} \mathrm{Cl}_{2} \mathrm{O}_{2} ;[51-44-5]$ & Quay, W. E. Acree, Jr., and M. H. \\
\hline $\begin{array}{l}\text { (2) 2-Methyl-1-butanol; } \mathrm{C}_{5} \mathrm{H}_{12} \mathrm{O} \text {; } \\
\text { [137-32-6] }\end{array}$ & $\begin{array}{l}\text { Abraham, Phys. Chem. Liq. 50, } 324 \\
\text { (2012). }\end{array}$ \\
\hline Variables: & Prepared by: \\
\hline$T / \mathrm{K}=298.15$ & W. E. Acree, Jr. \\
\hline
\end{tabular}

Experimental Values

\begin{tabular}{lc}
\hline \hline$x_{2}{ }^{\mathrm{a}}$ & $x_{1}{ }^{\mathrm{b}}$ \\
\hline 0.9817 & 0.01834 \\
\hline${ }^{\mathrm{a}} x_{2}:$ mole fraction of component 2 in the saturated solution. & \\
${ }^{\mathrm{b}} x_{1}:$ mole fraction solubility of the solute. &
\end{tabular}

${ }^{\mathrm{b}} x_{1}$ : mole fraction solubility of the solute.

\section{Auxiliary Information}

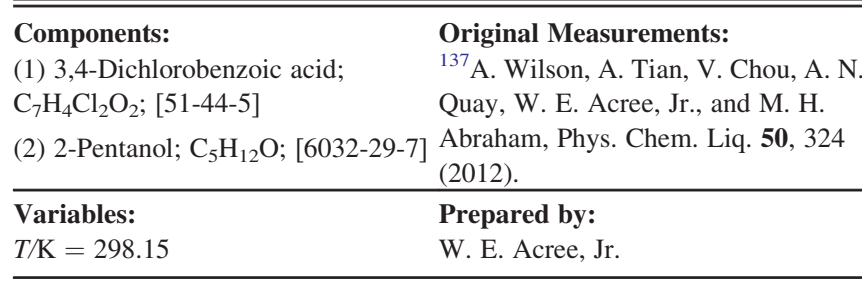

\section{Experimental Values}

\begin{tabular}{lc}
\hline \hline$x_{2}{ }^{\mathrm{a}}$ & $x_{1}{ }^{\mathrm{b}}$ \\
\hline 0.9752 & 0.02477 \\
\hline
\end{tabular}

${ }^{a_{2}} x_{2}$ mole fraction of component 2 in the saturated solution.

${ }^{b} x_{1}$ : mole fraction solubility of the solute.

\section{Method/Apparatus/Procedure:}

Constant-temperature bath, calorimetric thermometer, and an ultraviolet/ visible spectrophotometer.

Excess solute and solvent were placed in amber glass bottles and allowed to equilibrate for several days at constant temperature. Attainment of equilibrium was verified by several repetitive measurements and by approaching equilibrium from supersaturation. Aliquots of saturated solutions were transferred through a coarse filter into tared volumetric flasks, weighed, and diluted with 2-propanol. Concentrations were determined by spectrophotometric measurements at $280 \mathrm{~nm}$.

\section{Source and Purity of Chemicals:}

(1) $99 \%$, Aldrich Chemical Company, Milwaukee, WI, USA, was used as received.

(2) $99 \%$, Aldrich Chemical Company, stored over molecular sieves and distilled shortly before use.

\section{Estimated Error:}

Temperature: $\pm 0.1 \mathrm{~K}$.

$x_{1}: \pm 1.5 \%$ (relative error). 


\begin{tabular}{ll}
\hline \hline Components: & Original Measurements: \\
$\begin{array}{l}\text { (1) 3,4-Dichlorobenzoic acid; } \\
\mathrm{C}_{7} \mathrm{H}_{4} \mathrm{Cl}_{2} \mathrm{O}_{2} ; \text {; [51-44-5] }\end{array}$ & ${ }^{137}$ A. Wilson, A. Tian, V. Chou, A. N. \\
$\begin{array}{l}\text { (2) 3-Methyl-1-butanol; } \mathrm{C}_{5} \mathrm{H}_{12} \mathrm{O} ; \\
{[123-51-3]}\end{array}$ & $\begin{array}{l}\text { Abraham, W. E. Acree, Jr., and M. H. } \\
(2012) .\end{array}$ \\
\hline Variables: & Prepared by: \\
$T / \mathrm{K}=298.15$ & W. E. Acree, Jr. \\
\hline
\end{tabular}

Experimental Values

\begin{tabular}{lc}
\hline \hline$x_{2}{ }^{\mathrm{a}}$ & $x_{1}{ }^{\mathrm{b}}$ \\
\hline 0.9784 & 0.02157 \\
\hline${ }^{\mathrm{a}} x_{2}:$ mole fraction of component 2 in the saturated solution. &
\end{tabular}

${ }^{\mathrm{b}} x_{1}$ : mole fraction solubility of the solute.

\section{Auxiliary Information}

\section{Method/Apparatus/Procedure:}

Constant-temperature bath, calorimetric thermometer, and an ultraviolet/ visible spectrophotometer.

Excess solute and solvent were placed in amber glass bottles and allowed to equilibrate for several days at constant temperature. Attainment of equilibrium was verified by several repetitive measurements and by approaching equilibrium from supersaturation. Aliquots of saturated solutions were transferred through a coarse filter into tared volumetric flasks, weighed, and diluted with 2-propanol. Concentrations were determined by

spectrophotometric measurements at $280 \mathrm{~nm}$.

Source and Purity of Chemicals:

(1) $99 \%$, Aldrich Chemical Company, Milwaukee, WI, USA, was used as received.

(2) $99 \%$, anhydrous, Aldrich Chemical Company, stored over molecular sieves and distilled shortly before use.

Estimated Error:

Temperature: $\pm 0.1 \mathrm{~K}$.

$x_{1}: \pm 1.5 \%$ (relative error).

\begin{tabular}{ll}
\hline \hline Components: & Original Measurements: \\
$\begin{array}{l}\text { (1) 3,4-Dichlorobenzoic acid; } \\
\left.\mathrm{C}_{7} \mathrm{H}_{4} \mathrm{Cl}_{2} \mathrm{O}_{2} ; \text {; } 51-44-5\right]\end{array}$ & Quay, Wilson, A. Acree, Jr., and M. H. \\
(2) 1-Hexanol; $\mathrm{C}_{6} \mathrm{H}_{14} \mathrm{O} ;$ [111-27-3] & $\begin{array}{l}\text { Abraham, Phys. Chem. Liq. 50, 324 } \\
\text { (2012). }\end{array}$ \\
\hline Variables: & Prepared by: \\
$T / \mathrm{K}=298.15$ & W. E. Acree, Jr. \\
\hline
\end{tabular}

Experimental Values

\begin{tabular}{lc}
\hline \hline$x_{2}^{\mathrm{a}}$ & $x_{1}{ }^{\mathrm{b}}$ \\
\hline 0.9762 & 0.02376 \\
\hline${ }^{\mathrm{a}} x_{2}:$ mole fraction of component 2 in the saturated solution. & \\
${ }^{\mathrm{b}} x_{1}:$ mole fraction solubility of the solute. &
\end{tabular}

\section{Auxiliary Information}

Method/Apparatus/Procedure:

Constant-temperature bath, calorimetric thermometer, and an ultraviolet/ visible spectrophotometer.

Excess solute and solvent were placed in amber glass bottles and allowed to equilibrate for several days at constant temperature. Attainment of equilibrium was verified by several repetitive measurements and by approaching equilibrium from supersaturation. Aliquots of saturated solutions were transferred through a coarse filter into tared volumetric flasks, weighed, and diluted with 2-propanol. Concentrations were determined by spectrophotometric measurements at $280 \mathrm{~nm}$.

Source and Purity of Chemicals:

(1) $99 \%$, Aldrich Chemical Company, Milwaukee, WI, USA, was used as received.

(2) $99+\%$, Alfa Aesar, USA, stored over molecular sieves and distilled shortly before use.

Estimated Error:

Temperature: $\pm 0.1 \mathrm{~K}$.

$x_{1}: \pm 1.5 \%$ (relative error).

\begin{tabular}{|c|c|}
\hline $\begin{array}{l}\text { Components: } \\
\text { (1) 3,4-Dichlorobenzoic acid; } \\
\mathrm{C}_{7} \mathrm{H}_{4} \mathrm{Cl}_{2} \mathrm{O}_{2} ;[51-44-5] \\
\text { (2) } 1-\mathrm{Hexanol} ; \mathrm{C}_{6} \mathrm{H}_{14} \mathrm{O} ;[111-27-3]\end{array}$ & $\begin{array}{l}\text { Original Measurements: } \\
{ }^{76} \text { M. K. Chantooni and I. M. Kolthoff, } \\
\text { Anal. Chem. 51, } 133 \text { (1979). }\end{array}$ \\
\hline $\begin{array}{l}\text { Variables: } \\
T / \mathrm{K}=298.15\end{array}$ & $\begin{array}{l}\text { Prepared by: } \\
\text { W. E. Acree, Jr. }\end{array}$ \\
\hline
\end{tabular}

\section{Experimental Values}

The measured solubility was reported to be $0.271 \mathrm{~mol} \mathrm{dm}^{-3}$. The authors did not give the temperature at which the solubility was measured. Based on the experimental description given in an earlier paper [I. M. Kolthoff, J. J. Lingane, and W. Larson, J. Am. Chem. Soc. 60, 2512 (1938)], the compiler believes the temperature to be $298.15 \mathrm{~K}$.

\section{Auxiliary Information}

\section{Method/Apparatus/Procedure:}

Very little experimental details were provided. Solubility was determined by titrating a filtered aliquot of the saturated solution alkalimetrically in an aqueous-ethanol mixture using phenolphthalein as the acid-base indicator.

Source and Purity of Chemicals:

(1) Purity not given, synthesized by the authors by the oxidation of 3,4dichlorobenzaldehyde with hydrogen peroxide in dilute aqueous sodium hydroxide solution. The sample was further purified by recrystallization from either aqueous or aqueous-ethanol solution and dried in vacuo at $333 \mathrm{~K}$. (2) Yellow Label, Eastman Kodak Chemical Company, Rochester, NY, USA, was shaken with calcium hydride and distilled before use.

\section{Estimated Error:}

Temperature: $\pm 0.1 \mathrm{~K}$ (estimated by compiler). $c_{1}: \pm 2 \%$ (relative error, estimated by compiler). 


\begin{tabular}{ll}
\hline \hline Components: & Original Measurements: \\
$\begin{array}{l}\text { (1) 3,4-Dichlorobenzoic acid; } \\
\mathrm{C}_{7} \mathrm{H}_{4} \mathrm{Cl}_{2} \mathrm{O}_{2} ; \text {; [51-44-5] }\end{array}$ & ${ }^{137}$ A. Wilson, A. Tian, V. Chou, A. N. \\
$\begin{array}{l}\text { (2) 2-Methyl-1-pentanol; } \mathrm{C}_{6} \mathrm{H}_{14} \mathrm{O} ; \\
\text { [105-30-6] }\end{array}$ & $\begin{array}{l}\text { Abraham, W. Acree, Jr., and M. H. } \\
(2012) .\end{array}$ \\
\hline Variables: & Prepared by: \\
$T / \mathrm{K}=298.15$ & W. E. Acree, Jr. \\
\hline
\end{tabular}

\section{Experimental Values}

\begin{tabular}{lc}
\hline \hline$x_{2}{ }^{\mathrm{a}}$ & $x_{1}{ }^{\mathrm{b}}$ \\
\hline 0.9761 & 0.02388 \\
\hline${ }^{\mathrm{a}} x_{2}:$ mole fraction of component 2 in the saturated solution. & \\
${ }^{\mathrm{b}} x_{1}:$ mole fraction solubility of the solute. &
\end{tabular}

\section{Auxiliary Information}

\section{Method/Apparatus/Procedure:}

Constant-temperature bath, calorimetric thermometer, and an ultraviolet/ visible spectrophotometer.

Excess solute and solvent were placed in amber glass bottles and allowed to equilibrate for several days at constant temperature. Attainment of equilibrium was verified by several repetitive measurements and by approaching equilibrium from supersaturation. Aliquots of saturated solutions were transferred through a coarse filter into tared volumetric flasks, weighed, and diluted with 2-propanol. Concentrations were determined by spectrophotometric measurements at $280 \mathrm{~nm}$.

Source and Purity of Chemicals:

(1) $99 \%$, Aldrich Chemical Company, Milwaukee, WI, USA, was used as received.

(2) $99 \%$, Aldrich Chemical Company, stored over molecular sieves and distilled shortly before use.

Estimated Error:

Temperature: $\pm 0.1 \mathrm{~K}$.

$x_{1}: \pm 1.5 \%$ (relative error).

\begin{tabular}{|c|c|}
\hline $\begin{array}{l}\text { Components: } \\
\text { (1) 3,4-Dichlorobenzoic acid; } \\
\left.\mathrm{C}_{7} \mathrm{H}_{4} \mathrm{Cl}_{2} \mathrm{O}_{2} ; \text {; } 51-44-5\right] \\
\text { (2) 4-Methyl-2-pentanol; } \mathrm{C}_{6} \mathrm{H}_{14} \mathrm{O} \text {; } \\
\text { [108-11-2] }\end{array}$ & $\begin{array}{l}\text { Original Measurements: } \\
{ }^{137} \text { A. Wilson, A. Tian, V. Chou, A. N. } \\
\text { Quay, W. E. Acree, Jr., and M. H. } \\
\text { Abraham, Phys. Chem. Liq. 50, } 324 \\
\text { (2012). }\end{array}$ \\
\hline $\begin{array}{l}\text { Variables: } \\
T / \mathrm{K}=298.15\end{array}$ & $\begin{array}{l}\text { Prepared by: } \\
\text { W. E. Acree, Jr. }\end{array}$ \\
\hline
\end{tabular}

Experimental Values

\begin{tabular}{lc}
\hline \hline$x_{2}{ }^{\mathrm{a}}$ & $x_{1}{ }^{\mathrm{b}}$ \\
\hline 0.9744 & 0.02555 \\
\hline${ }^{\mathrm{a}} x_{2}:$ mole fraction of component 2 in the saturated solution. & \\
${ }^{\mathrm{b}} x_{1}:$ mole fraction solubility of the solute. &
\end{tabular}

\section{Auxiliary Information}

\section{Method/Apparatus/Procedure:}

Constant-temperature bath, calorimetric thermometer, and an ultraviolet/ visible spectrophotometer.

Excess solute and solvent were placed in amber glass bottles and allowed to equilibrate for several days at constant temperature. Attainment of equilibrium was verified by several repetitive measurements and by approaching equilibrium from supersaturation. Aliquots of saturated solutions were transferred through a coarse filter into tared volumetric flasks, weighed, and diluted with 2-propanol. Concentrations were determined by spectrophotometric measurements at $280 \mathrm{~nm}$.

\section{Source and Purity of Chemicals:}

(1) $99 \%$, Aldrich Chemical Company, Milwaukee, WI, USA, was used as received.

(2) $99+\%$, Acros Organics, USA, stored over molecular sieves and distilled shortly before use.

Estimated Error:

Temperature: $\pm 0.1 \mathrm{~K}$.

$x_{1}: \pm 1.5 \%$ (relative error)

\begin{tabular}{|c|c|}
\hline Components: & Original Measurements: \\
\hline $\begin{array}{l}\text { (1) 3,4-Dichlorobenzoic acid; } \\
\mathrm{C}_{7} \mathrm{H}_{4} \mathrm{Cl}_{2} \mathrm{O}_{2} ;[51-44-5]\end{array}$ & $\begin{array}{l}{ }^{137} \text { A. Wilson, A. Tian, V. Chou, A. N } \\
\text { Quay, W. E. Acree, Jr., and M. H. }\end{array}$ \\
\hline (2) $1-\mathrm{Heptanol} ; \mathrm{C}_{7} \mathrm{H}_{16} \mathrm{O}$; [111-70-6] & $\begin{array}{l}\text { Abraham, Phys. Chem. Liq. 50, } 324 \\
\text { (2012). }\end{array}$ \\
\hline Variables: & Prepared by: \\
\hline$T / \mathrm{K}=298.15$ & W. E. Acree, Jr. \\
\hline
\end{tabular}

Experimental Values

\begin{tabular}{lc}
\hline \hline$x_{2}{ }^{\mathrm{a}}$ & $x_{1}{ }^{\mathrm{b}}$ \\
\hline 0.9749 & 0.02506 \\
\hline${ }^{\mathrm{a}} x_{2}:$ mole fraction of component 2 in the saturated solution. \\
${ }^{\mathrm{b}} x_{1}:$ mole fraction solubility of the solute.
\end{tabular}

${ }^{\mathrm{b}} x_{1}$ : mole fraction solubility of the solute.

\section{Auxiliary Information}

\section{Method/Apparatus/Procedure:}

Constant-temperature bath, calorimetric thermometer, and an ultraviolet/ visible spectrophotometer.

Excess solute and solvent were placed in amber glass bottles and allowed to equilibrate for several days at constant temperature. Attainment of equilibrium was verified by several repetitive measurements and by approaching equilibrium from supersaturation. Aliquots of saturated solutions were transferred through a coarse filter into tared volumetric flasks, weighed, and diluted with 2-propanol. Concentrations were determined by spectrophotometric measurements at $280 \mathrm{~nm}$.

Source and Purity of Chemicals:

(1) $99 \%$, Aldrich Chemical Company, Milwaukee, WI, USA, was used as received.

(2) $99+\%$, Alfa Aesar, USA, stored over molecular sieves and distilled shortly before use.

\section{Estimated Error:}

Temperature: $\pm 0.1 \mathrm{~K}$.

$x_{1}: \pm 1.5 \%$ (relative error) 


\begin{tabular}{|c|c|}
\hline $\begin{array}{l}\text { Components: } \\
\text { (1) 3,4-Dichlorobenzoic acid; } \\
\mathrm{C}_{7} \mathrm{H}_{4} \mathrm{Cl}_{2} \mathrm{O}_{2} ;[51-44-5] \\
\text { (2) 1-Octanol; } \mathrm{C}_{8} \mathrm{H}_{18} \mathrm{O} ;[111-87-5]\end{array}$ & $\begin{array}{l}\text { Original Measurements: } \\
{ }^{137} \text { A. Wilson, A. Tian, V. Chou, A. N. } \\
\text { Quay, W. E. Acree, Jr., and M. H. } \\
\text { Abraham, Phys. Chem. Liq. 50, } 324 \\
\text { (2012). }\end{array}$ \\
\hline $\begin{array}{l}\text { Variables: } \\
T / \mathrm{K}=298.15\end{array}$ & $\begin{array}{l}\text { Prepared by: } \\
\text { W. E. Acree, Jr. }\end{array}$ \\
\hline
\end{tabular}

Experimental Values

\begin{tabular}{lc}
\hline \hline$x_{2}{ }^{\mathrm{a}}$ & $x_{1}{ }^{\mathrm{b}}$ \\
\hline 0.9758 & 0.02419 \\
\hline$x_{2}:$
\end{tabular}

${ }^{a} x_{2}$ : mole fraction of component 2 in the saturated solution.

${ }^{\mathrm{b}} x_{1}$ : mole fraction solubility of the solute.

\section{Auxiliary Information}

Method/Apparatus/Procedure:
Constant-temperature bath, calorimetric thermometer, and an ultraviolet/
visible spectrophotometer.
Excess solute and solvent were placed in amber glass bottles and allowed to
equilibrate for several days at constant temperature. Attainment of equilibrium
was verified by several repetitive measurements and by approaching
equilibrium from supersaturation. Aliquots of saturated solutions were
transferred through a coarse filter into tared volumetric flasks, weighed, and
diluted with 2-propanol. Concentrations were determined by
spectrophotometric measurements at 280 nm.
Source and Purity of Chemicals:
(1) $99 \%$, Aldrich Chemical Company, Milwaukee, WI, USA, was used as
received.
(2) $99+\%$, anhydrous, Aldrich Chemical Company, stored over molecular
sieves and distilled shortly before use.
Estimated Error:
Temperature: \pm 0.1 K.
$x_{1}: \pm 1.5 \%$ (relative error).

\subsection{3,4-Dichlorobenzoic acid solubility data in alkoxyalcohols}

\begin{tabular}{ll}
\hline \hline Components: & Original Measurements: \\
(1) 3,4-Dichlorobenzoic acid; & ${ }^{137}$ A. Wilson, A. Tian, V. Chou, A. N. \\
$\mathrm{C}_{7} \mathrm{H}_{4} \mathrm{Cl}_{2} \mathrm{O}_{2} ;$ [51-44-5] & Quay, W. E. Acree, Jr., and M. H. \\
(2) 2-Ethoxyethanol; $\mathrm{C}_{4} \mathrm{H}_{10} \mathrm{O}_{2} ;$ & Abraham, Phys. Chem. Liq. 50, 324 \\
{$[111-80-5]$} & $(2012)$. \\
\hline Variables: & Prepared by: \\
$T / \mathrm{K}=298.15$ & W. E. Acree, Jr. \\
\hline
\end{tabular}

Experimental Values

\begin{tabular}{lc}
\hline \hline$x_{2}{ }^{\mathrm{a}}$ & $x_{1}{ }^{\mathrm{b}}$ \\
\hline 0.9554 & 0.04455 \\
${ }^{\mathrm{a}} x_{2}:$ mole fraction of component 2 in the saturated solution. & \\
${ }^{\mathrm{b}} x_{1}:$ mole fraction solubility of the solute. &
\end{tabular}

\section{Auxiliary Information}

Method/Apparatus/Procedure:

Constant-temperature bath, calorimetric thermometer, and an ultraviolet/ visible spectrophotometer.

Excess solute and solvent were placed in amber glass bottles and allowed to equilibrate for several days at constant temperature. Attainment of equilibrium was verified by several repetitive measurements and by approaching equilibrium from supersaturation. Aliquots of saturated solutions were transferred through a coarse filter into tared volumetric flasks, weighed, and diluted with 2-propanol. Concentrations were determined by spectrophotometric measurements at $280 \mathrm{~nm}$.

Source and Purity of Chemicals:

(1) $99 \%$, Aldrich Chemical Company, Milwaukee, WI, USA, was used as received.

(2) $99 \%$, Aldrich Chemical Company, stored over molecular sieves and distilled shortly before use.

Estimated Error:

Temperature: $\pm 0.1 \mathrm{~K}$.

$x_{1}: \pm 1.5 \%$ (relative error)

\begin{tabular}{ll}
\hline \hline Components: & Original Measurements: \\
$(1)$ 3,4-Dichlorobenzoic acid; & ${ }^{137}$ A. Wilson, A. Tian, V. Chou, A. N. \\
$\mathrm{C}_{7} \mathrm{H}_{4} \mathrm{Cl}_{2} \mathrm{O}_{2} ;$ [51-44-5] & Quay, W. E. Acree, Jr., and M. H. \\
$\begin{array}{l}\text { (2) 2-Propoxyethanol; } \mathrm{C}_{5} \mathrm{H}_{12} \mathrm{O}_{2} ; \\
\text { [2807-30-9] }\end{array}$ & $\begin{array}{l}\text { Abraham, Phys. Chem. Liq. 50, 324 } \\
\text { (2012). }\end{array}$ \\
\hline Variables: & Prepared by: \\
$T / \mathrm{K}=298.15$ & W. E. Acree, Jr. \\
\hline
\end{tabular}

Experimental Values

\begin{tabular}{lc}
\hline \hline$x_{2}{ }^{\mathrm{a}}$ & $x_{1}{ }^{\mathrm{b}}$ \\
\hline 0.9532 & 0.04677
\end{tabular}

${ }^{\mathrm{a}} x_{2}$ : mole fraction of component 2 in the saturated solution.

${ }^{\mathrm{b}} x_{1}$ : mole fraction solubility of the solute.

\section{Auxiliary Information}

\section{Method/Apparatus/Procedure:}

Constant-temperature bath, calorimetric thermometer, and an ultraviolet/ visible spectrophotometer.

Excess solute and solvent were placed in amber glass bottles and allowed to equilibrate for several days at constant temperature. Attainment of equilibrium was verified by several repetitive measurements and by approaching equilibrium from supersaturation. Aliquots of saturated solutions were transferred through a coarse filter into tared volumetric flasks, weighed, and diluted with 2-propanol. Concentrations were determined by

spectrophotometric measurements at $280 \mathrm{~nm}$.

Source and Purity of Chemicals:

(1) $99 \%$, Aldrich Chemical Company, Milwaukee, WI, USA, was used as received.

(2) $99 \%$, Aldrich Chemical Company, stored over molecular sieves and distilled shortly before use.

Estimated Error:

Temperature: $\pm 0.1 \mathrm{~K}$.

$x_{1}: \pm 1.5 \%$ (relative error). 


\begin{tabular}{|c|c|}
\hline $\begin{array}{l}\text { Components: } \\
\text { (1) } 3,4-\text { Dichlorobenzoic acid; } \\
\mathrm{C}_{7} \mathrm{H}_{4} \mathrm{Cl}_{2} \mathrm{O}_{2} ;[51-44-5] \\
\text { (2) 2-Isopropoxyethanol; } \\
\mathrm{C}_{5} \mathrm{H}_{12} \mathrm{O}_{2} ;[109-59-1]\end{array}$ & $\begin{array}{l}\text { Original Measurements: } \\
{ }^{137} \text { A. Wilson, A. Tian, V. Chou, A. N. } \\
\text { Quay, W. E. Acree, Jr., and M. H. } \\
\text { Abraham, Phys. Chem. Liq. 50, } 324 \\
\text { (2012). }\end{array}$ \\
\hline $\begin{array}{l}\text { Variables: } \\
T / \mathrm{K}=298.15\end{array}$ & $\begin{array}{l}\text { Prepared by: } \\
\text { W. E. Acree, Jr. }\end{array}$ \\
\hline
\end{tabular}

\section{Experimental Values}

\begin{tabular}{lc}
\hline \hline$x_{2}{ }^{\mathrm{a}}$ & $x_{1}{ }^{\mathrm{b}}$ \\
\hline 0.9517 & 0.04831 \\
\hline
\end{tabular}

${ }^{\mathrm{a}} x_{2}$ : mole fraction of component 2 in the saturated solution.

${ }^{\mathrm{b}} x_{1}$ : mole fraction solubility of the solute.

\section{Auxiliary Information}

\section{Method/Apparatus/Procedure:}

Constant-temperature bath, calorimetric thermometer, and an ultraviolet/ visible spectrophotometer.

Excess solute and solvent were placed in amber glass bottles and allowed to equilibrate for several days at constant temperature. Attainment of equilibrium was verified by several repetitive measurements and by approaching equilibrium from supersaturation. Aliquots of saturated solutions were transferred through a coarse filter into tared volumetric flasks, weighed, and diluted with 2-propanol. Concentrations were determined by spectrophotometric measurements at $280 \mathrm{~nm}$.

\section{Source and Purity of Chemicals:}

(1) $99 \%$, Aldrich Chemical Company, Milwaukee, WI, USA, was used as received.

(2) $99 \%$, Aldrich Chemical Company, stored over molecular sieves and distilled shortly before use.

Estimated Error:

Temperature: $\pm 0.1 \mathrm{~K}$

$x_{1}: \pm 1.5 \%$ (relative error).

\begin{tabular}{ll}
\hline \hline Components: & Original Measurements: \\
$\begin{array}{l}\text { (1) 3,4-Dichlorobenzoic acid; } \\
\mathrm{C}_{7} \mathrm{H}_{4} \mathrm{Cl}_{2} \mathrm{O}_{2} ; \text { [51-44-5] }\end{array}$ & Quay, Wilson, A. Tian, V. Chou, A. N. \\
$\begin{array}{l}\text { (2) 2-Butoxyethanol; } \mathrm{C}_{6} \mathrm{H}_{14} \mathrm{O}_{2} ; \\
{[111-76-2]}\end{array}$ & $\begin{array}{l}\text { Abraham, and M. Phys. Chem. Liq. 50, } 324 \\
(2012) .\end{array}$ \\
\hline Variables: & Prepared by: \\
$T / \mathrm{K}=298.15$ & W. E. Acree, Jr. \\
\hline
\end{tabular}

Experimental Values

\begin{tabular}{lc}
\hline \hline$x_{2}{ }^{\mathrm{a}}$ & $x_{1}{ }^{\mathrm{b}}$ \\
\hline 0.9601 & 0.03991 \\
\hline${ }^{\mathrm{a}} x_{2}:$ mole fraction of component 2 in the saturated solution. & \\
${ }^{\mathrm{b}} x_{1}:$ mole fraction solubility of the solute. &
\end{tabular}

\section{Auxiliary Information}

\section{Method/Apparatus/Procedure:}

Constant-temperature bath, calorimetric thermometer, and an ultraviolet/ visible spectrophotometer.

Excess solute and solvent were placed in amber glass bottles and allowed to equilibrate for several days at constant temperature. Attainment of equilibrium was verified by several repetitive measurements and by approaching equilibrium from supersaturation. Aliquots of saturated solutions were transferred through a coarse filter into tared volumetric flasks, weighed, and diluted with 2-propanol. Concentrations were determined by spectrophotometric measurements at $280 \mathrm{~nm}$.

\section{Source and Purity of Chemicals:}

(1) $99 \%$, Aldrich Chemical Company, Milwaukee, WI, USA, was used as received.

(2) $99 \%$, Acros Organics, USA, stored over molecular sieves and distilled shortly before use.

Estimated Error:

Temperature: $\pm 0.1 \mathrm{~K}$.

$x_{1}: \pm 1.5 \%$ (relative error).

\begin{tabular}{ll}
\hline \hline Components: & Original Measurements: \\
(1) 3,4-Dichlorobenzoic acid; & ${ }^{137}$ A. Wilson, A. Tian, V. Chou, A. N. \\
$\mathrm{C}_{7} \mathrm{H}_{4} \mathrm{Cl}_{2} \mathrm{O}_{2} ;$ [51-44-5] & Quay, W. E. Acree, Jr., and M. H. \\
(2) 3-Methoxy-1-butanol; & Abraham, Phys. Chem. Liq. 50, 324 \\
$\mathrm{C}_{5} \mathrm{H}_{12} \mathrm{O}_{2} ;[2517-43-3]$ & $(2012)$. \\
\hline Variables: & Prepared by: \\
$T / \mathrm{K}=298.15$ & W. E. Acree, Jr. \\
\hline
\end{tabular}

Experimental Values

\begin{tabular}{lc}
\hline \hline$x_{2}{ }^{\mathrm{a}}$ & $x_{1}{ }^{\mathrm{b}}$ \\
\hline 0.9497 & 0.05033 \\
\hline${ }^{\mathrm{a}} x_{2}:$ mole fraction of component 2 in the saturated solution. & \\
${ }^{\mathrm{b}} x_{1}:$ mole fraction solubility of the solute. &
\end{tabular}

\section{Auxiliary Information}

\section{Method/Apparatus/Procedure:}

Constant-temperature bath, calorimetric thermometer, and an ultraviolet/ visible spectrophotometer.

Excess solute and solvent were placed in amber glass bottles and allowed to equilibrate for several days at constant temperature. Attainment of equilibrium was verified by several repetitive measurements and by approaching equilibrium from supersaturation. Aliquots of saturated solutions were transferred through a coarse filter into tared volumetric flasks, weighed, and diluted with 2-propanol. Concentrations were determined by spectrophotometric measurements at $280 \mathrm{~nm}$.

\section{Source and Purity of Chemicals:}

(1) $99 \%$, Aldrich Chemical Company, Milwaukee, WI, USA, was used as received.

(2) $99 \%$, Aldrich Chemical Company, stored over molecular sieves and distilled shortly before use.

Estimated Error:

Temperature: $\pm 0.1 \mathrm{~K}$.

$x_{1}: \pm 1.5 \%$ (relative error). 


\subsection{3,4-Dichlorobenzoic acid solubility data in miscellaneous organic solvents}

\begin{tabular}{|c|c|}
\hline $\begin{array}{l}\text { Components: } \\
\text { (1) 3,4-Dichlorobenzoic acid; } \\
\mathrm{C}_{7} \mathrm{H}_{4} \mathrm{Cl}_{2} \mathrm{O}_{2} ;[51-44-5] \\
\text { (2) Ethanenitrile; } \mathrm{C}_{2} \mathrm{H}_{3} \mathrm{~N} ;[75-05-8]\end{array}$ & $\begin{array}{l}\text { Original Measurements: } \\
{ }^{83} \text { M. K. Chantooni and I. M. Kolthoff, } \\
\text { J. Phys. Chem. 77, } 527 \text { (1973). }\end{array}$ \\
\hline $\begin{array}{l}\text { Variables: } \\
T / \mathrm{K}=298.15\end{array}$ & $\begin{array}{l}\text { Prepared by: } \\
\text { W. E. Acree, Jr. }\end{array}$ \\
\hline
\end{tabular}

\section{Experimental Values}

The measured solubility was reported to be $0.028 \mathrm{~mol} \mathrm{dm}^{-3}$. The authors did not give the temperature at which the solubility was measured. Based on the experimental description given in an earlier paper [I. M. Kolthoff, J. J. Lingane, and W. Larson, J. Am. Chem. Soc. 60, 2512 (1938)], the compiler believes the temperature to be $298.15 \mathrm{~K}$.

\section{Auxiliary Information}

\section{Method/Apparatus/Procedure:}

Very little experimental details were provided. Solubility was determined by titrating a filtered aliquot of the saturated solution alkalimetrically in an aqueous-ethanol mixture using phenolphthalein as the acid-base indicator.

\section{Source and Purity of Chemicals:}

(1) Purity not given, synthesized by the authors by the oxidation of 3,4dichlorobenzaldehyde with hydrogen peroxide in dilute aqueous sodium hydroxide solution. The sample was further purified by recrystallization from either aqueous or aqueous-ethanol solution and dried in vасио at $333 \mathrm{~K}$. (2) Purity not given, Chemical source not given, was purified by shaking with saturated potassium hydroxide, followed by activated alumina, and then anhydrous calcium chloride to remove water. Ethanenitrile was further dried over anhydrous magnesium sulfate and then phosphorous pentoxide. The sample was distilled shortly before use.

Estimated Error:

Temperature: $\pm 0.1 \mathrm{~K}$ (estimated by compiler).

$c_{1}: \pm 2 \%$ (relative error, estimated by compiler).

\begin{tabular}{|c|c|}
\hline $\begin{array}{l}\text { Components: } \\
\text { (1) } 3,4 \text {-Dichlorobenzoic acid; } \\
\mathrm{C}_{7} \mathrm{H}_{4} \mathrm{Cl}_{2} \mathrm{O}_{2} ;[51-44-5] \\
\text { (2) Dimethyl sulfoxide; } \mathrm{C}_{2} \mathrm{H}_{6} \mathrm{OS} \text {; } \\
\text { [67-68-5] }\end{array}$ & $\begin{array}{l}\text { Original Measurements: } \\
{ }^{83} \text { M. K. Chantooni and I. M. Kolthoff, } \\
\text { J. Phys. Chem. 77, } 527 \text { (1973). }\end{array}$ \\
\hline $\begin{array}{l}\text { Variables: } \\
T / \mathrm{K}=298.15\end{array}$ & $\begin{array}{l}\text { Prepared by: } \\
\text { W. E. Acree, Jr. }\end{array}$ \\
\hline
\end{tabular}

\section{Experimental Values}

The measured solubility was reported to be $2.14 \mathrm{~mol} \mathrm{dm}^{-3}$. The authors did not give the temperature at which the solubility was measured. Based on the experimental description given in an earlier paper [I. M. Kolthoff, J. J. Lingane, and W. Larson, J. Am. Chem. Soc. 60, 2512 (1938)], the compiler believes the temperature to be $298.15 \mathrm{~K}$.

\section{Auxiliary Information}

Method/Apparatus/Procedure:

Very little experimental details were provided. Solubility was determined by titrating a filtered aliquot of the saturated solution alkalimetrically in an aqueous-ethanol mixture using phenolphthalein as the acid-base indicator.

\section{Source and Purity of Chemicals:}

(1) Purity not given, synthesized by the authors by the oxidation of 3,4dichlorobenzaldehyde with hydrogen peroxide in dilute aqueous sodium hydroxide solution. The sample was further purified by recrystallization from either aqueous or aqueous-ethanol solution and dried in vacuo at $333 \mathrm{~K}$. (2) Purity not given, Chemical source not given, was shaken with activated alumina and then distilled before use.

\section{Estimated Error:}

Temperature: $\pm 0.1 \mathrm{~K}$ (estimated by compiler). $c_{1}: \pm 2 \%$ (relative error, estimated by compiler).

\begin{tabular}{|c|c|}
\hline $\begin{array}{l}\text { Components: } \\
\text { (1) 3,4-Dichlorobenzoic acid; } \\
\mathrm{C}_{7} \mathrm{H}_{4} \mathrm{Cl}_{2} \mathrm{O}_{2} ;[51-44-5] \\
\text { (2) } \mathrm{N}, \mathrm{N} \text {-Dimethylformamide; } \\
\mathrm{C}_{3} \mathrm{H}_{7} \mathrm{NO} ;[64-19-7]\end{array}$ & $\begin{array}{l}\text { Original Measurements: } \\
{ }^{83} \text { M. K. Chantooni and I. M. Kolthoff, } \\
\text { J. Phys. Chem. 77, } 527 \text { (1973). }\end{array}$ \\
\hline $\begin{array}{l}\text { Variables: } \\
T / \mathrm{K}=298.15\end{array}$ & $\begin{array}{l}\text { Prepared by: } \\
\text { W. E. Acree, Jr. }\end{array}$ \\
\hline
\end{tabular}

Experimental Values

The measured solubility was reported to be $2.86 \mathrm{~mol} \mathrm{dm}^{-3}$. The authors did not give the temperature at which the solubility was measured. Based on the experimental description given in an earlier paper [I. M. Kolthoff, J. J. Lingane, and W. Larson, J. Am. Chem. Soc. 60, 2512 (1938)], the compiler believes the temperature to be $298.15 \mathrm{~K}$.

\section{Auxiliary Information}

\section{Method/Apparatus/Procedure:}

Very little experimental details were provided. Solubility was determined by titrating a filtered aliquot of the saturated solution alkalimetrically in an aqueous-ethanol mixture using phenolphthalein as the acid-base indicator.

\section{Source and Purity of Chemicals:}

(1) Purity not given, synthesized by the authors by the oxidation of 3,4dichlorobenzaldehyde with hydrogen peroxide in dilute aqueous sodium hydroxide solution. The sample was further purified by recrystallization from either aqueous or aqueous-ethanol solution and dried in vacuo at $333 \mathrm{~K}$. (2) Purity not given, Chemical source not given, was shaken first with phosphorous pentoxide and then with potassium hydroxide pellets. Solvent was distilled shortly before use.

\section{Estimated Error:}

Temperature: $\pm 0.1 \mathrm{~K}$ (estimated by compiler). $c_{1}: \pm 2 \%$ (relative error, estimated by compiler). 


\section{Solubility of 3,5-Dichlorobenzoic Acid in Organic Solvents}

\subsection{Critical evaluation of experimental solubility data}

Only one laboratory has reported solubility data for 3,5dichlorobenzoic acid in organic solvents. Chantooni and Kolthoff $^{83,136}$ determined the solubility of 3,5-dichlorobenzoic acid in methanol and ethanenitrile at $298.15 \mathrm{~K}$. It is not possible to perform a critical evaluation of the experimental data as measurements were performed at only a single temperature, and there are no independent experimental solubility data for 3,5-dichlorobenzoic acid in either methanol or ethanenitrile.

The experimental solubility data for 3,5-dichlorobenzoic acid in organic solvents are in Secs. 28.2 and 28.3.

\subsection{3,5-Dichlorobenzoic acid solubility data in alcohols}

\begin{tabular}{|c|c|}
\hline $\begin{array}{l}\text { Components: } \\
\text { (1) } 3,5 \text {-Dichlorobenzoic acid; } \\
\mathrm{C}_{7} \mathrm{H}_{4} \mathrm{Cl}_{2} \mathrm{O}_{2} \text {; [51-36-5] } \\
\text { (2) Methanol; } \mathrm{CH}_{4} \mathrm{O} \text {; [67-56-1] }\end{array}$ & $\begin{array}{l}\text { Original Measurements: } \\
{ }^{83} \text { M. K. Chantooni and I. M. Kolthoff, } \\
\text { J. Phys. Chem. 77, } 527 \text { (1973). }\end{array}$ \\
\hline $\begin{array}{l}\text { Variables: } \\
T / \mathrm{K}=298.15\end{array}$ & $\begin{array}{l}\text { Prepared by: } \\
\text { W. E. Acree, Jr. }\end{array}$ \\
\hline
\end{tabular}

Experimental Values

The measured solubility was reported to be $1.25 \mathrm{~mol} \mathrm{dm}^{-3}$. The authors did not give the temperature at which the solubility was measured. Based on the experimental description given in an earlier paper [I. M. Kolthoff, J. J. Lingane, and W. Larson, J. Am. Chem. Soc. 60, 2512 (1938)], the compiler believes the temperature to be $298.15 \mathrm{~K}$.

\section{Auxiliary Information}

Method/Apparatus/Procedure:

Very little experimental details were provided. Solubility was determined by titrating a filtered aliquot of the saturated solution alkalimetrically in an aqueous-ethanol mixture using phenolphthalein as the acid-base indicator.

Source and Purity of Chemicals:

(1) Purity not given, Aldrich Chemical Company, Milwaukee, WI, USA, was recrystallized from either aqueous or aqueous-ethanol solution and dried in vacuo at $333 \mathrm{~K}$.

(2) Purity not given, Chemical source not given, was dehydrated and then distilled over sulfanilic acid to remove alkaline impurities.

\section{Estimated Error:}

Temperature: $\pm 0.1 \mathrm{~K}$ (estimated by compiler).

$c_{1}: \pm 2 \%$ (relative error, estimated by compiler).

\subsection{3,5-Dichlorobenzoic acid solubility data in} miscellaneous organic solvents

\begin{tabular}{ll}
\hline \hline Components: & Original Measurements: \\
(1) 3,5-Dichlorobenzoic acid; & ${ }^{136} \mathrm{M}$. K. Chantooni, Jr. and I. M. \\
$\mathrm{C}_{7} \mathrm{H}_{4} \mathrm{Cl}_{2} \mathrm{O}_{2} ;[51-36-5]$ & Kolthoff, J. Phys. Chem. 78, 839 \\
(2) Ethanenitrile; $\mathrm{C}_{2} \mathrm{H}_{3} \mathrm{~N} ;$ [75-05-8] & $(1974)$. \\
\hline Variables: & Prepared by: \\
$T / \mathrm{K}=298.15$ & W. E. Acree, Jr. \\
\hline
\end{tabular}

\section{Experimental Values}

The measured solubility was reported to be $0.085 \mathrm{~mol} \mathrm{dm}^{-3}$. The authors did not give the temperature at which the solubility was measured. Based on the experimental description given in an earlier paper [I. M. Kolthoff, J. J. Lingane, and W. Larson, J. Am. Chem. Soc. 60, 2512 (1938)], the compiler believes the temperature to be $298.15 \mathrm{~K}$.

\section{Auxiliary Information}

\section{Method/Apparatus/Procedure:}

Very little experimental details were provided. Solubility was determined by titrating a filtered aliquot of the saturated solution alkalimetrically in an aqueous-ethanol mixture using phenolphthalein as the acid-base indicator.

Source and Purity of Chemicals:

(1) Purity not given, Aldrich Chemical Company, Milwaukee, WI, USA, was recrystallized from either aqueous or aqueous-ethanol solution and dried in vacuo at $333 \mathrm{~K}$

(2) Purity not given, Chemical source not given, was purified by shaking with saturated potassium hydroxide, followed by activated alumina, and then anhydrous calcium chloride to remove water. Ethanenitrile was further dried over anhydrous magnesium sulfate and then phosphorous pentoxide. The sample was distilled shortly before use.

Estimated Error:

Temperature: $\pm 0.1 \mathrm{~K}$ (estimated by compiler). $c_{1}: \pm 2 \%$ (relative error, estimated by compiler).

\section{Solubility of 2,4-Dihydroxybenzoic Acid in Organic Solvents}

\subsection{Critical evaluation of experimental solubility data}

There is only a single study reporting solubility data for 2,4dihydroxybenzoic acid in organic solvents. Ongley ${ }^{56}$ determined the solubility of 2,4-dihydroxybenzoic acid in cyclohexane, benzene, trichloromethane and tetrachloromethane at $298.15 \mathrm{~K}$. It is not possible to perform a critical evaluation of the experimental data as measurements were performed at only a single temperature, and there are no independent experimental solubility data for 2,4-dihydroxybenzoic acid in the aforementioned four solvents.

The experimental solubility data for 2,4-dihydroxybenzoic acid dissolved in organic solvents are in Secs. 29.2-29.4. 


\subsection{2,4-Dihydroxybenzoic acid solubility data in saturated hydrocarbons (including cycloalkanes)}

\begin{tabular}{|c|c|}
\hline $\begin{array}{l}\text { Components: } \\
\text { (1) 2,4-Dihydroxybenzoic acid; } \\
\mathrm{C}_{7} \mathrm{H}_{6} \mathrm{O}_{4} ;[89-86-1] \\
\text { (2) Cyclohexane; } \mathrm{C}_{6} \mathrm{H}_{12} ;[110-82-7]\end{array}$ & $\begin{array}{l}\text { Original Measurements: } \\
{ }^{56} \text { P. A. Ongley, J. Chem. Soc. 1954, } \\
3634 \text {. }\end{array}$ \\
\hline $\begin{array}{l}\text { Variables: } \\
T / \mathrm{K}=298.15\end{array}$ & $\begin{array}{l}\text { Prepared by: } \\
\text { W. E. Acree, Jr. }\end{array}$ \\
\hline
\end{tabular}

\section{Experimental Values}

The measured solubility was reported to be $-\log _{10} c_{1}=5.000$, which corresponds to a solubility of $c_{1}=0.0000100 \mathrm{~mol} \mathrm{dm}^{-3}$.

\section{Auxiliary Information}

\section{Method/Apparatus/Procedure:}

Very little experimental details were provided. Excess solute and solvent were placed in tubes, which were rotated for at least $8 \mathrm{~h}$ at $298 \mathrm{~K}$. The solutions were then removed, concentrated if necessary, and titrated with standard alkali using a mixed (bromothymol blue + neutral red) indicator.

\section{Source and Purity of Chemicals:}

(1) Purity not given, Chemical source not given, solute was either "AnalaR" grade or recrystallized prior to use.

(2) Purity not given, Chemical source not given, no purification details were provided.

Estimated Error:

Temperature: No information was given. $c_{1}: \pm 4 \%$ (relative error, estimated by compiler).

\subsection{2,4-Dihydroxybenzoic acid solubility data in aromatic hydrocarbons}

\begin{tabular}{|c|c|}
\hline $\begin{array}{l}\text { Components: } \\
\text { (1) 2,4-Dihydroxybenzoic acid; } \\
\mathrm{C}_{7} \mathrm{H}_{6} \mathrm{O}_{4} ;[89-86-1] \\
\text { (2) Benzene; } \mathrm{C}_{6} \mathrm{H}_{6} ;[71-43-2]\end{array}$ & $\begin{array}{l}\text { Original Measurements: } \\
{ }^{56} \text { P. A. Ongley, J. Chem. Soc. 1954, } \\
3634 \text {. }\end{array}$ \\
\hline $\begin{array}{l}\text { Variables: } \\
T / \mathrm{K}=298.15\end{array}$ & $\begin{array}{l}\text { Prepared by: } \\
\text { W. E. Acree, Jr. }\end{array}$ \\
\hline
\end{tabular}

\section{Experimental Values}

The measured solubility was reported to be $-\log _{10} c_{1}=$ 2.412, which corresponds to a solubility of $c_{1}=0.00387 \mathrm{~mol}$ $\mathrm{dm}^{-3}$.

\section{Auxiliary Information}

\footnotetext{
Method/Apparatus/Procedure:

Very little experimental details were provided. Excess solute and solvent were placed in tubes, which were rotated for at least $8 \mathrm{~h}$ at $298 \mathrm{~K}$. The solutions were then removed, concentrated if necessary, and titrated with standard alkali using a mixed (bromothymol blue + neutral red) indicator.
}

Source and Purity of Chemicals:

(1) Purity not given, Chemical source not given, solute was either "AnalaR" grade or recrystallized prior to use.

(2) Purity not given, Chemical source not given, no purification details were provided.

\section{Estimated Error:}

Temperature: No information was given

$c_{1}: \pm 4 \%$ (relative error, estimated by compiler).

\subsection{2,4-Dihydroxybenzoic acid solubility data in haloalkanes, haloalkenes, and haloaromatic hydrocarbons}

\section{Components:}

(1) 2,4-Dihydroxybenzoic acid;

$\mathrm{C}_{7} \mathrm{H}_{6} \mathrm{O}_{4} ;$; $\left.89-86-1\right]$

(2) Trichloromethane; $\mathrm{CHCl}_{3}$; [67-66-3]

Variables:

$T / \mathrm{K}=298.15$

Prepared by:

W. E. Acree, Jr 3634.

Original Measurements:

${ }^{56}$ P. A. Ongley, J. Chem. Soc. 1954,

Experimental Values

The measured solubility was reported to be $-\log _{10} c_{1}=$ 1.897, which corresponds to a solubility of $c_{1}=0.0127 \mathrm{~mol}$ $\mathrm{dm}^{-3}$.

\section{Auxiliary Information}

\section{Method/Apparatus/Procedure:}

Very little experimental details were provided. Excess solute and solvent were placed in tubes, which were rotated for at least $8 \mathrm{~h}$ at $298 \mathrm{~K}$. The solutions were then removed, concentrated if necessary, and titrated with standard alkali using a mixed (bromothymol blue + neutral red) indicator.

Source and Purity of Chemicals:

(1) Purity not given, Chemical source not given, solute was either "AnalaR" grade or recrystallized prior to use.

(2) Purity not given, Chemical source not given, no purification details were provided.

Estimated Error:

Temperature: No information was given.

$c_{1}: \pm 4 \%$ (relative error, estimated by compiler).

\section{Components:}

(1) 2,4-Dihydroxybenzoic acid;

$\mathrm{C}_{7} \mathrm{H}_{6} \mathrm{O}_{4}$; [89-86-1]

(2) Tetrachloromethane; $\mathrm{CCl}_{4}$;

[56-23-5]

Variables:

$T / \mathrm{K}=298.15$

Experimental Values

The measured solubility was reported to be $-\log _{10} c_{1}=5.301$,

which corresponds to a solubility of $c_{1}=0.00000500 \mathrm{~mol} \mathrm{dm}^{-3}$.

\section{Original Measurements: \\ ${ }^{56}$ P. A. Ongley, J. Chem. Soc. 1954,} 3634.

\section{Prepared by:}

W. E. Acree, Jr. 


\section{Auxiliary Information}

\section{Method/Apparatus/Procedure:}

Very little experimental details were provided. Excess solute and solvent were placed in tubes, which were rotated for at least $8 \mathrm{~h}$ at $298 \mathrm{~K}$. The solutions were then removed, concentrated if necessary, and titrated with standard alkali using a mixed (bromothymol blue + neutral red) indicator.

Source and Purity of Chemicals:

(1) Purity not given, Chemical source not given, solute was either "AnalaR" grade or recrystallized prior to use.

(2) Purity not given, Chemical source not given, no purification details were provided.

Estimated Error:

Temperature: No information was given.

$c_{1}: \pm 4 \%$ (relative error, estimated by compiler).

\section{Solubility of 2,5-Dihydroxybenzoic Acid in Organic Solvents}

\subsection{Critical evaluation of experimental solubility data}

There are only two studies ${ }^{56,125}$ reporting solubility data for 2,5 -dihydroxybenzoic acid in organic solvents. Ongley ${ }^{56}$ determined the solubility of 2,5-dihydroxybenzoic acid in benzene and trichloromethane at $298.15 \mathrm{~K}$. Pinsuwan et al. ${ }^{125}$ measured the solubility of 2,5-dihydroxybenzoic acid in 1-octanol to examine correlations between experimental octanol/water solubility ratios and measured water-to-1-octanol practical partition coefficients. It is not possible to perform a critical evaluation of the experimental data as measurements were performed at only a single temperature, and there are no independent experimental solubility data for 2,5-dihydroxybenzoic acid in either benzene, trichloromethane or 1-octanol.

The experimental solubility data for 2,5-dihydroxybenzoic acid in organic solvents are in Secs. 30.2-30.4.

\subsection{2,5-Dihydroxybenzoic acid solubility data in aromatic hydrocarbons}

\begin{tabular}{|c|c|}
\hline $\begin{array}{l}\text { Components: } \\
\text { (1) 2,5-Dihydroxybenzoic acid; } \\
\mathrm{C}_{7} \mathrm{H}_{6} \mathrm{O}_{4} ;[470-79-9] \\
\text { (2) Benzene; } \mathrm{C}_{6} \mathrm{H}_{6} ;[71-43-2]\end{array}$ & $\begin{array}{l}\text { Original Measurements: } \\
\text { 56 P. A. Ongley, J. Chem. Soc. 1954, } \\
3634 \text {. }\end{array}$ \\
\hline $\begin{array}{l}\text { Variables: } \\
T / \mathrm{K}=298.15\end{array}$ & $\begin{array}{l}\text { Prepared by: } \\
\text { W. E. Acree, Jr. }\end{array}$ \\
\hline
\end{tabular}

Experimental Values

The measured solubility was reported to be $-\log _{10} c_{1}=$ 3.403 , which corresponds to a solubility of $c_{1}=0.000395 \mathrm{~mol}$ $\mathrm{dm}^{-3}$.
Auxiliary Information

Method/Apparatus/Procedure:

Very little experimental details were provided. Excess solute and solvent were placed in tubes, which were rotated for at least $8 \mathrm{~h}$ at $298 \mathrm{~K}$. The solutions were then removed, concentrated if necessary, and titrated with standard alkali using a mixed (bromothymol blue + neutral red) indicator.

Source and Purity of Chemicals:

(1) Purity not given, Chemical source not given, solute was either "AnalaR" grade or recrystallized prior to use.

(2) Purity not given, Chemical source not given, no purification details were provided.

Estimated Error:

Temperature: No information was given.

$c_{1}: \pm 4 \%$ (relative error, estimated by compiler).

\subsection{2,5-Dihydroxybenzoic acid solubility data in haloalkanes, haloalkenes, and haloaromatic hydrocarbons}

\begin{tabular}{|c|c|}
\hline $\begin{array}{l}\text { Components: } \\
\text { (1) } 2,5 \text {-Dihydroxybenzoic acid; } \\
\mathrm{C}_{7} \mathrm{H}_{6} \mathrm{O}_{4} ; \text { [470-79-9] } \\
\text { (2) Trichloromethane; } \mathrm{CHCl}_{3} \text {; } \\
{[67-66-3]}\end{array}$ & $\begin{array}{l}\text { Original Measurements: } \\
{ }^{56} \text { P. A. Ongley, J. Chem. Soc. 1954, } \\
3634 \text {. }\end{array}$ \\
\hline $\begin{array}{l}\text { Variables: } \\
T / \mathrm{K}=298.15\end{array}$ & $\begin{array}{l}\text { Prepared by: } \\
\text { W. E. Acree, Jr. }\end{array}$ \\
\hline
\end{tabular}

\section{Experimental Values}

The measured solubility was reported to be $-\log _{10} c_{1}=$ 1.900 , which corresponds to a solubility of $c_{1}=0.0126 \mathrm{~mol}$ $\mathrm{dm}^{-3}$

\section{Auxiliary Information}

\section{Method/Apparatus/Procedure:}

Very little experimental details were provided. Excess solute and solvent were placed in tubes, which were rotated for at least $8 \mathrm{~h}$ at $298 \mathrm{~K}$. The solutions were then removed, concentrated if necessary, and titrated with standard alkali using a mixed (bromothymol blue + neutral red) indicator.

Source and Purity of Chemicals:

(1) Purity not given, Chemical source not given, solute was either "AnalaR" grade or recrystallized prior to use.

(2) Purity not given, Chemical source not given, no purification details were provided.

Estimated Error:

Temperature: No information was given. $c_{1}: \pm 4 \%$ (relative error, estimated by compiler). 


\subsection{2,5-Dihydroxybenzoic acid solubility data in alcohols}

\begin{tabular}{ll}
\hline \hline Components: & Original Measurements: \\
(1) 2,5-Dihydroxybenzoic acid; & ${ }^{125}$ S. Pinsuwan, A. Li, and S. H. \\
$\mathrm{C}_{7} \mathrm{H}_{6} \mathrm{O}_{4} ;[$ [470-79-9] & Yalkowsky, J. Chem. Eng. Data 40, \\
(2) 1-Octanol; $\mathrm{C}_{8} \mathrm{H}_{18} \mathrm{O} ;[111-87-5]$ & 623 (1995). \\
\hline Variables: & Prepared by: \\
$T / \mathrm{K}=298.15$ & W. E. Acree, Jr. \\
\hline
\end{tabular}

Experimental Values

The measured solubility was reported to be $\log _{10} c_{1}=$ -0.13 , which corresponds to a molar solubility of $c_{1}=0.741$.

\section{Auxiliary Information}

Method/Apparatus/Procedure:

Constant-temperature bath, analytical balance, mechanical stirrer, and ultraviolet/visible spectrophotometer.

Excess solute and solvent were equilibrated at $298 \pm 2 \mathrm{~K}$ in a sealed vial for 48 $72 \mathrm{~h}$. The solution was mixed using an end-over-end mechanical stirrer. After equilibrium was obtained, the saturated sample was vacuum filtered through a $5.0 \mu \mathrm{m}$ fritted glass filter. An aliquot of the filtrate was diluted quantitatively with 2-propanol, and the absorbance of the diluted sample was recorded. The solubility was calculated from the measured absorbance.

Source and Purity of Chemicals:

(1) Purity not given, Sigma Chemical Company, St. Louis, MO, USA, used as received.

(2) Purity not given, Chemical source not given, used as received.

Estimated Error:

Temperature: $\pm 2 \mathrm{~K}$.

$c_{1}: \pm 10 \%$ (relative error, estimated by compiler given the large uncertainty in temperature).

\section{Solubility of 2,6-Dihydroxybenzoic Acid in Organic Solvents}

\subsection{Critical evaluation of experimental solubility data}

There is only a single study reporting solubility data for 2,6dihydroxybenzoic acid in organic solvents. Ongley ${ }^{56}$ determined the solubility of 2,6-dihydroxybenzoic acid in cyclohexane, benzene, trichloromethane, and tetrachloromethane at $298.15 \mathrm{~K}$. It is not possible to perform a critical evaluation of the experimental data as measurements were performed at only a single temperature, and there are no independent experimental solubility data for 2,6-dihydroxybenzoic acid in any of the four solvents listed above.

The experimental solubility data for 2,6-dihydroxybenzoic acid in organic solvents are in Secs. 31.2-31.4.

\subsection{2,6-Dihydroxybenzoic acid solubility data in saturated hydrocarbons (including cycloalkanes)}

\begin{tabular}{|c|c|}
\hline $\begin{array}{l}\text { Components: } \\
\text { (1) 2,6-Dihydroxybenzoic acid; } \\
\mathrm{C}_{7} \mathrm{H}_{6} \mathrm{O}_{4} ;[303-07-1] \\
\text { (2) Cyclohexane; } \mathrm{C}_{6} \mathrm{H}_{12} ;[110-82-7]\end{array}$ & $\begin{array}{l}\text { Original Measurements: } \\
{ }^{56} \text { P. A. Ongley, J. Chem. Soc. 1954, } \\
3634 \text {. }\end{array}$ \\
\hline $\begin{array}{l}\text { Variables: } \\
T / \mathrm{K}=298.15\end{array}$ & $\begin{array}{l}\text { Prepared by: } \\
\text { W. E. Acree, Jr. }\end{array}$ \\
\hline
\end{tabular}

\section{Experimental Values}

The measured solubility was reported to be $-\log _{10} c_{1}=$ 3.627 , which corresponds to a solubility of $c_{1}=0.000236 \mathrm{~mol}$ $\mathrm{dm}^{-3}$.

\section{Auxiliary Information}

\section{Method/Apparatus/Procedure:}

Very little experimental details were provided. Excess solute and solvent were placed in tubes, which were rotated for at least $8 \mathrm{~h}$ at $298 \mathrm{~K}$. The solutions were then removed, concentrated if necessary, and titrated with standard alkali using a mixed (bromothymol blue + neutral red) indicator.

\section{Source and Purity of Chemicals:}

(1) Purity not given, Chemical source not given, solute was either "AnalaR" grade or recrystallized prior to use.

(2) Purity not given, Chemical source not given, no purification details were provided.

Estimated Error:

Temperature: No information was given.

$c_{1}: \pm 4 \%$ (relative error, estimated by compiler).

\subsection{2,6-Dihydroxybenzoic acid solubility data in aromatic hydrocarbons}

\begin{tabular}{|c|c|}
\hline $\begin{array}{l}\text { Components: } \\
\text { (1) 2,6-Dihydroxybenzoic acid; } \\
\mathrm{C}_{7} \mathrm{H}_{6} \mathrm{O}_{4} ;[303-07-1] \\
\text { (2) Benzene; } \mathrm{C}_{6} \mathrm{H}_{6} ;[71-43-2]\end{array}$ & $\begin{array}{l}\text { Original Measurements: } \\
{ }^{56} \text { P. A. Ongley, J. Chem. Soc. 1954, } \\
3634 \text {. }\end{array}$ \\
\hline $\begin{array}{l}\text { Variables: } \\
T / \mathrm{K}=298.15\end{array}$ & $\begin{array}{l}\text { Prepared by: } \\
\text { W. E. Acree, Jr. }\end{array}$ \\
\hline
\end{tabular}

\section{Experimental Values}

The measured solubility was reported to be $-\log _{10} c_{1}=$ 1.896 , which corresponds to a solubility of $c_{1}=0.0127 \mathrm{~mol}$ $\mathrm{dm}^{-3}$.

\section{Auxiliary Information}

\section{Method/Apparatus/Procedure:}

Very little experimental details were provided. Excess solute and solvent were placed in tubes, which were rotated for at least $8 \mathrm{~h}$ at $298 \mathrm{~K}$. The solutions were then removed, concentrated if necessary, and titrated with standard alkali using a mixed (bromothymol blue + neutral red) indicator. 
Source and Purity of Chemicals:

(1) Purity not given, Chemical source not given, solute was either "AnalaR" grade or recrystallized prior to use.

(2) Purity not given, Chemical source not given, no purification details were provided.

\section{Estimated Error:}

Temperature: No information was given.

$c_{1}: \pm 4 \%$ (relative error, estimated by compiler).

\subsection{2,6-Dihydroxybenzoic acid solubility data in haloalkanes, haloalkenes, and haloaromatic hydrocarbons}

\begin{tabular}{ll}
\hline \hline Components: & Original Measurements: \\
(1) 2,6-Dihydroxybenzoic acid; & ${ }^{56} \mathrm{P}$. A. Ongley, J. Chem. Soc. 1954, \\
$\mathrm{C}_{7} \mathrm{H}_{6} \mathrm{O}_{4} ;[303-07-1]$ & 3634. \\
(2) Trichloromethane; $\mathrm{CHCl}_{3} ;$ & \\
67-66-3] & \\
\hline Variables: & Prepared by: \\
$T / \mathrm{K}=298.15$ & W. E. Acree, Jr. \\
\hline
\end{tabular}

\section{Experimental Values}

The measured solubility was reported to be $-\log _{10} c_{1}=0.539$, which corresponds to a solubility of $c_{1}=0.289 \mathrm{~mol} \mathrm{dm}^{-3}$.

\section{Auxiliary Information}

\section{Method/Apparatus/Procedure:}

Very little experimental details were provided. Excess solute and solvent were placed in tubes, which were rotated for at least $8 \mathrm{~h}$ at $298 \mathrm{~K}$. The solutions were then removed, concentrated if necessary, and titrated with standard alkali using a mixed (bromothymol blue + neutral red) indicator.

Source and Purity of Chemicals:

(1) Purity not given, Chemical source not given, solute was either "AnalaR" grade or recrystallized prior to use.

(2) Purity not given, Chemical source not given, no purification details were provided.

Estimated Error:

Temperature: No information was given.

$c_{1}: \pm 4 \%$ (relative error, estimated by compiler).

\begin{tabular}{|c|c|}
\hline $\begin{array}{l}\text { Components: } \\
\text { (1) 2,6-Dihydroxybenzoic acid; } \\
\left.\mathrm{C}_{7} \mathrm{H}_{6} \mathrm{O}_{4} ; \text {; } 303-07-1\right] \\
\text { (2) Tetrachloromethane; } \mathrm{CCl}_{4} \text {; } \\
\text { [56-23-5] }\end{array}$ & $\begin{array}{l}\text { Original Measurements: } \\
\text { 56 P. A. Ongley, J. Chem. Soc. 1954, } \\
3634 \text {. }\end{array}$ \\
\hline $\begin{array}{l}\text { Variables: } \\
T / \mathrm{K}=298.15\end{array}$ & $\begin{array}{l}\text { Prepared by: } \\
\text { W. E. Acree, Jr. }\end{array}$ \\
\hline
\end{tabular}

Experimental Values

The measured solubility was reported to be $-\log _{10} c_{1}=2.556$, which corresponds to a solubility of $c_{1}=0.00278 \mathrm{~mol} \mathrm{dm}^{-3}$.

\section{Auxiliary Information}

Method/Apparatus/Procedure:

Very little experimental details were provided. Excess solute and solvent were placed in tubes, which were rotated for at least $8 \mathrm{~h}$ at $298 \mathrm{~K}$. The solutions were then removed, concentrated if necessary, and titrated with standard alkali using a mixed (bromothymol blue + neutral red) indicator.

Source and Purity of Chemicals:

(1) Purity not given, Chemical source not given, solute was either "AnalaR" grade or recrystallized prior to use.

(2) Purity not given, Chemical source not given, no purification details were provided.

Estimated Error:

Temperature: No information was given

$c_{1}: \pm 4 \%$ (relative error, estimated by compiler).

\section{Solubility of 3,4-Dihydroxybenzoic Acid in Organic Solvents}

\subsection{Critical evaluation of experimental solubility data}

There is only a single study reporting solubility data for 3,4dihydroxybenzoic acid in organic solvents. Ongley ${ }^{56}$ determined the solubility of 3,4-dihydroxybenzoic acid in trichloromethane and tetrachloromethane at $298.15 \mathrm{~K}$. It is not possible to perform a critical evaluation of the experimental data as measurements were performed at only a single temperature, and there are no independent experimental solubility data for 3,4-dihydroxybenzoic acid in either trichloromethane or tetrachloromethane.

The experimental solubility data for 3,4-dihydroxybenzoic acid in organic solvents are in Sec. 32.2.

\subsection{3,4-Dihydroxybenzoic acid solubility data in haloalkanes, haloalkenes, and haloaromatic hydrocarbons}

\begin{tabular}{|c|c|}
\hline $\begin{array}{l}\text { Components: } \\
\text { (1) 3,4-Dihydroxybenzoic acid; } \\
\left.\mathrm{C}_{7} \mathrm{H}_{6} \mathrm{O}_{4} ; \text {; } 99-50-3\right] \\
\text { (2) Trichloromethane; } \mathrm{CHCl}_{3} \text {; } \\
\text { [67-66-3] }\end{array}$ & $\begin{array}{l}\text { Original Measurements: } \\
\text { 56 P. A. Ongley, J. Chem. Soc. 1954, } \\
3634 \text {. }\end{array}$ \\
\hline $\begin{array}{l}\text { Variables: } \\
T / \mathrm{K}=298.15\end{array}$ & $\begin{array}{l}\text { Prepared by: } \\
\text { W. E. Acree, Jr. }\end{array}$ \\
\hline
\end{tabular}

\section{Experimental Values}

The measured solubility was reported to be $-\log _{10} c_{1}=3.311$, which corresponds to a solubility of $c_{1}=0.000489 \mathrm{~mol} \mathrm{dm}^{-3}$. 


\section{Auxiliary Information}

\section{Method/Apparatus/Procedure:}

Very little experimental details were provided. Excess solute and solvent were placed in tubes, which were rotated for at least $8 \mathrm{~h}$ at $298 \mathrm{~K}$. The solutions were then removed, concentrated if necessary, and titrated with standard alkali using a mixed (bromothymol blue + neutral red) indicator.

Source and Purity of Chemicals:

(1) Purity not given, Chemical source not given, solute was either "AnalaR" grade or recrystallized prior to use.

(2) Purity not given, Chemical source not given, no purification details were provided.

Estimated Error:

Temperature: No information was given.

$c_{1}: \pm 4 \%$ (relative error, estimated by compiler).

\begin{tabular}{|c|c|}
\hline $\begin{array}{l}\text { Components: } \\
\text { (1) 3,4-Dihydroxybenzoic acid; } \\
\left.\mathrm{C}_{7} \mathrm{H}_{6} \mathrm{O}_{4} ; \text {; } 99-50-3\right] \\
\text { (2) Tetrachloromethane; } \mathrm{CCl}_{4} \text {; } \\
\text { [56-23-5] }\end{array}$ & $\begin{array}{l}\text { Original Measurements: } \\
\text { 56 P. A. Ongley, J. Chem. Soc. 1954, } \\
\text { 3634. }\end{array}$ \\
\hline $\begin{array}{l}\text { Variables: } \\
T / \mathrm{K}=298.15\end{array}$ & $\begin{array}{l}\text { Prepared by: } \\
\text { W. E. Acree, Jr. }\end{array}$ \\
\hline
\end{tabular}

Experimental Values

The measured solubility was reported to be $-\log _{10} c_{1}=4.398$, which corresponds to a solubility of $c_{1}=0.0000400 \mathrm{~mol} \mathrm{dm}^{-3}$.

\section{Auxiliary Information}

\section{Method/Apparatus/Procedure:}

Very little experimental details were provided. Excess solute and solvent were placed in tubes, which were rotated for at least $8 \mathrm{~h}$ at $298 \mathrm{~K}$. The solutions were then removed, concentrated if necessary, and titrated with standard alkali using a mixed (bromothymol blue + neutral red) indicator.

Source and Purity of Chemicals:

(1) Purity not given, Chemical source not given, solute was either "AnalaR" grade or recrystallized prior to use.

(2) Purity not given, Chemical source not given, no purification details were provided.

Estimated Error:

Temperature: No information was given.

$c_{1}: \pm 4 \%$ (relative error, estimated by compiler).

\section{Solubility of 2,6-Dimethoxybenzoic Acid in Organic Solvents}

\subsection{Critical evaluation of experimental solubility data}

There is only a single study reporting solubility data for 2,6dimethoxybenzoic acid in organic solvents. Ongley ${ }^{56}$ determined the solubility of 2,6-dimethoxybenzoic acid in cyclohexane, benzene, and trichloromethane at $298.15 \mathrm{~K}$. It is not possible to perform a critical evaluation of the experimental data as measurements were performed at only a single temperature, and there are no independent experimental solubility data for 2,6-dimethoxybenzoic acid in the aforementioned three solvents.

The experimental solubility data for 2,6-dimethoxybenzoic acid in organic solvents are in Secs. 33.2-33.4.

\subsection{2,6-Dimethoxybenzoic acid solubility data in saturated hydrocarbons (including cycloalkanes)}

\begin{tabular}{|c|c|}
\hline $\begin{array}{l}\text { Components: } \\
\text { (1) } 2,6 \text {-Dimethoxybenzoic acid; } \\
\mathrm{C}_{9} \mathrm{H}_{10} \mathrm{O}_{4} ;[1466-76-8] \\
\text { (2) Cyclohexane; } \mathrm{C}_{6} \mathrm{H}_{12} ;[110-82-7]\end{array}$ & $\begin{array}{l}\text { Original Measurements: } \\
\text { 56 P. A. Ongley, J. Chem. Soc. 1954, } \\
3634 \text {. }\end{array}$ \\
\hline $\begin{array}{l}\text { Variables: } \\
T / \mathrm{K}=298.15\end{array}$ & $\begin{array}{l}\text { Prepared by: } \\
\text { W. E. Acree, Jr. }\end{array}$ \\
\hline
\end{tabular}

\section{Experimental Values}

The measured solubility was reported to be $-\log _{10} c_{1}=4.000$, which corresponds to a solubility of $c_{1}=0.000100 \mathrm{~mol} \mathrm{dm}^{-3}$.

\section{Auxiliary Information}

\section{Method/Apparatus/Procedure:}

Very little experimental details were provided. Excess solute and solvent were placed in tubes, which were rotated for at least $8 \mathrm{~h}$ at $298 \mathrm{~K}$. The solutions were then removed, concentrated if necessary, and titrated with standard alkali using a mixed (bromothymol blue + neutral red) indicator.

Source and Purity of Chemicals:

(1) Purity not given, Chemical source not given, solute was either "AnalaR" grade or recrystallized prior to use.

(2) Purity not given, Chemical source not given, no purification details were provided.

Estimated Error:

Temperature: No information was given

$c_{1}: \pm 4 \%$ (relative error, estimated by compiler).

\subsection{2,6-Dimethoxybenzoic acid solubility data in aromatic hydrocarbons}

\begin{tabular}{ll}
\hline \hline Components: & Original Measurements: \\
(1) 2,6-Dimethoxybenzoic acid; & ${ }^{56} \mathrm{P}$. A. Ongley, J. Chem. Soc. 1954, \\
$\mathrm{C}_{9} \mathrm{H}_{10} \mathrm{O}_{4} ;[1466-76-8]$ & 3634. \\
$(2)$ Benzene; $\mathrm{C}_{6} \mathrm{H}_{6} ;[71-43-2]$ & \\
\hline Variables: & Prepared by: \\
$T / \mathrm{K}=298.15$ & W. E. Acree, Jr. \\
\hline
\end{tabular}

\section{Experimental Values}

The measured solubility was reported to be $-\log _{10} c_{1}=1.796$, which corresponds to a solubility of $c_{1}=0.0160 \mathrm{~mol} \mathrm{dm}^{-3}$. 


\section{Auxiliary Information}

\section{Method/Apparatus/Procedure:}

Very little experimental details were provided. Excess solute and solvent were placed in tubes, which were rotated for at least $8 \mathrm{~h}$ at $298 \mathrm{~K}$. The solutions were then removed, concentrated if necessary, and titrated with standard alkali using a mixed (bromothymol blue + neutral red) indicator.

Source and Purity of Chemicals:

(1) Purity not given, Chemical source not given, solute was either "AnalaR" grade or recrystallized prior to use.

(2) Purity not given, Chemical source not given, no purification details were provided.

Estimated Error:

Temperature: No information was given.

$c_{1}: \pm 4 \%$ (relative error, estimated by compiler).

\subsection{2,6-Dimethoxybenzoic acid solubility data in haloalkanes, haloalkenes, and haloaromatic hydrocarbons}

\begin{tabular}{|c|c|}
\hline $\begin{array}{l}\text { Components: } \\
\text { (1) 2,6-Dimethoxybenzoic acid; } \\
\mathrm{C}_{9} \mathrm{H}_{10} \mathrm{O}_{4} ;[1466-76-8] \\
\text { (2) Trichloromethane; } \mathrm{CHCl}_{3} \text {; } \\
\text { [67-66-3] }\end{array}$ & $\begin{array}{l}\text { Original Measurements: } \\
{ }^{56} \text { P. A. Ongley, J. Chem. Soc. 1954, } \\
\text { 3634. }\end{array}$ \\
\hline $\begin{array}{l}\text { Variables: } \\
T / \mathrm{K}=298.15\end{array}$ & $\begin{array}{l}\text { Prepared by: } \\
\text { W. E. Acree, Jr. }\end{array}$ \\
\hline
\end{tabular}

Experimental Values

The measured solubility was reported to be $-\log _{10} c_{1}=0.697$, which corresponds to a solubility of $c_{1}=0.201 \mathrm{~mol} \mathrm{dm}^{-3}$.

\section{Auxiliary Information}

\section{Method/Apparatus/Procedure:}

Very little experimental details were provided. Excess solute and solvent were placed in tubes, which were rotated for at least $8 \mathrm{~h}$ at $298 \mathrm{~K}$. The solutions were then removed, concentrated if necessary, and titrated with standard alkali using a mixed (bromothymol blue + neutral red) indicator.

\section{Source and Purity of Chemicals:}

(1) Purity not given, Chemical source not given, solute was either "AnalaR" grade or recrystallized prior to use.

(2) Purity not given, Chemical source not given, no purification details were provided.

\section{Estimated Error:}

Temperature: No information was given.

$c_{1}: \pm 4 \%$ (relative error, estimated by compiler).

\section{Solubility of 3,4-Dimethoxybenzoic Acid in Organic Solvents}

\subsection{Critical evaluation of experimental solubility data}

There have been two published studies ${ }^{138,139}$ investigating the solubility behavior of 3,4-dimethoxybenzoic acid in organic solvents of varying polarity and hydrogen-bonding capability. Bowen et al. ${ }^{138}$ measured the solubility of 3,4dimethoxybenzoic acid in 18 alcohols (methanol, ethanol, 1propanol, 2-propanol, 1-butanol, 2-butanol, 2-methyl-1-propanol, 2-methyl-2-propanol, 1-pentanol, 2-pentanol, 2methyl-1-butanol, 3-methyl-1-butanol, 1-hexanol, 2-methyl1-pentanol, 4-methyl-2-pentanol, 1-heptanol, 1-octanol, and 1-decanol), in three dialkyl ethers (1,1'-oxybisethane, 2,2'oxybispropane, and 1,1'-oxybisbutane) and two cyclic ethers (tetrahydrofuran and 1,4-dioxane), and in six alkyl alkanoates (methyl ethanoate, ethyl ethanoate, propyl ethanoate, butyl ethanoate, pentyl ethanoate, and methyl butanoate), in two alkanones (propanone and butanone), in four alkoxyalcohols (2-ethoxyethanol, 2-isopropoxyethanol, 2-butoxyethanol, and 3-methoxy-1-butanol), and in propylene carbonate at $298 \mathrm{~K}$. Results of the experimental measurements were used to calculate the Abraham solute descriptors of 3,4-dimethoxybenzoic acid. The authors were able to assemble a total of $52 \log _{10}$ (SR or $P$ ) and $\log _{10}(G S R$ or $K$ ) equations for which experimental partition coefficient data, solubility ratios, Abraham Model equation coefficients and aqueous molar solubility were available. The logarithm of the aqueous molar solubility of 3,4-dimethoxybenzoic acid is $\log _{10} c_{1, \mathrm{~W}}=-2.495$ (measured by the authors as part of the experimental study). The McGowan volume of 3,5-dimethoxybenzoic acid, $V=1.3309$, was calculated from the number of chemical bonds in the molecule and the individual atomic group volumes, $A V_{i}$, given in Sec. 1.3. The excess molar refraction solute descriptor was estimated as $E=0.950$. This left four solute descriptors ( $S, A, B$ and $L$ ) still to be determined. The 52 equations were then solved using the Microsoft "SOLVER" program to yield numerical values of the remaining four solute descriptors, $S=1.646$, $A=0.570, B=0.755$, and $L=6.746$, that best described the $\log _{10}(S R$ or $P)$ and $\log _{10}(G S R$ or $K)$ values. The computation treated $\log _{10} c_{1, \mathrm{G}}$ as a floating parameter to be determined as part of the regression analyses. The data analyses returned a value of $\log _{10} c_{1, \mathrm{G}}=-10.942$ for the gas-phase solute concentration that made the $\log _{10}\left(S R\right.$ or $P$ ) and $\log _{10}(G S R$ or $K)$ predictions internally consistent. The calculated molecular solute descriptors reproduced the $\log _{10}\left(S R\right.$ or $P$ ) and $\log _{10}$ ( GSR or $K$ ) values to within an average standard deviation of 0.084 and $0.082 \log _{10}$ units, respectively.

Table 29 compares the experimental $\log _{10} c_{1}$ values to calculated values based on Eqs. (20) and (21) of the Abraham model. For comparison purposes, the measured mole fraction solubilities of 3,4-dimethoxybenzoic acid, $x_{1}$, determined by Bowen et al. ${ }^{138}$ were converted into molar solubilities by dividing $x_{1}$ by the ideal molar volume of the saturated solution (i.e., $\left.c_{1}{ }^{\text {sat }}=x_{1} /\left[x_{1} V_{1}+\left(1-x_{1}\right) V_{\text {solvent }}\right]\right)$. The molar volume of the hypothetical subcooled liquid 3,4-dimethoxybenzoic acid 
TABLE 29. Comparison between observed and calculated molar solubilities of 3,4-dimethoxybenzoic acid based on the Abraham model, Eqs. (20) and (21)

\begin{tabular}{lccc}
\hline \hline Solvent & & $\log _{10} c_{1}{ }^{\text {calc}} ;$ & $\log _{10} c_{1}{ }^{\text {calc }}$ \\
Methanol & $\log _{10} c_{1}{ }^{\text {exp }}$ & Eq. $(20)$ & Eq. $(21)$ \\
Ethanol & -0.735 & -0.721 & -0.685 \\
1-Propanol & -0.916 & -0.924 & -0.892 \\
2-Propanol & -1.070 & -1.062 & -1.061 \\
1-Butanol & -1.097 & -1.085 & -1.064 \\
2-Butanol & -1.169 & -1.186 & -1.173 \\
2-Methyl-1-propanol & -1.154 & -1.099 & -1.104 \\
2-Methyl-2-propanol & -1.315 & -1.204 & -1.200 \\
1-Pentanol & -1.001 & -1.106 & -1.080 \\
2-Pentanol & -1.267 & -1.269 & -1.267 \\
3-Methyl-1-butanol & -1.293 & -1.256 & -1.230 \\
1-Hexanol & -1.309 & -1.182 & -1.258 \\
1-Heptanol & -1.362 & -1.302 & -1.296 \\
1-Octanol & -1.410 & -1.362 & -1.341 \\
1-Decanol & -1.501 & -1.379 & -1.443 \\
1,1'-Oxybisethane & -1.525 & -1.378 & -1.547 \\
Tetrahydrofuran & -0.328 & -0.520 & -1.443 \\
1,4-Dioxane & -0.480 & -0.608 & -0.546 \\
Propylene carbonate & -1.029 & -1.006 & -0.635 \\
Methyl ethanoate & -0.973 & -0.899 & -0.981 \\
Ethyl ethanoate & -1.085 & -1.128 & -1.101 \\
Propyl ethanoate & -1.229 & -1.205 & -1.182 \\
Butyl ethanoate & -1.303 & -1.283 & -1.263 \\
\hline
\end{tabular}

is $V_{\text {solute }}=143.8 \mathrm{~cm}^{3} \mathrm{~mol}^{-1}$. Examination of the numerical entries in Table 29 reveals that the Abraham model provides a reasonably accurate mathematical description of the observed solubility data, suggesting that there are no obvious outliers in the dataset.

Li et al. ${ }^{139}$ measured the solubility of 3,4-dimethoxybenzoic acid in five alcohols (ethanol, 1-propanol, 2-propanol, 1butanol, and 2-methyl-1-propanol), two alkyl ethanoates (methyl ethanoate and ethyl ethanoates) and one alkanone (2-butanone) over the temperature range from 278 to $323 \mathrm{~K}$. The internal consistency of the eight datasets was assessed by curve-fitting the measured mole fraction solubility data to the Modified Apelblat model [Eq. (8)]. The values of the equation coefficients $(A, B$, and $C$ ) are given in Table 30 , along with the mean relative deviation. Examination of the numerical values in the last column of Table 30 indicates that Eq. (8) provides a reasonably accurate mathematical description of how the solubility varies with temperature. The small mean relative

TABLe 30. Parameters of the Modified Apelblat equation for describing the solubility of 3,4-dimethoxybenzoic acid in various organic solvents

\begin{tabular}{lrrrrc}
\hline \hline Solvent & $T / \mathrm{K}$ & \multicolumn{1}{c}{$A$} & \multicolumn{1}{c}{$B$} & \multicolumn{1}{c}{$C$} & MRD (\%) \\
\hline Ethanol & $278-323$ & -179.892 & 4830.140 & 27.872 & 0.43 \\
1-Propanol & $278-323$ & -120.239 & 1951.116 & 19.067 & 0.35 \\
2-Propanol & $278-323$ & -108.899 & 1184.711 & 17.518 & 1.10 \\
1-Butanol & $278-323$ & -187.012 & 4848.020 & 29.062 & 1.00 \\
2-Methyl-1-propanol & $278-323$ & -69.072 & -831.534 & 11.649 & 0.77 \\
Methyl ethanoate & $278-323$ & -68.758 & 397.164 & 10.988 & 0.44 \\
Ethyl ethanoate & $278-323$ & -62.515 & 119.789 & 10.035 & 0.59 \\
2-Butanone & $279-323$ & -78.009 & 1085.742 & 12.277 & 0.20
\end{tabular}

${ }^{\mathrm{a}}$ Values of the coefficients and mean relative deviations were taken from $\mathrm{Li}$ et al. ${ }^{139}$ deviations suggest that the experimental values in each solvent dataset are internally consistent.

The experimental solubility data for 3,4-dimethoxybenzoic acid in organic solvents are in Secs. 34.2-34.8.

\subsection{3,4-Dimethoxybenzoic acid solubility data in esters}

\begin{tabular}{|c|c|}
\hline $\begin{array}{l}\text { Components: } \\
\text { (1) 3,4-Dimethoxybenzoic acid; } \\
\mathrm{C}_{9} \mathrm{H}_{10} \mathrm{O}_{4} \text {; [93-07-2] } \\
\text { (2) Methyl ethanoate; } \mathrm{C}_{3} \mathrm{H}_{6} \mathrm{O}_{2} \text {; } \\
\text { [79-20-9] }\end{array}$ & $\begin{array}{l}\text { Original Measurements: } \\
{ }^{138} \text { K. R. Bowen, T. W. Stephens, H. Lu, } \\
\text { K. Satish, D. Shan, W. E. Acree, Jr., } \\
\text { and M. H. Abraham, Eur. Chem. Bull. } \\
\text { 2, } 577 \text { (2013). }\end{array}$ \\
\hline $\begin{array}{l}\text { Variables: } \\
T / \mathrm{K}=298.15\end{array}$ & $\begin{array}{l}\text { Prepared by: } \\
\text { W. E. Acree, Jr. }\end{array}$ \\
\hline
\end{tabular}

\section{Experimental Values}

\begin{tabular}{lc}
\hline \hline$x_{2}{ }^{\mathrm{a}}$ & $x_{1}{ }^{\mathrm{b}}$ \\
\hline 0.9915 & 0.00854 \\
\hline${ }^{\mathrm{a}} x_{2}:$ mole fraction of component 2 in the saturated solution. &
\end{tabular}

${ }^{\mathrm{b}} x_{1}$ : mole fraction solubility of the solute.

\section{Auxiliary Information}

\section{Method/Apparatus/Procedure:}

Constant-temperature bath, calorimetric thermometer, and an ultraviolet/ visible spectrophotometer.

Excess solute and solvent were placed in amber glass bottles and allowed to equilibrate for several days at constant temperature. Attainment of equilibrium was verified by several repetitive measurements and by approaching equilibrium from supersaturation. Aliquots of saturated solutions were transferred through a coarse filter into tared volumetric flasks, weighed, and diluted with 2-propanol. Concentrations were determined by spectrophotometric measurements at $286 \mathrm{~nm}$.

\section{Source and Purity of Chemicals:}

(1) $99+\%$, Acros Organics, USA, was used as received.

(2) $99.5 \%$, anhydrous, Aldrich Chemical Company, Milwaukee, WI, USA, stored over molecular sieves and distilled shortly before use.

\section{Estimated Error:}

Temperature: $\pm 0.1 \mathrm{~K}$.

$x_{1}: \pm 2.0 \%$ (relative error).

\begin{tabular}{|c|c|}
\hline $\begin{array}{l}\text { Components: } \\
\text { (1) 3,4-Dimethoxybenzoic acid; } \\
\mathrm{C}_{9} \mathrm{H}_{10} \mathrm{O}_{4} \text {; [93-07-2] } \\
\text { (2) Methyl ethanoate; } \mathrm{C}_{3} \mathrm{H}_{6} \mathrm{O}_{2} \text {; } \\
\text { [79-20-9] }\end{array}$ & $\begin{array}{l}\text { Original Measurements: } \\
{ }^{139} \text { Q. Li, F. Lu, Y. Tian, S. Feng, Y. } \\
\text { Shen, and B. Wang, J. Chem. Eng. Data } \\
\text { 58, } 1020 \text { (2013). }\end{array}$ \\
\hline $\begin{array}{l}\text { Variables: } \\
\text { Temperature }\end{array}$ & $\begin{array}{l}\text { Prepared by: } \\
\text { W. E. Acree, Jr. }\end{array}$ \\
\hline
\end{tabular}


Experimental Values

\begin{tabular}{lcc}
\hline \hline$T / \mathrm{K}$ & $x_{2}{ }^{\mathrm{a}}$ & $x_{1}{ }^{\mathrm{b}}$ \\
\hline 278.30 & 0.9958 & 0.0042 \\
283.01 & 0.9951 & 0.0049 \\
288.05 & 0.9942 & 0.0058 \\
293.15 & 0.9932 & 0.0068 \\
298.00 & 0.9920 & 0.0080 \\
303.13 & 0.9905 & 0.0095 \\
308.07 & 0.9889 & 0.0111 \\
313.15 & 0.9869 & 0.0131 \\
318.06 & 0.9848 & 0.0152 \\
323.37 & 0.9823 & 0.0177
\end{tabular}

${ }^{\mathrm{a}} x_{2}$ : mole fraction of component 2 in the saturated solution.

${ }^{b} x_{1}$ : mole fraction solubility of the solute.

\section{Auxiliary Information}

\section{Method/Apparatus/Procedure:}

Water-jacketed equilibrium glass vessel, thermostated circulating water bath, magnetic stirrer, analytical balance, laser monitoring system.

Experimental solubilities were determined by a synthetic method. Preweighed amounts of solute and solvent and were placed in a water-jacketed equilibrium vessel, which was connected to a circulating constant-temperature water bath. The solution was stirred and small amounts of solute were incrementally added until no further solid dissolved. The dissolution of the solid was determined using laser monitoring. The total amount of solute dissolved was recorded. Experimental measurement was repeated three times.

Source and Purity of Chemicals:

(1) $99+\%$, Alfa Aesar China Co., Ltd., Tianjin, China, no purification details were provided.

(2) $99.5+\%$, Beijing Chemical Works, Beijing, China, no purification details were provided.

Estimated Error:

Temperature: $\pm 0.05 \mathrm{~K}$.

$x_{1}: \pm 4 \%$ (relative error).

\begin{tabular}{|c|c|}
\hline $\begin{array}{l}\text { Components: } \\
\text { (1) 3,4-Dimethoxybenzoic acid; } \\
\mathrm{C}_{9} \mathrm{H}_{10} \mathrm{O}_{4} \text {; [93-07-2] } \\
\text { (2) Ethyl ethanoate; } \mathrm{C}_{4} \mathrm{H}_{8} \mathrm{O}_{2} \text {; } \\
\text { [141-78-6] }\end{array}$ & $\begin{array}{l}\text { Original Measurements: } \\
{ }^{139} \text { Q. Li, F. Lu, Y. Tian, S. Feng, Y. } \\
\text { Shen, and B. Wang, J. Chem. Eng. Data } \\
\text { 58, } 1020 \text { (2013). }\end{array}$ \\
\hline $\begin{array}{l}\text { Variables: } \\
\text { Temperature }\end{array}$ & $\begin{array}{l}\text { Prepared by: } \\
\text { W. E. Acree, Jr. }\end{array}$ \\
\hline
\end{tabular}

Experimental Values

\begin{tabular}{lcc}
\hline \hline$T / \mathrm{K}$ & $x_{2}{ }^{\mathrm{a}}$ & $x_{1}{ }^{\mathrm{b}}$ \\
\hline 278.30 & 0.9963 & 0.0037 \\
283.01 & 0.9956 & 0.0044 \\
288.05 & 0.9948 & 0.0052 \\
293.15 & 0.9939 & 0.0061 \\
298.00 & 0.9928 & 0.0072 \\
303.13 & 0.9917 & 0.0083 \\
308.07 & 0.9901 & 0.0099 \\
313.15 & 0.9884 & 0.0116 \\
318.06 & 0.9867 & 0.0133 \\
323.37 & 0.9863 & 0.0137
\end{tabular}

${ }^{\mathrm{a}} x_{2}$ : mole fraction of component 2 in the saturated solution.

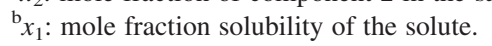

\section{Auxiliary Information}

\section{Method/Apparatus/Procedure:}

Water-jacketed equilibrium glass vessel, thermostated circulating water bath, magnetic stirrer, analytical balance, laser monitoring system.

Experimental solubilities were determined by a synthetic method. Preweighed amounts of solute and solvent and were placed in a water-jacketed equilibrium vessel, which was connected to a circulating constant-temperature water bath. The solution was stirred and small amounts of solute were incrementally added until no further solid dissolved. The dissolution of the solid was determined using laser monitoring. The total amount of solute dissolved was recorded. Experimental measurement was repeated three times.

Source and Purity of Chemicals:

(1) $99+\%$, Alfa Aesar China Co., Ltd., Tianjin, China, no purification details were provided.

(2) $99.5+\%$, Beijing Chemical Works, Beijing, China, no purification details were provided.

\section{Estimated Error:}

Temperature: $\pm 0.05 \mathrm{~K}$.

$x_{1}: \pm 4 \%$ (relative error).

\begin{tabular}{|c|c|}
\hline $\begin{array}{l}\text { Components: } \\
\text { (1) 3,4-Dimethoxybenzoic acid; } \\
\mathrm{C}_{9} \mathrm{H}_{10} \mathrm{O}_{4} \text {; [93-07-2] } \\
\text { (2) Ethyl ethanoate; } \mathrm{C}_{4} \mathrm{H}_{8} \mathrm{O}_{2} \text {; } \\
\text { [141-78-6] }\end{array}$ & $\begin{array}{l}\text { Original Measurements: } \\
{ }^{138} \text { K. R. Bowen, T. W. Stephens, H. Lu, } \\
\text { K. Satish, D. Shan, W. E. Acree, Jr., } \\
\text { and M. H. Abraham, Eur. Chem. Bull } \\
\text { 2, } 577 \text { (2013). }\end{array}$ \\
\hline $\begin{array}{l}\text { Variables: } \\
T / \mathrm{K}=298.15\end{array}$ & $\begin{array}{l}\text { Prepared by: } \\
\text { W. E. Acree, Jr. }\end{array}$ \\
\hline
\end{tabular}

\section{Experimental Values}

\begin{tabular}{lc}
\hline \hline$x_{2}{ }^{\mathrm{a}}$ & $x_{1}{ }^{\mathrm{b}}$ \\
\hline 0.9919 & 0.00813
\end{tabular}

${ }^{a} x_{2}$ : mole fraction of component 2 in the saturated solution.

${ }^{\mathrm{b}} x_{1}$ : mole fraction solubility of the solute.

\section{Auxiliary Information}

\section{Method/Apparatus/Procedure:}

Constant-temperature bath, calorimetric thermometer, and an ultraviolet/ visible spectrophotometer.

Excess solute and solvent were placed in amber glass bottles and allowed to equilibrate for several days at constant temperature. Attainment of equilibrium was verified by several repetitive measurements and by approaching equilibrium from supersaturation. Aliquots of saturated solutions were transferred through a coarse filter into tared volumetric flasks, weighed, and diluted with 2-propanol. Concentrations were determined by spectrophotometric measurements at $286 \mathrm{~nm}$.

\section{Source and Purity of Chemicals:}

(1) $99+\%$, Acros Organics, USA, was used as received.

(2) $99.8 \%$, anhydrous, Aldrich Chemical Company, Milwaukee, WI, USA, stored over molecular sieves and distilled shortly before use.

\section{Estimated Error:}

Temperature: $\pm 0.1 \mathrm{~K}$.

$x_{1}: \pm 2.0 \%$ (relative error). 


\begin{tabular}{|c|c|}
\hline $\begin{array}{l}\text { Components: } \\
\text { (1) 3,4-Dimethoxybenzoic acid; } \\
\mathrm{C}_{9} \mathrm{H}_{10} \mathrm{O}_{4} \text {; [93-07-2] } \\
\text { (2) Propyl ethanoate; } \mathrm{C}_{5} \mathrm{H}_{10} \mathrm{O}_{2} \text {; } \\
\text { [109-60-4] }\end{array}$ & $\begin{array}{l}\text { Original Measurements: } \\
{ }^{138} \text { K. R. Bowen, T. W. Stephens, H. Lu, } \\
\text { K. Satish, D. Shan, W. E. Acree, Jr., } \\
\text { and M. H. Abraham, Eur. Chem. Bull. } \\
\text { 2, } 577 \text { (2013). }\end{array}$ \\
\hline $\begin{array}{l}\text { Variables: } \\
T / \mathrm{K}=298.15\end{array}$ & $\begin{array}{l}\text { Prepared by: } \\
\text { W. E. Acree, Jr. }\end{array}$ \\
\hline
\end{tabular}

\section{Experimental Values}

\begin{tabular}{lc}
\hline \hline$x_{2}{ }^{\mathrm{a}}$ & $x_{1}{ }^{\mathrm{b}}$ \\
\hline 0.9932 & 0.00682 \\
\hline
\end{tabular}

${ }^{\mathrm{a}} x_{2}$ : mole fraction of component 2 in the saturated solution.

${ }^{\mathrm{b}} x_{1}$ : mole fraction solubility of the solute.

\section{Auxiliary Information}

\section{Method/Apparatus/Procedure:}

Constant-temperature bath, calorimetric thermometer, and an ultraviolet/ visible spectrophotometer.

Excess solute and solvent were placed in amber glass bottles and allowed to equilibrate for several days at constant temperature. Attainment of equilibrium was verified by several repetitive measurements and by approaching equilibrium from supersaturation. Aliquots of saturated solutions were transferred through a coarse filter into tared volumetric flasks, weighed, and diluted with 2-propanol. Concentrations were determined by

spectrophotometric measurements at $286 \mathrm{~nm}$.

Source and Purity of Chemicals:

(1) $99+\%$, Acros Organics, USA, was used as received.

(2) $99.5 \%$, Aldrich Chemical Company, Milwaukee, WI, USA, stored over molecular sieves and distilled shortly before use.

\section{Estimated Error:}

Temperature: $\pm 0.1 \mathrm{~K}$

$x_{1}: \pm 2.0 \%$ (relative error).

\begin{tabular}{ll}
\hline \hline Components: & Original Measurements: \\
(1) 3,4-Dimethoxybenzoic acid; & ${ }^{138}$ K. R. Bowen, T. W. Stephens, H. Lu, \\
$\mathrm{C}_{9} \mathrm{H}_{10} \mathrm{O}_{4} ;$ [93-07-2] & K. Satish, D. Shan, W. E. Acree, Jr., \\
$\begin{array}{l}\text { (2) Butyl ethanoate; } \mathrm{C}_{6} \mathrm{H}_{12} \mathrm{O}_{2} ; \\
{[123-86-4]}\end{array}$ & $\begin{array}{l}\text { and M. H. Abraham, Eur. Chem. Bull. } \\
\text { 2, 577 (2013). }\end{array}$ \\
\hline Variables: & Prepared by: \\
$T / \mathrm{K}=298.15$ & W. E. Acree, Jr. \\
\hline
\end{tabular}

Experimental Values

\begin{tabular}{lc}
\hline \hline$x_{2}{ }^{\mathrm{a}}$ & $x_{1}{ }^{\mathrm{b}}$ \\
\hline 0.9934 & 0.00660 \\
\hline${ }_{{ }^{\mathrm{a}} x_{2}: \text { mole fraction of component } 2 \text { in the saturated solution. }}$ & \\
${ }^{\mathrm{b}} x_{1}:$ mole fraction solubility of the solute. &
\end{tabular}

\section{Auxiliary Information}

\section{Method/Apparatus/Procedure:}

Constant-temperature bath, calorimetric thermometer, and an ultraviolet/ visible spectrophotometer.

Excess solute and solvent were placed in amber glass bottles and allowed to equilibrate for several days at constant temperature. Attainment of equilibrium was verified by several repetitive measurements and by approaching equilibrium from supersaturation. Aliquots of saturated solutions were transferred through a coarse filter into tared volumetric flasks, weighed, and diluted with 2-propanol. Concentrations were determined by spectrophotometric measurements at $286 \mathrm{~nm}$.

\section{Source and Purity of Chemicals:}

(1) $99+\%$, Acros Organics, USA, was used as received.

(2) $99.7 \%$, Aldrich Chemical Company, Milwaukee, WI, USA, stored over molecular sieves and distilled shortly before use.

\section{Estimated Error:}

Temperature: $\pm 0.1 \mathrm{~K}$.

$x_{1}: \pm 2.0 \%$ (relative error)

\begin{tabular}{ll}
\hline \hline Components: & Original Measurements: \\
(1) 3,4-Dimethoxybenzoic acid; & ${ }^{138}$ K. R. Bowen, T. W. Stephens, H. Lu, \\
$\mathrm{C}_{9} \mathrm{H}_{10} \mathrm{O}_{4} ;$ [ [93-07-2] & K. Satish, D. Shan, W. E. Acree, Jr., \\
$\begin{array}{l}\text { (2) Pentyl ethanoate; } \mathrm{C}_{7} \mathrm{H}_{14} \mathrm{O}_{2} ; \\
\text { [628-63-7] }\end{array}$ & and M. H. Abraham, Eur. Chem. Bull. \\
\hline Variables: & 2, 577 (2013). \\
$T / \mathrm{K}=298.15$ & Prepared by: \\
\hline
\end{tabular}

\section{Experimental Values}

\begin{tabular}{lc}
\hline \hline$x_{2}{ }^{\mathrm{a}}$ & $x_{1}{ }^{\mathrm{b}}$ \\
\hline 0.9935 & 0.00654 \\
\hline${ }^{\mathrm{a}} x_{2}:$ mole fraction of component 2 in the saturated solution. & \\
${ }^{\mathrm{b}} x_{1}:$ mole fraction solubility of the solute. &
\end{tabular}

\section{Auxiliary Information}

\section{Method/Apparatus/Procedure:}

Constant-temperature bath, calorimetric thermometer, and an ultraviolet/ visible spectrophotometer.

Excess solute and solvent were placed in amber glass bottles and allowed to equilibrate for several days at constant temperature. Attainment of equilibrium was verified by several repetitive measurements and by approaching equilibrium from supersaturation. Aliquots of saturated solutions were transferred through a coarse filter into tared volumetric flasks, weighed, and diluted with 2-propanol. Concentrations were determined by spectrophotometric measurements at $286 \mathrm{~nm}$.

\section{Source and Purity of Chemicals:}

(1) $99+\%$, Acros Organics, USA, was used as received.

(2) $99 \%$, Aldrich Chemical Company, Milwaukee, WI, USA, stored over molecular sieves and distilled shortly before use.

\section{Estimated Error:}

Temperature: $\pm 0.1 \mathrm{~K}$.

$x_{1}: \pm 2.0 \%$ (relative error) 


\begin{tabular}{|c|c|}
\hline $\begin{array}{l}\text { Components: } \\
\text { (1) 3,4-Dimethoxybenzoic acid; } \\
\mathrm{C}_{9} \mathrm{H}_{10} \mathrm{O}_{4} \text {; [93-07-2] } \\
\text { (2) Methyl butanoate; } \mathrm{C}_{5} \mathrm{H}_{10} \mathrm{O}_{2} \text {; } \\
\text { [623-42-7] }\end{array}$ & $\begin{array}{l}\text { Original Measurements: } \\
{ }^{138} \text { K. R. Bowen, T. W. Stephens, H. Lu, } \\
\text { K. Satish, D. Shan, W. E. Acree, Jr., } \\
\text { and M. H. Abraham, Eur. Chem. Bull. } \\
\text { 2, } 577 \text { (2013). }\end{array}$ \\
\hline $\begin{array}{l}\text { Variables: } \\
T / \mathrm{K}=298.15\end{array}$ & $\begin{array}{l}\text { Prepared by: } \\
\text { W. E. Acree, Jr. }\end{array}$ \\
\hline
\end{tabular}

\section{Experimental Values}

\begin{tabular}{lc}
\hline \hline$x_{2}{ }^{\mathrm{a}}$ & $x_{1}{ }^{\mathrm{b}}$ \\
\hline 0.9932 & 0.00678 \\
\hline
\end{tabular}

${ }^{\mathrm{a}} x_{2}$ : mole fraction of component 2 in the saturated solution.

${ }^{\mathrm{b}} x_{1}$ : mole fraction solubility of the solute.

\section{Auxiliary Information}

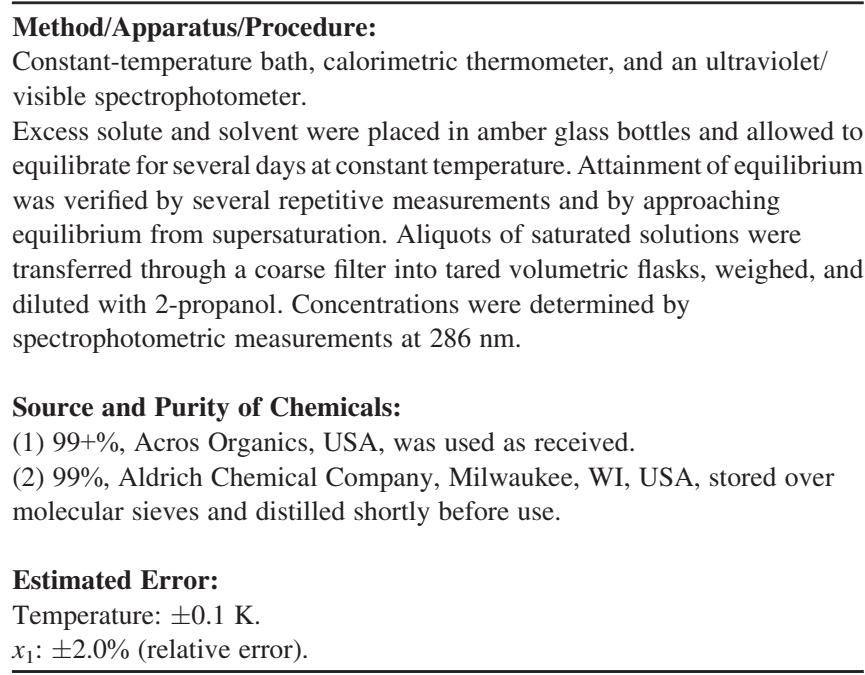

\subsection{3,4-Dimethoxybenzoic acid solubility data in ethers}

\begin{tabular}{|c|c|}
\hline $\begin{array}{l}\text { Components: } \\
\text { (1) 3,4-Dimethoxybenzoic acid; } \\
\left.\mathrm{C}_{9} \mathrm{H}_{10} \mathrm{O}_{4} ; \text {; } 93-07-2\right] \\
\text { (2) } 1,1^{\prime} \text {-Oxybisethane; } \mathrm{C}_{4} \mathrm{H}_{10} \mathrm{O} \text {; } \\
\text { [60-29-7] }\end{array}$ & $\begin{array}{l}\text { Original Measurements: } \\
{ }^{138} \text { K. R. Bowen, T. W. Stephens, H. Lu, } \\
\text { K. Satish, D. Shan, W. E. Acree, Jr., } \\
\text { and M. H. Abraham, Eur. Chem. Bull. } \\
\text { 2, } 577 \text { (2013). }\end{array}$ \\
\hline $\begin{array}{l}\text { Variables: } \\
T / \mathrm{K}=298.15\end{array}$ & $\begin{array}{l}\text { Prepared by: } \\
\text { W. E. Acree, Jr. }\end{array}$ \\
\hline
\end{tabular}

Experimental Values

\begin{tabular}{lc}
\hline \hline$x_{2}^{\mathrm{a}}$ & $x_{1}{ }^{\mathrm{b}}$ \\
\hline 0.9969 & 0.00313 \\
\hline${ }^{\mathrm{a}} x_{2}:$ mole fraction of component 2 in the saturated solution. \\
${ }^{\mathrm{b}} x_{1}:$ mole fraction solubility of the solute.
\end{tabular}

\section{Auxiliary Information}

\section{Method/Apparatus/Procedure:}

Constant-temperature bath, calorimetric thermometer, and an ultraviolet/ visible spectrophotometer.

Excess solute and solvent were placed in amber glass bottles and allowed to equilibrate for several days at constant temperature. Attainment of equilibrium was verified by several repetitive measurements and by approaching equilibrium from supersaturation. Aliquots of saturated solutions were transferred through a coarse filter into tared volumetric flasks, weighed, and diluted with 2-propanol. Concentrations were determined by spectrophotometric measurements at $286 \mathrm{~nm}$.

\section{Source and Purity of Chemicals:}

(1) $99+\%$, Acros Organics, USA, was used as received.

(2) $99+\%$, anhydrous Aldrich Chemical Company, Milwaukee, WI, USA, stored over molecular sieves and distilled shortly before use.

\section{Estimated Error:}

Temperature: $\pm 0.1 \mathrm{~K}$.

$x_{1}: \pm 2.0 \%$ (relative error).

\begin{tabular}{|c|c|}
\hline $\begin{array}{l}\text { Components: } \\
\text { (1) 3,4-Dimethoxybenzoic acid; } \\
\mathrm{C}_{9} \mathrm{H}_{10} \mathrm{O}_{4} \text {; [93-07-2] } \\
\text { (2) 2,2'-Oxybispropane; } \mathrm{C}_{6} \mathrm{H}_{14} \mathrm{O} \text {; } \\
\text { [108-20-3] }\end{array}$ & $\begin{array}{l}\text { Original Measurements: } \\
{ }^{138} \text { K. R. Bowen, T. W. Stephens, H. Lu, } \\
\text { K. Satish, D. Shan, W. E. Acree, Jr., } \\
\text { and M. H. Abraham, Eur. Chem. Bull. } \\
\text { 2, } 577 \text { (2013). }\end{array}$ \\
\hline $\begin{array}{l}\text { Variables: } \\
T / \mathrm{K}=298.15\end{array}$ & $\begin{array}{l}\text { Prepared by: } \\
\text { W. E. Acree, Jr. }\end{array}$ \\
\hline
\end{tabular}

\section{Experimental Values}

\begin{tabular}{lc}
\hline \hline$x_{2}{ }^{\mathrm{a}}$ & $x_{1}{ }^{\mathrm{b}}$ \\
\hline 0.9986 & 0.00143 \\
\hline${ }^{\mathrm{a}} x_{2}:$ mole fraction of component 2 in the saturated solution. \\
${ }^{\mathrm{b}} x_{1}:$ mole fraction solubility of the solute.
\end{tabular}

\section{Auxiliary Information}

\section{Method/Apparatus/Procedure:}

Constant-temperature bath, calorimetric thermometer, and an ultraviolet/ visible spectrophotometer.

Excess solute and solvent were placed in amber glass bottles and allowed to equilibrate for several days at constant temperature. Attainment of equilibrium was verified by several repetitive measurements and by approaching equilibrium from supersaturation. Aliquots of saturated solutions were transferred through a coarse filter into tared volumetric flasks, weighed, and diluted with 2-propanol. Concentrations were determined by spectrophotometric measurements at $286 \mathrm{~nm}$.

\section{Source and Purity of Chemicals:}

(1) $99+\%$, Acros Organics, USA, was used as received.

(2) $99 \%$, anhydrous, Aldrich Chemical Company, Milwaukee, WI, USA, stored over molecular sieves and distilled shortly before use.

\section{Estimated Error:}

Temperature: $\pm 0.1 \mathrm{~K}$.

$x_{1}: \pm 2.0 \%$ (relative error) 


\begin{tabular}{ll}
\hline \hline Components: & Original Measurements: \\
$(1)$ 3,4-Dimethoxybenzoic acid; & ${ }^{138}$ K. R. Bowen, T. W. Stephens, H. Lu, \\
$\mathrm{C}_{9} \mathrm{H}_{10} \mathrm{O}_{4} ;$ [93-07-2] & K. Satish, D. Shan, W. E. Acree, Jr., \\
$(2) 1,1^{\prime}-$ Oxybisbutane; $\mathrm{C}_{8} \mathrm{H}_{18} \mathrm{O} ;$ & and M. H. Abraham, Eur. Chem. Bull. \\
{$[142-96-1]$} & $\mathbf{2}, 577$ (2013). \\
\hline Variables: & Prepared by: \\
$T / \mathrm{K}=298.15$ & W. E. Acree, Jr. \\
\hline
\end{tabular}

Experimental Values

\begin{tabular}{lc}
\hline \hline$x_{2}{ }^{\mathrm{a}}$ & $x_{1}{ }^{\mathrm{b}}$ \\
\hline 0.9989 & 0.00112
\end{tabular}

${ }^{\mathrm{a}} x_{2}$ : mole fraction of component 2 in the saturated solution.

${ }^{\mathrm{b}} x_{1}$ : mole fraction solubility of the solute.

\section{Auxiliary Information}

\section{Method/Apparatus/Procedure:}

Constant-temperature bath, calorimetric thermometer, and an ultraviolet/ visible spectrophotometer.

Excess solute and solvent were placed in amber glass bottles and allowed to equilibrate for several days at constant temperature. Attainment of equilibrium was verified by several repetitive measurements and by approaching equilibrium from supersaturation. Aliquots of saturated solutions were transferred through a coarse filter into tared volumetric flasks, weighed, and diluted with 2-propanol. Concentrations were determined by

spectrophotometric measurements at $286 \mathrm{~nm}$.

Source and Purity of Chemicals:

(1) $99+\%$, Acros Organics, USA, was used as received.

(2) $99+\%$, Acros Organics, stored over molecular sieves and distilled shortly before use.

\section{Estimated Error:}

Temperature: $\pm 0.1 \mathrm{~K}$.

$x_{1}: \pm 2.0 \%$ (relative error).

\begin{tabular}{|c|c|}
\hline $\begin{array}{l}\text { Components: } \\
\text { (1) 3,4-Dimethoxybenzoic acid; } \\
\mathrm{C}_{9} \mathrm{H}_{10} \mathrm{O}_{4} ; \text { [93-07-2] } \\
\text { (2) Tetrahydrofuran; } \mathrm{C}_{4} \mathrm{H}_{8} \mathrm{O} \text {; } \\
\text { [109-99-9] }\end{array}$ & $\begin{array}{l}\text { Original Measurements: } \\
{ }^{138} \text { K. R. Bowen, T. W. Stephens, H. Lu, } \\
\text { K. Satish, D. Shan, W. E. Acree, Jr., } \\
\text { and M. H. Abraham, Eur. Chem. Bull. } \\
\text { 2, } 577 \text { (2013). }\end{array}$ \\
\hline $\begin{array}{l}\text { Variables: } \\
T / \mathrm{K}=298.15\end{array}$ & $\begin{array}{l}\text { Prepared by: } \\
\text { W. E. Acree, Jr. }\end{array}$ \\
\hline
\end{tabular}

\section{Experimental Values}

\begin{tabular}{lc}
\hline \hline$x_{2}{ }^{\mathrm{a}}$ & $x_{1}{ }^{\mathrm{b}}$ \\
\hline 0.9606 & 0.03937 \\
\hline$x_{2}: \mathrm{molefration}$
\end{tabular}

${ }^{\mathrm{a}} x_{2}$ : mole fraction of component 2 in the saturated solution.

${ }^{\mathrm{b}} x_{1}$ : mole fraction solubility of the solute.

\section{Auxiliary Information}

\section{Method/Apparatus/Procedure:}

Constant-temperature bath, calorimetric thermometer, and an ultraviolet/ visible spectrophotometer.

Excess solute and solvent were placed in amber glass bottles and allowed to equilibrate for several days at constant temperature. Attainment of equilibrium was verified by several repetitive measurements and by approaching equilibrium from supersaturation. Aliquots of saturated solutions were transferred through a coarse filter into tared volumetric flasks, weighed, and diluted with 2-propanol. Concentrations were determined by spectrophotometric measurements at $286 \mathrm{~nm}$.

\section{Source and Purity of Chemicals:}

(1) $99+\%$, Acros Organics, USA, was used as received.

(2) $99.9 \%$, anhydrous, Aldrich Chemical Company, Milwaukee, WI, USA, stored over molecular sieves and distilled shortly before use.

\section{Estimated Error:}

Temperature: $\pm 0.1 \mathrm{~K}$.

$x_{1}: \pm 2.0 \%$ (relative error)

\begin{tabular}{ll}
\hline \hline Components: & Original Measurements: \\
(1) 3,4-Dimethoxybenzoic acid; & ${ }^{138}$ K. R. Bowen, T. W. Stephens, H. Lu, \\
$\mathrm{C}_{9} \mathrm{H}_{10} \mathrm{O}_{4}$; [93-07-2] & K. Satish, D. Shan, W. E. Acree, Jr., \\
(2) 1,4-Dioxane; $\mathrm{C}_{4} \mathrm{H}_{8} \mathrm{O}_{2} ;$ & and M. H. Abraham, Eur. Chem. Bull. \\
[123-91-1] & 2, 577 (2013). \\
\hline Variables: & Prepared by: \\
$T / \mathrm{K}=298.15$ & W. E. Acree, Jr. \\
\hline
\end{tabular}

Experimental Values

\begin{tabular}{lc}
\hline \hline$x_{2}{ }^{\mathrm{a}}$ & $x_{1}{ }^{\mathrm{b}}$ \\
\hline 0.9710 & 0.02897 \\
\hline${ }^{\mathrm{a}} x_{2}:$ mole fraction of component 2 in the saturated solution. & \\
${ }^{\mathrm{b}} x_{1}:$ mole fraction solubility of the solute. &
\end{tabular}

${ }^{b} x_{1}$ : mole fraction solubility of the solute.

\section{Auxiliary Information}

\section{Method/Apparatus/Procedure:}

Constant-temperature bath, calorimetric thermometer, and an ultraviolet/ visible spectrophotometer.

Excess solute and solvent were placed in amber glass bottles and allowed to equilibrate for several days at constant temperature. Attainment of equilibrium was verified by several repetitive measurements and by approaching equilibrium from supersaturation. Aliquots of saturated solutions were transferred through a coarse filter into tared volumetric flasks, weighed, and diluted with 2-propanol. Concentrations were determined by spectrophotometric measurements at $286 \mathrm{~nm}$.

\section{Source and Purity of Chemicals:}

(1) $99+\%$, Acros Organics, USA, was used as received.

(2) $99.8 \%$, anhydrous, Aldrich Chemical Company, Milwaukee, WI, USA, stored over molecular sieves and distilled shortly before use.

\section{Estimated Error:}

Temperature: $\pm 0.1 \mathrm{~K}$.

$x_{1}: \pm 2.0 \%$ (relative error). 


\subsection{3,4-Dimethoxybenzoic acid solubility data in alcohols}

\begin{tabular}{ll}
\hline \hline Components: & Original Measurements: \\
(1) 3,4-Dimethoxybenzoic acid; & ${ }^{138}$ K. R. Bowen, T. W. Stephens, H. Lu, \\
$\mathrm{C}_{9} \mathrm{H}_{10} \mathrm{O}_{4} ;$ [93-07-2] & K. Satish, D. Shan, W. E. Acree, Jr., \\
(2) Methanol; $\mathrm{CH}_{4} \mathrm{O} ;[67-56-1]$ & and M. H. Abraham, Eur. Chem. Bull. \\
& 2, 577 (2013). \\
\hline Variables: & Prepared by: \\
$T / \mathrm{K}=298.15$ & W. E. Acree, Jr. \\
\hline
\end{tabular}

Experimental Values

\begin{tabular}{lc}
\hline \hline$x_{2}{ }^{\mathrm{a}}$ & $x_{1}{ }^{\mathrm{b}}$ \\
\hline 0.9924 & 0.00764 \\
\hline
\end{tabular}

${ }^{a} x_{2}$ : mole fraction of component 2 in the saturated solution.

${ }^{\mathrm{b}} x_{1}$ : mole fraction solubility of the solute.

\section{Auxiliary Information}

\section{Method/Apparatus/Procedure:}

Constant-temperature bath, calorimetric thermometer, and an ultraviolet/ visible spectrophotometer.

Excess solute and solvent were placed in amber glass bottles and allowed to equilibrate for several days at constant temperature. Attainment of equilibrium was verified by several repetitive measurements and by approaching equilibrium from supersaturation. Aliquots of saturated solutions were transferred through a coarse filter into tared volumetric flasks, weighed, and diluted with 2-propanol. Concentrations were determined by

spectrophotometric measurements at $286 \mathrm{~nm}$.

\section{Source and Purity of Chemicals:}

(1) $99+\%$, Acros Organics, USA, was used as received.

(2) $99.8 \%$, anhydrous, Aldrich Chemical Company, Milwaukee, WI, USA, stored over molecular sieves and distilled shortly before use.

\section{Estimated Error:}

Temperature: $\pm 0.1 \mathrm{~K}$.

$x_{1}: \pm 2.0 \%$ (relative error).

\begin{tabular}{|c|c|}
\hline $\begin{array}{l}\text { Components: } \\
\text { (1) 3,4-Dimethoxybenzoic acid; } \\
\mathrm{C}_{9} \mathrm{H}_{10} \mathrm{O}_{4} ;[93-07-2] \\
\text { (2) Ethanol; } \mathrm{C}_{2} \mathrm{H}_{6} \mathrm{O} ;[64-17-5]\end{array}$ & $\begin{array}{l}\text { Original Measurements: } \\
{ }^{138} \text { K. R. Bowen, T. W. Stephens, H. Lu, } \\
\text { K. Satish, D. Shan, W. E. Acree, Jr., } \\
\text { and M. H. Abraham, Eur. Chem. Bull. } \\
\text { 2, } 577 \text { (2013). }\end{array}$ \\
\hline Variables: & \\
\hline$T / \mathrm{K}=298.15$ & W. E. Acree, Jr. \\
\hline
\end{tabular}

\section{Experimental Values}

\begin{tabular}{lc}
\hline \hline$x_{2}{ }^{\mathrm{a}}$ & $x_{1}{ }^{\mathrm{b}}$ \\
\hline 0.9928 & 0.00720
\end{tabular}

${ }^{\mathrm{a}} x_{2}$ : mole fraction of component 2 in the saturated solution.

${ }^{\mathrm{b}} x_{1}$ : mole fraction solubility of the solute.

\section{Auxiliary Information}

\section{Method/Apparatus/Procedure:}

Constant-temperature bath, calorimetric thermometer, and an ultraviolet/ visible spectrophotometer.

Excess solute and solvent were placed in amber glass bottles and allowed to equilibrate for several days at constant temperature. Attainment of equilibrium was verified by several repetitive measurements and by approaching equilibrium from supersaturation. Aliquots of saturated solutions were transferred through a coarse filter into tared volumetric flasks, weighed, and diluted with 2-propanol. Concentrations were determined by spectrophotometric measurements at $286 \mathrm{~nm}$.

\section{Source and Purity of Chemicals:}

(1) $99+\%$, Acros Organics, USA, was used as received.

(2) Absolute, Aaper Alcohol and Chemical Company, USA, stored over molecular sieves and distilled shortly before use.

Estimated Error:

Temperature: $\pm 0.1 \mathrm{~K}$.

$x_{1}: \pm 2.0 \%$ (relative error)

\begin{tabular}{ll}
\hline \hline Components: & Original Measurements: \\
(1) 3,4-Dimethoxybenzoic acid; & ${ }^{139} \mathrm{Q}$. Li, F. Lu, Y. Tian, S. Feng, Y. \\
$\mathrm{C}_{9} \mathrm{H}_{10} \mathrm{O}_{4} ;[93-07-2]$ & Shen, and B. Wang, J. Chem. Eng. Data \\
(2) Ethanol; $\mathrm{C}_{2} \mathrm{H}_{6} \mathrm{O} ;[64-17-5]$ & $\mathbf{5 8 , 1 0 2 0}(2013)$. \\
\hline Variables: & Prepared by: \\
Temperature & W. E. Acree, Jr. \\
\hline
\end{tabular}

Experimental Values

\begin{tabular}{lcc}
\hline \hline$T / \mathrm{K}$ & $x_{2}{ }^{\mathrm{a}}$ & $x_{1}{ }^{\mathrm{b}}$ \\
\hline 278.15 & 0.9965 & 0.0035 \\
282.93 & 0.9958 & 0.0042 \\
288.21 & 0.9949 & 0.0051 \\
293.08 & 0.9938 & 0.0062 \\
298.00 & 0.9926 & 0.0074 \\
303.33 & 0.9907 & 0.0093 \\
307.90 & 0.9890 & 0.0110 \\
313.30 & 0.9864 & 0.0136 \\
317.15 & 0.9839 & 0.0161 \\
323.30 & 0.9798 & 0.0202 \\
\hline
\end{tabular}

${ }^{\mathrm{a}} x_{2}$ : mole fraction of component 2 in the saturated solution.

${ }^{b} x_{1}$ : mole fraction solubility of the solute.

\section{Auxiliary Information}

\section{Method/Apparatus/Procedure:}

Water-jacketed equilibrium glass vessel, thermostated circulating water bath, magnetic stirrer, analytical balance, laser monitoring system. Experimental solubilities were determined by a synthetic method. Preweighed amounts of solute and solvent and were placed in a water-jacketed equilibrium vessel, which was connected to a circulating constant-temperature water bath. The solution was stirred and small amounts of solute were incrementally added until no further solid dissolved. The dissolution of the solid was determined using laser monitoring. The total amount of solute dissolved was recorded. Experimental measurement was repeated three times. 
Source and Purity of Chemicals:

(1) $99+\%$, Alfa Aesar China Co., Ltd., Tianjin, China, no purification details were provided.

(2) $99.7+\%$, Beijing Chemical Works, Beijing, China, no purification details were provided.

\section{Estimated Error:}

Temperature: $\pm 0.05 \mathrm{~K}$.

$x_{1}: \pm 4 \%$ (relative error).

\section{Components:}

(1) 3,4-Dimethoxybenzoic acid; $\mathrm{C}_{9} \mathrm{H}_{10} \mathrm{O}_{4}$; [93-07-2]

(2) 1-Propanol; $\mathrm{C}_{3} \mathrm{H}_{8} \mathrm{O}$; [71-23-8]

Original Measurements:

${ }^{138}$ K. R. Bowen, T. W. Stephens, H. Lu, K. Satish, D. Shan, W. E. Acree, Jr., and M. H. Abraham, Eur. Chem. Bull. 2, 577 (2013).

\begin{tabular}{ll}
\hline Variables: & Prepared by: \\
$T / \mathrm{K}=298.15$ & W. E. Acree, Jr.
\end{tabular}

\section{Experimental Values}

\begin{tabular}{lc}
\hline \hline$x_{2}{ }^{\mathrm{a}}$ & $x_{1}{ }^{\mathrm{b}}$ \\
\hline 0.9936 & 0.00643 \\
\hline
\end{tabular}

${ }^{\mathrm{a}} x_{2}$ : mole fraction of component 2 in the saturated solution.

${ }^{b} x_{1}$ : mole fraction solubility of the solute.

\section{Auxiliary Information}

\section{Method/Apparatus/Procedure:}

Constant-temperature bath, calorimetric thermometer, and an ultraviolet/

Excess solute and solvent were placed in amber glass bottles and allowed to equilibrate for several days at constant temperature. Attainment of equilibrium was verified by several repetitive measurements and by approaching equilibrium from supersaturation. Aliquots of saturated solutions were transferred through a coarse filter into tared volumetric flasks, weighed, and diluted with 2-propanol. Concentrations were determined by spectrophotometric measurements at $286 \mathrm{~nm}$.

\section{Source and Purity of Chemicals:}

(1) $99+\%$, Acros Organics, USA, was used as received.

(2) $99+\%$, anhydrous, Aldrich Chemical Company, Milwaukee, WI, USA, stored over molecular sieves and distilled shortly before use.

\section{Estimated Error:}

Temperature: $\pm 0.1 \mathrm{~K}$.
Experimental Values

\begin{tabular}{lcc}
\hline \hline$T / \mathrm{K}$ & $x_{2}{ }^{\mathrm{a}}$ & $x_{1}{ }^{\mathrm{b}}$ \\
\hline 278.37 & 0.9973 & 0.0027 \\
283.05 & 0.9967 & 0.0033 \\
288.39 & 0.9958 & 0.0042 \\
293.30 & 0.9949 & 0.0051 \\
299.00 & 0.9934 & 0.0066 \\
303.17 & 0.9921 & 0.0079 \\
308.27 & 0.9907 & 0.0097 \\
313.13 & 0.9882 & 0.0118 \\
318.15 & 0.9854 & 0.0146 \\
323.28 & 0.9823 & 0.0177 \\
\hline
\end{tabular}

${ }^{\mathrm{a}} x_{2}$ : mole fraction of component 2 in the saturated solution.

${ }^{\mathrm{b}} x_{1}$ : mole fraction solubility of the solute.

\section{Auxiliary Information} visible spectrophotometer.

$x_{1}: \pm 2.0 \%$ (relative error)

\section{Method/Apparatus/Procedure:}

Water-jacketed equilibrium glass vessel, thermostated circulating water bath, magnetic stirrer, analytical balance, laser monitoring system.

Experimental solubilities were determined by a synthetic method. Preweighed amounts of solute and solvent and were placed in a water-jacketed equilibrium vessel, which was connected to a circulating constant-temperature water bath. The solution was stirred and small amounts of solute were incrementally added until no further solid dissolved. The dissolution of the solid was determined using laser monitoring. The total amount of solute dissolved was recorded. Experimental measurement was repeated three times.

Source and Purity of Chemicals:

(1) $99+\%$, Alfa Aesar China Co., Ltd., Tianjin, China, no purification details were provided.

(2) $99.8+\%$, Beijing Chemical Works, Beijing, China, no purification details were provided.

Estimated Error:

Temperature: $\pm 0.05 \mathrm{~K}$.

$x_{1}: \pm 4 \%$ (relative error)

\section{Components:}

(1) 3,4-Dimethoxybenzoic acid;

$\mathrm{C}_{9} \mathrm{H}_{10} \mathrm{O}_{4}$; [93-07-2]

(2) 2-Propanol; $\mathrm{C}_{3} \mathrm{H}_{8} \mathrm{O}$; [67-63-0]

Variables:
$T / \mathrm{K}=298.15$

\section{Original Measurements:}

${ }^{138}$ K. R. Bowen, T. W. Stephens, H. Lu, K. Satish, D. Shan, W. E. Acree, Jr., and M. H. Abraham, Eur. Chem. Bull. 2, 577 (2013).

Prepared by:
W. E. Acree, Jr.

\section{Experimental Values}

\begin{tabular}{|c|c|}
\hline $\begin{array}{l}\text { Components: } \\
\text { (1) 3,4-Dimethoxybenzoic acid; } \\
\mathrm{C}_{9} \mathrm{H}_{10} \mathrm{O}_{4} ;[93-07-2] \\
\text { (2) 1-Propanol; } \mathrm{C}_{3} \mathrm{H}_{8} \mathrm{O} ;[71-23-8]\end{array}$ & $\begin{array}{l}\text { Original Measurements: } \\
{ }^{139} \text { Q. Li, F. Lu, Y. Tian, S. Feng, Y. } \\
\text { Shen, and B. Wang, J. Chem. Eng. Data } \\
\text { 58, } 1020 \text { (2013). }\end{array}$ \\
\hline $\begin{array}{l}\text { Variables: } \\
\text { Temperature }\end{array}$ & $\begin{array}{l}\text { Prepared by: } \\
\text { W. E. Acree, Jr. }\end{array}$ \\
\hline
\end{tabular}

\begin{tabular}{lc}
\hline \hline$x_{2}{ }^{\mathrm{a}}$ & $x_{1}{ }^{\mathrm{b}}$ \\
\hline 0.9938 & 0.00618 \\
\hline${ }_{{ }_{2} x_{2}: \text { mole fraction of component } 2 \text { in the saturated solution. }}$ & \\
${ }^{\mathrm{b}} x_{1}:$ mole fraction solubility of the solute. &
\end{tabular}




\section{Auxiliary Information}

\section{Method/Apparatus/Procedure:}

Constant-temperature bath, calorimetric thermometer, and an ultraviolet/ visible spectrophotometer.

Excess solute and solvent were placed in amber glass bottles and allowed to equilibrate for several days at constant temperature. Attainment of equilibrium was verified by several repetitive measurements and by approaching equilibrium from supersaturation. Aliquots of saturated solutions were transferred through a coarse filter into tared volumetric flasks, weighed, and diluted with 2-propanol. Concentrations were determined by spectrophotometric measurements at $286 \mathrm{~nm}$.

Source and Purity of Chemicals:

(1) $99+\%$, Acros Organics, USA, was used as received.

(2) $99+\%$, anhydrous, Aldrich Chemical Company, Milwaukee, WI, USA, stored over molecular sieves and distilled shortly before use.

Estimated Error:

Temperature: $\pm 0.1 \mathrm{~K}$.

$x_{1}: \pm 2.0 \%$ (relative error).

\begin{tabular}{|c|c|}
\hline $\begin{array}{l}\text { Components: } \\
\text { (1) 3,4-Dimethoxybenzoic acid; } \\
\mathrm{C}_{9} \mathrm{H}_{10} \mathrm{O}_{4} ;[93-07-2] \\
\text { (2) 2-Propanol; } \mathrm{C}_{3} \mathrm{H}_{8} \mathrm{O} ;[67-63-0]\end{array}$ & $\begin{array}{l}\text { Original Measurements: } \\
{ }^{139} \text { Q. Li, F. Lu, Y. Tian, S. Feng, Y. } \\
\text { Shen, and B. Wang, J. Chem. Eng. Data } \\
\text { 58, } 1020 \text { (2013). }\end{array}$ \\
\hline $\begin{array}{l}\text { Variables: } \\
\text { Temperature }\end{array}$ & $\begin{array}{l}\text { Prepared by: } \\
\text { W. E. Acree, Jr. }\end{array}$ \\
\hline
\end{tabular}

Experimental Values

\begin{tabular}{lcc}
\hline \hline$T / \mathrm{K}$ & $x_{2}{ }^{\mathrm{a}}$ & $x_{1}{ }^{\mathrm{b}}$ \\
\hline 278.37 & 0.9976 & 0.0024 \\
283.05 & 0.9970 & 0.0030 \\
288.39 & 0.9961 & 0.0039 \\
294.07 & 0.9949 & 0.0051 \\
299.00 & 0.9937 & 0.0063 \\
303.17 & 0.9926 & 0.0074 \\
308.27 & 0.9905 & 0.0095 \\
313.13 & 0.9882 & 0.0118 \\
318.15 & 0.9850 & 0.0150 \\
323.20 & 0.9817 & 0.0183 \\
\hline
\end{tabular}

${ }^{a} x_{2}$ : mole fraction of component 2 in the saturated solution.

${ }^{\mathrm{b}} x_{1}$ : mole fraction solubility of the solute.

\section{Auxiliary Information}

\section{Method/Apparatus/Procedure:}

Water-jacketed equilibrium glass vessel, thermostated circulating water bath, magnetic stirrer, analytical balance, laser monitoring system.

Experimental solubilities were determined by a synthetic method. Preweighed amounts of solute and solvent and were placed in a water-jacketed equilibrium vessel, which was connected to a circulating constant-temperature water bath. The solution was stirred and small amounts of solute were incrementally added until no further solid dissolved. The dissolution of the solid was determined using laser monitoring. The total amount of solute dissolved was recorded. Experimental measurement was repeated three times.
Source and Purity of Chemicals:

(1) $99+\%$, Alfa Aesar China Co., Ltd., Tianjin, China, no purification details were provided.

(2) $99.7+\%$, Beijing Chemical Works, Beijing, China, no purification details were provided.

\section{Estimated Error:}

Temperature: $\pm 0.05 \mathrm{~K}$.

$x_{1}: \pm 4 \%$ (relative error)

\begin{tabular}{|c|c|}
\hline $\begin{array}{l}\text { Components: } \\
\text { (1) 3,4-Dimethoxybenzoic acid; } \\
\mathrm{C}_{9} \mathrm{H}_{10} \mathrm{O}_{4} ;[93-07-2] \\
\text { (2) 1-Butanol; } \mathrm{C}_{4} \mathrm{H}_{10} \mathrm{O} ;[71-36-3]\end{array}$ & $\begin{array}{l}\text { Original Measurements: } \\
{ }^{138} \text { K. R. Bowen, T. W. Stephens, H. Lu, } \\
\text { K. Satish, D. Shan, W. E. Acree, Jr., } \\
\text { and M. H. Abraham, Eur. Chem. Bull } \\
\text { 2, } 577 \text { (2013). }\end{array}$ \\
\hline $\begin{array}{l}\text { Variables: } \\
T / \mathrm{K}=298.15\end{array}$ & $\begin{array}{l}\text { Prepared by: } \\
\text { W. E. Acree, Jr. }\end{array}$ \\
\hline
\end{tabular}

\section{Experimental Values}

\begin{tabular}{lc}
\hline \hline$x_{2}{ }^{\mathrm{a}}$ & $x_{1}{ }^{\mathrm{b}}$ \\
\hline 0.9937 & 0.00625
\end{tabular}

${ }^{\mathrm{a}} x_{2}$ : mole fraction of component 2 in the saturated solution.

${ }^{\mathrm{b}} x_{1}$ : mole fraction solubility of the solute.

\section{Auxiliary Information}

\section{Method/Apparatus/Procedure:}

Constant-temperature bath, calorimetric thermometer, and an ultraviolet/ visible spectrophotometer.

Excess solute and solvent were placed in amber glass bottles and allowed to equilibrate for several days at constant temperature. Attainment of equilibrium was verified by several repetitive measurements and by approaching equilibrium from supersaturation. Aliquots of saturated solutions were transferred through a coarse filter into tared volumetric flasks, weighed, and diluted with 2-propanol. Concentrations were determined by spectrophotometric measurements at $286 \mathrm{~nm}$.

\section{Source and Purity of Chemicals:}

(1) $99+\%$, Acros Organics, USA, was used as received. (2) $99.8+\%$, HPLC grade, Aldrich Chemical Company, Milwaukee, WI, USA, stored over molecular sieves and distilled shortly before use.

\section{Estimated Error:}

Temperature: $\pm 0.1 \mathrm{~K}$.

$x_{1}: \pm 2.0 \%$ (relative error)

\begin{tabular}{|c|c|}
\hline $\begin{array}{l}\text { Components: } \\
\text { (1) 3,4-Dimethoxybenzoic acid; } \\
\mathrm{C}_{9} \mathrm{H}_{10} \mathrm{O}_{4} ;[93-07-2] \\
\text { (2) } 1 \text {-Butanol; } \mathrm{C}_{4} \mathrm{H}_{10} \mathrm{O} ;[71-36-3]\end{array}$ & $\begin{array}{l}\text { Original Measurements: } \\
{ }^{139} \text { Q. Li, F. Lu, Y. Tian, S. Feng, Y. } \\
\text { Shen, and B. Wang, J. Chem. Eng. Data } \\
\text { 58, } 1020 \text { (2013). }\end{array}$ \\
\hline $\begin{array}{l}\text { Variables: } \\
\text { Temperature }\end{array}$ & $\begin{array}{l}\text { Prepared by: } \\
\text { W. E. Acree, Jr. }\end{array}$ \\
\hline
\end{tabular}


Experimental Values

\begin{tabular}{lcc}
\hline \hline$T / \mathrm{K}$ & $x_{2}{ }^{\mathrm{a}}$ & $x_{1}{ }^{\mathrm{b}}$ \\
\hline 277.90 & 0.9976 & 0.0024 \\
283.25 & 0.9970 & 0.0030 \\
288.15 & 0.9963 & 0.0037 \\
293.04 & 0.9954 & 0.0046 \\
298.15 & 0.9943 & 0.0057 \\
302.94 & 0.9930 & 0.0070 \\
308.10 & 0.9912 & 0.0088 \\
313.15 & 0.9891 & 0.0109 \\
318.01 & 0.9865 & 0.0135 \\
323.33 & 0.9830 & 0.0170 \\
\hline
\end{tabular}

${ }^{\mathrm{a}} x_{2}$ : mole fraction of component 2 in the saturated solution.

${ }^{b} x_{1}$ : mole fraction solubility of the solute.

\section{Auxiliary Information}

\section{Method/Apparatus/Procedure:}

Water-jacketed equilibrium glass vessel, thermostated circulating water bath, magnetic stirrer, analytical balance, laser monitoring system.

Experimental solubilities were determined by a synthetic method. Preweighed amounts of solute and solvent and were placed in a water-jacketed equilibrium vessel, which was connected to a circulating constant-temperature water bath. The solution was stirred and small amounts of solute were incrementally added until no further solid dissolved. The dissolution of the solid was determined using laser monitoring. The total amount of solute dissolved was recorded. Experimental measurement was repeated three times.

Source and Purity of Chemicals:

(1) 99+\%, Alfa Aesar China Co., Ltd., Tianjin, China, no purification details were provided.

(2) $99.5+\%$, Beijing Chemical Works, Beijing, China, no purification details were provided.

Estimated Error:

Temperature: $\pm 0.05 \mathrm{~K}$.

$x_{1}: \pm 4 \%$ (relative error).

\begin{tabular}{ll}
\hline \hline Components: & Original Measurements: \\
(1) 3,4-Dimethoxybenzoic acid; & ${ }^{138}$ K. R. Bowen, T. W. Stephens, H. Lu, \\
$\mathrm{C}_{9} \mathrm{H}_{10} \mathrm{O}_{4} ;$; $\left.93-07-2\right]$ & K. Satish, D. Shan, W. E. Acree, Jr., \\
(2) 2-Butanol; $\mathrm{C}_{4} \mathrm{H}_{10} \mathrm{O} ;$ [78-92-2] & $\begin{array}{l}\text { and M. H. Abraham, Eur. Chem. Bull. } \\
\text { 2, 577 (2013). }\end{array}$ \\
\hline Variables: & Prepared by: \\
$T / \mathrm{K}=298.15$ & W. E. Acree, Jr. \\
\hline
\end{tabular}

Experimental Values

\begin{tabular}{lc}
\hline \hline$x_{2}^{\mathrm{a}}$ & $x_{1}{ }^{\mathrm{b}}$ \\
\hline 0.9935 & 0.00651 \\
\hline${ }^{\mathrm{a}} x_{2}:$ mole fraction of component 2 in the saturated solution. \\
${ }^{\mathrm{b}}{ }_{x_{1}: \text { mole fraction solubility of the solute. }}$
\end{tabular}

\section{Auxiliary Information}

Method/Apparatus/Procedure:

Constant-temperature bath, calorimetric thermometer, and an ultraviolet/ visible spectrophotometer.

Excess solute and solvent were placed in amber glass bottles and allowed to equilibrate for several days at constant temperature. Attainment of equilibrium was verified by several repetitive measurements and by approaching equilibrium from supersaturation. Aliquots of saturated solutions were transferred through a coarse filter into tared volumetric flasks, weighed, and diluted with 2-propanol. Concentrations were determined by spectrophotometric measurements at $286 \mathrm{~nm}$.

\section{Source and Purity of Chemicals:}

(1) $99+\%$, Acros Organics, USA, was used as received.

(2) $99+\%$, anhydrous, Aldrich Chemical Company, Milwaukee, WI, USA, stored over molecular sieves and distilled shortly before use.

\section{Estimated Error:}

Temperature: $\pm 0.1 \mathrm{~K}$.

$x_{1}: \pm 2.0 \%$ (relative error).

\begin{tabular}{|c|c|}
\hline $\begin{array}{l}\text { Components: } \\
\text { (1) 3,4-Dimethoxybenzoic acid; } \\
\mathrm{C}_{9} \mathrm{H}_{10} \mathrm{O}_{4} \text {; [93-07-2] } \\
\text { (2) 2-Methyl-1-propanol; } \mathrm{C}_{4} \mathrm{H}_{10} \mathrm{O} \text {; } \\
\text { [78-83-1] }\end{array}$ & $\begin{array}{l}\text { Original Measurements: } \\
{ }^{138} \text { K. R. Bowen, T. W. Stephens, H. Lu, } \\
\text { K. Satish, D. Shan, W. E. Acree, Jr., } \\
\text { and M. H. Abraham, Eur. Chem. Bull. } \\
\text { 2, } 577 \text { (2013). }\end{array}$ \\
\hline $\begin{array}{l}\text { Variables: } \\
T / \mathrm{K}=298.15\end{array}$ & $\begin{array}{l}\text { Prepared by: } \\
\text { W. E. Acree, Jr. }\end{array}$ \\
\hline
\end{tabular}

\section{Experimental Values}

\begin{tabular}{lc}
\hline \hline$x_{2}{ }^{\mathrm{a}}$ & $x_{1}{ }^{\mathrm{b}}$ \\
\hline 0.9955 & 0.00450 \\
\hline${ }^{\mathrm{a}} x_{2}:$ mole fraction of component 2 in the saturated solution. \\
${ }^{\mathrm{b}} x_{1}:$ mole fraction solubility of the solute.
\end{tabular}

\section{Auxiliary Information}

\section{Method/Apparatus/Procedure:}

Constant-temperature bath, calorimetric thermometer, and an ultraviolet/ visible spectrophotometer.

Excess solute and solvent were placed in amber glass bottles and allowed to equilibrate for several days at constant temperature. Attainment of equilibrium was verified by several repetitive measurements and by approaching equilibrium from supersaturation. Aliquots of saturated solutions were transferred through a coarse filter into tared volumetric flasks, weighed, and diluted with 2-propanol. Concentrations were determined by spectrophotometric measurements at $286 \mathrm{~nm}$.

\section{Source and Purity of Chemicals:}

(1) $99+\%$, Acros Organics, USA, was used as received.

(2) 99+\%, anhydrous, Aldrich Chemical Company, Milwaukee, WI, USA, stored over molecular sieves and distilled shortly before use.

\section{Estimated Error:}

Temperature: $\pm 0.1 \mathrm{~K}$.

$x_{1}: \pm 1.5 \%$ (relative error). 


\begin{tabular}{|c|c|}
\hline $\begin{array}{l}\text { Components: } \\
\text { (1) 3,4-Dimethoxybenzoic acid; } \\
\mathrm{C}_{9} \mathrm{H}_{10} \mathrm{O}_{4} \text {; [93-07-2] } \\
\text { (2) 2-Methyl-1-propanol; } \mathrm{C}_{4} \mathrm{H}_{10} \mathrm{O} \text {; } \\
\text { [78-83-1] }\end{array}$ & $\begin{array}{l}\text { Original Measurements: } \\
{ }^{139} \text { Q. Li, F. Lu, Y. Tian, S. Feng, Y. } \\
\text { Shen, and B. Wang, J. Chem. Eng. Data } \\
\mathbf{5 8 , ~} 1020 \text { (2013). }\end{array}$ \\
\hline $\begin{array}{l}\text { Variables: } \\
\text { Temperature }\end{array}$ & $\begin{array}{l}\text { Prepared by: } \\
\text { W. E. Acree Jr. }\end{array}$ \\
\hline
\end{tabular}

Experimental Values

\begin{tabular}{lcc}
\hline \hline$T / \mathrm{K}$ & $x_{2}{ }^{\mathrm{a}}$ & $x_{1}{ }^{\mathrm{b}}$ \\
\hline 277.90 & 0.9985 & 0.0015 \\
283.25 & 0.9980 & 0.0020 \\
288.15 & 0.9974 & 0.0026 \\
293.04 & 0.9968 & 0.0032 \\
298.15 & 0.9959 & 0.0041 \\
302.94 & 0.9948 & 0.0052 \\
308.10 & 0.9934 & 0.0066 \\
313.15 & 0.9917 & 0.0083 \\
318.01 & 0.9895 & 0.0105 \\
323.33 & 0.9868 & 0.0132 \\
\hline
\end{tabular}

${ }^{\mathrm{a}} x_{2}$ : mole fraction of component 2 in the saturated solution.

${ }^{\mathrm{b}} x_{1}$ : mole fraction solubility of the solute.

\section{Auxiliary Information}

\section{Method/Apparatus/Procedure:}

Water-jacketed equilibrium glass vessel, thermostated circulating water bath, magnetic stirrer, analytical balance, laser monitoring system.

Experimental solubilities were determined by a synthetic method. Preweighed amounts of solute and solvent and were placed in a water-jacketed equilibrium vessel, which was connected to a circulating constant-temperature water bath. The solution was stirred and small amounts of solute were incrementally added until no further solid dissolved. The dissolution of the solid was determined using laser monitoring. The total amount of solute dissolved was recorded. Experimental measurement was repeated three times.

\section{Source and Purity of Chemicals:}

(1) $99+\%$, Alfa Aesar China Co., Ltd., Tianjin, China, no purification details were provided.

(2) $99.5+\%$, Beijing Chemical Works, Beijing, China, no purification details were provided.

\section{Estimated Error:}

Temperature: $\pm 0.05 \mathrm{~K}$.

$x_{1}: \pm 4 \%$ (relative error).

\begin{tabular}{|c|c|}
\hline $\begin{array}{l}\text { Components: } \\
\text { (1) 3,4-Dimethoxybenzoic acid; } \\
\left.\mathrm{C}_{9} \mathrm{H}_{10} \mathrm{O}_{4} ; \text {; } 93-07-2\right] \\
\text { (2) 2-Methyl-2-propanol; } \mathrm{C}_{4} \mathrm{H}_{10} \mathrm{O} \text {; } \\
\text { [75-65-0] }\end{array}$ & $\begin{array}{l}\text { Original Measurements: } \\
{ }^{138} \text { K. R. Bowen, T. W. Stephens, H. Lu, } \\
\text { K. Satish, D. Shan, W. E. Acree, Jr., } \\
\text { and M. H. Abraham, Eur. Chem. Bull. } \\
\text { 2, } 577 \text { (2013). }\end{array}$ \\
\hline $\begin{array}{l}\text { Variables: } \\
T / \mathrm{K}=298.15\end{array}$ & $\begin{array}{l}\text { Prepared by: } \\
\text { W. E. Acree, Jr. }\end{array}$ \\
\hline
\end{tabular}

\section{Experimental Values}

\begin{tabular}{lc}
\hline \hline$x_{2}{ }^{\mathrm{a}}$ & $x_{1}{ }^{\mathrm{b}}$ \\
\hline 0.9905 & 0.00945
\end{tabular}

${ }^{a} x_{2}$ : mole fraction of component 2 in the saturated solution.

${ }^{\mathrm{b}} x_{1}$ : mole fraction solubility of the solute.

\section{Auxiliary Information}

\section{Method/Apparatus/Procedure:}

Constant-temperature bath, calorimetric thermometer, and an ultraviolet/ visible spectrophotometer.

Excess solute and solvent were placed in amber glass bottles and allowed to equilibrate for several days at constant temperature. Attainment of equilibrium was verified by several repetitive measurements and by approaching equilibrium from supersaturation. Aliquots of saturated solutions were transferred through a coarse filter into tared volumetric flasks, weighed, and diluted with 2-propanol. Concentrations were determined by spectrophotometric measurements at $286 \mathrm{~nm}$.

Source and Purity of Chemicals:

(1) $99+\%$, Acros Organics, USA, was used as received.

(2) $99+\%$, Arco Chemical Company, USA, stored over molecular sieves and distilled shortly before use.

Estimated Error:

Temperature: $\pm 0.1 \mathrm{~K}$.

$x_{1}: \pm 2.0 \%$ (relative error).

\begin{tabular}{ll}
\hline \hline Components: & Original Measurements: \\
(1) 3,4-Dimethoxybenzoic acid; & ${ }^{138}$ K. R. Bowen, T. W. Stephens, H. Lu, \\
$\mathrm{C}_{9} \mathrm{H}_{10} \mathrm{O}_{4}$; [93-07-2] & K. Satish, D. Shan, W. E. Acree, Jr., \\
(2) 1-Pentanol; $\mathrm{C}_{5} \mathrm{H}_{12} \mathrm{O} ;$ [71-41-0] & and M. H. Abraham, Eur. Chem. Bull. \\
& $\mathbf{2 , 5 7 7 ~ ( 2 0 1 3 ) .}$ \\
\hline Variables: & Prepared by: \\
$T / \mathrm{K}=298.15$ & W. E. Acree, Jr. \\
\hline
\end{tabular}

Experimental Values

\begin{tabular}{lc}
\hline \hline$x_{2}{ }^{\mathrm{a}}$ & $x_{1}{ }^{\mathrm{b}}$ \\
\hline 0.9938 & 0.00615 \\
\hline${ }^{\mathrm{a}} x_{2}:$ mole fraction of component 2 in the saturated solution. & \\
${ }^{\mathrm{b}} x_{1}:$ mole fraction solubility of the solute. &
\end{tabular}

\section{Auxiliary Information}

\section{Method/Apparatus/Procedure:}

Constant-temperature bath, calorimetric thermometer, and an ultraviolet/ visible spectrophotometer.

Excess solute and solvent were placed in amber glass bottles and allowed to equilibrate for several days at constant temperature. Attainment of equilibrium was verified by several repetitive measurements and by approaching equilibrium from supersaturation. Aliquots of saturated solutions were transferred through a coarse filter into tared volumetric flasks, weighed, and diluted with 2-propanol. Concentrations were determined by spectrophotometric measurements at $286 \mathrm{~nm}$. 
Source and Purity of Chemicals:

(1) $99+\%$, Acros Organics, USA, was used as received.

(2) $99+\%$, Aldrich Chemical Company, Milwaukee, WI, USA, stored over molecular sieves and distilled shortly before use.

Estimated Error:

Temperature: $\pm 0.1 \mathrm{~K}$.

$x_{1}: \pm 2.0 \%$ (relative error)

\begin{tabular}{ll}
\hline \hline Components: & Original Measurements: \\
(1) 3,4-Dimethoxybenzoic acid; & ${ }^{138}$ K. R. Bowen, T. W. Stephens, H. \\
$\mathrm{C}_{9} \mathrm{H}_{10} \mathrm{O}_{4} ;$ [93-07-2] & Lu, K. Satish, D. Shan, W. E. Acree, \\
(2) 2-Pentanol; $\mathrm{C}_{5} \mathrm{H}_{12} \mathrm{O} ;$ [6032-29-7] & Jr., and M. H. Abraham, Eur. Chem. \\
& Bull. 2, 577 (2013). \\
\hline Variables: & Prepared by: \\
$T / \mathrm{K}=298.15$ & W. E. Acree, Jr. \\
\hline
\end{tabular}

Experimental Values

\begin{tabular}{lc}
\hline \hline$x_{2}{ }^{\mathrm{a}}$ & $x_{1}{ }^{\mathrm{b}}$ \\
\hline 0.9941 & 0.00593 \\
\hline
\end{tabular}

${ }^{\mathrm{a}} x_{2}$ : mole fraction of component 2 in the saturated solution.

${ }^{\mathrm{b}} x_{1}$ : mole fraction solubility of the solute.

\section{Auxiliary Information}

\section{Method/Apparatus/Procedure:}

Constant-temperature bath, calorimetric thermometer, and an ultraviolet/ visible spectrophotometer.

Excess solute and solvent were placed in amber glass bottles and allowed to equilibrate for several days at constant temperature. Attainment of equilibrium was verified by several repetitive measurements and by approaching equilibrium from supersaturation. Aliquots of saturated solutions were transferred through a coarse filter into tared volumetric flasks, weighed, and diluted with 2-propanol. Concentrations were determined by

spectrophotometric measurements at $286 \mathrm{~nm}$.

Source and Purity of Chemicals:

(1) $99+\%$, Acros Organics, USA, was used as received.

(2) $99+\%$, Acros Organics, USA, stored over molecular sieves and distilled shortly before use.

Estimated Error:

Temperature: $\pm 0.1 \mathrm{~K}$

$x_{1}: \pm 2.0 \%$ (relative error).

\begin{tabular}{|c|c|}
\hline $\begin{array}{l}\text { Components: } \\
\text { (1) 3,4-Dimethoxybenzoic acid; } \\
\mathrm{C}_{9} \mathrm{H}_{10} \mathrm{O}_{4} \text {; [93-07-2] } \\
\text { (2) 3-Methyl-1-butanol; } \mathrm{C}_{5} \mathrm{H}_{12} \mathrm{O} \text {; } \\
\text { [123-51-3] }\end{array}$ & $\begin{array}{l}\text { Original Measurements: } \\
{ }^{138} \text { K. R. Bowen, T. W. Stephens, H. Lu, } \\
\text { K. Satish, D. Shan, W. E. Acree, Jr., } \\
\text { and M. H. Abraham, Eur. Chem. Bull. } \\
\text { 2, } 577 \text { (2013). }\end{array}$ \\
\hline $\begin{array}{l}\text { Variables: } \\
T / \mathrm{K}=298.15\end{array}$ & $\begin{array}{l}\text { Prepared by: } \\
\text { W. E. Acree, Jr. }\end{array}$ \\
\hline
\end{tabular}

Experimental Values

\begin{tabular}{lc}
\hline \hline$x_{2}{ }^{\mathrm{a}}$ & $x_{1}{ }^{\mathrm{b}}$ \\
\hline 0.9944 & 0.00560 \\
\hline${ }^{\mathrm{a}} x_{2}:$ mole fraction of component 2 in the saturated solution. \\
${ }^{\mathrm{b}}{ }_{x_{1}}:$ mole fraction solubility of the solute.
\end{tabular}

\section{Auxiliary Information}

\section{Method/Apparatus/Procedure:}

Constant-temperature bath, calorimetric thermometer, and an ultraviolet/ visible spectrophotometer.

Excess solute and solvent were placed in amber glass bottles and allowed to equilibrate for several days at constant temperature. Attainment of equilibrium was verified by several repetitive measurements and by approaching equilibrium from supersaturation. Aliquots of saturated solutions were transferred through a coarse filter into tared volumetric flasks, weighed, and diluted with 2-propanol. Concentrations were determined by spectrophotometric measurements at $286 \mathrm{~nm}$.

\section{Source and Purity of Chemicals:}

(1) $99+\%$, Acros Organics, USA, was used as received.

(2) $99 \%$, anhydrous, Aldrich Chemical Company, Milwaukee, WI, USA, stored over molecular sieves and distilled shortly before use.

Estimated Error:

Temperature: $\pm 0.1 \mathrm{~K}$.

$x_{1}: \pm 2.0 \%$ (relative error).

\begin{tabular}{|c|c|}
\hline $\begin{array}{l}\text { Components: } \\
\text { (1) 3,4-Dimethoxybenzoic acid; } \\
\mathrm{C}_{9} \mathrm{H}_{10} \mathrm{O}_{4} \text {; [93-07-2] } \\
\text { (2) 2-Methyl-1-butanol; } \mathrm{C}_{5} \mathrm{H}_{12} \mathrm{O} \text {; } \\
\text { [137-32-6] }\end{array}$ & $\begin{array}{l}\text { Original Measurements: } \\
{ }^{138} \text { K. R. Bowen, T. W. Stephens, H. Lu, } \\
\text { K. Satish, D. Shan, W. E. Acree, Jr., } \\
\text { and M. H. Abraham, Eur. Chem. Bull } \\
\text { 2, } 577 \text { (2013). }\end{array}$ \\
\hline $\begin{array}{l}\text { Variables: } \\
T / \mathrm{K}=298.15\end{array}$ & $\begin{array}{l}\text { Prepared by: } \\
\text { W. E. Acree, Jr. }\end{array}$ \\
\hline
\end{tabular}

\section{Experimental Values}

\begin{tabular}{lc}
\hline \hline$x_{2}{ }^{\mathrm{a}}$ & $x_{1}{ }^{\mathrm{b}}$ \\
\hline 0.9946 & 0.00541 \\
\hline${ }^{\mathrm{a}} x_{2}:$ mole fraction of component 2 in the saturated solution. & \\
${ }^{\mathrm{b}} x_{1}:$ mole fraction solubility of the solute. &
\end{tabular}

\section{Auxiliary Information}

\section{Method/Apparatus/Procedure:}

Constant-temperature bath, calorimetric thermometer, and an ultraviolet/ visible spectrophotometer.

Excess solute and solvent were placed in amber glass bottles and allowed to equilibrate for several days at constant temperature. Attainment of equilibrium was verified by several repetitive measurements and by approaching equilibrium from supersaturation. Aliquots of saturated solutions were transferred through a coarse filter into tared volumetric flasks, weighed, and diluted with 2-propanol. Concentrations were determined by spectrophotometric measurements at $286 \mathrm{~nm}$. 
Source and Purity of Chemicals:

(1) $99+\%$, Acros Organics, USA, was used as received.

(2) $99 \%$, Aldrich Chemical Company, Milwaukee, WI, USA, stored over molecular sieves and distilled shortly before use.

Estimated Error:

Temperature: $\pm 0.1 \mathrm{~K}$.

$x_{1}: \pm 2.0 \%$ (relative error).

\begin{tabular}{ll}
\hline \hline Components: & Original Measurements: \\
(1) 3,4-Dimethoxybenzoic acid; & ${ }^{138}$ K. R. Bowen, T. W. Stephens, H. Lu, \\
$\mathrm{C}_{9} \mathrm{H}_{10} \mathrm{O}_{4} ;$ [ [93-07-2] & K. Satish, D. Shan, W. E. Acree, Jr., \\
(2) 1-Hexanol; $\mathrm{C}_{6} \mathrm{H}_{14} \mathrm{O} ;$ [111-27-3] & $\begin{array}{l}\text { and M. H. Abraham, Eur. Chem. Bull. } \\
\text { 2, 577 (2013). }\end{array}$ \\
\hline Variables: & Prepared by: \\
$T / \mathrm{K}=298.15$ & W. E. Acree, Jr. \\
\hline
\end{tabular}

Experimental Values

\begin{tabular}{lc}
\hline \hline$x_{2}{ }^{\mathrm{a}}$ & $x_{1}{ }^{\mathrm{b}}$ \\
\hline 0.9938 & 0.00615 \\
\hline
\end{tabular}

${ }^{a} x_{2}$ : mole fraction of component 2 in the saturated solution.

${ }^{\mathrm{b}} x_{1}$ : mole fraction solubility of the solute.

\section{Auxiliary Information}

\section{Method/Apparatus/Procedure:}

Constant-temperature bath, calorimetric thermometer, and an ultraviolet/ visible spectrophotometer.

Excess solute and solvent were placed in amber glass bottles and allowed to equilibrate for several days at constant temperature. Attainment of equilibrium was verified by several repetitive measurements and by approaching equilibrium from supersaturation. Aliquots of saturated solutions were transferred through a coarse filter into tared volumetric flasks, weighed, and diluted with 2-propanol. Concentrations were determined by

spectrophotometric measurements at $286 \mathrm{~nm}$.

\section{Source and Purity of Chemicals:}

(1) $99+\%$, Acros Organics, USA, was used as received.

(2) $99+\%$, Alfa Aesar, USA, stored over molecular sieves and distilled shortly before use.

\section{Estimated Error:}

Temperature: $\pm 0.1 \mathrm{~K}$.

$x_{1}: \pm 2.0 \%$ (relative error).
Experimental Values

\begin{tabular}{lc}
\hline \hline$x_{2}{ }^{\mathrm{a}}$ & $x_{1}{ }^{\mathrm{b}}$ \\
\hline 0.9942 & 0.00573 \\
\hline${ }^{\mathrm{a}} x_{2}:$ mole fraction of component 2 in the saturated solution. & \\
${ }^{\mathrm{b}} x_{1}:$ mole fraction solubility of the solute. &
\end{tabular}

\section{Auxiliary Information}

\section{Method/Apparatus/Procedure:}

Constant-temperature bath, calorimetric thermometer, and an ultraviolet/ visible spectrophotometer.

Excess solute and solvent were placed in amber glass bottles and allowed to equilibrate for several days at constant temperature. Attainment of equilibrium was verified by several repetitive measurements and by approaching equilibrium from supersaturation. Aliquots of saturated solutions were transferred through a coarse filter into tared volumetric flasks, weighed, and diluted with 2-propanol. Concentrations were determined by spectrophotometric measurements at $286 \mathrm{~nm}$.

\section{Source and Purity of Chemicals:}

(1) $99+\%$, Acros Organics, USA, was used as received.

(2) $99 \%$, Aldrich Chemical Company, Milwaukee, WI, USA, stored over molecular sieves and distilled shortly before use.

Estimated Error:

Temperature: $\pm 0.1 \mathrm{~K}$.

$x_{1}: \pm 2.0 \%$ (relative error).

\begin{tabular}{|c|c|}
\hline $\begin{array}{l}\text { Components: } \\
\text { (1) 3,4-Dimethoxybenzoic acid; } \\
\left.\mathrm{C}_{9} \mathrm{H}_{10} \mathrm{O}_{4} ; \text {; } 93-07-2\right] \\
\text { (2) 4-Methyl-2-pentanol; } \mathrm{C}_{6} \mathrm{H}_{14} \mathrm{O} \text {; } \\
\text { [108-11-2] }\end{array}$ & $\begin{array}{l}\text { Original Measurements: } \\
{ }^{138} \text { K. R. Bowen, T. W. Stephens, H. Lu, } \\
\text { K. Satish, D. Shan, W. E. Acree, Jr., } \\
\text { and M. H. Abraham, Eur. Chem. Bull } \\
\text { 2, } 577 \text { (2013). }\end{array}$ \\
\hline $\begin{array}{l}\text { Variables: } \\
T / \mathrm{K}=298.15\end{array}$ & $\begin{array}{l}\text { Prepared by: } \\
\text { W. E. Acree, Jr. }\end{array}$ \\
\hline
\end{tabular}

\section{Experimental Values}

\begin{tabular}{lc}
\hline \hline$x_{2}{ }^{\mathrm{a}}$ & $x_{1}{ }^{\mathrm{b}}$ \\
\hline 0.9935 & 0.00646 \\
\hline${ }^{\mathrm{a}} x_{2}:$ mole fraction of component 2 in the saturated solution. \\
${ }^{\mathrm{b}} x_{1}:$ mole fraction solubility of the solute.
\end{tabular}

\section{Auxiliary Information}

(1) 3,4-Dimethoxybenzoic acid;

(2) 2-Methyl-1-pentanol; $\mathrm{C}_{6} \mathrm{H}_{14} \mathrm{O}$; [105-30-6]

\section{Variables:}

$T / \mathrm{K}=298.15$ $\mathrm{C}_{9} \mathrm{H}_{10} \mathrm{O}_{4}$; [93-07-2]
Original Measurements:

${ }^{138}$ K. R. Bowen, T. W. Stephens, H. Lu, K. Satish, D. Shan, W. E. Acree, Jr., and M. H. Abraham, Eur. Chem. Bull. 2, 577 (2013).

\section{Prepared by:}

W. E. Acree, Jr. 
Source and Purity of Chemicals:

(1) $99+\%$, Acros Organics, USA, was used as received.

(2) $99+\%$, Acros Organics, stored over molecular sieves and distilled shortly before use.

Estimated Error:

Temperature: $\pm 0.1 \mathrm{~K}$.

$x_{1}: \pm 2.0 \%$ (relative error).

\begin{tabular}{ll}
\hline \hline Components: & Original Measurements: \\
(1) 3,4-Dimethoxybenzoic acid; & ${ }^{138}$ K. R. Bowen, T. W. Stephens, H. \\
$\mathrm{C}_{9} \mathrm{H}_{10} \mathrm{O}_{4} ;$ [ [93-07-2] & Lu, K. Satish, D. Shan, W. E. Acree, \\
(2) 1-Heptanol; $\mathrm{C}_{7} \mathrm{H}_{16} \mathrm{O}$; [111-70-6] & Jr., and M. H. Abraham, Eur. Chem. \\
& Bull. 2, 577 (2013). \\
\hline Variables: & Prepared by: \\
$T / \mathrm{K}=298.15$ & W. E. Acree, Jr. \\
\hline
\end{tabular}

Experimental Values

\begin{tabular}{lc}
\hline \hline$x_{2}{ }^{\mathrm{a}}$ & $x_{1}{ }^{\mathrm{b}}$ \\
\hline 0.9938 & 0.00617 \\
\hline
\end{tabular}

${ }^{a} x_{2}$ : mole fraction of component 2 in the saturated solution.

${ }^{\mathrm{b}} x_{1}$ : mole fraction solubility of the solute.

\section{Auxiliary Information}

\section{Method/Apparatus/Procedure:}

Constant-temperature bath, calorimetric thermometer, and an ultraviolet/ visible spectrophotometer.

Excess solute and solvent were placed in amber glass bottles and allowed to equilibrate for several days at constant temperature. Attainment of equilibrium was verified by several repetitive measurements and by approaching equilibrium from supersaturation. Aliquots of saturated solutions were transferred through a coarse filter into tared volumetric flasks, weighed, and diluted with 2-propanol. Concentrations were determined by

spectrophotometric measurements at $286 \mathrm{~nm}$.

Source and Purity of Chemicals:

(1) $99+\%$, Acros Organics, USA, was used as received.

(2) $99+\%$, Alfa Aesar, USA, stored over molecular sieves and distilled shortly before use.

\section{Estimated Error:}

Temperature: $\pm 0.1 \mathrm{~K}$

$x_{1}: \pm 2.0 \%$ (relative error).

Experimental Values

\begin{tabular}{lc}
\hline \hline$x_{2}{ }^{\mathrm{a}}$ & $x_{1}{ }^{\mathrm{b}}$ \\
\hline 0.9938 & 0.00616 \\
\hline${ }^{\mathrm{a}} x_{2}:$ mole fraction of component 2 in the saturated solution. & \\
${ }^{\mathrm{b}}{ }_{x_{1}: \text { mole fraction solubility of the solute. }}$ &
\end{tabular}

\section{Auxiliary Information}

\section{Method/Apparatus/Procedure:}

Constant-temperature bath, calorimetric thermometer, and an ultraviolet/ visible spectrophotometer.

Excess solute and solvent were placed in amber glass bottles and allowed to equilibrate for several days at constant temperature. Attainment of equilibrium was verified by several repetitive measurements and by approaching equilibrium from supersaturation. Aliquots of saturated solutions were transferred through a coarse filter into tared volumetric flasks, weighed, and diluted with 2-propanol. Concentrations were determined by spectrophotometric measurements at $286 \mathrm{~nm}$.

\section{Source and Purity of Chemicals:}

(1) $99+\%$, Acros Organics, USA, was used as received.

(2) $99+\%$, anhydrous, Aldrich Chemical Company, Milwaukee, WI, USA, stored over molecular sieves and distilled shortly before use.

Estimated Error:

Temperature: $\pm 0.1 \mathrm{~K}$.

$x_{1}: \pm 2.0 \%$ (relative error).

\begin{tabular}{ll}
\hline \hline Components: & Original Measurements: \\
(1) 3,4-Dimethoxybenzoic acid; & ${ }^{138}$ K. R. Bowen, T. W. Stephens, H. Lu, \\
$\mathrm{C}_{9} \mathrm{H}_{10} \mathrm{O}_{4} ;$; $\left.93-07-2\right]$ & K. Satish, D. Shan, W. E. Acree, Jr., \\
(2) 1-Decanol; $\mathrm{C}_{10} \mathrm{H}_{22} \mathrm{O} ;$ [112-30-1] & and M. H. Abraham, Eur. Chem. Bull. \\
& 2, 577 (2013). \\
\hline Variables: & Prepared by: \\
$T / \mathrm{K}=298.15$ & W. E. Acree, Jr. \\
\hline
\end{tabular}

\section{Experimental Values}

\begin{tabular}{lc}
\hline \hline$x_{2}{ }^{\mathrm{a}}$ & $x_{1}{ }^{\mathrm{b}}$ \\
\hline 0.9942 & 0.00577 \\
\hline${ }_{{ }^{\mathrm{a}} x_{2}: \text { mole fraction of component } 2 \text { in the saturated solution. }}$ & \\
${ }^{\mathrm{b}} x_{1}:$ mole fraction solubility of the solute. &
\end{tabular}

\section{Components:}

(1) 3,4-Dimethoxybenzoic acid; $\mathrm{C}_{9} \mathrm{H}_{10} \mathrm{O}_{4}$; [93-07-2]

(2) 1-Octanol; $\mathrm{C}_{8} \mathrm{H}_{18} \mathrm{O}$; [111-87-5] and M. H. Abraham, Eur. Chem. Bull. 2, 577 (2013).

\begin{tabular}{ll}
\hline Variables: & Prepared by: \\
$T / \mathrm{K}=298.15$ & W. E. Acree, Jr.
\end{tabular}

Original Measurements:

${ }^{138}$ K. R. Bowen, T. W. Stephens, H. Lu, K. Satish, D. Shan, W. E. Acree, Jr.,

\section{Auxiliary Information}

\section{Method/Apparatus/Procedure:}

Constant-temperature bath, calorimetric thermometer, and an ultraviolet/ visible spectrophotometer.

Excess solute and solvent were placed in amber glass bottles and allowed to equilibrate for several days at constant temperature. Attainment of equilibrium was verified by several repetitive measurements and by approaching equilibrium from supersaturation. Aliquots of saturated solutions were transferred through a coarse filter into tared volumetric flasks, weighed, and diluted with 2-propanol. Concentrations were determined by spectrophotometric measurements at $286 \mathrm{~nm}$. 
Source and Purity of Chemicals:

(1) $99+\%$, Acros Organics, USA, was used as received.

(2) $99+\%$, Alfa Aesar, USA, stored over molecular sieves and distilled shortly before use.

Estimated Error:

Temperature: $\pm 0.1 \mathrm{~K}$

$x_{1}: \pm 2.0 \%$ (relative error).

Experimental Values

\begin{tabular}{lc}
\hline \hline$x_{2}{ }^{\mathrm{a}}$ & $x_{1}{ }^{\mathrm{b}}$ \\
\hline 0.9766 & 0.02335 \\
\hline${ }^{\mathrm{a}} x_{2}:$ mole fraction of component 2 in the saturated solution. & \\
${ }^{\mathrm{b}} x_{1}:$ mole fraction solubility of the solute. &
\end{tabular}

Auxiliary Information

\subsection{3,4-Dimethoxybenzoic acid solubility in alkoxyalcohols}

\begin{tabular}{ll}
\hline \hline Components: & Original Measurements: \\
(1) 3,4-Dimethoxybenzoic acid; & ${ }^{138}$ K. R. Bowen, T. W. Stephens, H. Lu, \\
$\mathrm{C}_{9} \mathrm{H}_{10} \mathrm{O}_{4} ;$ [93-07-2] & K. Satish, D. Shan, W. E. Acree, Jr., \\
$\begin{array}{l}\text { (2) 2-Ethoxyethanol; } \mathrm{C}_{4} \mathrm{H}_{10} \mathrm{O}_{2} ; \\
\text { [110-80-5] }\end{array}$ & $\begin{array}{l}\text { and M. H. Abraham, Eur. Chem. Bull. } \\
\text { 2, 577 (2013). }\end{array}$ \\
\hline Variables: & Prepared by: \\
$T / \mathrm{K}=298.15$ & W. E. Acree, Jr. \\
\hline
\end{tabular}

Experimental Values

\begin{tabular}{lc}
\hline \hline$x_{2}{ }^{\mathrm{a}}$ & $x_{1}{ }^{\mathrm{b}}$ \\
\hline 0.9696 & 0.03037 \\
\hline
\end{tabular}

${ }^{\mathrm{a}} x_{2}$ : mole fraction of component 2 in the saturated solution.

${ }^{b} x_{1}$ : mole fraction solubility of the solute.

\section{Auxiliary Information}

\section{Method/Apparatus/Procedure:}

Constant-temperature bath, calorimetric thermometer, and an ultraviolet/ visible spectrophotometer.

Excess solute and solvent were placed in amber glass bottles and allowed to equilibrate for several days at constant temperature. Attainment of equilibrium was verified by several repetitive measurements and by approaching equilibrium from supersaturation. Aliquots of saturated solutions were transferred through a coarse filter into tared volumetric flasks, weighed, and diluted with 2-propanol. Concentrations were determined by spectrophotometric measurements at $286 \mathrm{~nm}$.

\section{Source and Purity of Chemicals:}

(1) $99+\%$, Acros Organics, USA, was used as received.

(2) 99\%, Aldrich Chemical Company, Milwaukee, WI, USA, stored over molecular sieves and distilled shortly before use.

\section{Estimated Error:}

Temperature: $\pm 0.1 \mathrm{~K}$.

$x_{1}: \pm 2.0 \%$ (relative error).

\section{Components:}

(1) 3,4-Dimethoxybenzoic acid;

$\mathrm{C}_{9} \mathrm{H}_{10} \mathrm{O}_{4}$; [93-07-2]

(2) 2-Isopropoxyethanol; $\mathrm{C}_{5} \mathrm{H}_{12} \mathrm{O}_{2}$; [109-59-1]

\section{Variables:}

$T / \mathrm{K}=298.15$
Original Measurements:

${ }^{138}$ K. R. Bowen, T. W. Stephens, H. Lu, K. Satish, D. Shan, W. E. Acree, Jr., and M. H. Abraham, Eur. Chem. Bull. 2, 577 (2013).

\section{Prepared by:}

W. E. Acree, Jr.

\section{Auxiliary Information}

\section{Method/Apparatus/Procedure:}

Constant-temperature bath, calorimetric thermometer, and an ultraviolet/ visible spectrophotometer.

Excess solute and solvent were placed in amber glass bottles and allowed to equilibrate for several days at constant temperature. Attainment of equilibrium was verified by several repetitive measurements and by approaching equilibrium from supersaturation. Aliquots of saturated solutions were transferred through a coarse filter into tared volumetric flasks, weighed, and diluted with 2-propanol. Concentrations were determined by spectrophotometric measurements at $286 \mathrm{~nm}$. 
Source and Purity of Chemicals:

(1) $99+\%$, Acros Organics, USA, was used as received.

(2) $99 \%$, Acros Organics, stored over molecular sieves and distilled shortly before use.

Estimated Error:

Temperature: $\pm 0.1 \mathrm{~K}$.

$x_{1}: \pm 2.0 \%$ (relative error).

\begin{tabular}{|c|c|}
\hline $\begin{array}{l}\text { Components: } \\
\text { (1) 3,4-Dimethoxybenzoic acid; } \\
\left.\mathrm{C}_{9} \mathrm{H}_{10} \mathrm{O}_{4} ; \text {; } 93-07-2\right] \\
\text { (2) 3-Methoxy-1-butanol; } \mathrm{C}_{5} \mathrm{H}_{12} \mathrm{O}_{2} \text {; } \\
\text { [2517-43-3] }\end{array}$ & $\begin{array}{l}\text { Original Measurements: } \\
{ }^{138} \text { K. R. Bowen, T. W. Stephens, H. Lu, } \\
\text { K. Satish, D. Shan, W. E. Acree, Jr., } \\
\text { and M. H. Abraham, Eur. Chem. Bull. } \\
\text { 2, } 577 \text { (2013). }\end{array}$ \\
\hline $\begin{array}{l}\text { Variables: } \\
T / \mathrm{K}=298.15\end{array}$ & $\begin{array}{l}\text { Prepared by: } \\
\text { W. E. Acree, Jr. }\end{array}$ \\
\hline
\end{tabular}

Experimental Values

\begin{tabular}{lc}
\hline \hline$x_{2}{ }^{\mathrm{a}}$ & $x_{1}{ }^{\mathrm{b}}$ \\
\hline 0.9745 & 0.02547
\end{tabular}

${ }^{\mathrm{a}} x_{2}$ : mole fraction of component 2 in the saturated solution.

${ }^{\mathrm{b}} x_{1}$ : mole fraction solubility of the solute.

\section{Auxiliary Information}

\section{Method/Apparatus/Procedure:}

Constant-temperature bath, calorimetric thermometer, and an ultraviolet/ visible spectrophotometer.

Excess solute and solvent were placed in amber glass bottles and allowed to equilibrate for several days at constant temperature. Attainment of equilibrium was verified by several repetitive measurements and by approaching equilibrium from supersaturation. Aliquots of saturated solutions were transferred through a coarse filter into tared volumetric flasks, weighed, and diluted with 2-propanol. Concentrations were determined by

spectrophotometric measurements at $286 \mathrm{~nm}$.

Source and Purity of Chemicals:

(1) $99+\%$, Acros Organics, USA, was used as received.

(2) $99 \%$, Aldrich Chemical Company, Milwaukee, WI, USA, stored over molecular sieves and distilled shortly before use.

Estimated Error:

Temperature: $\pm 0.1 \mathrm{~K}$.

$x_{1}: \pm 2.0 \%$ (relative error).

\subsection{3,4-Dimethoxybenzoic acid solubility data in alkanones}

\begin{tabular}{|c|c|}
\hline $\begin{array}{l}\text { Components: } \\
\text { (1) 3,4-Dimethoxybenzoic acid; } \\
\left.\mathrm{C}_{9} \mathrm{H}_{10} \mathrm{O}_{4} ; \text {; } 93-07-2\right] \\
\text { (2) Propanone; } \mathrm{C}_{3} \mathrm{H}_{6} \mathrm{O} ;[67-64-1]\end{array}$ & $\begin{array}{l}\text { Original Measurements: } \\
{ }^{138} \text { K. R. Bowen, T. W. Stephens, H. Lu, } \\
\text { K. Satish, D. Shan, W. E. Acree, Jr., } \\
\text { and M. H. Abraham, Eur. Chem. Bull. } \\
\text { 2, } 577 \text { (2013). }\end{array}$ \\
\hline $\begin{array}{l}\text { Variables: } \\
T / \mathrm{K}=298.15\end{array}$ & $\begin{array}{l}\text { Prepared by: } \\
\text { W. E. Acree, Jr. }\end{array}$ \\
\hline
\end{tabular}

Experimental Values

\begin{tabular}{lc}
\hline \hline$x_{2}{ }^{\mathrm{a}}$ & $x_{1}{ }^{\mathrm{b}}$ \\
\hline 0.9882 & 0.01177 \\
\hline${ }^{\mathrm{a}} x_{2}:$ mole fraction of component 2 in the saturated solution. & \\
${ }^{\mathrm{b}}{ }_{x_{1}}:$ mole fraction solubility of the solute. &
\end{tabular}

Auxiliary Information

Method/Apparatus/Procedure:

Constant-temperature bath, calorimetric thermometer, and an ultraviolet/ visible spectrophotometer.

Excess solute and solvent were placed in amber glass bottles and allowed to equilibrate for several days at constant temperature. Attainment of equilibrium was verified by several repetitive measurements and by approaching equilibrium from supersaturation. Aliquots of saturated solutions were transferred through a coarse filter into tared volumetric flasks, weighed, and diluted with 2-propanol. Concentrations were determined by spectrophotometric measurements at $286 \mathrm{~nm}$.

\section{Source and Purity of Chemicals:}

(1) $99+\%$, Acros Organics, USA, was used as received.

(2) $99.9 \%$, HPLC grade, Aldrich Chemical Company, Milwaukee, WI, USA, stored over molecular sieves and distilled shortly before use.

Estimated Error:

Temperature: $\pm 0.1 \mathrm{~K}$.

$x_{1}: \pm 2.0 \%$ (relative error).

\begin{tabular}{|c|c|}
\hline $\begin{array}{l}\text { Components: } \\
\text { (1) 3,4-Dimethoxybenzoic acid; } \\
\mathrm{C}_{9} \mathrm{H}_{10} \mathrm{O}_{4} ;[93-07-2] \\
\text { (2) 2-Butanone; } \mathrm{C}_{4} \mathrm{H}_{8} \mathrm{O} ;[78-93-3]\end{array}$ & $\begin{array}{l}\text { Original Measurements: } \\
{ }^{138} \text { K. R. Bowen, T. W. Stephens, H. Lu, } \\
\text { K. Satish, D. Shan, W. E. Acree, Jr., } \\
\text { and M. H. Abraham, Eur. Chem. Bull. } \\
\text { 2, } 577 \text { (2013). }\end{array}$ \\
\hline $\begin{array}{l}\text { Variables: } \\
T / \mathrm{K}=298.15\end{array}$ & $\begin{array}{l}\text { Prepared by: } \\
\text { W. E. Acree, Jr. }\end{array}$ \\
\hline
\end{tabular}

Experimental Values

\begin{tabular}{lc}
\hline \hline$x_{2}{ }^{\mathrm{a}}$ & $x_{1}{ }^{\mathrm{b}}$ \\
\hline 0.9879 & 0.01206
\end{tabular}

${ }^{\mathrm{a}} x_{2}$ : mole fraction of component 2 in the saturated solution.

${ }^{\mathrm{b}} x_{1}$ : mole fraction solubility of the solute.

\section{Auxiliary Information}

\section{Method/Apparatus/Procedure:}

Constant-temperature bath, calorimetric thermometer, and an ultraviolet/ visible spectrophotometer.

Excess solute and solvent were placed in amber glass bottles and allowed to equilibrate for several days at constant temperature. Attainment of equilibrium was verified by several repetitive measurements and by approaching equilibrium from supersaturation. Aliquots of saturated solutions were transferred through a coarse filter into tared volumetric flasks, weighed, and diluted with 2-propanol. Concentrations were determined by

spectrophotometric measurements at $286 \mathrm{~nm}$. 
Source and Purity of Chemicals:

(1) $99+\%$, Acros Organics, USA, was used as received.

(2) $99.7 \%$, HPLC grade, Aldrich Chemical Company, Milwaukee, WI, USA, stored over molecular sieves and distilled shortly before use.

Estimated Error:

Temperature: $\pm 0.1 \mathrm{~K}$.

$x_{1}: \pm 2.0 \%$ (relative error).

\begin{tabular}{|c|c|}
\hline $\begin{array}{l}\text { Components: } \\
\text { (1) 3,4-Dimethoxybenzoic acid; } \\
\mathrm{C}_{9} \mathrm{H}_{10} \mathrm{O}_{4} \text {; [93-07-2] } \\
\text { (2) 2-Butanone; } \mathrm{C}_{4} \mathrm{H}_{8} \mathrm{O} ;[78-93-3]\end{array}$ & $\begin{array}{l}\text { Original Measurements: } \\
{ }^{139} \text { Q. Li, F. Lu, Y. Tian, S. Feng, Y. } \\
\text { Shen, and B. Wang, J. Chem. Eng. Data } \\
\text { 58, } 1020 \text { (2013). }\end{array}$ \\
\hline $\begin{array}{l}\text { Variables: } \\
\text { Temperature }\end{array}$ & $\begin{array}{l}\text { Prepared by: } \\
\text { W. E. Acree, Jr. }\end{array}$ \\
\hline
\end{tabular}

Experimental Values

\begin{tabular}{lcc}
\hline \hline$T / \mathrm{K}$ & $x_{2}{ }^{\mathrm{a}}$ & $x_{1}{ }^{\mathrm{b}}$ \\
\hline 278.95 & 0.9932 & 0.0068 \\
283.15 & 0.9923 & 0.0077 \\
287.95 & 0.9911 & 0.0089 \\
293.32 & 0.9896 & 0.0104 \\
298.13 & 0.9880 & 0.0120 \\
303.15 & 0.9860 & 0.0140 \\
308.37 & 0.9838 & 0.0162 \\
313.05 & 0.9816 & 0.0184 \\
318.15 & 0.9788 & 0.0212 \\
323.49 & 0.9754 & 0.0246 \\
\hline
\end{tabular}

${ }^{\mathrm{a}} x_{2}$ : mole fraction of component 2 in the saturated solution.

${ }^{\mathrm{b}} x_{1}$ : mole fraction solubility of the solute.

\section{Auxiliary Information}

\section{Method/Apparatus/Procedure:}

Water-jacketed equilibrium glass vessel, thermostated circulating water bath, magnetic stirrer, analytical balance, laser monitoring system.

Experimental solubilities were determined by a synthetic method. Preweighed amounts of solute and solvent and were placed in a water-jacketed equilibrium vessel, which was connected to a circulating constant-temperature water bath. The solution was stirred and small amounts of solute were incrementally added until no further solid dissolved. The dissolution of the solid was determined using laser monitoring. The total amount of solute dissolved was recorded. Experimental measurement was repeated three times.

Source and Purity of Chemicals:

(1) $99+\%$, Alfa Aesar China Co., Ltd., Tianjin, China, no purification details were provided.

(2) $99.7 \%$, Beijing Chemical Works, Beijing, China, no purification details were provided.

\section{Estimated Error:}

Temperature: $\pm 0.05 \mathrm{~K}$.

$x_{1}: \pm 4 \%$ (relative error).

\subsection{3,4-Dimethoxybenzoic acid solubility data in miscellaneous organic solvents}

\begin{tabular}{ll}
\hline \hline Components: & Original Measurements: \\
$\begin{array}{l}\text { (1) 3,4-Dimethoxybenzoic acid; } \\
\mathrm{C}_{9} \mathrm{H}_{10} \mathrm{O}_{4} ; \text {; [93-07-2] }\end{array}$ & ${ }^{138}$ K. R. Bowen, T. W. Stephens, H. Lu, \\
$\begin{array}{l}\text { (2) Propylene carbonate; } \mathrm{C}_{4} \mathrm{H}_{6} \mathrm{O}_{3} ; \\
\text { [108-32-7] }\end{array}$ & $\begin{array}{l}\text { K. Satish, D. Shan, W. E. Acree, Jr., } \\
\text { 2, } 577 \text { (2013). Abraham, Eur. Chem. Bull. }\end{array}$ \\
\hline Variables: & Prepared by: \\
$T / \mathrm{K}=298.15$ & W. E. Acree, Jr. \\
\hline
\end{tabular}

Experimental Values

\begin{tabular}{lc}
\hline \hline$x_{2}{ }^{\mathrm{a}}$ & $x_{1}{ }^{\mathrm{b}}$ \\
\hline 0.9920 & 0.00799
\end{tabular}

${ }^{a} x_{2}$ : mole fraction of component 2 in the saturated solution.

${ }^{\mathrm{b}} x_{1}$ : mole fraction solubility of the solute.

\section{Auxiliary Information}

\begin{abstract}
Method/Apparatus/Procedure:
Constant-temperature bath, calorimetric thermometer, and an ultraviolet/ visible spectrophotometer.

Excess solute and solvent were placed in amber glass bottles and allowed to equilibrate for several days at constant temperature. Attainment of equilibrium was verified by several repetitive measurements and by approaching equilibrium from supersaturation. Aliquots of saturated solutions were transferred through a coarse filter into tared volumetric flasks, weighed, and diluted with 2-propanol. Concentrations were determined by spectrophotometric measurements at $286 \mathrm{~nm}$.

\section{Source and Purity of Chemicals:}

(1) $99+\%$, Acros Organics, USA, was used as received.

(2) $99+\%$, anhydrous, Aldrich Chemical Company, Milwaukee, WI, USA, stored over molecular sieves and distilled shortly before use.
\end{abstract}

\section{Estimated Error:}

Temperature: $\pm 0.1 \mathrm{~K}$.

$x_{1}: \pm 2.0 \%$ (relative error).

\subsection{3,4-Dimethoxybenzoic acid solubility data in binary organic solvent mixtures}

\begin{tabular}{|c|c|}
\hline $\begin{array}{l}\text { Components: } \\
\text { (1) 3,4-Dimethoxybenzoic acid; } \\
\mathrm{C}_{9} \mathrm{H}_{10} \mathrm{O}_{4} ;[93-07-2] \\
\text { (2) Ethanol; } \mathrm{C}_{2} \mathrm{H}_{6} \mathrm{O} ;[64-17-5] \\
\text { (3) 1-Butanol; } \mathrm{C}_{4} \mathrm{H}_{10} \mathrm{O} ;[71-36-3]\end{array}$ & $\begin{array}{l}\text { Original Measurements: } \\
{ }^{139} \text { Q. Li, F. Lu, Y. Tian, S. Feng, Y. } \\
\text { Shen, and B. Wang, J. Chem. Eng. Data } \\
\text { 58, } 1020 \text { (2013). }\end{array}$ \\
\hline $\begin{array}{l}\text { Variables: } \\
\text { Temperature; Solvent Composition }\end{array}$ & W. E. Acree, Jr. \\
\hline
\end{tabular}


Experimental Values

\begin{tabular}{|c|c|c|}
\hline$T / \mathrm{K}$ & $m_{2}^{(\mathrm{s}) \mathrm{a}}$ & $x_{1}{ }^{\mathrm{b}}$ \\
\hline 277.90 & 0.0000 & 0.0024 \\
\hline 283.25 & 0.0000 & 0.0030 \\
\hline 288.15 & 0.0000 & 0.0037 \\
\hline 293.04 & 0.0000 & 0.0046 \\
\hline 298.15 & 0.0000 & 0.0057 \\
\hline 302.94 & 0.0000 & 0.0070 \\
\hline 308.10 & 0.0000 & 0.0088 \\
\hline 313.15 & 0.0000 & 0.0109 \\
\hline 318.01 & 0.0000 & 0.0135 \\
\hline 323.33 & 0.0000 & 0.0170 \\
\hline 278.75 & 0.1000 & 0.0028 \\
\hline 283.26 & 0.1000 & 0.0036 \\
\hline 288.15 & 0.1000 & 0.0045 \\
\hline 293.18 & 0.1000 & 0.0054 \\
\hline 298.41 & 0.1000 & 0.0067 \\
\hline 303.15 & 0.1000 & 0.0083 \\
\hline 308.01 & 0.1000 & 0.0102 \\
\hline 313.15 & 0.1000 & 0.0125 \\
\hline 317.94 & 0.1000 & 0.0152 \\
\hline 323.12 & 0.1000 & 0.0189 \\
\hline 278.65 & 0.2000 & 0.0030 \\
\hline 283.15 & 0.2000 & 0.0037 \\
\hline 288.21 & 0.2000 & 0.0045 \\
\hline 293.18 & 0.2000 & 0.0055 \\
\hline 298.41 & 0.2000 & 0.0069 \\
\hline 303.35 & 0.2000 & 0.0085 \\
\hline 308.01 & 0.2000 & 0.0103 \\
\hline 313.15 & 0.2000 & 0.0126 \\
\hline 317.94 & 0.2000 & 0.0152 \\
\hline 323.10 & 0.2000 & 0.0186 \\
\hline 277.94 & 0.3000 & 0.0029 \\
\hline 283.05 & 0.3000 & 0.0036 \\
\hline 288.38 & 0.3000 & 0.0045 \\
\hline 293.45 & 0.3000 & 0.0055 \\
\hline 298.25 & 0.3000 & 0.0067 \\
\hline 303.55 & 0.3000 & 0.0083 \\
\hline 308.13 & 0.3000 & 0.0101 \\
\hline 313.15 & 0.3000 & 0.0123 \\
\hline 318.29 & 0.3000 & 0.0150 \\
\hline 323.06 & 0.3000 & 0.0180 \\
\hline 279.20 & 0.4000 & 0.0029 \\
\hline 283.45 & 0.4000 & 0.0034 \\
\hline 288.03 & 0.4000 & 0.0043 \\
\hline 292.91 & 0.4000 & 0.0052 \\
\hline 298.02 & 0.4000 & 0.0064 \\
\hline 303.66 & 0.4000 & 0.0080 \\
\hline 307.94 & 0.4000 & 0.0095 \\
\hline 313.10 & 0.4000 & 0.0118 \\
\hline 318.15 & 0.4000 & 0.0143 \\
\hline 323.05 & 0.4000 & 0.0173 \\
\hline 279.20 & 0.5000 & 0.0034 \\
\hline 283.45 & 0.5000 & 0.0040 \\
\hline 288.03 & 0.5000 & 0.0048 \\
\hline 292.91 & 0.5000 & 0.0058 \\
\hline 298.02 & 0.5000 & 0.0071 \\
\hline 303.66 & 0.5000 & 0.0090 \\
\hline 307.94 & 0.5000 & 0.0106 \\
\hline 313.10 & 0.5000 & 0.0129 \\
\hline 318.15 & 0.5000 & 0.0156 \\
\hline 323.05 & 0.5000 & 0.0188 \\
\hline 278.04 & 0.6000 & 0.0032 \\
\hline 283.16 & 0.6000 & 0.0040 \\
\hline 288.15 & 0.6000 & 0.0049 \\
\hline 293.14 & 0.6000 & 0.0060 \\
\hline 298.08 & 0.6000 & 0.0073 \\
\hline
\end{tabular}

\begin{tabular}{|c|c|c|}
\hline$T / \mathrm{K}$ & $m_{2}{ }^{(\mathrm{s}) \mathrm{a}}$ & $x_{1}{ }^{\mathrm{b}}$ \\
\hline 303.13 & 0.6000 & 0.0089 \\
\hline 307.94 & 0.6000 & 0.0108 \\
\hline 313.05 & 0.6000 & 0.0130 \\
\hline 318.19 & 0.6000 & 0.0160 \\
\hline 323.33 & 0.6000 & 0.0195 \\
\hline 278.04 & 0.7000 & 0.0034 \\
\hline 283.16 & 0.7000 & 0.0040 \\
\hline 288.15 & 0.7000 & 0.0050 \\
\hline 293.14 & 0.7000 & 0.0061 \\
\hline 298.08 & 0.7000 & 0.0074 \\
\hline 303.13 & 0.7000 & 0.0090 \\
\hline 307.94 & 0.7000 & 0.0109 \\
\hline 313.05 & 0.7000 & 0.0132 \\
\hline 318.19 & 0.7000 & 0.0161 \\
\hline 323.33 & 0.7000 & 0.0194 \\
\hline 278.50 & 0.8000 & 0.0034 \\
\hline 283.15 & 0.8000 & 0.0041 \\
\hline 288.15 & 0.8000 & 0.0050 \\
\hline 293.16 & 0.8000 & 0.0061 \\
\hline 298.29 & 0.8000 & 0.0077 \\
\hline 303.22 & 0.8000 & 0.0093 \\
\hline 308.31 & 0.8000 & 0.0115 \\
\hline 312.99 & 0.8000 & 0.0137 \\
\hline 318.25 & 0.8000 & 0.0168 \\
\hline 323.42 & 0.8000 & 0.0207 \\
\hline 278.37 & 0.9000 & 0.0035 \\
\hline 283.05 & 0.9000 & 0.0043 \\
\hline 288.39 & 0.9000 & 0.0051 \\
\hline 293.16 & 0.9000 & 0.0062 \\
\hline 298.29 & 0.9000 & 0.0077 \\
\hline 303.22 & 0.9000 & 0.0091 \\
\hline 308.31 & 0.9000 & 0.0112 \\
\hline 312.99 & 0.9000 & 0.0133 \\
\hline 318.25 & 0.9000 & 0.0162 \\
\hline 323.42 & 0.9000 & 0.0196 \\
\hline 278.15 & 1.0000 & 0.0035 \\
\hline 282.93 & 1.0000 & 0.0042 \\
\hline 288.21 & 1.0000 & 0.0051 \\
\hline 293.08 & 1.0000 & 0.0062 \\
\hline 298.00 & 1.0000 & 0.0074 \\
\hline 303.33 & 1.0000 & 0.0093 \\
\hline 307.90 & 1.0000 & 0.0110 \\
\hline 313.30 & 1.0000 & 0.0136 \\
\hline 317.15 & 1.0000 & 0.0161 \\
\hline 323.30 & 1.0000 & 0.0202 \\
\hline
\end{tabular}

${ }^{\mathrm{a}} m_{2}{ }^{(\mathrm{s})}$ : mass fraction of component 2 in the initial binary solvent mixture.

${ }^{\mathrm{b}} x_{1}$ : mole fraction solubility of the solute.

\section{Auxiliary Information}

Method/Apparatus/Procedure:

Water-jacketed equilibrium glass vessel, thermostated circulating water bath, magnetic stirrer, analytical balance, laser monitoring system.

Experimental solubilities were determined by a synthetic method. Preweighed amounts of solute and solvent and were placed in a water-jacketed equilibrium vessel, which was connected to a circulating constant-temperature water bath. The solution was stirred and small amounts of solute were incrementally added until no further solid dissolved. The dissolution of the solid was determined using laser monitoring. The total amount of solute dissolved was recorded. Experimental measurement was repeated three times. 
Source and Purity of Chemicals:

(1) $99+\%$, Alfa Aesar China Co., Ltd., Tianjin, China, no purification details were provided.

(2) $99.7+\%$, Beijing Chemical Works, Beijing, China, no purification details were provided.

(3) $99.5+\%$, Beijing Chemical Works, Beijing, China, no purification details were provided.

Estimated Error:

Temperature: $\pm 0.05 \mathrm{~K}$.

$m_{2}{ }^{(\mathrm{s})}: 0.0001$ $x_{1}: \pm 4 \%$ (relative error).

\subsection{3,5-Dimethoxybenzoic acid solubility data} in alcohols

\begin{tabular}{|c|c|}
\hline $\begin{array}{l}\text { Components: } \\
\text { (1) 3,5-Dimethoxybenzoic acid; } \\
\mathrm{C}_{9} \mathrm{H}_{10} \mathrm{O}_{4} ;[1132-21-4] \\
\text { (2) Ethanol; } \mathrm{C}_{2} \mathrm{H}_{6} \mathrm{O} ;[64-17-5]\end{array}$ & $\begin{array}{l}\text { Original Measurements: } \\
{ }^{97} \text { H. Wang and W. Zhang, J. Chem. } \\
\text { Eng. Data 54, } 1942 \text { (2009). }\end{array}$ \\
\hline $\begin{array}{l}\text { Variables: } \\
\text { Temperature }\end{array}$ & $\begin{array}{l}\text { Prepared by: } \\
\text { W. E. Acree, Jr. }\end{array}$ \\
\hline
\end{tabular}

Experimental Values

\section{Solubility of 3,5-Dimethoxybenzoic Acid in Organic Solvents}

\subsection{Critical evaluation of experimental solubility data}

There is only a single published study regarding the solubility behavior of 3,5-dimethoxybenzoic acid in organic solvents. Wang and Zhang ${ }^{97}$ measured the mole fraction solubilities in ethanol as a function of temperature using a static and dynamic method. The internal consistency of the two datasets was assessed by curve-fitting the measured mole fraction solubility data to Eq. (8). The values of the equation coefficients $(A, B$, and $C$ ) are given in Table 31, along with the MRD calculated according to Eq. (24). Examination of the numerical entries in the last column of Table 31 reveals that the static experimental method yielded slightly more consistent experimental values as evidenced by the smaller MRD. In the case of the 3,5-dimethoxybenzoic acid solubility data, there is a slight difference between the two experimental methods. The relative difference can be greater than the reproducibility at times. For example, the static method gave a mole fraction solubility of $x_{1}=0.007464$ for 3,5-dimethoxybenzoic acid in ethanol at $308.15 \mathrm{~K}$, versus a value of $x_{1}=0.007934$ determined with the dynamic method. The calculated relative difference between the two reported values is about $6 \%$. The authors estimated the reproducibility of the measurements at $0.5 \%$.

The experimental solubility data for 3,5-dimethoxybenzoic acid in ethanol are in Sec. 33.2.

TABLE 31. Parameters of the Modified Apelblat equation for describing the solubility of 3,5-dimethoxybenzoic acid in ethanol ${ }^{\text {a }}$

\begin{tabular}{lccrc}
\hline \hline Solvent & $A$ & \multicolumn{1}{c}{$B$} & \multicolumn{1}{c}{$C$} & MRD (\%) \\
\hline Ethanol (dynamic method) & -0.1742 & -3316 & 1.865 & 2.160 \\
Ethanol (static method) & 40.45 & -5379 & -4.060 & 0.947
\end{tabular}

${ }^{\mathrm{a}}$ Values of the coefficients and the mean relative deviations were taken from Wang and Zhang. ${ }^{97}$
Experimental Values

\begin{tabular}{lcc}
\hline \hline$T / \mathrm{K}$ & $x_{2}{ }^{\mathrm{a}}$ & \multicolumn{1}{c}{$x_{1}{ }^{\mathrm{b}, \mathrm{c}}$} \\
\hline 298.2 & 0.9949 & 0.005137 \\
303.2 & 0.9939 & 0.006056 \\
308.2 & 0.9921 & 0.007934 \\
313.2 & 0.9902 & 0.009776 \\
318.2 & 0.9886 & 0.01143 \\
\hline
\end{tabular}

${ }^{\mathrm{a}} x_{2}$ : mole fraction of component 2 in the saturated solution.

${ }^{b} x_{1}$ : mole fraction solubility of the solute.

${ }^{\mathrm{c}}$ Solubility determined by the dynamic method.

\section{Auxiliary Information}

\section{Method/Apparatus/Procedure:}

Thermostated water-jacketed glass vessel, thermometer, analytical balance, laser monitoring system.

Solubilities were determined using both a static and dynamic method. In the static method, excess solute and solvent were placed in a water-jacketed vessel allowed to equilibrate with stirring for three days at constant temperature. Samples of the saturated liquid phase were decanted into Petri dishes of known mass and weighed. The samples were then evaporated to dryness and the solubility determined from the mass of the solid residue and the mass of the sample analyzed. In the dynamic method, an excess of carboxylic acid was added to a known mass of ethanol. The resulting suspension was stirred in a water-jacketed vessel for $1 \mathrm{~h}$ at constant temperature. A known mass of ethanol was added to the vessel through a buret until the solute completely dissolved. The dissolution was monitored with a laser monitoring system. When the last portion of solid solute disappeared, the penetrated light intensity reached its maximum value. The mass of the added solvent was recorded. The solubility of the carboxylic acid was calculated from the known masses of solute and ethanol.

Source and Purity of Chemicals:

(1) $98 \%$, Sigma-Aldrich Chemical Company, Milwaukee, WI, USA, no purification details provided.

(2) $99.55 \%$, Tianjin Kewei Chemical Reagent, China, no information given regarding any further purification. 
Estimated Error:

Temperature: $\pm 0.1 \mathrm{~K}$.

$x_{1}$ : Authors report that the uncertainty in the solubility values is estimated to be $\pm 0.5 \%$. The differences between the experimental values based on the static and dynamic solubility methods are considerably larger, and more on the order of $\pm 3 \%$ to $8 \%$ (relative error).

\section{Solubility of 3-(Dimethylamino)benzoic Acid in Organic Solvents}

\subsection{Critical evaluation of experimental solubility data}

There has been only a single study reporting the solubility of 3-(dimethylamino)benzoic acid in organic solvents. Hancock et al. ${ }^{54}$ determined the solubility of 3-(dimethylamino)benzoic acid in cyclohexane and benzene at $303 \mathrm{~K}$ based on a gravimetric method. It is not possible to perform a critical evaluation of the experimental data as measurements were performed at only a single temperature, and there are no independent experimental solubility data for 3-(dimethylamino)benzoic acid in either cyclohexane or benzene.

The experimental solubility data for 3-(dimethylamino) benzoic acid in organic solvents are in Secs. 36.2 and 36.3.

\subsection{3-(Dimethylamino)benzoic acid solubility data in saturated hydrocarbons (including cycloalkanes)}

\begin{tabular}{|c|c|}
\hline $\begin{array}{l}\text { Components: } \\
\text { (1) 3-(Dimethylamino)benzoic acid; } \\
\mathrm{C}_{9} \mathrm{H}_{11} \mathrm{NO}_{2} \text {; [99-64-9] } \\
\text { (2) Cyclohexane; } \mathrm{C}_{6} \mathrm{H}_{12} ;[110-82-7]\end{array}$ & $\begin{array}{l}\text { Original Measurements: } \\
{ }^{54} \text { C. K. Hancock, J. N. Pawloski, and J. } \\
\text { P. Idoux, J. Org. Chem. 31, } 3801 \\
\text { (1966). }\end{array}$ \\
\hline $\begin{array}{l}\text { Variables: } \\
T / \mathrm{K}=303.15\end{array}$ & $\begin{array}{l}\text { Prepared by: } \\
\text { W. E. Acree, Jr. }\end{array}$ \\
\hline
\end{tabular}

Experimental Values

\begin{tabular}{lc}
\hline \hline$x_{2}{ }^{\mathrm{a}}$ & $x_{1}{ }^{\mathrm{b}}$ \\
\hline 0.9996 & 0.000406 \\
\hline${ }^{\mathrm{a}} x_{2}:$ mole fraction of component 2 in the saturated solution. \\
${ }^{\mathrm{b}} x_{1}:$ mole fraction solubility of the solute.
\end{tabular}

Auxiliary Information

Method/Apparatus/Procedure:

Constant-temperature bath, Soxhlet thimble, conical flask, and analytical balance.

Excess solute and solvent were placed in a conical flask and allowed to equilibrate for several days at constant temperature. Attainment of equilibrium was verified by several repetitive measurements and by approaching equilibrium from supersaturation. Aliquots of saturated solutions were transferred using a Soxhlet thimble equipped with a one-hole cork stopper and an inverted U-shaped delivery tube to a second ground-glass stoppered flask suspended in the $303 \mathrm{~K}$ water bath. Successive portions of the contents were evaporated at room temperature in a tared aluminum foil weighing dish under a bell jar through which a slow stream of dry air was passed. The second flask plus unused saturated solution and the aluminum foil dish plus residue were weighed. The saturation solubility of the solute was calculated from the recorded mass data and molar masses of the solute and solvent.
Source and Purity of Chemicals:

(1) Purity not given, Chemical source not specified, was recrystallized several times from aqueous-ethanol mixture, and then dried under vacuum over phosphorous pentoxide.

(2) Reagent grade, Chemical source not specified, refluxed over phosphorous pentoxide for $24 \mathrm{~h}$, and then distilled through a $3 \times 80 \mathrm{~cm}$ column filled with $0.32-\mathrm{cm}$ glass helices.

Estimated Error:

Temperature: $\pm 0.02 \mathrm{~K}$

$x_{1}: \pm 3 \%$ (relative error).

\subsection{3-(Dimethylamino)benzoic acid solubility data} in aromatic hydrocarbons

\begin{tabular}{|c|c|}
\hline $\begin{array}{l}\text { Components: } \\
\text { (1) 3-(Dimethylamino)benzoic acid; } \\
\mathrm{C}_{9} \mathrm{H}_{11} \mathrm{NO}_{2} ;[99-64-9] \\
\text { (2) Benzene; } \mathrm{C}_{6} \mathrm{H}_{6} ;[71-43-2]\end{array}$ & $\begin{array}{l}\text { Original Measurements: } \\
{ }^{54} \text { C. K. Hancock, J. N. Pawloski, and J. } \\
\text { P. Idoux, J. Org. Chem. 31, } 3801 \\
\text { (1966). }\end{array}$ \\
\hline $\begin{array}{l}\text { Variables: } \\
T / \mathrm{K}=303.15\end{array}$ & $\begin{array}{l}\text { Prepared by: } \\
\text { W. E. Acree, Jr. }\end{array}$ \\
\hline
\end{tabular}

Experimental Values

\begin{tabular}{lc}
\hline \hline$x_{2}{ }^{\mathrm{a}}$ & $x_{1}{ }^{\mathrm{b}}$ \\
\hline 0.9894 & 0.0106
\end{tabular}

${ }^{a} x_{2}$ : mole fraction of component 2 in the saturated solution.

${ }^{\mathrm{b}} x_{1}$ : mole fraction solubility of the solute.

\section{Auxiliary Information}

\section{Method/Apparatus/Procedure:}

Constant-temperature bath, Soxhlet thimble, conical flask, and analytical balance.

Excess solute and solvent were placed in a conical flask and allowed to equilibrate for several days at constant temperature. Attainment of equilibrium was verified by several repetitive measurements and by approaching equilibrium from supersaturation. Aliquots of saturated solutions were transferred using a Soxhlet thimble equipped with a one-hole cork stopper and an inverted $\mathrm{U}$-shaped delivery tube to a second ground-glass stoppered flask suspended in the $303 \mathrm{~K}$ water bath. Successive portions of the contents were evaporated at room temperature in a tared aluminum foil weighing dish under a bell jar through which a slow stream of dry air was passed. The second flask plus unused saturated solution and the aluminum foil dish plus residue were weighed. The saturation solubility of the solute was calculated from the recorded mass data and molar masses of the solute and solvent.

Source and Purity of Chemicals:

(1) Purity not given, Chemical source not specified, was recrystallized several times from aqueous-ethanol mixture, and then dried under vacuum over phosphorous pentoxide.

(2) Reagent grade, Chemical source not specified, refluxed over phosphorous pentoxide for $24 \mathrm{~h}$, and then distilled through a $3 \times 80 \mathrm{~cm}$ column filled with $0.32-\mathrm{cm}$ glass helices.

\section{Estimated Error:}

Temperature: $\pm 0.02 \mathrm{~K}$

$x_{1}: \pm 3 \%$ (relative error). 


\section{Solubility of 4-(Dimethylamino)benzoic Acid in Organic Solvents}

\subsection{Critical evaluation of experimental solubility data}

There have been two studies ${ }^{54,83}$ reporting the solubility of 4-(dimethylamino)benzoic acid in organic solvents. Hancock et al. ${ }^{54}$ determined the solubility of 4-(dimethylamino)benzoic acid in cyclohexane and benzene at $303 \mathrm{~K}$ based on a gravimetric method. Chantooni and Kolthoff ${ }^{83}$ measured the solubility of 4-(dimethylamino)benzoic acid in methanol, ethanenitrile, dimethyl sulfoxide, and $N, N$-dimethylformamide at $298 \mathrm{~K}$. It is not possible to perform a critical evaluation of the experimental data as each research group performed measurements at only a single temperature, and there are no independent experimental solubility data for 4-(dimethylamino)benzoic acid in these six organic solvents.

The experimental solubility data for 4-(dimethylamino)benzoic acid in organic solvents are in Secs. 37.2-37.5.

\subsection{4-(Dimethylamino)benzoic acid solubility data in saturated hydrocarbons (including cycloalkanes)}

\begin{tabular}{ll}
\hline \hline Components: & Original Measurements: \\
(1) 4-(Dimethylamino)benzoic acid; & ${ }^{4} \mathrm{C}$. K. Hancock, J. N. Pawloski, and J. \\
$\mathrm{C}_{9} \mathrm{H}_{11} \mathrm{NO}_{2} ;[619-84-1]$ & P. Idoux, J. Org. Chem. 31, 3801 \\
$(2)$ Cyclohexane; $\mathrm{C}_{6} \mathrm{H}_{12} ;[110-82-7]$ & $(1966)$. \\
\hline Variables: & Prepared by: \\
$T / \mathrm{K}=303.15$ & W. E. Acree, Jr. \\
\hline
\end{tabular}

Experimental Values

\begin{tabular}{lc}
\hline \hline$x_{2}{ }^{\mathrm{a}}$ & $x_{1}{ }^{\mathrm{b}}$ \\
\hline 0.9996 & 0.000406
\end{tabular}

${ }^{a} x_{2}$ : mole fraction of component 2 in the saturated solution.

${ }^{\mathrm{b}} x_{1}$ : mole fraction solubility of the solute.

\section{Auxiliary Information}

\footnotetext{
Method/Apparatus/Procedure:

Constant-temperature bath, Soxhlet thimble, conical flask, and analytical balance.

Excess solute and solvent were placed in a conical flask and allowed to equilibrate for several days at constant temperature. Attainment of equilibrium was verified by several repetitive measurements and by approaching equilibrium from supersaturation. Aliquots of saturated solutions were transferred using a Soxhlet thimble equipped with a one-hole cork stopper and an inverted U-shaped delivery tube to a second ground-glass stoppered flask suspended in the $303 \mathrm{~K}$ water bath. Successive portions of the contents were evaporated at room temperature in a tared aluminum foil weighing dish under a bell jar through which a slow stream of dry air was passed. The second flask plus unused saturated solution and the aluminum foil dish plus residue were weighed. The saturation solubility of the solute was calculated from the recorded mass data and molar masses of the solute and solvent.
}

Source and Purity of Chemicals:

(1) Purity not given, Chemical source not specified, was recrystallized several times from aqueous-ethanol mixture, and then dried under vacuum over phosphorous pentoxide.

(2) Reagent grade, Chemical source not specified, refluxed over phosphorous pentoxide for $24 \mathrm{~h}$, and then distilled through a $3 \times 80 \mathrm{~cm}$ column filled with $0.32-\mathrm{cm}$ glass helices.

Estimated Error:

Temperature: $\pm 0.02 \mathrm{~K}$.

$x_{1}: \pm 3 \%$ (relative error).

\subsection{4-(Dimethylamino)benzoic acid solubility data in aromatic hydrocarbons}

\begin{tabular}{ll}
\hline \hline Components: & Original Measurements: \\
$\begin{array}{l}\text { (1) 4-(Dimethylamino)benzoic acid; } \\
\mathrm{C}_{9} \mathrm{H}_{11} \mathrm{NO}_{2} ;[\text { [619-84-1] } \mathrm{C} \text {. Hancock, J. N. Pawloski, and J. } \\
\text { (2) Benzene; } \mathrm{C}_{6} \mathrm{H}_{6} ;[71-43-2]\end{array}$ & $\begin{array}{l}\text { P. Idoux, J. Org. Chem. 31, 3801 } \\
(1966) .\end{array}$ \\
\hline Variables: & Prepared by: \\
$T / \mathrm{K}=303.15$ & W. E. Acree, Jr. \\
\hline
\end{tabular}

Experimental Values

\begin{tabular}{lc}
\hline \hline$x_{2}{ }^{\mathrm{a}}$ & $x_{1}{ }^{\mathrm{b}}$ \\
\hline 0.9994 & 0.000637 \\
\hline${ }_{{ }^{\mathrm{a}} x_{2}: \text { mole fraction of component } 2 \text { in the saturated solution. }}$ & \\
${ }^{\mathrm{b}}{ }_{x_{1}}:$ mole fraction solubility of the solute. &
\end{tabular}

${ }^{\mathrm{b}} \mathrm{x}_{1}$ : mole fraction solubility of the solute.

\section{Auxiliary Information}

\section{Method/Apparatus/Procedure:}

Constant-temperature bath, Soxhlet thimble, conical flask, and analytical balance.

Excess solute and solvent were placed in a conical flask and allowed to equilibrate for several days at constant temperature. Attainment of equilibrium was verified by several repetitive measurements and by approaching equilibrium from supersaturation. Aliquots of saturated solutions were transferred using a Soxhlet thimble equipped with a one-hole cork stopper and an inverted U-shaped delivery tube to a second ground-glass stoppered flask suspended in the $303 \mathrm{~K}$ water bath. Successive portions of the contents were evaporated at room temperature in a tared aluminum foil weighing dish under a bell jar through which a slow stream of dry air was passed. The second flask plus unused saturated solution and the aluminum foil dish plus residue were weighed. The saturation solubility of the solute was calculated from the recorded mass data and molar masses of the solute and solvent.

Source and Purity of Chemicals:

(1) Purity not given, Chemical source not specified, was recrystallized several times from aqueous-ethanol mixture, and then dried under vacuum over phosphorous pentoxide.

(2) Reagent grade, Chemical source not specified, refluxed over phosphorous pentoxide for $24 \mathrm{~h}$, and then distilled through a $3 \times 80 \mathrm{~cm}$ column filled with 0.32-cm glass helices.

\section{Estimated Error:}

Temperature: $\pm 0.02 \mathrm{~K}$.

$x_{1}: \pm 3 \%$ (relative error) 


\subsection{4-(Dimethylamino)benzoic acid solubility data in alcohols}

\begin{tabular}{|c|c|}
\hline $\begin{array}{l}\text { Components: } \\
\text { (1) 4-(Dimethylamino)benzoic acid; } \\
\mathrm{C}_{9} \mathrm{H}_{11} \mathrm{NO}_{2} ;[619-84-1] \\
\text { (2) Methanol; } \mathrm{CH}_{4} \mathrm{O} ;[67-56-1]\end{array}$ & $\begin{array}{l}\text { Original Measurements: } \\
{ }^{83} \text { M. K. Chantooni and I. M. Kolthoff, } \\
\text { J. Phys. Chem. 77, } 527 \text { (1973). }\end{array}$ \\
\hline $\begin{array}{l}\text { Variables: } \\
T / \mathrm{K}=298.15\end{array}$ & $\begin{array}{l}\text { Prepared by: } \\
\text { W. E. Acree, Jr. }\end{array}$ \\
\hline
\end{tabular}

\section{Experimental Values}

The measured solubility was reported to be $0.086 \mathrm{~mol} \mathrm{dm}^{-3}$. The authors did not give the temperature at which the solubility was measured. Based on the experimental description given in an earlier paper [I. M. Kolthoff, J. J. Lingane, and W. Larson, J. Am. Chem. Soc. 60, 2512 (1938)], the compiler believes the temperature to be $298.15 \mathrm{~K}$.

\section{Auxiliary Information}

\section{Method/Apparatus/Procedure:}

Very little experimental details were provided. Solubility was determined by titrating a filtered aliquot of the saturated solution alkalimetrically in an aqueous-ethanol mixture using phenolphthalein as the acid-base indicator.

Source and Purity of Chemicals:

(1) Purity not given, Aldrich Chemical Company, Milwaukee, WI, USA, was recrystallized from aqueous ethanol solution.

(2) Purity not given, Chemical source not given, was dehydrated and then distilled over sulfanilic acid to remove alkaline impurities.

Estimated Error:

Temperature: $\pm 0.1 \mathrm{~K}$ (estimated by compiler).

$c_{1}: \pm 2 \%$ (relative error, estimated by compiler).

\subsection{4-(Dimethylamino)benzoic acid solubility data in miscellaneous organic solvents}

\begin{tabular}{|c|c|}
\hline $\begin{array}{l}\text { Components: } \\
\text { (1) 4-(Dimethylamino)benzoic acid; } \\
\mathrm{C}_{9} \mathrm{H}_{11} \mathrm{NO}_{2} ;[619-84-1] \\
\text { (2) Ethanenitrile; } \mathrm{C}_{2} \mathrm{H}_{3} \mathrm{~N} ;[75-05-8]\end{array}$ & $\begin{array}{l}\text { Original Measurements: } \\
{ }^{83} \text { M. K. Chantooni and I. M. Kolthoff, } \\
\text { J. Phys. Chem. 77, } 527 \text { (1973). }\end{array}$ \\
\hline $\begin{array}{l}\text { Variables: } \\
T / \mathrm{K}=298.15\end{array}$ & $\begin{array}{l}\text { Prepared by: } \\
\text { W. E. Acree, Jr. }\end{array}$ \\
\hline
\end{tabular}

\section{Experimental Values}

The measured solubility was reported to be $0.023 \mathrm{~mol} \mathrm{dm}^{-3}$. The authors did not give the temperature at which the solubility was measured. Based on the experimental description given in an earlier paper [I. M. Kolthoff, J. J. Lingane, and W. Larson, J. Am. Chem. Soc. 60, 2512 (1938)], the compiler believes the temperature to be $298.15 \mathrm{~K}$.

\section{Auxiliary Information}

Method/Apparatus/Procedure:

Very little experimental details were provided. Solubility was determined by titrating a filtered aliquot of the saturated solution alkalimetrically in an aqueous-ethanol mixture using phenolphthalein as the acid-base indicator.

Source and Purity of Chemicals:

(1) Purity not given, Aldrich Chemical Company, Milwaukee, WI, USA, was recrystallized from aqueous ethanol solution.

(2) Purity not given, Chemical source not given, was purified by shaking with saturated potassium hydroxide, followed by activated alumina, and then anhydrous calcium chloride to remove water. Ethanenitrile was further dried over anhydrous magnesium sulfate and then phosphorous pentoxide. The sample was distilled shortly before use.

\section{Estimated Error:}

Temperature: $\pm 0.1 \mathrm{~K}$ (estimated by compiler). $c_{1}: \pm 2 \%$ (relative error, estimated by compiler).

\begin{tabular}{|c|c|}
\hline $\begin{array}{l}\text { Components: } \\
\text { (1) 4-(Dimethylamino)benzoic acid; } \\
\mathrm{C}_{9} \mathrm{H}_{11} \mathrm{NO}_{2} ; \text { [619-84-1] } \\
\text { (2) Dimethyl sulfoxide; } \mathrm{C}_{2} \mathrm{H}_{6} \mathrm{OS} \text {; } \\
\text { [67-68-5] }\end{array}$ & $\begin{array}{l}\text { Original Measurements: } \\
{ }^{83} \text { M. K. Chantooni and I. M. Kolthoff, } \\
\text { J. Phys. Chem. 77, } 527 \text { (1973). }\end{array}$ \\
\hline $\begin{array}{l}\text { Variables: } \\
T / \mathrm{K}=298.15\end{array}$ & $\begin{array}{l}\text { Prepared by: } \\
\text { W. E. Acree, Jr. }\end{array}$ \\
\hline
\end{tabular}

\section{Experimental Values}

The measured solubility was reported to be $1.95 \mathrm{~mol} \mathrm{dm}^{-3}$. The authors did not give the temperature at which the solubility was measured. Based on the experimental description given in an earlier paper [I. M. Kolthoff, J. J. Lingane, and W. Larson, J. Am. Chem. Soc. 60, 2512 (1938)], the compiler believes the temperature to be $298.15 \mathrm{~K}$.

\section{Auxiliary Information}

\section{Method/Apparatus/Procedure:}

Very little experimental details were provided. Solubility was determined by titrating a filtered aliquot of the saturated solution alkalimetrically in an aqueous-ethanol mixture using phenolphthalein as the acid-base indicator.

Source and Purity of Chemicals:

(1) Purity not given, Aldrich Chemical Company, Milwaukee, WI, USA, was recrystallized from aqueous ethanol solution.

(2) Purity not given, Chemical source not given, was shaken with activated alumina and then distilled before use.

Estimated Error:

Temperature: $\pm 0.1 \mathrm{~K}$ (estimated by compiler). $c_{1}: \pm 2 \%$ (relative error, estimated by compiler).

\begin{tabular}{|c|c|}
\hline $\begin{array}{l}\text { Components: } \\
\text { (1) 4-(Dimethylamino)benzoic acid; } \\
\mathrm{C}_{9} \mathrm{H}_{11} \mathrm{NO}_{2} ;[619-84-1] \\
\text { (2) } \mathrm{N}, \mathrm{N} \text {-Dimethylformamide; } \\
\mathrm{C}_{3} \mathrm{H}_{7} \mathrm{NO} ;[64-19-7]\end{array}$ & $\begin{array}{l}\text { Original Measurements: } \\
{ }^{83} \text { M. K. Chantooni and I. M. Kolthoff, } \\
\text { J. Phys. Chem. 77, } 527 \text { (1973). }\end{array}$ \\
\hline $\begin{array}{l}\text { Variables: } \\
T / \mathrm{K}=298.15\end{array}$ & $\begin{array}{l}\text { Prepared by: } \\
\text { W. E. Acree, Jr. }\end{array}$ \\
\hline
\end{tabular}




\section{Experimental Values}

The measured solubility was reported to be $1.10 \mathrm{~mol} \mathrm{dm}^{-3}$. The authors did not give the temperature at which the solubility was measured. Based on the experimental description given in an earlier paper [I. M. Kolthoff, J. J. Lingane, and W. Larson, J. Am. Chem. Soc. 60, 2512 (1938)], the compiler believes the temperature to be $298.15 \mathrm{~K}$.

\section{Auxiliary Information}

\section{Method/Apparatus/Procedure:}

Very little experimental details were provided. Solubility was determined by titrating a filtered aliquot of the saturated solution alkalimetrically in an aqueous-ethanol mixture using phenolphthalein as the acid-base indicator.

\section{Source and Purity of Chemicals:}

(1) Purity not given, Aldrich Chemical Company, Milwaukee, WI, USA, was recrystallized from aqueous ethanol solution.

(2) Purity not given, Chemical source not given, was shaken first with phosphorous pentoxide and then with potassium hydroxide pellets. Solvent was distilled shortly before use.

Estimated Error:

Temperature: $\pm 0.1 \mathrm{~K}$ (estimated by compiler). $c_{1}: \pm 2 \%$ (relative error, estimated by compiler).

\section{Solubility of 3,4-Dimethylbenzoic Acid in Organic Solvents}

\subsection{Critical evaluation of experimental solubility data}

There have been two studies ${ }^{76,83}$ reporting the solubility of 3,4-dimethylbenzoic acid in organic solvents. Chantooni and Kolthoff ${ }^{83}$ measured the solubility of 3,4-dimethylbenzoic acid in methanol, ethanenitrile, dimethyl sulfoxide, and $N$, $N$-dimethylformamide at $298 \mathrm{~K}$. In a followup study, the authors ${ }^{76}$ determined the solubility in three additional alcohol solvents (2-propanol, 2-methyl-2-propanol, and 1-hexanol). It is not possible to perform a critical evaluation of the experimental data as measurements were made at only a single temperature, and there are no independent experimental solubility data for 3,4-dimethylbenzoic acid in these seven organic solvents.

The experimental solubility data for 3,4-dimethylbenzoic acid in organic solvents are in Secs. 38.2 and 38.3.

\subsection{3,4-Dimethylbenzoic acid solubility data in alcohols}

\begin{tabular}{|c|c|}
\hline $\begin{array}{l}\text { Components: } \\
\text { (1) 3,4-Dimethylbenzoic acid; } \\
\mathrm{C}_{9} \mathrm{H}_{10} \mathrm{O}_{2} ;[619-04-5] \\
\text { (2) Methanol; } \mathrm{CH}_{4} \mathrm{O} ;[67-56-1]\end{array}$ & $\begin{array}{l}\text { Original Measurements: } \\
{ }^{83} \text { M. K. Chantooni and I. M. Kolthoff, } \\
\text { J. Phys. Chem. 77, } 527 \text { (1973). }\end{array}$ \\
\hline $\begin{array}{l}\text { Variables: } \\
T / \mathrm{K}=298.15\end{array}$ & $\begin{array}{l}\text { Prepared by: } \\
\text { W. E. Acree, Jr. }\end{array}$ \\
\hline
\end{tabular}

\section{Experimental Values}

The measured solubility was reported to be $0.595 \mathrm{~mol} \mathrm{dm}^{-3}$. The authors did not give the temperature at which the solubility was measured. Based on the experimental description given in an earlier paper [I. M. Kolthoff, J. J. Lingane, and W. Larson, J. Am. Chem. Soc. 60, 2512 (1938)], the compiler believes the temperature to be $298.15 \mathrm{~K}$.

\section{Auxiliary Information}

\section{Method/Apparatus/Procedure:}

Very little experimental details were provided. Solubility was determined by titrating a filtered aliquot of the saturated solution alkalimetrically in an aqueous-ethanol mixture using phenolphthalein as the acid-base indicator.

Source and Purity of Chemicals:

(1) Purity not given, K and K Chemical Company, USA, was recrystallized from either aqueous or aqueous-ethanol solution and dried in vacuo at $333 \mathrm{~K}$. (2) Purity not given, Chemical source not given, was dehydrated and then distilled over sulfanilic acid to remove alkaline impurities.

\section{Estimated Error:}

Temperature: $\pm 0.1 \mathrm{~K}$ (estimated by compiler). $c_{1}: \pm 2 \%$ (relative error, estimated by compiler).

\begin{tabular}{|c|c|}
\hline $\begin{array}{l}\text { Components: } \\
\text { (1) 3,4-Dimethylbenzoic acid; } \\
\mathrm{C}_{9} \mathrm{H}_{10} \mathrm{O}_{2} ;[619-04-5] \\
\text { (2) 2-Propanol; } \mathrm{C}_{3} \mathrm{H}_{8} \mathrm{O} ;[67-63-0]\end{array}$ & $\begin{array}{l}\text { Original Measurements: } \\
{ }^{76} \text { M. K. Chantooni and I. M. Kolthoff, } \\
\text { Anal. Chem. 51, } 133 \text { (1979). }\end{array}$ \\
\hline $\begin{array}{l}\text { Variables: } \\
T / \mathrm{K}=298.15\end{array}$ & $\begin{array}{l}\text { Prepared by: } \\
\text { W. E. Acree, Jr. }\end{array}$ \\
\hline
\end{tabular}

Experimental Values

The measured solubility was reported to be $0.513 \mathrm{~mol} \mathrm{dm}^{-3}$. The authors did not give the temperature at which the solubility was measured. Based on the experimental description given in an earlier paper [I. M. Kolthoff, J. J. Lingane, and W. Larson, J. Am. Chem. Soc. 60, 2512 (1938)], the compiler believes the temperature to be $298.15 \mathrm{~K}$.

\section{Auxiliary Information}

Method/Apparatus/Procedure:

Very little experimental details were provided. Solubility was determined by titrating a filtered aliquot of the saturated solution alkalimetrically in an aqueous-ethanol mixture using phenolphthalein as the acid-base indicator.

Source and Purity of Chemicals:

(1) Purity not given, K and K Chemical Company, USA, was recrystallized from either aqueous or aqueous-ethanol solution and dried in vacuo at $333 \mathrm{~K}$. (2) Purity not given, Fisher Scientific, USA, shaken with calcium hydride and distilled before use.

Estimated Error:

Temperature: $\pm 0.1 \mathrm{~K}$ (estimated by compiler).

$c_{1}: \pm 2 \%$ (relative error, estimated by compiler). 


\section{Components:}

(1) 3,4-Dimethylbenzoic acid;

$\mathrm{C}_{9} \mathrm{H}_{10} \mathrm{O}_{2}$; [619-04-5]

(2) 2-Methyl-2-propanol; $\mathrm{C}_{4} \mathrm{H}_{10} \mathrm{O}$;

[75-65-0]

\begin{tabular}{ll}
\hline Variables: & Prepared by: \\
$T / \mathrm{K}=298.15$ & W. E. Acree, Jr. \\
\hline
\end{tabular}

\section{Experimental Values}

The measured solubility was reported to be $0.616 \mathrm{~mol} \mathrm{dm}^{-3}$. The authors did not give the temperature at which the solubility was measured. Based on the experimental description given in an earlier paper [I. M. Kolthoff, J. J. Lingane, and W. Larson, J. Am. Chem. Soc. 60, 2512 (1938)], the compiler believes the temperature to be $298.15 \mathrm{~K}$.

\section{Auxiliary Information}

\section{Method/Apparatus/Procedure:}

Very little experimental details were provided. Solubility was determined by titrating a filtered aliquot of the saturated solution alkalimetrically in an aqueous-ethanol mixture using phenolphthalein as the acid-base indicator.

\section{Source and Purity of Chemicals:}

(1) Purity not given, K and K Chemical Company, USA, was recrystallized from either aqueous or aqueous-ethanol solution and dried in vacuo at $333 \mathrm{~K}$ (2) White Label, Eastman Kodak Chemical Company, Rochester, NY, USA, shaken with calcium hydride and distilled before use.

\section{Estimated Error:}

Temperature: $\pm 0.1 \mathrm{~K}$ (estimated by compiler).

$c_{1}: \pm 2 \%$ (relative error, estimated by compiler).

\begin{tabular}{|c|c|}
\hline $\begin{array}{l}\text { Components: } \\
\text { (1) 3,4-Dimethylbenzoic acid; } \\
\mathrm{C}_{9} \mathrm{H}_{10} \mathrm{O}_{2} ;[619-04-5] \\
\text { (2) 1-Hexanol; } \mathrm{C}_{6} \mathrm{H}_{14} \mathrm{O} ;[111-27-3]\end{array}$ & $\begin{array}{l}\text { Original Measurements: } \\
{ }^{76} \text { M. K. Chantooni and I. M. Kolthoff, } \\
\text { Anal. Chem. 51, } 133 \text { (1979). }\end{array}$ \\
\hline $\begin{array}{l}\text { Variables: } \\
T / \mathrm{K}=298.15\end{array}$ & $\begin{array}{l}\text { Prepared by: } \\
\text { W. E. Acree, Jr. }\end{array}$ \\
\hline
\end{tabular}

\section{Experimental Values}

The measured solubility was reported to be $0.408 \mathrm{~mol} \mathrm{dm}^{-3}$. The authors did not give the temperature at which the solubility was measured. Based on the experimental description given in an earlier paper [I. M. Kolthoff, J. J. Lingane, and W. Larson, J. Am. Chem. Soc. 60, 2512 (1938)], the compiler believes the temperature to be $298.15 \mathrm{~K}$.

\section{Auxiliary Information}

\footnotetext{
Method/Apparatus/Procedure:

Very little experimental details were provided. Solubility was determined by titrating a filtered aliquot of the saturated solution alkalimetrically in an aqueous-ethanol mixture using phenolphthalein as the acid-base indicator.

Source and Purity of Chemicals:

(1) Purity not given, K and K Chemical Company, USA, was recrystallized from either aqueous or aqueous-ethanol solution and dried in vacuo at $333 \mathrm{~K}$ (2) Yellow Label, Eastman Kodak Chemical Company, Rochester, NY, USA, shaken with calcium hydride and distilled before use.
}

Estimated Error:

Temperature: $\pm 0.1 \mathrm{~K}$ (estimated by compiler).

$c_{1}: \pm 2 \%$ (relative error, estimated by compiler).

\subsection{3,4-Dimethylbenzoic acid solubility data in miscellaneous organic solvents}

\begin{tabular}{|c|c|}
\hline $\begin{array}{l}\text { Components: } \\
\text { (1) 3,4-Dimethylbenzoic acid; } \\
\mathrm{C}_{9} \mathrm{H}_{10} \mathrm{O}_{2} ;[619-04-5] \\
\text { (2) Ethanenitrile; } \mathrm{C}_{2} \mathrm{H}_{3} \mathrm{~N} ;[75-05-8]\end{array}$ & $\begin{array}{l}\text { Original Measurements: } \\
{ }^{83} \text { M. K. Chantooni and I. M. Kolthoff } \\
\text { J. Phys. Chem. 77, } 527 \text { (1973). }\end{array}$ \\
\hline $\begin{array}{l}\text { Variables: } \\
T / \mathrm{K}=298.15\end{array}$ & $\begin{array}{l}\text { Prepared by: } \\
\text { W. E. Acree, Jr. }\end{array}$ \\
\hline
\end{tabular}

\section{Experimental Values}

The measured solubility was reported to be $0.091 \mathrm{~mol} \mathrm{dm}^{-3}$. The authors did not give the temperature at which the solubility was measured. Based on the experimental description given in an earlier paper [I. M. Kolthoff, J. J. Lingane, and W. Larson, J. Am. Chem. Soc. 60, 2512 (1938)], the compiler believes the temperature to be $298.15 \mathrm{~K}$.

\section{Auxiliary Information}

\section{Method/Apparatus/Procedure:}

Very little experimental details were provided. Solubility was determined by titrating a filtered aliquot of the saturated solution alkalimetrically in an aqueous-ethanol mixture using phenolphthalein as the acid-base indicator.

Source and Purity of Chemicals:

(1) Purity not given, K and K Chemical Company, USA, was recrystallized from either aqueous or aqueous-ethanol solution and dried in vacuo at $333 \mathrm{~K}$. (2) Purity not given, Chemical source not given, was purified by shaking with saturated potassium hydroxide, followed by activated alumina, and then anhydrous calcium chloride to remove water. Ethanenitrile was further dried over anhydrous magnesium sulfate and then phosphorous pentoxide. The sample was distilled shortly before use.

Estimated Error:

Temperature: $\pm 0.1 \mathrm{~K}$ (estimated by compiler). $c_{1}: \pm 2 \%$ (relative error, estimated by compiler).

\begin{tabular}{|c|c|}
\hline $\begin{array}{l}\text { Components: } \\
\text { (1) 3,4-Dimethylbenzoic acid; } \\
\mathrm{C}_{9} \mathrm{H}_{10} \mathrm{O}_{2} ; \text { [619-04-5] } \\
\text { (2) Dimethyl sulfoxide; } \mathrm{C}_{2} \mathrm{H}_{6} \mathrm{OS} \text {; } \\
\text { [67-68-5] }\end{array}$ & $\begin{array}{l}\text { Original Measurements: } \\
{ }^{83} \text { M. K. Chantooni and I. M. Kolthoff, } \\
\text { J. Phys. Chem. 77, } 527 \text { (1973). }\end{array}$ \\
\hline $\begin{array}{l}\text { Variables: } \\
T / \mathrm{K}=298.15\end{array}$ & $\begin{array}{l}\text { Prepared by: } \\
\text { W. E. Acree, Jr. }\end{array}$ \\
\hline
\end{tabular}




\section{Experimental Values}

The measured solubility was reported to be $3.07 \mathrm{~mol} \mathrm{dm}^{-3}$. The authors did not give the temperature at which the solubility was measured. Based on the experimental description given in an earlier paper [I. M. Kolthoff, J. J. Lingane, and W. Larson, J. Am. Chem. Soc. 60, 2512 (1938)], the compiler believes the temperature to be $298.15 \mathrm{~K}$.

\section{Auxiliary Information}

\begin{abstract}
Method/Apparatus/Procedure:
Very little experimental details were provided. Solubility was determined by titrating a filtered aliquot of the saturated solution alkalimetrically in an aqueous-ethanol mixture using phenolphthalein as the acid-base indicator.
\end{abstract}

Source and Purity of Chemicals:

(1) Purity not given, K and K Chemical Company, USA, was recrystallized from either aqueous or aqueous-ethanol solution and dried in vacuo at $333 \mathrm{~K}$. (2) Purity not given, Chemical source not given, was shaken with activated alumina and then distilled before use.

Estimated Error:

Temperature: $\pm 0.1 \mathrm{~K}$ (estimated by compiler).

$c_{1}: \pm 2 \%$ (relative error, estimated by compiler).

\begin{tabular}{|c|c|}
\hline $\begin{array}{l}\text { Components: } \\
\text { (1) 3,4-Dimethylbenzoic acid; } \\
\mathrm{C}_{9} \mathrm{H}_{10} \mathrm{O}_{2} \text {; [619-04-5] } \\
\text { (2) } \mathrm{N}, \mathrm{N} \text {-Dimethylformamide; } \\
\mathrm{C}_{3} \mathrm{H}_{7} \mathrm{NO} ;[64-19-7]\end{array}$ & $\begin{array}{l}\text { Original Measurements: } \\
{ }^{83} \text { M. K. Chantooni and I. M. Kolthoff, } \\
\text { J. Phys. Chem. 77, } 527 \text { (1973). }\end{array}$ \\
\hline $\begin{array}{l}\text { Variables: } \\
T / \mathrm{K}=298.15\end{array}$ & $\begin{array}{l}\text { Prepared by: } \\
\text { W. E. Acree, Jr. }\end{array}$ \\
\hline
\end{tabular}

Experimental Values

The measured solubility was reported to be $1.19 \mathrm{~mol} \mathrm{dm}^{-3}$. The authors did not give the temperature at which the solubility was measured. Based on the experimental description given in an earlier paper [I. M. Kolthoff, J. J. Lingane, and W. Larson, J. Am. Chem. Soc. 60, 2512 (1938)], the compiler believes the temperature to be $298.15 \mathrm{~K}$.

\section{Auxiliary Information}

\section{Method/Apparatus/Procedure: \\ Very little experimental details were provided. Solubility was determined by titrating a filtered aliquot of the saturated solution alkalimetrically in an aqueous-ethanol mixture using phenolphthalein as the acid-base indicator.}

\section{Source and Purity of Chemicals:}

(1) Purity not given, K and K Chemical Company, USA, was recrystallized from either aqueous or aqueous-ethanol solution and dried in vacuo at $333 \mathrm{~K}$. (2) Purity not given, Chemical source not given, was shaken first with phosphorous pentoxide and then with potassium hydroxide pellets. Solvent was distilled shortly before use.

\section{Estimated Error:}

Temperature: $\pm 0.1 \mathrm{~K}$ (estimated by compiler).

$c_{1}: \pm 2 \%$ (relative error, estimated by compiler).

\section{Solubility of 2,4-Dinitrobenzoic Acid in Organic Solvents}

\subsection{Critical evaluation of experimental solubility data}

There has been one study reporting the solubility of 2,4dinitrobenzoic acid in organic solvents. Chantooni and Kolthoff $^{83}$ determined the solubility of 2,4-dinitrobenzoic acid in methanol and ethanenitrile at $298 \mathrm{~K}$. It is not possible to perform a critical evaluation of the experimental data as the authors performed measurements at only a single temperature, and there are no independent experimental solubility data for 2,4-dinitrobenzoic acid in either cyclohexane or benzene.

The experimental solubility data for 2,4-dinitrobenzoic acid in organic solvents are in Secs. 39.2 and 39.3.

\subsection{2,4-Dinitrobenzoic acid solubility data in alcohols}

\begin{tabular}{|c|c|}
\hline $\begin{array}{l}\text { Components: } \\
\text { (1) 2,4-Dinitrobenzoic acid; } \\
\mathrm{C}_{7} \mathrm{H}_{4} \mathrm{~N}_{2} \mathrm{O}_{6} ;[610-30-0] \\
\text { (2) Methanol; } \mathrm{CH}_{4} \mathrm{O} ;[67-56-1]\end{array}$ & $\begin{array}{l}\text { Original Measurements: } \\
{ }^{83} \text { M. K. Chantooni and I. M. Kolthoff, } \\
\text { J. Phys. Chem. 77, } 527 \text { (1973). }\end{array}$ \\
\hline Variables: & Prepared by: \\
\hline
\end{tabular}

\section{Experimental Values}

The measured solubility was reported to be $3.23 \mathrm{~mol} \mathrm{dm}^{-3}$. The authors did not give the temperature at which the solubility was measured. Based on the experimental description given in an earlier paper [I. M. Kolthoff, J. J. Lingane, and W. Larson, J. Am. Chem. Soc. 60, 2512 (1938)], the compiler believes the temperature to be $298.15 \mathrm{~K}$.

\section{Auxiliary Information}

\section{Method/Apparatus/Procedure:}

Very little experimental details were provided. Solubility was determined by titrating a filtered aliquot of the saturated solution alkalimetrically in an aqueous-ethanol mixture using phenolphthalein as the acid-base indicator.

\section{Source and Purity of Chemicals:}

(1) Purity not given, Aldrich Chemical Company, Milwaukee, WI, USA, was recrystallized from either aqueous or aqueous-ethanol solution and dried in vacuo at $333 \mathrm{~K}$.

(2) Purity not given, Chemical source not given, was dehydrated and then distilled over sulfanilic acid to remove alkaline impurities.

\section{Estimated Error:}

Temperature: $\pm 0.1 \mathrm{~K}$ (estimated by compiler).

$c_{1}: \pm 2 \%$ (relative error, estimated by compiler). 


\subsection{2,4-Dinitrobenzoic acid solubility data in miscellaneous organic solvents}

\begin{tabular}{|c|c|}
\hline $\begin{array}{l}\text { Components: } \\
\text { (1) 2,4-Dinitrobenzoic acid; } \\
\mathrm{C}_{7} \mathrm{H}_{4} \mathrm{~N}_{2} \mathrm{O}_{6} ;[610-30-0] \\
\text { (2) Ethanenitrile; } \mathrm{C}_{2} \mathrm{H}_{3} \mathrm{~N} ;[75-05-8]\end{array}$ & $\begin{array}{l}\text { Original Measurements: } \\
{ }^{83} \text { M. K. Chantooni and I. M. Kolthoff, } \\
\text { J. Phys. Chem. 77, } 527 \text { (1973). }\end{array}$ \\
\hline $\begin{array}{l}\text { Variables: } \\
T / \mathrm{K}=298.15\end{array}$ & $\begin{array}{l}\text { Prepared by: } \\
\text { W. E. Acree, Jr. }\end{array}$ \\
\hline
\end{tabular}

\section{Experimental Values}

The measured solubility was reported to be $1.02 \mathrm{~mol} \mathrm{dm}^{-3}$. The authors did not give the temperature at which the solubility was measured. Based on the experimental description given in an earlier paper [I. M. Kolthoff, J. J. Lingane, and W. Larson, J. Am. Chem. Soc. 60, 2512 (1938)] the compiler believes the temperature to be $298.15 \mathrm{~K}$.

\section{Auxiliary Information}

\begin{abstract}
Method/Apparatus/Procedure:
Very little experimental details were provided. Solubility was determined by titrating a filtered aliquot of the saturated solution alkalimetrically in an aqueous-ethanol mixture using phenolphthalein as the acid-base indicator.
\end{abstract}

\section{Source and Purity of Chemicals:}

(1) Purity not given, Aldrich Chemical Company, Milwaukee, WI, USA, was recrystallized from either aqueous or aqueous-ethanol solution and dried in vacuo $333 \mathrm{~K}$.

(2) Purity not given, Chemical source not given, was purified by shaking with saturated potassium hydroxide, followed by activated alumina, and then anhydrous calcium chloride to remove water. Ethanenitrile was further dried over anhydrous magnesium sulfate and then phosphorous pentoxide. The sample was distilled shortly before use.

\section{Estimated Error:}

Temperature: $\pm 0.1 \mathrm{~K}$ (estimated by compiler).

$c_{1}: \pm 2 \%$ (relative error, estimated by compiler).

\section{Solubility of 3,5-Dinitrobenzoic Acid in Organic Solvents}

\subsection{Critical evaluation of experimental solubility data}

There have been several published studies $54,63,76,83,140$ investigating the solubility behavior of 3,5-dinitrobenzoic acid in organic solvents of varying polarity and hydrogen-bonding capability. Hoover et al. ${ }^{140}$ measured the solubility of 3,5dinitrobenzoic acid in 13 alcohols (ethanol, 1-propanol, 2propanol, 1-butanol, 2-butanol, 2-methyl-1-propanol, 1-pentanol, 2-pentanol, 3-methyl-1-butanol, 1-hexanol, 1-heptanol, 1-octanol, and 1-decanol), in three dialkyl ethers (1,1'-oxybisethane, 2,2'-oxybispropane, and 1,1'-oxybisbutane) and two cyclic ethers (tetrahydrofuran and 1,4-dioxane), and in three alkyl alkanoates (ethyl ethanoate, butyl ethanoate, and pentyl ethanoate) at $298 \mathrm{~K}$. Results of the experimental measurements were used to calculate the Abraham solute descriptors of 3,5-dinitrobenzoic acid. The authors were able to assemble a total of $38 \log _{10}\left(S R\right.$ or $P$ ) and $\log _{10}(G S R$ or $K$ ) equations for which experimental partition coefficient data, solubility ratios, Abraham Model equation coefficients, and aqueous molar solubility were available. The logarithm of the aqueous molar solubility of 3,5-dinitrobenzoic acid is $\log _{10} c_{1, \mathrm{~W}}=$ -2.417 . $^{141,142}$ The McGowan volume of 3,5-dinitrobenzoic acid, $V=1.2801$, was calculated from the number of chemical bonds in the molecule and the individual atomic group volumes, $A V_{i}$, given in Sec. 1.3. The excess molar refraction solute descriptor was estimated as $E=1.250$. This left four solute descriptors ( $S, A, B$, and $L$ ) still to be determined. The 38 equations were then solved using the Microsoft "SOLVER" program to yield numerical values of the remaining four solute descriptors, $S=1.630, A=0.700, B=0.590$, and $L=6.9837$, that best described the $\log _{10}\left(S R\right.$ or $P$ ) and $\log _{10}(G S R$ or $K$ ) values. The computation treated $\log _{10} c_{1, \mathrm{G}}$ as a floating parameter to be determined as part of the regression analyses. The data analyses returned a value of $\log _{10} c_{1, \mathrm{G}}=-10.717$ for the gas-phase solute concentration that made the $\log _{10}(S R$ or $P$ ) and $\log _{10}(G S R$ or $K)$ predictions internally consistent. The calculated molecular solute descriptors reproduced the $\log _{10}$ $\left(S R\right.$ or $P$ ) and $\log _{10}(G S R$ or $K$ ) values to within an average standard deviation of 0.091 and $0.114 \log _{10}$ units, respectively.

After the 3,5-dinitrobenzoic acid solubility study was published, Abraham model correlations have been developed for 2-pentanol, 3-methyl-1-butanol, and butyl ethanoate, and equation coefficients for a few solvents were updated based on additional experimental data. The new correlations (listed in Tables 1 and 2) will be used in illustrating the ability of the Abraham model to correlate the experimental 3,5-dinitrobenzoic acid solubility data. Table 32 compares the experimental $\log _{10} c_{1}$ values to calculated values based on Eqs. (20) and (21) of the Abraham model. For comparison purposes, the measured mole fraction solubilities of 3,5-dinitrobenzoic acid, $x_{1}$, determined by Hoover et al. ${ }^{140}$ were converted into molar solubilities by dividing $x_{1}$ by the ideal molar volume of the

TABLE 32. Comparison between observed and calculated molar solubilities of 3,5-dinitrobenzoic acid based on the Abraham model, Eqs. (20) and (21)

\begin{tabular}{lccc}
\hline \hline Solvent & & $\log _{10} c_{1}{ }^{\text {calc }} ;$ & $\log _{10} c_{1}{ }^{\text {calc }}$ \\
\hline Ethanol & $\log _{10} c_{1}{ }^{\text {exp }}$ & Eq. $(20)$ & Eq. $(21)$ \\
1-Propanol & -0.128 & -0.249 & -0.239 \\
2-Propanol & -0.326 & -0.396 & -0.385 \\
1-Butanol & -0.366 & -0.409 & -0.387 \\
2-Butanol & -0.469 & -0.518 & -0.498 \\
2-Methyl-1-propanol & -0.496 & -0.478 & -0.485 \\
1-Pentanol & -0.655 & -0.615 & -0.622 \\
2-Pentanol & -0.540 & -0.562 & -0.552 \\
3-Methyl-1-butanol & -0.523 & -0.590 & -0.564 \\
1-Hexanol & -0.574 & -0.660 & -0.627 \\
1-Heptanol & -0.621 & -0.605 & -0.600 \\
1-Octanol & -0.653 & -0.651 & -0.633 \\
1-Decanol & -0.683 & -0.657 & -0.735 \\
1,1'-Oxybisethane & -0.746 & -0.809 & -0.786 \\
Tetrahydrofuran & -0.555 & -0.723 & -0.608 \\
1,4-Dioxane & 0.209 & 0.254 & 0.236 \\
Ethyl ethanoate & -0.005 & 0.102 & 0.104 \\
Butyl ethanoate & -0.311 & -0.426 & -0.409 \\
\hline
\end{tabular}


saturated solution (i.e., $\left.c_{1}{ }^{\text {sat }}=x_{1} /\left[x_{1} V_{1}+\left(1-x_{1}\right) V_{\text {solvent }}\right]\right)$. The molar volume of the hypothetical subcooled liquid 3,5-dinitrobenzoic acid is $V_{\text {solute }}=131.0 \mathrm{~cm}^{3} \mathrm{~mol}^{-1}$. Examination of the numerical entries in Table 32 reveals that the Abraham model provides a reasonably accurate mathematical description of the observed solubility data, suggesting that there are no obvious outliers in the dataset.

Hancock et al. ${ }^{54,63}$ measured the solubility of 3,5-dinitrobenzoic acid in cyclohexane, benzene, tetrahydrofuran and 1,4-dioxane at $303.15 \mathrm{~K}$, which is at a slightly higher temperature than solubility data of Hoover et al. ${ }^{140} \mathrm{~A}$ direct comparison is not possible since the two sets of measurements were performed a different temperatures.

The experimental solubility data for 3,5-dinitrobenzoic acid in organic solvents are in Secs. 40.2-40.7.

\subsection{3,5-Dinitrobenzoic acid solubility data in saturated hydrocarbons (including cycloalkanes)}

\begin{tabular}{ll}
\hline \hline Components: & Original Measurements: \\
(1) 3,5-Dinitrobenzoic acid; & ${ }^{54} \mathrm{C}$. K. Hancock, J. N. Pawloski, and \\
$\mathrm{C}_{7} \mathrm{H}_{4} \mathrm{~N}_{2} \mathrm{O}_{6} ;$ [99-34-3] & J. P. Idoux, J. Org. Chem. 31, 3801 \\
(2) Cyclohexane; $\mathrm{C}_{6} \mathrm{H}_{12} ;[110-82-7]$ & $(1966)$. \\
\hline
\end{tabular}

\begin{tabular}{ll}
\hline Variables: & Prepared by: \\
$T / \mathrm{K}=303.15$ & W. E. Acree, Jr.
\end{tabular}

Experimental Values

\begin{tabular}{lc}
\hline \hline$x_{2}{ }^{\mathrm{a}}$ & $x_{1}{ }^{\mathrm{b}}$ \\
\hline 0.9999 & 0.00000698 \\
\hline${ }^{\mathrm{a}} x_{2}:$ mole fraction of component 2 in the saturated solution. \\
${ }^{\mathrm{b}} x_{1}:$ mole fraction solubility of the solute.
\end{tabular}

\section{Auxiliary Information}

\section{Method/Apparatus/Procedure:}

Constant-temperature bath, Soxhlet thimble, conical flask, and analytical balance.

Excess solute and solvent were placed in a conical flask and allowed to equilibrate for several days at constant temperature. Attainment of equilibrium was verified by several repetitive measurements and by approaching equilibrium from supersaturation. Aliquots of saturated solutions were transferred using a Soxhlet thimble equipped with a one-hole cork stopper and an inverted U-shaped delivery tube to a second ground-glass stoppered flask suspended in the $303 \mathrm{~K}$ water bath. Successive portions of the contents were evaporated at room temperature in a tared aluminum foil weighing dish under a bell jar through which a slow stream of dry air was passed. The second flask plus unused saturated solution and the aluminum foil dish plus residue were weighed. The saturation solubility of the solute was calculated from the recorded mass data and molar masses of the solute and solvent.

\section{Source and Purity of Chemicals:}

(1) Purity not given, Chemical source not specified, was recrystallized several times from aqueous-ethanol mixture, and then dried under vacuum over phosphorous pentoxide.

(2) Reagent grade, Chemical source not specified, refluxed over phosphorous pentoxide for $24 \mathrm{~h}$, and then distilled through a $3 \times 80 \mathrm{~cm}$ column filled with $0.32-\mathrm{cm}$ glass helices.
Estimated Error:

Temperature: $\pm 0.02 \mathrm{~K}$

$x_{1}: \pm 10 \%$ (relative error, by compiler).

\subsection{3,5-Dinitrobenzoic acid solubility data in aromatic hydrocarbons}

\begin{tabular}{ll}
\hline \hline Components: & Original Measurements: \\
$\begin{array}{l}\text { (1) 3,5-Dinitrobenzoic acid; } \\
\mathrm{C}_{7} \mathrm{H}_{4} \mathrm{~N}_{2} \mathrm{O}_{6} ;[99-34-3]\end{array}$ & ${ }^{54} \mathrm{C}$. K. Hancock, J. N. Pawloski, and J. \\
(2) Benzene; $\mathrm{C}_{6} \mathrm{H}_{6} ;[71-43-2]$ & $(1966)$. \\
\hline Variables: & Prepared by: \\
$T / \mathrm{K}=303.15$ & W. E. Acree, Jr. \\
\hline
\end{tabular}

\section{Experimental Values}

\begin{tabular}{lc}
\hline \hline$x_{2}{ }^{\mathrm{a}}$ & $x_{1}{ }^{\mathrm{b}}$ \\
\hline 0.9995 & 0.000454 \\
\hline${ }^{\mathrm{a}} x_{2}:$ mole fraction of component 2 in the saturated solution. & \\
${ }^{\mathrm{b}}{ }_{x_{1}}:$ mole fraction solubility of the solute. &
\end{tabular}

\section{Auxiliary Information}

\section{Method/Apparatus/Procedure:}

Constant-temperature bath, Soxhlet thimble, conical flask, and analytical balance.

Excess solute and solvent were placed in a conical flask and allowed to equilibrate for several days at constant temperature. Attainment of equilibrium was verified by several repetitive measurements and by approaching equilibrium from supersaturation. Aliquots of saturated solutions were transferred using a Soxhlet thimble equipped with a one-hole cork stopper and an inverted U-shaped delivery tube to a second ground-glass stoppered flask suspended in the $303 \mathrm{~K}$ water bath. Successive portions of the contents were evaporated at room temperature in a tared aluminum foil weighing dish under a bell jar through which a slow stream of dry air was passed. The second flask plus unused saturated solution and the aluminum foil dish plus residue were weighed. The saturation solubility of the solute was calculated from the recorded mass data and molar masses of the solute and solvent.

Source and Purity of Chemicals:

(1) Purity not given, Chemical source not specified, was recrystallized several times from aqueous-ethanol mixture, and then dried under vacuum over phosphorous pentoxide

(2) Reagent grade, Chemical source not specified, refluxed over phosphorous pentoxide for $24 \mathrm{~h}$, and then distilled through a $3 \times 80 \mathrm{~cm}$ column filled with $0.32-\mathrm{cm}$ glass helices.

\section{Estimated Error:}

Temperature: $\pm 0.02 \mathrm{~K}$.

$x_{1}: \pm 3 \%$ (relative error, by compiler). 


\subsection{3,5-Dinitrobenzoic acid solubility data in esters}

\begin{tabular}{ll}
\hline \hline Components: & Original Measurements: \\
(1) 3,5-Dinitrobenzoic acid; & ${ }^{140}$ K. R. Hoover, R. Coaxum, E. \\
$\mathrm{C}_{7} \mathrm{H}_{4} \mathrm{~N}_{2} \mathrm{O}_{6} ;$ [99-34-3] & Pustejovsky, W. E. Acree, Jr., and \\
$\begin{array}{l}\text { (2) Ethyl ethanoate; } \mathrm{C}_{4} \mathrm{H}_{8} \mathrm{O}_{2} ; \\
{[141-78-6]}\end{array}$ & M. H. Abraham, Phys. Chem. Liq. 42, \\
\hline Variables: & 457 (2004). \\
$T / \mathrm{K}=298.15$ & Prepared by: \\
\hline
\end{tabular}

Experimental Values

\begin{tabular}{lc}
\hline \hline$x_{2}{ }^{\mathrm{a}}$ & $x_{1}{ }^{\mathrm{b}}$ \\
\hline 0.9511 & 0.04892 \\
\hline${ }^{\mathrm{a}} x_{2}:$ mole fraction of component 2 in the saturated solution. \\
${ }^{\mathrm{b}} x_{1}:$ mole fraction solubility of the solute.
\end{tabular}

\section{Auxiliary Information}

\section{Method/Apparatus/Procedure:}

Constant-temperature bath, calorimetric thermometer, and an ultraviolet/ visible spectrophotometer.

Excess solute and solvent were placed in amber glass bottles and allowed to equilibrate for several days at constant temperature. Attainment of equilibrium was verified by several repetitive measurements and by approaching equilibrium from supersaturation. Aliquots of saturated solutions were transferred through a coarse filter into tared volumetric flasks, weighed, and diluted with methanol. Concentrations were determined by

spectrophotometric measurements at $267 \mathrm{~nm}$.

Source and Purity of Chemicals:

(1) $99+\%$, Aldrich Chemical Company, Milwaukee, WI, USA, was used as received.

(2) $99.9 \%$, HPLC grade, Aldrich Chemical Company, stored over molecular sieves and distilled shortly before use.

\section{Estimated Error:}

Temperature: $\pm 0.1 \mathrm{~K}$.

$x_{1}: \pm 1.5 \%$ (relative error).

\begin{tabular}{ll}
\hline \hline Components: & Original Measurements: \\
(1) 3,5-Dinitrobenzoic acid; & ${ }^{140}$ K. R. Hoover, R. Coaxum, E. \\
$\mathrm{C}_{7} \mathrm{H}_{4} \mathrm{~N}_{2} \mathrm{O}_{6}$; [99-34-3] & Pustejovsky, W. E. Acree, Jr., and \\
$\begin{array}{l}\text { (2) Butyl ethanoate; } \mathrm{C}_{6} \mathrm{H}_{12} \mathrm{O}_{2} ; \\
\text { [123-86-4] }\end{array}$ & $\begin{array}{l}\text { M. H. Abraham, Phys. Chem. Liq. 42, } \\
457 \text { (2004). }\end{array}$ \\
\hline Variables: & Prepared by: \\
$T / \mathrm{K}=298.15$ & W. E. Acree, Jr. \\
\hline
\end{tabular}

Experimental Values

\begin{tabular}{lc}
\hline \hline$x_{2}{ }^{\mathrm{a}}$ & $x_{1}{ }^{\mathrm{b}}$ \\
\hline 0.9621 & 0.03787
\end{tabular}

${ }^{\mathrm{a}} x_{2}$ : mole fraction of component 2 in the saturated solution.

${ }^{\mathrm{b}} x_{1}$ : mole fraction solubility of the solute.

\section{Auxiliary Information}

Method/Apparatus/Procedure:

Constant-temperature bath, calorimetric thermometer, and an ultraviolet/ visible spectrophotometer.

Excess solute and solvent were placed in amber glass bottles and allowed to equilibrate for several days at constant temperature. Attainment of equilibrium was verified by several repetitive measurements and by approaching equilibrium from supersaturation. Aliquots of saturated solutions were transferred through a coarse filter into tared volumetric flasks, weighed, and diluted with methanol. Concentrations were determined by spectrophotometric measurements at $267 \mathrm{~nm}$.

Source and Purity of Chemicals:

(1) $99+\%$, Aldrich Chemical Company, Milwaukee, WI, USA, was used as received.

(2) $99.7 \%$, HPLC grade, Aldrich Chemical Company, stored over molecular sieves and distilled shortly before use.

\section{Estimated Error:}

Temperature: $\pm 0.1 \mathrm{~K}$.

$x_{1}: \pm 1.5 \%$ (relative error).

\begin{tabular}{ll}
\hline \hline Components: & Original Measurements: \\
(1) 3,5-Dinitrobenzoic acid; & ${ }^{140}$ K. R. Hoover, R. Coaxum, E. \\
$\mathrm{C}_{7} \mathrm{H}_{4} \mathrm{~N}_{2} \mathrm{O}_{6} ;$ [99-34-3] & Pustejovsky, W. E. Acree, Jr., and \\
$\begin{array}{l}\text { (2) Pentyl ethanoate; } \mathrm{C}_{7} \mathrm{H}_{14} \mathrm{O}_{2} ; \\
\text { [628-63-7] }\end{array}$ & $\begin{array}{l}\text { M. H. Abraham, Phys. Chem. Liq. 42, } \\
\text { 457 (2004). }\end{array}$ \\
\hline Variables: & Prepared by: \\
$T / \mathrm{K}=298.15$ & W. E. Acree, Jr. \\
\hline
\end{tabular}

Experimental Values

\begin{tabular}{|c|c|}
\hline$x_{2}{ }^{\mathrm{a}}$ & $x_{1}{ }^{\mathrm{b}}$ \\
\hline 0.9691 & 0.03088 \\
\hline
\end{tabular}

\section{Auxiliary Information}

\section{Method/Apparatus/Procedure:}

Constant-temperature bath, calorimetric thermometer, and an ultraviolet/ visible spectrophotometer.

Excess solute and solvent were placed in amber glass bottles and allowed to equilibrate for several days at constant temperature. Attainment of equilibrium was verified by several repetitive measurements and by approaching equilibrium from supersaturation. Aliquots of saturated solutions were transferred through a coarse filter into tared volumetric flasks, weighed, and diluted with methanol. Concentrations were determined by spectrophotometric measurements at $267 \mathrm{~nm}$.

Source and Purity of Chemicals:

(1) $99+\%$, Aldrich Chemical Company, Milwaukee, WI, USA, was used as received.

(2) $99 \%$, Aldrich Chemical Company, stored over molecular sieves and distilled shortly before use.

\section{Estimated Error:}

Temperature: $\pm 0.1 \mathrm{~K}$.

$x_{1}: \pm 1.5 \%$ (relative error). 


\section{Components:}

(1) 3,5-Dinitrobenzoic acid;

$\mathrm{C}_{7} \mathrm{H}_{4} \mathrm{~N}_{2} \mathrm{O}_{6}$; [99-34-3]

(2) 1,2,3-Triacetoxypropane

(Triacetin); $\mathrm{C}_{9} \mathrm{H}_{14} \mathrm{O}_{6} ;[102-76-1]$

\begin{tabular}{ll}
\hline Variables: & Prepared by: \\
$T / \mathrm{K}=298.15$ & W. E. Acree, Jr. \\
\hline
\end{tabular}

\section{Experimental Values}

\begin{tabular}{lc}
\hline \hline$x_{2}{ }^{\mathrm{a}}$ & $x_{1}{ }^{\mathrm{b}}$ \\
\hline 0.9338 & 0.06616
\end{tabular}

${ }^{a_{2}} x_{2}$ mole fraction of component 2 in the saturated solution.

${ }^{\mathrm{b}} x_{1}$ : mole fraction solubility of the solute.

\section{Auxiliary Information}

\section{Method: Apparatus/Procedure:}

Constant-temperature bath, calorimetric thermometer, and an ultraviolet/ visible spectrophotometer

Excess solute and solvent placed in amber glass bottles and allowed to equilibrate for several days at constant temperature. Attainment of equilibrium was verified by several repetitive measurements and by approaching equilibrium from supersaturation. Aliquots of saturated solutions transferred through a coarse filter into tared volumetric flasks, weighed, and diluted with 2 propanol. Concentrations determined by spectrophotometric measurements at $267 \mathrm{~nm}$.

Source and Purity of Chemicals:

(1) $99+\%$, Aldrich Chemical Company, Milwaukee, WI, USA, was used as received.

(2) $99 \%$, Acros Organics, USA, stored over molecular sieves before use.

Estimated Error:

Temperature: $\pm 0.1 \mathrm{~K}$.

$x_{1}: \pm 1.5 \%$ (relative error)

\subsection{3,5-Dinitrobenzoic acid solubility data in ethers}

\begin{tabular}{ll}
\hline \hline Components: & Original Measurements: \\
(1) 3,5-Dinitrobenzoic acid; & ${ }^{140}$ K. R. Hoover, R. Coaxum, E. \\
$\mathrm{C}_{7} \mathrm{H}_{4} \mathrm{~N}_{2} \mathrm{O}_{6} ;$ [ [99-34-3] & Pustejovsky, W. E. Acree, Jr., and \\
$\begin{array}{l}\text { (2) } 1,1^{\prime} \text {-Oxybisethane; } \mathrm{C}_{4} \mathrm{H}_{10} \mathrm{O} ; \\
{[60-29-7]}\end{array}$ & M. H. Abraham, Phys. Chem. Liq. 42, \\
\hline Variables: & 457 (2004). \\
$T / \mathrm{K}=298.15$ & Prepared by: \\
\hline
\end{tabular}

Experimental Values

\begin{tabular}{lc}
\hline \hline$x_{2}{ }^{\mathrm{a}}$ & $x_{1}{ }^{\mathrm{b}}$ \\
\hline 0.9706 & 0.02938 \\
\hline${ }^{\mathrm{a}} x_{2}:$ mole fraction of component 2 in the saturated solution. \\
${ }^{\mathrm{b}} x_{1}:$ mole fraction solubility of the solute.
\end{tabular}

\section{Auxiliary Information}

Method/Apparatus/Procedure:

Constant-temperature bath, calorimetric thermometer, and an ultraviolet/ visible spectrophotometer.

Excess solute and solvent were placed in amber glass bottles and allowed to equilibrate for several days at constant temperature. Attainment of equilibrium was verified by several repetitive measurements and by approaching equilibrium from supersaturation. Aliquots of saturated solutions were transferred through a coarse filter into tared volumetric flasks, weighed, and diluted with methanol. Concentrations were determined by spectrophotometric measurements at $267 \mathrm{~nm}$.

Source and Purity of Chemicals:

(1) $99+\%$, Aldrich Chemical Company, Milwaukee, WI, USA, was used as received.

(2) 99+\%, anhydrous, Aldrich Chemical Company, stored over molecular sieves and distilled shortly before use.

Estimated Error:

Temperature: $\pm 0.1 \mathrm{~K}$.

$x_{1}: \pm 1.5 \%$ (relative error)

\begin{tabular}{ll}
\hline \hline Components: & Original Measurements: \\
(1) 3,5-Dinitrobenzoic acid; & ${ }^{140}$ K. R. Hoover, R. Coaxum, E. \\
$\mathrm{C}_{7} \mathrm{H}_{4} \mathrm{~N}_{2} \mathrm{O}_{6} ;$ [99-34-3] & Pustejovsky, W. E. Acree, Jr., and \\
$\begin{array}{l}\text { (2) 2,2'-Oxybispropane; } \mathrm{C}_{6} \mathrm{H}_{14} \mathrm{O} ; \\
{[108-20-3]}\end{array}$ & $\begin{array}{l}\text { M. H. Abraham, Phys. Chem. Liq. 42, } \\
457 \text { (2004). }\end{array}$ \\
\hline Variables: & Prepared by: \\
$T / \mathrm{K}=298.15$ & W. E. Acree, Jr. \\
\hline
\end{tabular}

\section{Experimental Values}

\begin{tabular}{lc}
\hline \hline$x_{2}{ }^{\mathrm{a}}$ & $x_{1}{ }^{\mathrm{b}}$ \\
\hline 0.9909 & 0.009087 \\
\hline${ }^{\mathrm{a}} x_{2}:$ mole fraction of component 2 in the saturated solution. & \\
${ }^{\mathrm{b}} x_{1}:$ mole fraction solubility of the solute. &
\end{tabular}

\section{Auxiliary Information}

\section{Method/Apparatus/Procedure:}

Constant-temperature bath, calorimetric thermometer, and an ultraviolet/ visible spectrophotometer.

Excess solute and solvent were placed in amber glass bottles and allowed to equilibrate for several days at constant temperature. Attainment of equilibrium was verified by several repetitive measurements and by approaching equilibrium from supersaturation. Aliquots of saturated solutions were transferred through a coarse filter into tared volumetric flasks, weighed, and diluted with methanol. Concentrations were determined by spectrophotometric measurements at $267 \mathrm{~nm}$.

Source and Purity of Chemicals:

(1) $99+\%$, Aldrich Chemical Company, Milwaukee, WI, USA, was used as received.

(2) $99 \%$, anhydrous, Aldrich Chemical Company, stored over molecular sieves and distilled shortly before use.

\section{Estimated Error:}

Temperature: $\pm 0.1 \mathrm{~K}$.

$x_{1}: \pm 1.5 \%$ (relative error) 


\section{Components:}

(1) 3,5-Dinitrobenzoic acid;

$\mathrm{C}_{7} \mathrm{H}_{4} \mathrm{~N}_{2} \mathrm{O}_{6}$; [99-34-3]

(2) $1,1^{\prime}$-Oxybisbutane; $\mathrm{C}_{8} \mathrm{H}_{18} \mathrm{O}$; [142-96-1]

\begin{tabular}{ll}
\hline Variables: & Prepared by: \\
$T / \mathrm{K}=298.15$ & W. E. Acree, Jr.
\end{tabular}

Experimental Values

\begin{tabular}{lc}
\hline \hline$x_{2}{ }^{\mathrm{a}}$ & $x_{1}{ }^{\mathrm{b}}$ \\
\hline 0.9942 & 0.005787
\end{tabular}

${ }^{\mathrm{a}} x_{2}$ : mole fraction of component 2 in the saturated solution.

${ }^{\mathrm{b}} x_{1}$ : mole fraction solubility of the solute.

\section{Auxiliary Information}

\section{Method/Apparatus/Procedure:}

Constant-temperature bath, calorimetric thermometer, and an ultraviolet/ visible spectrophotometer.

Excess solute and solvent were placed in amber glass bottles and allowed to equilibrate for several days at constant temperature. Attainment of equilibrium was verified by several repetitive measurements and by approaching equilibrium from supersaturation. Aliquots of saturated solutions were transferred through a coarse filter into tared volumetric flasks, weighed, and diluted with methanol. Concentrations were determined by spectrophotometric measurements at $267 \mathrm{~nm}$.

Source and Purity of Chemicals:

(1) $99+\%$, Aldrich Chemical Company, Milwaukee, WI, USA, was used as received.

(2) $99.3 \%$, anhydrous, Aldrich Chemical Company, stored over molecular sieves and distilled shortly before use.

\section{Estimated Error:}

Temperature: $\pm 0.1 \mathrm{~K}$.

$x_{1}: \pm 1.5 \%$ (relative error).

\begin{tabular}{|c|c|}
\hline $\begin{array}{l}\text { Components: } \\
\text { (1) 3,5-Dinitrobenzoic acid; } \\
\left.\mathrm{C}_{7} \mathrm{H}_{4} \mathrm{~N}_{2} \mathrm{O}_{6} ; \text {; } 99-34-3\right] \\
\text { (2) Tetrahydrofuran; } \mathrm{C}_{4} \mathrm{H}_{8} \mathrm{O} ; \\
\text { [109-99-9] }\end{array}$ & $\begin{array}{l}\text { Original Measurements: } \\
{ }^{140} \text { K. R. Hoover, R. Coaxum, E. } \\
\text { Pustejovsky, W. E. Acree, Jr., and M. } \\
\text { H. Abraham, Phys. Chem. Liq. 42, } 457 \\
\text { (2004). }\end{array}$ \\
\hline $\begin{array}{l}\text { Variables: } \\
T / \mathrm{K}=298.15\end{array}$ & $\begin{array}{l}\text { Prepared by: } \\
\text { W. E. Acree, Jr. }\end{array}$ \\
\hline
\end{tabular}

\section{Experimental Values}

\begin{tabular}{lc}
\hline \hline$x_{2}{ }^{\mathrm{a}}$ & $x_{1}{ }^{\mathrm{b}}$ \\
\hline 0.8569 & 0.1431 \\
\hline${ }^{\mathrm{a}} x_{2}:$ mole fraction of component 2 in the saturated solution. & \\
${ }^{\mathrm{b}} x_{1}:$ mole fraction solubility of the solute. &
\end{tabular}

\section{Auxiliary Information}

\section{Method/Apparatus/Procedure:}

Constant-temperature bath, calorimetric thermometer, and an ultraviolet/ visible spectrophotometer.

Excess solute and solvent were placed in amber glass bottles and allowed to equilibrate for several days at constant temperature. Attainment of equilibrium was verified by several repetitive measurements and by approaching equilibrium from supersaturation. Aliquots of saturated solutions were transferred through a coarse filter into tared volumetric flasks, weighed, and diluted with methanol. Concentrations were determined by spectrophotometric measurements at $267 \mathrm{~nm}$.

Source and Purity of Chemicals:

(1) $99+\%$, Aldrich Chemical Company, Milwaukee, WI, USA, was used as received.

(2) $99.9 \%$, anhydrous, Aldrich Chemical Company, stored over molecular sieves and distilled shortly before use.

Estimated Error:

Temperature: $\pm 0.1 \mathrm{~K}$.

$x_{1}: \pm 2.0 \%$ (relative error).

\section{Components:}

(1) 3,5-Dinitrobenzoic acid;

$\mathrm{C}_{7} \mathrm{H}_{4} \mathrm{~N}_{2} \mathrm{O}_{6}$; [99-34-3]

(2) Tetrahydrofuran; $\mathrm{C}_{4} \mathrm{H}_{8} \mathrm{O}$; [109-99-9]

\begin{tabular}{ll}
\hline Variables: & Prepared by: \\
$T / \mathrm{K}=303.15$ & W. E. Acree, Jr. \\
\hline
\end{tabular}

\begin{tabular}{lc}
\hline \hline$x_{2}{ }^{\mathrm{a}}$ & $x_{1}{ }^{\mathrm{b}}$ \\
\hline 0.827 & 0.173
\end{tabular}

${ }^{\mathrm{a}} x_{2}$ : mole fraction of component 2 in the saturated solution.

${ }^{\mathrm{b}} x_{1}$ : mole fraction solubility of the solute.

\section{Auxiliary Information}

\section{Method/Apparatus/Procedure:}

Constant-temperature bath, Soxhlet thimble, conical flask, and analytical balance.

Excess solute and solvent were placed in a conical flask and allowed to equilibrate for several days at constant temperature. Attainment of equilibrium was verified by several repetitive measurements and by approaching equilibrium from supersaturation. Aliquots of saturated solutions were transferred using a Soxhlet thimble equipped with a one-hole cork stopper and an inverted U-shaped delivery tube to a second ground-glass stoppered flask suspended in the $303 \mathrm{~K}$ water bath. Successive portions of the contents were evaporated at room temperature in a tared aluminum foil weighing dish under a bell jar through which a slow stream of dry air was passed. The second flask plus unused saturated solution and the aluminum foil dish plus residue were weighed. The saturation solubility of the solute was calculated from the recorded mass data and molar masses of the solute and solvent.

\section{Source and Purity of Chemicals:}

(1) Purity not given, Chemical source not specified, was recrystallized several times from aqueous-ethanol mixture, and then dried under vacuum over phosphorous pentoxide.

(2) Practical grade, Chemical source not specified, stored over sodium hydroxide pellets for $24 \mathrm{~h}$, and then passed through $2 \times 70-\mathrm{cm}$ chromatographic adsorption columns containing activated alumina. After this treatment, the purified solvent was stored over copper in a nitrogen atmosphere. 
Estimated Error:

Temperature: $\pm 0.02 \mathrm{~K}$

$x_{1}: \pm 1.0 \%$ (relative error).

\begin{tabular}{|c|c|}
\hline $\begin{array}{l}\text { Components: } \\
\text { (1) 3,5-Dinitrobenzoic acid; } \\
\left.\mathrm{C}_{7} \mathrm{H}_{4} \mathrm{~N}_{2} \mathrm{O}_{6} ; \text {; } 99-34-3\right] \\
\text { (2) 1,4-Dioxane; } \mathrm{C}_{4} \mathrm{H}_{8} \mathrm{O}_{2} \text {; } \\
\text { [123-91-1] }\end{array}$ & $\begin{array}{l}\text { Original Measurements: } \\
{ }^{140} \text { K. R. Hoover, R. Coaxum, E. } \\
\text { Pustejovsky, W. E. Acree, Jr., and } \\
\text { M. H. Abraham, Phys. Chem. Liq. 42, } \\
457 \text { (2004). }\end{array}$ \\
\hline $\begin{array}{l}\text { Variables: } \\
T / \mathrm{K}=298.15\end{array}$ & $\begin{array}{l}\text { Prepared by: } \\
\text { W. E. Acree, Jr. }\end{array}$ \\
\hline
\end{tabular}

Experimental Values

\begin{tabular}{lc}
\hline \hline$x_{2}{ }^{\mathrm{a}}$ & $x_{1}{ }^{\mathrm{b}}$ \\
\hline 0.9112 & 0.08878
\end{tabular}

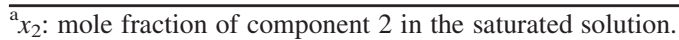

${ }^{\mathrm{b}} x_{1}$ : mole fraction solubility of the solute.

\section{Auxiliary Information}

\section{Method/Apparatus/Procedure:}

Constant-temperature bath, calorimetric thermometer, and an ultraviolet/ visible spectrophotometer.

Excess solute and solvent were placed in amber glass bottles and allowed to equilibrate for several days at constant temperature. Attainment of equilibrium was verified by several repetitive measurements and by approaching equilibrium from supersaturation. Aliquots of saturated solutions were transferred through a coarse filter into tared volumetric flasks, weighed, and diluted with methanol. Concentrations were determined by spectrophotometric measurements at $267 \mathrm{~nm}$.

Source and Purity of Chemicals:

(1) $99+\%$, Aldrich Chemical Company, Milwaukee, WI, USA, was used as received.

(2) $99.8 \%$, anhydrous, Aldrich Chemical Company, stored over molecular sieves and distilled shortly before use.

Estimated Error:

Temperature: $\pm 0.1 \mathrm{~K}$.

$x_{1}: \pm 2.0 \%$ (relative error).

\begin{tabular}{ll}
\hline \hline Components: & Original Measurements: \\
$\begin{array}{l}\text { (1) 3,5-Dinitrobenzoic acid; } \\
\mathrm{C}_{7} \mathrm{H}_{4} \mathrm{~N}_{2} \mathrm{O}_{6} ; \text { [99-34-3] }\end{array}$ & $\begin{array}{l}{ }^{63} \mathrm{C} \text {. K. Hancock, J. N. Pawloski, } \\
\text { and J. P. Idoux, J. Org. Chem. 32, }\end{array}$ \\
\hline (2) 1,4-Dioxane; $\mathrm{C}_{4} \mathrm{H}_{8} \mathrm{O}_{2} ;[$ [123-91-1] & 1931 (1967). \\
\hline Variables: & Prepared by: \\
$T / \mathrm{K}=303.15$ & W. E. Acree, Jr. \\
\hline
\end{tabular}

Experimental Values

\begin{tabular}{lc}
\hline \hline$x_{2}^{\mathrm{a}}$ & $x_{1}{ }^{\mathrm{b}}$ \\
\hline 0.900 & 0.100 \\
\hline${ }^{\mathrm{a}} x_{2}:$ mole fraction of component 2 in the saturated solution. \\
${ }^{\mathrm{b}} x_{1}:$ mole fraction solubility of the solute.
\end{tabular}

\section{Auxiliary Information}

Method/Apparatus/Procedure:

Constant-temperature bath, Soxhlet thimble, conical flask, and analytical balance.

Excess solute and solvent were placed in a conical flask and allowed to equilibrate for several days at constant temperature. Attainment of equilibrium was verified by several repetitive measurements and by approaching equilibrium from supersaturation. Weighed aliquots of saturated solutions were removed and titrated with a standardized sodium hydroxide solution (carbonate free) using a $\mathrm{pH}$ meter. The endpoint of the titration was determined by computing the second derivative in the $\mathrm{pH}$ versus volume of sodium hydroxide added.

\section{Source and Purity of Chemicals:}

(1) Purity not given, Chemical source not specified, was recrystallized several times from aqueous-ethanol mixture, and then dried under vacuum over phosphorous pentoxide.

(2) Practical grade, Chemical source not specified, stored over sodium hydroxide pellets for $24 \mathrm{~h}$, and then passed through $2 \times 70-\mathrm{cm}$ chromatographic adsorption columns containing activated alumina. After this treatment, the purified solvent was stored over copper in a nitrogen atmosphere.

\section{Estimated Error:}

Temperature: $\pm 0.02 \mathrm{~K}$

$x_{1}: \pm 1.0 \%$ (relative error)

\subsection{3,5-Dinitrobenzoic acid solubility data in alcohols}

\begin{tabular}{|c|c|}
\hline $\begin{array}{l}\text { Components: } \\
\text { (1) } 3,5 \text {-Dinitrobenzoic acid; } \\
\mathrm{C}_{7} \mathrm{H}_{4} \mathrm{~N}_{2} \mathrm{O}_{6} \text {; [99-34-3] } \\
\text { (2) Methanol; } \mathrm{CH}_{4} \mathrm{O} ;[67-56-1]\end{array}$ & $\begin{array}{l}\text { Original Measurements: } \\
{ }^{83} \text { M. K. Chantooni and I. M. Kolthoff, } \\
\text { J. Phys. Chem. 77, } 527 \text { (1973). }\end{array}$ \\
\hline $\begin{array}{l}\text { Variables: } \\
T / \mathrm{K}=298.15\end{array}$ & $\begin{array}{l}\text { Prepared by: } \\
\text { W. E. Acree, Jr. }\end{array}$ \\
\hline
\end{tabular}

\section{Experimental Values}

The measured solubility was reported to be $1.23 \mathrm{~mol} \mathrm{dm}^{-3}$. The authors did not give the temperature at which the solubility was measured. Based on the experimental description given in an earlier paper [I. M. Kolthoff, J. J. Lingane, and W. Larson, J. Am. Chem. Soc. 60, 2512 (1938)], the compiler believes the temperature to be $298.15 \mathrm{~K}$.

\section{Auxiliary Information}

\section{Method/Apparatus/Procedure:}

Very little experimental details were provided. Solubility was determined by titrating a filtered aliquot of the saturated solution alkalimetrically in an aqueous-ethanol mixture using phenolphthalein as the acid-base indicator.

\section{Source and Purity of Chemicals:}

(1) White Label, Eastman Kodak Chemical Company, Rochester, NY, USA, was recrystallized from aqueous ethanol solution and dried in vacuo over barium oxide at $343 \mathrm{~K}$.

(2) Purity not given, Chemical source not given, was dehydrated and then distilled over sulfanilic acid to remove alkaline impurities.

\section{Estimated Error:}

Temperature: $\pm 0.1 \mathrm{~K}$ (estimated by compiler).

$c_{1}: \pm 2 \%$ (relative error, estimated by compiler). 


\begin{tabular}{ll}
\hline \hline Components: & Original Measurements: \\
(1) 3,5-Dinitrobenzoic acid; & ${ }^{140}$ K. R. Hoover, R. Coaxum, E. \\
$\mathrm{C}_{7} \mathrm{H}_{4} \mathrm{~N}_{2} \mathrm{O}_{6} ;[$ [99-34-3] & Pustejovsky, W. E. Acree, Jr., and \\
(2) Ethanol; $\mathrm{C}_{2} \mathrm{H}_{6} \mathrm{O} ;$ [64-17-7] & $\begin{array}{l}\text { M. H. Abraham, Phys. Chem. Liq. 42, } \\
\end{array}$ \\
\hline Variables: & Prepared by: \\
$T / \mathrm{K}=298.15$ & W. E. Acree, Jr. \\
\hline
\end{tabular}

\section{Experimental Values}

\begin{tabular}{lc}
\hline \hline$x_{2}{ }^{\mathrm{a}}$ & $x_{1}{ }^{\mathrm{b}}$ \\
\hline 0.9538 & 0.04620 \\
\hline
\end{tabular}

${ }^{\mathrm{a}} x_{2}$ : mole fraction of component 2 in the saturated solution.

${ }^{\mathrm{b}} x_{1}$ : mole fraction solubility of the solute.

\section{Auxiliary Information}

\section{Method/Apparatus/Procedure:}

Constant-temperature bath, calorimetric thermometer, and an ultraviolet/ visible spectrophotometer.

Excess solute and solvent were placed in amber glass bottles and allowed to equilibrate for several days at constant temperature. Attainment of equilibrium was verified by several repetitive measurements and by approaching equilibrium from supersaturation. Aliquots of saturated solutions were transferred through a coarse filter into tared volumetric flasks, weighed, and diluted with methanol. Concentrations were determined by spectrophotometric measurements at $267 \mathrm{~nm}$.

Source and Purity of Chemicals:

(1) $99+\%$, Aldrich Chemical Company, Milwaukee, WI, USA, was used as received.

(2) Absolute, Aaper Alcohol and Chemical Company, USA, stored over molecular sieves and distilled shortly before use.

Estimated Error:

Temperature: $\pm 0.1 \mathrm{~K}$.

$x_{1}: \pm 1.5 \%$ (relative error).

\begin{tabular}{ll}
\hline \hline Components: & Original Measurements: \\
(1) 3,5-Dinitrobenzoic acid; & ${ }^{140}$ K. R. Hoover, R. Coaxum, E. \\
$\mathrm{C}_{7} \mathrm{H}_{4} \mathrm{~N}_{2} \mathrm{O}_{6} ;$ [99-34-3] & Pustejovsky, W. E. Acree, Jr., and \\
(2) 1-Propanol; $\mathrm{C}_{3} \mathrm{H}_{8} \mathrm{O} ;[71-23-8]$ & $\begin{array}{l}\text { M. H. Abraham, Phys. Chem. Liq. 42, } \\
457(2004) .\end{array}$ \\
\hline Variables: & Prepared by: \\
$T / \mathrm{K}=298.15$ & W. E. Acree, Jr. \\
\hline
\end{tabular}

Experimental Values

\begin{tabular}{lc}
\hline \hline$x_{2}{ }^{\mathrm{a}}$ & $x_{1}{ }^{\mathrm{b}}$ \\
\hline 0.9636 & 0.03637 \\
\hline${ }^{\mathrm{a}} x_{2}:$ mole fraction of component 2 in the saturated solution. & \\
${ }^{\mathrm{b}} x_{1}:$ mole fraction solubility of the solute. &
\end{tabular}

\section{Auxiliary Information}

Method/Apparatus/Procedure:

Constant-temperature bath, calorimetric thermometer, and an ultraviolet/ visible spectrophotometer.

Excess solute and solvent were placed in amber glass bottles and allowed to equilibrate for several days at constant temperature. Attainment of equilibrium was verified by several repetitive measurements and by approaching equilibrium from supersaturation. Aliquots of saturated solutions were transferred through a coarse filter into tared volumetric flasks, weighed, and diluted with methanol. Concentrations were determined by spectrophotometric measurements at $267 \mathrm{~nm}$.

Source and Purity of Chemicals:

(1) $99+\%$, Aldrich Chemical Company, Milwaukee, WI, USA, was used as received.

(2) $99+\%$, anhydrous, Aldrich Chemical Company, stored over molecular sieves and distilled shortly before use.

Estimated Error:

Temperature: $\pm 0.1 \mathrm{~K}$.

$x_{1}: \pm 1.5 \%$ (relative error).

\begin{tabular}{ll}
\hline \hline Components: & Original Measurements: \\
(1) 3,5-Dinitrobenzoic acid; & ${ }^{140}$ K. R. Hoover, R. Coaxum, E. \\
$\mathrm{C}_{7} \mathrm{H}_{4} \mathrm{~N}_{2} \mathrm{O}_{6} ;$ [99-34-3] & Pustejovsky, W. E. Acree, Jr., and \\
(2) 2-Propanol; $\mathrm{C}_{3} \mathrm{H}_{8} \mathrm{O} ;$ [67-63-0] & M. H. Abraham, Phys. Chem. Liq. 42, \\
& 457 (2004). \\
\hline Variables: & Prepared by: \\
$T / \mathrm{K}=298.15$ & W. E. Acree, Jr. \\
\hline
\end{tabular}

Experimental Values

\begin{tabular}{lc}
\hline \hline$x_{2}{ }^{\mathrm{a}}$ & $x_{1}{ }^{\mathrm{b}}$ \\
\hline 0.9661 & 0.03390 \\
\hline${ }^{\mathrm{a}} x_{2}:$ mole fraction of component 2 in the saturated solution. & \\
${ }^{\mathrm{b}} x_{1}:$ mole fraction solubility of the solute. &
\end{tabular}

\section{Auxiliary Information}

\section{Method/Apparatus/Procedure:}

Constant-temperature bath, calorimetric thermometer, and an ultraviolet/ visible spectrophotometer.

Excess solute and solvent were placed in amber glass bottles and allowed to equilibrate for several days at constant temperature. Attainment of equilibrium was verified by several repetitive measurements and by approaching equilibrium from supersaturation. Aliquots of saturated solutions were transferred through a coarse filter into tared volumetric flasks, weighed, and diluted with methanol. Concentrations were determined by

spectrophotometric measurements at $267 \mathrm{~nm}$.

Source and Purity of Chemicals:

(1) $99+\%$, Aldrich Chemical Company, Milwaukee, WI, USA, was used as received.

(2) $99+\%$, anhydrous, Aldrich Chemical Company, stored over molecular sieves and distilled shortly before use.

Estimated Error:

Temperature: $\pm 0.1 \mathrm{~K}$.

$x_{1}: \pm 1.5 \%$ (relative error). 


\begin{tabular}{|c|c|}
\hline $\begin{array}{l}\text { Components: } \\
\text { (1) 3,5-Dinitrobenzoic acid; } \\
\mathrm{C}_{7} \mathrm{H}_{4} \mathrm{~N}_{2} \mathrm{O}_{6} \text {; }[99-34-3] \\
\text { (2) 2-Propanol; } \mathrm{C}_{3} \mathrm{H}_{8} \mathrm{O} ;[67-63-0]\end{array}$ & $\begin{array}{l}\text { Original Measurements: } \\
{ }^{76} \text { M. K. Chantooni and I. M. Kolthoff, } \\
\text { Anal. Chem. 51, } 133 \text { (1979). }\end{array}$ \\
\hline $\begin{array}{l}\text { Variables: } \\
T / \mathrm{K}=298.15\end{array}$ & $\begin{array}{l}\text { Prepared by: } \\
\text { W. E. Acree, Jr. }\end{array}$ \\
\hline
\end{tabular}

Experimental Values

The measured solubility was reported to be $0.451 \mathrm{~mol} \mathrm{dm}^{-3}$. The authors did not give the temperature at which the solubility was measured. Based on the experimental description given in an earlier paper [I. M. Kolthoff, J. J. Lingane, and W. Larson, J. Am. Chem. Soc. 60, 2512 (1938)], the compiler believes the temperature to be $298.15 \mathrm{~K}$.

\section{Auxiliary Information}

\section{Method/Apparatus/Procedure:}

Very little experimental details were provided. Solubility was determined by titrating a filtered aliquot of the saturated solution alkalimetrically in an aqueous-ethanol mixture using phenolphthalein as the acid-base indicator.

\section{Source and Purity of Chemicals:}

(1) White Label, Eastman Kodak Chemical Company, Rochester, NY, USA, was recrystallized from aqueous ethanol solution and dried in vacuo over barium oxide at $343 \mathrm{~K}$.

(2) Purity not given, Fisher Scientific Chemical Company, USA, shaken with calcium hydride and distilled before use.

\section{Estimated Error:}

Temperature: $\pm 0.1 \mathrm{~K}$ (estimated by compiler).

$c_{1}: \pm 2 \%$ (relative error, estimated by compiler).

\begin{tabular}{ll}
\hline \hline Components: & Original Measurements: \\
(1) 3,5-Dinitrobenzoic acid; & ${ }^{140}$ K. R. Hoover, R. Coaxum, E. \\
$\mathrm{C}_{7} \mathrm{H}_{4} \mathrm{~N}_{2} \mathrm{O}_{6} ;$ [ [99-34-3] & Pustejovsky, W. E. Acree, Jr., and \\
(2) 1-Butanol; $\mathrm{C}_{4} \mathrm{H}_{10} \mathrm{O} ;$ [71-36-3] & $\begin{array}{l}\text { M. H. Abraham, Phys. Chem. Liq. 42, } \\
457 \text { (2004). }\end{array}$ \\
\hline Variables: & Prepared by: \\
$T / \mathrm{K}=298.15$ & W. E. Acree, Jr. \\
\hline
\end{tabular}

Experimental Values

\begin{tabular}{lc}
\hline \hline$x_{2}{ }^{\mathrm{a}}$ & $x_{1}{ }^{\mathrm{b}}$ \\
\hline 0.9683 & 0.03169
\end{tabular}

${ }^{\mathrm{a}} x_{2}$ : mole fraction of component 2 in the saturated solution.

${ }^{\mathrm{b}} x_{1}$ : mole fraction solubility of the solute.

\section{Auxiliary Information}

\section{Method/Apparatus/Procedure:}

Constant-temperature bath, calorimetric thermometer, and an ultraviolet/ visible spectrophotometer.

Excess solute and solvent were placed in amber glass bottles and allowed to equilibrate for several days at constant temperature. Attainment of equilibrium was verified by several repetitive measurements and by approaching equilibrium from supersaturation. Aliquots of saturated solutions were transferred through a coarse filter into tared volumetric flasks, weighed, and diluted with methanol. Concentrations were determined by spectrophotometric measurements at $267 \mathrm{~nm}$.
Source and Purity of Chemicals:

(1) $99+\%$, Aldrich Chemical Company, Milwaukee, WI, USA, was used as received.

(2) $99.8+\%$, HPLC grade, Aldrich Chemical Company, stored over molecular sieves and distilled shortly before use.

\section{Estimated Error:}

Temperature: $\pm 0.1 \mathrm{~K}$.

$x_{1}: \pm 1.5 \%$ (relative error).

\begin{tabular}{ll}
\hline \hline Components: & Original Measurements: \\
(1) 3,5-Dinitrobenzoic acid; & ${ }^{140}$ K. R. Hoover, R. Coaxum, E. \\
$\mathrm{C}_{7} \mathrm{H}_{4} \mathrm{~N}_{2} \mathrm{O}_{6} ;$ [99-34-3] & Pustejovsky, W. E. Acree, Jr., and M. \\
(2) 2-Butanol; $\mathrm{C}_{4} \mathrm{H}_{10} \mathrm{O} ;$ [78-92-2] & $\begin{array}{l}\text { H. Abraham, Phys. Chem. Liq. 42, 457 } \\
\text { (2004). }\end{array}$ \\
\hline Variables: & Prepared by: \\
$T / \mathrm{K}=298.15$ & W. E. Acree, Jr. \\
\hline
\end{tabular}

\section{Experimental Values}

\begin{tabular}{lc}
\hline \hline$x_{2}{ }^{\mathrm{a}}$ & $x_{1}{ }^{\mathrm{b}}$ \\
\hline 0.9702 & 0.02984 \\
\hline${ }^{\mathrm{a}} x_{2}:$ mole fraction of component 2 in the saturated solution. & \\
${ }^{\mathrm{b}} x_{1}:$ mole fraction solubility of the solute. &
\end{tabular}

\section{Auxiliary Information}

\section{Method/Apparatus/Procedure:}

Constant-temperature bath, calorimetric thermometer, and an ultraviolet/ visible spectrophotometer.

Excess solute and solvent were placed in amber glass bottles and allowed to equilibrate for several days at constant temperature. Attainment of equilibrium was verified by several repetitive measurements and by approaching equilibrium from supersaturation. Aliquots of saturated solutions were transferred through a coarse filter into tared volumetric flasks, weighed, and diluted with methanol. Concentrations were determined by spectrophotometric measurements at $267 \mathrm{~nm}$.

\section{Source and Purity of Chemicals:}

(1) $99+\%$, Aldrich Chemical Company, Milwaukee, WI, USA, was used as received.

(2) $99+\%$, anhydrous, Aldrich Chemical Company, stored over molecular sieves and distilled shortly before use.

\section{Estimated Error:}

Temperature: $\pm 0.1 \mathrm{~K}$.

$x_{1}: \pm 1.5 \%$ (relative error).

\begin{tabular}{|c|c|}
\hline $\begin{array}{l}\text { Components: } \\
\text { (1) 3,5-Dinitrobenzoic acid; } \\
\mathrm{C}_{7} \mathrm{H}_{4} \mathrm{~N}_{2} \mathrm{O}_{6} \text {; [99-34-3] } \\
\text { (2) 2-Methyl-1-propanol; } \mathrm{C}_{4} \mathrm{H}_{10} \mathrm{O} \text {; } \\
\text { [78-83-1] }\end{array}$ & $\begin{array}{l}\text { Original Measurements: } \\
{ }^{140} \text { K. R. Hoover, R. Coaxum, E. } \\
\text { Pustejovsky, W. E. Acree, Jr., and } \\
\text { M. H. Abraham, Phys. Chem. Liq. 42, } \\
457 \text { (2004). }\end{array}$ \\
\hline $\begin{array}{l}\text { Variables: } \\
T / \mathrm{K}=298.15\end{array}$ & $\begin{array}{l}\text { Prepared by: } \\
\text { W. E. Acree, Jr. }\end{array}$ \\
\hline
\end{tabular}


Experimental Values

\begin{tabular}{lc}
\hline \hline$x_{2}{ }^{\mathrm{a}}$ & $x_{1}{ }^{\mathrm{b}}$ \\
\hline 0.9783 & 0.02169 \\
${ }^{\mathrm{a}} x_{2}:$ mole fraction of component 2 in the saturated solution. & \\
${ }^{\mathrm{b}} x_{1}:$ mole fraction solubility of the solute. &
\end{tabular}

\section{Auxiliary Information}

\section{Method/Apparatus/Procedure:}

Constant-temperature bath, calorimetric thermometer, and an ultraviolet/ visible spectrophotometer.

Excess solute and solvent were placed in amber glass bottles and allowed to equilibrate for several days at constant temperature. Attainment of equilibrium was verified by several repetitive measurements and by approaching equilibrium from supersaturation. Aliquots of saturated solutions were transferred through a coarse filter into tared volumetric flasks, weighed, and diluted with methanol. Concentrations were determined by spectrophotometric measurements at $267 \mathrm{~nm}$.

\section{Source and Purity of Chemicals:}

(1) $99+\%$, Aldrich Chemical Company, Milwaukee, WI, USA, was used as received.

(2) $99+\%$, anhydrous, Aldrich Chemical Company, stored over molecular sieves and distilled shortly before use.

\section{Estimated Error:}

Temperature: $\pm 0.1 \mathrm{~K}$.

$x_{1}: \pm 1.5 \%$ (relative error).

\begin{tabular}{|c|c|}
\hline $\begin{array}{l}\text { Components: } \\
\text { (1) 3,5-Dinitrobenzoic acid; } \\
\left.\mathrm{C}_{7} \mathrm{H}_{4} \mathrm{~N}_{2} \mathrm{O}_{6} ; \text {; } 99-34-3\right] \\
\text { (2) 2-Methyl-2-propanol; } \mathrm{C}_{4} \mathrm{H}_{10} \mathrm{O} \text {; } \\
\text { [75-65-0] }\end{array}$ & $\begin{array}{l}\text { Original Measurements: } \\
{ }^{76} \text { M. K. Chantooni and I. M. Kolthoff, } \\
\text { Anal. Chem. 51, } 133 \text { (1979). }\end{array}$ \\
\hline $\begin{array}{l}\text { Variables: } \\
T / \mathrm{K}=298.15\end{array}$ & $\begin{array}{l}\text { Prepared by: } \\
\text { W. E. Acree, Jr. }\end{array}$ \\
\hline
\end{tabular}

\section{Experimental Values}

The measured solubility was reported to be $0.625 \mathrm{~mol} \mathrm{dm}^{-3}$. The authors did not give the temperature at which the solubility was measured. Based on the experimental description given in an earlier paper [I. M. Kolthoff, J. J. Lingane, and W. Larson, J. Am. Chem. Soc. 60, 2512 (1938)], the compiler believes the temperature to be $298.15 \mathrm{~K}$.

\section{Auxiliary Information}

\section{Method/Apparatus/Procedure:}

Very little experimental details were provided. Solubility was determined by titrating a filtered aliquot of the saturated solution alkalimetrically in an aqueous-ethanol mixture using phenolphthalein as the acid-base indicator.

\section{Source and Purity of Chemicals:}

(1) White Label, Eastman Kodak Chemical Company, Rochester, NY, USA, was recrystallized from aqueous ethanol solution and dried in vacuo over barium oxide at $343 \mathrm{~K}$.

(2) White Label, Eastman Kodak Chemical Company, Rochester, NY, USA, shaken with calcium hydride and distilled before use.
Estimated Error:

Temperature: $\pm 0.1 \mathrm{~K}$ (estimated by compiler).

$c_{1}: \pm 2 \%$ (relative error, estimated by compiler).

\begin{tabular}{ll}
\hline \hline Components: & Original Measurements: \\
(1) 3,5-Dinitrobenzoic acid; & ${ }^{140}$ K. R. Hoover, R. Coaxum, E. \\
$\mathrm{C}_{7} \mathrm{H}_{4} \mathrm{~N}_{2} \mathrm{O}_{6} ;$ [99-34-3] & Pustejovsky, W. E. Acree, Jr., and \\
(2) 1-Pentanol; $\mathrm{C}_{5} \mathrm{H}_{12} \mathrm{O} ;$ [71-41-0] & $\begin{array}{l}\text { M. H. Abraham, Phys. Chem. Liq. 42, } \\
457 \text { (2004). }\end{array}$ \\
\hline Variables: & Prepared by: \\
$T / \mathrm{K}=298.15$ & W. E. Acree, Jr. \\
\hline
\end{tabular}

Experimental Values

\begin{tabular}{lc}
\hline \hline$x_{2}{ }^{\mathrm{a}}$ & $x_{1}{ }^{\mathrm{b}}$ \\
\hline 0.9685 & 0.03153 \\
\hline${ }^{\mathrm{a}} x_{2}:$ mole fraction of component 2 in the saturated solution. \\
${ }^{\mathrm{b}}{ }_{x_{1}: \text { mole fraction solubility of the solute. }}$
\end{tabular}

\section{Auxiliary Information}

\section{Method/Apparatus/Procedure:}

Constant-temperature bath, calorimetric thermometer, and an ultraviolet/ visible spectrophotometer.

Excess solute and solvent were placed in amber glass bottles and allowed to equilibrate for several days at constant temperature. Attainment of equilibrium was verified by several repetitive measurements and by approaching equilibrium from supersaturation. Aliquots of saturated solutions were transferred through a coarse filter into tared volumetric flasks, weighed, and diluted with methanol. Concentrations were determined by spectrophotometric measurements at $267 \mathrm{~nm}$.

\section{Source and Purity of Chemicals:}

(1) $99+\%$, Aldrich Chemical Company, Milwaukee, WI, USA, was used as received.

(2) $99+\%$, Aldrich Chemical Company, stored over molecular sieves and distilled shortly before use.

\section{Estimated Error:}

Temperature: $\pm 0.1 \mathrm{~K}$.

$x_{1}: \pm 1.5 \%$ (relative error).

\begin{tabular}{ll}
\hline \hline Components: & Original Measurements: \\
(1) 3,5-Dinitrobenzoic acid; & ${ }^{140}$ K. R. Hoover, R. Coaxum, E. \\
$\mathrm{C}_{7} \mathrm{H}_{4} \mathrm{~N}_{2} \mathrm{O}_{6} ;$ [ [99-34-3] & Pustejovsky, W. E. Acree, Jr., and \\
$\begin{array}{l}\text { (2) 2-Pentanol; } \mathrm{C}_{5} \mathrm{H}_{10} \mathrm{O} ; \\
\text { [6032-29-7] }\end{array}$ & M. H. Abraham, Phys. Chem. Liq. 42, \\
\hline Variables: & 457 (2004). \\
$T / \mathrm{K}=298.15$ & Prepared by: \\
\hline
\end{tabular}

Experimental Values

\begin{tabular}{lc}
\hline \hline$x_{2}{ }^{\mathrm{a}}$ & $x_{1}{ }^{\mathrm{b}}$ \\
\hline 0.9669 & 0.03308 \\
\hline${ }^{\mathrm{a}} x_{2}:$ mole fraction of component 2 in the saturated solution. \\
${ }^{\mathrm{b}} x_{1}:$ mole fraction solubility of the solute.
\end{tabular}




\section{Auxiliary Information}

\section{Method/Apparatus/Procedure:}

Constant-temperature bath, calorimetric thermometer, and an ultraviolet/ visible spectrophotometer.

Excess solute and solvent were placed in amber glass bottles and allowed to equilibrate for several days at constant temperature. Attainment of equilibrium was verified by several repetitive measurements and by approaching equilibrium from supersaturation. Aliquots of saturated solutions were transferred through a coarse filter into tared volumetric flasks, weighed, and diluted with methanol. Concentrations were determined by spectrophotometric measurements at $267 \mathrm{~nm}$.

\section{Source and Purity of Chemicals:}

(1) $99+\%$, Aldrich Chemical Company, Milwaukee, WI, USA, was used as received.

(2) $99+\%$, Acros Organics, USA, stored over molecular sieves and distilled shortly before use.

\section{Estimated Error:}

Temperature: $\pm 0.1 \mathrm{~K}$.

$x_{1}: \pm 1.5 \%$ (relative error)

\begin{tabular}{ll}
\hline \hline Components: & Original Measurements: \\
(1) 3,5-Dinitrobenzoic acid; & ${ }^{140}$ K. R. Hoover, R. Coaxum, E. \\
$\mathrm{C}_{7} \mathrm{H}_{4} \mathrm{~N}_{2} \mathrm{O}_{6} ;$ [99-34-3] & Pustejovsky, W. E. Acree, Jr., and \\
$\begin{array}{l}\text { (2) 3-Methyl-1-butanol; } \mathrm{C}_{5} \mathrm{H}_{10} \mathrm{O} ; \\
\text { [123-51-3] }\end{array}$ & $\begin{array}{l}\text { M. H. Abraham, Phys. Chem. Liq. 42, } \\
457 \text { (2004). }\end{array}$ \\
\hline Variables: & Prepared by: \\
$T / \mathrm{K}=298.15$ & W. E. Acree, Jr. \\
\hline
\end{tabular}

\section{Experimental Values}

\begin{tabular}{lc}
\hline \hline$x_{2}{ }^{\mathrm{a}}$ & $x_{1}{ }^{\mathrm{b}}$ \\
\hline 0.9705 & 0.02948
\end{tabular}

${ }^{\mathrm{a}} x_{2}$ : mole fraction of component 2 in the saturated solution.

${ }^{\mathrm{b}} x_{1}$ : mole fraction solubility of the solute.

\section{Auxiliary Information}

\footnotetext{
Method/Apparatus/Procedure:

Constant-temperature bath, calorimetric thermometer, and an ultraviolet/ visible spectrophotometer.

Excess solute and solvent were placed in amber glass bottles and allowed to equilibrate for several days at constant temperature. Attainment of equilibrium was verified by several repetitive measurements and by approaching equilibrium from supersaturation. Aliquots of saturated solutions were transferred through a coarse filter into tared volumetric flasks, weighed, and diluted with methanol. Concentrations were determined by spectrophotometric measurements at $267 \mathrm{~nm}$.

Source and Purity of Chemicals:

(1) $99+\%$, Aldrich Chemical Company, Milwaukee, WI, USA, was used as received.

(2) $99 \%$, anhydrous, Aldrich Chemical Company, stored over molecular sieves and distilled shortly before use.
}

\section{Estimated Error:}

Temperature: $\pm 0.1 \mathrm{~K}$.

$x_{1}: \pm 1.5 \%$ (relative error)

\begin{tabular}{ll}
\hline \hline Components: & Original Measurements: \\
(1) 3,5-Dinitrobenzoic acid; & ${ }^{140}$ K. R. Hoover, R. Coaxum, E. \\
$\mathrm{C}_{7} \mathrm{H}_{4} \mathrm{~N}_{2} \mathrm{O}_{6}$; [99-34-3] & Pustejovsky, W. E. Acree, Jr., and \\
(2) 1-Hexanol; $\mathrm{C}_{6} \mathrm{H}_{14} \mathrm{O} ;$ [111-27-3] & $\begin{array}{l}\text { M. H. Abraham, Phys. Chem. Liq. 42, } \\
457 \text { (2004). }\end{array}$ \\
\hline Variables: & Prepared by: \\
$T / \mathrm{K}=298.15$ & W. E. Acree, Jr. \\
\hline
\end{tabular}

\section{Experimental Values}

\begin{tabular}{lc}
\hline \hline$x_{2}{ }^{\mathrm{a}}$ & $x_{1}{ }^{\mathrm{b}}$ \\
\hline 0.9700 & 0.02997 \\
\hline
\end{tabular}

${ }^{\mathrm{a}} x_{2}$ : mole fraction of component 2 in the saturated solution.

${ }^{b} x_{1}$ : mole fraction solubility of the solute.

\section{Auxiliary Information}

\section{Method/Apparatus/Procedure:}

Constant-temperature bath, calorimetric thermometer, and an ultraviolet/ visible spectrophotometer.

Excess solute and solvent were placed in amber glass bottles and allowed to equilibrate for several days at constant temperature. Attainment of equilibrium was verified by several repetitive measurements and by approaching equilibrium from supersaturation. Aliquots of saturated solutions were transferred through a coarse filter into tared volumetric flasks, weighed, and diluted with methanol. Concentrations were determined by spectrophotometric measurements at $267 \mathrm{~nm}$.

\section{Source and Purity of Chemicals:}

(1) $99+\%$, Aldrich Chemical Company, Milwaukee, WI, USA, was used as received.

(2) $99+\%$, Alfa Aesar, USA, stored over molecular sieves and distilled shortly before use.

\section{Estimated Error:}

Temperature: $\pm 0.1 \mathrm{~K}$.

$x_{1}: \pm 1.5 \%$ (relative error)

\begin{tabular}{ll}
\hline \hline Components: & Original Measurements: \\
(1) 3,5-Dinitrobenzoic acid; & ${ }^{76}$ M. K. Chantooni and I. M. Kolthoff, \\
$\mathrm{C}_{7} \mathrm{H}_{4} \mathrm{~N}_{2} \mathrm{O}_{6} ;[99-34-3]$ & Anal. Chem. 51, 133 (1979). \\
(2) 1-Hexanol; $\mathrm{C}_{6} \mathrm{H}_{14} \mathrm{O} ;[111-27-3]$ & \\
\hline Variables: & Prepared by: \\
$T / \mathrm{K}=298.15$ & W. E. Acree, Jr. \\
\hline
\end{tabular}

\section{Experimental Values}

The measured solubility was reported to be $0.357 \mathrm{~mol} \mathrm{dm}^{-3}$. The authors did not give the temperature at which the solubility was measured. Based on the experimental description given in an earlier paper [I. M. Kolthoff, J. J. Lingane, and W. Larson, J. Am. Chem. Soc. 60, 2512 (1938)], the compiler believes the temperature to be $298.15 \mathrm{~K}$. 


\section{Auxiliary Information}

\section{Method/Apparatus/Procedure:}

Very little experimental details were provided. Solubility was determined by titrating a filtered aliquot of the saturated solution alkalimetrically in an aqueous-ethanol mixture using phenolphthalein as the acid-base indicator.

Source and Purity of Chemicals:

(1) White Label, Eastman Kodak Chemical Company, Rochester, NY, USA, was recrystallized from aqueous ethanol solution and dried in vacuo over barium oxide at $343 \mathrm{~K}$.

(2) Yellow Label, Eastman Kodak Chemical Company, Rochester, NY, USA, shaken with calcium hydride and distilled before use.

\section{Estimated Error:}

Temperature: $\pm 0.1 \mathrm{~K}$ (estimated by compiler). $c_{1}: \pm 2 \%$ (relative error, estimated by compiler).

\begin{tabular}{|c|c|}
\hline $\begin{array}{l}\text { Components: } \\
\text { (1) 3,5-Dinitrobenzoic acid; } \\
\mathrm{C}_{7} \mathrm{H}_{4} \mathrm{~N}_{2} \mathrm{O}_{6} ;[99-34-3] \\
\text { (2) 1-Heptanol; } \mathrm{C}_{7} \mathrm{H}_{16} \mathrm{O} \text {; } \\
\text { [111-70-6] }\end{array}$ & $\begin{array}{l}\text { Original Measurements: } \\
{ }^{140} \text { K. R. Hoover, R. Coaxum, E. } \\
\text { Pustejovsky, W. E. Acree, Jr., and } \\
\text { M. H. Abraham, Phys. Chem. Liq. 42, } \\
457 \text { (2004). }\end{array}$ \\
\hline $\begin{array}{l}\text { Variables: } \\
T / \mathrm{K}=298.15\end{array}$ & $\begin{array}{l}\text { Prepared by: } \\
\text { W. E. Acree, Jr. }\end{array}$ \\
\hline
\end{tabular}

\section{Experimental Values}

\begin{tabular}{lc}
\hline \hline$x_{2}{ }^{\mathrm{a}}$ & $x_{1}{ }^{\mathrm{b}}$ \\
\hline 0.9685 & 0.03147 \\
\hline${ }^{\mathrm{a}} x_{2}:$ mole fraction of component 2 in the saturated solution. \\
${ }^{\mathrm{b}}{ }_{x_{1}: \text { mole fraction solubility of the solute. }}$
\end{tabular}

\section{Auxiliary Information}

\section{Method/Apparatus/Procedure:}

Constant-temperature bath, calorimetric thermometer, and an ultraviolet/ visible spectrophotometer.

Excess solute and solvent were placed in amber glass bottles and allowed to equilibrate for several days at constant temperature. Attainment of equilibrium was verified by several repetitive measurements and by approaching equilibrium from supersaturation. Aliquots of saturated solutions were transferred through a coarse filter into tared volumetric flasks, weighed, and diluted with methanol. Concentrations were determined by spectrophotometric measurements at $267 \mathrm{~nm}$.

Source and Purity of Chemicals:

(1) $99+\%$, Aldrich Chemical Company, Milwaukee, WI, USA, was used as received.

(2) $99+\%$, Alfa Aesar, USA, stored over molecular sieves and distilled shortly before use.

\section{Estimated Error:}

Temperature: $\pm 0.1 \mathrm{~K}$.

$x_{1}: \pm 1.5 \%$ (relative error).

\begin{tabular}{ll}
\hline \hline Components: & Original Measurements: \\
(1) 3,5-Dinitrobenzoic acid; & ${ }^{140}$ K. R. Hoover, R. Coaxum, E. \\
$\mathrm{C}_{7} \mathrm{H}_{4} \mathrm{~N}_{2} \mathrm{O}_{6} ;$ [99-34-3] & Pustejovsky, W. E. Acree, Jr., and \\
(2) 1-Octanol; $\mathrm{C}_{8} \mathrm{H}_{18} \mathrm{O} ;$ & M. H. Abraham, Phys. Chem. Liq. 42, \\
{$[111-87-5]$} & $457(2004)$. \\
\hline Variables: & Prepared by: \\
$T / \mathrm{K}=298.15$ & W. E. Acree, Jr. \\
\hline
\end{tabular}

Experimental Values

\begin{tabular}{lc}
\hline \hline$x_{2}^{\mathrm{a}}$ & $x_{1}{ }^{\mathrm{b}}$ \\
\hline 0.9673 & 0.03267
\end{tabular}

${ }^{\mathrm{a}} x_{2}$ : mole fraction of component 2 in the saturated solution.

${ }^{\mathrm{b}} x_{1}$ : mole fraction solubility of the solute.

\section{Auxiliary Information}

\section{Method/Apparatus/Procedure:}

Constant-temperature bath, calorimetric thermometer, and an ultraviolet visible spectrophotometer.

Excess solute and solvent were placed in amber glass bottles and allowed to equilibrate for several days at constant temperature. Attainment of equilibrium was verified by several repetitive measurements and by approaching equilibrium from supersaturation. Aliquots of saturated solutions were transferred through a coarse filter into tared volumetric flasks, weighed, and diluted with methanol. Concentrations were determined by spectrophotometric measurements at $267 \mathrm{~nm}$.

\section{Source and Purity of Chemicals:}

(1) $99+\%$, Aldrich Chemical Company, Milwaukee, WI, USA, was used as received.

(2) $99+\%$, anhydrous, Aldrich Chemical Company, stored over molecular sieves and distilled shortly before use.

\section{Estimated Error:}

Temperature: $\pm 0.1 \mathrm{~K}$.

$x_{1}: \pm 1.5 \%$ (relative error)

\begin{tabular}{ll}
\hline \hline Components: & Original Measurements: \\
(1) 3,5-Dinitrobenzoic acid; & ${ }^{140}$ K. R. Hoover, R. Coaxum, E. \\
$\mathrm{C}_{7} \mathrm{H}_{4} \mathrm{~N}_{2} \mathrm{O}_{6} ;$ [99-34-3] & Pustejovsky, W. E. Acree, Jr., and \\
(2) 1-Decanol; $\mathrm{C}_{10} \mathrm{H}_{22} \mathrm{O} ;$ & M. H. Abraham, Phys. Chem. Liq. 42, \\
{$[112-30-1]$} & 457 (2004). \\
\hline Variables: & Prepared by: \\
$T / \mathrm{K}=298.15$ & W. E. Acree, Jr. \\
\hline
\end{tabular}

\section{Experimental Values}

\begin{tabular}{lc}
\hline \hline$x_{2}{ }^{\mathrm{a}}$ & $x_{1}{ }^{\mathrm{b}}$ \\
\hline 0.9660 & 0.03402 \\
\hline${ }^{\mathrm{a}} x_{2}:$ mole fraction of component 2 in the saturated solution. & \\
${ }^{\mathrm{b}} x_{1}:$ mole fraction solubility of the solute. &
\end{tabular}




\section{Auxiliary Information}

Method/Apparatus/Procedure:

Constant-temperature bath, calorimetric thermometer, and an ultraviolet/ visible spectrophotometer.

Excess solute and solvent were placed in amber glass bottles and allowed to equilibrate for several days at constant temperature. Attainment of equilibrium was verified by several repetitive measurements and by approaching equilibrium from supersaturation. Aliquots of saturated solutions were transferred through a coarse filter into tared volumetric flasks, weighed, and diluted with methanol. Concentrations were determined by spectrophotometric measurements at $267 \mathrm{~nm}$.

Source and Purity of Chemicals:

(1) $99+\%$, Aldrich Chemical Company, Milwaukee, WI, USA, was used as received.

(2) $99+\%$, Alfa Aesar, USA, stored over molecular sieves and distilled shortly before use.

Estimated Error:

Temperature: $\pm 0.1 \mathrm{~K}$.

$x_{1}: \pm 1.5 \%$ (relative error).

\begin{tabular}{|c|c|}
\hline $\begin{array}{l}\text { Components: } \\
\text { (1) 3,5-Dinitrobenzoic acid; } \\
\mathrm{C}_{7} \mathrm{H}_{4} \mathrm{~N}_{2} \mathrm{O}_{6} \text {; [99-34-3] } \\
\text { (2) } 2,2,2 \text {-Trifluoroethanol; } \\
\mathrm{C}_{2} \mathrm{H}_{3} \mathrm{~F}_{3} \mathrm{O} \text {; [75-89-8] }\end{array}$ & $\begin{array}{l}\text { Original Measurements: } \\
{ }^{76} \text { M. K. Chantooni and I. M. Kolthoff, } \\
\text { Anal. Chem. 51, } 133 \text { (1979). }\end{array}$ \\
\hline $\begin{array}{l}\text { Variables: } \\
T / \mathrm{K}=298.15\end{array}$ & $\begin{array}{l}\text { Prepared by: } \\
\text { W. E. Acree, Jr. }\end{array}$ \\
\hline
\end{tabular}

\section{Experimental Values}

The measured solubility was reported to be $0.0212 \mathrm{~mol}$ $\mathrm{dm}^{-3}$. The authors did not give the temperature at which the solubility was measured. Based on the experimental description given in an earlier paper [I. M. Kolthoff, J. J. Lingane, and W. Larson, J. Am. Chem. Soc. 60, 2512 (1938)], the compiler believes the temperature to be $298.15 \mathrm{~K}$.

\section{Auxiliary Information}

\section{Method/Apparatus/Procedure:}

Very little experimental details were provided. Solubility was determined by titrating a filtered aliquot of the saturated solution alkalimetrically in an aqueous-ethanol mixture using phenolphthalein as the acid-base indicator.

\section{Source and Purity of Chemicals:}

(1) White Label, Eastman Kodak Chemical Company, Rochester, NY, USA, was recrystallized from aqueous ethanol solution and dried in vacuo over barium oxide at $343 \mathrm{~K}$.

(2) Purity not given, Chemical source not given, no purification details were provided.

\section{Estimated Error:}

Temperature: $\pm 0.1 \mathrm{~K}$ (estimated by compiler).

$c_{1}: \pm 2 \%$ (relative error, estimated by compiler).

\subsection{3,5-Dinitrobenzoic acid solubility data in miscellaneous organic solvents}

\begin{tabular}{|c|c|}
\hline $\begin{array}{l}\text { Components: } \\
\text { (1) 3,5-Dinitrobenzoic acid; } \\
\mathrm{C}_{7} \mathrm{H}_{4} \mathrm{~N}_{2} \mathrm{O}_{6} ;[99-34-3] \\
\text { (2) Ethanenitrile; } \mathrm{C}_{2} \mathrm{H}_{3} \mathrm{~N} ;[75-05-8]\end{array}$ & $\begin{array}{l}\text { Original Measurements: } \\
{ }^{83} \text { M. K. Chantooni and I. M. Kolthoff } \\
\text { J. Phys. Chem. 77, } 527 \text { (1973). }\end{array}$ \\
\hline $\begin{array}{l}\text { Variables: } \\
T / \mathrm{K}=298.15\end{array}$ & $\begin{array}{l}\text { Prepared by: } \\
\text { W. E. Acree, Jr. }\end{array}$ \\
\hline
\end{tabular}

Experimental Values

The measured solubility was reported to be $0.23 \mathrm{~mol} \mathrm{dm}^{-3}$. The authors did not give the temperature at which the solubility was measured. Based on the experimental description given in an earlier paper [I. M. Kolthoff, J. J. Lingane, and W. Larson, J. Am. Chem. Soc. 60, 2512 (1938)], the compiler believes the temperature to be $298.15 \mathrm{~K}$.

\section{Auxiliary Information}

\section{Method/Apparatus/Procedure:}

Very little experimental details were provided. Solubility was determined by titrating a filtered aliquot of the saturated solution alkalimetrically in an aqueous-ethanol mixture using phenolphthalein as the acid-base indicator.

Source and Purity of Chemicals:

(1) White Label, Eastman Kodak Chemical Company, Rochester, NY, USA, was recrystallized from aqueous ethanol solution and dried in vacuo over barium oxide at $343 \mathrm{~K}$.

(2) Purity not given, Chemical source not given, was purified by shaking with saturated potassium hydroxide, followed by activated alumina, and then anhydrous calcium chloride to remove water. Ethanenitrile was further dried over anhydrous magnesium sulfate and then phosphorous pentoxide. The sample was distilled shortly before use.

\section{Estimated Error:}

Temperature: $\pm 0.1 \mathrm{~K}$ (estimated by compiler).

$c_{1}: \pm 2 \%$ (relative error, estimated by compiler).

\section{Solubility of 3,5-Dinitro-2- methylbenzoic Acid in Organic Solvents}

\subsection{Critical evaluation of experimental solubility data}

There have been two published studies ${ }^{18,143}$ investigating the solubility behavior of 3,5-dinitro-2-methylbenzoic acid in organic solvents of varying polarity and hydrogen-bonding capability. Saifullah et al. ${ }^{18}$ measured the solubility of 3,5dinitro-2-methylbenzoic acid in tetrahydrofuran and 1,4-dioxane at $298 \mathrm{~K}$. Ye et al. ${ }^{143}$ measured the solubility of 3,5dinitro-2-methylbenzoic acid in 14 alcohols (1-propanol, 2propanol, 1-butanol, 2-butanol, 2-methyl-1-propanol, 2methyl-2-propanol, 1-pentanol, 2-pentanol, 3-methyl-1-butanol, 1-hexanol, 4-methyl-2-pentanol, 1-heptanol, 1-octanol, and 1-decanol) and in five alkyl alkanoates (methyl ethanoate, ethyl ethanoate, propyl ethanoate, butyl ethanoate, and pentyl ethanoate) at $298 \mathrm{~K}$. Results of the experimental measurements were used to calculate the Abraham solute descriptors of 
TABLE 33. Comparison between observed and calculated molar solubilities of 3,5-dinitro-2-methylbenzoic acid based on the Abraham model, Eqs. (20) and (21)

\begin{tabular}{|c|c|c|c|}
\hline Solvent & $\log _{10} c_{1}^{\exp }$ & $\begin{array}{c}\log _{10} c_{1}{ }^{\text {calc }} ; \\
\text { Eq. (20) }\end{array}$ & $\begin{array}{c}\log _{10} c_{1}{ }^{\text {calc }} \\
\text { Eq. (21) }\end{array}$ \\
\hline 1-Propanol & -0.661 & -0.711 & -0.690 \\
\hline 2-Propanol & -0.712 & -0.726 & -0.697 \\
\hline 1-Butanol & -0.786 & -0.837 & -0.800 \\
\hline 2-Butanol & -0.784 & -0.769 & -0.787 \\
\hline 2-Methyl-1-propanol & -1.054 & -0.981 & -0.955 \\
\hline 2-Methyl-2-propanol & -0.820 & -0.741 & -0.716 \\
\hline 1-Pentanol & -0.823 & -0.966 & -0.941 \\
\hline 2-Pentanol & -0.864 & -1.003 & -0.942 \\
\hline 3-Methyl-1-butanol & -0.962 & -1.065 & -0.955 \\
\hline 1-Hexanol & -0.969 & -0.983 & -0.967 \\
\hline 1-Heptanol & -0.925 & -0.984 & -1.000 \\
\hline 1-Octanol & -1.103 & -0.984 & -1.095 \\
\hline 1-Decanol & -1.336 & -1.243 & -1.223 \\
\hline Methyl ethanoate & -0.345 & -0.349 & -0.288 \\
\hline Ethyl ethanoate & -0.498 & -0.550 & -0.512 \\
\hline Butyl ethanoate & -0.703 & -0.747 & -0.703 \\
\hline
\end{tabular}

3,5-dinitro-2-methylbenzoic acid. The authors were able to assemble a total of $36 \log _{10}\left(S R\right.$ or $P$ ) and $\log _{10}(G S R$ or $K)$ equations for which experimental partition coefficient data, solubility ratios, Abraham model equation coefficients, and aqueous molar solubility were available. The logarithm of the aqueous molar solubility of 3,5-dinitro-2-methylbenzoic acid, $\log _{10} c_{1, \mathrm{~W}}=-2.60$, was determined by the authors as part of the experimental measurements. The McGowan volume of 3,5-dinitro-2-methylbenzoic acid, $V=1.4210$, was calculated from the number of chemical bonds in the molecule and the individual atomic group volumes, $A V_{i}$, given in Sec. 1.3. The excess molar refraction solute descriptor was estimated as $E=$ 1.310. This left four solute descriptors ( $S, A, B$, and $L$ ) still to be determined. The 36 equations were then solved using the Microsoft "SOLVER" program to yield numerical values of the remaining four solute descriptors, $S=2.120, A=0.750$, $B=0.650$, and $L=8.040$, that best described the $\log _{10}(S R$ or $P)$ and $\log _{10}(G S R$ or $K)$ values. The computation treated $\log _{10} c_{1, \mathrm{G}}$ as a floating parameter to be determined as part of the regression analyses. The data analyses returned a value of $\log _{10} c_{1, \mathrm{G}}=-12.556$ for the gas-phase solute concentration that made the $\log _{10}(S R$ or $P)$ and $\log _{10}(G S R$ or $K$ ) predictions internally consistent. The calculated molecular solute descriptors reproduced the $\log _{10}\left(S R\right.$ or $P$ ) and $\log _{10}(G S R$ or $K$ ) values to within an average standard deviation of 0.081 and 0.064 $\log _{10}$ units, respectively.

Table 33 compares the experimental $\log _{10} c_{1}$ values to calculated values based on Eqs. (20) and (21) of the Abraham model. For comparison purposes, the measured mole fraction solubilities of 3,5-dinitro-2-methylbenzoic acid, $x_{1}$, determined by Ye et al. ${ }^{143}$ were converted into molar solubilities by dividing $x_{1}$ by the ideal molar volume of the saturated solution (i.e., $\left.c_{1}{ }^{\text {sat }}=x_{1} /\left[x_{1} V_{1}+\left(1-x_{1}\right) V_{\text {solvent }}\right]\right)$. The molar volume of the hypothetical subcooled liquid 3,5-dinitro-2methylbenzoic acid is $V_{\text {solute }}=148.4 \mathrm{~cm}^{3} \mathrm{~mol}^{-1}$. Examination of the numerical entries in Table 33 reveals that the Abraham model provides a reasonably accurate mathematical description of the observed solubility data, suggesting that there are no obvious outliers in the dataset.

The experimental solubility data for 3,5-dinitro-2-methylbenzoic acid in organic solvents are in Secs. 41.2-41.4.

\subsection{3,5-Dinitro-2-methylbenzoic acid solubility data in esters}

\begin{tabular}{ll}
\hline \hline Components: & Original Measurements: \\
(1) 3,5-Dinitro-2-methylbenzoic acid; & ${ }^{143}$ S. Ye, M. Saifullah, L. M. \\
$\mathrm{C}_{8} \mathrm{H}_{6} \mathrm{~N}_{2} \mathrm{O}_{6} ;$ [28169-46-2] & Grubbs, M. C. McMillan-Wiggins, \\
(2) Methyl ethanoate; $\mathrm{C}_{3} \mathrm{H}_{6} \mathrm{O}_{2} ;$ & P. Acosta, D. Mejorado, I. Flores, \\
[79-20-9] & W. E. Acree, Jr., and M. H. \\
& Abraham, Phys. Chem. Liq. 49, 821 \\
& $(2011)$. \\
\hline Variables: & Prepared by: \\
$T / \mathrm{K}=298.15$ & W. E. Acree, Jr. \\
\hline
\end{tabular}

Experimental Values

\begin{tabular}{lc}
\hline \hline$x_{2}{ }^{\mathrm{a}}$ & $x_{1}{ }^{\mathrm{b}}$ \\
\hline 0.9629 & 0.0371 \\
\hline${ }^{\mathrm{a}} x_{2}:$ mole fraction of component 2 in the saturated solution. & \\
${ }^{\mathrm{b}} x_{1}:$ mole fraction solubility of the solute. &
\end{tabular}

Auxiliary Information

Method/Apparatus/Procedure:

Constant-temperature bath, calorimetric thermometer, and an ultraviolet/ visible spectrophotometer.

Excess solute and solvent were placed in amber glass bottles and allowed to equilibrate for several days at constant temperature. Attainment of equilibrium was verified by several repetitive measurements and by approaching equilibrium from supersaturation. Aliquots of saturated solutions were transferred through a coarse filter into tared volumetric flasks, weighed, and diluted with 2-propanol. Concentrations were determined by spectrophotometric measurements at $280 \mathrm{~nm}$.

Source and Purity of Chemicals:

(1) $99+\%$, Aldrich Chemical Company, Milwaukee, WI, USA, was used as received.

(2) $99.5 \%$, anhydrous, Aldrich Chemical Company, stored over molecular sieves and distilled shortly before use.

Estimated Error:

Temperature: $\pm 0.1 \mathrm{~K}$.

$x_{1}: \pm 1.5 \%$ (relative error). 


\section{Components:}

(1) 3,5-Dinitro-2-methylbenzoic acid;

$\mathrm{C}_{8} \mathrm{H}_{6} \mathrm{~N}_{2} \mathrm{O}_{6}$; [28169-46-2]

(2) Ethyl ethanoate; $\mathrm{C}_{4} \mathrm{H}_{8} \mathrm{O}_{2}$;

[141-78-6]
Original Measurements:

${ }^{143}$ S. Ye, M. Saifullah, L. M.

Grubbs, M. C. McMillan-Wiggins,

P. Acosta, D. Mejorado, I. Flores,

W. E. Acree, Jr., and M. H.

Abraham, Phys. Chem. Liq. 49, 821 (2011).

\section{Variables:}

$T / \mathrm{K}=298.15$
Prepared by:

W. E. Acree, Jr.
Experimental Values

\begin{tabular}{lc}
\hline \hline$x_{2}{ }^{\mathrm{a}}$ & $x_{1}{ }^{\mathrm{b}}$ \\
\hline 0.9683 & 0.0317 \\
\hline$x_{2}:$ mole fraction of compong 2 in
\end{tabular}

${ }^{\mathrm{a}} x_{2}$ : mole fraction of component 2 in the saturated solution.

${ }^{b} x_{1}$ : mole fraction solubility of the solute.

\section{Auxiliary Information}

\section{Method/Apparatus/Procedure:}

Constant-temperature bath, calorimetric thermometer, and an ultraviolet/ visible spectrophotometer.

Excess solute and solvent were placed in amber glass bottles and allowed to equilibrate for several days at constant temperature. Attainment of equilibrium was verified by several repetitive measurements and by approaching equilibrium from supersaturation. Aliquots of saturated solutions were transferred through a coarse filter into tared volumetric flasks, weighed, and diluted with 2-propanol. Concentrations were determined by spectrophotometric measurements at $280 \mathrm{~nm}$.

\section{Source and Purity of Chemicals:}

(1) $99+\%$, Aldrich Chemical Company, Milwaukee, WI, USA, was used as received.

(2) $99.9 \%$, HPLC grade, Aldrich Chemical Company, stored over molecular sieves and distilled shortly before use.

\section{Estimated Error:}

Temperature: $\pm 0.1 \mathrm{~K}$.

$x_{1}: \pm 1.5 \%$ (relative error).

\section{Auxiliary Information}

\section{Method/Apparatus/Procedure:}

Constant-temperature bath, calorimetric thermometer, and an ultraviolet/ visible spectrophotometer.

Excess solute and solvent were placed in amber glass bottles and allowed to equilibrate for several days at constant temperature. Attainment of equilibrium was verified by several repetitive measurements and by approaching equilibrium from supersaturation. Aliquots of saturated solutions were transferred through a coarse filter into tared volumetric flasks, weighed, and diluted with 2-propanol. Concentrations were determined by spectrophotometric measurements at $280 \mathrm{~nm}$.

Source and Purity of Chemicals:

(1) $99+\%$, Aldrich Chemical Company, Milwaukee, WI, USA, was used as received.

(2) $99.5 \%$, Aldrich Chemical Company, stored over molecular sieves and distilled shortly before use.

Estimated Error:

Temperature: $\pm 0.1 \mathrm{~K}$.

$x_{1}: \pm 1.5 \%$ (relative error)

\begin{tabular}{ll}
\hline \hline Components: & Original Measurements: \\
(1) 3,5-Dinitro-2-methylbenzoic acid; & ${ }^{143}$ S. Ye, M. Saifullah, L. M. \\
$\mathrm{C}_{8} \mathrm{H}_{6} \mathrm{~N}_{2} \mathrm{O}_{6} ;$ [28169-46-2] & Grubbs, M. C. McMillan-Wiggins, \\
(2) $\mathrm{Butyl}$ ethanoate; $\mathrm{C}_{6} \mathrm{H}_{12} \mathrm{O}_{2} ;$ & P. Acosta, D. Mejorado, I. Flores, \\
[123-86-4] & W. E. Acree, Jr., and M. H. \\
& Abraham, Phys. Chem. Liq. 49, 821 \\
& $(2011)$. \\
\hline Variables: & Prepared by: \\
$T / \mathrm{K}=298.15$ & W. E. Acree, Jr. \\
\hline
\end{tabular}

Experimental Values

\begin{tabular}{lc}
\hline \hline$x_{2}{ }^{\mathrm{a}}$ & $x_{1}{ }^{\mathrm{b}}$ \\
\hline 0.9737 & 0.0263
\end{tabular}

${ }^{\mathrm{a}} x_{2}$ : mole fraction of component 2 in the saturated solution.

${ }^{\mathrm{b}} x_{1}$ : mole fraction solubility of the solute.

\section{Auxiliary Information}

Components: Original Measurements:

(1) 3,5-Dinitro-2-methylbenzoic acid; ${ }^{143} \mathrm{~S}$. Ye, M. Saifullah, L. M.

$\mathrm{C}_{8} \mathrm{H}_{6} \mathrm{~N}_{2} \mathrm{O}_{6}$; [28169-46-2]

(2) Propyl ethanoate; $\mathrm{C}_{5} \mathrm{H}_{10} \mathrm{O}_{2}$;

[109-60-4]

Grubbs, M. C. McMillan-Wiggins, P. Acosta, D. Mejorado, I. Flores,

W. E. Acree, Jr., and M. H.

Abraham, Phys. Chem. Liq. 49, 821 (2011).

\begin{tabular}{ll}
\hline Variables: & Prepared by: \\
$T / \mathrm{K}=298.15$ & W. E. Acree, Jr. \\
\hline
\end{tabular}

Experimental Values

\begin{tabular}{lc}
\hline \hline$x_{2}^{\mathrm{a}}$ & $x_{1}{ }^{\mathrm{b}}$ \\
\hline 0.9707 & 0.0293
\end{tabular}

${ }^{\mathrm{a}} x_{2}$ : mole fraction of component 2 in the saturated solution.

${ }^{\mathrm{b}} x_{1}$ : mole fraction solubility of the solute.

\begin{abstract}
Method/Apparatus/Procedure:
Constant-temperature bath, calorimetric thermometer, and an ultraviolet/ visible spectrophotometer.

Excess solute and solvent were placed in amber glass bottles and allowed to equilibrate for several days at constant temperature. Attainment of equilibrium was verified by several repetitive measurements and by approaching equilibrium from supersaturation. Aliquots of saturated solutions were transferred through a coarse filter into tared volumetric flasks, weighed, and diluted with 2-propanol. Concentrations were determined by spectrophotometric measurements at $280 \mathrm{~nm}$.
\end{abstract}

Source and Purity of Chemicals:

(1) $99+\%$, Aldrich Chemical Company, Milwaukee, WI, USA, was used as received.

(2) 99.7\%, HPLC grade, Aldrich Chemical Company, stored over molecular sieves and distilled shortly before use.

\section{Estimated Error:}

Temperature: $\pm 0.1 \mathrm{~K}$.

$x_{1}: \pm 1.5 \%$ (relative error). 


\section{Components:}

(1) 3,5-Dinitro-2-methylbenzoic acid;

$\mathrm{C}_{8} \mathrm{H}_{6} \mathrm{~N}_{2} \mathrm{O}_{6}$; [28169-46-2]

(2) Pentyl ethanoate; $\mathrm{C}_{7} \mathrm{H}_{14} \mathrm{O}_{2}$; [628-63-7]
Original Measurements:

${ }^{143}$ S. Ye, M. Saifullah, L. M. Grubbs, M. C. McMillan-Wiggins, P. Acosta, D. Mejorado, I. Flores, W. E. Acree, Jr., and M. H. Abraham, Phys. Chem. Liq. 49, 821 (2011).

\section{Variables:}

$T / \mathrm{K}=298.15$

\section{Prepared by:}

W. E. Acree, Jr.
Experimental Values

\begin{tabular}{lc}
\hline \hline$x_{2}^{\mathrm{a}}$ & $x_{1}{ }^{\mathrm{b}}$ \\
\hline 0.9748 & 0.0252
\end{tabular}

${ }^{\mathrm{a}} x_{2}$ : mole fraction of component 2 in the saturated solution.

${ }^{\mathrm{b}} x_{1}$ : mole fraction solubility of the solute.

\section{Auxiliary Information}

\section{Method/Apparatus/Procedure:}

Constant-temperature bath, calorimetric thermometer, and an ultraviolet/ visible spectrophotometer.

Excess solute and solvent were placed in amber glass bottles and allowed to equilibrate for several days at constant temperature. Attainment of equilibrium was verified by several repetitive measurements and by approaching equilibrium from supersaturation. Aliquots of saturated solutions were transferred through a coarse filter into tared volumetric flasks, weighed, and diluted with 2-propanol. Concentrations were determined by

spectrophotometric measurements at $280 \mathrm{~nm}$.

\section{Source and Purity of Chemicals:}

(1) $99+\%$, Aldrich Chemical Company, Milwaukee, WI, USA, was used as received.

(2) $99.9 \%$, Aldrich Chemical Company, stored over molecular sieves and distilled shortly before use.

\section{Estimated Error:}

Temperature: $\pm 0.1 \mathrm{~K}$.

$x_{1}: \pm 1.5 \%$ (relative error)

\subsection{3,5-Dinitro-2-methylbenzoic acid solubility data in ethers}

\begin{tabular}{ll}
\hline \hline Components: & Original Measurements: \\
(1) 3,5-Dinitro-2-methylbenzoic acid; & ${ }^{18}$ M. Saifullah, S. Ye, L. M. Grubbs, \\
$\mathrm{C}_{8} \mathrm{H}_{6} \mathrm{~N}_{2} \mathrm{O}_{6} ;$ [28169-46-2] & N. E. De La Rosa, W. E. Acree, Jr., \\
(2) Tetrahydrofuran; $\mathrm{C}_{4} \mathrm{H}_{8} \mathrm{O} ;$ & and M. H. Abraham, J. Solution \\
[109-99-9] & Chem. 40, 2082 (2011). \\
\hline Variables: & Prepared by: \\
$T / \mathrm{K}=298.15$ & W. E. Acree, Jr. \\
\hline
\end{tabular}

Experimental Values

\begin{tabular}{lc}
\hline \hline$x_{2}^{\mathrm{a}}$ & $x_{1}^{\mathrm{b}}$ \\
\hline 0.8140 & 0.1860 \\
\hline${ }^{\mathrm{a}} x_{2}:$ mole fraction of component 2 in the saturated solution. \\
${ }^{\mathrm{b}} x_{1}:$ mole fraction solubility of the solute.
\end{tabular}

\section{Auxiliary Information}

\section{Method/Apparatus/Procedure:}

Constant-temperature bath, calorimetric thermometer, and an ultraviolet/ visible spectrophotometer.

Excess solute and solvent were placed in amber glass bottles and allowed to equilibrate for several days at constant temperature. Attainment of equilibrium was verified by several repetitive measurements and by approaching equilibrium from supersaturation. Aliquots of saturated solutions were transferred through a coarse filter into tared volumetric flasks, weighed, and diluted with 2-propanol. Concentrations were determined by spectrophotometric measurements at $280 \mathrm{~nm}$.

Source and Purity of Chemicals:

(1) $99+\%$, Aldrich Chemical Company, Milwaukee, WI, USA, was used as received.

(2) $99.9 \%$, anhydrous, Aldrich Chemical Company, stored over molecular sieves and distilled shortly before use.

Estimated Error:

Temperature: $\pm 0.1 \mathrm{~K}$.

$x_{1}: \pm 1.5 \%$ (relative error).

\begin{tabular}{ll}
\hline \hline Components: & Original Measurements: \\
(1) 3,5-Dinitro-2-methylbenzoic acid; & ${ }^{18}$ M. Saifullah, S. Ye, L. M. Grubbs, \\
$\mathrm{C}_{8} \mathrm{H}_{6} \mathrm{~N}_{2} \mathrm{O}_{6} ;[28169-46-2]$ & N. E. De La Rosa, W. E. Acree, Jr., \\
(2) 1,4-Dioxane; $\mathrm{C}_{4} \mathrm{H}_{8} \mathrm{O}_{2} ;$ [123-91-1] & $\begin{array}{l}\text { and M. H. Abraham, J. Solution } \\
\text { Chem. 40, 2082 (2011). }\end{array}$ \\
\hline Variables: & Prepared by: \\
$T / \mathrm{K}=298.15$ & W. E. Acree, Jr. \\
\hline
\end{tabular}

Experimental Values

\begin{tabular}{lc}
\hline \hline$x_{2}{ }^{\mathrm{a}}$ & $x_{1}{ }^{\mathrm{b}}$ \\
\hline 0.8196 & 0.1804 \\
\hline${ }^{a} x_{2}:$ mole fraction of component 2 in the saturated solution. & \\
${ }^{\mathrm{b}} x_{1}:$ mole fraction solubility of the solute. &
\end{tabular}

\section{Auxiliary Information}

\section{Method/Apparatus/Procedure:}

Constant-temperature bath, calorimetric thermometer, and an ultraviolet/ visible spectrophotometer.

Excess solute and solvent were placed in amber glass bottles and allowed to equilibrate for several days at constant temperature. Attainment of equilibrium was verified by several repetitive measurements and by approaching equilibrium from supersaturation. Aliquots of saturated solutions were transferred through a coarse filter into tared volumetric flasks, weighed, and diluted with 2-propanol. Concentrations were determined by spectrophotometric measurements at $280 \mathrm{~nm}$.

\section{Source and Purity of Chemicals:}

(1) $99+\%$, Aldrich Chemical Company, Milwaukee, WI, USA, was used as received.

(2) $99.8 \%$, anhydrous, Aldrich Chemical Company, stored over molecular sieves and distilled shortly before use.

\section{Estimated Error:}

Temperature: $\pm 0.1 \mathrm{~K}$.

$x_{1}: \pm 1.5 \%$ (relative error). 


\subsection{3,5-Dinitro-2-methylbenzoic acid solubility data in alcohols}

\begin{tabular}{l}
\hline \hline Components: \\
(1) 3,5-Dinitro-2-methylbenzoic acid; \\
$\mathrm{C}_{8} \mathrm{H}_{6} \mathrm{~N}_{2} \mathrm{O}_{6} ;[$ [28169-46-2] \\
(2) 1-Propanol; $\mathrm{C}_{3} \mathrm{H}_{8} \mathrm{O}$; [71-23-8]
\end{tabular}

(2) 1-Propanol; $\mathrm{C}_{3} \mathrm{H}_{8} \mathrm{O}$; [71-23-8]

Original Measurements:

${ }^{143}$ S. Ye, M. Saifullah, L. M.

Grubbs, M. C. McMillan-Wiggins,

P. Acosta, D. Mejorado, I. Flores,

W. E. Acree, Jr., and M. H.

Abraham, Phys. Chem. Liq. 49, 821 (2011).

\section{Variables:}

$T / \mathrm{K}=298.15$

Prepared by:

W. E. Acree, Jr.

Experimental Values

\begin{tabular}{lc}
\hline \hline$x_{2}^{\mathrm{a}}$ & $x_{1}^{\mathrm{b}}$ \\
\hline 0.9834 & 0.0166
\end{tabular}

${ }^{\mathrm{a}} x_{2}$ : mole fraction of component 2 in the saturated solution.

${ }^{b} x_{1}$ : mole fraction solubility of the solute.

\section{Auxiliary Information}

\section{Method/Apparatus/Procedure:}

Constant-temperature bath, calorimetric thermometer, and an ultraviolet/ visible spectrophotometer.

Excess solute and solvent were placed in amber glass bottles and allowed to equilibrate for several days at constant temperature. Attainment of equilibrium was verified by several repetitive measurements and by approaching equilibrium from supersaturation. Aliquots of saturated solutions were transferred through a coarse filter into tared volumetric flasks, weighed, and diluted with 2-propanol. Concentrations were determined by spectrophotometric measurements at $280 \mathrm{~nm}$.

\section{Source and Purity of Chemicals:}

(1) $99+\%$, Aldrich Chemical Company, Milwaukee, WI, USA, was used as received.

(2) $99+\%$, anhydrous, Aldrich Chemical Company, stored over molecular sieves and distilled shortly before use.

\section{Estimated Error:}

Temperature: $\pm 0.1 \mathrm{~K}$.

$x_{1}: \pm 1.5 \%$ (relative error).

\section{Components:}

(1) 3,5-Dinitro-2-methylbenzoic acid; $\mathrm{C}_{8} \mathrm{H}_{6} \mathrm{~N}_{2} \mathrm{O}_{6}$; [28169-46-2]

(2) 2-Propanol; $\mathrm{C}_{3} \mathrm{H}_{8} \mathrm{O}$; [67-63-0]

\section{Original Measurements:}

${ }^{143}$ S. Ye, M. Saifullah, L. M. Grubbs, M. C. McMillan-Wiggins, P. Acosta, D. Mejorado, I. Flores, W. E. Acree, Jr., and M. H. Abraham, Phys. Chem. Liq. 49, 821 (2011).

\begin{tabular}{ll}
\hline Variables: & Prepared by: \\
$T / \mathrm{K}=298.15$ & W. E. Acree, Jr.
\end{tabular}

Experimental Values

\begin{tabular}{lc}
\hline \hline$x_{2}{ }^{\mathrm{a}}$ & $x_{1}{ }^{\mathrm{b}}$ \\
\hline 0.9849 & 0.0151 \\
\hline${ }^{\mathrm{a}} x_{2}:$ mole fraction of component 2 in the saturated solution. & \\
${ }^{\mathrm{b}}{ }_{x_{1}}:$ mole fraction solubility of the solute. &
\end{tabular}

\section{Auxiliary Information}

Method/Apparatus/Procedure:

Constant-temperature bath, calorimetric thermometer, and an ultraviolet/ visible spectrophotometer.

Excess solute and solvent were placed in amber glass bottles and allowed to equilibrate for several days at constant temperature. Attainment of equilibrium was verified by several repetitive measurements and by approaching equilibrium from supersaturation. Aliquots of saturated solutions were transferred through a coarse filter into tared volumetric flasks, weighed, and diluted with 2-propanol. Concentrations were determined by spectrophotometric measurements at $280 \mathrm{~nm}$.

Source and Purity of Chemicals:

(1) $99+\%$, Aldrich Chemical Company, Milwaukee, WI, USA, was used as received.

(2) $99+\%$, anhydrous, Aldrich Chemical Company, stored over molecular sieves and distilled shortly before use.

\section{Estimated Error:}

Temperature: $\pm 0.1 \mathrm{~K}$.

$x_{1}: \pm 1.5 \%$ (relative error)

\begin{tabular}{ll}
\hline \hline Components: & Original Measurements: \\
(1) 3,5-Dinitro-2-methylbenzoic acid; & ${ }^{143}$ S. Ye, M. Saifullah, L. M. \\
$\mathrm{C}_{8} \mathrm{H}_{6} \mathrm{~N}_{2} \mathrm{O}_{6} ;[28169-46-2]$ & Grubbs, M. C. McMillan-Wiggins, \\
(2) 1-Butanol; $\mathrm{C}_{4} \mathrm{H}_{10} \mathrm{O} ;[$ [71-36-3] & P. Acosta, D. Mejorado, I. Flores, \\
& W. E. Acree, Jr., and M. H. \\
& Abraham, Phys. Chem. Liq. 49, 821 \\
& $(2011)$. \\
\hline Variables: & Prepared by: \\
$T / \mathrm{K}=298.15$ & W. E. Acree, Jr. \\
\hline
\end{tabular}

Experimental Values

\begin{tabular}{lc}
\hline \hline$x_{2}{ }^{\mathrm{a}}$ & $x_{1}{ }^{\mathrm{b}}$ \\
\hline 0.9848 & 0.0152 \\
\hline${ }^{\mathrm{a}} x_{2}:$ mole fraction of component 2 in the saturated solution. & \\
${ }^{\mathrm{b}} x_{1}:$ mole fraction solubility of the solute. &
\end{tabular}

\section{Auxiliary Information}

\section{Method/Apparatus/Procedure:}

Constant-temperature bath, calorimetric thermometer, and an ultraviolet/ visible spectrophotometer.

Excess solute and solvent were placed in amber glass bottles and allowed to equilibrate for several days at constant temperature. Attainment of equilibrium was verified by several repetitive measurements and by approaching equilibrium from supersaturation. Aliquots of saturated solutions were transferred through a coarse filter into tared volumetric flasks, weighed, and diluted with 2-propanol. Concentrations were determined by spectrophotometric measurements at $280 \mathrm{~nm}$. 
Source and Purity of Chemicals:

(1) $99+\%$, Aldrich Chemical Company, Milwaukee, WI, USA, was used as received.

(2) 99.8\%, HPLC grade, Aldrich Chemical Company, stored over molecular sieves and distilled shortly before use.

\section{Estimated Error:}

Temperature: $\pm 0.1 \mathrm{~K}$.

$x_{1}: \pm 1.5 \%$ (relative error).

\section{Components:}

(1) 3,5-Dinitro-2-methylbenzoic acid; $\mathrm{C}_{8} \mathrm{H}_{6} \mathrm{~N}_{2} \mathrm{O}_{6}$; [28169-46-2]

(2) 2-Butanol; $\mathrm{C}_{4} \mathrm{H}_{10} \mathrm{O}$; [78-92-2]

\section{Original Measurements:}

${ }^{143}$ S. Ye, M. Saifullah, L. M. Grubbs, M. C. McMillan-Wiggins, P. Acosta, D. Mejorado, I. Flores, W. E. Acree, Jr., and M. H. Abraham, Phys. Chem. Liq. 49, 821 (2011).

\begin{tabular}{ll}
\hline Variables: & Prepared by: \\
$T / \mathrm{K}=298.15$ & W. E. Acree, Jr.
\end{tabular}

Experimental Values

\begin{tabular}{lc}
\hline \hline$x_{2}{ }^{\mathrm{a}}$ & $x_{1}{ }^{\mathrm{b}}$ \\
\hline 0.9847 & 0.0153 \\
\hline
\end{tabular}

${ }^{\mathrm{a}} x_{2}$ : mole fraction of component 2 in the saturated solution.

${ }^{\mathrm{b}} x_{1}$ : mole fraction solubility of the solute.

\section{Auxiliary Information}

\section{Method/Apparatus/Procedure:}

Constant-temperature bath, calorimetric thermometer, and an ultraviolet/ visible spectrophotometer.

Excess solute and solvent were placed in amber glass bottles and allowed to equilibrate for several days at constant temperature. Attainment of equilibrium was verified by several repetitive measurements and by approaching equilibrium from supersaturation. Aliquots of saturated solutions were transferred through a coarse filter into tared volumetric flasks, weighed, and diluted with 2-propanol. Concentrations were determined by spectrophotometric measurements at $280 \mathrm{~nm}$.

\section{Source and Purity of Chemicals:}

(1) $99+\%$, Aldrich Chemical Company, Milwaukee, WI, USA, was used as received.

(2) 99+\%, anhydrous, Aldrich Chemical Company, stored over molecular sieves and distilled shortly before use.

\section{Estimated Error:}

Temperature: $\pm 0.1 \mathrm{~K}$.

$x_{1}: \pm 1.5 \%$ (relative error).

\section{Components:}

(1) 3,5-Dinitro-2-methylbenzoic acid; $\mathrm{C}_{8} \mathrm{H}_{6} \mathrm{~N}_{2} \mathrm{O}_{6}$; [28169-46-2]

(2) 2-Methyl-1-propanol; $\mathrm{C}_{4} \mathrm{H}_{10} \mathrm{O}$; [78-83-1]

\section{Variables:}

$T / \mathrm{K}=298.15$

\section{Original Measurements:}

${ }^{143}$ S. Ye, M. Saifullah, L. M. Grubbs, M. C. McMillan-Wiggins, P. Acosta, D. Mejorado, I. Flores, W. E. Acree, Jr., and M. H. Abraham, Phys. Chem. Liq. 49, 821 (2011).

\section{Prepared by:}

W. E. Acree, Jr.
Experimental Values

\begin{tabular}{lc}
\hline \hline$x_{2}{ }^{\mathrm{a}}$ & $x_{1}{ }^{\mathrm{b}}$ \\
\hline 0.9918 & 0.00822
\end{tabular}

${ }^{\mathrm{a}} x_{2}$ : mole fraction of component 2 in the saturated solution.

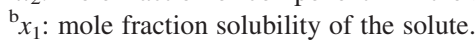

\section{Auxiliary Information}

\section{Method/Apparatus/Procedure:}

Constant-temperature bath, calorimetric thermometer, and an ultraviolet/ visible spectrophotometer.

Excess solute and solvent were placed in amber glass bottles and allowed to equilibrate for several days at constant temperature. Attainment of equilibrium was verified by several repetitive measurements and by approaching equilibrium from supersaturation. Aliquots of saturated solutions were transferred through a coarse filter into tared volumetric flasks, weighed, and diluted with 2-propanol. Concentrations were determined by spectrophotometric measurements at $280 \mathrm{~nm}$.

Source and Purity of Chemicals:

(1) $99+\%$, Aldrich Chemical Company, Milwaukee, WI, USA, was used as received.

(2) $99+\%$, anhydrous, Aldrich Chemical Company, stored over molecular sieves and distilled shortly before use.

\section{Estimated Error:}

Temperature: $\pm 0.1 \mathrm{~K}$.

$x_{1}: \pm 1.5 \%$ (relative error).

\begin{tabular}{|c|c|}
\hline $\begin{array}{l}\text { Components: } \\
\text { (1) 3,5-Dinitro-2-methylbenzoic acid; } \\
\left.\mathrm{C}_{8} \mathrm{H}_{6} \mathrm{~N}_{2} \mathrm{O}_{6} ; \text {; } 28169-46-2\right] \\
\text { (2) 2-Methyl-2-propanol; } \mathrm{C}_{4} \mathrm{H}_{10} \mathrm{O} \text {; } \\
\text { [75-65-0] }\end{array}$ & $\begin{array}{l}\text { Original Measurements: } \\
{ }^{143} \text { S. Ye, M. Saifullah, L. M. } \\
\text { Grubbs, M. C. McMillan-Wiggins, } \\
\text { P. Acosta, D. Mejorado, I. Flores, } \\
\text { W. E. Acree, Jr., and M. H. } \\
\text { Abraham, Phys. Chem. Liq. 49, } 821 \\
\text { (2011). }\end{array}$ \\
\hline $\begin{array}{l}\text { Variables: } \\
T / \mathrm{K}=298.15\end{array}$ & $\begin{array}{l}\text { Prepared by: } \\
\text { W. E. Acree, Jr. }\end{array}$ \\
\hline
\end{tabular}

Experimental Values

\begin{tabular}{lc}
\hline \hline$x_{2}{ }^{\mathrm{a}}$ & $x_{1}{ }^{\mathrm{b}}$ \\
\hline 0.9856 & 0.0144 \\
\hline${ }^{\mathrm{a}}{ }_{2}:$ mole fraction of component 2 in the saturated solution. & \\
${ }^{\mathrm{b}} x_{1}:$ mole fraction solubility of the solute. &
\end{tabular}

\section{Auxiliary Information}

\section{Method/Apparatus/Procedure:}

Constant-temperature bath, calorimetric thermometer, and an ultraviolet/ visible spectrophotometer.

Excess solute and solvent were placed in amber glass bottles and allowed to equilibrate for several days at constant temperature. Attainment of equilibrium was verified by several repetitive measurements and by approaching equilibrium from supersaturation. Aliquots of saturated solutions were transferred through a coarse filter into tared volumetric flasks, weighed, and diluted with 2-propanol. Concentrations were determined by spectrophotometric measurements at $280 \mathrm{~nm}$. 
Source and Purity of Chemicals:

(1) $99+\%$, Aldrich Chemical Company, Milwaukee, WI, USA, was used as received.

(2) $99+\%$, Arco Chemical Company, USA, stored over molecular sieves and distilled shortly before use.

\section{Estimated Error:}

Temperature: $\pm 0.1 \mathrm{~K}$.

$x_{1}: \pm 1.5 \%$ (relative error).

\section{Components:}

(1) 3,5-Dinitro-2-methylbenzoic acid; $\mathrm{C}_{8} \mathrm{H}_{6} \mathrm{~N}_{2} \mathrm{O}_{6}$; [28169-46-2]

(2) 1-Pentanol; $\mathrm{C}_{5} \mathrm{H}_{12} \mathrm{O}$; [71-41-0]

Original Measurements:

${ }^{143}$ S. Ye, M. Saifullah, L. M. Grubbs, M. C. McMillan-Wiggins, P. Acosta, D. Mejorado, I. Flores, W. E. Acree, Jr., and M. H. Abraham, Phys. Chem. Liq. 49, 821 (2011).

\begin{tabular}{ll}
\hline Variables: & Prepared by: \\
$T / \mathrm{K}=298.15$ & W. E. Acree, Jr. \\
\hline
\end{tabular}

Experimental Values

\begin{tabular}{lc}
\hline \hline$x_{2}{ }^{\mathrm{a}}$ & $x_{1}{ }^{\mathrm{b}}$ \\
\hline 0.9836 & 0.0164 \\
\hline
\end{tabular}

${ }^{\mathrm{a}} x_{2}$ : mole fraction of component 2 in the saturated solution.

${ }^{\mathrm{b}} x_{1}$ : mole fraction solubility of the solute.

\section{Auxiliary Information}

\section{Method/Apparatus/Procedure:}

Constant-temperature bath, calorimetric thermometer, and an ultraviolet/ visible spectrophotometer.

Excess solute and solvent were placed in amber glass bottles and allowed to equilibrate for several days at constant temperature. Attainment of equilibrium was verified by several repetitive measurements and by approaching equilibrium from supersaturation. Aliquots of saturated solutions were transferred through a coarse filter into tared volumetric flasks, weighed, and diluted with 2-propanol. Concentrations were determined by spectrophotometric measurements at $280 \mathrm{~nm}$.

\section{Source and Purity of Chemicals:}

(1) $99+\%$, Aldrich Chemical Company, Milwaukee, WI, USA, was used as received.

(2) $99+\%$, Aldrich Chemical Company, stored over molecular sieves and distilled shortly before use.

\section{Estimated Error:}

Temperature: $\pm 0.1 \mathrm{~K}$.

$x_{1}: \pm 1.5 \%$ (relative error)

\section{Components:}

(1) 3,5-Dinitro-2-methylbenzoic acid; $\mathrm{C}_{8} \mathrm{H}_{6} \mathrm{~N}_{2} \mathrm{O}_{6}$; [28169-46-2]

(2) 2-Pentanol; $\mathrm{C}_{5} \mathrm{H}_{12} \mathrm{O}$; [6032-29-7]
Original Measurements:

${ }^{143}$ S. Ye, M. Saifullah, L. M. Grubbs, M. C. McMillan-Wiggins, P. Acosta, D. Mejorado, I. Flores, W. E. Acree, Jr., and M. H. Abraham, Phys. Chem. Liq. 49, 821 (2011).

\section{Variables:}

$T / \mathrm{K}=298.15$

\section{Prepared by:}

W. E. Acree, Jr.
Experimental Values

\begin{tabular}{lc}
\hline \hline$x_{2}{ }^{\mathrm{a}}$ & $x_{1}{ }^{\mathrm{b}}$ \\
\hline 0.9850 & 0.0150 \\
${ }^{\mathrm{a}} x_{2}:$ mole fraction of component 2 in the saturated solution. \\
${ }^{\mathrm{b}} x_{1}:$ mole fraction solubility of the solute.
\end{tabular}

\section{Auxiliary Information}

Method/Apparatus/Procedure:

Constant-temperature bath, calorimetric thermometer, and an ultraviolet/ visible spectrophotometer.

Excess solute and solvent were placed in amber glass bottles and allowed to equilibrate for several days at constant temperature. Attainment of equilibrium was verified by several repetitive measurements and by approaching equilibrium from supersaturation. Aliquots of saturated solutions were transferred through a coarse filter into tared volumetric flasks, weighed, and diluted with 2-propanol. Concentrations were determined by spectrophotometric measurements at $280 \mathrm{~nm}$

Source and Purity of Chemicals:

(1) $99+\%$, Aldrich Chemical Company, Milwaukee, WI, USA, was used as received.

(2) $99+\%$, Acros Organics, USA, stored over molecular sieves and distilled shortly before use.

Estimated Error:

Temperature: $\pm 0.1 \mathrm{~K}$.

$x_{1}: \pm 1.5 \%$ (relative error)

\begin{tabular}{|c|c|}
\hline $\begin{array}{l}\text { Components: } \\
\text { (1) 3,5-Dinitro-2-methylbenzoic acid; } \\
\left.\mathrm{C}_{8} \mathrm{H}_{6} \mathrm{~N}_{2} \mathrm{O}_{6} ; \text {; } 28169-46-2\right] \\
\text { (2) 3-Methyl-1-butanol; } \mathrm{C}_{5} \mathrm{H}_{12} \mathrm{O} \text {; } \\
\text { [123-51-3] }\end{array}$ & $\begin{array}{l}\text { Original Measurements: } \\
{ }^{143} \text { S. Ye, M. Saifullah, L. M. } \\
\text { Grubbs, M. C. McMillan-Wiggins, } \\
\text { P. Acosta, D. Mejorado, I. Flores, } \\
\text { W. E. Acree, Jr., and M. H. } \\
\text { Abraham, Phys. Chem. Liq. 49, } 821 \\
\text { (2011). }\end{array}$ \\
\hline $\begin{array}{l}\text { Variables: } \\
T / \mathrm{K}=298.15\end{array}$ & $\begin{array}{l}\text { Prepared by: } \\
\text { W. E. Acree, Jr. }\end{array}$ \\
\hline
\end{tabular}

Experimental Values

\begin{tabular}{lc}
\hline \hline$x_{2}{ }^{\mathrm{a}}$ & $x_{1}{ }^{\mathrm{b}}$ \\
\hline 0.9880 & 0.0120 \\
${ }^{\mathrm{a}} x_{2}:$ mole fraction of component 2 in the saturated solution. & \\
${ }^{\mathrm{b}} x_{1}:$ mole fraction solubility of the solute. &
\end{tabular}

\section{Auxiliary Information}

\section{Method/Apparatus/Procedure:}

Constant-temperature bath, calorimetric thermometer, and an ultraviolet/ visible spectrophotometer.

Excess solute and solvent were placed in amber glass bottles and allowed to equilibrate for several days at constant temperature. Attainment of equilibrium was verified by several repetitive measurements and by approaching equilibrium from supersaturation. Aliquots of saturated solutions were transferred through a coarse filter into tared volumetric flasks, weighed, and diluted with 2-propanol. Concentrations were determined by spectrophotometric measurements at $280 \mathrm{~nm}$. 
Source and Purity of Chemicals:

(1) $99+\%$, Aldrich Chemical Company, Milwaukee, WI, USA, was used as received.

(2) $99 \%$, anhydrous, Aldrich Chemical Company, stored over molecular sieves and distilled shortly before use.

\section{Estimated Error:}

Temperature: $\pm 0.1 \mathrm{~K}$.

$x_{1}: \pm 1.5 \%$ (relative error).

\section{Components:}

(1) 3,5-Dinitro-2-methylbenzoic acid; $\mathrm{C}_{8} \mathrm{H}_{6} \mathrm{~N}_{2} \mathrm{O}_{6}$; [28169-46-2]

(2) 1-Hexanol; $\mathrm{C}_{6} \mathrm{H}_{14} \mathrm{O}$; [111-27-3]

Original Measurements:

${ }^{143}$ S. Ye, M. Saifullah, L. M. Grubbs, M. C. McMillan-Wiggins, P. Acosta, D. Mejorado, I. Flores, W. E. Acree, Jr., and M. H. Abraham, Phys. Chem. Liq. 49, 821 (2011).

\begin{tabular}{ll}
\hline Variables: & Prepared by: \\
$T / \mathrm{K}=298.15$ & W. E. Acree, Jr. \\
\hline
\end{tabular}

\section{Experimental Values}

\begin{tabular}{lc}
\hline \hline$x_{2}^{\mathrm{a}}$ & $x_{1}{ }^{\mathrm{b}}$ \\
\hline 0.9865 & 0.0135
\end{tabular}

${ }^{\mathrm{a}} x_{2}$ : mole fraction of component 2 in the saturated solution.

${ }^{\mathrm{b}} x_{1}$ : mole fraction solubility of the solute.

\section{Auxiliary Information}

\section{Method/Apparatus/Procedure:}

Constant-temperature bath, calorimetric thermometer, and an ultraviolet/ visible spectrophotometer.

Excess solute and solvent were placed in amber glass bottles and allowed to equilibrate for several days at constant temperature. Attainment of equilibrium was verified by several repetitive measurements and by approaching equilibrium from supersaturation. Aliquots of saturated solutions were transferred through a coarse filter into tared volumetric flasks, weighed, and diluted with 2-propanol. Concentrations were determined by spectrophotometric measurements at $280 \mathrm{~nm}$.

Source and Purity of Chemicals:

(1) $99+\%$, Aldrich Chemical Company, Milwaukee, WI, USA, was used as received.

(2) $99+\%$, Alfa Aesar, USA, stored over molecular sieves and distilled shortly before use.

Estimated Error:

Temperature: $\pm 0.1 \mathrm{~K}$.

$x_{1}: \pm 1.5 \%$ (relative error).

\section{Components:}

(1) 3,5-Dinitro-2-methylbenzoic acid; $\mathrm{C}_{8} \mathrm{H}_{6} \mathrm{~N}_{2} \mathrm{O}_{6}$; [28169-46-2]

(2) 4-Methyl-2-butanol; $\mathrm{C}_{6} \mathrm{H}_{14} \mathrm{O}$; [108-11-2]

Variables:

$T / \mathrm{K}=298.15$

\section{Original Measurements:}

${ }^{143}$ S. Ye, M. Saifullah, L. M. Grubbs, M. C. McMillan-Wiggins, P. Acosta, D. Mejorado, I. Flores, W. E. Acree, Jr., and M. H. Abraham, Phys. Chem. Liq. 49, 821 (2011).

\section{Prepared by:}

W. E. Acree, Jr.
Experimental Values

\begin{tabular}{lc}
\hline \hline$x_{2}{ }^{\mathrm{a}}$ & $x_{1}{ }^{\mathrm{b}}$ \\
\hline 0.9861 & 0.0139 \\
\hline${ }^{\mathrm{a}} x_{2}:$ mole fraction of component 2 in the saturated solution. & \\
${ }^{\mathrm{b}} x_{1}:$ mole fraction solubility of the solute. &
\end{tabular}

\section{Auxiliary Information}

Method/Apparatus/Procedure:

Constant-temperature bath, calorimetric thermometer, and an ultraviolet/ visible spectrophotometer.

Excess solute and solvent were placed in amber glass bottles and allowed to equilibrate for several days at constant temperature. Attainment of equilibrium was verified by several repetitive measurements and by approaching equilibrium from supersaturation. Aliquots of saturated solutions were transferred through a coarse filter into tared volumetric flasks, weighed, and diluted with 2-propanol. Concentrations were determined by spectrophotometric measurements at $280 \mathrm{~nm}$.

Source and Purity of Chemicals:

(1) $99+\%$, Aldrich Chemical Company, Milwaukee, WI, USA, was used as received.

(2) $99+\%$, Acros Organics, USA, stored over molecular sieves and distilled shortly before use.

Estimated Error:

Temperature: $\pm 0.1 \mathrm{~K}$.

$x_{1}: \pm 1.5 \%$ (relative error).

\begin{tabular}{ll}
\hline \hline Components: & Original Measurements: \\
(1) 3,5-Dinitro-2-methylbenzoic acid; & ${ }^{143}$ S. Ye, M. Saifullah, L. M. \\
$\mathrm{C}_{8} \mathrm{H}_{6} \mathrm{~N}_{2} \mathrm{O}_{6} ;[28169-46-2]$ & Grubbs, M. C. McMillan-Wiggins, \\
(2) 1-Heptanol; $\mathrm{C}_{7} \mathrm{H}_{16} \mathrm{O} ;[111-70-6]$ & P. Acosta, D. Mejorado, I. Flores, \\
& W. E. Acree, Jr., and M. H. \\
& Abraham, Phys. Chem. Liq. 49, 821 \\
& $(2011)$. \\
\hline Variables: & Prepared by: \\
$T / \mathrm{K}=298.15$ & W. E. Acree, Jr. \\
\hline
\end{tabular}

Experimental Values

\begin{tabular}{lc}
\hline \hline$x_{2}{ }^{\mathrm{a}}$ & $x_{1}{ }^{\mathrm{b}}$ \\
\hline 0.9832 & 0.0168 \\
\hline${ }^{\mathrm{a}} x_{2}:$ mole fraction of component 2 in the saturated solution. & \\
${ }^{\mathrm{b}} x_{1}:$ mole fraction solubility of the solute. &
\end{tabular}

\section{Auxiliary Information}

\section{Method/Apparatus/Procedure:}

Constant-temperature bath, calorimetric thermometer, and an ultraviolet/ visible spectrophotometer.

Excess solute and solvent were placed in amber glass bottles and allowed to equilibrate for several days at constant temperature. Attainment of equilibrium was verified by several repetitive measurements and by approaching equilibrium from supersaturation. Aliquots of saturated solutions were transferred through a coarse filter into tared volumetric flasks, weighed, and diluted with 2-propanol. Concentrations were determined by spectrophotometric measurements at $280 \mathrm{~nm}$. 
Source and Purity of Chemicals:

(1) $99+\%$, Aldrich Chemical Company, Milwaukee, WI, USA, was used as received.

(2) $99+\%$, Alfa Aesar, USA, stored over molecular sieves and distilled shortly before use.

Estimated Error:

Temperature: $\pm 0.1 \mathrm{~K}$.

$x_{1}: \pm 1.5 \%$ (relative error).

\section{Components:}

(1) 3,5-Dinitro-2-methylbenzoic acid; $\mathrm{C}_{8} \mathrm{H}_{6} \mathrm{~N}_{2} \mathrm{O}_{6}$; [28169-46-2]

(2) 1-Octanol; $\mathrm{C}_{8} \mathrm{H}_{18} \mathrm{O}$; [111-87-5]

Original Measurements:

${ }^{143}$ S. Ye, M. Saifullah, L. M. Grubbs, M. C. McMillan-Wiggins, P. Acosta, D. Mejorado, I. Flores, W. E. Acree, Jr., and M. H. Abraham, Phys. Chem. Liq. 49, 821 (2011).

\begin{tabular}{ll}
\hline Variables: & Prepared by: \\
$T / \mathrm{K}=298.15$ & W. E. Acree, Jr. \\
\hline
\end{tabular}

Experimental Values

\begin{tabular}{lc}
\hline \hline$x_{2}{ }^{\mathrm{a}}$ & $x_{1}{ }^{\mathrm{b}}$ \\
\hline 0.9875 & 0.0125 \\
\hline
\end{tabular}

${ }^{\mathrm{a}} x_{2}$ : mole fraction of component 2 in the saturated solution.

${ }^{\mathrm{b}} x_{1}$ : mole fraction solubility of the solute.

\section{Auxiliary Information}

\section{Method/Apparatus/Procedure:}

Constant-temperature bath, calorimetric thermometer, and an ultraviolet/ visible spectrophotometer.

Excess solute and solvent were placed in amber glass bottles and allowed to equilibrate for several days at constant temperature. Attainment of equilibrium was verified by several repetitive measurements and by approaching equilibrium from supersaturation. Aliquots of saturated solutions were transferred through a coarse filter into tared volumetric flasks, weighed, and diluted with 2-propanol. Concentrations were determined by spectrophotometric measurements at $280 \mathrm{~nm}$.

\section{Source and Purity of Chemicals:}

(1) $99+\%$, Aldrich Chemical Company, Milwaukee, WI, USA, was used as received.

(2) $99+\%$, anhydrous, Aldrich Chemical Company, stored over molecular sieves and distilled shortly before use.

Estimated Error:

Temperature: $\pm 0.1 \mathrm{~K}$.

$x_{1}: \pm 1.5 \%$ (relative error).

\begin{tabular}{ll}
\hline \hline Components: & Original Measurements: \\
(1) 3,5-Dinitro-2-methylbenzoic acid; & ${ }^{143}$ S. Ye, M. Saifullah, L. M. \\
$\mathrm{C}_{8} \mathrm{H}_{6} \mathrm{~N}_{2} \mathrm{O}_{6} ;[28169-46-2]$ & Grubbs, M. C. McMillan-Wiggins, \\
(2) 1-Decanol; $\mathrm{C}_{10} \mathrm{H}_{22} \mathrm{O} ;[112-30-1]$ & P. Acosta, D. Mejorado, I. Flores, \\
& W. E. Acree, Jr., and M. H. \\
& Abraham, Phys. Chem. Liq. 49, 821 \\
& $(2011)$. \\
\hline Variables: & Prepared by: \\
$T / \mathrm{K}=298.15$ & W. E. Acree, Jr.
\end{tabular}

Experimental Values

\begin{tabular}{lc}
\hline \hline$x_{2}{ }^{\mathrm{a}}$ & $x_{1}{ }^{\mathrm{b}}$ \\
\hline 0.9917 & 0.00833 \\
\hline${ }^{\mathrm{a}} x_{2}:$ mole fraction of component 2 in the saturated solution. & \\
${ }^{\mathrm{b}}{ }_{x_{1}}:$ mole fraction solubility of the solute. &
\end{tabular}

\section{Auxiliary Information}

Method/Apparatus/Procedure:

Constant-temperature bath, calorimetric thermometer, and an ultraviolet/ visible spectrophotometer.

Excess solute and solvent were placed in amber glass bottles and allowed to equilibrate for several days at constant temperature. Attainment of equilibrium was verified by several repetitive measurements and by approaching equilibrium from supersaturation. Aliquots of saturated solutions were transferred through a coarse filter into tared volumetric flasks, weighed, and diluted with 2-propanol. Concentrations were determined by spectrophotometric measurements at $280 \mathrm{~nm}$.

Source and Purity of Chemicals:

(1) $99+\%$, Aldrich Chemical Company, Milwaukee, WI, USA, was used as received.

(2) $99+\%$, Alfa Aesar, USA, stored over molecular sieves and distilled shortly before use.

\section{Estimated Error:}

Temperature: $\pm 0.1 \mathrm{~K}$.

$x_{1}: \pm 1.5 \%$ (relative error).

\section{Solubility of 2-Fluorobenzoic Acid in Organic Solvents}

\subsection{Critical evaluation of experimental solubility data}

There has been only a single publication reporting the solubility of 2-fluorobenzoic acid in organic solvents. Thuaire $^{72}$ determined the solubility of 2-fluorobenzoic acid in ethanol at $298 \mathrm{~K}$ as part of study involving solubilities of benzoic acids in binary aqueous-ethanol solvent mixtures. It is not possible to perform a critical evaluation of the experimental data as measurements were made at only a single temperature, and there are no independent experimental 2fluorobenzoic acid solubility data in ethanol.

The experimental solubility data for 2-fluorobenzoic acid in ethanol is given in Sec. 42.2.

\subsection{2-Fluorobenzoic acid solubility data in alcohols}

\begin{tabular}{|c|c|}
\hline $\begin{array}{l}\text { Components: } \\
\text { (1) 2-Fluorobenzoic acid; } \mathrm{C}_{7} \mathrm{H}_{5} \mathrm{FO}_{2} \text {; } \\
\text { [445-29-4] } \\
\text { (2) Ethanol; } \mathrm{C}_{2} \mathrm{H}_{6} \mathrm{O} ;[64-17-5]\end{array}$ & $\begin{array}{l}\text { Original Measurements: } \\
{ }^{72} \text { R. Thuaire, Bull. Soc. Chim. Fr. } \\
3815 \text { (1971). }\end{array}$ \\
\hline $\begin{array}{l}\text { Variables: } \\
T / \mathrm{K}=298.15\end{array}$ & $\begin{array}{l}\text { Prepared by: } \\
\text { W. E. Acree, Jr. }\end{array}$ \\
\hline
\end{tabular}


Experimental Values

The measured solubility was reported to be $2.994 \mathrm{~mol} / \mathrm{kg}$ of solvent, which corresponds to a mole fraction solubility of $x_{1}=0.1212$.

\section{Auxiliary Information}

\section{Method/Apparatus/Procedure:}

Very little experimental details were provided. Solubility was determined by a gravimetric method. The saturated solution was evaporated to dryness and the remaining solid residue was weighed. The solubility was calculated from the mass of the solid residue and mass of saturated solution analyzed.

Source and Purity of Chemicals:

(1) Purity not given, Chemical source not given, no purification details were provided.

(2) Purity not given, Chemical source not given, no purification details were provided.

\section{Estimated Error:}

Temperature: $\pm 0.05 \mathrm{~K}$.

$x_{1}: \pm 4 \%$ (relative error, estimated by compiler).

\section{Solubility of 3-Fluorobenzoic Acid in Organic Solvents}

\subsection{Critical evaluation of experimental solubility data}

There has been only a single publication reporting the solubility of 3-fluorobenzoic acid in organic solvents. Thuaire $^{72}$ determined the solubility of 3-fluorobenzoic acid in ethanol at $298 \mathrm{~K}$ as part of study involving solubilities of benzoic acids in binary aqueous-ethanol solvent mixtures. It is not possible to perform a critical evaluation of the experimental data as measurements were made at only a single temperature, and there are no independent experimental 3fluorobenzoic acid solubility data in ethanol.

The experimental solubility data for 3-fluorobenzoic acid in ethanol is given in Sec. 43.2.

\subsection{3-Fluorobenzoic acid solubility data in alcohols}

\begin{tabular}{|c|c|}
\hline $\begin{array}{l}\text { Components: } \\
\text { (1) 3-Fluorobenzoic acid; } \mathrm{C}_{7} \mathrm{H}_{5} \mathrm{FO}_{2} \text {; } \\
\text { [455-38-9] } \\
\text { (2) Ethanol; } \mathrm{C}_{2} \mathrm{H}_{6} \mathrm{O} ;[64-17-5]\end{array}$ & $\begin{array}{l}\text { Original Measurements: } \\
{ }^{72} \mathrm{R} \text {. Thuaire, Bull. Soc. Chim. Fr. } \\
3815 \text { (1971). }\end{array}$ \\
\hline $\begin{array}{l}\text { Variables: } \\
T / \mathrm{K}=298.15\end{array}$ & $\begin{array}{l}\text { Prepared by: } \\
\text { W. E. Acree, Jr. }\end{array}$ \\
\hline
\end{tabular}

\section{Experimental Values}

The measured solubility was reported to be $4.746 \mathrm{~mol} / \mathrm{kg}$ of solvent, which corresponds to a mole fraction solubility of $x_{1}=0.1794$.

\section{Auxiliary Information}

Method/Apparatus/Procedure:

Very little experimental details were provided. Solubility was determined by a gravimetric method. The saturated solution was evaporated to dryness and the remaining solid residue was weighed. The solubility was calculated from the mass of the solid residue and mass of saturated solution analyzed.

Source and Purity of Chemicals:

(1) Purity not given, Chemical source not given, no purification details were provided.

(2) Purity not given, Chemical source not given, no purification details were provided.

Estimated Error:

Temperature: $\pm 0.05 \mathrm{~K}$

$x_{1}: \pm 4 \%$ (relative error, estimated by compiler).

\section{Solubility of 4-Fluorobenzoic Acid in Organic Solvents}

\subsection{Critical evaluation of experimental solubility data}

There have been only two publications ${ }^{63,72}$ reporting the solubility of 4-fluorobenzoic acid in organic solvents. Hancock et al. ${ }^{63}$ determined the solubility of 4-fluorobenzoic acid in tetrahydrofuran and 1,4-dioxane at $303 \mathrm{~K}$ based on a gravimetric method. Thuaire ${ }^{72}$ determined the solubility in ethanol at $298 \mathrm{~K}$ as part of study involving solubilities of benzoic acids in binary aqueous-ethanol solvent mixtures. It is not possible to perform a critical evaluation of the experimental data as measurements were made at only a single temperature, and there are no independent experimental 4fluorobenzoic acid solubility data for these two organic solvents.

The experimental solubility data for 4-fluorobenzoic acid in organic solvents are given in Secs. 44.2 and 44.3.

\subsection{4-Fluorobenzoic acid solubility data in ethers}

\begin{tabular}{ll}
\hline \hline $\begin{array}{l}\text { Components: } \\
\text { (1) 4-Fluorobenzoic acid; } \mathrm{C}_{7} \mathrm{H}_{5} \mathrm{FO}_{2} ;\end{array}$ & $\begin{array}{l}\text { Original Measurements: } \\
\text { [456-22-4] }\end{array}$ \\
$\begin{array}{l}\text { C. K. Hancock, J. N. Pawloski, } \\
\text { and J. P. Idoux, J. Org. Chem. 32, } \\
{[109-99-9]}\end{array}$ & 1931 (1967). \\
\hline Variables: & \\
$T / \mathrm{K}=303.15$ & Prepared by: \\
\hline
\end{tabular}

Experimental Values

\begin{tabular}{lc}
\hline \hline$x_{2}{ }^{\mathrm{a}}$ & $x_{1}{ }^{\mathrm{b}}$ \\
\hline 0.811 & 0.189 \\
\hline${ }^{\mathrm{a}} x_{2}:$ mole fraction of component 2 in the saturated solution. & \\
${ }^{\mathrm{b}} x_{1}:$ mole fraction solubility of the solute. &
\end{tabular}




\section{Auxiliary Information}

\section{Method/Apparatus/Procedure:}

Constant-temperature bath, Soxhlet thimble, conical flask, and analytical balance.

Excess solute and solvent were placed in a conical flask and allowed to equilibrate for several days at constant temperature. Attainment of equilibrium was verified by several repetitive measurements and by approaching equilibrium from supersaturation. Aliquots of saturated solutions were transferred using a Soxhlet thimble equipped with a one-hole cork stopper and an inverted U-shaped delivery tube to a second ground-glass stoppered flask suspended in the $303 \mathrm{~K}$ water bath. Successive portions of the contents were evaporated at room temperature in a tared aluminum foil weighing dish under a bell jar through which a slow stream of dry air was passed. The second flask plus unused saturated solution and the aluminum foil dish plus residue were weighed. The saturation solubility of the solute was calculated from the recorded mass data and molar masses of the solute and solvent.

Source and Purity of Chemicals:

(1) Purity not given, Chemical source not specified, was recrystallized several times from aqueous-ethanol mixture, and then dried under vacuum over phosphorous pentoxide.

(2) Practical grade, Chemical source not specified, stored over sodium hydroxide pellets for $24 \mathrm{~h}$, and then passed through $2 \times 70-\mathrm{cm}$ chromatographic adsorption columns containing activated alumina. After this treatment, the purified solvent was stored over copper in a nitrogen atmosphere.

\section{Estimated Error:}

Temperature: $\pm 0.02 \mathrm{~K}$.

$x_{1}: \pm 1.0 \%$ (relative error).

\begin{tabular}{ll}
\hline \hline $\begin{array}{l}\text { Components: } \\
\text { (1) 4-Fluorobenzoic acid; } \mathrm{C}_{7} \mathrm{H}_{5} \mathrm{FO}_{2} ;\end{array}$ & $\begin{array}{l}\text { Original Measurements: } \\
{ }^{63} \mathrm{C} . \text { K. Hancock, J. N. Pawloski, } \\
\text { and J. P. Idoux, J. Org. Chem. 32, }\end{array}$ \\
$\begin{array}{l}\text { (2) 1,4-Dioxane; } \mathrm{C}_{4} \mathrm{H}_{8} \mathrm{O}_{2} ;[123-91-1] \\
1931 \text { (1967). }\end{array}$ \\
\hline Variables: & Prepared by: \\
$T / \mathrm{K}=303.15$ & W. E. Acree, Jr. \\
\hline
\end{tabular}

Experimental Values

\begin{tabular}{lc}
\hline \hline$x_{2}{ }^{\mathrm{a}}$ & $x_{1}{ }^{\mathrm{b}}$ \\
\hline 0.872 & 0.128 \\
\hline$x_{2}:$ mole fraction of component 2 in the satris
\end{tabular}

${ }^{a} x_{2}$ : mole fraction of component 2 in the saturated solution.

${ }^{\mathrm{b}} x_{1}$ : mole fraction solubility of the solute.

\section{Auxiliary Information}

\section{Method/Apparatus/Procedure:}

Constant-temperature bath, Soxhlet thimble, conical flask, and analytical balance.

Excess solute and solvent were placed in a conical flask and allowed to equilibrate for several days at constant temperature. Attainment of equilibrium was verified by several repetitive measurements and by approaching equilibrium from supersaturation. Weighed aliquots of saturated solutions were removed and titrated with a standardized sodium hydroxide solution (carbonate free) using a pH meter. The endpoint of the titration was determined by computing the second derivative in the $\mathrm{pH}$ versus volume of sodium hydroxide added.
Source and Purity of Chemicals:

(1) Purity not given, Chemical source not specified, was recrystallized several times from aqueous-ethanol mixture, and then dried under vacuum over phosphorous pentoxide.

(2) Practical grade, Chemical source not specified, stored over sodium hydroxide pellets for $24 \mathrm{~h}$, and then passed through $2 \times 70-\mathrm{cm}$ chromatographic adsorption columns containing activated alumina. After this treatment, the purified solvent was stored over copper in a nitrogen atmosphere.

Estimated Error:

Temperature: $\pm 0.02 \mathrm{~K}$.

$x_{1}: \pm 1.0 \%$ (relative error)

\subsection{4-Fluorobenzoic acid solubility data in alcohols}

\begin{tabular}{|c|c|}
\hline $\begin{array}{l}\text { Components: } \\
\text { (1) 4-Fluorobenzoic acid; } \mathrm{C}_{7} \mathrm{H}_{5} \mathrm{FO}_{2} \text {; } \\
\text { [456-22-4] } \\
\text { (2) Ethanol; } \mathrm{C}_{2} \mathrm{H}_{6} \mathrm{O} ;[64-17-5]\end{array}$ & $\begin{array}{l}\text { Original Measurements: } \\
{ }^{72} \text { R. Thuaire, Bull. Soc. Chim. Fr. } \\
3815 \text { (1971). }\end{array}$ \\
\hline $\begin{array}{l}\text { Variables: } \\
T / \mathrm{K}=298.15\end{array}$ & $\begin{array}{l}\text { Prepared by: } \\
\text { W. E. Acree, Jr. }\end{array}$ \\
\hline
\end{tabular}

\section{Experimental Values}

The measured solubility was reported to be $1.438 \mathrm{~mol} / \mathrm{kg}$ of solvent, which corresponds to a mole fraction solubility of $x_{1}=$ 0.06213 .

\section{Auxiliary Information}

\section{Method/Apparatus/Procedure:}

Very little experimental details were provided. Solubility was determined by a gravimetric method. The saturated solution was evaporated to dryness and the remaining solid residue was weighed. The solubility was calculated from the mass of the solid residue and mass of saturated solution analyzed.

Source and Purity of Chemicals:

(1) Purity not given, Chemical source not given, no purification details were provided.

(2) Purity not given, Chemical source not given, no purification details were provided.

Estimated Error:

Temperature: $\pm 0.05 \mathrm{~K}$.

$x_{1}: \pm 4 \%$ (relative error, estimated by compiler).

\section{Solubility of 4-Formylbenzoic Acid in Organic Solvents}

\subsection{Critical evaluation of experimental solubility data}

There have been three publications ${ }^{82,84,144}$ reporting solubility data for 4-formylbenzoic acid. Sun et al. ${ }^{144}$ measured the solubility of 4-formylbenzoic acid in ethanoic acid at 14 temperatures covering the range of 303-473 K. The internal consistency of the dataset was assessed by curve-fitting the measured mole fraction solubilities according to the Modified 
Apelblat model to yield the following correlation:

$$
\ln x_{1}=-42.006+\frac{114.588}{T}+6.266 \ln T .
$$

Equation (47) provides a reasonably accurate mathematical description for how the solubility varies with temperature. The mean relative deviation between the observed and calculated values is $6.9 \%$.

Dian-Qing et al. ${ }^{82}$ determined the solubility of 4-formylbenzoic acid in $N, N$-dimethylformamide as a function of temperature using a dynamic method with laser monitoring to observe when dissolution was complete. The calculated curve-fit parameters from the Buchowski $\lambda$ h-model [Eq. (9)] of $\lambda=1.398 \times 10^{-7}$ and $h=2879.94$ described the observed solubility data from 314 to $370 \mathrm{~K}$ to within a mean relative deviation of $2.4 \%$.

Li et al. ${ }^{84}$ determined the solubility of 4-formylbenzoic acid dissolved in $N$-methyl-2-pyrrolidone from 296 to $341 \mathrm{~K}$ using a synthetic method with laser monitoring to determine when the last amount of solid solute dissolved. The authors employed a polynomial expression in temperature,

$$
\begin{aligned}
x= & -2.4515+0.02776 T-1.10430 \times 10^{-4} T \\
& +1.54936 \times 10^{-7} T^{2},
\end{aligned}
$$

to represent the measured mole fraction solubility data. The root-mean-square deviation between the observed $x_{1}$ data and calculated values from Eq. (48) was on the order of 0.0005 mole fraction.

The experimental solubility data for 4-formylbenzoic acid in organic solvents are given in Sec. 45.2.

\begin{tabular}{|c|c|}
\hline $\begin{array}{l}\text { Components: } \\
\text { (1) 4-Formylbenzoic acid; } \mathrm{C}_{8} \mathrm{H}_{6} \mathrm{O}_{3} \text {; } \\
\text { [619-66-9] } \\
\text { (2) Ethanoic acid; } \mathrm{C}_{2} \mathrm{H}_{4} \mathrm{O}_{2} ; \text { [64-19-7] }\end{array}$ & $\begin{array}{l}\text { Original Measurements: } \\
{ }^{144} \text { W. Sun, W. Qu, and L. Zhao, J. } \\
\text { Chem. Eng. Data 55, } 4476 \text { (2010). }\end{array}$ \\
\hline $\begin{array}{l}\text { Variables: } \\
\text { Temperature }\end{array}$ & $\begin{array}{l}\text { Prepared by: } \\
\text { W. E Acree Jr. }\end{array}$ \\
\hline
\end{tabular}

\subsection{4-Formylbenzoic acid solubility data in miscellaneous organic solvents}

Experimental Values

\begin{tabular}{lcc}
\hline \hline$T / \mathrm{K}$ & $x_{2}{ }^{\mathrm{a}}$ & $x_{1}{ }^{\mathrm{b}, \mathrm{c}}$ \\
\hline 303.2 & 0.9975 & 0.0025 \\
323.2 & 0.9958 & 0.0042 \\
333.2 & 0.9949 & 0.0051 \\
343.2 & 0.9937 & 0.0063 \\
353.2 & 0.9924 & 0.0076 \\
363.2 & 0.9905 & 0.0095 \\
373.2 & 0.9869 & 0.0131 \\
403.2 & 0.9838 & 0.0162 \\
423.2 & 0.9794 & 0.0206 \\
433.2 & 0.9766 & 0.0234 \\
443.2 & 0.9747 & 0.0253 \\
453.3 & 0.9691 & 0.0309
\end{tabular}

\begin{tabular}{lcc}
\hline \hline$T / \mathrm{K}$ & $x_{2}{ }^{\mathrm{a}}$ & $x_{1}{ }^{\mathrm{b}, \mathrm{c}}$ \\
\hline 463.3 & 0.9640 & 0.0360 \\
473.2 & 0.9555 & 0.0445
\end{tabular}

${ }^{\mathrm{a}} x_{2}$ : mole fraction of component 2 in the saturated solution.

${ }^{b} x_{1}$ : mole fraction solubility of the solute, calculated by the compiler.

${ }^{\mathrm{c}}$ Solubilities were given in units of grams of solute per $100 \mathrm{~g}$ of solvent.

\section{Auxiliary Information}

\section{Method/Apparatus/Procedure:}

Jacketed vessel made from titanium, and a high-performance liquid chromatograph equipped with an ion-exchange column.

Experimental solubilities were determined by a static method. Excess solute and solvent were placed in a jacketed vessel constructed of titanium. The solution was allowed to equilibrate several hours at constant temperature. An aliquot of the clear saturated solution was filtered and transferred to a tared volumetric flask. The concentration of the dissolved solute was determined by high-performance liquid chromatography using an ion-exchange column.

Source and Purity of Chemicals:

(1) Analytical Reagent grade, Sinopharm Chemical Reagent Co. Ltd., China, was used as received.

(2) Analytical Reagent grade, Sinopharm Chemical Reagent Co. Ltd., China, was used as received.

\section{Estimated Error:}

Temperature: $\pm 0.1 \mathrm{~K}$.

$x_{1}: \pm 3 \%$ (relative error, by compiler).

\begin{tabular}{ll}
\hline \hline Components: & Original Measurements: \\
(1) 4-Formylbenzoic acid; $\mathrm{C}_{8} \mathrm{H}_{6} \mathrm{O}_{3} ;$ & ${ }^{82}$ L. Dian-Qing, L. Jiang-Chu, L. \\
[619-66-9] & Da-Zhuang, and W. Fu-An, Fluid \\
(2) $N, N$-Dimethylformamide; & Phase Equilib. 200, 69 (2002). \\
$\mathrm{C}_{3} \mathrm{H}_{7} \mathrm{NO} ;[64-19-7]$ & \\
\hline Variables: & Prepared by: \\
Temperature & W. E. Acree, Jr. \\
\hline
\end{tabular}

Experimental Values

\begin{tabular}{lcc}
\hline \hline$T / \mathrm{K}$ & $x_{2}{ }^{\mathrm{a}}$ & $x_{1}{ }^{\mathrm{b}}$ \\
\hline 313.45 & 0.7761 & 0.2239 \\
316.55 & 0.7732 & 0.2268 \\
319.35 & 0.7703 & 0.2297 \\
322.55 & 0.7671 & 0.2329 \\
327.55 & 0.7608 & 0.2392 \\
330.85 & 0.7569 & 0.2431 \\
334.05 & 0.7530 & 0.2470 \\
336.35 & 0.7503 & 0.2497 \\
338.95 & 0.7473 & 0.2527 \\
344.55 & 0.7407 & 0.2593 \\
349.45 & 0.7338 & 0.2662 \\
351.25 & 0.7322 & 0.2678 \\
354.75 & 0.7278 & 0.2722 \\
358.25 & 0.2233 & 0.2767 \\
359.85 & 0.7209 & 0.2791 \\
364.35 & 0.7153 & 0.2847 \\
367.75 & 0.7106 & 0.2894 \\
370.35 & 0.7069 & 0.2931 \\
\hline
\end{tabular}

${ }^{a} x_{2}$ : mole fraction of component 2 in the saturated solution.

${ }^{\mathrm{b}} x_{1}$ : mole fraction solubility of the solute. 
Auxiliary Information

\begin{abstract}
Method/Apparatus/Procedure:
Jacketed solid-liquid equilibrium cell, analytical balance, magnetic stirring system, temperature controlling system, and a laser monitoring system. Solubilities were determined using a synthetic method. Weighed amounts of solute and solvent were sealed in a jacketed equilibrium vessel, and the temperature slowly increased until the solid phase completely disappeared. The disappearance of the solid solute was detected by a laser monitoring system. Measurements were repeated two or three times to check the reproducibility.
\end{abstract}

\section{Source and Purity of Chemicals:}

(1) $98 \%$ (starting purity), Chemical source not given, was an industrial product purified by dissolving in sodium hydroxide, followed by acidification, washing, and the recrystallized from water. Final purity was $99.8 \%$.

(2) Analytical Reagent, Shanghai Chemical Reagent Company, used as received.

\section{Estimated Error:}

Temperature: $\pm 0.05 \mathrm{~K}$ (estimated by compiler).

$x_{1}: \pm 0.0005$ or less.

\section{Auxiliary Information}

Method/Apparatus/Procedure:

Jacketed solid-liquid equilibrium cell, analytical balance, magnetic stirring system, temperature controlling system, and a laser monitoring system. Solubilities were determined using a synthetic method. Weighed amounts of solute and solvent were sealed in a jacketed equilibrium vessel, and the temperature slowly increased until the solid phase completely disappeared. The disappearance of the solid solute was detected by a laser monitoring system. Measurements were repeated two or three times to check the reproducibility.

Source and Purity of Chemicals:

(1) $98 \%$ (starting purity), Chemical source not given, was an industrial product purified by dissolving in sodium hydroxide, followed by acidification, washing, and the recrystallized from water. Final purity was $99.8 \%$. (2) $99.0 \%$, Analytical Reagent, Shanghai Chemical Reagent Company, used as received.

Estimated Error:

Temperature: $\pm 0.05 \mathrm{~K}$ (estimated by compiler). $x_{1}: \pm 0.0005$ or less.

\section{Solubility of 2-Hydroxybenzoic Acid in Organic Solvents \\ 46.1. Critical evaluation of experimental solubility data}

\author{
(1) 4-Formylbenzoic acid; $\mathrm{C}_{8} \mathrm{H}_{6} \mathrm{O}_{3}$; ${ }^{84}$ D.-Q. Li, D.-Z. Liu, and F.-A. \\ [619-66-9] Wang, J. Chem. Eng. Data 46, 172 \\ (2) $N$-Methyl-2-pyrrolidone; $\mathrm{C}_{5} \mathrm{H}_{9} \mathrm{NO} ;$ (2001). \\ [872-50-4]
}

Original Measurements:

\begin{tabular}{ll}
\hline Variables: & Prepared by: \\
Temperature & W. E. Acree, Jr. \\
\hline
\end{tabular}

Experimental Values

\begin{tabular}{lcc}
\hline \hline$T / \mathrm{K}$ & $x_{2}{ }^{\mathrm{a}}$ & $x_{1}{ }^{\mathrm{b}}$ \\
\hline 296.25 & 0.8909 & 0.1091 \\
300.15 & 0.8781 & 0.1219 \\
306.35 & 0.8575 & 0.1425 \\
308.85 & 0.8461 & 0.1539 \\
312.65 & 0.8321 & 0.1679 \\
317.85 & 0.8086 & 0.1914 \\
321.65 & 0.7914 & 0.2086 \\
322.45 & 0.7882 & 0.2118 \\
326.05 & 0.7688 & 0.2312 \\
329.15 & 0.7531 & 0.2469 \\
331.95 & 0.7379 & 0.2621 \\
335.05 & 0.7196 & 0.2804 \\
337.45 & 0.7056 & 0.2944 \\
338.65 & 0.6979 & 0.3021 \\
340.95 & 0.6825 & 0.3175 \\
341.75 & 0.6777 & 0.3223 \\
\hline
\end{tabular}

${ }^{\mathrm{a}} x_{2}$ : mole fraction of component 2 in the saturated solution.

${ }^{\mathrm{b}} x_{1}$ : mole fraction solubility of the solute.
There have been numerous studies $50,56,61,66,67,71,73,87,102,127,145-163$ involving the solubility of 2-hydroxybenzoic acid (commonly referred to as salicylic acid) in organic solvents, particularly at $298 \mathrm{~K}$. Most notably, Barra et al. ${ }^{102}$ published solubility data for 2-hydroxybenzoic acid in two alkanes (heptane and cyclohexane), in one aromatic hydrocarbon (benzene), in one alkyl alkanoate (ethyl ethanoate), in one dialkyl ether (1,1'-oxybisethane) and one cyclic ether (1,4-dioxane), in two chloroalkanes (trichloromethane and 1,2-dichloroethane) and one chloroaromatic hydrocarbon (chlorobenzene), in seven alcohols (methanol, ethanol, 1-pentanol, 1-octanol, 1,2-ethanediol, 1,2-propanediol, and 1,2,3-propanetriol), in one alkanone (propanone) and one aromatic ketone (acetophenone), and in four miscellaneous organic solvents (ethanoic acid, propanoic acid, formamide, and $N, N$-dimethylformamide) as part of study directed towards calculating partial solubility parameters of sodium salts. The authors measured the solubility of a series of acid/sodium salt pairs. Replacement of the acidic proton by sodium ion was found to increase the dipolar and basic partial solubility parameters, whereas the dispersion parameter remained unchanged by the sodium for hydrogen substitution. Solubility data for 2-hydroxybenzoic acid in several alkane solvents (hexane, heptane, decane, dodecane, hexadecane, cyclohexane, and 2,2,4-trimethylpentane) were also determined by Fung and Higuchi. ${ }^{146}$ De Fina et al. ${ }^{151}$ measured 
solubilities of 2-hydroxybenzoic acid in two alkyl alkanoates (ethyl ethanoate and butyl ethanoate), in one dialkyl ether (1,1'-oxybisbutane) and two cyclic ethers (tetrahydrofuran and 1,4-dioxane), in eight alcohols (1-propanol, 2-propanol, 1butanol, 2-butanol, 2-methyl-1-propanol, 2-methyl-2-propanol, 1-pentanol, and 1-octanol), and three alkanones (propanone, butanone, and cyclohexanone). Matsuda et al., ${ }^{152}$ Shalmashi and Eliassi, ${ }^{153}$ Gomaa et al., ${ }^{161}$ Ongley, ${ }^{56}$ Lim et al. ${ }^{159}$ Fahkree et al., ${ }^{162}$ and Perlovich et al. ${ }^{145}$ have also performed benzoic acid solubility measurements at $298 \mathrm{~K}$.

The Abraham solvation parameter model can provide an indication of the quality of experimental solubility data for 2hydroxybenzoic acid in a series of organic solvents of varying polarity and hydrogen bonding character. As discussed above, the evaluation will be restricted to those solvents where dimerization is not likely to occur and to solvents where 2hydroxybenzoic acid does not form a solid solvate. This condition will limit the evaluation to primarily the alkyl alkanoates, dialkyl ethers, and alcohols. Numerical values of the solute descriptors for 2-hydroxybenzoic acid are known ( $E$ $=0.900, S=0.850, A=0.730, B=0.370$, and $V=0.9904)$, so that combination of these descriptors with the coefficients listed in Table 1 allows the prediction of $\log _{10}\left(c_{1, \mathrm{~S}} / c_{1, \mathrm{~W}}\right)$. The molar solubility of molecular 2-hydroxybenzoic acid in water, $\log _{10} c_{1, \mathrm{~W}}=-1.92,{ }^{164}$ is available to convert the predicted $\left(c_{1, \mathrm{~S}} / c_{1, \mathrm{~W}}\right)$ solubility ratios to $c_{1, \mathrm{~S}}$ values. For carboxylic acid solutes, $c_{1, \mathrm{~W}}$ corresponds to the aqueous solubility of the molecular, nonionized form of the solute.

The predicted molar solubilities of 2-hydroxybenzoic acid in methanol, ethanol, 1-propanol, 1-butanol, 1-pentanol, 1octanol, 2-propanol, 2-butanol, 2-methyl-1-propanol, 2methyl-2-propanol, 1,2-ethanediol, 1,1'-oxybisethane, tetrahydrofuran, 1,4-dioxane, ethyl ethanoate, butyl ethanoate, propanone, butanone, and cyclohexanone based on Eq. (20) are listed in the second column of Table 34. The numerical values represent outright solubility predictions in that none of the experimental data was used in the determination of the molecular solute descriptors. For comparison purposes, the measured mole fraction solubilities of 2-hydroxybenzoic acid, $x_{1}$, given in Secs. 46.2-46.10 were converted into molar solubilities by dividing $x_{1}$ by the ideal molar volume of the saturated solution (i.e., $\left.c_{1}{ }^{\text {sat }}=x_{1} /\left[x_{1} V_{1}+\left(1-x_{1}\right) V_{\text {solvent }}\right]\right)$. The molar volume of the hypothetical subcooled liquid 2-hydroxybenzoic acid is taken to be $V_{\text {solute }}=104 \mathrm{~cm}^{3} \mathrm{~mol}^{-1}$, which is larger than the value that other researchers have used. Sloan et al. ${ }^{156}$ assumed a molar volume of 2-hydroxybenzoic acid of $V_{1}=93.9 \mathrm{~cm}^{3} \mathrm{~mol}^{-1}$. This latter value seems too small compared to the molar volume of benzaldehyde, which is estimated to be $V_{1} \approx 101.1 \mathrm{~cm}^{3} \mathrm{~mol}^{-1}$, based on a molar mass of $106.12 \mathrm{~g} \mathrm{~mol}^{-1}$ and an experimental density of $1.0499 \mathrm{~g}$ $\mathrm{cm}^{-3}$ at $296 \mathrm{~K}$. A molar volume on the order of $V_{1} \approx 104 \mathrm{~cm}^{3}$ $\mathrm{mol}^{-1}$ would seem more realistic given the molecular structures of 2-hydroxybenzoic acid compared to benzaldehyde. To get an idea of how much error might be introduced in the mole fraction to molarity conversion, the mole fraction solubility of 2-hydroxybenzoic acid in ethyl ethanoate of $x_{1}=0.1383$ (Ref. 152) is converted into molar solubilities using both $V_{1}$ $=93.9 \mathrm{~cm}^{3} \mathrm{~mol}^{-1}\left(c_{1}=1.41 \mathrm{~mol} \mathrm{dm}{ }^{-3}\right)$ and $V_{1} \approx 104 \mathrm{~cm}^{3}$
TABLE 34. Comparison between observed and predicted molar solubilities of 2hydroxybenzoic acid based on the Abraham model, Eq. (20)

\begin{tabular}{|c|c|c|c|c|c|}
\hline Solvent & $\begin{array}{c}\log _{10} c_{1}{ }^{\text {calc }} ; \\
\text { Eq. (20) }\end{array}$ & $\begin{array}{l}\log _{10} \\
c_{1} \exp \end{array}$ & $\begin{array}{l}\log _{10} \\
c_{1} \exp \end{array}$ & $\begin{array}{l}\log _{10} \\
c_{1} \exp \end{array}$ & $\begin{array}{l}\log _{10} \\
c_{1} \exp \end{array}$ \\
\hline Methanol & 0.514 & $0.430^{\mathrm{a}}$ & & & $0.402^{b}$ \\
\hline \multirow[t]{2}{*}{ Ethanol } & 0.574 & $0.237^{\mathrm{a}}$ & & $0.329^{c}$ & $0.347^{\mathrm{b}}$ \\
\hline & & $0.296^{\mathrm{d}}$ & $0.348^{\mathrm{e}}$ & & \\
\hline 1-Propanol & 0.443 & $0.256^{\mathrm{e}}$ & $0.312^{\mathrm{f}}$ & $0.262^{\mathrm{g}}$ & \\
\hline 2-Propanol & 0.472 & & $0.340^{f}$ & $0.253^{\mathrm{g}}$ & \\
\hline 1-Butanol & 0.328 & $0.199^{\mathrm{e}}$ & $0.243^{\mathrm{f}}$ & & \\
\hline 2-Butanol & 0.359 & & $0.297^{\mathrm{f}}$ & & \\
\hline $\begin{array}{l}\text { 2-Methyl-1- } \\
\text { propanol }\end{array}$ & 0.268 & & $0.180^{f}$ & & \\
\hline $\begin{array}{l}\text { 2-Methyl-2- } \\
\text { propanol }\end{array}$ & 0.440 & & $0.357^{\mathrm{f}}$ & & \\
\hline 1-Pentanol & 0.379 & $0.157^{\mathrm{a}}$ & $0.174^{\mathrm{f}}$ & $0.157^{\mathrm{e}}$ & \\
\hline 2-Pentanol & 0.399 & & & & \\
\hline 3-Methyl-1-butanol & 0.298 & & & & \\
\hline 1-Hexanol & 0.307 & $0.114^{\mathrm{e}}$ & & & \\
\hline 1-Heptanol & 0.240 & $0.072^{\mathrm{e}}$ & & & \\
\hline 1-Octanol & 0.189 & $0.014^{\mathrm{a}}$ & $0.165^{\mathrm{f}}$ & $0.099^{\mathrm{h}}$ & \\
\hline 1-Decanol & 0.176 & & & & \\
\hline 1,2-Ethanediol & 0.180 & $0.152^{\mathrm{a}}$ & & & \\
\hline $1,1^{\prime}$-Oxybisethane & 0.100 & $0.165^{\mathrm{a}}$ & & & \\
\hline Tetrahydrofuran & 0.712 & & $0.609^{f}$ & & \\
\hline 1,4-Dioxane & 0.353 & $0.514^{\mathrm{a}}$ & $0.509^{f}$ & $0.460^{\mathrm{b}}$ & $0.566^{\mathrm{i}}$ \\
\hline Methyl ethanoate & 0.008 & & & & \\
\hline Ethyl ethanoate & 0.146 & $0.091^{\mathrm{a}}$ & $0.157^{\mathrm{f}}$ & $0.143^{\mathrm{c}}$ & $0.143^{\mathrm{b}}$ \\
\hline Propyl ethanoate & 0.068 & & & & \\
\hline Butyl ethanoate & -0.010 & & $0.025^{\mathrm{f}}$ & & \\
\hline Propanone & 0.270 & $0.229^{\mathrm{a}}$ & $0.359^{f}$ & $0.244^{\mathrm{g}}$ & $0.376^{\mathrm{j}}$ \\
\hline Butanone & 0.240 & & $0.300^{f}$ & & \\
\hline Cyclohexanone & 0.139 & & $0.343^{\mathrm{f}}$ & & \\
\hline
\end{tabular}

${ }^{\mathrm{a}}$ Experimental value is taken from Barra et al. ${ }^{102}$

${ }^{\mathrm{b}}$ Experimental value is taken from Matsuda et al. ${ }^{152}$

${ }^{c}$ Experimental value is taken from Shalmashi and Eliassi. ${ }^{153}$

${ }^{\mathrm{d}}$ Experimental value is taken from Jouyban et al. ${ }^{160}$

${ }^{\mathrm{e}}$ Experimental value is taken from Lim et al. ${ }^{159}$

${ }^{\mathrm{f}}$ Experimental value is taken from De Fina et al. ${ }^{151}$

${ }^{\mathrm{g}}$ Experimental value is taken from Fakhree et al. ${ }^{162}$

${ }^{h}$ Experimental value is taken from Perlovich et al. ${ }^{145}$

${ }^{\mathrm{i}}$ Experimental value is taken from Peña et al. ${ }^{157}$

${ }^{\mathrm{j}}$ Experimental value is taken from Marden and Dover. ${ }^{149}$

$\mathrm{mol}^{-1}\left(c_{1}=1.39 \mathrm{~mol} \mathrm{dm}^{-3}\right)$. Results of the computation suggest that any errors resulting from the estimation of 2hydroxybenzoic acid's hypothetical subcooled liquid molar volume, $V_{\text {solute }}$, or the ideal molar volume approximation will have negligible effect of the calculated $c_{1}$ values because 2hydroxybenzoic acid is not overly soluble in many of the solvents considered. From a mathematical standpoint, the $x_{1}{ }^{\exp } V_{\text {solute }}$ term contributes very little to the molar volumes of the saturated solutions.

Solution models, like the Abraham solvation parameter model, prove useful in screening datasets for obvious outliers, particularly in cases where there are only one or two experimental data points for a given solute-solvent system. Such models are only able to identify those outliers, however, which fall outside of the model's expected predictive applicability. There is a sufficient number of data points for several of the solvents in Secs. 46.2-46.11 to compute a recommended value. The recommended mole fraction solubilities were calculated as an arithmetic average and are given in Table 35 
TABLE 35. Recommended mole fraction solubilites for 2-hydroxybenzoic acid in selected solvents

\begin{tabular}{lclll}
\hline \hline Solvent & Recommended Value & \multicolumn{1}{c}{ Individual Values } & Outliers \\
\hline 1,4-Dioxane & 0.291 & $0.2945,{ }^{151} 0.2610,{ }^{152} 0.2978,{ }^{102} 0.3117$ (Ref. 155) & \\
Tetrachloromethane & 0.00306 & $0.00293,{ }^{67} 0.00291,{ }^{66} 0.00333$ (Ref. 153) & \\
Ethanol & 0.141 & $0.1450,{ }^{152} 0.1386,{ }^{153} 0.1455,{ }^{159} 0.1397,{ }^{155} 0.1378,{ }^{154} 0.1369$ (Ref. 71) & 0.1100 (Ref. 102) \\
1-Butanol & 0.155 & $0.1646,{ }^{151} 0.152,{ }^{127} 0.1484$ (Ref. 159) & \\
1-Pentanol & 0.157 & $0.1611,{ }^{151} 0.1547,{ }^{102} 0.1550$ (Ref. 159) & \\
\hline
\end{tabular}

for the solvents that had three or more close solubility measurements. Included in the table are the individual mole fraction solubilities that went into the calculation, and any suspected outlier values. In the case of ethyl ethanoate, there are two different sets of three experimental values each $\left[x_{1}=0.1425,{ }^{151} 0.1383,{ }^{152}\right.$ and 0.1381 (Ref. 153) $]$ and $\left[x_{1}=0.1152,{ }^{154} 0.1223,{ }^{102}\right.$ and 0.1136 (Ref. 135)], and one clear outlier value of $x_{1}=0.195 .^{149}$ Since there is no way of knowing which of the two datasets is better, the recommended solubility in ethyl ethanoate of $x_{1}=0.128$ is calculated as the arithmetic average of all six experimental values [excluding the outlier). A similar situation occurs with 2-propanol, two different datasets of two values each, $\left(x_{1}=0.1320\right.$ (Ref. 102) and 0.137 (Ref. 162) $]$ and $\left[x_{1}=0.1817\right.$ (Ref. 151) and 0.1892 (Ref. 149)]. Situations like this is why the criterion for making a recommendation was set at three or more data points that differed from each other by no more than approximately 15 relative percent.

There have been a few experimental studies examining the solubility of 2-hydroxybenzoic acid in different organic solvents as a function of temperature. Shalmashi and Eliassi ${ }^{153}$ measured the solubility of 2-hydroxybenzoic acid in ethyl ethanoate, tetrachloromethane, and ethanol at eleven temperatures between 298 and $348 \mathrm{~K}$ using a gravimetric method. The authors described the observed mass fraction solubilities, $w_{1}$, with a simple linear relationship

$$
\ln w_{1}=A+B T,
$$

where $A$ and $B$ are the intercept and slope of Eq. (49). The calculated values of $A$ and $B$ are tabulated in Table 36, along with the root-mean-square deviation defined by

$$
\text { RMSD }=\sqrt{\frac{\sum_{i}^{N}\left(w_{i}^{\text {exp }}-w_{i}^{\text {calc }}\right)^{2}}{N-1}} .
$$

In Eq. (50), $N$ represents the number of experimental values in the given data set, which in the present case would be 11 . The two larger RMSDs of 0.0060 and 0.0082 occur in the 2hydroxybenzoic acid + ethyl ethanoate and 2-hydroxybenzoic acid + ethanol systems, and correspond to approximately a $3 \%$ to $4 \%$ mean relative deviation.

Fakhree et al. ${ }^{162}$ measured the solubility of 2-hydroxybenzoic acid in water, 1-propanol, 2-propanol, and propanone at five temperatures between 298 and $338 \mathrm{~K}$, and in the three binary solvent mixtures containing water with each of the three organic compounds at $298 \mathrm{~K}$. The authors described the variation in the molar solubility with temperature with a Modified Apelblat equation

$$
\log _{10} c_{1}=A+\frac{B}{T}+C \log _{10} T
$$

The calculated equation coefficients $(A, B$, and $C)$, along with the mean percent relative deviation in molar concentration, are given in Table 37. Examination of the numerical entries in the last column of Table 37 reveals that the Apelblat type equation provides a reasonably accurate mathematical description of the observed solubility data, suggesting that there are no obvious outliers in the dataset.

Lim et al. ${ }^{159}$ examined the solubility behavior of 2-hydroxybenzoic acid in six neat 1-alkanols (ethanol through 1heptanol) at temperatures from 278 to $318 \mathrm{~K}$. The experimental data were correlated with the Non-Random TwoLiquid (NRTL), UNIQUAC, and Wilson models. Interaction coefficients calculated from the experimental solid-liquid equilibrium data provided a reasonably accurate mathematical description of the measured values. The mean absolute relative deviation between calculated and observed values was $0.475 \%$ (NRTL), 0.588\% (Wilson model), and 0.429\% (UNIQUAC model).

The experimental solubility data for 2-hydroxybenzoic acid in organic solvents are given in Secs. 46.2-46.11.
TABLE 36. Parameters of Eq. (49) for describing the solubility of 2-hydroxybenzoic acid in various organic solvents

\begin{tabular}{lccc}
\hline \hline Solvent & $A$ & $B$ & RMSD \\
\hline Ethyl ethanoate $^{\mathrm{a}}$ & -5.14 & 0.02 & 0.0060 \\
Tetrachloromethane $^{\mathrm{a}}$ & -18.59 & 0.04 & 0.0010 \\
Ethanol $^{\mathrm{a}}$ & -4.30 & 0.01 & 0.0082
\end{tabular}

${ }^{\mathrm{a}}$ Values of the coefficients and the mean relative deviations were taken from Shalmashi and Eliassi. ${ }^{153}$
Table 37. Parameters of the Modified Apelblat-type equation for describing the solubility of 2-hydroxybenzoic acid in various organic solvents

\begin{tabular}{lcrccc}
\hline \hline Solvent & $T / \mathrm{K}$ & \multicolumn{1}{c}{$A$} & \multicolumn{1}{c}{$B$} & \multicolumn{1}{c}{$C$} & MRD (\%) \\
\hline 1-Propanol $^{\mathrm{a}}$ & $298-338$ & 1.514 & -384.958 & 0 & 0.5 \\
2-Propanol $^{\mathrm{a}}$ & $298-338$ & 1.578 & -396.006 & 0 & 0.8 \\
Propanone $^{\mathrm{a}}$ & $298-338$ & -272.035 & 12249.4 & 93.438 & 0.1 \\
\hline
\end{tabular}

${ }^{\mathrm{a}}$ Values of the coefficients and mean relative deviation were taken from Fakhree et al. ${ }^{162}$ 


\subsection{2-Hydroxybenzoic acid solubility data in saturated hydrocarbons (including cycloalkanes)}

\begin{tabular}{ll}
\hline \hline Components: & Original Measurements: \\
$\begin{array}{l}\text { (1) 2-Hydroxybenzoic acid; } \\
\mathrm{C}_{7} \mathrm{H}_{6} \mathrm{O}_{3} ;[69-72-7]\end{array}$ & ${ }^{145}$ G. L. Perlovich, T. V. Volkova, \\
(2) Hexane; $\mathrm{C}_{6} \mathrm{H}_{14} ;[110-54-3]$ & and A. Bauer-Brandl, J. Pharm. Sci. \\
\hline Variables: & $\mathbf{9 5}, 1448(2006)$. \\
Temperature & Prepared by: \\
\hline
\end{tabular}

Experimental Values

\begin{tabular}{lcc}
\hline \hline$T / \mathrm{K}$ & $x_{2}{ }^{\mathrm{a}}$ & $x_{1}{ }^{\mathrm{b}}$ \\
\hline 293 & 0.9996 & 0.000390 \\
298 & 0.9995 & 0.000491 \\
303 & 0.9994 & 0.000643 \\
310 & 0.9990 & 0.000959 \\
315 & 0.9988 & 0.00123 \\
\hline
\end{tabular}

${ }^{\mathrm{a}} x_{2}$ : mole fraction of component 2 in the saturated solution.

${ }^{b} x_{1}$ : mole fraction solubility of the solute.

\section{Auxiliary Information}

\section{Method/Apparatus/Procedure:}

Thermostated constant-temperature bath, centrifuge, and an ultraviolet/visible spectrophotometer.

Excess solute and solvent were placed in a glass ampoule and allowed to equilibrate in a thermostated temperature bath with mixing (at a speed of $25 \mathrm{rpm}$ ) for a minimum of four days. After suitable equilibration, the saturated solution was centrifuged, the supernatant liquid collected, quantitatively diluted, and the absorbance recorded using an ultraviolet/visible spectrophotometer. The solubility of the solute was calculated from the measured absorbance.

Source and Purity of Chemicals:

(1) $99 \%$, Sigma-Aldrich Inc., Oslo, Norway, no purification details provided. (2) Analytical Reagent grade, SDS, Peypin, France, no purification details were provided.

\section{Estimated Error:}

Temperature: $\pm 0.1 \mathrm{~K}$.

$x_{2}: \pm 2.5 \%$ (relative error).

\begin{tabular}{ll}
\hline \hline Components: & Original Measurements: \\
$(1)$ 2-Hydroxybenzoic acid; & ${ }^{146} \mathrm{H} .-\mathrm{L}$. Fung and T. Higuchi, J. \\
$\mathrm{C}_{7} \mathrm{H}_{6} \mathrm{O}_{3} ;[69-72-7]$ & Pharm. Sci. 60, 1782 (1971). \\
$(2) \mathrm{Hexane} ; \mathrm{C}_{6} \mathrm{H}_{14} ;[110-54-3]$ & \\
\hline Variables: & Prepared by: \\
$T / \mathrm{K}=298.15$ & W. E. Acree, Jr. \\
\hline
\end{tabular}

Experimental Values

\begin{tabular}{lc}
\hline \hline$x_{2}^{\mathrm{a}}$ & $x_{1}^{\mathrm{b}}$ \\
\hline 0.9995 & 0.00048 \\
\hline${ }^{\mathrm{a}} x_{2}:$ mole fraction of component 2 in the saturated solution. \\
${ }^{\mathrm{b}}{ }_{x_{1}: \text { mole fraction solubility of the solute. }}$
\end{tabular}

\section{Auxiliary Information}

Method/Apparatus/Procedure:

Wrist-action shaker, constant-temperature bath, and an ultraviolet/visible spectrophotometer.

Excess solute and solvent were allowed to equilibrate in a stoppered and sealed volumetric flask, which was continuously shaken (wrist-action shaken) for at least $24 \mathrm{~h}$ in a thermostated constant-temperature bath. An aliquot of saturated solution was transferred to a volumetric flask and diluted with trichloromethane. Concentrations were determined by spectrophotometric measurements at $306 \mathrm{~nm}$.

Source and Purity of Chemicals:

(1) Purity not given, Chemical source not given, was recrystallized from hot water before use.

(2) Reagent grade, Chemical source not given, used as received.

Estimated Error:

Temperature: $\pm 0.1 \mathrm{~K}$ (estimated by compiler).

$x_{1}: \pm 3 \%$ (relative error, estimated by compiler).

\begin{tabular}{|c|c|}
\hline $\begin{array}{l}\text { Components: } \\
\text { (1) 2-Hydroxybenzoic acid; } \\
\mathrm{C}_{7} \mathrm{H}_{6} \mathrm{O}_{3} ;[69-72-7] \\
\text { (2) Hexane; } \mathrm{C}_{6} \mathrm{H}_{14} ;[110-54-3]\end{array}$ & $\begin{array}{l}\text { Original Measurements: } \\
{ }^{50} \text { P. G. Desai and A. M. Patel, J. } \\
\text { Indian Chem. Soc. 12, } 131 \text { (1935). }\end{array}$ \\
\hline $\begin{array}{l}\text { Variables: } \\
T / \mathrm{K}=301.2\end{array}$ & $\begin{array}{l}\text { Prepared by: } \\
\text { W. E. Acree, Jr. }\end{array}$ \\
\hline
\end{tabular}

Experimental Values

\begin{tabular}{lc}
\hline \hline$x_{2}{ }^{\mathrm{a}}$ & $x_{1}{ }^{\mathrm{b}}$ \\
\hline 0.9989 & 0.00111
\end{tabular}

${ }^{a} x_{2}$ : mole fraction of component 2 in the saturated solution.

${ }^{\mathrm{b}} x_{1}$ : mole fraction solubility of the solute.

\section{Auxiliary Information}

\section{Method/Apparatus/Procedure:}

Excess solute and solvent were placed in a glass flask which was kept revolving on a wheel in an air thermostat for two days. After two days of equilibration, the solution was filtered. To minimize absorption of the solute onto the filter paper, a $100 \mathrm{ml}$ portion of fresh saturated solution was first filtered through the paper before filtering the sample to be analyzed. An aliquot of the filtrate was titrated with barium hydroxide using phenolphthalein as the endpoint indictor. The barium hydroxide titrant was previously standardized against a solution of succinic acid.

\section{Source and Purity of Chemicals:}

(1) Purity not given, Chemical source not specified, no information provided concerning purification.

(2) Purity not given, Chemical source not specified, no information provided concerning purification.

\section{Estimated Error:}

Temperature: No information given.

$x_{1}: \pm 3 \%$ (relative error, estimated by compiler). 


\begin{tabular}{|c|c|}
\hline $\begin{array}{l}\text { Components: } \\
\text { (1) 2-Hydroxybenzoic acid; } \\
\mathrm{C}_{7} \mathrm{H}_{6} \mathrm{O}_{3} ;[69-72-7] \\
\text { (2) Heptane; } \mathrm{C}_{7} \mathrm{H}_{16} ;[142-82-5]\end{array}$ & $\begin{array}{l}\text { Original Measurements: } \\
{ }^{146} \text { H.-L. Fung and T. Higuchi, J. } \\
\text { Pharm. Sci. 60, } 1782 \text { (1971). }\end{array}$ \\
\hline $\begin{array}{l}\text { Variables: } \\
T / \mathrm{K}=298.15\end{array}$ & $\begin{array}{l}\text { Prepared by: } \\
\text { W. E. Acree, Jr. }\end{array}$ \\
\hline
\end{tabular}

Experimental Values

\begin{tabular}{lc}
\hline \hline$x_{2}{ }^{\mathrm{a}}$ & $x_{1}{ }^{\mathrm{b}}$ \\
\hline 0.9995 & 0.00048 \\
\hline
\end{tabular}

${ }^{\mathrm{a}} x_{2}$ : mole fraction of component 2 in the saturated solution.

${ }^{\mathrm{b}} x_{1}$ : mole fraction solubility of the solute.

\section{Auxiliary Information}

\section{Method/Apparatus/Procedure:}

Wrist-action shaker, constant-temperature bath, and an ultraviolet/visible spectrophotometer.

Excess solute and solvent were allowed to equilibrate in a stoppered and sealed volumetric flask, which was continuously shaken (wrist-action shaken) for at least $24 \mathrm{~h}$ in a thermostated constant-temperature bath. An aliquot of saturated solution was transferred to a volumetric flask and diluted with trichloromethane. Concentrations were determined by spectrophotometric measurements at $306 \mathrm{~nm}$.

\section{Source and Purity of Chemicals:}

(1) Purity not given, Chemical source not given, was recrystallized from hot water before use.

(2) Reagent grade, Chemical source not given, was purified by passing the solvent through a column of silica gel and then distilled over sodium under reduced pressure.

Estimated Error:

Temperature: $\pm 0.1 \mathrm{~K}$ (estimated by compiler).

$x_{1}: \pm 3 \%$ (relative error, estimated by compiler).

\begin{tabular}{ll}
\hline \hline Components: & Original Measurements: \\
$\begin{array}{l}\text { (1) 2-Hydroxybenzoic acid; } \\
\mathrm{C}_{7} \mathrm{H}_{6} \mathrm{O}_{3} ;[69-72-7]\end{array}$ & $\begin{array}{l}\text { 102 J. Barra, M.-A. Peña, and P. } \\
\text { (2) Heptane; } \mathrm{C}_{7} \mathrm{H}_{16} ;[142-82-5]\end{array}$ \\
\hline Bariables: & $153(2000)$. \\
$T / \mathrm{K}=298.15$ & Prepared by: \\
\hline
\end{tabular}

Experimental Values

\begin{tabular}{lc}
\hline \hline$x_{2}{ }^{\mathrm{a}}$ & $x_{1}{ }^{\mathrm{b}}$ \\
\hline 0.9989 & 0.001116 \\
\hline${ }^{\mathrm{a}} x_{2}:$ mole fraction of component 2 in the saturated solution. & \\
${ }^{\mathrm{b}}{ }_{x_{1}: \text { mole fraction solubility of the solute. }}$ &
\end{tabular}

\section{Auxiliary Information}

\section{Method/Apparatus/Procedure:}

Constant-temperature bath, analytical balance, and an ultraviolet/visible spectrophotometer.

Excess solute and solvent were placed in a flask and allowed to equilibrate for several days at constant temperature. The solid phase was removed by filtration, and the clear solution was diluted quantitatively with $96 \%$ ethanol. The concentration of the diluted solution was determined by spectrophotometric measurement at the wavelength of maximum absorption. In instances where the solvent interfered with the spectrophotometric determination, the samples were evaporated to dryness and the residue diluted with $96 \%$ ethanol.

\section{Source and Purity of Chemicals:}

(1) Purity not given, Sigma Chemical Company, USA, no purification details were provided.

(2) Spectrophotometric or Analytical grade, Chemical source not specified, no purification details were provided.

\section{Estimated Error:}

Temperature: $\pm 0.2 \mathrm{~K}$.

$x_{1}: \pm 10.0 \%$ (relative error, estimated by compiler).

\begin{tabular}{ll}
\hline \hline Components: & Original Measurements: \\
(1) 2-Hydroxybenzoic acid; & ${ }^{127} \mathrm{~N}$. V. Sidgwick and E. K. \\
$\mathrm{C}_{7} \mathrm{H}_{6} \mathrm{O}_{3} ;[69-72-7]$ & Ewbank, J. Chem. Soc. Trans. 119, \\
(2) Heptane; $\mathrm{C}_{7} \mathrm{H}_{16} ;[142-82-5]$ & $979(1921)$. \\
\hline Variables: & Prepared by: \\
Temperature & W. E. Acree, Jr. \\
\hline
\end{tabular}

Experimental Values

\begin{tabular}{lcc}
\hline \hline$T / \mathrm{K}$ & $x_{2}{ }^{\mathrm{a}}$ & $x_{1}{ }^{\mathrm{b}}$ \\
\hline 365.4 & 0.985 & 0.0152 \\
385.6 & 0.960 & 0.0395 \\
397.9 & 0.923 & 0.0765 \\
407.5 & 0.845 & 0.155 \\
415.2 & 0.659 & 0.341 \\
418.7 & 0.477 & 0.523 \\
422.7 & 0.240 & 0.760 \\
\hline
\end{tabular}

${ }^{\mathrm{a}} x_{2}$ : mole fraction of component 2 in the saturated solution.

${ }^{b} x_{1}$ : mole fraction solubility of the solute. Solubility data reported in units of mass percent. Mole fraction values calculated by the compiler.

\section{Auxiliary Information}

\section{Method/Apparatus/Procedure:}

Preweighed quantities of solute and solvent were placed in bulbs, which were then sealed and heated in a suitable bath. At the higher temperatures, the concentration of solvent in the liquid solution was corrected for the amount of solvent vapor in the bulb. The correction assumed that the vapor pressure of the saturated solution was one half that of the pure solvent at the solution temperature. The temperature at which the solid completely dissolved was recorded, and was taken to be the solid-liquid equilibrium temperature.

Source and Purity of Chemicals:

(1) Purity not given, prepared and purified by the authors using a published synthetic procedure [see J. Walker and J. K. Wood, J. Chem. Soc. 117, 40 (1920) for synthetic details]. Melting point of the purified solute was $432.2 \mathrm{~K}$. (2) Purity not given, sample isolated from the resin of Pinus sabiniana, purified by treatment with sulfuric acid and nitric acid, and then distilled before use. 
Estimated Error:

Temperature: Not given in paper.

$x_{1}: \pm 10 \%$ (relative error, estimated by compiler).

\begin{tabular}{|c|c|}
\hline $\begin{array}{l}\text { Components: } \\
\text { (1) 2-Hydroxybenzoic acid; } \\
\mathrm{C}_{7} \mathrm{H}_{6} \mathrm{O}_{3} ;[69-72-7] \\
\text { (2) } 2,2,4-\text { Trimethylpentane; } \\
\mathrm{C}_{8} \mathrm{H}_{18} ;[540-84-1]\end{array}$ & $\begin{array}{l}\text { Original Measurements: } \\
{ }^{146} \text { H.-L. Fung and T. Higuchi, J. } \\
\text { Pharm. Sci. 60, } 1782 \text { (1971). }\end{array}$ \\
\hline $\begin{array}{l}\text { Variables: } \\
T / \mathrm{K}=298.15\end{array}$ & $\begin{array}{l}\text { Prepared by: } \\
\text { W. E. Acree, Jr. }\end{array}$ \\
\hline
\end{tabular}

Experimental Values

\begin{tabular}{lc}
\hline \hline$x_{2}{ }^{\mathrm{a}}$ & $x_{1}{ }^{\mathrm{b}}$ \\
\hline 0.9996 & 0.00038 \\
\hline
\end{tabular}

${ }^{a} x_{2}$ : mole fraction of component 2 in the saturated solution.

${ }^{\mathrm{b}} x_{1}$ : mole fraction solubility of the solute.

\section{Auxiliary Information}

\begin{abstract}
Method/Apparatus/Procedure:
Wrist-action shaker, constant-temperature bath, and an ultraviolet/visible spectrophotometer.

Excess solute and solvent were allowed to equilibrate in a stoppered and sealed volumetric flask, which was continuously shaken (wrist-action shaken) for at least $24 \mathrm{~h}$ in a thermostated constant-temperature bath. An aliquot of saturated solution was transferred to a volumetric flask and diluted with trichloromethane. Concentrations were determined by spectrophotometric measurements at $306 \mathrm{~nm}$.
\end{abstract}

\section{Source and Purity of Chemicals:}

(1) Purity not given, Chemical source not given, was recrystallized from hot water before use.

(2) Reagent grade, Chemical source not given, was distilled from sodium under reduced pressure shortly before use.

\section{Estimated Error:}

Temperature: $\pm 0.1 \mathrm{~K}$ (estimated by compiler).

$x_{1}: \pm 3 \%$ (relative error, estimated by compiler).

\section{Components:}

(1) 2-Hydroxybenzoic acid;

$\mathrm{C}_{7} \mathrm{H}_{6} \mathrm{O}_{3} ;[69-72-7]$

(2) Decane; $\mathrm{C}_{10} \mathrm{H}_{22} ;$ [124-18-5]

\section{Variables:}

$T / \mathrm{K}=298.15$

Prepared by:

W. E. Acree, Jr.

\section{Experimental Values}

\begin{tabular}{lc}
\hline \hline$x_{2}^{\mathrm{a}}$ & $x_{1}{ }^{\mathrm{b}}$ \\
\hline 0.9994 & 0.00062 \\
\hline${ }^{\mathrm{a}} x_{2}:$ mole fraction of component 2 in the saturated solution. \\
${ }^{\mathrm{b}}{ }_{x_{1}: \text { mole fraction solubility of the solute. }}$
\end{tabular}

\section{Auxiliary Information}

Method/Apparatus/Procedure:

Wrist-action shaker, constant-temperature bath, and an ultraviolet/visible spectrophotometer.

Excess solute and solvent were allowed to equilibrate in a stoppered and sealed volumetric flask, which was continuously shaken (wrist-action shaken) for at least $24 \mathrm{~h}$ in a thermostated constant-temperature bath. An aliquot of saturated solution was transferred to a volumetric flask and diluted with trichloromethane. Concentrations were determined by spectrophotometric measurements at $306 \mathrm{~nm}$.

\section{Source and Purity of Chemicals:}

(1) Purity not given, Chemical source not given, was recrystallized from hot water before use.

(2) Reagent grade, Chemical source not given, was purified by passing the solvent through a column of silica gel and then distilled over sodium under reduced pressure.

\section{Estimated Error:}

Temperature: $\pm 0.1 \mathrm{~K}$ (estimated by compiler). $x_{1}: \pm 3 \%$ (relative error, estimated by compiler).

\begin{tabular}{ll}
\hline \hline Components: & Original Measurements: \\
$(1)$ 2-Hydroxybenzoic acid; & ${ }^{146} \mathrm{H}$-L. Fung and T. Higuchi, J. \\
$\mathrm{C}_{7} \mathrm{H}_{6} \mathrm{O}_{3} ;[69-72-7]$ & Pharm. Sci. 60, 1782 (1971). \\
(2) Dodecane; $\mathrm{C}_{12} \mathrm{H}_{26} ;[112-40-3]$ & \\
\hline Variables: & Prepared by: \\
$T / \mathrm{K}=298.15$ & W. E. Acree, Jr. \\
\hline
\end{tabular}

\section{Experimental Values}

\begin{tabular}{lc}
\hline \hline$x_{2}{ }^{\mathrm{a}}$ & $x_{1}{ }^{\mathrm{b}}$ \\
\hline 0.9994 & 0.00064 \\
\hline${ }^{\mathrm{a}} x_{2}:$ mole fraction of component 2 in the saturated solution. & \\
${ }^{\mathrm{b}}{ }_{x_{1}}:$ mole fraction solubility of the solute. &
\end{tabular}

\section{Auxiliary Information}

\section{Method/Apparatus/Procedure:}

Wrist-action shaker, constant-temperature bath, and an ultraviolet/visible spectrophotometer.

Excess solute and solvent were allowed to equilibrate in a stoppered and sealed volumetric flask, which was continuously shaken (wrist-action shaken) for at least $24 \mathrm{~h}$ in a thermostated constant-temperature bath. An aliquot of saturated solution was transferred to a volumetric flask and diluted with trichloromethane. Concentrations were determined by spectrophotometric measurements at $306 \mathrm{~nm}$.

\section{Source and Purity of Chemicals:}

(1) Purity not given, Chemical source not given, was recrystallized from hot water before use.

(2) Reagent grade, Chemical source not given, was purified by passing the solvent through a column of silica gel.

\section{Estimated Error:}

Temperature: $\pm 0.1 \mathrm{~K}$ (estimated by compiler). $x_{1}: \pm 3 \%$ (relative error, estimated by compiler). 


\begin{tabular}{|c|c|}
\hline $\begin{array}{l}\text { Components: } \\
\text { (1) 2-Hydroxybenzoic acid; } \\
\mathrm{C}_{7} \mathrm{H}_{6} \mathrm{O}_{3} ;[69-72-7] \\
\text { (2) Hexadecane; } \mathrm{C}_{16} \mathrm{H}_{34} ;[544-76-3]\end{array}$ & $\begin{array}{l}\text { Original Measurements: } \\
{ }^{146} \text { H.-L. Fung and T. Higuchi, J. } \\
\text { Pharm. Sci. 60, } 1782 \text { (1971). }\end{array}$ \\
\hline $\begin{array}{l}\text { Variables: } \\
T / \mathrm{K}=298.15\end{array}$ & $\begin{array}{l}\text { Prepared by: } \\
\text { W. E. Acree, Jr. }\end{array}$ \\
\hline
\end{tabular}

Experimental Values

\begin{tabular}{lc}
\hline \hline$x_{2}^{\mathrm{a}}$ & $x_{1}{ }^{\mathrm{b}}$ \\
\hline 0.9990 & 0.00104
\end{tabular}

${ }^{a_{X_{2}}}$ : mole fraction of component 2 in the saturated solution.

${ }^{\mathrm{b}} x_{1}$ : mole fraction solubility of the solute.

\section{Auxiliary Information}

\section{Method/Apparatus/Procedure:}

Wrist-action shaker, constant-temperature bath, and an ultraviolet/visible spectrophotometer.

Excess solute and solvent were allowed to equilibrate in a stoppered and sealed volumetric flask, which was continuously shaken (wrist-action shaken) for at least $24 \mathrm{~h}$ in a thermostated constant-temperature bath. An aliquot of saturated solution was transferred to a volumetric flask and diluted with trichloromethane. Concentrations were determined by spectrophotometric measurements at $306 \mathrm{~nm}$.

\section{Source and Purity of Chemicals:}

(1) Purity not given, Chemical source not given, was recrystallized from ho water before use.

(2) Reagent grade, Chemical source not given, was purified by passing the solvent through a column of silica gel and then distilled over sodium under reduced pressure.

Estimated Error:

Temperature: $\pm 0.1 \mathrm{~K}$ (estimated by compiler). $x_{1}: \pm 3 \%$ (relative error, estimated by compiler).

\begin{tabular}{ll}
\hline \hline Components: & Original Measurements: \\
$(1)$ 2-Hydroxybenzoic acid; & ${ }^{146} \mathrm{H}$.-L. Fung and T. Higuchi, J. \\
$\mathrm{C}_{7} \mathrm{H}_{6} \mathrm{O}_{3} ;[69-72-7]$ & Pharm. Sci. 60, 1782 (1971). \\
$(2)$ Cyclohexane; $\mathrm{C}_{6} \mathrm{H}_{12} ;[110-82-7]$ & \\
\hline Variables: & Prepared by: \\
$T / \mathrm{K}=298.15$ & W. E. Acree, Jr. \\
\hline
\end{tabular}

Experimental Values

\begin{tabular}{lc}
\hline \hline$x_{2}{ }^{\mathrm{a}}$ & $x_{1}{ }^{\mathrm{b}}$ \\
\hline 0.9996 & 0.00043 \\
\hline$x_{2}$ m
\end{tabular}

${ }^{\mathrm{a}} x_{2}$ : mole fraction of component 2 in the saturated solution.

${ }^{\mathrm{b}} x_{1}$ : mole fraction solubility of the solute.

\section{Auxiliary Information}

\section{Method/Apparatus/Procedure:}

Wrist-action shaker, constant-temperature bath, and an ultraviolet/visible spectrophotometer.

Excess solute and solvent were allowed to equilibrate in a stoppered and sealed volumetric flask, which was continuously shaken (wrist-action shaken) for at least $24 \mathrm{~h}$ in a thermostated constant-temperature bath. An aliquot of saturated solution was transferred to a volumetric flask and diluted with trichloromethane. Concentrations were determined by spectrophotometric measurements at $306 \mathrm{~nm}$.

\section{Source and Purity of Chemicals:}

(1) Purity not given, Chemical source not given, was recrystallized from hot water before use.

(2) Reagent grade, Chemical source not given, was distilled over phosphorous pentoxide shortly before use.

\section{Estimated Error:}

Temperature: $\pm 0.1 \mathrm{~K}$ (estimated by compiler). $x_{1}: \pm 3 \%$ (relative error, estimated by compiler).

\begin{tabular}{ll}
\hline \hline Components: & Original Measurements: \\
$\begin{array}{l}\text { (1) 2-Hydroxybenzoic acid; } \\
\mathrm{C}_{7} \mathrm{H}_{6} \mathrm{O}_{3} ;[69-72-7]\end{array}$ & ${ }^{56} \mathrm{P}$. A. Ongley, J. Chem. Soc. 1954, \\
$(2)$ Cyclohexane; $\mathrm{C}_{6} \mathrm{H}_{12} ;[110-82-7]$ & 3634. \\
\hline Variables: & Prepared by: \\
$T / \mathrm{K}=298.15$ & W. E. Acree, Jr. \\
\hline
\end{tabular}

\section{Experimental Values}

The measured solubility was reported to be $-\log _{10} c_{1}=$ 2.912, which corresponds to a solubility of $c_{1}=0.00122$ mol $\mathrm{dm}^{-3}$.

\section{Auxiliary Information}

\section{Method/Apparatus/Procedure:}

Very little experimental details were provided. Excess solute and solvent were placed in tubes, which were rotated for at least $8 \mathrm{~h}$ at $298 \mathrm{~K}$. The solutions were then removed, concentrated if necessary, and titrated with standard alkali using a mixed (bromothymol blue + neutral red) indicator.

\section{Source and Purity of Chemicals:}

(1) Purity not given, Chemical source not given, solute was either "AnalaR" grade or recrystallized prior to use.

(2) Purity not given, Chemical source not given, no purification details were provided.

\section{Estimated Error:}

Temperature: No information was given.

$c_{1}: \pm 4 \%$ (relative error, estimated by compiler).

\begin{tabular}{ll}
\hline \hline Components: & Original Measurements: \\
$(1)$ 2-Hydroxybenzoic acid; & 102J. Barra, M.-A. Peña, and P. \\
$\mathrm{C}_{7} \mathrm{H}_{6} \mathrm{O}_{3} ;[69-72-7]$ & Bustamante, Eur. J. Pharm. Sci. 10, \\
$\begin{array}{l}(2) \mathrm{Cyclohexane} ; \mathrm{C}_{6} \mathrm{H}_{12} ; \\
{[110-82-7]}\end{array}$ & $153(2000)$. \\
\hline Variables: & \\
$T / \mathrm{K}=298.15$ & Prepared by: \\
\hline
\end{tabular}


Experimental Values

\begin{tabular}{lc}
\hline \hline$x_{2}{ }^{\mathrm{a}}$ & $x_{1}{ }^{\mathrm{b}}$ \\
\hline 0.9994 & 0.000551 \\
${ }^{\mathrm{a}} x_{2}:$ mole fraction of component 2 in the saturated solution. \\
${ }^{\mathrm{b}} x_{1}:$ mole fraction solubility of the solute.
\end{tabular}

\section{Auxiliary Information}

\section{Method/Apparatus/Procedure:}

Constant-temperature bath, analytical balance, and an ultraviolet/visible spectrophotometer.

Excess solute and solvent were placed in a flask and allowed to equilibrate for several days at constant temperature. The solid phase was removed by filtration, and the clear solution was diluted quantitatively with $96 \%$ ethanol. The concentration of the diluted solution was determined by spectrophotometric measurement at the wavelength of maximum absorption. In instances where the solvent interfered with the spectrophotometric determination, the samples were evaporated to dryness and the residue diluted with $96 \%$ ethanol.

Source and Purity of Chemicals:

(1) Purity not given, Sigma Chemical Company, USA, no purification details were provided.

(2) Spectrophotometric or Analytical grade, Chemical source not specified, no purification details were provided.

Estimated Error:

Temperature: $\pm 0.2 \mathrm{~K}$.

$x_{1}: \pm 10.0 \%$ (relative error, estimated by compiler).

\subsection{2-Hydroxybenzoic acid solubility data in aromatic hydrocarbons}

\begin{tabular}{ll}
\hline \hline Components: & Original Measurements: \\
$(1)$ 2-Hydroxybenzoic acid; & ${ }^{127}$ N. V. Sidgwick and E. K. \\
$\mathrm{C}_{7} \mathrm{H}_{6} \mathrm{O}_{3} ;[69-72-7]$ & Ewbank, J. Chem. Soc. Trans. 119, \\
$(2)$ Benzene; $\mathrm{C}_{6} \mathrm{H}_{6} ;[71-43-2]$ & $979(1921)$. \\
\hline Variables: & Prepared by: \\
Temperature & W. E. Acree, Jr. \\
\hline
\end{tabular}

Experimental Values

\begin{tabular}{lcc}
\hline \hline$T / \mathrm{K}$ & $x_{2}{ }^{\mathrm{a}}$ & $x_{1}{ }^{\mathrm{b}}$ \\
\hline 317.5 & 0.989 & 0.0109 \\
338.2 & 0.969 & 0.0305 \\
371.7 & 0.871 & 0.129 \\
387.7 & 0.717 & 0.283 \\
404.7 & 0.496 & 0.504 \\
413.2 & 0.289 & 0.711 \\
\hline
\end{tabular}

${ }^{\mathrm{a}} x_{2}$ : mole fraction of component 2 in the saturated solution.

${ }^{\mathrm{b}} \mathrm{x}_{1}$ : mole fraction solubility of the solute. Solubility data reported in units of mass percent. Mole fraction values calculated by the compiler.

\section{Auxiliary Information}

\section{Method/Apparatus/Procedure:}

Preweighed quantities of solute and solvent were placed in bulbs, which were then sealed and heated in a suitable bath. At the higher temperatures, the concentration of solvent in the liquid solution was corrected for the amount of solvent vapor in the bulb. The correction assumed that the vapor pressure of the saturated solution was one half that of the pure solvent at the solution temperature. The temperature at which the solid completely dissolved was recorded, and was taken to be the solid-liquid equilibrium temperature.

\section{Source and Purity of Chemicals:}

(1) Purity not given, prepared and purified by the authors using a published synthetic procedure [see J. Walker and J. K. Wood, J. Chem. Soc. 117, 40 (1920) for synthetic details]. Melting point of the purified solute was $432.2 \mathrm{~K}$. (2) Purity not given, Chemical source not given, thiophene was removed by treatment with sulfuric acid. Sample was further purified by freezing several times.

Estimated Error:

Temperature: Not given in paper.

$x_{1}: \pm 10 \%$ (relative error, estimated by compiler).

\begin{tabular}{|c|c|}
\hline $\begin{array}{l}\text { Components: } \\
\text { (1) 2-Hydroxybenzoic acid; } \\
\mathrm{C}_{7} \mathrm{H}_{6} \mathrm{O}_{3} ;[69-72-7] \\
\text { (2) Benzene; } \mathrm{C}_{6} \mathrm{H}_{6} ;[71-43-2]\end{array}$ & $\begin{array}{l}\text { Original Measurements: } \\
{ }^{147} \text { J. Walker and J. K. Wood, J. } \\
\text { Chem. Soc. Trans. 73, } 618 \text { (1898). }\end{array}$ \\
\hline $\begin{array}{l}\text { Variables: } \\
\text { Temperature }\end{array}$ & $\begin{array}{l}\text { Prepared by: } \\
\text { W. E. Acree, Jr. }\end{array}$ \\
\hline
\end{tabular}

Experimental Values

\begin{tabular}{lcc}
\hline \hline$T / \mathrm{K}$ & $x_{2}{ }^{\mathrm{a}}$ & $x_{1}{ }^{\mathrm{b}}$ \\
\hline 284.9 & 0.9974 & 0.00259 \\
291.4 & 0.9967 & 0.00326 \\
303.7 & 0.9944 & 0.00557 \\
307.8 & 0.9929 & 0.00708 \\
309.8 & 0.9920 & 0.00802 \\
322.6 & 0.9867 & 0.01328 \\
337.4 & 0.9757 & 0.02428
\end{tabular}

${ }^{\mathrm{a}} x_{2}$ : mole fraction of component 2 in the saturated solution.

${ }^{b} x_{1}$ : mole fraction solubility of the solute. Solubility data reported as grams of solute per $100 \mathrm{~g}$ of solvent. Numerical values calculated by the compiler.

\section{Auxiliary Information}

Method/Apparatus/Procedure:

Excess solute and solvent were equilibrated with agitation by stirrers driven by a small turbine. At higher temperatures agitation was occasionally by hand. Equilibrium was obtained by both undersaturation at lower temperature and by supersaturation by pre-equilibrating at a higher temperature. The concentration of the dissolved solute was determined by titration with barium hydroxide with Congo Red being the endpoint indicator. Prior to titration, the solvent was removed by evaporation under reduced pressure.

Source and Purity of Chemicals:

(1) Purity not given, Chemical source not given, no purification details were provided.

(2) Purity not given, Chemical source not given, no purification details were provided.

Estimated Error:

Temperature: Not given in paper.

$x_{1}: \pm 10 \%$ (relative error, estimated by compiler). 


\section{Components:}

(1) 2-Hydroxybenzoic acid;

$\mathrm{C}_{7} \mathrm{H}_{6} \mathrm{O}_{3} ;$; [69-72-7]

(2) Benzene; $\mathrm{C}_{6} \mathrm{H}_{6} ;[71-43-2]$

\section{Variables:}

Temperature

Original Measurements:

${ }^{148}$ I. L. Krupatkin, J. Gen. Chem.

USSR (Engl. Transl.) 26, 3609

(1956).

Prepared by:

W. E. Acree, Jr.

Experimental Values

\begin{tabular}{lcc}
\hline \hline$T / \mathrm{K}$ & $x_{2}{ }^{\mathrm{a}}$ & $x_{1}{ }^{\mathrm{b}}$ \\
\hline 375.7 & 0.9711 & 0.0289 \\
386.2 & 0.9501 & 0.0499 \\
400.2 & 0.8832 & 0.1168 \\
408.2 & 0.7963 & 0.2037 \\
412.7 & 0.6670 & 0.3330 \\
418.2 & 0.4311 & 0.5689 \\
421.7 & 0.2383 & 0.7617 \\
\hline
\end{tabular}

${ }^{a} x_{2}$ : mole fraction of component 2 in the saturated solution.

${ }^{\mathrm{b}} x_{1}$ : mole fraction solubility of the solute.

\section{Auxiliary Information}

\section{Method/Apparatus/Procedure:}

Solubility was determined by a fusion method, which basically involves complete dissolution of the solid, followed by rapid solidification with vigorous stirring. The solid mass is then ground into smaller pieces. The melting point temperature of the mixture is then determined.

\section{Source and Purity of Chemicals:}

(1) Chemical Pure grade, Chemical source not given, no purification details were provided. Melting point temperature of sample is $428.2 \mathrm{~K}$.

(2) Purity not given, Chemical source not given, no purification details were provided.

\section{Estimated Error:}

Temperature: Not given in paper.

$x_{1}: \pm 10 \%$ (relative error, estimated by compiler)

\begin{tabular}{ll}
\hline \hline Components: & Original Measurements: \\
$(1)$ 2-Hydroxybenzoic acid; & ${ }^{56} \mathrm{P}$. A. Ongley, J. Chem. Soc. 1954, \\
$\mathrm{C}_{7} \mathrm{H}_{6} \mathrm{O}_{3} ;[69-72-7]$ & 3634. \\
(2) Benzene; $\mathrm{C}_{6} \mathrm{H}_{6} ;[71-43-2]$ & \\
\hline Variables: & Prepared by: \\
$T / \mathrm{K}=298.15$ & W. E. Acree, Jr. \\
\hline
\end{tabular}

\section{Experimental Values}

The measured solubility was reported to be $-\log _{10} c_{1}=1.239$, which corresponds to a solubility of $c_{1}=0.0577 \mathrm{~mol} \mathrm{dm}^{-3}$.

\section{Auxiliary Information}

Method/Apparatus/Procedure:

Very little experimental details were provided. Excess solute and solvent were placed in tubes, which were rotated for at least $8 \mathrm{~h}$ at $298 \mathrm{~K}$. The solutions were then removed, concentrated if necessary, and titrated with standard alkali using a mixed (bromothymol blue + neutral red) indicator.
Source and Purity of Chemicals:

(1) Purity not given, Chemical source not given, solute was either "AnalaR" grade or recrystallized prior to use.

(2) Purity not given, Chemical source not given, no purification details were provided.

\section{Estimated Error:}

Temperature: No information was given $c_{1}: \pm 4 \%$ (relative error, estimated by compiler).

\begin{tabular}{ll}
\hline \hline Components: & Original Measurements: \\
$(1)$ 2-Hydroxybenzoic acid; & 102 J. Barra, M.-A. Peña, and P. \\
$\mathrm{C}_{7} \mathrm{H}_{6} \mathrm{O}_{3} ;[69-72-7]$ & Bustamante, Eur. J. Pharm. Sci. 10, \\
$(2)$ Benzene; $\mathrm{C}_{6} \mathrm{H}_{6} ;[71-43-2]$ & $153(2000)$. \\
\hline Variables: & Prepared by: \\
$T / \mathrm{K}=298.15$ & W. E. Acree, Jr. \\
\hline
\end{tabular}

\section{Experimental Values}

\begin{tabular}{lc}
\hline \hline$x_{2}^{\mathrm{a}}$ & $x_{1}{ }^{\mathrm{b}}$ \\
\hline 0.9945 & 0.00545 \\
\hline${ }^{\mathrm{a}} x_{2}:$ mole fraction of component 2 in the saturated solution. \\
${ }^{\mathrm{b}}{ }_{x_{1}: \text { mole fraction solubility of the solute. }}$
\end{tabular}

\section{Auxiliary Information}

\section{Method/Apparatus/Procedure:}

Constant-temperature bath, analytical balance, and an ultraviolet/visible spectrophotometer.

Excess solute and solvent were placed in a flask and allowed to equilibrate for several days at constant temperature. The solid phase was removed by filtration, and the clear solution was diluted quantitatively with $96 \%$ ethanol. The concentration of the diluted solution was determined by spectrophotometric measurement at the wavelength of maximum absorption. In instances where the solvent interfered with the spectrophotometric determination, the samples were evaporated to dryness and the residue diluted with $96 \%$ ethanol.

\section{Source and Purity of Chemicals:}

(1) Purity not given, Sigma Chemical Company, USA, no purification details were provided.

(2) Spectrophotometric or Analytical grade, Chemical source not specified, no purification details were provided.

\section{Estimated Error:}

Temperature: $\pm 0.2 \mathrm{~K}$.

$x_{1}: \pm 10.0 \%$ (relative error, estimated by compiler).

\begin{tabular}{ll}
\hline \hline Components: & Original Measurements: \\
$\begin{array}{l}\text { (1) 2-Hydroxybenzoic acid; } \\
\mathrm{C}_{7} \mathrm{H}_{6} \mathrm{O}_{3} ;[69-72-7]\end{array}$ & ${ }^{149} \mathrm{~J}$. W. Marden and M. V. Dover, J. \\
$(2)$ Benzene; $\mathrm{C}_{6} \mathrm{H}_{6} ;[71-43-2]$ & Am. Chem. Soc. 39, 1 (1917). \\
\hline Variables: & \\
$T / \mathrm{K}=298.15$ & Prepared by: \\
\hline
\end{tabular}


Experimental Values

\begin{tabular}{lc}
\hline \hline$x_{2}^{\mathrm{a}}$ & $x_{1}^{\mathrm{b}}$ \\
\hline 0.995 & 0.00518
\end{tabular}

${ }^{\mathrm{a}} x_{2}$ : mole fraction of component 2 in the saturated solution.

${ }^{\mathrm{b}} x_{1}$ : mole fraction solubility of the solute. Experimental solubility data were given as grams of solute per $100 \mathrm{~g}$ of solvent. Mole fraction solubility was calculated by the compiler.

\section{Auxiliary Information}

\section{Method/Apparatus/Procedure:}

Constant-temperature thermostat, analytical balance and a steam bath. Excess solute and solvent were placed in a bottle with ground glass stopper which was protected by a coating of beeswax and rosin, over which was tied a piece of rubber sheeting. The sealed mixture was shaken in a constanttemperature thermostat for 8-20 h. An aliquot of the saturated solution was withdrawn through a glass wool filter into a weighing pipet from which the solutions were weighed directly into small glass evaporating dishes. The solvent was removed on a steam bath, and the sample further dried in a sulfuric acid desiccator. The solubility was calculated from the mass of the solid residue and the mass of the saturated solution removed for analysis.

\section{Source and Purity of Chemicals:}

(1) Purity not given, Chemical source not given, no purification details were provided.

(2) Purity not given, Chemical source not given, no purification details were provided.

\section{Estimated Error:}

Temperature: $\pm 0.1 \mathrm{~K}$.

$x_{1}: \pm 10.0 \%$ (relative error, estimated by compiler).

\begin{tabular}{ll}
\hline \hline Components: & $\begin{array}{l}\text { Original Measurements: } \\
\text { (1) 2-Hydroxybenzoic acid; }\end{array}$ \\
${ }^{150}$ A. N. Paruta, B. J. Sciarrone, and \\
$\mathrm{C}_{6} \mathrm{O}_{3} ;[69-72-7]$ & N. G. Lordi, J. Pharm. Sci. 53, 1349 \\
$(2)$ Benzene; $\mathrm{C}_{6} \mathrm{H}_{6} ;[71-43-2]$ & $(1964)$. \\
\hline Variables: & Prepared by: \\
$T / \mathrm{K}=303.8$ & W. E. Acree, Jr. \\
\hline
\end{tabular}

\section{Experimental Values}

The measured solubility was reported to be $7 \mathrm{mg} / \mathrm{ml}$ of solution, which corresponds to a solubility of $c_{1}=0.0507 \mathrm{~mol}$ $\mathrm{dm}^{-3}$.

\section{Auxiliary Information}

\section{Method/Apparatus/Procedure:}

Excess solute and solvent were placed in 15-ml screw-capped vials fitted with Teflon liners. The vials were attached to a rotating wheel in a constanttemperature water bath and allowed to equilibrate for $24 \mathrm{~h}$. An aliquot of the saturated solution was withdrawn from the vial with a pipet fitted with a glass wool filtering plug. The solubility was determined by titration using a freshly prepared sodium hydroxide solution. The endpoint of the titration was detected with phenolphthalein.

\section{Source and Purity of Chemicals:}

(1) Purity not given, Chemical source not given, no purification details were provided.

(2) Purity not given, Chemical source not given, no purification details were provided.
Estimated Error:

Temperature: $\pm 0.2 \mathrm{~K}$.

$x_{1}: \pm 5 \%$ (relative error, estimated by compiler).

\begin{tabular}{|c|c|}
\hline $\begin{array}{l}\text { Components: } \\
\text { (1) 2-Hydroxybenzoic acid; } \\
\mathrm{C}_{7} \mathrm{H}_{6} \mathrm{O}_{3} ;[69-72-7] \\
\text { (2) Benzene; } \mathrm{C}_{6} \mathrm{H}_{6} ;[71-43-2]\end{array}$ & $\begin{array}{l}\text { Original Measurements: } \\
{ }^{50} \text { P. G. Desai and A. M. Patel, J. } \\
\text { Indian Chem. Soc. 12, } 131 \text { (1935). }\end{array}$ \\
\hline $\begin{array}{l}\text { Variables: } \\
T / \mathrm{K}=301.2\end{array}$ & $\begin{array}{l}\text { Prepared by: } \\
\text { W. E. Acree, Jr. }\end{array}$ \\
\hline
\end{tabular}

Experimental Values

\begin{tabular}{lc}
\hline \hline$x_{2}{ }^{\mathrm{a}}$ & $x_{1}{ }^{\mathrm{b}}$ \\
\hline 0.9946 & 0.005441 \\
\hline$x_{2}:{ }^{2}$
\end{tabular}

${ }^{a} x_{2}$ : mole fraction of component 2 in the saturated solution.

${ }^{\mathrm{b}} x_{1}$ : mole fraction solubility of the solute.

\section{Auxiliary Information}

Method/Apparatus/Procedure:

Excess solute and solvent were placed in a glass flask which was kept revolving on a wheel in an air thermostat for two days. After two days of equilibration, the solution was filtered. To minimize absorption of the solute onto the filter paper, a $100 \mathrm{ml}$ portion of fresh saturated solution was first filtered through the paper before filtering the sample to be analyzed. An aliquot of the filtrate was titrated with barium hydroxide using phenolphthalein as the endpoint indictor. The barium hydroxide titrant was previously standardized against a solution of succinic acid.

\section{Source and Purity of Chemicals:}

(1) Purity not given, Chemical source not specified, no information provided concerning purification.

(2) Purity not given, Chemical source not specified, no information provided concerning purification.

\section{Estimated Error:}

Temperature: No information given.

$x_{1}: \pm 3 \%$ (relative error, estimated by compiler).

\begin{tabular}{|c|c|}
\hline $\begin{array}{l}\text { Components: } \\
\text { (1) 2-Hydroxybenzoic acid; } \\
\mathrm{C}_{7} \mathrm{H}_{6} \mathrm{O}_{3} ; \text { [69-72-7] } \\
\text { (2) Methylbenzene; } \mathrm{C}_{7} \mathrm{H}_{8} \text {; } \\
\text { [108-88-8] }\end{array}$ & $\begin{array}{l}\text { Original Measurements: } \\
{ }^{50} \text { P. G. Desai and A. M. Patel, J. } \\
\text { Indian Chem. Soc. 12, } 131 \text { (1935). }\end{array}$ \\
\hline $\begin{array}{l}\text { Variables: } \\
T / \mathrm{K}=301.2\end{array}$ & $\begin{array}{l}\text { Prepared by: } \\
\text { W. E. Acree, Jr. }\end{array}$ \\
\hline
\end{tabular}

\section{Experimental Values}

\begin{tabular}{lc}
\hline \hline$x_{2}{ }^{\mathrm{a}}$ & $x_{1}{ }^{\mathrm{b}}$ \\
\hline 0.9939 & 0.006052 \\
\hline${ }^{\mathrm{a}} x_{2}:$ mole fraction of component 2 in the saturated solution. \\
${ }^{\mathrm{b}_{x_{1}}: \text { mole fraction solubility of the solute. }}$ &
\end{tabular}




\section{Auxiliary Information}

\section{Method/Apparatus/Procedure:}

Excess solute and solvent were placed in a glass flask which was kept revolving on a wheel in an air thermostat for two days. After two days of equilibration, the solution was filtered. To minimize absorption of the solute onto the filter paper, a $100 \mathrm{ml}$ portion of fresh saturated solution was first filtered through the paper before filtering the sample to be analyzed. An aliquot of the filtrate was titrated with barium hydroxide using phenolphthalein as the endpoint indictor. The barium hydroxide titrant was previously standardized against a solution of succinic acid.

Source and Purity of Chemicals:

(1) Purity not given, Chemical source not specified, no information provided concerning purification.

(2) Purity not given, Chemical source not specified, no information provided concerning purification.

Estimated Error:

Temperature: No information given.

$x_{1}: \pm 3 \%$ (relative error, estimated by compiler).

\begin{tabular}{|c|c|}
\hline $\begin{array}{l}\text { Components: } \\
\text { (1) 2-Hydroxybenzoic acid; } \\
\mathrm{C}_{7} \mathrm{H}_{6} \mathrm{O}_{3} ;[69-72-7] \\
\text { (2) } 1,3 \text {-Dimethylbenzene; } \mathrm{C}_{8} \mathrm{H}_{10} \text {; } \\
\text { [108-38-3] }\end{array}$ & $\begin{array}{l}\text { Original Measurements: } \\
\text { 50 P. G. Desai and A. M. Patel, J. } \\
\text { Indian Chem. Soc. 12, } 131 \text { (1935). }\end{array}$ \\
\hline $\begin{array}{l}\text { Variables: } \\
T / \mathrm{K}=301.2\end{array}$ & $\begin{array}{l}\text { Prepared by: } \\
\text { W. E. Acree, Jr. }\end{array}$ \\
\hline
\end{tabular}

Experimental Values

\begin{tabular}{lc}
\hline \hline$x_{2}{ }^{\mathrm{a}}$ & $x_{1}{ }^{\mathrm{b}}$ \\
\hline 0.9934 & 0.006554
\end{tabular}

${ }^{a} x_{2}$ : mole fraction of component 2 in the saturated solution.

${ }^{\mathrm{b}} x_{1}$ : mole fraction solubility of the solute.

\section{Auxiliary Information}

\section{Method/Apparatus/Procedure:}

Excess solute and solvent were placed in a glass flask which was kept revolving on a wheel in an air thermostat for two days. After two days of equilibration, the solution was filtered. To minimize absorption of the solute onto the filter paper, a $100 \mathrm{ml}$ portion of fresh saturated solution was first filtered through the paper before filtering the sample to be analyzed. An aliquot of the filtrate was titrated with barium hydroxide using phenolphthalein as the endpoint indictor. The barium hydroxide titrant was previously standardized against a solution of succinic acid.

\section{Source and Purity of Chemicals:}

(1) Purity not given, Chemical source not specified, no information provided concerning purification.

(2) Purity not given, Chemical source not specified, no information provided concerning purification.

\section{Estimated Error:}

Temperature: No information given.

$x_{1}: \pm 3 \%$ (relative error, estimated by compiler).

\subsection{2-Hydroxybenzoic acid solubility data in esters}

\begin{tabular}{|c|c|}
\hline $\begin{array}{l}\text { Components: } \\
\text { (1) 2-Hydroxybenzoic acid; } \\
\mathrm{C}_{7} \mathrm{H}_{6} \mathrm{O}_{3} ; \text {;69-72-7] } \\
\text { (2) Ethyl ethanoate; } \mathrm{C}_{4} \mathrm{H}_{8} \mathrm{O}_{2} \text {; } \\
\text { [141-78-6] }\end{array}$ & $\begin{array}{l}\text { Original Measurements: } \\
{ }^{151} \text { K. M. De Fina, T. L. Sharp, L. E } \\
\text { Roy, and W. E. Acree, Jr., J. Chem } \\
\text { Eng. Data 44, } 1262 \text { (1999). }\end{array}$ \\
\hline $\begin{array}{l}\text { Variables: } \\
T / \mathrm{K}=298.15\end{array}$ & $\begin{array}{l}\text { Prepared by: } \\
\text { W. E. Acree, Jr. }\end{array}$ \\
\hline
\end{tabular}

\section{Experimental Values}

\begin{tabular}{lc}
\hline \hline$x_{2}{ }^{\mathrm{a}}$ & $x_{1}{ }^{\mathrm{b}}$ \\
\hline 0.8575 & 0.1425 \\
\hline${ }^{\mathrm{a}} x_{2}:$ mole fraction of component 2 in the saturated solution. \\
${ }^{\mathrm{b}} x_{1}:$ mole fraction solubility of the solute.
\end{tabular}

\section{Auxiliary Information}

\section{Method/Apparatus/Procedure:}

Constant-temperature bath, calorimetric thermometer, and an ultraviolet/ visible spectrophotometer.

Excess solute and solvent were placed in amber glass bottles and allowed to equilibrate for several days at constant temperature. Attainment of equilibrium was verified by several repetitive measurements and by approaching equilibrium from supersaturation. Aliquots of saturated solutions were transferred through a coarse filter into tared volumetric flasks, weighed, and diluted with methanol. Concentrations were determined by spectrophotometric measurements at $304 \mathrm{~nm}$.

\section{Source and Purity of Chemicals:}

(1) 99+\%, ACS Reagent grade, Aldrich Chemical Company, Milwaukee, WI, USA, was dried for several hours at $353 \mathrm{~K}$ before use and used without further purification

(2) $99.9 \%$, HPLC grade, Aldrich Chemical Company, stored over molecular sieves before use.

\section{Estimated Error:}

Temperature: $\pm 0.1 \mathrm{~K}$.

$x_{1}: \pm 1.5 \%$ (relative error)

\begin{tabular}{ll}
\hline \hline Components: & Original Measurements: \\
$(1)$ 2-Hydroxybenzoic acid; & ${ }^{152} \mathrm{H}$. Matsuda, K. Kaburagi, S. \\
$\mathrm{C}_{7} \mathrm{H}_{6} \mathrm{O}_{3} ;[69-72-7]$ & Matsumoto, K. Kurihara, K. \\
$(2)$ Ethyl ethanoate; $\mathrm{C}_{4} \mathrm{H}_{8} \mathrm{O}_{2} ;$ & Tochigi, and K. Tomono, J. Chem. \\
{$[141-78-6]$} & Eng. Data 54, 480 (2009). \\
\hline Variables: & Prepared by: \\
$T / \mathrm{K}=298.15$ & W. E. Acree, Jr. \\
\hline
\end{tabular}

Experimental Values

\begin{tabular}{lc}
\hline \hline$x_{2}{ }^{\mathrm{a}}$ & $x_{1}{ }^{\mathrm{b}}$ \\
\hline 0.8617 & 0.1383 \\
\hline${ }^{\mathrm{a}} x_{2}:$ mole fraction of component 2 in the saturated solution. & \\
${ }^{\mathrm{b}} x_{1}:$ mole fraction solubility of the solute. &
\end{tabular}




\section{Auxiliary Information}

\section{Method/Apparatus/Procedure:}

Thermostated constant-temperature water bath and high-performance liquid chromatograph with an uv detector.

Excess solute and solvent were allowed to equilibrate for $24 \mathrm{~h}$ in a constanttemperature thermostated water bath. Aliquots of saturated solutions were removed and filtered through a membrane filter of $0.45 \mu \mathrm{m}$ pore size (Millipore, USA). Concentrations were determined by high-performance liquid chromatography equipped with an uv detector (254 $\mathrm{nm}$ detection). Benzene was added to the sample as an internal standard.

\section{Source and Purity of Chemicals:}

(1) $99.5 \%$, Wako Pure Chemical Industries, Ltd., Japan, was used without further purification.

(2) $99.5 \%$, Wako Pure Chemical Industries, Ltd., Japan, was used without further purification.

Estimated Error:

Temperature: $\pm 0.05 \mathrm{~K}$.

$x_{1}: \pm 6.2 \%$ (relative error).

\begin{tabular}{|c|c|}
\hline $\begin{array}{l}\text { Components: } \\
\text { (1) 2-Hydroxybenzoic acid; } \\
\mathrm{C}_{7} \mathrm{H}_{6} \mathrm{O}_{3} ;[69-72-7] \\
\text { (2) Ethyl ethanoate; } \mathrm{C}_{4} \mathrm{H}_{8} \mathrm{O}_{2} \text {; } \\
\text { [141-78-6] }\end{array}$ & $\begin{array}{l}\text { Original Measurements: } \\
{ }^{153} \text { A. Shalmashi and A. Eliassi, J. } \\
\text { Chem. Eng. Data 53, } 199 \text { (2008). }\end{array}$ \\
\hline $\begin{array}{l}\text { Variables: } \\
\text { Temperature }\end{array}$ & $\begin{array}{l}\text { Prepared by: } \\
\text { W. E. Acree, Jr. }\end{array}$ \\
\hline
\end{tabular}

Experimental Values

\begin{tabular}{lcc}
\hline \hline$T / \mathrm{K}$ & $x_{2}{ }^{\mathrm{a}}$ & $x_{1}{ }^{\mathrm{b}}$ \\
\hline 298 & 0.8619 & 0.1381 \\
303 & 0.8491 & 0.1509 \\
308 & 0.8356 & 0.1644 \\
313 & 0.8227 & 0.1773 \\
318 & 0.8097 & 0.1903 \\
323 & 0.7965 & 0.2035 \\
328 & 0.7845 & 0.2155 \\
333 & 0.7707 & 0.2293 \\
338 & 0.7570 & 0.2430 \\
343 & 0.7431 & 0.2569 \\
348 & 0.7281 & 0.2719
\end{tabular}

${ }^{\mathrm{a}} x_{2}$ : mole fraction of component 2 in the saturated solution.

${ }^{b} x_{1}$ : mole fraction solubility of the solute. The solubility data were reported as mass fractions. The mole fraction values were calculated by the compiler.

\section{Auxiliary Information}

\section{Method/Apparatus/Procedure:}

Magnetically stirred jacketed equilibrium cell, constant-temperature circulating water bath, and an analytical balance.

Excess solute and solvent were placed in a magnetically stirred jacketed equilibrium cell and allowed to equilibrate at constant temperature for $2 \mathrm{~h}$ with continuous stirring. After $2 \mathrm{~h}$ the stirring was discontinued and the suspended solid was allowed to settle to the lower portion of the equilibrium cell. An aliquot of the saturated solution was withdrawn by a warmed pipet and transferred to a weighed vial. The vial was tightly closed and weighed to determine the mass of the sample analyzed. The vial was then placed in an oven and the solvent was allowed to evaporate. The vial was reweighed, and the solubility calculated from the mass of the solid residue and sample analyzed.
Source and Purity of Chemicals:

(1) Purity not given, Synthesized in the authors' department, dried under vacuum at $323 \mathrm{~K}$ for $8 \mathrm{~h}$ and then stored in a desiccator.

(2) Analytical grade, Merck Chemicals, Darmstadt, Germany, used as received.

\section{Estimated Error:}

Temperature: $\pm 0.05 \mathrm{~K}$

$x_{1}: \pm 3 \%$ (relative error, estimated by compiler).

\begin{tabular}{ll}
\hline \hline Components: & Original Measurements: \\
(1) 2-Hydroxybenzoic acid; & 102J. Barra, M.-A. Peña, and P. \\
$\mathrm{C}_{7} \mathrm{H}_{6} \mathrm{O}_{3} ;[69-72-7]$ & Bustamante, Eur. J. Pharm. Sci. 10, \\
$\begin{array}{l}\text { (2) Ethyl ethanoate; } \mathrm{C}_{4} \mathrm{H}_{8} \mathrm{O}_{2} ; \\
{[141-78-6]}\end{array}$ & $153(2000)$. \\
\hline Variables: & Prepared by: \\
$T / \mathrm{K}=298.15$ & W. E. Acree, Jr. \\
\hline
\end{tabular}

\section{Experimental Values}

\begin{tabular}{lc}
\hline \hline$x_{2}{ }^{\mathrm{a}}$ & $x_{1}{ }^{\mathrm{b}}$ \\
\hline 0.8777 & 0.1223 \\
\hline$x_{2}:$ mole fraction of compont 2 in the saturated
\end{tabular}

${ }^{a} x_{2}$ : mole fraction of component 2 in the saturated solution.

${ }^{\mathrm{b}} x_{1}$ : mole fraction solubility of the solute.

\section{Auxiliary Information}

\section{Method/Apparatus/Procedure:}

Constant-temperature bath, analytical balance, and an ultraviolet/visible spectrophotometer.

Excess solute and solvent were placed in a flask and allowed to equilibrate for several days at constant temperature. The solid phase was removed by filtration, and the clear solution was diluted quantitatively with $96 \%$ ethanol. The concentration of the diluted solution was determined by spectrophotometric measurement at the wavelength of maximum absorption. In instances where the solvent interfered with the spectrophotometric determination, the samples were evaporated to dryness and the residue diluted with $96 \%$ ethanol.

\section{Source and Purity of Chemicals:}

(1) Purity not given, Sigma Chemical Company, USA, no purification details were provided.

(2) Spectrophotometric or Analytical grade, Chemical source not specified, no purification details were provided.

\section{Estimated Error:}

Temperature: $\pm 0.2 \mathrm{~K}$.

$x_{1}: \pm 3.0 \%$ (relative error, estimated by compiler).

\begin{tabular}{ll}
\hline \hline Components: & Original Measurements: \\
$(1)$ 2-Hydroxybenzoic acid; & ${ }^{154}$ M. A. Peña, B. Escalera, A. \\
$\mathrm{C}_{7} \mathrm{H}_{6} \mathrm{O}_{3} ;[69-72-7]$ & Reíllo, A. B. Sánchez, and P. \\
$\begin{array}{l}\text { (2) Ethyl ethanoate; } \mathrm{C}_{4} \mathrm{H}_{8} \mathrm{O}_{2} ; \\
\text { [141-78-6] }\end{array}$ & Bustamante, J. Pharm. Sci. 98, 1129 \\
\hline Variables: & $(2009)$. \\
Temperature & Prepared by: \\
\hline
\end{tabular}


Experimental Values

\begin{tabular}{lcc}
\hline \hline$T / \mathrm{K}$ & $x_{2}{ }^{\mathrm{a}}$ & $x_{1}{ }^{\mathrm{b}}$ \\
\hline 293 & 0.8915 & 0.1085 \\
303 & 0.8781 & 0.1219 \\
308 & 0.8707 & 0.1293 \\
313 & 0.8671 & 0.1329
\end{tabular}

${ }^{a} x_{2}$ : mole fraction of component 2 in the saturated solution.

${ }^{\mathrm{b}} x_{1}$ : mole fraction solubility of the solute.

\section{Auxiliary Information}

\section{Method/Apparatus/Procedure:}

Constant temperature-controlled shaking bath and an uv/visible spectrophotometer.

Excess solute and solvent were allowed to equilibrate in a constant temperature-controlled shaking bath for four days at $313 \mathrm{~K}$, and then one day at the lower temperatures. The authors used the same samples for all temperatures. The authors started with $313 \mathrm{~K}$, and once the solution was saturated at the higher temperature they lowered the temperature for the next set of measurements. Aliquots of the saturated solution were removed and filtered (Durapore membrane filter, $0.2 \mu \mathrm{m}$ pore size). The filtered aliquot was then diluted with ethanol $(96 \% \mathrm{v} / \mathrm{v})$, and the concentration of the dissolved solute determined by spectrophotometric analysis at a wavelength of $302 \mathrm{~nm}$ The authors converted the molar solubilities to mole fractions using the measured densities of the saturated solutions.

\section{Source and Purity of Chemicals:}

(1) Purity not given, Sigma-Aldrich, Munich, Germany, was used as received. (2) UV grade, Panreac, Monplet and Esteban, Barcelona, Spain, no purification details were provided.

Estimated Error:

Temperature: \pm 0.1 .

$x_{1}: \pm 3 \%$ (relative error).

\begin{tabular}{ll}
\hline \hline Components: & Original Measurements: \\
(1) 2-Hydroxybenzoic acid; & ${ }^{155} \mathrm{M}$. A. Peña, A. Reíllo, B. \\
$\mathrm{C}_{7} \mathrm{H}_{6} \mathrm{O}_{3} ;[69-72-7]$ & Escalera, and P. Bustamante, Int. J. \\
$\begin{array}{l}\text { (2) Ethyl ethanoate; } \mathrm{C}_{4} \mathrm{H}_{8} \mathrm{O}_{2} ; \\
{[141-78-6]}\end{array}$ & Pharm. 321, 155 (2006). \\
\hline Variables: & \\
$T / \mathrm{K}=298.15$ & Prepared by: \\
\hline
\end{tabular}

\section{Experimental Values}

\begin{tabular}{lc}
\hline \hline$x_{2}{ }^{\mathrm{a}}$ & $x_{1}{ }^{\mathrm{b}}$ \\
\hline 0.8864 & 0.1136 \\
\hline${ }^{\mathrm{a}} x_{2}:$ mole fraction of component 2 in the saturated solution. \\
${ }^{\mathrm{b}} x_{1}:$ mole fraction solubility of the solute.
\end{tabular}

\section{Auxiliary Information}

\section{Method/Apparatus/Procedure:}

Constant temperature-controlled shaking bath and an uv/visible spectrophotometer.

Binary solvent mixtures were prepared by volume. Excess solute and solvent were allowed to equilibrate in a constant temperature-controlled shaking bath for at least five days. Aliquots of the saturated solution were removed and filtered (Durapore membrane filter, $0.2 \mu \mathrm{m}$ pore size). The filtered aliquot was then diluted with ethanol $(96 \% \mathrm{v} / \mathrm{v})$, and the concentration of the dissolved solute determined by spectrophotometric analysis at a wavelength of $302 \mathrm{~nm}$. The authors converted the molar solubilities to mole fractions using the measured densities of the saturated solutions.

\section{Source and Purity of Chemicals:}

(1) Purity not given, Sigma-Aldrich, Germany, no purification details were provided.

(2) Spectrophotometric grade, Panreac, Monplet and Esteban, Spain, no purification details were provided.

\section{Estimated Error:}

Temperature: $\pm 0.1 \mathrm{~K}$.

$x_{1}: \pm 2.0 \%$ (relative error, estimated by compiler).

\begin{tabular}{|c|c|}
\hline $\begin{array}{l}\text { Components: } \\
\text { (1) 2-Hydroxybenzoic acid; } \\
\mathrm{C}_{7} \mathrm{H}_{6} \mathrm{O}_{3} ; \text { [69-72-7] } \\
\text { (2) Ethyl ethanoate; } \mathrm{C}_{4} \mathrm{H}_{8} \mathrm{O}_{2} \text {; } \\
\text { [141-78-6] }\end{array}$ & $\begin{array}{l}\text { Original Measurements: } \\
{ }^{149} \text { J. W. Marden and M. V. Dover, J } \\
\text { Am. Chem. Soc. 39, } 1 \text { (1917). }\end{array}$ \\
\hline $\begin{array}{l}\text { Variables: } \\
T / \mathrm{K}=298.15\end{array}$ & $\begin{array}{l}\text { Prepared by: } \\
\text { W. E. Acree, Jr. }\end{array}$ \\
\hline
\end{tabular}

Experimental Values

\begin{tabular}{lc}
\hline \hline$x_{2}{ }^{\mathrm{a}}$ & $x_{1}{ }^{\mathrm{b}}$ \\
\hline 0.805 & 0.195 \\
${ }^{a} x_{2}:$ mole fraction of component 2 in the saturated solution. \\
${ }^{\mathrm{b}} x_{1}:$ mole fraction solubility of the solute. Experimental solubility data were \\
given as grams of solute per $100 \mathrm{~g}$ of solvent. Mole fraction solubility was \\
calculated by the compiler.
\end{tabular}

\section{Auxiliary Information}

\section{Method/Apparatus/Procedure:}

Constant-temperature thermostat, analytical balance, and a steam bath. Excess solute and solvent were placed in a bottle with ground glass stopper which was protected by a coating of beeswax and rosin, over which was tied a piece of rubber sheeting. The sealed mixture was shaken in a constanttemperature thermostat for 8-20 h. An aliquot of the saturated solution was withdrawn through a glass wool filter into a weighing pipet from which the solutions were weighed directly into small glass evaporating dishes. The solvent was removed on a steam bath, and the sample further dried in a sulfuric acid desiccator. The solubility was calculated from the mass of the solid residue and the mass of the saturated solution removed for analysis.

\section{Source and Purity of Chemicals:}

(1) Purity not given, Chemical source not given, no purification details were provided.

(2) Purity not given, Chemical source not given, no purification details were provided.

Estimated Error:

Temperature: $\pm 0.1 \mathrm{~K}$.

$x_{1}: \pm 10.0 \%$ (relative error, estimated by compiler). 


\section{Components:}

(1) 2-Hydroxybenzoic acid;

$\mathrm{C}_{7} \mathrm{H}_{6} \mathrm{O}_{3}$; [69-72-7]

(2) Ethyl ethanoate; $\mathrm{C}_{4} \mathrm{H}_{8} \mathrm{O}_{2}$;

[141-78-6]

\begin{tabular}{ll}
\hline Variables: & Prepared by: \\
$T / \mathrm{K}=303.8$ & W. E. Acree, Jr. \\
\hline
\end{tabular}

\section{Experimental Values}

The measured solubility was reported to be $23 \mathrm{mg} / \mathrm{ml}$ of solution, which corresponds to a solubility of $c_{1}=0.167 \mathrm{~mol}$ $\mathrm{dm}^{-3}$.

\section{Auxiliary Information}

\section{Method/Apparatus/Procedure:}

Excess solute and solvent were placed in 15-ml screw-capped vials fitted with Teflon liners. The vials were attached to a rotating wheel in a constanttemperature water bath and allowed to equilibrate for $24 \mathrm{~h}$. An aliquot of the saturated solution was withdrawn from the vial with a pipet fitted with a glass wool filtering plug. The solubility was determined by titration using a freshly prepared sodium hydroxide solution. The endpoint of the titration was detected with phenolphthalein.

\section{Source and Purity of Chemicals:}

(1) Purity not given, Chemical source not given, no purification details were provided.

(2) Purity not given, Chemical source not given, no purification details were provided.

\section{Estimated Error:}

Temperature: $\pm 0.2 \mathrm{~K}$

$x_{1}: \pm 5 \%$ (relative error, estimated by compiler).

\begin{tabular}{ll}
\hline \hline Components: & Original Measurements: \\
(1) 2-Hydroxybenzoic acid; & ${ }^{151}$ K. M. De Fina, T. L. Sharp, L. E. \\
$\mathrm{C}_{7} \mathrm{H}_{6} \mathrm{O}_{3} ;$ [69-72-7] & Roy, and W. E. Acree, Jr., J. Chem. \\
$\begin{array}{l}\text { (2) Butyl ethanoate; } \mathrm{C}_{6} \mathrm{H}_{12} \mathrm{O}_{2} ; \\
{[123-86-4]}\end{array}$ & Eng. Data 44, 1262 (1999). \\
\hline Variables: & Prepared by: \\
$T / \mathrm{K}=298.15$ & W. E. Acree, Jr. \\
\hline
\end{tabular}

Experimental Values

\begin{tabular}{lc}
\hline \hline$x_{2}{ }^{\mathrm{a}}$ & $x_{1}^{\mathrm{b}}$ \\
\hline 0.8637 & 0.1363 \\
\hline${ }^{\mathrm{a}} x_{2}:$ mole fraction of component 2 in the saturated solution. \\
${ }^{\mathrm{b}} x_{1}:$ mole fraction solubility of the solute.
\end{tabular}

\section{Auxiliary Information}

Method/Apparatus/Procedure:

Constant-temperature bath, calorimetric thermometer, and an ultraviolet/ visible spectrophotometer.

Excess solute and solvent were placed in amber glass bottles and allowed to equilibrate for several days at constant temperature. Attainment of equilibrium was verified by several repetitive measurements and by approaching equilibrium from supersaturation. Aliquots of saturated solutions were transferred through a coarse filter into tared volumetric flasks, weighed, and diluted with methanol. Concentrations were determined by spectrophotometric measurements at $304 \mathrm{~nm}$.

Source and Purity of Chemicals:

(1) 99+\%, ACS Reagent grade, Aldrich Chemical Company, Milwaukee, WI, USA, was dried for several hours at $353 \mathrm{~K}$ before use and used without further purification.

(2) $99.7 \%$, HPLC grade, Aldrich Chemical Company, stored over molecular sieves before use.

\section{Estimated Error:}

Temperature: $\pm 0.1 \mathrm{~K}$.

$x_{1}: \pm 1.5 \%$ (relative error)

\begin{tabular}{ll}
\hline \hline Components: & Original Measurements: \\
(1) 2-Hydroxybenzoic acid; & ${ }^{87}$ M. Dias, J. Hadgraft, and M. E. \\
$\mathrm{C}_{7} \mathrm{H}_{6} \mathrm{O}_{3} ;[69-72-7]$ & Lane, Int. J. Pharm. 336, 108 (2007). \\
$\begin{array}{l}\text { (2) } \mathrm{Butyl} \text { ethanoate; } \mathrm{C}_{6} \mathrm{H}_{12} \mathrm{O}_{2} ; \\
{[123-86-4]}\end{array}$ & \\
\hline Variables: & Prepared by: \\
$T / \mathrm{K}=305.2$ & W. E. Acree, Jr. \\
\hline
\end{tabular}

\section{Experimental Values}

The measured solubility was reported to be $218.0 \mathrm{mg} / \mathrm{ml}$, which corresponds to a molar solubility of $c_{1}=1.578 \mathrm{~mol}$ $\mathrm{dm}^{-3}$.

\section{Auxiliary Information}

\section{Method/Apparatus/Procedure:}

Constant-temperature bath, magnetic stirrer, centrifuge, and an ultraviolet/ visible spectrophotometer.

Excess solute and solvent were equilibrated in a constant-temperature bath with stirring for $48 \mathrm{~h}$. The saturated solution was then centrifuged for $10 \mathrm{~min}$, and an aliquot of the supernatant solution was removed and diluted quantitatively for spectrophotometric analysis. The concentration of the dissolved solute was determined from the measured absorbance.

\section{Source and Purity of Chemicals:}

(1) Purity not given, Fisher Scientific Equipment, UK, no purification details were provided.

(2) Purity not given, Aldrich Chemical Company, UK, no purification details were provided.

Estimated Error:

Temperature: Insufficient experimental details to estimate. $c_{1}: \pm 0.054$ 


\section{Components:}

(1) 2-Hydroxybenzoic acid;

$\mathrm{C}_{7} \mathrm{H}_{6} \mathrm{O}_{3}$; [69-72-7]

(2) 1-Methylethyl tetradecanoate;

$\mathrm{C}_{17} \mathrm{H}_{34} \mathrm{O}_{2} ;[110-27-0]$

\begin{tabular}{ll}
\hline Variables: & Prepared by: \\
$T / \mathrm{K}=296$ & W. E. Acree, Jr. \\
\hline
\end{tabular}

Experimental Values

The measured solubility was reported to be $41.4 \mathrm{mg} / \mathrm{ml}$, which corresponds to a solubility of $c_{1}=0.300 \mathrm{~mol} \mathrm{dm}^{-3}$. The authors calculated a mole fraction solubility of $x_{1}=0.089$. The mole fraction solubility was calculated by the authors assuming a molar volume of 2-hydroxybenzoic acid of $V_{1}=93.9$ $\mathrm{cm}^{3} \mathrm{~mol}^{-1}$, which seems too small compared to the molar volume of benzaldehyde, which is estimated to be $V_{1} \approx 101.1$ $\mathrm{cm}^{3} \mathrm{~mol}^{-1}$, based on a molar mass of $106.12 \mathrm{~g} \mathrm{~mol}^{-1}$ and an experimental density of $1.0499 \mathrm{~g} \mathrm{~cm}^{-3}$ at $296 \mathrm{~K}$. A molar volume on the order of $V_{1} \approx 104 \mathrm{~cm}^{3} \mathrm{~mol}^{-1}$ would seem more realistic given the molecular structures of 2-hydroxybenzoic acid compared to benzaldehyde.

\section{Auxiliary Information}

\section{Method/Apparatus/Procedure:}

Magnetic stirrer and an ultraviolet/visible spectrophotometer.

Solubility was determined by stirring an excess of solute in the solvent with a magnetic stirrer at ambient room temperature for $24 \mathrm{~h}$ in sealed flasks that were thermally insulated from the stirrer. The suspension was gravity filtered through Whatman \#1 (qualitative) filter paper. The filtrate was then quantitatively diluted with methanol, and the absorbance of the diluted solution recorded at $306 \mathrm{~nm}$.

Source and Purity of Chemicals:

(1) Purity not given, Aldrich Chemical Company, Milwaukee, WI, USA, no purification details were provided.

(2) Purity not given, Givauden Corporation, Clifton, NJ, USA, used as received.

Estimated Error:

Temperature: $\pm 1 \mathrm{~K}$.

$x_{1}: \pm 5 \%$ (relative error, estimated by compiler).

\begin{tabular}{ll}
\hline \hline Components: & Original Measurements: \\
(1) 2-Hydroxybenzoic acid; & 61 E. R. Cooper, J. Controlled \\
$\mathrm{C}_{7} \mathrm{H}_{6} \mathrm{O}_{3} ;[69-72-7]$ & Release 1, 153 (1984). \\
$(2) 1-\mathrm{Methylethyl} \mathrm{tetradecanoate;}$ & \\
$\mathrm{C}_{17} \mathrm{H}_{34} \mathrm{O}_{2} ;[110-27-0]$ & \\
\hline Variables: & Prepared by: \\
$T / \mathrm{K}=295$ & W. E. Acree, Jr. \\
\hline
\end{tabular}

Experimental Values

\begin{tabular}{lc}
\hline \hline$x_{2}^{\mathrm{a}}$ & $x_{1}^{\mathrm{b}}$ \\
\hline 0.925 & 0.075 \\
\hline${ }^{\mathrm{a}} x_{2}:$ mole fraction of component 2 in the saturated solution. \\
${ }^{\mathrm{b}} x_{1}$ : mole fraction solubility of the solute. Solubility data reported in terms of \\
mass percent. Mole fraction solubility calculated by the compiler.
\end{tabular}

\section{Auxiliary Information}

\section{Method/Apparatus/Procedure:}

Very little experimental details provided. Solubility determinations were made by stirring the solvent with excess solute for two days, centrifuging the sample, and analyzing the supernatant.

\section{Source and Purity of Chemicals:}

(1) Reagent grade, Fisher Scientific, Cincinnati, OH, USA, no purification details provided.

(2) Reagent grade, Wickhen Corporation, Huguenot, NJ, USA, no purification details provided.

\section{Estimated Error:}

Temperature: No information given.

$x_{1}$ : No information given.

\begin{tabular}{ll}
\hline \hline Components: & Original Measurements: \\
(1) 2-Hydroxybenzoic acid; & ${ }^{87}$ M. Dias, J. Hadgraft, and M. E. \\
$\mathrm{C}_{7} \mathrm{H}_{6} \mathrm{O}_{3} ;[69-72-7]$ & Lane, Int. J. Pharm. 336, 108 (2007). \\
$(2) 1-$ Methylethyl tetradecanoate; & \\
$\mathrm{C}_{17} \mathrm{H}_{34} \mathrm{O}_{2} ;[110-27-0]$ & \\
\hline Variables: & Prepared by: \\
$T / \mathrm{K}=305.2$ & W. E. Acree, Jr. \\
\hline
\end{tabular}

\section{Experimental Values}

The measured solubility was reported to be $66.0 \mathrm{mg} / \mathrm{ml}$, which corresponds to a molar solubility of $c_{1}=0.478 \mathrm{~mol}$ $\mathrm{dm}^{-3}$

\section{Auxiliary Information}

\section{Method/Apparatus/Procedure:}

Constant-temperature bath, magnetic stirrer, centrifuge, and an ultraviolet/ visible spectrophotometer.

Excess solute and solvent were equilibrated in a constant-temperature bath with stirring for $48 \mathrm{~h}$. The saturated solution was then centrifuged for $10 \mathrm{~min}$, and an aliquot of the supernatant solution was removed and diluted quantitatively for spectrophotometric analysis. The concentration of the dissolved solute was determined from the measured absorbance.

Source and Purity of Chemicals:

(1) Purity not given, Fisher Scientific Equipment, UK, no purification details were provided.

(2) Purity not given, Croda Universal Ltd., no purification details were provided.

Estimated Error:

Temperature: Insufficient experimental details to estimate. $c_{1}: \pm 0.095$

\subsection{2-Hydroxybenzoic acid solubility data in ethers}

\section{Components:}

(1) 2-Hydroxybenzoic acid;

$\mathrm{C}_{7} \mathrm{H}_{6} \mathrm{O}_{3} ;[69-72-7]$

(2) $1,1^{\prime}$-Oxybisethane;

$\mathrm{C}_{4} \mathrm{H}_{10} \mathrm{O}$; [60-29-7]

Variables:

$T / \mathrm{K}=298.15$

\section{Original Measurements:}

${ }^{102}$ J. Barra, M.-A. Peña, and P. Bustamante, Eur. J. Pharm. Sci. 10, 153 (2000)

Prepared by: W. E. Acree, Jr. 
Experimental Values

\begin{tabular}{lc}
\hline \hline$x_{2}{ }^{\mathrm{a}}$ & $x_{1}{ }^{\mathrm{b}}$ \\
\hline 0.8479 & 0.1521
\end{tabular}

${ }^{\mathrm{a}} x_{2}$ : mole fraction of component 2 in the saturated solution.

${ }^{\mathrm{b}} x_{1}$ : mole fraction solubility of the solute.

\section{Auxiliary Information}

\section{Method/Apparatus/Procedure:}

Constant-temperature bath, analytical balance, and an ultraviolet/visible spectrophotometer.

Excess solute and solvent were placed in a flask and allowed to equilibrate for several days at constant temperature. The solid phase was removed by filtration, and the clear solution was diluted quantitatively with $96 \%$ ethanol. The concentration of the diluted solution was determined by spectrophotometric measurement at the wavelength of maximum absorption. In instances where the solvent interfered with the spectrophotometric determination, the samples were evaporated to dryness and the residue diluted with $96 \%$ ethanol.

\section{Source and Purity of Chemicals:}

(1) Purity not given, Sigma Chemical Company, USA, no purification details were provided.

(2) Spectrophotometric or Analytical grade, Chemical source not specified, no purification details were provided.

\section{Estimated Error:}

Temperature: $\pm 0.2 \mathrm{~K}$.

$x_{1}: \pm 3.0 \%$ (relative error, estimated by compiler).

\begin{tabular}{ll}
\hline \hline Components: & Original Measurements: \\
(1) 2-Hydroxybenzoic acid; & ${ }^{147}$ J. Walker and J. K. Wood, J. \\
$\mathrm{C}_{7} \mathrm{H}_{6} \mathrm{O}_{3} ;[69-72-7]$ & Chem. Soc. Trans. 73, 618 (1898). \\
$(2) 1,1^{\prime}-$-Oxybisethane; $\mathrm{C}_{4} \mathrm{H}_{10} \mathrm{O} ;$ & \\
{$[60-29-7]$} & \\
\hline Variables: & Prepared by: \\
$T / \mathrm{K}=290$ & W. E. Acree, Jr. \\
\hline
\end{tabular}

\section{Experimental Values}

The measured solubility was reported to be $23.4 \mathrm{~g} / 100 \mathrm{ml}$ of solution, which corresponds to a molar concentration of 1.694 mol dm ${ }^{-3}$.

\section{Auxiliary Information}

\footnotetext{
Method/Apparatus/Procedure:

Excess solute and solvent were equilibrated with agitation by stirrers driven by a small turbine. At higher temperatures agitation was occasionally by hand. Equilibrium was obtained by both undersaturation at lower temperature and by supersaturation by pre-equilibrating at a higher temperature. The concentration of the dissolved solute was determined by titration with barium hydroxide with Congo Red being the endpoint indicator.
}

\section{Source and Purity of Chemicals:}

(1) Purity not given, Chemical source not given, no purification details were provided.

(2) Purity not given, Chemical source not given, no purification details were provided.
Estimated Error:

Temperature: Not given in paper.

$c_{1}: \pm 10 \%$ (relative error, estimated by compiler).

\begin{tabular}{|c|c|}
\hline $\begin{array}{l}\text { Components: } \\
\text { (1) 2-Hydroxybenzoic acid; } \\
\mathrm{C}_{7} \mathrm{H}_{6} \mathrm{O}_{3} ;[69-72-7] \\
\text { (2) } 1,1^{\prime} \text {-Oxybisbutane; } \mathrm{C}_{8} \mathrm{H}_{18} \mathrm{O} \text {; } \\
\text { [142-96-1] }\end{array}$ & $\begin{array}{l}\text { Original Measurements: } \\
{ }^{151} \text { K. M. De Fina, T. L. Sharp, L. E. } \\
\text { Roy, and W. E. Acree, Jr., J. Chem. } \\
\text { Eng. Data 44, } 1262 \text { (1999). }\end{array}$ \\
\hline $\begin{array}{l}\text { Variables: } \\
T / \mathrm{K}=298.15\end{array}$ & $\begin{array}{l}\text { Prepared by: } \\
\text { W. E. Acree, Jr. }\end{array}$ \\
\hline
\end{tabular}

Experimental Values

\begin{tabular}{lc}
\hline \hline$x_{2}{ }^{\mathrm{a}}$ & $x_{1}{ }^{\mathrm{b}}$ \\
\hline 0.9081 & 0.09185 \\
\hline${ }^{\mathrm{a}} x_{2}:$ mole fraction of component 2 in the saturated solution. \\
${ }^{\mathrm{b}} x_{1}:$ mole fraction solubility of the solute.
\end{tabular}

Auxiliary Information

\section{Method/Apparatus/Procedure:}

Constant-temperature bath, calorimetric thermometer, and an ultraviolet/ visible spectrophotometer.

Excess solute and solvent were placed in amber glass bottles and allowed to equilibrate for several days at constant temperature. Attainment of equilibrium was verified by several repetitive measurements and by approaching equilibrium from supersaturation. Aliquots of saturated solutions were transferred through a coarse filter into tared volumetric flasks, weighed, and diluted with methanol. Concentrations were determined by spectrophotometric measurements at $304 \mathrm{~nm}$.

\section{Source and Purity of Chemicals:}

(1) $99+\%$, ACS Reagent grade, Aldrich Chemical Company, Milwaukee, WI, USA, was dried for several hours at $353 \mathrm{~K}$ before use and used without further purification.

(2) $99 \%$, Aldrich Chemical Company, stored over molecular sieves before use.

\section{Estimated Error:}

Temperature: $\pm 0.1 \mathrm{~K}$.

$x_{1}: \pm 1.5 \%$ (relative error).

\begin{tabular}{ll}
\hline \hline Components: & Original Measurements: \\
$(1)$ 2-Hydroxybenzoic acid; & ${ }^{151}$ K. M. De Fina, T. L. Sharp, L. E. \\
$\mathrm{C}_{7} \mathrm{H}_{6} \mathrm{O}_{3} ;$ [69-72-7] & Roy, and W. E. Acree, Jr., J. Chem. \\
$\begin{array}{l}\text { (2) Tetrahydrofuran; } \mathrm{C}_{4} \mathrm{H}_{8} \mathrm{O} ; \\
{[109-99-9]}\end{array}$ & Eng. Data 44, 1262 (1999). \\
\hline Variables: & Prepared by: \\
$T / \mathrm{K}=298.15$ & W. E. Acree, Jr. \\
\hline
\end{tabular}

\section{Experimental Values}

\begin{tabular}{lc}
\hline \hline$x_{2}{ }^{\mathrm{a}}$ & $x_{1}{ }^{\mathrm{b}}$ \\
\hline 0.6358 & 0.3642 \\
\hline${ }^{\mathrm{a}} x_{2}:$ mole fraction of component 2 in the saturated solution. & \\
${ }^{\mathrm{b}}{ }_{x_{1}}:$ mole fraction solubility of the solute.
\end{tabular}




\section{Auxiliary Information}

\section{Method/Apparatus/Procedure:}

Constant-temperature bath, calorimetric thermometer, and an ultraviolet/ visible spectrophotometer.

Excess solute and solvent were placed in amber glass bottles and allowed to equilibrate for several days at constant temperature. Attainment of equilibrium was verified by several repetitive measurements and by approaching equilibrium from supersaturation. Aliquots of saturated solutions were transferred through a coarse filter into tared volumetric flasks, weighed, and diluted with methanol. Concentrations were determined by spectrophotometric measurements at $304 \mathrm{~nm}$.

\section{Source and Purity of Chemicals:}

(1) $99+\%$, ACS Reagent grade, Aldrich Chemical Company, Milwaukee, WI, USA, was dried for several hours at $353 \mathrm{~K}$ before use and used without further purification.

(2) $99.9 \%$, anhydrous, Aldrich Chemical Company, stored over molecular sieves before use.

Estimated Error:

Temperature: $\pm 0.1 \mathrm{~K}$.

$x_{1}: \pm 1.5 \%$ (relative error).

\begin{tabular}{ll}
\hline \hline Components: & Original Measurements: \\
$(1)$ 2-Hydroxybenzoic acid; & ${ }^{151}$ K. M. De Fina, T. L. Sharp, L. E. \\
$\mathrm{C}_{7} \mathrm{H}_{6} \mathrm{O}_{3} ;$ [69-72-7] & Roy, and W. E. Acree, Jr., J. Chem. \\
$(2) 1,4-D i o x a n e ; \mathrm{C}_{4} \mathrm{H}_{8} \mathrm{O}_{2} ;$ & Eng. Data 44, 1262 (1999). \\
{$[123-91-1]$} & \\
\hline Variables: & Prepared by: \\
$T / \mathrm{K}=298.15$ & W. E. Acree, Jr. \\
\hline
\end{tabular}

Experimental Values

\begin{tabular}{lc}
\hline \hline$x_{2}^{\mathrm{a}}$ & $x_{1}^{\mathrm{b}}$ \\
\hline 0.7055 & 0.2945
\end{tabular}

${ }^{\mathrm{a}} x_{2}$ : mole fraction of component 2 in the saturated solution.

${ }^{\mathrm{b}} x_{1}$ : mole fraction solubility of the solute.

\section{Auxiliary Information}

\footnotetext{
Method/Apparatus/Procedure:

Constant-temperature bath, calorimetric thermometer, and an ultraviolet/ visible spectrophotometer.

Excess solute and solvent were placed in amber glass bottles and allowed to equilibrate for several days at constant temperature. Attainment of equilibrium was verified by several repetitive measurements and by approaching equilibrium from supersaturation. Aliquots of saturated solutions were transferred through a coarse filter into tared volumetric flasks, weighed, and diluted with methanol. Concentrations were determined by spectrophotometric measurements at $304 \mathrm{~nm}$.
}

\section{Source and Purity of Chemicals:}

(1) $99+\%$, ACS Reagent grade, Aldrich Chemical Company, Milwaukee, WI, USA, was dried for several hours at $353 \mathrm{~K}$ before use and used without further purification.

(2) $99.8 \%$, anhydrous, Aldrich Chemical Company, stored over molecular sieves before use.

\section{Estimated Error:}

Temperature: $\pm 0.1 \mathrm{~K}$.

$x_{1}: \pm 1.5 \%$ (relative error).

\begin{tabular}{|c|c|}
\hline $\begin{array}{l}\text { Components: } \\
\text { (1) 2-Hydroxybenzoic acid; } \\
\mathrm{C}_{7} \mathrm{H}_{6} \mathrm{O}_{3} ;[69-72-7] \\
\text { (2) 1,4-Dioxane; } \mathrm{C}_{4} \mathrm{H}_{8} \mathrm{O}_{2} \text {; } \\
{[123-91-1]}\end{array}$ & $\begin{array}{l}\text { Original Measurements: } \\
{ }^{152} \mathrm{H} \text {. Matsuda, K. Kaburagi, S. } \\
\text { Matsumoto, K. Kurihara, K. } \\
\text { Tochigi, and K. Tomono, J. Chem. } \\
\text { Eng. Data 54, } 480 \text { (2009). }\end{array}$ \\
\hline $\begin{array}{l}\text { Variables: } \\
T / \mathrm{K}=298.15\end{array}$ & $\begin{array}{l}\text { Prepared by: } \\
\text { W. E. Acree, Jr. }\end{array}$ \\
\hline
\end{tabular}

Experimental Values

\begin{tabular}{lc}
\hline \hline$x_{2}{ }^{\mathrm{a}}$ & $x_{1}{ }^{\mathrm{b}}$ \\
\hline 0.7390 & 0.2610
\end{tabular}

${ }^{\mathrm{a}} x_{2}$ : mole fraction of component 2 in the saturated solution.

${ }^{\mathrm{b}} x_{1}$ : mole fraction solubility of the solute.

\section{Auxiliary Information}

\section{Method/Apparatus/Procedure:}

Thermostated constant-temperature water bath and high-performance liquid chromatograph with uv detector.

Excess solute and solvent were allowed to equilibrate for $24 \mathrm{~h}$ in a constanttemperature thermostated water bath. Aliquots of saturated solutions were removed and filtered through a membrane filter of $0.45 \mu \mathrm{m}$ pore size (Millipore, USA). Concentrations were determined by high-performance liquid chromatography equipped with an uv detector (254 nm detection). Benzene was added to the sample as an internal standard.

\section{Source and Purity of Chemicals:}

(1) $99.5 \%$, Wako Pure Chemical Industries, Ltd., Japan, was used without further purification.

(2) $99 \%$, Wako Pure Chemical Industries, Ltd., Japan, was used without further purification.

\section{Estimated Error:}

Temperature: $\pm 0.05 \mathrm{~K}$.

$x_{1}: \pm 6.2 \%$ (relative error).

\begin{tabular}{|c|c|}
\hline $\begin{array}{l}\text { Components: } \\
\text { (1) 2-Hydroxybenzoic acid; } \\
\mathrm{C}_{7} \mathrm{H}_{6} \mathrm{O}_{3} \text {; [69-72-7] } \\
\text { (2) 1,4-Dioxane; } \mathrm{C}_{4} \mathrm{H}_{8} \mathrm{O}_{2} \text {; } \\
\text { [123-91-1] }\end{array}$ & $\begin{array}{l}\text { Original Measurements: } \\
{ }^{102} \text { J. Barra, M.-A. Peña, and P. } \\
\text { Bustamante, Eur. J. Pharm. Sci. 10, } \\
153 \text { (2000). }\end{array}$ \\
\hline $\begin{array}{l}\text { Variables: } \\
T / \mathrm{K}=298.15\end{array}$ & $\begin{array}{l}\text { Prepared by: } \\
\text { W. E. Acree, Jr. }\end{array}$ \\
\hline
\end{tabular}

\section{Experimental Values}

\begin{tabular}{lc}
\hline \hline$x_{2}{ }^{\mathrm{a}}$ & $x_{1}{ }^{\mathrm{b}}$ \\
\hline 0.7022 & 0.2978 \\
\hline${ }^{\mathrm{a}} x_{2}:$ mole fraction of component 2 in the saturated solution. & \\
${ }^{\mathrm{b}} x_{1}:$ mole fraction solubility of the solute. &
\end{tabular}




\section{Auxiliary Information}

\section{Method/Apparatus/Procedure:}

Constant-temperature bath, analytical balance, and an ultraviolet/visible spectrophotometer.

Excess solute and solvent were placed in a flask and allowed to equilibrate for several days at constant temperature. The solid phase was removed by filtration, and the clear solution was diluted quantitatively with $96 \%$ ethanol. The concentration of the diluted solution was determined by spectrophotometric measurement at the wavelength of maximum absorption. In instances where the solvent interfered with the spectrophotometric determination, the samples were evaporated to dryness and the residue diluted with $96 \%$ ethanol.

\section{Source and Purity of Chemicals:}

(1) Purity not given, Sigma Chemical Company, USA, no purification details were provided.

(2) Spectrophotometric or Analytical grade, Chemical source not specified, no purification details were provided.

\section{Estimated Error:}

Temperature: $\pm 0.2 \mathrm{~K}$

$x_{1}: \pm 3.0 \%$ (relative error, estimated by compiler).

\section{Components:}

(1) 2-Hydroxybenzoic acid;

$\mathrm{C}_{7} \mathrm{H}_{6} \mathrm{O}_{3}$; [69-72-7]

(2) 1,4-Dioxane; $\mathrm{C}_{4} \mathrm{H}_{8} \mathrm{O}_{2}$;

[123-91-1]

\begin{tabular}{ll}
\hline Variables: & Prepared by: \\
$T / \mathrm{K}=298.15$ & W. E. Acree, Jr. \\
\hline
\end{tabular}

Experimental Values

\begin{tabular}{lc}
\hline \hline$x_{2}^{\mathrm{a}}$ & $x_{1}{ }^{\mathrm{b}}$ \\
\hline 0.6883 & 0.3117 \\
\hline
\end{tabular}

${ }^{\mathrm{a}} x_{2}$ : mole fraction of component 2 in the saturated solution.

${ }^{b} x_{1}$ : mole fraction solubility of the solute.

\section{Auxiliary Information}

\section{Method/Apparatus/Procedure:}

Constant temperature-controlled shaking bath and an uv/visible spectrophotometer.

Binary solvent mixtures were prepared by volume. Excess solute and solvent were allowed to equilibrate in a constant temperature-controlled shaking bath for at least five days. Aliquots of the saturated solution were removed and filtered (Durapore membrane filter, $0.2 \mu \mathrm{m}$ pore size). The filtered aliquot was then diluted with ethanol $(96 \% \mathrm{v} / \mathrm{v})$, and the concentration of the dissolved solute determined by spectrophotometric analysis at a wavelength of $302 \mathrm{~nm}$ The authors converted the molar solubilities to mole fractions using the measured densities of the saturated solutions.

\section{Source and Purity of Chemicals:}

(1) Purity not given, Sigma-Aldrich, Germany, no purification details were provided.

(2) Spectrophotometric grade, Panreac, Monplet and Esteban, Spain, no purification details were provided.

\section{Estimated Error:}

Temperature: $\pm 0.1 \mathrm{~K}$.

$x_{1}: \pm 2.0 \%$ (relative error, estimated by compiler).

\begin{tabular}{ll}
\hline \hline Components: & Original Measurements: \\
$(1)$ 2-Hydroxybenzoic acid; & ${ }^{157}$ M. A. Peña, P. Bustamante, B. \\
$\mathrm{C}_{7} \mathrm{H}_{6} \mathrm{O}_{3} ;$ [69-72-7] & Escalera, A. Reíllo, and J. M. \\
$(2) 1,4-D i o x a n e ; \mathrm{C}_{4} \mathrm{H}_{8} \mathrm{O}_{2} ;$ & Bosque-Sendra, J. Pharm. Biomed. \\
[123-91-1] & Anal. 36, 571 (2004). \\
\hline Variables: & Prepared by: \\
Temperature & W. E. Acree, Jr. \\
\hline
\end{tabular}

Experimental Values

\begin{tabular}{lc}
\hline \hline$T / \mathrm{K}$ & $c_{1}{ }^{\mathrm{a}}$ \\
\hline 283 & 2.596 \\
293 & 3.065 \\
298 & 3.680 \\
303 & 3.974 \\
308 & 4.340 \\
313 & 4.659 \\
\hline${ }^{\mathrm{a}} c_{1}:$ molar solubility of the solute expressed in units of $\mathrm{mol} \mathrm{dm}^{-3}$. &
\end{tabular}

Auxiliary Information

\section{Method/Apparatus/Procedure:}

Constant temperature-controlled shaking bath and an uv/visible spectrophotometer.

Excess solute and solvent were allowed first to equilibrate in a constant temperature-controlled shaking bath at $313 \mathrm{~K}$, and then at the lower temperatures. The authors used the same samples for all temperatures. The authors started with $313 \mathrm{~K}$, and once the solution was saturated at the higher temperature they lowered the temperature for the next set of measurements. Aliquots of the saturated solution were removed and filtered (Durapore membrane filter, $0.2 \mu \mathrm{m}$ pore size). The filtered aliquot was then diluted with ethanol $(96 \% \mathrm{v} / \mathrm{v})$, and the concentration of the dissolved solute determined by spectrophotometric analysis at a wavelength of $298 \mathrm{~nm}$.

\section{Source and Purity of Chemicals:}

(1) $99+\%$, Sigma Chemical Company, St. Louis, MO, USA, was used as received.

(2) $99.5+\%$, anhydrous, Panreac, Monplet and Esteban, Barcelona, Spain, no purification details were provided.

\section{Estimated Error:}

Temperature: \pm 0.1

$c_{1}: \pm 3 \%$ (relative error).

\begin{tabular}{|c|c|}
\hline $\begin{array}{l}\text { Components: } \\
\text { (1) 2-Hydroxybenzoic acid; } \\
\mathrm{C}_{7} \mathrm{H}_{6} \mathrm{O}_{3} \text {; [69-72-7] } \\
\text { (2) 1,4-Dioxane; } \mathrm{C}_{4} \mathrm{H}_{8} \mathrm{O}_{2} \text {; } \\
\text { [123-91-1] }\end{array}$ & $\begin{array}{l}\text { Original Measurements: } \\
{ }^{150} \text { A. N. Paruta, B. J. Sciarrone, and } \\
\text { N. G. Lordi, J. Pharm. Sci. 53, } 1349 \\
\text { (1964). }\end{array}$ \\
\hline $\begin{array}{l}\text { Variables: } \\
T / \mathrm{K}=303.8\end{array}$ & $\begin{array}{l}\text { Prepared by: } \\
\text { W. E. Acree, Jr. }\end{array}$ \\
\hline
\end{tabular}

\section{Experimental Values}

The measured solubility was reported to be $40 \mathrm{mg} / \mathrm{ml}$ of solution, which corresponds to a solubility of $c_{1}=$ $0.290 \mathrm{~mol} \mathrm{dm}^{-3}$. 


\section{Auxiliary Information}

\section{Method/Apparatus/Procedure:}

Excess solute and solvent were placed in 15-ml screw-capped vials fitted with Teflon liners. The vials were attached to a rotating wheel in a constanttemperature water bath and allowed to equilibrate for $24 \mathrm{~h}$. An aliquot of the saturated solution was withdrawn from the vial with a pipet fitted with a glass wool filtering plug. The solubility was determined by titration using a freshly prepared sodium hydroxide solution. The endpoint of the titration was detected with phenolphthalein.

Source and Purity of Chemicals:

(1) Purity not given, Chemical source not given, no purification details were provided.

(2) Purity not given, Chemical source not given, no purification details were provided.

Estimated Error:

Temperature: $\pm 0.2 \mathrm{~K}$

$x_{1}: \pm 5 \%$ (relative error, estimated by compiler).

\subsection{2-Hydroxybenzoic acid solubility data in haloalkanes, haloalkenes, and haloaromatic hydrocarbons}

\begin{tabular}{ll}
\hline \hline Components: & Original Measurements: \\
(1) 2-Hydroxybenzoic acid; & ${ }^{56} \mathrm{P}$. A. Ongley, J. Chem. Soc. 1954, \\
$\mathrm{C}_{7} \mathrm{H}_{6} \mathrm{O}_{3} ;[69-72-7]$ & 3634. \\
$\begin{array}{l}\text { (2) Trichloromethane; } \mathrm{CHCl}_{3} ; \\
{[67-66-3]}\end{array}$ & \\
\hline Variables: & Prepared by: \\
$T / \mathrm{K}=298.15$ & W. E. Acree, Jr. \\
\hline
\end{tabular}

\section{Experimental Values}

The measured solubility was reported to be $-\log _{10} c_{1}=0.680$, which corresponds to a solubility of $c_{1}=0.209 \mathrm{~mol} \mathrm{dm}^{-3}$.

\section{Auxiliary Information}

\section{Method/Apparatus/Procedure:}

Very little experimental details were provided. Excess solute and solvent were placed in tubes, which were rotated for at least $8 \mathrm{~h}$ at $298 \mathrm{~K}$. The solutions were then removed, concentrated if necessary, and titrated with standard alkali using a mixed (bromothymol blue + neutral red) indicator.

\section{Source and Purity of Chemicals:}

(1) Purity not given, Chemical source not given, solute was either "AnalaR" grade or recrystallized prior to use.

(2) Purity not given, Chemical source not given, no purification details were provided.

\section{Estimated Error:}

Temperature: No information was given. $c_{1}: \pm 4 \%$ (relative error, estimated by compiler).

\begin{tabular}{|c|c|}
\hline $\begin{array}{l}\text { Components: } \\
\text { (1) 2-Hydroxybenzoic acid; } \\
\mathrm{C}_{7} \mathrm{H}_{6} \mathrm{O}_{3} ; \text { [69-72-7] } \\
\text { (2) Trichloromethane; } \mathrm{CHCl}_{3} \text {; } \\
\text { [67-66-3] }\end{array}$ & $\begin{array}{l}\text { Original Measurements: } \\
\text { 66 W. Herz and W. Rathmann, Z. } \\
\text { Elektrochem. 19, } 887 \text { (1913). }\end{array}$ \\
\hline $\begin{array}{l}\text { Variables: } \\
T / \mathrm{K}=298.15\end{array}$ & $\begin{array}{l}\text { Prepared by: } \\
\text { W. E. Acree, Jr. }\end{array}$ \\
\hline
\end{tabular}

\section{Experimental Values}

The measured molar solubility was $c_{1}=0.157 \mathrm{~mol} \mathrm{dm}^{-3}$.

Auxiliary Information

\section{Method/Apparatus/Procedure:}

Very little experimental details were provided. Excess solute and solvent were shaken in a thermostat at $298 \mathrm{~K}$. After equilibrium was reached and the solid phase settled to the bottom of the container, an aliquot of the saturated clear solution was removed by pipet. The concentration of the solute in the saturated solution was determined by titration.

Source and Purity of Chemicals:

(1) Purity not given, Chemical source not given, no purification details were provided.

(2) Purity not given, Chemical source not given, no purification details were provided.

Estimated Error:

Temperature: No information was given

$c_{1}: \pm 4 \%$ (relative error, estimated by compiler)

\begin{tabular}{ll}
\hline \hline Components: & Original Measurements: \\
(1) 2-Hydroxybenzoic acid; & 102J. Barra, M.-A. Peña, and P. \\
$\mathrm{C}_{7} \mathrm{H}_{6} \mathrm{O}_{3} ;[69-72-7]$ & Bustamante, Eur. J. Pharm. Sci. 10, \\
$\begin{array}{l}\text { (2) Trichloromethane; } \mathrm{CHCl}_{3} ; \\
{[67-66-3]}\end{array}$ & $153(2000)$. \\
\hline Variables: & Prepared by: \\
$T / \mathrm{K}=298.15$ & W. E. Acree, Jr. \\
\hline
\end{tabular}

\section{Experimental Values}

\begin{tabular}{lc}
\hline \hline$x_{2}{ }^{\mathrm{a}}$ & $x_{1}{ }^{\mathrm{b}}$ \\
\hline 0.9985 & 0.001458 \\
\hline${ }^{\mathrm{a}} x_{2}:$ mole fraction of component 2 in the saturated solution. \\
${ }^{\mathrm{b}} x_{1}:$ mole fraction solubility of the solute.
\end{tabular}

\section{Auxiliary Information}

\footnotetext{
Method/Apparatus/Procedure:

Constant-temperature bath, analytical balance, and an ultraviolet/visible spectrophotometer.

Excess solute and solvent were placed in a flask and allowed to equilibrate for several days at constant temperature. The solid phase was removed by filtration, and the clear solution was diluted quantitatively with $96 \%$ ethanol. The concentration of the diluted solution was determined by spectrophotometric measurement at the wavelength of maximum absorption. In instances where the solvent interfered with the spectrophotometric determination, the samples were evaporated to dryness and the residue diluted with $96 \%$ ethanol.
} 
Source and Purity of Chemicals:

(1) Purity not given, Sigma Chemical Company, USA, no purification details were provided.

(2) Spectrophotometric or Analytical grade, Chemical source not specified, no purification details were provided.

\section{Estimated Error:}

Temperature: $\pm 0.2 \mathrm{~K}$.

$x_{1}: \pm 3.0 \%$ (relative error, estimated by compiler).

\begin{tabular}{|c|c|}
\hline $\begin{array}{l}\text { Components: } \\
\text { (1) 2-Hydroxybenzoic acid; } \\
\left.\mathrm{C}_{7} \mathrm{H}_{6} \mathrm{O}_{3} ; \text {; } 69-72-7\right] \\
\text { (2) Trichloromethane; } \mathrm{CHCl}_{3} \text {; } \\
\text { [67-66-3] }\end{array}$ & $\begin{array}{l}\text { Original Measurements: } \\
{ }^{150} \text { A. N. Paruta, B. J. Sciarrone, and } \\
\text { N. G. Lordi, J. Pharm. Sci. 53, } 1349 \\
(1964) \text {. }\end{array}$ \\
\hline $\begin{array}{l}\text { Variables: } \\
T / \mathrm{K}=303.8\end{array}$ & $\begin{array}{l}\text { Prepared by: } \\
\text { W. E. Acree, Jr. }\end{array}$ \\
\hline
\end{tabular}

\section{Experimental Values}

The measured solubility was reported to be $2 \mathrm{mg} / \mathrm{ml}$ of solution, which corresponds to a solubility of $c_{1}=$ $0.0145 \mathrm{~mol} \mathrm{dm}^{-3}$.

\section{Auxiliary Information}

\section{Method/Apparatus/Procedure:}

Excess solute and solvent were placed in 15-ml screw-capped vials fitted with Teflon liners. The vials were attached to a rotating wheel in a constanttemperature water bath and allowed to equilibrate for $24 \mathrm{~h}$. An aliquot of the saturated solution was withdrawn from the vial with a pipet fitted with a glass wool filtering plug. The solubility was determined by titration using a freshly prepared sodium hydroxide solution. The endpoint of the titration was detected with phenolphthalein.

\section{Source and Purity of Chemicals:}

(1) Purity not given, Chemical source not given, no purification details were provided.

(2) Purity not given, Chemical source not given, no purification details were provided.

\section{Estimated Error:}

Temperature: $\pm 0.2 \mathrm{~K}$.

$c_{1}: \pm 5 \%$ (relative error, estimated by compiler).

\begin{tabular}{ll}
\hline \hline Components: & Original Measurements: \\
(1) 2-Hydroxybenzoic acid; & ${ }^{50} \mathrm{P}$. G. Desai and A. M. Patel, J. \\
$\mathrm{C}_{7} \mathrm{H}_{6} \mathrm{O}_{3} ;[69-72-7]$ & Indian Chem. Soc. 12, 131 (1935). \\
(2) Trichloromethane; $\mathrm{CHCl}_{3} ;$ & \\
67-66-3] & \\
\hline Variables: & Prepared by: \\
$T / \mathrm{K}=301.2$ & W. E. Acree, Jr. \\
\hline
\end{tabular}

\section{Experimental Values}

\begin{tabular}{lc}
\hline \hline$x_{2}{ }^{\mathrm{a}}$ & $x_{1}{ }^{\mathrm{b}}$ \\
\hline 0.9732 & 0.02679
\end{tabular}

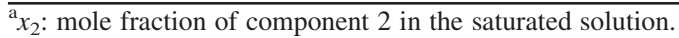

${ }^{\mathrm{b}} x_{1}$ : mole fraction solubility of the solute.

\section{Auxiliary Information}

\section{Method/Apparatus/Procedure:}

Excess solute and solvent were placed in a glass flask which was kept revolving on a wheel in an air thermostat for two days. After two days of equilibration, the solution was filtered. To minimize absorption of the solute onto the filter paper, a $100 \mathrm{ml}$ portion of fresh saturated solution was first filtered through the paper before filtering the sample to be analyzed. An aliquot of the filtrate was titrated with barium hydroxide using phenolphthalein as the endpoint indictor. The barium hydroxide titrant was previously standardized against a solution of succinic acid.

\section{Source and Purity of Chemicals:}

(1) Purity not given, Chemical source not specified, no information provided concerning purification.

(2) Purity not given, Chemical source not specified, no information provided concerning purification.

\section{Estimated Error:}

Temperature: No information given.

$x_{1}: \pm 3 \%$ (relative error, estimated by compiler).

\begin{tabular}{ll}
\hline \hline Components: & Original Measurements: \\
(1) 2-Hydroxybenzoic acid; & ${ }^{153}$ A. Shalmashi and A. Eliassi, J. \\
$\mathrm{C}_{7} \mathrm{H}_{6} \mathrm{O}_{3} ;[69-72-7]$ & Chem. Eng. Data 53, 199 (2008). \\
$\begin{array}{l}\text { (2) Tetrachloromethane; } \mathrm{CCl}_{4} ; \\
{[56-23-5]}\end{array}$ & \\
\hline Variables: & Prepared by: \\
Temperature & W. E. Acree, Jr. \\
\hline
\end{tabular}

Experimental Values

\begin{tabular}{lcc}
\hline \hline$T / \mathrm{K}$ & $x_{2}{ }^{\mathrm{a}}$ & $x_{1}{ }^{\mathrm{b}}$ \\
\hline 298 & 0.9967 & 0.00333 \\
303 & 0.9958 & 0.00420 \\
308 & 0.9946 & 0.00544 \\
313 & 0.9930 & 0.00702 \\
318 & 0.9914 & 0.00856 \\
323 & 0.9887 & 0.01134 \\
328 & 0.9860 & 0.01396 \\
333 & 0.9832 & 0.01681 \\
338 & 0.9798 & 0.02002 \\
343 & 0.9764 & 0.02363 \\
348 & 0.9722 & 0.02778 \\
\hline
\end{tabular}

${ }^{a} x_{2}$ : mole fraction of component 2 in the saturated solution.

${ }^{b} x_{1}$ : mole fraction solubility of the solute. The solubility data were reported as mass fractions. The mole fraction values were calculated by the compiler.

\section{Auxiliary Information}

\section{Method/Apparatus/Procedure:}

Magnetically stirred jacketed equilibrium cell, constant-temperature circulating water bath, and an analytical balance.

Excess solute and solvent were placed in a magnetically stirred jacketed equilibrium cell and allowed to equilibrate at constant temperature for $2 \mathrm{~h}$ with continuous stirring. After $2 \mathrm{~h}$ the stirring was discontinued and the suspended solid was allowed to settle to the lower portion of the equilibrium cell. An aliquot of the saturated solution was withdrawn by a warmed pipet and transferred to a weighed vial. The vial was tightly closed and weighed to determine the mass of the sample analyzed. The vial was then placed in an oven and the solvent was allowed to evaporate. The vial was reweighed, and the solubility calculated from the mass of the solid residue and sample analyzed. 
Source and Purity of Chemicals:

(1) Purity not given, Synthesized in the authors' department, dried under vacuum at $323 \mathrm{~K}$ for $8 \mathrm{~h}$ and then stored in a desiccator.

(2) Analytical grade, Merck Chemicals, Darmstadt, Germany, used as received.

\section{Estimated Error:}

Temperature: $\pm 0.05 \mathrm{~K}$.

$x_{1}: \pm 3 \%$ (relative error, estimated by compiler).

\section{Components:}

(1) 2-Hydroxybenzoic acid;

$\mathrm{C}_{7} \mathrm{H}_{6} \mathrm{O}_{3}$; [69-72-7]

(2) Tetrachloromethane; $\mathrm{CCl}_{4}$;

[56-23-5]

\begin{tabular}{ll}
\hline Variables: & Prepared by: \\
$T / \mathrm{K}=298.15$ & W. E. Acree, Jr. \\
\hline
\end{tabular}

\section{Experimental Values}

The measured solubility was reported to be $-\log _{10} c_{1}=$ 1.644, which corresponds to a solubility of $c_{1}=0.0227$ mol dm ${ }^{-3}$.

\section{Auxiliary Information}

\section{Method/Apparatus/Procedure:}

Very little experimental details were provided. Excess solute and solvent were placed in tubes, which were rotated for at least $8 \mathrm{~h}$ at $298 \mathrm{~K}$. The solutions were then removed, concentrated if necessary, and titrated with standard alkali using a mixed (bromothymol blue + neutral red) indicator.

\section{Source and Purity of Chemicals:}

(1) Purity not given, Chemical source not given, solute was either "AnalaR" grade or recrystallized prior to use.

(2) Purity not given, Chemical source not given, no purification details were provided.

\section{Estimated Error:}

Temperature: No information was given.

$c_{1}: \pm 4 \%$ (relative error, estimated by compiler).

\section{Components:}

(1) 2-Hydroxybenzoic acid;

$\mathrm{C}_{7} \mathrm{H}_{6} \mathrm{O}_{3}$; [69-72-7]

(2) Tetrachloromethane; $\mathrm{CCl}_{4}$;

[56-23-5]

\begin{tabular}{ll}
\hline Variables: & Prepared by: \\
$T / \mathrm{K}=298.15$ & W. E. Acree, Jr. \\
\hline
\end{tabular}

Experimental Values

\begin{tabular}{lc}
\hline \hline$x_{2}{ }^{\mathrm{a}}$ & $x_{1}{ }^{\mathrm{b}}$ \\
\hline 0.9971 & 0.00293
\end{tabular}

${ }^{a} x_{2}$ : mole fraction of component 2 in the saturated solution.

${ }^{b} x_{1}$ : mole fraction solubility of the solute.

\section{Auxiliary Information}

Method/Apparatus/Procedure:

Experimental solubility was determined by percolating the solvent at an adjustable rate through a column of solute in one limb of a U-shaped tube, a plug of cotton wool serving as support and filter. The saturated solution collects in the parallel arm and the whole assembly is immersed in a thermostat bath except for the stoppered ends. Samples of the saturated solutions were removed and titrated with standardized carbonate-free alkali using phenolphthalein as the indicator.

\section{Source and Purity of Chemicals:}

(1) Purity not given, Chemical source not specified, no information provided concerning purification.

(2) Purity not given, Chemical source not specified, no information provided concerning purification.

\section{Estimated Error:}

Temperature: $\pm 0.02 \mathrm{~K}$

$x_{1}: \pm 3 \%$ (relative error, estimated by compiler).

\begin{tabular}{ll}
\hline \hline Components: & Original Measurements: \\
(1) 2-Hydroxybenzoic acid; & ${ }^{50} \mathrm{P} . \mathrm{G}$. Desai and A. M. Patel, J. \\
$\mathrm{C}_{7} \mathrm{H}_{6} \mathrm{O}_{3} ;[69-72-7]$ & Indian Chem. Soc. 12, 131 (1935). \\
$\begin{array}{l}\text { (2) Tetrachloromethane; } \mathrm{CCl}_{4} ; \\
{[56-23-5]}\end{array}$ & \\
\hline Variables: & Prepared by: \\
$T / \mathrm{K}=301.2$ & W. E. Acree, Jr. \\
\hline
\end{tabular}

Experimental Values

\begin{tabular}{lc}
\hline \hline$x_{2}{ }^{\mathrm{a}}$ & $x_{1}{ }^{\mathrm{b}}$ \\
\hline 0.9962 & 0.003803
\end{tabular}

${ }^{a} x_{2}$ : mole fraction of component 2 in the saturated solution.

${ }^{\mathrm{b}} x_{1}$ : mole fraction solubility of the solute.

\section{Auxiliary Information}

\section{Method/Apparatus/Procedure:}

Excess solute and solvent were placed in a glass flask which was kept revolving on a wheel in an air thermostat for two days. After two days of equilibration, the solution was filtered. To minimize absorption of the solute onto the filter paper, a $100 \mathrm{ml}$ portion of fresh saturated solution was first filtered through the paper before filtering the sample to be analyzed. An aliquot of the filtrate was titrated with barium hydroxide using phenolphthalein as the endpoint indictor. The barium hydroxide titrant was previously standardized against a solution of succinic acid.

\section{Source and Purity of Chemicals:}

(1) Purity not given, Chemical source not specified, no information provided concerning purification.

(2) Purity not given, Chemical source not specified, no information provided concerning purification.

\section{Estimated Error:}

Temperature: No information given.

$x_{1}: \pm 3 \%$ (relative error, estimated by compiler). 


\section{Components:}

(1) 2-Hydroxybenzoic acid; $\mathrm{C}_{7} \mathrm{H}_{6} \mathrm{O}_{3}$;

[69-72-7]

(2) Tetrachloromethane; $\mathrm{CCl}_{4}$;

[56-23-5]

\begin{tabular}{ll}
\hline Variables: & Prepared by: \\
$T / \mathrm{K}=298.15$ & W. E. Acree, Jr. \\
\hline
\end{tabular}

\section{Experimental Values}

The measured molar solubility was $c_{1}=0.030 \mathrm{~mol} \mathrm{dm}^{-3}$.

\section{Auxiliary Information}

\section{Method/Apparatus/Procedure:}

Very little experimental details were provided. Excess solute and solvent were shaken in a thermostat at $298 \mathrm{~K}$. After equilibrium was reached and the solid phase settled to the bottom of the container, an aliquot of the saturated clear solution was removed by pipet. The concentration of the solute in the saturated solution was determined by titration.

Source and Purity of Chemicals:

(1) Purity not given, Chemical source not given, no purification details were provided.

(2) Purity not given, Chemical source not given, no purification details were provided.

\section{Estimated Error:}

Temperature: No information was given. $x_{1}: \pm 4 \%$ (relative error, estimated by compiler).

\begin{tabular}{|c|c|}
\hline $\begin{array}{l}\text { Components: } \\
\text { (1) 2-Hydroxybenzoic acid; } \mathrm{C}_{7} \mathrm{H}_{6} \mathrm{O}_{3} \text {; } \\
\text { [69-72-7] } \\
\text { (2) 1,2-Dichloroethane; } \mathrm{C}_{2} \mathrm{H}_{4} \mathrm{Cl}_{2} \text {; } \\
\text { [107-06-2] }\end{array}$ & $\begin{array}{l}\text { Original Measurements: } \\
{ }^{102} \text { J. Barra, M.-A. Peña, and P. } \\
\text { Bustamante, Eur. J. Pharm. Sci. 10, } \\
153 \text { (2000). }\end{array}$ \\
\hline $\begin{array}{l}\text { Variables: } \\
T / \mathrm{K}=298.15\end{array}$ & $\begin{array}{l}\text { Prepared by: } \\
\text { W. E. Acree, Jr. }\end{array}$ \\
\hline
\end{tabular}

\section{Experimental Values}

\begin{tabular}{lc}
\hline \hline$x_{2}{ }^{\mathrm{a}}$ & $x_{1}{ }^{\mathrm{b}}$ \\
\hline 0.9924 & 0.00758 \\
\hline
\end{tabular}

${ }^{a_{2}}$ : mole fraction of component 2 in the saturated solution.

${ }^{\mathrm{b}} x_{1}$ : mole fraction solubility of the solute.

\section{Auxiliary Information}

\section{Method/Apparatus/Procedure:}

Constant-temperature bath, analytical balance, and an ultraviolet/visible spectrophotometer.

Excess solute and solvent were placed in a flask and allowed to equilibrate for several days at constant temperature. The solid phase was removed by filtration, and the clear solution was diluted quantitatively with $96 \%$ ethanol. The concentration of the diluted solution was determined by spectrophotometric measurement at the wavelength of maximum absorption. In instances where the solvent interfered with the spectrophotometric determination, the samples were evaporated to dryness and the residue diluted with $96 \%$ ethanol.
Source and Purity of Chemicals:

(1) Purity not given, Sigma Chemical Company, USA, no purification details were provided.

(2) Spectrophotometric or Analytical grade, Chemical source not specified, no purification details were provided.

\section{Estimated Error:}

Temperature: $\pm 0.2 \mathrm{~K}$.

$x_{1}: \pm 5.0 \%$ (relative error, estimated by compiler).

\begin{tabular}{ll}
\hline \hline Components: & Original Measurements: \\
(1) 2-Hydroxybenzoic acid; $\mathrm{C}_{7} \mathrm{H}_{6} \mathrm{O}_{3} ;$ & ${ }^{66} \mathrm{~W}$. Herz and W. Rathmann, Z. \\
[69-72-7] & Elektrochem. 19, 887 (1913). \\
$(2) 1,1,2,2-$ Tetrachloroethane; & \\
$\mathrm{C}_{2} \mathrm{H}_{2} \mathrm{Cl}_{4} ;[79-34-5]$ & \\
\hline Variables: & Prepared by: \\
$T / \mathrm{K}=298.15$ & W. E. Acree, Jr. \\
\hline
\end{tabular}

\section{Experimental Values}

The measured molar solubility was $c_{1}=0.131 \mathrm{~mol} \mathrm{dm}^{-3}$.

Auxiliary Information

\section{Method/Apparatus/Procedure:}

Very little experimental details were provided. Excess solute and solvent were shaken in a thermostat at $298 \mathrm{~K}$. After equilibrium was reached and the solid phase settled to the bottom of the container, an aliquot of the saturated clear solution was removed by pipet. The concentration of the solute in the saturated solution was determined by titration.

Source and Purity of Chemicals:

(1) Purity not given, Chemical source not given, no purification details were provided.

(2) Purity not given, Chemical source not given, no purification details were provided.

\section{Estimated Error:}

Temperature: No information was given. $c_{1}: \pm 4 \%$ (relative error, estimated by compiler).

\begin{tabular}{|c|c|}
\hline $\begin{array}{l}\text { Components: } \\
\text { (1) 2-Hydroxybenzoic acid; } \mathrm{C}_{7} \mathrm{H}_{6} \mathrm{O}_{3} \text {; } \\
\text { [69-72-7] } \\
\text { (2) } 1,1,1,2,2-\text { Pentachloroethane; } \\
\mathrm{C}_{2} \mathrm{HCl}_{5} ;[76-01-7]\end{array}$ & $\begin{array}{l}\text { Original Measurements: } \\
{ }^{66} \text { W. Herz and W. Rathmann, Z. } \\
\text { Elektrochem. 19, } 887 \text { (1913). }\end{array}$ \\
\hline $\begin{array}{l}\text { Variables: } \\
T / \mathrm{K}=298.15\end{array}$ & $\begin{array}{l}\text { Prepared by: } \\
\text { W. E. Acree, Jr. }\end{array}$ \\
\hline
\end{tabular}

\section{Experimental Values}

The measured molar solubility was $c_{1}=0.077 \mathrm{~mol} \mathrm{dm}^{-3}$.

\section{Auxiliary Information}

\section{Method/Apparatus/Procedure:}

Very little experimental details were provided. Excess solute and solvent were shaken in a thermostat at $298 \mathrm{~K}$. After equilibrium was reached and the solid phase settled to the bottom of the container, an aliquot of the saturated clear solution was removed by pipet. The concentration of the solute in the saturated solution was determined by titration. 
Source and Purity of Chemicals:

(1) Purity not given, Chemical source not given, no purification details were provided.

(2) Purity not given, Chemical source not given, no purification details were provided.

\section{Estimated Error:}

Temperature: No information was given. $c_{1}: \pm 4 \%$ (relative error, estimated by compiler).

\section{Components:}

(1) 2-Hydroxybenzoic acid; $\mathrm{C}_{7} \mathrm{H}_{6} \mathrm{O}_{3}$; [69-72-7]

(2) Dichloroethene; $\mathrm{C}_{2} \mathrm{H}_{2} \mathrm{Cl}_{2}$; isomer was not given

\begin{tabular}{ll}
\hline Variables: & Prepared by: \\
$T / \mathrm{K}=288.15$ & W. E. Acree, Jr. \\
\hline
\end{tabular}

\section{Experimental Values}

\begin{tabular}{lc}
\hline \hline$x_{2}{ }^{\mathrm{a}}$ & $x_{1}{ }^{\mathrm{b}}$ \\
\hline 0.9947 & 0.00529
\end{tabular}

${ }^{\mathrm{a}} x_{2}$ : mole fraction of component 2 in the saturated solution.

${ }^{\mathrm{b}} x_{1}$ : mole fraction solubility of the solute.

\section{Auxiliary Information}

\section{Method/Apparatus/Procedure:}

Excess solute and solvent were continuously shaken in a water bath at $303 \mathrm{~K}$ for $1 \mathrm{~h}$. The solution was then transferred to a cellar which was maintained at a constant temperature of $288 \mathrm{~K}$. The solution was allowed to equilibrate in the cellar for at least two days with repeated shaking. The concentration of the dissolved solute was determined; however, the analytical method was not described.

\section{Source and Purity of Chemicals:}

(1) Purity not given, Chemical source not specified, no information provided concerning purification.

(2) Purity not given, Chemical source not specified, no information provided concerning purification.

\section{Estimated Error:}

Temperature: $\pm 0.2 \mathrm{~K}$.

$x_{1}$ : Not enough information in paper to estimate an uncertainty.

\begin{tabular}{ll}
\hline \hline Components: & Original Measurements: \\
$\begin{array}{l}\text { (1) 2-Hydroxybenzoic acid; } \mathrm{C}_{7} \mathrm{H}_{6} \mathrm{O}_{3} ; \\
{[69-72-7]}\end{array}$ & ${ }^{66} \mathrm{~W}$. Herz and W. Rathmann, Z. \\
$\begin{array}{l}\text { Elektrochem. 19, 887 (1913). } \\
\text { (2) Trichloroethene; } \mathrm{C}_{2} \mathrm{HCl}_{3} ;\end{array}$ \\
\\
\hline Variables: & \\
$T / \mathrm{K}=298.15$ & Prepared by: \\
\hline
\end{tabular}

\section{Experimental Values}

The measured molar solubility was $c_{1}=0.110 \mathrm{~mol} \mathrm{dm}^{-3}$.

\section{Auxiliary Information}

\section{Method/Apparatus/Procedure:}

Very little experimental details were provided. Excess solute and solvent were shaken in a thermostat at $298 \mathrm{~K}$. After equilibrium was reached and the solid phase settled to the bottom of the container, an aliquot of the saturated clear solution was removed by pipet. The concentration of the solute in the saturated solution was determined by titration.

Source and Purity of Chemicals:

(1) Purity not given, Chemical source not given, no purification details were provided.

(2) Purity not given, Chemical source not given, no purification details were provided.

Estimated Error:

Temperature: No information was given

$c_{1}: \pm 4 \%$ (relative error, estimated by compiler).

\begin{tabular}{ll}
\hline \hline Components: & Original Measurements: \\
$\begin{array}{l}\text { (1) 2-Hydroxybenzoic acid; } \mathrm{C}_{7} \mathrm{H}_{6} \mathrm{O}_{3} ; \\
{[69-72-7]}\end{array}$ & ${ }^{86} \mathrm{D}$. H. Wester and A. Bruins, \\
Pharm. Weekbl. 51, 1443 (1914). \\
[79-06-1]
\end{tabular}

Experimental Values

\begin{tabular}{lc}
\hline \hline$x_{2}{ }^{\mathrm{a}}$ & $x_{1}{ }^{\mathrm{b}}$ \\
\hline 0.9973 & 0.00268
\end{tabular}

${ }^{\mathrm{a}} x_{2}$ : mole fraction of component 2 in the saturated solution.

${ }^{\mathrm{b}} x_{1}$ : mole fraction solubility of the solute.

\section{Auxiliary Information}

\section{Method/Apparatus/Procedure:}

Excess solute and solvent were continuously shaken in a water bath at $303 \mathrm{~K}$ for $1 \mathrm{~h}$. The solution was then transferred to a cellar which was maintained at a constant temperature of $288 \mathrm{~K}$. The solution was allowed to equilibrate in the cellar for at least two days with repeated shaking. The concentration of the dissolved solute was determined; however, the analytical method was not described.

\section{Source and Purity of Chemicals:}

(1) Purity not given, Chemical source not specified, no information provided concerning purification.

(2) Purity not given, Chemical source not specified, no information provided concerning purification.

\section{Estimated Error:}

Temperature: $\pm 0.2 \mathrm{~K}$.

$x_{1}$ : Not enough information in paper to estimate an uncertainty. 


\section{Components:}

(1) 2-Hydroxybenzoic acid; $\mathrm{C}_{7} \mathrm{H}_{6} \mathrm{O}_{3}$;

[69-72-7]

(2) Tetrachloroethene; $\mathrm{C}_{2} \mathrm{Cl}_{4}$;

[127-18-4]

\begin{tabular}{ll}
\hline Variables: & Prepared by: \\
$T / \mathrm{K}=298.15$ & W. E. Acree, Jr. \\
\hline
\end{tabular}

\section{Experimental Values}

The measured molar solubility was $c_{1}=0.080 \mathrm{~mol} \mathrm{dm}^{-3}$.

\section{Auxiliary Information}

\section{Method/Apparatus/Procedure:}

Very little experimental details were provided. Excess solute and solvent were shaken in a thermostat at $298 \mathrm{~K}$. After equilibrium was reached and the solid phase settled to the bottom of the container, an aliquot of the saturated clear solution was removed by pipet. The concentration of the solute in the saturated solution was determined by titration.

Source and Purity of Chemicals:

(1) Purity not given, Chemical source not given, no purification details were provided.

(2) Purity not given, Chemical source not given, no purification details were provided.

\section{Estimated Error:}

Temperature: No information was given. $c_{1}: \pm 4 \%$ (relative error, estimated by compiler).

\begin{tabular}{ll}
\hline \hline Components: & Original Measurements: \\
$\begin{array}{l}\text { (1) 2-Hydroxybenzoic acid; } \mathrm{C}_{7} \mathrm{H}_{6} \mathrm{O}_{3} ; \\
{[69-72-7]}\end{array}$ & $\begin{array}{l}{ }^{102} \text { J. Barra, M.-A. Peña, and P. } \\
\text { Bustamante, Eur. J. Pharm. Sci. 10, } \\
\text { (2) Chlorobenzene; } \mathrm{C}_{6} \mathrm{H}_{5} \mathrm{Cl} ;\end{array}$ \\
$\begin{array}{ll}153(2000) . \\
\text { Variables: }\end{array}$ & \\
\hline$T / \mathrm{K}=298.15$ & Prepared by: \\
\hline
\end{tabular}

\section{Experimental Values}

\begin{tabular}{lc}
\hline \hline$x_{2}{ }^{\mathrm{a}}$ & $x_{1}{ }^{\mathrm{b}}$ \\
\hline 0.9933 & 0.00670 \\
\hline
\end{tabular}

${ }^{\mathrm{a}} x_{2}$ : mole fraction of component 2 in the saturated solution.

${ }^{\mathrm{b}} x_{1}$ : mole fraction solubility of the solute.

\section{Auxiliary Information}

\footnotetext{
Method/Apparatus/Procedure:

Constant-temperature bath, analytical balance, and an ultraviolet/visible spectrophotometer.

Excess solute and solvent were placed in a flask and allowed to equilibrate for several days at constant temperature. The solid phase was removed by filtration, and the clear solution was diluted quantitatively with $96 \%$ ethanol. The concentration of the diluted solution was determined by spectrophotometric measurement at the wavelength of maximum absorption. In instances where the solvent interfered with the spectrophotometric determination, the samples were evaporated to dryness and the residue diluted with $96 \%$ ethanol.
}

Source and Purity of Chemicals:

(1) Purity not given, Sigma Chemical Company, USA, no purification details were provided.

(2) Spectrophotometric or Analytical grade, Chemical source not specified, no purification details were provided.

\section{Estimated Error:}

Temperature: $\pm 0.2 \mathrm{~K}$.

$x_{1}: \pm 5.0 \%$ (relative error, estimated by compiler).

\begin{tabular}{|c|c|}
\hline $\begin{array}{l}\text { Components: } \\
\text { (1) 2-Hydroxybenzoic acid; } \mathrm{C}_{7} \mathrm{H}_{6} \mathrm{O}_{3} \text {; } \\
\text { [69-72-7] } \\
\text { (2) Chlorobenzene; } \mathrm{C}_{6} \mathrm{H}_{5} \mathrm{Cl} ; \\
\text { [108-90-7] }\end{array}$ & $\begin{array}{l}\text { Original Measurements: } \\
{ }^{50} \text { P. G. Desai and A. M. Patel, J. } \\
\text { Indian Chem. Soc. 12, } 131 \text { (1935). }\end{array}$ \\
\hline $\begin{array}{l}\text { Variables: } \\
T / \mathrm{K}=301.2\end{array}$ & $\begin{array}{l}\text { Prepared by: } \\
\text { W. E. Acree, Jr. }\end{array}$ \\
\hline
\end{tabular}

Experimental Values

\begin{tabular}{lc}
\hline \hline$x_{2}{ }^{\mathrm{a}}$ & $x_{1}{ }^{\mathrm{b}}$ \\
\hline 0.9921 & 0.007903 \\
\hline${ }^{\mathrm{a}} x_{2}:$ mole fraction of component 2 in the saturated solution. \\
${ }^{\mathrm{b}}{ }_{x_{1}: \text { mole fraction solubility of the solute. }}$
\end{tabular}

\section{Auxiliary Information}

\section{Method/Apparatus/Procedure:}

Excess solute and solvent were placed in a glass flask which was kept revolving on a wheel in an air thermostat for two days. After two days of equilibration, the solution was filtered. To minimize absorption of the solute onto the filter paper, a $100 \mathrm{ml}$ portion of fresh saturated solution was first filtered through the paper before filtering the sample to be analyzed. An aliquot of the filtrate was titrated with barium hydroxide using phenolphthalein as the endpoint indictor. The barium hydroxide titrant was previously standardized against a solution of succinic acid.

\section{Source and Purity of Chemicals:}

(1) Purity not given, Chemical source not specified, no information provided concerning purification.

(2) Purity not given, Chemical source not specified, no information provided concerning purification.

\section{Estimated Error:}

Temperature: No information given.

$x_{1}: \pm 3 \%$ (relative error, estimated by compiler).

\subsection{2-Hydroxybenzoic acid solubility data in alcohols}

\begin{tabular}{ll}
\hline \hline Components: & Original Measurements: \\
(1) 2-Hydroxybenzoic acid; $\mathrm{C}_{7} \mathrm{H}_{6} \mathrm{O}_{3} ;$ & ${ }^{152} \mathrm{H}$. Matsuda, K. Kaburagi, S. \\
[69-72-7] & Matsumoto, K. Kurihara, K. \\
(2) Methanol; $\mathrm{CH}_{4} \mathrm{O} ;[67-56-1]$ & Tochigi, and K. Tomono, J. Chem. \\
& Eng. Data 54, 480 (2009). \\
\hline Variables: & Prepared by: \\
$T / \mathrm{K}=298.15$ & W. E. Acree, Jr. \\
\hline
\end{tabular}


Experimental Values

\begin{tabular}{lc}
\hline \hline$x_{2}{ }^{\mathrm{a}}$ & $x_{1}{ }^{\mathrm{b}}$ \\
\hline 0.8777 & 0.1223
\end{tabular}

${ }^{\mathrm{a}} x_{2}$ : mole fraction of component 2 in the saturated solution.

${ }^{\mathrm{b}} x_{1}$ : mole fraction solubility of the solute.

\section{Auxiliary Information}

Method/Apparatus/Procedure:

Thermostated constant-temperature water bath and high-performance liquid chromatograph with uv detector.

Excess solute and solvent were allowed to equilibrate for $24 \mathrm{~h}$ in a constanttemperature thermostated water bath. Aliquots of saturated solutions were removed and filtered through a membrane filter of $0.45 \mu \mathrm{m}$ pore size (Millipore, USA). Concentrations were determined by high-performance liquid chromatography equipped with an uv detector (254 $\mathrm{nm}$ detection). Benzene was added to the sample as an internal standard.

Source and Purity of Chemicals:

(1) $99.5 \%$, Wako Pure Chemical Industries, Ltd., Japan, was used without further purification.

(2) $99.8 \%$, Wako Pure Chemical Industries, Ltd., Japan, was used without further purification.

Estimated Error:

Temperature: $\pm 0.05 \mathrm{~K}$.

$x_{1}: \pm 6.2 \%$ (relative error)

\begin{tabular}{ll}
\hline \hline Components: & Original Measurements: \\
$\begin{array}{l}\text { (1) 2-Hydroxybenzoic acid; } \mathrm{C}_{7} \mathrm{H}_{6} \mathrm{O}_{3} ; \\
\text { [69-72-7] }\end{array}$ & $\begin{array}{l}{ }^{\text {J. }} \text {. Barra, M.-A. Peña, and P. } \\
\text { Bustamante, Eur. J. Pharm. Sci. 10, } \\
\text { (2) Methanol; } \mathrm{CH}_{4} \mathrm{O} ;[67-56-1]\end{array}$ \\
\hline Variables: & 153 (2000). \\
$T / \mathrm{K}=298.15$ & Prepared by: \\
\hline
\end{tabular}

\section{Experimental Values}

\begin{tabular}{lc}
\hline \hline$x_{2}{ }^{\mathrm{a}}$ & $x_{1}{ }^{\mathrm{b}}$ \\
\hline 0.8679 & 0.1321 \\
\hline$x_{2}$ m
\end{tabular}

${ }^{\mathrm{a}} x_{2}$ : mole fraction of component 2 in the saturated solution.

${ }^{\mathrm{b}} x_{1}$ : mole fraction solubility of the solute.

\section{Auxiliary Information}

\footnotetext{
Method/Apparatus/Procedure:

Constant-temperature bath, analytical balance, and an ultraviolet/visible spectrophotometer.

Excess solute and solvent were placed in a flask and allowed to equilibrate for several days at constant temperature. The solid phase was removed by filtration, and the clear solution was diluted quantitatively with $96 \%$ ethanol The concentration of the diluted solution was determined by spectrophotometric measurement at the wavelength of maximum absorption In instances where the solvent interfered with the spectrophotometric determination, the samples were evaporated to dryness and the residue diluted with $96 \%$ ethanol.
}

Source and Purity of Chemicals:

(1) Purity not given, Sigma Chemical Company, USA, no purification details were provided.

(2) Spectrophotometric or Analytical grade, Chemical source not specified, no purification details were provided.

\section{Estimated Error:}

Temperature: $\pm 0.2 \mathrm{~K}$.

$x_{1}: \pm 3.0 \%$ (relative error, estimated by compiler).

\begin{tabular}{|c|c|}
\hline $\begin{array}{l}\text { Components: } \\
\text { (1) 2-Hydroxybenzoic acid; } \mathrm{C}_{7} \mathrm{H}_{6} \mathrm{O}_{3} \text {; } \\
\text { [69-72-7] } \\
\text { (2) Methanol; } \mathrm{CH}_{4} \mathrm{O} ;[67-56-1]\end{array}$ & $\begin{array}{l}\text { Original Measurements: } \\
{ }^{158} \text { E. Bergroth, Farm. Aikak. 70, } 91 \\
(1961) .\end{array}$ \\
\hline $\begin{array}{l}\text { Variables: } \\
T / \mathrm{K}=293.15\end{array}$ & $\begin{array}{l}\text { Prepared by: } \\
\text { W. E. Acree, Jr. }\end{array}$ \\
\hline
\end{tabular}

\section{Experimental Values}

The measured solubility was reported to be $33.13 \mathrm{~g} / 100 \mathrm{ml}$ of solution, which corresponds to a molar concentration of $2.398 \mathrm{~mol} \mathrm{dm}^{-3}$.

\section{Auxiliary Information}

\section{Method/Apparatus/Procedure:}

Solubility was determined using a gravimetric method. Excess solute and solvent were placed in a solubility vessel. The apparatus was closed tightly with rubber stoppers and allowed to equilibrate in a constant-temperature water bath with stirring. A known aliquot of the saturated solution was removed, filtered, and transferred to an evaporating dish. The solvent was carefully removed over a steam bath. The evaporating dish was then placed in a drying oven, and later removed and weighed. The solubility was calculated from the mass of the solid residue and volume of saturated sample analyzed.

\section{Source and Purity of Chemicals:}

(1) Purity not given, Schuchardt Chemicals, no purification details were provided.

(2) Purity not given, Chemical source not given, no purification details were provided.

Estimated Error:

Temperature: $\pm 0.2 \mathrm{~K}$ (estimated by compiler).

$c_{1}: \pm 5 \%$ (relative error, estimated by compiler).

\begin{tabular}{|c|c|}
\hline $\begin{array}{l}\text { Components: } \\
\text { (1) 2-Hydroxybenzoic acid; } \mathrm{C}_{7} \mathrm{H}_{6} \mathrm{O}_{3} \text {; } \\
\text { [69-72-7] } \\
\text { (2) Methanol; } \mathrm{CH}_{4} \mathrm{O} ;[67-56-1]\end{array}$ & $\begin{array}{l}\text { Original Measurements: } \\
{ }^{150} \text { A. N. Paruta, B. J. Sciarrone, and } \\
\text { N. G. Lordi, J. Pharm. Sci. 53, } 1349 \\
(1964) \text {. }\end{array}$ \\
\hline $\begin{array}{l}\text { Variables: } \\
T / \mathrm{K}=303.8\end{array}$ & $\begin{array}{l}\text { Prepared by: } \\
\text { W. E. Acree, Jr. }\end{array}$ \\
\hline
\end{tabular}

\section{Experimental Values}

The measured solubility was reported to be $299 \mathrm{mg} / \mathrm{ml}$ of solution, which corresponds to a solubility of $c_{1}=2.16 \mathrm{~mol}$ $\mathrm{dm}^{-3}$. 


\section{Auxiliary Information}

\section{Method/Apparatus/Procedure:}

Excess solute and solvent were placed in 15-ml screw-capped vials fitted with Teflon liners. The vials were attached to a rotating wheel in a constanttemperature water bath and allowed to equilibrate for $24 \mathrm{~h}$. An aliquot of the saturated solution was withdrawn from the vial with a pipet fitted with a glass wool filtering plug. The solubility was determined by titration using a freshly prepared sodium hydroxide solution. The endpoint of the titration was detected with phenolphthalein.

Source and Purity of Chemicals:

(1) Purity not given, Chemical source not given, no purification details were provided.

(2) Purity not given, Chemical source not given, no purification details were provided.

\section{Estimated Error:}

Temperature: $\pm 0.2 \mathrm{~K}$

$c_{1}: \pm 5 \%$ (relative error, estimated by compiler).

\begin{tabular}{|c|c|}
\hline $\begin{array}{l}\text { Components: } \\
\text { (1) 2-Hydroxybenzoic acid; } \mathrm{C}_{7} \mathrm{H}_{6} \mathrm{O}_{3} \text {; } \\
\text { [69-72-7] } \\
\text { (2) Methanol; } \mathrm{CH}_{4} \mathrm{O} ;[67-56-1]\end{array}$ & $\begin{array}{l}\text { Original Measurements: } \\
{ }^{50} \text { P. G. Desai and A. M. Patel, J. } \\
\text { Indian Chem. Soc. 12, } 131 \text { (1935). }\end{array}$ \\
\hline $\begin{array}{l}\text { Variables: } \\
T / \mathrm{K}=301.2\end{array}$ & $\begin{array}{l}\text { Prepared by: } \\
\text { W. E. Acree, Jr. }\end{array}$ \\
\hline
\end{tabular}

Experimental Values

\begin{tabular}{lc}
\hline \hline$x_{2}^{\mathrm{a}}$ & $x_{1}{ }^{\mathrm{b}}$ \\
\hline 0.8748 & 0.1252
\end{tabular}

${ }^{a} x_{2}$ : mole fraction of component 2 in the saturated solution.

${ }^{\mathrm{b}} x_{1}$ : mole fraction solubility of the solute.

\section{Auxiliary Information}

\section{Method/Apparatus/Procedure:}

Excess solute and solvent were placed in a glass flask which was kept revolving on a wheel in an air thermostat for two days. After two days of equilibration, the solution was filtered. To minimize absorption of the solute onto the filter paper, a $100 \mathrm{ml}$ portion of fresh saturated solution was first filtered through the paper before filtering the sample to be analyzed. An aliquot of the filtrate was titrated with barium hydroxide using phenolphthalein as the endpoint indictor. The barium hydroxide titrant was previously standardized against a solution of succinic acid.

Source and Purity of Chemicals:

(1) Purity not given, Chemical source not specified, no information provided concerning purification.

(2) Purity not given, Chemical source not specified, no information provided concerning purification.

\section{Estimated Error:}

Temperature: No information given.

$x_{1}: \pm 3 \%$ (relative error, estimated by compiler).

\begin{tabular}{ll}
\hline \hline Components: & Original Measurements: \\
(1) 2-Hydroxybenzoic acid; $\mathrm{C}_{7} \mathrm{H}_{6} \mathrm{O}_{3} ;$ & $\begin{array}{l}{ }^{152} \mathrm{H} \text {. Matsuda, K. Kaburagi, S. } \\
\text { [69-72-7] }\end{array}$ \\
Matsumoto, K. Kurihara, K. \\
(2) Ethanol; $\mathrm{C}_{2} \mathrm{H}_{6} \mathrm{O} ;[64-17-5]$ & Tochigi, and K. Tomono, J. Chem. \\
\hline Variables: & Eng. Data 54, 480 (2009). \\
$T / \mathrm{K}=298.15$ & Prepared by: \\
\hline
\end{tabular}

Experimental Values

\begin{tabular}{|c|c|}
\hline$x_{2}{ }^{\mathrm{a}}$ & $x_{1}{ }^{\mathrm{b}}$ \\
\hline 0.8550 & 0.1450 \\
\hline
\end{tabular}

\section{Auxiliary Information}

\section{Method/Apparatus/Procedure:}

Thermostated constant-temperature water bath and high-performance liquid chromatograph with uv detector.

Excess solute and solvent were allowed to equilibrate for $24 \mathrm{~h}$ in a constanttemperature thermostated water bath. Aliquots of saturated solutions were removed and filtered through a membrane filter of $0.45 \mu \mathrm{m}$ pore size (Millipore, USA). Concentrations were determined by high-performance liquid chromatography equipped with an uv detector (254 nm detection). Benzene was added to the sample as an internal standard.

Source and Purity of Chemicals:

(1) $99.5 \%$, Wako Pure Chemical Industries, Ltd., Japan, was used without further purification.

(2) $99.5 \%$, Wako Pure Chemical Industries, Ltd., Japan, was used without further purification.

\section{Estimated Error:}

Temperature: $\pm 0.05 \mathrm{~K}$.

$x_{1}: \pm 6.2 \%$ (relative error).

\begin{tabular}{|c|c|}
\hline $\begin{array}{l}\text { Components: } \\
\text { (1) 2-Hydroxybenzoic acid; } \mathrm{C}_{7} \mathrm{H}_{6} \mathrm{O}_{3} \text {; } \\
\text { [69-72-7] } \\
\text { (2) Ethanol; } \mathrm{C}_{2} \mathrm{H}_{6} \mathrm{O} ;[64-17-5]\end{array}$ & $\begin{array}{l}\text { Original Measurements: } \\
{ }^{153} \text { A. Shalmashi and A. Eliassi, J. } \\
\text { Chem. Eng. Data 53, } 199 \text { (2008). }\end{array}$ \\
\hline $\begin{array}{l}\text { Variables: } \\
\text { Temperature }\end{array}$ & $\begin{array}{l}\text { Prepared by: } \\
\text { W. E. Acree, Jr. }\end{array}$ \\
\hline
\end{tabular}

t:


Experimental Values

\begin{tabular}{|c|c|c|}
\hline$T / \mathrm{K}$ & $x_{2}{ }^{\mathrm{a}}$ & $x_{1}^{\mathrm{b}}$ \\
\hline 298 & 0.8614 & 0.1386 \\
\hline 303 & 0.8438 & 0.1562 \\
\hline 308 & 0.8272 & 0.1728 \\
\hline 313 & 0.8146 & 0.1854 \\
\hline 318 & 0.8007 & 0.1993 \\
\hline 323 & 0.7717 & 0.2283 \\
\hline 328 & 0.7661 & 0.2339 \\
\hline 333 & 0.7502 & 0.2498 \\
\hline 338 & 0.7291 & 0.2709 \\
\hline 343 & 0.7143 & 0.2857 \\
\hline 348 & 0.6936 & 0.3064 \\
\hline
\end{tabular}

\section{Auxiliary Information}

\section{Method/Apparatus/Procedure:}

Magnetically stirred jacketed equilibrium cell, constant-temperature circulating water bath, and an analytical balance.

Excess solute and solvent were placed in a magnetically stirred jacketed equilibrium cell and allowed to equilibrate at constant temperature for $2 \mathrm{~h}$ with continuous stirring. After $2 \mathrm{~h}$ the stirring was discontinued and the suspended solid was allowed to settle to the lower portion of the equilibrium cell. An aliquot of the saturated solution was withdrawn by a warmed pipet and transferred to a weighed vial. The vial was tightly closed and weighed to determine the mass of the sample analyzed. The vial was then placed in an oven and the solvent was allowed to evaporate. The vial was reweighed, and the solubility calculated from the mass of the solid residue and sample analyzed.

\section{Source and Purity of Chemicals:}

(1) Purity not given, Synthesized in the authors' department, dried under vacuum at $323 \mathrm{~K}$ for $8 \mathrm{~h}$ and then stored in a desiccator.

(2) Analytical grade, Merck Chemicals, Darmstadt, Germany, used as received.

\section{Estimated Error:}

Temperature: $\pm 0.05 \mathrm{~K}$.

$x_{1}: \pm 3 \%$ (relative error, estimated by compiler).

\begin{tabular}{|c|c|}
\hline $\begin{array}{l}\text { Components: } \\
\text { (1) 2-Hydroxybenzoic acid; } \mathrm{C}_{7} \mathrm{H}_{6} \mathrm{O}_{3} \text {; } \\
\text { [69-72-7] } \\
\text { (2) Ethanol; } \mathrm{C}_{2} \mathrm{H}_{6} \mathrm{O} ;[64-17-5]\end{array}$ & $\begin{array}{l}\text { Original Measurements: } \\
{ }^{159} \text { J. Lim, S. Jang, H. K. Cho, M. S. } \\
\text { Shin, and H. Kim, J. Chem. } \\
\text { Thermodyn. 57, } 295 \text { (2013). }\end{array}$ \\
\hline $\begin{array}{l}\text { Variables: } \\
\text { Temperature }\end{array}$ & $\begin{array}{l}\text { Prepared by: } \\
\text { W. E. Acree, Jr. }\end{array}$ \\
\hline
\end{tabular}

Experimental Values

\begin{tabular}{lcc}
\hline \hline$T / \mathrm{K}$ & $x_{2}{ }^{\mathrm{a}}$ & $x_{1}{ }^{\mathrm{b}}$ \\
\hline 278.15 & 0.8916 & 0.1084 \\
283.15 & 0.8831 & 0.1169 \\
288.15 & 0.8745 & 0.1255 \\
293.15 & 0.8642 & 0.1358 \\
298.15 & 0.8545 & 0.1455 \\
303.15 & 0.8434 & 0.1566 \\
308.15 & 0.8303 & 0.1697
\end{tabular}

\begin{tabular}{lcc}
\hline \hline$T / \mathrm{K}$ & $x_{2}{ }^{\mathrm{a}}$ & $x_{1}{ }^{\mathrm{b}}$ \\
\hline 313.15 & 0.8179 & 0.1821 \\
318.15 & 0.8031 & 0.1969 \\
\hline
\end{tabular}

${ }^{\mathrm{a}} x_{2}$ : mole fraction of component 2 in the saturated solution.

${ }^{b} x_{1}$ : mole fraction solubility of the solute. The solubility data were reported as mass fractions. The mole fraction values were calculated by the compiler.

\section{Auxiliary Information}

\section{Method/Apparatus/Procedure:}

Magnetic stirrer, drying oven and an analytical balance.

Excess solute and solvent were placed in 50-ml glass tubes and agitated with a magnetic stirrer at constant temperature for at least $48 \mathrm{~h}$. A clear aliquot of the saturated solution was withdrawn using a syringe through a filter. The mass of the filtered clear solution was recorded. The sample was dried in a vacuum oven for at least seven days, and the mass of the solid residue recorded. The solution was calculated from the mass of the solid residue and mass of sample analyzed.

Source and Purity of Chemicals:

(1) $99 \%$, Samchen Pure Chemical Company, Seoul Korea, was used as received.

(2) $99.9 \%$, Sigma Aldrich, St. Louis, MO, USA, used as received.

\section{Estimated Error:}

Temperature: $\pm 0.05 \mathrm{~K}$

$x_{1}: \pm 1 \%$ (relative error)

\begin{tabular}{ll}
\hline \hline Components: & Original Measurements: \\
(1) 2-Hydroxybenzoic acid; $\mathrm{C}_{7} \mathrm{H}_{6} \mathrm{O}_{3} ;$ & ${ }^{160}$ A. Jouyban, V. Panahi-Azar, and \\
[69-72-7] & F. Khonsari, J. Mol. Liq. 160, 14 \\
(2) Ethanol; $\mathrm{C}_{2} \mathrm{H}_{6} \mathrm{O} ;[64-17-5]$ & $(2011)$. \\
\hline Variables: & Prepared by: \\
Temperature & W. E. Acree, Jr. \\
\hline
\end{tabular}

Experimental Values

\begin{tabular}{lc}
\hline \hline$T / \mathrm{K}$ & $c_{1}{ }^{\mathrm{a}}$ \\
\hline 298.2 & 1.975 \\
308.2 & 2.500 \\
318.2 & 3.542 \\
328.2 & 4.148 \\
\hline${ }^{\mathrm{a}} c_{1}:$ molar solubility of the solute in units of moles per liter.
\end{tabular}

Auxiliary Information

\section{Method/Apparatus/Procedure:}

Constant-temperature incubator, temperature controlling system, and ultraviolet/visible spectrophotometer.

Excess solute and solvent were placed in sealed bottles and allowed to equilibrate at constant temperature in an incubator equipped with a temperature controlling system for at least two days. Solutions of the saturated solution were withdrawn and filtered using hydrophilic Durapore filters $(0.45 \mu \mathrm{m})$. The filtered solutions were quantitatively diluted. Absorbances of the diluted solutions were measured. Concentrations of the diluted solutions were computed using a Beer-Law calibration curve based on measured absorbances for standard solutions of known concentration.

\section{Source and Purity of Chemicals:}

(1) $98+\%$, Merck Chemical Company, Germany, was used as received.

(2) $99.5 \%$, Merck Chemical Company, no purification details were provided. 
Estimated Error:

Temperature: $\pm 0.2 \mathrm{~K}$.

$c_{1}: \pm 3 \%$ (relative error).

\begin{tabular}{|c|c|}
\hline $\begin{array}{l}\text { Components: } \\
\text { (1) 2-Hydroxybenzoic acid; } \mathrm{C}_{7} \mathrm{H}_{6} \mathrm{O}_{3} \text {; } \\
\text { [69-72-7] } \\
\text { (2) Ethanol; } \mathrm{C}_{2} \mathrm{H}_{6} \mathrm{O} ;[64-17-5]\end{array}$ & $\begin{array}{l}\text { Original Measurements: } \\
{ }^{127} \text { N. V. Sidgwick and E. K. } \\
\text { Ewbank, J. Chem. Soc. Trans. 119 } \\
979 \text { (1921). }\end{array}$ \\
\hline Variables: & Prepared by: \\
\hline Temperature & W. E. Acree, Jr. \\
\hline
\end{tabular}

Experimental Values

\begin{tabular}{lcc}
\hline \hline$T / \mathrm{K}$ & $x_{2}{ }^{\mathrm{a}}$ & $x_{1}{ }^{\mathrm{b}}$ \\
\hline 314.2 & 0.814 & 0.186 \\
358.4 & 0.663 & 0.337 \\
398.4 & 0.410 & 0.590
\end{tabular}

${ }^{\mathrm{a}} x_{2}$ : mole fraction of component 2 in the saturated solution.

${ }^{b} x_{1}$ : mole fraction solubility of the solute. Solubility data reported in units of mass percent. Mole fraction values calculated by the compiler.

\section{Auxiliary Information}

Method/Apparatus/Procedure:

Preweighed quantities of solute and solvent were placed in bulbs, which were then sealed and heated in a suitable bath. At the higher temperatures, the concentration of solvent in the liquid solution was corrected for the amount of solvent vapor in the bulb. The correction assumed that the vapor pressure of the saturated solution was one half that of the pure solvent at the solution temperature. The temperature at which the solid completely dissolved was recorded, and was taken to be the solid-liquid equilibrium temperature.

\section{Source and Purity of Chemicals:}

(1) Purity not given, prepared and purified by the authors using a published synthetic procedure [see J. Walker and J. K. Wood, J. Chem. Soc. 117, 40 (1920) for synthetic details]. Melting point of the purified solute was $432.2 \mathrm{~K}$. (2) $99 \%$, Chemical source not given, distilled over calcium oxide shortly before use.

Estimated Error:

Temperature: Not given in paper.

$x_{1}: \pm 10 \%$ (relative error, estimated by compiler).

\begin{tabular}{|c|c|}
\hline $\begin{array}{l}\text { Components: } \\
\text { (1) 2-Hydroxybenzoic acid; } \mathrm{C}_{7} \mathrm{H}_{6} \mathrm{O}_{3} \text {; } \\
\text { [69-72-7] } \\
\text { (2) Ethanol; } \mathrm{C}_{2} \mathrm{H}_{6} \mathrm{O} ;[64-17-5]\end{array}$ & $\begin{array}{l}\text { Original Measurements: } \\
{ }^{161} \text { E. A. Gomaa, M. A. Mousa, and } \\
\text { A. A. El-Khouly, Thermochim. } \\
\text { Acta 86, } 351 \text { (1985). }\end{array}$ \\
\hline $\begin{array}{l}\text { Variables: } \\
T / \mathrm{K}=298.15\end{array}$ & $\begin{array}{l}\text { Prepared by: } \\
\text { W. E. Acree, Jr. }\end{array}$ \\
\hline
\end{tabular}

\section{Experimental Values}

The authors' description regarding the experimental value is not clear. In the written text, the authors state that the concentration is in terms of the molal scale; however, in the footnote to Table 1 the unit is given as molal concentration/ liter. The compiler has assumed that the numerical value is given in terms of molality, in which case the solubility is $2.874 \mathrm{~mol} / \mathrm{kg}$ of solvent.

\section{Auxiliary Information}

\section{Method/Apparatus/Procedure:}

Experimental details are not definitive. Saturated solution was prepared by placing excess solute and solvent in test tubes. The sealed test tubes were allowed to equilibrate in a constant-temperature thermostated water bath for one week with shaking, followed by another day without shaking. Solubility was determined either gravimetrically or volumetrically. In the case of the gravimetric method, a $1 \mathrm{ml}$ aliquot of saturated solution was evaporated to dryness in a small aluminum disk heated by an infrared lamp. In the volumetric method, 3-5 $\mathrm{ml}$ of the saturated solution was titrated with a standardized sodium hydroxide solution. The authors studied the solubility of three carboxylic acid solutes in seven different organic solvents, and did not specify which method was used for each solute-solvent pair.

\section{Source and Purity of Chemicals:}

(1) Analytical grade, Merck Chemical Company, Germany, no purification details were provided.

(2) Spectroscopic grade, Merck Chemical Company, no purification details were provided.

\section{Estimated Error:}

Temperature: $\pm 0.1 \mathrm{~K}$ (estimated by compiler). $c_{1}: \pm 2 \%$ (relative error, estimated by compiler).

\begin{tabular}{|c|c|}
\hline $\begin{array}{l}\text { Components: } \\
\text { (1) 2-Hydroxybenzoic acid; } \mathrm{C}_{7} \mathrm{H}_{6} \mathrm{O}_{3} \text {; } \\
\text { [69-72-7] } \\
\text { (2) Ethanol; } \mathrm{C}_{2} \mathrm{H}_{6} \mathrm{O} ;[64-17-5]\end{array}$ & $\begin{array}{l}\text { Original Measurements: } \\
\text { }{ }^{158} \text { E. Bergroth, Farm. Aikak. 70, } 91 \\
(1961) .\end{array}$ \\
\hline $\begin{array}{l}\text { Variables: } \\
T / \mathrm{K}=293.15\end{array}$ & $\begin{array}{l}\text { Prepared by: } \\
\text { W. E. Acree, Jr. }\end{array}$ \\
\hline
\end{tabular}

\section{Experimental Values}

The measured solubility was reported to be $28.19 \mathrm{~g} / 100 \mathrm{ml}$ of solution, which corresponds to a molar concentration of $2.041 \mathrm{~mol} \mathrm{dm}^{-3}$.

\section{Auxiliary Information}

\section{Method/Apparatus/Procedure:}

Solubility was determined using a gravimetric method. Excess solute and solvent were placed in a solubility vessel. The apparatus was closed tightly with rubber stoppers and allowed to equilibrate in a constant-temperature water bath with stirring. A known aliquot of the saturated solution was removed, filtered, and transferred to an evaporating dish. The solvent was carefully removed over a steam bath. The evaporating dish was then placed in a drying oven, and later removed and weighed. The solubility was calculated from the mass of the solid residue and volume of saturated sample analyzed.

\section{Source and Purity of Chemicals:}

(1) Purity not given, Schuchardt Chemicals, no purification details were provided.

(2) Purity not given, Chemical source not given, no purification details were provided.

\section{Estimated Error:}

Temperature: $\pm 0.2 \mathrm{~K}$ (estimated by compiler). $c_{1}: \pm 5 \%$ (relative error, estimated by compiler). 


\section{Components:}

(1) 2-Hydroxybenzoic acid; $\mathrm{C}_{7} \mathrm{H}_{6} \mathrm{O}_{3}$;

[69-72-7]

(2) Ethanol; $\mathrm{C}_{2} \mathrm{H}_{6} \mathrm{O}$; [64-17-5]

\section{Variables:}

$T / \mathrm{K}=298.15$

W. E. Acree, Jr.

\section{Experimental Values}

\begin{tabular}{lc}
\hline \hline$x_{2}{ }^{\mathrm{a}}$ & $x_{1}{ }^{\mathrm{b}}$ \\
\hline 0.8900 & 0.1100
\end{tabular}

${ }^{\mathrm{a}} x_{2}$ : mole fraction of component 2 in the saturated solution.

${ }^{\mathrm{b}} x_{1}$ : mole fraction solubility of the solute.

\section{Auxiliary Information}

\section{Method/Apparatus/Procedure:}

Constant-temperature bath, analytical balance, and an ultraviolet/visible spectrophotometer.

Excess solute and solvent were placed in a flask and allowed to equilibrate for several days at constant temperature. The solid phase was removed by filtration, and the clear solution was diluted quantitatively with $96 \%$ ethanol. The concentration of the diluted solution was determined by spectrophotometric measurement at the wavelength of maximum absorption. In instances where the solvent interfered with the spectrophotometric determination, the samples were evaporated to dryness and the residue diluted with $96 \%$ ethanol.

\section{Source and Purity of Chemicals:}

(1) Purity not given, Sigma Chemical Company, USA, no purification details were provided.

(2) Spectrophotometric or Analytical grade, Chemical source not specified, no purification details were provided.

\section{Estimated Error:}

Temperature: $\pm 0.2 \mathrm{~K}$.

$x_{1}: \pm 3.0 \%$ (relative error, estimated by compiler).

\begin{tabular}{|c|c|}
\hline $\begin{array}{l}\text { Components: } \\
\text { (1) 2-Hydroxybenzoic acid; } \mathrm{C}_{7} \mathrm{H}_{6} \mathrm{O}_{3} \text {; } \\
\text { [69-72-7] } \\
\text { (2) Ethanol; } \mathrm{C}_{2} \mathrm{H}_{6} \mathrm{O} ;[64-17-5]\end{array}$ & $\begin{array}{l}\text { Original Measurements: } \\
{ }^{155} \text { M. A. Peña, A. Reíllo, B. } \\
\text { Escalera, and P. Bustamante, Int. J. } \\
\text { Pharm. 321, } 155 \text { (2006). }\end{array}$ \\
\hline $\begin{array}{l}\text { Variables: } \\
T / \mathrm{K}=298.15\end{array}$ & $\begin{array}{l}\text { Prepared by: } \\
\text { W. E. Acree, Jr. }\end{array}$ \\
\hline
\end{tabular}

Experimental Values

\begin{tabular}{lc}
\hline \hline$x_{2}{ }^{\mathrm{a}}$ & $x_{1}{ }^{\mathrm{b}}$ \\
\hline 0.8603 & 0.1397 \\
\hline
\end{tabular}

${ }^{\mathrm{a}} x_{2}$ : mole fraction of component 2 in the saturated solution.

${ }^{\mathrm{b}} x_{1}$ : mole fraction solubility of the solute.

\section{Auxiliary Information}

\section{Method/Apparatus/Procedure:}

Constant temperature-controlled shaking bath and an uv/visible spectrophotometer.

Binary solvent mixtures were prepared by volume. Excess solute and solvent were allowed to equilibrate in a constant temperature-controlled shaking bath for at least five days. Aliquots of the saturated solution were removed and filtered (Durapore membrane filter, $0.2 \mu \mathrm{m}$ pore size). The filtered aliquot was then diluted with ethanol $(96 \% \mathrm{v} / \mathrm{v})$, and the concentration of the dissolved solute determined by spectrophotometric analysis at a wavelength of $302 \mathrm{~nm}$. The authors converted the molar solubilities to mole fractions using the measured densities of the saturated solutions.

\section{Source and Purity of Chemicals:}

(1) Purity not given, Sigma-Aldrich, Germany, no purification details were provided.

(2) Spectrophotometric grade, Panreac, Monplet and Esteban, Spain, no purification details were provided.

\section{Estimated Error:}

Temperature: $\pm 0.1 \mathrm{~K}$.

$x_{1}: \pm 2.0 \%$ (relative error, estimated by compiler).

\begin{tabular}{ll}
\hline \hline Components: & Original Measurements: \\
(1) 2-Hydroxybenzoic acid; $\mathrm{C}_{7} \mathrm{H}_{6} \mathrm{O}_{3} ;$ & $\begin{array}{l}{ }^{154} \text { M. A. Peña, B. Escalera, A. } \\
\text { [69-72-7] }\end{array}$ \\
$\begin{array}{l}\text { Reíllo, A. B. Sánchez, and P. } \\
\text { (2) Ethanol; } \mathrm{C}_{2} \mathrm{H}_{6} \mathrm{O} ;[64-17-5]\end{array}$ & $\begin{array}{l}\text { Bustamante, J. Pharm. Sci. 98, 1129 } \\
(2009) .\end{array}$ \\
\hline Variables: & Prepared by: \\
Temperature & W. E. Acree, Jr. \\
\hline
\end{tabular}

Experimental Values

\begin{tabular}{lcc}
\hline \hline$T / \mathrm{K}$ & $x_{2}{ }^{\mathrm{a}}$ & $x_{1}{ }^{\mathrm{b}}$ \\
\hline 293 & 0.8652 & 0.1348 \\
303 & 0.8595 & 0.1405 \\
308 & 0.8515 & 0.1485 \\
313 & 0.8395 & 0.1605 \\
\hline
\end{tabular}

${ }^{a} x_{2}$ : mole fraction of component 2 in the saturated solution.

${ }^{b} x_{1}$ : mole fraction solubility of the solute.

\section{Auxiliary Information}

\section{Method/Apparatus/Procedure:}

Constant temperature-controlled shaking bath and an uv/visible spectrophotometer.

Excess solute and solvent were allowed to equilibrate in a constant temperature-controlled shaking bath for four days at $313 \mathrm{~K}$, and then one day at the lower temperatures. The authors used the same samples for all temperatures. The authors started with $313 \mathrm{~K}$, and once the solution was saturated at the higher temperature they lowered the temperature for the next set of measurements. Aliquots of the saturated solution were removed and filtered (Durapore membrane filter, $0.2 \mu \mathrm{m}$ pore size). The filtered aliquot was then diluted with ethanol $(96 \% \mathrm{v} / \mathrm{v})$, and the concentration of the dissolved solute determined by spectrophotometric analysis at a wavelength of $302 \mathrm{~nm}$. The authors converted the molar solubilities to mole fractions using the measured densities of the saturated solutions.

\section{Source and Purity of Chemicals:}

(1) Purity not given, Sigma-Aldrich, Munich, Germany, was used as received. (2) UV grade, Panreac, Monplet and Esteban, Barcelona, Spain, no purification details were provided. 
Estimated Error:

Temperature: \pm 0.1 .

$x_{1}: \pm 3 \%$ (relative error).

\begin{tabular}{|c|c|}
\hline $\begin{array}{l}\text { Components: } \\
\text { (1) 2-Hydroxybenzoic acid; } \mathrm{C}_{7} \mathrm{H}_{6} \mathrm{O}_{3} \text {; } \\
\text { [69-72-7] } \\
\text { (2) Ethanol; } \mathrm{C}_{2} \mathrm{H}_{6} \mathrm{O} ;[64-17-5]\end{array}$ & $\begin{array}{l}\text { Original Measurements: } \\
{ }^{71} \text { A. Seidell, Trans. Am. } \\
\text { Electrochem. Soc. 13, } 319 \text { (1908). }\end{array}$ \\
\hline $\begin{array}{l}\text { Variables: } \\
T / \mathrm{K}=298.15\end{array}$ & $\begin{array}{l}\text { Prepared by: } \\
\text { W. E. Acree, Jr. }\end{array}$ \\
\hline
\end{tabular}

Experimental Values

\begin{tabular}{lc}
\hline \hline$x_{2}{ }^{\mathrm{a}}$ & $x_{1}{ }^{\mathrm{b}}$ \\
\hline 0.8631 & 0.1369 \\
\hline${ }^{\mathrm{a}} x_{2}:$ mole fraction of component 2 in the saturated solution. & \\
${ }^{\mathrm{b}} x_{1}:$ mole fraction solubility of the solute. &
\end{tabular}

\section{Auxiliary Information}

\section{Method/Apparatus/Procedure:}

Excess solute and solvent were placed in a test tube and sealed with a rubber stopper. The test tube was attached to a rotating frame immersed in a constanttemperature water bath and revolved upon the axis parallel to the diameter of the test tube at the rate of approximately six revolutions per minute. The duration of the rotation was between four and seven days. At the end of the rotation period, the test tube was placed in an upright position in the bath with the stopper and a very small part of the test tube protruding from the bath, until the undissolved solid settled to the bottom of the test tube. A portion of the clear supernatant solution was withdrawn into a pipette having a cotton plug attached. The cotton plug served as a coarse filter. The concentration of the solution was determined by titration with standard alkali solution. The author determined the solubility of 2-hydroxybenzoic acid in several aqueous-alcohol solvent mixtures having ethanol concentrations between $0.00 \%$ and $99.8 \%$ by mass. The value for $100 \%$ ethanol was obtained from the curve of solubility versus alcohol concentration.

Source and Purity of Chemicals:

(1) Purity not given, Chemical source not specified, no information provided concerning purification.

(2) Purity not given, Chemical source not specified, no information provided concerning purification.

\section{Estimated Error:}

Temperature: $\pm 0.2 \mathrm{~K}$ (estimated by compiler). $x_{1}: \pm 3 \%$ (relative error, estimated by compiler).

\begin{tabular}{|c|c|}
\hline $\begin{array}{l}\text { Components: } \\
\text { (1) 2-Hydroxybenzoic acid; } \mathrm{C}_{7} \mathrm{H}_{6} \mathrm{O}_{3} \text {; } \\
\text { [69-72-7] } \\
\text { (2) Ethanol; } \mathrm{C}_{2} \mathrm{H}_{6} \mathrm{O} ;[64-17-5]\end{array}$ & $\begin{array}{l}\text { Original Measurements: } \\
{ }^{73} \text { M. Dias, S. L. Raghavan, and J. } \\
\text { Hadgraft, Int. J. Pharm. 216, } 51 \\
\text { (2001). }\end{array}$ \\
\hline $\begin{array}{l}\text { Variables: } \\
T / \mathrm{K}=305.2\end{array}$ & $\begin{array}{l}\text { Prepared by: } \\
\text { W. E. Acree, Jr. }\end{array}$ \\
\hline
\end{tabular}

\section{Experimental Values}

The measured solubility was reported to be $368.2 \mathrm{mg} / \mathrm{ml}$, which corresponds to a molar solubility of $c_{1}=2.666 \mathrm{~mol}$ $\mathrm{dm}^{-3}$.

\section{Auxiliary Information}

Method/Apparatus/Procedure:

Constant-temperature bath, magnetic stirrer, centrifuge, and an ultraviolet/ visible spectrophotometer.

Excess solute and solvent were equilibrated in a constant-temperature bath with stirring for $48 \mathrm{~h}$. The saturated solution was then centrifuged for $10 \mathrm{~min}$, and an aliquot of the supernatant solution was removed and diluted quantitatively for spectrophotometric analysis. The concentration of the dissolved solute was determined from the measured absorbance.

Source and Purity of Chemicals:

(1) Purity not given, Fisher Scientific, UK, no purification details were provided.

(2) Purity not given, Fisher Scientific, no purification details were provided.

\section{Estimated Error:}

Temperature: Insufficient experimental details to estimate. $c_{1}: \pm 0.033$.

\begin{tabular}{|c|c|}
\hline Components: & Original Measurements: \\
\hline $\begin{array}{l}\text { (1) 2-Hydroxybenzoic acid; } \mathrm{C}_{7} \mathrm{H}_{6} \mathrm{O}_{3} \text {; } \\
\text { [69-72-7] }\end{array}$ & $\begin{array}{l}{ }^{150} \text { A. N. Paruta, B. J. Sciarrone, and } \\
\text { N. G. Lordi, J. Pharm. Sci. 53, } 1349\end{array}$ \\
\hline (2) Ethanol; $\mathrm{C}_{2} \mathrm{H}_{6} \mathrm{O} ;[64-17-5]$ & $(1964)$ \\
\hline Variables: & Prepared by: \\
\hline$T / \mathrm{K}=303.8$ & W. E. Acree, Jr. \\
\hline
\end{tabular}

\section{Experimental Values}

The measured solubility was reported to be $377 \mathrm{mg} / \mathrm{ml}$ of solution, which corresponds to a solubility of $c_{1}=2.73 \mathrm{~mol}$ $\mathrm{dm}^{-3}$.

\section{Auxiliary Information}

\section{Method/Apparatus/Procedure:}

Excess solute and solvent were placed in 15-ml screw-capped vials fitted with Teflon liners. The vials were attached to a rotating wheel in a constanttemperature water bath and allowed to equilibrate for $24 \mathrm{~h}$. An aliquot of the saturated solution was withdrawn from the vial with a pipet fitted with a glass wool filtering plug. The solubility was determined by titration using a freshly prepared sodium hydroxide solution. The endpoint of the titration was detected with phenolphthalein.

\section{Source and Purity of Chemicals:}

(1) Purity not given, Chemical source not given, no purification details were provided.

(2) Purity not given, Chemical source not given, no purification details were provided.

\section{Estimated Error:}

Temperature: $\pm 0.2 \mathrm{~K}$.

$c_{1}: \pm 5 \%$ (relative error, estimated by compiler).

\begin{tabular}{|c|c|}
\hline $\begin{array}{l}\text { Components: } \\
\text { (1) 2-Hydroxybenzoic acid; } \mathrm{C}_{7} \mathrm{H}_{6} \mathrm{O}_{3} \text {; } \\
\text { [69-72-7] } \\
\text { (2) Ethanol; } \mathrm{C}_{2} \mathrm{H}_{6} \mathrm{O} ;[64-17-5]\end{array}$ & $\begin{array}{l}\text { Original Measurements: } \\
\text { }{ }^{50} \text { P. G. Desai and A. M. Patel, J. } \\
\text { Indian Chem. Soc. 12, } 131 \text { (1935). }\end{array}$ \\
\hline $\begin{array}{l}\text { Variables: } \\
T / \mathrm{K}=301.2\end{array}$ & $\begin{array}{l}\text { Prepared by: } \\
\text { W. E. Acree, Jr. }\end{array}$ \\
\hline
\end{tabular}


Experimental Values

\begin{tabular}{lc}
\hline \hline$x_{2}{ }^{\mathrm{a}}$ & $x_{1}{ }^{\mathrm{b}}$ \\
\hline 0.8521 & 0.1479
\end{tabular}

${ }^{\mathrm{a}} x_{2}$ : mole fraction of component 2 in the saturated solution.

${ }^{\mathrm{b}} \mathrm{x}_{1}$ : mole fraction solubility of the solute.

\section{Auxiliary Information}

\section{Method/Apparatus/Procedure:}

Excess solute and solvent were placed in a glass flask which was kept revolving on a wheel in an air thermostat for two days. After two days of equilibration, the solution was filtered. To minimize absorption of the solute onto the filter paper, a $100 \mathrm{ml}$ portion of fresh saturated solution was first filtered through the paper before filtering the sample to be analyzed. An aliquot of the filtrate was titrated with barium hydroxide using phenolphthalein as the endpoint indictor. The barium hydroxide titrant was previously standardized against a solution of succinic acid.

\section{Source and Purity of Chemicals:}

(1) Purity not given, Chemical source not specified, no information provided concerning purification.

(2) Purity not given, Chemical source not specified, no information provided concerning purification.

\section{Estimated Error:}

Temperature: No information given.

$x_{1}: \pm 3 \%$ (relative error, estimated by compiler).

\begin{tabular}{|c|c|}
\hline $\begin{array}{l}\text { Components: } \\
\text { (1) 2-Hydroxybenzoic acid; } \mathrm{C}_{7} \mathrm{H}_{6} \mathrm{O}_{3} \text {; } \\
\text { [69-72-7] } \\
\text { (2) 1-Propanol; } \mathrm{C}_{3} \mathrm{H}_{8} \mathrm{O} ;[71-23-8]\end{array}$ & $\begin{array}{l}\text { Original Measurements: } \\
{ }^{162} \text { M. A. A. Fakhree, S. Ahmadian, } \\
\text { V. Panahi-Azar, W. E. Acree, Jr., } \\
\text { and A. Jouyban, J. Chem. Eng. Data } \\
\text { 57, } 3303 \text { (2012). }\end{array}$ \\
\hline $\begin{array}{l}\text { Variables: } \\
\text { Temperature }\end{array}$ & $\begin{array}{l}\text { Prepared by: } \\
\text { W. E. Acree, Jr. }\end{array}$ \\
\hline
\end{tabular}

Experimental Values

\begin{tabular}{lcc}
\hline \hline$T / \mathrm{K}$ & $x_{2}{ }^{\mathrm{a}}$ & $x_{1}{ }^{\mathrm{b}}$ \\
\hline 298.2 & 0.868 & 0.132 \\
308.2 & 0.850 & 0.150 \\
318.2 & 0.837 & 0.163 \\
328.2 & 0.821 & 0.179 \\
338.2 & 0.805 & 0.195 \\
\hline
\end{tabular}

${ }^{\mathrm{a}} x_{2}$ : mole fraction of component 2 in the saturated solution.

${ }^{b} x_{1}$ : mole fraction solubility of the solute.

\section{Auxiliary Information}

\footnotetext{
Method/Apparatus/Procedure:

Constant temperature oven, temperature controlling system, and ultraviolet/ visible spectrophotometer.

Excess solute and solvent were placed in sealed bottles and allowed to equilibrate at constant temperature in an oven for three days. Samples were shaken manually three times per day to ensure that equilibrium had been achieved. Solutions of the saturated solution were withdrawn and filtered using hydrophilic Durapore filters $(0.45 \mu \mathrm{m})$. The filtered solutions were quantitatively diluted with ethanol. Absorbances of the diluted solutions were measured at $304 \mathrm{~nm}$. Concentrations of the diluted solutions were computed using a Beer-Law calibration curve based on measured absorbances for standard solutions of known concentration.
}

Source and Purity of Chemicals:

(1) $99 \%$, Merck Chemical Company, Germany, was used as received.

(2) $99.9 \%$, Scharlau Chemie, Spain, was used as received.

\section{Estimated Error:}

Temperature: $\pm 0.2 \mathrm{~K}$ to $\pm 0.6 \mathrm{~K}$; the higher temperatures have greater uncertainty.

$x_{1}: \pm 1 \%$ (relative error)

\begin{tabular}{|c|c|}
\hline $\begin{array}{l}\text { Components: } \\
\text { (1) 2-Hydroxybenzoic acid; } \mathrm{C}_{7} \mathrm{H}_{6} \mathrm{O}_{3} \text {; } \\
\text { [69-72-7] } \\
\text { (2) 1-Propanol; } \mathrm{C}_{3} \mathrm{H}_{8} \mathrm{O} ;[71-23-8]\end{array}$ & $\begin{array}{l}\text { Original Measurements: } \\
{ }^{151} \text { K. M. De Fina, T. L. Sharp, L. E } \\
\text { Roy, and W. E. Acree, Jr., J. Chem } \\
\text { Eng. Data 44, } 1262 \text { (1999). }\end{array}$ \\
\hline $\begin{array}{l}\text { Variables: } \\
T / \mathrm{K}=298.15\end{array}$ & $\begin{array}{l}\text { Prepared by: } \\
\text { W. E. Acree, Jr. }\end{array}$ \\
\hline
\end{tabular}

\section{Experimental Values}

\begin{tabular}{lc}
\hline \hline$x_{2}{ }^{\mathrm{a}}$ & $x_{1}{ }^{\mathrm{b}}$ \\
\hline 0.8364 & 0.1636 \\
\hline${ }^{\mathrm{a}} x_{2}:$ mole fraction of component 2 in the saturated solution. \\
${ }^{\mathrm{b}}{ }_{x_{1}}$ : mole fraction solubility of the solute.
\end{tabular}

\section{Auxiliary Information}

\section{Method/Apparatus/Procedure:}

Constant-temperature bath, calorimetric thermometer, and an ultraviolet/ visible spectrophotometer.

Excess solute and solvent were placed in amber glass bottles and allowed to equilibrate for several days at constant temperature. Attainment of equilibrium was verified by several repetitive measurements and by approaching equilibrium from supersaturation. Aliquots of saturated solutions were transferred through a coarse filter into tared volumetric flasks, weighed, and diluted with methanol. Concentrations were determined by spectrophotometric measurements at $304 \mathrm{~nm}$.

\section{Source and Purity of Chemicals:}

(1) $99+\%$, ACS Reagent grade, Aldrich Chemical Company, Milwaukee, WI, USA, was dried for several hours at $353 \mathrm{~K}$ before use and used without further purification.

(2) $99+\%$, anhydrous, Aldrich Chemical Company, stored over molecular sieves before use.

\section{Estimated Error:}

Temperature: $\pm 0.1 \mathrm{~K}$.

$x_{1}: \pm 1.5 \%$ (relative error)

\begin{tabular}{|c|c|}
\hline $\begin{array}{l}\text { Components: } \\
\text { (1) 2-Hydroxybenzoic acid; } \mathrm{C}_{7} \mathrm{H}_{6} \mathrm{O}_{3} \text {; } \\
\text { [69-72-7] } \\
\text { (2) 1-Propanol; } \mathrm{C}_{3} \mathrm{H}_{8} \mathrm{O} ;[71-23-8]\end{array}$ & $\begin{array}{l}\text { Original Measurements: } \\
\text { (158 E. Bergroth, Farm. Aikak. 70, } 91 \\
\text { (1961). }\end{array}$ \\
\hline $\begin{array}{l}\text { Variables: } \\
T / \mathrm{K}=293.15\end{array}$ & $\begin{array}{l}\text { Prepared by: } \\
\text { W. E. Acree, Jr. }\end{array}$ \\
\hline
\end{tabular}

\section{Experimental Values}

The measured solubility was reported to be $22.25 \mathrm{~g} / 100 \mathrm{ml}$ of solution, which corresponds to a molar concentration of $1.611 \mathrm{~mol} \mathrm{dm}^{-3}$. 


\section{Auxiliary Information}

\section{Method/Apparatus/Procedure:}

Solubility was determined using a gravimetric method. Excess solute and solvent were placed in a solubility vessel. The apparatus was closed tightly with rubber stoppers and allowed to equilibrate in a constant-temperature water bath with stirring. A known aliquot of the saturated solution was removed, filtered, and transferred to an evaporating dish. The solvent was carefully removed over a steam bath. The evaporating dish was then placed in a drying oven, and later removed and weighed. The solubility was calculated from the mass of the solid residue and volume of saturated sample analyzed.

Source and Purity of Chemicals:

(1) Purity not given, Schuchardt Chemicals, no purification details were provided.

(2) Purity not given, Chemical source not given, no purification details were provided.

Estimated Error:

Temperature: $\pm 0.2 \mathrm{~K}$ (estimated by compiler). $c_{1}: \pm 5 \%$ (relative error, estimated by compiler).

\begin{tabular}{|c|c|}
\hline $\begin{array}{l}\text { Components: } \\
\text { (1) 2-Hydroxybenzoic acid; } \mathrm{C}_{7} \mathrm{H}_{6} \mathrm{O}_{3} \text {; } \\
\text { [69-72-7] } \\
\text { (2) 1-Propanol; } \mathrm{C}_{3} \mathrm{H}_{8} \mathrm{O} ;[71-23-8]\end{array}$ & $\begin{array}{l}\text { Original Measurements: } \\
{ }^{150} \text { A. N. Paruta, B. J. Sciarrone, and } \\
\text { N. G. Lordi, J. Pharm. Sci. 53, } 1349 \\
(1964) \text {. }\end{array}$ \\
\hline $\begin{array}{l}\text { Variables: } \\
T / \mathrm{K}=303.8\end{array}$ & $\begin{array}{l}\text { Prepared by: } \\
\text { W. E. Acree, Jr. }\end{array}$ \\
\hline
\end{tabular}

\section{Experimental Values}

The measured solubility was reported to be $287 \mathrm{mg} / \mathrm{ml}$ of solution, which corresponds to a solubility of $c_{1}=2.08 \mathrm{~mol}$ $\mathrm{dm}^{-3}$.

\section{Auxiliary Information}

\footnotetext{
Method/Apparatus/Procedure:

Excess solute and solvent were placed in 15-ml screw-capped vials fitted with Teflon liners. The vials were attached to a rotating wheel in a constanttemperature water bath and allowed to equilibrate for $24 \mathrm{~h}$. An aliquot of the saturated solution was withdrawn from the vial with a pipet fitted with a glass wool filtering plug. The solubility was determined by titration using a freshly prepared sodium hydroxide solution. The endpoint of the titration was detected with phenolphthalein.
}

Source and Purity of Chemicals:

(1) Purity not given, Chemical source not given, no purification details were provided.

(2) Purity not given, Chemical source not given, no purification details were provided.

Estimated Error:

Temperature: $\pm 0.2 \mathrm{~K}$.

$c_{1}: \pm 5 \%$ (relative error, estimated by compiler).

\begin{tabular}{|c|c|}
\hline $\begin{array}{l}\text { Components: } \\
\text { (1) 2-Hydroxybenzoic acid; } \mathrm{C}_{7} \mathrm{H}_{6} \mathrm{O}_{3} \text {; } \\
\text { [69-72-7] } \\
\text { (2) 1-Propanol; } \mathrm{C}_{3} \mathrm{H}_{8} \mathrm{O} ;[71-23-8]\end{array}$ & $\begin{array}{l}\text { Original Measurements: } \\
{ }^{156} \text { K. B. Sloan, K. G. Siver, and } \\
\text { S. A. M. Koch, J. Pharm. Sci. 75, } \\
744 \text { (1986). }\end{array}$ \\
\hline $\begin{array}{l}\text { Variables: } \\
T / \mathrm{K}=296\end{array}$ & $\begin{array}{l}\text { Prepared by: } \\
\text { W. E. Acree, Jr. }\end{array}$ \\
\hline
\end{tabular}

\section{Experimental Values}

The measured solubility was reported to be $228 \mathrm{mg} / \mathrm{ml}$, which corresponds to a solubility of $c_{1}=1.651 \mathrm{~mol} \mathrm{dm}^{-3}$. The authors calculated a mole fraction solubility of $x_{1}=0.140$. The mole fraction solubility was calculated by the authors assuming a molar volume of 2-hydroxybenzoic acid of $V_{1}=93.9 \mathrm{~cm}^{3} \mathrm{~mol}^{-1}$, which seems too small compared to the molar volume of benzaldehyde, which is estimated to be $V_{1} \approx 101.1 \mathrm{~cm}^{3} \mathrm{~mol}^{-1}$, based on a molar mass of $106.12 \mathrm{~g} \mathrm{~mol}^{-1}$ and an experimental density of $1.0499 \mathrm{~g} \mathrm{~cm}^{-3}$ at $296 \mathrm{~K}$. A molar volume on the order of $V_{1} \approx 104 \mathrm{~cm}^{3} \mathrm{~mol}^{-1}$ would seem more realistic given the molecular structures of 2-hydroxybenzoic acid compared to benzaldehyde.

\section{Auxiliary Information}

\section{Method/Apparatus/Procedure:}

Magnetic stirrer and an ultraviolet/visible spectrophotometer.

Solubility was determined by stirring an excess of solute in the solvent with a magnetic stirrer at ambient room temperature for $24 \mathrm{~h}$ in sealed flasks that were thermally insulated from the stirrer. The suspension was gravity filtered through Whatman \#1 (qualitative) filter paper. The filtrate was then quantitatively diluted with methanol, and the absorbance of the diluted solution recorded at $306 \mathrm{~nm}$.

Source and Purity of Chemicals:

(1) Purity not given, Aldrich Chemical Company, Milwaukee, WI, USA, no purification details were provided.

(2) $99+\%$, Aldrich Chemical Company, used as received.

\section{Estimated Error:}

Temperature: $\pm 1 \mathrm{~K}$.

$x_{1}: \pm 5 \%$ (relative error, estimated by compiler).

\begin{tabular}{|c|c|}
\hline $\begin{array}{l}\text { Components: } \\
\text { (1) 2-Hydroxybenzoic acid; } \mathrm{C}_{7} \mathrm{H}_{6} \mathrm{O}_{3} \text {; } \\
\text { [69-72-7] } \\
\text { (2) 1-Propanol; } \mathrm{C}_{3} \mathrm{H}_{8} \mathrm{O} ;[71-23-8]\end{array}$ & $\begin{array}{l}\text { Original Measurements: } \\
{ }^{50} \text { P. G. Desai and A. M. Patel, J. } \\
\text { Indian Chem. Soc. 12, } 131 \text { (1935). }\end{array}$ \\
\hline $\begin{array}{l}\text { Variables: } \\
T / \mathrm{K}=301.2\end{array}$ & $\begin{array}{l}\text { Prepared by: } \\
\text { W. E. Acree, Jr. }\end{array}$ \\
\hline
\end{tabular}

Experimental Values

\begin{tabular}{lc}
\hline \hline$x_{2}{ }^{\mathrm{a}}$ & $x_{1}{ }^{\mathrm{b}}$ \\
\hline 0.8562 & 0.1438 \\
\hline${ }^{\mathrm{a}} x_{2}:$ mole fraction of component 2 in the saturated solution. \\
${ }^{\mathrm{b}} x_{1}:$ mole fraction solubility of the solute.
\end{tabular}

Auxiliary Information

Method/Apparatus/Procedure:

Excess solute and solvent were placed in a glass flask which was kept revolving on a wheel in an air thermostat for two days. After two days of equilibration, the solution was filtered. To minimize absorption of the solute onto the filter paper, a $100 \mathrm{ml}$ portion of fresh saturated solution was first filtered through the paper before filtering the sample to be analyzed. An aliquot of the filtrate was titrated with barium hydroxide using phenolphthalein as the endpoint indictor. The barium hydroxide titrant was previously standardized against a solution of succinic acid. 
Source and Purity of Chemicals:

(1) Purity not given, Chemical source not specified, no information provided concerning purification.

(2) Purity not given, Chemical source not specified, no information provided concerning purification.

\section{Estimated Error:}

Temperature: No information given.

$x_{1}: \pm 3 \%$ (relative error, estimated by compiler).

\section{Components:}

(1) 2-Hydroxybenzoic acid; $\mathrm{C}_{7} \mathrm{H}_{6} \mathrm{O}_{3}$;

[69-72-7]

(2) 1-Propanol; $\mathrm{C}_{3} \mathrm{H}_{8} \mathrm{O}$; [71-23-8]

\section{Variables:}

Temperature

Original Measurements:

${ }^{159}$ J. Lim, S. Jang, H. K. Cho, M. S.

Shin, and H. Kim, J. Chem.

Thermodyn. 57, 295 (2013).

Prepared by:

W. E. Acree, Jr.

Experimental Values

\begin{tabular}{lcc}
\hline \hline$T / \mathrm{K}$ & $x_{2}{ }^{\mathrm{a}}$ & $x_{1}{ }^{\mathrm{b}}$ \\
\hline 278.15 & 0.8983 & 0.1017 \\
283.15 & 0.8892 & 0.1108 \\
288.15 & 0.8796 & 0.1204 \\
293.15 & 0.8686 & 0.1314 \\
298.15 & 0.8572 & 0.1428 \\
303.15 & 0.8457 & 0.1543 \\
308.15 & 0.8320 & 0.1680 \\
313.15 & 0.8194 & 0.1806 \\
318.15 & 0.8040 & 0.1960 \\
\hline
\end{tabular}

${ }^{\mathrm{a}} x_{2}$ : mole fraction of component 2 in the saturated solution.

${ }^{b} x_{1}$ : mole fraction solubility of the solute. The solubility data were reported as mass fractions. The mole fraction values were calculated by the compiler.

\section{Auxiliary Information}

\section{Method/Apparatus/Procedure:}

Magnetic stirrer, drying oven and an analytical balance.

Excess solute and solvent were placed in 50-ml glass tubes and agitated with a magnetic stirrer at constant temperature for at least $48 \mathrm{~h}$. A clear aliquot of the saturated solution was withdrawn using a syringe through a filter. The mass of the filtered clear solution was recorded. The sample was dried in a vacuum oven for at least seven days, and the mass of the solid residue recorded. The solution was calculated from the mass of the solid residue and mass of sample analyzed.

Source and Purity of Chemicals:

(1) $99 \%$, Samchen Pure Chemical Company, Seoul Korea, was used as received.

(2) $99.9 \%$, Sigma Aldrich, St. Louis, MO, USA, used as received.

Estimated Error:

Temperature: $\pm 0.05 \mathrm{~K}$.

$x_{1}: \pm 1 \%$ (relative error).

\begin{tabular}{|c|c|}
\hline $\begin{array}{l}\text { Components: } \\
\text { (1) 2-Hydroxybenzoic acid; } \mathrm{C}_{7} \mathrm{H}_{6} \mathrm{O}_{3} \text {; } \\
\text { [69-72-7] } \\
\text { (2) 2-Propanol; } \mathrm{C}_{3} \mathrm{H}_{8} \mathrm{O} ;[67-63-0]\end{array}$ & $\begin{array}{l}\text { Original Measurements: } \\
{ }^{162} \text { M. A. A. Fakhree, S. Ahmadian, } \\
\text { V. Panahi-Azar, W. E. Acree, Jr., } \\
\text { and A. Jouyban, J. Chem. Eng. Data } \\
\mathbf{5 7 ,} 3303 \text { (2012). }\end{array}$ \\
\hline $\begin{array}{l}\text { Variables: } \\
\text { Temperature }\end{array}$ & $\begin{array}{l}\text { Prepared by: } \\
\text { W. E. Acree, Jr. }\end{array}$ \\
\hline
\end{tabular}

Experimental Values

\begin{tabular}{lcc}
\hline \hline$T / \mathrm{K}$ & $x_{2}{ }^{\mathrm{a}}$ & $x_{1}{ }^{\mathrm{b}}$ \\
\hline 298.2 & 0.856 & 0.144 \\
308.2 & 0.839 & 0.161 \\
318.2 & 0.827 & 0.173 \\
328.2 & 0.805 & 0.195 \\
338.2 & 0.786 & 0.214 \\
\hline
\end{tabular}

${ }^{\mathrm{a}} x_{2}$ : mole fraction of component 2 in the saturated solution.

${ }^{\mathrm{b}} x_{1}$ : mole fraction solubility of the solute.

\section{Auxiliary Information}

\section{Method/Apparatus/Procedure:}

Constant temperature oven, temperature controlling system, and ultraviolet/ visible spectrophotometer.

Excess solute and solvent were placed in sealed bottles and allowed to equilibrate at constant temperature in an oven for three days. Samples were shaken manually three times per day to ensure that equilibrium had been achieved. Solutions of the saturated solution were withdrawn and filtered using hydrophilic Durapore filters $(0.45 \mu \mathrm{m})$. The filtered solutions were quantitatively diluted with ethanol. Absorbances of the diluted solutions were measured at $304 \mathrm{~nm}$. Concentrations of the diluted solutions were computed using a Beer-Law calibration curve based on measured absorbances for standard solutions of known concentration.

\section{Source and Purity of Chemicals:}

(1) $99 \%$, Merck Chemical Company, Germany, was used as received.

(2) $99.9 \%$, Scharlau Chemie, Spain, was used as received.

\section{Estimated Error:}

Temperature: $\pm 0.2 \mathrm{~K}$ to $\pm 0.6 \mathrm{~K}$; the higher temperatures have greater uncertainty.

$x_{1}: \pm 1 \%$ (relative error)

\begin{tabular}{|c|c|}
\hline $\begin{array}{l}\text { Components: } \\
\text { (1) 2-Hydroxybenzoic acid; } \mathrm{C}_{7} \mathrm{H}_{6} \mathrm{O}_{3} \text {; } \\
\text { [69-72-7] } \\
\text { (2) 2-Propanol; } \mathrm{C}_{3} \mathrm{H}_{8} \mathrm{O} ;[67-63-0]\end{array}$ & $\begin{array}{l}\text { Original Measurements: } \\
{ }^{151} \text { K. M. De Fina, T. L. Sharp, L. E. } \\
\text { Roy, and W. E. Acree, Jr., J. Chem. } \\
\text { Eng. Data 44, } 1262 \text { (1999). }\end{array}$ \\
\hline $\begin{array}{l}\text { Variables: } \\
T / \mathrm{K}=298.15\end{array}$ & $\begin{array}{l}\text { Prepared by: } \\
\text { W. E. Acree, Jr. }\end{array}$ \\
\hline
\end{tabular}

\section{Experimental Values}

\begin{tabular}{lc}
\hline \hline$x_{2}{ }^{\mathrm{a}}$ & $x_{1}{ }^{\mathrm{b}}$ \\
\hline 0.8211 & 0.1789 \\
\hline${ }^{\mathrm{a}} x_{2}:$ mole fraction of component 2 in the saturated solution. &
\end{tabular}

${ }^{b} x_{1}$ : mole fraction solubility of the solute.

\section{Auxiliary Information}

Method/Apparatus/Procedure:

Constant-temperature bath, calorimetric thermometer, and an ultraviolet/ visible spectrophotometer.

Excess solute and solvent were placed in amber glass bottles and allowed to equilibrate for several days at constant temperature. Attainment of equilibrium was verified by several repetitive measurements and by approaching equilibrium from supersaturation. Aliquots of saturated solutions were transferred through a coarse filter into tared volumetric flasks, weighed, and diluted with methanol. Concentrations were determined by spectrophotometric measurements at $304 \mathrm{~nm}$. 
Source and Purity of Chemicals:

(1) $99+\%$, ACS Reagent grade, Aldrich Chemical Company, Milwaukee, WI, USA, was dried for several hours at $353 \mathrm{~K}$ before use and used without further purification.

(2) $99+\%$, anhydrous, Aldrich Chemical Company, stored over molecular sieves before use.

Estimated Error:

Temperature: $\pm 0.1 \mathrm{~K}$.

$x_{1}: \pm 1.5 \%$ (relative error).

\begin{tabular}{|c|c|}
\hline $\begin{array}{l}\text { Components: } \\
\text { (1) 2-Hydroxybenzoic acid; } \mathrm{C}_{7} \mathrm{H}_{6} \mathrm{O}_{3} \text {; } \\
\text { [69-72-7] } \\
\text { (2) 1-Butanol; } \mathrm{C}_{4} \mathrm{H}_{10} \mathrm{O} ;[71-36-3]\end{array}$ & $\begin{array}{l}\text { Original Measurements: } \\
{ }^{151} \text { K. M. De Fina, T. L. Sharp, L. E. } \\
\text { Roy, and W. E. Acree, Jr., J. Chem. } \\
\text { Eng. Data 44, } 1262 \text { (1999). }\end{array}$ \\
\hline $\begin{array}{l}\text { Variables: } \\
T / \mathrm{K}=298.15\end{array}$ & $\begin{array}{l}\text { Prepared by: } \\
\text { W. E. Acree, Jr. }\end{array}$ \\
\hline
\end{tabular}

Experimental Values

\begin{tabular}{lc}
\hline \hline$x_{2}{ }^{\mathrm{a}}$ & $x_{1}{ }^{\mathrm{b}}$ \\
\hline 0.8354 & 0.1646 \\
\hline$x_{2}:$ mole fraction
\end{tabular}

${ }^{\mathrm{a}} x_{2}$ : mole fraction of component 2 in the saturated solution.

${ }^{\mathrm{b}} x_{1}$ : mole fraction solubility of the solute.

\section{Auxiliary Information}

\section{Method/Apparatus/Procedure:}

Constant-temperature bath, calorimetric thermometer, and an ultraviolet/ visible spectrophotometer.

Excess solute and solvent were placed in amber glass bottles and allowed to equilibrate for several days at constant temperature. Attainment of equilibrium was verified by several repetitive measurements and by approaching equilibrium from supersaturation. Aliquots of saturated solutions were transferred through a coarse filter into tared volumetric flasks, weighed, and diluted with methanol. Concentrations were determined by

spectrophotometric measurements at $304 \mathrm{~nm}$.

\section{Source and Purity of Chemicals:}

(1) $99+\%$, ACS Reagent grade, Aldrich Chemical Company, Milwaukee, WI USA, was dried for several hours at $353 \mathrm{~K}$ before use and used without further purification.

(2) $99.8+\%$, HPLC grade, Aldrich Chemical Company, stored over molecular sieves before use.

Estimated Error:

Temperature: $\pm 0.1 \mathrm{~K}$.

$x_{1}: \pm 1.5 \%$ (relative error).

\begin{tabular}{|c|c|}
\hline $\begin{array}{l}\text { Components: } \\
\text { (1) 2-Hydroxybenzoic acid; } \mathrm{C}_{7} \mathrm{H}_{6} \mathrm{O}_{3} \text {; } \\
\text { [69-72-7] } \\
\text { (2) 1-Butanol; } \mathrm{C}_{4} \mathrm{H}_{10} \mathrm{O} ;[71-36-3]\end{array}$ & $\begin{array}{l}\text { Original Measurements: } \\
{ }^{127} \text { N. V. Sidgwick and E. K. } \\
\text { Ewbank, J. Chem. Soc. Trans. 119, } \\
979 \text { (1921). }\end{array}$ \\
\hline $\begin{array}{l}\text { Variables: } \\
\text { Temperature }\end{array}$ & $\begin{array}{l}\text { Prepared by: } \\
\text { W. E. Acree, Jr. }\end{array}$ \\
\hline
\end{tabular}

Experimental Values

\begin{tabular}{lcc}
\hline \hline$T / \mathrm{K}$ & $x_{2}{ }^{\mathrm{a}}$ & $x_{1}{ }^{\mathrm{b}}$ \\
\hline 297.2 & 0.852 & 0.148 \\
311.2 & 0.821 & 0.179 \\
358.8 & 0.661 & 0.339 \\
394.8 & 0.329 & 0.671
\end{tabular}

${ }^{\mathrm{a}} x_{2}$ : mole fraction of component 2 in the saturated solution.

${ }^{b} x_{1}$ : mole fraction solubility of the solute. Solubility data reported in units of mass percent. Mole fraction values calculated by the compiler.

\section{Auxiliary Information}

\section{Method/Apparatus/Procedure:}

Preweighed quantities of solute and solvent were placed in bulbs, which were then sealed and heated in a suitable bath. At the higher temperatures, the concentration of solvent in the liquid solution was corrected for the amount of solvent vapor in the bulb. The correction assumed that the vapor pressure of the saturated solution was one half that of the pure solvent at the solution temperature. The temperature at which the solid completely dissolved was recorded, and was taken to be the solid-liquid equilibrium temperature.

\section{Source and Purity of Chemicals:}

(1) Purity not given, prepared and purified by the authors using a published synthetic procedure [see J. Walker and J. K. Wood, J. Chem. Soc. 117, 40 (1920) for synthetic details]. Melting point of the purified solute was $432.2 \mathrm{~K}$. (2) Purity not given, Chemical source not given, fractionated and distilled several times before use.

\section{Estimated Error:}

Temperature: Not given in paper.

$x_{1}: \pm 10 \%$ (relative error, estimated by compiler).

\begin{tabular}{|c|c|}
\hline $\begin{array}{l}\text { Components: } \\
\text { (1) 2-Hydroxybenzoic acid; } \mathrm{C}_{7} \mathrm{H}_{6} \mathrm{O}_{3} \text {; } \\
\text { [69-72-7] } \\
\text { (2) 1-Butanol; } \mathrm{C}_{4} \mathrm{H}_{10} \mathrm{O} ;[71-36-3]\end{array}$ & $\begin{array}{l}\text { Original Measurements: } \\
{ }^{73} \text { M. Dias, S. L. Raghavan, and J. } \\
\text { Hadgraft, Int. J. Pharm. 216, } 51 \\
(2001) \text {. }\end{array}$ \\
\hline $\begin{array}{l}\text { Variables: } \\
T / \mathrm{K}=305.2\end{array}$ & $\begin{array}{l}\text { Prepared by: } \\
\text { W. E. Acree, Jr. }\end{array}$ \\
\hline
\end{tabular}

\section{Experimental Values}

The measured solubility was reported to be $283.7 \mathrm{mg} / \mathrm{ml}$, which corresponds to a molar solubility of $c_{1}=2.053 \mathrm{~mol}$ $\mathrm{dm}^{-3}$.

\section{Auxiliary Information}

\section{Method/Apparatus/Procedure:}

Constant-temperature bath, magnetic stirrer, centrifuge, and an ultraviolet/ visible spectrophotometer.

Excess solute and solvent were equilibrated in a constant-temperature bath with stirring for $48 \mathrm{~h}$. The saturated solution was then centrifuged for $10 \mathrm{~min}$, and an aliquot of the supernatant solution was removed and diluted quantitatively for spectrophotometric analysis. The concentration of the dissolved solute was determined from the measured absorbance.

\section{Source and Purity of Chemicals:}

(1) Purity not given, Fisher Scientific, UK, no purification details were provided.

(2) Purity not given, Fisher Scientific, no purification details were provided.

\section{Estimated Error:}

Temperature: Insufficient experimental details to estimate.

$c_{1}: \pm 0.03$ (estimated by compiler, value in the paper seemed too small given the analytical method). 


\section{Components:}

(1) 2-Hydroxybenzoic acid; $\mathrm{C}_{7} \mathrm{H}_{6} \mathrm{O}_{3}$; [69-72-7]

(2) 1-Butanol; $\mathrm{C}_{4} \mathrm{H}_{10} \mathrm{O} ;[$ [71-36-3]

\section{Variables:}

$T / \mathrm{K}=303.8$

Original Measurements:

${ }^{150}$ A. N. Paruta, B. J. Sciarrone, and N. G. Lordi, J. Pharm. Sci. 53, 1349 (1964).

Prepared by:

W. E. Acree, Jr.

\section{Experimental Values}

The measured solubility was reported to be $251 \mathrm{mg} / \mathrm{ml}$ of solution, which corresponds to a solubility of $c_{1}=1.82 \mathrm{~mol}$ $\mathrm{dm}^{-3}$.

\section{Auxiliary Information}

\section{Method/Apparatus/Procedure:}

Excess solute and solvent were placed in 15-ml screw-capped vials fitted with Teflon liners. The vials were attached to a rotating wheel in a constanttemperature water bath and allowed to equilibrate for $24 \mathrm{~h}$. An aliquot of the saturated solution was withdrawn from the vial with a pipet fitted with a glass wool filtering plug. The solubility was determined by titration using a freshly prepared sodium hydroxide solution. The endpoint of the titration was detected with phenolphthalein.

\section{Source and Purity of Chemicals:}

(1) Purity not given, Chemical source not given, no purification details were provided.

(2) Purity not given, Chemical source not given, no purification details were provided.

\section{Estimated Error:}

Temperature: $\pm 0.2 \mathrm{~K}$

$c_{1}: \pm 5 \%$ (relative error, estimated by compiler).

\begin{tabular}{|c|c|}
\hline $\begin{array}{l}\text { Components: } \\
\text { (1) 2-Hydroxybenzoic acid; } \mathrm{C}_{7} \mathrm{H}_{6} \mathrm{O}_{3} \text {; } \\
\text { [69-72-7] } \\
\text { (2) 1-Butanol; } \mathrm{C}_{4} \mathrm{H}_{10} \mathrm{O} ;[71-36-3]\end{array}$ & $\begin{array}{l}\text { Original Measurements: } \\
\text { }{ }^{50} \text { P. G. Desai and A. M. Patel, J. } \\
\text { Indian Chem. Soc. 12, } 131 \text { (1935). }\end{array}$ \\
\hline $\begin{array}{l}\text { Variables: } \\
T / \mathrm{K}=301.2\end{array}$ & $\begin{array}{l}\text { Prepared by: } \\
\text { W. E. Acree, Jr. }\end{array}$ \\
\hline
\end{tabular}

Experimental Values

\begin{tabular}{lc}
\hline \hline$x_{2}{ }^{\mathrm{a}}$ & $x_{1}{ }^{\mathrm{b}}$ \\
\hline 0.8412 & 0.1588 \\
\hline${ }^{\mathrm{a}} x_{2}:$ mole fraction of component 2 in the saturated solution. \\
${ }^{\mathrm{b}} x_{1}:$ mole fraction solubility of the solute.
\end{tabular}

\section{Auxiliary Information}

\footnotetext{
Method/Apparatus/Procedure:

Excess solute and solvent were placed in a glass flask which was kept revolving on a wheel in an air thermostat for two days. After two days of equilibration, the solution was filtered. To minimize absorption of the solute onto the filter paper, a $100 \mathrm{ml}$ portion of fresh saturated solution was first filtered through the paper before filtering the sample to be analyzed. An aliquot of the filtrate was titrated with barium hydroxide using phenolphthalein as the endpoint indictor. The barium hydroxide titrant was previously standardized against a solution of succinic acid.
}

Source and Purity of Chemicals:

(1) Purity not given, Chemical source not specified, no information provided concerning purification.

(2) Purity not given, Chemical source not specified, no information provided concerning purification.

\section{Estimated Error:}

Temperature: No information given.

$x_{1}: \pm 3 \%$ (relative error, estimated by compiler).

\begin{tabular}{|c|c|}
\hline Components: & Original Measurements: \\
\hline $\begin{array}{l}\text { (1) 2-Hydroxybenzoic acid; } \mathrm{C}_{7} \mathrm{H}_{6} \mathrm{O}_{3} \text {; } \\
\text { [69-72-7] }\end{array}$ & $\begin{array}{l}{ }^{159} \text { J. Lim, S. Jang, H. K. Cho, M. S } \\
\text { Shin, and H. Kim, J. Chem. }\end{array}$ \\
\hline (2) 1-Butanol; $\mathrm{C}_{4} \mathrm{H}_{10} \mathrm{O}$; [71-36-3] & Thermodyn. 57, 295 (2013). \\
\hline Variables: & Prepared by: \\
\hline Temperature & W. E. Acree, Jr. \\
\hline
\end{tabular}

Experimental Values

\begin{tabular}{lcc}
\hline \hline$T / \mathrm{K}$ & $x_{2}{ }^{\mathrm{a}}$ & $x_{1}{ }^{\mathrm{b}}$ \\
\hline 278.15 & 0.8937 & 0.1063 \\
283.15 & 0.8842 & 0.1158 \\
288.15 & 0.8745 & 0.1255 \\
293.15 & 0.8633 & 0.1367 \\
298.15 & 0.8516 & 0.1484 \\
303.15 & 0.8403 & 0.1597 \\
308.15 & 0.8264 & 0.1736 \\
313.15 & 0.8137 & 0.1863 \\
318.15 & 0.7988 & 0.2012 \\
\hline
\end{tabular}

${ }^{a} x_{2}$ : mole fraction of component 2 in the saturated solution.

${ }^{b} x_{1}$ : mole fraction solubility of the solute. The solubility data were reported as mass fractions. The mole fraction values were calculated by the compiler.

\section{Auxiliary Information}

\section{Method/Apparatus/Procedure:}

Magnetic stirrer, drying oven and an analytical balance.

Excess solute and solvent were placed in 50-ml glass tubes and agitated with a magnetic stirrer at constant temperature for at least $48 \mathrm{~h}$. A clear aliquot of the saturated solution was withdrawn using a syringe through a filter. The mass of the filtered clear solution was recorded. The sample was dried in a vacuum oven for at least seven days, and the mass of the solid residue recorded. The solution was calculated from the mass of the solid residue and mass of sample analyzed.

Source and Purity of Chemicals:

(1) $99 \%$, Samchen Pure Chemical Company, Seoul Korea, was used as received.

(2) 99.7\%, Sigma Aldrich, St. Louis, MO, USA, used as received.

\section{Estimated Error:}

Temperature: $\pm 0.05 \mathrm{~K}$.

$x_{1}: \pm 1 \%$ (relative error).

\begin{tabular}{|c|c|}
\hline $\begin{array}{l}\text { Components: } \\
\text { (1) 2-Hydroxybenzoic acid; } \mathrm{C}_{7} \mathrm{H}_{6} \mathrm{O}_{3} \text {; } \\
\text { [69-72-7] } \\
\text { (2) 2-Butanol; } \mathrm{C}_{4} \mathrm{H}_{10} \mathrm{O} ;[78-92-2]\end{array}$ & $\begin{array}{l}\text { Original Measurements: } \\
{ }^{151} \text { K. M. De Fina, T. L. Sharp, L. E. } \\
\text { Roy, and W. E. Acree, Jr., J. Chem. } \\
\text { Eng. Data 44, } 1262 \text { (1999). }\end{array}$ \\
\hline $\begin{array}{l}\text { Variables: } \\
T / \mathrm{K}=298.15\end{array}$ & $\begin{array}{l}\text { Prepared by: } \\
\text { W. E. Acree, Jr. }\end{array}$ \\
\hline
\end{tabular}


Experimental Values

\begin{tabular}{lc}
\hline \hline$x_{2}{ }^{\mathrm{a}}$ & $x_{1}{ }^{\mathrm{b}}$ \\
\hline 0.8131 & 0.1869 \\
${ }^{\mathrm{a}} x_{2}:$ mole fraction of component 2 in the saturated solution. \\
${ }^{\mathrm{b}} x_{1}:$ mole fraction solubility of the solute.
\end{tabular}

\section{Auxiliary Information}

\section{Method/Apparatus/Procedure:}

Constant-temperature bath, calorimetric thermometer, and an ultraviolet/ visible spectrophotometer.

Excess solute and solvent were placed in amber glass bottles and allowed to equilibrate for several days at constant temperature. Attainment of equilibrium was verified by several repetitive measurements and by approaching equilibrium from supersaturation. Aliquots of saturated solutions were transferred through a coarse filter into tared volumetric flasks, weighed, and diluted with methanol. Concentrations were determined by

spectrophotometric measurements at $304 \mathrm{~nm}$.

\section{Source and Purity of Chemicals:}

(1) $99+\%$, ACS Reagent grade, Aldrich Chemical Company, Milwaukee, WI, USA, was dried for several hours at $353 \mathrm{~K}$ before use and used without further purification.

(2) $99+\%$, anhydrous, Aldrich Chemical Company, stored over molecular sieves before use.

\section{Estimated Error:}

Temperature: $\pm 0.1 \mathrm{~K}$.

$x_{1}: \pm 1.5 \%$ (relative error).

\begin{tabular}{|c|c|}
\hline $\begin{array}{l}\text { Components: } \\
\text { (1) 2-Hydroxybenzoic acid; } \mathrm{C}_{7} \mathrm{H}_{6} \mathrm{O}_{3} \text {; } \\
\text { [69-72-7] } \\
\text { (2) 2-Methyl-1-propanol; } \mathrm{C}_{4} \mathrm{H}_{10} \mathrm{O} \text {; } \\
\text { [78-83-1] }\end{array}$ & $\begin{array}{l}\text { Original Measurements: } \\
{ }^{151} \text { K. M. De Fina, T. L. Sharp, L. E. } \\
\text { Roy, and W. E. Acree, Jr., J. Chem. } \\
\text { Eng. Data 44, } 1262 \text { (1999). }\end{array}$ \\
\hline $\begin{array}{l}\text { Variables: } \\
T / \mathrm{K}=298.15\end{array}$ & $\begin{array}{l}\text { Prepared by: } \\
\text { W. E. Acree, Jr. }\end{array}$ \\
\hline
\end{tabular}

\section{Experimental Values}

\begin{tabular}{lc}
\hline \hline$x_{2}{ }^{\mathrm{a}}$ & $x_{1}{ }^{\mathrm{b}}$ \\
\hline 0.8570 & 0.1430 \\
\hline${ }^{\mathrm{a}} x_{2}:$ mole fraction of component 2 in the saturated solution. \\
${ }^{\mathrm{b}} x_{1}:$ mole fraction solubility of the solute.
\end{tabular}

\section{Auxiliary Information}

\section{Method/Apparatus/Procedure:}

Constant-temperature bath, calorimetric thermometer, and an ultraviolet/ visible spectrophotometer.

Excess solute and solvent were placed in amber glass bottles and allowed to equilibrate for several days at constant temperature. Attainment of equilibrium was verified by several repetitive measurements and by approaching equilibrium from supersaturation. Aliquots of saturated solutions were transferred through a coarse filter into tared volumetric flasks, weighed, and diluted with methanol. Concentrations were determined by spectrophotometric measurements at $304 \mathrm{~nm}$.
Source and Purity of Chemicals:

(1) $99+\%$, ACS Reagent grade, Aldrich Chemical Company, Milwaukee, WI, USA, was dried for several hours at $353 \mathrm{~K}$ before use and used without further purification.

(2) $99+\%$, anhydrous, Aldrich Chemical Company, stored over molecular sieves before use.

\section{Estimated Error:}

Temperature: $\pm 0.1 \mathrm{~K}$.

$x_{1}: \pm 1.5 \%$ (relative error).

\begin{tabular}{|c|c|}
\hline $\begin{array}{l}\text { Components: } \\
\text { (1) 2-Hydroxybenzoic acid; } \mathrm{C}_{7} \mathrm{H}_{6} \mathrm{O}_{3} \text {; } \\
\text { [69-72-7] } \\
\text { (2) 2-Methyl-2-propanol; } \mathrm{C}_{4} \mathrm{H}_{10} \mathrm{O} \text {; } \\
\text { [75-65-0] }\end{array}$ & $\begin{array}{l}\text { Original Measurements: } \\
{ }^{151} \text { K. M. De Fina, T. L. Sharp, L. E. } \\
\text { Roy, and W. E. Acree, Jr., J. Chem. } \\
\text { Eng. Data 44, } 1262 \text { (1999). }\end{array}$ \\
\hline $\begin{array}{l}\text { Variables: } \\
T / \mathrm{K}=298.15\end{array}$ & $\begin{array}{l}\text { Prepared by: } \\
\text { W. E. Acree, Jr. }\end{array}$ \\
\hline
\end{tabular}

Experimental Values

\begin{tabular}{lc}
\hline \hline$x_{2}{ }^{\mathrm{a}}$ & $x_{1}{ }^{\mathrm{b}}$ \\
\hline 0.7807 & 0.2193 \\
\hline${ }^{\mathrm{a}} x_{2}:$ mole fraction of component 2 in the saturated solution. & \\
${ }^{\mathrm{b}} x_{1}:$ mole fraction solubility of the solute. &
\end{tabular}

\section{Auxiliary Information}

\section{Method/Apparatus/Procedure:}

Constant-temperature bath, calorimetric thermometer, and an ultraviolet/ visible spectrophotometer.

Excess solute and solvent were placed in amber glass bottles and allowed to equilibrate for several days at constant temperature. Attainment of equilibrium was verified by several repetitive measurements and by approaching equilibrium from supersaturation. Aliquots of saturated solutions were transferred through a coarse filter into tared volumetric flasks, weighed, and diluted with methanol. Concentrations were determined by spectrophotometric measurements at $304 \mathrm{~nm}$.

\section{Source and Purity of Chemicals:}

(1) $99+\%$, ACS Reagent grade, Aldrich Chemical Company, Milwaukee, WI, USA, was dried for several hours at $353 \mathrm{~K}$ before use and used without further purification.

(2) $99+\%$, Arco Chemical Company, USA, stored over molecular sieves before use.

Estimated Error:

Temperature: $\pm 0.1 \mathrm{~K}$.

$x_{1}: \pm 1.5 \%$ (relative error).

\begin{tabular}{|c|c|}
\hline Components: & Original Measurements: \\
\hline $\begin{array}{l}\text { (1) 2-Hydroxybenzoic acid; } \mathrm{C}_{7} \mathrm{H}_{6} \mathrm{O}_{3} \text {; } \\
\text { [69-72-7] }\end{array}$ & $\begin{array}{l}{ }^{151} \text { K. M. De Fina, T. L. Sharp, L. E } \\
\text { Roy, and W. E. Acree, Jr., J. Chem }\end{array}$ \\
\hline (2) 1-Pentanol; $\mathrm{C}_{5} \mathrm{H}_{12} \mathrm{O} ;[$ [71-41-0] & Eng. Data 44, 1262 (1999). \\
\hline Variables: & Prepared by: \\
\hline$T / \mathrm{K}=298.15$ & W. E. Acree, Jr. \\
\hline
\end{tabular}


Experimental Values

\begin{tabular}{lc}
\hline \hline$x_{2}{ }^{\mathrm{a}}$ & $x_{1}{ }^{\mathrm{b}}$ \\
\hline 0.8389 & 0.1611
\end{tabular}

${ }^{\mathrm{a}} x_{2}$ : mole fraction of component 2 in the saturated solution.

${ }^{\mathrm{b}} x_{1}$ : mole fraction solubility of the solute.

\section{Auxiliary Information}

\section{Method/Apparatus/Procedure:}

Constant-temperature bath, calorimetric thermometer, and an ultraviolet/ visible spectrophotometer.

Excess solute and solvent were placed in amber glass bottles and allowed to equilibrate for several days at constant temperature. Attainment of equilibrium was verified by several repetitive measurements and by approaching equilibrium from supersaturation. Aliquots of saturated solutions were transferred through a coarse filter into tared volumetric flasks, weighed, and diluted with methanol. Concentrations were determined by

spectrophotometric measurements at $304 \mathrm{~nm}$.

\section{Source and Purity of Chemicals:}

(1) $99+\%$, ACS Reagent grade, Aldrich Chemical Company, Milwaukee, WI, USA, was dried for several hours at $353 \mathrm{~K}$ before use and used without further purification.

(2) $99+\%$, Aldrich Chemical Company, stored over molecular sieves before use.

\section{Estimated Error:}

Temperature: $\pm 0.1 \mathrm{~K}$.

$x_{1}: \pm 1.5 \%$ (relative error)

\begin{tabular}{|c|c|}
\hline $\begin{array}{l}\text { Components: } \\
\text { (1) 2-Hydroxybenzoic acid; } \mathrm{C}_{7} \mathrm{H}_{6} \mathrm{O}_{3} \text {; } \\
\text { [69-72-7] } \\
\text { (2) 1-Pentanol; } \mathrm{C}_{5} \mathrm{H}_{12} \mathrm{O} ;[71-41-0]\end{array}$ & $\begin{array}{l}\text { Original Measurements: } \\
{ }^{102} \text { J. Barra, M.-A. Peña, and P. } \\
\text { Bustamante, Eur. J. Pharm. Sci. 10, } \\
153 \text { (2000). }\end{array}$ \\
\hline $\begin{array}{l}\text { Variables: } \\
T / \mathrm{K}=298.15\end{array}$ & $\begin{array}{l}\text { Prepared by: } \\
\text { W. E. Acree, Jr. }\end{array}$ \\
\hline
\end{tabular}

\section{Experimental Values}

\begin{tabular}{lc}
\hline \hline$x_{2}^{\mathrm{a}}$ & $x_{1}{ }^{\mathrm{b}}$ \\
\hline 0.8453 & 0.1547
\end{tabular}

${ }^{\mathrm{a}} x_{2}$ : mole fraction of component 2 in the saturated solution.

${ }^{\mathrm{b}} x_{1}$ : mole fraction solubility of the solute.

\section{Auxiliary Information}

\section{Method/Apparatus/Procedure:}

Constant-temperature bath, analytical balance, and an ultraviolet/visible spectrophotometer.

Excess solute and solvent were placed in a flask and allowed to equilibrate for several days at constant temperature. The solid phase was removed by filtration, and the clear solution was diluted quantitatively with $96 \%$ ethanol. The concentration of the diluted solution was determined by spectrophotometric measurement at the wavelength of maximum absorption In instances where the solvent interfered with the spectrophotometric determination, the samples were evaporated to dryness and the residue diluted with $96 \%$ ethanol.
Source and Purity of Chemicals:

(1) Purity not given, Sigma Chemical Company, USA, no purification details were provided.

(2) Spectrophotometric or Analytical grade, Chemical source not specified, no purification details were provided.

\section{Estimated Error:}

Temperature: $\pm 0.2 \mathrm{~K}$.

$x_{1}: \pm 3.0 \%$ (relative error, estimated by compiler).

\begin{tabular}{|c|c|}
\hline Components: & Original Measurements: \\
\hline $\begin{array}{l}\text { (1) 2-Hydroxybenzoic acid; } \mathrm{C}_{7} \mathrm{H}_{6} \mathrm{O}_{3} \text {; } \\
\text { [69-72-7] }\end{array}$ & $\begin{array}{l}{ }^{159} \text { J. Lim, S. Jang, H. K. Cho, M. S } \\
\text { Shin, and H. Kim, J. Chem. }\end{array}$ \\
\hline (2) 1-Pentanol; $\mathrm{C}_{5} \mathrm{H}_{12} \mathrm{O} ;[$ [1-41-0] & Thermodyn. 57, 295 (2013). \\
\hline Variables: & Prepared by: \\
\hline Temperature & W. E. Acree, Jr. \\
\hline
\end{tabular}

Experimental Values

\begin{tabular}{lcc}
\hline \hline$T / \mathrm{K}$ & $x_{2}{ }^{\mathrm{a}}$ & $x_{1}{ }^{\mathrm{b}}$ \\
\hline 278.15 & 0.8872 & 0.1128 \\
283.15 & 0.8773 & 0.1227 \\
288.15 & 0.8677 & 0.1323 \\
293.15 & 0.8560 & 0.1440 \\
298.15 & 0.8450 & 0.1550 \\
303.15 & 0.8337 & 0.1663 \\
308.15 & 0.8198 & 0.1802 \\
313.15 & 0.8081 & 0.1919 \\
318.15 & 0.7941 & 0.2059 \\
\hline
\end{tabular}

${ }^{a} x_{2}$ : mole fraction of component 2 in the saturated solution.

${ }^{b} x_{1}$ : mole fraction solubility of the solute. The solubility data were reported as mass fractions. The mole fraction values were calculated by the compiler.

\section{Auxiliary Information}

\section{Method/Apparatus/Procedure:}

Magnetic stirrer, drying oven and an analytical balance.

Excess solute and solvent were placed in 50-ml glass tubes and agitated with a magnetic stirrer at constant temperature for at least $48 \mathrm{~h}$. A clear aliquot of the saturated solution was withdrawn using a syringe through a filter. The mass of the filtered clear solution was recorded. The sample was dried in a vacuum oven for at least seven days, and the mass of the solid residue recorded. The solution was calculated from the mass of the solid residue and mass of sample analyzed.

\section{Source and Purity of Chemicals:}

(1) $99 \%$, Samchen Pure Chemical Company, Seoul Korea, was used as received.

(2) $99 \%$, Sigma Aldrich, St. Louis, MO, USA, used as received.

\section{Estimated Error:}

Temperature: $\pm 0.05 \mathrm{~K}$.

$x_{1}: \pm 1 \%$ (relative error).

\begin{tabular}{|c|c|}
\hline $\begin{array}{l}\text { Components: } \\
\text { (1) 2-Hydroxybenzoic acid; } \mathrm{C}_{7} \mathrm{H}_{6} \mathrm{O}_{3} \text {; } \\
\text { [69-72-7] } \\
\text { (2) } 1-\mathrm{Hexanol} ; \mathrm{C}_{6} \mathrm{H}_{14} \mathrm{O} ;[111-27-3]\end{array}$ & $\begin{array}{l}\text { Original Measurements: } \\
{ }^{159} \text { J. Lim, S. Jang, H. K. Cho, M. S. } \\
\text { Shin, and H. Kim, J. Chem. } \\
\text { Thermodyn. 57, } 295 \text { (2013). }\end{array}$ \\
\hline $\begin{array}{l}\text { Variables: } \\
\text { Temperature }\end{array}$ & $\begin{array}{l}\text { Prepared by: } \\
\text { W. E. Acree, Jr. }\end{array}$ \\
\hline
\end{tabular}


Experimental Values

\begin{tabular}{lcc}
\hline \hline$T / \mathrm{K}$ & $x_{2}{ }^{\mathrm{a}}$ & $x_{1}{ }^{\mathrm{b}}$ \\
\hline 278.15 & 0.8837 & 0.1163 \\
283.15 & 0.8731 & 0.1269 \\
288.15 & 0.8633 & 0.1367 \\
293.15 & 0.8542 & 0.1458 \\
298.15 & 0.8416 & 0.1584 \\
303.15 & 0.8309 & 0.1691 \\
308.15 & 0.8180 & 0.1820 \\
313.15 & 0.8050 & 0.1950 \\
318.15 & 0.7911 & 0.2089
\end{tabular}

${ }^{a_{x_{2}}}$ : mole fraction of component 2 in the saturated solution.

${ }^{b} x_{1}$ : mole fraction solubility of the solute. The solubility data were reported as mass fractions. The mole fraction values were calculated by the compiler.

\section{Auxiliary Information}

\section{Method/Apparatus/Procedure:}

Magnetic stirrer, drying oven and an analytical balance.

Excess solute and solvent were placed in 50-ml glass tubes and agitated with a magnetic stirrer at constant temperature for at least $48 \mathrm{~h}$. A clear aliquot of the saturated solution was withdrawn using a syringe through a filter. The mass of the filtered clear solution was recorded. The sample was dried in a vacuum oven for at least seven days, and the mass of the solid residue recorded. The solution was calculated from the mass of the solid residue and mass of sample analyzed.

Source and Purity of Chemicals:

(1) $99 \%$, Samchen Pure Chemical Company, Seoul Korea, was used as received.

(2) $99 \%$, Sigma Aldrich, St. Louis, MO, USA, used as received.

Estimated Error:

Temperature: $\pm 0.05 \mathrm{~K}$.

$x_{1}: \pm 1 \%$ (relative error).

\begin{tabular}{|c|c|}
\hline $\begin{array}{l}\text { Components: } \\
\text { (1) 2-Hydroxybenzoic acid; } \mathrm{C}_{7} \mathrm{H}_{6} \mathrm{O}_{3} \text {; } \\
\text { [69-72-7] } \\
\text { (2) 1-Heptanol; } \mathrm{C}_{7} \mathrm{H}_{16} \mathrm{O} ;[111-70-6]\end{array}$ & $\begin{array}{l}\text { Original Measurements: } \\
{ }^{159} \text { J. Lim, S. Jang, H. K. Cho, M. S. } \\
\text { Shin, and H. Kim, J. Chem. } \\
\text { Thermodyn. 57, } 295 \text { (2013). }\end{array}$ \\
\hline $\begin{array}{l}\text { Variables: } \\
\text { Temperature }\end{array}$ & $\begin{array}{l}\text { Prepared by: } \\
\text { W. E. Acree, Jr. }\end{array}$ \\
\hline
\end{tabular}

Experimental Values

\begin{tabular}{lcc}
\hline \hline$T / \mathrm{K}$ & $x_{2}{ }^{\mathrm{a}}$ & $x_{1}{ }^{\mathrm{b}}$ \\
\hline 278.15 & 0.8828 & 0.1172 \\
283.15 & 0.8720 & 0.1280 \\
288.15 & 0.8623 & 0.1377 \\
293.15 & 0.8515 & 0.1485 \\
298.15 & 0.8396 & 0.1604 \\
303.15 & 0.8286 & 0.1714 \\
308.15 & 0.8154 & 0.1846 \\
313.15 & 0.8031 & 0.1969 \\
318.15 & 0.7898 & 0.2102
\end{tabular}

${ }^{a} x_{2}$ : mole fraction of component 2 in the saturated solution.

${ }^{b} x_{1}$ : mole fraction solubility of the solute. The solubility data were reported as mass fractions. The mole fraction values were calculated by the compiler.

\section{Auxiliary Information}

\section{Method/Apparatus/Procedure:}

Magnetic stirrer, drying oven and an analytical balance.

Excess solute and solvent were placed in 50-ml glass tubes and agitated with a magnetic stirrer at constant temperature for at least $48 \mathrm{~h}$. A clear aliquot of the saturated solution was withdrawn using a syringe through a filter. The mass of the filtered clear solution was recorded. The sample was dried in a vacuum oven for at least seven days, and the mass of the solid residue recorded. The solution was calculated from the mass of the solid residue and mass of sample analyzed.

\section{Source and Purity of Chemicals:}

(1) $99 \%$, Samchen Pure Chemical Company, Seoul Korea, was used as received.

(2) $98 \%$, Tokyo Chemical Industry, Tokyo, Japan, used as received.

\section{Estimated Error:}

Temperature: $\pm 0.05 \mathrm{~K}$.

$x_{1}: \pm 1 \%$ (relative error).

\begin{tabular}{|c|c|}
\hline $\begin{array}{l}\text { Components: } \\
\text { (1) 2-Hydroxybenzoic acid; } \mathrm{C}_{7} \mathrm{H}_{6} \mathrm{O}_{3} \text {; } \\
\text { [69-72-7] } \\
\text { (2) } 1 \text {-Octanol; } \mathrm{C}_{8} \mathrm{H}_{18} \mathrm{O} ;[111-87-5]\end{array}$ & $\begin{array}{l}\text { Original Measurements: } \\
{ }^{151} \text { K. M. De Fina, T. L. Sharp, L. E. } \\
\text { Roy, and W. E. Acree, Jr., J. Chem. } \\
\text { Eng. Data 44, } 1262 \text { (1999). }\end{array}$ \\
\hline $\begin{array}{l}\text { Variables: } \\
T / \mathrm{K}=298.15\end{array}$ & $\begin{array}{l}\text { Prepared by: } \\
\text { W. E. Acree, Jr. }\end{array}$ \\
\hline
\end{tabular}

Experimental Values

\begin{tabular}{lc}
\hline \hline$x_{2}{ }^{\mathrm{a}}$ & $x_{1}{ }^{\mathrm{b}}$ \\
\hline 0.7857 & 0.2143
\end{tabular}

${ }^{a} x_{2}$ : mole fraction of component 2 in the saturated solution.

${ }^{\mathrm{b}} x_{1}$ : mole fraction solubility of the solute.

\section{Auxiliary Information}

\section{Method/Apparatus/Procedure:}

Constant-temperature bath, calorimetric thermometer, and an ultraviolet/ visible spectrophotometer.

Excess solute and solvent were placed in amber glass bottles and allowed to equilibrate for several days at constant temperature. Attainment of equilibrium was verified by several repetitive measurements and by approaching equilibrium from supersaturation. Aliquots of saturated solutions were transferred through a coarse filter into tared volumetric flasks, weighed, and diluted with methanol. Concentrations were determined by spectrophotometric measurements at $304 \mathrm{~nm}$.

\section{Source and Purity of Chemicals:}

(1) $99+\%$, ACS Reagent grade, Aldrich Chemical Company, Milwaukee, WI, USA, was dried for several hours at $353 \mathrm{~K}$ before use and used without further purification.

(2) $99+\%$, anhydrous, Aldrich Chemical Company, stored over molecular sieves before use.

\section{Estimated Error:}

Temperature: $\pm 0.1 \mathrm{~K}$.

$x_{1}: \pm 1.5 \%$ (relative error). 


\section{Components:}

(1) 2-Hydroxybenzoic acid; $\mathrm{C}_{7} \mathrm{H}_{6} \mathrm{O}_{3}$;

[69-72-7]

(2) 1-Octanol; $\mathrm{C}_{8} \mathrm{H}_{18} \mathrm{O} ;$ [111-87-5]

\section{Variables:}

$T / \mathrm{K}=298.15$

W. E. Acree, Jr.

\section{Experimental Values}

\begin{tabular}{lc}
\hline \hline$x_{2}^{\mathrm{a}}$ & $x_{1}{ }^{\mathrm{b}}$ \\
\hline 0.8452 & 0.1548
\end{tabular}

${ }^{\mathrm{a}} x_{2}$ : mole fraction of component 2 in the saturated solution.

${ }^{\mathrm{b}} x_{1}$ : mole fraction solubility of the solute.

\section{Auxiliary Information}

\section{Method/Apparatus/Procedure:}

Constant-temperature bath, analytical balance, and an ultraviolet/visible spectrophotometer.

Excess solute and solvent were placed in a flask and allowed to equilibrate for several days at constant temperature. The solid phase was removed by filtration, and the clear solution was diluted quantitatively with $96 \%$ ethanol. The concentration of the diluted solution was determined by spectrophotometric measurement at the wavelength of maximum absorption. In instances where the solvent interfered with the spectrophotometric determination, the samples were evaporated to dryness and the residue diluted with $96 \%$ ethanol.

\section{Source and Purity of Chemicals:}

(1) Purity not given, Sigma Chemical Company, USA, no purification details were provided.

(2) Spectrophotometric or Analytical grade, Chemical source not specified, no purification details were provided.

\section{Estimated Error:}

Temperature: $\pm 0.2 \mathrm{~K}$.

$x_{1}: \pm 3.0 \%$ (relative error, estimated by compiler).

\begin{tabular}{ll}
\hline \hline Components: & Original Measurements: \\
$\begin{array}{l}\text { (1) 2-Hydroxybenzoic acid; } \mathrm{C}_{7} \mathrm{H}_{6} \mathrm{O}_{3} ; \\
\text { [69-72-7] }\end{array}$ & ${ }^{145} \mathrm{G}$. L. Perlovich, T. V. Volkova, \\
and A. Bauer-Brandl, J. Pharm. Sci. \\
(2) 1-Octanol; $\mathrm{C}_{8} \mathrm{H}_{18} \mathrm{O} ;[111-87-5]$ & $\mathbf{9 5}, 1448$ (2006). \\
\hline Variables: & Prepared by: \\
Temperature & W. E. Acree, Jr. \\
\hline
\end{tabular}

Experimental Values

\begin{tabular}{lcc}
\hline \hline$T / \mathrm{K}$ & $x_{2}{ }^{\mathrm{a}}$ & $x_{1}{ }^{\mathrm{b}}$ \\
\hline 293 & 0.824 & 0.176 \\
298 & 0.814 & 0.186 \\
303 & 0.794 & 0.206 \\
310 & 0.765 & 0.235 \\
315 & 0.748 & 0.252
\end{tabular}

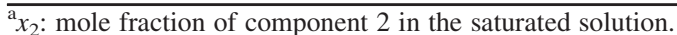

${ }^{\mathrm{b}} x_{1}$ : mole fraction solubility of the solute.

\section{Auxiliary Information}

\section{Method/Apparatus/Procedure:}

Thermostated constant-temperature bath, centrifuge, and an ultraviolet/visible spectrophotometer.

Excess solute and solvent were placed in a glass ampoule and allowed to equilibrate in a thermostated temperature bath with mixing (at a speed of $25 \mathrm{rpm}$ ) for a minimum of four days. After suitable equilibration, the saturated solution was centrifuged, the supernatant liquid collected, quantitatively diluted, and the absorbance recorded using an ultraviolet/visible spectrophotometer. The solubility of the solute was calculated from the measured absorbance.

Source and Purity of Chemicals:

(1) $99 \%$, Sigma-Aldrich Inc., Oslo, Norway, no purification details provided. (2) Analytical Reagent grade, Sigma-Aldrich Inc., no purification details were provided.

Estimated Error:

Temperature: $\pm 0.1 \mathrm{~K}$.

$x_{2}: \pm 2.5 \%$ (relative error)

\begin{tabular}{|c|c|}
\hline $\begin{array}{l}\text { Components: } \\
\text { (1) 2-Hydroxybenzoic acid; } \mathrm{C}_{7} \mathrm{H}_{6} \mathrm{O}_{3} \text {; } \\
\text { [69-72-7] } \\
\text { (2) 1-Octanol; } \mathrm{C}_{8} \mathrm{H}_{18} \mathrm{O} ;[111-87-5]\end{array}$ & $\begin{array}{l}\text { Original Measurements: } \\
{ }^{73} \text { M. Dias, S. L. Raghavan, and J. } \\
\text { Hadgraft, Int. J. Pharm. 216, } 51 \\
(2001) \text {. }\end{array}$ \\
\hline $\begin{array}{l}\text { Variables: } \\
T / \mathrm{K}=305.2\end{array}$ & $\begin{array}{l}\text { Prepared by: } \\
\text { W. E. Acree, Jr. }\end{array}$ \\
\hline
\end{tabular}

\section{Experimental Values}

The measured solubility was reported to be $186.1 \mathrm{mg} / \mathrm{ml}$, which corresponds to a molar solubility of $c_{1}=1.347 \mathrm{~mol}$ $\mathrm{dm}^{-3}$.

\section{Auxiliary Information}

\section{Method/Apparatus/Procedure:}

Constant-temperature bath, magnetic stirrer, centrifuge, and an ultraviolet visible spectrophotometer.

Excess solute and solvent were equilibrated in a constant-temperature bath with stirring for $48 \mathrm{~h}$. The saturated solution was then centrifuged for $10 \mathrm{~min}$, and an aliquot of the supernatant solution was removed and diluted quantitatively for spectrophotometric analysis. The concentration of the dissolved solute was determined from the measured absorbance.

\section{Source and Purity of Chemicals:}

(1) Purity not given, Fisher Scientific, UK, no purification details were provided

(2) Purity not given, Fisher Scientific, no purification details were provided.

\section{Estimated Error:}

Temperature: Insufficient experimental details to estimate. $c_{1}: \pm 0.02$

\begin{tabular}{|c|c|}
\hline $\begin{array}{l}\text { Components: } \\
\text { (1) 2-Hydroxybenzoic acid; } \mathrm{C}_{7} \mathrm{H}_{6} \mathrm{O}_{3} \text {; } \\
\text { [69-72-7] } \\
\text { (2) } 1-\text { Octanol; } \mathrm{C}_{8} \mathrm{H}_{18} \mathrm{O} ;[111-87-5]\end{array}$ & $\begin{array}{l}\text { Original Measurements: } \\
{ }^{156} \text { K. B. Sloan, K. G. Siver, and S. A. } \\
\text { M. Koch, J. Pharm. Sci. 75, } 744 \\
\text { (1986). }\end{array}$ \\
\hline $\begin{array}{l}\text { Variables: } \\
T / \mathrm{K}=296\end{array}$ & $\begin{array}{l}\text { Prepared by: } \\
\text { W. E. Acree, Jr. }\end{array}$ \\
\hline
\end{tabular}




\section{Experimental Values}

The measured solubility was reported to be $145 \mathrm{mg} / \mathrm{ml}$, which corresponds to a solubility of $c_{1}=1.050 \mathrm{~mol} \mathrm{dm}^{-3}$. The authors calculated a mole fraction solubility of $x_{1}=0.157$. The mole fraction solubility was calculated by the authors assuming a molar volume of 2-hydroxybenzoic acid of $V_{1}=93.9 \mathrm{~cm}^{3} \mathrm{~mol}^{-1}$, which seems too small compared to the molar volume of benzaldehyde, which is estimated to be $V_{1} \approx 101.1 \mathrm{~cm}^{3} \mathrm{~mol}^{-1}$, based on a molar mass of $106.12 \mathrm{~g} \mathrm{~mol}^{-1}$ and an experimental density of $1.0499 \mathrm{~g} \mathrm{~cm}^{-3}$ at $296 \mathrm{~K}$. A molar volume on the order of $V_{1} \approx 104 \mathrm{~cm}^{3} \mathrm{~mol}^{-1}$ would seem more realistic given the molecular structures of 2-hydroxybenzoic acid compared to benzaldehyde.

\section{Auxiliary Information}

Method/Apparatus/Procedure:

Magnetic stirrer and an ultraviolet/visible spectrophotometer.

Solubility was determined by stirring an excess of solute in the solvent with a magnetic stirrer at ambient room temperature for $24 \mathrm{~h}$ in sealed flasks that were thermally insulated from the stirrer. The suspension was gravity filtered through Whatman \#1 (qualitative) filter paper. The filtrate was then quantitatively diluted with methanol, and the absorbance of the diluted solution recorded at $306 \mathrm{~nm}$.

\section{Source and Purity of Chemicals:}

(1) Purity not given, Aldrich Chemical Company, Milwaukee, WI, USA, no purification details were provided.

(2) $99+\%$, Aldrich Chemical Company, used as received.

Estimated Error:

Temperature: $\pm 1 \mathrm{~K}$.

$x_{1}: \pm 5 \%$ (relative error, estimated by compiler).

\begin{tabular}{|c|c|}
\hline $\begin{array}{l}\text { Components: } \\
\text { (1) 2-Hydroxybenzoic acid; } \mathrm{C}_{7} \mathrm{H}_{6} \mathrm{O}_{3} \text {; } \\
\text { [69-72-7] } \\
\text { (2) 1-Decanol; } \mathrm{C}_{10} \mathrm{H}_{22} \mathrm{O} ;[112-30-1]\end{array}$ & $\begin{array}{l}\text { Original Measurements: } \\
{ }^{73} \text { M. Dias, S. L. Raghavan, and J. } \\
\text { Hadgraft, Int. J. Pharm. 216, } 51 \\
\text { (2001). }\end{array}$ \\
\hline $\begin{array}{l}\text { Variables: } \\
T / \mathrm{K}=305.2\end{array}$ & $\begin{array}{l}\text { Prepared by: } \\
\text { W. E. Acree, Jr. }\end{array}$ \\
\hline
\end{tabular}

\section{Experimental Values}

The measured solubility was reported to be $136.9 \mathrm{mg} / \mathrm{ml}$, which corresponds to a molar solubility of $c_{1}=0.991 \mathrm{~mol}$ $\mathrm{dm}^{-3}$.

\section{Auxiliary Information}

\section{Method/Apparatus/Procedure:}

Constant-temperature bath, magnetic stirrer, centrifuge, and an ultraviolet/ visible spectrophotometer.

Excess solute and solvent were equilibrated in a constant-temperature bath with stirring for $48 \mathrm{~h}$. The saturated solution was then centrifuged for $10 \mathrm{~min}$, and an aliquot of the supernatant solution was removed and diluted quantitatively for spectrophotometric analysis. The concentration of the dissolved solute was determined from the measured absorbance.

Source and Purity of Chemicals:

(1) Purity not given, Fisher Scientific, UK, no purification details were provided.

(2) Purity not given, Fisher Scientific, no purification details were provided.
Estimated Error:

Temperature: Insufficient experimental details to estimate. $c_{1}: \pm 0.02$.

\begin{tabular}{|c|c|}
\hline $\begin{array}{l}\text { Components: } \\
\text { (1) 2-Hydroxybenzoic acid; } \mathrm{C}_{7} \mathrm{H}_{6} \mathrm{O}_{3} \text {; } \\
\text { [69-72-7] } \\
\text { (2) Cyclohexanol; } \mathrm{C}_{6} \mathrm{H}_{12} \mathrm{O} ;[108-93-0]\end{array}$ & $\begin{array}{l}\text { Original Measurements: } \\
{ }^{150} \text { A. N. Paruta, B. J. Sciarrone, and } \\
\text { N. G. Lordi, J. Pharm. Sci. 53, } 1349 \\
\text { (1964). }\end{array}$ \\
\hline $\begin{array}{l}\text { Variables: } \\
T / \mathrm{K}=303.8\end{array}$ & $\begin{array}{l}\text { Prepared by: } \\
\text { W. E. Acree, Jr. }\end{array}$ \\
\hline
\end{tabular}

\section{Experimental Values}

The measured solubility was reported to be $217 \mathrm{mg} / \mathrm{ml}$ of solution, which corresponds to a solubility of $c_{1}=1.571 \mathrm{~mol}$ $\mathrm{dm}^{-3}$.

\section{Auxiliary Information}

\section{Method/Apparatus/Procedure:}

Excess solute and solvent were placed in 15-ml screw-capped vials fitted with Teflon liners. The vials were attached to a rotating wheel in a constanttemperature water bath and allowed to equilibrate for $24 \mathrm{~h}$. An aliquot of the saturated solution was withdrawn from the vial with a pipet fitted with a glass wool filtering plug. The solubility was determined by titration using a freshly prepared sodium hydroxide solution. The endpoint of the titration was detected with phenolphthalein.

Source and Purity of Chemicals:

(1) Purity not given, Chemical source not given, no purification details were provided.

(2) Purity not given, Chemical source not given, no purification details were provided.

Estimated Error:

Temperature: $\pm 0.2 \mathrm{~K}$.

$c_{1}: \pm 5 \%$ (relative error, estimated by compiler).

\begin{tabular}{ll}
\hline \hline Components: & Original Measurements: \\
$\begin{array}{l}\text { (1) 2-Hydroxybenzoic acid; } \mathrm{C}_{7} \mathrm{H}_{6} \mathrm{O}_{3} ; \\
\text { [69-72-7] }\end{array}$ & ${ }^{150}$ A. N. Paruta, B. J. Sciarrone, and \\
$\begin{array}{l}\text { (2) Benzenemethanol; } \mathrm{C}_{7} \mathrm{H}_{8} \mathrm{O} ; \\
\text { [100-51-6] }\end{array}$ & $(1964)$. \\
\hline Variables: & \\
$T / \mathrm{K}=303.8$ & Prepared by: \\
\hline
\end{tabular}

\section{Experimental Values}

The measured solubility was reported to be $203 \mathrm{mg} / \mathrm{ml}$ of solution, which corresponds to a solubility of $c_{1}=1.47 \mathrm{~mol}$ $\mathrm{dm}^{-3}$.

\section{Auxiliary Information}

\section{Method/Apparatus/Procedure:}

Excess solute and solvent were placed in 15-ml screw-capped vials fitted with Teflon liners. The vials were attached to a rotating wheel in a constanttemperature water bath and allowed to equilibrate for $24 \mathrm{~h}$. An aliquot of the saturated solution was withdrawn from the vial with a pipet fitted with a glass wool filtering plug. The solubility was determined by titration using a freshly prepared sodium hydroxide solution. The endpoint of the titration was detected with phenolphthalein 
Source and Purity of Chemicals:

(1) Purity not given, Chemical source not given, no purification details were provided.

(2) Purity not given, Chemical source not given, no purification details were provided.

Estimated Error:

Temperature: $\pm 0.2 \mathrm{~K}$.

$c_{1}: \pm 5 \%$ (relative error, estimated by compiler).

\section{Components:}

(1) 2-Hydroxybenzoic acid; $\mathrm{C}_{7} \mathrm{H}_{6} \mathrm{O}_{3}$;

[69-72-7]

(2) 1,2-Ethanediol; $\mathrm{C}_{2} \mathrm{H}_{6} \mathrm{O}_{2}$;

[107-21-1]

\begin{tabular}{ll}
\hline Variables: & Prepared by: \\
$T / \mathrm{K}=298.15$ & W. E. Acree, Jr. \\
\hline
\end{tabular}

Experimental Values

\begin{tabular}{lc}
\hline \hline$x_{2}{ }^{\mathrm{a}}$ & $x_{1}{ }^{\mathrm{b}}$ \\
\hline 0.9149 & 0.08509 \\
\hline$x_{2}$.
\end{tabular}

${ }^{\mathrm{a}} x_{2}$ : mole fraction of component 2 in the saturated solution.

${ }^{\mathrm{b}} x_{1}$ : mole fraction solubility of the solute.

\section{Auxiliary Information}

\section{Method/Apparatus/Procedure:}

Constant-temperature bath, analytical balance, and an ultraviolet/visible spectrophotometer.

Excess solute and solvent were placed in a flask and allowed to equilibrate for several days at constant temperature. The solid phase was removed by filtration, and the clear solution was diluted quantitatively with $96 \%$ ethanol. The concentration of the diluted solution was determined by spectrophotometric measurement at the wavelength of maximum absorption. In instances where the solvent interfered with the spectrophotometric determination, the samples were evaporated to dryness and the residue diluted with $96 \%$ ethanol.

\section{Source and Purity of Chemicals:}

(1) Purity not given, Sigma Chemical Company, USA, no purification details were provided.

(2) Spectrophotometric or Analytical grade, Chemical source not specified, no purification details were provided.

\section{Estimated Error:}

Temperature: $\pm 0.2 \mathrm{~K}$.

$x_{1}: \pm 3.0 \%$ (relative error, estimated by compiler).

\begin{tabular}{|c|c|}
\hline $\begin{array}{l}\text { Components: } \\
\text { (1) 2-Hydroxybenzoic acid; } \mathrm{C}_{7} \mathrm{H}_{6} \mathrm{O}_{3} \text {; } \\
\text { [69-72-7] } \\
\text { (2) } 1,2-\text { Ethanediol; } \mathrm{C}_{2} \mathrm{H}_{6} \mathrm{O}_{2} \text {; } \\
\text { [107-21-1] }\end{array}$ & $\begin{array}{l}\text { Original Measurements: } \\
{ }^{150} \text { A. N. Paruta, B. J. Sciarrone, and } \\
\text { N. G. Lordi, J. Pharm. Sci. 53, } 1349 \\
\text { (1964). }\end{array}$ \\
\hline $\begin{array}{l}\text { Variables: } \\
T / \mathrm{K}=303.8\end{array}$ & $\begin{array}{l}\text { Prepared by: } \\
\text { W. E. Acree, Jr. }\end{array}$ \\
\hline
\end{tabular}

\section{Experimental Values}

The measured solubility was reported to be $42 \mathrm{mg} / \mathrm{ml}$ of solution, which corresponds to a solubility of $c_{1}=0.304 \mathrm{~mol}$ $\mathrm{dm}^{-3}$.

\section{Auxiliary Information}

Method/Apparatus/Procedure:

Excess solute and solvent were placed in 15-ml screw-capped vials fitted with Teflon liners. The vials were attached to a rotating wheel in a constanttemperature water bath and allowed to equilibrate for $24 \mathrm{~h}$. An aliquot of the saturated solution was withdrawn from the vial with a pipet fitted with a glass wool filtering plug. The solubility was determined by titration using a freshly prepared sodium hydroxide solution. The endpoint of the titration was detected with phenolphthalein.

\section{Source and Purity of Chemicals:}

(1) Purity not given, Chemical source not given, no purification details were provided.

(2) Purity not given, Chemical source not given, no purification details were provided.

\section{Estimated Error:}

Temperature: $\pm 0.2 \mathrm{~K}$.

$c_{1}: \pm 5 \%$ (relative error, estimated by compiler).

\begin{tabular}{|c|c|}
\hline $\begin{array}{l}\text { Components: } \\
\text { (1) 2-Hydroxybenzoic acid; } \mathrm{C}_{7} \mathrm{H}_{6} \mathrm{O}_{3} \text {; } \\
\text { [69-72-7] } \\
\text { (2) } 1,2 \text {-Propanediol; } \mathrm{C}_{3} \mathrm{H}_{8} \mathrm{O}_{2} ;[57-55-6]\end{array}$ & $\begin{array}{l}\text { Original Measurements: } \\
{ }^{102} \text { J. Barra, M.-A. Peña, and P. } \\
\text { Bustamante, Eur. J. Pharm. Sci. 10, } \\
153 \text { (2000). }\end{array}$ \\
\hline $\begin{array}{l}\text { Variables: } \\
T / \mathrm{K}=298.15\end{array}$ & $\begin{array}{l}\text { Prepared by: } \\
\text { W. E. Acree, Jr. }\end{array}$ \\
\hline
\end{tabular}

Experimental Values

\begin{tabular}{lc}
\hline \hline$x_{2}{ }^{\mathrm{a}}$ & $x_{1}{ }^{\mathrm{b}}$ \\
\hline 0.8864 & 0.1136 \\
\hline${ }^{\mathrm{a}} x_{2}:$ mole fraction of component 2 in the saturated solution. & \\
${ }^{\mathrm{b}} x_{1}:$ mole fraction solubility of the solute. &
\end{tabular}

\section{Auxiliary Information}

\section{Method/Apparatus/Procedure:}

Constant-temperature bath, analytical balance, and an ultraviolet/visible spectrophotometer.

Excess solute and solvent were placed in a flask and allowed to equilibrate for several days at constant temperature. The solid phase was removed by filtration, and the clear solution was diluted quantitatively with $96 \%$ ethanol. The concentration of the diluted solution was determined by spectrophotometric measurement at the wavelength of maximum absorption. In instances where the solvent interfered with the spectrophotometric determination, the samples were evaporated to dryness and the residue diluted with $96 \%$ ethanol.

\section{Source and Purity of Chemicals:}

(1) Purity not given, Sigma Chemical Company, USA, no purification details were provided.

(2) Spectrophotometric or Analytical grade, Chemical source not specified, no purification details were provided.

Estimated Error:

Temperature: $\pm 0.2 \mathrm{~K}$.

$x_{1}: \pm 3.0 \%$ (relative error, estimated by compiler). 


\section{Components:}

(1) 2-Hydroxybenzoic acid; $\mathrm{C}_{7} \mathrm{H}_{6} \mathrm{O}_{3}$; [69-72-7]

(2) 1,2-Propanediol; $\mathrm{C}_{3} \mathrm{H}_{8} \mathrm{O}_{2} ;$ [57-55-6]

\section{Variables:}

Temperature
Original Measurements:

${ }^{160}$ A. Jouyban, V. Panahi-Azar, and F. Khonsari, J. Mol. Liq. 160, 14 (2011)

Prepared by:

W. E. Acree, Jr.

Experimental Values

\begin{tabular}{lc}
\hline \hline$T / \mathrm{K}$ & $c_{1}{ }^{\mathrm{a}}$ \\
\hline 298.2 & 1.592 \\
308.2 & 2.144 \\
318.2 & 3.061 \\
328.2 & 3.382 \\
\hline${ }^{\mathrm{a}} c_{1}$ : molar solubility of the solute in units of moles per liter. &
\end{tabular}

\section{Auxiliary Information}

\section{Method/Apparatus/Procedure:}

Constant-temperature incubator, temperature controlling system, and ultraviolet/visible spectrophotometer.

Excess solute and solvent were placed in sealed bottles and allowed to equilibrate at constant temperature in an incubator equipped with a temperature controlling system for at least two days. Solutions of the saturated solution were withdrawn and filtered using hydrophilic Durapore filters $(0.45 \mu \mathrm{m})$. The filtered solutions were quantitatively diluted. Absorbances of the diluted solutions were measured. Concentrations of the diluted solutions were computed using a Beer-Law calibration curve based on measured absorbances for standard solutions of known concentration.

\section{Source and Purity of Chemicals:}

(1) $98+\%$, Merck Chemical Company, Germany, was used as received.

(2) $99.5 \%$, Merck Chemical Company, no purification details were provided.

\section{Estimated Error:}

Temperature: $\pm 0.2 \mathrm{~K}$.

$c_{1}: \pm 3 \%$ (relative error).

\begin{tabular}{|c|c|}
\hline $\begin{array}{l}\text { Components: } \\
\text { (1) 2-Hydroxybenzoic acid; } \mathrm{C}_{7} \mathrm{H}_{6} \mathrm{O}_{3} \text {; } \\
\text { [69-72-7] } \\
\text { (2) 1,2-Propanediol; } \mathrm{C}_{3} \mathrm{H}_{8} \mathrm{O}_{2} ;[57-55-6]\end{array}$ & $\begin{array}{l}\text { Original Measurements: } \\
{ }^{73} \text { M. Dias, S. L. Raghavan, and } \\
\text { J. Hadgraft, Int. J. Pharm. 216, } \\
51 \text { (2001). }\end{array}$ \\
\hline $\begin{array}{l}\text { Variables: } \\
T / \mathrm{K}=305.2\end{array}$ & $\begin{array}{l}\text { Prepared by: } \\
\text { W. E. Acree, Jr. }\end{array}$ \\
\hline
\end{tabular}

\section{Experimental Values}

The measured solubility was reported to be $192.8 \mathrm{mg} / \mathrm{ml}$, which corresponds to a molar solubility of $c_{1}=1.396 \mathrm{~mol} \mathrm{dm}^{-3}$.

\section{Auxiliary Information}

\section{Method/Apparatus/Procedure:}

Constant-temperature bath, magnetic stirrer, centrifuge, and an ultraviolet/ visible spectrophotometer.

Excess solute and solvent were equilibrated in a constant-temperature bath with stirring for $48 \mathrm{~h}$. The saturated solution was then centrifuged for $10 \mathrm{~min}$, and an aliquot of the supernatant solution was removed and diluted quantitatively for spectrophotometric analysis. The concentration of the dissolved solute was determined from the measured absorbance.

Source and Purity of Chemicals:

(1) Purity not given, Fisher Scientific, UK, no purification details were provided.

(2) Purity not given, Fisher Scientific, no purification details were provided.

\section{Estimated Error:}

Temperature: Insufficient experimental details to estimate. $c_{1}: \pm 0.14$

\begin{tabular}{ll}
\hline \hline Components: & Original Measurements: \\
$\begin{array}{l}\text { (1) 2-Hydroxybenzoic acid; } \mathrm{C}_{7} \mathrm{H}_{6} \mathrm{O}_{3} ; \\
\text { [69-72-7] }\end{array}$ & $\begin{array}{l}{ }^{150} \text { A. N. Paruta, B. J. Sciarrone, } \\
\text { and N. G. Lordi, J. Pharm. Sci. }\end{array}$ \\
$\begin{array}{ll}\text { (2) 1,2-Propanediol; } \mathrm{C}_{3} \mathrm{H}_{8} \mathrm{O}_{2} ;[57-55-6] & \mathbf{5 3}, 1349(1964) .\end{array}$ \\
\hline Variables: & Prepared by: \\
$T / \mathrm{K}=303.8$ & W. E. Acree, Jr. \\
\hline
\end{tabular}

\section{Experimental Values}

The measured solubility was reported to be $118 \mathrm{mg} / \mathrm{ml}$ of solution, which corresponds to a solubility of $c_{1}=0.854 \mathrm{~mol}$ $\mathrm{dm}^{-3}$.

\section{Auxiliary Information}

\section{Method/Apparatus/Procedure:}

Excess solute and solvent were placed in 15-ml screw-capped vials fitted with Teflon liners. The vials were attached to a rotating wheel in a constanttemperature water bath and allowed to equilibrate for $24 \mathrm{~h}$. An aliquot of the saturated solution was withdrawn from the vial with a pipet fitted with a glass wool filtering plug. The solubility was determined by titration using a freshly prepared sodium hydroxide solution. The endpoint of the titration was detected with phenolphthalein.

Source and Purity of Chemicals:

(1) Purity not given, Chemical source not given, no purification details were provided.

(2) Purity not given, Chemical source not given, no purification details were provided.

Estimated Error:

Temperature: $\pm 0.2 \mathrm{~K}$.

$c_{1}: \pm 5 \%$ (relative error, estimated by compiler)

\begin{tabular}{|c|c|}
\hline $\begin{array}{l}\text { Components: } \\
\text { (1) 2-Hydroxybenzoic acid; } \mathrm{C}_{7} \mathrm{H}_{6} \mathrm{O}_{3} \text {; } \\
\text { [69-72-7] } \\
\text { (2) 1,2-Propanediol; } \mathrm{C}_{3} \mathrm{H}_{8} \mathrm{O}_{2} ;[57-55-6]\end{array}$ & $\begin{array}{l}\text { Original Measurements: } \\
{ }^{156} \text { K. B. Sloan, K. G. Siver, and } \\
\text { S. A. M. Koch, J. Pharm. Sci. 75, } \\
744 \text { (1986). }\end{array}$ \\
\hline $\begin{array}{l}\text { Variables: } \\
T / \mathrm{K}=296\end{array}$ & $\begin{array}{l}\text { Prepared by: } \\
\text { W. E. Acree, Jr. }\end{array}$ \\
\hline
\end{tabular}

\section{Experimental Values}

The measured solubility was reported to be $207 \mathrm{mg} / \mathrm{ml}$, which corresponds to a solubility of $c_{1}=1.499 \mathrm{~mol} \mathrm{dm}^{-3}$. The authors calculated a mole fraction solubility of $x_{1}=0.116$. The mole fraction solubility was calculated by the authors assuming a molar volume of 2-hydroxybenzoic acid of $V_{1}=93.9 \mathrm{~cm}^{3} \mathrm{~mol}^{-1}$, which seems too small compared to the molar volume of benzaldehyde, which is estimated to be $V_{1} \approx 101.1 \mathrm{~cm}^{3} \mathrm{~mol}^{-1}$, based on a molar mass of $106.12 \mathrm{~g} \mathrm{~mol}^{-1}$ and an experimental 
density of $1.0499 \mathrm{~g} \mathrm{~cm}^{-3}$ at $296 \mathrm{~K}$. A molar volume on the order of $V_{1} \approx 104 \mathrm{~cm}^{3} \mathrm{~mol}^{-1}$ would seem more realistic given the molecular structures of 2-hydroxybenzoic acid compared to benzaldehyde.

\section{Auxiliary Information}

\section{Method/Apparatus/Procedure:}

Magnetic stirrer and an ultraviolet/visible spectrophotometer.

Solubility was determined by stirring an excess of solute in the solvent with a magnetic stirrer at ambient room temperature for $24 \mathrm{~h}$ in sealed flasks that were thermally insulated from the stirrer. The suspension was gravity filtered through Whatman \#1 (qualitative) filter paper. The filtrate was then quantitatively diluted with methanol, and the absorbance of the diluted solution recorded at $306 \mathrm{~nm}$.

\section{Source and Purity of Chemicals:}

(1) Purity not given, Aldrich Chemical Company, Milwaukee, WI, USA, no purification details were provided.

(2) $99+\%$, Aldrich Chemical Company, used as received.

\section{Estimated Error:}

Temperature: $\pm 1 \mathrm{~K}$.

$x_{1}: \pm 5 \%$ (relative error, estimated by compiler).

\begin{tabular}{ll}
\hline \hline Components: & Original Measurements: \\
$\begin{array}{l}\text { (1) 2-Hydroxybenzoic acid; } \mathrm{C}_{7} \mathrm{H}_{6} \mathrm{O}_{3} ; \\
\text { [69-72-7] }\end{array}$ & $\begin{array}{l}{ }^{6} \text { R. Cooper, J. Controlled } \\
\text { (2) 1,2-Propanediol; } \mathrm{C}_{3} \mathrm{H}_{8} \mathrm{O}_{2} ;[57-55-6]\end{array}$ \\
\hline Variables & \\
$T / \mathrm{K}=295$ & Prepared by: \\
\hline
\end{tabular}

\section{Experimental Values}

\begin{tabular}{lc}
\hline \hline$x_{2}^{\mathrm{a}}$ & $x_{1}{ }^{\mathrm{b}}$ \\
\hline 0.872 & 0.128
\end{tabular}

${ }^{\mathrm{a}} x_{2}$ : mole fraction of component 2 in the saturated solution.

${ }^{b} x_{1}$ : mole fraction solubility of the solute. Solubility data reported in terms of mass percent. Mole fraction solubility calculated by the compiler.

\section{Auxiliary Information}

\section{Method/Apparatus/Procedure:}

Very little experimental details provided. Solubility determinations were made by stirring the solvent with excess solute for two days, centrifuging the sample, and analyzing the supernatant.

\section{Source and Purity of Chemicals:}

(1) Reagent grade, Fisher Scientific, Cincinnati, OH, USA, no purification details provided.

(2) Reagent grade, J.T. Baker Chemical Company, Phillipsburg, NJ, USA, no purification details provided.

\section{Estimated Error:}

Temperature: No information given.

$x_{1}$ : No information given.

\begin{tabular}{|c|c|}
\hline $\begin{array}{l}\text { Components: } \\
\text { (1) 2-Hydroxybenzoic acid; } \mathrm{C}_{7} \mathrm{H}_{6} \mathrm{O}_{3} \text {; } \\
\text { [69-72-7] } \\
\text { (2) } 1,2,3-\text { Propanetriol (Glycerol); } \\
\mathrm{C}_{3} \mathrm{H}_{8} \mathrm{O}_{3} ;[60-29-7]\end{array}$ & $\begin{array}{l}\text { Original Measurements: } \\
{ }^{102} \text { J. Barra, M.-A. Peña, and P. } \\
\text { Bustamante, Eur. J. Pharm. Sci. 10, } \\
153 \text { (2000). }\end{array}$ \\
\hline $\begin{array}{l}\text { Variables: } \\
T / \mathrm{K}=298.15\end{array}$ & $\begin{array}{l}\text { Prepared by: } \\
\text { W. E. Acree, Jr. }\end{array}$ \\
\hline
\end{tabular}

Experimental Values

\begin{tabular}{lc}
\hline \hline$x_{2}{ }^{\mathrm{a}}$ & $x_{1}{ }^{\mathrm{b}}$ \\
\hline 0.9253 & 0.07465
\end{tabular}

${ }^{\mathrm{a}} x_{2}$ : mole fraction of component 2 in the saturated solution.

${ }^{b} x_{1}$ : mole fraction solubility of the solute.

\section{Auxiliary Information}

\section{Method/Apparatus/Procedure:}

Constant-temperature bath, analytical balance, and an ultraviolet/visible spectrophotometer.

Excess solute and solvent were placed in a flask and allowed to equilibrate for several days at constant temperature. The solid phase was removed by filtration, and the clear solution was diluted quantitatively with $96 \%$ ethanol. The concentration of the diluted solution was determined by spectrophotometric measurement at the wavelength of maximum absorption. In instances where the solvent interfered with the spectrophotometric determination, the samples were evaporated to dryness and the residue diluted with $96 \%$ ethanol.

\section{Source and Purity of Chemicals:}

(1) Purity not given, Sigma Chemical Company, USA, no purification details were provided.

(2) Spectrophotometric or Analytical grade, Chemical source not specified, no purification details were provided.

\section{Estimated Error:}

Temperature: $\pm 0.2 \mathrm{~K}$.

$x_{1}: \pm 3.0 \%$ (relative error, estimated by compiler).

\begin{tabular}{|c|c|}
\hline $\begin{array}{l}\text { Components: } \\
\text { (1) 2-Hydroxybenzoic acid; } \mathrm{C}_{7} \mathrm{H}_{6} \mathrm{O}_{3} \text {; } \\
\text { [69-72-7] } \\
\text { (2) 1,2,3-Propanetriol (Glycerol); } \\
\mathrm{C}_{3} \mathrm{H}_{8} \mathrm{O}_{3} ;[60-29-7]\end{array}$ & $\begin{array}{l}\text { Original Measurements: } \\
{ }^{150} \text { A. N. Paruta, B. J. Sciarrone, and } \\
\text { N. G. Lordi, J. Pharm. Sci. 53, } 1349 \\
(1964) \text {. }\end{array}$ \\
\hline $\begin{array}{l}\text { Variables: } \\
T / \mathrm{K}=303.8\end{array}$ & $\begin{array}{l}\text { Prepared by: } \\
\text { W. E. Acree, Jr. }\end{array}$ \\
\hline
\end{tabular}

\section{Experimental Values}

The measured solubility was reported to be $15 \mathrm{mg} / \mathrm{ml}$ of solution, which corresponds to a solubility of $c_{1}=0.109 \mathrm{~mol}$ $\mathrm{dm}^{-3}$. 


\section{Auxiliary Information}

\section{Method/Apparatus/Procedure:}

Excess solute and solvent were placed in 15-ml screw-capped vials fitted with Teflon liners. The vials were attached to a rotating wheel in a constanttemperature water bath and allowed to equilibrate for $24 \mathrm{~h}$. An aliquot of the saturated solution was withdrawn from the vial with a pipet fitted with a glass wool filtering plug. The solubility was determined by titration using a freshly prepared sodium hydroxide solution. The endpoint of the titration was detected with phenolphthalein.

\section{Source and Purity of Chemicals:}

(1) Purity not given, Chemical source not given, no purification details were provided.

(2) Purity not given, Chemical source not given, no purification details were provided.

\section{Estimated Error:}

Temperature: $\pm 0.2 \mathrm{~K}$

$c_{1}: \pm 5 \%$ (relative error, estimated by compiler).

\begin{tabular}{ll}
\hline \hline Components: & Original Measurements: \\
$\begin{array}{l}\text { (1) 2-Hydroxybenzoic acid; } \mathrm{C}_{7} \mathrm{H}_{6} \mathrm{O}_{3} ; \\
\text { [69-72-7] }\end{array}$ & ${ }^{87}$ M. Dias, J. Hadgraft, and M. E. \\
Lane, Int. J. Pharm. 336, 108 (2007). \\
(2) $1,2,3$-Propanetriol (Glycerol); & \\
$\mathrm{C}_{3} \mathrm{H}_{8} \mathrm{O}_{3} ;$ [56-81-5] & \\
\hline Variables: & Prepared by: \\
$T / \mathrm{K}=305.2$ & W. E. Acree, Jr. \\
\hline
\end{tabular}

Experimental Values

The measured solubility was reported to be $24.99 \mathrm{mg} / \mathrm{ml}$, which corresponds to a molar solubility of $c_{1}=0.181 \mathrm{~mol}$ $\mathrm{dm}^{-3}$.

\section{Auxiliary Information}

\section{Method/Apparatus/Procedure:}

Constant-temperature bath, magnetic stirrer, centrifuge, and an ultraviolet/ visible spectrophotometer.

Excess solute and solvent were equilibrated in a constant-temperature bath with stirring for $48 \mathrm{~h}$. The saturated solution was then centrifuged for $10 \mathrm{~min}$ and an aliquot of the supernatant solution was removed and diluted quantitatively for spectrophotometric analysis. The concentration of the dissolved solute was determined from the measured absorbance.

\section{Source and Purity of Chemicals:}

(1) Purity not given, Fisher Scientific Equipment, UK, no purification details were provided.

(2) Purity not given, ICN Biochemicals, no purification details were provided.

\section{Estimated Error:}

Temperature: Insufficient experimental details to estimate. $c_{1}: \pm 0.022$.

\subsection{2-Hydroxybenzoic acid solubility data in alkoxyalcohols}

\begin{tabular}{|c|c|}
\hline $\begin{array}{l}\text { Components: } \\
\text { (1) 2-Hydroxybenzoic acid; } \\
\mathrm{C}_{7} \mathrm{H}_{6} \mathrm{O}_{3} ; \text {;69-72-7] } \\
\text { (2) 2-Ethoxyethanol; } \mathrm{C}_{4} \mathrm{H}_{10} \mathrm{O}_{2} \text {; } \\
\text { [110-80-5] }\end{array}$ & $\begin{array}{l}\text { Original Measurements: } \\
{ }^{150} \text { A. N. Paruta, B. J. Sciarrone, and } \\
\text { N. G. Lordi, J. Pharm. Sci. 53, } 1349 \\
\text { (1964). }\end{array}$ \\
\hline $\begin{array}{l}\text { Variables: } \\
T / \mathrm{K}=303.8\end{array}$ & $\begin{array}{l}\text { Prepared by: } \\
\text { W. E. Acree, Jr. }\end{array}$ \\
\hline
\end{tabular}

\section{Experimental Values}

The measured solubility was reported to be $425 \mathrm{mg} / \mathrm{ml}$ of solution, which corresponds to a solubility of $c_{1}=3.08 \mathrm{~mol}$ $\mathrm{dm}^{-3}$.

\section{Auxiliary Information}

\section{Method/Apparatus/Procedure:}

Excess solute and solvent were placed in 15-ml screw-capped vials fitted with Teflon liners. The vials were attached to a rotating wheel in a constanttemperature water bath and allowed to equilibrate for $24 \mathrm{~h}$. An aliquot of the saturated solution was withdrawn from the vial with a pipet fitted with a glass wool filtering plug. The solubility was determined by titration using a freshly prepared sodium hydroxide solution. The endpoint of the titration was detected with phenolphthalein.

Source and Purity of Chemicals:

(1) Purity not given, Chemical source not given, no purification details were provided.

(2) Purity not given, Chemical source not given, no purification details were provided.

\section{Estimated Error:}

Temperature: $\pm 0.2 \mathrm{~K}$.

$c_{1}: \pm 5 \%$ (relative error, estimated by compiler).

\subsection{2-Hydroxybenzoic acid solubility data in ketones}

\begin{tabular}{|c|c|}
\hline $\begin{array}{l}\text { Components: } \\
\text { (1) 2-Hydroxybenzoic acid; } \mathrm{C}_{7} \mathrm{H}_{6} \mathrm{O}_{3} \text {; } \\
\text { [69-72-7] } \\
\text { (2) Propanone; } \mathrm{C}_{3} \mathrm{H}_{6} \mathrm{O} ;[67-64-1]\end{array}$ & $\begin{array}{l}\text { Original Measurements: } \\
{ }^{162} \text { M. A. A. Fakhree, S. Ahmadian, } \\
\text { V. Panahi-Azar, W. E. Acree, Jr., } \\
\text { and A. Jouyban, J. Chem. Eng. Data } \\
\text { 57, } 3303 \text { (2012). }\end{array}$ \\
\hline $\begin{array}{l}\text { Variables: } \\
\text { Temperature }\end{array}$ & $\begin{array}{l}\text { Prepared by: } \\
\text { W. E. Acree, Jr. }\end{array}$ \\
\hline
\end{tabular}

Experimental Values

\begin{tabular}{lcc}
\hline \hline$T / \mathrm{K}$ & $x_{2}{ }^{\mathrm{a}}$ & $x_{1}{ }^{\mathrm{b}}$ \\
\hline 298.2 & 0.863 & 0.137 \\
308.2 & 0.861 & 0.139 \\
318.2 & 0.844 & 0.156 \\
328.2 & 0.804 & 0.196 \\
\hline
\end{tabular}

${ }^{a} x_{2}$ : mole fraction of component 2 in the saturated solution.

${ }^{\mathrm{b}} x_{1}$ : mole fraction solubility of the solute. 


\section{Auxiliary Information}

\section{Method/Apparatus/Procedure:}

Constant temperature oven, temperature controlling system, and ultraviolet/ visible spectrophotometer.

Excess solute and solvent were placed in sealed bottles and allowed to equilibrate at constant temperature in an oven for three days. Samples were shaken manually three times per day to ensure that equilibrium had been achieved. Solutions of the saturated solution were withdrawn and filtered using hydrophilic Durapore filters $(0.45 \mu \mathrm{m})$. The filtered solutions were quantitatively diluted with ethanol. Absorbances of the diluted solutions were measured at $304 \mathrm{~nm}$. Concentrations of the diluted solutions were computed using a Beer-Law calibration curve based on measured absorbances for standard solutions of known concentration.

\section{Source and Purity of Chemicals:}

(1) $99 \%$, Merck Chemical Company, Germany, was used as received.

(2) $99.5 \%$, Scharlau Chemie, Spain, was used as received.

\section{Estimated Error:}

Temperature: $\pm 0.2 \mathrm{~K}$ to $\pm 0.6 \mathrm{~K}$, the higher temperatures have greater uncertainty.

$x_{1}: \pm 1 \%$ (relative error)

\section{Components:}

(1) 2-Hydroxybenzoic acid; $\mathrm{C}_{7} \mathrm{H}_{6} \mathrm{O}_{3}$; [69-72-7]

(2) Propanone; $\mathrm{C}_{3} \mathrm{H}_{6} \mathrm{O}$; [67-64-1]

Original Measurements:

${ }^{151}$ K. M. De Fina, T. L. Sharp, L. E. Roy, and W. E. Acree, Jr., J. Chem. Eng. Data 44, 1262 (1999).

\section{Variables:}

$T / \mathrm{K}=298.15$

Prepared by:

W. E. Acree, Jr.

\section{Experimental Values}

\begin{tabular}{lc}
\hline \hline$x_{2}{ }^{\mathrm{a}}$ & $x_{1}{ }^{\mathrm{b}}$ \\
\hline 0.8183 & 0.1817 \\
\hline${ }^{\mathrm{a}} x_{2}:$ mole fraction of component 2 in the saturated solution. & \\
${ }^{\mathrm{b}} x_{1}:$ mole fraction solubility of the solute. &
\end{tabular}

\section{Auxiliary Information}

\section{Method/Apparatus/Procedure:}

Constant-temperature bath, calorimetric thermometer, and an ultraviolet/ visible spectrophotometer.

Excess solute and solvent were placed in amber glass bottles and allowed to equilibrate for several days at constant temperature. Attainment of equilibrium was verified by several repetitive measurements and by approaching equilibrium from supersaturation. Aliquots of saturated solutions were transferred through a coarse filter into tared volumetric flasks, weighed, and diluted with methanol. Concentrations were determined by spectrophotometric measurements at $304 \mathrm{~nm}$.

\section{Source and Purity of Chemicals:}

(1) $99+\%$, ACS Reagent grade, Aldrich Chemical Company, Milwaukee, WI, USA, was dried for several hours at $353 \mathrm{~K}$ before use and used without further purification.

(2) $99.9+\%$, HPLC grade, Aldrich Chemical Company, stored over molecular sieves before use.

\section{Estimated Error:}

Temperature: $\pm 0.1 \mathrm{~K}$.

$x_{1}: \pm 1.5 \%$ (relative error).

\begin{tabular}{ll}
\hline \hline $\begin{array}{l}\text { Components: } \\
\text { (1) 2-Hydroxybenzoic acid; } \mathrm{C}_{7} \mathrm{H}_{6} \mathrm{O}_{3} ;\end{array}$ & $\begin{array}{l}\text { Original Measurements: } \\
\text { [69-72-7] }\end{array}$ \\
$\begin{array}{l}\text { (2) Propanone; } \mathrm{C}_{3} \mathrm{H}_{6} \mathrm{O} ;[67-64-1] \\
\text { Bustamante, Eur. J. Pharm. Sci. 10, }\end{array}$ & $153(2000)$. \\
\hline Variables: & Prepared by: \\
$T / \mathrm{K}=298.15$ & W. E. Acree, Jr. \\
\hline
\end{tabular}

\section{Experimental Values}

\begin{tabular}{lc}
\hline \hline$x_{2}{ }^{\mathrm{a}}$ & $x_{1}{ }^{\mathrm{b}}$ \\
\hline 0.8680 & 0.1320 \\
\hline${ }^{a} x_{2}:$ mole fraction of component 2 in the saturated solution. & \\
${ }^{\mathrm{b}} x_{1}:$ mole fraction solubility of the solute. &
\end{tabular}

\section{Auxiliary Information}

\section{Method/Apparatus/Procedure:}

Constant-temperature bath, analytical balance, and an ultraviolet/visible spectrophotometer.

Excess solute and solvent were placed in a flask and allowed to equilibrate for several days at constant temperature. The solid phase was removed by filtration, and the clear solution was diluted quantitatively with $96 \%$ ethanol. The concentration of the diluted solution was determined by spectrophotometric measurement at the wavelength of maximum absorption. In instances where the solvent interfered with the spectrophotometric determination, the samples were evaporated to dryness and the residue diluted with $96 \%$ ethanol.

\section{Source and Purity of Chemicals:}

(1) Purity not given, Sigma Chemical Company, USA, no purification details were provided.

(2) Spectrophotometric or Analytical grade, Chemical source not specified, no purification details were provided.

\section{Estimated Error:}

Temperature: $\pm 0.2 \mathrm{~K}$.

$x_{1}: \pm 3.0 \%$ (relative error, estimated by compiler).

\begin{tabular}{|c|c|}
\hline $\begin{array}{l}\text { Components: } \\
\text { (1) 2-Hydroxybenzoic acid; } \mathrm{C}_{7} \mathrm{H}_{6} \mathrm{O}_{3} \text {; } \\
\text { [69-72-7] } \\
\text { (2) Propanone; } \mathrm{C}_{3} \mathrm{H}_{6} \mathrm{O} ;[67-64-1]\end{array}$ & $\begin{array}{l}\text { Original Measurements: } \\
{ }^{158} \text { E. Bergroth, Farm. Aikak. 70, } 91 \\
\text { (1961). }\end{array}$ \\
\hline $\begin{array}{l}\text { Variables: } \\
T / \mathrm{K}=293.15\end{array}$ & $\begin{array}{l}\text { Prepared by: } \\
\text { W. E. Acree, Jr. }\end{array}$ \\
\hline
\end{tabular}

\section{Experimental Values}

The measured solubility was reported to be $29.44 \mathrm{~g} / 100 \mathrm{ml}$ of solution, which corresponds to a molar concentration of $2.131 \mathrm{~mol} \mathrm{dm}^{-3}$.

\section{Auxiliary Information}

\section{Method/Apparatus/Procedure:}

Solubility was determined using a gravimetric method. Excess solute and solvent were placed in a solubility vessel. The apparatus was closed tightly with rubber stoppers and allowed to equilibrate in a constant-temperature water bath with stirring. A known aliquot of the saturated solution was removed, filtered, and transferred to an evaporating dish. The solvent was carefully removed over a steam bath. The evaporating dish was then placed in a drying oven, and later removed and weighed. The solubility was calculated from the mass of the solid residue and volume of saturated sample analyzed. 
Source and Purity of Chemicals:

(1) Purity not given, Schuchardt Chemicals, no purification details were provided.

(2) Purity not given, Chemical source not given, no purification details were provided.

\section{Estimated Error:}

Temperature: $\pm 0.2 \mathrm{~K}$ (estimated by compiler).

$c_{1}: \pm 5 \%$ (relative error, estimated by compiler).

\section{Components:}

(1) 2-Hydroxybenzoic acid; $\mathrm{C}_{7} \mathrm{H}_{6} \mathrm{O}_{3}$;

[69-72-7]

(2) Propanone; $\mathrm{C}_{3} \mathrm{H}_{6} \mathrm{O} ;[67-64-1]$

Variables:

$T / \mathrm{K}=303.8$

\section{Original Measurements:}

${ }^{150}$ A. N. Paruta, B. J. Sciarrone, and N. G. Lordi, J. Pharm. Sci. 53, 1349 (1964).

Prepared by:

W. E. Acree, Jr.

\section{Experimental Values}

The measured solubility was reported to be $381 \mathrm{mg} / \mathrm{ml}$ of solution, which corresponds to a solubility of $c_{1}=2.76 \mathrm{~mol}$ $\mathrm{dm}^{-3}$.

\section{Auxiliary Information}

\section{Method/Apparatus/Procedure:}

Excess solute and solvent were placed in 15-ml screw-capped vials fitted with Teflon liners. The vials were attached to a rotating wheel in a constanttemperature water bath and allowed to equilibrate for $24 \mathrm{~h}$. An aliquot of the saturated solution was withdrawn from the vial with a pipet fitted with a glass wool filtering plug. The solubility was determined by titration using a freshly prepared sodium hydroxide solution. The endpoint of the titration was detected with phenolphthalein.

\section{Source and Purity of Chemicals:}

(1) Purity not given, Chemical source not given, no purification details were provided.

(2) Purity not given, Chemical source not given, no purification details were provided.

Estimated Error:

Temperature: $\pm 0.2 \mathrm{~K}$.

$c_{1}: \pm 5 \%$ (relative error, estimated by compiler).

\begin{tabular}{|c|c|}
\hline $\begin{array}{l}\text { Components: } \\
\text { (1) 2-Hydroxybenzoic acid; } \mathrm{C}_{7} \mathrm{H}_{6} \mathrm{O}_{3} \text {; } \\
\text { [69-72-7] } \\
\text { (2) Propanone; } \mathrm{C}_{3} \mathrm{H}_{6} \mathrm{O} ;[67-64-1]\end{array}$ & $\begin{array}{l}\text { Original Measurements: } \\
\text { 50 P. G. Desai and A. M. Patel, J. Ind. } \\
\text { Chem. Soc. 12, } 131 \text { (1935). }\end{array}$ \\
\hline $\begin{array}{l}\text { Variables: } \\
T / \mathrm{K}=301.2\end{array}$ & $\begin{array}{l}\text { Prepared by: } \\
\text { W. E. Acree, Jr. }\end{array}$ \\
\hline
\end{tabular}

Experimental Values

\begin{tabular}{lc}
\hline \hline$x_{2}{ }^{\mathrm{a}}$ & $x_{1}{ }^{\mathrm{b}}$ \\
\hline 0.8094 & 0.1906 \\
\hline
\end{tabular}

${ }^{\mathrm{a}} x_{2}$ : mole fraction of component 2 in the saturated solution.

${ }^{\mathrm{b}} x_{1}$ : mole fraction solubility of the solute.

\section{Auxiliary Information}

\section{Method/Apparatus/Procedure:}

Excess solute and solvent were placed in a glass flask which was kept revolving on a wheel in an air thermostat for two days. After two days of equilibration, the solution was filtered. To minimize absorption of the solute onto the filter paper, a $100 \mathrm{ml}$ portion of fresh saturated solution was first filtered through the paper before filtering the sample to be analyzed. An aliquot of the filtrate was titrated with barium hydroxide using phenolphthalein as the endpoint indictor. The barium hydroxide titrant was previously standardized against a solution of succinic acid.

Source and Purity of Chemicals:

(1) Purity not given, Chemical source not specified, no information provided concerning purification.

(2) Purity not given, Chemical source not specified, no information provided concerning purification.

Estimated Error:

Temperature: No information given

$x_{1}: \pm 3 \%$ (relative error, estimated by compiler).

\begin{tabular}{|c|c|}
\hline $\begin{array}{l}\text { Components: } \\
\text { (1) 2-Hydroxybenzoic acid; } \mathrm{C}_{7} \mathrm{H}_{6} \mathrm{O}_{3} \text {; } \\
\text { [69-72-7] } \\
\text { (2) Propanone; } \mathrm{C}_{3} \mathrm{H}_{6} \mathrm{O} ;[67-64-1]\end{array}$ & $\begin{array}{l}\text { Original Measurements: } \\
{ }^{149} \text { J. W. Marden and M. V. Dover, J. } \\
\text { Am. Chem. Soc. 39, } 1 \text { (1917). }\end{array}$ \\
\hline $\begin{array}{l}\text { Variables: } \\
T / \mathrm{K}=298.15\end{array}$ & $\begin{array}{l}\text { Prepared by: } \\
\text { W. E. Acree, Jr. }\end{array}$ \\
\hline
\end{tabular}

Experimental Values

\begin{tabular}{lc}
\hline \hline$x_{2}{ }^{\mathrm{a}}$ & $x_{1}{ }^{\mathrm{b}}$ \\
\hline 0.8108 & 0.1892 \\
${ }^{\mathrm{a}}{ }_{x_{2}: \text { mole fraction of component } 2 \text { in the saturated solution. }}$ \\
${ }^{\mathrm{b}}{ }_{x_{1}}:$ mole fraction solubility of the solute. Experimental solubility data were \\
given as grams of solute per $100 \mathrm{~g}$ of solvent. Mole fraction solubility was \\
calculated by the compiler.
\end{tabular}

\section{Auxiliary Information}

\section{Method/Apparatus/Procedure:}

Constant-temperature thermostat, analytical balance, and a steam bath. Excess solute and solvent were placed in a bottle with ground glass stopper which was protected by a coating of beeswax and rosin, over which was tied a piece of rubber sheeting. The sealed mixture was shaken in a constanttemperature thermostat for 8-20 h. An aliquot of the saturated solution was withdrawn through a glass wool filter into a weighing pipet from which the solutions were weighed directly into small glass evaporating dishes. The solvent was removed on a steam bath, and the sample further dried in a sulfuric acid desiccator. The solubility was calculated from the mass of the solid residue and the mass of the saturated solution removed for analysis.

\section{Source and Purity of Chemicals:}

(1) Purity not given, Chemical source not given, no purification details were provided.

(2) Purity not given, Chemical source not given, no purification details were provided.

\section{Estimated Error:}

Temperature: $\pm 0.1 \mathrm{~K}$.

$x_{1}: \pm 10.0 \%$ (relative error, estimated by compiler). 


\begin{tabular}{|c|c|}
\hline $\begin{array}{l}\text { Components: } \\
\text { (1) 2-Hydroxybenzoic acid; } \mathrm{C}_{7} \mathrm{H}_{6} \mathrm{O}_{3} \text {; } \\
\text { [69-72-7] } \\
\text { (2) Propanone; } \mathrm{C}_{3} \mathrm{H}_{6} \mathrm{O} ;[67-64-1]\end{array}$ & $\begin{array}{l}\text { Original Measurements: } \\
\text { 147 J. Walker and J. K. Wood, J. } \\
\text { Chem. Soc. Trans. 73, } 618 \text { (1898). }\end{array}$ \\
\hline $\begin{array}{l}\text { Variables: } \\
T / \mathrm{K}=296\end{array}$ & $\begin{array}{l}\text { Prepared by: } \\
\text { W. E. Acree, Jr. }\end{array}$ \\
\hline
\end{tabular}

Experimental Values

The measured solubility was reported to be $31.3 \mathrm{~g} / 100 \mathrm{ml}$ of solution, which corresponds to a molar concentration of $2.266 \mathrm{~mol} \mathrm{dm}^{-3}$.

\section{Auxiliary Information}

\section{Method/Apparatus/Procedure:}

Excess solute and solvent were equilibrated with agitation by stirrers driven by a small turbine. At higher temperatures agitation was occasionally by hand. Equilibrium was obtained by both undersaturation at lower temperature and by supersaturation by pre-equilibrating at a higher temperature. The concentration of the dissolved solute was determined by titration with barium hydroxide with Congo Red being the endpoint indicator.

\section{Source and Purity of Chemicals:}

(1) Purity not given, Chemical source not given, no purification details were provided.

(2) Purity not given, Chemical source not given, no purification details were provided.

\section{Estimated Error:}

Temperature: Not given in paper.

$c_{1}: \pm 10 \%$ (relative error, estimated by compiler).

\begin{tabular}{ll}
\hline \hline Components: & Original Measurements: \\
$\begin{array}{l}\text { (1) 2-Hydroxybenzoic acid; } \mathrm{C}_{7} \mathrm{H}_{6} \mathrm{O}_{3} ; \\
{[69-72-7]}\end{array}$ & ${ }^{151}$ K. M. De Fina, T. L. Sharp, L. E. \\
(2) Butanone; $\mathrm{C}_{4} \mathrm{H}_{8} \mathrm{O} ;$ [78-93-3] & Eng. Data 44, 1262 (1999). \\
\hline Variables: & Prepared by: \\
$T / \mathrm{K}=298.15$ & W. E. Acree, Jr. \\
\hline
\end{tabular}

Experimental Values

\begin{tabular}{lc}
\hline \hline$x_{2}{ }^{\mathrm{a}}$ & $x_{1}{ }^{\mathrm{b}}$ \\
\hline 0.8148 & 0.1852 \\
\hline${ }^{\mathrm{a}} x_{2}:$ mole fraction of component 2 in the saturated solution. & \\
${ }^{\mathrm{b}} x_{1}:$ mole fraction solubility of the solute. &
\end{tabular}

\section{Auxiliary Information}

\footnotetext{
Method/Apparatus/Procedure:

Constant-temperature bath, calorimetric thermometer, and an ultraviolet/ visible spectrophotometer.

Excess solute and solvent were placed in amber glass bottles and allowed to equilibrate for several days at constant temperature. Attainment of equilibrium was verified by several repetitive measurements and by approaching equilibrium from supersaturation. Aliquots of saturated solutions were transferred through a coarse filter into tared volumetric flasks, weighed, and diluted with methanol. Concentrations were determined by

spectrophotometric measurements at $304 \mathrm{~nm}$.
}

Source and Purity of Chemicals:

(1) $99+\%$, ACS Reagent grade, Aldrich Chemical Company, Milwaukee, WI, USA, was dried for several hours at $353 \mathrm{~K}$ before use and used without further purification.

(2) $99.5+\%$, HPLC grade, Aldrich Chemical Company, stored over molecular sieves before use.

Estimated Error:

Temperature: $\pm 0.1 \mathrm{~K}$.

$x_{1}: \pm 1.5 \%$ (relative error).

\begin{tabular}{|c|c|}
\hline $\begin{array}{l}\text { Components: } \\
\text { (1) 2-Hydroxybenzoic acid; } \mathrm{C}_{7} \mathrm{H}_{6} \mathrm{O}_{3} \text {; } \\
\text { [69-72-7] } \\
\text { (2) Butanone; } \mathrm{C}_{4} \mathrm{H}_{8} \mathrm{O} ;[78-93-3]\end{array}$ & $\begin{array}{l}\text { Original Measurements: } \\
{ }^{158} \text { E. Bergroth, Farm. Aikak. 70, } 91 \\
\text { (1961). }\end{array}$ \\
\hline $\begin{array}{l}\text { Variables: } \\
T / \mathrm{K}=293.15\end{array}$ & $\begin{array}{l}\text { Prepared by: } \\
\text { W. E. Acree, Jr. }\end{array}$ \\
\hline
\end{tabular}

\section{Experimental Values}

The measured solubility was reported to be $23.56 \mathrm{~g} / 100 \mathrm{ml}$ of solution, which corresponds to a molar concentration of $1.706 \mathrm{~mol} \mathrm{dm}^{-3}$.

\section{Auxiliary Information}

\section{Method/Apparatus/Procedure:}

Solubility was determined using a gravimetric method. Excess solute and solvent were placed in a solubility vessel. The apparatus was closed tightly with rubber stoppers and allowed to equilibrate in a constant-temperature water bath with stirring. A known aliquot of the saturated solution was removed, filtered, and transferred to an evaporating dish. The solvent was carefully removed over a steam bath. The evaporating dish was then placed in a drying oven, and later removed and weighed. The solubility was calculated from the mass of the solid residue and volume of saturated sample analyzed.

Source and Purity of Chemicals:

(1) Purity not given, Schuchardt Chemicals, no purification details were provided.

(2) Purity not given, Chemical source not given, no purification details were provided.

\section{Estimated Error:}

Temperature: $\pm 0.2 \mathrm{~K}$ (estimated by compiler). $c_{1}: \pm 5 \%$ (relative error, estimated by compiler).

\begin{tabular}{ll}
\hline \hline Components: & Original Measurements: \\
$\begin{array}{l}\text { (1) 2-Hydroxybenzoic acid; } \mathrm{C}_{7} \mathrm{H}_{6} \mathrm{O}_{3} ; \\
{[69-72-7]}\end{array}$ & ${ }^{158}$ E. Bergroth, Farm. Aikak. 70, 91 \\
$(1961)$. & \\
\hline Variables: & Prepared by: \\
$T / \mathrm{K}=293.15$ & W. E. Acree, Jr. \\
\hline
\end{tabular}

\section{Experimental Values}

The measured solubility was reported to be $20.92 \mathrm{~g} / 100 \mathrm{ml}$ of solution, which corresponds to a molar concentration of $1.515 \mathrm{~mol} \mathrm{dm}^{-3}$. 


\section{Auxiliary Information}

\section{Method/Apparatus/Procedure:}

Solubility was determined using a gravimetric method. Excess solute and solvent were placed in a solubility vessel. The apparatus was closed tightly with rubber stoppers and allowed to equilibrate in a constant-temperature water bath with stirring. A known aliquot of the saturated solution was removed, filtered, and transferred to an evaporating dish. The solvent was carefully removed over a steam bath. The evaporating dish was then placed in a drying oven, and later removed and weighed. The solubility was calculated from the mass of the solid residue and volume of saturated sample analyzed.

Source and Purity of Chemicals:

(1) Purity not given, Schuchardt Chemicals, no purification details were provided.

(2) Purity not given, Chemical source not given, no purification details were provided.

\section{Estimated Error:}

Temperature: $\pm 0.2 \mathrm{~K}$ (estimated by compiler). $c_{1}: \pm 5 \%$ (relative error, estimated by compiler).

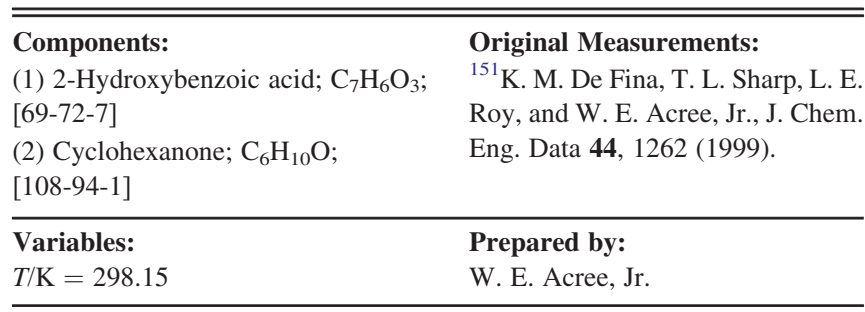

\section{Experimental Values}

\begin{tabular}{lc}
\hline \hline$x_{2}{ }^{\mathrm{a}}$ & $x_{1}{ }^{\mathrm{b}}$ \\
\hline 0.7699 & 0.2301 \\
\hline${ }^{\mathrm{a}} x_{2}:$ mole fraction of component 2 in the saturated solution. & \\
${ }^{\mathrm{b}}{ }_{x_{1}}:$ mole fraction solubility of the solute. &
\end{tabular}

\section{Auxiliary Information}

\section{Method/Apparatus/Procedure:}

Constant-temperature bath, calorimetric thermometer, and an ultraviolet/ visible spectrophotometer.

Excess solute and solvent were placed in amber glass bottles and allowed to equilibrate for several days at constant temperature. Attainment of equilibrium was verified by several repetitive measurements and by approaching equilibrium from supersaturation. Aliquots of saturated solutions were transferred through a coarse filter into tared volumetric flasks, weighed, and diluted with methanol. Concentrations were determined by spectrophotometric measurements at $304 \mathrm{~nm}$.

Source and Purity of Chemicals:

(1) $99+\%$, ACS Reagent grade, Aldrich Chemical Company, Milwaukee, WI, USA, was dried for several hours at $353 \mathrm{~K}$ before use and used without further purification.

(2) $99.8 \%$, anhydrous, Aldrich Chemical Company, stored over molecular sieves before use.

\section{Estimated Error:}

Temperature: $\pm 0.1 \mathrm{~K}$.

$x_{1}: \pm 1.5 \%$ (relative error).

\begin{tabular}{ll}
\hline \hline Components: & $\begin{array}{l}\text { Original Measurements: } \\
\text { (1) 2-Hydroxybenzoic acid; } \mathrm{C}_{7} \mathrm{H}_{6} \mathrm{O}_{3} ;\end{array}$ \\
$\begin{array}{l}{ }^{102} \text { J. Barra, M.-A. Peña, and P. } \\
\text { Bustamante, Eur. J. Pharm. Sci. 10, }\end{array}$ \\
(2) Acetophenone; $\mathrm{C}_{8} \mathrm{H}_{8} \mathrm{O} ;[98-86-2]$ & 153 (2000).
\end{tabular}

Variables: Prepared by:

$T / \mathrm{K}=298.15 \quad$ W. E. Acree, Jr.

\section{Experimental Values}

\begin{tabular}{lc}
\hline \hline$x_{2}{ }^{\mathrm{a}}$ & $x_{1}{ }^{\mathrm{b}}$ \\
\hline 0.8473 & 0.1527
\end{tabular}

${ }^{a} x_{2}$ : mole fraction of component 2 in the saturated solution.

${ }^{\mathrm{b}} x_{1}$ : mole fraction solubility of the solute.

\section{Auxiliary Information}

Method/Apparatus/Procedure:

Constant-temperature bath, analytical balance, and an ultraviolet/visible spectrophotometer.

Excess solute and solvent were placed in a flask and allowed to equilibrate for several days at constant temperature. The solid phase was removed by filtration, and the clear solution was diluted quantitatively with $96 \%$ ethanol. The concentration of the diluted solution was determined by spectrophotometric measurement at the wavelength of maximum absorption. In instances where the solvent interfered with the spectrophotometric determination, the samples were evaporated to dryness and the residue diluted with $96 \%$ ethanol.

Source and Purity of Chemicals:

(1) Purity not given, Sigma Chemical Company, USA, no purification details were provided.

(2) Spectrophotometric or Analytical grade, Chemical source not specified, no purification details were provided.

\section{Estimated Error:}

Temperature: $\pm 0.2 \mathrm{~K}$.

$x_{1}: \pm 3.0 \%$ (relative error, estimated by compiler).

\subsection{2-Hydroxybenzoic acid solubility data in miscellaneous organic solvents}

\begin{tabular}{|c|c|}
\hline Components: & \multirow{3}{*}{$\begin{array}{l}\text { Original Measurements: } \\
{ }^{160} \text { A. Jouyban, V. Panahi-Azar, and } \\
\text { F. Khonsari, J. Mol. Liq. 160, } 14 \\
(2011) \text {. }\end{array}$} \\
\hline $\begin{array}{l}\text { (1) 2-Hydroxybenzoic acid; } \mathrm{C}_{7} \mathrm{H}_{6} \mathrm{O}_{3} \text {; } \\
\text { [69-72-7] }\end{array}$ & \\
\hline $\begin{array}{l}\text { (2) } \mathrm{N} \text {-Methyl-2-pyrrolidone; } \mathrm{C}_{5} \mathrm{H}_{9} \mathrm{NO} \text {; } \\
\text { [872-50-4] }\end{array}$ & \\
\hline & \\
\hline Temperature & W. E. Acree, Jr. \\
\hline
\end{tabular}

\section{Experimental Values}

\begin{tabular}{lc}
\hline \hline$T / \mathrm{K}$ & $c_{1}{ }^{\mathrm{a}}$ \\
\hline 298.2 & 3.934 \\
308.2 & 5.749 \\
318.2 & 8.296 \\
328.2 & 10.258 \\
\hline
\end{tabular}

${ }^{\mathrm{a}} c_{1}$ : molar solubility of the solute in units of moles per liter. 


\section{Auxiliary Information}

\section{Method/Apparatus/Procedure:}

Constant-temperature incubator, temperature controlling system, and ultraviolet/visible spectrophotometer.

Excess solute and solvent were placed in sealed bottles and allowed to equilibrate at constant temperature in an incubator equipped with a temperature controlling system for at least two days. Solutions of the saturated solution were withdrawn and filtered using hydrophilic Durapore filters $(0.45 \mu \mathrm{m})$. The filtered solutions were quantitatively diluted. Absorbances of the diluted solutions were measured. Concentrations of the diluted solutions were computed using a Beer-Law calibration curve based on measured absorbances for standard solutions of known concentration.

\section{Source and Purity of Chemicals:}

(1) $98+\%$, Merck Chemical Company, Germany, was used as received.

(2) $99.5 \%$, Merck Chemical Company, no purification details were provided.

Estimated Error:

Temperature: $\pm 0.2 \mathrm{~K}$.

$c_{1}: \pm 3 \%$ (relative error).

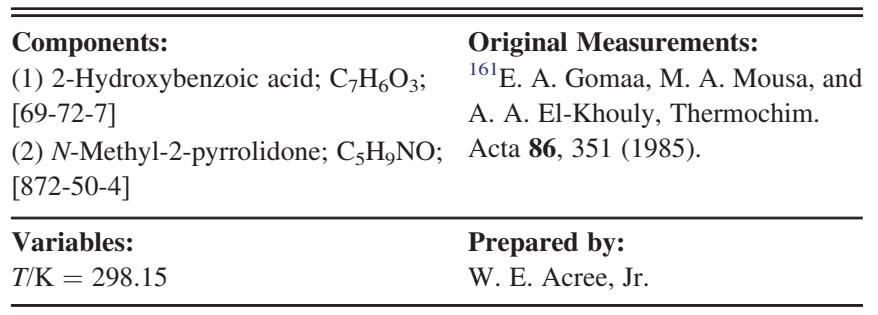

\section{Experimental Values}

The authors' description regarding the experimental value is not clear. In the written text, the authors state that the concentration is in terms of the molal scale; however, in the footnote to Table 1 the unit is given as molal concentration/ liter. The compiler has assumed that the numerical value is given in terms of molality, in which case the solubility is $5.526 \mathrm{~mol} / \mathrm{kg}$ of solvent.

\section{Auxiliary Information}

\section{Method/Apparatus/Procedure:}

Experimental details are not definitive. Saturated solution was prepared by placing excess solute and solvent in test tubes. The sealed test tubes were allowed to equilibrate in a constant-temperature thermostated water bath for one week with shaking, followed by another day without shaking. Solubility was determined either gravimetrically or volumetrically. In the case of the gravimetric method, a $1 \mathrm{ml}$ aliquot of saturated solution was evaporated to dryness in a small aluminum disk heated by an infrared lamp. In the volumetric method, 3-5 $\mathrm{ml}$ of the saturated solution was titrated with a standardized sodium hydroxide solution. The authors studied the solubility of three carboxylic acid solutes in seven different organic solvents, and did not specify which method was used for each solute-solvent pair.

\section{Source and Purity of Chemicals:}

(1) Analytical grade, Merck Chemical Company, Germany, no purification details were provided.

(2) Spectroscopic grade, Merck Chemical Company, no purification details were provided.

\section{Estimated Error:}

Temperature: $\pm 0.1 \mathrm{~K}$ (estimated by compiler).

$c_{1}: \pm 2 \%$ (relative error, estimated by compiler).

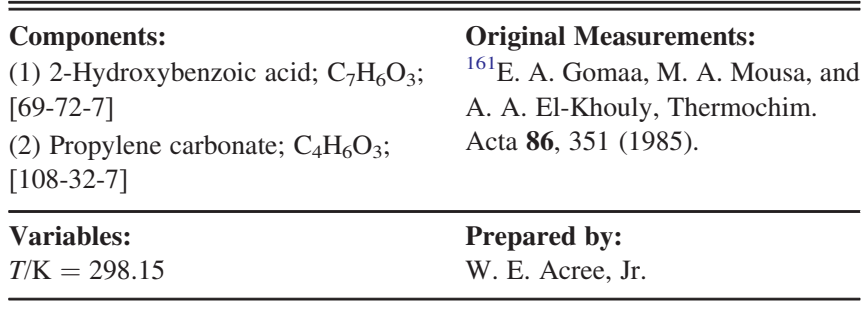

\section{Experimental Values}

The authors' description regarding the experimental value is not clear. In the written text, the authors state that the concentration is in terms of the molal scale; however, in the footnote to Table 1 the unit is given as molal concentration/ liter. The compiler has assumed that the numerical value is given in terms of molality, in which case the solubility is 0.283 $\mathrm{mol} / \mathrm{kg}$ of solvent.

\section{Auxiliary Information}

\section{Method/Apparatus/Procedure:}

Experimental details are not definitive. Saturated solution was prepared by placing excess solute and solvent in test tubes. The sealed test tubes were allowed to equilibrate in a constant-temperature thermostated water bath for one week with shaking, followed by another day without shaking. Solubility was determined either gravimetrically or volumetrically. In the case of the gravimetric method, a $1 \mathrm{ml}$ aliquot of saturated solution was evaporated to dryness in a small aluminum disk heated by an infrared lamp. In the volumetric method, 3-5 $\mathrm{ml}$ of the saturated solution was titrated with a standardized sodium hydroxide solution. The authors studied the solubility of three carboxylic acid solutes in seven different organic solvents, and did not specify which method was used for each solute-solvent pair.

Source and Purity of Chemicals:

(1) Analytical grade, Merck Chemical Company, Germany, no purification details were provided.

(2) Spectroscopic grade, Merck Chemical Company, no purification details were provided.

\section{Estimated Error:}

Temperature: $\pm 0.1 \mathrm{~K}$ (estimated by compiler). $c_{1}: \pm 2 \%$ (relative error, estimated by compiler).

\begin{tabular}{|c|c|}
\hline $\begin{array}{l}\text { Components: } \\
\text { (1) 2-Hydroxybenzoic acid; } \mathrm{C}_{7} \mathrm{H}_{6} \mathrm{O}_{3} \text {; } \\
\text { [69-72-7] }\end{array}$ & $\begin{array}{l}\text { Original Measurements: } \\
{ }^{161} \text { E. A. Gomaa, M. A. Mousa, and } \\
\text { A. A. El-Khouly, Thermochim. }\end{array}$ \\
\hline $\begin{array}{l}\text { (2) Dimethyl sulfoxide; } \mathrm{C}_{2} \mathrm{H}_{6} \mathrm{OS} \text {; } \\
{[67-68-5]}\end{array}$ & Acta 86, $351(1985)$ \\
\hline $\begin{array}{l}\text { Variables: } \\
T / \mathrm{K}=298.15\end{array}$ & $\begin{array}{l}\text { Prepared by: } \\
\text { W. E. Acree, Jr. }\end{array}$ \\
\hline
\end{tabular}

\section{Experimental Values}

The authors' description regarding the experimental value is not clear. In the written text, the authors state that the concentration is in terms of the molal scale; however, in the footnote to Table 1 the unit is given as molal concentration/ liter. The compiler has assumed that the numerical value is given in terms of molality, in which case the solubility is $4.911 \mathrm{~mol} / \mathrm{kg}$ of solvent. 


\section{Auxiliary Information}

\section{Method/Apparatus/Procedure:}

Experimental details are not definitive. Saturated solution was prepared by placing excess solute and solvent in test tubes. The sealed test tubes were allowed to equilibrate in a constant-temperature thermostated water bath for one week with shaking, followed by another day without shaking. Solubility was determined either gravimetrically or volumetrically. In the case of the gravimetric method, a $1 \mathrm{ml}$ aliquot of saturated solution was evaporated to dryness in a small aluminum disk heated by an infrared lamp. In the volumetric method, 3-5 $\mathrm{ml}$ of the saturated solution was titrated with a standardized sodium hydroxide solution. The authors studied the solubility of three carboxylic acid solutes in seven different organic solvents, and did not specify which method was used for each solute-solvent pair.

\section{Source and Purity of Chemicals:}

(1) Analytical grade, Merck Chemical Company, Germany, no purification details were provided.

(2) Spectroscopic grade, Merck Chemical Company, no purification details were provided.

\section{Estimated Error:}

Temperature: $\pm 0.1 \mathrm{~K}$ (estimated by compiler). $c_{1}: \pm 2 \%$ (relative error, estimated by compiler).

\begin{tabular}{|c|c|}
\hline $\begin{array}{l}\text { Components: } \\
\text { (1) 2-Hydroxybenzoic acid; } \mathrm{C}_{7} \mathrm{H}_{6} \mathrm{O}_{3} \text {; } \\
\text { [69-72-7] } \\
\text { (2) Formamide; } \mathrm{CH}_{3} \mathrm{NO} ;[75-12-7]\end{array}$ & $\begin{array}{l}\text { Original Measurements: } \\
{ }^{102} \text { J. Barra, M.-A. Peña, and P. } \\
\text { Bustamante, Eur. J. Pharm. Sci. 10, } \\
153 \text { (2000). }\end{array}$ \\
\hline $\begin{array}{l}\text { Variables: } \\
T / \mathrm{K}=298.15\end{array}$ & $\begin{array}{l}\text { Prepared by: } \\
\text { W. E. Acree, Jr. }\end{array}$ \\
\hline
\end{tabular}

Experimental Values

\begin{tabular}{lc}
\hline \hline$x_{2}{ }^{\mathrm{a}}$ & $x_{1}{ }^{\mathrm{b}}$ \\
\hline 0.9574 & 0.04264 \\
\hline
\end{tabular}

${ }^{\mathrm{a}} x_{2}$ : mole fraction of component 2 in the saturated solution.

${ }^{b} x_{1}$ : mole fraction solubility of the solute.

\section{Auxiliary Information}

\section{Method/Apparatus/Procedure:}

Constant-temperature bath, analytical balance, and an ultraviolet/visible spectrophotometer.

Excess solute and solvent were placed in a flask and allowed to equilibrate for several days at constant temperature. The solid phase was removed by filtration, and the clear solution was diluted quantitatively with $96 \%$ ethanol. The concentration of the diluted solution was determined by spectrophotometric measurement at the wavelength of maximum absorption. In instances where the solvent interfered with the spectrophotometric determination, the samples were evaporated to dryness and the residue diluted with $96 \%$ ethanol.

\section{Source and Purity of Chemicals:}

(1) Purity not given, Sigma Chemical Company, USA, no purification details were provided.

(2) Spectrophotometric or Analytical grade, Chemical source not specified, no purification details were provided.

\section{Estimated Error:}

Temperature: $\pm 0.2 \mathrm{~K}$.

$x_{1}: \pm 3.0 \%$ (relative error, estimated by compiler).

\begin{tabular}{ll}
\hline \hline Components: & Original Measurements: \\
$\begin{array}{l}\text { (1) 2-Hydroxybenzoic acid; } \mathrm{C}_{7} \mathrm{H}_{6} \mathrm{O}_{3} ; \\
{[69-72-7]}\end{array}$ & ${ }^{156} \mathrm{~K}$. B. Sloan, K. G. Siver, and S. A. \\
(2) Formamide; $\mathrm{CH}_{3} \mathrm{NO} ;$ [75-12-7] & $(1986)$. \\
\hline Variables: & Prepared by: \\
$T / \mathrm{K}=296$ & W. E. Acree, Jr. \\
\hline
\end{tabular}

\section{Experimental Values}

The measured solubility was reported to be $145 \mathrm{mg} / \mathrm{ml}$, which corresponds to a solubility of $c_{1}=1.050 \mathrm{~mol} \mathrm{dm}^{-3}$. The authors calculated a mole fraction solubility of $x_{1}=0.045$. The mole fraction solubility was calculated by the authors assuming a molar volume of 2-hydroxybenzoic acid of $V_{1}=93.9 \mathrm{~cm}^{3} \mathrm{~mol}^{-1}$, which seems too small compared to the molar volume of benzaldehyde, which is estimated to be $V_{1} \approx 101.1 \mathrm{~cm}^{3} \mathrm{~mol}^{-1}$, based on a molar mass of $106.12 \mathrm{~g} \mathrm{~mol}^{-1}$ and an experimental density of $1.0499 \mathrm{~g} \mathrm{~cm}^{-3}$ at $296 \mathrm{~K}$. A molar volume on the order of $V_{1} \approx 104 \mathrm{~cm}^{3} \mathrm{~mol}^{-1}$ would seem more realistic given the molecular structures of 2-hydroxybenzoic acid compared to benzaldehyde.

\section{Auxiliary Information}

\section{Method/Apparatus/Procedure:}

Magnetic stirrer and an ultraviolet/visible spectrophotometer.

Solubility was determined by stirring an excess of solute in the solvent with a magnetic stirrer at ambient room temperature for $24 \mathrm{~h}$ in sealed flasks that were thermally insulated from the stirrer. The suspension was gravity filtered through Whatman \#1 (qualitative) filter paper. The filtrate was then quantitatively diluted with methanol, and the absorbance of the diluted solution recorded at $306 \mathrm{~nm}$.

\section{Source and Purity of Chemicals:}

(1) Purity not given, Aldrich Chemical Company, Milwaukee, WI, USA, no purification details were provided.

(2) $99+\%$, Aldrich Chemical Company, used as received.

\section{Estimated Error:}

Temperature: $\pm 1 \mathrm{~K}$.

$x_{1}: \pm 5 \%$ (relative error, estimated by compiler).

\begin{tabular}{|c|c|}
\hline $\begin{array}{l}\text { Components: } \\
\text { (1) 2-Hydroxybenzoic acid; } \mathrm{C}_{7} \mathrm{H}_{6} \mathrm{O}_{3} \text {; } \\
\text { [69-72-7] } \\
\text { (2) } \mathrm{N} \text {-Methylformamide; } \mathrm{C}_{2} \mathrm{H}_{5} \mathrm{NO} ; \\
\text { [123-39-7] }\end{array}$ & $\begin{array}{l}\text { Original Measurements: } \\
{ }^{161} \text { E. A. Gomaa, M. A. Mousa, and } \\
\text { A. A. El-Khouly, Thermochim. } \\
\text { Acta 86, } 351 \text { (1985). }\end{array}$ \\
\hline $\begin{array}{l}\text { Variables: } \\
T / \mathrm{K}=298.15\end{array}$ & $\begin{array}{l}\text { Prepared by: } \\
\text { W. E. Acree, Jr. }\end{array}$ \\
\hline
\end{tabular}

\section{Experimental Values}

The authors' description regarding the experimental value is not clear. In the written text, the authors state that the concentration is in terms of the molal scale; however, in the footnote to Table 1 the unit is given as molal concentration/ liter. The compiler has assumed that the numerical value is given in terms of molality, in which case the solubility is $3.807 \mathrm{~mol} / \mathrm{kg}$ of solvent. 


\section{Auxiliary Information}

\section{Method/Apparatus/Procedure:}

Experimental details are not definitive. Saturated solution was prepared by placing excess solute and solvent in test tubes. The sealed test tubes were allowed to equilibrate in a constant-temperature thermostated water bath for one week with shaking, followed by another day without shaking. Solubility was determined either gravimetrically or volumetrically. In the case of the gravimetric method, a $1 \mathrm{ml}$ aliquot of saturated solution was evaporated to dryness in a small aluminum disk heated by an infrared lamp. In the volumetric method, 3-5 $\mathrm{ml}$ of the saturated solution was titrated with a standardized sodium hydroxide solution. The authors studied the solubility of three carboxylic acid solutes in seven different organic solvents, and did not specify which method was used for each solute-solvent pair.

\section{Source and Purity of Chemicals:}

(1) Analytical grade, Merck Chemical Company, Germany, no purification details were provided.

(2) Spectroscopic grade, Merck Chemical Company, no purification details were provided.

\section{Estimated Error:}

Temperature: $\pm 0.1 \mathrm{~K}$ (estimated by compiler). $c_{1}: \pm 2 \%$ (relative error, estimated by compiler).

Estimated Error:

Temperature: $\pm 0.1 \mathrm{~K}$ (estimated by compiler).

$c_{1}: \pm 2 \%$ (relative error, estimated by compiler).

\begin{tabular}{ll}
\hline \hline Components: & Original Measurements: \\
(1) 2-Hydroxybenzoic acid; $\mathrm{C}_{7} \mathrm{H}_{6} \mathrm{O}_{3} ;$ & $\begin{array}{l}{ }^{102} \text { J. Barra, M.-A. Peña, and P. } \\
\text { Bus-72-7] }\end{array}$ \\
$\begin{array}{ll}\text { (2) } N, N \text {-Dimethylformamide, Eur. J. Pharm. Sci. 10, } \\
\mathrm{C}_{3} \mathrm{H}_{7} \mathrm{NO} ;[68-12-2]\end{array}$ & $153(2000)$. \\
\hline Variables: & Prepared by: \\
$T / \mathrm{K}=298.15$ & W. E. Acree, Jr. \\
\hline
\end{tabular}

Experimental Values

\begin{tabular}{lc}
\hline \hline$x_{2}{ }^{\mathrm{a}}$ & $x_{1}{ }^{\mathrm{b}}$ \\
\hline 0.6160 & 0.3840 \\
\hline${ }^{\mathrm{a}} x_{2}:$ mole fraction of component 2 in the saturated solution. & \\
${ }^{\mathrm{b}} x_{1}:$ mole fraction solubility of the solute. &
\end{tabular}

\section{Auxiliary Information}

\section{Components:} [69-72-7]

(2) $\mathrm{N}, \mathrm{N}$-Dimethylformamide; $\mathrm{C}_{3} \mathrm{H}_{7} \mathrm{NO}$; [68-12-2]
(1) 2-Hydroxybenzoic acid; $\mathrm{C}_{7} \mathrm{H}_{6} \mathrm{O}_{3}$;

Original Measurements:

${ }^{161}$ E. A. Gomaa, M. A. Mousa, and A. A. El-Khouly,

Thermochim. Acta 86, 351 (1985).

\begin{tabular}{ll}
\hline Variables: & Prepared by: \\
$T / \mathrm{K}=298.15$ & W. E. Acree, Jr.
\end{tabular}

Experimental Values

The authors' description regarding the experimental value is not clear. In the written text, the authors state that the concentration is in terms of the molal scale; however, in the footnote to Table 1 the unit is given as molal concentration/ liter. The compiler has assumed that the numerical value is given in terms of molality, in which case the solubility is $4.459 \mathrm{~mol} / \mathrm{kg}$ of solvent.

\section{Auxiliary Information}

\section{Method/Apparatus/Procedure:}

Experimental details are not definitive. Saturated solution was prepared by placing excess solute and solvent in test tubes. The sealed test tubes were allowed to equilibrate in a constant-temperature thermostated water bath for one week with shaking, followed by another day without shaking. Solubility was determined either gravimetrically or volumetrically. In the case of the gravimetric method, a $1 \mathrm{ml}$ aliquot of saturated solution was evaporated to dryness in a small aluminum disk heated by an infrared lamp. In the volumetric method, 3-5 $\mathrm{ml}$ of the saturated solution was titrated with a standardized sodium hydroxide solution. The authors studied the solubility of three carboxylic acid solutes in seven different organic solvents, and did not specify which method was used for each solute-solvent pair.

Source and Purity of Chemicals:

(1) Analytical grade, Merck Chemical Company, Germany, no purification details were provided.

(2) Spectroscopic grade, Merck Chemical Company, no purification details were provided.

\section{Method/Apparatus/Procedure: \\ Constant-temperature bath, analytical balance, and an ultraviolet/visible spectrophotometer. \\ Excess solute and solvent were placed in a flask and allowed to equilibrate for several days at constant temperature. The solid phase was removed by filtration, and the clear solution was diluted quantitatively with $96 \%$ ethanol. The concentration of the diluted solution was determined by spectrophotometric measurement at the wavelength of maximum absorption. In instances where the solvent interfered with the spectrophotometric determination, the samples were evaporated to dryness and the residue diluted} with $96 \%$ ethanol.

\section{Source and Purity of Chemicals:}

(1) Purity not given, Sigma Chemical Company, USA, no purification details were provided.

(2) Spectrophotometric or Analytical grade, Chemical source not specified, no purification details were provided.

\section{Estimated Error:}

Temperature: $\pm 0.2 \mathrm{~K}$.

$x_{1}: \pm 3.0 \%$ (relative error, estimated by compiler).

\begin{tabular}{|c|c|}
\hline $\begin{array}{l}\text { Components: } \\
\text { (1) 2-Hydroxybenzoic acid; } \mathrm{C}_{7} \mathrm{H}_{6} \mathrm{O}_{3} \text {; } \\
\text { [69-72-7] } \\
\text { (2) Ethanenitrile; } \mathrm{C}_{2} \mathrm{H}_{3} \mathrm{~N} ;[75-05-8]\end{array}$ & $\begin{array}{l}\text { Original Measurements: } \\
{ }^{161} \text { E. A. Gomaa, M. A. Mousa, and } \\
\text { A. A. El-Khouly, Thermochim. } \\
\text { Acta 86, } 351 \text { (1985). }\end{array}$ \\
\hline $\begin{array}{l}\text { Variables: } \\
T / \mathrm{K}=298.15\end{array}$ & $\begin{array}{l}\text { Prepared by: } \\
\text { W. E. Acree, Jr. }\end{array}$ \\
\hline
\end{tabular}

\section{Experimental Values}

The authors' description regarding the experimental value is not clear. In the written text, the authors state that the concentration is in terms of the molal scale; however, in the footnote to Table 1 the unit is given as molal concentration/ liter. The compiler has assumed that the numerical value is 
given in terms of molality, in which case the solubility is $0.621 \mathrm{~mol} / \mathrm{kg}$ of solvent.

\section{Auxiliary Information}

\section{Method/Apparatus/Procedure:}

Experimental details are not definitive. Saturated solution was prepared by placing excess solute and solvent in test tubes. The sealed test tubes were allowed to equilibrate in a constant-temperature thermostated water bath for one week with shaking, followed by another day without shaking. Solubility was determined either gravimetrically or volumetrically. In the case of the gravimetric method, a $1 \mathrm{ml}$ aliquot of saturated solution was evaporated to dryness in a small aluminum disk heated by an infrared lamp. In the volumetric method, 3-5 $\mathrm{ml}$ of the saturated solution was titrated with a standardized sodium hydroxide solution. The authors studied the solubility of three carboxylic acid solutes in seven different organic solvents, and did not specify which method was used for each solute-solvent pair.

\section{Source and Purity of Chemicals:}

(1) Analytical grade, Merck Chemical Company, Germany, no purification details were provided.

(2) Spectroscopic grade, Merck Chemical Company, no purification details were provided.

\section{Estimated Error:}

Temperature: $\pm 0.1 \mathrm{~K}$ (estimated by compiler).

$c_{1}: \pm 2 \%$ (relative error, estimated by compiler).

\begin{tabular}{ll}
\hline \hline Components: & Original Measurements: \\
$\begin{array}{l}\text { (1) 2-Hydroxybenzoic acid; } \mathrm{C}_{7} \mathrm{H}_{6} \mathrm{O}_{3} ; \\
\text { 69-72-7] }\end{array}$ & $\begin{array}{l}{ }^{50} \mathrm{P} . \mathrm{G} \text {. Desai and A. M. Patel, J. } \\
\text { Indian Chem. Soc. 12, 131 }\end{array}$ \\
(2) Nitrobenzene; $\mathrm{C}_{6} \mathrm{H}_{5} \mathrm{NO}_{2} ;[98-95-3]$ & $(1935)$. \\
\hline Variables: & Prepared by: \\
$T / \mathrm{K}=301.2$ & W. E. Acree, Jr. \\
\hline
\end{tabular}

\section{Experimental Values}

\begin{tabular}{lc}
\hline$x_{2}{ }^{\mathrm{a}}$ & $x_{1}{ }^{\mathrm{b}}$ \\
\hline 0.9749 & 0.02509
\end{tabular}

${ }^{a} x_{2}$ : mole fraction of component 2 in the saturated solution.

${ }^{b} x_{1}$ : mole fraction solubility of the solute.

\section{Auxiliary Information}

\footnotetext{
Method/Apparatus/Procedure:

Excess solute and solvent were placed in a glass flask which was kept revolving on a wheel in an air thermostat for two days. After two days of equilibration, the solution was filtered. To minimize absorption of the solute onto the filter paper, a $100 \mathrm{ml}$ portion of fresh saturated solution was first filtered through the paper before filtering the sample to be analyzed. An aliquot of the filtrate was titrated with barium hydroxide using phenolphthalein as the endpoint indictor. The barium hydroxide titrant was previously standardized against a solution of succinic acid.
}

\section{Source and Purity of Chemicals:}

(1) Purity not given, Chemical source not specified, no information provided concerning purification.

(2) Purity not given, Chemical source not specified, no information provided concerning purification.
Estimated Error:

Temperature: No information given.

$x_{1}: \pm 3 \%$ (relative error, estimated by compiler).

\begin{tabular}{ll}
\hline \hline Components: & Original Measurements: \\
$\begin{array}{l}\text { (1) 2-Hydroxybenzoic acid; } \mathrm{C}_{7} \mathrm{H}_{6} \mathrm{O}_{3} ; \\
\text { [69-72-7] }\end{array}$ & $\begin{array}{l}{ }^{102} \text { J. Barra, M.-A. Peña, and P. } \\
\text { Bustamante, Eur. J. Pharm. Sci. 10, } \\
\text { (2) Ethanoic acid; } \mathrm{C}_{2} \mathrm{H}_{4} \mathrm{O}_{2} ; \text { [64-19-7] }\end{array}$ \\
\hline $153(2000)$. \\
\hline Variables: & Prepared by: \\
$T / \mathrm{K}=298.15$ & W. E. Acree, Jr. \\
\hline
\end{tabular}

\section{Experimental Values}

\begin{tabular}{lc}
\hline \hline$x_{2}{ }^{\mathrm{a}}$ & $x_{1}{ }^{\mathrm{b}}$ \\
\hline 0.9402 & 0.05979 \\
\hline${ }^{\mathrm{a}} x_{2}:$ mole fraction of component 2 in the saturated solution. & \\
${ }^{\mathrm{b}} x_{1}:$ mole fraction solubility of the solute. &
\end{tabular}

\section{Auxiliary Information}

\section{Method/Apparatus/Procedure:}

Constant-temperature bath, analytical balance, and an ultraviolet/visible spectrophotometer.

Excess solute and solvent were placed in a flask and allowed to equilibrate for several days at constant temperature. The solid phase was removed by filtration, and the clear solution was diluted quantitatively with $96 \%$ ethanol. The concentration of the diluted solution was determined by spectrophotometric measurement at the wavelength of maximum absorption. In instances where the solvent interfered with the spectrophotometric determination, the samples were evaporated to dryness and the residue diluted with $96 \%$ ethanol.

Source and Purity of Chemicals:

(1) Purity not given, Sigma Chemical Company, USA, no purification details were provided.

(2) Spectrophotometric or Analytical grade, Chemical source not specified, no purification details were provided.

\section{Estimated Error:}

Temperature: $\pm 0.2 \mathrm{~K}$.

$x_{1}: \pm 3.0 \%$ (relative error, estimated by compiler).

\begin{tabular}{|c|c|}
\hline $\begin{array}{l}\text { Components: } \\
\text { (1) 2-Hydroxybenzoic acid; } \mathrm{C}_{7} \mathrm{H}_{6} \mathrm{O}_{3} \text {; } \\
\text { [69-72-7] } \\
\text { (2) Propanoic acid; } \mathrm{C}_{3} \mathrm{H}_{6} \mathrm{O}_{2} ;[79-09-4]\end{array}$ & $\begin{array}{l}\text { Original Measurements: } \\
{ }^{102} \text { J. Barra, M.-A. Peña, and P. } \\
\text { Bustamante, Eur. J. Pharm. Sci. 10, } \\
153 \text { (2000). }\end{array}$ \\
\hline $\begin{array}{l}\text { Variables: } \\
T / \mathrm{K}=298.15\end{array}$ & $\begin{array}{l}\text { Prepared by: } \\
\text { W. E. Acree, Jr. }\end{array}$ \\
\hline
\end{tabular}

Experimental Values

\begin{tabular}{lc}
\hline \hline$x_{2}{ }^{\mathrm{a}}$ & $x_{1}{ }^{\mathrm{b}}$ \\
\hline 0.9339 & 0.06614 \\
\hline${ }^{\mathrm{a}} x_{2}:$ mole fraction of component 2 in the saturated solution. \\
${ }^{\mathrm{b}} x_{1}:$ mole fraction solubility of the solute.
\end{tabular}




\section{Auxiliary Information}

\section{Method/Apparatus/Procedure:}

Constant-temperature bath, analytical balance, and an ultraviolet/visible spectrophotometer.

Excess solute and solvent were placed in a flask and allowed to equilibrate for several days at constant temperature. The solid phase was removed by filtration, and the clear solution was diluted quantitatively with $96 \%$ ethanol. The concentration of the diluted solution was determined by spectrophotometric measurement at the wavelength of maximum absorption. In instances where the solvent interfered with the spectrophotometric determination, the samples were evaporated to dryness and the residue diluted with $96 \%$ ethanol.

\section{Source and Purity of Chemicals:}

(1) Purity not given, Sigma Chemical Company, USA, no purification details were provided.

(2) Spectrophotometric or Analytical grade, Chemical source not specified, no purification details were provided.

\section{Estimated Error:}

Temperature: $\pm 0.2 \mathrm{~K}$

$x_{1}: \pm 3.0 \%$ (relative error, estimated by compiler).

\section{Components:}

(1) 2-Hydroxybenzoic acid; $\mathrm{C}_{7} \mathrm{H}_{6} \mathrm{O}_{3}$; [69-72-7]

Original Measurements:

${ }^{156}$ K. B. Sloan, K. G. Siver, and

(2) 9 (Z)-Octadecenoic acid (Oleic acid); S. A. M. Koch, J. Pharm. Sci. 75 $\mathrm{C}_{18} \mathrm{H}_{34} \mathrm{O}_{2} ;[112-80-1]$

\begin{tabular}{ll}
\hline Variables: & Prepared by: \\
$T / \mathrm{K}=296$ & W. E. Acree, Jr. \\
\hline
\end{tabular}

\section{Experimental Values}

The measured solubility was reported to be $30.3 \mathrm{mg} / \mathrm{ml}$, which corresponds to a solubility of $c_{1}=0.219 \mathrm{~mol} \mathrm{dm}^{-3}$. The authors calculated a mole fraction solubility of $x_{1}=0.066$. The mole fraction solubility was calculated by the authors assuming a molar volume of 2-hydroxybenzoic acid of $V_{1}=93.9 \mathrm{~cm}^{3} \mathrm{~mol}^{-1}$, which seems too small compared to the molar volume of benzaldehyde, which is estimated to be $V_{1} \approx 101.1 \mathrm{~cm}^{3} \mathrm{~mol}^{-1}$, based on a molar mass of $106.12 \mathrm{~g} \mathrm{~mol}^{-1}$ and an experimental density of $1.0499 \mathrm{~g} \mathrm{~cm}^{-3}$ at $296 \mathrm{~K}$. A molar volume on the order of $V_{1} \approx 104 \mathrm{~cm}^{3} \mathrm{~mol}^{-1}$ would seem more realistic given the molecular structures of 2-hydroxybenzoic acid compared to benzaldehyde.

\section{Auxiliary Information}

\section{Method/Apparatus/Procedure:}

Magnetic stirrer and an ultraviolet/visible spectrophotometer.

Solubility was determined by stirring an excess of solute in the solvent with a magnetic stirrer at ambient room temperature for $24 \mathrm{~h}$ in sealed flasks that were thermally insulated from the stirrer. The suspension was gravity filtered through Whatman \#1 (qualitative) filter paper. The filtrate was then quantitatively diluted with methanol, and the absorbance of the diluted solution recorded at $306 \mathrm{~nm}$.

Source and Purity of Chemicals:

(1) Purity not given, Aldrich Chemical Company, Milwaukee, WI, USA, no purification details were provided.

(2) Purity not given, Fisher Scientific, Orlando, FL, USA, used as received.
Estimated Error:

Temperature: $\pm 1 \mathrm{~K}$.

$x_{1}: \pm 5 \%$ (relative error, estimated by compiler).

\begin{tabular}{|c|c|}
\hline $\begin{array}{l}\text { Components: } \\
\text { (1) 2-Hydroxybenzoic acid; } \mathrm{C}_{7} \mathrm{H}_{6} \mathrm{O}_{3} \text {; } \\
\text { [69-72-7] } \\
\text { (2) Ethyl 2-hydroxypropanoate; } \\
\mathrm{C}_{5} \mathrm{H}_{10} \mathrm{O}_{3} ; \text { [97-64-3] }\end{array}$ & $\begin{array}{l}\text { Original Measurements: } \\
{ }^{87} \text { M. Dias, J. Hadgraft, and M. E. } \\
\text { Lane, Int. J. Pharm. 336, } 108 \text { (2007). }\end{array}$ \\
\hline $\begin{array}{l}\text { Variables: } \\
T / \mathrm{K}=305.2\end{array}$ & $\begin{array}{l}\text { Prepared by: } \\
\text { W. E. Acree, Jr. }\end{array}$ \\
\hline
\end{tabular}

Experimental Values

The measured solubility was reported to be $194.4 \mathrm{mg} / \mathrm{ml}$, which corresponds to a molar solubility of $c_{1}=1.407 \mathrm{~mol}$ $\mathrm{dm}^{-3}$.

\section{Auxiliary Information}

\section{Method/Apparatus/Procedure:}

Constant-temperature bath, magnetic stirrer, centrifuge, and an ultraviolet/ visible spectrophotometer.

Excess solute and solvent were equilibrated in a constant-temperature bath with stirring for $48 \mathrm{~h}$. The saturated solution was then centrifuged for $10 \mathrm{~min}$, and an aliquot of the supernatant solution was removed and diluted quantitatively for spectrophotometric analysis. The concentration of the dissolved solute was determined from the measured absorbance.

\section{Source and Purity of Chemicals:}

(1) Purity not given, Fisher Scientific Equipment, UK, no purification details were provided.

(2) Purity not given, Purac Biochem, Gorinchem, Netherlands, no purification details were provided.

\section{Estimated Error:}

Temperature: Insufficient experimental details to estimate. $c_{1}: \pm 0.046$.

\begin{tabular}{|c|c|}
\hline $\begin{array}{l}\text { Components: } \\
\text { (1) 2-Hydroxybenzoic acid; } \mathrm{C}_{7} \mathrm{H}_{6} \mathrm{O}_{3} ; \\
\text { [69-72-7] } \\
\text { (2) 1-Methylethyl 2-hydroxypropanoate; } \\
\mathrm{C}_{6} \mathrm{H}_{12} \mathrm{O}_{3} ; \text {;617-64-3] }\end{array}$ & $\begin{array}{l}\text { Original Measurements: } \\
{ }^{87} \text { M. Dias, J. Hadgraft, and M. E. } \\
\text { Lane, Int. J. Pharm. 336, } 108 \\
\text { (2007). }\end{array}$ \\
\hline $\begin{array}{l}\text { Variables: } \\
T / \mathrm{K}=305.2\end{array}$ & $\begin{array}{l}\text { Prepared by: } \\
\text { W. E. Acree, Jr. }\end{array}$ \\
\hline
\end{tabular}

\section{Experimental Values}

The measured solubility was reported to be $222.34 \mathrm{mg} / \mathrm{ml}$, which corresponds to a molar solubility of $c_{1}=1.610 \mathrm{~mol}$ $\mathrm{dm}^{-3}$. 


\section{Auxiliary Information}

\section{Method/Apparatus/Procedure:}

Constant-temperature bath, magnetic stirrer, centrifuge, and an ultraviolet/ visible spectrophotometer.

Excess solute and solvent were equilibrated in a constant-temperature bath with stirring for $48 \mathrm{~h}$. The saturated solution was then centrifuged for $10 \mathrm{~min}$, and an aliquot of the supernatant solution was removed and diluted quantitatively for spectrophotometric analysis. The concentration of the dissolved solute was determined from the measured absorbance.

Source and Purity of Chemicals:

(1) Purity not given, Fisher Scientific Equipment, UK, no purification details were provided.

(2) Purity not given, Purac Biochem, Gorinchem, Netherlands, no purification details were provided.

Estimated Error:

Temperature: Insufficient experimental details to estimate. $c_{1}: \pm 0.29$.

\begin{tabular}{ll}
\hline \hline Components: & Original Measurements: \\
$\begin{array}{l}\text { (1) 2-Hydroxybenzoic acid; } \mathrm{C}_{7} \mathrm{H}_{6} \mathrm{O}_{3} ; \\
\text { 69-72-7] }\end{array}$ & $\begin{array}{l}{ }^{87} \mathrm{M} . \text { Dias, J. Hadgraft, and M. E. } \\
\text { Lane, Int. J. Pharm. 336, 108 (2007). } \\
\text { (2) Butyl 2-hydroxypropanoate; }\end{array}$ \\
$\mathrm{C}_{7} \mathrm{H}_{14} \mathrm{O}_{3} ;[138-22-7]$ & \\
\hline Variables: & Prepared by: \\
$T / \mathrm{K}=305.2$ & W. E. Acree, Jr. \\
\hline
\end{tabular}

Experimental Values

The measured solubility was reported to be $234.1 \mathrm{mg} / \mathrm{ml}$, which corresponds to a molar solubility of $c_{1}=1.695 \mathrm{~mol}$ $\mathrm{dm}^{-3}$.

\section{Auxiliary Information}

\section{Method/Apparatus/Procedure:}

Constant-temperature bath, magnetic stirrer, centrifuge, and an ultraviolet/ visible spectrophotometer.

Excess solute and solvent were equilibrated in a constant-temperature bath with stirring for $48 \mathrm{~h}$. The saturated solution was then centrifuged for $10 \mathrm{~min}$, and an aliquot of the supernatant solution was removed and diluted quantitatively for spectrophotometric analysis. The concentration of the dissolved solute was determined from the measured absorbance.

\section{Source and Purity of Chemicals:}

(1) Purity not given, Fisher Scientific Equipment, UK, no purification details were provided.

(2) Purity not given, Purac Biochem, Gorinchem, Netherlands, no purification details were provided.

Estimated Error:

Temperature: Insufficient experimental details to estimate. $c_{1}: \pm 0.11$.

\section{Components:}

(1) 2-Hydroxybenzoic acid; $\mathrm{C}_{7} \mathrm{H}_{6} \mathrm{O}_{3}$;

[69-72-7]

(2) 9(Z)-Octadecenoic acid (Oleic acid);

$\mathrm{C}_{18} \mathrm{H}_{34} \mathrm{O}_{2}$; [112-80-1]

\section{Original Measurements:}

${ }^{87}$ M. Dias, J. Hadgraft, and M. E. Lane, Int. J. Pharm. 336, 108 (2007).
$T / \mathrm{K}=305.2$

Prepared by:

W. E. Acree, Jr.

\section{Experimental Values}

The measured solubility was reported to be $121.20 \mathrm{mg} / \mathrm{ml}$, which corresponds to a molar solubility of $c_{1}=0.877 \mathrm{~mol}$ $\mathrm{dm}^{-3}$.

\section{Auxiliary Information}

\section{Method/Apparatus/Procedure:}

Constant-temperature bath, magnetic stirrer, centrifuge, and an ultraviolet/ visible spectrophotometer.

Excess solute and solvent were equilibrated in a constant-temperature bath with stirring for $48 \mathrm{~h}$. The saturated solution was then centrifuged for $10 \mathrm{~min}$, and an aliquot of the supernatant solution was removed and diluted quantitatively for spectrophotometric analysis. The concentration of the dissolved solute was determined from the measured absorbance.

Source and Purity of Chemicals:

(1) Purity not given, Fisher Scientific Equipment, UK, no purification details were provided.

(2) Purity not given, Chemical source not given, no purification details were provided.

Estimated Error:

Temperature: Insufficient experimental details to estimate. $c_{1}: \pm 0.024$.

\subsection{2-Hydroxybenzoic acid solubility data in binary organic solvent mixtures}

\begin{tabular}{|c|c|}
\hline Components: & Original Measurements: \\
\hline $\begin{array}{l}\text { (1) 2-Hydroxybenzoic acid; } \mathrm{C}_{7} \mathrm{H}_{6} \mathrm{O}_{3} \text {; } \\
\text { [69-72-7] }\end{array}$ & $\begin{array}{l}{ }^{152} \text { H. Matsuda, K. Kaburagi, S. } \\
\text { Matsumoto, K. Kurihara, K. }\end{array}$ \\
\hline (2) Ethanol; $\mathrm{C}_{2} \mathrm{H}_{6} \mathrm{O} ;[64-17-5]$ & Tochigi, and K. Tomono, J. Chem. \\
\hline $\begin{array}{l}\text { (3) Ethyl ethanoate; } \mathrm{C}_{4} \mathrm{H}_{8} \mathrm{O}_{2} \text {; } \\
\text { [141-78-6] }\end{array}$ & Eng. Data 54, 480 (2009). \\
\hline Variables: & \\
\hline$T / \mathrm{K}=298.15$; Solvent Composition & W. E. Acree, Jr. \\
\hline
\end{tabular}

Experimental Values

\begin{tabular}{lcc}
\hline \hline$x_{2}{ }^{(\mathrm{s}) \mathrm{a}}$ & $x_{2}{ }^{\mathrm{b}}$ & $x_{1}{ }^{\mathrm{c}}$ \\
\hline 0.0000 & 0.0000 & 0.1383 \\
0.1613 & 0.1346 & 0.1653 \\
0.2991 & 0.2462 & 0.1767 \\
0.4201 & 0.3441 & 0.1810 \\
0.5262 & 0.4311 & 0.1808 \\
0.6277 & 0.5123 & 0.1839 \\
0.7134 & 0.5894 & 0.1738 \\
0.7947 & 0.6553 & 0.1754 \\
0.8701 & 0.7295 & 0.1616 \\
0.9389 & 0.8031 & 0.1446 \\
1.0000 & 0.8550 & 0.1450 \\
\hline
\end{tabular}

${ }^{\mathrm{a}} x_{2}{ }^{(\mathrm{s})}$ : initial mole fraction of component 2 in the binary solvent mixture.

${ }^{\mathrm{b}} x_{2}$ : mole fraction of component 2 in the saturated solution.

${ }^{\mathrm{c}} x_{1}$ : mole fraction solubility of the solute. 


\section{Auxiliary Information}

\section{Method/Apparatus/Procedure:}

Thermostated constant-temperature water bath and high-performance liquid chromatograph with an uv detector.

Excess solute and solvent were allowed to equilibrate for $24 \mathrm{~h}$ in a constanttemperature thermostated water bath. Aliquots of saturated solutions were removed and filtered through a membrane filter of $0.45 \mu \mathrm{m}$ pore size (Millipore, USA). Concentrations were determined by high-performance liquid chromatography equipped with an uv detector (254 $\mathrm{nm}$ detection). Benzene was added to the sample as an internal standard.

\section{Source and Purity of Chemicals:}

(1) $99.5 \%$, Wako Pure Chemical Industries, Ltd., Japan, was used without further purification.

(2) $99.5 \%$, Wako Pure Chemical Industries, Ltd., Japan, was used without further purification.

(3) $99.5 \%$, Wako Pure Chemical Industries, Ltd., Japan, was used without further purification.

\section{Estimated Error:}

Temperature: $\pm 0.05 \mathrm{~K}$.

$x_{2}{ }^{(\mathrm{s})}: \pm 0.0001$

$x_{1}: \pm 6.2 \%$ (relative error)

\begin{tabular}{|c|c|}
\hline $\begin{array}{l}\text { Components: } \\
\text { (1) 2-Hydroxybenzoic acid; } \mathrm{C}_{7} \mathrm{H}_{6} \mathrm{O}_{3} \text {; } \\
\text { [69-72-7] } \\
\text { (2) Ethyl ethanoate; } \mathrm{C}_{4} \mathrm{H}_{8} \mathrm{O}_{2} ;[141-78-6] \\
\text { (3) Ethanol; } \mathrm{C}_{2} \mathrm{H}_{6} \mathrm{O} ;[64-17-5]\end{array}$ & $\begin{array}{l}\text { Original Measurements: } \\
{ }^{155} \text { M. A. Peña, A. Reíllo, B. } \\
\text { Escalera, and P. Bustamante, } \\
\text { Int. J. Pharm. 321, } 155 \text { (2006). }\end{array}$ \\
\hline $\begin{array}{l}\text { Variables: } \\
T / \mathrm{K}=298.15 \text {; Solvent Composition }\end{array}$ & $\begin{array}{l}\text { Prepared by: } \\
\text { W. E. Acree, Jr. }\end{array}$ \\
\hline
\end{tabular}

Experimental Values

\begin{tabular}{llc}
\hline \hline$T / \mathrm{K}$ & $v_{2}^{(\mathrm{s}) \mathrm{a}}$ & $x_{1}{ }^{\mathrm{c}}$ \\
\hline 298 & 0.00 & 0.1397 \\
298 & 0.10 & 0.1458 \\
298 & 0.20 & 0.1579 \\
298 & 0.30 & 0.1653 \\
298 & 0.40 & 0.1749 \\
298 & 0.50 & 0.1830 \\
298 & 0.60 & 0.1893 \\
298 & 0.70 & 0.1854 \\
298 & 0.80 & 0.1658 \\
298 & 0.90 & 0.1309 \\
298 & 1.00 & 0.1136 \\
\hline
\end{tabular}

${ }^{\mathrm{a}} v_{2}^{(\mathrm{s})}$ : initial volume fraction of component 2 in the binary solvent mixture.

${ }^{\mathrm{b}} x_{1}$ : mole fraction solubility of the solute.

\section{Auxiliary Information}

\section{Method/Apparatus/Procedure:}

Constant temperature-controlled shaking bath and an uv/visible spectrophotometer.

Binary solvent mixtures were prepared by volume. Excess solute and solven were allowed to equilibrate in a constant temperature-controlled shaking bath for at least five days. Aliquots of the saturated solution were removed and filtered (Durapore membrane filter, $0.2 \mu \mathrm{m}$ pore size). The filtered aliquot was then diluted with ethanol $(96 \% \mathrm{v} / \mathrm{v})$, and the concentration of the dissolved solute determined by spectrophotometric analysis at a wavelength of $302 \mathrm{~nm}$. The authors converted the molar solubilities to mole fractions using the measured densities of the saturated solutions.
Source and Purity of Chemicals:

(1) Purity not given, Sigma-Aldrich, Munich, Germany, was used as received. (2) UV grade, anhydrous, Panreac, Monplet and Esteban, Barcelona, Spain, was used as received.

(3) UV grade, anhydrous, Panreac, Monplet and Esteban, Barcelona, Spain, was used as received.

\section{Estimated Error:}

Temperature: $\pm 0.1 \mathrm{~K}$.

$v_{2}^{(\mathrm{s})}: \pm 0.01$.

$x_{1}: \pm 3 \%$ (relative error, most of the experimental values had uncertainties of $3 \%$ or less)

\begin{tabular}{ll}
\hline \hline Components: & Original Measurements: \\
$\begin{array}{l}\text { (1) 2-Hydroxybenzoic acid; } \mathrm{C}_{7} \mathrm{H}_{6} \mathrm{O}_{3} ; \\
\text { [69-72-7] }\end{array}$ & $\begin{array}{l}{ }^{154} \mathrm{M} \text {. A. Peña, B. Escalera, A. } \\
\text { Reíllo, A. B. Sánchez, and P. } \\
\text { (2) Ethyl ethanoate; } \mathrm{C}_{4} \mathrm{H}_{8} \mathrm{O}_{2} ;\end{array}$ \\
$\begin{array}{l}\text { Bustamante, J. Pharm. Sci. 98, 1129 } \\
\text { [141-78-6] }\end{array}$ & $(2009)$. \\
(3) Ethanol; $\mathrm{C}_{2} \mathrm{H}_{6} \mathrm{O} ;[64-17-5]$ & Prepared by: \\
\hline Variables: & W. E. Acree, Jr. \\
Temperature; Solvent Composition &
\end{tabular}

Experimental Values

\begin{tabular}{llc}
\hline \hline$T / \mathrm{K}$ & $v_{2}^{(\mathrm{s}) \mathrm{a}}$ & $x_{1}{ }^{\mathrm{c}}$ \\
\hline 293 & 0.00 & 0.1348 \\
293 & 0.10 & 0.1408 \\
293 & 0.20 & 0.1529 \\
293 & 0.30 & 0.1602 \\
293 & 0.40 & 0.1697 \\
293 & 0.50 & 0.1780 \\
293 & 0.60 & 0.1844 \\
293 & 0.70 & 0.1784 \\
293 & 0.80 & 0.1608 \\
293 & 0.90 & 0.1259 \\
293 & 1.00 & 0.1085 \\
303 & 0.00 & 0.1405 \\
303 & 0.10 & 0.1550 \\
303 & 0.20 & 0.1673 \\
303 & 0.30 & 0.1750 \\
303 & 0.40 & 0.1859 \\
303 & 0.50 & 0.1948 \\
303 & 0.60 & 0.1984 \\
303 & 0.70 & 0.1950 \\
303 & 0.80 & 0.1750 \\
303 & 0.90 & 0.1435 \\
303 & 1.00 & 0.1219 \\
308 & 0.00 & 0.1485 \\
308 & 0.10 & 0.1655 \\
308 & 0.20 & 0.1747 \\
308 & 0.30 & 0.1851 \\
308 & 0.40 & 0.1948 \\
308 & 0.50 & 0.1980 \\
308 & 0.60 & 0.2031 \\
308 & 0.70 & 0.2019 \\
308 & 0.80 & 0.1807 \\
308 & 0.90 & 0.1554 \\
308 & 1.00 & 0.1293 \\
313 & 0.00 & 0.1605 \\
313 & 0.10 & 0.1726 \\
313 & 0.20 & 0.1873
\end{tabular}




\begin{tabular}{lcc}
\hline \hline$T / \mathrm{K}$ & $v_{2}^{(\mathrm{s}) \mathrm{a}}$ & $x_{1}^{\mathrm{c}}$ \\
\hline 313 & 0.30 & 0.1948 \\
313 & 0.40 & 0.2019 \\
313 & 0.50 & 0.2071 \\
313 & 0.60 & 0.2104 \\
313 & 0.70 & 0.2100 \\
313 & 0.80 & 0.1918 \\
313 & 0.90 & 0.1609 \\
313 & 1.00 & 0.1329 \\
\hline
\end{tabular}

${ }^{\mathrm{a}} v_{2}^{(\mathrm{s})}$ : initial volume fraction of component 2 in the binary solvent mixture. ${ }^{\mathrm{b}} x_{1}$ : mole fraction solubility of the solute.

\section{Auxiliary Information}

\section{Method/Apparatus/Procedure:}

Constant temperature-controlled shaking bath and an uv/visible spectrophotometer.

Binary solvent mixtures were prepared by volume. Excess solute and solvent were allowed to equilibrate in a constant temperature-controlled shaking bath for four days at $313 \mathrm{~K}$, and then one day at the lower temperatures. The authors used the same samples for all temperatures. The authors started with $313 \mathrm{~K}$, and once the solution was saturated at the higher temperature they lowered the temperature for the next set of measurements. Aliquots of the saturated solution were removed and filtered (Durapore membrane filter, $0.2 \mu \mathrm{m}$ pore size). The filtered aliquot was then diluted with ethanol $(96 \% \mathrm{v} / \mathrm{v})$, and the concentration of the dissolved solute determined by spectrophotometric analysis at a wavelength of $302 \mathrm{~nm}$. The authors converted the molar solubilities to mole fractions using the measured densities of the saturated solutions.

\section{Source and Purity of Chemicals:}

(1) Purity not given, Sigma-Aldrich, Munich, Germany, was used as received. (2) UV grade, anhydrous, Panreac, Monplet and Esteban, Barcelona, Spain, was used as received.

(3) UV grade, anhydrous, Panreac, Monplet and Esteban, Barcelona, Spain, was used as received.

\section{Estimated Error:}

Temperature: $\pm 0.1 \mathrm{~K}$.

$v_{2}^{(\mathrm{s})}: \pm 0.01$.

$x_{1}: \pm 3 \%$ (relative error, most of the experimental values had uncertainties of $3 \%$ or less).

\section{Components:}

(1) 2-Hydroxybenzoic acid; $\mathrm{C}_{7} \mathrm{H}_{6} \mathrm{O}_{3}$;

[69-72-7]

(2) Propanone; $\mathrm{C}_{3} \mathrm{H}_{6} \mathrm{O}$; [67-64-1]

(3) Benzene; $\mathrm{C}_{6} \mathrm{H}_{6} ;[71-43-2]$

\begin{tabular}{ll}
\hline Variables: & Prepared by: \\
$T / \mathrm{K}=298.15 ;$ Solvent Composition & W. E. Acree, Jr. \\
\hline
\end{tabular}

Experimental Values

\begin{tabular}{lcc}
\hline \hline$w_{2}^{(\mathrm{s}) \mathrm{a}}$ & $s_{1}{ }^{\mathrm{b}}$ & $m_{1}{ }^{\mathrm{c}}$ \\
\hline 0.00 & 0.92 & 0.067 \\
0.10 & 7.1 & 0.514 \\
0.20 & 15.0 & 1.086 \\
0.40 & 25.3 & 1.832 \\
0.60 & 36.7 & 2.657
\end{tabular}

\begin{tabular}{lcc}
\hline \hline$w_{2}^{(\mathrm{s}) \mathrm{a}}$ & $s_{1}{ }^{\mathrm{b}}$ & $m_{1}{ }^{\mathrm{c}}$ \\
\hline 0.80 & 46.4 & 3.359 \\
0.90 & 51.1 & 3.700 \\
1.00 & 55.5 & 4.018 \\
\hline
\end{tabular}

$\mathrm{a}_{w_{2}(\mathrm{~s})}$ : initial mass fraction of component 2 in the binary solvent mixture.

$\mathrm{b}_{s_{1}}$ : solubility of the solute given as grams of solute per $100 \mathrm{~g}$ of solvent.

${ }^{c} m_{1}$ : solubility of the solute given as moles of solute per kilogram of solvent.

\section{Auxiliary Information}

\section{Method/Apparatus/Procedure:}

Constant-temperature thermostat, analytical balance, and a steam bath. Binary solvent mixtures were prepared by mass. Excess solute and solvent were placed in a bottle with ground glass stopper which was protected by a coating of beeswax and rosin, over which was tied a piece of rubber sheeting. The sealed mixture was shaken in a constant-temperature thermostat for 8-20 h. An aliquot of the saturated solution was withdrawn through a glass wool filter into a weighing pipet from which the solutions were weighed directly into small glass evaporating dishes. The solvent was removed on a steam bath, and the sample further dried in a sulfuric acid desiccator. The solubility was calculated from the mass of the solid residue and the mass of the saturated solution removed for analysis.

\section{Source and Purity of Chemicals:}

(1) Purity not given, Chemical source not given, purification details were not provided.

(2) Purity not given, Chemical source not given, purification details were not provided.

(3) Purity not given, Chemical source not given, purification details were not provided.

\section{Estimated Error:}

Temperature: $\pm 0.1 \mathrm{~K}$.

$w_{2}^{(\mathrm{s})}: \pm 0.01$.

$m_{1}: \pm 10 \%$ (relative error).

\begin{tabular}{ll}
\hline \hline Components: & Original Measurements: \\
$\begin{array}{l}\text { (1) 2-Hydroxybenzoic acid; } \mathrm{C}_{7} \mathrm{H}_{6} \mathrm{O}_{3} ; \\
\text { [69-72-7] }\end{array}$ & $\begin{array}{l}\text { 149 J. W. Marden and M. V. } \\
\text { Dover, J. Am. Chem. Soc. 39, 1 } \\
\text { (2) Ethyl ethanoate; } \mathrm{C}_{4} \mathrm{H}_{8} \mathrm{O}_{2} ;[141-78-6]\end{array}$ \\
$\begin{array}{ll}(1917) . \\
\text { (3) Benzene; } \mathrm{C}_{6} \mathrm{H}_{6} ;[71-43-2]\end{array}$ & Prepared by: \\
\hline Variables: & W. E. Acree, Jr. \\
$T / \mathrm{K}=298.15 ;$ Solvent Composition & \\
\hline
\end{tabular}

Experimental Values

\begin{tabular}{lcc}
\hline \hline$w_{2}^{(\mathrm{s}) \mathrm{a}}$ & $s_{1}{ }^{\mathrm{b}}$ & $m_{1}{ }^{\mathrm{c}}$ \\
\hline 0.00 & 0.92 & 0.067 \\
0.10 & 3.42 & 0.248 \\
0.20 & 6.2 & 0.449 \\
0.40 & 12.8 & 0.927 \\
0.60 & 16.6 & 1.202 \\
0.80 & 22.7 & 1.643 \\
0.90 & 24.2 & 1.752 \\
1.00 & 38.0 & 2.751 \\
\hline
\end{tabular}

${ }^{\mathrm{a}} w_{2}^{(\mathrm{s})}:$ initial mass fraction of component 2 in the binary solvent mixture.

${ }^{\mathrm{b}} s_{1}$ : solubility of the solute given as grams of solute per $100 \mathrm{~g}$ of solvent.

${ }^{c} m_{1}$ : solubility of the solute given as moles of solute per kilogram of solvent. 


\section{Auxiliary Information}

\section{Method/Apparatus/Procedure:}

Constant-temperature thermostat, analytical balance, and a steam bath. Binary solvent mixtures were prepared by mass. Excess solute and solvent were placed in a bottle with ground glass stopper which was protected by a coating of beeswax and rosin, over which was tied a piece of rubber sheeting. The sealed mixture was shaken in a constant-temperature thermostat for 8-20 h. An aliquot of the saturated solution was withdrawn through a glass wool filter into a weighing pipet from which the solutions were weighed directly into small glass evaporating dishes. The solvent was removed on a steam bath, and the sample further dried in a sulfuric acid desiccator. The solubility was calculated from the mass of the solid residue and the mass of the saturated solution removed for analysis.

\section{Source and Purity of Chemicals:}

(1) Purity not given, Chemical source not given, purification details were not provided.

(2) Purity not given, Chemical source not given, purification details were not provided.

(3) Purity not given, Chemical source not given, purification details were not provided.

Estimated Error:

Temperature: $\pm 0.1 \mathrm{~K}$

$w_{2}^{(\mathrm{s})}: \pm 0.01$.

$m_{1}: \pm 10 \%$ (relative error).

\begin{tabular}{|c|c|}
\hline $\begin{array}{l}\text { Components: } \\
\text { (1) 2-Hydroxybenzoic acid; } \mathrm{C}_{7} \mathrm{H}_{6} \mathrm{O}_{3} \text {; } \\
\text { [69-72-7] } \\
\text { (2) Heptane; } \mathrm{C}_{7} \mathrm{H}_{16} ;[142-82-5] \\
\text { (3) Ethanol; } \mathrm{C}_{2} \mathrm{H}_{6} \mathrm{O} ;[64-17-5]\end{array}$ & $\begin{array}{l}\text { Original Measurements: } \\
{ }^{163} \text { B. Bouillot, S. Teychené, and B. } \\
\text { Biscans, Ind. Eng. Chem. Res. 52, } \\
9276 \text { (2013). }\end{array}$ \\
\hline $\begin{array}{l}\text { Variables: } \\
T / \mathrm{K}=293 \text {; Solvent Composition }\end{array}$ & $\begin{array}{l}\text { Prepared by: } \\
\text { W. E. Acree, Jr. }\end{array}$ \\
\hline
\end{tabular}

Experimental Values

\begin{tabular}{lll}
\hline \hline$T / \mathrm{K}$ & $v_{2}^{(\mathrm{s}) \mathrm{a}}$ & \multicolumn{1}{c}{$x_{1}^{\mathrm{c}}$} \\
\hline 293 & 0.20 & 0.128 \\
293 & 0.40 & 0.116 \\
293 & 0.60 & 0.101 \\
293 & 0.80 & 0.0679 \\
293 & 1.00 & 0.00168 \\
\hline
\end{tabular}

$v_{2}^{(s)}:$ initial volume fraction of component 2 in the binary solvent mixture.

${ }^{b} x_{1}$ : mole fraction solubility of the solute.

\section{Auxiliary Information}

\section{Method/Apparatus/Procedure:}

Very little experimental details were provided in the manuscript. The authors simply state that the solubility measurements were carried out using the classical analytical shake flask method.

Source and Purity of Chemicals:

(1) $99.5 \%$, Fisher Scientific, used as received.

(2) $99+\%$, Analytical Reagent, Fischer Scientific, used as received.

(3) $99+\%$, Analytical Reagent, Fischer Scientific, used as received.

Estimated Error:

Temperature: Insufficient experimental detail provided in paper to estimate. $v_{2}^{(\mathrm{s})}: \pm 0.01$.

$x_{1}$ : Insufficient experimental detail provided in paper to estimate.

\section{Solubility of 3-Hydroxybenzoic Acid in Organic Solvents}

\subsection{Critical evaluation of experimental solubility data}

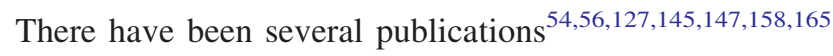
reporting the solubility of 3-hydroxybenzoic acid in organic solvents. Hancock et al. ${ }^{54}$ measured the solubility of 3-hydroxybenzoic acid in cyclohexane and benzene at $303 \mathrm{~K}$ based on a gravimetric method. Ongley ${ }^{56}$ measured the solubility of 3hydroxybenzoic acid in benzene, trichloromethane and tetrachloromethane at $298 \mathrm{~K}$. Bergroth ${ }^{158}$ reported solubility data for 3-hydroxybenzoic acid dissolved in methanol, ethanol, 1propanol, propanone, butanone, and 2-pentanone at $293 \mathrm{~K}$. Benzene is the only solvent for which there are two independent experimental data points; however, it is not possible to directly compare the experimental values as the measurements were performed at different temperatures.

Sidgwick and Ewbank ${ }^{127}$ measured the solubility of 3hydroxybenzoic acid in benzene, ethanol, and 1-butanol as a function of temperature. The internal consistency of the benzene dataset was assessed by curve-fitting the measured mole fraction solubility data to the Modified Apelblat model to yield the following mathematical representation:

$$
\ln x_{1}=-208.672+\frac{111.81}{T}+33.861 \ln T .
$$

The first experimental solubility value of $x_{1}=0.0070$ at $395.7 \mathrm{~K}$ was removed from the regression analysis in order to get a better curve-fit. There were too few data points in the ethanol and 1-butanol datasets to perform a meaningful regression analysis. The average absolute deviations between the observed experimental data and back-calculated values based on Eq. (52) of $15.9 \%$ is larger than desired. The large deviation may be due in part to the fact that the solution temperature is above the solvent's normal boiling point temperature, to uncertainties in estimating the amount of solvent that remained in the liquid phase, and the large range covered by the experimental values, $x_{1}=0.0169$ to $x_{1}=0.738$. It is more difficult to curve-fit experimental solubility data covering large mole fraction ranges.

The experimental solubility data for 3-hydroxybenzoic acid in organic solvents are in Secs. 47.2-47.9.

\subsection{3-Hydroxybenzoic acid solubility data in saturated hydrocarbons (including cycloalkanes)}

\begin{tabular}{|c|c|}
\hline $\begin{array}{l}\text { Components: } \\
\text { (1) 3-Hydroxybenzoic acid; } \mathrm{C}_{7} \mathrm{H}_{6} \mathrm{O}_{3} \text {; } \\
\text { [99-06-9] } \\
\text { (2) Hexane; } \mathrm{C}_{6} \mathrm{H}_{14} ;[110-54-3]\end{array}$ & $\begin{array}{l}\text { Original Measurements: } \\
{ }^{145} \text { G. L. Perlovich, T. V. Volkova, } \\
\text { and A. Bauer-Brandl, J. Pharm. Sci. } \\
\text { 95, } 1448 \text { (2006). }\end{array}$ \\
\hline $\begin{array}{l}\text { Variables: } \\
\text { Temperature }\end{array}$ & $\begin{array}{l}\text { Prepared by: } \\
\text { W. E. Acree, Jr. }\end{array}$ \\
\hline
\end{tabular}


Experimental Values

\begin{tabular}{lcc}
\hline \hline$T / \mathrm{K}$ & $x_{2}{ }^{\mathrm{a}}$ & $x_{1}{ }^{\mathrm{b}}$ \\
\hline 293 & 0.9999 & 0.00000614 \\
298 & 0.9999 & 0.00000917 \\
303 & 0.9999 & 0.0000112 \\
310 & 0.9999 & 0.0000174 \\
315 & 0.9999 & 0.0000262 \\
\hline
\end{tabular}

${ }^{\mathrm{a}} x_{2}$ : mole fraction of component 2 in the saturated solution.

${ }^{\mathrm{b}} x_{1}$ : mole fraction solubility of the solute.

\section{Auxiliary Information}

\section{Method/Apparatus/Procedure:}

Thermostated constant-temperature bath, centrifuge, and an ultraviolet/visible spectrophotometer.

Excess solute and solvent were placed in a glass ampoule and allowed to equilibrate in a thermostated temperature bath with mixing (at a speed of 25 $\mathrm{rpm}$ ) for a minimum of four days. After suitable equilibration, the saturated solution was centrifuged, the supernatant liquid collected, quantitatively diluted, and the absorbance recorded using an ultraviolet/visible spectrophotometer. The solubility of the solute was calculated from the measured absorbance.

Source and Purity of Chemicals:

(1) $99 \%$, Merck Chemicals, Darmstadt, Germany, no purification details provided.

(2) Analytical Reagent grade, SDS, Peypin, France, no purification details were provided.

Estimated Error:

Temperature: $\pm 0.1 \mathrm{~K}$.

$x_{2}: \pm 2.5 \%$ (relative error).

\begin{tabular}{ll}
\hline \hline Components: & Original Measurements: \\
$\begin{array}{l}\text { (1) 3-Hydroxybenzoic acid; } \mathrm{C}_{7} \mathrm{H}_{6} \mathrm{O}_{3} ; \\
\text { [99-06-9] }\end{array}$ & $\begin{array}{l}{ }^{54} \mathrm{C} . \text { K. Hancock, J. N. Pawloski, } \\
\text { and J. P. Idoux, J. Org. Chem. 31, }\end{array}$ \\
$\begin{array}{l}\text { (2) Cyclohexane; } \mathrm{C}_{6} \mathrm{H}_{12} ;[110-82-7] \\
3801 \text { (1966). }\end{array}$ \\
\hline Variables: & Prepared by: \\
$T / \mathrm{K}=303.15$ & W. E. Acree, Jr. \\
\hline
\end{tabular}

Experimental Values

\begin{tabular}{lc}
\hline \hline$x_{2}^{\mathrm{a}}$ & $x_{1}{ }^{\mathrm{b}}$ \\
\hline 0.9999 & 0.00000973 \\
${ }^{\mathrm{a}} x_{2}:$ mole fraction of component 2 in the saturated solution. \\
${ }^{\mathrm{b}} x_{1}:$ mole fraction solubility of the solute.
\end{tabular}

\section{Auxiliary Information}

\footnotetext{
Method/Apparatus/Procedure:

Constant-temperature bath, Soxhlet thimble, conical flask, analytical balance. Excess solute and solvent were placed in a conical flask and allowed to equilibrate for several days at constant temperature. Attainment of equilibrium was verified by several repetitive measurements and by approaching equilibrium from supersaturation. Aliquots of saturated solutions were transferred using a Soxhlet thimble equipped with a one-hole cork stopper and an inverted U-shaped delivery tube to a second ground-glass stoppered flask suspended in the $303 \mathrm{~K}$ water bath. Successive portions of the contents were evaporated at room temperature in a tared aluminum foil weighing dish under a bell jar through which a slow stream of dry air was passed. The second flask plus unused saturated solution and the aluminum foil dish plus residue were weighed. The saturation solubility of the solute was calculated from the recorded mass data and molar masses of the solute and solvent.
}

Source and Purity of Chemicals:

(1) Purity not given, Chemical source not specified, was recrystallized several times from aqueous-ethanol mixture, and then dried under vacuum over phosphorous pentoxide.

(2) Reagent grade, Chemical source not specified, refluxed over phosphorous pentoxide for $24 \mathrm{~h}$, and then distilled through a $3 \times 80 \mathrm{~cm}$ column filled with $0.32-\mathrm{cm}$ glass helices.

Estimated Error:

Temperature: $\pm 0.02 \mathrm{~K}$

$x_{1}: \pm 33 \%$ (relative error).

\subsection{3-Hydroxybenzoic acid solubility data in aromatic hydrocarbons}

\begin{tabular}{|c|c|}
\hline $\begin{array}{l}\text { Components: } \\
\text { (1) 3-Hydroxybenzoic acid; } \mathrm{C}_{7} \mathrm{H}_{6} \mathrm{O}_{3} \text {; } \\
\text { [99-06-9] } \\
\text { (2) Benzene; } \mathrm{C}_{6} \mathrm{H}_{6} ;[71-43-2]\end{array}$ & $\begin{array}{l}\text { Original Measurements: } \\
{ }^{54} \text { C. K. Hancock, J. N. Pawloski, } \\
\text { and J. P. Idoux, J. Org. Chem. 31, } \\
3801 \text { (1966). }\end{array}$ \\
\hline $\begin{array}{l}\text { Variables: } \\
T / \mathrm{K}=303.15\end{array}$ & $\begin{array}{l}\text { Prepared by: } \\
\text { W. E. Acree, Jr. }\end{array}$ \\
\hline
\end{tabular}

Experimental Values

\begin{tabular}{lc}
\hline \hline$x_{2}{ }^{\mathrm{a}}$ & $x_{1}{ }^{\mathrm{b}}$ \\
\hline 0.9999 & 0.0000504 \\
\hline$x_{2}:$ mole fraction of component 2 in
\end{tabular}

${ }^{\mathrm{a}} x_{2}$ : mole fraction of component 2 in the saturated solution.

${ }^{b} x_{1}$ : mole fraction solubility of the solute.

\section{Auxiliary Information}

\section{Method/Apparatus/Procedure:}

Constant-temperature bath, Soxhlet thimble, conical flask, analytical balance. Excess solute and solvent were placed in a conical flask and allowed to equilibrate for several days at constant temperature. Attainment of equilibrium was verified by several repetitive measurements and by approaching equilibrium from supersaturation. Aliquots of saturated solutions were transferred using a Soxhlet thimble equipped with a one-hole cork stopper and an inverted U-shaped delivery tube to a second ground-glass stoppered flask suspended in the $303 \mathrm{~K}$ water bath. Successive portions of the contents were evaporated at room temperature in a tared aluminum foil weighing dish under a bell jar through which a slow stream of dry air was passed. The second flask plus unused saturated solution and the aluminum foil dish plus residue were weighed. The saturation solubility of the solute was calculated from the recorded mass data and molar masses of the solute and solvent.

Source and Purity of Chemicals:

(1) Purity not given, Chemical source not specified, was recrystallized several times from aqueous-ethanol mixture, and then dried under vacuum over phosphorous pentoxide.

(2) Reagent grade, Chemical source not specified, refluxed over phosphorous pentoxide for $24 \mathrm{~h}$, and then distilled through a $3 \times 80 \mathrm{~cm}$ column filled with $0.32-\mathrm{cm}$ glass helices.

\section{Estimated Error:}

Temperature: $\pm 0.02 \mathrm{~K}$.

$x_{1}: \pm 10 \%$ (relative error, by compiler). 


\begin{tabular}{|c|c|}
\hline $\begin{array}{l}\text { Components: } \\
\text { (1) 3-Hydroxybenzoic acid; } \mathrm{C}_{7} \mathrm{H}_{6} \mathrm{O}_{3} \text {; } \\
\text { [99-06-9] } \\
\text { (2) Benzene; } \mathrm{C}_{6} \mathrm{H}_{6} ;[71-43-2]\end{array}$ & $\begin{array}{l}\text { Original Measurements: } \\
{ }^{127} \text { N. V. Sidgwick and E. K. } \\
\text { Ewbank, J. Chem. Soc. Trans. 119, } \\
979 \text { (1921). }\end{array}$ \\
\hline $\begin{array}{l}\text { Variables: } \\
\text { Temperature }\end{array}$ & $\begin{array}{l}\text { Prepared by: } \\
\text { W. E. Acree, Jr. }\end{array}$ \\
\hline
\end{tabular}

Experimental Values

\begin{tabular}{lcc}
\hline \hline$T / \mathrm{K}$ & $x_{2}{ }^{\mathrm{a}}$ & \multicolumn{1}{c}{$x_{1}{ }^{\mathrm{b}}$} \\
\hline 395.7 & 0.993 & 0.0070 \\
414.2 & 0.983 & 0.0169 \\
427.7 & 0.970 & 0.0299 \\
435.2 & 0.938 & 0.0624 \\
446.2 & 0.860 & 0.140 \\
455.7 & 0.713 & 0.287 \\
458.7 & 0.517 & 0.483 \\
465.7 & 0.262 & 0.738 \\
\hline
\end{tabular}

${ }^{a} x_{2}$ : mole fraction of component 2 in the saturated solution.

${ }^{b} x_{1}$ : mole fraction solubility of the solute. Solubility data reported in units of mass percent. Mole fraction values calculated by the compiler.

\section{Auxiliary Information}

\section{Method/Apparatus/Procedure:}

Preweighed quantities of solute and solvent were placed in bulbs, which were then sealed and heated in a suitable bath. At the higher temperatures, the concentration of solvent in the liquid solution was corrected for the amount of solvent vapor in the bulb. The correction assumed that the vapor pressure of the saturated solution was one half that of the pure solvent at the solution temperature. The temperature at which the solid completely dissolved was recorded, and was taken to be the solid-liquid equilibrium temperature.

\section{Source and Purity of Chemicals:}

(1) Purity not given, prepared and purified by the authors using a published synthetic procedure [see J. Walker and J. K. Wood, J. Chem. Soc. 117, 40 (1920) for synthetic details]. Melting point of the purified solute was $474.5 \mathrm{~K}$ (2) Purity not given, Chemical source not given, thiophene was removed by treatment with sulfuric acid. Sample was further purified by freezing several times.

\section{Estimated Error:}

Temperature: Not given in paper.

$x_{1}: \pm 10 \%$ (relative error, estimated by compiler).

\begin{tabular}{|c|c|}
\hline $\begin{array}{l}\text { Components: } \\
\text { (1) 3-Hydroxybenzoic acid; } \mathrm{C}_{7} \mathrm{H}_{6} \mathrm{O}_{3} \text {; } \\
\text { [99-06-9] } \\
\text { (2) Benzene; } \mathrm{C}_{6} \mathrm{H}_{6} ;[71-43-2]\end{array}$ & $\begin{array}{l}\text { Original Measurements: } \\
\text { 56 P. A. Ongley, J. Chem. Soc. 1954, } \\
3634 \text {. }\end{array}$ \\
\hline $\begin{array}{l}\text { Variables: } \\
T / \mathrm{K}=298.15\end{array}$ & $\begin{array}{l}\text { Prepared by: } \\
\text { W. E. Acree, Jr. }\end{array}$ \\
\hline
\end{tabular}

\section{Experimental Values}

The measured solubility was reported to be $-\log _{10} c_{1}=3.198$, which corresponds to a solubility of $c_{1}=0.000634 \mathrm{~mol} \mathrm{dm}^{-3}$.

\section{Auxiliary Information}

\section{Method/Apparatus/Procedure:}

Very little experimental details were provided. Excess solute and solvent were placed in tubes, which were rotated for at least $8 \mathrm{~h}$ at $298 \mathrm{~K}$. The solutions were then removed, concentrated if necessary, and titrated with standard alkali using a mixed (bromothymol blue + neutral red) indicator.

\section{Source and Purity of Chemicals:}

(1) Purity not given, Chemical source not given, solute was either "AnalaR" grade or recrystallized prior to use.

(2) Purity not given, Chemical source not given, no purification details were provided.

Estimated Error:

Temperature: No information was given.

$c_{1}: \pm 4 \%$ (relative error, estimated by compiler).

\begin{tabular}{|c|c|}
\hline $\begin{array}{l}\text { Components: } \\
\text { (1) 3-Hydroxybenzoic acid; } \mathrm{C}_{7} \mathrm{H}_{6} \mathrm{O}_{3} \text {; } \\
\text { [99-06-9] } \\
\text { (2) Benzene; } \mathrm{C}_{6} \mathrm{H}_{6} ;[71-43-2]\end{array}$ & $\begin{array}{l}\text { Original Measurements: } \\
{ }^{147} \text { J. Walker and J. K. Wood, J. } \\
\text { Chem. Soc. Trans. 73, } 618 \text { (1898). }\end{array}$ \\
\hline $\begin{array}{l}\text { Variables: } \\
\text { Temperature }\end{array}$ & $\begin{array}{l}\text { Prepared by: } \\
\text { W. E. Acree, Jr. }\end{array}$ \\
\hline
\end{tabular}

Experimental Values

\begin{tabular}{lcc}
\hline \hline$T / \mathrm{K}$ & $x_{2}{ }^{\mathrm{a}}$ & $x_{1}{ }^{\mathrm{b}}$ \\
\hline 298.2 & 0.9999 & 0.0000571 \\
307.3 & 0.9999 & 0.0000797 \\
319.2 & 0.9999 & 0.000132 \\
331.2 & 0.9998 & 0.000245 \\
337.2 & 0.9997 & 0.000335 \\
\hline
\end{tabular}

${ }^{a} x_{2}$ : mole fraction of component 2 in the saturated solution.

${ }^{b} x_{1}$ : mole fraction solubility of the solute. Solubility data reported as grams of solute per $100 \mathrm{~g}$ of solvent. Numerical values calculated by the compiler.

\section{Auxiliary Information}

\section{Method/Apparatus/Procedure:}

Excess solute and solvent were equilibrated with agitation by stirrers driven by a small turbine. At higher temperatures agitation was occasionally by hand. Equilibrium was obtained by both undersaturation at lower temperature and by supersaturation by pre-equilibrating at a higher temperature. The concentration of the dissolved solute was determined by titration with barium hydroxide with Congo Red being the endpoint indicator. Prior to titration, the solvent was removed by evaporation under reduced pressure.

\section{Source and Purity of Chemicals:}

(1) Purity not given, Chemical source not given, no purification details were provided.

(2) Purity not given, Chemical source not given, no purification details were provided.

\section{Estimated Error:}

Temperature: Not given in paper.

$x_{1}: \pm 10 \%$ (relative error, estimated by compiler). 


\subsection{3-Hydroxybenzoic acid solubility data in esters}

\begin{tabular}{|c|c|}
\hline $\begin{array}{l}\text { Components: } \\
\text { (1) 3-Hydroxybenzoic acid; } \mathrm{C}_{7} \mathrm{H}_{6} \mathrm{O}_{3} \text {; } \\
\text { [99-06-9] } \\
\text { (2) Ethyl ethanoate; } \mathrm{C}_{4} \mathrm{H}_{8} \mathrm{O}_{2} ;[141-78-6]\end{array}$ & $\begin{array}{l}\text { Original Measurements: } \\
{ }^{165} \text { F. L. Nordström and A. C. } \\
\text { Rasmuson, Eur. J. Pharm. Sci. } \\
\text { 28, } 377 \text { (2006). }\end{array}$ \\
\hline $\begin{array}{l}\text { Variables: } \\
\text { Temperature }\end{array}$ & $\begin{array}{l}\text { Prepared by: } \\
\text { W. E. Acree, Jr. }\end{array}$ \\
\hline
\end{tabular}

Experimental Values

\begin{tabular}{lcc}
\hline \hline$T / \mathrm{K}$ & $x_{2}{ }^{\mathrm{a}}$ & $x_{1}{ }^{\mathrm{b}}$ \\
\hline 283.2 & 0.9604 & 0.0396 \\
288.2 & 0.9569 & 0.0431 \\
293.2 & 0.9532 & 0.0468 \\
298.2 & 0.9493 & 0.0507 \\
303.2 & 0.9444 & 0.0556 \\
308.2 & 0.9398 & 0.0602 \\
313.2 & 0.9342 & 0.0658 \\
318.2 & 0.9283 & 0.0717 \\
323.2 & 0.9227 & 0.0773
\end{tabular}

${ }^{a} x_{2}$ : mole fraction of component 2 in the saturated solution.

${ }^{b} x_{1}$ : mole fraction solubility of the solute. The authors noted that the solid existed in different polymorphic forms. The solubility data are for monoclinic form. Solubilities were reported as grams of solute per kilogram of solvent. Mole fraction values were calculated by the compiler.

Experimental Values

\begin{tabular}{lcc}
\hline \hline$T / \mathrm{K}$ & $x_{2}{ }^{\mathrm{a}}$ & $x_{1}{ }^{\mathrm{b}}$ \\
\hline 283.2 & 0.9483 & 0.0517 \\
288.2 & 0.9450 & 0.0550 \\
293.2 & 0.9409 & 0.0591 \\
298.2 & 0.9358 & 0.0642 \\
303.2 & 0.9307 & 0.0693 \\
308.2 & 0.9248 & 0.0752 \\
313.2 & 0.9186 & 0.0814 \\
318.2 & 0.9115 & 0.0885 \\
323.2 & 0.9042 & 0.0958
\end{tabular}

${ }^{\mathrm{a}} x_{2}$ : mole fraction of component 2 in the saturated solution.

${ }^{b} x_{1}$ : mole fraction solubility of the solute. The authors noted that the solid existed in different polymorphic forms. The solubility data are for the orthorhombic form. Solubilities were reported as grams of solute per kilogram of solvent. Mole fraction values were calculated by the compiler.

\section{Auxiliary Information}

\section{Method/Apparatus/Procedure:}

Constant-temperature bath, magnetic stirrer, analytical balance, and ventilated laboratory hood.

Solubilities were determined by a gravimetric method. Excess solute and solvent were placed in sealed bottles and allowed to equilibrate in a constanttemperature bath for at least $24 \mathrm{~h}$ with stirring. The stirring was discontinued and the solid was allowed to settle to the bottom of the container. An aliquot of the clear solution was removed by syringe, filtered through a $0.2 \mu \mathrm{m}$ PTFE filter, and transferred to a preweighed glass vial. The glass vial was then weighed, and the solvent was allowed to evaporate in a ventilated laboratory hood at ambient room temperature. Once the solvent had evaporated, the vial with solid residue was weighed until constant weight was obtained. The solubility was calculated from the mass of the solid residue and mass of sample analyzed.
Source and Purity of Chemicals:

(1) $99 \%$, Sigma-Aldrich Chemical Company, USA, was used as received.

(2) $99.8+\%$, HiperSolv, VWR Scientific, USA, no purification details were provided.

Estimated Error:

Temperature: $\pm 0.01 \mathrm{~K}$.

$x_{1}: \pm 2 \%$ (relative error)

\subsection{3-Hydroxybenzoic acid solubility data in ethers}

\begin{tabular}{ll}
\hline \hline Components: & Original Measurements: \\
$\begin{array}{l}\text { (1) 3-Hydroxybenzoic acid; } \mathrm{C}_{7} \mathrm{H}_{6} \mathrm{O}_{3} ; \\
{[99-06-9]}\end{array}$ & $\begin{array}{l}{ }^{447} \text { J. Walker and J. K. Wood, J. } \\
\text { Chem. Soc. Trans. 73, 618 (1898). } \\
\text { (2) } 1,1^{\prime}-\text { Oxybisethane; } \mathrm{C}_{4} \mathrm{H}_{10} \mathrm{O} ;\end{array}$ \\
{$[60-29-7]$} & \\
\hline Variables: & Prepared by: \\
$T / \mathrm{K}=290$ & W. E. Acree, Jr. \\
\hline
\end{tabular}

\section{Experimental Values}

The measured solubility was reported to be $9.73 \mathrm{~g} / 100 \mathrm{ml}$ of solution, which corresponds to a molar concentration of $0.704 \mathrm{~mol} \mathrm{dm}^{-3}$.

\section{Auxiliary Information}

\section{Method/Apparatus/Procedure:}

Excess solute and solvent were equilibrated with agitation by stirrers driven by a small turbine. At higher temperatures agitation was occasionally by hand. Equilibrium was obtained by both undersaturation at lower temperature and by supersaturation by pre-equilibrating at a higher temperature. The concentration of the dissolved solute was determined by titration with barium hydroxide with Congo Red being the endpoint indicator.

\section{Source and Purity of Chemicals:}

(1) Purity not given, Chemical source not given, no purification details were provided.

(2) Purity not given, Chemical source not given, no purification details were provided.

Estimated Error:

Temperature: Not given in paper.

$c_{1}: \pm 10 \%$ (relative error, estimated by compiler).

\subsection{3-Hydroxybenzoic acid solubility data in haloalkanes, haloalkenes, and haloaromatic hydrocarbons}

\begin{tabular}{ll}
\hline \hline $\begin{array}{l}\text { Components: } \\
\text { (1) 3-Hydroxybenzoic acid; } \mathrm{C}_{7} \mathrm{H}_{6} \mathrm{O}_{3} ;\end{array}$ & $\begin{array}{l}\text { Original Measurements: } \\
\text { [99-06-9] }\end{array}$ \\
$\begin{array}{l}\text { (2) Trichloromethane; } \mathrm{CHCl}_{3} ; \\
\text { [67-66-3] }\end{array}$ & \\
\hline Variables: & \\
$T / \mathrm{K}=298.15$ & Prepared by: \\
\hline
\end{tabular}




\section{Experimental Values}

The measured solubility was reported to be $-\log _{10} c_{1}=2.629$, which corresponds to a solubility of $c_{1}=0.00235 \mathrm{~mol} \mathrm{dm}^{-3}$.

\section{Auxiliary Information}

\section{Method/Apparatus/Procedure:}

Very little experimental details were provided. Excess solute and solvent were placed in tubes, which were rotated for at least $8 \mathrm{~h}$ at $298 \mathrm{~K}$. The solutions were then removed, concentrated if necessary, and titrated with standard alkali using a mixed (bromothymol blue + neutral red) indicator.

Source and Purity of Chemicals:

(1) Purity not given, Chemical source not given, solute was either "AnalaR" grade or recrystallized prior to use.

(2) Purity not given, Chemical source not given, no purification details were provided.

\section{Estimated Error:}

Temperature: No information was given $c_{1}: \pm 4 \%$ (relative error, estimated by compiler).

\begin{tabular}{|c|c|}
\hline $\begin{array}{l}\text { Components: } \\
\text { (1) 3-Hydroxybenzoic acid; } \mathrm{C}_{7} \mathrm{H}_{6} \mathrm{O}_{3} \text {; } \\
\text { [99-06-9] } \\
\text { (2) Tetrachloromethane; } \mathrm{CCl}_{4} ;[56-23-5]\end{array}$ & $\begin{array}{l}\text { Original Measurements: } \\
56 \text { P. A. Ongley, J. Chem. Soc. } \\
\text { 1954, } 3634 \text {. }\end{array}$ \\
\hline $\begin{array}{l}\text { Variables: } \\
T / \mathrm{K}=298.15\end{array}$ & $\begin{array}{l}\text { Prepared by: } \\
\text { W. E. Acree, Jr. }\end{array}$ \\
\hline
\end{tabular}

\section{Experimental Values}

The measured solubility was reported to be $-\log _{10} c_{1}=$ 4.699, which corresponds to a solubility of $c_{1}=0.0000200$ $\mathrm{mol} \mathrm{dm}^{-3}$

\section{Auxiliary Information}

\section{Method/Apparatus/Procedure:}

Very little experimental details were provided. Excess solute and solvent were placed in tubes, which were rotated for at least $8 \mathrm{~h}$ at $298 \mathrm{~K}$. The solutions were then removed, concentrated if necessary, and titrated with standard alkali using a mixed (bromothymol blue + neutral red) indicator

\section{Source and Purity of Chemicals:}

(1) Purity not given, Chemical source not given, solute was either "AnalaR" grade or recrystallized prior to use.

(2) Purity not given, Chemical source not given, no purification details were provided.

\section{Estimated Error:}

Temperature: No information was given. $c_{1}: \pm 4 \%$ (relative error, estimated by compiler).

\subsection{3-Hydroxybenzoic acid solubility data in alcohols}

\begin{tabular}{|c|c|}
\hline $\begin{array}{l}\text { Components: } \\
\text { (1) 3-Hydroxybenzoic acid; } \mathrm{C}_{7} \mathrm{H}_{6} \mathrm{O}_{3} \text {; } \\
\text { [99-06-9] } \\
\text { (2) Methanol; } \mathrm{CH}_{4} \mathrm{O} ;[67-56-1]\end{array}$ & $\begin{array}{l}\text { Original Measurements: } \\
{ }^{158} \text { E. Bergroth, Farm. Aikak. 70, } 91 \\
\text { (1961). }\end{array}$ \\
\hline $\begin{array}{l}\text { Variables: } \\
T / \mathrm{K}=293.15\end{array}$ & $\begin{array}{l}\text { Prepared by: } \\
\text { W. E. Acree, Jr. }\end{array}$ \\
\hline
\end{tabular}

\section{Experimental Values}

The measured solubility was reported to be $27.86 \mathrm{~g} / 100 \mathrm{ml}$ of solution, which corresponds to a molar concentration of $2.017 \mathrm{~mol} \mathrm{dm}^{-3}$.

\section{Auxiliary Information}

Method/Apparatus/Procedure:

Solubility was determined using a gravimetric method. Excess solute and solvent were placed in a solubility vessel. The apparatus was closed tightly with rubber stoppers and allowed to equilibrate in a constant-temperature water bath with stirring. A known aliquot of the saturated solution was removed, filtered, and transferred to an evaporating dish. The solvent was carefully removed over a steam bath. The evaporating dish was then placed in a drying oven, and later removed and weighed. The solubility was calculated from the mass of the solid residue and volume of saturated sample analyzed.

\section{Source and Purity of Chemicals:}

(1) Purity not given, Schuchardt Chemicals, no purification details were provided.

(2) Purity not given, Chemical source not given, no purification details were provided.

\section{Estimated Error:}

Temperature: $\pm 0.2 \mathrm{~K}$ (estimated by compiler). $c_{1}: \pm 5 \%$ (relative error, estimated by compiler).

\begin{tabular}{|c|c|}
\hline $\begin{array}{l}\text { Components: } \\
\text { (1) 3-Hydroxybenzoic acid; } \mathrm{C}_{7} \mathrm{H}_{6} \mathrm{O}_{3} \text {; } \\
\text { [99-06-9] } \\
\text { (2) Methanol; } \mathrm{CH}_{4} \mathrm{O} ;[67-56-1]\end{array}$ & $\begin{array}{l}\text { Original Measurements: } \\
{ }^{165} \text { F. L. Nordström and A. C. } \\
\text { Rasmuson, Eur. J. Pharm. Sci. 28, } \\
377 \text { (2006). }\end{array}$ \\
\hline $\begin{array}{l}\text { Variables: } \\
\text { Temperature }\end{array}$ & $\begin{array}{l}\text { Prepared by: } \\
\text { W. E. Acree, Jr. }\end{array}$ \\
\hline
\end{tabular}

Experimental Values

\begin{tabular}{lcc}
\hline \hline$T / \mathrm{K}$ & \multicolumn{1}{c}{$x_{2}{ }^{\mathrm{a}}$} & $x_{1}{ }^{\mathrm{b}}$ \\
\hline 283.2 & 0.9153 & 0.0847 \\
288.2 & 0.9107 & 0.0893 \\
293.2 & 0.9057 & 0.0943 \\
298.2 & 0.9002 & 0.0998 \\
303.2 & 0.8950 & 0.1050 \\
308.2 & 0.8891 & 0.1109 \\
313.2 & 0.8823 & 0.1177 \\
318.2 & 0.8749 & 0.1251 \\
323.2 & 0.8683 & 0.1317 \\
\hline${ }^{\mathrm{a}}{ }_{2}$ : mole fraction of component 2 in the saturated solution. \\
${ }^{\mathrm{b}}{ }_{x_{1}}$ : mole fraction solubility of the solute. The authors noted that the solid \\
existed in different polymorphic forms. The solubility data are for monoclinic \\
form. Solubilities were reported as grams of solute per kilogram of solvent.
\end{tabular}




\section{Auxiliary Information}

\section{Method/Apparatus/Procedure:}

Constant-temperature bath, magnetic stirrer, analytical balance, and ventilated laboratory hood.

Solubilities were determined by a gravimetric method. Excess solute and solvent were placed in sealed bottles and allowed to equilibrate in a constanttemperature bath for at least $24 \mathrm{~h}$ with stirring. The stirring was discontinued and the solid was allowed to settle to the bottom of the container. An aliquot of the clear solution was removed by syringe, filtered through a $0.2 \mu \mathrm{m}$ PTFE filter, and transferred to a preweighed glass vial. The glass vial was then weighed, and the solvent was allowed to evaporate in a ventilated laboratory hood at ambient room temperature. Once the solvent had evaporated, the vial with solid residue was weighed until constant weight was obtained. The solubility was calculated from the mass of the solid residue and mass of sample analyzed.

\section{Source and Purity of Chemicals:}

(1) $99.8 \%$, Sigma-Aldrich Chemical Company, USA, was used as received. (2) $99.8 \%$, HiperSolv, VWR Scientific, USA, no purification details were provided.

\section{Estimated Error:}

Temperature: $\pm 0.01 \mathrm{~K}$.

$x_{1}: \pm 2 \%$ (relative error).

\begin{tabular}{|c|c|}
\hline $\begin{array}{l}\text { Components: } \\
\text { (1) 3-Hydroxybenzoic acid; } \mathrm{C}_{7} \mathrm{H}_{6} \mathrm{O}_{3} \text {; } \\
\text { [99-06-9] } \\
\text { (2) Ethanol; } \mathrm{C}_{2} \mathrm{H}_{6} \mathrm{O} ;[64-17-5]\end{array}$ & $\begin{array}{l}\text { Original Measurements: } \\
{ }^{127} \text { N. V. Sidgwick and E. K. } \\
\text { Ewbank, J. Chem. Soc. Trans. 119, } \\
979 \text { (1921). }\end{array}$ \\
\hline $\begin{array}{l}\text { Variables: } \\
\text { Temperature }\end{array}$ & $\begin{array}{l}\text { Prepared by: } \\
\text { W. E. Acree, Jr. }\end{array}$ \\
\hline
\end{tabular}

Experimental Values

\begin{tabular}{lcc}
\hline \hline$T / \mathrm{K}$ & $x_{2}{ }^{\mathrm{a}}$ & $x_{1}{ }^{\mathrm{b}}$ \\
\hline 338.2 & 0.821 & 0.179 \\
405.2 & 0.654 & 0.346 \\
442.2 & 0.402 & 0.598 \\
\hline
\end{tabular}

${ }^{\mathrm{a}} x_{2}$ : mole fraction of component 2 in the saturated solution.

${ }^{b} x_{1}$ : mole fraction solubility of the solute. Solubility data reported in units of mass percent. Mole fraction values calculated by the compiler.

\section{Auxiliary Information}

\begin{abstract}
Method/Apparatus/Procedure:
Preweighed quantities of solute and solvent were placed in bulbs, which were then sealed and heated in a suitable bath. At the higher temperatures, the concentration of solvent in the liquid solution was corrected for the amount of solvent vapor in the bulb. The correction assumed that the vapor pressure of the saturated solution was one half that of the pure solvent at the solution temperature. The temperature at which the solid completely dissolved was recorded, and was taken to be the solid-liquid equilibrium temperature.
\end{abstract}

\section{Source and Purity of Chemicals:}

(1) Purity not given, prepared and purified by the authors using a published synthetic procedure [see J. Walker and J. K. Wood, J. Chem. Soc. 117, 40 (1920) for synthetic details]. Melting point of the purified solute was $474.5 \mathrm{~K}$. (2) $99 \%$, Chemical source not given, distilled over calcium oxide shortly before use.

\section{Estimated Error:}

Temperature: Not given in paper.

$x_{1}: \pm 10 \%$ (relative error, estimated by compiler).

\begin{tabular}{|c|c|}
\hline $\begin{array}{l}\text { Components: } \\
\text { (1) 3-Hydroxybenzoic acid; } \mathrm{C}_{7} \mathrm{H}_{6} \mathrm{O}_{3} \text {; } \\
\text { [99-06-9] } \\
\text { (2) Ethanol; } \mathrm{C}_{2} \mathrm{H}_{6} \mathrm{O} ;[64-17-5]\end{array}$ & $\begin{array}{l}\text { Original Measurements: } \\
{ }^{158} \text { E. Bergroth, Farm. Aikak. 70, } 91 \\
\text { (1961). }\end{array}$ \\
\hline $\begin{array}{l}\text { Variables: } \\
T / \mathrm{K}=293.15\end{array}$ & $\begin{array}{l}\text { Prepared by: } \\
\text { W. E. Acree, Jr. }\end{array}$ \\
\hline
\end{tabular}

\section{Experimental Values}

The measured solubility was reported to be $22.54 \mathrm{~g} / 100 \mathrm{ml}$ of solution, which corresponds to a molar concentration of $1.632 \mathrm{~mol} \mathrm{dm}^{-3}$.

\section{Auxiliary Information}

\section{Method/Apparatus/Procedure:}

Solubility was determined using a gravimetric method. Excess solute and solvent were placed in a solubility vessel. The apparatus was closed tightly with rubber stoppers and allowed to equilibrate in a constant-temperature water bath with stirring. A known aliquot of the saturated solution was removed, filtered, and transferred to an evaporating dish. The solvent was carefully removed over a steam bath. The evaporating dish was then placed in a drying oven, and later removed and weighed. The solubility was calculated from the mass of the solid residue and volume of saturated sample analyzed.

Source and Purity of Chemicals:

(1) Purity not given, Schuchardt Chemicals, no purification details were provided.

(2) Purity not given, Chemical source not given, no purification details were provided.

\section{Estimated Error:}

Temperature: $\pm 0.2 \mathrm{~K}$ (estimated by compiler). $c_{1}: \pm 5 \%$ (relative error, estimated by compiler).

\begin{tabular}{|c|c|}
\hline $\begin{array}{l}\text { Components: } \\
\text { (1) 3-Hydroxybenzoic acid; } \mathrm{C}_{7} \mathrm{H}_{6} \mathrm{O}_{3} \text {; } \\
\text { [99-06-9] } \\
\text { (2) 1-Propanol; } \mathrm{C}_{3} \mathrm{H}_{8} \mathrm{O} ;[71-23-8]\end{array}$ & $\begin{array}{l}\text { Original Measurements: } \\
{ }^{158} \text { E. Bergroth, Farm. Aikak. 70, } 91 \\
(1961) \text {. }\end{array}$ \\
\hline $\begin{array}{l}\text { Variables: } \\
T / \mathrm{K}=293.15\end{array}$ & $\begin{array}{l}\text { Prepared by: } \\
\text { W. E. Acree, Jr. }\end{array}$ \\
\hline
\end{tabular}

\section{Experimental Values}

The measured solubility was reported to be $16.34 \mathrm{~g} / 100 \mathrm{ml}$ of solution, which corresponds to a molar concentration of $1.183 \mathrm{~mol} \mathrm{dm}^{-3}$.

\section{Auxiliary Information}

\footnotetext{
Method/Apparatus/Procedure:

Solubility was determined using a gravimetric method. Excess solute and solvent were placed in a solubility vessel. The apparatus was closed tightly with rubber stoppers and allowed to equilibrate in a constant-temperature water bath with stirring. A known aliquot of the saturated solution was removed, filtered, and transferred to an evaporating dish. The solvent was carefully removed over a steam bath. The evaporating dish was then placed in a drying oven, and later removed and weighed. The solubility was calculated from the mass of the solid residue and volume of saturated sample analyzed.

Source and Purity of Chemicals:

(1) Purity not given, Schuchardt Chemicals, no purification details were provided.

(2) Purity not given, Chemical source not given, no purification details were provided.
} 
Estimated Error:

Temperature: $\pm 0.2 \mathrm{~K}$ (estimated by compiler). $c_{1}: \pm 5 \%$ (relative error, estimated by compiler).

\begin{tabular}{|c|c|}
\hline $\begin{array}{l}\text { Components: } \\
\text { (1) 3-Hydroxybenzoic acid; } \mathrm{C}_{7} \mathrm{H}_{6} \mathrm{O}_{3} \text {; } \\
\text { [99-06-9] } \\
\text { (2) 1-Butanol; } \mathrm{C}_{4} \mathrm{H}_{10} \mathrm{O} ;[71-36-3]\end{array}$ & $\begin{array}{l}\text { Original Measurements: } \\
{ }^{127} \text { N. V. Sidgwick and E. K. } \\
\text { Ewbank, J. Chem. Soc. Trans. 119, } \\
979 \text { (1921). }\end{array}$ \\
\hline $\begin{array}{l}\text { Variables: } \\
\text { Temperature }\end{array}$ & $\begin{array}{l}\text { Prepared by: } \\
\text { W. E. Acree, Jr. }\end{array}$ \\
\hline
\end{tabular}

Experimental Values

\begin{tabular}{lcc}
\hline \hline$T / \mathrm{K}$ & $x_{2}{ }^{\mathrm{a}}$ & $x_{1}{ }^{\mathrm{b}}$ \\
\hline 309.7 & 0.877 & 0.123 \\
388.2 & 0.730 & 0.270 \\
424.4 & 0.562 & 0.438 \\
453.5 & 0.252 & 0.748
\end{tabular}

${ }^{\mathrm{a}} x_{2}$ : mole fraction of component 2 in the saturated solution.

${ }^{\mathrm{b}} x_{1}$ : mole fraction solubility of the solute. Solubility data reported in units of mass percent. Mole fraction values calculated by the compiler.

\section{Auxiliary Information}

\begin{abstract}
Method/Apparatus/Procedure:
Preweighed quantities of solute and solvent were placed in bulbs, which were then sealed and heated in a suitable bath. At the higher temperatures, the concentration of solvent in the liquid solution was corrected for the amount of solvent vapor in the bulb. The correction assumed that the vapor pressure of the saturated solution was one half that of the pure solvent at the solution temperature. The temperature at which the solid completely dissolved was recorded, and was taken to be the solid-liquid equilibrium temperature.
\end{abstract}

Source and Purity of Chemicals:

(1) Purity not given, prepared and purified by the authors using a published synthetic procedure [see J. Walker and J. K. Wood, J. Chem. Soc. 117, 40 (1920) for synthetic details]. Melting point of the purified solute was $474.5 \mathrm{~K}$. (2) Purity not given, Chemical source not given, fractionated and distilled several times before use.

\section{Estimated Error:}

Temperature: Not given in paper.

$x_{1}: \pm 10 \%$ (relative error, estimated by compiler).

\begin{tabular}{|c|c|}
\hline $\begin{array}{l}\text { Components: } \\
\text { (1) 3-Hydroxybenzoic acid; } \mathrm{C}_{7} \mathrm{H}_{6} \mathrm{O}_{3} \text {; } \\
\text { [99-06-9] } \\
\text { (2) 1-Octanol; } \mathrm{C}_{8} \mathrm{H}_{18} \mathrm{O} ;[111-87-5]\end{array}$ & $\begin{array}{l}\text { Original Measurements: } \\
{ }^{145} \text { G. L. Perlovich, T. V. Volkova, } \\
\text { and A. Bauer-Brandl, J. Pharm. Sci. } \\
\text { 95, } 1448 \text { (2006). }\end{array}$ \\
\hline Variables: & Prepared by: \\
\hline Temperature & W. E. Acree, Jr. \\
\hline
\end{tabular}

Experimental Values

\begin{tabular}{lcc}
\hline \hline$T / \mathrm{K}$ & $x_{2}{ }^{\mathrm{a}}$ & $x_{1}{ }^{\mathrm{b}}$ \\
\hline 293 & 0.9163 & 0.0837 \\
298 & 0.9056 & 0.0944 \\
303 & 0.9007 & 0.0993
\end{tabular}

\begin{tabular}{lcc}
\hline \hline$T / \mathrm{K}$ & $x_{2}{ }^{\mathrm{a}}$ & $x_{1}{ }^{\mathrm{b}}$ \\
\hline 310 & 0.887 & 0.113 \\
315 & 0.878 & 0.122 \\
\hline
\end{tabular}

${ }^{\mathrm{a}} x_{2}$ : mole fraction of component 2 in the saturated solution.

${ }^{\mathrm{b}} x_{1}$ : mole fraction solubility of the solute.

\section{Auxiliary Information}

\section{Method/Apparatus/Procedure:}

Thermostated constant-temperature bath, centrifuge, and an ultraviolet/visible spectrophotometer.

Excess solute and solvent were placed in a glass ampoule and allowed to equilibrate in a thermostated temperature bath with mixing (at a speed of $25 \mathrm{rpm}$ ) for a minimum of four days. After suitable equilibration, the saturated solution was centrifuged, the supernatant liquid collected, quantitatively diluted, and the absorbance recorded using an ultraviolet/visible spectrophotometer. The solubility of the solute was calculated from the measured absorbance.

Source and Purity of Chemicals:

(1) $99 \%$, Merck Chemicals, Darmstadt, Germany, no purification details provided.

(2) Analytical Reagent grade, Sigma-Aldrich, Inc., Oslo, Norway, no purification details were provided.

\section{Estimated Error:}

Temperature: $\pm 0.1 \mathrm{~K}$.

$x_{2}: \pm 2.5 \%$ (relative error).

\subsection{3-Hydroxybenzoic acid solubility data in ketones}

\begin{tabular}{|c|c|}
\hline $\begin{array}{l}\text { Components: } \\
\text { (1) 3-Hydroxybenzoic acid; } \mathrm{C}_{7} \mathrm{H}_{6} \mathrm{O}_{3} \text {; } \\
\text { [99-06-9] } \\
\text { (2) Propanone; } \mathrm{C}_{3} \mathrm{H}_{6} \mathrm{O} ;[67-64-1]\end{array}$ & $\begin{array}{l}\text { Original Measurements: } \\
{ }^{158} \text { E. Bergroth, Farm. Aikak. 70, } 91 \\
\text { (1961). }\end{array}$ \\
\hline $\begin{array}{l}\text { Variables: } \\
T / \mathrm{K}=293.15\end{array}$ & $\begin{array}{l}\text { Prepared by: } \\
\text { W. E. Acree, Jr. }\end{array}$ \\
\hline
\end{tabular}

\section{Experimental Values}

The measured solubility was reported to be $20.72 \mathrm{~g} / 100 \mathrm{ml}$ of solution, which corresponds to a molar concentration of $1.500 \mathrm{~mol} \mathrm{dm}^{-3}$.

\section{Auxiliary Information}

\section{Method/Apparatus/Procedure:}

Solubility was determined using a gravimetric method. Excess solute and solvent were placed in a solubility vessel. The apparatus was closed tightly with rubber stoppers and allowed to equilibrate in a constant-temperature water bath with stirring. A known aliquot of the saturated solution was removed, filtered, and transferred to an evaporating dish. The solvent was carefully removed over a steam bath. The evaporating dish was then placed in a drying oven, and later removed and weighed. The solubility was calculated from the mass of the solid residue and volume of saturated sample analyzed.

Source and Purity of Chemicals:

(1) Purity not given, Schuchardt Chemicals, no purification details were provided.

(2) Purity not given, Chemical source not given, no purification details were provided. 
Estimated Error:

Temperature: $\pm 0.2 \mathrm{~K}$ (estimated by compiler). $c_{1}: \pm 5 \%$ (relative error, estimated by compiler).

\begin{tabular}{|c|c|}
\hline $\begin{array}{l}\text { Components: } \\
\text { (1) 3-Hydroxybenzoic acid; } \mathrm{C}_{7} \mathrm{H}_{6} \mathrm{O}_{3} \text {; } \\
\text { [99-06-9] } \\
\text { (2) Propanone; } \mathrm{C}_{3} \mathrm{H}_{6} \mathrm{O} ;[67-64-1]\end{array}$ & $\begin{array}{l}\text { Original Measurements: } \\
{ }^{147} \text { J. Walker and J. K. Wood, J. } \\
\text { Chem. Soc. Trans. 73, } 618 \text { (1898). }\end{array}$ \\
\hline $\begin{array}{l}\text { Variables: } \\
T / \mathrm{K}=296\end{array}$ & $\begin{array}{l}\text { Prepared by: } \\
\text { W. E. Acree, Jr. }\end{array}$ \\
\hline
\end{tabular}

\section{Experimental Values}

The measured solubility was reported to be $22.7 \mathrm{~g} / 100 \mathrm{ml}$ of solution, which corresponds to a molar concentration of $1.643 \mathrm{~mol} \mathrm{dm}^{-3}$.

\section{Auxiliary Information}

\section{Method/Apparatus/Procedure:}

Excess solute and solvent were equilibrated with agitation by stirrers driven by a small turbine. At higher temperatures agitation was occasionally by hand. Equilibrium was obtained by both undersaturation at lower temperature and by supersaturation by pre-equilibrating at a higher temperature. The concentration of the dissolved solute was determined by titration with barium hydroxide with Congo Red being the endpoint indicator.

Source and Purity of Chemicals:

(1) Purity not given, Chemical source not given, no purification details were provided.

(2) Purity not given, Chemical source not given, no purification details were provided.

Estimated Error:

Temperature: Not given in paper.

$c_{1}: \pm 10 \%$ (relative error, estimated by compiler)

\begin{tabular}{|c|c|}
\hline $\begin{array}{l}\text { Components: } \\
\text { (1) 3-Hydroxybenzoic acid; } \mathrm{C}_{7} \mathrm{H}_{6} \mathrm{O}_{3} \text {; } \\
\text { [99-06-9] } \\
\text { (2) Propanone; } \mathrm{C}_{3} \mathrm{H}_{6} \mathrm{O} ;[67-64-1]\end{array}$ & $\begin{array}{l}\text { Original Measurements: } \\
{ }^{165} \text { F. L. Nordström and A. C. } \\
\text { Rasmuson, Eur. J. Pharm. Sci. 28, } \\
377 \text { (2006). }\end{array}$ \\
\hline $\begin{array}{l}\text { Variables: } \\
\text { Temperature }\end{array}$ & $\begin{array}{l}\text { Prepared by: } \\
\text { W. E. Acree, Jr. }\end{array}$ \\
\hline
\end{tabular}

Experimental Values

\begin{tabular}{lcc}
\hline \hline$T / \mathrm{K}$ & $x_{2}{ }^{\mathrm{a}}$ & $x_{1}{ }^{\mathrm{b}}$ \\
\hline 283.2 & 0.8994 & 0.1006 \\
288.2 & 0.8942 & 0.1058 \\
293.2 & 0.8883 & 0.1117 \\
298.2 & 0.8827 & 0.1173 \\
303.2 & 0.8759 & 0.1241 \\
308.2 & 0.8691 & 0.1309 \\
313.2 & 0.8614 & 0.1386 \\
318.2 & 0.8549 & 0.1451 \\
323.2 & 0.8468 & 0.1532
\end{tabular}

${ }^{a} x_{2}$ : mole fraction of component 2 in the saturated solution.

${ }^{b} x_{1}$ : mole fraction solubility of the solute. The authors noted that the solid existed in different polymorphic forms. The solubility data are for the monoclinic form. Solubilities were reported as grams of solute per kilogram of solvent. Mole fraction values were calculated by the compiler.
Experimental Values

\begin{tabular}{lcc}
\hline \hline$T / \mathrm{K}$ & $x_{2}{ }^{\mathrm{a}}$ & $x_{1}{ }^{\mathrm{b}}$ \\
\hline 283.2 & 0.8812 & 0.1188 \\
288.2 & 0.8760 & 0.1240 \\
293.2 & 0.8702 & 0.1298 \\
298.2 & 0.8631 & 0.1369 \\
303.2 & 0.8554 & 0.1446 \\
308.2 & 0.8473 & 0.1527 \\
313.2 & 0.8392 & 0.1608 \\
318.2 & 0.8298 & 0.1702 \\
323.2 & 0.8185 & 0.1815 \\
\hline $\mathrm{a}$
\end{tabular}

${ }^{\mathrm{a}} x_{2}$ : mole fraction of component 2 in the saturated solution.

${ }^{\mathrm{b}} x_{1}$ : mole fraction solubility of the solute. The authors noted that the solid existed in different polymorphic forms. The solubility data are for the orthorhombic form. Solubilities were reported as grams of solute per kilogram of solvent. Mole fraction values were calculated by the compiler.

\section{Auxiliary Information}

\section{Method/Apparatus/Procedure:}

Constant-temperature bath, magnetic stirrer, analytical balance, and ventilated laboratory hood.

Solubilities were determined by a gravimetric method. Excess solute and solvent were placed in sealed bottles and allowed to equilibrate in a constanttemperature bath for at least $24 \mathrm{~h}$ with stirring. The stirring was discontinued and the solid was allowed to settle to the bottom of the container. An aliquot of the clear solution was removed by syringe, filtered through a $0.2 \mu \mathrm{m}$ PTFE filter, and transferred to a preweighed glass vial. The glass vial was then weighed, and the solvent was allowed to evaporate in a ventilated laboratory hood at ambient room temperature. Once the solvent had evaporated, the vial with solid residue was weighed until constant weight was obtained. The solubility was calculated from the mass of the solid residue and mass of sample analyzed.

Source and Purity of Chemicals:

(1) $99 \%$, Sigma-Aldrich Chemical Company, USA, was used as received. (2) $99.8+\%$, HiperSolv, VWR Scientific, USA, no purification details were provided.

\section{Estimated Error:}

Temperature: $\pm 0.01 \mathrm{~K}$.

$x_{1}: \pm 2 \%$ (relative error).

\begin{tabular}{|c|c|}
\hline $\begin{array}{l}\text { Components: } \\
\text { (1) 3-Hydroxybenzoic acid; } \mathrm{C}_{7} \mathrm{H}_{6} \mathrm{O}_{3} \text {; } \\
\text { [99-06-9] } \\
\text { (2) 2-Butanone; } \mathrm{C}_{4} \mathrm{H}_{8} \mathrm{O} ;[78-93-3]\end{array}$ & $\begin{array}{l}\text { Original Measurements: } \\
{ }^{158} \text { E. Bergroth, Farm. Aikak. 70, } 91 \\
(1961) .\end{array}$ \\
\hline $\begin{array}{l}\text { Variables: } \\
T / \mathrm{K}=293.15\end{array}$ & $\begin{array}{l}\text { Prepared by: } \\
\text { W. E. Acree, Jr. }\end{array}$ \\
\hline
\end{tabular}

\section{Experimental Values}

The measured solubility was reported to be $15.69 \mathrm{~g} / 100 \mathrm{ml}$ of solution, which corresponds to a molar concentration of $1.136 \mathrm{~mol} \mathrm{dm}^{-3}$. 


\section{Auxiliary Information}

\section{Method/Apparatus/Procedure:}

Solubility was determined using a gravimetric method. Excess solute and solvent were placed in a solubility vessel. The apparatus was closed tightly with rubber stoppers and allowed to equilibrate in a constant-temperature water bath with stirring. A known aliquot of the saturated solution was removed, filtered, and transferred to an evaporating dish. The solvent was carefully removed over a steam bath. The evaporating dish was then placed in a drying oven, and later removed and weighed. The solubility was calculated from the mass of the solid residue and volume of saturated sample analyzed.

Source and Purity of Chemicals:

(1) Purity not given, Schuchardt Chemicals, no purification details were provided.

(2) Purity not given, Chemical source not given, no purification details were provided.

Estimated Error:

Temperature: $\pm 0.2 \mathrm{~K}$ (estimated by compiler). $c_{1}: \pm 5 \%$ (relative error, estimated by compiler).

\begin{tabular}{|c|c|}
\hline $\begin{array}{l}\text { Components: } \\
\text { (1) 3-Hydroxybenzoic acid; } \mathrm{C}_{7} \mathrm{H}_{6} \mathrm{O}_{3} \text {; } \\
\text { [99-06-9] } \\
\text { (2) 2-Pentanone; } \mathrm{C}_{5} \mathrm{H}_{10} \mathrm{O} ;[107-87-9]\end{array}$ & $\begin{array}{l}\text { Original Measurements: } \\
{ }^{158} \text { E. Bergroth, Farm. Aikak. 70, } 91 \\
(1961) .\end{array}$ \\
\hline $\begin{array}{l}\text { Variables: } \\
T / \mathrm{K}=293.15\end{array}$ & $\begin{array}{l}\text { Prepared by: } \\
\text { W. E. Acree, Jr. }\end{array}$ \\
\hline
\end{tabular}

\section{Experimental Values}

The measured solubility was reported to be $12.63 \mathrm{~g} / 100 \mathrm{ml}$ of solution, which corresponds to a molar concentration of $0.914 \mathrm{~mol} \mathrm{dm}^{-3}$.

\section{Auxiliary Information}

\section{Method/Apparatus/Procedure:}

Solubility was determined using a gravimetric method. Excess solute and solvent were placed in a solubility vessel. The apparatus was closed tightly with rubber stoppers and allowed to equilibrate in a constant-temperature water bath with stirring. A known aliquot of the saturated solution was removed, filtered, and transferred to an evaporating dish. The solvent was carefully removed over a steam bath. The evaporating dish was then placed in a drying oven, and later removed and weighed. The solubility was calculated from the mass of the solid residue and volume of saturated sample analyzed.

\section{Source and Purity of Chemicals:}

(1) Purity not given, Schuchardt Chemicals, no purification details were provided.

(2) Purity not given, Chemical source not given, no purification details were provided.

\section{Estimated Error:}

Temperature: $\pm 0.2 \mathrm{~K}$ (estimated by compiler). $c_{1}: \pm 5 \%$ (relative error, estimated by compiler).

\subsection{3-Hydroxybenzoic acid solubility data in miscellaneous organic solvents}

\begin{tabular}{ll}
\hline \hline Components: & Original Measurements: \\
$\begin{array}{l}\text { (1) 3-Hydroxybenzoic acid; } \mathrm{C}_{7} \mathrm{H}_{6} \mathrm{O}_{3} ; \\
\text { [99-06-9] }\end{array}$ & $\begin{array}{l}{ }^{165} \mathrm{~F} \text {. L. Nordström and A. C. } \\
\text { Rasmuson, Eur. J. Pharm. Sci. 28, }\end{array}$ \\
\hline 2) Ethanoic acid; $\mathrm{C}_{2} \mathrm{H}_{4} \mathrm{O}_{2} ;$ [64-19-7] & 377 (2006). \\
\hline Variables: & Prepared by: \\
Temperature & W. E. Acree, Jr. \\
\hline
\end{tabular}

Experimental Values

\begin{tabular}{lcc}
\hline \hline$T / \mathrm{K}$ & $x_{2}{ }^{\mathrm{a}}$ & $x_{1}{ }^{\mathrm{b}}$ \\
\hline 283.2 & 0.9780 & 0.0220 \\
288.2 & 0.9756 & 0.0244 \\
293.2 & 0.9727 & 0.0273 \\
298.2 & 0.9697 & 0.0303 \\
303.2 & 0.9665 & 0.0335 \\
308.2 & 0.9624 & 0.0376 \\
313.2 & 0.9585 & 0.0415 \\
318.2 & 0.9538 & 0.0462 \\
323.2 & 0.9488 & 0.0512
\end{tabular}

${ }^{\mathrm{a}} x_{2}$ : mole fraction of component 2 in the saturated solution.

${ }^{b} x_{1}$ : mole fraction solubility of the solute. The authors noted that the solid existed in different polymorphic forms. The solubility data are for the monoclinic form. Solubilities were reported as grams of solute per kilogram of solvent. Mole fraction values were calculated by the compiler.

Experimental Values

\begin{tabular}{lcc}
\hline \hline$T / \mathrm{K}$ & $x_{2}{ }^{\mathrm{a}}$ & $x_{1}{ }^{\mathrm{b}}$ \\
\hline 283.2 & 0.9709 & 0.0291 \\
288.2 & 0.9689 & 0.0311 \\
293.2 & 0.9659 & 0.0341 \\
298.2 & 0.9625 & 0.0375 \\
303.2 & 0.9585 & 0.0415 \\
308.2 & 0.9541 & 0.0459 \\
313.2 & 0.9489 & 0.0511 \\
318.2 & 0.9439 & 0.0561 \\
323.2 & 0.9386 & 0.0614
\end{tabular}

${ }^{a} x_{2}$ : mole fraction of component 2 in the saturated solution.

${ }^{b} x_{1}$ : mole fraction solubility of the solute. The authors noted that the solid existed in different polymorphic forms. The solubility data are for the orthorhombic form. Solubilities were reported as grams of solute per kilogram of solvent. Mole fraction values were calculated by the compiler.

\section{Auxiliary Information}

\section{Method/Apparatus/Procedure:}

Constant-temperature bath, magnetic stirrer, analytical balance, and ventilated laboratory hood.

Solubilities were determined by a gravimetric method. Excess solute and solvent were placed in sealed bottles and allowed to equilibrate in a constanttemperature bath for at least $24 \mathrm{~h}$ with stirring. The stirring was discontinued and the solid was allowed to settle to the bottom of the container. An aliquot of the clear solution was removed by syringe, filtered through a $0.2 \mu \mathrm{m}$ PTFE filter, and transferred to a preweighed glass vial. The glass vial was then weighed, and the solvent was allowed to evaporate in a ventilated laboratory hood at ambient room temperature. Once the solvent had evaporated, the vial with solid residue was weighed until constant weight was obtained. The solubility was calculated from the mass of the solid residue and mass of sample analyzed. 
Source and Purity of Chemicals:

(1) $99 \%$, Sigma-Aldrich Chemical Company, USA, was used as received.

(2) $96 \%$, Pro Analysis, Merck Chemical Company, Germany, no purification details were provided.

Estimated Error:

Temperature: $\pm 0.01 \mathrm{~K}$.

$x_{1}: \pm 2 \%$ (relative error).

\begin{tabular}{ll}
\hline \hline Components: & Original Measurements: \\
$\begin{array}{l}\text { (1) 3-Hydroxybenzoic acid; } \mathrm{C}_{7} \mathrm{H}_{6} \mathrm{O}_{3} ; \\
\text { [99-06-9] }\end{array}$ & $\begin{array}{l}{ }^{165} \mathrm{~F} \text {. L. Nordström and A. C. } \\
\text { Rasmuson, Eur. J. Pharm. Sci. 28, }\end{array}$ \\
$\begin{array}{l}\text { (2) Ethanenitrile; } \mathrm{C}_{2} \mathrm{H}_{3} \mathrm{~N} ;[\text { [75-05-8] } \\
\text { Variables: }\end{array}$ & 377 (2006). \\
Temperature & Prepared by: \\
\hline
\end{tabular}

Experimental Values

\begin{tabular}{lcc}
\hline \hline$T / \mathrm{K}$ & $x_{2}{ }^{\mathrm{a}}$ & $x_{1}{ }^{\mathrm{b}}$ \\
\hline 283.2 & 0.9929 & 0.0071 \\
288.2 & 0.9918 & 0.0082 \\
293.2 & 0.9904 & 0.0096 \\
298.2 & 0.9889 & 0.0111 \\
303.2 & 0.9870 & 0.0130 \\
308.2 & 0.9846 & 0.0154 \\
313.2 & 0.9821 & 0.0179 \\
318.2 & 0.9799 & 0.0201 \\
323.2 & 0.9766 & 0.0234
\end{tabular}

${ }^{\mathrm{a}} x_{2}$ : mole fraction of component 2 in the saturated solution.

${ }^{b} x_{1}$ : mole fraction solubility of the solute. The authors noted that the solid existed in different polymorphic forms. The solubility data are for the monoclinic form. Solubilities were reported as grams of solute per kilogram of solvent. Mole fraction values were calculated by the compiler.

Experimental Values

\begin{tabular}{lcc}
\hline \hline$T / \mathrm{K}$ & $x_{2}{ }^{\mathrm{a}}$ & $x_{1}{ }^{\mathrm{b}}$ \\
\hline 283.2 & 0.9910 & 0.0090 \\
288.2 & 0.9897 & 0.0103 \\
293.2 & 0.9881 & 0.0119 \\
298.2 & 0.9861 & 0.0139 \\
303.2 & 0.9839 & 0.0161 \\
308.2 & 0.9811 & 0.0189 \\
313.2 & 0.9781 & 0.0219 \\
318.2 & 0.9745 & 0.0255 \\
323.2 & 0.9706 & 0.0294
\end{tabular}

${ }^{a} x_{2}$ : mole fraction of component 2 in the saturated solution.

${ }^{b} x_{1}$ : mole fraction solubility of the solute. The authors noted that the solid existed in different polymorphic forms. The solubility data are for the orthorhombic form. Solubilities were reported as grams of solute per kilogram of solvent. Mole fraction values were calculated by the compiler.

\section{Auxiliary Information}

Method/Apparatus/Procedure:

Constant-temperature bath, magnetic stirrer, analytical balance, and ventilated laboratory hood.

Solubilities were determined by a gravimetric method. Excess solute and solvent were placed in sealed bottles and allowed to equilibrate in a constanttemperature bath for at least $24 \mathrm{~h}$ with stirring. The stirring was discontinued and the solid was allowed to settle to the bottom of the container. An aliquot of the clear solution was removed by syringe, filtered through a $0.2 \mu \mathrm{m}$ PTFE filter, and transferred to a preweighed glass vial. The glass vial was then weighed, and the solvent was allowed to evaporate in a ventilated laboratory hood at ambient room temperature. Once the solvent had evaporated, the vial with solid residue was weighed until constant weight was obtained. The solubility was calculated from the mass of the solid residue and mass of sample analyzed.

Source and Purity of Chemicals:

(1) $99 \%$, Sigma-Aldrich Chemical Company, USA, was used as received. (2) $99.8+\%$, LiChroSolv, VWR Scientific, USA, no purification details were provided.

Estimated Error:

Temperature: $\pm 0.01 \mathrm{~K}$.

$x_{1}: \pm 2 \%$ (relative error).

\section{Solubility of 4-Hydroxybenzoic Acid in Organic Solvents}

\subsection{Critical evaluation of experimental solubility data}

There have been several published studies ${ }^{54,56,77,127,147,166-}$ 168 involving the solubility of 4-hydroxybenzoic acid in organic solvent mixtures. Most notably, Martin et al. ${ }^{166}$ performed solubility measurements at $298 \mathrm{~K}$ for 4-hydroxybenzoic acid in two aromatic hydrocarbons (benzene and methylbenzene), in two alkyl alkanoates (ethyl ethanoate and butyl ethanoate) in one dialkyl ether (1,1'-oxybisethane) and one cyclic ether (1,4-dioxane), in two chloroalkanes (trichloromethane and 1,2-dichloroethane), in 13 alcohols (methanol, ethanol, 1-propanol, 2-propanol, 1-butanol, 2-methyl-1-propanol, 1-pentanol, 1-hexanol, 1-octanol, benzenemethanol, 1,2-ethanediol, 1,2-propanediol, and 1,2,3-propanetriol), in one alkanone (propanone) and one aromatic ketone (acetophenone), and in eight miscellaneous organic solvents (ethanoic acid, propanoic acid, dimethyl sulfoxide, pyridine, formamide, $\mathrm{N}$-methylformamide, $\mathrm{N}, \mathrm{N}$-dimethylformamide, and $N, N$-dimethylacetamide). Gracin and Rasmuson ${ }^{167}$ determined the solubility of 4-hydroxybenzoic acid in ethyl ethanoate, methanol, ethanol, 2-propanol, 1-octanol, and propanone at $298 \mathrm{~K}$.

The Abraham solvation parameter model can provide an indication of the quality of experimental solubility data for 4-hydroxybenzoic acid dissolved in a series of organic solvents of varying polarity and hydrogen bonding character. As discussed above, the evaluation will be restricted to those solvents where dimerization is not likely to occur and to solvents where 4-hydroxybenzoic acid does not form a solid solvate. This condition will limit the evaluation to primarily the alkyl alkanoates, dialkyl ethers, and alcohols. 
TABLE 38. Comparison between observed and predicted molar solubilities of 4 hydroxybenzoic acid based on the Abraham model, Eqs. (20) and (21)

\begin{tabular}{lrrrr}
\hline \hline & $\log _{10} c_{1}^{\text {calc }}$ & $\log _{10} c_{1}^{\text {calc }}$ & & \\
Solvent & Eq. $(20)$ & Eq. $(21)$ & $\log _{10} c_{1}{ }^{\exp }$ & $\log _{10} c_{1}{ }^{\text {exp }}$ \\
\hline Methanol & 0.327 & 0.225 & $0.377^{\mathrm{a}}$ & $0.377^{\mathrm{b}}$ \\
Ethanol & 0.329 & 0.271 & $0.276^{\mathrm{a}}$ & $0.292^{\mathrm{b}}$ \\
1-Propanol & 0.158 & 0.159 & $0.142^{\mathrm{a}}$ & \\
2-Propanol & 0.185 & 0.205 & $0.208^{\mathrm{a}}$ & $0.228^{\mathrm{b}}$ \\
1-Butanol & -0.008 & -0.003 & $0.092^{\mathrm{a}}$ & \\
2-Butanol & 0.042 & 0.043 & & \\
2-Methyl-1-propanol & -0.004 & -0.058 & $-0.016^{\mathrm{a}}$ & \\
2-Methyl-2-propanol & 0.098 & 0.189 & & \\
1-Pentanol & 0.061 & 0.058 & $0.025^{\mathrm{a}}$ & \\
2-Pentanol & 0.101 & 0.054 & & \\
3-Methyl-1-butanol & -0.014 & -0.089 & & \\
1-Hexanol & -0.038 & -0.054 & $-0.040^{\mathrm{a}}$ & \\
1-Heptanol & -0.214 & -0.105 & & \\
1-Octanol & -0.202 & -0.241 & $-0.170^{\mathrm{a}}$ & $-0.141^{\mathrm{b}}$ \\
1-Decanol & -0.209 & -0.210 & & \\
1,2-Ethanediol & 0.219 & 0.263 & $0.266^{\mathrm{a}}$ & \\
1,1'-Oxybisethane & -0.468 & -0.405 & $-0.300^{\mathrm{a}}$ & \\
Tetrahydrofuran & 0.198 & 0.174 & & \\
1,4-Dioxane & -0.148 & -0.186 & $-0.014^{\mathrm{a}}$ & \\
Methyl ethanoate & -0.486 & -0.553 & & \\
Ethyl ethanoate & -0.403 & -0.411 & $-0.128^{\mathrm{a}}$ & $-0.151^{\mathrm{b}}$ \\
Propyl ethanoate & -0.496 & -0.520 & & \\
Butyl ethanoate & -0.589 & -0.629 & $-0.358^{\mathrm{a}}$ & \\
Propanone & -0.226 & -0.230 & $0.184^{\mathrm{a}}$ & $0.187^{\mathrm{b}}$ \\
\hline a Expering & & & \\
\hline
\end{tabular}

${ }^{\mathrm{a}}$ Experimental value is taken from Martin et al. ${ }^{166}$

${ }^{\mathrm{b}}$ Gracin and Rasmuson. ${ }^{167}$

Numerical values of the solute descriptors for 4-hydroxybenzoic acid are known $(E=0.930, S=0.900, A=0.810, B$ $=0.570, V=0.9904$, and $L=4.867$ ), so that combination of these descriptors with the coefficients listed in Table 1 allows the prediction of $\log _{10}\left(c_{1, \mathrm{~S}}^{\mathrm{sat}} / c_{1, \mathrm{~W}}^{\mathrm{sat}}\right)$. The molar solubility of molecular 4-hydroxybenzoic acid in water, $\log _{10}$ $c_{1, \mathrm{~W}}^{\mathrm{sat}}=-1.47$ (Ref. 164), is available to convert the predicted $\left(c_{1, \mathrm{~S}}^{\mathrm{sat}} / c_{1, \mathrm{~W}}^{\mathrm{sat}}\right)$ solubility ratios to $c_{1, \mathrm{~S}}^{\mathrm{sat}}$ values. For carboxylic acid solutes, $c_{1, \mathrm{~W}}^{\mathrm{sat}}$ corresponds to the aqueous solubility of the molecular, nonionized form of the solute. A numerical value of $\log _{10} \quad c_{1, \mathrm{G}}=-8.25$ is used for the gas-phase molar concentration of 4-hydroxybenzoic acid.

The predicted molar solubilities of 4-hydroxybenzoic acid in methanol, ethanol, 1-propanol, 1-butanol, 1-pentanol, 1-octanol, 2-propanol, 2-butanol, 2-methyl-1-propanol, 2-methyl-2-propanol, 1,2-ethanediol, 1,1'-oxybisethane, tetrahydrofuran, 1,4dioxane, ethyl ethanoate, butyl ethanoate, propanone, butanone, and cyclohexanone based on Eqs. (20) and (21) are listed in the second and third columns of Table 38. The numerical values represent outright solubility predictions in that none of the experimental data was used in the determination of the molecular solute descriptors. For comparison purposes, the measured mole fraction solubilities of 4-hydroxybenzoic acid, $x_{1}$, given in Secs. 48.2-48.9 were converted into molar solubilities by dividing $x_{1}$ by the ideal molar volume of the saturated solution (i.e., $c_{1}{ }^{\text {sat }}=x_{1} /$ $\left.\left[x_{1} V_{1}+\left(1-x_{1}\right) V_{\text {solvent }}\right]\right)$. The molar volume of the hypothetical subcooled liquid 4-hydroxybenzoic acid is taken to be $V_{\text {solute }}$ $=104 \mathrm{~cm}^{3} \mathrm{~mol}^{-1}$, which is larger than the value that other researchers have used. Martin et al. ${ }^{166}$ assumed a molar volume of 4-hydroxybenzoic acid of $V_{1}=94.3 \mathrm{~cm}^{3} \mathrm{~mol}^{-1}$. This latter value seems too small compared to the molar volume of benzaldehyde, which is estimated to be $V_{1} \approx 101.7 \mathrm{~cm}^{3} \mathrm{~mol}^{-1}$, based on a molar mass of $106.12 \mathrm{~g} \mathrm{~mol}^{-1}$ and an experimental density of $1.0436 \mathrm{~g} \mathrm{~cm}^{-3}$ at $298 \mathrm{~K}$. A molar volume on the order of $V_{1} \approx$ $104 \mathrm{~cm}^{3} \mathrm{~mol}^{-1}$ would seem more realistic given the molecular structures of 4-hydroxybenzoic acid compared to benzaldehyde. To get an idea of how much error might be introduced in the mole fraction to molarity conversion, the mole fraction solubility of 4hydroxybenzoic acid in methanol of $x_{1}=0.1142$ (value from Martin et al. ${ }^{166}$ ) is converted into molar solubilities using both $V_{1}$ $=94.3 \mathrm{~cm}^{3} \mathrm{~mol}^{-1}\left(c_{1}=2.439 \mathrm{~mol} \mathrm{dm}{ }^{-3}\right)$ and $V_{1} \approx 104 \mathrm{~cm}^{3}$ $\mathrm{mol}^{-1}\left(c_{1}=2.383 \mathrm{~mol} \mathrm{dm}^{-3}\right)$. Results of the computation suggest that any errors resulting from the estimation of 4hydroxybenzoic acid's hypothetical subcooled liquid molar volume, $V_{\text {solute }}$, or the ideal molar volume approximation will have negligible effect of the calculated $c_{1}$ values because 4hydroxybenzoic acid is not overly soluble in many of the solvents considered. From a mathematical standpoint, the $x_{1}{ }^{\exp } V_{\text {solute }}$ term contributes very little to the molar volumes of the saturated solutions.

There is a sufficient number of data points for several of the solvents in Secs. 48.2-48.9 to compute a recommended value. The recommended mole fraction solubilities at $298 \mathrm{~K}$ are: $x_{1}=0.0719$ for ethyl ethanoate [arithmetic average of $x_{1}=0.0737,{ }^{166} 0.0698,{ }^{167}$ and 0.0722 (Ref. 168)]; $x_{1}=$ 0.114 for methanol [arithmetic average of $x_{1}=0.1142,{ }^{166}$ $0.1142,{ }^{167}$ and 0.1141 (Ref. 168)]; and $x_{1}=0.106$ for 1 octanol [arithmetic average of $0.1031,{ }^{166} 0.1102,{ }^{167}$ and $0.106,{ }^{77}$ excluding a suspected outlier value of $x_{1}=$ 0.139 (Ref. 145)].

Nordström and Rasmuson ${ }^{168}$ measured the solubility of 4hydroxybenzoic acid in ethyl ethanoate, methanol, propanone, ethanenitrile, and ethanoic acid at several temperatures between $283 \mathrm{~K}$ and $323 \mathrm{~K}$ in order to calculate thermodynamic data pertaining to the dissolution process. The authors computed the enthalpy of solution of 4-hydroxybenzoic acid in the five organic solvents through a Van't Hoff treatment of the measured solubility data. The natural logarithms of the mole fraction solubilities were curve-fit to a second degree polynomial in $1 / T$

$$
\ln x_{1}=A+\frac{B}{T}+\frac{C}{T^{2}},
$$

with the enthalpy of solution calculated as the temperature derivative, i.e., $\Delta H_{\text {soln }}=R T^{2}\left(\mathrm{~d} \ln x_{1} / \mathrm{d} T\right)$. The equation coefficients $(A, B$, and $C$ ), along with the squared correlation coefficients, $R^{2}$, are given in Table 39. The authors did note in the discussion the morphologies of crystals obtained by evaporation crystallization from the different solvents, and some of the difficulties encountered in making reproducible solubility measurements. Solubility analyses and FTIR-ATR spectroscopic measurements, in combination with the solubility analyses and microscopic examinations, did suggest indications of an enantiotropic polymorphism in ethanenitrile and ethanoic acid. The transition point was estimated to fall between 298 and $303 \mathrm{~K}$. An acetone solvate was found and 
TABLE 39. Parameters of Eq. (53) for describing the solubility of 4-hydroxybenzoic acid in various organic solvents

\begin{tabular}{lcccc}
\hline \hline Solvent & $A$ & $B$ & $C$ & $R^{2}$ \\
\hline Ansolvate $^{\mathrm{a}}$ & & & & \\
Methanol & 5.3439 & -3688.6 & 432010 & 0.9981 \\
Ethanenitrile & 8.1099 & -4864.5 & 351270 & 0.9993 \\
Ethanoic acid & 6.7801 & -4499.5 & 466550 & 0.9999 \\
Propanone & 13.414 & -8727.2 & 124350 & 0.9985 \\
Ethyl ethanoate $^{\text {Stu }}$ & 6.0428 & -4137.8 & 462870 & 0.9999 \\
Solvate $^{\mathrm{a}}$ & & & & \\
Acetone solvate & 10.286 & -5873.3 & 647790 & 0.9999 \\
\hline
\end{tabular}

${ }^{\mathrm{a}}$ Values of the coefficients and squared correlation coefficients were taken from Nordström and Rasmuson. ${ }^{168}$

shown to be the stable phase up to approximately $323 \mathrm{~K}$. The solubility of the ansolvate could be determined at higher temperature by dissolution up to equilibrium. Both propanone and 4-hydroxybenzoic acid had to be preheated to $323 \mathrm{~K}$ in order to inhibit phase conversion into the solvate. The authors were able to measure the solubility of the ansolvate down to $303 \mathrm{~K}$. Indication of the presence of further solvates in ethanenitrile, ethyl ethanoate, and methanol was found but could not be verified.

Qingzhu et $a l .{ }^{77}$ measured the solubility of 4-hydroxybenzoic acid in 1-octanol as a function of temperature using a dynamic method with laser monitoring to observe when dissolution was complete. The calculated curve-fit parameters from the Buchowski $\lambda$ h-model [Eq. (9)] of $\lambda=0.0248$ and $h=$ 5861.21 described the observed solubility data to within a mean relative deviation of $0.2 \%$.

The experimental solubility data for 4-hydroxybenzoic acid in organic solvents are in Secs. 48.2-48.9.

\subsection{4-Hydroxybenzoic acid solubility data in saturated hydrocarbons (including cycloalkanes)}

\begin{tabular}{|c|c|}
\hline $\begin{array}{l}\text { Components: } \\
\text { (1) 4-Hydroxybenzoic acid; } \mathrm{C}_{7} \mathrm{H}_{6} \mathrm{O}_{3} \text {; } \\
\text { [99-96-7] } \\
\text { (2) Hexane; } \mathrm{C}_{6} \mathrm{H}_{14} ;[110-54-3]\end{array}$ & $\begin{array}{l}\text { Original Measurements: } \\
{ }^{145} \text { G. L. Perlovich, T. V. Volkova, } \\
\text { and A. Bauer-Brandl, J. Pharm. Sci. } \\
\text { 95, } 1448 \text { (2006). }\end{array}$ \\
\hline Variables: & Prepared by: \\
\hline Temperature & W. E. Acree, Jr. \\
\hline
\end{tabular}

Experimental Values

\begin{tabular}{lcc}
\hline \hline$T / \mathrm{K}$ & $x_{2}{ }^{\mathrm{a}}$ & $x_{1}{ }^{\mathrm{b}}$ \\
\hline 293 & 0.9999 & 0.00000205 \\
294 & 0.9999 & 0.00000245 \\
298 & 0.9999 & 0.00000305 \\
303 & 0.9999 & 0.00000376 \\
310 & 0.9999 & 0.00000596 \\
315 & 0.9999 & 0.00000714 \\
\hline${ }^{\mathrm{a}} x_{2}:$ mole fraction of component 2 in the saturated solution. & \\
${ }^{\mathrm{b}}{ }_{1}:$ mole fraction solubility of the solute.
\end{tabular}

\section{Auxiliary Information}

\section{Method/Apparatus/Procedure:}

Thermostated constant-temperature bath, centrifuge, and an ultraviolet/visible spectrophotometer.

Excess solute and solvent were placed in a glass ampoule and allowed to equilibrate in a thermostated temperature bath with mixing (at a speed of $25 \mathrm{rpm}$ ) for a minimum of four days. After suitable equilibration, the saturated solution was centrifuged, the supernatant liquid collected, quantitatively diluted, and the absorbance recorded using an ultraviolet/visible spectrophotometer. The solubility of the solute was calculated from the measured absorbance.

Source and Purity of Chemicals:

(1) $99 \%$, Merck Chemicals, Darmstadt, Germany, no purification details provided.

(2) Analytical Reagent grade, Sigma-Aldrich Inc., Oslo, Norway, no purification details were provided.

Estimated Error:

Temperature: $\pm 0.1 \mathrm{~K}$.

$x_{2}: \pm 2.5 \%$ (relative error).

\begin{tabular}{|c|c|}
\hline Components: & Original Measurements: \\
\hline $\begin{array}{l}\text { (1) 4-Hydroxybenzoic acid; } \mathrm{C}_{7} \mathrm{H}_{6} \mathrm{O}_{3} \text {; } \\
\text { [99-96-7] }\end{array}$ & $\begin{array}{l}{ }^{54} \text { C. K. Hancock, J. N. Pawloski, } \\
\text { and J. P. Idoux, J. Org. Chem. 31, }\end{array}$ \\
\hline (2) Cyclohexane; $\mathrm{C}_{6} \mathrm{H}_{12} ;[110-82-7]$ & 3801 (1966). \\
\hline Variables: & Prepared by: \\
\hline$T / \mathrm{K}=303.15$ & W. E. Acree, Jr. \\
\hline
\end{tabular}

Experimental Values

\begin{tabular}{lc}
\hline \hline$x_{2}{ }^{\mathrm{a}}$ & $x_{1}{ }^{\mathrm{b}}$ \\
\hline 0.9999 & 0.0000370 \\
\hline${ }^{\mathrm{a}} x_{2}:$ mole fraction of component 2 in the saturated solution. \\
${ }^{\mathrm{b}} x_{1}:$ mole fraction solubility of the solute. \\
$\qquad \begin{array}{l}\text { Auxiliary Information } \\
\end{array}$
\end{tabular}

\section{Method/Apparatus/Procedure:}

Constant-temperature bath, Soxhlet thimble, conical flask, and analytical balance.

Excess solute and solvent were placed in a conical flask and allowed to equilibrate for several days at constant temperature. Attainment of equilibrium was verified by several repetitive measurements and by approaching equilibrium from supersaturation. Aliquots of saturated solutions were transferred using a Soxhlet thimble equipped with a one-hole cork stopper and an inverted U-shaped delivery tube to a second ground-glass stoppered flask suspended in the $303 \mathrm{~K}$ water bath. Successive portions of the contents were evaporated at room temperature in a tared aluminum foil weighing dish under a bell jar through which a slow stream of dry air was passed. The second flask plus unused saturated solution and the aluminum foil dish plus residue were weighed. The saturation solubility of the solute was calculated from the recorded mass data and molar masses of the solute and solvent.

\section{Source and Purity of Chemicals:}

(1) Purity not given, Chemical source not specified, was recrystallized several times from aqueous-ethanol mixture, and then dried under vacuum over phosphorous pentoxide.

(2) Reagent grade, Chemical source not specified, refluxed over phosphorous pentoxide for $24 \mathrm{~h}$, and then distilled through a $3 \times 80 \mathrm{~cm}$ column filled with 0.32 -cm glass helices. 
Estimated Error:

Temperature: $\pm 0.02 \mathrm{~K}$.

$x_{1}: \pm 10 \%$ (relative error, by compiler).

\begin{tabular}{|c|c|}
\hline $\begin{array}{l}\text { Components: } \\
\text { (1) 4-Hydroxybenzoic acid; } \mathrm{C}_{7} \mathrm{H}_{6} \mathrm{O}_{3} \text {; } \\
\text { [99-96-7] } \\
\text { (2) Cyclohexane; } \mathrm{C}_{6} \mathrm{H}_{12} ;[110-82-7]\end{array}$ & $\begin{array}{l}\text { Original Measurements: } \\
\text { 56 P. A. Ongley, J. Chem. Soc. 1954, } \\
3634 \text {. }\end{array}$ \\
\hline $\begin{array}{l}\text { Variables: } \\
T / \mathrm{K}=298.15\end{array}$ & $\begin{array}{l}\text { Prepared by: } \\
\text { W. E. Acree, Jr. }\end{array}$ \\
\hline
\end{tabular}

\section{Experimental Values}

The measured solubility was reported to be $-\log _{10} c_{1}=$ 4.398, which corresponds to a solubility of $c_{1}=0.0000400$ mol dm ${ }^{-3}$.

\section{Auxiliary Information}

\section{Method/Apparatus/Procedure:}

Very little experimental details were provided. Excess solute and solvent were placed in tubes, which were rotated for at least $8 \mathrm{~h}$ at $298 \mathrm{~K}$. The solutions were then removed, concentrated if necessary, and titrated with standard alkali using a mixed (bromothymol blue + neutral red) indicator.

\section{Source and Purity of Chemicals:}

(1) Purity not given, Chemical source not given, solute was either "AnalaR" grade or recrystallized prior to use.

(2) Purity not given, Chemical source not given, no purification details were provided.

\section{Estimated Error:}

Temperature: No information was given.

$c_{1}: \pm 4 \%$ (relative error, estimated by compiler).

\subsection{4-Hydroxybenzoic acid solubility data in aromatic hydrocarbons}

\begin{tabular}{ll}
\hline \hline Components: & Original Measurements: \\
$\begin{array}{l}\text { (1) 4-Hydroxybenzoic acid; } \mathrm{C}_{7} \mathrm{H}_{6} \mathrm{O}_{3} ; \\
\text { [99-96-7] }\end{array}$ & $\begin{array}{l}{ }^{166} \text { A. Martin, P. L. Wu, and A. } \\
\text { Beerbower, J. Pharm. Sci. 73, 188 } \\
\text { (2) Benzene; } \mathrm{C}_{6} \mathrm{H}_{6} ;[71-43-2]\end{array}$ \\
\hline Variables: & Prepared by: \\
$T / \mathrm{K}=298.15$ & W. E. Acree, Jr. \\
\hline
\end{tabular}

\section{Experimental Values}

\begin{tabular}{lc}
\hline \hline$x_{2}{ }^{\mathrm{a}}$ & $x_{1}{ }^{\mathrm{b}}$ \\
\hline 0.9999 & 0.000038 \\
\hline${ }^{\mathrm{a}} x_{2}:$ mole fraction of component 2 in the saturated solution. \\
${ }^{\mathrm{b}} x_{1}:$ mole fraction solubility of the solute.
\end{tabular}

Auxiliary Information

Method/Apparatus/Procedure:

Constant-temperature bath and ultraviolet/visible spectrophotometer.

Excess solute and solvent were placed in screw-capped vials. The vials were sealed and submerged in a constant-temperature water bath and shaken at 100 cycles/min for $24 \mathrm{~h}$. After the $24 \mathrm{~h}$ equilibration period, the vial was removed, wiped dry, and the contents analyzed. The solutions were transferred to a syringe and filtered through a $0.1 \mu \mathrm{m}$ pore size filter. The solutions were diluted and the absorbances recorded at the maximum absorption wavelength of benzoic acid. The solubility was determined at least six times.

Source and Purity of Chemicals:

(1) Purity not given, Chemical source not specified, recrystallized from aqueous ethanol before use.

(2) Spectrophotometric or ACS Reagent grade, Chemical source not specified, redistilled before use.

\section{Estimated Error:}

Temperature: $\pm 0.2 \mathrm{~K}$. $x_{1}: \pm 3 \%$ (relative error).

\begin{tabular}{|c|c|}
\hline $\begin{array}{l}\text { Components: } \\
\text { (1) 4-Hydroxybenzoic acid; } \mathrm{C}_{7} \mathrm{H}_{6} \mathrm{O}_{3} \text {; } \\
\text { [99-96-7] } \\
\text { (2) Benzene; } \mathrm{C}_{6} \mathrm{H}_{6} ;[71-43-2]\end{array}$ & $\begin{array}{l}\text { Original Measurements: } \\
{ }^{54} \text { C. K. Hancock, J. N. Pawloski, } \\
\text { and J. P. Idoux, J. Org. Chem. 31, } \\
3801 \text { (1966). }\end{array}$ \\
\hline $\begin{array}{l}\text { Variables: } \\
T / \mathrm{K}=303.15\end{array}$ & $\begin{array}{l}\text { Prepared by: } \\
\text { W. E. Acree, Jr. }\end{array}$ \\
\hline
\end{tabular}

\section{Experimental Values}

\begin{tabular}{lc}
\hline \hline$x_{2}{ }^{\mathrm{a}}$ & $x_{1}{ }^{\mathrm{b}}$ \\
\hline 0.9999 & 0.0000588 \\
\hline${ }^{\mathrm{a}} x_{2}:$ mole fraction of component 2 in the saturated solution. & \\
${ }^{\mathrm{b}}{ }_{x_{1}: \text { mole fraction solubility of the solute. }}$ &
\end{tabular}

\section{Auxiliary Information}

\begin{abstract}
Method/Apparatus/Procedure:
Constant-temperature bath, Soxhlet thimble, conical flask, and analytical balance.

Excess solute and solvent were placed in a conical flask and allowed to equilibrate for several days at constant temperature. Attainment of equilibrium was verified by several repetitive measurements and by approaching equilibrium from supersaturation. Aliquots of saturated solutions were transferred using a Soxhlet thimble equipped with a one-hole cork stopper and an inverted $\mathrm{U}$-shaped delivery tube to a second ground-glass stoppered flask suspended in the $303 \mathrm{~K}$ water bath. Successive portions of the contents were evaporated at room temperature in a tared aluminum foil weighing dish under a bell jar through which a slow stream of dry air was passed. The second flask plus unused saturated solution and the aluminum foil dish plus residue were weighed. The saturation solubility of the solute was calculated from the recorded mass data and molar masses of the solute and solvent.
\end{abstract}

\section{Source and Purity of Chemicals:}

(1) Purity not given, Chemical source not specified, was recrystallized several times from aqueous-ethanol mixture, and then dried under vacuum over phosphorous pentoxide.

(2) Reagent grade, Chemical source not specified, refluxed over phosphorous pentoxide for $24 \mathrm{~h}$, and then distilled through a $3 \times 80 \mathrm{~cm}$ column filled with $0.32-\mathrm{cm}$ glass helices.

\section{Estimated Error:}

Temperature: $\pm 0.02 \mathrm{~K}$

$x_{1}: \pm 10 \%$ (relative error, by compiler). 


\begin{tabular}{|c|c|}
\hline $\begin{array}{l}\text { Components: } \\
\text { (1) 4-Hydroxybenzoic acid; } \mathrm{C}_{7} \mathrm{H}_{6} \mathrm{O}_{3} \text {; } \\
\text { [99-96-7] }\end{array}$ & $\begin{array}{l}\text { Original Measurements: } \\
{ }^{127} \text { N. V. Sidgwick and E. K. } \\
\text { Ewbank, J. Chem. Soc. Trans. 119 }\end{array}$ \\
\hline (2) Benzene; $\mathrm{C}_{6} \mathrm{H}_{6} ;[$ [1-43-2] & $979(1921)$ \\
\hline $\begin{array}{l}\text { Variables: } \\
\text { Temperature }\end{array}$ & $\begin{array}{l}\text { Prepared by: } \\
\text { W. E. Acree, Jr. }\end{array}$ \\
\hline
\end{tabular}

Experimental Values

\begin{tabular}{lcl}
\hline \hline$T / \mathrm{K}$ & $x_{2}{ }^{\mathrm{a}}$ & $x_{1}{ }^{\mathrm{b}}$ \\
\hline 405.4 & 0.994 & 0.0059 \\
430.1 & 0.983 & 0.0174 \\
438.5 & 0.975 & 0.0248 \\
451.2 & 0.939 & 0.0610 \\
464.7 & 0.869 & 0.131 \\
468.9 & 0.721 & 0.279 \\
472.0 & 0.527 & 0.473 \\
479.2 & 0.266 & 0.734 \\
\hline
\end{tabular}

${ }^{a} x_{2}$ : mole fraction of component 2 in the saturated solution.

${ }^{b} x_{1}$ : mole fraction solubility of the solute. Solubility data reported in units of mass percent. Mole fraction values calculated by the compiler.

\section{Auxiliary Information}

\section{Method/Apparatus/Procedure:}

Preweighed quantities of solute and solvent were placed in bulbs, which were then sealed and heated in a suitable bath. At the higher temperatures, the concentration of solvent in the liquid solution was corrected for the amount of solvent vapor in the bulb. The correction assumed that the vapor pressure of the saturated solution was one half that of the pure solvent at the solution temperature. The temperature at which the solid completely dissolved was recorded, and was taken to be the solid-liquid equilibrium temperature.

\section{Source and Purity of Chemicals:}

(1) Purity not given, prepared and purified by the authors using a published synthetic procedure [see J. Walker and J. K. Wood, J. Chem. Soc. 117, 40 (1920) for synthetic details]. Melting point of the purified solute was $486.2 \mathrm{~K}$. (2) Purity not given, Chemical source not given, thiophene was removed by treatment with sulfuric acid. Sample was further purified by freezing several times.

Estimated Error:

Temperature: Not given in paper.

$x_{1}: \pm 10 \%$ (relative error, estimated by compiler).

\begin{tabular}{|c|c|}
\hline $\begin{array}{l}\text { Components: } \\
\text { (1) 4-Hydroxybenzoic acid; } \mathrm{C}_{7} \mathrm{H}_{6} \mathrm{O}_{3} \text {; } \\
\text { [99-96-7] } \\
\text { (2) Benzene; } \mathrm{C}_{6} \mathrm{H}_{6} ;[71-43-2]\end{array}$ & $\begin{array}{l}\text { Original Measurements: } \\
\text { 56 P. A. Ongley, J. Chem. Soc. 1954, } \\
3634 \text {. }\end{array}$ \\
\hline $\begin{array}{l}\text { Variables: } \\
T / \mathrm{K}=298.15\end{array}$ & $\begin{array}{l}\text { Prepared by: } \\
\text { W. E. Acree, Jr. }\end{array}$ \\
\hline
\end{tabular}

\section{Experimental Values}

The measured solubility was reported to be $-\log _{10} c_{1}=3.432$, which corresponds to a solubility of $c_{1}=0.000370 \mathrm{~mol} \mathrm{dm}^{-3}$.

\section{Auxiliary Information}

\section{Method/Apparatus/Procedure:}

Very little experimental details were provided. Excess solute and solvent were placed in tubes, which were rotated for at least $8 \mathrm{~h}$ at $298 \mathrm{~K}$. The solutions were then removed, concentrated if necessary, and titrated with standard alkali using a mixed (bromothymol blue + neutral red) indicator.

Source and Purity of Chemicals:

(1) Purity not given, Chemical source not given, solute was either "AnalaR" grade or recrystallized prior to use.

(2) Purity not given, Chemical source not given, no purification details were provided.

Estimated Error:

Temperature: No information was given.

$c_{1}: \pm 4 \%$ (relative error, estimated by compiler).

\begin{tabular}{|c|c|}
\hline $\begin{array}{l}\text { Components: } \\
\text { (1) 4-Hydroxybenzoic acid; } \mathrm{C}_{7} \mathrm{H}_{6} \mathrm{O}_{3} \text {; } \\
\text { [99-96-7] } \\
\text { (2) Benzene; } \mathrm{C}_{6} \mathrm{H}_{6} ;[71-43-2]\end{array}$ & $\begin{array}{l}\text { Original Measurements: } \\
{ }^{147} \text { J. Walker and J. K. Wood, J. } \\
\text { Chem. Soc. Trans. 73, } 618 \text { (1898). }\end{array}$ \\
\hline $\begin{array}{l}\text { Variables: } \\
\text { Temperature }\end{array}$ & $\begin{array}{l}\text { Prepared by: } \\
\text { W. E. Acree, Jr. }\end{array}$ \\
\hline
\end{tabular}

Experimental Values

\begin{tabular}{lcl}
\hline \hline$T / \mathrm{K}$ & $x_{2}{ }^{\mathrm{a}}$ & \multicolumn{1}{c}{$x_{1}{ }^{\mathrm{b}}$} \\
\hline 283.2 & 0.9999 & 0.0000111 \\
306.2 & 0.9999 & 0.0000323 \\
322.2 & 0.9999 & 0.0000876 \\
337.2 & 0.9998 & 0.000198 \\
353.2 & 0.9996 & 0.000373
\end{tabular}

${ }^{a} x_{2}$ : mole fraction of component 2 in the saturated solution.

${ }^{b} x_{1}$ : mole fraction solubility of the solute. Solubility data reported as grams of solute per $100 \mathrm{~g}$ of solvent. Numerical values calculated by the compiler.

\section{Auxiliary Information}

\section{Method/Apparatus/Procedure:}

Excess solute and solvent were equilibrated with agitation by stirrers driven by a small turbine. At higher temperatures agitation was occasionally by hand. Equilibrium was obtained by both undersaturation at lower temperature and by supersaturation by pre-equilibrating at a higher temperature. The concentration of the dissolved solute was determined by titration with barium hydroxide with Congo Red being the endpoint indicator. Prior to titration, the solvent was removed by evaporation under reduced pressure.

Source and Purity of Chemicals:

(1) Purity not given, Chemical source not given, no purification details were provided.

(2) Purity not given, Chemical source not given, no purification details were provided.

\section{Estimated Error:}

Temperature: Not given in paper.

$x_{1}: \pm 10 \%$ (relative error, estimated by compiler). 


\section{Components:}

(1) 4-Hydroxybenzoic acid; $\mathrm{C}_{7} \mathrm{H}_{6} \mathrm{O}_{3}$; [99-96-7]

(2) Toluene; $\mathrm{C}_{7} \mathrm{H}_{8}$; [108-88-3]

\section{Variables:}

$T / \mathrm{K}=298.15$

Original Measurements:

${ }^{166}$ A. Martin, P. L. Wu, and A.

Beerbower, J. Pharm. Sci. 73, 188 (1984).

Prepared by:

W. E. Acree, Jr.

Experimental Values

\begin{tabular}{lc}
\hline \hline$x_{2}{ }^{\mathrm{a}}$ & $x_{1}{ }^{\mathrm{b}}$ \\
\hline 0.9999 & 0.000027
\end{tabular}

${ }^{\mathrm{a}} x_{2}$ : mole fraction of component 2 in the saturated solution.

${ }^{\mathrm{b}} x_{1}$ : mole fraction solubility of the solute.

\section{Auxiliary Information}

\section{Method/Apparatus/Procedure:}

Constant-temperature bath and ultraviolet/visible spectrophotometer.

Excess solute and solvent were placed in screw-capped vials. The vials were sealed and submerged in a constant-temperature water bath and shaken at 100 cycles/min for $24 \mathrm{~h}$. After the $24 \mathrm{~h}$ equilibration period, the vial was removed, wiped dry, and the contents analyzed. The solutions were transferred to a syringe and filtered through a $0.1 \mu \mathrm{m}$ pore size filter. The solutions were diluted and the absorbances recorded at the maximum absorption wavelength of benzoic acid. The solubility was determined at least six times.

Source and Purity of Chemicals:

(1) Purity not given, Chemical source not specified, recrystallized from aqueous ethanol before use.

(2) Spectrophotometric or ACS Reagent grade, Chemical source not specified, redistilled before use.

Estimated Error:

Temperature: $\pm 0.2 \mathrm{~K}$.

$x_{1}: \pm 3 \%$ (relative error).

\begin{tabular}{ll}
\hline \hline Components: & Original Measurements: \\
$\begin{array}{l}\text { (1) 4-Hydroxybenzoic acid; } \mathrm{C}_{7} \mathrm{H}_{6} \mathrm{O}_{3} ; \\
\text { [99-96-7] }\end{array}$ & $\begin{array}{l}{ }^{167} \text { S. Gracin and A. C. Rasmuson, J. } \\
\text { Chem. Eng. Data 47, 1379 (2002). } \\
\text { (2) Toluene; } \mathrm{C}_{7} \mathrm{H}_{8} ;[108-88-3]\end{array}$ \\
\hline $\begin{array}{l}\text { Variables: } \\
T / \mathrm{K}=298.15\end{array}$ & $\begin{array}{l}\text { Prepared by: } \\
\text { W. E. Acree, Jr. }\end{array}$ \\
\hline
\end{tabular}

Experimental Values

\begin{tabular}{lc}
\hline$x_{2}{ }^{\mathrm{a}}$ & $x_{1}{ }^{\mathrm{b}}$ \\
\hline 0.9990 & 0.00100 \\
${ }^{\mathrm{a}} x_{2}:$ mole fraction of component 2 in the saturated solution. \\
${ }^{\mathrm{b}} x_{1}:$ mole fraction solubility of the solute. The solubility data were reported as \\
the grams of solute that dissolved per kilogram of solvent. Mole fraction \\
solubility calculated by the compiler.
\end{tabular}

solubility calculated by the compiler.

\section{Auxiliary Information}

\section{Method/Apparatus/Procedure:}

Thermostated water bath, analytical balance, magnetic stirrer, and vacuum drying oven.

Solubility was determined by the gravimetric method. Excess solute and solvent were placed in Erlenmeyer flasks and allowed to equilibrate for $72 \mathrm{~h}$ in a thermostatic water bath sitting on a multiple-position magnetic stirrer. The solutions were stirred during the equilibration period. The stirring was then stopped for $4 \mathrm{~h}$ to allow the suspended solid to settle to the bottom of the flask. A sample of the clear saturated solution was transferred with a heated syringe into a previously weighed sample vial. The vial containing the saturated solution was then weighed, and the solvent was allowed to evaporate in a vacuum oven at $293 \mathrm{~K}$ for approximately one week until a constant weight was obtained. The solubility was calculated from the mass of the solid residue and mass of the saturated solution analyzed.

Source and Purity of Chemicals:

(1) $99 \%$, Sigma-Aldrich, Sweden, no purification details were provided.

(2) $99.5 \%$, Kemetyl AB, Sweden, no purification details were provided.

\section{Estimated Error:}

Temperature: $\pm 0.02 \mathrm{~K}$.

$x_{1}: \pm 60 \%$ (relative error, based on standard deviation given in the paper).

\subsection{4-Hydroxybenzoic acid solubility data in esters}

\begin{tabular}{|c|c|}
\hline $\begin{array}{l}\text { Components: } \\
\text { (1) 4-Hydroxybenzoic acid; } \mathrm{C}_{7} \mathrm{H}_{6} \mathrm{O}_{3} \text {; } \\
\text { [99-96-7] } \\
\text { (2) Ethyl ethanoate; } \mathrm{C}_{4} \mathrm{H}_{8} \mathrm{O}_{2} \text {; } \\
\text { [141-78-6] }\end{array}$ & $\begin{array}{l}\text { Original Measurements: } \\
{ }^{166} \text { A. Martin, P. L. Wu, and A. } \\
\text { Beerbower, J. Pharm. Sci. 73, } 188 \\
\text { (1984). }\end{array}$ \\
\hline $\begin{array}{l}\text { Variables: } \\
T / \mathrm{K}=298.15\end{array}$ & $\begin{array}{l}\text { Prepared by: } \\
\text { W. E. Acree, Jr. }\end{array}$ \\
\hline
\end{tabular}

\section{Experimental Values}

\begin{tabular}{lc}
\hline \hline$x_{2}{ }^{\mathrm{a}}$ & $x_{1}{ }^{\mathrm{b}}$ \\
\hline 0.9263 & 0.0737
\end{tabular}

${ }^{a} x_{2}$ : mole fraction of component 2 in the saturated solution.

${ }^{\mathrm{b}} x_{1}$ : mole fraction solubility of the solute.

\section{Auxiliary Information}

\section{Method/Apparatus/Procedure:}

Constant-temperature bath and ultraviolet/visible spectrophotometer.

Excess solute and solvent were placed in screw-capped vials. The vials were sealed and submerged in a constant-temperature water bath and shaken at 100 cycles/min for $24 \mathrm{~h}$. After the $24 \mathrm{~h}$ equilibration period, the vial was removed, wiped dry, and the contents analyzed. The solutions were transferred to a syringe and filtered through a $0.1 \mu \mathrm{m}$ pore size filter. The solutions were diluted and the absorbances recorded at the maximum absorption wavelength of benzoic acid. The solubility was determined at least six times.

Source and Purity of Chemicals:

(1) Purity not given, Chemical source not specified, recrystallized from aqueous ethanol before use.

(2) Spectrophotometric or ACS Reagent grade, Chemical source not specified, redistilled before use. 
Estimated Error:

Temperature: $\pm 0.2 \mathrm{~K}$

$x_{1}: \pm 3 \%$ (relative error).

\begin{tabular}{ll}
\hline \hline Components: & Original Measurements: \\
$\begin{array}{l}\text { (1) 4-Hydroxybenzoic acid; } \mathrm{C}_{7} \mathrm{H}_{6} \mathrm{O}_{3} ; \\
\text { [99-96-7] }\end{array}$ & $\begin{array}{l}{ }^{167} \text { S. Gracin and A. C. Rasmuson, J. } \\
\text { Chem. Eng. Data 47, 1379 (2002). } \\
\text { [141-78-6] }\end{array}$ \\
\hline $\begin{array}{l}\text { Variables: } \\
T / \mathrm{K}=298.15\end{array}$ & $\begin{array}{l}\text { Prepared by: } \\
\mathrm{C}_{4} \mathrm{H}_{8} \mathrm{O}_{2} ;\end{array}$ \\
\hline
\end{tabular}

Experimental Values

\begin{tabular}{lc}
\hline \hline$x_{2}{ }^{\mathrm{a}}$ & $x_{1}{ }^{\mathrm{b}}$ \\
\hline 0.9302 & 0.0698 \\
\hline
\end{tabular}

${ }^{\mathrm{a}} x_{2}$ : mole fraction of component 2 in the saturated solution.

${ }^{b} x_{1}$ : mole fraction solubility of the solute. The solubility data were reported as the grams of solute that dissolved per kilogram of solvent. Mole fraction solubility calculated by the compiler.

\section{Auxiliary Information}

\section{Method/Apparatus/Procedure:}

Thermostated water bath, analytical balance, magnetic stirrer, and vacuum drying oven.

Solubility was determined by the gravimetric method. Excess solute and solvent were placed in Erlenmeyer flasks and allowed to equilibrate for $72 \mathrm{~h}$ in a thermostatic water bath sitting on a multiple-position magnetic stirrer. The solutions were stirred during the equilibration period. The stirring was then stopped for $4 \mathrm{~h}$ to allow the suspended solid to settle to the bottom of the flask. A sample of the clear saturated solution was transferred with a heated syringe into a previously weighed sample vial. The vial containing the saturated solution was then weighed, and the solvent was allowed to evaporate in a vacuum oven at $293 \mathrm{~K}$ for approximately one week until a constant weight was obtained. The solubility was calculated from the mass of the solid residue and mass of the saturated solution analyzed.

Source and Purity of Chemicals:

(1) $99 \%$, Sigma-Aldrich, Sweden, no purification details were provided. (2) Pro Analyse, Merck Chemical Company, no purification details were provided.

Estimated Error:

Temperature: $\pm 0.02 \mathrm{~K}$.

$x_{1}: \pm 1 \%$ (relative error).

\begin{tabular}{ll}
\hline \hline Components: & Original Measurements: \\
(1) 4-Hydroxybenzoic acid; $\mathrm{C}_{7} \mathrm{H}_{6} \mathrm{O}_{3} ;$ & $\begin{array}{l}168 \\
\text { F. Nordström and A. C. } \\
\text { [99-96-7] }\end{array}$ \\
$\begin{array}{ll}\text { Rasmuson, J. Pharm. Sci. 95, 748 } \\
\text { (2006). }\end{array}$ \\
\hline $141-78-6]$ & \\
\hline Variables: & Prepared by: \\
Temperature & W. E. Acree, Jr. \\
\hline
\end{tabular}

Experimental Values

\begin{tabular}{lcc}
\hline \hline$T / \mathrm{K}$ & $x_{2}{ }^{\mathrm{a}}$ & $x_{1}{ }^{\mathrm{b}}$ \\
\hline 283.2 & 0.9389 & 0.0611 \\
288.2 & 0.9356 & 0.0644 \\
293.2 & 0.9320 & 0.0680 \\
298.2 & 0.9278 & 0.0722 \\
303.2 & 0.9233 & 0.0767 \\
308.2 & 0.9189 & 0.0811 \\
313.2 & 0.9135 & 0.0865 \\
318.2 & 0.9084 & 0.0916 \\
323.2 & 0.9027 & 0.0973
\end{tabular}

${ }^{\mathrm{a}} x_{2}$ : mole fraction of component 2 in the saturated solution.

${ }^{b} x_{1}$ : mole fraction solubility of the solute. Solubility was reported as grams of solute per kilogram of solvent. Mole fraction values were calculated by the compiler.

\section{Auxiliary Information}

\section{Method/Apparatus/Procedure:}

Constant-temperature bath, magnetic stirrer, analytical balance, and ventilated laboratory hood.

Solubilities were determined by a gravimetric method. Excess solute and solvent were placed in sealed bottles and allowed to equilibrate in a constanttemperature bath. An aliquot of the clear solution was removed by syringe, filtered through a $0.2 \mu \mathrm{m}$ PTFE filter, and transferred to a preweighed glass vial. The glass vial was then weighed, and the solvent was allowed to evaporate in a ventilated laboratory hood at ambient room temperature. Once the solvent had evaporated, the vial with solid residue was weighed until constant weight was obtained. The solubility was calculated from the mass of the solid residue and mass of sample analyzed.

\section{Source and Purity of Chemicals:}

(1) $99+\%$, Sigma-Aldrich Chemical Company, USA, was used as received. (2) $99.8 \%$, HiperSolv, VWR Scientific, USA, used as received.

\section{Estimated Error:}

Temperature: $\pm 0.01 \mathrm{~K}$

$x_{1}: \pm 3 \%$ (relative error, estimated by compiler).

\begin{tabular}{|c|c|}
\hline $\begin{array}{l}\text { Components: } \\
\text { (1) 4-Hydroxybenzoic acid; } \mathrm{C}_{7} \mathrm{H}_{6} \mathrm{O}_{3} \text {; } \\
\text { [99-96-7] } \\
\text { (2) Butyl ethanoate; } \mathrm{C}_{6} \mathrm{H}_{12} \mathrm{O}_{2} \text {; } \\
\text { [123-86-4] }\end{array}$ & $\begin{array}{l}\text { Original Measurements: } \\
{ }^{166} \text { A. Martin, P. L. Wu, and A. } \\
\text { Beerbower, J. Pharm. Sci. 73, } 188 \\
\text { (1984). }\end{array}$ \\
\hline $\begin{array}{l}\text { Variables: } \\
T / \mathrm{K}=298.15\end{array}$ & $\begin{array}{l}\text { Prepared by: } \\
\text { W. E. Acree, Jr. }\end{array}$ \\
\hline
\end{tabular}

Experimental Values

\begin{tabular}{lc}
\hline \hline$x_{2}{ }^{\mathrm{a}}$ & $x_{1}{ }^{\mathrm{b}}$ \\
\hline 0.9426 & 0.0574 \\
\hline
\end{tabular}

${ }^{\mathrm{a}} x_{2}$ : mole fraction of component 2 in the saturated solution.

${ }^{\mathrm{b}} x_{1}$ : mole fraction solubility of the solute. 


\section{Auxiliary Information}

\section{Method/Apparatus/Procedure:}

Constant-temperature bath and ultraviolet/visible spectrophotometer.

Excess solute and solvent were placed in screw-capped vials. The vials were sealed and submerged in a constant-temperature water bath and shaken at 100 cycles/min for $24 \mathrm{~h}$. After the $24 \mathrm{~h}$ equilibration period, the vial was removed, wiped dry, and the contents analyzed. The solutions were transferred to a syringe and filtered through a $0.1 \mu \mathrm{m}$ pore size filter. The solutions were diluted and the absorbances recorded at the maximum absorption wavelength of benzoic acid. The solubility was determined at least six times.

Source and Purity of Chemicals:

(1) Purity not given, Chemical source not specified, recrystallized from aqueous ethanol before use.

(2) Spectrophotometric or ACS Reagent grade, Chemical source not specified, redistilled before use.

Estimated Error:

Temperature: $\pm 0.2 \mathrm{~K}$. $x_{1}: \pm 3 \%$ (relative error).

\subsection{4-Hydroxybenzoic acid solubility data in ethers}

\begin{tabular}{ll}
\hline \hline Components: & Original Measurements: \\
$\begin{array}{l}\text { (1) 4-Hydroxybenzoic acid; } \mathrm{C}_{7} \mathrm{H}_{6} \mathrm{O}_{3} ; \\
\text { [99-96-7] }\end{array}$ & $\begin{array}{l}{ }^{166} \text { A. Martin, P. L. Wu, and A. } \\
\text { Beerbower, J. Pharm. Sci. 73, } 188 \\
(2) 1,1^{\prime}-\text {-Oxybisethane; } \mathrm{C}_{4} \mathrm{H}_{10} \mathrm{O} ;\end{array}$ \\
$\begin{array}{ll}(1984) . \\
\text { 60-29-7] }\end{array}$ & Prepared by: \\
\hline Variables: & W. E. Acree, Jr. \\
$T / \mathrm{K}=298.15$ &
\end{tabular}

\section{Experimental Values}

\begin{tabular}{lc}
\hline \hline$x_{2}{ }^{\mathrm{a}}$ & $x_{1}{ }^{\mathrm{b}}$ \\
\hline 0.9479 & 0.0521 \\
\hline${ }^{\mathrm{a}} x_{2}:$ mole fraction of component 2 in the saturated solution. & \\
${ }^{\mathrm{b}} x_{1}:$ mole fraction solubility of the solute. &
\end{tabular}

\section{Auxiliary Information}

\section{Components:}

(1) 4-Hydroxybenzoic acid; $\mathrm{C}_{7} \mathrm{H}_{6} \mathrm{O}_{3}$;

[99-96-7]

(2) 1-Methylethyl tetradecanoate; $\mathrm{C}_{17} \mathrm{H}_{34} \mathrm{O}_{2} ;[110-27-0]$

\begin{tabular}{ll}
\hline Variables: & Prepared by: \\
$T / \mathrm{K}=295$ & W. E. Acree, Jr.
\end{tabular}

Experimental Values

\begin{tabular}{lc}
\hline \hline$x_{2}^{\mathrm{a}}$ & $x_{1}{ }^{\mathrm{b}}$ \\
\hline 0.984 & 0.016
\end{tabular}

${ }^{a} x_{2}$ : mole fraction of component 2 in the saturated solution.

${ }^{b} x_{1}$ : mole fraction solubility of the solute. Solubility data reported in terms of mass percent. Mole fraction solubility calculated by the compiler.

\section{Auxiliary Information}

\section{Method/Apparatus/Procedure:}

Very little experimental details provided. Solubility determinations were made by stirring the solvent with excess solute for two days, centrifuging the sample, and analyzing the supernatant.

\section{Source and Purity of Chemicals:}

(1) Reagent grade, Aldrich Chemical Company, Metuchen, NJ, USA, no purification details provided.

(2) Reagent grade, Wickhen Corporation, Huguenot, NJ, USA, no purification details provided.

\section{Estimated Error:}

Temperature: No information given.

$x_{1}$ : No information given.
Original Measurements:

Release 1, 153 (1984).

\section{Method/Apparatus/Procedure:}

Constant-temperature bath and ultraviolet/visible spectrophotometer.

Excess solute and solvent were placed in screw-capped vials. The vials were sealed and submerged in a constant-temperature water bath and shaken at 100 cycles/min for $24 \mathrm{~h}$. After the $24 \mathrm{~h}$ equilibration period, the vial was removed, wiped dry, and the contents analyzed. The solutions were transferred to a syringe and filtered through a $0.1 \mu \mathrm{m}$ pore size filter. The solutions were diluted and the absorbances recorded at the maximum absorption wavelength of benzoic acid. The solubility was determined at least six times.

Source and Purity of Chemicals:

(1) Purity not given, Chemical source not specified, recrystallized from aqueous ethanol before use.

(2) Spectrophotometric or ACS Reagent grade, Chemical source not specified, redistilled before use.

Estimated Error:

Temperature: $\pm 0.2 \mathrm{~K}$.

$x_{1}: \pm 3 \%$ (relative error).

\begin{tabular}{ll}
\hline \hline $\begin{array}{l}\text { Components: } \\
\text { (1) 4-Hydroxybenzoic acid; } \mathrm{C}_{7} \mathrm{H}_{6} \mathrm{O}_{3} ;\end{array}$ & $\begin{array}{l}\text { Original Measurements: } \\
\text { [99-96-7] }\end{array}$ \\
$\begin{array}{ll}\text { C. Wem. Walker and J. K. Wood, J. Trans. 73, 618 (1898). } \\
\text { Che-Oxybisethane; } \mathrm{C}_{4} \mathrm{H}_{10} \mathrm{O} ;\end{array}$ \\
\hline $60-29-7]$ & \\
\hline Variables: & Prepared by: \\
$T / \mathrm{K}=290$ & W. E. Acree, Jr. \\
\hline
\end{tabular}

\section{Experimental Values}

The measured solubility was reported to be $9.43 \mathrm{~g} / 100 \mathrm{ml}$ of solution, which corresponds to a molar concentration of $0.683 \mathrm{~mol} \mathrm{dm}^{-3}$. 


\section{Auxiliary Information}

\section{Method/Apparatus/Procedure:}

Excess solute and solvent were equilibrated with agitation by stirrers driven by a small turbine. At higher temperatures agitation was occasionally by hand. Equilibrium was obtained by both undersaturation at lower temperature and by supersaturation by pre-equilibrating at a higher temperature. The concentration of the dissolved solute was determined by titration with barium hydroxide with Congo Red being the endpoint indicator.

\section{Source and Purity of Chemicals:}

(1) Purity not given, Chemical source not given, no purification details were provided.

(2) Purity not given, Chemical source not given, no purification details were provided.

\section{Estimated Error:}

Temperature: Not given in paper.

$x_{1}: \pm 10 \%$ (relative error, estimated by compiler).

\begin{tabular}{|c|c|}
\hline $\begin{array}{l}\text { Components: } \\
\text { (1) 4-Hydroxybenzoic acid; } \mathrm{C}_{7} \mathrm{H}_{6} \mathrm{O}_{3} \text {; } \\
\text { [99-96-7] }\end{array}$ & $\begin{array}{l}\text { Original Measurements: } \\
{ }^{63} \text { C. K. Hancock, J. N. Pawloski, } \\
\text { and J. P. Idoux, J. Org. Chem. 32, }\end{array}$ \\
\hline $\begin{array}{l}\text { (2) Tetrahydrofuran; } \mathrm{C}_{4} \mathrm{H}_{8} \mathrm{O} \\
\text { [109-99-9] }\end{array}$ & 1931 (1967) \\
\hline $\begin{array}{l}\text { Variables: } \\
T / \mathrm{K}=303.15\end{array}$ & $\begin{array}{l}\text { Prepared by: } \\
\text { W. E. Acree, Jr. }\end{array}$ \\
\hline
\end{tabular}

Experimental Values

\begin{tabular}{lc}
\hline \hline$x_{2}{ }^{\mathrm{a}}$ & $x_{1}{ }^{\mathrm{b}}$ \\
\hline 0.707 & 0.293
\end{tabular}

${ }^{\mathrm{a}} x_{2}$ : mole fraction of component 2 in the saturated solution.

${ }^{\mathrm{b}} \mathrm{x}_{1}$ : mole fraction solubility of the solute.

\section{Auxiliary Information}

\section{Method/Apparatus/Procedure:}

Constant-temperature bath, Soxhlet thimble, conical flask, and analytical balance.

Excess solute and solvent were placed in a conical flask and allowed to equilibrate for several days at constant temperature. Attainment of equilibrium was verified by several repetitive measurements and by approaching equilibrium from supersaturation. Aliquots of saturated solutions were transferred using a Soxhlet thimble equipped with a one-hole cork stopper and an inverted U-shaped delivery tube to a second ground-glass stoppered flask suspended in the $303 \mathrm{~K}$ water bath. Successive portions of the contents were evaporated at room temperature in a tared aluminum foil weighing dish under a bell jar through which a slow stream of dry air was passed. The second flask plus unused saturated solution and the aluminum foil dish plus residue were weighed. The saturation solubility of the solute was calculated from the recorded mass data and molar masses of the solute and solvent.

\section{Source and Purity of Chemicals:}

(1) Purity not given, Chemical source not specified, was recrystallized several times from aqueous-ethanol mixture, and then dried under vacuum over phosphorous pentoxide.

(2) Practical grade, Chemical source not specified, stored over sodium hydroxide pellets for $24 \mathrm{~h}$, and then passed through $2 \times 70-\mathrm{cm}$ chromatographic adsorption columns containing activated alumina. After this treatment, the purified solvent was stored over copper in a nitrogen atmosphere.
Estimated Error:

Temperature: $\pm 0.02 \mathrm{~K}$

$x_{1}: \pm 1.0 \%$ (relative error)

\begin{tabular}{|c|c|}
\hline $\begin{array}{l}\text { Components: } \\
\text { (1) 4-Hydroxybenzoic acid; } \mathrm{C}_{7} \mathrm{H}_{6} \mathrm{O}_{3} \text {; } \\
\text { [99-96-7] } \\
\text { (2) 1,4-Dioxane; } \mathrm{C}_{4} \mathrm{H}_{8} \mathrm{O}_{2} ;[123-91-1]\end{array}$ & $\begin{array}{l}\text { Original Measurements: } \\
{ }^{166} \text { A. Martin, P. L. Wu, and A. } \\
\text { Beerbower, J. Pharm. Sci. 73, } 188 \\
\text { (1984). }\end{array}$ \\
\hline $\begin{array}{l}\text { Variables: } \\
T / \mathrm{K}=298.15\end{array}$ & $\begin{array}{l}\text { Prepared by: } \\
\text { W. E. Acree, Jr. }\end{array}$ \\
\hline
\end{tabular}

\section{Experimental Values}

\begin{tabular}{lc}
\hline \hline$x_{2}{ }^{\mathrm{a}}$ & $x_{1}{ }^{\mathrm{b}}$ \\
\hline 0.9156 & 0.0844 \\
\hline${ }^{\mathrm{a}} x_{2}:$ mole fraction of component 2 in the saturated solution. & \\
${ }^{\mathrm{b}} x_{1}:$ mole fraction solubility of the solute. &
\end{tabular}

\section{Auxiliary Information}

\section{Method/Apparatus/Procedure:}

Constant-temperature bath and ultraviolet/visible spectrophotometer Excess solute and solvent were placed in screw-capped vials. The vials were sealed and submerged in a constant-temperature water bath and shaken at 100 cycles/min for $24 \mathrm{~h}$. After the $24 \mathrm{~h}$ equilibration period, the vial was removed, wiped dry, and the contents analyzed. The solutions were transferred to a syringe and filtered through a $0.1 \mu \mathrm{m}$ pore size filter. The solutions were diluted and the absorbances recorded at the maximum absorption wavelength of benzoic acid. The solubility was determined at least six times.

\section{Source and Purity of Chemicals:}

(1) Purity not given, Chemical source not specified, recrystallized from aqueous ethanol before use.

(2) Spectrophotometric or ACS Reagent grade, Chemical source not specified, redistilled before use.

\section{Estimated Error:}

Temperature: $\pm 0.2 \mathrm{~K}$.

$x_{1}: \pm 3 \%$ (relative error).

\begin{tabular}{|c|c|}
\hline $\begin{array}{l}\text { Components: } \\
\text { (1) 4-Hydroxybenzoic acid; } \mathrm{C}_{7} \mathrm{H}_{6} \mathrm{O}_{3} \text {; } \\
\text { [99-96-7] } \\
\text { (2) 1,4-Dioxane; } \mathrm{C}_{4} \mathrm{H}_{8} \mathrm{O}_{2} ;[123-91-1]\end{array}$ & $\begin{array}{l}\text { Original Measurements: } \\
{ }^{63} \text { C. K. Hancock, J. N. Pawloski, } \\
\text { and J. P. Idoux, J. Org. Chem. 32, } \\
1931 \text { (1967). }\end{array}$ \\
\hline $\begin{array}{l}\text { Variables: } \\
T / \mathrm{K}=303.15\end{array}$ & $\begin{array}{l}\text { Prepared by: } \\
\text { W. E. Acree, Jr. }\end{array}$ \\
\hline
\end{tabular}

Experimental Values

\begin{tabular}{lc}
\hline \hline$x_{2}{ }^{\mathrm{a}}$ & $x_{1}{ }^{\mathrm{b}}$ \\
\hline 0.851 & 0.149 \\
\hline${ }^{\mathrm{a}} x_{2}:$ mole fraction of component 2 in the saturated solution. & \\
${ }^{\mathrm{b}} x_{1}:$ mole fraction solubility of the solute. &
\end{tabular}




\section{Auxiliary Information}

\section{Method/Apparatus/Procedure:}

Constant-temperature bath, Soxhlet thimble, conical flask, and analytical balance.

Excess solute and solvent were placed in a conical flask and allowed to equilibrate for several days at constant temperature. Attainment of equilibrium was verified by several repetitive measurements and by approaching equilibrium from supersaturation. Weighed aliquots of saturated solutions were removed and titrated with a standardized sodium hydroxide solution (carbonate free) using a pH meter. The endpoint of the titration was determined by computing the second derivative in the $\mathrm{pH}$ versus volume of sodium hydroxide added.

\section{Source and Purity of Chemicals:}

(1) Purity not given, Chemical source not specified, was recrystallized several times from aqueous-ethanol mixture, and then dried under vacuum over phosphorous pentoxide.

(2) Practical grade, Chemical source not specified, stored over sodium hydroxide pellets for $24 \mathrm{~h}$, and then passed through $2 \times 70-\mathrm{cm}$ chromatographic adsorption columns containing activated alumina. After this treatment, the purified solvent was stored over copper in a nitrogen atmosphere.

Estimated Error:

Temperature: $\pm 0.02 \mathrm{~K}$.

$x_{1}: \pm 1.0 \%$ (relative error).

\subsection{4-Hydroxybenzoic acid solubility data in haloalkanes, haloalkenes, and haloaromatic hydrocarbons}

\section{Components: \\ (1) 4-Hydroxybenzoic acid; $\mathrm{C}_{7} \mathrm{H}_{6} \mathrm{O}_{3}$; [99-96-7] \\ (2) Trichloromethane; $\mathrm{CHCl}_{3}$; [67-66-3]}

\begin{tabular}{ll}
\hline Variables: & Prepared by: \\
$T / \mathrm{K}=298.15$ & W. E. Acree, Jr.
\end{tabular}

Experimental Values

\begin{tabular}{lc}
\hline \hline$x_{2}{ }^{\mathrm{a}}$ & $x_{1}{ }^{\mathrm{b}}$ \\
\hline 0.9998 & 0.00015 \\
\hline$x_{2}$ m
\end{tabular}

${ }^{\mathrm{a}} x_{2}$ : mole fraction of component 2 in the saturated solution.

${ }^{\mathrm{b}} x_{1}$ : mole fraction solubility of the solute.

\section{Auxiliary Information}

\footnotetext{
Method/Apparatus/Procedure:

Constant-temperature bath and ultraviolet/visible spectrophotometer.

Excess solute and solvent were placed in screw-capped vials. The vials were sealed and submerged in a constant-temperature water bath and shaken at 100 cycles/min for $24 \mathrm{~h}$. After the $24 \mathrm{~h}$ equilibration period, the vial was removed, wiped dry, and the contents analyzed. The solutions were transferred to a syringe and filtered through a $0.1 \mu \mathrm{m}$ pore size filter. The solutions were diluted and the absorbances recorded at the maximum absorption wavelength of benzoic acid. The solubility was determined at least six times.
}

Source and Purity of Chemicals:

(1) Purity not given, Chemical source not specified, recrystallized from aqueous ethanol before use.

(2) Spectrophotometric or ACS Reagent grade, Chemical source not specified, redistilled before use.

\section{Estimated Error:}

Temperature: $\pm 0.2 \mathrm{~K}$.

$x_{1}: \pm 3 \%$ (relative error)

\begin{tabular}{ll}
\hline \hline Components: & Original Measurements: \\
$\begin{array}{l}\text { (1) 4-Hydroxybenzoic acid; } \mathrm{C}_{7} \mathrm{H}_{6} \mathrm{O}_{3} ; \\
\text { [99-96-7] }\end{array}$ & $\begin{array}{l}56 \mathrm{P} \text {. A. Ongley, J. Chem. Soc. 1954, } \\
\text { (2) Trichloromethane; } \mathrm{CHCl}_{3} ;\end{array}$ \\
$\begin{array}{l}3634 . \\
\text { [67-66-3] }\end{array}$ & \\
\hline Variables: & Prepared by: \\
$T / \mathrm{K}=298.15$ & W. E. Acree, Jr. \\
\hline
\end{tabular}

\section{Experimental Values}

The measured solubility was reported to be $-\log _{10} c_{1}=2.991$, which corresponds to a solubility of $c_{1}=0.00102 \mathrm{~mol} \mathrm{dm}^{-3}$.

\section{Auxiliary Information}

Method/Apparatus/Procedure:

Very little experimental details were provided. Excess solute and solvent were placed in tubes, which were rotated for at least $8 \mathrm{~h}$ at $298 \mathrm{~K}$. The solutions were then removed, concentrated if necessary, and titrated with standard alkali using a mixed (bromothymol blue + neutral red) indicator.

Source and Purity of Chemicals:

(1) Purity not given, Chemical source not given, solute was either "AnalaR" grade or recrystallized prior to use.

(2) Purity not given, Chemical source not given, no purification details were provided.

\section{Estimated Error:}

Temperature: No information was given $c_{1}: \pm 4 \%$ (relative error, estimated by compiler).

\begin{tabular}{ll}
\hline \hline Components: & Original Measurements: \\
$\begin{array}{l}\text { (1) 4-Hydroxybenzoic acid; } \mathrm{C}_{7} \mathrm{H}_{6} \mathrm{O}_{3} ; \\
\text { [99-96-7] }\end{array}$ & $\begin{array}{l}56 \mathrm{P} \text {. A. Ongley, J. Chem. Soc. 1954, } \\
\text { (2) Tetrachloromethane; } \mathrm{CCl}_{4} ;\end{array}$ \\
$\begin{array}{l}3634 . \\
\text { [56-23-5] }\end{array}$ & \\
\hline Variables: & Prepared by: \\
$T / \mathrm{K}=298.15$ & W. E. Acree, Jr. \\
\hline
\end{tabular}

\section{Experimental Values}

The measured solubility was reported to be $-\log _{10} c_{1}=5.000$, which corresponds to a solubility of $c_{1}=0.0000100 \mathrm{~mol} \mathrm{dm}^{-3}$. 


\section{Auxiliary Information}

\section{Method/Apparatus/Procedure:}

Very little experimental details were provided. Excess solute and solvent were placed in tubes, which were rotated for at least $8 \mathrm{~h}$ at $298 \mathrm{~K}$. The solutions were then removed, concentrated if necessary, and titrated with standard alkali using a mixed (bromothymol blue + neutral red) indicator.

Source and Purity of Chemicals:

(1) Purity not given, Chemical source not given, solute was either "AnalaR" grade or recrystallized prior to use.

(2) Purity not given, Chemical source not given, no purification details were provided.

Estimated Error:

Temperature: No information was given. $c_{1}: \pm 4 \%$ (relative error, estimated by compiler).

\begin{tabular}{|c|c|}
\hline $\begin{array}{l}\text { Components: } \\
\text { (1) 4-Hydroxybenzoic acid; } \mathrm{C}_{7} \mathrm{H}_{6} \mathrm{O}_{3} \text {; } \\
\text { [99-96-7] } \\
\text { (2) } 1,2-\text {-Dichloroethane; } \mathrm{C}_{2} \mathrm{H}_{4} \mathrm{Cl}_{2} \text {; } \\
\text { [107-60-2] }\end{array}$ & $\begin{array}{l}\text { Original Measurements: } \\
{ }^{166} \text { A. Martin, P. L. Wu, and A. } \\
\text { Beerbower, J. Pharm. Sci. 73, } 188 \\
\text { (1984). }\end{array}$ \\
\hline $\begin{array}{l}\text { Variables: } \\
T / \mathrm{K}=298.15\end{array}$ & $\begin{array}{l}\text { Prepared by: } \\
\text { W. E. Acree, Jr. }\end{array}$ \\
\hline
\end{tabular}

Experimental Values

\begin{tabular}{lc}
\hline \hline$x_{2}{ }^{\mathrm{a}}$ & $x_{1}{ }^{\mathrm{b}}$ \\
\hline 0.9999 & 0.00011 \\
\hline${ }^{\mathrm{a}} x_{2}:$ mole fraction of component 2 in the saturated solution. & \\
${ }^{\mathrm{b}} x_{1}:$ mole fraction solubility of the solute. &
\end{tabular}

\section{Auxiliary Information}

\section{Method/Apparatus/Procedure:}

Constant-temperature bath and ultraviolet/visible spectrophotometer.

Excess solute and solvent were placed in screw-capped vials. The vials were sealed and submerged in a constant-temperature water bath and shaken at 100 cycles/min for $24 \mathrm{~h}$. After the $24 \mathrm{~h}$ equilibration period, the vial was removed, wiped dry, and the contents analyzed. The solutions were transferred to a syringe and filtered through a $0.1 \mu \mathrm{m}$ pore size filter. The solutions were diluted and the absorbances recorded at the maximum absorption wavelength of benzoic acid. The solubility was determined at least six times.

Source and Purity of Chemicals:

(1) Purity not given, Chemical source not specified, recrystallized from aqueous ethanol before use.

(2) Spectrophotometric or ACS Reagent grade, Chemical source not specified, redistilled before use.

Estimated Error:

Temperature: $\pm 0.2 \mathrm{~K}$.

$x_{1}: \pm 3 \%$ (relative error).

\subsection{4-Hydroxybenzoic acid solubility data in alcohols}

\begin{tabular}{|c|c|}
\hline $\begin{array}{l}\text { Components: } \\
\text { (1) 4-Hydroxybenzoic acid; } \mathrm{C}_{7} \mathrm{H}_{6} \mathrm{O}_{3} \text {; } \\
\text { [99-96-7] } \\
\text { (2) Methanol; } \mathrm{CH}_{4} \mathrm{O} ;[67-56-1]\end{array}$ & $\begin{array}{l}\text { Original Measurements: } \\
{ }^{166} \text { A. Martin, P. L. Wu, and A. } \\
\text { Beerbower, J. Pharm. Sci. 73, } 188 \\
\text { (1984). }\end{array}$ \\
\hline $\begin{array}{l}\text { Variables: } \\
T / \mathrm{K}=298.15\end{array}$ & $\begin{array}{l}\text { Prepared by: } \\
\text { W. E. Acree, Jr. }\end{array}$ \\
\hline
\end{tabular}

\section{Experimental Values}

\begin{tabular}{lc}
\hline \hline$x_{2}{ }^{\mathrm{a}}$ & $x_{1}{ }^{\mathrm{b}}$ \\
\hline 0.8858 & 0.1142 \\
\hline${ }^{\mathrm{a}} x_{2}:$ mole fraction of component 2 in the saturated solution. & \\
${ }^{\mathrm{b}} x_{1}:$ mole fraction solubility of the solute. &
\end{tabular}

\section{Auxiliary Information}

\section{Method/Apparatus/Procedure:}

Constant-temperature bath and ultraviolet/visible spectrophotometer.

Excess solute and solvent were placed in screw-capped vials. The vials were sealed and submerged in a constant-temperature water bath and shaken at 100 cycles/min for $24 \mathrm{~h}$. After the $24 \mathrm{~h}$ equilibration period, the vial was removed, wiped dry, and the contents analyzed. The solutions were transferred to a syringe and filtered through a $0.1 \mu \mathrm{m}$ pore size filter. The solutions were diluted and the absorbances recorded at the maximum absorption wavelength of benzoic acid. The solubility was determined at least six times.

\section{Source and Purity of Chemicals:}

(1) Purity not given, Chemical source not specified, recrystallized from aqueous ethanol before use.

(2) Spectrophotometric or ACS Reagent grade, Chemical source not specified, redistilled before use.

\section{Estimated Error:}

Temperature: $\pm 0.2 \mathrm{~K}$.

$x_{1}: \pm 3 \%$ (relative error).

\begin{tabular}{ll}
\hline \hline $\begin{array}{l}\text { Components: } \\
\text { (1) 4-Hydroxybenzoic acid; } \mathrm{C}_{7} \mathrm{H}_{6} \mathrm{O}_{3} ;\end{array}$ & $\begin{array}{l}\text { Original Measurements: } \\
\text { [99-96-7] }\end{array}$ \\
$\begin{array}{ll}\text { Chem. Gracin and A. C. Rasmuson, J. } \\
\text { (2) Methanol; } \mathrm{CH}_{4} \mathrm{O} ;[67-56-1]\end{array}$ \\
\hline Variables: & Prepared by: \\
$T / \mathrm{K}=298.15$ & W. E. Acree, Jr. \\
\hline
\end{tabular}

\section{Experimental Values}

\begin{tabular}{lc}
\hline \hline$x_{2}{ }^{\mathrm{a}}$ & $x_{1}{ }^{\mathrm{b}}$ \\
\hline 0.8858 & 0.1142 \\
${ }^{\mathrm{a}} x_{2}:$ mole fraction of component 2 in the saturated solution. \\
${ }^{\mathrm{b}} x_{1}:$ mole fraction solubility of the solute. The solubility data were reported as \\
the grams of solute that dissolved per kilogram of solvent. Mole fraction \\
solubility calculated by the compiler.
\end{tabular}




\section{Auxiliary Information}

\section{Method/Apparatus/Procedure:}

Thermostated water bath, analytical balance, magnetic stirrer, and vacuum drying oven.

Solubility was determined by the gravimetric method. Excess solute and solvent were placed in Erlenmeyer flasks and allowed to equilibrate for $72 \mathrm{~h}$ in a thermostatic water bath sitting on a multiple-position magnetic stirrer. The solutions were stirred during the equilibration period. The stirring was then stopped for $4 \mathrm{~h}$ to allow the suspended solid to settle to the bottom of the flask. A sample of the clear saturated solution was transferred with a heated syringe into a previously weighed sample vial. The vial containing the saturated solution was then weighed, and the solvent was allowed to evaporate in a vacuum oven at $293 \mathrm{~K}$ for approximately one week until a constant weight was obtained. The solubility was calculated from the mass of the solid residue and mass of the saturated solution analyzed.

\section{Source and Purity of Chemicals:}

(1) $99 \%$, Sigma-Aldrich, Sweden, no purification details were provided.

(2) Pro Analyse, Merck Chemical Company, no purification details were provided.

\section{Estimated Error:}

Temperature: $\pm 0.02 \mathrm{~K}$.

$x_{1}: \pm 1 \%$ (relative error).

\begin{tabular}{|c|c|}
\hline $\begin{array}{l}\text { Components: } \\
\text { (1) 4-Hydroxybenzoic acid; } \mathrm{C}_{7} \mathrm{H}_{6} \mathrm{O}_{3} \text {; } \\
\text { [99-96-7] } \\
\text { (2) Methanol; } \mathrm{CH}_{4} \mathrm{O} ;[67-56-1]\end{array}$ & $\begin{array}{l}\text { Original Measurements: } \\
{ }^{168} \text { F. Nordström and A. C. } \\
\text { Rasmuson, J. Pharm. Sci. 95, } 748 \\
(2006) .\end{array}$ \\
\hline $\begin{array}{l}\text { Variables: } \\
\text { Temperature }\end{array}$ & $\begin{array}{l}\text { Prepared by: } \\
\text { W E Acree Jr }\end{array}$ \\
\hline
\end{tabular}

Experimental Values

\begin{tabular}{lcc}
\hline \hline$T / \mathrm{K}$ & $x_{2}{ }^{\mathrm{a}}$ & $x_{1}{ }^{\mathrm{b}}$ \\
\hline 283.2 & 0.8987 & 0.1013 \\
288.2 & 0.8961 & 0.1039 \\
293.2 & 0.8902 & 0.1098 \\
298.2 & 0.8859 & 0.1141 \\
303.2 & 0.8795 & 0.1205 \\
308.2 & 0.8746 & 0.1254 \\
313.2 & 0.8693 & 0.1307 \\
318.2 & 0.8617 & 0.1383 \\
323.2 & 0.8556 & 0.1444
\end{tabular}

${ }^{\mathrm{a}} x_{2}$ : mole fraction of component 2 in the saturated solution.

${ }^{b} x_{1}$ : mole fraction solubility of the solute. Solubility was reported as grams of solute per kilogram of solvent. Mole fraction values were calculated by the compiler.

\section{Auxiliary Information}

\section{Method/Apparatus/Procedure:}

Constant-temperature bath, magnetic stirrer, analytical balance, and ventilated laboratory hood.

Solubilities were determined by a gravimetric method. Excess solute and solvent were placed in sealed bottles and allowed to equilibrate in a constanttemperature bath. An aliquot of the clear solution was removed by syringe, filtered through a $0.2 \mu \mathrm{m}$ PTFE filter, and transferred to a preweighed glass vial. The glass vial was then weighed, and the solvent was allowed to evaporate in a ventilated laboratory hood at ambient room temperature. Once the solvent had evaporated, the vial with solid residue was weighed until constant weight was obtained. The solubility was calculated from the mass of the solid residue and mass of sample analyzed.

\section{Source and Purity of Chemicals:}

(1) $99+\%$, Sigma-Aldrich Chemical Company, USA, was used as received. (2) $99.8 \%$, HiperSolv, VWR Scientific, USA, used as received.

\section{Estimated Error:}

Temperature: $\pm 0.01 \mathrm{~K}$

$x_{1}: \pm 3 \%$ (relative error, estimated by compiler).

\begin{tabular}{ll}
\hline \hline Components: & Original Measurements: \\
$\begin{array}{l}\text { (1) 4-Hydroxybenzoic acid; } \mathrm{C}_{7} \mathrm{H}_{6} \mathrm{O}_{3} ; \\
\text { [99-96-7] }\end{array}$ & $\begin{array}{l}{ }^{158} \mathrm{E} \text {. Bergroth, Farm. Aikak. 70, } 91 \\
(1961) .\end{array}$ \\
\hline 2) Methanol; $\mathrm{CH}_{4} \mathrm{O} ;[67-56-1]$ & \\
\hline Variables: & Prepared by: \\
$T / \mathrm{K}=293.15$ & W. E. Acree, Jr. \\
\hline
\end{tabular}

\section{Experimental Values}

The measured solubility was reported to be $32.45 \mathrm{~g} / 100 \mathrm{ml}$ of solution, which corresponds to a molar concentration of $2.349 \mathrm{~mol} \mathrm{dm}^{-3}$.

\section{Auxiliary Information}

\section{Method/Apparatus/Procedure:}

Solubility was determined using a gravimetric method. Excess solute and solvent were placed in a solubility vessel. The apparatus was closed tightly with rubber stoppers and allowed to equilibrate in a constant-temperature water bath with stirring. A known aliquot of the saturated solution was removed, filtered, and transferred to an evaporating dish. The solvent was carefully removed over a steam bath. The evaporating dish was then placed in a drying oven, and later removed and weighed. The solubility was calculated from the mass of the solid residue and volume of saturated sample analyzed.

\section{Source and Purity of Chemicals:}

(1) $99.5 \%$, Schuchardt Chemicals, no purification details were provided. (2) Purity not given, Chemical source not given, no purification details were provided.

\section{Estimated Error:}

Temperature: $\pm 0.2 \mathrm{~K}$ (estimated by compiler). $c_{1}: \pm 5 \%$ (relative error, estimated by compiler).

\begin{tabular}{ll}
\hline \hline Components: & Original Measurements: \\
$\begin{array}{l}\text { (1) 4-Hydroxybenzoic acid; } \mathrm{C}_{7} \mathrm{H}_{6} \mathrm{O}_{3} ; \\
\text { [99-96-7] }\end{array}$ & $\begin{array}{l}{ }^{166} \text { A. Martin, P. L. Wu, and A. } \\
\text { Beerbower, J. Pharm. Sci. 73, 188 } \\
\text { (2) Ethanol; } \mathrm{C}_{2} \mathrm{H}_{6} \mathrm{O} ;[64-17-5]\end{array}$ \\
\hline Variables: & Prepared by: \\
$T / \mathrm{K}=298.15$ & W. E. Acree, Jr. \\
\hline
\end{tabular}


Experimental Values

\begin{tabular}{lc}
\hline \hline$x_{2}{ }^{\mathrm{a}}$ & $x_{1}{ }^{\mathrm{b}}$ \\
\hline 0.8787 & 0.1213 \\
\hline${ }^{\mathrm{a}} x_{2}:$ mole fraction of component 2 in the saturated solution. \\
${ }^{\mathrm{b}} x_{1}:$ mole fraction solubility of the solute.
\end{tabular}

Auxiliary Information

Method/Apparatus/Procedure:

Constant-temperature bath and ultraviolet/visible spectrophotometer.

Excess solute and solvent were placed in screw-capped vials. The vials were sealed and submerged in a constant-temperature water bath and shaken at 100 cycles/min for $24 \mathrm{~h}$. After the $24 \mathrm{~h}$ equilibration period, the vial was removed, wiped dry, and the contents analyzed. The solutions were transferred to a syringe and filtered through a $0.1 \mu \mathrm{m}$ pore size filter. The solutions were diluted and the absorbances recorded at the maximum absorption wavelength of benzoic acid. The solubility was determined at least six times.

Source and Purity of Chemicals:

(1) Purity not given, Chemical source not specified, recrystallized from aqueous ethanol before use.

(2) Spectrophotometric or ACS Reagent grade, Chemical source not specified, redistilled before use.

Estimated Error:

Temperature: $\pm 0.2 \mathrm{~K}$.

$x_{1}: \pm 3 \%$ (relative error).

\section{Components:}

(1) 4-Hydroxybenzoic acid; $\mathrm{C}_{7} \mathrm{H}_{6} \mathrm{O}_{3}$;

[99-96-7]

(2) Ethanol; $\mathrm{C}_{2} \mathrm{H}_{6} \mathrm{O} ;[64-17-5]$

\begin{tabular}{ll}
\hline Variables: & Prepared by: \\
$T / \mathrm{K}=298.15$ & W. E. Acree, Jr. \\
\hline
\end{tabular}

Experimental Values

\begin{tabular}{lc}
\hline \hline$x_{2}{ }^{\mathrm{a}}$ & $x_{1}{ }^{\mathrm{b}}$ \\
\hline 0.8739 & 0.1261 \\
\hline
\end{tabular}

${ }^{\mathrm{a}} x_{2}$ : mole fraction of component 2 in the saturated solution.

${ }^{b} x_{1}$ : mole fraction solubility of the solute. The solubility data were reported as the grams of solute that dissolved per kilogram of solvent. Mole fraction solubility calculated by the compiler.

\section{Auxiliary Information}

\section{Method/Apparatus/Procedure:}

Thermostated water bath, analytical balance, magnetic stirrer, and vacuum drying oven.

Solubility was determined by the gravimetric method. Excess solute and solvent were placed in Erlenmeyer flasks and allowed to equilibrate for $72 \mathrm{~h}$ in a thermostatic water bath sitting on a multiple-position magnetic stirrer. The solutions were stirred during the equilibration period. The stirring was then stopped for $4 \mathrm{~h}$ to allow the suspended solid to settle to the bottom of the flask. A sample of the clear saturated solution was transferred with a heated syringe into a previously weighed sample vial. The vial containing the saturated solution was then weighed, and the solvent was allowed to evaporate in a vacuum oven at $293 \mathrm{~K}$ for approximately one week until a constant weight was obtained. The solubility was calculated from the mass of the solid residue and mass of the saturated solution analyzed.
Source and Purity of Chemicals:

(1) $99 \%$, Sigma-Aldrich, Sweden, no purification details were provided.

(2) $99.5 \%$, Kemetyl AB, Sweden, no purification details were provided.

Estimated Error:

Temperature: $\pm 0.02 \mathrm{~K}$.

$x_{1}: \pm 1 \%$ (relative error).

\begin{tabular}{|c|c|}
\hline $\begin{array}{l}\text { Components: } \\
\text { (1) 4-Hydroxybenzoic acid; } \mathrm{C}_{7} \mathrm{H}_{6} \mathrm{O}_{3} \text {; } \\
\text { [99-96-7] } \\
\text { (2) Ethanol; } \mathrm{C}_{2} \mathrm{H}_{6} \mathrm{O} ;[64-17-5]\end{array}$ & $\begin{array}{l}\text { Original Measurements: } \\
{ }^{127} \text { N. V. Sidgwick and E. K. } \\
\text { Ewbank, J. Chem. Soc. Trans. 119, } \\
979 \text { (1921). }\end{array}$ \\
\hline $\begin{array}{l}\text { Variables: } \\
\text { Temperature }\end{array}$ & $\begin{array}{l}\text { Prepared by: } \\
\text { W. E. Acree, Jr. }\end{array}$ \\
\hline
\end{tabular}

Experimental Values

\begin{tabular}{lcc}
\hline \hline$T / \mathrm{K}$ & $x_{2}{ }^{\mathrm{a}}$ & $x_{1}{ }^{\mathrm{b}}$ \\
\hline 340.2 & 0.826 & 0.174 \\
409.7 & 0.658 & 0.342 \\
457.2 & 0.382 & 0.618 \\
\hline${ }^{\mathrm{a}} x_{2}:$ mole fraction of component 2 in the saturated solution. \\
${ }^{\mathrm{b}}{ }_{x_{1}: \text { mole fraction solubility of the solute. Solubility data reported in units of }}$ \\
mass percent. Mole fraction values calculated by the compiler.
\end{tabular}

Auxiliary Information

\section{Method/Apparatus/Procedure:}

Preweighed quantities of solute and solvent were placed in bulbs, which were then sealed and heated in a suitable bath. At the higher temperatures, the concentration of solvent in the liquid solution was corrected for the amount of solvent vapor in the bulb. The correction assumed that the vapor pressure of the saturated solution was one half that of the pure solvent at the solution temperature. The temperature at which the solid completely dissolved was recorded, and was taken to be the solid-liquid equilibrium temperature.

Source and Purity of Chemicals:

(1) Purity not given, prepared and purified by the authors using a published synthetic procedure [see J. Walker and J. K. Wood, J. Chem. Soc. 117, 40 (1920) for synthetic details]. Melting point of the purified solute was $486.2 \mathrm{~K}$. (2) $99 \%$, Chemical source not given, distilled over calcium oxide shortly before use.

\section{Estimated Error:}

Temperature: Not given in paper.

$x_{1}: \pm 10 \%$ (relative error, estimated by compiler).

\begin{tabular}{|c|c|}
\hline $\begin{array}{l}\text { Components: } \\
\text { (1) 4-Hydroxybenzoic acid; } \mathrm{C}_{7} \mathrm{H}_{6} \mathrm{O}_{3} \text {; } \\
\text { [99-96-7] } \\
\text { (2) Ethanol; } \mathrm{C}_{2} \mathrm{H}_{6} \mathrm{O} ;[64-17-5]\end{array}$ & $\begin{array}{l}\text { Original Measurements: } \\
{ }^{158} \text { E. Bergroth, Farm. Aikak. 70, } 91 \\
(1961) \text {. }\end{array}$ \\
\hline $\begin{array}{l}\text { Variables: } \\
T / \mathrm{K}=293.15\end{array}$ & $\begin{array}{l}\text { Prepared by: } \\
\text { W. E. Acree, Jr. }\end{array}$ \\
\hline
\end{tabular}

Experimental Values

The measured solubility was reported to be $24.36 \mathrm{~g} / 100 \mathrm{ml}$ of solution, which corresponds to a molar concentration of $1.699 \mathrm{~mol} \mathrm{dm}^{-3}$. 


\section{Auxiliary Information}

\section{Method/Apparatus/Procedure:}

Solubility was determined using a gravimetric method. Excess solute and solvent were placed in a solubility vessel. The apparatus was closed tightly with rubber stoppers and allowed to equilibrate in a constant-temperature water bath with stirring. A known aliquot of the saturated solution was removed, filtered, and transferred to an evaporating dish. The solvent was carefully removed over a steam bath. The evaporating dish was then placed in a drying oven, and later removed and weighed. The solubility was calculated from the mass of the solid residue and volume of saturated sample analyzed.

\section{Source and Purity of Chemicals:}

(1) $99.5 \%$, Schuchardt Chemicals, no purification details were provided.

(2) Purity not given, Chemical source not given, no purification details were provided.

\section{Estimated Error:}

Temperature: $\pm 0.2 \mathrm{~K}$ (estimated by compiler). $c_{1}: \pm 5 \%$ (relative error, estimated by compiler).

\begin{tabular}{ll}
\hline \hline Components: & Original Measurements: \\
$\begin{array}{l}\text { (1) 4-Hydroxybenzoic acid; } \mathrm{C}_{7} \mathrm{H}_{6} \mathrm{O}_{3} ; \\
\text { [99-96-7] }\end{array}$ & $\begin{array}{l}{ }^{166} \text { A. Martin, P. L. Wu, and A. } \\
\text { Beerbower, J. Pharm. Sci. 73, 188 } \\
\text { (2) 1-Propanol; } \mathrm{C}_{3} \mathrm{H}_{8} \mathrm{O} ; \text { [71-23-8] }\end{array}$ \\
\hline (1984). \\
\hline Variables: & Prepared by: \\
$T / \mathrm{K}=298.15$ & W. E. Acree, Jr. \\
\hline
\end{tabular}

Experimental Values

\begin{tabular}{lc}
\hline \hline$x_{2}{ }^{\mathrm{a}}$ & $x_{1}{ }^{\mathrm{b}}$ \\
\hline 0.8916 & 0.1084 \\
\hline${ }^{\mathrm{a}} x_{2}:$ mole fraction of component 2 in the saturated solution. & \\
${ }^{{ } x_{1}: \text { mole fraction solubility of the solute. }}$ &
\end{tabular}

\section{Auxiliary Information}

\section{Method/Apparatus/Procedure:}

Constant-temperature bath and ultraviolet/visible spectrophotometer.

Excess solute and solvent were placed in screw-capped vials. The vials were sealed and submerged in a constant-temperature water bath and shaken at 100 cycles/min for $24 \mathrm{~h}$. After the $24 \mathrm{~h}$ equilibration period, the vial was removed, wiped dry, and the contents analyzed. The solutions were transferred to a syringe and filtered through a $0.1 \mu \mathrm{m}$ pore size filter. The solutions were diluted and the absorbances recorded at the maximum absorption wavelength of benzoic acid. The solubility was determined at least six times.

\section{Source and Purity of Chemicals:}

(1) Purity not given, Chemical source not specified, recrystallized from aqueous ethanol before use.

(2) Spectrophotometric or ACS Reagent grade, Chemical source not specified, redistilled before use.

\section{Estimated Error:}

Temperature: $\pm 0.2 \mathrm{~K}$.

$x_{1}: \pm 3 \%$ (relative error)

\begin{tabular}{|c|c|}
\hline $\begin{array}{l}\text { Components: } \\
\text { (1) 4-Hydroxybenzoic acid; } \mathrm{C}_{7} \mathrm{H}_{6} \mathrm{O}_{3} \text {; } \\
\text { [99-96-7] } \\
\text { (2) 1-Propanol; } \mathrm{C}_{3} \mathrm{H}_{8} \mathrm{O} ;[71-23-8]\end{array}$ & $\begin{array}{l}\text { Original Measurements: } \\
{ }^{158} \text { E. Bergroth, Farm. Aikak. 70, } 91 \\
\text { (1961). }\end{array}$ \\
\hline $\begin{array}{l}\text { Variables: } \\
T / \mathrm{K}=293.15\end{array}$ & $\begin{array}{l}\text { Prepared by: } \\
\text { W. E. Acree, Jr. }\end{array}$ \\
\hline
\end{tabular}

\section{Experimental Values}

The measured solubility was reported to be $19.46 \mathrm{~g} / 100 \mathrm{ml}$ of solution, which corresponds to a molar concentration of $1.409 \mathrm{~mol} \mathrm{dm}^{-3}$.

\section{Auxiliary Information}

\section{Method/Apparatus/Procedure:}

Solubility was determined using a gravimetric method. Excess solute and solvent were placed in a solubility vessel. The apparatus was closed tightly with rubber stoppers and allowed to equilibrate in a constant-temperature water bath with stirring. A known aliquot of the saturated solution was removed, filtered, and transferred to an evaporating dish. The solvent was carefully removed over a steam bath. The evaporating dish was then placed in a drying oven, and later removed and weighed. The solubility was calculated from the mass of the solid residue and volume of saturated sample analyzed.

\section{Source and Purity of Chemicals:}

(1) $99.5 \%$, Schuchardt Chemicals, no purification details were provided.

(2) Purity not given, Chemical source not given, no purification details were provided.

\section{Estimated Error:}

Temperature: $\pm 0.2 \mathrm{~K}$ (estimated by compiler).

$c_{1}: \pm 5 \%$ (relative error, estimated by compiler).

\begin{tabular}{|c|c|}
\hline $\begin{array}{l}\text { Components: } \\
\text { (1) 4-Hydroxybenzoic acid; } \mathrm{C}_{7} \mathrm{H}_{6} \mathrm{O}_{3} \text {; } \\
\text { [99-96-7] } \\
\text { (2) 2-Propanol; } \mathrm{C}_{3} \mathrm{H}_{8} \mathrm{O} ;[67-63-0]\end{array}$ & $\begin{array}{l}\text { Original Measurements: } \\
{ }^{166} \text { A. Martin, P. L. Wu, and A. } \\
\text { Beerbower, J. Pharm. Sci. 73, } 188 \\
(1984) \text {. }\end{array}$ \\
\hline $\begin{array}{l}\text { Variables: } \\
T / \mathrm{K}=298.15\end{array}$ & $\begin{array}{l}\text { Prepared by: } \\
\text { W. E. Acree, Jr. }\end{array}$ \\
\hline
\end{tabular}

Experimental Values

\begin{tabular}{lc}
\hline \hline$x_{2}{ }^{\mathrm{a}}$ & $x_{1}{ }^{\mathrm{b}}$ \\
\hline 0.8703 & 0.1297 \\
\hline
\end{tabular}

${ }^{a} x_{2}$ : mole fraction of component 2 in the saturated solution.

${ }^{\mathrm{b}} x_{1}$ : mole fraction solubility of the solute.

\section{Auxiliary Information}

\section{Method/Apparatus/Procedure:}

Constant-temperature bath and ultraviolet/visible spectrophotometer. Excess solute and solvent were placed in screw-capped vials. The vials were sealed and submerged in a constant-temperature water bath and shaken at 100 cycles/min for $24 \mathrm{~h}$. After the $24 \mathrm{~h}$ equilibration period, the vial was removed, wiped dry, and the contents analyzed. The solutions were transferred to a syringe and filtered through a $0.1 \mu \mathrm{m}$ pore size filter. The solutions were diluted and the absorbances recorded at the maximum absorption wavelength of benzoic acid. The solubility was determined at least six times. 
Source and Purity of Chemicals:

(1) Purity not given, Chemical source not specified, recrystallized from aqueous ethanol before use.

(2) Spectrophotometric or ACS Reagent grade, Chemical source not specified, redistilled before use.

\section{Estimated Error:}

Temperature: $\pm 0.2 \mathrm{~K}$.

$x_{1}: \pm 3 \%$ (relative error).

\section{Components:}

(1) 4-Hydroxybenzoic acid; $\mathrm{C}_{7} \mathrm{H}_{6} \mathrm{O}_{3}$;

[99-96-7]

(2) 2-Propanol; $\mathrm{C}_{3} \mathrm{H}_{8} \mathrm{O}$; [67-63-0]

\begin{tabular}{ll}
\hline Variables: & Prepared by: \\
$T / \mathrm{K}=298.15$ & W. E. Acree, Jr. \\
\hline
\end{tabular}

Experimental Values

\begin{tabular}{lc}
\hline \hline$x_{2}{ }^{\mathrm{a}}$ & $x_{1}{ }^{\mathrm{b}}$ \\
\hline 0.8639 & 0.1361 \\
\hline$x_{2}: \mathrm{mole} \mathrm{faction}$
\end{tabular}

${ }^{a} x_{2}$ : mole fraction of component 2 in the saturated solution.

${ }^{b} x_{1}$ : mole fraction solubility of the solute. The solubility data were reported as the grams of solute that dissolved per kilogram of solvent. Mole fraction solubility calculated by the compiler.

\section{Auxiliary Information}

\section{Method/Apparatus/Procedure:}

Thermostated water bath, analytical balance, magnetic stirrer, and vacuum drying oven.

Solubility was determined by the gravimetric method. Excess solute and solvent were placed in Erlenmeyer flasks and allowed to equilibrate for $72 \mathrm{~h}$ in a thermostatic water bath sitting on a multiple-position magnetic stirrer. The solutions were stirred during the equilibration period. The stirring was then stopped for $4 \mathrm{~h}$ to allow the suspended solid to settle to the bottom of the flask. A sample of the clear saturated solution was transferred with a heated syringe into a previously weighed sample vial. The vial containing the saturated solution was then weighed, and the solvent was allowed to evaporate in a vacuum oven at $293 \mathrm{~K}$ for approximately one week until a constant weight was obtained. The solubility was calculated from the mass of the solid residue and mass of the saturated solution analyzed.

Source and Purity of Chemicals:

(1) $99 \%$, Sigma-Aldrich, Sweden, no purification details were provided. (2) Pro Analyse, Merck Chemical Company, no purification details were provided.

\section{Estimated Error:}

Temperature: $\pm 0.02 \mathrm{~K}$. $x_{1}: \pm 1 \%$ (relative error).
Experimental Values

\begin{tabular}{lc}
\hline \hline$x_{2}{ }^{\mathrm{a}}$ & $x_{1}{ }^{\mathrm{b}}$ \\
\hline 0.8846 & 0.1154 \\
\hline${ }^{\mathrm{a}} x_{2}:$ mole fraction of component 2 in the saturated solution. & \\
${ }^{\mathrm{b}}{ }_{x_{1}}:$ mole fraction solubility of the solute. &
\end{tabular}

Auxiliary Information

Method/Apparatus/Procedure:

Constant-temperature bath and ultraviolet/visible spectrophotometer.

Excess solute and solvent were placed in screw-capped vials. The vials were sealed and submerged in a constant-temperature water bath and shaken at 100 cycles/min for $24 \mathrm{~h}$. After the $24 \mathrm{~h}$ equilibration period, the vial was removed, wiped dry, and the contents analyzed. The solutions were transferred to a syringe and filtered through a $0.1 \mu \mathrm{m}$ pore size filter. The solutions were diluted and the absorbances recorded at the maximum absorption wavelength of benzoic acid. The solubility was determined at least six times.

Source and Purity of Chemicals:

(1) Purity not given, Chemical source not specified, recrystallized from aqueous ethanol before use.

(2) Spectrophotometric or ACS Reagent grade, Chemical source not specified, redistilled before use.

Estimated Error:

Temperature: $\pm 0.2 \mathrm{~K}$.

$x_{1}: \pm 3 \%$ (relative error)

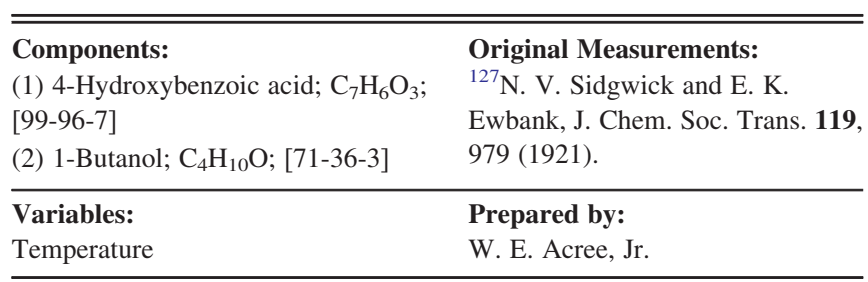

Experimental Values

\begin{tabular}{lcc}
\hline \hline$T / \mathrm{K}$ & $x_{2}{ }^{\mathrm{a}}$ & $x_{1}{ }^{\mathrm{b}}$ \\
\hline 305.7 & 0.885 & 0.115 \\
335.2 & 0.848 & 0.152 \\
389.3 & 0.741 & 0.259 \\
440.2 & 0.529 & 0.471 \\
467.0 & 0.240 & 0.760
\end{tabular}

${ }^{a} x_{2}$ : mole fraction of component 2 in the saturated solution.

${ }^{\mathrm{b}} x_{1}$ : mole fraction solubility of the solute. Solubility data reported in units of mass percent. Mole fraction values calculated by the compiler.

\section{Auxiliary Information}

(1) 4-Hydroxybenzoic acid; $\mathrm{C}_{7} \mathrm{H}_{6} \mathrm{O}_{3}$;

[99-96-7]

(2) 1-Butanol; $\mathrm{C}_{4} \mathrm{H}_{10} \mathrm{O} ;[$ [71-36-3]

Variables:

$T / \mathrm{K}=298.15$

\section{Components:}

\section{Original Measurements:}

${ }^{166}$ A. Martin, P. L. Wu, and A.

Beerbower, J. Pharm. Sci. 73, 188 (1984).

Prepared by:

W. E. Acree, Jr.

\footnotetext{
Method/Apparatus/Procedure:

Preweighed quantities of solute and solvent were placed in bulbs, which were then sealed and heated in a suitable bath. At the higher temperatures, the concentration of solvent in the liquid solution was corrected for the amount of solvent vapor in the bulb. The correction assumed that the vapor pressure of the saturated solution was one half that of the pure solvent at the solution temperature. The temperature at which the solid completely dissolved was recorded, and was taken to be the solid-liquid equilibrium temperature.
} 
Source and Purity of Chemicals:

(1) Purity not given, prepared and purified by the authors using a published synthetic procedure [see J. Walker and J. K. Wood, J. Chem. Soc. 117, 40

(1920) for synthetic details]. Melting point of the purified solute was $486.2 \mathrm{~K}$. (2) Purity not given, Chemical source not given, fractionated and distilled several times before use.

\section{Estimated Error:}

Temperature: Not given in paper.

$x_{1}: \pm 10 \%$ (relative error, estimated by compiler).

\begin{tabular}{|c|c|}
\hline $\begin{array}{l}\text { Components: } \\
\text { (1) 4-Hydroxybenzoic acid; } \mathrm{C}_{7} \mathrm{H}_{6} \mathrm{O}_{3} \text {; } \\
\text { [99-96-7] } \\
\text { (2) 2-Methyl-1-propanol; } \mathrm{C}_{4} \mathrm{H}_{10} \mathrm{O} \text {; } \\
\text { [78-83-1] }\end{array}$ & $\begin{array}{l}\text { Original Measurements: } \\
{ }_{166} \text { A. Martin, P. L. Wu, and A. } \\
\text { Beerbower, J. Pharm. Sci. 73, } 188 \\
\text { (1984). }\end{array}$ \\
\hline $\begin{array}{l}\text { Variables: } \\
T / \mathrm{K}=298.15\end{array}$ & $\begin{array}{l}\text { Prepared by: } \\
\text { W. E. Acree, Jr. }\end{array}$ \\
\hline
\end{tabular}

Experimental Values

\begin{tabular}{lc}
\hline \hline$x_{2}{ }^{\mathrm{a}}$ & $x_{1}{ }^{\mathrm{b}}$ \\
\hline 0.9099 & 0.0901 \\
\hline${ }^{\mathrm{a}} x_{2}:$ mole fraction of component 2 in the saturated solution. & \\
${ }^{{ } x_{1}: \text { mole fraction solubility of the solute. }}$ &
\end{tabular}

\section{Auxiliary Information}

\section{Method/Apparatus/Procedure:}

Constant-temperature bath and ultraviolet/visible spectrophotometer.

Excess solute and solvent were placed in screw-capped vials. The vials were sealed and submerged in a constant-temperature water bath and shaken at 100 cycles/min for $24 \mathrm{~h}$. After the $24 \mathrm{~h}$ equilibration period, the vial was removed, wiped dry, and the contents analyzed. The solutions were transferred to a syringe and filtered through a $0.1 \mu \mathrm{m}$ pore size filter. The solutions were diluted and the absorbances recorded at the maximum absorption wavelength of benzoic acid. The solubility was determined at least six times.

\section{Source and Purity of Chemicals:}

(1) Purity not given, Chemical source not specified, recrystallized from aqueous ethanol before use.

(2) Spectrophotometric or ACS Reagent grade, Chemical source not specified, redistilled before use.

\section{Estimated Error:}

Temperature: $\pm 0.2 \mathrm{~K}$.

$x_{1}: \pm 3 \%$ (relative error).

\begin{tabular}{|c|c|}
\hline $\begin{array}{l}\text { Components: } \\
\text { (1) 4-Hydroxybenzoic acid; } \mathrm{C}_{7} \mathrm{H}_{6} \mathrm{O}_{3} \text {; } \\
\text { [99-96-7] } \\
\text { (2) 1-Pentanol; } \mathrm{C}_{5} \mathrm{H}_{12} \mathrm{O} ;[71-41-0]\end{array}$ & $\begin{array}{l}\text { Original Measurements: } \\
{ }^{166} \text { A. Martin, P. L. Wu, and A. } \\
\text { Beerbower, J. Pharm. Sci. 73, } 188 \\
\text { (1984). }\end{array}$ \\
\hline $\begin{array}{l}\text { Variables: } \\
T / \mathrm{K}=298.15\end{array}$ & $\begin{array}{l}\text { Prepared by: } \\
\text { W. E. Acree, Jr. }\end{array}$ \\
\hline
\end{tabular}

Experimental Values

\begin{tabular}{lc}
\hline \hline$x_{2}{ }^{\mathrm{a}}$ & $x_{1}{ }^{\mathrm{b}}$ \\
\hline 0.8855 & 0.1145 \\
\hline${ }^{\mathrm{a}} x_{2}:$ mole fraction of component 2 in the saturated solution. & \\
${ }^{\mathrm{b}} x_{1}:$ mole fraction solubility of the solute. &
\end{tabular}

\section{Auxiliary Information}

Method/Apparatus/Procedure:

Constant-temperature bath and ultraviolet/visible spectrophotometer.

Excess solute and solvent were placed in screw-capped vials. The vials were sealed and submerged in a constant-temperature water bath and shaken at 100 cycles/min for $24 \mathrm{~h}$. After the $24 \mathrm{~h}$ equilibration period, the vial was removed, wiped dry, and the contents analyzed. The solutions were transferred to a syringe and filtered through a $0.1 \mu \mathrm{m}$ pore size filter. The solutions were diluted and the absorbances recorded at the maximum absorption wavelength of benzoic acid. The solubility was determined at least six times.

\section{Source and Purity of Chemicals:}

(1) Purity not given, Chemical source not specified, recrystallized from aqueous ethanol before use.

(2) Spectrophotometric or ACS Reagent grade, Chemical source not specified, redistilled before use.

\section{Estimated Error:}

Temperature: $\pm 0.2 \mathrm{~K}$.

$x_{1}: \pm 3 \%$ (relative error).

\begin{tabular}{ll}
\hline \hline Components: & Original Measurements: \\
$\begin{array}{l}\text { (1) 4-Hydroxybenzoic acid; } \mathrm{C}_{7} \mathrm{H}_{6} \mathrm{O}_{3} ; \\
\text { [99-96-7] }\end{array}$ & $\begin{array}{l}{ }^{166} \mathrm{~A} \text {. Martin, P. L. Wu, and A. } \\
\text { Beerbower, J. Pharm. Sci. 73, 188 } \\
\text { (2) 1-Hexanol; } \mathrm{C}_{6} \mathrm{H}_{14} \mathrm{O} ;[111-27-3]\end{array}$ \\
\hline (1984). \\
\hline Variables: & Prepared by: \\
$T / \mathrm{K}=298.15$ & W. E. Acree, Jr. \\
\hline
\end{tabular}

\section{Experimental Values}

\begin{tabular}{lc}
\hline \hline$x_{2}{ }^{\mathrm{a}}$ & $x_{1}{ }^{\mathrm{b}}$ \\
\hline 0.8879 & 0.1121
\end{tabular}

${ }^{a} x_{2}$ : mole fraction of component 2 in the saturated solution.

${ }^{\mathrm{b}} x_{1}$ : mole fraction solubility of the solute.

\section{Auxiliary Information}

\section{Method/Apparatus/Procedure:}

Constant-temperature bath and ultraviolet/visible spectrophotometer.

Excess solute and solvent were placed in screw-capped vials. The vials were sealed and submerged in a constant-temperature water bath and shaken at 100 cycles/min for $24 \mathrm{~h}$. After the $24 \mathrm{~h}$ equilibration period, the vial was removed, wiped dry, and the contents analyzed. The solutions were transferred to a syringe and filtered through a $0.1 \mu \mathrm{m}$ pore size filter. The solutions were diluted and the absorbances recorded at the maximum absorption wavelength of benzoic acid. The solubility was determined at least six times.

Source and Purity of Chemicals:

(1) Purity not given, Chemical source not specified, recrystallized from aqueous ethanol before use.

(2) Spectrophotometric or ACS Reagent grade, Chemical source not specified, redistilled before use. 
Estimated Error:

Temperature: $\pm 0.2 \mathrm{~K}$.

$x_{1}: \pm 3 \%$ (relative error).

\begin{tabular}{ll}
\hline \hline Components: & Original Measurements: \\
$\begin{array}{l}\text { (1) 4-Hydroxybenzoic acid; } \mathrm{C}_{7} \mathrm{H}_{6} \mathrm{O}_{3} ; \\
\text { [99-96-7] }\end{array}$ & $\begin{array}{l}{ }^{166} \text { A. Martin, P. L. Wu, and A. } \\
\text { Beerbower, J. Pharm. Sci. 73, } 188\end{array}$ \\
(2) 1-Octanol; $\mathrm{C}_{8} \mathrm{H}_{18} \mathrm{O} ;$ [111-87-5] & $(1984)$. \\
\hline Variables: & Prepared by: \\
$T / \mathrm{K}=298.15$ & W. E. Acree, Jr. \\
\hline
\end{tabular}

\section{Experimental Values}

\begin{tabular}{lc}
\hline \hline$x_{2}{ }^{\mathrm{a}}$ & $x_{1}{ }^{\mathrm{b}}$ \\
\hline 0.8968 & 0.1032 \\
\hline
\end{tabular}

${ }^{\mathrm{a}} x_{2}$ : mole fraction of component 2 in the saturated solution.

${ }^{\mathrm{b}} x_{1}$ : mole fraction solubility of the solute.

\section{Auxiliary Information}

\section{Method/Apparatus/Procedure:}

Constant-temperature bath and ultraviolet/visible spectrophotometer.

Excess solute and solvent were placed in screw-capped vials. The vials were sealed and submerged in a constant-temperature water bath and shaken at 100 cycles/min for $24 \mathrm{~h}$. After the $24 \mathrm{~h}$ equilibration period, the vial was removed, wiped dry, and the contents analyzed. The solutions were transferred to a syringe and filtered through a $0.1 \mu \mathrm{m}$ pore size filter. The solutions were diluted and the absorbances recorded at the maximum absorption wavelength of benzoic acid. The solubility was determined at least six times.

Source and Purity of Chemicals:

(1) Purity not given, Chemical source not specified, recrystallized from aqueous ethanol before use.

(2) Spectrophotometric or ACS Reagent grade, Chemical source not specified, redistilled before use.

\section{Estimated Error:}

Temperature: $\pm 0.2 \mathrm{~K}$.

$x_{1}: \pm 3 \%$ (relative error).

\begin{tabular}{|c|c|}
\hline $\begin{array}{l}\text { Components: } \\
\text { (1) 4-Hydroxybenzoic acid; } \mathrm{C}_{7} \mathrm{H}_{6} \mathrm{O}_{3} \text {; } \\
\text { [99-96-7] } \\
\text { (2) 1-Octanol; } \mathrm{C}_{8} \mathrm{H}_{18} \mathrm{O} ;[111-87-5]\end{array}$ & $\begin{array}{l}\text { Original Measurements: } \\
\text { }{ }^{167} \text { S. Gracin and A. C. Rasmuson, J. } \\
\text { Chem. Eng. Data 47, } 1379 \text { (2002). }\end{array}$ \\
\hline $\begin{array}{l}\text { Variables: } \\
T / \mathrm{K}=298.15\end{array}$ & $\begin{array}{l}\text { Prepared by: } \\
\text { W. E. Acree, Jr. }\end{array}$ \\
\hline
\end{tabular}

\section{Experimental Values}

\begin{tabular}{lc}
\hline \hline$x_{2}^{\mathrm{a}}$ & ${ }_{1}{ }^{\mathrm{b}}$ \\
\hline 0.8898 & 0.1102 \\
${ }^{\mathrm{a}} x_{2}:$ mole fraction of component 2 in the saturated solution. \\
${ }^{\mathrm{b}} x_{1}:$ mole fraction solubility of the solute. The solubility data were reported as \\
the grams of solute that dissolved per kilogram of solvent. Mole fraction \\
solubility calculated by the compiler.
\end{tabular}

\section{Auxiliary Information}

\section{Method/Apparatus/Procedure:}

Thermostated water bath, analytical balance, magnetic stirrer, and vacuum drying oven.

Solubility was determined by the gravimetric method. Excess solute and solvent were placed in Erlenmeyer flasks and allowed to equilibrate for $72 \mathrm{~h}$ in a thermostatic water bath sitting on a multiple-position magnetic stirrer. The solutions were stirred during the equilibration period. The stirring was then stopped for $4 \mathrm{~h}$ to allow the suspended solid to settle to the bottom of the flask. A sample of the clear saturated solution was transferred with a heated syringe into a previously weighed sample vial. The vial containing the saturated solution was then weighed, and the solvent was allowed to evaporate in a vacuum oven at $293 \mathrm{~K}$ for approximately one week until a constant weight was obtained. The solubility was calculated from the mass of the solid residue and mass of the saturated solution analyzed.

Source and Purity of Chemicals:

(1) $99 \%$, Sigma-Aldrich, Sweden, no purification details were provided.

(2) Pro Analyse, Merck Chemical Company, no purification details were provided.

Estimated Error:

Temperature: $\pm 0.02 \mathrm{~K}$

$x_{1}: \pm 1 \%$ (relative error).

\begin{tabular}{ll}
\hline \hline Components: & Original Measurements: \\
(1) 4-Hydroxybenzoic acid; $\mathrm{C}_{7} \mathrm{H}_{6} \mathrm{O}_{3} ;$ & ${ }^{77}$ J. Qingzhu, M. Peisheng, Y. \\
[99-96-7] & Shouzhi, W. Qiang, W. Chang, and \\
(2) 1-Octanol; $\mathrm{C}_{8} \mathrm{H}_{18} \mathrm{O} ;[111-87-5]$ & L. Guiju, J. Chem. Eng. Data 53, \\
& 1278 (2008). \\
\hline Variables: & Prepared by: \\
Temperature & W. E. Acree, Jr. \\
\hline
\end{tabular}

Experimental Values

\begin{tabular}{lcc}
\hline \hline$T / \mathrm{K}$ & $x_{2}{ }^{\mathrm{a}}$ & $x_{1}{ }^{\mathrm{b}}$ \\
\hline 297.65 & 0.8947 & 0.1053 \\
299.95 & 0.8928 & 0.1072 \\
301.45 & 0.8911 & 0.1089 \\
302.85 & 0.8897 & 0.1103 \\
304.25 & 0.8885 & 0.1115 \\
305.85 & 0.8869 & 0.1131 \\
306.95 & 0.8854 & 0.1146 \\
308.15 & 0.8841 & 0.1159 \\
309.85 & 0.8828 & 0.1172 \\
311.25 & 0.8812 & 0.1188 \\
313.15 & 0.8794 & 0.1206 \\
315.45 & 0.8776 & 0.1224 \\
317.25 & 0.8757 & 0.1243 \\
318.65 & 0.8740 & 0.1260 \\
320.55 & 0.8721 & 0.1279 \\
322.25 & 0.8701 & 0.1299 \\
\hline
\end{tabular}

${ }^{a} x_{2}$ : mole fraction of component 2 in the saturated solution.

${ }^{\mathrm{b}} x_{1}$ : mole fraction solubility of the solute. 


\section{Auxiliary Information}

\section{Method/Apparatus/Procedure:}

Circulating water bath, analytical balance, laser monitoring system. Pre-weighed amounts of solute and solvent and were placed in an equilibrium vessel, which was connected to a circulating water bath. The solution was stirred and the temperature gradually increased at a rate of $0.5 \mathrm{~K} / 20 \mathrm{~min}(0.2 \mathrm{~K} /$ $20 \mathrm{~min}$ or slower near saturation temperature) until all of the solid solute dissolved. The temperature at which all of the solute dissolved was determined using laser monitoring.

Source and Purity of Chemicals:

(1) $<99 \%$, Chemical source not specified, used as received.

(2) $<99 \%$, Chemical source not specified, used as received.

\section{Estimated Error:}

Temperature: $\pm 0.05 \mathrm{~K}$.

$x_{1}: \pm 0.0005$.

\section{Components:}

(1) 4-Hydroxybenzoic acid; $\mathrm{C}_{7} \mathrm{H}_{6} \mathrm{O}_{3}$; [99-96-7]

(2) 1-Octanol; $\mathrm{C}_{8} \mathrm{H}_{18} \mathrm{O}$; [111-87-5]

\section{Variables:}

Temperature

Original Measurements:

${ }^{145}$ G. L. Perlovich, T. V. Volkova, and A. Bauer-Brandl, J. Pharm. Sci 95, 1448 (2006).

Prepared by:

W. E. Acree, Jr.

Experimental Values

\begin{tabular}{lcc}
\hline \hline$T / \mathrm{K}$ & $x_{2}{ }^{\mathrm{a}}$ & $x_{1}{ }^{\mathrm{b}}$ \\
\hline 293 & 0.871 & 0.129 \\
298 & 0.861 & 0.139 \\
303 & 0.846 & 0.154 \\
310 & 0.823 & 0.177 \\
315 & 0.814 & 0.186 \\
\hline
\end{tabular}

${ }^{\mathrm{a}} x_{2}$ : mole fraction of component 2 in the saturated solution.

${ }^{b} x_{1}$ : mole fraction solubility of the solute.

\section{Auxiliary Information}

\section{Method/Apparatus/Procedure:}

Thermostated constant-temperature bath, centrifuge, and an ultraviolet/visible spectrophotometer.

Excess solute and solvent were placed in a glass ampoule and allowed to equilibrate in a thermostated temperature bath with mixing (at a speed of $25 \mathrm{rpm}$ ) for a minimum of four days. After suitable equilibration the saturated solution was centrifuged, the supernatant liquid collected, quantitatively diluted, and the absorbance recorded using an ultraviolet/visible spectrophotometer. The solubility of the solute was calculated from the measured absorbance.

Source and Purity of Chemicals:

(1) $99 \%$, Merck Chemicals, Darmstadt, Germany, no purification details provided.

(2) Analytical Reagent grade, Sigma-Aldrich Inc., Oslo, Norway, no purification details were provided.

\section{Estimated Error:}

Temperature: $\pm 0.1 \mathrm{~K}$

$x_{2}: \pm 2.5 \%$ (relative error)

\begin{tabular}{ll}
\hline \hline Components: & Original Measurements: \\
$\begin{array}{l}\text { (1) 4-Hydroxybenzoic acid; } \mathrm{C}_{7} \mathrm{H}_{6} \mathrm{O}_{3} ; \\
\text { [99-96-7] }\end{array}$ & $\begin{array}{l}{ }^{166} \text { A. Martin, P. L. Wu, and A. } \\
\text { Beerbower, J. Pharm. Sci. 73, 188 } \\
\text { (2) Benzenemethanol; } \mathrm{C}_{7} \mathrm{H}_{8} \mathrm{O} ;\end{array}$ \\
$\begin{array}{ll}(1984) . \\
\text { [100-51-6] }\end{array}$ & Prepared by: \\
\hline Variables: & W. E. Acree, Jr. \\
$T / \mathrm{K}=298.15$ &
\end{tabular}

Experimental Values

\begin{tabular}{lc}
\hline \hline$x_{2}{ }^{\mathrm{a}}$ & $x_{1}{ }^{\mathrm{b}}$ \\
\hline 0.9216 & 0.0784
\end{tabular}

${ }^{\mathrm{a}} x_{2}$ : mole fraction of component 2 in the saturated solution.

${ }^{b} x_{1}$ : mole fraction solubility of the solute.

\section{Auxiliary Information}

\section{Method/Apparatus/Procedure:}

Constant-temperature bath and ultraviolet/visible spectrophotometer.

Excess solute and solvent were placed in screw-capped vials. The vials were sealed and submerged in a constant-temperature water bath and shaken at 100 cycles/min for $24 \mathrm{~h}$. After the $24 \mathrm{~h}$ equilibration period, the vial was removed, wiped dry, and the contents analyzed. The solutions were transferred to a syringe and filtered through a $0.1 \mu \mathrm{m}$ pore size filter. The solutions were diluted and the absorbances recorded at the maximum absorption wavelength of benzoic acid. The solubility was determined at least six times.

Source and Purity of Chemicals:

(1) Purity not given, Chemical source not specified, recrystallized from aqueous ethanol before use.

(2) Spectrophotometric or ACS Reagent grade, Chemical source not specified, redistilled before use.

Estimated Error:

Temperature: $\pm 0.2 \mathrm{~K}$.

$x_{1}: \pm 3 \%$ (relative error).

\section{Components:}

(1) 4-Hydroxybenzoic acid; $\mathrm{C}_{7} \mathrm{H}_{6} \mathrm{O}_{3}$; [99-96-7]

(2) 1,2-Ethanediol; $\mathrm{C}_{2} \mathrm{H}_{6} \mathrm{O}_{2} ;[107-21-1]$

Variables:

$T / \mathrm{K}=298.15$
Original Measurements:

${ }^{166}$ A. Martin, P. L. Wu, and A. Beerbower, J. Pharm. Sci. 73, 188 (1984).

Prepared by:

W. E. Acree, Jr.

\section{Experimental Values}

\begin{tabular}{lc}
\hline \hline$x_{2}{ }^{\mathrm{a}}$ & $x_{1}{ }^{\mathrm{b}}$ \\
\hline 0.8868 & 0.1132 \\
\hline${ }^{\mathrm{a}} x_{2}:$ mole fraction of component 2 in the saturated solution. & \\
${ }^{\mathrm{b}} x_{1}:$ mole fraction solubility of the solute. &
\end{tabular}




\section{Auxiliary Information}

\section{Method/Apparatus/Procedure:}

Constant-temperature bath and ultraviolet/visible spectrophotometer.

Excess solute and solvent were placed in screw-capped vials. The vials were sealed and submerged in a constant-temperature water bath and shaken at 100 cycles/min for $24 \mathrm{~h}$. After the $24 \mathrm{~h}$ equilibration period, the vial was removed, wiped dry, and the contents analyzed. The solutions were transferred to a syringe and filtered through a $0.1 \mu \mathrm{m}$ pore size filter. The solutions were diluted and the absorbances recorded at the maximum absorption wavelength of benzoic acid. The solubility was determined at least six times.

Source and Purity of Chemicals:

(1) Purity not given, Chemical source not specified, recrystallized from aqueous ethanol before use.

(2) Spectrophotometric or ACS Reagent grade, Chemical source not specified, redistilled before use.

\section{Estimated Error:}

Temperature: $\pm 0.2 \mathrm{~K}$. $x_{1}: \pm 3 \%$ (relative error).

\begin{tabular}{ll}
\hline \hline Components: & Original Measurements: \\
(1) 4-Hydroxybenzoic acid; $\mathrm{C}_{7} \mathrm{H}_{6} \mathrm{O}_{3} ;$ & $\begin{array}{l}{ }^{166} \text { A. Martin, P. L. Wu, and A. } \\
\text { [99-96-7] }\end{array}$ \\
$\begin{array}{ll}\text { Beerbower, J. Pharm. Sci. 73, 188 } \\
\text { [57-2-P5-6] }\end{array}$ & $(1984)$. \\
\hline Variables: & Prepared by: \\
$T / \mathrm{K}=298.15$ & W. E. Acree, Jr. \\
\hline
\end{tabular}

\section{Experimental Values}

\begin{tabular}{lc}
\hline \hline$x_{2}{ }^{\mathrm{a}}$ & $x_{1}{ }^{\mathrm{b}}$ \\
\hline 0.8692 & 0.1308 \\
\hline${ }^{\mathrm{a}} x_{2}:$ mole fraction of
\end{tabular}

${ }^{\mathrm{a}} x_{2}$ : mole fraction of component 2 in the saturated solution.

${ }^{b} x_{1}$ : mole fraction solubility of the solute.

\section{Auxiliary Information}

\section{Method/Apparatus/Procedure:}

Constant-temperature bath and ultraviolet/visible spectrophotometer.

Excess solute and solvent were placed in screw-capped vials. The vials were sealed and submerged in a constant-temperature water bath and shaken at 100 cycles/min for $24 \mathrm{~h}$. After the $24 \mathrm{~h}$ equilibration period, the vial was removed, wiped dry, and the contents analyzed. The solutions were transferred to a syringe and filtered through a $0.1 \mu \mathrm{m}$ pore size filter. The solutions were diluted and the absorbances recorded at the maximum absorption wavelength of benzoic acid. The solubility was determined at least six times.

Source and Purity of Chemicals:

(1) Purity not given, Chemical source not specified, recrystallized from aqueous ethanol before use.

(2) Spectrophotometric or ACS Reagent grade, Chemical source not specified, redistilled before use.

\section{Estimated Error:}

Temperature: $\pm 0.2 \mathrm{~K}$

$x_{1}: \pm 3 \%$ (relative error).

\begin{tabular}{ll}
\hline \hline Components: & Original Measurements: \\
(1) 4-Hydroxybenzoic acid; $\mathrm{C}_{7} \mathrm{H}_{6} \mathrm{O}_{3} ;$ & $\begin{array}{l}{ }^{61} \text { E. R. Cooper, J. Controlled } \\
\text { [99-96-7] }\end{array}$ \\
$\begin{array}{ll}\text { Release 1, 153 (1984). } \\
\text { [57-55-6] }\end{array}$ \\
\hline Variables: & \\
$T / \mathrm{K}=295$ & Prepared by: \\
\hline
\end{tabular}

\section{Experimental Values}

\begin{tabular}{lc}
\hline \hline$x_{2}{ }^{\mathrm{a}}$ & $x_{1}{ }^{\mathrm{b}}$ \\
\hline 0.845 & 0.155 \\
${ }^{\mathrm{a}} x_{2}:$ mole fraction of component 2 in the saturated solution. \\
${ }^{\mathrm{b}} x_{1}:$ mole fraction solubility of the solute. Solubility data reported in terms of \\
mass percent. Mole fraction solubility calculated by the compiler.
\end{tabular}

\section{Auxiliary Information}

\section{Method/Apparatus/Procedure:}

Very little experimental details provided. Solubility determinations were made by stirring the solvent with excess solute for two days, centrifuging the sample, and analyzing the supernatant.

\section{Source and Purity of Chemicals:}

(1) Reagent grade, Aldrich Chemical Company, Metuchen, NJ, USA, no purification details provided.

(2) Reagent grade, J.T. Baker Chemical Company, Phillipsburg, NJ, USA, no purification details provided.

Estimated Error:

Temperature: No information given.

$x_{1}$ : No information given.

\begin{tabular}{|c|c|}
\hline $\begin{array}{l}\text { Components: } \\
\text { (1) 4-Hydroxybenzoic acid; } \mathrm{C}_{7} \mathrm{H}_{6} \mathrm{O}_{3} \text {; } \\
\text { [99-96-7] } \\
\text { (2) 1,2,3-Propanetriol (Glycerol); } \\
\mathrm{C}_{3} \mathrm{H}_{8} \mathrm{O}_{3} ;[60-29-7]\end{array}$ & $\begin{array}{l}\text { Original Measurements: } \\
{ }^{166} \text { A. Martin, P. L. Wu, and A. } \\
\text { Beerbower, J. Pharm. Sci. 73, } 188 \\
\text { (1984). }\end{array}$ \\
\hline $\begin{array}{l}\text { Variables: } \\
T / \mathrm{K}=298.15\end{array}$ & $\begin{array}{l}\text { Prepared by: } \\
\text { W. E. Acree, Jr. }\end{array}$ \\
\hline
\end{tabular}

\section{Experimental Values}

\begin{tabular}{lc}
\hline \hline$x_{2}{ }^{\mathrm{a}}$ & $x_{1}{ }^{\mathrm{b}}$ \\
\hline 0.9699 & 0.0301
\end{tabular}

${ }^{a} x_{2}$ : mole fraction of component 2 in the saturated solution.

${ }^{\mathrm{b}} x_{1}$ : mole fraction solubility of the solute. 


\section{Auxiliary Information}

\section{Method/Apparatus/Procedure:}

Constant-temperature bath and ultraviolet/visible spectrophotometer. Excess solute and solvent were placed in screw-capped vials. The vials were sealed and submerged in a constant-temperature water bath and shaken at 100 cycles/min for $24 \mathrm{~h}$. After the $24 \mathrm{~h}$ equilibration period, the vial was removed, wiped dry, and the contents analyzed. The solutions were transferred to a syringe and filtered through a $0.1 \mu \mathrm{m}$ pore size filter. The solutions were diluted and the absorbances recorded at the maximum absorption wavelength of benzoic acid. The solubility was determined at least six times.

Source and Purity of Chemicals:

(1) Purity not given, Chemical source not specified, recrystallized from aqueous ethanol before use.

(2) Spectrophotometric or ACS Reagent grade, Chemical source not specified, redistilled before use.

Estimated Error:

Temperature: $\pm 0.2 \mathrm{~K}$.

$x_{1}: \pm 3 \%$ (relative error).

\subsection{4-Hydroxybenzoic acid solubility data in ketones}

\begin{tabular}{|c|c|}
\hline $\begin{array}{l}\text { Components: } \\
\text { (1) 4-Hydroxybenzoic acid; } \mathrm{C}_{7} \mathrm{H}_{6} \mathrm{O}_{3} \text {; } \\
\text { [99-96-7] } \\
\text { (2) Propanone; } \mathrm{C}_{3} \mathrm{H}_{6} \mathrm{O} ;[67-64-1]\end{array}$ & $\begin{array}{l}\text { Original Measurements: } \\
{ }^{166} \text { A. Martin, P. L. Wu, and A. } \\
\text { Beerbower, J. Pharm. Sci. 73, } 188 \\
\text { (1984). }\end{array}$ \\
\hline $\begin{array}{l}\text { Variables: } \\
T / \mathrm{K}=298.15\end{array}$ & $\begin{array}{l}\text { Prepared by: } \\
\text { W. E. Acree, Jr. }\end{array}$ \\
\hline
\end{tabular}

\section{Experimental Values}

\begin{tabular}{lc}
\hline \hline$x_{2}{ }^{\mathrm{a}}$ & $x_{1}{ }^{\mathrm{b}}$ \\
\hline 0.8815 & 0.1185 \\
\hline
\end{tabular}

${ }^{\mathrm{a}} x_{2}$ : mole fraction of component 2 in the saturated solution.

${ }^{\mathrm{b}} x_{1}$ : mole fraction solubility of the solute.

\section{Auxiliary Information}

Method/Apparatus/Procedure:
Constant-temperature bath and ultraviolet/visible spectrophotometer.
Excess solute and solvent were placed in screw-capped vials. The vials were
sealed and submerged in a constant-temperature water bath and shaken at 100
cycles/min for $24 \mathrm{~h}$. After the 24 h equilibration period, the vial was removed,
wiped dry, and the contents analyzed. The solutions were transferred to a
syringe and filtered through a $0.1 \mu \mathrm{m}$ pore size filter. The solutions were diluted
and the absorbances recorded at the maximum absorption wavelength of
benzoic acid. The solubility was determined at least six times.
Source and Purity of Chemicals:
(1) Purity not given, Chemical source not specified, recrystallized from
aqueous ethanol before use.
(2) Spectrophotometric or ACS Reagent grade, Chemical source not specified,
redistilled before use.
Estimated Error:
Temperature: $\pm 0.2 \mathrm{~K}$.
$x_{1}: \pm 3 \%$ (relative error).

\begin{tabular}{|c|c|}
\hline $\begin{array}{l}\text { Components: } \\
\text { (1) 4-Hydroxybenzoic acid; } \mathrm{C}_{7} \mathrm{H}_{6} \mathrm{O}_{3} \text {; } \\
\text { [99-96-7] } \\
\text { (2) Propanone; } \mathrm{C}_{3} \mathrm{H}_{6} \mathrm{O} ;[67-64-1]\end{array}$ & $\begin{array}{l}\text { Original Measurements: } \\
{ }^{167} \text { S. Gracin and A. C. Rasmuson, J } \\
\text { Chem. Eng. Data 47, } 1379 \text { (2002). }\end{array}$ \\
\hline $\begin{array}{l}\text { Variables: } \\
T / \mathrm{K}=298.15\end{array}$ & $\begin{array}{l}\text { Prepared by: } \\
\text { W. E. Acree, Jr. }\end{array}$ \\
\hline
\end{tabular}

Experimental Values

\begin{tabular}{lc}
\hline \hline$x_{2}{ }^{\mathrm{a}}$ & $x_{1}{ }^{\mathrm{b}}$ \\
\hline 0.8806 & 0.1194 \\
${ }^{\mathrm{a}} x_{2}:$ mole fraction of component 2 in the saturated solution. \\
${ }^{\mathrm{b}} x_{1}:$ mole fraction solubility of the solute. The solubility data were reported as \\
the grams of solute that dissolved per kilogram of solvent. Mole fraction \\
solubility calculated by the compiler.
\end{tabular}

Auxiliary Information

Method/Apparatus/Procedure:

Thermostated water bath, analytical balance, magnetic stirrer, and vacuum drying oven.

Solubility was determined by the gravimetric method. Excess solute and solvent were placed in Erlenmeyer flasks and allowed to equilibrate for $72 \mathrm{~h}$ in a thermostatic water bath sitting on a multiple-position magnetic stirrer. The solutions were stirred during the equilibration period. The stirring was then stopped for $4 \mathrm{~h}$ to allow the suspended solid to settle to the bottom of the flask. A sample of the clear saturated solution was transferred with a heated syringe into a previously weighed sample vial. The vial containing the saturated solution was then weighed, and the solvent was allowed to evaporate in a vacuum oven at $293 \mathrm{~K}$ for approximately one week until a constant weight was obtained. The solubility was calculated from the mass of the solid residue and mass of the saturated solution analyzed.

Source and Purity of Chemicals:

(1) $99 \%$, Sigma-Aldrich, Sweden, no purification details were provided. (2) Pro Analyse, Merck Chemical Company, no purification details were provided.

\section{Estimated Error:}

Temperature: $\pm 0.02 \mathrm{~K}$.

$x_{1}: \pm 1 \%$ (relative error)

\begin{tabular}{|c|c|}
\hline $\begin{array}{l}\text { Components: } \\
\text { (1) 4-Hydroxybenzoic acid; } \mathrm{C}_{7} \mathrm{H}_{6} \mathrm{O}_{3} \text {; } \\
\text { [99-96-7] } \\
\text { (2) Propanone; } \mathrm{C}_{3} \mathrm{H}_{6} \mathrm{O} ;[67-64-1]\end{array}$ & $\begin{array}{l}\text { Original Measurements: } \\
{ }^{168} \text { F. Nordström and A. C. } \\
\text { Rasmuson, J. Pharm. Sci. 95, } 748 \\
\text { (2006). }\end{array}$ \\
\hline $\begin{array}{l}\text { Variables: } \\
\text { Temperature }\end{array}$ & $\begin{array}{l}\text { Prepared by: } \\
\text { W. E. Acree, Jr. }\end{array}$ \\
\hline
\end{tabular}

Experimental Values

\begin{tabular}{lcc}
\hline \hline$T / \mathrm{K}$ & $x_{2}{ }^{\mathrm{a}}$ & $x_{1}{ }^{\mathrm{b}}$ \\
\hline 303.2 & 0.8417 & 0.1583 \\
308.2 & 0.8371 & 0.1629 \\
313.2 & 0.8299 & 0.1701 \\
318.2 & 0.8235 & 0.1765 \\
323.2 & 0.8145 & 0.1855 \\
\hline${ }^{\mathrm{a}}{ }_{2}:$ mole fraction of component 2 in the saturated solution. \\
${ }^{\mathrm{b}}{ }_{x_{1}: \text { mole fraction solubility of the solute. Solubility was reported as grams of }}$ \\
solute per kilogram of solvent. Mole fraction values were calculated by the \\
compiler.
\end{tabular}


Auxiliary Information

\section{Method/Apparatus/Procedure:}

Constant-temperature bath, magnetic stirrer, analytical balance, and ventilated laboratory hood.

Solubilities were determined by a gravimetric method. Excess solute and solvent were placed in sealed bottles and allowed to equilibrate in a constanttemperature bath. An aliquot of the clear solution was removed by syringe, filtered through a $0.2 \mu \mathrm{m}$ PTFE filter, and transferred to a preweighed glass vial. The glass vial was then weighed, and the solvent was allowed to evaporate in a ventilated laboratory hood at ambient room temperature. Once the solvent had evaporated, the vial with solid residue was weighed until constant weight was obtained. The solubility was calculated from the mass of the solid residue and mass of sample analyzed.

Source and Purity of Chemicals:

(1) $99+\%$, Sigma-Aldrich Chemical Company, USA, was used as received.

(2) $99.8 \%$, HiperSolv, VWR Scientific, USA, used as received.

\section{Estimated Error:}

Temperature: $\pm 0.01 \mathrm{~K}$.

$x_{1}: \pm 3 \%$ (relative error, estimated by compiler).

\begin{tabular}{|c|c|}
\hline $\begin{array}{l}\text { Components: } \\
\text { (1) 4-Hydroxybenzoic acid; } \mathrm{C}_{7} \mathrm{H}_{6} \mathrm{O}_{3} \text {; } \\
\text { [99-96-7] } \\
\text { (2) Propanone; } \mathrm{C}_{3} \mathrm{H}_{6} \mathrm{O} ;[67-64-1]\end{array}$ & $\begin{array}{l}\text { Original Measurements: } \\
\text { (158 E. Bergroth, Farm. Aikak. 70, } 91 \\
(1961) \text {. }\end{array}$ \\
\hline $\begin{array}{l}\text { Variables: } \\
T / \mathrm{K}=293.15\end{array}$ & $\begin{array}{l}\text { Prepared by: } \\
\text { W. E. Acree, Jr. }\end{array}$ \\
\hline
\end{tabular}

\section{Experimental Values}

The measured solubility was reported to be $20.55 \mathrm{~g} / 100 \mathrm{ml}$ of solution, which corresponds to a molar concentration of $1.488 \mathrm{~mol} \mathrm{dm}^{-3}$.

\section{Auxiliary Information}

\section{Method/Apparatus/Procedure:}

Solubility was determined using a gravimetric method. Excess solute and solvent were placed in a solubility vessel. The apparatus was closed tightly with rubber stoppers and allowed to equilibrate in a constant-temperature water bath with stirring. A known aliquot of the saturated solution was removed, filtered, and transferred to an evaporating dish. The solvent was carefully removed over a steam bath. The evaporating dish was then placed in a drying oven, and later removed and weighed. The solubility was calculated from the mass of the solid residue and volume of saturated sample analyzed.

\section{Source and Purity of Chemicals:}

(1) $99.5 \%$, Schuchardt Chemicals, no purification details were provided.

(2) Purity not given, Chemical source not given, no purification details were provided.

\section{Estimated Error:}

Temperature: $\pm 0.2 \mathrm{~K}$ (estimated by compiler). $c_{1}: \pm 5 \%$ (relative error, estimated by compiler).

\begin{tabular}{|c|c|}
\hline $\begin{array}{l}\text { Components: } \\
\text { (1) 4-Hydroxybenzoic acid; } \mathrm{C}_{7} \mathrm{H}_{6} \mathrm{O}_{3} \text {; } \\
\text { [99-96-7] } \\
\text { (2) Propanone; } \mathrm{C}_{3} \mathrm{H}_{6} \mathrm{O} ;[67-64-1]\end{array}$ & $\begin{array}{l}\text { Original Measurements: } \\
{ }^{147} \text { J. Walker and J. K. Wood, J. } \\
\text { Chem. Soc. Trans. 73, } 618 \text { (1898). }\end{array}$ \\
\hline $\begin{array}{l}\text { Variables: } \\
T / \mathrm{K}=296\end{array}$ & $\begin{array}{l}\text { Prepared by: } \\
\text { W. E. Acree, Jr. }\end{array}$ \\
\hline
\end{tabular}

\section{Experimental Values}

The measured solubility was reported to be $22.7 \mathrm{~g} / 100 \mathrm{ml}$ of solution, which corresponds to a molar concentration of $1.643 \mathrm{~mol} \mathrm{dm}^{-3}$.

\section{Auxiliary Information}

\section{Method/Apparatus/Procedure:}

Excess solute and solvent were equilibrated with agitation by stirrers driven by a small turbine. At higher temperatures agitation was occasionally by hand. Equilibrium was obtained by both undersaturation at lower temperature and by supersaturation by pre-equilibrating at a higher temperature. The concentration of the dissolved solute was determined by titration with barium hydroxide with Congo Red being the endpoint indicator.

Source and Purity of Chemicals:

(1) Purity not given, Chemical source not given, no purification details were provided.

(2) Purity not given, Chemical source not given, no purification details were provided.

\section{Estimated Error:}

Temperature: Not given in paper. $c_{1}: \pm 10 \%$ (relative error, estimated by compiler).

\begin{tabular}{|c|c|}
\hline $\begin{array}{l}\text { Components: } \\
\text { (1) 4-Hydroxybenzoic acid; } \mathrm{C}_{7} \mathrm{H}_{6} \mathrm{O}_{3} \text {; } \\
\text { [99-96-7] } \\
\text { (2) 2-Butanone; } \mathrm{C}_{4} \mathrm{H}_{8} \mathrm{O} ;[78-93-3]\end{array}$ & $\begin{array}{l}\text { Original Measurements: } \\
\text { (158 E. Bergroth, Farm. Aikak. 70, } 91 \\
\text { (1961). }\end{array}$ \\
\hline $\begin{array}{l}\text { Variables: } \\
T / \mathrm{K}=293.15\end{array}$ & $\begin{array}{l}\text { Prepared by: } \\
\text { W. E. Acree, Jr. }\end{array}$ \\
\hline
\end{tabular}

\section{Experimental Values}

The measured solubility was reported to be $20.58 \mathrm{~g} / 100 \mathrm{ml}$ of solution, which corresponds to a molar concentration of $1.490 \mathrm{~mol} \mathrm{dm}^{-3}$.

\section{Auxiliary Information}

\section{Method/Apparatus/Procedure:}

Solubility was determined using a gravimetric method. Excess solute and solvent were placed in a solubility vessel. The apparatus was closed tightly with rubber stoppers and allowed to equilibrate in a constant-temperature water bath with stirring. A known aliquot of the saturated solution was removed, filtered, and transferred to an evaporating dish. The solvent was carefully removed over a steam bath. The evaporating dish was then placed in a drying oven, and later removed and weighed. The solubility was calculated from the mass of the solid residue and volume of saturated sample analyzed.

\section{Source and Purity of Chemicals:}

(1) $99.5 \%$, Schuchardt Chemicals, no purification details were provided. (2) Purity not given, Chemical source not given, no purification details were provided.

\section{Estimated Error:}

Temperature: $\pm 0.2 \mathrm{~K}$ (estimated by compiler). $c_{1}: \pm 5 \%$ (relative error, estimated by compiler). 


\section{Components:}

(1) 4-Hydroxybenzoic acid; $\mathrm{C}_{7} \mathrm{H}_{6} \mathrm{O}_{3}$; [99-96-7]

(2) 2-Pentanone; $\mathrm{C}_{5} \mathrm{H}_{10} \mathrm{O}$; [107-87-9]

\section{Variables:}

$T / \mathrm{K}=293.15$

Prepared by:

W. E. Acree, Jr

\section{Experimental Values}

The measured solubility was reported to be $17.09 \mathrm{~g} / 100 \mathrm{ml}$ of solution, which corresponds to a molar concentration of $1.237 \mathrm{~mol} \mathrm{dm}^{-3}$.

\section{Auxiliary Information}

\section{Method/Apparatus/Procedure:}

Solubility was determined using a gravimetric method. Excess solute and solvent were placed in a solubility vessel. The apparatus was closed tightly with rubber stoppers and allowed to equilibrate in a constant-temperature water bath with stirring. A known aliquot of the saturated solution was removed, filtered, and transferred to an evaporating dish. The solvent was carefully removed over a steam bath. The evaporating dish was then placed in a drying oven, and later removed and weighed. The solubility was calculated from the mass of the solid residue and volume of saturated sample analyzed.

\section{Source and Purity of Chemicals:}

(1) $99.5 \%$, Schuchardt Chemicals, no purification details were provided.

(2) Purity not given, Chemical source not given, no purification details were provided.

\section{Estimated Error:}

Temperature: $\pm 0.2 \mathrm{~K}$ (estimated by compiler).

$c_{1}: \pm 5 \%$ (relative error, estimated by compiler).

\section{Components:}

(1) 4-Hydroxybenzoic acid; $\mathrm{C}_{7} \mathrm{H}_{6} \mathrm{O}_{3}$; [99-96-7]

(2) 4-Methyl-2-pentanone; $\mathrm{C}_{6} \mathrm{H}_{12} \mathrm{O}$;

[108-10-1]

\begin{tabular}{ll}
\hline Variables: & Prepared by: \\
$T / \mathrm{K}=298.15$ & W. E. Acree, Jr. \\
\hline
\end{tabular}

\section{Experimental Values}

\begin{tabular}{lr}
\hline \hline$x_{2}^{\mathrm{a}}$ & \multicolumn{1}{c}{$x_{1}{ }^{\mathrm{b}}$} \\
\hline 0.8999 & 0.1001 \\
${ }^{\mathrm{a}} x_{2}:$ mole fraction of component 2 in the saturated solution. \\
${ }^{\mathrm{b}} x_{1}:$ mole fraction solubility of the solute. The solubility data were reported as \\
the grams of solute that dissolved per kilogram of solvent. Mole fraction \\
solubility calculated by the compiler.
\end{tabular}

\section{Auxiliary Information}

\section{Method/Apparatus/Procedure:}

Thermostated water bath, analytical balance, magnetic stirrer, and vacuum drying oven.

Solubility was determined by the gravimetric method. Excess solute and solvent were placed in Erlenmeyer flasks and allowed to equilibrate for $72 \mathrm{~h}$ in a thermostatic water bath sitting on a multiple-position magnetic stirrer. The solutions were stirred during the equilibration period. The stirring was then stopped for $4 \mathrm{~h}$ to allow the suspended solid to settle to the bottom of the flask. A sample of the clear saturated solution was transferred with a heated syringe into a previously weighed sample vial. The vial containing the saturated solution was then weighed, and the solvent was allowed to evaporate in a vacuum oven at $293 \mathrm{~K}$ for approximately one week until a constant weight was obtained. The solubility was calculated from the mass of the solid residue and mass of the saturated solution analyzed.

\section{Source and Purity of Chemicals:}

(1) $99 \%$, Sigma-Aldrich, Sweden, no purification details were provided.

(2) Pro Analyse, Merck Chemical Company, no purification details were provided.

\section{Estimated Error:}

Temperature: $\pm 0.02 \mathrm{~K}$

$x_{1}: \pm 1 \%$ (relative error).

\begin{tabular}{|c|c|}
\hline $\begin{array}{l}\text { Components: } \\
\text { (1) 4-Hydroxybenzoic acid; } \mathrm{C}_{7} \mathrm{H}_{6} \mathrm{O}_{3} \text {; } \\
\text { [99-96-7] } \\
\text { (2) Acetophenone; } \mathrm{C}_{8} \mathrm{H}_{8} \mathrm{O} ;[98-86-2]\end{array}$ & $\begin{array}{l}\text { Original Measurements: } \\
{ }_{166} \text { A. Martin, P. L. Wu, and A. } \\
\text { Beerbower, J. Pharm. Sci. 73, } 188 \\
\text { (1984). }\end{array}$ \\
\hline $\begin{array}{l}\text { Variables: } \\
T / \mathrm{K}=298.15\end{array}$ & $\begin{array}{l}\text { Prepared by: } \\
\text { W. E. Acree, Jr. }\end{array}$ \\
\hline
\end{tabular}

\section{Experimental Values}

\begin{tabular}{lc}
\hline \hline$x_{2}{ }^{\mathrm{a}}$ & $x_{1}{ }^{\mathrm{b}}$ \\
\hline 0.9777 & 0.0223 \\
\hline${ }^{\mathrm{a}} x_{2}:$ mole fraction of component 2 in the saturated solution. & \\
${ }^{\mathrm{b}} x_{1}:$ mole fraction solubility of the solute. &
\end{tabular}

\section{Auxiliary Information}

\section{Method/Apparatus/Procedure:}

Constant-temperature bath and ultraviolet/visible spectrophotometer. Excess solute and solvent were placed in screw-capped vials. The vials were sealed and submerged in a constant-temperature water bath and shaken at 100 cycles/min for $24 \mathrm{~h}$. After the $24 \mathrm{~h}$ equilibration period, the vial was removed, wiped dry, and the contents analyzed. The solutions were transferred to a syringe and filtered through a $0.1 \mu \mathrm{m}$ pore size filter. The solutions were diluted and the absorbances recorded at the maximum absorption wavelength of benzoic acid. The solubility was determined at least six times.

\section{Source and Purity of Chemicals:}

(1) Purity not given, Chemical source not specified, recrystallized from aqueous ethanol before use.

(2) Spectrophotometric or ACS Reagent grade, Chemical source not specified, redistilled before use

\section{Estimated Error:}

Temperature: $\pm 0.2 \mathrm{~K}$.

$x_{1}: \pm 3 \%$ (relative error) 


\subsection{4-Hydroxybenzoic acid solubility data in miscellaneous organic solvents}

\begin{tabular}{|c|c|}
\hline $\begin{array}{l}\text { Components: } \\
\text { (1) 4-Hydroxybenzoic acid; } \mathrm{C}_{7} \mathrm{H}_{6} \mathrm{O}_{3} \text {; } \\
\text { [99-96-7] } \\
\text { (2) Dimethyl sulfoxide; } \mathrm{C}_{2} \mathrm{H}_{6} \mathrm{OS} \text {; } \\
\text { [67-68-5] }\end{array}$ & $\begin{array}{l}\text { Original Measurements: } \\
{ }^{166} \text { A. Martin, P. L. Wu, and A. } \\
\text { Beerbower, J. Pharm. Sci. 73, } 188 \\
\text { (1984). }\end{array}$ \\
\hline $\begin{array}{l}\text { Variables: } \\
T / \mathrm{K}=298.15\end{array}$ & $\begin{array}{l}\text { Prepared by: } \\
\text { W. E. Acree, Jr. }\end{array}$ \\
\hline
\end{tabular}

Experimental Values

\begin{tabular}{lc}
\hline \hline$x_{2}{ }^{\mathrm{a}}$ & $x_{1}{ }^{\mathrm{b}}$ \\
\hline 0.6326 & 0.3674 \\
\hline$x_{2}{ }^{2} \mathrm{~mole}$
\end{tabular}

${ }^{\mathrm{a}} x_{2}$ : mole fraction of component 2 in the saturated solution.

${ }^{b} x_{1}$ : mole fraction solubility of the solute.

\section{Auxiliary Information}

\section{Method/Apparatus/Procedure:}

Constant-temperature bath and ultraviolet/visible spectrophotometer.

Excess solute and solvent were placed in screw-capped vials. The vials were sealed and submerged in a constant-temperature water bath and shaken at 100 cycles/min for $24 \mathrm{~h}$. After the $24 \mathrm{~h}$ equilibration period, the vial was removed, wiped dry, and the contents analyzed. The solutions were transferred to a syringe and filtered through a $0.1 \mu \mathrm{m}$ pore size filter. The solutions were diluted and the absorbances recorded at the maximum absorption wavelength of benzoic acid. The solubility was determined at least six times.

Source and Purity of Chemicals:

(1) Purity not given, Chemical source not specified, recrystallized from aqueous ethanol before use.

(2) Spectrophotometric or ACS Reagent grade, Chemical source not specified, redistilled before use.

Estimated Error:

Temperature: $\pm 0.2 \mathrm{~K}$

$x_{1}: \pm 3 \%$ (relative error).

\begin{tabular}{|c|c|}
\hline $\begin{array}{l}\text { Components: } \\
\text { (1) 4-Hydroxybenzoic acid; } \mathrm{C}_{7} \mathrm{H}_{6} \mathrm{O}_{3} \text {; } \\
\text { [99-96-7] } \\
\text { (2) Pyridine; } \mathrm{C}_{5} \mathrm{H}_{5} \mathrm{~N} \text {; [110-86-1] }\end{array}$ & $\begin{array}{l}\text { Original Measurements: } \\
{ }^{166} \text { A. Martin, P. L. Wu, and A. } \\
\text { Beerbower, J. Pharm. Sci. 73, } 188 \\
\text { (1984). }\end{array}$ \\
\hline $\begin{array}{l}\text { Variables: } \\
T / \mathrm{K}=298.15\end{array}$ & $\begin{array}{l}\text { Prepared by: } \\
\text { W. E. Acree, Jr. }\end{array}$ \\
\hline
\end{tabular}

Experimental Values

\begin{tabular}{lc}
\hline \hline$x_{2}{ }^{\mathrm{a}}$ & $x_{1}{ }^{\mathrm{b}}$ \\
\hline 0.8956 & 0.1044 \\
\hline${ }^{\mathrm{a}} x_{2}:$ mole fraction of component 2 in the saturated solution. \\
${ }^{\mathrm{b}} x_{1}:$ mole fraction solubility of the solute.
\end{tabular}

\section{Auxiliary Information}

Method/Apparatus/Procedure:

Constant-temperature bath and ultraviolet/visible spectrophotometer. Excess solute and solvent were placed in screw-capped vials. The vials were sealed and submerged in a constant-temperature water bath and shaken at 100 cycles/min for $24 \mathrm{~h}$. After the $24 \mathrm{~h}$ equilibration period, the vial was removed, wiped dry, and the contents analyzed. The solutions were transferred to a syringe and filtered through a $0.1 \mu \mathrm{m}$ pore size filter. The solutions were diluted and the absorbances recorded at the maximum absorption wavelength of benzoic acid. The solubility was determined at least six times.

Source and Purity of Chemicals:

(1) Purity not given, Chemical source not specified, recrystallized from aqueous ethanol before use.

(2) Spectrophotometric or ACS Reagent grade, Chemical source not specified, redistilled before use.

Estimated Error:

Temperature: $\pm 0.2 \mathrm{~K}$.

$x_{1}: \pm 3 \%$ (relative error)

\begin{tabular}{ll}
\hline \hline Components: & Original Measurements: \\
(1) 4-Hydroxybenzoic acid; $\mathrm{C}_{7} \mathrm{H}_{6} \mathrm{O}_{3} ;$ & $\begin{array}{l}{ }^{166} \text { A. Martin, P. L. Wu, and A. } \\
\text { [99-96-7] }\end{array}$ \\
Beerbower, J. Pharm. Sci. 73, 188 \\
(2) Formamide; $\mathrm{CH}_{3} \mathrm{NO} ;$ [75-12-7] & $(1984)$. \\
\hline Variables: & Prepared by: \\
$T / \mathrm{K}=298.15$ & W. E. Acree, Jr. \\
\hline
\end{tabular}

Experimental Values

\begin{tabular}{lc}
\hline \hline$x_{2}{ }^{\mathrm{a}}$ & $x_{1}{ }^{\mathrm{b}}$ \\
\hline 0.9659 & 0.0341 \\
\hline${ }^{\mathrm{a}} x_{2}:$ mole fraction of component 2 in the saturated solution. & \\
${ }^{\mathrm{b}} x_{1}:$ mole fraction solubility of the solute. &
\end{tabular}

${ }^{\mathrm{b}} x_{1}$ : mole fraction solubility of the solute.

\section{Auxiliary Information}

\section{Method/Apparatus/Procedure:}

Constant-temperature bath and ultraviolet/visible spectrophotometer. Excess solute and solvent were placed in screw-capped vials. The vials were sealed and submerged in a constant-temperature water bath and shaken at 100 cycles/min for $24 \mathrm{~h}$. After the $24 \mathrm{~h}$ equilibration period, the vial was removed, wiped dry, and the contents analyzed. The solutions were transferred to a syringe and filtered through a $0.1 \mu \mathrm{m}$ pore size filter. The solutions were diluted and the absorbances recorded at the maximum absorption wavelength of benzoic acid. The solubility was determined at least six times.

Source and Purity of Chemicals:

(1) Purity not given, Chemical source not specified, recrystallized from aqueous ethanol before use.

(2) Spectrophotometric or ACS Reagent grade, Chemical source not specified, redistilled before use.

\section{Estimated Error:}

Temperature: $\pm 0.2 \mathrm{~K}$.

$x_{1}: \pm 3 \%$ (relative error). 


\section{Components:}

(1) 4-Hydroxybenzoic acid; $\mathrm{C}_{7} \mathrm{H}_{6} \mathrm{O}_{3}$; [99-96-7]

(2) $\mathrm{N}$-Methylformamide; $\mathrm{C}_{2} \mathrm{H}_{5} \mathrm{NO}$; [123-39-7]

\section{Variables:}

$T / \mathrm{K}=298.15$
Original Measurements:

${ }^{166}$ A. Martin, P. L. Wu, and A.

Beerbower, J. Pharm. Sci. 73, 188 (1984).

\section{Prepared by:}

W. E. Acree, Jr.

Experimental Values

\begin{tabular}{lc}
\hline \hline$x_{2}{ }^{\mathrm{a}}$ & $x_{1}{ }^{\mathrm{b}}$ \\
\hline 0.8975 & 0.1025
\end{tabular}

${ }^{\mathrm{a}} x_{2}$ : mole fraction of component 2 in the saturated solution.

${ }^{b} x_{1}$ : mole fraction solubility of the solute.

\section{Auxiliary Information}

\section{Method/Apparatus/Procedure:}

Constant-temperature bath and ultraviolet/visible spectrophotometer.

Excess solute and solvent were placed in screw-capped vials. The vials were sealed and submerged in a constant-temperature water bath and shaken at 100 cycles/min for $24 \mathrm{~h}$. After the $24 \mathrm{~h}$ equilibration period, the vial was removed, wiped dry, and the contents analyzed. The solutions were transferred to a syringe and filtered through a $0.1 \mu \mathrm{m}$ pore size filter. The solutions were diluted and the absorbances recorded at the maximum absorption wavelength of benzoic acid. The solubility was determined at least six times.

\section{Source and Purity of Chemicals:}

(1) Purity not given, Chemical source not specified, recrystallized from aqueous ethanol before use.

(2) Spectrophotometric or ACS Reagent grade, Chemical source not specified, redistilled before use.

\section{Estimated Error:}

Temperature: $\pm 0.2 \mathrm{~K}$

$x_{1}: \pm 3 \%$ (relative error).

\section{Components:}

(1) 4-Hydroxybenzoic acid; $\mathrm{C}_{7} \mathrm{H}_{6} \mathrm{O}_{3}$; [99-96-7]

(2) $N, N$-Dimethylformamide; $\mathrm{C}_{3} \mathrm{H}_{7} \mathrm{NO}$; [68-12-2]

\begin{tabular}{ll}
\hline Variables: & Prepared by: \\
$T / \mathrm{K}=298.15$ & W. E. Acree, Jr. \\
\hline
\end{tabular}

Experimental Values

\begin{tabular}{lc}
\hline \hline$x_{2}{ }^{\mathrm{a}}$ & $x_{1}{ }^{\mathrm{b}}$ \\
\hline 0.7863 & 0.2137 \\
\hline$x_{2}$.
\end{tabular}

${ }^{\mathrm{a}} x_{2}$ : mole fraction of component 2 in the saturated solution.

${ }^{\mathrm{b}} x_{1}$ : mole fraction solubility of the solute.

\section{Auxiliary Information}

Method/Apparatus/Procedure:

Constant-temperature bath and ultraviolet/visible spectrophotometer.

Excess solute and solvent were placed in screw-capped vials. The vials were sealed and submerged in a constant-temperature water bath and shaken at 100 cycles/min for $24 \mathrm{~h}$. After the $24 \mathrm{~h}$ equilibration period, the vial was removed, wiped dry, and the contents analyzed. The solutions were transferred to a syringe and filtered through a $0.1 \mu \mathrm{m}$ pore size filter. The solutions were diluted and the absorbances recorded at the maximum absorption wavelength of benzoic acid. The solubility was determined at least six times.

Source and Purity of Chemicals:

(1) Purity not given, Chemical source not specified, recrystallized from aqueous ethanol before use.

(2) Spectrophotometric or ACS Reagent grade, Chemical source not specified, redistilled before use.

\section{Estimated Error:}

Temperature: $\pm 0.2 \mathrm{~K}$. $x_{1}: \pm 3 \%$ (relative error).

\begin{tabular}{|c|c|}
\hline $\begin{array}{l}\text { Components: } \\
\text { (1) 4-Hydroxybenzoic acid; } \mathrm{C}_{7} \mathrm{H}_{6} \mathrm{O}_{3} \text {; } \\
\text { [99-96-7] } \\
\text { (2) } N, N \text {-Dimethylacetamide; } \mathrm{C}_{4} \mathrm{H}_{9} \mathrm{NO} \text {; } \\
\text { [127-19-5] }\end{array}$ & $\begin{array}{l}\text { Original Measurements: } \\
{ }^{166} \text { A. Martin, P. L. Wu, and A. } \\
\text { Beerbower, J. Pharm. Sci. 73, } 188 \\
\text { (1984). }\end{array}$ \\
\hline $\begin{array}{l}\text { Variables: } \\
T / \mathrm{K}=298.15\end{array}$ & $\begin{array}{l}\text { Prepared by: } \\
\text { W. E. Acree, Jr. }\end{array}$ \\
\hline
\end{tabular}

\section{Experimental Values}

\begin{tabular}{lc}
\hline \hline$x_{2}{ }^{\mathrm{a}}$ & $x_{1}{ }^{\mathrm{b}}$ \\
\hline 0.7646 & 0.2354 \\
\hline${ }^{\mathrm{a}} x_{2}:$ mole fraction of component 2 in the saturated solution. & \\
${ }^{\mathrm{b}} x_{1}:$ mole fraction solubility of the solute. &
\end{tabular}

\section{Auxiliary Information}

\begin{abstract}
Method/Apparatus/Procedure:
Constant-temperature bath and ultraviolet/visible spectrophotometer.

Excess solute and solvent were placed in screw-capped vials. The vials were sealed and submerged in a constant-temperature water bath and shaken at 100 cycles/min for $24 \mathrm{~h}$. After the $24 \mathrm{~h}$ equilibration period, the vial was removed, wiped dry, and the contents analyzed. The solutions were transferred to a syringe and filtered through a $0.1 \mu \mathrm{m}$ pore size filter. The solutions were diluted and the absorbances recorded at the maximum absorption wavelength of benzoic acid. The solubility was determined at least six times.
\end{abstract}

\section{Source and Purity of Chemicals:}

(1) Purity not given, Chemical source not specified, recrystallized from aqueous ethanol before use.

(2) Spectrophotometric or ACS Reagent grade, Chemical source not specified, redistilled before use.

\section{Estimated Error:}

Temperature: $\pm 0.2 \mathrm{~K}$.

$x_{1}: \pm 3 \%$ (relative error). 


\section{Components:}

(1) 4-Hydroxybenzoic acid; $\mathrm{C}_{7} \mathrm{H}_{6} \mathrm{O}_{3}$; [99-96-7]

(2) Ethanoic acid; $\mathrm{C}_{2} \mathrm{H}_{4} \mathrm{O}_{2}$; [64-19-7]

\section{Variables:}

$T / \mathrm{K}=298.15$

W. E. Acree, Jr

\section{Experimental Values}

\begin{tabular}{lc}
\hline \hline$x_{2}^{\mathrm{a}}$ & $x_{1}{ }^{\mathrm{b}}$ \\
\hline 0.9556 & 0.0444
\end{tabular}

${ }^{a} x_{2}$ : mole fraction of component 2 in the saturated solution.

${ }^{\mathrm{b}} x_{1}$ : mole fraction solubility of the solute.

\section{Auxiliary Information}

\section{Method/Apparatus/Procedure:}

Constant-temperature bath and ultraviolet/visible spectrophotometer.

Excess solute and solvent were placed in screw-capped vials. The vials were sealed and submerged in a constant-temperature water bath and shaken at 100 cycles/min for $24 \mathrm{~h}$. After the $24 \mathrm{~h}$ equilibration period, the vial was removed, wiped dry, and the contents analyzed. The solutions were transferred to a syringe and filtered through a $0.1 \mu \mathrm{m}$ pore size filter. The solutions were diluted and the absorbances recorded at the maximum absorption wavelength of benzoic acid. The solubility was determined at least six times.

Source and Purity of Chemicals:

(1) Purity not given, Chemical source not specified, recrystallized from aqueous ethanol before use.

(2) Spectrophotometric or ACS Reagent grade, Chemical source not specified, redistilled before use.

Estimated Error:

Temperature: $\pm 0.2 \mathrm{~K}$.

$x_{1}: \pm 3 \%$ (relative error).

\begin{tabular}{|c|c|}
\hline $\begin{array}{l}\text { Components: } \\
\text { (1) 4-Hydroxybenzoic acid; } \mathrm{C}_{7} \mathrm{H}_{6} \mathrm{O}_{3} \text {; } \\
\text { [99-96-7] } \\
\text { (2) Ethanoic acid; } \mathrm{C}_{2} \mathrm{H}_{4} \mathrm{O}_{2} ; \text { [64-19-7] }\end{array}$ & $\begin{array}{l}\text { Original Measurements: } \\
{ }^{168} \text { F. Nordström and A. C. } \\
\text { Rasmuson, J. Pharm. Sci. } 95,748 \\
\text { (2006). }\end{array}$ \\
\hline $\begin{array}{l}\text { Variables: } \\
\text { Temperature }\end{array}$ & $\begin{array}{l}\text { Prepared by: } \\
\text { W. E. Acree, Jr. }\end{array}$ \\
\hline
\end{tabular}

Experimental Values

\begin{tabular}{lcc}
\hline \hline$T / \mathrm{K}$ & $x_{2}{ }^{\mathrm{a}}$ & $x_{1}{ }^{\mathrm{b}}$ \\
\hline 283.2 & 0.9628 & 0.0372 \\
288.2 & 0.9599 & 0.0401 \\
293.2 & 0.9568 & 0.0432 \\
298.2 & 0.9531 & 0.0469 \\
303.2 & 0.9494 & 0.0506 \\
308.2 & 0.9455 & 0.0545 \\
313.2 & 0.9412 & 0.0588 \\
318.2 & 0.9363 & 0.0637 \\
323.2 & 0.9311 & 0.0689
\end{tabular}

${ }^{\mathrm{a}} x_{2}$ : mole fraction of component 2 in the saturated solution.

${ }^{b} x_{1}$ : mole fraction solubility of the solute. Solubility was reported as grams of solute per kilogram of solvent. Mole fraction values were calculated by the compiler.

\section{Auxiliary Information}

\section{Method/Apparatus/Procedure:}

Constant-temperature bath, magnetic stirrer, analytical balance, and ventilated laboratory hood.

Solubilities were determined by a gravimetric method. Excess solute and solvent were placed in sealed bottles and allowed to equilibrate in a constanttemperature bath. An aliquot of the clear solution was removed by syringe, filtered through a $0.2 \mu \mathrm{m}$ PTFE filter, and transferred to a preweighed glass vial. The glass vial was then weighed, and the solvent was allowed to evaporate in a ventilated laboratory hood at ambient room temperature. Once the solvent had evaporated, the vial with solid residue was weighed until constant weight was obtained. The solubility was calculated from the mass of the solid residue and mass of sample analyzed.

\section{Source and Purity of Chemicals:}

(1) $99.7 \%$, Merck Chemical Company, Germany, was used as received. (2) $96 \%$, Pro Analysi, Merck Chemicals, Darmstadt, Germany, used as received.

\section{Estimated Error:}

Temperature: $\pm 0.01 \mathrm{~K}$

$x_{1}: \pm 3 \%$ (relative error, estimated by compiler).

\begin{tabular}{ll}
\hline \hline Components: & Original Measurements: \\
$\begin{array}{l}\text { (1) 4-Hydroxybenzoic acid; } \mathrm{C}_{7} \mathrm{H}_{6} \mathrm{O}_{3} ; \\
\text { [99-96-7] }\end{array}$ & $\begin{array}{l}{ }^{166} \text { A. Martin, P. L. Wu, and A. } \\
\text { Beerbower, J. Pharm. Sci. 73, 188 }\end{array}$ \\
(2) Propanoic acid; $\mathrm{C}_{3} \mathrm{H}_{6} \mathrm{O}_{2} ;$ [79-09-4] & $(1984)$. \\
\hline Variables: & Prepared by: \\
$T / \mathrm{K}=298.15$ & W. E. Acree, Jr. \\
\hline
\end{tabular}

Experimental Values

\begin{tabular}{lc}
\hline \hline$x_{2}{ }^{\mathrm{a}}$ & $x_{1}{ }^{\mathrm{b}}$ \\
\hline 0.9653 & 0.0347 \\
\hline${ }^{\mathrm{a}} x_{2}:$ mole fraction of component 2 in the saturated solution. & \\
${ }^{\mathrm{b}} x_{1}:$ mole fraction solubility of the solute. &
\end{tabular}

\section{Auxiliary Information}

\section{Method/Apparatus/Procedure:}

Constant-temperature bath and ultraviolet/visible spectrophotometer.

Excess solute and solvent were placed in screw-capped vials. The vials were sealed and submerged in a constant-temperature water bath and shaken at 100 cycles/min for $24 \mathrm{~h}$. After the $24 \mathrm{~h}$ equilibration period, the vial was removed, wiped dry, and the contents analyzed. The solutions were transferred to a syringe and filtered through a $0.1 \mu \mathrm{m}$ pore size filter. The solutions were diluted and the absorbances recorded at the maximum absorption wavelength of benzoic acid. The solubility was determined at least six times.

\section{Source and Purity of Chemicals:}

(1) Purity not given, Chemical source not specified, recrystallized from aqueous ethanol before use.

(2) Spectrophotometric or ACS Reagent grade, Chemical source not specified, redistilled before use.

\section{Estimated Error:}

Temperature: $\pm 0.2 \mathrm{~K}$.

$x_{1}: \pm 3 \%$ (relative error). 


\section{Components:}

(1) 4-Hydroxybenzoic acid; $\mathrm{C}_{7} \mathrm{H}_{6} \mathrm{O}_{3}$; [99-96-7]

(2) Ethanenitrile; $\mathrm{C}_{2} \mathrm{H}_{3} \mathrm{~N} ;[$ [75-05-8]

\section{Variables:}

Temperature

W. E. Acree, Jr

Experimental Values

\begin{tabular}{lcc}
\hline \hline$T / \mathrm{K}$ & $x_{2}{ }^{\mathrm{a}}$ & \multicolumn{1}{c}{$x_{1}{ }^{\mathrm{b}}$} \\
\hline 283.2 & 0.9907 & 0.00927 \\
288.2 & 0.9894 & 0.01057 \\
293.2 & 0.9878 & 0.0122 \\
298.2 & 0.9857 & 0.0143 \\
303.2 & 0.9833 & 0.0167 \\
308.2 & 0.9815 & 0.0185 \\
313.2 & 0.9786 & 0.0214 \\
318.2 & 0.9755 & 0.0245 \\
323.2 & 0.9721 & 0.0279 \\
\hline
\end{tabular}

${ }^{a} x_{2}$ : mole fraction of component 2 in the saturated solution.

${ }^{b} x_{1}$ : mole fraction solubility of the solute. Solubility was reported as grams of solute per kilogram of solvent. Mole fraction values were calculated by the compiler.

\section{Auxiliary Information}

\section{Method/Apparatus/Procedure:}

Constant-temperature bath, magnetic stirrer, analytical balance, and ventilated laboratory hood.

Solubilities were determined by a gravimetric method. Excess solute and solvent were placed in sealed bottles and allowed to equilibrate in a constanttemperature bath. An aliquot of the clear solution was removed by syringe, filtered through a $0.2 \mu \mathrm{m}$ PTFE filter, and transferred to a preweighed glass vial. The glass vial was then weighed, and the solvent was allowed to evaporate in a ventilated laboratory hood at ambient room temperature. Once the solvent had evaporated, the vial with solid residue was weighed until constant weight was obtained. The solubility was calculated from the mass of the solid residue and mass of sample analyzed.

Source and Purity of Chemicals:

(1) $99+\%$, Sigma-Aldrich Chemical Company, USA, was used as received.

(2) $99.8+\%$, LiChroSolv, VWR Scientific, USA, used as received.

\section{Solubility of 2-Hydroxy-3- methylbenzoic Acid in Organic Solvents}

\subsection{Critical evaluation of experimental solubility data}

There has been only a single study involving the solubility of 2-hydroxy-3-methylbenzoic acid in organic solvents. Sidgwick and Ewbank ${ }^{127}$ measured the solubility of 2-hydroxy-3methylbenzoic acid in both heptane and benzene at elevated temperatures. The observed mole fraction solubilities were curve-fit to Eq. (8) to yield the following mathematical representations:

$$
\begin{aligned}
& \ln x_{1}=-140.489-\frac{2.700}{T}+23.144 \ln T \\
& \ln x_{1}=-91.627-\frac{1.853}{T}+15.108 \ln T
\end{aligned}
$$

for heptane and benzene, respectively. The experimental data point at $392.2 \mathrm{~K}$ had to be excluded from the regression analysis for heptane in order to get a reasonable mathematical correlation. The mean absolute deviations between experimental and calculated values are on the order of $14.5 \%$ [Eq. (54)] and 7.6\% [Eq. (55)], which are larger than desired. The large deviation may be due in part to the fact that the solution temperature is above the solvent's normal boiling point temperature, to uncertainties in estimating the amount of solvent that remained in the liquid phase, and the large range covered by the experimental values, $x_{1}=0.0114$ to $x_{1}=0.8275$ for heptane and $x_{1}=0.0094$ to $x_{1}=0.8127$ for benzene. It is more difficult to curve-fit experimental solubility data covering large mole fraction ranges.

The experimental solubility data for 2-hydroxy-3-methylbenzoic acid in organic solvents are in Secs. 49.2 and 49.3.

\subsection{2-Hydroxy-3-methylbenzoic acid solubility data in saturated hydrocarbons (including cycloalkanes)}

\begin{tabular}{ll}
\hline \hline Components: & Original Measurements: \\
(1) 2-Hydroxy-3-methylbenzoic acid; & ${ }^{127}$ N. V. Sidgwick and E. K. \\
$\mathrm{C}_{8} \mathrm{H}_{8} \mathrm{O}_{3} ;[83-40-9]$ & Ewbank, J. Chem. Soc. Trans. 119, \\
(2) $\mathrm{Heptane}_{\mathrm{C}_{7} \mathrm{H}_{16} ;[142-82-5]}$ & $979(1921)$. \\
\hline Variables: & Prepared by: \\
Temperature & W. E. Acree, Jr. \\
\hline
\end{tabular}

Experimental Values

\begin{tabular}{lcc}
\hline \hline$T / \mathrm{K}$ & $x_{2}{ }^{\mathrm{a}}$ & $x_{1}{ }^{\mathrm{b}}$ \\
\hline 354.2 & 0.9886 & 0.0114 \\
374.2 & 0.9705 & 0.0295 \\
392.2 & 0.9382 & 0.0618 \\
406.1 & 0.8360 & 0.1640 \\
414.1 & 0.6485 & 0.3515 \\
415.1 & 0.6074 & 0.3926 \\
419.8 & 0.4178 & 0.5822 \\
427.5 & 0.1727 & 0.8275 \\
\hline
\end{tabular}

${ }^{\mathrm{a}} x_{2}$ : mole fraction of component 2 in the saturated solution.

${ }^{b} x_{1}$ : mole fraction solubility of the solute. Solubility data reported in units of mass percent. Mole fraction values calculated by the compiler. 


\section{Auxiliary Information}

\section{Method/Apparatus/Procedure:}

Preweighed quantities of solute and solvent were placed in bulbs, which were then sealed and heated in a suitable bath. At the higher temperatures, the concentration of solvent in the liquid solution was corrected for the amount of solvent vapor in the bulb. The correction assumed that the vapor pressure of the saturated solution was one half that of the pure solvent at the solution temperature. The temperature at which the solid completely dissolved was recorded, and was taken to be the solid-liquid equilibrium temperature.

Source and Purity of Chemicals:

(1) Purity not given, prepared and purified by the authors using a published synthetic procedure [see J. Walker and J. K. Wood, J. Chem. Soc. 117, 40 (1920) for synthetic details]. Melting point of the purified solute was $440.2 \mathrm{~K}$. (2) Purity not given, sample isolated from the resin of Pinus sabiniana, purified by treatment with sulfuric acid and nitric acid, and then distilled before use

Estimated Error:

Temperature: Not given in paper.

$x_{1}: \pm 10 \%$ (relative error, estimated by compiler).

\subsection{2-Hydroxy-3-methylbenzoic acid solubility data in aromatic hydrocarbons}

\begin{tabular}{|c|c|}
\hline $\begin{array}{l}\text { Components: } \\
\text { (1) 2-Hydroxy-3-methylbenzoic acid; } \\
\mathrm{C}_{8} \mathrm{H}_{8} \mathrm{O}_{3} ;[83-40-9] \\
\text { (2) Benzene; } \mathrm{C}_{6} \mathrm{H}_{6} ;[71-43-2]\end{array}$ & $\begin{array}{l}\text { Original Measurements: } \\
{ }^{127} \text { N. V. Sidgwick and E. K. } \\
\text { Ewbank, J. Chem. Soc. Trans. 119, } \\
979 \text { (1921). }\end{array}$ \\
\hline $\begin{array}{l}\text { Variables: } \\
\text { Temperature }\end{array}$ & $\begin{array}{l}\text { Prepared by: } \\
\text { W. E. Acree, Jr. }\end{array}$ \\
\hline
\end{tabular}

Experimental Values

\begin{tabular}{lcc}
\hline \hline$T / \mathrm{K}$ & $x_{2}{ }^{\mathrm{a}}$ & $x_{1}{ }^{\mathrm{b}}$ \\
\hline 318.4 & 0.9906 & 0.0094 \\
335.6 & 0.9750 & 0.0250 \\
352.3 & 0.9526 & 0.0474 \\
380.4 & 0.8342 & 0.1658 \\
396.2 & 0.6899 & 0.3101 \\
411.5 & 0.4808 & 0.5192 \\
428.6 & 0.1873 & 0.8127
\end{tabular}

${ }^{\mathrm{a}} x_{2}$ : mole fraction of component 2 in the saturated solution.

${ }^{b} x_{1}$ : mole fraction solubility of the solute. Solubility data reported in units of mass percent. Mole fraction values calculated by the compiler.

\section{Auxiliary Information}

\section{Method/Apparatus/Procedure:}

Preweighed quantities of solute and solvent were placed in bulbs, which were then sealed and heated in a suitable bath. At the higher temperatures, the concentration of solvent in the liquid solution was corrected for the amount of solvent vapor in the bulb. The correction assumed that the vapor pressure of the saturated solution was one half that of the pure solvent at the solution temperature. The temperature at which the solid completely dissolved was recorded, and was taken to be the solid-liquid equilibrium temperature.
Source and Purity of Chemicals:

(1) Purity not given, prepared and purified by the authors using a published synthetic procedure [see J. Walker and J. K. Wood, J. Chem. Soc. 117, 40 (1920) for synthetic details]. Melting point of the purified solute was $440.2 \mathrm{~K}$. (2) Purity not given, Chemical source not given, thiophene was removed by treatment with sulfuric acid. Sample was further purified by freezing several times.

Estimated Error:

Temperature: Not given in paper.

$x_{1}: \pm 10 \%$ (relative error, estimated by compiler).

\section{Solubility of 2-Hydroxy-4- methylbenzoic Acid in Organic Solvents}

\subsection{Critical evaluation of experimental solubility data}

There has been only a single study involving the solubility of 2-hydroxy-4-methylbenzoic acid in organic solvents. Sidgwick and Ewbank ${ }^{127}$ measured the solubility of 2-hydroxy-4methylbenzoic acid in both heptane and benzene at elevated temperatures. The observed mole fraction solubilities were curve-fit to Eq. (8) to yield the following mathematical representations:

$$
\begin{gathered}
\ln x_{1}=-146.021-\frac{2.939}{T}+23.876 \ln T, \\
\ln x_{1}=-89.757-\frac{1.876}{T}+14.728 \ln T
\end{gathered}
$$

for heptane and benzene, respectively. The mean absolute deviations between experimental and calculated values are on the order of $19.9 \%$ [Eq. (56)] and 7.1\% [Eq. (57)], which are larger than desired. The large deviation may be due in part to the fact that the solution temperature is above the solvent's normal boiling point temperature, to uncertainties in estimating the amount of solvent that remained in the liquid phase, and the large range covered by the experimental values, $x_{1}=0.0127$ to $x_{1}=0.6953$ for heptane and $x_{1}=$ 0.0095 to $x_{1}=0.8351$ for benzene. It is more difficult to curvefit experimental solubility data covering large mole fraction ranges.

The experimental solubility data for 2-hydroxy-4-methylbenzoic acid in organic solvents are in Secs. 50.2 and 50.3.

\subsection{2-Hydroxy-4-methylbenzoic acid solubility data in saturated hydrocarbons (including cycloalkanes)}

\begin{tabular}{ll}
\hline \hline Components: & Original Measurements: \\
$\begin{array}{l}\text { (1) 2-Hydroxy-4-methylbenzoic acid; } \\
\mathrm{C}_{8} \mathrm{H}_{8} \mathrm{O}_{3} ;[50-85-1]\end{array}$ & ${ }^{127} \mathrm{~N}$. V. Sidgwick and E. K. \\
(2) Heptane; $\mathrm{C}_{7} \mathrm{H}_{16} ;[142-82-5]$ & $979(1921)$. \\
\hline Variables: & Prepared by: \\
Temperature & W. E. Acree, Jr. \\
\hline
\end{tabular}


Experimental Values

\begin{tabular}{lcc}
\hline \hline$T / \mathrm{K}$ & $x_{2}{ }^{\mathrm{a}}$ & $x_{1}{ }^{\mathrm{b}}$ \\
\hline 373.8 & 0.9873 & 0.0127 \\
389.9 & 0.9726 & 0.0274 \\
408.8 & 0.9379 & 0.0621 \\
420.3 & 0.8693 & 0.1307 \\
429.8 & 0.7466 & 0.2534 \\
435.4 & 0.5172 & 0.4828 \\
441.9 & 0.3047 & 0.6953 \\
\hline
\end{tabular}

${ }^{a} x_{2}$ : mole fraction of component 2 in the saturated solution.

${ }^{b} x_{1}$ : mole fraction solubility of the solute. Solubility data reported in units of mass percent. Mole fraction values calculated by the compiler.

\section{Auxiliary Information}

\section{Method/Apparatus/Procedure:}

Preweighed quantities of solute and solvent were placed in bulbs, which were then sealed and heated in a suitable bath. At the higher temperatures, the concentration of solvent in the liquid solution was corrected for the amount of solvent vapor in the bulb. The correction assumed that the vapor pressure of the saturated solution was one half that of the pure solvent at the solution temperature. The temperature at which the solid completely dissolved was recorded, and was taken to be the solid-liquid equilibrium temperature.

\section{Source and Purity of Chemicals:}

(1) Purity not given, prepared and purified by the authors using a published synthetic procedure [see J. Walker and J. K. Wood, J. Chem. Soc. 117, 40 (1920) for synthetic details]. Melting point of the purified solute was $451.0 \mathrm{~K}$. (2) Purity not given, sample isolated from the resin of Pinus sabiniana, purified by treatment with sulfuric acid and nitric acid, and then distilled before use

Estimated Error:

Temperature: Not given in paper.

$x_{1}: \pm 10 \%$ (relative error, estimated by compiler).

\subsection{2-Hydroxy-4-methylbenzoic acid solubility data in aromatic hydrocarbons}

\begin{tabular}{ll}
\hline \hline Components: & Original Measurements: \\
$\begin{array}{l}\text { (1) 2-Hydroxy-4-methylbenzoic acid; } \\
\mathrm{C}_{8} \mathrm{H}_{8} \mathrm{O}_{3} ;[50-85-1]\end{array}$ & Ewbank, J. Chem. Soc. Trans. 119, \\
$(2)$ Benzene; $\mathrm{C}_{6} \mathrm{H}_{6} ;[71-43-2]$ & $979(1921)$. \\
\hline Variables: & Prepared by: \\
Temperature & W. E. Acree, Jr. \\
\hline
\end{tabular}

Experimental Values

\begin{tabular}{lcc}
\hline \hline$T / \mathrm{K}$ & $x_{2}{ }^{\mathrm{a}}$ & $x_{1}{ }^{\mathrm{b}}$ \\
\hline 322.0 & 0.9905 & 0.0095 \\
344.9 & 0.9764 & 0.0236 \\
363.4 & 0.9523 & 0.0477 \\
390.8 & 0.8384 & 0.1616 \\
408.3 & 0.6767 & 0.3233 \\
423.5 & 0.4594 & 0.5406 \\
440.8 & 0.1649 & 0.8351 \\
${ }^{a}{ }_{x_{2}: \text { mole fraction of component } 2 \text { in the saturated solution. }}$ \\
${ }^{\mathrm{b}}{ }_{x_{1}: \text { mole fraction solubility of the solute. Solubility data reported in units of }}$ \\
mass percent. Mole fraction values calculated by the compiler.
\end{tabular}

Auxiliary Information

\section{Method/Apparatus/Procedure:}

Preweighed quantities of solute and solvent were placed in bulbs, which were then sealed and heated in a suitable bath. At the higher temperatures, the concentration of solvent in the liquid solution was corrected for the amount of solvent vapor in the bulb. The correction assumed that the vapor pressure of the saturated solution was one half that of the pure solvent at the solution temperature. The temperature at which the solid completely dissolved was recorded, and was taken to be the solid-liquid equilibrium temperature.

Source and Purity of Chemicals:

(1) Purity not given, prepared and purified by the authors using a published synthetic procedure [see J. Walker and J. K. Wood, J. Chem. Soc. 117, 40 (1920) for synthetic details]. Melting point of the purified solute was $451.0 \mathrm{~K}$. (2) Purity not given, Chemical source not given, thiophene was removed by treatment with sulfuric acid. Sample was further purified by freezing several times.

\section{Estimated Error:}

Temperature: Not given in paper.

$x_{1}: \pm 10 \%$ (relative error, estimated by compiler).

\section{Solubility of 2-Hydroxy-5-} methylbenzoic Acid in Organic Solvents

\subsection{Critical evaluation of experimental solubility data}

There has been only a single study involving the solubility of 2-hydroxy-5-methylbenzoic acid in organic solvents. Sidgwick and Ewbank ${ }^{127}$ measured the solubility of 2-hydroxy-5methylbenzoic acid in both heptane and benzene at elevated temperatures. The observed mole fraction solubilities were curve-fit to Eq. (8) to yield the following mathematical representations:

$$
\begin{gathered}
\ln x_{1}=-152.882-\frac{3.209}{T}+25.256 \ln T, \\
\ln x_{1}=-89.710-\frac{1.955}{T}+14.876 \ln T,
\end{gathered}
$$

for heptane and benzene, respectively. The mean absolute deviations between experimental and calculated values are on the order of $25.2 \%$ [Eq. (58)] and $7.1 \%$ [Eq. (59)], which are larger than desired. The large deviation may be due in part to the fact that the solution temperature is above the solvent's normal boiling point temperature, to uncertainties in estimating the amount of solvent that remained in the liquid phase, and the large range covered by the experimental values, $x_{1}=0.0110$ to $x_{1}=0.8414$ for heptane and $x_{1}=0.0083$ to $x_{1}=$ 0.8262 for benzene. It is more difficult to curve-fit experimental solubility data covering large mole fraction ranges.

The experimental solubility data for 2-hydroxy-5-methylbenzoic acid in organic solvents are in Secs. 51.2 and 51.3. 


\subsection{2-Hydroxy-5-methylbenzoic acid solubility data in saturated hydrocarbons (including cycloalkanes)}

\begin{tabular}{|c|c|}
\hline $\begin{array}{l}\text { Components: } \\
\text { (1) 2-Hydroxy-5-methylbenzoic acid; } \\
\mathrm{C}_{8} \mathrm{H}_{8} \mathrm{O}_{3} ;[89-56-5] \\
\text { (2) Heptane; } \mathrm{C}_{7} \mathrm{H}_{16} ;[142-82-5]\end{array}$ & $\begin{array}{l}\text { Original Measurements: } \\
{ }^{127} \text { N. V. Sidgwick and E. K. } \\
\text { Ewbank, J. Chem. Soc. Trans. 119, } \\
979 \text { (1921). }\end{array}$ \\
\hline $\begin{array}{l}\text { Variables: } \\
\text { Temperature }\end{array}$ & $\begin{array}{l}\text { Prepared by: } \\
\text { W. E. Acree, Jr. }\end{array}$ \\
\hline
\end{tabular}

Experimental Values

\begin{tabular}{lcc}
\hline \hline$T / \mathrm{K}$ & $x_{2}{ }^{\mathrm{a}}$ & $x_{1}{ }^{\mathrm{b}}$ \\
\hline 352.2 & 0.9890 & 0.0110 \\
370.3 & 0.9721 & 0.0279 \\
389.4 & 0.9352 & 0.0648 \\
404.3 & 0.7936 & 0.2064 \\
408.7 & 0.6181 & 0.3819 \\
411.9 & 0.4195 & 0.5805 \\
419.1 & 0.1586 & 0.8414
\end{tabular}

${ }^{a} x_{2}$ : mole fraction of component 2 in the saturated solution.

${ }^{\mathrm{b}} x_{1}$ : mole fraction solubility of the solute. Solubility data reported in units of mass percent. Mole fraction values calculated by the compiler.

\section{Auxiliary Information}

\begin{abstract}
Method/Apparatus/Procedure:
Preweighed quantities of solute and solvent were placed in bulbs, which were then sealed and heated in a suitable bath. At the higher temperatures, the concentration of solvent in the liquid solution was corrected for the amount of solvent vapor in the bulb. The correction assumed that the vapor pressure of the saturated solution was one half that of the pure solvent at the solution temperature. The temperature at which the solid completely dissolved was recorded, and was taken to be the solid-liquid equilibrium temperature.
\end{abstract}

Source and Purity of Chemicals:

(1) Purity not given, prepared and purified by the authors using a published synthetic procedure [see J. Walker and J. K. Wood, J. Chem. Soc. 117, 40 (1920) for synthetic details]. Melting point of the purified solute was $425.7 \mathrm{~K}$. (2) Purity not given, sample isolated from the resin of Pinus sabiniana, purified by treatment with sulfuric acid and nitric acid, and then distilled before use.

Estimated Error:

Temperature: Not given in paper.

$x_{1}: \pm 10 \%$ (relative error, estimated by compiler).

\subsection{2-Hydroxy-5-methylbenzoic acid solubility data in aromatic hydrocarbons}

\begin{tabular}{|c|c|}
\hline $\begin{array}{l}\text { Components: } \\
\text { (1) 2-Hydroxy-5-methylbenzoic acid; } \\
\mathrm{C}_{8} \mathrm{H}_{8} \mathrm{O}_{3} ;[89-56-5] \\
\text { (2) Benzene; } \mathrm{C}_{6} \mathrm{H}_{6} ;[71-43-2]\end{array}$ & $\begin{array}{l}\text { Original Measurements: } \\
{ }^{127} \text { N. V. Sidgwick and E. K. } \\
\text { Ewbank, J. Chem. Soc. Trans. 119, } \\
979 \text { (1921). }\end{array}$ \\
\hline Variables: & Prepared by: \\
\hline Temperature & W. E. Acree, Jr. \\
\hline
\end{tabular}

Experimental Values

\begin{tabular}{lcc}
\hline \hline$T / \mathrm{K}$ & $x_{2}{ }^{\mathrm{a}}$ & $x_{1}{ }^{\mathrm{b}}$ \\
\hline 303.2 & 0.9917 & 0.0083 \\
322.0 & 0.9775 & 0.0225 \\
341.2 & 0.9482 & 0.0518 \\
366.5 & 0.8341 & 0.1659 \\
383.8 & 0.6648 & 0.3352 \\
397.9 & 0.4756 & 0.5244 \\
415.2 & 0.1738 & 0.8262
\end{tabular}

${ }^{\mathrm{a}} x_{2}$ : mole fraction of component 2 in the saturated solution.

${ }^{\mathrm{b}} x_{1}$ : mole fraction solubility of the solute. Solubility data reported in units of mass percent. Mole fraction values calculated by the compiler.

\section{Auxiliary Information}

\begin{abstract}
Method/Apparatus/Procedure:
Preweighed quantities of solute and solvent were placed in bulbs, which were then sealed and heated in a suitable bath. At the higher temperatures, the concentration of solvent in the liquid solution was corrected for the amount of solvent vapor in the bulb. The correction assumed that the vapor pressure of the saturated solution was one half that of the pure solvent at the solution temperature. The temperature at which the solid completely dissolved was recorded, and was taken to be the solid-liquid equilibrium temperature.
\end{abstract}

Source and Purity of Chemicals:

(1) Purity not given, prepared and purified by the authors using a published synthetic procedure [see J. Walker and J. K. Wood, J. Chem. Soc. 117, 40 (1920) for synthetic details]. Melting point of the purified solute was $425.7 \mathrm{~K}$. (2) Purity not given, Chemical source not given, thiophene was removed by treatment with sulfuric acid. Sample was further purified by freezing several times.

Estimated Error:

Temperature: Not given in paper.

$x_{1}: \pm 10 \%$ (relative error, estimated by compiler).

\section{Solubility of 3-Hydroxy-4- methylbenzoic Acid in Organic Solvents}

\subsection{Critical evaluation of experimental solubility data}

There has been only a single study involving the solubility of 3-hydroxy-4-methylbenzoic acid in organic solvents. Sidgwick and Ewbank ${ }^{127}$ measured the solubility of 3-hydroxy-4methylbenzoic acid in benzene at elevated temperatures. The observed mole fraction solubilities were curve-fit to Eq. (8) to yield the following correlation:

$$
\ln x_{1}=-178.244-\frac{3.1001}{T}+28.896 \ln T .
$$

The mean absolute deviation between experimental and calculated values is on the order of $14.1 \%$, which is larger than desired. The large deviation may be due in part to the fact that the solution temperature is above the solvent's normal boiling point temperature, to uncertainties in estimating the amount of solvent that remained in the liquid phase, and the large range covered by the experimental values, $x_{1}=0.0102$ to 
$x_{1}=0.8369$. It is more difficult to curve-fit experimental solubility data covering large mole fraction ranges.

The experimental solubility data for 3-hydroxy-4-methylbenzoic acid in organic solvents are in Sec. 52.2.

\subsection{3-Hydroxy-4-methylbenzoic acid solubility data in aromatic hydrocarbons}

\begin{tabular}{|c|c|}
\hline $\begin{array}{l}\text { Components: } \\
\text { (1) 3-Hydroxy-4-methylbenzoic acid; } \\
\mathrm{C}_{8} \mathrm{H}_{8} \mathrm{O}_{3} ;[586-30-1] \\
\text { (2) Benzene; } \mathrm{C}_{6} \mathrm{H}_{6} ;[71-43-2]\end{array}$ & $\begin{array}{l}\text { Original Measurements: } \\
{ }^{127} \text { N. V. Sidgwick and E. K. } \\
\text { Ewbank, J. Chem. Soc. Trans. 119, } \\
979 \text { (1921). }\end{array}$ \\
\hline Variables: & Prepared by: \\
\hline Temperature & W. E. Acree, Jr. \\
\hline
\end{tabular}

Experimental Values

\begin{tabular}{lcc}
\hline \hline$T / \mathrm{K}$ & $x_{2}{ }^{\mathrm{a}}$ & $x_{1}{ }^{\mathrm{b}}$ \\
\hline 404.8 & 0.9898 & 0.0102 \\
420.6 & 0.9768 & 0.0232 \\
433.7 & 0.9520 & 0.0480 \\
449.7 & 0.8353 & 0.1647 \\
456.9 & 0.6828 & 0.3172 \\
465.2 & 0.4470 & 0.5530 \\
475.7 & 0.1631 & 0.8369
\end{tabular}

${ }^{\mathrm{a}} x_{2}$ : mole fraction of component 2 in the saturated solution.

${ }^{\mathrm{b}} x_{1}$ : mole fraction solubility of the solute. Solubility data reported in units of mass percent. Mole fraction values calculated by the compiler.

\section{Auxiliary Information}

Method/Apparatus/Procedure:

Preweighed quantities of solute and solvent were placed in bulbs, which were then sealed and heated in a suitable bath. At the higher temperatures, the concentration of solvent in the liquid solution was corrected for the amount of solvent vapor in the bulb. The correction assumed that the vapor pressure of the saturated solution was one half that of the pure solvent at the solution temperature. The temperature at which the solid completely dissolved was recorded, and was taken to be the solid-liquid equilibrium temperature.

\section{Source and Purity of Chemicals:}

(1) Purity not given, prepared and purified by the authors using a published synthetic procedure [see J. Walker and J. K. Wood, J. Chem. Soc. 117, 40 (1920) for synthetic details]. Melting point of the purified solute was $481.7 \mathrm{~K}$ (2) Purity not given, Chemical source not given, thiophene was removed by treatment with sulfuric acid. The sample was further purified by freezing several times.

\section{Estimated Error:}

Temperature: Not given in paper.

$x_{1}: \pm 10 \%$ (relative error, estimated by compiler).

\section{Solubility of 4-Hydroxy-3- methoxybenzoic Acid in Organic Solvents}

\subsection{Critical evaluation of experimental solubility data}

There has been only a single study involving the solubility of 4-hydroxy-3-methoxybenzoic acid (commonly referred to as vanillic acid) in organic solvents. Manic et al. ${ }^{169}$ measured the solubility of 4-hydroxy-3-methoxybenzoic acid in ethyl 2hydroxypropanoate at five temperatures between $296 \mathrm{~K}$ and $333 \mathrm{~K}$. The observed mole fraction solubilities were curve-fit to Eq. (8) to yield the following correlation:

$$
\ln x_{1}=-37.631+\frac{114.97}{T}+5.916 \ln T .
$$

The mean absolute deviation between experimental and calculated values is on the order of $0.9 \%$, indicating that the experimental data are internally consistent.

The experimental solubility data for 4-hydroxy-3-methoxybenzoic acid in ethyl 2-hydroxypropanoate are in Sec. 53.2.

\subsection{4-Hydroxy-3-methoxybenzoic acid solubility data in miscellaneous organic solvents}

\begin{tabular}{ll}
\hline \hline Components: & Original Measurements: \\
(1) 4-Hydroxy-3-methoxybenzoic acid; & ${ }^{169}$ M. S. Manic, D. Villanueva, \\
$\mathrm{C}_{8} \mathrm{H}_{8} \mathrm{O}_{4} ;[121-34-6]$ & T. Fornari, A. J. Queimada, E. A. \\
$(2)$ Ethyl 2-hydroxypropanoate, & Macedo, and V. Najdanovic- \\
$\mathrm{C}_{5} \mathrm{H}_{10} \mathrm{O}_{3} ;$ [687-47-8] & Visak, J. Chem. Thermodyn. 48, \\
& $93(2012)$. \\
\hline Variables: & Prepared by: \\
Temperature & W. E. Acree, Jr. \\
\hline
\end{tabular}

Experimental Values

\begin{tabular}{lcc}
\hline \hline$T / \mathrm{K}$ & $x_{2}{ }^{\mathrm{a}}$ & $x_{1}{ }^{\mathrm{b}}$ \\
\hline 296.2 & 0.9721 & 0.0279 \\
303.1 & 0.9679 & 0.0321 \\
312.7 & 0.9621 & 0.0379 \\
323.0 & 0.9556 & 0.0444 \\
333.3 & 0.9455 & 0.0545 \\
\hline
\end{tabular}

${ }^{\mathrm{a}} x_{2}$ : mole fraction of component 2 in the saturated solution.

${ }^{\mathrm{b}} x_{1}$ : mole fraction solubility of the solute. 


\section{Auxiliary Information}

\section{Method/Apparatus/Procedure:}

Constant-temperature water bath, stirring plate, and analytical balance. Solubilities were determined using a gravimetric method. Excess solute and solvent were placed in a glass vessel with stirrer. The vessel was placed in a constant-temperature water bath and allowed to equilibrate with stirring for $48 \mathrm{~h}$. After equilibrium had been attained, the stirring was discontinued and the sample was allowed to set for more than $48 \mathrm{~h}$. Samples of the clear saturated solution were removed by micropipette, transferred to a weighed empty vial, and the mass of the vial plus sample were recorded. The sample was placed in a vacuum oven, and the solvent was evaporated at $338 \mathrm{~K}$. Once the solvent had completely evaporated, the mass of the vial and solid residue was determined. The solubility was calculated from the mass of the solid residue and mass of the saturated sample analyzed.

Source and Purity of Chemicals:

(1) $97+\%$, Fluka Chemical Company, used as received.

(2) $98 \%$, Sigma-Aldrich Chemical Company, dried in a vacuum oven at room temperature for several days prior to use.

Estimated Error:

Temperature: $\pm 0.1 \mathrm{~K}$.

$x_{1}: \pm 0.0007$

\section{Solubility of 4-Hydroxy-3- methylbenzoic Acid in Organic Solvents}

\subsection{Critical evaluation of experimental solubility data}

There has been only a single study involving the solubility of 4-hydroxy-3-methylbenzoic acid in organic solvents. Sidgwick and Ewbank ${ }^{127}$ measured the solubility of 4-hydroxy-3methylbenzoic acid in benzene at elevated temperatures. The observed mole fraction solubilities were curve-fit to Eq. (8) to yield the following correlation:

$$
\ln x_{1}=-217.192-\frac{4.4130}{T}+35.778 \ln T .
$$

The experimental mole fraction solubility at $439 \mathrm{~K}$ had to be excluded in order to obtain a reasonable mathematical correlation. The mean absolute deviation between experimental and calculated values is on the order of $8.3 \%$, which is larger than desired. The large deviation may be due in part to the fact that the solution temperature is above the solvent's normal boiling point temperature, to uncertainties in estimating the amount of solvent that remained in the liquid phase, and the large range covered by the experimental values, $x_{1}=0.0131$ to $x_{1}=$ 0.5406 . The derived equation should not be used to predict 4 hydroxy-3-methylbenzoic acid solubilities at temperatures greater than $426 \mathrm{~K}$, as the calculated $x_{1}$ values will exceed unity. It is more difficult to curve-fit experimental solubility data covering large mole fraction ranges.

The experimental solubility data for 4-hydroxy-3-methylbenzoic acid in benzene are in Sec. 54.2.

\subsection{4-Hydroxy-3-methylbenzoic acid solubility data in aromatic hydrocarbons}

\begin{tabular}{ll}
\hline \hline Components: & Original Measurements: \\
$\begin{array}{l}\text { (1) 4-Hydroxy-3-methylbenzoic acid; } \\
\mathrm{C}_{8} \mathrm{H}_{8} \mathrm{O}_{3} ;[499-76-3]\end{array}$ & ${ }^{127} \mathrm{~N}$. V. Sidgwick and E. K. \\
$(2)$ Benzene; $\mathrm{C}_{6} \mathrm{H}_{6} ;[71-43-2]$ & $979(1921)$. \\
\hline Variables: & Prepared by: \\
Temperature & W. E. Acree, Jr. \\
\hline
\end{tabular}

Experimental Values

\begin{tabular}{lcc}
\hline \hline$T / \mathrm{K}$ & $x_{2}{ }^{\mathrm{a}}$ & $x_{1}{ }^{\mathrm{b}}$ \\
\hline 382.7 & 0.9869 & 0.0131 \\
389.9 & 0.9772 & 0.0228 \\
399.4 & 0.9503 & 0.0497 \\
412.7 & 0.8353 & 0.1647 \\
418.2 & 0.6599 & 0.3401 \\
426.0 & 0.4594 & 0.5406 \\
439.2 & 0.1808 & 0.8192
\end{tabular}

${ }^{a} x_{2}$ : mole fraction of component 2 in the saturated solution.

${ }^{\mathrm{b}} x_{1}$ : mole fraction solubility of the solute. Solubility data reported in units of mass percent. Mole fraction values calculated by the compiler.

\section{Auxiliary Information}

\section{Method/Apparatus/Procedure:}

Preweighed quantities of solute and solvent were placed in bulbs, which were then sealed and heated in a suitable bath. At the higher temperatures, the concentration of solvent in the liquid solution was corrected for the amount of solvent vapor in the bulb. The correction assumed that the vapor pressure of the saturated solution was one half that of the pure solvent at the solution temperature. The temperature at which the solid completely dissolved was recorded, and was taken to be the solid-liquid equilibrium temperature.

Source and Purity of Chemicals:

(1) Purity not given, prepared and purified by the authors using a published synthetic procedure [see J. Walker and J. K. Wood, J. Chem. Soc. 117, 40 (1920) for synthetic details]. Melting point of the purified solute was 445.6 K. (2) Purity not given, Chemical source not given, thiophene was removed by treatment with sulfuric acid. Sample was further purified by freezing several times.

Estimated Error:

Temperature: Not given in paper.

$x_{1}: \pm 10 \%$ (relative error, estimated by compiler).

\section{Solubility of 4-lodobenzoic Acid in Organic Solvents}

\subsection{Critical evaluation of experimental solubility data}

There has been only one published paper $^{83}$ reporting the solubility of 4-iodobenzoic acid in organic solvents. Chantooni and Kolthoff ${ }^{83}$ determined the solubility of 4-iodobenzoic acid in methanol, ethanenitrile, dimethyl sulfoxide, and $N$, $N$-dimethylformamide at $298 \mathrm{~K}$. It is not possible to perform a critical evaluation of the experimental data as the authors 
performed measurements at only a single temperature, and there are no independent experimental 4-iodobenzoic acid solubility data in any of the four organic solvents.

The experimental solubility data for 4-iodobenzoic acid in organic solvents are given in Secs. 55.2 and 55.3.

\subsection{4-lodobenzoic acid solubility data in alcohols}

\begin{tabular}{ll}
\hline \hline Components: & Original Measurements: \\
(1) 4-Iodobenzoic acid; $\mathrm{C}_{7} \mathrm{H}_{5} \mathrm{IO}_{2} ;$ & $\begin{array}{l}{ }^{83} \mathrm{M} \text {. K. Chantooni and I. M. } \\
\text { 6619-58-9] }\end{array}$ \\
Kolthoff, J. Phys. Chem. 77, 527 \\
(2) Methanol; $\mathrm{CH}_{4} \mathrm{O} ;[67-56-1]$ & $(1973)$. \\
\hline Variables: & Prepared by: \\
$T / \mathrm{K}=298.15$ & W. E. Acree, Jr. \\
\hline
\end{tabular}

\section{Experimental Values}

The measured solubility was reported to be $0.068 \mathrm{~mol} \mathrm{dm}^{-3}$. The authors did not give the temperature at which the solubility was measured. Based on the experimental description given in an earlier paper [I. M. Kolthoff, J. J. Lingane, and W. Larson, J. Am. Chem. Soc. 60, 2512 (1938)], the compiler believes the temperature to be $298.15 \mathrm{~K}$.

\section{Auxiliary Information}

\section{Method/Apparatus/Procedure:}

Very little experimental details were provided. Solubility was determined by titrating a filtered aliquot of the saturated solution alkalimetrically in an aqueous-ethanol mixture using phenolphthalein as the acid-base indicator.

\section{Source and Purity of Chemicals:}

(1) Purity not given, Chemical source not given, was recrystallized from either aqueous or aqueous-ethanol solution and dried in vacuo at $333 \mathrm{~K}$.

(2) Purity not given, Chemical source not given, was dehydrated and then distilled over sulfanilic acid to remove alkaline impurities.

\section{Estimated Error:}

Temperature: $\pm 0.1 \mathrm{~K}$ (estimated by compiler).

$c_{1}: \pm 2 \%$ (relative error, estimated by compiler).

\subsection{4-lodobenzoic acid solubility data in miscellaneous organic solvents}

\begin{tabular}{|c|c|}
\hline $\begin{array}{l}\text { Components: } \\
\text { (1) 4-Iodobenzoic acid; } \mathrm{C}_{7} \mathrm{H}_{5} \mathrm{IO}_{2} \text {; } \\
\text { [619-58-9] } \\
\text { (2) Ethanenitrile; } \mathrm{C}_{2} \mathrm{H}_{3} \mathrm{~N} ;[75-05-8]\end{array}$ & $\begin{array}{l}\text { Original Measurements: } \\
{ }^{83} \text { M. K. Chantooni and I. M. } \\
\text { Kolthoff, J. Phys. Chem. 77, } 527 \\
\text { (1973). }\end{array}$ \\
\hline $\begin{array}{l}\text { Variables: } \\
T / \mathrm{K}=298.15\end{array}$ & $\begin{array}{l}\text { Prepared by: } \\
\text { W. E. Acree, Jr. }\end{array}$ \\
\hline
\end{tabular}

\section{Experimental Values}

The measured solubility was reported to be $0.0098 \mathrm{~mol}$ $\mathrm{dm}^{-3}$. The authors did not give the temperature at which the solubility was measured. Based on the experimental description given in an earlier paper [I. M. Kolthoff, J. J. Lingane, and
W. Larson, J. Am. Chem. Soc. 60, 2512 (1938)], the compiler believes the temperature to be $298.15 \mathrm{~K}$.

\section{Auxiliary Information}

Method/Apparatus/Procedure:
Very little experimental details were provided. Solubility was determined by
titrating a filtered aliquot of the saturated solution alkalimetrically in an
aqueous-ethanol mixture using phenolphthalein as the acid-base indicator.

Source and Purity of Chemicals:

(1) Purity not given, Chemical source not given, was recrystallized from either aqueous or aqueous-ethanol solution and dried in vacuo at $333 \mathrm{~K}$.

(2) Purity not given, Chemical source not given, was purified by shaking with saturated potassium hydroxide, followed by activated alumina, and then anhydrous calcium chloride to remove water. Ethanenitrile was further dried over anhydrous magnesium sulfate and then phosphorous pentoxide. The sample was distilled shortly before use.

\section{Estimated Error:}

Temperature: $\pm 0.1 \mathrm{~K}$ (estimated by compiler).

$c_{1}: \pm 2 \%$ (relative error, estimated by compiler).

\begin{tabular}{|c|c|}
\hline $\begin{array}{l}\text { Components: } \\
\text { (1) 4-Iodobenzoic acid; } \mathrm{C}_{7} \mathrm{H}_{5} \mathrm{IO}_{2} \text {; } \\
\text { [619-58-9] } \\
\text { (2) Dimethyl sulfoxide; } \mathrm{C}_{2} \mathrm{H}_{6} \mathrm{OS} \text {; } \\
\text { [67-68-5] }\end{array}$ & $\begin{array}{l}\text { Original Measurements: } \\
{ }^{83} \text { M. K. Chantooni and I. M. } \\
\text { Kolthoff, J. Phys. Chem. 77, } 527 \\
\text { (1973). }\end{array}$ \\
\hline $\begin{array}{l}\text { Variables: } \\
T / \mathrm{K}=298.15\end{array}$ & $\begin{array}{l}\text { Prepared by: } \\
\text { W. E. Acree, Jr. }\end{array}$ \\
\hline
\end{tabular}

\section{Experimental Values}

The measured solubility was reported to be $2.14 \mathrm{~mol} \mathrm{dm}^{-3}$. The authors did not give the temperature at which the solubility was measured. Based on the experimental description given in an earlier paper [I. M. Kolthoff, J. J. Lingane, and W. Larson, J. Am. Chem. Soc. 60, 2512 (1938)], the compiler believes the temperature to be $298.15 \mathrm{~K}$.

\section{Auxiliary Information}

\section{Method/Apparatus/Procedure:}

Very little experimental details were provided. Solubility was determined by titrating a filtered aliquot of the saturated solution alkalimetrically in an aqueous-ethanol mixture using phenolphthalein as the acid-base indicator.

Source and Purity of Chemicals:

(1) Purity not given, Chemical source not given, was recrystallized from either aqueous or aqueous-ethanol solution and dried in vacuo at $333 \mathrm{~K}$.

(2) Purity not given, Chemical source not given, was shaken with activated alumina and then distilled before use.

\section{Estimated Error:}

Temperature: $\pm 0.1 \mathrm{~K}$ (estimated by compiler).

$c_{1}: \pm 2 \%$ (relative error, estimated by compiler). 


\section{Components:}

(1) 4-Iodobenzoic acid; $\mathrm{C}_{7} \mathrm{H}_{5} \mathrm{IO}_{2}$; [619-58-9]

(2) $\mathrm{N}, \mathrm{N}$-Dimethylformamide; $\mathrm{C}_{3} \mathrm{H}_{7} \mathrm{NO}$; [64-19-7]

\begin{tabular}{ll}
\hline Variables: & Prepared by: \\
$T / \mathrm{K}=298.15$ & W. E. Acree, Jr. \\
\hline
\end{tabular}

Experimental Values

The measured solubility was reported to be $0.72 \mathrm{~mol} \mathrm{dm}^{-3}$. The authors did not give the temperature at which the solubility was measured. Based on the experimental description given in an earlier paper [I. M. Kolthoff, J. J. Lingane, and W. Larson, J. Am. Chem. Soc. 60, 2512 (1938)], the compiler believes the temperature to be $298.15 \mathrm{~K}$.

\section{Auxiliary Information}

\section{Method/Apparatus/Procedure:}

Very little experimental details were provided. Solubility was determined by titrating a filtered aliquot of the saturated solution alkalimetrically in an aqueous-ethanol mixture using phenolphthalein as the acid-base indicator.

\section{Source and Purity of Chemicals:}

(1) Purity not given, Chemical source not given, was recrystallized from either aqueous or aqueous-ethanol solution and dried in vacuo at $333 \mathrm{~K}$.

(2) Purity not given, Chemical source not given, was shaken first with phosphorous pentoxide and then with potassium hydroxide pellets. Solvent was distilled shortly before use.

\section{Estimated Error:}

Temperature: $\pm 0.1 \mathrm{~K}$ (estimated by compiler).

$c_{1}: \pm 2 \%$ (relative error, estimated by compiler).

\section{Solubility of 2-Methoxybenzoic Acid in Organic Solvents}

\subsection{Critical evaluation of experimental solubility data}

There have been four published studies ${ }^{9,56,72,130}$ investigating the solubility behavior of 2-methoxybenzoic acid in organic solvents of varying polarity and hydrogen-bonding capability. Hoover et al. ${ }^{9}$ measured the solubility of 2-methoxybenzoic acid in 18 alcohols (methanol, ethanol, 1-propanol, 2-propanol, 1-butanol, 2-butanol, 2-methyl-1-propanol, 2methyl-2-propanol, 1-pentanol, 2-pentanol, 2-methyl-1-butanol, 3-methyl-1-butanol, 1-hexanol, 2-methyl-1-pentanol, 4methyl-2-pentanol, 1-heptanol, 1-octanol, and 1-decanol), in three dialkyl ethers (1,1'-oxybisethane, 2,2'-oxybispropane, and 1,1'-oxybisbutane) and two cyclic ethers (tetrahydrofuran and 1,4-dioxane), in four alkyl alkanoates (methyl ethanoate, ethyl ethanoate, butyl ethanoate, and pentyl ethanoate), and in propylene carbonate at $298 \mathrm{~K}$. Results of the experimental measurements were used to calculate the Abraham solute descriptors of 2-methoxybenzoic acid. The authors were able to assemble a total of $47 \log _{10}\left(S R\right.$ or $P$ ) and $\log _{10}(G S R$ or $K)$ equations for which experimental partition coefficient data, solubility ratios, Abraham model equation coefficients and aqueous molar solubility were available. The logarithm of the aqueous molar solubility of 2-methoxybenzoic acid is $\log _{10} c_{1, \mathrm{~W}}=-1.554 .{ }^{56,72}$ The McGowan volume of 2-methoxybenzoic acid, $V=1.1313$, was calculated from the number of chemical bonds in the molecule and the individual atomic group volumes, $A V_{i}$, given in Sec. 1.3. The excess molar refraction solute descriptor was estimated as $E=0.899$. This left four solute descriptors ( $S, A, B$, and $L$ ) still to be determined. The 47 equations were then solved using the Microsoft "SOLVER" program to yield numerical values of the remaining four solute descriptors, $S=1.410, A=0.450, B=0.620$, and $L$ $=5.636$, that best described the $\log _{10}(S R$ or $P)$ and $\log _{10}(G S R$ or $K$ ) values. The computation treated $\log _{10} c_{1, \mathrm{G}}$ as a floating parameter to be determined as part of the regression analyses. The data analyses returned a value of $\log _{10} c_{1, \mathrm{G}}=-8.354$ for the gas-phase solute concentration that made the $\log _{10}(S R$ or $P)$ and $\log _{10}(G S R$ or $K)$ predictions internally consistent. The calculated molecular solute descriptors reproduced the $\log _{10}$ (SR or $P$ ) and $\log _{10}(G S R$ or $K$ ) values to within an average standard deviation of 0.146 and $0.146 \log _{10}$ units, respectively.

After the 2-methoxybenzoic acid solubility study was published, Abraham model correlations have been developed for 2-pentanol, 3-methyl-1-butanol, methyl ethanoate, and butyl ethanoate, and equation coefficients for a few solvents were updated based on additional experimental data. The new correlations (listed in Tables 1 and 2) will be used in illustrating the ability of the Abraham model to correlate the experimental 2-methoxybenzoic acid solubility data. Table 40 compares the experimental $\log _{10} c_{1}$ values to calculated values based on Eqs. (20) and (21) of the Abraham model. For comparison purposes, the measured mole fraction solubilities of 2-methoxybenzoic acid, $x_{1}$, determined by Hoover et al. ${ }^{9}$ were converted into molar solubilities by dividing $x_{1}$ by the

TABLE 40. Comparison between observed and calculated molar solubilities of 2-methoxybenzoic acid based on the Abraham model, Eqs. (20) and (21)

\begin{tabular}{lccr}
\hline \hline Solvent & & $\log _{10} c_{1}{ }^{\text {calc }} ;$ & $\log _{10} c_{1}{ }^{\text {calc }}$; \\
Methanol & 0.267 & 0.081 & Eq. (21) \\
Ethanol & 0.074 & -0.087 & -0.064 \\
1-Propanol & -0.120 & -0.217 & -0.218 \\
2-Propanol & -0.392 & -0.252 & -0.235 \\
1-Butanol & -0.247 & -0.308 & -0.303 \\
2-Butanol & -0.299 & -0.257 & -0.266 \\
2-Methyl-1-propanol & -0.392 & -0.332 & -0.336 \\
2-Methyl-2-propanol & -0.286 & -0.260 & -0.243 \\
1-Pentanol & -0.327 & -0.375 & -0.379 \\
2-Pentanol & -0.411 & -0.383 & -0.373 \\
3-Methyl-1-butanol & -0.402 & -0.415 & -0.401 \\
1-Hexanol & -0.430 & -0.407 & -0.403 \\
1-Heptanol & -0.471 & -0.467 & -0.449 \\
1-Octanol & -0.518 & -0.485 & -0.545 \\
1-Decanol & -0.658 & -0.640 & -0.626 \\
1,1'-Oxybisethane & -0.638 & -0.455 & -0.354 \\
Tetrahydrofuran & 0.299 & 0.316 & 0.309 \\
1,4-Dioxane & 0.197 & 0.240 & 0.235 \\
Methyl ethanoate & -0.054 & 0.007 & -0.040 \\
Ethyl ethanoate & -0.187 & -0.184 & -0.169 \\
Butyl ethanoate & -0.447 & -0.323 & -0.313 \\
\hline
\end{tabular}


ideal molar volume of the saturated solution (i.e., $c_{1}{ }^{\text {sat }}=x_{1} /$ $\left.\left[x_{1} V_{1}+\left(1-x_{1}\right) V_{\text {solvent }}\right]\right)$. The molar volume of the hypothetical subcooled liquid 2-methoxybenzoic acid is $V_{\text {solute }}=$ $124.1 \mathrm{~cm}^{3} \mathrm{~mol}^{-1}$. Examination of the numerical entries in Table 40 reveals that the Abraham model provides a reasonably accurate mathematical description of the observed solubility data, suggesting that there are no obvious outliers in the dataset.

Ongley ${ }^{56}$ measured the solubility of 2-methoxybenzoic acid in cyclohexane, benzene, trichloromethane, and tetrachloromethane at $298.15 \mathrm{~K}$. Abraham model predictions would not be applicable to these solvents because 2-methoxybenzoic acid would likely dimerize to an appreciable extent. In such cases, the measured solubility would represent the sum of both the monomeric and dimeric forms of the solute. The solute descriptors that have been calculated for 2-methoxybenzoic acid pertain only to the monomeric form. Thuaire ${ }^{72}$ reported a mole fraction solubility for 2-methoxybenzoic acid in ethanol of $x_{1}=0.0758$, which differs by less than $1 \%$ from the value of $x_{1}=0.0765$ determined by Hoover et al. ${ }^{9}$

The experimental solubility data for 2-methoxybenzoic acid in organic solvents are in Secs. 56.2-56.9.

\subsection{2-Methoxybenzoic acid solubility data in saturated hydrocarbons (including cycloalkanes)}

\begin{tabular}{|c|c|}
\hline $\begin{array}{l}\text { Components: } \\
\text { (1) 2-Methoxybenzoic acid; } \mathrm{C}_{8} \mathrm{H}_{8} \mathrm{O}_{3} \text {; } \\
\text { [579-75-9] } \\
\text { (2) Cyclohexane; } \mathrm{C}_{6} \mathrm{H}_{12} ;[110-82-7]\end{array}$ & $\begin{array}{l}\text { Original Measurements: } \\
\text { 56 P. A. Ongley, J. Chem. Soc. 1954, } \\
3634 \text {. }\end{array}$ \\
\hline $\begin{array}{l}\text { Variables: } \\
T / \mathrm{K}=298.15\end{array}$ & $\begin{array}{l}\text { Prepared by: } \\
\text { W. E. Acree, Jr. }\end{array}$ \\
\hline
\end{tabular}

\section{Experimental Values}

The measured solubility was reported to be $-\log _{10} c_{1}=1.241$, which corresponds to a solubility of $c_{1}=0.0574 \mathrm{~mol} \mathrm{dm}^{-3}$.

\section{Auxiliary Information}

\section{Method/Apparatus/Procedure:}

Very little experimental details were provided. Excess solute and solvent were placed in tubes, which were rotated for at least $8 \mathrm{~h}$ at $298 \mathrm{~K}$. The solutions were then removed, concentrated if necessary, and titrated with standard alkali using a mixed (bromothymol blue + neutral red) indicator.

Source and Purity of Chemicals:

(1) Purity not given, Chemical source not given, solute was either "AnalaR" grade or recrystallized prior to use.

(2) Purity not given, Chemical source not given, no purification details were provided.

\section{Estimated Error:}

Temperature: No information was given.

$c_{1}: \pm 4 \%$ (relative error, estimated by compiler).

\subsection{2-Methoxybenzoic acid solubility data in aromatic hydrocarbons}

\begin{tabular}{|c|c|}
\hline $\begin{array}{l}\text { Components: } \\
\text { (1) 2-Methoxybenzoic acid; } \mathrm{C}_{8} \mathrm{H}_{8} \mathrm{O}_{3} \text {; } \\
\text { [579-75-9] } \\
\text { (2) Benzene; } \mathrm{C}_{6} \mathrm{H}_{6} ;[71-43-2]\end{array}$ & $\begin{array}{l}\text { Original Measurements: } \\
{ }^{56} \text { P. A. Ongley, J. Chem. Soc. } 1954 \\
3634 .\end{array}$ \\
\hline $\begin{array}{l}\text { Variables: } \\
T / \mathrm{K}=298.15\end{array}$ & $\begin{array}{l}\text { Prepared by: } \\
\text { W. E. Acree, Jr. }\end{array}$ \\
\hline
\end{tabular}

\section{Experimental Values}

The measured solubility was reported to be $-\log _{10} c_{1}=0.674$, which corresponds to a solubility of $c_{1}=0.212 \mathrm{~mol} \mathrm{dm}^{-3}$.

\section{Auxiliary Information}

\section{Method/Apparatus/Procedure:}

Very little experimental details were provided. Excess solute and solvent were placed in tubes, which were rotated for at least $8 \mathrm{~h}$ at $298 \mathrm{~K}$. The solutions were then removed, concentrated if necessary, and titrated with standard alkali using a mixed (bromothymol blue + neutral red) indicator.

Source and Purity of Chemicals:

(1) Purity not given, Chemical source not given, solute was either "AnalaR" grade or recrystallized prior to use.

(2) Purity not given, Chemical source not given, no purification details were provided.

Estimated Error:

Temperature: No information was given.

$c_{1}: \pm 4 \%$ (relative error, estimated by compiler).

\subsection{2-Methoxybenzoic acid solubility data in esters}

\begin{tabular}{ll}
\hline \hline Components: & Original Measurements: \\
(1) 2-Methoxybenzoic acid; $\mathrm{C}_{8} \mathrm{H}_{8} \mathrm{O}_{3} ;$ & ${ }^{9}$ K. R. Hoover, D. M. Stovall, E. \\
[579-75-9] & Pustejovsky, R. Coaxum, K. Pop, \\
(2) Methyl ethanoate; $\mathrm{C}_{3} \mathrm{H}_{6} \mathrm{O}_{2} ;$ & W. E. Acree, Jr., and M. H. \\
[79-20-9] & Abraham, Can. J. Chem. 82, 1353 \\
& $(2004)$. \\
\hline Variables: & Prepared by: \\
$T / \mathrm{K}=298.15$ & W. E. Acree, Jr. \\
\hline
\end{tabular}

Experimental Values

\begin{tabular}{lc}
\hline \hline$x_{2}{ }^{\mathrm{a}}$ & $x_{1}{ }^{\mathrm{b}}$ \\
\hline 0.9267 & 0.0733 \\
\hline${ }^{a} x_{2}:$ mole fraction of component 2 in the saturated solution. & \\
${ }^{\mathrm{b}} x_{1}:$ mole fraction solubility of the solute. &
\end{tabular}




\section{Auxiliary Information}

\section{Method/Apparatus/Procedure:}

Constant-temperature bath, calorimetric thermometer, and an ultraviolet/ visible spectrophotometer.

Excess solute and solvent were placed in amber glass bottles and allowed to equilibrate for several days at constant temperature. Attainment of equilibrium was verified by several repetitive measurements and by approaching equilibrium from supersaturation. Aliquots of saturated solutions were transferred through a coarse filter into tared volumetric flasks, weighed, and diluted with methanol. Concentrations were determined by

spectrophotometric measurements at $295 \mathrm{~nm}$.

Source and Purity of Chemicals:

(1) $99 \%$, Aldrich Chemical Company, Milwaukee, WI, USA, was used as received.

(2) $99.5 \%$, anhydrous, Aldrich Chemical Company, stored over molecular sieves and distilled shortly before use.

Estimated Error:

Temperature: $\pm 0.1 \mathrm{~K}$

$x_{1}: \pm 1.5 \%$ (relative error).

\begin{tabular}{ll}
\hline \hline Components: & Original Measurements: \\
(1) 2-Methoxybenzoic acid; $\mathrm{C}_{8} \mathrm{H}_{8} \mathrm{O}_{3} ;$ & ${ }^{9}$ K. R. Hoover, D. M. Stovall, E. \\
[579-75-9] & Pustejovsky, R. Coaxum, K. Pop, \\
(2) Ethyl ethanoate; $\mathrm{C}_{4} \mathrm{H}_{8} \mathrm{O}_{2} ;$ & W. E. Acree, Jr., and M. H. \\
[141-78-6] & Abraham, Can. J. Chem. 82, 1353 \\
& $(2004)$. \\
\hline Variables: & Prepared by: \\
$T / \mathrm{K}=298.15$ & W. E. Acree, Jr. \\
\hline
\end{tabular}

\section{Experimental Values}

\begin{tabular}{|c|c|}
\hline$x_{2}{ }^{\mathrm{a}}$ & $x_{1}{ }^{\mathrm{b}}$ \\
\hline 0.9345 & 0.0655 \\
\hline
\end{tabular}

${ }^{\mathrm{a}} x_{2}$ : mole fraction of component 2 in the saturated solution.

${ }^{\mathrm{b}} x_{1}$ : mole fraction solubility of the solute.

\section{Auxiliary Information}

\section{Method/Apparatus/Procedure:}

Constant-temperature bath, calorimetric thermometer, and an ultraviolet/ visible spectrophotometer.

Excess solute and solvent were placed in amber glass bottles and allowed to equilibrate for several days at constant temperature. Attainment of equilibrium was verified by several repetitive measurements and by approaching equilibrium from supersaturation. Aliquots of saturated solutions were transferred through a coarse filter into tared volumetric flasks, weighed, and diluted with methanol. Concentrations were determined by

spectrophotometric measurements at $295 \mathrm{~nm}$.

Source and Purity of Chemicals:

(1) $99 \%$, Aldrich Chemical Company, Milwaukee, WI, USA, was used as received.

(2) $99.9 \%$, HPLC grade, Aldrich Chemical Company, stored over molecular sieves and distilled shortly before use.

Estimated Error:

Temperature: $\pm 0.1 \mathrm{~K}$.

$x_{1}: \pm 1.5 \%$ (relative error).

\begin{tabular}{ll}
\hline \hline Components: & Original Measurements: \\
(1) 2-Methoxybenzoic acid; $\mathrm{C}_{8} \mathrm{H}_{8} \mathrm{O}_{3} ;$ & ${ }^{9}$ K. R. Hoover, D. M. Stovall, E. \\
[579-75-9] & Pustejovsky, R. Coaxum, K. Pop, \\
(2) Butyl ethanoate; $\mathrm{C}_{6} \mathrm{H}_{12} \mathrm{O}_{2} ;$ & W. E. Acree, Jr., and M. H. \\
[123-86-4] & $\begin{array}{l}\text { Abraham, Can. J. Chem. 82, 1353 } \\
(2004) .\end{array}$ \\
\hline Variables: & Prepared by: \\
$T / \mathrm{K}=298.15$ & W. E. Acree, Jr. \\
\hline
\end{tabular}

Experimental Values

\begin{tabular}{lc}
\hline \hline$x_{2}{ }^{\mathrm{a}}$ & $x_{1}{ }^{\mathrm{b}}$ \\
\hline 0.9555 & 0.0445 \\
\hline${ }^{\mathrm{a}} x_{2}:$ mole fraction of component 2 in the saturated solution. & \\
${ }^{\mathrm{b}} x_{1}:$ mole fraction solubility of the solute. &
\end{tabular}

\section{Auxiliary Information}

\section{Method/Apparatus/Procedure:}

Constant-temperature bath, calorimetric thermometer, and an ultraviolet/ visible spectrophotometer.

Excess solute and solvent were placed in amber glass bottles and allowed to equilibrate for several days at constant temperature. Attainment of equilibrium was verified by several repetitive measurements and by approaching equilibrium from supersaturation. Aliquots of saturated solutions were transferred through a coarse filter into tared volumetric flasks, weighed, and diluted with methanol. Concentrations were determined by spectrophotometric measurements at $295 \mathrm{~nm}$.

Source and Purity of Chemicals:

(1) $99 \%$, Aldrich Chemical Company, Milwaukee, WI, USA, was used as received.

(2) 99.7\%, HPLC grade, Aldrich Chemical Company, stored over molecular sieves and distilled shortly before use.

\section{Estimated Error:}

Temperature: $\pm 0.1 \mathrm{~K}$.

$x_{1}: \pm 1.5 \%$ (relative error)

\begin{tabular}{ll}
\hline \hline Components: & Original Measurements: \\
(1) 2-Methoxybenzoic acid; $\mathrm{C}_{8} \mathrm{H}_{8} \mathrm{O}_{3} ;$ & ${ }^{9}$ K. R. Hoover, D. M. Stovall, E. \\
[579-75-9] & Pustejovsky, R. Coaxum, K. Pop, \\
(2) Pentyl ethanoate; $\mathrm{C}_{7} \mathrm{H}_{14} \mathrm{O}_{2} ;$ & W. E. Acree, Jr., and M. H. \\
[628-63-7] & $\begin{array}{l}\text { Abraham, Can. J. Chem. 82, 1353 } \\
\end{array}$ \\
\hline (2004). \\
\hline Variables: & Prepared by: \\
$T / \mathrm{K}=298.15$ & W. E. Acree, Jr. \\
\hline
\end{tabular}

Experimental Values

\begin{tabular}{lc}
\hline \hline$x_{2}{ }^{\mathrm{a}}$ & $x_{1}{ }^{\mathrm{b}}$ \\
\hline 0.9621 & 0.0379 \\
\hline${ }^{\mathrm{a}} x_{2}:$ mole fraction of component 2 in the saturated solution. & \\
${ }^{\mathrm{b}} x_{1}:$ mole fraction solubility of the solute. &
\end{tabular}




\section{Auxiliary Information}

\section{Method/Apparatus/Procedure:}

Constant-temperature bath, calorimetric thermometer, and an ultraviolet/ visible spectrophotometer.

Excess solute and solvent were placed in amber glass bottles and allowed to equilibrate for several days at constant temperature. Attainment of equilibrium was verified by several repetitive measurements and by approaching equilibrium from supersaturation. Aliquots of saturated solutions were transferred through a coarse filter into tared volumetric flasks, weighed, and diluted with methanol. Concentrations were determined by

spectrophotometric measurements at $295 \mathrm{~nm}$.

Source and Purity of Chemicals:

(1) $99 \%$, Aldrich Chemical Company, Milwaukee, WI, USA, was used as received.

(2) $99 \%$, Aldrich Chemical Company, stored over molecular sieves and distilled shortly before use.

Estimated Error:

Temperature: $\pm 0.1 \mathrm{~K}$

$x_{1}: \pm 1.5 \%$ (relative error).

\subsection{2-Methoxybenzoic acid solubility data in ethers}

\begin{tabular}{|c|c|}
\hline $\begin{array}{l}\text { Components: } \\
\text { (1) 2-Methoxybenzoic acid; } \mathrm{C}_{8} \mathrm{H}_{8} \mathrm{O}_{3} \text {; } \\
\text { [579-75-9] } \\
\text { (2) } 1,1^{\prime}-\text { Oxybisethane; } \mathrm{C}_{4} \mathrm{H}_{10} \mathrm{O} \text {; } \\
\text { [60-29-7] }\end{array}$ & $\begin{array}{l}\text { Original Measurements: } \\
{ }^{9} \text { K. R. Hoover, D. M. Stovall, E. } \\
\text { Pustejovsky, R. Coaxum, K. Pop, } \\
\text { W. E. Acree, Jr., and M. H. } \\
\text { Abraham, Can. J. Chem. 82, } 1353 \\
\text { (2004). }\end{array}$ \\
\hline $\begin{array}{l}\text { Variables: } \\
T / \mathrm{K}=298.15\end{array}$ & $\begin{array}{l}\text { Prepared by: } \\
\text { W. E. Acree, Jr. }\end{array}$ \\
\hline
\end{tabular}

Experimental Values

\begin{tabular}{lc}
\hline \hline$x_{2}{ }^{\mathrm{a}}$ & $x_{1}^{\mathrm{b}}$ \\
\hline 0.9757 & 0.0243 \\
\hline${ }^{\mathrm{a}} x_{2}:$ mole fraction of component 2 in the saturated solution. \\
${ }^{\mathrm{b}} x_{1}:$ mole fraction solubility of the solute.
\end{tabular}

\section{Auxiliary Information}

\section{Method/Apparatus/Procedure:}

Constant-temperature bath, calorimetric thermometer, and an ultraviolet/ visible spectrophotometer.

Excess solute and solvent were placed in amber glass bottles and allowed to equilibrate for several days at constant temperature. Attainment of equilibrium was verified by several repetitive measurements and by approaching equilibrium from supersaturation. Aliquots of saturated solutions were transferred through a coarse filter into tared volumetric flasks, weighed, and diluted with methanol. Concentrations were determined by

spectrophotometric measurements at $295 \mathrm{~nm}$.

Source and Purity of Chemicals:

(1) $99 \%$, Aldrich Chemical Company, Milwaukee, WI, USA, was used as received.

(2) $99+\%$, anhydrous, Aldrich Chemical Company, stored over molecular sieves and distilled shortly before use.

\section{Estimated Error:}

Temperature: $\pm 0.1 \mathrm{~K}$.

$x_{1}: \pm 1.5 \%$ (relative error)

\begin{tabular}{ll}
\hline \hline Components: & Original Measurements: \\
(1) 2-Methoxybenzoic acid; $\mathrm{C}_{8} \mathrm{H}_{8} \mathrm{O}_{3} ;$ & ${ }^{9}$ K. R. Hoover, D. M. Stovall, E. \\
[579-75-9] & Pustejovsky, R. Coaxum, K. Pop, \\
$\begin{array}{l}\text { (2) 2,2'-Oxybispropane; } \mathrm{C}_{6} \mathrm{H}_{14} \mathrm{O} ; \\
{[108-20-3]}\end{array}$ & W. E. Acree, Jr., and M. H. \\
& $\begin{array}{l}\text { Abraham, Can. J. Chem. 82, 1353 } \\
(2004) .\end{array}$ \\
\hline Variables: & Prepared by: \\
$T / \mathrm{K}=298.15$ & W. E. Acree, Jr. \\
\hline
\end{tabular}

Experimental Values

\begin{tabular}{lc}
\hline \hline$x_{2}{ }^{\mathrm{a}}$ & $x_{1}{ }^{\mathrm{b}}$ \\
\hline 0.9916 & 0.00838 \\
\hline${ }^{\mathrm{a}} x_{2}:$ mole fraction of component 2 in the saturated solution. & \\
${ }^{\mathrm{b}} x_{1}:$ mole fraction solubility of the solute. &
\end{tabular}

Auxiliary Information

Method/Apparatus/Procedure:

Constant-temperature bath, calorimetric thermometer, and an ultraviolet/ visible spectrophotometer.

Excess solute and solvent were placed in amber glass bottles and allowed to equilibrate for several days at constant temperature. Attainment of equilibrium was verified by several repetitive measurements and by approaching equilibrium from supersaturation. Aliquots of saturated solutions were transferred through a coarse filter into tared volumetric flasks, weighed, and diluted with methanol. Concentrations were determined by spectrophotometric measurements at $295 \mathrm{~nm}$.

Source and Purity of Chemicals:

(1) $99 \%$, Aldrich Chemical Company, Milwaukee, WI, USA, was used as received.

(2) $99 \%$, anhydrous, Aldrich Chemical Company, stored over molecular sieves and distilled shortly before use.

\section{Estimated Error:}

Temperature: $\pm 0.1 \mathrm{~K}$.

$x_{1}: \pm 1.5 \%$ (relative error).

\begin{tabular}{ll}
\hline \hline Components: & Original Measurements: \\
(1) 2-Methoxybenzoic acid; $\mathrm{C}_{8} \mathrm{H}_{8} \mathrm{O}_{3} ;$ & ${ }^{9}$ K. R. Hoover, D. M. Stovall, E. \\
[579-75-9] & Pustejovsky, R. Coaxum, K. Pop, \\
(2) 1,1'-Oxybisbutane; $\mathrm{C}_{8} \mathrm{H}_{18} \mathrm{O} ;$ & W. E. Acree, Jr., and M. H. \\
[142-96-1] & Abraham, Can. J. Chem. 82, 1353 \\
& $(2004)$. \\
\hline Variables: & Prepared by: \\
$T / \mathrm{K}=298.15$ & W. E. Acree, Jr. \\
\hline
\end{tabular}

Experimental Values

\begin{tabular}{lc}
\hline \hline$x_{2}{ }^{\mathrm{a}}$ & $x_{1}{ }^{\mathrm{b}}$ \\
\hline 0.9940 & 0.00603 \\
\hline${ }^{\mathrm{a}} x_{2}:$ mole fraction of component 2 in the saturated solution. & \\
${ }^{\mathrm{b}}{ }_{x_{1}}$ : mole fraction solubility of the solute. &
\end{tabular}




\section{Auxiliary Information}

\section{Method/Apparatus/Procedure:}

Constant-temperature bath, calorimetric thermometer, and an ultraviolet/ visible spectrophotometer.

Excess solute and solvent were placed in amber glass bottles and allowed to equilibrate for several days at constant temperature. Attainment of equilibrium was verified by several repetitive measurements and by approaching equilibrium from supersaturation. Aliquots of saturated solutions were transferred through a coarse filter into tared volumetric flasks, weighed, and diluted with methanol. Concentrations were determined by

spectrophotometric measurements at $295 \mathrm{~nm}$.

Source and Purity of Chemicals:

(1) $99 \%$, Aldrich Chemical Company, Milwaukee, WI, USA, was used as received.

(2) $99.3 \%$, anhydrous, Aldrich Chemical Company, stored over molecular sieves and distilled shortly before use.

Estimated Error:

Temperature: $\pm 0.1 \mathrm{~K}$

$x_{1}: \pm 1.5 \%$ (relative error).

\begin{tabular}{ll}
\hline \hline Components: & Original Measurements: \\
(1) 2-Methoxybenzoic acid; $\mathrm{C}_{8} \mathrm{H}_{8} \mathrm{O}_{3} ;$ & ${ }^{9}$ K. R. Hoover, D. M. Stovall, E. \\
[579-75-9] & Pustejovsky, R. Coaxum, K. Pop, \\
$\begin{array}{l}\text { (2) Tetrahydrofuran; } \mathrm{C}_{4} \mathrm{H}_{8} \mathrm{O} ; \\
\text { [109-99-9] }\end{array}$ & W. E. Acree, Jr., and M. H. \\
& Abraham, Can. J. Chem. 82, 1353 \\
& $(2004)$. \\
\hline Variables: & Prepared by: \\
$T / \mathrm{K}=298.15$ & W. E. Acree, Jr. \\
\hline
\end{tabular}

Experimental Values

\begin{tabular}{lc}
\hline \hline$x_{2}{ }^{\mathrm{a}}$ & $x_{1}{ }^{\mathrm{b}}$ \\
\hline 0.8226 & 0.1774 \\
\hline
\end{tabular}

${ }^{\mathrm{a}} x_{2}$ : mole fraction of component 2 in the saturated solution.

${ }^{\mathrm{b}} x_{1}$ : mole fraction solubility of the solute.

\section{Auxiliary Information}

\section{Method/Apparatus/Procedure:}

Constant-temperature bath, calorimetric thermometer, and an ultraviolet/ visible spectrophotometer.

Excess solute and solvent were placed in amber glass bottles and allowed to equilibrate for several days at constant temperature. Attainment of equilibrium was verified by several repetitive measurements and by approaching equilibrium from supersaturation. Aliquots of saturated solutions were transferred through a coarse filter into tared volumetric flasks, weighed, and diluted with methanol. Concentrations were determined by spectrophotometric measurements at $295 \mathrm{~nm}$.

Source and Purity of Chemicals:

(1) $99 \%$, Aldrich Chemical Company, Milwaukee, WI, USA, was used as received.

(2) $99.9 \%$, anhydrous, Aldrich Chemical Company, stored over molecular sieves and distilled shortly before use.

Estimated Error:

Temperature: $\pm 0.1 \mathrm{~K}$.

$x_{1}: \pm 1.5 \%$ (relative error).

\begin{tabular}{ll}
\hline \hline Components: & Original Measurements: \\
(1) 2-Methoxybenzoic acid; $\mathrm{C}_{8} \mathrm{H}_{8} \mathrm{O}_{3} ;$ & ${ }^{9}$ K. R. Hoover, D. M. Stovall, E. \\
[579-75-9] & Pustejovsky, R. Coaxum, K. Pop, \\
(2) 1,4-Dioxane; $\mathrm{C}_{4} \mathrm{H}_{8} \mathrm{O}_{2} ;$ [123-91-1] & W. E. Acree, Jr., and M. H. \\
& $\begin{array}{l}\text { Abraham, Can. J. Chem. 82, 1353 } \\
(2004) .\end{array}$ \\
\hline Variables: & Prepared by: \\
$T / \mathrm{K}=298.15$ & W. E. Acree, Jr. \\
\hline
\end{tabular}

Experimental Values

\begin{tabular}{lc}
\hline \hline$x_{2}{ }^{\mathrm{a}}$ & $x_{1}{ }^{\mathrm{b}}$ \\
\hline 0.8559 & 0.1441 \\
\hline${ }^{\mathrm{a}} x_{2}:$ mole fraction of component 2 in the saturated solution. & \\
${ }^{\mathrm{b}} x_{1}:$ mole fraction solubility of the solute. &
\end{tabular}

Auxiliary Information

Method/Apparatus/Procedure:

Constant-temperature bath, calorimetric thermometer, and an ultraviolet/ visible spectrophotometer.

Excess solute and solvent were placed in amber glass bottles and allowed to equilibrate for several days at constant temperature. Attainment of equilibrium was verified by several repetitive measurements and by approaching equilibrium from supersaturation. Aliquots of saturated solutions were transferred through a coarse filter into tared volumetric flasks, weighed, and diluted with methanol. Concentrations were determined by spectrophotometric measurements at $295 \mathrm{~nm}$.

Source and Purity of Chemicals:

(1) $99 \%$, Aldrich Chemical Company, Milwaukee, WI, USA, was used as received.

(2) $99.8 \%$, anhydrous, Aldrich Chemical Company, stored over molecular sieves and distilled shortly before use.

\section{Estimated Error:}

Temperature: $\pm 0.1 \mathrm{~K}$.

$x_{1}: \pm 1.5 \%$ (relative error).

\subsection{2-Methoxybenzoic acid solubility data in haloalkanes, haloalkenes, and haloaromatic hydrocarbons}

\begin{tabular}{ll}
\hline \hline Components: & Original Measurements: \\
$\begin{array}{l}\text { (1) 2-Methoxybenzoic acid; } \mathrm{C}_{8} \mathrm{H}_{8} \mathrm{O}_{3} ; \\
\text { [579-75-9] }\end{array}$ & $\begin{array}{l}56 \\
\mathrm{P} . \text { A. Ongley, J. Chem. Soc. 1954, } \\
\text { (2) Trichloromethane; } \mathrm{CHCl}_{3} ;\end{array}$ \\
$\begin{array}{l}3634 . \\
\text { [67-66-3] }\end{array}$ & \\
\hline Variables: & Prepared by: \\
$T / \mathrm{K}=298.15$ & W. E. Acree, Jr. \\
\hline
\end{tabular}

\section{Experimental Values}

The measured solubility was reported to be $-\log _{10} c_{1}=$ -0.225 , which corresponds to a solubility of $c_{1}=$ $1.679 \mathrm{~mol} \mathrm{dm}^{-3}$. 


\section{Auxiliary Information}

\section{Method/Apparatus/Procedure:}

Very little experimental details were provided. Excess solute and solvent were placed in tubes, which were rotated for at least $8 \mathrm{~h}$ at $298 \mathrm{~K}$. The solutions were then removed, concentrated if necessary, and titrated with standard alkali using a mixed (bromothymol blue + neutral red) indicator.

Source and Purity of Chemicals:

(1) Purity not given, Chemical source not given, solute was either "AnalaR" grade or recrystallized prior to use.

(2) Purity not given, Chemical source not given, no purification details were provided.

Estimated Error:

Temperature: No information was given. $c_{1}: \pm 4 \%$ (relative error, estimated by compiler).

\begin{tabular}{|c|c|}
\hline $\begin{array}{l}\text { Components: } \\
\text { (1) 2-Methoxybenzoic acid; } \mathrm{C}_{8} \mathrm{H}_{8} \mathrm{O}_{3} \text {; } \\
\text { [579-75-9] } \\
\text { (2) Tetrachloromethane; } \mathrm{CCl}_{4} \text {; } \\
\text { [56-23-5] }\end{array}$ & $\begin{array}{l}\text { Original Measurements: } \\
{ }^{56} \text { P. A. Ongley, J. Chem. Soc. 1954, } \\
3634 .\end{array}$ \\
\hline $\begin{array}{l}\text { Variables: } \\
T / \mathrm{K}=298.15\end{array}$ & $\begin{array}{l}\text { Prepared by: } \\
\text { W. E. Acree, Jr. }\end{array}$ \\
\hline
\end{tabular}

\section{Experimental Values}

The measured solubility was reported to be $-\log _{10} c_{1}=$ 1.605, which corresponds to a solubility of $c_{1}=$ $0.0248 \mathrm{~mol} \mathrm{dm}^{-3}$.

\section{Auxiliary Information}

\footnotetext{
Method/Apparatus/Procedure:

Very little experimental details were provided. Excess solute and solvent were placed in tubes, which were rotated for at least $8 \mathrm{~h}$ at $298 \mathrm{~K}$. The solutions were then removed, concentrated if necessary, and titrated with standard alkali using a mixed (bromothymol blue + neutral red) indicator.
}

\section{Source and Purity of Chemicals:}

(1) Purity not given, Chemical source not given, solute was either "AnalaR" grade or recrystallized prior to use.

(2) Purity not given, Chemical source not given, no purification details were provided.

Estimated Error:

Temperature: No information was given.

$c_{1}: \pm 4 \%$ (relative error, estimated by compiler).

\subsection{2-Methoxybenzoic acid solubility data in alcohols}

\section{Components:}

(1) 2-Methoxybenzoic acid; $\mathrm{C}_{8} \mathrm{H}_{8} \mathrm{O}_{3}$; [579-75-9]

(2) Methanol; $\mathrm{CH}_{4} \mathrm{O} ;[67-56-1]$
Original Measurements:

${ }^{9}$ K. R. Hoover, D. M. Stovall, E. Pustejovsky, R. Coaxum, K. Pop, W. E. Acree, Jr., and M. H. Abraham, Can. J. Chem. 82, 1353 (2004).

Prepared by:

W. E. Acree, Jr
Experimental Values

\begin{tabular}{lc}
\hline \hline$x_{2}{ }^{\mathrm{a}}$ & $x_{1}{ }^{\mathrm{b}}$ \\
\hline 0.9089 & 0.0911 \\
\hline${ }^{\mathrm{a}} x_{2}:$ mole fraction of component 2 in the saturated solution. & \\
${ }^{\mathrm{b}} x_{1}:$ mole fraction solubility of the solute. &
\end{tabular}

\section{Auxiliary Information}

\section{Method/Apparatus/Procedure:}

Constant-temperature bath, calorimetric thermometer, and an ultraviolet/ visible spectrophotometer.

Excess solute and solvent were placed in amber glass bottles and allowed to equilibrate for several days at constant temperature. Attainment of equilibrium was verified by several repetitive measurements and by approaching equilibrium from supersaturation. Aliquots of saturated solutions were transferred through a coarse filter into tared volumetric flasks, weighed, and diluted with methanol. Concentrations were determined by spectrophotometric measurements at $295 \mathrm{~nm}$.

\section{Source and Purity of Chemicals:}

(1) $99 \%$, Aldrich Chemical Company, Milwaukee, WI, USA, was used as received.

(2) $99.8 \%$, anhydrous, Aldrich Chemical Company, stored over molecular sieves and distilled shortly before use.

\section{Estimated Error:}

Temperature: $\pm 0.1 \mathrm{~K}$.

$x_{1}: \pm 1.5 \%$ (relative error).

\begin{tabular}{ll}
\hline \hline Components: & Original Measurements: \\
(1) 2-Methoxybenzoic acid; $\mathrm{C}_{8} \mathrm{H}_{8} \mathrm{O}_{3} ;$ & ${ }^{9}$ K. R. Hoover, D. M. Stovall, E. \\
[579-75-9] & Pustejovsky, R. Coaxum, K. Pop, \\
(2) Ethanol; $\mathrm{C}_{2} \mathrm{H}_{6} \mathrm{O} ;$ [64-17-5] & W. E. Acree, Jr., and M. H. \\
& Abraham, Can. J. Chem. 82, 1353 \\
& $(2004)$. \\
\hline Variables: & Prepared by: \\
$T / \mathrm{K}=298.15$ & W. E. Acree, Jr. \\
\hline
\end{tabular}

Experimental Values

\begin{tabular}{lc}
\hline \hline$x_{2}{ }^{\mathrm{a}}$ & $x_{1}{ }^{\mathrm{b}}$ \\
\hline 0.9235 & 0.0765 \\
\hline${ }^{\mathrm{a}} x_{2}:$ mole fraction of component 2 in the saturated solution. & \\
${ }^{\mathrm{b}} x_{1}:$ mole fraction solubility of the solute. &
\end{tabular}

\section{Auxiliary Information}

\section{Method/Apparatus/Procedure:}

Constant-temperature bath, calorimetric thermometer, and an ultraviolet/ visible spectrophotometer.

Excess solute and solvent were placed in amber glass bottles and allowed to equilibrate for several days at constant temperature. Attainment of equilibrium was verified by several repetitive measurements and by approaching equilibrium from supersaturation. Aliquots of saturated solutions were transferred through a coarse filter into tared volumetric flasks, weighed, and diluted with methanol. Concentrations were determined by spectrophotometric measurements at $295 \mathrm{~nm}$.

\section{Variables:}

$T / \mathrm{K}=298.15$ 
Source and Purity of Chemicals:

(1) $99 \%$, Aldrich Chemical Company, Milwaukee, WI, USA, was used as received.

(2) Absolute, Aaper Alcohol and Chemical Company, stored over molecular sieves and distilled shortly before use.

\section{Estimated Error:}

Temperature: $\pm 0.1 \mathrm{~K}$.

$x_{1}: \pm 1.5 \%$ (relative error).

\begin{tabular}{|c|c|}
\hline $\begin{array}{l}\text { Components: } \\
\text { (1) 2-Methoxybenzoic acid; } \mathrm{C}_{8} \mathrm{H}_{8} \mathrm{O}_{3} \text {; } \\
\text { [579-75-9] } \\
\text { (2) Ethanol; } \mathrm{C}_{2} \mathrm{H}_{6} \mathrm{O} ;[64-17-5]\end{array}$ & $\begin{array}{l}\text { Original Measurements: } \\
{ }^{72} \text { R. Thuaire, Bull. Soc. Chim. Fr. } \\
3815 \text { (1971). }\end{array}$ \\
\hline $\begin{array}{l}\text { Variables: } \\
T / \mathrm{K}=298.15\end{array}$ & $\begin{array}{l}\text { Prepared by: } \\
\text { W. E. Acree, Jr. }\end{array}$ \\
\hline
\end{tabular}

Experimental Values

The measured solubility was reported to be $1.780 \mathrm{~mol} / \mathrm{kg}$ of solvent, which corresponds to a mole fraction solubility of $x_{1}=0.07579$.

\section{Auxiliary Information}

\section{Method/Apparatus/Procedure:}

Very little experimental details were provided. Solubility was determined by a gravimetric method. The saturated solution was evaporated to dryness and the remaining solid residue was weighed. The solubility was calculated from the mass of the solid residue and mass of saturated solution analyzed.

Source and Purity of Chemicals:

(1) Purity not given, Chemical source not given, no purification details were provided.

(2) Purity not given, Chemical source not given, no purification details were provided.

\section{Estimated Error:}

Temperature: $\pm 0.05 \mathrm{~K}$.

$x_{1}: \pm 4 \%$ (relative error, estimated by compiler).

\begin{tabular}{|c|c|}
\hline $\begin{array}{l}\text { Components: } \\
\text { (1) 2-Methoxybenzoic acid; } \mathrm{C}_{8} \mathrm{H}_{8} \mathrm{O}_{3} \text {; } \\
\text { [579-75-9] } \\
\text { (2) 1-Propanol; } \mathrm{C}_{3} \mathrm{H}_{8} \mathrm{O} ;[71-23-8]\end{array}$ & $\begin{array}{l}\text { Original Measurements: } \\
{ }^{9} \text { K. R. Hoover, D. M. Stovall, E. } \\
\text { Pustejovsky, R. Coaxum, K. Pop, } \\
\text { W. E. Acree, Jr., and M. H. } \\
\text { Abraham, Can. J. Chem. 82, } 1353 \\
\text { (2004). }\end{array}$ \\
\hline $\begin{array}{l}\text { Variables: } \\
T / \mathrm{K}=298.15\end{array}$ & $\begin{array}{l}\text { Prepared by: } \\
\text { W. E. Acree, Jr. }\end{array}$ \\
\hline
\end{tabular}

\section{Experimental Values}

\begin{tabular}{lc}
\hline \hline$x_{2}{ }^{\mathrm{a}}$ & $x_{1}{ }^{\mathrm{b}}$ \\
\hline 0.9405 & 0.0595 \\
\hline
\end{tabular}

${ }^{\mathrm{a}} x_{2}$ : mole fraction of component 2 in the saturated solution.

${ }^{b} x_{1}$ : mole fraction solubility of the solute.

\section{Auxiliary Information}

Method/Apparatus/Procedure:

Constant-temperature bath, calorimetric thermometer, and an ultraviolet/ visible spectrophotometer.

Excess solute and solvent were placed in amber glass bottles and allowed to equilibrate for several days at constant temperature. Attainment of equilibrium was verified by several repetitive measurements and by approaching equilibrium from supersaturation. Aliquots of saturated solutions were transferred through a coarse filter into tared volumetric flasks, weighed, and diluted with methanol. Concentrations were determined by spectrophotometric measurements at $295 \mathrm{~nm}$.

\section{Source and Purity of Chemicals:}

(1) $99 \%$, Aldrich Chemical Company, Milwaukee, WI, USA, was used as received.

(2) $99+\%$, anhydrous, Aldrich Chemical Company, stored over molecular sieves and distilled shortly before use.

Estimated Error:

Temperature: $\pm 0.1 \mathrm{~K}$.

$x_{1}: \pm 1.5 \%$ (relative error)

\begin{tabular}{ll}
\hline \hline Components: & Original Measurements: \\
(1) 2-Methoxybenzoic acid; $\mathrm{C}_{8} \mathrm{H}_{8} \mathrm{O}_{3} ;$ & ${ }^{9}$ K. R. Hoover, D. M. Stovall, E. \\
[579-75-9] & Pustejovsky, R. Coaxum, K. Pop, \\
(2) 2-Propanol; $\mathrm{C}_{3} \mathrm{H}_{8} \mathrm{O} ;$ [67-63-0] & W. E. Acree, Jr., and M. H. \\
& Abraham, Can. J. Chem. 82, 1353 \\
& $(2004)$. \\
\hline Variables: & Prepared by: \\
$T / \mathrm{K}=298.15$ & W. E. Acree, Jr. \\
\hline
\end{tabular}

\section{Experimental Values}

\begin{tabular}{lc}
\hline \hline$x_{2}{ }^{\mathrm{a}}$ & $x_{1}{ }^{\mathrm{b}}$ \\
\hline 0.9537 & 0.0463 \\
\hline${ }^{{ }^{a} x_{2}: \text { mole fraction of component } 2 \text { in the saturated solution. }}$ \\
${ }^{\mathrm{b}} x_{1}:$ mole fraction solubility of the solute.
\end{tabular}

\section{Auxiliary Information}

\section{Method/Apparatus/Procedure:}

Constant-temperature bath, calorimetric thermometer, and an ultraviolet/ visible spectrophotometer.

Excess solute and solvent were placed in amber glass bottles and allowed to equilibrate for several days at constant temperature. Attainment of equilibrium was verified by several repetitive measurements and by approaching equilibrium from supersaturation. Aliquots of saturated solutions were transferred through a coarse filter into tared volumetric flasks, weighed, and diluted with methanol. Concentrations were determined by spectrophotometric measurements at $295 \mathrm{~nm}$.

\section{Source and Purity of Chemicals:}

(1) $99 \%$, Aldrich Chemical Company, Milwaukee, WI, USA, was used as received.

(2) $99+\%$, anhydrous, Aldrich Chemical Company, stored over molecular sieves and distilled shortly before use.

\section{Estimated Error:}

Temperature: $\pm 0.1 \mathrm{~K}$.

$x_{1}: \pm 1.5 \%$ (relative error) 


\section{Components:}

(1) 2-Methoxybenzoic acid; $\mathrm{C}_{8} \mathrm{H}_{8} \mathrm{O}_{3}$; [579-75-9]

(2) 1-Butanol; $\mathrm{C}_{4} \mathrm{H}_{10} \mathrm{O} ;[$ [11-36-3]

\section{Variables:}

W. E. Acree, Jr. (2004).

Prepared by:
$T / \mathrm{K}=298.15$

Original Measurements:

${ }^{9}$ K. R. Hoover, D. M. Stovall, E. Pustejovsky, R. Coaxum, K. Pop, W. E. Acree, Jr., and M. H. Abraham, Can. J. Chem. 82, 1353

Experimental Values

\begin{tabular}{lc}
\hline \hline$x_{2}{ }^{\mathrm{a}}$ & $x_{1}{ }^{\mathrm{b}}$ \\
\hline 0.9466 & 0.0534 \\
\hline${ }^{\mathrm{a}} x_{2}:$ mole fraction of component 2 in the saturated solution. \\
${ }^{\mathrm{b}}{ }_{x_{1}}:$ mole fraction solubility of the solute.
\end{tabular}

\section{Auxiliary Information}

\section{Method/Apparatus/Procedure:}

Constant-temperature bath, calorimetric thermometer, and an ultraviolet/ visible spectrophotometer.

Excess solute and solvent were placed in amber glass bottles and allowed to equilibrate for several days at constant temperature. Attainment of equilibrium was verified by several repetitive measurements and by approaching equilibrium from supersaturation. Aliquots of saturated solutions were transferred through a coarse filter into tared volumetric flasks, weighed, and diluted with methanol. Concentrations were determined by

spectrophotometric measurements at $295 \mathrm{~nm}$.

\section{Source and Purity of Chemicals:}

(1) $99 \%$, Aldrich Chemical Company, Milwaukee, WI, USA, was used as received.

(2) $99.8+\%$, HPLC grade, Aldrich Chemical Company, stored over molecular sieves and distilled shortly before use.

\section{Estimated Error:}

Temperature: $\pm 0.1 \mathrm{~K}$.

$x_{1}: \pm 1.5 \%$ (relative error).

\section{Components:}

(1) 2-Methoxybenzoic acid; $\mathrm{C}_{8} \mathrm{H}_{8} \mathrm{O}_{3}$; [579-75-9]

(2) 2-Butanol; $\mathrm{C}_{4} \mathrm{H}_{10} \mathrm{O}$; [78-92-2]

\section{Variables:}

$T / \mathrm{K}=298.15$
Original Measurements:

${ }^{9}$ K. R. Hoover, D. M. Stovall, E. Pustejovsky, R. Coaxum, K. Pop, W. E. Acree, Jr., and M. H. Abraham, Can. J. Chem. 82, 1353 (2004).

\section{Prepared by:}

W. E. Acree, Jr.

\section{Experimental Values}

\begin{tabular}{lc}
\hline \hline$x_{2}^{\mathrm{a}}$ & $x_{1}^{\mathrm{b}}$ \\
\hline 0.9526 & 0.0474 \\
\hline${ }^{\mathrm{a}} x_{2}:$ mole fraction of component 2 in the saturated solution. \\
${ }^{\mathrm{b}} x_{1}:$ mole fraction solubility of the solute.
\end{tabular}

\section{Auxiliary Information}

\section{Method/Apparatus/Procedure:}

Constant-temperature bath, calorimetric thermometer, and an ultraviolet/ visible spectrophotometer.

Excess solute and solvent were placed in amber glass bottles and allowed to equilibrate for several days at constant temperature. Attainment of equilibrium was verified by several repetitive measurements and by approaching equilibrium from supersaturation. Aliquots of saturated solutions were transferred through a coarse filter into tared volumetric flasks, weighed, and diluted with methanol. Concentrations were determined by spectrophotometric measurements at $295 \mathrm{~nm}$.

Source and Purity of Chemicals:

(1) $99 \%$, Aldrich Chemical Company, Milwaukee, WI, USA, was used as received.

(2) 99+\%, anhydrous, Aldrich Chemical Company, stored over molecular sieves and distilled shortly before use.

Estimated Error:

Temperature: $\pm 0.1 \mathrm{~K}$.

$x_{1}: \pm 1.5 \%$ (relative error)

\begin{tabular}{|c|c|}
\hline $\begin{array}{l}\text { Components: } \\
\text { (1) 2-Methoxybenzoic acid; } \mathrm{C}_{8} \mathrm{H}_{8} \mathrm{O}_{3} \text {; } \\
\text { [579-75-9] } \\
\text { (2) 2-Methyl-1-propanol; } \mathrm{C}_{4} \mathrm{H}_{10} \mathrm{O} \text {; } \\
\text { [78-83-1] }\end{array}$ & $\begin{array}{l}\text { Original Measurements: } \\
{ }^{9} \text { K. R. Hoover, D. M. Stovall, E. } \\
\text { Pustejovsky, R. Coaxum, K. Pop, } \\
\text { W. E. Acree, Jr., and M. H. } \\
\text { Abraham, Can. J. Chem. 82, } 1353 \\
\text { (2004). }\end{array}$ \\
\hline $\begin{array}{l}\text { Variables: } \\
T / \mathrm{K}=298.15\end{array}$ & $\begin{array}{l}\text { Prepared by: } \\
\text { W. E. Acree, Jr. }\end{array}$ \\
\hline
\end{tabular}

\section{Experimental Values}

\begin{tabular}{lc}
\hline \hline$x_{2}{ }^{\mathrm{a}}$ & $x_{1}{ }^{\mathrm{b}}$ \\
\hline 0.9617 & 0.0383 \\
\hline${ }^{\mathrm{a}} x_{2}:$ mole fraction of component 2 in the saturated solution. & \\
${ }^{\mathrm{b}} x_{1}:$ mole fraction solubility of the solute. &
\end{tabular}

\section{Auxiliary Information}

\section{Method/Apparatus/Procedure:}

Constant-temperature bath, calorimetric thermometer, and an ultraviolet/ visible spectrophotometer.

Excess solute and solvent were placed in amber glass bottles and allowed to equilibrate for several days at constant temperature. Attainment of equilibrium was verified by several repetitive measurements and by approaching equilibrium from supersaturation. Aliquots of saturated solutions were transferred through a coarse filter into tared volumetric flasks, weighed, and diluted with methanol. Concentrations were determined by spectrophotometric measurements at $295 \mathrm{~nm}$.

\section{Source and Purity of Chemicals:}

(1) $99 \%$, Aldrich Chemical Company, Milwaukee, WI, USA, was used as received.

(2) $99+\%$, anhydrous, Aldrich Chemical Company, stored over molecular sieves and distilled shortly before use.

Estimated Error:

Temperature: $\pm 0.1 \mathrm{~K}$.

$x_{1}: \pm 1.5 \%$ (relative error) 


\section{Components:}

(1) 2-Methoxybenzoic acid; $\mathrm{C}_{8} \mathrm{H}_{8} \mathrm{O}_{3}$; [579-75-9]

(2) 2-Methyl-2-propanol; $\mathrm{C}_{4} \mathrm{H}_{10} \mathrm{O}$; [75-65-0]

\section{Variables:}

$T / \mathrm{K}=298.15$

Original Measurements:

${ }^{9}$ K. R. Hoover, D. M. Stovall, E. Pustejovsky, R. Coaxum, K. Pop, W. E. Acree, Jr., and M. H. Abraham, Can. J. Chem. 82, 1353 (2004).

\section{Prepared by:}

W. E. Acree, Jr.

\section{Experimental Values}

\begin{tabular}{lc}
\hline \hline$x_{2}^{\mathrm{a}}$ & $x_{1}{ }^{\mathrm{b}}$ \\
\hline 0.9502 & 0.0498
\end{tabular}

${ }^{\mathrm{a}} x_{2}$ : mole fraction of component 2 in the saturated solution.

${ }^{\mathrm{b}} \mathrm{x}_{1}$ : mole fraction solubility of the solute.

\section{Auxiliary Information}

\section{Method/Apparatus/Procedure:}

Constant-temperature bath, calorimetric thermometer, and an ultraviolet/ visible spectrophotometer.

Excess solute and solvent were placed in amber glass bottles and allowed to equilibrate for several days at constant temperature. Attainment of equilibrium was verified by several repetitive measurements and by approaching equilibrium from supersaturation. Aliquots of saturated solutions were transferred through a coarse filter into tared volumetric flasks, weighed, and diluted with methanol. Concentrations were determined by spectrophotometric measurements at $295 \mathrm{~nm}$.

\section{Source and Purity of Chemicals:}

(1) $99 \%$, Aldrich Chemical Company, Milwaukee, WI, USA, was used as received.

(2) 99+\%, Arco Chemical Company, USA, stored over molecular sieves and distilled shortly before use.

\section{Estimated Error:}

Temperature: $\pm 0.1 \mathrm{~K}$.

$x_{1}: \pm 1.5 \%$ (relative error).

\section{Components:}

(1) 2-Methoxybenzoic acid; $\mathrm{C}_{8} \mathrm{H}_{8} \mathrm{O}_{3}$; [579-75-9]

(2) 1-Pentanol; $\mathrm{C}_{5} \mathrm{H}_{12} \mathrm{O}$; [71-41-0]

\section{Variables:}

$T / \mathrm{K}=298.15$
Original Measurements:

${ }^{9}$ K. R. Hoover, D. M. Stovall, E. Pustejovsky, R. Coaxum, K. Pop, W. E. Acree, Jr., and M. H. Abraham, Can. J. Chem. 82, 1353 (2004).

\section{Prepared by:}

W. E. Acree, Jr.

\section{Experimental Values}

\begin{tabular}{lc}
\hline \hline$x_{2}{ }^{\mathrm{a}}$ & $x_{1}{ }^{\mathrm{b}}$ \\
\hline 0.9481 & 0.0519 \\
\hline${ }^{\mathrm{a}} x_{2}:$ mole fraction of component 2 in the saturated solution. \\
${ }^{\mathrm{b}} x_{1}:$ mole fraction solubility of the solute.
\end{tabular}

\section{Auxiliary Information}

\section{Method/Apparatus/Procedure:}

Constant-temperature bath, calorimetric thermometer, and an ultraviolet/ visible spectrophotometer.

Excess solute and solvent were placed in amber glass bottles and allowed to equilibrate for several days at constant temperature. Attainment of equilibrium was verified by several repetitive measurements and by approaching equilibrium from supersaturation. Aliquots of saturated solutions were transferred through a coarse filter into tared volumetric flasks, weighed, and diluted with methanol. Concentrations were determined by spectrophotometric measurements at $295 \mathrm{~nm}$.

Source and Purity of Chemicals:

(1) $99 \%$, Aldrich Chemical Company, Milwaukee, WI, USA, was used as received.

(2) $99+\%$, Aldrich Chemical Company, stored over molecular sieves and distilled shortly before use.

Estimated Error:

Temperature: $\pm 0.1 \mathrm{~K}$.

$x_{1}: \pm 1.5 \%$ (relative error).

\begin{tabular}{ll}
\hline \hline Components: & Original Measurements: \\
(1) 2-Methoxybenzoic acid; $\mathrm{C}_{8} \mathrm{H}_{8} \mathrm{O}_{3} ;$ & ${ }^{9}$ K. R. Hoover, D. M. Stovall, E. \\
[579-75-9] & Pustejovsky, R. Coaxum, K. Pop, \\
(2) 2-Pentanol; $\mathrm{C}_{5} \mathrm{H}_{12} \mathrm{O} ;$ [6032-29-7] & W. E. Acree, Jr., and M. H. \\
& Abraham, Can. J. Chem. 82, 1353 \\
& $(2004)$. \\
\hline Variables: & Prepared by: \\
$T / \mathrm{K}=298.15$ & W. E. Acree, Jr. \\
\hline
\end{tabular}

\section{Experimental Values}

\begin{tabular}{lc}
\hline \hline$x_{2}{ }^{\mathrm{a}}$ & $x_{1}{ }^{\mathrm{b}}$ \\
\hline 0.9573 & 0.0427 \\
\hline$x_{2}:$ mole fraction of componen 2 in
\end{tabular}

${ }^{\mathrm{a}} x_{2}$ : mole fraction of component 2 in the saturated solution.

${ }^{\mathrm{b}} x_{1}$ : mole fraction solubility of the solute.

\section{Auxiliary Information}

\section{Method/Apparatus/Procedure:}

Constant-temperature bath, calorimetric thermometer, and an ultraviolet/ visible spectrophotometer.

Excess solute and solvent were placed in amber glass bottles and allowed to equilibrate for several days at constant temperature. Attainment of equilibrium was verified by several repetitive measurements and by approaching equilibrium from supersaturation. Aliquots of saturated solutions were transferred through a coarse filter into tared volumetric flasks, weighed, and diluted with methanol. Concentrations were determined by spectrophotometric measurements at $295 \mathrm{~nm}$.

\section{Source and Purity of Chemicals:}

(1) $99 \%$, Aldrich Chemical Company, Milwaukee, WI, USA, was used as received.

(2) $99+\%$, Acros Organics, USA, stored over molecular sieves and distilled shortly before use.

Estimated Error:

Temperature: $\pm 0.1 \mathrm{~K}$.

$x_{1}: \pm 1.5 \%$ (relative error) 


\section{Components:}

(1) 2-Methoxybenzoic acid; $\mathrm{C}_{8} \mathrm{H}_{8} \mathrm{O}_{3}$; [579-75-9]

(2) 2-Methyl-1-butanol; $\mathrm{C}_{5} \mathrm{H}_{12} \mathrm{O}$; [137-32-6]

\section{Variables:}

$T / \mathrm{K}=298.15$

W. E. Acree, Jr.

Experimental Values

\begin{tabular}{lc}
\hline \hline$x_{2}{ }^{\mathrm{a}}$ & $x_{1}{ }^{\mathrm{b}}$ \\
\hline 0.9573 & 0.0427
\end{tabular}

${ }^{a} x_{2}$ : mole fraction of component 2 in the saturated solution.

${ }^{\mathrm{b}} x_{1}$ : mole fraction solubility of the solute.

\section{Auxiliary Information}

\section{Method/Apparatus/Procedure:}

Constant-temperature bath, calorimetric thermometer, and an ultraviolet/ visible spectrophotometer.

Excess solute and solvent were placed in amber glass bottles and allowed to equilibrate for several days at constant temperature. Attainment of equilibrium was verified by several repetitive measurements and by approaching equilibrium from supersaturation. Aliquots of saturated solutions were transferred through a coarse filter into tared volumetric flasks, weighed, and diluted with methanol. Concentrations were determined by

spectrophotometric measurements at $295 \mathrm{~nm}$.

Source and Purity of Chemicals:

(1) $99 \%$, Aldrich Chemical Company, Milwaukee, WI, USA, was used as received.

(2) $99 \%$, Aldrich Chemical Company, stored over molecular sieves and distilled shortly before use.

\section{Estimated Error:}

Temperature: $\pm 0.1 \mathrm{~K}$.

$x_{1}: \pm 1.5 \%$ (relative error).

\section{Components:}

(1) 2-Methoxybenzoic acid; $\mathrm{C}_{8} \mathrm{H}_{8} \mathrm{O}_{3}$; [579-75-9]

(2) 3-Methyl-1-butanol; $\mathrm{C}_{5} \mathrm{H}_{12} \mathrm{O}$; [123-51-3]

\section{Variables:}

$T / \mathrm{K}=298.15$
Original Measurements:

${ }^{9}$ K. R. Hoover, D. M. Stovall, E. Pustejovsky, R. Coaxum, K. Pop, W. E. Acree, Jr., and M. H. Abraham, Can. J. Chem. 82, 1353 (2004).

Prepared by:

W. E. Acree, Jr.
Experimental Values

\begin{tabular}{lc}
\hline \hline$x_{2}{ }^{\mathrm{a}}$ & $x_{1}{ }^{\mathrm{b}}$ \\
\hline 0.9600 & 0.0400 \\
\hline${ }^{\mathrm{a}} x_{2}:$ mole fraction of component 2 in the saturated solution. \\
${ }^{\mathrm{b}} x_{1}:$ mole fraction solubility of the solute.
\end{tabular}

\section{Auxiliary Information}

\section{Method/Apparatus/Procedure:}

Constant-temperature bath, calorimetric thermometer, and an ultraviolet/ visible spectrophotometer.

Excess solute and solvent were placed in amber glass bottles and allowed to equilibrate for several days at constant temperature. Attainment of equilibrium was verified by several repetitive measurements and by approaching equilibrium from supersaturation. Aliquots of saturated solutions were transferred through a coarse filter into tared volumetric flasks, weighed, and diluted with methanol. Concentrations were determined by spectrophotometric measurements at $295 \mathrm{~nm}$.

\section{Source and Purity of Chemicals:}

(1) $99 \%$, Aldrich Chemical Company, Milwaukee, WI, USA, was used as received.

(2) $99 \%$, anhydrous, Aldrich Chemical Company, stored over molecular sieves and distilled shortly before use.

Estimated Error:

Temperature: $\pm 0.1 \mathrm{~K}$.

$x_{1}: \pm 1.5 \%$ (relative error).

\begin{tabular}{ll}
\hline \hline Components: & Original Measurements: \\
(1) 2-Methoxybenzoic acid; $\mathrm{C}_{8} \mathrm{H}_{8} \mathrm{O}_{3} ;$ & ${ }^{9}$ K. R. Hoover, D. M. Stovall, E. \\
[579-75-9] & Pustejovsky, R. Coaxum, K. Pop, \\
(2) 1-Hexanol; $\mathrm{C}_{6} \mathrm{H}_{14} \mathrm{O} ;[111-27-3]$ & W. E. Acree, Jr., and M. H. \\
& Abraham, Can. J. Chem. 82, 1353 \\
& $(2004)$. \\
\hline Variables: & Prepared by: \\
$T / \mathrm{K}=298.15$ & W. E. Acree, Jr. \\
\hline
\end{tabular}

\section{Experimental Values}

\begin{tabular}{lc}
\hline \hline$x_{2}{ }^{\mathrm{a}}$ & $x_{1}{ }^{\mathrm{b}}$ \\
\hline 0.9533 & 0.0467 \\
\hline
\end{tabular}

${ }^{\mathrm{a}} x_{2}$ : mole fraction of component 2 in the saturated solution.

${ }^{\mathrm{b}} x_{1}$ : mole fraction solubility of the solute.

\section{Auxiliary Information}

\section{Method/Apparatus/Procedure:}

Constant-temperature bath, calorimetric thermometer, and an ultraviolet/ visible spectrophotometer.

Excess solute and solvent were placed in amber glass bottles and allowed to equilibrate for several days at constant temperature. Attainment of equilibrium was verified by several repetitive measurements and by approaching equilibrium from supersaturation. Aliquots of saturated solutions were transferred through a coarse filter into tared volumetric flasks, weighed, and diluted with methanol. Concentrations were determined by spectrophotometric measurements at $295 \mathrm{~nm}$.

\section{Source and Purity of Chemicals:}

(1) $99 \%$, Aldrich Chemical Company, Milwaukee, WI, USA, was used as received.

(2) $99+\%$, Alfa Aesar, USA, stored over molecular sieves and distilled shortly before use.

Estimated Error:

Temperature: $\pm 0.1 \mathrm{~K}$.

$x_{1}: \pm 1.5 \%$ (relative error) 


\section{Components:}

(1) 2-Methoxybenzoic acid; $\mathrm{C}_{8} \mathrm{H}_{8} \mathrm{O}_{3}$; [579-75-9]

(2) 2-Methyl-1-pentanol; $\mathrm{C}_{6} \mathrm{H}_{14} \mathrm{O}$; [105-30-6]

\section{Variables:}

$T / \mathrm{K}=298.15$

Original Measurements:

${ }^{9}$ K. R. Hoover, D. M. Stovall, E. Pustejovsky, R. Coaxum, K. Pop, W. E. Acree, Jr., and M. H. Abraham, Can. J. Chem. 82, 1353 (2004).

\section{Prepared by:}

W. E. Acree, Jr

Experimental Values

\begin{tabular}{lc}
\hline \hline$x_{2}{ }^{\mathrm{a}}$ & $x_{1}{ }^{\mathrm{b}}$ \\
\hline 0.9562 & 0.0438 \\
\hline
\end{tabular}

${ }^{\mathrm{a}} x_{2}$ : mole fraction of component 2 in the saturated solution.

${ }^{\mathrm{b}} x_{1}$ : mole fraction solubility of the solute.

\section{Auxiliary Information}

\section{Method/Apparatus/Procedure:}

Constant-temperature bath, calorimetric thermometer, and an ultraviolet/ visible spectrophotometer.

Excess solute and solvent were placed in amber glass bottles and allowed to equilibrate for several days at constant temperature. Attainment of equilibrium was verified by several repetitive measurements and by approaching equilibrium from supersaturation. Aliquots of saturated solutions were transferred through a coarse filter into tared volumetric flasks, weighed, and diluted with methanol. Concentrations were determined by spectrophotometric measurements at $295 \mathrm{~nm}$.

\section{Source and Purity of Chemicals:}

(1) $99 \%$, Aldrich Chemical Company, Milwaukee, WI, USA, was used as received.

(2) $99 \%$, Aldrich Chemical Company, stored over molecular sieves and distilled shortly before use.

\section{Estimated Error:}

Temperature: $\pm 0.1 \mathrm{~K}$.

$x_{1}: \pm 1.5 \%$ (relative error).

\begin{tabular}{|c|c|}
\hline $\begin{array}{l}\text { Components: } \\
\text { (1) 2-Methoxybenzoic acid; } \mathrm{C}_{8} \mathrm{H}_{8} \mathrm{O}_{3} \text {; } \\
\text { [579-75-9] } \\
\text { (2) 4-Methyl-2-pentanol; } \mathrm{C}_{6} \mathrm{H}_{14} \mathrm{O} \text {; } \\
\text { [108-11-2] }\end{array}$ & $\begin{array}{l}\text { Original Measurements: } \\
{ }^{9} \text { K. R. Hoover, D. M. Stovall, E. } \\
\text { Pustejovsky, R. Coaxum, K. Pop, } \\
\text { W. E. Acree, Jr., and M. H. } \\
\text { Abraham, Can. J. Chem. 82, } 1353 \\
\text { (2004). }\end{array}$ \\
\hline $\begin{array}{l}\text { Variables: } \\
T / \mathrm{K}=298.15\end{array}$ & $\begin{array}{l}\text { Prepared by: } \\
\text { W. E. Acree, Jr. }\end{array}$ \\
\hline
\end{tabular}

\section{Experimental Values}

\begin{tabular}{lc}
\hline \hline$x_{2}^{\mathrm{a}}$ & $x_{1}^{\mathrm{b}}$ \\
\hline 0.9591 & 0.0409 \\
\hline${ }^{\mathrm{a}} x_{2}:$ mole fraction of component 2 in the saturated solution. \\
${ }^{\mathrm{b}} x_{1}:$ mole fraction solubility of the solute.
\end{tabular}

\section{Auxiliary Information}

\section{Method/Apparatus/Procedure:}

Constant-temperature bath, calorimetric thermometer, and an ultraviolet/ visible spectrophotometer.

Excess solute and solvent were placed in amber glass bottles and allowed to equilibrate for several days at constant temperature. Attainment of equilibrium was verified by several repetitive measurements and by approaching equilibrium from supersaturation. Aliquots of saturated solutions were transferred through a coarse filter into tared volumetric flasks, weighed, and diluted with methanol. Concentrations were determined by spectrophotometric measurements at $295 \mathrm{~nm}$.

Source and Purity of Chemicals:

(1) $99 \%$, Aldrich Chemical Company, Milwaukee, WI, USA, was used as received.

(2) $99+\%$, Acros Organics, USA, stored over molecular sieves and distilled shortly before use.

Estimated Error:

Temperature: $\pm 0.1 \mathrm{~K}$.

$x_{1}: \pm 1.5 \%$ (relative error)

\begin{tabular}{ll}
\hline \hline Components: & Original Measurements: \\
(1) 2-Methoxybenzoic acid; $\mathrm{C}_{8} \mathrm{H}_{8} \mathrm{O}_{3} ;$ & ${ }^{9}$ K. R. Hoover, D. M. Stovall, E. \\
[579-75-9] & Pustejovsky, R. Coaxum, K. Pop, \\
(2) 1-Heptanol; $\mathrm{C}_{7} \mathrm{H}_{16} \mathrm{O} ;$ [111-70-6] & W. E. Acree, Jr., and M. H. \\
& Abraham, Can. J. Chem. 82, 1353 \\
& $(2004)$. \\
\hline Variables: & Prepared by: \\
$T / \mathrm{K}=298.15$ & W. E. Acree, Jr. \\
\hline
\end{tabular}

\section{Experimental Values}

\begin{tabular}{lc}
\hline \hline$x_{2}{ }^{\mathrm{a}}$ & $x_{1}{ }^{\mathrm{b}}$ \\
\hline 0.9522 & 0.0478
\end{tabular}

${ }^{\mathrm{a}} x_{2}$ : mole fraction of component 2 in the saturated solution.

${ }^{\mathrm{b}} x_{1}$ : mole fraction solubility of the solute.

\section{Auxiliary Information}

\section{Method/Apparatus/Procedure:}

Constant-temperature bath, calorimetric thermometer, and an ultraviolet/ visible spectrophotometer.

Excess solute and solvent were placed in amber glass bottles and allowed to equilibrate for several days at constant temperature. Attainment of equilibrium was verified by several repetitive measurements and by approaching equilibrium from supersaturation. Aliquots of saturated solutions were transferred through a coarse filter into tared volumetric flasks, weighed, and diluted with methanol. Concentrations were determined by spectrophotometric measurements at $295 \mathrm{~nm}$.

\section{Source and Purity of Chemicals:}

(1) $99 \%$, Aldrich Chemical Company, Milwaukee, WI, USA, was used as received.

(2) $99+\%$, Alfa Aesar, USA, stored over molecular sieves and distilled shortly before use.

Estimated Error:

Temperature: $\pm 0.1 \mathrm{~K}$.

$x_{1}: \pm 1.5 \%$ (relative error) 


\section{Components:}

(1) 2-Methoxybenzoic acid; $\mathrm{C}_{8} \mathrm{H}_{8} \mathrm{O}_{3}$; [579-75-9]

(2) 1-Octanol; $\mathrm{C}_{8} \mathrm{H}_{18} \mathrm{O} ;[111-87-5]$

Original Measurements:

${ }^{9}$ K. R. Hoover, D. M. Stovall, E. Pustejovsky, R. Coaxum, K. Pop, W. E. Acree, Jr., and M. H. Abraham, Can. J. Chem. 82, 1353 (2004).

\section{Variables:}

$T / \mathrm{K}=298.15$

Prepared by:

W. E. Acree, Jr.

Experimental Values

\begin{tabular}{lc}
\hline \hline$x_{2}{ }^{\mathrm{a}}$ & $x_{1}{ }^{\mathrm{b}}$ \\
\hline 0.9523 & 0.0477
\end{tabular}

${ }^{a} x_{2}$ : mole fraction of component 2 in the saturated solution.

${ }^{\mathrm{b}} \mathrm{x}_{1}$ : mole fraction solubility of the solute.

\section{Auxiliary Information}

\section{Method/Apparatus/Procedure:}

Constant-temperature bath, calorimetric thermometer, and an ultraviolet/ visible spectrophotometer.

Excess solute and solvent were placed in amber glass bottles and allowed to equilibrate for several days at constant temperature. Attainment of equilibrium was verified by several repetitive measurements and by approaching equilibrium from supersaturation. Aliquots of saturated solutions were transferred through a coarse filter into tared volumetric flasks, weighed, and diluted with methanol. Concentrations were determined by spectrophotometric measurements at $295 \mathrm{~nm}$.

\section{Source and Purity of Chemicals:}

(1) $99 \%$, Aldrich Chemical Company, Milwaukee, WI, USA, was used as received.

(2) $99+\%$, anhydrous, Aldrich Chemical Company, stored over molecular sieves and distilled shortly before use.

\section{Estimated Error:}

Temperature: $\pm 0.1 \mathrm{~K}$.

$x_{1}: \pm 1.5 \%$ (relative error).

\section{Components:}

(1) 2-Methoxybenzoic acid; $\mathrm{C}_{8} \mathrm{H}_{8} \mathrm{O}_{3}$; [579-75-9]

(2) 1-Decanol; $\mathrm{C}_{10} \mathrm{H}_{22} \mathrm{O}$; [112-30-1]

Variables:
$T / \mathrm{K}=298.15$
Original Measurements:

${ }^{9}$ K. R. Hoover, D. M. Stovall, E. Pustejovsky, R. Coaxum, K. Pop, W. E. Acree, Jr., and M. H. Abraham, Can. J. Chem. 82, 1353 (2004).

W. E. Acree, Jr.

\section{Prepared by:}

\section{Experimental Values}

\begin{tabular}{lc}
\hline \hline$x_{2}^{\mathrm{a}}$ & $x_{1}{ }^{\mathrm{b}}$ \\
\hline 0.9584 & 0.0416 \\
\hline${ }^{\mathrm{a}} x_{2}:$ mole fraction of component 2 in the saturated solution. \\
${ }^{\mathrm{b}} x_{1}:$ mole fraction solubility of the solute.
\end{tabular}

\section{Auxiliary Information}

\section{Method/Apparatus/Procedure:}

Constant-temperature bath, calorimetric thermometer, and an ultraviolet/ visible spectrophotometer.

Excess solute and solvent were placed in amber glass bottles and allowed to equilibrate for several days at constant temperature. Attainment of equilibrium was verified by several repetitive measurements and by approaching equilibrium from supersaturation. Aliquots of saturated solutions were transferred through a coarse filter into tared volumetric flasks, weighed, and diluted with methanol. Concentrations were determined by spectrophotometric measurements at $295 \mathrm{~nm}$.

Source and Purity of Chemicals:

(1) $99 \%$, Aldrich Chemical Company, Milwaukee, WI, USA, was used as received.

(2) $99+\%$, Alfa Aesar, USA, stored over molecular sieves and distilled shortly before use.

Estimated Error:

Temperature: $\pm 0.1 \mathrm{~K}$.

$x_{1}: \pm 1.5 \%$ (relative error).

\subsection{2-Methoxybenzoic acid solubility data in alkoxyalcohols}

\begin{tabular}{|c|c|}
\hline Components: & Original Measurements: \\
\hline (1) 2-Methoxybenzoic acid; $\mathrm{C}_{8} \mathrm{H}_{8} \mathrm{O}_{3}$; & ${ }^{130}$ L. M. Grubbs, M. Saifullah, N. E. \\
\hline$[579-75-9]$ & De La Rosa, S. Ye, S. S. Achi, W. E. \\
\hline $\begin{array}{l}\text { (2) 2-Ethoxyethanol; } \mathrm{C}_{4} \mathrm{H}_{10} \mathrm{O}_{2} \text {; } \\
\text { [111-80-5] }\end{array}$ & $\begin{array}{l}\text { Acree, Jr., and M. H. Abraham, } \\
\text { Fluid Phase Equilib. 298, } 48 \text { (2010). }\end{array}$ \\
\hline Variables: & \\
\hline$T / \mathrm{K}=298.15$ & W. E. Acree, Jr. \\
\hline
\end{tabular}

\section{Experimental Values}

\begin{tabular}{lc}
\hline \hline$x_{2}{ }^{\mathrm{a}}$ & $x_{1}{ }^{\mathrm{b}}$ \\
\hline 0.8807 & 0.1193
\end{tabular}

${ }^{\mathrm{a}} x_{2}$ : mole fraction of component 2 in the saturated solution.

${ }^{\mathrm{b}} x_{1}$ : mole fraction solubility of the solute.

\section{Auxiliary Information}

\section{Method/Apparatus/Procedure:}

Constant-temperature bath, calorimetric thermometer, and an ultraviolet/ visible spectrophotometer.

Excess solute and solvent were placed in amber glass bottles and allowed to equilibrate for several days at constant temperature. Attainment of equilibrium was verified by several repetitive measurements and by approaching equilibrium from supersaturation. Aliquots of saturated solutions were transferred through a coarse filter into tared volumetric flasks, weighed, and diluted with methanol. Concentrations were determined by spectrophotometric measurements at $295 \mathrm{~nm}$.

\section{Source and Purity of Chemicals:}

(1) $99 \%$, Aldrich Chemical Company, Milwaukee, WI, USA, was used as received.

(2) 99\%, Aldrich Chemical Company, stored over molecular sieves and distilled shortly before use. 
Estimated Error:

Temperature: $\pm 0.1 \mathrm{~K}$.

$x_{1}: \pm 1.5 \%$ (relative error).

\begin{tabular}{|c|c|}
\hline $\begin{array}{l}\text { Components: } \\
\text { (1) 2-Methoxybenzoic acid; } \mathrm{C}_{8} \mathrm{H}_{8} \mathrm{O}_{3} \text {; } \\
\text { [579-75-9] } \\
\text { (2) 2-Propoxyethanol; } \mathrm{C}_{5} \mathrm{H}_{12} \mathrm{O}_{2} \text {; } \\
\text { [2807-30-9] }\end{array}$ & $\begin{array}{l}\text { Original Measurements: } \\
{ }^{130} \text { L. M. Grubbs, M. Saifullah, N. E. } \\
\text { De La Rosa, S. Ye, S. S. Achi, W. E. } \\
\text { Acree, Jr., and M. H. Abraham, } \\
\text { Fluid Phase Equilib. 298, } 48 \text { (2010). }\end{array}$ \\
\hline $\begin{array}{l}\text { Variables: } \\
T / \mathrm{K}=298.15\end{array}$ & $\begin{array}{l}\text { Prepared by: } \\
\text { W. E. Acree, Jr. }\end{array}$ \\
\hline
\end{tabular}

Experimental Values

\begin{tabular}{lc}
\hline \hline$x_{2}{ }^{\mathrm{a}}$ & $x_{1}{ }^{\mathrm{b}}$ \\
\hline 0.8948 & 0.1052 \\
\hline$x_{2}: \mathrm{mole}$
\end{tabular}

${ }^{a} x_{2}$ : mole fraction of component 2 in the saturated solution.

${ }^{\mathrm{b}} x_{1}$ : mole fraction solubility of the solute.

\section{Auxiliary Information}

\section{Method/Apparatus/Procedure:}

Constant-temperature bath, calorimetric thermometer, and an ultraviolet/ visible spectrophotometer.

Excess solute and solvent were placed in amber glass bottles and allowed to equilibrate for several days at constant temperature. Attainment of equilibrium was verified by several repetitive measurements and by approaching equilibrium from supersaturation. Aliquots of saturated solutions were transferred through a coarse filter into tared volumetric flasks, weighed, and diluted with methanol. Concentrations were determined by

spectrophotometric measurements at $295 \mathrm{~nm}$.

Source and Purity of Chemicals:

(1) $99 \%$, Aldrich Chemical Company, Milwaukee, WI, USA, was used as received.

(2) $99+\%$, Aldrich Chemical Company, stored over molecular sieves and distilled shortly before use.

Estimated Error:

Temperature: $\pm 0.1 \mathrm{~K}$.

$x_{1}: \pm 1.5 \%$ (relative error)

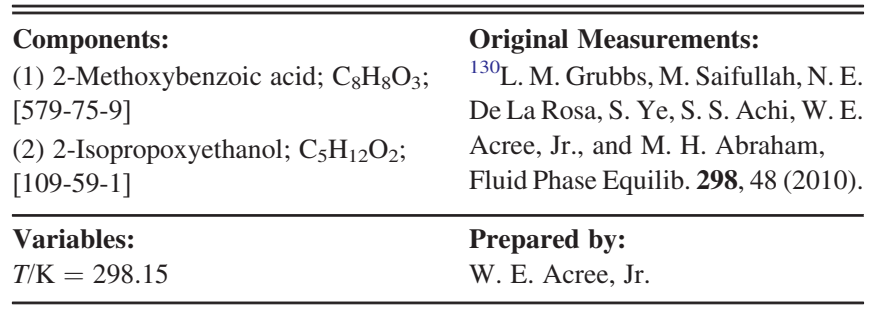

Experimental Values

\begin{tabular}{lc}
\hline \hline$x_{2}{ }^{\mathrm{a}}$ & $x_{1}^{\mathrm{b}}$ \\
\hline 0.9150 & 0.0850 \\
\hline${ }^{\mathrm{a}} x_{2}:$ mole fraction of component 2 in the saturated solution. & \\
${ }^{\mathrm{b}}{ }_{x_{1}: \text { mole fraction solubility of the solute. }}$ &
\end{tabular}

Auxiliary Information

Method/Apparatus/Procedure:

Constant-temperature bath, calorimetric thermometer, and an ultraviolet/ visible spectrophotometer.

Excess solute and solvent were placed in amber glass bottles and allowed to equilibrate for several days at constant temperature. Attainment of equilibrium was verified by several repetitive measurements and by approaching equilibrium from supersaturation. Aliquots of saturated solutions were transferred through a coarse filter into tared volumetric flasks, weighed, and diluted with methanol. Concentrations were determined by

spectrophotometric measurements at $295 \mathrm{~nm}$.

\section{Source and Purity of Chemicals:}

(1) $99 \%$, Aldrich Chemical Company, Milwaukee, WI, USA, was used as received.

(2) $99 \%$, Aldrich Chemical Company, stored over molecular sieves and distilled shortly before use.

\section{Estimated Error:}

Temperature: $\pm 0.1 \mathrm{~K}$.

$x_{1}: \pm 1.5 \%$ (relative error)

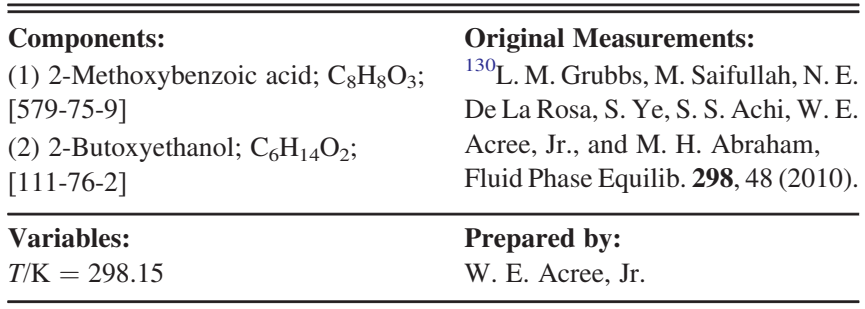

Experimental Values

\begin{tabular}{lc}
\hline \hline$x_{2}{ }^{\mathrm{a}}$ & $x_{1}{ }^{\mathrm{b}}$ \\
\hline 0.8699 & 0.1301 \\
\hline${ }^{\mathrm{a}} x_{2}:$ mole fraction of component 2 in the saturated solution. \\
${ }^{\mathrm{b}} x_{1}:$ mole fraction solubility of the solute.
\end{tabular}

\section{Auxiliary Information}

\section{Method/Apparatus/Procedure:}

Constant-temperature bath, calorimetric thermometer, and an ultraviolet/ visible spectrophotometer.

Excess solute and solvent were placed in amber glass bottles and allowed to equilibrate for several days at constant temperature. Attainment of equilibrium was verified by several repetitive measurements and by approaching equilibrium from supersaturation. Aliquots of saturated solutions were transferred through a coarse filter into tared volumetric flasks, weighed, and diluted with methanol. Concentrations were determined by spectrophotometric measurements at $295 \mathrm{~nm}$.

Source and Purity of Chemicals:

(1) $99 \%$, Aldrich Chemical Company, Milwaukee, WI, USA, was used as received.

(2) $99+\%$, Acros Organics, USA, stored over molecular sieves and distilled shortly before use.

\section{Estimated Error:}

Temperature: $\pm 0.1 \mathrm{~K}$.

$x_{1}: \pm 1.5 \%$ (relative error). 


\section{Components:}

(1) 2-Methoxybenzoic acid; $\mathrm{C}_{8} \mathrm{H}_{8} \mathrm{O}_{3}$; [579-75-9]

(2) 3-Methoxy-1-butanol; $\mathrm{C}_{5} \mathrm{H}_{12} \mathrm{O}_{2}$; [2517-43-3]

\section{Variables:}

$T / \mathrm{K}=298.15$
Original Measurements:

${ }^{130}$ L. M. Grubbs, M. Saifullah, N. E. De La Rosa, S. Ye, S. S. Achi, W. E. Acree, Jr., and M. H. Abraham, Fluid Phase Equilib. 298, 48 (2010).

Prepared by:

W. E. Acree, Jr.

Experimental Values

\begin{tabular}{lc}
\hline \hline$x_{2}^{\mathrm{a}}$ & $x_{1}{ }^{\mathrm{b}}$ \\
\hline 0.8908 & 0.1092 \\
\hline
\end{tabular}

${ }^{\mathrm{a}} x_{2}$ : mole fraction of component 2 in the saturated solution.

${ }^{b} x_{1}$ : mole fraction solubility of the solute.

\section{Auxiliary Information}

\section{Method/Apparatus/Procedure:}

Constant-temperature bath, calorimetric thermometer, and an ultraviolet/ visible spectrophotometer.

Excess solute and solvent were placed in amber glass bottles and allowed to equilibrate for several days at constant temperature. Attainment of equilibrium was verified by several repetitive measurements and by approaching equilibrium from supersaturation. Aliquots of saturated solutions were transferred through a coarse filter into tared volumetric flasks, weighed, and diluted with methanol. Concentrations were determined by spectrophotometric measurements at $295 \mathrm{~nm}$.

Source and Purity of Chemicals:

(1) $99 \%$, Aldrich Chemical Company, Milwaukee, WI, USA, was used as received.

(2) $99 \%$, Aldrich Chemical Company, stored over molecular sieves and distilled shortly before use.

Estimated Error:

Temperature: $\pm 0.1 \mathrm{~K}$.

$x_{1}: \pm 1.5 \%$ (relative error).

\begin{tabular}{|c|c|}
\hline $\begin{array}{l}\text { Components: } \\
\text { (1) 2-Methoxybenzoic acid; } \mathrm{C}_{8} \mathrm{H}_{8} \mathrm{O}_{3} \text {; } \\
\text { [579-75-9] } \\
\text { (2) 1-Methyl-2-tert-butoxyethanol; } \\
\mathrm{C}_{7} \mathrm{H}_{16} \mathrm{O}_{2} ; \text { [57018-52-7] }\end{array}$ & $\begin{array}{l}\text { Original Measurements: } \\
{ }^{130} \text { L. M. Grubbs, M. Saifullah, N. E. } \\
\text { De La Rosa, S. Ye, S. S. Achi, W. E. } \\
\text { Acree, Jr., and M. H. Abraham, } \\
\text { Fluid Phase Equilib. 298, } 48 \text { (2010). }\end{array}$ \\
\hline $\begin{array}{l}\text { Variables: } \\
T / \mathrm{K}=298.15\end{array}$ & $\begin{array}{l}\text { Prepared by: } \\
\text { W. E. Acree, Jr. }\end{array}$ \\
\hline
\end{tabular}

Experimental Values

\begin{tabular}{lc}
\hline \hline$x_{2}^{\mathrm{a}}$ & $x_{1}{ }^{\mathrm{b}}$ \\
\hline 0.9236 & 0.0764 \\
\hline${ }^{\mathrm{a}} x_{2}:$ mole fraction of component 2 in the saturated solution. \\
${ }^{\mathrm{b}} x_{1}:$ mole fraction solubility of the solute.
\end{tabular}

\section{Auxiliary Information}

Method/Apparatus/Procedure:

Constant-temperature bath, calorimetric thermometer, and an ultraviolet/ visible spectrophotometer.

Excess solute and solvent were placed in amber glass bottles and allowed to equilibrate for several days at constant temperature. Attainment of equilibrium was verified by several repetitive measurements and by approaching equilibrium from supersaturation. Aliquots of saturated solutions were transferred through a coarse filter into tared volumetric flasks, weighed, and diluted with methanol. Concentrations were determined by spectrophotometric measurements at $295 \mathrm{~nm}$.

Source and Purity of Chemicals:

(1) $99 \%$, Aldrich Chemical Company, Milwaukee, WI, USA, was used as received.

(2) $99 \%$, Aldrich Chemical Company, stored over molecular sieves and distilled shortly before use.

Estimated Error:

Temperature: $\pm 0.1 \mathrm{~K}$.

$x_{1}: \pm 1.5 \%$ (relative error).

\subsection{2-Methoxybenzoic acid solubility data in miscellaneous organic solvents}

\begin{tabular}{ll}
\hline \hline Components: & Original Measurements: \\
(1) 2-Methoxybenzoic acid; $\mathrm{C}_{8} \mathrm{H}_{8} \mathrm{O}_{3} ;$ & ${ }^{9}$ K. R. Hoover, D. M. Stovall, E. \\
[579-75-9] & Pustejovsky, R. Coaxum, K. Pop, \\
(2) Propylene carbonate; $\mathrm{C}_{4} \mathrm{H}_{6} \mathrm{O}_{3} ;$ & W. E. Acree, Jr., and M. H. \\
{$[108-32-7]$} & $\begin{array}{l}\text { Abraham, Can. J. Chem. 82, 1353 } \\
(2004) .\end{array}$ \\
\hline Variables: & Prepared by: \\
$T / \mathrm{K}=298.15$ & W. E. Acree, Jr. \\
\hline
\end{tabular}

Experimental Values

\begin{tabular}{lc}
\hline \hline$x_{2}{ }^{\mathrm{a}}$ & $x_{1}{ }^{\mathrm{b}}$ \\
\hline 0.9105 & 0.0895 \\
\hline${ }^{\mathrm{a}} x_{2}:$ mole fraction of component 2 in the saturated solution. \\
${ }^{\mathrm{b}}{ }_{x_{1}: \text { mole fraction solubility of the solute. }}$
\end{tabular}

\section{Auxiliary Information}

\section{Method/Apparatus/Procedure:}

Constant-temperature bath, calorimetric thermometer, and an ultraviolet/ visible spectrophotometer.

Excess solute and solvent were placed in amber glass bottles and allowed to equilibrate for several days at constant temperature. Attainment of equilibrium was verified by several repetitive measurements and by approaching equilibrium from supersaturation. Aliquots of saturated solutions were transferred through a coarse filter into tared volumetric flasks, weighed, and diluted with methanol. Concentrations were determined by spectrophotometric measurements at $295 \mathrm{~nm}$.

Source and Purity of Chemicals:

(1) $99 \%$, Aldrich Chemical Company, Milwaukee, WI, USA, was used as received.

(2) 99.7\%, anhydrous, Aldrich Chemical Company, stored over molecular sieves and distilled shortly before use. 
Estimated Error:

Temperature: $\pm 0.1 \mathrm{~K}$

$x_{1}: \pm 1.5 \%$ (relative error)

\section{Solubility of 3-Methoxybenzoic Acid in Organic Solvents}

\subsection{Critical evaluation of experimental solubility data}

There have been four published studies ${ }^{54,56,63,72}$ investigating the solubility behavior of 3-methoxybenzoic acid in organic solvents of varying polarity and hydrogen-bonding capability. Thuaire ${ }^{72}$ reported a mole fraction solubility for 3methoxybenzoic acid dissolved in ethanol. Hancock et al. ${ }^{54,63}$ measured the solubility of 3-methoxybenzoic acid in cyclohexane, benzene, tetrahydrofuran, and 1,4-dioxane at $303 \mathrm{~K}$ based on a gravimetric method. Ongley ${ }^{56}$ measured the solubility of 3-methoxybenzoic acid in cyclohexane, benzene, trichloromethane, and tetrachloromethane at $298 \mathrm{~K}$. It is not possible to directly compare the two sets of solubility data as the measurements were performed at different temperatures.

The experimental solubility data for 3-methoxybenzoic acid in organic solvents are in Secs. 57.2-57.6.

57.2. 3-Methoxybenzoic acid solubility data in saturated hydrocarbons (including cycloalkanes)

\begin{tabular}{|c|c|}
\hline $\begin{array}{l}\text { Components: } \\
\text { (1) 3-Methoxybenzoic acid; } \mathrm{C}_{8} \mathrm{H}_{8} \mathrm{O}_{3} \text {; } \\
\text { [586-38-9] } \\
\text { (2) Cyclohexane; } \mathrm{C}_{6} \mathrm{H}_{12} ;[110-82-7]\end{array}$ & $\begin{array}{l}\text { Original Measurements: } \\
{ }^{54} \text { C. K. Hancock, J. N. Pawloski, } \\
\text { and J. P. Idoux, J. Org. Chem. 31, } \\
3801 \text { (1966). }\end{array}$ \\
\hline $\begin{array}{l}\text { Variables: } \\
T / \mathrm{K}=303.15\end{array}$ & $\begin{array}{l}\text { Prepared by: } \\
\text { W. E. Acree, Jr. }\end{array}$ \\
\hline
\end{tabular}

Experimental Values

\begin{tabular}{lc}
\hline \hline$x_{2}{ }^{\mathrm{a}}$ & $x_{1}{ }^{\mathrm{b}}$ \\
\hline 0.9951 & 0.00493 \\
\hline${ }^{\mathrm{a}} x_{2}:$ mole fraction of component 2 in the saturated solution. \\
${ }^{\mathrm{b}} x_{1}:$ mole fraction solubility of the solute.
\end{tabular}

\section{Auxiliary Information}

Method/Apparatus/Procedure:

Constant-temperature bath, Soxhlet thimble, conical flask, and analytical balance.

Excess solute and solvent were placed in a conical flask and allowed to equilibrate for several days at constant temperature. Attainment of equilibrium was verified by several repetitive measurements and by approaching equilibrium from supersaturation. Aliquots of saturated solutions were transferred using a Soxhlet thimble equipped with a one-hole cork stopper and an inverted U-shaped delivery tube to a second ground-glass stoppered flask suspended in the $303 \mathrm{~K}$ water bath. Successive portions of the contents were evaporated at room temperature in a tared aluminum foil weighing dish under a bell jar through which a slow stream of dry air was passed. The second flask plus unused saturated solution and the aluminum foil dish plus residue were weighed. The saturation solubility of the solute was calculated from the recorded mass data and molar masses of the solute and solvent.

\section{Source and Purity of Chemicals:}

(1) Purity not given, Chemical source not specified, was recrystallized several times from aqueous-ethanol mixture, and then dried under vacuum over phosphorous pentoxide.

(2) Reagent grade, Chemical source not specified, refluxed over phosphorous pentoxide for $24 \mathrm{~h}$, and then distilled through a $3 \times 80 \mathrm{~cm}$ column filled with $0.32-\mathrm{cm}$ glass helices.

\section{Estimated Error:}

Temperature: $\pm 0.02 \mathrm{~K}$.

$x_{1}: \pm 3 \%$ (relative error).

\begin{tabular}{|c|c|}
\hline $\begin{array}{l}\text { Components: } \\
\text { (1) 3-Methoxybenzoic acid; } \mathrm{C}_{8} \mathrm{H}_{8} \mathrm{O}_{3} \text {; } \\
\text { [586-38-9] } \\
\text { (2) Cyclohexane; } \mathrm{C}_{6} \mathrm{H}_{12} ;[110-82-7]\end{array}$ & $\begin{array}{l}\text { Original Measurements: } \\
\text { 56 P. A. Ongley, J. Chem. Soc. 1954, } \\
\text { 3634. }\end{array}$ \\
\hline $\begin{array}{l}\text { Variables: } \\
T / \mathrm{K}=298.15\end{array}$ & $\begin{array}{l}\text { Prepared by: } \\
\text { W. E. Acree, Jr. }\end{array}$ \\
\hline
\end{tabular}

\section{Experimental Values}

The measured solubility was reported to be $-\log _{10} c_{1}=1.851$, which corresponds to a solubility of $c_{1}=0.0141 \mathrm{~mol} \mathrm{dm}^{-3}$.

\section{Auxiliary Information}

\section{Method/Apparatus/Procedure:}

Very little experimental details were provided. Excess solute and solvent were placed in tubes, which were rotated for at least $8 \mathrm{~h}$ at $298 \mathrm{~K}$. The solutions were then removed, concentrated if necessary, and titrated with standard alkali using a mixed (bromothymol blue + neutral red) indicator.

Source and Purity of Chemicals:

(1) Purity not given, Chemical source not given, solute was either "AnalaR" grade or recrystallized prior to use.

(2) Purity not given, Chemical source not given, no purification details were provided.

Estimated Error:

Temperature: No information was given

$c_{1}: \pm 4 \%$ (relative error, estimated by compiler). 


\subsection{3-Methoxybenzoic acid solubility data in aromatic hydrocarbons}

\begin{tabular}{|c|c|}
\hline $\begin{array}{l}\text { Components: } \\
\text { (1) 3-Methoxybenzoic acid; } \mathrm{C}_{8} \mathrm{H}_{8} \mathrm{O}_{3} \text {; } \\
\text { [586-38-9] } \\
\text { (2) Benzene; } \mathrm{C}_{6} \mathrm{H}_{6} ;[71-43-2]\end{array}$ & $\begin{array}{l}\text { Original Measurements: } \\
{ }^{54} \text { C. K. Hancock, J. N. Pawloski, } \\
\text { and J. P. Idoux, J. Org. Chem. 31, } \\
3801 \text { (1966). }\end{array}$ \\
\hline $\begin{array}{l}\text { Variables: } \\
T / \mathrm{K}=303.15\end{array}$ & $\begin{array}{l}\text { Prepared by: } \\
\text { W. E. Acree, Jr. }\end{array}$ \\
\hline
\end{tabular}

Experimental Values

\begin{tabular}{lc}
\hline \hline$x_{2}^{\mathrm{a}}$ & $x_{1}{ }^{\mathrm{b}}$ \\
\hline 0.9558 & 0.0442
\end{tabular}

${ }^{\mathrm{a}} x_{2}$ : mole fraction of component 2 in the saturated solution.

${ }^{\mathrm{b}} x_{1}$ : mole fraction solubility of the solute.

\section{Auxiliary Information}

\section{Method/Apparatus/Procedure:}

Constant-temperature bath, Soxhlet thimble, conical flask, and analytical balance.

Excess solute and solvent were placed in a conical flask and allowed to equilibrate for several days at constant temperature. Attainment of equilibrium was verified by several repetitive measurements and by approaching equilibrium from supersaturation. Aliquots of saturated solutions were transferred using a Soxhlet thimble equipped with a one-hole cork stopper and an inverted U-shaped delivery tube to a second ground-glass stoppered flask suspended in the $303 \mathrm{~K}$ water bath. Successive portions of the contents were evaporated at room temperature in a tared aluminum foil weighing dish under a bell jar through which a slow stream of dry air was passed. The second flask plus unused saturated solution and the aluminum foil dish plus residue were weighed. The saturation solubility of the solute was calculated from the recorded mass data and molar masses of the solute and solvent.

\section{Source and Purity of Chemicals:}

(1) Purity not given, Chemical source not specified, was recrystallized several times from aqueous-ethanol mixture, and then dried under vacuum over phosphorous pentoxide.

(2) Reagent grade, Chemical source not specified, refluxed over phosphorous pentoxide for $24 \mathrm{~h}$, and then distilled through a $3 \times 80 \mathrm{~cm}$ column filled with 0.32 -cm glass helices.

\section{Estimated Error:}

Temperature: $\pm 0.02 \mathrm{~K}$.

$x_{1}: \pm 3 \%$ (relative error).

\begin{tabular}{|c|c|}
\hline $\begin{array}{l}\text { Components: } \\
\text { (1) 3-Methoxybenzoic acid; } \mathrm{C}_{8} \mathrm{H}_{8} \mathrm{O}_{3} \text {; } \\
\text { [586-38-9] } \\
\text { (2) Benzene; } \mathrm{C}_{6} \mathrm{H}_{6} ;[71-43-2]\end{array}$ & $\begin{array}{l}\text { Original Measurements: } \\
\text { 56 P. A. Ongley, J. Chem. Soc. 1954, } \\
3634 \text {. }\end{array}$ \\
\hline $\begin{array}{l}\text { Variables: } \\
T / \mathrm{K}=298.15\end{array}$ & $\begin{array}{l}\text { Prepared by: } \\
\text { W. E. Acree, Jr. }\end{array}$ \\
\hline
\end{tabular}

\section{Experimental Values}

The measured solubility was reported to be $-\log _{10} c_{1}=0.398$, which corresponds to a solubility of $c_{1}=0.400 \mathrm{~mol} \mathrm{dm}^{-3}$.

\section{Auxiliary Information}

\section{Method/Apparatus/Procedure:}

Very little experimental details were provided. Excess solute and solvent were placed in tubes, which were rotated for at least $8 \mathrm{~h}$ at $298 \mathrm{~K}$. The solutions were then removed, concentrated if necessary, and titrated with standard alkali using a mixed (bromothymol blue + neutral red) indicator.

\section{Source and Purity of Chemicals:}

(1) Purity not given, Chemical source not given, solute was either "AnalaR" grade or recrystallized prior to use.

(2) Purity not given, Chemical source not given, no purification details were provided.

Estimated Error:

Temperature: No information was given.

$c_{1}: \pm 4 \%$ (relative error, estimated by compiler).

\subsection{3-Methoxybenzoic acid solubility data in ethers}

\begin{tabular}{|c|c|}
\hline Components: & Original Measurements: \\
\hline $\begin{array}{l}\text { (1) 3-Methoxybenzoic acid; } \mathrm{C}_{8} \mathrm{H}_{8} \mathrm{O}_{3} \text {; } \\
\text { [586-38-9] }\end{array}$ & $\begin{array}{l}{ }^{63} \text { C. K. Hancock, J. N. Pawloski, } \\
\text { and J. P. Idoux, J. Org. Chem. }\end{array}$ \\
\hline (2) Tetrahydrofuran; $\mathrm{C}_{4} \mathrm{H}_{8} \mathrm{O}$; [109-99-9] & 32, 1931 (1967) \\
\hline Variables: & Prepared by: \\
\hline$T / \mathrm{K}=303.15$ & W. E. Acree, Jr. \\
\hline
\end{tabular}

Experimental Values

\begin{tabular}{lc}
\hline \hline$x_{2}{ }^{\mathrm{a}}$ & $x_{1}{ }^{\mathrm{b}}$ \\
\hline 0.691 & 0.309 \\
\hline${ }^{\mathrm{a}} x_{2}:$ mole fraction of component 2 in the saturated solution. & \\
${ }^{\mathrm{b}} x_{1}:$ mole fraction solubility of the solute. &
\end{tabular}

Auxiliary Information

Method/Apparatus/Procedure:

Constant-temperature bath, Soxhlet thimble, conical flask, and analytical balance.

Excess solute and solvent were placed in a conical flask and allowed to equilibrate for several days at constant temperature. Attainment of equilibrium was verified by several repetitive measurements and by approaching equilibrium from supersaturation. Aliquots of saturated solutions were transferred using a Soxhlet thimble equipped with a one-hole cork stopper and an inverted U-shaped delivery tube to a second ground-glass stoppered flask suspended in the $303 \mathrm{~K}$ water bath. Successive portions of the contents were evaporated at room temperature in a tared aluminum foil weighing dish under a bell jar through which a slow stream of dry air was passed. The second flask plus unused saturated solution and the aluminum foil dish plus residue were weighed. The saturation solubility of the solute was calculated from the recorded mass data and molar masses of the solute and solvent.

Source and Purity of Chemicals:

(1) Purity not given, Chemical source not specified, was recrystallized several times from aqueous-ethanol mixture, and then dried under vacuum over phosphorous pentoxide

(2) Practical grade, Chemical source not specified, stored over sodium hydroxide pellets for $24 \mathrm{~h}$, and then passed through $2 \times 70-\mathrm{cm}$ chromatographic adsorption columns containing activated alumina. After this treatment, the purified solvent was stored over copper in a nitrogen atmosphere. 
Estimated Error:

Temperature: $\pm 0.02 \mathrm{~K}$

$x_{1}: \pm 1.0 \%$ (relative error).

\begin{tabular}{ll}
\hline \hline $\begin{array}{l}\text { Components: } \\
\text { (1) 3-Methoxybenzoic acid; } \mathrm{C}_{8} \mathrm{H}_{8} \mathrm{O}_{3} ;\end{array}$ & $\begin{array}{l}\text { Original Measurements: } \\
\text { [586-38-9] } \mathrm{C} \text {. K. Hancock, J. N. Pawloski, } \\
\text { and J. P. Idoux, J. Org. Chem. 32, } \\
\text { (2) 1,4-Dioxane; } \mathrm{C}_{4} \mathrm{H}_{8} \mathrm{O}_{2} ;[123-91-1]\end{array}$ \\
\hline Variables: & Prepared by: \\
$T / \mathrm{K}=303.15$ & W. E. Acree, Jr. \\
\hline
\end{tabular}

\section{Experimental Values}

\begin{tabular}{lc}
\hline \hline$x_{2}{ }^{\mathrm{a}}$ & $x_{1}{ }^{\mathrm{b}}$ \\
\hline 0.754 & 0.246
\end{tabular}

${ }^{\mathrm{a}} x_{2}$ : mole fraction of component 2 in the saturated solution.

${ }^{\mathrm{b}} x_{1}$ : mole fraction solubility of the solute.

\section{Auxiliary Information}

\section{Method/Apparatus/Procedure:}

Constant-temperature bath, Soxhlet thimble, conical flask, and analytical balance.

Excess solute and solvent were placed in a conical flask and allowed to equilibrate for several days at constant temperature. Attainment of equilibrium was verified by several repetitive measurements and by approaching equilibrium from supersaturation. Weighed aliquots of saturated solutions were removed and titrated with a standardized sodium hydroxide solution (carbonate free) using a $\mathrm{pH}$ meter. The endpoint of the titration was determined by computing the second derivative in the $\mathrm{pH}$ versus volume of sodium hydroxide added.

\section{Source and Purity of Chemicals:}

(1) Purity not given, Chemical source not specified, was recrystallized several times from aqueous-ethanol mixture, and then dried under vacuum over phosphorous pentoxide.

(2) Practical grade, Chemical source not specified, stored over sodium hydroxide pellets for $24 \mathrm{~h}$, and then passed through $2 \times 70-\mathrm{cm}$ chromatographic adsorption columns containing activated alumina. After this treatment, the purified solvent was stored over copper in a nitrogen atmosphere.

\section{Estimated Error:}

Temperature: $\pm 0.02 \mathrm{~K}$

$x_{1}: \pm 1.0 \%$ (relative error).

\subsection{3-Methoxybenzoic acid solubility data in haloalkanes, haloalkenes, and haloaromatic hydrocarbons}

\begin{tabular}{|c|c|}
\hline $\begin{array}{l}\text { Components: } \\
\text { (1) 3-Methoxybenzoic acid; } \mathrm{C}_{8} \mathrm{H}_{8} \mathrm{O}_{3} \text {; } \\
\text { [586-38-9] } \\
\text { (2) Trichloromethane; } \mathrm{CHCl}_{3} ;[67-66-3]\end{array}$ & $\begin{array}{l}\text { Original Measurements: } \\
{ }^{56} \mathrm{P} . \text { A. Ongley, J. Chem. Soc. } \\
\text { 1954, } 3634 .\end{array}$ \\
\hline Variables: & Prepared by: \\
\hline$T / \mathrm{K}=298.15$ & W. E. Acree, Jr. \\
\hline
\end{tabular}

\section{Experimental Values}

The measured solubility was reported to be $-\log _{10} c_{1}=$ -0.049 , which corresponds to a solubility of $c_{1}=$ $1.119 \mathrm{~mol} \mathrm{dm}^{-3}$.

\section{Auxiliary Information}

\section{Method/Apparatus/Procedure:}

Very little experimental details were provided. Excess solute and solvent were placed in tubes, which were rotated for at least $8 \mathrm{~h}$ at $298 \mathrm{~K}$. The solutions were then removed, concentrated if necessary, and titrated with standard alkali using a mixed (bromothymol blue + neutral red) indicator.

Source and Purity of Chemicals:

(1) Purity not given, Chemical source not given, solute was either "AnalaR" grade or recrystallized prior to use.

(2) Purity not given, Chemical source not given, no purification details were provided.

Estimated Error:

Temperature: No information was given.

$c_{1}: \pm 4 \%$ (relative error, estimated by compiler).

\begin{tabular}{|c|c|}
\hline $\begin{array}{l}\text { Components: } \\
\text { (1) 3-Methoxybenzoic acid; } \mathrm{C}_{8} \mathrm{H}_{8} \mathrm{O}_{3} \text {; } \\
\text { [586-38-9] } \\
\text { (2) Tetrachloromethane; } \mathrm{CCl}_{4} ;[56-23-5]\end{array}$ & $\begin{array}{l}\text { Original Measurements: } \\
{ }^{56} \text { P. A. Ongley, J. Chem. Soc. } \\
\text { 1954, } 3634 .\end{array}$ \\
\hline $\begin{array}{l}\text { Variables: } \\
T / \mathrm{K}=298.15\end{array}$ & $\begin{array}{l}\text { Prepared by: } \\
\text { W. E. Acree, Jr. }\end{array}$ \\
\hline
\end{tabular}

\section{Experimental Values}

The measured solubility was reported to be $-\log _{10} c_{1}=0.737$, which corresponds to a solubility of $c_{1}=0.183 \mathrm{~mol} \mathrm{dm}^{-3}$.

\section{Auxiliary Information}

\section{Method/Apparatus/Procedure:}

Very little experimental details were provided. Excess solute and solvent were placed in tubes, which were rotated for at least $8 \mathrm{~h}$ at $298 \mathrm{~K}$. The solutions were then removed, concentrated if necessary, and titrated with standard alkali using a mixed (bromothymol blue + neutral red) indicator.

Source and Purity of Chemicals:

(1) Purity not given, Chemical source not given, solute was either "AnalaR" grade or recrystallized prior to use.

(2) Purity not given, Chemical source not given, no purification details were provided.

\section{Estimated Error:}

Temperature: No information was given

$c_{1}: \pm 4 \%$ (relative error, estimated by compiler). 


\subsection{3-Methoxybenzoic acid solubility data in alcohols}

\begin{tabular}{|c|c|}
\hline $\begin{array}{l}\text { Components: } \\
\text { (1) 3-Methoxybenzoic acid; } \mathrm{C}_{8} \mathrm{H}_{8} \mathrm{O}_{3} \text {; } \\
\text { [586-38-9] } \\
\text { (2) Ethanol; } \mathrm{C}_{2} \mathrm{H}_{6} \mathrm{O} ;[64-17-5]\end{array}$ & $\begin{array}{l}\text { Original Measurements: } \\
\text { 72 R. Thuaire, Bull. Soc. Chim. Fr. } \\
3815 \text { (1971). }\end{array}$ \\
\hline $\begin{array}{l}\text { Variables: } \\
T / \mathrm{K}=298.15\end{array}$ & $\begin{array}{l}\text { Prepared by: } \\
\text { W. E. Acree, Jr. }\end{array}$ \\
\hline
\end{tabular}

Experimental Values

The measured solubility was reported to be $4.216 \mathrm{~mol} / \mathrm{kg}$ of solvent, which corresponds to a mole fraction solubility of $x_{1}=0.1626$.

\section{Auxiliary Information}

\section{Method/Apparatus/Procedure:}

Very little experimental details were provided. Solubility was determined by a gravimetric method. The saturated solution was evaporated to dryness and the remaining solid residue was weighed. The solubility was calculated from the mass of the solid residue and mass of saturated solution analyzed.

\section{Source and Purity of Chemicals:}

(1) Purity not given, Chemical source not given, no purification details were provided.

(2) Purity not given, Chemical source not given, no purification details were provided.

\section{Estimated Error:}

Temperature: $\pm 0.05 \mathrm{~K}$.

$x_{1}: \pm 4 \%$ (relative error, estimated by compiler).

\section{Solubility of 4-Methoxybenzoic Acid in Organic Solvents}

\subsection{Critical evaluation of experimental solubility data}

There have been several published studies ${ }^{9,54,56,72,130}$ investigating the solubility behavior of 4-methoxybenzoic acid in organic solvents of varying polarity and hydrogen-bonding capability. Hoover et al. ${ }^{9}$ measured the solubility of 4-methoxybenzoic acid in 14 alcohols (ethanol, 1-propanol, 2-propanol, 1-butanol, 2-butanol, 2-methyl-1-propanol, 2-methyl2-propanol, 1-pentanol, 2-pentanol, 3-methyl-1-butanol, 1hexanol, 1-heptanol, 1-octanol, and 1-decanol), in three dialkyl ethers (1,1'-oxybisethane, 2,2'-oxybispropane, and 1,1'oxybisbutane) and two cyclic ethers (tetrahydrofuran and 1,4dioxane), and in three alkanoates (ethyl ethanoate, butyl ethanoate, and pentyl ethanoate) at $298 \mathrm{~K}$. Results of the experimental measurements were used to calculate the Abraham solute descriptors of 4-methoxybenzoic acid. The authors were able to assemble a total of $44 \log _{10}\left(S R\right.$ or $P$ ) and $\log _{10}$ (GSR or $K$ ) equations for which experimental partition coefficient data, solubility ratios, Abraham model equation coefficients and aqueous molar solubility were available. The logarithm of the aqueous molar solubility of 4-methoxyben- zoic acid is $\log _{10} c_{1, \mathrm{w}}=-2.800{ }^{72,170}$ The McGowan volume of 4-methoxybenzoic acid, $V=1.1313$, was calculated from the number of chemical bonds in the molecule and the individual atomic group volumes, $A V_{i}$, given in Sec. 1.3. The excess molar refraction solute descriptor was estimated as $E=$ 0.899 . This left four solute descriptors $(S, A, B$, and $L$ ) still to be determined. The 44 equations were then solved using the Microsoft "SOLVER" program to yield numerical values of the remaining four solute descriptors, $S=1.250, A=0.620$, $B=0.520$, and $L=5.741$, that best described the $\log _{10}(S R$ or $P$ ) and $\log _{10}(G S R$ or $K)$ values. The computation treated $\log _{10} c_{1, \mathrm{G}}$ as a floating parameter to be determined as part of the regression analyses. The data analyses returned a value of $\log _{10} c_{1, \mathrm{G}}=-9.500$ for the gas-phase solute concentration that made the $\log _{10}\left(S R\right.$ or $P$ ) and $\log _{10}(G S R$ or $K$ ) predictions internally consistent. The calculated molecular solute descriptors reproduced the $\log _{10}\left(S R\right.$ or $P$ ) and $\log _{10}(G S R$ or $K)$ values to within an average standard deviation of 0.119 and $0.110 \log _{10}$ units, respectively.

After the 4-methoxybenzoic acid solubility study was published, Abraham model correlations have been developed for 2-pentanol, 3-methyl-1-butanol, and butyl ethanoate, and equation coefficients for a few solvents were updated based on additional experimental data. The new correlations (listed in Tables 1 and 2) will be used in illustrating the ability of the Abraham model to correlate the experimental 4-methoxybenzoic acid solubility data. Table 41 compares the experimental $\log _{10} c_{1}$ values to calculated values based on Eqs. (20) and (21) of the Abraham model. For comparison purposes, the measured mole fraction solubilities of 4-methoxybenzoic acid, $x_{1}$, determined by Hoover et al. ${ }^{9}$ were converted into molar solubilities by dividing $x_{1}$ by the ideal molar volume of the saturated solution (i.e., $\left.c_{1}{ }^{\text {sat }}=x_{1} /\left[x_{1} V_{1}+\left(1-x_{1}\right) V_{\text {solvent }}\right]\right)$. The molar volume of the hypothetical subcooled liquid 4-methoxybenzoic acid is $V_{\text {solute }}=124.1 \mathrm{~cm}^{3} \mathrm{~mol}^{-1}$. Examination of the

TABLE 41. Comparison between observed and calculated molar solubilities of 4-methoxybenzoic acid based on the Abraham model, Eqs. (20) and (21)

\begin{tabular}{|c|c|c|c|}
\hline Solvent & $\log _{10} c_{1}^{\exp }$ & $\begin{array}{c}\log _{10} c_{1}{ }^{\text {alc }} ; \\
\text { Eq. (20) }\end{array}$ & $\begin{array}{c}\log _{10} c_{1}{ }^{\text {calc }} \text {; } \\
\text { Eq. (21) }\end{array}$ \\
\hline Ethanol & -0.702 & -0.753 & -0.717 \\
\hline 1-Propanol & -0.875 & -0.879 & -0.839 \\
\hline 2-Propanol & -0.864 & -0.879 & -0.822 \\
\hline 1-Butanol & -0.929 & -0.987 & -0.937 \\
\hline 2-Butanol & -0.898 & -0.932 & -0.905 \\
\hline 2-Methyl-1-propanol & -1.100 & -1.039 & -1.015 \\
\hline 2-Methyl-2-propanol & -0.783 & -0.889 & -0.810 \\
\hline 1-Pentanol & -0.984 & -1.014 & -0.970 \\
\hline 2-Pentanol & -0.977 & -1.007 & -0.958 \\
\hline 3-Methyl-1-butanol & -1.079 & -1.065 & -1.020 \\
\hline 1-Hexanol & -1.022 & -1.059 & -1.020 \\
\hline 1-Heptanol & -1.060 & -1.109 & -1.054 \\
\hline 1-Octanol & -1.093 & -1.145 & -1.161 \\
\hline 1-Decanol & -1.174 & -1.256 & -1.198 \\
\hline 1,1'-Oxybisethane & -1.043 & -1.157 & -1.044 \\
\hline Tetrahydrofuran & -0.162 & -0.409 & -0.378 \\
\hline 1,4-Dioxane & -0.401 & -0.619 & -0.582 \\
\hline Ethyl ethanoate & -0.879 & -0.987 & -0.938 \\
\hline Butyl ethanoate & -1.086 & -1.139 & -1.107 \\
\hline
\end{tabular}


numerical entries in Table 41 reveals that the Abraham model provides a reasonably accurate mathematical description of the observed solubility data, suggesting that there are no obvious outliers in the dataset.

Ongley ${ }^{56}$ measured the solubility of 4-methoxybenzoic acid in cyclohexane, benzene, trichloromethane, and tetrachloromethane at $298.15 \mathrm{~K}$. Abraham model predictions would not be applicable to these solvents because 4-methoxybenzoic acid would likely dimerize to an appreciable extent. In such cases, the measured solubility would represent the sum of both the monomeric and dimeric forms of the solute. The solute descriptors that have been calculated for 4-methoxybenzoic acid pertain only to the monomeric form. Thuaire ${ }^{72}$ reported a mole fraction solubility for 4-methoxybenzoic acid in ethanol of $x_{1}=0.01242$, which differs by less than $6 \%$ from the value of $x_{1}=0.01185$ determined by Hoover et al. ${ }^{9}$ Differences in chemical purities and experimental methodologies can lead to differences of a few percent between values determined by two different research groups.

The experimental solubility data for 4-methoxybenzoic acid in organic solvents are in Secs. 58.2-58.8.

\subsection{4-Methoxybenzoic acid solubility data in saturated hydrocarbons (including cycloalkanes)}

\begin{tabular}{|c|c|}
\hline $\begin{array}{l}\text { Components: } \\
\text { (1) 4-Methoxybenzoic acid; } \mathrm{C}_{8} \mathrm{H}_{8} \mathrm{O}_{3} \text {; } \\
{[100-09-4]} \\
\text { (2) Cyclohexane; } \mathrm{C}_{6} \mathrm{H}_{12} ;[110-82-7]\end{array}$ & $\begin{array}{l}\text { Original Measurements: } \\
{ }^{54} \text { C. K. Hancock, J. N. Pawloski, } \\
\text { and J. P. Idoux, J. Org. Chem. 31, } \\
3801 \text { (1966). }\end{array}$ \\
\hline $\begin{array}{l}\text { Variables: } \\
T / \mathrm{K}=303.15\end{array}$ & $\begin{array}{l}\text { Prepared by: } \\
\text { W. E. Acree, Jr. }\end{array}$ \\
\hline
\end{tabular}

Experimental Values

\begin{tabular}{lc}
\hline \hline$x_{2}{ }^{\mathrm{a}}$ & $x_{1}{ }^{\mathrm{b}}$ \\
\hline 0.9999 & 0.0000640 \\
\hline
\end{tabular}

${ }^{a} x_{2}$ : mole fraction of component 2 in the saturated solution.

${ }^{\mathrm{b}} x_{1}$ : mole fraction solubility of the solute.

\section{Auxiliary Information}

Method/Apparatus/Procedure:

Constant-temperature bath, Soxhlet thimble, conical flask, and analytical balance.

Excess solute and solvent were placed in a conical flask and allowed to equilibrate for several days at constant temperature. Attainment of equilibrium was verified by several repetitive measurements and by approaching equilibrium from supersaturation. Aliquots of saturated solutions were transferred using a Soxhlet thimble equipped with a one-hole cork stopper and an inverted U-shaped delivery tube to a second ground-glass stoppered flask suspended in the $303 \mathrm{~K}$ water bath. Successive portions of the contents were evaporated at room temperature in a tared aluminum foil weighing dish under a bell jar through which a slow stream of dry air was passed. The second flask plus unused saturated solution and the aluminum foil dish plus residue were weighed. The saturation solubility of the solute was calculated from the recorded mass data and molar masses of the solute and solvent.
Source and Purity of Chemicals:

(1) Purity not given, Chemical source not specified, was recrystallized several times from aqueous-ethanol mixture, and then dried under vacuum over phosphorous pentoxide.

(2) Reagent grade, Chemical source not specified, refluxed over phosphorous pentoxide for $24 \mathrm{~h}$, and then distilled through a $3 \times 80 \mathrm{~cm}$ column filled with $0.32-\mathrm{cm}$ glass helices.

Estimated Error:

Temperature: $\pm 0.02 \mathrm{~K}$

$x_{1}: \pm 10 \%$ (relative error, by compiler).

\subsection{4-Methoxybenzoic acid solubility data in aromatic hydrocarbons}

\begin{tabular}{|c|c|}
\hline $\begin{array}{l}\text { Components: } \\
\text { (1) 4-Methoxybenzoic acid; } \mathrm{C}_{8} \mathrm{H}_{8} \mathrm{O}_{3} \text {; } \\
\text { [100-09-4] } \\
\text { (2) Benzene; } \mathrm{C}_{6} \mathrm{H}_{6} ;[71-43-2]\end{array}$ & $\begin{array}{l}\text { Original Measurements: } \\
{ }^{54} \text { C. K. Hancock, J. N. Pawloski, } \\
\text { and J. P. Idoux, J. Org. Chem. 31, } \\
3801 \text { (1966). }\end{array}$ \\
\hline $\begin{array}{l}\text { Variables: } \\
T / \mathrm{K}=303.15\end{array}$ & $\begin{array}{l}\text { Prepared by: } \\
\text { W. E. Acree, Jr. }\end{array}$ \\
\hline
\end{tabular}

Experimental Values

\begin{tabular}{lc}
\hline \hline$x_{2}{ }^{\mathrm{a}}$ & $x_{1}{ }^{\mathrm{b}}$ \\
\hline 0.9983 & 0.00166 \\
\hline${ }^{\mathrm{a}} x_{2}:$ mole fraction of component 2 in the saturated solution. & \\
${ }^{\mathrm{b}} x_{1}:$ mole fraction solubility of the solute. &
\end{tabular}

Auxiliary Information

\section{Method/Apparatus/Procedure:}

Constant-temperature bath, Soxhlet thimble, conical flask, and analytical balance.

Excess solute and solvent were placed in a conical flask and allowed to equilibrate for several days at constant temperature. Attainment of equilibrium was verified by several repetitive measurements and by approaching equilibrium from supersaturation. Aliquots of saturated solutions were transferred using a Soxhlet thimble equipped with a one-hole cork stopper and an inverted $\mathrm{U}$-shaped delivery tube to a second ground-glass stoppered flask suspended in the $303 \mathrm{~K}$ water bath. Successive portions of the contents were evaporated at room temperature in a tared aluminum foil weighing dish under a bell jar through which a slow stream of dry air was passed. The second flask plus unused saturated solution and the aluminum foil dish plus residue were weighed. The saturation solubility of the solute was calculated from the recorded mass data and molar masses of the solute and solvent.

Source and Purity of Chemicals:

(1) Purity not given, Chemical source not specified, was recrystallized several times from aqueous-ethanol mixture, and then dried under vacuum over phosphorous pentoxide.

(2) Reagent grade, Chemical source not specified, refluxed over phosphorous pentoxide for $24 \mathrm{~h}$, and then distilled through a $3 \times 80 \mathrm{~cm}$ column filled with 0.32-cm glass helices.

\section{Estimated Error:}

Temperature: $\pm 0.02 \mathrm{~K}$

$x_{1}: \pm 3 \%$ (relative error, by compiler). 


\begin{tabular}{|c|c|}
\hline $\begin{array}{l}\text { Components: } \\
\text { (1) 4-Methoxybenzoic acid; } \mathrm{C}_{8} \mathrm{H}_{8} \mathrm{O}_{3} \text {; } \\
\text { [100-09-4] } \\
\text { (2) Benzene; } \mathrm{C}_{6} \mathrm{H}_{6} ;[71-43-2]\end{array}$ & $\begin{array}{l}\text { Original Measurements: } \\
\text { 56 P. A. Ongley, J. Chem. Soc. 1954, } \\
3634 \text {. }\end{array}$ \\
\hline Variables: & Prepared by: \\
\hline$T / \mathrm{K}=298.15$ & W. E. Acree, Jr. \\
\hline
\end{tabular}

\section{Experimental Values}

The measured solubility was reported to be $-\log _{10} c_{1}=1.714$, which corresponds to a solubility of $c_{1}=0.0193 \mathrm{~mol} \mathrm{dm}^{-3}$.

\section{Auxiliary Information}

\section{Method/Apparatus/Procedure:}

Very little experimental details were provided. Excess solute and solvent were placed in tubes, which were rotated for at least $8 \mathrm{~h}$ at $298 \mathrm{~K}$. The solutions were then removed, concentrated if necessary, and titrated with standard alkali using a mixed (bromothymol blue + neutral red) indicator.

Source and Purity of Chemicals:

(1) Purity not given, Chemical source not given, solute was either "AnalaR" grade or recrystallized prior to use.

(2) Purity not given, Chemical source not given, no purification details were provided.

Estimated Error:

Temperature: No information was given.

$c_{1}: \pm 4 \%$ (relative error, estimated by compiler).

\subsection{4-Methoxybenzoic acid solubility data in esters}

\begin{tabular}{|c|c|}
\hline $\begin{array}{l}\text { Components: } \\
\text { (1) 4-Methoxybenzoic acid; } \mathrm{C}_{8} \mathrm{H}_{8} \mathrm{O}_{3} \text {; } \\
\text { [100-09-4] } \\
\text { (2) Ethyl ethanoate; } \mathrm{C}_{4} \mathrm{H}_{8} \mathrm{O}_{2} \text {; } \\
\text { [141-78-6] }\end{array}$ & $\begin{array}{l}\text { Original Measurements: } \\
{ }^{9} \text { K. R. Hoover, D. M. Stovall, E. } \\
\text { Pustejovsky, R. Coaxum, K. Pop, } \\
\text { W. E. Acree, Jr., and M. H. } \\
\text { Abraham, Can. J. Chem. 82, } 1353 \\
\text { (2004). }\end{array}$ \\
\hline $\begin{array}{l}\text { Variables: } \\
T / \mathrm{K}=298.15\end{array}$ & $\begin{array}{l}\text { Prepared by: } \\
\text { W. E. Acree, Jr. }\end{array}$ \\
\hline
\end{tabular}

\section{Experimental Values}

\begin{tabular}{|c|c|}
\hline$x_{2}{ }^{a}$ & $x_{1}{ }^{\mathrm{b}}$ \\
\hline 0.9869 & 0.01308 \\
\hline
\end{tabular}

${ }^{a} x_{2}$ : mole fraction of component 2 in the saturated solution.

${ }^{\mathrm{b}} x_{1}$ : mole fraction solubility of the solute.

\section{Auxiliary Information}

\section{Method/Apparatus/Procedure:}

Constant-temperature bath, calorimetric thermometer, and an ultraviolet/ visible spectrophotometer.

Excess solute and solvent were placed in amber glass bottles and allowed to equilibrate for several days at constant temperature. Attainment of equilibrium was verified by several repetitive measurements and by approaching equilibrium from supersaturation. Aliquots of saturated solutions were transferred through a coarse filter into tared volumetric flasks, weighed, and diluted with methanol. Concentrations were determined by spectrophotometric measurements at $273 \mathrm{~nm}$.

Source and Purity of Chemicals:

(1) $99 \%$, Aldrich Chemical Company, Milwaukee, WI, USA, was used as received.

(2) $99.9 \%$, HPLC grade, Aldrich Chemical Company, stored over molecular sieves and distilled shortly before use.

Estimated Error:

Temperature: $\pm 0.1 \mathrm{~K}$.

$x_{1}: \pm 1.5 \%$ (relative error)

\begin{tabular}{ll}
\hline \hline Components: & Original Measurements: \\
(1) 4-Methoxybenzoic acid; $\mathrm{C}_{8} \mathrm{H}_{8} \mathrm{O}_{3} ;$ & ${ }^{9}$ K. R. Hoover, D. M. Stovall, E. \\
[100-09-4] & Pustejovsky, R. Coaxum, K. Pop, \\
(2) Butyl ethanoate; $\mathrm{C}_{6} \mathrm{H}_{12} \mathrm{O}_{2} ;$ & W. E. Acree, Jr., and M. H. \\
{$[123-864]$} & Abraham, Can. J. Chem. 82, 1353 \\
& $(2004)$. \\
\hline Variables: & Prepared by: \\
$T / \mathrm{K}=298.15$ & W. E. Acree, Jr. \\
\hline
\end{tabular}

\section{Experimental Values}

\begin{tabular}{lc}
\hline \hline$x_{2}{ }^{\mathrm{a}}$ & $x_{1}{ }^{\mathrm{b}}$ \\
\hline 0.9891 & 0.01087 \\
\hline${ }^{\mathrm{a}} x_{2}:$ mole fraction of component 2 in the saturated solution. & \\
${ }^{\mathrm{b}} x_{1}:$ mole fraction solubility of the solute. &
\end{tabular}

\section{Auxiliary Information}

\section{Method/Apparatus/Procedure:}

Constant-temperature bath, calorimetric thermometer, and an ultraviolet/ visible spectrophotometer.

Excess solute and solvent were placed in amber glass bottles and allowed to equilibrate for several days at constant temperature. Attainment of equilibrium was verified by several repetitive measurements and by approaching equilibrium from supersaturation. Aliquots of saturated solutions were transferred through a coarse filter into tared volumetric flasks, weighed, and diluted with methanol. Concentrations were determined by spectrophotometric measurements at $273 \mathrm{~nm}$.

Source and Purity of Chemicals:

(1) $99 \%$, Aldrich Chemical Company, Milwaukee, WI, USA, was used as received.

(2) 99.7\%, HPLC grade, Aldrich Chemical Company, stored over molecular sieves and distilled shortly before use.

Estimated Error:

Temperature: $\pm 0.1 \mathrm{~K}$.

$x_{1}: \pm 1.5 \%$ (relative error) 


\section{Components:}

(1) 4-Methoxybenzoic acid; $\mathrm{C}_{8} \mathrm{H}_{8} \mathrm{O}_{3}$; [100-09-4]

(2) Pentyl ethanoate; $\mathrm{C}_{7} \mathrm{H}_{14} \mathrm{O}_{2}$; [628-63-7]

\section{Variables:}

$T / \mathrm{K}=298.15$

Original Measurements:

${ }^{9}$ K. R. Hoover, D. M. Stovall, E. Pustejovsky, R. Coaxum, K. Pop, W. E. Acree, Jr., and M. H. Abraham, Can. J. Chem. 82, 1353 (2004).

Prepared by:

W. E. Acree, Jr.

Experimental Values

\begin{tabular}{lc}
\hline \hline$x_{2}{ }^{\mathrm{a}}$ & $x_{1}{ }^{\mathrm{b}}$ \\
\hline 0.9905 & 0.00949
\end{tabular}

${ }^{\mathrm{a}} x_{2}$ : mole fraction of component 2 in the saturated solution.

${ }^{\mathrm{b}} \mathrm{x}_{1}$ : mole fraction solubility of the solute.

\section{Auxiliary Information}

\section{Method/Apparatus/Procedure:}

Constant-temperature bath, calorimetric thermometer, and an ultraviolet/ visible spectrophotometer.

Excess solute and solvent were placed in amber glass bottles and allowed to equilibrate for several days at constant temperature. Attainment of equilibrium was verified by several repetitive measurements and by approaching equilibrium from supersaturation. Aliquots of saturated solutions were transferred through a coarse filter into tared volumetric flasks, weighed, and diluted with methanol. Concentrations were determined by

spectrophotometric measurements at $273 \mathrm{~nm}$.

Source and Purity of Chemicals:

(1) $99 \%$, Aldrich Chemical Company, Milwaukee, WI, USA, was used as received.

(2) $99 \%$, Aldrich Chemical Company, stored over molecular sieves and distilled shortly before use.

\section{Estimated Error:}

Temperature: $\pm 0.1 \mathrm{~K}$

$x_{1}: \pm 1.5 \%$ (relative error).

\subsection{4-Methoxybenzoic acid solubility data in ethers}

\begin{tabular}{ll}
\hline \hline Components: & Original Measurements: \\
$\begin{array}{l}\text { (1) 4-Methoxybenzoic acid; } \mathrm{C}_{8} \mathrm{H}_{8} \mathrm{O}_{3} ; \\
{[100-09-4]}\end{array}$ & ${ }^{9}$ K. R. Hoover, D. M. Stovall, E. \\
Pustejovsky, R. Coaxum, K. Pop, \\
(2) $1,1^{\prime}-$ Oxybisethane; $\mathrm{C}_{4} \mathrm{H}_{10} \mathrm{O} ;$ & W. E. Acree, Jr., and M. H. \\
{$[60-29-7]$} & $\begin{array}{l}\text { Abraham, Can. J. Chem. 82, 1353 } \\
(2004) .\end{array}$ \\
\hline Variables: & Prepared by: \\
$T / \mathrm{K}=298.15$ & W. E. Acree, Jr. \\
\hline
\end{tabular}

Experimental Values

\begin{tabular}{lc}
\hline \hline$x_{2}{ }^{\mathrm{a}}$ & $x_{1}{ }^{\mathrm{b}}$ \\
\hline 0.9905 & 0.00952 \\
\hline${ }^{\mathrm{a}} x_{2}:$ mole fraction of component 2 in the saturated solution. & \\
${ }^{\mathrm{b}} x_{1}:$ mole fraction solubility of the solute. &
\end{tabular}

\section{Auxiliary Information}

Method/Apparatus/Procedure:

Constant-temperature bath, calorimetric thermometer, and an ultraviolet/ visible spectrophotometer.

Excess solute and solvent were placed in amber glass bottles and allowed to equilibrate for several days at constant temperature. Attainment of equilibrium was verified by several repetitive measurements and by approaching equilibrium from supersaturation. Aliquots of saturated solutions were transferred through a coarse filter into tared volumetric flasks, weighed, and diluted with methanol. Concentrations were determined by spectrophotometric measurements at $273 \mathrm{~nm}$.

\section{Source and Purity of Chemicals:}

(1) $99 \%$, Aldrich Chemical Company, Milwaukee, WI, USA, was used as received.

(2) $99+\%$, anhydrous, Aldrich Chemical Company, stored over molecular sieves and distilled shortly before use.

\section{Estimated Error:}

Temperature: $\pm 0.1 \mathrm{~K}$.

$x_{1}: \pm 1.5 \%$ (relative error).

\begin{tabular}{|c|c|}
\hline $\begin{array}{l}\text { Components: } \\
\text { (1) 4-Methoxybenzoic acid; } \mathrm{C}_{8} \mathrm{H}_{8} \mathrm{O}_{3} \text {; } \\
\text { [100-09-4] } \\
\text { (2) } 2,2^{\prime}-\text {-Oxybispropane; } \mathrm{C}_{6} \mathrm{H}_{14} \mathrm{O} \text {; } \\
\text { [108-20-3] }\end{array}$ & $\begin{array}{l}\text { Original Measurements: } \\
{ }^{9} \text { K. R. Hoover, D. M. Stovall, E. } \\
\text { Pustejovsky, R. Coaxum, K. Pop, } \\
\text { W. E. Acree, Jr., and M. H. } \\
\text { Abraham, Can. J. Chem. 82, } 1353 \\
\text { (2004). }\end{array}$ \\
\hline $\begin{array}{l}\text { Variables: } \\
T / \mathrm{K}=298.15\end{array}$ & $\begin{array}{l}\text { Prepared by: } \\
\text { W. E. Acree, Jr. }\end{array}$ \\
\hline
\end{tabular}

Experimental Values

\begin{tabular}{lc}
\hline \hline$x_{2}{ }^{\mathrm{a}}$ & $x_{1}{ }^{\mathrm{b}}$ \\
\hline 0.9962 & 0.00377
\end{tabular}

${ }^{\mathrm{a}} x_{2}$ : mole fraction of component 2 in the saturated solution.

${ }^{\mathrm{b}} x_{1}$ : mole fraction solubility of the solute.

\section{Auxiliary Information}

\section{Method/Apparatus/Procedure:}

Constant-temperature bath, calorimetric thermometer, and an ultraviolet/ visible spectrophotometer.

Excess solute and solvent were placed in amber glass bottles and allowed to equilibrate for several days at constant temperature. Attainment of equilibrium was verified by several repetitive measurements and by approaching equilibrium from supersaturation. Aliquots of saturated solutions were transferred through a coarse filter into tared volumetric flasks, weighed, and diluted with methanol. Concentrations were determined by spectrophotometric measurements at $273 \mathrm{~nm}$.

Source and Purity of Chemicals:

(1) $99 \%$, Aldrich Chemical Company, Milwaukee, WI, USA, was used as received.

(2) $99 \%$, anhydrous, Aldrich Chemical Company, stored over molecular sieves and distilled shortly before use.

\section{Estimated Error:}

Temperature: $\pm 0.1 \mathrm{~K}$.

$x_{1}: \pm 1.5 \%$ (relative error). 


\section{Components:}

(1) 4-Methoxybenzoic acid; $\mathrm{C}_{8} \mathrm{H}_{8} \mathrm{O}_{3}$; [100-09-4]

(2) 1,1'-Oxybisbutane; $\mathrm{C}_{8} \mathrm{H}_{18} \mathrm{O}$; [142-96-1]

\section{Variables:}

$T / \mathrm{K}=298.15$
${ }^{9}$ K. R. Hoover, D. M. Stovall, E. Pustejovsky, R. Coaxum, K. Pop, W. E. Acree, Jr., and M. H. Abraham, Can. J. Chem. 82, 1353 (2004).

Prepared by:

W. E. Acree, Jr.
Original Measurements:

Experimental Values

\begin{tabular}{lc}
\hline \hline$x_{2}{ }^{\mathrm{a}}$ & $x_{1}{ }^{\mathrm{b}}$ \\
\hline 0.9972 & 0.00275 \\
${ }^{\mathrm{a}} x_{2}:$ mole fraction of component 2 in the saturated solution. \\
${ }^{\mathrm{b}} x_{1}:$ mole fraction solubility of the solute.
\end{tabular}

\section{Auxiliary Information}

\section{Method/Apparatus/Procedure:}

Constant-temperature bath, calorimetric thermometer, and an ultraviolet/ visible spectrophotometer.

Excess solute and solvent were placed in amber glass bottles and allowed to equilibrate for several days at constant temperature. Attainment of equilibrium was verified by several repetitive measurements and by approaching equilibrium from supersaturation. Aliquots of saturated solutions were transferred through a coarse filter into tared volumetric flasks, weighed, and diluted with methanol. Concentrations were determined by

spectrophotometric measurements at $273 \mathrm{~nm}$.

Source and Purity of Chemicals:

(1) $99 \%$, Aldrich Chemical Company, Milwaukee, WI, USA, was used as received.

(2) $99.3 \%$, anhydrous, Aldrich Chemical Company, stored over molecular sieves and distilled shortly before use.

\section{Estimated Error:}

Temperature: $\pm 0.1 \mathrm{~K}$.

$x_{1}: \pm 1.5 \%$ (relative error).

\section{Components:}

Original Measurements:

(1) 4-Methoxybenzoic acid; $\mathrm{C}_{8} \mathrm{H}_{8} \mathrm{O}_{3}$;

[100-09-4]

(2) Tetrahydrofuran; $\mathrm{C}_{4} \mathrm{H}_{8} \mathrm{O}$; [109-99-9]

K. R. Hoover, D. M. Stovall, E. Pustejovsky, R. Coaxum, K. Pop, W. E. Acree, Jr., and M. H. Abraham, Can. J. Chem. 82, 1353 (2004).

\section{Variables:}

$T / \mathrm{K}=298.15$

Prepared by:

W. E. Acree, Jr.

\section{Experimental Values}

\begin{tabular}{lc}
\hline \hline$x_{2}{ }^{\mathrm{a}}$ & $x_{1}{ }^{\mathrm{b}}$ \\
\hline 0.9419 & 0.05811 \\
\hline${ }^{\mathrm{a}} x_{2}:$ mole fraction of component 2 in the saturated solution. \\
${ }^{\mathrm{b}} x_{1}:$ mole fraction solubility of the solute.
\end{tabular}

\section{Auxiliary Information}

\section{Method/Apparatus/Procedure:}

Constant-temperature bath, calorimetric thermometer, and an ultraviolet/ visible spectrophotometer.

Excess solute and solvent were placed in amber glass bottles and allowed to equilibrate for several days at constant temperature. Attainment of equilibrium was verified by several repetitive measurements and by approaching equilibrium from supersaturation. Aliquots of saturated solutions were transferred through a coarse filter into tared volumetric flasks, weighed, and diluted with methanol. Concentrations were determined by spectrophotometric measurements at $273 \mathrm{~nm}$.

\section{Source and Purity of Chemicals:}

(1) $99 \%$, Aldrich Chemical Company, Milwaukee, WI, USA, was used as received.

(2) $99.9 \%$, anhydrous, Aldrich Chemical Company, stored over molecular sieves and distilled shortly before use.

Estimated Error:

Temperature: $\pm 0.1 \mathrm{~K}$.

$x_{1}: \pm 1.5 \%$ (relative error)

\section{Components:}

(1) 4-Methoxybenzoic acid; $\mathrm{C}_{8} \mathrm{H}_{8} \mathrm{O}_{3}$; [100-09-4]

Original Measurements:

(2) Tetrahydrofuran; $\mathrm{C}_{4} \mathrm{H}_{8} \mathrm{O}$; [109-99-9]

${ }^{63}$ C. K. Hancock, J. N. Pawloski, and J. P. Idoux, J. Org. Chem. 32, 1931 (1967).

Variables:

$T / \mathrm{K}=303.15$

Prepared by:

W. E. Acree, Jr.

\section{Experimental Values}

\begin{tabular}{lc}
\hline \hline$x_{2}{ }^{\mathrm{a}}$ & $x_{1}{ }^{\mathrm{b}}$ \\
\hline 0.934 & 0.066 \\
\hline${ }^{\mathrm{a}} x_{2}:$ mole fraction of component 2 in the saturated solution. & \\
${ }^{\mathrm{b}} x_{1}:$ mole fraction solubility of the solute. &
\end{tabular}

\section{Auxiliary Information}

\section{Method/Apparatus/Procedure:}

Constant-temperature bath, Soxhlet thimble, conical flask, and analytical balance.

Excess solute and solvent were placed in a conical flask and allowed to equilibrate for several days at constant temperature. Attainment of equilibrium was verified by several repetitive measurements and by approaching equilibrium from supersaturation. Aliquots of saturated solutions were transferred using a Soxhlet thimble equipped with a one-hole cork stopper and an inverted U-shaped delivery tube to a second ground-glass stoppered flask suspended in the $303 \mathrm{~K}$ water bath. Successive portions of the contents were evaporated at room temperature in a tared aluminum foil weighing dish under a bell jar through which a slow stream of dry air was passed. The second flask plus unused saturated solution and the aluminum foil dish plus residue were weighed. The saturation solubility of the solute was calculated from the recorded mass data and molar masses of the solute and solvent.

\section{Source and Purity of Chemicals:}

(1) Purity not given, Chemical source not specified, was recrystallized several times from aqueous-ethanol mixture, and then dried under vacuum over phosphorous pentoxide.

(2) Practical grade, Chemical source not specified, stored over sodium hydroxide pellets for $24 \mathrm{~h}$, and then passed through $2 \times 70-\mathrm{cm}$ chromatographic adsorption columns containing activated alumina. After this treatment, the purified solvent was stored over copper in a nitrogen atmosphere. 
Estimated Error:

Temperature: $\pm 0.02 \mathrm{~K}$.

$x_{1}: \pm 1.0 \%$ (relative error).

\begin{tabular}{ll}
\hline \hline Components: & Original Measurements: \\
(1) 4-Methoxybenzoic acid; $\mathrm{C}_{8} \mathrm{H}_{8} \mathrm{O}_{3} ;$ & $\begin{array}{l}9 \text { K. R. Hoover, D. M. Stovall, E. } \\
\text { Pustejovsky, R. Coaxum, K. Pop, }\end{array}$ \\
(100-09-4] 1 (1,4-Dioxane; $\mathrm{C}_{4} \mathrm{H}_{8} \mathrm{O}_{2} ;$ [123-91-1] & W. E. Acree, Jr., and M. H. \\
& $\begin{array}{l}\text { Abraham, Can. J. Chem. 82, 1353 } \\
(2004) .\end{array}$ \\
\hline Variables: & Prepared by: \\
$T / \mathrm{K}=298.15$ & W. E. Acree, Jr. \\
\hline
\end{tabular}

Experimental Values

\begin{tabular}{lc}
\hline \hline$x_{2}{ }^{\mathrm{a}}$ & $x_{1}{ }^{\mathrm{b}}$ \\
\hline 0.9652 & 0.03476 \\
\hline${ }^{\mathrm{a}} x_{2}:$ mole fraction of component 2 in the saturated solution. \\
${ }^{\mathrm{b}}{ }_{x_{1}: \text { mole fraction solubility of the solute. }}$
\end{tabular}

\section{Auxiliary Information}

Method/Apparatus/Procedure:

Constant-temperature bath, calorimetric thermometer, and an ultraviolet/ visible spectrophotometer.

Excess solute and solvent were placed in amber glass bottles and allowed to equilibrate for several days at constant temperature. Attainment of equilibrium was verified by several repetitive measurements and by approaching equilibrium from supersaturation. Aliquots of saturated solutions were transferred through a coarse filter into tared volumetric flasks, weighed, and diluted with methanol. Concentrations were determined by

spectrophotometric measurements at $273 \mathrm{~nm}$.

Source and Purity of Chemicals:

(1) $99 \%$, Aldrich Chemical Company, Milwaukee, WI, USA, was used as received.

(2) $99.8 \%$, anhydrous, Aldrich Chemical Company, stored over molecular sieves and distilled shortly before use.

Estimated Error:

Temperature: $\pm 0.1 \mathrm{~K}$.

$x_{1}: \pm 1.5 \%$ (relative error).

\begin{tabular}{|c|c|}
\hline $\begin{array}{l}\text { Components: } \\
\text { (1) 4-Methoxybenzoic acid; } \mathrm{C}_{8} \mathrm{H}_{8} \mathrm{O}_{3} \text {; } \\
\text { [100-09-4] } \\
\text { (2) 1,4-Dioxane; } \mathrm{C}_{4} \mathrm{H}_{8} \mathrm{O}_{2} ;[123-91-1]\end{array}$ & $\begin{array}{l}\text { Original Measurements: } \\
{ }^{63} \text { C. K. Hancock, J. N. Pawloski, } \\
\text { and J. P. Idoux, J. Org. Chem. 32, } \\
1931 \text { (1967). }\end{array}$ \\
\hline $\begin{array}{l}\text { Variables: } \\
T / \mathrm{K}=303.15\end{array}$ & $\begin{array}{l}\text { Prepared by: } \\
\text { W. E. Acree, Jr. }\end{array}$ \\
\hline
\end{tabular}

Experimental Values

\begin{tabular}{lc}
\hline \hline$x_{2}{ }^{\mathrm{a}}$ & $x_{1}{ }^{\mathrm{b}}$ \\
\hline 0.959 & 0.041 \\
\hline${ }_{{ }^{\mathrm{a}} x_{2}: \text { mole fraction of component } 2 \text { in the saturated solution. }}$ & \\
${ }^{\mathrm{b}} x_{1}:$ mole fraction solubility of the solute. &
\end{tabular}

Auxiliary Information

Method/Apparatus/Procedure:

Constant-temperature bath, Soxhlet thimble, conical flask, and analytical balance.

Excess solute and solvent were placed in a conical flask and allowed to equilibrate for several days at constant temperature. Attainment of equilibrium was verified by several repetitive measurements and by approaching equilibrium from supersaturation. Weighed aliquots of saturated solutions were removed and titrated with a standardized sodium hydroxide solution (carbonate free) using a pH meter. The endpoint of the titration was determined by computing the second derivative in the $\mathrm{pH}$ versus volume of sodium hydroxide added.

Source and Purity of Chemicals:

(1) Purity not given, Chemical source not specified, was recrystallized several times from aqueous-ethanol mixture, and then dried under vacuum over phosphorous pentoxide.

(2) Practical grade, Chemical source not specified, stored over sodium hydroxide pellets for $24 \mathrm{~h}$, and then passed through $2 \times 70-\mathrm{cm}$ chromatographic adsorption columns containing activated alumina. After this treatment, the purified solvent was stored over copper in a nitrogen atmosphere.

Estimated Error:

Temperature: $\pm 0.02 \mathrm{~K}$.

$x_{1}: \pm 1.0 \%$ (relative error).

\subsection{4-Methoxybenzoic acid solubility data in haloalkanes, haloalkenes, and haloaromatic hydrocarbons}

\begin{tabular}{|c|c|}
\hline $\begin{array}{l}\text { Components: } \\
\text { (1) 4-Methoxybenzoic acid; } \mathrm{C}_{8} \mathrm{H}_{8} \mathrm{O}_{3} \text {; } \\
\text { [100-09-4] } \\
\text { (2) Trichloromethane; } \mathrm{CHCl}_{3} ;[67-66-3]\end{array}$ & $\begin{array}{l}\text { Original Measurements: } \\
{ }^{56} \text { P. A. Ongley, J. Chem. Soc. } \\
\text { 1954, } 3634 .\end{array}$ \\
\hline $\begin{array}{l}\text { Variables: } \\
T / \mathrm{K}=298.15\end{array}$ & $\begin{array}{l}\text { Prepared by: } \\
\text { W. E. Acree, Jr. }\end{array}$ \\
\hline
\end{tabular}

\section{Experimental Values}

The measured solubility was reported to be $-\log _{10} c_{1}=1.130$, which corresponds to a solubility of $c_{1}=0.0741 \mathrm{~mol} \mathrm{dm}^{-3}$.

\section{Auxiliary Information}

\section{Method/Apparatus/Procedure:}

Very little experimental details were provided. Excess solute and solvent were placed in tubes, which were rotated for at least $8 \mathrm{~h}$ at $298 \mathrm{~K}$. The solutions were then removed, concentrated if necessary, and titrated with standard alkali using a mixed (bromothymol blue + neutral red) indicator.

\section{Source and Purity of Chemicals:}

(1) Purity not given, Chemical source not given, solute was either "AnalaR" grade or recrystallized prior to use.

(2) Purity not given, Chemical source not given, no purification details were provided.

\section{Estimated Error:}

Temperature: No information was given.

$c_{1}: \pm 4 \%$ (relative error, estimated by compiler). 


\subsection{4-Methoxybenzoic acid solubility data in alcohols}

\begin{tabular}{ll}
\hline \hline Components: & Original Measurements: \\
(1) 4-Methoxybenzoic acid; $\mathrm{C}_{8} \mathrm{H}_{8} \mathrm{O}_{3} ;$ & ${ }^{9}$ K. R. Hoover, D. M. Stovall, E. \\
[100-09-4] & Pustejovsky, R. Coaxum, K. Pop, \\
(2) Ethanol; $\mathrm{C}_{2} \mathrm{H}_{6} \mathrm{O} ;$ [64-17-5] & W. E. Acree, Jr., and M. H. \\
& $\begin{array}{l}\text { Abraham, Can. J. Chem. 82, 1353 } \\
\text { (2004). }\end{array}$ \\
\hline Variables: & Prepared by: \\
$T / \mathrm{K}=298.15$ & W. E. Acree, Jr. \\
\hline
\end{tabular}

\section{Experimental Values}

\begin{tabular}{lc}
\hline \hline$x_{2}{ }^{\mathrm{a}}$ & $x_{1}{ }^{\mathrm{b}}$ \\
\hline 0.9881 & 0.01185 \\
\hline
\end{tabular}

${ }^{a} x_{2}$ : mole fraction of component 2 in the saturated solution.

${ }^{\mathrm{b}} \mathrm{x}_{1}$ : mole fraction solubility of the solute.

\section{Auxiliary Information}

\section{Method/Apparatus/Procedure:}

Constant-temperature bath, calorimetric thermometer, and an ultraviolet/ visible spectrophotometer.

Excess solute and solvent were placed in amber glass bottles and allowed to equilibrate for several days at constant temperature. Attainment of equilibrium was verified by several repetitive measurements and by approaching equilibrium from supersaturation. Aliquots of saturated solutions were transferred through a coarse filter into tared volumetric flasks, weighed, and diluted with methanol. Concentrations were determined by spectrophotometric measurements at $273 \mathrm{~nm}$.

\section{Source and Purity of Chemicals:}

(1) $99 \%$, Aldrich Chemical Company, Milwaukee, WI, USA, was used as received.

(2) Absolute, Aaper Alcohol and Chemical Company, USA, stored over molecular sieves and distilled shortly before use.

\section{Estimated Error:}

Temperature: $\pm 0.1 \mathrm{~K}$.

$x_{1}: \pm 1.5 \%$ (relative error).

\begin{tabular}{|c|c|}
\hline $\begin{array}{l}\text { Components: } \\
\text { (1) 4-Methoxybenzoic acid; } \mathrm{C}_{8} \mathrm{H}_{8} \mathrm{O}_{3} \text {; } \\
\text { [100-09-4] } \\
\text { (2) Ethanol; } \mathrm{C}_{2} \mathrm{H}_{6} \mathrm{O} ;[64-17-5]\end{array}$ & $\begin{array}{l}\text { Original Measurements: } \\
{ }^{72} \text { R. Thuaire, Bull. Soc. Chim. Fr. } \\
3815 \text { (1971). }\end{array}$ \\
\hline $\begin{array}{l}\text { Variables: } \\
T / \mathrm{K}=298.15\end{array}$ & $\begin{array}{l}\text { Prepared by: } \\
\text { W. E. Acree, Jr. }\end{array}$ \\
\hline
\end{tabular}

\section{Experimental Values}

The measured solubility was reported to be $0.273 \mathrm{~mol} / \mathrm{kg}$ of solvent, which corresponds to a mole fraction solubility of $x_{1}=0.01242$.

\section{Auxiliary Information}

\section{Method/Apparatus/Procedure:}

Very little experimental details were provided. Solubility was determined by a gravimetric method. The saturated solution was evaporated to dryness and the remaining solid residue was weighed. The solubility was calculated from the mass of the solid residue and mass of saturated solution analyzed.

\section{Source and Purity of Chemicals:}

(1) Purity not given, Chemical source not given, no purification details were provided.

(2) Purity not given, Chemical source not given, no purification details were provided.

Estimated Error:

Temperature: $\pm 0.05 \mathrm{~K}$.

$x_{1}: \pm 4 \%$ (relative error, estimated by compiler).

\begin{tabular}{ll}
\hline \hline Components: & Original Measurements: \\
(1) 4-Methoxybenzoic acid; $\mathrm{C}_{8} \mathrm{H}_{8} \mathrm{O}_{3} ;$ & ${ }^{9}$ K. R. Hoover, D. M. Stovall, E. \\
[100-09-4] & Pustejovsky, R. Coaxum, K. Pop, \\
(2) 1-Propanol; $\mathrm{C}_{3} \mathrm{H}_{8} \mathrm{O} ;$ [71-23-8] & W. E. Acree, Jr., and M. H. \\
& Abraham, Can. J. Chem. 82, 1353 \\
& $(2004)$. \\
\hline Variables: & Prepared by: \\
$T / \mathrm{K}=298.15$ & W. E. Acree, Jr. \\
\hline
\end{tabular}

\section{Experimental Values}

\begin{tabular}{lc}
\hline \hline$x_{2}{ }^{\mathrm{a}}$ & $x_{1}{ }^{\mathrm{b}}$ \\
\hline 0.9899 & 0.01008 \\
\hline${ }^{\mathrm{a}} x_{2}:$ mole fraction of component 2 in the saturated solution. \\
${ }^{\mathrm{b}} x_{1}:$ mole fraction solubility of the solute.
\end{tabular}

\section{Auxiliary Information}

Method/Apparatus/Procedure:

Constant-temperature bath, calorimetric thermometer, and an ultraviolet/ visible spectrophotometer.

Excess solute and solvent were placed in amber glass bottles and allowed to equilibrate for several days at constant temperature. Attainment of equilibrium was verified by several repetitive measurements and by approaching equilibrium from supersaturation. Aliquots of saturated solutions were transferred through a coarse filter into tared volumetric flasks, weighed, and diluted with methanol. Concentrations were determined by spectrophotometric measurements at $273 \mathrm{~nm}$.

Source and Purity of Chemicals:

(1) $99 \%$, Aldrich Chemical Company, Milwaukee, WI, USA, was used as received.

(2) $99+\%$, anhydrous, Aldrich Chemical Company, stored over molecular sieves and distilled shortly before use.

Estimated Error:

Temperature: $\pm 0.1 \mathrm{~K}$.

$x_{1}: \pm 1.5 \%$ (relative error) 


\section{Components:}

(1) 4-Methoxybenzoic acid; $\mathrm{C}_{8} \mathrm{H}_{8} \mathrm{O}_{3}$; [100-09-4]

(2) 2-Propanol; $\mathrm{C}_{3} \mathrm{H}_{8} \mathrm{O}$; [67-63-0]

Variables:

$T / \mathrm{K}=298.15$

Original Measurements:

${ }^{9}$ K. R. Hoover, D. M. Stovall, E. Pustejovsky, R. Coaxum, K. Pop, W. E. Acree, Jr., and M. H. Abraham, Can. J. Chem. 82, 1353 (2004).

Prepared by:

W. E. Acree, Jr

Experimental Values

\begin{tabular}{lc}
\hline \hline$x_{2}{ }^{\mathrm{a}}$ & $x_{1}{ }^{\mathrm{b}}$ \\
\hline 0.9894 & 0.01060
\end{tabular}

${ }^{a} x_{2}$ : mole fraction of component 2 in the saturated solution.

${ }^{\mathrm{b}} x_{1}$ : mole fraction solubility of the solute.

\section{Auxiliary Information}

\section{Method/Apparatus/Procedure:}

Constant-temperature bath, calorimetric thermometer, and an ultraviolet/ visible spectrophotometer.

Excess solute and solvent were placed in amber glass bottles and allowed to equilibrate for several days at constant temperature. Attainment of equilibrium was verified by several repetitive measurements and by approaching equilibrium from supersaturation. Aliquots of saturated solutions were transferred through a coarse filter into tared volumetric flasks, weighed, and diluted with methanol. Concentrations were determined by

spectrophotometric measurements at $273 \mathrm{~nm}$.

Source and Purity of Chemicals:

(1) $99 \%$, Aldrich Chemical Company, Milwaukee, WI, USA, was used as received.

(2) $99+\%$, anhydrous, Aldrich Chemical Company, stored over molecular sieves and distilled shortly before use.

\section{Estimated Error:}

Temperature: $\pm 0.1 \mathrm{~K}$.

$x_{1}: \pm 1.5 \%$ (relative error).

\section{Components:}

Original Measurements:

(1) 4-Methoxybenzoic acid; $\mathrm{C}_{8} \mathrm{H}_{8} \mathrm{O}_{3}$; [100-09-4]

(2) 1-Butanol; $\mathrm{C}_{4} \mathrm{H}_{10} \mathrm{O}$; [71-36-3]

K. R. Hoover, D. M. Stovall, E. Pustejovsky, R. Coaxum, K. Pop, W. E. Acree, Jr., and M. H. Abraham, Can. J. Chem. 82, 1353 (2004).

\section{Variables:}

$T / \mathrm{K}=298.15$

Prepared by:

W. E. Acree, Jr.

Experimental Values

\begin{tabular}{lc}
\hline \hline$x_{2}{ }^{\mathrm{a}}$ & $x_{1}{ }^{\mathrm{b}}$ \\
\hline 0.9891 & 0.01088 \\
\hline${ }^{\mathrm{a}} x_{2}:$ mole fraction of component 2 in the saturated solution. \\
${ }^{\mathrm{b}} x_{1}:$ mole fraction solubility of the solute.
\end{tabular}

\section{Auxiliary Information}

\section{Method/Apparatus/Procedure:}

Constant-temperature bath, calorimetric thermometer, and an ultraviolet/ visible spectrophotometer.

Excess solute and solvent were placed in amber glass bottles and allowed to equilibrate for several days at constant temperature. Attainment of equilibrium was verified by several repetitive measurements and by approaching equilibrium from supersaturation. Aliquots of saturated solutions were transferred through a coarse filter into tared volumetric flasks, weighed, and diluted with methanol. Concentrations were determined by spectrophotometric measurements at $273 \mathrm{~nm}$.

Source and Purity of Chemicals:

(1) $99 \%$, Aldrich Chemical Company, Milwaukee, WI, USA, was used as received.

(2) $99.8+\%$, HPLC grade, Aldrich Chemical Company, stored over molecular sieves and distilled shortly before use.

Estimated Error:

Temperature: $\pm 0.1 \mathrm{~K}$.

$x_{1}: \pm 1.5 \%$ (relative error)

\begin{tabular}{ll}
\hline \hline Components: & Original Measurements: \\
(1) 4-Methoxybenzoic acid; $\mathrm{C}_{8} \mathrm{H}_{8} \mathrm{O}_{3} ;$ & ${ }^{9}$ K. R. Hoover, D. M. Stovall, E. \\
[100-09-4] & Pustejovsky, R. Coaxum, K. Pop, \\
(2) 2-Butanol; $\mathrm{C}_{4} \mathrm{H}_{10} \mathrm{O} ;$ [78-92-2] & W. E. Acree, Jr., and M. H. \\
& Abraham, Can. J. Chem. 82, 1353 \\
& $(2004)$. \\
\hline Variables: & Prepared by: \\
$T / \mathrm{K}=298.15$ & W. E. Acree, Jr. \\
\hline
\end{tabular}

\section{Experimental Values}

\begin{tabular}{lc}
\hline \hline$x_{2}{ }^{\mathrm{a}}$ & $x_{1}{ }^{\mathrm{b}}$ \\
\hline 0.9882 & 0.01175 \\
\hline
\end{tabular}

${ }^{a} x_{2}$ : mole fraction of component 2 in the saturated solution.

${ }^{\mathrm{b}} x_{1}$ : mole fraction solubility of the solute.

\section{Auxiliary Information}

\section{Method/Apparatus/Procedure:}

Constant-temperature bath, calorimetric thermometer, and an ultraviolet visible spectrophotometer.

Excess solute and solvent were placed in amber glass bottles and allowed to equilibrate for several days at constant temperature. Attainment of equilibrium was verified by several repetitive measurements and by approaching equilibrium from supersaturation. Aliquots of saturated solutions were transferred through a coarse filter into tared volumetric flasks, weighed, and diluted with methanol. Concentrations were determined by spectrophotometric measurements at $273 \mathrm{~nm}$.

Source and Purity of Chemicals:

(1) $99 \%$, Aldrich Chemical Company, Milwaukee, WI, USA, was used as received.

(2) $99+\%$, anhydrous, Aldrich Chemical Company, stored over molecular sieves and distilled shortly before use.

Estimated Error:

Temperature: $\pm 0.1 \mathrm{~K}$.

$x_{1}: \pm 1.5 \%$ (relative error) 


\section{Components:}

(1) 4-Methoxybenzoic acid; $\mathrm{C}_{8} \mathrm{H}_{8} \mathrm{O}_{3}$; [100-09-4]

(2) 2-Methyl-1-propanol; $\mathrm{C}_{4} \mathrm{H}_{10} \mathrm{O}$; [78-83-1]

\section{Variables:}

$T / \mathrm{K}=298.15$

Original Measurements:

${ }^{9}$ K. R. Hoover, D. M. Stovall, E. Pustejovsky, R. Coaxum, K. Pop, W. E. Acree, Jr., and M. H. Abraham, Can. J. Chem. 82, 1353 (2004).

\section{Prepared by:}

W. E. Acree, Jr.

\section{Experimental Values}

\begin{tabular}{lc}
\hline \hline$x_{2}{ }^{\mathrm{a}}$ & $x_{1}{ }^{\mathrm{b}}$ \\
\hline 0.9926 & 0.00740
\end{tabular}

${ }^{a} x_{2}$ : mole fraction of component 2 in the saturated solution.

${ }^{\mathrm{b}} \mathrm{x}_{1}$ : mole fraction solubility of the solute.

\section{Auxiliary Information}

\section{Method/Apparatus/Procedure:}

Constant-temperature bath, calorimetric thermometer, and an ultraviolet/ visible spectrophotometer.

Excess solute and solvent were placed in amber glass bottles and allowed to equilibrate for several days at constant temperature. Attainment of equilibrium was verified by several repetitive measurements and by approaching equilibrium from supersaturation. Aliquots of saturated solutions were transferred through a coarse filter into tared volumetric flasks, weighed, and diluted with methanol. Concentrations were determined by spectrophotometric measurements at $273 \mathrm{~nm}$.

\section{Source and Purity of Chemicals:}

(1) $99 \%$, Aldrich Chemical Company, Milwaukee, WI, USA, was used as received.

(2) $99+\%$, anhydrous, Aldrich Chemical Company, stored over molecular sieves and distilled shortly before use.

\section{Estimated Error:}

Temperature: $\pm 0.1 \mathrm{~K}$.

$x_{1}: \pm 1.5 \%$ (relative error).

\section{Components:}

Original Measurements:

(1) 4-Methoxybenzoic acid; $\mathrm{C}_{8} \mathrm{H}_{8} \mathrm{O}_{3}$; [100-09-4]

(2) 2-Methyl-2-propanol; $\mathrm{C}_{4} \mathrm{H}_{10} \mathrm{O}$; [75-65-0]

K. R. Hoover, D. M. Stovall, E. Pustejovsky, R. Coaxum, K. Pop, W. E. Acree, Jr., and M. H. Abraham, Can. J. Chem. 82, 1353 (2004).

\section{Variables:}

$T / \mathrm{K}=298.15$

Prepared by:

W. E. Acree, Jr.

\section{Experimental Values}

\begin{tabular}{lc}
\hline \hline$x_{2}{ }^{\mathrm{a}}$ & $x_{1}{ }^{\mathrm{b}}$ \\
\hline 0.9844 & 0.01561 \\
\hline${ }^{\mathrm{a}} x_{2}:$ mole fraction of component 2 in the saturated solution. & \\
${ }^{\mathrm{b}} x_{1}:$ mole fraction solubility of the solute. &
\end{tabular}

\section{Auxiliary Information}

\section{Method/Apparatus/Procedure:}

Constant-temperature bath, calorimetric thermometer, and an ultraviolet/ visible spectrophotometer.

Excess solute and solvent were placed in amber glass bottles and allowed to equilibrate for several days at constant temperature. Attainment of equilibrium was verified by several repetitive measurements and by approaching equilibrium from supersaturation. Aliquots of saturated solutions were transferred through a coarse filter into tared volumetric flasks, weighed, and diluted with methanol. Concentrations were determined by spectrophotometric measurements at $273 \mathrm{~nm}$.

\section{Source and Purity of Chemicals:}

(1) $99 \%$, Aldrich Chemical Company, Milwaukee, WI, USA, was used as received.

(2) $99+\%$, Arco Chemical Company, USA, stored over molecular sieves and distilled shortly before use.

Estimated Error:

Temperature: $\pm 0.1 \mathrm{~K}$.

$x_{1}: \pm 1.5 \%$ (relative error).

\begin{tabular}{ll}
\hline \hline Components: & Original Measurements: \\
(1) 4-Methoxybenzoic acid; $\mathrm{C}_{8} \mathrm{H}_{8} \mathrm{O}_{3} ;$ & ${ }^{9}$ K. R. Hoover, D. M. Stovall, E. \\
[100-09-4] & Pustejovsky, R. Coaxum, K. Pop, \\
(2) 1-Pentanol; $\mathrm{C}_{5} \mathrm{H}_{12} \mathrm{O} ;$ [71-41-0] & W. E. Acree, Jr., and M. H. \\
& Abraham, Can. J. Chem. 82, 1353 \\
& $(2004)$. \\
\hline Variables: & Prepared by: \\
$T / \mathrm{K}=298.15$ & W. E. Acree, Jr. \\
\hline
\end{tabular}

\section{Experimental Values}

\begin{tabular}{lc}
\hline \hline$x_{2}{ }^{\mathrm{a}}$ & $x_{1}{ }^{\mathrm{b}}$ \\
\hline 0.9887 & 0.01130 \\
\hline${ }^{a} x_{2}:$ mole fraction of component 2 in the saturated solution. & \\
${ }^{\mathrm{b}} x_{1}:$ mole fraction solubility of the solute. &
\end{tabular}

\section{Auxiliary Information}

\section{Method/Apparatus/Procedure:}

Constant-temperature bath, calorimetric thermometer, and an ultraviolet/ visible spectrophotometer.

Excess solute and solvent were placed in amber glass bottles and allowed to equilibrate for several days at constant temperature. Attainment of equilibrium was verified by several repetitive measurements and by approaching equilibrium from supersaturation. Aliquots of saturated solutions were transferred through a coarse filter into tared volumetric flasks, weighed, and diluted with methanol. Concentrations were determined by spectrophotometric measurements at $273 \mathrm{~nm}$.

\section{Source and Purity of Chemicals:}

(1) $99 \%$, Aldrich Chemical Company, Milwaukee, WI, USA, was used as received.

(2) $99+\%$, Aldrich Chemical Company, stored over molecular sieves and distilled shortly before use.

\section{Estimated Error:}

Temperature: $\pm 0.1 \mathrm{~K}$.

$x_{1}: \pm 1.5 \%$ (relative error) 


\section{Components:}

(1) 4-Methoxybenzoic acid; $\mathrm{C}_{8} \mathrm{H}_{8} \mathrm{O}_{3}$; [100-09-4]

(2) 2-Pentanol; $\mathrm{C}_{5} \mathrm{H}_{12} \mathrm{O}$; [6032-29-7]

Original Measurements:

${ }^{9}$ K. R. Hoover, D. M. Stovall, E. Pustejovsky, R. Coaxum, K. Pop, W. E. Acree, Jr., and M. H. Abraham, Can. J. Chem. 82, 1353 (2004).

\section{Variables:}

$T / \mathrm{K}=298.15$

Prepared by:

W. E. Acree, Jr.

Experimental Values

\begin{tabular}{lc}
\hline \hline$x_{2}{ }^{\mathrm{a}}$ & $x_{1}{ }^{\mathrm{b}}$ \\
\hline 0.9884 & 0.01156
\end{tabular}

${ }^{a} x_{2}$ : mole fraction of component 2 in the saturated solution.

${ }^{\mathrm{b}} \mathrm{x}_{1}$ : mole fraction solubility of the solute.

\section{Auxiliary Information}

\section{Method/Apparatus/Procedure:}

Constant-temperature bath, calorimetric thermometer, and an ultraviolet/ visible spectrophotometer.

Excess solute and solvent were placed in amber glass bottles and allowed to equilibrate for several days at constant temperature. Attainment of equilibrium was verified by several repetitive measurements and by approaching equilibrium from supersaturation. Aliquots of saturated solutions were transferred through a coarse filter into tared volumetric flasks, weighed, and diluted with methanol. Concentrations were determined by

spectrophotometric measurements at $273 \mathrm{~nm}$.

Source and Purity of Chemicals:

(1) $99 \%$, Aldrich Chemical Company, Milwaukee, WI, USA, was used as received.

(2) $99+\%$, Acros Organics, USA, stored over molecular sieves and distilled shortly before use.

\section{Estimated Error:}

Temperature: $\pm 0.1 \mathrm{~K}$

$x_{1}: \pm 1.5 \%$ (relative error).

\section{Components:}

Original Measurements:

(1) 4-Methoxybenzoic acid; $\mathrm{C}_{8} \mathrm{H}_{8} \mathrm{O}_{3}$; [100-09-4]

(2) 3-Methyl-1-butanol; $\mathrm{C}_{5} \mathrm{H}_{12} \mathrm{O}$; [123-51-3]

K. R. Hoover, D. M. Stovall, E. Pustejovsky, R. Coaxum, K. Pop, W. E. Acree, Jr., and M. H. Abraham, Can. J. Chem. 82, 1353 (2004).

\section{Variables:}

$T / \mathrm{K}=298.15$

\section{Prepared by:}

W. E. Acree, Jr.

\section{Experimental Values}

\begin{tabular}{lc}
\hline \hline$x_{2}^{\mathrm{a}}$ & $x_{1}{ }^{\mathrm{b}}$ \\
\hline 0.9908 & 0.00916 \\
\hline${ }^{\mathrm{a}} x_{2}:$ mole fraction of component 2 in the saturated solution. \\
${ }^{\mathrm{b}} x_{1}:$ mole fraction solubility of the solute.
\end{tabular}

\section{Auxiliary Information}

\section{Method/Apparatus/Procedure:}

Constant-temperature bath, calorimetric thermometer, and an ultraviolet/ visible spectrophotometer.

Excess solute and solvent were placed in amber glass bottles and allowed to equilibrate for several days at constant temperature. Attainment of equilibrium was verified by several repetitive measurements and by approaching equilibrium from supersaturation. Aliquots of saturated solutions were transferred through a coarse filter into tared volumetric flasks, weighed, and diluted with methanol. Concentrations were determined by spectrophotometric measurements at $273 \mathrm{~nm}$.

Source and Purity of Chemicals:

(1) $99 \%$, Aldrich Chemical Company, Milwaukee, WI, USA, was used as received.

(2) $99 \%$, anhydrous, Aldrich Chemical Company, stored over molecular sieves and distilled shortly before use.

Estimated Error:

Temperature: $\pm 0.1 \mathrm{~K}$.

$x_{1}: \pm 1.5 \%$ (relative error).

\begin{tabular}{ll}
\hline \hline Components: & Original Measurements: \\
(1) 4-Methoxybenzoic acid; $\mathrm{C}_{8} \mathrm{H}_{8} \mathrm{O}_{3} ;$ & ${ }^{9}$ K. R. Hoover, D. M. Stovall, E. \\
[100-09-4] & Pustejovsky, R. Coaxum, K. Pop, \\
(2) 1-Hexanol; $\mathrm{C}_{6} \mathrm{H}_{14} \mathrm{O} ;[111-27-3]$ & W. E. Acree, Jr., and M. H. \\
& Abraham, Can. J. Chem. 82, 1353 \\
& $(2004)$. \\
\hline Variables: & Prepared by: \\
$T / \mathrm{K}=298.15$ & W. E. Acree, Jr. \\
\hline
\end{tabular}

\section{Experimental Values}

\begin{tabular}{lc}
\hline \hline$x_{2}{ }^{\mathrm{a}}$ & $x_{1}{ }^{\mathrm{b}}$ \\
\hline 0.9881 & 0.01190 \\
\hline${ }^{a} x_{2}:$ mole fraction of component 2 in the saturated solution. & \\
${ }^{\mathrm{b}} x_{1}:$ mole fraction solubility of the solute. &
\end{tabular}

\section{Auxiliary Information}

\section{Method/Apparatus/Procedure:}

Constant-temperature bath, calorimetric thermometer, and an ultraviolet/ visible spectrophotometer.

Excess solute and solvent were placed in amber glass bottles and allowed to equilibrate for several days at constant temperature. Attainment of equilibrium was verified by several repetitive measurements and by approaching equilibrium from supersaturation. Aliquots of saturated solutions were transferred through a coarse filter into tared volumetric flasks, weighed, and diluted with methanol. Concentrations were determined by spectrophotometric measurements at $273 \mathrm{~nm}$.

\section{Source and Purity of Chemicals:}

(1) $99 \%$, Aldrich Chemical Company, Milwaukee, WI, USA, was used as received.

(2) $99+\%$, Alfa Aesar, USA, stored over molecular sieves and distilled shortly before use.

Estimated Error:

Temperature: $\pm 0.1 \mathrm{~K}$.

$x_{1}: \pm 1.5 \%$ (relative error) 


\section{Components:}

(1) 4-Methoxybenzoic acid; $\mathrm{C}_{8} \mathrm{H}_{8} \mathrm{O}_{3}$; [100-09-4]

(2) 1-Heptanol; $\mathrm{C}_{7} \mathrm{H}_{16} \mathrm{O}$; [111-70-6]

Original Measurements:

${ }^{9}$ K. R. Hoover, D. M. Stovall, E. Pustejovsky, R. Coaxum, K. Pop, W. E. Acree, Jr., and M. H. Abraham, Can. J. Chem. 82, 1353 (2004).

\section{Variables:}

$T / \mathrm{K}=298.15$

Prepared by:

W. E. Acree, Jr.

Experimental Values

\begin{tabular}{lc}
\hline \hline$x_{2}{ }^{\mathrm{a}}$ & $x_{1}{ }^{\mathrm{b}}$ \\
\hline 0.9877 & 0.01234
\end{tabular}

${ }^{\mathrm{a}} x_{2}$ : mole fraction of component 2 in the saturated solution.

${ }^{\mathrm{b}} \mathrm{x}_{1}$ : mole fraction solubility of the solute.

\section{Auxiliary Information}

\section{Method/Apparatus/Procedure:}

Constant-temperature bath, calorimetric thermometer, and an ultraviolet/ visible spectrophotometer.

Excess solute and solvent were placed in amber glass bottles and allowed to equilibrate for several days at constant temperature. Attainment of equilibrium was verified by several repetitive measurements and by approaching equilibrium from supersaturation. Aliquots of saturated solutions were transferred through a coarse filter into tared volumetric flasks, weighed, and diluted with methanol. Concentrations were determined by

spectrophotometric measurements at $273 \mathrm{~nm}$.

Source and Purity of Chemicals:

(1) $99 \%$, Aldrich Chemical Company, Milwaukee, WI, USA, was used as received.

(2) $99+\%$, Alfa Aesar, USA, stored over molecular sieves and distilled shortly before use.

\section{Estimated Error:}

Temperature: $\pm 0.1 \mathrm{~K}$.

$x_{1}: \pm 1.5 \%$ (relative error).

\section{Components:}

(1) 4-Methoxybenzoic acid; $\mathrm{C}_{8} \mathrm{H}_{8} \mathrm{O}_{3}$; [100-09-4]

(2) 1-Octanol; $\mathrm{C}_{8} \mathrm{H}_{18} \mathrm{O}$; [111-87-5]

\section{Variables:}

$T / \mathrm{K}=298.15$
Original Measurements:

${ }^{9}$ K. R. Hoover, D. M. Stovall, E. Pustejovsky, R. Coaxum, K. Pop, W. E. Acree, Jr., and M. H. Abraham, Can. J. Chem. 82, 1353 (2004).

\section{Prepared by:}

W. E. Acree, Jr.

\section{Experimental Values}

\begin{tabular}{lc}
\hline \hline$x_{2}^{\mathrm{a}}$ & $x_{1}{ }^{\mathrm{b}}$ \\
\hline 0.9872 & 0.01275 \\
\hline${ }^{\mathrm{a}} x_{2}:$ mole fraction of component 2 in the saturated solution. & \\
${ }^{\mathrm{b}} x_{1}:$ mole fraction solubility of the solute. &
\end{tabular}

\section{Auxiliary Information}

\section{Method/Apparatus/Procedure:}

Constant-temperature bath, calorimetric thermometer, and an ultraviolet/ visible spectrophotometer.

Excess solute and solvent were placed in amber glass bottles and allowed to equilibrate for several days at constant temperature. Attainment of equilibrium was verified by several repetitive measurements and by approaching equilibrium from supersaturation. Aliquots of saturated solutions were transferred through a coarse filter into tared volumetric flasks, weighed, and diluted with methanol. Concentrations were determined by spectrophotometric measurements at $273 \mathrm{~nm}$.

\section{Source and Purity of Chemicals:}

(1) $99 \%$, Aldrich Chemical Company, Milwaukee, WI, USA, was used as received.

(2) 99+\%, anhydrous, Aldrich Chemical Company, stored over molecular sieves and distilled shortly before use.

Estimated Error:

Temperature: $\pm 0.1 \mathrm{~K}$.

$x_{1}: \pm 1.5 \%$ (relative error)

\begin{tabular}{ll}
\hline \hline Components: & Original Measurements: \\
(1) 4-Methoxybenzoic acid; $\mathrm{C}_{8} \mathrm{H}_{8} \mathrm{O}_{3} ;$ & ${ }^{9} \mathrm{~K}$. R. Hoover, D. M. Stovall, E. \\
[100-09-4] & Pustejovsky, R. Coaxum, K. Pop, \\
(2) 1-Decanol; $\mathrm{C}_{10} \mathrm{H}_{22} \mathrm{O} ;[112-30-1]$ & W. E. Acree, Jr., and M. H. \\
& Abraham, Can. J. Chem. 82, 1353 \\
& $(2004)$. \\
\hline Variables: & Prepared by: \\
$T / \mathrm{K}=298.15$ & W. E. Acree, Jr. \\
\hline
\end{tabular}

\section{Experimental Values}

\begin{tabular}{lc}
\hline \hline$x_{2}{ }^{\mathrm{a}}$ & $x_{1}{ }^{\mathrm{b}}$ \\
\hline 0.9872 & 0.01278 \\
\hline
\end{tabular}

${ }^{\mathrm{a}} x_{2}$ : mole fraction of component 2 in the saturated solution.

${ }^{\mathrm{b}} x_{1}$ : mole fraction solubility of the solute.

\section{Auxiliary Information}

\section{Method/Apparatus/Procedure:}

Constant-temperature bath, calorimetric thermometer, and an ultraviolet/ visible spectrophotometer.

Excess solute and solvent were placed in amber glass bottles and allowed to equilibrate for several days at constant temperature. Attainment of equilibrium was verified by several repetitive measurements and by approaching equilibrium from supersaturation. Aliquots of saturated solutions were transferred through a coarse filter into tared volumetric flasks, weighed, and diluted with methanol. Concentrations were determined by spectrophotometric measurements at $273 \mathrm{~nm}$.

\section{Source and Purity of Chemicals:}

(1) $99 \%$, Aldrich Chemical Company, Milwaukee, WI, USA, was used as received.

(2) $99+\%$, Alfa Aesar, USA, stored over molecular sieves and distilled shortly before use.

Estimated Error:

Temperature: $\pm 0.1 \mathrm{~K}$.

$x_{1}: \pm 1.5 \%$ (relative error) 


\subsection{4-Methoxybenzoic acid solubility data in alkoxyalcohols}

\begin{tabular}{|c|c|}
\hline $\begin{array}{l}\text { Components: } \\
\text { (1) 4-Methoxybenzoic acid; } \mathrm{C}_{8} \mathrm{H}_{8} \mathrm{O}_{3} \text {; } \\
\text { [100-09-4] } \\
\text { (2) 2-Ethoxyethanol; } \mathrm{C}_{4} \mathrm{H}_{10} \mathrm{O}_{2} \text {; } \\
\text { [110-80-5] }\end{array}$ & $\begin{array}{l}\text { Original Measurements: } \\
{ }^{130} \text { L. M. Grubbs, M. Saifullah, N. E. } \\
\text { De La Rosa, S. Ye, S. S. Achi, W. E. } \\
\text { Acree, Jr., and M. H. Abraham, } \\
\text { Fluid Phase Equilib. 298, } 48 \text { (2010). }\end{array}$ \\
\hline $\begin{array}{l}\text { Variables: } \\
T / \mathrm{K}=298.15\end{array}$ & $\begin{array}{l}\text { Prepared by: } \\
\text { W. E. Acree, Jr. }\end{array}$ \\
\hline
\end{tabular}

Experimental Values

\begin{tabular}{lc}
\hline \hline$x_{2}{ }^{\mathrm{a}}$ & $x_{1}{ }^{\mathrm{b}}$ \\
\hline 0.9588 & 0.0412 \\
\hline$x_{2}$ m
\end{tabular}

${ }^{\mathrm{a}} x_{2}$ : mole fraction of component 2 in the saturated solution.

${ }^{\mathrm{b}} x_{1}$ : mole fraction solubility of the solute.

\section{Auxiliary Information}

\section{Method/Apparatus/Procedure:}

Constant-temperature bath, calorimetric thermometer, and an ultraviolet/ visible spectrophotometer.

Excess solute and solvent were placed in amber glass bottles and allowed to equilibrate for several days at constant temperature. Attainment of equilibrium was verified by several repetitive measurements and by approaching equilibrium from supersaturation. Aliquots of saturated solutions were transferred through a coarse filter into tared volumetric flasks, weighed, and diluted with methanol. Concentrations were determined by

spectrophotometric measurements at $273 \mathrm{~nm}$.

Source and Purity of Chemicals:

(1) $99 \%$, Aldrich Chemical Company, Milwaukee, WI, USA, was used as received.

(2) $99 \%$, Aldrich Chemical Company, stored over molecular sieves and distilled shortly before use.

Estimated Error:

Temperature: $\pm 0.1 \mathrm{~K}$.

$x_{1}: \pm 1.5 \%$ (relative error)

\begin{tabular}{ll}
\hline \hline Components: & Original Measurements: \\
(1) 4-Methoxybenzoic acid; $\mathrm{C}_{8} \mathrm{H}_{8} \mathrm{O}_{3} ;$ & ${ }^{130}$ L. M. Grubbs, M. Saifullah, N. E. \\
[100-09-4] & De La Rosa, S. Ye, S. S. Achi, W. E. \\
$\begin{array}{l}\text { (2) 2-Propoxyethanol; } \mathrm{C}_{5} \mathrm{H}_{12} \mathrm{O}_{2} ; \\
\text { [2807-30-9] }\end{array}$ & Acree, Jr., and M. H. Abraham, \\
\hline Variables: & Fluid Phase Equilib. 298, 48 (2010). \\
$T / \mathrm{K}=298.15$ & Prepared by: \\
\hline
\end{tabular}

\section{Experimental Values}

\begin{tabular}{lc}
\hline \hline$x_{2}^{\mathrm{a}}$ & $x_{1}^{\mathrm{b}}$ \\
\hline 0.9667 & 0.0333 \\
\hline${ }^{\mathrm{a}} x_{2}:$ mole fraction of component 2 in the saturated solution. & \\
${ }^{\mathrm{b}} x_{1}:$ mole fraction solubility of the solute. &
\end{tabular}

\section{Auxiliary Information}

Method/Apparatus/Procedure:

Constant-temperature bath, calorimetric thermometer, and an ultraviolet/ visible spectrophotometer.

Excess solute and solvent were placed in amber glass bottles and allowed to equilibrate for several days at constant temperature. Attainment of equilibrium was verified by several repetitive measurements and by approaching equilibrium from supersaturation. Aliquots of saturated solutions were transferred through a coarse filter into tared volumetric flasks, weighed, and diluted with methanol. Concentrations were determined by spectrophotometric measurements at $273 \mathrm{~nm}$.

Source and Purity of Chemicals:

(1) $99 \%$, Aldrich Chemical Company, Milwaukee, WI, USA, was used as received.

(2) $99+\%$, Aldrich Chemical Company, stored over molecular sieves and distilled shortly before use.

Estimated Error:

Temperature: $\pm 0.1 \mathrm{~K}$.

$x_{1}: \pm 1.5 \%$ (relative error)

\begin{tabular}{|c|c|}
\hline Components: & Original Measurements: \\
\hline $\begin{array}{l}\text { (1) 4-Methoxybenzoic acid; } \mathrm{C}_{8} \mathrm{H}_{8} \mathrm{O}_{3} \text {; } \\
\text { [100-09-4] }\end{array}$ & $\begin{array}{l}{ }^{130} \text { L. M. Grubbs, M. Saifullah, N. E } \\
\text { De La Rosa, S. Ye, S. S. Achi, W. E }\end{array}$ \\
\hline $\begin{array}{l}\text { (2) 2-Isopropoxyethanol; } \mathrm{C}_{5} \mathrm{H}_{12} \mathrm{O}_{2} \text {; } \\
\text { [109-59-1] }\end{array}$ & $\begin{array}{l}\text { Acree, Jr., and M. H. Abraham, } \\
\text { Fluid Phase Equilib. 298, } 48 \text { (2010) }\end{array}$ \\
\hline Variables: & \\
\hline$T / \mathrm{K}=298.15$ & W. E. Acree, Jr. \\
\hline
\end{tabular}

Experimental Values

\begin{tabular}{lc}
\hline \hline$x_{2}{ }^{\mathrm{a}}$ & $x_{1}{ }^{\mathrm{b}}$ \\
\hline 0.9639 & 0.0361
\end{tabular}

${ }^{\mathrm{a}} x_{2}$ : mole fraction of component 2 in the saturated solution.

${ }^{\mathrm{b}} x_{1}$ : mole fraction solubility of the solute.

\section{Auxiliary Information}

\section{Method/Apparatus/Procedure:}

Constant-temperature bath, calorimetric thermometer, and an ultraviolet/ visible spectrophotometer.

Excess solute and solvent were placed in amber glass bottles and allowed to equilibrate for several days at constant temperature. Attainment of equilibrium was verified by several repetitive measurements and by approaching equilibrium from supersaturation. Aliquots of saturated solutions were transferred through a coarse filter into tared volumetric flasks, weighed, and diluted with methanol. Concentrations were determined by spectrophotometric measurements at $273 \mathrm{~nm}$.

Source and Purity of Chemicals:

(1) $99 \%$, Aldrich Chemical Company, Milwaukee, WI, USA, was used as received.

(2) $99 \%$, Aldrich Chemical Company, stored over molecular sieves and distilled shortly before use.

\section{Estimated Error:}

Temperature: $\pm 0.1 \mathrm{~K}$.

$x_{1}: \pm 1.5 \%$ (relative error). 


\section{Components:} [100-09-4]

(2) 2-Butoxyethanol; $\mathrm{C}_{6} \mathrm{H}_{14} \mathrm{O}_{2}$; [111-76-2]

\begin{tabular}{ll}
\hline Variables: & Prepared by: \\
$T / \mathrm{K}=298.15$ & W. E. Acree, Jr. \\
\hline
\end{tabular}

Experimental Values

\begin{tabular}{lc}
\hline \hline$x_{2}{ }^{\mathrm{a}}$ & $x_{1}{ }^{\mathrm{b}}$ \\
\hline 0.9616 & 0.0384
\end{tabular}

${ }^{\mathrm{a}} x_{2}$ : mole fraction of component 2 in the saturated solution.

${ }^{\mathrm{b}} x_{1}$ : mole fraction solubility of the solute.

\section{Auxiliary Information}

\section{Method/Apparatus/Procedure:}

Constant-temperature bath, calorimetric thermometer, and an ultraviolet/ visible spectrophotometer.

Excess solute and solvent were placed in amber glass bottles and allowed to equilibrate for several days at constant temperature. Attainment of equilibrium was verified by several repetitive measurements and by approaching equilibrium from supersaturation. Aliquots of saturated solutions were transferred through a coarse filter into tared volumetric flasks, weighed, and diluted with methanol. Concentrations were determined by spectrophotometric measurements at $273 \mathrm{~nm}$.

\section{Source and Purity of Chemicals:}

(1) $99 \%$, Aldrich Chemical Company, Milwaukee, WI, USA, was used as received.

(2) $99 \%$, Acros Organics, USA, stored over molecular sieves and distilled shortly before use.

\section{Estimated Error:}

$x_{1}: \pm 1.5 \%$ (relative error).
(1) 4-Methoxybenzoic acid; $\mathrm{C}_{8} \mathrm{H}_{8} \mathrm{O}_{3}$;

Temperature: $\pm 0.1 \mathrm{~K}$.

\section{Auxiliary Information}

\section{Method/Apparatus/Procedure:}

Constant-temperature bath, calorimetric thermometer, and an ultraviolet/ visible spectrophotometer.

Excess solute and solvent were placed in amber glass bottles and allowed to equilibrate for several days at constant temperature. Attainment of equilibrium was verified by several repetitive measurements and by approaching equilibrium from supersaturation. Aliquots of saturated solutions were transferred through a coarse filter into tared volumetric flasks, weighed, and diluted with methanol. Concentrations were determined by spectrophotometric measurements at $273 \mathrm{~nm}$.

\section{Source and Purity of Chemicals:}

(1) $99 \%$, Aldrich Chemical Company, Milwaukee, WI, USA, was used as received.

(2) $99 \%$, Aldrich Chemical Company, stored over molecular sieves and distilled shortly before use.

Estimated Error:

Temperature: $\pm 0.1 \mathrm{~K}$.

$x_{1}: \pm 1.5 \%$ (relative error).

\begin{tabular}{|c|c|}
\hline Components: & Original Measurements: \\
\hline (1) 4-Methoxybenzoic acid; $\mathrm{C}_{8} \mathrm{H}_{8} \mathrm{O}_{3}$; & ${ }^{130}$ L. M. Grubbs, M. Saifullah, N. E \\
\hline [100-09-4] & De La Rosa, S. Ye, S. S. Achi, W. E \\
\hline $\begin{array}{l}\text { (2) 1-Methyl-2-tert-butoxyethanol; } \\
\mathrm{C}_{7} \mathrm{H}_{16} \mathrm{O}_{2} ; \text { [57018-52-7] }\end{array}$ & Acree, Jr., and M. H. Abraham, \\
\hline $\mathrm{C}_{7} \mathrm{H}_{16} \mathrm{O}_{2} ;[57018-52-7]$ & Fluid Phase Equilib. 298, 48 (2010) \\
\hline Variables & \\
\hline$T / \mathrm{K}=298.15$ & W. E. Acree, Jr. \\
\hline
\end{tabular}

Experimental Values

\begin{tabular}{lc}
\hline \hline$x_{2}{ }^{\mathrm{a}}$ & $x_{1}{ }^{\mathrm{b}}$ \\
\hline 0.9727 & 0.0273
\end{tabular}

${ }^{\mathrm{a}} x_{2}$ : mole fraction of component 2 in the saturated solution.

${ }^{\mathrm{b}} x_{1}$ : mole fraction solubility of the solute.

\section{Auxiliary Information}

\section{Components:} [100-09-4]

(2) 3-Methoxy-1-butanol; $\mathrm{C}_{5} \mathrm{H}_{12} \mathrm{O}_{2}$; [2517-43-3]

\section{Variables:}

$T / \mathrm{K}=298.15$

Original Measurements:

${ }^{130}$ L. M. Grubbs, M. Saifullah, N. E. De La Rosa, S. Ye, S. S. Achi, W. E Acree, Jr., and M. H. Abraham, Fluid Phase Equilib. 298, 48 (2010).

\section{Prepared by:}

W. E. Acree, Jr.
(1) 4-Methoxybenzoic acid; $\mathrm{C}_{8} \mathrm{H}_{8} \mathrm{O}_{3}$;

Experimental Values

\begin{tabular}{lc}
\hline \hline$x_{2}{ }^{\mathrm{a}}$ & $x_{1}{ }^{\mathrm{b}}$ \\
\hline 0.9629 & 0.0371 \\
\hline${ }^{\mathrm{a}} x_{2}:$ mole fraction of component 2 in the saturated solution. & \\
${ }^{\mathrm{b}}{ }_{x_{1}: \text { mole fraction solubility of the solute. }}$ &
\end{tabular}

\author{
Method/Apparatus/Procedure: \\ Constant-temperature bath, calorimetric thermometer, and an ultraviolet/ \\ visible spectrophotometer. \\ Excess solute and solvent were placed in amber glass bottles and allowed to \\ equilibrate for several days at constant temperature. Attainment of equilibrium \\ was verified by several repetitive measurements and by approaching \\ equilibrium from supersaturation. Aliquots of saturated solutions were \\ transferred through a coarse filter into tared volumetric flasks, weighed, and \\ diluted with methanol. Concentrations were determined by \\ spectrophotometric measurements at $273 \mathrm{~nm}$.
}

\section{Source and Purity of Chemicals:}

(1) $99 \%$, Aldrich Chemical Company, Milwaukee, WI, USA, was used as received.

(2) $99 \%$, Aldrich Chemical Company, stored over molecular sieves and distilled shortly before use.

Estimated Error:

Temperature: $\pm 0.1 \mathrm{~K}$.

$x_{1}: \pm 1.5 \%$ (relative error). 


\section{Solubility of 2-Methylbenzoic Acid in Organic Solvents}

\subsection{Critical evaluation of experimental solubility data}

There have been several published studies ${ }^{72,77,171-174}$ investigating the solubility behavior of 2-methylbenzoic acid in organic solvents of varying polarity and hydrogen-bonding capability. Coaxum et al. ${ }^{171}$ measured the solubility of 2methylbenzoic acid in 16 alcohols (ethanol, 1-propanol, 2propanol, 1-butanol, 2-butanol, 2-methyl-1-propanol, 2methyl-2-propanol, 1-pentanol, 2-pentanol, 2-methyl-1-butanol, 3-methyl-1-butanol, 1-hexanol, 4-methyl-2-pentanol, 1heptanol, 1-octanol, and 1-decanol), in three dialkyl ethers (1,1'-oxybisethane, 2,2'-oxybispropane, and 1,1'-oxybisbutane) and two cyclic ethers (tetrahydrofuran and 1,4-dioxane), and in four alkanoates (methyl ethanoate, ethyl ethanoate, butyl ethanoate, and pentyl ethanoate), and in propylene carbonate at $298 \mathrm{~K}$. Results of the experimental measurements were used to calculate the Abraham solute descriptors of 2methylbenzoic acid. The authors were able to assemble a total of $47 \log _{10}(S R$ or $P)$ and $\log _{10}(G S R$ or $K)$ equations for which experimental partition coefficient data, solubility ratios, Abraham model equation coefficients and aqueous molar solubility were available. The logarithm of the aqueous molar solubility of 2-methylbenzoic acid is $\log _{10} c_{1, \mathrm{w}}=-2.06 .{ }^{131,175}$ The McGowan volume of 2-methylbenzoic acid, $V=1.0726$, was calculated from the number of chemical bonds in the molecule and the individual atomic group volumes, $A V_{i}$, given in Sec. 1.3. The excess molar refraction solute descriptor was estimated as $E=0.730$. This left four solute descriptors ( $S, A$, $B$, and $L$ ) still to be determined. The 47 equations were then solved using the Microsoft "sOLVER" program to yield numerical values of the remaining four solute descriptors, $S=0.840$, $A=0.420, B=0.440$, and $L=4.6770$, that best described the $\log _{10}(S R$ or $P)$ and $\log _{10}(G S R$ or $K)$ values. The computation treated $\log _{10} c_{1, \mathrm{G}}$ as a floating parameter to be determined as part of the regression analyses. The data analyses returned a value of $\log _{10} c_{1, \mathrm{G}}=-6.360$ for the gas-phase solute concentration that made the $\log _{10}\left(S R\right.$ or $P$ ) and $\log _{10}(G S R$ or $K)$ predictions internally consistent. The calculated molecular solute descriptors reproduced the $\log _{10}\left(S R\right.$ or $P$ ) and $\log _{10}$ ( $G S R$ or $K$ ) values to within an average standard deviation of 0.080 and $0.067 \log _{10}$ units, respectively.

After the 2-methylbenzoic acid solubility study was published, Abraham model correlations have been developed for 2-pentanol, 3-methyl-1-butanol, methyl ethanoate, and butyl ethanoate, and equation coefficients for a few solvents were updated based on additional experimental data. The new correlations (listed in Tables 1 and 2) will be used in illustrating the ability of the Abraham model to correlate the experimental 2-methylbenzoic acid solubility data. Table 42 compares the experimental $\log _{10} c_{1}$ values to calculated values based on Eqs. (20) and (21) of the Abraham model. For comparison purposes, the measured mole fraction solubilities of 2-methylbenzoic acid, $x_{1}$, determined by Coaxum et al. ${ }^{171}$ were converted into molar solubilities by dividing $x_{1}$ by the
TABLE 42. Comparison between observed and calculated molar solubilities of 2-methylbenzoic acid based on the Abraham model, Eqs. (20) and (21)

\begin{tabular}{lccc}
\hline \hline Solvent & & $\log _{10} c_{1}{ }^{\text {calc }} ;$ & $\log _{10} c_{1}{ }^{\text {calc}}$; \\
Ethanol & 0.325 & Eq. $(20)$ & Eq. $(21)$ \\
1-Propanol & 0.248 & 0.238 & 0.333 \\
2-Propanol & 0.282 & 0.232 & 0.242 \\
1-Butanol & 0.205 & 0.221 & 0.248 \\
2-Butanol & 0.242 & 0.168 & 0.178 \\
2-Methyl-1-propanol & 0.106 & 0.203 & 0.208 \\
2-Methyl-2-propanol & 0.329 & 0.152 & 0.143 \\
1-Pentanol & 0.172 & 0.229 & 0.278 \\
2-Pentanol & 0.220 & 0.181 & 0.188 \\
3-Methyl-1-butanol & 0.121 & 0.214 & 0.200 \\
1-Hexanol & 0.137 & 0.169 & 0.132 \\
1-Heptanol & 0.103 & 0.140 & 0.149 \\
1-Octanol & 0.063 & 0.093 & 0.119 \\
1-Decanol & 0.016 & 0.037 & 0.021 \\
1,1'-Oxybisethane & 0.203 & -0.003 & 0.025 \\
Tetrahydrofuran & 0.456 & 0.101 & 0.172 \\
1,4-Dioxane & 0.427 & 0.608 & 0.609 \\
Methyl ethanoate & 0.185 & 0.336 & 0.338 \\
Ethyl ethanoate & 0.170 & 0.162 & 0.117 \\
Butyl ethanoate & 0.063 & 0.162 & 0.169 \\
Expermental & 0.067 & 0.066 \\
\hline
\end{tabular}

${ }^{\mathrm{a}}$ Experimental solubility data were taken from Coaxum et al. ${ }^{17}$

ideal molar volume of the saturated solution (i.e., $c_{1}{ }^{\text {sat }}=x_{1} /$ $\left.\left[x_{1} V_{1}+\left(1-x_{1}\right) V_{\text {solvent }}\right]\right)$. The molar volume of the hypothetical subcooled liquid 2-methylbenzoic acid is $V_{\text {solute }}=121.8$ $\mathrm{cm}^{3} \mathrm{~mol}^{-1}$. Examination of the numerical entries in Table 42 reveals that the Abraham model provides a reasonably accurate mathematical description of the observed solubility data, suggesting that there are no obvious outliers in the dataset.

There are three close independent experimental values in Sec. 59.7 for the solubility of 2-methylbenzoic acid in ethanol. The recommended solubility in ethanol is $x_{1}=0.143$, which is calculated as the arithmetic average of the values of $x_{1}=$ $0.1401,{ }^{171} 0.1460,{ }^{72}$ and $0.142 .{ }^{173}$

Two research groups have studied the solubility behavior of 2-methylbenzoic acid as a function of temperature. Domańska ${ }^{172,173}$ determined the solubility of 2-methylbenzoic acid in hexane, heptane, cyclohexane, benzene, 1,2-dimethylbenzene, tetrachloromethane, diiodomethane, chlorobenzene, 1,2-dichlorobenzene, bromobenzene, ethanol, 2-propanol, and nitrobenzene at several temperatures from about 295-345 K based on a dynamic solubility method. The internal consistency of the 13 datasets can be assessed by curve-fitting the measured mole fraction solubilities to Buchowski $\lambda \mathrm{h}$ Model [see Eq. (9)]. Table 43 tabulates the equation coefficients $(h$ and $\lambda$ ) calculated by the author, along with the corresponding mean standard temperature deviations, $\sigma_{\mathrm{T}}$,

$$
\sigma_{\mathrm{T}}=\sqrt{\frac{\sum\left(T_{i}^{\mathrm{calc}}-T_{i}^{\mathrm{exp}}\right)^{2}}{n-1}},
$$

that describe the goodness-of-fit. The summation in Eq. (63) extends over the number of experimental data points, $n$. 
TABLE 43. Parameters of the Buchowski $\lambda$ h model for describing the solubility of 2-methylbenzoic acid in various organic solvents ${ }^{\mathrm{a}}$

\begin{tabular}{lcccc}
\hline \hline Solvent & $T / \mathrm{K}$ & $h$ & $\lambda$ & $\sigma_{\mathrm{T}}(\mathrm{K})$ \\
\hline Hexane & $298-332$ & 19550 & 0.55 & 0.36 \\
Heptane & $292-341$ & 9370 & 0.52 & 0.83 \\
Cyclohexane & $295-338$ & 5700 & 1.02 & 0.87 \\
Benzene & $294-345$ & 5200 & 1.62 & 0.57 \\
1,3-Dimethylbenzene & $297-340$ & 5120 & 1.61 & 0.36 \\
Tetrachloromethane & $296-336$ & 5270 & 1.72 & 0.17 \\
Diiodomethane & $294-345$ & 9040 & 0.83 & 1.27 \\
Chlorobenzene & $299-354$ & 3210 & 2.44 & 0.38 \\
1,2-Dichlorobenzene & $296-335$ & 4710 & 1.59 & 0.33 \\
Bromobenzene & $298-352$ & 4850 & 1.52 & 0.39 \\
Ethanol & $291-337$ & 3450 & 0.62 & 0.25 \\
2-Propanol & $293-345$ & 3090 & 0.68 & 0.42 \\
Nitrobenzene & $298-338$ & 5280 & 1.55 & 0.22 \\
\hline
\end{tabular}

${ }^{\mathrm{a}}$ Values of the coefficients and the mean standard temperature deviations were taken from Domańska. ${ }^{172}$

Qingzhu et al. ${ }^{77}$ measured the solubility of 2-methylbenzoic acid in 1-octanol from 297 to $322 \mathrm{~K}$ employing a laser monitoring method that determined the temperature at which the solid solute was completely dissolved. The authors curvefit the mole fraction solubilities to Eq. (8) (with $C=0$ ). The derived mathematical expression

$$
\ln x_{1}=5.432-\frac{2127}{T}
$$

described the experimental $x_{1}$ values to within an average absolute relative deviation of $0.8 \%$.

The experimental solubility data for 2-methylbenzoic acid in organic solvents are in Secs. 59.2-59.9.

\subsection{2-Methylbenzoic acid solubility data in saturated hydrocarbons (including cycloalkanes)}

\begin{tabular}{|c|c|}
\hline $\begin{array}{l}\text { Components: } \\
\text { (1) 2-Methylbenzoic acid; } \mathrm{C}_{8} \mathrm{H}_{8} \mathrm{O}_{2} \text {; } \\
\text { [118-90-1] } \\
\text { (2) Hexane; } \mathrm{C}_{6} \mathrm{H}_{14} ;[110-54-3]\end{array}$ & $\begin{array}{l}\text { Original Measurements: } \\
\text { }{ }^{172} \text { U. Domańska, Pol. J. Chem. 60, } \\
847 \text { (1986). }\end{array}$ \\
\hline Variables: & Prepared by: \\
\hline Temperature & W. E. Acree, Jr. \\
\hline
\end{tabular}

Experimental Values

\begin{tabular}{lcc}
\hline \hline$T / \mathrm{K}$ & $x_{2}{ }^{\mathrm{a}}$ & $x_{1}{ }^{\mathrm{b}}$ \\
\hline 298.10 & 0.9872 & 0.0128 \\
301.95 & 0.9843 & 0.0157 \\
306.20 & 0.9841 & 0.0199 \\
311.45 & 0.9731 & 0.0269 \\
319.65 & 0.9574 & 0.0426 \\
322.65 & 0.9501 & 0.0499 \\
331.85 & 0.9145 & 0.0855 \\
\hline
\end{tabular}

${ }^{\mathrm{a}} x_{2}$ : mole fraction of component 2 in the saturated solution.

${ }^{\mathrm{b}} x_{1}$ : mole fraction solubility of the solute.

\section{Auxiliary Information}

\section{Method/Apparatus/Procedure:}

Analytical balance and constant-temperature bath.

Solubilities were determined by a dynamic method. Weighed amounts of solute and solvent were placed in sample container and placed in a constanttemperature bath. The temperature of the bath was slowly increased. The temperature at which the last crystals disappeared was taken as the temperature of the solution-crystal equilibrium.

Source and Purity of Chemicals:

(1) $99+\%$, Analytical grade, Fluka Chemicals, Switzerland, purified by double vacuum sublimation.

(2) Purity not given, POCh Gliwice, Poland, dried over phosphorous pentoxide and then fractionally distilled before use.

Estimated Error:

Temperature: $\pm 0.1 \mathrm{~K}$.

$x_{1}: \pm 2.0 \%$ (relative error, estimated by compiler).

\begin{tabular}{|c|c|}
\hline $\begin{array}{l}\text { Components: } \\
\text { (1) 2-Methylbenzoic acid; } \mathrm{C}_{8} \mathrm{H}_{8} \mathrm{O}_{2} \text {; } \\
\text { [118-90-1] } \\
\text { (2) Heptane; } \mathrm{C}_{7} \mathrm{H}_{16} ;[142-82-5]\end{array}$ & $\begin{array}{l}\text { Original Measurements: } \\
{ }^{173} \text { U. Domańska and T. Hofman, J. } \\
\text { Solution Chem. 14, } 531 \text { (1985). } \\
{ }^{172} \text { U. Domańska, Pol. J. Chem. 60, } \\
847 \text { (1986). }\end{array}$ \\
\hline $\begin{array}{l}\text { Variables: } \\
\text { Temperature }\end{array}$ & $\begin{array}{l}\text { Prepared by: } \\
\text { W. E. Acree, Jr. }\end{array}$ \\
\hline
\end{tabular}

Experimental Values

\begin{tabular}{lcc}
\hline \hline$T / \mathrm{K}$ & $x_{2}{ }^{\mathrm{a}}$ & $x_{1}{ }^{\mathrm{b}}$ \\
\hline 292.15 & 0.9886 & 0.0114 \\
296.75 & 0.9851 & 0.0149 \\
302.05 & 0.9803 & 0.0197 \\
307.35 & 0.9741 & 0.0259 \\
311.85 & 0.9676 & 0.0324 \\
315.45 & 0.9605 & 0.0395 \\
319.15 & 0.9523 & 0.0477 \\
323.55 & 0.9415 & 0.0585 \\
329.45 & 0.9271 & 0.0729 \\
334.85 & 0.9047 & 0.0953 \\
341.45 & 0.8618 & 0.1382 \\
341.85 & 0.8449 & 0.1551
\end{tabular}

${ }^{a} x_{2}$ : mole fraction of component 2 in the saturated solution.

${ }^{\mathrm{b}} x_{1}$ : mole fraction solubility of the solute.

\section{Auxiliary Information}

\section{Method/Apparatus/Procedure:}

Analytical balance and constant-temperature bath

Solubilities were determined by a dynamic method. Weighed amounts of solute and solvent were placed in sample container and placed in a constanttemperature bath. The temperature of the bath was slowly increased $(2 \mathrm{~K} / \mathrm{h}$ near the equilibrium temperature). The temperature at which the last crystals disappeared was taken as the temperature of the solution-crystal equilibrium.

Source and Purity of Chemicals:

(1) $99+\%$, Analytical grade, Fluka Chemicals, Switzerland, purified by double vacuum sublimation.

(2) Purity not given, VEB Jena Pharm, dried over molecular sieves and then fractionally distilled before use. 
Estimated Error:

Temperature: $\pm 0.1 \mathrm{~K}$

$x_{1}: \pm 2.0 \%$ (relative error, estimated by compiler).

\begin{tabular}{ll}
\hline \hline Components: & Original Measurements: \\
(1) 2-Methylbenzoic acid; $\mathrm{C}_{8} \mathrm{H}_{8} \mathrm{O}_{2} ;$ & ${ }^{173}$ U. Domańska and T. Hofman, J. \\
[118-90-1] & $\begin{array}{l}\text { Solution Chem. 14, 531 (1985). } \\
\text { (2) Cyclohexane; } \mathrm{C}_{6} \mathrm{H}_{12} ;[110-82-7]\end{array}$ \\
& ${ }^{172} \mathrm{U}$. Domańska, Pol. J. Chem. 60, \\
& 847 (1986). \\
\hline Variables: & Prepared by: \\
Temperature & W. E. Acree, Jr. \\
\hline
\end{tabular}

Experimental Values

\begin{tabular}{lcc}
\hline \hline$T / \mathrm{K}$ & $x_{2}{ }^{\mathrm{a}}$ & $x_{1}{ }^{\mathrm{b}}$ \\
\hline 295.45 & 0.9854 & 0.0146 \\
304.95 & 0.9721 & 0.0279 \\
316.65 & 0.9485 & 0.0515 \\
319.40 & 0.9385 & 0.0615 \\
325.30 & 0.9115 & 0.0885 \\
329.35 & 0.8941 & 0.1059 \\
333.45 & 0.8619 & 0.1381 \\
338.15 & 0.8152 & 0.1848
\end{tabular}

${ }^{\mathrm{a}} x_{2}$ : mole fraction of component 2 in the saturated solution.

${ }^{\mathrm{b}} x_{1}$ : mole fraction solubility of the solute.

\section{Auxiliary Information}

\section{Method/Apparatus/Procedure:}

Analytical balance and constant-temperature bath.

Solubilities were determined by a dynamic method. Weighed amounts of solute and solvent were placed in sample container and placed in a constanttemperature bath. The temperature of the bath was slowly increased $(2 \mathrm{~K} / \mathrm{h}$ near the equilibrium temperature). The temperature at which the last crystals disappeared was taken as the temperature of the solution-crystal equilibrium.

Source and Purity of Chemicals:

(1) $99+\%$, Analytical grade, Fluka Chemicals, Switzerland, purified by double vacuum sublimation.

(2) Purity not given, POCh Gliwice, Poland, dried over phosphorous pentoxide and then fractionally distilled before use.

\section{Estimated Error:}

Temperature: $\pm 0.1 \mathrm{~K}$

$x_{1}: \pm 2.0 \%$ (relative error, estimated by compiler).

\subsection{2-Methylbenzoic acid solubility data in aromatic hydrocarbons}

\begin{tabular}{|c|c|}
\hline $\begin{array}{l}\text { Components: } \\
\text { (1) 2-Methylbenzoic acid; } \mathrm{C}_{8} \mathrm{H}_{8} \mathrm{O}_{2} \text {; } \\
\text { [118-90-1] } \\
\text { (2) Benzene; } \mathrm{C}_{6} \mathrm{H}_{6} ;[71-43-2]\end{array}$ & $\begin{array}{l}\text { Original Measurements: } \\
{ }^{172} \text { U. Domańska, Pol. J. Chem. 60, } \\
847 \text { (1986). }\end{array}$ \\
\hline $\begin{array}{l}\text { Variables: } \\
\text { Temperature }\end{array}$ & $\begin{array}{l}\text { Prepared by: } \\
\text { W. E. Acree, Jr. }\end{array}$ \\
\hline
\end{tabular}

Experimental Values

\begin{tabular}{lcc}
\hline \hline$T / \mathrm{K}$ & $x_{2}{ }^{\mathrm{a}}$ & $x_{1}{ }^{\mathrm{b}}$ \\
\hline 294.25 & 0.9352 & 0.0648 \\
301.85 & 0.9049 & 0.0951 \\
311.85 & 0.8471 & 0.1529 \\
323.75 & 0.7721 & 0.2279 \\
329.25 & 0.7186 & 0.2814 \\
334.85 & 0.6559 & 0.3441 \\
338.75 & 0.6113 & 0.3887 \\
345.25 & 0.5389 & 0.4611 \\
\hline
\end{tabular}

${ }^{\mathrm{a}} x_{2}$ : mole fraction of component 2 in the saturated solution.

${ }^{b} x_{1}$ : mole fraction solubility of the solute.

\section{Auxiliary Information}

Method/Apparatus/Procedure:

Analytical balance and constant-temperature bath.

Solubilities were determined by a dynamic method. Weighed amounts of solute and solvent were placed in sample container and placed in a constanttemperature bath. The temperature of the bath was slowly increased. The temperature at which the last crystals disappeared was taken as the temperature of the solution-crystal equilibrium.

Source and Purity of Chemicals:

(1) $99+\%$, Analytical grade, Fluka Chemicals, Switzerland, purified by double vacuum sublimation.

(2) Purity not given, POCh Gliwice, Poland, dried over molecular sieves and then fractionally distilled before use.

Estimated Error:

Temperature: $\pm 0.1 \mathrm{~K}$.

$x_{1}: \pm 2.0 \%$ (relative error, estimated by compiler).

\begin{tabular}{|c|c|}
\hline $\begin{array}{l}\text { Components: } \\
\text { (1) 2-Methylbenzoic acid; } \mathrm{C}_{8} \mathrm{H}_{8} \mathrm{O}_{2} \text {; } \\
\text { [118-90-1] } \\
\text { (2) } 1,3-\text { Dimethylbenzene; } \mathrm{C}_{8} \mathrm{H}_{10} \text {; } \\
\text { [108-38-3] }\end{array}$ & $\begin{array}{l}\text { Original Measurements: } \\
{ }^{172} \text { U. Domańska, Pol. J. Chem. 60, } \\
847 \text { (1986). }\end{array}$ \\
\hline $\begin{array}{l}\text { Variables: } \\
\text { Temperature }\end{array}$ & $\begin{array}{l}\text { Prepared by: } \\
\text { W. E. Acree, Jr. }\end{array}$ \\
\hline
\end{tabular}

Experimental Values

\begin{tabular}{lcc}
\hline \hline$T / \mathrm{K}$ & $x_{2}{ }^{\mathrm{a}}$ & $x_{1}{ }^{\mathrm{b}}$ \\
\hline 297.55 & 0.9175 & 0.0825 \\
300.70 & 0.9063 & 0.0937 \\
306.60 & 0.8765 & 0.1235 \\
311.55 & 0.8476 & 0.1524 \\
316.10 & 0.8224 & 0.1776 \\
321.90 & 0.7812 & 0.2188 \\
323.30 & 0.7593 & 0.2407 \\
331.00 & 0.6954 & 0.3046 \\
339.90 & 0.5916 & 0.4084 \\
\hline
\end{tabular}

${ }^{a} x_{2}$ : mole fraction of component 2 in the saturated solution.

${ }^{\mathrm{b}} x_{1}$ : mole fraction solubility of the solute. 


\section{Auxiliary Information}

\section{Method/Apparatus/Procedure:}

Analytical balance and constant-temperature bath.

Solubilities were determined by a dynamic method. Weighed amounts of solute and solvent were placed in sample container and placed in a constanttemperature bath. The temperature of the bath was slowly increased. The temperature at which the last crystals disappeared was taken as the temperature of the solution-crystal equilibrium.

\section{Source and Purity of Chemicals:}

(1) $99+\%$, Analytical grade, Fluka Chemicals, Switzerland, purified by double vacuum sublimation.

(2) Purity not given, Reakhim, Poland, dried over molecular sieves and then fractionally distilled before use.

\section{Estimated Error:}

Temperature: $\pm 0.1 \mathrm{~K}$.

$x_{1}: \pm 2.0 \%$ (relative error, estimated by compiler).

\subsection{2-Methylbenzoic acid solubility data in esters}

\begin{tabular}{ll}
\hline \hline Components: & Original Measurements: \\
(1) 2-Methylbenzoic acid; $\mathrm{C}_{8} \mathrm{H}_{8} \mathrm{O}_{2} ;$ & $\begin{array}{l}{ }^{171} \text { R. Coaxum, K. R. Hoover, E. } \\
\text { [118-90-1] }\end{array}$ \\
$\begin{array}{l}\text { Pustejovsky, D. M. Stovall, W. E. } \\
\text { (2) Methyl ethanoate; } \mathrm{C}_{3} \mathrm{H}_{6} \mathrm{O}_{2} ;\end{array}$ & $\begin{array}{l}\text { Acree, Jr., and M. H. Abraham, } \\
\text { 79-20-9] }\end{array}$ \\
\hline Phys. Chem. Liq. 42, 313 (2004). \\
$T / \mathrm{K}=298.15$ & Prepared by: \\
\hline
\end{tabular}

Experimental Values

\begin{tabular}{lc}
\hline \hline$x_{2}^{\mathrm{a}}$ & $x_{1}{ }^{\mathrm{b}}$ \\
\hline 0.8694 & 0.1306 \\
\hline${ }^{\mathrm{a}} x_{2}:$ mole fraction of component 2 in the saturated solution. \\
${ }^{\mathrm{b}} x_{1}:$ mole fraction solubility of the solute.
\end{tabular}

\section{Auxiliary Information}

\section{Method/Apparatus/Procedure:}

Constant-temperature bath, calorimetric thermometer, and an ultraviolet/ visible spectrophotometer.

Excess solute and solvent were placed in amber glass bottles and allowed to equilibrate for several days at constant temperature. Attainment of equilibrium was verified by several repetitive measurements and by approaching equilibrium from supersaturation. Aliquots of saturated solutions were transferred through a coarse filter into tared volumetric flasks, weighed, and diluted with methanol. Concentrations were determined by spectrophotometric measurements at $279 \mathrm{~nm}$.

\section{Source and Purity of Chemicals:}

(1) $99 \%$, Aldrich Chemical Company, Milwaukee, WI, USA, was used as received.

(2) $99.5 \%$, anhydrous, Aldrich Chemical Company, stored over molecular sieves and distilled shortly before use.

\begin{tabular}{ll}
\hline \hline Components: & Original Measurements: \\
(1) 2-Methylbenzoic acid; $\mathrm{C}_{8} \mathrm{H}_{8} \mathrm{O}_{2} ;$ & $\begin{array}{l}{ }^{171} \text { R. Coaxum, K. R. Hoover, E. } \\
\text { [118-90-1] }\end{array}$ \\
$\begin{array}{l}\text { Pustejovsky, D. M. Stovall, W. E. } \\
\text { (2) Ethyl ethanoate; } \mathrm{C}_{4} \mathrm{H}_{8} \mathrm{O}_{2} ;\end{array}$ & Acree, Jr., and M. H. Abraham, \\
[141-78-6] & Phys. Chem. Liq. 42, 313 (2004). \\
\hline Variables: & Prepared by: \\
$T / \mathrm{K}=298.15$ & W. E. Acree, Jr. \\
\hline
\end{tabular}

Experimental Values

\begin{tabular}{lc}
\hline \hline$x_{2}{ }^{\mathrm{a}}$ & $x_{1}{ }^{\mathrm{b}}$ \\
\hline 0.8491 & 0.1509 \\
\hline${ }^{\mathrm{a}} x_{2}:$ mole fraction of component 2 in the saturated solution. & \\
${ }^{\mathrm{b}} x_{1}:$ mole fraction solubility of the solute. &
\end{tabular}

\section{Auxiliary Information}

\section{Method/Apparatus/Procedure:}

Constant-temperature bath, calorimetric thermometer, and an ultraviolet/ visible spectrophotometer.

Excess solute and solvent were placed in amber glass bottles and allowed to equilibrate for several days at constant temperature. Attainment of equilibrium was verified by several repetitive measurements and by approaching equilibrium from supersaturation. Aliquots of saturated solutions were transferred through a coarse filter into tared volumetric flasks, weighed, and diluted with methanol. Concentrations were determined by spectrophotometric measurements at $279 \mathrm{~nm}$.

\section{Source and Purity of Chemicals:}

(1) $99 \%$, Aldrich Chemical Company, Milwaukee, WI, USA, was used as received.

(2) $99.9 \%$, HPLC grade, Aldrich Chemical Company, stored over molecular sieves and distilled shortly before use.

\section{Estimated Error:}

Temperature: $\pm 0.1 \mathrm{~K}$.

$x_{1}: \pm 1.5 \%$ (relative error).

\begin{tabular}{ll}
\hline \hline Components: & Original Measurements: \\
(1) 2-Methylbenzoic acid; $\mathrm{C}_{8} \mathrm{H}_{8} \mathrm{O}_{2} ;$ & $\begin{array}{l}{ }^{171} \text { R. Coaxum, K. R. Hoover, E. } \\
\text { [118-90-1] }\end{array}$ \\
$\begin{array}{l}\text { Pustejovsky, D. M. Stovall, W. E. } \\
\text { (2) Butyl ethanoate; } \mathrm{C}_{6} \mathrm{H}_{12} \mathrm{O}_{2} ;\end{array}$ & $\begin{array}{l}\text { Acree, Jr., and M. H. Abraham, } \\
{[123-86-4]}\end{array}$ \\
\hline Phys. Chem. Liq. 42, 313 (2004). \\
\hline$T / \mathrm{K}=298.15$ & Prepared by: \\
\hline
\end{tabular}

Experimental Values

\begin{tabular}{lc}
\hline \hline$x_{2}{ }^{\mathrm{a}}$ & $x_{1}{ }^{\mathrm{b}}$ \\
\hline 0.8486 & 0.1514 \\
\hline${ }^{\mathrm{a}} x_{2}:$ mole fraction of component 2 in the saturated solution. & \\
${ }^{\mathrm{b}} x_{1}:$ mole fraction solubility of the solute. &
\end{tabular}

\section{Estimated Error:}

Temperature: $\pm 0.1 \mathrm{~K}$.

$x_{1}: \pm 1.5 \%$ (relative error). 


\section{Auxiliary Information}

\section{Method/Apparatus/Procedure:}

Constant-temperature bath, calorimetric thermometer, and an ultraviolet/ visible spectrophotometer.

Excess solute and solvent were placed in amber glass bottles and allowed to equilibrate for several days at constant temperature. Attainment of equilibrium was verified by several repetitive measurements and by approaching equilibrium from supersaturation. Aliquots of saturated solutions were transferred through a coarse filter into tared volumetric flasks, weighed, and diluted with methanol. Concentrations were determined by

spectrophotometric measurements at $279 \mathrm{~nm}$.

Source and Purity of Chemicals:

(1) $99 \%$, Aldrich Chemical Company, Milwaukee, WI, USA, was used as received.

(2) $99.7 \%$, HPLC grade, Aldrich Chemical Company, stored over molecular sieves and distilled shortly before use.

Estimated Error:

Temperature: $\pm 0.1 \mathrm{~K}$.

$x_{1}: \pm 1.5 \%$ (relative error).

\begin{tabular}{ll}
\hline \hline $\begin{array}{l}\text { Components: } \\
\text { (1) 2-Methylbenzoic acid; } \mathrm{C}_{8} \mathrm{H}_{8} \mathrm{O}_{2} ;\end{array}$ & $\begin{array}{l}\text { Original Measurements: } \\
{ }^{171} \text { R. Coaxum, K. R. Hoover, E. } \\
\text { [118-90-1] }\end{array}$ \\
$\begin{array}{l}\text { Pustejovsky, D. M. Stovall, W. E. } \\
\text { [628-63-7] }\end{array}$ & Acree, Jr., and M. H. Abraham, \\
\hline Variables: & Phys. Chem. Liq. 42, 313 (2004). \\
$T / \mathrm{K}=298.15$ & Prepared by: \\
\hline
\end{tabular}

Experimental Values

\begin{tabular}{lc}
\hline \hline$x_{2}{ }^{\mathrm{a}}$ & $x_{1}{ }^{\mathrm{b}}$ \\
\hline 0.8506 & 0.1494 \\
\hline${ }^{\mathrm{a}} x_{2}:$ mole fraction of component 2 in the saturated solution. & \\
${ }^{\mathrm{b}} x_{1}:$ mole fraction solubility of the solute. &
\end{tabular}

\section{Auxiliary Information}

\section{Method/Apparatus/Procedure:}

Constant-temperature bath, calorimetric thermometer, and an ultraviolet/ visible spectrophotometer.

Excess solute and solvent were placed in amber glass bottles and allowed to equilibrate for several days at constant temperature. Attainment of equilibrium was verified by several repetitive measurements and by approaching equilibrium from supersaturation. Aliquots of saturated solutions were transferred through a coarse filter into tared volumetric flasks, weighed, and diluted with methanol. Concentrations were determined by

spectrophotometric measurements at $279 \mathrm{~nm}$.

Source and Purity of Chemicals:

(1) $99 \%$, Aldrich Chemical Company, Milwaukee, WI, USA, was used as received.

(2) $99 \%$, Aldrich Chemical Company, stored over molecular sieves and distilled shortly before use.

Estimated Error:

Temperature: $\pm 0.1 \mathrm{~K}$.

$x_{1}: \pm 1.5 \%$ (relative error).

\begin{tabular}{|c|c|}
\hline $\begin{array}{l}\text { Components: } \\
\text { (1) 2-Methylbenzoic acid; } \mathrm{C}_{8} \mathrm{H}_{8} \mathrm{O}_{2} \text {; } \\
\text { [118-90-1] } \\
\text { (2) } 1,2,3-\text { Triacetoxypropane (Triacetin); } \\
\mathrm{C}_{9} \mathrm{H}_{14} \mathrm{O}_{6} ;[102-76-1]\end{array}$ & $\begin{array}{l}\text { Original Measurements: } \\
\text { W. E. Acree, Jr., unpublished } \\
\text { data. }\end{array}$ \\
\hline $\begin{array}{l}\text { Variables: } \\
T / \mathrm{K}=298.15\end{array}$ & $\begin{array}{l}\text { Prepared by: } \\
\text { W. E. Acree, Jr. }\end{array}$ \\
\hline
\end{tabular}

\section{Experimental Values}

\begin{tabular}{lc}
\hline \hline$x_{2}{ }^{\mathrm{a}}$ & $x_{1}{ }^{\mathrm{b}}$ \\
\hline 0.8876 & 0.1124 \\
\hline${ }^{\mathrm{a}} x_{2}:$ mole fraction of component 2 in the saturated solution. & \\
${ }^{\mathrm{b}} x_{1}:$ mole fraction solubility of the solute. &
\end{tabular}

\section{Auxiliary Information}

\section{Method: Apparatus/Procedure:}

Constant-temperature bath, calorimetric thermometer, and an ultraviolet/ visible spectrophotometer

Excess solute and solvent were placed in amber glass bottles and allowed to equilibrate for several days at constant temperature. Attainment of equilibrium was verified by several repetitive measurements and by approaching equilibrium from supersaturation. Aliquots of saturated solutions were transferred through a coarse filter into tared volumetric flasks, weighed, and diluted with 2-propanol. Concentrations were determined by spectrophotometric measurements at $279 \mathrm{~nm}$.

Source and Purity of Chemicals:

(1) $99 \%$, Aldrich Chemical Company, Milwaukee, WI, USA, was used as received.

(2) $99 \%$, Acros Organics, USA, stored over molecular sieves before use.

Estimated Error:

Temperature: $\pm 0.1 \mathrm{~K}$.

$x_{1}: \pm 1.5 \%$ (relative error).

\subsection{2-Methylbenzoic acid solubility data in ethers}

\begin{tabular}{|c|c|}
\hline $\begin{array}{l}\text { Components: } \\
\text { (1) 2-Methylbenzoic acid; } \mathrm{C}_{8} \mathrm{H}_{8} \mathrm{O}_{2} \text {; } \\
\text { [118-90-1] } \\
\text { (2) } 1,1^{\prime} \text {-Oxybisethane; } \mathrm{C}_{4} \mathrm{H}_{10} \mathrm{O} \text {; } \\
\text { [60-29-7] }\end{array}$ & $\begin{array}{l}\text { Original Measurements: } \\
{ }^{171} \text { R. Coaxum, K. R. Hoover, E. } \\
\text { Pustejovsky, D. M. Stovall, W. E. } \\
\text { Acree, Jr., and M. H. Abraham, } \\
\text { Phys. Chem. Liq. 42, } 313 \text { (2004). }\end{array}$ \\
\hline $\begin{array}{l}\text { Variables: } \\
T / \mathrm{K}=298.15\end{array}$ & $\begin{array}{l}\text { Prepared by: } \\
\text { W. E. Acree, Jr. }\end{array}$ \\
\hline
\end{tabular}

Experimental Values

\begin{tabular}{lc}
\hline \hline$x_{2}{ }^{\mathrm{a}}$ & $x_{1}{ }^{\mathrm{b}}$ \\
\hline 0.8282 & 0.1718 \\
\hline${ }^{\mathrm{a}} x_{2}:$ mole fraction of component 2 in the saturated solution. \\
${ }^{\mathrm{b}} x_{1}:$ mole fraction solubility of the solute.
\end{tabular}




\section{Auxiliary Information}

\section{Method/Apparatus/Procedure:}

Constant-temperature bath, calorimetric thermometer, and an ultraviolet/ visible spectrophotometer.

Excess solute and solvent were placed in amber glass bottles and allowed to equilibrate for several days at constant temperature. Attainment of equilibrium was verified by several repetitive measurements and by approaching equilibrium from supersaturation. Aliquots of saturated solutions were transferred through a coarse filter into tared volumetric flasks, weighed, and diluted with methanol. Concentrations were determined by spectrophotometric measurements at $279 \mathrm{~nm}$.

Source and Purity of Chemicals:

(1) $99 \%$, Aldrich Chemical Company, Milwaukee, WI, USA, was used as received.

(2) 99+\%, anhydrous, Aldrich Chemical Company, stored over molecular sieves and distilled shortly before use.

Estimated Error:

Temperature: $\pm 0.1 \mathrm{~K}$.

$x_{1}: \pm 1.5 \%$ (relative error).

\begin{tabular}{ll}
\hline \hline $\begin{array}{l}\text { Components: } \\
\text { (1) 2-Methylbenzoic acid; } \mathrm{C}_{8} \mathrm{H}_{8} \mathrm{O}_{2} ;\end{array}$ & $\begin{array}{l}\text { Original Measurements: } \\
{ }^{171} \text { R. Coaxum, K. R. Hoover, E. } \\
\text { [118-90-1] }\end{array}$ \\
$\begin{array}{l}\text { Pustejovsky, D. M. Stovall, W. E. } \\
\text { (2,2'-Oxybispropane; } \mathrm{C}_{6} \mathrm{H}_{14} \mathrm{O} ;\end{array}$ & $\begin{array}{l}\text { Acree, Jr., and M. H. Abraham, } \\
\text { Phys- } 20-3]\end{array}$ \\
\hline Variables: & Prepared by: \\
$T / \mathrm{K}=298.15$ & W. E. Acree, Jr. \\
\hline
\end{tabular}

Experimental Values

\begin{tabular}{lc}
\hline \hline$x_{2}{ }^{\mathrm{a}}$ & $x_{1}{ }^{\mathrm{b}}$ \\
\hline 0.8852 & 0.1148
\end{tabular}

${ }^{\mathrm{a}} x_{2}$ : mole fraction of component 2 in the saturated solution.

${ }^{\mathrm{b}} x_{1}$ : mole fraction solubility of the solute.

\section{Auxiliary Information}

\section{Method/Apparatus/Procedure:}

Constant-temperature bath, calorimetric thermometer, and an ultraviolet/ visible spectrophotometer.

Excess solute and solvent were placed in amber glass bottles and allowed to equilibrate for several days at constant temperature. Attainment of equilibrium was verified by several repetitive measurements and by approaching equilibrium from supersaturation. Aliquots of saturated solutions were transferred through a coarse filter into tared volumetric flasks, weighed, and diluted with methanol. Concentrations were determined by

spectrophotometric measurements at $279 \mathrm{~nm}$.

\section{Source and Purity of Chemicals:}

(1) $99 \%$, Aldrich Chemical Company, Milwaukee, WI, USA, was used as received.

(2) $99 \%$, anhydrous, Aldrich Chemical Company, stored over molecular sieves and distilled shortly before use.

\section{Estimated Error:}

Temperature: $\pm 0.1 \mathrm{~K}$

$x_{1}: \pm 1.5 \%$ (relative error).

\begin{tabular}{|c|c|}
\hline $\begin{array}{l}\text { Components: } \\
\text { (1) 2-Methylbenzoic acid; } \mathrm{C}_{8} \mathrm{H}_{8} \mathrm{O}_{2} \text {; } \\
\text { [118-90-1] } \\
\text { (2) } 1,1^{\prime}-\text { Oxybisbutane; } \mathrm{C}_{8} \mathrm{H}_{18} \mathrm{O} \text {; } \\
\text { [142-96-1] }\end{array}$ & $\begin{array}{l}\text { Original Measurements: } \\
{ }^{171} \text { R. Coaxum, K. R. Hoover, E. } \\
\text { Pustejovsky, D. M. Stovall, W. E. } \\
\text { Acree, Jr., and M. H. Abraham, } \\
\text { Phys. Chem. Liq. 42, } 313 \text { (2004). }\end{array}$ \\
\hline $\begin{array}{l}\text { Variables: } \\
T / \mathrm{K}=298.15\end{array}$ & $\begin{array}{l}\text { Prepared by: } \\
\text { W. E. Acree, Jr. }\end{array}$ \\
\hline
\end{tabular}

Experimental Values

\begin{tabular}{lc}
\hline \hline$x_{2}{ }^{\mathrm{a}}$ & $x_{1}{ }^{\mathrm{b}}$ \\
\hline 0.9061 & 0.0939
\end{tabular}

${ }^{\mathrm{a}} x_{2}$ : mole fraction of component 2 in the saturated solution.

${ }^{b} x_{1}$ : mole fraction solubility of the solute.

\section{Auxiliary Information}

\section{Method/Apparatus/Procedure:}

Constant-temperature bath, calorimetric thermometer, and an ultraviolet/ visible spectrophotometer.

Excess solute and solvent were placed in amber glass bottles and allowed to equilibrate for several days at constant temperature. Attainment of equilibrium was verified by several repetitive measurements and by approaching equilibrium from supersaturation. Aliquots of saturated solutions were transferred through a coarse filter into tared volumetric flasks, weighed, and diluted with methanol. Concentrations were determined by spectrophotometric measurements at $279 \mathrm{~nm}$.

\section{Source and Purity of Chemicals:}

(1) $99 \%$, Aldrich Chemical Company, Milwaukee, WI, USA, was used as received.

(2) $99.3 \%$, anhydrous, Aldrich Chemical Company, stored over molecular sieves and distilled shortly before use.

\section{Estimated Error:}

Temperature: $\pm 0.1 \mathrm{~K}$.

$x_{1}: \pm 1.5 \%$ (relative error).

\begin{tabular}{ll}
\hline \hline Components: & Original Measurements: \\
(1) 2-Methylbenzoic acid; $\mathrm{C}_{8} \mathrm{H}_{8} \mathrm{O}_{2} ;$ & $\begin{array}{l}{ }^{171} \text { R. Coaxum, K. R. Hoover, E. } \\
\text { [118-90-1] }\end{array}$ \\
$\begin{array}{l}\text { Pustejovsky, D. M. Stovall, W. E. } \\
\text { (2) Tetrahydrofuran; } \mathrm{C}_{4} \mathrm{H}_{8} \mathrm{O} ;\end{array}$ & $\begin{array}{l}\text { Acree, Jr., and M. H. Abraham, } \\
\text { [109-99-9] }\end{array}$ \\
\hline Pariables: & Phys. Chem. Liq. 42, 313 (2004). \\
$T / \mathrm{K}=298.15$ & Prepared by: \\
\hline
\end{tabular}

Experimental Values

\begin{tabular}{lc}
\hline \hline$x_{2}{ }^{\mathrm{a}}$ & $x_{1}{ }^{\mathrm{b}}$ \\
\hline 0.7371 & 0.2629 \\
\hline${ }^{\mathrm{a}} x_{2}:$ mole fraction of component 2 in the saturated solution. \\
${ }^{\mathrm{b}}{ }_{x_{1}: \text { mole fraction solubility of the solute. }}$
\end{tabular}




\section{Auxiliary Information}

\section{Method/Apparatus/Procedure:}

Constant-temperature bath, calorimetric thermometer, and an ultraviolet/ visible spectrophotometer.

Excess solute and solvent were placed in amber glass bottles and allowed to equilibrate for several days at constant temperature. Attainment of equilibrium was verified by several repetitive measurements and by approaching equilibrium from supersaturation. Aliquots of saturated solutions were transferred through a coarse filter into tared volumetric flasks, weighed, and diluted with methanol. Concentrations were determined by spectrophotometric measurements at $279 \mathrm{~nm}$.

Source and Purity of Chemicals:

(1) $99 \%$, Aldrich Chemical Company, Milwaukee, WI, USA, was used as received.

(2) $99.9 \%$, anhydrous, Aldrich Chemical Company, stored over molecular sieves and distilled shortly before use.

Estimated Error:

Temperature: $\pm 0.1 \mathrm{~K}$.

$x_{1}: \pm 2.0 \%$ (relative error)

\begin{tabular}{ll}
\hline \hline Components: & Original Measurements: \\
(1) 2-Methylbenzoic acid; $\mathrm{C}_{8} \mathrm{H}_{8} \mathrm{O}_{2} ;$ & $\begin{array}{l}{ }^{171} \text { R. Coaxum, K. R. Hoover, E. } \\
\text { [118-90-1] }\end{array}$ \\
$\begin{array}{l}\text { Pustejovsky, D. M. Stovall, W. E. } \\
\text { (2) 1,4-Dioxane; } \mathrm{C}_{4} \mathrm{H}_{8} \mathrm{O}_{2} ; \text { [123-91-1] }\end{array}$ & $\begin{array}{l}\text { Acree, Jr., and M. H. Abraham, } \\
\text { Phys. Chem. Liq. 42, 313 (2004). }\end{array}$ \\
\hline Variables: & Prepared by: \\
$T / \mathrm{K}=298.15$ & W. E. Acree, Jr. \\
\hline
\end{tabular}

Experimental Values

\begin{tabular}{|c|c|}
\hline$x_{2}{ }^{\mathrm{a}}$ & $x_{1}{ }^{\mathrm{b}}$ \\
\hline 0.7466 & 0.2534 \\
\hline
\end{tabular}

${ }^{\mathrm{a}} x_{2}$ : mole fraction of component 2 in the saturated solution.

${ }^{\mathrm{b}} x_{1}$ : mole fraction solubility of the solute.

\section{Auxiliary Information}

\section{Method/Apparatus/Procedure:}

Constant-temperature bath, calorimetric thermometer, and an ultraviolet/ visible spectrophotometer.

Excess solute and solvent were placed in amber glass bottles and allowed to equilibrate for several days at constant temperature. Attainment of equilibrium was verified by several repetitive measurements and by approaching equilibrium from supersaturation. Aliquots of saturated solutions were transferred through a coarse filter into tared volumetric flasks, weighed, and diluted with methanol. Concentrations were determined by spectrophotometric measurements at $279 \mathrm{~nm}$.

Source and Purity of Chemicals:

(1) $99 \%$, Aldrich Chemical Company, Milwaukee, WI, USA, was used as received.

(2) $99.8 \%$, anhydrous, Aldrich Chemical Company, stored over molecular sieves and distilled shortly before use.

Estimated Error:

Temperature: $\pm 0.1 \mathrm{~K}$.

$x_{1}: \pm 2.0 \%$ (relative error).

\subsection{2-Methylbenzoic acid solubility data in haloalkanes, haloalkenes, and haloaromatic hydrocarbons}

\begin{tabular}{|c|c|}
\hline $\begin{array}{l}\text { Components: } \\
\text { (1) 2-Methylbenzoic acid; } \mathrm{C}_{8} \mathrm{H}_{8} \mathrm{O}_{2} \text {; } \\
\text { [118-90-1] } \\
\text { (2) Tetrachloromethane; } \mathrm{CCl}_{4} \text {; } \\
\text { [56-23-5] }\end{array}$ & $\begin{array}{l}\text { Original Measurements: } \\
\text { }{ }^{172} \text { U. Domańska, Pol. J. Chem. 60, } \\
847 \text { (1986). }\end{array}$ \\
\hline $\begin{array}{l}\text { Variables: } \\
\text { Temperature }\end{array}$ & $\begin{array}{l}\text { Prepared by: } \\
\text { W. E. Acree, Jr. }\end{array}$ \\
\hline
\end{tabular}

Experimental Values

\begin{tabular}{lcc}
\hline \hline$T / \mathrm{K}$ & $x_{2}{ }^{\mathrm{a}}$ & $x_{1}{ }^{\mathrm{b}}$ \\
\hline 296.20 & 0.9383 & 0.0617 \\
305.85 & 0.9017 & 0.0983 \\
312.05 & 0.8692 & 0.1308 \\
318.15 & 0.8273 & 0.1727 \\
328.05 & 0.7492 & 0.2508 \\
336.35 & 0.6602 & 0.3398 \\
\hline
\end{tabular}

${ }^{a} x_{2}$ : mole fraction of component 2 in the saturated solution.

${ }^{\mathrm{b}} x_{1}$ : mole fraction solubility of the solute.

\section{Auxiliary Information}

\section{Method/Apparatus/Procedure:}

Analytical balance and constant-temperature bath.

Solubilities were determined by a dynamic method. Weighed amounts of solute and solvent were placed in sample container and placed in a constanttemperature bath. The temperature of the bath was slowly increased. The temperature at which the last crystals disappeared was taken as the temperature of the solution-crystal equilibrium.

Source and Purity of Chemicals:

(1) $99+\%$, Analytical grade, Fluka Chemicals, Switzerland, purified by double vacuum sublimation.

(2) Purity not given, POCh Gliwice, Poland, dried over phosphorous pentoxide and then fractionally distilled before use.

\section{Estimated Error:}

Temperature: $\pm 0.1 \mathrm{~K}$.

$x_{1}: \pm 2.0 \%$ (relative error, estimated by compiler).

\begin{tabular}{|c|c|}
\hline $\begin{array}{l}\text { Components: } \\
\text { (1) 2-Methylbenzoic acid; } \mathrm{C}_{8} \mathrm{H}_{8} \mathrm{O}_{2} \text {; } \\
\text { [118-90-1] } \\
\text { (2) Diiodomethane; } \mathrm{CH}_{2} \mathrm{I}_{2} ;[75-11-6]\end{array}$ & $\begin{array}{l}\text { Original Measurements: } \\
{ }^{172} \text { U. Domańska, Pol. J. Chem. 60, } \\
847 \text { (1986). }\end{array}$ \\
\hline $\begin{array}{l}\text { Variables: } \\
\text { Temperature }\end{array}$ & $\begin{array}{l}\text { Prepared by: } \\
\text { W. E. Acree, Jr. }\end{array}$ \\
\hline
\end{tabular}


Experimental Values

\begin{tabular}{lcc}
\hline \hline$T / \mathrm{K}$ & $x_{2}{ }^{\mathrm{a}}$ & $x_{1}{ }^{\mathrm{b}}$ \\
\hline 294.45 & 0.9962 & 0.00378 \\
301.05 & 0.9941 & 0.00588 \\
303.75 & 0.9926 & 0.00740 \\
307.45 & 0.9913 & 0.00870 \\
308.55 & 0.9905 & 0.00948 \\
312.15 & 0.9886 & 0.0114 \\
316.45 & 0.9851 & 0.0149 \\
321.15 & 0.9787 & 0.0213 \\
324.85 & 0.9733 & 0.0267 \\
328.35 & 0.9664 & 0.0336 \\
332.45 & 0.9562 & 0.0438 \\
336.45 & 0.9401 & 0.0599 \\
340.45 & 0.9175 & 0.0825 \\
343.85 & 0.8823 & 0.1177 \\
345.55 & 0.8614 & 0.1386 \\
\hline
\end{tabular}

${ }^{\mathrm{a}} x_{2}$ : mole fraction of component 2 in the saturated solution.

${ }^{\mathrm{b}} x_{1}$ : mole fraction solubility of the solute.

\section{Auxiliary Information}

\section{Method/Apparatus/Procedure:}

Analytical balance and constant-temperature bath.

Solubilities were determined by a dynamic method. Weighed amounts of solute and solvent were placed in sample container and placed in a constanttemperature bath. The temperature of the bath was slowly increased. The temperature at which the last crystals disappeared was taken as the temperature of the solution-crystal equilibrium.

\section{Source and Purity of Chemicals:}

(1) $99+\%$, Analytical grade, Fluka Chemicals, Switzerland, purified by double vacuum sublimation.

(2) Purity not given, Chemapol, CSRS, used as received.

Estimated Error:

Temperature: $\pm 0.1 \mathrm{~K}$.

$x_{1}: \pm 2.0 \%$ (relative error, estimated by compiler).

\begin{tabular}{|c|c|}
\hline $\begin{array}{l}\text { Components: } \\
\text { (1) 2-Methylbenzoic acid; } \mathrm{C}_{8} \mathrm{H}_{8} \mathrm{O}_{2} \text {; } \\
\text { [118-90-1] } \\
\text { (2) Chlorobenzene; } \mathrm{C}_{6} \mathrm{H}_{5} \mathrm{Cl} ;[108-90-7]\end{array}$ & $\begin{array}{l}\text { Original Measurements: } \\
{ }^{172} \text { U. Domańska, Pol. J. Chem. } \\
\text { 60, } 847 \text { (1986). }\end{array}$ \\
\hline Variables: & Prepared by: \\
\hline Temperature & W. E. Acree, Jr. \\
\hline
\end{tabular}

Experimental Values

\begin{tabular}{lcc}
\hline \hline$T / \mathrm{K}$ & $x_{2}{ }^{\mathrm{a}}$ & $x_{1}{ }^{\mathrm{b}}$ \\
\hline 299.05 & 0.8517 & 0.1483 \\
301.05 & 0.8429 & 0.1571 \\
305.95 & 0.8120 & 0.1880 \\
309.10 & 0.7796 & 0.2204 \\
314.70 & 0.7439 & 0.2561 \\
319.15 & 0.7011 & 0.2989 \\
323.70 & 0.6582 & 0.3418 \\
326.85 & 0.6213 & 0.3787 \\
334.00 & 0.5389 & 0.4611 \\
342.85 & 0.4295 & 0.5705 \\
354.15 & 0.2799 & 0.7201 \\
\hline
\end{tabular}

${ }^{\mathrm{a}} x_{2}$ : mole fraction of component 2 in the saturated solution.

${ }^{\mathrm{b}} x_{1}$ : mole fraction solubility of the solute.
Auxiliary Information

\section{Method/Apparatus/Procedure:}

Analytical balance and constant-temperature bath.

Solubilities were determined by a dynamic method. Weighed amounts of solute and solvent were placed in sample container and placed in a constanttemperature bath. The temperature of the bath was slowly increased. The temperature at which the last crystals disappeared was taken as the temperature of the solution-crystal equilibrium.

Source and Purity of Chemicals:

(1) $99+\%$, Analytical grade, Fluka Chemicals, Switzerland, purified by double vacuum sublimation.

(2) Purity not given, Intern. Enzymes Limited, dried over molecular sieves and then fractionally distilled before use.

Estimated Error:

Temperature: $\pm 0.1 \mathrm{~K}$.

$x_{1}: \pm 2.0 \%$ (relative error, estimated by compiler).

\begin{tabular}{|c|c|}
\hline $\begin{array}{l}\text { Components: } \\
\text { (1) 2-Methylbenzoic acid; } \mathrm{C}_{8} \mathrm{H}_{8} \mathrm{O}_{2} \text {; } \\
\text { [118-90-1] } \\
\text { (2) } 1,2-\text {-Dichlorobenzene; } \mathrm{C}_{6} \mathrm{H}_{4} \mathrm{Cl}_{2} \text {; } \\
\text { [95-50-1] }\end{array}$ & $\begin{array}{l}\text { Original Measurements: } \\
{ }^{172} \text { U. Domańska, Pol. J. Chem. 60, } \\
847 \text { (1986). }\end{array}$ \\
\hline $\begin{array}{l}\text { Variables: } \\
\text { Temperature }\end{array}$ & $\begin{array}{l}\text { Prepared by: } \\
\text { W. E. Acree, Jr. }\end{array}$ \\
\hline
\end{tabular}

Experimental Values

\begin{tabular}{lcc}
\hline \hline$T / \mathrm{K}$ & $x_{2}{ }^{\mathrm{a}}$ & $x_{1}{ }^{\mathrm{b}}$ \\
\hline 296.05 & 0.9031 & 0.0969 \\
302.35 & 0.8720 & 0.1280 \\
307.70 & 0.8410 & 0.1590 \\
310.80 & 0.8205 & 0.1795 \\
318.65 & 0.7681 & 0.2319 \\
328.50 & 0.6828 & 0.3172 \\
335.25 & 0.6121 & 0.3879
\end{tabular}

${ }^{\mathrm{a}} x_{2}$ : mole fraction of component 2 in the saturated solution.

${ }^{\mathrm{b}} x_{1}$ : mole fraction solubility of the solute.

\section{Auxiliary Information}

\section{Method/Apparatus/Procedure:}

Analytical balance and constant-temperature bath.

Solubilities were determined by a dynamic method. Weighed amounts of solute and solvent were placed in sample container and placed in a constanttemperature bath. The temperature of the bath was slowly increased. The temperature at which the last crystals disappeared was taken as the temperature of the solution-crystal equilibrium.

Source and Purity of Chemicals:

(1) $99+\%$, Analytical grade, Fluka Chemicals, Switzerland, purified by double vacuum sublimation.

(2) Purity not given, POCh Gliwice, Poland, dried over molecular sieves and then fractionally distilled before use.

\section{Estimated Error:}

Temperature: $\pm 0.1 \mathrm{~K}$.

$x_{1}: \pm 2.0 \%$ (relative error, estimated by compiler). 


\begin{tabular}{|c|c|}
\hline $\begin{array}{l}\text { Components: } \\
\text { (1) 2-Methylbenzoic acid; } \mathrm{C}_{8} \mathrm{H}_{8} \mathrm{O}_{2} \text {; } \\
\text { [118-90-1] } \\
\text { (2) Bromobenzene; } \mathrm{C}_{6} \mathrm{H}_{5} \mathrm{Br} \text {; } \\
\text { [108-86-1] }\end{array}$ & $\begin{array}{l}\text { Original Measurements: } \\
{ }^{172} \text { U. Domańska, Pol. J. Chem. 60, } \\
847 \text { (1986). }\end{array}$ \\
\hline $\begin{array}{l}\text { Variables: } \\
\text { Temperature }\end{array}$ & $\begin{array}{l}\text { Prepared by: } \\
\text { W. E. Acree, Jr. }\end{array}$ \\
\hline
\end{tabular}

Experimental Values

\begin{tabular}{lcc}
\hline \hline$T / \mathrm{K}$ & $x_{2}{ }^{\mathrm{a}}$ & $x_{1}{ }^{\mathrm{b}}$ \\
\hline 298.35 & 0.9043 & 0.0957 \\
301.95 & 0.8884 & 0.1116 \\
308.20 & 0.8526 & 0.1474 \\
312.65 & 0.8237 & 0.1763 \\
317.55 & 0.7867 & 0.2133 \\
330.95 & 0.6773 & 0.3227 \\
334.60 & 0.6371 & 0.3629 \\
344.15 & 0.5176 & 0.4824 \\
352.10 & 0.4063 & 0.5937 \\
\hline
\end{tabular}

${ }^{\mathrm{a}} x_{2}$ : mole fraction of component 2 in the saturated solution.

${ }^{\mathrm{b}} x_{1}$ : mole fraction solubility of the solute.

\section{Auxiliary Information}

\section{Method/Apparatus/Procedure:}

Analytical balance and constant-temperature bath.

Solubilities were determined by a dynamic method. Weighed amounts of solute and solvent were placed in sample container and placed in a constanttemperature bath. The temperature of the bath was slowly increased. The temperature at which the last crystals disappeared was taken as the temperature of the solution-crystal equilibrium.

Source and Purity of Chemicals:

(1) $99+\%$, Analytical grade, Fluka Chemicals, Switzerland, purified by double vacuum sublimation.

(2) Purity not given, POCh Gliwice, Poland, dried over molecular sieves and then fractionally distilled before use.

\section{Estimated Error:}

Temperature: $\pm 0.1 \mathrm{~K}$.

$x_{1}: \pm 2.0 \%$ (relative error, estimated by compiler).

\begin{tabular}{lc}
\multicolumn{2}{c}{ Experimental Values } \\
\hline \hline$x_{2}{ }^{\mathrm{a}}$ & $x_{1}{ }^{\mathrm{b}}$ \\
\hline 0.8599 & 0.1401 \\
\hline${ }^{\mathrm{a}} x_{2}:$ mole fraction of component 2 in the saturated solution. \\
${ }^{\mathrm{b}}{ }_{x_{1}}:$ mole fraction solubility of the solute.
\end{tabular}

\section{Auxiliary Information}

Method/Apparatus/Procedure:

Constant-temperature bath, calorimetric thermometer, and an ultraviolet/ visible spectrophotometer.

Excess solute and solvent were placed in amber glass bottles and allowed to equilibrate for several days at constant temperature. Attainment of equilibrium was verified by several repetitive measurements and by approaching equilibrium from supersaturation. Aliquots of saturated solutions were transferred through a coarse filter into tared volumetric flasks, weighed, and diluted with methanol. Concentrations were determined by spectrophotometric measurements at $279 \mathrm{~nm}$.

Source and Purity of Chemicals:

(1) $99 \%$, Aldrich Chemical Company, Milwaukee, WI, USA, was used as received.

(2) Absolute, Aaper Alcohol and Chemical Company, USA, stored over molecular sieves and distilled shortly before use.

Estimated Error:

Temperature: $\pm 0.1 \mathrm{~K}$.

$x_{1}: \pm 1.5 \%$ (relative error).

\begin{tabular}{|c|c|}
\hline $\begin{array}{l}\text { Components: } \\
\text { (1) 2-Methylbenzoic acid; } \mathrm{C}_{8} \mathrm{H}_{8} \mathrm{O}_{2} \text {; } \\
{[118-90-1]} \\
\text { (2) Ethanol; } \mathrm{C}_{2} \mathrm{H}_{6} \mathrm{O} ;[64-17-5]\end{array}$ & $\begin{array}{l}\text { Original Measurements: } \\
{ }^{72} \text { R. Thuaire, Bull. Soc. Chim. Fr. } \\
3815 \text { (1971). }\end{array}$ \\
\hline $\begin{array}{l}\text { Variables: } \\
T / \mathrm{K}=298.15\end{array}$ & $\begin{array}{l}\text { Prepared by: } \\
\text { W. E. Acree, Jr. }\end{array}$ \\
\hline
\end{tabular}

\section{Experimental Values}

The measured solubility was reported to be $3.712 \mathrm{~mol} / \mathrm{kg}$ of solvent, which corresponds to a mole fraction solubility of $x_{1}=0.1460$.

\section{Auxiliary Information}

\section{Method/Apparatus/Procedure:}

Very little experimental details were provided. Solubility was determined by a gravimetric method. The saturated solution was evaporated to dryness and the remaining solid residue was weighed. The solubility was calculated from the mass of the solid residue and mass of saturated solution analyzed.

\section{Source and Purity of Chemicals:}

(1) Purity not given, Chemical source not given, no purification details were provided.

(2) Purity not given, Chemical source not given, no purification details were provided.

\section{Estimated Error:}

Temperature: $\pm 0.05 \mathrm{~K}$.

$x_{1}: \pm 4 \%$ (relative error, estimated by compiler). 


\section{Components:}

(1) 2-Methylbenzoic acid; $\mathrm{C}_{8} \mathrm{H}_{8} \mathrm{O}_{2}$;

[118-90-1]

(2) Ethanol; $\mathrm{C}_{2} \mathrm{H}_{6} \mathrm{O} ;[64-17-5]$
Original Measurements:

${ }^{173}$ U. Domańska and T. Hofman, J. Solution Chem. 14, 531 (1985).

${ }^{172}$ U. Domańska, Pol. J. Chem. 60 847 (1986).

\begin{tabular}{ll}
\hline Variables: & Prepared by: \\
Temperature & W. E. Acree, Jr.
\end{tabular}

Experimental Values

\begin{tabular}{lcc}
\hline \hline$T / \mathrm{K}$ & $x_{2}{ }^{\mathrm{a}}$ & $x_{1}{ }^{\mathrm{b}}$ \\
\hline 291.55 & 0.8791 & 0.1209 \\
296.05 & 0.8665 & 0.1335 \\
301.65 & 0.8442 & 0.1558 \\
306.25 & 0.8228 & 0.1772 \\
312.05 & 0.7973 & 0.2027 \\
316.05 & 0.7749 & 0.2251 \\
319.35 & 0.7560 & 0.2440 \\
323.35 & 0.7301 & 0.2699 \\
329.35 & 0.6874 & 0.3126 \\
336.85 & 0.6349 & 0.3651
\end{tabular}

${ }^{\mathrm{a}} x_{2}$ : mole fraction of component 2 in the saturated solution.

${ }^{\mathrm{b}} x_{1}$ : mole fraction solubility of the solute.

\section{Auxiliary Information}

\section{Method/Apparatus/Procedure:}

Analytical balance and constant-temperature bath.

Solubilities were determined by a dynamic method. Weighed amounts of solute and solvent were placed in sample container and placed in a constanttemperature bath. The temperature of the bath was slowly increased $(2 \mathrm{~K} / \mathrm{h}$ near the equilibrium temperature). The temperature at which the last crystals disappeared was taken as the temperature of the solution-crystal equilibrium.

\section{Source and Purity of Chemicals:}

(1) $99+\%$, Analytical grade, Fluka Chemicals, Switzerland, purified by double vacuum sublimation.

(2) Purity not given, POCh Gliwice, Poland, dried over molecular sieves and then fractionally distilled before use.

\section{Estimated Error:}

Temperature: $\pm 0.1 \mathrm{~K}$.

$x_{1}: \pm 2.0 \%$ (relative error, estimated by compiler).

\begin{tabular}{ll}
\hline \hline Components: & Original Measurements: \\
(1) 2-Methylbenzoic acid; $\mathrm{C}_{8} \mathrm{H}_{8} \mathrm{O}_{2} ;$ & $\begin{array}{l}{ }^{171} \text { R. Coaxum, K. R. Hoover, E. } \\
\text { [118-90-1] }\end{array}$ \\
$\begin{array}{ll}\text { Pustejovsky, D. M. Stovall, W. E. } \\
\text { (2) 1-Propanol; } \mathrm{C}_{3} \mathrm{H}_{8} \mathrm{O} ;[\text { [11-23-8] }\end{array}$ & $\begin{array}{l}\text { Acree, Jr., and M. H. Abraham, } \\
\text { Phys. Chem. Liq. 42, 313 (2004). }\end{array}$ \\
\hline Variables: & Prepared by: \\
$T / \mathrm{K}=298.15$ & W. E. Acree, Jr. \\
\hline
\end{tabular}

Experimental Values

\begin{tabular}{lc}
\hline \hline$x_{2}^{\mathrm{a}}$ & $x_{1}{ }^{\mathrm{b}}$ \\
\hline 0.8550 & 0.1450
\end{tabular}

${ }^{\mathrm{a}} x_{2}$ : mole fraction of component 2 in the saturated solution.

${ }^{\mathrm{b}} x_{1}$ : mole fraction solubility of the solute.

\section{Auxiliary Information}

\section{Method/Apparatus/Procedure:}

Constant-temperature bath, calorimetric thermometer, and an ultraviolet/ visible spectrophotometer.

Excess solute and solvent were placed in amber glass bottles and allowed to equilibrate for several days at constant temperature. Attainment of equilibrium was verified by several repetitive measurements and by approaching equilibrium from supersaturation. Aliquots of saturated solutions were transferred through a coarse filter into tared volumetric flasks, weighed, and diluted with methanol. Concentrations were determined by spectrophotometric measurements at $279 \mathrm{~nm}$.

\section{Source and Purity of Chemicals:}

(1) $99 \%$, Aldrich Chemical Company, Milwaukee, WI, USA, was used as received.

(2) $99+\%$, anhydrous, Aldrich Chemical Company, stored over molecular sieves and distilled shortly before use.

\section{Estimated Error:}

Temperature: $\pm 0.1 \mathrm{~K}$.

$x_{1}: \pm 1.5 \%$ (relative error).

\begin{tabular}{ll}
\hline \hline Components: & Original Measurements: \\
(1) 2-Methylbenzoic acid; $\mathrm{C}_{8} \mathrm{H}_{8} \mathrm{O}_{2} ;$ & ${ }^{171}$ R. Coaxum, K. R. Hoover, E. \\
[118-90-1] & Pustejovsky, D. M. Stovall, W. E. \\
(2) 2-Propanol; $\mathrm{C}_{3} \mathrm{H}_{8} \mathrm{O} ;$ [67-63-0] & $\begin{array}{l}\text { Acree, Jr., and M. H. Abraham, } \\
\text { Phys. Chem. Liq. 42, 313 (2004). }\end{array}$ \\
\hline Variables: & Prepared by: \\
$T / \mathrm{K}=298.15$ & W. E. Acree, Jr. \\
\hline
\end{tabular}

\section{Experimental Values}

\begin{tabular}{lc}
\hline \hline$x_{2}{ }^{\mathrm{a}}$ & $x_{1}{ }^{\mathrm{b}}$ \\
\hline 0.8388 & 0.1612 \\
\hline${ }^{\mathrm{a}} x_{2}:$ mole fraction of component 2 in the saturated solution. &
\end{tabular}

${ }^{b_{1}}$ : mole fraction solubility of the solute.

\section{Auxiliary Information}

\section{Method/Apparatus/Procedure:}

Constant-temperature bath, calorimetric thermometer, and an ultraviolet/ visible spectrophotometer.

Excess solute and solvent were placed in amber glass bottles and allowed to equilibrate for several days at constant temperature. Attainment of equilibrium was verified by several repetitive measurements and by approaching equilibrium from supersaturation. Aliquots of saturated solutions were transferred through a coarse filter into tared volumetric flasks, weighed, and diluted with methanol. Concentrations were determined by spectrophotometric measurements at $279 \mathrm{~nm}$.

Source and Purity of Chemicals:

(1) $99 \%$, Aldrich Chemical Company, Milwaukee, WI, USA, was used as received.

(2) $99+\%$, anhydrous, Aldrich Chemical Company, stored over molecular sieves and distilled shortly before use.

\section{Estimated Error:}

Temperature: $\pm 0.1 \mathrm{~K}$.

$x_{1}: \pm 1.5 \%$ (relative error). 


\section{Components:}

(1) 2-Methylbenzoic acid; $\mathrm{C}_{8} \mathrm{H}_{8} \mathrm{O}_{2}$;

[118-90-1]

(2) 2-Propanol; $\mathrm{C}_{3} \mathrm{H}_{8} \mathrm{O} ;[67-63-0]$
Original Measurements:

${ }^{173}$ U. Domańska and T. Hofman, J. Solution Chem. 14, 531 (1985).

${ }^{172}$ U. Domańska, Pol. J. Chem. 60, 847 (1986).

Variables: $\quad$ Prepared by:

Temperature
Experimental Values

\begin{tabular}{lcc}
\hline \hline$T / \mathrm{K}$ & $x_{2}{ }^{\mathrm{a}}$ & $x_{1}{ }^{\mathrm{b}}$ \\
\hline 292.65 & 0.8643 & 0.1357 \\
296.75 & 0.8457 & 0.1543 \\
301.95 & 0.8262 & 0.1738 \\
306.75 & 0.8019 & 0.1981 \\
312.40 & 0.7723 & 0.2277 \\
317.75 & 0.7388 & 0.2612 \\
324.90 & 0.6957 & 0.3043 \\
333.35 & 0.6337 & 0.3663 \\
340.15 & 0.5798 & 0.4202 \\
344.75 & 0.5344 & 0.4656 \\
\hline
\end{tabular}

${ }^{\mathrm{a}} x_{2}$ : mole fraction of component 2 in the saturated solution.

${ }^{\mathrm{b}} x_{1}$ : mole fraction solubility of the solute.

\section{Auxiliary Information}

\section{Method/Apparatus/Procedure:}

Analytical balance and constant-temperature bath.

Solubilities were determined by a dynamic method. Weighed amounts of solute and solvent were placed in sample container and placed in a constanttemperature bath. The temperature of the bath was slowly increased $(2 \mathrm{~K} / \mathrm{h}$ near the equilibrium temperature). The temperature at which the last crystals disappeared was taken as the temperature of the solution-crystal equilibrium.

\section{Source and Purity of Chemicals:}

(1) $99+\%$, Analytical grade, Fluka Chemicals, Switzerland, purified by double vacuum sublimation.

(2) Purity not given, POCh Gliwice, Poland, dried over molecular sieves and then fractionally distilled before use.

\section{Estimated Error:}

Temperature: $\pm 0.1 \mathrm{~K}$

$x_{1}: \pm 2.0 \%$ (relative error, estimated by compiler).

\begin{tabular}{|c|c|}
\hline $\begin{array}{l}\text { Components: } \\
\text { (1) 2-Methylbenzoic acid; } \mathrm{C}_{8} \mathrm{H}_{8} \mathrm{O}_{2} \text {; } \\
\text { [118-90-1] } \\
\text { (2) 1-Butanol; } \mathrm{C}_{4} \mathrm{H}_{10} \mathrm{O} \text {; [71-36-3] }\end{array}$ & $\begin{array}{l}\text { Original Measurements: } \\
{ }^{171} \text { R. Coaxum, K. R. Hoover, E. } \\
\text { Pustejovsky, D. M. Stovall, W. E. } \\
\text { Acree, Jr., and M. H. Abraham, } \\
\text { Phys. Chem. Liq. 42, } 313 \text { (2004). }\end{array}$ \\
\hline $\begin{array}{l}\text { Variables: } \\
T / \mathrm{K}=298.15\end{array}$ & $\begin{array}{l}\text { Prepared by: } \\
\text { W. E. Acree, Jr. }\end{array}$ \\
\hline
\end{tabular}

Experimental Values

\begin{tabular}{lc}
\hline \hline$x_{2}^{\mathrm{a}}$ & $x_{1}{ }^{\mathrm{b}}$ \\
\hline 0.8449 & 0.1551
\end{tabular}

${ }^{\mathrm{a}} x_{2}$ : mole fraction of component 2 in the saturated solution.

${ }^{\mathrm{b}} x_{1}$ : mole fraction solubility of the solute.

\section{Auxiliary Information}

Method/Apparatus/Procedure:

Constant-temperature bath, calorimetric thermometer, and an ultraviolet/ visible spectrophotometer.

Excess solute and solvent were placed in amber glass bottles and allowed to equilibrate for several days at constant temperature. Attainment of equilibrium was verified by several repetitive measurements and by approaching equilibrium from supersaturation. Aliquots of saturated solutions were transferred through a coarse filter into tared volumetric flasks, weighed, and diluted with methanol. Concentrations were determined by spectrophotometric measurements at $279 \mathrm{~nm}$.

\section{Source and Purity of Chemicals:}

(1) $99 \%$, Aldrich Chemical Company, Milwaukee, WI, USA, was used as received.

(2) $99.8 \%$, HPLC grade, Aldrich Chemical Company, stored over molecular sieves and distilled shortly before use.

\section{Estimated Error:}

Temperature: $\pm 0.1 \mathrm{~K}$.

$x_{1}: \pm 1.5 \%$ (relative error).

\begin{tabular}{ll}
\hline \hline Components: & Original Measurements: \\
(1) 2-Methylbenzoic acid; $\mathrm{C}_{8} \mathrm{H}_{8} \mathrm{O}_{2} ;$ & $\begin{array}{l}{ }^{171} \text { R. Coaxum, K. R. Hoover, E. } \\
\text { [118-90-1] }\end{array}$ \\
$\begin{array}{ll}\text { Pustejovsky, D. M. Stovall, W. E. } \\
\text { (2) 2-Butanol; } \mathrm{C}_{4} \mathrm{H}_{10} \mathrm{O} ; \text { [78-92-2] }\end{array}$ & $\begin{array}{l}\text { Acree, Jr., and M. H. Abraham, } \\
\text { Phys. Chem. Liq. 42, 313 (2004). }\end{array}$ \\
\hline Variables: & Prepared by: \\
$T / \mathrm{K}=298.15$ & W. E. Acree, Jr. \\
\hline
\end{tabular}

\section{Experimental Values}

\begin{tabular}{lc}
\hline \hline$x_{2}{ }^{\mathrm{a}}$ & $x_{1}{ }^{\mathrm{b}}$ \\
\hline 0.8300 & 0.1700
\end{tabular}

${ }^{\mathrm{a}} x_{2}$ : mole fraction of component 2 in the saturated solution.

${ }^{\mathrm{b}} x_{1}$ : mole fraction solubility of the solute.

\section{Auxiliary Information}

\section{Method/Apparatus/Procedure:}

Constant-temperature bath, calorimetric thermometer, and an ultraviolet/ visible spectrophotometer.

Excess solute and solvent were placed in amber glass bottles and allowed to equilibrate for several days at constant temperature. Attainment of equilibrium was verified by several repetitive measurements and by approaching equilibrium from supersaturation. Aliquots of saturated solutions were transferred through a coarse filter into tared volumetric flasks, weighed, and diluted with methanol. Concentrations were determined by spectrophotometric measurements at $279 \mathrm{~nm}$.

Source and Purity of Chemicals:

(1) $99 \%$, Aldrich Chemical Company, Milwaukee, WI, USA, was used as received.

(2) $99+\%$, anhydrous, Aldrich Chemical Company, stored over molecular sieves and distilled shortly before use.

\section{Estimated Error:}

Temperature: $\pm 0.1 \mathrm{~K}$.

$x_{1}: \pm 1.5 \%$ (relative error). 


\section{Components:}

(1) 2-Methylbenzoic acid; $\mathrm{C}_{8} \mathrm{H}_{8} \mathrm{O}_{2}$;

[118-90-1]

(2) 2-Methyl-1-propanol; $\mathrm{C}_{4} \mathrm{H}_{10} \mathrm{O}$;

[78-83-1]

\begin{tabular}{ll}
\hline Variables: & Prepared by: \\
$T / \mathrm{K}=298.15$ & W. E. Acree, Jr. \\
\hline
\end{tabular}

Experimental Values

\begin{tabular}{lc}
\hline \hline$x_{2}{ }^{\mathrm{a}}$ & $x_{1}{ }^{\mathrm{b}}$ \\
\hline 0.8771 & 0.1229
\end{tabular}

${ }^{\mathrm{a}} x_{2}$ : mole fraction of component 2 in the saturated solution.

${ }^{\mathrm{b}} x_{1}$ : mole fraction solubility of the solute.

\section{Auxiliary Information}

\section{Method/Apparatus/Procedure:}

Constant-temperature bath, calorimetric thermometer, and an ultraviolet/ visible spectrophotometer.

Excess solute and solvent were placed in amber glass bottles and allowed to equilibrate for several days at constant temperature. Attainment of equilibrium was verified by several repetitive measurements and by approaching equilibrium from supersaturation. Aliquots of saturated solutions were transferred through a coarse filter into tared volumetric flasks, weighed, and diluted with methanol. Concentrations were determined by spectrophotometric measurements at $279 \mathrm{~nm}$.

\section{Source and Purity of Chemicals:}

(1) $99 \%$, Aldrich Chemical Company, Milwaukee, WI, USA, was used as received.

(2) $99+\%$, anhydrous, Aldrich Chemical Company, stored over molecular sieves and distilled shortly before use.

\section{Estimated Error:}

Temperature: $\pm 0.1 \mathrm{~K}$.

$x_{1}: \pm 1.5 \%$ (relative error).

\section{Auxiliary Information}

\section{Method/Apparatus/Procedure:}

Constant-temperature bath, calorimetric thermometer, and an ultraviolet/ visible spectrophotometer.

Excess solute and solvent were placed in amber glass bottles and allowed to equilibrate for several days at constant temperature. Attainment of equilibrium was verified by several repetitive measurements and by approaching equilibrium from supersaturation. Aliquots of saturated solutions were transferred through a coarse filter into tared volumetric flasks, weighed, and diluted with methanol. Concentrations were determined by spectrophotometric measurements at $279 \mathrm{~nm}$.

Source and Purity of Chemicals:

(1) $99 \%$, Aldrich Chemical Company, Milwaukee, WI, USA, was used as received.

(2) $99+\%$, Arco Chemical Company, USA, stored over molecular sieves and distilled shortly before use.

Estimated Error:

Temperature: $\pm 0.1 \mathrm{~K}$.

$x_{1}: \pm 2.0 \%$ (relative error)

\begin{tabular}{ll}
\hline \hline Components: & Original Measurements: \\
(1) 2-Methylbenzoic acid; $\mathrm{C}_{8} \mathrm{H}_{8} \mathrm{O}_{2} ;$ & $\begin{array}{l}{ }^{171} \text { R. Coaxum, K. R. Hoover, E. } \\
\text { [118-90-1] }\end{array}$ \\
$\begin{array}{ll}\text { Pustejovsky, D. M. Stovall, W. E. } \\
\text { (2) 1-Pentanol; } \mathrm{C}_{5} \mathrm{H}_{12} \mathrm{O} ; \text { [71-41-0] }\end{array}$ & $\begin{array}{l}\text { Acree, Jr., and M. H. Abraham, } \\
\text { Phys. Chem. Liq. 42, 313 (2004). }\end{array}$ \\
\hline Variables: & Prepared by: \\
$T / \mathrm{K}=298.15$ & W. E. Acree, Jr. \\
\hline
\end{tabular}

Experimental Values

\begin{tabular}{lc}
\hline \hline$x_{2}{ }^{\mathrm{a}}$ & $x_{1}{ }^{\mathrm{b}}$ \\
\hline 0.8354 & 0.1646 \\
\hline${ }^{\mathrm{a}} x_{2}:$ mole fraction of component 2 in the saturated solution. & \\
${ }^{\mathrm{b}} x_{1}:$ mole fraction solubility of the solute. &
\end{tabular}

${ }^{\mathrm{b}} x_{1}$ : mole fraction solubility of the solute.

\section{Auxiliary Information}

\section{Components:}

[118-90-1]

(2) 2-Methyl-2-propanol; $\mathrm{C}_{3} \mathrm{H}_{8} \mathrm{O}$; [75-65-0]

\section{Variables:}

$T / \mathrm{K}=298.15$

Original Measurements:

${ }^{171}$ R. Coaxum, K. R. Hoover, E. Pustejovsky, D. M. Stovall, W. E. Acree, Jr., and M. H. Abraham, Phys. Chem. Liq. 42, 313 (2004).

\section{Prepared by:}

W. E. Acree, Jr.
(1) 2-Methylbenzoic acid; $\mathrm{C}_{8} \mathrm{H}_{8} \mathrm{O}_{2}$;

Experimental Values

\begin{tabular}{lc}
\hline \hline$x_{2}{ }^{\mathrm{a}}$ & $x_{1}{ }^{\mathrm{b}}$ \\
\hline 0.7862 & 0.2138 \\
\hline${ }^{\mathrm{a}} x_{2}:$ mole fraction of component 2 in the saturated solution. \\
${ }^{\mathrm{b}} x_{1}:$ mole fraction solubility of the solute.
\end{tabular}

\author{
Method/Apparatus/Procedure: \\ Constant-temperature bath, calorimetric thermometer, and an ultraviolet/ \\ visible spectrophotometer. \\ Excess solute and solvent were placed in amber glass bottles and allowed to \\ equilibrate for several days at constant temperature. Attainment of equilibrium \\ was verified by several repetitive measurements and by approaching \\ equilibrium from supersaturation. Aliquots of saturated solutions were \\ transferred through a coarse filter into tared volumetric flasks, weighed, and \\ diluted with methanol. Concentrations were determined by \\ spectrophotometric measurements at $279 \mathrm{~nm}$.
}

\section{Source and Purity of Chemicals:}

(1) $99 \%$, Aldrich Chemical Company, Milwaukee, WI, USA, was used as received.

(2) $99+\%$, Aldrich Chemical Company, stored over molecular sieves and distilled shortly before use.

Estimated Error:

Temperature: $\pm 0.1 \mathrm{~K}$.

$x_{1}: \pm 1.5 \%$ (relative error). 


\section{Components:}

(1) 2-Methylbenzoic acid; $\mathrm{C}_{8} \mathrm{H}_{8} \mathrm{O}_{2}$;

[118-90-1]

(2) 2-Pentanol; $\mathrm{C}_{5} \mathrm{H}_{12} \mathrm{O}$; [6032-29-7]

\begin{tabular}{ll}
\hline Variables: & Prepared by: \\
$T / \mathrm{K}=298.15$ & W. E. Acree, Jr. \\
\hline
\end{tabular}

\section{Experimental Values}

\begin{tabular}{lc}
\hline \hline$x_{2}{ }^{\mathrm{a}}$ & $x_{1}{ }^{\mathrm{b}}$ \\
\hline 0.8144 & 0.1856 \\
\hline${ }^{\mathrm{a}} x_{2}:$ mole fraction of component 2 in the saturated solution. & \\
${ }^{\mathrm{b}} x_{1}:$ mole fraction solubility of the solute. &
\end{tabular}

\section{Auxiliary Information}

\begin{abstract}
Method/Apparatus/Procedure:
Constant-temperature bath, calorimetric thermometer, and an ultraviolet/ visible spectrophotometer.

Excess solute and solvent were placed in amber glass bottles and allowed to equilibrate for several days at constant temperature. Attainment of equilibrium was verified by several repetitive measurements and by approaching equilibrium from supersaturation. Aliquots of saturated solutions were transferred through a coarse filter into tared volumetric flasks, weighed, and diluted with methanol. Concentrations were determined by spectrophotometric measurements at $279 \mathrm{~nm}$.
\end{abstract}

Source and Purity of Chemicals:

(1) $99 \%$, Aldrich Chemical Company, Milwaukee, WI, USA, was used as received.

(2) $99+\%$, Acros Organics, USA, stored over molecular sieves and distilled shortly before use.

Estimated Error:

Temperature: $\pm 0.1 \mathrm{~K}$.

$x_{1}: \pm 1.5 \%$ (relative error).

\begin{tabular}{ll}
\hline \hline Components: & Original Measurements: \\
(1) 2-Methylbenzoic acid; $\mathrm{C}_{8} \mathrm{H}_{8} \mathrm{O}_{2} ;$ & ${ }^{171}$ R. Coaxum, K. R. Hoover, E. \\
[118-90-1] & Pustejovsky, D. M. Stovall, W. E. \\
(2) 2-Methyl-1-butanol; $\mathrm{C}_{5} \mathrm{H}_{12} \mathrm{O} ;$ & Acree, Jr., and M. H. Abraham, \\
[137-32-6] & Phys. Chem. Liq. 42, 313 (2004). \\
\hline Variables: & Prepared by: \\
$T / \mathrm{K}=298.15$ & W. E. Acree, Jr. \\
\hline
\end{tabular}

Experimental Values

\begin{tabular}{lc}
\hline \hline$x_{2}{ }^{\mathrm{a}}$ & $x_{1}{ }^{\mathrm{b}}$ \\
\hline 0.8619 & 0.1381 \\
\hline${ }^{\mathrm{a}} x_{2}:$ mole fraction of component 2 in the saturated solution. & \\
${ }^{\mathrm{b}} x_{1}:$ mole fraction solubility of the solute. &
\end{tabular}

\section{Auxiliary Information}

Method/Apparatus/Procedure:

Constant-temperature bath, calorimetric thermometer, and an ultraviolet/ visible spectrophotometer.

Excess solute and solvent were placed in amber glass bottles and allowed to equilibrate for several days at constant temperature. Attainment of equilibrium was verified by several repetitive measurements and by approaching equilibrium from supersaturation. Aliquots of saturated solutions were transferred through a coarse filter into tared volumetric flasks, weighed, and diluted with methanol. Concentrations were determined by spectrophotometric measurements at $279 \mathrm{~nm}$.

Source and Purity of Chemicals:

(1) $99 \%$, Aldrich Chemical Company, Milwaukee, WI, USA, was used as received.

(2) $99 \%$, Aldrich Chemical Company, stored over molecular sieves and distilled shortly before use.

Estimated Error:

Temperature: $\pm 0.1 \mathrm{~K}$.

$x_{1}: \pm 1.5 \%$ (relative error).

\begin{tabular}{ll}
\hline \hline Components: & Original Measurements: \\
(1) 2-Methylbenzoic acid; $\mathrm{C}_{8} \mathrm{H}_{8} \mathrm{O}_{2} ;$ & ${ }^{171}$ R. Coaxum, K. R. Hoover, E. \\
[118-90-1] & Pustejovsky, D. M. Stovall, W. E. \\
$\begin{array}{l}\text { (2) 3-Methyl-1-butanol; } \mathrm{C}_{5} \mathrm{H}_{12} \mathrm{O} ; \\
\text { [123-51-3] }\end{array}$ & $\begin{array}{l}\text { Acree, Jr., and M. H. Abraham, } \\
\text { Phys. Chem. Liq. 42, } 313 \text { (2004). }\end{array}$ \\
\hline Variables: & Prepared by: \\
$T / \mathrm{K}=298.15$ & W. E. Acree, Jr. \\
\hline
\end{tabular}

Experimental Values

\begin{tabular}{lc}
\hline \hline$x_{2}{ }^{\mathrm{a}}$ & $x_{1}{ }^{\mathrm{b}}$ \\
\hline 0.8527 & 0.1473
\end{tabular}

${ }^{\mathrm{a}} x_{2}$ : mole fraction of component 2 in the saturated solution.

${ }^{\mathrm{b}} x_{1}$ : mole fraction solubility of the solute.

\section{Auxiliary Information}

\section{Method/Apparatus/Procedure:}

Constant-temperature bath, calorimetric thermometer, and an ultraviolet/ visible spectrophotometer.

Excess solute and solvent were placed in amber glass bottles and allowed to equilibrate for several days at constant temperature. Attainment of equilibrium was verified by several repetitive measurements and by approaching equilibrium from supersaturation. Aliquots of saturated solutions were transferred through a coarse filter into tared volumetric flasks, weighed, and diluted with methanol. Concentrations were determined by spectrophotometric measurements at $279 \mathrm{~nm}$.

Source and Purity of Chemicals:

(1) $99 \%$, Aldrich Chemical Company, Milwaukee, WI, USA, was used as received.

(2) $99 \%$, anhydrous, Aldrich Chemical Company, stored over molecular sieves and distilled shortly before use.

Estimated Error:

Temperature: $\pm 0.1 \mathrm{~K}$.

$x_{1}: \pm 1.5 \%$ (relative error). 


\section{Components:}

(1) 2-Methylbenzoic acid; $\mathrm{C}_{8} \mathrm{H}_{8} \mathrm{O}_{2}$; [118-90-1]

(2) 1-Hexanol; $\mathrm{C}_{6} \mathrm{H}_{14} \mathrm{O}$; [111-27-3]

\begin{tabular}{ll}
\hline Variables: & Prepared by: \\
$T / \mathrm{K}=298.15$ & W. E. Acree, Jr. \\
\hline
\end{tabular}

\section{Experimental Values}

\begin{tabular}{lc}
\hline \hline$x_{2}{ }^{\mathrm{a}}$ & $x_{1}{ }^{\mathrm{b}}$ \\
\hline 0.8293 & 0.1707 \\
\hline
\end{tabular}

${ }^{\mathrm{a}} x_{2}$ : mole fraction of component 2 in the saturated solution.

${ }^{\mathrm{b}} x_{1}$ : mole fraction solubility of the solute.

\section{Auxiliary Information}

\begin{abstract}
Method/Apparatus/Procedure:
Constant-temperature bath, calorimetric thermometer, and an ultraviolet/ visible spectrophotometer.

Excess solute and solvent were placed in amber glass bottles and allowed to equilibrate for several days at constant temperature. Attainment of equilibrium was verified by several repetitive measurements and by approaching equilibrium from supersaturation. Aliquots of saturated solutions were transferred through a coarse filter into tared volumetric flasks, weighed, and diluted with methanol. Concentrations were determined by spectrophotometric measurements at $279 \mathrm{~nm}$.
\end{abstract}

Source and Purity of Chemicals:

(1) $99 \%$, Aldrich Chemical Company, Milwaukee, WI, USA, was used as received.

(2) $99+\%$, Alfa Aesar, USA, stored over molecular sieves and distilled shortly before use.

Estimated Error:

Temperature: $\pm 0.1 \mathrm{~K}$.

$x_{1}: \pm 1.5 \%$ (relative error).

\begin{tabular}{ll}
\hline \hline Components: & Original Measurements: \\
(1) 2-Methylbenzoic acid; $\mathrm{C}_{8} \mathrm{H}_{8} \mathrm{O}_{2} ;$ & $\begin{array}{l}{ }^{171} \text { R. Coaxum, K. R. Hoover, E. } \\
\text { [118-90-1] }\end{array}$ \\
$\begin{array}{l}\text { Pustejovsky, D. M. Stovall, W. E. } \\
\text { (2) 4-Methyl-2-pentanol; } \mathrm{C}_{6} \mathrm{H}_{14} \mathrm{O} ;\end{array}$ & $\begin{array}{l}\text { Acree, Jr., and M. H. Abraham, } \\
\text { [108-11-2] }\end{array}$ \\
\hline Phys. Chem. Liq. 42, 313 (2004). \\
$T / \mathrm{K}=298.15$ & Prepared by: \\
\hline
\end{tabular}

Experimental Values

\begin{tabular}{lc}
\hline \hline$x_{2}^{\mathrm{a}}$ & $x_{1}{ }^{\mathrm{b}}$ \\
\hline 0.8265 & 0.1735 \\
\hline${ }^{\mathrm{a}} x_{2}:$ mole fraction of component 2 in the saturated solution. \\
${ }^{\mathrm{b}} x_{1}:$ mole fraction solubility of the solute.
\end{tabular}

\section{Auxiliary Information}

\section{Method/Apparatus/Procedure:}

Constant-temperature bath, calorimetric thermometer, and an ultraviolet/ visible spectrophotometer.

Excess solute and solvent were placed in amber glass bottles and allowed to equilibrate for several days at constant temperature. Attainment of equilibrium was verified by several repetitive measurements and by approaching equilibrium from supersaturation. Aliquots of saturated solutions were transferred through a coarse filter into tared volumetric flasks, weighed, and diluted with methanol. Concentrations were determined by spectrophotometric measurements at $279 \mathrm{~nm}$.

Source and Purity of Chemicals:

(1) $99 \%$, Aldrich Chemical Company, Milwaukee, WI, USA, was used as received.

(2) $99 \%$, Acros Organics, USA, stored over molecular sieves and distilled shortly before use.

Estimated Error:

Temperature: $\pm 0.1 \mathrm{~K}$.

$x_{1}: \pm 1.5 \%$ (relative error).

\begin{tabular}{ll}
\hline \hline Components: & Original Measurements: \\
(1) 2-Methylbenzoic acid; $\mathrm{C}_{8} \mathrm{H}_{8} \mathrm{O}_{2} ;$ & ${ }^{171}$ R. Coaxum, K. R. Hoover, E. \\
[118-90-1] & Pustejovsky, D. M. Stovall, W. E. \\
(2) 1-Heptanol; $\mathrm{C}_{7} \mathrm{H}_{16} \mathrm{O} ;$ [111-70-6] & $\begin{array}{l}\text { Acree, Jr., and M. H. Abraham, } \\
\text { Phys. Chem. Liq. 42, 313 (2004). }\end{array}$ \\
\hline Variables: & Prepared by: \\
$T / \mathrm{K}=298.15$ & W. E. Acree, Jr. \\
\hline
\end{tabular}

Experimental Values

\begin{tabular}{lc}
\hline \hline$x_{2}{ }^{\mathrm{a}}$ & $x_{1}{ }^{\mathrm{b}}$ \\
\hline 0.8245 & 0.1755
\end{tabular}

${ }^{\mathrm{a}} x_{2}$ : mole fraction of component 2 in the saturated solution.

${ }^{\mathrm{b}} x_{1}$ : mole fraction solubility of the solute.

\section{Auxiliary Information}

\section{Method/Apparatus/Procedure:}

Constant-temperature bath, calorimetric thermometer, and an ultraviolet/ visible spectrophotometer.

Excess solute and solvent were placed in amber glass bottles and allowed to equilibrate for several days at constant temperature. Attainment of equilibrium was verified by several repetitive measurements and by approaching equilibrium from supersaturation. Aliquots of saturated solutions were transferred through a coarse filter into tared volumetric flasks, weighed, and diluted with methanol. Concentrations were determined by spectrophotometric measurements at $279 \mathrm{~nm}$.

\section{Source and Purity of Chemicals:}

(1) $99 \%$, Aldrich Chemical Company, Milwaukee, WI, USA, was used as received.

(2) $99+\%$, Alfa Aesar, USA, stored over molecular sieves and distilled shortly before use.

\section{Estimated Error:}

Temperature: $\pm 0.1 \mathrm{~K}$.

$x_{1}: \pm 1.5 \%$ (relative error). 


\begin{tabular}{|c|c|}
\hline $\begin{array}{l}\text { Components: } \\
\text { (1) 2-Methylbenzoic acid; } \mathrm{C}_{8} \mathrm{H}_{8} \mathrm{O}_{2} \text {; } \\
\text { [118-90-1] } \\
\text { (2) 1-Octanol; } \mathrm{C}_{8} \mathrm{H}_{18} \mathrm{O} ;[111-87-5]\end{array}$ & $\begin{array}{l}\text { Original Measurements: } \\
{ }^{171} \text { R. Coaxum, K. R. Hoover, E. } \\
\text { Pustejovsky, D. M. Stovall, W. E. } \\
\text { Acree, Jr., and M. H. Abraham, } \\
\text { Phys. Chem. Liq. 42, } 313 \text { (2004). }\end{array}$ \\
\hline $\begin{array}{l}\text { Variables: } \\
T / \mathrm{K}=298.15\end{array}$ & $\begin{array}{l}\text { Prepared by: } \\
\text { W. E. Acree, Jr. }\end{array}$ \\
\hline
\end{tabular}

\section{Experimental Values}

\begin{tabular}{lc}
\hline \hline$x_{2}{ }^{\mathrm{a}}$ & $x_{1}{ }^{\mathrm{b}}$ \\
\hline 0.8242 & 0.1758 \\
\hline
\end{tabular}

${ }^{\mathrm{a}} x_{2}$ : mole fraction of component 2 in the saturated solution.

${ }^{\mathrm{b}} x_{1}$ : mole fraction solubility of the solute.

\section{Auxiliary Information}

\footnotetext{
Method/Apparatus/Procedure:

Constant-temperature bath, calorimetric thermometer, and an ultraviolet/ visible spectrophotometer.

Excess solute and solvent were placed in amber glass bottles and allowed to equilibrate for several days at constant temperature. Attainment of equilibrium was verified by several repetitive measurements and by approaching equilibrium from supersaturation. Aliquots of saturated solutions were transferred through a coarse filter into tared volumetric flasks, weighed, and diluted with methanol. Concentrations were determined by spectrophotometric measurements at $279 \mathrm{~nm}$.
}

Source and Purity of Chemicals:

(1) $99 \%$, Aldrich Chemical Company, Milwaukee, WI, USA, was used as received.

(2) $99+\%$, anhydrous, Aldrich Chemical Company, stored over molecular sieves and distilled shortly before use.

Estimated Error:

Temperature: $\pm 0.1 \mathrm{~K}$.

$x_{1}: \pm 1.5 \%$ (relative error).

\begin{tabular}{ll}
\hline \hline Components: & Original Measurements: \\
(1) 2-Methylbenzoic acid; $\mathrm{C}_{8} \mathrm{H}_{8} \mathrm{O}_{2} ;$ & ${ }_{77}$ J. Qingzhu, M. Peisheng, Y. \\
[118-90-1] & Shouzhi, W. Qiang, W. Chang, and \\
(2) 1-Octanol; $\mathrm{C}_{8} \mathrm{H}_{18} \mathrm{O} ;[111-87-5]$ & L. Guiju, J. Chem. Eng. Data 53, \\
& 1278 (2008). \\
\hline Variables: & Prepared by: \\
Temperature & W. E. Acree, Jr. \\
\hline
\end{tabular}

Experimental Values

\begin{tabular}{lcc}
\hline \hline$T / \mathrm{K}$ & $x_{2}{ }^{\mathrm{a}}$ & $x_{1}{ }^{\mathrm{b}}$ \\
\hline 297.15 & 0.8452 & 0.1548 \\
299.65 & 0.8354 & 0.1646 \\
301.15 & 0.8195 & 0.1805 \\
302.65 & 0.8105 & 0.1895 \\
304.75 & 0.8044 & 0.1956 \\
305.35 & 0.8033 & 0.1967 \\
306.25 & 0.7998 & 0.2002 \\
306.95 & 0.7924 & 0.2076 \\
308.95 & 0.7806 & 0.2194 \\
310.65 & 0.7704 & 0.2296
\end{tabular}

\begin{tabular}{lcc}
\hline \hline$T / \mathrm{K}$ & $x_{2}{ }^{\mathrm{a}}$ & $x_{1}{ }^{\mathrm{b}}$ \\
\hline 312.25 & 0.7606 & 0.2394 \\
314.05 & 0.7501 & 0.2499 \\
315.75 & 0.7399 & 0.2601 \\
316.95 & 0.7295 & 0.2705 \\
318.75 & 0.7193 & 0.2807 \\
320.35 & 0.7060 & 0.2940 \\
321.55 & 0.6954 & 0.3046 \\
\hline
\end{tabular}

${ }^{a} x_{2}$ : mole fraction of component 2 in the saturated solution.

${ }^{\mathrm{b}} x_{1}$ : mole fraction solubility of the solute.

\section{Auxiliary Information}

\section{Method/Apparatus/Procedure:}

Circulating water bath, analytical balance, and laser monitoring system. Pre-weighed amounts of solute and solvent and were placed in an equilibrium vessel, which was connected to a circulating water bath. The solution was stirred and the temperature gradually increased at a rate $0.5 \mathrm{~K} / 20 \mathrm{~min}(0.2 \mathrm{~K} / 20$ $\mathrm{min}$ or slower near saturation temperature) until all of the solid solute dissolved. The temperature at which all of the solute dissolved was determined using laser monitoring.

Source and Purity of Chemicals:

(1) $<99 \%$, Chemical source not specified, used as received.

(2) $<99 \%$, Chemical source not specified, used as received.

Estimated Error:

Temperature: $\pm 0.05 \mathrm{~K}$.

$x_{1}: \pm 0.0005$.

\begin{tabular}{ll}
\hline \hline Components: & Original Measurements: \\
(1) 2-Methylbenzoic acid; $\mathrm{C}_{8} \mathrm{H}_{8} \mathrm{O}_{2} ;$ & $\begin{array}{l}{ }^{171} \text { R. Coaxum, K. R. Hoover, E. } \\
\text { [118-90-1] }\end{array}$ \\
$\begin{array}{l}\text { Pustejovsky, D. M. Stovall, W. E. } \\
\text { (2) 1-Decanol; } \mathrm{C}_{10} \mathrm{H}_{22} \mathrm{O} ; \text { [112-30-1] }\end{array}$ & $\begin{array}{l}\text { Acree, Jr., and M. H. Abraham, } \\
\text { Phys. Chem. Liq. 42, } 313 \text { (2004). }\end{array}$ \\
\hline Variables: & Prepared by: \\
$T / \mathrm{K}=298.15$ & W. E. Acree, Jr. \\
\hline
\end{tabular}

Experimental Values

\begin{tabular}{lc}
\hline \hline$x_{2}{ }^{\mathrm{a}}$ & $x_{1}{ }^{\mathrm{b}}$ \\
\hline 0.8147 & 0.1853
\end{tabular}

${ }^{\mathrm{a}} x_{2}$ : mole fraction of component 2 in the saturated solution.

${ }^{\mathrm{b}} x_{1}$ : mole fraction solubility of the solute.

\section{Auxiliary Information}

\section{Method/Apparatus/Procedure:}

Constant-temperature bath, calorimetric thermometer, and an ultraviolet/ visible spectrophotometer.

Excess solute and solvent were placed in amber glass bottles and allowed to equilibrate for several days at constant temperature. Attainment of equilibrium was verified by several repetitive measurements and by approaching equilibrium from supersaturation. Aliquots of saturated solutions were transferred through a coarse filter into tared volumetric flasks, weighed, and diluted with methanol. Concentrations were determined by spectrophotometric measurements at $279 \mathrm{~nm}$. 
Source and Purity of Chemicals:

(1) $99 \%$, Aldrich Chemical Company, Milwaukee, WI, USA, was used as received.

(2) $99+\%$, Alfa Aesar, USA, stored over molecular sieves and distilled shortly before use.

\section{Estimated Error:}

Temperature: $\pm 0.1 \mathrm{~K}$

$x_{1}: \pm 1.5 \%$ (relative error).

\subsection{2-Methylbenzoic acid solubility data in miscellaneous organic solvents}

\begin{tabular}{|c|c|}
\hline Components: & Original Measurements: \\
\hline $\begin{array}{l}\text { (1) 2-Methylbenzoic acid; } \mathrm{C}_{8} \mathrm{H}_{8} \mathrm{O}_{2} \text {; } \\
\text { [118-90-1] }\end{array}$ & $\begin{array}{l}{ }^{171} \text { R. Coaxum, K. R. Hoover, E. } \\
\text { Pustejovsky, D. M. Stovall, W. E. }\end{array}$ \\
\hline $\begin{array}{l}\text { (2) Propylene carbonate; } \mathrm{C}_{4} \mathrm{H}_{6} \mathrm{O}_{3} \text {; } \\
\text { [108-32-7] }\end{array}$ & $\begin{array}{l}\text { Acree, Jr., and M. H. Abraham, } \\
\text { Phys. Chem. Liq. 42, } 313 \text { (2004). }\end{array}$ \\
\hline Variables: & Prepared by: \\
\hline$T / \mathrm{K}=298.15$ & W. E. Acree, Jr. \\
\hline
\end{tabular}

Experimental Values

\begin{tabular}{lc}
\hline \hline$x_{2}{ }^{\mathrm{a}}$ & $x_{1}{ }^{\mathrm{b}}$ \\
\hline 0.9592 & 0.04081 \\
\hline$x_{2}:$ mole fraction
\end{tabular}

${ }^{\mathrm{a}} x_{2}$ : mole fraction of component 2 in the saturated solution.

${ }^{\mathrm{b}} x_{1}$ : mole fraction solubility of the solute.

\section{Auxiliary Information}

\section{Method/Apparatus/Procedure:}

Constant-temperature bath, calorimetric thermometer, and an ultraviolet/ visible spectrophotometer.

Excess solute and solvent were placed in amber glass bottles and allowed to equilibrate for several days at constant temperature. Attainment of equilibrium was verified by several repetitive measurements and by approaching equilibrium from supersaturation. Aliquots of saturated solutions were transferred through a coarse filter into tared volumetric flasks, weighed, and diluted with methanol. Concentrations were determined by spectrophotometric measurements at $279 \mathrm{~nm}$.

\section{Source and Purity of Chemicals:}

(1) $99 \%$, Aldrich Chemical Company, Milwaukee, WI, USA, was used as received.

(2) $99.7 \%$, anhydrous, Aldrich Chemical Company, stored over molecular sieves and distilled shortly before use.

Estimated Error:

Temperature: $\pm 0.1 \mathrm{~K}$.

$x_{1}: \pm 1.5 \%$ (relative error)

\section{Components:}

(1) 2-Methylbenzoic acid; $\mathrm{C}_{8} \mathrm{H}_{8} \mathrm{O}_{2}$;

[118-90-1]

(2) Nitrobenzene; $\mathrm{C}_{6} \mathrm{H}_{5} \mathrm{NO}_{2} ;[98-95-3]$

\section{Original Measurements:}

${ }^{172}$ U. Domańska, Pol. J. Chem. 60, 847 (1986).

\section{Variables:}

Temperature

Prepared by:

W. E. Acree, Jr.
Experimental Values

\begin{tabular}{lcc}
\hline \hline$T / \mathrm{K}$ & $x_{2}{ }^{\mathrm{a}}$ & $x_{1}{ }^{\mathrm{b}}$ \\
\hline 298.35 & 0.9170 & 0.0830 \\
299.95 & 0.9092 & 0.0908 \\
305.25 & 0.8853 & 0.1147 \\
315.00 & 0.8283 & 0.1717 \\
321.65 & 0.7827 & 0.2173 \\
327.15 & 0.7373 & 0.2627 \\
338.35 & 0.6172 & 0.3828
\end{tabular}

${ }^{a} x_{2}$ : mole fraction of component 2 in the saturated solution.

${ }^{\mathrm{b}} x_{1}$ : mole fraction solubility of the solute.

\section{Auxiliary Information}

\section{Method/Apparatus/Procedure:}

Analytical balance and constant-temperature bath.

Solubilities were determined by a dynamic method. Weighed amounts of solute and solvent were placed in sample container and placed in a constanttemperature bath. The temperature of the bath was slowly increased. The temperature at which the last crystals disappeared was taken as the temperature of the solution-crystal equilibrium.

Source and Purity of Chemicals:

(1) $99+\%$, Analytical grade, Fluka Chemicals, Switzerland, purified by double vacuum sublimation.

(2) Purity not given, J. E. London, dried over molecular sieves and then fractionally distilled before use.

Estimated Error:

Temperature: $\pm 0.1 \mathrm{~K}$.

$x_{1}: \pm 2.0 \%$ (relative error, estimated by compiler).

\subsection{2-Methylbenzoic acid solubility data in binary organic solvent mixtures}

\begin{tabular}{|c|c|}
\hline $\begin{array}{l}\text { Components: } \\
\text { (1) 2-Methylbenzoic acid; } \mathrm{C}_{8} \mathrm{H}_{8} \mathrm{O}_{2} \text {; } \\
\text { [118-90-1] } \\
\text { (2) Heptane; } \mathrm{C}_{7} \mathrm{H}_{16} ;[142-82-5] \\
\text { (3) Cyclohexane; } \mathrm{C}_{6} \mathrm{H}_{12} ;[110-82-7]\end{array}$ & $\begin{array}{l}\text { Original Measurements: } \\
{ }^{173} \text { U. Domańska and T. Hofman, J } \\
\text { Solution Chem. 14, } 531 \text { (1985). }\end{array}$ \\
\hline $\begin{array}{l}\text { Variables: } \\
\text { Temperature; Solvent Composition }\end{array}$ & $\begin{array}{l}\text { Prepared by: } \\
\text { W. E. Acree, Jr. }\end{array}$ \\
\hline
\end{tabular}

Experimental Values

\begin{tabular}{lccc}
\hline \hline$T / \mathrm{K}$ & $x_{2}{ }^{(\mathrm{s}) \mathrm{a}}$ & $x_{2}{ }^{\mathrm{b}}$ & $x_{1}{ }^{\mathrm{c}}$ \\
\hline 295.45 & 0.0000 & 0.0000 & 0.0146 \\
304.95 & 0.0000 & 0.0000 & 0.0279 \\
316.65 & 0.0000 & 0.0000 & 0.0515 \\
319.40 & 0.0000 & 0.0000 & 0.0615 \\
325.30 & 0.0000 & 0.0000 & 0.0885 \\
329.35 & 0.0000 & 0.0000 & 0.1059 \\
333.45 & 0.0000 & 0.0000 & 0.1381 \\
338.15 & 0.0000 & 0.0000 & 0.1848 \\
292.05 & 0.1999 & 0.1976 & 0.0117 \\
294.85 & 0.1999 & 0.1971 & 0.0140 \\
300.55 & 0.1999 & 0.1960 & 0.0194 \\
304.95 & 0.1999 & 0.1948 & 0.0254 \\
310.95 & 0.1999 & 0.1932 & 0.0336
\end{tabular}




\begin{tabular}{|c|c|c|c|}
\hline$T / \mathrm{K}$ & $x_{2}{ }^{(\mathrm{s}) \mathrm{a}}$ & $x_{2}{ }^{\mathrm{b}}$ & $x_{1}{ }^{\mathrm{c}}$ \\
\hline 314.55 & 0.1999 & 0.1912 & 0.0436 \\
\hline 319.55 & 0.1999 & 0.1888 & 0.0556 \\
\hline 327.15 & 0.1999 & 0.1838 & 0.0805 \\
\hline 333.05 & 0.1999 & 0.1773 & 0.1130 \\
\hline 338.35 & 0.1999 & 0.1699 & 0.1501 \\
\hline 342.55 & 0.1999 & 0.1628 & 0.1855 \\
\hline 292.55 & 0.3988 & 0.3944 & 0.0111 \\
\hline 297.05 & 0.3988 & 0.3931 & 0.0144 \\
\hline 302.85 & 0.3988 & 0.3908 & 0.0200 \\
\hline 306.45 & 0.3988 & 0.3889 & 0.0248 \\
\hline 311.15 & 0.3988 & 0.3859 & 0.0324 \\
\hline 319.55 & 0.3988 & 0.3792 & 0.0491 \\
\hline 325.45 & 0.3988 & 0.3719 & 0.0675 \\
\hline 330.95 & 0.3988 & 0.3621 & 0.0921 \\
\hline 336.65 & 0.3988 & 0.3478 & 0.1280 \\
\hline 340.95 & 0.3988 & 0.3336 & 0.1634 \\
\hline 292.65 & 0.5938 & 0.5867 & 0.0119 \\
\hline 297.85 & 0.5938 & 0.5842 & 0.0161 \\
\hline 302.95 & 0.5938 & 0.5810 & 0.0216 \\
\hline 308.95 & 0.5938 & 0.5759 & 0.0301 \\
\hline 316.25 & 0.5938 & 0.5671 & 0.0449 \\
\hline 322.55 & 0.5938 & 0.5569 & 0.0622 \\
\hline 329.05 & 0.5938 & 0.5427 & 0.0861 \\
\hline 334.85 & 0.5938 & 0.5247 & 0.1163 \\
\hline 339.15 & 0.5938 & 0.5062 & 0.1476 \\
\hline 343.15 & 0.5938 & 0.4861 & 0.1814 \\
\hline 292.95 & 0.8000 & 0.7841 & 0.0119 \\
\hline 299.95 & 0.8000 & 0.7862 & 0.0172 \\
\hline 305.55 & 0.8000 & 0.7811 & 0.0236 \\
\hline 311.35 & 0.8000 & 0.7738 & 0.0327 \\
\hline 318.75 & 0.8000 & 0.7621 & 0.0474 \\
\hline 324.45 & 0.8000 & 0.7500 & 0.0638 \\
\hline 328.95 & 0.8000 & 0.7334 & 0.0832 \\
\hline 334.65 & 0.8000 & 0.7174 & 0.1032 \\
\hline 337.25 & 0.8000 & 0.6964 & 0.1295 \\
\hline 341.35 & 0.8000 & 0.6702 & 0.1622 \\
\hline 344.45 & 0.8000 & 0.6454 & 0.1933 \\
\hline 292.15 & 1.0000 & 0.9886 & 0.0114 \\
\hline 296.75 & 1.0000 & 0.9851 & 0.0149 \\
\hline 302.05 & 1.0000 & 0.9803 & 0.0197 \\
\hline 307.35 & 1.0000 & 0.9741 & 0.0259 \\
\hline 311.85 & 1.0000 & 0.9676 & 0.0324 \\
\hline 315.45 & 1.0000 & 0.9605 & 0.0395 \\
\hline 319.15 & 1.0000 & 0.9523 & 0.0477 \\
\hline 323.55 & 1.0000 & 0.9415 & 0.0585 \\
\hline 329.45 & 1.0000 & 0.9271 & 0.0729 \\
\hline 334.85 & 1.0000 & 0.9047 & 0.0953 \\
\hline 341.45 & 1.0000 & 0.8618 & 0.1382 \\
\hline 341.85 & 1.0000 & 0.8449 & 0.1551 \\
\hline
\end{tabular}

${ }^{\mathrm{a}} x_{2}{ }^{(\mathrm{s})}$ : initial mole fraction of component 2 in the binary solvent mixture.

${ }^{\mathrm{b}} x_{2}$ : mole fraction of component 2 in the saturated solution.

${ }^{c} x_{1}$ : mole fraction solubility of the solute.

\section{Auxiliary Information}

Method/Apparatus/Procedure:

Analytical balance and constant-temperature bath.

Solubilities were determined by a dynamic method. Weighed amounts of solute and solvent were placed in sample container and placed in a constanttemperature bath. The temperature of the bath was slowly increased $(2 \mathrm{~K} / \mathrm{h}$ near the equilibrium temperature). The temperature at which the last crystals

disappeared was taken as the temperature of the solution-crystal equilibrium.
Source and Purity of Chemicals:

(1) $99+\%$, Analytical grade, Fluka Chemicals, Switzerland, purified by double vacuum sublimation.

(2) Purity not given, Chemical source not given, dried over molecular sieves and then fractionally distilled before use.

(3) Purity not given, Chemical source not given, dried over molecular sieves and then fractionally distilled before use.

\section{Estimated Error:}

Temperature: $\pm 0.1 \mathrm{~K}$.

$x_{2}{ }^{(s)}: \pm 0.0001$.

$x_{1}: \pm 2.0 \%$ (relative error, estimated by compiler).

\begin{tabular}{|c|c|}
\hline $\begin{array}{l}\text { Components: } \\
\text { (1) 2-Methylbenzoic acid; } \mathrm{C}_{8} \mathrm{H}_{8} \mathrm{O}_{2} \text {; } \\
\text { [118-90-1] } \\
\text { (2) Ethanol; } \mathrm{C}_{2} \mathrm{H}_{6} \mathrm{O} ;[64-17-5] \\
\text { (3) Cyclohexane; } \mathrm{C}_{6} \mathrm{H}_{12} ;[110-82-7]\end{array}$ & $\begin{array}{l}\text { Original Measurements: } \\
{ }^{173} \text { U. Domańska and T. Hofman, J. } \\
\text { Solution Chem. 14, } 531 \text { (1985). }\end{array}$ \\
\hline $\begin{array}{l}\text { Variables: } \\
\text { Temperature; Solvent Composition }\end{array}$ & $\begin{array}{l}\text { Prepared by: } \\
\text { W. E. Acree, Jr. }\end{array}$ \\
\hline
\end{tabular}

Experimental Values

\begin{tabular}{lccc}
\hline \hline$T / \mathrm{K}$ & $x_{2}{ }^{(\mathrm{s}) \mathrm{a}}$ & $x_{2}{ }^{\mathrm{b}}$ & $x_{1}{ }^{\mathrm{c}}$ \\
\hline 295.45 & 0.0000 & 0.0000 & 0.0146 \\
304.95 & 0.0000 & 0.0000 & 0.0279 \\
316.65 & 0.0000 & 0.0000 & 0.0515 \\
319.40 & 0.0000 & 0.0000 & 0.0615 \\
325.30 & 0.0000 & 0.0000 & 0.0885 \\
329.35 & 0.0000 & 0.0000 & 0.1059 \\
333.45 & 0.0000 & 0.0000 & 0.1381 \\
338.15 & 0.0000 & 0.0000 & 0.1848 \\
287.15 & 0.1999 & 0.1885 & 0.0569 \\
294.15 & 0.1999 & 0.1852 & 0.0735 \\
303.15 & 0.1999 & 0.1804 & 0.0975 \\
311.15 & 0.1999 & 0.1749 & 0.1250 \\
313.85 & 0.1999 & 0.1719 & 0.1399 \\
317.65 & 0.1999 & 0.1684 & 0.1576 \\
320.75 & 0.1999 & 0.1658 & 0.1708 \\
325.35 & 0.1999 & 0.1603 & 0.1980 \\
331.15 & 0.1999 & 0.1526 & 0.2366 \\
332.15 & 0.1999 & 0.1485 & 0.2570 \\
286.15 & 0.4007 & 0.3675 & 0.0828 \\
290.15 & 0.4007 & 0.3628 & 0.0946 \\
294.15 & 0.4007 & 0.3578 & 0.1070 \\
297.15 & 0.4007 & 0.3533 & 0.1183 \\
298.45 & 0.4007 & 0.3518 & 0.1221 \\
308.15 & 0.4007 & 0.3372 & 0.1585 \\
311.15 & 0.4007 & 0.3294 & 0.1780 \\
317.05 & 0.4007 & 0.3180 & 0.2064 \\
321.35 & 0.4007 & 0.3054 & 0.2379 \\
324.35 & 0.4007 & 0.2968 & 0.2594 \\
289.35 & 0.5902 & 0.5252 & 0.1101 \\
298.85 & 0.5902 & 0.5061 & 0.1425 \\
303.65 & 0.5902 & 0.4955 & 0.1604 \\
314.65 & 0.5902 & 0.4646 & 0.2128 \\
318.15 & 0.5902 & 0.4599 & 0.2207 \\
319.95 & 0.5902 & 0.4466 & 0.2433 \\
325.15 & 0.5902 & 0.4270 & 0.2766 \\
327.75 & 0.5902 & 0.4164 & 0.2945 \\
288.05 & 0.6718 & 0.1190 \\
292.15 & 0.1324 \\
295.75 & & 0.1446 \\
299.45 & 0.1603 \\
& 0.801 &
\end{tabular}




\begin{tabular}{lccc}
\hline \hline$T / \mathrm{K}$ & $x_{2}{ }^{(\mathrm{s}) \mathrm{a}}$ & $x_{2}{ }^{\mathrm{b}}$ & $x_{1}{ }^{\mathrm{c}}$ \\
\hline 304.05 & 0.8001 & 0.6590 & 0.1763 \\
306.95 & 0.8001 & 0.6470 & 0.1914 \\
312.25 & 0.8001 & 0.6315 & 0.2107 \\
315.85 & 0.8001 & 0.6172 & 0.2286 \\
319.65 & 0.8001 & 0.6021 & 0.2475 \\
323.45 & 0.8001 & 0.5626 & 0.2968 \\
329.55 & 0.8001 & 0.5611 & 0.2987 \\
333.95 & 0.8001 & 0.5370 & 0.3288 \\
291.55 & 1.0000 & 0.8791 & 0.1209 \\
296.05 & 1.0000 & 0.8665 & 0.1335 \\
301.65 & 1.0000 & 0.8442 & 0.1558 \\
306.25 & 1.0000 & 0.8228 & 0.1772 \\
312.05 & 1.0000 & 0.7973 & 0.2027 \\
316.05 & 1.0000 & 0.7749 & 0.2251 \\
319.35 & 1.0000 & 0.7560 & 0.2440 \\
323.35 & 1.0000 & 0.7301 & 0.2699 \\
329.35 & 1.0000 & 0.6874 & 0.3126 \\
336.85 & 1.0000 & 0.6349 & 0.3651 \\
\hline
\end{tabular}

$\overline{{ }^{a} x_{2}{ }^{(\mathrm{s})} \text { : initial mole fraction of component } 2 \text { in the binary solvent mixture. }}$

${ }^{\mathrm{b}} \mathrm{x}_{2}$ : mole fraction of component 2 in the saturated solution.

${ }^{c} x_{1}$ : mole fraction solubility of the solute.

\section{Auxiliary Information}

Method/Apparatus/Procedure:

Analytical balance and constant-temperature bath.

Solubilities were determined by a dynamic method. Weighed amounts of solute and solvent were placed in sample container and placed in a constanttemperature bath. The temperature of the bath was slowly increased $(2 \mathrm{~K} / \mathrm{h}$ near the equilibrium temperature). The temperature at which the last crystals

disappeared was taken as the temperature of the solution-crystal equilibrium.

Source and Purity of Chemicals:

(1) $99+\%$, Analytical grade, Fluka Chemicals, Switzerland, purified by double vacuum sublimation.

(2) Purity not given, Chemical source not given, dried over molecular sieves and then fractionally distilled before use.

(3) Purity not given, Chemical source not given, dried over molecular sieves and then fractionally distilled before use.

Estimated Error:

Temperature: $\pm 0.1 \mathrm{~K}$.

$x_{2}{ }^{(\mathrm{s})}: \pm 0.0001$.

$x_{1}: \pm 2 \%$ (relative error, estimated by compiler).

\begin{tabular}{|c|c|}
\hline $\begin{array}{l}\text { Components: } \\
\text { (1) 2-Methylbenzoic acid; } \mathrm{C}_{8} \mathrm{H}_{8} \mathrm{O}_{2} \text {; } \\
\text { [118-90-1] } \\
\text { (2) 2-Propanol; } \mathrm{C}_{3} \mathrm{H}_{8} \mathrm{O} ;[67-63-0] \\
\text { (3) Cyclohexane; } \mathrm{C}_{6} \mathrm{H}_{12} ;[110-82-7]\end{array}$ & $\begin{array}{l}\text { Original Measurements: } \\
{ }^{173} \text { U. Domańska and T. Hofman, J. } \\
\text { Solution Chem. 14, } 531 \text { (1985). }\end{array}$ \\
\hline $\begin{array}{l}\text { Variables: } \\
\text { Temperature; Solvent Composition }\end{array}$ & $\begin{array}{l}\text { Prepared by: } \\
\text { W. E. Acree, Jr. }\end{array}$ \\
\hline
\end{tabular}

Experimental Values

\begin{tabular}{lccc}
\hline \hline$T / \mathrm{K}$ & $x_{2}{ }^{(\mathrm{s}) \mathrm{a}}$ & $x_{2}{ }^{\mathrm{b}}$ & $x_{1}{ }^{\mathrm{c}}$ \\
\hline 295.45 & 0.0000 & 0.0000 & 0.0146 \\
304.95 & 0.0000 & 0.0000 & 0.0279 \\
316.65 & 0.0000 & 0.0000 & 0.0515 \\
319.40 & 0.0000 & 0.0000 & 0.0615 \\
325.30 & 0.0000 & 0.0000 & 0.0885
\end{tabular}

\begin{tabular}{|c|c|c|c|}
\hline$T / \mathrm{K}$ & $x_{2}^{(\mathrm{s}) \mathrm{a}}$ & $x_{2}{ }^{\mathrm{b}}$ & $x_{1}^{\mathrm{c}}$ \\
\hline 329.35 & 0.0000 & 0.0000 & 0.1059 \\
\hline 333.45 & 0.0000 & 0.0000 & 0.1381 \\
\hline 338.15 & 0.0000 & 0.0000 & 0.1848 \\
\hline 292.45 & 0.2000 & 0.1849 & 0.0757 \\
\hline 296.65 & 0.2000 & 0.1828 & 0.0862 \\
\hline 301.75 & 0.2000 & 0.1804 & 0.0982 \\
\hline 306.95 & 0.2000 & 0.1773 & 0.1136 \\
\hline 311.55 & 0.2000 & 0.1735 & 0.1325 \\
\hline 317.25 & 0.2000 & 0.1683 & 0.1585 \\
\hline 323.45 & 0.2000 & 0.1612 & 0.1940 \\
\hline 328.55 & 0.2000 & 0.1535 & 0.2326 \\
\hline 334.55 & 0.2000 & 0.1433 & 0.2834 \\
\hline 339.45 & 0.2000 & 0.1339 & 0.3303 \\
\hline 343.35 & 0.2000 & 0.1258 & 0.3712 \\
\hline 290.95 & 0.4000 & 0.3566 & 0.1085 \\
\hline 293.25 & 0.4000 & 0.3538 & 0.1156 \\
\hline 298.45 & 0.4000 & 0.3479 & 0.1303 \\
\hline 301.85 & 0.4000 & 0.3416 & 0.1459 \\
\hline 307.95 & 0.4000 & 0.3337 & 0.1658 \\
\hline 312.65 & 0.4000 & 0.3248 & 0.1881 \\
\hline 318.85 & 0.4000 & 0.3120 & 0.2199 \\
\hline 325.75 & 0.4000 & 0.2950 & 0.2625 \\
\hline 332.45 & 0.4000 & 0.2757 & 0.3108 \\
\hline 338.35 & 0.4000 & 0.2564 & 0.3590 \\
\hline 343.15 & 0.4000 & 0.2412 & 0.3970 \\
\hline 291.15 & 0.6000 & 0.5236 & 0.1274 \\
\hline 295.75 & 0.6000 & 0.5129 & 0.1452 \\
\hline 300.75 & 0.6000 & 0.5003 & 0.1662 \\
\hline 306.55 & 0.6000 & 0.4859 & 0.1901 \\
\hline 311.35 & 0.6000 & 0.4712 & 0.2147 \\
\hline 318.05 & 0.6000 & 0.4516 & 0.2473 \\
\hline 324.45 & 0.6000 & 0.4283 & 0.2863 \\
\hline 331.25 & 0.6000 & 0.3997 & 0.3339 \\
\hline 336.55 & 0.6000 & 0.3761 & 0.3731 \\
\hline 342.55 & 0.6000 & 0.3452 & 0.4246 \\
\hline 291.25 & 0.8001 & 0.6940 & 0.1326 \\
\hline 298.45 & 0.8001 & 0.6794 & 0.1509 \\
\hline 302.45 & 0.8001 & 0.6594 & 0.1759 \\
\hline 307.45 & 0.8001 & 0.6407 & 0.1992 \\
\hline 312.45 & 0.8001 & 0.6214 & 0.2234 \\
\hline 317.85 & 0.8001 & 0.5988 & 0.2516 \\
\hline 323.25 & 0.8001 & 0.5749 & 0.2815 \\
\hline 329.65 & 0.8001 & 0.5411 & 0.3237 \\
\hline 337.15 & 0.8001 & 0.5006 & 0.3743 \\
\hline 341.15 & 0.8001 & 0.4768 & 0.4041 \\
\hline 292.65 & 1.0000 & 0.8643 & 0.1357 \\
\hline 296.75 & 1.0000 & 0.8457 & 0.1543 \\
\hline 301.95 & 1.0000 & 0.8262 & 0.1738 \\
\hline 306.75 & 1.0000 & 0.8019 & 0.1981 \\
\hline 312.40 & 1.0000 & 0.7723 & 0.2277 \\
\hline 317.75 & 1.0000 & 0.7388 & 0.2612 \\
\hline 324.90 & 1.0000 & 0.6957 & 0.3043 \\
\hline 333.35 & 1.0000 & 0.6337 & 0.3663 \\
\hline 340.15 & 1.0000 & 0.5798 & 0.4202 \\
\hline 344.75 & 1.0000 & 0.5344 & 0.4656 \\
\hline
\end{tabular}

${ }^{\mathrm{a}} x_{2}{ }^{(\mathrm{s})}$ : initial mole fraction of component 2 in the binary solvent mixture.

${ }^{b} x_{2}$ : mole fraction of component 2 in the saturated solution.

${ }^{c} x_{1}$ : mole fraction solubility of the solute. 


\section{Auxiliary Information}

Method/Apparatus/Procedure:

Analytical balance and constant-temperature bath.

Solubilities were determined by a dynamic method. Weighed amounts of

solute and solvent were placed in sample container and placed in a constanttemperature bath. The temperature of the bath was slowly increased $(2 \mathrm{~K} / \mathrm{h}$ near the equilibrium temperature). The temperature at which the last crystals

disappeared was taken as the temperature of the solution-crystal equilibrium.

Source and Purity of Chemicals:

(1) $99+\%$, Analytical grade, Fluka Chemicals, Switzerland, purified by double vacuum sublimation.

(2) Purity not given, Chemical source not given, dried over molecular sieves and then fractionally distilled before use.

(3) Purity not given, Chemical source not given, dried over molecular sieves and then fractionally distilled before use.

Estimated Error:

Temperature: $\pm 0.1 \mathrm{~K}$.

$x_{2}{ }^{(\mathrm{s})}: \pm 0.0001$.

$x_{1}: \pm 2 \%$ (relative error, estimated by compiler).

\begin{tabular}{|c|c|}
\hline $\begin{array}{l}\text { Components: } \\
\text { (1) 2-Methylbenzoic acid; } \mathrm{C}_{8} \mathrm{H}_{8} \mathrm{O}_{2} \text {; } \\
\text { [118-90-1] } \\
\text { (2) Diiodomethane; } \mathrm{CH}_{2} \mathrm{I}_{2} ;[75-11-6] \\
\text { (3) Cyclohexane; } \mathrm{C}_{6} \mathrm{H}_{12} ;[110-82-7]\end{array}$ & $\begin{array}{l}\text { Original Measurements: } \\
{ }^{174} \text { U. Domańska, J. Solution Chem. } \\
\text { 18, } 1153 \text { (1989). }\end{array}$ \\
\hline $\begin{array}{l}\text { Variables: } \\
\text { Temperature; Solvent Composition }\end{array}$ & $\begin{array}{l}\text { Prepared by: } \\
\text { W. E. Acree, Jr. }\end{array}$ \\
\hline
\end{tabular}

Experimental Values

\begin{tabular}{lccc}
\hline \hline$T / \mathrm{K}$ & $x_{2}{ }^{(\mathrm{s}) \mathrm{a}}$ & $x_{2}{ }^{\mathrm{b}}$ & $x_{1}{ }^{\mathrm{c}}$ \\
\hline 295.45 & 0.0000 & 0.0000 & 0.0146 \\
304.95 & 0.0000 & 0.0000 & 0.0279 \\
316.65 & 0.0000 & 0.0000 & 0.0515 \\
319.40 & 0.0000 & 0.0000 & 0.0615 \\
325.30 & 0.0000 & 0.0000 & 0.0885 \\
329.35 & 0.0000 & 0.0000 & 0.1059 \\
333.45 & 0.0000 & 0.0000 & 0.1381 \\
338.15 & 0.0000 & 0.0000 & 0.1848 \\
290.75 & 0.2000 & 0.1954 & 0.0228 \\
293.35 & 0.2000 & 0.1947 & 0.0265 \\
295.35 & 0.2000 & 0.1939 & 0.0306 \\
297.95 & 0.2000 & 0.1930 & 0.0350 \\
301.35 & 0.2000 & 0.1915 & 0.0425 \\
304.25 & 0.2000 & 0.1897 & 0.0516 \\
306.95 & 0.2000 & 0.1881 & 0.0595 \\
309.25 & 0.2000 & 0.1862 & 0.0691 \\
312.95 & 0.2000 & 0.1818 & 0.0909 \\
316.55 & 0.2000 & 0.1804 & 0.0979 \\
318.45 & 0.2000 & 0.1767 & 0.1164 \\
322.05 & 0.2000 & 0.1717 & 0.1416 \\
327.25 & 0.2000 & 0.1626 & 0.1869 \\
330.75 & 0.2000 & 0.1540 & 0.2299 \\
334.45 & 0.2000 & 0.1446 & 0.2769 \\
338.05 & 0.2000 & 0.1336 & 0.3318 \\
342.95 & 0.2000 & 0.1196 & 0.4021 \\
346.05 & 0.2000 & 0.0989 \\
349.45 & 0.2000 & 0.3853 & 0.04537 \\
294.85 & 0.3975 & 0.03054 \\
298.25 & 0.3975 & 0.0308 \\
301.55 & 0.3975 & &
\end{tabular}

\begin{tabular}{|c|c|c|c|}
\hline$T / \mathrm{K}$ & $x_{2}{ }^{(\mathrm{s}) \mathrm{a}}$ & $x_{2}{ }^{\mathrm{b}}$ & $x_{1}{ }^{\mathrm{c}}$ \\
\hline 304.95 & 0.3975 & 0.3746 & 0.0577 \\
\hline 307.65 & 0.3975 & 0.3705 & 0.0678 \\
\hline 310.45 & 0.3975 & 0.3661 & 0.0789 \\
\hline 313.55 & 0.3975 & 0.3616 & 0.0903 \\
\hline 318.25 & 0.3975 & 0.3524 & 0.1134 \\
\hline 322.65 & 0.3975 & 0.3426 & 0.1381 \\
\hline 327.45 & 0.3975 & 0.3259 & 0.1801 \\
\hline 332.15 & 0.3975 & 0.3080 & 0.2252 \\
\hline 336.35 & 0.3975 & 0.2894 & 0.2720 \\
\hline 341.75 & 0.3975 & 0.2647 & 0.3342 \\
\hline 351.15 & 0.3975 & 0.2152 & 0.4586 \\
\hline 294.35 & 0.5002 & 0.4862 & 0.0280 \\
\hline 298.55 & 0.5002 & 0.4812 & 0.0379 \\
\hline 302.55 & 0.5002 & 0.4759 & 0.0486 \\
\hline 307.95 & 0.5002 & 0.4687 & 0.0630 \\
\hline 311.65 & 0.5002 & 0.4604 & 0.0796 \\
\hline 316.15 & 0.5002 & 0.4494 & 0.1016 \\
\hline 320.85 & 0.5002 & 0.4374 & 0.1255 \\
\hline 325.35 & 0.5002 & 0.4210 & 0.1583 \\
\hline 329.05 & 0.5002 & 0.4049 & 0.1905 \\
\hline 332.85 & 0.5002 & 0.3873 & 0.2258 \\
\hline 335.55 & 0.5002 & 0.3701 & 0.2600 \\
\hline 342.15 & 0.5002 & 0.3305 & 0.3393 \\
\hline 296.85 & 0.6002 & 0.5830 & 0.0287 \\
\hline 302.45 & 0.6002 & 0.5755 & 0.0411 \\
\hline 308.35 & 0.6002 & 0.5649 & 0.0588 \\
\hline 312.75 & 0.6002 & 0.5550 & 0.0753 \\
\hline 316.85 & 0.6002 & 0.5440 & 0.0937 \\
\hline 318.85 & 0.6002 & 0.5349 & 0.1088 \\
\hline 323.25 & 0.6002 & 0.5235 & 0.1278 \\
\hline 323.75 & 0.6002 & 0.5121 & 0.1467 \\
\hline 328.05 & 0.6002 & 0.5002 & 0.1666 \\
\hline 331.35 & 0.6002 & 0.4821 & 0.1967 \\
\hline 334.75 & 0.6002 & 0.4606 & 0.2326 \\
\hline 337.45 & 0.6002 & 0.4395 & 0.2677 \\
\hline 342.45 & 0.6002 & 0.3988 & 0.3356 \\
\hline 346.95 & 0.6002 & 0.3595 & 0.4011 \\
\hline 293.85 & 0.7999 & 0.7877 & 0.0152 \\
\hline 299.35 & 0.7999 & 0.7838 & 0.0201 \\
\hline 304.05 & 0.7999 & 0.7777 & 0.0277 \\
\hline 311.65 & 0.7999 & 0.7673 & 0.0408 \\
\hline 314.65 & 0.7999 & 0.7589 & 0.0512 \\
\hline 320.05 & 0.7999 & 0.7477 & 0.0653 \\
\hline 324.45 & 0.7999 & 0.7335 & 0.0830 \\
\hline 328.55 & 0.7999 & 0.7152 & 0.1059 \\
\hline 333.65 & 0.7999 & 0.8587 & 0.1413 \\
\hline 337.55 & 0.7999 & 0.6562 & 0.1797 \\
\hline 342.95 & 0.7999 & 0.6029 & 0.2463 \\
\hline 348.55 & 0.7999 & 0.5223 & 0.3471 \\
\hline 294.45 & 1.0000 & 0.9962 & 0.00378 \\
\hline 301.05 & 1.0000 & 0.9941 & 0.00588 \\
\hline 303.75 & 1.0000 & 0.9926 & 0.00740 \\
\hline 307.45 & 1.0000 & 0.9913 & 0.00870 \\
\hline 308.55 & 1.0000 & 0.9905 & 0.00948 \\
\hline 312.15 & 1.0000 & 0.9886 & 0.0114 \\
\hline 316.45 & 1.0000 & 0.9851 & 0.0149 \\
\hline 321.15 & 1.0000 & 0.9787 & 0.0213 \\
\hline 324.85 & 1.0000 & 0.9733 & 0.0267 \\
\hline 328.35 & 1.0000 & 0.9664 & 0.0336 \\
\hline 332.45 & 1.0000 & 0.9562 & 0.0438 \\
\hline 336.45 & 1.0000 & 0.9401 & 0.0599 \\
\hline 340.45 & 1.0000 & 0.9175 & 0.0825 \\
\hline 343.85 & 1.0000 & 0.8823 & 0.1177 \\
\hline 345.55 & 1.0000 & 0.8614 & 0.1386 \\
\hline
\end{tabular}




\section{Auxiliary Information}

\section{Method/Apparatus/Procedure:}

Analytical balance and constant-temperature bath.

Solubilities were determined by a dynamic method. Weighed amounts of solute and solvent were placed in sample container and placed in a constanttemperature bath. The temperature of the bath was slowly increased $(2 \mathrm{~K} / \mathrm{h}$ near the equilibrium temperature). The temperature at which the last crystals disappeared was taken as the temperature of the solution-crystal equilibrium.

Source and Purity of Chemicals:

(1) Analytical grade, Fluka Chemicals, Switzerland, purified by double vacuum sublimation.

(2) Purity not given, Chemapol, Czechoslovakia, used as received.

(3) $99.9 \%$, Chemical source not given, dried over phosphorous pentoxide and then fractionally distilled before use.

Estimated Error:

Temperature: $\pm 0.1 \mathrm{~K}$.

$x_{2}{ }^{(\mathrm{s})}: \pm 0.0001$.

$x_{1}: \pm 0.0005$

\section{Solubility of 3-Methylbenzoic Acid in Organic Solvents}

\subsection{Critical evaluation of experimental solubility data}

There have been several published studies ${ }^{14,49,54,63,72,77,130}$ investigating the solubility behavior of 3-methylbenzoic acid in organic solvents of varying polarity and hydrogen-bonding capability. Daniels et al. ${ }^{14}$ measured the solubility of 3methylbenzoic acid in 18 alcohols (methanol, ethanol, 1propanol, 2-propanol, 1-butanol, 2-butanol, 2-methyl-1-propanol, 2-methyl-2-propanol, 1-pentanol, 2-pentanol, 2methyl-1-butanol, 3-methyl-1-butanol, 1-hexanol, 2-methyl1-pentanol, 4-methyl-2-pentanol, 1-heptanol, 1-octanol, and 1-decanol), in one dialkyl ether (1,1'-oxybisethane) and two cyclic ethers (tetrahydrofuran and 1,4-dioxane), and in three alkanoates (methyl ethanoate, ethyl ethanoate, and butyl ethanoate), and in propylene carbonate at $298 \mathrm{~K}$. Results of the experimental measurements were used to calculate the Abraham solute descriptors of 3-methylbenzoic acid. The authors were able to assemble a total of $42 \log _{10}(S R$ or $P$ ) and $\log _{10}(G S R$ or $K)$ equations for which experimental partition coefficient data, solubility ratios, Abraham model equation coefficients and aqueous molar solubility were available. The logarithm of the aqueous molar solubility of 3methylbenzoic acid is $\log _{10} c_{1, \mathrm{w}}=-2.14{ }^{131,132,176}$ The McGowan volume of 3-methylbenzoic acid, $V=1.0726$, was calculated from the number of chemical bonds in the molecule and the individual atomic group volumes, $A V_{i}$, given in Sec. 1.3. The excess molar refraction solute descriptor was estimated as $E=0.730$. This left four solute descriptors ( $S, A$, $B$, and $L$ ) still to be determined. The 42 equations were then solved using the Microsoft "SOLVER" program to yield numerical values of the remaining four solute descriptors, $S=0.890$, $A=0.600, B=0.400$, and $L=4.8187$, that best described the $\log _{10}(S R$ or $P)$ and $\log _{10}(G S R$ or $K)$ values. The computation
TABLE 44. Comparison between observed and calculated molar solubilities of 3-methylbenzoic acid based on the Abraham model, Eqs. (20) and (21)

\begin{tabular}{|c|c|c|c|}
\hline Solvent & $\log _{10} c_{1}{ }^{\text {exp,a }}$ & $\begin{array}{c}\log _{10} c_{1}{ }^{\text {calc }} ; \\
\text { Eq. (20) }\end{array}$ & $\begin{array}{c}\log _{10} c_{1}{ }^{\text {calc }} ; \\
\text { Eq. (21) }\end{array}$ \\
\hline Methanol & 0.437 & 0.369 & 0.345 \\
\hline Ethanol & 0.371 & 0.399 & 0.389 \\
\hline 1-Propanol & 0.292 & 0.296 & 0.300 \\
\hline 2-Propanol & 0.292 & 0.315 & 0.333 \\
\hline 1-Butanol & 0.217 & 0.206 & 0.215 \\
\hline 2-Butanol & 0.288 & 0.258 & 0.241 \\
\hline 2-Methyl-1-propanol & 0.145 & 0.161 & 0.140 \\
\hline 2-Methyl-2-propanol & 0.353 & 0.325 & 0.371 \\
\hline 1-Pentanol & 0.204 & 0.219 & 0.224 \\
\hline 2-Pentanol & 0.244 & 0.254 & 0.239 \\
\hline 3-Methyl-1-butanol & 0.164 & 0.192 & 0.158 \\
\hline 1-Hexanol & 0.166 & 0.170 & 0.164 \\
\hline 1-Heptanol & 0.141 & 0.130 & 0.134 \\
\hline 1-Octanol & 0.119 & 0.070 & 0.024 \\
\hline 1-Decanol & -0.044 & 0.022 & 0.027 \\
\hline 1,1'-Oxybisethane & 0.242 & 0.072 & 0.127 \\
\hline Tetrahydrofuran & 0.545 & 0.664 & 0.638 \\
\hline 1,4-Dioxane & 0.303 & 0.342 & 0.333 \\
\hline Methyl ethanoate & 0.203 & 0.069 & 0.050 \\
\hline Ethyl ethanoate & 0.136 & 0.130 & 0.125 \\
\hline Butyl ethanoate & -0.016 & 0.004 & -0.026 \\
\hline
\end{tabular}

${ }^{\mathrm{a}}$ Experimental data were taken from Daniels et al. ${ }^{14}$

treated $\log _{10} c_{1, \mathrm{G}}$ as a floating parameter to be determined as part of the regression analyses. The data analyses returned a value of $\log _{10} c_{1, \mathrm{G}}=-7.120$ for the gas-phase solute concentration that made the $\log _{10}\left(S R\right.$ or $P$ ) and $\log _{10}(G S R$ or $K$ ) predictions internally consistent. The calculated molecular solute descriptors reproduced the $\log _{10}\left(S R\right.$ or $P$ ) and $\log _{10}$ (GSR or $K$ ) values to within an average standard deviation of 0.077 and $0.083 \log _{10}$ units, respectively.

After the 3-methylbenzoic acid solubility study was published, Abraham model correlations have been developed for 2-pentanol, 3-methyl-1-butanol, methyl ethanoate, and butyl ethanoate, and equation coefficients for a few solvents were updated based on additional experimental data. The new correlations (listed in Tables 1 and 2) will be used in illustrating the ability of the Abraham model to correlate the experimental 3-methylbenzoic acid solubility data. Table 44 compares the experimental $\log _{10} c_{1}$ values to calculated values based on Eqs. (20) and (21) of the Abraham model. For comparison purposes, the measured mole fraction solubilities of 3-methylbenzoic acid, $x_{1}$, determined by Daniels et al. ${ }^{14}$ were converted into molar solubilities by dividing $x_{1}$ by the ideal molar volume of the saturated solution (i.e., $c_{1}{ }^{\text {sat }}=$ $\left.x_{1} /\left[x_{1} V_{1}+\left(1-x_{1}\right) V_{\text {solvent }}\right]\right)$. The molar volume of the hypothetical subcooled liquid 3-methylbenzoic acid is $V_{\text {solute }}=121.8 \mathrm{~cm}^{3}$ $\mathrm{mol}^{-1}$. Examination of the numerical entries in Table 44 reveals that the Abraham model provides a reasonably accurate mathematical description of the observed solubility data, suggesting that there are no obvious outliers in the dataset.

Qingzhu et al. ${ }^{77}$ measured the solubility of 3-methylbenzoic acid in 1-octanol as a function of temperature using a dynamic method with laser monitoring to observe when dissolution was complete. The calculated curve-fit parameters from the Buchowski $\lambda$ h-model [see Eq. (9)] of $\lambda=0.7617$ and 
$h=2624.62$ described the observed solubility data to within a mean relative deviation of $0.8 \%$.

The experimental solubility data for 3-methylbenzoic acid in organic solvents are in Secs. 60.2-60.9.

\subsection{3-Methylbenzoic acid solubility data in saturated hydrocarbons (including cycloalkanes)}

\begin{tabular}{|c|c|}
\hline $\begin{array}{l}\text { Components: } \\
\text { (1) 3-Methylbenzoic acid; } \mathrm{C}_{8} \mathrm{H}_{8} \mathrm{O}_{2} \text {; } \\
\text { [99-04-7] } \\
\text { (2) Hexane; } \mathrm{C}_{6} \mathrm{H}_{14} ;[110-54-3]\end{array}$ & $\begin{array}{l}\text { Original Measurements: } \\
{ }^{49} \text { W. E. Acree, Jr. and G. L. } \\
\text { Bertrand, J. Pharm. Sci. 70, } 1033 \\
\text { (1981). }\end{array}$ \\
\hline $\begin{array}{l}\text { Variables: } \\
T / \mathrm{K}=298.15\end{array}$ & $\begin{array}{l}\text { Prepared by: } \\
\text { W. E. Acree, Jr. }\end{array}$ \\
\hline
\end{tabular}

Experimental Values

\begin{tabular}{lc}
\hline \hline$x_{2}{ }^{\mathrm{a}}$ & $x_{1}{ }^{\mathrm{b}}$ \\
\hline 0.9883 & 0.0117 \\
\hline${ }^{\mathrm{a}} x_{2}:$ mole fraction of component 2 in the saturated solution. & \\
${ }^{\mathrm{b}} x_{1}:$ mole fraction solubility of the solute. &
\end{tabular}

\section{Auxiliary Information}

\section{Method/Apparatus/Procedure:}

Constant-temperature water bath and an analytical balance.

Excess solute and solvent and were placed in brown glass containers and allowed to equilibrate in a constant-temperature water bath for several days. Attainment of equilibrium was verified by repetitive measurements after several additional days. Solubility was determined by transferring a weighed aliquot through a coarse filter into a flask containing blank nonaqueous titration solvent. The solutions were titrated with freshly standardized sodium methoxide to the thymol blue endpoint.

\section{Source and Purity of Chemicals:}

(1) $99 \%$, Chemical source not given, was recrystallized twice from aqueousethanol and dried at $353 \mathrm{~K}$ before use.

(2) $99 \%$, Chemical source not given, stored over molecular sieves and distilled before use.

Estimated Error:

Temperature: $\pm 0.01 \mathrm{~K}$.

$x_{1}: \pm 1 \%$ (relative error).

\begin{tabular}{|c|c|}
\hline $\begin{array}{l}\text { Components: } \\
\text { (1) 3-Methylbenzoic acid; } \mathrm{C}_{8} \mathrm{H}_{8} \mathrm{O}_{2} \text {; } \\
\text { [99-04-7] } \\
\text { (2) Cyclohexane; } \mathrm{C}_{6} \mathrm{H}_{12} ;[110-82-7]\end{array}$ & $\begin{array}{l}\text { Original Measurements: } \\
{ }^{54} \text { C. K. Hancock, J. N. Pawloski, } \\
\text { and J. P. Idoux, J. Org. Chem. 31, } \\
3801 \text { (1966). }\end{array}$ \\
\hline $\begin{array}{l}\text { Variables: } \\
T / \mathrm{K}=303.15\end{array}$ & $\begin{array}{l}\text { Prepared by: } \\
\text { W. E. Acree, Jr. }\end{array}$ \\
\hline
\end{tabular}

Experimental Values

\begin{tabular}{lc}
\hline \hline$x_{2}{ }^{\mathrm{a}}$ & $x_{1}{ }^{\mathrm{b}}$ \\
\hline 0.9821 & 0.0179 \\
\hline${ }^{\mathrm{a}} x_{2}:$ mole fraction of component 2 in the saturated solution. & \\
${ }^{\mathrm{b}}{ }_{x_{1}}:$ mole fraction solubility of the solute. &
\end{tabular}

\section{Auxiliary Information}

\section{Method/Apparatus/Procedure:}

Constant-temperature bath, Soxhlet thimble, conical flask, and analytical balance.

Excess solute and solvent were placed in a conical flask and allowed to equilibrate for several days at constant temperature. Attainment of equilibrium was verified by several repetitive measurements and by approaching equilibrium from supersaturation. Aliquots of saturated solutions were transferred using a Soxhlet thimble equipped with a one-hole cork stopper and an inverted U-shaped delivery tube to a second ground-glass stoppered flask suspended in the $303 \mathrm{~K}$ water bath. Successive portions of the contents were evaporated at room temperature in a tared aluminum foil weighing dish under a bell jar through which a slow stream of dry air was passed. The second flask plus unused saturated solution and the aluminum foil dish plus residue were weighed. The saturation solubility of the solute was calculated from the recorded mass data and molar masses of the solute and solvent.

Source and Purity of Chemicals:

(1) Purity not given, Chemical source not specified, was recrystallized several times from aqueous-ethanol mixture, and then dried under vacuum over phosphorous pentoxide.

(2) Reagent grade, Chemical source not specified, refluxed over phosphorous pentoxide for $24 \mathrm{~h}$, and then distilled through a $3 \times 80 \mathrm{~cm}$ column filled with 0.32 -cm glass helices.

\section{Estimated Error:}

Temperature: $\pm 0.02 \mathrm{~K}$.

$x_{1}: \pm 3 \%$ (relative error)

\begin{tabular}{|c|c|}
\hline $\begin{array}{l}\text { Components: } \\
\text { (1) 3-Methylbenzoic acid; } \mathrm{C}_{8} \mathrm{H}_{8} \mathrm{O}_{2} \text {; } \\
\text { [99-04-7] } \\
\text { (2) Cyclohexane; } \mathrm{C}_{6} \mathrm{H}_{12} ;[110-82-7]\end{array}$ & $\begin{array}{l}\text { Original Measurements: } \\
\text { }{ }^{49} \text { W. E. Acree, Jr. and G. L. } \\
\text { Bertrand, J. Pharm. Sci. 70, } 1033 \\
\text { (1981). }\end{array}$ \\
\hline $\begin{array}{l}\text { Variables: } \\
T / \mathrm{K}=298.15\end{array}$ & $\begin{array}{l}\text { Prepared by: } \\
\text { W. E. Acree, Jr. }\end{array}$ \\
\hline
\end{tabular}

\section{Experimental Values}

\begin{tabular}{lc}
\hline \hline$x_{2}{ }^{\mathrm{a}}$ & $x_{1}{ }^{\mathrm{b}}$ \\
\hline 0.9873 & 0.0127 \\
\hline${ }^{\mathrm{a}}{ }_{2}:$ mole fraction of component 2 in the saturated solution. & \\
${ }^{\mathrm{b}}{ }_{x_{1}: \text { mole fraction solubility of the solute. }}$ &
\end{tabular}




\section{Auxiliary Information}

\section{Method/Apparatus/Procedure:}

Constant-temperature water bath and an analytical balance.

Excess solute and solvent and were placed in brown glass containers and allowed to equilibrate in a constant-temperature water bath for several days. Attainment of equilibrium was verified by repetitive measurements after several additional days. Solubility was determined by transferring a weighed aliquot through a coarse filter into a flask containing blank nonaqueous titration solvent. The solutions were titrated with freshly standardized sodium methoxide to the thymol blue endpoint.

\section{Source and Purity of Chemicals:}

(1) $99 \%$, Chemical source not given, was recrystallized twice from aqueousethanol and dried at $353 \mathrm{~K}$ before use.

(2) $99 \%$, Chemical source not given, stored over molecular sieves and distilled before use.

\section{Estimated Error:}

Temperature: $\pm 0.01 \mathrm{~K}$.

$x_{1}: \pm 1 \%$ (relative error).

\subsection{3-Methylbenzoic acid solubility data in aromatic hydrocarbons}

\begin{tabular}{ll}
\hline \hline $\begin{array}{l}\text { Components: } \\
\text { (1) 3-Methylbenzoic acid; } \mathrm{C}_{8} \mathrm{H}_{8} \mathrm{O}_{2} ;\end{array}$ & $\begin{array}{l}\text { Original Measurements: } \\
\text { [99-04-7] }\end{array}$ \\
$\begin{array}{l}{ }^{54} \text {. K. Hancock, J. N. Pawloski, } \\
\text { and J. P. Idoux, J. Org. Chem. 31, }\end{array}$ \\
\hline $\begin{array}{l}\text { Variables: } \\
T / \mathrm{K}=303.15\end{array}$ & $\begin{array}{l}\text { Prepared by: } \\
\mathrm{H}_{6} ;[71-43-2]\end{array}$ \\
\hline
\end{tabular}

Experimental Values

\begin{tabular}{lc}
\hline \hline$x_{2}{ }^{\mathrm{a}}$ & $x_{1}^{\mathrm{b}}$ \\
\hline 0.894 & 0.106 \\
\hline${ }^{\mathrm{a}} x_{2}:$ mole fraction of component 2 in the saturated solution. & \\
${ }^{\mathrm{b}} x_{1}:$ mole fraction solubility of the solute. &
\end{tabular}

\section{Auxiliary Information}

\section{Method/Apparatus/Procedure:}

Constant-temperature bath, Soxhlet thimble, conical flask, and analytical balance.

Excess solute and solvent were placed in a conical flask and allowed to equilibrate for several days at constant temperature. Attainment of equilibrium was verified by several repetitive measurements and by approaching equilibrium from supersaturation. Aliquots of saturated solutions were transferred using a Soxhlet thimble equipped with a one-hole cork stopper and an inverted U-shaped delivery tube to a second ground-glass stoppered flask suspended in the $303 \mathrm{~K}$ water bath. Successive portions of the contents were evaporated at room temperature in a tared aluminum foil weighing dish under a bell jar through which a slow stream of dry air was passed. The second flask plus unused saturated solution and the aluminum foil dish plus residue were weighed. The saturation solubility of the solute was calculated from the recorded mass data and molar masses of the solute and solvent.
Source and Purity of Chemicals:

(1) Purity not given, Chemical source not specified, was recrystallized several times from aqueous-ethanol mixture, and then dried under vacuum over phosphorous pentoxide.

(2) Reagent grade, Chemical source not specified, refluxed over phosphorous pentoxide for $24 \mathrm{~h}$, and then distilled through a $3 \times 80 \mathrm{~cm}$ column filled with 0.32-cm glass helices.

Estimated Error:

Temperature: $\pm 0.02 \mathrm{~K}$.

$x_{1}: \pm 3 \%$ (relative error).

\subsection{3-Methylbenzoic acid solubility data in esters}

\begin{tabular}{ll}
\hline \hline Components: & Original Measurements: \\
(1) 3-Methylbenzoic acid; $\mathrm{C}_{8} \mathrm{H}_{8} \mathrm{O}_{2} ;$ & ${ }^{14} \mathrm{C}$. R. Daniels, A. K. Charlton, R. \\
[99-04-7] & M. Wold, W. E. Acree, Jr., and M. \\
$\begin{array}{l}\text { (2) Methyl ethanoate; } \mathrm{C}_{3} \mathrm{H}_{6} \mathrm{O}_{2} ; \\
\text { [79-20-9] }\end{array}$ & $\begin{array}{l}\text { H. Abraham, Can. J. Chem. 81, 1492 } \\
(2003) .\end{array}$ \\
\hline Variables: & Prepared by: \\
$T / \mathrm{K}=298.15$ & W. E. Acree, Jr. \\
\hline
\end{tabular}

\section{Experimental Values}

\begin{tabular}{lc}
\hline \hline$x_{2}{ }^{\mathrm{a}}$ & $x_{1}{ }^{\mathrm{b}}$ \\
\hline 0.8636 & 0.1364 \\
\hline${ }^{\mathrm{a}} x_{2}:$ mole fraction of component 2 in the saturated solution. & \\
${ }^{\mathrm{b}} x_{1}:$ mole fraction solubility of the solute. &
\end{tabular}

Auxiliary Information

\section{Method/Apparatus/Procedure:}

Constant-temperature bath, calorimetric thermometer, and an ultraviolet/ visible spectrophotometer.

Excess solute and solvent were placed in amber glass bottles and allowed to equilibrate for several days at constant temperature. Attainment of equilibrium was verified by several repetitive measurements and by approaching equilibrium from supersaturation. Aliquots of saturated solutions were transferred through a coarse filter into tared volumetric flasks, weighed, and diluted with methanol. Concentrations were determined by spectrophotometric measurements at $279 \mathrm{~nm}$.

\section{Source and Purity of Chemicals:}

(1) $99 \%$, Aldrich Chemical Company, Milwaukee, WI, USA, was used as received.

(2) $99.5 \%$, anhydrous, Aldrich Chemical Company, stored over molecular sieves and distilled shortly before use.

\section{Estimated Error:}

Temperature: $\pm 0.1 \mathrm{~K}$.

$x_{1}: \pm 1.5 \%$ (relative error) 


\section{Components:}

(1) 3-Methylbenzoic acid; $\mathrm{C}_{8} \mathrm{H}_{8} \mathrm{O}_{2}$; [99-04-7]

(2) Ethyl ethanoate; $\mathrm{C}_{4} \mathrm{H}_{8} \mathrm{O}_{2}$; [141-78-6]

Variables:

$T / \mathrm{K}=298.15$
Original Measurements:

${ }^{14}$ C. R. Daniels, A. K. Charlton, R. M. Wold, W. E. Acree, Jr., and M. H. Abraham, Can. J. Chem. 81, 1492 (2003).

\section{Prepared by:}

W. E. Acree, Jr.

Experimental Values

\begin{tabular}{lc}
\hline \hline$x_{2}{ }^{\mathrm{a}}$ & $x_{1}{ }^{\mathrm{b}}$ \\
\hline 0.8609 & 0.1391 \\
\hline
\end{tabular}

${ }^{\mathrm{a}} x_{2}$ : mole fraction of component 2 in the saturated solution.

${ }^{b} x_{1}$ : mole fraction solubility of the solute.

\section{Auxiliary Information}

\section{Method/Apparatus/Procedure:}

Constant-temperature bath, calorimetric thermometer, and an ultraviolet/ visible spectrophotometer.

Excess solute and solvent were placed in amber glass bottles and allowed to equilibrate for several days at constant temperature. Attainment of equilibrium was verified by several repetitive measurements and by approaching equilibrium from supersaturation. Aliquots of saturated solutions were transferred through a coarse filter into tared volumetric flasks, weighed, and diluted with methanol. Concentrations were determined by spectrophotometric measurements at $279 \mathrm{~nm}$.

\section{Source and Purity of Chemicals:}

(1) $99 \%$, Aldrich Chemical Company, Milwaukee, WI, USA, was used as received.

(2) $99.9 \%$, HPLC grade, Aldrich Chemical Company, stored over molecular sieves and distilled shortly before use.

\section{Estimated Error:}

Temperature: $\pm 0.1 \mathrm{~K}$.

$x_{1}: \pm 1.5 \%$ (relative error).

\begin{tabular}{ll}
\hline \hline Components: & Original Measurements: \\
(1) 3-Methylbenzoic acid; $\mathrm{C}_{8} \mathrm{H}_{8} \mathrm{O}_{2} ;$ & ${ }^{14} \mathrm{C}$. R. Daniels, A. K. Charlton, R. \\
[99-04-7] & M. Wold, W. E. Acree, Jr., and M. \\
$\begin{array}{l}\text { (2) Butyl ethanoate; } \mathrm{C}_{6} \mathrm{H}_{12} \mathrm{O}_{2} ; \\
\text { [123-86-4] }\end{array}$ & $\begin{array}{l}\text { H. Abraham, Can. J. Chem. 81, 1492 } \\
\text { (2003). }\end{array}$ \\
\hline Variables: & Prepared by: \\
$T / \mathrm{K}=298.15$ & W. E. Acree, Jr. \\
\hline
\end{tabular}

Experimental Values

\begin{tabular}{lc}
\hline \hline$x_{2}{ }^{\mathrm{a}}$ & $x_{1}{ }^{\mathrm{b}}$ \\
\hline 0.8737 & 0.1263 \\
\hline${ }^{\mathrm{a}} x_{2}:$ mole fraction of component 2 in the saturated solution. \\
${ }^{\mathrm{b}}{ }_{x_{1}: \text { mole fraction solubility of the solute. }}$
\end{tabular}

\section{Auxiliary Information}

Method/Apparatus/Procedure:

Constant-temperature bath, calorimetric thermometer, and an ultraviolet/ visible spectrophotometer.

Excess solute and solvent were placed in amber glass bottles and allowed to equilibrate for several days at constant temperature. Attainment of equilibrium was verified by several repetitive measurements and by approaching equilibrium from supersaturation. Aliquots of saturated solutions were transferred through a coarse filter into tared volumetric flasks, weighed, and diluted with methanol. Concentrations were determined by spectrophotometric measurements at $279 \mathrm{~nm}$.

Source and Purity of Chemicals:

(1) $99 \%$, Aldrich Chemical Company, Milwaukee, WI, USA, was used as received.

(2) $99.7 \%$, HPLC grade, Aldrich Chemical Company, stored over molecular sieves and distilled shortly before use.

Estimated Error:

Temperature: $\pm 0.1 \mathrm{~K}$.

$x_{1}: \pm 1.5 \%$ (relative error).

\begin{tabular}{|c|c|}
\hline $\begin{array}{l}\text { Components: } \\
\text { (1) 3-Methylbenzoic acid; } \mathrm{C}_{8} \mathrm{H}_{8} \mathrm{O}_{2} \text {; } \\
\text { [99-04-7] } \\
\text { (2) } 1,2,3 \text {-Triacetoxypropane (Triacetin); } \\
\mathrm{C}_{9} \mathrm{H}_{14} \mathrm{O}_{6} ;[102-76-1]\end{array}$ & $\begin{array}{l}\text { Original Measurements: } \\
\text { W. E. Acree, Jr., unpublished } \\
\text { data. }\end{array}$ \\
\hline $\begin{array}{l}\text { Variables: } \\
T / \mathrm{K}=298.15\end{array}$ & $\begin{array}{l}\text { Prepared by: } \\
\text { W. E. Acree, Jr. }\end{array}$ \\
\hline
\end{tabular}

Experimental Values

\begin{tabular}{lc}
\hline \hline$x_{2}{ }^{\mathrm{a}}$ & $x_{1}{ }^{\mathrm{b}}$ \\
\hline 0.8820 & 0.1180 \\
\hline${ }^{\mathrm{a}} x_{2}:$ mole fraction of component 2 in the saturated solution. \\
${ }^{\mathrm{b}} x_{1}:$ mole fraction solubility of the solute.
\end{tabular}

${ }^{\mathrm{b}} x_{1}$ : mole fraction solubility of the solute.

\section{Auxiliary Information}

\section{Method: Apparatus/Procedure:}

Constant-temperature bath, calorimetric thermometer, and an ultraviolet/ visible spectrophotometer

Excess solute and solvent were placed in amber glass bottles and allowed to equilibrate for several days at constant temperature. Attainment of equilibrium was verified by several repetitive measurements and by approaching equilibrium from supersaturation. Aliquots of saturated solutions were transferred through a coarse filter into tared volumetric flasks, weighed, and diluted with 2-propanol. Concentrations were determined by spectrophotometric measurements at $279 \mathrm{~nm}$.

\section{Source and Purity of Chemicals:}

(1) $99 \%$, Aldrich Chemical Company, Milwaukee, WI, USA, was used as received.

(2) $99 \%$, Acros Organics, USA, stored over molecular sieves before use.

\section{Estimated Error:}

Temperature: $\pm 0.1 \mathrm{~K}$.

$x_{1}: \pm 1.5 \%$ (relative error). 


\subsection{3-Methylbenzoic acid solubility data in ethers}

\begin{tabular}{ll}
\hline \hline Components: & Original Measurements: \\
(1) 3-Methylbenzoic acid; $\mathrm{C}_{8} \mathrm{H}_{8} \mathrm{O}_{2} ;$ & ${ }^{14} \mathrm{C}$. R. Daniels, A. K. Charlton, R. \\
[99-04-7] & M. Wold, W. E. Acree, Jr., and M. \\
$\begin{array}{l}\text { (2) } 1,1^{\prime} \text {-Oxybisethane; } \mathrm{C}_{4} \mathrm{H}_{10} \mathrm{O} ; \\
\text { [60-29-7] }\end{array}$ & $\begin{array}{l}\text { H. Abraham, Can. J. Chem. 81, 1492 } \\
\text { (2003). }\end{array}$ \\
\hline Variables: & Prepared by: \\
$T / \mathrm{K}=298.15$ & W. E. Acree, Jr. \\
\hline
\end{tabular}

Experimental Values

\begin{tabular}{lc}
\hline \hline$x_{2}^{\mathrm{a}}$ & $x_{1}{ }^{\mathrm{b}}$ \\
\hline 0.8111 & 0.1889 \\
\hline${ }^{\mathrm{a}} x_{2}:$ mole fraction of component 2 in the saturated solution. \\
${ }^{\mathrm{b}}{ }_{x_{1}: \text { mole fraction solubility of the solute. }}$
\end{tabular}

\section{Auxiliary Information}

\section{Method/Apparatus/Procedure:}

Constant-temperature bath, calorimetric thermometer, and an ultraviolet/ visible spectrophotometer.

Excess solute and solvent were placed in amber glass bottles and allowed to equilibrate for several days at constant temperature. Attainment of equilibrium was verified by several repetitive measurements and by approaching equilibrium from supersaturation. Aliquots of saturated solutions were transferred through a coarse filter into tared volumetric flasks, weighed, and diluted with methanol. Concentrations were determined by

spectrophotometric measurements at $279 \mathrm{~nm}$.

Source and Purity of Chemicals:

(1) $99 \%$, Aldrich Chemical Company, Milwaukee, WI, USA, was used as received.

(2) $99+\%$, anhydrous, Aldrich Chemical Company, stored over molecular sieves and distilled shortly before use.

\section{Estimated Error:}

Temperature: $\pm 0.1 \mathrm{~K}$

$x_{1}: \pm 1.5 \%$ (relative error).

\begin{tabular}{ll}
\hline \hline Components: & Original Measurements: \\
(1) 3-Methylbenzoic acid; $\mathrm{C}_{8} \mathrm{H}_{8} \mathrm{O}_{2} ;$ & ${ }^{14} \mathrm{C}$. R. Daniels, A. K. Charlton, R. \\
[99-04-7] & M. Wold, W. E. Acree, Jr., and M. \\
$\begin{array}{l}\text { (2) Tetrahydrofuran; } \mathrm{C}_{4} \mathrm{H}_{8} \mathrm{O} ; \\
\text { [109-99-9] }\end{array}$ & $\begin{array}{l}\mathrm{H} \text {. Abraham, Can. J. Chem. 81, 1492 } \\
\text { (2003). }\end{array}$ \\
\hline Variables: & Prepared by: \\
$T / \mathrm{K}=298.15$ & W. E. Acree, Jr. \\
\hline
\end{tabular}

Experimental Values

\begin{tabular}{lc}
\hline \hline$x_{2}{ }^{\mathrm{a}}$ & $x_{1}{ }^{\mathrm{b}}$ \\
\hline 0.6671 & 0.3329 \\
\hline$x_{2}$ mole fration of compont
\end{tabular}

${ }^{\mathrm{a}} x_{2}$ : mole fraction of component 2 in the saturated solution.

${ }^{\mathrm{b}} x_{1}$ : mole fraction solubility of the solute.

\section{Auxiliary Information}

\section{Method/Apparatus/Procedure:}

Constant-temperature bath, calorimetric thermometer, and an ultraviolet/ visible spectrophotometer.

Excess solute and solvent were placed in amber glass bottles and allowed to equilibrate for several days at constant temperature. Attainment of equilibrium was verified by several repetitive measurements and by approaching equilibrium from supersaturation. Aliquots of saturated solutions were transferred through a coarse filter into tared volumetric flasks, weighed, and diluted with methanol. Concentrations were determined by spectrophotometric measurements at $279 \mathrm{~nm}$.

Source and Purity of Chemicals:

(1) $99 \%$, Aldrich Chemical Company, Milwaukee, WI, USA, was used as received.

(2) $99 \%$, anhydrous, Aldrich Chemical Company, stored over molecular sieves and distilled shortly before use.

Estimated Error:

Temperature: $\pm 0.1 \mathrm{~K}$.

$x_{1}: \pm 2.0 \%$ (relative error).

\begin{tabular}{|c|c|}
\hline Components: & Original Measurements: \\
\hline $\begin{array}{l}\text { (1) 3-Methylbenzoic acid; } \mathrm{C}_{8} \mathrm{H}_{8} \mathrm{O}_{2} \text {; } \\
\text { [99-04-7] }\end{array}$ & $\begin{array}{l}{ }^{63} \text { C. K. Hancock, J. N. Pawloski, } \\
\text { and J. P. Idoux, J. Org. Chem. 32, }\end{array}$ \\
\hline $\begin{array}{l}\text { (2) Tetrahydrofuran; } \mathrm{C}_{4} \mathrm{H}_{8} \mathrm{O} \\
\text { [109-99-9] }\end{array}$ & 1931 (1967). \\
\hline Variables: & Prepared by: \\
\hline$T / \mathrm{K}=303.15$ & W. E. Acree, Jr. \\
\hline
\end{tabular}

Experimental Values

\begin{tabular}{lc}
\hline \hline$x_{2}{ }^{\mathrm{a}}$ & $x_{1}{ }^{\mathrm{b}}$ \\
\hline 0.627 & 0.373
\end{tabular}

${ }^{\mathrm{a}} x_{2}$ : mole fraction of component 2 in the saturated solution.

${ }^{\mathrm{b}} x_{1}$ : mole fraction solubility of the solute.

\section{Auxiliary Information}

\section{Method/Apparatus/Procedure:}

Constant-temperature bath, Soxhlet thimble, conical flask, and analytical balance.

Excess solute and solvent were placed in a conical flask and allowed to equilibrate for several days at constant temperature. Attainment of equilibrium was verified by several repetitive measurements and by approaching equilibrium from supersaturation. Aliquots of saturated solutions were transferred using a Soxhlet thimble equipped with a one-hole cork stopper and an inverted U-shaped delivery tube to a second ground-glass stoppered flask suspended in the $303 \mathrm{~K}$ water bath. Successive portions of the contents were evaporated at room temperature in a tared aluminum foil weighing dish under a bell jar through which a slow stream of dry air was passed. The second flask plus unused saturated solution and the aluminum foil dish plus residue were weighed. The saturation solubility of the solute was calculated from the recorded mass data and molar masses of the solute and solvent. 
Source and Purity of Chemicals:

(1) Purity not given, Chemical source not specified, was recrystallized several times from aqueous-ethanol mixture, and then dried under vacuum over phosphorous pentoxide.

(2) Practical grade, Chemical source not specified, stored over sodium hydroxide pellets for $24 \mathrm{~h}$, and then passed through $2 \times 70$-cm chromatographic adsorption columns containing activated alumina. After this treatment, the purified solvent was stored over copper in a nitrogen atmosphere.

Estimated Error:

Temperature: $\pm 0.02 \mathrm{~K}$.

$x_{1}: \pm 1.0 \%$ (relative error).

\begin{tabular}{ll}
\hline \hline Components: & Original Measurements: \\
(1) 3-Methylbenzoic acid; $\mathrm{C}_{8} \mathrm{H}_{8} \mathrm{O}_{2} ;$ & ${ }^{14} \mathrm{C}$. R. Daniels, A. K. Charlton, R. \\
[99-04-7] & M. Wold, W. E. Acree, Jr., and M. \\
(2) 1,4-Dioxane; $\mathrm{C}_{4} \mathrm{H}_{8} \mathrm{O}_{2} ;$ [123-91-1] & $\begin{array}{l}\text { H. Abraham, Can. J. Chem. 81, 1492 } \\
(2003) .\end{array}$ \\
\hline Variables: & Prepared by: \\
$T / \mathrm{K}=298.15$ & W. E. Acree, Jr. \\
\hline
\end{tabular}

Experimental Values

\begin{tabular}{lc}
\hline \hline$x_{2}{ }^{\mathrm{a}}$ & $x_{1}{ }^{\mathrm{b}}$ \\
\hline 0.8140 & 0.1860 \\
\hline
\end{tabular}

${ }^{a} x_{2}$ : mole fraction of component 2 in the saturated solution.

${ }^{b} x_{1}$ : mole fraction solubility of the solute.

\section{Auxiliary Information}

\section{Method/Apparatus/Procedure:}

Constant-temperature bath, calorimetric thermometer, and an ultraviolet/ visible spectrophotometer.

Excess solute and solvent were placed in amber glass bottles and allowed to equilibrate for several days at constant temperature. Attainment of equilibrium was verified by several repetitive measurements and by approaching equilibrium from supersaturation. Aliquots of saturated solutions were transferred through a coarse filter into tared volumetric flasks, weighed, and diluted with methanol. Concentrations were determined by

spectrophotometric measurements at $279 \mathrm{~nm}$.

Source and Purity of Chemicals:

(1) $99 \%$, Aldrich Chemical Company, Milwaukee, WI, USA, was used as received.

(2) $99.8 \%$, anhydrous, Aldrich Chemical Company, stored over molecular sieves and distilled shortly before use.

Estimated Error:

Temperature: $\pm 0.1 \mathrm{~K}$.

$x_{1}: \pm 1.5 \%$ (relative error).

\begin{tabular}{|c|c|}
\hline $\begin{array}{l}\text { Components: } \\
\text { (1) 3-Methylbenzoic acid; } \mathrm{C}_{8} \mathrm{H}_{8} \mathrm{O}_{2} \text {; } \\
\text { [99-04-7] } \\
\text { (2) 1,4-Dioxane; } \mathrm{C}_{4} \mathrm{H}_{8} \mathrm{O}_{2} ;[123-91-1]\end{array}$ & $\begin{array}{l}\text { Original Measurements: } \\
{ }^{63} \text { C. K. Hancock, J. N. Pawloski, } \\
\text { and J. P. Idoux, J. Org. Chem. 32, } \\
1931 \text { (1967). }\end{array}$ \\
\hline $\begin{array}{l}\text { Variables: } \\
T / \mathrm{K}=303.15\end{array}$ & $\begin{array}{l}\text { Prepared by: } \\
\text { W. E. Acree, Jr. }\end{array}$ \\
\hline
\end{tabular}

Experimental Values

\begin{tabular}{lc}
\hline \hline$x_{2}{ }^{\mathrm{a}}$ & $x_{1}{ }^{\mathrm{b}}$ \\
\hline 0.709 & 0.291 \\
\hline${ }^{\mathrm{a}} x_{2}:$ mole fraction of component 2 in the saturated solution. & \\
${ }^{\mathrm{b}}{ }_{x_{1}}:$ mole fraction solubility of the solute. &
\end{tabular}

\section{Auxiliary Information}

Method/Apparatus/Procedure:

Constant-temperature bath, Soxhlet thimble, conical flask, and analytical balance.

Excess solute and solvent were placed in a conical flask and allowed to equilibrate for several days at constant temperature. Attainment of equilibrium was verified by several repetitive measurements and by approaching equilibrium from supersaturation. Weighed aliquots of saturated solutions were removed and titrated with a standardized sodium hydroxide solution (carbonate free) using a pH meter. The endpoint of the titration was determined by computing the second derivative in the $\mathrm{pH}$ versus volume of sodium hydroxide added.

\section{Source and Purity of Chemicals:}

(1) Purity not given, Chemical source not specified, was recrystallized several times from aqueous-ethanol mixture, and then dried under vacuum over phosphorous pentoxide.

(2) Practical grade, Chemical source not specified, stored over sodium hydroxide pellets for $24 \mathrm{~h}$, and then passed through $2 \times 70-\mathrm{cm}$ chromatographic adsorption columns containing activated alumina. After this treatment, the purified solvent was stored over copper in a nitrogen atmosphere.

\section{Estimated Error:}

Temperature: $\pm 0.02 \mathrm{~K}$

$x_{1}: \pm 1.0 \%$ (relative error).

\subsection{3-Methylbenzoic acid solubility data in alcohols}

\begin{tabular}{ll}
\hline \hline Components: & Original Measurements: \\
(1) 3-Methylbenzoic acid; $\mathrm{C}_{8} \mathrm{H}_{8} \mathrm{O}_{2} ;$ & ${ }^{14} \mathrm{C}$. R. Daniels, A. K. Charlton, R. \\
[99-04-7] & M. Wold, W. E. Acree, Jr., and M. \\
(2) Methanol; $\mathrm{CH}_{4} \mathrm{O} ;$ [67-56-1] & $\begin{array}{l}\text { H. Abraham, Can. J. Chem. 81, 1492 } \\
\text { (2003). }\end{array}$ \\
\hline Variables: & Prepared by: \\
$T / \mathrm{K}=298.15$ & W. E. Acree, Jr. \\
\hline
\end{tabular}

\section{Experimental Values}

\begin{tabular}{lc}
\hline \hline$x_{2}{ }^{\mathrm{a}}$ & $x_{1}{ }^{\mathrm{b}}$ \\
\hline 0.8570 & 0.1430 \\
\hline${ }^{\mathrm{a}} x_{2}:$ mole fraction of component 2 in the saturated solution. & \\
${ }^{\mathrm{b}} x_{1}:$ mole fraction solubility of the solute. &
\end{tabular}




\section{Auxiliary Information}

\section{Method/Apparatus/Procedure:}

Constant-temperature bath, calorimetric thermometer, and an ultraviolet/ visible spectrophotometer.

Excess solute and solvent were placed in amber glass bottles and allowed to equilibrate for several days at constant temperature. Attainment of equilibrium was verified by several repetitive measurements and by approaching equilibrium from supersaturation. Aliquots of saturated solutions were transferred through a coarse filter into tared volumetric flasks, weighed, and diluted with methanol. Concentrations were determined by spectrophotometric measurements at $279 \mathrm{~nm}$.

Source and Purity of Chemicals:

(1) $99 \%$, Aldrich Chemical Company, Milwaukee, WI, USA, was used as received.

(2) 99+\%, anhydrous, Aldrich Chemical Company, stored over molecular sieves and distilled shortly before use.

Estimated Error:

Temperature: $\pm 0.1 \mathrm{~K}$.

$x_{1}: \pm 1.5 \%$ (relative error).

\begin{tabular}{ll}
\hline \hline Components: & Original Measurements: \\
(1) 3-Methylbenzoic acid; $\mathrm{C}_{8} \mathrm{H}_{8} \mathrm{O}_{2} ;$ & ${ }^{14}$ C. R. Daniels, A. K. Charlton, R. \\
[99-04-7] & M. Wold, W. E. Acree, Jr., and M. \\
(2) Ethanol; $\mathrm{C}_{2} \mathrm{H}_{6} \mathrm{O} ;$ [64-17-5] & H. Abraham, Can. J. Chem. 81, 1492 \\
& (2003). \\
\hline Variables: & Prepared by: \\
$T / \mathrm{K}=298.15$ & W. E. Acree, Jr. \\
\hline
\end{tabular}

Experimental Values

\begin{tabular}{lc}
\hline \hline$x_{2}{ }^{\mathrm{a}}$ & $x_{1}{ }^{\mathrm{b}}$ \\
\hline 0.8383 & 0.1617 \\
\hline$x_{2}:$ mole fration
\end{tabular}

${ }^{\mathrm{a}} x_{2}$ : mole fraction of component 2 in the saturated solution.

${ }^{\mathrm{b}} x_{1}$ : mole fraction solubility of the solute.

\section{Auxiliary Information}

\section{Method/Apparatus/Procedure:}

Constant-temperature bath, calorimetric thermometer, and an ultraviolet/ visible spectrophotometer.

Excess solute and solvent were placed in amber glass bottles and allowed to equilibrate for several days at constant temperature. Attainment of equilibrium was verified by several repetitive measurements and by approaching equilibrium from supersaturation. Aliquots of saturated solutions were transferred through a coarse filter into tared volumetric flasks, weighed, and diluted with methanol. Concentrations were determined by

spectrophotometric measurements at $279 \mathrm{~nm}$.

\section{Source and Purity of Chemicals:}

(1) $99 \%$, Aldrich Chemical Company, Milwaukee, WI, USA, was used as received.

(2) Absolute, Aaper Alcohol and Chemical Company, USA, stored over molecular sieves and distilled shortly before use.

Estimated Error:

Temperature: $\pm 0.1 \mathrm{~K}$.

$x_{1}: \pm 1.5 \%$ (relative error)

\begin{tabular}{ll}
\hline \hline Components: & Original Measurements: \\
(1) 3-Methylbenzoic acid; $\mathrm{C}_{8} \mathrm{H}_{8} \mathrm{O}_{2} ;$ & ${ }^{72} \mathrm{R}$. Thuaire, Bull. Soc. Chim. Fr. \\
[99-04-7] & 3815 (1971). \\
(2) Ethanol; $\mathrm{C}_{2} \mathrm{H}_{6} \mathrm{O} ;[64-17-5]$ & \\
\hline Variables: & Prepared by: \\
$T / \mathrm{K}=298.15$ & W. E. Acree, Jr. \\
\hline
\end{tabular}

\section{Experimental Values}

The measured solubility was reported to be $4.187 \mathrm{~mol} / \mathrm{kg}$ of solvent, which corresponds to a mole fraction solubility of $x_{1}=0.1616$.

\section{Auxiliary Information}

\section{Method/Apparatus/Procedure:}

Very little experimental details were provided. Solubility was determined by a gravimetric method. The saturated solution was evaporated to dryness and the remaining solid residue was weighed. The solubility was calculated from the mass of the solid residue and mass of saturated solution analyzed.

\section{Source and Purity of Chemicals:}

(1) Purity not given, Chemical source not given, no purification details were provided.

(2) Purity not given, Chemical source not given, no purification details were provided.

\section{Estimated Error:}

Temperature: $\pm 0.05 \mathrm{~K}$

$x_{1}: \pm 4 \%$ (relative error, estimated by compiler).

\begin{tabular}{ll}
\hline \hline Components: & Original Measurements: \\
(1) 3-Methylbenzoic acid; $\mathrm{C}_{8} \mathrm{H}_{8} \mathrm{O}_{2} ;$ & ${ }^{14} \mathrm{C}$. R. Daniels, A. K. Charlton, R. \\
[99-04-7] & M. Wold, W. E. Acree, Jr., and M. \\
(2) 1-Propanol; $\mathrm{C}_{3} \mathrm{H}_{8} \mathrm{O} ;$ [71-23-8] & $\begin{array}{l}\text { H. Abraham, Can. J. Chem. 81, 1492 } \\
\text { (2003). }\end{array}$ \\
\hline Variables: & Prepared by: \\
$T / \mathrm{K}=298.15$ & W. E. Acree, Jr. \\
\hline
\end{tabular}

Experimental Values

\begin{tabular}{lc}
\hline \hline$x_{2}{ }^{\mathrm{a}}$ & $x_{1}{ }^{\mathrm{b}}$ \\
\hline 0.8382 & 0.1618 \\
\hline${ }^{\mathrm{a}} x_{2}:$ mole fraction of component 2 in the saturated solution. & \\
${ }^{\mathrm{b}} x_{1}:$ mole fraction solubility of the solute. &
\end{tabular}

\section{Auxiliary Information}

\section{Method/Apparatus/Procedure:}

Constant-temperature bath, calorimetric thermometer, and an ultraviolet/ visible spectrophotometer.

Excess solute and solvent were placed in amber glass bottles and allowed to equilibrate for several days at constant temperature. Attainment of equilibrium was verified by several repetitive measurements and by approaching equilibrium from supersaturation. Aliquots of saturated solutions were transferred through a coarse filter into tared volumetric flasks, weighed, and diluted with methanol. Concentrations were determined by spectrophotometric measurements at $279 \mathrm{~nm}$. 
Source and Purity of Chemicals:

(1) $99 \%$, Aldrich Chemical Company, Milwaukee, WI, USA, was used as received.

(2) $99+\%$, anhydrous, Aldrich Chemical Company, stored over molecular sieves and distilled shortly before use.

\section{Estimated Error:}

Temperature: $\pm 0.1 \mathrm{~K}$.

$x_{1}: \pm 1.5 \%$ (relative error).

\section{Components:}

(1) 3-Methylbenzoic acid; $\mathrm{C}_{8} \mathrm{H}_{8} \mathrm{O}_{2}$; [99-04-7]

(2) 2-Propanol; $\mathrm{C}_{3} \mathrm{H}_{8} \mathrm{O} ;[67-63-0]$

Original Measurements:

${ }^{14}$ C. R. Daniels, A. K. Charlton, R. M. Wold, W. E. Acree, Jr., and M. H. Abraham, Can. J. Chem. 81, 1492 (2003).

\begin{tabular}{ll}
\hline Variables: & Prepared by: \\
$T / \mathrm{K}=298.15$ & W. E. Acree, Jr.
\end{tabular}

\section{Experimental Values}

\begin{tabular}{lc}
\hline \hline$x_{2}^{\mathrm{a}}$ & $x_{1}{ }^{\mathrm{b}}$ \\
\hline 0.8347 & 0.1653
\end{tabular}

${ }^{\mathrm{a}} x_{2}$ : mole fraction of component 2 in the saturated solution.

${ }^{\mathrm{b}} x_{1}$ : mole fraction solubility of the solute.

\section{Auxiliary Information}

\section{Method/Apparatus/Procedure:}

Constant-temperature bath, calorimetric thermometer, and an ultraviolet/ visible spectrophotometer.

Excess solute and solvent were placed in amber glass bottles and allowed to equilibrate for several days at constant temperature. Attainment of equilibrium was verified by several repetitive measurements and by approaching equilibrium from supersaturation. Aliquots of saturated solutions were transferred through a coarse filter into tared volumetric flasks, weighed, and diluted with methanol. Concentrations were determined by spectrophotometric measurements at $279 \mathrm{~nm}$.

Source and Purity of Chemicals:

(1) $99 \%$, Aldrich Chemical Company, Milwaukee, WI, USA, was used as received.

(2) $99+\%$, anhydrous, Aldrich Chemical Company, stored over molecular sieves and distilled shortly before use.

Estimated Error:

Temperature: $\pm 0.1 \mathrm{~K}$.

$x_{1}: \pm 1.5 \%$ (relative error).

\begin{tabular}{ll}
\hline \hline Components: & Original Measurements: \\
(1) 3-Methylbenzoic acid; $\mathrm{C}_{8} \mathrm{H}_{8} \mathrm{O}_{2} ;$ & ${ }^{14} \mathrm{C}$. R. Daniels, A. K. Charlton, R. \\
[99-04-7] & M. Wold, W. E. Acree, Jr., and M. \\
(2) 1-Butanol; $\mathrm{C}_{4} \mathrm{H}_{10} \mathrm{O} ;$ [71-36-3] & $\begin{array}{l}\text { H. Abraham, Can. J. Chem. 81, 1492 } \\
\text { (2003). }\end{array}$ \\
\hline $\begin{array}{l}\text { Variables: } \\
T / \mathrm{K}=298.15\end{array}$ & $\begin{array}{l}\text { Prepared by: } \\
\text { W. E. Acree, Jr. }\end{array}$ \\
\hline
\end{tabular}

Experimental Values

\begin{tabular}{lc}
\hline \hline$x_{2}{ }^{\mathrm{a}}$ & $x_{1}{ }^{\mathrm{b}}$ \\
\hline 0.8405 & 0.1595 \\
\hline${ }^{\mathrm{a}} x_{2}:$ mole fraction of component 2 in the saturated solution. & \\
${ }^{\mathrm{b}}{ }_{x_{1}}:$ mole fraction solubility of the solute. &
\end{tabular}

\section{Auxiliary Information}

Method/Apparatus/Procedure:

Constant-temperature bath, calorimetric thermometer, and an ultraviolet/ visible spectrophotometer.

Excess solute and solvent were placed in amber glass bottles and allowed to equilibrate for several days at constant temperature. Attainment of equilibrium was verified by several repetitive measurements and by approaching equilibrium from supersaturation. Aliquots of saturated solutions were transferred through a coarse filter into tared volumetric flasks, weighed, and diluted with methanol. Concentrations were determined by spectrophotometric measurements at $279 \mathrm{~nm}$.

Source and Purity of Chemicals:

(1) $99 \%$, Aldrich Chemical Company, Milwaukee, WI, USA, was used as received.

(2) $99.8+\%$, HPLC grade, Aldrich Chemical Company, stored over molecular sieves and distilled shortly before use.

\section{Estimated Error:}

Temperature: $\pm 0.1 \mathrm{~K}$.

$x_{1}: \pm 1.5 \%$ (relative error)

\begin{tabular}{ll}
\hline \hline Components: & Original Measurements: \\
(1) 3-Methylbenzoic acid; $\mathrm{C}_{8} \mathrm{H}_{8} \mathrm{O}_{2} ;$ & ${ }^{14} \mathrm{C}$. R. Daniels, A. K. Charlton, R. \\
[99-04-7] & M. Wold, W. E. Acree, Jr., and M. \\
(2) 2-Butanol; $\mathrm{C}_{4} \mathrm{H}_{10} \mathrm{O} ;$ [78-92-2] & $\begin{array}{l}\text { H. Abraham, Can. J. Chem. 81, 1492 } \\
\text { (2003). }\end{array}$ \\
\hline Variables: & Prepared by: \\
$T / \mathrm{K}=298.15$ & W. E. Acree, Jr. \\
\hline
\end{tabular}

\section{Experimental Values}

\begin{tabular}{lc}
\hline \hline$x_{2}{ }^{\mathrm{a}}$ & $x_{1}{ }^{\mathrm{b}}$ \\
\hline 0.8105 & 0.1895 \\
\hline${ }^{\mathrm{a}} x_{2}:$ mole fraction of component 2 in the saturated solution. & \\
${ }^{\mathrm{b}}{ }_{x_{1}}$ : mole fraction solubility of the solute. &
\end{tabular}

\section{Auxiliary Information}

\section{Method/Apparatus/Procedure:}

Constant-temperature bath, calorimetric thermometer, and an ultraviolet/ visible spectrophotometer.

Excess solute and solvent were placed in amber glass bottles and allowed to equilibrate for several days at constant temperature. Attainment of equilibrium was verified by several repetitive measurements and by approaching equilibrium from supersaturation. Aliquots of saturated solutions were transferred through a coarse filter into tared volumetric flasks, weighed, and diluted with methanol. Concentrations were determined by spectrophotometric measurements at $279 \mathrm{~nm}$. 
Source and Purity of Chemicals:

(1) $99 \%$, Aldrich Chemical Company, Milwaukee, WI, USA, was used as received.

(2) $99+\%$, anhydrous, Aldrich Chemical Company, stored over molecular sieves and distilled shortly before use.

\section{Estimated Error:}

Temperature: $\pm 0.1 \mathrm{~K}$.

$x_{1}: \pm 1.5 \%$ (relative error).

\section{Components:}

(1) 3-Methylbenzoic acid; $\mathrm{C}_{8} \mathrm{H}_{8} \mathrm{O}_{2}$;

[99-04-7]

(2) 2-Methyl-1-propanol; $\mathrm{C}_{4} \mathrm{H}_{10} \mathrm{O}$;

[78-83-1]

\begin{tabular}{ll}
\hline Variables: & Prepared by: \\
$T / \mathrm{K}=298.15$ & W. E. Acree, Jr. \\
\hline
\end{tabular}

\begin{tabular}{lc}
\hline \hline$x_{2}{ }^{\mathrm{a}}$ & $x_{1}{ }^{\mathrm{b}}$ \\
\hline 0.8655 & 0.1345
\end{tabular}

${ }^{a} x_{2}$ : mole fraction of component 2 in the saturated solution.

${ }^{\mathrm{b}} x_{1}$ : mole fraction solubility of the solute.

\section{Auxiliary Information}

\section{Method/Apparatus/Procedure:}

Constant-temperature bath, calorimetric thermometer, and an ultraviolet/ visible spectrophotometer.

Excess solute and solvent were placed in amber glass bottles and allowed to equilibrate for several days at constant temperature. Attainment of equilibrium was verified by several repetitive measurements and by approaching equilibrium from supersaturation. Aliquots of saturated solutions were transferred through a coarse filter into tared volumetric flasks, weighed, and diluted with methanol. Concentrations were determined by spectrophotometric measurements at $279 \mathrm{~nm}$.

\section{Source and Purity of Chemicals:}

(1) $99 \%$, Aldrich Chemical Company, Milwaukee, WI, USA, was used as received.

(2) $99+\%$, anhydrous, Aldrich Chemical Company, stored over molecular sieves and distilled shortly before use.

\section{Estimated Error:}

Temperature: $\pm 0.1 \mathrm{~K}$.

$x_{1}: \pm 1.5 \%$ (relative error).
Experimental Values

Experimental Values

\begin{tabular}{lc}
\hline \hline$x_{2}{ }^{\mathrm{a}}$ & $x_{1}{ }^{\mathrm{b}}$ \\
\hline 0.7733 & 0.2267 \\
\hline${ }^{\mathrm{a}} x_{2}:$ mole fraction of component 2 in the saturated solution. & \\
${ }^{\mathrm{b}} x_{1}:$ mole fraction solubility of the solute. &
\end{tabular}

\section{Auxiliary Information}

Method/Apparatus/Procedure:

Constant-temperature bath, calorimetric thermometer, and an ultraviolet/ visible spectrophotometer.

Excess solute and solvent were placed in amber glass bottles and allowed to equilibrate for several days at constant temperature. Attainment of equilibrium was verified by several repetitive measurements and by approaching equilibrium from supersaturation. Aliquots of saturated solutions were transferred through a coarse filter into tared volumetric flasks, weighed, and diluted with methanol. Concentrations were determined by spectrophotometric measurements at $279 \mathrm{~nm}$.

Source and Purity of Chemicals:

(1) $99 \%$, Aldrich Chemical Company, Milwaukee, WI, USA, was used as received.

(2) $99+\%$, Arco Chemical Company, USA, stored over molecular sieves and distilled shortly before use.

\section{Estimated Error:}

Temperature: $\pm 0.1 \mathrm{~K}$.

$x_{1}: \pm 2.0 \%$ (relative error)

\begin{tabular}{ll}
\hline \hline Components: & Original Measurements: \\
(1) 3-Methylbenzoic acid; $\mathrm{C}_{8} \mathrm{H}_{8} \mathrm{O}_{2} ;$ & ${ }^{14} \mathrm{C}$. R. Daniels, A. K. Charlton, R. \\
[99-04-7] & M. Wold, W. E. Acree, Jr., and M. \\
(2) 1-Pentanol; $\mathrm{C}_{5} \mathrm{H}_{12} \mathrm{O} ;$ [71-41-0] & $\begin{array}{l}\text { H. Abraham, Can. J. Chem. 81, 1492 } \\
\text { (2003). }\end{array}$ \\
\hline Variables: & Prepared by: \\
$T / \mathrm{K}=298.15$ & W. E. Acree, Jr. \\
\hline
\end{tabular}

\section{Experimental Values}

\begin{tabular}{lc}
\hline \hline$x_{2}{ }^{\mathrm{a}}$ & $x_{1}{ }^{\mathrm{b}}$ \\
\hline 0.8227 & 0.1773 \\
\hline${ }^{\mathrm{a}} x_{2}:$ mole fraction of component 2 in the saturated solution. & \\
${ }^{\mathrm{b}}{ }_{x_{1}}$ : mole fraction solubility of the solute. &
\end{tabular}

\section{Auxiliary Information}

\begin{tabular}{|c|c|}
\hline $\begin{array}{l}\text { Components: } \\
\text { (1) 3-Methylbenzoic acid; } \mathrm{C}_{8} \mathrm{H}_{8} \mathrm{O}_{2} \text {; } \\
\text { [99-04-7] } \\
\text { (2) 2-Methyl-2-propanol; } \mathrm{C}_{4} \mathrm{H}_{10} \mathrm{O} \text {; } \\
\text { [75-65-0] }\end{array}$ & $\begin{array}{l}\text { Original Measurements: } \\
{ }^{14} \text { C. R. Daniels, A. K. Charlton, R. } \\
\text { M. Wold, W. E. Acree, Jr., and M. } \\
\text { H. Abraham, Can. J. Chem. 81, } 1492 \\
(2003) \text {. }\end{array}$ \\
\hline $\begin{array}{l}\text { Variables: } \\
T / \mathrm{K}=298.15\end{array}$ & $\begin{array}{l}\text { Prepared by: } \\
\text { W. E. Acree, Jr. }\end{array}$ \\
\hline
\end{tabular}

\section{Method/Apparatus/Procedure:} visible spectrophotometer.

Excess solute and solvent were placed in amber glass bottles and allowed to equilibrate for several days at constant temperature. Attainment of equilibrium was verified by several repetitive measurements and by approaching equilibrium from supersaturation. Aliquots of saturated solutions were transferred through a coarse filter into tared volumetric flasks, weighed, and diluted with methanol. Concentrations were determined by spectrophotometric measurements at $279 \mathrm{~nm}$.
Constant-temperature bath, calorimetric thermometer, and an ultraviolet/ 
Source and Purity of Chemicals:

(1) $99 \%$, Aldrich Chemical Company, Milwaukee, WI, USA, was used as received.

(2) $99+\%$, Aldrich Chemical Company, stored over molecular sieves and distilled shortly before use.

\section{Estimated Error:}

Temperature: $\pm 0.1 \mathrm{~K}$.

$x_{1}: \pm 1.5 \%$ (relative error).

\section{Components:}

(1) 3-Methylbenzoic acid; $\mathrm{C}_{8} \mathrm{H}_{8} \mathrm{O}_{2}$;

[99-04-7]

(2) 2-Pentanol; $\mathrm{C}_{5} \mathrm{H}_{12} \mathrm{O}$; [6032-29-7]

Original Measurements:

${ }^{14}$ C. R. Daniels, A. K. Charlton, R.

M. Wold, W. E. Acree, Jr., and M.

H. Abraham, Can. J. Chem. 81, 1492 (2003).

\begin{tabular}{ll}
\hline Variables: & Prepared by: \\
$T / \mathrm{K}=298.15$ & W. E. Acree, Jr.
\end{tabular}

\section{Experimental Values}

\begin{tabular}{lc}
\hline \hline$x_{2}{ }^{\mathrm{a}}$ & $x_{1}{ }^{\mathrm{b}}$ \\
\hline 0.8035 & 0.1965
\end{tabular}

${ }^{\mathrm{a}} x_{2}$ : mole fraction of component 2 in the saturated solution.

${ }^{\mathrm{b}} x_{1}$ : mole fraction solubility of the solute.

\section{Auxiliary Information}

\section{Method/Apparatus/Procedure:}

Constant-temperature bath, calorimetric thermometer, and an ultraviolet/ visible spectrophotometer.

Excess solute and solvent were placed in amber glass bottles and allowed to equilibrate for several days at constant temperature. Attainment of equilibrium was verified by several repetitive measurements and by approaching equilibrium from supersaturation. Aliquots of saturated solutions were transferred through a coarse filter into tared volumetric flasks, weighed, and diluted with methanol. Concentrations were determined by spectrophotometric measurements at $279 \mathrm{~nm}$.

Source and Purity of Chemicals:

(1) $99 \%$, Aldrich Chemical Company, Milwaukee, WI, USA, was used as received.

(2) $99+\%$, Acros Organics, USA, stored over molecular sieves and distilled shortly before use.

Estimated Error:

Temperature: $\pm 0.1 \mathrm{~K}$

$x_{1}: \pm 1.5 \%$ (relative error).

\begin{tabular}{|c|c|}
\hline $\begin{array}{l}\text { Components: } \\
\text { (1) 3-Methylbenzoic acid; } \mathrm{C}_{8} \mathrm{H}_{8} \mathrm{O}_{2} \text {; } \\
\text { [99-04-7] } \\
\text { (2) 2-Methyl-1-butanol; } \mathrm{C}_{5} \mathrm{H}_{12} \mathrm{O} \text {; } \\
\text { [137-32-6] }\end{array}$ & $\begin{array}{l}\text { Original Measurements: } \\
{ }^{14} \text { C. R. Daniels, A. K. Charlton, R. } \\
\text { M. Wold, W. E. Acree, Jr., and M. } \\
\text { H. Abraham, Can. J. Chem. 81, } 1492 \\
\text { (2003). }\end{array}$ \\
\hline $\begin{array}{l}\text { Variables: } \\
T / \mathrm{K}=298.15\end{array}$ & $\begin{array}{l}\text { Prepared by: } \\
\text { W. E. Acree, Jr. }\end{array}$ \\
\hline
\end{tabular}

Experimental Values

\begin{tabular}{lc}
\hline \hline$x_{2}{ }^{\mathrm{a}}$ & $x_{1}{ }^{\mathrm{b}}$ \\
\hline 0.8530 & 0.1470 \\
\hline${ }^{\mathrm{a}} x_{2}:$ mole fraction of component 2 in the saturated solution. & \\
${ }^{\mathrm{b}} x_{1}:$ mole fraction solubility of the solute. &
\end{tabular}

\section{Auxiliary Information}

Method/Apparatus/Procedure:

Constant-temperature bath, calorimetric thermometer, and an ultraviolet/ visible spectrophotometer.

Excess solute and solvent were placed in amber glass bottles and allowed to equilibrate for several days at constant temperature. Attainment of equilibrium was verified by several repetitive measurements and by approaching equilibrium from supersaturation. Aliquots of saturated solutions were transferred through a coarse filter into tared volumetric flasks, weighed, and diluted with methanol. Concentrations were determined by spectrophotometric measurements at $279 \mathrm{~nm}$.

Source and Purity of Chemicals:

(1) $99 \%$, Aldrich Chemical Company, Milwaukee, WI, USA, was used as received.

(2) $99 \%$, Aldrich Chemical Company, stored over molecular sieves and distilled shortly before use.

\section{Estimated Error:}

Temperature: $\pm 0.1 \mathrm{~K}$.

$x_{1}: \pm 1.5 \%$ (relative error).

\begin{tabular}{ll}
\hline \hline Components: & Original Measurements: \\
(1) 3-Methylbenzoic acid; $\mathrm{C}_{8} \mathrm{H}_{8} \mathrm{O}_{2} ;$ & ${ }^{14} \mathrm{C}$. R. Daniels, A. K. Charlton, R. \\
[99-04-7] & M. Wold, W. E. Acree, Jr., and M. \\
$\begin{array}{l}\text { (2) 3-Methyl-1-butanol; } \mathrm{C}_{5} \mathrm{H}_{12} \mathrm{O} ; \\
\text { [123-51-3] }\end{array}$ & $\begin{array}{l}\text { H. Abraham, Can. J. Chem. 81, 1492 } \\
(2003) .\end{array}$ \\
\hline Variables: & Prepared by: \\
$T / \mathrm{K}=298.15$ & W. E. Acree, Jr. \\
\hline
\end{tabular}

\section{Experimental Values}

\begin{tabular}{lc}
\hline \hline$x_{2}{ }^{\mathrm{a}}$ & $x_{1}{ }^{\mathrm{b}}$ \\
\hline 0.8368 & 0.1632 \\
\hline${ }^{{ }^{a}} x_{2}:$ mole fraction of component 2 in the saturated solution. & \\
${ }^{\mathrm{b}} x_{1}:$ mole fraction solubility of the solute. &
\end{tabular}

\section{Auxiliary Information}

\section{Method/Apparatus/Procedure:}

Constant-temperature bath, calorimetric thermometer, and an ultraviolet/ visible spectrophotometer.

Excess solute and solvent were placed in amber glass bottles and allowed to equilibrate for several days at constant temperature. Attainment of equilibrium was verified by several repetitive measurements and by approaching equilibrium from supersaturation. Aliquots of saturated solutions were transferred through a coarse filter into tared volumetric flasks, weighed, and diluted with methanol. Concentrations were determined by spectrophotometric measurements at $279 \mathrm{~nm}$. 
Source and Purity of Chemicals:

(1) $99 \%$, Aldrich Chemical Company, Milwaukee, WI, USA, was used as received.

(2) $99+\%$, anhydrous, Aldrich Chemical Company, stored over molecular sieves and distilled shortly before use.

\section{Estimated Error:}

Temperature: $\pm 0.1 \mathrm{~K}$.

$x_{1}: \pm 1.5 \%$ (relative error).

\section{Components:}

(1) 3-Methylbenzoic acid; $\mathrm{C}_{8} \mathrm{H}_{8} \mathrm{O}_{2}$; [99-04-7]

(2) 1-Hexanol; $\mathrm{C}_{6} \mathrm{H}_{14} \mathrm{O}$; [111-27-3]

Original Measurements:

${ }^{14}$ C. R. Daniels, A. K. Charlton, R. M. Wold, W. E. Acree, Jr., and M. H. Abraham, Can. J. Chem. 81, 1492 (2003).

\begin{tabular}{ll}
\hline Variables: & Prepared by: \\
$T / \mathrm{K}=298.15$ & W. E. Acree, Jr.
\end{tabular}

\section{Experimental Values}

\begin{tabular}{lc}
\hline \hline$x_{2}{ }^{\mathrm{a}}$ & $x_{1}{ }^{\mathrm{b}}$ \\
\hline 0.8172 & 0.1828
\end{tabular}

${ }^{\mathrm{a}} x_{2}$ : mole fraction of component 2 in the saturated solution.

${ }^{\mathrm{b}} x_{1}$ : mole fraction solubility of the solute.

\section{Auxiliary Information}

\section{Method/Apparatus/Procedure:}

Constant-temperature bath, calorimetric thermometer, and an ultraviolet/ visible spectrophotometer.

Excess solute and solvent were placed in amber glass bottles and allowed to equilibrate for several days at constant temperature. Attainment of equilibrium was verified by several repetitive measurements and by approaching equilibrium from supersaturation. Aliquots of saturated solutions were transferred through a coarse filter into tared volumetric flasks, weighed, and diluted with methanol. Concentrations were determined by spectrophotometric measurements at $279 \mathrm{~nm}$.

Source and Purity of Chemicals:

(1) $99 \%$, Aldrich Chemical Company, Milwaukee, WI, USA, was used as received.

(2) $99+\%$, Alfa Aesar, USA, stored over molecular sieves and distilled shortly before use.

Estimated Error:

Temperature: $\pm 0.1 \mathrm{~K}$.

$x_{1}: \pm 1.5 \%$ (relative error).

\begin{tabular}{|c|c|}
\hline $\begin{array}{l}\text { Components: } \\
\text { (1) 3-Methylbenzoic acid; } \mathrm{C}_{8} \mathrm{H}_{8} \mathrm{O}_{2} \text {; } \\
\text { [99-04-7] } \\
\text { (2) 2-Methyl-1-pentanol; } \mathrm{C}_{6} \mathrm{H}_{14} \mathrm{O} \text {; } \\
\text { [105-30-6] }\end{array}$ & $\begin{array}{l}\text { Original Measurements: } \\
{ }^{14} \text { C. R. Daniels, A. K. Charlton, R. } \\
\text { M. Wold, W. E. Acree, Jr., and M. } \\
\text { H. Abraham, Can. J. Chem. 81, } 1492 \\
\text { (2003). }\end{array}$ \\
\hline $\begin{array}{l}\text { Variables: } \\
T / \mathrm{K}=298.15\end{array}$ & $\begin{array}{l}\text { Prepared by: } \\
\text { W. E. Acree, Jr. }\end{array}$ \\
\hline
\end{tabular}

Experimental Values

\begin{tabular}{lc}
\hline \hline$x_{2}{ }^{\mathrm{a}}$ & $x_{1}{ }^{\mathrm{b}}$ \\
\hline 0.8332 & 0.1668 \\
\hline${ }^{\mathrm{a}} x_{2}:$ mole fraction of component 2 in the saturated solution. & \\
${ }^{\mathrm{b}} x_{1}:$ mole fraction solubility of the solute. &
\end{tabular}

\section{Auxiliary Information}

Method/Apparatus/Procedure:

Constant-temperature bath, calorimetric thermometer, and an ultraviolet/ visible spectrophotometer.

Excess solute and solvent were placed in amber glass bottles and allowed to equilibrate for several days at constant temperature. Attainment of equilibrium was verified by several repetitive measurements and by approaching equilibrium from supersaturation. Aliquots of saturated solutions were transferred through a coarse filter into tared volumetric flasks, weighed, and diluted with methanol. Concentrations were determined by spectrophotometric measurements at $279 \mathrm{~nm}$.

Source and Purity of Chemicals:

(1) $99 \%$, Aldrich Chemical Company, Milwaukee, WI, USA, was used as received.

(2) $99 \%$, Aldrich Chemical Company, stored over molecular sieves and distilled shortly before use.

\section{Estimated Error:}

Temperature: $\pm 0.1 \mathrm{~K}$.

$x_{1}: \pm 1.5 \%$ (relative error)

\begin{tabular}{ll}
\hline \hline Components: & Original Measurements: \\
(1) 3-Methylbenzoic acid; $\mathrm{C}_{8} \mathrm{H}_{8} \mathrm{O}_{2} ;$ & ${ }^{14} \mathrm{C}$. R. Daniels, A. K. Charlton, R. \\
[99-04-7] & M. Wold, W. E. Acree, Jr., and M. \\
$\begin{array}{l}\text { (2) 4-Methyl-2-pentanol; } \mathrm{C}_{6} \mathrm{H}_{14} \mathrm{O} ; \\
\text { [108-11-2] }\end{array}$ & $\begin{array}{l}\text { H. Abraham, Can. J. Chem. 81, 1492 } \\
(2003) .\end{array}$ \\
\hline Variables: & Prepared by: \\
$T / \mathrm{K}=298.15$ & W. E. Acree, Jr. \\
\hline
\end{tabular}

\section{Experimental Values}

\begin{tabular}{lc}
\hline \hline$x_{2}{ }^{\mathrm{a}}$ & $x_{1}{ }^{\mathrm{b}}$ \\
\hline 0.7867 & 0.2133 \\
\hline${ }^{\mathrm{a}} x_{2}:$ mole fraction of component 2 in the saturated solution. & \\
${ }^{\mathrm{b}} x_{1}:$ mole fraction solubility of the solute. &
\end{tabular}

\section{Auxiliary Information}

\section{Method/Apparatus/Procedure:}

Constant-temperature bath, calorimetric thermometer, and an ultraviolet/ visible spectrophotometer.

Excess solute and solvent were placed in amber glass bottles and allowed to equilibrate for several days at constant temperature. Attainment of equilibrium was verified by several repetitive measurements and by approaching equilibrium from supersaturation. Aliquots of saturated solutions were transferred through a coarse filter into tared volumetric flasks, weighed, and diluted with methanol. Concentrations were determined by spectrophotometric measurements at $279 \mathrm{~nm}$. 
Source and Purity of Chemicals:

(1) $99 \%$, Aldrich Chemical Company, Milwaukee, WI, USA, was used as received.

(2) $99+\%$, Acros Organics, USA, stored over molecular sieves and distilled shortly before use.

\section{Estimated Error:}

Temperature: $\pm 0.1 \mathrm{~K}$

$x_{1}: \pm 1.5 \%$ (relative error).

\section{Components:}

(1) 3-Methylbenzoic acid; $\mathrm{C}_{8} \mathrm{H}_{8} \mathrm{O}_{2}$; [99-04-7]

(2) 1-Heptanol; $\mathrm{C}_{7} \mathrm{H}_{16} \mathrm{O} ;[111-70-6]$

Original Measurements:

${ }^{14}$ C. R. Daniels, A. K. Charlton, R. M. Wold, W. E. Acree, Jr., and M. H. Abraham, Can. J. Chem. 81, 1492 (2003).

\begin{tabular}{ll}
\hline Variables: & Prepared by: \\
$T / \mathrm{K}=298.15$ & W. E. Acree, Jr.
\end{tabular}

\section{Experimental Values}

\begin{tabular}{lc}
\hline \hline$x_{2}{ }^{\mathrm{a}}$ & $x_{1}{ }^{\mathrm{b}}$ \\
\hline 0.8090 & 0.1910 \\
\hline$x_{2}:$ mole fraction of componen 2 in
\end{tabular}

${ }^{\mathrm{a}} x_{2}$ : mole fraction of component 2 in the saturated solution.

${ }^{\mathrm{b}} x_{1}$ : mole fraction solubility of the solute.

\section{Auxiliary Information}

\section{Method/Apparatus/Procedure:}

Constant-temperature bath, calorimetric thermometer, and an ultraviolet/ visible spectrophotometer.

Excess solute and solvent were placed in amber glass bottles and allowed to equilibrate for several days at constant temperature. Attainment of equilibrium was verified by several repetitive measurements and by approaching equilibrium from supersaturation. Aliquots of saturated solutions were transferred through a coarse filter into tared volumetric flasks, weighed, and diluted with methanol. Concentrations were determined by spectrophotometric measurements at $279 \mathrm{~nm}$.

Source and Purity of Chemicals:

(1) $99 \%$, Aldrich Chemical Company, Milwaukee, WI, USA, was used as received.

(2) $99+\%$, Alfa Aesar, USA, stored over molecular sieves and distilled shortly before use.

Estimated Error:

Temperature: $\pm 0.1 \mathrm{~K}$.

$x_{1}: \pm 1.5 \%$ (relative error)

\begin{tabular}{ll}
\hline \hline Components: & Original Measurements: \\
(1) 3-Methylbenzoic acid; $\mathrm{C}_{8} \mathrm{H}_{8} \mathrm{O}_{2} ;$ & ${ }^{14} \mathrm{C}$. R. Daniels, A. K. Charlton, R. \\
[99-04-7] & M. Wold, W. E. Acree, Jr., and M. \\
(2) 1-Octanol; $\mathrm{C}_{8} \mathrm{H}_{18} \mathrm{O} ;$ [111-87-5] & $\begin{array}{l}\text { H. Abraham, Can. J. Chem. 81, 1492 } \\
\text { (2003). }\end{array}$ \\
\hline Variables: & Prepared by: \\
$T / \mathrm{K}=298.15$ & W. E. Acree, Jr. \\
\hline
\end{tabular}

Experimental Values

\begin{tabular}{lc}
\hline \hline$x_{2}{ }^{\mathrm{a}}$ & $x_{1}{ }^{\mathrm{b}}$ \\
\hline 0.8015 & 0.1985 \\
\hline${ }^{\mathrm{a}} x_{2}:$ mole fraction of component 2 in the saturated solution. & \\
${ }^{\mathrm{b}} x_{1}:$ mole fraction solubility of the solute. &
\end{tabular}

\section{Auxiliary Information}

Method/Apparatus/Procedure:

Constant-temperature bath, calorimetric thermometer, and an ultraviolet/ visible spectrophotometer.

Excess solute and solvent were placed in amber glass bottles and allowed to equilibrate for several days at constant temperature. Attainment of equilibrium was verified by several repetitive measurements and by approaching equilibrium from supersaturation. Aliquots of saturated solutions were transferred through a coarse filter into tared volumetric flasks, weighed, and diluted with methanol. Concentrations were determined by spectrophotometric measurements at $279 \mathrm{~nm}$.

Source and Purity of Chemicals:

(1) $99 \%$, Aldrich Chemical Company, Milwaukee, WI, USA, was used as received.

(2) $99+\%$, anhydrous, Aldrich Chemical Company, stored over molecular sieves and distilled shortly before use.

\section{Estimated Error:}

Temperature: $\pm 0.1 \mathrm{~K}$.

$x_{1}: \pm 1.5 \%$ (relative error)

\begin{tabular}{ll}
\hline \hline Components: & Original Measurements: \\
(1) 3-Methylbenzoic acid; $\mathrm{C}_{8} \mathrm{H}_{8} \mathrm{O}_{2} ;$ & ${ }^{77}$ J. Qingzhu, M. Peisheng, Y. \\
[99-04-7] & Shouzhi, W. Qiang, W. Chang, and \\
(2) 1-Octanol; $\mathrm{C}_{8} \mathrm{H}_{18} \mathrm{O} ;$ [111-87-5] & L. Guiju, J. Chem. Eng. Data 53, \\
& 1278 (2008). \\
\hline Variables: & Prepared by: \\
Temperature & W. E. Acree, Jr. \\
\hline
\end{tabular}

Experimental Values

\begin{tabular}{lcc}
\hline \hline$T / \mathrm{K}$ & $x_{2}{ }^{\mathrm{a}}$ & $x_{1}{ }^{\mathrm{b}}$ \\
\hline 296.25 & 0.8259 & 0.1741 \\
297.25 & 0.8212 & 0.1788 \\
299.15 & 0.8144 & 0.1856 \\
300.25 & 0.8063 & 0.1937 \\
302.90 & 0.8011 & 0.1989 \\
303.80 & 0.7906 & 0.2094 \\
305.85 & 0.7806 & 0.2194 \\
308.45 & 0.7687 & 0.2313 \\
311.75 & 0.7543 & 0.2470 \\
313.80 & 0.7379 & 0.2621 \\
316.25 & 0.7251 & 0.2749 \\
317.65 & 0.7133 & 0.2867 \\
322.15 & 0.6945 & 0.3055
\end{tabular}

${ }^{a} x_{2}$ : mole fraction of component 2 in the saturated solution.

${ }^{\mathrm{b}} x_{1}$ : mole fraction solubility of the solute. 


\section{Auxiliary Information}

\section{Method/Apparatus/Procedure:}

Circulating water bath, analytical balance, and laser monitoring system. Preweighed amounts of solute and solvent and were placed in an equilibrium vessel, which was connected to a circulating water bath. The solution was stirred and the temperature gradually increased at a rate of $0.5 \mathrm{~K} / 20 \mathrm{~min}(0.2 \mathrm{~K} /$ $20 \mathrm{~min}$ or slower near saturation temperature) until all of the solid solute dissolved. The temperature at which all of the solute dissolved was determined using laser monitoring.

\section{Source and Purity of Chemicals:}

(1) $<99 \%$, Chemical source not specified, used as received.

(2) $<99 \%$, Chemical source not specified, used as received.

\section{Estimated Error:}

Temperature: $\pm 0.05 \mathrm{~K}$. $x_{1}: \pm 0.0005$

\section{Components:}

(1) 3-Methylbenzoic acid; $\mathrm{C}_{8} \mathrm{H}_{8} \mathrm{O}_{2}$; [99-04-7]

(2) 1-Decanol; $\mathrm{C}_{10} \mathrm{H}_{22} \mathrm{O}$; [112-30-1]

Original Measurements:

${ }^{14}$ C. R. Daniels, A. K. Charlton, R. M. Wold, W. E. Acree, Jr., and M. H. Abraham, Can. J. Chem. 81, 1492 (2003).

\begin{tabular}{ll}
\hline Variables: & Prepared by: \\
$T / \mathrm{K}=298.15$ & W. E. Acree, Jr.
\end{tabular}

\section{Experimental Values}

\begin{tabular}{lc}
\hline \hline$x_{2}{ }^{\mathrm{a}}$ & $x_{1}{ }^{\mathrm{b}}$ \\
\hline 0.8370 & 0.1630 \\
\hline$x_{2}:$ mole fraction
\end{tabular}

${ }^{\mathrm{a}} x_{2}$ : mole fraction of component 2 in the saturated solution.

${ }^{b} x_{1}$ : mole fraction solubility of the solute.

\section{Auxiliary Information}

\section{Method/Apparatus/Procedure:}

Constant-temperature bath, calorimetric thermometer, and an ultraviolet/ visible spectrophotometer.

Excess solute and solvent were placed in amber glass bottles and allowed to equilibrate for several days at constant temperature. Attainment of equilibrium was verified by several repetitive measurements and by approaching equilibrium from supersaturation. Aliquots of saturated solutions were transferred through a coarse filter into tared volumetric flasks, weighed, and diluted with methanol. Concentrations were determined by spectrophotometric measurements at $279 \mathrm{~nm}$.

Source and Purity of Chemicals:

(1) $99 \%$, Aldrich Chemical Company, Milwaukee, WI, USA, was used as received.

(2) $99+\%$, Alfa Aesar, USA, stored over molecular sieves and distilled shortly before use.

Estimated Error:

Temperature: $\pm 0.1 \mathrm{~K}$

$x_{1}: \pm 1.5 \%$ (relative error).

\subsection{3-Methylbenzoic acid solubility data in alkoxyalcohols}

\begin{tabular}{|c|c|}
\hline Components: & Original Measurements: \\
\hline (1) 3-Methylbenzoic acid; $\mathrm{C}_{8} \mathrm{H}_{8} \mathrm{O}_{2}$; & ${ }^{130}$ L. M. Grubbs, M. Saifullah, N. E \\
\hline$[99-04-7]$ & De La Rosa, S. Ye, S. S. Achi, W. E \\
\hline $\begin{array}{l}\text { (2) 2-Ethoxyethanol; } \mathrm{C}_{4} \mathrm{H}_{10} \mathrm{O}_{2} \text {; } \\
\text { [110-80-5] }\end{array}$ & $\begin{array}{l}\text { Acree, Jr., and M. H. Abraham, } \\
\text { Fluid Phase Equilib. 298, } 48 \text { (2010) }\end{array}$ \\
\hline Variables: & Prepare \\
\hline
\end{tabular}

Experimental Values

\begin{tabular}{lc}
\hline \hline$x_{2}{ }^{\mathrm{a}}$ & $x_{1}{ }^{\mathrm{b}}$ \\
\hline 0.7894 & 0.2106 \\
\hline${ }^{\mathrm{a}}{ }_{2}:$ mole fraction of component 2 in the saturated solution. &
\end{tabular}

${ }^{\mathrm{b}} x_{1}$ : mole fraction solubility of the solute.

\section{Auxiliary Information}

\section{Method/Apparatus/Procedure:}

Constant-temperature bath, calorimetric thermometer, and an ultraviolet/ visible spectrophotometer.

Excess solute and solvent were placed in amber glass bottles and allowed to equilibrate for several days at constant temperature. Attainment of equilibrium was verified by several repetitive measurements and by approaching equilibrium from supersaturation. Aliquots of saturated solutions were transferred through a coarse filter into tared volumetric flasks, weighed, and diluted with methanol. Concentrations were determined by spectrophotometric measurements at $279 \mathrm{~nm}$.

Source and Purity of Chemicals:

(1) $99 \%$, Aldrich Chemical Company, Milwaukee, WI, USA, was used as received.

(2) $99 \%$, Aldrich Chemical Company, stored over molecular sieves and distilled shortly before use.

Estimated Error:

Temperature: $\pm 0.1 \mathrm{~K}$.

$x_{1}: \pm 1.5 \%$ (relative error)

\begin{tabular}{|c|c|}
\hline Components: & Original Measurements: \\
\hline $\begin{array}{l}\text { (1) 3-Methylbenzoic acid; } \mathrm{C}_{8} \mathrm{H}_{8} \mathrm{O}_{2} \text {; } \\
\text { [99-04-7] }\end{array}$ & $\begin{array}{l}{ }^{130} \text { L. M. Grubbs, M. Saifullah, N. E. } \\
\text { De La Rosa, S. Ye, S. S. Achi, W. E. }\end{array}$ \\
\hline $\begin{array}{l}\text { (2) 2-Propoxyethanol; } \mathrm{C}_{5} \mathrm{H}_{12} \mathrm{O}_{2} \text {; } \\
\text { [2807-30-9] }\end{array}$ & $\begin{array}{l}\text { Acree, Jr., and M. H. Abraham, } \\
\text { Fluid Phase Equilib. 298, } 48 \text { (2010). }\end{array}$ \\
\hline $\begin{array}{l}\text { Variables: } \\
T / \mathrm{K}=298.15\end{array}$ & $\begin{array}{l}\text { Prepared by: } \\
\text { W. E. Acree, Jr. }\end{array}$ \\
\hline
\end{tabular}

\section{Experimental Values}

\begin{tabular}{lc}
\hline \hline$x_{2}{ }^{\mathrm{a}}$ & $x_{1}{ }^{\mathrm{b}}$ \\
\hline 0.7894 & 0.2106 \\
\hline${ }^{\mathrm{a}} x_{2}:$ mole fraction of component 2 in the saturated solution. \\
${ }^{\mathrm{b}} x_{1}:$ mole fraction solubility of the solute.
\end{tabular}




\section{Auxiliary Information}

\section{Method/Apparatus/Procedure:}

Constant-temperature bath, calorimetric thermometer, and an ultraviolet/ visible spectrophotometer.

Excess solute and solvent were placed in amber glass bottles and allowed to equilibrate for several days at constant temperature. Attainment of equilibrium was verified by several repetitive measurements and by approaching equilibrium from supersaturation. Aliquots of saturated solutions were transferred through a coarse filter into tared volumetric flasks, weighed, and diluted with methanol. Concentrations were determined by spectrophotometric measurements at $279 \mathrm{~nm}$.

Source and Purity of Chemicals:

(1) $99 \%$, Aldrich Chemical Company, Milwaukee, WI, USA, was used as received.

(2) $99 \%$, Aldrich Chemical Company, stored over molecular sieves and distilled shortly before use.

Estimated Error:

Temperature: $\pm 0.1 \mathrm{~K}$. $x_{1}: \pm 1.5 \%$ (relative error).

\begin{tabular}{|c|c|}
\hline Components: & Original Measurements: \\
\hline $\begin{array}{l}\text { (1) 3-Methylbenzoic acid; } \mathrm{C}_{8} \mathrm{H}_{8} \mathrm{O}_{2} \text {; } \\
\text { [99-04-7] }\end{array}$ & $\begin{array}{l}{ }^{130} \text { L. M. Grubbs, M. Saifullah, N. E. } \\
\text { De La Rosa, S. Ye, S. S. Achi, W. E. }\end{array}$ \\
\hline $\begin{array}{l}\text { (2) 2-Isopropoxyethanol; } \mathrm{C}_{5} \mathrm{H}_{12} \mathrm{O}_{2} \text {; } \\
\text { [109-59-1] }\end{array}$ & $\begin{array}{l}\text { Acree, Jr., and M. H. Abraham, } \\
\text { Fluid Phase Equilib. 298, } 48 \text { (2010). }\end{array}$ \\
\hline Variables: & Prepared by: \\
\hline$T / \mathrm{K}=298.15$ & W. E. Acree, Jr. \\
\hline
\end{tabular}

Experimental Values

\begin{tabular}{lc}
\hline \hline$x_{2}^{\mathrm{a}}$ & $x_{1}{ }^{\mathrm{b}}$ \\
\hline 0.7726 & 0.2274
\end{tabular}

${ }^{\mathrm{a}} x_{2}$ : mole fraction of component 2 in the saturated solution.

${ }^{\mathrm{b}} \mathrm{x}_{1}$ : mole fraction solubility of the solute.

\section{Auxiliary Information}

\section{Method/Apparatus/Procedure:}

Constant-temperature bath, calorimetric thermometer, and an ultraviolet/ visible spectrophotometer.

Excess solute and solvent were placed in amber glass bottles and allowed to equilibrate for several days at constant temperature. Attainment of equilibrium was verified by several repetitive measurements and by approaching equilibrium from supersaturation. Aliquots of saturated solutions were transferred through a coarse filter into tared volumetric flasks, weighed, and diluted with methanol. Concentrations were determined by

spectrophotometric measurements at $279 \mathrm{~nm}$.

\section{Source and Purity of Chemicals:}

(1) $99 \%$, Aldrich Chemical Company, Milwaukee, WI, USA, was used as received.

(2) $99 \%$, Aldrich Chemical Company, stored over molecular sieves and distilled shortly before use.

\section{Estimated Error:}

Temperature: $\pm 0.1 \mathrm{~K}$.

$x_{1}: \pm 1.5 \%$ (relative error).

\begin{tabular}{|c|c|}
\hline $\begin{array}{l}\text { Components: } \\
\text { (1) 3-Methylbenzoic acid; } \mathrm{C}_{8} \mathrm{H}_{8} \mathrm{O}_{2} \text {; } \\
\text { [99-04-7] } \\
\text { (2) 2-Butoxyethanol; } \mathrm{C}_{6} \mathrm{H}_{14} \mathrm{O}_{2} \text {; } \\
\text { [111-76-2] }\end{array}$ & $\begin{array}{l}\text { Original Measurements: } \\
{ }^{130} \text { L. M. Grubbs, M. Saifullah, N. E. } \\
\text { De La Rosa, S. Ye, S. S. Achi, W. E. } \\
\text { Acree, Jr., and M. H. Abraham, } \\
\text { Fluid Phase Equilib. 298, } 48 \text { (2010). }\end{array}$ \\
\hline $\begin{array}{l}\text { Variables: } \\
T / \mathrm{K}=298.15\end{array}$ & $\begin{array}{l}\text { Prepared by: } \\
\text { W. E. Acree, Jr. }\end{array}$ \\
\hline
\end{tabular}

Experimental Values

\begin{tabular}{lc}
\hline \hline$x_{2}{ }^{\mathrm{a}}$ & $x_{1}{ }^{\mathrm{b}}$ \\
\hline 0.7835 & 0.2165 \\
\hline${ }^{\mathrm{a}} x_{2}:$ mole fraction of component 2 in the saturated solution. & \\
${ }^{\mathrm{b}} x_{1}:$ mole fraction solubility of the solute. &
\end{tabular}

Auxiliary Information

\section{Method/Apparatus/Procedure:}

Constant-temperature bath, calorimetric thermometer, and an ultraviolet/ visible spectrophotometer.

Excess solute and solvent were placed in amber glass bottles and allowed to equilibrate for several days at constant temperature. Attainment of equilibrium was verified by several repetitive measurements and by approaching equilibrium from supersaturation. Aliquots of saturated solutions were transferred through a coarse filter into tared volumetric flasks, weighed, and diluted with methanol. Concentrations were determined by

spectrophotometric measurements at $279 \mathrm{~nm}$.

Source and Purity of Chemicals:

(1) $99 \%$, Aldrich Chemical Company, Milwaukee, WI, USA, was used as received.

(2) $99+\%$, Acros Organics, USA, stored over molecular sieves and distilled shortly before use.

\section{Estimated Error:}

Temperature: $\pm 0.1 \mathrm{~K}$.

$x_{1}: \pm 1.5 \%$ (relative error).

\begin{tabular}{|c|c|}
\hline Components: & Original Measurements: \\
\hline (1) 3-Methylbenzoic acid; $\mathrm{C}_{8} \mathrm{H}_{8} \mathrm{O}_{2}$; & ${ }^{130}$ L. M. Grubbs, M. Saifullah, N. E. \\
\hline$[99-04-7]$ & De La Rosa, S. Ye, S. S. Achi, W. E. \\
\hline $\begin{array}{l}\text { (2) 3-Methoxy-1-butanol; } \mathrm{C}_{5} \mathrm{H}_{12} \mathrm{O}_{2} \text {; } \\
\text { [2517-43-3] }\end{array}$ & $\begin{array}{l}\text { Acree, Jr., and M. H. Abraham, } \\
\text { Fluid Phase Equilib. 298, } 48 \text { (2010). }\end{array}$ \\
\hline Variable & \\
\hline$T / \mathrm{K}=298.15$ & W. E. Acree, Jr. \\
\hline
\end{tabular}

Experimental Values

\begin{tabular}{lc}
\hline \hline$x_{2}{ }^{\mathrm{a}}$ & $x_{1}{ }^{\mathrm{b}}$ \\
\hline 0.7945 & 0.2055 \\
${ }^{\mathrm{a}} x_{2}:$ mole fraction of component 2 in the saturated solution. & \\
${ }^{\mathrm{b}} x_{1}:$ mole fraction solubility of the solute. &
\end{tabular}




\section{Auxiliary Information}

Method/Apparatus/Procedure:

Constant-temperature bath, calorimetric thermometer, and an ultraviolet/ visible spectrophotometer.

Excess solute and solvent were placed in amber glass bottles and allowed to equilibrate for several days at constant temperature. Attainment of equilibrium was verified by several repetitive measurements and by approaching equilibrium from supersaturation. Aliquots of saturated solutions were transferred through a coarse filter into tared volumetric flasks, weighed, and diluted with methanol. Concentrations were determined by spectrophotometric measurements at $279 \mathrm{~nm}$.

Source and Purity of Chemicals:

(1) $99 \%$, Aldrich Chemical Company, Milwaukee, WI, USA, was used as received.

(2) $99 \%$, Aldrich Chemical Company, stored over molecular sieves and distilled shortly before use.

Estimated Error:

Temperature: $\pm 0.1 \mathrm{~K}$.

$x_{1}: \pm 1.5 \%$ (relative error).

\subsection{3-Methylbenzoic acid solubility data in miscellaneous organic solvents}

\begin{tabular}{ll}
\hline \hline Components: & Original Measurements: \\
(1) 3-Methylbenzoic acid; $\mathrm{C}_{8} \mathrm{H}_{8} \mathrm{O}_{2} ;$ & ${ }^{14} \mathrm{C}$. R. Daniels, A. K. Charlton, R. \\
[99-04-7] & M. Wold, W. E. Acree, Jr., and M. \\
$\begin{array}{l}\text { (2) Propylene carbonate; } \mathrm{C}_{4} \mathrm{H}_{6} \mathrm{O}_{3} ; \\
\text { [108-32-7] }\end{array}$ & $\begin{array}{l}\text { H. Abraham, Can. J. Chem. 81, 1492 } \\
(2003) .\end{array}$ \\
\hline Variables: & Prepared by: \\
$T / \mathrm{K}=298.15$ & W. E. Acree, Jr. \\
\hline
\end{tabular}

Experimental Values

\begin{tabular}{lc}
\hline \hline$x_{2}{ }^{\mathrm{a}}$ & $x_{1}{ }^{\mathrm{b}}$ \\
\hline 0.9611 & 0.03892
\end{tabular}

${ }^{a} x_{2}$ : mole fraction of component 2 in the saturated solution.

${ }^{\mathrm{b}} x_{1}$ : mole fraction solubility of the solute.

\section{Auxiliary Information}

\section{Components:}

(1) 3-Methylbenzoic acid; $\mathrm{C}_{8} \mathrm{H}_{8} \mathrm{O}_{2}$; [99-04-7]

(2) 1-Methyl-2-tert-butoxyethanol; $\mathrm{C}_{7} \mathrm{H}_{16} \mathrm{O}_{2} ;$; $\left.57018-52-7\right]$

\section{Variables:}

$T / \mathrm{K}=298.15$

Original Measurements:

${ }^{130}$ L. M. Grubbs, M. Saifullah, N. E. De La Rosa, S. Ye, S. S. Achi, W. E. Acree, Jr., and M. H. Abraham, Fluid Phase Equilib. 298, 48 (2010).

Prepared by:

W. E. Acree, Jr.

Experimental Values

\begin{tabular}{lc}
\hline \hline$x_{2}^{\mathrm{a}}$ & $x_{1}{ }^{\mathrm{b}}$ \\
\hline 0.7743 & 0.2257
\end{tabular}

${ }^{\mathrm{a}} x_{2}$ : mole fraction of component 2 in the saturated solution.

${ }^{\mathrm{b}} x_{1}$ : mole fraction solubility of the solute.

\section{Auxiliary Information}

\section{Method/Apparatus/Procedure:}

Constant-temperature bath, calorimetric thermometer, and an ultraviolet/ visible spectrophotometer.

Excess solute and solvent were placed in amber glass bottles and allowed to equilibrate for several days at constant temperature. Attainment of equilibrium was verified by several repetitive measurements and by approaching equilibrium from supersaturation. Aliquots of saturated solutions were transferred through a coarse filter into tared volumetric flasks, weighed, and diluted with methanol. Concentrations were determined by spectrophotometric measurements at $279 \mathrm{~nm}$.

Source and Purity of Chemicals:

(1) $99 \%$, Aldrich Chemical Company, Milwaukee, WI, USA, was used as received.

(2) $99 \%$, Aldrich Chemical Company, stored over molecular sieves and distilled shortly before use.

\section{Estimated Error:}

Temperature: $\pm 0.1 \mathrm{~K}$

$x_{1}: \pm 1.5 \%$ (relative error).

\begin{abstract}
Method/Apparatus/Procedure:
Constant-temperature bath, calorimetric thermometer, and an ultraviolet/ visible spectrophotometer.

Excess solute and solvent were placed in amber glass bottles and allowed to equilibrate for several days at constant temperature. Attainment of equilibrium was verified by several repetitive measurements and by approaching equilibrium from supersaturation. Aliquots of saturated solutions were transferred through a coarse filter into tared volumetric flasks, weighed, and diluted with methanol. Concentrations were determined by spectrophotometric measurements at $279 \mathrm{~nm}$.
\end{abstract}

Source and Purity of Chemicals:

(1) $99 \%$, Aldrich Chemical Company, Milwaukee, WI, USA, was used as received.

(2) $99+\%$, anhydrous, Aldrich Chemical Company, stored over molecular sieves and distilled shortly before use.

Estimated Error:

Temperature: $\pm 0.1 \mathrm{~K}$.

$x_{1}: \pm 1.5 \%$ (relative error).

\subsection{3-Methylbenzoic acid solubility data in binary organic solvent mixtures}

\begin{tabular}{|c|c|}
\hline $\begin{array}{l}\text { Components: } \\
\text { (1) 3-Methylbenzoic acid; } \mathrm{C}_{8} \mathrm{H}_{8} \mathrm{O}_{2} \text {; } \\
\text { [99-04-7] } \\
\text { (2) Cyclohexane; } \mathrm{C}_{6} \mathrm{H}_{12} ;[110-82-7] \\
\text { (3) Hexane; } \mathrm{C}_{6} \mathrm{H}_{14} ;[110-54-3]\end{array}$ & $\begin{array}{l}\text { Original Measurements: } \\
{ }^{49} \text { W. E. Acree, Jr. and G. L. } \\
\text { Bertrand, J. Pharm. Sci. 70, } 1033 \\
\text { (1981). }\end{array}$ \\
\hline $\begin{array}{l}\text { Variables: } \\
T / \mathrm{K}=298.15 \text {; Solvent Composition }\end{array}$ & $\begin{array}{l}\text { Prepared by: } \\
\text { W. E. Acree, Jr. }\end{array}$ \\
\hline
\end{tabular}


Experimental Values

\begin{tabular}{lcc}
\hline \hline$x_{2}{ }^{(\mathrm{s}) \mathrm{a}}$ & $x_{2}{ }^{\mathrm{b}}$ & $x_{1}{ }^{\mathrm{c}}$ \\
\hline 0.0000 & 0.0000 & 0.0117 \\
0.2380 & 0.2350 & 0.0127 \\
0.4028 & 0.3974 & 0.0133 \\
0.6121 & 0.6037 & 0.0137 \\
0.8239 & 0.8127 & 0.0136 \\
1.0000 & 0.9873 & 0.0127 \\
${ }^{\mathrm{a}}{ }_{x_{2}}{ }^{(\mathrm{s})}:$ initial mole fraction of component 2 in the binary solvent mixture. \\
${ }^{\mathrm{b}}{ }_{x_{2}: \text { mole fraction of component 2 in the saturated solution. }}$ \\
${ }^{{ }^{c}} x_{1}:$ mole fraction solubility of the solute.
\end{tabular}

\section{Auxiliary Information}

\section{Method/Apparatus/Procedure:}

Constant-temperature water bath and an analytical balance.

Excess solute and solvent and were placed in brown glass containers and allowed to equilibrate in a constant-temperature water bath for several days. Attainment of equilibrium was verified by repetitive measurements after several additional days. Solubility was determined by transferring a weighed aliquot through a coarse filter into a flask containing blank nonaqueous titration solvent. The solutions were titrated with freshly standardized sodium methoxide to the thymol blue endpoint.

Source and Purity of Chemicals:

(1) $99 \%$, Chemical source not given, was recrystallized twice from aqueous ethanol and dried at $353 \mathrm{~K}$ before use.

(2) $99+\%$, Chemical source not given, was dried over molecular sieves and distilled before use.

(3) $99 \%$, Chemical source not given, was dried over molecular sieves and distilled before use.

Estimated Error:

Temperature: $\pm 0.01 \mathrm{~K}$.

$x_{2}{ }^{(\mathrm{s})}: \pm 0.0001$

$x_{1}: \pm 1 \%$ (relative error)

\section{Solubility of 4-Methylbenzoic Acid in Organic Solvents}

\subsection{Critical evaluation of experimental solubility data}

There have been several published stu$\operatorname{dies}^{54,60,63,72,77,82,84,85,177-179}$ investigating the solubility behavior of 4-methylbenzoic acid in organic solvents of varying polarity and hydrogen-bonding capability. Hancock et al. ${ }^{54,63}$ measured the solubility of 4-methylbenzoic acid in cyclohexane, benzene, tetrahydrofuran, and 1,4-dioxane at $303 \mathrm{~K}$. Thuaire ${ }^{72}$ determined the solubility of 4-methylbenzoic acid in ethanol as part of study involving solubilities of benzoic acids in binary aqueous-ethanol solvent mixtures. It is not possible to perform a critical evaluation of the experimental data as both research groups performed measurements at only a single temperature, and there are no independent experimental solubility data for 4-methylbenzoic acid in these five solvents.

There have been several experimental studies ${ }^{60,77,84,177-179}$ examining the solubility of 4-methylbenzoic acid in different organic solvents as a function of temperature. Luo et al. ${ }^{179}$ determined the solubility of 4-methylbenzoic acid in ethanoic acid and in binary aqueous-ethanoic acid mixtures at several temperatures between 303 and $363 \mathrm{~K}$ as part of an experimental study aimed at calculating UNIFAC model group parameters for the $\mathrm{ArCOOH}$ group (carboxylic acid group connected to an aromatic ring) interacting with an aliphatic $\mathrm{COOH}$ group, with aliphatic $\mathrm{CH}_{3}$ and $\mathrm{CH}_{2}$ groups, with an aromatic $\mathrm{CH}_{3}$ group and with an aromatic ArH group. The authors used the Modified Apelblat equation [Eq. (8); $A=$ $-11.717, B=-1729.0$, and $C=3.2722]$ to describe the variation in the mole fraction solubility with temperature to within a mean relative deviation of $2.75 \%$. Zhao et al. ${ }^{60}$ measured the solubility of 4-methylbenzoic acid in isobutyl ethanoate at 22 temperatures between 300 and $348 \mathrm{~K}$. The experimental data were correlated with the Wilson and UNIQUAC models. Interaction coefficients calculated from the experimental solid-liquid equilibrium data provided a reasonably accurate mathematical description of the measured values. The mean absolute relative deviation between calculated and observed values was $4.76 \%$ (Wilson model) and 5.74\% (UNIQUAC model).

Qingzhu et al. ${ }^{77}$ measured the solubility of 4-methylbenzoic acid in 1-octanol using a dynamic method with laser monitoring to observe when dissolution was complete. The calculated curve-fit parameters from the Buchowski $\lambda \mathrm{h}$-model [see Eq. (9)] of $\lambda=0.7326$ and $h=2940.14$ described the observed solubility data to within a mean relative deviation of $0.7 \%$.

Li et al. ${ }^{84}$ determined the solubility of 4-methylbenzoic acid in $N$-methyl-2-pyrrolidone from 296 to $370 \mathrm{~K}$ using a synthetic method with laser monitoring to determine when the last amount of solid solute dissolved. The authors employed a polynomial expression in temperature

$$
\begin{aligned}
x_{1}= & -32.6862+0.30878 T-9.78125 \times 10^{-4} T \\
& +10.4307 \times 10^{-7} T^{2}
\end{aligned}
$$

to represent the measured mole fraction solubility data. The root-mean-square deviation between the observed $x_{1}$ data and calculated values from Eq. (65) was on the order of 0.0052 mole fraction.

The experimental solubility data for 4-methylbenzoic acid in different organic solvents are in Secs. 61.2-61.7.

\subsection{4-Methylbenzoic acid solubility data in

\begin{tabular}{|c|c|}
\hline $\begin{array}{l}\text { Components: } \\
\text { (1) 4-Methylbenzoic acid; } \mathrm{C}_{8} \mathrm{H}_{8} \mathrm{O}_{2} \text {; } \\
{[100-09-4]} \\
\text { (2) Cyclohexane; } \mathrm{C}_{6} \mathrm{H}_{12} ;[110-82-7]\end{array}$ & $\begin{array}{l}\text { Original Measurements: } \\
{ }^{54} \text { C. K. Hancock, J. N. Pawloski, } \\
\text { and J. P. Idoux, J. Org. Chem. 31, } \\
3801 \text { (1966). }\end{array}$ \\
\hline $\begin{array}{l}\text { Variables: } \\
T / \mathrm{K}=303.15\end{array}$ & $\begin{array}{l}\text { Prepared by: } \\
\text { W. E. Acree, Jr. }\end{array}$ \\
\hline
\end{tabular} saturated hydrocarbons (including cycloalkanes)}


Experimental Values

\begin{tabular}{lc}
\hline \hline$x_{2}{ }^{\mathrm{a}}$ & $x_{1}{ }^{\mathrm{b}}$ \\
\hline 0.9975 & 0.00249 \\
${ }^{\mathrm{a}} x_{2}:$ mole fraction of component 2 in the saturated solution. \\
${ }^{\mathrm{b}} x_{1}:$ mole fraction solubility of the solute.
\end{tabular}

\section{Auxiliary Information}

Method/Apparatus/Procedure:

Constant-temperature bath, Soxhlet thimble, conical flask, and analytical balance.

Excess solute and solvent were placed in a conical flask and allowed to equilibrate for several days at constant temperature. Attainment of equilibrium was verified by several repetitive measurements and by approaching equilibrium from supersaturation. Aliquots of saturated solutions were transferred using a Soxhlet thimble equipped with a one-hole cork stopper and an inverted U-shaped delivery tube to a second ground-glass stoppered flask suspended in the $303 \mathrm{~K}$ water bath. Successive portions of the contents were evaporated at room temperature in a tared aluminum foil weighing dish under a bell jar through which a slow stream of dry air was passed. The second flask plus unused saturated solution and the aluminum foil dish plus residue were weighed. The saturation solubility of the solute was calculated from the recorded mass data and molar masses of the solute and solvent.

\section{Source and Purity of Chemicals:}

(1) Purity not given, Chemical source not specified, was recrystallized severa times from aqueous-ethanol mixture, and then dried under vacuum over phosphorous pentoxide.

(2) Reagent grade, Chemical source not specified, refluxed over phosphorous pentoxide for $24 \mathrm{~h}$, and then distilled through a $3 \times 80 \mathrm{~cm}$ column filled with 0.32 -cm glass helices.

Estimated Error:

Temperature: $\pm 0.02 \mathrm{~K}$.

$x_{1}: \pm 3 \%$ (relative error).

\subsection{4-Methylbenzoic acid solubility data in aromatic hydrocarbons}

\begin{tabular}{ll}
\hline \hline Components: & Original Measurements: \\
(1) 4-Methylbenzoic acid; $\mathrm{C}_{8} \mathrm{H}_{8} \mathrm{O}_{2} ;$ & ${ }^{54} \mathrm{C}$. K. Hancock, J. N. Pawloski, \\
[100-09-4] & and J. P. Idoux, J. Org. Chem. 31, \\
(2) Benzene; $\mathrm{C}_{6} \mathrm{H}_{6} ;[71-43-2]$ & 3801 (1966).
\end{tabular}

(2) Benzene; $\mathrm{C}_{6} \mathrm{H}_{6} ;[71-43-2]$

Prepared by:

$T / \mathrm{K}=303.15$

W. E. Acree, Jr.

Experimental Values

\begin{tabular}{lc}
\hline \hline$x_{2}{ }^{\mathrm{a}}$ & $x_{1}{ }^{\mathrm{b}}$ \\
\hline 0.9908 & 0.00920
\end{tabular}

${ }^{a} x_{2}$ : mole fraction of component 2 in the saturated solution.

${ }^{\mathrm{b}} \mathrm{x}_{1}$ : mole fraction solubility of the solute.

\section{Auxiliary Information}

\section{Method/Apparatus/Procedure:}

Constant-temperature bath, Soxhlet thimble, conical flask, and analytical balance.

Excess solute and solvent were placed in a conical flask and allowed to equilibrate for several days at constant temperature. Attainment of equilibrium was verified by several repetitive measurements and by approaching equilibrium from supersaturation. Aliquots of saturated solutions were transferred using a Soxhlet thimble equipped with a one-hole cork stopper and an inverted U-shaped delivery tube to a second ground-glass stoppered flask suspended in the $303 \mathrm{~K}$ water bath. Successive portions of the contents were evaporated at room temperature in a tared aluminum foil weighing dish under a bell jar through which a slow stream of dry air was passed. The second flask plus unused saturated solution and the aluminum foil dish plus residue were weighed. The saturation solubility of the solute was calculated from the recorded mass data and molar masses of the solute and solvent.

\section{Source and Purity of Chemicals:}

(1) Purity not given, Chemical source not specified, was recrystallized several times from aqueous-ethanol mixture, and then dried under vacuum over phosphorous pentoxide.

(2) Reagent grade, Chemical source not specified, refluxed over phosphorous pentoxide for $24 \mathrm{~h}$, and then distilled through a $3 \times 80 \mathrm{~cm}$ column filled with 0.32 -cm glass helices.

\section{Estimated Error:}

Temperature: $\pm 0.02 \mathrm{~K}$.

$x_{1}: \pm 3 \%$ (relative error)

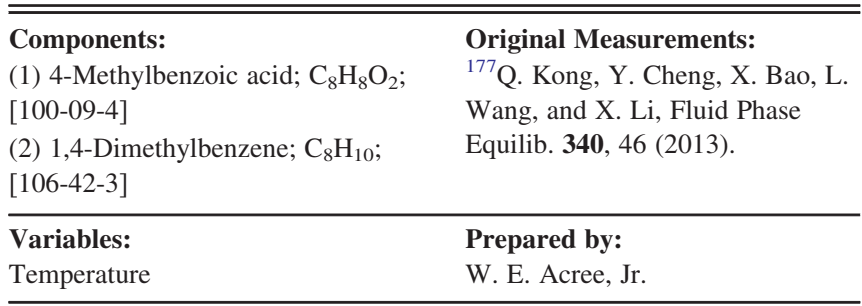

Experimental Values

\begin{tabular}{lcc}
\hline \hline$T / \mathrm{K}$ & $x_{2}{ }^{\mathrm{a}}$ & $x_{1}{ }^{\mathrm{b}}$ \\
\hline 303.2 & 0.9867 & 0.01332 \\
308.2 & 0.9847 & 0.01525 \\
313.2 & 0.9822 & 0.01776 \\
318.2 & 0.9766 & 0.02338 \\
323.2 & 0.9716 & 0.02840 \\
328.2 & 0.9646 & 0.03544 \\
333.2 & 0.9578 & 0.04217 \\
338.2 & 0.9467 & 0.05326 \\
343.2 & 0.9308 & 0.06917 \\
348.2 & 0.9217 & 0.07825 \\
353.2 & 0.9144 & 0.08555
\end{tabular}

${ }^{\mathrm{a}} x_{2}$ : mole fraction of component 2 in the saturated solution.

${ }^{\mathrm{b}} x_{1}$ : mole fraction solubility of the solute. 


\section{Auxiliary Information}

\section{Method/Apparatus/Procedure:}

Thermostatic circulating water bath, analytical balance, jacketed equilibrium glass vessel, and a high-performance liquid chromatographic system equipped with uv absorbance detector.

Solubility was measured by a static analytical method. Excess solute and solvent were placed in a jacketed equilibrium glass vessel and allowed to equilibrate at constant temperature for at least $36 \mathrm{~h}$. Samples of the clear saturated solution was removed by syringe and transferred to a tared test tube containing $3 \mathrm{ml}$ of methanol. The test tube with sample was then weighed. The concentration of the 4-methylbenzoic acid in the saturated solution was determined in triplicate by chromatographic analysis (hplc).

Source and Purity of Chemicals:

(1) $99+\%$, Shanghai Fe Xiang Chemical Company, Shanghai, China, used as received.

(2) 99\%, Sinopharm Chemical Reagent Co., Ltd., China, used as received.

Estimated Error:

Temperature: $\pm 0.1 \mathrm{~K}$.

$x_{1}: \pm 3 \%$ (relative error).

\subsection{4-Methylbenzoic acid solubility data in esters}

\begin{tabular}{|c|c|}
\hline $\begin{array}{l}\text { Components: } \\
\text { (1) 4-Methylbenzoic acid; } \mathrm{C}_{8} \mathrm{H}_{8} \mathrm{O}_{2} \text {; } \\
\text { [100-09-4] } \\
\text { (2) Isobutyl ethanoate; } \mathrm{C}_{6} \mathrm{H}_{12} \mathrm{O}_{2} \text {; } \\
\text { [110-19-0] }\end{array}$ & $\begin{array}{l}\text { Original Measurements: } \\
{ }^{60} \text { S. Zhao, X. Chen, Q. Dai, and L. } \\
\text { Wang, J. Chem. Eng. Data 56, } 2399 \\
(2011) \text {. }\end{array}$ \\
\hline Variables: & \\
\hline Temperature & W. E. Acree, Jr. \\
\hline
\end{tabular}

Experimental Values

\begin{tabular}{lcc}
\hline \hline$T / \mathrm{K}$ & $x_{2}{ }^{\mathrm{a}}$ & $x_{1}{ }^{\mathrm{b}}$ \\
\hline 300.19 & 0.9672 & 0.0328 \\
303.04 & 0.9662 & 0.0338 \\
306.43 & 0.9644 & 0.0356 \\
310.28 & 0.9579 & 0.0421 \\
312.70 & 0.9566 & 0.0434 \\
315.36 & 0.9525 & 0.0475 \\
319.97 & 0.9475 & 0.0525 \\
323.16 & 0.9427 & 0.0573 \\
324.84 & 0.9388 & 0.0612 \\
325.76 & 0.9347 & 0.0653 \\
329.67 & 0.9308 & 0.0692 \\
332.48 & 0.9265 & 0.0735 \\
333.91 & 0.9226 & 0.0774 \\
335.96 & 0.9187 & 0.0813 \\
337.49 & 0.9152 & 0.0848 \\
339.46 & 0.9115 & 0.0885 \\
341.07 & 0.9082 & 0.0918 \\
342.68 & 0.9046 & 0.0954 \\
344.69 & 0.9006 & 0.0994 \\
345.82 & 0.8969 & 0.1031 \\
347.26 & 0.8933 & 0.1067 \\
348.41 & 0.8900 & 0.1100 \\
& &
\end{tabular}

${ }^{\mathrm{a}} x_{2}$ : mole fraction of component 2 in the saturated solution.

${ }^{b} x_{1}$ : mole fraction solubility of the solute.

\section{Auxiliary Information}

Method/Apparatus/Procedure:

Equilibrium jacketed glass vessel, thermoelectric circulating water temperature controller, electromagnetic stirrer, analytical balance, and laser monitoring system.

Experimental solubilities were determined by a dynamic method. Pre-weighed amounts of solute and solvent and were placed in an equilibrium vessel, which was connected to a circulating water bath. The solution was stirred and the temperature gradually increased at a rate of $1-2 \mathrm{~K} / \mathrm{h}(0.5-1.0 \mathrm{~K} / \mathrm{h}$ or slower near saturation temperature) until all of the solid solute dissolved. The temperature at which all of the solute dissolved was determined using laser monitoring.

Source and Purity of Chemicals:

(1) Analytical Reagent grade, Sinopharm Chemical Reagent Co. Ltd., China, was used as received.

(2) Analytical Reagent grade, Sinopharm Chemical Reagent Co. Ltd., China, was used as received.

Estimated Error:

Temperature: $\pm 0.05 \mathrm{~K}$

$x_{1}: \pm 0.0006$.

\subsection{4-Methylbenzoic acid solubility data in ethers}

\begin{tabular}{|c|c|}
\hline $\begin{array}{l}\text { Components: } \\
\text { (1) 4-Methylbenzoic acid; } \mathrm{C}_{8} \mathrm{H}_{8} \mathrm{O}_{2} \text {; } \\
\text { [100-09-4] } \\
\text { (2) Tetrahydrofuran; } \mathrm{C}_{4} \mathrm{H}_{8} \mathrm{O} \text {; } \\
\text { [109-99-9] }\end{array}$ & $\begin{array}{l}\text { Original Measurements: } \\
{ }^{63} \text { C. K. Hancock, J. N. Pawloski, } \\
\text { and J. P. Idoux, J. Org. Chem. 32, } \\
1931 \text { (1967). }\end{array}$ \\
\hline $\begin{array}{l}\text { Variables: } \\
T / \mathrm{K}=303.15\end{array}$ & $\begin{array}{l}\text { Prepared by: } \\
\text { W. E. Acree, Jr. }\end{array}$ \\
\hline
\end{tabular}

Experimental Values

\begin{tabular}{lc}
\hline \hline$x_{2}{ }^{\mathrm{a}}$ & $x_{1}{ }^{\mathrm{b}}$ \\
\hline 0.834 & 0.166 \\
\hline${ }^{{ }^{a}} x_{2}:$ mole fraction of component 2 in the saturated solution. & \\
${ }^{\mathrm{b}} x_{1}:$ mole fraction solubility of the solute. &
\end{tabular}

\section{Auxiliary Information}

\footnotetext{
Method/Apparatus/Procedure:

Constant-temperature bath, Soxhlet thimble, conical flask, and analytical balance.

Excess solute and solvent were placed in a conical flask and allowed to equilibrate for several days at constant temperature. Attainment of equilibrium was verified by several repetitive measurements and by approaching equilibrium from supersaturation. Aliquots of saturated solutions were transferred using a Soxhlet thimble equipped with a one-hole cork stopper and an inverted U-shaped delivery tube to a second ground-glass stoppered flask suspended in the $303 \mathrm{~K}$ water bath. Successive portions of the contents were evaporated at room temperature in a tared aluminum foil weighing dish under a bell jar through which a slow stream of dry air was passed. The second flask plus unused saturated solution and the aluminum foil dish plus residue were weighed. The saturation solubility of the solute was calculated from the recorded mass data and molar masses of the solute and solvent.
} 
Source and Purity of Chemicals:

(1) Purity not given, Chemical source not specified, was recrystallized several times from aqueous-ethanol mixture, and then dried under vacuum over phosphorous pentoxide.

(2) Practical grade, Chemical source not specified, stored over sodium hydroxide pellets for $24 \mathrm{~h}$, and then passed through $2 \times 70-\mathrm{cm}$ chromatographic adsorption columns containing activated alumina. After this treatment, the purified solvent was stored over copper in a nitrogen atmosphere.

Estimated Error:

Temperature: $\pm 0.02 \mathrm{~K}$.

$x_{1}: \pm 1.0 \%$ (relative error).

\begin{tabular}{|c|c|}
\hline $\begin{array}{l}\text { Components: } \\
\text { (1) 4-Methylbenzoic acid; } \mathrm{C}_{8} \mathrm{H}_{8} \mathrm{O}_{2} \text {; } \\
\text { [100-09-4] } \\
\text { (2) 1,4-Dioxane; } \mathrm{C}_{4} \mathrm{H}_{8} \mathrm{O}_{2} ;[123-91-1]\end{array}$ & $\begin{array}{l}\text { Original Measurements: } \\
{ }^{63} \text { C. K. Hancock, J. N. Pawloski, } \\
\text { and J. P. Idoux, J. Org. Chem. 32, } \\
1931 \text { (1967). }\end{array}$ \\
\hline $\begin{array}{l}\text { Variables: } \\
T / \mathrm{K}=303.15\end{array}$ & $\begin{array}{l}\text { Prepared by: } \\
\text { W. E. Acree, Jr. }\end{array}$ \\
\hline
\end{tabular}

\section{Experimental Values}

\begin{tabular}{lc}
\hline \hline$x_{2}^{\mathrm{a}}$ & $x_{1}^{\mathrm{b}}$ \\
\hline 0.904 & 0.096 \\
\hline${ }^{\mathrm{a}} x_{2}:$ mole fraction of component 2 in the saturated solution. & \\
${ }^{\mathrm{b}}{ }_{x_{1}: \text { mole fraction solubility of the solute. }}$ &
\end{tabular}

\section{Auxiliary Information}

\section{Method/Apparatus/Procedure:}

Constant-temperature bath, Soxhlet thimble, conical flask, and analytical balance.

Excess solute and solvent were placed in a conical flask and allowed to equilibrate for several days at constant temperature. Attainment of equilibrium was verified by several repetitive measurements and by approaching equilibrium from supersaturation. Weighed aliquots of saturated solutions were removed and titrated with a standardized sodium hydroxide solution (carbonate free) using a $\mathrm{pH}$ meter. The endpoint of the titration was determined by computing the second derivative in the $\mathrm{pH}$ versus volume of sodium hydroxide added.

\section{Source and Purity of Chemicals:}

(1) Purity not given, Chemical source not specified, was recrystallized several times from aqueous-ethanol mixture, and then dried under vacuum over phosphorous pentoxide.

(2) Practical grade, Chemical source not specified, stored over sodium hydroxide pellets for $24 \mathrm{~h}$, and then passed through $2 \times 70-\mathrm{cm}$ chromatographic adsorption columns containing activated alumina. After this treatment, the purified solvent was stored over copper in a nitrogen atmosphere.

Estimated Error:

Temperature: $\pm 0.02 \mathrm{~K}$.

$x_{1}: \pm 1.0 \%$ (relative error).

\subsection{4-Methylbenzoic acid solubility data in alcohols}

\begin{tabular}{|c|c|}
\hline $\begin{array}{l}\text { Components: } \\
\text { (1) 4-Methylbenzoic acid; } \mathrm{C}_{8} \mathrm{H}_{8} \mathrm{O}_{2} \text {; } \\
\text { [100-09-4] } \\
\text { (2) Ethanol; } \mathrm{C}_{2} \mathrm{H}_{6} \mathrm{O} ;[64-17-5]\end{array}$ & $\begin{array}{l}\text { Original Measurements: } \\
{ }^{72} \text { R. Thuaire, Bull. Soc. Chim. Fr } \\
3815 \text { (1971). }\end{array}$ \\
\hline $\begin{array}{l}\text { Variables: } \\
T / \mathrm{K}=298.15\end{array}$ & $\begin{array}{l}\text { Prepared by: } \\
\text { W. E. Acree, Jr. }\end{array}$ \\
\hline
\end{tabular}

\section{Experimental Values}

The measured solubility was reported to be $1.202 \mathrm{~mol} / \mathrm{kg}$ of solvent, which corresponds to a mole fraction solubility of $x_{1}=$ 0.05247 .

\section{Auxiliary Information}

\section{Method/Apparatus/Procedure:}

Very little experimental details were provided. Solubility was determined by a gravimetric method. The saturated solution was evaporated to dryness and the remaining solid residue was weighed. The solubility was calculated from the mass of the solid residue and mass of saturated solution analyzed.

\section{Source and Purity of Chemicals:}

(1) Purity not given, Chemical source not given, no purification details were provided.

(2) Purity not given, Chemical source not given, no purification details were provided.

\section{Estimated Error:}

Temperature: $\pm 0.05 \mathrm{~K}$.

$x_{1}: \pm 4 \%$ (relative error, estimated by compiler).

\begin{tabular}{ll}
\hline \hline Components: & Original Measurements: \\
(1) 4-Methylbenzoic acid; $\mathrm{C}_{8} \mathrm{H}_{8} \mathrm{O}_{2} ;$ & ${ }^{77}$ J. Qingzhu, M. Peisheng, Y. \\
[100-09-4] & Shouzhi, W. Qiang, W. Chang, and \\
(2) 1-Octanol; $\mathrm{C}_{8} \mathrm{H}_{18} \mathrm{O} ;$ [111-87-5] & L. Guiju, J. Chem. Eng. Data 53, \\
& 1278 (2008). \\
\hline Variables: & Prepared by: \\
Temperature & W. E. Acree, Jr. \\
\hline
\end{tabular}

Experimental Values

\begin{tabular}{lcc}
\hline \hline$T / \mathrm{K}$ & $x_{2}{ }^{\mathrm{a}}$ & $x_{1}{ }^{\mathrm{b}}$ \\
\hline 297.35 & 0.9405 & 0.0595 \\
298.05 & 0.9392 & 0.0608 \\
298.95 & 0.9382 & 0.0618 \\
301.65 & 0.9337 & 0.0663 \\
307.25 & 0.9233 & 0.0767 \\
308.65 & 0.9211 & 0.0789 \\
309.65 & 0.9184 & 0.0816 \\
312.95 & 0.9129 & 0.0871 \\
314.35 & 0.9104 & 0.0896 \\
315.80 & 0.9075 & 0.0925 \\
317.65 & 0.9038 & 0.0962 \\
319.65 & 0.9003 & 0.0997 \\
320.40 & 0.8990 & 0.1010 \\
321.55 & 0.8971 & 0.1029 \\
322.15 & 0.8960 & 0.1040 \\
\hline${ }^{a} x_{2}:$ mole fraction of component 2 in the saturated solution. \\
${ }^{\mathrm{b}} x_{1}:$ mole fraction solubility of the solute.
\end{tabular}




\section{Auxiliary Information}

\section{Method/Apparatus/Procedure:}

Circulating water bath, analytical balance, and laser monitoring system. Preweighed amounts of solute and solvent and were placed in an equilibrium vessel, which was connected to a circulating water bath. The solution was stirred and the temperature gradually increased at a rate $0.5 \mathrm{~K} / 20 \mathrm{~min}(0.2 \mathrm{~K} / 20$ min or slower near saturation temperature) until all of the solid solute dissolved. The temperature at which all of the solute dissolved was determined using laser monitoring.

Source and Purity of Chemicals:

(1) $<99 \%$, Chemical source not specified, used as received.

(2) $<99 \%$, Chemical source not specified, used as received.

\section{Estimated Error:}

Temperature: $\pm 0.05 \mathrm{~K}$.

$x_{1}: \pm 0.0005$.

\subsection{4-Methylbenzoic acid solubility data in miscellaneous organic solvents}

\begin{tabular}{|c|c|}
\hline $\begin{array}{l}\text { Components: } \\
\text { (1) 4-Methylbenzoic acid; } \mathrm{C}_{8} \mathrm{H}_{8} \mathrm{O}_{2} \text {; } \\
\text { [100-09-4] } \\
\text { (2) Ethanoic acid; } \mathrm{C}_{2} \mathrm{H}_{4} \mathrm{O}_{2} ;[64-19-7]\end{array}$ & $\begin{array}{l}\text { Original Measurements: } \\
{ }^{178} \text { M. Chen and P. Ma, J. Chem. } \\
\text { Eng. Data 49, } 756(2004) .\end{array}$ \\
\hline $\begin{array}{l}\text { Variables: } \\
\text { Temperature }\end{array}$ & Prepared by: \\
\hline
\end{tabular}

Experimental Values

\begin{tabular}{lcc}
\hline \hline$T / \mathrm{K}$ & $x_{2}{ }^{\mathrm{a}}$ & $x_{1}{ }^{\mathrm{b}}$ \\
\hline 291.95 & 0.9658 & 0.03421 \\
292.45 & 0.9656 & 0.03440 \\
297.35 & 0.9628 & 0.03724 \\
301.65 & 0.9599 & 0.04006 \\
307.75 & 0.9551 & 0.04488 \\
311.95 & 0.9517 & 0.04829 \\
318.25 & 0.9449 & 0.05505 \\
324.95 & 0.9360 & 0.06397 \\
328.25 & 0.9313 & 0.06868 \\
335.05 & 0.9177 & 0.08225 \\
340.45 & 0.9037 & 0.09625 \\
344.45 & 0.8907 & 0.1093 \\
348.85 & 0.8730 & 0.1270 \\
349.75 & 0.8697 & 0.1303
\end{tabular}

${ }^{\mathrm{a}} x_{2}$ : mole fraction of component 2 in the saturated solution.

${ }^{\mathrm{b}} x_{1}$ : mole fraction solubility of the solute.

\section{Auxiliary Information}

\section{Method/Apparatus/Procedure:}

Titanium solid-liquid equilibrium cell, analytical balance, magnetic stirring system, temperature controlling system, and a laser monitoring system. Solubilities were determined using a dynamic method. Weighed amounts of solute and solvent were sealed in a titanium solid-liquid equilibrium cell, and the temperature slowly increased until the solid phase completely disappeared. Near the solid-liquid equilibrium temperature, the rate of temperature increase was $0.1 \mathrm{~K} / 10 \mathrm{~min}$. The disappearance of the solid solute was detected by a laser monitoring system.
Source and Purity of Chemicals:

(1) Purity not given, Chemical source not given, no purification details were provided.

(2) Purity not given, Chemical source not given, no purification details were provided.

\section{Estimated Error:}

Temperature: $\pm 0.1 \mathrm{~K}$ (estimated by compiler).

$x_{1}: \pm 3 \%$ (relative error, estimated by compiler).

\begin{tabular}{|c|c|}
\hline $\begin{array}{l}\text { Components: } \\
\text { (1) 4-Methylbenzoic acid; } \mathrm{C}_{8} \mathrm{H}_{8} \mathrm{O}_{2} \text {; } \\
\text { [100-09-4] } \\
\text { (2) Ethanoic acid; } \mathrm{C}_{2} \mathrm{H}_{4} \mathrm{O}_{2} ;[64-19-7]\end{array}$ & $\begin{array}{l}\text { Original Measurements: } \\
{ }^{179} \text { W. Luo, Q. Wang, L. Fu, W. } \\
\text { Deng, X. Zhang, and C. Guo, Ind. } \\
\text { Eng. Chem. Res. 50, } 4099 \text { (2011). }\end{array}$ \\
\hline $\begin{array}{l}\text { Variables: } \\
\text { Temperature }\end{array}$ & $\begin{array}{l}\text { Prepared by: } \\
\text { W. E. Acree, Jr. }\end{array}$ \\
\hline
\end{tabular}

Experimental Values

\begin{tabular}{lcc}
\hline \hline$T / \mathrm{K}$ & $x_{2}{ }^{\mathrm{a}}$ & $x_{1}{ }^{\mathrm{b}}$ \\
\hline 303.2 & 0.9616 & 0.03839 \\
313.2 & 0.9529 & 0.04710 \\
323.2 & 0.9383 & 0.06174 \\
333.2 & 0.9182 & 0.08177 \\
343.2 & 0.8935 & 0.1065 \\
353.2 & 0.8649 & 0.1351 \\
363.2 & 0.8333 & 0.1667
\end{tabular}

${ }^{\mathrm{a}} x_{2}$ : mole fraction of component 2 in the saturated solution.

${ }^{\mathrm{b}} x_{1}$ : mole fraction solubility of the solute.

\section{Auxiliary Information}

\section{Method/Apparatus/Procedure:}

Jacketed equilibrium glass bottle, analytical balance and thermostatic watercirculator bath.

Excess solute and solvent were placed in a jacketed equilibrium glass bottle and allowed to equilibrate for at least $24 \mathrm{~h}$ at constant temperature. For the experimental methodology for determining the concentration of the dissolved solute, the authors reference a paper involving the solubility of 1,4benzenedicarboxylic acid [Q. B. Wang, H. B. Xu, and X. Li, J. Chem. Eng. Data 50, 258 (2005)]. In the referenced paper the concentration of the solute was determined by a high-performance liquid chromatographic method of analysis.

Source and Purity of Chemicals:

(1) $99.9 \%$, Shanghai Fine Chemical Reagent Company, China, no purification details were provided.

(2) Analytical Reagent grade, Hangzhou Chemical Reagent Company, China, was used as received.

\section{Estimated Error:}

Temperature: $\pm 0.1 \mathrm{~K}$.

$x_{1}: \pm 3 \%$ (relative error) 


\section{Components:}

(1) 4-Methylbenzoic acid; $\mathrm{C}_{8} \mathrm{H}_{8} \mathrm{O}_{2}$; [100-09-4]

(2) $N, N$-Dimethylformamide; $\mathrm{C}_{3} \mathrm{H}_{7} \mathrm{NO}$; [64-19-7]

\begin{tabular}{ll}
\hline Variables: & Prepared by: \\
Temperature & W. E. Acree, Jr. \\
\hline
\end{tabular}

Experimental Values

\begin{tabular}{lcc}
\hline \hline$T / \mathrm{K}$ & $x_{2}{ }^{\mathrm{a}}$ & $x_{1}{ }^{\mathrm{b}}$ \\
\hline 295.65 & 0.8815 & 0.1185 \\
298.75 & 0.8691 & 0.1309 \\
303.05 & 0.8509 & 0.1491 \\
307.55 & 0.8311 & 0.1689 \\
311.05 & 0.8131 & 0.1869 \\
315.55 & 0.7895 & 0.2105 \\
320.45 & 0.7592 & 0.2408 \\
324.55 & 0.7325 & 0.2675 \\
326.85 & 0.7142 & 0.2858 \\
328.95 & 0.6989 & 0.3011 \\
331.65 & 0.6759 & 0.3241 \\
333.05 & 0.6631 & 0.3369 \\
335.05 & 0.6435 & 0.3565 \\
335.55 & 0.6364 & 0.3636 \\
\hline
\end{tabular}

${ }^{\mathrm{a}} x_{2}$ : mole fraction of component 2 in the saturated solution.

${ }^{\mathrm{b}} x_{1}$ : mole fraction solubility of the solute.

\section{Auxiliary Information}

\section{Method/Apparatus/Procedure:}

Jacketed solid-liquid equilibrium cell, analytical balance, magnetic stirring system, temperature controlling system, and a laser monitoring system. Solubilities were determined using a synthetic method. Weighed amounts of solute and solvent were sealed in a jacketed equilibrium vessel, and the temperature slowly increased until the solid phase completely disappeared. The disappearance of the solid solute was detected by a laser monitoring system. Measurements were repeated two or three times to check the reproducibility.

\section{Source and Purity of Chemicals:}

(1) $98.5 \%$ (starting purity), Chemical source not given, was an industrial product purified by extracting with trichloromethane, and then recrystallized successively from acetic acid and water. Final purity was $99.7 \%$.

(2) Analytical Reagent, Shanghai Chemical Reagent Company, used as received

\section{Estimated Error:}

Temperature: $\pm 0.05 \mathrm{~K}$ (estimated by compiler). $x_{1}: \pm 0.0005$ or less.

\begin{tabular}{|c|c|}
\hline $\begin{array}{l}\text { Components: } \\
\text { (1) 4-Methylbenzoic acid; } \mathrm{C}_{8} \mathrm{H}_{8} \mathrm{O}_{2} \text {; } \\
\text { [100-09-4] } \\
\text { (2) } \mathrm{N} \text {-Methyl-2-pyrrolidone; } \mathrm{C}_{5} \mathrm{H}_{9} \mathrm{NO} \text {; } \\
\text { [872-50-4] }\end{array}$ & $\begin{array}{l}\text { Original Measurements: } \\
{ }^{84} \text { D.-Q. Li, D.-Z. Liu, and F.-A. } \\
\text { Wang, J. Chem. Eng. Data 46, } 172 \\
\text { (2001). }\end{array}$ \\
\hline $\begin{array}{l}\text { Variables: } \\
\text { Temperature }\end{array}$ & $\begin{array}{l}\text { Prepared by: } \\
\text { W. E. Acree, Jr. }\end{array}$ \\
\hline
\end{tabular}

Experimental Values

\begin{tabular}{lcc}
\hline \hline$T / \mathrm{K}$ & $x_{2}{ }^{\mathrm{a}}$ & $x_{1}{ }^{\mathrm{b}}$ \\
\hline 295.65 & 0.9321 & 0.0679 \\
298.65 & 0.9255 & 0.0745 \\
303.35 & 0.9134 & 0.0866 \\
308.55 & 0.8997 & 0.1013 \\
313.75 & 0.8825 & 0.1175 \\
319.55 & 0.8612 & 0.1388 \\
323.45 & 0.8458 & 0.1542 \\
328.75 & 0.8253 & 0.1747 \\
334.85 & 0.7962 & 0.2038 \\
340.25 & 0.7699 & 0.2301 \\
343.35 & 0.7526 & 0.2474 \\
346.65 & 0.7337 & 0.2663 \\
351.45 & 0.7034 & 0.2966 \\
355.75 & 0.6721 & 0.3279 \\
360.65 & 0.6295 & 0.3705 \\
364.05 & 0.5900 & 0.4100 \\
366.55 & 0.5547 & 0.4453 \\
369.25 & 0.5149 & 0.4851 \\
370.05 & 0.4995 & 0.5005 \\
\hline
\end{tabular}

${ }^{\mathrm{a}} x_{2}$ : mole fraction of component 2 in the saturated solution.

${ }^{\mathrm{b}} x_{1}$ : mole fraction solubility of the solute.

\section{Auxiliary Information}

\section{Method/Apparatus/Procedure:}

Jacketed solid-liquid equilibrium cell, analytical balance, magnetic stirring system, temperature controlling system, and a laser monitoring system. Solubilities were determined using a synthetic method. Weighed amounts of solute and solvent were sealed in a jacketed equilibrium vessel, and the temperature slowly increased until the solid phase completely disappeared. The disappearance of the solid solute was detected by a laser monitoring system. Measurements were repeated two or three times to check the reproducibility.

\section{Source and Purity of Chemicals:}

(1) $98.5 \%$ (starting purity), Chemical source not given, was an industrial product purified by extracting with trichloromethane, and then recrystallized successively from acetic acid and water. Final purity was $99.7 \%$.

(2) $99.0 \%$, Analytical Reagent, Shanghai Chemical Reagent Company, used as received.

\section{Estimated Error:}

Temperature: $\pm 0.05 \mathrm{~K}$ (estimated by compiler). $x_{1}: \pm 0.0005$ or less.

\begin{tabular}{|c|c|}
\hline $\begin{array}{l}\text { Components: } \\
\text { (1) 4-Methylbenzoic acid; } \mathrm{C}_{8} \mathrm{H}_{8} \mathrm{O}_{2} \text {; } \\
\text { [100-09-4] } \\
\text { (2) 5-Methyl-2-thiophenecarboxylic acid; } \\
\mathrm{C}_{6} \mathrm{H}_{6} \mathrm{O}_{2} \mathrm{~S} ;[\text { [1918-79-2] }\end{array}$ & $\begin{array}{l}\text { Original Measurements: } \\
{ }^{85} \text { K. Mislow, J. Phys. Coll. } \\
\text { Chem. 52, } 729 \text { (1948). }\end{array}$ \\
\hline $\begin{array}{l}\text { Variables: } \\
\text { Temperature }\end{array}$ & $\begin{array}{l}\text { Prepared by: } \\
\text { W. E. Acree, Jr. }\end{array}$ \\
\hline
\end{tabular}


Experimental Values

\begin{tabular}{lcc}
\hline \hline$t /{ }^{\circ} \mathrm{C}$ (thawing) & $t /{ }^{\circ} \mathrm{C}($ melting) & $w_{1}^{\mathrm{a}}$ \\
\hline 138 & 139 & 0.000 \\
116.6 & 133.2 & 0.094 \\
117.0 & 130.4 & 0.192 \\
116.2 & 127.4 & 0.270 \\
115.6 & 144.4 & 0.450 \\
116.2 & 147.0 & 0.500 \\
116.4 & 151.8 & 0.600 \\
116.2 & 159.0 & 0.703 \\
116.8 & 165.4 & 0.795 \\
154.2 & 173.4 & 0.900 \\
177 & 178 & 1.000 \\
\hline
\end{tabular}

$\overline{{ }^{\mathrm{a}} w_{1}}$ : mass fraction solubility of the solute.

The author reports that the binary system forms a simple eutectic at $w_{1}=0.250$ and $t=116^{\circ} \mathrm{C}$, with an indication of solid solution formation at $w_{1}>0.80$.

\section{Auxiliary Information}

\section{Method/Apparatus/Procedure:}

Experimental method involved observing the thawing-melting behavior of binary mixtures of known composition. Thoroughly mixed samples were placed in capillaries and sealed at one end. The capillaries were placed next to the junction of a thermocouple in a hot stage, which was mounted on a polarizing microscope. Heating rates were kept at $(2 \pm 1)^{\circ} \mathrm{C} / \mathrm{min}$. The thawing temperature was observed by reflected light, while the melting points were observed by transmitted light. Accuracy of the method, as judged by the reproducibility of the measured data, was estimated to be $\pm 2{ }^{\circ} \mathrm{C}$ for thawing and melting temperatures.

\section{Source and Purity of Chemicals:}

(1) Purity not given, Chemical source not given, recrystallized from aqueous solution.

(2) Purity not given, Prepared by the authors, synthesized by heating levulinic acid with phosphorous trisulfide to give 2-methylthiophene. Iodination of 2methylthiophene yielded 5-iodo-2-methylthiophene, which was converted to 5-methyl-2-thiophenecarboxylic acid by the Grignard synthesis.

Estimated Error:

Temperature: $2{ }^{\circ} \mathrm{C}$.

$w_{1}: \pm 0.002$ (estimated by compiler).

\begin{tabular}{|c|c|}
\hline $\begin{array}{l}\text { Components: } \\
\text { (1) 4-Methylbenzoic acid; } \mathrm{C}_{8} \mathrm{H}_{8} \mathrm{O}_{2} \text {; } \\
\text { [100-09-4] } \\
\text { (2) 5-Bromo-2-thiophenecarboxylic acid; } \\
\mathrm{C}_{5} \mathrm{H}_{3} \mathrm{BrO}_{2} \mathrm{~S} \text {; [7311-63-9] }\end{array}$ & $\begin{array}{l}\text { Original Measurements: } \\
{ }^{85} \text { K. Mislow, J. Phys. Coll. } \\
\text { Chem. 52, } 729 \text { (1948). }\end{array}$ \\
\hline $\begin{array}{l}\text { Variables: } \\
\text { Temperature }\end{array}$ & $\begin{array}{l}\text { Prepared by: } \\
\text { W. E. Acree, Jr. }\end{array}$ \\
\hline
\end{tabular}

Experimental Values

\begin{tabular}{lcc}
\hline \hline$t /{ }^{\circ} \mathrm{C}$ (thawing) & $t /{ }^{\circ} \mathrm{C}$ (melting) & $w_{1}{ }^{\mathrm{a}}$ \\
\hline 140 & 141 & 0.000 \\
134.2 & 139.6 & 0.066 \\
134.2 & 138.2 & 0.190 \\
133.6 & 146.2 & 0.285
\end{tabular}

\begin{tabular}{lcc}
\hline \hline$t /{ }^{\circ} \mathrm{C}$ (thawing) & $t /{ }^{\circ} \mathrm{C}$ (melting) & $w_{1}{ }^{\mathrm{a}}$ \\
\hline 133.8 & 150.6 & 0.398 \\
135.2 & 157.0 & 0.491 \\
135.4 & 162.0 & 0.580 \\
134.6 & 168.8 & 0.696 \\
154.2 & 175.8 & 0.869 \\
177 & 178 & 1.000 \\
\hline
\end{tabular}

${ }^{a} w_{1}$ : mass fraction solubility of the solute.

The author reports that the binary system forms a simple eutectic at $w_{1}=0.140$ and $\mathrm{t}=116^{\circ} \mathrm{C}$, with an indication of solid solution formation at $w_{1}>0.75$.

\section{Auxiliary Information}

Method/Apparatus/Procedure:

Experimental method involved observing the thawing-melting behavior of binary mixtures of known composition. Thoroughly mixed samples were placed in capillaries and sealed at one end. The capillaries were placed next to the junction of a thermocouple in a hot stage, which was mounted on a polarizing microscope. Heating rates were kept at $(2 \pm 1)^{\circ} \mathrm{C} / \mathrm{min}$. The thawing temperature was observed by reflected light, while the melting points were observed by transmitted light. Accuracy of the method, as judged by the reproducibility of the measured data, was estimated to be $\pm 2{ }^{\circ} \mathrm{C}$ for thawing and melting temperatures.

Source and Purity of Chemicals:

(1) Purity not given, Chemical source not given, recrystallized from aqueous solution.

(2) Purity not given, Prepared by the authors, synthesized by direct bromination of 2-thiophene-carboxylic acid in glacial acetic acid.

\section{Estimated Error:}

Temperature: $2^{\circ} \mathrm{C}$

$w_{1}: \pm 0.002$ (estimated by compiler).

\section{Solubility of 2-Nitrobenzoic Acid in Organic Solvents}

\subsection{Critical evaluation of experimental solubility data}

There have been several published stu$\operatorname{dies}^{72,77,83,126,127,180,181}$ investigating the solubility behavior of 2-nitrobenzoic acid in organic solvents of varying polarity and hydrogen-bonding capability. Kolthoff and co-work$\mathrm{ers}^{83,181}$ measured the solubility of 2-nitrobenzoic acid in both methanol and ethanenitrile at $298 \mathrm{~K}$. Biswas et al. ${ }^{126}$ and Thuaire $^{72}$ both determined the solubility in ethanol at $298 \mathrm{~K}$. Sidgwick and Ewbank ${ }^{127}$ measured the solubility of 2-nitrobenzoic acid in benzene. Collett and Lazzell ${ }^{180}$ later performed solubility measurements for 2-nitrobenzoic acid in benzene, 1,1'-oxybisethane, trichloromethane, and propanone. The internal consistency of the five datasets was assessed by curve-fitting the measured mole fraction solubility data to Eq. (8). The values of the equation coefficients $(A, B$, and $C)$ are given in Table 45, along with the MRD calculated according to Eq. (24). The largest MRD occurs in the 2-nitrobenzoic acid - benzene system, and corresponds to about a $11.5 \%$ error in back-calculating the observed mole fraction solubilities. 
TABLE 45. Parameters of the Modified Apelblat equation for describing the solubility of 2-nitrobenzoic acid in various organic solvents

\begin{tabular}{lrlrc}
\hline \hline Solvent & \multicolumn{1}{c}{$A$} & \multicolumn{1}{c}{$B$} & \multicolumn{1}{c}{$C$} & MRD (\%) \\
\hline Benzene $^{\mathrm{a}}$ & -149.976 & 112.67 & 24.905 & 11.44 \\
Benzene $^{\mathrm{b}}$ & -172.585 & 112.116 & 28.700 & 9.46 \\
1,1'-Oxybisethane $^{\mathrm{b}}$ & -38.774 & -0.878 & 6.420 & 3.23 \\
Trichloromethane $^{\mathrm{b}}$ & -123.562 & -7.489 & 20.558 & 7.19 \\
Propanone $^{\mathrm{b}}$ & -26.429 & -0.573 & 4.190 & 2.22 \\
\hline
\end{tabular}

${ }^{\mathrm{a}}$ Calculated from the dataset of Sidgwick and Ewbank.

${ }^{\mathrm{b}} \mathrm{Calculated}$ from the dataset of Collett and Lazzell. ${ }^{180}$ For benzene, the first and last data points were removed from the regression analysis in order to obtain a reasonable mathematical representation.

The large deviation may be due in part to the fact that the solution temperature is above the solvent's normal boiling point, to changes in the liquid phase composition due to solvent evaporation into the vapor phase, and the large range covered by the experimental values, $x_{1}=0.00142$ to $x_{1}=0.8501$. It is more difficult to curve-fit experimental solubility data covering large mole fraction ranges.

The experimental solubility data for 2-nitrobenzoic acid in organic solvents are in Secs. 62.2-62.7.

\subsection{2-Nitrobenzoic acid solubility data in aromatic hydrocarbons}

\begin{tabular}{|c|c|}
\hline $\begin{array}{l}\text { Components: } \\
\text { (1) 2-Nitrobenzoic acid; } \mathrm{C}_{7} \mathrm{H}_{5} \mathrm{NO}_{4} \text {; } \\
\text { [552-16-9] }\end{array}$ & $\begin{array}{l}\text { Original Measurements: } \\
{ }^{127} \text { N. V. Sidgwick and E. K. } \\
\text { Ewbank, J. Chem. Soc. Trans. 119 }\end{array}$ \\
\hline (2) Benzene; $\mathrm{C}_{6} \mathrm{H}_{6} ;[71-43-2]$ & $979(1921)$ \\
\hline Variables: & Prepared by: \\
\hline Temperature & W. E. Acree, Jr. \\
\hline
\end{tabular}

Experimental Values

\begin{tabular}{lcl}
\hline \hline$T / \mathrm{K}$ & $x_{2}{ }^{\mathrm{a}}$ & \multicolumn{1}{c}{$x_{1}{ }^{\mathrm{b}}$} \\
\hline 336.2 & 0.990 & 0.00993 \\
351.8 & 0.976 & 0.0242 \\
363.7 & 0.951 & 0.0494 \\
379.0 & 0.844 & 0.156 \\
386.2 & 0.679 & 0.321 \\
401.5 & 0.334 & 0.666 \\
\hline
\end{tabular}

${ }^{\mathrm{a}} x_{2}$ : mole fraction of component 2 in the saturated solution.

${ }^{b} x_{1}$ : mole fraction solubility of the solute. Solubility data reported in units of mass percent. Mole fraction values calculated by the compiler.

\section{Auxiliary Information}

\section{Method/Apparatus/Procedure:}

Preweighed quantities of solute and solvent were placed in bulbs, which were then sealed and heated in a suitable bath. At the higher temperatures, the concentration of solvent in the liquid solution was corrected for the amount of solvent vapor in the bulb. The correction assumed that the vapor pressure of the saturated solution was one half that of the pure solvent at the solution temperature. The temperature at which the solid completely dissolved was recorded, and was taken to be the solid-liquid equilibrium temperature.
Source and Purity of Chemicals:

(1) Purity not given, prepared and purified by the authors using a published synthetic procedure [see J. Walker and J. K. Wood, J. Chem. Soc. 117, 40 (1920) for synthetic details]. Melting point of the purified solute was $420.0 \mathrm{~K}$. (2) Purity not given, Chemical source not given, thiophene was removed by treatment with sulfuric acid. Sample was further purified by freezing several times.

Estimated Error:

Temperature: Not given in paper.

$x_{1}: \pm 10 \%$ (relative error, estimated by compiler).

\begin{tabular}{|c|c|}
\hline $\begin{array}{l}\text { Components: } \\
\text { (1) 2-Nitrobenzoic acid; } \mathrm{C}_{7} \mathrm{H}_{5} \mathrm{NO}_{4} \text {; } \\
\text { [552-16-9] } \\
\text { (2) Benzene; } \mathrm{C}_{6} \mathrm{H}_{6} ;[71-43-2]\end{array}$ & $\begin{array}{l}\text { Original Measurements: } \\
{ }^{180} \text { A. R. Collett and C. L. Lazzell, J. } \\
\text { Phys. Chem. 34, } 1838 \text { (1930). }\end{array}$ \\
\hline $\begin{array}{l}\text { Variables: } \\
\text { Temperature }\end{array}$ & $\begin{array}{l}\text { Prepared by: } \\
\text { W. E. Acree, Jr. }\end{array}$ \\
\hline
\end{tabular}

Experimental Values

\begin{tabular}{lcl}
\hline \hline$T / \mathrm{K}$ & $x_{2}{ }^{\mathrm{a}}$ & \multicolumn{1}{c}{$x_{1}{ }^{\mathrm{b}}$} \\
\hline 297.7 & 0.9986 & 0.00142 \\
365.4 & 0.9501 & 0.0499 \\
372.4 & 0.9130 & 0.0870 \\
381.9 & 0.7906 & 0.2094 \\
387.9 & 0.6608 & 0.3392 \\
392.9 & 0.5415 & 0.4585 \\
396.4 & 0.4539 & 0.5461 \\
401.4 & 0.3574 & 0.6426 \\
412.0 & 0.1499 & 0.8501
\end{tabular}

${ }^{a} x_{2}$ : mole fraction of component 2 in the saturated solution.

${ }^{b} x_{1}$ : mole fraction solubility of the solute. The solubility is given as molal percentage, which, based on the description of 100 times the mole fraction, is taken to be mole percentage.

\section{Auxiliary Information}

Method/Apparatus/Procedure:

Preweighed quantities of solute and solvent were placed in bulbs, which were then sealed and heated in a suitable bath. The temperature at which the solid completely dissolved was recorded, and was taken to be the solid-liquid equilibrium temperature. In a few instances near room temperature, the solubility was determined by agitating the solvent in a thermostat with an excess of solute. After equilibrium had been established, an aliquot of the saturated solution was pipetted, weighed, and the amount of dissolved solute determined with a standard solution of titanium (III) chloride.

\section{Source and Purity of Chemicals:}

(1) Certified Pure, Eastman Kodak Chemical Company, Rochester, NY, USA, was recrystallized twice from aqueous solution. Melting point temperature of purified sample was $420.9 \mathrm{~K}$.

(2) Thiophene-free, Kahlbaum, was dried over sodium and distilled before use.

\section{Estimated Error:}

Temperature: Not given in paper.

$x_{1}: \pm 10 \%$ (relative error, estimated by compiler). 


\subsection{2-Nitrobenzoic acid solubility data in ethers}

\begin{tabular}{|c|c|}
\hline $\begin{array}{l}\text { Components: } \\
\text { (1) 2-Nitrobenzoic acid; } \mathrm{C}_{7} \mathrm{H}_{5} \mathrm{NO}_{4} \text {; } \\
\text { [552-16-9] } \\
\text { (2) } 1,1^{\prime}-\text { Oxybisethane; } \mathrm{C}_{4} \mathrm{H}_{10} \mathrm{O} \text {; } \\
\text { [60-29-7] }\end{array}$ & $\begin{array}{l}\text { Original Measurements: } \\
{ }^{180} \text { A. R. Collett and C. L. Lazzell, J. } \\
\text { Phys. Chem. 34, } 1838 \text { (1930). }\end{array}$ \\
\hline $\begin{array}{l}\text { Variables: } \\
\text { Temperature }\end{array}$ & $\begin{array}{l}\text { Prepared by: } \\
\text { W. E. Acree, Jr. }\end{array}$ \\
\hline
\end{tabular}

Experimental Values

\begin{tabular}{lcc}
\hline \hline$T / \mathrm{K}$ & $x_{2}{ }^{\mathrm{a}}$ & $x_{1}{ }^{\mathrm{b}}$ \\
\hline 305.9 & 0.8775 & 0.1225 \\
329.0 & 0.7719 & 0.2281 \\
377.3 & 0.4919 & 0.5081 \\
381.1 & 0.4648 & 0.5352 \\
396.7 & 0.3183 & 0.6817 \\
408.4 & 0.1710 & 0.8290 \\
\hline
\end{tabular}

${ }^{\mathrm{a}} x_{2}$ : mole fraction of component 2 in the saturated solution.

${ }^{b} x_{1}$ : mole fraction solubility of the solute. The solubility is given as molal percentage, which, based on the description of 100 times the mole fraction, is taken to be mole percentage.

Auxiliary Information

Method/Apparatus/Procedure:

Preweighed quantities of solute and solvent were placed in bulbs, which were then sealed and heated in a suitable bath. The temperature at which the solid completely dissolved was recorded, and was taken to be the solid-liquid equilibrium temperature.

Source and Purity of Chemicals:

(1) Certified Pure, Eastman Kodak Chemical Company, Rochester, NY, USA, was recrystallized twice from aqueous solution. Melting point temperature of purified sample was $420.9 \mathrm{~K}$.

(2) U.S.P. grade, Chemical source not given, was washed three times with distilled water, dried over calcium chloride, and distilled twice before use.

\section{Estimated Error:}

Temperature: Not given in paper.

$x_{1}: \pm 10 \%$ (relative error, estimated by compiler)

\subsection{2-Nitrobenzoic acid solubility data in haloalkanes, haloalkenes, and haloaromatic hydrocarbons}

\begin{tabular}{ll}
\hline \hline Components: & Original Measurements: \\
(1) 2-Nitrobenzoic acid; $\mathrm{C}_{7} \mathrm{H}_{5} \mathrm{NO}_{4} ;$ & ${ }^{180}$ A. R. Collett and C. L. Lazzell, J. \\
[552-16-9] & Phys. Chem. 34, 1838 (1930). \\
$\begin{array}{l}\text { (2) Trichloromethane; } \mathrm{CHCl}_{3} ; \\
\text { 67-66-3] }\end{array}$ & \\
\hline Variables: & Prepared by: \\
Temperature & W. E. Acree, Jr. \\
\hline
\end{tabular}

Experimental Values

\begin{tabular}{lcc}
\hline \hline$T / \mathrm{K}$ & $x_{2}{ }^{\mathrm{a}}$ & $x_{1}{ }^{\mathrm{b}}$ \\
\hline 337.4 & 0.9789 & 0.0211 \\
357.4 & 0.9450 & 0.0550 \\
374.5 & 0.8306 & 0.1694 \\
379.4 & 0.7626 & 0.2374 \\
387.3 & 0.6165 & 0.3835 \\
392.1 & 0.5137 & 0.4863 \\
394.4 & 0.4573 & 0.5427 \\
398.2 & 0.4081 & 0.5919 \\
399.2 & 0.3928 & 0.6072 \\
404.0 & 0.2888 & 0.7112 \\
405.1 & 0.2760 & 0.7240 \\
413.1 & 0.1199 & 0.8801 \\
\hline
\end{tabular}

${ }^{a} x_{2}$ : mole fraction of component 2 in the saturated solution.

${ }^{\mathrm{b}} x_{1}$ : mole fraction solubility of the solute. The solubility is given as molal percentage, which, based on the description of 100 times the mole fraction, is taken to be mole percentage.

\section{Auxiliary Information}

\section{Method/Apparatus/Procedure:}

Preweighed quantities of solute and solvent were placed in bulbs, which were then sealed and heated in a suitable bath. The temperature at which the solid completely dissolved was recorded, and was taken to be the solid-liquid equilibrium temperature.

Source and Purity of Chemicals:

(1) Certified Pure, Eastman Kodak Chemical Company, Rochester, NY, USA, was recrystallized twice from aqueous solution. Melting point temperature of purified sample was $420.9 \mathrm{~K}$.

(2) Certified Pure, Merck Chemical Company, dried over calcium chloride and distilled twice before use.

Estimated Error:

Temperature: Not given in paper.

$x_{1}: \pm 10 \%$ (relative error, estimated by compiler).

\subsection{2-Nitrobenzoic acid solubility data in alcohols}

\begin{tabular}{ll}
\hline \hline Components: & Original Measurements: \\
$\begin{array}{l}\text { (1) 2-Nitrobenzoic acid; } \mathrm{C}_{7} \mathrm{H}_{5} \mathrm{NO}_{4} ; \\
\text { [552-16-9] }\end{array}$ & ${ }^{181}$ I. M. Kolthoff, J. J. Lingane, and \\
(2) Methanol; $\mathrm{CH}_{4} \mathrm{O} ;[67-56-1]$ & 2512 (1938). \\
\hline $\begin{array}{l}\text { Variables: } \\
T / \mathrm{K}=298.15\end{array}$ & Prepared by: \\
\hline
\end{tabular}




\section{Experimental Values}

The measured solubility was reported to be $2.99 \mathrm{~mol} \mathrm{dm}^{-3}$.

\section{Auxiliary Information}

Method/Apparatus/Procedure:

Very little experimental details were provided. Solubility was determined by the conductance method.

\section{Source and Purity of Chemicals:}

(1) Purity not given, Chemical source not given, no purification details were provided.

(2) Purity not given, Chemical source not given, refluxed over silver oxide, distilled and dehydrated with magnesium.

\section{Estimated Error:}

Temperature: $\pm 0.1 \mathrm{~K}$ (estimated by compiler). $c_{1}: \pm 2 \%$ (relative error, estimated by compiler).

\begin{tabular}{ll}
\hline \hline Components: & Original Measurements: \\
$\begin{array}{l}\text { (1) 2-Nitrobenzoic acid; } \mathrm{C}_{7} \mathrm{H}_{5} \mathrm{NO}_{4} ; \\
\text { [552-16-9] }\end{array}$ & $\begin{array}{l}\text { 126. } \mathrm{P} \text {. Biswas, S. C. Lahiri, and B. } \\
\text { (2) Ethanol; } \mathrm{C}_{2} \mathrm{H}_{6} \mathrm{O} ;[64-17-5]\end{array}$ \\
\hline Variables: & 2785 (1993). \\
$T / \mathrm{K}=298.15$ & Prepared by: \\
\hline
\end{tabular}

Experimental Values

\begin{tabular}{|c|c|c|}
\hline$c_{1}{ }^{\mathrm{a}}$ & $x_{2}{ }^{\mathrm{b}}$ & $x_{1}{ }^{\mathrm{c}}$ \\
\hline 1.6261 & 0.8945 & 0.1055 \\
\hline
\end{tabular}

\section{Auxiliary Information}

\section{Method/Apparatus/Procedure:}

Analytical balance and an ultraviolet/visible spectrophotometer.

Solutions containing excess solute and solvent were allowed to equilibrate at constant temperature for at least $24 \mathrm{~h}$. An aliquot of the saturated solution was removed, filtered, and the absorbance recorded. Solubility was calculated from the measured absorbance.

\section{Source and Purity of Chemicals:}

(1) Puris grade, Fluka Chemicals, recrystallized from aqueous ethanol mixture and dried in an air oven at $390 \mathrm{~K}$. The purified compound was stored in vacuum desiccators.

(2) Absolute, B.C.P.W., Calcutta, India, was distilled twice before use.

\section{Estimated Error:}

Temperature: $\pm 0.2 \mathrm{~K}$ (estimated by compiler).

$c_{1}: \pm 1.0 \%$ (relative error, estimated by compiler).

$x_{1}: \pm 2.5 \%$ (relative error, estimated by compiler).

\begin{tabular}{|c|c|}
\hline $\begin{array}{l}\text { Components: } \\
\text { (1) 2-Nitrobenzoic acid; } \mathrm{C}_{7} \mathrm{H}_{5} \mathrm{NO}_{4} \text {; } \\
\text { [552-16-9] } \\
\text { (2) Ethanol; } \mathrm{C}_{2} \mathrm{H}_{6} \mathrm{O} ;[64-17-5]\end{array}$ & $\begin{array}{l}\text { Original Measurements: } \\
{ }^{72} \mathrm{R} \text {. Thuaire, Bull. Soc. Chim. Fr. } \\
3815 \text { (1971). }\end{array}$ \\
\hline $\begin{array}{l}\text { Variables: } \\
T / \mathrm{K}=298.15\end{array}$ & $\begin{array}{l}\text { Prepared by: } \\
\text { W. E. Acree, Jr. }\end{array}$ \\
\hline
\end{tabular}

\section{Experimental Values}

The measured solubility was reported to be $4.516 \mathrm{~mol} / \mathrm{kg}$ of solvent, which corresponds to a mole fraction solubility of $x_{1}=0.1722$.

\section{Auxiliary Information}

\begin{abstract}
Method/Apparatus/Procedure:
Very little experimental details were provided. Solubility was determined by a gravimetric method. The saturated solution was evaporated to dryness and the remaining solid residue was weighed. The solubility was calculated from the mass of the solid residue and mass of saturated solution analyzed.
\end{abstract}

\section{Source and Purity of Chemicals:}

(1) Purity not given, Chemical source not given, no purification details were provided.

(2) Purity not given, Chemical source not given, no purification details were provided.

\section{Estimated Error:}

Temperature: $\pm 0.05 \mathrm{~K}$.

$x_{1}: \pm 4 \%$ (relative error, estimated by compiler).

\begin{tabular}{|c|c|}
\hline $\begin{array}{l}\text { Components: } \\
\text { (1) 2-Nitrobenzoic acid; } \mathrm{C}_{7} \mathrm{H}_{5} \mathrm{NO}_{4} \text {; } \\
\text { [552-16-9] } \\
\text { (2) } 1-\text { Octanol; } \mathrm{C}_{8} \mathrm{H}_{18} \mathrm{O} ;[111-87-5]\end{array}$ & $\begin{array}{l}\text { Original Measurements: } \\
{ }^{77} \text { J. Qingzhu, M. Peisheng, Y. } \\
\text { Shouzhi, W. Qiang, W. Chang, and } \\
\text { L. Guiju, J. Chem. Eng. Data 53, } \\
1278 \text { (2008). }\end{array}$ \\
\hline $\begin{array}{l}\text { Variables: } \\
\text { Temperature }\end{array}$ & $\begin{array}{l}\text { Prepared by: } \\
\text { W. E. Acree, Jr. }\end{array}$ \\
\hline
\end{tabular}

Experimental Values

\begin{tabular}{lcc}
\hline \hline$T / \mathrm{K}$ & $x_{2}{ }^{\mathrm{a}}$ & $x_{1}{ }^{\mathrm{b}}$ \\
\hline 297.15 & 0.9239 & 0.0761 \\
299.65 & 0.9194 & 0.0806 \\
300.15 & 0.9160 & 0.0840 \\
302.05 & 0.9105 & 0.0895 \\
303.15 & 0.9072 & 0.0928 \\
306.15 & 0.8989 & 0.1011 \\
307.15 & 0.8955 & 0.1045 \\
308.35 & 0.8921 & 0.1079 \\
309.25 & 0.8873 & 0.1127 \\
310.15 & 0.8828 & 0.1172 \\
311.75 & 0.8781 & 0.1219 \\
313.15 & 0.8710 & 0.1290 \\
314.85 & 0.8649 & 0.1351 \\
317.15 & 0.8585 & 0.1415 \\
318.65 & 0.8525 & 0.1475 \\
319.85 & 0.8459 & 0.1541 \\
321.15 & 0.8393 & 0.1607
\end{tabular}

${ }^{\mathrm{a}} x_{2}$ : mole fraction of component 2 in the saturated solution.

${ }^{b} x_{1}$ : mole fraction solubility of the solute. 


\section{Auxiliary Information}

Method/Apparatus/Procedure:

Circulating water bath, analytical balance, and laser monitoring system. Preweighed amounts of solute and solvent and were placed in an equilibrium vessel, which was connected to a circulating water bath. The solution was stirred and the temperature gradually increased at a rate $0.5 \mathrm{~K} / 20 \mathrm{~min}(0.2 \mathrm{~K} / 20$ min or slower near saturation temperature) until all of the solid solute dissolved. The temperature at which all of the solute dissolved was determined using laser monitoring.

Source and Purity of Chemicals:

(1) $<99 \%$, Chemical source not specified, used as received.

(2) $<99 \%$, Chemical source not specified, used as received.

Estimated Error:

Temperature: $\pm 0.05 \mathrm{~K}$.

$x_{1}: \pm 0.0005$

\subsection{2-Nitrobenzoic acid solubility data in ketones}

\begin{tabular}{|c|c|}
\hline $\begin{array}{l}\text { Components: } \\
\text { (1) 2-Nitrobenzoic acid; } \mathrm{C}_{7} \mathrm{H}_{5} \mathrm{NO}_{4} \text {; } \\
\text { [552-16-9] } \\
\text { (2) Propanone; } \mathrm{C}_{3} \mathrm{H}_{6} \mathrm{O} ;[67-64-1]\end{array}$ & $\begin{array}{l}\text { Original Measurements: } \\
{ }^{180} \text { A. R. Collett and C. L. Lazzell, J } \\
\text { Phys. Chem. 34, } 1838 \text { (1930). }\end{array}$ \\
\hline $\begin{array}{l}\text { Variables: } \\
\text { Temperature }\end{array}$ & $\begin{array}{l}\text { Prepared by: } \\
\text { W. E. Acree, Jr. }\end{array}$ \\
\hline
\end{tabular}

Experimental Values

\begin{tabular}{lcc}
\hline \hline$T / \mathrm{K}$ & $x_{2}{ }^{\mathrm{a}}$ & $x_{1}{ }^{\mathrm{b}}$ \\
\hline 310.8 & 0.7415 & 0.2585 \\
341.4 & 0.6334 & 0.3666 \\
348.4 & 0.6068 & 0.3932 \\
370.8 & 0.4868 & 0.5132 \\
375.1 & 0.4616 & 0.5384 \\
386.7 & 0.3745 & 0.6255 \\
403.1 & 0.2238 & 0.7762
\end{tabular}

${ }^{a} x_{2}$ : mole fraction of component 2 in the saturated solution.

${ }^{b} x_{1}$ : mole fraction solubility of the solute. The solubility is given as molal percentage, which, based on the description of 100 times the mole fraction, is taken to be mole percentage.

\section{Auxiliary Information}

\footnotetext{
Method/Apparatus/Procedure:

Preweighed quantities of solute and solvent were placed in bulbs, which were then sealed and heated in a suitable bath. The temperature at which the solid completely dissolved was recorded, and was taken to be the solid-liquid equilibrium temperature. In a few instances near room temperature, the solubility was determined by agitating the solvent in a thermostat with an excess of solute. After equilibrium had been established, an aliquot of the saturated solution was pipetted, weighed, and the amount of dissolved solute determined with a standard solution of titanium (III) chloride.
}

Source and Purity of Chemicals:

(1) Certified Pure, Eastman Kodak Chemical Company, Rochester, NY, USA, was recrystallized twice from aqueous solution. Melting point temperature of purified sample was $420.9 \mathrm{~K}$

(2) Certified Pure, Merck Chemical Company, was dried over calcium chloride and distilled twice before use.
Estimated Error:

Temperature: Not given in paper.

$x_{2}: \pm 10 \%$ (relative error, estimated by compiler).

\subsection{2-Nitrobenzoic acid solubility data in miscellaneous organic solvents}

\begin{tabular}{|c|c|}
\hline $\begin{array}{l}\text { Components: } \\
\text { (1) 2-Nitrobenzoic acid; } \mathrm{C}_{7} \mathrm{H}_{5} \mathrm{NO}_{4} \text {; } \\
\text { [552-16-9] } \\
\text { (2) Ethanenitrile; } \mathrm{C}_{2} \mathrm{H}_{3} \mathrm{~N} ;[75-05-8]\end{array}$ & $\begin{array}{l}\text { Original Measurements: } \\
{ }^{83} \text { M. K. Chantooni and I. M. } \\
\text { Kolthoff, J. Phys. Chem. 77, } 527 \\
\text { (1973). }\end{array}$ \\
\hline $\begin{array}{l}\text { Variables: } \\
T / \mathrm{K}=298.15\end{array}$ & $\begin{array}{l}\text { Prepared by: } \\
\text { W. E. Acree, Jr. }\end{array}$ \\
\hline
\end{tabular}

\section{Experimental Values}

The measured solubility was reported to be $0.98 \mathrm{~mol} \mathrm{dm}^{-3}$. The authors did not give the temperature at which the solubility was measured. Based on the experimental description given in an earlier paper [I. M. Kolthoff, J. J. Lingane, and W. Larson, J. Am. Chem. Soc. 60, 2512 (1938)], the compiler believes the temperature to be $298.15 \mathrm{~K}$.

\section{Auxiliary Information}

\section{Method/Apparatus/Procedure:}

Very little experimental details were provided. Solubility was determined by titrating a filtered aliquot of the saturated solution alkalimetrically in an aqueous-ethanol mixture using phenolphthalein as the acid-base indicator.

Source and Purity of Chemicals:

(1) Purity not given, Baker Chemical Company, USA, was recrystallized from either aqueous or aqueous-ethanol solution and dried in vacuo at $333 \mathrm{~K}$.

(2) Purity not given, Chemical source not given, was purified by shaking with saturated potassium hydroxide, followed by activated alumina, and then anhydrous calcium chloride to remove water. Ethanenitrile was further dried over anhydrous magnesium sulfate and then phosphorous pentoxide. The sample was distilled shortly before use.

\section{Estimated Error:}

Temperature: $\pm 0.1 \mathrm{~K}$ (estimated by compiler). $c_{1}: \pm 2 \%$ (relative error, estimated by compiler).

\section{Solubility of 3-Nitrobenzoic Acid in Organic Solvents}

\subsection{Critical evaluation of experimental solubility data}

There have been several published stu$\operatorname{dies}^{10,54,72,83,99,126,127,180,181}$ investigating the solubility behavior of 3-nitrobenzoic acid in organic solvents of varying polarity and hydrogen-bonding capability. Charlton et al. ${ }^{10}$ measured the solubility of 3-nitrobenzoic acid in 17 alcohols (methanol, ethanol, 1-propanol, 2-propanol, 1-butanol, 2butanol, 2-methyl-1-propanol, 2-methyl-2-propanol, 1-pentanol, 2-pentanol, 2-methyl-1-butanol, 3-methyl-1-butanol, 1hexanol, 2-methyl-1-pentanol, 1-heptanol, 1-octanol, and 
1-decanol), in three dialkyl ethers (1,1'-oxybisethane, 2,2'oxybispropane, and 1,1'-oxybisbutane) and two cyclic ethers (tetrahydrofuran and 1,4-dioxane), in three alkyl alkanoates (methyl ethanoate, ethyl ethanoate, and butyl ethanoate), and in propylene carbonate at $298 \mathrm{~K}$. Results of the experimental measurements were used to revise and update the existing values that the authors had for the Abraham solute descriptors of 3-nitrobenzoic acid. The authors were able to assemble a total of $48 \log _{10}\left(S R\right.$ or $P$ ) and $\log _{10}(G S R$ or $K$ ) equations for which experimental partition coefficient data, solubility ratios, Abraham model equation coefficients and aqueous molar solubility were available. The logarithm of the aqueous molar solubility of 3-nitrobenzoic acid is $\log _{10} c_{1, \mathrm{w}}=-1.68 .{ }^{176}$ The McGowan volume of 3-nitrobenzoic acid, $V=1.1059$, was calculated from the number of chemical bonds in the molecule and the individual atomic group volumes, $A V_{i}$, given in Sec. 1.3. The excess molar refraction solute descriptor was estimated as $E=0.990$. This left four solute descriptors $(S, A$, $B$, and $L$ ) still to be determined. The 48 equations were then solved using the Microsoft "SOLVER" program to yield numerical values of the remaining four solute descriptors, $S=1.180$, $A=0.730, B=0.520$, and $L=5.6011$, that best described the $\log _{10}(S R$ or $P)$ and $\log _{10}(G S R$ or $K)$ values. The computation treated $\log _{10} c_{1, \mathrm{G}}$ as a floating parameter to be determined as part of the regression analyses. The data analyses returned a value of $\log _{10} c_{1, \mathrm{G}}=-8.610$ for the gas-phase solute concentration that made the $\log _{10}\left(S R\right.$ or $P$ ) and $\log _{10}(G S R$ or $K)$ predictions internally consistent. The calculated molecular solute descriptors reproduced the $\log _{10}\left(S R\right.$ or $P$ ) and $\log _{10}$ ( $G S R$ or $K$ ) values to within an average standard deviation of 0.072 and $0.094 \log _{10}$ units, respectively.

After the 3-nitrobenzoic acid solubility study was published, Abraham model correlations have been developed for 2-pentanol, 3-methyl-1-butanol, methyl ethanoate, ethyl ethanoate, and butyl ethanoate, and equation coefficients for a few solvents were updated based on additional experimental data. The new correlations (listed in Tables 1 and 2) will be used in illustrating the ability of the Abraham model to correlate the experimental 3-nitrobenzoic acid solubility data. Table 46 compares the experimental $\log _{10} c_{1}$ values to calculated values based on Eqs. (20) and (21) of the Abraham model. For comparison purposes, the measured mole fraction solubilities of 3-nitrobenzoic acid, $x_{1}$, determined by Charlton et al. ${ }^{10}$ were converted into molar solubilities by dividing $x_{1}$ by the ideal molar volume of the saturated solution (i.e., $c_{1}^{\text {sat }}=$ $\left.x_{1} /\left[x_{1} V_{1}+\left(1-x_{1}\right) V_{\text {solvent }}\right]\right)$. The molar volume of the hypothetical subcooled liquid 3-nitrobenzoic acid, $V_{\text {solute }}=117.7 \mathrm{~cm}^{3}$ $\mathrm{mol}^{-1}$, was estimated as the molar volume of benzoic acid $\left(V_{\text {benzoic acid }}=104.4 \mathrm{~cm}^{3} \mathrm{~mol}^{-1}\right)+$ molar volume of nitrobenzene $\left(V_{\text {nitrobenzene }}=102.7 \mathrm{~cm}^{3} \mathrm{~mol}^{-1}\right)-$ molar volume of benzene $\left(V_{\text {benzene }}=89.40 \mathrm{~cm}^{3} \mathrm{~mol}^{-1}\right)$. Any errors resulting from the estimation of 3-nitrobenzoic acid's hypothetical subcooled liquid molar volume, $V_{\text {solute }}$, or the ideal molarvolume approximation should have only a very small effect on the calculated $c_{1}$ values. 3-Nitrobenzoic acid is not very soluble in many of the solvents considered, and the $x_{1} V_{\text {solute }}$ term contributes very little to the molar volume of the saturated solution. Examination of the numerical entries in Table 46
TABLE 46. Comparison between observed and calculated molar solubilities of 3-nitrobenzoic acid based on the Abraham model, Eqs. (20) and (21)

\begin{tabular}{lccc}
\hline \hline Solvent & & $\log _{10} c_{1}{ }^{\text {calc }} ;$ & $\log _{10} c_{1}{ }^{\text {calc }}$ \\
Eq. & $(20)$ & Eq. 21$)$ \\
\hline Methanol & 0.568 & 0.460 & 0.419 \\
Ethanol & 0.408 & 0.421 & 0.401 \\
1-Propanol & 0.262 & 0.275 & 0.281 \\
2-Propanol & 0.306 & 0.288 & 0.309 \\
1-Butanol & 0.184 & 0.144 & 0.157 \\
2-Butanol & 0.286 & 0.192 & 0.183 \\
2-Methyl-1-propanol & 0.071 & 0.093 & 0.066 \\
2-Methyl-2-propanol & 0.339 & 0.244 & 0.307 \\
1-Pentanol & 0.114 & 0.153 & 0.158 \\
2-Pentanol & 0.200 & 0.164 & 0.155 \\
3-Methyl-1-butanol & 0.116 & 0.078 & 0.059 \\
1-Hexanol & 0.047 & 0.088 & 0.081 \\
1-Heptanol & -0.007 & 0.012 & 0.042 \\
1-Octanol & -0.026 & -0.017 & -0.075 \\
1-Decanol & -0.115 & -0.093 & -0.085 \\
1,1'-Oxybisethane & 0.076 & -0.139 & -0.061 \\
Tetrahydrofuran & 0.581 & 0.632 & 0.608 \\
1,4-Dioxane & 0.322 & 0.367 & 0.351 \\
Methyl ethanoate & 0.236 & -0.002 & -0.023 \\
Ethyl ethanoate & 0.135 & 0.015 & 0.017 \\
Butyl ethanoate & -0.084 & -0.155 & -0.184 \\
\hline Experimental data & & & \\
\hline
\end{tabular}

${ }^{\mathrm{a}}$ Experimental data were taken from Charlton et al. ${ }^{10}$

reveals that the Abraham model provides a reasonably accurate mathematical description of the observed solubility data, suggesting that there are no obvious outliers in the dataset.

There have also been a few published papers reporting 3nitrobenzoic acid solubilities in organic solvents as a function of temperature. Sidgwick and Ewbank ${ }^{127}$ measured the solubility of 3-nitrobenzoic acid in benzene. Collett and Lazzel1 ${ }^{180}$ later performed solubility measurements for 3-nitrobenzoic acid in benzene, 1,1'-oxybisethane, trichloromethane, tetrachloromethane and propanone. The internal consistency of the six datasets was assessed by curve-fitting the measured mole fraction solubility data to Eq. (8). The values of the equation coefficients $(A, B$, and $C)$ are given in Table 47 , along with the MRD, calculated according to Eq. (24). The largest MRD occurs in the 3-nitrobenzoic acid - tetrachloromethane system, and corresponds to about a $14 \%$ error in back-calculating the observed mole fraction solubilities. As noted in the table

TABle 47. Parameters of the Modified Apelblat equation for describing the solubility of 3-nitrobenzoic acid in various organic solvents

\begin{tabular}{lrcrc}
\hline \hline Solvent & \multicolumn{1}{c}{$A$} & \multicolumn{1}{c}{$B$} & \multicolumn{1}{c}{$C$} & MRD (\%) \\
\hline Benzene $^{\mathrm{a}}$ & -101.165 & -2.046 & 16.864 & 7.15 \\
Benzene $^{\mathrm{b}}$ & -103.906 & -2.100 & 17.329 & 10.34 \\
1,1'-Oxybisethane $^{\mathrm{b}}$ & -32.134 & -0.681 & 5.310 & 7.25 \\
Trichloromethane $^{\mathrm{b}}$ & -71.525 & -1.467 & 11.907 & 6.19 \\
Tetrachloromethane $^{\mathrm{b}}$ & -249.472 & -4.655 & 41.634 & 13.81 \\
Propanone $^{\mathrm{b}}$ & -28.705 & -0.424 & 4.760 & 2.68 \\
\hline
\end{tabular}

${ }^{\mathrm{a} C a l c u l a t e d ~ f r o m ~ t h e ~ d a t a s e t ~ o f ~ S i d g w i c k ~ a n d ~ E w b a n k . ~}$

${ }^{\mathrm{b}}$ Calculated from the dataset of Collett and Lazzell. ${ }^{180}$ For benzene, the last data point was removed from the regression analysis in order to obtain a reasonable representation. For tetrachloromethane, the first and last data points were removed from the regression analysis in order to obtain a reasonable correlation. 
footnote, the first and last data points had to be removed from the regression analysis in order to obtain a reasonable mean relative deviation. The large deviation may be due in part to the fact that the solution temperature is above the solvent's normal boiling point temperature, to changes in the liquid phase composition due to solvent evaporation into the vapor phase, and the large range covered by the experimental values, $x_{1}=$ 0.00135 to $x_{1}=0.833$. It is more difficult to curve-fit experimental solubility data covering large mole fraction ranges.

Jia et al. ${ }^{99}$ determined the solubility of 3-nitrobenzoic acid in 1-octanol in the temperature range from 293 to $323 \mathrm{~K}$. The calculated curve-fit parameters from the Buchowski $\lambda$ h-model (see Eq. (9)) of $\lambda=1.866$ and $h=1274.18$ described the observed solubility data to within a mean relative deviation of $0.7 \%$.

The experimental solubility data for 3-nitrobenzoic acid in organic solvents are in Secs. 63.2-63.9.

\subsection{3-Nitrobenzoic acid solubility data in saturated hydrocarbons (including cycloalkanes)}

\begin{tabular}{|c|c|}
\hline $\begin{array}{l}\text { Components: } \\
\text { (1) 3-Nitrobenzoic acid; } \mathrm{C}_{7} \mathrm{H}_{5} \mathrm{NO}_{4} \text {; } \\
\text { [121-92-6] } \\
\text { (2) Cyclohexane; } \mathrm{C}_{6} \mathrm{H}_{12} ;[110-82-7]\end{array}$ & $\begin{array}{l}\text { Original Measurements: } \\
{ }^{54} \text { C. K. Hancock, J. N. Pawloski, } \\
\text { and J. P. Idoux, J. Org. Chem. 31, } \\
3801 \text { (1966). }\end{array}$ \\
\hline $\begin{array}{l}\text { Variables: } \\
T / \mathrm{K}=303.15\end{array}$ & $\begin{array}{l}\text { Prepared by: } \\
\text { W. E. Acree, Jr. }\end{array}$ \\
\hline
\end{tabular}

Experimental Values

\begin{tabular}{lc}
\hline \hline$x_{2}{ }^{\mathrm{a}}$ & $x_{1}{ }^{\mathrm{b}}$ \\
\hline 0.9966 & 0.00340 \\
\hline${ }^{\mathrm{a}} x_{2}:$ mole fraction of component 2 in the saturated solution. & \\
${ }^{\mathrm{b}} x_{1}:$ mole fraction solubility of the solute. &
\end{tabular}

\section{Auxiliary Information}

\section{Method/Apparatus/Procedure:}

Constant-temperature bath, Soxhlet thimble, conical flask, and analytical balance.

Excess solute and solvent were placed in a conical flask and allowed to equilibrate for several days at constant temperature. Attainment of equilibrium was verified by several repetitive measurements and by approaching equilibrium from supersaturation. Aliquots of saturated solutions were transferred using a Soxhlet thimble equipped with a one-hole cork stopper and an inverted U-shaped delivery tube to a second ground-glass stoppered flask suspended in the $303 \mathrm{~K}$ water bath. Successive portions of the contents were evaporated at room temperature in a tared aluminum foil weighing dish under a bell jar through which a slow stream of dry air was passed. The second flask plus unused saturated solution and the aluminum foil dish plus residue were weighed. The saturation solubility of the solute was calculated from the recorded mass data and molar masses of the solute and solvent.
Source and Purity of Chemicals:

(1) Purity not given, Chemical source not specified, was recrystallized several times from aqueous-ethanol mixture, and then dried under vacuum over phosphorous pentoxide.

(2) Reagent grade, Chemical source not specified, refluxed over phosphorous pentoxide for $24 \mathrm{~h}$, and then distilled through a $3 \times 80 \mathrm{~cm}$ column filled with 0.32-cm glass helices.

Estimated Error:

Temperature: $\pm 0.02 \mathrm{~K}$.

$x_{1}: \pm 3 \%$ (relative error).

\subsection{3-Nitrobenzoic acid solubility data in aromatic hydrocarbons}

\begin{tabular}{|c|c|}
\hline $\begin{array}{l}\text { Components: } \\
\text { (1) 3-Nitrobenzoic acid; } \mathrm{C}_{7} \mathrm{H}_{5} \mathrm{NO}_{4} \text {; } \\
{[121-92-6]} \\
\text { (2) Benzene; } \mathrm{C}_{6} \mathrm{H}_{6} ;[71-43-2]\end{array}$ & $\begin{array}{l}\text { Original Measurements: } \\
{ }^{54} \text { C. K. Hancock, J. N. Pawloski, } \\
\text { and J. P. Idoux, J. Org. Chem. 31, } \\
3801 \text { (1966). }\end{array}$ \\
\hline $\begin{array}{l}\text { Variables: } \\
T / \mathrm{K}=303.15\end{array}$ & $\begin{array}{l}\text { Prepared by: } \\
\text { W. E. Acree, Jr. }\end{array}$ \\
\hline
\end{tabular}

Experimental Values

\begin{tabular}{lc}
\hline \hline$x_{2}{ }^{\mathrm{a}}$ & $x_{1}{ }^{\mathrm{b}}$ \\
\hline 0.9918 & 0.00824 \\
\hline${ }^{\mathrm{a}} x_{2}:$ mole fraction of component 2 in the saturated solution. & \\
${ }^{\mathrm{b}} x_{1}:$ mole fraction solubility of the solute. &
\end{tabular}

Auxiliary Information

\section{Method/Apparatus/Procedure:}

Constant-temperature bath, Soxhlet thimble, conical flask, and analytical balance.

Excess solute and solvent were placed in a conical flask and allowed to equilibrate for several days at constant temperature. Attainment of equilibrium was verified by several repetitive measurements and by approaching equilibrium from supersaturation. Aliquots of saturated solutions were transferred using a Soxhlet thimble equipped with a one-hole cork stopper and an inverted $\mathrm{U}$-shaped delivery tube to a second ground-glass stoppered flask suspended in the $303 \mathrm{~K}$ water bath. Successive portions of the contents were evaporated at room temperature in a tared aluminum foil weighing dish under a bell jar through which a slow stream of dry air was passed. The second flask plus unused saturated solution and the aluminum foil dish plus residue were weighed. The saturation solubility of the solute was calculated from the recorded mass data and molar masses of the solute and solvent.

Source and Purity of Chemicals:

(1) Purity not given, Chemical source not specified, was recrystallized several times from aqueous-ethanol mixture, and then dried under vacuum over phosphorous pentoxide.

(2) Reagent grade, Chemical source not specified, refluxed over phosphorous pentoxide for $24 \mathrm{~h}$, and then distilled through a $3 \times 80 \mathrm{~cm}$ column filled with 0.32-cm glass helices.

\section{Estimated Error:}

Temperature: $\pm 0.02 \mathrm{~K}$

$x_{1}: \pm 3 \%$ (relative error) 


\begin{tabular}{|c|c|}
\hline $\begin{array}{l}\text { Components: } \\
\text { (1) 3-Nitrobenzoic acid; } \mathrm{C}_{7} \mathrm{H}_{5} \mathrm{NO}_{4} \text {; } \\
\text { [121-92-6] } \\
\text { (2) Benzene; } \mathrm{C}_{6} \mathrm{H}_{6} ;[71-43-2]\end{array}$ & $\begin{array}{l}\text { Original Measurements: } \\
{ }^{127} \text { N. V. Sidgwick and E. K. } \\
\text { Ewbank, J. Chem. Soc. Trans. 119 } \\
979 \text { (1921). }\end{array}$ \\
\hline $\begin{array}{l}\text { Variables: } \\
\text { Temperature }\end{array}$ & $\begin{array}{l}\text { Prepared by: } \\
\text { W. E. Acree, Jr. }\end{array}$ \\
\hline
\end{tabular}

Experimental Values

\begin{tabular}{lcl}
\hline \hline$T / \mathrm{K}$ & $x_{2}{ }^{\mathrm{a}}$ & \multicolumn{1}{c}{$x_{1}{ }^{\mathrm{b}}$} \\
\hline 306.2 & 0.991 & 0.00921 \\
321.2 & 0.976 & 0.0238 \\
338.4 & 0.951 & 0.0487 \\
362.7 & 0.830 & 0.170 \\
378.2 & 0.632 & 0.368 \\
394.2 & 0.354 & 0.646 \\
\hline
\end{tabular}

${ }^{\mathrm{a}} x_{2}$ : mole fraction of component 2 in the saturated solution.

${ }^{b} x_{1}$ : mole fraction solubility of the solute. Solubility data reported in units of mass percent. Mole fraction values calculated by the compiler.

\section{Auxiliary Information}

\section{Method/Apparatus/Procedure:}

Preweighed quantities of solute and solvent were placed in bulbs, which were then sealed and heated in a suitable bath. At the higher temperatures, the concentration of solvent in the liquid solution was corrected for the amount of solvent vapor in the bulb. The correction assumed that the vapor pressure of the saturated solution was one half that of the pure solvent at the solution temperature. The temperature at which the solid completely dissolved was recorded, and was taken to be the solid-liquid equilibrium temperature.

\section{Source and Purity of Chemicals:}

(1) Purity not given, prepared and purified by the authors using a published synthetic procedure [see J. Walker and J. K. Wood, J. Chem. Soc. 117, 40 (1920) for synthetic details]. Melting point of the purified solute was $414.6 \mathrm{~K}$. (2) Purity not given, Chemical source not given, thiophene was removed by treatment with sulfuric acid. Sample was further purified by freezing several times.

\section{Estimated Error:}

Temperature: Not given in paper.

$x_{1}: \pm 10 \%$ (relative error, estimated by compiler).

\begin{tabular}{|c|c|}
\hline $\begin{array}{l}\text { Components: } \\
\text { (1) 3-Nitrobenzoic acid; } \mathrm{C}_{7} \mathrm{H}_{5} \mathrm{NO}_{4} \text {; } \\
\text { [121-92-6] } \\
\text { (2) Benzene; } \mathrm{C}_{6} \mathrm{H}_{6} ;[71-43-2]\end{array}$ & $\begin{array}{l}\text { Original Measurements: } \\
{ }^{180} \text { A. R. Collett and C. L. Lazzell, J. } \\
\text { Phys. Chem. 34, } 1838 \text { (1930). }\end{array}$ \\
\hline $\begin{array}{l}\text { Variables: } \\
\text { Temperature }\end{array}$ & $\begin{array}{l}\text { Prepared by: } \\
\text { W. E. Acree, Jr. }\end{array}$ \\
\hline
\end{tabular}

Experimental Values

\begin{tabular}{lcc}
\hline \hline$T / \mathrm{K}$ & $x_{2}{ }^{\mathrm{a}}$ & \multicolumn{1}{c}{$x_{1}{ }^{\mathrm{b}}$} \\
\hline 297.7 & 0.9937 & 0.00628 \\
323.8 & 0.9773 & 0.0227 \\
333.9 & 0.9664 & 0.0336 \\
345.0 & 0.9355 & 0.0645 \\
353.5 & 0.8975 & 0.1025 \\
366.6 & 0.7755 & 0.2245
\end{tabular}

\begin{tabular}{lcc}
\hline \hline$T / \mathrm{K}$ & $x_{2}{ }^{\mathrm{a}}$ & $x_{1}{ }^{\mathrm{b}}$ \\
\hline 377.4 & 0.6336 & 0.3664 \\
382.6 & 0.5242 & 0.4758 \\
385.6 & 0.4670 & 0.5330 \\
398.0 & 0.2761 & 0.7239 \\
408.0 & 0.1115 & 0.8885
\end{tabular}

${ }^{\mathrm{a}} x_{2}$ : mole fraction of component 2 in the saturated solution.

${ }^{\mathrm{b}} x_{1}$ : mole fraction solubility of the solute. The solubility is given as molal percentage, which, based on the description of 100 times the mole fraction, is taken to be mole percentage.

\section{Auxiliary Information}

\begin{abstract}
Method/Apparatus/Procedure:
Preweighed quantities of solute and solvent were placed in bulbs, which were then sealed and heated in a suitable bath. The temperature at which the solid completely dissolved was recorded, and was taken to be the solid-liquid equilibrium temperature. In a few instances near room temperature, the solubility was determined by agitating the solvent in a thermostat with an excess of solute. After equilibrium had been established, an aliquot of the saturated solution was pipetted, weighed, and the amount of dissolved solute determined with a standard solution of titanium (III) chloride.
\end{abstract}

Source and Purity of Chemicals:

(1) Purity not given, Synthesized by the authors according to the published method of Holleman [Recl. Trav. Chim. 18, 267 (1899)], was washed with water, recrystallized from trichloromethane, and recrystallized again from water to give a purified sample having a melting point temperature of $414.6 \mathrm{~K}$. (2) Thiophene-free, Kahlbaum, was dried over sodium and distilled before use.

\section{Estimated Error:}

Temperature: Not given in paper.

$x_{1}: \pm 10 \%$ (relative error, estimated by compiler).

\subsection{3-Nitrobenzoic acid solubility data in esters}

\begin{tabular}{ll}
\hline \hline Components: & Original Measurements: \\
(1) 3-Nitrobenzoic acid; $\mathrm{C}_{7} \mathrm{H}_{5} \mathrm{NO}_{4} ;$ & ${ }^{10}$ A. K. Charlton, C. R. Daniels, R. \\
[121-92-6] & M. Wold, E. Pustejovsky, W. E. \\
$\begin{array}{l}\text { (2) Methyl ethanoate; } \mathrm{C}_{3} \mathrm{H}_{6} \mathrm{O}_{2} ; \\
\text { [79-20-9] }\end{array}$ & Acree, Jr., and M. H. Abraham, J. \\
\hline Variables: & Mol. Liq. 116, 19 (2005). \\
$T / \mathrm{K}=298.15$ & Prepared by: \\
\hline
\end{tabular}

\section{Experimental Values}

\begin{tabular}{lc}
\hline \hline$x_{2}{ }^{\mathrm{a}}$ & $x_{1}{ }^{\mathrm{b}}$ \\
\hline 0.853 & 0.147 \\
\hline${ }^{\mathrm{a}} x_{2}:$ mole fraction of component 2 in the saturated solution. & \\
${ }^{\mathrm{b}} x_{1}:$ mole fraction solubility of the solute. &
\end{tabular}




\section{Auxiliary Information}

\section{Method/Apparatus/Procedure:}

Constant-temperature bath, calorimetric thermometer, and an ultraviolet/ visible spectrophotometer.

Excess solute and solvent were placed in amber glass bottles and allowed to equilibrate for several days at constant temperature. Attainment of equilibrium was verified by several repetitive measurements and by approaching equilibrium from supersaturation. Aliquots of saturated solutions were transferred through a coarse filter into tared volumetric flasks, weighed, and diluted with methanol. Concentrations were determined by

spectrophotometric measurements at $280 \mathrm{~nm}$.

Source and Purity of Chemicals:

(1) $99 \%$, Acros Organics, USA, and also from Aldrich Chemical Company, Milwaukee, WI, USA, was used as received.

(2) $99.5 \%$, anhydrous, Aldrich Chemical Company, stored over molecular sieves and distilled shortly before use.

Estimated Error:

Temperature: $\pm 0.1 \mathrm{~K}$.

$x_{1}: \pm 1.5 \%$ (relative error).

\begin{tabular}{ll}
\hline \hline Components: & Original Measurements: \\
$\begin{array}{l}\text { (1) 3-Nitrobenzoic acid; } \mathrm{C}_{7} \mathrm{H}_{5} \mathrm{NO}_{4} ; \\
\text { [121-92-6] }\end{array}$ & ${ }^{10}$ A. K. Charlton, C. R. Daniels, R. \\
$\begin{array}{l}\text { (2) Ethyl ethanoate; } \mathrm{C}_{4} \mathrm{H}_{8} \mathrm{O}_{2} ; \\
\text { [141-78-6] }\end{array}$ & Acree, Jr., and M. H. Abraham, J. \\
\hline Variables: & Mol. Liq. 116, 19 (2005). \\
$T / \mathrm{K}=298.15$ & Prepared by: \\
\hline
\end{tabular}

Experimental Values

\begin{tabular}{lc}
\hline \hline$x_{2}^{\mathrm{a}}$ & $x_{1}^{\mathrm{b}}$ \\
\hline 0.862 & 0.138 \\
\hline${ }^{\mathrm{a}} x_{2}:$ mole fraction of component 2 in the saturated solution. & \\
${ }^{\mathrm{b}}{ }_{x_{1}: \text { mole fraction solubility of the solute. }}$ &
\end{tabular}

\section{Auxiliary Information}

\section{Method/Apparatus/Procedure:}

Constant-temperature bath, calorimetric thermometer, and an ultraviolet/ visible spectrophotometer.

Excess solute and solvent were placed in amber glass bottles and allowed to equilibrate for several days at constant temperature. Attainment of equilibrium was verified by several repetitive measurements and by approaching equilibrium from supersaturation. Aliquots of saturated solutions were transferred through a coarse filter into tared volumetric flasks, weighed, and diluted with methanol. Concentrations were determined by

spectrophotometric measurements at $280 \mathrm{~nm}$.

\section{Source and Purity of Chemicals:}

(1) $99 \%$, Acros Organics, USA, and also from Aldrich Chemical Company, Milwaukee, WI, USA, was used as received.

(2) $99.9 \%$, HPLC grade, Aldrich Chemical Company, stored over molecular sieves and distilled shortly before use.

\section{Estimated Error:}

Temperature: $\pm 0.1 \mathrm{~K}$.

$x_{1}: \pm 1.5 \%$ (relative error).

\begin{tabular}{ll}
\hline \hline Components: & Original Measurements: \\
(1) 3-Nitrobenzoic acid; $\mathrm{C}_{7} \mathrm{H}_{5} \mathrm{NO}_{4} ;$ & ${ }^{10}$ A. K. Charlton, C. R. Daniels, R. \\
[121-92-6] & M. Wold, E. Pustejovsky, W. E. \\
$\begin{array}{l}\text { (2) Butyl ethanoate; } \mathrm{C}_{6} \mathrm{H}_{12} \mathrm{O}_{2} ; \\
\text { [123-86-4] }\end{array}$ & Acree, Jr., and M. H. Abraham, J. \\
\hline Variables: & Mol. Liq. 116, 19 (2005). \\
$T / \mathrm{K}=298.15$ & Prepared by: \\
\hline
\end{tabular}

Experimental Values

\begin{tabular}{lc}
\hline \hline$x_{2}{ }^{\mathrm{a}}$ & $x_{1}{ }^{\mathrm{b}}$ \\
\hline 0.892 & 0.108 \\
\hline${ }^{\mathrm{a}} x_{2}:$ mole fraction of component 2 in the saturated solution. \\
${ }^{\mathrm{b}}{ }_{x_{1}: \text { mole fraction solubility of the solute. }}$
\end{tabular}

\section{Auxiliary Information}

\section{Method/Apparatus/Procedure:}

Constant-temperature bath, calorimetric thermometer, and an ultraviolet/ visible spectrophotometer.

Excess solute and solvent were placed in amber glass bottles and allowed to equilibrate for several days at constant temperature. Attainment of equilibrium was verified by several repetitive measurements and by approaching equilibrium from supersaturation. Aliquots of saturated solutions were transferred through a coarse filter into tared volumetric flasks, weighed, and diluted with methanol. Concentrations were determined by

spectrophotometric measurements at $280 \mathrm{~nm}$.

\section{Source and Purity of Chemicals:}

(1) $99 \%$, Acros Organics, USA, and also from Aldrich Chemical Company, Milwaukee, WI, USA, was used as received.

(2) $99.7 \%$, HPLC grade, Aldrich Chemical Company, stored over molecular sieves and distilled shortly before use.

\section{Estimated Error:}

Temperature: $\pm 0.1 \mathrm{~K}$.

$x_{1}: \pm 1.5 \%$ (relative error).

\begin{tabular}{|c|c|}
\hline $\begin{array}{l}\text { Components: } \\
\text { (1) 3-Nitrobenzoic acid; } \mathrm{C}_{7} \mathrm{H}_{5} \mathrm{NO}_{4} \text {; } \\
\text { [121-92-6] } \\
\text { (2) 1,2,3-Triacetoxypropane (Triacetin); } \\
\mathrm{C}_{9} \mathrm{H}_{14} \mathrm{O}_{6} ;[102-76-1]\end{array}$ & $\begin{array}{l}\text { Original Measurements: } \\
\text { W. E. Acree, Jr., unpublished } \\
\text { data. }\end{array}$ \\
\hline $\begin{array}{l}\text { Variables: } \\
T / \mathrm{K}=298.15\end{array}$ & $\begin{array}{l}\text { Prepared by: } \\
\text { W. E. Acree, Jr. }\end{array}$ \\
\hline
\end{tabular}

Experimental Values

\begin{tabular}{lc}
\hline \hline$x_{2}{ }^{\mathrm{a}}$ & $x_{1}{ }^{\mathrm{b}}$ \\
\hline 0.8539 & 0.1461 \\
\hline${ }^{\mathrm{a}} x_{2}:$ mole fraction of component 2 in the saturated solution. \\
${ }^{\mathrm{b}}{ }_{x_{1}: \text { mole fraction solubility of the solute. }}$
\end{tabular}




\section{Auxiliary Information}

\section{Method: Apparatus/Procedure:}

Constant-temperature bath, calorimetric thermometer, and an ultraviolet/ visible spectrophotometer.

Excess solute and solvent were placed in amber glass bottles and allowed to equilibrate for several days at constant temperature. Attainment of equilibrium was verified by several repetitive measurements and by approaching equilibrium from supersaturation. Aliquots of saturated solutions were transferred through a coarse filter into tared volumetric flasks, weighed, and diluted with 2-propanol. Concentrations were determined by

spectrophotometric measurements at $280 \mathrm{~nm}$.

Source and Purity of Chemicals:

(1) $99 \%$, Acros Organics, USA, and also from Aldrich Chemical Company, Milwaukee, WI, USA, was used as received.

(2) $99 \%$, Acros Organics, USA, stored over molecular sieves before use.

Estimated Error:

Temperature: $\pm 0.1 \mathrm{~K}$.

$x_{1}: \pm 1.5 \%$ (relative error).

\subsection{3-Nitrobenzoic acid solubility data in ethers}

\begin{tabular}{|c|c|}
\hline $\begin{array}{l}\text { Components: } \\
\text { (1) 3-Nitrobenzoic acid; } \mathrm{C}_{7} \mathrm{H}_{5} \mathrm{NO}_{4} \text {; } \\
\text { [121-92-6] } \\
\text { (2) } 1,1^{\prime}-\text { Oxybisethane; } \mathrm{C}_{4} \mathrm{H}_{10} \mathrm{O} \text {; } \\
\text { [60-29-7] }\end{array}$ & $\begin{array}{l}\text { Original Measurements: } \\
{ }^{10} \text { A. K. Charlton, C. R. Daniels, R. } \\
\text { M. Wold, E. Pustejovsky, W. E. } \\
\text { Acree, Jr., and M. H. Abraham, J. } \\
\text { Mol. Liq. 116, } 19 \text { (2005). }\end{array}$ \\
\hline $\begin{array}{l}\text { Variables: } \\
T / \mathrm{K}=298.15\end{array}$ & $\begin{array}{l}\text { Prepared by: } \\
\text { W. E. Acree, Jr. }\end{array}$ \\
\hline
\end{tabular}

Experimental Values

\begin{tabular}{lc}
\hline \hline$x_{2}^{\mathrm{a}}$ & $x_{1}^{\mathrm{b}}$ \\
\hline 0.873 & 0.127
\end{tabular}

${ }^{\mathrm{a}} x_{2}$ : mole fraction of component 2 in the saturated solution.

${ }^{\mathrm{b}} \mathrm{x}_{1}$ : mole fraction solubility of the solute.

\section{Auxiliary Information}

\section{Method/Apparatus/Procedure:}

Constant-temperature bath, calorimetric thermometer, and an ultraviolet/ visible spectrophotometer.

Excess solute and solvent were placed in amber glass bottles and allowed to equilibrate for several days at constant temperature. Attainment of equilibrium was verified by several repetitive measurements and by approaching equilibrium from supersaturation. Aliquots of saturated solutions were transferred through a coarse filter into tared volumetric flasks, weighed, and diluted with methanol. Concentrations were determined by spectrophotometric measurements at $280 \mathrm{~nm}$.

\section{Source and Purity of Chemicals:}

(1) $99 \%$, Acros Organics, USA, and also from Aldrich Chemical Company, Milwaukee, WI, USA, was used as received.

(2) 99+\%, anhydrous, Aldrich Chemical Company, stored over molecular sieves and distilled shortly before use.

\begin{tabular}{|c|c|}
\hline $\begin{array}{l}\text { Components: } \\
\text { (1) 3-Nitrobenzoic acid; } \mathrm{C}_{7} \mathrm{H}_{5} \mathrm{NO}_{4} \text {; } \\
\text { [121-92-6] } \\
\text { (2) } 1,1^{\prime}-\text { Oxybisethane; } \mathrm{C}_{4} \mathrm{H}_{10} \mathrm{O} \text {; } \\
\text { [60-29-7] }\end{array}$ & $\begin{array}{l}\text { Original Measurements: } \\
{ }^{180} \text { A. R. Collett and C. L. Lazzell, J. } \\
\text { Phys. Chem. 34, } 1838 \text { (1930). }\end{array}$ \\
\hline $\begin{array}{l}\text { Variables: } \\
\text { Temperature }\end{array}$ & $\begin{array}{l}\text { Prepared by: } \\
\text { W. E. Acree, Jr. }\end{array}$ \\
\hline
\end{tabular}

Experimental Values

\begin{tabular}{lcc}
\hline \hline$T / \mathrm{K}$ & $x_{2}{ }^{\mathrm{a}}$ & $x_{1}{ }^{\mathrm{b}}$ \\
\hline 283.2 & 0.8658 & 0.1342 \\
325.2 & 0.7867 & 0.2133 \\
345.1 & 0.7020 & 0.2980 \\
355.1 & 0.6404 & 0.3596 \\
362.8 & 0.5820 & 0.4180 \\
371.6 & 0.5118 & 0.4882 \\
386.2 & 0.3849 & 0.6151 \\
395.4 & 0.2802 & 0.7198 \\
406.2 & 0.1364 & 0.8636 \\
\hline
\end{tabular}

${ }^{a} x_{2}$ : mole fraction of component 2 in the saturated solution.

${ }^{\mathrm{b}} x_{1}$ : mole fraction solubility of the solute. The solubility is given as molal percentage, which, based on the description of 100 times the mole fraction, is taken to be mole percentage.

\section{Auxiliary Information}

\section{Method/Apparatus/Procedure:}

Preweighed quantities of solute and solvent were placed in bulbs, which were then sealed and heated in a suitable bath. The temperature at which the solid completely dissolved was recorded, and was taken to be the solid-liquid equilibrium temperature. In a few instances near room temperature, the solubility was determined by agitating the solvent in a thermostat with an excess of solute. After equilibrium had been established, an aliquot of the saturated solution was pipetted, weighed, and the amount of dissolved solute determined with a standard solution of titanium (III) chloride.

\section{Source and Purity of Chemicals:}

(1) Purity not given, Synthesized by the authors according to the published method of Holleman [Recl. Trav. Chim. 18, 267 (1899)], was washed with water, recrystallized from trichloromethane, and recrystallized again from water to give a purified sample having a melting point temperature of $414.6 \mathrm{~K}$. (2) U.S.P. grade, Chemical source not given, washed three times with distilled water, dried over calcium chloride, and distilled twice before use.

\section{Estimated Error:}

Temperature: Not given in paper.

$x_{2}: \pm 10 \%$ (relative error, estimated by compiler).

\begin{tabular}{ll}
\hline \hline Components: & Original Measurements: \\
(1) 3-Nitrobenzoic acid; $\mathrm{C}_{7} \mathrm{H}_{5} \mathrm{NO}_{4} ;$ & ${ }^{10}$ A. K. Charlton, C. R. Daniels, R. \\
[121-92-6] & M. Wold, E. Pustejovsky, W. E. \\
$\begin{array}{l}\text { (2) } 2,2^{\prime}-\text {-Oxybispropane; } \mathrm{C}_{6} \mathrm{H}_{14} \mathrm{O} ; \\
{[108-20-3]}\end{array}$ & $\begin{array}{l}\text { Acree, Jr., and M. H. Abraham, J. } \\
\text { Mol. Liq. 116, 19 (2005). }\end{array}$ \\
\hline Variables: & Prepared by: \\
$T / \mathrm{K}=298.15$ & W. E. Acree, Jr. \\
\hline
\end{tabular}


Experimental Values

\begin{tabular}{lc}
\hline \hline$x_{2}^{\mathrm{a}}$ & $x_{1}{ }^{\mathrm{b}}$ \\
\hline 0.9409 & 0.0591 \\
\hline
\end{tabular}

${ }^{\mathrm{a}} x_{2}$ : mole fraction of component 2 in the saturated solution.

${ }^{\mathrm{b}} \mathrm{x}_{1}$ : mole fraction solubility of the solute.

\section{Auxiliary Information}

\section{Method/Apparatus/Procedure:}

Constant-temperature bath, calorimetric thermometer, and an ultraviolet/ visible spectrophotometer.

Excess solute and solvent were placed in amber glass bottles and allowed to equilibrate for several days at constant temperature. Attainment of equilibrium was verified by several repetitive measurements and by approaching equilibrium from supersaturation. Aliquots of saturated solutions were transferred through a coarse filter into tared volumetric flasks, weighed, and diluted with methanol. Concentrations were determined by

spectrophotometric measurements at $280 \mathrm{~nm}$.

\section{Source and Purity of Chemicals:}

(1) $99 \%$, Acros Organics, USA, and also from Aldrich Chemical Company, Milwaukee, WI, USA, was used as received.

(2) $99 \%$, anhydrous, Aldrich Chemical Company, stored over molecular sieves and distilled shortly before use.

\section{Estimated Error:}

Temperature: $\pm 0.1 \mathrm{~K}$

$x_{1}: \pm 1.5 \%$ (relative error).

\section{Components:}

(1) 3-Nitrobenzoic acid; $\mathrm{C}_{7} \mathrm{H}_{5} \mathrm{NO}_{4}$;

[121-92-6]

(2) 1,1'-Oxybisbutane; $\mathrm{C}_{8} \mathrm{H}_{18} \mathrm{O}$; [142-96-1]

\section{Variables:}

$T / \mathrm{K}=298.15$

Original Measurements:

${ }^{10}$ A. K. Charlton, C. R. Daniels, R. M. Wold, E. Pustejovsky, W. E. Acree, Jr., and M. H. Abraham, J. Mol. Liq. 116, 19 (2005).

\section{Prepared by:}

W. E. Acree, Jr.
Experimental Values

\begin{tabular}{lc}
\hline \hline$x_{2}{ }^{\mathrm{a}}$ & $x_{1}{ }^{\mathrm{b}}$ \\
\hline 0.9654 & 0.0346 \\
\hline$x_{2}$ mole faction of compont 2 inthe satrated
\end{tabular}

${ }^{\mathrm{a}} x_{2}$ : mole fraction of component 2 in the saturated solution.

${ }^{\mathrm{b}} x_{1}$ : mole fraction solubility of the solute.

\section{Auxiliary Information}

\section{Method/Apparatus/Procedure:}

Constant-temperature bath, calorimetric thermometer, and an ultraviolet/ visible spectrophotometer.

Excess solute and solvent were placed in amber glass bottles and allowed to equilibrate for several days at constant temperature. Attainment of equilibrium was verified by several repetitive measurements and by approaching equilibrium from supersaturation. Aliquots of saturated solutions were transferred through a coarse filter into tared volumetric flasks, weighed, and diluted with methanol. Concentrations were determined by spectrophotometric measurements at $280 \mathrm{~nm}$.
Source and Purity of Chemicals:

(1) $99 \%$, Acros Organics, USA, and also from Aldrich Chemical Company, Milwaukee, WI, USA, was used as received.

(2) $99.3 \%$, anhydrous, Aldrich Chemical Company, stored over molecular sieves and distilled shortly before use.

\section{Estimated Error:}

Temperature: $\pm 0.1 \mathrm{~K}$.

$x_{1}: \pm 1.5 \%$ (relative error).

\begin{tabular}{ll}
\hline \hline Components: & Original Measurements: \\
(1) 3-Nitrobenzoic acid; $\mathrm{C}_{7} \mathrm{H}_{5} \mathrm{NO}_{4} ;$ & ${ }^{10}$ A. K. Charlton, C. R. Daniels, R. \\
[121-92-6] & M. Wold, E. Pustejovsky, W. E. \\
$\begin{array}{l}\text { (2) Tetrahydrofuran; } \mathrm{C}_{4} \mathrm{H}_{8} \mathrm{O} ; \\
\text { [109-99-9] }\end{array}$ & Acree, Jr., and M. H. Abraham, J. \\
\hline Variables: & Mol. Liq. 116, 19 (2005). \\
$T / \mathrm{K}=298.15$ & Prepared by: \\
\hline
\end{tabular}

\section{Experimental Values}

\begin{tabular}{lc}
\hline \hline$x_{2}{ }^{\mathrm{a}}$ & $x_{1}{ }^{\mathrm{b}}$ \\
\hline 0.640 & 0.360 \\
\hline${ }^{a} x_{2}:$ mole fraction of component 2 in the saturated solution. & \\
${ }^{\mathrm{b}} x_{1}:$ mole fraction solubility of the solute. &
\end{tabular}

\section{Auxiliary Information}

\section{Method/Apparatus/Procedure:}

Constant-temperature bath, calorimetric thermometer, and an ultraviolet/ visible spectrophotometer.

Excess solute and solvent were placed in amber glass bottles and allowed to equilibrate for several days at constant temperature. Attainment of equilibrium was verified by several repetitive measurements and by approaching equilibrium from supersaturation. Aliquots of saturated solutions were transferred through a coarse filter into tared volumetric flasks, weighed, and diluted with methanol. Concentrations were determined by spectrophotometric measurements at $280 \mathrm{~nm}$.

\section{Source and Purity of Chemicals:}

(1) $99 \%$, Acros Organics, USA, and also from Aldrich Chemical Company, Milwaukee, WI, USA, was used as received.

(2) $99.9 \%$, anhydrous, Aldrich Chemical Company, stored over molecular sieves and distilled shortly before use.

\section{Estimated Error:}

Temperature: $\pm 0.1 \mathrm{~K}$.

$x_{1}: \pm 2.0 \%$ (relative error).

\begin{tabular}{ll}
\hline \hline Components: & Original Measurements: \\
(1) 3-Nitrobenzoic acid; $\mathrm{C}_{7} \mathrm{H}_{5} \mathrm{NO}_{4} ;$ & ${ }^{10}$ A. K. Charlton, C. R. Daniels, R. \\
[121-92-6] & M. Wold, E. Pustejovsky, W. E. \\
(2) 1,4-Dioxane; $\mathrm{C}_{4} \mathrm{H}_{8} \mathrm{O}_{2} ;[123-91-1]$ & $\begin{array}{l}\text { Acree, Jr., and M. H. Abraham, J. } \\
\end{array}$ \\
\hline Mol. Liq. 116, 19 (2005). \\
\hline Variables: & Prepared by: \\
$T / \mathrm{K}=298.15$ & W. E. Acree, Jr. \\
\hline
\end{tabular}


Experimental Values

\begin{tabular}{lc}
\hline \hline$x_{2}{ }^{\mathrm{a}}$ & $x_{1}{ }^{\mathrm{b}}$ \\
\hline 0.807 & 0.193 \\
\hline${ }^{\mathrm{a}} x_{2}:$ mole fraction of component 2 in the saturated solution. & \\
${ }^{\mathrm{b}} x_{1}:$ mole fraction solubility of the solute. &
\end{tabular}

\section{Auxiliary Information}

Method/Apparatus/Procedure:

Constant-temperature bath, calorimetric thermometer, and an ultraviolet/ visible spectrophotometer.

Excess solute and solvent were placed in amber glass bottles and allowed to equilibrate for several days at constant temperature. Attainment of equilibrium was verified by several repetitive measurements and by approaching equilibrium from supersaturation. Aliquots of saturated solutions were transferred through a coarse filter into tared volumetric flasks, weighed, and diluted with methanol. Concentrations were determined by

spectrophotometric measurements at $280 \mathrm{~nm}$.

Source and Purity of Chemicals:

(1) $99 \%$, Acros Organics, USA, and also from Aldrich Chemical Company, Milwaukee, WI, USA, was used as received.

(2) $99.8 \%$, anhydrous, Aldrich Chemical Company, stored over molecular sieves and distilled shortly before use.

Estimated Error:

Temperature: $\pm 0.1 \mathrm{~K}$

$x_{1}: \pm 2.0 \%$ (relative error)

Auxiliary Information

\section{Method/Apparatus/Procedure:}

Preweighed quantities of solute and solvent were placed in bulbs, which were then sealed and heated in a suitable bath. The temperature at which the solid completely dissolved was recorded, and was taken to be the solid-liquid equilibrium temperature.

\section{Source and Purity of Chemicals:}

(1) Purity not given, Synthesized by the authors according to the published method of Holleman [Recl. Trav. Chim. 18, 267 (1899)], was washed with water, recrystallized from trichloromethane, and recrystallized again from water to give a purified sample having a melting point temperature of $414.6 \mathrm{~K}$. (2) Certified Pure, Merck Chemical Company, dried over calcium chloride and distilled twice before use.

Estimated Error:

Temperature: Not given in paper.

$x_{2}: \pm 10 \%$ (relative error, estimated by compiler).

\begin{tabular}{|c|c|}
\hline $\begin{array}{l}\text { Components: } \\
\text { (1) 3-Nitrobenzoic acid; } \mathrm{C}_{7} \mathrm{H}_{5} \mathrm{NO}_{4} \text {; } \\
\text { [121-92-6] } \\
\text { (2) Tetrachloromethane; } \mathrm{CCl}_{4} \text {; } \\
\text { [56-23-5] }\end{array}$ & $\begin{array}{l}\text { Original Measurements: } \\
{ }^{180} \text { A. R. Collett and C. L. Lazzell, J. } \\
\text { Phys. Chem. 34, } 1838 \text { (1930). }\end{array}$ \\
\hline $\begin{array}{l}\text { Variables: } \\
\text { Temperature }\end{array}$ & $\begin{array}{l}\text { Prepared by: } \\
\text { W. E. Acree, Jr. }\end{array}$ \\
\hline
\end{tabular}

\subsection{3-Nitrobenzoic acid solubility data in haloalkanes, haloalkenes, and haloaromatic hydrocarbons}

\begin{tabular}{|c|c|}
\hline $\begin{array}{l}\text { Components: } \\
\text { (1) 3-Nitrobenzoic acid; } \mathrm{C}_{7} \mathrm{H}_{5} \mathrm{NO}_{4} \text {; } \\
\text { [121-92-6] } \\
\text { (2) Trichloromethane; } \mathrm{CHCl}_{3} \text {; } \\
\text { [67-66-3] }\end{array}$ & $\begin{array}{l}\text { Original Measurements: } \\
{ }^{180} \text { A. R. Collett and C. L. Lazzell, J. } \\
\text { Phys. Chem. 34, } 1838 \text { (1930). }\end{array}$ \\
\hline $\begin{array}{l}\text { Variables: } \\
\text { Temperature }\end{array}$ & Prepared by: \\
\hline Temperature & W. E. Acree, Jr. \\
\hline
\end{tabular}

Experimental Values

\begin{tabular}{lcc}
\hline \hline$T / \mathrm{K}$ & $x_{2}{ }^{\mathrm{a}}$ & $x_{1}{ }^{\mathrm{b}}$ \\
\hline 320.9 & 0.9431 & 0.0569 \\
340.7 & 0.8836 & 0.1164 \\
358.5 & 0.7718 & 0.2282 \\
373.0 & 0.6173 & 0.3827 \\
373.2 & 0.6139 & 0.3861 \\
373.7 & 0.6106 & 0.3894 \\
379.0 & 0.5294 & 0.4706 \\
387.1 & 0.4168 & 0.5832 \\
397.5 & 0.2807 & 0.7193 \\
405.3 & 0.1503 & 0.8497 \\
\hline
\end{tabular}

${ }^{a_{2}}$ : mole fraction of component 2 in the saturated solution.

${ }^{b} x_{1}$ : mole fraction solubility of the solute. The solubility is given as molal percentage, which, based on the description of 100 times the mole fraction, is taken to be mole percentage.
Experimental Values

\begin{tabular}{lcc}
\hline \hline$T / \mathrm{K}$ & $x_{2}{ }^{\mathrm{a}}$ & $x_{1}{ }^{\mathrm{b}}$ \\
\hline 302.6 & 0.9986 & 0.00135 \\
368.8 & 0.9663 & 0.0337 \\
380.4 & 0.9048 & 0.0952 \\
385.5 & 0.7963 & 0.2037 \\
389.1 & 0.6468 & 0.3532 \\
390.2 & 0.6035 & 0.3965 \\
392.5 & 0.4854 & 0.5146 \\
398.9 & 0.3132 & 0.6862 \\
405.5 & 0.1670 & 0.8330 \\
\hline
\end{tabular}

${ }^{a} x_{2}$ : mole fraction of component 2 in the saturated solution.

${ }^{\mathrm{b}} x_{1}$ : mole fraction solubility of the solute. The solubility is given as molal percentage, which, based on the description of 100 times the mole fraction, is taken to be mole percentage.

\section{Auxiliary Information}

\section{Method/Apparatus/Procedure:}

Preweighed quantities of solute and solvent were placed in bulbs, which were then sealed and heated in a suitable bath. The temperature at which the solid completely dissolved was recorded, and was taken to be the solid-liquid equilibrium temperature. In a few instances near room temperature, the solubility was determined by agitating the solvent in a thermostat with an excess of solute. After equilibrium had been established, an aliquot of the saturated solution was pipetted, weighed, and the amount of dissolved solute determined with a standard solution of titanium (III) chloride. 
Source and Purity of Chemicals:

(1) Purity not given, Synthesized by the authors according to the published method of Holleman [Recl. Trav. Chim. 18, 267 (1899)], was washed with water, recrystallized from trichloromethane, and recrystallized again from water to give a purified sample having a melting point temperature of $414.6 \mathrm{~K}$. (2) Certified Pure, Merck Chemical Company, dried over calcium chloride and distilled twice before use.

Estimated Error:

Temperature: Not given in paper.

$x_{2}: \pm 10 \%$ (relative error, estimated by compiler).

\subsection{3-Nitrobenzoic acid solubility data in alcohols}

\begin{tabular}{ll}
\hline \hline Components: & Original Measurements: \\
(1) 3-Nitrobenzoic acid; $\mathrm{C}_{7} \mathrm{H}_{5} \mathrm{NO}_{4} ;$ & ${ }^{10} \mathrm{~A}$. K. Charlton, C. R. Daniels, R. \\
[121-92-6] & M. Wold, E. Pustejovsky, W. E. \\
(2) Methanol; $\mathrm{CH}_{4} \mathrm{O} ;$ [67-56-1] & Acree, Jr., and M. H. Abraham, J. \\
& Mol. Liq. 116, 19 (2005). \\
\hline Variables: & Prepared by: \\
$T / \mathrm{K}=298.15$ & W. E. Acree, Jr. \\
\hline
\end{tabular}

Experimental Values

\begin{tabular}{lc}
\hline \hline$x_{2}^{\mathrm{a}}$ & $x_{1}{ }^{\mathrm{b}}$ \\
\hline 0.789 & 0.211
\end{tabular}

${ }^{\mathrm{a}} x_{2}$ : mole fraction of component 2 in the saturated solution.

${ }^{\mathrm{b}} x_{1}$ : mole fraction solubility of the solute.

\section{Auxiliary Information}

\section{Method/Apparatus/Procedure:}

Constant-temperature bath, calorimetric thermometer, and an ultraviolet/ visible spectrophotometer.

Excess solute and solvent were placed in amber glass bottles and allowed to equilibrate for several days at constant temperature. Attainment of equilibrium was verified by several repetitive measurements and by approaching equilibrium from supersaturation. Aliquots of saturated solutions were transferred through a coarse filter into tared volumetric flasks, weighed, and diluted with methanol. Concentrations were determined by

spectrophotometric measurements at $280 \mathrm{~nm}$.

\section{Source and Purity of Chemicals:}

(1) $99 \%$, Acros Organics, USA, and also from Aldrich Chemical Company, Milwaukee, WI, USA, was used as received.

(2) $99.8 \%$, anhydrous, Aldrich Chemical Company, stored over molecular sieves and distilled shortly before use.

\section{Estimated Error:}

Temperature: $\pm 0.1 \mathrm{~K}$

$x_{1}: \pm 1.5 \%$ (relative error).

\begin{tabular}{l}
\hline \hline Components: \\
(1) 3-Nitrobenzoic acid; $\mathrm{C}_{7} \mathrm{H}_{5} \mathrm{NO}_{4}$; \\
[121-92-6] \\
(2) Methanol; $\mathrm{CH}_{4} \mathrm{O} ;[67-56-1]$
\end{tabular}

Original Measurements:

${ }^{83}$ M. K. Chantooni and I. M. Kolthoff, J. Phys. Chem. 77, 527 (1973).

${ }^{181}$ I. M. Kolthoff, J. J. Lingane, and W. Larson, J. Am. Chem. Soc. 60, 2512 (1938).

\section{Variables:}

$T / \mathrm{K}=298.15$

\section{Prepared by:}

W. E. Acree, Jr.
Experimental Values

The measured solubility was reported to be $3.46 \mathrm{~mol} \mathrm{dm}^{-3}$.

\section{Auxiliary Information}

\section{Method/Apparatus/Procedure:}

Very little experimental details were provided. Solubility was determined by the conductance method.

Source and Purity of Chemicals:

(1) Purity not given, Chemical source not given, no purification details were provided.

(2) Purity not given, Chemical source not given, refluxed over silver oxide, distilled and dehydrated with magnesium.

\section{Estimated Error:}

Temperature: $\pm 0.1 \mathrm{~K}$ (estimated by compiler). $c_{1}: \pm 2 \%$ (relative error, estimated by compiler).

\begin{tabular}{ll}
\hline \hline Components: & Original Measurements: \\
(1) 3-Nitrobenzoic acid; $\mathrm{C}_{7} \mathrm{H}_{5} \mathrm{NO}_{4} ;$ & ${ }^{10}$ A. K. Charlton, C. R. Daniels, R. \\
[121-92-6] & M. Wold, E. Pustejovsky, W. E. \\
(2) Ethanol; $\mathrm{C}_{2} \mathrm{H}_{6} \mathrm{O} ;$ [64-17-5] & Acree, Jr., and M. H. Abraham, J. \\
& Mol. Liq. 116, 19 (2005). \\
\hline Variables: & Prepared by: \\
$T / \mathrm{K}=298.15$ & W. E. Acree, Jr. \\
\hline
\end{tabular}

\section{Experimental Values}

\begin{tabular}{lc}
\hline \hline$x_{2}{ }^{\mathrm{a}}$ & $x_{1}{ }^{\mathrm{b}}$ \\
\hline 0.825 & 0.175 \\
\hline${ }^{\mathrm{a}} x_{2}:$ mole fraction of component 2 in the saturated solution. &
\end{tabular}

${ }^{b_{1}}$ : mole fraction solubility of the solute.

\section{Auxiliary Information}

\section{Method/Apparatus/Procedure:}

Constant-temperature bath, calorimetric thermometer, and an ultraviolet/ visible spectrophotometer.

Excess solute and solvent were placed in amber glass bottles and allowed to equilibrate for several days at constant temperature. Attainment of equilibrium was verified by several repetitive measurements and by approaching equilibrium from supersaturation. Aliquots of saturated solutions were transferred through a coarse filter into tared volumetric flasks, weighed, and diluted with methanol. Concentrations were determined by spectrophotometric measurements at $280 \mathrm{~nm}$.

Source and Purity of Chemicals:

(1) $99 \%$, Acros Organics, USA, and also from Aldrich Chemical Company, Milwaukee, WI, USA, was used as received.

(2) Absolute, Aaper Alcohol and Chemical Company, USA, stored over molecular sieves and distilled shortly before use.

\section{Estimated Error:}

Temperature: $\pm 0.1 \mathrm{~K}$.

$x_{1}: \pm 1.5 \%$ (relative error). 


\section{Components:}

(1) 3-Nitrobenzoic acid; $\mathrm{C}_{7} \mathrm{H}_{5} \mathrm{NO}_{4}$;

[121-92-6]

(2) Ethanol; $\mathrm{C}_{2} \mathrm{H}_{6} \mathrm{O} ;[64-17-5]$

\section{Variables:}

$T / \mathrm{K}=298.15$

Original Measurements:

${ }^{126}$ P. K. Biswas, S. C. Lahiri, and B.

P. Dey, Bull. Chem. Soc. Jpn. 66, 2785 (1993).

Prepared by:

W. E. Acree, Jr.

Experimental Values

\begin{tabular}{lcc}
\hline \hline$c_{1}^{\mathrm{a}}$ & $x_{2}{ }^{\mathrm{b}}$ & $x_{1}{ }^{\mathrm{c}}$ \\
\hline 1.6157 & 0.8952 & 0.1048
\end{tabular}

${ }^{a} c_{1}$ : solubility of the solute expressed in units of $\mathrm{mol} \mathrm{dm}^{-3}$.

${ }^{b} x_{2}$ : mole fraction of component 2 in the saturated solution.

${ }^{c} x_{1}$ : mole fraction solubility of the solute calculated by the compiler.

\section{Auxiliary Information}

\section{Method/Apparatus/Procedure:}

Analytical balance and an ultraviolet/visible spectrophotometer.

Solutions containing excess solute and solvent were allowed to equilibrate at constant temperature for at least $24 \mathrm{~h}$. An aliquot of the saturated solution was removed, filtered, and the absorbance recorded. Solubility was calculated from the measured absorbance.

\section{Source and Purity of Chemicals:}

(1) Puris grade, Fluka Chemicals, recrystallized from aqueous ethanol mixture and dried in an air oven at $390 \mathrm{~K}$. The purified compound was stored in vacuum desiccators.

(2) Absolute, B.C.P.W., Calcutta, India, was distilled twice before use.

\section{Estimated Error:}

Temperature: $\pm 0.2 \mathrm{~K}$ (estimated by compiler).

$c_{1}: \pm 1.0 \%$ (relative error, estimated by compiler).

$x_{1}: \pm 2.5 \%$ (relative error, estimated by compiler).

\begin{tabular}{|c|c|}
\hline $\begin{array}{l}\text { Components: } \\
\text { (1) 3-Nitrobenzoic acid; } \mathrm{C}_{7} \mathrm{H}_{5} \mathrm{NO}_{4} \text {; } \\
\text { [121-92-6] } \\
\text { (2) Ethanol; } \mathrm{C}_{2} \mathrm{H}_{6} \mathrm{O} ;[64-17-5]\end{array}$ & $\begin{array}{l}\text { Original Measurements: } \\
\text { }{ }^{72} \mathrm{R} \text {. Thuaire, Bull. Soc. Chim. Fr } \\
3815 \text { (1971). }\end{array}$ \\
\hline Variables: & Prepared by: \\
\hline$T / \mathrm{K}=298.15$ & W. E. Acree, Jr. \\
\hline
\end{tabular}

\section{Experimental Values}

The measured solubility was reported to be $4.795 \mathrm{~mol} / \mathrm{kg}$ of solvent, which corresponds to a mole fraction solubility of $x_{1}=0.1809$.

\section{Auxiliary Information}

\section{Method/Apparatus/Procedure:}

Very little experimental details were provided. Solubility was determined by a gravimetric method. The saturated solution was evaporated to dryness and the remaining solid residue was weighed. The solubility was calculated from the mass of the solid residue and mass of saturated solution analyzed.

\section{Source and Purity of Chemicals:}

(1) Purity not given, Chemical source not given, no purification details were provided.

(2) Purity not given, Chemical source not given, no purification details were provided.

\section{Estimated Error:}

Temperature: $\pm 0.05 \mathrm{~K}$

$x_{1}: \pm 4 \%$ (relative error, estimated by compiler).

\begin{tabular}{ll}
\hline \hline Components: & Original Measurements: \\
(1) 3-Nitrobenzoic acid; $\mathrm{C}_{7} \mathrm{H}_{5} \mathrm{NO}_{4} ;$ & ${ }^{10}$ A. K. Charlton, C. R. Daniels, R. \\
[121-92-6] & M. Wold, E. Pustejovsky, W. E. \\
(2) 1-Propanol; $\mathrm{C}_{3} \mathrm{H}_{8} \mathrm{O} ;$ [71-23-8] & Acree, Jr., and M. H. Abraham, J. \\
& Mol. Liq. 116, 19 (2005). \\
\hline Variables: & Prepared by: \\
$T / \mathrm{K}=298.15$ & W. E. Acree, Jr. \\
\hline
\end{tabular}

Experimental Values

\begin{tabular}{lc}
\hline \hline$x_{2}{ }^{\mathrm{a}}$ & $x_{1}{ }^{\mathrm{b}}$ \\
\hline 0.851 & 0.149 \\
\hline${ }^{\mathrm{a}} x_{2}:$ mole fraction of component 2 in the saturated solution. & \\
${ }^{\mathrm{b}} x_{1}:$ mole fraction solubility of the solute. &
\end{tabular}

\section{Auxiliary Information}

\section{Method/Apparatus/Procedure:}

Constant-temperature bath, calorimetric thermometer, and an ultraviolet/ visible spectrophotometer.

Excess solute and solvent were placed in amber glass bottles and allowed to equilibrate for several days at constant temperature. Attainment of equilibrium was verified by several repetitive measurements and by approaching equilibrium from supersaturation. Aliquots of saturated solutions were transferred through a coarse filter into tared volumetric flasks, weighed, and diluted with methanol. Concentrations were determined by spectrophotometric measurements at $280 \mathrm{~nm}$.

\section{Source and Purity of Chemicals:}

(1) $99 \%$, Acros Organics, USA, and also from Aldrich Chemical Company, Milwaukee, WI, USA, was used as received.

(2) $99+\%$, anhydrous, Aldrich Chemical Company, stored over molecular sieves and distilled shortly before use.

\section{Estimated Error:}

Temperature: $\pm 0.1 \mathrm{~K}$.

$x_{1}: \pm 1.5 \%$ (relative error).

\begin{tabular}{ll}
\hline \hline Components: & Original Measurements: \\
(1) 3-Nitrobenzoic acid; $\mathrm{C}_{7} \mathrm{H}_{5} \mathrm{NO}_{4} ;$ & ${ }^{10}$ A. K. Charlton, C. R. Daniels, R. \\
[121-92-6] & M. Wold, E. Pustejovsky, W. E. \\
(2) 2-Propanol; $\mathrm{C}_{3} \mathrm{H}_{8} \mathrm{O} ;$ [ [67-63-0] & Acree, Jr., and M. H. Abraham, J. \\
& Mol. Liq. 116, 19 (2005). \\
\hline Variables: & Prepared by: \\
$T / \mathrm{K}=298.15$ & W. E. Acree, Jr. \\
\hline
\end{tabular}

Experimental Values

\begin{tabular}{lc}
\hline \hline$x_{2}{ }^{\mathrm{a}}$ & $x_{1}{ }^{\mathrm{b}}$ \\
\hline 0.830 & 0.170 \\
\hline${ }^{\mathrm{a}} x_{2}:$ mole fraction of component 2 in the saturated solution. & \\
${ }^{\mathrm{b}} x_{1}:$ mole fraction solubility of the solute. &
\end{tabular}




\section{Auxiliary Information}

\section{Method/Apparatus/Procedure:}

Constant-temperature bath, calorimetric thermometer, and an ultraviolet/ visible spectrophotometer.

Excess solute and solvent were placed in amber glass bottles and allowed to equilibrate for several days at constant temperature. Attainment of equilibrium was verified by several repetitive measurements and by approaching equilibrium from supersaturation. Aliquots of saturated solutions were transferred through a coarse filter into tared volumetric flasks, weighed, and diluted with methanol. Concentrations were determined by spectrophotometric measurements at $280 \mathrm{~nm}$.

\section{Source and Purity of Chemicals:}

(1) $99 \%$, Acros Organics, USA, and also from Aldrich Chemical Company, Milwaukee, WI, USA, was used as received.

(2) $99+\%$, anhydrous, Aldrich Chemical Company, stored over molecular sieves and distilled shortly before use.

\section{Estimated Error:}

Temperature: $\pm 0.1 \mathrm{~K}$.

$x_{1}: \pm 1.5 \%$ (relative error).

\begin{tabular}{ll}
\hline \hline Components: & Original Measurements: \\
(1) 3-Nitrobenzoic acid; $\mathrm{C}_{7} \mathrm{H}_{5} \mathrm{NO}_{4} ;$ & ${ }^{10}$ A. K. Charlton, C. R. Daniels, R. \\
[121-92-6] & M. Wold, E. Pustejovsky, W. E. \\
(2) 1-Butanol; $\mathrm{C}_{4} \mathrm{H}_{10} \mathrm{O} ;$ [71-36-3] & $\begin{array}{l}\text { Acree, Jr., and M. H. Abraham, J. } \\
\text { Mol. Liq. 116, 19 (2005). }\end{array}$ \\
\hline Variables: & Prepared by: \\
$T / \mathrm{K}=298.15$ & W. E. Acree, Jr. \\
\hline
\end{tabular}

Experimental Values

\begin{tabular}{lc}
\hline \hline$x_{2}{ }^{\mathrm{a}}$ & $x_{1}{ }^{\mathrm{b}}$ \\
\hline 0.854 & 0.146 \\
\hline${ }^{\mathrm{a}} x_{2}:$ mole fraction of component 2 in the saturated solution. & \\
${ }^{\mathrm{b}} x_{1}:$ mole fraction solubility of the solute. &
\end{tabular}

\section{Auxiliary Information}

\section{Method/Apparatus/Procedure:}

Constant-temperature bath, calorimetric thermometer, and an ultraviolet/ visible spectrophotometer.

Excess solute and solvent were placed in amber glass bottles and allowed to equilibrate for several days at constant temperature. Attainment of equilibrium was verified by several repetitive measurements and by approaching equilibrium from supersaturation. Aliquots of saturated solutions were transferred through a coarse filter into tared volumetric flasks, weighed, and diluted with methanol. Concentrations were determined by

spectrophotometric measurements at $280 \mathrm{~nm}$.

\section{Source and Purity of Chemicals:}

(1) $99 \%$, Acros Organics, USA, and also from Aldrich Chemical Company, Milwaukee, WI, USA, was used as received.

(2) $99.8+\%$, HPLC grade, Aldrich Chemical Company, stored over molecular sieves and distilled shortly before use.

\section{Estimated Error:}

Temperature: $\pm 0.1 \mathrm{~K}$.

$x_{1}: \pm 1.5 \%$ (relative error).

\begin{tabular}{ll}
\hline \hline Components: & Original Measurements: \\
(1) 3-Nitrobenzoic acid; $\mathrm{C}_{7} \mathrm{H}_{5} \mathrm{NO}_{4} ;$ & ${ }^{10}$ A. K. Charlton, C. R. Daniels, R. \\
[121-92-6] & M. Wold, E. Pustejovsky, W. E. \\
(2) 2-Butanol; $\mathrm{C}_{4} \mathrm{H}_{10} \mathrm{O} ;$ [78-92-2] & Acree, Jr., and M. H. Abraham, J. \\
& Mol. Liq. 116, 19 (2005). \\
\hline Variables: & Prepared by: \\
$T / \mathrm{K}=298.15$ & W. E. Acree, Jr. \\
\hline
\end{tabular}

\section{Experimental Values}

\begin{tabular}{lc}
\hline \hline$x_{2}{ }^{\mathrm{a}}$ & $x_{1}{ }^{\mathrm{b}}$ \\
\hline 0.812 & 0.188 \\
\hline${ }^{\mathrm{a}} x_{2}:$ mole fraction of component 2 in the saturated solution. & \\
${ }^{\mathrm{b}} x_{1}:$ mole fraction solubility of the solute. &
\end{tabular}

\section{Auxiliary Information}

\section{Method/Apparatus/Procedure:}

Constant-temperature bath, calorimetric thermometer, and an ultraviolet/ visible spectrophotometer.

Excess solute and solvent were placed in amber glass bottles and allowed to equilibrate for several days at constant temperature. Attainment of equilibrium was verified by several repetitive measurements and by approaching equilibrium from supersaturation. Aliquots of saturated solutions were transferred through a coarse filter into tared volumetric flasks, weighed, and diluted with methanol. Concentrations were determined by spectrophotometric measurements at $280 \mathrm{~nm}$.

\section{Source and Purity of Chemicals:}

(1) $99 \%$, Acros Organics, USA, and also from Aldrich Chemical Company, Milwaukee, WI, USA, was used as received.

(2) $99+\%$, anhydrous, Aldrich Chemical Company, stored over molecular sieves and distilled shortly before use.

\section{Estimated Error:}

Temperature: $\pm 0.1 \mathrm{~K}$.

$x_{1}: \pm 1.5 \%$ (relative error)

\begin{tabular}{ll}
\hline \hline Components: & Original Measurements: \\
(1) 3-Nitrobenzoic acid; $\mathrm{C}_{7} \mathrm{H}_{5} \mathrm{NO}_{4} ;$ & ${ }^{10}$ A. K. Charlton, C. R. Daniels, R. \\
[121-92-6] & M. Wold, E. Pustejovsky, W. E. \\
$\begin{array}{l}\text { (2) 2-Methyl-1-propanol; } \mathrm{C}_{4} \mathrm{H}_{10} \mathrm{O} ; \\
\text { [78-83-1] }\end{array}$ & Acree, Jr., and M. H. Abraham, J. \\
\hline Variables: & Mol. Liq. 116, 19 (2005). \\
$T / \mathrm{K}=298.15$ & Prepared by: \\
\hline
\end{tabular}

Experimental Values

\begin{tabular}{lc}
\hline \hline$x_{2}{ }^{\mathrm{a}}$ & $x_{1}{ }^{\mathrm{b}}$ \\
\hline 0.887 & 0.113 \\
\hline${ }^{\mathrm{a}} x_{2}:$ mole fraction of component 2 in the saturated solution. & \\
${ }^{\mathrm{b}}{ }_{x_{1}: \text { mole fraction solubility of the solute. }}$ &
\end{tabular}




\section{Auxiliary Information}

\section{Method/Apparatus/Procedure:}

Constant-temperature bath, calorimetric thermometer, and an ultraviolet/ visible spectrophotometer.

Excess solute and solvent were placed in amber glass bottles and allowed to equilibrate for several days at constant temperature. Attainment of equilibrium was verified by several repetitive measurements and by approaching equilibrium from supersaturation. Aliquots of saturated solutions were transferred through a coarse filter into tared volumetric flasks, weighed, and diluted with methanol. Concentrations were determined by

spectrophotometric measurements at $280 \mathrm{~nm}$.

Source and Purity of Chemicals:

(1) $99 \%$, Acros Organics, USA, and also from Aldrich Chemical Company, Milwaukee, WI, USA, was used as received.

(2) $99+\%$, anhydrous, Aldrich Chemical Company, stored over molecular sieves and distilled shortly before use.

Estimated Error:

Temperature: $\pm 0.1 \mathrm{~K}$.

$x_{1}: \pm 1.5 \%$ (relative error).

\begin{tabular}{ll}
\hline \hline Components: & Original Measurements: \\
$\begin{array}{l}\text { (1) 3-Nitrobenzoic acid; } \mathrm{C}_{7} \mathrm{H}_{5} \mathrm{NO}_{4} ; \\
\text { [121-92-6] }\end{array}$ & ${ }^{10}$ A. K. Charlton, C. R. Daniels, R. \\
$\begin{array}{l}\text { M. Wold, E. Pustejovsky, W. E. } \\
\text { [75-65-0] }\end{array}$ & Acree, Jr., and M. H. Abraham, J. \\
\hline Variables: & Mol. Liq. 116, 19 (2005). \\
$T / \mathrm{K}=298.15$ & Prepared by: \\
\hline
\end{tabular}

Experimental Values

\begin{tabular}{lc}
\hline \hline$x_{2}^{\mathrm{a}}$ & $x_{1}{ }^{\mathrm{b}}$ \\
\hline 0.783 & 0.217
\end{tabular}

${ }^{\mathrm{a}} x_{2}$ : mole fraction of component 2 in the saturated solution.

${ }^{\mathrm{b}} x_{1}$ : mole fraction solubility of the solute.

\section{Auxiliary Information}

\section{Method/Apparatus/Procedure:}

Constant-temperature bath, calorimetric thermometer, and an ultraviolet/ visible spectrophotometer.

Excess solute and solvent were placed in amber glass bottles and allowed to equilibrate for several days at constant temperature. Attainment of equilibrium was verified by several repetitive measurements and by approaching equilibrium from supersaturation. Aliquots of saturated solutions were transferred through a coarse filter into tared volumetric flasks, weighed, and diluted with methanol. Concentrations were determined by

spectrophotometric measurements at $280 \mathrm{~nm}$.

\section{Source and Purity of Chemicals:}

(1) $99 \%$, Acros Organics, USA, and also from Aldrich Chemical Company, Milwaukee, WI, USA, was used as received.

(2) $99+\%$, Arco Chemical Company, USA, stored over molecular sieves and distilled shortly before use.

\section{Estimated Error:}

Temperature: $\pm 0.1 \mathrm{~K}$

$x_{1}: \pm 2.0 \%$ (relative error).

\begin{tabular}{ll}
\hline \hline Components: & Original Measurements: \\
(1) 3-Nitrobenzoic acid; $\mathrm{C}_{7} \mathrm{H}_{5} \mathrm{NO}_{4} ;$ & ${ }^{10}$ A. K. Charlton, C. R. Daniels, R. \\
[121-92-6] & M. Wold, E. Pustejovsky, W. E. \\
(2) 1-Pentanol; $\mathrm{C}_{5} \mathrm{H}_{12} \mathrm{O} ;$ [71-41-0] & Acree, Jr., and M. H. Abraham, J. \\
& Mol. Liq. 116, 19 (2005). \\
\hline Variables: & Prepared by: \\
$T / \mathrm{K}=298.15$ & W. E. Acree, Jr. \\
\hline
\end{tabular}

\section{Experimental Values}

\begin{tabular}{lc}
\hline \hline$x_{2}{ }^{\mathrm{a}}$ & $x_{1}{ }^{\mathrm{b}}$ \\
\hline 0.857 & 0.143 \\
\hline${ }^{\mathrm{a}} x_{2}:$ mole fraction of component 2 in the saturated solution. & \\
${ }^{\mathrm{b}} x_{1}:$ mole fraction solubility of the solute. &
\end{tabular}

\section{Auxiliary Information}

\section{Method/Apparatus/Procedure:}

Constant-temperature bath, calorimetric thermometer, and an ultraviolet/ visible spectrophotometer.

Excess solute and solvent were placed in amber glass bottles and allowed to equilibrate for several days at constant temperature. Attainment of equilibrium was verified by several repetitive measurements and by approaching equilibrium from supersaturation. Aliquots of saturated solutions were transferred through a coarse filter into tared volumetric flasks, weighed, and diluted with methanol. Concentrations were determined by spectrophotometric measurements at $280 \mathrm{~nm}$.

\section{Source and Purity of Chemicals:}

(1) $99 \%$, Acros Organics, USA, and also from Aldrich Chemical Company, Milwaukee, WI, USA, was used as received.

(2) $99+\%$, Aldrich Chemical Company, stored over molecular sieves and distilled shortly before use.

\section{Estimated Error:}

Temperature: $\pm 0.1 \mathrm{~K}$.

$x_{1}: \pm 1.5 \%$ (relative error)

\begin{tabular}{ll}
\hline \hline Components: & Original Measurements: \\
(1) 3-Nitrobenzoic acid; $\mathrm{C}_{7} \mathrm{H}_{5} \mathrm{NO}_{4} ;$ & ${ }^{10}$ A. K. Charlton, C. R. Daniels, R. \\
[121-92-6] & M. Wold, E. Pustejovsky, W. E. \\
(2) 2-Pentanol; $\mathrm{C}_{5} \mathrm{H}_{12} \mathrm{O} ;$ [6032-29-7] & Acree, Jr., and M. H. Abraham, J. \\
& Mol. Liq. 116, 19 (2005). \\
\hline Variables: & Prepared by: \\
$T / \mathrm{K}=298.15$ & W. E. Acree, Jr. \\
\hline
\end{tabular}

\section{Experimental Values}

\begin{tabular}{lc}
\hline \hline$x_{2}{ }^{\mathrm{a}}$ & $x_{1}{ }^{\mathrm{b}}$ \\
\hline 0.824 & 0.176 \\
\hline${ }^{\mathrm{a}} x_{2}:$ mole fraction of component 2 in the saturated solution. & \\
${ }^{\mathrm{b}} x_{1}:$ mole fraction solubility of the solute. &
\end{tabular}




\section{Auxiliary Information}

\section{Method/Apparatus/Procedure:}

Constant-temperature bath, calorimetric thermometer, and an ultraviolet/ visible spectrophotometer.

Excess solute and solvent were placed in amber glass bottles and allowed to equilibrate for several days at constant temperature. Attainment of equilibrium was verified by several repetitive measurements and by approaching equilibrium from supersaturation. Aliquots of saturated solutions were transferred through a coarse filter into tared volumetric flasks, weighed, and diluted with methanol. Concentrations were determined by spectrophotometric measurements at $280 \mathrm{~nm}$.

Source and Purity of Chemicals:

(1) $99 \%$, Acros Organics, USA, and also from Aldrich Chemical Company, Milwaukee, WI, USA, was used as received.

(2) $99+\%$, Acros Organic, USA, stored over molecular sieves and distilled shortly before use.

Estimated Error:

Temperature: $\pm 0.1 \mathrm{~K}$. $x_{1}: \pm 1.5 \%$ (relative error).

\begin{tabular}{|c|c|}
\hline Components: & Original Measurements: \\
\hline $\begin{array}{l}\text { (1) 3-Nitrobenzoic acid; } \mathrm{C}_{7} \mathrm{H}_{5} \mathrm{NO}_{4} \text {; } \\
\text { [121-92-6] }\end{array}$ & $\begin{array}{l}{ }^{10} \text { A. K. Charlton, C. R. Daniels, R. } \\
\text { M. Wold, E. Pustejovsky, W. E. }\end{array}$ \\
\hline $\begin{array}{l}\text { (2) 2-Methyl-1-butanol; } \mathrm{C}_{5} \mathrm{H}_{12} \mathrm{O} \text {; } \\
\text { [137-32-6] }\end{array}$ & $\begin{array}{l}\text { Acree, Jr., and M. H. Abraham, J. } \\
\text { Mol. Liq. 116, } 19 \text { (2005). }\end{array}$ \\
\hline Variables: & Prepared by: \\
\hline$T / \mathrm{K}=298.15$ & W. E. Acree, Jr. \\
\hline
\end{tabular}

Experimental Values

\begin{tabular}{lc}
\hline \hline$x_{2}^{\mathrm{a}}$ & $x_{1}^{\mathrm{b}}$ \\
\hline 0.890 & 0.110 \\
\hline${ }^{\mathrm{a}} x_{2}:$ mole fraction of component 2 in the saturated solution. & \\
${ }^{\mathrm{b}}{ }_{x_{1}: \text { mole fraction solubility of the solute. }}$ &
\end{tabular}

\section{Auxiliary Information}

\section{Method/Apparatus/Procedure:}

Constant-temperature bath, calorimetric thermometer, and an ultraviolet/ visible spectrophotometer.

Excess solute and solvent were placed in amber glass bottles and allowed to equilibrate for several days at constant temperature. Attainment of equilibrium was verified by several repetitive measurements and by approaching equilibrium from supersaturation. Aliquots of saturated solutions were transferred through a coarse filter into tared volumetric flasks, weighed, and diluted with methanol. Concentrations were determined by

spectrophotometric measurements at $280 \mathrm{~nm}$.

\section{Source and Purity of Chemicals:}

(1) $99 \%$, Acros Organics, USA, and also from Aldrich Chemical Company, Milwaukee, WI, USA, was used as received.

(2) $99 \%$, Aldrich Chemical Company, stored over molecular sieves and distilled shortly before use.

\section{Estimated Error:}

Temperature: $\pm 0.1 \mathrm{~K}$

$x_{1}: \pm 1.5 \%$ (relative error).

\begin{tabular}{ll}
\hline \hline Components: & Original Measurements: \\
(1) 3-Nitrobenzoic acid; $\mathrm{C}_{7} \mathrm{H}_{5} \mathrm{NO}_{4} ;$ & ${ }^{10}$ A. K. Charlton, C. R. Daniels, R. \\
[121-92-6] & M. Wold, E. Pustejovsky, W. E. \\
$\begin{array}{l}\text { (2) 3-Methyl-1-butanol; } \mathrm{C}_{5} \mathrm{H}_{12} \mathrm{O} ; \\
\text { [123-51-3] }\end{array}$ & Acree, Jr., and M. H. Abraham, J. \\
\hline Mol. Liq. 116, 19 (2005). \\
\hline Variables: & Prepared by: \\
\hline
\end{tabular}

Experimental Values

\begin{tabular}{lc}
\hline \hline$x_{2}{ }^{\mathrm{a}}$ & $x_{1}{ }^{\mathrm{b}}$ \\
\hline 0.855 & 0.145
\end{tabular}

${ }^{\mathrm{a}} x_{2}$ : mole fraction of component 2 in the saturated solution.

${ }^{b} x_{1}$ : mole fraction solubility of the solute.

\section{Auxiliary Information}

\section{Method/Apparatus/Procedure:}

Constant-temperature bath, calorimetric thermometer, and an ultraviolet/ visible spectrophotometer.

Excess solute and solvent were placed in amber glass bottles and allowed to equilibrate for several days at constant temperature. Attainment of equilibrium was verified by several repetitive measurements and by approaching equilibrium from supersaturation. Aliquots of saturated solutions were transferred through a coarse filter into tared volumetric flasks, weighed, and diluted with methanol. Concentrations were determined by spectrophotometric measurements at $280 \mathrm{~nm}$.

Source and Purity of Chemicals:

(1) $99 \%$, Acros Organics, USA, and also from Aldrich Chemical Company, Milwaukee, WI, USA, was used as received.

(2) $99 \%$, anhydrous, Aldrich Chemical Company, stored over molecular sieves and distilled shortly before use.

\section{Estimated Error:}

Temperature: $\pm 0.1 \mathrm{~K}$.

$x_{1}: \pm 1.5 \%$ (relative error).

\begin{tabular}{ll}
\hline \hline Components: & Original Measurements: \\
(1) 3-Nitrobenzoic acid; $\mathrm{C}_{7} \mathrm{H}_{5} \mathrm{NO}_{4} ;$ & ${ }^{10}$ A. K. Charlton, C. R. Daniels, R. \\
[121-92-6] & M. Wold, E. Pustejovsky, W. E. \\
(2) 1-Hexanol; $\mathrm{C}_{6} \mathrm{H}_{14} \mathrm{O} ;[111-27-3]$ & Acree, Jr., and M. H. Abraham, J. \\
& Mol. Liq. 116, 19 (2005). \\
\hline Variables: & Prepared by: \\
$T / \mathrm{K}=298.15$ & W. E. Acree, Jr. \\
\hline
\end{tabular}

Experimental Values

\begin{tabular}{lc}
\hline \hline$x_{2}{ }^{\mathrm{a}}$ & $x_{1}{ }^{\mathrm{b}}$ \\
\hline 0.861 & 0.139 \\
\hline${ }^{\mathrm{a}} x_{2}:$ mole fraction of component 2 in the saturated solution. & \\
${ }^{\mathrm{b}} x_{1}:$ mole fraction solubility of the solute. &
\end{tabular}




\section{Auxiliary Information}

\section{Method/Apparatus/Procedure:}

Constant-temperature bath, calorimetric thermometer, and an ultraviolet/ visible spectrophotometer.

Excess solute and solvent were placed in amber glass bottles and allowed to equilibrate for several days at constant temperature. Attainment of equilibrium was verified by several repetitive measurements and by approaching equilibrium from supersaturation. Aliquots of saturated solutions were transferred through a coarse filter into tared volumetric flasks, weighed, and diluted with methanol. Concentrations were determined by spectrophotometric measurements at $280 \mathrm{~nm}$.

Source and Purity of Chemicals:

(1) $99 \%$, Acros Organics, USA, and also from Aldrich Chemical Company, Milwaukee, WI, USA, was used as received.

(2) $99+\%$, Alfa Aesar, USA, stored over molecular sieves and distilled shortly before use.

Estimated Error:

Temperature: $\pm 0.1 \mathrm{~K}$.

$x_{1}: \pm 1.5 \%$ (relative error).

\begin{tabular}{ll}
\hline \hline Components: & Original Measurements: \\
$\begin{array}{l}\text { (1) 3-Nitrobenzoic acid; } \mathrm{C}_{7} \mathrm{H}_{5} \mathrm{NO}_{4} ; \\
\text { [121-92-6] }\end{array}$ & ${ }^{10}$ A. K. Charlton, C. R. Daniels, R. \\
$\begin{array}{l}\text { M. Wold, E. Pustejovsky, W. E. } \\
\text { [105-30-6] }\end{array}$ & Acree, Jr., and M. H. Abraham, J. \\
\hline Variables: & Mol. Liq. 116, 19 (2005). \\
$T / \mathrm{K}=298.15$ & Prepared by: \\
\hline
\end{tabular}

Experimental Values

\begin{tabular}{lc}
\hline \hline$x_{2}{ }^{\mathrm{a}}$ & $x_{1}{ }^{\mathrm{b}}$ \\
\hline 0.866 & 0.134 \\
\hline${ }^{\mathrm{a}} x_{2}:$ mole fraction of component 2 in the saturated solution. & \\
${ }^{\mathrm{b}} x_{1}:$ mole fraction solubility of the solute. &
\end{tabular}

\section{Auxiliary Information}

\section{Method/Apparatus/Procedure:}

Constant-temperature bath, calorimetric thermometer, and an ultraviolet/ visible spectrophotometer.

Excess solute and solvent were placed in amber glass bottles and allowed to equilibrate for several days at constant temperature. Attainment of equilibrium was verified by several repetitive measurements and by approaching equilibrium from supersaturation. Aliquots of saturated solutions were transferred through a coarse filter into tared volumetric flasks, weighed, and diluted with methanol. Concentrations were determined by

spectrophotometric measurements at $280 \mathrm{~nm}$.

\section{Source and Purity of Chemicals:}

(1) $99 \%$, Acros Organics, USA, and also from Aldrich Chemical Company, Milwaukee, WI, USA, was used as received.

(2) $99 \%$, Aldrich Chemical Company, stored over molecular sieves and distilled shortly before use.

\section{Estimated Error:}

Temperature: $\pm 0.1 \mathrm{~K}$.

$x_{1}: \pm 1.5 \%$ (relative error).

\begin{tabular}{ll}
\hline \hline Components: & Original Measurements: \\
(1) 3-Nitrobenzoic acid; $\mathrm{C}_{7} \mathrm{H}_{5} \mathrm{NO}_{4} ;$ & ${ }^{10}$ A. K. Charlton, C. R. Daniels, R. \\
[121-92-6] & M. Wold, E. Pustejovsky, W. E. \\
(2) 1-Heptanol; $\mathrm{C}_{7} \mathrm{H}_{16} \mathrm{O} ;$ [111-70-6] & Acree, Jr., and M. H. Abraham, J. \\
& Mol. Liq. 116, 19 (2005). \\
\hline Variables: & Prepared by: \\
$T / \mathrm{K}=298.15$ & W. E. Acree, Jr. \\
\hline
\end{tabular}

\section{Experimental Values}

\begin{tabular}{lc}
\hline \hline$x_{2}{ }^{\mathrm{a}}$ & $x_{1}{ }^{\mathrm{b}}$ \\
\hline 0.864 & 0.136 \\
\hline${ }^{\mathrm{a}} x_{2}:$ mole fraction of component 2 in the saturated solution. & \\
${ }^{\mathrm{b}} x_{1}:$ mole fraction solubility of the solute. &
\end{tabular}

\section{Auxiliary Information}

\section{Method/Apparatus/Procedure:}

Constant-temperature bath, calorimetric thermometer, and an ultraviolet/ visible spectrophotometer.

Excess solute and solvent were placed in amber glass bottles and allowed to equilibrate for several days at constant temperature. Attainment of equilibrium was verified by several repetitive measurements and by approaching equilibrium from supersaturation. Aliquots of saturated solutions were transferred through a coarse filter into tared volumetric flasks, weighed, and diluted with methanol. Concentrations were determined by spectrophotometric measurements at $280 \mathrm{~nm}$.

\section{Source and Purity of Chemicals:}

(1) $99 \%$, Acros Organics, USA, and also from Aldrich Chemical Company, Milwaukee, WI, USA, was used as received.

(2) $99+\%$, Alfa Aesar, USA, stored over molecular sieves and distilled shortly before use.

\section{Estimated Error:}

Temperature: $\pm 0.1 \mathrm{~K}$.

$x_{1}: \pm 1.5 \%$ (relative error)

\begin{tabular}{ll}
\hline \hline Components: & Original Measurements: \\
(1) 3-Nitrobenzoic acid; $\mathrm{C}_{7} \mathrm{H}_{5} \mathrm{NO}_{4} ;$ & ${ }^{10}$ A. K. Charlton, C. R. Daniels, R. \\
[121-92-6] & M. Wold, E. Pustejovsky, W. E. \\
(2) 1-Octanol; $\mathrm{C}_{8} \mathrm{H}_{18} \mathrm{O} ;$ [111-87-5] & Acree, Jr., and M. H. Abraham, J. \\
& Mol. Liq. 116, 19 (2005). \\
\hline Variables: & Prepared by: \\
$T / \mathrm{K}=298.15$ & W. E. Acree, Jr. \\
\hline
\end{tabular}

\section{Experimental Values}

\begin{tabular}{lc}
\hline \hline$x_{2}{ }^{\mathrm{a}}$ & $x_{1}{ }^{\mathrm{b}}$ \\
\hline 0.856 & 0.144 \\
\hline${ }^{\mathrm{a}} x_{2}:$ mole fraction of component 2 in the saturated solution. & \\
${ }^{\mathrm{b}} x_{1}:$ mole fraction solubility of the solute. &
\end{tabular}




\section{Auxiliary Information}

\section{Method/Apparatus/Procedure:}

Constant-temperature bath, calorimetric thermometer, and an ultraviolet/ visible spectrophotometer.

Excess solute and solvent were placed in amber glass bottles and allowed to equilibrate for several days at constant temperature. Attainment of equilibrium was verified by several repetitive measurements and by approaching equilibrium from supersaturation. Aliquots of saturated solutions were transferred through a coarse filter into tared volumetric flasks, weighed, and diluted with methanol. Concentrations were determined by spectrophotometric measurements at $280 \mathrm{~nm}$.

Source and Purity of Chemicals:

(1) $99 \%$, Acros Organics, USA, and also from Aldrich Chemical Company, Milwaukee, WI, USA, was used as received.

(2) $99+\%$, anhydrous, Aldrich Chemical Company, stored over molecular sieves and distilled shortly before use.

Estimated Error:

Temperature: $\pm 0.1 \mathrm{~K}$.

$x_{1}: \pm 1.5 \%$ (relative error)

\begin{tabular}{|c|c|}
\hline $\begin{array}{l}\text { Components: } \\
\text { (1) 3-Nitrobenzoic acid; } \mathrm{C}_{7} \mathrm{H}_{5} \mathrm{NO}_{4} \text {; } \\
\text { [121-92-6] } \\
\text { (2) 1-Octanol; } \mathrm{C}_{8} \mathrm{H}_{18} \mathrm{O} \text {; [111-87-5] }\end{array}$ & $\begin{array}{l}\text { Original Measurements: } \\
\text { 99 Q. Jia, P. Ma, S. Ma, and C. Wang, } \\
\text { Chin. J. Chem. Eng. 15, } 710 \text { (2007). }\end{array}$ \\
\hline Variables: & Prepared by: \\
\hline Temperature & W. E. Acree, Jr. \\
\hline
\end{tabular}

Experimental Values

\begin{tabular}{lcc}
\hline \hline$T / \mathrm{K}$ & $x_{2}{ }^{\mathrm{a}}$ & $x_{1}{ }^{\mathrm{b}}$ \\
\hline 296.25 & 0.8259 & 0.1741 \\
297.25 & 0.8213 & 0.1787 \\
299.15 & 0.8144 & 0.1856 \\
300.25 & 0.8063 & 0.1937 \\
302.90 & 0.8012 & 0.1988 \\
303.38 & 0.7904 & 0.2094 \\
305.85 & 0.7806 & 0.2194 \\
308.45 & 0.7866 & 0.2312 \\
311.75 & 0.7530 & 0.2470 \\
313.80 & 0.7379 & 0.2621 \\
316.25 & 0.7251 & 0.2749 \\
317.65 & 0.7133 & 0.2867 \\
322.15 & 0.6945 & 0.3055 \\
\hline
\end{tabular}

${ }^{\mathrm{a}} x_{2}$ : mole fraction of component 2 in the saturated solution.

${ }^{\mathrm{b}} x_{1}$ : mole fraction solubility of the solute.

\section{Auxiliary Information}

\section{Method/Apparatus/Procedure:}

Constant-temperature circulating bath, stirrer, analytical balance, and laser monitoring system.

Solubilities were determined by a dynamic method. Pre-weighed amounts of solute and solvent were placed in a stoppered equilibrium vessel, which was connected to a circulating constant-temperature water bath. The solution was stirred and the temperature slowly increased until all of the solid dissolved. Near the dissolution temperature, the temperature was increased at a rate of 0.2 $\mathrm{K} / 20 \mathrm{~min}$. Complete dissolution was determined using a laser monitoring system.
Source and Purity of Chemicals:

(1) $99+\%$, Chemical source not specified, no purification details were provided.

(2) $99+\%$, Chemical source not specified, no purification details were provided.

\section{Estimated Error:}

Temperature: $\pm 0.05 \mathrm{~K}$

$x_{1}: \pm 1 \%$ (relative error)

\begin{tabular}{ll}
\hline \hline Components: & Original Measurements: \\
(1) 3-Nitrobenzoic acid; $\mathrm{C}_{7} \mathrm{H}_{5} \mathrm{NO}_{4} ;$ & ${ }^{10}$ A. K. Charlton, C. R. Daniels, R. \\
[121-92-6] & M. Wold, E. Pustejovsky, W. E. \\
(2) 1-Decanol; $\mathrm{C}_{10} \mathrm{H}_{22} \mathrm{O} ;[112-30-1]$ & Acree, Jr., and M. H. Abraham, J. \\
& Mol. Liq. 116, 19 (2005). \\
\hline Variables: & Prepared by: \\
$T / \mathrm{K}=298.15$ & W. E. Acree, Jr. \\
\hline
\end{tabular}

\section{Experimental Values}

\begin{tabular}{lc}
\hline \hline$x_{2}{ }^{\mathrm{a}}$ & $x_{1}{ }^{\mathrm{b}}$ \\
\hline 0.861 & 0.139 \\
\hline${ }^{\mathrm{a}} x_{2}:$ mole fraction of component 2 in the saturated solution. & \\
${ }^{\mathrm{b}} x_{1}:$ mole fraction solubility of the solute. &
\end{tabular}

\section{Auxiliary Information}

\section{Method/Apparatus/Procedure:}

Constant-temperature bath, calorimetric thermometer, and an ultraviolet/ visible spectrophotometer.

Excess solute and solvent were placed in amber glass bottles and allowed to equilibrate for several days at constant temperature. Attainment of equilibrium was verified by several repetitive measurements and by approaching equilibrium from supersaturation. Aliquots of saturated solutions were transferred through a coarse filter into tared volumetric flasks, weighed, and diluted with methanol. Concentrations were determined by spectrophotometric measurements at $280 \mathrm{~nm}$.

Source and Purity of Chemicals:

(1) $99 \%$, Acros Organics, USA, and also from Aldrich Chemical Company, Milwaukee, WI, USA, was used as received.

(2) $99+\%$, Alfa Aesar, USA, stored over molecular sieves and distilled shortly before use.

Estimated Error:

Temperature: $\pm 0.1 \mathrm{~K}$.

$x_{1}: \pm 1.5 \%$ (relative error).

\subsection{3-Nitrobenzoic acid solubility data in ketones}

\begin{tabular}{|c|c|}
\hline $\begin{array}{l}\text { Components: } \\
\text { (1) 3-Nitrobenzoic acid; } \mathrm{C}_{7} \mathrm{H}_{5} \mathrm{NO}_{4} \text {; } \\
\text { [121-92-6] } \\
\text { (2) Propanone; } \mathrm{C}_{3} \mathrm{H}_{6} \mathrm{O} ;[67-64-1]\end{array}$ & $\begin{array}{l}\text { Original Measurements: } \\
{ }^{180} \text { A. R. Collett and C. L. Lazzell, J. } \\
\text { Phys. Chem. 34, } 1838 \text { (1930). }\end{array}$ \\
\hline $\begin{array}{l}\text { Variables: } \\
\text { Temperature }\end{array}$ & $\begin{array}{l}\text { Prepared by: } \\
\text { W. E. Acree, Jr. }\end{array}$ \\
\hline
\end{tabular}


Experimental Values

\begin{tabular}{lcc}
\hline \hline$T / \mathrm{K}$ & $x_{2}{ }^{\mathrm{a}}$ & $x_{1}{ }^{\mathrm{b}}$ \\
\hline 283.2 & 0.8477 & 0.1523 \\
301.2 & 0.7730 & 0.2270 \\
338.0 & 0.6168 & 0.3832 \\
361.2 & 0.4892 & 0.5108 \\
377.2 & 0.3847 & 0.6153 \\
393.9 & 0.2317 & 0.7683 \\
\hline
\end{tabular}

${ }^{a_{2}}$ : mole fraction of component 2 in the saturated solution.

${ }^{\mathrm{b}} x_{1}$ : mole fraction solubility of the solute. The solubility is given as molal percentage, which, based on the description of 100 times the mole fraction, is taken to be mole percentage.

\section{Auxiliary Information}

\section{Method/Apparatus/Procedure:}

Preweighed quantities of solute and solvent were placed in bulbs, which were then sealed and heated in a suitable bath. The temperature at which the solid completely dissolved was recorded, and was taken to be the solid-liquid equilibrium temperature. In a few instances near room temperature, the solubility was determined by agitating the solvent in a thermostat with an excess of solute. After equilibrium had been established, an aliquot of the saturated solution was pipetted, weighed, and the amount of dissolved solute determined with a standard solution of titanium (III) chloride.

\section{Source and Purity of Chemicals:}

(1) Purity not given, Synthesized by the authors according to the published method of Holleman [Recl. Trav. Chim. 18, 267 (1899)], was washed with water, recrystallized from trichloromethane, and recrystallized again from water to give a purified sample having a melting point temperature of $414.6 \mathrm{~K}$. (2) Certified Pure, Merck Chemical Company, was dried over calcium chloride and distilled twice before use.

\section{Estimated Error:}

Temperature: Not given in paper.

$x_{2}: \pm 10 \%$ (relative error, estimated by compiler).

\subsection{3-Nitrobenzoic acid solubility data in miscellaneous organic solvents}

\begin{tabular}{ll}
\hline \hline Components: & Original Measurements: \\
(1) 3-Nitrobenzoic acid; $\mathrm{C}_{7} \mathrm{H}_{5} \mathrm{NO}_{4} ;$ & $\begin{array}{l}{ }^{10} \text { A. K. Charlton, C. R. Daniels, R. } \\
\text { [121-92-6] }\end{array}$ \\
$\begin{array}{l}\text { M. Wold, E. Pustejovsky, W. E. } \\
\text { (2) Propylene carbonate; } \mathrm{C}_{4} \mathrm{H}_{6} \mathrm{O}_{3} ;\end{array}$ & $\begin{array}{l}\text { Acree, Jr., and M. H. Abraham, J. } \\
\text { [108-32-7] }\end{array}$ \\
\hline Mol. Liq. 116, 19 (2005). \\
$T / \mathrm{K}=298.15$ & Prepared by: \\
\hline
\end{tabular}

Experimental Values

\begin{tabular}{lc}
\hline \hline$x_{2}{ }^{\mathrm{a}}$ & $x_{1}{ }^{\mathrm{b}}$ \\
\hline 0.9166 & 0.0834 \\
\hline${ }^{\mathrm{a}} x_{2}:$ mole fraction of component 2 in the saturated solution. & \\
${ }^{\mathrm{b}}{ }_{x_{1}}:$ mole fraction solubility of the solute. &
\end{tabular}

\section{Auxiliary Information}

\section{Method/Apparatus/Procedure:}

Constant-temperature bath, calorimetric thermometer, and an ultraviolet/ visible spectrophotometer.

Excess solute and solvent were placed in amber glass bottles and allowed to equilibrate for several days at constant temperature. Attainment of equilibrium was verified by several repetitive measurements and by approaching equilibrium from supersaturation. Aliquots of saturated solutions were transferred through a coarse filter into tared volumetric flasks, weighed, and diluted with methanol. Concentrations were determined by spectrophotometric measurements at $280 \mathrm{~nm}$.

\section{Source and Purity of Chemicals:}

(1) $99 \%$, Acros Organics, USA, and also from Aldrich Chemical Company, Milwaukee, WI, USA, was used as received.

(2) $99.7 \%$, anhydrous, Aldrich Chemical Company, stored over molecular sieves and distilled shortly before use.

\section{Estimated Error:}

Temperature: $\pm 0.1 \mathrm{~K}$.

$x_{1}: \pm 1.5 \%$ (relative error)

\begin{tabular}{|c|c|}
\hline $\begin{array}{l}\text { Components: } \\
\text { (1) 3-Nitrobenzoic acid; } \mathrm{C}_{7} \mathrm{H}_{5} \mathrm{NO}_{4} \text {; } \\
\text { [121-92-6] } \\
\text { (2) Ethanenitrile; } \mathrm{C}_{2} \mathrm{H}_{3} \mathrm{~N} \text {; [75-05-8] }\end{array}$ & $\begin{array}{l}\text { Original Measurements: } \\
{ }^{83} \text { M. K. Chantooni and I. M. } \\
\text { Kolthoff, J. Phys. Chem. 77, } 527 \\
(1973) \text {. }\end{array}$ \\
\hline $\begin{array}{l}\text { Variables: } \\
T / \mathrm{K}=298.15\end{array}$ & $\begin{array}{l}\text { Prepared by: } \\
\text { W. E. Acree, Jr. }\end{array}$ \\
\hline
\end{tabular}

\section{Experimental Values}

The measured solubility was reported to be $0.78 \mathrm{~mol} \mathrm{dm}^{-3}$. The authors did not give the temperature at which the solubility was measured. Based on the experimental description given in an earlier paper [I. M. Kolthoff, J. J. Lingane, and W. Larson, J. Am. Chem. Soc. 60, 2512 (1938)], the compiler believes the temperature to be $298.15 \mathrm{~K}$.

\section{Auxiliary Information}

\section{Method/Apparatus/Procedure:}

Very little experimental details were provided. Solubility was determined by titrating a filtered aliquot of the saturated solution alkalimetrically in an aqueous-ethanol mixture using phenolphthalein as the acid-base indicator.

\section{Source and Purity of Chemicals:}

(1) Purity not given, Baker Chemical Company, USA, was recrystallized from either aqueous or aqueous-ethanol solution and dried in vacuo at $333 \mathrm{~K}$.

(2) Purity not given, Chemical source not given, was purified by shaking with saturated potassium hydroxide, followed by activated alumina, and then anhydrous calcium chloride to remove water. Ethanenitrile was further dried over anhydrous magnesium sulfate and then phosphorous pentoxide. The sample was distilled shortly before use.

\section{Estimated Error:}

Temperature: $\pm 0.1 \mathrm{~K}$ (estimated by compiler). $c_{1}: \pm 2 \%$ (relative error, estimated by compiler). 


\section{Solubility of 4-Nitrobenzoic Acid in Organic Solvents}

\subsection{Critical evaluation of experimental solubility data}

There have been several published studies $11,54,63,72,76,126,127,129,130,136,180,181$ investigating the solubility behavior of 4-nitrobenzoic acid in organic solvents of varying polarity and hydrogen-bonding capability. Hoover et $a l .{ }^{11}$ measured the solubility of 4-nitrobenzoic acid in 19 alcohols (methanol, ethanol, 1-propanol, 2-propanol, 1-butanol, 2-butanol, 2-methyl-1-propanol, 2-methyl-2-propanol, 1pentanol, 2-pentanol, 2-methyl-1-butanol, 3-methyl-1-butanol, 2-methyl-2-butanol, 1-hexanol, 2-methyl-1-pentanol, 4methyl-2-pentanol, 1-heptanol, 1-octanol, and 1-decanol), in three dialkyl ethers (1,1'-oxybisethane, 2,2'-oxybispropane, and 1,1'-oxybisbutane) and two cyclic ethers (tetrahydrofuran and 1,4-dioxane), in four alkyl alkanoates (methyl ethanoate, ethyl ethanoate, butyl ethanoate, and pentyl ethanoate), and in propylene carbonate at $298 \mathrm{~K}$. Results of the experimental measurements were used to calculate Abraham solute descriptors of 4-nitrobenzoic acid. The authors were able to assemble a total of $51 \log _{10}(S R$ or $P)$ and $\log _{10}(G S R$ or $K)$ equations for which experimental partition coefficient data, solubility ratios, Abraham model equation coefficients and aqueous molar solubility were available. The logarithm of the aqueous molar solubility of 4-nitrobenzoic acid is $\log _{10} c_{1, \mathrm{~W}}=-2.98 .^{72}$ The McGowan volume of 4-nitrobenzoic acid, $V=1.1059$, was calculated from the number of chemical bonds in the molecule and the individual atomic group volumes, $A V_{i}$, given in Sec. 1.3. The excess molar refraction solute descriptor was estimated as $E=0.990$. This left four solute descriptors ( $S, A$, $B$, and $L$ ) still to be determined. The 48 equations were then solved using the Microsoft "SOLVER" program to yield numerical values of the remaining four solute descriptors, $S=1.520$, $A=0.680, B=0.440$, and $L=5.7699$, that best described the $\log _{10}(S R$ or $P)$ and $\log _{10}(G S R$ or $K)$ values. The computation treated $\log _{10} c_{1, \mathrm{G}}$ as a floating parameter to be determined as part of the regression analyses. The data analyses returned a value of $\log _{10} c_{1, \mathrm{G}}=-9.880$ for the gas-phase solute concentration that made the $\log _{10}(S R$ or $P)$ and $\log _{10}(G S R$ or $K)$ predictions internally consistent. The calculated molecular solute descriptors reproduced the $\log _{10}\left(S R\right.$ or $P$ ) and $\log _{10}$ ( $G S R$ or $K$ ) values to within an average standard deviation of 0.058 and $0.088 \log _{10}$ units, respectively.

After the 4-nitrobenzoic acid solubility study was published, Abraham model correlations have been developed for 2-pentanol, 3-methyl-1-butanol, methyl ethanoate, and butyl ethanoate, and equation coefficients for a few solvents were updated based on additional experimental data. The new correlations (listed in Tables 1 and 2) will be used in illustrating the ability of the Abraham model to correlate the experimental 4-nitrobenzoic acid solubility data. Table 48 compares the experimental $\log _{10} c_{1}$ values to calculated values based on Eqs. (20) and (21) of the Abraham model. For comparison purposes, the measured mole fraction solubilities of 4-nitrobenzoic acid, $x_{1}$, determined by Hoover et al. ${ }^{11}$ were converted into molar solubilities by dividing
TABLE 48. Comparison between observed and calculated molar solubilities of 4-nitrobenzoic acid based on the Abraham model, Eqs. (20) and (21)

\begin{tabular}{|c|c|c|c|}
\hline Solvent & $\log _{10} c_{1}{ }^{\exp , \mathrm{a}}$ & $\begin{array}{c}\log _{10} c_{1}{ }^{\text {calc }} ; \\
\text { Eq. (20) }\end{array}$ & $\begin{array}{c}\log _{10} c_{1}{ }^{\text {calc. }} ; \\
\text { Eq. }(21)\end{array}$ \\
\hline Methanol & -0.719 & -0.697 & -0.632 \\
\hline Ethanol & -0.841 & -0.816 & -0.769 \\
\hline 1-Propanol & -0.975 & -0.935 & -0.910 \\
\hline 2-Propanol & -0.956 & -0.930 & -0.898 \\
\hline 1-Butanol & -1.063 & -1.028 & -0.993 \\
\hline 2-Butanol & -1.017 & -0.982 & -1.007 \\
\hline 2-Methyl-1-propanol & -1.183 & -1.163 & -1.147 \\
\hline 2-Methyl-2-propanol & -0.821 & -0.904 & -0.879 \\
\hline 1-Pentanol & -1.067 & -1.108 & -1.082 \\
\hline 2-Pentanol & -1.086 & -1.150 & -1.102 \\
\hline 3-Methyl-1-butanol & -1.178 & -1.207 & -1.134 \\
\hline 1-Hexanol & -1.076 & -1.133 & -1.124 \\
\hline 1-Heptanol & -1.121 & -1.128 & -1.158 \\
\hline 1-Octanol & -1.209 & -1.162 & -1.261 \\
\hline 1-Decanol & -1.271 & -1.344 & -1.337 \\
\hline 1,1'-Oxybisethane & -0.902 & -1.094 & -1.008 \\
\hline Tetrahydrofuran & -0.117 & -0.195 & -0.234 \\
\hline 1,4-Dioxane & -0.317 & -0.338 & -0.326 \\
\hline Methyl ethanoate & -0.837 & -0.758 & -0.684 \\
\hline Ethyl ethanoate & -0.902 & -0.813 & -0.795 \\
\hline Butyl ethanoate & -1.119 & -0.985 & -1.012 \\
\hline
\end{tabular}

${ }^{\mathrm{a}}$ Experimental data were taken from Hoover et al. ${ }^{1}$

$x_{1}$ by the ideal molar volume of the saturated solution (i.e., $c_{1}{ }^{\text {sat }}=$ $\left.x_{1} /\left[x_{1} V_{1}+\left(1-x_{1}\right) V_{\text {solvent }}\right]\right)$. The molar volume of the hypothetical subcooled liquid 4-nitrobenzoic acid, $V_{\text {solute }}=$ $117.7 \mathrm{~cm}^{3} \mathrm{~mol}^{-1}$, was estimated as the molar volume of benzoic acid $\left(V_{\text {benzoic acid }}=104.4 \mathrm{~cm}^{3} \mathrm{~mol}^{-1}\right)+$ molar volume of nitrobenzene $\left(V_{\text {nitrobenzene }}=102.7 \mathrm{~cm}^{3} \mathrm{~mol}^{-1}\right)-$ molar volume of benzene $\left(V_{\text {benzene }}=89.40 \mathrm{~cm}^{3} \mathrm{~mol}^{-1}\right)$. Any errors resulting from the estimation of 4-nitrobenzoic acid's hypothetical subcooled liquid molar volume, $V_{\text {solute }}$, or the ideal molar-volume approximation should have only a very small effect on the calculated $c_{1}$ values. 4-Nitrobenzoic acid is not very soluble in many of the solvents considered, and the $x_{1} V_{\text {solute }}$ term contributes very little to the molar volume of the saturated solution. Examination of the entries in Table 48 reveals that the Abraham model provides a reasonably accurate mathematical description of the observed solubility data, suggesting that there are no obvious outliers in the dataset.

There have also been a few published papers reporting 4nitrobenzoic acid solubilities in organic solvents as a function of temperature. Sidgwick and Ewbank ${ }^{127}$ measured the solubility of 4-nitrobenzoic acid in benzene. Collett and Lazzell ${ }^{180}$ later performed solubility measurements for 4-nitrobenzoic acid in benzene, tetrachloromethane, and propanone. The internal consistency of the two of the four datasets was assessed by curve-fitting the measured mole fraction solubility data to Eq. (8). The limited number of data points in both the tetrachloromethane and propanone datasets was too small to perform a meaningful regression analysis. The values of the equation coefficients $(A, B$, and $C$ ) for the two benzene datasets are given in Table 49, along with the MRD, calculated according to Eq. (24). The largest MRD occurs for the experimental values determined by Sidgwick and Ewbank, ${ }^{127}$ 
TABLE 49. Parameters of the Modified Apelblat equation for describing the solubility of 4-nitrobenzoic acid in various organic solvents

\begin{tabular}{lcccc}
\hline \hline Solvent & $A$ & $B$ & $C$ & MRD (\%) \\
\hline Benzene $^{\mathrm{a}}$ & -139.082 & 2.458 & 22.363 & 10.40 \\
Benzene $^{\mathrm{b}}$ & -128.582 & -2.054 & 20.658 & 4.92 \\
\hline
\end{tabular}

${ }^{\mathrm{a}}$ Calculated from the dataset of Sidgwick and Ewbank. ${ }^{12}$

${ }^{\mathrm{b}}$ Calculated from the dataset of Collett and Lazzell. ${ }^{180}$ For benzene, the first data point was removed from the regression analysis in order to obtain a reasonable representation.

and corresponds to about a $10.4 \%$ error in back-calculating the observed mole fraction solubilities. The large deviation may be due in part to the fact that the solution temperature is above the solvent's normal boiling point temperature, to changes in the liquid phase composition due to solvent evaporation into the vapor phase, and the large range covered by the experimental values, $x_{1}=0.0465$ to $x_{1}=0.682$. It is more difficult to curve-fit experimental solubility data covering large mole fraction ranges.

The experimental solubility data for 4-nitrobenzoic acid in organic solvents are in Secs. 64.2-64.10.

\subsection{4-Nitrobenzoic acid solubility data in saturated hydrocarbons (including cycloalkanes)}

\begin{tabular}{|c|c|}
\hline $\begin{array}{l}\text { Components: } \\
\text { (1) 4-Nitrobenzoic acid; } \mathrm{C}_{7} \mathrm{H}_{5} \mathrm{NO}_{4} \text {; } \\
\text { [62-23-7] } \\
\text { (2) Cyclohexane; } \mathrm{C}_{6} \mathrm{H}_{12} ;[110-82-7]\end{array}$ & $\begin{array}{l}\text { Original Measurements: } \\
{ }^{54} \text { C. K. Hancock, J. N. Pawloski, } \\
\text { and J. P. Idoux, J. Org. Chem. 31, } \\
3801 \text { (1966). }\end{array}$ \\
\hline $\begin{array}{l}\text { Variables: } \\
T / \mathrm{K}=303.15\end{array}$ & $\begin{array}{l}\text { Prepared by: } \\
\text { W. E. Acree, Jr. }\end{array}$ \\
\hline
\end{tabular}

Experimental Values

\begin{tabular}{lc}
\hline \hline$x_{2}{ }^{\mathrm{a}}$ & $x_{1}{ }^{\mathrm{b}}$ \\
\hline 0.9999 & 0.0000118 \\
\hline
\end{tabular}

${ }^{a_{2}}$ : mole fraction of component 2 in the saturated solution.

${ }^{\mathrm{b}} x_{1}$ : mole fraction solubility of the solute.

\section{Auxiliary Information}

\section{Method/Apparatus/Procedure:}

Constant-temperature bath, Soxhlet thimble, conical flask, and analytical balance.

Excess solute and solvent were placed in a conical flask and allowed to equilibrate for several days at constant temperature. Attainment of equilibrium was verified by several repetitive measurements and by approaching equilibrium from supersaturation. Aliquots of saturated solutions were transferred using a Soxhlet thimble equipped with a one-hole cork stopper and an inverted U-shaped delivery tube to a second ground-glass stoppered flask suspended in the $303 \mathrm{~K}$ water bath. Successive portions of the contents were evaporated at room temperature in a tared aluminum foil weighing dish under a bell jar through which a slow stream of dry air was passed. The second flask plus unused saturated solution and the aluminum foil dish plus residue were weighed. The saturation solubility of the solute was calculated from the recorded mass data and molar masses of the solute and solvent.
Source and Purity of Chemicals:

(1) Purity not given, Chemical source not specified, was recrystallized several times from aqueous-ethanol mixture, and then dried under vacuum over phosphorous pentoxide.

(2) Reagent grade, Chemical source not specified, refluxed over phosphorous pentoxide for $24 \mathrm{~h}$, and then distilled through a $3 \times 80 \mathrm{~cm}$ column filled with 0.32-cm glass helices.

Estimated Error:

Temperature: $\pm 0.02 \mathrm{~K}$

$x_{1}: \pm 10 \%$ (relative error, by compiler).

\subsection{4-Nitrobenzoic acid solubility data in aromatic hydrocarbons}

\begin{tabular}{|c|c|}
\hline $\begin{array}{l}\text { Components: } \\
\text { (1) 4-Nitrobenzoic acid; } \mathrm{C}_{7} \mathrm{H}_{5} \mathrm{NO}_{4} \text {; } \\
\text { [62-23-7] } \\
\text { (2) Benzene; } \mathrm{C}_{6} \mathrm{H}_{6} ;[71-43-2]\end{array}$ & $\begin{array}{l}\text { Original Measurements: } \\
{ }^{54} \text { C. K. Hancock, J. N. Pawloski, } \\
\text { and J. P. Idoux, J. Org. Chem. 31, } \\
3801 \text { (1966). }\end{array}$ \\
\hline $\begin{array}{l}\text { Variables: } \\
T / \mathrm{K}=303.15\end{array}$ & $\begin{array}{l}\text { Prepared by: } \\
\text { W. E. Acree, Jr. }\end{array}$ \\
\hline
\end{tabular}

Experimental Values

\begin{tabular}{lc}
\hline \hline$x_{2}{ }^{\mathrm{a}}$ & $x_{1}{ }^{\mathrm{b}}$ \\
\hline 0.9998 & 0.000166 \\
\hline${ }_{x_{2}: \text { mole fraction of component } 2 \text { in the saturated solution. }}$ & \\
${ }^{\mathrm{b}} x_{1}:$ mole fraction solubility of the solute. &
\end{tabular}

\section{Auxiliary Information}

\section{Method/Apparatus/Procedure:}

Constant-temperature bath, Soxhlet thimble, conical flask, and analytical balance.

Excess solute and solvent were placed in a conical flask and allowed to equilibrate for several days at constant temperature. Attainment of equilibrium was verified by several repetitive measurements and by approaching equilibrium from supersaturation. Aliquots of saturated solutions were transferred using a Soxhlet thimble equipped with a one-hole cork stopper and an inverted $\mathrm{U}$-shaped delivery tube to a second ground-glass stoppered flask suspended in the $303 \mathrm{~K}$ water bath. Successive portions of the contents were evaporated at room temperature in a tared aluminum foil weighing dish under a bell jar through which a slow stream of dry air was passed. The second flask plus unused saturated solution and the aluminum foil dish plus residue were weighed. The saturation solubility of the solute was calculated from the recorded mass data and molar masses of the solute and solvent.

Source and Purity of Chemicals:

(1) Purity not given, Chemical source not specified, was recrystallized several times from aqueous-ethanol mixture, and then dried under vacuum over phosphorous pentoxide.

(2) Reagent grade, Chemical source not specified, refluxed over phosphorous pentoxide for $24 \mathrm{~h}$, and then distilled through a $3 \times 80 \mathrm{~cm}$ column filled with 0.32 -cm glass helices.

\section{Estimated Error:}

Temperature: $\pm 0.02 \mathrm{~K}$

$x_{1}: \pm 5 \%$ (relative error, by compiler). 


\begin{tabular}{ll}
\hline \hline Components: & Original Measurements: \\
$\begin{array}{l}\text { (1) 4-Nitrobenzoic acid; } \mathrm{C}_{7} \mathrm{H}_{5} \mathrm{NO}_{4} ; \\
{[62-23-7]}\end{array}$ & ${ }^{127}$ N. V. Sidgwick and E. K. \\
(2) Benzene; $\mathrm{C}_{6} \mathrm{H}_{6} ;[71-43-2]$ & $979(1921)$. \\
\hline Variables: & Prepared by: \\
Temperature & W. E. Acree, Jr. \\
\hline
\end{tabular}

Experimental Values

\begin{tabular}{lcc}
\hline \hline$T / \mathrm{K}$ & $x_{2}{ }^{\mathrm{a}}$ & \multicolumn{1}{c}{$x_{1}{ }^{\mathrm{b}}$} \\
\hline 437.7 & 0.953 & 0.0465 \\
456.6 & 0.889 & 0.111 \\
469.7 & 0.810 & 0.190 \\
474.8 & 0.638 & 0.362 \\
495.2 & 0.318 & 0.682 \\
\hline
\end{tabular}

${ }^{\mathrm{a}} x_{2}$ : mole fraction of component 2 in the saturated solution.

${ }^{b} x_{1}$ : mole fraction solubility of the solute. Solubility data reported in units of mass percent. Mole fraction values calculated by the compiler.

\section{Auxiliary Information}

\section{Method/Apparatus/Procedure:}

Preweighed quantities of solute and solvent were placed in bulbs, which were then sealed and heated in a suitable bath. At the higher temperatures, the concentration of solvent in the liquid solution was corrected for the amount of solvent vapor in the bulb. The correction assumed that the vapor pressure of the saturated solution was one half that of the pure solvent at the solution temperature. The temperature at which the solid completely dissolved was recorded, and was taken to be the solid-liquid equilibrium temperature.

\section{Source and Purity of Chemicals:}

(1) Purity not given, prepared and purified by the authors using a published synthetic procedure [see J. Walker and J. K. Wood, J. Chem. Soc. 117, 40 (1920) for synthetic details]. Melting point of the purified solute was $515.6 \mathrm{~K}$ (2) Purity not given, Chemical source not given, thiophene was removed by treatment with sulfuric acid. Sample was further purified by freezing several times.

\section{Estimated Error:}

Temperature: Not given in paper.

$x_{1}: \pm 10 \%$ (relative error, estimated by compiler).

\begin{tabular}{|c|c|}
\hline $\begin{array}{l}\text { Components: } \\
\text { (1) 4-Nitrobenzoic acid; } \mathrm{C}_{7} \mathrm{H}_{5} \mathrm{NO}_{4} \text {; } \\
\text { [62-23-7] } \\
\text { (2) Benzene; } \mathrm{C}_{6} \mathrm{H}_{6} ;[71-43-2]\end{array}$ & $\begin{array}{l}\text { Original Measurements: } \\
{ }^{180} \text { A. R. Collett and C. L. Lazzell, } \\
\text { J. Phys. Chem. 34, } 1838 \text { (1930). }\end{array}$ \\
\hline $\begin{array}{l}\text { Variables: } \\
\text { Temperature }\end{array}$ & $\begin{array}{l}\text { Prepared by: } \\
\text { W. E. Acree, Jr. }\end{array}$ \\
\hline
\end{tabular}

Experimental Values

\begin{tabular}{lll}
\hline \hline$T / \mathrm{K}$ & \multicolumn{1}{c}{$x_{2}{ }^{\mathrm{a}}$} & \multicolumn{1}{c}{$x_{1}{ }^{\mathrm{b}}$} \\
\hline 297.7 & 0.9998 & 0.000156 \\
412.1 & 0.9842 & 0.0158 \\
442.1 & 0.9424 & 0.0576 \\
451.1 & 0.9022 & 0.0978 \\
470.4 & 0.7737 & 0.2263 \\
479.6 & 0.6357 & 0.3643 \\
\hline
\end{tabular}

${ }^{a} x_{2}$ : mole fraction of component 2 in the saturated solution.

${ }^{\mathrm{b}} x_{1}$ : mole fraction solubility of the solute. The solubility is given as molal percentage, which, based on the description of 100 times the mole fraction, is taken to be mole percentage.

\section{Auxiliary Information}

\section{Method/Apparatus/Procedure:}

Preweighed quantities of solute and solvent were placed in bulbs, which were then sealed and heated in a suitable bath. The temperature at which the solid completely dissolved was recorded, and was taken to be the solid-liquid equilibrium temperature. In a few instances near room temperature, the solubility was determined by agitating the solvent in a thermostat with an excess of solute. After equilibrium had been established, an aliquot of the saturated solution was pipetted, weighed, and the amount of dissolved solute determined with a standard solution of titanium (III) chloride.

Source and Purity of Chemicals:

(1) Technical grade, Eastman Kodak Chemical Company, Rochester, NY, USA, was dissolved in dilute aqueous sodium hydroxide, precipitated by addition of hydrochloric acid, and then recrystallized twice from aqueous solution. Purified sample had a melting point temperature of $515.6 \mathrm{~K}$.

(2) Thiophene-free, Kahlbaum, was dried over sodium and distilled before use.

Estimated Error:

Temperature: Not given in paper.

$x_{1}: \pm 10 \%$ (relative error, estimated by compiler).

\subsection{4-Nitrobenzoic acid solubility data in esters}

\begin{tabular}{ll}
\hline \hline Components: & Original Measurements: \\
$\begin{array}{l}\text { (1) 4-Nitrobenzoic acid; } \mathrm{C}_{7} \mathrm{H}_{5} \mathrm{NO}_{4} ; \\
\text { [62-23-7] }\end{array}$ & $\begin{array}{l}{ }^{11} \text { K. R. Hoover, R. Coaxum, E. } \\
\text { (2) Methyl ethanoate; } \mathrm{C}_{3} \mathrm{H}_{6} \mathrm{O}_{2} ;\end{array}$ \\
$\begin{array}{l}\text { Pustejovsky, D. M. Stovall, W. E. } \\
\text { [79-20-9] }\end{array}$ & Acree, Jr., and M. H. Abraham, \\
\hline Variables: & Phys. Chem. Liq. 42, 339 (2004). \\
$T / \mathrm{K}=298.15$ & Prepared by: \\
\hline
\end{tabular}

Experimental Values

\begin{tabular}{lc}
\hline \hline$x_{2}{ }^{\mathrm{a}}$ & $x_{1}{ }^{\mathrm{b}}$ \\
\hline 0.9883 & 0.01168 \\
\hline${ }^{\mathrm{a}} x_{2}:$ mole fraction of component 2 in the saturated solution. & \\
${ }^{\mathrm{b}} x_{1}:$ mole fraction solubility of the solute. &
\end{tabular}




\section{Auxiliary Information}

\section{Method/Apparatus/Procedure:}

Constant-temperature bath, calorimetric thermometer, and an ultraviolet/ visible spectrophotometer.

Excess solute and solvent were placed in amber glass bottles and allowed to equilibrate for several days at constant temperature. Attainment of equilibrium was verified by several repetitive measurements and by approaching equilibrium from supersaturation. Aliquots of saturated solutions were transferred through a coarse filter into tared volumetric flasks, weighed, and diluted with methanol. Concentrations were determined by spectrophotometric measurements at $272 \mathrm{~nm}$.

Source and Purity of Chemicals:

(1) $99+\%$, Acros Organics, USA, was used as received.

(2) $99.5 \%$, anhydrous, Aldrich Chemical Company, Milwaukee, WI, USA, stored over molecular sieves and distilled shortly before use.

\section{Estimated Error:}

Temperature: $\pm 0.1 \mathrm{~K}$.

$x_{1}: \pm 1.5 \%$ (relative error)

\begin{tabular}{ll}
\hline \hline Components: & Original Measurements: \\
(1) 4-Nitrobenzoic acid; $\mathrm{C}_{7} \mathrm{H}_{5} \mathrm{NO}_{4} ;$ & ${ }^{11}$ K. R. Hoover, R. Coaxum, E. \\
[62-23-7] & Pustejovsky, D. M. Stovall, W. E. \\
(2) Ethyl ethanoate; $\mathrm{C}_{4} \mathrm{H}_{8} \mathrm{O}_{2} ;$ & Acree, Jr., and M. H. Abraham, \\
[141-78-6] & Phys. Chem. Liq. 42, 339 (2004). \\
\hline Variables: & Prepared by: \\
$T / \mathrm{K}=298.15$ & W. E. Acree, Jr. \\
\hline
\end{tabular}

\section{Experimental Values}

\begin{tabular}{|c|c|}
\hline$\underline{x_{2}{ }^{\mathrm{a}}}$ & $x_{1}{ }^{\mathrm{b}}$ \\
\hline 0.9876 & 0.01237 \\
\hline
\end{tabular}

${ }^{\mathrm{a}} x_{2}$ : mole fraction of component 2 in the saturated solution.

${ }^{b} x_{1}$ : mole fraction solubility of the solute.

\section{Auxiliary Information}

\section{Method/Apparatus/Procedure:}

Constant-temperature bath, calorimetric thermometer, and an ultraviolet/ visible spectrophotometer.

Excess solute and solvent were placed in amber glass bottles and allowed to equilibrate for several days at constant temperature. Attainment of equilibrium was verified by several repetitive measurements and by approaching equilibrium from supersaturation. Aliquots of saturated solutions were transferred through a coarse filter into tared volumetric flasks, weighed, and diluted with methanol. Concentrations were determined by

spectrophotometric measurements at $272 \mathrm{~nm}$.

Source and Purity of Chemicals:

(1) $99+\%$, Acros Organics, USA, was used as received.

(2) $99.9 \%$, HPLC grade, Aldrich Chemical Company, Milwaukee, WI, USA, stored over molecular sieves and distilled shortly before use.

\section{Estimated Error:}

Temperature: $\pm 0.1 \mathrm{~K}$

$x_{1}: \pm 1.5 \%$ (relative error)

\begin{tabular}{ll}
\hline \hline Components: & Original Measurements: \\
(1) 4-Nitrobenzoic acid; $\mathrm{C}_{7} \mathrm{H}_{5} \mathrm{NO}_{4} ;$ & ${ }^{11} \mathrm{~K}$. R. Hoover, R. Coaxum, E. \\
[62-23-7] & Pustejovsky, D. M. Stovall, W. E. \\
$\begin{array}{l}\text { (2) Butyl ethanoate; } \mathrm{C}_{6} \mathrm{H}_{12} \mathrm{O}_{2} ; \\
{[123-86-4]}\end{array}$ & Acree, Jr., and M. H. Abraham, \\
\hline Pariables: & Phys. Chem. Liq. 42, 339 (2004). \\
$T / \mathrm{K}=298.15$ & Prepared by: \\
\hline
\end{tabular}

Experimental Values

\begin{tabular}{lc}
\hline \hline$x_{2}{ }^{\mathrm{a}}$ & $x_{1}{ }^{\mathrm{b}}$ \\
\hline 0.9899 & 0.01007 \\
\hline${ }^{\mathrm{a}} x_{2}:$ mole fraction of component 2 in the saturated solution. & \\
${ }^{\mathrm{b}} x_{1}:$ mole fraction solubility of the solute. &
\end{tabular}

Auxiliary Information

\section{Method/Apparatus/Procedure:}

Constant-temperature bath, calorimetric thermometer, and an ultraviolet/ visible spectrophotometer.

Excess solute and solvent were placed in amber glass bottles and allowed to equilibrate for several days at constant temperature. Attainment of equilibrium was verified by several repetitive measurements and by approaching equilibrium from supersaturation. Aliquots of saturated solutions were transferred through a coarse filter into tared volumetric flasks, weighed, and diluted with methanol. Concentrations were determined by spectrophotometric measurements at $272 \mathrm{~nm}$.

\section{Source and Purity of Chemicals:}

(1) $99+\%$, Acros Organics, USA, was used as received.

(2) $99.7 \%$, HPLC grade, Aldrich Chemical Company, Milwaukee, WI, USA, stored over molecular sieves and distilled shortly before use.

\section{Estimated Error:}

Temperature: $\pm 0.1 \mathrm{~K}$.

$x_{1}: \pm 1.5 \%$ (relative error).

\begin{tabular}{ll}
\hline \hline Components: & Original Measurements: \\
(1) 4-Nitrobenzoic acid; $\mathrm{C}_{7} \mathrm{H}_{5} \mathrm{NO}_{4} ;$ & ${ }^{11}$ K. R. Hoover, R. Coaxum, E. \\
[62-23-7] & Pustejovsky, D. M. Stovall, W. E. \\
$\begin{array}{l}\text { (2) Pentyl ethanoate; } \mathrm{C}_{7} \mathrm{H}_{14} \mathrm{O}_{2} ; \\
\text { [628-63-7] }\end{array}$ & Acree, Jr., and M. H. Abraham, \\
\hline Variables: & Phys. Chem. Liq. 42, 339 (2004). \\
$T / \mathrm{K}=298.15$ & Prepared by: \\
\hline
\end{tabular}

Experimental Values

\begin{tabular}{lc}
\hline \hline$x_{2}{ }^{\mathrm{a}}$ & $x_{1}{ }^{\mathrm{b}}$ \\
\hline 0.9925 & 0.007469 \\
\hline${ }^{a} x_{2}:$ mole fraction of component 2 in the saturated solution. \\
${ }^{\mathrm{b}}{ }_{x_{1}}$ : mole fraction solubility of the solute.
\end{tabular}




\section{Auxiliary Information}

\section{Method/Apparatus/Procedure:}

Constant-temperature bath, calorimetric thermometer, and an ultraviolet/ visible spectrophotometer.

Excess solute and solvent were placed in amber glass bottles and allowed to equilibrate for several days at constant temperature. Attainment of equilibrium was verified by several repetitive measurements and by approaching equilibrium from supersaturation. Aliquots of saturated solutions were transferred through a coarse filter into tared volumetric flasks, weighed, and diluted with methanol. Concentrations were determined by spectrophotometric measurements at $272 \mathrm{~nm}$.

Source and Purity of Chemicals:

(1) $99+\%$, Acros Organics, USA, was used as received.

(2) $99 \%$, Aldrich Chemical Company, Milwaukee, WI, USA, stored over molecular sieves and distilled shortly before use.

Estimated Error:

Temperature: $\pm 0.1 \mathrm{~K}$.

$x_{1}: \pm 1.5 \%$ (relative error)

\section{Components:}

(1) 4-Nitrobenzoic acid; $\mathrm{C}_{7} \mathrm{H}_{5} \mathrm{NO}_{4}$;

[62-23-7]

(2) 1,2,3-Triacetoxypropane (Triacetin); $\mathrm{C}_{9} \mathrm{H}_{14} \mathrm{O}_{6} ;[102-76-1]$

\begin{tabular}{ll}
\hline Variables: & Prepared by: \\
$T / \mathrm{K}=298.15$ & W. E. Acree, Jr.
\end{tabular}

Experimental Values

\begin{tabular}{lc}
\hline \hline$x_{2}{ }^{\mathrm{a}}$ & $x_{1}{ }^{\mathrm{b}}$ \\
\hline 0.9848 & 0.01542 \\
\hline${ }^{\mathrm{a}} x_{2}:$ mole fraction of component 2 in the saturated solution. & \\
${ }^{\mathrm{b}} x_{1}:$ mole fraction solubility of the solute. &
\end{tabular}

\section{Auxiliary Information}

\section{Method: Apparatus/Procedure:}

Constant-temperature bath, calorimetric thermometer, and an ultraviolet/ visible spectrophotometer.

Excess solute and solvent were placed in amber glass bottles and allowed to equilibrate for several days at constant temperature. Attainment of equilibrium was verified by several repetitive measurements and by approaching equilibrium from supersaturation. Aliquots of saturated solutions were transferred through a coarse filter into tared volumetric flasks, weighed, and diluted with 2-propanol. Concentrations were determined by

spectrophotometric measurements at $272 \mathrm{~nm}$.

Source and Purity of Chemicals:

(1) $99+\%$, Acros Organics, USA, was used as received.

(2) $99 \%$, Acros Organics, USA, stored over molecular sieves before use.

\section{Estimated Error:}

Temperature: $\pm 0.1 \mathrm{~K}$

$x_{1}: \pm 1.5 \%$ (relative error).

\subsection{4-Nitrobenzoic acid solubility data in ethers}

\begin{tabular}{ll}
\hline \hline Components: & Original Measurements: \\
$\begin{array}{l}\text { (1) 4-Nitrobenzoic acid; } \mathrm{C}_{7} \mathrm{H}_{5} \mathrm{NO}_{4} ; \\
{[62-23-7]}\end{array}$ & ${ }^{11}$ K. R. Hoover, R. Coaxum, E. \\
$\begin{array}{l}\text { (2) } 1,1^{\prime}-\text { Oxybisethane; } \mathrm{C}_{4} \mathrm{H}_{10} \mathrm{O} ; \\
{[60-29-7]}\end{array}$ & Acree, Jr., and M. H. Abraham, \\
\hline Variables: & Phys. Chem. Liq. 42, 339 (2004). \\
$T / \mathrm{K}=298.15$ & Prepared by: \\
\hline
\end{tabular}

\section{Experimental Values}

\begin{tabular}{lc}
\hline \hline$x_{2}{ }^{\mathrm{a}}$ & $x_{1}{ }^{\mathrm{b}}$ \\
\hline 0.9901 & 0.009861 \\
\hline${ }^{\mathrm{a}} x_{2}:$ mole fraction of component 2 in the saturated solution. & \\
${ }^{\mathrm{b}} x_{1}:$ mole fraction solubility of the solute. &
\end{tabular}

\section{Auxiliary Information}

\section{Method/Apparatus/Procedure:}

Constant-temperature bath, calorimetric thermometer, and an ultraviolet/ visible spectrophotometer.

Excess solute and solvent were placed in amber glass bottles and allowed to equilibrate for several days at constant temperature. Attainment of equilibrium was verified by several repetitive measurements and by approaching equilibrium from supersaturation. Aliquots of saturated solutions were transferred through a coarse filter into tared volumetric flasks, weighed, and diluted with methanol. Concentrations were determined by spectrophotometric measurements at $272 \mathrm{~nm}$.

\section{Source and Purity of Chemicals:}

(1) $99+\%$, Acros Organics, USA, was used as received.

(2) $99+\%$, anhydrous, Aldrich Chemical Company, Milwaukee, WI, USA, stored over molecular sieves and distilled shortly before use.

\section{Estimated Error:}

Temperature: $\pm 0.1 \mathrm{~K}$.

$x_{1}: \pm 2.0 \%$ (relative error).

\begin{tabular}{|c|c|}
\hline $\begin{array}{l}\text { Components: } \\
\text { (1) 4-Nitrobenzoic acid; } \mathrm{C}_{7} \mathrm{H}_{5} \mathrm{NO}_{4} \text {; } \\
\text { [62-23-7] } \\
\text { (2) } 1,1^{\prime}-\text { Oxybisethane; } \mathrm{C}_{4} \mathrm{H}_{10} \mathrm{O} \text {; } \\
\text { [60-29-7] }\end{array}$ & $\begin{array}{l}\text { Original Measurements: } \\
{ }^{180} \text { A. R. Collett and C. L. Lazzell, J. } \\
\text { Phys. Chem. 34, } 1838 \text { (1930). }\end{array}$ \\
\hline $\begin{array}{l}\text { Variables: } \\
\text { Temperature }\end{array}$ & $\begin{array}{l}\text { Prepared by: } \\
\text { W. E. Acree, Jr. }\end{array}$ \\
\hline
\end{tabular}

\section{Experimental Values}

\begin{tabular}{lcc}
\hline \hline$T / \mathrm{K}$ & $x_{2}{ }^{\mathrm{a}}$ & $x_{1}{ }^{\mathrm{b}}$ \\
\hline 460.8 & 0.9463 & 0.0537 \\
466.8 & 0.7454 & 0.2546
\end{tabular}

${ }^{\mathrm{a}} x_{2}$ : mole fraction of component 2 in the saturated solution.

${ }^{b} x_{1}$ : mole fraction solubility of the solute. The solubility is given as molal percentage, which, based on the description of 100 times the mole fraction, is taken to be mole percentage. 


\section{Auxiliary Information}

\section{Method/Apparatus/Procedure:}

Preweighed quantities of solute and solvent were placed in bulbs, which were then sealed and heated in a suitable bath. The temperature at which the solid completely dissolved was recorded, and was taken to be the solid-liquid equilibrium temperature.

Source and Purity of Chemicals:

(1) Technical grade, Eastman Kodak Chemical Company, Rochester, NY, USA, was dissolved in dilute aqueous sodium hydroxide, precipitated by addition of hydrochloric acid, and then recrystallized twice from aqueous solution. Purified sample had a melting point temperature of $515.6 \mathrm{~K}$.

(2) U.S.P., Chemical Source not given, was dried over calcium chloride and distilled twice before use.

\section{Estimated Error:}

Temperature: Not given in paper.

$x_{2}: \pm 10 \%$ (relative error, estimated by compiler).

\begin{tabular}{ll}
\hline \hline Components: & Original Measurements: \\
(1) 4-Nitrobenzoic acid; $\mathrm{C}_{7} \mathrm{H}_{5} \mathrm{NO}_{4} ;$ & $\begin{array}{l}{ }^{11} \mathrm{~K} . \text { R. Hoover, R. Coaxum, E. } \\
\text { [62-23-7] }\end{array}$ \\
$\begin{array}{l}\text { Pustejovsky, D. M. Stovall, W. E. } \\
\text { (2) 2,2'-Oxybispropane; } \mathrm{C}_{6} \mathrm{H}_{14} \mathrm{O} ;\end{array}$ & $\begin{array}{l}\text { Acree, Jr., and M. H. Abraham, } \\
{[108-20-3]}\end{array}$ \\
\hline Vhys. Chem. Liq. 42, 339 (2004). \\
$T / \mathrm{K}=298.15$ & Prepared by: \\
\hline
\end{tabular}

Experimental Values

\begin{tabular}{lc}
\hline \hline$x_{2}{ }^{\mathrm{a}}$ & $x_{1}{ }^{\mathrm{b}}$ \\
\hline 0.9964 & 0.003621 \\
\hline${ }^{\mathrm{a}} x_{2}:$ mole fraction of component 2 in the saturated solution. \\
${ }^{\mathrm{b}}{ }_{x_{1}}:$ mole fraction solubility of the solute.
\end{tabular}

\section{Auxiliary Information}

\section{Method/Apparatus/Procedure:}

Constant-temperature bath, calorimetric thermometer, and an ultraviolet/ visible spectrophotometer.

Excess solute and solvent were placed in amber glass bottles and allowed to equilibrate for several days at constant temperature. Attainment of equilibrium was verified by several repetitive measurements and by approaching equilibrium from supersaturation. Aliquots of saturated solutions were transferred through a coarse filter into tared volumetric flasks, weighed, and diluted with methanol. Concentrations were determined by spectrophotometric measurements at $272 \mathrm{~nm}$.

\section{Source and Purity of Chemicals:}

(1) $99+\%$, Acros Organics, USA, was used as received.

(2) $99 \%$, anhydrous, Aldrich Chemical Company, Milwaukee, WI, USA, stored over molecular sieves and distilled shortly before use

\section{Estimated Error:}

Temperature: $\pm 0.1 \mathrm{~K}$

$x_{1}: \pm 2.0 \%$ (relative error)

\begin{tabular}{ll}
\hline \hline Components: & Original Measurements: \\
(1) 4-Nitrobenzoic acid; $\mathrm{C}_{7} \mathrm{H}_{5} \mathrm{NO}_{4} ;$ & ${ }^{11}$ K. R. Hoover, R. Coaxum, E. \\
[62-23-7] & Pustejovsky, D. M. Stovall, W. E. \\
$\begin{array}{l}\text { (2) } 1,1^{\prime}-\text {-Oxybisbutane; } \mathrm{C}_{8} \mathrm{H}_{18} \mathrm{O} ; \\
\text { [142-96-1] }\end{array}$ & Acree, Jr., and M. H. Abraham, \\
\hline Variables: & Phys. Chem. Liq. 42, 339 (2004). \\
$T / \mathrm{K}=298.15$ & Prepared by: \\
\hline
\end{tabular}

Experimental Values

\begin{tabular}{lc}
\hline \hline$x_{2}{ }^{\mathrm{a}}$ & $x_{1}{ }^{\mathrm{b}}$ \\
\hline 0.9972 & 0.002806 \\
\hline${ }^{\mathrm{a}} x_{2}:$ mole fraction of component 2 in the saturated solution. \\
${ }^{\mathrm{b}} x_{1}:$ mole fraction solubility of the solute.
\end{tabular}

Auxiliary Information

\section{Method/Apparatus/Procedure:}

Constant-temperature bath, calorimetric thermometer, and an ultraviolet/ visible spectrophotometer.

Excess solute and solvent were placed in amber glass bottles and allowed to equilibrate for several days at constant temperature. Attainment of equilibrium was verified by several repetitive measurements and by approaching equilibrium from supersaturation. Aliquots of saturated solutions were transferred through a coarse filter into tared volumetric flasks, weighed, and diluted with methanol. Concentrations were determined by spectrophotometric measurements at $272 \mathrm{~nm}$.

\section{Source and Purity of Chemicals:}

(1) $99+\%$, Acros Organics, USA, was used as received.

(2) $99.3 \%$, anhydrous, Aldrich Chemical Company, Milwaukee, WI, USA, stored over molecular sieves and distilled shortly before use.

\section{Estimated Error:}

Temperature: $\pm 0.1 \mathrm{~K}$.

$x_{1}: \pm 2.0 \%$ (relative error).

\begin{tabular}{ll}
\hline \hline Components: & Original Measurements: \\
$\begin{array}{l}\text { (1) 4-Nitrobenzoic acid; } \mathrm{C}_{7} \mathrm{H}_{5} \mathrm{NO}_{4} ; \\
\text { [62-23-7] }\end{array}$ & ${ }^{129} \mathrm{~S}$. H. Ghosh and D. K. Hazra, J. \\
$\begin{array}{l}\text { Indian Chem. Soc. 65, 620 (1988). } \\
{[110-71-4]}\end{array}$ & \\
\hline Variables: & Prepared by: \\
$T / \mathrm{K}=298.15$ & W. E. Acree, Jr. \\
\hline
\end{tabular}

\section{Experimental Values}

The measured solubility was reported to be $c_{1}=$ $0.8324 \mathrm{~mol} \mathrm{dm}^{-3}$.

\section{Auxiliary Information}

\section{Method/Apparatus/Procedure:}

Mechanical shaker and a constant-temperature thermostat.

Excess solute and solvent were placed in a bottle and stoppered. The solution was shaken in a mechanical shaker for $24 \mathrm{~h}$ at ambient room temperature. The solution was then thermostated at $298 \mathrm{~K}$ for $24 \mathrm{~h}$ with occasional shaking. An aliquot of the saturated solution was then removed and filtered. The concentration of the dissolved solute was determined by titration with standard caustic soda using phenolphthalein as indicator. 
Source and Purity of Chemicals:

(1) G.R., Merck Chemical Company, was recrystallized from aqueous ethanol mixture.

(2) Purum, Fluka, was shaken with ferrous sulfate and then distilled. The distillate was further purified by refluxing for $12 \mathrm{~h}$ and then distilling over metallic sodium.

\section{Estimated Error:}

Temperature: $\pm 0.01 \mathrm{~K}$.

$c_{1}: \pm 1.5 \%$ (relative error, estimated by compiler).

\begin{tabular}{ll}
\hline \hline Components: & Original Measurements: \\
(1) 4-Nitrobenzoic acid; $\mathrm{C}_{7} \mathrm{H}_{5} \mathrm{NO}_{4} ;$ & $\begin{array}{l}{ }^{11} \mathrm{~K} . \text { R. Hoover, R. Coaxum, E. } \\
\text { [62-23-7] }\end{array}$ \\
$\begin{array}{l}\text { Pustejovsky, D. M. Stovall, W. E. } \\
\text { (2) Tetrahydrofuran; } \mathrm{C}_{4} \mathrm{H}_{8} \mathrm{O} ;\end{array}$ & $\begin{array}{l}\text { Acree, Jr., and M. H. Abraham, } \\
{[109-99-9]}\end{array}$ \\
\hline Phys. Chem. Liq. 42, 339 (2004). \\
\hline$T / \mathrm{K}=298.15$ & Prepared by: \\
\hline
\end{tabular}

Experimental Values

\begin{tabular}{lc}
\hline \hline$x_{2}^{\mathrm{a}}$ & $x_{1}{ }^{\mathrm{b}}$ \\
\hline 0.9361 & 0.06393 \\
\hline${ }^{\mathrm{a}} x_{2}:$ mole fraction of component 2 in the saturated solution. & \\
${ }^{\mathrm{b}}{ }_{x_{1}: \text { mole fraction solubility of the solute. }}$ &
\end{tabular}

\section{Auxiliary Information}

\section{Method/Apparatus/Procedure:}

Constant-temperature bath, calorimetric thermometer, and an ultraviolet/ visible spectrophotometer.

Excess solute and solvent were placed in amber glass bottles and allowed to equilibrate for several days at constant temperature. Attainment of equilibrium was verified by several repetitive measurements and by approaching equilibrium from supersaturation. Aliquots of saturated solutions were transferred through a coarse filter into tared volumetric flasks, weighed, and diluted with methanol. Concentrations were determined by spectrophotometric measurements at $272 \mathrm{~nm}$.

\section{Source and Purity of Chemicals:}

(1) $99+\%$, Acros Organics, USA, was used as received.

(2) $99.9 \%$, anhydrous, Aldrich Chemical Company, Milwaukee, WI, USA, stored over molecular sieves and distilled shortly before use.

\section{Estimated Error:}

Temperature: $\pm 0.1 \mathrm{~K}$

$x_{1}: \pm 2.0 \%$ (relative error)

\begin{tabular}{|c|c|}
\hline $\begin{array}{l}\text { Components: } \\
\text { (1) 4-Nitrobenzoic acid; } \mathrm{C}_{7} \mathrm{H}_{5} \mathrm{NO}_{4} \text {; } \\
\text { [62-23-7] } \\
\text { (2) Tetrahydrofuran; } \mathrm{C}_{4} \mathrm{H}_{8} \mathrm{O} \text {; } \\
\text { [109-99-9] }\end{array}$ & $\begin{array}{l}\text { Original Measurements: } \\
{ }^{63} \text { C. K. Hancock, J. N. Pawloski, } \\
\text { and J. P. Idoux, J. Org. Chem. 32, } \\
1931 \text { (1967). }\end{array}$ \\
\hline $\begin{array}{l}\text { Variables: } \\
T / \mathrm{K}=303.15\end{array}$ & $\begin{array}{l}\text { Prepared by: } \\
\text { W. E. Acree, Jr. }\end{array}$ \\
\hline
\end{tabular}

Experimental Values

\begin{tabular}{lc}
\hline \hline$x_{2}{ }^{\mathrm{a}}$ & $x_{1}{ }^{\mathrm{b}}$ \\
\hline 0.929 & 0.071 \\
\hline${ }^{\mathrm{a}} x_{2}:$ mole fraction of component 2 in the saturated solution. & \\
${ }^{\mathrm{b}} x_{1}:$ mole fraction solubility of the solute. &
\end{tabular}

\section{Auxiliary Information}

Method/Apparatus/Procedure:

Constant-temperature bath, Soxhlet thimble, conical flask, and analytical balance.

Excess solute and solvent were placed in a conical flask and allowed to equilibrate for several days at constant temperature. Attainment of equilibrium was verified by several repetitive measurements and by approaching equilibrium from supersaturation. Aliquots of saturated solutions were transferred using a Soxhlet thimble equipped with a one-hole cork stopper and an inverted U-shaped delivery tube to a second ground-glass stoppered flask suspended in the $303 \mathrm{~K}$ water bath. Successive portions of the contents were evaporated at room temperature in a tared aluminum foil weighing dish under a bell jar through which a slow stream of dry air was passed. The second flask plus unused saturated solution and the aluminum foil dish plus residue were weighed. The saturation solubility of the solute was calculated from the recorded mass data and molar masses of the solute and solvent.

\section{Source and Purity of Chemicals:}

(1) Purity not given, Chemical source not specified, was recrystallized several times from aqueous-ethanol mixture, and then dried under vacuum over phosphorous pentoxide.

(2) Practical grade, Chemical source not specified, stored over sodium hydroxide pellets for $24 \mathrm{~h}$, and then passed through $2 \times 70-\mathrm{cm}$ chromatographic adsorption columns containing activated alumina. After this treatment, the purified solvent was stored over copper in a nitrogen atmosphere.

\section{Estimated Error:}

Temperature: $\pm 0.02 \mathrm{~K}$.

$x_{1}: \pm 1.0 \%$ (relative error)

\begin{tabular}{ll}
\hline \hline Components: & Original Measurements: \\
(1) 4-Nitrobenzoic acid; $\mathrm{C}_{7} \mathrm{H}_{5} \mathrm{NO}_{4} ;$ & ${ }^{11} \mathrm{~K}$. R. Hoover, R. Coaxum, E. \\
[62-23-7] & Pustejovsky, D. M. Stovall, W. E. \\
(2) 1,4-Dioxane; $\mathrm{C}_{4} \mathrm{H}_{8} \mathrm{O}_{2} ;$ [123-91-1] & Acree, Jr., and M. H. Abraham, \\
& Phys. Chem. Liq. 42, 339 (2004). \\
\hline Variables: & Prepared by: \\
$T / \mathrm{K}=298.15$ & W. E. Acree, Jr. \\
\hline
\end{tabular}

\section{Experimental Values}

\begin{tabular}{lc}
\hline \hline$x_{2}{ }^{\mathrm{a}}$ & $x_{1}{ }^{\mathrm{b}}$ \\
\hline 0.9580 & 0.04203 \\
\hline${ }_{{ }^{\mathrm{a}} x_{2}: \text { mole fraction of component } 2 \text { in the saturated solution. }}$ & \\
${ }^{\mathrm{b}} x_{1}:$ mole fraction solubility of the solute. &
\end{tabular}




\section{Auxiliary Information}

\section{Method/Apparatus/Procedure:}

Constant-temperature bath, calorimetric thermometer, and an ultraviolet/ visible spectrophotometer.

Excess solute and solvent were placed in amber glass bottles and allowed to equilibrate for several days at constant temperature. Attainment of equilibrium was verified by several repetitive measurements and by approaching equilibrium from supersaturation. Aliquots of saturated solutions were transferred through a coarse filter into tared volumetric flasks, weighed, and diluted with methanol. Concentrations were determined by spectrophotometric measurements at $272 \mathrm{~nm}$.

Source and Purity of Chemicals:

(1) $99+\%$, Acros Organics, USA, was used as received.

(2) $99.8 \%$, anhydrous, Aldrich Chemical Company, Milwaukee, WI, USA, stored over molecular sieves and distilled shortly before use.

Estimated Error:

Temperature: $\pm 0.1 \mathrm{~K}$.

$x_{1}: \pm 2.0 \%$ (relative error)

\begin{tabular}{|c|c|}
\hline $\begin{array}{l}\text { Components: } \\
\text { (1) 4-Nitrobenzoic acid; } \mathrm{C}_{7} \mathrm{H}_{5} \mathrm{NO}_{4} \text {; } \\
\text { [62-23-7] } \\
\text { (2) 1,4-Dioxane; } \mathrm{C}_{4} \mathrm{H}_{8} \mathrm{O}_{2} ;[123-91-1]\end{array}$ & $\begin{array}{l}\text { Original Measurements: } \\
{ }^{63} \text { C. K. Hancock, J. N. Pawloski, } \\
\text { and J. P. Idoux, J. Org. Chem. 32, } \\
\text { 1931 (1967). }\end{array}$ \\
\hline $\begin{array}{l}\text { Variables: } \\
T / \mathrm{K}=303.15\end{array}$ & $\begin{array}{l}\text { Prepared by: } \\
\text { W. E. Acree, Jr. }\end{array}$ \\
\hline
\end{tabular}

\section{Experimental Values}

\begin{tabular}{lc}
\hline \hline$x_{2}{ }^{\mathrm{a}}$ & $x_{1}{ }^{\mathrm{b}}$ \\
\hline 0.950 & 0.050 \\
\hline
\end{tabular}

${ }^{a_{2}}$ : mole fraction of component 2 in the saturated solution.

${ }^{b} x_{1}$ : mole fraction solubility of the solute.

\section{Auxiliary Information}

\section{Method/Apparatus/Procedure:}

Constant-temperature bath, Soxhlet thimble, conical flask, and analytical balance.

Excess solute and solvent were placed in a conical flask and allowed to equilibrate for several days at constant temperature. Attainment of equilibrium was verified by several repetitive measurements and by approaching equilibrium from supersaturation. Weighed aliquots of saturated solutions were removed and titrated with a standardized sodium hydroxide solution (carbonate free) using a pH meter. The endpoint of the titration was determined by computing the second derivative in the $\mathrm{pH}$ versus volume of sodium hydroxide added.

\section{Source and Purity of Chemicals:}

(1) Purity not given, Chemical source not specified, was recrystallized several times from aqueous-ethanol mixture, and then dried under vacuum over phosphorous pentoxide.

(2) Practical grade, Chemical source not specified, stored over sodium hydroxide pellets for $24 \mathrm{~h}$, and then passed through $2 \times 70$-cm chromatographic adsorption columns containing activated alumina. After this treatment, the purified solvent was stored over copper in a nitrogen atmosphere.

Estimated Error:

Temperature: $\pm 0.02 \mathrm{~K}$.

$x_{1}: \pm 1.0 \%$ (relative error)

\subsection{4-Nitrobenzoic acid solubility data in haloalkanes, haloalkenes, and haloaromatic hydrocarbons}

\begin{tabular}{ll}
\hline \hline Components: & Original Measurements: \\
$\begin{array}{l}\text { (1) 4-Nitrobenzoic acid; } \mathrm{C}_{7} \mathrm{H}_{5} \mathrm{NO}_{4} ; \\
{[62-23-7]}\end{array}$ & $\begin{array}{l}{ }^{180} \text { A. R. Collett and C. L. Lazzell, J. } \\
\text { Phys. Chem. 34, 1838 (1930). } \\
\text { [56-23-5] }\end{array}$ \\
\hline Variables: & \\
Temperature & Prepared by: \\
\hline
\end{tabular}

Experimental Values

\begin{tabular}{lll}
\hline \hline$T / \mathrm{K}$ & $x_{2}{ }^{\mathrm{a}}$ & \multicolumn{1}{c}{$x_{1}{ }^{\mathrm{b}}$} \\
\hline 302.6 & 0.9999 & 0.000101 \\
439.8 & 0.9818 & 0.0182 \\
471.4 & 0.9337 & 0.0663 \\
\hline
\end{tabular}

${ }^{a_{X_{2}}}$ : mole fraction of component 2 in the saturated solution.

${ }^{b} x_{1}$ : mole fraction solubility of the solute. The solubility is given as molal percentage, which, based on the description of 100 times the mole fraction, is taken to be mole percentage.

\section{Auxiliary Information}

Method/Apparatus/Procedure:

Preweighed quantities of solute and solvent were placed in bulbs, which were then sealed and heated in a suitable bath. The temperature at which the solid completely dissolved was recorded, and was taken to be the solid-liquid equilibrium temperature. In a few instances near room temperature, the solubility was determined by agitating the solvent in a thermostat with an excess of solute. After equilibrium had been established, an aliquot of the saturated solution was pipetted, weighed, and the amount of dissolved solute determined with a standard solution of titanium (III) chloride.

Source and Purity of Chemicals:

(1) Technical grade, Eastman Kodak Chemical Company, Rochester, NY, USA, was dissolved in dilute aqueous sodium hydroxide, precipitated by addition of hydrochloric acid, and then recrystallized twice from aqueous solution. Purified sample had a melting point temperature of $515.6 \mathrm{~K}$. (2) Certified Pure, Merck Chemical Company, was dried over calcium chloride and distilled twice before use.

\section{Estimated Error:}

Temperature: Not given in paper.

$x_{1}: \pm 10 \%$ (relative error, estimated by compiler).

\subsection{4-Nitrobenzoic acid solubility data in alcohols}

\begin{tabular}{ll}
\hline \hline Components: & Original Measurements: \\
(1) 4-Nitrobenzoic acid; $\mathrm{C}_{7} \mathrm{H}_{5} \mathrm{NO}_{4} ;$ & ${ }^{11} \mathrm{~K}$. R. Hoover, R. Coaxum, E. \\
[62-23-7] & Pustejovsky, D. M. Stovall, W. E. \\
(2) Methanol; $\mathrm{CH}_{4} \mathrm{O} ;$ [67-56-1] & $\begin{array}{l}\text { Acree, Jr., and M. H. Abraham, } \\
\end{array}$ \\
& Phys. Chem. Liq. 42, 339 (2004). \\
\hline Variables: & Prepared by: \\
$T / \mathrm{K}=298.15$ & W. E. Acree, Jr. \\
\hline
\end{tabular}


Experimental Values

\begin{tabular}{lc}
\hline \hline$x_{2}{ }^{\mathrm{a}}$ & $x_{1}{ }^{\mathrm{b}}$ \\
\hline 0.9921 & 0.007881 \\
${ }^{\mathrm{a}} x_{2}:$ mole fraction of component 2 in the saturated solution. \\
${ }^{\mathrm{b}} x_{1}:$ mole fraction solubility of the solute.
\end{tabular}

Auxiliary Information

Method/Apparatus/Procedure:

Constant-temperature bath, calorimetric thermometer, and an ultraviolet/ visible spectrophotometer.

Excess solute and solvent were placed in amber glass bottles and allowed to equilibrate for several days at constant temperature. Attainment of equilibrium was verified by several repetitive measurements and by approaching equilibrium from supersaturation. Aliquots of saturated solutions were transferred through a coarse filter into tared volumetric flasks, weighed, and diluted with methanol. Concentrations were determined by spectrophotometric measurements at $272 \mathrm{~nm}$.

Source and Purity of Chemicals:

(1) $99+\%$, Acros Organics, USA, was used as received.

(2) $99.8 \%$, anhydrous, Aldrich Chemical Company, Milwaukee, WI, USA, stored over molecular sieves and distilled shortly before use.

Estimated Error:

Temperature: $\pm 0.1 \mathrm{~K}$.

$x_{1}: \pm 1.5 \%$ (relative error).

\begin{tabular}{|c|c|}
\hline $\begin{array}{l}\text { Components: } \\
\text { (1) 4-Nitrobenzoic acid; } \mathrm{C}_{7} \mathrm{H}_{5} \mathrm{NO}_{4} \text {; } \\
\text { [62-23-7] } \\
\text { (2) Methanol; } \mathrm{CH}_{4} \mathrm{O} ;[67-56-1]\end{array}$ & $\begin{array}{l}\text { Original Measurements: } \\
{ }^{181} \text { I. M. Kolthoff, J. J. Lingane, and } \\
\text { W. Larson, J. Am. Chem. Soc. 60, } \\
2512 \text { (1938). }\end{array}$ \\
\hline $\begin{array}{l}\text { Variables: } \\
T / \mathrm{K}=298.15\end{array}$ & $\begin{array}{l}\text { Prepared by: } \\
\text { W. E. Acree, Jr. }\end{array}$ \\
\hline
\end{tabular}

\section{Experimental Values}

The measured solubility was reported to be $0.200 \mathrm{~mol} \mathrm{dm}^{-3}$.

\section{Auxiliary Information}

\section{Method/Apparatus/Procedure:}

Very little experimental details were provided. Solubility was determined by the conductance method.

\section{Source and Purity of Chemicals:}

(1) Purity not given, Chemical source not given, no purification details were provided.

(2) Purity not given, Chemical source not given, refluxed over silver oxide, distilled and dehydrated with magnesium.

\section{Estimated Error:}

Temperature: $\pm 0.1 \mathrm{~K}$ (estimated by compiler).

$c_{1}: \pm 2 \%$ (relative error, estimated by compiler).

\begin{tabular}{ll}
\hline \hline Components: & Original Measurements: \\
(1) 4-Nitrobenzoic acid; $\mathrm{C}_{7} \mathrm{H}_{5} \mathrm{NO}_{4} ;$ & ${ }^{11}$ K. R. Hoover, R. Coaxum, E. \\
[62-23-7] & Pustejovsky, D. M. Stovall, W. E. \\
(2) Ethanol; $\mathrm{C}_{2} \mathrm{H}_{6} \mathrm{O} ;[64-17-5]$ & Acree, Jr., and M. H. Abraham, \\
& Phys. Chem. Liq. 42, 339 (2004). \\
\hline Variables: & Prepared by: \\
$T / \mathrm{K}=298.15$ & W. E. Acree, Jr. \\
\hline
\end{tabular}

\section{Experimental Values}

\begin{tabular}{lc}
\hline \hline$x_{2}{ }^{\mathrm{a}}$ & $x_{1}{ }^{\mathrm{b}}$ \\
\hline 0.9915 & 0.008544 \\
\hline${ }^{\mathrm{a}} x_{2}:$ mole fraction of component 2 in the saturated solution. \\
${ }^{\mathrm{b}} x_{1}:$ mole fraction solubility of the solute.
\end{tabular}

\section{Auxiliary Information}

\section{Method/Apparatus/Procedure:}

Constant-temperature bath, calorimetric thermometer, and an ultraviolet/ visible spectrophotometer.

Excess solute and solvent were placed in amber glass bottles and allowed to equilibrate for several days at constant temperature. Attainment of equilibrium was verified by several repetitive measurements and by approaching equilibrium from supersaturation. Aliquots of saturated solutions were transferred through a coarse filter into tared volumetric flasks, weighed, and diluted with methanol. Concentrations were determined by spectrophotometric measurements at $272 \mathrm{~nm}$.

\section{Source and Purity of Chemicals:}

(1) $99+\%$, Acros Organics, USA, was used as received.

(2) Absolute, Aaper Alcohol and Chemical Company, USA, stored over molecular sieves and distilled shortly before use.

\section{Estimated Error:}

Temperature: $\pm 0.1 \mathrm{~K}$.

$x_{1}: \pm 1.5 \%$ (relative error).

\begin{tabular}{ll}
\hline \hline Components: & Original Measurements: \\
$\begin{array}{l}\text { (1) 4-Nitrobenzoic acid; } \mathrm{C}_{7} \mathrm{H}_{5} \mathrm{NO}_{4} ; \\
\text { [62-23-7] }\end{array}$ & $\begin{array}{l}{ }^{126} \mathrm{P} . \text { K. Biswas, S. C. Lahiri, and B. } \\
\text { (2) Ethanol; } \mathrm{C}_{2} \mathrm{H}_{6} \mathrm{O} ;[64-17-5]\end{array}$ \\
\hline Pariables: & 2785 (1993). \\
$T / \mathrm{K}=298.15$ & Prepared by: \\
\hline
\end{tabular}

Experimental Values

\begin{tabular}{lcc}
\hline \hline$c_{1}^{\mathrm{a}}$ & $x_{2}{ }^{\mathrm{b}}$ & $x_{1}{ }^{\mathrm{c}}$ \\
\hline 0.05445 & 0.9968 & 0.00321
\end{tabular}

${ }^{\mathrm{a}} c_{1}$ : solubility of the solute expressed in units of $\mathrm{mol} \mathrm{dm}^{-3}$.

${ }^{\mathrm{b}} x_{2}$ : mole fraction of component 2 in the saturated solution.

${ }^{c} x_{1}$ : mole fraction solubility of the solute calculated by the compiler. 


\section{Auxiliary Information}

\section{Method/Apparatus/Procedure:}

Analytical balance and an ultraviolet/visible spectrophotometer.

Solutions containing excess solute and solvent were allowed to equilibrate at constant temperature for at least $24 \mathrm{~h}$. An aliquot of the saturated solution was removed, filtered, and the absorbance recorded. Solubility was calculated from the measured absorbance.

\section{Source and Purity of Chemicals:}

(1) Puris grade, Fluka Chemicals, recrystallized from aqueous ethanol mixture and dried in an air oven at $390 \mathrm{~K}$. The purified compound was stored in a vacuum desiccators.

(2) Absolute, B.C.P.W., Calcutta, India, was distilled twice before use.

\section{Estimated Error:}

Temperature: $\pm 0.2 \mathrm{~K}$ (estimated by compiler). $c_{1}: \pm 1.0 \%$ (relative error, estimated by compiler). $x_{1}: \pm 2.5 \%$ (relative error, estimated by compiler).

\begin{tabular}{|c|c|}
\hline $\begin{array}{l}\text { Components: } \\
\text { (1) 4-Nitrobenzoic acid; } \mathrm{C}_{7} \mathrm{H}_{5} \mathrm{NO}_{4} \text {; } \\
\text { [62-23-7] } \\
\text { (2) Ethanol; } \mathrm{C}_{2} \mathrm{H}_{6} \mathrm{O} ;[64-17-5]\end{array}$ & $\begin{array}{l}\text { Original Measurements: } \\
\text { }{ }^{72} \text { R. Thuaire, Bull. Soc. Chim. Fr. } \\
3815 \text { (1971). }\end{array}$ \\
\hline $\begin{array}{l}\text { Variables: } \\
T / \mathrm{K}=298.15\end{array}$ & $\begin{array}{l}\text { Prepared by: } \\
\text { W. E. Acree, Jr. }\end{array}$ \\
\hline
\end{tabular}

\section{Experimental Values}

The measured solubility was reported to be $0.190 \mathrm{~mol} / \mathrm{kg}$ of solvent, which corresponds to a mole fraction solubility of $x_{1}=0.00868$.

\section{Auxiliary Information}

\section{Method/Apparatus/Procedure:}

Very little experimental details were provided. Solubility was determined by a gravimetric method. The saturated solution was evaporated to dryness and the remaining solid residue was weighed. The solubility was calculated from the mass of the solid residue and mass of saturated solution analyzed.

\section{Source and Purity of Chemicals:}

(1) Purity not given, Chemical source not given, no purification details were provided.

(2) Purity not given, Chemical source not given, no purification details were provided.

\section{Estimated Error:}

Temperature: $\pm 0.05 \mathrm{~K}$.

$x_{1}: \pm 4 \%$ (relative error, estimated by compiler).

\begin{tabular}{ll}
\hline \hline Components: & Original Measurements: \\
(1) 4-Nitrobenzoic acid; $\mathrm{C}_{7} \mathrm{H}_{5} \mathrm{NO}_{4} ;$ & $\begin{array}{l}{ }^{11} \text { K. R. Hoover, R. Coaxum, E. } \\
\text { [62-23-7] }\end{array}$ \\
$\begin{array}{l}\text { Pustejovsky, D. M. Stovall, W. E. } \\
\text { (2) 1-Propanol; } \mathrm{C}_{3} \mathrm{H}_{8} \mathrm{O} ;[\text { [71-23-8] }\end{array}$ & $\begin{array}{l}\text { Acree, Jr., and M. H. Abraham, } \\
\text { Phys. Chem. Liq. 42, 339 (2004). }\end{array}$ \\
\hline Variables: & Prepared by: \\
$T / \mathrm{K}=298.15$ & W. E. Acree, Jr. \\
\hline
\end{tabular}

Experimental Values

\begin{tabular}{lc}
\hline \hline$x_{2}{ }^{\mathrm{a}}$ & $x_{1}{ }^{\mathrm{b}}$ \\
\hline 0.9920 & 0.007990 \\
\hline${ }^{\mathrm{a}} x_{2}:$ mole fraction of component 2 in the saturated solution. \\
${ }^{\mathrm{b}} x_{1}:$ mole fraction solubility of the solute.
\end{tabular}

\section{Auxiliary Information}

\section{Method/Apparatus/Procedure:}

Constant-temperature bath, calorimetric thermometer, and an ultraviolet/ visible spectrophotometer.

Excess solute and solvent were placed in amber glass bottles and allowed to equilibrate for several days at constant temperature. Attainment of equilibrium was verified by several repetitive measurements and by approaching equilibrium from supersaturation. Aliquots of saturated solutions were transferred through a coarse filter into tared volumetric flasks, weighed, and diluted with methanol. Concentrations were determined by spectrophotometric measurements at $272 \mathrm{~nm}$.

\section{Source and Purity of Chemicals:}

(1) $99+\%$, Acros Organics, USA, was used as received.

(2) $99+\%$, anhydrous, Aldrich Chemical Company, Milwaukee, WI, USA, stored over molecular sieves and distilled shortly before use.

\section{Estimated Error:}

Temperature: $\pm 0.1 \mathrm{~K}$.

$x_{1}: \pm 1.5 \%$ (relative error)

\begin{tabular}{ll}
\hline \hline Components: & Original Measurements: \\
(1) 4-Nitrobenzoic acid; $\mathrm{C}_{7} \mathrm{H}_{5} \mathrm{NO}_{4} ;$ & ${ }^{11} \mathrm{~K}$. R. Hoover, R. Coaxum, E. \\
[62-23-7] & Pustejovsky, D. M. Stovall, W. E. \\
(2) 2-Propanol; $\mathrm{C}_{3} \mathrm{H}_{8} \mathrm{O} ;$ [67-63-0] & $\begin{array}{l}\text { Acree, Jr., and M. H. Abraham, } \\
\text { Phys. Chem. Liq. 42, 339 (2004). }\end{array}$ \\
\hline Variables: & Prepared by: \\
$T / \mathrm{K}=298.15$ & W. E. Acree, Jr. \\
\hline
\end{tabular}

\section{Experimental Values}

\begin{tabular}{lc}
\hline \hline$x_{2}{ }^{\mathrm{a}}$ & $x_{1}{ }^{\mathrm{b}}$ \\
\hline 0.9914 & 0.008550 \\
\hline${ }^{\mathrm{a}} x_{2}:$ mole fraction of component 2 in the saturated solution. \\
${ }^{\mathrm{b}} x_{1}:$ mole fraction solubility of the solute.
\end{tabular}

\section{Auxiliary Information}

\section{Method/Apparatus/Procedure:}

Constant-temperature bath, calorimetric thermometer, and an ultraviolet/ visible spectrophotometer.

Excess solute and solvent were placed in amber glass bottles and allowed to equilibrate for several days at constant temperature. Attainment of equilibrium was verified by several repetitive measurements and by approaching equilibrium from supersaturation. Aliquots of saturated solutions were transferred through a coarse filter into tared volumetric flasks, weighed, and diluted with methanol. Concentrations were determined by spectrophotometric measurements at $272 \mathrm{~nm}$.

Source and Purity of Chemicals:

(1) $99+\%$, Acros Organics, USA, was used as received.

(2) $99+\%$, anhydrous, Aldrich Chemical Company, Milwaukee, WI, USA, stored over molecular sieves and distilled shortly before use. 
Estimated Error:

Temperature: $\pm 0.1 \mathrm{~K}$

$x_{1}: \pm 1.5 \%$ (relative error).

\begin{tabular}{ll}
\hline \hline Components: & $\begin{array}{l}\text { Original Measurements: } \\
\text { (1) 4-Nitrobenzoic acid; } \mathrm{C}_{7} \mathrm{H}_{5} \mathrm{NO}_{4} ;\end{array}$ \\
$\begin{array}{l}{ }^{76} \mathrm{M} \text {. K. Chantooni and I. M. } \\
\text { 62-23-7] }\end{array}$ & $\begin{array}{l}\text { Kolthoff, Anal. Chem. 51, 133 } \\
\left.\text { (2) 2-Propanol; } \mathrm{C}_{3} \mathrm{H}_{8} \mathrm{O} ;[679) .63-0\right]\end{array}$ \\
\hline Variables: & Prepared by: \\
$T / \mathrm{K}=298.15$ & W. E. Acree, Jr. \\
\hline
\end{tabular}

Experimental Values

The measured solubility was reported to be $0.111 \mathrm{~mol} \mathrm{dm}^{-3}$. The authors did not give the temperature at which the solubility was measured. Based on the experimental description given in an earlier paper [I. M. Kolthoff, J. J. Lingane, and W. Larson, J. Am. Chem. Soc. 60, 2512 (1938)], the compiler believes the temperature to be $298.15 \mathrm{~K}$.

\section{Auxiliary Information}

\section{Method/Apparatus/Procedure:}

Very little experimental details were provided. Solubility was determined by titrating a filtered aliquot of the saturated solution alkalimetrically in an aqueous-ethanol mixture using phenolphthalein as the acid-base indicator.

Source and Purity of Chemicals:

(1) White Label, Eastman Kodak Chemical Company, Rochester, NY, USA, was recrystallized from aqueous solution and dried in vacuo at $343 \mathrm{~K}$.

(2) No Purity given, Fisher Scientific, USA, shaken with calcium hydride and distilled before use.

\section{Estimated Error:}

Temperature: $\pm 0.1 \mathrm{~K}$ (estimated by compiler).

$c_{1}: \pm 2 \%$ (relative error, estimated by compiler).

\begin{tabular}{ll}
\hline \hline Components: & Original Measurements: \\
(1) 4-Nitrobenzoic acid; $\mathrm{C}_{7} \mathrm{H}_{5} \mathrm{NO}_{4} ;$ & $\begin{array}{l}{ }^{11} \text { K. R. Hoover, R. Coaxum, E. } \\
\text { [62-23-7] }\end{array}$ \\
$\begin{array}{ll}\text { Pustejovsky, D. M. Stovall, W. E. } \\
\text { (2) 1-Butanol; } \mathrm{C}_{4} \mathrm{H}_{10} \mathrm{O} ; \text { [71-36-3] }\end{array}$ & $\begin{array}{l}\text { Acree, Jr., and M. H. Abraham, } \\
\text { Phys. Chem. Liq. 42, 339 (2004). }\end{array}$ \\
\hline Variables: & Prepared by: \\
$T / \mathrm{K}=298.15$ & W. E. Acree, Jr. \\
\hline
\end{tabular}

\section{Experimental Values}

\begin{tabular}{lc}
\hline \hline$x_{2}{ }^{\mathrm{a}}$ & $x_{1}{ }^{\mathrm{b}}$ \\
\hline 0.9920 & 0.007973 \\
\hline${ }^{\mathrm{a}} x_{2}:$ mole fraction of component 2 in the saturated solution. \\
${ }^{\mathrm{b}} x_{1}:$ mole fraction solubility of the solute.
\end{tabular}

\section{Auxiliary Information}

Method/Apparatus/Procedure:

Constant-temperature bath, calorimetric thermometer, and an ultraviolet/ visible spectrophotometer.

Excess solute and solvent were placed in amber glass bottles and allowed to equilibrate for several days at constant temperature. Attainment of equilibrium was verified by several repetitive measurements and by approaching equilibrium from supersaturation. Aliquots of saturated solutions were transferred through a coarse filter into tared volumetric flasks, weighed, and diluted with methanol. Concentrations were determined by spectrophotometric measurements at $272 \mathrm{~nm}$.

\section{Source and Purity of Chemicals:}

(1) $99+\%$, Acros Organics, USA, was used as received.

(2) $99.8+\%$, HPLC grade, Aldrich Chemical Company, Milwaukee, WI, USA, stored over molecular sieves and distilled shortly before use.

\section{Estimated Error:}

Temperature: $\pm 0.1 \mathrm{~K}$.

$x_{1}: \pm 1.5 \%$ (relative error)

\begin{tabular}{ll}
\hline \hline Components: & Original Measurements: \\
(1) 4-Nitrobenzoic acid; $\mathrm{C}_{7} \mathrm{H}_{5} \mathrm{NO}_{4} ;$ & ${ }^{11}$ K. R. Hoover, R. Coaxum, E. \\
[62-23-7] & Pustejovsky, D. M. Stovall, W. E. \\
(2) 2-Butanol; $\mathrm{C}_{4} \mathrm{H}_{10} \mathrm{O} ;$ [78-92-2] & $\begin{array}{l}\text { Acree, Jr., and M. H. Abraham, } \\
\text { Phys. Chem. Liq. 42, 339 (2004). }\end{array}$ \\
\hline Variables: & Prepared by: \\
$T / \mathrm{K}=298.15$ & W. E. Acree, Jr. \\
\hline
\end{tabular}

\section{Experimental Values}

\begin{tabular}{lc}
\hline \hline$x_{2}{ }^{\mathrm{a}}$ & $x_{1}{ }^{\mathrm{b}}$ \\
\hline 0.9911 & 0.008898 \\
\hline${ }^{\mathrm{a}} x_{2}:$ mole fraction of component 2 in the saturated solution. & \\
${ }^{\mathrm{b}}{ }_{x_{1}}:$ mole fraction solubility of the solute. &
\end{tabular}

\section{Auxiliary Information}

\section{Method/Apparatus/Procedure:}

Constant-temperature bath, calorimetric thermometer, and an ultraviolet/ visible spectrophotometer.

Excess solute and solvent were placed in amber glass bottles and allowed to equilibrate for several days at constant temperature. Attainment of equilibrium was verified by several repetitive measurements and by approaching equilibrium from supersaturation. Aliquots of saturated solutions were transferred through a coarse filter into tared volumetric flasks, weighed, and diluted with methanol. Concentrations were determined by spectrophotometric measurements at $272 \mathrm{~nm}$.

\section{Source and Purity of Chemicals:}

(1) $99+\%$, Acros Organics, USA, was used as received.

(2) $99+\%$, anhydrous, Aldrich Chemical Company, Milwaukee, WI, USA, stored over molecular sieves and distilled shortly before use.

\section{Estimated Error:}

Temperature: $\pm 0.1 \mathrm{~K}$.

$x_{1}: \pm 1.5 \%$ (relative error) 


\begin{tabular}{ll}
\hline \hline Components: & Original Measurements: \\
(1) 4-Nitrobenzoic acid; $\mathrm{C}_{7} \mathrm{H}_{5} \mathrm{NO}_{4} ;$ & $\begin{array}{l}{ }^{11} \text { K. R. Hoover, R. Coaxum, E. } \\
\text { [62-23-7] }\end{array}$ \\
$\begin{array}{l}\text { Pustejovsky, D. M. Stovall, W. E. } \\
\text { (2) 2-Methyl-1-propanol; } \mathrm{C}_{4} \mathrm{H}_{10} \mathrm{O} ;\end{array}$ & $\begin{array}{l}\text { Acree, Jr., and M. H. Abraham, } \\
\text { [78-83-1] }\end{array}$ \\
\hline Phys. Chem. Liq. 42, 339 (2004). \\
\hline Variables: & Prepared by: \\
\hline$/ \mathrm{K}=298.15$ & W. E. Acree, Jr. \\
\hline
\end{tabular}

Experimental Values

\begin{tabular}{lc}
\hline \hline$x_{2}{ }^{\mathrm{a}}$ & $x_{1}{ }^{\mathrm{b}}$ \\
\hline 0.9939 & 0.006093 \\
\hline
\end{tabular}

${ }^{\mathrm{a}} x_{2}$ : mole fraction of component 2 in the saturated solution.

${ }^{b} x_{1}$ : mole fraction solubility of the solute.

\section{Auxiliary Information}

\section{Method/Apparatus/Procedure:}

Constant-temperature bath, calorimetric thermometer, and an ultraviolet/ visible spectrophotometer.

Excess solute and solvent were placed in amber glass bottles and allowed to equilibrate for several days at constant temperature. Attainment of equilibrium was verified by several repetitive measurements and by approaching equilibrium from supersaturation. Aliquots of saturated solutions were transferred through a coarse filter into tared volumetric flasks, weighed, and diluted with methanol. Concentrations were determined by spectrophotometric measurements at $272 \mathrm{~nm}$.

\section{Source and Purity of Chemicals:}

(1) $99+\%$, Acros Organics, USA, was used as received.

(2) $99+\%$, anhydrous, Aldrich Chemical Company, Milwaukee, WI, USA, stored over molecular sieves and distilled shortly before use.

\section{Estimated Error:}

Temperature: $\pm 0.1 \mathrm{~K}$

$x_{1}: \pm 1.5 \%$ (relative error).

\begin{tabular}{ll}
\hline \hline Components: & Original Measurements: \\
(1) 4-Nitrobenzoic acid; $\mathrm{C}_{7} \mathrm{H}_{5} \mathrm{NO}_{4} ;$ & ${ }^{11} \mathrm{~K}$. R. Hoover, R. Coaxum, E. \\
[62-23-7] & Pustejovsky, D. M. Stovall, W. E. \\
$\begin{array}{l}\text { (2) 2-Methyl-2-propanol; } \mathrm{C}_{4} \mathrm{H}_{10} \mathrm{O} ; \\
\text { [75-65-0] }\end{array}$ & Acree, Jr., and M. H. Abraham, \\
\hline Variables: & Phys. Chem. Liq. 42, 339 (2004). \\
$T / \mathrm{K}=298.15$ & Prepared by: \\
\hline
\end{tabular}

Experimental Values

\begin{tabular}{lc}
\hline \hline$x_{2}{ }^{\mathrm{a}}$ & $x_{1}{ }^{\mathrm{b}}$ \\
\hline 0.9857 & 0.01430
\end{tabular}

${ }^{\mathrm{a}} x_{2}$ : mole fraction of component 2 in the saturated solution.

${ }^{\mathrm{b}} x_{1}$ : mole fraction solubility of the solute.

\section{Auxiliary Information}

\section{Method/Apparatus/Procedure:}

Constant-temperature bath, calorimetric thermometer, and an ultraviolet/ visible spectrophotometer.

Excess solute and solvent were placed in amber glass bottles and allowed to equilibrate for several days at constant temperature. Attainment of equilibrium was verified by several repetitive measurements and by approaching equilibrium from supersaturation. Aliquots of saturated solutions were transferred through a coarse filter into tared volumetric flasks, weighed, and diluted with methanol. Concentrations were determined by spectrophotometric measurements at $272 \mathrm{~nm}$.

\section{Source and Purity of Chemicals:}

(1) $99+\%$, Acros Organics, USA, was used as received.

(2) $99+\%$, Arco Chemical Company, USA, stored over molecular sieves and distilled shortly before use.

\section{Estimated Error:}

Temperature: $\pm 0.1 \mathrm{~K}$.

$x_{1}: \pm 1.5 \%$ (relative error).

\begin{tabular}{|c|c|}
\hline $\begin{array}{l}\text { Components: } \\
\text { (1) 4-Nitrobenzoic acid; } \mathrm{C}_{7} \mathrm{H}_{5} \mathrm{NO}_{4} \text {; } \\
\text { [62-23-7] } \\
\text { (2) 2-Methyl-2-propanol; } \mathrm{C}_{4} \mathrm{H}_{10} \mathrm{O} \text {; } \\
\text { [75-65-0] }\end{array}$ & $\begin{array}{l}\text { Original Measurements: } \\
{ }^{76} \text { M. K. Chantooni and I. M. } \\
\text { Kolthoff, Anal. Chem. 51, } 133 \\
\text { (1979). }\end{array}$ \\
\hline $\begin{array}{l}\text { Variables: } \\
T / \mathrm{K}=298.15\end{array}$ & $\begin{array}{l}\text { Prepared by: } \\
\text { W. E. Acree, Jr. }\end{array}$ \\
\hline
\end{tabular}

\section{Experimental Values}

The measured solubility was reported to be $0.144 \mathrm{~mol} \mathrm{dm}^{-3}$. The authors did not give the temperature at which the solubility was measured. Based on the experimental description given in an earlier paper [I. M. Kolthoff, J. J. Lingane, and W. Larson, J. Am. Chem. Soc. 60, 2512 (1938)], the compiler believes the temperature to be $298.15 \mathrm{~K}$.

\section{Auxiliary Information}

\section{Method/Apparatus/Procedure:}

Very little experimental details were provided. Solubility was determined by titrating a filtered aliquot of the saturated solution alkalimetrically in an aqueous-ethanol mixture using phenolphthalein as the acid-base indicator.

\section{Source and Purity of Chemicals:}

(1) White Label, Eastman Kodak Chemical Company, Rochester, NY, USA, was recrystallized from aqueous solution and dried in vacuo at $343 \mathrm{~K}$.

(2) White Label, Eastman Kodak Chemical Company, Rochester, NY, USA, shaken with calcium hydride and distilled before use.

\section{Estimated Error:}

Temperature: $\pm 0.1 \mathrm{~K}$ (estimated by compiler).

$c_{1}: \pm 2 \%$ (relative error, estimated by compiler). 


\section{Components:}

(1) 4-Nitrobenzoic acid; $\mathrm{C}_{7} \mathrm{H}_{5} \mathrm{NO}_{4}$; [62-23-7]

(2) 1-Pentanol; $\mathrm{C}_{5} \mathrm{H}_{12} \mathrm{O}$; [71-41-0]

\begin{tabular}{ll}
\hline Variables: & Prepared by: \\
$T / \mathrm{K}=298.15$ & W. E. Acree, Jr. \\
\hline
\end{tabular}

\section{Experimental Values}

\begin{tabular}{lc}
\hline \hline$x_{2}{ }^{\mathrm{a}}$ & $x_{1}{ }^{\mathrm{b}}$ \\
\hline 0.9907 & 0.009317 \\
\hline$x_{2}$.
\end{tabular}

${ }^{\mathrm{a}} x_{2}$ : mole fraction of component 2 in the saturated solution.

${ }^{\mathrm{b}} x_{1}$ : mole fraction solubility of the solute.

\section{Auxiliary Information}

Method/Apparatus/Procedure:
Constant-temperature bath, calorimetric thermometer, and an ultraviolet/
visible spectrophotometer.
Excess solute and solvent were placed in amber glass bottles and allowed to
equilibrate for several days at constant temperature. Attainment of equilibrium
was verified by several repetitive measurements and by approaching
equilibrium from supersaturation. Aliquots of saturated solutions were
transferred through a coarse filter into tared volumetric flasks, weighed, and
diluted with methanol. Concentrations were determined by
spectrophotometric measurements at 272 nm.
Source and Purity of Chemicals:
(1) $99+\%$, Acros Organics, USA, was used as received.
(2) $99+\%$, Aldrich Chemical Company, Milwaukee, WI, USA, stored over
molecular sieves and distilled shortly before use.
Estimated Error:
Temperature: $\pm 0.1 \mathrm{~K}$.
$x_{1}: \pm 1.5 \%$ (relative error).

\begin{tabular}{ll}
\hline \hline Components: & Original Measurements: \\
(1) 4-Nitrobenzoic acid; $\mathrm{C}_{7} \mathrm{H}_{5} \mathrm{NO}_{4} ;$ & ${ }^{11}$ K. R. Hoover, R. Coaxum, E. \\
[62-23-7] & Pustejovsky, D. M. Stovall, W. E. \\
(2) 2-Pentanol; $\mathrm{C}_{5} \mathrm{H}_{12} \mathrm{O} ;$ [6032-29-7] & $\begin{array}{l}\text { Acree, Jr., and M. H. Abraham, } \\
\text { Phys. Chem. Liq. 42, 339 (2004). }\end{array}$ \\
\hline Variables: & Prepared by: \\
$T / \mathrm{K}=298.15$ & W. E. Acree, Jr. \\
\hline
\end{tabular}

\section{Experimental Values}

\begin{tabular}{lc}
\hline \hline$x_{2}{ }^{\mathrm{a}}$ & $x_{1}{ }^{\mathrm{b}}$ \\
\hline 0.9910 & 0.008967 \\
\hline${ }_{{ }_{2}}:$ mole fraction of component 2 in the saturated solution. \\
${ }^{\mathrm{b}} x_{1}:$ mole fraction solubility of the solute.
\end{tabular}

\section{Auxiliary Information}

\section{Method/Apparatus/Procedure:}

Constant-temperature bath, calorimetric thermometer, and an ultraviolet/ visible spectrophotometer.

Excess solute and solvent were placed in amber glass bottles and allowed to equilibrate for several days at constant temperature. Attainment of equilibrium was verified by several repetitive measurements and by approaching equilibrium from supersaturation. Aliquots of saturated solutions were transferred through a coarse filter into tared volumetric flasks, weighed, and diluted with methanol. Concentrations were determined by spectrophotometric measurements at $272 \mathrm{~nm}$.

\section{Source and Purity of Chemicals:}

(1) $99+\%$, Acros Organics, USA, was used as received.

(2) $99+\%$, Acros Organics, stored over molecular sieves and distilled shortly before use.

\section{Estimated Error:}

Temperature: $\pm 0.1 \mathrm{~K}$.

$x_{1}: \pm 1.5 \%$ (relative error).

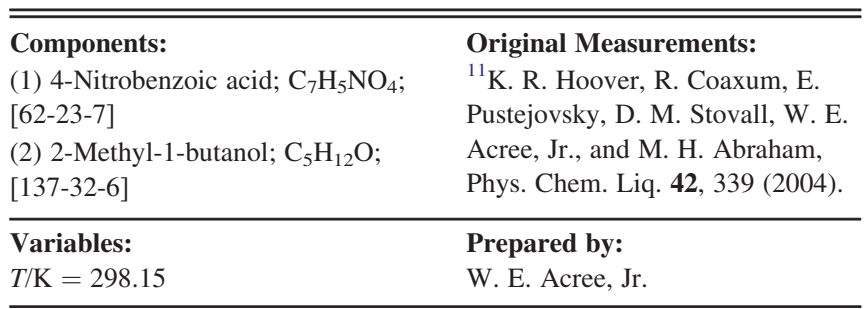

\section{Experimental Values}

\begin{tabular}{lc}
\hline \hline$x_{2}{ }^{\mathrm{a}}$ & $x_{1}{ }^{\mathrm{b}}$ \\
\hline 0.9941 & 0.005897 \\
\hline${ }^{\mathrm{a}} x_{2}:$ mole fraction of component 2 in the saturated solution. \\
${ }^{\mathrm{b}}{ }_{x_{1}: \text { mole fraction solubility of the solute. }}$
\end{tabular}

\section{Auxiliary Information}

\section{Method/Apparatus/Procedure:}

Constant-temperature bath, calorimetric thermometer, and an ultraviolet/ visible spectrophotometer.

Excess solute and solvent were placed in amber glass bottles and allowed to equilibrate for several days at constant temperature. Attainment of equilibrium was verified by several repetitive measurements and by approaching equilibrium from supersaturation. Aliquots of saturated solutions were transferred through a coarse filter into tared volumetric flasks, weighed, and diluted with methanol. Concentrations were determined by spectrophotometric measurements at $272 \mathrm{~nm}$.

\section{Source and Purity of Chemicals:}

(1) $99+\%$, Acros Organics, USA, was used as received. (2) $99 \%$, Aldrich Chemical Company, Milwaukee, WI, USA, stored over molecular sieves and distilled shortly before use.

\section{Estimated Error:}

Temperature: $\pm 0.1 \mathrm{~K}$.

$x_{1}: \pm 1.5 \%$ (relative error). 


\section{Components:}

(1) 4-Nitrobenzoic acid; $\mathrm{C}_{7} \mathrm{H}_{5} \mathrm{NO}_{4}$; [62-23-7]

(2) 2-Methyl-2-butanol; $\mathrm{C}_{5} \mathrm{H}_{12} \mathrm{O}$; [75-85-4]

\section{Variables:}

$T / \mathrm{K}=298.15$
Original Measurements:

${ }^{11}$ K. R. Hoover, R. Coaxum, E. Pustejovsky, D. M. Stovall, W. E. Acree, Jr., and M. H. Abraham, Phys. Chem. Liq. 42, 339 (2004).

Prepared by:

W. E. Acree, Jr.

Experimental Values

\begin{tabular}{lc}
\hline \hline$x_{2}{ }^{\mathrm{a}}$ & $x_{1}{ }^{\mathrm{b}}$ \\
\hline 0.9819 & 0.01814
\end{tabular}

${ }^{\mathrm{a}} x_{2}$ : mole fraction of component 2 in the saturated solution.

${ }^{b} x_{1}$ : mole fraction solubility of the solute.

\section{Auxiliary Information}

\section{Method/Apparatus/Procedure:}

Constant-temperature bath, calorimetric thermometer, and an ultraviolet/ visible spectrophotometer.

Excess solute and solvent were placed in amber glass bottles and allowed to equilibrate for several days at constant temperature. Attainment of equilibrium was verified by several repetitive measurements and by approaching equilibrium from supersaturation. Aliquots of saturated solutions were transferred through a coarse filter into tared volumetric flasks, weighed, and diluted with methanol. Concentrations were determined by spectrophotometric measurements at $272 \mathrm{~nm}$.

Source and Purity of Chemicals:

(1) $99+\%$, Acros Organics, USA, was used as received.

(2) $99 \%$, Aldrich Chemical Company, Milwaukee, WI, USA, stored over molecular sieves and distilled shortly before use.

\section{Estimated Error:}

Temperature: $\pm 0.1 \mathrm{~K}$

$x_{1}: \pm 1.5 \%$ (relative error).

\section{Components:}

(1) 4-Nitrobenzoic acid; $\mathrm{C}_{7} \mathrm{H}_{5} \mathrm{NO}_{4}$; [62-23-7]

(2) 3-Methyl-1-butanol; $\mathrm{C}_{5} \mathrm{H}_{12} \mathrm{O}$; [123-51-3]

\begin{tabular}{ll}
\hline Variables: & Prepared by: \\
$T / \mathrm{K}=298.15$ & W. E. Acree, Jr. \\
\hline
\end{tabular}

Experimental Values

\begin{tabular}{lc}
\hline \hline$x_{2}{ }^{\mathrm{a}}$ & $x_{1}{ }^{\mathrm{b}}$ \\
\hline 0.9927 & 0.007285 \\
\hline${ }^{\mathrm{a}} x_{2}:$ mole fraction of component 2 in the saturated solution. \\
${ }^{\mathrm{b}}{ }_{x_{1}: \text { mole fraction solubility of the solute. }}$
\end{tabular}

\section{Auxiliary Information}

\section{Method/Apparatus/Procedure:}

Constant-temperature bath, calorimetric thermometer, and an ultraviolet/ visible spectrophotometer.

Excess solute and solvent were placed in amber glass bottles and allowed to equilibrate for several days at constant temperature. Attainment of equilibrium was verified by several repetitive measurements and by approaching equilibrium from supersaturation. Aliquots of saturated solutions were transferred through a coarse filter into tared volumetric flasks, weighed, and diluted with methanol. Concentrations were determined by spectrophotometric measurements at $272 \mathrm{~nm}$.

\section{Source and Purity of Chemicals:}

(1) $99+\%$, Acros Organics, USA, was used as received.

(2) $99 \%$, anhydrous, Aldrich Chemical Company, Milwaukee, WI, USA, stored over molecular sieves and distilled shortly before use.

\section{Estimated Error:}

Temperature: $\pm 0.1 \mathrm{~K}$.

$x_{1}: \pm 1.5 \%$ (relative error).

\begin{tabular}{ll}
\hline \hline Components: & Original Measurements: \\
(1) 4-Nitrobenzoic acid; $\mathrm{C}_{7} \mathrm{H}_{5} \mathrm{NO}_{4} ;$ & $\begin{array}{l}{ }^{11} \mathrm{~K} . \text { R. Hoover, R. Coaxum, E. } \\
\text { [62-23-7] }\end{array}$ \\
$\begin{array}{l}\text { Pustejovsky, D. M. Stovall, W. E. } \\
\text { (2) 1-Hexanol; } \mathrm{C}_{6} \mathrm{H}_{14} \mathrm{O} ;[111-27-3]\end{array}$ & $\begin{array}{l}\text { Acree, Jr., and M. H. Abraham, } \\
\text { Phys. Chem. Liq. 42, } 339 \text { (2004). }\end{array}$ \\
\hline Variables: & Prepared by: \\
$T / \mathrm{K}=298.15$ & W. E. Acree, Jr. \\
\hline
\end{tabular}

\section{Experimental Values}

\begin{tabular}{lc}
\hline \hline$x_{2}{ }^{\mathrm{a}}$ & $\frac{x_{1}{ }^{\mathrm{b}}}{}$ \\
\hline 0.9895 & 0.01051 \\
${ }^{\mathrm{a}} x_{2}:$ mole fraction of component 2 in the saturated solution. \\
${ }^{\mathrm{b}} x_{1}:$ mole fraction solubility of the solute.
\end{tabular}

\section{Auxiliary Information}

\section{Method/Apparatus/Procedure:}

Constant-temperature bath, calorimetric thermometer, and an ultraviolet/ visible spectrophotometer.

Excess solute and solvent were placed in amber glass bottles and allowed to equilibrate for several days at constant temperature. Attainment of equilibrium was verified by several repetitive measurements and by approaching equilibrium from supersaturation. Aliquots of saturated solutions were transferred through a coarse filter into tared volumetric flasks, weighed, and diluted with methanol. Concentrations were determined by spectrophotometric measurements at $272 \mathrm{~nm}$.

\section{Source and Purity of Chemicals:}

(1) $99+\%$, Acros Organics, USA, was used as received.

(2) $99+\%$, Alfa Aesar, USA, stored over molecular sieves and distilled shortly before use.

\section{Estimated Error:}

Temperature: $\pm 0.1 \mathrm{~K}$.

$x_{1}: \pm 1.5 \%$ (relative error). 


\section{Components:}

(1) 4-Nitrobenzoic acid; $\mathrm{C}_{7} \mathrm{H}_{5} \mathrm{NO}_{4}$; [62-23-7]

(2) 1-Hexanol; $\mathrm{C}_{6} \mathrm{H}_{14} \mathrm{O}$; [111-27-3]

\section{Variables:}

W. E. Acree, Jr. (1979).

Prepared by:
$T / \mathrm{K}=298.15$

Original Measurements:

${ }^{76} \mathrm{M}$. K. Chantooni and I. M. Kolthoff, Anal. Chem. 51, 133

\section{Experimental Values}

The measured solubility was reported to be $0.084 \mathrm{~mol} \mathrm{dm}^{-3}$. The authors did not give the temperature at which the solubility was measured. Based on the experimental description given in an earlier paper [I. M. Kolthoff, J. J. Lingane, and W. Larson, J. Am. Chem. Soc. 60, 2512 (1938)], the compiler believes the temperature to be $298.15 \mathrm{~K}$.

\section{Auxiliary Information}

\footnotetext{
Method/Apparatus/Procedure:

Very little experimental details were provided. Solubility was determined by titrating a filtered aliquot of the saturated solution alkalimetrically in an aqueous-ethanol mixture using phenolphthalein as the acid-base indicator.

Source and Purity of Chemicals:

(1) White Label, Eastman Kodak Chemical Company, Rochester, NY, USA, was recrystallized from aqueous solution and dried in vacuo at $343 \mathrm{~K}$. (2) Yellow Label, Eastman Kodak Chemical Company, Rochester, NY, USA, shaken with calcium hydride and distilled before use.
}

Estimated Error:

Temperature: $\pm 0.1 \mathrm{~K}$ (estimated by compiler). $c_{1}: \pm 2 \%$ (relative error, estimated by compiler).

\begin{tabular}{ll}
\hline \hline Components: & Original Measurements: \\
(1) 4-Nitrobenzoic acid; $\mathrm{C}_{7} \mathrm{H}_{5} \mathrm{NO}_{4} ;$ & ${ }^{11}$ K. R. Hoover, R. Coaxum, E. \\
[62-23-7] & Pustejovsky, D. M. Stovall, W. E. \\
(2) 2-Methyl-1-pentanol; $\mathrm{C}_{6} \mathrm{H}_{14} \mathrm{O} ;$ & Acree, Jr., and M. H. Abraham, \\
[105-30-6] & Phys. Chem. Liq. 42, 339 (2004). \\
\hline Variables: & Prepared by: \\
$T / \mathrm{K}=298.15$ & W. E. Acree, Jr. \\
\hline
\end{tabular}

\section{Experimental Values}

\begin{tabular}{lc}
\hline \hline$x_{2}^{\mathrm{a}}$ & $x_{1}{ }^{\mathrm{b}}$ \\
\hline 0.9935 & 0.006460 \\
\hline${ }^{\mathrm{a}} x_{2}:$ mole fraction of component 2 in the saturated solution. \\
${ }^{\mathrm{b}} x_{1}:$ mole fraction solubility of the solute.
\end{tabular}

\section{Auxiliary Information}

\section{Method/Apparatus/Procedure:}

Constant-temperature bath, calorimetric thermometer, and an ultraviolet/ visible spectrophotometer.

Excess solute and solvent were placed in amber glass bottles and allowed to equilibrate for several days at constant temperature. Attainment of equilibrium was verified by several repetitive measurements and by approaching equilibrium from supersaturation. Aliquots of saturated solutions were transferred through a coarse filter into tared volumetric flasks, weighed, and diluted with methanol. Concentrations were determined by spectrophotometric measurements at $272 \mathrm{~nm}$.
Source and Purity of Chemicals:

(1) $99+\%$, Acros Organics, USA, was used as received.

(2) $99 \%$, Aldrich Chemical Company, Milwaukee, WI, USA, stored over molecular sieves and distilled shortly before use.

Estimated Error:

Temperature: $\pm 0.1 \mathrm{~K}$.

$x_{1}: \pm 1.5 \%$ (relative error).

\begin{tabular}{|c|c|}
\hline $\begin{array}{l}\text { Components: } \\
\text { (1) 4-Nitrobenzoic acid; } \mathrm{C}_{7} \mathrm{H}_{5} \mathrm{NO}_{4} \text {; } \\
\text { [62-23-7] } \\
\text { (2) 4-Methyl-2-pentanol; } \mathrm{C}_{6} \mathrm{H}_{14} \mathrm{O} \text {; } \\
\text { [108-11-2] }\end{array}$ & $\begin{array}{l}\text { Original Measurements: } \\
{ }^{11} \text { K. R. Hoover, R. Coaxum, E. } \\
\text { Pustejovsky, D. M. Stovall, W. E. } \\
\text { Acree, Jr., and M. H. Abraham, } \\
\text { Phys. Chem. Liq. 42, } 339 \text { (2004). }\end{array}$ \\
\hline $\begin{array}{l}\text { Variables: } \\
T / \mathrm{K}=298.15\end{array}$ & $\begin{array}{l}\text { Prepared by: } \\
\text { W. E. Acree, Jr. }\end{array}$ \\
\hline
\end{tabular}

Experimental Values

\begin{tabular}{lc}
\hline \hline$x_{2}{ }^{\mathrm{a}}$ & $x_{1}{ }^{\mathrm{b}}$ \\
\hline 0.9915 & 0.008513
\end{tabular}

${ }^{\mathrm{a}} x_{2}$ : mole fraction of component 2 in the saturated solution.

${ }^{b} x_{1}$ : mole fraction solubility of the solute.

\section{Auxiliary Information}

\section{Method/Apparatus/Procedure:}

Constant-temperature bath, calorimetric thermometer, and an ultraviolet/ visible spectrophotometer.

Excess solute and solvent were placed in amber glass bottles and allowed to equilibrate for several days at constant temperature. Attainment of equilibrium was verified by several repetitive measurements and by approaching equilibrium from supersaturation. Aliquots of saturated solutions were transferred through a coarse filter into tared volumetric flasks, weighed, and diluted with methanol. Concentrations were determined by spectrophotometric measurements at $272 \mathrm{~nm}$.

Source and Purity of Chemicals:

(1) $99+\%$, Acros Organics, USA, was used as received.

(2) $99+\%$, Acros Organics, stored over molecular sieves and distilled shortly before use.

Estimated Error:

Temperature: $\pm 0.1 \mathrm{~K}$.

$x_{1}: \pm 1.5 \%$ (relative error).

\begin{tabular}{ll}
\hline \hline Components: & Original Measurements: \\
(1) 4-Nitrobenzoic acid; $\mathrm{C}_{7} \mathrm{H}_{5} \mathrm{NO}_{4} ;$ & ${ }^{11}$ K. R. Hoover, R. Coaxum, E. \\
[62-23-7] & Pustejovsky, D. M. Stovall, W. E. \\
(2) 1-Heptanol; $\mathrm{C}_{7} \mathrm{H}_{16} \mathrm{O} ;$ [111-70-6] & $\begin{array}{l}\text { Acree, Jr., and M. H. Abraham, } \\
\text { Phys. Chem. Liq. 42, } 339 \text { (2004). }\end{array}$ \\
\hline Variables: & Prepared by: \\
$T / \mathrm{K}=298.15$ & W. E. Acree, Jr. \\
\hline
\end{tabular}


Experimental Values

\begin{tabular}{lc}
\hline \hline$x_{2}{ }^{\mathrm{a}}$ & $x_{1}{ }^{\mathrm{b}}$ \\
\hline 0.9893 & 0.01071 \\
\hline${ }^{\mathrm{a}}{ }_{x_{2}}:$ mole fraction of component 2 in the saturated solution. \\
${ }^{\mathrm{b}}{ }_{x_{1}: \text { mole fraction solubility of the solute. }}$
\end{tabular}

\section{Auxiliary Information}

\section{Method/Apparatus/Procedure:}

Constant-temperature bath, calorimetric thermometer, and an ultraviolet/ visible spectrophotometer.

Excess solute and solvent were placed in amber glass bottles and allowed to equilibrate for several days at constant temperature. Attainment of equilibrium was verified by several repetitive measurements and by approaching equilibrium from supersaturation. Aliquots of saturated solutions were transferred through a coarse filter into tared volumetric flasks, weighed, and diluted with methanol. Concentrations were determined by spectrophotometric measurements at $272 \mathrm{~nm}$.

Source and Purity of Chemicals:

(1) $99+\%$, Acros Organics, USA, was used as received.

(2) $99+\%$, Alfa Aesar, USA, stored over molecular sieves and distilled shortly before use.

Estimated Error:

Temperature: $\pm 0.1 \mathrm{~K}$.

$x_{1}: \pm 1.5 \%$ (relative error).

\begin{tabular}{ll}
\hline \hline Components: & Original Measurements: \\
(1) 4-Nitrobenzoic acid; $\mathrm{C}_{7} \mathrm{H}_{5} \mathrm{NO}_{4} ;$ & $\begin{array}{l}{ }^{11} \text { K. R. Hoover, R. Coaxum, E. } \\
\text { [62-23-7] }\end{array}$ \\
Pustejovsky, D. M. Stovall, W. E. \\
(2) 1-Octanol; $\mathrm{C}_{8} \mathrm{H}_{18} \mathrm{O} ;$ [111-87-5] & $\begin{array}{l}\text { Acree, Jr., and M. H. Abraham, } \\
\text { Phys. Chem. Liq. 42, 339 (2004). }\end{array}$ \\
\hline Variables: & Prepared by: \\
$T / \mathrm{K}=298.15$ & W. E. Acree, Jr. \\
\hline
\end{tabular}

Experimental Values

\begin{tabular}{lc}
\hline \hline$x_{2}{ }^{\mathrm{a}}$ & $x_{1}{ }^{\mathrm{b}}$ \\
\hline 0.9902 & 0.009758
\end{tabular}

${ }^{\mathrm{a}} x_{2}$ : mole fraction of component 2 in the saturated solution.

${ }^{\mathrm{b}} x_{1}$ : mole fraction solubility of the solute.

\section{Auxiliary Information}

\footnotetext{
Method/Apparatus/Procedure:

Constant-temperature bath, calorimetric thermometer, and an ultraviolet/ visible spectrophotometer.

Excess solute and solvent were placed in amber glass bottles and allowed to equilibrate for several days at constant temperature. Attainment of equilibrium was verified by several repetitive measurements and by approaching equilibrium from supersaturation. Aliquots of saturated solutions were transferred through a coarse filter into tared volumetric flasks, weighed, and diluted with methanol. Concentrations were determined by spectrophotometric measurements at $272 \mathrm{~nm}$.
}

Source and Purity of Chemicals:

(1) $99+\%$, Acros Organics, USA, was used as received.

(2) $99+\%$, anhydrous, Aldrich Chemical Company, Milwaukee, WI, USA, stored over molecular sieves and distilled shortly before use.
Estimated Error:

Temperature: $\pm 0.1 \mathrm{~K}$.

$x_{1}: \pm 1.5 \%$ (relative error)

\begin{tabular}{ll}
\hline \hline Components: & Original Measurements: \\
(1) 4-Nitrobenzoic acid; $\mathrm{C}_{7} \mathrm{H}_{5} \mathrm{NO}_{4} ;$ & ${ }^{11} \mathrm{~K}$. R. Hoover, R. Coaxum, E. \\
[62-23-7] & Pustejovsky, D. M. Stovall, W. E. \\
(2) 1-Decanol; $\mathrm{C}_{10} \mathrm{H}_{22} \mathrm{O} ;[112-30-1]$ & $\begin{array}{l}\text { Acree, Jr., and M. H. Abraham, } \\
\text { Phys. Chem. Liq. 42, } 339 \text { (2004). }\end{array}$ \\
\hline Variables: & Prepared by: \\
$T / \mathrm{K}=298.15$ & W. E. Acree, Jr. \\
\hline
\end{tabular}

Experimental Values

\begin{tabular}{lc}
\hline \hline$x_{2}^{\mathrm{a}}$ & $x_{1}^{\mathrm{b}}$ \\
\hline 0.9898 & 0.01022 \\
\hline${ }^{\mathrm{a}}{ }_{2}:$ mole fraction of component 2 in the saturated solution. \\
${ }^{\mathrm{b}}{ }_{x_{1}: \text { mole fraction solubility of the solute. }}$
\end{tabular}

\section{Auxiliary Information}

\section{Method/Apparatus/Procedure:}

Constant-temperature bath, calorimetric thermometer, and an ultraviolet/ visible spectrophotometer.

Excess solute and solvent were placed in amber glass bottles and allowed to equilibrate for several days at constant temperature. Attainment of equilibrium was verified by several repetitive measurements and by approaching equilibrium from supersaturation. Aliquots of saturated solutions were transferred through a coarse filter into tared volumetric flasks, weighed, and diluted with methanol. Concentrations were determined by spectrophotometric measurements at $272 \mathrm{~nm}$.

\section{Source and Purity of Chemicals:}

(1) $99+\%$, Acros Organics, USA, was used as received.

(2) $99+\%$, Alfa Aesar, USA, stored over molecular sieves and distilled shortly before use.

\section{Estimated Error:}

Temperature: $\pm 0.1 \mathrm{~K}$.

$x_{1}: \pm 1.5 \%$ (relative error).

\subsection{4-Nitrobenzoic acid solubility data in alkoxyalcohols}

\begin{tabular}{|c|c|}
\hline $\begin{array}{l}\text { Components: } \\
\text { (1) 4-Nitrobenzoic acid; } \mathrm{C}_{7} \mathrm{H}_{5} \mathrm{NO}_{4} \text {; } \\
\text { [62-23-7] } \\
\text { (2) 2-Methoxyethanol; } \mathrm{C}_{3} \mathrm{H}_{8} \mathrm{O}_{2} \text {; } \\
\text { [109-86-4] }\end{array}$ & $\begin{array}{l}\text { Original Measurements: } \\
{ }^{129} \text { S. H. Ghosh and D. K. Hazra, J. } \\
\text { Indian Chem. Soc. 65, } 620 \text { (1988). }\end{array}$ \\
\hline $\begin{array}{l}\text { Variables: } \\
T / \mathrm{K}=298.15\end{array}$ & $\begin{array}{l}\text { Prepared by: } \\
\text { W. E. Acree, Jr. }\end{array}$ \\
\hline
\end{tabular}




\section{Experimental Values}

The measured solubility was reported to be $c_{1}=$ $0.4884 \mathrm{~mol} \mathrm{dm}^{-3}$.

\section{Auxiliary Information}

\section{Method/Apparatus/Procedure:}

Mechanical shaker and a constant-temperature thermostat.

Excess solute and solvent were placed in a bottle and stoppered. The solution was shaken in a mechanical shaker for $24 \mathrm{~h}$ at ambient room temperature. The solution was then thermostated at $298 \mathrm{~K}$ for $24 \mathrm{~h}$ with occasional shaking. An aliquot of the saturated solution was then removed and filtered. The concentration of the dissolved solute was determined by titration with standard caustic soda using phenolphthalein as indicator.

\section{Source and Purity of Chemicals:}

(1) G.R., Merck Chemical Company, was recrystallized from aqueous ethanol mixture.

(2) G.R., Merck Chemical Company, was distilled twice before use.

\section{Estimated Error:}

Temperature: $\pm 0.01 \mathrm{~K}$.

$c_{1}: \pm 1.5 \%$ (relative error, estimated by compiler).

\section{Components:}

(1) 4-Nitrobenzoic acid; $\mathrm{C}_{7} \mathrm{H}_{5} \mathrm{NO}_{4}$; [62-23-7]

(2) 2-Ethoxyethanol; $\mathrm{C}_{4} \mathrm{H}_{10} \mathrm{O}_{2}$; [110-80-5]

\begin{tabular}{ll}
\hline Variables: & Prepared by: \\
$T / \mathrm{K}=298.15$ & W. E. Acree, Jr. \\
\hline
\end{tabular}

Experimental Values

\begin{tabular}{lc}
\hline \hline$x_{2}^{\mathrm{a}}$ & $x_{1}{ }^{\mathrm{b}}$ \\
\hline 0.9699 & 0.0301 \\
\hline
\end{tabular}

${ }^{\mathrm{a}} x_{2}$ : mole fraction of component 2 in the saturated solution.

${ }^{\mathrm{b}} x_{1}$ : mole fraction solubility of the solute.

\section{Auxiliary Information}

\section{Method/Apparatus/Procedure:}

Constant-temperature bath, calorimetric thermometer, and an ultraviolet/ visible spectrophotometer.

Excess solute and solvent were placed in amber glass bottles and allowed to equilibrate for several days at constant temperature. Attainment of equilibrium was verified by several repetitive measurements and by approaching equilibrium from supersaturation. Aliquots of saturated solutions were transferred through a coarse filter into tared volumetric flasks, weighed, and diluted with methanol. Concentrations were determined by spectrophotometric measurements at $272 \mathrm{~nm}$.

Source and Purity of Chemicals:

(1) $99+\%$, Acros Organics, USA, was used as received.

(2) 99\%, Aldrich Chemical Company, Milwaukee, WI, USA, stored over molecular sieves and distilled shortly before use.

Estimated Error:

Temperature: $\pm 0.1 \mathrm{~K}$.

$x_{1}: \pm 1.5 \%$ (relative error).

\begin{tabular}{ll}
\hline \hline Components: & Original Measurements: \\
(1) 4-Nitrobenzoic acid; $\mathrm{C}_{7} \mathrm{H}_{5} \mathrm{NO}_{4} ;$ & ${ }^{130}$ L. M. Grubbs, M. Saifullah, N. E. \\
[62-23-7] & De La Rosa, S. Ye, S. S. Achi, W. E. \\
$\begin{array}{l}\text { (2) 2-Propoxyethanol; } \mathrm{C}_{5} \mathrm{H}_{12} \mathrm{O}_{2} ; \\
\text { [2807-30-9] }\end{array}$ & $\begin{array}{l}\text { Acree, Jr., and M. H. Abraham, } \\
\text { Fariables: }\end{array}$ \\
\hline$T / \mathrm{K}=298.15$ & Prepared byase Equilib. 298, 48 (2010). \\
\hline
\end{tabular}

Experimental Values

\begin{tabular}{lc}
\hline \hline$x_{2}{ }^{\mathrm{a}}$ & $x_{1}{ }^{\mathrm{b}}$ \\
\hline 0.9753 & 0.0247
\end{tabular}

${ }^{\mathrm{a}} x_{2}$ : mole fraction of component 2 in the saturated solution.

${ }^{b} x_{1}$ : mole fraction solubility of the solute.

\section{Auxiliary Information}

\section{Method/Apparatus/Procedure:}

Constant-temperature bath, calorimetric thermometer, and an ultraviolet/ visible spectrophotometer.

Excess solute and solvent were placed in amber glass bottles and allowed to equilibrate for several days at constant temperature. Attainment of equilibrium was verified by several repetitive measurements and by approaching equilibrium from supersaturation. Aliquots of saturated solutions were transferred through a coarse filter into tared volumetric flasks, weighed, and diluted with methanol. Concentrations were determined by spectrophotometric measurements at $272 \mathrm{~nm}$.

\section{Source and Purity of Chemicals:}

(1) $99+\%$, Acros Organics, USA, was used as received.

(2) $99+\%$, Aldrich Chemical Company, Milwaukee, WI, USA, stored over molecular sieves and distilled shortly before use.

\section{Estimated Error:}

Temperature: $\pm 0.1 \mathrm{~K}$.

$x_{1}: \pm 1.5 \%$ (relative error).

\begin{tabular}{ll}
\hline \hline Components: & Original Measurements: \\
$\begin{array}{l}\text { (1) 4-Nitrobenzoic acid; } \mathrm{C}_{7} \mathrm{H}_{5} \mathrm{NO}_{4} ; \\
\text { [62-23-7] }\end{array}$ & $\begin{array}{l}130 \text { L. M. Grubbs, M. Saifullah, N. E. } \\
\text { (2) 2-Isopropoxyethanol; } \mathrm{C}_{5} \mathrm{H}_{12} \mathrm{O}_{2} ;\end{array}$ \\
$\begin{array}{l}\text { De La Rosa, S. Ye, S. S. Achi, W. E. } \\
\text { [109-59-1] }\end{array}$ & Free, Jr., and M. H. Abraham, \\
\hline Variables: & Prepared by: \\
$T / \mathrm{K}=298.15$ & W. E. Acree, Jr. \\
\hline
\end{tabular}

Experimental Values

\begin{tabular}{lc}
\hline \hline$x_{2}{ }^{\mathrm{a}}$ & $x_{1}{ }^{\mathrm{b}}$ \\
\hline 0.9719 & 0.0281 \\
\hline${ }^{\mathrm{a}} x_{2}:$ mole fraction of component 2 in the saturated solution. & \\
${ }^{\mathrm{b}} x_{1}:$ mole fraction solubility of the solute. &
\end{tabular}




\section{Auxiliary Information}

\section{Method/Apparatus/Procedure:}

Constant-temperature bath, calorimetric thermometer, and an ultraviolet/ visible spectrophotometer.

Excess solute and solvent were placed in amber glass bottles and allowed to equilibrate for several days at constant temperature. Attainment of equilibrium was verified by several repetitive measurements and by approaching equilibrium from supersaturation. Aliquots of saturated solutions were transferred through a coarse filter into tared volumetric flasks, weighed, and diluted with methanol. Concentrations were determined by spectrophotometric measurements at $272 \mathrm{~nm}$.

Source and Purity of Chemicals:

(1) $99+\%$, Acros Organics, USA, was used as received.

(2) $99 \%$, Aldrich Chemical Company, Milwaukee, WI, USA, stored over molecular sieves and distilled shortly before use.

Estimated Error:

Temperature: $\pm 0.1 \mathrm{~K}$.

$x_{1}: \pm 1.5 \%$ (relative error)

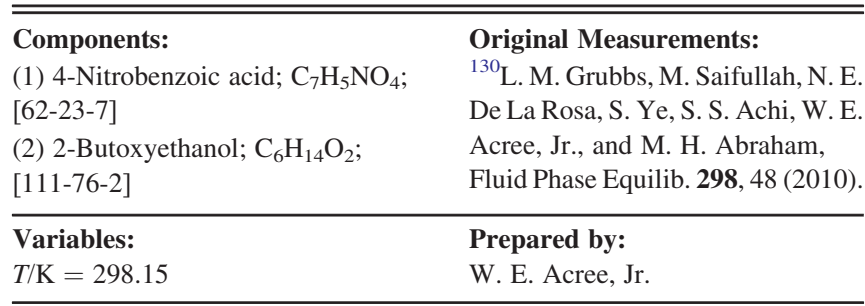

\section{Experimental Values}

\begin{tabular}{lc}
\hline \hline$x_{2}{ }^{\mathrm{a}}$ & $x_{1}{ }^{\mathrm{b}}$ \\
\hline 0.9750 & 0.0250 \\
\hline
\end{tabular}

${ }^{\mathrm{a}} x_{2}$ : mole fraction of component 2 in the saturated solution.

${ }^{b} x_{1}$ : mole fraction solubility of the solute.

\section{Auxiliary Information}

\section{Method/Apparatus/Procedure:}

Constant-temperature bath, calorimetric thermometer, and an ultraviolet/ visible spectrophotometer.

Excess solute and solvent were placed in amber glass bottles and allowed to equilibrate for several days at constant temperature. Attainment of equilibrium was verified by several repetitive measurements and by approaching equilibrium from supersaturation. Aliquots of saturated solutions were transferred through a coarse filter into tared volumetric flasks, weighed, and diluted with methanol. Concentrations were determined by

spectrophotometric measurements at $272 \mathrm{~nm}$.

Source and Purity of Chemicals:

(1) $99+\%$, Acros Organics, USA, was used as received.

(2) $99+\%$, Acros Organics, stored over molecular sieves and distilled shortly before use.

\section{Estimated Error:}

Temperature: $\pm 0.1 \mathrm{~K}$.

$x_{1}: \pm 1.5 \%$ (relative error).

\begin{tabular}{|c|c|}
\hline $\begin{array}{l}\text { Components: } \\
\text { (1) 4-Nitrobenzoic acid; } \mathrm{C}_{7} \mathrm{H}_{5} \mathrm{NO}_{4} \text {; } \\
\text { [62-23-7] } \\
\text { (2) 3-Methoxy-1-butanol; } \mathrm{C}_{5} \mathrm{H}_{12} \mathrm{O}_{2} \text {; } \\
\text { [2517-43-3] }\end{array}$ & $\begin{array}{l}\text { Original Measurements: } \\
{ }^{130} \text { L. M. Grubbs, M. Saifullah, N. E. } \\
\text { De La Rosa, S. Ye, S. S. Achi, W. E. } \\
\text { Acree, Jr., and M. H. Abraham, } \\
\text { Fluid Phase Equilib. 298, } 48 \text { (2010). }\end{array}$ \\
\hline $\begin{array}{l}\text { Variables: } \\
T / \mathrm{K}=298.15\end{array}$ & $\begin{array}{l}\text { Prepared by: } \\
\text { W. E. Acree, Jr. }\end{array}$ \\
\hline
\end{tabular}

Experimental Values

\begin{tabular}{lc}
\hline \hline$x_{2}{ }^{\mathrm{a}}$ & $x_{1}{ }^{\mathrm{b}}$ \\
\hline 0.9739 & 0.0261
\end{tabular}

${ }^{\mathrm{a}} x_{2}$ : mole fraction of component 2 in the saturated solution.

${ }^{b} x_{1}$ : mole fraction solubility of the solute.

\section{Auxiliary Information}

\section{Method/Apparatus/Procedure:}

Constant-temperature bath, calorimetric thermometer, and an ultraviolet/ visible spectrophotometer.

Excess solute and solvent were placed in amber glass bottles and allowed to equilibrate for several days at constant temperature. Attainment of equilibrium was verified by several repetitive measurements and by approaching equilibrium from supersaturation. Aliquots of saturated solutions were transferred through a coarse filter into tared volumetric flasks, weighed, and diluted with methanol. Concentrations were determined by spectrophotometric measurements at $272 \mathrm{~nm}$.

\section{Source and Purity of Chemicals:}

(1) $99+\%$, Acros Organics, USA, was used as received.

(2) $99 \%$, Aldrich Chemical Company, Milwaukee, WI, USA, stored over molecular sieves and distilled shortly before use.

\section{Estimated Error:}

Temperature: $\pm 0.1 \mathrm{~K}$.

$x_{1}: \pm 1.5 \%$ (relative error).

\begin{tabular}{ll}
\hline \hline Components: & Original Measurements: \\
(1) 4-Nitrobenzoic acid; $\mathrm{C}_{7} \mathrm{H}_{5} \mathrm{NO}_{4} ;$ & ${ }^{130}$ L. M. Grubbs, M. Saifullah, N. E. \\
[62-23-7] & De La Rosa, S. Ye, S. S. Achi, W. E. \\
$\begin{array}{l}\text { (2) 1-Methyl-2-tert-butoxyethanol; } \\
\mathrm{C}_{7} \mathrm{H}_{16} \mathrm{O}_{2} ; \text { [57018-52-7] }\end{array}$ & $\begin{array}{l}\text { Acree, Jr., and M. H. Abraham, } \\
\text { Variables: }\end{array}$ \\
$T / \mathrm{K}=298.15$ & Pluid Phase Equilib. 298, 48 (2010). \\
\hline
\end{tabular}

\section{Experimental Values}

\begin{tabular}{lc}
\hline \hline$x_{2}{ }^{\mathrm{a}}$ & $x_{1}{ }^{\mathrm{b}}$ \\
\hline 0.9676 & 0.0324 \\
\hline${ }^{\mathrm{a}} x_{2}:$ mole fraction of component 2 in the saturated solution. & \\
${ }^{\mathrm{b}} x_{1}:$ mole fraction solubility of the solute. &
\end{tabular}




\section{Auxiliary Information}

Method/Apparatus/Procedure:

Constant-temperature bath, calorimetric thermometer, and an ultraviolet/ visible spectrophotometer.

Excess solute and solvent were placed in amber glass bottles and allowed to equilibrate for several days at constant temperature. Attainment of equilibrium was verified by several repetitive measurements and by approaching equilibrium from supersaturation. Aliquots of saturated solutions were transferred through a coarse filter into tared volumetric flasks, weighed, and diluted with methanol. Concentrations were determined by spectrophotometric measurements at $272 \mathrm{~nm}$.

Source and Purity of Chemicals:

(1) $99+\%$, Acros Organics, USA, was used as received.

(2) $99 \%$, Aldrich Chemical Company, Milwaukee, WI, USA, stored over molecular sieves and distilled shortly before use.

Estimated Error:

Temperature: $\pm 0.1 \mathrm{~K}$.

$x_{1}: \pm 1.5 \%$ (relative error)

\subsection{4-Nitrobenzoic acid solubility data in ketones}

\begin{tabular}{|c|c|}
\hline $\begin{array}{l}\text { Components: } \\
\text { (1) 4-Nitrobenzoic acid; } \mathrm{C}_{7} \mathrm{H}_{5} \mathrm{NO}_{4} \text {; } \\
\text { [62-23-7] } \\
\text { (2) Propanone; } \mathrm{C}_{3} \mathrm{H}_{6} \mathrm{O} ;[67-64-1]\end{array}$ & $\begin{array}{l}\text { Original Measurements: } \\
{ }^{180} \text { A. R. Collett and C. L. Lazzell, J. } \\
\text { Phys. Chem. 34, } 1838 \text { (1930). }\end{array}$ \\
\hline $\begin{array}{l}\text { Variables: } \\
\text { Temperature }\end{array}$ & $\begin{array}{l}\text { Prepared by: } \\
\text { W. E. Acree, Jr. }\end{array}$ \\
\hline
\end{tabular}

Experimental Values

\begin{tabular}{lcc}
\hline \hline$T / \mathrm{K}$ & $x_{2}{ }^{\mathrm{a}}$ & $x_{1}{ }^{\mathrm{b}}$ \\
\hline 345.7 & 0.9626 & 0.0374 \\
378.5 & 0.8952 & 0.1048 \\
420.5 & 0.7809 & 0.2191 \\
437.3 & 0.7122 & 0.2878 \\
\hline
\end{tabular}

${ }^{\mathrm{a}} x_{2}$ : mole fraction of component 2 in the saturated solution.

${ }^{\mathrm{b}} x_{1}$ : mole fraction solubility of the solute. The solubility is given as molal percentage, which, based on the description of 100 times the mole fraction, is taken to be mole percentage.

\section{Auxiliary Information}

Method/Apparatus/Procedure:

Preweighed quantities of solute and solvent were placed in bulbs, which were then sealed and heated in a suitable bath. The temperature at which the solid completely dissolved was recorded, and was taken to be the solid-liquid equilibrium temperature.

Source and Purity of Chemicals:

(1) Technical grade, Eastman Kodak Chemical Company, Rochester, NY, USA, was dissolved in dilute aqueous sodium hydroxide, precipitated by addition of hydrochloric acid, and then recrystallized twice from aqueous solution. Purified sample had a melting point temperature of $515.6 \mathrm{~K}$.

(2) Certified Pure, Merck Chemical Company, was dried over calcium chloride and distilled twice before use.

Estimated Error:

Temperature: Not given in paper.

$x_{2}: \pm 10 \%$ (relative error, estimated by compiler).

\subsection{4-Nitrobenzoic acid solubility data in miscellaneous organic solvents}

\begin{tabular}{ll}
\hline \hline Components: & Original Measurements: \\
$\begin{array}{l}\text { (1) 4-Nitrobenzoic acid; } \mathrm{C}_{7} \mathrm{H}_{5} \mathrm{NO}_{4} ; \\
\text { [62-23-7] }\end{array}$ & $\begin{array}{l}{ }^{11} \text { K. R. Hoover, R. Coaxum, E. } \\
\text { Pustejovsky, D. M. Stovall, W. E. } \\
\text { [108-32-7] }\end{array}$ \\
\hline Variables: & Acree, Jr., and M. H. Abraham, \\
$T / \mathrm{K}=298.15$ & Phys. Chem. Liq. 42, 339 (2004). \\
\hline
\end{tabular}

Experimental Values

\begin{tabular}{lc}
\hline \hline$x_{2}{ }^{\mathrm{a}}$ & $x_{1}{ }^{\mathrm{b}}$ \\
\hline 0.9951 & 0.004850 \\
\hline${ }^{\mathrm{a}}{ }_{2}:$ mole fraction of component 2 in the saturated solution. \\
${ }^{\mathrm{b}} x_{1}:$ mole fraction solubility of the solute.
\end{tabular}

Auxiliary Information

\section{Method/Apparatus/Procedure:}

Constant-temperature bath, calorimetric thermometer, and an ultraviolet/ visible spectrophotometer.

Excess solute and solvent were placed in amber glass bottles and allowed to equilibrate for several days at constant temperature. Attainment of equilibrium was verified by several repetitive measurements and by approaching equilibrium from supersaturation. Aliquots of saturated solutions were transferred through a coarse filter into tared volumetric flasks, weighed, and diluted with methanol. Concentrations were determined by spectrophotometric measurements at $272 \mathrm{~nm}$.

Source and Purity of Chemicals:

(1) $99+\%$, Acros Organics, USA, was used as received.

(2) $99.7 \%$, anhydrous, Aldrich Chemical Company, Milwaukee, WI, USA, stored over molecular sieves and distilled shortly before use.

Estimated Error:

Temperature: $\pm 0.1 \mathrm{~K}$.

$x_{1}: \pm 1.5 \%$ (relative error).

\begin{tabular}{ll}
\hline \hline Components: & Original Measurements: \\
$\begin{array}{l}\text { (1) 4-Nitrobenzoic acid; } \mathrm{C}_{7} \mathrm{H}_{5} \mathrm{NO}_{4} ; \\
\text { [62-23-7] }\end{array}$ & $\begin{array}{l}{ }^{136} \mathrm{M} \text {. K. Chantooni, Jr. and I. M. } \\
\text { Kolthoff, J. Phys. Chem. 78, 839 } \\
(2) \text { Ethanenitrile; } \mathrm{C}_{2} \mathrm{H}_{3} \mathrm{~N} ; \text { [75-05-8] }\end{array}$ \\
\hline Variables: & Prepared by: \\
$T / \mathrm{K}=298.15$ & W. E. Acree, Jr. \\
\hline
\end{tabular}

\section{Experimental Values}

The measured solubility was reported to be $0.041 \mathrm{~mol} \mathrm{dm}^{-3}$. The authors did not give the temperature at which the solubility was measured. Based on the experimental description given in an earlier paper [I. M. Kolthoff, J. J. Lingane, and W. Larson, J. Am. Chem. Soc. 60, 2512 (1938)], the compiler believes the temperature to be $298.15 \mathrm{~K}$. 


\section{Auxiliary Information}

\section{Method/Apparatus/Procedure:}

Very little experimental details were provided. Solubility was determined by titrating a filtered aliquot of the saturated solution alkalimetrically in an aqueous ethanol mixture using phenolphthalein as the acid-base indicator.

Source and Purity of Chemicals:

(1) White Label, Eastman Kodak Chemical Company, Rochester, NY, USA, recrystallized from aqueous solution and dried in vacuo at $343 \mathrm{~K}$.

(2) Purity not given, Chemical source not given, was purified by shaking with saturated potassium hydroxide, followed by activated alumina, and then anhydrous calcium chloride to remove water. Ethanenitrile was further dried over anhydrous magnesium sulfate and then phosphorous pentoxide. The sample was distilled shortly before use.

\section{Estimated Error:}

Temperature: $\pm 0.1 \mathrm{~K}$ (estimated by compiler). $c_{1}: \pm 2 \%$ (relative error, estimated by compiler).

\section{Solubility of 3-Nitro-1,2- benzenedicarboxylic Acid in Organic Solvents}

\subsection{Critical evaluation of experimental solubility data}

There has been only a single study reporting the solubility of 3-nitro-1,2-benzenedicarboxylic acid in organic solvents. Wang et al. ${ }^{182}$ used a dynamic method with laser monitoring to determine the solubility of 3-nitro-1,2-benzenedicarboxylic acid in four alkyl alkanoates (ethyl methanoate, methyl ethanoate, ethyl ethanoate, and butyl ethanoate), in two cyclic ethers (tetrahydrofuran and 1,4-dioxane), and in one alkanone (propanone) solvent as a function of temperature. The internal consistency of the seven datasets was assessed by curve-fitting the measured mole fraction solubility data to Eq. (8). The values of the equation coefficients $(A, B$, and $C)$ are given in Table 50, along with the RMSD, calculated according to Eq. (35). The largest RMSD occurs in the 3-nitro-1,2-benzenedicarboxylic acid - dioxane system, and corresponds to about a $5 \%$ error in back-calculating the observed mole fraction solubilities. Results of the mathematical representation analyses indicate that the experimental data for all seven 3-nitro-1,2-benzenedicarboxylic acid - organic solvent systems are internally consistent.

TABLE 50. Parameters of the Modified Apelblat equation for describing the solubility of 3-nitro-1,2-benzenedicarboxylic acid in various organic solvents ${ }^{\mathrm{a}}$

\begin{tabular}{lcccc}
\hline \hline Solvent & $A$ & $B$ & $C$ & $10^{4}$ RMSD \\
\hline Ethyl methanoate & -147.71 & 4843.3 & 21.02 & 0.10 \\
Methyl ethanoate & -54.07 & 1746 & 7.8873 & 0.97 \\
Ethyl ethanoate & -103.18 & 3427.5 & 15.275 & 0.42 \\
Butyl ethanoate & 148.35 & -9015.2 & -21.697 & 0.87 \\
Tetrahydrofuran & -19.492 & 735.44 & 2.7106 & 0.42 \\
1,4-Dioxane & 178.46 & -9552.9 & -26.11 & 8.71 \\
Propanone & -79.296 & 2807.6 & 11.772 & 0.21
\end{tabular}

${ }^{\mathrm{a}}$ Values of the coefficients and root-mean-square deviations were taken from Wang et al. ${ }^{182}$
The experimental solubility data for 3-nitro-1,2-benzenedicarboxylic acid in different organic solvents are in Secs. 65.2-65.4.

\subsection{3-Nitro-1,2-benzenedicarboxylic acid solubility data in esters}

\begin{tabular}{ll}
\hline \hline Components: & Original Measurements: \\
$\begin{array}{l}\text { (1) 3-Nitro-1,2-benzenedicarboxylic } \\
\text { acid; } \mathrm{C}_{8} \mathrm{H}_{5} \mathrm{NO}_{6} ; \text { [603-11-2] }\end{array}$ & R. Wang, Q.-S. Li, X. Z. Lin, H. \\
$\begin{array}{l}\text { (2) Ethyl methanoate; } \mathrm{C}_{3} \mathrm{H}_{6} \mathrm{O}_{2} ; \\
\text { [109-94-4] }\end{array}$ & Data 52, 876 (2007)..$~$ \\
\hline Variables: & Prepared by: \\
Temperature & W. E. Acree, Jr. \\
\hline
\end{tabular}

Experimental Values

\begin{tabular}{lcc}
\hline \hline$T / \mathrm{K}$ & $x_{2}{ }^{\mathrm{a}}$ & $x_{1}{ }^{\mathrm{b}}$ \\
\hline 283.17 & 0.9973 & 0.002679 \\
288.18 & 0.9971 & 0.002866 \\
293.21 & 0.9969 & 0.003088 \\
298.26 & 0.9967 & 0.003346 \\
303.16 & 0.9964 & 0.003631 \\
308.19 & 0.9960 & 0.003956 \\
313.27 & 0.9957 & 0.004329 \\
318.25 & 0.9953 & 0.004729 \\
323.29 & 0.9948 & 0.005173 \\
\hline
\end{tabular}

${ }^{\mathrm{a}} x_{2}$ : mole fraction of component 2 in the saturated solution.

${ }^{b} x_{1}$ : mole fraction solubility of the solute.

\section{Auxiliary Information}

\begin{abstract}
Method/Apparatus/Procedure:
Equilibrium jacketed glass vessel, circulating water temperature controller, stirrer, analytical balance, laser monitoring system.

Experimental solubilities were determined by a dynamic method. Pre-weighed amounts of solute and solvent and were placed in an equilibrium vessel, which was connected to a circulating water bath. The solution was stirred and small quantities of solid solute were added until the saturation point was reached. The saturation point was determined using laser monitoring.
\end{abstract}

Source and Purity of Chemicals:

(1) $99.4 \%$, Zhe Jiang Lianhe Chemical Technology Company, Ltd., China, no purification details were provided.

(2) Analytical Reagent grade, Shanghai Chemical Reagent Company, China, no purification details were provided.

Estimated Error:

Temperature: $\pm 0.05 \mathrm{~K}$.

$x_{1}: \pm 3 \%$ (relative error, estimated by compiler).

\begin{tabular}{|c|c|}
\hline $\begin{array}{l}\text { Components: } \\
\text { (1) 3-Nitro-1,2-benzenedicarboxylic } \\
\text { acid; } \mathrm{C}_{8} \mathrm{H}_{5} \mathrm{NO}_{6} ; \text { [603-11-2] } \\
\text { (2) Methyl ethanoate; } \mathrm{C}_{3} \mathrm{H}_{6} \mathrm{O}_{2} \text {; } \\
\text { [79-20-9] }\end{array}$ & $\begin{array}{l}\text { Original Measurements: } \\
{ }^{182} \text { S. Wang, Q.-S. Li, X. Z. Lin, H } \\
\text { R. Wang, and L. Liu, J. Chem. Eng } \\
\text { Data 52, } 876 \text { (2007). }\end{array}$ \\
\hline $\begin{array}{l}\text { Variables: } \\
\text { Temperature }\end{array}$ & $\begin{array}{l}\text { Prepared by: } \\
\text { W. E. Acree, Jr. }\end{array}$ \\
\hline
\end{tabular}


Experimental Values

\begin{tabular}{lcc}
\hline \hline$T / \mathrm{K}$ & $x_{2}{ }^{\mathrm{a}}$ & $x_{1}{ }^{\mathrm{b}}$ \\
\hline 278.17 & 0.9666 & 0.03344 \\
283.26 & 0.9657 & 0.03431 \\
288.27 & 0.9646 & 0.03535 \\
293.21 & 0.9635 & 0.03652 \\
298.24 & 0.9622 & 0.03783 \\
303.16 & 0.9608 & 0.03922 \\
308.22 & 0.9593 & 0.04071 \\
313.28 & 0.9578 & 0.04224 \\
318.17 & 0.9563 & 0.04373 \\
323.19 & 0.9548 & 0.04524 \\
\hline
\end{tabular}

${ }^{\mathrm{a}} x_{2}$ : mole fraction of component 2 in the saturated solution.

${ }^{b} x_{1}$ : mole fraction solubility of the solute.

\section{Auxiliary Information}

\section{Method/Apparatus/Procedure:}

Equilibrium jacketed glass vessel, circulating water temperature controller, stirrer, analytical balance, laser monitoring system.

Experimental solubilities were determined by a dynamic method. Pre-weighed amounts of solute and solvent and were placed in an equilibrium vessel, which was connected to a circulating water bath. The solution was stirred and small quantities of solid solute were added until the saturation point was reached. The saturation point was determined using laser monitoring.

Source and Purity of Chemicals:

(1) 99.4\%, Zhe Jiang Lianhe Chemical Technology Company, Ltd., China, no purification details were provided.

(2) Analytical Reagent grade, Shanghai Chemical Reagent Company, China, no purification details were provided.

Estimated Error:

Temperature: $\pm 0.05 \mathrm{~K}$.

$x_{1}: \pm 3 \%$ (relative error, estimated by compiler).

\begin{tabular}{ll}
\hline \hline Components: & Original Measurements: \\
(1) 3-Nitro-1,2-benzenedicarboxylic acid; & ${ }^{182}$ S. Wang, Q.-S. Li, X. Z. Lin, \\
$\mathrm{C}_{8} \mathrm{H}_{5} \mathrm{NO}_{6} ;[603-11-2]$ & H. R. Wang, and L. Liu, J. \\
(2) Ethyl ethanoate; $\mathrm{C}_{4} \mathrm{H}_{8} \mathrm{O}_{2} ;[141-78-6]$ & Chem. Eng. Data 52, 876 \\
& (2007). \\
\hline Variables: & Prepared by: \\
Temperature & W. E. Acree, Jr. \\
\hline
\end{tabular}

Experimental Values

\begin{tabular}{lcc}
\hline \hline$T / \mathrm{K}$ & $x_{2}{ }^{\mathrm{a}}$ & $x_{1}{ }^{\mathrm{b}}$ \\
\hline 283.20 & 0.9921 & 0.007926 \\
288.26 & 0.9915 & 0.008467 \\
293.28 & 0.9910 & 0.009015 \\
298.19 & 0.9904 & 0.009577 \\
303.21 & 0.9898 & 0.01019 \\
308.30 & 0.9891 & 0.01088 \\
313.16 & 0.9884 & 0.01160 \\
318.22 & 0.9876 & 0.01244 \\
323.16 & 0.9866 & 0.01337 \\
328.23 & 0.9856 & 0.01443 \\
333.17 & 0.9844 & 0.01561 \\
\hline
\end{tabular}

${ }^{\mathrm{a}} x_{2}$ : mole fraction of component 2 in the saturated solution.

${ }^{\mathrm{b}} x_{1}$ : mole fraction solubility of the solute.
Auxiliary Information

\section{Method/Apparatus/Procedure:}

Equilibrium jacketed glass vessel, circulating water temperature controller, stirrer, analytical balance, laser monitoring system.

Experimental solubilities were determined by a dynamic method. Pre-weighed amounts of solute and solvent and were placed in an equilibrium vessel, which was connected to a circulating water bath. The solution was stirred and small quantities of solid solute were added until the saturation point was reached. The saturation point was determined using laser monitoring.

Source and Purity of Chemicals:

(1) $99.4 \%$, Zhe Jiang Lianhe Chemical Technology Company, Ltd., China, no purification details were provided.

(2) Analytical Reagent grade, Shanghai Chemical Reagent Company, China, no purification details were provided.

\section{Estimated Error:}

Temperature: $\pm 0.05 \mathrm{~K}$

$x_{1}: \pm 3 \%$ (relative error, estimated by compiler).

\begin{tabular}{ll}
\hline \hline Components: & Original Measurements: \\
(1) 3-Nitro-1,2-benzenedicarboxylic acid; & ${ }^{182}$ S. Wang, Q.-S. Li, X. Z. Lin, \\
$\mathrm{C}_{8} \mathrm{H}_{5} \mathrm{NO}_{6} ;$ [603-11-2] & H. R. Wang, and L. Liu, J. \\
(2) Butyl ethanoate; $\mathrm{C}_{6} \mathrm{H}_{12} \mathrm{O}_{2} ;[123-86-4]$ & $\begin{array}{l}\text { Chem. Eng. Data 52, 876 } \\
(2007) .\end{array}$ \\
\hline Variables: & Prepared by: \\
Temperature & W. E. Acree, Jr.
\end{tabular}

Experimental Values

\begin{tabular}{lcc}
\hline \hline$T / \mathrm{K}$ & $x_{2}{ }^{\mathrm{a}}$ & $x_{1}{ }^{\mathrm{b}}$ \\
\hline 283.18 & 0.9976 & 0.002433 \\
288.16 & 0.9970 & 0.002970 \\
293.21 & 0.9965 & 0.003532 \\
298.19 & 0.9959 & 0.004103 \\
303.22 & 0.9953 & 0.004698 \\
308.26 & 0.9947 & 0.005312 \\
313.27 & 0.9941 & 0.005939 \\
318.18 & 0.9936 & 0.006570 \\
323.22 & 0.9928 & 0.007236 \\
328.24 & 0.9921 & 0.007917 \\
333.19 & 0.9914 & 0.008605 \\
338.28 & 0.9907 & 0.009330 \\
343.25 & 0.9899 & 0.01006 \\
348.24 & 0.9892 & 0.01080 \\
353.26 & 0.9884 & 0.01157 \\
\hline
\end{tabular}

${ }^{a_{x_{2}}}$ : mole fraction of component 2 in the saturated solution.

${ }^{\mathrm{b}} x_{1}$ : mole fraction solubility of the solute.

\section{Auxiliary Information}

\section{Method/Apparatus/Procedure:}

Equilibrium jacketed glass vessel, circulating water temperature controller, stirrer, analytical balance, laser monitoring system.

Experimental solubilities were determined by a dynamic method. Pre-weighed amounts of solute and solvent and were placed in an equilibrium vessel, which was connected to a circulating water bath. The solution was stirred and small quantities of solid solute were added until the saturation point was reached. The saturation point was determined using laser monitoring. 
Source and Purity of Chemicals:

(1) $99.4 \%$, Zhe Jiang Lianhe Chemical Technology Company, Ltd., China, no purification details were provided.

(2) Analytical Reagent grade, Shanghai Chemical Reagent Company, China, no purification details were provided.

Estimated Error:

Temperature: $\pm 0.05 \mathrm{~K}$. $x_{1}: \pm 3 \%$ (relative error, estimated by compiler).

\begin{tabular}{ll}
\hline \hline Components: & Original Measurements: \\
(1) 3-Nitro-1,2-benzenedicarboxylic acid; & ${ }^{182} \mathrm{~S}$. Wang, Q.-S. Li, X. Z. Lin, \\
$\mathrm{C}_{8} \mathrm{H}_{5} \mathrm{NO}_{6} ;[603-11-2]$ & H. R. Wang, and L. Liu, J. \\
(2) 1,4-Dioxane; $\mathrm{C}_{4} \mathrm{H}_{8} \mathrm{O}_{2} ;$ [123-91-1] & Chem. Eng. Data 52, 876 \\
& (2007). \\
\hline Variables: & Prepared by: \\
Temperature & W. E. Acree, Jr. \\
\hline
\end{tabular}

Experimental Values

\subsection{3-Nitro-1,2-benzenedicarboxylic acid solubility data in ethers}

\begin{tabular}{ll}
\hline \hline Components: & Original Measurements: \\
(1) 3-Nitro-1,2-benzenedicarboxylic acid; & ${ }^{182}$ S. Wang, Q.-S. Li, X. Z. Lin, \\
$\mathrm{C}_{8} \mathrm{H}_{5} \mathrm{NO}_{6} ;$ [603-11-2] & H. R. Wang, and L. Liu, J. \\
(2) Tetrahydrofuran; $\mathrm{C}_{4} \mathrm{H}_{8} \mathrm{O} ;[109-99-9]$ & Chem. Eng. Data 52, 876 \\
& (2007). \\
\hline Variables: & Prepared by: \\
Temperature & W. E. Acree, Jr. \\
\hline
\end{tabular}

Experimental Values

\begin{tabular}{lcc}
\hline \hline$T / \mathrm{K}$ & $x_{2}{ }^{\mathrm{a}}$ & $x_{1}{ }^{\mathrm{b}}$ \\
\hline 278.28 & 0.7966 & 0.2034 \\
283.17 & 0.7962 & 0.2038 \\
288.16 & 0.7957 & 0.2043 \\
293.21 & 0.7950 & 0.2050 \\
298.29 & 0.7943 & 0.2057 \\
303.28 & 0.7934 & 0.2066 \\
308.19 & 0.7924 & 0.2076 \\
313.23 & 0.7912 & 0.2088 \\
318.24 & 0.7900 & 0.2100 \\
323.16 & 0.7886 & 0.2114 \\
328.19 & 0.7871 & 0.2129 \\
333.17 & 0.7855 & 0.2145 \\
\hline
\end{tabular}

${ }^{\mathrm{a}} x_{2}$ : mole fraction of component 2 in the saturated solution.

${ }^{\mathrm{b}} x_{1}$ : mole fraction solubility of the solute.

\section{Auxiliary Information}

\section{Method/Apparatus/Procedure:}

Equilibrium jacketed glass vessel, circulating water temperature controller, stirrer, analytical balance, laser monitoring system.

Experimental solubilities were determined by a dynamic method. Pre-weighed amounts of solute and solvent and were placed in an equilibrium vessel, which was connected to a circulating water bath. The solution was stirred and small quantities of solid solute were added until the saturation point was reached. The saturation point was determined using laser monitoring.

Source and Purity of Chemicals:

(1) $99.4 \%$, Zhe Jiang Lianhe Chemical Technology Company, Ltd., China, no purification details were provided.

(2) Analytical Reagent grade, Shanghai Chemical Reagent Company, China, no purification details were provided.

\section{Estimated Error:}

Temperature: $\pm 0.05 \mathrm{~K}$.

$x_{1}: \pm 3 \%$ (relative error, estimated by compiler).

\begin{tabular}{lcc}
\hline \hline$T / \mathrm{K}$ & $x_{2}{ }^{\mathrm{a}}$ & $x_{1}{ }^{\mathrm{b}}$ \\
\hline 288.17 & 0.9242 & 0.07583 \\
293.28 & 0.9132 & 0.08682 \\
298.31 & 0.9029 & 0.09705 \\
303.29 & 0.8934 & 0.1066 \\
308.26 & 0.8844 & 0.1156 \\
313.20 & 0.8760 & 0.1240 \\
318.21 & 0.8681 & 0.1319 \\
323.29 & 0.8607 & 0.1393 \\
328.19 & 0.8541 & 0.1459 \\
333.18 & 0.8479 & 0.1521 \\
338.22 & 0.8422 & 0.1578 \\
343.16 & 0.8373 & 0.1627 \\
348,23 & 0.8327 & 0.1673 \\
353.32 & 0.8288 & 0.1712 \\
\hline
\end{tabular}

${ }^{\mathrm{a}} x_{2}$ : mole fraction of component 2 in the saturated solution.

${ }^{b_{x_{1}}}$ : mole fraction solubility of the solute.

\section{Auxiliary Information}

\section{Method/Apparatus/Procedure:}

Equilibrium jacketed glass vessel, circulating water temperature controller, stirrer, analytical balance, laser monitoring system.

Experimental solubilities were determined by a dynamic method. Pre-weighed amounts of solute and solvent and were placed in an equilibrium vessel, which was connected to a circulating water bath. The solution was stirred and small quantities of solid solute were added until the saturation point was reached. The saturation point was determined using laser monitoring.

Source and Purity of Chemicals:

(1) $99.4 \%$, Zhe Jiang Lianhe Chemical Technology Company, Ltd., China, no purification details were provided.

(2) Analytical Reagent grade, Shanghai Chemical Reagent Company, China, no purification details were provided.

Estimated Error:

Temperature: $\pm 0.05 \mathrm{~K}$

$x_{1}: \pm 3 \%$ (relative error, estimated by compiler).

\subsection{3-Nitro-1,2-benzenedicarboxylic acid solubility data in ketones}

\begin{tabular}{|c|c|}
\hline $\begin{array}{l}\text { Components: } \\
\text { (1) 3-Nitro-1,2-benzenedicarboxylic } \\
\text { acid; } \mathrm{C}_{8} \mathrm{H}_{5} \mathrm{NO}_{6} ;[603-11-2] \\
\text { (2) Propanone; } \mathrm{C}_{3} \mathrm{H}_{6} \mathrm{O} ;[67-64-1]\end{array}$ & $\begin{array}{l}\text { Original Measurements: } \\
{ }^{182} \text { S. Wang, Q.-S. Li, X. Z. Lin, H. } \\
\text { R. Wang, and L. Liu, J. Chem. Eng. } \\
\text { Data 52, } 876 \text { (2007). }\end{array}$ \\
\hline Variables: & Prepared by: \\
\hline Temperature & W. E. Acree, Jr. \\
\hline
\end{tabular}


Experimental Values

\begin{tabular}{lcc}
\hline \hline$T / \mathrm{K}$ & $x_{2}{ }^{\mathrm{a}}$ & $x_{1}{ }^{\mathrm{b}}$ \\
\hline 278.16 & 0.9475 & 0.05249 \\
283.18 & 0.9459 & 0.05414 \\
288.27 & 0.9440 & 0.05604 \\
293.33 & 0.4918 & 0.05815 \\
298.24 & 0.9396 & 0.06041 \\
303.19 & 0.9371 & 0.06290 \\
308.32 & 0.9343 & 0.06570 \\
313.16 & 0.9314 & 0.06855 \\
318.20 & 0.9283 & 0.07173 \\
\hline
\end{tabular}

${ }^{\mathrm{a}} x_{2}$ : mole fraction of component 2 in the saturated solution.

${ }^{\mathrm{b}} x_{1}$ : mole fraction solubility of the solute.

\section{Auxiliary Information}

\section{Method/Apparatus/Procedure:}

Equilibrium jacketed glass vessel, circulating water temperature controller, stirrer, analytical balance, laser monitoring system.

Experimental solubilities were determined by a dynamic method. Pre-weighed amounts of solute and solvent and were placed in an equilibrium vessel, which was connected to a circulating water bath. The solution was stirred and small quantities of solid solute were added until the saturation point was reached. The saturation point was determined using laser monitoring.

\section{Source and Purity of Chemicals:}

(1) 99.4\%, Zhe Jiang Lianhe Chemical Technology Company, Ltd., China, no purification details were provided.

(2) Analytical Reagent grade, Shanghai Chemical Reagent Company, China, no purification details were provided.

\section{Estimated Error:}

Temperature: $\pm 0.05 \mathrm{~K}$.

$x_{1}: \pm 3 \%$ (relative error, estimated by compiler).

\section{References}

${ }^{1}$ A. Goto, H. Miyamoto, M. Salomon, R. Goto, H. Fukuda, E. Königsberger, and L.-C. Königsberger, J. Phys. Chem. Ref. Data 40, 013101 (2011)

${ }^{2}$ A. Goto, H. Miyamoto, M. Salomon, R. Goto, H. Fukuda, E. Königsberger, L.-C. Königsberger, and P. Scharlin, J. Phys. Chem. Ref. Data 40, 023102 (2011).

${ }^{3}$ J. M. Prausnitz, Molecular Thermodynamics of Fluid Phase Equilibria (Prentice-Hall, Englewood Cliffs, NJ, 1969).

${ }^{4}$ H. Buchowski, A. Ksizczak, and S. J. Pietrzyk, J. Phys. Chem. 84, 975 (1980).

${ }^{5}$ H. Buchowski and A. Khiat, Fluid Phase Equilib. 25, 273 (1986).

${ }^{6}$ M. H. Abraham, Chem. Soc. Rev. 22, 73 (1993).

${ }^{7}$ M. H. Abraham, A. Ibrahim, and A. M. Zissimos, J. Chromatogr. A 1037, 29 (2004).

${ }^{8}$ K. R. Hoover, K. Pop, W. E. Acree, Jr., and M. H. Abraham, S. Afr. J. Chem. 58, 25 (2005).

${ }^{9}$ K. R. Hoover, D. M. Stovall, E. Pustejovsky, R. Coaxum, K. Pop, W. E. Acree, Jr., and M. H. Abraham, Can. J. Chem. 82, 1353 (2004).

${ }^{10}$ A. K. Charlton, C. R. Daniels, R. M. Wold, E. Pustejovsky, W. E. Acree, Jr., and M. H. Abraham, J. Mol. Liq. 116, 19 (2005).

${ }^{11}$ K. R. Hoover, R. Coaxum, E. Pustejovsky, D. M. Stovall, W. E. Acree, Jr., and M. H. Abraham, Phys. Chem. Liq. 42, 339 (2004).

${ }^{12}$ A. K. Charlton, C. R. Daniels, W. E. Acree, Jr., and M. H. Abraham, J. Solution Chem. 32, 1087 (2003).

${ }^{13}$ D. M. Stovall, C. Givens, S. Keown, K. R. Hoover, R. Barnes, C. Harris, J. Lozano, M. Nguyen, E. Rodriguez, W. E. Acree, Jr., and M. H. Abraham, Phys. Chem. Liq. 43, 351 (2005).
${ }^{14}$ C. R. Daniels, A. K. Charlton, R. M. Wold, W. E. Acree, Jr., and M. H. Abraham, Can. J. Chem. 81, 1492 (2003).

${ }^{15}$ M. H. Abraham, R. E. Smith, R. Luchtefeld, A. J. Boorem, R. Luo, and W. E. Acree, Jr., J. Pharm. Sci. 99, 1500 (2010).

${ }^{16}$ T. W. Stephens, A. N. Quay, V. Chou, M. Loera, C. Shen, A. Wilson, W. E. Acree, Jr., and M. H. Abraham, Global J. Phys. Chem. 3, 1/1 (2012).

${ }^{17}$ T. W. Stephens, A. Wilson, N. Dabadge, A. Tian, H. J. Hensley, M. Zimmerman, W. E. Acree, Jr., and M. H. Abraham, Global J. Phys. Chem. 3, 9/1 (2012).

${ }^{18}$ M. Saifullah, S. Ye, L. M. Grubbs, N. E. La Rosa, W. E. Acree, Jr., and M. H. Abraham, J. Solution Chem. 40, 2082 (2011).

${ }^{19}$ T. W. Stephens, M. Loera, A. N. Quay, V. Chou, C. Shen, A. Wilson, W. E. Acree, Jr., and M. H. Abraham, Open Thermodyn. J. 5, 104 (2011).

${ }^{20}$ T. W. Stephens, N. E. De La Rosa, M. Saiffullah, S. Ye, V. Chou, A. N. Quay, W. E. Acree, Jr., and M. H. Abraham, Fluid Phase Equilib. 309, 30 (2011).

${ }^{21}$ T. W. Stephens, N. E. De La Rosa, M. Saifullah, S. Ye, V. Chou, A. N. Quay, W. E. Acree, Jr., and M. H. Abraham, Fluid Phase Equilib. 308, 64 (2011).

${ }^{22}$ L. M. Sprunger, S. S. Achi, R. Pointer, W. E. Acree, Jr., and M. H. Abraham, Fluid Phase Equilib. 288, 121 (2010).

${ }^{23}$ M. H. Abraham and W. E. Acree, Jr., Phys. Chem. Chem. Phys. 12, 13182 (2010).

${ }^{24}$ M. H. Abraham, A. Nasezadeh, and W. E. Acree, Jr., Ind. Eng. Chem. Res. 47, 3990 (2008).

${ }^{25}$ L. M. Sprunger, J. Gibbs, W. E. Acree, Jr., and M. H. Abraham, QSAR Comb. Sci. 28, 72 (2009).

${ }^{26}$ L. M. Sprunger, W. E. Acree, Jr., and M. H. Abraham, J. Chem. Inf. Model. 47, 1808 (2007).

${ }^{27}$ C. Mintz, T. Ladlie, K. Burton, M. Clark, W. E. Acree, Jr., and M. H. Abraham, QSAR Comb. Sci. 27, 483 (2008).

${ }^{28}$ L. M. Sprunger, J. Gibbs, W. E. Acree, Jr., and M. H. Abraham, QSAR Comb. Sci. 27, 1130 (2008).

${ }^{29}$ M. H. Abraham, A. Ibrahim, and W. E. Acree, Jr., Eur. J. Med. Chem. 42, 743 (2007).

${ }^{30}$ M. H. Abraham, A. Ibrahim, and W. E. Acree, Jr., Eur. J. Med. Chem. 43, 478 (2008).

${ }^{31}$ M. H. Abraham, A. Ibrahim, Y. Zhao, and W. E. Acree, Jr., J. Pharm. Sci. 95, 2091 (2006).

${ }^{32}$ M. H. Abraham, A. Ibrahim, and W. E. Acree, Jr., Eur. J. Med. Chem. 41, 494 (2006).

${ }^{33}$ M. H. Abraham, A. Ibrahim, and W. E. Acree, Jr., Chem. Res. Toxicol. 19, 801 (2006).

${ }^{34}$ M. H. Abraham, A. Ibrahim, and W. E. Acree, Jr., Chem. Res. Toxicol. 18, 904 (2005).

${ }^{35}$ W. E. Acree, Jr. and M. H. Abraham, J. Solution Chem. 31, 293 (2002).

${ }^{36}$ A. M. Zissimos, M. H. Abraham, C. M. Du, K. Valko, B. Klara, C. Bevan, D. Reynolds, J. Wood, and K. Y. Tam, J. Chem. Soc., Perkin Trans. 2 2002, 2001.

${ }^{37}$ A. M. Zissimos, M. H. Abraham, M. C. Barker, K. J. Box, and K. Y. Tam, J. Chem. Soc., Perkin Trans. 2 2002, 470.

${ }^{38}$ M. H. Abraham and J. C. McGowan, Chromatographia 23, 243 (1987).

${ }^{39}$ Advanced Chemistry Development, 110 Yonge St., 14th Floor, Toronto, Ontario M5C 1T4, Canada. The ACD Freeware can be accessed at http:// www.acdlabs.com/.

${ }^{40}$ J. A. Platts, D. Butina, M. H. Abraham, and A. Hersey, J. Chem. Inf. Comput. Sci. 39, 835 (1999).

${ }^{41}$ PharmaAlgorithms, ADME Boxes, Version 3.0, PharmaAlgorithms Inc., 591 Indian Road, Toronto, Ontario M6P 2C4, Canada.

${ }^{42}$ W. E. Acree, Jr. and A. I. Zvaigzne, Thermochim. Acta 178, 151 (1991).

${ }^{43}$ W. E. Acree, Jr., J. W. McCargar, A. I. Zvaigzne, and I.-L. Teng, Phys. Chem. Liq. 23, 27 (1991).

${ }^{44}$ W. E. Acree, Jr., J. Phys. Chem. Ref. Data 42, 013103 (2013).

${ }^{45}$ W. E. Acree, Jr., J. Phys. Chem. Ref. Data 42, 013104 (2013).

${ }^{46}$ W. E. Acree, Jr., J. Phys. Chem. Ref. Data 42, 013105 (2013).

${ }^{47}$ A. Beerbower, P. L. Wu, and A. Martin, J. Pharm. Sci. 73, 179 (1984).

${ }^{48}$ J. Thati, F. L. Nordström, and A. C. Rasmuson, J. Chem. Eng. Data 55, $5124(2010)$

${ }^{49}$ W. E. Acree, Jr. and G. L. Bertrand, J. Pharm. Sci. 70, 1033 (1981).

${ }^{50}$ P. G. Desai and A. M. Patel, J. Indian Chem. Soc. 12, 131 (1935).

${ }^{51}$ H.-M. Lin and R. A. Nash, J. Pharm. Sci. 82, 1018 (1993).

${ }^{52}$ A. Ksiazczak, Fluid Phase Equilib. 28, 57 (1986).

${ }^{53}$ G. L. Perlovich and A. Bauer-Brandl, Pharm. Res. 20, 471 (2003). 
${ }^{54}$ C. K. Hancock, J. N. Pawloski, and J. P. Idoux, J. Org. Chem. 31, 3801 (1966).

${ }^{55}$ B. Long, J. Li, R. Zhang, and L. Wan, Fluid Phase Equilib. 297, 113 (2010).

${ }^{56}$ P. A. Ongley, J. Chem. Soc. 1954, 3634.

${ }^{57}$ J. W. Marden and M. V. Dover, J. Am. Chem. Soc. 38, 1235 (1916).

${ }^{58}$ J. Chipman, J. Am. Chem. Soc. 46, 2445 (1924).

${ }^{59}$ H. Buchowski, J. Solution Chem. 20, 139 (1991).

${ }^{60}$ S. Zhao, X. Chen, Q. Dai, and L. Wang, J. Chem. Eng. Data 56, 2399 (2011).

${ }^{61}$ E. R. Cooper, J. Controlled Release 1, 153 (1984).

${ }^{62}$ S. H. Ghosh and D. K. Hazra, J. Chem. Soc., Perkin Trans. 2 1989, 1021

${ }^{63}$ C. K. Hancock, J. N. Pawloski, and J. P. Idoux, J. Org. Chem. 32, 1931 (1967).

${ }^{64}$ E. A. Gomaa, Phys. Chem. Liq. 50, 279 (2012).

${ }^{65}$ A. F. Lagalante, A. Abdulagatov, and T. J. Bruno, J. Chem. Eng. Data 47, 47 (2002).

${ }^{66}$ W. Herz and W. Rathmann, Z. Elektrochem. 19, 887 (1913).

${ }^{67}$ M. Davies and D. M. L. Griffiths, J. Chem. Soc. 1955, 132.

${ }^{68}$ K. K. Kundu, A. L. De, and M. N. Das, J. Chem. Soc. Dalton Trans. 1972 386.

${ }^{69}$ C. M. McLoughlin, W. A. M. McMinn, and T. R. A. Magee, Powder Technol. 134, 40 (2003).

${ }^{70}$ J. M. Jessy, J. Indian Chem. Soc. 75, 352 (1998)

${ }^{71}$ A. Seidell, Trans. Am. Electrochem. Soc. 13, 319 (1908).

${ }^{72}$ R. Thuaire, Bull. Soc. Chim. Fr. 3815 (1971).

${ }^{73}$ M. Dias, S. L. Raghavan, and J. Hadgraft, Int. J. Pharm. 216, 51 (2001)

${ }^{74}$ A. Pal and S. C. Lahiri, Indian J. Chem. 28A, 276 (1989).

${ }^{75}$ F. A. Restaino and A. N. Martin, J. Pharm. Sci. 53, 636 (1964).

${ }^{76}$ M. K. Chantooni and I. M. Kolthoff, Anal. Chem. 51, 133 (1979).

${ }^{77}$ J. Qingzhu, M. Peisheng, Y. Shouzhi, W. Qiang, W. Chang, and L. Guiju, J. Chem. Eng. Data 53, 1278 (2008).

${ }^{78}$ A. Yurquina, M. E. Manzur, P. Brito, R. Manzo, and M. A. A. Molina, J. Mol. Liq. 108, 119 (2003)

${ }^{79}$ Q. Wang, L. Hou, Y. Cheng, and X. Li, J. Chem. Eng. Data 52, 936 (2007) ${ }^{80}$ P. Ma and Q. Xia, Chin. J. Chem. Eng. 9, 39 (2001).

${ }^{81}$ A. G. Zakharov, M. I. Voronova, D. V. Batov, and K. V. Smirnova, Russ. J. Phys. Chem. 85, 408 (2011).

${ }^{82}$ L. Dian-Qing, L. Jiang-Chu, L. Da-Zhuang, and W. Fu-An, Fluid Phase Equilib. 200, 69 (2002).

${ }^{83}$ M. K. Chantooni and I. M. Kolthoff, J. Phys. Chem. 77, 527 (1973).

${ }^{84}$ D.-Q. Li, D.-Z. Liu, and F.-A. Wang, J. Chem. Eng. Data 46, 172 (2001)

${ }^{85}$ K. Mislow, J. Phys. Chem. 52, 729 (1948).

${ }^{86}$ D. H. Wester and A. Bruins, Pharm. Weekbl. 51, 1443 (1914)

${ }^{87}$ M. Dias, J. Hadgraft, and M. E. Lane, Int. J. Pharm. 336, 108 (2007).

${ }^{88}$ B. J. Aungst, J. A. Blake, and A. Hussain, Pharm. Res. 7, 712 (1990).

${ }^{89}$ S. J. Lloyd, J. Phys. Chem. 22, 300 (1918).

${ }^{90}$ W. Cheng, S. Feng, X. Cui, and F. Cheng, Adv. Mater. Res. 518-523, 3975 (2012).

${ }^{91}$ J. Bradil, J. Malek, and V. Bazant, Chem. Prumysl 20, 117 (1970).

${ }^{92}$ G. D. Maia and M. Giulietti, J. Chem. Eng. Data 53, 256 (2008).

${ }^{93}$ C. Lindenberg, M. Krättli, J. Cornel, M. Mazzotti, and J. Brozio, Cryst. Growth Des. 9, 1124 (2009).

${ }^{94}$ S. L. Cassidy, P. A. Lympany, and J. A. Henry, J. Pharm. Pharmacol. 40, 130 (1988).

${ }^{95}$ R. Castro-Carela, C. Rey-Castro, T. Viaremo, and M. E. Sastre de Vicente, J. Chem. Eng. Data 47, 1432 (2002).

${ }^{96}$ A. F. Diaz and D. L. Drogos, Oxygenates in Gasoline, ACS Symposium Series 799 (American Chemical Society, Washington, DC, 2002), pp. 138 152

${ }^{97}$ H. Wang and W. Zhang, J. Chem. Eng. Data 54, 1942 (2009).

${ }^{98}$ N. Sunsandee, M. Hronec, M. Stolcova, N. Leepipatpiboon, and U. Pancharoen, J. Mol. Liq. 180, 252 (2013).

${ }^{99}$ Q. Jia, P. Ma, S. Ma, and C. Wang, Chin. J. Chem. Eng. 15, 710 (2007).

${ }^{100}$ C. L. Lazzell and J. Johnston, J. Phys. Chem. 32, 1331 (1928).

${ }^{101}$ M. Svärd, F. L. Nordström, and A. C. Rasmuson, Cryst. Growth Des. 10 195 (2010).

${ }^{102}$ J. Barra, M.-A. Peña, and P. Bustamante, Eur. J. Pharm. Sci. 10, 153 (2000).

${ }^{103}$ C. R. Daniels, A. K. Charlton, R. M. Wold, R. J. Moreno, W. E. Acree, Jr., and M. H. Abraham, Phys. Chem. Liq. 42, 633 (2004).

${ }^{104}$ S. Gracin and A. C. Rasmuson, Cryst. Growth Des. 4, 1013 (2004).
${ }^{105}$ P. K. Takayama, N. Nambu, and T. Nagai, Chem. Pharm. Bull. 25, 879 (1977).

${ }^{106}$ X. Q. Chen, S. J. Cho, Y. Li, and S. Venkatesh, J. Pharm. Sci. 91, 1838 (2002).

${ }^{107}$ A. Patel, A. Vaghasiya, R. Gajera, and S. Baluja, J. Chem. Eng. Data 55, 1453 (2010).

${ }^{108}$ B.-Z. Ren, C.-H. Hou, H.-G. Chong, W.-R. Li, and H.-J. Song, J. Chem. Eng. Data 51, 2022 (2006).

${ }^{109}$ Y.-K. Che, Y.-X. Qu, and S. Wang, J. Chem. Eng. Data 54, 3130 (2009).

${ }^{110}$ M. K. Chantooni, Jr. and I. M. Kolthoff, J. Phys. Chem. 79, 1176 (1975).

${ }^{111}$ B. K. Dolui, S. K. Bhattacharya, and K. K. Kundu, J. Solution Chem. 37, 987 (2008).

${ }^{112}$ B. Long, Y. Wang, R. Zhang, and J. Xu, J. Chem. Eng. Data 54, 1764 (2009).

${ }^{113}$ L. Feng, L. Wang, G. Peng, X. Guo, and X. Li, J. Chem. Eng. Data 55, 500 (2010).

${ }^{114}$ G. N. Friedlin and V. N. Davydov, Zh. Prikl. Khim. 35, 2530 (1962)

${ }^{115}$ G. N. Freidlin and V. N. Davydov, Dopov. Akad. Nauk Ukr. RSR 622 (1962).

${ }^{116}$ B. Long and Z. Yang, Fluid Phase Equilib. 266, 38 (2008).

${ }^{117}$ L. Feng, Q. Wang, and X. Li, J. Chem. Eng. Data 53, 2501 (2008).

${ }^{118}$ J. J. Harper and P. Janik, J. Chem. Eng. Data 15, 439 (1970).

${ }^{119}$ M.-M. Chen, P.-S. Ma, L. Wang, and F. Chen, Acta Phys. Chim. Sin. 20, 445 (2004).

${ }^{120}$ Q. Wang, H. Xu, and X. Li, J. Chem. Eng. Data 50, 258 (2005).

${ }^{121}$ P. Ma and M. Chen, Chin. J. Chem. Eng. 11, 334 (2003).

${ }^{122}$ Q. Wang, H. Xu, and X. Li, J. Chem. Eng. Data 50, 719 (2005).

${ }^{123}$ X. Guo, Y.-W. Cheng, L.-J. Wang, and X. Li, J. Chem. Eng. Data 53, 1421 (2008).

${ }^{124}$ Q. Wang, H. Xu, and X. Li, J. Chem. Eng. Data 50, 243 (2005).

${ }^{125}$ S. Pinsuwan, A. Li, and S. H. Yalkowsky, J. Chem. Eng. Data 40, 623 (1995)

${ }^{126}$ P. K. Biswas, S. C. Lahiri, and B. P. Dey, Bull. Chem. Soc. Jpn. 66, 2785 (1993).

${ }^{127}$ N. V. Sidgwick and E. K. Ewbank, J. Chem. Soc. Trans. 119, 979 (1921).

${ }^{128}$ G. S. Krasil'nikova, E. G. Freidlin, and Y. N. Pirig, Zh. Prikl. Khim. 60, 2519 (1987).

${ }^{129}$ S. H. Ghosh and D. K. Hazra, J. Indian Chem. Soc. 65, 620 (1988).

${ }^{130}$ L. M. Grubbs, M. Saifullah, N. E. De La Rosa, S. Ye, S. S. Achi, W. E. Acree, Jr., and M. H. Abraham, Fluid Phase Equilib. 298, 48 (2010).

${ }^{131}$ M. H. Abraham and J. Le, J. Pharm. Sci. 88, 868 (1999).

${ }^{132}$ G. Yang, Y. Ran, and S. H. Yalkowsky, J. Pharm. Sci. 91, 517 (2002).

${ }^{133}$ A. Osol and M. Kilpatrick, J. Am. Chem. Soc. 55, 4430 (1933).

${ }^{134}$ P. V. Phatak and V. K. Gaikar, J. Chem. Eng. Data 38, 217 (1993).

${ }^{135}$ S. S. Laddha and M. M. Sharma, J. Appl. Chem. Biotechnol. 28, 69 (1978).

${ }^{136}$ M. K. Chantooni, Jr. and I. M. Kolthoff, J. Phys. Chem. 78, 839 (1974).

${ }^{137}$ A. Wilson, A. Tian, V. Chou, A. N. Quay, W. E. Acree, Jr., and M. H. Abraham, Phys. Chem. Liq. 50, 324 (2012).

${ }^{138}$ K. R. Bowen, T. W. Stephens, H. Lu, K. Satish, D. Shan, W. E. Acree, Jr., and M. H. Abraham, Eur. Chem. Bull. 2, 577 (2013).

${ }^{139}$ Q. Li, F. Lu, Y. Tian, S. Feng, Y. Shen, and B. Wang, J. Chem. Eng. Data 58, 1020 (2013).

${ }^{140}$ K. R. Hoover, R. Coaxum, E. Pustejovsky, W. E. Acree, Jr., and M. H. Abraham, Phys. Chem. Liq. 42, 457 (2004).

${ }^{141}$ A. Seidell, Solubilities of Organic Compounds, 3rd ed. (Van Nostrand, New York, 1941).

${ }^{142}$ J. C. Philip and F. B. Garner, J. Chem. Soc. Trans. 95, 1466 (1909).

${ }^{143}$ S. Ye, M. Saifullah, L. M. Grubbs, M. C. McMillan-Wiggins, P. Acosta, D. Mejorado, I. Flores, W. E. Acree, Jr., and M. H. Abraham, Phys. Chem. Liq. 49, 821 (2011)

${ }^{144}$ W. Sun, W. Qu, and L. Zhao, J. Chem. Eng. Data 55, 4476 (2010).

${ }^{145}$ G. L. Perlovich, T. V. Volkova, and A. Bauer-Brandl, J. Pharm. Sci. 95, 1448 (2006).

${ }^{146}$ H.-L. Fung and T. Higuchi, J. Pharm. Sci. 60, 1782 (1971).

${ }^{147}$ J. Walker and J. K. Wood, J. Chem. Soc. Trans. 73, 618 (1898).

${ }^{148}$ I. L. Krupatkin, J. Gen. Chem. USSR (Engl. Transl.) 26, 3609 (1956).

${ }^{149}$ J. W. Marden and M. V. Dover, J. Am. Chem. Soc. 39, 1 (1917).

${ }^{150}$ A. N. Paruta, B. J. Sciarrone, and N. G. Lordi, J. Pharm. Sci. 53, 1349 (1964).

${ }^{151}$ K. M. De Fina, T. L. Sharp, L. E. Roy, and W. E. Acree, Jr., J. Chem. Eng. Data 44, 1262 (1999). 
${ }^{152}$ H. Matsuda, K. Kaburagi, S. Matsumoto, K. Kurihara, K. Tochigi, and K. Tomono, J. Chem. Eng. Data 54, 480 (2009).

${ }^{153}$ A. Shalmashi and A. Eliassi, J. Chem. Eng. Data 53, 199 (2008).

${ }^{154}$ M. A. Peña, B. Escalera, A. Reíllo, A. B. Sánchez, and P. Bustamante, J. Pharm. Sci. 98, 1129 (2009).

${ }^{155}$ M. A. Peña, A. Reíllo, B. Escalera, and P. Bustamante, Int. J. Pharm. 321, 155 (2006)

${ }^{156}$ K. B. Sloan, K. G. Siver, and S. A. M. Koch, J. Pharm. Sci. 75, 744 (1986).

${ }^{157}$ M. A. Peña, P. Bustamante, B. Escalera, A. Reíllo, and J. M. BosqueSendra, J. Pharm. Biomed. Anal. 36, 571 (2004).

${ }^{158}$ E. Bergroth, Farm. Aikak. 70, 91 (1961).

${ }^{159}$ J. Lim, S. Jang, H. K. Cho, M. S. Shin, and H. Kim, J. Chem. Thermodyn. 57, 295 (2013).

${ }^{160}$ A. Jouyban, V. Panahi-Azar, and F. Khonsari, J. Mol. Liq. 160, 14 (2011).

${ }^{161}$ E. A. Gomaa, M. A. Mousa, and A. A. El-Khouly, Thermochim. Acta 86, 351 (1985).

${ }^{162}$ M. A. A. Fakhree, S. Ahmadian, V. Panahi-Azar, W. E. Acree, Jr., and A. Jouyban, J. Chem. Eng. Data 57, 3303 (2012).

${ }^{163}$ B. Bouillot, S. Teychené, and B. Biscans, Ind. Eng. Chem. Res. 52, 9276 (2013).

${ }^{164}$ M. H. Abraham, W. E. Acree, Jr., and J. E. Cometto-Muñiz, New J. Chem. 33, 2034 (2009).

${ }^{165}$ F. L. Nordström and A. C. Rasmuson, Eur. J. Pharm. Sci. 28, 377 (2006).
${ }^{166}$ A. Martin, P. L. Wu, and A. Beerbower, J. Pharm. Sci. 73, 188 (1984). ${ }^{167}$ S. Gracin and A. C. Rasmuson, J. Chem. Eng. Data 47, 1379 (2002).

${ }^{168}$ F. Nordström and A. C. Rasmuson, J. Pharm. Sci. 95, 748 (2006).

${ }^{169}$ M. S. Manic, D. Villanueva, T. Fornari, A. J. Queimada, E. A. Macedo, and V. Najdanovic-Visak, J. Chem. Thermodyn. 48, 93 (2012).

${ }^{170}$ A. Apelblat and E. Manzurola, J. Chem. Thermodyn. 29, 1527 (1997).

${ }^{171}$ R. Coaxum, K. R. Hoover, E. Pustejovsky, D. M. Stovall, W. E. Acree, Jr., and M. H. Abraham, Phys. Chem. Liq. 42, 313 (2004).

${ }^{172}$ U. Domańska, Pol. J. Chem. 60, 847 (1986).

${ }^{173}$ U. Domańska and T. Hofman, J. Solution Chem. 14, 531 (1985).

${ }^{174}$ U. Domańska, J. Solution Chem. 18, 1153 (1989).

${ }^{175}$ L. E. Strong, R. M. Neff, and I. Whitesel, J. Solution Chem. 18, 101 (1989).

${ }^{176}$ Y. Ran and S. H. Yalkowsky, J. Chem. Inf. Comput. Sci. 41, 354 (2001).

${ }^{177}$ Q. Kong, Y. Cheng, X. Bao, L. Wang, and X. Li, Fluid Phase Equilib. 340, 46 (2013).

${ }^{178}$ M. Chen and P. Ma, J. Chem. Eng. Data 49, 756 (2004).

${ }^{179}$ W. Luo, Q. Wang, L. Fu, W. Deng, X. Zhang, and C. Guo, Ind. Eng. Chem. Res. 50, 4099 (2011).

${ }^{180}$ A. R. Collett and C. L. Lazzell, J. Phys. Chem. 34, 1838 (1930).

${ }^{181}$ I. M. Kolthoff, J. J. Lingane, and W. Larson, J. Am. Chem. Soc. 60, 2512 (1938).

${ }^{182}$ S. Wang, Q.-S. Li, X. Z. Lin, H. R. Wang, and L. Liu, J. Chem. Eng. Data 52, 876 (2007). 